

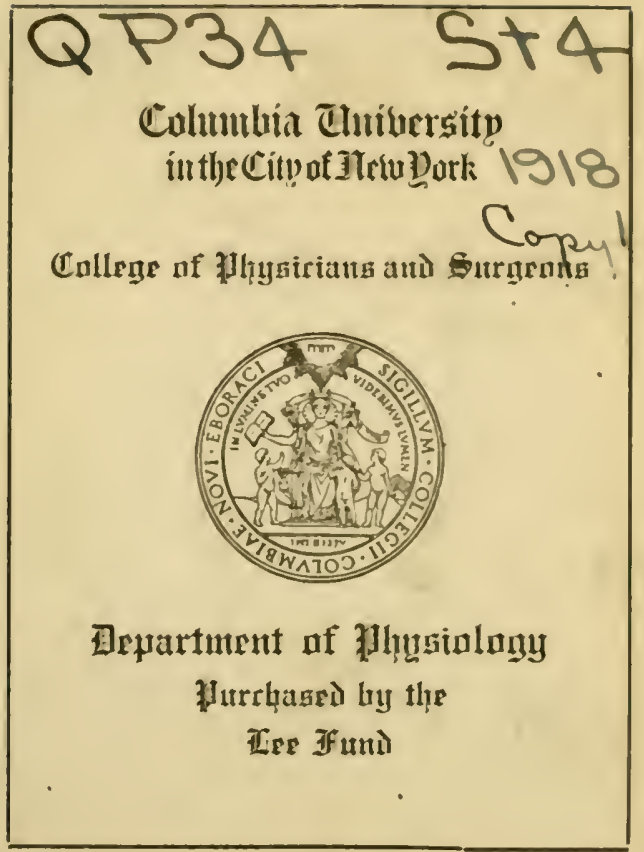



A MANUAL OF PHYSIOLOGY 


\section{THE 'UNIVERSITY' SERIES OF MANUALS}

\section{FOR STUDENTS AND PRACTITIONERS.}

\section{Manual of Surgery (Rose and Carless). (Ninth Edition.)}

Revised by Al.rert Cakt.rss, M.K., M.S. LON1., F.R.C.S., ('lemp.) Colonel, A.M.S., Consulting Surgeon, liststern ('ommand; Protessor of Surgery in, and Surgeon to, King's College Hosprital, London; Formerly Examiner in Surgery to the Universities of Jondon, Glasgow, Manchester, Livwrpool, and Leeds: Consulting Surgeon to the King Edward's Alemorial Hospital, Ealing; 10 the St. Johr's Ilospital, Twickenham, etc.

Price 2as. od. net.

Manual of Anatomy. (Third Edition.) By A. M. Bochanan, M.A., M.1)., C.M., F.F.P.S. GLAs., Professor of Anatony, Anderson's College, Glasgow; Examiner in Anatoniy to the Faculty of Phyaicians and Surgeons of Glasgow; Ex-Examiner in Anatony tothe University or Glasgow. P'rice 22s. 6d. net.

Manual of Medicine. (Fourth Edition.) By T. KIRKPATRICK Monko, M.A., M.D., F.F.P.S. Gl.As., Profersor of Medicine, University of Glasgow, and St. Mungo's College, Glasgow : Physician to the Glasgow Royal Infirmary: Fellow of and Examiner to the Faculiy of Plyysicians and Surgeons, Glasgow; formerly Examiner in the University of Glasgow.

Price 18 s. net.

Manual of Physiology. (Eighth Edition.) By G. N. Stewart, M.A., D.Sc., M.D. EDIN., D.P.H. CAM13, Professor of Experimental Medicine in Western Reserve University ; Clinical Pbysiologist to Lakevide Hospital, Cleveland; formerly Professor of Physiology in the University of Chicago ; Examiner in Physiology, University of Aherdeen; Senior Detnonstrator of Physiology, Owens College, and Victoria University.

Price ats. net.

Manual of Midwifery. (Third Edition.) By Henry Jeleetr, 13.A., M.D. DUB., F.R.C.P.1., Master of the Rotunda Haspital; formerly King's Professar of Midwifery, Trinity College, Dublin; Gynetcologist to Sir Patrick Dun's Ilospital ; Extern Exanniner in Midwifery and Gyniecology, Royal University of Ireland : Examiner in Midwifery and Ginnatcology, Royal College of Physicians of Ireland.

Irsproparation.

Manual of Tropical Medicine. (Third Edition.) By ALDO Castel.lani, M.D. Flokence, Pr. Joc. Naples, Professor of Tropical Medicine, University of Ninles, and Director of the Royal Clinic for Tropica: 1)iseases, Naples; and AlinerT J. Cinat.neks, M.D. Vict. and Livert., F.R C.S. Einr., D.P.H. Cask., Director Wellcome Tropical Resesrch Laboratories, Khartoum; Ceylon Medical Service, rgo:-s 8 .

[lu preparation.

\section{Manual of Pathology and Morbid Anatomy (Cireen's).} ('Twelfth lidition.) Revisel and lidited by W. C. Busanqurt, M.A. Oxon., M D., F.R.C.P. LoND., Physician to Charing Cross Hospital, and W: W: C. Tul, Y, M.13., B.C. Cantas., M.R.C.P. Lond., Bacteriologist and Chemical Pathologist to Charing Cross Hospital. P'rice 18 s. net. For further farticulars see complote catalogus. 
Digitized by the Internet Archive in 2010 with funding from Columbia University Libraries 

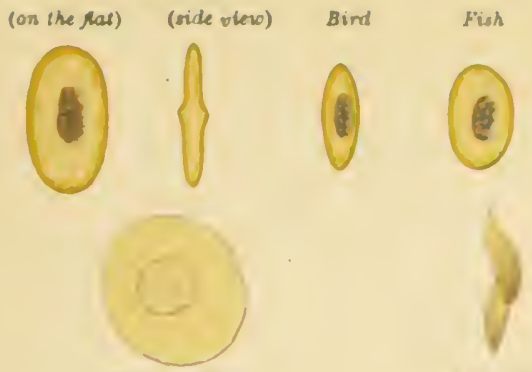

Sammal

lamel

Blood of mammal

Frog's Ciorpucke

afle- adilition of water
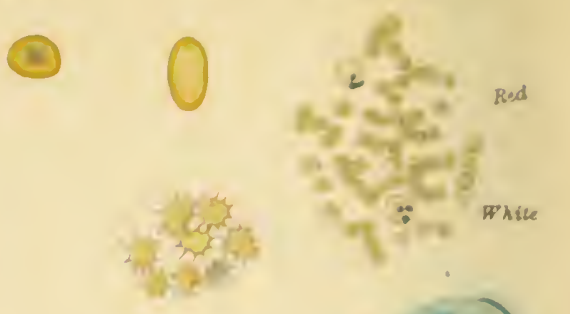

Mammaliun

afser culdition of syrup

Mammaluan

a/ber addution of salt

1. Red bloor-corpuscles.
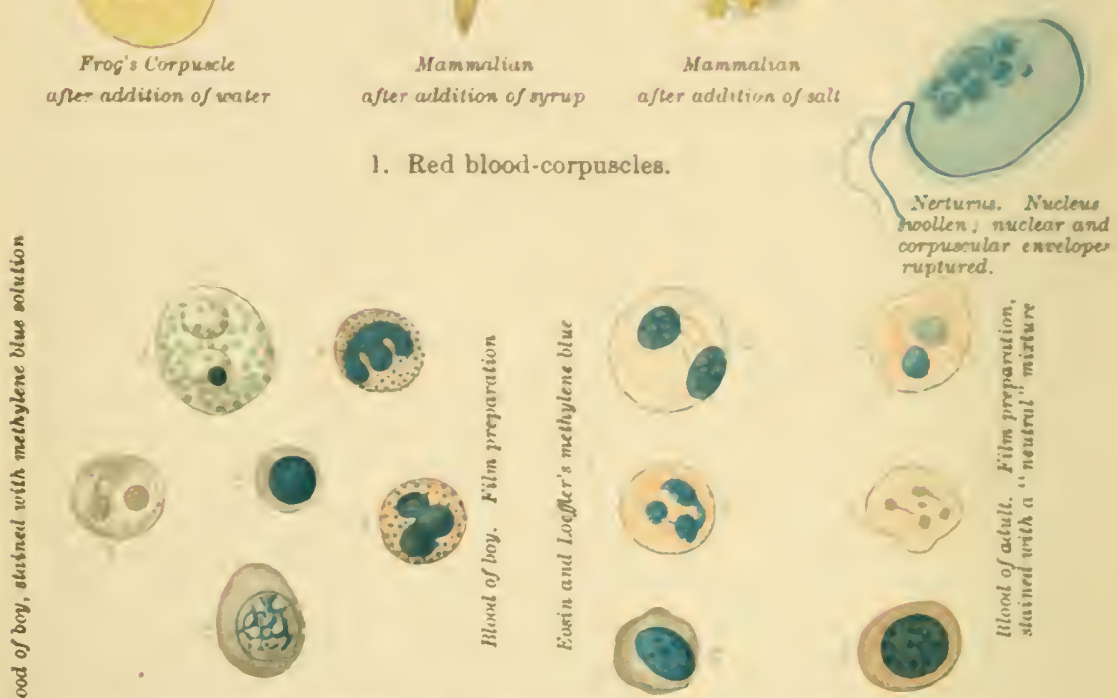

2. The colourless corpuscles of human blood, $\times 1000 . \quad a, 60$ inophile cells ;

$b$, finely granular oxyphile cells; $c$, hysline cells : $d$, lymphocyte;

$e$, polymorphonuclear nentrophile cells (Kanthack and Hardy). The magnification is much greater than in 1.

3. Cover-glass preparation of spinal cord of $0 x, \times 250$.

(Strined with methylene blut).

Lendritic proceses
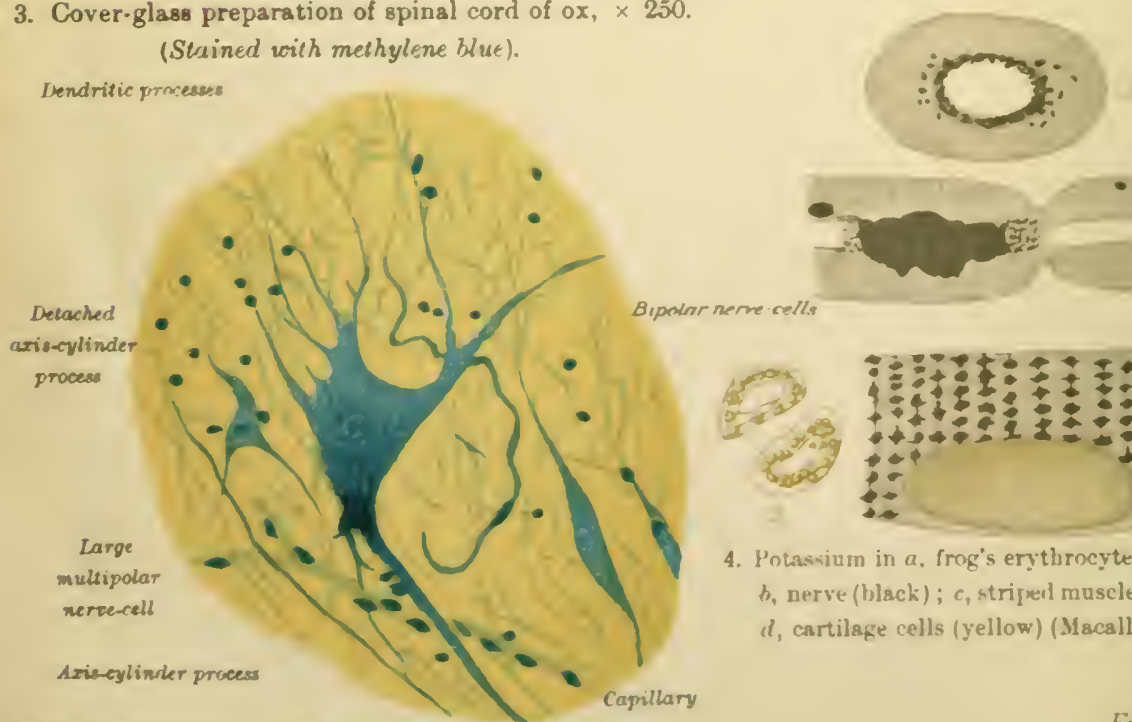

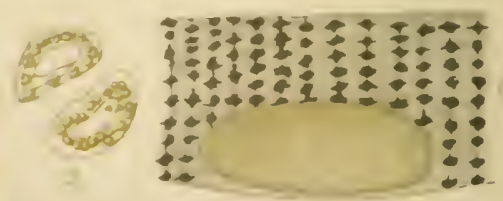

4. Potaskium in $a$. frog's erythrocyte (black); b, nerve (black); $c$, striperl muscle (black): d, cartilage cells (yellow) (Macallum). 
A

\section{MANUAL OF PHYSIOLOGY}

\section{V(Titb Dractical Ererciscs}

BY

G. N. STEWART, M.A, D.Sc., M.D. EDIN., D.P.H. CAMB. PROFESSOR OF EXPERIMENTAL MEDICINE IN WESTERN RESERVE UNIYERSITY, CIINICAL FHYSIOLOGIST TO LAKFSIDF HOSPITAL, CLEVELAND; FORMERLY PROFFSSOR OH JHYSIOLORY IN THE UNIVERSITV OF CHICAGO ; PROFESSOR OF PHYSIOLOGY IN TIIR WHSTEKN RESERVE UNIVERSITY; GEORGE HENRY LEWFS STUDENT ; EXAMINEK IN PHYSIOLOGY IN THE UNIVEKSITY OF ABERDI EN ; SENIOR DEMONSTRATON OF PHVSIOLOGY IN THE OWENS COLLEGE, VICTORIA UNIVERSITY, ETC.

WITH COLOURED PLATE AND 492 OTHER ILLUSTRATIONS

EIGHTH EDITION

UNIVERSITY SERIES

NEW YORK

WILLIAM WOOD AND COMPANY MDCCCCXVIII

All rights reserved 


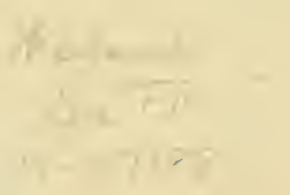

Printed in Great Brituin

$$
\begin{aligned}
& Q P 34 \\
& \text { ot4 } \\
& \text { isit } \\
& \text { axyl }
\end{aligned}
$$




\section{PREFACE TO THE EIGHTH EDITION}

Ix the midst of arms the laboratories are silent, and if the book has not been quite so extensively revised in this as in the previous edition there is only too valid an excuse in the withering influence of the war upon the output of new work. Nevertheless, considerable clianges and additions have been made, especially in the portions dealing with the chemical phenomena of respiration, the functions of the endocrine organs and metabolism. Some of the newer calorimetric work has been more adequately taken account of. The filtration-reabsorption theory of urine formation, as recently formulated by Professor Cushny, is discussed, although necessarily in less detail than its importance merits. Some of the more recent results of the examination of the mechanics of the circulation by optical methods of recording have been noticed. Several of the old illustrations have been omitted and a considerable number of new ones added. In deference to the opinion of a number of teachers a bibliography has been inserted in the form of an appendix. Most of the references are to papers written in English, as these will necessarily be of the most general use, and in any case will usually contain references to the most important paper; in other languages. An exception is made in favour of monographs which are themselves provided with extensive bibliographies. Recent communications are often cited in preference to older ones on the same subject, not because the new work is necessarily better or more important than the old, but because recent papers will, as a matter of course, refer to previous publications.

Despite suggestions made from time to time by critical friends and friendly critics (it is curious how little there is of cross division liere), the Practical Exercises have been retained in their original place at the end of the related chapters. The author has been asked more than once whether it would not be better to collect them into a separate small volume, for greater convenience of use in the laboratory. Apart from the fact that this would entail a not inconsiderable duplication of material, including illustrations, the exercises in their present form and position being supplemented treely by cross-references to the text, the suggested change would run counter to all the ideas of the author as to the relation between textbook and practical work in the study of a science like physiology. But for the exigencies of curricula, which necessarily, having to rcckon 
with the brevity of life, tend to sunder things which should be joined, and in the sane measure, the law of action and reaction holling eren in schedules and time tables, to join things which were better apart, it would have seemed to the author even more appropriate to place the exercises at the beginning of the chapters, or best of all to interweare them with the text till the "phase boundaries" between the two disappeared as far as might be-a process which might perhaps often be applied with advantage to the phase boundaries (of lath and plaster, still more of mental attitude) between lecture-room and laboratory. The book woukt then become, if the design could be adequately catried out, a text-book of physiology, in the perusal of which the student was not permitted to forget for a moment that the value of the so-called 'factdescribed was in the last analysis dependent wholly upon the accuracy of innumerable observations carried out in the laboraturs by methods and apparatus with which he had gained, or was gaining. some first-hand acquaintance in his practical work. When a statement in the text is followed by a page reference to the Practical Exercises this is for the precise purpose of reminding the student that the laboratory is the seed-bed of the whole science. l.est he forget this the exercises are not eren placed all at the end of the book, still less in a separate volume.

The question has been sometimes asked whether the methods. and especially the apparatus described in the exercises, will exactly. suit most laboratories. Ther will not, and ther ought not to be expected to do so. This is also necessarily true of all laboratory guides. All must be adapted in some nieasure not only to the material equipment of any particular laboratory in which they art being used, but quite as much to the ideas and, what is by no means unimportant, to the habits of the teacher. To a certain extent in America a standardization of plursiological apparatus for students: has been realized, and this has been of considerable benefit to the teaching of physiology. Individual teachers and liboriatories have nevertheless gone on develnpins their own ideas with marked advantage, and nothing would be less desirable than the uniformity of machine-made physiological teaching. could this be achieved which luckily, in the nature of things, it cannot be.

\section{CLEVELAND,} April, I9IS. 


\section{EXTRAC" $\Gamma$ FROM THE PREFACE TO THE FIRST EDITION}

Is this book an attempt has been made to interweave formal exposition with practical work, according to a programme which I have followed for some time past in teaching Physiology to medical students on the other side of the Atlantic, and which has, it is believed, proved to be well adapted to their needs and opportunities. It ought, however, to be explained that, for various reasons, a somewhat wider range of experiment is open to the student in America than in this country. But as nobody will use this book except in a regular laboratory and under responsible guidance, it has not been thought necessary to mark in any special manner the parts of the exercises which the English student must do by proxy (that is, learn from demonstrations), and the parts he ought to perform for himself.

An arrangement of the exercises with reference to the systematic course has this advantage-that by a little care it is possible to secure that practical work on a given subject shall actually be going on at the time it is being expounded in the lectures. Cross-reference from lecture-room to laboratory, and from laboratory to lecture-room, from the detailed discussion of the relations of a phenomenon to the living fact itself, is thus rendered easy, natural, and fruitful.

As some teachers may wish to know how a course such as that described in the Practical Exercises may be conducted for a fairly large class, a few words on the method we have followed may not be out of place. It is obvious that many of the exercises require more than one person for their performance; and it may be said 
that, except in the case of the simpler experiments and the chemical work as a whole, which each student does for himself, it has been found convenient to divide the class into groups of four, each group remaining together throughout the session. It is possible that some may find a group of four too large a unit, and it is certain that three, or perhaps even two, would be better; but in a large school so minute a subdivision is hardly possible, without entailing cxcessive labour on the teachers.

The systematic portion of the book is so arranged that it can equally well be used independently of the practical work, and aims at being in itself a complete exposition of the subject, adapted to the requirements of the student of medicinc.

As to the matter of the text, it is hardly necessary to say that this book does not aspire to the dubious distinction of originality; and it is literally impossible to acknowledge all the sources from which information has been derived. In many cases names have been quoted, but names no less worthy of mention have often been of necessity omitted.

G. N. STEWART.

\section{CAMBRIDGE,}

September, I 895 .

\section{ERRATA}

On p. 6r7, line 5 from bottom, for 'McCollom 'read 'McCollum'

.. p. 632, line 28, for 'McCollom ' read 'McCollum.'

'. P. 647, line 1.3. for 'are' read ' is.'

.. p. 648 , line 11 , for 'and 'rad ' the.'

.. pp. 667 and 659 (headline), for 'adrenals ' read 'pituitary.'

.. p. 67I (headline), for 'adrenals ' rad 'pineal gland.'

.. p. 673 (lieadline), for 'adremals ' cad 'spleen,' 


\title{
CONTENTS
}

\author{
CHAPTER I.
}

I NTRODUCTION.

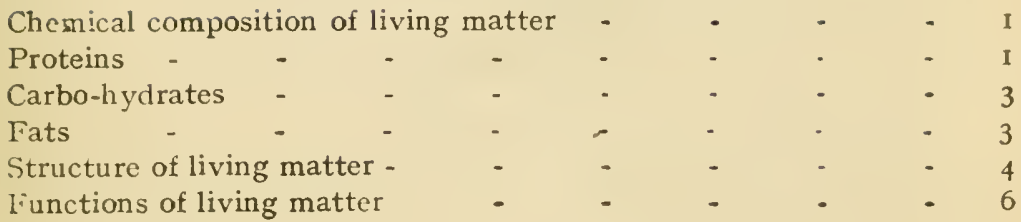

CHAPTER II.

THE CIRCULATING LIQUIDS OF THE BODY.

Section I.-Morphology OF THE BLoOD

Blood-corpuscles

Life-history of the corpuscles

Section II.-General Physical and Cinemical Properties of THE BLOOD

Viscosity of blood

Reaction of blood

Specific gravity of blood

Electrical conductivity of blood

Relative volume of corpuscles and plasma

Hæmolysis

Agglutination -

Precipitins

Anaphylaxis -

Coagulation of blood -

Vaso-constrictor property of shed blood 
SECTION V.-.J. YMPH AND CHYLE - - -

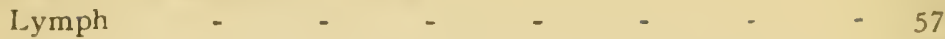

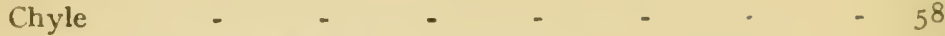

Section VI.--Functions of Blood and Lymph - $\quad 59$

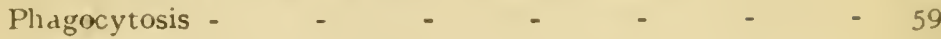

Diapedesis - $\quad$ - $\quad$ - $\quad$ -

CHAPTER III.

THE CIRCULATION OF THE BLOOD AND LYMPH.

Section I.-Prelumnary Anatomical and Physical Data - 80 Physiological anatomy of the vascular system - $\quad$ - 1

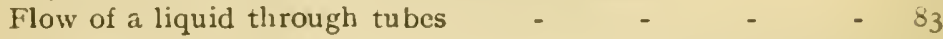

Section II.-The Beat of the Heart in its Pirysical or

ilechanical Relations - $\quad$ - $\quad$ - $\quad$ - $\quad-85$

Events in the cardiac cycle - $\quad-\quad-\quad-\quad-85$

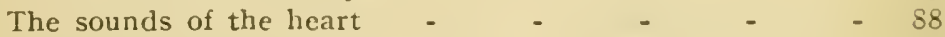

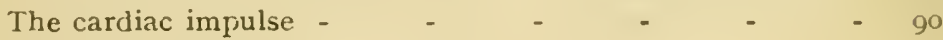

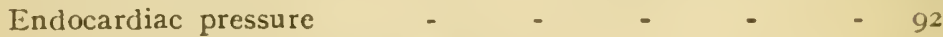

The ventricular pressure-curve $\quad$ - $\quad$ - $\quad$ - $\quad$ - 94

The auricular and venous pressure-curve - - $\quad 98$

Section III.-Physical or Mechanical Phenomena of the

Circulation IN THE Bloodvessels - $\quad$ - $\quad$ - 101

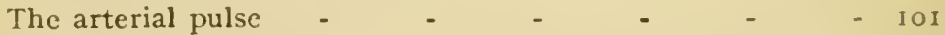

Arterial blood-pressure - $\quad$ - $\quad$ - $\quad$ - $\quad$ - 109

Mcasurement of the blood-pressure in man - - $\quad$ - II3

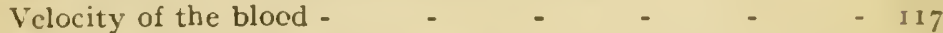

Measurement of velocity of blood - - - - $\quad$ - 120

The volume-pulse - $\quad$ - $\quad$ - $\quad$ - $\quad$ - $\quad$ - 127

The circulation in the capillaries - $\quad$ - $\quad-\quad$ - 129

The circulation in the veins - - - - $\quad$ - 132

The circulation-time - - - - - - $\quad$ - 135

Work and output of heart - _ - _

Section IV.-The Heart-Beat in its Physiological Rela-

\section{IIONS}

Intrinsic nerves of the heart -

Cause of the heart-beat

Conduction and co-ordination in heart

Auriculo-ventricular bundle -

Fibrillary contractions

Chemical conditions of heart-beat

Resuscitation of the heart

Refractory period of heart
- 140

- 141

- 141

- 146

- 147

- 151

- 152

I 53

- 155 
Strction V.-THE Nervous Reglilation of the Heart (I:XTRINSIC NeRvol's MFCHA.ISM OF THE HLART) - - i50́

Action of poisons on the heart - - - - 106

Normal excitation of cardiac nervous mechanism - - rijs

Section Vi.--The Nervous Regllation of the Bloonyfestls

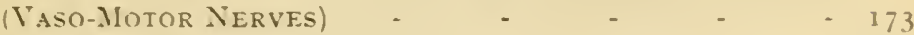

The chief vaso-motor nerves - $\quad$ - $\quad$ - $\quad$ - $\quad-175$

Vaso-dilator fibres - $\quad$ - $\quad$ - $\quad$ - $\quad$ - 179

Course of the vaso-motor nerves - - _ - ISI

Vaso-motor centres - $\quad \cdot \quad \cdot \quad \cdot \quad \cdot \quad-182$

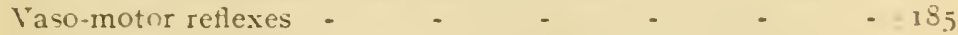

Influence of gravity on the circulation - $\quad$ - 190

Section Vil.-The Limphatic Circlilation • • • 192

CHAPTER IV.

\section{RESPIRATION.}

Section I.-Preiminary ANatomical Data - - - 228

Phrsiological anatomy of the respiratory apparatus - - 223

Bloor-supply of the lungs - $\quad$ - $\quad$ - $\quad$ - $\quad$ - 223

Section 1I.-Mechanical Phenomexa of Extersal RfspiraTION

Types of respiration

Artificial respiration -

Respiratory sounds

Frecuency of respiration

Vital capacity -

The dead space

Intrathoracic pressure

Respiratory pressure -

-
$:$
$:$
$:$
$:$
$\cdot$

$-225$

- 229

$-230$

$-231$

$-23.4$

$-\quad 236$

- 236

- 230

- 238

Section III.-The Chesistri of Extersal Rf.spiratiox - 239

Inspired and expired air $\quad-\quad \cdot \quad \cdot \quad \cdot \quad-239$

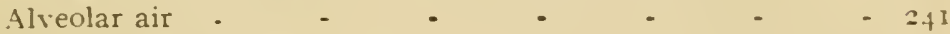

Respiratory quotient - $\quad$ - $\quad$ -

Ventilation - $\quad$ - $\quad$ - $\quad$ - $\quad$ - $\quad$ - $\quad-242$

The quantity of carbon dioxide given off and of oxygen

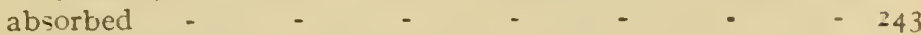

Section IV.-The Gases of the Blood - • - - 245

Phrsical introduction - $\quad$ - $\quad$ - $\quad$ - $\quad$ - $\quad$ - 245

Quantity of the bloorl-gases - - - - - - 250

Distribution and condition of oxygen in the blood - - 252

Distribution and condition of carbon dioxide in the blood - 255

The tension of the blond-gases - - - - 258

Mechanism $n f$ the gaseous excharge in the lungs - - 263 
Seats of oxidation-

Passage of oxven from the blood into the tissues

Respiration of musele - -

Nature of the oxidative process - - - $\quad 271$

Passage of carbon dioxicle from the tissues into the blood - 272

SFCtion VI.--lielation OF Respiration to THE Neriot's

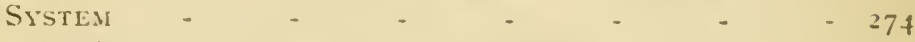

The respiratory centre and its connections - $\quad$ - 274

Regulation of respiration through the vagus - - $\quad 276$

Action of other afferent fibres on the respiration - $\quad 280$

The chenical regulation of the respiration - - $28 \mathrm{I}$

Aproea $\quad-\quad$ - $\quad$ - $\quad$ - $\quad$ - $\quad$ - $\quad-283$

Automaticity of the respiratory centre - - - $\quad 28+$

Special modifications of the respiratory movements - $\quad 287$

Sectiox VII.-The Ixfluexcr of Respiration on tife BLnod-

PRESSLRE - $\quad$ - $\quad$ - $\quad$ - $\quad$ -

SECTION VIII. ThE FFFECTS OF BREATHING CONDIXSED AND

RAREFIED AIR - $\quad$ - $\quad$ - $\quad$ - $\quad$ - $\quad-\quad 295$

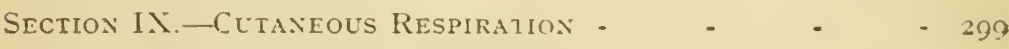

CHAPTER V.

VOICE AND SPEECH.

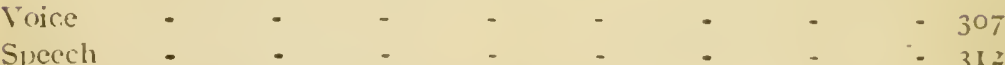

CHAPTER V'I.

DIGESTION.

Section 1.--Prelmixary ANatomical aNh Chemical Data - 3I8

Anatomy of alimentary canal - - - - $\quad 319$

Section II.-Mechanical Pifexomina of Digestion - $\quad 32$ I

Mastication - - - - - - - - $\quad$ - 321

Deglutition - $\quad-\quad$ - $\quad$ - $\quad$ - $\quad-322$

Movements of strmach - - - - $\quad$ - 320

Movements of intestine - - - - - - - $\quad 328$

Influence of central nervous system on gastro-intestinal

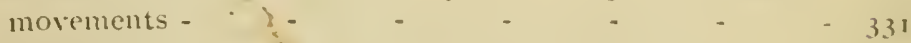

Defrecation -

lomiting - - - - - - - - - 334

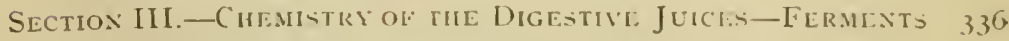

Saliva - $\quad$ - $\quad$ - $\quad$ - $\quad$ - $\quad-3.41$

Crastric juice - $\quad$ - $\quad$ - $\quad$ - $\quad-\quad-349$

Antiseptic functions of gastric juice - - $\quad-\quad 3.56$ 
Srction III. (continued)-

Pancreatic juice

Bile

Succus entericus

Section IV.-Secretion of the Digestive Jéices-MicroScopical Cha.iges ix the Giandells - - - 374

Changes in pancreas and parotid during secretion - $\quad 375$

Changes in gastric glands during secretion - - - $\quad 37$ -

Changes in nucous glands during secretion - - $\quad 381$

Mode of formation of the digestive juices _ _ _ $\quad 3_{3}$

Why are the tissues of digestion not affected by the digestive

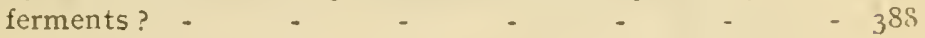

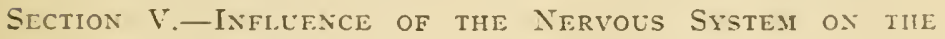

Digestive Gland - $\quad$ - $\quad$ - $\quad$ - $\quad$ - $\quad 391$

Influence of nervous system on salivary glands $\quad-\quad$ - 391

Influence of nervous system on gastric gland; - $\quad 40$

Influence of nervous system on the pancreas $\quad-40 . \overline{7}$

Secretin - $\quad$ - $\quad$ - $\quad$ - $\quad$ - $\quad . \quad 4 \quad 40_{i}^{-}$

Influence of nervous system on secretion of bile - - 411

Influence of nervous system on the secretion of intestinal

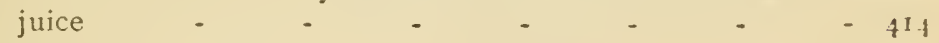

Secretion of the digestive juices (summary) - _ - $\quad$ - 415

Section VI.-Sirver of Digestion as A Whore - - 4 Ir,

Reaction of intestinal contents - . - . + fic

Bacterial digestion - - $\quad$ - $\quad$ - $\quad$ - 422

Frces - $\quad$ - $\quad$ - $\quad$ - $\quad$ - $\quad$ - 423

\section{CHAPTFR VII.}

\section{ABSORPTION.}

SECtion I.-PRELTMiNARY PHYSICO-ChEMical Data

Imbibition, diffusion, and osmosis

Flectrolytes -

Surface tension

Adsorption

Section II.-MechanisM of ABsorption

Theories of absorption

- 426

- 428

- 429

- 430

Permeability of intestinal epithelium

Absorption from the peritoneal cavity

Section III.-Absorption of the VARIols FoOd Sl̈bStances - $44^{\prime}$

Absorption of fat

Absorption of carbo-hydrates 
CIIAPTER VIII.

\section{FORMATION OF LYMPH.}

Differen kinds of lymph -

$-4 \quad-\quad-406$

Fa tors concerned in lymph formation - - - - - 467

The contribution of the tissue-cells to the lymph - - - 472

\section{CHAITER IS.}

\section{EXCRETION.}

Section I.-EAcretion by the libseys-The CheMistry of THE LRINE -

The urine in disease

Section II. - The Secretiox of the Urine

Bloodvessels and tubules of the kidney

Theories of renal secretion

Excretion of pigments by the lidney -

Infuence of the circulation on the secretion of urine

Section III.-Expulsion of the Urine -

$-470$

- $4^{85}$

- 489

- 489

- 491

- 495

- 506

Section IV.-Exchetion by the Srin -

- 510

- $51 \mathrm{I}$

\section{CHAPTER X.}

\section{METABOLISM, NUTRITION AND DIETETICS.}

Sectiox I.-Metabolism of Carbo-Hrdrates-Glycogen - 533

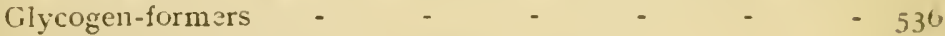

Function and fate of the glycogen - $\quad$ - $\quad-\quad-\quad-539$

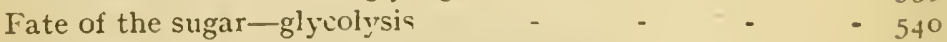

Intermediary metabolism of carbo-hydrates - - $\quad 54^{2}$

The experimental glycosurias - - - - $\quad 54^{\circ}$

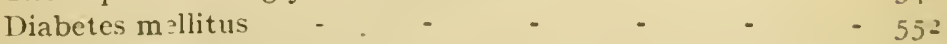

Section II.-Metabolism of Fat - - - - 550

Form ition of fat from carbo-hydrates - - - $\quad 500$

Formation of fat from protein - - - - $\quad 502$

Interm zdiary metabolism of fat - - - - $\quad-505$

Non-nutritive functions of fat - - - - $\quad-508$

Obesity

Ietabolism of sterins

Metabolism of phosphatides .

$-5^{1) 8}$

- 570

$-571$

Section III.-Metabolism of Proteins - - - - $\quad 572$

Living and dead proteins - $\quad$ - $\quad-\quad 570$

Formation of amino-acids from tissue-proteins - $\quad 578$

Form $x$ tion of hippuric acid - $\quad$ - $\quad$ - $\quad$ - 580

Fate of amino-acids in the boly $\quad \cdot \quad \cdot \quad \cdot 582$ 
SEction 111. (continued)-

formation of urea -

Formation of uric acid

Metabolism of nucleic acids and purin bases - _ $\quad 592$

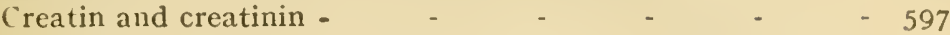

Autolysis

SECTION IV.-STATISTICS OF NUTRITION-IxCOME AÑD EXPENDI-

TURE OF THE BODY IN TERMS OF MATTER - _ - - 601

The nitrogen balance-sheet-nitrogenous equilibrium - 602

Relation of nitrogenous metabolism to muscular work - 110

Relative value of different proteins in nutrition - - 672

The carbon balance-sheet - - - -

The oxygen deficit - $\quad$ - $\quad$ - $\quad .620$

Inorganic salts in nutrition - . - . - . 620

Section V.Dietetics - $\quad$ - $\quad$ - $\quad$ - $\quad 622$

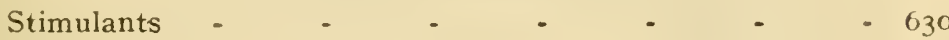

Vitamines $\quad . \quad \ldots 32$

CHAPTER XI.

INTERNAL SECRETION-ENDOCRINE GLANDS.

Pancreas

Sexual organs

Thymus

Thyroid and parathyroid -

Adrenals

Pituitary -

Spleen

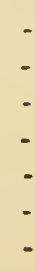

CHAPTER XII.

ANIMAL HEAT.

Section I. - Thermometry aNd Calorimetry - - - 674 Body-temperature - $\quad$ - $\quad$ - $\quad$ - $\quad$ - $\quad$ - 680

Section II.---Ixcome and Expenditure of the Body in Terms

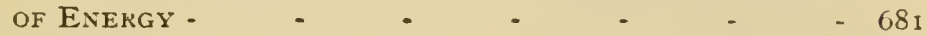

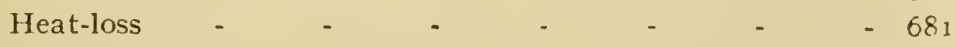

Heat-production - $\quad$ - $\quad$ - $\quad$ - $\quad-\quad-6 \delta_{3}$

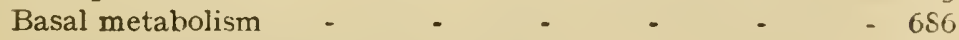

Seats of heat-production - - - . - - 686

Section III.--Thermotaxis - - - - - - $\quad$ - 690

Relations between heat-production, surface and blood-flow - 696

The nervous system and thermotaxis - - $\quad-700$

Fever - - - - - - - - $\quad$ - 703

Section IV.-Temperature Topography - - - - $\quad$ - 709

Normal variations in body temperature $\quad-\quad 712$ 
CHAPTER IIII.

THE PHYSIOLOGY OF THE CONTRACTILE TISSUES.

SECTION I.-PRFLIMINARY OBSERVATIONS-PHYSICAL AND TICHNical Data

Cilia

Section It.--Pliysical Propertils and Strallation of

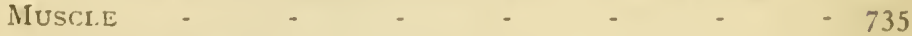

Elasticity of muscle - $\quad$ - $\quad$ - $\quad$ - $\quad-\quad 435$

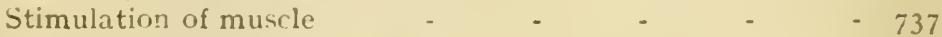

Direct excitability of muscle - _ - _ - - 738

Siction IIt-Piryical ant Mechanicat, Pilfiomexa of time

Muscutar Contractiox - - - - - - $\quad$ - $7 f^{2}$

Optical phenomena-structure of muscle - - - $74^{2}$

Meclianical phenomena $\quad$ - $\quad$ - $\quad$ - $\quad$ - 745

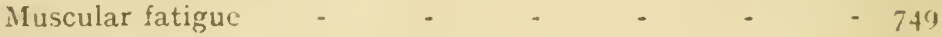

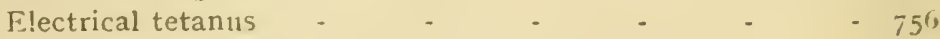

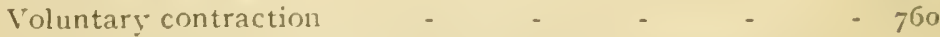

Thermal phenomena and transformation of energy in mus-

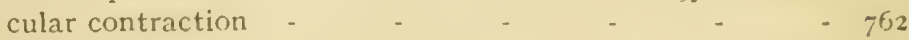

Relation between mechanical energy and heat-production in

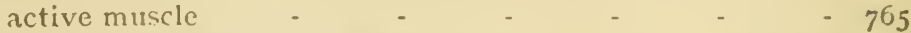

Section IV.-Chemical Phenomexa ol Muscular Contraction 767

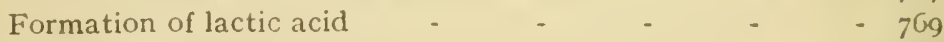

The substances metabolized in muscular contraction $\quad-771$

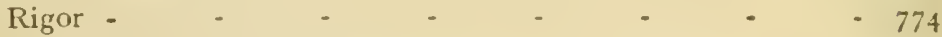

\section{CHAPTER XIV.}

NERVE.

Section I.-Tht: Nerve-Iupulse or Propagatin DistcisANCE: ITS INITIATION AND CONDECTION - - - 791

Stimulation of nerve - - - - - - - $\quad-783$

Excitability of nerve - $\quad$ - $\quad$ - $\quad-784$

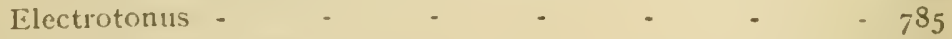

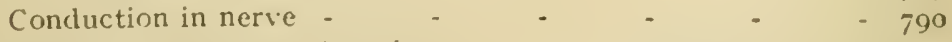

Velocity of the nerve-impulse $\quad-\quad-\quad-\quad-793$

Section II,-Cimmistry, Degeneration, and Regexeration

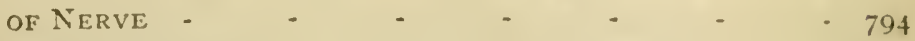

Chemistry of nerve - $\quad$ - $\quad$ - $\quad$ - $\quad$ - $\quad-794$

Degeneration of nerve $\quad-\quad$ - $\quad-\quad-\quad 495$

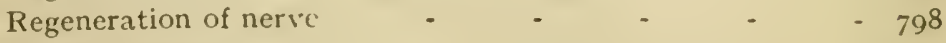

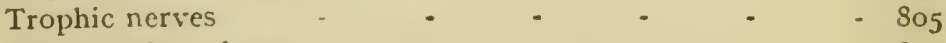

Classification of nerves $\quad-\quad \cdot \quad \cdot \quad-\quad+807$ 
CHATER IV.

\section{ELECTRO-PHYSIOLOGY.}

Currents of rest and action

PAGE

Relation between action current and functional activity -

Polarization of muscle and nerve -

Filectrotonic currents

Heart-currents

Human electro-cardiogram

Glandular currents

Eye-currents

Electric fishes

CHAPTER IVI.

\section{THE CENTRAL NERVOUS SYSTEM.}

Section I.-Structure-Histological Flemexits - - 847

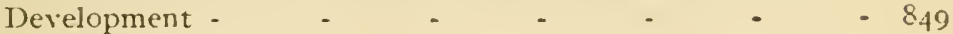

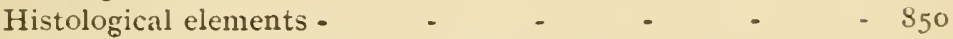

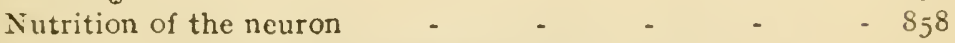

Sectiox II.-Gexeral ArRa.jgenext of the Grey aÑ White

Matier ix lue Central Nervous System - - 861

Section III.-Arrangemext of Grey axd White Matter in

SPINAT. CORD

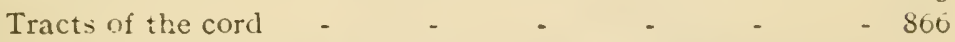

Section IV.-ArRaNgemext of Grey aNd White Matter in

THE UPPER Portiox of tHe CEREBRo-SPINAI. Axis - 809

Section V-Coxnections of the Iong PATHs of the CoRd - 870

Section VI.-Paths FROM AND to ThE CORTEx - - 878

Section VII.-Coniections of Brain Stem wil CoRd-

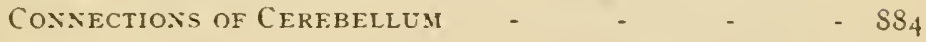

Section ViII.-Fexctions of the Cf.xtral Nervous System

- THE SPINAL CORD

Decussation of the sensory paths - _ _ _ _ $\quad$ - 894

Reflex action - $\quad$ - $\quad$ - $\quad$ - $\quad$ - $\quad$ - $\quad$ - 897

Principle of the common path - - _ $\quad$ - $\quad$ - 899

Rôle of the receptor in reflex action - . - . - 900

Characteristic properties of the reflex arc - - - 902

Irradiation of reflex action - - _ - $\quad$ - 905

Co-ordination of reflexes - $\quad$ - $\quad$ - $\quad$ - $\quad$ - gns

Influence of the brain on spinal reflexes - _ - $\quad$ - 910

Antomatism of the spinal cord - . - - 916

Muscular tonus-postural reflexes - _ _ - $\quad$ - 917 
Section 1X.-The Cranial Ñervies

Siction X.-Functions of TII: BRAIN -

Functions of the cerchellum -

- 93 I

Equilibration and orientation

- 933

Forced movements

Functions of the cercbral cortex

- 936

- 944

Motor areas

- 947

Histological differentiation of the cortex - - $\quad-9 j^{8}$

Sensory areas -

Aphasia

Localization of function in central nervous system

- 965

Influence of anastomosis of nerves on function

Co-ordination of voluntary movements

Reaction time

- $9^{5} x y$

- 975

- 976

- 981

- $9^{82}$

Section XI.- Fatigue and Steep-Hypnosis

-,$S_{3}$

Section XII.-Size of Braty and Intelligence-Cerebral. Circulation-Chemistry of Nervous ACtivity-CerlBRO-SPINAL FLUID

CHAPTIR XIII.

CHAPTER IVIII.

THE SENSES.

The Senses in General -

SECTION I.-Tision

Physical introduction

Structure of the eye -

Chemistry of the refractive modia

Refraction in the eye -

Accommodation

Functions of the iris -

Defects of the eye -

Ophthalmoscope

Skiascopy

Diplopia

Steroscopic vision

Visual judgments and illusiors

Purkinje's figures

Blind spot

Rods and cones in vision

'Talbot's law

Colour vision -

Contrast

Perimetry

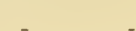

- 1006

- 1008

- 1008

- 1014

- IOIt

- 1017

- 1020

- 1026

- 1027

- 1031

- 1034

- 1037

- 1039

- 10.70

$-10.42$

- 104.1

- 1045

- 1050

- $105 \mathrm{I}$

- 1056

- 1058 
SEction I. (contimued)-

Colour-blindness

Movements of the eyes

SICTION H.- HEARING

Section ili. - Simel.l axd Taste -

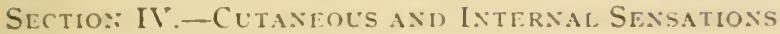

lantile senses -

Sensations of warmtl and cold

Pain

Phencmena after section of cutaneous nerves

Muscular sense

Sensations of hunger and thirst

\section{CHAPTER XII.}

\section{REPRODUCTION.}

\section{Regeneration of tissues}

Reproduction in the higher animals

Menstruation

Derelopment of the orum

Parthenogenesis

Formation of the embryo -

Development of the connections -

Exchange of materials in the placenta

Metabolism of the embryo

Par:urition

Milk

Cultivation of tissues outside of the bor'y -

Transplantation of tissues

Parabiosis .

Appendix A. - Comparison of Metrical with Exglish

\section{MEASURES}

APPENDIX B.-BIBLIOGRAPHY

INDEX

\section{PRACTICAL EXERCISES.}

\section{CHAPTER I.}

General reactions of proteins

Colour reactions of proteins

Precipitation reactions of proteins

Special reactions of groups of proteilis

Reartions of clerivatives of pr: teins

Carbo-hỹdrates

Fats

Scheme for testing for proteins and carbo-liydratos 
CHAPTIER II.

1. Reaction of blood

2. Specific gravity of blood

3. Coagulation of blood -

4. Preparation of fibrin-ferment -

5. Preparation of extracts containing thrombolinase

5. Serum -

7. Action of serum on artery rings

8. Comparison of action of serum and epinephrin un artery rings

9. Comparison of action of serum and plasma on artery rings

10. Enumeration of the blood-corpuscles -

11. Hámatocrite -

12. Electrical conductivity of blood

13. Opacity of blood

14. Laking of blood

15. Hamolysis and agglutination -

16. Osmotic resistance of coloured corpuscles

17. Blond-pigment

(1) Preparation of hamoglobin crystals.

(2) Spectroscopic examination of hamoglobin and its derivatives

(3) Guaiacum test for blood

(4) Quantitative estimation of hamoglobin

(5) Hamin test for blood-pigment

\section{CHAPTER III.}

3. Microscopic examination of the circulating blood

2. Anatomy of the frog's heart.

3. Beat of the heart

4. Apex of the heart

5. Heart tracings

6. Dissection of vagus and cardiac sympathetic in frog -

7. Stimulation of the vagus in the frog -

8. Stimulation of the junction of the sinus and auricles -

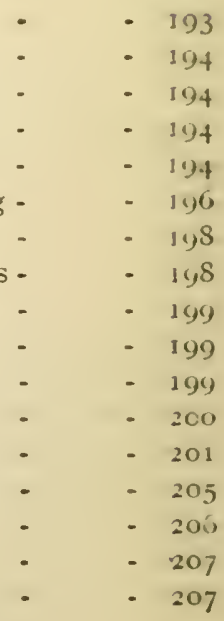

9. Action of muscarine and atropine on the heart - $\quad$ - 199

10. Stannius' experiment - - $\quad-\quad-\quad-\quad-199$

11. Stimulation of cardiac sympathetic in frog - - - 199

12. Action of inorganic salts on heart-muscle . -

13. Action of the mammalian heart - -

14. Perfusion of the isolater mammalian heart -

15. Action of the valves of the heart - -

16. Sounds of the heart - - - - - - $\quad-207$

17. Cardiogram - $\quad$ - $\quad$ - $\quad . \quad-207$ 
IS. Sphygmographic tracings

I9. Venous pulse tracing from jugular

2o. Polygraph tracings

21. Plethysmographic tracings

22. I'ulse-rate

23. Blood-pressure tracing

24. Estimation of arterial pressure in man

25. Influence of position of the body on blood-pressure -

26. Effects of hremorrhage and transfusion on blood-pressure

27. Influence of proteoses on blood-pressure

28. Effect of suprarenal extract on blood-pressure

29. Action of epinephrin on artery rings -

30. Determination of the circulation-time

3r. Measurement of the blood-How in the hands -

32. Vaso-motor reflexes

\section{CHAPTER IV.}

I. Tracing of the respiratory movements in man

- 300

2. Production of apnœa and periodic breathing in man -

- 300

3. Tracing of the respiratory movements in animals -

- 300

4. Heat dyspnca -

5. Measurement of volume of air inspired and expired -

- 302

6. Cardio-pneumatic movements -

- 303

7. Auscultation of the lungs

- 303

8. Neasurement of the respiratory pressure

9. Estimation of carbon dioxide and water given off by an animal 305

Io. Muscular contraction in the absence of free oxygen - $\quad 306$

II. Oxidizing ferments

- 306

\section{CHAPTERS VI. AND VII.}

I. Contraction of isolated intestines in Ringer's solution

$-452$

2. Effect of serum on the contractions of intestinal segments

3. Action of epinephrin on intestinal segments -

- 453

4. Quantitative estimation of ferment action -

- 453

5. Chemistry and digestive action of saliva

6. Stimulation of the chorda tympani

7. Effect of drugs on the secretion of saliva

$-454$

$-456$

8. Digestive action of gastric juice

- 457

9. To obtain chyme and gastric juice

- $45^{8}$

10. Digestive action of pancreatic juice

I I. Chemistry of bile

I2. Microscopical examination of freces

$-459$

- 460

$-462$

- 463 
13. Absorption of fat

14. Time required for digestion and absorption of food substances

15. Quantity of cane-sugar inverted and absorbed in a given time

16. Auto-digestion of the stomach

CHAPTER IX.

I. Specific gravity of urine

2. Reaction of urine

3. Chlorides in urine

4. Phosphates in urine -

5. Sulphates in urine

6. Indoxyl in urine

7. Urea

8. Ammonia in urine

9. Total nitrogen in urine

Io. Uric acid

I1. Creatinin

12. Hippuric acid -

13. Proteins in urine

I 4. Sugar in urine

15. Pentoses in urine

I6. Acetone in urine

17. Determination of the freczing-point of urine -

I8. Examination of urine -

I9. Urinary sediments

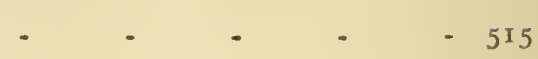

- 515

- $\quad 5 \mathrm{I} .5$

- $5 \mathrm{I}(\mathrm{)}$

- 517

$-\quad 517$

- 518

- 521

- 521

- 523

- 523

- 524

- 524

- 525

- 528

- 529

- 529

- 531

- 531

CHAPTERS X., XI., AND XII.

I. Glycogen

2. Catheterism

3. Experimental glycosuria

(I) Injection of sugar into the blood

(2) Phlorhizin glycosuria

(3) Alimentary glycosuria

(4) Estimation of the sugar in blood

4. Milk

5. Cheesc -

6. Flour

7. Bread

8. Excretion of urea (and total nitrogen) and proteins in food

9. Action of epinephrin

ro. Measurement of the heat given off in respiration 
CHAPTERS XIII. AND KIV.

1. Differcnce of make and brcak induction shocks

2. Stimulation by the voltaic current - -

3. Ciliary motion

- 810

4. Dircct excitability of muscle-curara -

- 811

5. Graphic record of ' twitch' -

(). Influence of temperature on the muscle-curve

7. Influence of load on the musclc-curve

- 811

8. Influcnce of fatiguc on the muscle-curve

9. Seat of exhaustion in fatigue of the muscle-nerve preparation $\mathrm{S}_{13}$

Io. Influence of veratrine on muscular contraction _ $\quad$ - 814

11. Measurement of the latent period of muscular contracticn - 815

12. Summation of stimuli - - $\quad$ - $\quad$ - $\quad$ - $\quad$ - 810

13. Supcrposition of contractions - - - - - $\quad$ - 810

14. Composition of tetanus - - _ _ - $\quad$ - 810

15. Contraction of smooth muscles - $\quad-\quad+\quad-817$

10. Velocity of the nerve-impulse - $\quad-\quad+\quad-\quad-\quad-818$

17. Chemistry of muscle - $\quad$ - $\quad-\quad$ - $\quad-\quad$ - 819

18. Reaction of muscle in rest, activity, and rigor $\quad$ - $\quad$ - 820

CHAPTER XV.
I. Galvani's experiment -
$-\quad-\quad-\quad-842$
2. Contraction without metals - $\quad$ - $\quad$ - $\quad-\quad 842$
3. Secondary contraction
4. Demarcation and action currents with capillary electrometer $\mathrm{S}_{42}$
5. Action current of the heart - $\quad$ - $\quad$ - $\quad-\quad$ - $\quad 844$

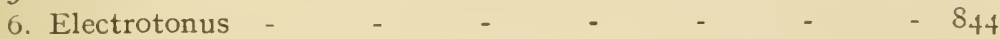
7. Paradoxical contraction - $\quad$ - $\quad$ - $\quad-\quad$ - 844

8. Alterations in excitability and conductivity produced in nerve

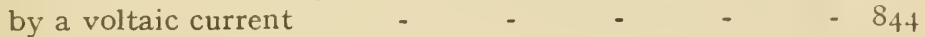

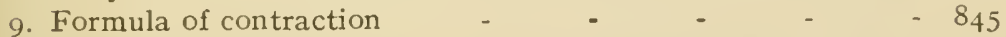

10. Formula of contraction for (human) nerves in situ - _ - 846

I I. Ritter's tetanus

- 846

\section{CHAPTER XVI.}

i. Section and stimulation of nerve-roots

2. Reflex action in the 'spinal' frog - . - . $\quad$ - 995

3. Reflex time - $\quad$ - $\quad$ - $\quad$ - $\quad$ - $\quad$ - $\quad$ - 995

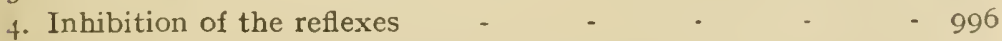

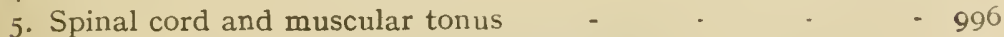

6. Spinal cord and tonus of the bloodvessels . - . $\quad$ - 996

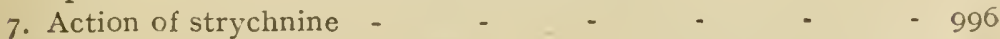

8. Mammalian spinal preparation $\quad$ - $\quad$ - $\quad$ - $\quad$ - $\quad$ - 996

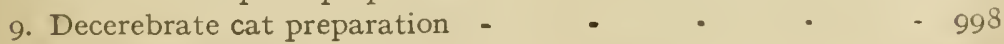

Io. Swallowing reflex 
I I. Reflex postural tonus

12. Reflexes in man

13. Excision of cerebral hemispheres in the frug -

14. Excision of cerebral hemispheres in the pigeon

15. Stimulation of the motor areas in the degr -

1. Dissection of the eye

2. Formation of inverted image on the retina

3. Helinholtz's phakoscope

4. Scheiner's experiment

5. Kühne's artificial cye -

6. Astigmatism (ophthalmumeter)

7. Spherical aberration

8. Chromatic aberration -

9. Measurement of the field of vision

o. Mapping the blind spot

[1. The yellow spot

12. Ophthalmoscope

13. Retinoscopy

I4. Pupillo-dilator and constrictor fibres -

15. Colour-mixing -

16. After-images

17. Retinal fatigue -

18. Visual acuity

19. Colour-blindness

20. Talbot's law -

21. Purkinje's figures

22. Relation of pitch and vibration frequency

23. Beats -

24. Sympathetic vibration

25. Galton's whistle

26. Cranial conduction of sound

27. Taste

28. Smell

29. Touch and pressure

30. Temperature sensations

31. Pain

\section{CHAPTER XIX.}

I. Contractions of isolated uterine rings -

2. Comparison of clianges of tone produced in uterus segments by difierent concentrations of adrenalin

3. Partition of adrenalin between serum and corpuscles 


\section{A MANUAL OF PHYSIOLOGY'}

\section{CHAPTER I}

\section{INTRODUCTION}

LIviNG matter, whether it is studied in plants or in animals, has certain peculiarities of chemical composition and structure, but especially certain peculiarities of action or function, which mark it off from the unorganized material of the dead world around it.

Chemical Composition of Living Matter.-Although we cannot analyze the living substance as such, we can to a certain, but limited, extent reconstruct it, so to speak, from its ruins. When subjected to analytical processes, which necessarily kill it, living matter invariably yields bodies of the class of proteins, exceedingly complex substances, which have approximately the following composition: Carbon, $5 I \cdot 5$ to 54.5 per cent.; oxygen, $20 \cdot 9$ to 23.5 per cent.; nitrogen, I5.2 to I 7 per cent.; hydrogen, 6.9 to 7.3 per cent., with small quantities of sulphur. Nucleo-proteins, which are compounds of ordinary proteins with nucleic acids, a series of sulphurfree organic acids rich in phosphorus, are constantly met with. Certain carbo-hydrates, composed of carbon, hydrogen, and oxygen (the last two in the proportions necessary to form water), of which glycogen $\left(\mathrm{C}_{6} \mathrm{H}_{10} \mathrm{O}_{5}\right)_{n}$ may be taken as a type, appear to be always present. Fats, which consist of carbon, hydrogen, and oxygen, and of which tristearin, a compound of stearic acid with glycerin, of the formula $\mathrm{C}_{3} \mathrm{H}_{5}, 3\left(\mathrm{C}_{18} \mathrm{H}_{35} \mathrm{O}_{2}\right)$, may be given as an cxample, are often, and certain lipoids, e.g., lecithin (p. f), are always, found. Finally, water and certain inorganic salts, such as the chlorides and phosphates of sodium, potassium, and calcium, are constantly present.

The Proteins.-The constitution of the protein molecule is still unknown; but when proteins are broken down by the action of ferments, such as exist in gastric and in pancreatic juice, or by chemical methods -for example, by boiling with dilute acids-the most important of the cleavage products are various amino-acids (p. 360). It has thcrefore been suggested that proteins are built up by the linking together of amino-acids, the different protcins differing quantitativcly or qualitatively as regards the amino-acids present (E. Fischer). Thus serumalbumin and egg-albumin yield no glycin or glycocoll (amino-acetic acid, $\mathrm{CH}_{2} . \mathrm{NH}_{2} . \mathrm{COOH}$ ), while glycin is constantly found among the cleavage products of serum-globulin. And while leucin ( $a$-amino- 
isobutylacetic acicl) is present to the extent of about $20^{\circ} 5$ per cent. in the cleavage products of (horse's) serun-albumin, (hen's) egg-albumin yiclds only $7^{\circ}$ I per cent.

On the other hand, egg-albumin yields $8 \cdot 1$ per cent. of alánin (aminopropionic acid, $\left.\mathrm{C}_{2} \mathrm{H}_{4} \cdot \mathrm{NH}_{2} \cdot \mathrm{COOIL}\right)$, while serum-albumin yields only 27 per cent. Of the aromatic amino-acids-that is, amino-acids united to the benzene ring-phenyl-alanin (amino-propionic acid in which one atom of $\mathrm{H}$ is replaced by phenyl, $\mathrm{C}_{6} \mathrm{H}_{5}$ ) is obtaincd to the extent of $f+$ per cent. from egg-albumin, and a little over 3 per cent. from serumalbumin. Tyrosin or oxyphenyl-alanin (amino-propionic acid in which a $\mathrm{H}$ atom is replaced by oxyphenyl, $\mathrm{C}_{6} \mathrm{H}_{4} . \mathrm{OH}$ ) appears to the amount of $I^{\prime} 5$ per cent. among the cleavage products of cgg-albumin, and to the amount of 2 I per cent. among those of sermm-albumin. It is an interesting point in this connection that gelatin, which yiclds 16.5 per cent. of glycin, yields no tyrosin at all; tryptophane, an aromatic amino-acid still more complex than tyrosin, is also absent. These facts afford an explanation of certain colour reactions of proteins long known cmpirically, but only recently understood (p. 8). The process by which the protein moleculc is thus decomposed is called hydrolysis - that is, the molecule takes up water, and then splits into smaller molecules. The hydrolysis occurs in various stages, bodics like acid-or alkalialbumin (meta- or infra-proteins) being first formed, then proteoses, then peptones. The peptones are further split into bodies containing a relatively small number of amino-acids linked together. These bodies are called polypeptides, which finally are decomposed so as to yield the individual amino-acids, also called in this connection the peptides or monopeptides, the "building-stones" out of whicls the protein molccule is constructed. The inverse process can also be carried on to a certain extent, and Fischer has taken an important step towards the cventual synthesis of proteins by showing how polypeptides of increasing compiexity can be built up by linking amino-acicls together. When two amino-acids are so united, the resulting compound is called a dipeptide; with three amino-acids we get tripeptides, etc. Still more complicated polypeptides may thus be formed in the laboratory, which give some of the cliaracteristic reactions of peptones.

The numerous substances included in the group of proteins may be classified as follows, beginning with the simplest:

1. Protamins, such as the bodies called salmin and sturin present in fish-sperm.

2. Histones, bodies separated from blood-corpuscles. Globin, the protein constituent of hamoglobin, is onc of them. Unlike the other groups of proteins, they are precipitated by ammonia.

3. Albumins.

4. Globulins.

5. Sclero-proteins or albuminoids, sucls as gelatin and keratin.

6. Phospho-proteins, including such substances as vitellin, a body obtainable from egg-yolk, and cascinogen, the chief protein of milk. They are rich in phosphorus, but are to be distinguished from nucleoproteins, which also contain a relatively large amount of phosphorus, by the fact that they do not yicld the purin bases, the characteristic products of the decomposition of nuclco-proteins.

7. Conjugated protcins, substances in which the protein molecule is united to another constituent, usually spoken of as a "prosthetic ' group. Thus the nuclco-proteins consist of protein united with nncleic acid, the chromo-proteins (e.g., hamoglobin) of protcin united with a pigment, and the gluco-proteins (e.g, mucin) of protein united with a carbo-hydrate group. 
Among the derivatives of proteins, the most important are those already mentioned as being produced in protein-lıydrolysis, viz.:

(a) Mcta-proteins.

(b) Proteoses, including albumose, the proteose derived from albumin; globulose, that derived from globulin; gelatose, that derived from gelatin, etc. The proteoses may be further subdivided, according to the order in which they are formed in digestion into proto-proteoses, hetero-proteoses, and deutero-proteoses.

(c) Peptones.

(d) Polypeptides. The majority of these are artificial products, formed by the synthesis of amino-acids, although some can be obtained from proteins by hydrolysis. Only a few of those hitherto prepared give the biuret test.

However formidable the above list may appear to the student, it gives an inadequate idea of the extreme complexity of the protein class and its richness in individuals. For, apart from the fact that the list has been purposely left incomplete, especially as regards the numerous vegetable proteins, there is the best evidence that proteins of the same name from different animal species have certain propertics which distinguish them from each other. The serum-albumins can be crystallized much more easily in some animals than in others. The same is conspicuously true of the hæmoglobins, which differ also in certain animals in the relative proportion of sulphur and iron in the molecule, as well as in the crystalline form. Even when no chemical or physical differences have as yet been made out, proteins of the same name from the blood or organs of different species show notable 'specific' differences when subjected to certain biological tests (sce, e.g., the paragraph on Precipitins, p. $3 \mathbf{I}$; and that on Anaphylaxis, p. 32).

Carbo-Hydrates. - The most important carbo-hydrates in their physiological relations are dextrose, levulose, galactose, lactose, maltose, sucrose (cane-sugar), starch, and glycogen. As regards their chemical constitution, the simplest carbo-hydrates are aldehydes or ketonesthat is, the first oxidation products of primary and secondary alcohols respectively. Thus dextrose is the aldehyde of sorbite, a hexatomic alcohol (an alcohol containing six OH groups), while levulose is the ketone of the isomeric alcohol called mannite, and galactose the aldehyde of the isomeric alcohol called dulcite. The sugars containing six carbon atoms are termed hexoses. They include dextrose, levulose, and galactose. The empirical formula of these three simple sugars (or monosaccharides) is the same $\left(\mathrm{C}_{6} \mathrm{H}_{12} \mathrm{O}_{6}\right)$, but, owing to the different arrangement of the atoms or groups of atoms, they have each their characteristic properties by which they can be easily distinguished. For example, dextrose rotates the plane of polarization to the right, levulose to the left. By the union or 'condensation' of two molecules of a monosaccharide, with loss of a molecule of water, a disaccharide is formed. Cane-sugar, maltose, and lactose, all with the same empirical formula, $\left(\mathrm{C}_{12} \mathrm{H}_{22} \mathrm{O}_{11}\right)$, are disaccharides. Cane-sugar yields on hydrolysis a mixture of equal parts of dextrose and levulose; lactose, a mixture of dextrose and galactose; while maltose is converted into dextrose. By the condensation of more than two molecules of monosaccharide polysaccharides are formed, such as starch, dextrin, and glycogen. The exact molecular weights of these substances are unknown. Their general formula can be written $\left(\mathrm{C}_{6} \mathrm{H}_{10} \mathrm{O}_{5}\right)_{n}$, where $n$ represents the number of monosaccharide molecules condensed to form the polysaccharide, in the case of starch probably some hundreds.

Fats and Lipoids. - The fats are compounds of higher fatty acids with glycerin (glycerin esters). The ordinary body-fat consi:ts of a 
mixture of three neutral fats (palmitin, stearin, and olein) which differ both chemically and physically from each other-e.g., in melting-point and in the so-called iodine value, the number which represents the amount of iodine taken up from a standard solution. Olein melts at $-5^{\circ} \mathrm{C}$., palmitin at $45^{\circ} \mathrm{C}$, and stearin at a still higher temperature. It is, therefore, the presence of olein which keeps the body-fat liquid at the temperature of the body. The fats are soluble in ether, in hot alcohol, and in many other liquids, but insoluble in water. Besides the ordinary fats, the tissues and liquids of the body contain phosphatides, a group of compounds which stand in close relation to the fats. but differ in containing phosphoric acid and nitrogenous bases. The most important representative of this group is lecithin $\left(\mathrm{C}_{\mathbf{4}} \mathrm{H}_{84} \mathrm{NPO}_{9}\right)$. a fat-like compound which yields on decomposition, in addition to glycerin and a fatty acid, phosphoric acid and a nitrogen-containing substance called cholin (p. 366). Lecithin, though found in all cells, is especially abundant in nervous tissues. It is associated with cholesterin and with other substances which, like lecithin and cholesterin, are soluble in ether and similar solvents of fat. For this reason these substances are often grouped together as lipoids, although some of them are chemically different from fat. Cholesterin, for instance, is an alcohol. Although usually present only in small amount, the lipoids play a very important part in the structure and in the economy of the cell.

Structure of Living Matter-The Cell.*-Bioplasm is the name given to the living matter of cells. The portion of the bioplasm differentiated as the nucleus is distinguished by the term karyoplasm, and the portion outside the nucleus by the term protoplasm or cytoplasm. Protoplasm, when examined in its most primitire undifferentiated condition in such cells as the amœba or the white blood-corpuscles, appears on first view a homogencous, structureless mass, except for certain granules embedded in it, and consisting either of products formed by its activity or of food materials. But even here more careful study reveals a certain complexity of structure. At the very least, an external layer, or ectoplasm, can be distinguished from the interior mass, or endoplasm. There is reason to believe that even where no histological demonstration of an ectoplasmic layer or a definite envelope is possible, the surface of the cell is physiologically different from its interior. In many cells the protoplasm presents the appearance of a honeycomb or network, with granules usually situated at the nodes, and holding in its vesicles or meshes a fluid, perhaps containing pabulum, from which the waste of the living framework is made good, or material upon which it works, and which it is its business to transform. Some observers, however, maintain that the network is an artificial appearance produced by the precipitation of the colloid constituents of the protoplasm by the fixing reagent, or even by the coagulative processes associated with the act of dying, and that the unaltered living substance is a homogencous fluid or jelly. It is known that

- Space permits only the slightest sketch of this subject here. For de. tailed information the student is referred to textbooks of histology. 
changes of reaction occur when the living substance dies, and slight changes of reaction, i.e., clianges in the relative concentration of hydrogen ions $(\mathrm{H}+)$ and hydroxyl ions $(\mathrm{OH}-)$, can bring about similar precipitates in colloid solutions. Nevertheless in some cells a certain differentiation in the structure of the protoplasm can be seen during life and before the addition of any reagent, and in such cases there can be no doubt that the structural details pre-exist and are not aite-facts. In certain respects protoplasm behaves like a liquid, and in others like a solid, a peculiarity which is undoubtedly associated with the fact that its chief constituents exist in the colloid state, as expcriments with such substances as gelatin and agar have shown. In building up our typical cell we start witli a piece of protoplasm. Somewhere in the midst of this we find a body which, if not absolutely different in kind from the protoplasm of the rest of the cell or cytoplasm, is yet marked off from it by very definite morphological and chemical characters.

This is the nucleus, generally of round or oval shape, and bounded by an envelope. Within the envelope lies a second network of fine threads, which do not themselves stain with nuclear dyes such as hæmatoxylin. But in or on these 'achromatic' filaments lie small, highly refractive particles, staining readily and deeply with dyes, and therefore described as consisting of chromatin. This chromatin is either made up of nucleins (conjugated proteins particularly rich in nucleic acid, and therefore in phosphorus), or yields nucleins by its decomposition; and it seems to owe its affinity for certain staining substances to the presence of nucleic acid. The meshes of the nuclear reticulum contain a semi-fluid material, which does not readily stain. The nucleus is distinguished from the cytoplasm, even as regards its inorganic constituents, by the absence of potassium.* Besides the nucleus, another much smaller structure, the centrosome, is differentiated from the protoplasm of many cells. This is a minute dot staining deeply with such dyes as hæmatoxylin, and generally situated near the nucleus. Surrounding it is a clear area, the attraction sphere, in and beyond which fine fibrils radiate out into the cytoplasm. Both the attrac. tion sphere and the nucleus play an important part in division of the cell by the process known as karyokinesis, or mitosis, or indirect division, which is by far the most common mode.

When the nucleus is about to divide, the chromatin granules arrange themselves into one or more coiled filaments or skeins, which then break up into a number of separate portions called

* This has been shown microchemically. The potassium is precipitated by a solution of hexanitrite of sodium and cobalt as orange-yellow crystals of the triple salt, hexanitrite of potassium, sodium, and cobalt. Where very minute traces of potassium are present, ammonium sulphide must be added, after washing out the excess of the cobalt reagent. Black cobalt sulphide is thus formed from the triple salt (Macallum, Frontispiece). 
chromosomes. These undergo a remarkable series of transformations, leading eventually to the segregation of the nuclear chromatin in two separate daughter nuclei, each surrounded by a portion of the original cytoplasm. Apart from its rôle in the division, and therefore in the multiplication, of the cell, the nucleus is now known to exert an infuence perhaps not less important upon those chemical changes in the cytoplasm which are necessary for its normal nutrition and function.* It is doubtful whether any portion of protoplasm can permanently survive the loss of its nuclear material. It must be remembered, however, that nuclear material may sometimes be present in diffuse form in cells which do not show a nucleus in the histological sense.

When we carry back the analysis of an organized body as far as we can, we find that every organ of it is made up of cells, which upon the whole conform to the type we liave been describing, although there are many differences in details. Some organisms there are, low down in the scale, whose whole activity is confined within the narrow limits of a single cell. The amœba sets up in life as a cell split off from its parent. It divides in its turn, and each half is a complete amœba. When we come a little higher than the amœba, we find organisms which consist of several cells, and 'specialization of function' begins to appear. Thus the hydra, the ' common fresh-water polyp' of our ponds and marshes, has an outer set of cells, the ectoderm, and an inner set, the endoderm. Through the superficial portions of the former it learns what is going on in the-world; by the contraction of their deeply placed processes it shapes its life to its environment. As we mount in the animal scale, specialization of structure and of function are found continually advancing, and the various kinds of cells are grouped together into colonies or organs. In some organs and tissues the bond of union is simple juxtaposition and similarity of function of the constituent cells. But in others the union is protoplasmic, processes of the cytoplasm actually passing from cell to cell. This is seen in certain epithelial tissues, and conspicuously in the cardiac muscle.

The Functions of Living Matter.-The peculiar functions of living matter as exhibited in the animal body will form the subject of the main portion of this book; and we need only say here: (I) That in all living organisms certain chemical changes go on, the sum total of which constitutes the metabolism of the body. These may be divided into (a) integrative or anabolic changes, by which complex substances (including the living matter itself) are built up from

* According to Hertwig, a precursor of chromatin, 'prochromatin,' a substance without characteristic staining reaction, is formed in the cytoplasm. taken up by the nucleus, and there elaborated into chromatin. From the nucleus chromatin and its derivatives return to the cytoplasm to be used in its function. 
simpler materials; and $(b)$ disintegrative or kutubolic changes, in which complex bodies (including the living substance) are broken down into comparatively simple products. In plants, upon the whole, it is integration which predominates; from substances so simple as the carbon dioxide of the air and the nitrates of the soil the plant builds up its carbo-hydrates and its proteins. In animals the main drift of the metabolic current is from the complex to the simple; no animal can construct its own protoplasm from the inorganic materials that lie around it; it must have ready-made frotein in its food. But in all plants there is some disintegration; in all animals there is some synthesis. The progress of biochemistry in recent years has indeed shown that the synthetic powers of animal cells have been greatly underestimated. (2) The living substance is excitable - that is, it responds to certain external impressions, or stimuli, by actions peculiar to each kind of cell. (3) The living substance reproduces itself. All the manifold activities included under these three heads have but one source, the transformation of the energy of the food. It is not, however, upon the whole, peculiarities in food, but in molecular structure, that underlie the peculiarities of function of different living cells. A locomotive is fed with coal; a steam-pump is fed with coal. The one carries the mail, and the other keeps a mine from being flooded. Wherein lies the difference of action? Clearly in the build, the structure of the mechanism, which determines the manner in which energy shall be transformed within it, not in any difference in the source of the energy. So one animal cell, whrin it is stimulated, shortens or contracts; another, fed perhaps with the same food, selects certain constituents from the blood or lymph, and passes thein through its substance, changing them, it may be, on the way; and a third sets up impulses which, when transmitted to the other two, initiate the contraction or secretion. In the living body the cell is the machine; the transformation of the energy of the food is the process which 'runs' it. The structure and arrangement of cells and the steps by which energy is transformed within them sum up the whole of biology.

\section{PRACTICAL EXERCISES ON CHAPTER I.}

\section{Reactions of Proteins.}

I. General Reactions of Proteins.-Egg-albumin may be taken as a type. Prepare a solution of it by adding water to white of egg, which consists mainly of egg-albumin with a little globulin. In breaking the egg, take care that none of the yolk gets mixed with the white. Snip the white up with scissors in a large capsule, then add ten or fifteen times its volume of distilled water. The solution becomes turbid from the precipitation of traces of globulin, since globulins are insoluble in distilled water. Stir thoroughly, strain through several layers of muslin, and then filter through paper. 


\section{Colour Reactions.}

(1) . Id to a little of tlie solution in a test-tube a few rlrops of strong nitric alcid. A precipitate is tlurown down, which becomes yellow on boiling. Cool, and add strong ammonia: the colour clianges to orange (xantho-proteic reaction). The reaction depends upon tlie presence of aromatic groups in the protein (in phenylalanin, tyrosin, tryptophane, oxytryptophane), wlicli are converted into nitro-compounds.

(2) To a third portion add a drop or two of very dilute cupric sulphate and excess of sodium or potassium hydroxide; a violet colour appears (Piotrowski's tesi). Peptones and proteoses (albumoses) give a pink (biuret reaction).* Sec p. 458

(3) To another portion add Millon's reagent; $\dagger$ a white precipitate comes down, which is turned reddish on boiling. If only traces of protein are present, no precipitate is caused, but the liquid takes on a red tinge. The reaction is due to ty-rosin. It is given by all aromatic substances which contain the group $\mathrm{C}_{6} \mathrm{H}_{6}$ with at least one $\mathrm{H}$ replaced by $\mathrm{OH}$, i.e., the hydroxyphenyl group $\mathrm{C}_{6} \mathrm{H}_{4} \mathrm{OH}$.

(4) Adamkiewicz's Reaction (Hopkins's modification).-To a small quantity of the albumin solution add the same bulk of dilute glyoxylic acid.f Mix, and to the mixture add an equal volume of strong pure sulphuric acid. A purple colour is obtained. The substance in the protein molecule which gives the reaction is tryptophane (p. 360).

(5) The Formaldehyde Reaction.-Add to the albumin solution a few drops of a very dilute solution of formaldehyde $(\mathbf{I}: 2,500)$, and then allow some strong (commercial) sulphuric acid to run from a pipette into the bottom of the test-tube. A purple ring appears at the surface of contact. This reaction depends on the presence of tryptophanc in the protein.

\section{Precipitation Reactions.}

(6) Acidify another portion strongly with acetic acid, and add a few drops of a solution of potassium ferrocyanide. A white precipitate is obtained. Peptones do not give this reaction.

(7) Heat a portion to $30^{\circ} \mathrm{C}$. on a water-bath. Saturate with crystals of ammonium sulphate; the albumin is precipitated. Filter, and test the filtrate for proteins by (2). None, or only slight traces, will be found. The sodium hydroxide must be added in more than sufficient quantity to decompose all the ammonium sulphatc. It will be best to add a piece of the solid hydroxide. Peptones are not precipitated by ammonium sulphate, but all other protcins are.

(8) Add alcolol to a small quantity of the solution. The protein is

- The reaction is also given, although more faintly, with the hydroxides of lithium, strontium, and barium. It is given by all substances containing at least two $\mathrm{CONH}_{2}$ groups attached to one another (as in oxamide), or to the nitrogen atom (as in biuret), or to the same carbon atom.

+ IIIllon's reagent consists of a mixture of the nitrates of mercury with nitric acid in excess, anrl some nitrous acid. To make it, dissolve mercury in its own weight of strong nitric acid, and add to the solution thus obtained twice its volume of water. Let it stand for a short time, and then decant the clear liquid, which is the reagent.

¥ A solution containing glyoxylic acjd in the requisite strength can be prepared by treating half a litre of a saturated solution of oxalic acid with 40 grammes of 2 per cent. sodjum amalgam in a tall cylinder. When all the hydrogen has been evolved, the solution is filtered, and diluted with twice its volume of water. Oxalic acid and sodium binoxalate are also present in the solution. 
precipitated. It can be redissolved at first, but rapidly becomes in. soluble.

2. Special Reactions of Certain Proteins-(I) Heat-Coagulable Proteins: (a) Albumins. - (a) Heat a little of the solution of egg-albumin in a test-tube; it coagulates. With another sample determine the temperature of coagulation, first very slightly acidulating witlı a 2 per cent. solution of acctic acid.

To determine the Temperature of Coagulation.-Support a beaker by a ring which just grips it at the rim. Nearly fili the beaker with water, and slide the ring on the stand till the lower part of the beaker is immersed in a small water-bath (a tin can will do quite wcll). In this beaker place a test-tube, and in the test-tube a thermometer, botli supported by rings or clamps attached to the same stand. Put into the test-tube at least cnough of the albumin solution to completely cover the bulb of the thermometer, and heat the bath, stirring the water in the beaker occasionally with a feather or a splinter of wood, or a glass rod, the end of which is guarded with a picce of indiarubber tubing. Note the temperature at which the solution becomes turbid, and then the temperature at, which a distinct coagulum or precipitate is formed. Repeat with the unacidulated albumin solution.

$(\beta)$ A similar experiment may be performed with serum-albumin obtained as on p. 65 .

(b) Globulins.-Use serum-globulin (p. 65), or myosinogen (p. 819). Fibrinogen is also a globulin, but cannot easily be obtained in quantity. Verify the following properties of globulins:

(a) They coagulate on heating.

( $\beta$ ) They are insoluble in distilled water (p. 65).

( $\gamma$ ) They are precipitated by saturation with magnesium sulphate or sodium chloride (p. 65).

They give the general protein tests $(\mathrm{I})$ to $(8)$.

Both the heat-coagulated proteins and such proteins as the solid fibrin which is formed from fibrinogen in the clotting of blood give such of the general protein tests, (I), (2), (3) (p.8), as with suitable modifications can be instituted on solid substances. Thus, in performing (2), a flake of fibrin or a small piece of the boiled egg-white should be soaked for a few minutes in a dilute solution of cupric sulphate. Then the excess of the cupric sulphate should be poured off, and sodium hydroxide added, when the coagulated protein will become violet. Heat-coagulated proteins are insoluble in water, weak acids and alkalies, and saline solutions; fibrin is slightly soluble in the latter.

(2) Gelatin.-Add some pieces of gelatin to cold water in a test-tubc. It does not dissolve. Immerse the tube in a boiling water-bath till the gelatin goes into solution. Then cool the test-tube under the tap; the solution sets into a jelly. On heating it redissolves.

Try the general protein reactions (p. 8) on a dilute solution. In Piotrowski's test a violet colour is obtained. The tests which depend on the presence of tyrosin or tryptophane are not given by a solution of pure gelatin, since these amino-acids are absent from the gelatin inolecule. Commercial gelatin may give a slight reaction due to traces of other protcins.

3. Reactions of Certain Derivatives of Native Proteins-(I) MetaProteins: (a) Acid-Albumin.-To a solution of egg-albumin add a little $\mathrm{O}_{4} 4$ per cent. hydrochloric acid, and heat to about body temperaturesay $40^{\circ} \mathrm{C}$. - for a few minutes. Acid-albumin is formed. It can be produced from all albumins and globulins by the action of dilute acid. Make the following tests:

(a) Add to a portion of the solution in a test-tube a few drops of a 
sulution of litmus, the colour becomes red. Now add drop by drop sodium carbonate or dilute sodium hydroxide solution till the tint just begins to change to blue. A precipitate of acid-albumin is thrown down. Add a little more of the alkali, and the precipitate is redissolved. It can be again brought down by neutralizing with acid.

$(\beta)$ Heat a portion of the solution to boiling; no precipitate is formed.

( $\gamma)$ Add strong nitric acid; a precipitate appears, which dissolves on heating, and the liquid becomes yellow.

(b) Alkali-albumin.-To a solution of egg-albumin add a little sodium hydroxide, and heat gently for a few minutes. Alkali-albumin is produced. It can be derived by similar treatment from any albumin or globulin.

(a) Neutralize, after colouring with litmus solution, by the arldition of dilute hydrochloric or acetic acid. Alkali-albumin is precipitated when neutralization has been reached. It is redissolved in excess of the acid.

$(\beta)$ To another portion of the solution of alkali-albumin add a few drops of sodium phosphate solution, then litmus, and then dilute acid till the alkali-albumin is precipitated. More of the dilute acid should now be required to precipitate the alkali-albumin, since the sodium phosphate must first be clianged into acid sodium phosphate.

$(\gamma)$ On heating the solution of alkali-albumin there is no coagulation.

(2) Proteoses.-For preparation and reactions, see p. 458. They differ from albumins and globulins in not being coagulated by heat, and from meta-proteins in not being precipitated by nuutralization. They are soluble (with the exception of hetero-albumose) in distilled water, and are not precipitated by saturation of their solutions with magnesium sulphate or sodium chloride. Saturation with ammonium sulphate precipitates them. With a solution of 'commercial peptone,' which consists chiefly of albumoses, and contains only a little true peptone, perform the following tests:

(a) Boil the slightly acidulated solution; there is no coagulation.

( $\beta$ ) Biuret reaction, p. 8.

$(\gamma)$ To a portion of the solution add its own volume of saturated ammonium sulphate solution. The primary albumoses (proto- and hetero-albumose) are precipitated. Filter. Add a drop of sulphuric acid to the filtrate and saturate it with ammonium sulphate crystals. The secondary or deutero-albumoses are precipitated. Filter. The filtrate still contains peptones. U'se it for (3).

(3) Peptones.-For preparation and tests, sec p. 459. They differ from heat-coagulable proteins and meta-proteins in the same way as proteoses, and they differ from proteoses in not being precipitated by ammonium sulphate. On the filtrate from (2) perform the biuret test, as described in (7), p. 8 ; and note that the pink colour is the same as that given by proteoses.

\section{Carbo-Hydrates.}

1. Glucose or Dextrose.-Make a solution of dextrose in water, and apply- to it Trommer's test for reducing sugar. Put some of the dextrose solution in a test-tube, then a few drops of cupric sulphate, and then excess of sodium or potassium hydroxide. The blue precipitate of cupric hydroxide which is first thrown down is immediately dissolved in the presence of dextrose and many other organic substances. Now boil the blue liquid, and a yellow or red precipitate (cuprous hydroxide or oxide) is formed. 
2. Cane-Sugar.-Perforın Tromminr's test with ál sample of a solution. A bluc liquid is obtained, which is not changed on boiling. Now put the rest of the solution in a flask. Add $\frac{1}{20}$ th of its bulk of strong hydrochloric acid, and boil for a quarter of an hour. Agdin perform Troniner's test. Remember that excess of alkali must be present after the acid is neutralized. The test now shows much reducing sugar. The cane-sugar has been 'inverted'-i.e., changed into a mixture of dextrose and levulose.

3. Starch.-(I) Cut a slice from a well-washed potato; takc a scraping from it with a knife, and examine with the microscope. Note the starch granules with their concentric markings, using a small diaphragm. Run a drop of dilute iodine solution under the cover-slip, and observe that the granules become bluish. Examine also with a polarization inicroscope. (2) Rub up a little starch in a mortar with cold water. then add boiling water and stir thoroughly. Decant into a capsule or beaker, and boil for a few ininutes. After the liquid has cooled, perform the following experiments:

(a) Add a few drops of iodine solution to a little of the thin starch mucilage in a test-tube. A blue colour is produced, which disappears on heating, returns on cooling, is bleached by the addition of a little sodium hydroxide, and restored by dilute acid.

(b) Test the starch solution for reducing sugar by Trommer's test. If none is found, boil some of the mucilage with a little dilute sulphuric acid in a flask for twenty minutes, and again perform Trommer's test. Abundance of reducing sugar will now be present.

4. Dextrin.-Dissolve some dextrin in boiling water. Cool. Add iodine solution to a portion; a reddish-brown (port-wine) colour results, which disappears on heating. As a control, the same amount of iodine should be added to an equal quantity of water in another test-tubc. The colour returns on cooling. The colour is also bleached by alkali, restored by acid. Excess of iodine should be added for the bleaching experiment (i.e., more than enough to give the maximum depth of tint). If too little iodine has been added, there may be no restoration of the colour by the acid. The addition of a little more iodine to the acid solution will then cause the port-wine colour to return, and this may be again bleached by alkali, and wili now be restored by acid.

5. Glycogen.-See p. 7 I 5 .

6. Molisch's Test for Carbo-Hydrates.-This is a general test for carbohydrates. It is also given by proteins which contain a carbo-hydrate group. Put a drop of dextrose solution in a test-tube. Add a drop of a ro per cent. solution of a-naphthol in methyl alcohol, and then $0^{\circ} 5$ c.c. of water. Then cautiously allow I c.c. of pure concentrated sulphuric acid to run under the mixture, and shake gently. A violet or reddish colour appears.

\section{Fats.}

I. Take a little lard or olive-oil, and observe that fat is soluble in ether or warm alcohol, but not in water. Put a drop of the ethereal solution of fat on a piece of paper, and note that it leaves a greasy stain.

2. Put a little alcohol in a test-tube, and then a drop of phenolphthalein solution and a drop or two of dilute sodium hydroxide to give the solution a red colour. Add a few drops of an ethereal solution of the lard or olive-oil. If the red colour persists, the fat is neutral; if it disappears, the fat contains free fatty acids.

3. Saponification.-Melt some lard in a porcelain dish, and pour it 
into an alcoholic solution of potassium hydroxide previously heated on a water-bath nearly to boiling. Mix well, and keep the mixture gently boiling on the bath till saponification is complcte. This only takes is short time. Remove a little of the soap solution, and droy it into dis. tilled water in a test-tube. If unsaponified fat is present, it will rise to the top as drops of oil. In this case boiling should be continued. If all the fat has been saponified, the soap solution will mix with the water and no oil-drops will separate.

4. Fatty Acids.-Heat some 20 per cent. sulphuric acid in a small flask nearly to boiling, and drop into it some of the soap obtained in 3 . The fatty acids separate out and rise to the top as an oily layer. Cool skim off the fatty acid, and wash it with distilled water till the washwater is no longer acid.

(a) Dissolve a little of the washed fatty acid in ether. Add a few drops of an alkaline solution of phenolphthalein to a few c.c. of water in a test-tube. Drop into this the ethereal solution of fatty acid. The red colour is discharged.

(b) Put a small portion of the fatty acid on a glass slide resting on a piece of white paper. Place on it a drop or two of a I per cent. solution of osmic acid (osmium tetroxide). The osmic acid is reduced to a lower oxide (which is black) by the action of oleic acid present in the fatty acid mixture, which abstracts some of the oxygen. Any fat which contains olein or oleic acid, as body-fat does, is therefore blackened by osmic acid.

(c) Add to a portion of the fatty acid some sodium hydroxide solution, and warm. Sodium soap is formed. Add warm water and shake up. A lather is produced. Keep the soap solution for 6 . Keep a little of the fatty acid for $5(b)$ and $6(b)$.

5. Glycerin.-(a) Add to a little glycerin in a dry test-tube a few crystals of potassium bisulphate $\left(\mathrm{KHSO}_{4}\right)$, and heat over the free flame. Acrolein is given off, which is recognized by its pungent odour, and by blackening a piece of filter-paper moistened with ammoniacal silver nitrate solution, and held over the mouth of the test-tube. The paper is blackened owing to the reducing action of the vapour on the silver nitrate.

(b) Repeat this test with lard, and with a portion of the fatty acid from 4. Acrolein will be given off by the lard because glycerin is contained in neutral fat, but not by the fatty acid if it has been properly. separated from the glycerin.

6. Emulsification.-(a) Take three test-tubes and label them A, B. and $C$. Put a few c.c. of water in $A$, a solution of soap in $B$, and it dilute solution of sodium carbonate or sodium hydroxide in C. To each add a few drops of fresh olive-oil and shake. An emulsion will be formed in $\mathrm{B}$, but not in A. Probably there will be some emulsification in Calso, owing to the presence in the oil of some fatty acid, which forms soap with the alkali. But if the oil is free from fatty acid, no emulsion will be formed.

(b) Repeat (a) with rancid olive-oil, which contains much fatty acid, or with fresh olive-oil to which some of the fatty acid obtained in 4 lias been added. A good emulsion will be produced in $\mathrm{C}$ as well as in B.

7. Melting-Point of Fat.-Put into a very narrow test-tube or a sliort piece of narrow glass tubing some finely divided mutton fat, freed, as far as possible, from connective tissue. Fasten the test-tube on to the bulb of a thermometer with a rubber band, and immerse the thermometer and tube in a beaker filled with water and standing on a waterbath, which is gradually heated. Observe the temperature at which the fat melts. Repeat the experiment with hog's lard and dog's fat. 
SCHEME FOR TESTING A SOLUTION FOR THE MORE COMMON PROTEINS AND PROTEIN-DERIVATIVES, AND FOR CARBOHYDRATES

I. Note the reaction, and whether the liquid is coloured or colourless, clear or opalescent. A reddish colour suggests blood; opalescence suggests glycogen or starch. Try one or more of the general protein tests (e.g., the xantho. protcic or biuret). If the result is positive, proceed as in 2; if negative, pass to 3 .

2. Test for Proteins. - (I) If the reaction is acid or alkaline, neutralize with very dilute sodium carbonate or sulphuric acid. A precipitate=acid- or alkali-albumin, according as the original reaction is acid or alkaline. If the original reaction is neutral, no acid- or alkali-albumin can be present in solution. Filter off the precipitate, if any.

(2) Boil some of the filtrate from (r) (or some of the original solution if it is neutral), acidulating slightly with dilute acetic acid. A precipitate= albumin or globulin. Filter, and keep the filtrate.

(3) If a precipitate has becn obtained in (2), (a) saturate some of the original solution with magnesium sulphate, or half saturate it with ammonium sulphate (i.e., add to it an equal volume of saturated ammonium sulphate solution). If there is no precipitate, globulin is absent, and therefore the precipitate obtained in (2) must be albumin. A precipitate=globulin. But albumin may also be present in the solution. To see whether this is so, filter off the globulin and boil the filtrate after acidulation with acetic acid. A precipitate =albumin.

(b) Half saturate the filtrate from (2) with ammonium sulphate (i.e., add its own volume of a saturated solution of the salt). A precipitate=primary proteoses. Filter.

(c) Saturate the filtrate from (b) with ammonium sulphate crystals. A precipitate $=$ secondary proteoses. Filter.

(d) To the filtrate from (c) add excess of solid sodium hydroxide in small pieces at a time. Much ammonia is given off. Allow the test-tube to stand fifteen minutes, shaking it at intervals. Then add dilute cupric sulphate, and if much of the sodium sulphate formed remains undissolved, add water to dissolve it. A well-marked rose colour $=$ peptone.

(4) If no precipitate has been obtained in (2), the solution contains neither albumin nor globulin. To test whether primary or secondary proteose or peptone is present, apply $(3)(b),(c)$, and $(d)$.

3. Test for Carbo-Hydrates. - Use the original solution, freed from coagulable proteins, if such have been found, by acidulation and boiling.

(I) Add iodine. If the solution is alkaline neutralize it before adding the iodine. A blue colour=starch. Confirm by boiling with dilute sulphuric acid and testing for reducing sugar. A reddish-brown colour with iodine= glycogen or dextrin.

Glycogen gives an opalescent, dextrin a clear, solution. Glycogen is pre. cipitated by basic lead acetate, dextrin is not (p.7r5). Both are changed into reducing sugar by boiling with dilute acid.

(2) Add to some of the original solution cupric sulphate and excess of sodium hydroxide, and boil. Yellow or red precipitate=reducing sugar.

(3) If (I) and (2) are negative, boil some of the liquid with one-twentieth of its volume of strong hydrochloric acid for fifteen minutes, and test as in (2). A red or yellow precipitate indicates that a disaccharide like cane-sugar was originally present, and has been inverted. 


\section{CHAPTER II}

\section{THE CIRCULATING LIQUIDS OF THE BODY}

In the living cells of the animal body chemical changes are constantly going on; energy, on the whole, is running down; complex substances are being broken up into simpler combinations. So long as life lasts, food must be brought to the tissues, and waste products carried away from them. In lowly forms like the amœba these functions are performed by interchange at the surface of the animal without any special mechanism; but in all complex organisms they are the business of special liquids, which circulate in finely branching channels, and are brought into close relation at various parts of their course with absorbing organs, with eliminating organs, and with the tissue elements in general.

In the higher animals three circulating liquids have been distinguished: blood, lymph, and chyle. But it is to be remarked that chyle is only lymph derived from the walls of the alimentary canal, and therefore, during digestion, containing certain freshlyabsorbed constituents of the food; while both ordinary lymph and chyle ultimately find their way into the blood, and are in their turn recruited from it. The blood contains at one time or another everything which is about to become part of the tissues, and everything which has ceased to belong to them. It is at once the scavenger and the food-provider of the cell. But no bloodvessel enters any cell;* and if we could unravel the complex mass of tissue elements which essentially constitute what we call an organ, we should see a sheet of cells, with capillaries in very close relation to them, but everywhere separated from them by a thin layer of lymph. And to describe in a word the circulation of the food substances we may say that the blood feeds the lymph, and the lymph feeds the cell.

\section{SECtion I.-MoRphology of THE Blood.}

The blood consists essentially of a liquid part, the plasma, in which are suspended cellular elements, the corpuscles. When the circulation in a frog's web or lung or in the tail of a tadpole is

* Fine intracellular canaliculi, communicating with the blood-capillaries, and probably performing a nutritive function, since they seem to contain blood-plasma, have been described by Schäfer and others in the liver cells. 
examined under the microscope, the bloodvessels are seen to be crowded with oval bodies - of a yellowish tinge in a thin layer, but in thick layers crimson-which move with varying velocity, now in single file, now jostling each other two or three abreast, as they are borne along in the axis of an apparently scanty stream of transparent liquid. Nearer the walls of the vessels, sometimes clinging to them for a little and then being washed away again, may be seen, especially as the blood-flow slackens, a few comparatively small, round, colourless cells. The oval bodies are the red or coloured corpuscles, or erythrocytes; the colourless elements are the white blood-corpuscles, or leucocytes; the liquid in which they float is the plasma (Practical Exercises, p. I93).

The Red Blood-Corpuscles, or Erythrocytes, differ in shape and size and in other respects in different animal groups. In amphibians, such as the frog and the newt, they are flattened ellipsoids containing a nucleus, and the same is true of nearly all the other ver. tebrates, except mammals. In mammals they are discs, hollowed out on both the flat surfaces, or biconcave, and possess no nucleus. But the red corpuscles of the llama and the camel, although nonnucleated, are ellipsoidal in shape, like those of the lower vertebrates. As to size, the average diameter

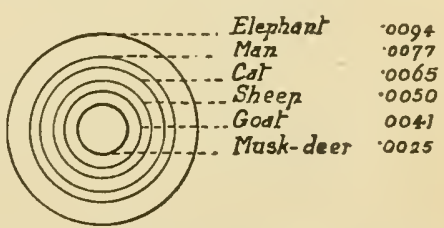

Fig. I.-Diagram showing Relative Size of Red Corpuscles of Various Animals. in man is between 7 and $8 \mu{ }^{*}$ In the frog the long diameter is about $22 \mu$, while in Proteus it is as much as $60 \mu$, and in Amphiuma, the corpuscles of which can be seen with the naked eye, nearly So $\mu$ (Frontispiece).

As regards the structure of the red corpuscles, the most probable view is that they are solid bodies, with a spongy and elastic structureless framework, denser at the surface of the corpuscle than in its centre, but continuous throughout its whole mass (Rollett). The denser peripheral layer constitutes a physiological envelope which permits the passage of certain substances into or out of the corpuscles, and hinders the passage of others. In the large oval corpuscles of Necturus (see Frontispiece) the envelope can be clearly demonstrated as a detachable membrane comparable to the membrane surrounding the nucleus.

Envelope and spongework are sometimes spoken of as the stroma of the corpuscle, in contradistinction to its most important constituent, a highly complex pigment, the hæmoglobin. This pigment is not in solution as such, for its solubility is not nearly great enough to permit this, but either in solution as a compound with

* A micro-millimetre, represented by symbol $\mu$, is $\frac{1}{1000}$ millimetre. 
some other unknown substance, or more probably bound in some solid or semi-solid combination to the stroma, and filling up the space within the envelope in the interstices of the spongework. Since there is good reason to believe that the hamoglobin as obtained artificially from the corpuscles is not quite the same substance as the native blood-pigment within them, the latter is sometimes distinguished by a separate name-hremochrome. To the physical properties of the stroma it is usual to attribute the great elasticity of the corpuscles-that is, the power of recovering their original shape after distortion-for their elasticity is in no wise impaired by the removal of the hæmoglobin.

Rouleaux Formation.-When blood with disc-shaped corpuscles is shed, there is a great tendency for the corpuscles to run together into groups resembling rouleaux, or piles of coin. No satisfactory explanation of this curious fact has yet been given.

Crenation of the corpuscles, a condition in which they become studded witl fine projections, is caused by the addition of moderately strong salt solution, by the passage of slocks of clectricity at high potential, as from a Leyden jar, or by simple exposure to the air. Concentrated saline solutions, which abstract water from the corpuscles and cause them to shrink, make the colour of blood a brighter red, because more light is now reflected from the crumpled surfaces. On the other hand, the addition of water renders the corpuscles spherical; more of the light passes through them, less is reflected, and the colour becomes dark crimson (Frontispiece).

The White Blood-Corpuscles, or Leucocytes.-The red corpuscles are peculiar to blood. The white corpuscles may be looked upon as peripatetic portions of the mesoderm (see Cliap. XIX.), and some of them ought not in strictness to be called blood-corpuscles. They are more truly body corpuscles. Similar cells are found in many

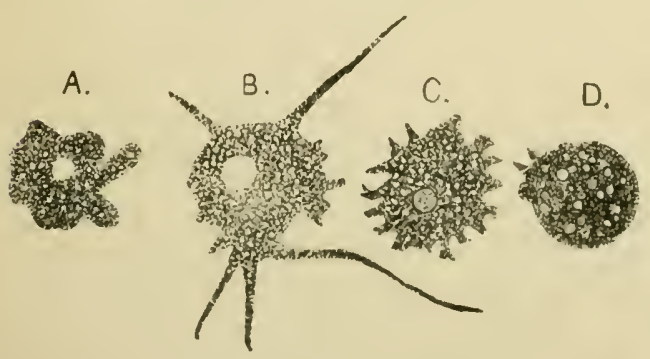

Fig. 2.-Amœboid Movement. A, B, C, D, succes. sive changes in the form of an amœba. situations, and wander everywhere in the spaces of the connective tissue. They pass into the bloodvessels with the lymph, and may pass out of them again in virtue of their amneboid power. They consist of protoplasm, less differentiated than that of any other cells in the body, and under the microscope appear as granular, colourless, transparent bodies, spherical in form when at rest, and containing a nucleus, often tri- or multi-lobed. Many of the leuco cytes of frog's blood at the ordinary temperature, and of mammalian blood when artificially heated on the warm stage, may be 
seen to undergo slow changes of form. Processes called psendopodia are pushed out at one portion of the surface, retracted at another, and thus the corpuscle gradually moves or 'flows' from place to place, and envelopes or eats up substances, such as grains of carmine, which come in its way. This kind of motion was first observed in the amcba, and is therefore called amceboid. It is perhaps due to local alterations of surface tension; at any rate, similar phenomena can be thus produced artificially. The leucocytes of human blood are not all of the same size, and differ also in other respects. They may be classified according to the presence or absence of granules in their protoplasm, and the fineness or coarseness of the granules; according to the chemical nature of the dyes with which the granules most readily stain, and according to the form of the nucleus. Five or six varieties of leucocytes may thus be distinguished in normal blood (Frontispiece):

I. Polymorphomuclear Neutrophile Cells.-.The nucleus assumes a great variety of forms, often contorted or deeply lobed, the lobes being united by fine strands of chromatin. The cytoplasm contains numerous fine refractive granules, which stain best neither with simple acid dyes like eosin nor with simple basic dyes like methylene blue, but with mixtures which raust be assumed to contain 'neutral' stains, like Ehrlich's so-called triacid stain.* These cells make up 65 to 75 per cent. of the total number of leucocytes. Their diameter is Io to I2 $\mu$.

2. Eosinophile Cells (I2 to I5 $\mu$ in diameter), much less numerous in normal blood than the neutrophiles (less than 5 per cent. of the whole), but found in considerable numbers in the serous cavities, the connective tissue, and the bone-marrow. The granules in the cytoplasm are coarser than the neutrophile granules, and stain much more deeply with eosin. The nucleus may be simple, lobed, or even divided into fragments between which no connection can be traced. It is less rich in chromatin, and stains less easily with basic dyes, like methylene blue, than the nucleus of the first variety.

3. Large Mononuclear (also called Transitional) Leucocytes, with a diameter of I $_{2}$ to $\mathrm{I}_{5} \mu$. They possess a large simple or slightly lobed nucleus, poor in chromatin, surrounded by a relatively great amount of cytoplasm, with faint neutrophile granules-i.e., granules which stain with neutral dyes. They constitute 3 to 5 per cent. of the total number of leucocytes.

4. Lymphocytes of Two Varieties - (a) Small Lymphocytes.-Smaller cells than any of the preceding (diameter $6 \mu$ ), possessing a single large nucleus, surrounded by a comparatively small amount of non-granular cytoplasm; 20 to 25 per cent. of the leucocytes of the blood belong to this group. The lymphocytes are markedly deficient in the power of amoboid motion in comparison with the other varieties of colourless corpuscles.

(b) Large Lymphocytes. - The largest of all the white cells of the blood, and at least twice as large as the small lymphocytes. They possess a relatively great proportion of cytoplasm, which is devoid of Ganules. They constitute no more than I per cent. of the total number of the colourless corpuscles.

* A mixture of orange G., acid fuchsin, and methyl green. 
5. 'Mast Cells,' or 'Basophiles,' the least numerous variety (0'5 per cent. of the total number). Very few are to be found in the normal blood of adults, but more in children. They are somewhat smaller than the neutrophiles (average diameter about $10 \mu$ ). The nucleus is irregularly trilobed. The protoplasm shows coarse granules, which do not glitter like the granules of the eosinophile cells, and are therefore less conspicuous in the unstained condition. Unlike the eosinophile granules, they stain with basic dyes, such as methylene blue.

Blood-Plates, or Thrombocytes.-When blood is cxamined immediately after being shed, small colourless bodies ( $\mathrm{I}$ to $3 \mu$ in diameter) of various shapes, but usually round or oval, may be seen. These are the blood-plates or platelets, also called thrombucytes, on account of their function in the coagulation of blood. If the blood is not at unce subjected to some procedure which prevents clotting, the platelets swell and then break up. There is reason to believe that in most of the methods of preventing coagulation the essential action is to hinder the break-up of the platelets (p. 37). They can be isolated by receiving a drop of blood from the finger upon a well-cleaned cover-slip, which is then laid, supported by two thin glass fibres, on a carefully cleaned slide. The plasma with the coloured corpuscles and leucocytes are washed away by irrigating the space between slide and slip with a suitable solution, e.g., a salt solution containing a certain proportion of manganese sulphate, which prevents disintegration of the platelets. The platelets stich to the cover-slip (Deetjen). They can then be fixed and stained. The blood-plates can even, like leucocytes, be kept alive on the warm stage in an appropriate medium (agar, to which certain salts have been added), and then show lively amueboid movements (I)etjen). They have been described as nucleated cells, although the nucleus is not easy to stain, and with the ultra-microscope, a delicate means of testing whether such an object as a platelet is optically homogeneous, no evidence of the presence of a nucleus has been obtained. The origin of the platelets has been a matter of lively controversy. They are not produced by the breaking up of other elements of the shed blood, for they have been observed within the freshly excised, and therefore still living, capillariesin the mesentery of the guinea-pig and rat (Osler). According to the best evidence, they are derivatives neither of the erythrocytes nor of the leucocytes of the blood, but are dereloped from special elements (so-called megakaryocytes) of the blood-forming organs (bone-marrow) (J. H. Wright).

Enumeration of the Blood-Corpuscles. - This is done by taking a measured quantity of blood, diluting it to a known extent with a liquid which does not destroy the corpuscles, and counting tlic number in a given volume of the diluted blood (p. 67).

The average number of red corpuscles in a cubic millimetre of blood is about $5,000,000$ in a healthy man, and about $4,500,000$ in 
a healthy woman, but a variation of $1,000,000$ up or duwn can hardly be considered abnormal. In persons suffering from profound ancemia the number may sink to I,000,000 per cubic millimetre, or even less. In one case of pernicious anæmia, only $I+3,000$ corpuscles

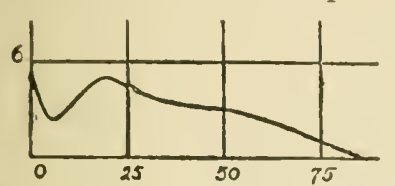

Fig. 3.-Curve showing the Number of Red Corpuscles at Different Ages (after Sörensen's Estimations). The figures along the horizontal axis are vears of age, those along the vertical axis millions of corpuscles per cubic millimetre of blood. per cubic millimetre were present, the lowest number recorded. In new-born children the average is over $6,000,000$ and in the inhabitants of high plateau or mountains it may rise to 7,000,000 or even more. In the latter instance a residence of a fortnight in the rarefied air is sufficient to bring about the increase, and a subsequent residence of a fortnight in the lowlands to annul it.* In certain pathological conditions a great increase in the relative number of corpuscles (polycythamia) is found. Over 13,000,000 erythrocyte: to the cubic millimetre have been counted in a case of cyanosis (imperfect oxygenation of the blood, with blueness of the lips, etc.), dur to congenital disease of the heart. An interesting form of experimental polycythæmia is caused by injection of adrenalin.

The number of white blood-corpuscles is on the average about I0,000 per cubic millimetre of blood, or one leucocyte for every 500 red bloodcorpuscles. But if the count is made when digestion is relatively inactive, four to five hours after a meal, it gives no more than 7,000 to the cubic millimetre. In new-born children the average number is over I8,000 per cubic millimetre. The total leucocyte count, and still more the so-called differential count, i.e., the determination of the relative number of the different kinds of leucocytes, is often resorted to in the study of pathological conditions. A distinct increase in the number is designated leucocytosis. In leukæmia the number of white corpuscles is enormously increased-on the average to about 300,000 , but in extreme cases

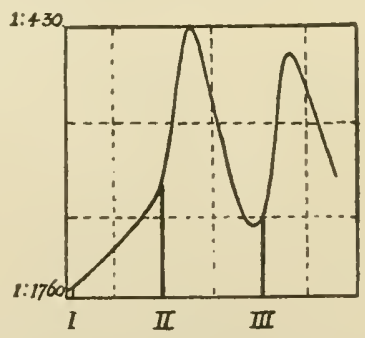

Fig. 4--Curve showing Propor tion of White Corpuscles to Red at Different Times of the Day (after the Results of Hirt). At I the morning meal was taken; at II the midday meal; at III the evening ineal. During active digestion the number of lymphocytes in the blood is greatly increased, both absolutely and relatively to the number of the other leucocytes. to 600,000 per cubic millimetre-while at the same time the number

* In II 3 apparently healthy students (male) the average number of red corpuscles was 5,190,000 per cubic millimetre. In 104 of these, the number ranged from $4,000,000$ to $6,400,000$; in 71 (or 63 per cent. of the whole), from $4,400,000$ to $5,500,000$; in 3 , from $3,500,000$ to $3,900,000$; in 5 , from $6,500,000$ to $7,000,000$. In one observation the number reached $7,300,000$. 
of the red corpuscles is diminished; and the ratio of white to red may approach $x: 4$. As the anæmia rapidly advances towards the fatal tormination of an acute case, and the erythrocyte count falls to $\mathrm{I}, 000,000$, or even less, the ratio may come still nearer to unity. An increase in the number of leucocytes has also been observed in cer tain infective diseases as part of the inflammatory reaction. There are also physiological variations, even within short periods of time; for example, the number of lymphocytes is increased when digestion is going on (digestive lymphocytosis). The normal number of blood-plates varies from a quarter to half a million to the cubic millimetre, but may be greater in clisease and at high levels (Kemp).

Life-History of the Corpuscles.-The corpuscles of the blood, like the body itself, fulfil the allotted round of life, and then die. They arise, perform their functions for a time, and disappear. But although the place and mode of their origin, the seat of their destruction or decay, and the average length of their life, have been the subject of active research and still more active discussion for many years, much yet remains unsettled.

Origin of the Erythrocytes.-In the embryo the red corpuscles, even of those forms (mammals) which have non-nucleated corpuscles in adult life, are at first possessed of nuclei, and approximately spherical in form. In the human fœtus, at the fourth week all the red corpuscles are nucleated. Later on the nucleated corpuscles gradually diminish in number, and at birth they have almost or altogether disappeared, some of them, at least, having been converted by a shrivelling of the nucleus into the ordinary non-nucleated form. In the newly born rat, which comes into the world in a comparatively immature state, many of the red corpuscles may be seen to be still nucleated. The first corpuscles formed in embryonic life are developed outside of the embryo altogether. Even before the heart has as yet begun to beat, certain cells of the mesoderm (see Chapter XIX.) in a zone ("vascular area ") around the growing embryo begin to sprout into long, anastomosing processes, which afterwards become hollowed out to form capillary bloodvessels. At the same time clumps of nuclei, formed by division of the original nuclei of the cells, gather at the nodes of the network. Around each nucleus clings a little lump of protoplasm, which soon devclops hrmoglobin in its substance; and the new-made corpuscles float away within the new-made vessels, where they rapidly multiply by mitosis. In later embryonic life the nucleated corpuscles continuc in part to be developed within the bloodvessels in the liver, allantois, spleen, and red bone-marrow, and in certain localities in the connective tissue, by mitotic division of previously existing nucleated corpuscles, in part to be formed endogenously within special cells in the liver and perhaps other organs. Still later the nucleated corpuscles give place in the blood of the mammai to non-nucleated erythrocytes. Many of these are doubtless derived from the nucleated corpuscles, but some appear to be produced in the interior of certain cells of the connective tissue, and are non-nucleated from the start.

In the mammal in extra-uterine life the chief seat of formation of the red blood-corpuscles, or hæmatopoiesis, is the red marrow of 
the bones of the skull and trunk, and of the ends of the long bones of the limbs. Special nucleated cells in the marrow, originally colourless, multiply by karyolinesis, take up hamoglobin or, what is much more likely, form it within their protoplasm, and are transformed by various stages into the ordinary non-nucleated red corpuscles, which then pass into the blood-stream. These bloodforming cells have received the name of erythroblasts or hiematoblasts. According to their size, erythroblasts have been distinguished as normoblasts, megaloblasts, and microblasts. The normo. blasts are most numerous, and have about the same diameter as the full-formed erythrocytes, into which they are belicved to develop. The megaloblasts are larger, and the microblasts smaller, and they are thought to be the precursors of those aberrant forms of erythrocytes sometimes found in the blood in certain diseases. After hæmorrhage rapid regeneration of the blood takes place, so that in a few weeks the loss of even as much as a third of the total blood is made good. The plasma is much sooner restored to its normal amount than the corpuscles. Microscopical examination shows in the red marrow the tokens of increased production of coloured corpuscles, and nucleated erythrocytes appear in the blood, the normoblasts being, as it were, hurried into the circulation before the transformation which normally results in the disappearance of the nucleus is complete. The same is true in severe pathological anæmias, e.g., pernicious anæmia. It is a matter of interest that other organs also, which in embryonic life perform a hæmatopoietic function, particularly the spleen, may, in such emergencies, again take on the office of forming blood-corpuscles.

A constant destruction of red blood-corpuscles must go on, for the bile-pigment and the pigments of the urine are derived from blood-pigment. The bile-pigment is formed in the liver. It contains no iron; but the liver cells are rich in iron, and on treatment with hydrochloric acid and potassium ferrocyanide, a section of liver is coloured by Prussian blue. Iron must therefore be removed by the liver from the blood-pigment or from one of its derivatives; and there is other evidence that the liver is either one of the places in which red corpuscles are actually destroyed, or receives blood charged with the products of their destruction. Although it cannot be doubted that in all animals whose blood contains hæmoglobin the iron found in the liver bears an important relation to the building up or breaking down of the blood-pigment, the injection of hæmoglobin or hæmin, indeed, increasing markedly the amount of iron in the liver, as well as in the spleen, bone-marrow and other tissues, this does not scem to be the only function of the hepatic iron, for the liver of the crayfish and the lobster, which have no hæmoglobin in their blood, is rich in iron. Destruction of 
erythrocytes may also take place in the spleen and bone-marrow. Although the statement that free blood-pigment exists in demonstrable amount in the plasma of the splenic vein is incorrect, red corpuscles have been seen in various stages of decomposition within large amoboid cells in the splenic pulp; and deposits containing iron have been found there and in the red bone-marrow in certain pathological conditions. But there is no good foundation for the statement sometimes ratlıer fancifully made that the spleen is in any special sense the 'graveyard of the red corpuscles.' Some of the coloured corpuscles may break up in the blood itself, forming granules of pigment, which may then be taken up by the liver, spleen, and lymph glands. Indeed, it is probable that a large proportion of the worn-out erythrocytes are finally destroyed in the bloodstream. The portal circulation may be more than other vascular tracts a seat of this natural decay, perhaps in virtue of the presence of substances with a hremolytic action (p. 28 ) absorbed from the alimentary canal.

It has been argued that the erytnrocytes must be short-lived, since they are devoid of nuclei (p. 6), and attempts have been made to calculate the average time for which they survive in the circulation from the amount of hæmoglobin (or of its derivative, hæmatin) required to furnish the daily excretion of bile-pigment. The results arrived at, however, are not sufficiently trustworthy to warrant their citation.

Origin and Fate of the Leucocytes.--There has been much discussion as to the origin of the white blood-corpuscles. The numerous theories fall into two groups, which have been designated somewhat pompously the monistic and the dualistic. According to the first, all the colourless corpuscles arise from a single type of parent cell, namely, the lymphocyte type, in its small or large variety. According to the dualistic school, a fundamental distinction exists between the lymphocytes, or cells peculiar to lymphoid tissues, and to the blood on the one hand, and the remaining varieties of leucocytes on the other. The former are supposed to be derived from the lymphoblasts of lymphoid tissue, and the latter from the myeloblasts, the forerunners of the myelocytes of bone-marrow. The question has recently been studied by Foot by a new method, namely, by cultivating chicken marrow outside of the body, and watching the transformation of certain of its cells. He concludes in favour of the development of the polymorphonuclear leucocyte from a lymphoid type of cell existing in the marrow, a conclusion in harmony with the monistic view. As regards their immediate source, the small lymphocytes of the blood are undoubtedly derived from the lymph, and are identical with the lymph-corpuscles. That they are formed largely in the lymphatic glands is shown by the fact that the lymph coming to the glands is much poorer 
in corpuscles than that which leaves them. The lymphatic glands, however, although the principal, are not the only seat of formation of lymphocytes, for lympl contains some corpuscles before it has passed through any gland; and although a certain number of these may have found their way by diapedesis from the blood, others are developed in the diffuse adenoid tissue, or in special collections of it, such as the thymus, the tonsils, the Peyer's patches and solitary follicles of the intestine, and the splenic corpuscles. To a very small extent white blood-corpuscles may multiply by karyokinesis or indirect division in the blood.

The fate of the leucocytes is even less known than that of the red corpuscles, for they contain no characteristic substance, like the blood-pigment, by which their destruction may be traced. That they are constantly disappcaring is certain, for they are constantly being produced. Not a few of them actually escape from the mucous membranes of the respiratory, digestive, and urinary tracts. The remnants of broken-down leucocytes have been found in the spleen and lymph glands. It must be assumed that many break up in the blood-plasma itself.

\section{Section II.-General Physical and Chemical Properties of THE BLOOD.}

Fresh blood varies in colour, from scarlet in the arteries to purple-red in the veins. It is a somewhat viscid liquid, with a saline taste and a peculiar odour.

Viscosity of Blood.-The viscosity of normal dog's blood is about six times greater than that of distilled water at body temperature. It can be determined by allowing the blood to flow through a capillary tube of known dimensions under a definite pressure, and measuring the amount which escapes in a given time. In general the viscosity and specific gravity of the blood vary in the same direction, although there is not an exact proportionality between them. Thus, sweating, which causes a diminution of the water of the blood, causes also an increase in its viscosity. With increasing temperature the viscosity of the blood diminishes, as is the case with other liquids (Burton-Opitz).

In polycythæmia, where the number of erythrocytes in proportion to plasma is greatly increased, the viscosity of the blood increases in an equal degree. In one case of polycythæmia, with a blood-count of $\$, 300,000$, the viscosity was 9.4 times that of water; in a case of marked chlorosis it, was only $2 \cdot$ I4. But the importance of this factor in causing an abnormal blood-pressure by increasing or diminishing the resistance to the blood-flow has been exaggerated. Although it has been shown that in the living vessels, so long as 
their calibre remains constant, the flow is affected by changes in the viscosity of the bloud, just as in glatss tubes, compensation by adjustment of the vascular calibre is so ample and so easy tha: even the greatest alterations of viscosity produce little effect on the mean blood-pressure.

Reaction of Blood.--In the sense in which the term is used in phrsical chemistry, the reaction of a solution depends on the proportion between its content of hydrogen $(\mathrm{H}+)$ and hydroxyl $(\mathrm{OH}-)$ ions, an excess of hydrogen ions corresponding to an acid and an excess of hydroxyl ions to an alkaline reaction. It has been shown by a physical method (the determination of the electromotive force of a cell containing blood or serum as one liquid) that hydroxyl ions are present only in small excess, and that blood is really but a little more alkaline than distilled water. Practically, it may be regarded as a neutral liquid. Under a great variety of conditions, phrsiological and patholngical, its reaction remains almost unchanged. Yet it is known that acids (carbon dioxide, lactic, phosphoric, and sulphuric acids) are constantly being produced in the normal metabolism of the tissues. The administration of large quantities of acid or alkali causes a surprisingly small effect. In diabetes, even when it can be proved that an abnormal production of acid substances is taking place, the blood shows little, if any, diminution in the proportion of hydroxyl ions; it remains to all intents and purposes a neutral liquid. In diabetic coma, where the blood may in extreme cases turn blue litmus red, the true reaction is only slightly altered.

The manner in which the reaction of the blood, the tissue liquids, and probably the protoplasm itself, is regulated within such narrow limits is a subject of great interest. For there is reason to believe that it is of the utmost moment that the equilibrium should be maintained not only in order that the functions of the tissues may be properly performed, but that danger to life may be averted. To be sure, the excretory organs, the lungs and the kidneys, provide the means by which the excess of acid (or of alkali) is finally, under normal circumstances, eliminated. Other regulative mechanisms also exist. For example, it has been shown that when an excessive production of acids (acidosis) occurs in conditions of disordered metabolism, or when acids are purposely administered in large amount, a greater quantity of ammonia, split off from the proteins, is mobilized to aid in neutralizing the acids. But very simple experiments on blood in vitro are sufficient to show that the blood itself has a great capacity, as compared with water, to resist a change in its reaction even when large amounts of acid or alkali are added to it. The secret of the reaction-regulating power lies, therefore, to a large extent in the blood itself. Two factors have been shown to be of importance: (I) The power of the proteins 
in virtue of their amphoteric character, to combine either with acids or with bases, so that, when excess of base is added to blood, the proteins act as acids, and neutralize the base; when excess of acid is added, the proteins act as bases, and neutralize the acid. (2) The equilibrium of certain of the inorganic constituents of the blood (carbon dioxicle, the carbonates, and the phosphates) is such that even great variations in the concentration of any of these, such as may normally occur, produce scarcely any effect upon the concentrations of the hydrogen and hydroxyl ions.

Thus, when phosplıoric acid and sodium hydroxide are added to water in certain proportions, and the solution placed under a certain tension of carbon clioxide (which is kept constant), we get a morc or less accurate imitation of blood as regards the inorganic substances concerned in the regulation of its reaction, sodium bicarbonate $\left(\mathrm{NaHCO}_{3}\right)$ and disodium plosphate $\left(\mathrm{Na}_{2} \mathrm{HPO}_{4}\right)$ being present in the solution as in blood. It is found that when the quantities are so chosen that the $\mathrm{H}+$ concentration lies within the limits of variation of the normal blood reaction, relatively large quantities of alkalies can be added or withdrawn without causing inuch cliange in the $\mathrm{H}+$ concentration. It can be shown both theoretically and cxperimentally that precisely those weak acids present in blood $\left(\mathrm{CO}_{2}, \mathrm{NaH}_{2} \mathrm{PO}_{4}\right)$ require the largest addition of alkali to alter the reaction to a given extent, and are therefore particularly suited to give stability to the reaction. Thus carbon dioxide requires twenty-four times, and monosodium phosphate thirty-three times, as mucli alkali as an equivalent solution of acetic acid to cause a given alteration of colour in rosolic acid (E. Henderson).

The so-called 'titratable' alkalinity of blood or serum, measured by the amount of standard acid which must be added before the colour of the indicator used changes from alkaline to acid, bears no necessary or fixed proportion to the actual alkalinity. When blood, for instance, is titrated with hydrochloric acid, with methyl orange as indicator, at the point where the red colour appears all the disodium phosplate and sodium bicarbonate will have been changed into monosodium phosphate and carbon dioxide, all the alkali removed from combination with proteins, a certain amount of acid-protein compounds formed, and other minor reactions produced (Henderson). It is difficult to correlate the quantity decuced from such a titration with any physiological condition, although undoubtedly it bears some relation to the acid-neutralizing power of the blood, and some relation to its real reaction. Still, by titration information of value can be obtained which is not yielded by the physico-chemical method in regard to the potential ' acid capacity' of the blood and its power of resistance against acid-poisoning.

What is estimated here is the quantity of acid required to satisfy the proteins and to react with the carbonates and phosphates before that concentration of hydrogen and hydroxyl ions just necessary to cause the change of colour is established. This is not the same for different indicators, since there is a certain minimum ratio in the concentration of these ions at which each indicator turns in one or the other direction, none turning precisely at the neutral point. Thus serum appears to be acid when tested with phenolphthalein, and alkali must be added to the serum before the pink colour indicating alkalinity is produced. On the other hand, with litmus or methyl orange it gives the alkaline 
reaction, and a considerable amount of acid must be added before the colour of the indicator which denotes acidity appears. The true reaction of the serum is not, of course, at one and the same time both alkaline and acid; but it is so near neutrality that it falls just below the degrec of alkalinity necessary to give the pink colour with phenolpluthalein, and just below the degrec of acidity which gives the pink colour corresponding to an acid reaction with metleyl orange. Certain indicitors-for example, rosolic acid-turn so as to give slarp colour reactions at about the concentration of hydrogen and hydroxyl ions in the blood, and these may possibly be of use in determining the changes in the truc reaction for clinical purposes (Adler).

IIore closely related to the true alkalinity of the blood than the titratable alkalinity is the carbon dioxide content. The estimation of the total carbon dioxide in a sample of blood throws light upon the capacity of the blood to perform one of its most important functions - the transportation of carbon dioxide-and to preserve one of its essential properties-an almost neutral reaction-in the presence of an excessive intake or production of acid substances. In herbivorous animals the carbon dioxide content of the blood is easily lessened by the administration of acids, but in carnivora and in man it is much more difficult to bring about such a decided effect, for the reason already mentioned, the acid being neutralized by ammonia. In many diseases, however, and particularly in those accompanied by fever, this protective mechanism breaks down.

Specific Gravity of Blood.- The average specific gravity of blood is about 1066 at birth. It falls during infancy to about 1050 in the third year, then rises till puberty is reached to about 1058 in males (at the seventeenth year), and 1055 in females (at the fourteenth year). It remains at this level during middle life in males, but falls somewhat in females. In chlorotic anæmia of young women it may be as low as I030 or 1035. It rises in starvation. Sleep and regular exercise increase it (Lloyd Jones).* The specific gravity of the serum or plasma varies from 1026 to 1032 .

The Electrical Conductivity of Blood.-The liquid portion of the blood conducts the current entirely by means of the electrolytes dissolved in it, the most important of these being the inorganic salts; and the conductivity of the serum varies, in different specimens of blood, within a comparatively narrow range. The conductivity of entire (defibrinated) blood, on the contrary, varies within wide limits. For instance, in a case of pernicious anæmia the conductivity of the blood was found to be almost double that of normal human blood, while the conductivity of the serum was normal. The most influential factor which governs this variation

* In 165 students (male) the average specific gravity of the blood, as determined by Hammerschlag's method (p. 62) was 1054. In I 49 of these the variation was from 1050 to 1065 ; in 94 (or 57 per cent. of the whole), from $105+$ to 1060 ; in 4 , from 1046 to 1049 ; in 9, from 1066 to 1070 . In 3 the specific gravity was only 1040 to 1042 . 
is the relative volume of the corpuscles and serum. When the blood is relatively rich in corpuscles and poor in serum, its conductivity is low; when it is poor in corpuscles and rich in serum, its conductivity is ligh. The explanation is that the corpuscle: refuses passage to the ions of the dissociated molecules, which, in virtue of their electrical charges, render a liquid like blood a conductor (p. 428), or permits them only to pass very slowly, so that the intact red corpuscles lave an electrical conductivity so many. times less than that of serum, that they may, in comparison, be looked upon as non-conductors (Practical Exercises, p. 69).

The Relative Volume of Corpuscles and Plasma in Unclotted Blood, or, what can be converted into this by a small correction, the relative volume of corpuscles and serum in defibrinated blood, can be easily determined, with approximate accuracy, by comparing the electrical conductivity of entire blood with that of its serum.* Another method, more suitable for clinical work, though not so accurate, is the so-called hæmatocrite method. A small quantity of blood is centrifugalized in a graduated glass tube of narrow bore until the corpuscles have been collected into a solid 'thread' at the outer extremity of the tube. Their volume and that of the clear plasma which has been separated from them are then read off on the scale. The hæmatocrite must rotate at such a high speed (I0,000 turns a minute) that separation of the corpuscles from the plasma is accomplished before clotting has occurred. Dilution of the blood with liquids which prevent clotting is not permissible for exact work (Practical Exercises, p. 68). By these and other methods too elaborate for description here, it has been shown that the plasma or serum usually makes up rather less than two-thirds, and the corpuscles rather more than one-third, of the blood. But this proportion is, of course, liable to the same variations as the number of corpuscles in a cubic millimetre of blood. It depends, further, the number of corpuscles being given, on the average volume of each corpuscle. For instance, when the molecular concentration, and therefore the osmotic pressure (p. 427), of the plasma is reduced, as by the addition of water or the abstraction of salts, water passes into the corpuscles and they swell; when the molecular concentration of the plasma is increased, by the abstraction of water or the addition of salts, water passes out of the corpuscles, and they shrink. In human serum the average

* The formula $p=\frac{K(b)}{K(s)}\left(\mathrm{r}_{74}-K(b)\right)$, where $p$ is the number of c.c. of serum in roo c.c. of blood; $K(b), K(s)$, the specific conductivities respectively of the blood and serum (both measured at or reduced to $5^{\circ} \mathrm{C}$., and, to obtain whole numbers, multiplied by $\left.10^{4}\right)$, may be used in the calculation. $\mathrm{K}$ is the specific conductivity of the liquid-i.e., the conductivity of a cube of the liquid of I centimetre side. The conductivity of a similar cube of mercury is 10,630. 
depression of the freezing-point below that of distilled water, which is a measure of the molecular concentration and of the osmotic pressure, is about $0.56^{\circ} \mathrm{C}$. (Practical Exercises, p. 73). For clinical purposes, the determination of the relative volume of corpuscles and plasma is most useful in cases where the average size of the erythrocytes departs from the normal, and where, accordingly, the enumeration of the corpuscles would give an erroneous idea of their total mass.

Laking of Blood, or Hæmolysis.-Even in thin layers blood is opaque, owing to reflection of the light by the red corpuscles. It becomes transparent or 'laky' when by any means the pigment is brought out of the corpuscles and goes into true solution. Repeated freezing and thawing of the blood, the addition of water, the passage of electrical currents, constant and induced,* putrefaction, heating the blood to $60^{\circ} \mathrm{C}$., and many chemical agents (as bile-salts, ether, saponin), cause this change. Certain complex poisons of animal origin, such as snake-venoms, bee-poison, spiderpoison or arachnolysin, and certain toxins produced by pathogenic bacteria-for instance, tetanolysin, formed by the tetanus bacillus -also possess decided hæmolytic power. The blood-serum of certain animals acts on the coloured corpuscles of others, and sets free their pigment-for example, the serum of the dog or ox causes hæmolysis of rabbit's corpuscles; the serum of the ox, goat, dog, or rabbit lakes guinea-pig's corpuscles. But rabbit's serum does not lake dog's corpuscles, and guinea-pig's serum is inactive towards the corpuscles both of the rabbit and the dog. It has been shown that in hæmolysis by forcign serum two bodies are concerned: one, which is easily destroyed by heating to about $56^{\circ} \mathrm{C}$., the so-called complement, and another, the intermediary body or amboceptor, which is not affected by being heated to this temperature. 'T hus if dog's serum be heated to $56^{\circ} \mathrm{C}$. for twenty minutes, no amount of it will lake rabbit's washed corpuscles-that is, rabbit's corpuscles freed from their own serum by repeated washing with salt solution and centrifugalization. If, however, serum which is not itself hæmolytic for rabbit's blood (e.g., rabbit's or guinea-pig's serum) be added to the washed rabbit's corpuscles, they will be laked by the heated dog's serum. Unheated dog's serum will lake rabbit's corpuscles, whether they have been washed free from their own serum or not (Practical Exercises, p. 7I).

The hypothesis which best explains these facts and many similar ones is that dog's serum contains both of the bodies necessary for hæmolysis of rabbit's corpuscles. When the complement has been rendered inactive by heating, the amboceptor cannot cause laking

- The laking action of induced currents is due simply to the heating of the blood. Condenser discharges, which cause liberation of the hrmoglobin without raising the temperature of the blood as a whole to the point at which heat-laking occurs, possibly act in the same way by causing local heating of the corpuscles owing to their high resistance. 
by itself. Rabbit's serum contains complement, but not the specific amboceptor necessary for the laking of rabbit's corpuscles. Accordingly, the addition of fresh rabbit's serum to heated dog's serum restores complement to the latter, and thus it is again rendered active for rabbit's corpuscles. The amboceptor is supposed to unite on the one hand with certain groups in the corpuscle and on the other with the complement, which is thus enabled to develop its hæmolytic action upon the envelope or the stroma. The complement is incapable of acting, even in the presence of amboceptor, if the temperature is reduced to $0^{\circ} \mathrm{C}$. Nevertheless, the corpuscles take up amboceptor at this temperature, and on this fact is based a method of freeing serum from amboceptor. For example, if dog's serum and excess of rabbit's washed corpuscles, both previously cooled to $0^{\circ} \mathrm{C}$., be mixed and placed at $0^{\circ} \mathrm{C}$. for some hours, and the serum then removed, it will be found that it has lost the power of laking rabbit's corpuscles, washed or unwashed, at air or body temperature, although it will still do so on the addition of dog's serum in which the complement has been destroyed by heating it to $56^{\circ} \mathrm{C}$. The real nature and mode of action of complements and amboceptors are not yet satisfactorily determined. The laws of chemical equivalents and definite proportions do not seem to be observed in the reactions into which they enter. It has therefore been suspected that the bodies in question belong to the group of ferments, or are closely related thereto, and there is some evidence that a fat-splitting enzyme, or lipase, is concerned in the complement action (Jobling).

As to the manner in which hæmolytic agents cause the liberation of the blood-pigment, the fact that in so many forms of laking the corpuscles swell up before the hrmoglobin escapes indicates that the entrance of water is an important step. The entrance of water is favoured by changes produced in the chemical and physical condition of certain constituents of the superficial layer (envelope) of the corpuscle, as well as by changes in its interior. Saponin and ether, for example, are known to be solvents of cholesterin and lecithin, and cholesterin and lecithin are important constituents of the stroma and envelope of the erythrocyte. It is easy to understand that if a portion of one or both of these substances is dissolved, or altered without being actually dissolved, profound changes may be produced in the permeability of the corpuscle to water and to the salts dissolved in the liquid in which the erythrocytes are suspended. In addition to this change of permeability, many laking agents, perhaps all, exert also a more direct influence on the normal relations of the native blood-pigment to the stroma. Ether and saponin, for instance, seem to act in two waysby disorganizing the envelope through solution of its lipoids, and thus increasing its permeability to water; and by helping to dissociate the blood-pigment-stroma complex by exerting a pull on the lipoids of the stroma, while the water simultaneously exerts a pull on the pigment.

The conclusion follows from this view of hæmolysis, that the erythrocytes, normally so perfectly adapted to the plasma in which they float, may, when the conditions on which their equilibrium with it depends 
are altered, be rapidly and inevitably destroyed by that very plasma itself. It is, indecd, the very fact of the exquisite adaptation of liquid and cell for a strictly regulated exchange of material which constitutes the danger when the regulation is upset. A liquid like mercury, which is not adapted either to give anything to erythrocytes in contact with it or to take anything from them, would not cause haenolysis, even if the permeability of the corpuscles for water or sodium chloride were increased to any extent. The continued survival of the erythrocytes in an aqueous solution of salts and proteins like the plasma-nay, more, the protection of the corpuscles up to a certain point by the plasma against the attack of extrancous hremolytic agents-are facts we are prone to take so mucl for granted as to forget that they depend entirely upon a most delicate adjustment of the permeability of the corpuscles for essential constituents of the plasma. Disturb these relations to a sufficient degree, and the plasma becomes a poison to tie erythrocytes not much less deadly than distilled water.

When we add to blood a hæmolytic substance, and see that presently the blood-pigment has left the corpuscles, we are apt to attribute the whole effect to the foreign material added, and to say that the saponin, the ether, the alien serum, has laked the blood. In a certain sense this is true, but it is not the whole truth. In reality the hemolytic agent has acted in an essential degree, although not exclusively, by overthrowing the equilibrium between the corpuscles and the aqueous solution of certain substances in which they are suspended. To say that the foreign substance alone causes the hæmolysis is no more accurate than it would be to say that a man swimming strongly in a rough sea, who sinks when hit and stunned by a piece of wreckage, was drowned by: the blow, and not by the sea. No doubt it is true that, but for the blow, he would have continued to swim; yet, in reality, he loses his life bccause he is environed by a medium deadly to him as soon as his power of adjustment to it has been too much diminished. On land, the blow would have stunned, but would not have killed him. In like manner, to glance at one phase of the natural decay of the corpuscles within the body, an erythrocyte may float secure in its watery environment through many rounds of the circulation. But its security is not static, like that of a log floating on the water. It is dynamic, a triumph of perfect physico-chemical poise, as the security of the swimmer, still more of the tight-rope dancer, is dynamic, a triumph of perfect neuromuscular poise. The time, however, arrives when, either through changes in the corpuscle itself (the changes of cellular senility, as we may call them), or through changes in the environing medium, or through a combination of the two, the adjustment is upset, and the erytlirocyte is now destroyed by the plasma in which it has so long lived.

In general hæmolysis by foreign serum is preceded by agglutination or aggregation of the corpuscles into groups. Agglutination may be obtained without hamolysis by heating the hamolytic serum to the temperature at which the complement is destroyed, since the agglutinating agents, or agglutimins, are relatively resistant to heat. Besides the amboceptors naturally present in the blood of certain animals, and capable, in conjunction with complement, of hæmolyzing the corpuscles of certain other animals, amboceptors may be produced in much greater strength by artificial means. 
When the corpuscles of one animal are injected intraperitoneally or subcutaneously into an animal of a different kind, the serum of the latter acquires the property of agglutinating and laking the corpuscles of an animal of the same kind as that whose corpuscles have been injected. This is especially marked if the injection is several times repeated at intervals of a few days. If, for instance, dog's corpuscles are injected into a rabbit, the rabbit's serum after a time becomes strongly hæmolytic for dog's corpuscles. It also agglutinates them. This is due to the appearance in the rabbit's serum of an amboceptor and an agglutinin which have a specific action on dog's corpuscles. Such a serum is often termed an immune serum, and the animal which has received the injections is spoken of as immunized in regard to this particular kind of corpuscles. For the reaction involved in the production of the amboceptor and agglutinin is a particular case of the peculiar and specific response which the body makes to the presence of foreign juices or cells, including bacteria, and which constitutes an attempt to render itself 'immune' to them.

Many other animal cells besides the coloured blood-corpuscles give rise, when injected, to similar specific substances (cytolysins), which cause destruction of cells of the same kind-e.g., leucocytes and spermatozoa.* The process of hæmolysis is more easily followed than the cytolysis of ordinary cells. Yet in its main features it is essentially similar.

In each case the specific antibody seems to be produced in response to the presence of some particular constituent of the foreign cell. The suijstances which on injection give rise to antibodies are spoken of as antigens. In the case of the erythrocytes there is evidence that the antigens (both the hremolysinogen, which causes the production of specific amboceptor, and the agglutininogen, the substance which gives rise to specific agglutinin) are lipoids, or are so closely associated with the lipoids of the corpuscles that they are extracted by the same solvents. Thus ethereal extracts of erythrocytes cause the production of hæmolysin and agglutinin, just as the entire corpuscles do. The group of antibodies known as precipitins is of special interest.

Precipitins.-When the serum of one animal is injected into another of a different group, the serum of the latter acquires the property of causing a precipitate in the normal serum of animals of the same group as that whose serum was injected, but not

* Recent studies have tended to modify the view that the cytotoxins formed after the introduction of different foreign tissues into animals are quite specific for each tissue. Thus Lambert, using tissues cultivated on media outside of the body for testing the toxic action, finds that the plasma of guinca-pigs which have received injections of either chick embryo heart or intestine becomes toxir for both of these tissues. In like manner the plasma. of guinea-pigs treated by injection of rat sarcoma, a tumour which can be propagated by inoculation in rats, acquires a toxic action on cultures of both rat sarcoma and the skin of embryo rats. And the plasma of guinea-pigs treated with rat embryo skin is also toxic for cells of both types. 
in the serum of any other kind of animal. Thus, if human blood or serum is repeatedly injected at short intervals into a rabbit, the serum of the rabbit will cause a precipitate in diluted human blood or serum, but not in the blood or serum of other animals, except that of monkeys, where a slight reaction may be obtained. Tlie specific bodies which cause the precipitation are termed precipitins. The plienomenon has been made the basis of a method of distinguishing luman blood for forensic purposes. Other animal Huids and solutions containing tissue proteins likewise give rise to the production of precipitins. Thus, when cow's milk is injected into a rabbit, the rabbit's serum acquires the power of precipitating the caseinogen of cow's milk. Indeed, the response of the animal body to the presence of foreign proteins is so catholic, and at the same time so approximately specific, that many artificially isolated proteins, even those of regetable origin, after as careful purification as possible, occasion, when injected, the production of antibodies which will precipitate from a solution only the variety of protein injected, or sometimes also, though in slighter degree, proteins nearly related to it.

Anaphylaxis.-Under certain conditions the injection of a toxin, a serum, or a protein solution, instead of eliciting an immunity reaction which tends to combat the effects of a subsequent injection of the same matcrial, produces the opposite result-namely, a sensitization of the animal which renders the second dose far more harmful than the first. Thus, Richet found that animals into which eel serum, or the poison contained in the tentacles of Actinaria, was subcutaneously introduced became much more sensitive to the toxic action of a second injection. This phenomenon he designated anaphylaxis, as being the opposite of the propliylaxis or protection afforded by previous treatment with the toxins hitherto studied. Later on it was discovered that the subcutaneous injection of a great variety of proteins alicn to the animal into which they are introduced causes anaphylaxis. Only very minute amounts are necessary for the first or sensitizing dose, and at interval considerably greater than that employed in the production of an immune serum (p. $3 \mathrm{I}$ ) is allowed to elapse before the second injection is made. The symptoms induced in the scnsitized animal by a subsequent dose of the same material used in sensitization differ somewhat in different animals, but may be designated in general terms as those of collapse or shock (anapliylactic shock). They have been especially studied in the rabbit and guinea-pig, the heart being particularly affecteil in the former, and the lungs in the latter. The symptoms are very severe, and manifest themselves within a very sloort time (a few minutes) of the injection. A large proportion of the animals die. If an animal recovers, it docs so suddenly, and for some time afterwards it is insensitive to the particular protcin. While the real nature of protein sensitization or anaphylaxis is not as yet understood, it affords a new and delicate test for the detection and di:crimination of proteins, ancl has already been utilized in a number of practical applications. For instance, the soplistication of sausages with other than the orthodox ingredients-e.g., with horseflesh — can be thus exposed, since an animal sensitized by horseflesh will exhibit anaphylaxis to horseflesh, but not to beef or pork. In like manner, the anaphylactic reaction may be 
used for the ilentification of luman blood. It is probable that anaplıylaxis plàys an important rôle in certain pathologicàl reactions. It is well linown, for example, that some persons are so susceptible to particular foods that the slightest indugence in them brings on in attack of urticaria or nettlerash. It has been suggested that tliese: persons have become sensitized to certain foreign proteins-such as those existing in eggs, veal, pork, strawberries, shellfish, or whatever the peccant article of diet may be-possibly by absorption at sonc previons time, owing to gastro-intestinal disturbance, of small quan. tities of the proteins which have escaped complete digestion.

It is only when proteins are introduced parenterally (i.e., by some other route than the alimentary canal, such as the subcutaneous tissue, the blood, or the scrous cavities) that the immunity reactions already described and the phenomenon of anaphylaxis can be experimentally produced. For in digestion the protein molecule is decomposed, and although, as will be seen later on, the decomposition products are not the same for each kind of protein, the factor on which the specificity of the molecule depends does not survive the hvdrolysis.

\section{CoAgulation of the Blood.}

Since changes begin in the blood as soon as it is shed, having for their outcome clotting or coagulation, we have to gather from the composition of the stable factors of clotted blood, or of blood which has been artificially prevented from clotting, some notion of the composition of the unaltered fluid as it circulates within the vessels. The first step, therefore, in the study of the chemistry of blood is the study of coagulation.

When blood is shed, its viscidity soon begins to increase, and after an interval, varying with the kind of blood, the temperature of the air, and other conditions, but in man seldom exceeding ten, or falling below three, minutes, it sets into a firm jelly. This jelly gradually shrinks and squeezes out a straw-coloured liquid, the serum. Under the microscope the serum is seen to contain few or no red corpuscles; these are nearly all in the clot, entangled in the meshes of a kind of network of fine fibrils composed of fibrin. In uncoagulated blood no such fibrils are present; they have accordingly been formed by a change in some constituent or constituents of the normal blood. Now, it has been shown that there exists in the plasma- the liquid portion of unclotted blood-a substance from which fibrin can be derived, while no such substance is present in the corpuscles. In various ways coagulation can be prevented or delayed, and the plasma separated from the corpuscles. For example, the blood of the horse clots very slowly, and a low temperature lessens the rapidity of coagulation of every kind of blood. If horse's blood is run into a vessel surrounded by ice and allowed to stand, the corpuscles, being of greater specific gravity than the plasma, gradually sink to the bottom, and the clear straw-yellow plasma can be pipetted off. Or the addition of neutral salts to 
blood may be used to delay coagulation, the blood being run direct from the animal into, say, a third of its volume of saturated magnesium sulphate solution. The plasma may then be conveniently separated from the corpuscles by means of a centrifugal machine. Again, two ligatures may be placed on a large bloodvessel, so that a portion of it can be excised full of blood and suspended vertically (the so-called experiment of the 'living test-tube'); coagulation is long delayed, and the corpuscles sink to the lower end. In these and many other ways plasma free from corpuscles can be got; and it is found that when the conditions which restrain coagulation are removed-when, for instance, the temperature of the horse's plasma is allowed to rise or the magnesium sulphate plasma is diluted with several times its bulk of water-clotting takes place, with formation of fibrin in all respects similar to that of ordinary blood-clot. The corpuscles themselves cannot form a clot.* From this we conclude that the essential process in coagulation of the blood is the formation of fibrin from some constituent of the plasma, and that the presence of corpuscles in ordinary blood-clot is accidental. In accordance with this conclusion, we find that lymph entirely free from red corpuscles clots spontaneously, with formation of fibrin; and when fibrin is removed from newly shed blood by whipping it with a bundle of twigs or a piece of wood, it will no longer coagulate, although all the corpuscles are stiil there.

What, now, is the substance in the plasma which is changed into fibrin when blood coagulates? If plasma, obtained in any of the way's described, be saturated with sodium chloride, a precipitate is thrown down. The filtrate separated from this precipitate does not coagulate on dilution with water; but the precipitate itself-the so-called plasmine of Denis-on being dissolved in a little water, does form a clot. Fibrin is therefore derived from something in this precipitate. Now, 'plasmine' contains two protein bodiesfibrinogen, which coagulates by heat at about $56^{\circ} \mathrm{C}$., and serumglobulin, which coagulates at about $75^{\circ} \mathrm{C}$., and it was at one time believed that both of these entered into the formation of fibrin (Schmidt). Hammersten, however, has shown that fibrinogen alone is a precursor of fibrin; pure serum-globulin neither helps nor hinders its formation. This observer isolated fibrinogen from bloodplasma by adding sodium chloride till about I3 per cent. was present. With this amount the fibrinogen is precipitated, while serum-globulin is not precipitated till 20 per cent. of salt is reached. After precipitation of the fibrinogen, the plasma no longer coagulates; and a solution of pure fibrinogen can be made to clot and to form fibrin, while a solution of serum-globulin cannot. Blood-

- Bird's corpuscles, however, washel free from plasma, will form a clot when laked in various ways, as by addition of water or by freczing and thawing. 
serum, too, which contains abundance of serum-globulin, but no fibrinogen, will not coagulate.

So tar, then, we have reached the conclusion that fibrin is formed by a change in a substance, fibrinogen, which can be obtained by certain metlods from blood-plasmia. It may be added that there is evidence that fibrinogen exists as sucl in the circulating blood; for if unclotted blood be suddenly heated to about $56^{\circ} \mathrm{C}$., the temperature of heat-coagulation of fibrinogen, the blood for ever loses its power of clotting. The liver seems to be an important place of origin of fibrinogen, which may also be formed in the bone-marrow. That the liver is intimately concerned in the production of fibrinogen is indicated by a number of facts. In phosphorus poisoning, and notably in poisoning by chloroform, which causes necrosis, especially of the central portions of the hepatic lobules, the amount of fibrinogen in the blood is quickly diminished. The diminution is proportional to the extent of the injury to the liver, and the blood loses more or less completely its power of clotting. If the injury is repaired, the fibrinogen is rapidly regenerated (Whipple). If the blood is allowed to circulate for a time in the head and thorax of an animal without passing into the rest of the animal's body, it becomes incoagulable, and the fibrinogen is found to be markedly deficient in amount. When the blood of an animal is defibrinated by whipping, and reinjected, regeneration of the fibrinogen does not occur if the liver has been eliminated, whereas it takes place rapidly if the liver is intact (Meek).

Since fibrinogen is readily soluble in dilute saline solutions, and fibrin only soluble with great difficulty, we may say that in coagulation of the blood a substance soluble in the plasma passes into an insoluble form. How is this change determined when blood is shed? We have said that a solution of pure fibrinogen can be made to coagulate, but it does not coagulate of itself. The addition of another substance in minute quantity is necessary. This substance, to which the name thrombin has been applied, can be obtained in various ways, although not in a state of purity; for example, by precipitating blood-serum, or defibrinated blood, with fifteen to twenty times its bulk of alcohol, letting the whole stand for a month or more, and then extracting the precipitate with water. All the ordinary proteins of the blood having been rendered insoluble by the alcohol, the thrombin passes into solution in the water, and the addition of a trace of the extract to a solution of fibrinogen causes coagulation. When purified as well as possible, thrombin still gives protein reactions, but it is not known whether it is really a protein.

The action of thrombin on fibrinogen helps to explain many experiments in coagulation. Thus, transudations like hydrocele fluid do not clot spontaneously, although they contain fibrinogen, 
which can be precipitated from them by a stream of carbon dioxide or by sodium chloride. But the addition of a little thrombin causes hydrocele fluid to coagulate. So does the addition of scrum, not because of the serum-globulin which it contains, as was once believed, but because of the thrombin in it. The addition of bloodclot, either before or after the corpuscles have been washed away, or of serum-globulin obtained from serum, also causes coagulation of hydrocele fluid, and for a similar reason, the thrombin having a tendency to cling to everything derived from a liquid containing it. On the other hand, serum which, although thrombin is present in it, does not of itself clot, because the fibrinogen has all been changed into fibrin during coagulation of the blood, can be made to coagulate by the addition of hydrocele fluid, which contains fibrinogen. We have thus arrived a step farther in our attempt to explain the coagulation of the blood: it is essentially due to the formation of fibrin from the fibrinogen of the plasma under the infuence of thrombin. Up to this point there is agreement between physiologists. Some difference of opinion exists, however, as to the manner in which thrombin is formed or activated when blood is shed, al d $\dot{d}$ as to the nature of its action upon fibrinogen once it is fully formed.

The Formation of Thrombin from its Precursors.--There is good reason to believe that thrombin is formed by the interaction of three factors: (I) A substance which, since it is a precursor of thrombin, is called thrombogen, or prothrombin. It is already present in the circulating plasma. (2) A substance liberated from the formed elements of the shed blood, but which can be obtained also from the cells of all tissues. Since it has been supposed to act upon thrombogen, changing it into fully formed thrombin, much in the same way as enterokinase (p. $37 \mathrm{c}$ ) acts upon trypsinogen, changing it into fully formed trypsin, it is called thrombokinase (Morawitz). (3) Calcium ions. The following experiments illustrate the rôle of these three factors:

The plasma obtained by drawing off bird's blood-e.g., the blood of a fowl or goose - through a perfectly clean cannula into a perfectly clean vessel, without contact with the tissues, and then rapidly centrifugalizing off the formed elements, can be kept unclotted for days and even weeks. The addition of a small amount of tissuc extract (procured by rubbing up blood-free liver, thymus, muscle. or other organs with sand, and extracting for several hours with salt solution) to the bird's plasma causes rapid coagulation. The plasma contains thrombogen and calcium salts, but is lacking in thrombokinase, which is supplied by the tissue extract. A solution of fibrinogen containing calcium will clot if serum, in which fibrin-ferment is always present, be added. It will not clot on addition of tissue-extract alone, nor on addition of bird's plasma alone (obtained as above), but will readily coagulate if both tissue extract and bird's plasma be added. Therefore, something in the bird's plasma (thrombogen), plus something in the tissue extract (thrombokinase), produce in the presence of calcium the same effect as the thrombin of serum. It can be shown that calcium is only necessary 
for the formation of the thrombin, but not for its action on fibrinogen. For instance, a calcium-free solution of fibrinogen can be made to clot by serum from which the calcimm has been removed.

If a soluble oxalate (potassium or ammonium oxalate) is mixed with freslily drawn dog's blood to the amount of $0^{\circ} 2$ or $0^{\circ} 3$ per cent., the blord remains unclotted. The plasma separated from this oxalated blood contains both thrombogen and thrombokinase, but it does not coagulate, because the calcium has bcen precipitated out in the form of insoluble calcium oxalate. In the absence of calcium the reaction of the thrombogen and thrombohinase which leads to the formation of thrombin does not take place. All that is necessary to bring about coagulation is to add calcium chloride in somewliat greater quantity than is required to combine witl any cxcess of oxalate present. If more than a certain amount of calcium be added, clotting is hindered instead of being lielped, so that it is only within certain limits of concentration that calcium favours coagulation. From oxalate plasma a nucleoprotein or a mixture of nucleo-proteins can be separated which contains thrombogen and thrombokinase, but little or no calcium, and does not cause clotting, but which on treatment with a calcium salt acquires the properties of thrombin.

When sodium fluoride is added to freshly drawn blood to the amount of $0^{\circ} 3$ per cent., coagulation is also prevented. But there is this difference between oxalate and fluoride plasma - that, altlough the calcium has been precipitated in both, the addition of calcium chloride to fluoride plasma is not sufficient to induce clotting. Tissue extract containing thrombokinase must be supplied as well. In some way or other sodium fluoride interferes with the liberation of thrombokinase from the formed elements of the blood, although in the concentration mentioned it does not hinder the action of fully formed thrombin, as is shown by the fact that fluoride plasma coagulates on the addition of a little serum, which supplies thrombin. The fluoride blood clots readily if it is diluted with water, and at the same time mixed with calcium chloride solution, for the water damages the formed elements, and thus favours the liberation of thrombokinase.

Sodium citrate solution prevents the coagulation of blood run into it, although there is no precipitation of the calcium. The addition of calcium chloride to citrate plasma induces clotting, and the action of the citrate is assumed to be due to the formation of a compound with the calcium of the blood, which does not dissociate so as to yield calcium ions. It ought to be remarked, however, that in all so-called decalcified plasmas, as ordinarily obtained, blood-platelets are present, and that platelets disintegrate under the influence of calcium salts. It has been shown, indeed, that many of the reagents and procedures which hinder the clotting of shed blood also prevent the breaking up of the platelets. Thus, the cooling of the blood, the addition of hirudin, sodium oxalate, sodium citrate, manganese salts, etc., which are classical methods used in obtaining platelets for microscopical study, are also classical methods of hindering coagulation. These facts have not hitherto been sufficiently taken account of in interpreting experiments on decalcified blood. They indicate that the decalcifying agents may hinder clotting by interfering with the liberation of essential substances from the platelets, and that this may be the decisive factor, and not merely the withdrawal of the calcium from the field where the already liberated thrombokinase and thrombogen would otherwise react to form thrombin.

When proteoses (or peptones) are injected into the circulation of a dog or goose, the blood is deprived of the power of coagulation. The 
peptone plasma must be assumed to contain both thrombogen and thrombokinase, since it can be made to clot in various ways (e.g., by dilution with water or by slight acidulation with acctic acid) without the addition of anything which could supply either of these factors. Yet a little tissuc extract causes it to clot much more rapidly than simple dilution or acidulation, and more rapidly than the addition of serum. So that cither the thrombolinase already present in peptone plasma is present in an unavailable form, or in some way the formation of thrombin from its precursors is hindered. But this is not the only cause of the incoagulability of peptone plasma. It may be shown to contain an antithrombin, a body which antagonizes the action of fully formecl thrombin, and which does not seem to be a ferment, since it acts quantitatively in proportion to the amount present. This is the reason why, although peptone plasma can always be made to clot by the addition of fibrin ferment, in serum, for instance, relatively large quantities of it must be supplied (Practical Excrcises, pp. 64,65 ).

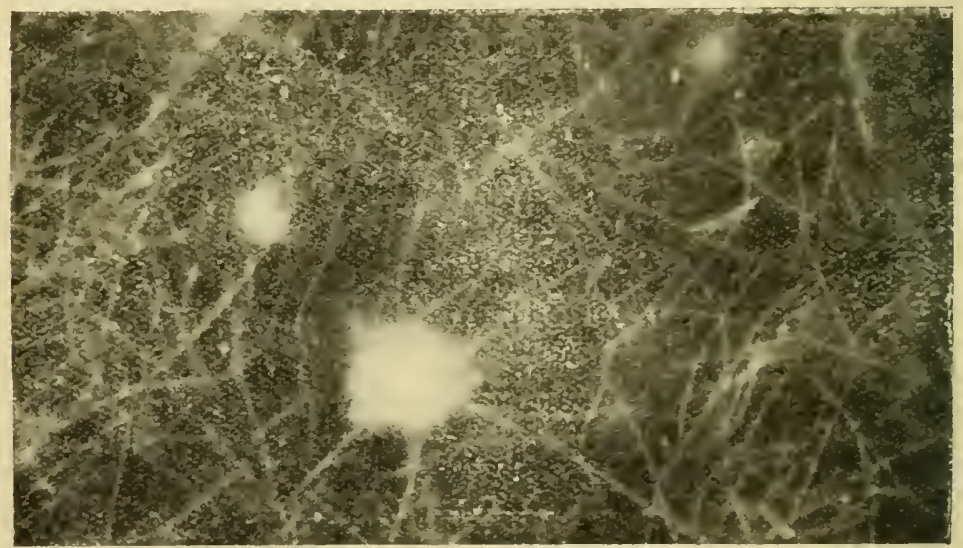

Fig. 5.-Fibrin Formation in Horse's Plasma (Ultramicroscope) (Stübel). Several clumps of disintegrated platelets from which the fibrin filaments radiate.

An extract of the head of the medicinal leech in salt solution prevents the clotting of blood both in the test-tube and when injected into the circulation. The plasma obtained differs from peptone plasma in refusing to coagulate unless tissue extract is added. It is therefore deficient in thrombolinase, or, rather, as lias been shown, the kinase present is unable to act, because neutralized by antikinase present in the leecli extract. Leech extract also contains an antithrombin, which can be neutralized by a sufficient amount of thrombin. In the small wound from whic. the leech sucks blood this sufficient amount is not present, and the blood.remains unclotted, as it also does in the alimentary canal of the lecch. The anticoagulant substance, hirudin, has been isolated, and gives the reactions of an albumose.

Sources of Thrombogen and Thrombokinase.-It has already been stated that thrombogen exists in the circulating plasma. This is shown by the fact that fluoride plasma obtained from blood drawn directly through a wide cannula into sodium fluoride solution, with 
all precautions to prevent alteration of the blood, and immediately separated from the formed elements by the centrifuge, will clot on the addition of tissuc extract. The source of the thrombogen has been thought to be the blood-plates, but this has not been proved. Thrombokinase is not present in the circulating plasma. In shed and clotting blood which has not been allowed to come into contact with cut tissues, the only possible sources of thrombokinase, so far as we know, are the corpuscles and the blood-plates. The red corpuscles we may at once dismiss, for although the stromata, especially of nucleated corpuscles, contain thrombokinase, or can under artificial conditions be made to develop that action on coagulation by which we recognize its presence, not only do they remain intact under ordinary circumstances during coagulation, but there is strong evidence, as has already been pointed out, that they do not make any essential contribution to the process. We have left over the leucocytes and the platelets, and it is lighly probable that from the platelets thrombokinase is liberated in the first moments after blood is drawn, and, acting on the thrombogen already present in the plasma, changes it into actual thrombin. This surmise is strengthened by the fact that in freshly shed mammalian blood extensive destruction of blood-plates takes place. Viewed with the ultramicroscope, the blood-platelets,

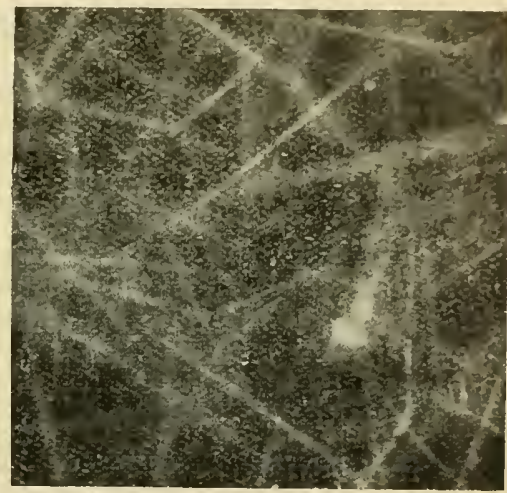

Fig. 6.-Fibrin Formation in Plasma from a Case of Hæmophilia (Ultramicroscope) (Stiibel). The needles of fibrin are slowly formed, and very large. in a drop of clotting plasma, which are at first homogeneous in appearance (optically empty), become granular. Then the platelets begin to agglutinate and swell up, and the agglutinated platelets are transformed into clumps of granules, from which needles of fibrin shoot out. Other needles and filaments of fibrin form in contact with the glass or free in the plasma, and soon the field is occupied by a felt-work of fibrin. The leucocytes have not been observed to be related to the process, at least, in the blood of mammals (Stübel). It is true that the white layer or 'buffy coat' which tops the tardily formed clot of horse's blood, and consists of the lighter, and therefore more slowly sinking colourless cells, causes clotting in otherwise incoagulable liquids like hydrocele fluid much more readily than the red portion of the clot, and yields far more thrombin on treatment with alcohol. It can also be easily verified that in mammalian 
blood collected in paraffined vessels, so as to delay clotting, and mmediately centrifugalized, coagulation begins in and around the layer of white elements, and then spreads upwards in the stratum of plasma and downwards in the stratum of erytlirocytes. But in this white upper layer platelets are always intermingled with leucocytes. It has been shown, however, that the blnod of the crayfish, which coagulates with extreme rapidity, contains certain colourless corpuscles, which immediately it is withdrawn, break up with explosive suddenness, and that substances which hinder the breaking up of these corpuscles restrain coagulation (Hardy). In the blood of another crustacean Limulus, the kingcrab, coagulation is preceded by an agglutination of the leucocytes which exhibit amoboid movements. They become entangled by the interlacing of the psendopodia which they protrude (L. Loeb).

The disintegration of the platelets in shed blood has been attributed by Deetjen to an increase in the alkalinity of the blood, by escape of carbon dioxide, it may be. When blood is placed on a quartz slide and covered with a quartz cover-slip, the platelets, according to this observer, do not break up ; but if they are brought into contact with a medium whose $\mathrm{OH}$ - concentration is raised ten times or more above that of freshly drawn blood (still only a weak alkaline reaction), disintegration ensues. He supposes that the contact of glass acts harmfully on account of the alkali in it. It is impossible to say at present whether this observation has any bearing on normal coagulation.

Thrombokinase has been shown to exist not only in the leucocytes, the platelets, and the stromata of the coloured curpuscles, but, as already stated, in all tissues hitherto examined. Under ordinary circumstances it appears that a larger amount of thrombogen is liberated or is already present in shed blood than can be changed into thrombin by the thrombokinase set free, since serum contains a surplus of thrombogen in addition to the fully formed ferment. This is shown by the fact that the activity of a given quantity of serum in causing the coagulation of a plasma not spontaneously coagulable or of a fibrinogen solution is increased by the addition of tissue extract (containing thrombokinase).

The thrombin of any particular kind of vertebrate blood has no marked specific action - that is, will cause coagulation in solutions of fibrinogen or plasma of very different origin. For example, the sera of all vertebrates hitherto investigated induce clotting in goose's plasma. On the other hand, it appears tlat a greater degree of specificity exists in the case of the thrombokinase and thrombogen, the specificity being absolute in some cases, relative in others. That is to say, the thrombokinase of one animal may activate the thrombogen of an animal of another gromp, while it may fail to activate the thrombogen of an animal belonging to a third group. 
But it will always activate the thrombogen of an animal of the sarrie kind.

To sum up, we may say that rehcn blood is shed, thrombin is rapidly formed by the action of thrombokinase, liberated from the lencocytes, the blood-plates, and possibly to some extent from the erythrocyles, upon thrombogen, already present in the circulating plasma. Further -and this is of great practical importance-since no vessel is opened ander ordinary circumstances except through a wound in the overlying structures, the cut lissues supply a store of thrombokinase al the point where il is required to aid in the stanching of the wound. Calcium is essential to the reaction by which thrombogen and thrombokinase form thrombin, but is not necessary for that action of thrombin on fibrinoges by which fibrin is produced (Practical Exercises, pp. $62-65)$.

The Nature of the Action of Thrombin on Fibrinogen.-The usual view, first advanced by Schmidt many years ago, is that thrombin acts as an enzyme. Hence it is often spoken of as fibrin-ferment. In support of this theory it has been stated that the thrombin does not itself seem to be used up in the process, nor to enter bodily into the fibrin formed; that a small quantity of it can cause an indefinitely large amount of fibrinogen to clot; and that its power is abolished by boiling (p. 33j). There has been a disposition among more recent observers to question this evidence. According to Rettger, the quantity of fibrin formed when a small amount of thrombin is added to a fibrinogen solution tends to a fixed maximum, which does not increase with the time of action.* Under certain conditions, also, it is said that thrombin is not destroyed at the temperature of boiling water. Whatever the precise nature of the reaction which leads to the precipitation of the fibrinogen in the form of fibrils, thrombin is very loosely combined if combined at all in the fibrin, since it is readily extracted by an $\delta$ per cent. solution of sodium chloride. This, indeed, is one of the best ways of obtaining an active thrombin solution.

The view which we have followed above, in accordance with Morawitz, that the substances in tissue extracts which favour coagulation do so by activating prothrombin to fully formed thrombin, has also been opposed by a number of the more recent workers. Some consider that they exert a direct action upon fibrinogen similar to, although not necessarily identical with, that of thrombin, and speak of them as coagulins (L. Loeb). Howell holds that these substances, which he prefers to term thromboplastic substances, since this makes no assumption as to their mode of action, play a quite different. rôle, namely, that of neutralizing antithrombin. His observations have led him to the conclusion that

* The inquiry is complicated by the fact that fibrin, once formed, tends to adsorb the remaining thrombin and so to interfere with its further action. 
the effective thromboplastic substance in the tissues is a pliosphatide, probably kephalin, united with protein.

Intravascular Coagulation Regulation of the Clotting Process, or Thrombotaxis.-So far we liave been considering the problem of coagulation as if all the data for its solution could be obtained by a study of the blood itself. In other words, our main business up to this point has been the explanation of coagulation in the shed blood; it has been only incidentally, and with the object of casting light on the question of extravascular clotting, that we have touched on the coagulation of the blood within the living vessels. It is not possible here to adequately discuss, nor even to define, the differences between the two problems. All we can do is to warn the student, and to emphasize the warning by one or two illustrations, that valuable as is the knowledge derived from experiments on extravascular coagulation, it would be totally misleading if applied without modification to the circulating blood. For instance, we have recognized in the blood-plates an important source of the thrombokinase which plays so great a part in the clotting of shed blood; but we may be sure that blood-plates are constantly breaking down in the lymph and the blood, and we have to inquire how it is that coagulation does not occur, except in disease, within the vessels. Calcium is not wanting to the circulating plasma, fibrinogen is not wanting, and it has already been mentioned that thrombogen exists in perfectly fresh and, as we may say, still living blood. Why, then, does it not coagulate? Some have said that coagulation is 'restrained' by the contact of the living walls of the bloodvessels; but although it is certain that the contact of foreign matter-and all dead matter is foreign to living cells-does hasten the destruction of blood-plates or that alteration in them on which the liberation of the precursors of the ferment depends, it is evident that it is just this 'restraining' influence of the vessels, if it is due to anything more than the mere smoothness of their endothelial lining, which has to be explained. The best answer which can be given to the question is: First, that the quantity of thrombokinase free in the plasma at any given time must be small, since no evidence of its presence in fluoride plasma can be obtained. If thrombokinase is liberated in the circulating blood, we may assume that it is changed into some inactive substance, or quickly eliminated. And it appears that, unlike the true ferments, thrombokinase acts quantitatively-i.e., in proportion to its amount-upon thrombogen. Second, an antithrombin exists in the circulating plasma, and even if fully formed fibrin-ferment were present, it could not cause coagulation until the antithrombin had been neutralized. This antithrombin is probably not manufactured in the blood, or at least not exclusively in the blood, but in the tissues, and there is no reason to deny the vessels themselves 
a share in its production, even if its presence has not hitherto been demonstrated in the internal coat (L. Loeb). So that living blood within the living vessels may be said to be acted upon by two sets of influences, one tending to favour coagulation, the other to oppose it. In the clotting of extravascular plasma, free from corpuscles, we may indeed see the continuation, under modified conditions, of a normal process always going on within the bloodvessels. Under normal conditions, the processes that make for coagulation never obtain the upper hand.

Indeed the margin of safety within which what may be called the thrombo-regulative mechanism works seems to be surprisingly wide, and the equilibrium in the circulating blood far more stable than observations on clotting outside of the body might lead us te suppose. Very considerable quantities of thrombin or of defibrinated blood or serum containing thrombin can be injected into the blood-stream without ill effect. Arcording to Howell, the presence of the abnormally great amount of thrombin causes the formation of sufficient antithrombin to neutralize it, probably by a protective reflex secretion. In like manner the injection of tissue extracts or a solution of thrombo plastic substance (thrombokinasc) prepared from them by precipitation does not necessarily induce coagulation in the vessels. On the contrary, when injected slowly or in small amount into the veins of an animal, it abolishes for a time the power of coagulation of the blood; and when this 'negative phase,' as it is called, has been once established, even a very large and rapid injection produces no further effect, possibly because an antibody which neutralizes the action of thrombokinase has been produced. In both cases the limits of safety can be overstepped, and intravascular clotting induced by the injection eithes of thrombin or of thrombokinase. When a considerable quantity of the active substance in tissue extract is introduced at the first injection, extensive coagulation in the vessels instantly ensues; the animal dies in a few minutes; and the right side of the heart, the venæ cavæ, the portal vein, and perhaps the pulmonary arteries, may be found choked with thrombi. Here the injected thrombokinase is responsible for the clotting, thrombogen and calcium being already present. Curiously enough, intravascular coagulation fails to be produced in a certain proportion of cases when albino animals are injected with material from pigmented animals, while there is no absolute failure of coagulation when albinos are injected witl material from albinos, and no failure when pigmented animals are injected with material either from other pigmented animals or from albinos. Intravascular coagulation on injection of tissue extracts is especially striking in birds.

To a certain extent the action of tissue extracts in coagulation can be imitated by other substances of animal origin, such as the 
venoms of some vipers (Martin). It is not known whether these substances act on the blood-plates, leucocytes, or other cells, and thus cause an increased production or an increased liberation of one or more of the precursors of thrombin, or whether they take part directly in its formation. But there is some evidence that the venoms which favour coagulation do so in virtue of their containing a kinase. On the other hand, cobra-renom prevents coagulation by means of an antikinase-that is, a substance which antagonizes the action of kinase, and so hinders the formation of thrombin. It does not contain an antithrombin-that is, a body wlich will prevent the action of thrombin already formed (Alellanby).

Relation of the Liver to Coagulation.--It is not known with any degree of certainty whether the thrombo-regulative processes are especially associated with any particular organ. But there are facts which suggest that the relations of the liver to the coagulation of the blood are peculiarly close. Not only, as previously shown, loes it take an important share in the formation of fibrinogen, but there is some evidence that it is closely related to the formation of antithrombin. We have already mentioned that the injection of commercial peptone, which consists chicfly of proteoses, into the blood of dogs causes it to lose its coagulability. The effect gradually passes away, till after some hours the original power of coagulation is restored (p. 63). The liver is known to be intimately concerned in the production of this remarkable result, for if the circulation throngh it be interrupted, the injection of proteose is ineffective. Further, if a solution of proteose is artificially circulated through an excised liver, a substance (perhaps an antithrombin) is formed which is capable of suspending the coagulation of blood outside of the body, a property which proteoses themselves do not possess, or possess only in slight degree. It is not believed that the proteose is actually changed intu this anticoagulant substance, but rather that the liver cells produce it as a 'reaction' to the presence of the foreign substance, being perhaps stimulated in some way by the circulating proteose. In part the abnormally great alkalinity of the peptone blood, due to the excess of alkali secreted by the liver, is responsible for its slow coagulation. Under certain conditions, some of which are known and others not, the injection even of one or other of the purified proteoses causes not retardation, but hastening, of coagulation; and if this has been the result of a first injection, a second is equally unsuccessful. It is possible that by an effort of the organisn to restore the normal coagulability of the blood, on which its very existẹnce depends, substances which favour coagulation are produced, and that the result of an injection of proteose is determined by the relative amount of coagulant and anticoagulant secreted in a given time. Protamins (products obtained from the ripe milt of certain fishes, 
and believed to be the simplest proteins) exert, when injected intravenously, a retarding influence on coagulation, and lower the blood-pressure, just as albumoses do (Thompson). Even scrumalbumin and serum-globulin possess this property in some degree. All these substances also cause a diminution in the number of leucocytes in the blood owing, in the case of albumose at any rate, to their accumulation in the abdominal vessels, and not to any actual destruction of them.

it has been lately announced that the adrenal glands have a relation to the coagulation of the blood. Stimulation of the splanchnic nerve, which supplies secretory fibres to the adrenal, greatly hastens coagulation, but lias no such effect if the adrenal on the corresponding side has been previously removed (Cannon). It is possible that this effect is exerted through the liver, since it is known that one important function of the liver, the regulation of the sugar content of the blood, is intimately dependent upon the adrenal, and is affected by excitation of its splanchnic nerve-supply.

In certain pathological conditions the normal balance of the factors that make for clotting and prevent it may be upset, and the scales may tip in cither direction. In patients suffering from the formation of spontaneous clots in the veins (thrombosis) it is stated that the antithrombin in the blood is diminished, the amount of prothrombin being normal. The mere slowing of the blood-stream in conditions where the circulatory mechanism is enfeebled may favour thrombosis. For anything which cripples the circulation, and consequently limits the free interchange between blood and tissues, interferes with the elimination or neutralization of the precursors of thrombin, and with the entrance of the substances that render the fully formed thrombin inactive. This, as well as the injury caused by the ligature, which may favour the passage of thromboplastic substances into the lumen of the occluded vessel, is a possible factor in the formation of the clot on which the surgeon relies for the permanent sealing of ligated vessels.

In hremophilia, a disease in which the coagulation of the blood is characteristically slow, and in which even slight wounds may occasion severe or fatal hæmorrhage, the thrombogen (prothrombin) has been found deficient in amount, and the injection of normal serum or the transfusion of normal blood has been used with temporary advantage in the treatment of the condition. In certain cases of purpura, however, where hæemorrhage also occurs with abnormal ease, no variation from the normal could be detected in the content of either antithrombin or prothrombin (Howcll). Some have supposed that in such conditions the fault is an unnatural fragility of the small vessels rather than a deficiency in the power of the blood to clot, but of this also no actual evidence has becil adduced. Another factor on which the promptness and completeness of the sealing of wounded vessels may depend lias been recently brought into notice, namely-

The Vaso-Constrictor Property of Shed Blood.- It has been shown that when blood is shed and no precautions are taken to prevent clotting, it very quickly develops the power of causing marked constriction of bloodvessels. This can be demonstrated by allow. ing the serum to act on rings cut from arteries (Practical Exercises, 
p. 66), or by perfusing the hind-legs of frogs with a saline solution containing serum. Plasma derived from blood in which the platelets have been prevented from breaking down, and which therefore remains unclotted, has no such effect, or a much slighter

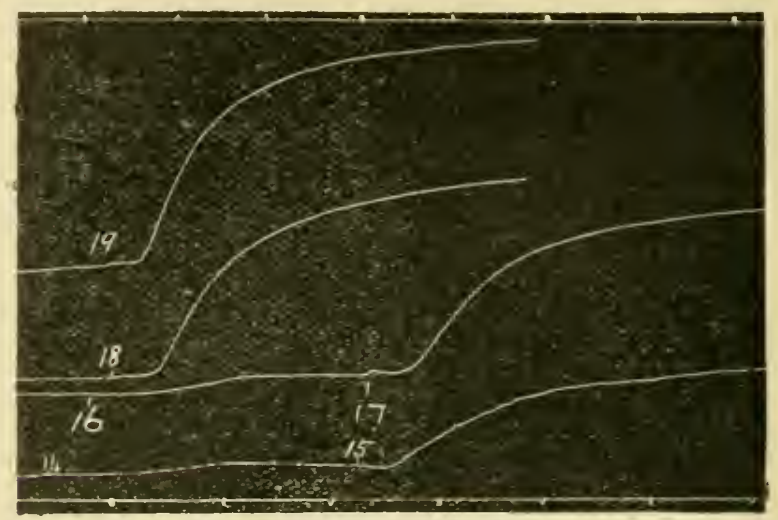

Fig. 7.-Sheep Artery Rings. At 14 and 16 Ringer's solution was replaced by citrate plasma (two different specimens). At 15 and $I 7$ the plasmas were replaced by the corresponding sera. At I 8 and 19 the sera replaced Ringer's solution directly. Time-trace, half-minutes. Tracings reduced to $\frac{1}{2}$.

effect. But when the platelets are separated from the plasma and then decomposed, the resulting extracts of platelets are rich in vaso-constrictor material. In the sealing of wounded vessels the platelets would therefore appear to play a double rôle, yielding

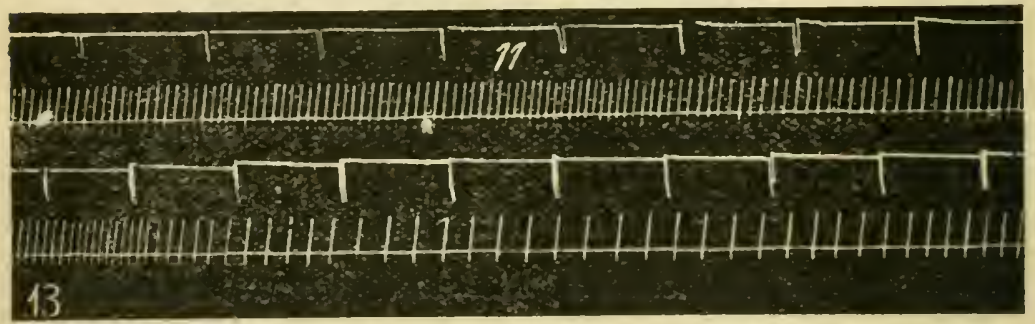

lig 8.-Frog lerfusion Experinent with Serum. The drops of liquid flowing through the preparation are recorded. At II citrate plasma was injected; at $\mathbf{I}_{3}$ the curresponding citrate serum. The tracing is to be read from left to right. Time is marked in half-minutes

a substance which causes constriction of the vessel in the neighbourhood of the wound while a plug of clot is being formed, thanks to other substances liberated from the platelets, which take an essential part in coagulation. The vaso-constriction may perhaps 
be looked upon as a form of 'first aid' to climinish the hiemorrhage, and also to make it less easy for the beginning clot to be washed away. It is obvious that the two processes would be mutually advantageous in dealing with those injuries of the vascular system on the prompt repair of which the very existence of the

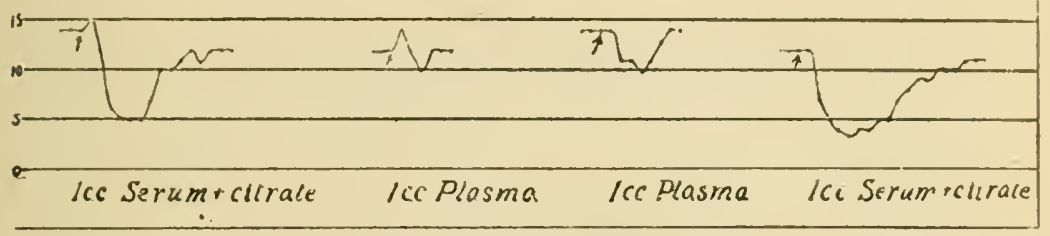

Fig. 9.-Frog Perfusion Experiment with Serum. Curves showing the flow. The number of drops per half-ininute is laid off along the vertical axis, and the time (in half-minutes) along the horizontal axis; $3^{8}$ drops correspond to I c.c.

organism at all times depends, and it is not without interest to find that special formed elements in the blood, the platelets, are pre-eminently associated with both processes.

\section{Section III.-The Chemical Composition of Blood.}

The serum of coagulated blood represents the plasma minus fibrinogen; the clot represents the corpuscles plus fibrin. Thus:

$$
\begin{aligned}
& \text { Plasma }- \text { Fibrin(ogen) = Serum. } \\
& \text { Corpuscles }+ \text { Fibrin }=\text { Clot. } \\
& \text { Plasma }+ \text { Corpuscles }=\text { Serum }+ \text { Clot }=\text { Blood. }
\end{aligned}
$$

Bulky as the clot is, the quantity of fibrin is trifling $(0.2$ to 0.4 per cent. in human blood). The plasma contains about ro per cent. of solids, the red corpuscles about 40 per cent., the entire blood about 20 per cent.

Serum contains 7 to 8 per cent. of proteins, about 0.8 per cent. of inorganic salts, and smiall quantities of neutral fats, soaps, cholesterin esters, lecithin, dextrose, urea, lactic acid, glycuronic

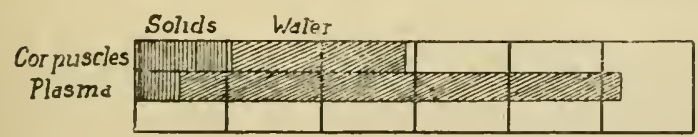

Fig. 10.-Diagram showing Relative Quantity of Solids and Water in Red Corpuscles and Plasma. acid, amino - acids, and other substances. The chief proteins are serumalbumin and sernmglobulin. In the rabbit the former, in the horse the latter, is the more abundant; in man they exist in not far from equal amount. A small quantity of nucleo-protein and of fibrino-globulin (which some consider a soluble product formed from 
librinogen in clotting) is also present. Ferments which cause hydrolysis of proteins and carboliydrates, a ferment (lipase) which acts upon fats, and certain oxidizing ferments (oxydases), have also been demonstrated. The chemical nature of the bodies which confer on serum or plasma its specific hxmolytic, agglutinating. precipitating, and bactericiclal properties has not been definitely determined.

The quantitative composition of serum, especially as regards the inorganic salts, is remarkably constant in animals of the same species, and even in animals of different species belonging to the same, or to not very widely scparated, natural groups. In cold-blooded animals the serum-albumin is scantier than in mammals, the globulin relatively more plentiful.

Serum-albumin bclongs to the class of native albumins. It has been obtaincd in a c1y'stalline form from the serum of horse's bloorl. It

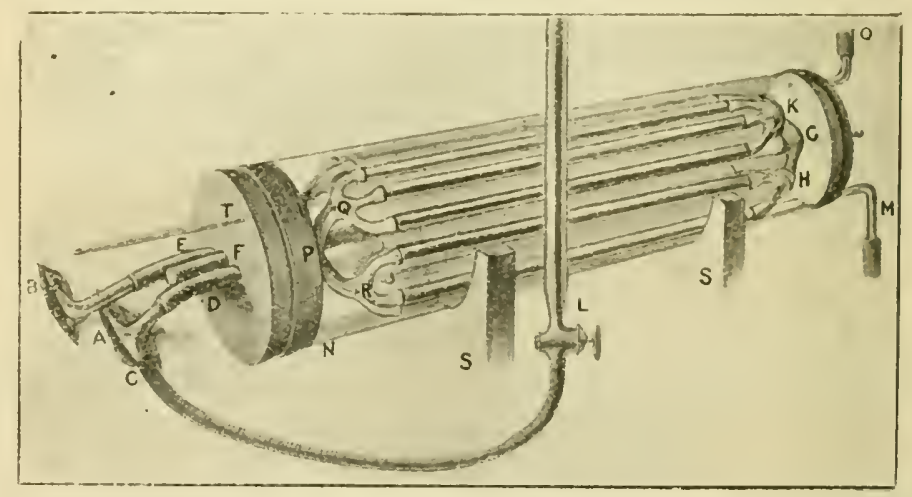

Fig. II.-Perspective View of Vivi-Diffusion Apparatus (Abel). This form of the apparatus contains sixteen tubes. A, arterial cannula; B, venous cannula; $\mathrm{C}$, side tube for introduction of hirudin; $\mathrm{D}$, inflow tube; E, outlet tube for the blood; F, G, supporting rod attached at $\mathrm{H}$ and $\mathrm{K}$ to branched $\mathrm{V}$-tubes; L. burette for hirndin; M, N, tube for filling and emptying liquid in onter jacket; $\mathrm{O}$, air outlet; $P$, dichotomous branching-point of inflow tube; $Q$ and $R$, quadruple branching-points of the same; S, S, wooden supports; T, thermometer. At each of the points $\mathrm{H}$ and $\mathrm{K}$ the blood is collected from four tubes into one, bending round to the back, and there redividing into four return flow tubes. Arrows show the direction of the flow.

is soluble in distilled water, and is not precipitated by saturating its solutions with certain neutral salts. Heated in neutral or slightly acid solution, it coagulates first at $73^{\circ}$, then at $77^{\circ}$, then at $84^{\circ} \mathrm{C}$. Although this is not of itself sufficient proof, there is other evidence that it consists of a mixture of protcins.

Serum-globulin, also called paraglobulin, belongs to the globulin group of proteins. When heated, it coagulates at about $75^{\circ} \mathrm{C}$. (p. ()). It is insoluble in distilled water, and is precipitated by saturation with such neutral salts as magnesium sulphate, or by half-saturation with ammonium sulphate. It appears that, as thus obtained, it is not a single substance, but a mixture of at least two proteins-en-globulin, 
which can be precipitated from its saline solution by dialyzing off the salts, and pseudo-globulin, which cannot be so precipitated.

In addition to the nitrogen represented as protein, serum (or plasma) contains non-protein nitrogen, the amount of which varies with tlic nature of the food and the stage of digestion. Part of this fraction is attributable to urea and other metabolites on their way to be excreted, but another portion, and an important one, is due to amino-acids absorbed from the intestine during the digestion of proteins and on their way to be utilized in the tissues.

Of the inorganic salts of serum, the most important are sodium chloride and sodium bicarbonate. Small amounts of potassium, calcium, and magnesium, united with phosphoric acid or chlorine, and a trace of fluoride, are also present. A portion of the salts is loosely combined with the proteins.

Our knowledge of the cliemistry of the circulating plasma is likely to be notably augmented by the method of vivi-diffusion recently introduced by Abel. An artery of an anæsthetized animal is connected by a cannula to a system of celloidin tubes immersed in a saline solution. Blood passes from the artery through the tubes, where it exchanges diffusible constituents with the solution, and is then returned to the animal's body by another cannula attached to a vein. Coagulation of the blood in the apparatus is prevented by hirudin, and under aseptic conditions the circulation may proceed through the tubes for a long time. The saline solution can then be analyzed for substances which have entered it from the blood-amino-acids, for example (Fig. I I).

The following tables give some details of the composition of blood:

I,000 Grammes of Pig's Blood (Corpuscles, 435o9; Serum, 564.9i) CONTAINED-

\begin{tabular}{|c|c|c|c|c|c|}
\hline & Corpuscles. & Serum. & & Corpuscles. & Serum. \\
\hline Water & $272 \cdot 20$ & $518 \cdot 36$ & $\mathrm{P}_{2} \mathrm{O}_{5}$ as nuclein & 0.0455 & 0.0123 \\
\hline Solids & $162 \cdot 89$ & $46 \cdot 54$ & $\mathrm{Na}_{2} \mathrm{O} \ldots$ & -* & $2 \cdot 401$ \\
\hline Hæmoglobin & $I+2 \cdot 20$ & - & $\mathrm{K}_{2} \mathrm{O} \quad \ldots$ & $2 \cdot 157$ & $0 \cdot 152$ \\
\hline Protein $\quad$. & $8 \cdot 35$ & $38 \cdot 26$ & $\mathrm{Fe}_{2} \mathrm{O}_{3} \ldots$ & 0.696 & - \\
\hline Sugar & - & $0.68_{4}$ & $\mathrm{CaO} \cdots$ & - & 0.0689 \\
\hline Cholesterin & 0.213 & $0 \cdot 23 I$ & $\mathrm{MgO}$ & $0 \cdot 0656$ & 0.0233 \\
\hline Lecithin . . & $I \cdot 504$ & $0 \cdot 80_{5}$ & $\mathrm{Cl} \quad \mathrm{C}$ & 0.642 & $2 \cdot 048$ \\
\hline Fat.. & 1 & $I \cdot x 0_{4}$ & $\mathrm{P}_{2} \mathrm{O}_{5} \quad \ldots$ & 0.895 & O.III \\
\hline Fatty acids & 0.027 & $0.44^{8}$ & Inorganic $\mathrm{P}_{2} \mathrm{O}_{5}$ & 0.719 & 0.296 \\
\hline
\end{tabular}

Proteins of Plasma in i, ooo Grammes.

\begin{tabular}{|c|c|c|c|c|c|c|}
\hline & & & Albumin. & Globulin. & Fibrinogen. & Total. \\
\hline Man & $\cdots$ & $\cdots$ & $40 \cdot I$ & $28 \cdot 3$ & $4 \cdot 2$ & $72 \cdot 6$ \\
\hline Dog & . & . & $3 I \cdot 7$ & $22 \cdot 6$ & $6 \cdot 0$ & $60 \cdot 3$ \\
\hline Sheep & . & .. & $38 \cdot 3$ & $30 \cdot 0$ & $4 \cdot 6$ & $72 \cdot 9$ \\
\hline Horse & . & . & $28 \cdot 0$ & $47^{\circ} 9$ & $4 \cdot 5$ & $80 \cdot 4$ \\
\hline Pig & $\cdots$ & . & $44^{\circ} 2$ & $29 \cdot 8$ & $6 \cdot 5$ & $80 \cdot 5$ \\
\hline
\end{tabular}

* The pig's erythrocytes are peculiar in that the sodium appears to be entirely confined to the plasma. 
The Coloured Corpuscles consist of rather less than 60 per cent. of water and rather more than 40 per cent. of solids. Of the solids the pigment hamoglobin makes up about 90 per cent.; the proteins and nucleo-protein of the stroma about 7 per cent.; lecithin and cholesterin 2 to 3 per cent.; inorganic salts (which vary greatly in their relative proportions in different animals, but in man consist chiefly of phosphates and chloride of potassium, with a much smaller amount of sodium chloride) about I per cent. Potassium has been demonstrated microchemically in frog's erythrocytes (Macallum) (Frontispiece). There is evidence that a portion of the salts is more firmly combined than the rest, so that, even after the action of the most energetic laking agents, this fraction remains at tached to the stroma. The erythrocytes of some animals-e.g., the dog-contain dextrose. When dextrose is added to human blood it rapidly distributes itself over corpuscles and plasma (Rona), although not exactly in proportion to their respective volumes (Masing). Hitherto the dextrose in blood has been reckoned as if it all belonged to the plasma.

Hamoglobin.-Of all the solid constituents of the blood, hæmoglobin is present in greatest amount, constituting as it does no less than I3 per cent., by weight, of that liquid. It is an exceedingly complex

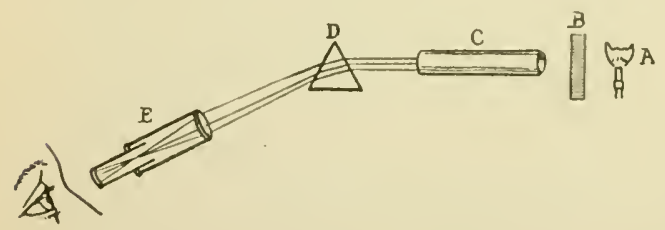

Irig. 12.-Diagram of Spectroscope. A, source of light; B, laver of blocel; C, collimator for rendering rays parallel; D, prisin; E, telescope. is made up of a protein element which contains all the sulphur and a pigment which contains all the iron, the protein constituting by far the larger portion of the gigantic molecule, whose weight has been estimated at more than 16,000 times that of a molecule of hydrogen. Since its percentage composition is still undetermined with absolute precision, it is impossible to give an empirical formula that is more than approximately correct. For dog's hamoglobin Jaquet gives $\left({ }_{75} \mathrm{H}_{1203} \mathrm{Y}_{195} \mathrm{~S}_{11} \mathrm{Fec}\right)_{218}$. which would malic the molecular weight 16,669 . Direct eleterminations of the molecular weight gave 15,155 for oxyhamoglobin of the horse, and 16,321 for that of the ox (Hüfner and Gansser). Whilethese numbers need not be taken as more than a rough approximation, they at least show that the hrmoglobin molecule is an excecelingly large one.

The most remarkable property of hamoglohin is its power of combining loosely witl oxygen when exposed to an atmospliere containing it, and of again giving it up in the presence of oxidizable substances or in an atmosphere in whicl the partial pressure of oxygen (pp. 248-253) has been reduced below a certain limit. It 
D E b F

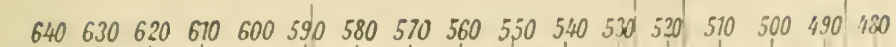
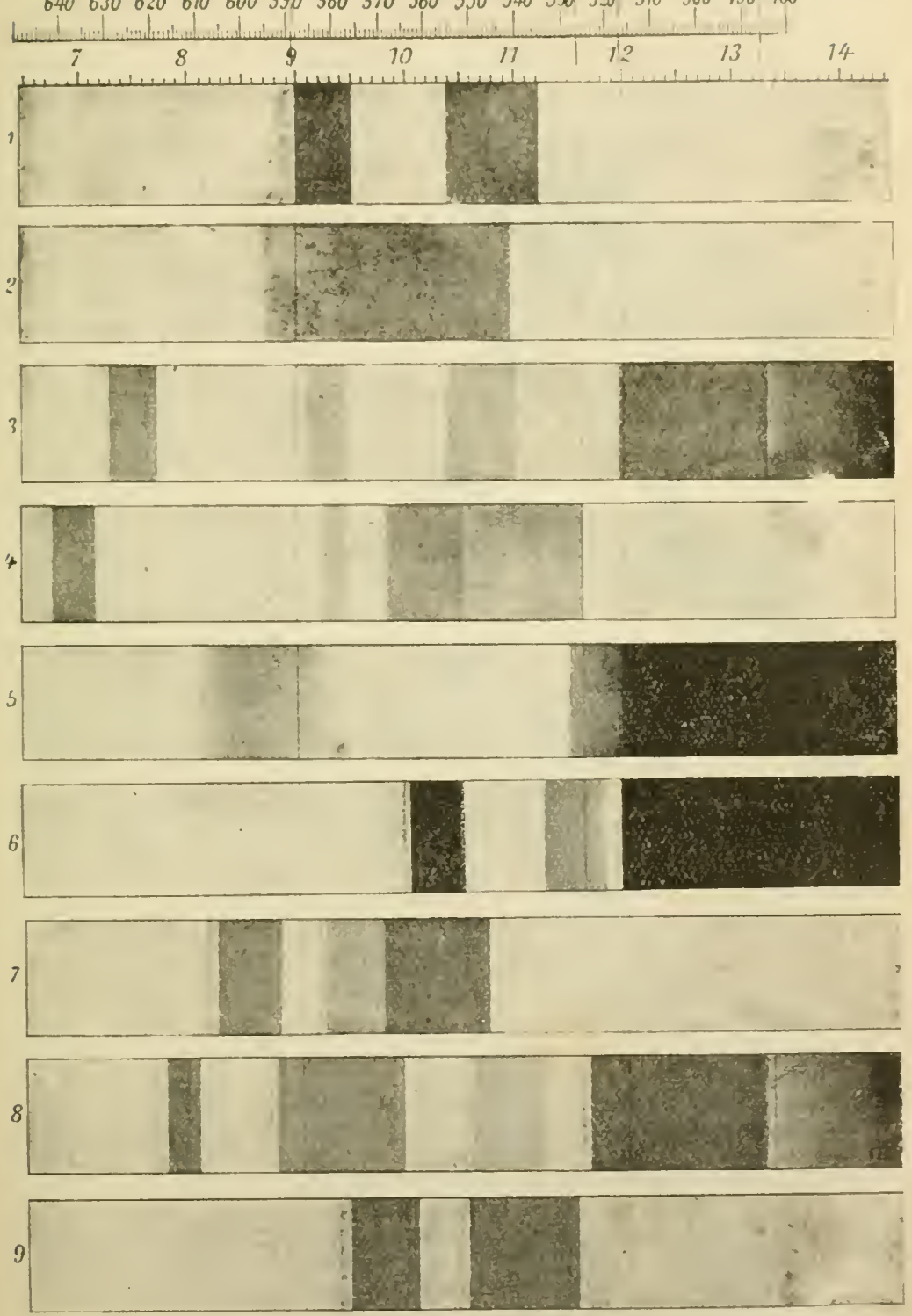

Fig. r3.-Table of Spectra of Hæmoglobin and its Derivatives (Ziemka and Muilier). I, Oxyh $x$ moglobin; 2 , reduced hamoglobin 3 , methxmoglobin: 4 , a' d 1 xmatin: 5, alkaline bæmatin; 6 , hæmochromogen; 7 , acid h.matoporphyrin; S, alkaline hæmatoporphyrin; 9 , carbon monoxide hæmoglobin. 
is this property that enables hæmoglobin to perform the part of an oxygen-carrier to the tissues, a function of the first importance, which will be more minutely considered when we come to deal with respiration.

The bright red colour of blood drawn from an artery or of venous blood after free exposure to air is due to the fact that the lixmo-

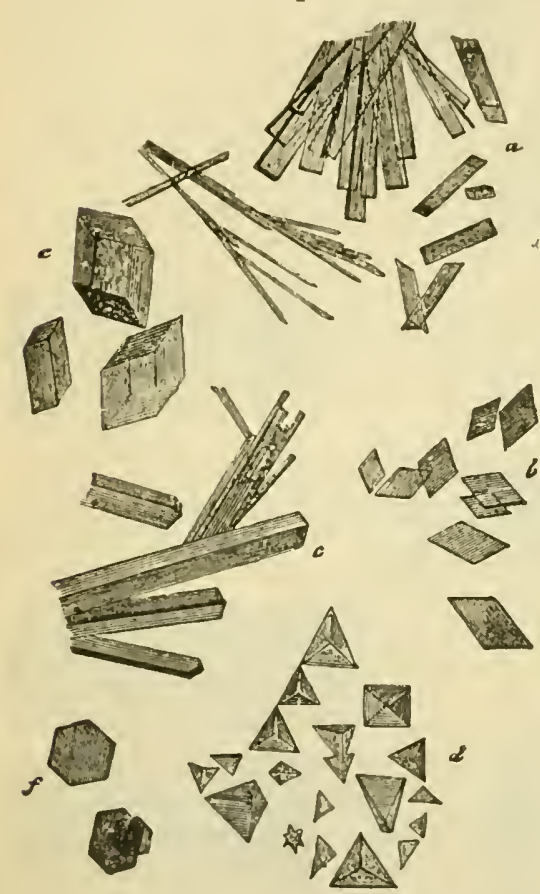

liig. I 4.-Oxyhæmoglobin Crystals (Frey). $a, b$, from man; $c$, from cat; $d$, from guineapig; $e$, from hainster; $f$, from squirrel. globin is in the oxidized state -in the state of oxylicmoglobin, as it is called. The amount of oxygen with which hamoglobin combines to form oxylumoglobin is such that one atom of iron corresponds to two atoms of oxygen. If the formula for hæmoglobin given on p. 50 be represented by the symbol $\mathrm{Hb}$, a molecule of oxyhæmoglobin would be represented as $\mathrm{HbO}_{2}$. If the oxygen is removed by means of reducing agents, such as ammonium sulphide, or by exposure to the vacuum of an airpump, the colour darkens, the blood-pigment being now in the form of reduced hæmoglobin. In ordinary venous blood a large proportion of the pigment is in this condition, but there is ahways oxyhæmoglobin present as well. In asphyxia (p. 28I), however, nearly the whole of the oxyhæmoglobin may disappear.

Crystallization of I' comoglobin.--In the circulating blood the hamoglobin is related in such a way to the stroma of the corpuscles that, although the latter are suspended in a liquid readily capable of dissolving the pigment, it yet remains under ordinary circumstances strictly within them. In a few invertebrates, however, it is normally in solution in the circulating liquid. As a rare occurrence himoglobin may form crystals inside the corpuscles (p. 7I). When it is in any way brought into solution outsicle the body, it shows in many animals, but not in the same degree in all, a tendency to crystallization; and the ease with which crystallization can be induced is in inverse proportion to the solubility of the hrmoglobin. Thus, it is far more difficult to obtain crystals of hremoglobin from human blood than from the blood of the rat, guineapig, or dog, whose blood-pigment is less soluble than that of man, and for a like reason the oxyhæmoglobin of the bird, the rabbit, or the frog crystallizes still less readily than that of human blood. 
As to the form of the crystals, in the vast majority of ani:sals they are rhombic prisms or needles, but in the guinea-pig they are sphenrorls belonging to the rhombic system, and in the squirrel six-sided plates of the hexagonal system (Fig. I 4 ). Careful study of the crystallography of lixmoglobin from a large number of animals has established differences and resemblances so constant and so clear-cut that they may be used for the purposes of classification and for the identification of the source of a specimen of blood (licichert and Brown).

Reduced laxmoglobin can also be caused to crystallize, though with more difficulty than oxyhamoglobin, since it is more soluble. Crystals of reduced haemoglobin were first prepared from luman blood by Hüfner, who allowed it to putrefy in sealed tubes for several weeks.

When a solution of oxyhæmoglobin of moderate strength is examined with the spectroscope, two well-marked absorption bands are seen, one a little to the right of Fraunhofer's line $D$, and the other a little to the left of $\mathrm{E}$. A third band exists in the extreme violet between $\mathrm{G}$ and $\mathrm{H}$. It cannot be detected with an ordinary spectroscope, but has been studied by the aid of a fluorescent eyepiece, by projecting the spectrum on a fluorescent screen, and by photographing the spectrum. The addition of a reducing agent, such as ammonium sulphide, causes the bands in the visible spectrum to clisappear, and they are replaced by a less sharply defined band, of which the centre is about equidistant from D and $E$. This is the characteristic band of reduced hæmoglobin. The spectrum of ordinary venous blood shows the bands of oxyhæmoglobin.

Carbonic oxide hamoglobin is a representative of a class of hæmoglobin compounds analogous to oxyhæmoglobin, in which the looselycombined oxygen has been replaced by other gases (carbon monoxide, nitric oxide) in firmer union. Its spectrum shows two bands very like those of oxyhæmoglobin, but a little nearer the violet end. Carbonic oxide hæmoglobin is formed in poisoning with coal-gas. Owing to the great stability of the compound, the hæmoglobin can no longer be oxidized in the lungs, and death may take place from aspliyxia. It is, however, gradually broken up, and therefore artificial respiration may be of use in such cases. Inhalation of oxygen and especially transfusion of blood are also of great value.

Methemoglobin is a derivative of oxyhæmoglobin which can be formed from it in various ways - e.g., by the addition of ferricyanide of potassium or nitrite of amyl (Gamgee), by electrolysis (in the neighbourhood of the anode), or by the action of the oxidizing ferment 'echidnase' in the poison of the viper (Phisalix). It very often appears in an oxyhzmoglobin solution which is exposed to the air. It has been found in the urine in cases of hamoglobinuria, in the fluid of ovarian cysts, and in hæmatoceles. The strongest band in its spectrum is in the red, between $C$ and $D$, but nearer C, nearly in the same position as the band of acid-hæmatin. Reducing agents, such as ammonium sulphicle, change methæmoglobin first into oxyhæmoglobin and then into reduced hæmoglobin. It has by some been regarded as a more highly oxidized hæmoglobin than oxyhæmoglobin. Rebutting evidence has, however, been offered to the effect that the same quantity of oxygen is required to saturate both pigments, and this evidence appears to be sound. The difference lies rather in the manner in which the oxygen is united to the hæmoglobin in the methæmoglobin molecule than in the quantity of oxygen which it contains. For methrmo- 
globin, mulike oxylixmoglobın, parts with no oxygen to the vacuum, while, on the other hand, in the presence of reducing agents it yelds up its oxygen even more readily tian oxyhamoglobin does (Hallane) (p. 248).

By the action of acids or alkalies oxyhemoglobin is split into a pigment, hamatin, and a protein, globin, belonging to the histon group. It is casily precipitated from solution by ammonia. On hydrolysis, it

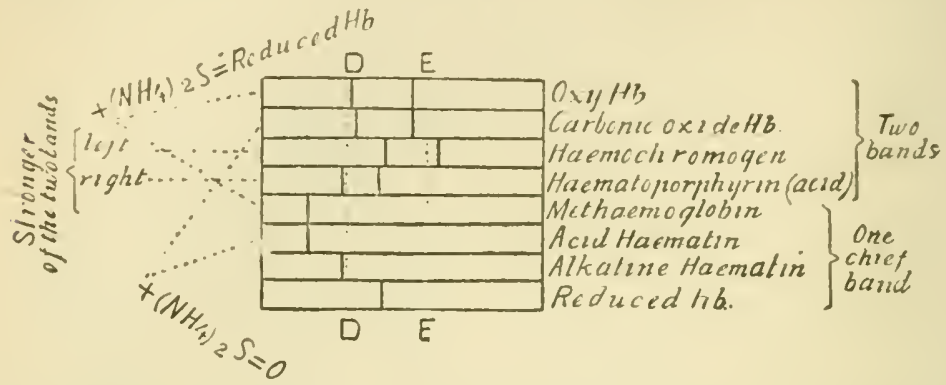

rig 15.-Diagram to show the Chuef Characteristics by which Hæmoglobu and some of its Derivatives may be recognized spectroscopically. The position of the iniddle of each band is indicated roughly by a vertical line

viclds a large amount of histidin, to which its basic properties are chicfly ilue From 100 grammes of oxyhæmoglobin about + grammes of ha matin are obtained. As to the pigment moiety, when hamoglobin is acted on by acids in the absence of oxygen, hemochromogen is first formed, which then gradually loses its iron and is changed into hæmato-

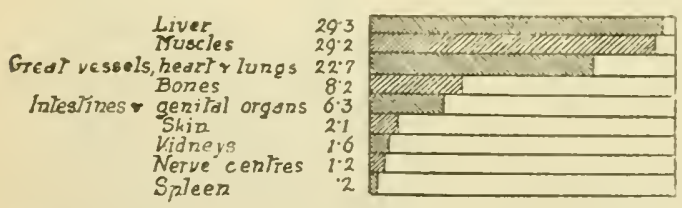

Fig. I6.-Diagram to illustrate the Distribution of the Blood in the Various Organs of a Rabbit (after Raske's Measurements). The numbers are percentages of the total blood.

mogen, which is stable in alkaline solution, and gives a beautiful spectrum with two bands, bearing some resemblance to those of oxyhamoglobin, but placed nearer the violet end. The band next the red end is much sharper than the other (p. $\tau^{(6))}$. Hemochromogen binds exactly the same amount of oxygen as the hermoglobin from which it is derived, and it is lue to the hamochromenen in its molecule that the blord-pigment fulfils its function of taking up and transporting oxygen.

$H$ amatin $\left(\mathrm{C}_{32} \mathrm{H}_{32}()_{4} \mathrm{~N}_{4}\right.$. Fe( $\left.) \mathrm{H}\right)$, the most frequent result of the splitting up of hemoglobin, is gencrally obtained as an amorphous substance with a bluish-black colour and a metallic lustre, insoluble in water, but soluble in dilute alkalies and acids, or in alcohol containing them. In ardition to the iron of the hemoglohin, hematin contain the four chief elements of proteins - carbon, hyclrogen, nitrogen, and oxgyen (Practical Excrciscs, p. 7.5)

Hamatoporphyrin $\left(\mathrm{C}_{33} \mathrm{H}_{36}()_{6} \mathrm{~N}_{4}\right)$, or iron-frec hamatin, nay be obtained from blood or hemuglobin by the action of strong sulphuric acid, from hæmatin or hxmin by the action of hydrobromic acid. It 
Is distinguished from these pigments by the fact that it comtains no, iron. When strong sulphuric acid is allowed to act on blood or hienn(s)globin solution, hamatoporphyrin is also produced, as may be casily shown by the spectroscope. Its spectrum in acid solution shows two bands, one just to the left of $D$, the other about midway between 1 ) and E. Like oxyhamoglobin, reduced hamoglobin, carbonic oxide hamoglobin, methamoglobin, and other derivatives of hanoglobin, it also has a band in the ultra-violet

$H$ cemin $\left(\mathrm{C}_{32} \mathrm{H}_{32} \mathrm{O}_{4} \mathrm{~N}_{4}\right.$. FeCl) is readily obtained from hamatin and also from hanoglobin by heating with dilute hydrochloric acid, and also directly from blood, as described in the Practical Exercises, p. 78 . It crystallizes in the form of small rhombic plates, of a brownish or brownish-black colour (Fig. 24, p. 78). They are insoluble in water, but readily soluble in dilute alkalies (Practical Exercises, p. 79).

Chemistry of the White Blood-Corpuscles.-The composition of puscells and the leucocytes of lymphatic glands has alone been investigated. The chief constituents of the latter are a globulin coagulating by heat at $48^{\circ}$ to $50^{\circ} \mathrm{C}$; ; a nucleo-protein coagulating in 5 per cent. magnesiun sulphate solution at $75^{\circ} \mathrm{C}$., and causing coagulation of the blood on injection into the veins of rabbits; an albumin coagulating at $73^{\circ} \mathrm{C}$. and a ferment with powers like the pepsin of the gastric juice. In puscells glycogen has been found, and it can be demonstrated microchemically in the leucocytes of blood by the iodine reaction in various conditions. Fats, cholesterol, and lecithin are also present, as well as the so-called protagon. The ordinary inorganic constituents have been demonstrated-namely, potassium, sodium, calcium, magnesium, and iron, united with chlorine and phosphoric acid. The total solids amount to I I to $\mathrm{I} 2$ per cent.

\section{Section IV.-Quantity and Distribution of the Blood.}

The Quantity of Blood.-The quantity of blood in an animal is most accurately determined by the method of Welcker. The animal is bled from the carotid into a weighed flask. When blood has ceased to flow the vessels are washed out with water or physiological saline solution, and the last traces of blood are removed by chopping up the body, after the intestinal contents have been cleared away; and extracting it with water. The extract and washings are mixed and weighed; a given quantity of the mixture is placed in a hæmatinometer (a glass trough with parallel sides, e.g.), and a weighed quantity of the unmixed blood diluted in a similar vessel till the tint is the same in both. From the amount of dilution required, the quantity of blood in the watery solution can be calculated. This is added to the amount of unmixed blood directly determined. Since hæmorrhage is immediately followed by the entrance of liquid into the bloodvessels from the lymph and tissue fluids, somewhat ton high a result will be obtained if the bleeding is at all prolonged. It is well, therefore, to take only a moderate amount of blood for direct estimation, and to compute the balance by the colorimetric method.

Many other methods have been devised on the principle of injecting a known quantity of some substance into the circulating blood, and then, after an interval has been allowed for mixture, determining the change produced in a sample. Thus, the specific 
gravity of a drop of blood having been measured, a certain quantity of a solution of sodium chloride isotonic with the plasma may be injected into is rein, and the specific gravity again determined. Or the electrical resistance of a small sample of blood may be measured before and after injection of a given quantity of isotonic salt solution.

The total mass of blood in a living man has been estimated by causing the person to inhale a mixture of carbon monoxide with oxygen or air. The amount of carbon monoxide taken 11 is determined and also, in a sample of blood taken from the finger the percentage amount to which the hemoglobin has become saturated with carbon monoxide. All that remains is to estimate the volume of carbon monoxide (or, what is precisely the same thing, the volume of oxygen) which roo c.c. of blood will take up. This latter quantity is called the percentage oxygen capacity. From these data the total volume of the blood can be calculated. If the volume is multiplied by the specific gravity the mass is obtained. Notwithstanding the elegance of this method in principle, it is by no means easy to obtain accurate results with it in practice. It unquestionably gives values for the total quantity of blood which are too low. A better method is to inject into a vein a measured quantity of a solution of a pigment (" vital red "), whicl is only slowly climinated and is not taken up to a sensible degree by the erythrocytes. Samples of blood are drawn before and a short time after the injection and the plasma separated from each by the centrifuge. The amount of pigment is then determined which must be added to the first sample of plasma to make its tint the same as that of the sccond. The total quantity of plasma can thus be calculated and from it, by determining the relative proportion of corpuscles and plasma in the blood, the total quantity of blood (Rowntree, etc.).

The quantity of blood in the body was greatly over-estimated by the ancient physicians. Avicenna put it at $25 \mathrm{lb}$., and many loose statements are on record of as much as $20 \mathrm{lb}$. being lost by a patient without causing death. By Welcker's method the proportion of blood to body-weight has been found to be in the dog I: 13 , cat I: I4, horse I: I5, frog $I: x 7$, rabbit $I: I 9$, fowl $I: 20$. In new-born children the proportion was I: 19 , in adult human beings (executed criminals) I : I3. By the 'vital red' method, the amount of plasma was found to be one-twentieth and that of blood oneeleventh to one-twelfth of the body-weight.

According to Dreyer, the blood volume is a function of the surface of the body, so that the smaller and lighter animals in any given species have a relatively greater blood volume than the larger and heavier individuals. Accordingly, he considers that the practice of expressing the volume of blood as a percentage of the body-weight should be abandoned.

Fig. I6 (p. 54) illustrates the distribution of the blood in the various organs of a rabbit. The liver and skeletal muscles each contain rather more than one-fourth; the heart, lungs, and great vessels 
rather less than one-fourth; and the rest of the budy about one-fifth, of the total blood. The kidney and spleen of the rabbit each contain one-eighth of their own weight of blood, the liver between one-third and one-fourth of its weight, the muscles only one-twentieth of their weight.

\section{Section V.-Lymph AND Chyi.e.}

Lymph has been defined as blood without its red corpuscles (Johannes Müller); it resembles, in fact, a dilute blood-plasma, containing leucocytes, some of which (lymphocytes) are common to lymph and blood, others (coarsely granular basophile cells, present only in small numbers) are absent from the blood. Lymph also contains thrombocytes. The reason of this similarity appears when it is recognized that the plasma of tissue-lymph (p. 460) is derived, in large part at any rate, from the plasma of blood by a process of physiological filtration (or secretion) through the walls of the capillaries into the lymph-spaces that everywhere occupy the interstices of areolar tissue, while the lymph of the lymphatic vessels is in turn derived from the tissue fluid. But in addition to the constituents of the plasma, lymph contains substances produced in the metabolism of the tissues which pass into it directly. As collected from one of the large lymphatic vessels of the limbs, or from the thoracic duct of a fasting animal, lymph is a colourless or sometimes yellowish or slightly reddish liquid of alkaline reaction. Its specific gravity is much less than that of the blood (IOI5 to Io30). It coagulates spontancously, but the clot is always less firm and less bulky than that of blood. The plasma contains fibrinogen, from which the fibrin of the clot is derived. Serum-albumin and serumglobulin are present in much the same relative proportion as in blood, although in smaller absolute amount. Neutral fats, urea, and sugar are also found in small quantities. The inorganic salts are the same as those of the blood-serum, and exist in about the same amount, sodium preponderating among the bases, as it does in serum. The following table shows the results of analyses of lymph from man and the horse (Munk):

\begin{tabular}{|c|c|c|c|c|c|c|c|}
\hline & & & & \multicolumn{2}{|c|}{ Man. } & \multicolumn{2}{|c|}{ Horse. } \\
\hline \multirow[t]{2}{*}{ Water } & Gibrin ${ }^{-}$ & - & - & \multicolumn{2}{|c|}{$95^{\circ}$ o per cent. } & \multicolumn{2}{|c|}{$95^{\circ} \mathrm{S}$ per cent. } \\
\hline & Other proteins & - & - & $4 \cdot 1$ & & $2 \cdot 9$ & \\
\hline \multirow[t]{3}{*}{ Solids } & Fat - - & - & - & trace & $5 \cdot 0$ & trace & $4 \cdot 2$ \\
\hline & Extractives* & - & - & $0 \cdot 3$ & & $0 \cdot \mathbf{I}$ & \\
\hline & (Salts - & - & - & 0.5 & & $I \cdot I$ & \\
\hline
\end{tabular}

* The term ' extractives ' is somewhat loosely applied to organic substances which exist in so small an amount, or have such indefinite chemical characters, that they cannot be separately estimated, and are extracted together from the residue by various solvents. 
Chyle is merely the name given to the lymph coming from the alimentary canal. The fat of the food is absorbed by the $1 y \mathrm{~m}$ phatics, and during digestion the chyle is crowded with fine fatty globules, which give it a milky appearance. There may also be in cliyle a few red blood-corpuscles, carried into the thoracic duct by a back-flow from the veins into which it opens. Chyic clots like ordinary lymph, the size of the clot varying according to the quantity of fat present and enmeshed by the fibrin. Wounds of the thoracic duct or of lymphatics opening into it are occasionally produced in operations on the neck, and when these remain open chyle may be readily collected. In samples obtained from a patient only a week after the section of a branch of the duct during an operation for the removal of tubercular glands, water constituted 928.90 parts in $\mathrm{I}, 000$, total solids $7 \mathrm{I} \cdot \mathrm{IO}$, inorganic solids $6 \cdot 04$, organic solids $65 \cdot 06$, proteins 18.52 , ether extract (fatty substances) 19.30 (Sollmann). The following is the composition of a sample analyzed by Paton, and obtained from a fistula of the thoracic duct in a man:

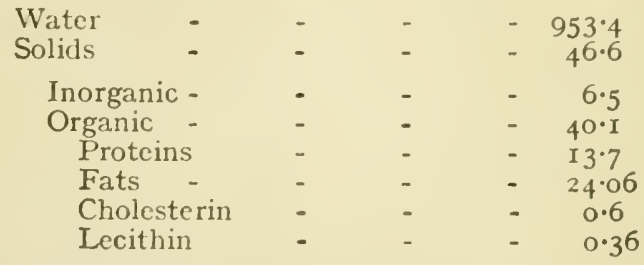

The quantity of chyle flowing from the fistula was estimated at as much as 3 to 4 kilos per twenty-four hours, or nearly as much as the whole of the blood. The flow has been calculated in various animals at one-eighteenth to one-seventh of the body-weight in the twentyfour hours. The quantity of lymph in the body is unknown, but it must be very great-perhaps two or three times that of the blood.

Allied to tissuc-lymph, but not identical with it, are the fluids present in health in very small amount in such serous cavities as the pericardium. The synovial fluid of the joints differs from lymph especially in containing a small amount of a mucin-like substancc.

The aqueous humour, and still more the cerebro-spinal fluid, arc characterized by a marked deficiency in solicls, especially protein. In the following table (from Spiro) the differences in the composition of lymph and allied fluids from different parts of the hody are illustrated.

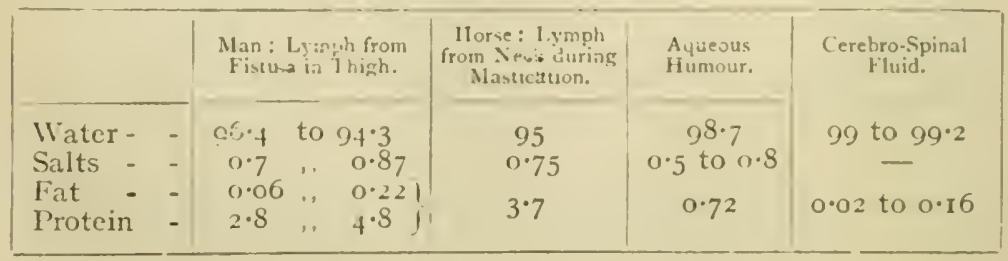


The gases of the blood and lympl will be treated of in Chapter IV., the formation of lymph in Clapter VIII., its circulation in Chapter III.

\section{Section VI.-The Functions of Blood and Lymph.}

We have already said that these liquids provide the tissues with the materials they require, and carry away from them materials which have served their turn and are done with. These materiais are gaseous, liquid, and solid. Oxygen is brought to the tissues in the red corpuscles; carbon dioxide is carried away from them partly in the erythrocytes, but chiefly in the plasma of the blood and lymph. The water and solids which the cells of the body take in and give out are also, at one time or another, constituents of the plasma. The heat produced in the tissues, too, is, to a large extent, conducted into the blood and distributed by it throughout the body. The leucocytes, as will be seen farther on, aid in some measure in the absorption of certain of the food substances from the intestine. It is not known whether, apart from this, they play any rôle in the normal nutrition of other cells, although it is probable that they exercise an influence on the plasma in which they live. But they have important functions of another kind, to which it is necessary to refer briefly here.

Phagocytosis.-Certain of the amœboid cells of blood and lymph, and the cells of the splenic pulp, are able to include or ' eat up ' foreign bodies with which they come in contact, in the same way as the amœba takes in its food. Such cells are called phagocytes; and it is to be remarked that this term neither comprises all leucocytes nor excludes all other cells, for some fixed cells, such as those of the endothelial lining of bloodvessels, are phagocytes in virtue of their power of sending out protoplasmic processes, while the small, relatively immobile lymphocyte is not a phagocyte.

Although it is not at present possible to assign a physiological value to all the phenomena of phagocytosis, either as regards the phagocytes themselves or as regards the organism of which they form a part, there seems little doubt that under certain circumstances the process is connected with the removal of structures which in the course of development have become obsolete, or with the neutralization or elimination of harmful substances introduced from without, or formed by the activity of bacteria within the tissues. During the metamorphosis of some larvæ, groups of cilia and muscle-fibres may be absorbed and eaten up by the leucocytes. In the metamorphosis of maggots, for example, the muscular fibres of the abdominal wall, which are used in creeping, and are therefore not required in the adult, degenerate, and are devoured by swarms of leucocytes which migrate into them. In the human subject an example of 
absorption of tissue by the aid of lencocytes is the removal of the necrosed decidua reflexa, the fold of uterine mucous membrane which envelops the ovum (Minot).

The behaviour of phagocytes towards pathogenic micro-organisms is of even greater interest and importance. Metchnikoff laid the foundation of our knowledge of this subject by his researches on Daphnia, a small crustacean with transparent tissues, which can be observed under the microscope. When this creature is fed with a fungus, Monospora, the spores of the latter find their way into the body-cavity. Here they are at once attacked by the leucocytes, ingested, and destroyed. But after a time so many spores get through that the leucocytes are unable to deal with them all; some of them develop into the first or " conidium 'stage of the fungus; the conidia poison the leucocytes, instead of being destroyed by them, and the animal generally dies. Occasionally, however, the leucocytes are able to destroy all the spores, and the life of the Daphnia is preserved. This battle, ending sometimes in victory, sometimes in defeat, is believed by Metchnikoff to be typical of the struggle which the phagocytes of higher animals and of man seem to engage in when the germs of disease are introduced into the organism. He supposes that the immunity to certain diseases possessed naturally by some animals, and which may be conferred on others by vaccination with various protective substances, is, to a large extent, due to the early and complete success of the phagocytes in the fight with the bacteria; and that in rapidly-fatal diseases-such as chickencholera in birds and rabbits, and anthrax in mice-the absence of " any effective phagocytosis is the factor which determines the result. Others have laid stress on the action of protective substances supposed to exist in the plasma itself. It is possible that such substances are manufactured by the leucocytes, and either given off by them to the plasma by a process of 'excretion,' or liberated by their complete solution.

The most recent investigations go to show that Metchnikoff's phagocytic theory of immunity requires modification, at any rate in the case of the higher animals and man, although the brilliant biological observations on which it was originally built retain all their value. He supposed that in the immunizing process the leucocytes underwent certain clianges, acquired, so to speak, a sort of 'education' that enabled them to cope with bacteria against which they were previously powerless. It seems more probable that in the presence of the substances that confer immunity; not only the leucocytes, but other cells, are stimulated to produce bodics which cut short the life, or inhibit the growth, of the bacteria (alexins), or prepare them for being taken up by the phagocytes (opsonins). It has been shown that bacteria which have been in contact with serum containing the appropriate opsonins are taken 
up readily by leucocytes washed free from serum constituents by pliysiological salt solution, whereas the washed leucocytes either do not ingest bacteria which lave not been acted on by serum, or take them up in much smaller numbers. There is some evidence that in certain bacterial infections - for example, chronic furunculosis, a condition in which crops of boils continue to appear - the grip of the bacteria on the body is perpetuated by a deficiency in the amount or in the activity of opsonins capable of acting specifically upon the micro-organisms in question. A numerical expression, which in certain cases, perhaps, gives a measure of the patient's resistance to the infection, has been worked out by Wright under the name 'opsonic index.' This index is the ratio between the average number of bacteria taken up, under certain fixed conditions, by each polymorphonuclear leucocyte in an emulsion made with the patient's serum, and the average number taken up by similar leucocytes in an emulsion made with normal serum. The significance of this index and even the practicability of the methods used to ascertain it, are still the subject of discussion.

Diapedesis.-The fact that leucocytes can pass out of the bloodvessels into the tissues has a very important bearing on the subject of phagocytosis. The phenomenon is called diapedesis, and is best seen when a transparent part, such as the mesentery of the frog, is irritated. The first effect of irritation is an increase in the flow of blood through the affected region. If the irritation continues, or if it was originally severe, the current soon begins to slacken, the corpuscles stagnate in the vessels, and inflammatory stasis is produced. The leucocytes adhere in large numbers to the walls of the capillaries, and particularly of the small veins, and then begin to pass slowly through them by amœboid movements, the passage taking place at the junctions between, or it may be through the substance of, the endothelial cells. Plasma is also poured out into the tissues, the whole iorming an inflammatory exudation. Even red bloodcorpuscles may pass out of the vessels in small numbers. The exudation may be gradually reabsorbed, or destruction of tissue may ensue, and a collection of pus be formed. The cells of pus are emigrated leucocytes (Practical Exercises, Chap. III., p. I93).

Their emigration is connected with the defence of the organism against the entrance of certain forms of bacteria at the seat of injury, and with the repair of the injured tissue, but the nature of the summons which gathers them there is not yet clearly understood. It is probably some sort of chemical attraction (chemiotaxis) between constituents of the bacteria or decomposition prod. ucts of the injured tissue on the one hand, and constituents of the leucocytes on the other.

As for the blood-plates, it will suffice to say by way of summary that their important function in the sealing of wounded vessels (p. 46) 
is the sole office which at present can be attributed to them. And if it is permissible to consider the leucocytes as a patrol for the defence of the tissues in general against invading micro-organisms, it may perhaps not be too far-fetched an idea to look upon the bloodplates as essentially a patrol in the interests of the anatomical integrity of the vascular system itself. This does not exclude the possibility that the cloiting of extravasated plasma may furnish a more favourable medium for the processes of repair in all injured tissues.

\section{PRACTICAL EXERCISES ON CHAPTER II.}

N.B.-In the following exercises all experiments on animals which would canse the slightest pain are to be done under complete ancesthesia.

1. Reaction of Blood.-(I) Put a drop of fresh dog's or ox blood on a piece of glazed neutral litmus paper (the litmus paper can be glazed by dipping it into a neutral solution of gelatin and allowing it to dry). Wash the blood off in Io to 30 seconds with distilled water. A bluish stain will be left, showing that fresh blood is alkaline. (2) Repeat with dog's or ox serum. It is not necessary to wash the serum off, as it does not obscure the change of colour. (3) Repeat (I) with human blood. With a clean suture-needle or a good-sized sewing-needle which has been sterilized in the flame of a Bunsen burner, prick one of the fingers behind the nail. Bandaging the finger with a handkerchief from above downwards, so as to render its tip congested, will often facilitate the getting of a good-sized drop, but for quantitative experiments, like 2 , io, and $17(4)$, this should not be done.

2. Specific Gravity of Blood-Hammerschlag's Method.-(I) Put a mixture of chloroform and benzol of specific gravity $\mathrm{I} \cdot 060$ into a small glass cylinder. Put a drop of dog's or ox defibrinated blood into the mixture by means of a small pipette. If the drop sinks add chloroform. if it rises add benzol, till it just remains suspended when the liquid has been well stirred. Then with a small hydrometer measure the specific gravity of the mixture, which is now equal to that of the blood. Filter the liquid to free it from blood, and put it back into the stock-bottle. (2) Obtain a drop of human blood as in I, and repeat the measurement of the specific gravity:

3. Coagulation of Blood.*-(I) Take three tumblers or beakers, label them $a, \zeta$, and $\%$ and measure into each roo c.c. of water. Mark the level of the water by strips of gummed paper, and pour it out. (If a sufficient number of graduated cylinders is available, they may of course be used, and this measurement aroided.) Into a put 25 c.c of a saturated solution of magnesium sulphate, into $\beta 25$ c.c. of a I per cent. solution of potassium or ammonium oxalate in 0.9 per cent. solution of sodium chloride, and into $\gamma 25$ c.c. of a $1 \cdot 2$ per cent. solution of sodium fluoride in $c .9$ per cent. salt solution if the dog provided is a large one, these quantities may be all doubled; for a small dog they may be all halved.

- This experiment requires two laboratory periods, the various blood mixtures being obtained during the first and worked up during the second. 
(2) Insert a cannula into the central end of the carotid artery of a dog anasthetized with morphine* and ether, or A.C.E. mixture. $†$

To put a Cammula into an Artery. - Salect a glass cannula of suitable size, fecl for the artery, make an incision in its course tlirough the skin, then isolate about an inch of it with forceps or a blunt needle, carefully clearing away the fascia or connective tissue. Next pass a small pair of forceps under the artery, and draw two ligatures through below it. If the canmula is to be inserted into the central end of the artery, tic the ligature which is farthest from the heart, and cut one end short. Then between the heart and the other ligature compress the artery with a small clamp (often spoken of as 'bulldog ' forceps). Now lift the artery by the distal ligature, make a transverse slit in it with a pair of finc scissors, insert the cannula, and tic the ligature over its neck. Cut the ends of the ligature short. If the cannula is to be put into the distal end of the artery, both ligatures must be between the clamp and the heart, and the bulldog must be put on before the first ligature (the one nearest the heart) is tied, so that the piece of bloodvessel between it and the ligature may be full of blood, as this facilitates the opening of the artery.

(3) Run into $a, \beta$, and $\gamma$ enough blood to fill them to the mark. Shake the vessels, or stir up once or twice with a glass rod, to mix the blood and solution.

(4) Take a small thin copper or brass vessel, and place it in a freezing mixture of ice and salt. Run into it some of the blood from the artery. It soon freezes to a hard mass. Now take the vessel out of the freezing mixture and allow the blood to thaw. It will be seen that it remains liquid for a short time and then clots.

(5) Run some of the blood into a porcelain capsule, stirring it vigorously with a glass rod. The fibrin collects on the rod; the blood is defibrinated and will no longer clot.

(6) Now let some blood run into a small beaker or jar. Notice that the blood begins to clot in a few minutes, and that soon the vessel can be tilted without spilling it. Note the time required for clotting to occur. Set the coagulated blood aside, and observe next day that clear yellow serum has separated from the clot.

(7) Weigh out a quantity of Witte's 'peptone' equivalent to 0.5 gramme for every kilo of body-weight of the dog. Dissolve the peptone in about twenty times its weight of 0.9 per cent. salt solution. Put a cannula into the central end of a crural vein (p. 2r2). Fill the cannula with the peptone solution and connect it with a burette. Put I 5 drops of the peptone solution into a test-tube labelled 'Peptone A.' Put the rest into the burette, and see that the connecting tube is filled with the solution and free from air. Run into the test-tube about 5 c.c. of blood from the cannula in the carotid. Now let the peptone solution flow from the burette into the vein. Feel the pulse over the heart as the solution is running in. If the heart becomes very weak, stop the injection; otherwise the animal may die from the great lowering of blood-pressure (p. $2 \mathrm{r}_{4}$ ). As soon as the injection is finished, draw off a sample of 5 c.c. of blood into a test-tube labelled 'Peptone B', and let it stand. In ten minutes collect five further samples of 5 c.c. ("Peptone C, D, E, F, G'), and a large one, $\mathrm{H}$; in half an hour

* One to 2 centigrammes of morphine hydrochlorate per kilogramme of body-weight should be injected subcutaneously about half an hour before the operation. Ten c.c. of a 2 per cent. solution is sufficient for a dog of good size. Note that diarrhœa and salivation are caused by such a dose. For directions for fastening the dog on the holder, see footnote on p. I 99 .

$\dagger$ A mixture of $\mathrm{I}$ part of alcohol, 2 of ether, and 3 of chloroform. 
another set of five small samples, and at as long an interval as possible thereafter five more. Now letting the dog bleed to death, olserve that the flow of blood is temporarily increased by pressure on the abdominal walls, which squeczes it towards the heart, by passive movements of the hind-legs, and also during the convulsions of asplyyia, which soon appear. Add to the peptone blood D 5 c.c. of serum, to li a little sodium chloride extract of liver, to $F$ a little extract of muscle, and to G I5 drops of a 2 per cent. solution of calcium chloride, and put $C, D$, $E, F$, and $G$ into a water-bath at $40^{\circ} \mathrm{C}$. Treat the other sets of small

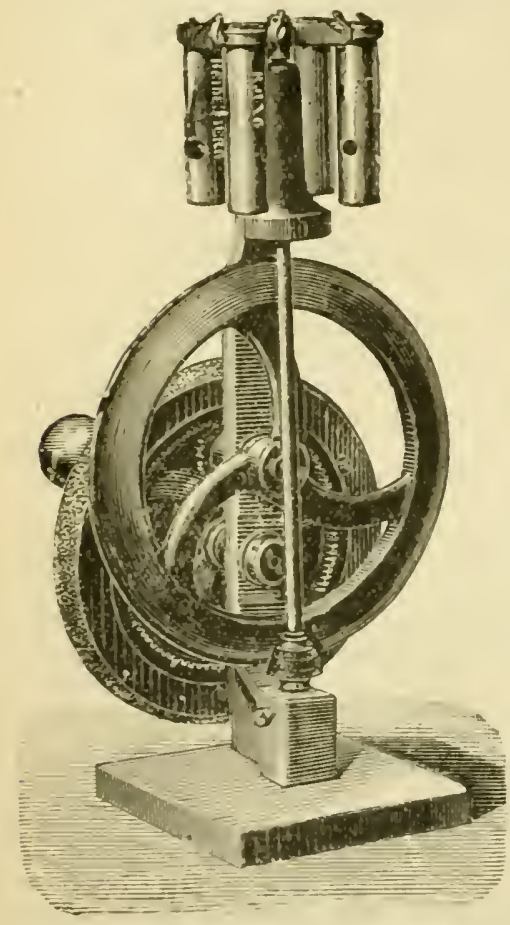

Fig. 17.-Centrifuge (Jung). The four cylinders shown at the top of the figure are so swung that they become horizontal as soon as speed is up. samples in the same way. Note liow long each specimen takes to clot, and report your results.*

(8) Observe that the blood in $a$. 8. and $\gamma$ has not coagulated. Label four test-tubes 'Oxalate A, B, C, $D$ ' and put into each about 5 C.c. of the oxalated blood. Add to $A$ and $B$, or 6 drops of a 2 per cent. solution of calcium chloride, to C I 2 drops, and to $\mathrm{D}$ as much as there is of the blood. Leave $A$ at the ordinary temperature, put the other test-tubes in a water-bath at $40^{\circ} \mathrm{C}$., and note when clotting occurs.

(9) To ro c.c. of the fluoride blood add a little more $\mathrm{CaCl}_{2}$ than is required to combine with the excess of fluoride present. Label four testtubes 'Fluoride A, B, C, D,' and into each put about 2 c.c. of this 'recalcified' fluoride blood. To B add I c.c. liver extract. to $C$ I c.c. muscle extract, and to $\mathrm{D} 4$ c.c. water. Label two more test-tubes 'Fluoride E and F!' Into each put 2 c.c. of the fluoride blood without $\mathrm{C} \mathrm{Cl}_{2}$. Add also to $\mathrm{E}$ I c.c. liver extract and to F I c.c. serum. Put all the tubes in a bath at about $40^{\circ} \mathrm{C}$, and observe in which and in what time coagulation takes place.

(Io) By means of a centrifuge (Fig. I 7) separate the plasma from the corpuscles in $a, \beta$, and $\gamma$, and also from the peptone blood.

With the oxalate plasma from $\beta$, and the fluoride plasma from $\gamma$, repeat the observations in (S) and (9), using smaller quantities of the plasma, if necessary, in small testtubes. With the plasma from a perform the following experiments: Put a small quantity of the plasma (I c.c.) into four test-tubes, labelling

- Sometimes the injection of peptone hastens coagulation instead of hindering it. It has been asserted that this is only the case when small doses are used (less than $0^{\circ} 02$ gramme per kilo of body-weight). But in 2 dogs out of I I dose of 0.5 gramme per kilo bas been seen to hasten coagulation, and in I out of $\mathbf{2}$ to leave it unaffected; in the other 9 coagulation was markedly retarded 
them 'Magnesium Sulphate A, B, C, D.' Dilute B with four times, C with eight times, and $D$ with twenty times as mucli distilled water as was taken of the plasma. Observe in which, if any, coagulation occurs, and the time of its occurrence, and report the result.

(I1) With peptone plasma from $H$ and from the peptone blood obtained later repeat the experiments done in (7). In addition dilute I c.c. of the plasma with three volumes of water and I c.c. of it with ten volumes of water, and put in the bath at $40^{\circ} \mathrm{C}$. Observe whether clotting occurs.

Instead of dog's blood, the blood of an ox or pig may be obtained at the slaughter-house.

4. Preparation of Fibrin-Ferment.-Precipitate blood-serum with ten times its volume of alcohol. Let it stand for several weeks, then extract the precipitate with water. The water dissolves out the fibrinferment, but not the coagulater serum proteins.

3. Preparation of Tissue Extracts containing Thrombokinase.-In a dog or rabbit killed by bleeding insert a cannula into the lower end of the thoracic aorta. Fill the cannula with 0.9 per cent. salt solution, and connect it with a bottle also containing salt solution. Wash out the vessels of the lower portion of the body, making an opening in the inferior vena cava above the diaphragm to allow the liquid to escape. For the sake of cleanliness, a cannula armed with a piece of rubber tubing should be inserted for this purpose into the inferior vena cava. Continue the injection till the liquid issues colourless. Then remove portions of liver and muscle. Mince each separately. Rub up with sand in a mortar. Add 0.9 per cent. sodium chloride solution and rub up again. Put into bottles and keep in the ice-chest. For use take off some of the liquid from the top with a pipette, or strain through cheese-cloth.

6. Serum. - Test the reaction, and determine, both by the hydrometer and the pycnometer, or specific gravity bottle, the specific gravity of the serum provided, or of the serum obtained in experi. ment 3 .

Serum Proteins.-(I) Saturate serum with magnesium sulphate crystals at $30^{\circ} \mathrm{C}$. The serum-globulin is precipitated. Filter off. Wash the precipitate on the filter with a saturated solution of magnesium sulphate. Dissolve the precipitate by the addition of a little distilled water, and perform the following tests for globulins: (a) Saturate with magnesium sulphate. A precipitate is obtainer. (b) Drop into a large quantity of water, and a flocculent precipitate falls down. (c) Heat. Coagulation occurs. Determine the temperature of coagulation (p. 9).

(2) To a portion of the filtrate from (I) add sodium sulphate to saturation. The serum-albumin is precipitated. (Neither magnesium sulphate nor sodium sulphate precipitates serum-albumin alone, but the double salt sodio-magnesium sulphate precipitates it, and this is formed when sodium sulphate is added to magnesium sulphate.)

(3) Dilute another portion of the filtrate from (I) with its own bulk of water. Very slightly acidulate with dilute acetic acid, and determine the temperature of heat coagulation.

(4) Precipitate the serum-globulin from another portion of serum by adding to it an equal volume of saturated solution of ammonium sulphate. Filte-. Precipitate the serum-albumin from the filtrate by saturating with ammonium sulphate crystals.

(5) Dilute serum with ten to twenty times its volume of distilled water, and pass through it a stream of carbon dioxide. The serumglobulin is partially precipitated. 
(6) Acidulate some serum with dilute acetic acid and boil. Filtes off the coagulum, and to the filtrate add silver nitrate. A non-protein precipitate insoluble in nitric acid, but soluble in ammonia, indicates the presence of chloricles.

7. Action of Serum on Artery Rings.-Cut a number of rings about I $\frac{1}{2}$ millimetres wide from a fresh carotid artery of the sheep, obtained from the slaughter-house. Keep the rings in a dish in Ringer's solution.* They should be as nearly as possible of uniform width. A small cylindrical glass vessel is supported on a stand in such a way that it can be casily lowered into a bath of water kept at a temperature of about $39^{\circ}$ to $40^{\circ}$. A stock of Ringer's solution is kept in a beaker or bottle immersed in the bath. A ring of the artery is put into the small cylinder, where it is held between two aluminium hooks, onc fastened to the bottom of the cylinder, the other (the upper one) connected with the sliort arm of a lever, the long arm of which is arranged to write on a slowly revolving drum. A time-trace, say in halfminutes, is recorded below. The small cylinder is now filled with warm Ringer's solution and lowered into the bath. Uxygen is bubbled through the solution by means of a side-tube near the bottom of the cylindrical vessel. The artery ring is now stretched for five minutes by a weight of 10 grammes attached to the long arm of the lever at the same distance from the axis as that at which the ring is attached. After the stretching period the weight is removed, and a little time allowed to elapse till the writing-point traces a horizontal line on the drum. Then a bent pipette is filled with serum already heated to bath temperature in a vessel immersed in the bath. The pipette is introduced in to the small cylinder so that its point is at the bottom, without disturbing the ring, and the serum is allowed to run in till the Ringer solution is displaced. The ring shortens under the influence of the constrictor substance in the serum, and tle tracing is continued till the shortening has reached its maximum and the trace is again horizontal (Fig. 3, p. 46).

Tarious dilutions of the serum are now made with Ringer's solution, and the greatest dilution in which the serum will still cause a perceptible constriction of the rings is determined. This affords a measure of comparison with other sera of the strength of the constrictor action. For each dilution of serum a separate ring must be used. It must be remembered that comparisons of this kind can only be made with arteries of the same sensitiveness, and different arteries vary much in this regard.

8. Comparison of the Action of Serum and Adrenalin (Epinephrin) on Artery Rings.-Tracings showing the effect of various dilutions of adrenalin cliloride on artery rings may now be taken for comparison with the serum effects. The adrenalin dilutions should be made just before use, as adrenalin is rapidly oxidized. Or a separate experiment on the action of adrenalin may be made under 'Circulation,' as on p. $2 \mathbf{I}$.

9. Comparison of the Action of Serum and Plasma on Artery Rings.Citrate plasina is obtained as follows: $\Lambda$ cannula and attached rubber tube are boiled, oiled inside with fresh olive-oil, and filled with a citrate

* This is the name given to a solution containing the most important of the inorganic constituents of blood-serum in approximately the normal proportions. The various 'Ringer's solutions' used by different workers have varied slightly. That recommended by Locke (for perfusion of the isolated heart) contains $\mathrm{NaCl}$, 0.9 per cent.; $\mathrm{KCl}, 0^{\circ} 0_{4} 2$ per cent.; $\mathrm{CaCl}_{2}, 0^{\circ} 024$ per cent. ; $\mathrm{NaHCO}_{3}$, O.OI to $0^{\circ} \mathrm{O}_{3}$ per cent.; with in addition $0^{*} \mathrm{I}$ per cent. of dextrose, which can be omitted for such experiments as 7 . 
solution made by dissolving sodium citrate in Ringer's solution to the extent of 2 per cent. The solution is prevented from escaping by a clip on the tube. The cannula is inserted into the carotid of a dog, the end of the rubber tubc dipped below a quantity of citratcRinger solution in a beaker, and a volume of blood equal to that of the: solution run in. Then the blood and solution are at once stirred gently, but sufficiently to insure proper admixturc.

Some blood is now run into another vessel, defibrinated, and measured. An equal volume of the citrate-Ringer solution is added to it while the mass of fibrin is still floating in the blood. After mixing, the fibrin is removed. Plasma is then separated by the centrifuge from the first specimen of blood, and serum from the second, and comparison experiments are made with each on artery rings. If the plasma has been properly obtained, it will have little constrictor effect on the rings in comparison with the serum. In making the comparison, arteries which give a decided effect with serum should be employed. The defibrinated blood and the unclotted citrate blood may also be used for the comparison.

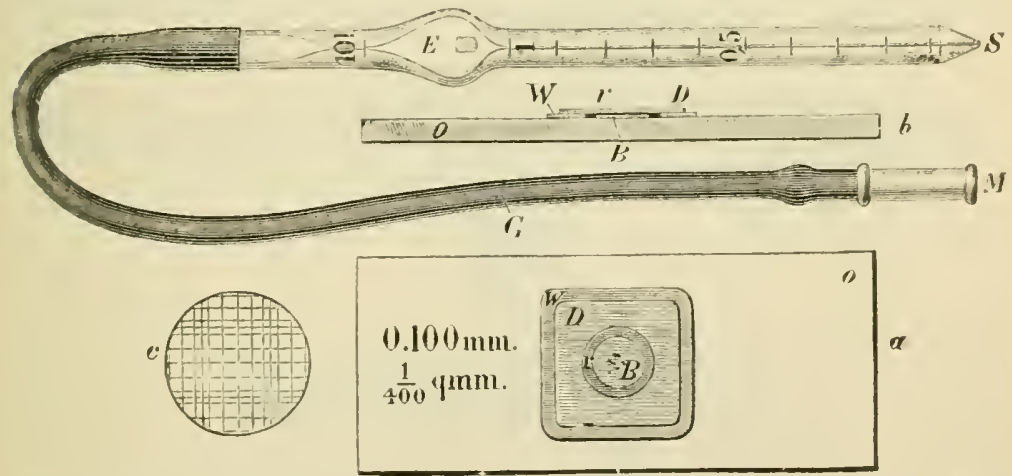

Fig. I $\delta$ - Thoma-Zeiss Hamocytometer. $M$, mouthpiece of tube $G$, by which blood is sucked into $S$; $E$, bead for mixing; $a$, view of slide from above; $b$, in section; $c$, squares in middle of $B$, as seen under microscope.

10. Enumeration of the Blood-Corpuscles. - Use the Thoma-Zeiss apparatus (Fig. I 8). (I) Suck a drop of ox or dog's blood up into the capillary tube $S$ to the mark 1 . Wipe off any blood which may adhere to the end of the tube. Then fill it with 3 per cent. sodium chloride to the mark ror. This represents a dilution of Ioo times. Mix the blood and solution thoroughly, then blow out a drop or two of the liquid to remove all the solution which remains in the capillary tube. Now fill the shallow cell $B$ with the blood mixture. Put the cover-glass on, taking care that it does not float on the liquid, but that the cell is exactly filled. Put the slide under the microscope (say Leitz's oc. III., obj. 5), and count the number of red corpuscles in not less than ten to twenty squares. Sixteen squares is a good routine number. The greater the number of squares counted, the nearer will be the approximation to the truth. Now take the average number in a square. The depth of the cell is ${ }_{10}^{1} \mathrm{~mm}$., the area of each square $\frac{1}{400} \mathrm{sq} . \mathrm{mm}$. The volume of the column of liquid standing upon a square is $\frac{1}{1000}$ cub. $\mathrm{mm}$. One cub. $\mathrm{mm}$. of the diluted blood would therefore contain 4,000 times as many corpuscles as one square. But the blood has been diluted Ioo times, therefore I cub. mm. of the 
undiluted blood would contain 400,000 times the number of corpuscles in one square. Suppose the average for a square is found to be 13 . This would correspond to 5,200,000 corpuscles in I cub. $\mathrm{mm}$. of blood. Compare your result with the true number supplied by the demonstrator. (2) Prick the finger to obtain a drop of blood, and repeat the crunt as in (I).*

To Sount the White Corpuscles.-Add to I part of blood 9 parts of $\frac{1}{3}$ per cent. acetic acid, in order to lake the coloured corpuscles and render it easy to see the leucocytes.

Ir. Relative Volume of Corpuscles and Plasma by Hæmatocrite.(I) For practice, fill the two graduated glass tubes with the defibrinated blood of an animal. The rubber tube with mouthpiece supplied with the apparatus is to be attached to the glass tube, and the blood sucked up. Press the tip of the index-finger against the pointed end, and carefully remove the rubber tube. Place the tube in the hrmatocrite frame, blunt end outwards-that is, farthest from the axis of rotation-and then slip the pointed end down into position against the spring. Instead of the rubber tube, a special suction pipette for automatically filling the graduated tubes may be employed (Daland). Attach the hæmatocrite frame to the centrifuge, and rotate till the volume of sediment

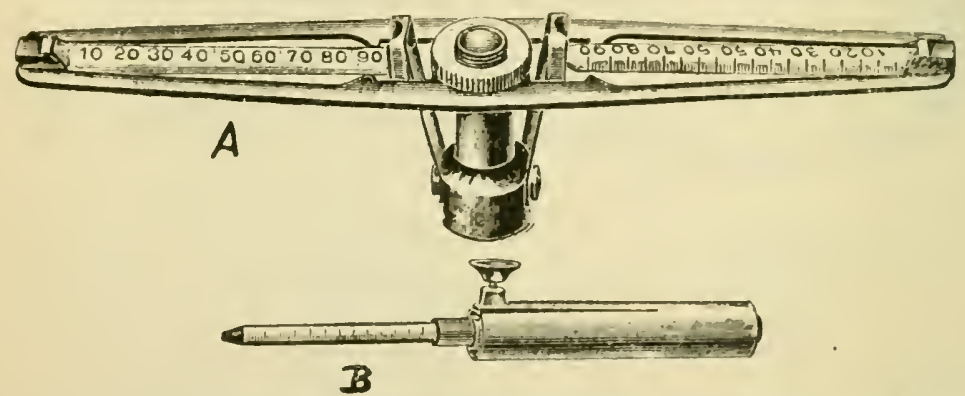

Fig. 19.-Hæmatocrite. $A$, hæmatocrite attachment with graduated tubes; $\boldsymbol{B}$, automatic pipette for filling the tubes (Daland).

(corpuscles) ceases to diminish. The graduations are best read with a hand lens. The leucocytes will be seen to form a thin whitish line proximal to the column of red corpuscles.

(2) Prick the finger or the lobe of the ear, fill the tubes as in (I), and centrifugalize. Everything must be donc as rapidly as possible, so that the blood may not clot till the separation of plasma and corpuscles is completed. The centrifuge must rotate very rapidly (about 10,000 revolutions a minute) for two or threc minutes. For clinical purposes it is best to rotate the centrifuge always at the same spced for the same length of time rather than to aim at rcaching a constant length of the column of corpuscles. In this way usciul comparative results can be obtained. It is well, to avoid the risk of accident, to rotate the centrifuge under a guard.

12. Electrical Conductivity of Blood.-(I) Fill a small U-tube with blood up to a mark. In each limb insert a platinum electrodet con-

* If the tube has not becn properly filled, blow the blood out immediately. On no account permit it to clot in the capillary tube.

+ If the platinum electrodes are of good size and the resistance of the tubc of liquid considerable, it is not necessary to platinize them-i.e., to cover them by electrolysis of a solution of platinic chloride with a layer of platinum-black. 
nected with a holder, which insures that the electrode shall always dip to the same depth into the tube. Arrange the $\mathbf{U}$-tube so that it is immersed at least to the mark in water of constant temperature. Water running frcely from the cold-water tap into and out of a large vessel will have a sufficiently constant temperature for the purpose. A thermometer must be fixed in the water with its bulb in contact with the $\mathrm{U}$-tube. Connect the electrodes with a resistance-box in the Wheatstone's bridge arrangement (Fig. 231, p. 726), so that the U-tube occupies the position of the unknown resistance CD. Instead of the battery $F$, connect the poles of the secondary of a small induction-coil, arranged for an interrupted current, with $\mathrm{A}$ and $\mathrm{C}$, and instead of the galvanometer $\mathrm{G}$ insert a telephone. The resistances $\mathrm{AB}$ and $\mathrm{AD}$ will be obtained by taking out two plugs from the appropriate part of the resistanoe-box. Whether $\mathrm{AB}$ and $\mathrm{AD}$ should be equal (say, Io : Io, I00: I00, or I,000: I,000 ohms) or unequal (say, Io: Ioo, or I00 : I, 000, or IO : I, O00 ohms) will depend upon the resistance of the tube of liquid to be measured. Take out from the part of the box corresponding to $\mathrm{BC}$ a plug representing a resistance something like that which the tube of blood is expected to have. Close the primary circuit of the induction-coil, and apply the telephone to the ear. A buzzing sound will be heard, which will be louder the farther from the true resistance of the tube the resistance taken out of the box is. Go on altering the resistance in the box by taking out or putting in plugs till the sound disappears, or is reduced to a minimum. The temperature of the water should now be read off. The resistance of the tube of blood for this temperature can easily be calculated from the formula on p. 726. It increases about 2 per cent. for each degree Centigrade of diminution of temperature. The conductivity is the reciprocal of the resistance. By determining once for all the resistance of the tube when filled with a standard solution of a salt whose conductivity is known, the specific conductivity of the blood can be expressed in definite units, but this is not necessary for the purposes of the student. Compare the resistances of defibrinated blood, serum, 0.9 per cent. sodium chloride solution, and a sediment of blood-corpuscles scparated by centrifugalization.

(2) Instead of the resistance-box a wire mounted on a scale may be used for the resistances $\mathrm{AB}, \mathrm{AD}$, the ends of the wire being connected at $B$ and D. A slider with an insulated handle moving along the graduated wire is joined by a flexible wire with one pole of the secondary coil, the other pole being connected at $\mathrm{C}$. The resistance $\mathrm{BC}$ is constituted by a rheostat from which a fixed resistance can be taken out. Instead of obtaining the minimum sound in the telephone by varying the resistance $\mathrm{BC}$ in the box, the measurement is made by varying the position of the slider; iu other words, by changing the ratio $\mathrm{AB}$ : AD.

(3) If no rheostat is available instructive comparative measurements may still be made with the graduated wire by substituting for the resistance $\mathrm{BC}$ a $\mathbf{U}$-tube of another liquid.

If the tubes are of the same dimensions, and the liquids with which they are filled are approximately at the same initial temperature, it is not necessary to immerse them in water at constant temperature. It is sufficient to place them side by side in the air. Perform the following experiments in this way:

(a) Label the tubes A and B. Fill them both to the mark with 0.9 per cent. $\mathrm{NaCl}$ solution. Connect as in the figure, and move the slider along the wire till the sound is a minimum. Probably the two tubes are not exactly of the same dimensions, and therefore the slider will not be exactly in the middle of the wire. Suppose it is at $49^{\circ} 0$ 
the total length of the wire being 100 . Then resistance of $\mathrm{A}$ : resistance of $\mathrm{B}:: 49^{\circ} \mathrm{O}: 5^{\mathrm{I}} \cdot \mathrm{O}$, i.e., resistance of $\mathrm{A}=\frac{49}{51}$ resistance of $\mathrm{B}$.

(b) Fill A with defibrinated blood, liceping $\mathrm{B}$ filled with $\mathrm{NaCl}$ solution, and repeat the measurement. The slicler must now be moved much farther away from the zero of the scalc. Suppose the minimum sound is obtained with the slider at $70^{\circ} 0$. Then resistance of blood $=$ $3 \times \frac{5 \mathrm{I}}{49}$ resistance of the $\mathrm{NaCl}$ solution.

749

(c) Compare in the same way the resistance of serum with that of the $\mathrm{NaCl}$ solution. It will be found much less than that of the blood.

(d) Centrifugalize some of the blood for as long as is convenient, and compare the resistance of the blood from the top of the tubes and from the bottom of the tubes with that of the $\mathrm{NaCl}$ solution. The resistance of the blood from the bottom of the tubes will be found much greater than that of the blood from the top.

I3. Opacity of Blood.-Smear a little fresh blood on a glass slide, and hold the slide above some printed matter. It will not be possible to read it, because the light is reflected from the corpuscles in all directions, and little of it passes throngh.

I 4. Laking of Blood by Chemical and Physical Agents.-(I) Put a little fresli blood into three test-tubes, A, B, and C. Dilute $A$ with an equal volume, $B$ with two volumes, and $C$ with three volumes, of distilled water, and repeat experiment 9. The print can now be read probably through a layer of $\mathrm{A}$, but certainly through $\mathrm{B}$ and $\mathrm{C}$, since the hæmoglobin is dissolved out of the corpuscles by the water and goes into solution, the blood becoming transparent or laked. That the difference is not due mercly to dilution can be shown by putting an equal quantity of blood in two test-tubes, and gradually diluting one with distilled water and the other with a 0.9 per cent. solution of sodium chloride, which does not dissolve out the hremoglobin. Print can be read through the first with a smaller degree of dilution than through the second. Examine the laked blood with the microscope for the ' ghosts,' or shadows of the red corpuscles. The addition of a drop or two of methylene blue will render them somewhat more distinct.

(2) Heat a little dog's or ox blood in a test-tube immersed in a waterbath. Put a thermometer in the test-tube, taking care that there is enough blood to cover the bulb. Keep the temperature about $60^{\circ} \mathrm{C}$. In a few minutes the blood becomes dark and laking occurs.

(3) (a) Put a little blood into each of four test-tubes. To one add a little ether, to another a little chloroform, to the third dilute acetic acid in 0.9 per cent. $\mathrm{NaCl}$, and to the fourth a dilute solution of bile salts (or of sodium taurocholate) in 0.9 per cent. $\mathrm{NaCl}$ solution. Laking occurs in all.

(b) To 5 c.c. of blood add 0.5 c.c. of a 3 per cent. solution of saponin in 0.9 per cent. $\mathrm{NaCl}$ solution, and put the mixture at $40^{\circ} \mathrm{C}$. Laking soon occurs.

(c) Using a ro per cent. dilution of blood (blood to which nine volumes of $\mathrm{NaCl}$ solution have been added) or a 5 per cent. suspension of washed corpuscles in $\mathrm{NaCl}$ solution (i.e., a suspension of corpuscles which have becn washed free from serum by being repeatedly mixed with $\mathrm{NaCl}$ solution and centrifugalized), determine the minimum dose of 0.3 per cent. saponin solution which will just cause complete laking. Keep the tubes at about $40^{\circ} \mathrm{C}$, and observe them from time to time. Now add to some of the 10 per cent. dilution or the 5 per cent. suspension of blood an equal volume of serum from the same kind of blood, and repeat the determination of the minimum dose of saponin necessary for laking. 
It will be found that more is now required. The cholesterin in the scrum neutralizes the action of some of the saponin.

(4) (a) Put I c.c. of blood into each of two test-tubes. To one add 1 c.c. of 2 per cent. aqueous solition of urea, and to the other 3 c.c. laking will take place in the second, whether this lias been the case in the first or not.

(b) Repeat the experiment with a 2 per cent. solution of urea in $0 \cdot \mathrm{g}$ per cent. $\mathrm{NaCl}$ solution. Laking does not occur. This shows that the urea in the first experiment did not act as a liamolytic agent. Laking occurred because urea penetrates the corpuscles easily, and therefore, although the freezing-point of the urea solution is not very different from that of the $\mathrm{NaCl}$ solution, its actual osmotic pressure, in relation to the envelopes of the corpuscles, is very much less, and the laking is really water-laking.

(5) Put some blood into a flask or test-tube, cork up, and let it stand till it begins to putrefy. It becomes laked. The same occurs when the blood is collected aseptically in a sterile tube and sealed up, although it takes a longer time for the laking to become complete.

(6) With blood containing nucleated corpuscles (necturus, frog or chicken) diluted with isotonic salt solution, perform the following experiments under the microscope:

(a) With a glass rod drawn to a fine point put a small drop of bloocl on a slide, and near it a drop of distilled water. Carefully lower the cover-slip and obscrve the interface with the microscope, first with the low and then with the high power. Then mix and sce complete laking. Add a little methylene blue. Note that the nuclei still stain.

(b) Place a small drop of a 3 per cent. solution of saponin in isotonic salt solution on a slide, and near it a small drop of blood. Observe as in (a). Repeat with a 2 per cent. solution of sodium taurocholate in salt solution. If necturus corpuscles, which are splendid objects for such experiments on account of their great size, have been used, intracorpuscular crystallization of the hæmoglobin may be observed.

(c) Repeat $(a)$ and $(b)$ with mammalian blood. Note that the corpuscles swell before being laked by the saponin. If any of the corpuscles are crenated, it may be seen that before being laked by the saponin the crenations disappear, the corpuscles becoming round, while in the taurocholate solution they may remain crenated till laking has occurred. This indicates that the permeability of the envelopes is not affected in the same way by the two laking agents.

I5. Hæmolysis and Agglutination by Foreign Serum.--(I) To a small quantity of rabbit's blood add an equal volume of dog's serum. Mix and let stand at $40^{\circ} \mathrm{C}$. The colour of the blood is soon darker than before, and it can be seen to be laked. Examine microscopically.

(2) Place a small drop of rabbit's blood and a somewhat larger drop of the dog's serum on a slide, near, but not quite in contact with, each other. Now put on a cover-slip, so that the drops just come together, and examine at once with the microscope with a moderately high power. Where the two drops mingle, the red corpuscles will be seen first to become agglutinated into groups, and then to fade out, leaving only their 'ghosts.' A few of the corpuscles which come into contact with the, as yet, undiluted serum may be entirely dissolved.

(3) Heat some of the dog's serum to $60^{\circ} \mathrm{C}$. for ten minutes, and repeat (I) and (2). No laking will now be produced in the rabbit's corpuscles, but agglutination may be observed as before.

(4) Repeat (I) and (2) with dog's blood and rabbit's serum. The blood will not be laked, although sometimes the dog's corpuscles may become crenated. There will be no agglutination. 
(5) With a 5 per cent. suspension of rabbit's washed corpuscles perform the following experiments:"

Put into each of six small test-tubes I c.c. of the suspension. Label the tubes $\mathrm{A}, \mathrm{A}^{\prime}, \mathrm{B}, \mathrm{B}^{\prime}, \mathrm{C}, \mathrm{C}^{\prime}$.

(a) To $A$ and $A^{\prime}$ add respectively 0.1 c.c. and 0.5 c.c. ox serum.

(b) To $\mathrm{B}$ and $\mathrm{B}^{\prime}$ add respectively 0.1 c.c. and 0.5 c.c. dog's serum.

(c) To $C$ and $C^{\prime}$ add respectively 0.I c.c. and 0.5 c.c. of 0.9 per cent. sodium chloride solution.

Put all the tubes in a bath at $40^{\circ} \mathrm{C}$. Compare the amount of laking and agglutination in the various tubes at intervals of two minutes or less. Repeat $(a),(b)$, and $(c)$ with guinea-pig's washed corpuscles and serum of ox and dog. Determine which of these sera has the strongest hremolytic power.t

(6) Heat I c.c. of ox and dog's serum respectively to $56^{\circ} \mathrm{C}$., keeping it at that temperature, or not more than a couple of degrees above it, for ten + minutes, and repeat experiment (5), labelling the tubes $D, D^{\prime}$, $E, E^{\prime}, F, F^{\prime}$. Save the rest of the leated sera for (8). There is no laking in any of the tubes, but probably agglutination in $\mathrm{D}, \mathrm{D}^{\prime}$, and $\mathrm{E}, \mathrm{E}^{\prime}$. (The complement is destroyed, but not the intermediary body or amboceptor, or the agglutinin-p. 28.)

(7) Put half of the contents of tubes D, D', E, E', into four separate test-tubes, and add to each 0.2 c.c. of rabbit's serum. If there is laking now it is because the rabbit's serum contains complement. Save the balance of $D, D^{\prime}, E$ and $E^{\prime}$ for (8).

(8) Allow 0.5 c.c. of ox serum to act at $0^{\circ} \mathrm{C}$. on the rabbit's washed corpuscles contained in 5 c.c. of the 5 per cent. suspension after removal of the sodium chloride solution. The ox serum and rabbit's corpuscles are separately cooled to $0^{\circ} \mathrm{C}$. before being mixed, and the mixture is then kept at $o^{\circ} \mathrm{C}$. for one hour. Centrifugalize the serum off rapidly. Label it 'Serum S.' To $0 \cdot 2$ c.c. of the original 5 per cent. suspension of rabbit's washed corpuscles add $0 \cdot I$ c.c. of this serum (labelling the tube $\mathrm{G}$ ), and put at $40^{\circ} \mathrm{C}$. with a control-tube containing the same amount of suspension plus salt solution instead of serum. Add the rest of the serum $\mathrm{S}$, cooled to $0^{\circ} \mathrm{C}$., to the same cooled rabbit's corpuscles, and leave for a further period at $0^{\circ} \mathrm{C}$. Then centrifugalize rapidly, and to 0.2 c.c. of the original suspension of washed rabbit's corpuscles add 0.1 c.c. of serum $S$ (labelling the tube $H$ ), and put at $40^{\circ} \mathrm{C}$. with a sodium chloride tube as control. There may be no

* The material obtained from one medium-sized dog, two rabbits, and one guinea-pig is enough for fifty or sixty students, working together in sets of two, to perform experiments (5) to (8). In order to obtain a serum more strongly hæmolytic for rabbit's corpuscles than normal dog's serum, a dog may be 'immunized' by previous injection of all the washed corpuscles obtainable from a rabbit. The injection should be made under the skin or, better, into the peritoneal cavity-of course, with aseptic precautions. It should be repeated not less than twice, with an interval of ten days between the successive injections, and the dog's blood should be drawn off about ten days after the last injection.

$\dagger$ To determine the amount of laking at any given moment, drop the small test-tubes in to the metallic centrifuge cups after shaking them up, and centrifugalize. A very short time is sufficient to separate a clear supernatant liquid, from the tint of which the extent of the hæmolysis can be deduced. Before replacing the tubes in the thermostat, they should, of course, be shaken up. Small test-tubes of about $8 \mathrm{~mm}$. internal diameter and short enough to go conveniently into the centrifuge cups are the most serviceable.

$\ddagger$ For exact work a longer time is recommended. But for the student the time is made as short as possible, and it is only in exceptional cases that ten minutes is not enough. 
laking in either $\mathrm{G}$ or $\mathrm{H}$, or if there is laking it may be greater in G than in $\mathrm{H}$. The amboceptor has been removed from serum S by the rabbit's corpuscles. Add O.I c.c. of this 'inactivated' serum to the balance of $\mathrm{D}, \mathrm{D}^{\prime}$, and $\mathrm{E}, \mathrm{E}^{\prime}$ (left from 6). Laking will occur because the serum $\mathrm{S}$ contains complement, and the heated serum added in (6) to these tubes contains amboceptor. Wash the rabbit's corpuscles which have been treated with ox serum at $0^{\circ} \mathrm{C}$. with cooled sodium chloride solution. Add to them some of serum $\mathrm{S}$ (that from the top of tube $\mathrm{H}$ will do if no more is left), and put at $40^{\circ} \mathrm{C}$. Laking will occur, showing that the amboceptor was fixed by the rabbit's corpuscles at $0^{\circ} \mathrm{C}$. To a further portion of the washed rabbit's corpuscles which were treated with ox serum at $0^{\circ} \mathrm{C}$. add normal rabbit's serum, and put at $40^{\circ} \mathrm{C}$. If laking occurs it is because the rabbit's serum contains complement.

Dog's serum may be used instcad of ox serum for experiment (8).

16. Osmotic Resistance of the Coloured Corpuscles.-Fill a burette with a I per cent. solution of sodium chloride and another with distilled water. Take a series of ten test-tubes and run into the first 6 c.c. of the $\mathrm{NaCl}$ solution, into the second 5.8 c.c., into the third 5.6 c.c., and so on, always making a difference of 0.2 c.c. between successive test-tubes. From the other burette run in enough distilled water to make up ro c.c. of solution in each tube-that is, 4 c.c. of distilled water for the first tube, $4^{\circ} 2$ c.c. for the second, and so on. Shake up. The tubes now contain a series of solutions of salt differing in strength by 0.02 per cent. in successive tubes, the strongest being 0.6 per cent., and the weakest 0.42 per cent. Number the tubes I to ro, beginning with the strongest solution. Put into each tube one drop of perfectly fresh blood. Shake moderately so as to mix the blood and salt solution, and allow the tubes to stand for ten to thirty minutes. Observe the colour of the clear liquid above the sediment of corpuscles. Determine in which tube the first tinge of hæmoglobin appears. The next higher concentration of the salt solution is that in which all the corpuscles are just able to retain their hæmoglobin, and is a measure of the minimum osmotic resistance of the corpuscles, or the resistance of the weakest corpuscles. Repeat with blood which has stood at room temperature for twelve to twenty-four hours. For clinical purposes tubes, each containing 5 c.c. of salt solution, may be used. A single drop of blood can then be distributed between the tubes with a fine pipette or a glass rod, beginning with the most concentrated solution. and passing down to the less concentrated. The blood must be distributed rapidly before coagulation occurs. Only such concentrations of the salt solution as are known to correspond to the possible variations of the osmotic resistance for any particular disease or for any particular variety of blood need be employed.

I7. Blood-Pigment-(I) Preparation of Hæmoglobin Crystals.-(a) To a little dog's blood in a narrow test-tube add its own volume or twice its volume of chloroform. Invert the tube ten or twelve times so as to allow the chloroform to act on the blood, but avoid violent shaking. When the tube is now allowed to stand for a few minutes the laked blood all rises to the top. Remove a little of the layer of blood without taking with it any of the chloroform layer, and examine the oxyhrmoglobin crystals with the microscope. They form long rhombic prisms and needles (Fig. I4, p. 52 ).

(b) Add a little crude saponin to dog's blood in a test-tube. Shake up well, and allow it to stand till the colour becomes dark. Then shake vigorously, and a mass of hæmoglobin crystals will be formed.

(c) Put a small drop of guinea-pig's blood on a slide. Mix with a 
drop of Canada balsam and cover. Tetrahedral crystals of oxyhrmoglobin will form after a time. The slide may be kept.

(2) Spectroscopic Examination of Hæmoglobin and its Derivatives. - (a) With a small, direct-vision spectroscope look at a bright part of the sky or a white cloud. Focus by pulling out or pushing in the eyepiece until the numerous fine dark lines (Fraunhofer's lines), running vertically across the spectrum, are seen. Narrow the slit by moving the milled edge till the lines are as sharp as they can be made. Note especially the line $D$ in the orange, the lines $E$ and $b$ in the green, and $F$ in the blue. Always hold the spectroscope so that the red is at the left of the field. Now dip an iron or platinum wire with a loop on the end of it into water, and then into some common salt or sodium carbonate, and fasten or hold it in the flame of a fishtail burner. On examining the flame with the spectroscope, a bright yellow line will be seen occupying the position of the dark line $D$ in the solar spectrum. This is a convenient line of reference in the spectrum, and in studying the spectra of hrmoglobin and its derivatives, the position of the absorption bands with regard to the $\mathrm{D}$ line should always be noted. The dark lines in the solar spectrum are due to the absorption of light of a definite range of wave-lengths by metals in a state of vapour in the sun's atmosphere, and of course no dark lines are seen in the spectrum of a gas-flame. Put some defibrinated blood into a test-tube.

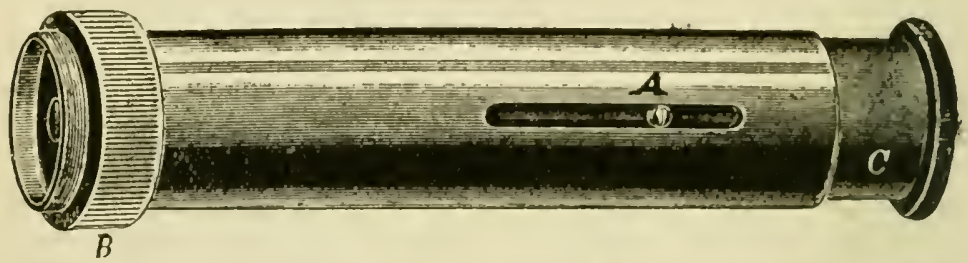

Fig. 20.-Direct Vision Spectroscope of Simple Type. A, slot in which 2 pin on the eyepiece $C$ slides in focussing the spectrum: $B$, milled head, by the rotation of which the slit is narrowed or widened.

Fasten it vertically in a clamp in front of the flame and examine it with the spectroscope, holding the latter in one hand with the slit close to the test-tube, and focussing the eyepiece with the other. Or arrange the spectroscope, test-tube and gas-flame on a stand as in Fig. 21 . Nothing can be seen till the blood is diluted. Pour a little of the blood into another test-tube, and go on diluting till, on focussing, two bands of oxyhamoglobin are seen in the position indicated in Fig. I3. p. 5I. Draw the spectrum; then dilute still more, and observe which of the bands first disappears. Now put 5 c.c. of the blood into another test-tube, and dilute it with four times its volume of water. Take 5 c.c. of this dilution, and again add four times as much water, and so on till the solution is only faintly coloured. Note with what degree of dilution the bands disappear. Then examine each of the solutions with the spectroscope and draw its spectrum.

(b) Make a solution of blood which shows the oxyhremoglobin bands sharply. Add some ammonium sulphide solution to reduce the oxyhamoglobin. Heat gently to about body temperature. A single, ill-defined band now appears, occupying a position midway between the oxyhrmoglobin bands, and the latter disappear. This is the band of reduced hamoglobin (Fig. 13).

(c) Carbonic Oxide Hamoglobin.-Pass coal-gas through blood for 
a considerable time. Examine some of the blood (after dilution) with the spectroscope. Two bands, almost in the position of the oxyhamoglobin bands, are scen; but no change is caused by the addition of ammonium sulphide, since carlonic oxide hæmoglobin is a more stable compound than oxyhemoglobin.

(d) Methemoglobin.-Put some blood into a test-tube, add a few drops of a solution of ferricyanicle of potassium, and heat gently. On diluting a well-marked band will be seen in the red. On auldition of ammonium sulphide this band disappears; the oxylıæmoglobin bands are seen for a moment, and then give place to the band of reduced hamoglobin (Fig. I3. p. $5^{1}$ ).

(e) Acid Hamatin.-To a little diluted blood ad'l strong acetic acid and heat gently. The colour becomes brownisli. The spectrum shows a band in the red between $\mathrm{C}$ and $\mathrm{D}$, not far from the position of the band of methamoglobin. The addition of a drop or two of ammonium sulphide causes no change in the spectrum, and this is a means of distinguishing acid hæmatin from methæmoglobin. If more ammonium sulphide be added, hrematin will be precipitated when the acid solution has been rendered neutral, and a further addition of ammonium sulplide or sodium hydroxide will cause the hamatin to be again dissolved, a solution of alkaline hæmatin being formed. This in its turn may be reduced by an excess of ammonium sulphide, and the spectrum of hremochromogen may be obtained (Fig. I3, p. 5I).

Since the watery solution of acid hrmatin obtained as above is usually somewhat turbid, a solution in acid ether is sometimes employerl for spectroscopic examination. Add to a little undiluted defibrinated blood about half its volume of glacial acetic acid, and then not less than an equal volume of

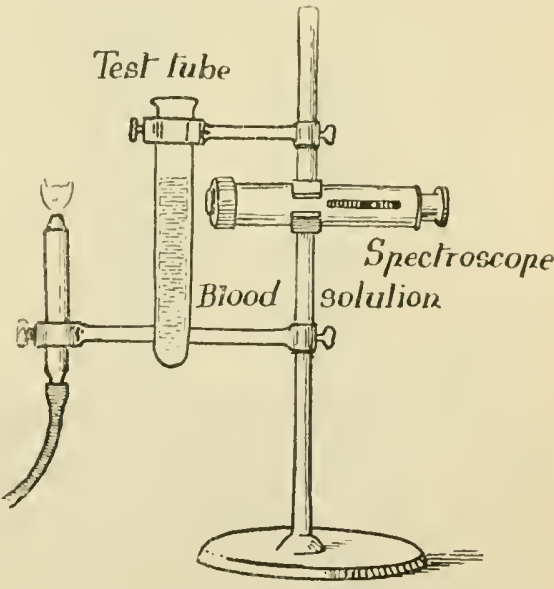

Fig. 21.-Spectroscopic Examination of Blood-Pigment. ether. Mix well, pour off the ethereal extract and examine it with the spectroscope, diluting, if necessary, with ether and glacial acetic acid. It shows a strong band in the red somewhat farther from the $D$ line than the methæmoglobin band. On dilution, three additional fainter bands may be seen.

(f) Alkaline Hamatin.-To diluted blood add strong acetic acid and warm gently for a few minutes. Then, when the spectroscopic examination of a sample shows that acid hæmatin has been formed, neutralize with sodium hydroxide. A brownish precipitate of hrematin is thrown down, which dissolves in an excess of sodium hydroxide, giving a solution of alkaline hæmatin (or alkali hæmatin).

Or add sodium hydroxide to blood directly, and warm for a couple of minutes after the colour has changed decided:y to brownish-black. The spectrum of alkaline hæmatin is a broad but ill-defined band just overlapping the D line, and situated chiefly to the red side of it (Fig. I3). The solution should be shaken up with air before being examined, as 
some of the alkali hrematin is changed into hæmochromogen by reducing substances formed by the action of the alkali on the blood.

(g) Hamochromogen.-To a solution of alkaline hæmatin add a drop or two of ammonium sulphide. The band near D disappears, and two bands make their appearance in the green (Fig. 13, p. 5I).

(h) Hamatoporphyrin.-Put some strong sulphuric acid into a testtubc. Add a few drops of blood, agitate the test-tube tiil the blood di-solves, and examine the purple liquid, diluting it, if necessary, with sulphuric acid. Its spectrum shows two well-marked bands, one just to the left of D, and the other midway between D and E (Fig. 13).

(3) Guaiacum Test for Blood.-A test for blood-mucl used in hospitals, and, indeed, a delicate one, but quite untrustwortly unless certain precautions be taken - is the guaiacum test. A drop of freshlyprepared tincture of guaiacum is added to the liquid to be tested, and then peroxide of hydrogen. If blood be present, the guaiacum strikes a blue or greenish-blue colour. The decomposition of the peroxide by the blood is due mainly to the hemoglobin of the corpuscles. Any derivative of hamoglobin which still contains the iron will act, and boiling does not abolish this power. On the other hand, oxydases or oxidizing ferments present, not only in the formed elements of blood, but elsewherc, e.g., in fresh vegetable protoplasm, milk, seminal fluid, and pus, will cause the same colour (p. 271), but not if they have been previously boiled.* The test has been considered chiefly of valuc as a negative test. When the blue colour is not obtainerl, we have good evidence that blood is absent. But, according to Buckmaster, if the precaution of first boiling the liquid suspected to contain blood be adopted, it is also a good positive test. It is, however, far inferior to the hrmin test (p. $7 \circlearrowleft$ ) where that can be obtained, and of course inferior to the identification of erythrocytes with the microscope, or to the spectrascopic identification of the blood-pigment where the material is suitable for this.

(4) Quantitative Estimation of Hæmoglobin-(a) By Haldane's Modification of Gowers' Hemoglotinometer.-Place in the graduated tube B (Fig. 22) an amount of water less than will ultimately be required to dilute the blood to the required tint. Puncture the finger or lobe of the ear with one of the small lancets in $F$, and fill the capillary pipette $D$ to a little beyond the mark 2o. IVipe the point of the pipette and dab it on a piece of filter-paper till the blood stands exactly at the mark. Blow the blood into the water in $B$, and rinse the pipette witl the water. Attacli the cap of tube $G$ to a gas-bumer. Introduce the rubber tube into B nearly to the level of the water, and allow gas to pass for a few seconds. Withdraw the tube while the gas is still passing. Immediately: close the end of $B$ with the finger, and move the tube so that the liquid passes from end to end of it at least a dozen times, to saturate the hremoglobin with carbonic oxide. While this is being done, the tube should be held in a cloth, otherwise it will become lieated, and liquid will spurt out when the finger is removed. Water is now adderl

* The formed elements of blood really contain no less than three ferments of interest in this connection: (I) A catalase which decomposes peroxide of hydrogen into water and molecular oxygen (i.e., oxygen not in the atomic or nascent state). This reaction is given by both blood and pus. (2) An oxydase (also spelled oxidase), which oxidizes guaiacum and similar substances without the presence of hydrogen peroxide. This reaction is obtainable even from aqueous extracts of leucocytes. (3) A peroxydase (also spelled peroxidase) which causes the oxidation of these substances only in the presence of hydrogen peroxide, a reaction also given by leucocytes. These ferments are all inactivated by boiling (Kastle). 
drop by drop with the pipette stopper of the bottle E, which is used for loolding the water, the tube being inverted after each addition, till the tint in B is the same as that in $\mathbf{A}$. In comparing the tubes. they should be held against the light from the sky or from an opal glass lamp-shade. It is necessary to transpose the tubes repeatedly. The level at which the tints are equal is read off on 13 half a minute after the addition of the last drop of water. Water is now again added by drops till the tint in $B$ is just roticeably weaker than in $A$, and the mean of the two readings is taken. The result is the percentage actually present of the average proportion of hrmoglobin in the blood of healthy adult males. Healthy women give an average of only 89 per cent., and healthy children an average of only 87 per cent., of the proportion in men. The liquid in $\Lambda$ is a $I$ per cent. solution of blood containing the average percentage of hrmoglobin found in the blood of healthy

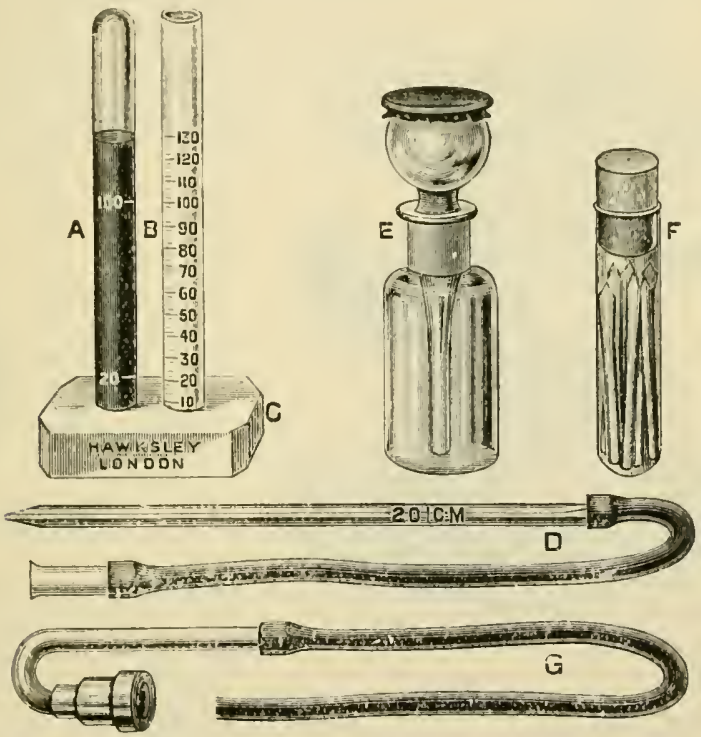

Fig. 22.-Haldane's Modification of Gowers' Hæmoglobinometer.

adult males, and having an oxygen capacity of $x^{8}, 5$ per cent.-i.e.. roo c.c. of the blood with which the standard was made would take up in combination from air 18.5 c.c. of oxygen. The solution in $A$ has been saturated with carbonic oxide.

This method is probably more accurate than any other !sed in clinical work, the error, in the hands of an experienced observer, not exceeding I per cent.

(b) By Fleischl's Hamometer (Fig. 23).-Fill with distilled water that compartment $a^{\prime}$ of the small cylinder (above the stage) which is over the tinted wedge. Put a little distilled water into the other compartment a. Now prick the finger and fill one of the small capillary tubes with blood. See that none of the blood is smeared on the outside of the tube. Then wash all the blood into the water in compartment a, and fill it to the brim with distilled water. By means of the milled 
licad $T$ move the tinted wodge $K$ till the depth of colour is the same in the two compartinents. 'The percentage of the normal quantity' of hamoglobin is given by the graduated scale $P$. For example, if the reading is 90 , the blood contains 90 per cent. of the normal amount; if roo, it contains the normal quantity. The observations should be made in a dark room, the white surface $\mathrm{S}$, arranged below the compartments $a$ and a', being illuminated by a lamp. Or the instrument may be placed in a small box, lighted by a candle. It is best that each result should be the mean of two readings, one just too large and the other just too small. In any case the instrument does not give readings accurate to less than 5 per cent.

(c) Hoppe-Seyler's Method.-Two parallel-sided glass troughs are used. In one is put a standard solution of oxyhamoglobin of known strength, in the other a measured quantity of the blood to be

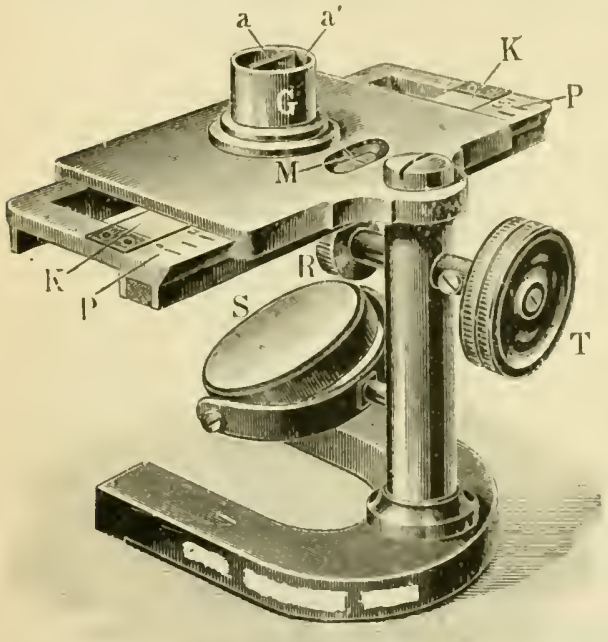

Fig. 23.-Fleischl's Hæmometer. tested. The latter is diluted with water until its tint appears the same as that of the standard solution. when the troughs are placed

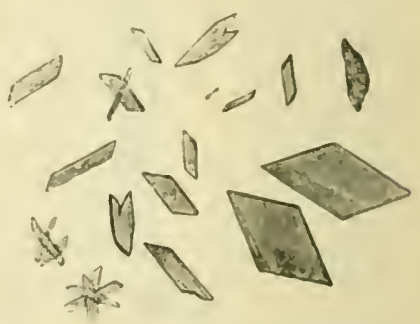

Fig. 24.-Crystals of Hæmin (Frey).

side by side on white paper. From the quantity of water added it is easy to calculate the proportion of hremoglobin in the undiluted blood. Greater accuracy is obtinined if the hemoglobin in the standard solution and that of the blood are converted into carbonic oxide hæmoglobin by passing a stream of coal-gas through them.

(d) Tallquist's Method.-In this method the tint produced by a drop of biood on a picce of white filter-paper is compared with a scale representing ro percentages of hæmoglobin (from 10 to roo per cent.). The standard filter-paper is supplied in the form of a book with the scale. To make an estimation, all that is nccessary is to touch a clrop of blood with a piece of the filter-paper, and allow the blood to diffuse slowly through the paper, so as to give an even stain. The position of the stain is then determined by the scale; e.g., it may be deeper than 90 , but fainter than 100 , in which case the percentage of hemoglobin lies between 90 and xoo. The method is by no means a very accurateone, but more accurate than it appears at first sight.

(5) Microscopic Test for Blood-Pigment.-Put a drop of blood on a slide. Allow the blood to dry, or heat it gently over a flame, so as to evaporate the water. Add a drop of glacial acetic acid; put on a cover- 
glass, and again heat slowly till the liquid just begins to boil. Take the slide away from the flame for a few seconds, then heat it again for a moment; and repeat this process two or three times. Now let the slide cool, and examine with the microscope (high power). The small black, or brownish-black, crystals of hiemin will be secn (Fig. 24, p. 78). This is an important test where only a minute trace of blood is to be examined, as in some medico-legal cases. If a blood-stain is old, a minute crystal of sodium chloride should be added along with the glacial acetic acid. Fresh blood contains enough sodium chloride.

A blood-stain on a piece of cloth may first of all be soaked in a small quantity of distilled water, and the liquid examined with the spectroscope or the micro-spectroscope (a microscope in which a small spectroscope is substituted for the eyepiece). Then evaporate the liquid to dryness on a water-bath, and apply the hæmin test. Or perform the hremin test directly on the piece of cloth. In a fresh stain the bloodcorpuscles might be recognized under the microscope. Very few liquids, however, are available for washing out the blood, as all ordinary solutions, and even serum itself, cause laking of dried corpuscles (Guthrie). Absolute alcohol, or 35 per cent. potassium hydroxide, may be used to soak and rub up the cloth in. 


\section{CHAPTER III}

\section{THE CIRCULATION OF THE BLOOD AND LYMPH}

THE blood can only fulfil its functions by continual movement. This movement implies a constant transformation of energy; and in the animal body the transformation of energy into mechanical work is almost entirely allotted to a special form of tissue, muscle. In most animals there exist one or more rhythmically contractile muscular organs, or hearts, upon which the chief share of the work of keeping up the circulation falls.

\section{Section I.-Preliminary Anatomical and Physical Data.}

Comparative.-In Echinus a contractile tube connects the two vascular rings that surround the beginning and end of the alimentary canal, and plays the part of a heart. In the lower crustacea and in insects the heart is simply the contractile and generally sacculated dorsal bloodvessel; in the higher crustacea, sucl as the lobster, it is a welldefined muscular sac situated dorsally. A closed vascular system is the exception among invertebrates. In most of them the blood passes from the arteries into irregular spaces or lacunæ in the tissues, and thence finds its way back to the heart. In the primitive vertebrate heart five parts can be distinguished as we proceed from the venous to the arterial end: (I) The sinus venosus, into which the great veins open; (2) the auricular canal, from the dorsal wall of which is developed - (3) the auricle; (4) the ventricle; (5) the bulbus arteriosus, from which the chief artery starts (Fig. 25, p. 8I). Amphioxus, the lowest vertebrate, has a primitive lacunar vascular system; a contractile dorsal bloodvessel serves as arterial or systemic heart, a contractile ventral vessel as venous or respiratory heart. From the latter, vessels go to the gills. Fishes possess only a respiratory heart, consisting of a venous sinus, auricle, ventricle, and bulbus arteriosus. This drives the blood to the gills, from which it is gathered into the aorta; it has thence to find its way without further propulsion through the systemic vessels. Amphibians have a venous sinus, two auricles, a single restricle, and an arterial bulb; reptiles, two auricles and two incompletely-separated ventricles. In birds and mammals the respiratory and systemic hearts are completely separated. The former, consisting of the right auricle and ventricle, propels the blood through the lungs; the latter, consisting of the left auricle and ventricle, receives it from the pulmonary veins, and sends it through the systemic vessels.

The sinus venosus seems to be represented in the mammalian lieart by certain small portions of tissue, especially the so-called sino-auricular node, a little knot of primitive fibres near the mouth of the superior 
vena cava. The auricular canal is probably represented by the auriculo-ventricular bundle (conveniently designated as the a.-v. bundle), which will again be referred to in relation to the conduction of the heartbeat from auricles to ventricles $\left(p . I_{47}\right)$. This bundle starts from in clump of primitive tissue, the aturiculo-ventricular node (a.-v. node) at the base of the interauricular septum on the right side, below and to the right of the coronary sinus, and rums down the interventricular scptum. The sino-auricular and the auriculo-ventricular nodes are connected by fibres which run in the interauricular septum, so that it may be considered that the primitive cardiac tube is still represented from base to apex of the adult mammalian heart, although only by very slender threads of tissuc, amidst the massive secondary developments of auricular and ventricular muscle (Keith and Flack).

General View of the Circulation in Man.-The whole circuit of the blood is divided into two portions, very distinct from each other, both anatomically and functionally-the respiratory or lesser circulation, and the systemic or greater circulation. Starting from the left ventricle, the blood passes along the systemic vessels-arteries, capillaries, veins-and, on returning to the heart, is poured into the right auricle, and thence into the right ventricle. From the latter it is driven through the pulmonary artery to the lungs, passes through the capillaries of these organs, and returns through the pulmonary veins to the left auricle and ventricle. The portal system, which gathers up the blood from the intestines, forms a kind of loop on the systemic circulation. The lymph-current is also in a sense a slow and stagnant side-stream of the bloodcirculation; for substances are

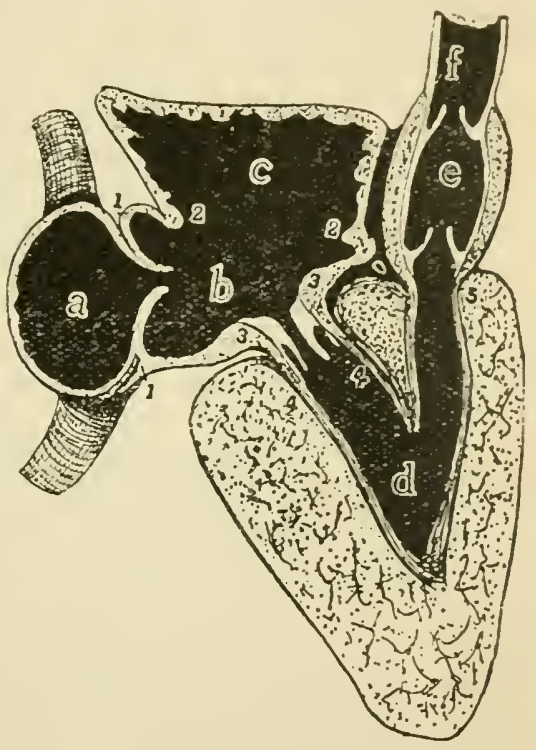

Fig. 25.-Diagram of Primitive Vertebrate Heart, combining Features found in the Eel, Dogfish, and Frog (Flack, after Keith). $a$, Sinus venosus; $b$, auricular canal; $c$, auricle; $d$, ventricle; $e$, bulbus cordis; $f$, aorta; I-I, sino-auricular junction and venous valves; $2 \cdot 2$, junction of canal and auricle; $3-3$, annular part of auricle; 5 , bulbo-ventricular junction. constantly passing from the bloodvessels into the lymph-spaces, and retuming, although after a comparatively long interval, into the blood by the great lymphatic trunks.

Physiological Anatomy of the Vascular System.-The heart is to be looked upon as a portion of a bloodvessel which has been modified to act as a fump for driving the blood in a definite direction. Morphologically it is a bloodvessel; and the physiological property of automatic rhythmical contraction which belongs to the heart in so eminent a degree is, as has been mentioned (p. So), an endowment of bloodvessels in many animals that possess no localized heart. Even in some mammals contractile bloodvessels occur; the veins of the bat's wing, for example, beat with a regular rhythm, and perform the function of accessory hearts. 
The whole vascular system is lined with a single layer of enctothelial cells. In the capillaries nothing else is present; the endothelial layer forms the whole thickness of the wall. In young animals, at any rate, the endothelial cells of the capillaries are capable of contracting when stimulated; and clianges in the calibre of these vessels can be brought about in this way. The walls of the arteries and veins are chiefly made up of two kinds of tissue, which render them distensible and clastic: non-striped muscular fibres and yellow clastic fibres. The muscular fibres are mainly arranged as a circular middle coat, which, especially in the smaller arteries, is relatively thick. One conspicuous layer of elastic fibres marks the boundary between the middle and inner coats. In the larger arteries clastic laminze are also scattered freely among the muscular fibres of the middle coat. The outer coat is composed chiefly of ordinary connective tissue. The veins differ from the arteries in having thinner walls, with the layers less distinctly marked, and containing a smaller proportion of non-striped muscle and elastic tissue; although in some veins, those of the pregnant uterus, for instance, and the cardiac ends of the large thoracic veins, there is a greater development of muscular tissue. Further, and this is of prime physiological importance, valves are present in many veins. These are semilunar folds of the internal coat projecting into the lumen in such a direction as to favour the flow of blood towards the heart, but to check its return. In some veins, as the venæ cavæ, the pulmonary veins, the veins of most internal organs, and of bone, there are no valves; in the portal system they are rudimentary in man and the great majority of mammals. The valves are especially well marked in the lower limbs, where the venous circulation is uphill. When a valve ceases to perform its function of supporting the column of blood between it and the valve next above, the foundation of varicose veins is laid; the valve immediately bclow the incompetent one, having to bear up too great a weight of blood, tends to yield in its turn, and so the condition spreads. The smallest veins, or venules, are very like the smallest arteries, or arterioles, but somewhat wider and less muscular. The transition from the capillaries to the arterioles and venules is not abrupt, but may be considered as marked by the appearance of the non-striped muscular fibres, at first scattered singly, but gradually becoming closer and more numerous as we pass away from the capillaries, until at lengtl they form a complete layer.

In the heart the muscular element is greatly developed and differentiated. Both histologically and physiologically the fibres seem to stand between the striated skelctal muscle and the smooth muscle. In the mammal the cardiac muscular fibres are generally described as made up of short oblong cells, devoid of a sarcolemma, often branched. and arranged in anastomosing rows, each cell liaving a single nucleus in the middle of it. But it has recently been showil that the muscle fibrils run right through the apparent cell boundaries, and form a continuous sheet of tissue anastomosing in every direction. The fibres are transversely striated, but the strix are not so distinct as in skeletal muscle. A sarcolemma is not absent, although it is more delicatc than in skeletal muscle, and perhaps of a different nature. Many fibres pass from one auricle to the other, and from one ventricle to the other.

In the frog's heart the muscular fibres are spindle-shaped, like those of smooth muscle, but transversely striated, like those of skeletal muscle. From the sinus to the apex of the ventricle there is a continuous sheet of muscular tissue. 
The problems of the circulation are partly physical, partly vital. Some of the phenomena observed in the blood-stream of a living animal can be reproduced on an artificial model ; and they may justly be called the physical or mechanical phenomena of the circulation. Others are essentially bound up with the properties of living tissues; and these may be classified as the vital or physiological phenomena of the circulation. The distinction, although by no means sharp and absolute, is a convenient one-at least, for purposes of description; and as such we shall use it. But it must not be forgotten that the physiological factors play into the sphere of the physical, and the physical factors modify the physiological. Considered in its physical relations, the circulation of the blood is the flow of a liquid. along a system of elastic tubes, the bloodvessels, under the influence of an intermittent pressure produced by the action of a central pump, the heart. But the branch of dynamics which treats of the movement of liquids, or hydrodynamics, is one of the most difficult parts of physics, and even in the physical portion of our subject we are forced to rely chiefly on empirical methods. It would, therefore, not be profitable to enter here into mathematical theory, but it may be well to recall to the mind of the reader one or two of the simplest data connected with the flow of liquids through tubes:

Torricelli's Theorem.-Suppose a vessel filled with water, the level of which is kept constant; the velocity with which the water will escape from a hole in the side of the vessel at a vertical depth $h$ below the surface will be $v=\sqrt{2 g h}$, where $g$ is the acceleration produced by gravity.* In other words, the velocity is that which the water would have acquired in falling in vacuo through the distance $h$. This formula was deduced experimentally by Torricelli, and holds only when the resistance to the outflow is so small as to be negligible. The reason of this restriction will be easily seen, if we consider that when a mass $m$ of water has flowed out of the opening, and an equal niass $m$ has flowed in at the top to maintain the old level, everything is the same as before, except that energy of position equal to that possessed by a mass $m$ at a height $h$ has disappeared. If this has all been changed into kinetic energy $\mathrm{E}$, in the form of visible motion of the escaping water, then $\mathrm{E}=\frac{1}{2} m v^{2}=m g h$, i.e., $v=\sqrt{2 g} h$. If, however, there has been any sensible resistance to the outflow, any sensible friction, some of the potential energy (energy of position) will have been spent in overcoming this, and will have ultimately been transformed into the kinetic energy of molecular motion, or heat.

Flow of a Liquid through Tubes. - Next let a horizontal tube of uniform cross-section be fitted on to the orifice. The velocity of outflow will be diminished, for resistances now come into play. When the liquid flowing through a tube wets it, the layer next the wall of the tube is prevented by adhesion from moving on. The particles next this stationary layer rub on it, so to speak, and are retarded, although not stopped altogether. The next layer rubs on the comparatively slowly moving particles outside it, and is alsc delayed, although not so much as that in contact with the immovable layer on the walls of

* I.e., the amount added per second to the velocity of a falling body $(g=32$ feet). 
the tube. In this way it comes about that every particle of the liquid is hindered by its friction against others - those in the axis of the tube least, those near the periphery most-and part of the energy of position of the water in the reservoir is used up i.l overcoming this resistance, only the remainder being transformed into the visible kinetic energy of the liquid escaping from the open end of the tube.

If vertical tubes be inserted at different points of the horizontal tube, it will be found that the water stands at continually decreasing heights as we pass away from the reservoir towards the open end of the tube. The height of the liquid in any of the vertical tubes indicates the lateral pressure at the point at which it is inserted; in other words, the excess of potential energy, or energy of position, which at that point the liquid possesses as compared witlı the water at the free end, where the pressure is zero. If the centre of the cross-section of the free end of the tube be joined to the centres of all the menisci, it will be found that the line is a straight line. The lateral pressure at any point of the tube is therefore proportional to its distance from the free end. Since the same quantity of water must pass through each crosssection of the horizontal tube in a given time as flows out at the open end, the kinetic energy of the liquid at every cross-section must be

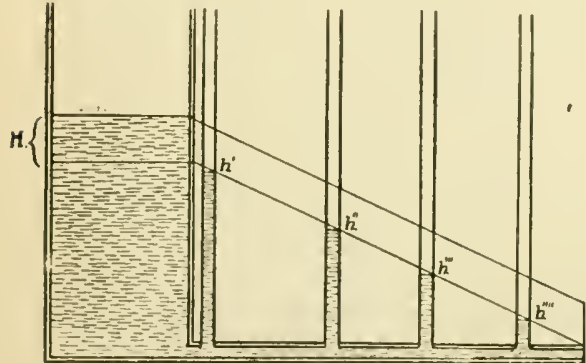

Fig. 26.-Diagram to illustrate Flow of Water along a Horizontal Tube connected with a Reservoir. constant and cqual to $\frac{1}{2} m v^{2}$, where $v$ is the mean velocity (the quantity which escapes in unit of cime divided by the cross-section) of the water at the free end.

Just inside the orifice the total energy of a mass $m$ of water is mgh; just beyond it at the first vertical tube, $m g h^{\prime}$ $+\frac{1}{2} m v^{2}$, where $h^{\prime}$ is the lateral pressure. On the assumption that between the inside of the orifice and the first tube no energy has been transformed into heat (an assumption the more nearly correct the smaller the distance between it and the inside of the orifice is made), we have $m g h=m g h^{\prime}+\frac{1}{2} m v^{2}$. i.e., $\frac{1}{2} m v^{2}=m g\left(h-h^{\prime}\right)$. In other words, the portion of the energy of position of the water in the rescrvoir which is transformed into the kinetic energy of the water flowing along the horizontal tube is measured by the difference between the lieight of the level of the reservoir and the lateral pressure at the beginning of the horizontal tube-that is, the height at which the straight line joining the menisci of the vertical tubes intersects the column of water in the reservoir. Let $H$ represent the height corresponding to that part of the energy of position which is transformed into the kinetic energy of the flowing water. $H$ is casily calculated when the mean velocity of cfflux is known. For $v=\sqrt{2 g} \mathrm{H}$ by Torricelli's theorem (since none of the energy corresponding to $\mathrm{H}$ is supposed to be used up in overcoming friction), or $\mathrm{H}=\frac{v^{2}}{2 g}$. At the second tube the lateral pressure is only $h^{\prime \prime}$. The sum of the visible kinetic and potential energy here is therefore $\frac{1}{2} m v^{2}+m g h^{\prime \prime}$. A quantity of energy $m g\left(h^{\prime}-h^{\prime \prime}\right)$ must have been transformed into heat owing to the resistance caused by fluid friction in the portion of the horizontal 
tube between the first two vertical tubes. In general the energy of position represented by the lateral pressure at any point is cyual to the energy used up in overcoming the resistance of the portion of the path beyond this point.

Velocity of Outflow.-It has been found by experiment that $v$, the mean velocity of outflow, when the tube is not of very small calibre, varies directly as the diameter, and therefore the volume of outflow as the cube of the diameter. In fine capillary tubes the mean velocity is proportional to the square, and the volume of outflow to the fourth power of the cliameter (Poise uille). If, for example, the linear velocity of the blood in a capillary of $I 0 \mu$ in diameter is $\frac{1}{2} \mathrm{~mm}$. per sec., it will be four times as great (or $2 \mathrm{~mm}$. per sec.) in a capillary of $20 \mu$ diameter, and one-fourtl as great (or $\frac{1}{8} \mathrm{~mm}$. per sec.) in a capillary of $5 \mu$ cliameter, the pressure being supposed equal in all. The volume of outflow per second is obtained by multiplying the cross-section by the linear velocity. The cross-scction of a circular capillary, so $\mu$ in diameter, is $\pi\left(5 \times \frac{1}{1000}\right)^{2}=$, say, $\frac{1}{12500}$ sq. mm. The outflow will be $\overline{1} \frac{1}{5} \overline{5} \overline{0} \times \frac{1}{2}$ $=\frac{1}{500}$ cub. mm. per sec. The outflow from the capillary of .?o $\mu$ diameter would be sixteen times as much, from the $5 \mu$ capillary only one-sixteenth as much. Some idea of the extremely minute scale on which the blood-flow through a single capillary takes place may be obtained if we consider that for the capillary of Io $\mu$ diameter a

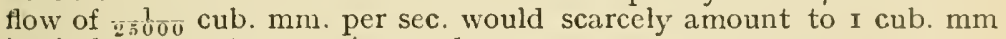
in six hours, or to I c.c. in 250 days.

When the initial energy is obtained in any other way than by means of a 'head' of water in a reservoir-say, by the descent of a piston which keeps up a constant pressure in a cylinder filled with liquidthe results are exactly the same. Even when the horizontal tube is distensible and elastic, there is no difference when once the tube has taken up its position of equilibrium for any given pressure, and that pressure does not vary.

Flow with Intermittent Pressure.-When this acts on a rigid tube, everything is the same as before. When the pressure alters, the flow at once comes to correspond with the new pressure. Water tlırown by a force-pump into a system of rigid tubes escapes at every stroke of the pump in exactly the quantity in which it enters, for water is practically incompressible, and the total quantity present at one time in the system callnot be sensibly altered. In the intervals between the strokes the flow ceases; in other words, it is intermittent. It is very different with a system of distensible and elastic tubes. During each stroke the tubes expand, and make room for a portion of the extra liquid thrown into them, so that a smaller quantity flows out than passes in. In the intervals between the strokes the distended tubes, in virtue of their elasticity, tend to regain their original calibre. Pressure is thus exerted upon the liquid, and it continues to be forced out, so that when the strokes of the pump succeed each other with sufficient rapidity, the outflow becomes continuous. This is the state of affairs in the vascular system. The intermittent action of the heart is toned down in the elastic vessels to a continuous steady flow.

\section{Section II.-The Beat of the Heart in its Physical or Mechanical Relations.}

Events in the Cardiac Cycle.-In the frog's heart the contraction can be seen to begin about the mouths of the great veins which open into the sinus venosus. Thence it spreads in succession over the 
sinus and auricles, hesitates for a moment at the auriculo-ventricular junction, and then with a certain suddenness invades the ventricle. In the mammalian heart the contraction likewise commences, so far as can be ascertained by inspection or the study of tracings, in the region near the mouths of the veins opening into the auricles. It will be seen, when the question of the origin of the rhythmical beat is being discussed (p. I4I), that the actual startingpoint is probably the sinus tissue of the right auricle (p. I42) near the opening of the superior vena cava, which is richly provided with muscular fibres ak $\mathrm{n}$ to those of the heart. But the wave advances so rapidly that it is difficult to trace in its course a regular progress from base to apex, although the ventricular beat undoubtedly follows that of the auricle, and in a heart beating normally the electrical change associated witl contraction of the ventricle begins at the base, then rcaches the apcx (p. 85.3), and finally passes towards the orifices of the great arteries.

The most conspicuous events in the beat of the heart, in their normal sequence, are: (I) the auricular contraction or systole, (2) the ventricular contraction or systole, each followed by relaxation, (3) the pause. The auricles, into which, and beyond which into the ventricles, blood has been flowing during the pause from the great thoracic veins, contract sharply, the right, perhaps, a little before the left. The contraction begins in the muscular tissue that surrounds the orifices of the veins, so that these, destitute of valves as they are, are functionally, at least, if not anatomically, sealed up for an instant, and regurgitation of blood into them is to a great extent, if not entirely, prevented. Apparently, complete closure of the inferior cava is unnecessary, the pressure of the blood in it being sufficiently high to hinder any important back-flow. The action of the circular fibres of the veins in closing their orifices is reinforced by the contraction of a band of muscle (the tania terminalis) in the roof of the right auricle. This band compresses especially the mouth of the superior vena cava. The filling of the ventricles is thus completed. The actual amount of extra blood injected into the ventricles by the auricular contraction is not large. The ventricles are already nearly charged, but the auricles, so to speak, ram the charge home. The ventricular contraction follows hard on the relaxation of the auricles. The mitral and tricuspid valves, whose strong but delicate curtains have during the diastole been hanging down into the ventricles and swinging frecly in the entering current of blood, are floated up as the intraventricular pressure begins to rise, so that, in the first moment of the sudden and powerful ventricular systole, the free edges of their segments come together, and the auriculo-ventricular orifices are completely closed (Fig. 98, p. 206). In thic measure in which the pressure in the contracting ventricles increases, the contact of the valvular seg- 
ments becomes closer and more extensive; and their tendency to belly into the auricles is opposed by the pull of the chorda tendineat, whose slender cords, inserted into tle valves from borcler to base, are kept taut, in spite of the shortening of the ventricles by the contraction of the papillary muscles. The arrangement and connections of the muscular fibres of the heart are such that lluring the auricular systole the auriculo-ventricular groove moves towards the base of the heart, while during the systole of the ventricles it moves towards the apex, which constitutes a relatively fixed point on account of the mutual action of the numerous fibres which converge here and constitute the 'whorl.' 'The line joining the apex and the origin of the aorta does not shorten when the ventricles contract, but all parts of the heart are drawn towards this line. The apex is, therefore, pushed forwards, while the rest of the ventricular surface is being drawn inwards. During the systole, the ventricles change their shape in such a way that their combined cross-section--wlich in the relaxed state is a rough ellipse with the major axis from right to left-becomes approximately circular, and they then form a right circular cone. As soon as the pressure of the blood within the contracting ventricles exceeds that in the aorta and pulmonary artery respectively, the semilunar valves, which at the beginning of the ventricular systole are closed, yield to the pressure, and blood is driven from the ventricles into these arteries.

The ventricles are more or less completely emptied during the contraction, which seems still to be maintained for a short time after the blood has ceased to pass out. The contraction is followed by sudden relaxation. The intraventricular pressure falls. The lunules of the semilunar valves slap together under the weight of the blood as it attempts to regurgitate, the corpora Arantii seal up the central chink, and the aorta and pulmonary artery are thus cut off from the heart. Then follows an interval during which the whole heart is at rest, namely, the interval between the end of the relaxation of the ventricles and the beginning of the systole of the auricles. This constitutes the pause. The whole series of events is called a cardiac cycle or revolution (see Practical Exercises, p. $20 \mathrm{I}$ ).

It will be easily understood that the time occupied by any one of the events of the cardiac cycle is not constant, for the rate of the heart is variable. If we take about 70 beats a minute as the average normal rate in a man, the ventricular systole will occupy about 0.3 second; the diastole, ${ }^{*}$ including the ventricular relaxation, about

" The term 'diastole' is variously used, as meaning the pause, the pause plus the period during which relaxation is occurring, or the period of relaxation alone. We shall employ it in the second sense. Henderson refers to the period during which the ventricular muscle is at rest, from the end of its relaxation to the onset of the auricular systole, as the 'diastasis' and the period during which the relaxation is taking place as the 'diastole,' a terminology which seems worthy of general adoption. 
0.5 second. The systole of the auricle is one-third as long as that of the ventricle.

This rhythmical beat of the heart is the ground phenomenon of the circulation. It reveals itself by certain tokens-sounds, surfacemovements or pulsations, alterations of the pressure and velocity of the blood, changes of volume in parts-all periodic phenomena, continually recurring with the same period as the heart-beat, and all fundamentally connected together. And if we hold fast the idea that when we take a pulse-tracing, or a blood-pressure curve, or a plethysmographic record, we are really investigating the same fact from clifferent sides, we shall be able, by following the cardiac rhythm and its consequences as far as we can trace them, to hang upon a single thread many of the most important of the physical phenomena of the circulation.

The Sounds of the Heart.-When the ear is applied to the chest, or to a stethoscope placed over the cardiac region, two sounds are heard with every beat of the heart. They follow each other closely, and are succeeded by a period of silence. The dull booming 'first sound 'is heard loudest in a region which we shall afterwards have to speak of as that of the ' cardiac impulse' (p. 90); the short, sharp, ' second sound ' over the junction of the second right costal cartilage with the sternum.

The heart-sounds can be registered by placing over the chest a microphone receiver connected with a string galvanometer. The magnified sounds are translated into electrical changes which cause movements of the fibre of the galvanometer, and the movements are photographed on a travelling plate (Einthoven). The record is called a cardiophonogram. When this is studied, a third sound can be detected, and it is probable that it is present in all persons, although it is as a rule inaudible to auscultation. It occurs early in diastole very shortly after the second sound. In those persons in whom it is audible it is nost distinct over the region of cardiac impulse. It is described as softer and of lower pitch than the second sound (Thayer).

There has been much discussion as to the cause of the first sound. That a sound corresponding with it in time is heard in an excised bloodless heart when it contracts is certain; and therefore the first sound cannot be exclusively due, as some have asserted, to vibrations of the auriculo-ventricular valves when they are suddenly rendered tense by the contraction of the ventricles, for in a bloodless heart the valves are not stretched. Part of the sound must accordingly be associated with the muscular contraction as such.

Again, the fact that the first sound is heard during the whole, or nearly the whole, of the ventricular systole is against the idea that it is exclusively due to the vibrations of membranes like the valves, which would speedily be damped by the blood and rendered inaudible. But while there is good reason to believe that the vibration of the suddenly-contracted ventricles is the fundamental factor, the shock sets up vibrations also in the blood, the chest-wall, and 
perhaps the resonant tissue of the lungs. Further, as we shall see later on (p. 760), the sound caused by a contracting muscle readily calls forth sympathetic resonance in the ear, and the peculiar booming character of the first sound may be due to the superposition of these various resonance tones upon the muscular note. But, in addition, the vibration of the auriculo-ventricular valves undoubtedly contributes to the production of the sound, and some observers have been able to distinguish in the first sound the valvular and the muscular elements, the former being higher in pitch than the latter, but a minor third below the second sound. In the excised empty heart the deeper tone of the first sound is alone heard, while the higher note is elicited when in a dead heart the auriculo-ventricular valves are suddenly put under tension (Haycraft). When the mitral valve is prevented from closing by experimental division of the chordæ tendineæ, or by pathological lesions, the first sound of the heart is altered or replaced by a 'murmur.' This evidence is not only important as regards the physiological question, but of great practical interest from its bearing on the diagnosis of cardiac disease. It may be added that the point of the chest-wall at which the first sound is most casily recognized is also the point at which a changed sound or murmur connected with disease of the mitral valve is most distinctly heard. The sound is, therefore, best conducted from the mitral valve along the heart to the point at which it comes in contact with the wall of the chest. Changes in the first sound connected with disease of the tricuspid valve are heard best, in the comparatively rare cases where they can be distinctly recognized, in the third to the fifth interspace, a little to the right of the sternum.

The second sound is caused by the vibrations of the semilunar valves when suddenly closed, "the recoiling blood forcing them back, as one unfurls an umbrella, and with an audible check as they tighten' (Watson). The sharpness of its note is lost, and nothing but a rushing noise or bruit can be heard, when the valves are hooked back and prevented from closing. It is altered, or replaced by a murmur, when the valves are diseased. As there is a mitral and a tricuspid factor in the first sound, so there is an aortic and a pulmonary factor in the second. The place where the second sound is best heard (over the junction of the second right costal cartilage and sternum) is that at which any change produced by disease of the aortic valves is most easily recognized. The sound is conducted up from the valves along the aorta, which comes nearest to the surface at this point. Changes connected with disease of the pulmonary valves are most readily detected over the second left intercostal space near the edge of the sternum, for here the pulmonary artery most nearly approaches the chest-wall. The first sound is 'systolic" -that is, it occurs during the ventricular systole; the second is ' diastolic,' beginning at the commencement of the diastole 
Various explanations of the third sound have been given, but, as the authors who have studied it are not even agreed as to whether it is produced at the auriculo-ventricular orifices or at the aortic and pulmonary orifices, it would not be useful to discuss them at present.

The Cardiac Impulse.-A surface-movement is seen, or an impulse felt, at every cardiac contraction in various situations where the heart or arteries approach the surface. The pulsation, or impulse, of the heart, often styled the apex-beat, is usually most distinct to sight and touch in a small area lying in the fifth left intercostal space, between the manmary and the parasternal line, ${ }^{*}$ and generally, in an adult, about an inch and a half to the sternal side of the former. It is due to the systolic hardening of the ventricles, which are here in contact with the chest-wall, the contact being at the same time rendered closer by their change of shape, and by a slight

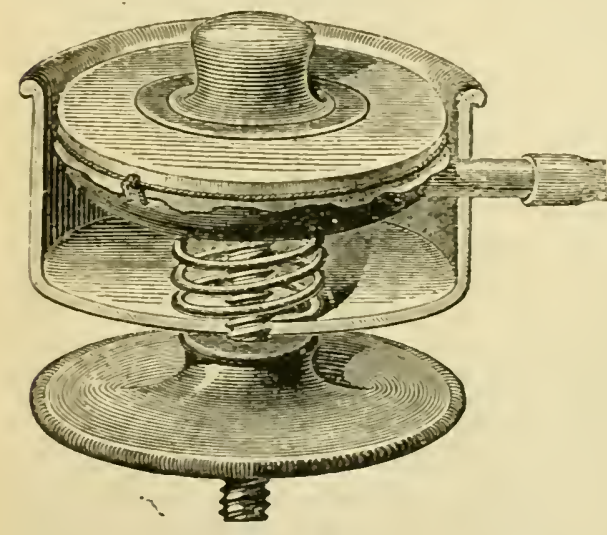

Fig. 27.-Diagram of Marey's Cardiograph. movement of rotation of the heart from left to right during the contraction (Practical Exercises, p. 207). When the left ventricle is in contact with the chest at the position of the apex-beat, as is usually the case, an important element in the impulse is the actual forward thrust of the apex. When the apex-beat corresponds in position with the right ventricle, there is no actual forward movement, although the hardening of the ventricle may be felt as a thrust by the finger. Even in health the position of the impulse varies somewhat with the position of the body and the respiratory movements. In children it is usually situated in the fourth intercostal space. In disease its displacement is an important diagnostic sign, and may be very marked, especially in cases of effusion of fluid into the pleural cavity. It is sometimes, though not invariably, a little lower in the standing than in the sitting position, and shifts an inch or two to the left or right when the person lies on the corresponding side.

Various instruments, called cardiographs, have been devised for magnifying and recording the movements produced by the cardiac impulse. Marey's cardiograph (Fig. 27) consists essentially of a small

- The mammary line is an imaginary vertical line supposed to be drawn on the chest through the middle point of the clavicle. It usually, but not necessarily, passes through the nipple. The parasternal line is the vertical line lying midway between the mammary line and the corresponding border of the sternum. 
chamber, or tambour, filled with air, and closed at one end by a flexible membrane carrying a button, which can be adjusted to the wall of the chest. This receiving tambour is connected by a tube with a recording tambour, the flexible plate of which acts upon a lever writing on a travelling surface-a uniformly-rotating drum, for cxample-covered with smoked paper. Any movement communicated to the button forces in the end of the tambour to which it is attached, and thus raises the pressure of the air in it and in the recording tambour; the flexible plate of the latter moves in response, and the lever transfers the movement to the paper. The tracing, or cardiogram, obtained in this way shows a small elevation corresponding to the auricular systole, succecded by a large abrupt rise corresponding to the beginning of the first sound, and caused by the ventricular systole. This ventricular elevation is the essential portion of the curve; it is alone felt by the palpating hand, and the auricular clevation is often absent from the cardiogram in man. The rise is maintained, with small secondary oscillations, for about 0.3 of a second in a tracing from a normal man, then gives way to a sudien descent, that marks the relaxation of the ventricles, the beginning of the second sound, and the closure of the semilunar valves. An interval of about 0.5 seconch clapses before the curve begins again to rise at the next auricula. contraction.

Such was the interpretation which Chauveau and Marey put upon their tracings. Although neither their results nor their deductions from them have escaped the criticism of succeeding investigators, it is doubtful whether any adequate reason has been brought forward for

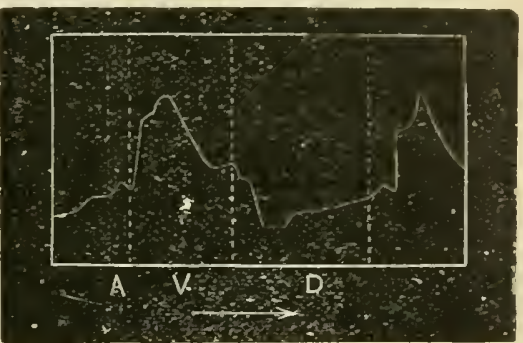

Fig. 23.-Cardiogram taken with Marey" Cardiograph. A, auricular systole ; $V$, ventricular systole; $D$, diastole. The arrow shows the direction in which the tracing is to be read.

discarding them, and Chauveau has furnished further proofs of their accuracy. The difficulties that beset the subject are great, for the cardiogram is a record of a complex series of events. The very rapid variation of pressure within the ventricles, the change of volume and of shape of the heart, the slight change of position of its apex, must all leave their mark upon the curye, which is besides distorted by the resistance of the elastic chest-wall, the inertia of the recording lever, and the compression of the air in the connecting tubes. It is only by comparing in animals the cardiographic record with the changes of blood-pressure in the heart and arteries that our present degree of knowledge of the human cardiogram has been attained. Could we register directly the fluctuations of pressure in the interior of the human heart, the cardiographic method would be rarely employed. For cl.nical purposes the receiving tambour can be advantageously replaced by a small glass funnel or a small metal cup, the open end of which is applied without a membrane over the cardiac impulse, the stem being connected with the recording tambour. In cases in which the right ventricle is in contact with the chest-wall at the position of the apexbeat the cardiogram is 'inverted '-that is to say, the chest-wall is drawn in during systole and protruded during diastole of the ventricles. Inversion of the cardiogram is, therefore, not an infallible sign of the pathological condition known as adherent pericardium (Mackenzie). 
Endocardiac Pressure.-The function of the heart is to maintain an excess of pressure in the aorta and pulmonary artery sufficient to overcome the friction of the whole vascular channel, and to keep up the flow of blood. So long as the semilunar valves are closed, most of the work of the contracting ventricles is expended in raising the pressure of the blood within them. At the moment when blood begins to pass into the arteries, nearly all the energy of this blood is potential; it is the energy of a liquid under pressure. During a cardiac cycle the pressure in the cavities of the heart, or the endocardiac pressure, varies from moment to moment, and its variations

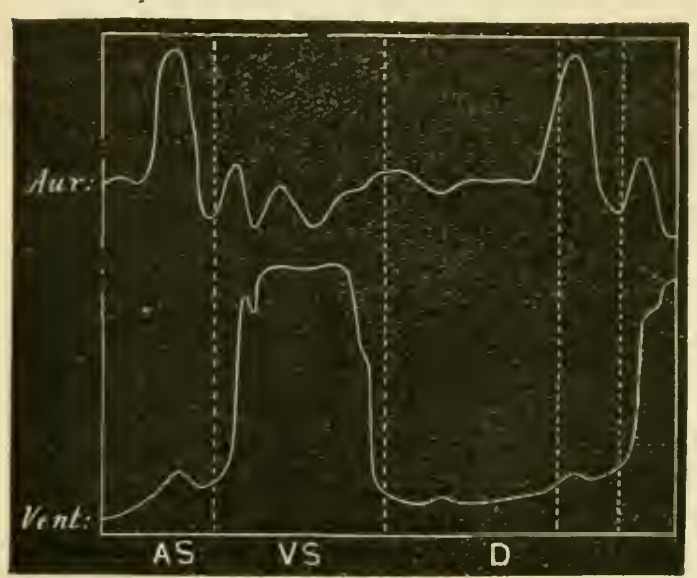

Fig. 29.-Curves of Endocardiac Pressure taken with Cardiac Sounds. Aur., auricular curve; Vent., ven. tricular curve; AS, period of auricular systole, in. cluding relaxation; VS, of ventricular systole, including relaxation; D, pause.

ded with the true oscillations of blood-pressure

aftord important datia for the study of the mechanics of the circulation.

Manometers. - For the study of the endocardiac pressure, the ordinary mercurial manometer (p. I Io) is unsuitable, since, owing to the relatively great amount of work required to produce a given displacement of the mercury, it does not readily follow rapid changes of pressure, and the mercurial column, once displaced, continues for a time to execute vibrations of its own, which are compounBut by introducing in the connection between the manometer and the heart a valve so arranged as to oppose the passage of blood towards the heart, while it favours its passage towards the manometer, the maximum pressurc attained in the cardiac cavities during the cycle may be measured with considerable accuracy. When the valve is reversed the apparatus becomes a minimum manometer. In this way it has been found that in large dogs the pressure in the left ventricle may risc as high as 230 to $240 \mathrm{~mm}$. of mercury, and sink as low as -30 to $-50 \mathrm{~mm}$.; while in the right ventricle it may be as much as $70 \mathrm{~mm}$., and as little as $-25 \mathrm{~mm}$. In the right auricle a maximum pressure of $20 \mathrm{~mm}$. of mercury has been recorded, and a mininum pressure of - $10 \mathrm{~mm}$. or even less. But these results were obtained under somewhat exceptional experimental conditions, and the normal maximum pressures in the heart cavities in man are probably not so high, especially in the right auricle and ventricle.

Our knowledge of the maximum and minimum pressure attained in the cavities of the heart, even if it were far more precise than it actually is, would only carry us a little way in the study of the endocardiac pressure-curve, for it would merely tell us how far above the 
base-line of atmospheric pressure the curve ascends, and how far below the base-line it sinks. To exhaust the problem, we require to have tracings of the exact forn of the curve for each of the cavities of the heart, and to know tlic time-relations of the curves so as to be able to compare them with each other, and with the pressure-curves of the great arterics and great veins. To obtain satisfactory tracings of the swiftly' changing endocardiac pressure is a task of the lighest technical difficulty, and it is only in very recent years that it lias bcen accomplished, with any approach to accuracy by the use of clastic manometers, in which the blood-pressure is counterbalanced, not by the weiglit of a column of liquid, as in the mercurial manometer, but by the resistance to compression of a small column of air or the tension of an elastic disc or of

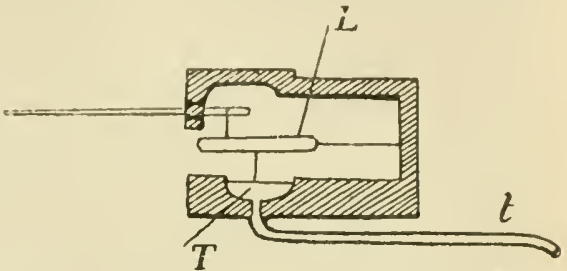

Fig. 30.-Diagram of Hurthle's Elastic Manometer. $T$, small chamber covered by mem. brane; $t$, tube coinmunicating with interior of heart; $L$, compound lever to magnify the movements of the membrane. a spring. Modifications in the nature and dimensions of the elastic resistance of the recording apparatus and of the size of the cavity have produced successive improvements, as, e.g., in the manometers of Hürthle (Fig. 30).

The penetrating analysis of the principles of manometer construction by Frank has recently stimulated renewed investigation of the whole subject with the aid of instruments whose movements are optically re-

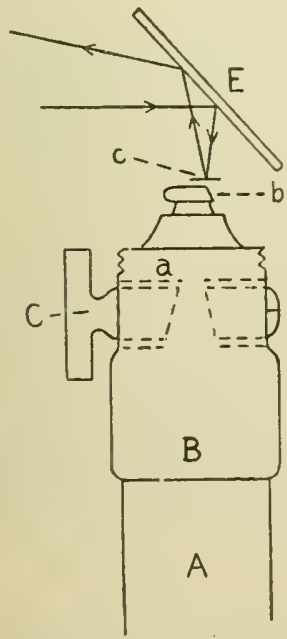

Fig. 31-Diagram of Optical Manometer (Wiggers). $A$ is a vertical glass tube surmounted by a hollow brass cylinder, B, which contains a stopcock, C, whose lumen comes into apposition with a plate, a, having a small opening in it. By opening the stopcock more or less, the pulsations will be "damped" to a smaller or greater extent. Above a the cylinder ends in a segment capsule b (i.e., a capsule cut away at one side) $3 \mathrm{~mm}$. in diameter, covered with rubber dam. Upon this a small piece of celluloid carrying 2 little mirror, c, is fastened, so that it pivots on the chord side of the capsule. Over the capsule and its recording mirror is mounted a support bearing an inclined reflecting mirror, E, adjustable about a hrsizontal axis by a screw, so that the image of the jecording mirror appears within it. Upon this image a strong light is focussed. The incident rays are doubly reflected, as shown in the figure, and the movements of the capsule are thus greatly magnified. The beam of light is photographed on a moving plate.

corded on a photographic plate, so as to eliminate all unnecessary friction. Fig. $3 \mathrm{I}$ is a diagram of the manometer devised by Wiggers on this principle.

Hürthle's spring manometer consists of a small drum covered with an indiarubber membrane, loosely arranged so as not to vibrate with a period of its own. The drum is connected with the heart or witl a vessel, and the blood-pressure is transmitted to a steel spring by means of a light metal disc fastencd on the membrane. The spring 
acts on a writing lever. The instrument is so constructed that for a given change of pressure the quantity of liquid displaced is as small as possible, and it is on this that its capacity to follow sudden variations of pressure chicfly depends. The manometer is connected with the cavity of the heart by an appropriately curved cannula of metal or glass, which, after being filled with some liquid that prevents coagulation (Practical Lxercises, p. $2 \mathrm{I}$ ), is pushed through the jugular vein into the right auricle or ventricle, or through the carotid artery and aorta into the left ventricle. Some observers fill only the cannula with fluid, and leave the capsule of the elastic manometer and as much of the connections as possible full of air. Others fill the whole system with liquid. Ancl around the question of the relative merits of 'transmission ' by liquid and by air has raged a controversy which, lowever, now shows signs of coming to an end. For there is reason to suppose that the character of the curves obtained is moditied among other circumstances by the manner in which the pressure is transmitted, as it is certainly modified by the dimensions and mass of the moving parts and the method of recording. As Wiggers has pointed out, the differences in the records obtained by different observers, even with the latest methods of optical registration, are determined largely by the sensitiveness and degree of damping of the manometer.

The Ventricular Pressure-Curve.-Thus, the pressure-curve of the ventricle, according to most of those who have employed manometers with liquid transmission and small inertia of the moving parts (Fig. 33), remains after the first abrupt rise, which undoubtedly corresponds to the ventricular systole, well above the abscissa line for a considerable time, and then descends somewhat less suddenly than it rose. 'This systolic 'plateau,' although usually broken by minor heights and hollows, which may be partly due to inertia oscillations of the liquid or the recording apparatus, would indicate that the ventricular pressure, after its first swift rise, maintained itself at a considerable height throughout the greater part of the systole. The tracings yielded by most of the manometers with air transmission show the same suddenness in the first part of the upstroke and the last part of the descent-that is, the same abruptness in the beginning of tile contraction and the end of the relaxation. But they differ totally in the intermediate portion of the curve, which, climbing ever more gradually as it nears its apex, remains but a moment at the maximum, then immediately descending forms a 'peak,' and not a plateau. It ought to be distinctly understood, however, that the use of the term 'plateau' must not be taken to imply that the pressure remains constant and the curve parallel to the abscissa during this interval.

Wiggers, using the optical method of recording the pressurecurve in the riglit ventricle (p. 93), finds that when the auricular pressure and the pressure in the pulmonary artery are normal the curve of intraventricular pressure may be divided into (I) an auricular period; (2) a period of rising pressure while the ventricle is contracting and its cavity is closed by the auriculo-ventricular and semilunar valves; (3) an ejection period during which the pressure 
still rises, reaches a summit, and then slowly falls; and (4) a relaxation period (Fig. 32).

Without cntering further into a technical discussion, we may say

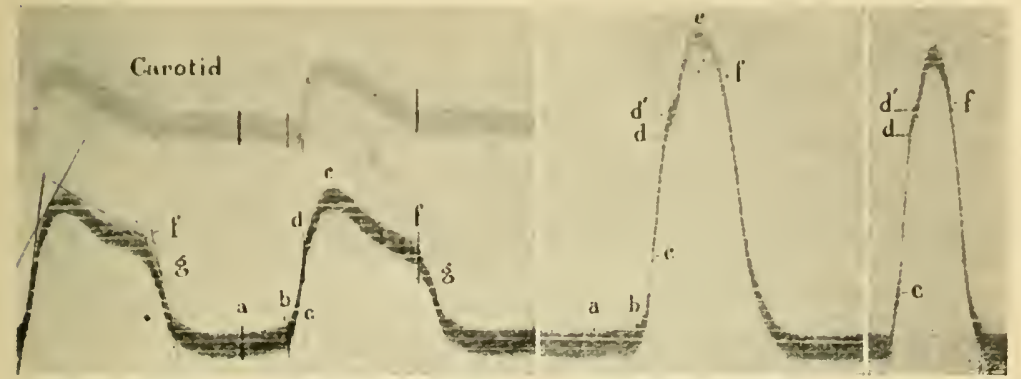

Fig. 32.- Intraventricular P'ressure Curves with Optical Recording (W 1ggers). Three types of normal curves are reproduced, taken with manometers of different degrees of sensitiveness. The second at the left-hand side was taken with the least sensitive. $a-b$, auricular systolic; $b-d$, isometric period, during which the auriculo-ventricular and the semilunar valves are both closed; $d-f$, ejection period; after $f$, diastole.

the bulk of the evidence goes to show that the plateau is not, as the advocates of the peak have claimed, an artificial phenomenon, but does in reality correspond to that continuation of the systole of the

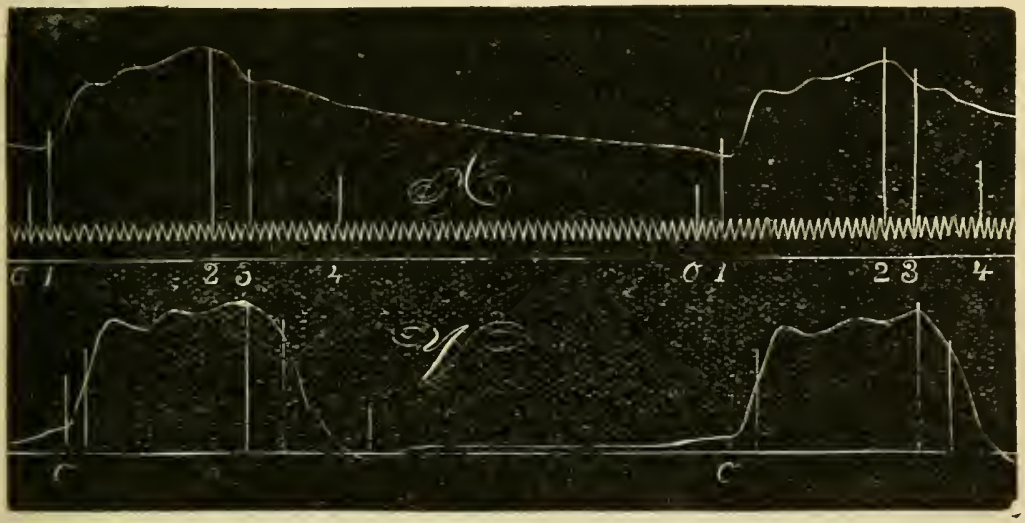

Fig. 33.- Simultaneous Record of Pressure in Left Ventricle (V) and Aorta (A). (Hürthle.) The tracings were taken with elastic manomcters; 0 indicates a point just before the closure of the mitral valve; $I$, the opening of the semilunar valve; 2 , beginning of the relaxation of the ventricle; 3 , the closure of the semi. lunar valve; 4 , the opening of the mitral valve. The ventricular curve shows a 'plateau.'

ventricle, that dogged grip, if we may so phrase it, which it seems to maintain upon the blood after the greater portion of it has been expelled. 
This conclusion is essentially in accordance with the results of Chauveau and Marey, obtained long ago by means of their carcliac sound,' which was in principle an elastic manometer.

It consisted of an ampulla of indiarubber, supported on a framework, and communicating with a long tube, whicli was connected with a recording tambour. The ampulla was introduced into the heart (of a horse) through the jugular vein or carotid artery in the way already described. Sometimes a double sound was employed, armed with two ampulla, placed at such a distance from each other that when one was in the right ventricle the other was in the auricle of the same side. Each ampulla communicated by a separate tube in the common stem of the instrument with a recording tambour, and the writing points of the two tambours were arranged in the same vertical line. When any change in the blood-pressure takes place, the degree of compression of the ampullx is altered, and the change is transmitted along the air-tight connections to the recording tambours.

On most of the endocardiac pressure tracings taken witl modern manometers, whether the curves belong to the type of the peak or of the plateau, no sudden change of curvature, no nick, or crease, or undulation reveals the moment of opening or closure of any valve. This has been considered by some writers a striking tribute to the smooth working of the cardiac pump. There is reason to think, however, that the smoothness of the curve is still in some degree artificial, and on some of the records obtained by optical methods (Fig. 32) indications of changes of curvature, associated with the action of the valves, may be observed. But even in the absence of such indications, by experimentally graduating a pair of elastic manometers, and obtaining with them simultaneous records of the pressure in auricle and ventricle, or by using a 'differential ' manometer, in which the pressures in two cavities are opposed to each other, so that the movement of the membrane corresponds to their difference, we can calculate at what points of the ventricular curve the pressure is just greater than and just less than the pressure in the auricle. The first point, it is evident, will correspond to the instant at which the mitral or tricuspid valve, as the case may be, is closed, and the second to the instant at which it is opened. And in like manner, by comparing the pressure-curve of the aorta with that of the left ventricle, the moment of opening and closure of the semilunar valves may be determined (Figs. 33 and 34). According to the best observations, the closure of the semilunar valves takes place at a time corresponding to a point on the upper portion of the descending limb of the intraventricular curve.

On the blood-pressure curve of the aorta, simultaneously registered, the corresponding point is near the bottom of the so-called "aortic' notch (p. 105) which precedes the dicrotic elevation. For clinical purposes, in man the moment of closure of the semilunar valves (denoted by the abbreviation S.C. point) may be taken as 0.03 second before the bottom of the aortic notcli in spliymographic tracings from the carotid, this being approximately the average time occupied 
by the pulse-wave in travelling from the aorta to the carotid. The S.C. point, the A.O. point, or moment of opening of the auriculoventricular valves, and the teginning of the ventricular systole, are three important points of reference in the measurement and interpretation of pulsc-tracings in clinical work. The A.O. point in man may be taken as a point 0.03 second in advance of the summit of the dicrotic wave' on the carotid pulse-tracing (Lewis). But this is the most difficult of the three standard points to determine clinically with anything like accuracy.

The study of the curves of endocardiac pressure enables us to add precision in certain points to the description of the events of the cardiac cycle which we have already given, and, as regards the ventricles, to divide the cycle into four periods:

(I) A period during which the pressure is lower in the ventricles than either in the auricles or the arteries, and the auriculo-ventricular valves are consequently open, and the semilunar valves closed. This is the period of "filling " of the heart, or the pause.

(2) A period, beginning with the ventricular systole, during which the pressure is increasing abruptly in the ventricles, while they are as yet completely cut off from the auricles on the one hand and the arteries on the other by the closure of both sets of valves. This is the period of ' rising pressure,' during which the ventricles are, so to say, 'getting up steam.' The interval between the beginning of the ventricular systole and the opening of the semilunar valves is termed the 'presphygmic' interval.

(3) A period during which the pressure in the ventricles overtops that in the arteries, and the semilumar vaives are open, while the auriculoventricular valves remain shut. This is the period of 'discharge' or 'sphygmic' period.

(4) A period during which the pressure in the ventricles is again less than the arterial, while it still exceeds the auricular pressure, and both sets of valves are closed. This is the period of rapid relaxation. The interval between the closure of the semilunar and the opening of the auriculo-ventricular valves is sometimes called the "post-sphygmic" interval.

Of the four periods, the second and fourth are exceedingly brief. The third is relatively long and constant, being but slightly dependent on either the pulse-rate or the pressure in the arteries. The duration of the first period varies inversely as the frequency of the heart; with the ordinary pulse-rate it is the longest of all.

From records taken in a person with a defect in the chest-wall which rendered the heart accessible the following results were obtained as to the duration of the various events of the cardiac cycle: First and fourth periods together, 0.445 ; third period, 0.254 ; second period (presphygmic interval), $0.05 \mathrm{I}$ second. the pulse-rate being 80 a minute (Tigerstedt). In another case with a similar defect the first period lasted 0.32 , the fourth period (post-sphygmic interval) 0.06 , the second and third periods together 0.4 , and the auricular systole 0.1 second, the pulse-rate being 66 . 
The Auricular (and Venous) Pressure-Curve.--The fluctuations of pressure in the auricles, although confined within narrower limits than in the ventricles, are of equal interest. They have been studied in considerable detail both in animals and by indirect methods in man. No fewer than three distinct elevations or 'positive waves,' separated or followed by three depressions or 'negative waves,' have been described on the curve of intra-auricular pressure.

The first elevation corresponds to the systole of the auricle. The second coincides with the onsct of the ventricular systole, and is

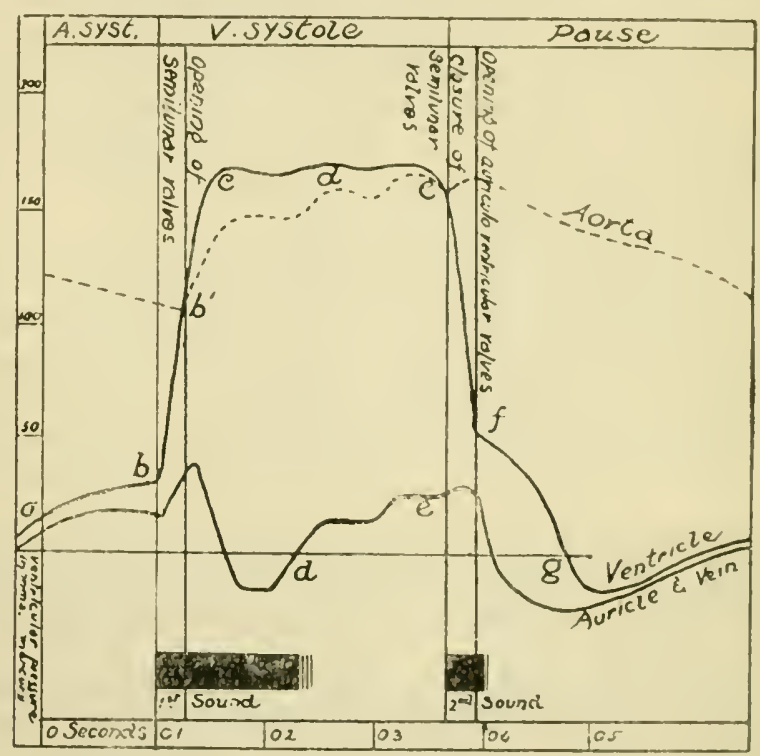

Fig. 34.-Schematic Comparison of Pressure Curves in the Auricle (or Superior Vena Cava), the Ventricle and the Aorta in 1 he 1)og (Fredericq). In the auricular curve are to be distinguished $a b$, the first positive or presystolic wave, corre. spouding to the auricular systole ( $a$ wave); $b b^{\prime}$ or $b c$, second positive wave or frst systolic wave, which corresponds with the beginning of the ventricular systole ( $c$ wave); $b^{\prime} c d$, the steep negative wave of which the heginning corresponds to the opening of the semilunar valves; def, the third positive wave ( $v$ wave), more or less serrated, ending at $f$, the point of opening of the auriculoventricular valves; $f g$, a luegative wave corresponding to the relaxation of the ventricle. The time is indicated along the abscissa in tenths of a second, the pressure along the vertical axis at the left in mm. of mercury.

probably due to the sudden bulging of the auriculo-ventricular valve into the auricle, or even to a slight regurgitation of blood from the ventricle through the valve before it has completely closed. The cause of the third elevation, which occurs during the period occupied in the ventricular pressure-curve by the platcau, is less clearly made out. In man, the events taking place in the right auricle during its 
systole can be followed to some extent by recording the venous pulse in the jugular veins, especially the internal jugular, at the root of the the neck (Fig. 36). Successful tracings can be obtained, not only in certain pathological conditions, but in many normal individuals, and it is probably only a matter of improved technique to obtain them in all. The jugular venous pulse-tracing, like the intra-auricular pressure-curve, shows in general three well-marked elevations and three depressions, and there is good evidence that, broadly speaking, these features of the jugular curve correspond as regards their origin with the changes of pressure in the auricle.

Identical features are observed on records of the normal venous pulse taken from veins of dogs near the heart, and on records of the pulse taken by a sound in the œsophagus. The œsophagus pulse is related to the pulsation of the left auricle, the venous pulse to the changes of pressure in the right auricle. The first elevation, called the $a$ (auricular) or $p$ (presystolic) wave, begins with, and is the result of, the auricular systole. It is probably produced by stasis in the veins due to the contraction of the auricle, as well as to the effect of the impact of the auricular systole. The downstroke on the curve which succeeds this first elevation corresponds to the first negative wave or presystolic fall, which is due to the auricular relaxation. This

Fig. 35.-Schema of Events in the Cardiac Cycle, in Relation to the Venous Pulse (Ewing). I, Tracing from Vena Cava, showing presystolic rise and fall, PR. PF ( $a$ wave); SR. systolic rise and fall (c wave); $\mathrm{O}^{\prime}$, first onflow wave and, DR, diastolic rise and fall ( $v$ wave); $O^{\prime}$, second onflow wave; 2. auricular myogram (tracing of contraction of auricle); 3, ventricular myogram (tracing of con. traction of ventricle); 4 , record of the movement of the auriculo-ventricular septum; 5 , ventricular volume curve (plethysmographic curve of dis. charge of the ventricles); 6 , curve of aortic pressure; 7 . intraventricular pressure-curve.

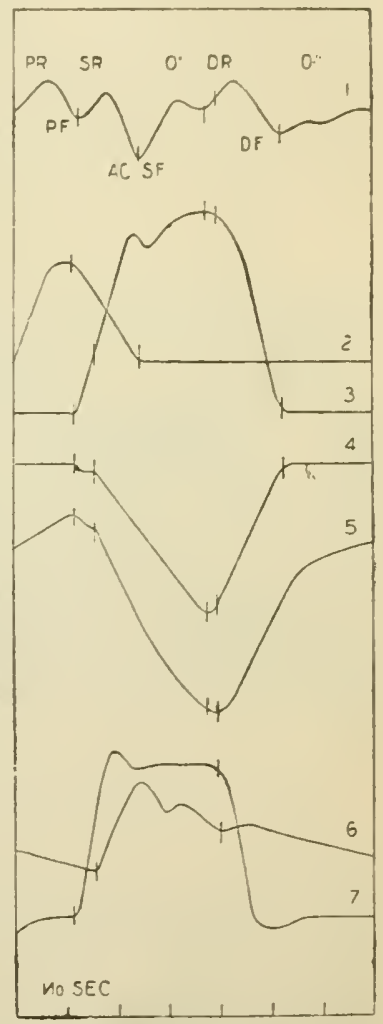

fall of pressure is terminated by a rise-the second positive wave-which begins at the same moment as the ventricular systole, and is the expression on the venous pulse-curve of that sccond elevation of the intra-auricular pressure whose probable cause has alreadybeen found in the sharp protrusion of the auriculo-ventricular valve into the auricular cavity under the stress of the ventricular systole while the semilunar valve are still closed. In addition to the actual bulging of the auriculo-ventricular valves, the impact of the sudden contraction of the ventricle on its contents transmitted through the valve to the contents of the auricle may aid in producing the rise of venous pressure. The second elevation has been termed the $c$ wave by certain 
writers, who studied it on jugular tracings, because they supposed it to be simply transmitted from the pulse in the adjacent carotid artery. This, however, has been shown to be erroneous, although it is truc enough that pulsations transmitted from the great arterics of the thorax and neck may angment or distort the second elevation of the venous pulse. It has been proposed that the second positive wave should be called the $s$ (systolic) wave. It lasts practically throughout the presphygmic period of the ventricular systole; the opening of the semilunar valves, as indicated by the appearance of the pulse in the innominate artery, occurs just before the end of the second clevation (Porter, Ewing, etc.). The rapid discharge of the ventricle through the open semilunar valves, and its consequent diminution in sizc, especially in its longitudinal diameter, is associated with a dilatation of the auricular cavity and a fall of intra-auricular pressure which is expressed on the venous pulsn-curve as the downstroke succeeding the second positive wave. Thus second negative wave gives place to the third positive wave, due to the steady inflow of blood into the auricle from the veins. According to Ewing, the third positive wave, the $v$

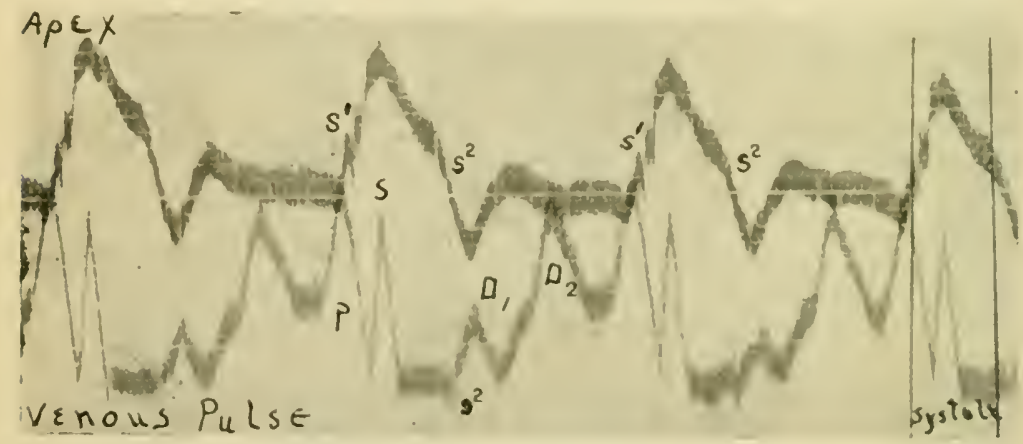

Fig. 36. -Normal ipex and Vennus Pulses, Photographically Recorded (reduced nine-tenths) (Niles and Wiggers). P, presystolic (or $a$ ) wave; $\mathrm{S}$, systolic (or $c$ ) wave; $D_{1}$, first diastolic $(v)$ wave. In this venous record a second diastolic wave, $D_{2}$, is present. $S^{1}$, vibrations corresponding to first sound; $S^{2}$, to second sound.

wave of Maclienzie, really consists of two waves, the "first onflow wave" and the "diastolic rise" or d wave. This last is terminated by the third negative wave or cliastolic fall of venous pressure coincident with the opening of the auriculo-ventricular valves. The re-examination of the venous pulse with apparatus of which the moving parts have an cxccedingly small mass and optical methods of recording (see p. 93) has confirmed the existence on the phlebogram of thrce cssential waves. A fourth is sometimes adcled when the cardiac cycle is long. The first wave is clearly presystolic, the second systolic, as agreed by all observers who have used polygraph tracings. The third wave, however, is diastolic, as is, of course, the fourth when it exists. The position of these waves can be definitely fixed by simultaneous heart apex tracings, since on such optically recorded cardiograms the heartsounds are represented by distinct vibrations, except where the chest wall is too thick or the heart overlaid by emphysematous lung. Sometimes heart-sound vibrations may be present also on the record of the venous pulse (Wiggers). 
The jugular curve, when properly interpreted, affords valuable information as to the action of the auricle, information of the same kind as that afforded by the arterial pulse-tracing and the cardiogram as to the action of the ventricle. In the interpretation of the venous pulse-tracings, a simultancous record of the radial or, better, the carotid pulse or of the apex-beat is always important, and often indispensable, for it enables the time of onset of the ventricular systole to be marked upon the phlebogram (the venous trace), and this facilitates the icientification of the $a$ wave, which must immediately precede, and the $c$ or $s$ wave, which should coincide with the beginning of the contraction of the ventricle. The student must, however, be warned that the proper interpretation of such tracings in the study of cardiac disease is often difficult and requires special knowledge and training.*

Suction Action of the Ventricle.-We have alrearly sair that a negative pressure may be detected in the cardiac cavities by means of a special form of mercurial manometer. This is confirmed by an examination of the tracings written by good elastic manometers, for the curves of both ventricles may often descend below the line of atmospheric pressure. The cause of this negative pressure has been much discussed. In part it may be ascribed to the aspiration of the thoracic cage when it expands during inspiration (p. 226). But since the pressure in a vigorously-beating heart may still become negative, when the thorax has been opened, and the influence of the respiratory movements eliminated, we must conclude that the recoil of the somewhat narrowed, or at least distorted, auriculo-ventricular rings, and of elastic structures in the walls of the ventricles, exerts of itself a certain suction upon the blood. This, however, is not an important factor in the maintenance of the circulation.

\section{Section III.-Physical or Mechanical Phenomena of the Circulation in the Bloodvessels.}

The Arterial Pulse.-At each contracton of the heart a quantity of blood, probably varying within rather wide limits (p. I39): is forced into the already full aorta. If the walls of the bloodvessels were rigid, it is evident (p. 85) that exactly the same quantity would pass at once from the veins into the right auricle. The work of the ventricle would all be spent within the time of the systole, and only while blood was being pumped out of the heart would any enter it. Since, however, the vessels are extensible, some of the blood forced into the aorta during the systole is heaped up in the arteries, beyond which, in the narrow arterioles and in the capillary tract, with its relatively great surface, the chief resistance lies. The arteries are accordingly distended to a greater extent than before the systole, and, being elastic, they keep contracting upon their contents until the next systole over-distends them again. In this way, during the pause, the walls of the arteries are executing a kind of elastic systole,

* The necessary details must be sought in such works as Mackenzie's 'Diseases of the Heart.' 
and driving the blood on into the capillaries. The work done by the ventricle is, in fact, partly stored up as potential encrgy in the tense arterial wall, and this energy is being continually transformed into work upon the blood during the pause, the heart continuing, as it were, to contract by proxy during its diastole. Thus, the blood progresses along the arteries in a series of waves, to which the name of 'blood-waves' or 'pulse-waves' may be given. W'herever the pulse-wave spreads it manifests itself in various ways-by an increase of blood-pressure, an increase in the mean velocity of the blood-flow, an increase in the volume of organs, and by the visible and palpable signs to which the name of pulse is commonly given in a restricted sense. The intermittence in the flow with which the pulse-wave is necessarily associated is at its height at the beginning of the aorta. In middle-sized arteries, such as the radial, it is still well marked, but it dies away as the capillaries are reached, and only under special conditions passes on into the veins, where, however, as has just been mentioned, pulsatory plenomena of a different origin may be detected.

The pulse was well known to the Greek physicians, and used by them to a certain extent as an indication in practical medicine. Harvey demonstrated with some clearness the relation of the pulse to the contraction of the heart, but Thomas Young was the first to form a proper conception of it as the outward token of a wave propagated from heart to periphery.

When the finger is placed over a superficial artery like the carotid, the radial, or the temporal, a throb or beat is felt, which, without measurement, seems to be exactly coincident with the cardiac impulse. In certain situations the pulse can be seen as a distinct rhythmical rise and fall of the skin over the vessel. The throbbing of the carotid, especially after exertion, is familiar to everyone, and the beat of the ulnar artery can be easily rendered visible by extending the hand sharply on the wrist. When the pulse is felt by the finger, it is not the expansion, but the harclening of the wall of the vessel, due to the increase of arterial pressure, that is perceived; and even a superficial artery, when embedded in soft tissues so that it cannot be compressed, gives no token of its presence to the sense of touch. Sometimes an artery is longitudinally extended by the pulse-wave, and this extension may be far more conspicuous than the lateral dilatation. This is particularly seen when one point of the vessel is fixed and a more distal point offers some obstruction to the blood-flow, as at a bifurcation or in an artery which has been ligatured and divided.

By means of the sphygmograph, the lateral movements of the arterial wall, or, rather, in man, the movements of the skin and other tissues lying over the bloodvessel, can be magnified and recorded.

It would be very unprofitable to enumerate all the sphygmographs which ingenuity has invented and found names for. The first attempt 
to magnify the movements of the pulse was made by loosely attaching a thin fibre of glass or wax to the slin with a little iard, in order to demonstrate the venous pulse which appears under certain conditions. In all modern sphygmograplis there is a part, usually button-shaped, which is pressed over the artery by means of a spring, as in Marey's and Dudgeon's sphygmographs, or by a weight, or by a column of liquid. In Marey's instrument, the button acts upon a toothed rod gearing into a toothed wheel, to which a lever, or a system of levers, is attached. The lever has a writing-point which records the movement on a smoked plate, or a plate covered with smoked paper, drawn uniformily along by clockwork (Figs. 37, I00). Special forms of sphygmographs (polygraphs) have been devised, which, by the addition of one or more

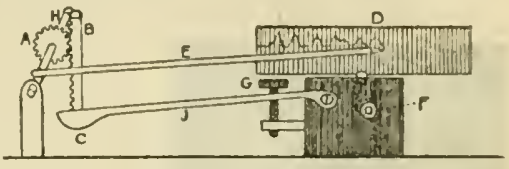

Fig. 37.-Scheme of Marey's Sphygmograph. A, toothed wheel connected with axle $\mathrm{H}$. and gearing into toothed upright B; C. ivory pad which rests over blood. vessel and is pressed on it by moving $\mathrm{G}$. a screw passing through the spriner $\mathrm{J}$; $\mathrm{E}$, writing-lever attached to axle $\mathrm{H}$, and moved by its rotation. E writes on $\mathrm{D}, \mathrm{a}$ travelling surface moved by clockwork $\mathrm{F}$. recording tambours, permit the simultaneous record of movements from two or more points of the vascular system-for example, the radial artery and the jugular vein, or the radial or carotid artery, jugular vein, and the apex of the heart.

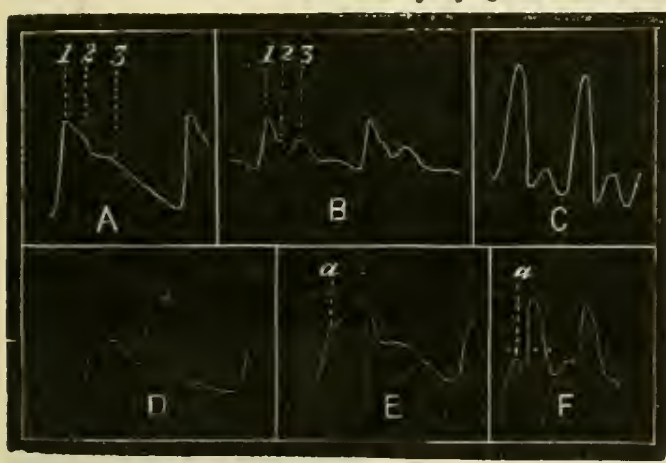

Fig. 38.-Pulse-Tracings. I, primary elevation; 2, predi. crotic or first tidal wave; 3 , dicrotic wave. The depression between 2 and 3 is the dicrotic or aortic notch; 3 is better marked in B than in A. C, dicrotic pulse with low arterial pressure; D, pulse with high arterial pressure - summit of primary elevation in the form of an ascending plateau. E, systolic anacrotic pulse; the secondary wavelet $a$ occurs during the upstroke corresponding to the ventricular systole. $\mathrm{F}$, presystolic anacrotic pulse; $\boldsymbol{a}$ occurs just before the systole of the ventricle. In this rarer form of anacrotism, a may sometimes be due to the auricular systole when the aortic valves are incompetent.
In rare cases, with defect of the chest wall, a tracing may be obtained even from the aorta (Fig. 4o).

In a normal arterial pulse-tracing (Fig. 38) the ascent or anacrotic limb is abrupt and unbroken; the descent or katacrotic limb is more gradual, and is interrupted by one, two, or even Liree or more, secondary wavelets. The most important and constant of these is the one marked 3 . which has received the name of the dicrotic wave. Usually less marked, and sometimes absent, is the wavelet 2 between the dicrotic elevation and the apex of the curve. It is generally termed the predicrotic wave. Oscillations, due to vibrations of the recording apparatus, appear on many pulse- 
tracings, and it is important to recognize their cause, so that no weight may be given to them.

In the explanation of the pulse-tracing, a fundamental fact to be borne in mind is the elasticity of the vessels. When an incompressible fluid like water is injected by an intermittent pump into one end of an clastic tube a wave is set up, which is transmitted to the other

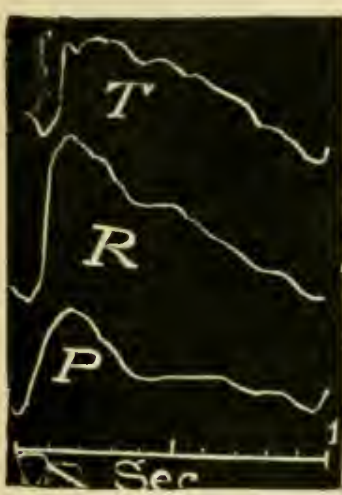

1ig. 36. - Pulse - Tracings from Different Arteries (v. Frey). $T$, temporal; $R$, radial: $P$, artery of foot. end of the tube. It is a positive wave-that is, it causes an increase of pressure and an expansion of the tube wherever it arrives; and if a series of levers be placed in contact with the tube, they will rise and sink in succession as the wave passes them. After the passage of this primary ware the walls of the tube, instead of coming instantly to rest in their original position, regain it by a series of oscillations, first slirinking too much, then expanding too much, but at each movement coming nearer to the position of equiiibrium. Each ribration of the clastic wall is of course accompanied by a change of pressure in the contents of the tube. This change of pressure runs along the tube as a wave; and sucls waves, succeeding the primary one, may be called secondary waves of oscillation. These secondary waves will be set up in an elastic system whether the distal end of the system be closed or open. But if it is closed, or sufficiently obstructed without being actually closed, secondary waves of another kind may also be generated, the primary wave on arriving at the distal end being reflected there. The reflected wave running back towards the central end may there again undergo reflexion, and pass out once more towards the distal end as a centrifugal, twice-reflected wave. When the liquid ceases to enter the tube at the end of the stroke, a wave of diminished pressure-a negative wave-is generated at the central end, and is propagated to the distal end, where it may be reflected just like the positive wave.

Although under certain conditions the dicrotic wave is so marked that the double beat of the pulse was discovered and named by physicians long before the invention of any sphygmograph, perhaps no physiological question has been more discussed or is less understood than the mechanism of its production. Two points, however, seem to be clear: (I) That

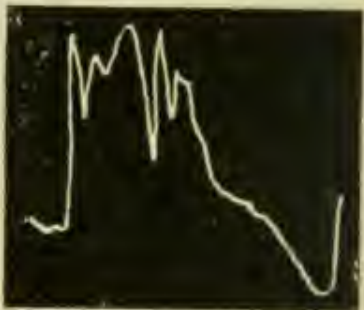

Fig. 40. - Pulse-Curve from Human Aorta (after Tiger. stedt). it is a centrifugal, and not a centripetal, wave-that is to say, it travels away from, and not towards, the heart; (2) that the aortic semilunar valves have something to do with its origin.

It is not a centripetal wave, for in tracings taken at all parts of the arterial path, no matter what the distance from the heart and the 
capillaries (e.g., the origin of the carotid and the radial at the wrist), the dicrotic wave is separated by the same interval from the beginning of the primary elevation. This can only be explained by supposing that it has the same point of origin, and travels with the same velocity and in the same direction as the primary wave. It is not, then, a wave reflected directly from the peripheral distribution of the artery from which the pulse-tracing is taken.

Some writers have contended that it is a centrifugal twice-reflected wave, and, indeed, traces of such waves may be detected in the vessels of newly-killed animals when changes of pressure of the same order of magnitude as the arterial pulse are artificially produced by a pump and recorded by elastic manometers connected with the interior of an artery. It has been supposed that these secondary waves are reflected first from peripheral points at which the blood-flow is particularly obstructed (the bifurcations of the larger arteries, and the small arteries and capillaries in general), and that, running towards the heart, they are again reflected outwards from the semilunar valves. It has been urged in support of this view that in very small animals (guineapigs) no dicrotic elevation occurs on the pulse-tracing, since the path which the reflected wave has to follow is so short that it arrives at the root of the aorta before the primary elevation is over. But this argument is by no means conclusive, and, indeed, the great difference in the distance from the heart of the numerous points at which reflection must take place is one of the chief difficulties of the hypothesis. For it is not easy to understand how the reflected fragments of the primary wave, arriving at different intervals at the heart, can be integrated into the single considerable dicrotic elevation.

The explanation that best takes account of the facts and renders most clear the rôle of the semilunar valves is somewhat as follows: When the systole abruptly comes to an end and the out flow from the ventricle ceases, the column of blood in the aorta tends still to move on in virtue of its inertia, and a diminution of pressure, accompanied by a corresponding contraction of the aorta, takes place behind it, just as a negative wave is set up in the central end of the elastic tube when the stroke of the pump is over. At the same moment, and while the semilunar valves are still for an instant incompletely closed, the diminution of pressure in the beginning of the aorta is intensified by the aspiration of the relaxing ventricle, which sucks the blood back against the valves, and draws them a little way into its cavity. A negative wave, therefore-a wave of diminished pressure, represented in the pulse-curve by the 'aortic notch'travels out towards the periphery. The diminution of pressure is quickly followed by a rebound, as always happens in an elastic system. The recoiling blood meets the closed semilunar valves. The aorta expands again, and the expansion is propagated once more along the arteries as the dicrotic elevation. It is possible that this elevation may be reinforced by a reflected wave produced in the manner described. 
SWen the semilunar valve becomes incompetent in disease, or is rendered insufficiont in animals by the artificial rupture of one or more of its segunents, the dicrotic wave, as will be readily unclerstood from the manner in which it is produced, either disippears altogether or is markedly enfecbled. But apart from any anatomical lesion or functional defect in the aortic valves, the prominence of the wave varies with a great number of circumstances, some of which are in a measure understood, while others remain obscure. It varies in particnlar with the abruptness of discharge of the ventricle and the extensibility of the arteries. The conditions are usually favourable when the arterial pressure is low, for the blood then passes quickly from the ventricle into the arteries, which, already only moderately tense, are easily dilated by the primary wave, then sharply collapse, and are igain abruptly distended when the dicrotic wave arrives. And, in fact, an exaggeration of the dicrotic wavelet may be artificially produced by nitrite of anyl (Fig. 102, p. 209), a drug wlich lessens the blooel-pressure by dilating the small arteries. Muscular exercise (Fig. Ior, p. 209), running or bicycling, for instance, has a similar effect on the sphygmogram, althougl the explanation can scarcely be the same, since the bloodpressure mounts rapidly when moderate exercise begins, and only gradually falls during its continuance, with an abrupt decline to normal or below it on cessation of work (Bowe11). The increase in the pulserate may have something to do in this case with the exaggeration of the dicrotism, which is very frequently, although by no means invariably, associated with a rapidly-beating heart, and therefore is often seen in fever. On the other hand, in certain diseases associated with a ligh arterial pressure, the dicrotic elevation almost disappears. Atheromatous arteries, being very inextensible, do not allow a dicrotic pulse.

Since the pulse represents a periodical increase and diminution in the amount of distension of an artery at any point, the line joining all the minima of the pulse-curve will vary in its height above the base-line, or line of no pressure, according to the amount of permanent distension, i.e., permanent blood-pressure, which the heart in any given circumstances is able to maintain. Any circumstance that tends to lessen the permanent distension will cause a fall of the line of minima. and any circumstance tencling to increase tlie distension will cause that line to rise. If, for example, the arm be raised while a pulse-tracing is being taken from the wrist, the line of minima falls because tle permanent pressure in the radial artery is diminished.

The form of the pulse-curve varies in the different arteries, and therefore in making comparisons the same artery should be used. When the wave of blood only enters an artery slowly, the ascending part of the curve will be oblique. This is normally the case in a pulse-curve of a distant artery, such as the posterior tibial. The height of the wave is also less than in an artery nearer the heart, such as the carotid, or even the axillary, where the primary elevation is relatively abrupt (Fig. 39 , p. I04).

Anacrotic Pulse.- As a rule, the ascent of the tracing is unbroken by secondary waves, but in certain circumstances these may appear on it. This condition, which, when well marked at any rate, may be considered pathological, is called anacrotism (Fig. 38). It is seen when the discharge of the left ventricle into the aorta is slow and difficult-e.g., in cases where the orifice of the aorta has been 
narrowed from disease of the semilumar valves (aortic stenosis). Since this condition is associated with hypertropliy and dilatation of the left ventricle, the slow emptying of the ventricle is partly due to the greater quantity of blood which it contains. In whatever way the delay in the emptying of the ventricle is brought about, the most probable explanation of the anacrotic pulse is that the delay affords time for one or more sccondary waves to be developed in the arterial system before the summit of the curve has been reached, and that these are superposed upon the long-drawn primary elevation. In artic insufficiency, where the left side of the heart is never cut off cntirely from the aorta, the auricular impulse is sometimes marked on the pulse-curve as a distinct elevation; and this gives rise to a peculiar kind of anacrotic pulse, especially in the arteries nearest the heart (Fig. 38, F, p. I03).

Frequency of the Pulse.--In health, the working of the cardiac pump is so smooth and apparently so self-directed that it needs a certain degree of attention to perceive that the rate of the stroke is not absolutely constant. It is, in reality, affected by many internal conditions and external influences.

At the end of foetal life the rate is given as I44 to $\mathrm{I} 33$; from birth till the end of the first year, I40 to I23; from Io to I 5 years, 9 I to 76 ; from 20 to 25 years, 73 to 69 . It remains at this till 60 years, and increases again somewhat in old age.* At all ages the pulse is somewhat quicker in the female than in the male, the excess amounting to about 8 beats a minute. So that if we take the average rate for a man (in the sitting position) as 72 , the average for a woman will be So. The difference is partly due to the fact that the average man is taller than the average woman; and it is known that in persons of the same sex and age the pulse-rate has an inverse relation to the stature. But there may be, in addition, a real sexual difference. It must not be forgotten that a certain number of perfectly healthy persons, who may even be noted for their powers of physical endurance, have an habitually slow pulse, not above 50 in the minute. The position of the body exercises a slight, but relatively constant, influence on the rate, which is greater in the standing than in the sitting posture, and greater in the latter than in the recumbent position. And this is true even when muscular action is as far as possible eliminated by fastening the person to a board. The pulse

* It must be remembered that these numbers are merely averages. Some healthy individuals have a much lower pulse-rate than 72 per minute, and some a rate consiclerably greater. Thus, while the average pulse-rate (taken in the sitting position) of 87 healthy (male) students, whose ages ranged from I 8 to 36 years, was 73 , the extreme variation was from 54 to 89 . In the standing position the average was 80 , and the variations 64 to 105 . In the supine position, average 69 , and variations $4^{S}$ to 98 . After a short spell of muscular exercise (generally running up and down some flights of stairs) the average in the standing position was 119 , the variations 75 to 164 , and the average increase 32 . 
is further affected by the respiratory movements, especially when they are exaggerated in forced breathing, being accelerated during each inspiration (p. 293). It is also increased by the taking of food, and especially of alcoholic stimulants, by muscular exercise, in fever and many other pathological conditions, and by a high external temperature. A warm bath, for example, causes a very distinct acceleration of the heart; and Delaroche found that in air at the temperature of $65^{\circ} \mathrm{C}$. his pulse went up to I6o. A cold bath may depress the pulse-rate to 60 , or even less. During sleep it may fall to 5o. It is greatly influenced by psychical events, and that in the direction either of an increase or a clecrease. Finally, it ought to be remembered as of some practical importance that the pulse-rate in women and children, but particularly in the latter, is less steady than in men, and more apt to be affected by trivial causes. And it is a good general rule to let a short interval elapse after the finger is laid on the artery before beginning to count the pulse, so that the acceleration due to the agitation of the patient may have time to subside.

Rate of Propagation of the Pulse-Wave.-When pulse-tracings are taken simultaneously at two points of the arterial system unequally distant from the heart, by two sphygmographs whose writing-points move in the same vertical straight line, it is found that the ascent of the curve begins later at the more distant than at the nearer point. Since waves like the pulse-wave travel with approximately the same velocity in different parts of an elastic system like the arterial ' tree,' this ' delay ' must be due to the difference in the length of the two paths. The difference in length can be measured; the time-value of the 'delay' can be deduced from the rate of movement of the recording surface; dividing the length by the time, we arrive at the rate of propagation of the pulse-wave. The average rate has been found to be about 7 metres per second in man in the arteries of the upper limb, and 8 metres in those of the lower limb, the difference being due to the smaller distensibility of the latter. In sleep the velocity diminishes almost a metre a second. It increases in arteriosclerosis, where the distensibility of the arteries is diminished, and in chronic nephritis with hypertrophy of the heart, in which the blood-pressure is increased. The mean velocity of the pulse-wave is about the same as the speed of a moderately fast steamship (say, I7 miles an hour), but less than that of a wave of the sea in a strong gale. The velocity of the pulse-wave must not be confounded with that of the blood-stream itself, which is not one-thirtieth as great. A ripple passes over the surface of a river at its own rate-a rate that is independent of the velocity of the current. The passage of the ripple is not a bodily transference of the particles of water of which at any given moment the wave is composed, but the propagation of a change of relative position of the particles. The mere fact 
that the ripple can pass upstream as well as down is sufficicnt to illustrate this. The pulse-wave does not, however, correspond in every respect to a ripple on a stream, for the bodily transfer of the blood depends upon the series of blood-waves which the heart sets travelling along the arteries. Every particle of blood is advanced, on the whole, by a certain distance with every pulse-wave in which for the time it takes its place. But no particle continues in the front of the pulse-wave from beginning to end of the arterial system. The ' delay ' or ' retardation ' of the pulse (the interval, say, between the beginning of the ascent of the carotid and radial curves) is practically constant in the same individual, not only in health, but also in most diseases. But the retardation is markedly increased when the pulse-wave has to pass through a portion of an artery whose lumen is either greatly widened (in aneurism) or greatly constricted (in endarteritis obliterans).

The Blood-Pressure Pulse in the Arteries.-In man it is only possible to trace the pulse-wave along the arteries by movements of the walls of the vessels transmitted through the overlying tissues. In animals the changes of pressure that occur in the blood itself can be directly registered, and these changes may be spoken of as the blood-pressure pulse. At bottom, as already pointed out, the phenomenon is exactly the same as that we have been dealing with in our study of the external pulse. We are only now to follow, by a more direct, and in some respects a more perfect method, the same wave of blood along the same channel.

Measurement of the Arterial Blood-Pressure.-Hales was the first to measure the blood-pressure. This he did by connecting a tall glass tube with the crural artery of a horse. The height to which the blood rose in the tube indicated the pressure in the vessel. Poiseuille, nearly half a century later, applied the mercury manometer, which had already been used in physics, to the measurement of blood-pressure. Ludwig and others improved this method by making the manometer selfregistering by means of a float in the open limb, supporting a style which writes on a revolving drum, or kymograph. (For the method of taking a blood-pressure tracing, see p. 2 ro.)

For reasons already mentioned, the mercurial manometer is better suited for measuring the mean blood-pressure, or for recording changes in the pressure which last for some time, than for following the rapid variations of the pulse-wave. For the latter purpose, one of the class of elastic manometers is required (p. 93).

A blood-pressure tracing taken from an artery with a manometer of this sort yields the truest picture of the pulse-wave which it is possible to obtain, because the reproduction of it is the most direct. The fact that such a tracing shows a close agreement with the trace of a good sphygmograph properly applied to the corresponding artery on the other side is a striking proof of the general accuracy of the sphygmographic method for physiological purposes, arid enables us to guide ourselves in transferring to man, in whom, of course, the sphygmograph can alone be used, the information derived from direct manometric observations in animals. 
For the same reason it is unnecessary to discuss the mdnumetric tracings, as regards the pulsatory phenomena, in all their details. It will be sufficient to say that, while the form of the blood-pressure pulse-curve varies with the mean blood-pressure, th. dicrotic wave is always marked on it, preceded by one or more oscillations falling within the period of the systole, and followed by one or more within the period of the diastole. When the blool-presure is low, the first or primary elevation is the highest of the whole curve (Fig. 42). When the blood-pressure is high, the maximum falls later coinciding with

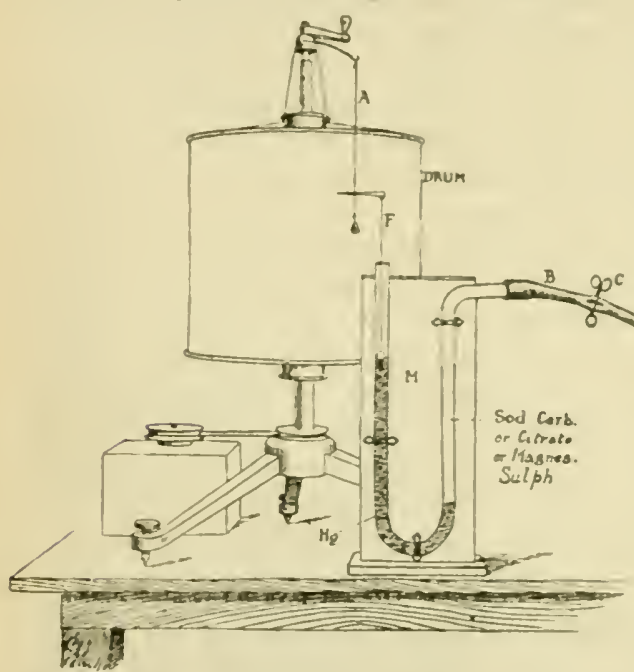

Fig. 41.--Arrangement for taking a Blnod-Pressure Tracing. M, manometer; $\mathrm{Hg}$, nercury ; F, float armed with writing-point; A. thread attached to a wire projecting from the drum and supporting a small weight. The thread keeps the writing. point in rontact with the smoked paper on the drum. $B$ is a strong rubber tube connecting the mannmeter with the artery; C. a pinchcock on the rubber tube, which is taken off when a tracing is to be obtained. rabbit this pulsatory variation is one-third to onc-fourth of the minimum pressure. In the dog it is still greater, owing to the slower rate of the heart, and often amounts to $50 \mathrm{~mm}$. of meriry. while under favourable conditions (low minimum pressure and slowly-beating lieart) the systolic increase of pressure may be actually more than double the minimum (Hürtlle). Fick found also, by means of his spring manometer, that the pulsatory variations of blood-pressure were greater than the respiratory variations (p. $2 S(1)$, although in the records of the mercury manometer the reverse appears often to be the case. Landois, too, in the course of experiments in which a divided artery was allowed to spout against a moving surface, and to trace on it a sort of pulse-curve painted in blood (a limmautogram as it is called) 
observed that the rate of escape of the blood was nearly 50 per cent. greater dirring the systole than during the pause of the heart. The existence of the dicrotic wave on this tracing was long looked on as the best proof that it was not an artificial phenomenon.

The wave of increased pressure, as it rums along the arterial system, carries with it wherever it arrives an increase of potential energy. But this excess of potential energy is continually being worn down, owing to the friction of the vascular bed; and although in the com paratively large arteries the loss of energy is not great, it rapidly increases as the arteries approach their termination, and begin to break up into the narrow arterioles which feed the capillary network. For not only is the ratio of the total surface to the total cross-section, and threfore the friction, increased with every bifurcation, but the mere change of direction and division of the wave camnot take place without loss of energy. For this reason the fluctuations of blood-pres-

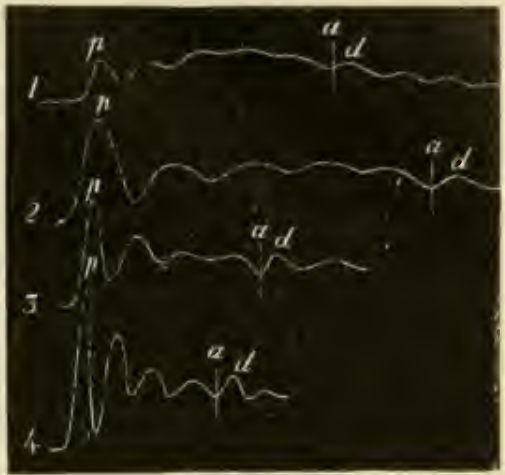

Fig. 42,-Curves of Blood-Pressure taken with a Spring Manometer from the Carotid Artery of a Dog (Hürthle). When I was taken the blood-pressure was high; 2 corresponds to a medium, 3 to a low, and 4 to a very low, blood. pressure; $p$ is the primary elevation - this and the succeeding elevations between $p$ and $a$ are called systolic waves; the systolic waves are followed by a marked elevation $d$, which corre. sponds to the dicrotic wave.

sure are greater in the large arteries near the heart than in arteries smaller and more remote. In the wide and much-branched capillary bed the pulse-wave disappears altogether, and the blood-pressure becomes relatively comstant or permanent. And it is for some

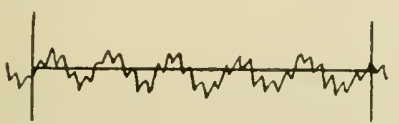

Fig. 43. - Blood-Pressure Tracing. The horizontal straight line inter. secting the curves is the line of mean pressure. purposes convenient to look upon the blood-pressure in the arteries as made up of a permanent element, with pulsatory oscillations superposed on it. Since no portion of the arterial system is more than partially emptied in the interval between two blood-waves, the minimum or permanent pressure is always positive -i.e., always above that of the atmosphere, the beats of the heart succeeding each other so rapidly that the successive waves overlap or 'interfere,' and are only separated at their crests.

If the heart is stopped while a blood-pressure tracing is being taken-and we shall see later on how this can be done (p. I57)-the minimum line of the tracing goes on falling towards the zero-line. 
When the heart begins beating again, the pressure-curve rises, not by a continuous ascent, but by successive leaps, each corresponding to a beat of the heart, and each overtopping its predecessor, till the old line of minimum or of mean pressure is again reached.

The mean arterial blood-pressure is the permanent pressure plus one-half of the average pulsatory oscillation. In a blood-pressure tracing the line of permanent pressure joins all the minima; the line of maximum pressure joins all the maxima; the line of mean pressurc is drawn between them in such a way that, of the area included between it and the blood-pressure curve, as much lies above as below it (Fig. 43). As has been said, a tracing taken with a mercury manometer gives approximately the mean blood-pressure. Each beat of the heart is represented on it by a single elevation of variable size, sometimes not amounting to more than one-twentieth of the height of the curve above the line of zero or at mospheric pressure, but sometimes much larger. The oscillations due to the heart-beat are superposed upon much longer, and often, as registered in this way, larger waves, caused by the movements of respiration. So much having been said by way of definition, we have now to consider the amount of the mean arterial pressure, the variations which it undergoes, and the factors on which its maintenance depends.

As to its amount, it will be sufficiently accurate to say that in the systemic arteries of warm-blooded animals in general (including birds), and of man in particular, the mean pressure does not, under ordinary conditions, descend much below Ioo $\mathrm{mm}$. of mercury, nor rise much above $200 \mathrm{~mm}$.; while in cold-blooded animals it seldom exceeds $50 \mathrm{~mm}$., and may fall as low as $20 \mathrm{~mm}$.

It does not seem possible, at least with our present data, to further subdivide these two great groups; nor do we know precisely whether the distinction depends mainly on morphological or mainly on physiological differences, whether, that is to say, the warm-blooded animal has a higher blood-pressure than the cold-blooded chicfly because its vascular system (and especially its heart) is anatomically more perfect. or because its heart beats faster and works harder. It may be that it is for both of these reasons that the birds, which in certain other respects are more nearly related to the reptiles than to the mammals, mount, as regards the pressure of the blood, into the mammalian class, and that a manometer in the carotid of a goose will rise as ligh, or almost as high, as in the carotid of a horse, a sheep, or a dog, while the pressure in the aorta of a tortoise is no ligher than in the aorta of a frog. But we know that the mere average rate of the heart has of itself comparatively little influence on the blood-pressure within either group, for the heart of a rabbit beats, on the average, very much faster than the heart of a dog, and yet the arterial pressure in the dog is ccrtainly at least as great as in the rabbit. Nor does the size of the body secm to have any definite relation to the mean pressure, even in animals of the same specics; and there is no reason to suppose that the pressure is materially less in the radial artery of a dwarf than in the radial artery of a giant. 
Measurement of the Blood-Pressure in Man.-In man the bloodpressure has been estimated by adjusting over an artery an instrument known as a sphygmomanometer or sphygmometer, which, in its most modern form, consists essentially of a hollow rubber pad or hag containing air, and connected with a metallic pressure gange or a mercurial manometer.

The simplest method is that devised by Riva-Rocei (Fig. 44). An armlet in the form of a broad rubber bag, supported externally by canvas or leather, is adjusted round the upper arm. The interior of the bag is connected with a mercury manometer, and also with a strong rubber bulb provided with a valve. By rhythmical compression of the bulb the pressure can be raised. Between the pessure bulb and the rest of the system is a thin rubber balloon, which by its distension renders the changes of pressure more gradual. The finger of the observer is placed over the radial artery, and the pressure is raised until the pulse disappears. Then the pressure is allowed to fall gradually, and the manometer reading at the moment when the pulse first reappears in the radial gives the maximum or systolic pressure in the brachial artery.

Instead of palpating the radial artery, a stethoscope may be placed over the brachial just below the edge of the armlet, according to the method of Korotkoff, by which, in addition to the systolic, the minimum or diastolic pressure may be determined. This is much the best of all the methods of measuring the arterial pressures in man. The pressure is raised somewhat above that necessary to obliterate the pulse, and then allowed to fall slowly. At the moment when pulsations first begin to break through below the armlet, a succession of sharp taps, synchronous with the pulse, is heard. The tapping sound grows rapidly louder as the artery opens up more and more, then abruptly diminishes and changes its character, and gradually disThe finger of the appears. Several phases have been distinguished after the first maximum. Everybody agrees that the pressure shown by the manometer when the sound is first heard is the systolic pressure. This corresponds closely with the systolic pressure as determined by palpating the radial; and it can be shown experimentally that at pressures in the armlet exceeding this the lumen of the brachial artery is actually obliterated and not merely narrowed to such a degree as to prevent the passage of the pulse wave, while still permitting the passage of some blood (see Practical Exercises, p. 2I 3).

The diastolic pressure is the pressure at which a sudden weakening and dulling of the sound occurs (beginning of the fourth phase), and not the pressure at which the sound becomes altogether inaudible (MacWilliam, etc.). The sound seems to be due to vibrations set up in the walls of the artery and the structures in contact with it when it is suddenly opened by the pulse waves. According to Erlanger, the essential thing is the 'water hammer' action of the blood when, moving through the artery under compression in the armlet, it strikes the stagnant blood in the uncompressed artery below and distends it. 
Various instrmments have been devised for determining the blood pressure from the changes in the oscillations communicated to the armlet by the artery as the pressure in the armlet is allowed to fall or caused to rise. The sphygmomanometer of Erlanger (Fig. 45) is arranged to obtain graphic records of the pulse (4) and the minimum blood-pressures
may be deducel. The mean pres- $\begin{aligned} & \text { sure cannot be directly measured, } \\ & \text { but must lie much nearer to the } \\ & \text { minimum than to the maximum, }\end{aligned}$ . $\begin{aligned} & \text { minimum than to the maximum, } \\ & \text { since the line of mean pressure bi- }\end{aligned}$

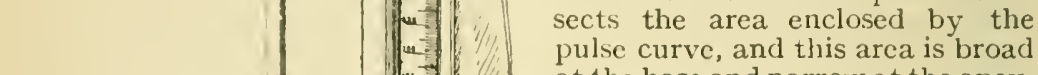
. 1.7 at the base and narrow at the apex.

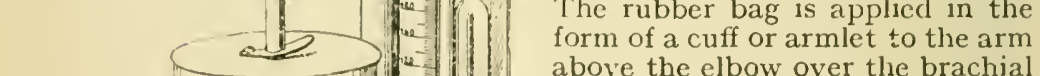
above the elbow over the brachial
artery. It communicates with a
mercury manometer, which gives . 1 mercury manometer, which gives It is also connected with a rubber
bulb, B, enclosed in

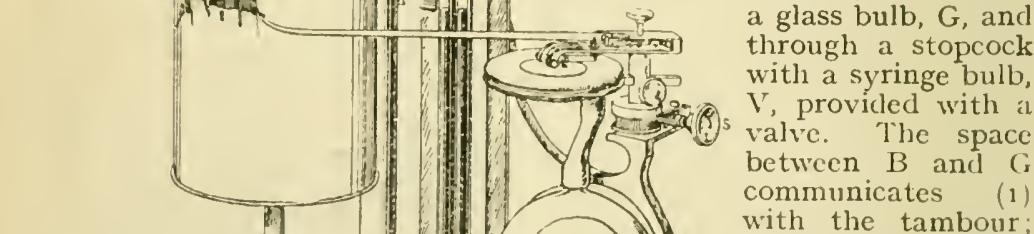
With the tambour:

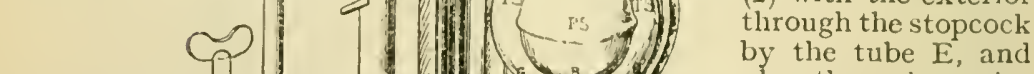
C- of 1 point opening in the 100 tambour. Whilethe 6 (2) being ad7 . that the rubber bag is $(\mathrm{V})$ in communication with Q. 15 the external air through the space TS in the Fig. 45.-Sphygmomanometer of Erlanger. glass bulb. The tambour is thus protected against undue strain during adjustment. The stopcock is now rotated so as to cut off the armlet from the exterior and to permit the entrance of air through $\mathrm{F}$ from $\mathrm{V}$, which is used as a pump to raise the pressure, the space TS and the tambour being still in communication with the exterior. When the desired pressure lias been reaclied, the stopeock is turned into an intermediate position, which cuts off both the armlet and the space 'TS from the exterior, and the pulse is then transmitted to the tambour and recorded on the drum. By certain adjustments of the 
stopeock air can be allowed to escape more or less rapidly from the armlet.

To determine the maximum or systolic blood-pressure, the airpressure in the armlet is raised considerably (about $50 \mathrm{~mm}$. Hg) aloove what it is expected to be. While the lever is writing on the drum the small oscillations flue to the impact on the bag of the pulse-waves in the central portion of the obliterated artery, the pressure is gradually diminished by allowing air to escape. At the moment when the pressure upon the arm falls below the maximum blood-pressure, and the pulse-wave is first able to break through the brachial artery, the oscillations of the lever will more or less abruptly increase in amplitude. The pressure shown by the manometer at this point is the systolic blood-pressure. To obtain the minimum or diastolic pressure, the airpressure in the armlet is raised somewhat (ro to $15 \mathrm{~mm}$. Hg) above the pressure expected. The pressure is diminished by $5 \mathrm{~mm}$. $\mathrm{Hg}$ at a time, records of the oscillations being taken on the drum. The inanometer reading at the point at which the oscillations, after reaching the maximum, begin abruptly to diminish, corresponds to the minimum bloodpressure.

According to Brooks and Luckhardt, the criteria which are supposed to fix the systolic and diastolic pressures in these and similar methods yield results which are too high. The personal equation also seems to introduce a considerable error in the selection of the points on the tracings at which the characteristic changes are supposed to occur (Kilgore).

In using the sphygmometer of Hill and Barnard (Fig. 46), the bag is inflated till the pulsation indicated by the gauge reaches a maxinum. The mean pressure shown at this point is assumed to be equalto, orsomewhat greater than, the diastolic pressure.

The effect of muscular exercise upon the pressure is influenced by the nature of the work. Such an effort as the lifting of a heavy weight causes a sudden and great increase, which is very

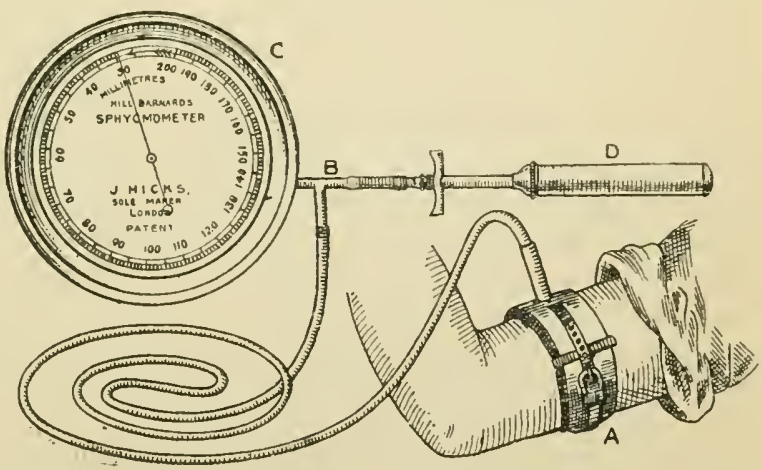

Fig. 46.-Sphygmometer of Hill and Barnard. It consists of a broad armlet, $\mathrm{A}$, connected by a $\mathrm{T}$-tube, $\mathrm{B}$, with a pressure gauge, C, and a small compressing air-pump, D, fitted with a valve.

transient. Thus,

the average arterial pressure in a number of men was II before, I So during, and I 10 two to three minutes after the lift (McCurdy). The rise of pressure in this case is due largely to the marked diminution of the calibre of the bloodvessels mechanically produced by the strong and sustained contraction of the muscles. This increases the resistance to the passage of the blood along the arteries, while the veins are emptier! by the pressure, and more blood thus reaches the right side of the heart. It is obvious that the heart and vessels may easily be exposed to an injurious strain during such efforts. In such an exercise as running, while the pressure mounts to some extent at first, as already mentioned, 
the rise is not maintained, owing to the dilatation of the cutancous vessels. In the anterior tibial artery of a boy whose leg was to be amputated, the blood-pressure, measured by means of a manometcr connected directly with the artery, was found to vary from 100 to ion mm., according to the position of the body and other circumstances. In a woman sixty vears old, in gorsl health, the following readings were of tained with a splyyermomanometer:

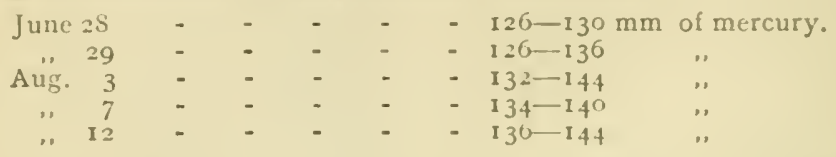

Such measurements on man show that the mean blood-pressure under similar conditions in one and the same artery, and in one and the same individual, may vary for a considerable time only within comparatively narrow limits.

This relative constancy of the general arterial pressure is the result of a delicate adjustment between the work of the heart, the resistance of the vessels, and the volume of the circulating liquid. The quantity of the blood is tolerably steady in health, and considerable changes may be artificially produced in it (p. IgI) without affecting the pressure in any great degree. On the other hand, the work of the heart and the peripheral resistance are highly variable and vastly influential. A narrowing of the arterioles throughout the body or in some extensive vascular tract increases the peripheral resistance; and if the heart continues to beat as before, the pressure must rise. If the arterioles are widened, while the heart's action remains unchanged, the pressure must fall. In like manner an increase or a decrease in the activity of the heart, in the absence of any change in the peripheral resistance, will cause a rise or a fall in the blood-pressure. But if a slowing of the heart is accompanied by an increase in the peripheral resistance, or a dilatation of the arterioles by an increase in the activity of the heart, the one change may be partially or completely balanced by the other, and the pressure may vary within narrow limits or not at all.

Not only is the mean pressure, as measured in a large artery, tolerably constant, but if recorded simultaneously in two arteries at different distances from the heart, it is seen to decrease very gradually so long as the arteries remain large enough to hold a cannula. It is nearly as high, for instance, in the crural artery of a dog as in the carotid. It is easy to see that this must be so, for the resistance of the arteries between the point where the arterioles are given ofi and the heart is only a small fraction of the total resistance of the vascular path; and we have said (p. $S_{4}$ ) that the lateral pressure at any cross-section of a system of tubes through which liquid is flowing is proportional to the resistance still to be overcome. This is also the reason why the pressure is always much lower in the pulmonary artery and right ventricle than in the aorta and left ventricle 
(only one-fiftl to one-sixtl as great), for the total resistance of the vascular path through the lungs is much less than that of the systemic circuit. In dogs with natural respiration the pressure in the pulmonary artery was found to vary between 14 and $26 \mathrm{~mm}$. of mercury, averaging about $20 \mathrm{~mm}$.

The Velocity-Pulse.-We liave seen that the blood is propelled through the arteries in a series of waves that travel from the heart towards the periphery. The particles in the front of the pulse-wave are constantly changing, but since every section of the arterial tree is successively distended, every section contains more blood while the pulse-wave is passing over it than it contained immediately before. And since there is always a fairly free passage for this blood towards the periphery, there is a bodily transfer on the whole of a certain quantity with every wave.

The translation of the blood, instead of being entirely intermittent, as it would be in a rigid tube or in an elastic system with a slow action of the central pump, is to some extent constantly going on; for a portion of a blood-wave is always passing through every section of the arterial channel. Thus, we arrive at the same distinction as to the onward movement of the blood itself as we previously reached in regard to the blood-pressure, the distinction between the constant or permanent factor of the velocity and the periodic factor, which we may call the velocity-pulse.

The Velocity of the Blood.-By the velocity or rate of flow of a river we should mean, if the flow were uniform throughout the whole crosssection, the rate of movement of any given portion or particle of the water. If we could identify a portion of the water, we could determine the velocity by measuring the distance travelled over by that portion in a given time. If the velocity was uniform over the channel, we could predict the actual time which would be required to traverse any fractional part of the measured distance. If, however, the velocity of the current changed from point to point, then we could only deduce from our observation the mean rate of the river for the measured distance. To determine the actual rate for any given portion of this distance over which the rate was uniform, we sliould have to make a separate observation for this portion alone.

But as soon as we pass from an ideal frictionless river to an actual stream, in which the water at the bottom and near the banks flows more slowly than that in the middle and on the surface, we are in every case restricted to the notion of mean velocity. We may distinguish between the velocity of different parts of the current, between that of the mid-stream and the side current, the bottom and the surface layers; but when we consider the river as a whole, we take cognizance only of the mean or average velocity. And at any cross-section this may be defined as the volume of water passing per hour, or whatever the unit of time may be, divided by the cross-section of the current. It is evident that this does not enable us to determine the actual velocity of any given particle of the water at any given moment within a measured interval; nor does it tell us whether or not the average velocity of the current has itself undergone variations within the period of observation. 
We have dwelt upon this point because the measurement of the velocity of the blood, to which we must now turn, involves the same considerations. Within the smaller arteries, as the microscope shows us, and as we should in any case expect from what we know of fluid motion, the blood-current, apart from the periodical variations in its velocity, due to the action of the heart, varies in speed from point to point of the same cross-section. The layer next the periphery of the vessel, the so-called peripheral plasma-layer or Poiseuille's space, moves more slowly than the central portion, the axial stream. In fact, we must suppose that in the large as well as in the small vessels the layer just in contact with the vessel-wall is at rest, while the stratum.internal to this slides on it and has its velocity diminished by the friction. The next layer again slides on the last, but since this is already in motion, its velocity is not so much diminished, and so on. The velocity must therefore increase as we pass towards the axis of the bloodvessel, and reach its maximum there (p. I93).

Again, the velocity must be altered wherever an alteration occurs in the width of the bed, that is, in the total cross-section of the vascular system; for since as much blood comes back in a given time to the right side of the heart as leaves the left side, the same quantity must pass in a given time through every cross-section of the circulation. Wherever the total section of the vascular tree increases, the blood-current must slacken; wherever it diminishes, the current must become more rapid. Now, the total section, increasing somewhat as we pass from the heart along the branching arteries, undergoes an abrupt augmentation, and reaches its maximum in the capillary region. It suddenly diminishes again at the venous end of the capillary tract, and then more gradually as we pass heartwards along the veins, but never becomes so small as in the arterial tract. We must, therefore, expect the mean velocity to be greatest in the large arteries, less in the veins, and least in the arterioles, capillaries, and venules. It must, of course, be remembered that the total section varies from time to time at any given distance from the heart. The capillary tract is especially variable in its area, and capillaries full of blood at one moment may be collapsed and empty at another, according to the changes of calibre and pressure in the arteries which feed and the veins which drain them.

Althougl in strictness we are only at present concerned with the arteries, it will be well to consider here what a change of velocity at any part of the vascular channel really implies. To say that when the channel widens the velocity diminishes is not to explain the meaning of this diminution. A diminution of velocity implics a diminution of kinetic energy, and it is necessary to know what becomes of the energy that disappears. The stock of energy imparted by the contraction of the heart to a given mass of blood constantly diminishes as it passes round from the aorta to the right side of the heart, for friction is constantly being overcome and heat gencrated. This energy, 
as we have seen, exists in a moving liquid in two forms, potential and kinetic, the former being measured by the lateral pressure, the latter varying directly as the square of the velocity. Whenever the velocity, and therefore the kinetic energy, of a given mass of the blood is diminished without a corresponding increase in the potential energy, some of the total stock of energy must liave been used up to overcome resistance (p. $\left.s_{4}\right)$.

In a uniform, rigid, horizontal tube, as has been already remarked, the velocity (and consequently the kinetic energy) is the same at every cross-section of the tube, while the potential energy, represented by the lateral pressure, diminishes regularly along the tube. When the calibre of the tube varies, it is different. Suppose, for instance, that the liquid passes from a narrower to a wider part, the velocity must diminish in the latter. The kinetic energy of visible motion wlich has disappeared must have left something in its room. Here there are three possibilities: (I) The kinetic energy that has disappeared may be just enough to overcome the extra friction in the wider part of the tube due to eddies and consequent cliange of direction of the lines of flow; in this case the potential energy of a given mass of the liquid will be the same at the beginning of the wider part as in the narrower part. The lost kinetic energy will have been transformed into heat. (2) The kinetic energy which has disappeared may be greater than is enough to overcome the extra resistance; a portion of it must, therefore, have gone to increase the potential energy, and the lateral pressure will be greater in the wide than in the narrow part. (3) The lost kinetic energy may be less than enough to overcome the extra resistance; in this case both the lateral pressure and the velocity will be less in the wide than in the narrow part. It has been experimentally shown that when a narrow portion of a tube is succeeded by a considerably wider portion, and this again by a narrow part, case (2) holds; and the liquid may, under these conditions, actually flow from a place of lower to a place of higher lateral pressure.

In the vascular system the conditions are not the same. The widening of tie bed which takes place as we proceed in the direction of the arterial current is not due to the widening of a single trunk, but to the branching of the channel into smaller and smaller tubes. In the larger arteries the increase of resistance is so gradual that both the potential and the kinetic energy diminish only slowly, and the lateral pressure and velocity are not much less in the femoral artery than in the aorta or carotid. But in the arterioles the friction increases so greatly that although the velocity, and therefore the kinetic energy, in the capillary region is much less than in the arteries, the amount of kinetic energy lost is not upon the whole equivalent to the energy consumed in overcoming the extra resistance; the potential energy of the blood is also drawn upon, and the lateral pressure falls sharply in the capillary region, as well as the velocity. Where the capillaries open into the veins, the lateral pressure again sinks abruptly, while the velocity begins to increase, till in the largest veins it is probably about half as great as in the aorta.

Where does the extra kinetic energy of the blood in the veins come from? To say that the vascular channel again contracts as the blood passes from the capillaries into the veins, and that, since the 
same quantity must flow through every cross-section of the channel, the velocity must necessarily be greater in the narrower than in the wider part, does not answer the question. The greater portion of the kinetic energy of the arterial blood is, as we have seen, destroyed, or, rather, changed into an unavailable form, into heat, in the capillary region. The mean velocity of the blood in the capillaries is not

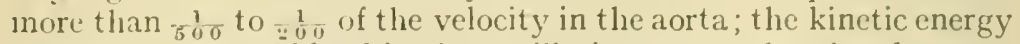
of a given mass of hlood in the capillaries cannot therefore be more than $\left(\frac{1}{20}\right)^{2}$, or $\frac{1}{4000}$ of its kinetic energy in the aorta. In the veins, taking the velocity at half the arterial velocity, the kinetic energy: of the mass would be one-fourth of that in the aorta, or at least Io,ooo times as great as in the capillary region. This extra kinctic. energy comes partly from the transformation of some of the potential energy of the blood. The resistance in the veins is very small compared with that in the capillaries; less of the potential energy represented by the lateral pressure at the end of the capillary tract is required to overcome this resistance, and some of it is converted into the kinetic energy of visible motion, the lateral pressure at the same time falling somewhat abruptly. Contributory sources of kinetic energy in the veins are the aspiration caused by the respiratory movements and the pressure caused by muscular contraction in general, which, thanks to the valves, always aids the flow towards the heart. From these two sources new energy is supplied, to reinforce the remnant due to the cardiac systole (p. I33).

Measurement of the Velocity of the Blood-I. Direct Observation.(a) This method can be applied to transparent parts by observing the rate of flow of the corpuscles under the microscope. But it is only where the blood moves slowly, as in the capillaries, that the method is of use. (b) Part of the path of the blood through a large vessel may be artificially rendered transparent by the introduction of a glass tube, of approximately the same bore as the vessel (Volkmann). The tube is filled with salt solution, and the blood admitted by means of a stopcock at the moment of observation. The time which the blood takes to pass from one end of the tube to the other is noted, and the length divided by the time gives the velocity of the blood in the tube. If the calibre of the tube is the same as that of the artery, this is also the velocity in the vessel; but if the calibre is different, a correction would have to be made. The method is not a good one, for the reason, among others, that the long tube introduces an extra resistance.

2. Ludwig's Stromuhr. - This instrument measures the quantity of blood which passes in a given time through the vessel at the crosssection where it is inserted. It consists of a $\mathbf{U}$-shaped tube, with the limbs widened into bulbs, but narrow at the free ends, which are connected with a metal disc. By rotating the instrument, these ends can be placed alternately in communication with a cannula in the central, and another in the peripheral, portion of a divided artery; or they can be placed so that none of the blood passes through the bulbs, but all goes by a short-cut. One limb of the instrument is filled with oil, and the other with defibrinated blood. The limb containing the oil is first put into communication with the central cnd, and that containing the blood with the peripheral end, of the artery. The blood 
from the artery rushes in and displaces the oil into the other limb, the defibrinated blood passing on into the circulation. As soon as the bloorl has reached a certain height, indicated by a mark, the instrument is reversed and the oil is again displaced into the limb it originally occupied. This process is repeated again and again, the time from

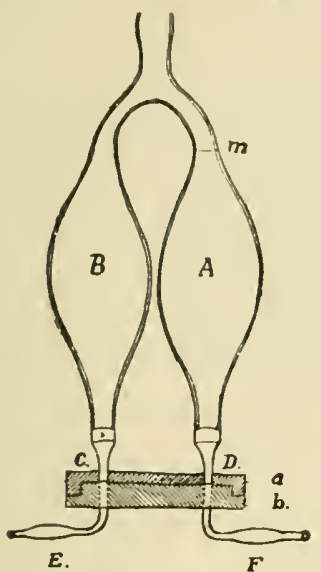

Fig. 47.-Stromuhr of Ludwig and Dogiel. $A, B$, glass bulbs; $a$, a metal disc, to which $A$ and $G$ are attached, and which can be rotated on the disc $b ; E, F$, cannule attached to $b$, and connected with the peripheral and central ends of a divided blood. vessel. At the beginning of the experiment, $A$ and the junction between $A$ and $B$ are filled with oil; $B$ is filled with physiological salt solution or defibrinated blood: $a$ being turned into the position shown in the figure, the blood passes through $F$ and $D$ into $A$, and the oil is forced into $B$. As soon as the blood has reached the mark $m$, the disc $a$, with the bulbs, is rapidly rotated, so that $C$ is now opposite $F$. The blood now passes into $B$, and the oil is again driven into $A$. When the oil has reached $D$, reversal is again made, and so on.

deflection bears a relation to the velocity of the stream (Vierordt's homatachometer; Chauveau and Lortet's much more perfect dromograph) (Fig. 49).

4. Pitot's Tubes. - If two vertical tubes, $a$ and $b$, of the form shown in Fig. 48 , be inserted into a horizontal tube in which liquid is flowing in the direction of the arrow, the level will be higher in a than would be the case in an ordinary side-tube without an elbow; in $b$ it will be lower. For the moving liquid will exert a push on the column in $a$, and a pull 
on that in $b$. The amount of this push and pull will vary with the velocity, so that a cliange in the latte $r$ will correspond to an alteration in the difference of level in the two tubes. Instruments on this prin. ciple have been constructed by Marey and Cybulski, the former registering the movements of the two columns of blood by connecting the tubes to tambours provided with writing levers, the latter by plioto-

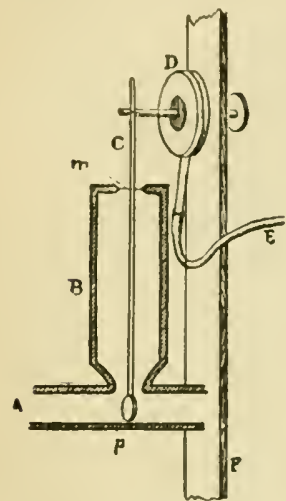

Fig. 49. - Chauveau's Dromograph. A, tube connected with blood. vessel; B, metal cylinder in communica. tion with $\mathrm{A}$. The upper cnd of $\mathrm{B}$ has a hole in the centre, which is covered by a mem. brane, $m$, through which a lever, $C$, passes; $C$ has a small disc, $p$, at its end, which projects into the lumen of $A$, and is de. flected in the direction of the blood-stream through A. The deflection is registered by a recording tambour in communication by the tube $\mathrm{E}$ with a tambour D, the flexible membrane of which is con. nected with the lever or pendulum $\mathrm{C}$. grapliy (Fig. 5o).

5. The electrical method, described on p. 135 , for the measurement of the circulation time, can also be applied to the estimation of the mean velocity of the blood between two cross-sections of the arterial path whicli are separated by a sufficient distance. For example, salt solution can be injected into the left ventricle or the beginning of the aorta, and the interval which it takes to reach a pair of electrodes in contact witl, say, the femoral artery determined. Knowing the distance between the point of injection and the electrodes, we can then calculate the mean velocity.

6. In the calorimetric method of measuring the quantity of blood which passes through such parts as the liands (or fect) in man, the flow is deduced from the quantity of heat given off by the part in a given time, and the difference between the temperatures of the blood entering and leaving the part. The hands are immersed in a large bath of water (a few degrees below arterial blood temperature) for a sufficient time to permit any change of temperature of the parts due to the difference in temperature between them and the water to be established. The hands are then rapidly transferred to calorimeters previously filled with water at the same temperature as that of the bath. All the heat henceforth given off can be assumed to be due to the cooling of the blood passing through the hands, since the small amount of lieat produced in the resting hands is negligible for this purpose. The temperature of the arterial blood at the wrist is taken as $0.5^{\circ} \mathrm{C}$. below that of the rectum, this being the relation actually found in a normal man.* Tlic temperature of the venous blood leaving the hand is taken as that of the calorimeter, since it has been found that blood withdrawn from the liand veins by puncture, and collected with suitable precautions to prevent loss of heat as far as possible and to permit the calculation of the unavoidable loss, has a temperature only a negligible fraction of a degree above that of the bath in which the hand is immersed. The flow in grammes per minute is obtained from the formula $\mathrm{Q}=m-\frac{\mathrm{H}}{\left.\mathrm{T}-\mathrm{T}^{1}\right)} \cdot \frac{\mathrm{I}}{\mathrm{s}}$, where $\mathrm{Q}$ is the quantity of blood, $\mathrm{H}$ the number

* The temperature of the arterial blood at the wrist was assumed to be the calorimeter temperature at which the calorimeter neither loses heat to the hand nor $g$ sins heat from it. If the heat production in the resting hand is negligible, this must correspond to the temperature of the entering blood. 
of small calories (gramme-calories) given off in $m$ minutes, $T$ the temperature of the blood entering the luand, $T^{2}$ the temperature of the blood leaving the laand, and $s$ the specific heat of lood $(0.9)$. For purposes of comparison the volume of the hands is measured, and the blood-flow expressed in grammes per $I$ on c.c. of liand per minute. Further details are given in tlie Practical Excrcises (p. 2 I9). Iig. 5 I shows one of the calorimeters on its adjustable stand. The collar of thick felt which fits closely around the wrist, and prevents loss of heat from the orifice through which the hand is inserted, is shown standing on the top of the calorimeter, as also the thermometer with the small sliding lens, or 'reader.' In Fig. 108, p. 220, the position of the subject with hands in the calorimeters is shown.

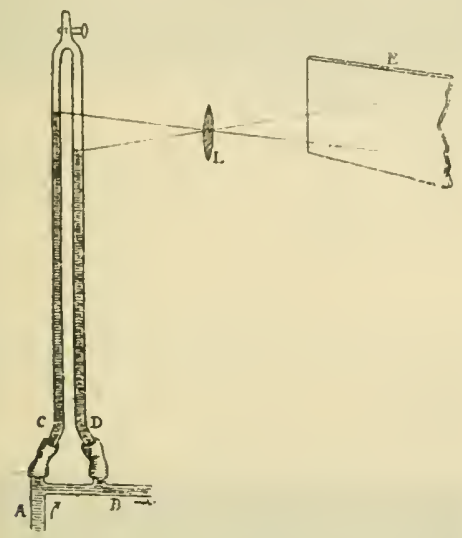

Fig. 50,-Cybulski's Arrangement for recording Variations in the Velocity of the Blood. A, tube connected with central, B with peripheral, end of divided bloodvessel. The blood stands higher in the tube $\mathrm{C}$ than in $\mathrm{D}$. A beam of light passing through the meniscus in both tubes is focussed by the lens $\mathrm{L}$ on the travelling photographic plate $\mathrm{E}$. The velocity at any moment is deduced from the height of the meniscus in the two tubes $C$ and $D$.

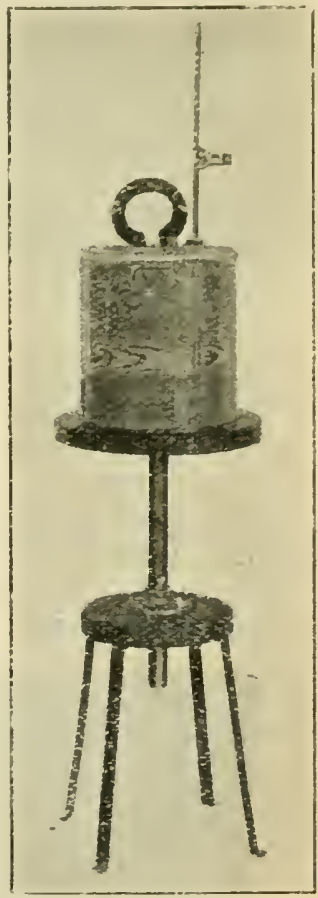

Fig. 51.-Calorimeter with stand for measuring blood-flow in hand.

Of these methods, 3 and 4 are alone suited for the study of the velocity-pulse, that is, the change of velocity occurring with every beat of the heart. The curves obtanied by Chauveau's dromograph show a general agreement with blood-pressure tracings taken by a spring manometer, and with records of the external pulse obtained by a sphygmograph. There is a primary increase of velocity corresponding with the ventricular systole, and a secondary increase corresponding with the dicrotic wave (Fig. 54). Like all the other pulsatory phenomena, the velocity-pulse disappears in the capillaries, and is only present under exceptional circumstances in the veins. 
Fick, from a comparison of sphygmographic and plethysmographic tracings (p. I2S), taken simultaneously from the radial artery and the hand, has demonstrated that in man the velocity-pulse exhibits

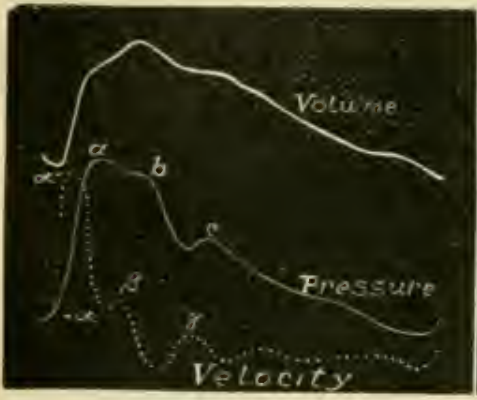

Fig. 52.

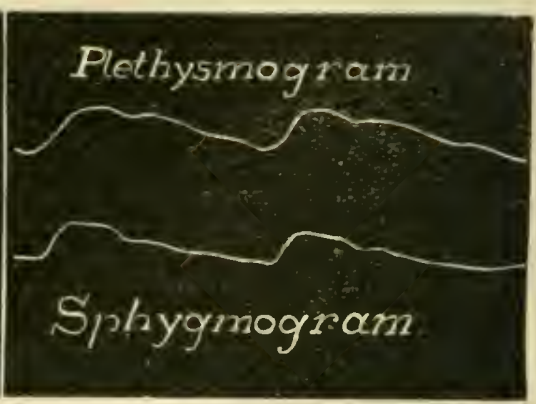

Fig. 53 .

Fig. 52.-The highest of the three curves is a plethysinographic record taken from the hand; the second curve is a sphygmogram taken simultaneously from the corresponding radial artery; the lowest (interrupted) curve is the curve of velocity deduced from a comparison of the first two (Fick).

Fig. 53.- Simultaneous plethysmographic and sphygmographic tracings.

the same general characters as in animals (Figs. 52 and 53). And v. Kries has confirmed Fick's conclusions by actual records of the velocity-pulse obtained by means of an arrangement called a gas tachograph (Fig. 55).

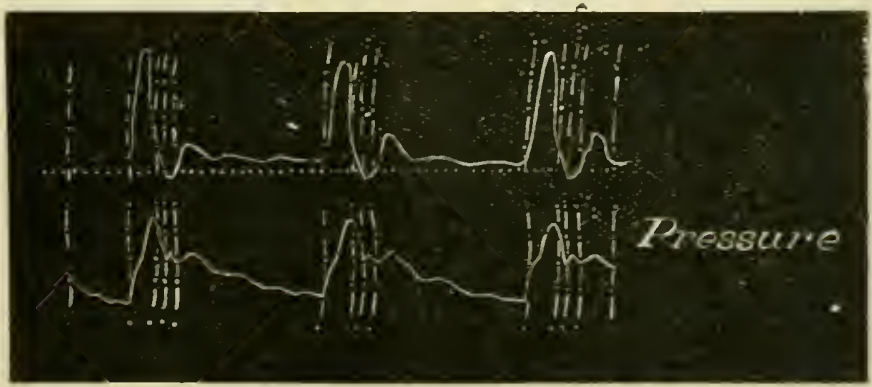

IFig. 54--Simultaneuus Tracings of the Velocity (Upper Curve) and l'ressure (Lower Curve) (Lortet). The tracings were taken from the carotid artery of a horse, The curve of velocity was obtained by the dromograph. The dicrotic wave is marked on it. The slightly curved ordinates drawn through the curves indicatc corresponding points.

This consists of a plethysmograph connecterl with the tube of a gasburner. When the part enclosed in the plethysmograph expands, air issues from the connecting tube, and causes an increase in the height of the flame. When the part shrinks, air is drawn in from the flame, which is depressed. Since the speed of the blood in the veins may be considered constant during the time of an experiment, the rate at which 
the volume of the part alters at any moment is a measure of the pulsatory change of velocity in the arteries of the part. And by pliotographing the movements of the flame on a travelling sensitive surface, the velocity-pulse is directly recorded.

The mean velocity, like the mean blood-pressure, is more variable in the large arteries near the heart than in the smaller and more distant arteries.

Dogiel found in measurements taken with the stromulir (a good instrument for the estimation of mean speed), within a period of $t w o$ minutes, velocities ranging from over $200 \mathrm{~mm}$. to under Ioo $\mathrm{mm}$. per second in the carotid of the

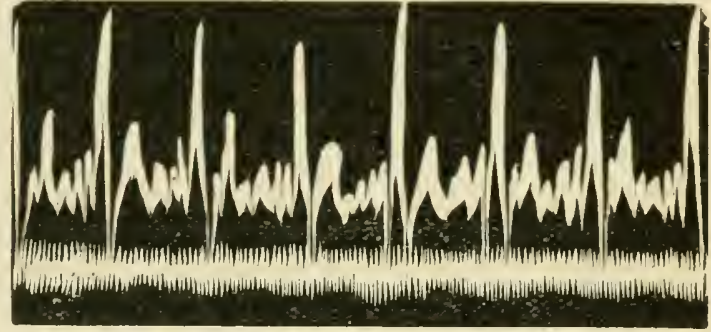

Fig. 55.-Photographic Record of the Velocity-Pulse obtained by the Gas Tachograph (v. Kries). The upper curve is the photographic representation of the move. inents of the flame, and corresponds to the curve of velocity.

rabbit, and from over $500 \mathrm{~mm}$. to less than $25^{\circ} \mathrm{mm}$. in the carotid of the dog. Chauveau, with the dromograph, found the velocity in the carotid of a horse to be $520 \mathrm{~mm}$. per second during systole, I50 $\mathrm{mm}$. during the pause, $220 \mathrm{~mm}$. during the period of the dicrotic wave.

It is probable, however, that, if these numbers are at all accurate for bloodvessels in the immediate neighbourhood of the heart, there must be a rapid diminution in the velocity even while the arteries are still of considerable calibre. For it has been found by the electrical method that, in anæsthetized dogs at any rate, as is shown in the following table, the mean velocity between the origin of the aorta and the crural artery in the middle of the thigh is usually less than Ioo $\mathrm{mm}$. per second.

\begin{tabular}{|c|c|c|c|c|c|c|}
\hline $\begin{array}{c}\text { No. of } \\
\text { Experi- } \\
\text { ment. }\end{array}$ & $\begin{array}{l}\text { Body- } \\
\text { weight } \\
\text { in Kilos. }\end{array}$ & $\begin{array}{l}\text { Distance } \\
\text { between Point } \\
\text { of lnjection and } \\
\text { Electrodes, } \\
\text { in Millimetres. }\end{array}$ & $\begin{array}{l}\text { Average Time be- } \\
\text { tween Injection } \\
\text { and Arrival of the } \\
\text { Salt Solution, in } \\
\text { Seconds. }\end{array}$ & $\begin{array}{c}\text { Average } \\
\text { Pulse-rate } \\
\text { per Minute. }\end{array}$ & $\begin{array}{l}\text { Average } \\
\text { Velocity } \\
\text { per } \\
\text { Secord, } \\
\text { in Milli- } \\
\text { metres. }\end{array}$ & $\begin{array}{c}\text { Average } \\
\text { Distance } \\
\text { traversed per } \\
\text { Heart-beat, } \\
\text { in Milli- } \\
\text { metres. }\end{array}$ \\
\hline $\begin{array}{l}\text { I. } \\
\text { II. } \\
\text { III. } \\
\text { IV. } \\
\text { V. }\end{array}$ & $\begin{array}{l}34: 55 \\
17 \cdot 5 \\
14 \cdot 99 \\
10.32 \\
7 \cdot 165\end{array}$ & $\begin{array}{l}420 \\
495 \\
400 \\
470 \\
330\end{array}$ & $\begin{array}{l}4 \cdot 62 \\
5 \cdot 7 \\
5 \cdot 0 \\
7 \cdot 12 \\
7 \cdot 83\end{array}$ & $\begin{array}{c}\text { ro5 } \\
69 \\
\text { Io } 2 \\
74.5 \\
46 \cdot 3 \\
\text { (weak beat) }\end{array}$ & $\begin{array}{l}90 \cdot 9 \\
86 \cdot 8 \\
80 \cdot 0 \\
72 \cdot 9 \\
42 \cdot 1\end{array}$ & $\begin{array}{l}51 \cdot 9 \\
75 \cdot 4 \\
47 \cdot 0 \\
58 \cdot 7 \\
54 \cdot 5\end{array}$ \\
\hline
\end{tabular}

In $\mathrm{I}$. the injecting cannula was in the descending part of the thoracic aorta, in V. at the very origin of the aorta, and in II., III., and IV. in the left ventricle. 
As to the speed of the blood in the arteries of inan, our data are insufficient for more than a loose estimate. But it does not seem likely that the mean velocity of a particle of blood in moving from the heart to the femoral artery can exceed $550 \mathrm{~mm}$. per second for the whole of its path. This would correspond to rather more than a third of a mile per hour. In the arch of the aorta the average speed may be twice as great. 'The rivers of the blood ' are, even at their fastest, no more rapid than a sluggish stream. A red corpuscle, even if it continued to move with the velocity with which it set out through the aorta, would only cover about 15 miles in twentyfour hours, and would require five years to go round the world.

The average flow through the hands of a healthy young man, as determined in eighteen experiments on different dates ranging over two years, at room temperatures varying from $19^{\circ}$ to $27^{\circ} \mathrm{C}$., was I2.8 grammes per roo c.c. of hand per minute for the right hand, and r 2.3 grammes for the left. Ten of the observations on this man are condensed in the table.

\begin{tabular}{|c|c|c|c|c|c|c|c|}
\hline \multirow{2}{*}{\multicolumn{2}{|c|}{ Date. }} & & \multicolumn{3}{|c|}{ Temperature of- } & \multicolumn{2}{|c|}{$\begin{array}{l}\text { Blood flow in Grammes } \\
\text { per roo c.c. of Hand } \\
\text { per Mlinute. }\end{array}$} \\
\hline & & & Room. & $\begin{array}{l}\text { Arterial } \\
\text { Blood. }\end{array}$ & Calorim. & Right. & Left. \\
\hline November 30 , xgro & - & - & $20 \cdot 2$ & $36 \cdot 6$ & $28 \cdot 0$ & $x 0 \cdot 1$ & 9.4 \\
\hline December 22 , I9ro & - & - & $2 I \cdot I$ & $36 \cdot 8$ & $29 \cdot 7$ & $\times 3.7$ & $12 \cdot 5$ \\
\hline February I, I9II & - & - & $22 \cdot 8$ & $36 \cdot 9$ & $30 \cdot 3$ & I 2.6 & $12 \cdot 7$ \\
\hline March I7, x9x & - & - & $2 I \cdot x$ & $36 \cdot 6$ & $29 \cdot 9$ & $\mathrm{II} \cdot 8$ & $I x \cdot 3$ \\
\hline May $24, x 9 x x-$ & - & - & $27 \cdot 0$ & $36 \cdot 8$ & $30 \cdot 9$ & I $8 \cdot 5$ & $17 \cdot 5$ \\
\hline November 3, I9II & - & - & $25 \cdot x$ & $36 \cdot 7$ & $30 \cdot 0$ & I $2 \cdot \mathrm{I}$ & $x+7$ \\
\hline November 9, I9I I & - & - & $24^{\circ} 1$ & $36 \cdot 7$ & $30 \cdot 8$ & $13 \cdot 7$ & $12 \cdot 5$ \\
\hline November I 5 , I 9 II & - & - & $25 \cdot 2$ & $36 \cdot 8$ & $30 \cdot 8$ & $14 \cdot 0$ & 13.8 \\
\hline December $x \mathrm{I}$, I9II & - & - & $24 \cdot 5$ & $36 \cdot 6$ & $3 I \cdot x$ & $x \geq \cdot 4$ & I $2 \cdot 0$ \\
\hline March 26, I9I 3 & - & - & $24 \cdot 5$ & $36 \cdot 6$ & $3 I \cdot 5$ & 14.7 & $x 5^{\cdot x}$ \\
\hline
\end{tabular}

Since the great function of the circulation in the skin is the regulation of the temperature of the body (see Chapter XII.), the blood-flow in the hands is, of course, much influenced by the external temperature. Thus, by far the greatest flow in the above table corresponds to the high room temperature of $27^{\circ} \mathrm{C}$. With a given external temperature, the degree of humidity of the air also affects the flow. Under similar conditions of external temperature and daily routine, including diet, the hand flow in one and the same individual does not vary greatly' when measured at about the same hour on different days. Different individuals, when tested under apparently similar conditions, show a greater range in the blood-flow. Some normal persons know and say that their hands are habitually cool or cold; others, like the man on whom the above results were obtained, that their hands are habitually warm. The former may be expected to show a relatively small, and the latter a relatively large, flow of blood through the hands. It is 
possible that such habitual differences are associated with differences in the total heat value of the food consumed or in the proportions of the various food substinces, especially of proteins (see p. 605). for it is well known that even persons engaged in the same work, and living under similar external conditions, may differ greatly in their dictetic habits, both as to quantity and quality of the food. And since tlic cutancous circulation is by far the most important factor in the loss of heat from the body, the hearty caters, other things being equal, may be expected to have the largest blood-flow through parts like the hands. The importance of the flow through the skin in the total hand flow is illustrated by the fact that the flow per unit of volume through the distal half of the hand, which, of course, has a large surface in proportion to its volume, is considerably greater than through the hand as a whole. For the forearm the flow per roo c.c. is, in its turn, much less than in the hand (Hiewlett). In the foot the blood-flow, as estimated by the calorimetric method, is smaller per unit of volume of the part than in the hand, the ratio of foot flow to hand flow per Ioo c.c. of the part usually lying in normal persons between $I$ to 3 and I to 2 . This is largely due to the proportionally greater proportion of skin in the hand, as well as to the smaller proportion of bone, which has not an active circulation. In the sitting position the following results were obtained for the flow in the feet on the person whose hand flows have been given above:

\begin{tabular}{|c|c|c|c|c|c|c|c|}
\hline \multirow{2}{*}{ Date. } & & & \multicolumn{3}{|c|}{ Temperature of - } & \multicolumn{2}{|c|}{$\begin{array}{l}\text { Blood-flow in Grammes } \\
\text { per too c.c. of Eoot } \\
\text { per Minute. }\end{array}$} \\
\hline & & & Room. & $\begin{array}{c}\text { Arterial } \\
\text { Blood. }\end{array}$ & Calorim.| & Right. & Left. \\
\hline May 2, I9I I & - & 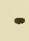 & $25^{\cdot 2}$ & $36 \cdot 8$ & $30 \cdot 4$ & $3 \cdot 5$ & 一 \\
\hline May I8, I9I I - & - & - & $26 \cdot 4$ & $36 \cdot 9$ & $3 I \cdot 4$ & $3 \cdot 5$ & - \\
\hline June I7,I91 I - & - & - & $2 I \cdot 8$ & $37^{\circ} \mathrm{O}$ & $30 \cdot 6$ & $4 \cdot 9$ & $4 \cdot 2$ \\
\hline $\operatorname{March} 26,1913$ & - & - & $24 \cdot 5$ & $3^{6 \cdot 5}$ & $3 I \cdot 4$ & $3 \cdot 9$ & $4^{\cdot} \cdot I$ \\
\hline
\end{tabular}

The great variations in the vascularity of different organs and parts, as revealed by the examination of injected specimens or by inspection of the organs during life, indicate that there must be great differences in the blood-flow. Observations with the stromuhr in animals have shown that this is the case. The following list gives the number of c.c. of blood passing per minute through roo grammes of organ, according to the results of Burton-Opitz, Tigerstedt, and other observers:

\begin{tabular}{|c|c|c|c|c|}
\hline & & \\
\hline \multicolumn{3}{|c|}{ Skeletal muscle } & ... & 12 \\
\hline Head & .. & .. & .. & 20 \\
\hline Stomach & & • & . & $2 I$ \\
\hline Liver (art & al) & •. & .. & 25 \\
\hline Intestines & .. & .. & .. & 31 \\
\hline Spleen & $\ldots$ & .. & . & $5^{8}$ \\
\hline
\end{tabular}

$\begin{array}{lllr}\text { Liver (venous) } & \ldots & 59 \\ \text { Liver (total) } & \ldots & \ldots & 84 \\ \text { Brain .. } & \ldots & \ldots & \text { I } 36 \\ \text { Kidney } & \ldots & \ldots & 150 \\ \text { Adrenal } & \ldots & \ldots & 500 \\ \text { Thyroid gland } & \ldots & 560\end{array}$

The Volume-Pulse.-When the pulse-wave reaches a part it distends its arteries, increases its volume, and gives rise to what may be called the volume-pulse. 
This may be readily recorded by means of a plethysmograpls, an instrument consisting essentially of a chamber with rigid walls which enclose the organ, the intervening space being filled up with liquid (Fig. 56). The movements of the liquid are transmitted either through a tube filled with air to a recording tambour, or directly to a piston or float acting upon a writing lever. Special names have been given to plethysmographs adapted to particular organs; for example, Roy's oncometer for the kidney. The method has been successfully applied to the investigation of circulatory changes in man, a finger, a hand or an entire limb being enclosed in the pletlysmograpls. With a fairly. sensitive arrangement, every beat of the heart is represented on the tracing by a primary clevation and a dicrotic wave (Fig. 57).

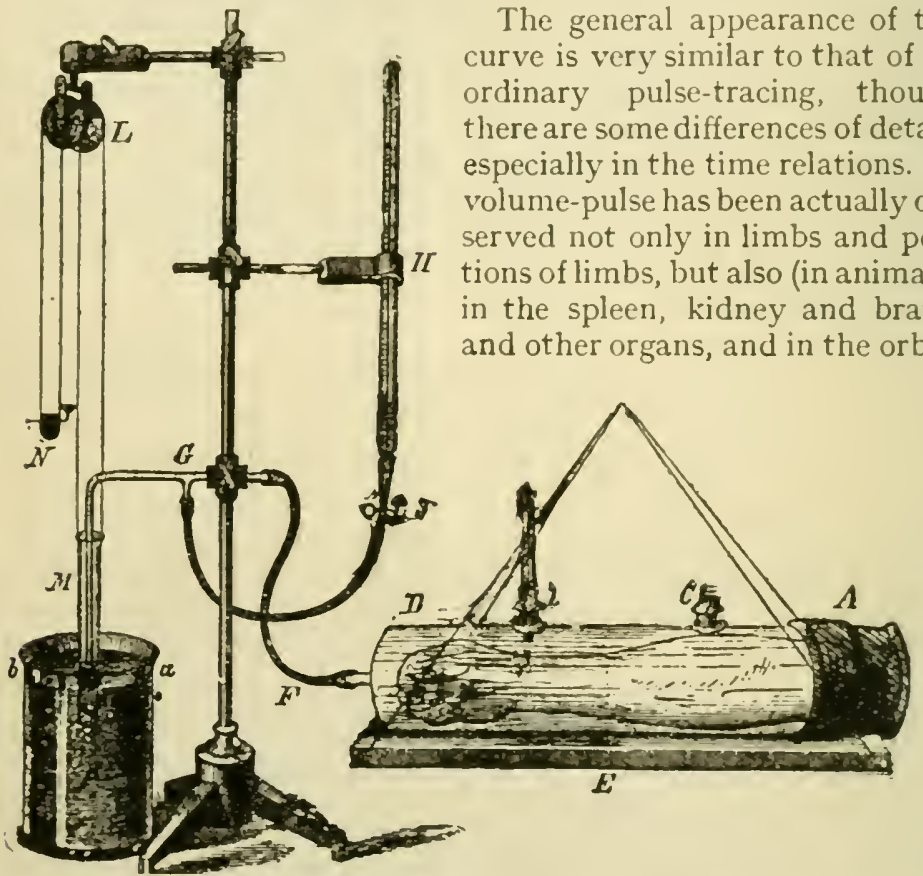

Fig. 56.-Plethysmograph (Mosso. M, balanced test-tube, in communication with the glass vessel, $D$, which contains the arm, escape of water being prevented by the rubber cuff, $A$. When water passes from vessel $D$ to $M$, or from $M$ to $D$, $M$ moves down or up, and its movements are recorded by the writing-point.$V$. $M$ is steadied by the liquid in $P$, into which it dips.

The so-called cardio-pneumatic movements also constitute a volume-pulse, although of complex origin. This name is given to the rhythmical changes of pressure accompanying the beat of the heart, which can be detected in the air of the respiratory passages when one nostril is connected with a recording tambour, or water manometer, the other nostril and the mouth being closed, and the respiration suspended in inspiration, with the glottis open. Or the 
mouth may be connected with the recording apparatus, the nostrils being closed. One factor in the production of these movements may be the change of blood-volume in the soft tissues of the mouth, nasopharynx, and perhaps also in the lower respiratory passages accompanying the heart-beat. Another factor, and a more influential one, is the rhythmical alteration of pressure caused directly by the alternate systole and diastole of the heart in the air contained in the lung-tissue surrounding it, which acts as a kind of air plethysmograph. One interesting way in which the cardio-pneumatic movements may reveal themselves is by a variation with each beat of the heart in the intensity of a note prolonged in singing, especially after fatigue has set in. Upon the whole, the air-pressure falls during systole, owing to the expulsion of blood from the chest, and rises during diastole. The main cardio-pneumatic movement is, therefore, a systolic inspiration and a diastolic expiration (Practical Exercises, p. 303).

Doubtless the weight of an organ would also show a pulse corresponding to the beat of the leart, and so would the temperature-at least. of the superficial parts. For the amount of heat given off by the blood

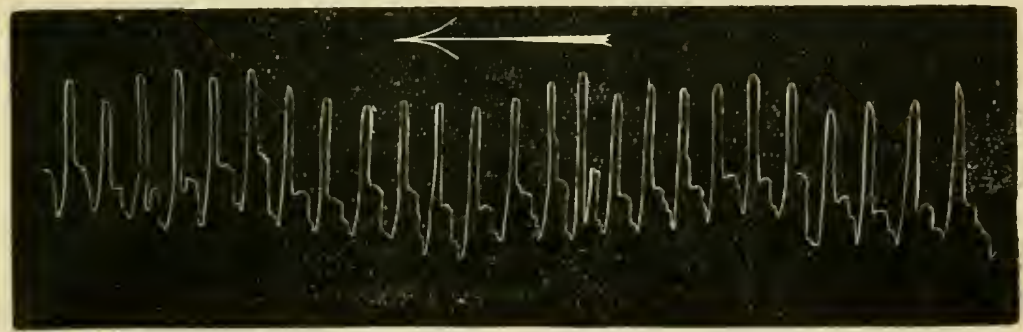

Fig. 57.-Plethysmograph Tracing frum Arm. The tracing was taken by means o1 a tambour connected with the plethysmograph. The dicrotic wave is distinctly marked.

to the skin increases with its mean velocity, and, therefore, although the difference may not in general be measurable, more heat is presumably given off during the systolic increase of velocity than during the diastolic slackening. And this, along with other considerations, suggests that, at any rate in certain situations and under certain conditions, there may even be a pulse of chemical change; that is, a slight and as yet doubtless inappreciable ebb and flow of metabolism corresponding to the rhythm of the heart.

The Circulation in the Capillaries.-From the arteries the blood passes into a network of narrow and thin-walled vessels, the capillaries, which in their turn are connected with the finest rootlets of the veins. Physiologically, the arterioles and venules must for many purposes be included in the capillary tract, but the great anatomical difference-the presence of circularly-arranged muscular fibres in the arterioles, their absence in the capillaries-has its 
physiological correlative. The calibre of the arterioles can be altered by contraction of these fibres under nervous influences; the calibre of the capillaries, although it varies passively with the bloodpressure, and is possibly to some extent affected by active contraction of the endothelial cells, cannot be under the control of vasomotor nerves acting on muscular fibres (but see p. I73).

Harvey had deduced from his observations the existence of channels between the arteries and the veins. Malpighi was the first to observe the capillary blood-stream with the microscope, and thus to give ocular demonstration of the truth of Harvey's brilliant reasoning. He used the lungs, mesentery and bladder of the frog. The web of the frog, the tail of the tadpole, the wing of the bat, the mesentery of the rabbit and rat, and other transparent parts, have also been frequently employed for such investigations. From the apparent velocity of the corpuscles and the degree of magnification,

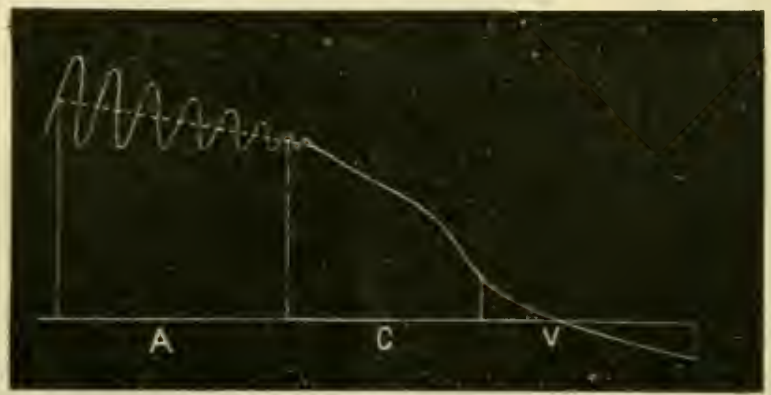

Fig. 53.-Diagram to Illustrate the Slope of Pressure along the Vascular System. A, arterial; C, capillary; $V$, venous tract. The interrupted line represents the line of mean pressure in the arteries, the wavy line indicating that the pressure varies with each heart-beat. The line passes below the abscissa axis (line of zcro or atmospheric pressure) in the veins, indicating that at the end of the venous system the pressure becomes negative.

it is easy to calculate the velocity of the capillary blood-stream. It has been estimated at from 0.2 to $0.8 \mathrm{~mm}$. per second in different parts and different animals.

The comparative slowness of the current and the disappearance of the pt:lse are the chief characteristics of the capillary circulation. The explanation we have already found in the great resistance of the narrow arterioles and the much-branched capillary vessels. Although the average diameter of a capillary is only about io $\mu$ ( 5 to $20 \mu$ in different parts of the body), the number of branches is so prodigious that the total cross-section of the systemic capillary tract has been estimated at 500 to 700 times that of the aorta. Such estimates are, of course, by no means exact.

The total cross-section of the vascular channel gradually widens as it passes away from the left ventricle. In the capillary region 
it undergoes a great and sudden increase. A part of this increase is to be attributed to the arterioles, which, although individually very narrow, have a total bed considerably greater than that of the arteries from which they spring. Where the arterioles pass into the capillaries proper, a further and a still greater and more abrupt increase in the bed occurs. At the venous end of this region the cross-section is again somewhat abruptly contracted, and then gradually lessens as the right side of the heart is approached; but the united sectional area of the large thoracic veins is greater than that of the aorta.

Attempts have bcen made to measure the blood-pressure in the capillaries by weighting a small plate of glass laid on the back of one of the fingers behind the nail, until the capillaries are just empticd, as shown by the paling of the skin (v. Kries), or by observing the height of a column of liquid that just stops the circulation in a transparent part (Roy and Graham Brown). The lastnamed observers found that a pressure of 100 to $150 \mathrm{~mm}$. of water (about 7 to II min. of $\mathrm{Hg}$ ) was needed to bring the blood to a standstill in the capillaries and veins of the frog's web; that is, about a third of the blood-pressure in the frog's aorta. The pressure in the capillaries at the root of the nail in man varies from

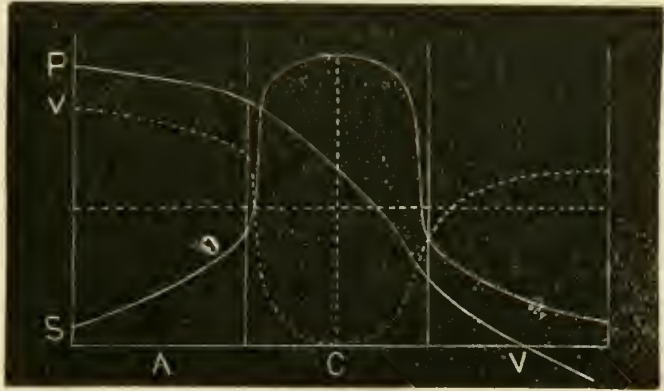

Fig. 59.-Relation of Blood-Pressure, Velocity, and Cross-Section. The curves $\mathrm{P}, \mathrm{V}$, and $\mathrm{S}$ represent the blood-pressure, velocity of the blood, and total crosssection respectively in the arteries $\mathrm{A}$, capillaries $\mathrm{C}$, and reins $V$.

30 to $50 \mathrm{~mm}$. of mercury, as estimated by the metliod of v. Kries. But the method is exposed to serious errors. The method of measuring the venous pressure described on p. 132 can also be applied to the eapillaries, and is somewhat more satisfactory.

Under certain conditions the pulse-wave may pass into the capillaries and appear beyond them as a venous pulse. Thus, we shall see that when the small arteries of the submaxillary gland are widened, and the vascular resistance lessened, by the stimulation of the chorda tympani nerve, the pulse passes through to the veins. And, normally, a pulse may be seen in the wide capillaries of the nail-bed-especially when they are partially emptied by pressureas a flicker of pink that comes and goes with every beat of the heart.

We have seen that the lateral pressure at any point of a uniform rigid tube through which water is flowing is proportional to the amount of resistance in the portion of the tube between this point and the outlet. In any system of tubes the sum of the potential and kinetic energy must diminish in the direction of the flow; and although the problem 
is complicated in the vascular system by the branching of the channel and the variation in the total cross-section, yet theory and experiment agree that in the larger arteries the lateral pressure diminishes but slowly from the heart to the periphery, the resistance bcing small compared with the resistance of the whole circuit. In the capillary region the vascular resistance abruptly increases; the velocity (and therefore the kinetic energy) abruptly diminishes, and the lateral pressure falls much more steeply between the beginning and the end of this region than between the heart and its commencement. In the veins only a small remnant of resistance remains to be overcome, and the lateral pressure must sink again rather suddenly about the end of the capillary tract. Fig. 59 shows by a rough diagram the manner in which the pressure, velocity and cross-section probably change from part to part of the vascular system.

The Circulation in the Veins.-The slope of pressure, as we have just explained, must fall rather suddenly near the beginning and near the end of the capillary tract. It continues falling as we pass along the veins, till the heart is again reached. In the right heart, and in the thoracic portions of the great veins which enter it, the pressure may be negative-that is, less than the atmospheric pressure. And since nowhere in the venous system is the pressure more than a small fraction of that in the arteries, its measurement in the veins is correspondingly difficult, because any obstruction to the normal flow is apt to artificially raise the pressure. A manometer containing some lighter liquid than mercury, such as water or a solution of sodium citrate or magnesium sulphate, is usually employed, so that the difference of level may be as great as possible. In the sheep the pressure was found to be $3 \mathrm{~mm}$. of mercury in the brachial, and about II $\mathrm{mm}$. in the crural vein. Burton-Opitz obtained the following pressures in dogs (of about ${ }_{5}$ kilos): left facial vein, 5.1 ; right external jugular, -0. I I ; central end of superior vena cava, -2.8 ; femoral vein, 5.4 ; renal vein, 10.9; portal vein, $8.9 \mathrm{~mm}$. of mercury.

Estimation of Venous Pressure in Man.--The venous pressure in man has been estimated by several observers with more or less satisfactory results. The best-known method is that of v. Recklinghausen. A circular rubber bag, with a central opening, is laid over the course of a vein, so that the vein can be observed through the opening, as in Fig. 6o. The bag is smeared with glycerin. A glass plate is laid over the opening and held firmly, so that the vein and the surrounding skin are in a closed chamber. The bag is provided with a side-tube, which connects it with a pump and a water manometer. By means of the pump air is forced into the bag till the vein is just scen to collapse. The pressure indicated on the manometer at this moment is taken as the pressure in the vein.

By means of a modification in this method, Eyster and Hooker have found that the pressure in the small veins of the arm or hand generally varies betwcen 3 and $10 \mathrm{~cm}$. of water. In conditions of congestion of the venous system the pressure may rise to $20 \mathrm{~cm}$. of water (say $15 \mathrm{~mm}$. of mercury) or more.

In making this measurement it is necessary to take account of the 
position of the vein, since the hydrostatic factor (p. 190) in the venous pressure is so important. Thus it is obvious that the pressure in the veins of the hand will be greater when it is hanging down than when it is raised to level of the heart or above it. Accordingly, the actual readings of the manometer inust always be corrected for the vertical distance between the vein and the heart, the height of a column of blood equal to this distance being deducted from or added to the manometer reading, according to whether the vein is below or above the heart level. For practical purposes the heart level is supposed to correspond to the lower end of the sternum (costal angle).

For the measurement of the pressure in the right auricle, the following simple and elegant method has been given by Gaertner, following a suggestion of Frey: He raises the arm of the sitting patient, and observes a small vein on the back of the hand. At the moment when the vein collapses the elevation of the arm is stopped, and the vertical distance between the vein and the heart measured. This expressed in millimetres of blood (i.e., approximately of water) is the pressure in the auricle, since the veins of the arm constitute manometer tubes connected with the auricle.

The venous pressure being so low, or, in other words, the potential energy which the systole of the heart imparts to the blood being so greatly exhausted before it reaches the veins, other influences begin here appreciably to affect the bloodstream:

I. Contraction of the Muscles. - This compresess the neighbouring veins,

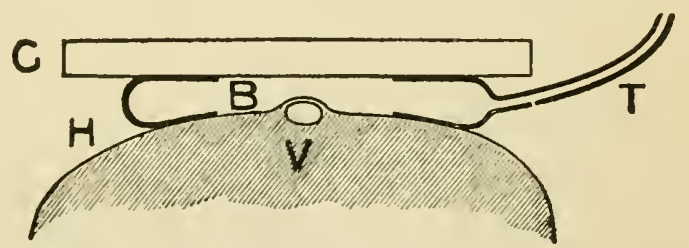

Fig. 60.-Diagram of Measurement of Venous Pressure (v. Recklinghausen). $\mathrm{H}$, back of hand, with $\mathrm{V}$, a vein; $B$, the rubber bag with central opening; $T$, tube leading from bag to manometer and pump; $G$, glass plate. and since the blood is compelled by the valves, if it moves at all, to move towards the heart, the venous circulation is in this way helped.

2. Aspiration of the Thorax.-In inspiration the intrathoracic pressure, and therefore the pressure in the great thoracic veins, is diminished, and blood is drawn from the more peripheral parts of the venous system into the right heart (p. 226).

3. Aspiration of the Heart. - When the heart after its contraction suddenly relaxes, the endocardiac pressure becomes negative, and blood is sucked into it, just as when the indiarubber ball of a syringe is compressed and then allowed to expand. But we cannot attribute any great importance to this; and, of course, it is only the relaxation of the right ventricle which could directly affect the venous circulation.

4. Every change of position of the limbs, as in walking, aids the venous circulation (Braune), and this independently of the muscular contraction. When the thigh of a dead body is rotated outwards, and 
at the same time extended, a manometer connected with the femoral vein shows a negative pressure of 5 to $10 \mathrm{~mm}$. of water. When the opposite movements are made, the pressure becomes positive.

It follows from the number of casually-acting influences which aftect the blood-flow in the veins that it cannot be very regular or constant. We have seen that in the great arteries there is a considerable variation of velocity and of pressure with every discharge of the ventricle, and although this variation is absent from the veins, since normally the pulse, due to the ventricular discharge, does not penetrate into them, the venous flow is, nevertheless, as a matter of fact, more irregular than the arterial. So that if it is difficult to give a useful definition of the term 'velocity of the blood ' in the case of the arteries, it is still more difficult to do so in the case of the veins. Where voluntary movement is prevented, one potent cause of variation in the venous flow is eliminated; and in curarized animals certain observers have found but little difference between the mean velocity in the veins and in the corresponding arteries. Others have found the velocity in the veins considerably less, which is indeed what we should expect from the fact that the average cross-section of the venous system is greater than that of the arterial system. Burton-Opitz, by means of a stromuhr, obtained a mean velocity of $\mathrm{I}_{47} \mathrm{~mm}$. per second in the external jugular vein of a r3-kilo dog.

To sum up, we may conclude that, upon the whole, the blood passes with gradually-diminishing velocity from the left ventricle along the arteries; it is greatly and somewhat suddenly slowed in the broad and branching capillary bed; but the stream gathers force again as it becomes more and more narrowed in the venous channel, although it never acquires the speed which it has in the aorta.

Venous Pulse.-To complete the account of the circulation in the veins, it may be recalled that, in addition to the venous pulse described on p. $13 \mathrm{r}$, which, as an occasional phenomenon, may travel through widened arterioles and capillaries from the arteries into the veins, and therefore in the direction of the blood-stream, a so-called venous pulse, travelling from the heart against the bloodstream and depending on variations of pressure in the right auricle, may be detected in the jugular veins in healthy persons, and more distinctly in certain disorders of the circulation, where indeed it may be evident at a greater distance from the heart-for example, over the liver as the so-called liver pulse. In animals a venous pulse of this nature has been demonstrated in the venæ cavæ, the jugular vein, and with a delicate manometer even in the large veins of the limbs. It moves with a speed of I to 3 metres a second (Morrow). It is most easily observed in the jugular veins in man, because of their proximity to the heart. We have already pointed out the significance of the study of this venous pulse for the analysis 
of cardiac events (p. 100).

A jugular venous pulse of a perfectly different origin is seen in cases of incompetence of the tricuspid valve. Here the chief elevation is synchronous with the ventricular systole, and is caused by the regurgitation of blood from the right ventricle through the auricle into the veins. The so-called 'communicated venous pulse 'is simply due to the proximity of some large artery, especially when enclosed in a common sheath, whose pulsations are directly transmitted to the vein. The changes of pressure in the great veins accompanying the respiratory movements (p. 290) are also sometimes spoken of as a venous pulse, but they are produced in a different way-namely, by the rhythmical alteration in the intrathoracic pressure, which alternately favours and hinders the venous return to the heart.

The Circulation-Time.-Hering was the first who attempted to measure the time required by the blood, or by a blood-corpuscle, to complete the circuit of the vascular system. He injected a solution of potassium ferrocyanide into a vein (generally the jugular), and collected blood at intervals from the corresponding vein of the opposite side. After the blood had clotted, he tested for the ferrocyanide by addition of ferric chloride to the serum. The first of the samples that gave the Prussian blue reaction corresponded to the time when the injected salt had just completed the circulation. This method was improved by Vierordt, who arranged a number of cups on a revolving disc below the vein from which the blood was to be taken. In these cups samples of the blood were received, and the rate of rotation of the disc being known, it was possible to measure the interval between the injection and appearance of the salt with considerable accuracy. Hermann made a further advance by allowing the blood to play upon a revolving drum covered with paper soaked in ferric chloride, and by using the less poisonous sodium ferrocyanide for injection.

Even as thus modified, the method laboured under serious defects. It was not possible to make more than a single observation on one animal, at least without allowing a considerable interval for the elimination of the ferrocyanide, and, further, the method was unsuited for the estimation of the circulation-time in individual organs. In both of these respects the more recently introduced electrical method presents considerable advantages; for by its aid we can not only obtain satisfactory measurements of the circulation-time in such organs as the lungs, liver, kidney, etc., but we can repeat them an indefinite number of times on the same animal.

A cannula, connected with a burette (or a Mariotte's bottle, or a syringe), containing a solution of sodium chloride (usually a $\mathbf{I}^{\cdot} 5$ to 2 per cent. solution), is tied into a vessel-say the jugular vein. Suppose that the time of the circulation from the jugular to the carotid is required-that is, practically the time of the lesser or pulmonary circulation. A small portion of one carotid artery is isolated, and laid on a pair of hook-shaped platinum electrodes, ${ }^{*}$ covered, except on the concave side of the hook, with a layer of insulating varnish. To further secure insulation, a bit of very thin sheet-indiarubber is slipped between the artery and the tissues. By means of the electrodes the

* The electrodes can easily be made by beating out one end of a piece of thick platinum wire to a breadth of 5 or $6 \mathrm{~mm}$., and then bending the flattened part into a hook, or by bending pieces of stout platinum foil. 
piece of artery lying between them, with the blood that flows in it, is connected up as one of the resistances in a Wheatstonc's bridge (p. 726). The secondary coil of a small inductorium, arranged for giving an interrupted current, and with a single Daniell or dry cell in its primary, is substituted for the hattery, and a telephone for the galvanometer, according to Kohlrausch's well-knowm method for the measurement of the resistance of electrolytes. It is well to have the induction machine set up in a separate room and connected to the resistance-box by long wires, so that the noise of the Neef's hammer may be inaudible. The bridge is balanced by adjusting the resistances until the sound heard in the telephone is at its mininum intensity, the secondary coil being placed at sucli a distance from the primary that there is no sign of stimulation of muscles or nerves in the neighbourhood of the electrodes

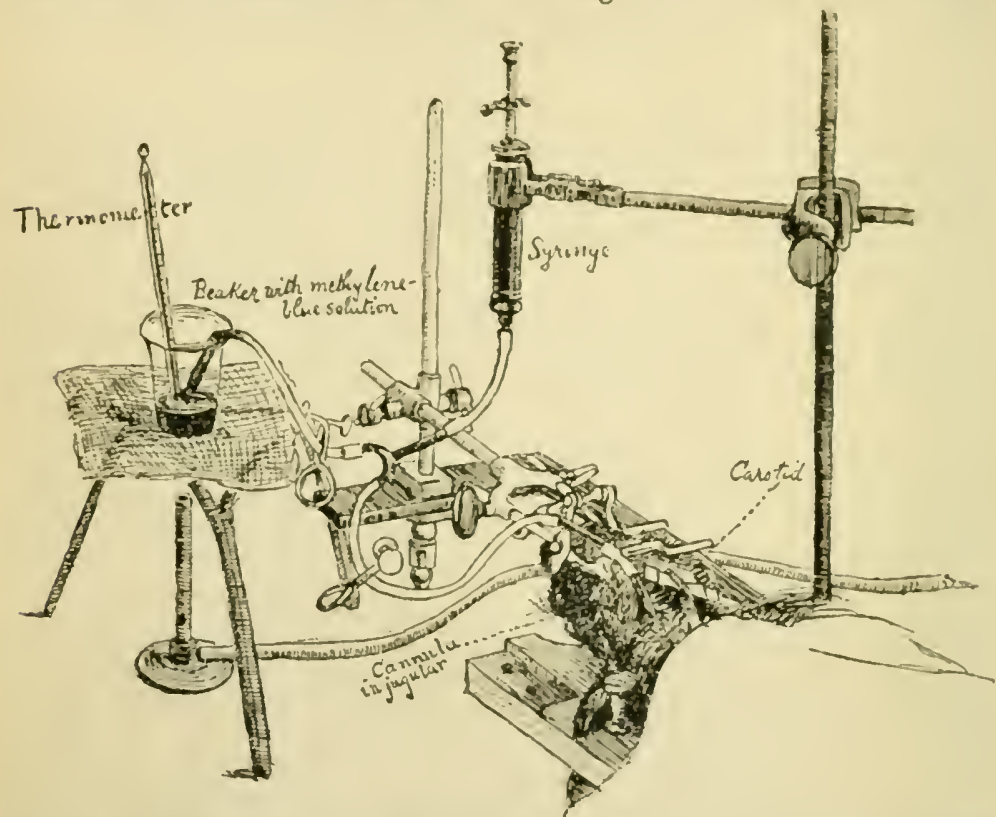

Fig. 61.-Measurement of the Pulmonary Circularion-Time in Rabbit by Injection of Methylene Blue.

when the current is closed. A definite, small quantity of the salt solution is now allowed to run into the vein by turning the stop-cock of the burette. It moves on with the velocity of the blood, and reaching the artery on the electrodes causes a diminution of its electrical resistance (p. 26). This disturbs the balance of the bridge, and the sound in the teleplione becomes louder. The time from the beginning of the injection to the alteration in the sound is the circulation-time between jugular and carotid. It can be read off by a stop-watch, or more accurately by an electric time-maker writing on a revolving drum (Fig. 62). Instead of the telephone a galvanometer may be used, the equal and oppositely directed induction slocks being replaced by a weak voltaic current, and the platinum by unpolarizable electrodes (p. 731). But this is less convenient. 
The circulation-time of an organ like tlie kidneycan be measured by adjusting a pair of electrodes under the renal artery and another under the renal vein, and reading off the interval required by the salt solution to pass from the point of injection first to the artery and then to the vein. The difference is the circulation-time througl the kidney.

For certain purposes, and particularly for measurements on small animals like the rabbit, or on organs whose vessels are too delicate to be placed on electrocles without the risk of scrious interference with the circulation, another method may be employed with advantage. It depends on the injection of a pigment, like methylene blue, which at first overpowers the colour of the blood and shows through the walls of the bloodvessels, but is soon reduced to a colourless substance (Fig. 6r). The details of the method are given in the Practical Exercises (p. 217.)

It may be said in general terms that in one and the same animal the time of the lesser circulation is short as compared with the total circulation-time, relatively constant, and but little affected by changes of temperature. In animals of the same species it increases with the size, but more slowly, and rather in proportion to the increase of surface than to the increase of weight.

Thus a dog weighing 2 kilogrammes had an average pulmonary circulation-time of $4^{\circ} 05$ seconds, while that of a dog weighing II 8 lilos was 8.7 seconds, and that of a dog with a body-weight of 18.2 kilos only $\mathrm{IO}^{\circ} 4$ seconds. It is probable that in a man the pulmonary circulationtime is not usually much less than 12 seconds, nor much more than I5 seconds.

The circulation-time in the kidney, spleen and liver is relatively long and much more variable than that of the lungs, being easily affected by exposure and changes of temperature (increased by cold, diminished by warmth).

In a dog of 13.3 kilos weight the average circulation-time of the spleen was I0.95 seconds; kidney, I3.3 seconds; lungs, 8.4 seconds. The circulation-time of the stomach and intestines is (in the rabbit) comparatively short, not exceeding very greatly that of the lungs, but it is lengthened by exposure. The circulation-time of the retina and that of the heart (coronary circulation) are the shortest of all.

The total circulation-time is properly the time required for the whole of the blood to complete the round of the pulmonary and systemic circulation. But there are many routes open to any given particle of blood in maling its systemic circuit. If it passes from the aorta through the coronary circulation it takes an exceedingly short route. If it passes through the intestines and liver, or through the kidney, or through the lower limbs, it takes a long route. So that to determine the total circulation-time by direct measurement we must know (I) the quantity of blood that passes on the average by each path in a given time, and (2) the average circulation-time of each path. If the average weight of blood in each organ be represented by $w_{1}, w_{2}, w_{3}$, etc.; and the average circulation-times by $t_{1}, t_{2}, t_{3}$, etc.; and $t$ be the total systemic circulation. time; then $w_{1} \frac{t}{t_{1}}, w_{\frac{1}{2} t_{2}}, w_{\frac{3}{3} t_{3}}$, etc., will represent the quantity of blood passing through each organ in $t$ seconds. since in the average circula- 
tion-time of an organ the whole of the blood in it at the beginning of the period of observation will have been exchanged for fresh blood. But the whole of the blood in the body, which we may call W. passes once round the systemic circulation in $t$ seconds. Therefore, $w_{1} \frac{t}{t_{1}}+w_{2} \frac{t}{t_{2}}+w_{3} \frac{t}{t_{3}}$, ctc., $=W$. In this equation everything can be de. termined by experiment except $t$, and therefore $t$ can le calculated. Adding $t$ to the pulmonary circulation-tinc, we arrive at the tota circulation-time.

Although our experimental data are as yet too meagre to make the calculation more than a rough approximation, it appears probable that in certain animals the total circulation-time is five or six times as great as the pulmonary circulation-time. If the same ratio holds good in man, the total circulation-time is unlikely to be much less than a minute

1

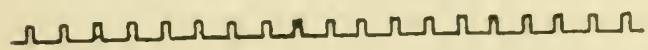
or much greater than a minute and a quarter. We shall see directly that this estimate is confirmed by data derived from a different source. In the meantime, we may use it provisionally to calculate the work done by the heart. Let us take for simplicity the total circulation-time as I minute in a 70 -kilo man, the quantity of blood as $4 \frac{1}{2}$ kilos, * and the mean pressure in the aorta as $150 \mathrm{~mm}$. of mercury. Lip to the time when the semilunarvalves areopened, the work done by the left ventricle is spent in raising the intraventricular pressure till it is sufficicnt to overcome the pressure in the aorta. If a vertical tube were connected with the left ventricle, the blood would rise till

the column was of the same weight as a column of mercury of equal section and $150 \mathrm{~mm}$. high. This column of blood would be about 192 metres in heiglit. If a reservoir were placed in communication with the tube at this height, a quantity of blood equal to that ejected from tlie ventricle would at each systole pass into the reservoir; and the work which the blood thus collected would be capable of doing, if it were allowed to fall to the level of the heart, would be equal to the work expended by the heart in forcing it up. Thus, in I minute the work of the left ventricle would be equal to that done in raising $4 \frac{1}{2}$ kilos of blood to a height

* The mean of the $5 \frac{1}{2}$ kilos given by most writers, and of the $3 \frac{1}{2}$ kilon obtained by Haldane and smith (p. 56). 
of 1.92 metres-that is, about 8.6 .4 kilogramme-metres; in 24 hours it would be, say, 12,450 kilogramme-metres. Taking the mean pressure in the pulmonary artery at one-third of the aortic pressure, we get for the daily work of the right ventricle about 4, I50 kilogramme-metres. The work of the two ventricles is thus about 16,600 kilogramme-metres, which is enough to raise a weight of nearly 4 pounds from the bottom of the decpest mine in the world to the top of its highest mountain, or to raise the man himself to $1 \frac{1}{2}$ times the lieight of the spire of Strasburg Catliedral, or twice the lieight of the loftiest 'sky'scraper' in New York. By friction in the bloodvessels this work is almost all changed into its equivalent of heat, nearly 40,000 gramme-calories (p. 688). Further, since the contraction of the lieart is always maximal (p. 154), and there is reason to believe that the quantity of blood ejected at a single systole by the left ventricle (being dependent upon the inflow from the pulmonary veins, and therefore upon the inflow into the right side of the heart from the systemic veins) varies widely, some of the mechanical effect of the contraction must be wasted when the quantity is less than the ventricle is capable of expelling.

Output of the Heart.--If $4 \frac{1}{2}$ kilos of blood pass through the heart in I minute with the average pulse-rate of 72 per minute, the quantity ejected by either ventricle with every systole will be $\frac{4500}{72}=62.5 \mathrm{grm}$., or a little less than 60 c.c. The output may be expressed in grammes or cubic centimetres per minute (the minute volume), or per second, or per beat. It has been measured in animals in several ways-e.g., by inserting a stromuhr (p. 12 I) on the course of the aorta, or by recording the variations in the volume of the heart, or, better, of the ventricles, by means of a plethysmograph (cardiometer of Henderson), in which the organ is enclosed. Another method, which does not entail the opening of the chest, is to allow a salt solution to run slowly, for a definite number of seconds, into the left ventricle through a tube passed into it from the carotid artery. A sample of the mixture of blood and salt solution is collected from a branch of the femoral artery, where its arrival is detected by the change of electrical resistance (p. 135). From the amount of salt solution which must be added to a normal sample of blood drawn before the injection to make its conductivity the same as that of the sample taken during the passage of the mixture, the quantity of blood with which the solution was mixed in the ventricle during the injection can be approximately determined. By this method it has been shown in a series of experiments on more than twenty dogs, ranging in weight from 5 to nearly 35 kilos, that the output of the left ventricle per kilo of body-weight per second diminishes as the size of the animal increases; and the relation between body-weight and output is such that in a man weighing 70 kilos we can hardly suppose that the ventricle discharges, during bodily rest, more than $105 \mathrm{grm}$. of blood per second, or 87 grm. (80 c.c.) per heart-beat with a pulse-rate of 72. Putting this result along with that deduced from the circulationtime, we can pretty safely conclude that the average amount of blood thrown out by each ventricle at each beat is not more than 70 or 80 c.c. Zuntz, from the quantity of oxygen absorbed by the blood in the lungs in a definite short time, and the difference between the oxygen content of samples of the arterial and venous blood, has estimated the output per beat at 60 c.c. But according to him this may be doubled during

- Since the blood on expulsion is moving with a certain velocity, an addition might be made for its kinetic energy. But this would only increase the total work by a small fraction (about I per cent.). 
severe muscular work, when, as a matter of fact, by the aid of the Röntgen-rays or by percussion of the chest, the volume of the lheart may be shown to be considerably increased. Tigerstedt, on the basis of stromuhr measurements in animals, puts the ventricular output per beat in man at 50 to 100 c.c.; Plesch, on the basis of gasometric observations on man, at 59 c.c. Recently Kroglı, using a gasometric method based on the absorption of nitrous oxide gas in the lungs, found that the minute volume during rest may vary between wide limits $(2.8$ to 8.7 litres of blood per minute, corresponding, with a pulse-rate of 70 , to 40 c.c. to 120 c.c. per beat). During muscular work there is a great and immediate increase, up to, it may be, $21 \cdot 6$ litres per minute. These great variations in the output of the ventricle depend primarily upon variations in the rate of return of the blood to the heart by the veins. According to Henderson, however, such great variations in the output per beat as are postulated by the majority of physiologists wlio have worked at the subject do not occur, and the fundamental variable is the rate of the beat.

In lealthy persons in whom the pulse-rate is permanently much below the normal (p. 107) the output of the ventricle per beat must, of course, be correspondingly increased. In a man with a pulse-rate always below 40 during rest in the sitting position, the flow in the hands was found to be normal in amount, and all the signs of a normal delivery of blood from the left ventricle were present. Here the output per beat must have been twice the usual amount during rest.

\section{Section IV.-The Heart-Beat in its Physiolog Cal RELATIONS.}

So far we have been considering the circulation as a purely physical problem. We have spoken of the action of the heart as that of a force-pump, and perhaps to a small extent that of a suctionpump too. We have spoken of the bloodvessels as a system of more or less elastic tubes through which the blood is propelled. We have spoken of the resistance which the blood experiences and the pressure which it exerts in this system of tubes, and we have considered the causes of this resistance, the interpretation of this pressure, and the physical changes in the vascular system that may lead to variations of both. But so far we have not at all, or only incidentally and very briefly, dealt with the physiological mechanism through which the physical changes are brought about. We have now to see that, although the heart is a pump, it is a living pump; that, although the vascular system is an arrangement of tubes, these tubes are alive; and that both leart and vessels are kept constantly in the most delicate poise and balance by impulses passing from the central nervous system along the nerves.

In many respects, and notably as regards the influence of nerves on it, we may look upon the heart as an expanded, thickened and rhythmically-contractile bloodvessel, so that an account of its innervation may fitly precede the description of vaso-motor action in general. 
'The Relation of the Heart to the Nervous System.-A very simple experiment is sufficient to prove that the beat of the heart does not depend on its connection witl the central nervous system, for an excised frog's heart may, under favourable conditions, of which the most important are a moderately low temperature, the presence of oxygen, and the prevention of evaporation, continue to beat for days. The maminalian heart also, after removal from the body, beats for a time, and indeed, if defibrinated blood be artificially circulated through the coronary vessels, for several or even many hours. But although this proves that the heart can beat when separated from the central nervous system, it does not prove that nervous influence is not essential to its action, for in the cardiac substance nervous elements, both cells and fibres, are to be found.

The Intrinsic Nerves of the Heart.-In the heart of the frog numerous nerve-cells occur in the sinus venosus, especially near its junction with the right auricle (Remak's ganglion). A branch from each vagus, or rather from each vago-sympathetic nerve (for in the frog the vagus is joined a little below its exit from the skull by the sympathetic), enters the heart along the superior vena cava

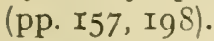

Running through the sinus, with whose ganglion-cells the true vagus fibres, or some of them, are believed to make physiological junction (p. 163), the nerves pursue their course to the auricular septum. Herc they form an intricate plexus, studded with ganglion-cells. From the plexus nerve-fibres issue in two main bundles, which pass down the anterior and posterior borders of the septum to end in two clumps of nerve-cells (Bidder's ganglia), situated at the auriculo-ventricular groove. These ganglia in turn give off fine nerve-bundles to the ventricle, which form three plexuses - one under the pericardium, another under the endocardium, and a third in the muscular wall itself, or myocardium. From the last of these plexuses numerous non-medullated fibres run in among the muscular fibres and end in close relation with them. Similar plexuses of nerve-fibres exist in the mammalian ventricle. But while scattered ganglion-cells are found in the upper part of the ventricular wall, most observers have been unable to demonstrate any either in the mammal or the frog in the apical half. In the rat's heart, according to the careful observations of Schwartz, true ganglion-cells are confined to an area on the posterior surface of the auricles, lying always under the visceral pericardium. Other writers, however, have stated that ganglion-cells do exist in the apex both of the cat's and of the frog's heart. In connection with the whole question it must be borne in mind that in other organs improved histological methods have brought typical nerve-cells to light in situations where they were not suspected or were denied to exist, and, further, that all investigators are not agreed upon the histological criteria by which ganglion-cells are to be distinguished.

Cause of the Rhythmical Beat of the Heart.-Scarcely any physiological question has excited greater interest for many years than the mechanism of the heart-beat. Several properties of the cardiac tissue ought to be distinguished in discussing this question: (I) Its 
automatism-i.e., its power of beating in the absence of external stimuli; (2) its rhythmicity-i.e., its power of responding to continuous stimulation by a series of rliythmically repeated contractions; (3) its conductivity-i.e., its power of conducting the contraction wave or the impulse to contraction once it has been set up; and (4) the power of co-ordination, in virtue of which the various parts of the heart beat in a regular sequence.

The excitability of the cardiac tissue-that is, its power of appropriate response (namely, by contraction) to a suitable stimulusdoes not particularly concern us here, since it is in no wise a property special to the heart. Only, as we shall see in the sequel, the timerelations of this excitability are of interest, for the existence of a refractory period-that is, an interval during which the cardiac muscle refuses to respond to excitation-throws light upon the rhythmicity of the heart-beat. The lonicity of the heart-i.e., its power of remaining contracted to a certain extent in the intervals between successive beats-is another property of great importance in certain aspects, but which only needs to be mentioned at present.

Automatism of the Heart-Beat-Neurogenic and Myogenic Hypotheses.-That the heart-beat is automatic is sufficiently shown by the fact that, as already mentioned, an excised and empty heart will go on beating for a time, for many hours or even for days in the case of cold-blooded animals. When blood, or even a suitable solution of such inorganic salts as exist in serum, is caused to circulate through the coronary vesscls of the excised heart of a warmblooded animal, it also continues to contract for a long time. In trying to understand the real significance of the automatic beat of the heart, physiologists have endea voured, first, to compare different portions of the heart as regards the degree in which they possess this property of automaticity; and, second, to associate, if possible, one or other of the active tissues that compose the organ, muscle, and nervous tissue, with this characteristic property. It cannot be pretended that a final answer to this question is possible at present. Nor is the historical controversy which it has occasioned perhaps as important in itself as the space usually devoted to it in textbooks might imply. Yet it is probable that the series of fundamental facts in the physiology of the heart elicited in the long discussion can be best presented, even for the purposes of the elementary student, as they were originally brought forward in the form of pros and cons, of arguments for and against the neurogenic or the myogenic hypothesis. There is good evidence that as in the amphibian heart the contraction starts in the sinus venosus, so in the mammalian heart it starts in the sinus tissue of the right auricle in the region of the sino-auricular node. Attempts have been made to demonstrate that the origination of the impulses which are afterwards conducted to all parts of the heart is normally confined to 
the node itself, and the sino-auricular node is by some authors denominated the pace-maker of the heart, the tissue which sets the pace for the rest of the organ and gives the time to auricles and ventricles alike. The experimental results, however, are by no means harmonious, some observers finding that destruction of the region of the node causes no change in the rate of the heart-beat, others that the beat is permanently slowed. But even were we in a position to sharply delimit a given region of the heart as the point at which the strong tendency to contraction inherent in the cardiac tissue as a whole first breaks into an actual beat, this would scarcel-y enable us to decide offhand where the cause of the automatism resides, in the muscular tissue or in the intrinsic nervous apparatus, because in nearly all animals hitherto investigated the muscular tissue, ganglion-cells, and nerve-fibres are inseparably intermingled. In Limulus, however, the horseshoe or king crab, the cardiac ganglion-cells are collected in a nerve-cord running longitudinally in the median line along the dorsai surface of the segmenter heart, and

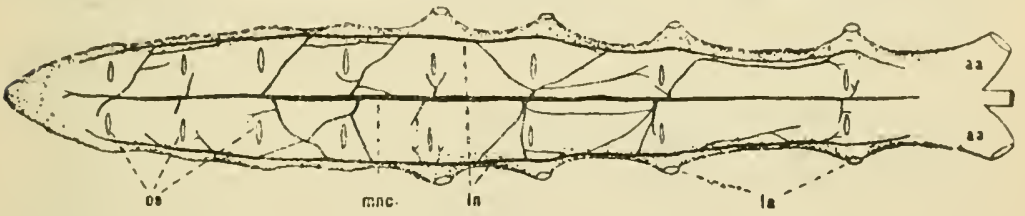

Fig. 63.-The Heart and the Heart Nerves of Limulus: Dorsal View (Carlson). (The heart is figured one-half the natural size of a large specimen.) $a a$, Anterior artery; $l a$, lateral arteries; ln, lateral nerves; mnc, median nerve-cord; os, ostia.

sending off at intervals branches to two lateral cords, and also branches which enter the heart muscle directly (Fig. 63). When the median nerve-cord is removed, as can be done without injuring the muscle, the heart ceases for ever to beat spontaneously. It still contracts when directly stimulated, mechanically or electrically, but the contraction never outlasts the stimulation. The automatic power therefore resides in the nerve-cord alone, and not in the muscle. The same is true of the rhythmical power, for excitation of the nerves that pass from the median cord to the muscle produces, ' not a rhythmical series of beats in the resting, and an acceleration of the rhyth $m$ in the pulsating heart, but a tetanus closely resembling that produced in skeletal muscle on stimulation of a motor nerve (Carlson). Conduction and co-ordination are also effected in this heart through the nervous mechanism, and essentially through the median nerve-cord; for section of the longitudinal nerves in any segment of the heart abolishes the co-ordination of the two ends of the heart on either side of the lesion, while division of the muscle in any segment does not affect the co-ordination. It is not permissible to transfer these results wholesale to higher hearts, and 
especially the conclusions as to rlyythm, conduction, and co-ordination. Nevertheless the Limulus heart affords one absolutely un ambiguous example of a heart whose rhythmical beat is sustained by nervous impulses. And in the case of the higher animals also facts may be adduced in favour of the neurogenic origin of the beat. The isolated auricular appendices of the mammalian heart, in which no ganglion-cells have been found, refuse to beat spontaneously. If in the frog we divide the sinus, which is conspicuously rich in ganglion-cells, from the lower portion of the heart, it continues to pulsate. A fragment from the base of the ventricle will go on contracting if it includes Bidder's ganglion, but not otherwise. We cut off the lower two-thirds of the frog's ventricle, the so-called apex preparation, which either contains no ganglion-cells or is relatively poor in them, and it remains obstinately at rest. Further, if, without actually cutting off the apex, we dissever it physiologically from the heart by crushing a narrow zone of tissue miclway between it and the auriculo-ventricular groove, we abolish for ever its power of spontaneous rhythmical contraction. The frog may live for many weeks, but in general the apex remains in permanent diastolc. It can be caused to contract by an artificial stimulus, but it neither takes part in the spontaneous contraction of the rest of the heart, nor does it start an independent beat of its own.

What can be simpler than to assume that the sinus beats because it has numerous ganglion-cells in its walls, and that the apex refuses to beat because it has comparatively few or none? Could we pick out the nerve-cells from the sinus, without injuring the muscular tissue, as easily as we can extirpate the median norve-cord in Limulus, we may well suppose that it would lose its power of automatic contraction. And althougls, if we pursue our investigations -a little farther, facts may emerge which seem to contradict the neurogenic hypothesis, the contradiction is usually only apparent. Let us inquire, for instance, what happens to the auricles and ventricle of the frog's heart when the sinus is cut off. The answer is that as a rule, while the sinus goes on beating, the rest of the heart comes to a standstill, in spite of the numerous ganglion-cells in the auricular septum and the auriculo-ventricular groove. Not only so, but if the ventricle be now severed from the auricles by a section carried through the groove, it is the former, poor in nerve-cells though it be, which will usually first begin to beat. We shall again have to discuss this experiment (p. I66). It, at any rate, cannot be interpreted as proving that the automaticity of the heart does not depend upon the presence of ganglion-cells. For although a portion of the heart rich in ganglion-cells may, under the circumstances mentioned, refuse for a time to beat, there is grood evidence that this is due either to a peculiar condition called inhibition into which the muscular tissue or the nerve-cells of the lower portions of the 
heart have been thrown by the first section, or more probably to the loss of the accustomed impulses from the sinus which normally: give the signal for the auricular contraction. A stronger argument in favour of the myogenic theory is the fact that the embryonic heart beats witl a regular rhythm at a time when as yet no ganglioncells have settled in its walls. But it may well be that this primitive automatic power of the cardiac muscle, absolutely necessary at first, since the early establishment of the circulation is essential for the development of the tissues in general and of the nervous system in particular, falls into abeyance when the intrinsic cardiac nervous mechanism is completed, or at least becomes subordinated to the latter. The advocates of the myogenic theory further state that the isolated bulbus aortæ of the frog, and even tiny fragments of it, will pulsate spontaneously, and that the same is true of small portions of the great veins which open into the sinus. The rhythmical contraction of the veins of the bat's wing has also been considered an argument in favour of myogenic automatism. In none of these cases, however, can the complete absence of ganglion-cells be considered satisfactorily demonstrated. The statement that a portion of the apex of the dog's ventricle continues for a considerable time to beat with a rhythm of its own when connected with the rest of the heart by nothing but its bloodvessels and the narrow isthmus of visceral pericardium and connective tissue in which they lie has not been confirmed by all observers. But even if it be accepted, it can hardly be used as a decisive argument against the neurogenic theory so long as the absence of ganglion-cells from such a ventricular strip has not been demonstrated.

The fact that under the influence of a constant stimulus portions of the heart can be made to beat rhythmically has been sometimes, though erroneously, brought forward as evidence of myogenic automatism. Thus the supposedly ganglion-free apex of the frog's heart, lifeless as it seems when left to itself, can be caused to execute a long and faultless series of pulsations when its cavity is distended with defibrinated blood or serum, or certain artificial nutritive fluids, or even physiological salt solution. The passage of a constant current through the preparation may also start a regular rhythm. But apart from the question whether nervous elements would not be subjected to the constant stimulus impartially with the muscular elements (and nerve-fibres, at any rate, are acknowledged to be present), the beat here produced ought not to be considered as an automatic beat, but as a contraction evoked by an external stimulus. Such experiments, in fact, throw no light upon the automatism of the heart, but prove clearly its rhythmicity-i.e., its power of responding to a continuous stimulus by regularly recurring contractions. While we are hardly at present in a position to discriminate sharply between the influence of constant stimulation 
upon the nervous and upon the muscular elements of the heart, and certainly not in a position to deny to the nervous dements the power of responding to such stimulation by rhythmical discharges, it cannot be doubted that the carcliac muscle itself possesses rhythmical power. One of the best proofs of this is derived from observations on the growth of tissue cultures containing fragments of heart muscle. A group of heart muscle cells which had beconc entirely separated from the rest of the culture continued to beat rhythmically although with a different rlythm from that of the original piece. No ganglion cells or nerve fibres could be seen in it. Rlythmical contraction was observed even in a single isolated heart muscle cell (Burrows). The power of rhythmical contraction is a property which also belongs to the smooth muscle of such tubes as the ureter, whose rhythmical contraction is affected by distension much as that of the lieart is, and in a smaller degree even to urdinary skeletal muscle, which can contract with a kind of rhythm under the stimulus of a certain tension and in certain saline solutions. But just as the primitive automatism of the cardiac muscle may lave become subordinated in the course of development to the antomatism of the nervous elements, so the primitive rhythmical power of the muscle may under ordinary conditions remain in abeyance and yet be capable of asscrting itself in favourable circumstances, and when the normal rhythmical impulses from the nervous apparatus are withdrawn. In any case, in the normally beating heart the opportunity for the exercise of the rhythmical power of the muscle does not arise, at least in the case of the lower portions of the heart. For the impulses which (in the frog's lreart), descending from the sinus, liberate the contraction of the auricles, and the impulses which, descending from the auricles, liberate the contraction of the sentricle, appear to be discrete, and not continuous; in uther words, the lower portions of the heart do not receive from the upper portions a continuous stream of stimuli to which they respond by rhythmical contractions, but a series of rhythmically repeated impulses, each of which erokes a single contraction. One of the best proofs of this is that if the sinus is heated the ventricle beats much more rapidly in unison with the rapidly beating sinus and auricles, while if the ventricle itself is heated no change takes place in its rhythm. Now, if the rentricle responds to a constant stimulus by rlyythmical beats, the condition of the ventricular tissue ought to affect the rate of its beat. In the mammalian heart, too, an alteration in the temperature of a definite area of the wall of the right auricle lying between the mouths of the vence cavæ produces a change in the rate of the whole heart, while no effect is caused by altering the temperature of any other portion of the heart. It has already been stated that the impulses from the nerve-cord which maintain the rhythm in the Limulus heart are also discontinuous.

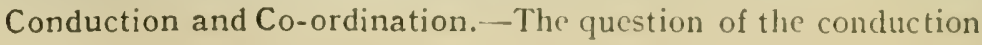


of the excitation over the heart and the co-ordination of its parts is in the same position as the question of the automatism and rhythmicity. In the horseshoe crab, as already remarked, the mechanism appears to be a nervous one. In ligher hearts, on the other hand, facts latve been discovered which favour each of the rival hypotheses. In the frog's heart the probability that the contraction wave is propagated from fibre to fibre of the muscle without the intervention of nerves has been much insisted upon, since the muscular tissue, although presenting certain variations in its character in the different divisions of the heart and at their junctions, forms a practically continuous shcet over the whole organ from base to apex. In support of this vicw has been brought forward the observation that the delay of the wave at the auriculo-ventricular groove is much greater than it ought to be if the excitation were transmitted by nerves, since the velocity of the nerve-impulse is exceedingly great (p. 793); and the further observation that, when the ventricle is caused to contract by artificial stimulation of the auricle, this delay is appreciably greater when the stimulus is applied as far from the ventricle as possible than when it is applied as near to it as possible. The delay has been attributed to the "embryonic ' character of the muscular tissue at the junction of the sinus with the auricles and of the auricles with the ventricles. But it has never been demonstrated that muscular fibres with the histological characters described do, as a matter of fact, conduct the contraction wave so much more slowly than the other cardiac muscular fibres It is just as probable, and indeed more so, that, whether the contraction travels in any particular division of the heart directly from muscle-fibre to muscle-fibre or not, the impulse to contraction is transferred from each division of the heart to the next by a nervous mechanism whose action is timed with the very object of securing a certain interval between the systoles of successive divisions. In any case, since we know that the velocity of the nerve-impulse is very different in different varieties of nerves, the question cannot be decided by general arguments of this kind. In Limulus, as a matter of fact, the velocity in the intrinsic heart nerves is only one-tenth as great as in the ordinary motor (limb) nerves of the animal (Carlson).

In the mammalian heart the alleged absence of muscular connection between the auricles and ventricles was long the foundation of the general belief that the link was a nervous one. Certainly there is no dearth of nerves which might serve as such a bridge. But it has been shown (Kent, His, etc.) that in the mammalian heart, too, a slender band of muscular fibres, arising at a definitc point (the auriculo-ventricular node) near the coronary sinus on the right side of the interauricular septum below the fossa ovalis, passes forwards and downwards through the fibrous ring between the auricles and ventricles under the septal cusp of the tricuspid valve. It then divides into two branches, one for each ventricle, which run 
down the interventricular septum towards the apex, spreading ont as the Purkinje fibres or their equivalent, to blend at last with the ordinary muscle of the ventricles, and particularly of the interventricular septum. The fibres of the bundle are narower than the other fibres of the auricles, very rich in nuclei, and only slightly differentiated into fibrillæ. They seem to represent the remains of the primitive cardiac tube, which by the development of certain pouches and twists becomes transformed into a multi-chambered heart. Their resemblance to embryonic fibres suggests that they may have retained the primitive capacity of the mesodermic tissue of the embryonis heart to conduct, and even to originate, the

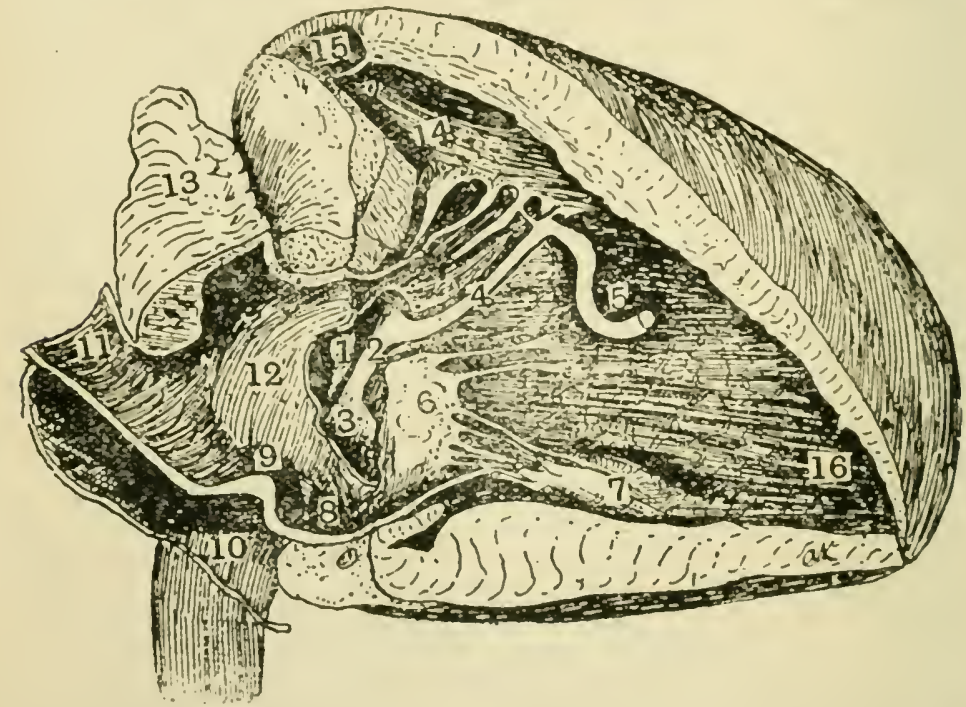

Fig. 64.- Right Auricle and Ventricle of Calf, to show Auriculo-Ventricular Band (Kieith). I, central cartilage; 2, inain auriculo-ventricular bundle; 3, auriculoventricular $(\mathrm{A}-\mathrm{V})$ node; 4 , right septal division of the bundle; 5 , moderator band; 6, medial or septal cusp of tricuspid valve; 8 , coronary sinus.

rhythmical contraction. But while there is no decisive evidence that they constitute an automatic cardio-motor centre, as some authors have supposed, they, or at least the narrow bridge of tissue in which they lie, do play an important part in the conduction of the contraction from the auricles to the ventricles. For compression of the band produces a block, just as the pressure of a clamp in the auriculo-ventricular groove does in the frog's heart (Kent). With a certain degree of pressure the ventricle beats only once for two beats of the auricle, with greater pressure only once for three or more auricular beats. With a still greater pressure or after crushing or section of the bundle conduction is abolished, and the ventricle either remains at rest for a time, as in the frog's heart, or 
what is much more common, inmediately starts beating with an independent rhythm, which is slower than that of the auricles (Erlang(r). It can be considered certain that in these observations nerves maty have been involved in the block as well as the muscle of the aturiculo-ventricular band, since this band is riclily provided

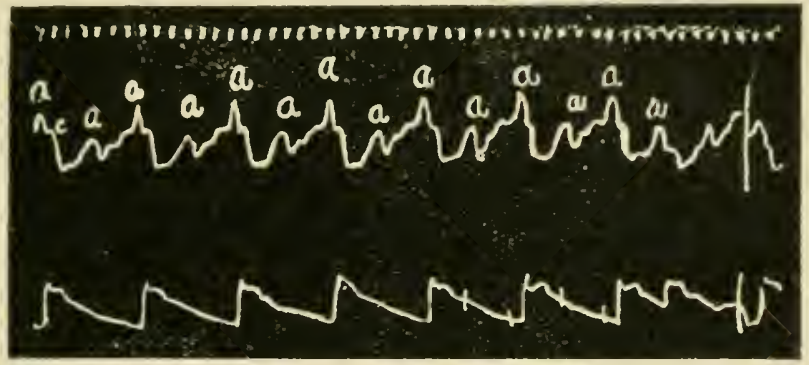

Fig. 65.-Jugular (Upper) and Carotid (Lower) Pulsc-Tracing from a Case of Arterio. sclerosis, showing Partial Failurc of Conduction in the Auriculo.Ventricular Bundle (Cushny and Grosh). The ventricle only beats once to two beats of the auricle. Time-trace, fifths of a second.

with nerve-fibres as well as ganglion-cells (Wilson). Yet it is unlikely that all the nerves capable of conducting the impulses to contraction should be gathered into such a narrow compass, and therefore the experiment supports the view that the conduction is carried out in the muscular tissue. And if the conduction of the

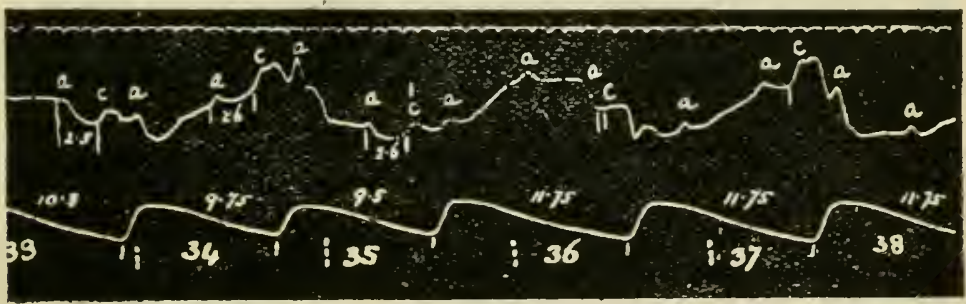

Fig. 66.-Tracing of Jugular (Upper) and Radial (Lower) Pulse from a Man with Heart-Block (Lewis and Macnalty). In the cycles marked 34, 35, and 36 the ventricular contraction, although less frequent than the auricular, was initiated from the auricle. In the last two cycles $(37$ and 38 ) and the pause of 36 complete heart-block was present. On the jugular trace the a.c interval (representing the interval bctween the onset of the auricular and ventricular contractions) is given, and on the radial trace the duration of a cardiac cycle, both in fifths of a second.

excitation from auricies to ventricles is accomplished by a muscular connection, it is natural to suppose that the co-ordination of symmetrical portions of the leart on either side of the longitudinal axis, the co-ordination in virtue of which the two auricles contract together and the two ventricles together, is also achieved by the passage of impulses through the muscular tissue. In accordance 
with this, it has been shown that the ventricles in the dog and cat continue in leat in unison, after the attempt has been made to sever any nerves connecting them by extensive zigzag incisions, so long as they are united by a narrow bridge of muscle (Porter).

In discase, interference with the conduction of the stimulus from auricles to ventricles along the atrio-ventricular bundle is a not uncommon phenomenon. According to the degree of interference, the ventricular contraction may be simply delayed, or only a certain proportion of the auricular contractions (every sccond, every third, or every fourth) may be conducted to the ventricle, or, finally, the block may be complete, and the ventricle then contracts quite independently of the auricle, the stimulus to contraction originating, perhaps, in the uninjured portion of the bundle below the scat of the block. These conditions are most easily recognized by comparing tracings simultaneously obtained from the jugular vein and the radial artery or apexbeat (p. 9o). When the block is complete the rate of the ventricle is very slow (about 30 in the minute, or less), the time of the ventricular beat is clearly unrelated to that of the auricular, and the stability of the ventricular rhythm is abnormally great, such circumstances as usually causc a marked increase in the pulse-rate-mental excitement, for instance-affecting it little or not at all. This is the condition in

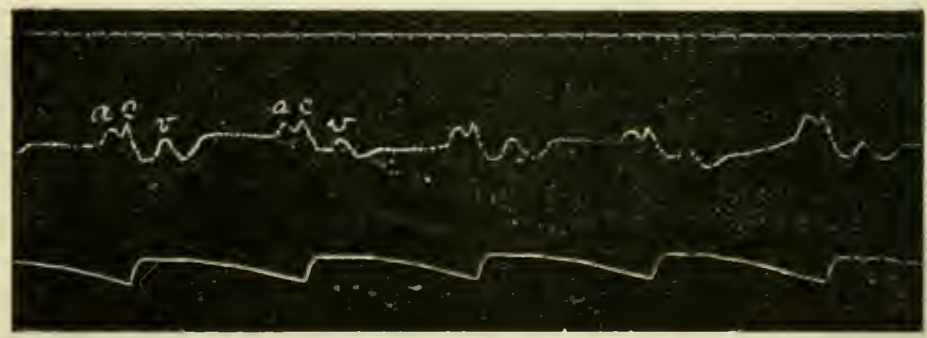

Fig. 67.- Polygraph Tracing in in a Case of True Bradycardia (Carter). The :ower trace is the radial, the upper the jugular. Time-trace, fifths of a second.

the so-called Stokes-Adams disease. In some of these cases pathological (syphilitic) clianges in the $\mathrm{A}-\mathrm{V}$ bundle have actually heen discovered at necropsy. In others there is some reason to believe that abnormal excitation of the cardio-inhibitory nerves may be responsible even for long-continued block, especially when the conductivity of the bundle has been already permanently diminished.

Cases of slow heart are also known in which there is no block in the conduction system, but the original rhythm of the auricle is siow (so-called true bradycardia. Fig. 67).

Kent has pointed out that the muscular connection between the auricles and ventricles is not single and confir.ed to the A-V bundle, but multiple, and that the co-ordinated action of the chambers of the heart is to some extent dependent upon the integrity of muscular connections other than that which exists in the A-V bundle. One of these he describes as the 'right lateral connection,' at the junction of the right auricle, the right ventricle, and the tricuspid valve, at the right-hand margin of the heart. The existence of this additional connection, the importance of which relatively to that of the $\mathrm{A}-\mathrm{V}$ bundle need not be the same in every heart, may explain otherwise puzzling 
results both clinical and experimental - e.g., that sometimes co-ordination between the ventricles and anricles las continued after destruction of the A-V bundle, while sometimes co-ordination has been upset by lesions not affecting the bundle.

Fibrillary Contraction.--In the case of the warm-blooded lieart a complete breakdown of co-ordination occurs under certain circumstances, producing the phenomenon known as fibrillary contraction, or delirium cordis, a condition in which each minute portion, perhaps each fibre, of the whole heart, or of a portion of it, goes on contracting in a disorderly manner, quite independently of the rest. The condition is often seen in a heart that has been exposed for some time, particularly in the ventricle, and can be induced by stimulating it with strong induction shocks or by ligation of the coronary arteries. According to the best evidence, the condition is due to the fact that the conductivity of the fibrillating muscle is interfered with so that the contraction wave is prevented from running its usual course. The consequence of this 'blocking' is that the normal co-ordinated action of the musculature gives place to the confused movement characteristic of fibrillation (Porter, Garrey). There is no reason to believe that fibrillary contraction is connected with the loss of impulses from any special co-ordinating centre, for it is not peculiar to the heart, but is typically seen in the tongue when the circulation after a long interruption is restored. The peculiar 'boiling' movement is exactly similar to that observed in the heart, probably because the tongue also contains fibres running in several directions.

The confused fibrillary contractions are quite ineffective for driving on the contents of the heart. Fibrillation of the ventricle is therefore incompatible with life. On the other hand, auricular fibrillation, far from being immediately fatal, is one of the most common of the chronic cardiac disorders in man. It is characterized by extreme irregularity of the pulse, due to the fact that the ventricles are played upon by an irregular stream of impulses from the fibrillating auricles to which they respond as they best can. The auricular wave $(a$, Figs. $65-67)$ is absent from the jugular pulse-tracing and the $P$ wave (p. 836), corresponding to the electrical change produced by the normally contracting auricles, is absent from the electrocardiogram. Auricular flutter is a condition which must be distinguished from auricular fibrillation. When a weak stimulus is applied to the auricle of a dog or cat, the auricular beats are grcatly increased in frequency up to 300 or 400 a minute. Although the beats are so rapid, they are otherwise normal beats. When the strength of the stimulus is increased, this condition of flutter (MaciVilliam) passes into fibrillation. Auricular flutter is also recognized clinically. In the majority of cases the ventricle does not respond to each beat of the auricle, and the arterial pulse is irregular; but each anricular contraction produces its appropriate effect upon the electrocardiogram and often also upon the jugular tracing (Mackenzie).

Without entering further into a discussion of the rival hypotheses, we may sum up by saying that for one heart (that of Limulus) the automatism and the rhythmical power have been clearly shown to reside 
in the local nerrous apparatus; for the hearts of other animals full and formal proof of the neurogenic theory, so far as those two properties of the cardiac tissue are concerned, has not been given. As regards the conduction and co-urdination of the contraction, the bulk of the evidence (learing the Limulus heart out of account) points to the muscular lissue as the channd through achich the cffective impulses pass. The normal order or sequence in which the different parts of the heart contract depends upon the fact that the automatism of the upper portions is more pronowned than that of the lower. so that under strictly phy'siological conditions the contraction is only propagated, and not originated, by the lower parts of the heart. When, however, the signal to contraction normally given by the basal region is prevented from reaching the lower parts, an indejendent automatic rhythm of the latter may be developed, as in the case of the mammalian ventricle mentioned above. Here we may suppose that the automatic mechanism of the lower portions of the heart discharges itself as soon as a sufficient accumulation of energy has taken place in it, although it requires a longer time to reach the point of discharge than the automatic mechanism of higher parts, and therefore is normally discharged from above. Under certain conditions the normal sequence can be reversed. In the heart of the skate it is easy, by stimulating the bulbus artcriosus, to cause a contraction passing from bulbus 10 sinus. The power of propagating the contraction may also be artificially altered. As already mentioned, it may be diminished or abolished by pressure. The same effect may be produced by fatigue or cold, while heating a portion of the heart in general increases its power of conducting the contraction.

Chemical Conditions of the Beat.--When we have localized the essential mechanism of the rhythmical beat in the nervous or in the muscular elements, the question may still be asked what the chemical and physical conditions are which are necessary to its maintenance. While it is known that a supply of arterial blood at or near body-temperature, and under a suflicient pressure, is required for permanent cardiac contraction, much simpler solutions will suffice to maintain the activity even of the isolated mammalian heart for a considerable time. One of the best of these is a solution containing sodium chloride, potassium chloride, calcium chloride, and sodium bicarbonate in the proportions in which they exist in blood-serum, with the addition of a small quantity of dextrose (Locke, p. 66). When this solution, properly oxygenated and warmed, is circulated through the coronary vessels of an excised rabbit's or cat's heart, strong and regular beats may be observed for many liours. Some investigators liave clinimed for sodium chloride, and even for sodium ions, others for calcium salts or calcium iuns, a special rôle in the origination or maintenance of the rhythmical beat. There is 110) doubt that strips from the ventricle of the tortoise or turtle, which 
after isolation have ceased beating, and if left to themselves in a moist chamber do not develop rhythmical contractions, begin after a while to beat when immersed in or irrigated with a solution of sodium chloricle or a solution of canc-sugar containing a little of that salt. They refuse to beat in any solution which does not contain sodium chloride (Lingle). The addition of calcium chloride to the sodium chloride solution, or preliminary treatment of the strip with a solution of a calcium salt before its immersion in the sodium chloride solution, hastens the onset of the contractions, and increases the length of time for which they are kept up (Erlanger). It is unquestionable that for the normal beat of the heart the presence of both salts is one of the necessary conditions, but there is at present no sufficient foundation for the view that either the one or the other acts as a special chemical excitant of the automatic contraction. Still less necessary is it to make this assumption for potassium. Certain potassium salts are, of course, beneficial to the heart as to other tissues. This might be assumed from their presence in blood and lymph, and it has been shown experimentally. But a terrapin's heart will continue beating, and beating well for a considerable time when irrigated with a solution containing sodium and calcium salts alone and free from potassium. That the reaction of the perfusive fluid is of great importance in connection with the origination of rhythm is well established, and it is an interesting fact that the limits of $\mathrm{H}$ ion concentration within which the development of spontaneous beats is possible differs for the hearts of different kinds of animals (Mines), and even for the different portions of the frog's heart (Dale and Thacker). While these facts illustrate the importance of the inorganic composition of the nutritive liquids for the action of the heart, they leave the old question of the existence and the nature of an inner stimulus to the rhythmic contraction very much where it was.

Resuscitation of the Heart.-Not only can the beat of the freshlyexcised mammalian heart be long maintained by artificial circulation, but many hours or even some days after somatic death pulsation may be restored by the perfusion of such a solution of inorganic salts as Locke's through the coronary vessels. Kuliabko in this way was able to restore a rabbit's heart which had been kept fortyfour hours in the ice-chest. Even after an interval of three to five days from the death of the animal, in other experiments, pulsation returned in certain parts of the heart, while twenty hours after death from double pneumonia the heart of a boy three months old was restored, and went on beating for over an hour. He obtained also more or less complete restoration of the beat in the hearts of persons dead from bronchitis combined with peritonitis or meningitis, and from cholera infantum, but was unsuccessful in cases of diphtheria complicated with septicæmia or erysipelas, and in cases 
of pleurisy with elfusion. It is to be remarked, however, that although beats of a kind can be obtained a long time after death, they are either confined to the auricles or to portions of them, or, if they involve the ventricles too, they are only shallow and local contractions, especially seen in the neighbourhood of the larger coronary vessels, and are utterly inadequate to the maintenance of an efficient circulation. The heart can also be resuscitated in situs for some time after complete stoppage without the injection of any solution by clamping the aorta in the thorax and practising direct cardiac massage, the lower end of the animal at the same time being clevated to allow blood to pass out of the engorged abdominal veins to the right auricle. The clamping of the aorta permits a sufficient pressure to be attained for the filling of the coronary arteries. The injection of adrenalin into the blood has also been recommended as a means of raising the blood-pressure by constricting the small arteries, and stimulating the action of the cardiac muscle. The possibility of restoration of the mammalian heart many hours after somatic death has been consiclered by some a strong argument for the myogenic theory of cardiac automatism, since, they say, it is improbable that ganglion-cells, elsewhere such physiologically fragile structures, should in the heart retain their vitality for so long a time. But it is easy to overdo this argument, and we must not assume without proof that ganglion-cells in all parts of the body have an equal capacity of survival. Indeed, we know that there are great differences, the nervous mechanism concerned in respiration, e.g., being capable of restoration when the circulation is renewed after total anæmia of the brain and cervical cord lasting for as much as an hour (in cats), while the nervous mechanism concerned in voluntary movements cannot be completely restored even after a much shorter interval. It is very probable that the cardiac ganglia, if the all-important automatic function of the heart depends upon them, are, like the cardiac muscle, cndowed with exceptional powers of resistance to those changes which constitute death. The possibility also must not be overlooked that the contractions obtained after such long intervals are not truly automatic, but similar rather to the rhytlinical beats developed under the influence of pressure in the frog's apex preparation or by immersion in salt solutions of tortoise ventricle strips.

In addition to its marked power of rhythmical contraction, the cardiac muscle is distinguished from ordinary skeletal muscle by other peculiarities. It used to be considered the most striking of these peculiarities that 'it is everything or nothing with the heart '; in other words, that the heart muscle, when it contracts, makes the best effort of which it is capable at the time; a weak stimulus, if it can just produce a beat, causing as great a contraction as a strong stimulus. Recent work, however, has indicated that this property 
is also possessed by the skeletal muscle-fibre. When a wliole skeletal muscle is excited either directly or through its motor nerve, it is true that througlout a considerable range increase of stimulus is accompanied by an apparent increase in the strength of contraction. But there is reason to believe that this is because a larger and larger number of fibres become involved in the excitation as the stimulus is increased, and not because cach fibre responds more and more strongly (Lucas). In skeletal muscle the fibres are completely isolated from each other, and the excitation does not spread from fibre to fibre, as happens in the heart.

Refractory Period and Extra Contraction of Hear! Muscle.-A more characteristic property of the cardiac muscle than the "all or nothing ' law is that a true tetanus of the heart cannot be obtained at all, or only under very special conditions. When the ventricle of a normally beating frog's heart is stimulated by a rapid series of induction shocks, its rate is gencrally increased, but there is no definite relation between the number of stimuli and the number of beats. Many of the stimuli are ineffective. In the same way a portion of the heart, such as the apex of the ventricle, when stimulated in the quiescent condition by an interrupted current, responds by a rhythmical series of beats, and not by a tetanus. It is evident that the cardiac muscle, like ordinary striped muscle, is for some time after excitation incapable of responding to a fresh stimulus-. i.e., there is a refractory period. But this is immensely longer in cardiac than in skeletal muscle. When the phenomenon is analyzed, it is found that a stimulus falling into the heart muscle between the moment at which the contraction begins and the moment at which it reaches its maximum produces no effect-is, so to speak, ignored. When the stimulus is thrown in at any point between the maximum of the systole and the beginning of the next contraction, it causes what is called an extra contraction. The extra contraction is followed by a longer pause than usual-a so-called compensatory pause-which just restores the rhythm, so that the succeeding systole falls in the curve where it would have fallen had there been no extra contraction (Fig. 68).

In man, extra systoles followed by compensatory pauses may occur under pathological conditions, giving rise to an important group of cardiac irregularities. These extra systoles may be cither auricular or ventricular, the auricle or the ventricle contracting prematurely without waiting for the signal of the sinus rhythm. The analysis of pulsetracings showing these irregularities has led to results of great physiological and clinical interest (Cushny, Mackenzie, etc.), but cannot be dwelt on here. When every second beat is an extra systole, generally weaker than the preceding and the succeeding normal beat, the condition is called pulsus bigeminus. The weaker beat is always followed by a compensatory pause of greater duration than that preceding it. From the pulsus bigeminus must be distinguished that form of altemating pulse termed pulsus alternans, in which every second beat is diminshed in size, but the intervals separating the beats are of uniform length. 
The refractory period is shorter for strong than for weak stimuli, and is markedly diminished by raising the temperature of the heart. So that stimulation of the heated heart with a series of strong induction shocks may cause a tetaniform condition, if not a typical

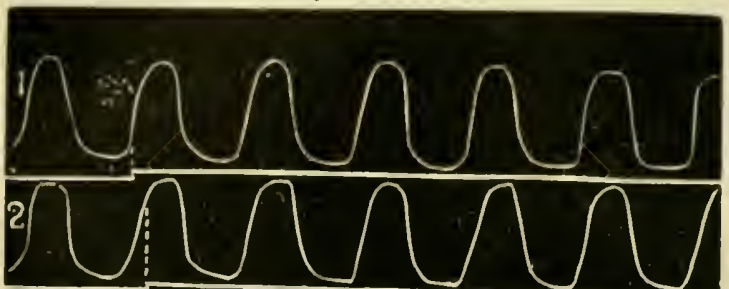

tetanus. The contraction of the normally beating heart is really a simple contraction, and not a tetanus. The clectrical changes

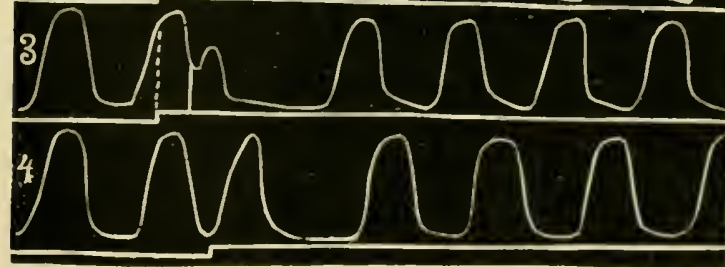

Fig. 68. - Refractory Period and Compensatory Pause (Marey). A frog's heart was stimulated at a point corre. sponding to the nick in the horizontal line below each curve. In I and 2 there was no response; in 3 and 4 there was an extra contraction, succeeded by a compensatory pause. correspond to a single contraction (p. 833); and when the nerve of a nerve-muscle preparation is laicl on the heart, the muscle responds to each beat by a simple twitch, and not by tetanus (p. 20.3). That the cardiac muscle itself, apart from the intrinsic nervous merhanism, shows the phenomenon of 'refractory state' has been shown in the l.imulus heart after extirpation of the ganglion (Carlson).

Like ordinary skeletal muscle, the cardiac muscle is at first benefited by contraction, perhaps by an ' augmenting ' action of fatigucproducts such as carbon dioxide (I.ee), so that when the apex is stimulated at regular intervals each contraction is somewliat stronger than the preceding one. To this phenomenon the name of the staircase or 'treppe ' has been given, from the appearance of the tracings (p. 749).

\section{Section V.-The Nervous Regulation of the Heart} (Extrinsic Nervous Meclianism of the Heart).

While, as we have seen, the essential cause of the rhythmical beat of the heart resides in the tissue of the heart itself, it is constantly affected by impulses that reach it from the central nervous system. These impulses are of two kinds, or, rather, produce two distinct effects: inhibition, shown by a diminution in the rate or force of the heart-beat, or in the ease with which the contraction is conducted over the heart-wall; and augmentation, or increase in the rate or 
force of the beat or in the conductivity. Both the inlibitory and th: augmentor impulses arise in the medulla oblongata, and perhaps a narrow zone of the neighbouring portion of the cord; and they can be artificially excited by stimulation in this region. They pursue their course to the heart by fibres which may in certain animals be mingled together, but are anatomically distinct. We may, therefore, divide the extrinsic or external nervous mechanism of the heart into a cardio-inhibitory centre with its efferent inhibitory nerve-fibres and a cardio-augmentor centre with its efferent accelerator or augmentor fibres. Both of those centres, as we shall see, have, in addition, extensive relations with afferent nerve-fibres from all parts of the body, including the heart itself.

It was in the vagus of the frog that inhibitory nerves were first discovered by the brothers Weber seventy years ago, and even now our knowledge of the cardiac nervous mechanism is more complete in this animal than in any other. We shall, therefore, first describe the phenomena of inhibition and augmentation as we see them in the heart of the frog, and then pass on to the mammal.

In the frog the inhibitory fibres leave the medulla oblongata in the vagus nerve. The augmentor fibres come off from the upper part of the spinal cord by a branch from the third nerve to the third sympathetic ganglion, and thence find their way along the sympathetic cord to its junction with the vagus, in which they run, mingled with the inhibitory fibres, down to the heart.

When the vago-sympathetic in the frog or toad is cut, and its peripheral end stimulated, the heart in the vast majority of cases is stopped or slowed,

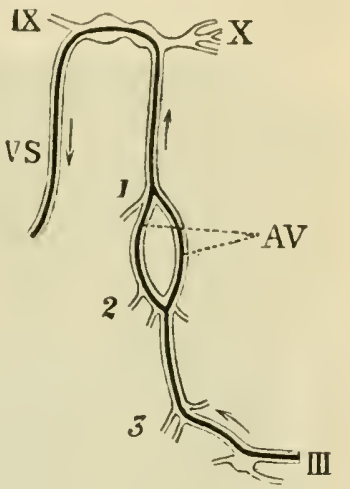

Fig. 69.-Diagram of Extrinsic Nerves of Frog's Heart (after Foster). III, 3rd spinal nerve; $A V$, annulus of Vieus. sens; $\mathrm{X}$, roots of vagus; IX, glosso-pharyngeal nerve; VS, combined vagus and sympathetic; I, 2, and 3 , the 1st. 2nd, and 3 rd sympathetic ganglia. The dark line indicates the course of the sympathetic fibres. The arrows show the direction of the aug. mentor impulses.

or its beat is distinctly weakened without, it may be, any marked slowing. In other words, the rate at which the heart was working before the stimulation is greatly diminished, or reduced to zero. Such an effect, a diminution of the rate of working, we call Inhibition. What precise form the inhibition shall take, whether the stoppage shall be complete or partial, and if partial whether the beats shall be simply weakened without being slowed or both slowed and weakened, appears to depend partly upon the strength of the stimulus used and partly upon the state of the heart itself. Some hearts it may be impossible to stop with weak stimulation, although other signs of inhibition may be distinct; 
while they are readily stopped by stronger stimulation. In other cases the strongest stimulation may not produce complete standstill. Again, the inhibitory effect produced on a heated heart by a given strength of stimulation of the vagus may be greater than that caused in a heart at the ordinary temperature or a cooled heart. This is especially evident on the auricular tracings when these are recorded separatcly from those of the ventricle. Even on the verge of heat standstill of the heart inhibition is easily obtained (Fig. 7I). Some writers have assumed that the different inhibitory effects produced by the vagus are due to the existence in it of separate groups of fibres, some affecting only the rate of the contraction, others its strength,

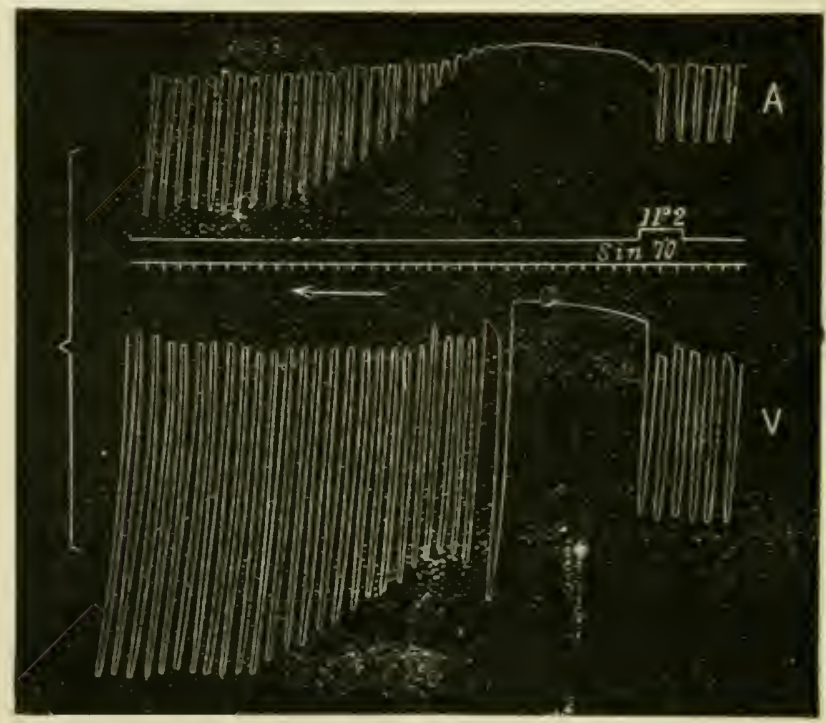

Fig. 70.-Traciug from Frog's Heart. A, auricular, V, ventricular tracing. Sinus stimulated (primary coil $70 \mathrm{~mm}$. from seconlary). Heart at temperature II $\cdot 2^{\circ} \mathrm{C}$. Complete standstill. The time-tracing between the curves marks intervals of two seconds.

others still the conductivity of the muscular tissue and its excitability. This theory has enriched the vocabulary of physiology with a number of sollurous terms derived from the Greek, but has not otherwive established itself, although it has been useful in emphasizing the fact that the inhibitory nerves can influence the heart-beat in several distinct ways.

But there are other points of importance to be noted in regard to this inhibition: (I) It does not begin for a little time after stimulation has begun. In other words, there is a distinct latent period; and the length of this latent period is related to the phase of the heart's 
contraction at which the stimulus is thrown in, and to the rate at which the heart is beating. As a general rule, the heart makes at least one beat before it stops.

(2) The inlibition does not continue indefinitely, even if stimulation of the nerve is kept up. Sooner or later, and usually, in fact, after an interval of a few seconds, the heart begins again to beat if it has been completely stopped, or to quicken its beat if it has only been slowed, or to strengthen it if the inhibition has only weakened the contraction, and it soon regains its old rate of working. Not only so, but very often there follows a longer or shorter period during which the heart works at a greater rate than it did before the inhibition, and this greater rate of working may be manifested by increased
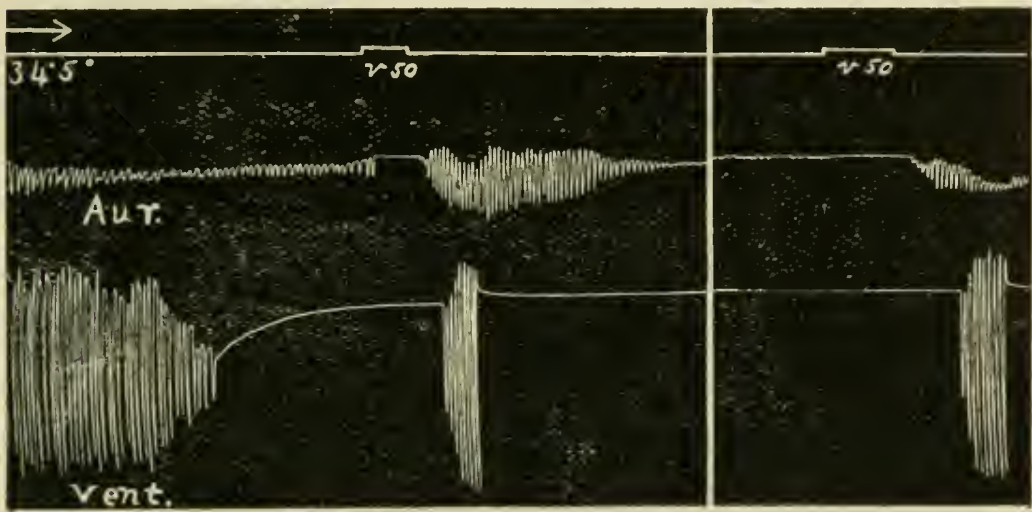

Fig. 71.-Activity of Vagus on Verge of Heat Standstill. Auricular and ventricular contractions of toad's heart recorded. Heart at $34.5^{\circ} \mathrm{C}$., $v 50$, stimulation of vagus (distance of coils $50 \mathrm{~mm}$.). The ventricle was already in heat standstill; the auricle was at once inhibited. Then follows secondary augmentation (due to the sympathetic fibres), during which the ventricle also resumes beating. An interval of a minute elapsed between the first and second parts of the tracing, during which the heart remained at $345^{\circ} \mathrm{C}$. The auricle was almost in standstill (contractions can still be seen on the curve with a lens), when the vagus was again stinulated at $v$ 5o with the same distance between the coils. Complete inhibition followed by secondary augmentation.

frequency of beat, or increased strength of beat, or by both. When the temperature of the heart is low, increased frequency; when it is high, increased strength, is generally seen during this period of secondary angmentation.* The cause of this secondary augmentation, and of the primary augmentation sometimes seen in fresh prepara. tions and often in hearts that have been long exposed (Fig. 73), excited much speculation before it was known that sympathetic fibres existed in the vagus. There is no longer any doubt that it is due to the stimulation of these accelerator or, as it is better to call

* Augmentation is termed 'secondary' when it is preceded by inhibition, primar.: ' when it is not so preceded. 
them (since mere acceleration is not the only consequence of their stimulation), angmentor fibres in the mixed nerve. For (I) excitation of the routs of the vagus proper within the skull, and therefore above the junction of the sympathetic fibres, causes no secondary augmentation, or very little, and the inhibition lasts far longer than when the mixed trunk is stimulated. (2) Excitation of the upper or cephalic end of the sympathetic cord before it has joined the vagus causes, after a relatively long latent period, marked augmentation. And if the contractions of the heart are registered, the tracing bears a close resemblance to the curve of secondary augmentation following excitation of the mixed nerve on the other side with an equally strong stimulus and for an equal time. (3) When the vago-sympathetic is stimulated weakly there is little or no

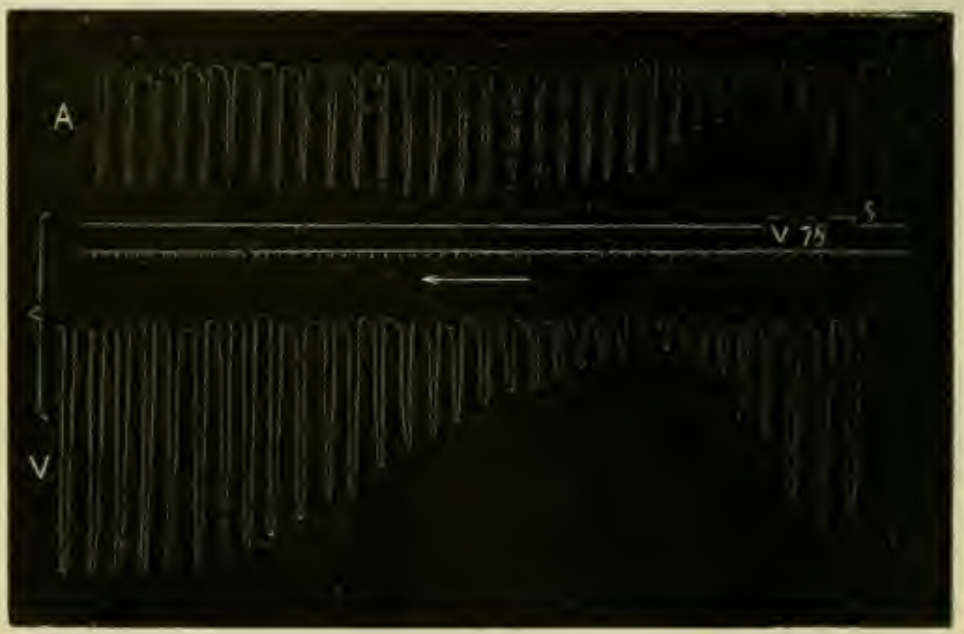

Fig. 72.-Frog's Heart: Vagus stimulated. Temperature of heart $8^{\circ} \mathrm{C}$; $73 \mathrm{~mm}$. between the coils. Diminution in force of auricle and ventricle, but not com. plete standstill. Time-tracing shows two-second intervals.

secondary augmentation. Now, it is known that the augmentor fibres require a comparatively strong stimulus to cause any effect when they are separately excited, whereas a weak stimulus will excite the inhibitory fibres.

The question arises at this point, why it is that, when the inhibitory and augmentor fibres are stimulated together in the mixed nerve (and the same is true when the sympathetic on one side and the vagus on the other are stimulated at the same time), the inhibitory effect always comes first, when there is any inhibitory effect, while the augmentation always lias to follow. The answer has sometimes been given, that the latent period of the augmentor fibres is longer than that of the inhibitory fibres. But although this is certainly the case, the answer is insufficient. For the period of postponement may be much greater than the 
latent perioul of the sympathetic fibres when stimulated by themselves. The inhibition apparently runs its course without being affecter by the simultancons angmentor effect, which, lying latent until the end of the inhibition, then bursts out and completes its own curve. It is not like the passing of two waves throngh each other, but rather like the stopping of one wave until the other has passed by. It seems as if augmentation cannot develop itself in the presence of inlibition-at least, until the litter is nearly spent. Iike a musical-box devised to play a series of melodies in a fixed order, and from which a particular tune cannot be obtained till those preceding it have been run through, the heart, in some way or other, is arranged, in the presence of competing impulses from its extrinsic nerves, to play the tune of inhibition before the tune

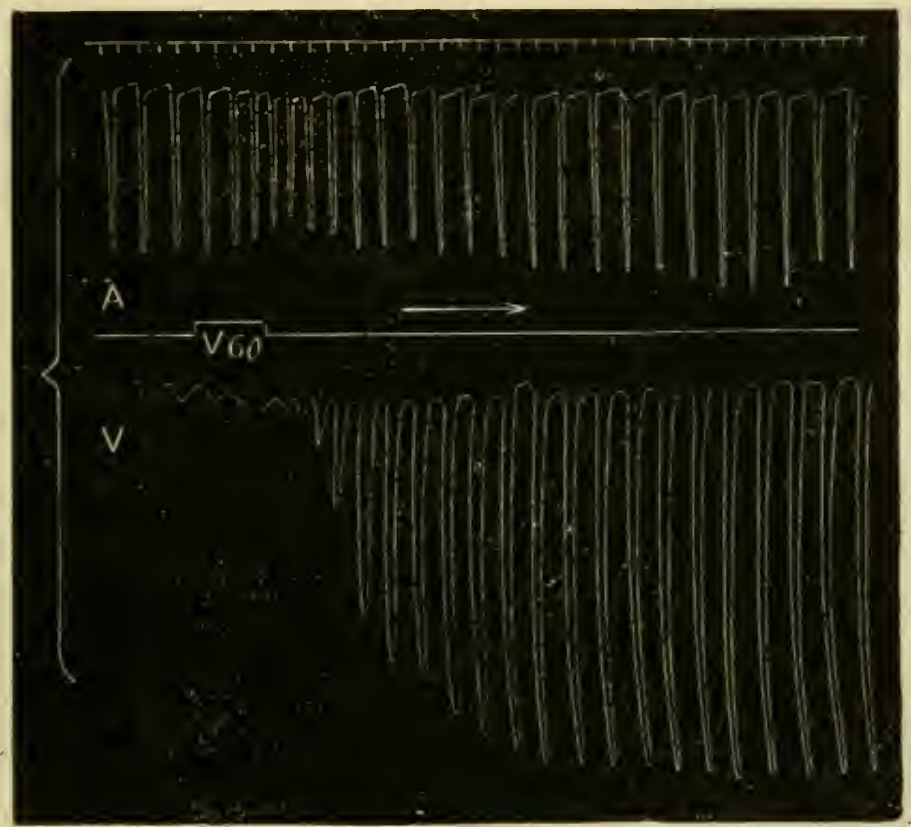

Fig. 73a.-Frog's Heart. A, auriciar, V, ventricular tracing. Ventricle beating very feebly. Vagus stimulated $(60 \mathrm{~mm}$. between coils). Marked augmentation of ventricular beat.

of a ugmentation. In the frog, at any rate, the two processes can hardly be considered as antagonistic, in the sense that a definite amount of augmentor excitation can overcome a definite amount of inhibitory excitation. Nor is it the case that, when tl?c heart is played upon at the same time by impulses of both kinds, it pits them ageinst each other and strikes the balance accurately between them. It is possible, lowever, that when the inhibitory fibres are very wcakly; and the augmentor fibres very strongly stimulated, the amount of inlibition may be somewhat diminished. In mammals, on the otlicr hand, a true antagonism scems to exist; and stimulation of the inlilitory nerves is less effective when the augmentors are excited at the sarne time. The cardiac nerves affect not only the rate and force of the contraction, but also the con- 
ductivity of the heart. 'Thus in the frog's heart during stimulation of the vagus, the contraction patsics more slowly, ansl during stimulation of the sympathetic more quickly, from aturicles to ventricle.

In mammals (and in whit follows we shall restrict ourselves chiefly to the clog. cat, and rabbit, as it is in these animals that the subject bas been most carefully sturlied) the inhibitory fibres run down the vagus in the neck and reach the heart by its cardiac branches. They are lerived from the bulbar roots ot the spinal accessory, whose inner branch joins the vagus. The augmentor ibres leave the spinal cord in the anterior routs of the second and thira thoracic nerves, and possibly to some extent by the fourth and fifth. Through the corresponding

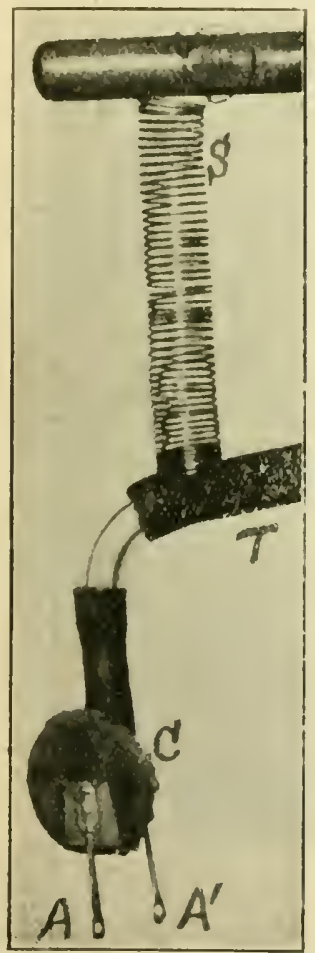

Fig. 7.3b.-Niniature Mycoardiograph (actual size) (Wiggers). C, light iluminium segunent capsule covered we tightly stretched rubber dam, upon which is cemented an aluminiun plate pivoling upon lhe chord side of the capsule. The plate carries a light am, $A$, with an evelet at its end. A similar arm, $A^{1}$, is rigidly fastened to the brody of the capsule. The arms can be bent so that the distance between the eyclets varies from 3 to 25 millimetres, and are comected by stitches through the evelets to points on the auricular surface. The approximation of the points causes a negative pressure in the capsule, and in the recording capsule (sec descrip)ti.n of fing. 3I, P. 93) connected with it by the tube 1. Hence, the down-stroke of the curse represents conraction, the up-stroke relixation. The ipparatus is supported by a very light spring, $S$. This enables it to follow varying degrees of distension and movement of the auricle without affecting the curve of contraction. The small mass (less than 2 gm.) and ligh vibration frequency of the instrument insure a more faithful record of the contraction than with older forms.

white rami communicantes they reach the sympathetic cord. and running up through the stellate ganglion (first thoracic), and the annulus of Vieussens, which surrounds the subclavian artery, to the inferior cervical ganglion, they pass off to the heart by separate "accelerator' branches, laking origin either from the ammulus or from the inferior cervical ganglion. Some augmentor fibres are often, if not always, present in the dog's vago-sympathetic in the neck. It is cspecially easy to demomstrate their presence five or six days after section of the nerve, when the excitability of the inhibitory fibres has disappeared.

In the dog the vagus and cervical sympathetic are, in the great majority of cases, contained it a strong common sheath, and pass 
together through the inferior cervical ganglion. Lpon opening this sheath they may with care be separated, the fibres running in rlistinct strands, and not mixed together as in the vago-sympathetic of the frog. For some distance below the superior cervical ganglion the cervical sympathetic is not connected with the vagus, and here the nerves may be separately stimulated without any artificial isolation. In the rabbit and some other mammals, including man, the vagus and sympathetic run a separate course in the neck.

In the mammal, the inhibitory fibres have a smaller direct action on the ventricle than in the frog. It indeed beats more slowly when the auricle is slowed, but this is only because in the normally beating heart the ventricle takes the time from the auricle. The strength of the ventricular contractions may be not at all diminished, even when the auricle is beating very feebly during inhibition. When the auricle is completely stopped, which does not occur so readily as in the frog, the ventricle also stops for a short time, but soon begins to beat again with an independent rlyythm of its own. In the frog the ventricle is directly affected by stimulation of the vagus, and the force of its beats is diminished independently of the inhibitory effects in the auricles (Practical Exercises, pp. I98, 203).

It has been shown by delicate optical methods of recording the contraction of small units of the auricular musculature (designated as the 'fractionate' contraction by Wiggers) by the method illustrated in Fig. $73 b$, that the diminution in the size of the contraction produced by stimulation of the vagus is essentially due to depression of the contractility of the muscle, and not, or at any rate not primarily, to a diminution in its excitability. In other words, the strength of stimulus needed to elicit a contraction of the muscle when under the influence of vagus excitation, in the early part of the inhibition at least, is not increased, whereas the size of the contraction evoked by a given stimulus is diminished (Fig. 74).

It is not doubted that the excitation of the vagus does reduce the excitability of the auricular muscle, but this reduction seems not to occur so early as the reduction in the amplitude of the beat, and cannot therefore be responsible for it. The duration of each (fractionate) contraction is not altered by vagus excitation. It can likewise be shown that the depression of contractility is not secondary to the depression of conductivity produced by the vagus. Finally, it is not dependent upon the slowing of the rhythm which accompanies the diminution in the contraction of the naturally beating auricle. For when the auricle is compelled to beat with an artificial rhythm by applying to it a series of regularly spaced electrical stimuli at a more rapid rate than the normal rhythm, stimulation of the vagus still causes reduction in the amplitude of the contraction without change in the rate (Fig. 74). One other point is worthy of note. Excitation of the vagus causes an increase in the size of the first, or occasionally of the first two subsequent 
contractions of the aturicular muscle when the rhythm is slowed, but not otherwise. It is probable that this is due to the beneficial influence of the longer period of rest associated with the diminution in frequency of the beat before the inlibitory action has had time to cause depression in the amplitude (Wiggers).
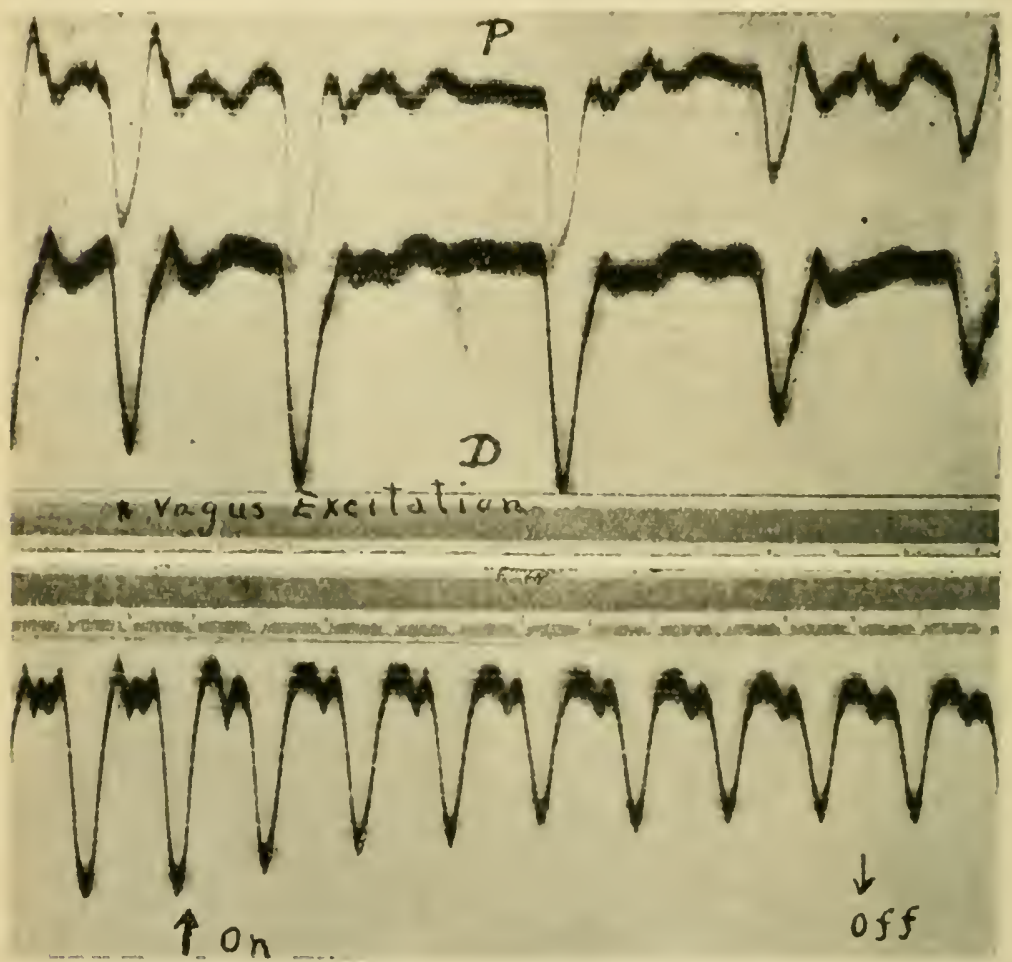

Fig. 74--Influence of the Vagus on the Contraction of the Dog's Right Auricle The two upper curves are simultaneous records of the contraction of very short portions of the auricle (s)-called fractionate contraction) taken from two regions, one near the sinus node $P$, and one far from $i t, D$. The contraction begins and ends slightly sooner in the proximal than in the distal region. The lowest curve shows the depressint effect of the vagus excitation inanifested when an artificial rhythm (a serit's of electrical break shocks), which is not altered by stimulation of the vagus, is substituted for the mormal rhythm sustained by "the" "pacemaker.'

The inhibitory fibres, then, influence the heart particularly through the auricles; they are par excellence auricular nerves. On the other hand, the accelerantes in all mummals which have been investigated not unly extend to the ventricles, but are even mainls. distributed to them. They are emphatically rentricular fibres, and 
in accordance with its greater mass the left ventricle receives more fibres than the right.

Stimulation of the accelerator nerves in the dog causes an increase in the force of both the auricular and ventricular contraction, and as a rule, in addition, some increase in the rate of the beat.

As to the nature of the physiological linkage between the cardiac nerves and the muscular tissue of the heart we know but little. Cranglion-cells lie on the course of the vagus fibres after they late entered the heart, and although the view has been advocated that they are simply stations where the inhibitory impulses pass from medullated to non-medullated fibres, and where possibly other

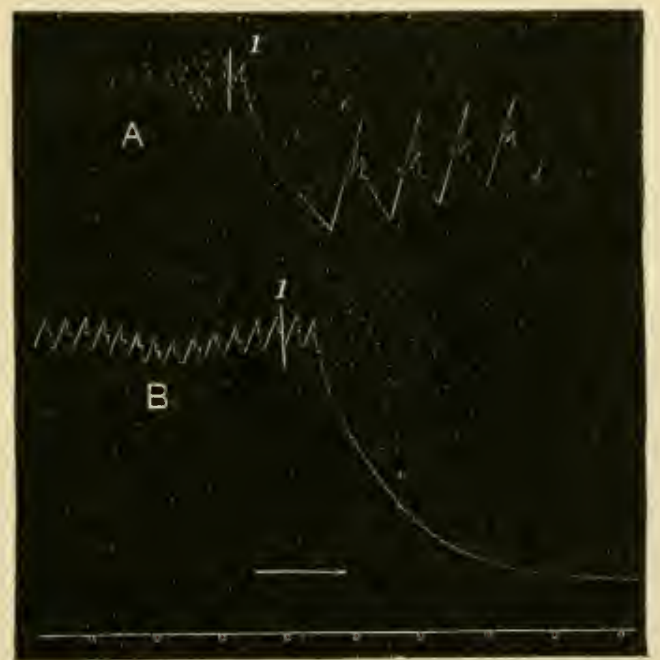

Iig. 75.-1 ood-Pres $u$ ? Tracings: Rabbit. Vagus stimulated at r. Stimulus strullet $111 \mathrm{~B}$ than in A (Hürthlc's spring manometer).

anatomical changes and rearrangements occur, they may be intermediate mechanisms which essentially modify the physiological impulses falling into them. It has been stated that in the dog the right vagus controls chiefly the rate of the heart, and the left vagus chicfly the conduction from auricles to ventricles, and the suggestion has been made that this is because the right vagus has a special relation to the sino-auricular nocke, in which impulses are supposed to arise, and the left vagus a special relation to the auriculo-ventricular node, the upper end of the $\mathrm{A}-\mathrm{V}$ bundle, the main conduction system (Cohn and Lewis).

The nervi accelerantes arc already non-medullated before they 
reach the heart. The fact that the action of the accelerantes can be restored by perfusing the heart with a nutrient solution at a much longer interval after somatic death than the action of the vagus strengthens the suggestion that ganglion-cells are interposed on the inhibitory though not on the angmentor path, without. however, proving of itself that such a difference exists in one experiment the heart of an anthropoid ape was reviveu when thice successive periods viz., four and a half, twenty-eiglit and a half, and fifty-three hours respectively-had elapsed after the death of the animal, although during the last period the heart had been twice frozen hard. The vagus was shown to be still capable of causing some inhibition six hours after death, and the accelerans some augnentation as late as fifty-three hours after death (Hering).

In the discussions over the relation of the extrinsic to the intrinsic cardiac nervous apparatus appeal has frequently becn made to the action of certain poisons on the heart. Thus, after nicotine stimulation of the vago-sympathetic causes no inhibition of the frog's heart; it may cause augmentation. But stimulation of the junction of the sinus and auricle still causes inhibition. Alropine not only abolishes the inhibitory effect of stimulation of the vagus trunk, but also that of stimulation of the junction of sinus and auricle. Muscarine causes diastolic arrest in a heart already poisoned with nicotine, but not in a heart under the influence of atropine. And a heart brought to a standstill by muscarine can be made to beat again by the application of atropine, although not by nicotine

These facts may be explained as follows: Nicotine paralyzes, not the very ends of the vagus, but the ganglia through which its fibres pass. Stimulation of the sinus, which is practically stimulation of the vagus fibres between the ganglion-cells and the muscular fibres, is therefore cffective, although stimulation of the nerve-trunk is not (Langley). On the other hand, the atropine group paralyzes the nerve-endings themselves, or interferes with the reception of the inhibitory impulses by acting on a so-called receptive substance in the muscle (p. I $S_{2}$ ), so that neither stinulation of the sinus nor of the nerve-trunk can cause inhibition. Muscarine, on the contrary, stimulates the vagus fibres between the nerve-cells and the muscle, or the actual nerve-endings, or cxerts an inhibitory action on the muscle itself through the appropriate receptive substance, and thus keeps the heart in a state of permanent inhibition, which is removed when atropine cuts out the nerve-endings, or combines with the receptive substance. It is quite in accordance with this that muscarine has no effect on a heart whose vagus nerves, as occasionally happens, have no inhibitory power. Pilocarpine has very mucli the same action as muscarine.

Stannius' Experiment.-Another series of phenomena, intimately related to our present subject, have excited, since they were first made known by Stannius, an enormous amount of discussion. The chief facts of this classical experiment we have already mentioned (p. 144), and they are also described in the Practical Exercises (p. 194). They are easy to verify, but difficult to inter $)^{2}$ et. The most probable explanation of the standstill caused by the first ligature is that the lower portion 
of the heat, when cut off from the simus in which the beat normally originate: necels some time for the elevelopment of its antomatic power to the point at which an independent rhythm can be mantained. The effects following the sccond Stannius ligature secm to depend upon the power of the ventricle to develop and matintain an independent rhythm, but the contractions are supposed by some to be started by stimulation of the muscular tissue in the auriculo-ventricular groove by the ligature.

Nature of Inhibition and Augmentation. - So far we have been cliscussing the phenomena of inhibition and augmentation as ultimate facts. We have not attempted to go behind them, nor to ask what it is that really happens when inhibitory impulses fall in to a heart, which from the first days of embryonic life has gone on beating with a regular rhythm, and in the space of a second or two bring it to a standstill. The question cannot fail to press itsclf ufun the mind of anyone who has ever witnessed this most beautiful of physiological experiments;

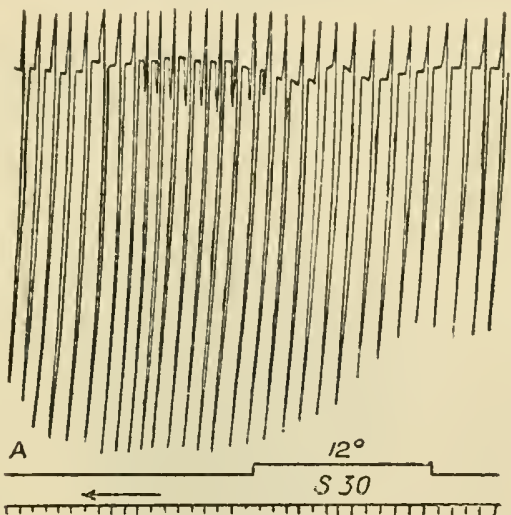

Fig. 76.-Frog's Heart. Sympathetic stimulated (30 $\mathrm{mm}$. between the coils). Temperature $12^{\circ}$. Marked increase in force. Only auricular tracing repro. duced. Time-trace, two-second intervals.

but as yet there is no answer except ingenious speculations. The most plausible of these is the trophic theory of Gaskell, who sees in the vagus a nerve which so acts upon the chemical changes going on in the heart as to give them a trophic, or anabolic, or constructive turn, and thus to lessen for the time the destructive changes underlying the muscular contraction. The augmentor nerves, on the other hand, are supposed to exert a katabolic influence, and to favour these destructive changes. And while, according to Gaskell, the natural consequence of inhibition is a stage of increased efficiency and working power when the inhibition has passed away, the natural complement of augmentation is a temporary exhaustion. It is very risky, however, to rely, as Gaskell did, upon a supposed change of sign in the electrical effects during vagus stimulation, and the only chemical test to which the theory has been subjected, the comparison of the oxygen consumption of the heart during and in the absence of inhibition, is adverse to it. The amount of oxygen used up relatively to the functional activity of the heart as measured by the product of the frequency of the beat and the maximal increase of pressure causcd by it, is not increased by stimulation of the ragus (Rohde). 
Whateve: the exact mechaniom of augmentation may be, there is no basis for the statement that the cardie-ingmentor nerves have an action on the heart so funclamentally different from the action of motor nerves on skcletal muscle that they cannot originate contractions in a heart entirely at rest. Excitation of the cardio-alugmentor nerves (iul cause flughmical contractions in the perfectly yuicacent heart of molluses, and a sudden and prolomged outburst of beats of great force in the frog's heart, which has been brought to a standstill by cautiously heating it to for to +3 C. (Practical Exercises, p. 194) for a minute or two, or to a considerably lower temperature, for a longer time (Jig. 77). A similar effect can be obtained on the quiescent mammalian heart by stimulation of the nervi accelerantes.
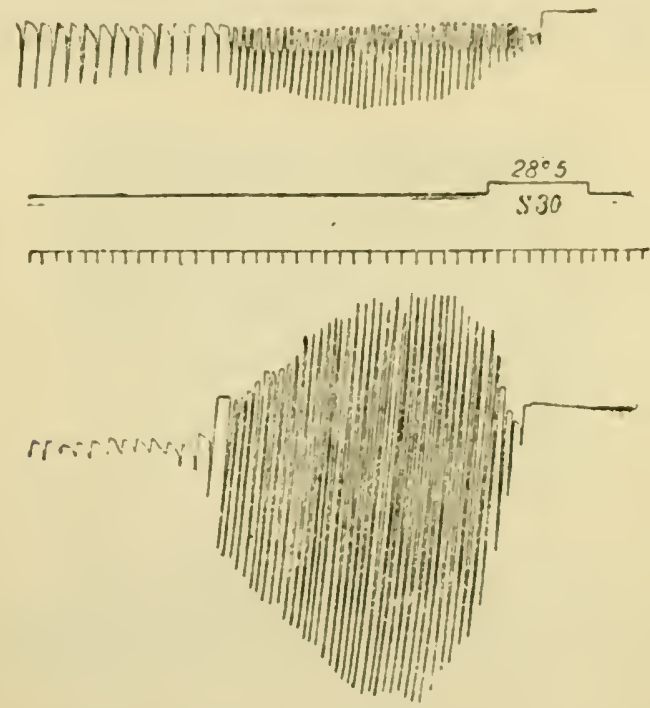

lin. 7;.-. Effect of Stimulation of lirog - Cardiac Sympathetic during complete Standstill of the Heart at $28.5^{\circ} \mathrm{C}$. Leper tracing, auricle; lower, vestricle. To be read from right to left. Tinse-trace, two-sucend intervials.

The Normal Excitation of the Cardiac Nervous Mechanism.We have now to inquire how this elaborate nervous mechanism is normally set into action. And we may say at once, that striking as are the effects of experimental stimulation of the ragus trunk or the nervi accelerantes in their course, it is unly under exceptional circumstances that the efferent nerve-fibres, at any rate before they have entered the heart, can be directly excited in the intact hody. In ecrtain cases the pressure of a tumour of an ancurism on the nerve-trunks, or, in the case of the accelerators the progress of a pathological change in the sympathetic ganglia through which the 
nerve-trunks, or, in the rase of the accelerators, the progress of a patlological cliange in the sympathetic ganglia through which the fibres pass, has been thought to bring about by direct stimulation a slowing or a quickening of the pulse. In some individuals the vagus has been cxicited by compressing it against a bony tumour in the neck; and by compressing the nerve against the vertebral column it is possible to cause inhibition in many normal persons, although it ought to be stated that the experiment is not free from danger. But it is from the cardio-inhibitory and cardio-augme $n^{2}$ or centres in the mcdulla oblongata that the impulses which regulate the activity of the heart are normally discharged. Inhibitory impulses are constantly passing out from the medulla, for section of both ragi causes almost invariably an increase in the rate of the lieart, at least in mammals, although the increase is less conspicuous in animals like the rabbit, whose normal pulse-rate is high, than in animals like the dog, whose pulse-rate is comparatively low. Section of one vagus usually causes only a comparatively slight increasc, for the other is able of itself to control the heart. It is not certainly known whether the augmentor centre in like manner discharges a continuous stream of impulses, or is only roused to occasional activity by special stimuli. For the results of section of the nervi accelerantes, or the extirpation of the inferior cervical and stellate ganglia, are dubious and conflicting. But if it does exert a tonic influence on the heart, this is feebler than the tone of the inhibitory centre. As to the nature of this inhibitory tone, and the manner in which it is maintained, we know but little. It may be that the chemical changes in the nerve-cells of the inhibitory centre lead of themselves to the discharge of impulses along the inhibitory nerves. But there is some evidence that, in the complete absence of stimulation from without, the activity of the centre would languish, and perhaps be ultimately extinguished. For when the greater number of the afferent impulses have been cut off from the medulla oblongata by a transverse section carried through its lower border, division of the vagi produces little effect on the rate of the heart. Also, when the upper cervical cord and the brain are resuscitated after a period of anæmia, the return of cardio-inhibitory tone is tardy in comparison with the return of the truly automatic function of respiration, and does not scem to precede the opening up of the afferent paths to the cardio-inhibitory centre. Indeed, reflex inhibition may be produced at a time when the inhibitory centre has regained none of its tone.

The suggestion is that the normal tone of the centre is largely depandent upon reflex impulses. Be this as it may, we know that the activity of the inhibitory centre is profoundly influenced-and that both in the direction of an increase and of a diminution-by impulses that fall into it through afferent nerves and by stimuli directly applied to it. And we may assume that the same is true of the augmentor centre. The common statement that stimulation 
of the central end of one vagus, the other being intact, produces distinet inhibition does not hold for all mammals. In dogs this is sumetimes the case, but often (under anæesthesia, at any rate) there is little or no inhibition, or even augmentation. In etherized cats, on the other hand, some inhibition is always seen. Of all the afferent fibres of the vagus, the pulmonary fibres produce the most marked reflex inhibition. The cardiac fibres are much less effective.

These pulmonary nerves also influence the respiratory and vasomotor centres. The respiration is temporarily arrested, and the blood-pressure falls through the dilatation of the small arteries when they are excited. It is of interest in connection with the subject of death during the administration of anæsthetics, that the afferent vagus fibres coming from the alveoli of the lungs can be chemically stimulated when irritant vapours, such as chloroform, hydrocliloric acid, ammonia, bromine, or formaldehyde are inhaled through a traclieal cannula, causing reflex arrest of the heart and of the respiratory movements and a fall of blood-pressure through vaso-dilatation (Brodie). At a certain stage in chloroform anesthesia, before it has become very deep, comparatively trifling causes may bring about great and sudden changes in the pulse-rate, owing to the abnormal mobility of the vagus centre (MacWilliam).

The depressor nerve, a branch of the vagus, which is easily found in the rabbit as a slender nerve running close to the sympathetic in the neck, and a little to its inner side, but in the dog is usually blended with the vago-sympathetic, falls into the same category with the vagus itself as regards its reflex action on the heart, to which it bears an important relation. In all mammals some of its fibres end in the wall of the aorta, but some of them may run down over the heart to the ventricle. Stimulation of its peripheral end has no effect, for the fibres in it which influence the circulation are afferent, not efferent. But excitation of its central end causes a marked fall of blood-pressure (p. I85), accompanied by, but not essentially due to, a distinct slowing of the heart. If the animal is not anæsthetized, there may be signs of pain, and for this reason the depressor has sometimes been spoken of, somewhat loosely, as the sensory nerve of the heart. The abdominal sympathetic (of the frog) also contains afferent fibres, through which reflex inhibition of the heart can be produced when they are excited mechanically by a rapid succession of light strokes on the abdomen with the handle of a scalpel.

On the other hand, when the central end of an ordinary peripheral ne : ve like the sciatic or brachial is excited, the common effect is pure augmentation (Fig. 78), which sometimes develops itself with even greater suddenness than when the accelerator nerves are directly stimulated. Occasionally, however, the augmentation is abruptly followed by a typical vagus action. Here the reflex inhibitory effect seems to break in upon and cut short the reflex augmentor effect. 
These examples show that certain afierent nerves are especially related to the cardio-inlibitory, and others to the cardio-augnentor, centre, or at least that the central connections of some nerves are such that inhibition is the usual effect of their reflex excitation, while the opposite is the case with other nerves. But it is improbable that the effect of a stream of afferent impulses reaching the cardiac centres by any given nerve is determined solely by anatomical relations. 'The intensity and the nature of the stimulus seem also to have something to do with the result. For when ordinary sensory nerves are weakly stimulated, augmentation is said to be more common than inhibition, and the opposite when they are strongly stimulated. And while a chemical stimulus, like the inhaled vapour of chloroform or aminonia, causes in the rabbit

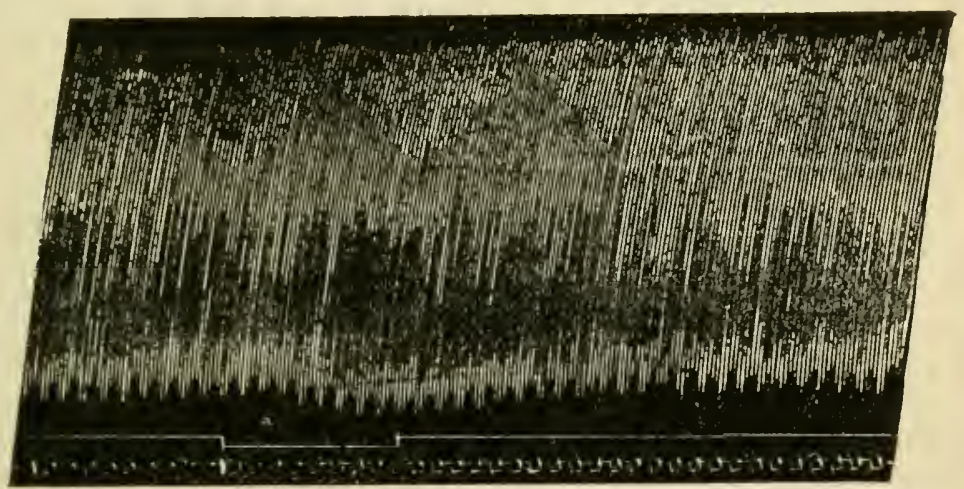

Fig. 78.-Nyocardiographic Tracing of Cat's Ventricle. The signal line shows the point at which the central end of the brachial nerve was stimulated during resuscitation of the animal after a period of cerebral anæmia. Some augmentation of the ventricular beat is seen. The notches in the ventricular tracing are due to the artificial respiration. Time-trace, seconds.

reflex inhibition of the heart through the fibres of the trigeminus that confer common sensation on the mucous membrane of the nose, the mechanical excitation of the sensory nerves of the pharynx and œesophagus when water is slowly sipped causes acceleration.* The stimulation of the nerves of special sense is followed sometimes by the one effect and sometimes by the other. To complete the catalogue of the nervous channels by which impulses may reach tlie cardiac centres in the medulla, we may add that there must be an extensive connection between them and the cerebral cortex, since every passing emotion leaves its trace upon the curve of cardiac action. The so-called 'reflex cardiac death,' which is an occasional consequence of intense psychical influences (anxiety, fright, etc.),

* In 78 healthy students the average pulse-rate (in the sitting position) was increased from 73 to $S_{5}$ per minute by sipping water. 
may be due to the prolonged excitation of the cardio-inhibitory centre, as well as to the disturbance of other centres in the bulb by the cortical storm. It is a remarkable fact, too, and one that can only be explained by such a connection, that although in the vast majority of individuals the will has no influence whatever on the rate or force of the heart, except, perhaps, indirectly through the respiration, some persons liave the power, by a voluntary effort, of markedly accelerating the pulse. In one case of this kind it was noticed that perspiration broke out on the hands and other parts of the body when the heart was voluntarily accelerated. A rise of blood-pressure due to constriction of the vessels has also been observed. The effort cannot be kept up for more than a short time, and the pulse-rate quickly goes back to normal. It has been recently shown that this peculiar power is more common than has been suppused, and that where it is present in rudinsent it can be cultivated, although it is a dangerous acquisition.

Is an example of the direct action on a cardiac centre of a changed chemical composition of the blood, we may cite the inhibition produced by injection of bile into a vein and revealed in the slow pulse of many cases of janndice; and as an instance of the direct action of a physical change, the slowing of the heart as the blood-pressure rises (p. I88) in asphyxia or on clamping the aorta. The variation in the pulse-rate associated with changes in the position of the body, to which we have already referred (p. 107), is brought about by direct stimulation of the inhibitory centre by the increase of blood-pressure in the medulla oblongata when a person who has been standing assumes the supine, or even the sitting, posture. But it is also due in part to clianges in the amount of muscular contraction, since muscular exercise causeacceleration of the heart either reflexly, through afferent muscular nerves, or by a direct effect of waste products of the metabolism of the muscles on the cardiac centres in the bulb or on the heart itself (p. 280).

Theoretically, quickening of the heart might be caused either by a diminution in the inhibitory tone or by an increase in the activity of the augmentor centre; and slowing of the heart might be due either to a diminution in the augmentor tone, if such exists, or to an increase in the activity of the inhibitory centre. So that it is not always easy to interpret such results as we have quoted above. But it would appear that under ordinary conditions the rate of the heart is mainly regulated by the inhibitory centre, which, within a cunsiderable range, can produce variations in either direction. The augmentor mechanism is perhaps merely auxiliary to the inhibitory, being called into action only in emergencies. 
Shction VI.-The Nervous Regulation of the Bloodvessels (VASO-Motor Nerves).

Just as the muscular walls of the heart are governed by two sets of nerve-fibres, a set which keeps down the rate of working and a set which may increase it, the muscular walls of the vessels are under the control of nerves which have the power of diminishing their calibre (vaso-constrictor), and of nerves which have the power of increasing it (vaso-dilator). All nerves that affect the calibre of the vessels, whether vaso-constrictor or vaso-dilator, are included under the general name vasn-motor. These vaso-motor nerves, like the augmentor and inhibitory fibres of the heart, are connected with a centre or centres, which in turn are in relation with numerous afferent nerves. It is convenient to distinguish the afferent nerves which cause on the whole a vaso-constriction and a consequent increase of arterial pressure as pressor nerves, and those which cause on the whole vaso-dilatation, with fall of pressure, as depressor nerves, reserving the terms vaso-constrictor and vaso-dilator for the efferent portions of the reflex arcs. It is through this reflex mechanism that the bloodvessels are mainly influenced, although the endings of the vaso-motor nerves in the smooth muscular fibres or the muscular fibres themselves are sometimes directly affected by substances circulating in the blood. Proteoses, for instance, cause by peripheral action dilatation of the vessels and a fall of blood-pressure (p. 215); suprarenal extract, or its active principle, adrenalin, or epinephrin, constriction, with a rise of pressure (pp. 2I6, 655). Apocodeine paralyzes the vaso-motor nerve-endings after a preliminary stimulation, and now adrenalin causes no constriction. Chrysotoxin, an active principle of ergot, causes a marked rise of bloodpressure by stimulating the sympathetic ganglion-cells or the preganglionic fibres of the vaso-constrictor path. Vaso-motor nerves control chiefly the small arteries. They have no direct influence on the capillaries.* Nor has the existence of an effective vaso-motor regulation of the calibre of the veins, except in the portal system, been proved up to this time by any clear and unambiguous experiment, although there are grounds on which it has been surmised that the nervous system does influence the 'tone' of the whole venous tract. These grounds will be mentioned in the proper place.

* It is usually taught that the capillaries, being devoid of muscular fibres in their walls, are not supplied with vaso-motor fibres, and that the only kind of active contraction of which they are capable is due to a process analogous to the turgescence of regetable cells, the thickness of the wall being increased at the expense of the lumen, while the total cross-section of the vessel remains unchanged. It has been asoerted, however, that a true contraction, in which both the total section and the lumen are diminished, may be caused in the capillaries of the nictitating membrane of the frog either by direct stimulation or by excitation of vaso-motor fibres in the sympathetic (Steinach and Kahn). 
Meanwhile, before describing the distribution of the best-known tracts of vaso-motor fibres and defining the position of the vasomotor centres, we must glance at the methods by which our knowledge has been attained.

(I) In translucent parts inspection is sufficient. Paling of the part indicates constriction; flushing, dilatation of the small vessels. This method has been much used, sometimes in conjunction with (2), in such parts as the balls of the toes of dogs or cats, the ear of the rabbit, the conjunctiva, the mucous membrane of the mouth and gums, the web of the frog, the wing of the bat, the intestines, etc.

(2) Observation of changes in the temperature of parts. This method has been chiefly employed in investigating the vaso-motor nerves of the limbs, the thermometer bulb being fixed between the toes. In such peripheral parts the temperature of the blood is normally less than that of the blood in the internal organs, because the opportunities of cooling are greater. The effect of a freer circulation of blood (dilatation of the arteries) is to raise the temperature; of a more restricted circulation (constriction of the arteries), to lower it.

(3) Ileasurement of the blood-pressure. If we measure the arterial blood-pressure at one point, and find that stimulation of certain nerves increases it without affecting the action of the heart, we can conclude that upon the whole the tone of the small vessels has been increased. But we cannot tell in what region or regions the increase has taken place; nor can we tell whether it has not been accompanicd by diminution of tone in other tracts.

But if we measure simultaneously the blood-pressure in the chief artery and chief vein of a part such as a limb, we can tell from the changes caused by section or stimulation of nerves whether, and in what sense, the tone of the small vessels within thisarea has been altered. For example, if we found that the lateral pressure in the artery was diminished, while at the same time it was increased in the vein, we should know that the 'resistance' between artery and vein had been lessened, and that the blood now found its way more readily from the artery into the vein. If, on the other hand, the venous pressure was diminished, and the arterial pressure simultaneously increased, we sliould have to conclude that the vascular resistance in the part was greater than before. If the pressure both in artery and vein was increased, we could not come to any conclusion as to local changes of resistance without knowing how the general blood-pressure had varied.

(4) The measurement of the velocity of the blood in the vessels of the part. This may be done by the stromuhr or dromograph, or by allowing the blood to escape from a small vein and measuring the outflow in a given time, or, without opening the vessels, by estimating the circulation-time (p. r 35). When changes in the general arterial pressure are eliminated, slowing of the blood-stream through a part corresponds to increase of vascular resistance in it; increase in the rate of flow implies diminished vascular resistance. Sometimes the red colour of the blood issuing from a cut vein, and the visible pulse in the strcam, indicate with certainty that the vessels of the organ have been dilated.

(5) Alterations in the volume of an organ or limb are often taken as indications of changes in the calibre of the small vessels in it. We have already seen how these alterations are recorded by means of a plethysmograph (p. 128 ). The brain is enclosed in the skull as in a natural pletlyysmograph, and changes in its volume may be registered by connecting a recording apparatus with a trephine hole. 
(6) For the separation of the effects of stimulation of vaso-constrictor and vaso-dilator fibres when they are mingled together, as is the case in many nerves, advantage is taken of certain differences between them. For example, the vaso-constrictors lose their excitability soower than the raso-dilators when cut off from the nerve-cells to which they belong. So that if a nerve is divided, and some days allowed to elapse before stimulation, only the dilators will be excited. The vaso-dilators are more sensitive to weak stimuli repeated at long intervals than to strong and frequent stimuli, and the opposite is true of the constrictors. When a nerve containing both kinds of fibres is heated, the excitability of the raso-constrictors is increased in a greater degree than that of the dilators; when the nerve is cooled, the dilators preserve their excitability at a temperature at which the constrictors liave ceased to respond to stimulation (Fig. 79).

The Chief Vaso-Motor Nerves.-The first discovery of vaso-motor nerves was made in the cervical sympathetic. When this nerve is

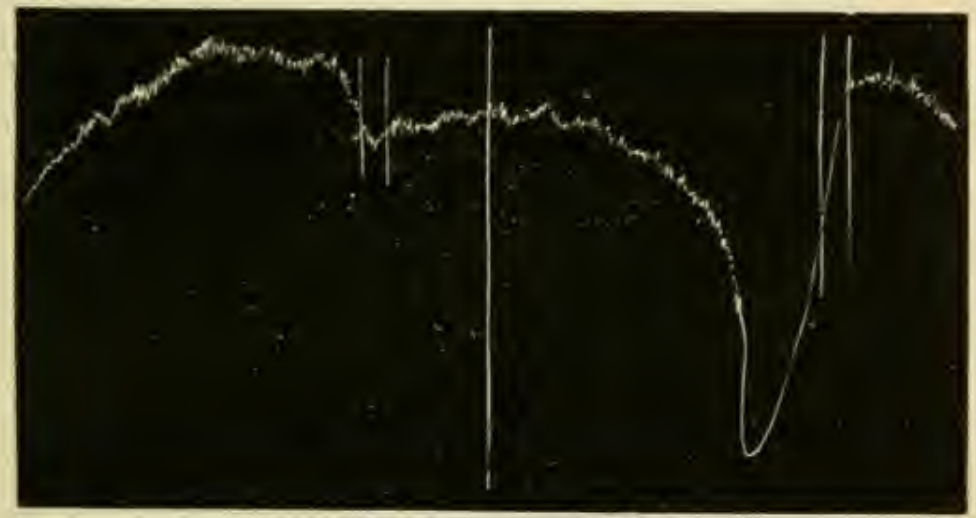

Fig. 79.-Plethysmograms: Hind-Limo of Cat (after Bowditch and Warren). To be read from right to left. On the left hand is shown the effect of slow stimulation of the sciatic (I per second); on the right hand the effect of rapid stimulation (64 per second). In the first case the limb swelled owing to excitation of the vaso-dilators; in the second, it shrank trough excitation of the vaso-constrictors.

cut, the corresponding side of the head, and especially the ear, become greatly injected owing to the dilatation of the vessels. This cxperiment can be very readily performed on the rabbit, and the changes are most easily followed in an albino. The ear on the side of the cut nerve is redder and hotter than the other; the main arteries and reins are swollen with blood, and many vessels formerly invisible come into view. The slow rhythmical changes of calibre, which in the normal rabbit are very characteristically seen in the middle artery of the ear, disappear for a time after section of the sympathetic, although they ultimately again become visible.

Stimulation of the cephalic end of the cut sympathetic causes a marked constriction of the vessels and a fall of temperature on the same side of the head. From these facts we know that the cervical 
sympathetic in maminals contains vaso-constrictur fibres for the side of the head and ear, and that these fibres are constuntly in action. Certain parts of the eyc, and the salivary glands, larynx, oesophagus, and thyroid gland, are also supplied with vaso-motor (constrictor) nerves from the cervical sympathetıc.

It has been asserted that the cervical svmpathetic contains some of the vaso-constrictor fibres that supply the correspondiris half of the brain and its membranes, but this has been disputed, and sone observers deny that the vessels of the brain have any vaso-motor nerves. Non-medullated nerve-fibies, however, may be seen in and around the walls of the cerebral and spinal bloodvessels, and it is difficult to believe that these have not a vaso-motor function, although this has not as yet been clearly demonstrated by experimental methods.

It has sometimes been argued that we ouglit not to expect the brain to be supplied witl raso-motors. For it is enclosed in a rigid box, and the quantity of blood in it can be increased or diminished only to the slight extent to which the cerebro-spinal liquid can be displaced into the vertebral canal. Important clianges in the cerebral blood-supply are therefore brought about, it is said, not by a widening or narrowing of the cerebral vessels, but by an alteration in the velocity of the blood in them as a result of a rise or fall of the systemic arterial pressure. This argument, however, leaves out of account the consideration that in general the brain does not function as a whole, but that certain parts of it may often become active and relatively hyperzmis, while other parts are inactive and relatively ancmic, and that important changes in the distribution of the blood in the encephalon may be effected, although the total mass of blood in the organ undergoes little or no alteration. It is, of course, true that it is not the absolute quantity of blood in an organ whicl is a function of its activity, but the rate at which it is renewed. And it is theoretically possible that an organ at rest should contain as much blood as when it is active, or even morc. But such cases, if they exist, are certainly rare. The fact that adrenalin generally constricts the vessels of a perfused brain (Wiggers) is in favour of the existence of vaso-motors. The retina, which from the standpoint of development is a portion of the brain, is undoubtedly supplied witl vaso-constrictor fibres which run in the cervical sympathetic.

That the cervical sympathetic contains some dilator fibres is proved by the fact that stimulation of the cephalic end in the dog causes flushing of the mucous membrane of the mouth on the same side. Further, after division of the nerve on one side in the rabbit it may be observed that when the animal is excited the ressels of the ear whose nerve is intact may become still more dilated than those whose constrictor fibres have been paralyzed. The only explanation is that vaso-dilators are being excited from the central nervous system.

The vaso-motor fibres of the head run up in the cervical sympathetic, and then pass into various cerebral nerves, of which the fifth or trigeminus is the most important.

The trigeminus nerve contains vaso-constrictor nerves for various parts of the eye (conjunctiva, sclerotic, iris), and for the mucous 
nembrane of the nose and gums, and section of it is followed by dilatation of the ressels of these regions. The lingual branch of the trigeninus supplie's vaso-motor fibres to the tongue, and apparently both vaso-constrictor and vaso-dilator.

In some animals-the rabbit, for instance-the ear derives part of its vaso-motor supply through the great auricular nerve, a branch of the third rervical nerve, which they reach as grey rami from the stellate ganglion.

Another great vaso-motor tract, the most influential in the body, is contained in the splanchnic nerves, which govern the vesscls of many of the abdominal organs. Section of these nerves causes an immediate and sharp fall of arterial pressure. The intestinal vessels are dilated and overfilled with blood. As a necessary consequence of their immense capacity, the rest of the vascular system is underfilled, and the blood-prcssure falls accordingly. Stimulation of the peripheral end of the splanchnic nerves causes a great rise of bloodpressure, owing to the constriction of vessels in the intestinal area. We therefore conclude that in the splanchnics there are vaso-motor fibres of the constrictor type, and that impulses are constantly passing down them to maintain the normal tone of the vascular tract which they command. When the splanchnic nerves are stimulated, the adrenal glands are so affected that adrenalin passes out by the veins into the blood-stream. It is clear that if the quan. tity thus liberated were sufficiently large and its liberation sufficiently prompt it might play a part in the rise of pressure (p. 66I) which follows stimulation of the nerves, whether they are excited directly or in the normal course of events reflexly. But it has not been demonstrated that this is an effective factor. Dilator fibres (for the intestines and the kidney. for example) have also been discovered in the splanchnic nerves, although the constrictors predominate, and special methods have to be employed for the detection of the dilators.

The same is true of the nerves of the extromities, which certainly contain vaso-dilator fibres in addition to vaso-constrictors, although the difficulty of demonstrating the presence of the former is fully as great as it is in the splanchnics. For the investigation is complicated by the fact that such nerves as the sciatic supply with vaso-motor fibres two leading tissues-skin and muscle; and these are not necessarily affected in the same direction or to the same extent by stimulation of their vaso-motor fibres. The vaso-constrictors under ordinary conditions preponderate, so that section of the sciatic or the brachial is generally followed by flushing of the balls of the toes and rise of temperature of the fect, stimulation by paling and fall of temperature. By taking advantage, however, of the unequal excitability of clilators and constrictors in a ilegenerating nerve, and of the differences between the two kinds of fibres in their 
reaction to electrical stimuli (p. 175), it has been shown that vasodilators are also present, and come to the front when the conditions are rendered favourable for them and unfavuurable for the constrictors.

Vaso-motor fibres for the fore-limb (dog) issue from the cord in the anterior roots of the third to the eleventh dorsal nerves, and for the hind-limb in the anterior roots of the eleventh dorsal to the third lumbar. Stimulation of most of lliese roots causes constriction of the vessels but stimulation of the eleventh dorsal may cause dilatation (Bayliss and Brálford).

The Taso-Motor. Veres of . Wuscle. When the motor nerve of the thin mylo-liyoid muscle of the frog, which can be observed under the microscope, is cut, and the peripheral end stimulated, the vessels are seen to clilate distinctly, and this effect is not abolished when contraction of the muscle is prevented by a dose of curara insufficient to paralyze the vaso-motor nerves. This indicates the existence in the nerve of vasodilator fibres. But we must be cautious in transferring this result to ordinary skeletal muscle, for the mylo-hyoid is more closely allied to the muscles of the tongue than, for cxample, to the muscles of the limbs. and in the mammal the tongue muscles are known to be better supplied with vaso-dilator fibres than the limb muscles. The average flow of blood through a mammalian muscle is indeed increased during volun tary contraction, and during rhythmically repeated artificial tetanization of its motor nerve. The outflow of blood from the main vein of the levator labii superioris, one of the muscles used in feeding in the horse, was found to be in one experiment nearly eight times, in an ther about seven times, and in a third three and a half times as great during voluntary work witl it (in chewing) as in rest. But as no increase in the blood-flow through the skeletal muscles of a completely curarized mammal during excitation of their nerves has ever been satisfactorily demonstrated, we must conclude that they are very scantily provided with vaso-dilator fibres or not at all. It is uncertain whether they are supplied with vaso-constrictors. The undoubted increase in the bloodflow in contraction may therefore be connected in some way with the mcchanical or chemical changes in the muscular fibres themselves.

It has been suggested that the muscular vessels are widened by the (lirect action of the acici products of the active muscle, since very dilute acids (lactic acid, e.g.) cause general dilatation of the small vessels. A similar cxplanation has been extended to the dilatation of the vessels of the brain during cercbral activity by some of those who deny the existence of vaso-motor nerves for that organ, but here the evidence is by no means satisfactory. The vagus lias been stated to contain vaso-constrictor, and the annulus of lieussens vaso-dilator, fibres for the coronary arteries of the heart. But this question is far from being settled. Adrenalin causes dilatation and not constriction of the coronary vessels. There is some reason to belicre that the metabolic products liberated in the heart-muscle, e.g., carbon dioxide, govern the changes in the calibre of the coronary arterioles. A close relationslip exists between the output of carbon dioxide and the rate of flow through the coronary circulation. In asplyyia the flow through the coronary ressals is notably increased; indeed, it is at its maximum just before the heart fails altogether, as if an effort were being made to keep the heart going to the last by making up to it in the yuantity of the blood supplied what it lacks in quality. As this increased flow is seen in the isolated heart-lung preparation, it has been concluded that metabolites produced in the carliac muscle itsclf cause an increased coronary flow when increased demand, are made on the heart, a local regulative 
mechanism bcing tlus constituted. There is some evidence that carbon clioxide is not the most potent of these substances.

Vaso-. Hotor Neres of the Lungs.-There lias been much discussion as to the course, and even as to the cxistence, of vaso-motor fibres for the lungs. The problem is perhaps the most difficult in the whole range of vaso-motor topography, for the pulmonary circulation is so related to other vascular tracts, that changes produced in the ressels of distant organs by the stimulation or section of nerves may affect the quantity of blood received by the right side of the heart, and therefore the quantity propelled through the lungs and the pressure in the pulmonary artery. And changes in the systemic arterial pressure may favour or hinder the discharge of the left ventricle, and therefore affect the pressure in the left auricle and the pulmonary veins. Nevertheless, evidence has been obtained from a number of sources that the lungs are supplied with vaso-constrictor fibres. Plumier, perfusing isolated, 'surviving' lungs with blood under constant pressure and measuring the outflow, showed that adrenalin and also stimulation of the annulus of Vieussens caused great diminution in the flow-that is, constriction of the vessels. Wiggers also obtained constriction with adrenalin. Fühner and Starling, working with a preparation including the heart as well as the lungs, found that adrenalin caused a rise of pressure in the pulmonary artery coupled with a fall of pressure in the left auricle, which could only be due to constriction of the vessels of the lungs. It is assumed that adrenalin produces vaso-constriction only in vessels supplied with vaso-constrictor nerves (p. 65.5), and that in organs where this substance does not cause vaso-constriction no such fibres are present. In mammals the vaso-constrictor fibres seem to pass out from the upper half of the dorsal spinal cord, and some of them can be detected nearer their destination in the annulus of Vieussens. The vago-sympathetic of the tortoise contains vaso-constrictors for the lung of the same side (Krogh).

Vaso-Dilator Fibres.-In most of the peripheral nerves these are mingled with vaso-constrictors; but in certain situations, for an anatomical reason that will be mentioned presently, nerves exist in which the only vaso-motor fibres are of the dilator type. Of these, the most conspicuous examples are the chorda tympani and the nervi erigentes or pelvic nerves; and, indeed, it was in the chorda that vaso-dilators were first discovered by Bernard. The chorda tympani contains vaso-dilator and secretory fibres for the submaxillary and sublingual salivary glands. With the secretory fibres we have at present nothing to do; and the whole subject will have to be returned to, and more fully discussed in Chapter VI. But a most marked vascular change is produced by stimulation of the peripheral end of the divided chorda tympani nerve. The glands flush red; more blood is evidently passing through their vessels. Allowed to escape from a divided vein, the blood is seen to be of a bright arterial colour and shows a clistinct pulse. The small arteries have been dilated by the action of the vaso-motor fibres in the nerve. The resistance being thus reduced, the blood passes in a fuller and more rapid stream through the capillaries into the veins, and on the way there is not time for it to become completely venous. These vaso-dilator fibres are not in constant action, for section of the 
nerve, as a rule, produces little or no change. Vaso-conntrietor fibres pass to the salivary glands from the cervical svmpathetiv along the arteries, and stimulation of that nerve causes narrowing of the ressels and diminution of the blood-flow, sometimes almost to complete stoppage.

The nervi crigentes are the nerves through which erection of the penis is caused. When they are divided there is no effect, but stimulation of the peripheral end causes dilatation of the vessels nf the erectile tissue of the organ, which becomes overfilled with blood. During stimulation of these nerves, the quantity of blood flowing from the cut dorsal vein of the penis may be fifteen times greater than in the absence of stimulation. It spurts out in a strong stream, and is $b_{i}$. hter than ordinary venous blond (Eckhard). Stimulation of the peripheral end of the nerius pudendus cause: constriction of the vessels of the penis, so that it contains vasoconstrictor fibres which are the antagonists of the nervi erigentes.

Vaso-Motor Nerves of Veins.-.-Like arteries, veins have plexuses of nerve-fibres in their walls, and contract in response to various stimuli. In some cases-e.g., in the wing of the bat-rhythmical contractions of the veins are strikingly displayed, but they do not depend on the central nervous system, as they persist after section of the brachial nerves. The existence of vaso-constrictor fibres for the venules given off in the liver by the portal vein is indicated by the fact that adrenalin diminislies the blood flow through the organ even when the hepatic artery has been tied (Burton-Opitz; Macleod and Pearce, etc.). Stimulation of the distal end of the hepatic plexus causes similar effects. The fibres issue from the spinal cord by the anterior roots of the third to the eleventh dorsal nerves, but chiefiy in the fifth to the ninth dorsal. The arterioles arising from the hepatic artery have their own vaso-motor supply. which is more complete than that of the portal vessels. When the liver is enclosed in a plethysmograph, and the central end of an ordinary sensory nerve. like the sciatic. excited, reflex vaso-constriction takes place in the portal area, the volume of the organ diminishes, and the blood-pressure rises in the portal vein (Irançois-Iranch).

The vena portæe and its branches are in the phrsiological sense arteries rather than veins, since they break up intri rapillaries, and it was to be expected that the regulation of the bloud-flow in them would be carried out in the same way as in ordinary arteries, namely. by means of vaso-motor nerves. But we must not, without special proof, extend the results obtained in the portal system to ordinary veins. A rertain amount of evidence, however, exists that even such veins as those of the extremities are supplied, though scantily, with vaso-constrictor (veno-motor) fibres. After ligation of the crural artery or aorta, stimulation of the peripheral ('nd of the sciatic has been scen tr cause contraction of short portions of the superficial veins of the leg. 
linally, adrenalin (epineplirin) causes constriction of rings of 'surviving ' veins just as of artery rings, altlıugh in correspondence with the smaller amount of muscular tissue in the former the contraction is not so strong. As adrenalin is assumed to act only upon muscle supplied by sympathetic nerve-fibres (p. 655), this would seem to indicate the existence of such a supply for veins. The question is an important one in connection with the regulation of the filling, and therefore of the discharge, of the heart (Henderson), but the experimental data are as yet too meagre to justify further discussion of the matter here.

Course of the Vaso-Motor Nerves.-In the dog the vaso-constrictors pass out as fine medullated fibres $(x \cdot 8$ to $3.6 \mu$ in diameter) in the anterior roots of the second dorsal to about the second lumbar nerves. They proceed by the white rami communicantes to the lateral sym. pathetic ganglia, where, or in more distal ganglia such as the inferior mesenteric, they lose their medulla, and their axis-cylinder processes (p. 851) break up into fibrils that come into close relation with the nerve-cells of the ganglia. These ganglion-cells in their turn send off axis-cylinder processes, which, enveloped by a neurilemma, pass as non-medullated fibres by various routes to their final destination, the unstriped muscular fibres of the bloodvessels. Their course to the head has been already described. To the limbs they are distributed in the great nerves (brachial plexus, sciatic, etc.), which they reach from the sympathetic ganglia by the grey rami communicantes.

The outflow of vaso-dilator fibies is not restricted to the same portion of the cord from which the outflow of constrictor fibres takes place. Their cxistence is indeed most easily demonstrated in nerves springing from those regions of the cerebro-spinal axis from which vaso-constrictor fibres do not arise, and where, therefore, we have not to contend with the difficulty of interpreting mixed effects. Vaso-dilators for the external generative organs and the mucous membrane of the lower end of the rectum pass out as small medullated fibres of the anterior roots. of certain of the sacral nerves (mainly the second and third in the cat) into the pelvic nerve (nervus erigens). They end in relation witl ganglion-cells in the neighbourhood of the organs which they supply. The seventh and ninth cranial nerves carry vaso-dilator fibres which are distributed by way of the lingual and other branches of the fiftl to the salivary glands, the tongue, the mucous membrane of the floor of the mouth, and part of the soft palate. Those in the lingual, passing through the chorda tympani, end in gangiion-cells near the submaxillary and sublingual glands, and the axons of these cells continue the path to the ressels of the glands. It is supposed that the vaso-dilators distributed in other branches of the fifth also have ganglion-cells on their course. In fact, there is good evidence that every efferent vaso-motor fibre is interrupted by one, and only by one, ganglion-cell between the cord and the bloodvessels. The statement has been made that for certain regions of the body, especially the skin of the limbs, the vasodilator nerves are contained, not in the anterior, but in the posterior roots. And these, it is claimed, are not aberrant efferent fibres which have strayed in the course of development into the wrong roots, but true posterior root-fibres whose cells of origin lic in the spinal ganglia, and which conduct efferent vaso-dilator impulses in the wrong direction, so to speak, from the cord to the periphery- 'antidromic' impulses (Bayliss). 
Effect of Nicotine on Nerve-Cells. - 1 method which has bcen found most fruitful in studying the relations of sympathetic ganglion-cells to the vaso-motor fibres, as well as to the pilo-motor* and secretory filores which in certain situations are so intricately iningled with them, must here be mentioned. It depends upon the fact that when is suitable dose of nicotine (ro milligrammes in a cat) is injected into a rein, or a solution is painted on a ganglion with a brush, the passage of nervempulses through the ganglion is blocked for a time (Langley). The nerve-fibres peripheral to the ganglion are not affected. The question whether efferent fibres are connected with nerve-cells between a given point and their peripheral distribution can, therefore, be answered by observing whether any effect of stimulation is abolished by nicotine. If, for instance, the excitation of a nerve caused constriction of certain bloodvessels before, and has no effect after, the application of nicotine to a ganglion, its vaso-constrictor fibres, or some of them, must be connected with nerve-cells in that ganglion. Langley has brought forward evidence that many of the bodies which are commonly supposed to act upon nerve-endings (as nicotine, curara, atropine, pilocarpine, adrenalin. etc.) really act upon 'receptive' substances of the cells in connection with which the nerve-fibres end. These receptive substances are conccived to be capable of being specifically affected by cliemical bodics and by nervous stimuli, and in their turn to be capable of influencing the metabolism $\mathrm{cf}$ the main cell substance on which its function depends. The recentive substances thus form beyond the histological link of the nerve-cnding a kind of cliemical link between the nerve-fibre and the cell which it supplies.

We have thus traced the vaso-motor nerves from the cerebrospinal axis to the bloodressels which they control; it still remains to define the portion of the central nervous system to which these scattered threads are related, which holds them in its hand and acts upon them as the needs of the organism may require.

Vaso-Motar Centres.-Now, experiment has shown that there is one very definite region of the spinal bulb which has a most intimate relation to the vaso-motor nerves. If while the blood-pressure in the carotid is being registered, say, in a curarized raboit, the central end of a peripheral nerve like the sciatic is stimulated, the pressure rises so long as the bulb is intact, this rise being largely due to the reflex constriction of the ressels in the splanchnic area. If a series of transverse sections be made through the brain, the rise of pressure caused by stimulation of the sciatic is not affected till the upper limit of the bulb is almost reached. If the slicing is still carried downwards, the blood-pressure sinks, and the rise following stimulation of the sciatic becomes less and less. When the medulla has been cut away to a certain level, only an insignificant rise or none at all can be obtained. The portion of the medulla the removal of which exerts an influence on the blood-pressurc, and its increase by reflex stimulation, extends from a level 4 to $5 \mathrm{~mm}$. above the point of the calamus scriptorius to within I to $2 \mathrm{~mm}$. of the corpora quadrigemina. Stimulation of the medulla causes a rise, destruc-

* Pilo-motor nerves supply the snooth arrector pili muscles, whose contrac. tion causes the hair to 'stand on end.' 
tion of this portion of it a severe fall, of general blood-pressure. There is evidently in this region a nervous ' centre' so intimately related, if not to all the vaso-motor nerves, at least to sucl very important tracts as to deserve the name of a vaso-motor centre. Experiment has shown that this is much the most influential centre, and it is usually called the chief or general vaso-motor centre. Some writers prefer to speak of it as the vaso-constrictor centre, since it is undoubtedly connected with most or all of the vaso-constrictor paths, and has not been shown to be similarly connected with the vaso-dilator paths. There is, indeed, not the same solid evidence for the existence of a general vaso-dilator centre in the bulb as for the existence of the general vaso-constrictor centre. Yet there are facts which indicate that the bulbar vaso-motor centre or centres, when reflexly stimulated, can, and often do, respond not merely by an increase or a remission of vaso-constrictor tone, but by a simultaneous inhibition of vaso-constrictor fibres and excitation of vascdilators leading to a fall of pressure, or by a simultaneous inlibition of vaso-dilators and excitation of vaso-constrictors leading to a rise of pressure.

The spinal cells of origin of the pre-ganglionic segments of the vaso-constrictor paths constitute subordinate centres which either normally support a certain degree of vascular tone, or come to do so after the chief vaso-motor centre has been cut off.

Thus, in the frog it is possible to go on destroying more and more of the cord from above downwards, and still to obtain reflex vasomotor effects, as seen in the vessels of the web, by stimulating the central end of the sciatic nerve. Although these effects indeed diminish in amount as the destruction of the cord proceeds, yet a distinct change can be caused when only a small portion of the cord remains intact.

Similarly, in the mammal evidence has been obtained of the existence of 'centres' at various levels of the cord, capable of acting eventually, if not at once, as vaso-constrictor centres after the loss of the controlling influence of the bulb. The best example of a vaso-dilator centre is that situated in the lumbar cord, which controls the erection of the penis. After total section of the cord at the upper limit of the lumbar region, erection, which is known to be due to a reflex dilatation of the arteries of the organ through the nervi erigentes, can still be caused (in dogs) by mechanical stimulation of the glans penis, so long as the afferent fibres of the reflex arc contained in the nervus pudendus are intact. Destruction of the lumbar cord abolishes the effect. It is impossible to avoid the conclusior that a vaso-dilator or erection centre, which is in relation on the one hand with the nervi erigentes, and on the other with the nervus pudendus, exists in the lower portion of the spinal cord. Vasomotor centres for the hind-limbs have also been located in the 
same region. When the brain, the bulb, and the upper purtion of the cord have been eliminated by ligation of all the arteries from which blood can possibly reach them, a sufficient vascular pressure persists to permit the circulation to go on in the low'r portion of the body for hours. And while section-or freezing (Fig. 80)-of the cord in the lower cervical region canses a marked fall of pressure. this : not permanent if the animal is allowed to survive. Forty-on:" days after total section of the cord at the seventh cervical segment in a dog an arterial pressure of $130 \mathrm{~mm}$. of mercury was found. A mechanism for the maintenance of vascular tone exists even beyond the limits of the central nervous systen. For when the lower portion of the cord is completely destroyed, the dilatation of the vessels of the hind-limbs, which is at first so conspicuous, passes away after a time, the functions of vaso-motor centres having perhaps been assumed by the sympathetic ganglia (Goltz and Ewald). When the lumbo-sacral sympathetic chain is extirpated,

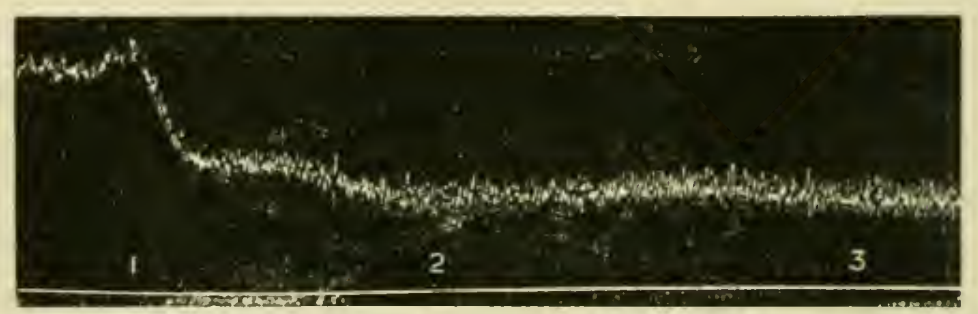

J'ig. 80.-Effect on Blood-Pressure of Freezing Spinal Cord (I'ike). At I the first or second dorsal segment of a dog's cord was frozen with liquid air; at 2 and 3 central end of sciatic stimulated without effect on pressure (respectively one and a half and three minutes after freezing of cord). (Four-fifths of original size.)

there is a further loss of vascular tone in the affected region. But even this is not irremediable. After a time recovery again occurs, although it may be more partial and tardy than before. This may take place either through the intervention of still more peripheral ganglia, or through the development of a certain tonus by the muscular fibres of the vessels when abandoned to themselves.

As to the nature of the tone of the general vaso-motor centre, the same question may bc asked which has been already discussed for the cardio-inhibitory centre. Is it ieflex, or does it depend upon direct excitation of the centre by some constituent of the blood or lymph, or some substance produced in the centre :tsclf? The best answer which can at present be made is that a constant central excitation by the carbon dioxide formed in the centre or circulating in the blood is a not unimportant factor in the maintenance of the vaso-motor tone. A marked diminution in the carbon dioxide tension of the blood, a condition which is termed 'acapnia,' may indeed contribute to the severe fall of blood-pressure associated with 
stergical shock (Henderson). In addition to the direct influence of carbun dioxide, and possibly of other substances, the arrival of afferent impulses at the centre secms to play a part in maintaining that continual discharge of efferent impulses along the vaso-motor nerves which constitutes its tone. In this regard, the vaso-motor centre occupies an intermediate position between the respiratory centre, the most purely automatic, and the cardio-inlibitory centre, the most purcly reflex of the three great bulbar mechanisms.

()f the anatomical relations of the nerve-cells that make up the bulbar and spinal vaso-motor centres, little more is known than mav be deduced from the physiological facts we have been $r$ citing. It has becn surmised on histological grounds that certain cells of small size scattered up and down the thoracic and upper lumbar regions of the cord in the lateral horn (intermedio-lateral tract), and perhaps cropping out also in the bulb, are vaso-motor cells. There is good evidence that the pre-ganglionic sympathetic fibres, including the vaso-motor fibres which we have already discovered emerging from the cord in the spinal ronts, are connected with these cells. And, indeed. tliere is reason to belicve that the conncetion is made without the intervention of any other nerve-cells, and that the axis-cylinders of these vaso-motor fibres are the axis-cylinder processes of the vaso-motor cells. So that the simplest efferent path along which vaso-motor impulses can pass may be considered as built up of two neurons, one with its cell-body in the cord, and the cther in a sympathctic ganglion. Less is known of the clements which constitute the bulbar centre and of their connections. But since it would appzar that the spinal vaso-motor centres are under the control of the chicf centre in the bulb, it is necessary to suppose that the axis-cylinder processes of some of the cells of the bulbar centre come into relation with the spinal vaso-motor cells, and that impulses passing, let us siby, irom the bulb to the vessals of the leg, would have to traverse three neurons (p. 852).

Vaso-Motor Reflexes.-We have already seen that the cardiac centres are constantly influenced by afferent impulses, and that in the direction either of augmentation or inhibition. The vaso-motor centre in the bulb is equally sensitive to such impulses. They reach it for the most part along the same nerves, and by increasing or diminishing its tone cause sometimes constriction and sometimes dilatation of the vessels, the result depending partly upon the anatomical connection of the afferent fibres, but apparently in part also upon the state of the centre.

Of the afferent nerves that cause vaso-dilatation, the most important is the depressor, whose reflex inhibitory action on the heart has been already described. The fall in the arterial pressure is due chiefly, not to the inhibition of the heart, but to inhibition of the vaso-constrictor tone of the bulbar vaso-motor centre, combined with stimulation of vaso-dilator nerves, and consequent general dilatation of the arterioles throughout the body. That the depressor action involves excitation of vaso-dilators follows from the fact that vaso-dilatation occurs in the limbs on stimulation of the depressor after their vaso-constrictor nerves have been cut. Stimulation of the central end of the depressor may also cause dilatation of the 
vessels of the submaxillary gland even on the opposite side, whether the sympathetic has been divided or not, so long as the chorda tympani is intact, and this dilatation is not accompanied by a flow of saliva. Stimulation of the depressor produces its usual result after section of the vagi. It has been suggested that the function of the nerve is to act as an automatic check upon the blood-pressure in the interest both of the heart and the vessels, its terminations in the aorta or the ventricular wall being mechanically stimulated when the pressure tends to rise towards the danger limit. In rare cases, efferent inhibitory fibres for the heart have been found in the depressor of the rabbit.

Many of the peripheral nerves contain fibres whose stimulation is folloved by clilatation of the

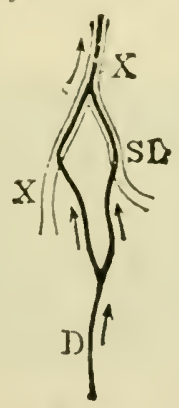

Fig. 81.-Diagram of Depressur in Rabbit. $\mathbf{X}$, vagus; $\mathrm{SL}$, superior laryngeal; $D$, depressor. Arrows sbow course of impulses.

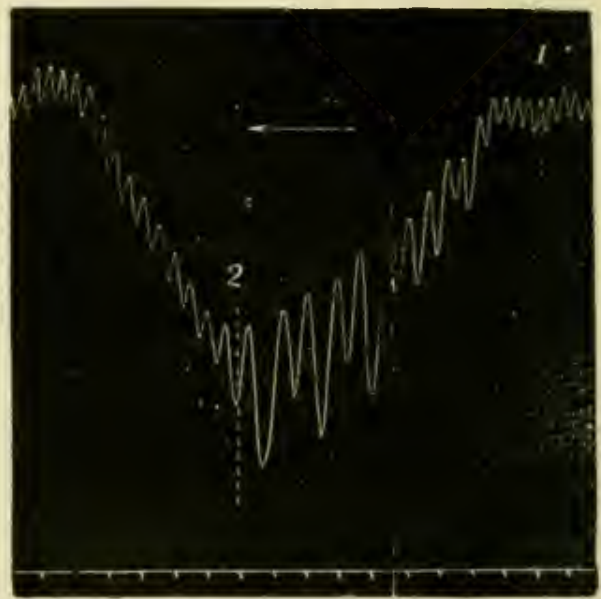

Fig. 82.-Blood-Pressure Tracing: Rabbit. Central end of depressor stimulated at I; stimulation stopped at 2. Time-trace, seconds.

bloodvessels in special regions, usually the areas to which they are themselves distributed, accompanied by constriction of distant and, it may be, more extensive vascular tracts. Thus, the usual local effect of stimulating the afferent fibres of the lowest three thoracic nerves, in whose anterior roots run the vaso-motor fibres for the kidney, is a dilatation of the renal vessels (Bradford), and the usual local effect of stimulating the infra-orbital or supra-orbital nerve a dilatation of the external maxillary artery. But the general effect in both cases is vaso-constriction in other regions of the body, which more than compensates the local dilatation, so that the arterial bloodpressure rises. It is not difficult to see that both of these changes render it easier for the part to obtain an increased supply of blood.

Sometimes the reciprocal relation between vaso-dilatation in one part of the body and vaso-constriction in another is only apparent. For example, stimulation of the cut end of the sciatic canses, as we have already seen, extensive vaso-constriction and a notable rise in the bloodpressure. The constriction certainly involves the splanchnic area; but 
superficial parts, as the lips, may be seen to be fluslied with blood. In asplayidi, when the vaso-motor centres are directly stimulated by the venous blood, this apparent antagonism is still better marked: the cutancous vessels are widely dilated and engorged, the face is livid, but the abdominal organs are pale and bloodless (Heidenhain). The blood-pressure rises rapidly, rcaches a maximum, and then gradually falls as the vaso-motor centre becomes paralyzed (Figs. 84 and 85 ). It has been shown that in both cases vaso-constriction of the skin is really produced as well as raso-constriction of the internal organs, but the increased blood-pressure mechanically overcomes the constriction of the cutaneous vessels.

The kind of stimulus seems to have something to do with the direction of the reflex vaso-motor change. For while electrical stimulation of every muscular nerve, even of the very finest twigs that can be isolated and laid on electrodes, provokes always, whether the shocks follow each other rapidly or slowly, a rise of general

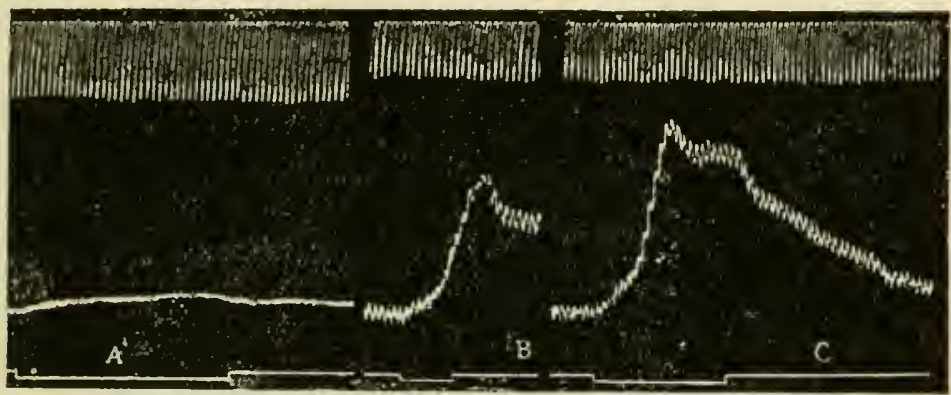

Fig. 83.-Pressor Effect of Stimulation of Central End of Vagus in a Cat during Resuscitation after Cerebral Anæmia. The depressions in the signal line ABC indicate the duration of three successive excitations of equal strength, sixty-five, seventy-three, and seventy-nine minutes respectively after restoration of the circulation. The pressor effect increases as resuscitation proceeds. Later on the original depressor effect was again obtained. The upper tracing is that of the artificial respiration. (Two-thirds original size.)

blood-pressure, mechanical stimulation of a muscle, as by kneading or massage, causes a fall. The condition of the afferent fibres also exerts an influence. For example, excitation of the central end of a sciatic nerve that has been cooled is followed by vaso-dilatation and fall of pressure, the opposite of the ordinary result. These and similar facts have led to the idea that most afferent nerves contain two kinds of fibres, whose stimulation can affect the actrity of the vaso-motor centres - 'reflex vaso-constrictor,' or 'pressor ' fibres, and 'reflex vaso-dilator,' or 'depressor ' fibres. The branch of the vagus, however, to which the name 'depressor ' has been specially given is usually described as the only peripheral nerve the excitation of which is in all circumstances followed by a general diminution of arterial pressure. But this is not strictly correct, for at an early period in the resuscitation of the brain after anæmia excitation of 
the rabbit's ' depressor ' causes a slight rise of pressure not followed br any fall. This, perhaps, indicates the presence in the ' depr.ssor' of a small number of pressor fibres, which are resuscitated sooner than the depressor fibres proper. The same phenomenon, only more marked, may be seen when the central end of the cat's vagus: containing the depressor fibres, is excited at intervals during resuscitation (Fig. 83). Or the result may depend upon a change in the. response of the altered vaso-motor centres to impulses reaching them along the depressor fibres. If specific 'depressor ' fibres exist in other nerves, they are so mingled with 'pressor ' fibres that their action is masked when both are stimulated together. The state of the vaso-motor centre is unquestionably a factor which lats some importance in determining the result of reflex vaso-motor stimulation. For instance, in an animal deeply anæsthetized with chloroform or chloral, excitation of pressor fibres (in an ordinary sensory

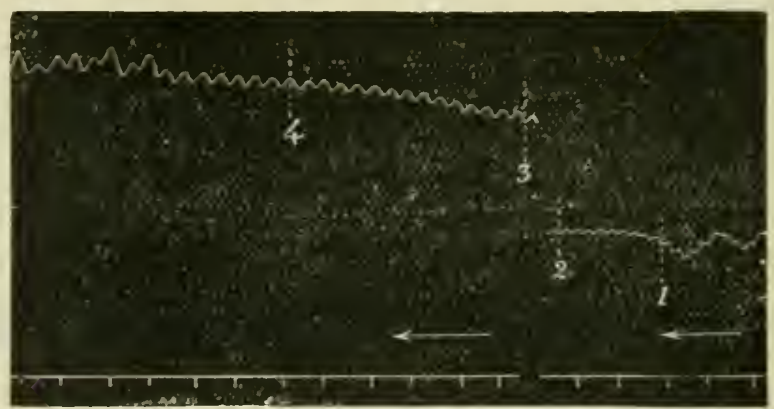

Fig. 84.-Rise of Blood.Pressure in Asphyxia: Rabbit. Respiration stopped at I. Interval between 2 and 3 (not reproduced) 44 seconds, during which the bloud. pressure steadily rose. At 4 , respiration resumed. Time-trace, seconds.

nerve) causes, not a rise, but a fall of blood-pressure; while in an animal fully under the influence of strychnine stimulation of the depressor nerve causes not a fall, but a rise.

The vaso-motor reflexes in man can be conveniently studied by the calorimetric method described on p. 221 . Onc of the most important of the vaso-motor reactions is that by which the vessels of the skin respond to the temperature of the environment so as to regulate the loss of heat from the body (p: 699). When one hand, $e g$. the left, is immorsed in cold water (say at about $\delta^{\circ} \mathrm{C}$.), the blood-flow in the right is at once reduced owing to reflex rasoconstriction. Other parts of the body are also affected, but not so readily as the contra-lateral hand, since the segments of the cord into which the afferent fibres from a given skin area run are at the same time the segments from which the efferent vaso-motor fibres for the symmetrically-placed area on the opposite side of the body arise. The reflex diminution in the flow persists for a time which 
varies with the individual, the external temperature, and other circumstances, and then as a rule rather suddenly the vaso-constriction gives way and the flow begins again to increase, even while the left hand is still kept in the cold water. When the left hand is transferred from the cold to warm water (at $43^{\circ}$ or $44^{\circ} \mathrm{C}$.), the first effect is a transient climinution in the blood-flow in the right hand. This soon gives place to an increase (vaso-dilatation). As an example, the following table gives the condensed results of three experiments on two young men. Experiments II. and III. were on the same man at an interval of three days.

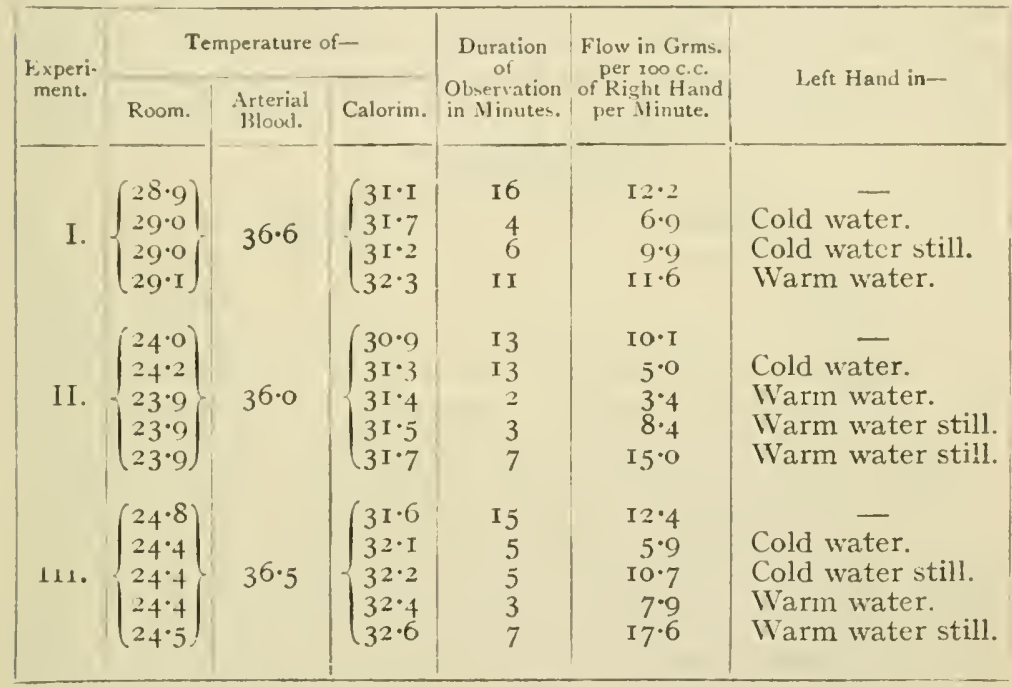

Such facts enable us to some extent to understand the manner in which the distribution of the blood is adjusted to the requirements of the different parts of the body, so that to a certain degree of approximation no organ has too much, and none too little. The blood-supply of the organs is always shifting with the calls upon them. Now, it is the actively-digesting stomach and the activelysecreting glands of the alimentary tract which must be fed with a full stream of blood, to supply waste and to carry away absorbed nutriment. Again, it is the working muscles of the legs or of the arms that need the chief blood-supply. But wherever the call may be, the vaso-motor mechanism is able, in health, to answer it by bringing about a widening of the small arteries of the part which needs more blood, and a compensatory narrowing of the vessels of other parts whose needs are not so great.

It is also through the vaso-motor system, and especially by the action of that portion of it which governs the abdominal vessels, and 
of the netves that regulate the work of the heart, that in animals to which the upright position is normal (monkey) and in man the influence of changes of posture on the circulation is almost conpletely compensated." The pressure in the upper part of the human brachial artery has been measured with a sphygmomanometer, first in the horizontal and then immediately afterwards in the standing posture, and in health it has been found to remain practically unchanged (Hill). But if the person was overworked or out of sorts, the compensation was less complete. It is well known that in debilitated persons, especially if long confined to bed, the sudden assumption of the upright position may cause vertigo, and even syncope, the normal compensatory mechanism being deranged. In such animals as the rabbit this compensation is totally inefficient. When a domesticated rabbit, which has been kept in a hutch, is suspended vertically with the feet down, the blood drains into the abdominal vessels, syncope speedily ensues, and in a period that ranges from less than a quarter to three-quarters of an hour the animal dies in the convulsions of acute cerebral anæmia (Salathé, IIill). The head-down position has no ill-effects. In wild rabbits, whose abdominal wall is more tense and elastic, these fatal symptoms are not easily produced, and the same is true of cats and dogs. But in all animals, when the compensation is destroyed, as in paralysis of the vaso-motor centre by chloroform, the circulation may be profoundly influenced by the position of the body: elevation of the head may lead to cerebral anæmia, syncope, and even death ; elevation of the legs, and particularly the abdomen, may restore the sinking pulse by filling the heart and the vessels of the brain. If a chloralized dog be fastened on a board which can be rotated about a horizontal axis passing under the neck, the blood-pressure in the carotid artery falls greatly when the animal is made to assume the vertical position with the head up, and either rises a little or remains practically unchanged when the head is made to hang down. So great may the fall of pressure be in the former position that death may occur if it be long maintained (Practical Exercises, p. 213).

* Two factors may be distinguished in the blood-pressure, the hydrustatic and the hydrolynamic elements. The hydrostatic portion of the pressure is due to the weight of the column of blood acting on the vessel; the bydrodynamic portion of the pressure is clue to the work of the heart. If a dog be securely fastened to a holder arrangel in such a way that the animal can be placed vertically, with the head up or down, and the mean blood-pressure in the crural artery be measured in the two positions. there will be a considerable lifference. For when the legs are uppermost the heart has to overcome the weight of the column of blood rising above it to the crural artery; when the head is uppermost the action of the heart is reinforced by the weight of the blood. And if no change were produced in the action of the heart, or in the general resistance of the vascular path, by the change of position, this difference would be equal to the pressure of a column of blood twice as high as the straight-line distance between the cannula and the point of the arterial system at which the pressure is the same with head up as with head down (indifferent point). 
Finally; it is in virtue of the amazing power of accummodation posscssed by the vascular system, as controlled by the vaso-motor and cardiac nerves, that so long as these are not disabled the total quantity of blood may be greatly climinished or greatly increased. without endangering life, or even causing more than a transicnt alteration in the arterial pressure. It is not until at least a quarter of the bleod has been withdrawn that there is any notable effect on the pressure, for the loss is quickly compensated by an increase in the activity of the heart and a constriction of the small arteries. An animal may recover after losing considerably more than half $j$ ts blood.* Conversely, the volume of the circulating liquid may be doubled by the injection of blood or physiological salt solution without causing death, and increased by 50 per cent. without any marked increase in the pressure. The excess is promptly stower

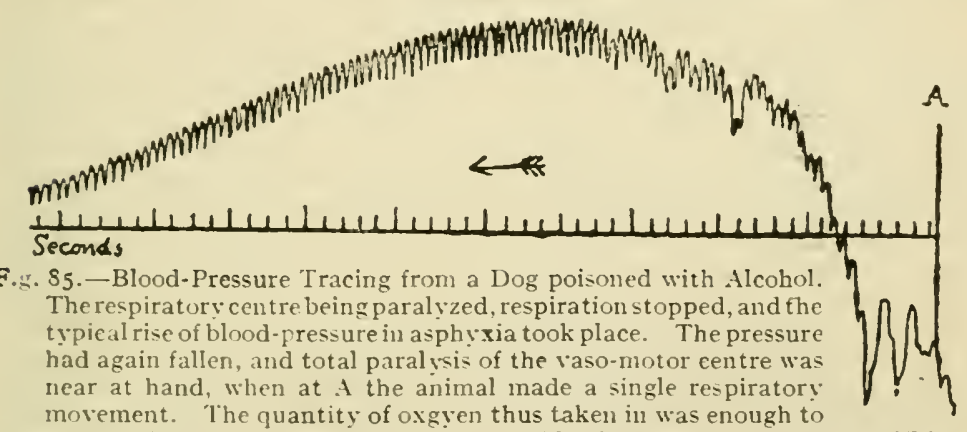
restore the vaso-mutor centre, and the blood-pressure again rose. This was repeated five or six times. (Three-fourths original size.)

away in the dilated vessels, especially those of the splanclunic area; the water passes rapidly into the lymph, and is then more gradually eliminated by the kidneys.

From these facts we can deduce the practical lesson, that bloodletting, unless fairly copious, is useless as a means of lowering the general arterial pressure, while it need not be feared that transfusion of a considerable quantity of blood, or of salt solution, in cases of severe hæmorrhage, will dangerously increase the pressure. And from the physiological point of view the term ' hæmorrhage 'includes more than it does in its ordinary sense. For as dirt to the sanitarian is 'matter in the wrong place,' hæmorrhage to the physiologist is blood in the wrong place. Not a drop of blood may be lost from the body, and yet death may occur from hænıorrhage into the pleural or the abdominal carity, into the stomach or intestines. Not only so, but a man may bleed to death into his own blood-

* It is not usually possible to obtain quite two-thirds of the total blood by bleeding a dog from a large artery. In seven dogs bled from the carotid, the ratio of the weight of the blood obtained to the body-weight was

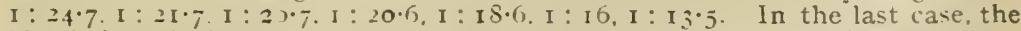
blood clotte 1 wi: h abnormal slo vnes; and the animal died in a few minutes. 
vessels; in surgical (also sometimes called traumatic or vascular) shock, it would appear that the blood which onght to be circulating through the brain, heart, and lungs may stagnate in the lilated reins.

The essential nature of 'vascular' shock, which should be distinguished from the spinal shock described in Chapter XVI. is so little understood, in spite of the large amount of work devoted to the subject, that it would not be profitable to discuss it here. It may be remarked, however, that there is no reason to suppose that exhaustion or loss of sensitiveness of the vaso-motor centre is concerned. IThile an undue proportion of the blood is accumulated in the great veins, the arterioles and capillaries of the splanchnic area, like thos' of the skin, are contracted and contain less blood than normal (Lyon, Janeway and Jackson, etc.).

\section{Section Vil.-The Lymphatic Circulation.}

As has already been stated, some of the constituents of the blood, instearl of passing back to the heart from the capillaries along the veins, find their way by a much more terlious route along the lymphatics. The blood capillaries are everywhere in very intimate relation with lymph capillaries, which, completely lined with epithelioid cells, lie in irregular spaces in the connective-tissue that everywhere accompanics and supports the bloodvessels. The constitnents of the blood-plasme are filtered through, or secreted by the capillary walls into these lymph spaces, and mingling there with waste products discharged by the cells of the tissues, form the liquid known as tissue liquid or tissue lymph. From the tissue liquid the lymph capillaries take up the constituents of the 'lymphatic' lymph, which then passes into larger lymphatic vessels, with lymphatic glands at intervals on their course. These fall in to still larger trunks, and finally the greater part of the lymph reaches the blood again by the thoracic duct, which opens into the venous system at the junction of the left subclavian and internal jugular veins. The lympla from the right side of the head and neck, the right extremity, and the right sicle of the thorax, with its viscera, is collected by the right lymphatic duct, which opens at the junction of the right subclavian and internal jugular veins. The openings of both ducts are guarded by semilunar valves, which prevent the reflux of blood from the veins. Scrous cavities like the plenral sacs, although differing from ordinary lymph spaces, are connected through small opening, called stomata, witl lynphatic vessels.

The rate of flow of the lymph in the thoracic duct is very small compared with that of the blond in the arteries-only about + mm. per second, according to one observer. Nercrtheless, a substance injected into the blood can be detected in the lymph of the duct in four to seven minutes (Tschirwinsky). The factors which contribute to the maintenance of the lymph flow are:

(I) The pressure uncler which it passes from the blood capillaries into the lymph spaces and from the lymph spaces into the lympla capillaries. The pressure in the thoracic duct of a liorse may be as high as $12 \mathrm{~mm}$. of mercury; in the dog it may be less than 1 mm. The difference is probably duc, in part at least, to a difference in the experimental conditions, dogs being usually anesthetized for such measurements, horses not. The pressure in the lymph capillaries must, of course, be higher than in the thoracic duct how much higher we do not know.

(2) The contraction of muscles increases the pressure of the lymph by compressing the channels in which it is contained, and the valves 
with which the lymplatics are even more richly provided than the veins, hinder a backward and favour an onward flow. The contractions of the intestines, and especially of the villi, aid the movenent of the chyle. By the contraction of the diaphragm, substances may be sucked from the peritoneal cavity into the lymphatics of its central tendon, through the stomata in the serous layer with which its lower surface is clad. It is even possible by passive movements of the diapluragin in a dead rabbit to inject its lymphatics witl a coloured liquld placed on its peritoneal surface. Passive movements of the limbs and massage of the museles are also known to hasten the sluggish current of the lymph, and are sometines employed with this object in the treatment of disease.

(3) The movements of respiration aid the flow. At every inspiration thic pressure in the great veins near the neart becomes negative, and lymph is sucked into then (p. 226).

(4) In some animals rhythmically-contracting muscular sacs or hearts exist on the course of the lyniplatic circulation. 'The frog has two pairs, an anterior and a posterior, of these lymph hearts, which pulsate, although not with any great regularity, at an average rate of sixty to seventy beats a minute, and are governed by motor and inhibitory centres situated in the spinal cord. The beat is not directly initiated from the cord, but the tonic influence of the cord is necessary in order that the lymph hearts may continue to beat (Tschermak). Such hearts are also found in reptiles. It is possible that in animals without localized lymph hearts the smooth muscle, which is so conspicuous an clement in the walls of the lymphatic vessels, may aid the flow by rhythmical contractions.

\section{PRACTICAL EXLRCISES ON CHAPTER III.}

I. Microscopic Examination of the Circulating Blood.-(I) Take a tadpole and lay it on a glass slide. Cover the tail with a large coverslip, and examine it with the low power (Leitz, oc. III., obj. 3). Generally the tail will stick so closely to the slide, and the animal will move so little, that a sufficiently good view of the circulation can be obtained. If there is any trouble, destroy the brain with a needle. Observe the current of the blood in the arteries, capillaries and veins. An artery may be easily distinguished from a vein by looking for a place at which the vessel bifurcates. In veins the blood flows in the two branches of the fork towards the point of bifurcation, in arteries away from it. Sketcli a part of a field.

To Pith a Frog.- IVrap the animal in a towel, bend the head forwards with the index-finger of one hand, feel with the other for the depression at the junction of the head and backbone, and push a narrow-bladed knife right down in the middle line. The spinal cord will thus be divided with little bleeding. Now push into the cavity of the skull a piece of pointed lucifer match. The brain will thus be destroyed. The spinal cord can be destroyed by passing a blunt needle down inside the vertebral canal.

(2) Take a frog and pith its brain only, inserting a match to prevent blceding. Pin the frog on a plate of cork into one end of which a glass slide has been fastened with sealing-wax. Lay the web of onc of the hind-legs on the glass and gently separate two of the toes, if necessary by threads attached to them and secured to the cork plate. Put the plate on the microscope-stage and fasten by the clips (see pp. I 5, I 18 ).

(3) After the normal circulation has been studied thoroughly put a very small drop of tincture of cantharides on the portion of the web 
which is in the field of the microscope, using a fine pipette. Observe the process of inflammation, including stasis and diapedesis (p. 61).

2. Anatomy of the Frog's Heart.-Expose the heart of a pithed frog by pinching up the skin over the abdomen in the middle line, dividing it with scissors up to the lower jaw, and then cutting through the abdominal muscles and the bony pectoral girdle. The external abdominal vein, which will be observed on reflecting the skin, can be casily avoided. The heart will now be seen enclosed in a thin membrane, the pericardium, which should be grasped with fine-pointed forceps and freely divided. Connecting the posterior surface of the heart and the pericardium is a slender band of connective tissue, the frænum. A silk ligature may be passed round this with a threaded curved needle, or curved fine-pointed forceps; and tied, and then the frenum may be divided posterior to the ligature. The anatomical arrangement of the various parts of the heart should now be studied. Note the single ventricle with the bulbus arteriosus, the two auricles, and the sinus venosus, turning the heart over to see the latter by means of the ligature. Observe the whitish crescent at the junction of the sinus venosus and the right auricle (Fig. S6).

3. The Beat of the Heart. - Note that the auricles beat first, and then the ventricle. The wentricle becomes smaller and paler during

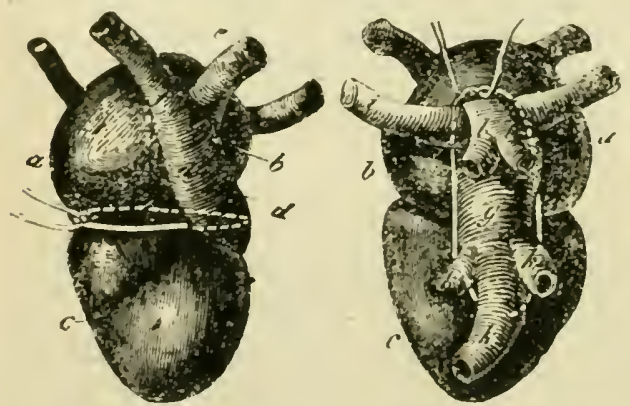

lig. 86.-Frog's Heart with Stannius' Ligatures in Position (Cyon). Anterior surface of heart shown on the left, posterior surface on the right. $a$, right auricle; $b$, left auricle; $c$, ventricle; $d$, bulbus arteriosus; $e, f$, antar; $g$, sinus venosus. its systolc, and bluslies red during diastole. Count the number of beats of the heart in a minute. Now excise the heart, lifting it by means of the ligature, and takingcare to cutwide of the sinus renosus. Place the licart in a small porcelain capsule on a little blotting-paper moist ened with physiological salt solution.* Observe that it goes on beating. Put a little ice or snow in contact with the heart and count the unmber of beats in a minute. The rate is greatly diminished. Now remove the ice and blotting-paper, cover the heart with the salt solution, and heat, noting the temperature with a thermometer. Observe that the heart beats faster and faster as the temperature rises. At $40^{\circ}$ to $43^{\circ} \mathrm{C}$. it stops beating in diastole (heat stanclstill). Now at once pour off the heated liquid, and rum in some cold salt solution. The heart will begin to beat again.

4. Cut off the apex of the ventricle a little below the auriculoventricular groove. The auricles, with the attached portions of the ventricle, go on beating. The apex does not contract spontaneously, but can be made to beat by stinulating it mechanically (by pricking it with a necdle) or electrically. Divide the still contracting portion of the heart by a longitudinal incision. The two lialves go on beating.

5. Heart Tracings.--(I) Fasten a myograph-plate (Fig. 87) on a stand. Take a long light lever, consisting of a straw or a piece of

* For frog's tissues this should be 0.7 to 0.75 per cent. sodium chloride solution, for mammalian tissues a little stronger (about o.9 per oent.). 
thin chip, armed at one end with a writing-point of parchment-paper, supported near the other end by a horizontal axis, and pierced not far from the axis by a neclle carrying on its point a small piece of cork or a ball of sealing-wax.

A counterpoise is adjusted on the short arm of the lever in the form of a small leaden weight. Cover a drum with glazed pajer and smoke it. The paper must be put on so tightly that it will not slip. To smoke the drum, hold it by the spindle in both hands over a fish-tail burner, depress the drum in the flame, and rotate rapidly. The speed of the drum can be varied by putting in or taking out a sinall vane. Arrange an electro-magnetic timemarker for writing seconds (Fig. 88). Pith a frog (brain only), expose the heart, and put under it a cover-slip to give it support. Pin the frog on the myographplate, and adjust the foot of the lever so that it rests on the ventricle or the auriculo-ventricular junction. Bring the writing-point of the lever and that of the timemarker vertically under each other on the surface of the drum. Set off the drum at the slow speed (say, a centimetre a second). When the

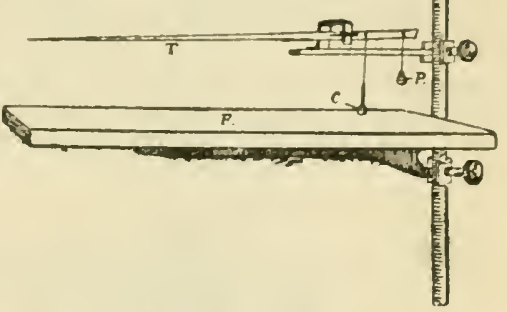

Pjg. 87.-Arrangement for obtaining a Heart Tracing from a lirng. lever rests on the auriculo-ventricular junction, the part of the tracing corresponding to the contraction of the heart will be broken into two

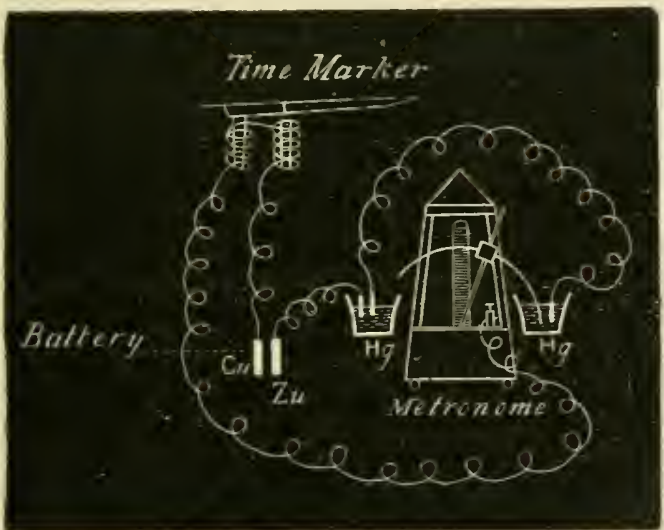

IFig. 88.-Electro-Magnetic Time-Marker connected with Metronome. The pendulum of the metronome carries a wire which closes the circuit when it dips into either of the mercury cups, $\mathrm{Hg}$. portions, representing the systole of the auricles and ventricle respectively. Cut the paper off the drum with a knife (keeping the back of the knife to the drum to avoid scoring it) and carry it to the varnishingtrough, holding the tracing by the ends with both hands, smoked side up. Immerse the middle of it in the varnish, draw first one end and then the other through the varnish, let it drip for a minute into the trough, and fasten it up with a pin to dry. (2) Heart Tracing, with Simultaneous $R e$ cord of Auricular and Ventricular Contractions.-(a) For this purpose two levers may be arranged, cne resting on the auricle, the other on the ventricle, the writing-points being placed in the same vertical straight line on the drum. A convenient form of apparatus is shown in Fig. 89 .

(b) Gaskell's Method (a modification of).-Attach a silk ligature to the very apex of the ventricle. Divide the franum, cut the aorta 
across close to the bulbus, pincls up a tiny portion of the auricle and ligature it. Renove the intestines, liver, lungs, etc., care being taken in cutting away tlic liver not to injure the sinus. Then remove tlie lower jaw, and cut away the whole of the body except the head, part of the osophagus, and the tissue connecting it with the heart. Fix the head in a clamp sliding on an ordinary stand. The heart is held at the auriculo-ventricular junction in a Gaskell's clanp supported on a separate stand. The thread connected witl the ventricle is brought round a pulley and attached to a lever above the heart. The auricle is connected with another lever. The writing-points of the two levers are arranged in a vertical line on the drum. The small pulley must be oiled from time to time to lessen the friction (Fig. 90).

If tortoises or turtles are available, the much larger heart of these animals may be used for Jixperiments $5(2)(a)$ and $(b)$. The animal having been killed by cutting off its liead, the ventral portion of the carapace is detache d by the saw. The pericardium can now be slit open, and the pads of the levers arranged on auricles and ventricle

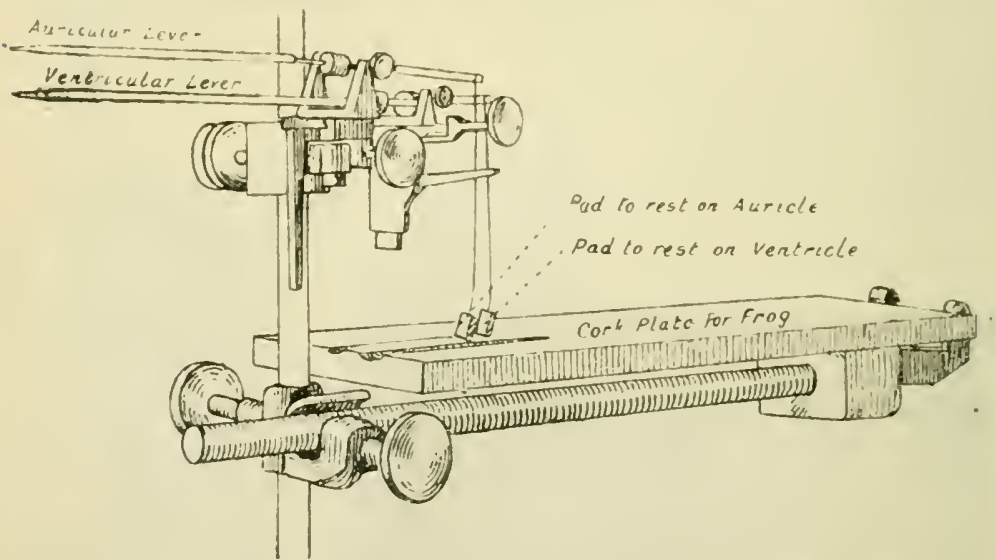

Fig. 89.-Apparatus for obtaining a Simultaneous Tracing of Auriculdr and Ventricular Contractions.

respectively, as in Experiment 5 (2) (a), without further disturbing the heart. Or the heart may be removed, togetlee with the upper portion of the body, the pericardium opened, and the liver cut away. The aortic trunk is then divided, and the portion of it attached to the heart grasped by a small forceps clamp. Fine silk ligatures are attached to the apex of the ventricle and the top of the right auricle The vagus nerves are exposed in the neck, ligaterl, and divided. The upper portion of the body is supported on a stancl. The forceps grasping the aorta is fixed in an ordinary holder, and the threads are attached to the levers, as in Experiment 5 (2) (b).

With the vagi, Experiment 7 may be performed. It must be remembered that the activity of the two vagi is unequal in the tortoise, the right being the nore active.

6. Dissection of the Vagus and Cardiac Sympathetic Nerves in the Frog.-(1) Fut the tissucs in the region of the neck on the stretch by passing into the gullet a nariow test-tube or a thick glass rod moistener with water, and by pinning ayart the anterior limbs. Expose the heart 
by cuttung through the pectoral girdle in the way described in 2 (p. I94). (in leatsing iwa) a little connective tissue and muscl. with a seeker. three large nerves will come into view. The upper is the glossopharyngeal, the lower the hypoglossal; the vagus irosses diagonally between them (Fig. aI). Above the ragus trunk, running parallel to it, and separated from it by a thin muscle and a bloodvessel (the carotid artery), lies its laryngeal branch. The vagus should be traced up to the ganglion situated on it near its exit from the skull.

(2) Then cut away tlic lower jaw, dividing and reflecting the membrane covering the roof of the mouth. At the junction of the skull and the backbone will be seen on each side the levator anguli scapula muscle (Fig. 92). Remove this muscle carefully with fine forceps. Clear away a little connective tissue lying just over the upper cervical vertebra, and the sympathetic chain, with its ganglia, will be seen. Pass a fine silk thread bencath the sympathetic about the level of the large brachial nerve, by means of a sewing-needle which has been slightly bent in a flame and fastened in a handle. Tie the ligature, divide the sympathetic below it, and isolate it carefully with fine scissors up to its junction with the vagus ganglion.

Batteries-To set $u p$ a Daniell Cell.-Fill the porous pot (Fig. 230, p. 724), previously well soaked in water, with dilute sulphuric acid ( $I$ part of commercial acid to Io or I5 parts of water) to within

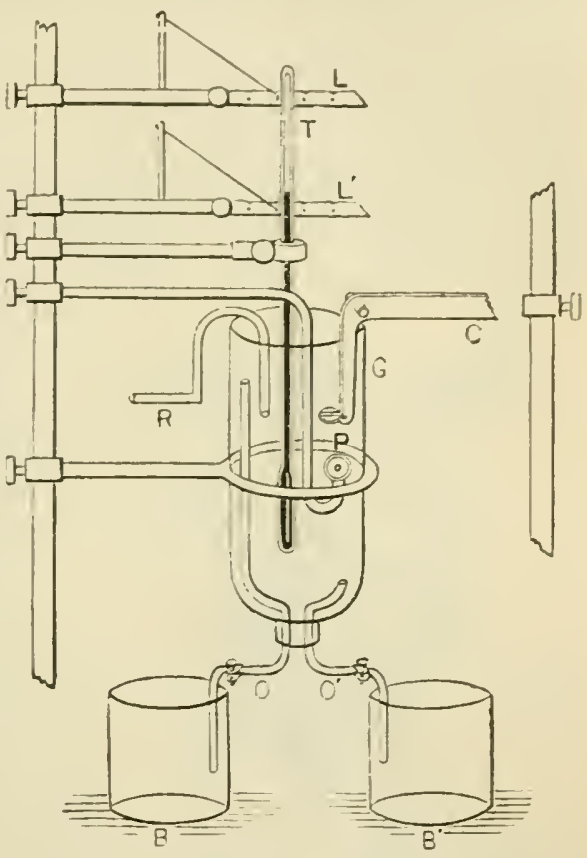

Fig. 9o.--Arrangement for recording Auricular and Ventricular Contractions (and studying the Influence of Temperature of the Heart). C, clamp holding the heart at the auriculo-ven. tricular groove; $\mathrm{P}$. pulley round which a thread attached to the apex of the rentricle passes to the lever L'; L, lever connected with auricle. (The rest of the arrangement is for studying the influence of temperature on the heart and its nerves, $G$ being a vessel filled with physiological salt solution in which the heart is immersed; $R$. an inflow tube from a reservoir containing salt solution at the temperature required; $\mathrm{O}^{\prime}$, an outflow tube by which $G$ may be emptied into the beaker $\mathrm{B}^{\prime}$; $\mathrm{O}$, a tube passing to the beaker $\mathrm{B}$ to prevent overflow from $G$; $T$, a thermometer.)

$\mathrm{r} \frac{1}{2}$ inches of the brim, and place in it the piece of amalgamated zinc. If the zinc is not properly amalgamated, leave it in the pot for a minute or two to clean its surface. Then lift it out, pour over it a little mercury. and rub the mercury thoroughly over it with a cloth. Put the pot into the outer vessel, which contains the copper plate, and is filled with a saturated solution of sulphate of copper, with some undissolved 
crystals to keep it saturated. After using the Daniell, it must always be taken down. The outer pot is lett with the copper plate and the sulphate solution in it. The zinc is washed and bruslied bright. The sulphuric acid is poured into the stock bottle, and the porous pot put into a large jar of water to soak.

The Bichromate Ciell contains only one liquid-a mixture of I part of sulphuric acid with 4 parts of a 10 per cent. solution of potassium biciromate. In this is placed one, or in some forms two, carbon plates and a plate of amalgamated zinc. After using the battery, take the zinc out of the liquid.

The Leclanché battery consists of a porous pot filled with a mixture of manganese dioxide and carbon packed around a carbon plate, which forms the positive pole. The pot stands in an outer jar of glass filled with a saturated solution of ammonium chloride, into which dips an amalgamated zinc rod, which constitutes the negative pole. Various forms of dry batteries can be conveniently used for running induction-

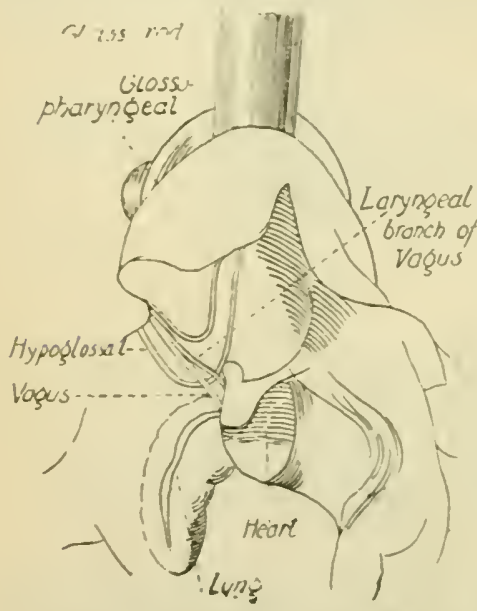

Fig. 91.-The Relations of the Vagus in the Frog. coils or time-markers, but are not adapted for yielding constant currents of long duration.

7. Stimulation of the Vagus in the Frog. - Make the same arrangements as in 5 (I) (p. I95), but in addition set up an induction machine arranged for an interrupted current (Fig. 93), with a Daniell, a bichromate, a Leclanché, or a dry cell in the primary circuit. which should also include a simple key. Insert a short-circuiting key in the secondary circuit. Attach the electrodes to the short-circuiting key, push the secondary coil up towards the primary until the shocks are distinctly felt on the tongue when the Neef's hammer is set going and the short-circuiting key opened. Pith the brain of a frog, cxpose the heart, dissect out the vagus on one side, ligature it as high up as possible, and divide above thr ligature. Fasten the electrodes on the cork plate by means of an indiarubber band, and lay the vagus on them. Set the drum off (at slow speed). After a dozen heart-beats liave been recorded. stimulate the vagus for two or three seconds by opening the shortcircuiting key. If the nerve is active, the heart will be slowed, weakened, or stopped. In the last case the lever will trace an unbrolien straight line; but even if the stimulation is continued the beats will again begin.

8. Stimulation of the Junction of the Sinus and Auricles.-After a sufficient number of the observations described in 7 have been taken with varying time and strength of stimulation, take the writing-points off the drum, apply the electrodes directly to the crescent at the junction of the sinus venosus with the right auricle, and stimulate. The heart will be affected very mucli in the same way as by stimulation of the vagus, except that during the actual stimulation its beats may be quickened and the inlibition may only begin after the electrodes have been removed (Fig. 70, p. ${ }_{5} 8$ ). 
9. Effect of Muscarine (or Pilocarpine) and Atropine.-Paint on the sinus venosus with a small camel's-hair brush a very dilute solution of muscarine (or of pilocarpine). The heart will soon be scen to beat more slowly, and will ultinately stop in diastole. Now apply a dilute solution of sulphate of atropine to the sinus. The heart will again begin to beat. Stimulation of the vagus will now cause no inhibition of the heart, because its endings have been paralyzed by atropine. (Muscarine or pilocarpinc has also been applicd to the heart, but it could be shown by a separate experiment that atropine by itself has the same effect on the vagus enclings-p. I6 .)

Io. Stannius' Experiment.-Pith a frog. Expose the heart in the way described under 2 (p. 194). I,igature the frenum with a fine silk thread, and use the thread to manipulate the heart. With a curved needle pass a moistened silk thread between the aorta and the superior vena cava, and tic it round the junction of the sinus and right auricle (Fig. 86). The auricles and ventricle stop beating as soon as the ligature is tightened. The sinus venosus goes on beating. Now separate the ventricle from the rest of the heart by an incision through the auriculo-ventricular groove, or tic a second ligature in the groove. The ventricle begins to beat again, the auricle remaining quiescent in diastole (p. I60). Occasionally both auricle and ventricle, or only the auricle, may begin to beat.

I1. Stimulation of Cardiac Sympathetic Fibres in the Frog -(I) In the vago-sympathetic after the inhibitory fibres haie been cut out by atropine.Arrange everything as in 7 (p. 193): Assure yourself, by stimulating the vagus, that it inhibits the heart, and take a tracing during stimulation. Then paint a dilute solution of atropine on the sinus.

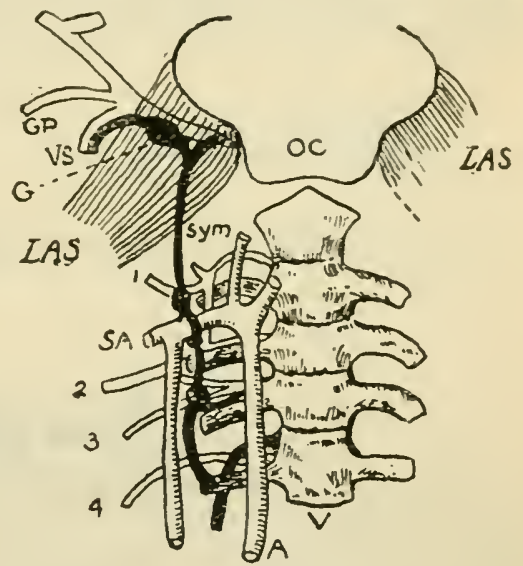

Fig. 92.-Relation of the Sympathetic to the Vagus in the Frog (after Gaskell). Sym, sympathetic chain; G, ganglion of the vagus; VS, vago-sympathetic; GP. glosso-pharyngeal nerve; LAS, levator anguli scapulæ muscle; S.A, subclavian artery; A, descending aorta; V, vertebral column; OC, occipital part of skull; I-4. spinal nerves.

Stimulation of the vagus, which is rcally the vago-sympathetic (see Fig. 92), will now cause, not inhibition, but augmentation (increase in rate or force, or both), since the endings of the inhibitory fibres have been paralyzed by atropine. The strength of the stimulating current required to bring out a typical augmentor effect is greater than that needed to stimulate the inhibitory fibres. Take a tracing to show augmentation produced by stimulating the nerve.

(2) By direct stimulation of the cervical sympathetic.-Make the same arrangements as in II (I), but, instead of isclating the vagus, dissect out the sympathetic on one side in the manner described in 6 (2) (p. I97). and do not apply atropine to the heart. Lay the upper (cephalic) end of the sympathetic on very fine and well-insulated electrodes, and stimulate (Fig. 76 , p. I67). (To insulate electrodes the points may be covered with melted paraffin. When the paraffin has cooled, a narrow 
gronve, just sufticient to lay bare the wires on the upper side, is macle in it, and the nerve is laid in this groove.)

Experiments 7, I I (I) and I I (2) will be rendered more cxact by connecting a second electro-magnetic signal with a Pohl's commutator without cross-wires (Fig. (9), in such a way that the circuit is interrupted at the instant when stimulation begins.

12. The Action of Inorganic Salts on Heart-Muscle.-Expose and remove the heart of a tortoise or turtle (p. I,6). Cut off the apical twothirds of the ventricle by an incision parallel to the auriculo-ventricular groove. By a second parallel cut remove a ring of tissue 2 or 3 millimetres wide from the upper end of this portion of the ventricle. Divide the ring at opposite cnds of a diameter, so as to form two strips. Tie a finc silk thread to each end of onc strip. Attach one of the threads to the short limb of a glass rod bent at right angles, so tlat it can be immersed at will in a beaker. The other end of the rod is fixed in a holder sliding on a stand. Attach the second thread to the short arm

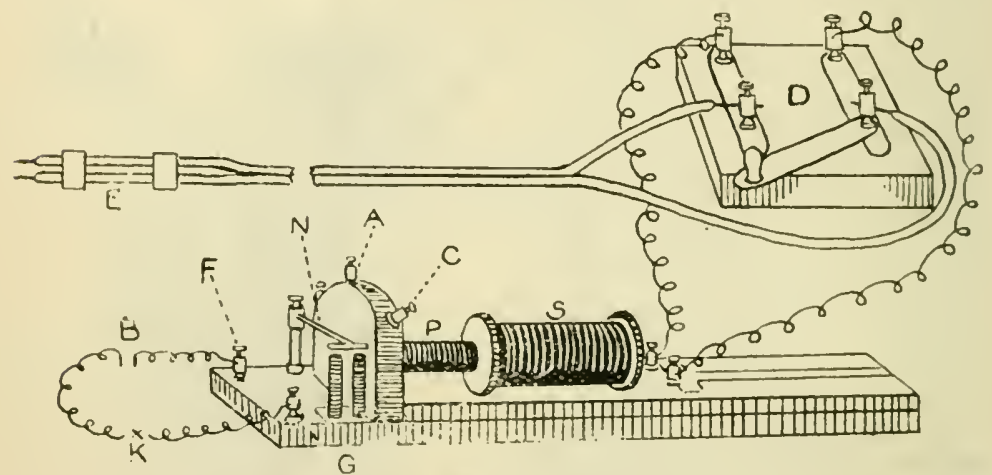

Fig. :3--Arrangement of Induction Machine for Tetanus. B, battery; $\mathbf{k}$, simple key; P, primary coil; S. secondary coil: A, C, binding screws to be connected with battery for single shocks; $F, G$, bincling screws for tetanizing current; $\mathbb{N}$, Neef's hammer; D, short-circuiting key in secondary; E, electrodes. D and E are drawn to a much larger scale than the rest of the ligure.

of a counterpoised lever arranged to write on a slowly-moving drum. If the strip is still beating, wait till the contractions lave ceased; then

(I) Immerse the strip in a beaker filled with $0^{\circ} 7$ per cent. solution of sodium chloride. After a time it begins to beat rhythmically. The contractions become rapidly stronger, and then after a while diminish, and gradually cease. The tone or tonus of the strip is diminished by the solution.

(2) Arrange the other strip in the same way, and immerse it in a solution of calcium chloride (about I per cent.) isotonic with the sodium chloride solution used in (I). If the strip is contracting, the contractions will cease. Rhythmical contractions will not appear as in the sodium chloride solution. The tone of the strip may be increased.

(3) Remove most of the calcium cluloride solution from the beaker, and fill it up with 0.7 per cent. sodium chloride solution. The rhythmi. cal contractions will appear after a longer or shorter latent period, and will be stronger and last for a longer time than in the sodium chloride solution alone.

(4) Immerse 2 fresh strip in a solution containing sodium chloride 
(0.7 per cent.), calcium chloride (10025 per cent.), and potassium chloride (0.03 per cent.) (a modified Ringer's solution). A longer series of rlythmical contractions will be obtiined than in either ( 1 ) or (3). That this is not due to the potassium chloride acting alone can be sliown by immersing a strip in a solution of potassium chloride (about 0.9 per cent.) isotonic with the sodium chloride solution used in (I). No contractions will be caused.

13. The Action of the Mammalian Heart. - Inject under the skin of a dog (preferably a small one) I c.c. of a 2 per cent. solution of morphine hydrochlorate for every kilo of body-weight. As soon as the morphine has taken effect (in I5 to 30 minutes, but better after an hour), fasten the animal back down on a holder (as in Fig. 135. p. 30I), pushing the mouth-pin behind the canine tecth and screwing the nut home.* In the meantime select a tracheal cannulat of suitable size, and get ready instruments for dissection-one or two pairs of artery-forceps, a pair of artery-clamps (bulldog pattern), two or three glass cannulac of

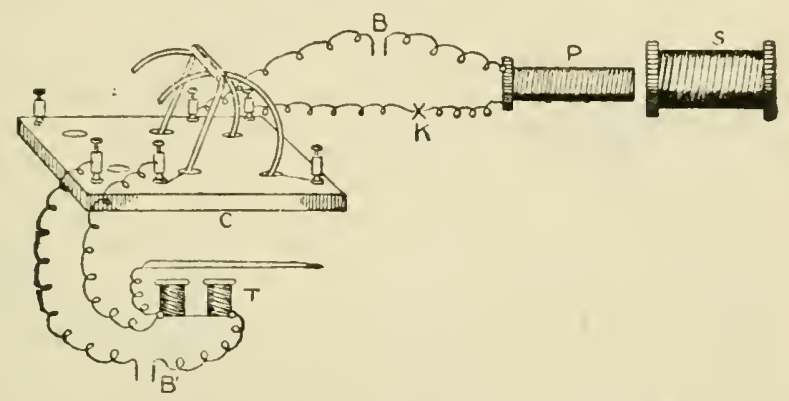

Ftg. 94.-Arrangement for recording the Beginning and End of Stimulation. C. Pohl's commutator without cross-wires; B, battery in circuit of primary coil P; $\mathrm{B}^{\prime}$, battery in circuit of electro-magnetic signal $\mathrm{T} ; \mathrm{K}$, simple key in primary circuit; S, secondary coil. When the bridge of the commutator is tilted into the position shown in the figure, the primary circuit is closed and the circuit of the signal broken.

various sizes for bloodvessels, ten strong waxed ligatures, sponges, hot water, a towel or two, and a pair of bellows to be connected with the tracheal cannula when the chest is opened. Arrange an induction-

* A simple but efficient and convenient holder for a dog may be easily constructed as follows: Take a board of the length required ( $2 \frac{1}{2}$ to 5 feet, according to the size of the dog). At one end fasten two short upright wooden pins, with a clear space of 4 to 6 inches between them. These are pierced from side to side with four or five holes at different heights. An iron pin passes behind the canine teeth of the animal through two corresponding holes in the uprights, and the muzzle is tied over this by a cord which secures the head. For a large dog an upper pair of holes is used, for a small dog a lower pair. The feet are fastened by cords to siaples inserted into the sides of the board, the fore-legs being drawn tailwards for all operations on the neck or head, headwards for operations on the thorax. A rabbit-holder can be made in exactly the same way.

$\dagger \mathrm{A}$ tracheal cannula is easily made by heating a piece of glass tubing, about 6 inches long, a short distance from one end, and drawing it out slightly so as to form a 'neck.' The tubing is then bent about its middle to an obtuse angle, and the end next the neck is ground obliquely on a stone. The diameter of the cannula should be about the same as that of the trachea, into which it is to be inserted by its oblique end. 
coil and elcctrocles for a tetanizing current (Fig. 93, p. $2 \mathrm{co}$ ). With scissors curvel on the flat clip away the hair from the front of the ncek. Put the hair carefully away, and remove all the loose hairs with a wet sponge so that they may not get in to the wounds. Give ether. or pour into the stomach by a tube 5 c.c. of a $0^{\circ} 5$ per cent. solution of chloroform in 10 per cent. alcohol per kilo of body-weight, diluted before administration with 3 or $\&$ volumes of water (Gréhant's method).

To put a Cannula in the Trachea. - The hair liaving been clipped in the middle line of the neck and the skin shaved, a mesial incision is to be made, beginning a little below the cricoid cartilage, which can be felt with the finger. The trachea is then cleared from its attachments by forceps or a blunt necdle, and two strong ligatures are passed beneath it. A single loop is placed on each of tlicse, but is not drawn tight. Raising the trachea by means of the upper ligature, the studeut makes a longitudinal incision through two or thrce of the cartilaginous rings, inserts the cannula, and ties the lower ligature firmly around its neck. The upper ligature can now be withdrawn.

Clip off the hair on cach side of the sternum. Nake an incision on each side through the skin and down to the costal cartilages about 2 inches from the edge of the breast-bone, and long enough to expose about four costal cartilages (say, 3rd to 6th). With a curved needle pass waxed ligatures round the cartilages, and tie firmly to compress the intercostal vessels. The bellows should now, or earlier if any symptoms of impeded respiration have appeared, be connected with one end of the horizontal limb of a glass $\mathbf{T}$-piece, the other end of which is similarly connected with the tracheal cannula. The stem of the $\mathbf{T}$-piece is provided with a short piece of rubber tubing, which, when artificial respiration is being carried on, is to be alternately closed and opened-closed during inflation of the lungs, and opened when the air is to be allowed to escape from them. Or a screw-clamp may be adjusted on the piece of tubing so that the opening is sufficiently narrow to permit the lungs to be properly inflated when the bellows are compressed, and yet sufficiently wide to permit easy escape of the air and collapse of the lungs at the end of each inflation. Ether may, when necessary, be adnninistered, by inserting betwcen the $\mathbf{T}$-piece and the tube from the bellows an ether bottle with two tubes passing through the cork to within an inch or two of the ether. If the cannula has a sicle-opening, as is usually the case with metal cannula, the $T$-piece may he dispensed with. One student should take sole charge of the artificial respiration, which ought to be begun as soon as the chest has been opened, and continued at the rate of about twenty inflations per minute. The costal cartilages are rapidly cut through with strong scissors just on the sternal side of the ligatures, the artificial respiration being suspended for an instant, as eacls cut is made, to avoicl wounding the lungs. The sternum is divided at its lower end and turned up. If there is much blceding a ligaturc should be tied rouncl its upper end. With a curved necdle a ligature is passed below tlic internal mammary artcries as thcy approach the sternum. That bone may now be removed, and the heart, enclosed in the pericardium, comes into view. A thread is passed with a suture-ncedle through each side of the pericardium, which is then stitched to the cliest-wall and opened.

(a) Note the various portions of the heart, right and left ventricles, right and left auricles, with the auricular app.ndices. Fcel the heart with the hand, and observe that the right ventricle is softer and has thinner walls than the left, and that the auricles are softer than the ventricles. Note how all the parts of the heart liarden in the banil during systole and soften during diastole (pp. 86, 90). 
(b) Dissect out the vago-sympathetic on one side in the neck of the a log. The guide to the nerve is the carotid artery. These two structures and the intemal jugular vein lie side by side in a common sheatli. Fcel for the artery a little $c x$ ternal to the trachea, cut down on it, open the slieath, isolate the vago-sympathetic for about an inch, pass two ligatures under it, tie them, and divide between the ligatures. The periplieral and central ends of the nerve may now be successively stimulated. Stimulation of the peripheral end causes slowing of the heart, or stoppage in diastole. Feel that it softens when it stops. It soon bcgins to beat again. Stimulation of the central end of the vago-sympathetic may or may not cause inhibition. If it does, expose the other vagosympathetic, divide it, and repeat the stimulation of the central end. There will now be no inhibition of the leart. Incidentally it may be seen that stimulation of the central end of the vago-sympathetic causes strong, though, of course, with opened chest, abortive, respiratory movements.

(c) Pith a frog (brain and cord), dissect out the sciatic nerve on one side up to the sacral plexus. Cut off the whole leg. Drop the cut end of the nerve on the heart, and hold the preparation so that the nerve touches the heart also by its longitudinal surface. At each cardiac beat the nerve is stimulated by the action current (p. 833), and the muscles of the leg contract.

(d) Raise the board so that the head of the animal is down and the lind-feet up, and note whether there is any effect on the action

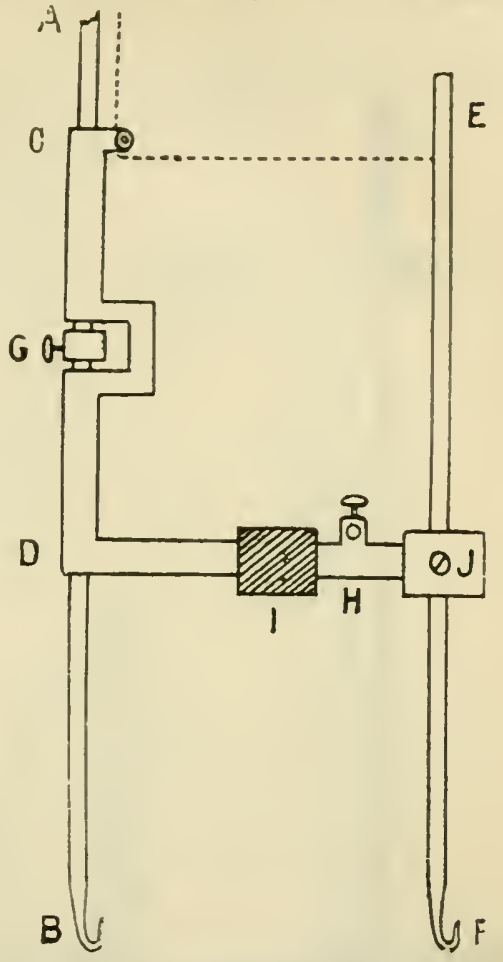

Fig. 95.-Myocardiograph of Adami and Roy. (modified by Cushny and Matthews). AB, a perpendicular rod descending from a universal joint, which is not shown in the figure; $C D$, a brass sheath, moving easily on the rod, and bearing on its upper end an ivory pulley, and at its lower end a horizontal bar, which is interrupted by a plate of hard rubber, I. The per pendicular rod $E F$ moves on the horizontal bal by the hinge-joint, J. EF is hooked at one end for attachment to the heart, and bored at the other for a thread which, passing over the pulley at $C$, passes through the universal joint and moves a writing lever not shown in the figure. $C D$ is prevented from moving up $A B$ by a ring of brass, $G$, which is screwed to $A B$, but is not attached to $\mathrm{CD}$; the hook $\mathrm{F}$ can therefore move to and from $A B$, and can rotate round it, while it cannot move up or down. The hooks $F$ and $B$ are insulated from each other by the hard rubber. I. $\mathrm{H}$ is a binding post through which, and through another connected with $A$, induction shocks may be sent at will into the portion of the heart lying between the hooks. 
and filling of the heart. Repeat the observation with head up and fect down.

(e) Compress the aorta with the fangers, and observe the ceffect on the degice of dilatation of the various cavities of the leart. Repeat the experinent with the inferior vena cava, and compare the results.

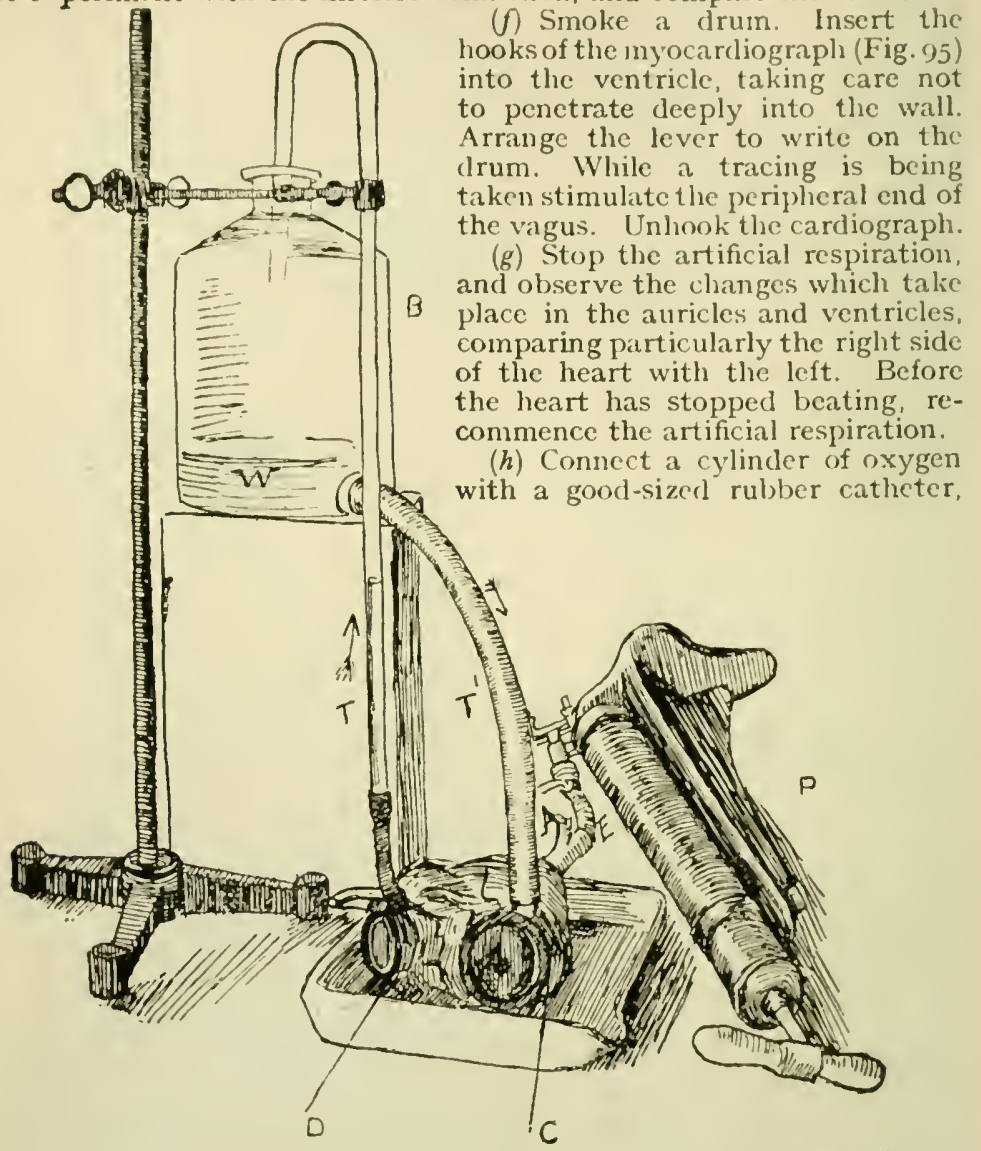

Fig. 96.-Arrangement to illustrate Action of Cardiac Valves in the Heart of all $O x$

(Gad). C, glass window in left auricle; D, window in aorta; E, tube inserted through apex of heart into left ventricic and counccted with pump $P$; $A$, side tube on $\mathrm{E}$, through which wires are connected with a tiny incandescent lamp in the ventricle; $W$, water in bottle $B ; T, T^{\prime}$, tubes.

and pass the catheter down the tracheal cannula or through a separate opening in the trachea. Allow a small stream of oxygen to flow into the lungs. Artificial respiration is now unnecessary. The lungs remain at rest, yet the blood is sufficiently oxygenated, and the heart goes on beating. The myocardiographic tracing thus goes on undisturbed by respiratory movements.

(i) Stop the oxygen, and resume the artificial respiration. Make a 
small penetrating wound with a scalpel in the left ventricle. Observe the course of the hivmorrhage, and note especially the difference in systole and diastole.

(j) Lay the electrodes on the heart, and stimulate it with a strong interrupted current. The character of the contraction soon becomes profoundly altered. Shallow, irregular contractions ficlier over the surface, with a lind of simmering movement suggestive of a boiling pot (delirium corclis, fibrillar contraction). Now kill the animal by stopping the artificial respiration . Observe how long the heart continues to beat, and which of its divisions stops last.

(k) Iake a dissection of the cervical sympathetic up to the superior cervical ganglion, and down throngh the inferior cervical ganglion to the stellate or first thoracic ganglion. Make out the annulus of Vienssens and the cardiac sympathetic (accelerator) branches going off from the annulus or the inferior cervical ganglion to the cardiac plexus.

I4. Perfusion of the Isolated Mammalian Heart. - The heart of a dog employed for some other experiment may be used. Or a rabbit may be killed by a blow on the back of the head, and the heart at once removed. The aorta should not be cut off too short. Tie a cannula into the aorta and attach it to a T-piece comnected by rubber tubes, which must be perfectly clean, with two bottles, one containing Ringer's solution (pp. 66, 20I), preferably that made with dextrose, the other containing defibrinated blood diluted with Ringer's solution. The defibrinated blood should be strained so as to remove any small pieces of fibrin. The bottles are supported on a high stand, so that the level of the bottles above the heart can be altered, and the pressure of the perfusion liquid thus varied. Perfusion may be begun with Ringer, to wash out any remaining blood and obviate the possible formation of clots in the small vessels. Oxygen is allowed to bubble through the Ringer's solution, but this is not necessary for the blood, since, if shaken up, it will retain far more oxygen than the Ringer's solution. The temperature of the liquids

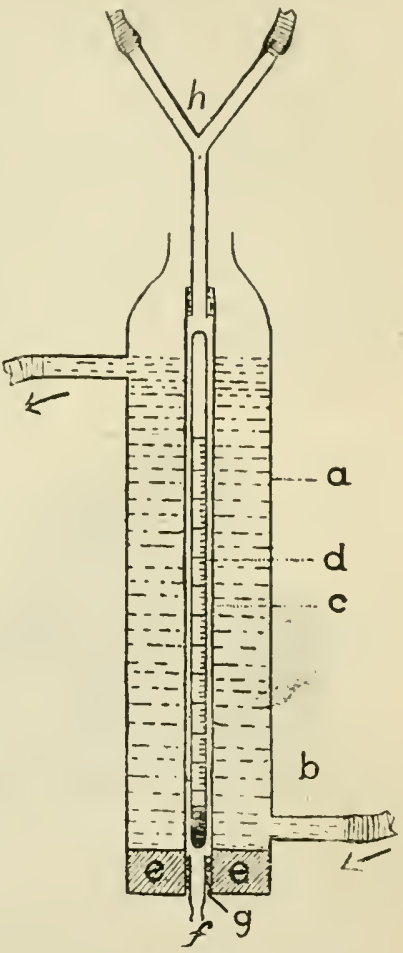

Fig. 97.-Mammalian Heart Per. fusion Apparatus (Gunn). $a$, Liebig condenser, cut off as shown; $b$, inlet for the warm water; $d$, thermometer almost. filling up the lumen of the thin glass tube $c$; $e$, cork ; $f$, cannula for aorta fitted with a collar of rubber tubing, $g$, in the end of the tube $c ; h$, Y-tube comnected with two reservoirs, one containing Ringer's solution, the other any other liquid which is to be perfused.

should be at about $40^{\circ} \mathrm{C}$. when nearing the heart. This can be most easily insured by interposing a worm immersed in a heated bath or other heating arrangement between the cannula and the $T$-tube, and for the study of its movements by inspcction the heart itself can he placed in a glass vessel immersed in the bath. When records of the contractions 
are to be olstimed, threads are attached to the auricle and to the itpex of the ventricl?. The heart is suspended by fastening the rannula in a holder on a stand, and the threads, after passing over pulleys to give them a convenient direction, are attached to writing-levers.

As the heart cannot now be easily kept immersed in the bath, it is suspended in the air, and can be kept warm by the following simple arrangement: A copper pipe about 4 inches long is slit on one side, anc] on the opposite side is screwed or riveted to a copper rod, under which is liung a spirit-lamp. The lamp is adjusted at such a point on the rod that when the copper tube is placed around the heart the lieat conducterl aloing the rod keeps the air around the heart at about body-temperature.

The perfusion liquid before it enters the heart may be heated thus: A I.iebig's condenser is cut through the middle, and the large end closed by a paraffined cork. A glass tube is run clown from the top through this cork, and the aorta is attached directly to this, so that the heart is very near the condenser. This tube is mostly filled up by a thermometer, so that the perfusion liquid passes through it in a thin strean which is easily heated by the water in the condenser, which

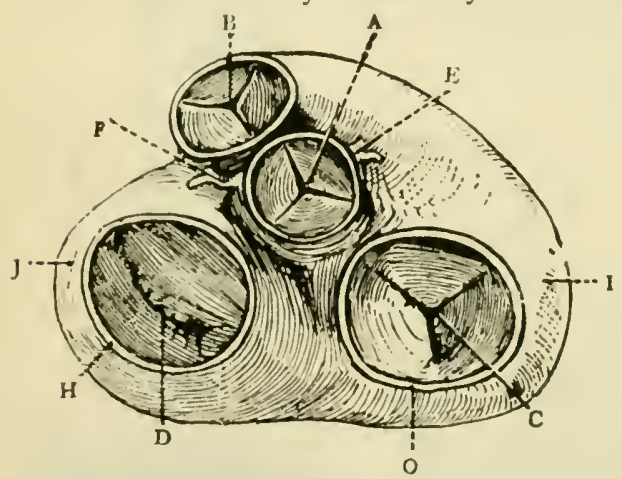

Fig. 98.-Diagram of Valves of the Heart. The valves are supposed to be viewed from above, the auricles having been partially removed. A, aurta with semilunar valve; $\mathbf{B}$, pulmonary artery and valve; $C$, tricuspid, and $[$, mitral valve; $E$, right, and $\mathrm{F}$, left coronary artery; $\mathrm{G}$, wall of right, and $\mathrm{H}$, of left auricle, $I$, wall of right, and $J$, of $l$ ft ventricle. contains a second thermometer. This water is kept constantly flowing through the condenser from a heated bath. The $\mathbf{T}$-piece comnecting with the perfusion bottles is attached to the upper end of the glass tube to which the heart is attached (Gunn and (ushny).

I5. Action of the Valves of the Heart.(I) Study the action of the valves of the oxheart, connected with the pump $\mathrm{P}$ and bottle 1 , in the artificial scheme, as shown in Fig. 96. The cavity of the heart is illuminated by means of a small electric lamp, the wires of which pass in at

A. When the piston of the pumpl is pusherl down, water is forcel through the arrta D along the tube 1 into the bottle, and flows back again into the left auricle by $\left\{h_{2}\right.$ tube ' $T$ '. During eacly stroke of the pump the anriculo-ventricular valve is seen through the glass disc inserted into $C$ to close, and the semilunar valve is seen througl the glass in D to open. When the piston is raised, the semilunar valve is seen to be closed and the auriculo-ventricular valve to be opened. for comparison, a human heart with a valvular lesion might be used.

(2) With the sheep's or dog's heart provided, perform the following cxperiments :

(a) Open the pericardium and notice how it is reflected around the great vessels at the base of the heart. Distinguisl the pulmonary artery, the aorta, the superior and inferior vena cavid, and the pulmonary veins. The tracliea and portions of the lungs may also be attaclicd. If so, remove them carefully without injuring the heart. 
(b) Take two wide glass tubes, drawn slightly into a neck at one end. One of the tubes should be abont ro $\mathrm{cm}$. long, and the other about $50 \mathrm{~cm}$. Tie the sliort tube A furmly by its neck into the superior vena ava, the long tube B iuto the pulmonary artery. Ligature the inferior vena cava. Connect $A$ by a small picce of rubber tubing with a funnel supported in a ring on is stand. Pour water into the funnel till the right side of the leart is full. It will escape from the left azygos vein, which must be tied. Put on any additional ligatures that may be needed to render the heart watcr-tight. Support B in the vertical position by a clamp. Fill the funnel with water, and it will rise in $B$ to the same level as in the funnel. Now compriss the right ventricle with the hand, and the water will rise higher in $B$. Relax the pressurc and notice that the water remains at the higher level in $B$, being prevented by the semilunar valves from flowing back into the ventricle. By alternately compressing the ventricle and allowing it to relax, water can be pumped into $\mathbf{B}$ till it cscapes from its upper end, and if this is so curved that the water falls into the funnel, a 'circulation' which imitates that of the blood can be established. Note that during the pumping the sinuses of Valsalva, behind the semilunar valves at the origin of the pulmonary artery, become prominent.

(c) Take out B and tear out one of the scgments of the semilunar valve. Replace $B$, and notice that, while compression of the ventricle has the same effect as before, the water no longer keeps its level on relaxation, but regurgitates into the ventricle. This illustrates the condition known as insufficiency or incompetence of the valves. But if the injury is not too extensive, it is still possible, by more vigorously and more rapidly compressing the heart, to pump water into the funnel. This illustrates the establishment of compensation in cases of valvular lesion.

(d) Now remove both tubes. Tie the puimonary artery. Cut away the greater part of the right auricle. Pour water into the auriculoventricular orifice, and notice that the segments of the tricuspid valve are floated up so as to close the orifice. Invert the lieart, and the ventricle will remain full of water. Open the right ventricle carefully, and study the papillary muscles and the chordæe tendinere, noting that the latter are inserted into the lower surface of the segments of the tricuspid valve, as well as into their free edges.

$(e)$ Repeat $(b),(c)$, and $(d)$ on tlic left side of the heart, tying tube B in to the aorta as far from the heart as possible, and $\mathrm{A}$ into the left auricle.

(f) Separate the aorta from the lett ventricle, cutting wide of its origin so as not to injure the semilunar valves, and tie a short wide tube into its distal end. Fill the tube with water, and notice that the valves support it. Cut open the aorta just between two adjacent segments of the valve, and notice the pockets behind the segments, and how they are related to each other, and connected to the wall of the vessel.

I6. Sounds of the Heart.- $(a)$ In a fellow-student notice the position of the cardiac impulse, the chest being well exposed. Use both a binaural and a single-tube stethoscopc. Place the chest-piece of the stethoscope over the impulse, and make out the two sounds and the pause. (b) With the hand over the radial or brachial artery, try to determine whether the beat of the pulse is felt in the period of the sounds or of the pause. (c) Listen with the stethoscope over the junction of the second right costal cartilage with the stemum, and compare the relative intensity of the two sounds as heard here with their : elative intensity as heard over the cardiac impulse.

I7. Cardiogram. - Smoke a drum, and arrange a recording tambour and a time-marker beating half or quarter scconds to writc on it (Fig. 88 , 
p. 195). Apply the button of a cardiograph (Fig. 2\%, p. 90) over your own cardiac impulsn and fasten it round the body by the bands attached to the instrument. Connect the cardiograph by an indiarubber tube with a recording tambour (Fig. y9). Set the drum off at a fast speed, take a tracing, and varnish it. Compare with Fig. 28 (p. 91), and if the tracing is sufficiently typical, as is often not the case with human cardiograms, measure out the time-value of the various events in the cardiac revolution.

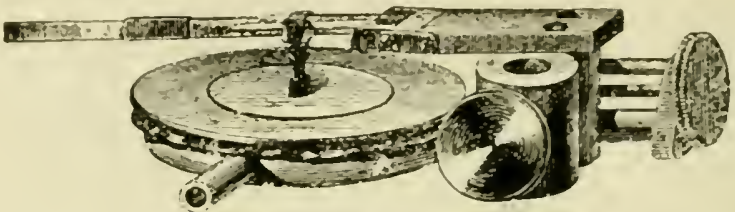

Fig. 99.-Marey's Tambour.

For the cardiograpl, a small glass funnel, or thistle-tube, the stem of which is connected with the recording tambour, may be substituted, the broad end of the funnel being pressed over the apex-beat.

18. Sphygmographic Tracings.-Attach a Marey's spliygmograph (Fig. 37, p. 103) to the arm. Fasten a smoked paper on the plate D. Apply the pad C of the splyygmograph to the wrist over the point

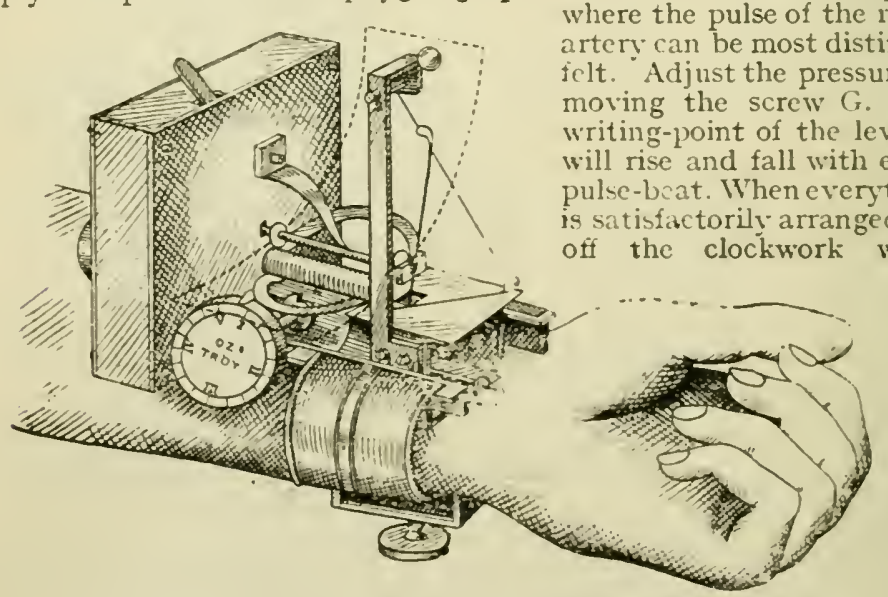

Fig. 100.-Dulgeon's Sphygmograph.

moves the plate D, and a pulse tracing will be obtained. Study the cliang whicls can be producel in the pulse curve- $(a)$ by altering the position of the body (sitting. standing, and lying down); (b) by exercise (Fig. IUI); (c) by inhalation of 2 drops of amyl nitrite poured on a handkerchicf by the demonstrator (Fig. 102); (d) by raising the arm above the head and letting it hang at the side; (e) by compression of the brachial artery at the bend of the elbow; $(f)$ by altering the pressure of the pad Vamish the tracings after marking on them the conditions under which they were obtained.

A Dudgeon's splyygmograph (Fig. 100) may also be employed. In this the clockwork carries the strip of blackened paper along beneath 
the needle which records the movements of the artery. (1) a small glass funuel or thistle-tube connerted with a recorcling tambour may be pressed over the carotid artery. The lever of the timbour writes on a drum, on which at the same time lablf or quarter seconds are marked by an electro-magnetic signal.

19. Venous Pulse Tracing from the Jugular Vein.-Arrange a recorling tambour to write on a drum. Connect the tambour with the stem of a snall glass thistlc-tube or funnd (or with a small metal cup) by a picce of narrow rubber tubing, and apply the cup-shaped end of the thistle-tube over the right jugular bulb of a fcllow-student. This lies about $x$ inch external to the right sterno-clavicular articulation, and a little above it. The receiver may liave to be moved about a little until the best pulsation is

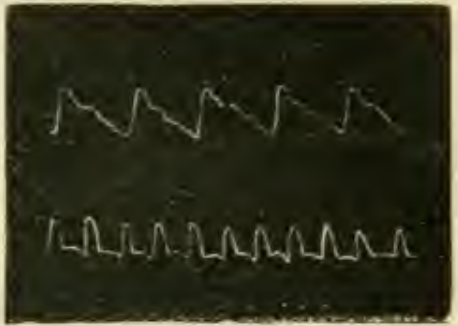

Fig. Ior.-Effect of Exercise on the Pulse (Marey). Lipper tracing. normal; lower, after running. obtained. The 'patient' should be lying down, the shoulders slightly raised. the head on a pillow and turned slightly to the right, in order to relax the right sterno-mastoid muscle (Mackenzie).

20. Polygraph Tracings.-Arrang? the polygraph over the radial artery, as with an ordinary sphygngraph, so that the lever will record the radial pulse when the strip of paper is set moving. If the instru-

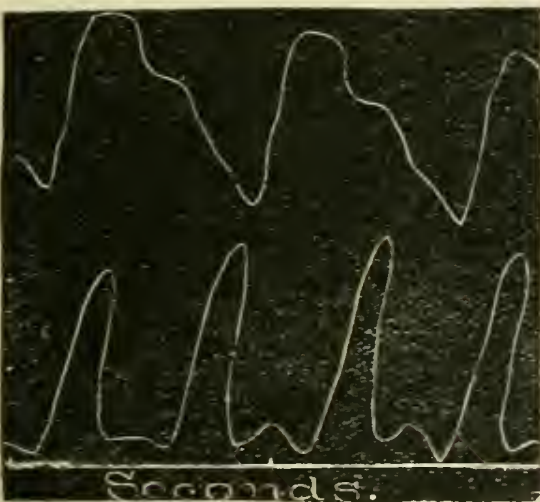

Fig. I02.-Effect of Amyl Nitrite on the Pulse (Marey). Upper tracing, normal; lower, after inhalation of amyl nitrite. ment has only one tambour, connect the tambour to a receiver or thistle-tube over the jugular bulb. The writing-point of the tambour is arrange $I$ so as to be immediately below the writingpoint connected with the radial. If the polygraph is provided with clockwork to record time, set off the time-marker writing fifths of a second. When it is seen that the writing-points are narking properiy, start the clockwork which moves the strip of smoked paper. Repeat the observation with the tambour connected with the apexbeat. Ietter the curves as far as possible as in Figs. 65 and 66 (p. I +9) without at present attempting their exact analysis.

If the polygraph lias two tambours, simultaneous tracing of the radial pulse, tlie jugular pulse, and the cardiac impulse, or of the carotid pulse, the jugular pulse, and the apexbeat, may be taken, and other combinations as well. If no polygrapl is available, a drum may be employed, the tracings being all taken with thistle-tubes connected with recording tambours. The levers of the tambours must be arranged to write on the drum in the same vertical straight line, or, without making the adjustment quite exact, vertical lines of reference may be drawn through cach curve, with the drum at rest, indicating the relative positions of the writing-points. 
21. Plechysmographic Tracings.-Comnect the vessel D (Fig. 36, p. 128 ), arrectly with a recorling tambour by the tube $F$, omitting for simpoicity the recording arrangement in the figure. Place the arm in the plethysmogreph, and adjust the incliarubber band to make a watertight connection. Support $D$ so that the arm rests easily within it, and fill it with water at body temperature. No water must get into the tambour, and it is well to insert a piece of glass tubing in the comnection between it and the plethysmograph. so that it may be seen when the water is rising too high. A $T$-piece with a short piece of rubber tubing on the stem should be inserted in the course of the tube leading to the tambour. All adjustments are made with the $T$-piece open, and when a tracing is to be taken the short rubber tube is closed by a clip. Arrange a time-marker to write half or quarter seconds (Fig. 88. p. 195). Adjust the writing-point to write on a drum, and close the upper tubulure $\mathrm{C}$ with a cork. The quantity of blood in the arm is increased with every systole of the left ventricle, diminished in diastole. The lever will therefore rise when the rentricle contracts, and sink when it relaxes.

(I) Take tracings with the arm (a) horizontal, (b) hanging down.

(2) With the arm horizontal, take tracings to show the effect $(a)$ of closing and opening the fist inside the plethysmograph;* $(b)$ of applying a iight bandage round the arm a little way above the indiarubber band; (c) of inhaling 2 drops of amyl nitrite.

Instead of the arm plethysmograph, a small plethysmograph to hold a finger may be employed. It consists of a glass tube drawn out at one end. The wide end is provided with a rubber collar. The narrow end is connected by a small rubber tube with a very small and sensitive recording tambour, a $\mathbf{T}$-piece being inserted on the connection as bx fore. With the T-piece closed fill the tube with water. Then, holding up the wide encl of the tube, the tip of the finger is put in so as just to close the tube. The $T$-piece is then raised and opened, and the finger pushedin as far as it will go. The collar must fit the finger so as to form a watertight joint. Now get the proper pressure in the tambour by blowing into the $\mathrm{T}$-piece, and close the clamp. A time-tracing can be taken as before.

22. Pulse-Rate.-(I) Count the radial pulse for a minute in the sitting, supine, and standing positions. Use a stop-watch, setting it off on a pulse-beat and counting the next beat as one. Make three observations in each position.

(2) Count the pulse in a person sitting at rest, and then again in the sitting position immediately after active muscular exertion. Note how long if takes before the pulse-rate comes back to normal.

(3) Count the pulse in a person sitting at rest. Repeat the observation while water is being slowly sipped, and note any change.

(4) With one hand over the thordx of a rabbit, count its pulse. Then notice the effect $(a)$ of suddenly closing its nostrils, $(b)$ of bringing a small piece of cotton-wool sprinkled with ammonia or chloroform in front of the nose (reflex inhibition of the heart).

23. Blood-Pressure Tracing.-(a) Put a dog under morphine (p. 63). Sot up an induction machine arranged for an intermpted current (Fig. 93. p. 200). Fill the $U$-shaped manometer tube (if this has not already been done) with clean mercury to the height of ro to $12 \mathrm{~cm}$. in each limb. If the float tends to stick, half an inch of oil may be put above the mercury in the distal (straight) limb before putting in the float. But where the mercury is clean and dry, and the

* Closing the fist causes a lall in the curve-i.e. a diminution in the volume of tise arm. On spenung the hand, the curve regains its level. 
size of the float properly adjusted to that of the tube, this is no necessary, and is to be isvoided. Then, tilting the tube carefully, fill the proximal limb (i.e., the limb which is to be connected with the bloodvessel) with a saturated solution of sodium carbonate or a half-saturated solution of magnesium sulphate, or, what is better for most purposes, a 2 per cent. solution of sodiun citrate. This is easily done by means of a pipette furnished with a long point. Now attach a strong rubber tube to the proximal end of the manometer, and fill it also with the solution. All air must be got out of the manometer and its connectingtube. Raise the end of the rubber tube and blow in to it, so as to cause a difference of about $10 \mathrm{~cm}$. in the height of the mercury in the two limbs of the manometer, and, without releasing the pressure, clamp the tube with a pinchcock or screw clamp (Fig. + , p. I10).

Now smoke a drum, and arrange the writing-point of the manometerfloat so that it will write on it. Suspend a small weight by a piece of silk thread from a support attached to the stand of the drum, so that it hangs down outside of the writing-point of the manometer-float and always keeps it in contact with the smoked surface without undue friction. Or a piece of glass rod drawn out to a fine thread in the blowpipe flame answers very well. Below the writing-point of the tloat, and in the same vertical line with it, adjust the writing-point of a time-marker beating seconds (Fig. 88, p. I95).

Next fasten the animal on a holder, back down. Give ether and insert a tracheal cannula (p. 202). (The tracheal cannula is not absolutely required for the experiment, but it is convenient, as the animal is more under control, and artificial respiration can be begun at any moment, should this be necessary.) Insert a glass cannula, armed with a short piece of rubber tubing. into the central (cardiac) end of the carotid artery (p. 63). Leaving the bulldog forceps on the artery, fill the cannula and tube with the sodium citrate or one of the other solutions. Slip the rubber tube over a short glass connecting-tube. Fill this also with the solution, and connect it with the manometer-tube, seeing that both are quite full of liquid, so that no air may be enclosed.

Where a permanent working place is provided for blood-pressiure experiments it is convenient to connect the cannula and manometer with a pressure-bottle containing the sodium citrate solution, and to use a three-way cannula for the bloodressels (Fig. 103). The cannula has a bulbous enlargement, which hinders clotting. The end of the cannula is connected witl the tube from the pressure-bottle, which is closed by a clip, and the side-tube is connected with one limb, E, of the manometer shown in Fig. Io4. $E$ is itself provided witl a sidetube, $F$, armed with a short piecc of rubber tubing. The cannula does not require to be filled with liquid before being inserted into the artery. By opening $F$ and releasing the clip on the tube from the pressurebottle the cannula and the tube connecting it with the manometer can be filled, and any blood-clots can be easily washed out in the course of an experiment. Before the bulldog forceps is taken off the artery to obtain a blood-pressure tracing. F must be closed, and the clip on the tube from the pressure-bottle opened. The bottle is attached to a strong cord passing over a pulley, by which it is raised to a height sufficient to balance approximately the pressure in the artery. I'luc tube to the pressure-bottle is then clipped. If no manometer with side-tube is available, a T-piece can be inserted in the connection between the cannula and the manometer, and the cannula can be washed out through this.

Now take the bulldog forceps off the artery, and allow the drum to revolve at slow speed. The writing-point of the manometer-float will 
trace a cuave slowing an cheletion for cas heart-beat, and longes waves due to the movements of respiration.

(b) lsoliste the vagu-sympithetic nerve in the neck. Ligature doubly, and cut between the ligatures. Stimulate the peripheral (lower) (nd; the leart will be s!cweil or stopped, and the blood-pressure will fall. Stimulate the central (upper) cnd; there may be inlibition of the licart or acceleration, and the pressure may fall or rise (p. 170$)$.

(c) Expose and divide the other vagosympathetic while a tracing is being taken. Igain stimulate the central end of the nerve and observe whether there is any effect.

(d) Expose the sciatic nerve in one leg: as follows: The leg having been loosened from the holder, the foot is seized by one hand and lifted straight up, so as to put

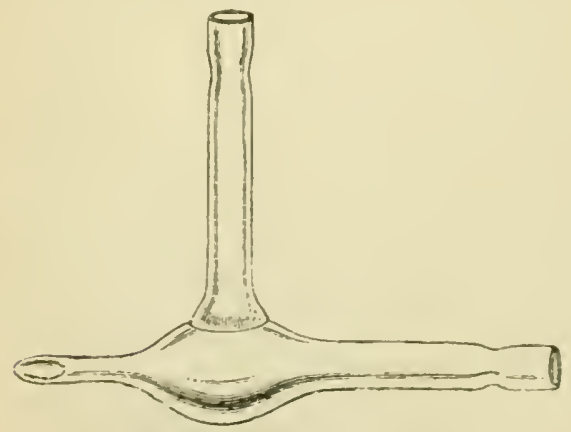

Fig. I03.--Three-way Camula.

the skin of the thigl on the streteli. An incision is now made in the micide line on the posterior aspect of the thigh, through the slin and subcutaneous tissue. The muscles are separated in the line of the incision with the fingers, and the sciatic nerve comes into view lying decply between them. Place a double ligature on it, and divide between the ligatures. Stimulate tle upper (central cud); the bloodpressure probably rises, and the heart may be acceleratcd. Stimulate the periphera! end of the nerve; there is litile change in the blood-pressure and none in the rate of the lieart.

(e) Note, incidentally, that stimulation of the central end of the sciatic or the upper

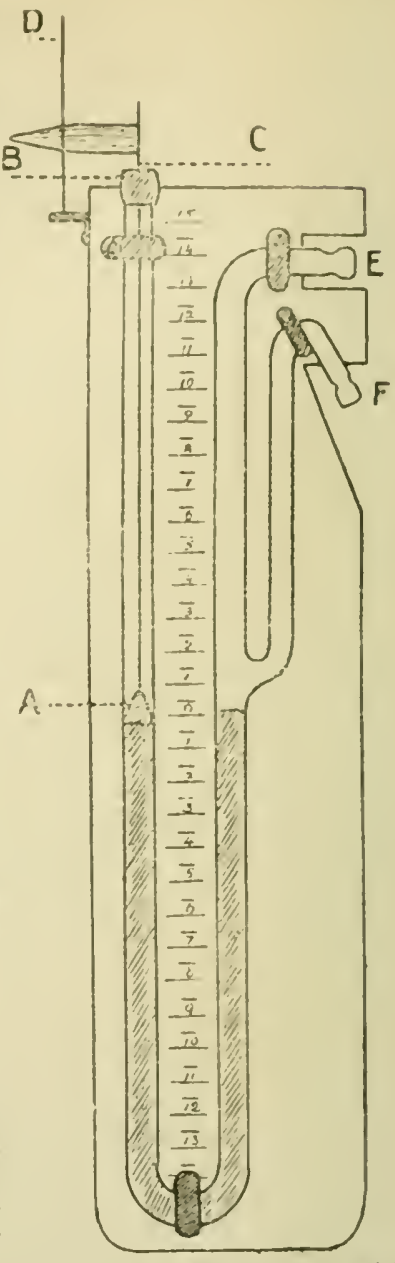

Fig. Iof.-Manometer with Side-tube (Guthrie). A, flozt; B. collar througl which the wire $C$ of the lloat inoves; $D$, vertical wire fixed to manometer-holder, which keeps the writing-point on the drum; $\mathrm{E}$, limb of manoineter connected with camnula, with its side-piece, F. (cephalic) end of the vago-sympathetic may cause increase in the rate and depth of the respiratory movenents. Dilatation of the pupil is also caused by stimulation of the upper end of 
the vago-sympathetic through the sympathetic (pupillo-dilator) fibres that supply tlic iris.

(f) Agcin stimulate the piripleral end of one vagus, or of both at the same time, while a tracing is being taken, and see how long it is possible to kecp the heart from beating. Sometines, but rarely in the dog, inhibition can be kept up so long that the animal dies.

(g) Close the tracheal cannula so that air can no longer enter the lungs. In a very short time the blood-pressure curve begins to rise (rise of asphyxia). After some minutes the pressure falls, and finally, when the circulation has stopped completely and the pressure has become equalized throughout the whole vascular system, a residual pressure of only a few $\mathrm{mm}$. (usually about ro $\mathrm{mm}$. $\mathrm{Hg}$ ) is indicated. In order to"rget the true zero pressure, disconnect the arterial cannula

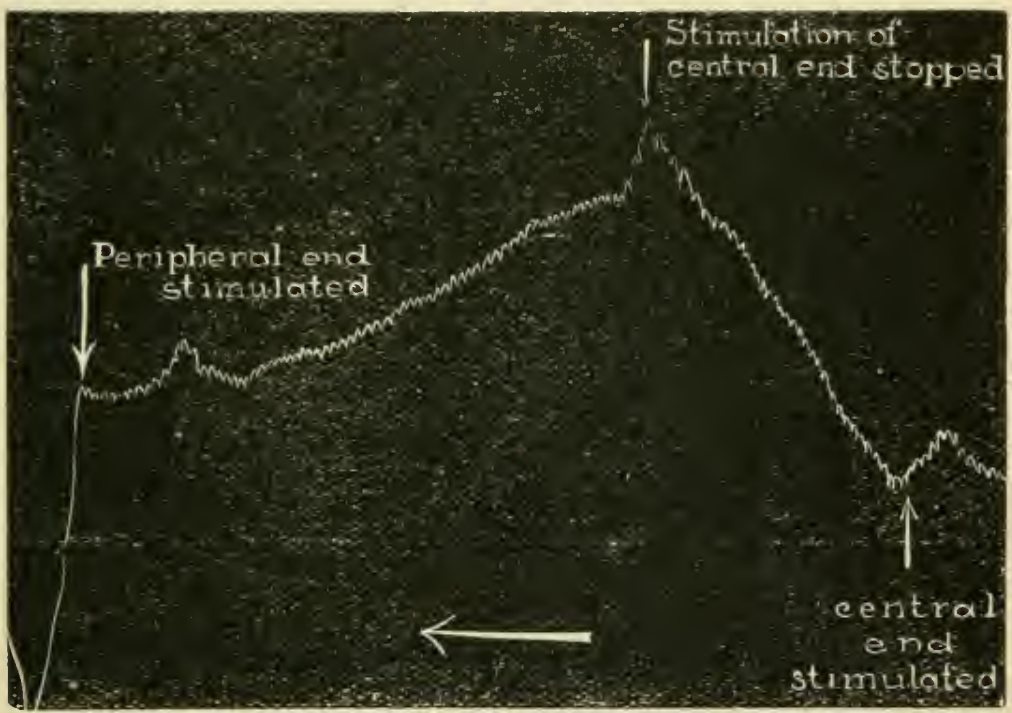

Fig. 105.-Blood.Pıessure Tracing from a Dog: Stimulation of Central and Peripheral Ends of Vagus. The other vagus was intact. Stimulation of the peripheral end caused stoppage of the heart and a marked fall of pressure. Stimulation of the central end produced a great rise of pressure, with, perhaps, a slight acceleration of the heart.

from the manometer, and allow the writing-point to trace a horizontal straight line (line of zero pressure) on the drum (Figs. 84 and 85 ).

24. Estimation of the Arterial Blood-Pressure in Man.-Use the RivaRocci apparatus, as described on p. I13. Begin with the subject in the sitting position. The observer's left hand may be used for palpating the pulse, and the right for working the bulb. Employ the auscultatory method as well as palpation, and determine the systolic and diastolic pressures. Repcat the observations with the person standing up and lying down. Investigate the effcet of muscular exercise on the blood-pressure.

25. The Influence of the Position of the Body on the Blood-Pressure. -Inject into the rectum of a $\log 3$ to $4 \mathrm{grm}$. of chloral hydrate dissolved in a little water. See chat it does not run out again immediately 
after injection. In ten minutes ancestletize the animal fully with a mixture of equal parts of alcohol, chloroform, and ether (one of the so-called A.C.E. mixtures), or with chloroform, and tie it very securely; back downward, on a board, which can be rotated around a horizontal axis, corresponding in position to the point at which the cannula is to be inserted.* Set up a drum and manometer as in 23 (p. 2 ro), but with a rubber connecting-tube of such length as will allow free rotation of the board. Put a cannula in the trachea. Insert a cannula into the central end of the carotid artery at a point immediately above the axis of rotation of the board, and connect it with the manometer.

(a) Take a blood-pressure tracing with the board horizontal.

(b) Whilst the tracing is being taken, rotate the board so that the position of the animal becomes vertical, with the feet down. Mark on the tracing the moment when the change of position takes place. The pressure falls. Replace the dog in the horizontal position. The manometer regains its former level. Now rotate the board, till the animal is again vertical, but with feet up and head down, and observe the effect on the blood-pressure. The respiratory variations in the pressure are usually greater with feet down than with head down. Notice in botl cases whether there is any change in the rate of the lieart.

(c) Take the board off the stands, lay it on a table, expose the femoral artery, and insert a cannula into it. Shift the axis so that it now lies below this cannula. IReplace the board on the stands, and repeat $(a)$ and $(b)$. The fall of pressure will now take place in the head-down position.t In the fect-down position (with the cannula in the femoral artery) a rise of pressure in general takes place. But sometimes this is very small, and lasts only a few seconds, being succeeded by a fall. during which the heart-beats on the tracing are much weaker than before, since enough blood is not reaching the heart to enable it to maintain the pressure. In the feet-down position sec whether the corneal reflex can be got. If not, as is likely, turn the animal into the head-down position. The reflex may now soon be obtained, and it may again disappear on putting the animal in the feet-down position. If the chloroform ancsthesia is light the reflex may not be abolished in the feet-down position, althougli strong respiratory movements may occur, owing to anamia of the medulla oblongata.

26. Effects of Hæmorrhage and Transfusion on the Blood-Pressure. - Anxsthetize a dog with morphine and ether, and insert a cannula into the trachea. Put a cannula into the central end of the carotid artery and another into the central end of the femoral artery. Then insert a cannula, which should have a piece of indiarubber tubing 2 to 3 inches in length on its wide end, into the central end of the femoral vein on the opposite side. In doing this more care is necessary than

* A simple arrangement for this purpose is a board with a number of staples fastened in pairs into its lower surface, so that an iron rod can be pushel through any pair, and form a horizontal axis at right angles to the length of the board. The dog laving been tied down, the rod is pushed through the pair of staples corresponding to the position of the cannula in the artery that is to be connected with the manometer. The projecting ends of the rod rest in two ordinary clamp-holders, fastened at a convenient lieight on two strong stands, whose bases are clamped to the end of a table. The other end of the board is supported by a piece of wood that rests on the floor, and can be removed when the board is to be rotated.

$t$ In 16 dogs the fall of pressure in the carotid in the feet-down position varied from 12 to $100 \mathrm{~mm}$. of mercury; average fall, $44^{\circ} 4 \mathrm{~mm}$. In 12 out of the 16 aninals the rise of pressure in the head-down position varied from 2 to $36 \mathrm{~mm}$.; in I there was no cliange; in 3 there was a fall of 5 to $24 \mathrm{~mm}$. 
in putting a cannuli into an artery. Feel for the femoral artery, cut lown over it, and with forceps or a blunt needle separate the femoral vein from it for about an incli. Pass two ligatures under the vein, and tie a loose loop on each. Put a pair of bulldog forceps on the vein between the ligatures and the heart. Now tic the lower (distal) ligature, and cut one end short. The picce of vein between it and the bulldog forceps is thus distended with blood, and this facilitates the next step. With fine-pointed scissors make a snip in the wall of the vein. The cannula is now pushed through the slit in the vein, and the upper ligature tied firmly round its neck. By the aid of a pipette, made by drawing a picce of glass tubing out to a long point, the cannula and rubber tube are then completely filled with $0^{\circ} 9$ per cent. salt solution. Be sure to pass the point of the pipette right down to the point of the cannula, so as to dislodge any bubble of air that may tend to cling there. Then, holding up the open end of the rubber tube, close it, without allowing any air to enter, by means of a screw clamp or bulldog forceps, or a small piece of glass rod. Connect the cannula in the carotid with a manometer, arranged to write on a drum as in experiment 23 (p. 210). Take the bulldog off the carotid, and measure the difference in the level of the mercury in the two limbs of the manometer with a millimetre scale.

(I) (a) While a tracing is being taken, draw off about ro c.c. of blood from the femoral artery, and obscrve whether there is any effect on the tracing. Mark on the tracing the moment when the removal of the blood begins and ends.

(b) Repeat $(a)$, but run off about roo c.c.* of blood, and let this be immediately defibrinated. Then draw off portions of roo c.c.* at short intervals until a distinct fall of blood-pressure has been produced. All the samples of blood should be defibrinated and strained through cheese-cloth.

(2) (a) Now, while a tracing is being taken, inject the whole of the defibrinated blood slowly through the cannula in the femoral vein by means of a funnel supported by a stand at such a height that the blood runs in easily. A pinchcock should be put on the tube connecting the funnel and the cannula, and this should be closed before the funnel is quite empty, so as to obviate any risk of air getting into the vein. Of course, the cannula and connecting-tubes must all be freed from air before injection is begun. Again measure the difference in the level of the mercury and compare the pressure with that observed before the first hæmorrhage.

(b) Inject into the vein, while a tracing is being obtained, about roo c.c. ${ }^{*}$ of $0^{\circ} 9$ per cent. salt solution heated to $40^{\circ} \mathrm{C}$., and go on injecting portions of 100 c.c. until a distinct rise of pressure has taken place, keeping a record of the total amount injected, and marking the time of each injection on the curve.

(c) After an interval of thirty minutes, again measure the lieight of the mercury in the manometer. Then bleed the dog to death while a tracing is being recorded.

27. The Influence of Proteoses (and Peptones) on the Blood-Pressure. - Set up the apparatus for taking a blood-pressure tracing as in experiment 23 (p. 2 ro), but omit the induction-coil. Weigh a dog. Weigh out a quantity of Witte's peptone equivalent to $0^{\circ} 5 \mathrm{grm}$. for evcry kilo of body-weight. Dissolve the peptone in about ten times its weight of $0^{\circ} 9$ per cent. salt solution. Anzsthetize the dog with morphine and ether or A.C.E. mixture. Insert a cannula into the trachea. Put cannule into the central end of one carotid and of one femoral vein

$$
\text { * } 200 \text { c.c. for a large dog. }
$$


(p. 21.t). Connect the carotid with the manonncter, and the femoral 'cin with a lurette or large syringe containing the peptone solution. Take care that the connecting-tube and canmula are free from air. Now commence to talic a blood-pressure tracing and while it is going un inject the peptone solution. The pressure falls owing largely to a dilatation of the small arteries through the direct action of the peptone on their muse viat tissuc or on the cndings of the vaso-motor nerves. *

28. Effect of Suprarenal Extract on the Blood-Pressure.-Make the arrangements for a blood-pressure tracing from a log ats in 23 (p. 210). Put a cannula in the carotid and another in the femoral vein or one of its branches (p. 21.t). Expose both vagi in the neck, and pass threads loosely under them. Connect the caroticl with the manometer and take a tracing. Then, while the tracing is continued, inject slowly into the femoral vein an amount of watery extract corresponding to about $0^{\circ} 2$ grm. of suprarcual, or, what is more convenient, a few c.c. of a solution of adrenalin cliforide of the strength of I to 50,000 in 0.9 per cent.? sodium chloride solution, the dose depending, of course, on the size of the animal. The blood-pressure risest owing to con-

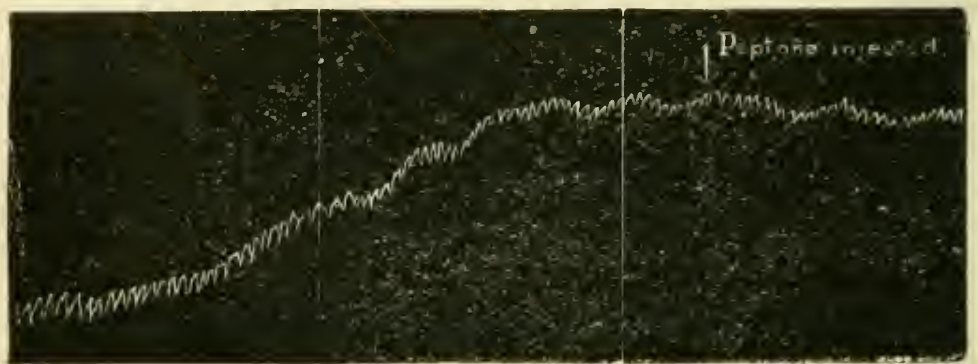

Fig. I06.-Eifect of Injection of Peptone on the Blood-Pressure in a Dog. (To be read from right to left.)

striction of the arterioles by fircet cxeitation of the junction between their vaso-constrictor ncrves and their muscular tissue. The heart is slowed, but its beat is strengthened. At once cut both vagi while a tracing is being taken; the blood-pressure rises still more (p. 655). The rise of pressure is sometimes so great that to prevent the mercury from being forced out of the manometer the tube must be clipped. The rise is not long maintained, but a second injection causes a renewed increase of pressurc.

29. Action of Epinephrin (Adrenalin) on Artery Rings.-The experiment (8) clescribed on $\mathrm{p} .06$ in connection with the constrictor action of serum may equally well be performed licre.

* In 12 (logs the blood-pressure always fell, the amount of the fall varying from 81 to $21 \mathrm{~mm}$. of mercury (average, $60 \mathrm{~mm}$.). It sometimes returned to normal in twenty to thirty minutes, but usually required a longer time. In some dogs, after the injection of the whole of this amount of peptone, deatl occurs before there has been any considerable recovery of the pressure.

$t$ The amount of the initial rise of pressure is very variable, since tlie slowing of the locart tends to diminish the pressure, while the constriction of the arterioles tends to increase it. Thus, in one experiment the increase of pressure on injection of the extract was only $6 \mathrm{~mm}$. of mercury, while in another it was $56 \mathrm{~mm}$. On section of the vagi in this second experiment, there was an alditional rise of $64 \mathrm{~mm}$., and after a secomel injection a further rise of $70 \mathrm{nmm}$., making an increase of $190 \mathrm{~mm}$. in all above the origmal pressure. 
30. Determination of the Circulation-Time.-(1) Br:gin with an artificial scheme (lig. 107). libll the syringe with a 0.2 per cent. Solution of methylene blue. Allow the water to flow from the bottle by loosening the clamp. Inject a definite quantity of the methylene-blue solution, and with a stop-watch observe low long it takes to pass from - the point of injection to the end of the glass tube filled with beads Make ten readings of this kind, and take the mean. Then raise the bottle so as to increase the ra $\mathrm{c}$ of flow of the water, and repeat the observations, The 'circulation-tine' will be found to be diminished.

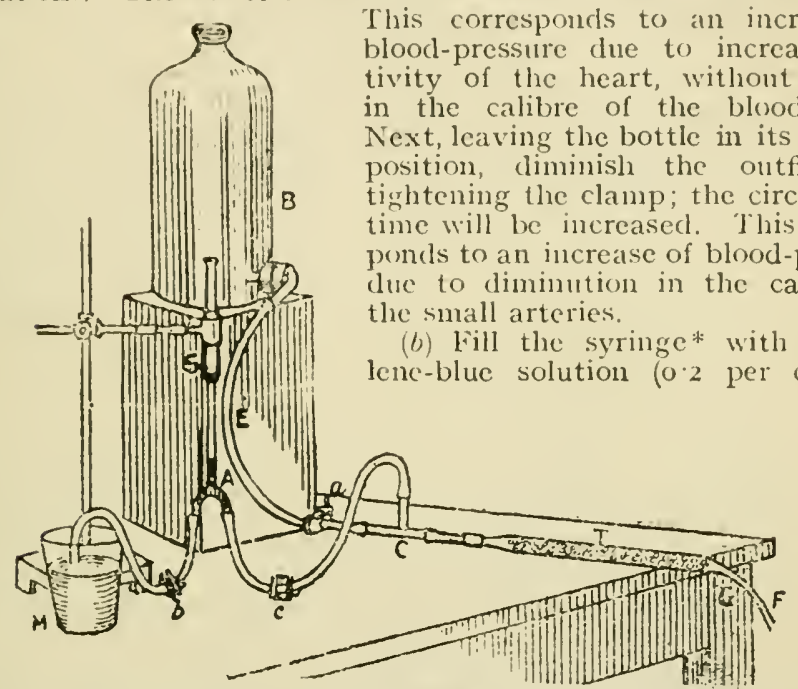

Fig. 107.-Artificial Scliente $t$ i illustrate a Method of measuring the CirculationTime. $\mathrm{B}$, bottle containing water, the rate of outflow of which is regulated by screw-clanp a; S, syringe filled with metlyylent-blue solution, connected with T-piece A; II, beaker containing methylene-blue solution; $b, c$, screw-clamps; $\mathrm{C}, \mathrm{T}$-piece, inserted in the course of the flexible tube $\mathrm{E}$, and connected with the glass tube $T$, which is filled with beads; 1 , outflow tube: The clamp $c$ having been closed and $b$ opened, the syringe is filled with the methylene-blue solution; $b$ is then closed, $c$ opened, and a definite quantity of the solution injected into the sy'stem. The time from the beginning of injection till the appearance of the blue at $\mathrm{G}$ is measured with the stop-watcl.

0.9 per cent. Salt solution), as in $(a)$. Keep the solution warmed to ${ }_{4} \mathrm{o}^{\circ} \mathrm{C}$. by immersing the snall beaker containing it in a water-bath, or heating it over a Eunsen with a small flame. Weigh a rabbit or cat. In the case of the rabbit, inject $\frac{1}{2} \mathrm{grm}$. chloral hydrate into the rectum, and later on give ether if necessary. If a cat, give ether alone, or urethane ( 1.5 grm. per kilo by stomach tube 1 to 2 hours before). Iiasten it on a holder, back downwards (Fig. 61, p. 136). Cover it with a towel to keep it warm. Clip off the hair on the front of the neck, and make an incision $\mathrm{I} \frac{1}{2}$ inches long in the middle line, beginning a little

* A burette, sloped so as to make a small angle with the horizontal, may be substituted for the syringe. The burette is supported on a stand at such a height (say ro-r $5 \mathrm{~cm}$. above the level of the cannula) that the methyleneblue solution runs without great force into the jugular. The danger of producing an abnormal result by suddenly raising the pressure in the right side of the heart is thus avoided. 
waty below the cricoid cartilage. Reflect the skin and isolate the exterual jugular veiu, which is quite superficial. Carefully separate about $\frac{3}{4}$ inch of the vein from the surrounding tissue, and pass two ligatures under it, but do not tie them. Compress the vein with a pair of bulldog forceps between the heart and the ligatures. Now tie the uppermost of the two ligatures (that next the head), but only put a single loose loop on the other. The piece of vein between the upper ligature and the bulldog is now distended with blood. With finepointed scissors make a small slit in the vein, taking great care not to divide it completely, insert the cannula, and tic tlic loose ligature firmly over its neck. Fill the cannula and the small picce of rubber tubing attached to it with o.9 per cent. salt solution by means of a pipette with a long point. Expose the carotid on the other side, isolate it tor 3 inch, clear it carefully from its sheath, slip under it a strip of thin sheet indiarubber, and between this and the artery a little piece of white glazed paper. Connect the cannula in the jugular with the $T$-piece attached to the syringe. Care must be taken that no air remains in the cannula or its connecting-tube, as a rabbit not unfrequently dies instantaneous!y when a bubble of air is injected into the right heart. although a considerable quantity of air can generally be injected into the jugular of a dog without killing it.

Now take off the bulldog from the vein, and make a series of observations on the pulmonary circulation-time. The animal must be so placed that a good light falls on the carotid. If necessary, the light of a gas-flame may be concentrated on it by a lens. The student holds the stop-watch in one hand, and injects a measured quantity of the methylene-blue solution with the other. Uniformity in the quantity injected is secured by fastening on the piston of the syringe a screwclamp, which stops the piston at the desired point. The observation consists in setting off the watch at the moment when injection begins and stopping it when the blue appears in the carotid. After eacli injection the screw-clamp or pincheock on the tube connected with the cannula must be tightened, the other opened, and the syringe refilled. Great care must be taken never to open the two clamps at the same time, as in that case blood may regurgitate through the jugular and fill the syringe, or methylene blue may be sucked into the circulation. As many observations as possible sliould be taken, and the mean determined. The circulation-time obscrved is approximately that of the lesser circulation, the time taken by the blood to pass from the left ventricle to the carotid being negligible for the purposes of the student.

The specific gravity of the blood may also be tested at the beginning and end of the experiment by Hammerschlag's method (p. 62). If a large number of injections have been made in quick succession, the specific gravity will be less than normal; but if a considerable interval has been allowed to elapse after tlic last injection, little or no difference may be found, as the surplus liquid readily passes out of the bloodvessels.

Necropsy.-Observe particularly the state of the lungs, whether the bladder is distended or not, and whether any of the serous cavities or the intestines contain much liquid; so as to determine, if possible, by what channel the water injected into the blood may liave been eliminated. Study the distribution of the methylene blue in such organs as the kidneys and the muscles immediately after death, and notice that the blue colour becomes more pronounced after exposure for a time to the air. Make a longitudinal section through a kidn?y, and observe that the pigment is found especially in the cortex and around the 
pelvis at the apices of the pyramids, or it may be only in the cortex. The urine is greenisli. If some metliylene blue has been injected after the heart ceased to beat, the bloodvessels, particularly in the mesentery, may be beautifully mapped out by the pigment. This is not the case if the last injection took place before deatl, since the methylene blue is rapidly reduced by living tissues to a colourless substance, leuco-methylene bluc.

31. Measurement of the Blood-Flow in the Hands.-Arrange the calorimeter's as in Fig. IC8. The thermometers in the calorimeters should be graduated in tenths of a legree, so that by means of the small lenses or 'readers' which slide on the stems hundredths of a degree can be estimated. Where it is desirable that a number of students should make observations in as short a time as possible, one calorimeter can be allotted to each subject, the other liand being kept in the pocket or covered with a glove if the room is cool, so as to avoid rellex vasomotor interference. A felt collar is chosen which fits the wrist closely. $\Lambda$ horizontal pencil-mark is made at the lower edge of the styloid process of the ulna, and another parallel mark at a distance above this slightly greater than the thickness of the collar. When this second mark is just kept in view above the collar with the hand in the calorimeter, the first (lower) mark will be jut below the level of the lid. A large bath holding 20 or 30 litres or more (a clean 'garbage' or 'offal' can is suitable) is filled with water at about $32^{\circ} \mathrm{C}$. The exact temperature is not important, but it should be about the same in all measurements which are to be compared. An ordinary thermometer graduated in degrees is all that is necessary for reading the temperature of the bath. The calorimeters are now filled from the batl. They are conveniently made of such a size that 3 litres of water and the hand can be contained in them without any slopping over when the water is stirred. Time is saved by having a metal flask which just holds the quantity of water that goes into cach calorimeter. The orifices of the calorimeters are closed by felt discs. The subject, sitting in a high chair placed between the calorimeters, now immerses his hands in the batl to a point between the two marks. The fingers are kept spread. The bath is occasionally stirred. An ordinary thermometer suspended at the back of the chair gives the room temperature. After ten minutes the hands are withdrawn from the bath, the wrists rapidly dried with a towel, the hands at once introduced into the calorimeters, and the felt collars adjusted round the wrists. The subject leans back comfortably in the chair, allowing the arms to hang down without cffort. The fingers are kept slightly spread. The observer sits on a low seat behind the subject, and reads the thermometers from time to time, always after stirring the water well with goosefeathers passing through the stirring-holes in the lid. The readings can be made at intervals of a minute, two minutes, or any interval which is convenient. At the end the hands are quickly withdrawn, the fe!t discs put over the orifices, and the water vigorously stirred for ten or fifteen seconds before the thermometers are read. In this way any errors due to imperfect stirring or to accidental contact of the hands with the thermometers are eliminated.

The volume of each hand is now measured by immersing it exactly to the lower mark in water contained in a glass douche-can connected by a short rubber tube with a pipette furnished with a side-tube at its lower end. The lowest graduation on the burette (50 on a 50 c.c. burette) is brought level with the water before the hand is immersed. While the hand is being held steadily and vertically in the water by an assistant, the level of the water in the burctte is read off. All that 
is necessary to git the volume of the hand is to pour watcr into the can from a graduatcol measure after withdrawal of the hand until the same level is reaclied. Or the value of a division of the burette can be determined once for all. The burette is simply used as a transparent scalc. When the two hands are successively measured, the small amount of water removed by the first is automatically restored by dipping the second into a separate vessel of water, and putting it wet into the douche-can. The rectal i mperature should now be obtained. The temperature of the arterial blood entering the hand is taken as $0^{\circ} 5^{\circ} \mathrm{C}$. below that of the rectum. If only the mouth temperature can be got, the thermometer should be put in a second time without shaking

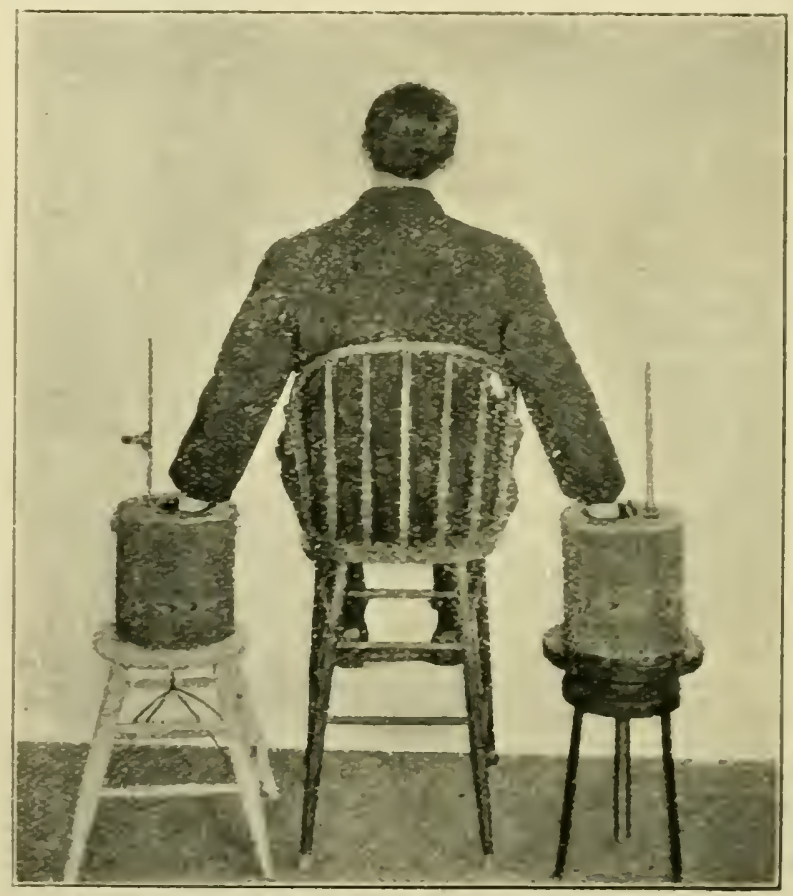

Fig. ro8.-Calorimetric Method of measuring Blood.Flow in Hands.

down to see if it rises any more. The mouth temperature is titien as equal to the arterial blood temperature.

After thorough stirring, the calorimeter temperatures can now be read again. The two being noted, the amount of cooling of the calorimeters can be determincd. This has to be added to the actually observed rise of the thermometers during immersion of the hands.

Suppose an experiment yielded the following data: Rise of thermometer in a calorimeter in twenty minutes during immersion of a hand in it, $\mathrm{r}^{\circ} \mathrm{O} \mathrm{C}$; temperature of calorimeter at beginning of the twenty minutes, $31^{\circ} 0^{\circ} \mathrm{C}$; ; at end of twenty minutes, $32^{\circ} 0^{\circ} \mathrm{C}$; cooling of calorimeter in twenty minutes, $0^{\circ} x^{\circ} \mathrm{C}$; water in calorimeter, 3,coo c.c.; 
volume of liand, +50 c.c.; rectal temperallure, $37^{\circ} 0^{\circ} \mathrm{C}$; water ecpuivalent of calorimeter, 100 c.c.

The water cquivaleut of the hand is $45^{\circ} \times 0^{\circ} 8^{*}=360$ c.c.

llic water ecjuivalent of the calnrimeter is - I0o c.c.

Water - - - - - $\quad$ - 3,000 c.c.

Total - $\quad-\quad \quad-3.460$ c.c.

$3,460 \times I$ I $I=3,806$ small calories given off by the hand in twenty ininutes.

Temperature of arterial blood $\left(36^{\circ} 5^{\circ}\right)$ minus temperature of venous blood $\left(31^{\circ} 5^{\circ}\right.$, the mean temperature of the calorimeter $)=5^{\circ} \mathrm{o}$.

Flow per minute tlırough hand $=\frac{3,806}{20 \times 5} \times \frac{\text { 1o }}{9}=42.3 \mathrm{grm}$.

Flow per 100 c.c. of hand per minute $=9^{\circ} 4$ grm.

The readings of the calorimeter thermometers for the first one or two minutes may not be usable, owing to disturbance caused by the introduction of the hands. As soon as they begin to rise steadily and uniformly, the readings can be utilized for the calculation of the flow.

32. Vasomotor Reflexes.-Begin as in 3I. Then, after the hands have been in the calorimeters for a sufficient period (say ten minutes) to allow satisfactory readings for the determination of the blood-flow to be obtained, rapidly transfer one hand to cold water (at about $8^{\circ} \mathrm{C}$.), while the other remains in the calorimeter. Continue reading the calorimeter thermometer. Its rise will be checked by reflex vasoconstriction. If the hand is kept for a few minutes in the calorimeter, the reflex vaso-constriction of the hand in the calorimeter will probably disappear, and the thermometer will rise faster. When a sufficient number of readings have been obtained for calculating the alteration in the flow, which will usually be the case in eight or ten minutes, transfer the hand from the cold water to warm water (at about $43^{\circ} \mathrm{C}$.), and continue reading the calorimeter thermometer. There is usually a reflex vaso-constriction followed by vaso-dilatation.

* This factor is the product of the specific gravity and the specific heat of the hand. The volume multiplied by the specific gravity gives the mass of the hand, which multiplied by the specific heat, gives the water equivalent of the hand.

+ The reciprocal of the specific heat of blood (see formula, p. I22). 


\section{CHAPTER IV}

\section{RE S P I R A I IO N}

RESPIRATION in its widest sense is the sum total of the processes by which the ultimate elements of the body gain the oxygen they require, and get rid of the carbon dioxide they produce.

\section{Section I.-Preliminary Anatomical Data.}

Comparative.-In a unicellular organism no special mechanism of respiration is needed; the oxygen diffuses in, and the carbon dioxide diffuses out, through the general surface. The simple wants of such multicellular animals as the cœlenterates, the group to which the seaanemone belongs, are also supplied by diffusion through the ectodern from and into the surrounding water, and through the endoderm from and into the contents of the body-cavity and its ramifications.

But in animals of more complex structure special arrangements become necessary, and respiration is divided into two stages: (I) External respiration, an interchange between the air or water and a circulating medium or blood as it passes through richly vascular skin, gills, tracheæ, or lungs; and (2) internal respiration, an interchange between the blood, or lymph, and the cells.

In the lower kinds of worms respiration goes on solely through the skin, under which plexuses of bloodvessels often exist, but in some higher worms there are special vascular appendages that play the part of gills. The crustacea also possess gills, while in the other arthropoda respiration is carried on either by the general surface of the body (in some low forms), or more commonly by means of trachea, or branched tubes surrounded by blood spaces and communicating externally with the air and internally by their finest twigs with the individual cells. IIost of the mollusca breathe by gills, but a few only by the skin.

Among vertebrates the fishes and larval amphibians breathe by gills. but most adult amphibians liave lungs. The skin, too, in such animals as the frog has a very important respiratory function, more of the gascous exchange taking place through it in some conditions than through the lungs.

One small group of fishes, the dipnoi, has the peculiarity of possessing both gills and a kind of lungs, the swim-bladder being surrounded with a plexus of bloodvessels and taking on a respiratory function.

In all the higher vertebrates the respiration is carricd on by lungs: the trifling amount of gaseous interchange which can possibly take place through the skin is not worth takeg into account. The lungs are to be regarded as developed from outgrowths of the alimentary ranal, beginning near the mouth. 
The ubject of all special respiratory arrangements being, in the first instance, to facilitate the gaseous exchange between the surrounding medimm (air or water) and the blood, a prime necessity of a respiratory organ, be it skin, gill, trachea, or lung, is a free supply of blood, in vesiels so fine and thin that diffusion readily takes place in fo them and out of them. But a free supply of blood would be of no avail if the nedium to which the blood gave up its carbon dioxicle and from which it drew its oxygen was not being constantly and sufficiently renewed.

Sometimes the natural currents of the water or the air are of themselves sufficient to secure this renewal; in other cases, artificial currents are set up by cilia, or special bailing organs, like the scaphognathites of the lobster. In all the higher animals, active movements by which air or water is brought into contact with the respiratory surfaces, are neccssary; and it is possible that such movements take place even in the trachex of insects and other air-breathing arthropoda. Fishes, by rhothmical swallowing movements, take in water through the mouth ancl pass it over the gills and out by the gill-slits, while the frog distends its lungs by swallowing air.

Physiological Anatomy of the Respiratory Apparatus.-In man the respiratory apparatus consists of a tube (the trachea) widened at its upper part into the larynx, which contains the special mechanism of voice, and communicates through the nose or mouth with the external air. Below, the trachea divides dendritically into inmumerable branches, the ultimate divisions of which are called bronchioles. Each bronchiole ends in several openings or vestibula, each of which in turn leads into a dilatation called an atrium. From each atrium are given off two, or more, often funnel-shaped diverticula, the infundibula, the walls of which are everywhere pitted with recesses or alcoves, called alveoli. The atria are also lined with alveoli. The infundibula (with the atria) constitute the essential distensible elements of the lung, by the alternate stretching and relaxation of which the respiratory changes in the volume of the organ are mainly brought about. The trachea and bronchi are strengthened by hyaline cartilage which renders them relatively rigid. But the fact that the cartilage does not form complete rings permits small changes of calibre to take place.

In the bronchioles, no cartilage is present, but the circularly-arranged muscular fibres still persist, and also form a thin layer in the infundibula. In the air-cells, or alveoli, however, there are no muscular fibres. Their walls consist essentially of a network of elastic fibres, continuous with a similar layer in the infundibula and bronchioles, and covered on the side next the lumen by a single layer of large, clear epithelial scales, with here and there a few smaller and more granular polyhedral cells.

From the larynx to the bronchioles the mucous membrane is ciliated on its free surface, the cilia lashing upwards so as to move the secretion towards the larynx and mouth. In the infundibula the ciliated epithelium begins to disappear, and is absent from the alveoli. Part of the nasal cavity and the upper part of the pharynx are also lined with ciliated epithelium. Mucous glands are present in abundance in the upper portic $n$; of the respiratory passages, but disappear in the smaller bronchi.

Blood-Supply of the Lungs.- The quantity of blood traversing the lungs bears no proportion to the amount required for their actual nourishment. Small, however, as this latter quantity is, it cannot apparently be derived from the vitiated blood of the right ventricle, but is obtained directly from the aortic system by the bronchial arteries. These are distributed with the bronchi, which they supply as well as the connective tissue of the interlobular septa running through the 
substance of the lung, the pleurit lining it and the walls of the large. bloodvessels. Most of the blood from the bronclial arteries is returncel by the bronchial veins into the systemic venous system, but some of it finds its way by anastomoses into the pulmonary veins.

The branches of the pulmonary artery are also distributed with the bronchi, and break up into a dense capillary network around the alvcoli. From the capillaries veins arise which, gradually uniting, form the large pulmonary veins that pour their blood into the left auricle.

The same quantity of blood must, on the whole, pass per unit of time through the lesser as through the greater circulation, otherwise equilibrium could not exist, and blood would accumulate either in the lungs or in the systemic vessels. But it does not follow that at each heart-beat the output of tlie two ventricles is exactly equal. If, indeed, the capacity of the lesser circulation were constant, the quantity driven out at one systole by the right ventricle would be the same as that ejected at the next by the left ventricle. But it is known that the capacity of the pulmonary vessels is altered by the movements of respiration and probably in other ways, so that it is only on the average of a number of beats that the output of the two ventricles can be supposed equal.

The time required by a given small portion of blood-e.g., by a single corpuscle-to complete the round of the lesser circulation, is, as we have seen (p. 137), much less than the average time needed to complete the systemic circulation. In man the ratio is probably about $I: 5$. Since all the blood in a vascular tract must pass out of it in a period equal to the circulation time, the average quantity of blood in the lungs and right heart of a man would thus be about one-fifth of that in the systemic vessels. That is to say, not less than $700 \mathrm{grm}$. out of the $4^{\frac{1}{2}}$ kilos* of blood in a 70-kilo man would be contained in the lesser circulation, and about $3 \frac{3}{4}$ kilos in the greater. This corresponds sufficiently well with calculations from other data.

For example, the average weight of the lungs in three persons executed by beheading, was $457 \mathrm{grm}$. (Gluge). The average weight of the lungs in a great number of persons who had died a natural death was I,024 grm. (Juncker). The weight of the pulmonary tissue alone in the first set of cases must be less than 457 grm., for the lungs of a person who has bled to deatlı are never bloodless. In a dog killed by bleeding from the carotid, one-quarter of the weiglit of the lungs consisted of blood. Assuming the same proportion for the decapitated individuals, we get $343 \mathrm{grm}$. as the net weight of the blood-free lungs. Deducting this from $\mathrm{I}, 024 \mathrm{grm}$., we arrive at $68 \mathrm{I} \mathrm{grm}$. as the average quantity of blood in the lungs. Adding to this the quantity in the right side of the lieart (p. I fo), we get, in round numbərs, 750 grm. as the amount in the lesser circulation. It is true that in the living body the conditions are not the same as after death; but it is probable that in a laige number of cases taken at random the differences would be approximately cqualized.

It has been further calculated that the total area of the alveolar surface of the lungs of a man is about I oo square metres (sixty times greater than the area of the skin), of which, perhaps, 75 square metres are occupied by capillaries. The average thickness of this immense sheet of blood has been reckoned to be equal to the diameter of a red blood-corpuscle, or, say, $8 \mu$. This would give 600 c.c. $(630 \mathrm{grm}$.) as the quantity of blood in the lungs, which is probably somewhat too low an estimate. 
If we take the pulmonary circulation-time as 13 seconds (p. 1 37). and the quantity of blood in the lungs as $7 m$ grme, then $\frac{07}{7} \frac{6}{3} \times(x)$ $=194$ kilos of blood will pass through the lungs in an hour, or 4,056 kilos (say, 4,40o litres) in twenty-four hours. This would fill a cubica! tank in which the man could almost stand upright witl the licl closed.

\section{Section II. Mechanical Phenomena oli Extiknal REspiration.}

The lungs are enclosed in an air-tight box, the thorax; or it may he said with equal truth that they form part of the wall of the thoracic cavity, and the part which has by far the greatest capacity of adjustment. The alveolar surface of the lungs is in contact with the air. The plema, which covers their internal surface, is reflected over the chest-walls and diapliragm, so as to form two lateral sacs, the pleural cavities. In health these are almost obliterated, and the visceral and parietal pleuræ, separated and lubricated by a few drops of lymph, glide on each other with every movement of respiration. But in disease the pleural cavities may be filled and their walls widely separated by exudation, as in pleurisy, or by blood, as in rupture of an aneurism, or by air in the condition known as pneumo-thorax. Between the two pleural sacs lies a mesial space, the mediastinum, commonly divided into an anterior mediastinum in front of the heart, and a posterior mediastinum belind it. The pleural and pericardial sacs and the mediastinum constitute together the thoracic cavity. The external surface of the chestwall and the alveolar surface of the lungs are subjected to the pressure of the atmosphere, to which the pressure in the thoracic cavity (intra-thoracic pressure) would be exactly equal if its boundaries were perfectly yielding. But in reality the intra-thoracic pressure is always normally something less than this. For even the lungs, the least rigid part of the boundary, oppose a certain resistance to distension, and so hold off, as it were, from the thoracic cavity a portion of the alveolar pressure; and in any given position of the chest the intra-thoracic pressure is equal to the atmospheric pressure minus this elastic tension of the lungs.

The object of the respiratory movements is the renewal of the air in contact with the alveolar membrane-in other words, the ventilation of the lungs. Two main methods are followed by sanitary engineers in the ventilation of buildings: they force air in, or they draw it in. In both cases the movement of the air depends on the establishment of a slope of pressure from the inlet to the interior. In the first method, this is done by increasing the pressure at the inlet; in the second, by diminishing the pressure at the outlet. In certain animals Nature, in solving its problem of ventilation, has made use of the first principle. Thus, the frog forces air into its 
iungs by a swallowing novement. In artificial respiration, as practised in pliysiological experiments, the same method is usually employed: air is driven into the lungs under pressure. But in the vast majority of air-breathing animals, including man, the opposite principle has been adopted; and the 'indraught' of air from nose and pharynx to alveoli is not set up by increasing the pressure in the former, but by diminishing it in the litter. 'This 'indranght,' or inspiration, is brought about by certain movements of the chestwall, which increase the capacity of the thoracic cage and lower the pressure in the thoracic cavity. The expansion of the highly-distensible lungs keeps pace with the diminution of pressure in the plemal sacs, and they follow at every point the retreating chest-wall and diaphritgm, although they do not expand equally in all directions. The dorsal surface in contact with the vertebral column, the mediastinal surface in contact with the pericardium and the contents of the mediastinum, and the surface of the apex, move but little. The surfaces in contact with the diaphragm, ribs, and sternum have the greatest range of movement. Intermediate portions of the parencliyma of the lungs expand in a degrec determined by their distance from the relatively stationary and mobile surfaces. The pressure of the air in the alveoli during the rapid expansion of the lungs necessarily sinks below that of the atimospliere, and air rushes in through the trachea and bronchi till the difference is equalized. Then commences the movement of expiration. The expanded chest falls back to its original limits; the pressure in the thoracic cavity increases; the distended

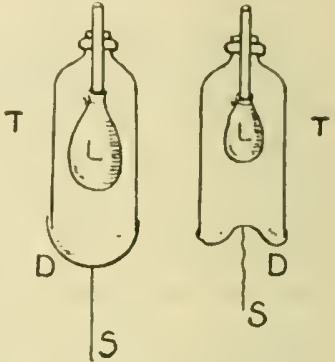

Fig. rug. - Scherne to illus. trate the Movements of the Iungs in the Chest. $T$ is a buttle: from which the brittom has been removed: 1), a llexible and clastic membrane lied on the bottle, anc! capable of being pulled out by the string $\$$ so as to increase the ca. pacity of the bottle. $L$ is a thin elastic bag repre. senting the lungs. It com. 1nunicateswith the external air by a glass tube fitted airtight through a cork in the neck of the botile. IVhen $D$ is drawn down, the pressure of the exterual air causes L to cxpand. When the string is let go. L contracts again, in virtue of its elasticity. lungs, in virtue of their elasticity, shrink to their former volume; the pressure of the air in the alveoli rises above that of the atmosphere, and with this reversal of the slope of pressure air streams out of the bronchi and trachea.

In inspiration the chest dilates in all its diameters. Its vertical diameter is increased by the contraction of the diapliragm, which, composed of a central tendon, a peripheral ring of muscular tissue, and the two muscular crura, bulges up into the thorax in the form of two flattened domes, one on each side, and thus closes its lower 
aperture. When the diaphragn contracts, even in orclinary quiet breathing, the central tendon descends distinctly (about half an inch) after the manner of a piston. The acute angle which the inuscular ring makes during relaxation with the thoracic wall opens out around its whole circumference, so as to form a groove of triangular section. But the most peripheral portion of the ring is always kept in close apposition to the chest-wall by the negative intrathoracic pressure. The lungs follow the descending diaphragm, their lower borders keeping accurately in contact with it. The descent of the diaplıragm is not directly downwards, but downwards and forwards. For it is compounded of two movements, the spinal segment of the muscle (the crura) causing a vertical elongation of the thorax, while the sterno-costal part (the muscular ring) pushes the abdominal viscera downwards and forwards (Keith). Since the diaphragm is attached to the lower ribs, there is a tendency luring its contraction for these to be drawn inwards and upwards; but this is opposed by the pressure of the abdominal viscera, and by the action of the quadratus lumborum, which fixes the twelfth rib, and of the serratus posticus inferior, which draws the lower four ribs backward. When these and the other inspiratory muscles that act especially upon the ribs are paralyzed by injury to the spinal cord, and respiration is carried on by the diaphragm alone, the line of its attachment to the ribs is distinctly marked during inspiration by a shallow circular groove.

The thorax is also enlarged by the action of certain muscles that act upon the ribs. Among the elevators of the ribs, as their name indicates, are usually reckoned, although erroneously, the levatores costarum-twelve in number on each side. They arise from the transverse processes of the last cervical and first eleven dorsal vertebræ, and passing obliquely downwards and outwards, are inserted between the tubercle and the angle into the first or second rib below their origin. They do not elevate the ribs, but take part in lateral movements of the spinal column. The scalene muscles, which may in a lean person be felt to be tense during inspiration, fix the first and second ribs (scalenus anticus and medius, the first; scalenus posticus, the second rib), and so afford a fixed line for the intercostal muscles to work from on the lower ribs.

The most important elevators of the ribs are the external intercostals. The intercartilaginous portions of the internal intercostals (the intercartilaginei muscles, as they are sometimes called) also contract simultaneously with the diaphragm, and may therefore be included in the list of inspiratory muscles; but instead of elevating the ribs they depress the costal cartilages, and thus help to widen the angles between them and the ribs. In addition to increasing the capacity of the chest, the contraction of the external intercostals and the intercartilaginous muscles aids in inspiration 
by augnenting the rigidity of the intercostal spaces, and so preventing them from being drawn in as easily as would otherwise be the case when the thorax is expanded by the action of the diaphragn and the other inspiratory muscles.

Leaving out of account the floating ribs, which functionally form a part of the abdominal wall, the ribs in relation to their respiratory functions may be divided into the following groups: (I) The first rib, which, moving itself very little, provides a fixed line towards which the next set of ribs may be raised.

(2) An upper costal series consisting of the ribs from the second to the fifth. These are raised in inspiration towards the fixed first rib by the contraction of the intercostal muscles. The movement of these ribs is, mainly at any rate, a rotation around a transverse axis, the axes on which they move corresponding to their necks. The manner in which they are articulated to the vertebræ prevents any sensible rotation around an antero-posterior axis or "buckethandle ' movement. Since these ribs slant downwards and forwards to their sternal attachments, the stcrnum is raised when they are elevated; or, rather, since the manubrium is practically immovable in ordinary breathing, the body of that bone is bent on the manubrium at the manubrio-sternal joint. This causes an increase in the antero-posterior diameter of the thorax. Further, since the arches formed by the ribs widen in regular progression from above downwards in the upper portion of the thoracic cage, so that the second rib is a segment of a larger circle than the first, and the third than the second, it is clear that a general elevation of the chest will tend to increase the transverse diameter at any given level. Such an increase is also favoured by the opening out of the angles between the bony ribs and the costal cartilages under the influence of the couple (or pair of oppositely directed forces) that acts on thein - viz., the upward pull of the external intercostals exerted on the ribs, and the downward pull of the intercartilaginei and the resistance of the sternum to further displacement exerted on the cartilages. The whole arrangement is perfectly aclapted to permit the expansion of the roughly conical upper lobes of the lungs.

(3) The lower costal series, consisting of the ribs from the sixth to the tenth. These ribs, with their muscles, form a mechanism which normally acts along with the diaphragm (Keith). They are so arranged that in inspiration the lateral and anterior part of each moves outwards to a greater extent than the one above it. There is not only a rotation around a transverse axis, by which the lower end of the sternum, connected to these ribs by the combined cartilages of the sixth to the ninth, is elevated, but also a rotation around an antero-posterior axis. The movement of the lower ribs results, therefore, in increasing both the back-to-front diameter and the transverse diameter of the lower portion of the thorax. The 
widening of the thorax from side to side may also be in a slight degree ascribed to a twisting movement of the ribs, which tends to evert their lower borders. With the diaphragm, these lower ribs arranged in a vertical series of not very difierent curvature constitute a mechanism for the inspiratory expansion of the roughly cylindrical lower lobes of the lungs.

Expiration in perfectly tranquil breathing is brought about with less aid from active muscular contraction. The sense of effort disappears as soon as the chest ceases to expand. The diaphragin and the elevators of the ribs relax. The structures that rave been stretched or twisted recoil into their original positions; the structures that have been raised against the force of gravity fall back by their weight, and in the measure in which the pressure increases in the thoracic cavity the elasticity of the lungs causes them to shrink. The pressure in the alveoli, which at the end of inspiration was just equal to that of the atmosphere, is thus increased, and the air expelled. It is probable that, even in man and in quiet respiration, the interosseous portions of the internal intercostals help by their contraction in depressing the ribs, and that a slight contraction of the abdominal muscles hastens the return of the diaphragm to its position of rest. In reptiles and birds, expiration is normally effected by an active muscular contraction. This is also true in some mammals-the rabbit, for instance, in which the external oblique muscles of the abdominal wall take an important share in the expiratory act.

Types of Respiration.-Differences exist also, not only between different groups of animals, but even between women and men, in the relative importance in inspiration of the diaphragm and the muscles that raise the lower ribs on the one hand, and the muscles that elevate the upper ribs on the other. W'hen the movements of the diaphragm predominate, the respiration is said to be of the abdominal or diaphragmatic type; when the movements of the upper ribs and sternum are most conspicuous, of the costal or thoracic type. In abdominal respiration, the inspiratory movement commences at the diaphragn, and then involves the lower ribs and the tip of the sternum. In costal respiration, the upper ribs initiate the movement, and are followed by the abdomen. In the rabbit, during quiet breathing, the respiration is purely diaphragmatic, the ribs remain motionless; and herbivorous animals in general conform more or less closely to this type. In the carnivora, on the contrary, the costal type prevails. Man allies himself as regards his respiration with the rabbit and the sheep; he uses his diaphragm more than his upper ribs. Civilized woman falls into the class of the wolf and the tiger; she uses her upper ribs more than her diaphragm. The cause of the difference between men and women has been much discussed. It is not a primitive sexual difference, for it is far from 
being umversal; in the uncivilized and semi-civilized races that have been investigated, the women breathe like the men. It is therefore probable that the predominance of the costal type among women of European race is a peculiarity developed by a mocle of dressing which hampers the movements of the diaphragm while permitting the elevation of the ribs. This conclusion is strengthened by the fact that in children no difference exists; both boys and girls show the abdominal trpe of respiration.

All this refers to ordinary breathing. In forced respiration, when the need for air becomes urgent, costal treathing always becomes prominent alike in men, in women, and in animals, for by elevation of the ribs the capacity of the chest can be increased to a greater degree than by any contraction of the diapliragm.

In forced inspiration, indeed, all the muscles that can elevate the ribs mav be thrown into contraction, as well as other muscles which give these fixed points to act from. During a paroxysm of asthma, for example, the patient may grasp the back of a chair with his hands, so as to fix the arms and shoulders and allow the pectorals and serratus magnus to raise the ribs. Similarly in forced expiration all the muscles are used which can depress the ribs, or increase the intra-abdominal pressure and push up the diaphragm.

Artificial Respiration.-An efficient pulmonary ventilation can be obtained by various methods when the natural breathing is in abeyance. In animals the method most commonly employed for experimental purposes is the rhythmical inflation of the hungs br a pump or bellows, or by a stream of compressed air which is regularly interrupted, the chest being allowed to collapse after each inflation. When the animal is tu be kept alive after the experiment the inflation is produced through a tube introduced through the glottis. If the animal is not to be kept alive, the apparatus is generally connected with a cannula in the trachea. When it is desired to avoid movements of the lungs, respiration may be maintained by a stream of uxygen through a catheter passed down the trachea (method o! insufflation). In man the exchange of air between the atmosphere and the lungs may be most readily accomplished by strong rhythmical compression of the lower part of the chest. This forces out some of the air from the lungs; on relaxing the pressure the chest expands again and air is drawn in. Schäfer has shown that this is the most efficient method of respiration in resuscitation of the apparently drowned. "The patient is placed face downwards on the ground, with a folded coat under the lower part of the chest. The operator puts himself athwart or at the side of the patient, facing his head and kneeling upon one or both knees (Fig. I Io), and places his hands on each side over the lower part of the back (lowest ribs). He then slowly throws the weight of his body forward to bear upon his own arms, and thus presses upon the thorax and forces air out of the lungs. He then gradually relaxes the pressure by bringing his 
own body up atgain to a moge crect prosition, but without moving the hands.' dis is thus drawne inte the lungs. The process is repeated fwclie to fifteen times a mimute.

Certain accessory phenomena (movements and sounds) are associated with the proper movements of respiration. The larynx rises in expiration, and sinks in inspiration. The glottis (and particularly its posterior portion, the glottis respiratoria) is widened during decp inspiration and narrowed during deep expiration. The same is the case with the nostrils, and, indeed, in some persons the alæe nasi move even in ordinary breathing. It has long been known that in deep respiration changes in the calibre of the bronchi synchronous with the respiratory movements may occur. In young persons it may be directly observed with the bronchoscope, an instrument used by laryngologists for exploring the larger bronchi,

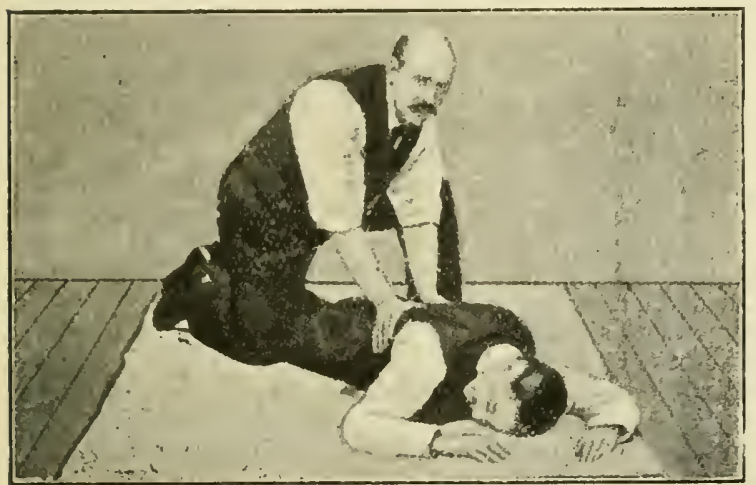

Fig. I10.-Artificial Respiration in Cases of Drowning (after Schäfer). that these dilate in inspiration and constrict in expiration (Ingalls). In part at least these movements are passively produced by the changes of intrathoracic pressure, but it has not been definitely determined whether they are not in part caused by alternate contraction and relaxation of the circular bronchial muscles. To these muscles has sometimes been attributed the function of regulating the flow of air into and out of the infundibula, as the muscle of the arterioles regulates the distribution of the blood in the organs.

As regards the respiratory sounds, all that is necessary to be said here is that when we listen over the greater portion of the lungs with the ear, or, much better, with a stethoscope, a soft breczy nurmur. that has been compared to the rustling of the wind through distant trees, is heard. This has been called the vesicular murmur. It is only heard in health during inspiration and the very beginning of expiration, and is louder in children than in adults. Around the larger bronchi and the trachea a blowillg sound is heard, which certainly originates at the glottis, and is strengthened by the resonance of the air-tubes. In health this is not recognized over the greater portion of the lung. But in certain diseases in which the alveoli are devoid of air, whether from compression or because they are filled up with exudation, and in other conditions, this bronchial or tubular brcathing 
may be heard over the affected area. The bronchi themselves, however, must still he patent and contain air. The most commonly accepted explanation is that the laryngeal sound is better conducted through the smaller bronchi towards the surface of the lungs when their walls have been rendered more rigid by the solidification of the parenchyma, in spite of the fact that the consolidatcel tissue as such does not conduct the sound so well as the air-containing alveoli. It scems paobable that, in addition, the columns of air in the bronchi, which are encaser in solid tissue, may actually increase the intensity of the transmitted laryngeal murmur by resonance.

It has been much debated whether the vesicular murmur also arises at the glottis, and is modified by transmission through the pulmonary tissue, or whether it arises somewhere in the terminal bronchi, the infundibula or the alveoli. Both views may be supported by certain arguments, and to both some objections nilay be raised. The fact appears to be that there are two elements in the inspiratory murmur-. a true vesicular sound, produced about the place where the terminal bronclioles give off the infundibula. and a resonance sound set up in the trachea and bronchi by the glottic murmur. This resonance sound as lieard over portions of the lung containing only small bronclii lias a different character from that heard over large bronchi, inasmuch as the fundamental note, and to a still greater extent the overtones tp. 310), are much weakened in those sinall and easily-distensible tubes. The true vesicular element is heard all over the lungs, but the resonant laryngeal element in large animals, like the horse and $\mathbf{o x}$. dies out as an audible murmur before it reaches the remotest lobules, and can only be distinguished over a portion of the pulmonary area. When the glottic sound is eliminated by causing an animal to breatle through a tracheal fistula, the vesicular murmur is still heard, and in the horse is even somewhat sharper than normal, although in the dog it is softer and weaker. The expiratory murmur does not scem to contain a true vesicular element, but is exclusively clue to the resonance of the expiratory glottic sound (Marck). It is generally admitted, and this is of great importance in practical medicine, that when the normal vesicular sound is heard over any portion of the lung tissue, it may bc inferred that this nortion is being properly distended, and that air is freely cntering its alvcoli.

Up to this point we have contented ourselves with a purely qualitative description of the mechanical plenomena of respiration. IVe have now to consider their quantitative relations, and the methods by which these have been studied.

The expansion of the lungs in inspiration may be easily demonstrated in man, and even a rough estimate of its amount obtained, by the clinical method of percussion. For example, the resonant note tlat is elicited when a finger laid on the chest at a part where it overlies the right lung is smartly struck can be followed down until it is lost in the "liver duliess.' If the lower limit of the resonant area be marked on the chest-wall first in full inspiration, and then in full expiration, the mark will be lower in the former than in the latter, and the difference will represent the difference in the vertical length of the shrunken and distended lung. A similar enlargement in the transverse direction may ba demonstrated in the same way, the inner borders of the lungs coming nearer to the middle line in inspiration, and receding from it in expiration. The examination of the cliest by the Röntgen rays has also 
yicleled results of importance in the study of normal respiratory conditions, and still more important results in pulmonary discase.

For most pliysiological purpeses, however, a faithful graphic record of the respiratory movements is indispensable. This may be obtained-

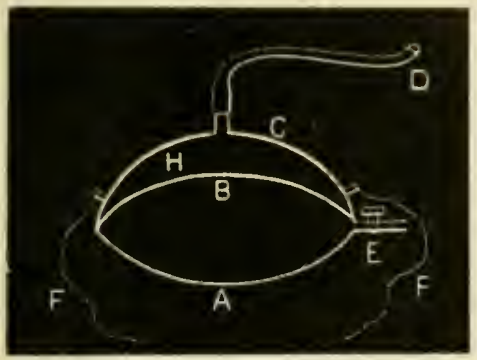

lig. III.-Scheme of Tambour for recording Respiratory Movements. $C$, a metal capsule connccted airtight with B, A, two caoutchouc men. branes, the chamber formed by which can be inflated by means of the tube and stopcock E. The tube D connects the space $H$ with a registering tambour provided with a lever. The membrane $\mathbf{A}$ is applied to the chest, round which the inextensible strings F are tied. At every expansion of the chest the pressure in $\mathrm{H}$ is increased. and the increase of pressure is trans. initted to the registering tambour.

(I) By registering the movements of a single point, or the variations in a single circumference, of the boundary of the thoracic cavity. In man clianges in the circumference of the thorax at any level can be recorded by means of a tambour adjusted to the chest (Figs. I 11 and 13.), and in communication with another, which is provided with a writing lever (Figs. 99 and 137). Or an clastic tube, with a spiral spring in its lumen, may be fastened around the thorax or abdomen and connected with a piston-rccorder (a small cylinder in which works a piston carrying a writing-point) (Fitz).

(2) By recording the changes of pressure produced in the air-passages by the respiratory movements. This can be done by connecting a cannula in the tracliea of an animal with a recording tambour in the manner described in the Practical Exercises (p. 30o). The variations of pressure may be mcasured by connecting a manometer with the trachea, or in man with the nostril.

(3) By writing off the changes of pressure which occur in the thoracic cavity during respiration. For this purpose a trocar (Fig. 113) is introduced through an intercostal sparc into one of the pleural sacs, without the admission of air, or into the pericardium, and then connected with a manometer or other recording apparatus. Or a $t u b e$, similar in construction to a cardiac soind (p. 96), may be pushed down the oesopliagus. The variations, in the intrathorcicic pressure are transmitted to the air in the elas. tic bag, and thence to a tambour.

(4) In the rabbit the part of the dia-

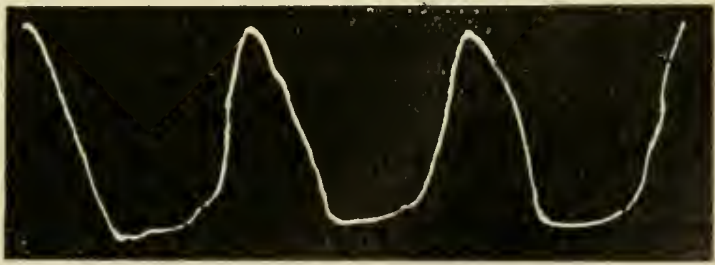

Fig. I 2.- - Respiratory Tracing from Man (Marey), Down stroke, inspiration; up stroke, expiration.

phragm attached to the ensiform cartilage may be isolated from the rest and its contractions recorded by a lever (Head). For some purposes this is the best method.

When the respiratory movements are studied in any of these ways, it is found that there is practically no pause between the end of 
inspiration and the lunimning of expiration. Nor, although the chest collapses more gradually than it expands, is there any distinct interval in ordinary breatling between the end of expiration and the beginning of the succeeding inspiration. Viliten, however, the respiration is unusually slow, an actual pause (expiratory pause) may occur at this point. Expiration takes somewhat longer time than inspiration, the ratio varying from $7: 6$ to $3: 2$, according to age, sex, and other circumstances.

The frequency of respiration is by no means constant even in health. All kinds of influences affect it. It is difficult even to clirect the attention to the respiratory act without hringing about a modification in its rhytlim. In the adult I5 to 20 respirations per minute may be taken as about the normal. In young children the frequency may be twice as great (new-born child, 50 to 70 ; child from I to 5 years old, 20 to 30 per minute). It is greater in a female than in a male of the same age. A rise of temperature increases it; I50 respirations per minute have been seen in a dog with a high temperature. Sudden cooling of the skin, exercise, and various emotional states, increase the rate, and sleep diminishes it. The will can alter the frequency and depth of respiration for a time, and even stop it altogether, but in less than a minute, in ordinary individuals, the desire to breathe becomes imperative. Cato's assertion that he could kill himself at any time 'merely by holding his breath' is only a proof that he was a better philosopher than physiologist. After a period of forced respiration the breath can be held for a much longer time. 'This is due to the 'washing out' of the carbon dioxide, the normal stimulus to the respiratory centre (p. 281). After six minutes of forced

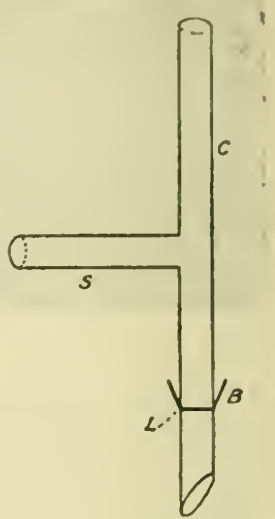

Fig. r13. - S i m ple Pleural Cannula. $B$. a line of sinall spurs which, after the cannula $C$ has been pushed without admission of air through an intercostal space into the pleural cavity, stick in the parietal pleura and securely fasten the cannula. Traction being made on the cannula, a ligature is tied at $L$ around the protruding tissue for greater security. $S$. side-tube by which the canmula is connected with a manometer or tambour. breathing the interval of voluntary inhibition can be extended beyond four minutes. A professional diver has remained uncler water in a tank for about four and three-quarter minutes. Whon oxygen is inhaled instead of air during the last few breaths of the forced respiration, the interval during which the breath can be held may be much increased (up to nine or ten minutes). In animals the rate of respiration can be greatly affected by drugs and by the section and stimulation of certain nerves; but to this we shall return when we come to consider the nervous mechanism of respiration. 
It cannot fail to be observed that to a great extent the rate of respiration is affected by the same circumstances as the frequency of the heart (p. I07), and in the same direction. And, indeed, in health, these two physiological quantities, amid all their absolute variations, maintain to each other a fairly constant ratio ( $\mathrm{I}$ to 4 or

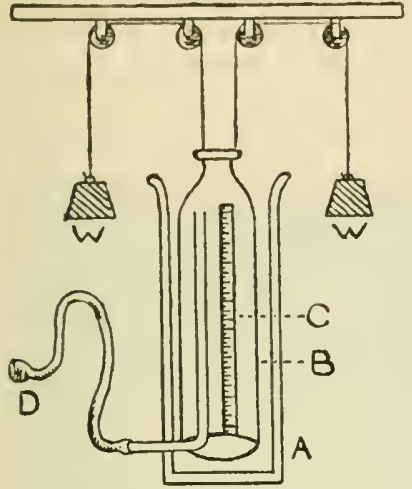

Fig. I I 4.-Diagram of Spirometer. $A$, vessel filled with water. B, glass cylinder with scale $C$, swung on pulleys and counterpoised by weights W. D, tube for breathing through. I to 5 in man). Even in many diseases this proportion remains tolerably stable, although in others it is disturbed.

The total quantity of air expired, or, what comes to the same thing, the alteration in the capacity of the chest during expiration, can be measured by means of a gas-meter or of a spirometer (Fig. II4), which consists of an inverted graduated glass cylinder dipping by its open mouth into water and balanced by weights. The vessel is sunk till it is full of water, the air being allowed to escape by a cock. The expired air is now permitted to enter it through a tube, and displaces some of the water. The spirometer is adjusted so that the level of the water inside and outside is the same, and then the volume of air contained in it is read off. This gives the volume of the expired air at atmospheric pressure. Similarly, by breathing air from the spirometer the amount inspired can be measured (p. 303).

From 400 to 500 c.c. of air* are taken in and given out at each respiration in quiet breathing. This is called tidal air. It amounts to 35 pounds by weight in twenty-four hours, or enough to fill, at atmospheric pressure, a cubical box with a side of 8 feet. With the deepest possible inspiration room can be

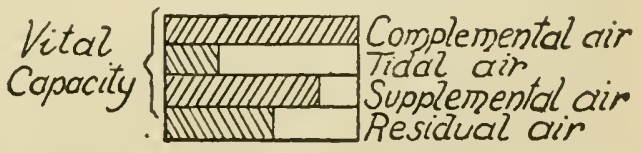

Fig. I I5.-Diagram to illustrate the Relative Amount of Complemental, Tidal, Supplemental, and Residual Air.

made for 2,000 c.c. more; this is called complemental air. By a forced expiration $I, 500$ c.c. can be expelled besides the tidal air; and to this

* The average for $8 \mathrm{I}$ healthy students, with an average body-weight of 66 kilos, was 460 c.c., or 7 c.c. per kilo. In 4 new-born children the tidal air varied from 20 to 30 c.c., and from $7^{\prime} 6$ to $7^{\prime} 3$ c.c. per kilo, which is not very different from the amount in the adult. The pulmonary ventilation must therefore be far more rapid in the child, since its respiratory frequency is so much greater. 
quantity the mane of supplemental or reserve air has been given. After the deepest expiration there always remains $\mathrm{I}, 000$ to I,200 c.c. of air in the lings (I)urig), and this is called the residual air. After a normal expiration following a normal inspiration the lungs still contain stalionary air to the amount of about 2,500 c.c.

The term vital or respiratory capacity is applied to the quantity of air which can be expelled by the deepest expiration following the deepest inspiration, and amounts in an adult of average height to 3,500 or 4,000 c.c. The maximum yuantity of air which the lungs can contain is evidently equal to vital capacity plus residual air. At one time the vital capacity was thought to be capable of affording valuable information in the diagnosis of chest diseases; but little stress is now laid upon it, as it varies from so many causes. For instance, it can be increased by practice with the spirometer. It is greater in mountaineers than in the inliabitants of lowland plains.

The Dead Space. - It is clear from the figures we have given that in ordinary breathing only a small proportion of the air in the lung. comes in direct at each inspiration from the atmosphere, and only a small proportion escapes into the atmosphere at each expiration. The greater part of the air in the lungs is simply moved a little farther from the upper respiratory passages, or a little nearer them; and fresh oxygen reaches the alveoli, as carbon dioxide leaves them. mainly by diffusion, aided by convection currents due to inequalities of temperature, and to the churning which the alternate expansion and shrinking of the lungs, and the pulsations of their arteries must produce. But that some of the tidal air strikes right down to the alveoli is evident enough. For the respiratory' 'dead space ' - that is, the capacity of the upper air-passages and the bronchial tree down to the infundibula - is only I 40 c.c., or one-third of the amount of the tidal air (Zuntz, Loewy). There is no direct way of determining whether any respiratory exchange goes on through the walls of the upper air-passages. But by indirect methods it has been estimated that about 30 per cent. of the volume of the tidal air is pure air (Haldane and Priestley). This, of course, corresponds to the 'effective' dead space. Taking the average tidal air at foo c.c. (p. 235), it is clear that the effective corresponds very closely with the anatomical dead space-that is to say, the respiratory function of the air-passages above the point where the infundibula are given off is negligible. Althongh such calculations can only b2 approximately correct, the agreement is of interest. Someobservers have stated that great variations occur in the size of the dead space with changes in the depth of respiration, its volume being increased four or even eight times by very deep breathing. This, however, is incorrect, although with maximum expansion of the lungs the increase may amount to Ioo c.c. (Krogh and Lindhard. Pearce).

The Amount and Variations of the Intrathoracic Pressure.-In the deepest expiration the lung are never completcly collapied: their 
clastic fibres are sti!l stretched; and the tension of these acts in the: opposite direction to the external atmospheric pressure, and dimin ishes by its amount the pressure inside the thoracic cavity. In tho dead body Donders measured the value of this tension, and therefore of the negative pressure of the thorax, by tying a manometer into the trachea, and then causing tlee lungs to collapse by opening the chest. It varied from $7.5 \mathrm{~mm}$. of mercury in the expiratory position to $9 \mathrm{~mm}$. in the inspiratory. So far as can be judged from observations made on persons suffering from various diseases of the respiratory organs, the alterations during ordinary breatling do not

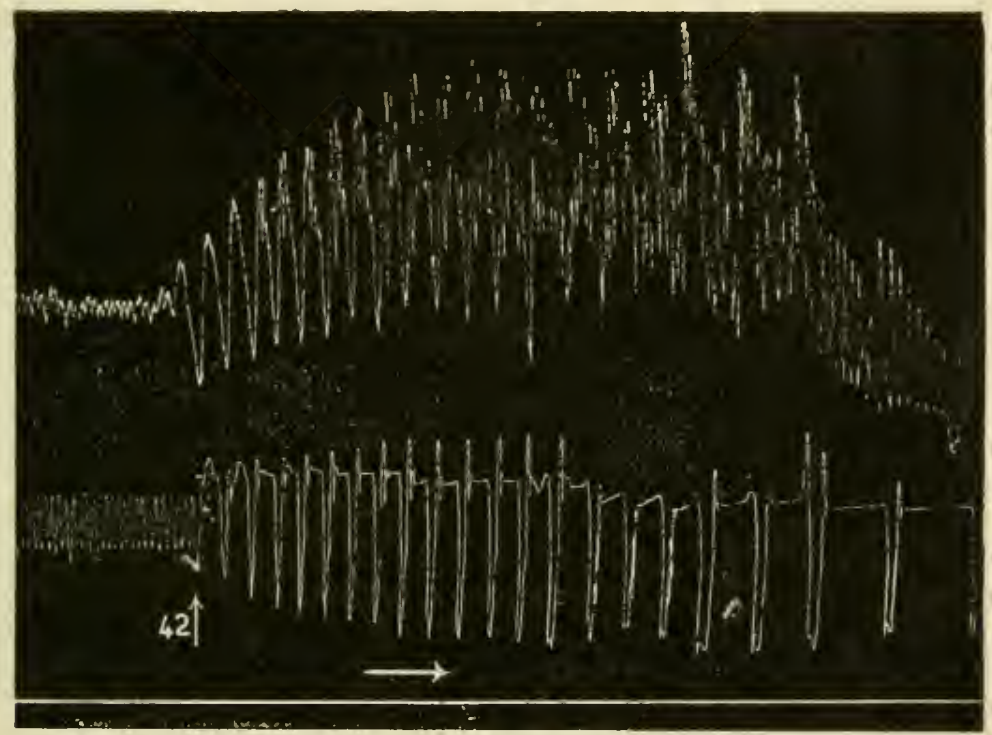

Fig. I16.-Variations of Intrathoracic Pressure. Upper curve, carotid blood-pressure (dog) lower curve, intrapleural pressure. At 42 the trachea was closed; the blond-pressure curve shows the rise of asphyxia, and the intrapleural curve, greatly exaggerated pressure variations due to the strong and slow but abortive respirations.

amount to more than 3 or $4 \mathrm{~mm}$. of mercury. But when an attempt is made in the dead body to imitate a deep inspiration by making traction on the chest walls so as to expand the lungs, the intrathoracic pressure may fall to $-30 \mathrm{~mm}$. of mercury; and in a living rabbit, during a deep natural inspiration, a pressure of $-20 \mathrm{~mm}$. has been seen.

The reason why the lungs collapse when the chest is opened is that the pressure is now equal on the pleural and alveolar surfaces, being in both cases that of the atmosphere. There is therefore nothing to oppose the elasticity of the lungs, which tends to contract them. So long as the chest is unopened, the pressure on the pleural surface of the lungs is less than that on the alveolar surface 
and the elastic tension can only cause them to shrink until it just balances this difference.

In intra-uterine life, and in stillborn children who have never breathed, the lings are completely collapsed (atelectatic), and there is no negative intrathoracic pressure. They are kept in this conlition by adhesion of the walls of the bronchioles and alveoli. If the lungs have been once inflated, this adhesion ceases to act, and they never completely collapse again.

Amount and Variations of the Respiratory or Intrapulmonary Pressure.-As we have already remarked, the pressure in the alveoli and air-passages is less than that of the atmosphere while the inspiratory movement js going on, greater than that of the atmosphere during the expiratory movement, and equal to that of the atmosphere when the chest-walls are at rest. When the external airpassages are closed-o.g., by connecting a manometer with the mouth and pinching the nostrils-the greatest possible variations of pressure are produced. In the deepest inspiration under thest conditions a negative pressure of about $75 \mathrm{~mm}$. of mercury (i.e., a pressure less than that of the atmosphere by this amount) has been found, and in deep expiration a somewhat greater positive pressure* (Practical Exercises, p. 304).

But with ordinary breathing, the variations of pressure as measured by this method do not exceed 5 to Io $\mathrm{mm}$. of mercury above or below the pressure of the atmospliere.

When the external openings are not obstructed, as, for example, when the lateral pressure is taken in the trachea of an animal by means of a cannula with a side-tube connected with a manometer, still smaller, and doubtless truer, values have been found (2-3 $\mathrm{mm}$. of inercury as the positive expiratory pressure and I $\mathrm{mm}$. as the negative inspiratory pressure in dogs). But since the respiratory passages are a.?ruptly narrowed at the glottis, the variations of pressure mut be greater below tl:an above it, and in general they must increase with the distance from that orifice, being greater, for instance, in the alveoli than in the bronchi.

The mechanical phenomena of respiration having been described, it might seem logical to consider next the nervous mechanism by which the respiratory movements are controlled; but the regulation of these movements through the nervous system is in so important a degree a chemical regulation that it cannot be properly understood without some knowledge of the chemical changes in the blood associated with external and internal respiration. We therefore pass to the consideration of-

* The maximum negative pressure in deepest inspiration averaged for 49 students $-73 \mathrm{~mm}$. (highest observation - $137 \mathrm{~mm}$.) of mercury; the maximum positive pressure in deepest expiration, + So $\mathrm{mm}$. (highest observation $+140 \mathrm{~mm}$.). 


\section{Section 111.-The Chemistry of External Respiration.}

Our knowledge of this subject has been entirely acquired in the last 200 years, and chicfly in the last century.

Boyle showed by means of the air-pump that animals die in a vacuum, and Bernouilli that fisl cannot live in water from which the air has been driven out by boiling.

Mayow, of Oxford, seems to a considerable extent to have anticipated Black, who in 1757 demonstrated the presence of carbonic acid (carbon dioxide) in expired air by the turbidity which it causes in lime-water.

A fundamental step was the discovery of oxygen by Priestley in I77I, and his proof that the venous blood could be made crimson, like arterial, by being shaken up with oxygen.

Lavoisier discovered the composition of carbonic acid, and applied lis discovery to the explanation of the respiratory processes in animals, the heat of which he showed to be generated, like l'1at of a candle, by the union of carbon and oxygen. He made many further important experiments on respiration, publishing some of his results in $\mathrm{I}_{\mathrm{S}} \mathrm{g}$, when the French Revolution, in which he was to be one of the most distinguished victims, was breaking out. He made the mistake, however, of supposing that the oxidation of the carbon takes place in the blood as it passes through the lesser circulation.

That some carbon dioxide is formed in the lungs there is no reason to doubt, and the quantity may even be considerable. But that they are not the chief seat of oxidation was sufficiently proved as soon as it: was known that the blood which comes to them from the right heart is rich in carbon dioxide, while the blood which leaves them through the pulmonary veins is comparatively poor.

There are two main lines on which research has gone in trying to solve the chemical problems of respiration: (I) The analysis and comparison of the inspired and expired air, or, in general, the investigation of the gaseous exchange between the blood and tl:2 air in the lungs. (2) The analysis and comparison of the gases of arterial and venous blood, of the other liquids, and of the solid tissues of the body, with a view to the determination of the gaseous exchange between the tissues and the blood. IVe shall take these up as far as possible in their order.

The methods which have been used for comparing the composition of inspired and expired air and estimating the respiratory exchange are very various.

(I) Breathing into one spirometer and sut of another, the inspired and expired air being directed by valves. The cuntents of the spirometers are analyzed at the end of the experiment (Speck). In the arrangement of Zuntz and Geppert, instead of the whole of the expired air, a sample is collected for analysis during the entire duration of the experiment, while the total volume expired is measured by a gas-meter. This is a very convenient method for observations on man, especially 
In cliscase, but each experiment can ouly be airried on at most for sitteen to twenty minutes.

(2) A small apparatus, much on the same principle, was used for rabbits by Pflüger and his pupils. A cannula in tlie trachea was connected with a balanced and self-adjusting spironeter containing oxygen, and the inspired and expired air separated by potassium liydroxide valves, which absorbed the carbon lioxide. The amount of oxygen used could be read off on the spirometer, and the amount of carbon dioxide produced estimated in the liquid of the valves.

(3) Elaborate arrangements, such as Pettenkofer's great respiration apparatus, and the still larger and more efficient modifications of it constructed since his time, in whicls a man, or even several men, can remain for an indefinite period, working, eating, and sleeping. Air is drawn out of the chamber by an engine, its volume being measured by a gas-meter. But as it would be far too troublesome to analyze the whole of the air, a sample stream of it is constantly drawn off, which also passes through a gas-meter, through drying-tubes containing sulphuric acid, and through tubes filled with baryta water. The baryta solution is titrated to determine the quantity of carbon dioxide; the increase in weight of the drying tubes gives the quantity of aqueous vapour. A similar sample stream of the air before it passes into the chamber is treated exactly in the same way, and from the data thus got the quantity of carbon dioxide and aqueous vapour given off can readily be ascertained. The oxygen can be calculated, as the difference between the final body-weight and the original body-weight plus the weight of the carbon dioxide and watr: eliminated, but may also be directly estimated by special methods.

(4) Haldane and Pembrey have elaborated a gravimetric method, which is very suitable for small animals. It deperids upon the absorption of carbon dioxide by soda lime. (See Practical Exercises, p. 30*5.) In Atwater's so-called respiration calorimeter, which will be referred to again under 'Animal Heat,' and by which, not only the gaseous netabolism, but the heat production can be measured in man, the carbon dioxide is estimated in the same way.

Inspired and Expired Air.- The expired air is at or near the body temperature, and is saturated with watery vapour. In ordinary breathing it contains about + per cent. of carbon dioxide, while the inspired air only contains a trace. The expired air contains 16 or I 7 per cent. of oxygen, the inspired air about 2 I per cent. There are in addition in expired air small quantities of hydrogen and marshgas derived from the alimentary canal, either directly from eructation or after absorption into the blood. Sometimes a trace of ammonia can be detected in the air of expiration, but this is due to decomposition of proteins taking place in the mouth, especially in carious teeth, or in the air-passages and lungs in disease of these organs. It has indeed been shown that the lungs are practically impermeable for ammonia. Expired air is entirely free from floating matter (dust), which is always present in the inspired air. The volume of the expired air, owing to its higher temperature and excess of watery vapour, is somewhat greater than that of the inspired air, but if it be measured at the temperature and degree of saturation of the latter, the volume is somewhat less. Since the oxygen of a given quantity of carbon dioxide would have exactly the same 
volume as the carbon rlioxide itself at a given temperature and pressure, it is clear that the deficiency is due to the fact that all the oxygen which is taken "1p in the hungs is not given ofi as carbon dioxide. Some of it, going to oxidize livelrogen, reappears at water.

Alveolar Air. - The pereentage of calbon clioxide in the air of the alveoli is of course greater and that of oxvgen less than in the ordinary expired air. For the relatively pure air of the dead-spare makes up) a substantial fraction of the tidal air. The mean of the oxygen or carbon dioxide percentages in samples taken from the last portions of the air of two deep expirations, one following an ordinary inspiration and the other following an ordinary expiration, corresponds fairly well with the mean percentage in the alveoli during ordinary breathing. This method becomes untrustworthy during muscular work. The average percentage of oxygen may be taken as $\mathrm{I}+\cdot 5$, corresponding to a partial pressure (p. 248) of $\mathrm{I} 0 \mathrm{~g}$ mm. of mercury. The percentage of carbon dioxide in the alveolar air with the body at rest is remarkably constant in one and the same individual at constant atmospheric pressure. It varies in different men from $4 \cdot 6$ to $6 \cdot 2$ (mean $5 \cdot 5$ ) per cent. of the dry alveolar air. In women and in children of both sexes it is less than in men. From this we conclude that in men the partial pressure of carbon dioxide in the alveoli may be at least one-eighteenth of an atmosphere, or $42 \mathrm{~mm}$. of mercury (Fitzgerald and Halclane).

Respiratory Quotient.-The quotient of the volume of oxygen given out as carbon dioxicle by the volume of oxygen taken in is the respiratory quotient. It shows what proportion of the oxygen is used to oxidize carbon. It may approach unity on a carbo-hydrate diet which contains enough oxgyen to oxidize all its own hydrogen to water. With a diet rich in fat it is least of all; with a dict of lean meat it is intermediate in amount. For ordinary fat contains no more than one-sixth, and proteins not one-half, of the oxygen needed to oxidize their hydrogen (p. 620). In man on a mixed diet the respiratory quotient may be taken as 0.8 or 0.9 . So long as the type of respiration is not changed, the respiratory quotient may remain constant for a wide range of metabolism. In hibernating animals, however, the respiratory quotient may become very small during winter slecp (as low as 0.25 ), both the output of carbon dioxide and the comsumption of oxygen falling enormously, but the former in general more than the latter. This has been explained on the assumption that oxigen is stored away in winter sleep in the form of incompletely oxidized substances. On the other hand, in dyspnœea accompanying muscular exertion the respiratory quotient has been found as high as $\mathbf{I} \cdot 2$. It must be remembered that even a voluntary increase in the respiratory movements causes an immediate temporary increase in the respiratory quotient, owing to the ' washing out ' of carbon dioxide from the blood and tissues. This change has no metabolic significance. Indeed, the determination 
of the respiratery quotient for short periods las only a limited value, and such obiervations must be interpreted with great care. In starvation the respiratory quotient diminishes, the production of carbon dioxide falling off at a greater rate than the consumption of oxygen, for the starving organism lives on its own fat and proteins, and has only a trifling carbo-hydrate stock to draw upon. In a cliabetic patient, fed on a diet of fat and protein alone, the respiratory quoticnt was only 0.6 to $0 \cdot 7$, just as in a starving man.

Total Respiratory Exchange.-The amount of oxygen absorbed in a man at rest has been determined under certain conditions as about 0.29 gramme per hour, and the discharge of carbon dioxide as abont 0.33 gramme per hour per kilogramme of body-weight. In an average man weighing 70 kilos the mean production of carbon dioxide is about 800 grammes (400 litres) in twenty-four hours, and the mean consumption of oxygen about 700 grammes ( 190 litres). But there are very great variations depending upon the state of the. body as regards rest or inuscular activity and on other circumstances. In hard work the production of carbon dioxide was found to rise to nearly $I, 300$ grammes, and in rest to sink to less than 700 grammes, the consumption of oxygen in the same circumstances increasing to nearly I, Ioo grammes and diminishing to 600 grammes. In rest, in moderate exertion, and in liard work, the production of carbon dioxide was found to be nearly proportionate to the numbers 2 , 3 , and 6 respectively. When unaccustomed work is performed, the increase in the carbon dioxide output (and oxygen intake) may be much greater. With training it diminishes. In a case of diabetes the consumption of oxygen was 50 per cent. greater than in a healthy man, corresponding to the higher heat-equivalent of the food of the diabetic patient.

Ventilation.- Taking foo litres per twenty-four hours, or 17 litres per hour, as the maan production of carbon dioxide by all average maic adult at rest or doing only light work, we can calculate the quantity of fresh air which must be supplied to a room in order to keep it properly ventilated.

It lias been found that when the carbon dioxide given off in respration amounts to no more than 2 parts in 10,000 in the air of an ordinary room, the air remains swect. When the carbon dioxide given off reaches 4 parts in 10,000 , the room feels distinctly, and at 6 in 10,000 disarecably, close, while at 9 parts in 10,000 it is oppressive and almost intolerable. This is not due to the carbon dioxide as such, for pure carbon dioxide added alone in similar proportions to the air of a room lias not the same bad effect, and the amount of this gas is only taken as an index of the extent to which the air lias been vitiated by some other products or processes connected with the occupation of the room. Very often the mere rise of temperature in a crowded and illventilated space is sufficient to induce disagreable symptoms, especially as it is inevitably associated with an increase in the humidity of the air, which reduces the capacity of the body to cool itself by increasing the secretion of sweat. Thus it has been found that persons in a respiratory cliamber fecl quite comfortable with only moderate rentilation when the carbon dioxide las risen to 1 per cent., if care is taken 
that the temperature and the proportion of watery vapour do not rise too high. In aldition, however, it has been supposed by some that it volatile poison exhaled from the lungs is peculiarly responsible for the evil effects. Certain observers, indeed, alleged that the condensed vapour of the breath, when injected into rabbits, produced fatal symy). toms. But this has been shown to be erroneous; and the most careful experiments have failed to detect in the air expired by healthy persons any trace of such a poison. It lias therefore been suggested that the odour and some of the other ill-effects of a close room are due to substances given off in the sweat and the sebum, and allowed by persons of uncleanly habits to accumulate on the skin, and also to the products of slow putrefactive processes constantly going on, under favourable conditions, on the walls, floor, or furniture, but only becoming perceptible to the sense of smell when ventilation is insufficient. In a small, newly-painted chamber, presumably free from such impurities, it was not until the carbon dioxide reached 3 to 4 per cent., an immensely greater proportion than occurs even in very badly ventilated rooms, that marked discomfort, with dyspnœa, began to be felt. No close odour could be detected.

Nevertheless, experience has shown that it is a good working rule for ventilation to take the limit of permissible respiratory impurity at 2 parts of carbon dioxide per 10,000; and the 17 litres of carbon dioxide given off in thie hour will require 85,000 litres (or 3,000 cubic feet) of air to dilute it to this extent. This is the average quantity required for the male adult per hour. For men engaged in active labour, as in factories or mines, twice this amount may not be too much. For women and children less is required than for men. If a room smells close, it needs ventilation, whatever be the proportion of carbon dioxide in the air. It must be remembered that in permanently occupied rooms mere increase in the size will not compensate for incomplete renewal of the air, although it may be easier to ventilate a large room than a small one without causing draughts and other inconveniences. But as few a partments are occupied during the whole twenty-four hours. a large room which can be thoroughly ventilated in the absence of its inmates has a distinct advantage over a small one in its great initial stock of fresh air. The cubic space per head in an ordinary dwellinghouse should be not less than 28 cubic metres or I, ooo cubic feet.

The quantity of carbon dioxide given off (and of oxygen consumed) is not only affected by muscular work, but also by everything which influences the general metabolism. In males it is greater on the average than in females (in the latter there is a temporary increase during pregnancy), but for the same body-weight and under similar external conditions there is no difference between the sexes. The gaseous exchange is greater in proportion to the body-weight in the child than in the adult. This depends largely on the fact that, other things being equal, the metabolism is relatively to the bodyweight more active in a small, than in a large organism, since the surface (and therefore the heat loss) is relatively greater in the former. But it has been shown that even in proportion to the surface the metabolism is greater in youth than in adult life, and greater in the vigorous adult than in the old man. So that the age of the organism has an influence apart from the extent of surface. The taking of food increases the gaseous exchange, partly from the 
increased nechanical and chemical work performed by the alimentary canal and the digestive glands. But that this is not the sole cause of the increase is shown by the fact that it varies with different kinds of food to a greater extent than can be explained by differences in the ease with which they are digested. For instance, maize produces a much greater increase than oats when given in equal amount, and a protein diet a greater increase than a diet of carbo-hydrate or fat. Sleep diminishes the production of carbon dioxide partly because the muscles are at rest, but also to some extent because the external stinuli that in waking life excite the nerves of special sense are absent or ineffective. Even a bright light is said to cause an increase in the amount of carbon dioxide produced and of oxjgen consumed; but probably only by increasing muscular movements, including the movements of respiration. The external temperature also has an influence. In poikilothermal animals (such as the frog), the temperature of which varies with that of the surrounding medium, the production of carbon dioxide, on the whole, diminishes as the external temperature falls, and increases as it rises. In homoiothermal animals, that is, animals with constant blood temperature, external cold increases the production of carbon dioxide and the consumption of oxygen But if the connection of the nervous system with the striated muscles has been cut out by curara, the warm-blooded animal behaves like the cold-blooded (Pflüger and his pupils in guinea-pig and rabbit). These interesting facts will be returned to under 'Animal Heat.'

Cold-blooded animals produce far less carbon dioxide, and consume far less oxygen, per kilo of body-weight than warm-blooded.

The following table shows the relation between the body-weight and the excretion of carbon dioxide in man:

\begin{tabular}{|c|c|c|c|c|}
\hline \multicolumn{2}{|c|}{ Age. } & \multirow{2}{*}{$\begin{array}{c}\text { Weight in Kilos. } \\
\\
84 \cdot 6 \\
76 \cdot 5 \\
65 \\
82 \\
57 \cdot 7 \\
22\end{array}$} & \multicolumn{2}{|c|}{$\begin{array}{c}\mathrm{CO}_{2} \text { excreted per Kilo } \\
\text { per Hour. }\end{array}$} \\
\hline Male & $\begin{array}{l}58 \\
14 \\
3.5 \\
28 \\
16 \\
9 \cdot 0\end{array}$ & & $\begin{array}{l}0.41 \\
0.48 \\
0.51 \\
0.49 \\
0.59 \\
0.92\end{array}$ & $\begin{array}{l}\text { amme } \\
, " \\
\text { ", } \\
\text { ", } \\
\text { ", }\end{array}$ \\
\hline Female & $\begin{array}{l}66 \\
30 \\
19 \\
10\end{array}$ & $\begin{array}{l}66 \cdot 9 \\
53 \cdot 9 \\
55 \cdot 7 \\
23\end{array}$ & $\begin{array}{l}0.39 \\
0.54 \\
0.45 \\
0.83\end{array}$ & 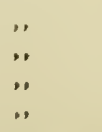 \\
\hline
\end{tabular}

The next table illustrates the difference in the intensity of metabolism in different kinds of animals, a difference, however, largely dependent upon relative size: 


\begin{tabular}{|c|c|c|c|c|c|c|}
\hline \multirow{2}{*}{\multicolumn{2}{|c|}{ Animal. }} & \multicolumn{2}{|c|}{$\begin{array}{l}\text { Oxygen ahsorbed per } \\
\text { Kilo per llour. }\end{array}$} & \multicolumn{2}{|c|}{$\begin{array}{l}\text { Cartoon Dioxide given of } \\
\text { per Kilo per Hour. }\end{array}$} & \multirow{2}{*}{$\begin{array}{c}\text { Respiratory Quotient } \\
\frac{\mathrm{CO}_{2}}{\mathrm{O}_{2}} \text { or } \frac{\mathrm{O}_{2}\left(\text { in } \mathrm{CO}_{2}\right)}{\mathrm{O}_{2}} \text {. }\end{array}$} \\
\hline & & In Grnus. & In C.C. & In Grms. & In C.C. & \\
\hline Greenfincl & - & 13.000 & $909 I$ & 13.590 & 6909 & $0 \cdot 7^{6}$ \\
\hline Hen - & - & $\mathrm{I} \cdot 05^{\mathrm{S}}$ & 740 & $I \cdot 327$ & 675 & $0.9 \mathrm{I}$ \\
\hline Dog - & - & $I \cdot 303$ & 9 I I & $x \cdot 325$ & 674 & $0 \cdot 74$ \\
\hline Ritbbit: & - & 0.187 & 690 & $I \cdot 244$ & 632 & $0.9 \mathrm{I}$ \\
\hline Slicep - & - & 0.490 & 343 & $0.67 \mathrm{I}$ & 341 & 0.90 \\
\hline Boar ${ }^{2}$ - & - & 0.391 & 273 & 0.443 & 225 & 0.82 \\
\hline Frog - & - & $0 \cdot 10_{5}$ & $73^{\circ} 4$ & 0.113 & $57 \cdot 7$ & $0 \cdot 78$ \\
\hline Crayfish & - & 0.054 & 38 & 0.064 & $32 \cdot 7$ & 0.86 \\
\hline
\end{tabular}

Forced respiration, although it will temporarily increase the quantity of carbon dioxide given off by the lungs, and thus raise for a short time the respiratory quotient, does not sensibly affect the production; it is only the store of already formed carbon dioxide in the body which is drawn upon. The amount of oxygen taken up is little altered by changes in the movements of respiration. Within wide limits the oxygen consumption of the organism is independent of the supply of oxygen offered to it.

How it is that the depth of the respiration may affect the rate at which carbon dioxide is eliminated, we can only understand when we have examined the process by which the gaseous interchange between the blood and the air of the alveoli is accomplished; and before doing this it is necessary to consider the condition of the oxygen and carbon dioxide in the blood.

\section{Section IV.-The Gases of the Blood.}

Physical Introduction.-Matter may be assumed to be made up of molecules beyond which it cannot be divided without altering its essential character. A molecule may consist of two or more particles of matter (atoms) bound to each other by chemical links. The kinetic theory of matter supposes the molecules of a substance to be in constant motion, frequently colliding with each other, and thus having the direction of their motion changed.

In a gas the mean free path, that is, the average distance which a molecule travels without striking another, is comparatively long, and far more time is passed by any molecule without an encounter than is taken up with collisions. Although the average velocity of the molecules is very great, these collisions will produce all sorts of differences in the actual velocity of different molecules at any given time. Some will be moving at a greater, some at a slower rate, than the average; while some may be for a moment at rest. If the gas is in a closed vessel, the molecules will be constantly striking its sides and rebounding from them. If a very small opening is made in the vessel, some molecules will occasionally hit on the opening and escape altogether. If the opening is made larger, or the experiment continued for a longer time 
with the small opening, all the molecules will in course of time have passed out of the vessel into the air, while molecules of the oxygen, nitrogen, and argon of the air will have passed in. In a gas, then, not enclosed by impenetrable boundaries, there is no restriction on the path which a molecule may take, no tendency for it to keep within any limits.

When two chemically indifferent gases are placed in contact with each other, diffusion will go on till they are uniformly mixed. The diffusion of gases may be illustrated thus. Suppose we have a perfectly level and in every way uniform field divided into two equal parts by a visible but intangible line, the well-known whitewash line, for instance. On one side of the line place 500 blind men in grcen, and on the other 500 blind men in red. At a given signal let them begin to move about in the field. Some of the men in green will pass over the line to the "red" side; some of the men in red will wander to the 'green' side. Some of the men may pass over the line and again come back to the side they started from. But, upon the whole, after a given interval has elapsed, as many green coats will be seen on the red side as red coats on the green. And if the interval is long enough there will be at lengtl about 250 men in red and 250 in green on each side of the boundaryline. When this state of equilibrium has once been reached, it will henceforth be maintained, for, upon the whole, as many red uniforms will pass across the line in one direction, as will recross it in the other.

In a liquid it is very different; the molecule has no frce path. In the depth of the liquid no molecule ever gets out of the reach of other molecules, although after an encounter there is no tendency to retum on the old path rather than to choose any other; so that any molecule may wander through the whole liquid. Although the average velocity of the molecules is much less in the liquid state than it would be for the same substance in the state of gas or yapour (gas in presence of its liquid), some of them may have velocities much above the average. If any of these happen to be moving near the surface and towards it, they may overcome the attraction of the neighbouring molecules and escape as vapour. But if in their further wanderings they strike the liquid again, they may again become bound down as liquid molecules. And so a constant interchange may take place between a liquid and its vapour, or between a liquid and any other gas, until the state of equilibrium is reached, in which on the average as many molecules leave the liquid to become vapour as are restored by the vapour to the liquid, or as many molecules of the dissolved gas escape from solution as enter into it.

For the sake of a simple illustration, let us take tlie case of a shallow vessel of water originally gas-free, standing exposed to the air. It will be found after a time that the water contains the atmospheric gases in certain proportions-in round numbers, about ifo of its volume of oxygen and $\frac{1}{50}$ of its volume of nitrogen (measured at $760 \mathrm{~mm}$. mercury and $0^{\circ} \mathrm{C}$.).

Now, let a similar vessel of gas-free water be placed in a large airtight box filled with air at atmospheric pressure, and let the oxygen be all absorbed before the water is exposed to the atmosphere of the box. The latter now consists practically only of the nitrogen of the air, and its pressure will be only about four-fifths that of the external atmosphere. Nevertheless, the quantity of nitrogen absorbed by the water will be exactly the same as was absorbed from the air. If the box was completely exhausted, and then a quantity of oxygen, equal to that in it at first, introduced before the water was exposed to it, the pressure would be found to be only about one-fifth that of the external atmosphere; but the quantity of oxygen taken up by the water would be exactly equal to that taken up in the first experiment. 
Two well-known physical laws are illustrated by our supposed experiments: (I) In a mixture of gases which do not act chemically on each other the pressure exerted by cach gas (called the partial pressure of the gas) is the sume as it would exert if the others were absent. (2) The yuantity (mass) of a gas absorbed by a liquid which does not act chemically upon it is proportional to the partial pressure of the gas. It also depends upon the nature of the gas and of the liquid, and on the temperature, increase of temperature in general dininishing the quantity of gas absorbed. It is to be noted that when the volume of the absorbed gas is measurecl at a pressure equal to the partial pressure under which i was absorbed. the same rolume of gas is taken up at cvery pressure.

The volume of a gas (reduced to $0^{\circ} \mathrm{C}$. and $760 \mathrm{~mm}$. pressure) pliysically absorbed or dissolved in I c.c. of a liquid cxposed to the gas at $760 \mathrm{~mm}$. pressure is called the absorption coefficient of the gas in that liquicl. The following table from Boln shows the absorption cocfficients of the three gases of physiological interest-oxygen, nitrogen, and carbon dioxide in water, blood-plasma, whole blood and blood-corpuscles at the body temperature $\left(38^{\circ} \mathrm{C}.\right)$ :

\begin{tabular}{|lll|l|l|l|}
\hline & & Oxygen. & Nitrogen. & Carbon Dioxide. \\
\hline Water - & - & - & 0.0237 & 0.0122 & 0.555 \\
Blood-plasmia & - & - & 0.023 & 0.012 & $0.54 \mathrm{I}$ \\
Blood - - & - & - & 0.022 & 0.011 & 0.5 I I \\
Blood-cells - & - & - & 0.019 & 0.010 & 0.450 \\
\hline
\end{tabular}

Suppose, now, that a vessel of water, saturated witl oxygen and nitrogen for the partial pressures under which these gases exist in the air, is placed in a box filled with pure nitrogin at full atmospheric pressure. As we have scen, there is a constant interchange going on betwcen a liquid which contains gas in solution and the atmosphere to which it is exposed. Oxygen and nitrogen molecules will therefore continue to leave the water; but if the box is large, few oxygen molecules will find their way back to the water, and ultimately little oxygen will remain in it. In other words, the quantity of oxygen absorbed by the water will become again propertional to the partial pressure of oxygen, which is not now much above zero. On the other hand, molecules of nitrogen will at first enter the water in larger number than they escape from it, for the pressure of the nitrogen is now that of the external atmosphere, of which its partial pressure was formerly only four-firths. In unit volume of the gas above the water there will be 5 molecules of nitrogen for every 4 molecules in the same volume of atmospheric air. Therefore, on the average 5 nitrogen molecules will in a given time get entangled by liquid molecules for every 4 which came within their sphere of attraction before. On the whole, then, the water will lose oxygen and gain nitrogen, while the atmosphere of the airtight box will gain oxygen and lose nitrogen.

In the case of water, in which oxygan and nitrogen are absorbed solely in solution, the partial pressures of these gases under which the water was originally saturated could, of course, be easily calculated from the amount dissolved and the ccefficient of absorption. But supposing that these partial pressures were unknown, it is evident that by exposing it to an atmosphere of known composition, and afterwaris determining the changes produced in the composition of that atmo. 
sphere by loss to, or giain from, the gases of the water, we could find out something about the original partial pressures. If, for example, the quantity of oxygen in the atmosphere or the chamber was increased, we could conclude that the partial prissure of oxygen under which the water had becn saturated was greater than that in the chamber at the beginning of the experment. And if we found that with a certain partial pressure of oxygen in the atmospliere of the chamber there was neither gain nor loss of this gas, we might be sure that the partial pressure (the temperature hing sty)poscd not to vary) was the same when the water was saturated. IVe shall see later on how this prmciple has been applied to determine the partial pressure of oxygen or carbon dioxidle which just suffices to prevent blooxl, or ally other of the liquids of the body, from losing or gaining these gases when they are not merely dissolved, but also combined in the form of dissociable compounds. This pressure is evidently equal to that exerted by the gases of the liquid at its surface, which is sometimes called their 'tension'; for if it were greater, gas would, upon the whole, pass into the bloorl; and if it were less, gas would escape from the blood. Thus, the tension of a gas in solution in a iiquid is equal to the partial pressure of that gas in an atmosphere to which the liquid is exposed, which is just sufficient to prevent gain or loss of the gas by the liquid (p. 258).

The following imaginary experiment may further illustrate the meaning of the tern 'tension' of a gas in a liquid in this connection.

Suppose a cylinder filled with a linuirl containing a gas in solution, and closed above by a piston moving airtight and without friction, in contact with the surface of the liquid (Fig. 1 17). Let the weiglit of the piston be balanced by a counterpoise. The pressure a the surface of the liquid is evidently that of the atmosphere. Now, let the whole be put into the receiver of an air-pump, and the air gradually exhausted. Let exhaustion proceed until gas begins to escape from the licpuid and lies in a thin layer between its surface and the piston, the quintity of gas which has become free being very small in proportion to that still in solution. At this point the piston is acted upon by two forces which balance each other, the pressure of the air in the receiver acting downwards, and the pressure of the gas escaping from the liquid acting upwards. If

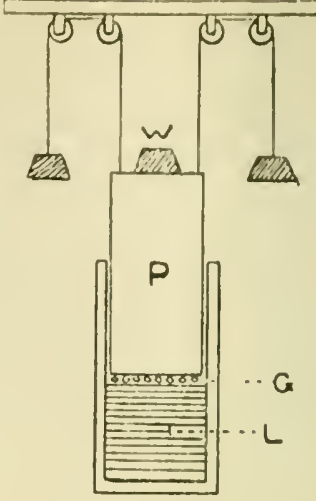

Fig. Ir7.-Imaginary Experiment to illustrate 'Tension' of a Gias in a Liquid. P. frictionless piston; L, liquid in cylinder; $\mathrm{G}$, gas beginuing to escape from liquid. $\mathrm{P}$ is exactly counter. poised. In addition to the manner described in the text, the experinent may be supposed to be performed thus: Let the weight, W, be determined which, when the receiver is completely exhausted, suffices just ti keep the pir on in contact with the liquid. The pressure of the gas is then just counter. balanced by $W$; and it $S$ is the area of the cross. section of the piston, the pressure of the gas per unit of area is $\frac{\mathrm{W}}{\mathrm{S}}$. Or, if the piston is hollow, and mercury is poured into it so as just to keep it in contact with the liquid, the height of the column of mercury required is also equal to the pressure or tension of the gas. the pressure in the receiver is now slightly increased, the gas is again absorbed. The pressure at which this just happens, and against which the piston is still supported by the impacts of gaseous molecules flying out of the linuul while no pressure is as yet 
exerted directly between the liquid and the piston, is obviously equal to the pressure or tension of the gas in the liquid.

From the above principles it follows that a gas held in solution may be extracted by exposure to an atmosphere in which the partial pressure of the gas is made as small as possible. Thus, oxygen can be obtained from liquids in which it is simply dissolved by putting them in an atmosphere of lyydrogen or nitrogen, in which the partial pressure of oxygen is zero, or in the vacuum of an air-pump, in wlich it is extremely small. Heat also aids the expulsion of dissolved gases. Some gases held in weak cliemical union. like the loosely-combined oxygen of uxyluemoglobin, can be obtained by dissociation of their compounds

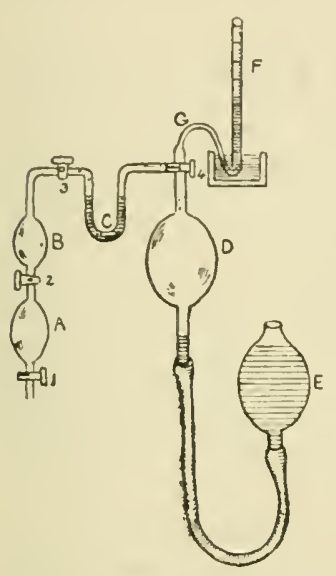

Fig. 118. Scheme of Gas-Pump. A, the bluod bulb; $B$, the froth chamber; $C$, the drying tube; D. fixed mercury bulb; $\mathbf{E}$, movable mercury bulb connected by a flexible tube with D; F, eudiometer; G, a narrow delivery tube; $I, 2,3,4$. taps, \& being a three-way tap. A is filled with blood by connecting the tap I by means of a tube with a bloodvessel. Taps $I$ and 2 are then closed. The rest of the apparatus from $B$ to D is now exhausted by raising $E$, with tap 4 turned so as to place $D$ only in communication with $G$, till the mercury fills D. Tap 4 is now turned so as to connect $C$ with $D$, and cut off $G$ from $D$, and $E$ is lowered. The mercury passes out of $\mathrm{D}$, and air passes into it from B and C. Tap 4 is again turned so as to cut off $C$ from $D$ and connect $G$ and $D$. E is raised and the mercury passes into $D$ and forces the air out through $G$, the end of which has not hitherto been placed under $F$. This alternate raising and lowering of $\mathrm{E}$ is continued till a manometer connected between $\mathrm{C}$ and 4 indicates that the pressure has been sufficiently reduced. The tap 2 is now opened; the gases of the blood bubble up into the froth chamber, pass through the drying-tube $\mathrm{C}$, which is filled with pumice-stone and sulphuric acid, and enter D. The end of $G$ is placed under the eudiometer $F$, and by raising $E$, with tap + turned so as to cut off $C$, the gases are forced out through $G$ and collected in $F$. The movements required for exhaustion can be repeated several times till no more gas comes off. The escape of gas from the blood is facilitated by immersing the bulb $\mathrm{A}$ in water at $40^{\circ}$ to $50^{\circ} \mathrm{C}$.

when the partial pressure is reduced. More stable combinations may require to be broken up by chemical agents-carbonates, for instance, by acids.

Extraction of the Blood-Gases.-This is best accomplished by exposing blood to a nearly perfect vacuum. The gas-pumps which have been most largely used in blood analysis are constructed on the principle of the Torricellian vacuum. A diagram of a simple form of Pfluger's gas-pump is given in Fig. I 18 . The gases obtained are ultimately dried and collected in a eudiometer, which is a graduated glass tube with its mouth dipping into mercury. The carbon dioxide is estimated by introducing a little potassium hydroxide to absorb it. The diminution in the volume of the gas contained in the eudiometer gives the volume of the carbon dioxide. The oxygen may be estimated by putting into the eudiometer more than enough hydrogen to unite with all the oxygen so as to form water, and then, after reading off the volume, exploding the mixture by means of an electric spark passed through two platinum wires fused into the glass. One-third of the diminution of volume represents the quantity of oxygen present. It can also be estimated 
by absorption with a solution of pyrogallic acid and potassium hydroxide, or an alkaline solution of sodium hydrosulphite, which is more cleanly. The remainder of the original mixture of blood-gases, aiter decluction of the carbon dioxide and oxygen, is put down as nitrogen (with, no doubt, a small proportion of argon). For the sake of easy comparison, the observed volume of gas is always stated in terms of its equivalent at a standard pressure and temperature $(7,10 \mathrm{~mm}$, or sometimes on the Continent I metre of mercury, and o (.).

It is also possible in various ways to estimate the amount of oxygen in blood without the use of the pump. Thus, since a definite volume of oxygen (1.338 c.c. at $0^{\circ} \mathrm{C}$. and $760 \mathrm{~mm}$. pressure) combines with a gramme of hamoghobin, we can calculate the total volume of oxygen present if we know how much of the blord-pigment is in the form of oxyhamoglobin; and this can be determined by means of the spectrophotometer. Or potassium ferricyanide may be added to the blood. This expels the oxygen from its combination with the hamoglobin, which then unites with an exactly equal amount of oxygen sbtained from the ferricyanide to form inethamoglobin (Haldane) (p. 75).

In the hands of Barcroft and his pupils this method has been highly developed, so that accurate results can be obtained with small quantities of blood ( I c.c., and with the smaller apparatus even 0.I c.c.). Barcroft's differential apparatus consists essentially of two small bottles, as nearly alike as possible, connected by a manometer filled with oil (of cloves). The amount of oxygen liberated in one of the bottles by potassium ferricyanide from a measured amount of blool can be estimated from the displacement of the liquid in the manometer. "The function of the second bottle is to automatically eliminate effects clue to changes in the temperature of the bath in which the apparatus is immersed, etc., since both bottles are affected alike.

The Quantity of the Blood-Gases.-In arterial and in venous blood oxygen, carbon dioxide, nitrogen, and argon are constantly found. Both the oxygen and the carbon dioxide vary considerably in amount in the arterial blood, even of individuals of the same animal group, and, of course, much more in the venous blood, as might naturally be expected, since even to the eye it varies greatly according to the vein it is obtained from, the rapidity of the circulation, and the activity of the tissues which it has just left. In one observation on blood obtained directly from a human artery, 2 I 6 c.c. of oxygen, $40 \cdot 3$ c.c. of carbon dioxicle, and I. 6 c.c. of nitrogen were found in roo c.c. of blood. The quantity of oxygen taken up outside of the body by specimens of human blood drawn from six normal persons, when shaken up with atmospheric air, varied from $I 7 \cdot 6$ c.c. to 22.5 c.c. per roo c.c. of blond, the variations depending mainly on the hemoglobin content. The arterial blood as it actually left the lungs of those persons must have contained somewhat less oxygen (about I c.c. less per roo c.c. of blood), since the partial pressure of oxygen in the alveolar air is decidedly below that in atmospheric air. In dogs the amount of carbon dioxide in arterial blood has been found to vary from 35 to 45 c.c. per roo c.c. of blood, the differences being due to variations in the extent of the pulmonary ventilation and to other factors. 
In a series of observations on the venous blood of dogs the oxygen content ranged from $5 \cdot 5$ to $16 \cdot 6$ c.c.(average I I.9 c.c.), and the carbon dioxide content from 38.8 c.c. to 47.5 (average $44 \cdot 3$ c.c.) per 100 c.c. of blood (Schöffer). It will be sufficiently accurate to assume that on the average,

roo volumes of arterial blood yield - ". "right heart) yield

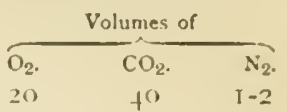

$$
\text { (reduced to } 0^{\circ} \overline{\mathrm{C}} \text {. and } 7^{60} \mathrm{~mm} \text {. of mercury). }
$$

Average venous blood contains 7 or 8 per cent. by volume less oxygen, and 7 or 8 per cent. more carbon dioxide, than arterial blood. Thus, in the lungs the blood gains about twice as many' volumes of oxygen per cent. as the air loses, and the air gains about half as many volumes of carbon dioxide per cent. as the blood loses. It is easy to see that this must be so, for the volume of air inspired in a given time is about twice as great as that of the blood which passes through the pulmonary circulation (pp. 223, 236). Even arterial blood is not quite saturated with oxygen; it can still take up a variable small amount. The percentage saturation with oxygen of the arterial blood of a normal woman from whom blood was being transfused into a patient was directly determined. The blood proved to be $9+$ per cent. saturated-i.e., it could stili have taken up about one-sixteenth of the quantity contained in it. Nor is venous blood nearly saturated with carbon dioxide; when shaken with the gas it can take up about I 50 volumes per cent.

The total oxygen capacity of the blood in any individual can be determined, if the volume of the blood is known (p. 56 ), by estimating the amount of oxygen needed to saturate a sample of the blood. This is most conveniently done by the ferricyanide method with Barcroft's apparatus. Suppose, for example, that I c.c. of blood, after being thoroughly shaken up with air or oxygen, gives off 0.2 c.c. of oxygen when acted upon by ferricyanide, and that the total volume of the blood has been determined to be 5 litres, then the total oxygen capacity of the blood will be I,000 c.c. This quantity, it is clear, is a measure of the power of the blood to transport oxygen. The oxygen capacity of a sample of blood is proportional to the amount of hæmoglobin in it, so that the cstimation of the percentage amount of hæmoglobin by a properly standardized hæmoglobinometer is really an estimation of the oxgyen capacity of the quantity of blood used for the determination. The total oxygen capacity of the body cannot, of course, be derived from such an observation, any more than the total quantity of hæmoglobin, unles: the amount of blood in the body is known.

When the gases are not removed from blood immediately after 
it is drawn, it yields more carbon dioxide and less oxygen than if it is evacuated at once (Pflïger). From this it is concluded llat oxidation goes on in the blood for some time after it is shed. The oxidizable substances are, however, confined to the corpuscles, which suggesti that ordinary metabolism simply continues for some time in the formed elements of the shed blood, and that the disappearance of oxygen is not due to the oxidation of substances which have reached the blood from the tissues. It is an interesting fact that the rate of oxygen consumption of nucleated (bird's) erythrocytes is much greater than that of non-nucleated mammalian corpuscles. The young non-nucleated ervthrocytes, which in experimental anemia in mammals (e.g., after hemorrhage) find their way in large numbers into the circulation, have a relatively intense metabolism, and therefore consume a relatively large amount of oxygen.

The Distribution and Condition of the Oxygen in the Blood.-The oxygen is nearly all contained in the corpuscles. A little oxygen ran be pumped out of serum $(0.2$ or 0.3 per cent. by volume), but this follows the Henry-Dalton law of pressures - that is, it comes off in proportion to the reduction of the partial pressure of the oxygen in the pump, and is simply in solution.

When blood at body temperature is shaken up with air at the ordinary pressure, corresponding to a partial pressure of oxygen of a little over one-fifth of an atmosphere (in round numbers $160 \mathrm{~mm}$. of mercury), the blood-pigment becomes saturated with oxygen or nearly so. When the blood is now pumped out, very little oxygen comes off till the pressure has been reduced to about half an atmosphere, corresponding to a pressure of oxygen of about $80 \mathrm{~mm}$. At about $70 \mathrm{~mm}$. partial pressure the dissociation is somewhat greater. At a third to a quarter of an atmosphere $(50$ to $40 \mathrm{~mm}$.; the amount of oxygen liberated is markedly increased, and the dis. sociation becomes more and more rapid as the pressure falls towards zero. This behaviour shows that the oxygen is not simply absorbed, but is united, as a dissociable compound, to some constituent of the blood. The same thing is, of course, seen when defibrinated blood is saturated at body temperature with oxygen at different pressures. As the partial pressure of the gas is increased from zero the first increments of pressure correspond to a much greater absorption of oxygen than further equal increments. Thus as is seen in Fig. I20, with an oxygen pressure of $10 \mathrm{~mm}$. IOO c.c. of blood took up 6 c.c. of oxygen, or 30 per cent. of the amount required to saturate it. When the pressure of oxygen was $30 \mathrm{~mm}$. over 16 c.c. of oxygen was absorbed, the blood being 80 per cent. saturated. A further increase of the oxygen pressure to $40 \mathrm{~mm}$. increased the quantity of the gas taken up by only 2 c.c. (to 90 per cent. saturation). The uext increment of $10 \mathrm{~mm}$. in the oxygen 
pressure only produced an additional absorption of I c.c., and above this increasing the pressure had very little effect.

We may suppose that at the ordinary temperature and pressure some oxygen is continually escaping from the bonds by which it is tied to the hamoglobin: but, on the whole, an equal number of free molecules of oxygen, coming within the range of the hamoglobin molecules, are entangled by them, and thus equilibrinm is kept up. If now the atmospheric pressure, ard therefore the partial pressure of oxygen is reduced, the tendency of the oxvgen to break off from the hæmoglobin will be unchanged, and as many molecules on the whole will escape as before; but even after a considerable reduction of pressure the hæmoglobin, such is its avidity for oxygen, will still be able to seize as much oxygen as it loses. The more, however, the partial pressure of the oxygen is diminished -that is to say, the fewer oxygen molecules there are in a given space above the hæmoglobinthe smaller will be the chance of the loss being made up by accidental captures. At a certain pressure the escapes will become conspicuously more numerous than the captures; and the gas-pump will give evidence of this. The higher the temperature of the hæmoglobin is, the greater will be the average velocity. of the molecules, and the

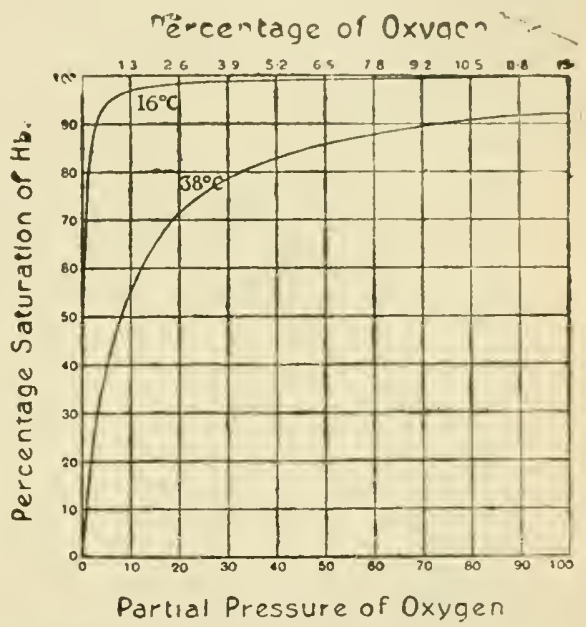

Fig. II9.-Curves of Dissociation of Oxyhæmoglobin freed from salts by dialysis (after Barcroft). Along the horizontal axis are plotted the partial pressures (numbers below the curve) of oxygen in air. to which a solution of hrmoglobin was exposed. The corresponding percentages of oxygen are given above the curve. Along the vertical axis is plotted the percentage saturation of the hæmoglobin with oxygen. greater the chance of escape of molecules of oxygen.

It is easily proved that the substance in the corpuscles which unites with oxygen is the blood-pigment. Although a solution of oxyhæmoglobin crystals behaves towards oxygen somewhat differently from blood containing the same proportion of the native pigment, the maximum amount of oxygen taken up is the same for each. Much labour has been spent in determining curves which express the relation between the partial pressure of oxygen to which blood or a hæmoglobin solution is exposed, and the proportion to which the blood pigment becomes saturated with oxygen under each pressure. The differences in the results of the various investigators who have worked out the curves of dissociation for hæmoglobin and for blood (Figs. I I9, I20, I2I) have been largely cleared up by the researches of Barcroft and his co-workers. One 
factor which was overlooked in the earlice observations is the intluence of salts.

The form of curve (a rectangular hyperbola, lig. Is which Hüfner put forware by an unjustifiable generalization, as the curve of dissociation of oxyhamoglobin is only found when the hamoglobin solution is thoroughly freed from salts, as by prolonged dialysis. When this condition is fulfilled it can be shown that the curve is always a rectangular hyperbola, but a different one for each temperature at which the observations are macle. lior hannglobin solutions not freed from salts or for blood or dilutions of blnod the curve is of a different order altogether, a curve with a double contour (S-shaped). (Figs. I20, 121). If to a solution of hxmoglobin freed from salts and yielding a rectangular hyperbola as its dissociation curve, salts be added in the quantities and of the kind known to exist in dog's blood, the curve of

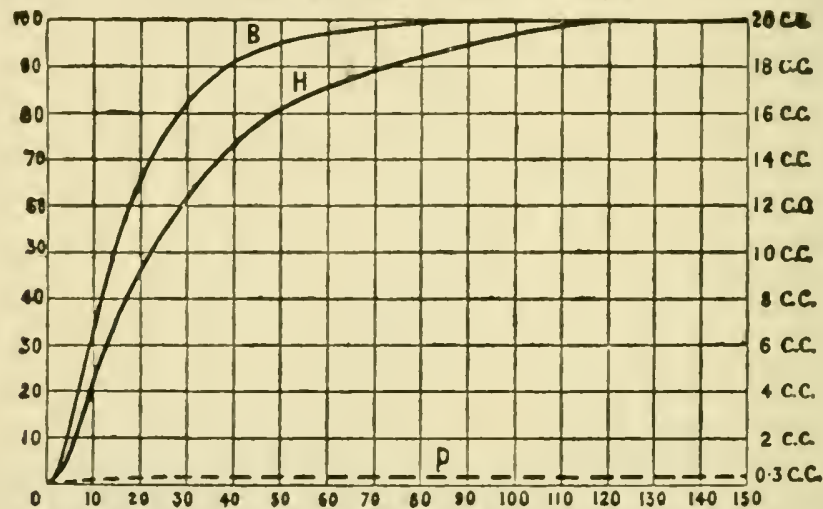

Fig. I 20.-Curves of Dissociation of Uxygen for Horse's Blood (B) and Jug's Hæmo globin solution $(\mathrm{H})$ at $38^{\circ} \mathrm{C}$. (Bolir). The figures along the basc-line are th partial pressures of oxygen to which the blood and hæmoglobin solution were exposed. Those along the vertical axis on the left are the percentage saturations with oxygen. The figures along the vertical at the right give the actual number of c.c. of oxygen chemically combined by roo c.c. of the blood for each pressure of oxygen. The interrupted line $P$ indicates the amount of oxygen dissolved in the plasma of the blood at each partial pressure on the assumption that the plasma is two-thirds of the volume of the blood. Thus, at $150 \mathrm{~mm}$. oxygen pressure the plasma of 100 c.c. of blood took up o.3 c.c. oxygen.

dissociation given by dog's blood is obtained. The addition of the salts appropriate to human blood in the proper amounts causes the hyperbolic curve of the pure hæmoglobin solution to change into an S-shaped curve, such as is given by human blood, and so on.

The foundation has thus heen removed from the theors that different animals possess hamoglobins ciffering in their capacity to take up oxygen. The salts are supposied to alter the dissociation curve, by changing the degree of aggregation of the hamoglobin molecules-i.c., by causing them to adhere to each other to a greater or less extent according to the quantity and kind of salts present.

Another factor which greatly influences the binding power of hemoglobin for oxygen, and therefore the dissociation curve, is the reaction of the blond (p. 24). When the hydrogen-ion concen- 
Iration is increased, as by addition of carbon diuxide (lig. 121) or lactic acid, the effect is to increase the dissociation tension of (xyhenoglobin, wr what is a different way of expressing the same thing. to diminish the amount of oxygen taken up under a given oxygen partial pressure. So that to obtain a given percentage saturation of the hiemoglobin, a greater partial pressure of oxygen must be employed than in the absence of the acid.

So sensitive is the dissociation curve to changes in the hydrogen-ion concentration, that a method of detecting and estimating alterations in the acidity of the blood has been ba-ed upon the determination of the percentage saturation of the blood with oxygen, at a definite partial pressire. The influence of the carbon dioxide in the patient's blood is eliminated by adlling to the gaseous mixture with which the blood is shaken up a percentage of carbon dioxide, corre. sponcling to the partial pressure of that gas in the blood, that is to say, a percentage cqual to the percentage of carbon dioxide in his alveolar air (p. $24 \mathrm{I}$ )

In blood hæmoglobin, of course, exists in the presence of salts and of carbon dioxide and wher acid metabolites, and therefore such curres as are given in Figs. I2O and $12 \mathrm{I}$, more nearly represent the dissociation curves of the blood

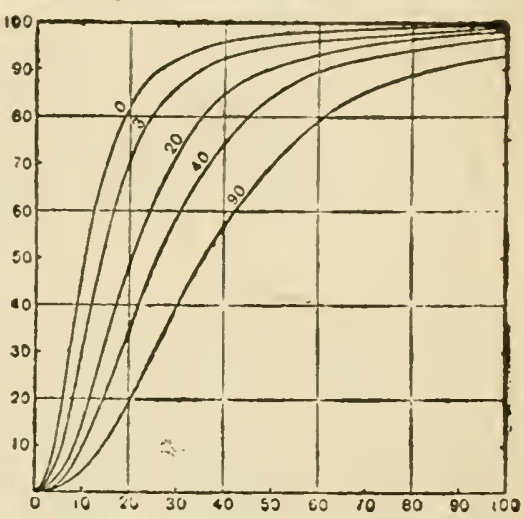

Fig. I $2 \mathrm{x} .-$ Dissociation Curves of Bluod, with Different Tensions of $\mathrm{CO}_{2}(0,3$, 20,40 , and 90 mm.). Ordinates = percentage saturation. Abscissie = oxygen pressure. (After Barcroft.) pigment under physiological conditions, than those reproduced in Fig. II 9 .

The Distribution and Condition of the Carbon Dioxide in the Blood. - The question is much more complicated than for the oxygen, which is practically confined to one of the morphological elements of the blood (the erythrocytes), and exists in the form of a single compound. Carbon dioxide is distributed over the entire blood in important amounts, and is present in several forms. The serum yields a larger percentage of carbon dioxide than the clot, but this percentage is not great enough to allow us to assume that the whole of the carbon dioxide is contained in the plasma. Somewhat more than a third of it belongs to the corpuscles.

As regards the condition of the carbon dioxide, it is known that some of it is simply dissolved in the plasma and corpuscles; but although this fraction, on account of the relatively high coefficient of absorption of the gas (p. 245), is much greater than the corresponding oxygen fraction, it is insignificant in comparison with the quantity chemically combincd. Carbon dioxide is united in dissociable 
combinations with a number of the constituents of the bluod, both inorganic and organic, and our knowledge of these combinations, especially of the compounds formed with organic substances, is far from complete. The inquiry is complicated by the circumstance that the proportion of the total combined carbon dioxide united with a given constituent or bound by plasma and corpuscles respectively is not constant, but varies with the varying tension of the gas, while the total amount of carbon dioxide is itself dependent upon the varying 'titratable alkalinity' (p. 25). There is no doubt that some of the carbon dioxide in blood is combined with alkali, but the amount of alkali available is not nearly sufficient to unite with all the carbon dioxide even in the form of licarbonate. Some of the dissociable carbon dio ide must therefore be combined witl। organic substances. The relations, even of that portion which exists as bicarbonate, are peculiar. 'This is sufficiently indicated by the fact that from defibrinated blood the whole of the carbon dioxide can in time be pumped out without the addition of an acid to displace it from the bases with which it is united. On the other hand, from a bicarbonate solution whose concentration corresponds to that of the blood, not much more than half of the loosely bound carbon dioxide (that is, the carbon dioxide which comes off according to the equation $\left.2 \mathrm{HNaCO}_{3}=\mathrm{Na}_{2} \mathrm{CO}_{3}+\mathrm{CO}_{2}+\mathrm{H}_{2} \mathrm{O}\right)$ can be obtained even when the evacuation is kept up for days. This is only about one-fourth of the total carbon dioxide in the bicarbonate; yet, when sodium bicarbonate is added to blood, even in consider"able amount, all the carbon dioxide in it can be obtained by the pump. From serum a great deal, but not the whole, of the carbon dioxide can be likewise pumped out, and the liberation of the gas does not stop, as in the case of the bicarbonate solution, when all the bicarbonate las been changed into carbonate. The residue (from Io to 18 per cent. of the whole) is set free on the addition of an acid-e.g., phosphoric acid.

The most satisfactory explanation is that in the scrmm there cxist substances which can act as wcak acids in gradually driving out the carbon dioxide, when its escape is rendered casier by the vacumn. The quantity of these, however, is not so large but that a portion of the carbon dioxide remains in the serun. The proteins of the serum, such as serum-globulin, beliave in certain rispcets like weak acids, and may contribute to the driving out of the carbon dioxide. When defibrinated blood is pumped out, the whole of the carbon lioxide cin be removed, apparently because substances of acid nature pass from the corpuscles into the serum and help to break up the cirbonates. The hamoglobin in the corpuscles acts ats a wicak acid, and as some corpuscles are hamolyzed during the evacuation, the hamoglobin may exert this action in the scrum as well as in the interior of the corpuscles.

The quantity of carbon dioxide combined with alkali (as bicarbonate) has not been exactly determined. Bohr estimated it by shaking blood with atmospheric air, which was supposed to leave 
the bicarbonate intact, while removing nearly all the rest of the carbon dioxide, the compounds of the gas with organic constituents of the blood being more easily dissociated than the bicarbonate. The best known of these compounds is that which carbon dioxide seems to form with hiemoglobin. A solution of hemoglobin absorbs more of the gas than water, and the quantity taken up is not preportional to the pressure. There is a!so evidence of the existence of dissociable combinations between carbon dioxide and the proteins of tle plasma, by which considerable amounts of the gas can be bound it such carbon dioxide tensions as normally exist in blood.

In the red corpuscles a portion of the carbon dioxide is in com. binstion with allialies. We know that the corpuscles contain more alkali than the serum, and the titratable alkalinity of "laked" blood (pp. 25, 28) is greater than that of unlaked blood, unless a long time is allowed in the case of the latter for the alkalies of the c rrpuscles to reach the acid used in titration. The hrmoglobin of the corpuscles holds a portion of the carbon dioxide in weak com bination.

Although the student is warned not to give too much weight to the actual numbers, the present position of our knowledge in regard to the distribution and condition of the carbon dioxide of the blood may be summed up by quoting the calculation of Loewy, that in roo c.c. of arterial blood containing (with a carbon dioxide tension of $30 \mathrm{~mm}$. of mercury) 40 c.c. of carbon dioxide, there are-

\begin{tabular}{|c|c|c|c|c|c|}
\hline & & & In Plasma. & In Corpuscles. & In Blood. \\
\hline $\begin{array}{l}\text { Physically absorbed - } \\
\text { Combined as bicarbonate } \\
\text { In organic combinations }\end{array}$ & - & - & $\begin{array}{l}\text { I· } 2 \text { C.C. } \\
\text { I } 2 \cdot 0, \\
\text { II.S }\end{array}$ & $\begin{array}{l}0.7 \text { c.c. } \\
6.8 \quad \text { ", } \\
7.5 \quad \text {," }\end{array}$ & $\begin{array}{l}\text { J'9 C.C. } \\
\text { I } 8 \cdot 8 \quad, \\
\text { I } 9 \cdot 3 \quad,\end{array}$ \\
\hline
\end{tabular}

When blood is saturated with carbon dioxide and then separated into serum and clot, the serum is found to yield more gas than the clot; but if the serum and clot are separately saturated, the latter takes up more carbon dioxide than the former. From this it is argued tlut a substance combined with carbon dioxide must in blood saturated with the gas pass out of the corpuscles into the serum. The corpuscles at the same time gain water and become larger. The molecular concentration (p. 426) of the serum of defibrinated blood, as measured by the lowering of the frcezing-point, increases when it is saturated with carbon dioxide. On the other hand, when blood is saturated with oxygen, the corpuscles lose water and shrink in volume, while the molecular concentration of the serum is diminished. Hamburger has extended these observations to the circulating blood, and has shown that the plasma of venous blood has a higher percentage of alkali, protein, sugar, and fat than the plasma of arterial blood. and that the corpuscles have a greater volume, thougl not a greater diameter. He therefore supposes that in the pulmonary capillaries, under the influence of oxygen, water passes into the plasma from the corpuscles. In the systemic capillaries the blood becomes loaded with carbon dioxide, and therefore the corpuscles 
take ip) water from the plasma, which accordingly has a more concentrated supply of food-substances to offer to the tissues than the plasma of arterial blood itself. Some writers sce in this intercliange an automatic arrangement by which oxidation is favoured. Whatever may be thought of this view-and objections to it are not wanting - the current theory, that the corpuscles are simply passive carricrs of oxygen, and exercise no further influznca on the plasma, breaks down in face of the facts. We must admit that an active and many-sided commerce exists between them and the liquid in which they float.

\section{The nitrogen of the blood is simply absorbed.*}

The Tension of the Blood-Gases.- - If the gases of the blood existed in simple solution, their tension or partial pressure could be deduced from the amount dissolved and the coefficient of absorption. We have seen that they are mainly combined, and it is characteristic of dissociable compounds of this kind that the relation between the partial pressure of the gas in contact with the liquid and the quantity of gas taken up is much more complicated than in the case of pure physical absorption. It is therefore necessary to determine the tension directly.

This can be done by means of arrangements called aevolonomeiers. There are various forms of aerotonometer, but the object of all is to bring the blood into contact with an atmosplere or gascous mixture into which the gases of the blood can diffuse and from which gases can enter the blood. When the composition of the mixture has ceased to change, the gases in it are under the same partial pressure as the corresponding gases in the blood, and all that is necessary in order to arrive at the tension of the blood-gases is to determine the final composition of the gaseous mixture by analysis. Tlie speed with which equilibrium is attained depends essentially upon the magnitude of the surface of contact between the blood and the gas mixture in proportion to the volume of the gas spacc. In the earlier observations with the aerotonometer it was found tr be very difficult to get complete equilibrium, and therefore the gas spacc was filled at the beginning with a mixture whose gases had partial pressures as nearly equal as possible to those expected in the blood. In one form of the apparatus the blood is made to pass directly from the vessel to glass tubes, which it traverses at the same time, the stream being divided between them; it then passes out agnin. The tubes are warmed by means of a water-jacliet to the body-temperature. Some of them are filled with gascous mixtures having 2 greater, and the others with mixtures having a smaller, partial pressure, say of carbon dioxide, than is expected to be found in the blood. As the latter runs in a thin sheet over the walls of the tubes, it loses carbon dioxide to some of them and takes up carbon dioxide from others. From the alteration in the proportion of the carbon dioxide in the tubes, the partial pressure of that gas in the blood is calculated-that is, the partial pressure which would be necessary in the tubes in order that the blood might pass through them without losing or gaining carbon dioxide (p. 248).

Bohr's acrotonometer, constructed and worked much in the same way as a stromulir (p. I2I), permits the blood after passing through the gas space to return to the circulation. A stream of blood can thus be

* But according to Buckmaster and Gardner, the volume of nitrogen in blood does not follow the ordinary physical laws of absorption with varying nitroge: pressures in the alveolar air. 
kept in contalct with the gas for a quarter of an hour or longer, so as to insure equilibrium. Finally, in Krogh's microtonomeler tl. gras space is reduced to the smallest pussible dimensions, being composed merely of an air-bubble $2 \mathrm{~mm}$. in diameter, which is exposed to the contact of a stream of blood from an artery or vein. Equilibrium is established so quickly that it is indifferent whether a bubble of air or of pure nitrogen is employed. The bubble is analyzed at the end of the observation, and its composition gives the tension of the blood gases.

Suppose that the gaseous mixture which is in equilibrium with thr blood contains ro per cent. of oxygen and 5 per cent. of carbon dioxide,
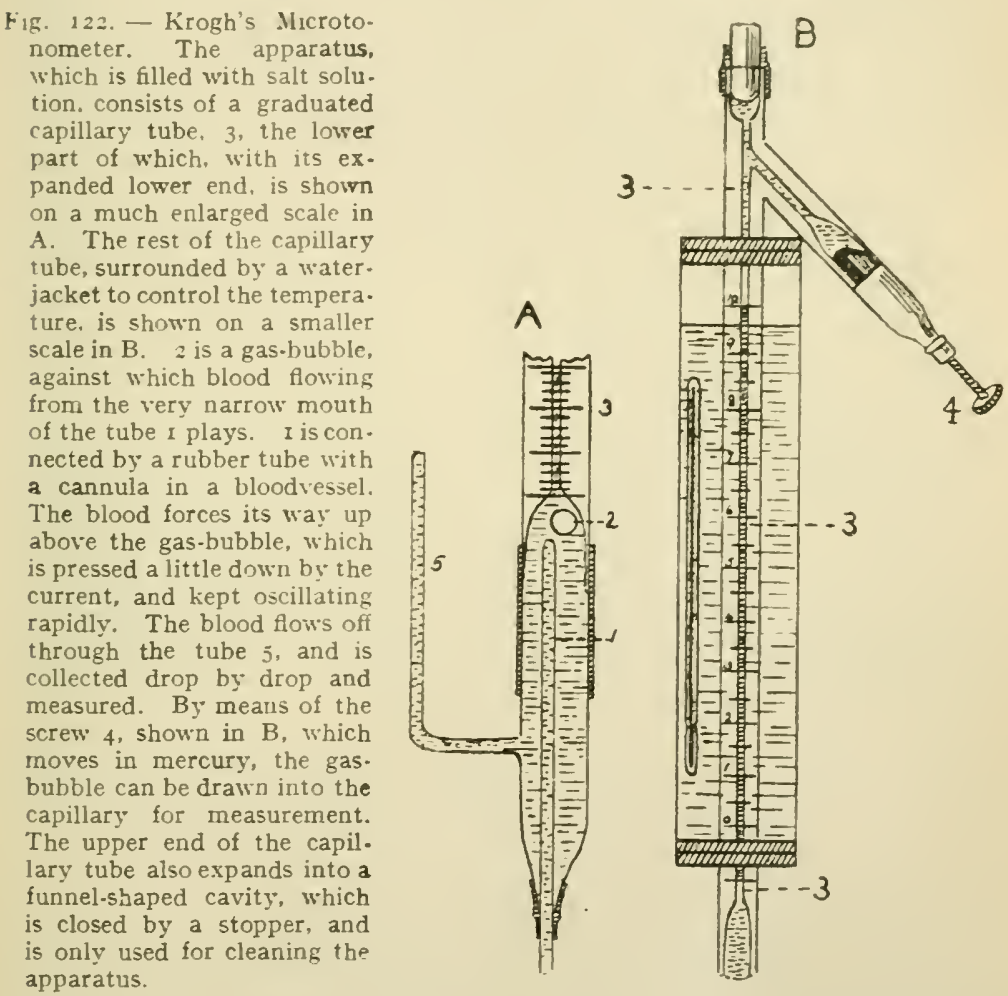

the tension of oxygen in the blood would be one-tenth of an atmosphere (i.e., of $760 \mathrm{~mm}$. of mercury), or $76 \mathrm{~mm}$., and the tension of the carbon dioxide in the blood one-twentieth of an atmosphere, or $38 \mathrm{~mm}$. of mercury.

Another method by which the tension of the gases in the venous blood passing from the right heart through the lungs has been estimated depends upon the use of the pulmonary cathe'er. This consists of two tubes, one within the other. The inner tube, which is a fine slastic catheter, projects free from the other for a little distance at its lower end. The outer tube terminates in a thin india-rubher balloon, throwgh which the inner tube passes without communicating with the balloon. 
The balloon can be inflated so as to block the bronchus into which it is passed, and cut off the corresponding portion of the lung from com. minication with the outer air. A sample of the air below the block can be drawn off through the inner tube, which opens free in the bronchus.

This method has been applied both to animals and to man. In ubservations on man the catheter was passed into the right bronchus so as to occlude at will any one of the lobes of the right lung. On the assumption that the gaseous exchange in the lungs depends essentially on the physical process of diffusion, the occluded alveoli will correspond to the gas space of an aerotonometer. W'hen the occlusion has lasted long enough for the gases in the alveoli and the blood gases to come completely into equilibrium-say half an hour-all that is necessary is to draw off the air, and from its composition to deduce the tensions in the blood. Since the respiratory function of the occluded lobe is in abeyance, the blood circulating in it is all unaitered venous blood, as it comes from the rigit ventricle, so that the gas tensions found can be considered those of the mixed renous blood.

For estimating the oxygen tension in the arterial blood a method was introduced by Haldane and Smith, which differs fundamentally from those described above, in that it does not depend upon the use of aerotonometers. They applied it not only to animals but also to man. The subject of the experiment breathes air containing a definitely. known very small percentage of carbon monoxide until the hæmoglobin has united with as much of that gas as it will take up for the given concentration of it in the air. Then the percentage amount to which the hremoglobin has become saturated with carbon monoxide is determined in a sample of blood taken, say, from the finger. Now, the final saturation with carbon monoxide of a hremoglobin solution bronght into contact with a gaseons mixture containing carbon monoxide and oxygen, depends on the relative tensions of the two gases in the liquid. But the tension of carbon monoxide in the blood leaving the lungs will (after absorption has ceased) be the same as that in the inspired air. Knowing this tension and the degree of saturation of the hæmoglobin with carbon monoxide, the oxygen tension in the blood leaving the lungs-i.e., in the arterial blood -is known.

Before proceeding to the consideration of the results obtained by these diver: methods, it may be well to point out that when a gas is stated to be under such and such a tension in the blood, no direct information is given as to the quantity of gas present. For instance. the oxygen tension in blnod exposed to atmospheric air will be the same for the erythrocytes as for the serum-namely, about $160 \mathrm{~mm}$. of mercury; but IOO c.c. of serum will scarcely contain $\mathbb{1}$ c.c. of oxygen, while roo c.c. of corpuscles will have absorbed about bo c.c. of the gas.

When we now turn to the actual blood-gan tensions obtained by different observers and by different methods, these, as displayed in such a table as appears on p. $26 \mathrm{I}$, seem to present, at first sight, nothing but a welter of widely diverging and contradictory figures.

This table, at the time it was drawn up a few years ago. represented the outcome of a vast amount of work by physiologists of the greatest ability, and specially skilled in such studies. To-day, the absolute numbers possess little value, especially in view of the fact that most 
of them could not be accurately compared with the corresponding gas tensions in the alveolar air. The discussion of the table is none the less instructive to the student who desires to learn how knowledge is won, and how frequently an advance in knowledge is dependent upon an advance in technique.

As regards the venous blood, we have already learnt that very considerable variations in the content of oxygen and of carbon dioxide are associated with the varying functional activity of the tissues from which the blood comes. This factor, of course, is also not without influence upon the gas tensions of the venons blood. The carbon dioxide tension of arterial blood is affected by variations in the amount of the pulmonary ventilation, which affect the partial pressure of the carbon dioxide in the alveolar air and thus alter the steepness of the slope of pressure between the two sides of the pulmonary membrane.

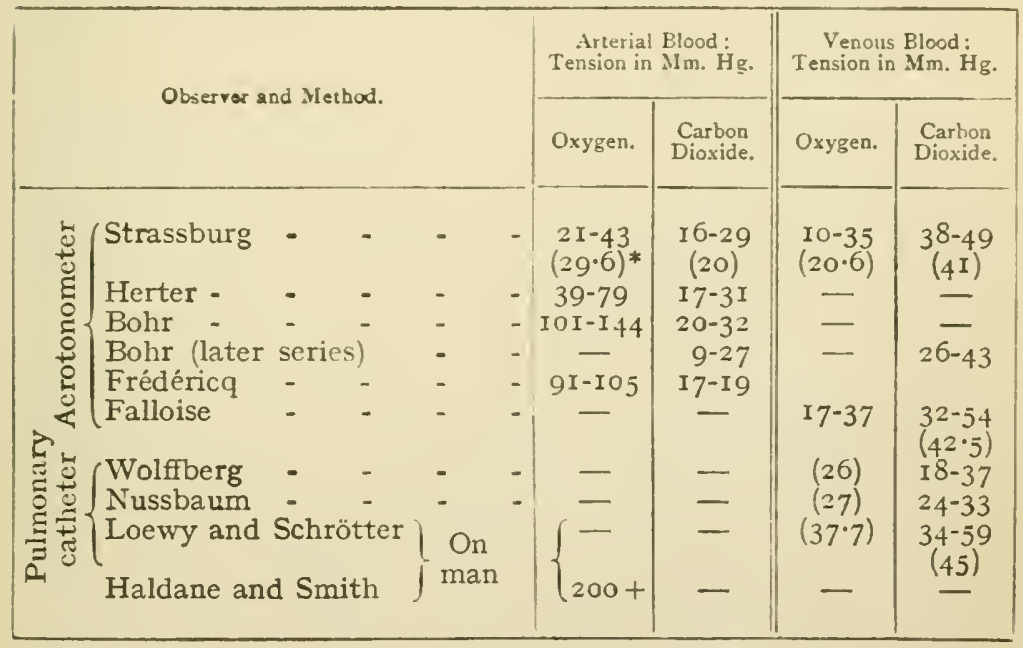

It is chiefly the enormous differences in the recorded oxygen tensions of the arterial blood which excite surprise. To some extent, indeed, these also may depend upon differences in the partial pressure of the oxygen in the alveoli, and it has been shown experimentally (by the aerotonometer) that with increasing oxygen tension of the inspired air the oxygen tension of the arterial blood increases (Frédéricq). Still, the differences which can possibly have existed in the partial pressure of the oxygen in the alveoli in the various series of observations can only to a small extent account for the differences in th results. The main reason for the great range of values lies unquestionably in the different experimental procedures

- The numbers in brackets are averages. 
by which they were obtained. There is no doubt that in the earlicr observations with the aerotonometer (Strassburg) the oxygen of the blool rould not have come into equilibrium with the mixture in the gas space, in which the oxygen pressure was at the beginning much lower than that in the blood; the results are therefore too low. The same is true for the oxygen tension of the venous blood, but as this is in any case considerably smaller than that of the arterial blood, the proportional error is not so great. The later experiments (of Herter), given in the second line of the table, yield much higher values, owing to improved technique, but the finclings are still to be regarded as minimal and not average results. At the other end of the scale stand the results of Haldane and smith, who found in man an oxygen tension in the arterial blood of over $200 \mathrm{~mm}$. of mercury equal to more than 26 per cent. of an atmosphere. This exceeds the partial pressure of oxygen in the external air, and is about twice as great as that of the air of the alveoli. In the bird they found an oxygen tension of between 300 and $400 \mathrm{~mm}$., equal to 45 per cent. of an atmosphere.

The chief interest of this discussion of the blood-gas tensions lies in their fundamental importance in the problem of the gaseous exchange in the lungs, on the one hand, and between the blood and tissues on the other. In the presence of such results Haldane and Smith necessarily adopted a secretion theory of gaseous exchange in the lungs. For it was manifestly imposible for oxygen to diffuse from the alveoli into the blood against the slope of pressure.

Their findings, however, differed so vastly from those of all observers who had used tonometric methods, and it seemed so difficult to assign an adequate physiological value to the slight increase in the percentage saturation of the hrmoglobin with oxygen (sec Figs. II9-I21), which could be brought about by even a great excess of oxygen tension above that of atmospheric air, that there was a general disposition to clistrust the accuracy of their method. At the same time the ordinary tonometric technique left so much to be desired that there seemed little hope of bringing the matter to a decisive test. The introduction a few years ago by Krogh, of better acrotonometric methods, and the remarkable series of researches carried out by their aid, have changed the whole aspect of the question. He showed that in the arterial blond of rabbits the oxygen tension was in all cases somewhat (usually 2 to 3 per cent. of the atmospheric pressure, or 15 to $23 \mathrm{~mm}$. of mercury") lower than the exygen tension in air from the bifurcation of the trachea. In animals it is not possible to obtain the actual alveolar air. These results agree very well with those of Frederica, who found in dogs oxygen tensions of 13 to $1+$ per cent. of an atmosphere-i.e., something like soo mm. of mercury, when the animals breathed atmospheric air. Krogh proved that the amount of oxygen lost by self-reduction of the blood (see p. 26I) in his acrotonometer was negligible, even in rabbits, although it is known that rabbit's blood nses up more oxygen than that of higher mammals. He also pointed on some sources of crror in the Haldane-Smith method. About the same time Haldane, after a careful re-examination of the question, came to the conclusion that his previous results were much too high; and that 
during rest an!l when normal air is breathed the oxygen tension 11 arterial blood never exceeds the tension in the alveolar air. He still believes, and brings forward evirence for his belief, that even the best arotonometric methods yicld with arterial blood oxygen pressures lower than the true oxygen pressure in the blool leitving the pulmonary capillaries. But he no Ionger doubts that a sufficient slope of pressure exists between the alveoli and the blood to explain the absorption of oxygen under ordinary conditions.

To all intents and purposes, then, we may look upon the controversy as closed, and with the happy result, too rare in physiological disputes, thit a practically unanimous conclusion lias been arrivect at. While the oxygen tension in arterial blood may fall only slighlly below that in alveolar air, the difference perhaps being even less than that found by Krogh, the slope of pressure is alway's, under ordinary conditions at least, from the alveoli to the blood.

Mechanism of the Gaseous Exchange in the Lungs.-Granting that this is so, it must still be asked whether the diffusion of oxygen from the lungs into the blood can take place rapidly enough to account for the quantities actually absorbed.

Calculations marle on the basis of such anatomical and physical data as are available (total surface of the lungs, thickness of the membrane which separates the air of the alveoli and the blood in the capillaries, rate of 'invasion,' or entrance of the gases into water; velocity of diffusion of oxygen and carbon dioxide), indicate that even with differences of oxygen tension between the blood and the alveolar air, which would lie within the limits of error of our present methods of measurement, enough oxygen could diffuse across the pulmonary membrane to cover the whole normal intake.

For example, it has been shown from determinations of the 'invasioncoefficient ' of oxygen that 300 c.c. of oxygen, the amount ordinarily absorbed in a minute, in an average man, can be carried from the alveolar air into the wet surface of the pulmonary epithelium with a difference of oxygen pressure of a little over $3 \mathrm{~mm}$. of mercury. If during muscular exercise six or seven times as much oxygen were absorbed, the necessary difference of oxygen pressure would still be only about $20 \mathrm{~mm}$. of mercury, less than 3 per cent. of an atmosphere.

It is one thing, however, to know that the necessary oxygen can be taken up into the surface of the pulmonary membrane by a purely pliysical process, but quite another to prove that it can also be trans: ported with sufficient speed across the thickness of the alveolar wall into the blood. A direct test of this question, made by Krogh, has also yielded an affirmative answer. He determined the amonnt of carbon monoxicle actually taken up in a given time in a human subject. It was about zo $\mathrm{c} \mathrm{c}$. per $\mathrm{mm}$. of partial pressure in the alveolar air per minute when the subject was at rest, and a little above 30 c.c. when breathing was forced, as it would be during heavy work. Now the speed of diffusion of oxygen is somewhat greater than that of carbon monoxide, so that at rest the subject could have absorbed between 200 and 300 c.c. of oxygen per minute with a difference of oxygen tension of only $10 \mathrm{~mm}$. of mercury between the alveolar air and the arterial blood. Even during hard muscular work the observed differences of oxygen tension seem adequate to the transport of the necessary amount of cxygen. 
So tar, then, as the absorption of oxyen is concerned, there is erery reason to conclude that it is managed by physical processe's alone. Haldane, it is truc, still contends that under exceptional conditions when the call for oxygen is much increased, as during active muscular exercise, or when an adequate intake is hampered by reduced atmospheric pressure, as at high altitudes, the oxygen tension in the arterial blood may materially exceed that in the alveolar air. Those who have worked with other methods do not, however, grant that even under these conditions there is any excess of oxygen pressure in the blood. It may, of course, be formally admitted that even if diffusion can account for the absorption of the whole of the oxygen, this is not of itself a proof that it is by diffusion that the thing is actually done; it is only a reason for refusing to call in the aid of a more recondite hypothesis, until the necessity for doing so is clearly denonstrated.

As regards the carbon dioxide the evidence is fully as clear. The speed of diffusion of carbon dioxide across such a membrane as the alveolar wall being much greater than that of oxygen, still smaller differences of tension would suffice to permit the whole normal output of that gas to be eliminated by diffusion.

According (1) the observations of Buhr, nearly 500 c.c. of carbon dioxide per minute could pass out of the blood by diffusion into the alveolar air iniler a dificrence of partial pressure of $1 \mathrm{~mm}$. of mercury. This is with the ordinary breathing of a man at rest. With the increased respiration associated with hard muscular work, this quantity would be increased to between 700 and soo c.c. The amount of carbon dioxide eliminated during rest in an average adult (300 c.c.) can therefore be easily excreted by diffusion with a tension-difference of $1 \mathrm{~mm}$. of mercury. Even during very hard work the necessary tension difference need not be more than $3 \mathrm{~mm}$. With a movement of carbon dioxide so free as this, it is obvious that if the excretion of that gas is solely a matter of diffusion its partial pressure in the arterial blood must be nearly identical with its partial pressure in the alveolar air.

A glance at the table on p. 26I show's that while the carbon dioxide tension of renous blood may sometimes, perhaps generally, exceed that of the alveolar air, the difference is quite small. The average for the obscrvations on man with the pulmonary catheter was $45 \mathrm{~mm}$., which compares with an average alveolar tension of $42 \mathrm{~mm}$.

Experiments made by more modern methods have placed the matter beyond doubt. Krogh proved, for example, that in rabbits the carbon dioxide tension in the arterial blood was always slightly (on the average 0.4 per cent. of an atmosphere, or $3 \mathrm{~mm}$. of mercury) higher than in air taken from the bifurcation of the trachea. Now this air is known to contain slightly less carbon dioxide than the alveolar air. Haldane's results on the regulation of the respiration (p. 28I) would be unintelligible unless the carbon dioxide pressure in the arterial blond adjusted itself with great rapidity, to changes 
in the tension of earbun dioxide in the alveolar air, so as always to preserve a definite relation. It is quite generally assumed that this relation is one of practical equality, and the classical method of measuring the carbon clioxide tension in the arterial blood in man is to determine it in the alveolar air.

Evidence in favour of the view that there is, besides diffusion, an element of selective secretion in the excliange of gases through the pulmonary mernbrane has been found by some writers in the results of a study of the gases of the swim-bladder in fishes. This study has demonstrated the cxistence of animal cells which actually secrete gases. This fact, however, even if it removes a presumption against, does not establish a presumption in favour of, the secretion theory of external respiration. These gases consist of oxygen, nitrogen, and usually a small quantity of carbon dioxide, but in very different proportions from those in which they exist in the air or the water. Thus, as much as 87 per cent. of oxygen has been found in the bladder of fishes taken at a considerable depth, but a smaller amount in those captured near the surface. When the gas is withdrawn by puncturing the bladder with a trocar, the organ rapidly refills, and the percentage of oxygen increases. Further, this process of gaseous secretion is under the influence of nerves, for gas ceases to accumulate in the organ when the branches of the vagi that supply it are cut. In the tortoise stimulation of the peripheral end of the vagus causes a fall of gaseous exchange in the corresponding lung, with an accompanying rise in the other lung. But this is a consequence of an alteration in the pulmonary circulation through the vasomotor fibres for the lungs which are known to run in the vagus in this animal. In the mammal, no such effect has been demonstrated, and the decisive proof that the lungs are gas-secreting glands which would be afforded by the discovery of secretory nerves is wanting.

We have now completed the description of the phenomena of external respiration, with the discussion of its contral fact, the exchange of gases between the blood and the air at the surface of the lungs. It remains to trace the fate of the absorbed oxygen, and to determine where and how the carbon dioxide arises.

\section{SEction V.-INTERNAL OR TISSUE RESPIRATION.}

Seats of Oxidation.-The suggestion which lies nearest at hand and which, as a matter of fact, was first put forward, is that the oxygen does not leave the blood at all, but that it meets with oxidizable substances in it, and unites with their carbon to form carbon dioxide. While there is a certain amount of truth in this view, oxygen, as already mentioned, being to some extent taken up by freshly shed blood, and also by blood under other conditions, to oxidize bodies, other than hremoglobin, either naturally contained 
in it or artificially added, there is no doubt that the cells of the loody are the busiest seats of oxidation. This is shown by the presence of carbon dioxide in large amount in lymph and other liquids whicle are, or have been, in intimate relation with tissue elements; by its presence, also in considerable amount, in the tissues themselvesin muscle, for instance; by its continued and scarcely lessened production not only in a frog whose blood has been replaced by physiological salt solution, and which continues to live in an atmosphere of pure oxygen, but in excised muscles; and by the remarkable connection between the amount of this production and the functional state of those tissues. In insects the finest twigs of the trachere, through which oxygen passes to the tissues, actually end in the cells; and in luminous insects, like the glow-worm, it has been noticed that the phosphorescence, which is certainly dependent on oxidation, begins and is most brilliant in those parts of the cells of the lightproducing organ that surround the ends of the tracher. Nicroscopic evidence has been obtained that intracellular oxidation proceeds most rapidly near surfaces like the nuclear and plasma membranes -e.g., in the indophenol (p. 272) and similar reactions the coloured oxidation products are deposited chiefly in and around the nuclei of such cells as liver and kidney cells and frog's red corpuscles (Lillie).

The Passage of Oxygen from the Blood into the Tissues. - A fundamental fact of tissue respiration is that the amount of oxygen taken up by the cells depends essentially upon their needs, and not upon the amount of oxygen offered to them in the blood. In every case studied, an increase of functional activity on the part of an organ leads to an increased call for oxygen by that organ, and an increased consumption of oxygen in it. The cells cannot be cajoled, so to say, into consuming more oxygen merely by increasing the available supply. Nor can they be prevented from absorbing and consuming more of whatever supply is available when they are caused to work harder.

For skcletal muscle at rest, Barcroft gives 0.004 c.c. per gramme per minute as the oxygen consumption; during maximal activity twenty times as much ( 0.08 gramme). In the heart of a small dog through which blood was pumped by a larger dog the oxygen intake when the heart was beating feebly was, on the average, about o.o I c.c. per gramme of heart-muscle per minute. When the heart was caused to beat very strongly under the influence of adrenalin, the oxygen intake rose in one case to 0.08 , and in two others to 0.04 . In the resting pancreas the oxygen intake has been found to be 0.03 to 0.05 C.c. per gramme per minute; in the active pancreas, $0 . I$ r.c. The corresponding number for the submaxillary gland at rest is 0.03 , and in activity 0.09 ; for the kidncy, 0.03 at rest or during scanty secretion, and 0.07 or even 0.09 during active serretion; for the liver in fasting animals 0.01 , in fed animals 0.035 . 
We are as yet less precisely informed as to the manner in which the tissues regulate the amount of oxigen which they take up from the bloud, than in the eatse of the passage of oxygen into the blood from the lungs. The problem, indecd, is superficially at least a different and probably a more complex one. In the lungs the task is to saturate, or nearly to saturate, the hremoglobin with oxygen, whether the blood passes fast or slow. It is a monotonous invariable process of 'oxygen-grabbing,' with no possibility of its cver being orerclone. In the tissu's, the task is to meet the widely varying demand from blood which is always charged with oxygen to approximately the same degree. How is this thing managed ? There is little doubt that the process is again fundamentally a matter of diffusion. The oxygen dissociated from the oxyhamoglobin in the capillaries must find its way through the plasma across the capillary walls into the tissue lympli, and thence into the interior of the cells. There is evidence that it can do so by physical diffusion. For the oxygen tension in the capillary blood is in general higher than the oxygen tension in the tissues. The common statement that the partial pressure of oxygen in the tissues is zero, or nearly zero, fits in very well with the conclusion that the oxygen is transported into the cells from the blood of the capillaries by a process of diffusion. For the slope of oxygen pressure thus maintained would ordinarily be grcater than in the lungs, since even at the end of the capillary tract the venous blood still has a considerable partial pressure of oxygen.

The fact that such liquids as lymph, bile, urine, the serous fluids, saliva, pancreatic juice and milk contain little or no oxygen was supposed to support the view that the pressure of oxygen must be very low in the tissues by which they are secreted, or with which they have been in intimate contact. From isolated muscles no free oxygen at all can be pumped out, and muscle being taken as a type of the other tissues in regard to the problems of internal respiration, it was concluded that the scarcity of oxygen in the parenchymatous liquids which bathe the tissues deepens in the tissues themselves into actual famine. The inference seemed plain. Either the tissues used up oxygen so rapidly. that with an average blood flow their wants could just be supplied, or without actually consuming it they 'fixed' and stowed it away in some compound in which it was still available for oxidation in the metabolic processes of the cell, but had lost the properties of free oxygen. On the first alternative, an increased consumption of oxygen could only be met by an increase in the blood flow; on the second assumption the stored oxygen would supply the means of temporarily increasing the metabolism even without an immediate augmentation of the blood flow. Although it was recognized that even the resting metabolism of certain organs was not inconsiderable, and required a good supply. of oxygen, it was always difficult to understand why inactive tissues should have such an avidity for oxygen that practically every molecule seemed to be captured as soon as it appeared. Nor did it help much to assume that some of the tissue oxidation might really take place in the tissue lymph, oxidizable products split off in the metabolism of the cells passing out into the lymph before being burnt, and thus diminishing the oxygen tension outside the capillary walls. 
Recent work has indicated, however, that the oxvgen pressure in some tissues is far from negligible. In the resting submaxillary gland, indeed, it seems to be practically equal to the oxygen pressure in the venous blood leaving the organ (over $40 \mathrm{~mm}$. of mercury in certain experiments). In general, the same was found to be the case for the kidney. This does not mean, of course, that in such organs there is no slope of oxygen pressure from blood to tissue, in virtue of which oxygen can be moved out of the blood by diffusion. For at the beginning of the capillary tract the whole difference in oxygen pressure between the arterial and the venous blood would be available. At the end of the capillary tract, the blood by the loss of oxygen to the tissues would have come approximately into equilibrium with them. These observations enable us to understand better the manner in which the tissues themselves can regulate their intake of oxygen. If the glands are using little oxygen their oxygen tension will be relatively high, and the passage of a comparatively small amount of oxygen from the blood in the capillaries will suffice to bring it into equilibrium with the tissues, after which the diffusion of oxygen will cease. If, on the other hand, the consumption of oxygen in the glands is suddenly increased, their oxygen pressure will fall, the pressure gradient between them and the blood will become steeper, and oxygen will diffuse more rapidly into them from the capillaries. It must be remarked, also, that up to a certain point the existence of a substantial oxygen pressure in the tissues permits them at once to increase their intake of oxygen from the blood without the necessity of an immediate increase in the blood flow. For, as the cells consume the oxygen already present in and between them, the tissue oxygen tension falls, and the gradient between the interior and the exterior of the capillaries is thus rendered more abrupt.

These results were not obtained by direct tonometric measurement of the oxygen pressure in the glands investigated, but were deduced from observations on the quantities of oxygen taken up by them when different oxygen pressures were produced in the capillaries by altering the oxygen tension in the inspired air. The quantity of oxygen which diffuses out of the capillaries in a given time will be proportional to the difference of oxygen pressure on the two sides of the capillary wall. If the pressure on the side of the tissues is always zero, then the quantity of oxygen passing out will vary directly as the intracapillary oxygen pressure. Far from doing this, the amount of oxygen taken up by the glands was found to be about the same with intra-capillary oxygen pressures as different as $6 \mathrm{r} \mathrm{mm}$. and $16 \mathrm{~mm}$. of mercury. It should be distinctly understood that no great importance must be attached to the actual numbers in experiments of this kind. But the gencral conclusion that in some tissues, at any rate in the resting condition, a sensible partial pressure of oxygen exists, seems to be established (Verzar). The application of the same method to muscle has led to a different result. Herc, the possible partial pressure of oxygen was found decidedly less than that of the venous blood, althougl at times a small oxygen pressure was detected in resting muscle (25 mm. or less) 
Muscle may accordingly be taken as a type of tissues in which the oxygen pressure is so low that the blood-flow through them cannot be much diminished without interference with their absorp. tion of oxygen, while in tissnes like the resting submaxillary gland a considerable diminution of the blood-flow can occur without diminution of the oxygen consumption of the tissue. Whether this difference is merely a quantitative one, depending upon the great oxygen requirement of active muscle, or a qualitative difference connected with the peculiar relations of oxygen to the muscular contraction, is unknown. These relations have so great an interest in connection with the problem of intracellular oxidation, and have been so much more minutely studied than the relations of oxygen to the functional work of other tissues, that a few words on the respiration of muscle may fitly be introduced here. The subject must be returned to later on (Chapters XII. and XIII.).

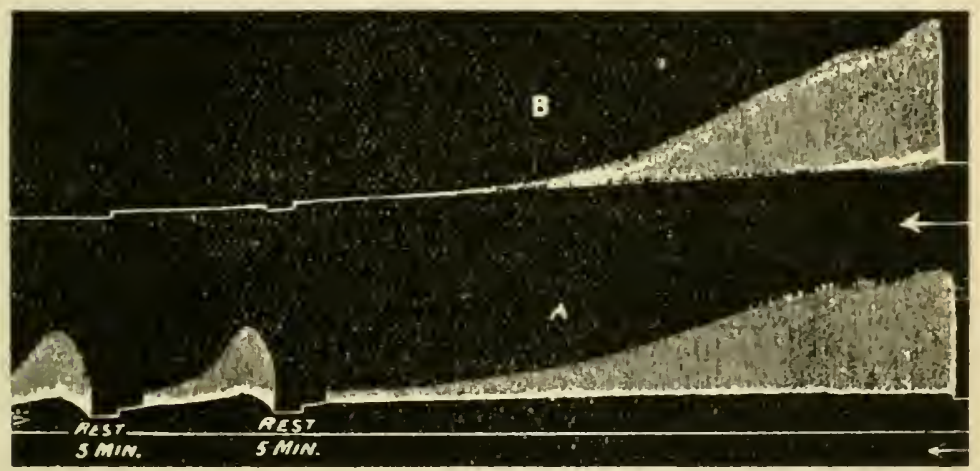

Fig. 123.-Fatigue of a Pair of Sartorius Muscles (Fletcher). A, in an atmosphere of oxygen; $\mathrm{B}$, in an atmosphere of nitrogen. A is partially restored by a rest of five minutes.

Respiration of Muscle.-It is a remarkable fact that an excised frog's muscle is capable of going on yielding carbon dioxide for a long time, in the entire absence of oxygen, in a chamber, for instance, filled with nitrogen or other indifferent gas. Not only so, but it can be made to contract many times in this oxygen-free atmosphere, although it loses its power of contraction sooner than in oxygen, and does not show the same capacity for recuperation during an interval of rest. In mammals the muscles can also be made to contract repeatedly when the dissociable oxygen has, as far as possible, been got rid of from the blood by asphyxiating the animal, and to give off a correspondingly large quantity of carbon dioxide, although they lose their contractibility much more rapidly than the muscles of the frog. This has usually been interpreted as meaning that the carbon dioxide does not arise, so to speak, on the spot, from 
the immediate union of carbon and oxigen, but that a stock of it is taken up by the muscle, and stored in some compound or compounds, which are broken down during eontraction, and more slowly during rest, carbon rlioxicle in both cases being one of the end-products. In a normal muscle with intact circulation, while carbon dioxide is given off, curtain wf the other decomposition products are supposed, in conjunction with oxygen and some substance rich in carbon, like sugar, to be regenerated into the material which breaks down in contraction. When oxygen is not available, as in an atmosphere of nitrogen, carbon dioxide is still given off, but the other decomposition products are supposed not to be regenerated to contractile substance, but to accumulate in the muscle, producing the phenomena of fatigue, and eventually of rigor.

When muscle goes into rigor (Chapter XIII.)-and this is most strikingly seen when the rigor is caused by raising the temperature of frog's muscle to about $40^{\circ}$ or $4 \mathrm{I}^{\circ} \mathrm{C}$. - there is a sudden increase in the quantity of carbon dioxide given off. Moreover, in an isolated muscle the total quantity of carbon dioxide obtainable during rigor is markedly less if the muscle has been previously tetanized. From this it has been argued that the hypothetical substance ("inogen '), th: decumposition of which yields carbon dioxide in contraction, is also the substance which decomposes so rapidly in rigor; that a given amount of it exists in the muscle at the time it is removed from the influcnce of the blood; and that this can all ' explode' cither in contraction or in rigor, or partly in the one and partly in the other. However, according to Fletcher, there is no increase in the amount of carbon dioxicle given off cluring tetanus by all excised frog's muscle unless the stimulation is so severe and prolonged as to hasten the onset of rigor. He therefore supposes that in the contraction the decomposition does not proceed quite to the formation of carbon dioxide, which in the intact body is afterwards liberated from some more complex carbon-containing waste-product. He considers that the carbon dioxide yiclded by excised muscles is really performed carbon dioxide, already existing in a state of loose combination, from which it is displaced by the lactic acid formed after cxcision. There is no reason to suppose that any independent new formation of carbon dioxide occurs within the isolated muscle in the absence of a good supply of oxygen. However this may be, there is good evidence that oxygen is used up in recovery processes after the contraction is over, and that these recovery processes are not completed when oxygen is lacking. Hill's work on the heat production of muscle (Chapter XIII.) has tended to rehabilitate the older conceptions, at least to this extent, tliat his results are in favour of the view that oxygen is used largely during the recovery after contraction in reactions "whereby the molecular machine--like a steam-engine charging an accumulator-builds up bodies contain 
ing considerable amounts of potential energy which (like the acenmulator) can be discharged by appropriate stimuli.'

The respiration of muscles in situ can be studied by collecting samples of the blood coming to and leaving then and analyzing the gases. The mere difference of colsur between the venous and arterial blood of a muscle, or other actire organ, is sufficient to show that oxygen is taken up and carbon dioxide given out by it to the blood. This is the case in muscle sat rest, and even in muscles with artificial circulation after they have become inexcitable. In active muscles more oxygen is used up and more carbon dioxide produced than in the resting state. Chauveau and Kaufmann, in their experiments on the levator labii superioris muscle of the horse in feeding, found that the consumption of oxygen and the production of carbon dioxide might be many times as great in activity as in rest.

Thus in one experiment the amount of oxygen taken in, expressed in c.c. per gramme of muscle per minute, was 0.008 during rest, and $0 \cdot r_{4}$ during work; the corresponding quantities for the carbon dioxide given off were 0.006 and 0.18 . The respiratory quotient rose to $\mathrm{I} \cdot 3$ in two experiments, and even to $1 \cdot 7$ in a third, showing that the increase in the production of carboll dioxide was relatively greater than the increase in the intake of oxygen. These experiments were performed under conditions so normal that the animal continued to eat its hay with sceming unconcern throughout the observations, although these involved the exposure of the main bloodvessels of the muscle, and the collection of samples of blood from them.

By means of the modern technique permitting the use of small quantities of blood for the gas analysis, similar experiments lave been performed on a muscle as small as the cat's gastrociemius. In one experiment it was found that as a result of stimulation of the sciatic lasting about 25 seconds the intake of oxygen was increased for at least 220 seconds. In this time the muscle used up 0.75 c.c. of oxygen, as compared with 0.26 c.c., which it would have consumed had it not been stimulated (Verzàr).

Nature of the Oxidative Process.-When we have recognized the cells as the seat of oxidation, the question immediately presents itself, How do they accomplish the feat of burning such masses of food substances as can only be rapidly oxidized in the laboratory at the temperature of the body by the most energetic chemical reagents? The researches of late years have furnished a key to the solution of this long-standing puzzle by demonstrating the existence in the tissues of oxidizing ferments or oxydases. Of these, one of the most widely distributed is a ferment which splits off oxygen from hydrogen peroxide. Since any oxidation produced is only secondary to this decomposition, ferments which decompose hydrogen peroxide are often spoken of as catalases, to distinguish them from the oxydases proper. A catalase is found in practically all the tissues of the body, as well as in vegetable cells, and we have already mentioned instances of its action in connection with the oxidation of the guaiaconic acid in tincture of guaiacum in the 
presence of the peroxide $(p, 76)$. As regards the activity of this ferment, blood comes first; then follow spleen, liver, pancreas, thymus, brain, muscle, and ovary. It is present in the blood-free organs as well as in the blood. Some tissues, both animal and vegetable, contain a ferment, an oxydase, which causes the oxidation of guaiaconic acid in the presence of atmospheric oxygen, and these do not need peroxide of hydrogen in order to render guaiacum blue. An allied ferment which also induces the blue colour in tincture of graiacum is the so-called laccase found in the most active form in the latex of the tree from which Japanese lacquer is obtained, but also in many other plants. Many fungi contain a ferment, tyrosinase, which oxidizes tyrosin, and in certain animals tyrosinases have also been demonstrated. Another well-known oxidizing ferment in fresh animal tissues is characterized by the property of forming indophenol by oxidation in an alkaline solution of paraphenylenediamin and $a$-11aphthol, and may therefore be termed indophenyloxydase. The colourless solution becomes reddish or violet. This ferment is contained in pancreas, salivary glands, spleen, thymus, and bone marrow; but has not been detected in muscle, lungs, brain, kidneys, and other organs. It is to be expected that other oxydases capable of favouring oxiclation of specific kinds of food substances or their decomposition products will be discovered, but it ought to be remarked that those at present known are only capable of attacking relatively. simple organic substances, and it would be rash to conclude that this is the only way in which living protoplasm can bring about the rapid, but at the same time the regulated, oxidation which is so characteristic a feature of its activity. Yet the capacity of the cell to regulate the intensity and the extent of the intra cellular oxidations would seem to find a simple explanation if we assign an important rôle to oxidizing ferments formed by the cell itself in accordance with its needs. In this connection we may mention a ferment, aldelyydase, which was formerly included among the oxydases, but is now known to be a hydrolytic enzyme. It splits aldehydes so as to yicld the corresponcling acid-e.g., salicylic aldehyde is split into salicylic acid and saligenin. Evidence of its presence in most organs has been obtained.

The Passage of Carbon Dioxide from the Tissues into the Blood. - Since nearly the whole of the carbon dioxide eventually found in the blood is formed in the tissues, and only a small amount in the blood itself, it might be supposed that the partial pressure of the gas in the tissues would necessarily be greater than in the blood. This would certainly be true if the whole of the carbon dioxide was transported in the form of dissolved gas. This, however, is not the case. Much of it is combined, and as the proportion of free to combined carbon dioxide in the blood is variable, it may be 
assumed that it is also variable in the tissues. These is no evidlence and little probability that the variations are always parallel. (Duintitative and even qualitative differences in the substances whicl can bind carbon dioxide are known to exist between the blood and the tissues, and it is uncertain how much interchange of sucl. compounds carrying with them combined carbon dioxide, which maty afterwards become dissociated, takes place between the blood and the tissue lymph or the cclls. However probable, then, it may be that the transportation of carbon dioxide from the cells to the lymph, and from the lymph to the blood is managed in the same way as that of the carbon dioxide from the blood to the alveolar air, namely, by diffusion of the gas in solution, it cannot be said that at present clear proof of this has been obtained. Results are, indeed, on record, which purport to show that the partial pressure of carbon dioxide in various tissues or in physiological liquids which have been in contact with them is higher than that of the arterial or even than that of the venous blood. But these results are of unequal and some of them of doubtful value for the solution of the problem under discussion.

Lymph, bile, urine, and the serous fluids contain so much carbon dioxide that the pressure of that gas in all of them is greater than in arterial blood, while in lymph alone (taken from the large thoracic duct) has it been found less than that of venous blood. And it is probable that lymph gathered nearer the primary seats of its production (the spaces of areolar tissue) would show a higher proportion of carbon dioxide. Strassburg found that with a pressure of carbon dioxide in the arterial blood of $21 \mathrm{~mm}$. of mercury, the pressure in bile was $50 \mathrm{~mm}$., in peritoneal fluid $58 \mathrm{~mm}$., in urine $68 \mathrm{~mm}$., in the surface of the empty intestine $58 \mathrm{~mm}$. Saliva, pancreatic juice, and milk, also contain mucl carbon dioxide. From muscle as much as 1,5 volumes per Ioo of carbon dioxide can be pumped out, some of which is free-that is, is given up to the vacuum alone-while some of it is fixed, and only comes off after the addition of an acid.

Before leaving the subject of the gaseous exchange between tissues and blood and between blood and air some important consequerices of the form of the dissociation curve of oxyhæmoglobin, and of the modifications produced in it by change of temperature, by salts and by acids, must be alluded to. They have been developed in masterly fashion by Barcroft. The flattening of the hyperbolic dissociation curve of dialyzed oxyhæmoglobin when the temperature is raised (Fig. IIg) makes it obvious that for any percentage saturation which can exist in blood as it leaves the systemic capillaries, the corresponding partial pressure of oxygen must be much greater at the body temperature $\left(38^{\circ} \mathrm{C}\right.$.) than at $16^{\circ} \mathrm{C}$. For hæmoglobin which is $5^{0}$ per cent. saturated it is twenty-five times as great. This favours the giving up of oxygen to the tissues by blood in which the percentage saturation is considfrably redured. On 
the other hand, the absorption of oxygen in the lungs is not seriously interfered with, because the available alveolar partial pressure of oxygen is so ligh (I00 $\mathrm{mm}$. or more) that it cen be considerably diminished without causing any appreciable diminution in the percentage saturation.

There is evidence that in dialyzed hæmoglobin solutions the hamoglobin is all in the form of single molecules. The addition of salts as they exist in blood produces a certain amount of aggregation or clumping of the molecules, and as already pointed out, this alters the shape of the dissociation curve. The S-shape of the curve of blood inclicates that blood will part with its oxygen at low oxygen pressures, as in passing througl the tissues, much more readily than a corresponding solution of salt-free oxyhamoglobin; and that it will take up oxygen more easily at high pressures, ats in passing through the lungs. As regards the effect of acids, it was observed by Bohr (p. 255) that an increase in the carbon dioxide tension of blood diminishes its combining power for oxygen, and therefore favours the giving up of oxygen to the lymph and tissues. This may have an important influence on internal respiration. The effect is much more marked where the oxygen tension is low than where it is high, so that in the lungs the taking up of oxygen is scarcely interfered with even by a high carbon dioxicle tension.

According to Barcroft, the combined effect of the increased temperature, the salts and the carbon dioxide of blood (at the partial pressure of $40 \mathrm{~mm}$. of mercury) is to make possible a diffusion of oxygen into the tissues at roo times the speed at which it would diffuse from a salt-free solution of oxyhemoglobin at a tempcrature of $16^{\circ} \mathrm{C}$. This astonishing feat is accomplished without sensibly decreasing the percentage saturation with oxygen of the blood passing throngh the lungs.

\section{SECTION VI.-REIATION OF Respiration to THE NERVOtS SISTEN.}

The Respiratory Centre and its Connections.--Unlike the beat of the heart, the respiratory movements are entirely dependent on the central nervous system. The 'centre ' which presides over them is situated in the spinal bulb. It is a bilateral centre-that is, it has two functionally symmetrical halves, one on sach side of the middle line. Each of these halves has to do more particularly with the respiratory muscles of its own side, for destruction of one-half of the spinal bulb causes paralysis of respiration only on that side. Anatomically the respiratory centre has not been sharply localized, but it lies lower than the vaso-motor centre, not far from the point of the calamus scriptorius. Stimulation of this region during apnoca (p. 28,3) is stated to cause co-ordinated inspiratory movements and 
widening of the opening of the glottis through abduction of the vocal cords. The centre is brought into relation with the muscles of respiration by efferent nerves. The phrenic nerves to the diapliragm, and the intercostal nerves to the muscles which elevate the ribs, are the most inportant of those concerned in ordinary breathing. The respiratory centre is further related to afferent nerves, of which the most influential are those which supply the respiratory tract itself, particularly the pulmonary fibres and superior laryngeal branch of the vagus. But almost any afferent nerve may powerfully affect the centre; and it is also influenced by fibres passing to it from the higher parts of the central nervous system.

Section of the spinal cord in animals above the origin of the phrenic nerves causes complcte paralysis of respiration, and consequent death. The phrenics arise from the third and fourth cervical nerves, and are joined by a branch from the fifth; and in man fracture of any of the four upper cervical vertebra is as a rule instantly fatal. But in one case respiration was carried on, and life maintained for thirty minutes, merely by the contraction of the muscles of the neck and shoulders in a man entirely paralyzed below this level (Bell). Section of the cord just below the origin of the phrenics leaves the diaphragm working, although the other respiratory muscles are paralyzed. A case has been recorded of a man in whom, from discase of the spine in the lower cervical region, all the ribs became completely immovable. He was able to lead an active life, and to carry on his business, although he breathed entirely by his diaphragm and abdominal muscles.

Section of one phrenic is followed by paralysis of the corresponding half of the diaphragm, section of both phrenics by complete paralysis of that muscle, and although respiration still goes on by means of the muscles which act upon the ribs, it is usually inadequate to the prolonged maintenance of life. In the horse, however, not only has survival been seen after this operation, but the animal, after the first temporary increase in the frequency of the breathing had disappeared, could be driven in a light vehicle without any marked dyspnœe. The phrenic nuclei in the two halves of the cord are connected across the middle line. For when a semisection of the cord is made between this level and the respiratory centre in the medulla, respiratory impulses are still able to reach both phrenic nerves. In some animals both halves of the diaphragm go on contracting. But when, as usually happens, this is not the case, and the diaphragm on the side of the semisection has ceased to act, it at once begins to contract again when the opposite phrenic nerve is cut, and the respiratory impulse, descending from the bulb, is blocked out from the direct, and forced to follow the crossed path. It has been shown that the crossing takes place at the level of the phrenic nuclei, and nowhere else (Porter). 
The Regulation of the Respiration through the Afferent Vagus Fibres. - When une vagus is divided, there is little or no change in the respiratory movements. Half an inch of one vagus nerve has been excised in removing a tumour, and the patient showed no symptoms whatever. But section of both vagi in such animals as the dog, cat and rabbit causes respiration to become much deeper and slower, the one change for a time compensating the other, so that the total amount of air taken in and given out, the amount of carbon dioxide eliminated, and the partial pressure of that gas in the pulmonary alveoli are not greatly altered. The relative duration of the two respiratory phases is completely changed, inspiration being much more prolonged than expiration. It has been shown that the effect is really due to the loss of impulses that normally ascend the vagi, not to any irritation of the cut ends. For a nerve can be frozen without exciting it; and when a portion of each vagus is frozen, the respiration is affected in preciscly the same way as when the nerves are divided.

After section of both vagi certain fibres coming from the brain above the respiratory centre appear to take a share in the regulation of the respiratory movements. The bloodvessels supplying these fibres, or the centres from which they come, can be blocked by injection of paraffin wax into the common or internal carotid, or the bulb can be severed with the knife above the level of the respiratory centre, withont any effect being produced upon the breathing, except that the rate is as a rule somewhat lessened. But when both the vagi and these upper paths are cut the character of the respiration is changed, exccedingly prolonged inspiratory spasms alternating with long periods of complete relaxation of the diaphragm till the animal dies.

From these facts it appears that the periodic automatic discharges of the respiratory centre are being continually controlled and modified by impulses passing up the vagus, and that in the absence of these impulses a certain degree of control is excrcised by the higher paths, which, however, do not appear to be normally in action, at any rate to the full measure of their capacity. When the vagi are severed, the control of the higher paths comes into play, and is sufficient still to keep the breathing regular, although it is slowed. When the higher paths are cut off, the vagus of itself is able to regulate the discharge. But when both are gone, the respiratory centre, freed from nervous control, passes into a condition of alternate spasm and exhaustion. Of the central connections of these upper paths but little is surely known. The corpora quarlrigemina, however, seem to contain centres which can affect the respiration. Certain areas on the cerebral cortex have also been described, the excitation of which modifies the respiratory movements. There is no question that the cortex is connected, and extensively connected, 
with the respiratory centre, since the rate and depth of the coordinated respiratory movenents, which are universally acknowledged to involve the activity of the centre, can be altered not only by the will, but by the most varied psychical events.

The rhythmical excitation of the regulating vagus fibres must be brought about by either mechanical stimulation of the nerveendings in the lungs, due to the alternate stretching and shrinking, or by chemical stimulation of these endings depending on the changes that occur with each respiration in the content of oxygen and carbon clioxide in the alveolar air, and therefore in their pressure ( $\rho .260$ ) in the blood. Both vicws have found advocates, but whatever influence the chemical changes in the blood niay exert, there is no doubt that the mechanical factors are the more important. That the vagus is really excited is shown by the fact that a negative variation (p. 824) is set up in the nerve when the lungs are inflated. An electrical change is also observed when air is sucked out of the lungs (Alcock and Secmann, Einthoven).

When the normal excitation of the vagus fibres by expansion of the lungs is exaggerated by closing the trachea at the end of inspiration, the diaphragm inmediately relaxes, and a long expiratory pause ensues, broken at last by a series of inspirations much deeper and more prolonged than those which were taking place before occlusion. When the trachea is occluded at the end of expiration, a series of deep and long-drawn inspirations occurs, the first of which begins at the moment when the next normal inspiration ought to have taken place had the windpipe been left free. The most obvious explanation of these results is that the expansion of the lungs sets up impulses in the vagi which cut short the inspiratory activity of the respiratory centre (inspiration-inhibiting fibres), while in collapse impulses are set up which excite it to renewed inspiratory discharge (inspiration-exciting fibres). Since ordinary expiration is in the main not associated with active muscular contraction, the inspiration-inhibiting fibres would be at the same time expiration-exciting. Clearly this would constitute a so-called 'self-steering' arrangement, each inspiration leading inevitably to the succeeding expiration, and each expiration providing the necessary stimulus for the succeeding inspiration. On this hypothesis section of the vagi must necessarily be followed by slowing of the respiratory movements, and we have seen that this is the case.

A rival hypothesis is that the automatic activity of the respiratory centre leads normally to the discharge of motor impulses to the inspiratory muscles, which are cut short at each expansion of the lungs by the inhibitory action of the vagus, the nerve not being excited during pulmonary collapse, and therefore carrying no inspiratory impulses to the centre. On this assumption, we may think of the centre as being 'wound up ' like a clock, the periodic 
arrival of regulating impulses acting like an escapement movenent, and allowing a certain amount of discharge. When the vagi are cut, the inspirations are greatly prolonged and deepened, because the check on the discharge of the centre has been removed.

Attempts have been made by experimental stimulation of the vagus trunk to determine whether, as a matter of fact, it contains both inspiratory and expiratory fibres. But the results are neither so clear nor so constant that we can confidently appeal to them in making a decision, and even some of the investigators who maintain the existence of but one anatomical set of fibres believe that these are affected differently by different kinds of stimulationmomentary stimuli, for example, setting up in them impulses which we may call inspiratory, and long-lasting stimuli impulses which we may call expiratory.

Excitation of the central end of the cut vagus below the origin of its superior laryngeal branch, with induction shocks of moderate

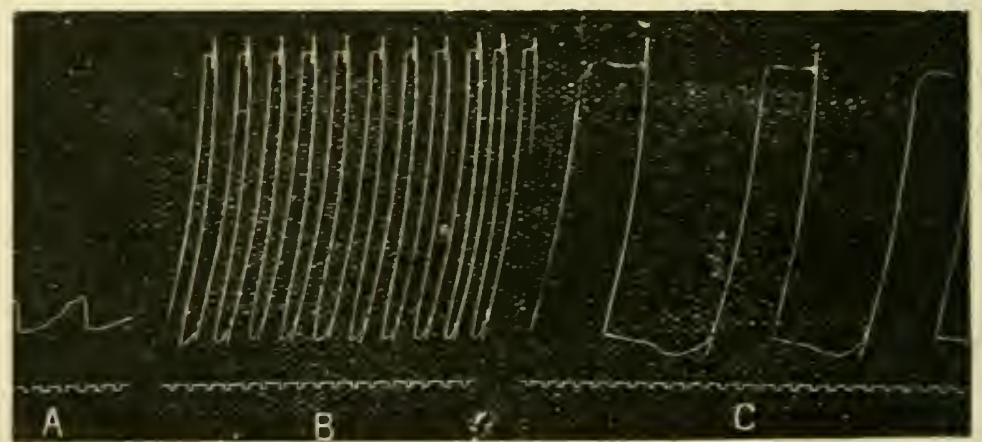

Fig. I24.-Kespiratory Tracings: $\mathrm{Nog}$. A, normal; B, eftect of stimulation of the central end of vagus; $C$, effect of section of both vagi. (Tracing takeu as in Fig. 135, p. 301.) Time-tracing, seconds.

strength, certainly causes quickening of respiration. If the excitation be strong, there is arrest in the inspiratory phase. A brief mechanical stimulus, or a series of such, has a similar effect. But chemical stimulation (e.g., with a strong solution of potassium chloride) or long-continued mechanical excitation like that produced by stretching or compression of the nerve, or certain kinds of electrical stimulation-for instance, the very weakest induction shocks, or the closure of an ascending voltaic current*-cause slowing of the respiratory movements or expiratory standstill. This is also the usual, though not the invariable result of stimulating the superior laryngeal, even when weak induction shocks are employed. With stronger stimulation energetic contractions of the expiratory muscles may occur. These facts undoubtedly suggest the existence in the vagus of two kinds of afferent nerve-fibres that affect the

- I.e., a current passing towards the head in the nerve. 
respiratory centro in "pposite ways-inspiratory fibres, which stimulate it to rreater activity of discharge, and expiratory fibres, which inhibit it action. The latter variety we may suppose to be more numerous in thr superior laryngeal, the former in the pulmonary branches of the vagus. And there is nothing forced in the hypothesis that certain kinds of stimuli act particularly on the one set of fibres, and certain kinds on the other, for we have already scen an instance of this in studying the differences between the vasoconstrictor and the vaso-dilator nerves (p. I7j).

The most probable conclusion, and the one uhich best reconciles the conflicting hypotheses, is that two sets of fibres are present: (I) Fibres which inhibit inspiration (and cause expiration), and are excited in ordinary inspiration by the expansion of the lungs. (2) Fibres which
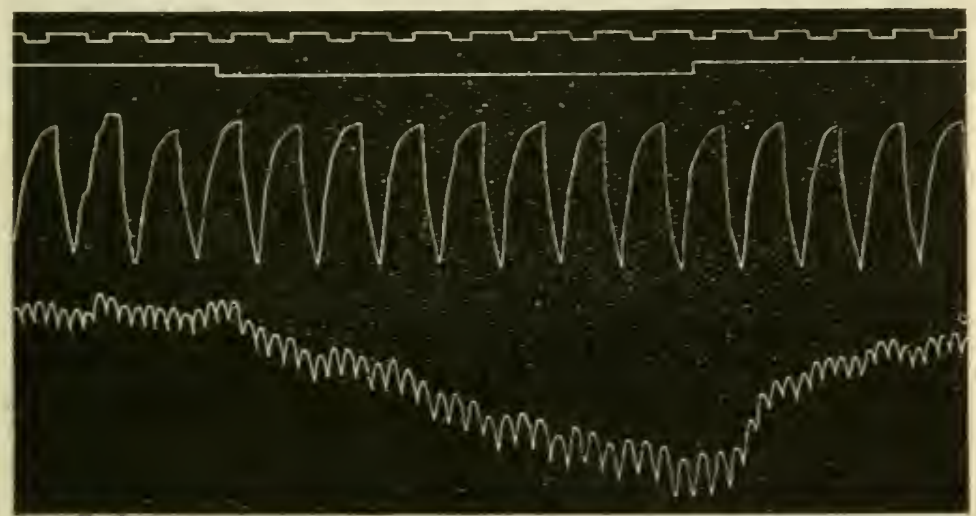

Fig. 125.-Effect of S:imulation of Central End of Vagus in a Cat. Upper Trac3, Respiration; Lower Trace, Blood-Pressure. At the top are the time-trace (seconds), and below it the signal line, the depression in which indicates the duration of the excitation. Practically no effect was produced on the respira. tion, but a fall of blood-pressure with slowing of the heart.

causc inspiration (and inhibit expiration), and are excited in strong expiration, as in dyspncen, by the collapse of the lungs, but are not active in ordinary expiration.

However this may be, the facts we have been discussing have an importance of their own, apart from any hypothetical explanations of them. Some of them have been more than once unintentionally illustrated on man. In one case the left vagus trunk was included in a ligiture with the common carotid. The respiratory movements immediately stopped, the pulse was slowed, and death occurred in thirty minutes (Rouse). The superior laryngeal fibres, unlike those of the vagus proper, are not constantly in action, as section of both nerves has no effect on respiration. Any source of irritation in the larynx may stimulate these fibres and produce a 
cough, which may atso be caused by irritation of the pulmonary fibres of the vagus.

Action of Other Afferent Fibres on the Respiration.- The cutaneous nerves, and eiperially those of the face (fiftl nerve), abdomen and chest, have a marked influence on respiration. They can be easily excited in the intact body by thermal and mechanical stimulation. A cold bath, for instance, usually causes acceleration and deepening of the respiratory movements; and the efficacy of mechanical stimulation of sensory nerves in stirring up a sluggish respiratory centre is well known to midwives, who sometimes slap the buttocks of a new-

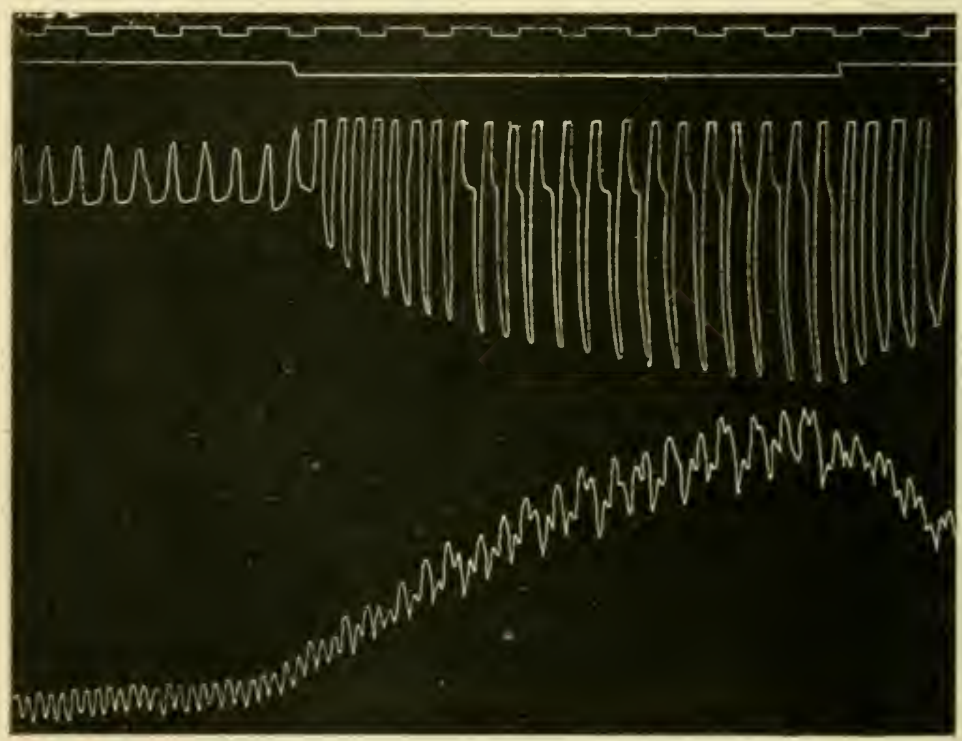

Fig. 126.-- Effect of Stimulation of Central End of Brachial Nerve on Respiration (Upper Tracing) and Blood-Pressure (Lower Tracing) in the Cat. At the top of the figure are the time-trace (seconds) and the signal line, showing beginning and end of stimulation.

born child to start its breathing. The reflex expiratory standstill caused in rabbits by inhalation of such sharp-smelling substances as ammonia, acetic acid, and tobacco-smoke is due to afferent impulses passing up the trigeminus fibres from the mucous membrane of the nose, and is still obtained after section of the olfactory nerves.

Another set of afferent nerves which have been supposed by some to bear an important relation to the respiratory centre are those which supply the muscles. W'e have already noticed that the frequency of respiration is greatly augmented by muscular exercise. The simplest "xplanation would serm to be that afferent muscular nerves are stimulated either by mechanical compression of their 
terminal 'spindles,' or by the chemical action on them of certain waste products produced in contraction. It is quite likely that this is one way in which the adjustment is achieved. But this is not the only, and perhaps not the most important, way. For an increase in the respiratory movements is caused by tetanizing the muscles of a limb whose nerves have been completely severed, and which is indeed connected with the rest of the body by no ather structures than its bloodvessels. This can only be due to two things: a direct action on the respiratory centre by the blood that has passed through, and been altered in, the contracting muscles, or an action exerted by the blood indirectly on the centre through the excitation of afferent respiratory nerves whose connection with it is still intact--for example, the other muscular nerves or the pulunonary branches of the vagus. That the action is direct is shown by the fact that after section of the vagi, the sympathetic, and the spinal cord below the origin of the phrenics, an increase in the respiratory movements is still produced by tetanizing a limb.

The Chemical Regulation of the Respiration.-However inportant the regulation of respiration by afferent nervous impulses may be, the normal discharge of the respiratory centre is intimately associated with the gases of the blood.

It is generally acknowledged that the centre may be excited both by blood that is rich in carbon dioxide and by blood that is poor in oxygen. Stimulation by deficiency of oxygen has to some minds presented a metaphysical difficulty-namely, that it is not easy to sec how the absence of a thing could cause stimulation. The difficulty does not exist, but none the less there is some eridence that when oxygen is lacking the respiratory centre can be excited by substances like lactic acid, which are easily oxidizable and rapidly disappear from properly oxygenated blood.

Be that as it may, it has been the subject of long-continued discussion whether excess of carbon diovide or deficiency of oxygen is the more potent stimulus for the respiratory centre. The best evidence points to the conclusion that comparatively small alterations in the amount of carbon dioxide in the inspired air cause a relatively great increase in the respiration, while in the case of the oxygen the departure from the normal proportion must be much more decided to bring about any notable effect. Nor is it at all out of harmony with this that, when very large quantities of carbon dioxide (30 per cent. and upwards in rabbits) are inhaled, a condition of narcosis comes on without any previous respiratory distress. For many substances act differently in large and in small doses. Haldane has pointed out how cxquisitely sensitive the respiratory centre is to even small changes in the partial pressure of carbon dioxide in the alveolar air, and therefore in the arterial blood and the centre itself, and has demonstrated that this is the way in which the amount of the 
pulnonary ventilation (the volume of air breathed per unit of time) is rhiefly regulated in ordinary breathing.

For instance, an increase of as little as 0.2 per cent. of carbon dioxide in the alveolar air, corresponding to an increase of $\mathrm{I} \cdot \mathrm{mm}$. of mercury in the partial pressure (p. 247) of the gas, caused an increase in the pulmonary ventilation of 100 per cent. The alveolar oxygen pressure had to be diminished to I3 per cent. of an atmosphere before any decided increase in the respiration occurred. During moderate muscular work the percentage of carbon dioxide in the alveolar air, and therefore in the blood, increases slightly, causing an increase in the ventilation, and this is one of the way in which the liyperpncea associated with muscular exercise is brought about. In serere work lack of oxygen, with accumulation of lactir acid and other metabolic products, which increase the hydrogen-ion concentration in the blood and thus stimulate the respiratory centre or render it excitable by smaller pressures of carbon dioxicle, also plays a part. There is some evidence that in the case of carbon dioxide also the actual excitation of the respiratory centre is due to the increased hydrogen-ion concentration. Some physiologists hold the view that the respiratory centre reacts in the same way to a given increase in hydrogen-ion concentration no matter what the acid may be, and that there is nothing spccific in the action of carbon dioxide. If it be assumed that changes in the hydrogen-ion concentration of the blood are only causcd by substances like carbon dioxide, which are eliminated by the lungs, or by substances like lactic acid, which are either got ricl of by oxidation or are not liberated into the blood in the presence of a good oxygen supply, no serious theoretica! objection can be urged against this conclusion. For increased activity of the respiratory centre would favour the elimination of carbon dioxide and absorption of oxygen, and in both ways would tend to maintain the normal hydrogen-ion concentration in the blood. If, however, the hydrogenion concentration can be influenced by substances which are not directly affected by the respiratory process, it would seem unlikely. that the respiratory centre should blindly respond to such changes just as if they had been produced by substances, the amount of which it can effectively control.

To sum up, the regulation of normal breathing is twolold-a chemical regulation (through the carbon dioxide, possibly' by changes produced in the hydrogen-ion concentration in the blood) of the amount of air moved into and out of the lungs per unit of time; and a nervous regulation (chiefly through the vagi) of the rate and depth of the movements necessary to effect the given amount of ventilation.

When the vagi have been divided, an increase in the carbon dioxide pressure within certain limits is responded to by an increasc in the total ventilation, just as in the normal animal, but the form of the response is different. Whereas in the normal animal both 
the rate and the deptl of respiration are increased, in the vagotumized animal there is a marked increase in depth, with little or no increase in rate (Scott).

When the gaseous exchange in the lungs from any cause becomes insufficient, the respiratory movements are exaggerated, and ultimately every muscle which can clirectly or indirectly act upon the chest-wall is called into play in the struggle to pass more air into and out of the lungs. To a lesser and greater degree of this exaggeration of breathing the terms Hyperpuce and Dyspnoea have been respectively applied. If the gaseous interchange remains insuffcient, or is altogether prevented, asphyxia sets in. Sometimes in man impending asphyxia from loss of function by a part of the lungs (with crippling of the lesser circulation), as in pneumonia, may be warded off by inhalations of oxygen. Increase in the temperature of the blood circulating through the spinal bulb, as when the carotid arteries of a dog are laid on metal boxes through which hot water is kept flowing, also causes dyspnœa (heat-dyspncex) (p. 302). But if the temperature be too high, the respiratory movements may be slowed, perhaps by a partial paralysis or inhibition of the respiratory centre. When the blood is cooled the respiration becomes deeper and slower, but if the temperature is greatly and suddenly lowered, the centre may be stimulated and the breathing quickened. In man the increased temperature of the blood in fever is a cause, though not the only one, of the increase in the rate of respiration.

Apnœa.-The physiological opposite of dyspnœa is apnœe. This condition may be produced in an animal by rapid or prolonged artificial respiration. It is especially easy to obtain in an animal in which the circulation through the brain and bulb is interrupted for a time and then restored, while artificial respiration is being kept up. Spontaneous respiration returns after a longer or shorter interval, but if the artificial respiration be still maintained, it again ceases. In a successful experiment the animal remains without breathing for many seconds after the artificial respiration is stopped. In apnoea the chest remains at rest in the expiratory phase if the lungs have been inflated by the artificial respiration and then allowed to collapse of themselves (expiratory apnœa), but in the inspiratory phase if they have been emptied by suction and then permitted of themselves to expand (inspiratory apnœea). The apnœa is not produced, as some have thought, by the accumulation of an excess of oxygen in the blood, for rapid and repeated inflation of the lungs with hydrogen may cause the condition. Indeed, towards the end of the apnœic period the venous blood may be very distinctly poorer in oxygen than normal venous blood. Apnœa is easily caused in man by a period of deep and rapid breathing and in other ways. The essential thing in this chemical or true apnœea (apncex vera) is the lowering of the partial pressure of carbon dioxide in the alveolar air, and therefore in the arterial blood and the respiratory 
centre. The carbon dioxide is waslicel out of the body, so to say, by the excessive pulmonary ventilation.

In addition to chemical apnoea, which is obtainable whether the vagi are intact or not, a so-called mechanical apnœa, or apncx vagi, exists-that is to say, a stoppage of the respiration due to an inhibitory effect produced through the vagi on the respiratory centre when the vagus endings in the lungs are excited mechanically by inflation. Some observers state that this vagus apnoea does not outlast the inflation. Others believe that the results of successive inflations can be 'summated ' in the centre, giving rise to an apnœa which persists after stoppage of the artificial respiration. That a ' memory' of a prolonged rhytlimical inflation of the lungs call impress itself in some way on the respiratory centre is shown by the: curious phenomenon that in resuscitation of the bulb after a period of anemia the natural respiration, when it returns, may have for a short time exactly the same rhythm as the artificial respiration which has just been stopped.

That the blood when the gascous exchange in the lungs is interferea with produces dyspncea by arting on some portion of the brain may be shown in an interesting manner by establishing what is called a cross-circulation in two rabbits or dogs. The vertebral arteries and one carotid are tied in both animals; the remaining carotids are divided and comnected crosswise by glass tubes, or, what is better, as it avoids the risk of clotting, they are crossed by suturing the cut ends, so that the brain of each is supplied by blood from the other. When the respiration is artificially hindered or stopped in one of the animals, it shows no dyspnoca; it is in the other, whose brain is being fed with improperly ventilated blood, that the respiratory movements become exaggerated. The point of attack of the 'venous' blood has been further localized in the spinal bulb by the observation that when the brain has been cut away above it, the cord severed below the origin of the phrenics, and all other nerves connected with the region between the two planes of section divided, any interference with the gaseous exchange in the lungs is at once followed by dyspnœa.*

Automaticity of the Respiratory Centre.-The question has been raised whether, in the absence of this 'natural' stimulation by the blood, and of the impulses that constantly reach the centre along its afferent nerves, it would continue to discharge itself, or whether it would sink into inaction. We have already discussed a similar question in regard to the cardiac and vaso-motor centres, and the subject must again present itself when we come to examine the functions of the central nervous system. In the meantime it is only: necessary to say that there is evidence that it is not the mere

* The conclusion is doubtless correct, but this experiment is not decisive. For the phrenic nerves themselves contain afferent fibres, the stimulation of which can influence the respiration after section of the vagi. 
presence of carbon dioxicle (or other substances) in the blood circulating through the respiratory centre which determines the constant excitation of the centre, but rather the accumulation of carbon dioxide, or the increase of hydrogen-ion concentration, in the centre itself when the partial pressure of that gas in the blood is raised. The idea that the conti unous excitation of the centre is 'autochthonous '-in other words, that it is due to an internal stimulating substance or substances manufactured in the centre itself, as well as carried to it in the blood-renders it easy to understand that the discharge of the respiratory centre, although modified by the quality of the blood which circulates in it, is not essentially dependent on it. Indeed, in cold-blooded animals whose blood has been replaced by physiological salt solution, and (in frogs) even after the circulation has been stopped altogether by excision of the heart, quiet, regular breathing may be seen for a considerable time. Of course, blood is essential for the continued nutrition of the centre and its connections, and it eventually breaks down and ceases to discharge. The respiratory discharge is still less dependent for its initiation upon the arrival of afferent impulses. For after section of the bulb above the centre, of the cord below the origin of the phrenics, of the vagi and of the posterior roots of all the upper cervical nerves, the spasmodic respiration which we have already described as occurring when the vagi and the higher paths have been severed continues without essential modification. It has also been observed that during resuscitation of the bulb and upper cervical cord after a period of anæmia, stimulation of afferent nerves, including the vagi, is entirely without influence on the respiratory movements for some time after respiration has returned, presumably because the synapses (p. 852) on the afferent paths lying within the previously anæmic area are as yet unable to conduct the nerve impulses. Nevertheless, the respiratory centre continues steadily to discharge itself along the efferent paths, whose synapses are situated beyond the anæmic region. Section of the bulb above the level of the respiratory centre, and of the cord below the origin of the phrenic nerves, in addition to the anæmia, makes no essential difference in the result. The initial rate of discharge of the centre thus isolated from afferent impulses is approximately constant in different experiments (about four a minute in cats).

Spinal Respiratory Centres.-Although the chief respiratory centre lies in the medulla oblongata, under certain conditions impulses to the respiratory muscles may originate in the spinal cord. Thus, in young mammals (kittens, puppies), especially when the excitability of the cord has been increased by strychnine, in birds and in alligators, movements, apparently respiratory, have been seen after destruction of the brain and spinal bulb. In adult cats, when the functions of the brain, medulla, and cervical cord have been abolished by occlusion of their vessels, similar movements of the 
thoracic and abdominal muscles may be seen, but they are not suffi cient for effective respiration. No proof has ever been given that in the intact organism the spinal cord below the level of the bulb takes any other part in respiration than that of a mere conductor of nerve impulses; and it is not justifiable to assume the existence of automatic spinal respiratory centres on the strength of such experiments as these.

Death after Double Vagotomy.-Alterations in the rhythm of respiration are not the only effects that follow division of both vagi (or vagosympathetics) in the neck. In certain animals, at least, this operation is incompatible with life. In the rabbit, as a rule, death takes place in twenty-four hours. A sheep may live three days, and a horse five or six. Dogs often live a week, occasionally a montli or even two, and in rare instances they survive indefinitely. The most prominent symptoms (in the $d o g$ ), in addition to the marked and permanent slowing of respiration, quickening of the pulse and contraction of the pupils, are difficult deglutition, accompanied by frequent vomiting and progressive emaciation. The appetite is sometimes ravenous, but no sooner is the food swallowed than it is rejected; and this is particularly true of water or liquid food. Sometimes the rejected food is simply regurgitated after having reached the lower end of the oesophagus, without entering the stomach. The fatal result is usually caused, or at least preceded, by changes of a pneumonic nature in the lungs. The precise significance of the pulmonary lesion is obscure. But it would seem that paralysis of the laryngeal and œesophageal muscles, with the consequent entrance of saliva, food, or foreign bodies, carrying bacteria into the lungs, is responsible to a great extent. And when only a partial palsy of the glottis is produced, by dividing the right vagus below the origin of the recurrent laryngeal, and the left as usual in the neck, pneumonia either does not occur or is long delayed. It may be that the tissue of the lungs is rendered particularly susceptible to such insults in consequence of trophic or vascular changes induced by section of the pulmonary and cardiac fibres in the vagi. It may be quite clearly demonstrated, however, in animals which live for some weeks, that, notwithstanding the paralysis of the glottis associated with aphonia, no pulmonary symptoms may be present till a day or two before death. The picture presented in these cases is that of an animal suffering. above all, from alimentary disturbances. The respiration is, to be sure, very different from the normal in frequency, depth, and type, but there is nothing to suggest that the lungs are the seat of any pathological process. Suddenly the picture changes. Pulmonary symptoms obtrude themselves. The pliysical signs of consolidation of the lungs may be detected, and in a short time the animal is inevitably dead. Occasionally the determining cause of the pulmonary lesion seems to be some external circumstance, as a sudden fall of the air temperature. The idea is exccedingly apt to present itself to the observer that the pneumonia is an accident, an acute intercurrent affection breaking the course of a chronic malnutrition, which in any case must have ended in death. Of course, the vagotomized animal is predisposed to this accident, but there is no definite time after section of the nerves at which it must take place. The vomiting is certainly connected with the paralysis and consequent dilatation of the œsophagus; and by previously making an artificial opening into the stomach or by a surgical prophylaxis still more heroic, the establishment of a double gastric and osophageal fistula (p. 40I), deatl may be prevented for many months. Elimination of all the pulnonary fibres of the vagi, by extirpation of 
one lung, followed after an interval by section of the opposite vangus in the neck, is not fatal in rabbits. This is also in favour of the viev, that in double vagotomy the stress falls mainly on the digestive system

Innervation of the Bronchial Muscles.-Both constrictor and dilator fibres for the bronchi are contained in the vagus. They are not constantly in action, but can be reflexly excited, most easily (in the dog and cat) by stimulating the nasal mucous membrane, and particularly a small area well back upon the nasal septum. Cauterization of the corresponding area in man is said to give permanent relief in certain cases of spasmodic asthma, a condition in which the recurrent attacks of dyspnca seem, according to the most generally accepted view, to be associated with spasm of the bronchial muscles.

Special Modifications of the Respiratory Movements.-CheyneStokes Respiration is the name given to a peculiar type of breathing, marked by pauses of many seconds alternating with groups of respirations. In each group the movements gradually increase to a maximum amplitude, and then become gradually shallower again, till they cease for the next pause. The phenomenon often occurs in certain diseases of the brain and of the circulation, and pressure on the spinal bulb may produce it. In cats in which the circulation in the brain and medulla oblongata has been interrupted for a time and then restored it is often noticed at a certain stage of resuscitation of the respiratory centre. In frogs, Cheyne-Stokes breathing has been observed as the result of interference with the circulation in the spinal bulb, 'drowning,' or ligature of the aorta, and also as a consequence of removal of the krain, or parts of it (hemispheres and optic thalami). But it is not peculiar to pathological conditions, being also seen, more or less perfectly, in normal sleep, especially in children, in healthy men at high altitudes, in hibernating animals, and in morphine and chloral poisoning.

Well-marked Cheyne-Stokes breathing can be obtained experimentally in normal persons in a variety of ways. If, for example, the subject is caused to breathe deeply and frequently for about two minutes, so as to produce a prolonged apnœa, the respiration, when it is resumed spontaneously, is of the Cheyne-Stokes type (Haldane). The explanation given by Haldane is that the fall in the partial pressure of the oxygen in the pulmonary alveoli (p. 283) during the primary apnœe, with the consequent fall of oxygen pressure in the arterial blood and the respiratory centre, leads to the production of lactic acid in the respiratory centre and elsewhere, which stimulates the centre in the same way as carbon dioxide, and thus permits it to be excited by a smaller partial pressure of carbon dioxide than that normally necessary. As soon as the pressure of carbon dioxide, which is increasing during the period of apnoea, has reached the exciting value breathing is resumed. The respirations, beginning as very feeble movements, rapidly increase in strength till the 
brenthing becomes quitr decp or actually dyspnoic. The store of oxygen is replenished by this thorough ventilation of the lungs, the changes in the excitability of the respiratory centre due to lack of oxygen disappear (perhaps by oxidation of the lactic acid), and the centre relapses into a period of repose. During this period of apnceit the oxygen pressure sinks once more to the point at which the change in the excitability of the respiratory centre by carbon dioxide occurs, and the breathing again starts. In pathological cases the want of oxygen may be associated either with deficient circulation through the bulb-centre or with deficient intake by the lungs. The administration of oxygen through a mask has been shown in such cases to abolish the periodicity in the respiration, and to render it more normal.

Peculiarly modified, but more or less normal, respiratory acts are coughing, sneezing, yawning, sighing, and hiccup.

A cough is an abrupt expiration with open mouth, which forces open the previously closed glottis. It may be excited reflexly from the mucous membrane of the respiratory tract or stomach through the afferent fibres of the vagus, from the back of the tongue or mouth, and (by cold) from the skin.

Sneezing is a violent expiration in which the air is chiefly expelled through the nose. It is usually excited reflexly from the nasal mucous membrane through the branch of the fifth nerve which supplies it. Pressure on the course of the nasal nerve will often stop a sneeze. A bright light sometimes causes a sneeze, and so in some individuals does pressure on the supra-orbital nerve, when the skin over it is slightly inflamed.

Yawning is a prolonged and very deep inspiration, sometimes accompanied with stretching of the arms and the whole body. It is a sign of mental or physical weariness.

A sigh is a long-drawn inspiration, followed by a deep expiration.

Hiccup, or hiccough, is due to a spasmodic contraction of the diaphragm, which causes a sudden inspiration. The abrupt closure of the glottis cuts this short and gives rise to the characteristic sound. The following readings of the intervals between successive spasms were obtained in one attack: 13 secs., I2 secs., 15 secs., 9 secs., I4 secs., etc.-i.e., one-fourth or one-fifth of the frequency of the ordinary respiratory movements. The mere fixing of the attention on the observations soon stopped the hiccup.

Hiccup is generally considered to be a reflex movement, brought about through the respiratory centre by afferent impulses originating in the stomach. The irritation may be merely due to some slight digestive disturbance set up by overfilling of the stomach, perhaps. This is exceedingly common in infants. But persistent hiccup may also be a distressing symptom of very formidable diseases-for example, carcinoma of the pylorus. Experimentally, reflex contractions of the diaphragm can sometimes be elicited by stimulation 
of the central end of the vignts at at time when no spmintureus respiratory movements are going on. This las been ubserved, for instance, in cats during resuscitation of the brain after a periud of aniemia. In man also, in a case of Cheyne-Stokes respiration accom. panied by hiccup, it was seen that the hiceup persisted during the periods of apnoa. If the respiratory centre is the centre for the hiccup reflex, it can therefore be excited hy afferent nervous im. pulses at a time when it is not excited by the normal clemical stimulus (Mackenzic and Cushny).

Section Vil.-The Influexce of Respikation on the BloOdPRESSURE.

We have already stated, in treating of arterial blood-pressure (p. I I ), that a normal tracing shows a series of waves corresponding with the respiratory novements.

The relationship between the respiratory phases and the rise and fall of the blood-pressure is not by any means a simple and invariabl. one. It depends upon a number of factors, which need not $x$ equally influential under different conditions or in different animals (Lewis). Something depends upon the rate, something upon the relative preponderance of costal and abdominal respiration, and something probably upon the size of the animal. For instance, an inspiratory rise of blood-pressure occurs in man with pure dia-

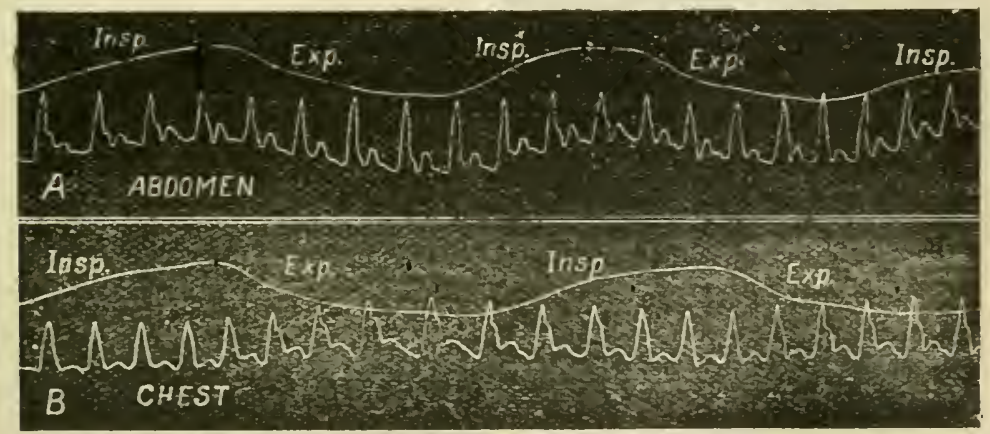

Fig. 127.-Respiratory II aves in the Blood-Pressure: Simultaneous Tracings of Movements of Respiration and of Radial Pulse in Human Subject (Lewis). In A the respiration was diaphragmatic; in $\mathrm{B}$, costal. In A the respiratory tracing was taken from the abdominal wall; in $\mathrm{B}$, from the chest.

phragmatic, and a fall with pure thoracic, breathing (Fig. I27). In cats with fairly fast and not very deep respiration the blood-pressure rises in expiration and sinks in inspiration. With deep and slow respiration the opposite effect may, upon the whole, be seen. In dogs, according to Einbrodt," although the mean blood-pressure is falling for a short time at the beginning of inspiration, it soon reaches its minimum, then begins to rise, and continues rising during the 
rest of this priog. At the commencement of expiration it is still mounting, but soon reaches its maximum, begins to fall, and continues falling through the remainder of the expiratory pliase.

A partial explanation is afforded by a consideration of the mechanical changes produced in the thorax by the respiratory movements. Of these, the influence of variations in the intrathoracic pressure on the filling of the heart is of special importance. With deep abdominal breathing the changes of intra-abdominal pressure also affect the filling of the heart, an increase of pressure (in inspiration) tending to cause more blood to be squeczed from the abdominal veins towards the chest. The changes of vascular resistance in the lungs, due to the alteration in the calibre of the pulmonary vessels, may also contribute, but, for such variations of intrathoracic pressure as normally occur, only in a minor degree. The changes in the vascular capacity of the lungs-that is, in the amount of blood contained in the pulmonary vessels-are of importance especially in delaying or accelerating the alterations of blood-pressure in the systemic arteries due to the other factors.

The intrathoracic pressure, which, as we have scen, is always less than that of the atmosphere, unless cluring a forced expiration when the free escape of air from the lungs is obstructed, diminishes in inspiration and increases in expiration. The great veins outside the chest, the jugular veins in the neck, for cxample, are under the atmospheric pressure, which is readily transmitted through their

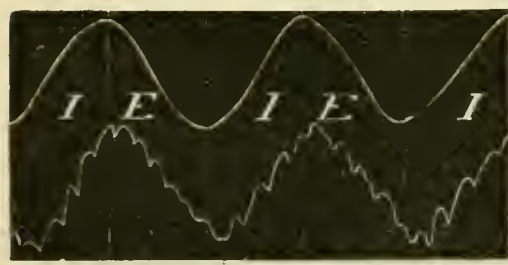

Fig. I28.-The upper tracing shows the respiratory movements in a rabbit with rather deep and slow diapluragmatic breathing; the lower tracing is the blood-pressure curve; $I$, inspiration; $E$, expiration, including the pause.

thin walls, while the heart and thoracic veins are under a smaller pressure. The venous blood both in inspiration and $\mathrm{cx}^{-}$ piration will, accordingly, tend to be drawn into the right auricle. In inspiration the venous flow will be increased, since the pressure in the thorax, and therefore in the pericardial cavity, is diminished; and upon the whole more venous blood will pass into the right heart during inspiration than during expiration. Now, the right ventricle is not in general working as hard as it can work. Hence, the excess of blood which reaches it during an inspiration is at once sent into the lungs, although not even the first of it can have passed through to the left side of the heart at the end of the inspiration, since the pulmonary circulation-time (four to five seconds in a small dog, two to three seconds in a rabbit) is longer than the time of a complete inspiration at any ordinary rate. The increase in the quantity of blood pumped into the pulmonary artery will, if not counteracted by other circumstances, tend to raise the blood-pressure in the 
artery and its branches, and therefore at once to accelcrate the out flow through the pulmonary veins. This will be aided if at the same time the vascular resistance in the lungs is reduced, as is generally stated to be the case. The left ventricle, like the right, is capable of discharging more blood than it or linarily receives. The excess of blood coming to it is easily and promptly ejected. The systemic arteries are better filled and the arterial pressure rises.

In expiration the contrary will happen. The return of blood to the thorax will be checked. This is well showil by the swelling of the veins at the root of the neck in expiration, their shrinking in inspiration, the so-called respiratory venous pulse. Less blood being drawn into the right heart, less will be pumped into the pulmonary artery, in which the pressure will, of course, fall. The outflow into the left auricle will thus be diminished-all the more if in the expiratory phase the vascular resistance in the lungs is increased -and the systemic arterial pressure will be lowered. In both cases, however, the change seen in the blood-pressure curve will be belated, and will not coincide exactly with the commencement of the inspiration or the expiration. If it is delayed for a period about equal to the length of an inspiration or an expiration, the blood-pressure will be seen to sink in inspiration and to rise in expiration. If the period of delay is less than this, the pressure will be mounting during a part of each respiratory phase and falling during the rest. As to the explanation of the delay, several factors may be concerned.

The negative pressure of the thorax acts on the aorta, as well as on the thoracic veins, although, on account of the greater thickness of its walls, to a smaller extent than on the veins. The diminution of pressure in inspiration tends to expand the thoracic aorta, and to draw blood back out of the systemic arteries, while expiration has the opposite effect. And although the hindrance caused in this way to the flow of blood into the arteries during inspiration, and the accleration of the flow during expiration may not be great, they will, of course, be better marked in small animals with comparatively yielding arteries than in large animals. Yet, whether great or small, the tendency will be to diminish the pressure in the one phase and increase it in the other. As soon as the changes of pressure produced by alterations in the flow of venous blood into the chest and through the lungs are thoroughly established, the arterial effect will be overborne; but before this happens-that is, at the beginning of inspiration and expiration-it will be in evidence, and will help to delay the main change.

Another factor in this delay is found in the changes of vascular capacity which take place in the lungs when they pass from the expanded to the collapsed condition. The expansion of the lungs in natural respiration causes a widening of the pulmonary capillaries, with a consequent increase of their capacity and diminution of their 
resistance. When the vessels at the base of the heart are ligatured either at the height of inspiration or the end of expiration, so as to obtain the whole of the blood in the lungs, it is found that they invariably contain more blood in inspiration than in expiration. During inspiration, as we have seen, the right ventricle is sending an increased supply of blood into the pulmonary artery; but before any increase in the outflow through the pulmonary veins can take place, the vessels of the lung must be filled to their new capacity. The first effect, then, of the lessened vascular resistance of the lungs in inspiration is a temporary falling off in the outflow through the aorta, and therefore a fall of arterial pressure. As soon as a more copious stream begins to flow through the lungs, this is succeeded by a rise. In like manner the first effect of expiration, which increases the resistance and diminishes the capacity of the pulmonary vessels, is to force out of the lungs into the left auricle the blood for which there is no room. This causes a rise of arterial bloodpressure, succeeded by a fall as soon as the lessened blood-flow through the lungs is established.

The changes in the diastolic capacity of the chambers of the heart itself, with the changes of pericardial pressure, must also act in the

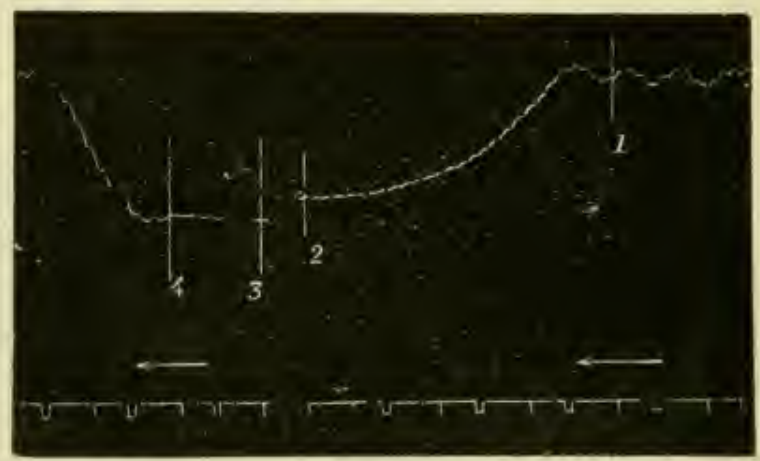

Fig. 129.-Effect on Blood-Pressure of Inflation of the Lungs: Rabbit. Artificial respiration stopped in inflation at $\mathbf{I}$. Interval between 2 and 3 (not reproduced) 5 I seconds, during which the curve was almost a straight line. Time tracing shows seconds.

same direction. It is obvious, then, how greatly the rate and depth of respiration in relation to the size of the animal and the other circumstances already mentioned may influence the time relations of the respiratory oscillations in the arterial pressure curve, so that we ought not to expect them to be absolutely constant.

In artificial respiration oscillations of blood-pressure, synchronous with the movements of the lungs, are also scen. During inflation (inspiration) the arterial pressure rises; during deflation (expiration) it falls. When artificial respiration is stopped at the height of inflation and the lungs kept inflated (Fig. I 29), the arterial blood-pressure falls rapidly, and continues low until the rise of asphyxia begins. In the 
fall of pressure the increased intrathoracic pressure due to the inflation is an important factor. When the respiration is stopped in collapse, instead of a fall a steady rise of pressure occurs (as in Fig. 84, p. 188). This ultimately merges in the elevation due to asphyxia, which show: itself soomer than in inflation. When the tracheal cannula is closed in natural respiration, no initial fall of pressure takes place (Fig. 130).

Besides the mechanical effects of the respiratory movements on the circulation, it may be influenced by changes in the cardioinhibitory and vaso-motor centres synchronous with the rhythm of the respiratory centre. In many animals (the dog, for instance) and in man, it can be very easily made out that the rate of the heart is greater during inspiration, especially towards its end, than in expiration. The phenomenon is especially distinct in deep and slow respiration. It is caused by a rhythmical rise and fall in the activity of the cardio-inhibitory centre, synchronous with the respiratory movements, for the difference disappears after division of both vagi. The normal respiratory oscillations of blood-pressure are not due in any great degree to such changes in the rate of the heart, for they persist after section of the vagi, and they are seen in animals like the rabbit, in which in ordinary breathing little or no variation in the rate of the heart is connected with the phases of respiration. The most probable explanation of the respiratory

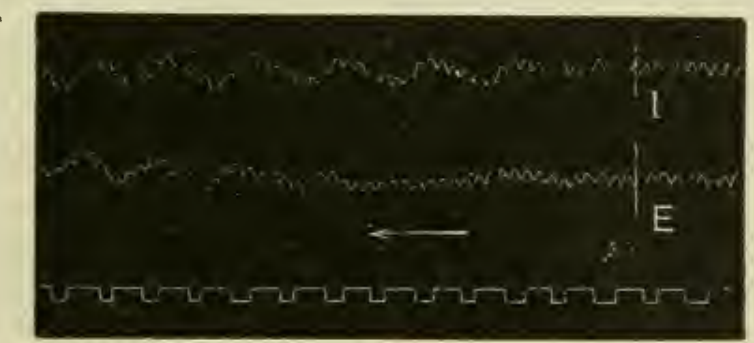

Fig. I30.-Blood-Pressure Tracing: Rabbit, under Chloral. Natural respiration stopped at $I$ in inspiration, at $\mathrm{E}$ in expiration. The mean blood-pressure is scarcely altered; but the respiratory waves become much larger owing to the abortive efforts at breathing. Time tracing shows seconds.

variations in the pulse-rate is that the respiratory centre, when it is discharging itself in inspiration, sends out impulses as a sort of overflow along fibres connecting it with the cardio-inhibitory centre. These increase the tone of that centre, but, owing to the long latent period of the cardio-inhibitory apparatus, the inhibition does not reveal itself till the succeeding expiration. It may be, however, that the impulses discharged from the respiratory centre in inspiration diminish the tone of the cardio-inhibitory centre, and thus lead to acceleration of the heart towards the end of the inspiratory phase. In certain pathological conditions the influence of the respiration on the pulse-rate is exaggerated (so-called 'respiratory arhythmia ').

Traube-Hering Curves.-Rhythmical changes in the activity of the vaso-motor centre, also associated with periodic discharges from 
the vaso-motor centre, also associated with periodic discharges from the respiratory centre, may be observed under certain conditionse.g., when in an animal paralyzed by curara, and therefore unable to breathe spontaneously, the artificial respiration is stopped for a time. If such a dose of curara be given as will still permit slight spontaneous respiration to go on, and both vagi be cut, it can be seen on stopping the artificial respiration that the waves on the blood-pressure curve are exactly synchronous with the slow respiratory movements. The Traube-Hering waves sink in inspiration and rise in expiration.

The fact that they have invariably a longer period than the natural respiratory movements indicates that they are not concerned in the production of the normal respiratory oscillations of arterial pressure. Probably the reason why the Traube waves appear after section of the vagi is the increased vigour of the slow respiratory discharges, coupled with a liyperexcitability of the vaso-motor centre, due to the long pauses in the aeration of the blood. In the asphyxial rise of pressure in a curarized dog they are constantly seen, and are often observed when the circulation in the medulla

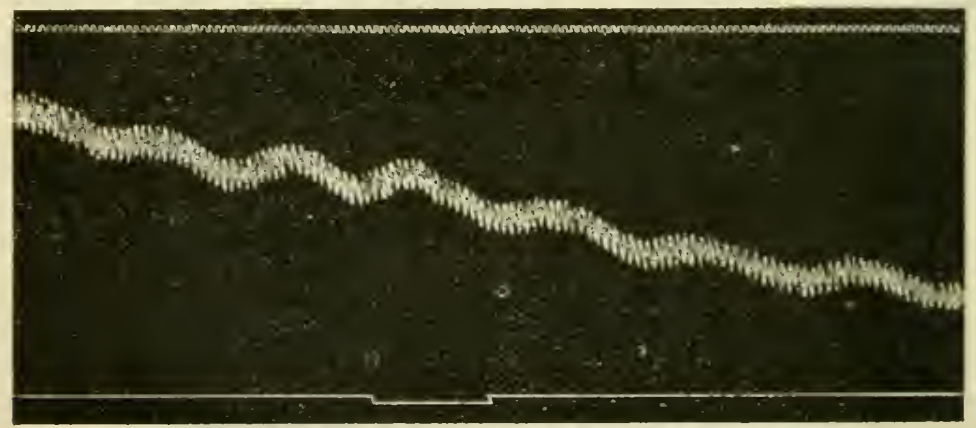

Fig. I31.-Traube-Hering Waves as the Blood-Pressure is falling during Occlusion of the Cerebral Arteries in a Cat.

oblongata is in any way interfered with (Fig. I3I). In addition to the true Traube-Hering waves, other and much longer periodic variations in the blood-pressure are sometimes noticed. If spontaneous respiration is going on, their long sweeping curves then show the ordinary respiratory waves superposed on them.

The normal respiratory oscillations in the veins, as might be expected, run precisely in the opposite direction to those in the arteries, and so do the Traube-Hering curves. The increased flow from the veins to the thorax during inspiration lowers the pressure in the jugular vein, while it increases the pressure in the carotid. The constriction of the small bloodvessels to which the TraubeHering curves are due increases the blood-pressure in the arteries, because it increases the peripheral resistance to the blond-flow; in 
the veins it lowers the pressure, because less blood gets through to them. Accordingly, when the Tranbe-Hering curve is ascending in the carotid, it is descending in the jugular.

The respiratory variations in the volume of the brain, which are so striking a phenomenon when a trephine hole is made in the skull, but which can also take place, thanks to the displacement of cerebro-spinal fluid (p. I74), when the cranium is intact, have by some been attributed to interference with the venous outflow from the cranial cavity during expiration, and by others to those changes in the arterial pressure whose causes we have just been discussing. The truth is that neither factor is exclusively concerned. The question turns largely upon the time-relations of the movements. The swelling of the brain is sometimes synchronous with expiration, and the shrinking with inspiration. Here the damming back of the blood in the sinuses when the outflow is checked by the expiratory rise of pressure in the thoracic veins either conspires with an expiratory rise of arterial pressure or is more than enough to counterbalance an expiratory fall of pressure in the cerebral arteries if the respiratory conditions are such as to lead to an expiratory fall. But sometimes the dura mater bulges into the trephine hole in inspiration and sinks down in expiration. Here the increase in the volume of the brain produced by the increased pressure in the arteries and capillaries in inspiration is more than sufficient to counterbalance the quickened escape of blood from the cerebral veins.

\section{Section VIII.-The Effects of Breathing Condensed and RAREFIED AIR.}

These are-(I) mechanical, shown chicfly by changes in the circulation, in the blood-pressure, for instance; (2) chemical.

The mechanical effects differ according to whether the whole body; or only the respiratory tract, is exposed to the altered pressure. When the trachea of an animal is comnected with a chamber in which the pressure can be raised or lowered, it is found that at first the arterial blood-pressure rises as the pressure of the air of respiration is increased above that of the atmosphere. But a maximum is soon reached; and when respiration begins to be impeded, the pressure falls in the arteries and increases in the veins. When the pressure of the air in the chamber is diminished a little below that of the atmosphere, there is a slight sinking of the arterial bloodpressure, which rises if the air-pressure is further diminished.

It is clear that any change of the air-pressure which tends to diminish the intrathoracic pressure will favour the venous return to the heart, and therefore, if the exit of blood from the thorax is not proportionally impeded, the filling of the arterias. An increase in the intra-alveolar pressure must tend on the whole to increase, and a diminution in it to lessen, the pressure inside the thorax, which always remains equal to the intra-alveolar pressure, minus the elastic tension of the lungs. 
Breathing compressed air should, therefore, under the conditions described, be upon the whole unfivourable to the venous return to the heart and to the filling of the arteries, and the arterial pressure should fall; while loreathing rarefied air should have the opposite effect. But a very gleat diminution of the intrathoracic pressure is not necessarily favourable to the circulation, since the auricles are then unable to contract perfectly.

Certain chest diseases liave been treated hy the use of apparatus by which the patient is made to breatle cither compressed or rareficd air; or to inspire air at one pressure and to expire into air at another pressure. And it has, upon the whole, lecen found, in agrecment with theory, that condensed air cannot help the circulation however it is applied, but always hinders it; while rarefied air aids the circulation both in inspira-

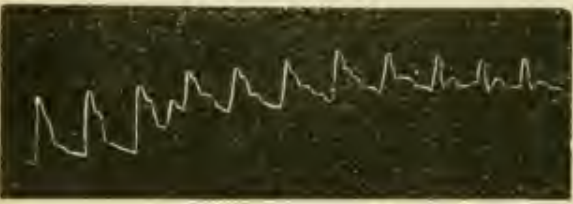

Fig. 132.-Pulse Tracing in Valsalva's Experi. ment (Rollett). tion and in expiration. But the increased work of the inspiratory muscles may counterbalance the advantage.

Yalsalva's experiment. which is performed by closing the mouth and nostrils after a previous inspiration, and then forcibly trying to expire, is an imitation of breathing into compressed air. The intrathoracic pressure is raised, it may be, to considerably more than that of the atmosphere; the venous retmo to the heart is impeded, and may bo stopped; and the pulse curve is altered in such a way as to inclicate first an increase and then a decrease of the arterial bloodpressure succecded by a sacond risa (Fig. 132).

Müller's experiment, which should be braclicted witl Valsalva's, consists in making, after a previons expiration, a strong inspiratory effort with mouth and nostrils closed. Here the intrathoracic pressure is greatly diminished, more blood is drawn into the chest, and upon the whole cffects opposite to those of Valsalva's experiment are produced (Fig. I 3.3). Neither cxperiment is quite free from danger. In botl the dicrotism of the pulse becomes more marked.

When the whole body is subjected to the clianged pressure, as in a balloon or on a mountain, in a diving-bell or a caisson used

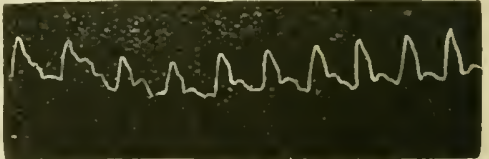

Fig. I33.-Pulse Tracing in Müller's Experiment (Rollett).

in building the piers of a bridge, the conditions are very different For the blood-pressure, the intrathoracic pressure, and the intraalveolar pressure, all fall together when the pressure of the atmosphere is diminished, and all rise together when it is increased. It is possible not only to live, but to do hard manual labour, at very different atmospheric pressures.

As regards the chemical effects of condensed and rarefied air, Loewy found that the quantity of oxygen absorbed by a man breathing air in the pneumatic cabinct remained constant at all pressures between about two atmospheres and half an atmosphere. At $440 \mathrm{~mm}$. of mercury dyspnoea became evident; but if the person was now made to work, the dyspmea passed away, and did not again manifest itself 
till the pressure was reduced to fio mm. There are towns on the higli tablelands of the Indes, and in the Himalayas, where the barometric pressure is not more than $I 6$ to 20 inclies, yet the in habitants feel no ill eflects. And in the caissons of the Fiorth Bridge the workmen were cugaged in severe toil under a maximum pressure of over three atmospheres, while in the caissons of the St. Louis Bridge in America a maximum pressure of over four atmosplices (i.c., more than three atmospheres in addition to the ordinary airpressure) was reached.

lnside the caissons the men sometimes suffer from pain and noise in the ears, due to excessive pressure on the external surface of the tym. panic membrane. If the pressure in the tympanum is raised by a swallowing movement, which opens the Eustachian tube and permits atir to enter it, the symptoms generally disappear. The suddenness of the change of pressure has much to do with its effects, and it is found that the men are most liable to dangerous symptoms while passing through the air-lock from the caissons to the external air. It may be concluded, from experiments on animals, that some of the most serious of these-tlie localized paralysis usually affecting the legs (paraplegia) and the circulatory disturbances - are due to the formation of gaseous cmboli, by the liberation of nitrogen in the blood and other bodyfluids when the pressure is abruptly reduced. And, indeed, it is found that the symptoms can often be caused to disappear, both in animals and men, by promptly subjecting them again to compressed air. To avoid g.1s cmbolism on decompression, the shift should be so short that the body-fluids do not become fully saturated with nitrogen, and the decompression should be slow. Even with a rate of decompression of twenty minutes for eacl atmosphere of excess pressure, the equilibrium between the dissolved and the atmospheric nitrogen is not entirely established fifteen minutes after decompression.

But that the action of air under a high pressure is not merely mechanical follows from the singular fact that in pure oxygen at a pressure of + to 5 atmospheres, which corresponds to air at 20 to 25 atmospheres, convulsions are often produced in vertebrate animals, while exposure to 6 to 25 atmospheres of oxygen causes dyspnœa and coma, usually without convulsions. All animals, so far as investigated, are instantly convulsed and killed under a pressure of $5^{0}$ atmospheres of oxygen (Hill and Macleod). Even sceds and vegetable orgamisms in general are killed in a short time in oxygen at 3 to 5 atmospheres; and an atmosphere of pure oxygen, equal to 5 atmosplicres of air, hinders the development of cggs. Lorrain Smith has shown that in small birds and mice exposure for inany hours to a pressure of between $I$ and 2 atmospheres of pure oxygen causes pneumonia. Hc confirms Bert's observations on the acute toxic cffects produced by higher pressures, and supposes that in the production of caisson disease the special action of the oxygen at high pressure may play a part as well as the rapid Iccompression. Even atmospheres containing 8o to 96 per cent. of oxygen under normal barometric pressure produce in rabbits, in 24 to 48 hours, congestion and œdema of the lungs, and finally a fibrinous broncho-pncumonia. Still smaller oxygen pressures may cause similar effects in a longer time (Karsner).

When the air-pressure is diminished below a certain limit, death takes place from asplıyxia, more or less gradual according to the rate at which the pressure is reduced. Tlie hrmoglobin cannot get 
or retain enough oxigen to enable it to perform its respiratury function; its dissociation tension is no longer balanced by an equal or greater partial pressure of oxygen in the air. The tencion of carbon diuxide in the blood is also lessened, owing to the dyspnoa and the consequent increase of pulmonary ventilation.

To sucli changes, as well as to the cold, some of the deaths in high balloon ascents nuust be attributed. Messrs. Glaisher and Coxwcll supposed that they reached the height of 37,000 feet; the former became unconscious at 29,000 feet $(8,800$ metres), at which height the amount of oxygen in the arterial blood would probably not exceed 10 volumes per cent., but recovererl during the clescent. The symptoms of the 'mountain sickness' so familiar to Alpine climbers (nausea, headache, and marked depression), the undue hyperpnoea produced by muscular exertion, and the slcep disturbed by irregular breathing, are also mainly due to deficiency of oxygen in the blood. The most rational prophylaxis is to leave the high peaks severely alone. But for the enthusiasts who cannot do this a portable apparatus for generating oxygen has been clevised. Experiments in the pneumatic cabinet indicate that the hyperpnoea is due to the indirect action of want of oxygen already referred to in discussing the normal regulation of respiration (p. 28r) - that is, to the formation, in consequence of the insufficient oxygen supply, of lactic acid or other substances which have the same influence as carbon dioxide on the respiratory centre-so that less carbon dioxide is required to excite the centre. Although the hyperpnoa leads to a diminution in the partial pressure of carbon dioxide in the pulmonary alveoli, there is no evidence that lack of carbon dioxide ('acapnia') is the primary cause of mountain sickness (Haldane). It must be remembered, however, that here the influence of the low barometric pressure is complicated by other conditions. lior example, while in the pneumatic cabinet, as already stated, rliminution of the pressure does not affect the oxygen consumption, it is relatively much grater on the high mountain levels both during rest and diring work than on the plains. This is not the case in balloon ascents. And evidence has been brought forward that changes in the mechanics as well as in the chemistry of respiration are concerned (the breathing, for instance, taking on a periodic character, with some approach to the Cherne-Stokes type - p. 287), and that there is something not connected with the want of oxygen which diminishes the capacity for muscular work. This "something' is perhaps a peculiar excitation of the nervous system in the fierce light of those high levels, which acts not only on the retina, but on the skin, and may even affect the distribution of the blood. It is said that a so-called light bath, as used in the treatment of ccrtain diseases, may increase the quantity of blood in rabbits by 25 per cent. in four hours. The shorter wavelengths which are relatively more intense in the mountain light are most effective. Recent investigations of the effects of high mountain climates have becn conducted by Haldane, Henderson, and others, on Pike's Peak, and by Barcroft and his associates on the Peak of Teneriffe and in the Alps As already mentioned, Haldane concluded that there was evidence of oxygen secretion by the lungs which became more marked witl the duration of residence on the mountain (14,000 feet above sea-level). The total oxygen capacity, and therefore the total amount of hrmoglobin, grarlually increased. The total volume of the blood was but slightly augmented, and the percentage of hrmoglobin rose decidedly. The changes in the circulation have been especially studied by Schncider, 
who found a marked increase in the rate of blood-flow through the fiands, associated with an accelcration of the heart beat, dilatation of the arterioles and a fall in the venous pressure. In 'aviator's sickness ' the essential factor is also oxygen deficiency.

\section{SECTION IX.-Cutaneous REspiRation.}

It has already been remarlied that a frog survives the loss of its lungs for some time, respiration going on througl the skin. Indeed, it has been calculated that in the intact frog, under ordinary conditions, as much as threc-quarters of the total gaseous exchange may be cutaneous. Two frogs were seen to live thirty-three days, and one even forty days, after excision of the lungs. The effect of exclusion of the pulmonary respiration on the gaseous exchange depends on the previous intensity of the metabolism. If this is high the gaseous excliange sinks markedly: if it is low there is scarcely any alteration. At their maximum efficiency, the trog's lungs are capable of sustaining a much greater exchange than the skin. Besides this quantitative, there is a qualitative difference, the carbon dioxide passing more easily through the skin than the oxygen, so tlat the respiratory quotient is increased by elimination of the lungs. In maminals the structure of the skin is different, and respiration can only go on through it to a very slight extent. The ainount of carbon dioxide cxcreted in man, altlough only about $4 \mathrm{grm}$. or 2 litres in twenty-four hours, is mucl greater than corresponds to the quantity of oxygen absorbed through the slin. It has been asserted, and no doubt with justice, that some at least of the carbon clioxide given off is due to putrefactive processes taking place on the surface of the body. Such processes, as lias already been pointed out, seem also responsible in part for the heavy odour of a 'close' room. For no harmful products appear to be exhaled from the skin when it is properly cleansed. In spite of the romantic statements to the contrary in ancient and modern books (for instance, the story of the cliild that was gilded to play the part of an angel at the coronation of a medieval pope, but died before the ceremony began), the whole of the human skin may be coated with an impermeable varnish without any ill effects. The entire surface of the body of a patient with cutaneous disease was covered with tar, and kept covered for ten days. There was not the least disturbance of any normal function. The serious effects of varnishing the skin in animals are duc, not to retention of poisonous substances, but to increased heat loss. Varnishing is not so rapidly harmful in large animals like dogs as in rabbits, which have a relatively great surface and a delicate slin. The danger of widespread superficial burns is well known. But it is not due to diminished excretion by the skin, for death occurs when large cutaneous areas remain uninjured. The patient nearly always dies when a quarter of the whole skin is burnt; yet the remaining $t_{\mathrm{L}}$.ree-quarters may surely be considered capable, from all analogy, of making up the loss by increased activity. One kidney is enough to eliminate the products of the nitrogenous metabolism of the whole body. It is difficult to see why the excretion of the trifling amount of solid matter in the perspiration should be interfered with by the loss of 25 per cent. of the sweat-glands. The real explanation of the serious effects of extensive superficial burns is perhaps the excessive irritation of the sensory nerves, which may lead to changes in the nervous centres, or reflexly in other organs, or the chemical changes in the damaged tissue, for example, in the bloodcorpuscles, or the transudation of lymph at the injured part, and consequent increase in the concentration of the blood. 


\section{PIRACTICAL EXERCISES ON CHAPTER $1 V^{\prime}$.}

1. Tracing of the Respiratory Movements in Man.-Pass a tape through the rings B of the stethograph shown in Fig. 134, and then

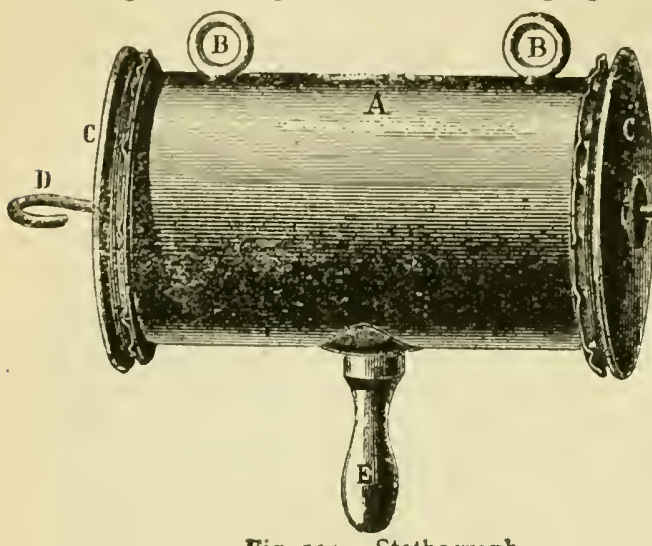

Fig. 134,-Stethograph. around the neck or over the shoulders, so as to support the instrument on the chest at a convenient height. Fasten tapes to the hooks and tie them by a slip-knot round the chest. The tube $\mathrm{E}$ is connected to a recording tambour, writing on a clrum. Or use the belt stethograph or spirograph of Fitz (p. 233), fastening the elastic tube round the chest with the chain, and connecting it with a tambour or the bellows recorder shown in Fig. 137. Compare the extent of the excursion when the $t$ ube is adjusted at different levels over the thorax and abdomen.

2.* Production of Apnca and Periodic Breathing in Man.-Arrange for taking tracings of the respiratory movements from a fellow-student as in $I$. Let the subject of the experiment recline in a perfectly easy position in an armchair. Let him then breathe deeply and frequently for about two minutes, so as to produce a prolonged apnœa of about two minutes' duration. Whenever any desire to breathe returns, the breathing is to be allowed to take its own course. It may be expected at first to be of the periodic (Cheyne-Stokes) type.

3. Tracing of the Respiratory Movements in Animals.-(a) Set up the arrangement shown in Fig. 135, and test whether it is air-tight. Have also in readiness an induction machine and electrodes arranged for an interrupted current. Anæsthetize a rabbit with chloral or ether (p. 217), or a small dog $\dagger$ with morphine and ether, or A.C.E. mixture. Insert a cannula into the trachea (p. 20I), and connect it with the large bottle by a tube. Connect the bottle with a recording tambour adjusted to write on a drum, and regulate the amount of the excursion of the lever by slackening or tightening the screw-clainp. Set the drum off at slow speed, and take a tracing.

(b) Then disconnect the cannula from its tube. Dissect out the vagus in the lower part of the neck, pass a ligature under it, but do not tie it. Connect the cannula again with the bottle, and while a tracing is being taken ligature the vagus. Cut below the ligature and stimulate its central end with weak shocks, marking the time of stimulation on the drum. Repeat the stimulation with strong shocks, and observe the results.

- This experiment is only to be attempted under the direct supervision of the demonstrator.

+ If a large dog is used the bottle should be omitted, the tracheal cannula being connected with the stem of a T-tube. One end of the horizontal limb of the T-tube is connected with the tambour; the other is provided with a rubber tube, which can be partially closed by a screw-clamp to regulate the excursion. 'Ether may be given when required by connecting the horizontal limb of the T-tube with a bottle with two glass tubes in the cork (p. 20I). 
(c) Apply a strong solution of potassium chloride witl a canc!'slair brush to the central end of the vagus while a tracing is being taken, and observe the effect.

(d) Isolate the sciatic nerve (p. 2 I 2), ligature it, and cut below the ligature. Stimulate its central end while a tracing is being taken. The respiratory movements will be increased.

(e) Disconnect the cannula, and isolate the vagus on the other side. While a tracing is being taken, divide it. The respiratory movements will probably at once become decper and less frequent.

(f) Again disconnect the cannula. Isolate the superior laryngeal branch of the vagus. This will be found entering the larymx at the point where the laryngeal horn of the hyoid bone is connected with the thyroid cartilage. If the finger is passed back along the upper border

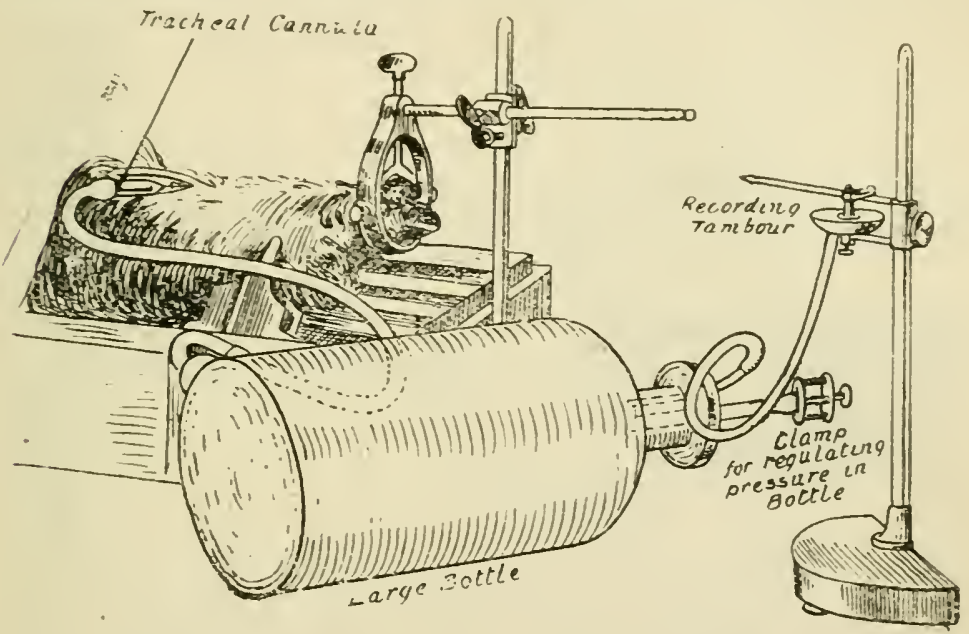

Fig. I35.-Arrangement for Respiratory Tracing. Two glass tubes are inserted through a cork in the mouth of the large bottle. One of thein has a small piece of indiarubber tubing on it, which is closed or opened, as may be required, by a screw-clamp. The other is connected by a rubber tube with a recording tambour. The tubulure at the bottom of the bottle is closed by a cork, through which passes a glass tube, connected by a rubber tube with the tracheal cannula. If no bottle with tubulure is available, it is only necessary to pass through the cork. down to the bottom of the bottle, a third glass tube, which is connected with the tracheal cannula. While a tracing is being taken the animal breathes the air contained in the bottle. When this becomes vitiated the respiratory movements are exaggerated and a normal tracing is no longer obtained. For this reason the tracheal cannula must be connected with the bottle only at the moment when a tracing is to be taken. The arrangement is most suitable for a small animal.

of the thyroid cartilage, this point will easily be felt. Ligature the nerve, and divide it between the larynx and the ligature. Reconnect the cannula. Take a tracing first with weak, and then with strong stimulation of the central end of the superior laryngeal.

(g) Make an incision through the abdominal wall in the linea alba, and study the movements of the diaphragm. Find the nerves from which the phrenics take origin in the neck. In the dog they arise from the fifth, sixth, and seventh cervical nerves. Divide the phrenic fibres 
on one side, and oloserve that the diaphragm on the corresponding side is now paralyzed.

(h) Insert a cannula into the carotid artery. While a respiratory tracing is being taken, allow blood to flow from the artery. Dyspnoa and exaggeration of the respiratory movements will be seen when a considerable quantity of blood has been lost. Nark and varnish the tracings.

In the whole of this experiment the tracheal cannula is to be disconnected, except when the lever is actually writing on the drum, in order that the period during which the animal must breathe into the confined space of the bottle may be diminished as much as possible. Instead of the method described, the stetlograph shown in Fig. I36 may be used to obtain respiratory tracings from animals, a broad canvas band being put round the animal's chest. 'To each end of this band is clamped with sufficient tension a strong thread (F), fastened to a small metal disc on the inside of the rubber dam closing the obliquelycut ends of the metal cylinder D. The tube $G$ is connected with a tambour or with a bellows recorder (Fig. I 37).

4. The Effect of Temperature on th $\geq$ Respiratory Centre-Heat Dyspnoea.- -Set up an arrangement for

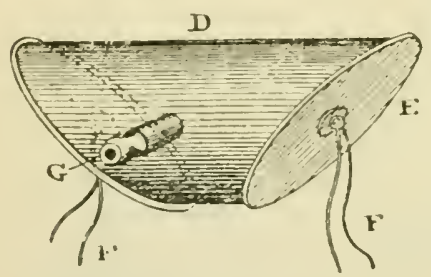

Fig. 136.-Stethograph (Crile).

taking a respiratory tracing as in 2 (footnote, p. 300). Anasthetize a dog, and fasten it, back downward, on a holder. Make an incision in the middle line of the neck, commencing a little below the cricoid cartilage, and extending lown for 4 or 5 inches. Insert a cannula into the trachea. Isolate both carotid arteri:.: for as great a distance as possible, and arrange them on the brass iubes shown in Fig. I38. Connect two adjacent cnds of the tubes liy a short rubber tube. Connect one of the remaining ends to a funnel, supported on a stand, and the other to a rubber tube laanging over ti: table above a large jar. Slip two or three folds of paper betwer: the tubes and the vagus nerves. Heat two or three litres of water to about $65^{\circ} \mathrm{C}$. (a) Now connect the tracheal cannula with the tambo::" As soon as the tracing is under way, let the hot water rum throug! the funnel and tubes into the jar. Mark on the tracing the point a which the flow of the lot water was begun, and go on passing it until it has produced an effect. Then stop the drum, and circulate water at the ordinary temperature till the breathing is again normal. Then, while a tracing is being taken, pass ice-cold water through the tubes, and again notice the effect. 
(b) Expose the sciatic. Pass ice-water through the tubes, and while a respiratory tracing is being taken stimulate its central end with induction shocks so weak as just to cause an effect. Pass water at air temperature through the tubes, and repeat the stimulation with the coils at the same distance. Do the same while hot water is being passed through the tubes, and compare the results. Always allow the water to pass for a time before making an observation.

5. Measurement of Volume of Air inspired or expired-Vital Capacity. -A spirometer (Fig. I 4 , p. 235) of sufficient accuracy for this cxperiment can be made by removing the bottom of a large bottle witli a capacity of not less than 4 litres. A good cork, through which passes a glass tube connected with a rubber tube, is fitted into the neck. The bottle is fixed vertically, mouth downwards, the glass tube being closed for the time, and graduated, by pouring in measured quantities of water, say roo c.c. at a time, and marking the lerel. The divisions are then etched in. If the cork does not fit air-tight, it is covered with wax. The bottle is swung on two pulleys, counterpoised and immersed, bottom down, in a large glass jar or a small cask nearly full of water. A smaller bottle may be used for the determination of the tidal air, so as to reduce the error of reading.

(I) Submerge the bottle to the stopper, after opening the pinclicock on the rubber tube. Breathe into the bottle, close the cock, adjust the bottle so that the level of the water is the same inside and outside, and then read off the level. Determine the volume of air expired in-

(a) A normal expiration after a normal inspiration (tidal air);

(b) The greatest possible expiration after a normal inspiration (supplemental air plus tidal air);

(c) The greatest possible expiration after the greatest possible inspiration (vital capacity).

(2) Open the cock and raise the bottle till it is nearly full of air. Determine the volume of air inspired in -

(a) A normal inspiration after a normal expiration (tidal air);

(b) The greatest possible inspiration after a normal expiration (complemental air plus tidal air):

(c) The greatest possible inspiration after the greatest possible expiration (vital capacity).

Make several observations of each quantity, and take the mean.

(3) Count the rate of respiration for three minutes, keeping the breathing as nearly normal as possible; repeat the observation; and from the mean result and the amount of the tidal air calculate the quantity of air taken into the lungs in twenty-four hours (pulmonary ventilation).

6. Cardio-Pneumatic Movements. - Fill a U-tube with tobaccosmoke. One end of the tube is placed in the nostril of a fellow-student. 
and made tight with a little cotton-wool. The other nostril and the mouth are closed, and respiration suspended. The colum of smoke moves in and out at cach beat of the heart. By feeling the apex-beat, try to verify the fact that during systole the cardin-pneumatic movement is inspiratory, and in diastole expiratory.

7. Auscultation of the Lungs. - This is taught in the course of pliysical diagnosis, but in conmection with the subject the student may perform the following experiment on a dog used for some other purpose: Open the trachea as described on p. 201. Insert into it the cross-piece of a glass $T$-tube of as large a bore as possible, tying the tracliea over it on each side of the stem. The stem projecting from the wound is armed with a short piece of rubber tubing, which can be closed at will with a clip. When the tube is thus closed the animal breathes through the glottis in the ordinary way. When the tube is open, and the moutli and nose covered tiglitly with a cloth, no air goes through the glottis. The tube being closed, listen with the stethoscope or the ear alone over a part of the chest where the vesicular munmur is well heard. If the

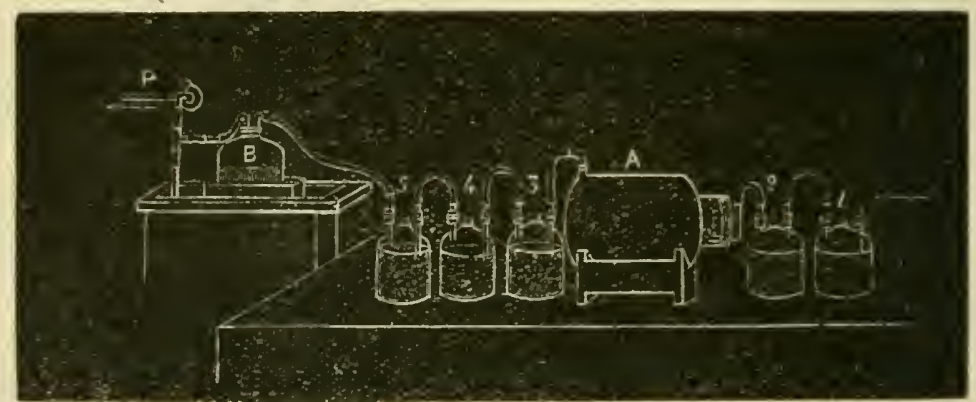

Fig. 139.-Haldane's Apparatus for measuring the Quantity of $\mathrm{CO}_{2}$ and Aquevis Vapour given off by an Animal. A. chamber into which the animal is put: I and 4 , Woulff's bottles filled with soda-lime to absorb carbon dioxicle; 2,3 , and 5, Woulff's bottles filled with pumice-stone soaked in sulphuric acid to absorl, watery vapour; $\mathbf{B}$, glass bell-jar suspended in water, by means of which the negative pressure is known; 1 , water-pump which sucks air through the appar. atus; $I$ and 2 are simply for absorbing the carbon dioxide and water of the inging air.

rubbing of the hairs below the stethoscope cituses clisturbing sounds, sliave a portion of the skin. Continue listening while an assistant closes the tube and covers up the animal's muzzle. Determine whether any change takes place in the vesicular sound.

Repeat the observation while listening over the lower part of the trachea, and determine whether any change takes place in the bronchial breathing sound.

8. Respiratory Pressure.-Connect a strong rubber tube with a glass bulb, and the bulb with a mercurial manometer provided with a scale. (r) Fasten the tube with a little cotton-wool in one nostril, breatlic through the other with closed mouth, and observe the anount by which the level of the mercury is altered in ordinary inspiration and expiraton.

(2) Repeat the observation with forced breathing, pinching the tube at the height of inspiration and expiration, and reading off the maximum inspiratory and expiratory pressure. 
(3) Repeat (I) with the tube connected to the mouth by a glass tube held between the lips, and the nostrils open.

(4) Repeat (2) with the tube in the mouth and the nostrils closed.

9. Estimation of the Quantity of Water and of Carbon Dioxide given off by an Animal (Haldane's Melhod).-(I) Connect the apparatus shown in Fig. I 39 with the water-pump. Allow a negative pressure of 5 or 6 inches of water to be established in it, as shown by the rise of water in the bell-jar $B$. Then close the open tube of carbon dioxide bottle $I$, and clamp the tube between the water-pump and the bell-jar. If the negative pressure is maintained, the arrangenent is air-tight. Now weigh bottle 3 and bottles 4 and 5 . the last two together. Place a cat in the respiratory chamber $A$, connect the chamber directly with the water-puinp, and test whether it is tight. Then take the stopper out of bottle $\mathbf{r}$, and adjust the rate at which air is drawn through the: apparatus. Let the ventilation go on for a few minutes, then insert bottles 3,4 , and 5 again. Note the time exactly at this point, alld after an hour disconnect 3,4 , and 5 , and again weigh. The difference of the two weighings of 3 shows the quantity of water given off by the animal in an hour; the difference in the combined weight of 4 and 5 , the quantity of carbon dioxicle. Weigh the cat. and calculate the amount of water and of carbon dioxide given off per kilo per hour.

(2) For the student it is more convenient to use smaller animals. The mouse may be taken as an cxample of a warm-blooded animal, and the frog of a cold-blooded. Instead of the Woulff's bottles use wide test-tubes connected as in Fig. I 40 , and for the animal chamber a small beaker, closed with a very carefuliy fitted

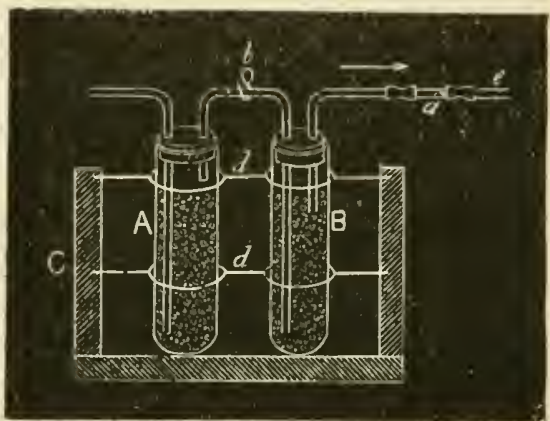

Fig. 140.-Absorption Tubes for $\mathrm{CO}_{2}$ and Moisture. A, soda-lime tube; $\mathrm{B}$, 'sulphuric acid tube; C, wooden frame, in which $\mathrm{A}$ and $\mathrm{B}$ are supported by wires $d$ : ;, wire hook, which grips the glass tube firmly, and by means of which the tubes are lifted out of the frame in order to be weighed; $a$, short piece of glass tubing. by taking out which the absorption tubes are disconnected from the rest of the apparatus; $e$, glass tube going off to animal chamber.

cork which has been boiled in paraffin. The inlet and outlet tubes of the chamber are to be introduccd through this cork. The holes for thesc are to be bored with the greatest care, and the tubes to be put in while the cork is still hot from boiling in paraffin. Also insert a thermometer about 6 inches long registering from $0^{\circ} \mathrm{C}$. to $45^{\circ} \mathrm{C}$. Modeller's wax is to be used finally to render all the junctions air-tight.

Add to the series of tubes described in the apparatus a single tube containing baryta-water. This is placed to the left of tube 5 , and so arranged that the air-current bubbles through the water. As long as the absorption of carbon dioxide is complete, the baryta-ivater remains clear. Beyond this a water-bottle should be placed to act as a valve and to indicate the negative pressure in the apparatus. It can be most simply constructed by using a cylinder of stout glass tubing in a wide-mouthed bottle containing some water, the inlet and outlet tubes passing through a paraffined cork which seals the upper end of the cylinder. 
Before making an obscrvation, test whether the apparatus is airtight, as explained above, after introducing the aninal into the chamber, sealing the latter with wax and connecting it wh the absorptiontubes. But a negative pressure of 2 or 3 inches of water is a sufficient test for the small apparatus.

To make an observation, set the air-current going at the desired rate. Allow it to run for a few minutes till the carbon clioxide, which has accumulated during the testing, has been swept out. At a time which has been decided on and noted, stop the current by disconnecting the water-pump. Disconnect and stopper up the animal chamber, and weigh it as quickly as possible. Connect up again, using only recentlyweighed absorption-tubes, and finally connect with the water-pump' and allow the current to pass for a definite period, say an hour.

The soda-lime sliould not be too dry, or absorption is not sufficiently rapid. The following facts are made out: $(a)$ Loss of weight by the animal chamber (chiefly loss by the animal); (b) gain of the sulphuric acid tube in water; (c) gain of the soda-lime tubes in carbon dioxide.

The total loss and total gain do not correspond, the gain being always greater than the loss. The surplis can only be oxygen absorbed by the animal and added to the hydrogen and carbon of its substance to form water and carbon dioxide. Calculate the respiratory quotient (p. 24 I).

Io. Muscular Contraction in the Absence of Free Oxygen (sce p. 267). - Pith a frog (brain and cord). Cut off one hind-leg at the middle of the thigh, and strip the skin from it. Pass a thread under the tendo Achillis, tic it, and divide the tendon below it. Free the tendon and the gastrocnemius muscle from the loose connective tissue lying between them and the bones of the leg. and divide the latter just below the lenee. Remove superfluous thigh muscles, and fasten the gastrocnemius in a moist chamber by means of the femur. Attach the thread on the tendon to a lever. Connect the poles of the secondary coil of an induction machine by fine copper wircs to the femur and the tendon. Put a battery and simple key in the primary, and arrange it for single shocks. Stimulate the muscle and observe the lieight of the contraction. Now pass into the chamber a current of washed hydrogen gas from a botile containing granulated zinc, upon which a little dilute sulphuric acid is poured from time to time. The air in the moist chamber will soon be entirely displaced by the hydrogen, but the muscle will contract on being stimulated, and the stimulation can be repeated many times.

II. Oxidizing Ferments.-Wash out the bloodvessels of a dog or rabbit (Practical Exercises, p. 65). Chop up fincly portions of pancreas, spleen, muscle, lungs, and kidney, kecping each separate, and avoiding any contamination of one by another. Grind up half of each portion with sand in a small mortar, and cxtract with a small quantity of water, keeping all the extracts separate. Into each of cleven test-tubes put Io c.c. of a colourless dilute alkaline solution of paraphenylenediamin and a-naphthol (freslily made by mixing solutions of the two substances in cquimolecular proportions* and adding a little sodium carbonate). To five of the tubes add the chopped organs, to five the watery extracts of the organs, and enough water to make the volume equal in all the tubes. To the remaining tube add the same amount of water. Observe in which tube a cliange of colour takes place (p. 272).

* I.e., the weight of each of the two substances in the mixture should be proportional to its molecular weight. A convenient solution contains o I $_{44}$ per cent. of $\alpha$-naphthol and o ros per cent. of paraplienylencliamin. These quantities are one-hundredth-molecular. Sod um carbonate is added to the amount of $0^{\circ} 25$ per cent. The $a$-naphthol can be kept as a 1 per cent. solution in 50 per cent. alcohol. 


\section{CHAPTER V}

\section{VOICE AND SPEECH}

Voice.-Sounds of various kinds are frequently produced by the movements of animals as a whole, or of individual organs. The muscular sound, the sounds of the heart and of respiration, we have already had to speak of. Such sounds may be considered as purely accidental as the footfall of a man or the buzzing of a fly. The wings of an insect beat the air, not to cause sound, but to produce motion; the respiratory murmur is a mere indication that air is finding its way into the lungs, it is in no way related to the oxidation of the blood in the pulmonary capillaries. But in many of the higher animals mechanisms exist which are specially devoted to the utterance of sounds as their prime and proper end. In man the voice-producing mechanism consists of a triple series of tubes and chambers: (I) The trachea, through which a blast of air is blown; (2) the larynx, with the vocal cords, by the vibrations of which sound-waves are set up; and (3) the upper resonance chambers, the pharynx, mouth, and nasal cavities, in which the sounds produced in the larynx are modified and intensified, and in which independent notes and noises arise.

The larynx is a cartilaginous box, across which are stretched, from front to back, two thin and sharp-edged membranes, the (true) vocal cords. In front the cords are attached to the thyroid cartilage, one a little to each side of the middle line; behind they are connected to the vocal or anterior processes of the pyramidal arytenoid cartilages. The thyroid and the two arytenoids are mounted upon a cartilaginous ring, the cricoid. The arytenoids can rotate on the cricoid about a vertical axis, while the cricoid can rotate on the thyroid cartilage around a transverse horizontal axis. The cricoid can thus be raised by the contraction of the cricothyroid muscle, and the vocal cords stretched. By the pull of the posterior crico-arytenoid muscles, attached to the external or muscular processes of the arytenoid cartilages, the vocal processes are rotated outwards, the cords separated from each other or abducted, and the chink between them, the rima glottidis, widened. IVhen the vocal processes are approximated $b_{y}$ contraction of the lateral 
crico-arytenoid muscles and the consequent forward movement of the muscular processes, the vocal cords are brought close together, or adducted, and the rima is narrowed. The transverse or posterior arytenoid muscle, which connects the two arytenoid cartilages behind, also helps, by its contraction, to narrow the glottis by shifting the cartilages on their articular surfaces somewhat nearer thic middle line. Running in each vocal cord, and, in fact, incorporated with its elastic tissue, is a muscle, the thyro-arytenoid, the external portion of which may to some extent cause inward rotation of the vocal processes and adduction of the cords; but the main function, at least of its inner part, is to alter the tension of the cords. The diagrams in Figs. I4I and I42 illustrate the action of the abductors and adductors of the vocal cords.

The crico-thyroid muscle and the deflectors of the epiglottis are supplied by the superior laryngeal branch of the vagus, which also

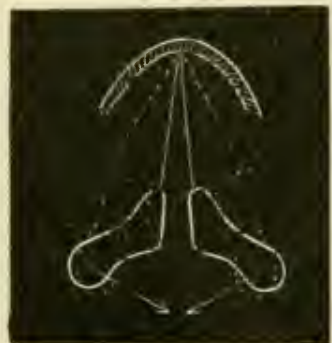

Fig. I4x,-Diagrammatic Horizontal Section of Larynx to show the Direction of Pull of the Posterior Crico-Arytenoid Muscles, which abduct the Vocal Cords. Dotted lines show position in abduction.

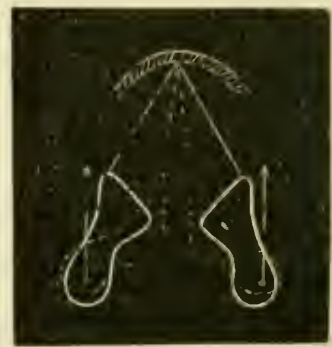

Iig. I42.-Direction of Pull- of the Lateral Crico-Arytenoids, which adduct the Vocal Cords. Dotted lines show position in adduction.

contains the sensory fibres for the mucous membrane of the larynx above the vocal cords. In the dog and rabbit motor fibres also reach the crico-thyroid by the so-called middle laryngeal nerve which arises from the superior pharyngeal branch of the vagus. All the other intrinsic muscles are supplied by the recurrent laryngeal branch of the vagus. It receives these motor fibres from the spinal accessory, and supplies sensory fibres to the mucous membrane of the larynx below the vocal cords and to the trachea.

The voice is produced, like the sounds of a reed instrument, by the rhythmical interruption of an expiratory blast of air by the vibrating vocal cords. When a bell is struck, vibrations are set up in the metal, which are communicated to the air. It is not the same with the vibrations of the vocal cords; if they were plucked or struck, they would only produce a feeble note. The air in the mouth, pharynx, larynx, trachea, and lungs is the real sounding 
body; a pulse of alternate rarefaction and condensation is set up in it by the interference, at regular intervals, of the vocal cords with the expiratory blast. Forced abruptly from their position of equilibrium as the blast begins, they almost immediately regain and pass below it, in virtue of their elasticity, and continue to vibrate as long as the stream of air continues to issue in sufficient strength. Not only do they vibrate up and down, but also towards and away from the middle line, so that, at least in the chest voice, they come into contact with each other at each swing. The sound-waves thus set up spread out on every side, impinge on the tympanic membrane, set it quivering in response, and give rise to the sensation of sound.

We may say, in a word, that the whole exquisite mechanism of cartilages, ligaments, and muscles, has for its object the production of a sufficient pressure in the blast of air driven through the windpipe by an expiratory act, and of a suitable tension in the vibrating cords. An approximation of the cords, a narrowing of the glottis, is essential to the production of voice; with a widely-opened glottis the air escapes too easily, and the necessary pressure cannot be attained. The pressure in the windpipe was found in a woman with a tracheal fistula to be about $12 \mathrm{~mm}$. of mercury for a note of medium height, about $\mathrm{I}_{5} \mathrm{~mm}$. for a high note, and about $72 \mathrm{~mm}$. for the highest possible note. The period of vibration of structures like the vocal cords depends on their length, thickness, density and tension; the shorter, thinner, more dense and less tense a stretched string is, the greater is the vibration frequency, the higher the note. In the child the cords are short (6 to $8 \mathrm{~mm}$.), in woman longer (Io to $\mathrm{I} 2 \mathrm{~mm}$. when slack, I3 to $15 \mathrm{~mm}$. when stretched), in man longest of all ( $I_{4}$ to $\mathrm{I} 8 \mathrm{~mm}$. in the relaxed, and $\mathrm{r} 8$ to $22 \mathrm{~mm}$. in the stretched position); and the lower limit of the voice is fixed by the maximum length of the relaxed cords. A boy or a woman cannot utter a deep bass note, because their vocal cords are relatively short, and do not vibrate with sufficient slowness. It is true that by the action of the crico-thyroid muscle the cords can be lengthened, and that the maximum length in a woman approaches or exceeds the minimum length in a man. But the lengthening of the vocal cords in one and the same individual is always accompanied by other changes -increase of tension, decrease of breadth and thickness-which tell upon the vibration frequency in the opposite way, and more than compensate the effect of the increase of length, so that for high notes the cords are longer than for low. The contraction of the thyro-arytenoid muscle is a more influential factor in altering the tension of the cords than the contraction of the cricothyroid. It is probable that, when the highest notes are uttered, only the anterior portions of the cords are free to vibrate, their posterior portions being damped by the approximation of the vocal processes of the arytenoid cartilages by the contraction of the 
lateral crico-arytenoid and transverse arytenoid muscles. The range of an ordinary voice is 2 octaves; by training $2 \frac{1}{2}$ octaves can be reached; but in exceptional cases a range of 3 , and even $3 \frac{1}{2}$, octaves (as in the celebrated singer Catalini) has been known.

The development of the voice in children is of great interest. At the age of six years the boy's voice has a rather narrower range than the girl's in both directions. The boy's voice reaches its full height in the twelfth and its full depth in the thirteenth year, when the range is almost 3 octaves, its upper limit being a semitone higher than the girl's, but its lower limit a whole tone deeper. When the voice 'breaks" in boys at the age of puberty it falls about an octave. The control of the vocal organs becomes so incomplete that only in one-fourth of the cases can notes of sufficient steadiness to be used in music be produced. The vocal cords, as may be seen with the laryngoscope, are frequently, though not always, congested.

The pilch of a note, while it depends chiefly, as has been said, on the tension of the vocal cords, rises and falls somewhat with the strength of the expiratory blast; the highest notes are only reached with a strong expiratory effort. The intensity of all. vocal sounds is determined by the strength of the blast, for the amplitude of vibration of the cords is proportional to this. Besides pitch and intensity, the ear can still distinguish the quality or timbre of sounds; and the explanation is as follows: Two simple tones of the same pitch and intensity, that is, the sounds caused by two series of airwaves of the same period and amplitude-of the same frequency and height, to use less technical terms-would appear absolutely identical to the sense of hearing; just as the aerial disturbances on which they depend would be absolutely alike to any physical test that could be applied. But no musical instrument ever produces sound-waves of one definite period, and one only; and the same is true of the voice. When a stretched string is displaced in any way from its position of rest, it is set into vibration; and not only does the string vibrate as a whole, but portions of it vibrate independently and give out separate tones. The tone corresponding to the vibration period of the whole string is the lowest of all. It is also the loudest, for it is more difficult to set up quick than slow vibrations. The ear therefore picks it out from all the rest; and the pitch of the compound note is taken to be the pitch of this, its fundamental tone. The others are called partial or overtones, or harmonics of the fundamental tone, their vibration frequency being twice, three times, four times, etc., that of the latter. Now, the fundamental tone of a compound note or clang produced by two musical instruments may be the same, while the number, period, and intensity of the harmonics are different; and this difference the ear recognizes as a difference of timbre or quality. The timbre of the voice depends for the most part on partial tones produced or intensified in the upper resonance chambers. 
A great deal of our knowledge as to the mode and meclianism of the production of voice has been acyuired by means of the laryngoscope (Fig. I43). This consists of a small plane mirror mounted on a handle, which is held at the back of the mouth in such a position that a beam of light, reflected from a larger concave mirror fastened on the forehead of the observer, is thrown into the larynx of the patient. The observer looks through a hole in the centre of the large mirror; and an image of the interior of the larynx is seen in the small mirror, in which the parts that are anterior appear as posterior, the arytenoid cartilages in front, the thyroid behind, and the vocal cords stretching between. The small mirror is warmed to body-temperature before being introduced, so as to prevent the condensation of moisture on it. The tendency to retch, which is

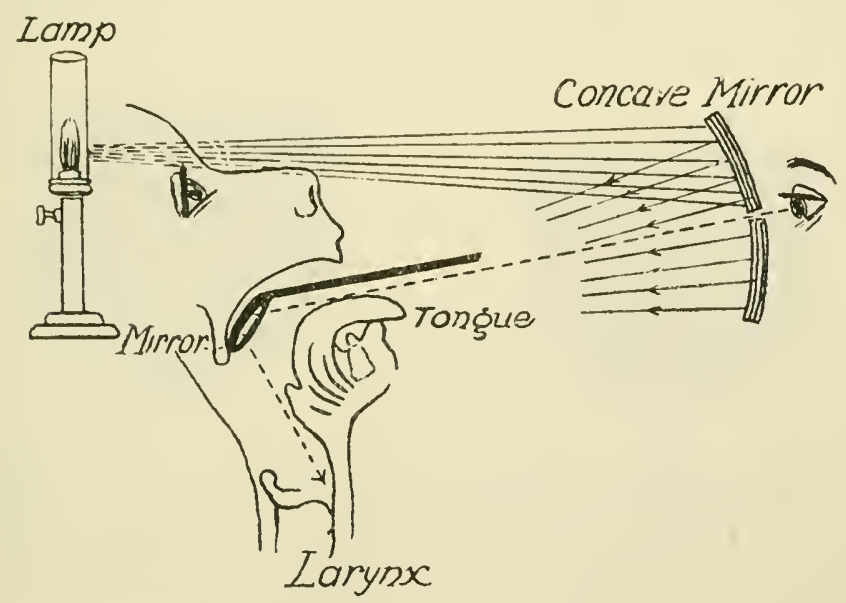

Fig. I43--Diagram of Laryngoscope.

caused by contact of the instrument with the soft palate, may be removed or lessened by the application of a solution of cocaine.

Examined with the laryngoscope during quiet respiration, the glottis is seen to be moderately, though not widely, open, and the vocal cords almost motionless. Although the portion between the arytenoid cartilages has received the name of glottis respiratoria, in contradistinction to the glottis vocalis between the vocal cords, the rima in its whole extent from front to back is really concerned in the respiratory act. In deep expiration the vocal cords come nearer to the middle line, and the glottis is narrowed; in deep inspiration they are widely separated, and the rings of the trachea, and even its bifurcation, may be disclosed to view. When a sound is produced -a note sung, for example--the cords are approximated (Figs. I44 and 145 ); and with a high note more than with a low. 
The essential difference between the production of notes in the lower register, or chest voice, and in the higher register, or falsetto, has been inuch debated. The lowest notes which can be uttered by any given voice are chest notes, the highest are falsetto notes; but there is a debatable land common to both registers, and medium notes can be sung cither from the cliest or from the head. Chest notes impart a vibration or fremitus to the thoracic walls, from the resonance of the lower airchambers, the trachea and bronchi; and this can be distinctly felt by the hand. In head notes or falsetto the resonance is chicfly in the upper cavities, the plarynx, mouth, and nose. As to the mechanical conditions in the larynx, there is a pretty general agreement that during the production of falsetto notes the vocal cords are less closely approximated than in the sounding of chest notes. The escape of air is consequently more rapid in the head voice, and a falsetto note cannot be maintained so long as a note sung from the chest. But it is only the anterior part of the rima glottidis that is wider in the falsetto voice; the whole of the glottis respiratoria, and even the posterior portion of the glottis vocalis, are closed during the emission of falsctto notes.

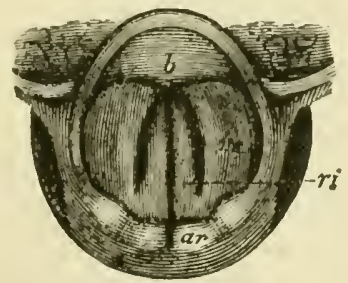

Fig. I44. - Position of the Glottis preliminary to the Utterance of Sound. $r s$, false vocal cord; $r i$, true vocal cord; ar, arytenoid cartilage; $b$. pad of the epiglottis.

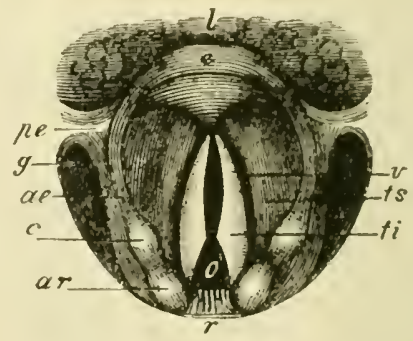

Fig. I45.-Position of Open Glottis. $l$, tongue; $e$, epiglottis; ae, ary. epiglottidean fold; $c$, cartilage of Wrisberg; ar, arytenoid cartilage; $o$, glottis; $v$, ventricle of Mor. gagni; $t i$, true vocal cord; $t s$, false vocal cord.

Oertel has stated, and the statement has been confirmed by others, that the free edge of the vocal cord alone vibrates in the falsetto voice, one or more nodes or motionless lines parallel to the edge being formed by the contraction of the internal part of the thyro-arytenoid muscle, which thus acts like a stop upon the cord.

Approximation of the vocal cords may take place in certain acts unconnected with the production of voice. Thus, a cough, as has already been mentioned, is initiated by closure of the glottis. During a strong muscular effort, too, the chink of the glottis is obliterated, and respiration and phonation both arrested. The object of this is to fix the thorax, and so afford points of support for the action of the muscles of the limbs and abdomen. But considerable efforts can be made even by persons with a tracheal fistula.

Speech.-Ordinary speech is articulated voice-voice shaped and fashioned by the resonance of the upper air-cavities, and jointed 
together by the sounds or noises to which the varying form of these cavities gives rise. Here we conte upon the fundamental distinction between vowels and consonants. Vowels are musical sounds; consonants are not musical sounds, but noises-that is to say, they are due to irregular vibrations, not to regularly recurring waves, the frequency of which the ear can appreciate as a definite pitch. This difference of character corresponds to a difference of origin: the vowels are produced by the vibrations of the vocal cords; the consonants are due to the rushing of the expiratory blast through certain constricted portions of the buccal cliamber, where a kind of temporary glottis is established by the approximation of its walls. One of these 'positions of articulation' is the orifice of the lips; the consonants formed there, such as $p$ and $b$, are called labials. A second articulation position is between the anterior part of the tongue and the teeth and hard palate. Here are formed the dentals, $t$, $d$, etc. The ordinary English $r$, and the $r$ of the Berwickshire and East Prussian 'burr,' also arise in this position througl a vibratory motion of the point of the tongue. The third position of articul:tion is the narrow strait formed between the posterior portion of th arched tongue and the soft palate. To the consonants arising hero the name of gutturals has been given. They include $k, g$, the Scottish $c h$, and the uvular German $r$. The latter is produced by a vibration of the uvula. The aspirated $h$ is a noise set up by the air rushing through a moderately wide glottis, and some have thercfore included the glottis as a fourth articulation position for consonants. Certain sounds like $n, m$, and $n g$, when final (as in pen, dam, ring), although produced at the glottis, are intensified by the resonance of the air in the nose and pharynx, and are sometimes spoken of as nasal consonants.

As we have said, the vowels are produced by vibrations of the vocal cords, but to what they owe their special timbre or quality has been much discussed. According to the view with which Helmholtz's name is particularly connected this is due to the reinforcement of certain overtones by the resonating cavities, the shape and fundamental tone of which are different for each vowel.

When a vowel is whispered, the mouth assumes a characteristic shape, and emits the fundamental tone proper to the form and size of the particular 'vowel-cavity,' not as a reinforcement of a tone set up by the vibrations of the vocal cords, but in response to the rush of air through the cavity; just as a bottle of given shape and size gives out a definite note when the air which it contains is set in vibration, by blowing across its mouth. A whisper, in fact, is speech without voice; the larynx takes scarcely any part in the production of the sound; the vocal cords remain apart and comparatively slack; and the expiratory blast rushes through without setting them in vibration.

The fundamental tone of the 'vowel-cavity' may be found for each vowel by placing the mouth in the position necessary for uttering it, then bringing tuning-forks of different period in front of it, and noting 
which of them sr.ts up sympathetic resonance in the air of the mouth, and so causes its sound to be intensified. The fundamental tone is lowest for $u$ (as in lute). Next comes 0 ; then $a$ (as in path); then $a$ (as in $f(a n e)$; then $i$; while $e$ is highest of all. A simple illustration of this may be found in the fact that when the vowels are whispered in the order given, the pitch rises. When $u$ or 0 is sounded, the buccal cavity has the form of a wide-bellied flask, with a short and narrow neck for $u$, a still shorter but wider neck for $o$. For $e$ the tongue is raised and almost in contact with the palate, and the cavity of the moutl is shaped iike a flask with a long narrow neck and a very short belly. For $i$ the shape is similar, but the neck is not so narrow. For a (as in path) the vowel-cavity is intermediate in form between that of $u$ and $e$, being roughly funnel-shaped, and the mouth is rather widely opened. For $u(00)$ the resonating cavity is made as long as possible, the larynx being depressed and the lips protruded; for $e$ the resonating cavity is at its shortest, the larynx being raised as much as possible and the lips retracted (Figs. I 46 to 148 ).

According to Helmholtz, all that the resonating cavity does is to strengtlen certain of the partials or overtones of the laryngeal note.

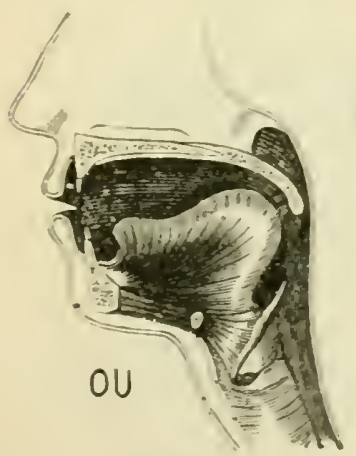

Fig. 146.

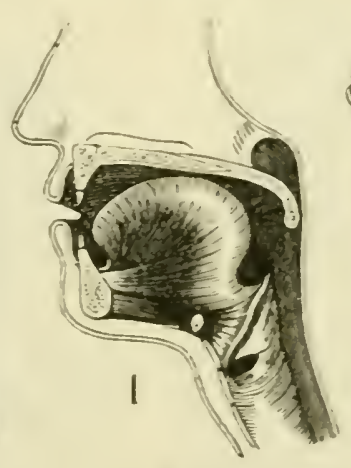

Fig. 147 .

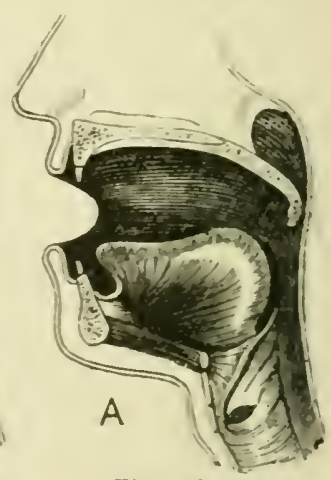

Fig. 148.

If this is true, the partials which give a vowel-sound the timbre by which we recognize it as different from other vowel-sounds cannot preserve the same numerical relation to the fundamental tone when the pitch of the latter is altered. Suppose, for example, that a given vowel is sounded with a pitch corresponding to roo ribrations a second, and that the partial which is particularly strengthened by the resonance of the mouth cavity is the fifth overtone, corresponding to 600 vibrations. Then when the same vowel is sounded with a pitcl of 200 vibrations, the reinforced partial which will now give the quality to the sound will still correspond to 600 vibrations a second. since this is the rate which most easily elicits the resonance, but it will not now be the fifth but the second overtone.

Universally accepted for a time, the Helmholtz theory has been in recent years assailed. especially by Hermann, who bases his criticism on microscopic examination of curves obtained by the Edison phonograph, and on reproductions of such records obtained by photographing on a moving drum covered with sensitive paper a beam of light reHected from a small mirror attached to a system of levers whose move. ments follow the curves faithfully and greatly magnify them. Hermann 
luas come to the conclusion that the mouth does not act as a mere resonator, but that for each vowel, in addition to the fundamental note due to the vibration of the vocal cords, the pitch of which is, of course, variable, one or, it may be, two other notes (formants, as he calls them), not necessarily harmonics of the laryngeal note, but separated from it by a constant or nearly constant musical interval, are directly produced by the passage of the regularly intcrrupted expiratory blast through the mouth, the air contained in that cavity being for an instant set into vibration at each interruption. On this view it is the musical effect produced by the oscillation or continual recurrence, in short series, of these vibrations which gives the vowels their quality. The fact that it is by no means difficult to sing (with the larynx) and whistle (with the moutli) at the same time, shows the possibility of Hermann's view, that a fixed tone can be gencrated in the mouth by the intermittent stream of air issuing from between the vibrating vocal cords, just as a tone is generated in a pipe by blowing into or over it, and his records do show continually recurring groups of vibrations as lis theory requires. Mc Kendrick takes up a middle position, believing that both theories are partially true, and this seems to be the best conclusion which can at present be arrived at. It scems clear, at any ratc, that more than one factor is concerned in the timbre of the vowel sounds.

When the vowels are being uttered, the soft palate closes the entrance to the nasal chambers completely, as may be shown by holding a candle in front of the nose, or trying to inject water through the nares. If the cavities of the nose are not completely blocked off, the voice assumes a nasal character in pronouncing certain of the vowels; and in some languages this is the ordinary and correct pronunciation.

Many animals have the power of emitting articulated sounds; a few have risen, like man, to the dignity of sentences, but these only by imitation of the human voice. Both vowels and consonants can be distinguished in the notes of birds, the vocal powers of which are in general higher than those of mammalian animals. The latter, as a rule, produce only vowels, though some are able to form consonants too.

The nervous mechanism of voice and speech will have to be again considered when we come to study the physiology of the brain and spinal cord. But the curious physiological antithesis between the functions of abduction and of adduction of the vocal cords may be mentioned here. The abductor muscles are not employed in the production of voice; they are associated with the less specialized, the less skilled and purposive function of respiration. The adductor muscles are not brought into action in respiration; they are associated with the highly specialized function of speech. Corresponding to this difference of function, we find that adduction is preponderatingly represented in the cortex of the brain, abduction in the medulla oblongata. Stimulation of an area in the lower part of the ascending frontal convolution, near the fissure of Rolando, in the macaque monkey, causes adduction of the vocal cords, never 
abductioli. In the cat, however, abduction of the cords may also be obtained by stimulation of the cortex. The same is true of the dog, but only when tire peripheral adductor nerves have been divided. Stimulation of $\mathrm{i}$ ii medulla oblongata (accessory nucleus) causes abduction, never adduction. The skilled adductor function is, therefore, placed under cont lol of the cortex. The vitally important, but more mechanical, abductor function is governed by the medulla. The abductor movements are more likely to be affected by organic disease, the adductor movements by functional changes. But the distinction between the two groups of muscles is not entirely due to a difference of central connections, since by altering the strength of the stimulus and the external conditions the one or the other may be separately excited through the inferior laryngeal nerve. Thus, strong stimulation of the inferior laryngeal causes closure of the glottis, for although it supplies both abductors

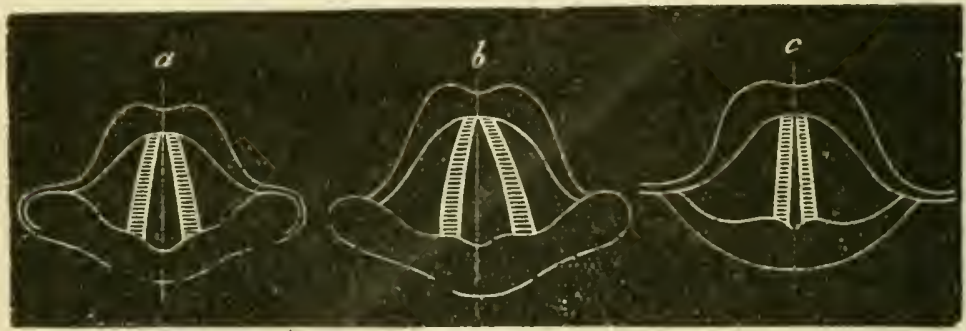

Fig. 149.-Diagram of Vocal Cords in Paralyses of the Larynx. a, Paralysis of both inferior laryngeal nerves. The vocal cords have taken up the 'mean' position. $b$, Paralysis of right inferior laryngeal nerve. An attempt is being made to narrow the glottis for the utterance of sound. The right cord remains in its 'mearı ' position. 'c, Paralysis of the abductor muscles only, on both sides. The cords are approximated beyond the 'mean' position by the action of the adductors.

and adductors, the latter, as the stronger muscles, prevail. With weak stimulation, and in young animals, the abductors, owing to the greater excitability of the neuro-muscular apparatus, carry off the victory, and the glottis is opened (Russell).

When the nerve is cooled the abductors give way before the adductoi : The same is true when it is allowed to become dry: And after death in a cholera patient it was observed that the posterior crico-arytenoid, an abductor muscle, was the first of the intrinsic laryngeal muscles to lose its excitability. Lesions of the medulla oblongata are often accompanied by marked changes in the character of the voice and the power of articulation.

Section or paralysis of the superior laryngeal nerve causes the voice to become lioarse, and renders the sounding of high notes an inpossibility, owing to the want of power to make the vocal cords tense. Stimulation of the vagus within the skull causes contraction 
of the crico-thyroid muscle and increased tension of the cords. Section or paralysis of the inferior laryngeal nerves leads to loss of voice or aphonia, and dyspnœea (Fig. I 49). Both adductor and abductor muscles are paralyzed; the vocal cords assume their mean positionthe position they have in the dead body-and the glottis can neither be narrowed to allow of the production of a note, nor widened during inspiration. It is said, however, that young animals, in which the structures around the glottis are more yielding than in adults, can still utter shrill cries after section of the inferior laryngeals, the contraction of the crico-thyroid muscle alone being able, while increasing the tension of the cords, to draw them together.

Interference with the connections on one side between the higher cerebral centres and the medulla oblongata, as by rupture of an artery and effusion of blood into the posterior portion of the internal capsule (giving rise to hemiplegia, or paralysis of the osposite side of the body), is not followed by loss of voice; the larjingeal muscles on both sides are still able to act. 


\section{H A P TER VI}

\section{DIGESTION}

IN the last chapter we have described the manner in which the interchange of gases between the tissues and the air is carried out. We have now to consider the digestion and absorption of the solid and liquid food, its further fate in relation to the chemical changes or metabolism of the tissues, and finally the excretion of the waste products by other channels than the lung.

Logically, we ought to take metabolism after absorption and before excretion, tracing the food through all its vicissitudes from the moment when it enters the blood or lymph till it is cast out as useless matter by the various excretory organs. Unfortunately, however, many of the intermediate steps of the process are as yet hidden from us; we know best the beginning and the end. We can follow the food from the time it enters the alimentary canal till it is taken up by the tissues of absorption; and we have really a fair knowledge of this part of its course. We can collect the end-products as they escape in the urine, or in the breath, or in the sweat; and our knowledge of them and of the manner in which they are excreted is considerable. But of the wonderful pathway by which the dead molecules of the food mount up into life, and then descend again into death, we catch only a glimpse here and there. Only the introduction and the conclusion of the story of metabolism are at present in our possession in fairly continuous and legible form. We will read these before we try to decipher the handful of torn leaves which represents the rest.

\section{Section I.-Preliminary Anatomical and Chemical Data.}

Comparative.-In the lowest kinds of animals, such as the amøba, there is neither mouth, nor alimentary canal, nor anus: the food, wrapped round by pseudopodia, is taken in at any part of the animal with which it happens to come in contact. A vacuole is formed around it. Acid is secreted into the vacuole, the food is digested within the cell-substance, and the part of it which is useless for nutrition is cast out again at any part of the surface.

Conning a little higher, we find in the Cœlenterates a mouth and alimentary tube, which opens into the body-cavity, where a ccrtain 
amount of digestion seems to take place, and from which the food is absorbed cither through the cells of the endoderm, or, as in Medusa, by means of fine canals, which radiate from the body-cavity into its walls, and form part of the so-called gastro-vascular system. In the Echinodermata we have a further development, a complete alimentary canal with mouth and anus, and entircly shut off from the body-cavity. In many Arthropods it is possible already to distinguish parts corresponding to the stomarh, and the small and large intestines of higher forms, the digestive glands being represented by organs which in some groups seem to be homologous witl the liver, and in others with the salivary glands of the higher Vertebrates. A few Molluses scem in addition to possess a pancreas.

Among Vertebrates fishes have the simplest, and lirds and mammals the most complicated, alimentary system. In the lowest fishes tlie stomach is only indicated by a slight widening of the anterior part of the digestive tube. In water-living Vertebrates there are no salivary glands. In birds the œsopliagus is generally dilated to form a crop. from which the food passes into a stomacli consisting of two parts, one pre-eminently glandular (proventriculus), the other pre-eminently muscular (ventriculus). Among mammals a twofold division of the stomacl is distinctly indicated in rodents and cetacex, but this organ reaches its greatest complexity in ruminants, which possess no fewer than four gastric pouches. The differentiation of the intestine into small and large intestine and rectum is more distinct, both anatomically and functionally, in mammals than in lower forms; but there are marked differences between the various mammalian groups both in the relative size of the several parts of the digestive tube, and in the proportion between the total length of the alimentary canal and the length of the body. In general, the canal is longest in herbivora, shortest in carnivora. Thus, the ratio between length of body and length of intestine is in the cat I : $4, \operatorname{dog} I: 6$, man I : 5 or 6 , horse $I: I 2$, cow I : 20 , sheep I : 27 . The relative capacity of the stomach, small intestine, and large intestine, is in the $\operatorname{dog} 6: 2: 1 \cdot 5$, in the horse $1: 3.5: 7$, in the cow $7: 2: I$. The area of the mucous surface of the alimentary canal is very considerable, in the dog more than half that of the skin, the surface of the small intestine being three times that of the stomach and four times tliat of the large intestine. In the horse the mueous surface has twice the area of the skin.

Anatomy of the Alimentary Canal in Man.-The alimentary canal is a muscular tube, which, beginning at the mouth, runs under the various names of pharynx, œsophagus, stomach, small intestine, large intestine, and rectum, till it ends at the anus. Its walls are largely composed of muscular fibres; its lumen is clad with epithelium, and into it open the ducts of glands, which, morphologically speaking, are involutions or diverticula formed in its course. In virtue of its muscular fibres it is a contractile tube; in virtue of its epithelial lining and its special glands it is a secreting tube; in virtue of both it is fitted to perform those mechanical and chemical actions upon the food which are necessary for digestion. Its inner surface is in most parts richly supplied with bloodvessels, and in special regions beset with peculiarlyarranged lymphatics; by both of these channels the alimentary tube performs its function of absorption. From the beginning of the œsophagus to the end of the rectum the muscular wall consists, broadly speaking, of an outer coat of longitudinally-arranged fibres, and a thicker inner coat of fibres running eircularly or transversely around the tube. Between the layers lies a plexus of non-medullated nerves and nerve-cells (Auerbach's plexus). In the stomach the longitudinal 
fibres are found only on the two curvatures, and a third incomplete coat of oblique fibres makes its appearance internal to the circular layer. In the large intestine, again, the longitudinal fibres are chiefly collected into three isolated strands. In the pharynx the typical arrangement is departed from, inasmuch as there is no regular longitudinal layer; but the three constrictor muscles represent to a certain extent the great circular coat. The muscles of the mouth and of the pharynx are of the striped variety. So is the muscle of the upper half of the œsophagus in man and the cat, and of the whole œesophagus in the dog and the rabbit. In the rest of the alimentary canal the muscle is smooth, except at the very end, where the external sphincter of the anus is striped. In certain situations the circular coat is developed into a regular anatomical sphincter, a definite muscular ring, whose function it is to shut one part of the tube off from another (sphincter pylori, ileo-colic sphincter), or to help to close the external opening of the tube (intemal sphincter of anus). Elscwhere a tonic contraction of a portion of the circular coat, not anatomically developed beyond the rest, creates a functional sphincter (cardiac sphincter of stomach).

Throughout the greater part of the digestive tract the peritoneum forms a thin serous layer, external to the muscular coat. Internally the muscular coat is separated from the mucous membrane, the lining of the canal, by some loose areolar tissue containing bloodressels, lymphatics, and nerves (Meissner's plexus), and called the submucous coat. Between the mucous and submucous layers. but belonging to the former, in the whole canal below the beginning of the osophagus, is a thin coat of smooth muscular fibres, the muscularis mucosæ, consisting in some parts, e.g., in the stomach, of two, or even threc, layers. Between this and the lumen of the canal lie the ducts and alveoli of glands. surrounded by bloodvessels and embedded in adenoid or lymphoid tissue, which in particular regions is collected into welldefined masses (solitary follicles, Peyer's patches, tonsils), extending, it may be, into the submucous tissue. In the mouth, pharynx, and œsophagus, the glands lie in the submucosa, as do the glands of Brunner in the duodenum; everywhere else they are confined to the mucous membrane proper. Between the openings of the glands the mucous membrane is lined with a single layer of columnar epithelial cells, sometimes (in the small intestine) arranged along the sides of tiny projections or villi. When the intestine is contracted the villi are long and cylindrical in shape, when it is relaxed or distended they are flat and conical. At the ends of the alimentary canal, viz., in the mouth, pharynx, and œsophagus, and at the anus, the epithelium is stratified sipuamous, and not columnar.

The purpose of food is to supply the waste of the tissues, to replenish the stores of material from the oxidation of which the energy required for the running of the bodily machine is derived, and thus to maintain the normal composition of the body. In the body we find a inultitude of substances marked off from each other, some by the sharpest chemical differences, others by characters much less distinct, but falling upon the whole into the few fairly definite groups already described (p. I).

Now, although it is by no means necessary that a substance in the body beionging to one of these great groups should be derived from a substance of the same group in the food, it has been found 
that upon the whole no diet is sufficient for man unless it contains representatives of all; a proper diet must include proteins, carbohydrates, fats, inorganic salts, and water. These proximate principles have to be obtained from the raw material of the foodstuffsthat is, as regards the first three groups, which can alone yield energy in the body, from the tissues and juices of other living things, plants or animals; it is the business of digestion to sift them out and to prepare thein for absorption. This preparation is partly mechanical, partly chemical.

The water and salts and some carbo-hydrates, such as dextrose, are ready for absorption without change. Fats are split into glycerin and fatty acids before absorption. Indiffusible colloidal carbo-hydrates, like starch and dextrin, are changer into diffusible and readily soluble sugars, and the natural proteins into diffusible peptones, and eventually into much simpler decomposition products. These changes are obviously favourable to absorption. But this is not their whole significance. For disaccharides, such as cane-sugar, maltose, or lactose, although easily soluble in the contents of the gut, and in themselves perfectly capable of being absorbed without change, are, unless present in unusually large amount, all converted into monosaccharides, such as dextrose, levulose, or galactose, either in the lumen or in the wall of the alimentary tube. The reason is that the disaccharides are unsuitable as pabulum for the cells. Digestion is not only a preparation of the food for absorption by: the gut, but for assimilation by the tissues after absorption. An equally important instance of this double function is seen in the digestion of proteins. The complete shattering of the protein molecule into amino-acids and the other groups yielded by its decomposition (p. 360) is required, in the case of that portion of the protein which goes to build up the tissues, because of the high degree of specificity of the tissue proteins. The myosinogen of beef cannot be cobbled into the myosinogen of human muscle, still less we may suppose into the serum-albumin of human blood. It is necessary that the food protein should be completely 'wrecked ' in digestion so that protein which is to take its place in protoplasm may be built exactly to order from the bricks. A satisfactory ' fit ' cannot be obtained with ready-made protein. Mechanical division of the food is an important aid to the chemical action of the digestive juices. We shall see that this mechanical division forms a great part of the work of the stomach, but it is normally begun in the mouth, and it is of consequence that this preliminary stage should be properly performed.

\section{Section II.-The Mechanical Phexomexa of Digestion.}

Mastication.-It is among the mammalia that regular mastication of the food first makes its appearance as an important aid to digestion. The amphibian bolts its fly, the bird its grain, and the fish 
its brotler, witlout the ceremony of chewing. In ruminating mammals we see mastication carried to its highest point; the teeth work all day long, and most of them are specially adapted for grinding the food. The carnivora spend but a short time in mastication; their teeth are in general adapted rather for tearing and cutting than for grinding. Where the diet is partly animal and partly vegetable, as in man, the teeth are fitted for all kinds of work; and the process of mastication is in general neither so long as in the purely vegetable feeders, nor so short as in the carnivora.

In man there are two sets of teeth: the temporary or milk teeth, and the permanent teeth. The milk teeth are twenty in number, and consist on each side of four incisors or cutting-tecth, two canines or tearing-teetl, and four molars or grinding-tecth. The central incisors emerge at the seventl month from birth, the other incisors at the ninth month, the canines at the eighteenth, and the molars at the twelfth and twenty-fourth month respectively. Each tooth in the lower jaw appears a little before the corresponding one in the upper jaw. Each of the milk teeth is in course of time replaced by a permanent tooth, and in addition the vacant portion of the gums behind the milk set is now filled up by twelve teeth, six on each side, three above and three below. These twelve are the permanent molars; they raise the number of the permanent teeth to thirty-two. The permanent teeth which occupy the position of the milk molars now receive the name of premolars. The first tooth of the permanent set (the first true molar) appears at the age of $6 \frac{1}{2}$ years; the last molar, or wisdom-tooth, does not emerge till the seventeenth to the twenty-fifth year.

In mastication the lower jaw is moved up and down, so as to alternately separate and approximate the two rows of teeth. It has also a certain amount of movement from side to side, and from front to back. The masseter, temporal and internal pterygoid muscles raise, and the digastric, with the assistance of the mylo- and geniohyoid, depresses, the lower jaw, but its downward movement is mainly a passive one. The external pterygoids pull it forward when both contract, forward and to one side when only one contracts. The lower fibres of the temporal inuscle retract the jaw. The buccinator and orbicularis oris muscles prevent the food from passing between the teeth and the cheeks and lips. The tongue keeps the food in motion, works it up with the saliva, and finally gathers it into a bolus ready for deglutition.

Deglutition. - This act consists of a voluntary and an involuntary stage. Just before the begiuning of the voluntary stage mastication is suspended, and a slight contraction of the diaphragm generally takes place. The anterior part of the tongue is suddenly elevated and pressed against the hard palate, and the elevation travels back from the tip towards the root, as the mylo- 
hyoir muscles in the floor of the mouth contract sharply so as to thrust the bolus through the isthmus of the fauces. As soon as this has happened, and the food lias reached the posterior portion of the tongue, it has passed beyond the control of the will, and the second or involuntary stage of the process begins.

This stage may be divided into two parts: (I) Pharyngeal, (2) oesophageal-both being reflex acts. During the first the food has to pass through the pharynx, the upper portion of which forms a part of the respiratory tract, and is in free communication with the larynx during ordinary breathing. It is therefore necessary that respiration should be interrupted and the larynx closed while the food is being moved through the pharynx. But that the interruption may be short, the food must be rapidly passed over this perilous portion of its descent. The main propelling force under which the bolus is shot through the back of the pharynx is derived from the contraction of the mylo-hyoid muscles already mentioned, assisted to some extent by the stylo- and palato-glossi; and that none of the purchase may be lost, the pharyngeal cavity is cut off from the nose and mouth as soon as the bolus has entered it. The soft palate is raised by the levator palati and palato-pharyngei muscles; at the same time the upper part of the pharynx, narrowed by the contraction of the superior constrictor, comes forward to meet the soft palate, closes in upon it, and so prevents the food from passing into the nasal cavities. The pharynx is cut off from the mouth by the closure of the fauces through the contraction of the palato-pharyngeal muscles which lie in their posterior pillars. The upper free end of the epiglottis (the so-called pharyngeal part) aids the back of the tongue in completing a movable partition across the pharynx, which keeps close to the bolus as it passes down between the posterior surface of the epiglottis and the posterior wall of the pharynx. Almost immediately after the contraction of the mylo-hyoids the larynx is pulled upwards and forwards by the contraction of the thyro-hyoid muscle, and the elevation of the hyoid bone by the muscles which connect it to the lower jaw. The base of the tongue is simultaneously drawn backwards by the stylo- and palato-glossus. The lower or laryngeal portion of the epiglottis is thus caused to come into contact with the upper orifice of the larynx, occluding it completely, but the pharyngeal portion projects beyond the larynx, and takes no share in its closure (Eykman). The glottis is closed by the approximation of the vocal cords and the arytenoid cartilages. The epiglottis, however, is not absolutely indispensable for closing the larynx, since swallowing proceeds in the ordinary way when it is absent. The morsel of food, grasped by the middle and lower constrictors as it leaves the back of the tongue, passes rapidly and safely over the closed larynx, the process being accelerated by the pulling up of the lower portion 
of the pharynx over the bolus by the action of the palato- and stylopharyngei.

The second or œsophageal portion of the involuntary stage is a more leisurely performance. The bolus is carried along by a peculiar 'peristaltic' contraction of the muscular wall of the œsophagus, which travels down as a wave, constricting the tube and pushing the food before it. In front of the constricting wave moves a wave of inhibition, so that the part of the œsophagus into which the bolus is about to pass is always relaxed, while the part behind it is contracted. This exact co-ordination of inhibition and contraction is the essential thing in peristalsis. When the food reaches the lower end of the gullet the tonic contraction of that part of the tube is for a moment relaxed by reflex inhibition, and the morsel passes irto the stomach. Beammont saw, in the case of St. Martin, that the œsophageal orifice of the stomach contracted firmly after each morsel was swallowed, and so did the gastric walls in the neighbourhood of the fistula when food was introduced by this opening. In the dog the whole process of swallowing from mouth to stomach has been shown to occupy four to five seconds, but the time is by no means constant. In man the peristaltic wave requires about five to six seconds to travel from the level of the glottis to the cardiac orifice. The rate of movement is greater in the upper than in the lower portion of the œsophagus.

Such is the mechanism of deglutition when the bolus is of such consistence and size that it actually distends the osophagus. But it has been shown that liquid food is swallowed in a different way. The food lying on the dorsum of the tongue, suddenly put under pressure by the sharp contraction of the mylo-hyoid muscles, is shot rapidly down to the lower part of the lax œsophagus, or, occasionally, some of it even into the stomach. So far the process has only occupied one-tenth of a second. After several seconds, the food, or the portion which still remains in the œsophagus, is forced through the cardiac sphincter into the stomach by the arrival of the tardy peristaltic contraction of the œsophageal wall (Kronecker and Meltzer). Two sounds may be heard in man on listening in the region of the stomach or œsophagus during deglutition of liquids, especially when, as generally happens, they are mixed with air. The first sound occurs at once, and is due to the sudden squirt of the liquid along the gullet; the second, which is heard after a distinct interval (about six seconds), is caused by the forcing of the fluid through the cardiac orifice of the stomach by the contraction of the œsophagus.

There are certain peculiarities which distinguish this peristaltic movement of the oesophagus from that of other parts of the alimentary canal. It is far more closely related to the central nervous system, and, unlike the peristaltic contraction of the intestine, can 
pass over any muscular bluck caused by ligature, section, or crush. ing, so long as the nervuus connections are intact. But division of the cesophageal nerves causes, as a rule, stoppage of oesophageal movements; although an excised portion of the tube retains its vitality for a long time, and may, under certain circumstances, go on contracting in the characteristic way after removal from the body (p. $8 I \%$ ). Stimulation of the mucous membrane of the pharynx will cause reflex movements of the cesophagus, while stimulation of its own mucous membrane is ineffective. From these facts we learn that although the osophageal wall may possess a feeble power of spontaneous peristaltic contraction, yet this is usually in abeyance, or at least overmastered by central nervous control; so that impulses discharged as a 'fusillade' from successive portions of the vagus centre, and travelling down the œesophageal nerves, excite the inuscular fibres in regular order from the upper to the lower end of the tube.

Nervous Mechanism of Deglutition.-The centre for the whole involuntary stage (both pharyngeal and cesophageal) lies in the upper part of the medulla oblongata. When the brain is sliced away above the medulla, deglutition is not affected; but if the upper part of the medull 2 is removed, the power of swallowing is abolished. In man, disease of the spinal bulb interferes far more with deglutition than disease of the brain proper.

Normally, the afferent impulses to the centre are set up by the contact of food or saliva with the mucous membrane of the posterior part of the tongue, the soft palate and the fauces, the nervechannels being the superior laryngeal, the pharyngeal branches of the vagus, and the palatal branches of the fifth nerve.* A feather has sometimes been swallowed involuntarily by a reflex movement of deglutition set up while the soft palate or pharynx was being tickled to produce vomiting. Artificial stimulation of the central end of the superior laryngeal will cause the movements of deglutition independently of the presence of food or liquid; but if the central end of the glosso-pliaryngeal nerve be stimulated at the same time, the movements do not occur. The glosso-pharyngeal is therefore able to inhibit tle deglutition centre, and it is owing to the action of this nerve that in a series of efforts at swallowing, repeated within less than a certiin short interval (about a second), only the last is successful. It is also through the glosso-pharyngeal nerve that the respiratory movements are inhibited during deglutition. When the central end of this nerve is stimulated, respiration is stopped

* It appears that the most influential reflex paths may differ in different animals. In the rabbit, e.g., the retlex is set up by excitation of the trigeminal fibres which supply the mucous membrane anterior to the tonsils, in the dog and cat by excitation of the glosso-pharyngeal fibres in the posterior wall of the pharynx, and in monkeys by excitation of the trigeminal branches dis. tributed to the mucous membrane over the tonsils (Kahn). 
for four or five seconds, and this cessation is distinguished from that produced by any other afferent nerve by the circumstance that it occurs not in expiration exclusively or in inspiration exclusively, but with the respiratory muscles in the precise degree of contraction in which they happened to be at the moment of stimulation. The efferent nerves of the reflex act of deglutition are the hypoglossal to the tongue and the thyro-hyoid and other muscles concerned in raising the larynx; the glosso-pharyngeal, vagus, facial and fifth to the muscles of the palate, fauces, and pharynx; the fifth to the mylo-hyoid; and the vagus to the larynx and œsophagus. Section of the vagus interferes with the passage of food along the œesophagus; stimulation of its peripheral end causes œsophageal movements.

Movements of the Stomach. - The whole of the stomach does not take part equally in the movements associated with digestion. We may divide the organ, both anatomically and functionally, into two portions-a pyloric portion, or antrum pylori, comprising about a fifth of the stomach, and a larger cardiac portion, or fundus.* At the junction of the antrum and the fundus the circular muscular coat is slightly thickened into a ring called the 'transverse band,' or 'sphincter of the antrum.' In the living stomach the region of the transverse band is usually contracted so strongly and continuously that a distinct groove is seen to separate the tubular antrum from the bag-like cardiac end. The suggestion of a massive constricting ring of muscle is belied by an examination of the dead viscus. The transverse band is really little more than a pliysiological sphincter. The empty stomach is contracted and at rest. A few minutes after food is taken contractions begin in the antrum, and run on in constricting undulations (in the cat at the rate of six in the minute) towards the pyloric sphincter. Each wave takes about twenty seconds (in the cat) to pass from the middle of the stomach to the pylorus. Fecble at first, they become stronger and stronger as digestion proceeds, and gradually come to involve the portion of the fundus next the splincter of the antrum, but their direction is always towards the pylorus, never, in normal digestion, away from it. The food is thus subjected to energetic churning movements in the pyloric end of the stomach, and worked up thoroughly with the gastric juice. Kept in constant circulation, it gradually becomes reduced to a semi-liquid mass, the chyme, which is at intervals driven against the pylorus by strong and regular peristaltic contractions of the lower end of the stomach,

* Here 'fundus ' is used in the sense in which it is generally employed in speaking of the stomach of the dog oi cat as signifying the wnole of the organ with the exception of the antruin pyıri. By the fundus of the human stomach most writers mean only the cul-'ie-sac at the cardiac end; the portion intervening between it and the a:acrum pylori is often terms 1 the boly of the stomach. 
the sphincter relaxing from time to time by a reflex inhibition to admit the better-digested portions into the duodenum, but tightening more stubbornly at the impact of a hard and undigested morsel. The nature, as well as the consistence of the food, influences the length of its sojourn in the stomach. Carbo-hydrate food passes more rapidly through the pylorus than fatty food, and fat more rapidly than protein. The reason is that the acidity of the gastric juice varies with the different kinds of food, hydrochloric acid being secreted in abundance in the presence of proteins, and to a much smaller extent in the presence of fats and carbo-hydrates. Now, dilute hydrochloric acid when introduced into the stomach remains there for a much longer time than water. This depends upon the fact that sucl portions of the acid as get into the duodenum stimulate afferent fibres in its mucous membrane, and so cause reflex spasm of the pyloric sphincter. When the acid chyme becomes neutralized to a certain point by the bile and pancreatic juice, inhibitory impulses pass up from the duodenum and cause the sphincter to relax. The cardiac division of the stomach, with the exception of the portion that borders the transverse band, takes no share in the peristaltic movements. And, indeed, it is far more difficult to cause such contractions by artificial stimulation in the fundus* than in the pylorus. The two portions of the stomach are partially, or in certain animals from time to time completely, cut off from each other by the contraction of the sphincter of the antrum. The fundus, so far as its mechanical functions are concerned, acts chiefly as a reservoir for the food, which, like a hopper, it gradually passes into

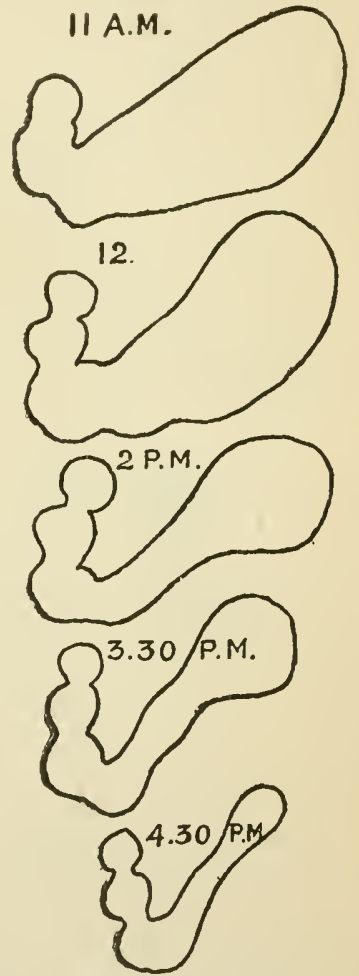

Fig. I50.-Cat's Stomach seen by Röntgen Rays (Cannon). The outlines of the stomach containing food mixed with bismuth subnitrate were drawn at intervals from I I a.m. to 4.30 p.m.

the pyloric mill as digestion goes on by a tonic contraction of its walls. The existence of this reservoir enables larger quantities of food to be taken at one meal, which can then be digested gradually.

* The common idea, however, that the fundus is completely inactive is erroneous. Rhythmical variations in tone with a period of $\mathrm{I}$ to 3 minutes have been observed during digestion. In hunger vigorous peristaltic contractions sweep over the whole stomach, beginning at the cardiac end. 
These facts lave been mainly ascertained by observations on animals, such as the dog and the cat, wither by clirect inspection after opening the abdomen (Rossbach), or in the intact body, under absolutely physiological conditions, by means of the Röntgen rays (Cannon). In the latter method the food is mixed with subnitrate of bismuth, which is opaque to these rays, so that when the animal is looked at through a fluorescent screen the stomach appears as a dark shadow in the field (lig. I50). This method has even been atpplied with success to the study of the passage of the food along the luman alimentary canal from deglutition to defacation (Hertz). It has been shown in this way that in the living body in the erect position the long axis of the stomach is much more nearly vertical than had been supposed. When food is taken it sinks into the lower (pyloric) end, and at the upper end gas collects.

When the person lies down the lower end of the stomach passes more towards the left, so that the long axis lies more transversely. Other methods have thrown light on the gastric movements- - .g., direct inspection through a fistula of the stomach, and the study

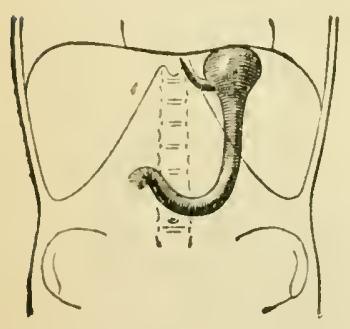

$a$

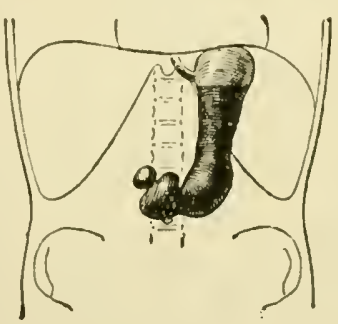

$b$

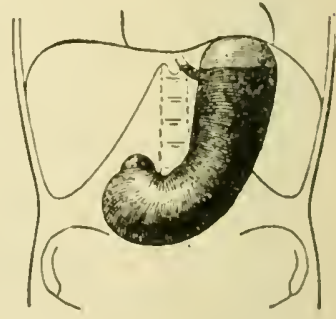

$c$

Fig. I5I.-Human Stomach studied by Rüntgen Rays. a. Empty stomach in ver. tical pusitjon; $b$, shortly after a meal (peristaltir contrartious are occurring at the pyloric end); $c$, full stomach in vertical position. (Halliburton after Hertz.)

of records showing the changes of pressure in the viscus obtained by means of small balloons introduced into it. Such balloons attached to a rubber tube liave been swallowed by normal men and kept for long periods in the stomach (Carlson). Even in the excised stomach, kept in salt solution at the body-temperature, the typical movements can be observed proceeding for some time.

Movements of the Small Intestine.-In the small intestine two kinds of movements are to be seen: (1) Gentle, swaying, 'pendulum ' movements, sometimes irregular, but often recurring rhytlimically. at the rate (in the $\mathrm{dog}$ ) of 10 or 12 in the minute. Both the longitudinal and the circular muscular coats contract, causing slight waves of constriction, which may originate at any part of the gut, but, under normal circumstances, nearly always travel from above downwards, with a velocity of 2 to 5 centimetres per second. These movements cause the coils of the intestine to swily gently from side 
to side. Ender abnormal conditions, as in the exposed 'surviving intestines of the rabbit, contractions, probably similar to the pendulum movements, but rumning indifferently in buth directions, can be set up by local stimulation. The function of these pendulum movements seems to be the thorough mixing of the food with the digestive juices in the intestine. When an animal is fed with food containing bismuth subnitrate and observed with the liöntgen rays, it is seen that the food in a coil is often divided into small segments, which then join together to form longer masses, these being in turn again divided. This segmentation is rhythnitically repeated (in the cat at the rate of thirty times a minute). Although of itself it insures only the mixing of the contents of the gut, and not their onward progress, it is usually accompanied by peristalsis, so that while the food is undergoing segmentation it is also slowly passing down the intestine. Often, however, a column of food remains for a considerable time, dividing, uniting, and dividing again, without sensibly shifting its position. In addition to the relatively rapid pendulum movements, much slower periodic variations of tone of the whole musculature may be normally observed.

(2) True peristaltic movements, in which a ring of constriction, obliterating the lumen, moves slowly down the tube, with a speed, it may bc, no greater than I $\mathrm{mm}$. per second. The portion of the intestine immediately below the advancing constriction is relaxed and motionless, so that we may say that a wave of inhibition precedes the wave of contraction. The peristaltic movements of the small intestine, the most typical of their kind, are most easily excited by mechanical stimulation of the mucous membrane, as by the contact of a morsel of food or an artificial bolus of cottonwool. Travelling, under normal conditions, always downwards, the constriction squeezes the contents of the tube before it, and the wave usually ends at the ileo-cæcal valve, which separates the small intestine from the large. The cause of the definite direction of the peristaltic wave is grounded in the anatomical relations of the intestinal wall. For when a portion of the intestine is resected. turned round in its place and sutured, so that what was before its upper is now its lower end, the contraction wave is unable to pass, and the obstruction to the onward flow of the intestinal contents causes marked dilatation of the gut, and sometimes serious disturbance of nutrition. The most probable explanation is that the peristalsis is governed by a local reflex nervous mechanism (Auerbach's plexus), the stimulation of which by the contact of the food with the mucous membranc or by the distension of the gut causes excitation of the circular muscular fibres above the point of stimulation, and inlibition of them below it. The automatic pendulum movements, and also the slow, rhythmical variations of tone, have a different relation to the local nervous mechanism, for they behave differently to poisons like cocaine and nicotine, which act on that 
mechanism. The pendulum movements are, if anything, increased in intensity and made more regular. But the pe-istaltic waves, although they can be locally excited by direct stimulation of the muscular fibres, are no longer propagated, and a bolus introduced into the intestine remains at rest where it is placed. Some have interpreted these facts as indicating that the pendulum movements are myogenic in origin. But evidence has lately been obtained that, although they are not reflex movements elicited by afferent impulses from the mucous membrane, since they continue in unaltered intensity, in isolated loops of intestine immersed in Ringer's (or Locke's) solution (p. 66) after removal of both mucosa and submucosa, they are nevertheless dependent upon Auerbach's plexus. For when the circular muscular coat is separated from this plexus, the automatic movements of this coat are abolished, although the

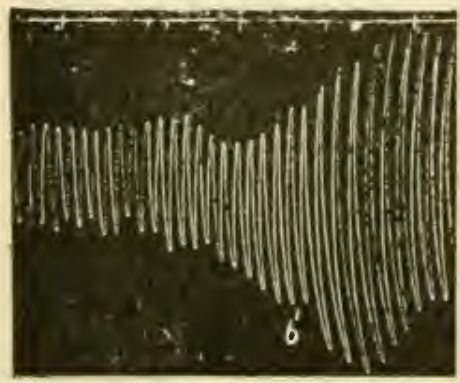

Fig. 152.-Intestine Segment beating in Ringer's Solution. At 6 the oxygen stream was increased. To be read from left to right. Time trace, half-minutes. (Reduced to one-half.) excitability of the musculature to direct stimulation is not affected. The longitudinal coat, which is still in connection with Auerbach's plexus, goes on contracting spontaneously (Magnus). Under certain conditions a movement of food or secretions in the reverse of the normal direction can be set up in the small intestine in the intact body-e.g., in the case of obstruction of the intestine leading to vomiting of its contents. But this does not necessarily indicate a reversal of the normal direction of the peristalsis. Such a reversal, if it occurs at all, is not easy to realize by artificial stimulation, and even when an antiperistaltic wave is apparently started, it travels up the intestine only for a short distance and then dies out. A third variety of intestinal movement has sometimes been described. the so-called 'peristaltic rush' (Meltzer, etc.). It consists of a rapidly moving peristaltic contraction, preceded by relaxation of a long portion of the tube. Such a contraction may even sweep down without pause from the duodenum to the end of the ileum.

The Movements of the Large Intestine differ from those of the small mainly in the great frequency of antiperistalsis. This, indeed, seems to be the usual movement of the transverse and ascending colon. The antiperistalsis recurs in periods about every fifteen minutes, and each period generally lasts about five minutes. The constrictions, running towards the cæcum, thoroughly churn and mix the remnants of the food, a considerable absorption of which may take place in the upper part of the large intestine. Regurgitation into the ileum in man is prevented partly by the oblique entry 
of the ileum through the wall of the colon (so-called ileo-cæcal valve), but essentially by the tonic contraction of the ileo-colic sphincter. The sphincter usually permits the passage of material only in the direction from small to large intestine. But as an occasional phenomenon, a reverse movement may occur. Thus food may actually pass back through the ileo-colic sphincter into the small intestine under the action of a long-continued and vigorous antiperistalsis, and in this way a considerable portion of a bulky enema may be eventually disposed of (Cannon). This so-called antiperistalsis is not precisely the same kind of movement, leaving out of account its direction, as the peristalsis already described in the small intestine, since it is not preceded by a wave of inhibition. True peristaltic contractions preceded by relaxation of the gut may also be observed to start in the cæcum, and to travel down the large intestine. They are not very frequent in comparison with those of the small intestine, and they die away before reaching the end of the colon, allowing the food to be driven back again towards the cæcum by the antiperistalsis. A true downward peristalsis is more commonly seen in the descending colon, and is here associated with the propulsion and collection of the fæces, which are mainly stored in the sigmoid flexure. These peristaltic contractions do not normally reach the rectum, which, except during defæcation, remains at rest.

Influence of the Central Nervous System on the Gastro-Intestinal Movements.-As we have already said, these movements are much less closely dependent on the central nervous system than are those of the œsophagus. They can not only go on, but are in general better marked when the extrinsic nervous connections are cut; they cannot spread when the continuity of the tube is destroyed, and the mere presence of food will excite them when other than local reflex action has been excluded by section of the nerves. Nevertheless, the central nervous system does exercise some influence in the way of regulation and control, if not in the way of direct initiation of the movements, and the swallowing or even the smell of food has been observed to strengthen the contractions of a loop of intestine severed from the rest, but with its nerves still intact. The vagus is the efferent channel of this reflex action: stimulation of its peripheral end may cause movements of all parts of the alimentary canal from œsophagus to large intestine, and may strengthen movements already going on; but section of it does not stop them, nor hinder the food from causing peristalsis wherever it comes. The vagus also contains inhibitory fibres for the lower end of the œsophagus and the whole of the stomach. Stimulation of it is followed first by inhibition, and then, after an interval, by an increase of tone and augmentation of the contraction of the whole stomach, including the cardiac and pyloric sphincters. The 
splanchnic nerves contain fibres by which the intestinal movements can be inhibited, and they appear to be always in action, for after section of these nerves the movements are strengthened. On the other hand, stimulation of the peripheral end of the cut splanchnic causes arrest of the movements. Occasionally, however, it has the opposite effect. Contractions of the small intestine are more easily caused by excitation of the vagus after the inhibitory splanchnic nerves have been cut. The splanchnics also contain inhibitory fibres for the stomach, and it is only when these are intact that complete reflex inhibition of the organ can be obtained in the rabbit (Auer). The gastric movements are not permanently affected by section of these nerves alone, or even by simultaneous section of the splanchnics and the gastric branches of the vagi. But if the vagi are cut while the splanchnics remain intact, the peristalsis of the stomach is weakened, its onset delayed, and the proper emptying of the viscus through the pylorus interfered with. In all probability these results are due to the uncontrolled action of the inhibitory libres. The splanchnics have a special relation to the ileo-colic sphincter, which closes when they are stimulated, and becomes insufficient when they are cut. The vagus does not affect it.

The lower part of the large intestine is influenced by the sacral nerves (second, third, and fourth sacral in the rabbit), and by certain lumbar nerves, in the same way as the higher parts of the alimentary canal, and particularly the small intestine, are influenced by the vagus and the splanchnics. Stimulation of these sacral nerves within the spinal canal, or of the pelvic nerves (nervi erigentes) into which they pass, causes contraction of the parts of the large intestine concerned in defæcation - that is, in the dog, of the whole colon, with the exception of the cæcum; in the cat, of the distal two-thirds of the colon. The colon first undergoes rapid shortening due to the contraction of the longitudinal fibres and the recto-coccygeus muscle. After a few seconds this is followed by contraction of the circular fibres, beginning at the lower limit of the region in which antiperistalsis can occur, and spreading downwards, so as to empty the portion of the bowel involved in the contraction. This is a very close imitation of what occurs in natural defæcation. In man the parts involved in these movements are probably the sigmoid flexure and rectum. In addition to these characteristic motor effects on the lower part of the large intestine, stimulation of the pelvic nerves causes an increase in the antiperistalsis of its upper portions. Stimulation of the lumbar nerves or of the portions of the sympathetic into which their visceral fibres pass lumbar sympathetic chain from second to sixtl ganglia, or the rami from it to the inferior mesenteric ganglia) causes inlibition of the movements of the cæcum and the whole colon, including the antiperistaltic movements. Excitation of the sacral nerves initiates or increases the contraction of both coats of the portions of the large intestine on whicli they act, excitation of the lumbar nerves inhibits both. And in the small intestine the same law holds good; the two coats are contracted together by the action of the vagus or inhibited together by that of the splanchnics.

Defæcation is partly a voluntary and partly a reflex act. But in the infant the voluntary control has not yet been developed; 
in the adult it may be lost by discase; in an animal it may be abolished by operation, and in each case the action becomes wholly reflex. Owing to the tonic contraction of the rectum and the acute angle formed at the pelvi-rectal flexure, the faces are arrested at this point. In consequence the pelvic colon becomes filled with freces from below upwards, and the rectum remains empty till immediately before defacation. This has been verified in man by observations with the Röntgen rays (1 Lertz). In persons whose bowels are opened regularly after breakfast, the passage of faeces into the rectum gives rise to the characteristic sensation which may be termed the "call to defacation.' It is the distension of the rectum, and of the rectum alone, which is associated with this sensation, for in persons from whom the entire rectum lias been removed for malignant disease the sensation is absent, and it may be elicited by artificially distending the rectum, though not any other part of the alimentary canal. The minimum pressure required to elicit the sensation is smaller the greater the length of the gut exposed to it, varying in one individual from 32 to $48 \mathrm{~mm}$. of mercury, according to the length of a balloon introduced into the rectum. The passage of the fres from the pelvic colon into the rectum is due to the discharge of that reflex contraction of the lower portion of the bowel already described (p. 332), of which the pelvic nerves constitute the efferent path. This reflex peristalsis is elicited by various causes, among which one of the most important is the taking of food at bieakfast into the empty stomacli, and another the muscular activity associated with getting up and dressing. The desire to defæcate may for a time be resisted by the will, or it may be yielded to. In the latter case the abdominal muscles, and, according to Hertz, the diaphragm also, are forcibly contracted; and the glottis being closed, the whole effect of their contraction is expended in raising the pressure within the abdomen and pelvis, and so aiding the muscular wall of the bowel itself in driving the fæces from the sigmoid flexure to the rectum. The two sphincters which close the anus-the internal sphincter of smooth muscle, and the external of striated-are now relaxed by the inhibition of a centre in the lumbar portion of the spinal cord, through the activity of which the tonic contraction of the sphincters is normally maintained. This relaxation is partly voluntary, the impulses that come from the brain acting probably through the medium of the lumbar centre. But in the dog, after section of the cord in the dorsal region, the whole act of defæcation, including contraction of the abdominal muscles and relaxation of the sphincters, still takes place, and here the process must be purely reflex. Even after complete destruction of the lumbar and sacral portions of the spinal cord the tone of the sphincters returns after a time, and defæcation is carried on as in a normal animal, the control of the sphincters 
being due either to a property of the muscular tissue itself or to local ganglia. The contraction of the levatores ani helps to resist overdistension of the pelvic floor and to pull the anus up over the fæces as they escape. The nervi erigentes carry efferent constrictor fibres, and the hypogastrics, as a rule, efferent dilator fibres, to the splincters. While the internal sphincter is by itself capable of maintaining a tonus of considerable strength, the external sphincter contributes an important share (30 to 60 per cent.) to the closure of the rectum. If the call to defacation is neglected, the desire passes away. This is not due to the fxes being carried back into the pelvic colon by antiperistalsis, as has generally been stated. The fæces which have passed into the rectum remain there, as can be shown by examination with the finger after the desire to empty the bowels has disappeared. The reason for the disappearance of the sensation is the relaxation of tone which occurs in the muscular coat of the rectum after a period of distension. It is not till it has been again clistended by the entrance of a further portion of frees that the call to defæcation is again experienced. When the call is repeatedly neglected, the sensibility of the rectum to distension becomes blunted, and this is a common cause of constipation.

The time of passage of substances through the alimentary canal has been studied by administering collodion capsules filled with subnitrate of bismuth to human beings, and observing their progress by taking shadow pictures of them at intervals with the Röntgen rays. During the first twenty minutes two such capsules swallowed at the same time by a healthy young man were clearly scen in the greater curvature of the stomach, but in the interval between the first half-hour and the seventh or eighth hour no further trace of them was detected. About the eighth hour they reappeared in the cxecum, where they remained with little or no onward movement till the fourteenth hour. From the fourteenth to the sixteenth hour they travelled along the ascending colon, and tarried a long time at the left angle of the colon. From the nineteenth to the twenty-second or twenty-fourth hour they slowly passed downward in the descending colon, and stopped at the sigmoid flexure till their expulsion in defacation. In some subjects the entire passage of the capsules was complete in sixteen hours, in others not until after thirty hours. A one cent piece swallowed by a healthy child four years old was recovered in the fæces 52 hours later, and a button, slightly larger, swallowed by the same child, appeared after almost exactly the same interval.

Vomiting.-We have seen that under normal conditions the movements of the alimentary canal always tend to carry the food in one definite direction, along the tube from the mouth to the rectum. The peristaltic waves generally run only in this direction, and, further, regurgitation is prevented at three points by the 
cardiac and pyloric sphincters of the stomach and the ileo-colic sphincter and valve. But in certain circumstances the peristalsis may be reversed, one or more of the guarded orifices forced, and the onvard stream of the intestinal contents turned back. In obstruction of the bowel, the frecal contents of the large intestine may pass up beyond the ilco-crecal valve, and, reaching the stomach. be driven by an act of voniting through the cardiac orifice; in what is called a 'bilious attack,' the contents of the duodenum may pass back through the pylorus and be ejected in a sinilar way; or, what is by far the most common case, the contents of the stomach alone may be expelled.

Vomiting is ustually preceded by a feeling of nausea and a rapid secretion of saliva, which perhaps serves, by means of the air carricd down with it when swallowed, to dilate the cardiac orifice of the stomach, but may be a mere by-jlay of the reflex stimulation bringing about the act, since evidence of stimulation of other bulbar centres (vaso-motor and cardio-inhibitory) has also been obtained. The diaphragm is now forced down upon the abdominal viscera, first with open and then with closed glottis. The thoracic portion of the œsophagus is thus placed under diminished pressure, and therefore widened, while saliva and air are aspirated into it out of the mouth. The abdominal muscles strongly contract. At the same time the stomach itself, and particularly the antrum pylori, contracts, the cardiac orifice relaxes, and the gastric contents are slot up into the lax osophagus, and through it into the pharynx, and issue by the mouth or nose. The movements of the stomach during vomiting induced by apomorphine have been studied in the cat by the Röntgen ray method. There is first observed extreme relaxation of the cardiac end; then a deep constriction appears a little below the cardiac orifice, and runs towards the pylorus, increasing in depth as it goes. When the transverse band is reached, this contracts firmly and remains contracted, and the constriction passes on over the antrum pylori. Ten or twelve similar waves follow, at the end of which time the constriction in the region of the transverse band divides the stomach into the firmly-contracted antrum and the relaxed fundus. Now follows a sudden contraction of the diaphragm and abdominal muscles accompanied by the opening of the cardiac orifice. Either the diaphragm and abdominal muscles alone, without the stomach, or the diaphragm and stomach together, without the abdominal muscles, can carry out the act of vomiting. For an animal whose stomach has been replaced by a bladder filled with water can be made to romit by the administration of an emetic (Magendie); and Hilton saw that a man who lived fourtcen years after an injury to the spinal cord at the height of the sixtl cervical nerve, which caused complete paralysis below that level, could vomit, though with great difficulty. In a young child in which very slight causes 
will induce vomiting, the stomach alone contracts during the act. But in the adult such a contraction is ineffectual, and the same iy the case in animals, for a dog under the influence of a moderate dose of curara, which paralyzes the voluntary muscles but not the stomach, cannot vomit.

The nerve-centre is in the medulla oblongata. It may be excited by many afferent channels: the sensory nerves of the fauces or pharynx, of the stomach or intestines (as in strangulated hernia). of the liver or kidney (as in cases of gall-stone or renal calculi), of the uterus or ovary, and of the brain (as in cerebral tumour), are all capable, when irritated, of causing vomiting by impulses passing along them to the vomiting centre.

The vagus nerve in man certainly contains afferent fibres by the stimulation of which this centre can be excited, for it has been noticed that when the vagus was exposed in the neck in the course of an operation, the patient vomited whenever the nerve was touched (Boinet, quoted by Gowers). In meningitis, vomiting is often a prominent symptom, and is sometimes due to irritation of the vagus nerve by the inflammatory process.

Some drugs act as emetics by irritating surfaces in which efficient afferent impulses may be set up, the gastric mucous membrane, for example; sulphate of zinc and sulphate of copper act mainly in this way. Apomorphine, on the other hand, stimulates the centre directly, and this is also the mode in which vomiting is produced in certain diseases of the medulla oblongata. The efferent nerves for the diaphragm are the phrenics, for the abdominal muscles the intercostals. The impulses which cause contraction of the stomach pass along the vagi. Dilatation of the cardiac orifice is brought about by the inhibitory fibres in the vagus already mentioned.

\section{Section III.-The Chemistry of the / igestive Juices.}

Ferments.-The chemical changes wrought in the food as it passes along the alimentary canal are due to the secretions of various glands which line its cavities or pour their juices into it through special ducts. These secretions owe their power for the most part to substances present in them in very small amount, but which, nevertheless, act with extraordinary encrgy upon the various constituents of the food, causing profound changes without, upon the whole, being themselves used up, or their digestive power affected. The active agents are the enzymes, sometimes. spoken of as unformed or unorganized ferments-unorganized because their action does not depend upon the growth of living cells, which was long supposed to be the case for some other ferments, such as yeast. Since it has been shown that specific enzymes can be separated from cells which were formerly believed to act 
by their mere growth, the distinction between formed and unformed ferments has lost its significance, and has to a great extent been superseded by the distinction between intra- and extra-cellular enzymes (also called cudo- and exo-enzymes) - i.e., between ferments which normally act in the interior of the cells where they are produced and ferments which act outsidc of the cells that secrete them. From yeast cultures, for instance, by crushing the cells, a ferment (zymase*) can be obtained which in the complete absence of living reast-cells, and, indeed, of any living micro-organism, forms alcohol and carbon dioxide from sugar, just as living yeast does. There is every reason to believe that it is by the intracellular action of this endoenzyme that the yeast-cell normally causes alcoholic fermentation. The digestive ferments are typical extracellular enzymes. Their chemical nature has not been exactly made out; some of them at least do not appear to be proteins, or to contain a protcin group. Many of them apparently exist in the colloidal condition, although this has not been shown for all. In certain cases the more or less stable union of a definite inorganic substance with the ferment, or its actual inclusion in the ferment molecule, seems to be a condition of its action. Thus there is reason to believe that in gastric digestion hydrochloric acid is loosely combined with the pepsin. In the plant oxydase, laccase (p. 272), manganese is present. And the fact that manganese salts oxidize certain substances as laccase does suggests that it is the manganese in combination with some protein or other organic compound in the ferment molecule which confeîs upon laccase its oxidizing power. A similar relation between iron and some animal oxydases is possible, though not definitely proved. But none of the ferments of the digestive juices has as yet been satisfactorily isolated, and at present it is only by their effects that we recognize them. The difficulty of isolating them is increased by the fact that, like other colloids, they readily adhere to surfaces, and are carried down by the most diverse precipitates of substances to which they are chemically indifferent. On the other hand, this very property is taken advantage of to procure more concentrated, although still impure, solutions of them than cxist in the natural secretions. Thus in the preparation of many ferments the first step is to produce an inert precipitate, such as calcinm phosphate, in the juice or extract. Some of the ferments act best in an alkaline, some in an acid medium. They all agree in having an 'optimum' temperature, which is more favourable to their action than any other; a low temperature suspends their activity, and boiling abolishes it for ever. The optimum temperatures of the majority of enzymes lie between

* Ferments are usually designated by names with the termination ' ase,' and indicating the kind of substances on which they act, or sometimes their source. Thus proteases are ferments acting on proteins, amylases ferments acting on starch, etc. 
$37^{\circ}$ and $53^{\circ} \mathrm{C}$; the ' killing' temperatures between $60^{\circ}$ and $75^{\circ} \mathrm{C}$. when they are heated in solutions, but considerably higher when they are heated dry. The action of the digestive enzymes is hydrolytic-i.e., it is accompanied with the taking up of the elements of water by the substance acted upon. The accumulation of the products of the action first checks and then arrests it. In many calses this seems to be due to combination of the ferment with one or other of the end products, and the consequent segregation of the ferment from the reaction mixture. The enzyme is not affected indiscriminately by any of the end products. On the contrary, their action is curiously selective. Thus the hydrolysis of lactose by lactase is retarded by galactuse, but not by the other end product dextrose. The hydrolysis of cane-sugar by invertase is retarded by levulose, but not by dextrose. The splitting of the dipeptid (p. 2) glycyl-l-tyrosin by a ferment in the expressed juice of yeast-cells is greatly delayed by one of the products ( $l$-tyrosin), but not by the other (glycocoll). Combination of the ferment with an end product is not, however, the only way in which the reaction may stop before the whole of the substrate, as the substance acted on by the ferment is termed, has been changed. It has been demonstrated in some cases that this is due to the action of the enzymes being reversible. For example, lipase (p. 363) not only decomposes the esters ethyl butyrate or glycerin butyrate, but also builds them up again from the decomposition products-ethyl butyrate from ethyl alcohol and butyric acid, glycerin butyrate from glycerin and butyric acid (Kastle and Loevenhart, Hanriot). Thus:

$$
\underset{3}{\mathrm{C}_{3} \mathrm{H}_{7} \mathrm{COOC}_{2} \mathrm{H}_{5}}+\underset{\text { Withyl butyrate. }}{\mathrm{H}_{2} \mathrm{O}} \underset{\text { Water. }}{\longrightarrow} \underset{3}{\mathrm{C}_{3} \mathrm{H}_{7} \mathrm{COOH}}+\underset{\text { Butyric acid. Ethyl alcohol. }}{\mathrm{C}_{2} \mathrm{H}_{5} \mathrm{OH} .}
$$

The action of the enzyme is merely to accelerate the establishment of the proportions in which the four bodies entering into the reaction are in equilibrium, and the point of equilibrium is the same whether we start from one or the other side of the equation representing the reaction. Such reversible reactions in the presence of enzymes seem to afford the key to the explanation of many of the syntheses which are known to occur in the body. Sometimes the action is not strictly reversible in the sense that precisely the original material is reconstructed, but from the products of the hydrolysis substances are synthesized or condensed, which are then incapable of being split by the ferment. When a concentrated solution of dextrose is acted on for a long time by yeast maltase, a ferment obtained from yeast which changes maltose into dextrose, some of the dextrose is reconverted into isomaltose and dextrin-like bodies. Isomaltose is not again hydrolysed by maltase. The ferment emulsin contained in almonds behaves in the converse way. It liydrolyses isomaltose so as to form dextrose, and then condenses dextrose to maltuse (Armstrong). 
Many of the ordinary substances of the laboratory will accelerate a reaction which goes on slowly in their absence. These are called catalysers. Some writers also speak of catalysers which retard a reaction progressing quickly in their absence. The process by which the reaction is accelerated (or retarded) is termed catalysis. A typical catalyser can exert its action when it is present in exceedingly small amount in comparison with the substance acted upon. However it may enter into the reaction, it does not take part in the formation of the final products nor contribute to the energy changes, and for this reason is often apparently unaltered at the end of the process. The catalysers have therefore been compared to the lubricants used for machinery as contrasted with the coal or other source of energy. If it be remembered that the expression is a purely metaphorical one, we may say that the catalyst oils the reaction so that it slips on smoothly and swiftly to an end-point which would, however, have been reached just the same in time. A classical instance of catalysis is the inversion of cane-sugar by weak acids, i.e., the change of the cane-sugar into a mixture of equal quantities of dextrose and levulose-a reaction which may be represented by the equation

$$
\underset{\text { Cane-sugar. Water. Dextrose. }}{\mathrm{C}_{12} \mathrm{H}_{22} \mathrm{O}_{11}}+\underset{\mathrm{H}_{2} \mathrm{O}}{\mathrm{H}_{6} \mathrm{H}_{12} \mathrm{O}_{6}}+\underset{\mathrm{C}_{6} \mathrm{H}_{12} \mathrm{O}_{6} \text {. }}{\mathrm{C}_{2}}
$$

This is a reaction which occurs also when the sugar is simply dissolved in water, but with extreme slowness at the ordinary temperature, although more rapidly at $100^{\circ} \mathrm{C}$. The effect of the acid is to catalyse the reaction, to markedly accelerate it. The hydrogen ions of the free acid are responsible for the catalysis, and they are not used up in the process, for the reaction at the end is unaltered. The same action upon cane-sugar is exerted by an enzyme, invertase, found in intestinal juice, although the laws governing the reaction are somewhat different. Reversibility of the reaction can be even more clearly demonstrated for catalysers than for enzymes. For example, the condensation of acetone to diacetone-alcohol, which is accelerated by hydroxyl ions (as by the addition of sodium hydroxide, ammonia, etc.), only proceeds to a certain point, at which equilibrium is established between the proportions of acetone and the condensation product. Henceforth as much of the latter is decomposed as is condensed. Thus:

\section{${ }_{2} \mathrm{CH}_{3} \cdot \mathrm{CO} \cdot \mathrm{CH}_{3} \rightleftarrows \mathrm{CH}_{3} \cdot \mathrm{CO} \cdot \mathrm{CH}_{2} \cdot \mathrm{C}\left(\mathrm{CH}_{3}\right)_{2} \mathrm{OH}$. \\ Acetone. \\ Acetone alcohol.}

On the other hand, the final equilibrium point need not be the same for a catalyser and an enzyme. For example, amyl butyrate is formed and decomposed according to the equation

$$
\underset{\text { Amyl alcohol. }}{\mathrm{C}_{5} \mathrm{H}_{11} \mathrm{OH}}+\underset{\text { Butyric acid. }}{\mathrm{C}_{3} \mathrm{H}_{7} \mathrm{COOH}} \underset{\text { Amyl butyrate }}{\mathrm{C}_{3} \mathrm{H}_{7} \mathrm{COO} . \mathrm{C}_{5}} \mathrm{H}_{11}+\underset{\text { Water. }}{\mathrm{H}_{2} \mathrm{O} .}
$$


The reaction can be accelerated either by a catalyst-e.g., H ionsas by addition of free hydrochloric or picric acid, or by pancreas lipase. When the concentrations of the reacting substances are appropriately chosen, the same equilibrium point will be reached from either side of the equation-i.e., the same percentage of the butyric acid will be converted into the ester if we start with the alcohol and acid, as will remain combined as ester if we start with the amyl butyrate. But the proportion will not be the same when the reaction is acclerated by $H+$ as when it is accelerated by the enzyme. And although it is probable that there is no fundamental difference between the action of the digestive enzymes and that of the inorganic catalysers, it is much too early to dogmatize.

Not even the $m$ rkedly specific action of the digestive ferments can be considered an essential distinction. It is true that invertase will act upon dextrose, and not at all upon maltose or lactase. But there are other sugars, e.g., raffinose, a trisaccharide with the formula $\mathrm{C}_{18} \mathrm{H}_{32} \mathrm{O}_{16}$, obtained from beet-sugar residues, which it will hydrolyse. Raffinose is made up of one molecule each of dextrose, levulose, and galactose. On heating with dilute acids, it is decomposed into these substances. Invertase, however, only splits off the levulose molecule, leaving a disaccharide isomeric, but not identical with lactose. Similarly lactase, which is without action upon cane-sugar or maltose, will hydrolyse the $\beta$-galactosicles, and maltase, inert as regards cane-sugar or lactose, will hydrolyse the a-glucosides. On the other hand, emulsin decomposes the $\hat{\beta}$-glucosides, to which group most of the natural glucosides belong, as well as the $\beta$-galactosides and lactose. From raffinose emulsin splits off galactose, leaving cane-sugar. Since the $a$ and $\beta$ compounds are isomeric, and differ not in their composition but in their structure, it has been concluded that the structure of the molecule of a substance must be related to the structure of the enzyme which can act on it, in some sucl way as a lock is related to its proper key. Thus the key lactase fits in the lock lactose, but not in the lock dextrose or the lozk maltose. Although the same specificity is not to be observed in the action of catalysers as in the action of enzymes, it is not difficult to find many instances in which inorganic substances show a marked limitation of their catalytic effects to particular reastions. Thus hydriodic acid is slowly oxidized in presence of liydrogen peroxide, with formation of iodine and water. This reaction is accelerated by the addition of many substances, c.g., tungstic acid. But tungstic acid has no catalytic effect on the oxidation of hydriodic acid by bromic acid.

The existence of an optimum temperature for ferment action, above which it rapidly decreases, and eventually comes to a complete stop, is also in all probability only a superficial distinction between enzymes and catalysers. For enzymes are casily altered, 
or even destroyed, at temperatures which very likely would favour their action were they as thermostable as the majority of eatalysing agents. And inorganic catalysts are known which also show the phenomenom of an optimum temperature depending on changes produced in their physical condition when the temperature is raised above this point. Thus a colloidal solution (or 'sol,' as it is called) of platinum, prepared by passing electric sparks between two platinum electrodes immersed in distilled water, and containing the metal in the form of ultra-microscopic particles, acts as a catalyser of a number of reactions. As the temperature is increased Ip to a certain 'optimum,' the velocity of the catalysed reaction is increased. But beyoud this, as the boiling-point is approached, the colloidal platinum is precipitated, and ceases to influence the reaction.

As to the manner in which an enzyme increases the velocity of its appropriate reaction, it is not easy to make any very positive statement. Several possibilities are recognized, of which two have been especially discussed. (I) The existence of the enzyme in colloidal solution may be important. It is characteristic of colloidal solutions, in which the dissolved substance is present in the form of extremely fine particles, that the total surface of the particles is very great in proportion to the mass of the substance in solution. Thus, a spliere of about the same volume as the eyeball, with a diameter of, say, 2 centimetres, would have a surface of $12^{\circ} 5$ square centimetres. If this material were subdivided into spheres of about the same volume as a leucocyte, with a diameter of, say, Io $\mu$, it would form eight thousand million of these spheres, with a total surface of over $2 \frac{1}{2}$ square metres. If the small spheres were further subdivided into spherical particles, with a diameter only the thousandth part of that of a leucocyte, say $\frac{\mu}{100}$, each would form a thousand million of these particles, and the total surface of all the particles would be about 2,500 square metres.

Now, it is known that the intensity of action of some of the inorganic catalysers is proportional to the surface exposed. For example, hydrogen peroxide, if left to itself, is slowly decomposed into water and oxygen. The addition of finely divided platinum, in the form of platinum black, greatly hastens the decomposition, and the oxygen bubbles off. The colloidal platinum sol is still more effective. The nature of the surface effect is not entirely clear. One factor has been thought to be an increase in the concentration of dissolved substances or condensation of gases at the surface, and the better opportunity for mutual action thus afforded to the ferment and the substrate. The great extension of the surface cannot be the only factor in the catalysis; otherwise any fine powder or suspension would have a catalytic action. But kaolin, or fine sand, or colloidal solutions of ordinary proteins or gelatin, have little, if any, effect on the decomposition of hydrogen peroxide.

(2) Enzymes may produce their effects by contributing to the formation of bodies intermediate between the substrate and the endproducts. If the time required for the formation of a given quantity of the intermediate compound and the time required for the decomposition of this compound into the final products of the ferment action are in sum less than the time required for the direct cliange of the 
substrate into the end-products, the enzyme will clearly act as a catalyser of the reaction. It has becn shown that in the case of certain inorganic catalysers this docs occur. Thus, in the oxidatior of hydriodic acid by hydrogen peroxide, which has been already referred to, molybdic acid has the power of acting as a catalyser. It has been proved that the reaction occurs in two stages, permolybdic acid being first formed by the action of the peroxide on inolybdic acid. The permolybdic acid then acts on hydriodic acid, producing iodine and water, and being itself reduced again to molybdic acid, which therefore comes out at the end of the reaction unchanged. The velocity of the double reaction is much greater than that of the direct oxidation of hydriodic acid by hydrogen peroxide.

There is evidence that the ferment actually combines with the substrate, the combination then breaking up to form the end-products. For instance, it has been shown that the amount of lactose hydrolysed by lactase in a given time, when the ferment is present in very small (juantity in comparison with the substrate, is proportional to the concentration of the ferment. and independent of the concentration of the lactose. Also with a given small concentration of ferment the amount of lactose hydrolysed is at first the sanie for successive equal intervals of time. These facts can only be cxplained by the assumption that the ferment first combines witlı a portion of the substrate, the rest of which remains inactive as regards the reaction, and that this combination then takes up water and decomposes into the endproducts, in this case dextrose and galactose, setting free the ferment to combine with another portion of the substrate.

The Quantitative Estimation of Ferment Action.-Since we have as yet no certain method of freeing the digestive ferments from impurities, our only quantitative test is their digestive activity. And since a very small quantity of ferment can act upon a practically indefinite amount of material if allowed sufficient time, we can only make comparisons when the time of digestion and all other conditions are the same. If we find that a given quantity of one gastric extract, acting on a given weight of fibrin, dissolves it in half the time required by an equal amount of another gastric extract, or dissolves twice as much of it in a given time, we conclude that the digestive activity of the pepsin is twice as great in the first extract as in the second. But this does not permit us to say that the onc contains twice as much pepsin as the other. For it has been found that the amount of digestion in a given time is not directly proportional to the quantity of ferment present, but to the square root of the quantity of ferment (Schütz's law). This law was deduced by Schütz for pepsin, but is said to hold also for trypsin, steapsin, and ptyalin (Pawlow, Vernon). To determine the amount of proteolysis the nitrogen of the protein which has gone into solution may be estimated (p. 52I). The following table shows the results of one cxperiment:

\begin{tabular}{|c|c|c|}
\hline \multirow{2}{*}{$\begin{array}{c}\text { Pepsin Solution used } \\
\text { in C.C. }\end{array}$} & \multicolumn{2}{|c|}{ Digested Nitrogen in Grammes. } \\
\cline { 2 - 3 } & Found. & Calculated. \\
\hline I & 0.0230 & 0.0223 \\
4 & $0^{\circ} 0427$ & $0^{\circ} 0440$ \\
9 & 0.0686 & 0.0669 \\
16 & 0.0889 & 0.0892 \\
\hline
\end{tabular}


Or a piece of a glass capillary-tube filled with licat-coagulated egg-white may be cut off and pliced in the digestive mixture (Mett's tubes). At the end of the period of digestion the lengtli of the piece of tube and that of the undigested remnant of the column of coagulated protein are measured with a millimetre scale under a low-power microscope. The difference gives the length of the column digested. If I c.c. of gastric juice caused in a given time digestion of $2 \mathrm{~mm}$. of the egg-white, 4 C.c. of the same juice would digest in the same time and under identical conditions about $4 \mathrm{~mm}$., and 9 c.c. about $6 \mathrm{~mm}$. As a test of the activity of a diastatic ferment, we take the amount of sugar formed in a given time in a given quantity of a standard starch solution. To determine the activity of a licuud, say, the pancreat ic juice, as regards fat-splitting ferment, the acidity of the emulsion fermed by the juice and fat after standing for a definite time at a given temperature (with occasional sliaking) can be estimated by titration with baryta solution.

In addition to the ferments of the digestive juices which act extracellularly in the lumen of the alimentary canal, and those which do thcir work intracellularly in its walls, micro-organisms are present in the gut, and even in normal digestion contribute to the changes brought about in the food; while under abnormal conditions they may awaken into troublesome, and even dangerous, activity. It is now known that these act by producing intracellular enzymes.

It may be noted here, althougl the subject must be again referred to (p. 390), that specific substances capable of inhibiting the action of ferments exist. Some of these antiferments are normally present in the body-an antitrypsin, for instance, in normal blood-serum. Numerous antiferments may be artificially obtained by inmmunizing animals with the original ferments. Thus an antilipase is found in the serum of rabbits after injection of pancreatic lipase, and an antiemulsin after injection of emulsin. Injection of rennin causes the formation of antirennin, which can be demonstrated in the blood-serum and milk of the immunized animal. Besides the antiferments, bodies, sometimes spoken of as 'co-enzymes,' are known which aid the action of certain enzymes, not in the general way in which, for instance, increase of temperature up to the optimun does, but in some quite special manner. Thus, as we shall see, bile salts greatly facilitate the fat-splitting action of lipase. This co-operation is not to be confounded with the activation of the proferment or zymogen which in many cases represents the inactive form of the enzyme, while it is still within the secreting cells. For, once activated, the fully formed enzyme cannot be made to revert to the zymogen stage. For example, the active trypsin of the pancreatic juice cannot be changed into inactive trypsinogen, whereas substances which simply co-operate or co-act with enzymes leave the latter unaltered when they are removed. Thus lipase does not preserve the increased activity conferred upon it by bile salts when the bile salts are again separated from the digestive mixture.

It is now necessary to consider in detail the nature of the various 
juices yickled by the digestive glands, and the inechanism of their secretion. Since it is along the digestive tract that glanclular action is seen on the greatest scale, this discussion will practically embrace the nature of secretion in general. And here it may be well to say that, although in describing digestion it is necessary to break it up into sections, a true view is only got when we look upon it as a single, though complex, process, one part of which fits into the other from beginning to end. It is, incleed, the business of the physiologist, wherever it is possible to insert a cannula into a duct and to drain off an unmixed secretion, to investigate the properties of each juice upon its own basis; but it must not be forgotten that in the body digestion is the joint result of the chemical work of five or six secretions, the grrater number of which are actually mixed together in the alimentary canal, and of the mechanical work of the gastrointestinal walls.

Saliva. - The saliva of the mouth is a mixture of the secretions of three large glands on each side, and of many small ones. The large glands are the parotid, which opens by Stenson's duct opposite the second upper molar tooth; the submaxillary, which opens by Wharton's duct under the tongue; and the sublingual, opening by a number of ducts near and into Wharton's. The small glands are scattered over the sides, floor, and roof of the mouth, and over the tongue.

Two types of salivary glands, the serous or albuminous and the mucous, are distinguished by structural characters and by the nature of their secretion; and the distinction has been extended to other glands. The parotid of many, if not all, mammals is a purely serous gland; it secretes a watery juice with a general resemblance in composition to dilute bloorl-serum. The submaxillary of the dog and cat is a typical mucous gland; its secretion is viscid, and contains mucin. The submaxillary gland of man is a mixed gland; mucous and serous alveoli, and even mucous and serous cells, are intermingled in it. The submaxillary of the rabbit is purely serous. The sublingual is, in general, a mixed gland, but witl far more mucous than serous alveoli. Some of the small glands are serous, others mucous in type.

The mixed saliva of man is a somewhat viscous, colourless liquid of low specific gravity (1002 to 1008, average about 1005), alkaline to litmus, acid to phenolphthalein, but when tested by the electrical method (p. 24) almost neutral. Besides water and salts, it contains mucin (entirely from the submaxillary, the sublingual and the small mucous glands of the mouth), to which its viscidity is due, traces of sermm-albumin and serum-globulin (chiefly from the parotid), and a ferment, which hyclrolyses starch, and therefore belongs to the group of amlyases or diastases. It differs somewhat from the amylase of pancreatic juice. But the small differences 


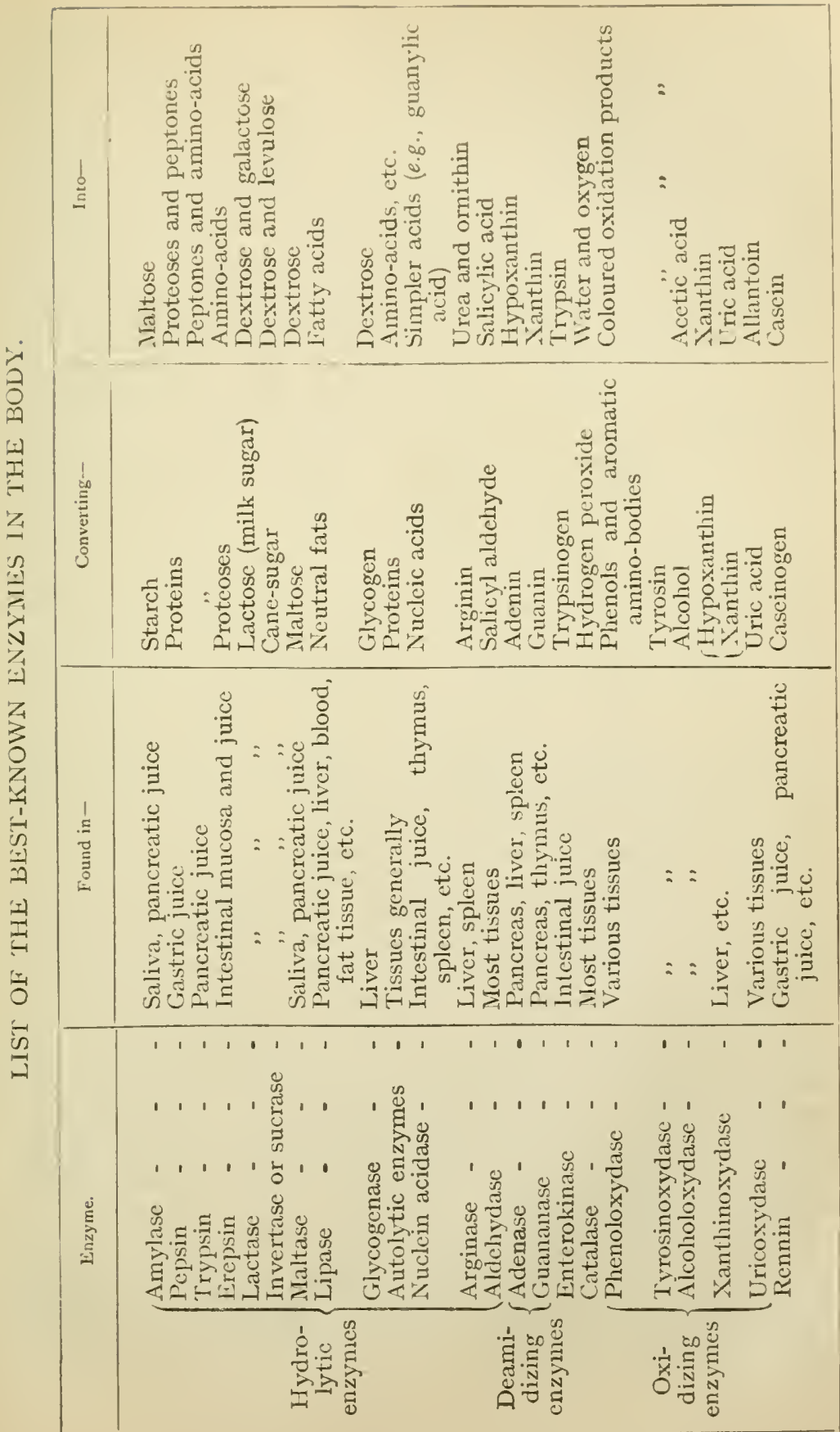


usually found between f(rments of the same kind derived from different sources may be due to the presence of other substances, and do not necessarily indicate that the ferments are distinct. For the present it may be assumed that the amylase of saliva is the same ferment encountered in the pancreatic juice, and in many, or all, of the tissues. An oxyclase or oxidizing ferment is also present in saliva. The salts are calcium carbonate and phosphate (often deposited as 'tartar' around the tecth, occasionally as salivary calculi in the glands and ducts), sodium bicarbonate, sodium and potassium chloride, and almost always a trace of sulphocyanide of potassium, detected by the red colour which it strikes with ferric chloride.* The total solids amount only to five or six parts in the thousand. A great deal of carbon dioxide can be pumped out from saliva, as much as 60 to 70 c.c. from 100 c.c. of the secretion-i.e., more than can be obtained from venous blood. Only a small proportion of this is in solution, the rest existing as carbonates. Oxygen is also present even in saliva which has not come into contact with the air, and, indeed, in somewhat greater quantity than in serum (about 0.6 volume per cent. in dog's saliva). Under the microscope epithelial scales, dead and swollen leucocytes (the so-called salivary corpuscles), bacteria, and portions of food, may be found. All these things are as accidental as the lastthey are mere flotsam and jetsam, washed by the saliva from the inside of the mouth. But greater significance attaches to certain peculiar bodies, either spherical or of irregular shape, that are seen in the viscid submaxillary saliva of the dog or cat. They appear to be masses of secreted material. The quantity of saliva secreted in the twenty-four hours varies a good deal. On an average it is from I to 2 litres (Practical Exercises, p. 454).

Besides its functions of dissolving sapid substances, and so allowing them to excite sensations of taste, of moistening the food for deglutition and the mouth for speech, and of cleansing the teeth after a meal, saliva, in virtue of its ferment, amylase, has the power of digesting starch and converting it into the disaccharide maltose, a reducing sugar $\left(\mathrm{C}_{12} \mathrm{H}_{22} \mathrm{O}_{11}\right)$. In man the secretion of any of the three great salivary glands has this power, although that of the parotid is most active. In the dog, on the other hand, parotid saliva has little action on starch, and submaxillary none at all; while in animals like the rat and the rabbit the parotid secretion is lighly active. In the horse, sheep, and ox, the saliva secreted by all the glands seems equally inert.

When starch is boiled, the granules are ruptured, and the starch

* In Ioo students the saliva only once failed to give the reaction, and in this individual a trace of sulphocyanicle was present 3 days later. It is absent from the saliva of many animals. In 25 dogs submaxillary saliva obtained by stimulation of the chorda tympani only once gave the ferric chloricle reaction, and then faintly. 
passes into colloidal solution, yielding an opalescent liquid. If a little saliva be added to some boiled starch solution which is free from sugar, and the mixture be set to digest at a suitable temperature (s.y $40^{\circ} \mathrm{C}$.), the solution in a very sliort time loses its opalesience and becomes clear. It still, however, givos the blue reaction witl iodine; and Trommer's test (p. Io) shows that no sugar has as yet been formed. Iater on the iodine reaction passes gradually through violet into red; and finally iodine causes no colour change at all, while maltose is found in large amount, along with some isomaltose (p. 338), a sugar having the same formula as maltose, but differing from it in the melting-point of the crystalline compound formed by it with phenyl-hydrazine (p. 525). Traces of dextrose, a sugar which rotates the plane of polarization less than maltose, but has greater reducing power, may be found among the end-products when the digestion is conducted in vitro. It is probable that this is produced from the maltose by another enzyme (maltase), present in small amount in saliva. In any case it is well known that maltose may be a stage in the hydrolysis of starch to dextrose, and can be detected among the intermediate products when the starch is acted on by dilute acid under conditions which permit a gradual decomposition of the fragments into which the polysaccharide molecule is successively split. But the observation has also been made that the saliva itself (in the cat) may contain a trace of dextrose (Carlson).

The red colour indicates the presence of a kind, or a group, of dextrins sometimes called erythrodextrin, because of this colour reaction; the violet colour shows that at first this is still mixed with some unchanged starch. Soon the dextrins which give the red colour disappear, and are succeeded by other dextrins, which give no colour with iodine, and are therefore called achrodextrins. These are partly, but in artificial digestion never completely, converted into maltose, and can always at the end be precipitated in greater or less amount by the addition of alcohol to the liquid. It is probable that a whole series of dextrins is formed during the digestion of starch. But little is known of their chemical nature. Recently, however, some of these intermediate bodies have been obtained in crystalline form. One of these appears to be a hexa-amylosei.e., it consists of $\operatorname{six} \mathrm{C}_{6} \mathrm{H}_{10} \mathrm{O}_{5}$ groups, and would therefore be capable of yielding, on the assumption of water, three molecules of maltose or six of dextrose. This is accordingly a relatively small fragment of the big original molecule of starch. When the sugar is removed as it is formed, as is approximately the case when the digestion is performed in a dialyser, the residue of unchanged dextrin is less than when the sugar is allowed to accumulate (Lea). In ordinary artificial digestion, for instance, under the most favourable circumstances at least I2 to I5 per cent. of the starch is left 
as dextrin; in dialyser digestions the residue of dextrin may be little more than 4 per cent. This goes far to explain the complete digestion of starch which takes place in the alimentary canal, a digestion so exhaustive that, although dextrins may be found in the stomach after a starchy meal, they do not occur in the intestine, or only in minute traces. Here the amylolytic ferment of the pancreatic juice, which is essentially the same in its action as the amylase of saliva, only more powerful, must effect a very complete conversion of the starch molecules accessible to its attack. It is not inconsistent with this, that unchanged starch granules may sometimes be excreted in the fæces, especially when imbedded in raw vegetable structures. For it may be easily shown that unboiled starch is digested by amylase with far greater difficulty than boiled starch, an illustration of the important part played by cooking in the preparation of the food for digestion.

It is a notable fact that amylases, also called diastases, are not confined to the animal body, but are widely distributed in plants.

The polysaccharide starch forms the great reserve of carbohydrate material in plant nutrition, and is mobilized for the use of the vegctable cells by being hydrolysed to simple sugars under the influence of these enzymes, just as the polysaccharide glycogen, the great carbohydrate reserve of animal nutrition (p. 533), is mobilized in the form of dextrose under the influence of the diastase of the liver. A diastase, which is present in all sprouting seeds, and may be readily extracted by water from malt, forms dextrin and maltose from starch. The optimum temperature of malt diastase, however, is about $55^{\circ} \mathrm{C}$., while that of ptyalin is about $40^{\circ} \mathrm{C}$.

While a neutral or weakly alkaline reaction is not unfavourable to salivary digestion, it goes on best in a slightly acid medium. It has been shown that the activity of ptyalin on starch, both having been previously dialysed to get rid as far as possible of salts, is increased by the addition of very small amounts of acids and of the neutral salts of strong monobasic acids. The action is decreased by larger amounts of acid (0.0007 to 0.0012 per cent. of hydrochloric acid) and by neutral salts of weak acids. An acidity equal to that of a O.I per cent. solution of hydrochloric acid stops salivary digestion completely, although the ferment is still for a time able to act when the acidity is sufficiently reduced. Strong acids or alkalies permanently destroy it. These facts indicate that in the mouth, where the reaction is weakly alkaline, the conditions are comparatively favourable to the action of the ptyalin. They are still more favourable in the stomach for some time after the beginning of a meal, while the reaction is yet weakly acid. It has been observed that (in cats) salivary digestion may go on for an hour or more in the cardiac end of the stomach, since free hydrochloric acid does not appear here before that time. Since the con- 
tents of the cardiac end are not freely intermixed with those of the pyloric end, a greater proportion of sugar is found in the former, and the difference is more marked with solid than with liquid fond (Cannon and Day). But during the greater part of gastric digestion the degree of acidity is such that the ptyalin must be hindererl. Althongh the food stays but a short time in the mouth, there is no doubt that, in man at least, some of the starch is there changed into sugar (p. 350). But this is not the case in all animals. Something depends on the amylolytic activity of the saliva, and something upon the form in which the starchy food is taken, whether it is cooked or raw, enclosed in vegetable fibres, or exposed to free admixture with the serretions of the mouth.

The fact already mentioned that hydrolytic changes of the same nature as those produced by enzymes can be brought about in other ways holds good for the salivary amylase. If starch is heated for a time with dilute hydrochloric or sulphuric acid, it is changed first into dextrin, and then into a sugar, which, lowwever, is not the disaccharide maltose, but the monosaccharicie dextrose-that is to say, the hydrolysis with acid proceeds a step farther than the hydrolysis in the presence of ptyalin. If maltose is treated with acid in the same way, it is also changed into dextrose. When glycogen (p. I) is boiled with dilute oxalic acid at a pressure of three atmospheres, isomaltose and dextrose are formed (Cremer). Facts already mentioned, and others to be cited later on, show that the action of the other digestive ferments can also be imitated by purely artificial means. Indeed, we may say that the ferments accomplish at a comparatively low temperature what can be done in the laboratory at a higher temperature, and by the aid of what may be called more violent methods.

Gastric Juice.-The Abbé Spallanzani, although not, perhaps, the first to recognize, was the first to study systematically, the chemical powers of the gastric juice, but it was by the careful and convincing experiments of Beaumont that the foundation of our exact knowledge of its composition and action was laid.

It is difficult to speak without enthusiasm of the work of Beaumont, if we consider the difficulties under which it was carried on. An army surgeon stationed in a lonely post in the wilderness that was then called the territory of Michigan, a thousand miles from a University, and four thousand from anything like a physiological laboratory, he was accidentally called upon to treat a gun-shot wound of the stomach. in a Canadian voyageur, Alexis St. Martin. When the wound healed, a permanent fistulous opening was left, by means of which food could be introduced into the stomach and gastric juice obtained from it. Beaumont at once perceived the possibilities of such a case for physiological research, and began a series of experiments on digzstion. After a while, St. Martin, with the wandering spirit of the voyageur, returned to Canada without Dr. Beaumont's consent and in his absence. Beaumont traced him, with great difficulty, by the help of the agents of a fur-trading company, induced him to come back, provided for his 
fannily as well as for himself, and proceeded with his imvestigations. A second time St. Martin went back to his native country, and a second time the zealous investigator of the gastric juice, at heavy expens?, secured his return. And although his experiments were necessarily less exact than would be permissible in a modern rescarch, the modest book in which he published his results is still counted among the classics of physiology. The production of artificial fistula in animals, a method that has since proved so fruitful. was first suggested by his work.

Gastric juice when obtained pure, as it can be from an accidental fistula in man, or, better, by giving a dog with an oesopliageal as well as a gastric fistula a 'sham-meal ' (p. 402), is a clear, thin, colourless liquid of low specific gravity (in the dog I003 to I006) and distinctly acid reaction. The total solids average about 5 parts per thousand, of which the ash (chiefly sodium and potassium chloride, with small quantities of calcium and magnesium phosphate) represents about a fourth, and heat-coagulable substances (proteins, nucleoprotein) about a third. None of these has any special importance in digestion. Of quite a different significance are the three ferments present: pepsin, which changes proteins into peptones; rennin, which curdles milk; and a fat-splitting ferment or lipase which, under certain conditions at least, splits up emulsified neutral fats-e.g., the fat of milk-into the alcohol (glycerin) and the fatty acids linked with it, but has so little action upon non-emulsified fat, that when this is taken into the stomach, it eventually passes into the duodenum practically unchanged. The acidity is due to free hydrochloric acid, the other important constituent of the juice. In the dog the proportion of this acid varies from 0.46 to 0.58 per cent. In such analyses as have been made of approximately pure human gastric juice a smaller percentage of hydrochloric acid has usually been obtained (at most 0.35 to 0.4 per cent.). But there is some reason to believe that if the human juice could be collected in a faultless manner, and especially free from any admixture with saliva or with a pathological secretion of mucus, it would show as high a percentage of acid as the dog's juice.

In cases of cancer, whether the growth is situated in the stomach or not, the free hydrochloric acid of the gastric juice is usually much reduced, and often absent. Under such conditions some lactic acid may be present in the stomach, being produced from the carbo-hydrates by the action of bacteria (Bacillus acidi lactici), which are normally held in clieck by the hydrochloric acid, although not rendered incapable of growth when they have passed on into the intestine. Even in the strength of 0.07 to 0.08 per cent. hydrochloric acid prevents the formation of lactic acid from dextrose. Indeed, when all the hydrochloric acid of the gastric juice is combined with proteins, the protein-acid compound still inhibits the growth of bacteria in the stomach, although not so efficiently as the 
same amount of free acid. That in normal gastric juice the acidity is not due to lactic acid can be slown by sliaking the juice with ther which takes up lactic acid, and lhen applying Uffelmann's test to the ethereal extract (Practical Exercises, p. 460 ).

IIore than this, it is not duc to an organic, but to an inorganic acid, for healthy gastric juice causes such an alteration in the colour of aniline dyes like congo-red and methyl violet as would be produced by dilute inineral acids, and not by organic acids, even when present in much greater strength.* Finally, when the bases and acid radicles of the juice are quantitatively comparid, it is found that there is more chlorine than is required to combine with the bases; the excess must be present as free hydrochloric acid In the pure gastric juice of fishes like the dogfish and skate, however, the acid is said not to be hydrochloric but an organic acid. The quantity of gastric juice secreted depends upon the nature and amount of the food. It has been estimated at as much as 5 litres in twenty-four hours, or several times the quantity of saliva secreted in the same time. With sham feeding a dog may yield 200-300 c.c. in an hour.

The great action of gastric juice is upon proteins. In this two of its constituents have a share, the pepsin and the free acid. One member of this chemical copartnery cannot act without the other; peptic digestion requires the presence both of pepsin and of acid; and, indeed, an active artificial juice can be obtained by digesting the gastric mucous membrane with dilute $(0.2$ to 0.4 per cent.) hydrochloric acid. A glycerin extract of a stomach which is not too fresh also possesses peptic power, the zymogen or mother substance, pepsinogen, having been activated to pepsin. Free acid very readily effects this activation, but this is far from being the only function of the hydrochloric acid, for active pepsin still requires the addition of a sufficient quantity of acid to render its proteolytic power available.

Well-washed fibrin obtained from blood is a convenient protein for use in experiments on digestion; although, of course, for many purposes only isolated purified proteins can be employed. Since the blood contains traces of pepsin, the fibrin should be boiled to destroy any which may be present (see also p. 453).

If we place a little fibrin in a beaker, cover it with gastric juice obtained from a dog or with 0.4 per cent. hydrochloric acid, to which a small quantity of pepsin or of a gastric extract has been added, and put the beaker in a water-bath at $f 0^{\circ} \mathrm{C}$., the fibrin soon swells up and becomes translucent, then begins to be dissolved, and in a short time has disappeared (sec Practical Exercises, p. 458). If we examine the

* A dilute solution of congo-red is turned violet by organic and blue by in organic acids; the gastric juice turns it blue. Methyl violet is rendered blue by an inorganic acid like hydrochloric acid, and green if more of the acid be adder. It is not altered by organic acids. Gastric juice turns it blue. 
liquic before digestion las proceeled very far. we shall find chicfly so-called acid-albumin in solution; later on, clicfly albumoses; and of these some authors distinguish the primary albumoses (proto-allmimose and hetero-albumose), the first to appear in quantity, followed by secondary or deutrro-albumoses (p. 1o).* Still later, peptones in large and always relatively increasing amounts will he present along with the albumoses. From this we conclude that acid-albunin is a stage in the conversion of fibrin into albumose, and albumose a half-way house between acid-albumin and peptone. It must not be supposed, however, that all the protein is first changed into acid-albumin before any of the acid-albuinin is changed into albumose, or that all the protein has already reached the albumose stage before peptone begins to appear. On the contrary, a certain amount of albumoses and of peptones are present very early in peptic digestion, while the greater part of the original protein is still unaltered. Among the somewhat vaguely characterized group of bodies comprised under the term peptones, there are no doubt decomposition products of the proteins in which the hydrolysis has bon carried to different degrees. Similar, but not identical, intermediate substances occur in the digestion of the other proteins, including that of bodies like gelatin, which are not ordinary proteins, but which pepsin can digest. The generic name of proteose properly includes all bodies of the albumose type, the term "albumose" itself being sometimes reserved for such intermediate products of the digestion of albumin; while those of fibrin are called fibrinoses; of globulin, globuloses; of cascin, caseoses; and so on. The peptones produced from different protcins are also not absolutely identical. If the digestion is prolonged, the peptones first formed are in turn further hydroiysed, so that eventually a considerable proportion of the original protein is converted into bodies which no longer give the biuret reaction.

In the stomach, during the four or five hours for which gastric digestion ordinarily lasts, none of the protein passes beyond the stage of proteose and peptone, including those relatively simple ' abiuret ' compounds which still consist of several 'building-stones,' chiefly, it would seem, the amino-arids, phenylalanin, and prolin, linked together. When precautions are taken to prevent the passage of any portion of the contents of the duoden:im into the stomach, no amino-acids can be detected in the gastic contents during the digestion of protein. In this connection it is interesting to note that none of the polypeptides hitherto prepared (p. 2) are decomposed by pepsin. It is not known at what points in the linkage of the groups that compose the complex protein molecule the pepsin ruptures the chain, but the points of attack are different from those of trypsin. The pancreatic juice, as we shall see later on, not

* In the light of modern investigation the results of fractional precipitation by salts of the products of proteolysis have lost a good deal of their interest, and it is seen that undue importance has often been attached to them. The student should be warned that such terms as 'albumose' and 'peptone' do not indicate precise chemical differences between the products separated in this way, nor even invariably such differences in molecular weight as the current schemata of the digestive processes ase apt to imply. Some so-called 'peptones' may indeed have a higher molecular weight and be more nearly related to the original protein than some so-called ' albumoses.' 
only effects a more complete conversion into peptones, but can split up the whole or a very large proportion of the peptones themselves into amino-acids and the other 'building-stones' of the original protein. Since the subject of protein digestion must come up again, it will be well to postpone any closer discussion of the process till we can view it as a whole. In the meantime it is only necessary to repeat that pepsin alone cannot digest proteins at all. Its action requires the presence of an acid; in a neutral or alkaline medium peptic digestion stops. The precise mode of action of the acid is by 110 means clear.

Dilute acid alone does not dissolve coagulated proteins like boiled fibrin, or does so only with extreme slowness. But it causes them to swell up by imbibition of water, and probably in this way facilitates the entrance of the ferment. Uncoagulated proteins are readily changed by acid into acid-albumin; and by the prolonged action of acids, especially at a high temperature, further changes of much the same nature as those produced in peptic digestion may be caused in all proteins. But under the ordinary conditions of natural gastric digestion, it may be said that the acid alone does little until it is aided by the ferment, just as the ferment alone does nothing without the aid of the acid. The acid enters into a temporary combination with the protein, the more highly hydrolysed proteins, such as peptones, combining with a greater proportion of acid than such proteins as fibrin or albumin. These compounds so easily undergo hydrolytic dissociation that, in spite of its union with the proteins, the hydrochloric acid is able to act along with the pepsin, so that peptic digestion goes on even when enough protein is present to combine with all the acid. There is some evidence that in the gastric juice the pepsin exists in the form of an unstable compound with hydrochloric acid, and it is probably this pepsin-hydrochloric acid compound wlich is the actual catalytic agent in peptic digestion. Although hydrochloric acid acts most powerfully, other acids, such as nitric, phosphoric, sulphuric, or lactic (arranged in the order of their efficacy), can replace it.

The Milk-Curdling Action of Gastric Juice.-The milk-curdling ferment, rennin, or chymosin, is contained in large amount in an extract of the fourth stomach of the calf, which, as rennet, has long been used in the manufacture of cheese. It exists in the healthy gastric juice of man, but disappears in cancer of the stomach and in chronic gastric catarrh. It has been stated by a number of observers that the properties of rennin are never found in gastric juice or any preparation obtained from it or from the gastric mucous membrane unless pepsin is present. This has suggested that there is no separate milk-curdling ferment, but that the clotting or precipitation of caseinogen is merely an associated action of the pepsin. Ferments of the most varied origin will curdle milk. Pawlow has maintained 
that the milk-curdling property not only of the gastric juice, but also of the pancreatic juice and of the secretion of Brunner's glands, is associated with the proteolytic ferment.

He asserts that when the comparison is instituted under proper conditions there is an exact parallelism between the proteolytic and the milk-curdling power of these secretions, no matter what the circumstances may be in which they are collected, or the influences to which they are exposed after collection. He has found it impossible to separate from any one of them a fraction whicli has milk-curdling power without proteolytic power. On the other hand, the majority of investigators maintain the separate identity of rennin. Hammarsten especially states that he can destroy the peptic activity without destroving the milk-curdling power of gastric extracts, and vice versa. According to Burge, when a solution displaying both peptic and milk-curdling power is electrolyzed, the pepsin action is abolished at a certain stage, while the rennet action is unaffected. It would seem, then, that the balance of evidence is in favour of the separate identity of the rennet enzyme.

However this may be, the curdling of milk by the gastric ferment includes two processes: (I) An action on caseinogen in the course of which it acquires new properties, becoming changed into casein. This substance is not capable of being converted into casein, and remains in solution in the whey. (2) The altered caseinogen or casein combines with soluble calcium salts and in the presence of these is precipitated as the curd. The change which occurs in the caseinogen has been the subject of mucl discussion, which has not yet led to a definite conclusion. According to some observers, the cliange consists in a decomposition of caseinogen, in the course of which a new substance, whey-protein, not previously present in the milk, is split off. This substance is not capable of being precipitated by lime salts, and remains in solution in the whey. According to others, the molecule of caseinogen is simply changed into two molecules of casein (van Slyke). Dilute acid will of itself precipitate caseinogen, and the presence of acid, and particularly hydrochloric acid, in the gastric juice lielps its milk-curdling action. But that a ferment is really concerned is indicated by the fact that the juice, after being made neutral or alkaline, still curdles milk, and that this power is destroyed by boiling. The optimum temperature is the same as that of the other ferments of the digestive tract, about f $0^{\circ} \mathrm{C}$. (p. 337). The persistence of the milk-curdling activity in the presence of $\mathrm{OH}$ ions, while for peptic activity free $\mathrm{H}$ ions are necessary, is a further and, indeed, a strong argument in favour of the separate existence of the rennet ferment.

As to the exact function of the milk-curdling ferment of the gastric juice in digestion, we have no precise knowledge. It seems s'iperfluous if we suppose that the free acid is able of itself to do all 
that the ferment does along with it. But there is evidence that the eurd produced by the ferment is more profoundly changed than the precipitate caused by dilute acids; for the latter may be redissolved, and then again curclled by renuin in the presence of calcium salts, while this cannot be done with the former. WVe may suppose, then, that the ferment is capable of effecting changes more favourable to the subsequent action of the pepsin upon the cascin than those which the acid alone would effect. Or it may be that the ferment acts in the early stages of digestion before much acid has been secreted. The curdling of milk probably plays a part in ensuring the retention of this food, the proper digestion of which is all-important for the suckling, for a sufficient length of time in the stomach. Otherwise, like water and watery liquids in general, it would be rapidly passed into the duodenum. Even if this were not the case, there is another reason for early curdling. Milk is a very dilute food, and the immense proportion of water in it might weaken the gastric juice too much for rapid digestion of the proteins. The separation of the whey and its prompt escape through the pylorus would obviate this. But caution should be exercised in giving a physiological value to all the details of the milk-curdling action of the gastric juice. Nilk-curdling ferments, or, at any rate, ferments with a milk-curdling influence, have an extremely wide distribution, both in secretions which in normal circumstances can never come into contact with milk, and in the tissues of animals and plants. Many bacteria produce them. And it appears that in the suckling, where it might be expected, if anywhere, to have a definite and important office, the rennet action of the gastric juice is distinctly less than in the aduit. It is worthy of note that the curd formed by rennet from human milk is more finely divided than that formed from cow's milk, and therefore is more easily digested. The addition of lime-water or barley-water to cow's milk keeps the curd from adhering in large masses, and thus aids its digestion-a fact which is sometimes usefully applied in the artificial feeding of infants.

Gastric Lipase.-On fats gastric juice has usually been supposed to have no action, although everybody admits that it will dissolve the protein constituents of fat-cells and the protein substances which keep the fat-globules of milk apart from each other. It has, however, been recently shown that both in the stomach and in vitro (with glycerin extracts of the gastric mucous membrane) a considerable amount of well-emulsified fat may be split up, and that this is due to a ferment which is different in several respects from the lipase of pancreatic juice. Gastric juice splits up tat, both in neutral and in weakly acid solutions. The slightest excess of alkali checks the action. The glycerin extract is much more resistant to alkali, while very sensitive to hydrochloric acid. This indicates that the fat-splitting ferment exists in the mucous membrane in a 
different form from that in which it exists in tlı juice-namely, as a zymogen or mother-substance. But while the zymogen of the pancreatic lipase is activated by bile, this is not the case with the mother-substance of the gastric lipase. It appears that in the suckling the lipase of the gastric juice plays a more important part than in later life. This is obviously in accordance with the fact that the specific food of the suckling-milk-contains as an essential constituent a large proportion of emulsified fat. The conditions for the emulsification of fat do not exist in the gastric juice, and this is the reason why the gastric lipase has so slight an effect upon uncmulsified fat, which presents a surface of contact proportionally so small. In any case the amount of fat hydrolysed in the stomacli under ordinary con litions is small in comparison with the amount split in the intestine, although it has been shown that with a diet rich in fat some of the intestinal contents, including pancreatic lipase, may pass back into the stomach.

As regards the carbo-hydrates, the swallowed saliva will continue to act on starch in the stomach, so long as the acidity is not too great; while the hydrochloric acid of the gastric juice is able to invert canesugar, changing it into a mixture of dextrose and levulose, ${ }^{*}$ and also, doubtless, to hydrolyse to dextrose a portion of the maltose formed by the saliva. Altogether, there is no doubt that the proportion of the carbo-hydrates of the food digested in the stomach is far from insignificant.

The Antiseptic Function of the Gastric Juice.-The stomach, with its acid contents, forms during the greater part of gastric digestion a valve or trap to cut off the upper end of the intestine from the bacteria-infested regions of the inouth and pharynx, and to destroy or inhibit the micro-organisms swallowed with the food and saliva. The occasional presence in vomited matter of sarcinæ or regularly arranged groups of micrococci, generally four to a group, shows that under abnormal conditions the gastric contents are not perfectly aseptic; and even from a normal stomach active micro-organisms of various kinds can be obtained. But upon the whole there is no doubt that the acidity of the gastric juice is an important check on bacterial activity during the first part of digestion, and in the upper portion of the alimentary canal. Koch has shown that the acidity of the gastric juice of a guinea-pig is sufficient to kill the comma bacillus of cholera. Normal guinea-pigs fed with cholera bacilli

* These are both reducing sugars, but, as their names indicate, they rotate the plane of polarization in opposite directions. The specific rotatory power of levulose is greater than that of dextrose, so that when cane-sugar is completely inverted, although equal quantities of dextrose and levulose are produced, the plane of polarization is rotated to the left. Cane-sugar itself rotates it to the right. The term 'inversion' has been extended to include the similar hydrolysis of other sugars of the disaccharicle group-e.g., maltose to dextrose, and lactose to a mixture of dextrose and galactose, even although the products are not levo-rotatory. 
were unaffected. But if the gastric juice was neutralized by an alkali before the aclninistration of the bacilli the guinea-pigs died. ('harrin found, too, that digestion with pepsin and hydrochloric acid causes an appreciable destruction or attenuation of diphtheria toxin. Bacteria, like tle lactic acid bacillus, which form acid products, may be less profoundly affected by the acid gastric juice than the putrefactive bacteria, which, on the whole, form alkalies, and are therefore accustomed to an alkaline medium. Yet ive have seen that the growth of even the lactic acid bacillus is very strictly controlled when the gastric juice contains the normal amount of hydrochloric acid.

It has been supposed by some that this bactericidal action is the chief function of the stomach, and the question has been asked why we should attribute any digestive importance to the secretion of that viscus, since the pancreatic juice can do all that the gastric juice does, and some things which it cannot do. Further, it has been shown that a dog may live five years after complete cxcision of the stomach, comport himself in all respects like a normal dog, and when killed for necropsy show every organ in perfect health (Czerny). In man, too, the stomach has been excised witl a successful result. But if this is to be admitted as evidence against the digestive function of the stomach, it is just as good evidence against the bactericidal function, particularly as it has in addition been shown that even putrid flesh lias no harmful effect on a dog after excision of the stomach, any more than on a normal dog. And, indeed, the reasoning is fallacious which assumes that what may happen under abnormal conditions must happen when the conditions are normal. For nothing is impressed more often on the physiological observer than the extraordinary power of adaptation, of making the best of everything, which the animal organism possesses. Doubtless a dog without a stomach will use to the best advantage the digestive fluids that remain to him; and the pancreatic juice, with the aid of the bile and the succus entericus, may be adequate to the complete task of digestion. So, too, a man from whom the surgeon has removed a kidney, or a testicle, or a lobe of the thyroid gland, may be in no respect worse off than the man who possesses a pair of these organs. But what do we deduce from this? Not, surely, that the excised thyroid, or testicle, or kidney was useless, or the gastric juice inactive, but that the organism has been able to compensate itself for their loss. Further, it would seem that the fate of the protein or of part of the protein digested and absorbed by the stomach is different from that digested and absorbed by the intestine. For after the operation of gastro-enterostomy (the establishment of an artificial opening between the stomach and the small intestine through which the food passes rapidly without having to submit to the challenge of the pyloric splincter), the ingested nitrogen is more quickly eliminated than when the protein is first subjected to full gastric 
digestion. So that when the quantity of protein in the food is increased above that necessary for nitrugen equilibrium (p. 602), none of the excess is assimilated and stored up, as is the case in a normal animal (Levin, etc.).

Pancreatic Juice--Pancreatic juice, bile, and intestinal juice are all mingled together in the small intestine, and act upon the food, not in succession, but simultaneonsly. But by artificial fistula in animals they can be obtained separately; and occasionally some of then can be procured through acciclental fistula in man. It is said that uncler certain conditions, especially when fat or oil is introduced into the stomach, the pyloris may remain open long enough to permit the passage of pancreatic juice or bile from the duodenum into the stomach, and this has been recommended as a practical method of obtaining these secretions in man.

Human pancreatic juice, as obtained from a fistula, is a clear, only slightly viscid liquid of distinctly alkaline reaction to litmus. Its specific gravity is about 1007 to ro Io. The total solids constitute about I. 5 or 2 per cent., of which a little less than I per cent. is made up of inorganic salts, chiefly sodium carbonate, with small quantities of chlorides. The balance of the solids consists mainly of proteins. The alkaline reaction is due to the sodium carbonate, and it is worthy of remark, as showing the important part taken by this secretion in the neutralization of the chyme, that when titrated against standard acid the alkalinity of the pancreatic juice is not much less than the acidity of the gastric juice when titrated against standard alkali. The quantity of pancreatic juice secreted during the twenty-four hours in an average man has been estimated at 500 to Soo c.c. from observations on cases of fistula. Probably under perfectly normal conditions it is greater. A so-called artificial pancreatic juice can be made by extracting the pancreas with water or glycerin. Since better methods of obtaining the natural juice have been developed, these extracts have lost some of their importance.

Fresh pancreatic juice contains four ferments: (I) The zymogen or mother-substance, trypsinogen, of a proteolytic or protein-digesting ferment, trypsin; (2) an amylolytic ferment, or amylase; (3) a fatsplitting or lipolytic ferment, steapsin; (4) a milk-curdling ferment. The question whether the last is a different body from the proteolytic ferment has been discussed just as in the case of the gastric rennin (see p. 353). In any case, it cannot be considered as taking any practical share in digestion, since it can hardly ever happen that milk passes through the stomach without heing curdled.

Trypsinogen has no action upon proteins, but in normal digestion it is changed into active trypsin by the enterokinase of the intestinal juice (p. 372). Pancreatic juice collected without contact with intestinal contents or with the mucous membrane of the intestine 
does not digest proteins. The silme is true of extracts of perfectly fresh pancreas, but if the pancreas is allowed to stand for a time, the extracts contain active trypsin, perhaps because some decomposition product has activated the trypsinogen. Some writers, however, state that when contamination of the gland with intestinal contents or contact with the mucosa has been avoided in its removal from the borly, such extracts will remain inactive for months, although the trypsinogen can at once be activated to trypsin by the aclilition of enterokinase.

Trypsin, to a certain extent, corresponds with pepsin in its action on proteins. But it acts energetically in an alkaline as well as in a not too acid medium (a very slight amount of digestion may go on in (listilled water); and its action, unlike that of pepsin-at least in digestions of moderate duration-does not stop at the peptone stage, but goes on rapidly to the production of the amino-acids, the basic substances arginin, lysin, and histidin, known as the hexone bases, and most of the other decomposition products obtained by boiling proteins with dilute acids. The most important of these products, so far as they have been isolated and identified, are enumerated in the table on p. 360 (see also pp. I-3).

As to the chemical nomenclature of these bodies, the student should refer to his textbook of organic chemistry. It need only be remarked here, by way of illustration. that when, e.g., leucin is designated as a-amino-isolutylacetic acid, this indicates that it can be derived from the fatty acid isobutylacetic acid by the substitution of an anino group, $\mathrm{NH}_{2}$, for a hydrogen atom in the a-carbon group (see p. $55^{(1)}$ ) of the fatty acid-i.e., the group next the carboxyl (COOH) group. Thus,

$$
\underset{\substack{a \\ \mathrm{CH}_{3}}}{\mathrm{CH}_{3}}, \underset{\text { Isobutylacetic acid. }}{\mathrm{CH}} \cdot \underset{\mathrm{CH}_{2}}{\mathrm{CH}_{2}} \cdot \mathrm{COOH}, \underset{\mathrm{CH}_{3}}{\mathrm{CH}_{3}}>\mathrm{CH} \cdot \mathrm{CH}_{2} \cdot \mathrm{CH} \cdot \mathrm{COOH} .
$$

When norleucin, an amino-acid found especially in the proteins of nervous tissue, is termed a-amino-caproic acid, it is indicated that $\mathrm{NH}_{2}$ replaces one $\mathrm{H}$ in the $\mathrm{CCH}_{2}$ group of caproic acid $\left(\mathrm{CH}_{3} \cdot \mathrm{CH}_{2} \cdot \mathrm{CH}_{2} \cdot \mathrm{CH}_{2}\right.$. $\mathrm{CH}_{2} \cdot \mathrm{COOH}$ ). The long chenical name of isoleucin (a compound also derived from the proteins of nervous tissue and from some plant proteins; indicates that in propionic acici,

$$
{ }_{\beta} \mathrm{CH}_{3} \cdot \mathrm{CH}_{2} \cdot \mathrm{COOH} \text {, }
$$

$\mathrm{NH}_{2}$ is introduced into the $a$ group, yiclding

$$
{ }_{\beta} \mathrm{CH}_{3} \cdot \mathrm{CH} . \mathrm{COOH} \text {, }
$$

or amino-propionic acid (alanin); while in the $\beta$ group one $H$ is rejlatied by a methyl $\left(\mathrm{CH}_{3}\right)$ group, and another $\mathrm{H}$ by an erliyl $\left(\mathrm{C}_{2} \mathrm{H}_{5}\right)$ gouup, yielding finally isonuclein:<smiles>CCC(C)C(N)C(=O)O</smiles> 


\section{CHIEF DECOMIOSI'TION PKODUC'TS OF PROTEINS.}

\section{MONOAMINO-ACIDS AND THEIR COMPOUNDS.}
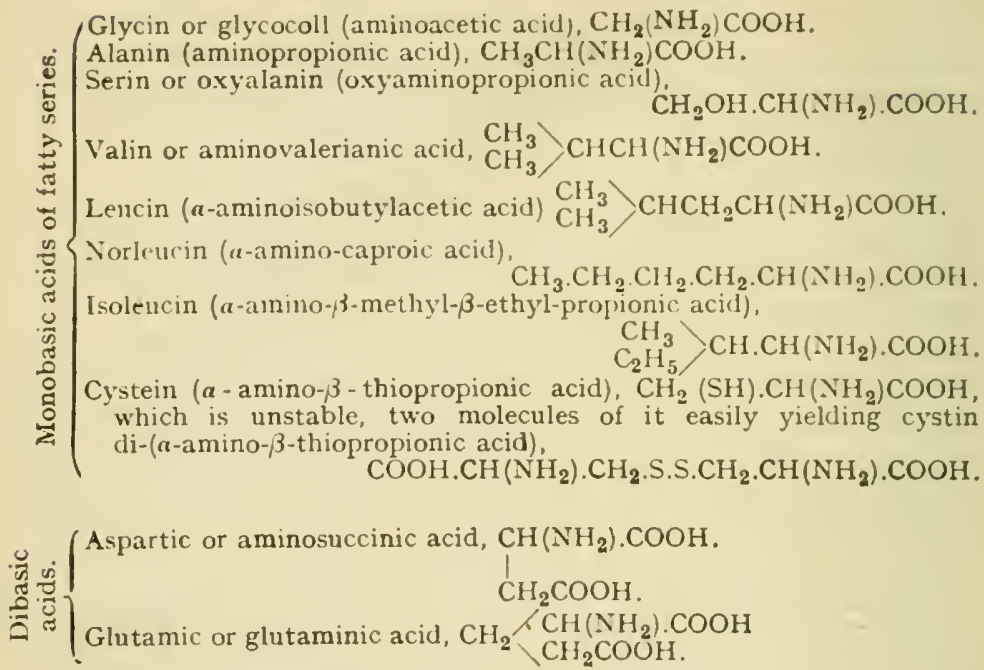

Tyrosin (para-oxyphenylaminopropionic acid),
$\mathrm{C}_{6} \mathrm{H}_{4} \mathrm{OH} . \mathrm{CH}_{2} \cdot \mathrm{CH}\left(\mathrm{NH}_{2}\right) \cdot \mathrm{COOH}$.

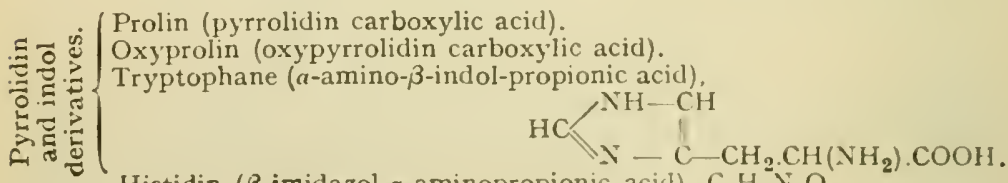

Histidin ( $\beta$-imidazol- $\alpha$-aminopropionic acid), $\mathrm{C}_{6} \mathrm{H}_{9} \mathrm{~N}_{3} \mathrm{O}_{2}$.

\section{Diamino-Acids and thFir Compotnds.} E. Arginin (guanidinaminovalerianic acid).
$\mathrm{HN}=\mathrm{C} / \mathrm{NH}_{2}$ $\mathrm{NH}^{2} \mathrm{CH}_{2}\left(\mathrm{CH}_{2}\right)_{2} \mathrm{CH}\left(\mathrm{NH}_{2}\right) \mathrm{COOH}$.

Ammonia (representing the so-called 'amide-nitrogen,' and liberated from the products of acid hydrolysis of proteins by heating the mixture after addition of alkali). It has not been shown that ammonia is itself one of the 'Bansteine' of the proteins. 
In the artificial compounds of two or more amuo-acids which have been synthesizad by firscher and named by him polypeptides (p. 2), the carboxyl group of one amino-acid is linked with the amino group of another. For example, a molecule of alanin and a molecule of glycin form, with loss of a molecule of water, a molecule of alanyl-glycin, according to the equation

\section{$\mathrm{NH}_{2} \cdot \mathrm{CH}_{2} \cdot \mathrm{CO} \mathrm{OH}+\mathrm{H} \mathrm{NH} \cdot \mathrm{CH} \cdot \mathrm{CH}_{3}-\mathrm{H}_{2} \mathrm{O}=\mathrm{NH}_{2} \cdot \mathrm{CH}_{2} \cdot \mathrm{CO} \cdot \mathrm{NH} \cdot \mathrm{CH} \cdot \mathrm{CH}_{3}$.

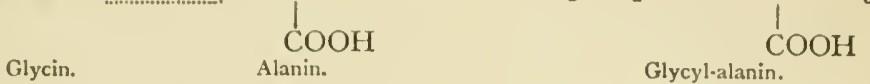

Two or more molecules of the same amino-acid can be linked in the same way; e.g., two molecules of glycin yicld a molecule of glycyl-glycin, and so on. It has been proved that polypepticles identical with some of these synthetic bodies are present in the peptone mixtures derived from the native proteins, so that it must be assumed that one of the ways at least in which amino-acids are linked in the protein molecule is that described.

It has been suggested that the early appearance of some of these amino-acids in pancreatic digestion is not really due to trypsin, but to other ferments, peptases, which act upon the peptones formed by the trypsin. There is, however, no clear evidence of the existence of a separate peptone-splitting enzyme in pancreatic juice, like the erepsin of intestinal juice, and it is therefore most natural to suppose that under the influence of trypsin the protein molecule breaks at different points from those at which it ruptures under the influence of pepsin.

After the most prolonged artificial digestion with trypsin, a residue of the protein remains unconverted into these relatively simple substances. But even this small portion of the original protein has undergone a great change, for it no longer gives the biuret reaction. It can be split into amino-acids, etc., by heating with acid, and also by the action of the erepsin of the intestinal juice, and then yields mainly prolin and phenylalanin, substances which are generally not to be detected among the decomposition products of protein after digestion with pancreatic juice. This illustrates the important fact that some of the 'building stones' of the protein molecule can be separated from it with far greater ease than others. Tyrosin, tryptophane, and cystin appear very early in the digestive fluid, and tyrosin, as shown in the following example from Abderhalden, may be completely liberated at a time when glutaminic acid is scarcely more than beginning to appear.

The plant protein edestin, obtained from cottonseed, was digested with pancreatic juice or an extract containing trypsin. The quantities of tyrosin and glutaminic acid liberated at different periods of the experiment are expressed as percentages of the total amounts of these substances contained in the edestin. 


\begin{tabular}{|c|c|c|c|c|c|c|}
\hline Duration of Digestion & & I Nay. & 21 .ay:. & 3 Days. & 7 Days. & 16 Days. \\
\hline $\begin{array}{l}\text { Tyrosin - } \\
\text { Glutaminic acid }\end{array}$ & - & $\begin{array}{r}7^{8}+4 \\
43\end{array}$ & $\begin{array}{r}97^{\circ} \\
7+4\end{array}$ & $\begin{array}{l}97^{\circ} 6 \\
10.9\end{array}$ & $\begin{array}{l}100 \\
3 I^{1} 1\end{array}$ & $\begin{array}{l}100 \\
6002\end{array}$ \\
\hline
\end{tabular}

When trypsin acts upon protein already digested by pepsin, this partially hyclrolysed residue is smaller than when the trypsin acts alone, no matter for low long a time. Also the decomposition of a given quantity of protein by trypsin is accomplished in a notably shorter time if it has been previously subjected to the action of pepsin. This illustrates the cu-operative relation of these two ferments-a relation still more clearly implied in the fact that, although trypsin readily forms albumoses and peptones from native protein when such is offered to it, yet in natural digestion the great albumose- and peptone-forming ferment is pepsin. In the lumen of the intestine the trypsin is confronted mainly with protein already hydrolysed to the albumose and peptone stage in the stomach. In other words, instead of the very large molecules of the original protein food with a weight of perhaps 5,000 to 7,000 , the trypsin begins its action on a much larger number of much smaller molecules of only one-twentieth the initial weight or less. The statement is sometimes made that trypsin is a stronger proteolytic ferment than pepsin. This may be true in the sense that trypsin carries the decomposition down to bodies of smaller molecular weight than pepsin. But within the range of its hydrolytic action pepsin decomposes certain proteins and allied bodies more readily than trypsin-e.g., the serum proteins, and especially elastin and the constituents of connective tissue.

In all that we have hitherto said regarding tryptic digestion we have supposed that putrefaction has been entirely prevented-e.y., by the addition of toluol. If no antiseptic is added to a tryptic digest, it rapidly becomes filled with micro-organisms, and emits a very disagreeable facal odour; and now various bodies which are not found in the absence of putrefaction make their appearance, such as indol, skatol, and other substances, to which the frecal odour is due. They are not true products of tryptic digestion, but are formed by the putrefactive micro-organisms, which can themselves split off from proteins numerous decomposition products, including tyrosin, and change tyrosin into indol.

Amylase or pancreatic amylase, the diastatic or sugar-forming ferment of pancreatic juice, changes starch into dextrin and maltose, just as the ptyalin of saliva does. The two fermients are possibly identical, but under the conditions of action of the pancreatic juice its diastatic power is greater than that of saliva, and it readily acts 
on raw starch as well as boiled. Pincreatic amylase is mainly, perhaps entirely, present in the juice in the form of active ferment. If a zymogen stage exists, the mother-substance is less stable or less easily extracted from the gland than is trypsinogen. In this respect imylase also resembles ptyalin. A small amount of malta: is contained in pancreatic juice, and further hyclrolyses to dextrose a portion of the maltose formed by the amylase.

Steapsin or pancreatic lipasc splits up nentral fats into glycerin and the corresponding fatty acids. The latter mite witl the alkalies of the pancreatic juice and the bile to form soaps. In this important process bile acts as the helpmate of pancreatic juice; together they effect much more than either or both can accomplish by separate action. Many tissues contain fat-splitting ferments or lipases, some of which are perlaps identical with the pancreatic lipase. The lipase exists as active ferment in the pancreatic juice, but there is reason to believe that a portion of it may be present as a zymogen in the gland, and probably in the secretion as well. It is clianged into active ferment by the bile salts. Active lipase can also be extracted from the pancreas by glycerin or water. It is to be noted that it is only the proteolytic enzyme which is totally inactive till it reaches the intestine. The significance of this will be discussed later on.

Bile.-Bile is a liquid the colour of which varies in different groups of animals, and even in the same species is not constant, dependingr on the length of time the fluid has remained in the gall-bladder and other circumistances. When it is recognized that the colour is due to a series of pigments, which are by no means stable, and of which one can be caused to pass into another by oxidation or reduction, this want of uniformity will be easily intelligible. The fresh bile of carnivora is golden-red. The bile of herbivorous animals is in general of a green tint, but, when it has been retained long in the gall-bladder, may incline to reddish-brown. Fresh human bile, as it flows from a fistula just established, is of a reddish-brown, goldenyellow or yellow colour. Beaumont speaks of the yellowish bile which he could press into the stomach of St. Martin by manipulating the abdomen. In a case observed by the writer, it was seen that when the bile flowing from a fistula was allowed to spread out in a dressing, it became greenish, because of oxidation of a part of the bilirubin to biliverdin, although as it actually escaped from the fistula it was yellow. The bile of a monkey taken from the gall-bladder immediately after deatl is dark green, but if left a few hours in the gall-bladder it is brown, the green pigment having been reduced. It should be remembered that human bile from the post-mortem room may alter its colour in the interval which must elapse before it can usually be procured after death. Bile, as obtained from fistulæ in otherwise healthy persons, has a specific gravity of about Ioos to IOIO. In the gall-bladder water is absorbed from the bile and 
mucin added to it, so that the specific gravity of bladder bile is as high as rozo to Iofo. The reaction is feebly alkaline to litmus.

The composition of two specimens of human bile-one from a fistula, the other from the gall-bladder-is shown in the following table:

\begin{tabular}{|c|c|c|c|c|}
\hline & & & Bladder Bile. & Fistula Bile. \\
\hline Water - & - & - & $898 \cdot 1$ & $977^{\circ} 4$ \\
\hline Solids - & - & - & $101 \cdot 9$ & $22 \cdot 6$ \\
\hline Mucin and otl & ler subst: & insoluble & & \\
\hline in alcohol & - $\quad-$ & - $\quad-$ & I 4.5 & $2 \cdot 3$ \\
\hline Sodium taur & ocholate & sodium & & \\
\hline glycocholate & - & - & $56 \cdot 5$ & $10 \cdot 1$ \\
\hline Inorganic silts & - & - & $6 \cdot 3$ & $8 \cdot 5$ \\
\hline Fat I & & & & \\
\hline $\left.\begin{array}{l}\text { Lecithin } \\
\text { Cholesterin }\end{array}\right\}$ & - & - & $30 \cdot 9$ & $\begin{array}{l}0 \cdot 05 \\
0 \cdot 56\end{array}$ \\
\hline
\end{tabular}

The substance which renders bladder bile viscid, but which is present in much smaller amount in bile from a fistula, and is probably entirely absent from the fluid as it is secreted by the liver-cells, is commonly termed 'mucin.' It has been shown, however, that in many animalsfor cxample, the ox, dog, sheep, ctc.-the substance is not a true mucin. It does not yield, like mucin, on boiling with dilute acid, a carboliydrate group (viz,, glucosamine, $\mathrm{C}_{6} \mathrm{H}_{11} \mathrm{O}_{5} \mathrm{NH}_{2}$, corresponding to dextrose in which $\mathrm{OH}$ is replaced by $\mathrm{NH}_{2}$ ). It is relatively rich in phosphorus, and consists-mainly, at any rate-of a phosplo-protein (p. 2). The mucilaginous substance of human bile consists largely of true mucin.

Mucin is scarcely to be looked upon as an essential constitucnt of bile; it is not formed by the actual bile-secreting cells, but by mucous glands in the walls and goblet-cells in the epithelial lining of the larger bile-ducts, and especially of the gall-bladder.

Bile-Pigments. - It has been said that these form a series, but only two of the pigments of that series are present in normal bile, bilirubin, and biliverdin. In human bile, the former, in herbivorous bile and that of some cold-blonded animals, such as the frog, the latter is the clief pigment. But bilirubin can be extracted in large amount from the gall-stones of cattle; while the placenta of the bitch contains biliverdin in quantity, althougl, as in all carnivora, it is either absent from the bile or exists in it in comparatively small amount. These facts show that the two pigments are readily interchangeable, but there is no question that bilirubin is the pigment which is formed by the liver-cells.

Bilinubin $\left(\mathrm{C}_{32} \mathrm{H}_{36} \mathrm{~N}_{4} \mathrm{O}_{6}\right)$ can be prepared from powdered red gallstones by dissolving the chalk with hydrochloric acid, and extracting the resiclue with chloroform, which takes up the pigment. From this solution, on evaporation, or from hot dimethyl anilin, beautiful rhombic tables or prisms of bilirubin separate out.

Biliverdin $\left(\mathrm{C}_{32} \mathrm{H}_{36} \mathrm{~N}_{4} \mathrm{O}_{8}\right)$ can be obtained from the placenta of the bitch by extraction with alcohol. It is insoluble in chloroform, and by means of this property it may be separated from bilirubin wlien the two happen to be present together in bile. Biliverdin can also be formed from bilirubin by oxidation. By the aid of active oxidizing agents, 
such as yellow nitric acid (which contains some nitrous acid), a whole. series of oxidation products of bilirubin is obtained, beginning with biliverdin, and passing throngh bilicyanin, a blue pigment, and other intermediate bodies, to choletelin, a ycllow substance. It is questionable whether these are all definite compounds. This is the foundation of Gmelin's test for bile-pigments (sce Practical Exercises, p. 462). The same colours are produced, and in the same order, when a solution of bilirubin in chloroform is treated with a dilute alcoholic solution of iodine.

The positive pole of a galvanic current canses the same oxiclative changes, the same play of colours, while the reducing action of the negative pole reverses the effect, if the action of the positive electrode lias not gone too far. These reactions can also be used for the detection of bile-pigments.

By the reducing action of sodium amalgam on bilirubin, hemibilirubin $\left(\mathrm{C}_{33} \mathrm{H}_{44} \mathrm{~N}_{4} \mathrm{O}_{6}\right)$ is obtained. It gives a beautiful red colour with p-dimethylaminobenzaldehyde (Elurlich's reaction). Hemibilirubin is identical with the urobilinogen of urine from which urobilin is derived. Urobilinogen and urobilin (often called in this connection stercobilin) are also found in the fæees from birth onwards, although not in the meconium (p. 424). Urobilinogen is derived from the normal bilepigment by reduction in the intestine itself, where reducing substances due to the action of micro-organisms are never absent in extra-uterine life.

The bile of most animals shows no characteristic absorption spectrum. But the fresh bile of certain animals, the ox, for instance, does show bands. These, however, are not due to the normal bile-pigment, and they are not essentially changed when this is oxidized or reduced by electrolysis. MacMunn attributes the spectrum of the bile of the ox and sheep to a body which he calls cholohæmatin, and which does not belong to the bile-pigments proper.

The Bile-Salts. -These are the sodium salts of certain acids, of which glycocholic and taurocholic are the chief. In the bile of omnivora, including man, both are in general present, and in various proportions; in human bile there is more glycocholic than taurocholic acid; sometimes taurocholic acid is entirely absent. In the bile of many carnivora - e.g., the dog and cat-only taurocholic acid is found; in that of the carnivora generally, it is by far the more important of the two acids. In the bile of most herbivora there is much more glycocholic than taurocholic acid. The bile acids are paired acids: glycocholic acid (better named cholyl-glycin) formed by the union of glycin and cholic acid, and taurocholic acid (or cholyl-taurin), consisting of cholic acid united with taurin.

The decomposition of the bile-acids into these substances is effected by boiling them with dilute acid or alkali, a molecule of water being taken up; thus-

$$
\underset{\text { Glycocholic acid. }}{\mathrm{C}_{26} \mathrm{H}_{43} \mathrm{NO}_{6}}+\mathrm{H}_{2} \mathrm{O}=\underset{\text { Glycin. }}{\mathrm{CH}_{2}\left(\mathrm{NH}_{2}\right) \cdot \mathrm{COOH}}+\underset{\text { Cholic acid. }}{\mathrm{C}_{24} \mathrm{H}_{40} \mathrm{O}_{5}} \text {; }
$$

$$
\underset{\text { Taurocholic acid. }}{\mathrm{C}_{26} \mathrm{H}_{45} \mathrm{NSO}_{7}}+\mathrm{H}_{2} \mathrm{O}=\underset{\text { Taurin. }}{\mathrm{CH}_{2}\left(\mathrm{NH}_{2}\right) \cdot \mathrm{CH}_{2} \cdot \mathrm{SO}_{2} \cdot \mathrm{OH}}+\underset{23}{\mathrm{C}_{23} \mathrm{H}_{40} \mathrm{O}_{5}} \text {. }
$$

A notable difference between glycocholic and taurocholic acid is that the latter contains sulphur. The whole of this belongs to the taurin. Both glycin and taurin are derived from the disintegration of proteins. We have already seen that among the products of protein hydrolysis a sulphur-containing body, cystein, which is readily changed into cystin, is found, and there is good evidence that taurin is derived from cystein 
or cystin. In certan pathological conditions cystin apjears in the urine (cystinuria). The source of the cholnc acid which geses to formu the bile acids is unknown, but it las been surmised that it may be derived from cholesterin. Thus,

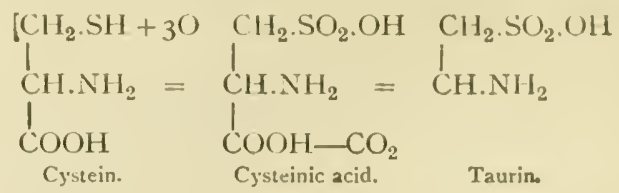

Traces of cholic acid, formed by liydrolysis from the bile-acids by the action of putrefactive bacteria, are found in the intestines, especially in the lower portion.

Pettenkofer's test for bile-acids (Practical lixercises, p. 462), accidentally discovered in examining the action of bile upon sugar, depends upon three facts: (1) That cholic acisl and furfuraldehyde grive a purple colour when brought together; (2) that the bile-salts yicld cholic acid when acted upon by sulphuric acid; (3) that when cane-sugar is decomposecl by strong sulphuric acid, furfuraldeliycle is formed.

Since a similar colour is given when the same reagents are added to a solution containing aibumin, it is necessary to remove this, if present, from any liquid which is to be tested for bile-acids.

Lecithin and cholesterin, or cholesterol, are by no means peculiar to bile (p. 4). They are very widely distributed in the body. Iceithin $\left(\mathrm{C}_{41} \mathrm{H}_{90} \mathrm{NPO}_{9}\right)$ belongs to the group of phosphatieles, fat-like phosphoruscontaining substances present in all cells. It is a compound of glycerin with two molecules of fatty acid and one of plosploric acid. The phosphoric acid is at the same time united with a base cholin $\left(\mathrm{C}_{5} \mathrm{H}_{15} \mathrm{NO} \mathrm{O}_{2}\right)$. The fatty acid (stearic, palmitic, oleic, etc.) varies in lifferent varictics of lecithin. Heated with haryta-water, lecithin yiclds the corresponding fatty acid in the form of a soap, along with cholin and glyccryl-phosphoric acid. Glyceryl-phosphoric acid can be further split so as to yield a molecule of glycerin and one of phosplıoric acicl.

Cholesterin is a substance with the empirical formula $\mathrm{C}_{27} \mathrm{H}_{46} \mathrm{O}$. It contains an alcohol group in virtuc of whicls fatty acids can be linked to it, forming esters. It is best obtainel from white gall-stones, of which it is the chief, and sometimes almost the sole constituent (see Practical Excrcises, p. 463).

All the compounds related to cholesterin are grrouped together under the name of sterins. The sterins are very wiclely distributed botls in animals (zoosterins) and in plants (phytosterins). livery cell secms to contain sterins and sterin esters (compounds of the sime nature as the compounds of fatty acids with the alcohol gryecrin which constitute the nentral fats). In the vertebrates cholesterin and its product co ustitute the chicf, perhaps the only sterins, but in invertebrate animals and plants tlicre is a mucl greater varicty of these sulbstances.

The chief inorganic salts of bile are sodium chloride, sollium carbonate, and alkaline sodium plospliate. The phosplioric acid of the ash comes lartly from the phosphorus of organic compounds (lecitlin and bileunucin), the sulphuric acid from the sulphur of tanocholic aciel, the sodium largely from the bile-salts. Iron is a notable inorganic constituent of bile, although it exists only in traces, in the form of phosphate of iron. Manganese is also present in minute amount. Ioo c.c. of fresh bile yields 50 to Ioo c.c. of carbon dioxide, part of which is in solution and part combined with alkalies. 
The quantity of bile secreted in twenty-four hours in an average man is probably from 750 c.c. to a litre. In nine cases of fistula ol the gall-bladder in patients operated on for gall-stones or cchinococcus the daily (puantity varied from 500 to I, 100 c.c. (Brand).

Digestive Functions of Bile.-The great action of the bile in digestion is undoubtedly the preparation of the fats for absorption. In this preparation four processes are important: two chemical actions, hydrolysis of nentral fats to glycerin and fatty acids, and saponification, or the formation of soaps by the union of fatty acids with bases, especially sodium; and two physical processes, cmulsification, or the formation of a mechanical suspension of such fme globules of unaltered neutral fat as exist in milk, and solution of soaps and fatty acids. While there has been much discussion as to the relative share taken by these processes, and especially by saponification and emulsification in the absorption of fat (p. $4+\mathrm{I}$ ), there is no doubt that they are all concerned in the digestion of fat or the preparation of it for absorption and assimilation. In this, indeed, the processes are complementary to each other, for an essential preliminary to emulsification in the intestine seems to be the formation of a certain amount of soaps, soluble in the intestinal contents, while the formation of an emulsion enormously increases the surface of contact between the unaltered fat and the digestive juices, and so favours more rapid hydrolysis, saponification, and solution. In the whole series of changes the bile plays a part, though not an independent one; it acts always in conjunction with the pancreatic juice.

While no complete explanation has been given of the precise nature of this partnership, it is certain that the fat-splitting ferment of the pancreatic juice on the one land, and the bile-salts on the: other, contribute largely to the total action. An alkaline solution. a solution of sodium carbonate, e.g., is unable of itself to emulsify a perfectly neutral oil; but if some free fatty acid be added, emulsification is rapid and complete (p. I2). Now, there is no doubt that here a soap is formed by the action of the alkali on the fatty acid, and there is equally little doubt that the formation of the soap is an essential part of the emulsification. But it is not clear in what manner the soap acts, whether by forming a coating round the oilglobules, or by so altering the surface-tension, or other physical properties of the solution in which it is dissolved, that they no longer tend to run together. However this may be, in pancreatic juice we have the two factors present which this simple experiment shows to be necessary and sufficient for emulsification; we have a ferment which can split up neutral fats and set free fatty acids, and an alkali which can combine with those acids to form soaps. Accordingly, pancreatic juice is able of itself to form emulsions with perfectly neutral oils. It is possible that the protein constituents of pancreatic juice may have a share in emulsification, since the addition of protein 
-c.g., egg-white-to a soap solution increases the stability of the emulsions formed by the soap. In bile, on the contrary, although the alkali is present, there is no fat-splitting ferment, and, according to the best experiments, bile alone has no emulsifying power on perfectly neutral fat. But we now come to a remarkable fact: this inert bile when added to pancreatic juice greatly intensifies its emulsifying action, and a solution of bile-salts has much the same effect as bile itself. The fact is undoubted, but the explanation is obscure. What it is that the bile or bile-salts can add to the pancreatic juice which so increases its power of emulsification, we do not know. It has been surmised that a characteristic pliysical property of bile, the diminution of the surface-tension of watery liquids to which it is added, may play an important part, perhaps, in enabling the fat-splitting ferments or the emulsifying soaps to get into closer contact with the unaltered fat. It is also true that bile, presumably in virtue of the chemical action of its allkaline salts, can, in presence of a free fatty acid, rapidly form an emulsion. But the pancreatic juice itself contains so considerable a quantity of sodium carbonate that it would scarcely seem to require the relatively fecble reinforcement of the alkaline salts of the bile.*

An important part of the effect of the bile is certainly due to its favouring the fat-splitting action of the pancreatic juice. By the addition of bile, the quantity of fat split up by a definite amount of dog's pancreatic juice may be increased two to threefold. It has been shown that this is an action of the bile-salts. The sodium salts of synthetically-obtained glycocholic and taurocholic acids produce the same effect. It is in virtue of this action that the bilesalts are sometimes spoken of as the co-ferment of the lipase. As already pointed out, this action is exerted in presence of the fully. formed enzyme, and should not be confounded with the effect of the bile-salts in activating the lipase zymogen. The capacity of dissolving soaps, which is a property of the bile-salts, is also of great importance in supplementing the solvent power of the intestinal liquids for the products formed by the pancreatic juice. The solution of soaps in the bile-salts has the power in its turn of dissolving free fatty acids. The significance of this in fat absorption will be referred to again. A further illustration of the nutual adaptation of the various digestive juices, of the remarkably precise manner in which the action of each dovetails into the action of otlers, is afforded by the facts already mentioned in connection with the lipase of the stomach. It is highly probable that the fatty acids formed by the gastric lipase, cven if formed only in small amount, may exertan important influence in emulsifying the fat as soon as it enters the intestine. The intestinal juice itself also unquestionably takes a share in the digestion of fat along with the pancreatic

* It has lately been shown that bile-sa'ts accelerate the formation of soap from oleic acid by sodium bicarbonate (Kingsbury). 
secretion and the bile. There exists also, as will be seen later on, a certan adajtation between the fool and the digestive secretions. Not the best illustration of this, but one which suits the present topic, is the fact that the food itself probably always contains some free fatty acids when it contains fat at all. Althougl our knowledge of the mutual action of the pancreatic juice and the bile on the digestion of fats is still incomplete, there is no doubt that they are cqually necessary. For in some diseases of the pancreas fat or fatty acid often appears in the stools, and this token of imperfect digestion of the fatty food may be confirmerl by the wasting of the patient. The sane may occur when the bile is prevented by obstruction of the duct or by a biliary fistula from entering the intestine. Yet in some cases of fistula, where there is every reason to believe that all the bile is escaping externally, the nutrition of the patient-at any rate, on a diet not abnormally rich in fat-is unaffected. The mere deficiency of bile in the intestine is, of course, complicated in obstructive jaundice by the harmful effects of the biliary constituents circulating in the blood.

The white stools of jaundice owe their colour, not merely to the absence of bile-pigment, but also to the presence of fat. Their highly offensive odour used to be adduced as evidence that bile is the "natural antiseptic ' of the intestine. It seems rather to be due to the coating of the particles of food with undigested fat, which shields the proteins from the action of the digestive juices, while permitting the putrefactive bacteria to revel in them unchecked. As a matter of fact, the bile itself has little, if any, power of hindering the growth of micro-organisms, although the free bile-acids are tolerably active antiseptics. In suckling children it is not uncommon to see the frecs white with fat. This is a less serious symptom than in adults, and perliaps betokens merely that the milk in the feeding-bottle is undiluted cow's milk, which is richer in fat than human milk, and ought to be mixed with water.

Bidder and Schmidt found that the chyle in the thoracic duct of a normal dog contained $3 \cdot 2$ per cent. of fat. In a dog with the bile-duct ligatured the proportion fell to 0.2 per cent. It is an instance of the extraordinarily exact adaptation of the digestive juices to the nature of the food, the mechanism of which will present itself for discussion later on, that the reinforcing action of the bile upon the fat-splitting ferment of the pancreatic juice is said to be greater when the food is rich in fat $(\mathrm{p} .4 \mathrm{I} 4)$.

Bile has been credited with a physical power of aiding the passage of fat through membranes molstened with it by diminishing the surface tension, and it has been inferred that this has an important bearing on the absorption of fat from the intestine. But the inference does not follow from the statement, and the statement has been itself denied. There is at present no evidence that the digestive function of the bile extends beyond the preparation of the food for absorption to the preparation of the mucosa for absorbing it.

On proteins bile has either no digestive action, or only a feeble one. Fibrin is slightly digested by the bile of the dog and of man. But the addition of it to fresh pancreatic juice considerably increases the proteolytic power of that secretion (Rachford), although not so decidedly as in the case of the fat-splitting action. The amylolytic 
action of the pancreatic juice is also favoured by the bile, and in about the same degree as its protenlytic effect. Although bile sometimes exerts by itself a feebly amylolytic action, this is not to be included among its specific powers, for a diastatic ferment in small quantities is widely diffused in the body:

The addition of bile or bile-salts to a gastric digest causes the precipitation of any unaltered native protein, acid-albumin, albuinose, and pepsin. The precipitate, which is a salt-like compound of protein with taurocholic acid, is redissolved when the liquid is rendered allaline, and therefore in excess of bile, or of a solution of bile-salts, but the pepsin has no longer any power of digesting proteins. Pait of the bile-acids and bile-mucin is also thrown down by the acid of the digest. It has been suggested that by thus precipitating the constituents of the chyme which have not been carried to the peptone stage bile prepares them for the action of the pancreatic juice. But it is difficult to see how the precipitation of a substance can prepare the way for its digestion, and it is more probable that if any physiological value is to be given to this reaction, it has the function of preventing the absorption of proteins which have not been sufficiently split up. There is little doubt, however, that the rendering of the pepsin inactive has physiological significance, for pepsin exerts an injurious influence upon the ferments of the pancreatic juice. In digestion, then, the bile has a tïofold function, favouring greatly the activity of the pancreatic ferments, especially the fat-splitting ferment, and aiding in establishing the conditions necessary for the transition of gastric into intestinal digestion.

Succus Entericus.-This is the name given to the special secretion of the small intestine, which is supposed to be a product of the Lieberkiihn's crypts or intestinal glands. In order to obtain it pure, it is of course necessary to prevent admixture with the bile, the pancreatic juice, and the food." This can be done by dividing a loop of intestine from the rest by two transverse cuts, the abdomen having been opened in the linea alba. The continuity of the digestive tube is restored by stitching the portion below the isolated loop to the part above it. One end of the loop is serred into the lips of the wound in the linea alba, and the other being closed by sutures, the whole forms a sort of test-tube opening externally (Thiry's fistula). Or both ends are made to open through the abdominal wound (Vella's fistula). Another method is to make a single opening in the intestine, and by means of two indiarubber balls, one of which is pushed down, and the other up through the opening, and which are afterwards inflated, to block off a piece of gut from communication with the rest. Or several openings may be made at different levels in the intestine, each being allowed to heal into a wound in the abdominal wall. When pure juice is required it is collected from the lower fistulæ, while the upper fistulæ are opened to permit the escape of the 
sccretions which enter the higher portions of the alimentary canal (gastric juice, pancreatic juice, and bile). The intestinal juice so obtained is a thin yellowish liquid of alkaline reaction, generally somewhat turbid from the presence of a certain number of leucocytes and epithelial cells. Its specific gravity is about IoIo, the total solids about 1.5 per cent. It contains a small amount of proteins, including serum albumin and serum globulin, and about the same proportion of inorganic salts as most of the liquids and solids of the body, namely, 0.7 or 0.8 per cent., chiefly sodium carbonate and sodium chloride; but, like the other digestive liquids, it is adapted to the nature of the food, and therefore its composition is not quite constant. Like bile, intestinal juice acts but fecbly on the food substances by itself, and if we contented ourselves with examining the pure and isolated secretion, we should greatly underestimate its importance. The sodium carbonate, in which it is relatively rich, will, to be sure, form soaps with fatty acids produce 1 by the action of the pancreatic juice or of the fat-splitting bacteria in which the intestine abounds, and thus aid in the digestion of fats. A lipase, feebler than that of the pancreatic juice, or present in smaller concentration, is also a constituent of the succus entericus. That a great deal of fat may be split up in the alimentary canal in the absence both of bile and pancreatic juice is well ascertained. The alkali of the succus entericus must at the same time aid in neutralizing the original acidity of the chyme, and in preserving the proper reaction of the intestinal contents. A ferment called invertase, or sucrase - which is not introduced with the food or formed by bacterial action as has been suggested, since it occurs in the aseptic intestine of the new-born child-will invert cane-sugar. The ferments maltase and lactase will cause a corresponding change in maltose and lactose (see footnote, p. $35^{6}$ ). It is worthy of remark that these inverting enzymes are present in the intestinal mucosa as well as in the intestinal juice, and extracts of the mucosa are usually distinctly more active than the juice itself. So that there is reason to believe that hydrolysis of the disaccharides may take place both in the lumen of the gut before absorption and in the wall of the gut during absorption. Inverting enzymes appear in the intestine early in embryonic life. Maltase is the most generally distributed of all these enzymes, and it is found along with lactase in the intestine of the embryo pig, while invertase is missing till after birth (Mendel). On native proteins and starch the isolated succus entericus has little or no action. But it contains a ferment, crepsin, which, although it does not affect native proteins like serum- and egg-albumin (fibrin and caseinogen may be slightly digested), exerts a powerful action on the first products of protein hydrolysis, albumoses, and peptones, breaking them up into bodies which no longer give the biuret reaction (ammonia, mono-amino acids, hexone bases, etc.). It 
destroys the diphtheria toxin, which is also rendered innucuous by trypsin. Erupsin, however, is not specific to the secreted intestinal juice, for it occurs also, not only in the mucous membrane of the intestine, which, indeed, contains a greater quantity of it than the succus entericus, but in all animal tissues hitherto investigated. It is said even to be sometimes present in pancreatic juice, since inactivated pancreatic juice, which does not digest uther proteins, will sometimes digest casein. But the matter is far from being settled, and the presence of erepsin in the pancreatic tissue is a complicating circumstance. For under abnormal conditions, most glands provided with artificial fistule have an increased liability to injuries of various kinds, which might permit constituents not normally present in the secretion to pass into it from the cells. The kidney in mammals is even richer in erepsin than the intestinal mucous membrane. Next to these come the pancreas, spleen, and liver, then at a long interval the heart muscle, while skeletal muscle and brain-tissue are poorest of all in the ferment. The intestinal mucosa varies in its erepsin content at different levels and on different diets. In cats on a meat or a mixed diet the duodenum is about five times richer in the ferment than the stomach. The ileum is about half as rich as the duodenum, and the jejunum occupies an intermediate position between the duodenum and ileum (Vernon). The secretion of Brunner's glands in the duodenum, which resemble in structure the pyloric glands of the stomach, digests coagulated albumin, although its proteolytic powers are feebler than those of the pancreatic juice.

Enterokinase.-The most characteristic constituent of succus entericus is a ferment, enterokinase, which differs from all the ferments we have hitherto described in acting not directly upon the foodstuffs, but upon the trypsinogen of the pancreatic juice, changing it into the active enzyme trypsin. It may therefore be spoken of as a ferment of ferments. It has been previously stated that freshly secreted pancreatic juice is without action upon proteins. The addition of succus entericus immediately confers upon it a high degree of proteolytic power. In one experiment pancreatic juice, obtained by a temporary fistula, required four to six hours to dissolve fibrin, and did not attack coagulated albumin even in ten hours. On addition of succus entericus, the same pancreatic juice dissolved fibrin in three to seven minutes, and rapidly digested coagulated albumin (Pawlow). In like manner a glycerin extract of a fresh pancreas has hardly any effect on proteins; a similar extract of a stale pancreas is active. The fresh pancreas contains trypsinogen, which is suluble in glycerin, for the inert extract becomes active when it is treated with dilute acetic acid, or even when it is diluted with water and kept at the body-temperature. If the fresh pancreas be first treated with dilute acetic acid, and then with glycerin, the 
extract is at once active. The trypsing gen can therefore be activated within the pancreatic cells, gradually when the pancreas is simply allowed to stand after excision, more rapidly in presence of the dilute acid. The ordinary tests for ferment action (destruction by boiling, activity in very small amounts, etc.) have shown that this property of the intestinal juice is due to a ferment, although it differs in certain respects from most ferments-for instance, in requiring a relatively high temperature to inactivate it. The smallest trace of enterokinase will convert a large quantity of trypsinogen into trypsin if time be given. At the same time, although to a much smaller extent, tle fat-splitting and starch-digesting activity of the pancreatic juice is increased. The secretion of the duodenum causes a greater increase in the proteolytic power than that of the other portions of the small intestine, while no such difference has been made out in the casc of the amylolytic and lipolytic functions. It is probable that the enterokinase, which is secreted mainly in the upper two-sevenths of the small intestine, and solely by the intestinal epithelium, acts only on the trypsinogen, and that the amylopsin and steapsin are aided in some other way. Enterokinase is only found in the intestinal juice when pancreatic juice is present in the gut. It is therefore secreted in response to the presence of trypsinogen or of some other constituent of the pancreatic juice.

Delezenne has attempted to explain the interaction of enterokinase and trypsinogen as an adaptive phenomenon of the same kind as the formation of antitoxins and lhamolysins (p. 3i). According to him, cnterokinase acts like a complement in lizemolysis, while trypsinogen plays the part of an intermediary body or amboceptor which enables the enterokinase to attack the protein molecule. $\mathrm{He}$ asserts that enterokinase, or a substance which produces a similar effect on trypsinogen, is contained not only in the mucous membrane of the intestine, but also in leucocytes, in fibrin (one of whose properties it is to pick out ferments from liquids containing them), in lympl-glands, in snake venom, and even in certain anaerobic bacteria. On this view trypsin would not be a definite substance produced by the interaction of enterokinase and trypsinogen, but only an expression for these two bodics acting tngether. Strong evidence against this view, and in favour cf the independent existence of trypsin, has been brought forward by Bayliss and Starling, and it does not seem to merit further consideration. According to Mellanby, enterokinase is really a proteolytic ferment, and trypsinogen contains a protein moiety with which trypsin is firmly combined. The conversion of trypsinogen into trypsin depends on the digestion of this protein moietr: and the consequent liberation of trypsin. Ternon has put forward the view that, while cnterokinase starts the activation of trypsinogen in the intestine, and can no doubt in time complete it, the trypsin as it is formed aids in the activation of more trypsinogen to trypsin, and so on by a process of so-called auto-catalysis of the trypsinogen. This idea can be harmonized with Mellanby's conception by assuming that the trypsin formed from trypsinogen can itself digest the protein moiety of a further portion of trypsinogen. 
According to Pawlow, the reason why the trvpsin is not secreted in the active form is that active trypsin readily destroys the amylolytic and lipolytic ferments. In the intestine, where trypsin is rendered active by enterokinase, these ferments are protected from its attack by the proteins of the food and by the bile. Enterokinase is itself iminediately destroyed in the presence of free acid (centinormal hydrochloric acid).

Having now finished our review of the chemistry of the digestive juices, our next task is to describe what is known as to their secretion-the nature of the cells by which it is effected and their histological appearance in activity and repose, and the manner in which it is called forth and controlled.

\section{Section IV.-Tine Secretion of the Digestive Juices- Microscopical Ghanges in the Gland Celds.}

The ligestive glands are formed originally from involutions of the mucous membrane of the alimentary canal, the salivary glands from the ectoderm, the others from the endoderm (Chap. XIX.). Some are simple unbranched tubes, in which there is either no distinction into bocly and duct, as in Licberkïhn's crypts in the intestines, or in which one or more of the tubes open into a duct, as in the glands of the fundus of the stomach. Some are branched tubes, several of which may end in a common duct; such are the glands of the pyloric end of the stomach and the Brunner's glands in the duodenum. In others the main duct ramifies into a more or less complex system of small channels, into each of the ultimate branches of which one or more (11sually several) of the secreting tubules or alveoli open. The salivary glands and the pancreas belong to this class of compound tubular or racemose glands, and so cloes the liver of such animals as the frog. But in the latter organ the typical arrangement is obscured in the higher vertebrates by the predominance of the portal bloodvessels over the system of bile-channels as a groundwork for the grouping of the cells.

In every secreting gland there is a vascular plexus nutside the cclls of the gland-tubes, and a system of collecting chanuels on their inner surface; and in a certain sense the cells of ivery gland are arranged with reference to the blondvessels on the one hand, and the ducts on the other. But in the ordinary racemose gland the blood-supply is mainly required to feed the secretion; the cells of the alveoln lave either no nther function than to secrete, or if they have other functions, they are not such as to entail a great disproportion between the size of the cells and the lumen of the channels into which they pour their products. For both reasons the relation of the grouping of the cells to the ductsystem is very obvious, to the blood-system very obscure. In the liver, the conditions are precisely reversed. We cannot suppose that the manufacture of a quantity of bile less in volume than the secretion of the salivary glands, though doubtless containing far more solids, requires an immense organ like the liver, and a tide of blood like that which passes through the portal rein. And, as we shall see, the liver has other functions, some of them certainly of at least equal importance with the secretion of bile, and one of them evidently requiring from its very nature a bulliy organ. Accordingly, both the richness of the blood-supply and the size of the secreting cells are out of proportion to 
the calibre of the ultinate clinnels that carry the secretion away. The so-called bile-citpilliries, which represent the lumen of the secreting tubules, are mere grooves in the surface of adjoining cells; and the architectural lines on which the liver lobule is built are: (1) the interlobular veins which carry blood to it; (2) the rich capillary network whicil separates its cells and feeds them; and (3) the central intralobular vein which drains it. Thus a network of cells lying in the meshes of a network of blood-capillaries takes the place of a regular dendritic arrangement of ducts and tubules; and in accordance witl this the bile-capillaries, instead of opening separately into the ducts, form a plexus with each other within the hepatic lobule (see also footnote, p. I. 4 ).

The ducts and secreting tubules of all glands are lined by cells of columnar epithelial type, but the type is most closely preserved in the ducts. In none of the digestive glands is there more than a single complete layer of secreting cells. But the alveoli of the mucous salivary glands show here at:ıl there a crescent-shaped group of small deeply-staining cells (crescents of Gianuzzi) outside the columnar layer (Fig. I $\left.5 S, A^{\prime \prime}, B^{\prime \prime}\right)$, and between it and the basement membrane, while the gland-tubes of the fundus of the stomach have in the same situation a discontinuous layer of larse ovoid cells, termed parietal from their position, oxyntic (or acid-secreting) from their supposed function (Figs. $155-157$ ). Access to the lumen of the glands is provided for these deeply-placed parietal cells and for the cells of the crescents by finc branching channels which enter and surround the cells. The serous salivary glands, the pyloric glands of the stomach, and the Licberkühn's crypts, have but a single layer of epithelium; and since there is no hepatic cell which is not in contact with at least one bile capillary, the liver may be regarded as having no morc. The same is truc of the pancreatic alveoli, except that in the centre of many of the acini a few spindle-shaped cells (centro-acinar cells), apparently continued from the lining of the smallest ducts, may be seen. Remarkable histological changes, evidently connected with changes in functional activity, have been noticed in most of the digestive glands. In discussing these, it will be best to omit for the present any detailed reference to the liver, since, although there are histological marks of secretive activity in this gland as well as in others, and of the same general character, they are accompanied, and to some extent overlaid, by the microscopic evidences of other functions ( $p .534)$. The serous salivary glands and the pancreas can be taken together; so can the glands of the various regions of the stomach; the mucous salivary glands must be considered separately.

Changes in the Pancreas and Parotid during Secretion.-The cells of the alveoli of the pancreas or parotid during rest, as can be seen by examining thin lobules of the former between the folds of the mesentery in the living rabbit, or fresh teased preparations of the latter, are filled with fine granules to such an extent as to obscure the nucleus. In the parotid the whole cell is granular, in the pancreas there is still a narrow clear zone at the outer edge of the cell which contains few granules or none; in both, the divisions between the cells are very indistinct, and the lumen of the alveolus cannot be mide out. During activity the granules seem to be carried from the outer portion of the cell towards the lumen, and 
there discharged. The ciear outer zone of the pancreatic cell grows broader and broader at the expense of the inner granular zone, until at last the granular zone may in its turn be reduced to a narrow contour line around the lumen (Fig. I53). In the uniformly clouded parotid cell a similar change takes place; a transparent outer zone
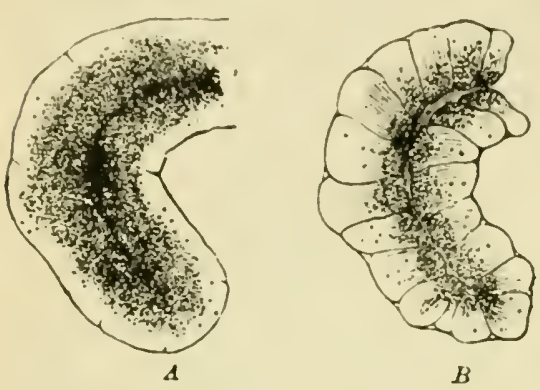

Fig. 153.- A, alveolus of ralıit's pancreas, 'Inaded' (resting): $B$, 'discharged' (active), observed in the living animal (Kühne and Lea). arises; and, after prolonged secretion, only a thin edging of granules may remain at the immer portion of the cell (Fig. I5t). In both glands the outlines of the cells become more clearly indicated, and a distinct lumen can now be recognized. The cells are smaller than they are during rest.

The disappearance of granules from without inwards during activity suggests that these are manufactured products eliminated in the secretion, and they are generally spoken of as zymogen granules.

Bensley, who has made a careful study of the pancreas in the guinea-pig, has been able to distinguish, even in fresh preparations examined in the animal's own serum, but better after staining with such a dye as neutral red, another kind of granules, which he regards
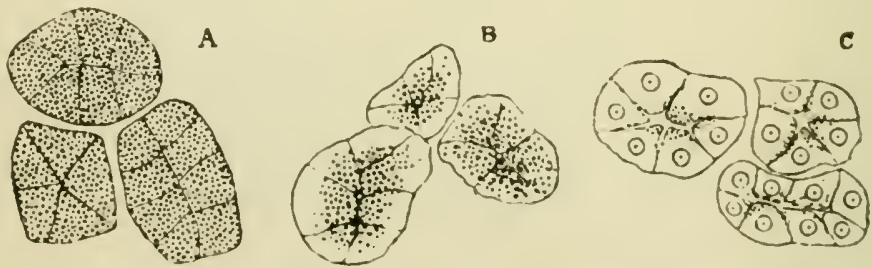

Fig. 154.-Alreoli of Parotid Gland: A at Rest: B, after a Short Period of Aclivity; C. after a Prolonged Perind of Activity (Fresh Preparations) (Langley). In A and 13 the nuclei are obscured by the granules of zymogen.

as zymugen granules in the course of formation, and therefore designates prozymogen granules. The resting acini show a clear basal zone which is unstiained, and a zome next the lumen containing coarse zymogen granules which are faintly stained. In the active gland-c.g. after a meal or after the injection of secretin (p. 407)prozymogen granules which stain much more intensely than the zymogen granules with neutral red make their appearance between the zymogen granules, now much reduced in number and size, and 
the clear outer zone. After prolonged secretion the zymogen granules may be entirely absent from the cells, and only a narrow rim of prozymogen granules can be seen around the lumen.

In one respect the pancreas differs remarkably from the salivary glands-namely, in the presence of the islets of Langerhanscharacteristic groups of small polygonal cells, richly supplied with bloodvessels, but not arranged in the form of alveoli. Some observers state that they are remarkably increased in size, and even in number when the pancreas is caused to secrete actively by repeated injections of secretin, and also in starvation. But it has been shown that this conclusion was based upon faulty methods of counting the islets, and even of identifying the islet cells. There appears to be no foundation for the view that they are derived from the ordinary secreting cells, and that they can, in turn, give rise to new alveoli by a process of proliferation. It is far more probable that they are independent structures, with a different function from the pancreatic alveoli (p. 6,8 ).

Changes in the Glands of the Stomach during Secretion.- The mucous membrane of the stomach is covered with a single layer of columnar epithelium, largely consisting of mucigenous gobletcells. It is studded with

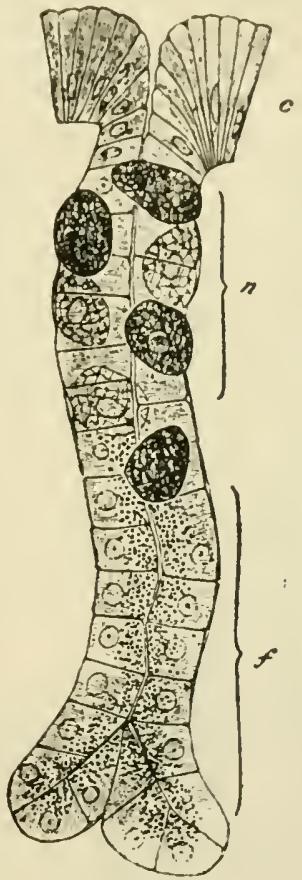

Fig. 155.

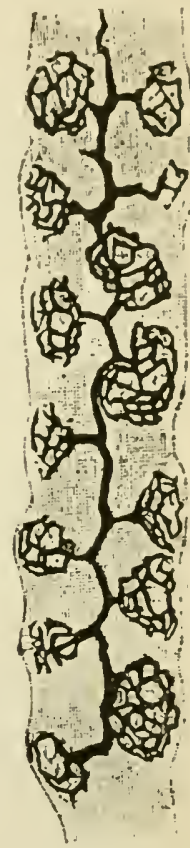

Fig. 156.

Fig. 155.-A Fundus Gland of Simple Form from the Bat's Stomach (Osmic Acid Preparation) (Langley). $c$, Columnar epithe. lium of the surface; $n$, neck of the gland with chief or central and parietal cells: $f$, base, occupied only by chief cells, which show the granules accumulated tuwards the lumen of the gland.

Fig. I56.-A Fundus Gland prepared by Golgi's Method, showing the Mode of Com. munication of the Parietal Cells with the Gland-Lumen (Schäfer, after E. Muller). minute pits, into which open the ducts of the peptic and pyloric glands, the ducts being lined with cells just like those of the general gastric surface. Three varieties of gastric glands have been distinguished: (I) The glands of the cardia. In man these occupy a small portion of the mucous membrane at the cardiac end, near the orifice of 
the asopliagus. Some of the glands are single tubules, but others have two or more tubules opening into a common duct. Both are lined by a single layer of short columnar epithelium, which contains granules. (2) The glands of the pyloric canal or antrum. These consist of short, branched tubules, which oren by twos and threes into long ducts. (3) The glands of the fundus or oxyntic glands, occupying the intermediate and greater portion of the organ. The gland tubules are long and seldom branched, and the ducts, into each of which open from one to three tubules, are relatively short. The secreting parts of both kinds of glands are lined by short columnar granular cells; and in the pyloric tubules no others are present. But, as we have said, in the glands of the fundus there are besides large ovoid cells scatterecl at intervals like beads between the basement membrane and the lining or chief cells. The cells of the pyloric glands have a general resemblance to the chief cells of the fundus glands, but they are not quite the same. For example, the granules are less distinct in the pyloric glands. In the human stomach it is only quite near the pylorus that the parictal cells disappear altogether. The parietal cells also contain granules, but they are smaller and less numerous than those of the chief cells, so that the deeper portions of the fundus glands are much darker in appearance than the more superficial portions, since the oxyntic or parietal cells are more numerous in the neighbourhood of the ducts (Bensley).

The histological changes connected with gastric secretion do not differ essentially from those described in the pancreas and the parotid, but there is much greater difficulty in making observations on the living, or at least but slightly altered, cells. For the mammal the best method is to use animals with a permanent gastric fistula, and to remove from time to time small portions of the mucous membrane for examination in the fresh condition. During digestion the granules disappear from the outer part of the chief cells of the fundus glands, leaving a clear zone, the lmmen being bordered by a granular layer. Or, more rarely, there may be a uniform decrease in the number of granules througliout the cell. The total volume of the cell is less than in the fasting condition. The parietal cells, which are small in the fasting animal, swell up, so as to bulge out the mombrana propria. They reach their maximum size (in the dog) very late in digestion (the thirtecnth to the fifteenth hour). No such definite changes in their contents as those observed in the other cells have been made out. The granules in the ovoid cells during and after activity seem to be as large and as numerous as in the resting cell, or even larger. After sham feeding in dogs the histological changes in the gastric glands are very slight, even when considerable amounts of gastric juice have been secreted (Noll and Sokoloff). 
The chief cells of the nxyntic, and the similar if not identical cells of the pyloric glands, are believed to manufacture the pepsin-forming substance. The ovoid cills of the former are supposed to secrete the hydrochloric acid. The evidence on which this belief is based is as follows:

The glands of the antrum pylori in the dog, in which in most siturtions no ovoid cells are to be scen, secrete pepsin, but no acid. The pyloric end of the stomach or a portion of it has been isolated,

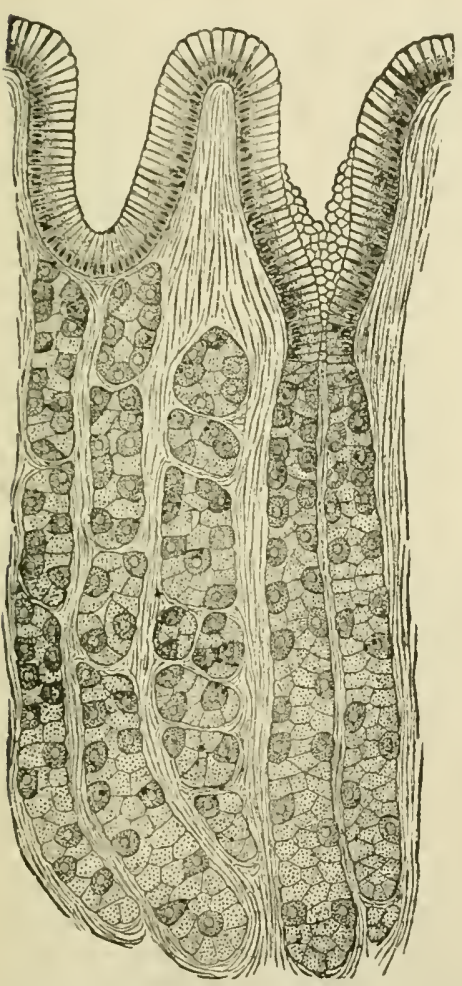

Fig. 15\%.-The Gastric Glands (Ebstein).

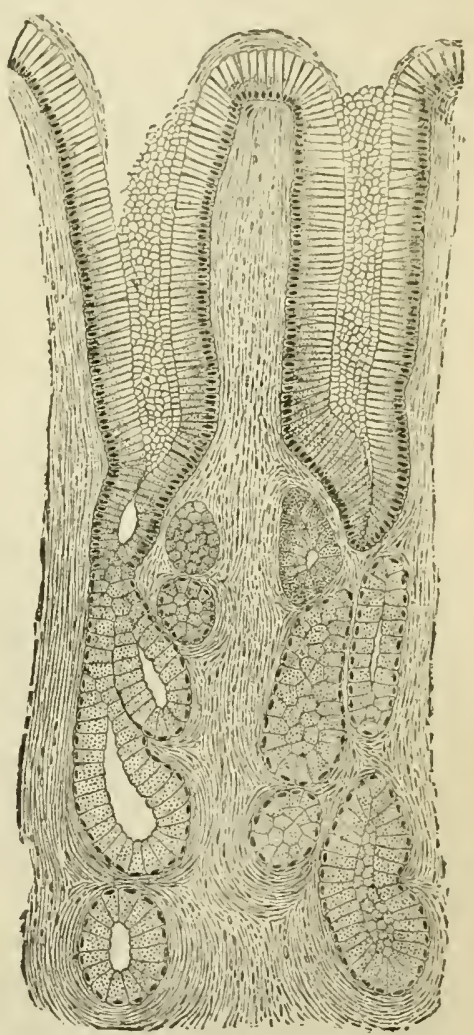

On the left, ox yntic; right, pyloric.

the continuity of the alimentary canal restored by sutures, and the secretion of the pyloric pocket collected. It was found to be alkaline, and contained pepsin. The glands of the frog's œesophagus, which contain only chief cells, secrete pepsin, but no acid. It seems fair to conclude that the chief cells of the fundus glands in the mammal secrete none of the free hydrochloric acid, but certainly some of pepsin. But it does not follow that all the pepsin is formed by these cells, although it would seem that all the hydrochloric acid 
must be secreted by the only other glandular elements present, the parietal or 'border' cells. And, indeed, the glands in the funclus of the frog's stomach, which are composed only of ovoid cells, whilst secreting much acid, also form some pepsin, although far less than the œesophageal glands. During winter sleep (in the marmot) the production of liydrochloric acid in the parietal cells stops altogether, while the chief cells continue to accumulate granules of pepsinogen.

That some pepsin is secreted by the pyloric end of the stomach is not difficult to prove. The secretion collected from the isolated pyloric portion is, indeed, like the secretion of the Brunner's glands in the duodenum, quite unable to digest protein until dilute hydrochloric acid is added. But this is because in both cases the juice as it flows from the glands is slightly alkaline, and, as we have already seen, pepsin only acts in the presence of an acid. The milk-curdling action of these two juices also unfolds itself only when the secretions are first acidulated, and later on again neutralized; in other words, the ferments must be activated by the addition of an acid. In normal digestion the pepsin of the (in itself) alkaline secretion of the pyloric end of the stomach becomes a constituent of the acid gastric juice; and it may perhaps be considered a morphological accident, so to speak, that the oxyntic cells of the fundus should mingle their acid products with the (presumedly) alkaline secretion of the chicf cells in the lumen of each gland-tube, instead of being massed as a separate organ with a special duct.

We are not without other examples of digestive juices fitted or destined to act in a medium with an opposite reaction to their own. The 'saliva' of the cephalopod Octopus macropus, strongly acid though it is, contains a proteolytic ferment which in vitro acts, like trypsin, better in an alkaline than in an acid solution. And trypsin, whose precursor is a constituent of the alkaline pancreatic juice, while the enterokinase which activates it is a constituent of the alkaline succus entericus, performs a part at least of its work in an acid medium.

Attempts made to demonstrate an acid reaction in the border cells have hitherto failed. Harvey and Bensley on the basis of experiments with dyes (cyanimin and neutral red), which give different colours according to whether the reaction is acid, alkaline, or neutral, have concluded that free acid exists only on the internal surface of the stomach, or at most also in the necks of the glands. The parietal cells they find alkaline. They suggest that these cells form in some way, of course ultimately from the chloricles of the blood, a cliloride of an organic base which does not decompose so as to yield free liydrochloric acid until it reaches the mouth of the gland. The nature of this decomposition, if it occurs, is unknown. It may be mentionerl, although only as a matter of historical interest, that some observers have denied that the acid is secreted in the depths of any cell from the chlorides of the blood, and liave asserted that it is formed at the surface of contact of the stomach-wall with the gastric contents from the sodium cliloride of the food by an exchange of sodium ions (p. 428 ) for hydrogen ions from 
the blood or lympli. It was pointed ont in favour of this view that when, insteal of solium chloride. solimm bromide is given in the food, the hydrochloric acid in the stomich is to a large extent replaced by lyydrobromic acid. And it was argued that this cannot be due to the clecomposition of the bromide by hydrochloric acid, since it occurs in animals deprived for a considerable time of salts, and in 'salt-hunger' the stomach contains no acid (Kocppe). It may be, however, that even in 'salt-hunger' the presence of sodium bromide in the stomach stimulates the secretion of liydrochloric acid, which then decomposes the bromide, with the formation of hydrobromic acid. The sodium cliloricle formed in the double decomposition might be re-absorbed, and the stock of clilorides in the blood remain undiminished. It is in any case a decisive objection to this now defunct theory that a copious secretion of gastric juice, containing hydrochloric acid in almudance, can be olstained, without the introduction of any food into the stomach. either by the process of sham fecding (p.40I) or by psyclical st.mulation of the gastric glands when food is slown to an animal.

Changes in Mucous Glands during Secretion.-In the mucous salivary and other mucous glands similar, but apparently more complex, changes occur. During rest the cells which line the lumen may be secn in fresh, teased preparations to be filled with granules or 'splierules.' After active secretion there is a great diminution in the number of the granules. Those that remain are chiefly collected around the lumen, although some may also be seen in the peripheral portion of the cell; and there is no very distinct differentiation into two zones. That a discharge of material takes place from these cells is shown by their smaller size in the active gland. That the material thus discharged is not protoplasmic is indicated by the behaviour of the cells to protoplasmic stains such as carmine. The resting cells around the lumen stain but feebly, in contrast to the deep stain of the demilunes, while the discharged cells take on the carmine stain much more readily. Further, when a resting gland is treated with various reagents (water, dilute acids, or alkalies), the granules swell up into a transparent substance identical with mucin, which fills the meshes of a fine protoplasmic network.

In ordinary alcohol-carmine preparations only the network and nucleus are stained; the nucleus, small and shrivelled, is situated close to the outer border of the cell. When a discharged gland is treated in the same way there is proportionally more 'protoplasm ' (or ' bioplasm ') and less of the clear material, what remains of the latter being chiefly in the inner portion of the cell, while the nucleus is now large and spherical, and not so near the basement membranc (Fig. I $5^{8}$ ).

Everything, therefore, points to the granules in what we may now call the mucin-forming cells as being in some way or other precursors of the fully-formed mucin; manufactured during " rest 'by the protoplasm and partly at its expense, moved towards the lumen in activity, discharged as mucin in the secretion. It has been asserted that not only is the protoplasm lessened in the loaded cell and renewed after activity, but that many of the mucigenous cells may be altogether broken down and discharged, their place being supplied by proliferation of the small cells of the demilunes. This conclusion, however, is not supported by sufficient evidence. The cells of the crescents contain fine granules, but none which can be changed into 
mucin. They are of serous and not of mucous type. But the fact on which we would specially insist is that the granules of the resting mucigenous cell may be looked upon as a mother-substance from which the mucin of the secretion is derived; they are not actual, but potential, mucin.

So in the pancreas, the serous or albuminous salivary glands, and the glands of the stomach, there is every reason to believe that the granules which appear in the intervals of rest, and are moved towards the lumen and discharged during activity, are the precursors, the mother-substances, of important constituents of the secretion. These granules are sharply marked off from the protoplasm in which they lie and by which they are built up. By every mark, by their reaction to stains, for instance, they are non-living substance, formed in the bosom of the living cell from the raw material which it culls from the blood, or, what is more likely, formed from its own protoplasm, then shed out in granular form and
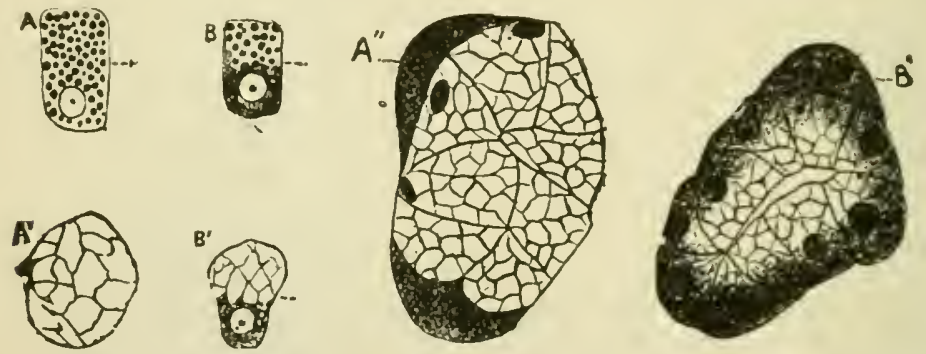

Fig. 158.-Mucous Cells (from Submaxillary of Dog) in Rest and Activity (Langlev). $A, B$, fresh $A^{\prime}, B^{\prime}$, after treatment with dilute acetic acid; $A^{n}, B^{n}$, alveoli hardened in alcohol and stained with carmine. $A, A^{\prime}$, and $A^{\prime \prime}$ represent the loaded; $B, B^{\prime}$, and $B^{\prime \prime}$, the discharged condition.

secluded from further change. The granules in the ferment-forming glands are not in general composed of the actual ferments, and, indeed, in several instances it has been shown that the actual ferments are not present in the secreting cells at all.

We have already seen that the pancreas and even the fresh pancreatic juice are devoid of active trypsin. Similarly, a glycerin extract of a fresh gastric mucous membrane is inert as regards proteins, or nearly so. But if the nucous membrane has been previously treated with dilute hydrochloric acid, the glycerin extract is active, as is an extract made with acidulated glycerin. Here we must assume the existence in the gastric glands of a mother-substance, pepsinogen, from which pepsin is formed. The rennin of the gastric juice, which is formed in the chief cells, also has a precursor. which, if the ferment is identical with pepsin (p. 353), must be pepsinogen. The proteolytic power of an extract of the pancreas, 
when the trypsinogen has heen activated into trypsin, or of the gastric mucous membrane, when the pepsinogen has been changed into pepsin, seems to be, rougluly speaking, in proportion to the quantity of granules present in the cells. Therefore it is concluded that the granules represent mother-substances of the ferments or zymogens. Some observers believe they have obtained evidence of stages in the elaboration of the ferments still further back than the mother-substances, grandmother-substances so to speak, or prozymogens. Bensley, e.g., concludes that the nuclei of the chief cells in the fundus glands of the stomach take part in the formation of a prozymogen, the precursor of the zymogen or pepsinogen, as pepsinogen is the precursor of the enzyme pepsin.

A glycerin or watery extract of the salivary glands always contains active amylolytic fernent, if the natural secretion is active. So that if ptyalin is preceded by a zymogen in the cells, it must be very easily changed into the actual ferment.

But we should greatly deceive ourselves if we supposed that granules of this nature in gland-cells are necessarily related to the production of ferments. The micigenous granules have no such significance. Most digestive secretions contain protein constituents, with which the granules may have to do as well as with ferments. And bile, a secretion which contains no mucin, no proteins, and either no ferments or mere traces, as essential and original constituents, is formed in cells with granules so disposed and so affected by the activity of the gland as to suggest some relation between them and the process of secretion. In the liver-cells of the frog, in addition to glycogen, and oil-globules small granules may be seen, especially near the lumen of the gland tubules; they diminish in number during digestion, when the secretion of bile is active and increase when food is withheld and secretion slow. And in fasting dogsthe secreting cells of Brunner's glands, the pyloric glands and the pancreas, as well as the lining epithelium of the bile-ducts, have been found to contain many fatty granules. Possibly some of these represent the fat which is known to be excreted into the alimentary canal (pp. $443,4+4$ ).

The Nature of the Process by which the Digestive Secretions are Formed.- We have spoken more than once of the gland-cells as manufacturing their secretions. It is an idea that rises naturally in the mind as we follow with the microscope the traces of their functional activity. And when we compare the composition of the digestive juices with that of the blood-plasma and lymph, the suggestion that the glands which produce them are not merely passive filters, but living laboratories, acquires additional strength. It is evident that everything in the secretion must, in some form or other, exist in the blood which comes to the gland, and in the lymph which bathes its cells. No glandular cell, if we except the leucocytes, which in some respects are to be considered as unicellular glands, dips directly into the blood; everything a gland-cell receives must pass through the walls of the bloodvessels. (But see footnote 
on p. If). So that anything which we find in the secretion and do not find in the blood must liave been elaborated by the gland epithelium (or by the capillary endothelium) from raw material brought to it by the blood.

Take, for example, the saliva or gastric juice. These liquids bot $l_{1}$ contain certain things that also exist in the blood, but in addition they contain certain things specific to themselves: mucin in saliva, hydrochloric acid in gastric juice, ferments in both. It is true that a trace of pepsin and a trace of a diastatic ferment may be discovered in blood; but there is no reason whatever to believe that this is the source of the pepsin of the gastric juice, or the ptyalin of the salivary glands, except, perhaps, in animals like the cat. whose saliva contains a diastase in still smaller concentration than the serum (Carlson). On the contrary, it is possible that the ferments of the blood may be in part absorbed from the digestive glands, the rest being formed by the leucocytes and liberated when they break down.

Formation of Bile.-The liver affords an even better example of this ' manufacturing' activity of gland-cells, and many facts may be brought forward to prove that the characteristic constituents of the bile, the bile-pigments and bile-acids, are formed in the liver, and not merely separated from the blood. Bile-pigment has indeed been recognized in the normal serum of the horse, and bile-acids in the chyle of the dog, but only in such minute traces as are easily accounted for by absorption from the intestine. Frogs live for some time after excision of the liver, but no bile-acids are found in the blood or tissues. But if the bile-duct be ligatured, bile-acids and pigments accumulate in the body, being absorbed by the lymphatics of the liver (Ludwig and Fleischl). If the thoracic duct and the bile-duct are both ligatured in the dog, no bile-acids or pigments appear in the blood or tissues. Wertheimer and Lepage state that bile or bilirubin injected into a bile-duct appears sooner in the urine than in the lymph of the thoracic duct, and therefore conclude that the bloodvessels are the most important channel of absorption. This conclusion, however, cannot be accepted until it is shown that in these experiments the injection did not cause rupture of some of the hepatic capillaries and direct entrance of the bile-pigment into the blood. It is not improbable that the pressure attained by' the bile in the bile-capillaries is a factor in determining the path by which it is absorbed, and that when the pressure rises beyond a certain limit it may pass both into the bloodvessels and into the lymphatics. In mammals life cannot be maintained for any length of time after ligature of the portal vein, since this throws the whole intestinal tract out of gear. But after an artificial communication has been made between the portal and the left renal vein or th inferior cava, the portal may be tied and the animal live for months 
(Eck). The liver ean now be completely removed, but deati follows in a few hours. A good method of establishing an Eck's fistula is to make a longitudinal incision in the inferior vena cava and the portal or superior mesenteric vein, and to suture the edges of the two openings togetluer witls a very fine sewing-needle and thread (Carrel and Guthrie). In birds there exists a commmicating branch between the portal vein and a voin (therenal-portal) which passes from the posterior portion of the body to the kidney, and there breaks up into capillaries: and not only may the portal be tied, but the liver may be completely destroyed without immediately killing the animal. In the hours of life that still remain to it no accumulation of biliary substances (acids or pigments) takes place in the blood or tissues. A further indication that bile-pigment is produced in the liver is the fact that the liver contains iron in relative abundance in its cells (p. $2 \mathrm{I}$ ), and eliminates small quantities of iron in its sccretion. Now, bile-pigment, which contains no iron, is certainly formed from blood-pigment, which is rich in iron. For hamatin, when injected under the skin, has been found to appear almost quantitatively in the form of bile-pigment in the bile, and hamatoidin (Fig. I59), a crystalline derivative of hæmoglobin found in old extravasations of blood, especially in the brain and in the corpus luteum, is identical with bilirubin. The fact that one of the derivatives of hæmatin, hæmatoporphyrin $\left(\mathrm{C}_{33} \mathrm{H}_{38} \mathrm{~N}_{4} \mathrm{O}_{6}\right)$, contains no iron, and is probably nearly related to bilirubin $\left(\mathrm{C}_{32} \mathrm{H}_{36} \mathrm{~N}_{4} \mathrm{O}_{6}\right)$, suggests that hxmatoporphyrin may be an intermediate step in the formation of bile-pigment

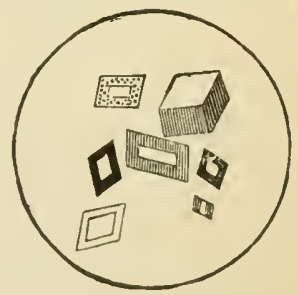

Fig. 159.-Hæmatoidin. from blood-pigment. In any case, the seat of formation of bilepigment might be expected to be an organ peculiarly rich in iron. The existence of haematoidin, however, shows that bile-pigment may, under certain conditions, be former outside of the hepatic cells. The occurrence of biliverdin in the placenta of the bitch points in the same direction. But the pathological evidence in favour of the pre-formation of the biliary constituents tends ratler to shrink than to increase. For many cases of what used to be considered 'idiopathic' or 'hæmatogenic ' jaundice, i.e., an accumulation of bile-pigments and bile-acids in the tissues, due to defective elimination by the liver, are now known to be caused by obstruction of the bile-ducts and consequent re-absorption of bile ("obstructive" or 'hepatogenic' jaundice).

But if substances such as the ferments, mucin, hydrochloric acid, the bile-salts and bile-pigments, are undoubtedly manufactured in the gland-cells, it is different with the water and inorganic salts which form so large a part of every secretion. No tissue lacks them; no 
physiological process gues on without them; they are not high and special products. As we breathe nitrogen which we do not need because it is mixed with the oxygen we require, the secreting cell passes through its substance water and salts as a sort of by-play or adjunct to its specific work. But this is not the whole truth. The gland-cell is not a mere filter througly which water and salts pass in the same proportions in which they exist in the liquids that the cell draws them from. When, e.g., the salivary glands secrete against the resistance of an abnormally high pressure in the ducts, the percentage of salts in the saliva increases. The secretions of different glands differ in the nature, and especially in the relative proportions, of their inorganic constituents. They differ also in their osmotic pressure and electrical conductivity, which depend so largely upon those constituents, notwithstanding tlie fact that the osmotic pressure and conductivity of the blood-serum (p. 26) vary only within narrow limits. Even the secretion of one and the same gland is by no means constant in this respect, as we shall have to note more especially when we come to deal with the influence of the nerrous system on secretion (p. 395). The following tables illustrate this point:

\begin{tabular}{|c|c|c|c|c|}
\hline \multirow{2}{*}{ Dog. } & \multicolumn{2}{|c|}{ Hlood-Serum." } & \multicolumn{2}{|c|}{ Filtra:e of Gastric Contents. } \\
\hline & $\Delta t$ & $K:\left(5^{\circ} \mathrm{C}.\right) \times 10^{4}$ & $\Delta$ & $K !\left(5^{\circ}\right.$ C. $) \times 10^{4}$ \\
\hline $\begin{array}{l}\text { I. } \\
\text { II. } \\
\text { III. }\end{array}$ & $\begin{array}{l}0.643^{\circ} \\
0.628^{\circ} \\
0.602^{\circ}\end{array}$ & $\begin{array}{l}92 \cdot 0 \\
87 \cdot 6 \\
87 \cdot 7\end{array}$ & $\begin{array}{l}0.585^{\circ} \\
0.585^{\circ} \\
0.642^{\circ}\end{array}$ & $\begin{array}{l}312 \cdot 5 \\
179 \cdot 4 \\
351 \cdot 7\end{array}$ \\
\hline $\begin{array}{l}\text { Vomit } \\
\text { tinal }\end{array}$ & $\begin{array}{l}\text { n with } \\
\text { uction }\end{array}$ & $\begin{array}{c}\text { plete intes- } \\
-\end{array}$ & $0.433^{\circ}$ & $84 \cdot 7$ \\
\hline
\end{tabular}

Pancreatic Juice of Dog (Pincussohn).

\begin{tabular}{|c|c|c|c|c|}
\hline \multicolumn{4}{|c|}{ Diet. } & $\Delta$ \\
\hline $\begin{array}{l}\text { Milk - } \\
\text { Cauliflower } \\
\text { Horseflesh }\end{array}$ & - & $\overline{-}$ & - & $\begin{array}{l}0.57^{\circ}-0.63^{\circ} \\
0.58^{\circ}-0.63^{\circ} \\
0.02^{\circ}-0.63^{\circ}\end{array}$ \\
\hline
\end{tabular}

* The blood and gastric contents were obtained from the animals twenty. four hours after the last meal.

* The depression of the freezing-point below that of distilled water

* See footnote on $\mathrm{p} .27$. The number in brackets is the temperature at which the measurement was made. 
Gastric Juice from Miniature Stomach in a Dog in Different Experiments (Bickel).

\begin{tabular}{|c|ccc|}
\hline \multicolumn{2}{|c|}{ Milk Diet. } & \multicolumn{2}{c|}{ Meat Dict. } \\
\hline 1 & $K\left(25^{\circ} \mathrm{C}.\right) \times 10^{\circ}$ & 1 & $K\left(25^{\circ} \mathrm{C}.\right) \times 10^{\circ}$. \\
\hline & & & \\
\hline $0.52^{\circ}$ & 195.9 & $0.60^{\circ}$ & 310.3 \\
$0.65^{\circ}$ & 402.6 & $0.71^{\circ}$ & 473.5 \\
$0.64^{\circ}$ & 436.5 & $1.21^{\circ}$ & 483.3 \\
$0.69^{\circ}$ & 40.2 & $0.79^{\circ}$ & 514.1 \\
$0.81^{\circ}$ & 436.5 & $0.70^{\circ}$ & 514.1 \\
\hline
\end{tabular}

$\Delta$ of Blood and Saliva Compared (Jappelli).

\begin{tabular}{|c|c|}
\hline s of Blood. & د of Submaxillary Saliva of Dog. \\
\hline $0.570^{\circ}$ & $0.410^{\circ}$ \\
$0.610^{\circ}$ & $0.350^{\circ}$ \\
$0.600^{\circ}$ & $0.430^{\circ}$ \\
$0.590^{\circ}$ & $0.410^{\circ}$ \\
$0.580^{\circ}$ & $0.450^{\circ}$ \\
$0.605^{\circ}$ & $0.425^{\circ}$ \\
$0.650^{\circ}$ & $0.380^{\circ}$ \\
$0.610^{\circ}$ & $0.475^{\circ}$ \\
& \\
\hline
\end{tabular}

$\Delta$ of Human Fistula Bile.

$$
\begin{aligned}
& 0.56^{\circ} \\
& 0.547^{\circ} \\
& 0.615^{\circ} \\
& 0.60^{\circ} \\
& 0.545^{\circ}
\end{aligned}
$$

$د$ of Human Bladder Bile.

$$
\begin{aligned}
& 0.65^{\circ} \\
& 0.965^{\circ} \\
& 0.78^{\circ} \\
& 0.92^{\circ}
\end{aligned}
$$

$\Delta$ of Dog's Submaxillary Saliva.

Chorda stimulated:
Left submaxi
Both glands
Spontaneous secretion:

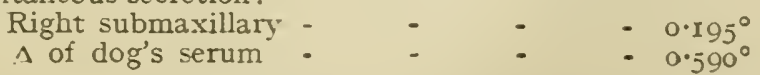

The protein substances, such as serum-albumin and globulin, common to blood and to some of the digestive secretions, take a middle place between the constituents that are undoubtedly manu- 
factured in the cell and those which seem by a less special and laborious, though a selective, process to be passed through it from the blood. Their practical absence from bile, and, as we shall see, from urine, their relative abundance in pancreatic and scantiness in gastric juice, point to a closer dependence upon the special activity of the gland-cell than we can suppose necessary in the case of the salts.

Although it is in the cells of the digestive glands that the power of forming ferments is most conspicuous, it is by no means confined to them. It seems to be a primitive, a native power of protoplasm. Lowly animals, like the amœba, lowly plants, like bacteria, form ferments within the single cell which serves for all the purposes of their life. The ferment-secreting gland-cells of higher forms are perhaps only lopsided amœbæ, not so much endowed with new properties as disproportionately developed in one direction. The contractility has been lost or lessened, the digestive power has been retained or increased; just as in muscle the power of contraction has been developed, and that of digestion has fallen behind. The muscle-cell and the cartilagecell are parasites, if we look to the function of digestion alone. They live on food already more or less prepared by the labours of other cells; and it is a universal law that in the measure in which a power becomes uscless it disappears. But the presence of pepsin in the white bloodcorpuscles, the parasites as well as the scavengers of the blood, and of amylnlytic, proteolytic and lipolytic ferments in many tissues, should warn us not to conclude that the power of forming ferments belongs exclusively to any class of cells. There is good and growing evidence that food-substances absorbed from the blood are further decomposed and, in turn, elaborated by ferment action within the tissues themselves; while many facts show that the power of contraction is widely diffused among structures whose special function is very different, and a few point to its possession in some degree even by glandular epithelium. On the other hand, it must be remembered that none of the digestive glands absorb food directly from the alimentary canal to be then digested within their own cell-substance; the ferments which they form do their work outside of them; their cells feed also upon the blood.

Why are the Tissues of Digestion not affected by the Digestive Ferments?-This is the place to mention a point which has been very much debated. Why is it that the stomach or the small intestine does not digest itself ? This is really a part of a wider question : Why is it that living tissues resist all kinds of influences, which attack dead tissues with success? And we have to inquire whether the immunity of the alimentary canal to the digestive juices is an example of a general resistance of all living tissues to destructive agencies, or a specific resistance of certain tissues to certain influences.

That all living tissues cannot withstand the action of the gastric juice has been shown by putting the leg of a living frog inside the stomach of a dog; the leg is gradually eaten away (Bernard).

It is true that it has first been killed and then digested, but the question is, why the stomach-wall is not first killed and then 
digested? When the wall has been injured by caustics or by an embolus, the gastric juice acts on it. But the living epithelium that covers it is able to resist the action of the acid and pepsin, which destroys the tissues of the frog's leg. The explanation is not to be found in the alkalinity of the blood, for the frog's blood is also alkaline, and the cells that line the intestine are preserved from the pancreatic juice, which is intensely active in an alkaline medium, while the living frog's leg is not harmed by a weakly alkaline pancreatic extract, which does not digest the epithelium because it cannot kill it. A certain amount of protection may be afforded to the walls of the stomach by the thin layer of mucus which covers the whole cavity, for mucin is not affected by peptic digestion. And a mucous secretion seems in some other cases to act as a protective covering to the walls of hollow viscera, whose contents are such as would certainly be harmful to more delicate membranes, e.g., in the urinary bladder, large intestine, and gall-bladder. Still, however important such a mechanical protection may be, it does not explain the whole matter, and it is necessary to suppose that the gastric epithelium has some special power of resisting the gastric juice, either by turning any of the ferment which may invade it into an inert substance and neutralizing any intrusive acid, or by opposing their entrance as the epithelium of the bladder opposes the absorption of urea. There is reason to believe that, as a matter of fact, free hydrochloric acid cannot penetrate the living cells, and it is to be noted that both active pepsin and free acid must be present at the same point within the cells before digestion of them can take place. In the gland-cells of the pancreas the protoplasm is, no doubt, shielded from digestion by the existence of the ferment in an inert form as zymogen; and it is possible that this is one of the reasons for the existence of the mother-substance. But no such explanation is, of course, available for the intestinal epithelium. Trypsin when injected below the skin causes the tissue to break down and ulcerate. And while an active solution of trypsin can be allowed to remain a long time in an isolated loop of small intes. tine without producing any ill effect, damage is soon caused not only to the intestinal wall, but also to the liver, when the mucous membrane of the loop has been injured before the introduction of the trypsin. IVe must suppose, then, that the normal mucous membrane of the intestine prevents the absorption of trypsin, or, if it absorbs any of it, renders it harmless. On the other hand, the intestinal mucosa is injured by the natural gastric juice when introduced directly into it unless the animal takes food simultaneously or a little earlier. But for reasons already given (p. 370) injury to the intestine cannot be produced in this way in normal digestion. It is impossible to escape the conclusion that each nembrane becomes accustomed, and, so to speak, 'immune,' to the secretion normally 
in contact with it, although not necessarily to other secretions. It is easy to multiply illustrations of this principle.

The mucosa of the dog's urinary bladder is digested by the natural activated pancreatic juice of the dog, and still more readily by the natural gastric juice. Yet few tissues but the lining of the urinary tract or of tlıc large intestine could bear the constant contact of urine or fæces. When urine is extravasated under the skin, or the contents of the alimentary canal burst into the peritoneal cavity, they come into contact with tissues which, although alive, are much less fitted to resist them than the surfaces by which they are normally enclosed; and the consequences, which are not wholly lue to infection, are often disastrous. Ieucocytes thrive in the blood, but perish in urine. Blood does not harm the endothelial cells of the vessels, but kills a muscle whose cross-section is dipped into it. The defensive or, in some cases, offensive liquids secreted by many animals are harmless to the tissues which produce and enclose them. A caterpillar investigated by Poulton secretes a liquid so rich in formic acid that the mere contact of it would kill most cells. The so-called saliva of Octopus macropus contains a substance fatal to the crabs and other animals on which it preys. The blood of the viper contains an active principle similar to that secreted by its poison-glands, but its tissues are not affected by this substance, so deadly to other animals.

A step in the solution of our problem has been taken by Weinland. Starting with the idea that if special protective mechanisms against the digestive juices were anywhere to be found, it would be in the intestinal parasites whose whole existence is passed among them, he has made the important discovery that in these parasitic worms specific antiferments exist-i.e., substances which inhibit the action either of pepsin or of trypsin or of both. These substances can be precipitated from the expressed juice of the worms by alcohol, without completely losing their activity. Fibrin can be impregnated with them, and it is then, just like the 'living tissue,' rendered for a longer or shorter time unassailable by the proteolytic ferments. These facts are full of suggestion for future work, although the supposed proof that similar antiferments are contained in the cells of the mucous membrane of the stomach and intestines of the higher animals appears to have broken down. Substances can indeed be obtained by Weinland's method from the gastric and intestinal mucosa which, when added to a digestive mixture, strongly inhibit the digestion of proteins. But there is no clear proof that these substances are specific antiferments. They are probably merely some of the split products of protein (Langenskjöld). There is, however, some evidence of the existence of an antipepsin in many tissues including the mucous membrane of the stomach. As already mentioned, it is known that an antitrypsin exists in the blood, with the 
sane properties as the antitrypsin in the intestinal worms (Hamill). This explains the resistance of blood-serum to the digestive action of trypsin. In addition to this body, which hinders the action of fully-formed trypsin, and has no effect upon enterokinase, the serum of some animals contains an antikinase-i.c., a substance which hinders the action, not of trypsin, but of enterokinase, preventing it from activating the trypsinogen into trypsin.

\section{Section V.-The Influexce of the Nervous Syste.M oN the Digestive Glands.}

The Influence of Nerves on the Salivary Glands.-All the salivary glands have a double nerve-supply, from the medulla oblongata tlirough some of the cranial nerves, and from the spinal cord through the cervical sympathetic (Fig. I60).

In the dog the chorda tympani branch of the facial nerve carries the cranial supply of the sublingual and submaxillary glands. It joins the lingual branch of the fifth nerve, runs in company with it for a little way, and then, breaking off, after giving some fibres to the lingual, passes, as the chorda tympani proper, along Wharton's duct to the submaxillary gland. In the hilus of this gland most of its fibres break up into fibrils around nerve-cells situated there, and lose their medulla in doing so. A few fibres terminate in a similar manner before entering the hilus, and a few deeper in the gland. The nervous path is continued by the axis-cylinder processes (p. 85 I) of these nerve-cells, which. passing in as non-medullated fibres, end in a plexus on the basement membrane of the alyeoli. From the plexus fibrils run in among the gland-cells, but do not seem to penetrate them. The lingual, the chorda tympani proper, and Wharton's duct form the sides of what is called the chordo-lingual trianglc. Within this triangle are situated many ganglion cells, a special accumulation of which has received the name of the submaxillary ganglion. This, however, should rather be called the sublingual ganglion, since its cells, as well as the others in the chordo-lingual triangle, are the cells of origin of axons which proceed as non-medullated fibres to the sublingual gland. The sublingual gland receives its cerebral fibres partly from branches given off from the lingual in the chordo-lingual triangle after the chorda tympani proper has separated from it, and ending around the nerve-cells within that triangle, partly from the chorda itself in the terminal portion of its course. These statements rest on anatomical and physiological evidence. The latter we shall return to.

The cerebral fibres for the parotid (in the dog) pass from the tympanic branch of the glosso-pharyngeal (Jacobson's nerve) through connecting filaments to the small superficial petrosal branch of the facial, with this nerve to the otic ganglion, and thence by the auriculo-temporal branch of the fifth to the gland.

The sympathetic fibres for all the salivary glands appear to arise from nerve-cells in the upper dorsal portion of the spinal cord. Issuing from the cord in the anterior roots of the upper thoracic nerves (first to fifth, but mainly second thoracic for the submaxillary), they enter the sympathetic chain, in which they run up to the superior cervical ganglion. Here they break up into terminal twigs, and thus come into 
r.lation with ganglion colls, whose axons pass ont as non-medullated filres, and, surroumding the external carotid, reach the salivary glands along its brancles. Langley has shown, by means of nicotine (1). 182). that the sympathetic fibres for the submaxillary and sublingual, and, indecel, for the head in general in the dog and cat, are connected with nerve-cells in this ganglion, but not between it and their termination, or between it and their origin from the spinal cord.

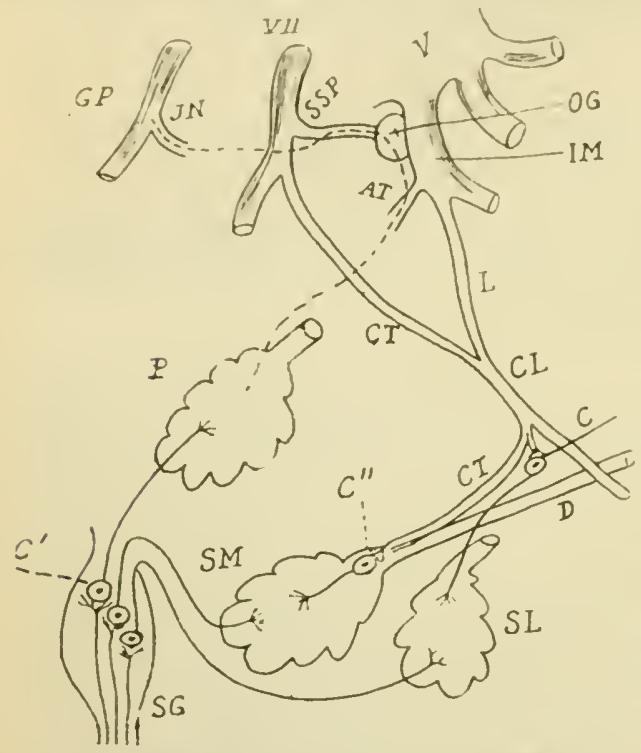

I:ig. I bo -Nerves of the Salivary Glands. S.M and SI. submaxillary and sublingual glands; $P$, parotid; V. fifth nerve; VII, facial; GP, glosso-pharyngeal; L, lingual; CT, chorda tympani; CL, chordo-lingual; 1). submaxillary (Wharton's) duct; C, ganglion cell of so-called submaxillary ganglion in the chordo. lingual triangle, connected with a nerve fibre going

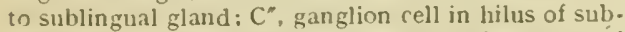
maxillary gland; SSP, small superficial petrosal l,ranch of the facial; OG. otic ganglion; I.M. inferior maxillary division of fifth nerve: AT, auriculo. Imuporal brauch of fifth: JN, Jacobson's nerve: $C^{\prime}$, ganglion cells in superior cervical ganglion (SG) connected with sympallietic fibres going to parotid. submaxillary and sublingual glands. The figure is schematic.

the lymph. That the increased secretion is not lue merely to the greater hlond-supply, and the consequent increase of capillary prossure, is shown by the injection of atropine, after which stimulation of the nerve, although it still causes dilatation of the ressels, is not followed by a flow of saliva. This can be shown fully as well by injecting a small quantity of yhimbin juto the submaxillary artery. Creat dilatation of the vessels is produced, but no saliva is secreted; 
nor is ilie amount of oxvigun consuned by the gland increased. Nere increase of pressure i ould not in any case of itself account for the secretion, since it has been found that the maximum pressure in the salivary duct when inc outflow of saliva from the duct is prevented may, during stimulation of the chorda, much exceed the artrial blood-pressure (I-urlwig). In one experiment, for example, the pressure in the carotid of a dog was $125 \mathrm{~mm}$., in Wharton's duct $195 \mathrm{~mm}$. of mercury.

Even in the head of a decapitated animal a certain amount of saliva may be caused to flow by stimulation of the chorda, but too much may easily be made of this. And since the blood is the ultimate soilce of the secretion, we could not expect a permanent or copious How in the absence of the sirculation, even if the gland-cells could continue to live. In fact, when the circulation is almost stopped by strong stimulation of the sympathetic, the flow of saliva caused by excitation of the chorda is at the same time greatly lessened or arrested, even though the sympathetic itself possesses secretory fibres. So that, while there is no doubt that the chorda tympani contains fibres whose function is to increase the activity of the gland-cells, its vaso-dilator action is, under normal conditions, closely connected with, and, indeed, auxiliary to, its secretory action. although the dilatation of the vessels does not directly produce the secretion. This is only a particular case of a physiological law of wide application, that an organ in action in general receives more blood than the same organ in repose, or, in other words, that the tissues are fed according to their needs. The contracting muscle, the secreting gland, is flushed with blood, not because an increased bloodflow can of itself cause contraction or secretion, but because these high efforts require for their continuance a rich supply of what blood brings to an organ, and a ready remoral of what it takes away. Evidence exists that in the salivary glands, as in muscle (p. $1-\delta$ ), metabolic products given off during functional activity contribute to the dilatation of the ressels. This is the simplest explanation of the fact that the dilatation caused by chorda stimulation lasts longer when saliva is being secreted than when the secretion has been abolished by atropine.

The quantity of blood passing through the parotid of a horse when it is actively secreting during mastication may be quadrupled (Chauveau). The parallel between the muscle and the gland is drawn closer when it is stated that electrical changes accompany secretion ( $\left.p . S_{3} S\right)$, and that the rate of production of carbon dioxide and consumption of oxygen (in the submaxillary gland) is three or four times greater during activity than during rest. The temperature of the saliva flowing from the dog's submaxillary during stimulation of the chorda has been found to be as much as $1.5^{\circ} \mathrm{C}$. above that of the blood of the carotid, although with the gland at rest no constant difference could be detected between the arterial blood and 
the interior of Wharton's duct. But such measurements are open to many fallacies; and while there is no doubt that more heat is produced in the active than in the passive gland, it will not be surprising, when the vastly-increased blood-flow is remembered, that no difference of temperature between the incoming and outgoing blood has been satisfactorily demonstrated.

It has already been mentioned that most of the fibres of the chorda tympani proper become connected with ganglion-cells, and lose their medulla inside the submaxillary gland, only a few having already lost it by a sinnilar connection with ganglion-cells in the chordo-lingual triangle. These facts have been made out by means of the nicotine method previously described (p. 182). Thus, it is found that, after the injection of nicotine $(5$ to $10 \mathrm{mg}$. in a rabbit or cat, 40 or $50 \mathrm{mg}$. in a dog), stimulation of the chorda tympani proper or of the chordolingual nerve causes no secretion from the submaxillary gland; but stimulation of the lililus of the gland is followed by a copious secretionas much, if the stimulation is fairly strong, as was caused by excitation of the nerve before injection of nicotine. That this is due neither to any dircct action on the gland-cells, nor to stimulation of the sympathetic plexus on the submaxillary artery, but to stimulation of chorda fibres beyond the hilus, is shown by the fact that after atropine has been injected in sufficient amount to paralyze the nerve endings of the chorda, but not of the sympathetic, stimulation of the hilus causes little or no flow of saliva. The application of nicotine solution to the chordolingual triangle does not affect the submaxillary secretion caused by stimulation of the chordo-lingual nerve, even in cases where a few secretory fibres for the submaxillary do not leave the chordo-lingual nerve in the chorda tympani proper, but are given off to the chordolingual triangle. This shows that none of the ganglion-cells in the triangle are connected with the secretory fibres of the submaxillary gland. By observations of the same kind they are known to be connected with fibres going to the sublingual. In a sinilar way, by observing the effects of stimulation of the chorda on the bloodvessels before and after the application of nicotine, it has been found that the vasodilator fibres are connected with ganglion-cells in the same positions as the secretory fibres (Langley).

Stimulation of the Sympathetic Fibres.-The sympathetic, as has been already indicated, contains both vaso-constrictor and secretory fibres for the salivary glands. If the cervical sympathetic in the dog is divided, and the cephalic end moderately stimulated, a few drops of a thick, viscid and scanty saliva flow from the submaxillary and sublingual ducts, while the current of blood through the glands is diminished. As a rule, no visible secretion escapes from the parotid, but microscopic examination shows that many of the ductules are filled with fluid, which is apparently so thick as to plug them up (Langley); while the cells show signs of 'activity' (p. 376).

Simultaneous Stimulation of Cranial and Sympathetic Fibres.When the chorda and sympathetic are stimulated together, the former prevails so far, with moderate stimulation of the iatter, that the submaxillary saliva is secreted in considerable quantity, and is not particularly viscid. It is, however, richer in organic matter than is the chorda saliva itself. When the chorda is weakly, and 
the sympathetic strongly, excited, the scanty secretion (if there is any) is of sympathetic type, thick and rich in organic matter. With strong stimulation of both nerves, the secretion, at first plentiful and watery, soon diminishes, even below the amount obtained by stimulation of the chorda alone, because of the diminution in the blood-flow, and therefore in the oxygen-supply, produced by the vaso-constrictors of the sympathetic (Heidenhain). With stimulation just strong enough to cause secretion when applied separately to either nerve, there is no secretion when it is applied simultaneously to both.

All this refers to the dog. In this animal, then, there seems to be a certain anount of physiological antagonism between the secretory action of the two nerves. But it differs in one respect from the antagonism between their vaso-motor fibres; for with strong stimulation the constrictors of the sympathetic always swamp the dilators of the chorda, while the secretory fibres of the chorda appear upon the whole to prevail over those of the sympathetic. And in all probability this apparent secretory antagonism is very superficial, and is due largely to the difference in the vaso-motor effects of the two nerves. For it has been shown that, when the blood-flow through the submaxillary gland is artificially diminished by graduated compression of its artery, stimulation of the chorda gives rise to a thick, viscid and scanty saliva, relatively rich in organic solids (Heidenhain). When the amount of blood passing through the gland is made approximately the same as during stimulation of the sympathetic, the chorda saliva becomes practically identical in composition with the sympathetic saliva. This is one reason, perhaps the chief one, why the sympathetic, when both nerves are stimulated together, without artificial interference with the bloodsupply, always appears to add something to the common secretion when there is a secretion at all, this something being represented by an increase in the percentage of organic matter. The observation that the sympathetic effect persists after stimulation has been stopped, and that excitation of the chorda after previous stimulation of the sympathetic causes a flow of saliva richer in organic matter than would have been the case if the sympathetic had not been stimulated, has long been considered a proof that the secretory fibres of the two nerves are widely different in function. To explain this result, Heidenhain assumed the existence in the sympathetic of a preponderance of fibres concerned in the building up in the cells of the organic constituents of the saliva (so-called 'trophic,' or, better, since the word 'trophic' is usually associated with the building up of the bioplasm itself, 'trophic-secretory' fibres). It would seem, however, that the increase in organic constituents is only realized when a sufficient time has not been allowed, after stimulation of the sympathetic, for the normal circulation to become re-established in the gland. The saliva obtained by stimulation of 
the chorda immediately after a period of artificially diminished blood-flow, without any previous excitation of the sympathetic, also contains a surplus of organic matter (Carlson).

Indeed, the distinction between clorda and sympathetic saliva, which, by taking account of the parotid as well as the submaxillary and sublingual glands, has been generalized into a distinction between cerebral and sympathetic saliva, and which, when the vaso-motor conditions are left out of account, appears to hold good in the dog and the rabbit, breaks down before a wider induction. For in the cat the sympathetic saliva of the submaxillary gland, although more scanty, is more watery than the chorda saliva (Langley). which, however, is by no means viscid; and the two secretions differ far less than in the dog. The discovery of Carlson that the usual effect of stimulation of the cat's cervical sympathetic with a weak interrupted current is a marked augmentation in the blood-flow* through the submaxillary gland affords an explanation. In accordance with this functional similarity, there is a much smaller difference in the action of atropine on the two sets of fibres in the cat than in the dog, although even in the cat the sympathetic is less readily paralyzed than the chorda.

In their secretory action there is not even an apparent antagonism in the cat, with minimal stimulation of both nerves. which causes as much secretion as would be produced if both were separately excited. Further, even in the dog, after prolonged stimulation of the sympathetic, the submaxillary saliva is no longer viscid, but watery, the proportion of solids, and especially of organic solids, being much lessened, as it is also in chorda saliva after long excitation. When the cerebral nerve of the resting gland is strongly excited, it is found that up to a certain limit the percentage of organic matter in a small sample of saliva subsequently collected during a bricf weak excitation increases with the strength of the previous stimulation; this is also true of the inorganic solids. But there is a striking difference when the experiment is made on a gland after a long period of activity; here increase of stimulation causes 110 increase in the percentage of organic material, while the inorganic solids are still increased. In both cases the absolute quantity of water, and therefore the rate of flow of the secretion, is augmented.

All this points to the same conclusion as the microscopic appearances in the gland-cells, that the cells during rest manufacture the organic constituents of the secretion, or some of them, and store them up, to be discharged during activity. The water and the inorganic salts, on the other hand. seem rather to be secreted on the spur of the moment, so to speak, and not to require such elaborate preparation. And it has been stated that when the

* The increased blood-flow, which can be even better clicited by-injection of adrenalin, immediately succeeds the secretion of saliva, and seems to be due not to the presence of vaso-dilator fibres in the sympathetic, but to metabolic products. 
chorda tympani is stimulated with currents of varying strength, the quantity of organic substances in small samples of saliva collected from a fresh gland is more nearly proportional to the rate of secretion than is the quantity of water and salts, which varies also with the blood-supply.

Lest the apparently insignificant result of artificial stimulation of the sympathetic in such animals as the dog should cause its secretory action to be appraised at too low a value, it should be remembered that in rne intact body the sympathetic secretory fibres, when they are excited, are, it may be assumed, excited independently of the vaso-constrictors, and even in conjunction with the vasodilators of the salivary glands.

It is conceivable that such differences between chorda and sympathetic saliva as are not accounted for by the differences in the blood-flow during their stimulation are due, not to the nerve fibres, but to the end organs with which they are connected; that is, the two nerves may supply, not the same, but different glandcells. And it is well known that even after prolonged stimulation of the chorda or chordo-lingual alone, some alveoli of the dog's submaxillary gland remain in the 'resting' state; after stimulation of the sympathetic alone, the number of unaffected alveoli is much greater; while after stimulation of both nerves, few alveoli seem to have escaped change. If there is no essential difference between the cranial and sympathetic secretory fibres, it is easy to understand that they will be distributed to different secreting elements. The supposed proof that there must be some overlapping in the nervesupply-i.e., that some cells must be supplied from both sources, since excitation of the sympathetic influences the amount of organic material in the saliva obtained by subsequent stimulation of the chorda-is, as we have just seen, by no means so cogent as has been assumed. And, indeed, we know nothing of a division of labour between the cells of a gland, except when there are obvious anatomical distinctions. Thus, the submaxillary gland in man contains both serous and mucous acini, and mucin-making cells are scattered over the ducts of most glands, and, indeed, on nearly every surface which is clad with columnar epithelium. In these cases we cannot doubt that one constituent-mucin-of the entire secretion is manufactured by a portion only of the cells. In the cardiac glands of the stomach, too, the ovoid cells, in all probability, vield the whole of the acid of the gastric juice. But, so far as we know, every hepatic cell is a liver in little. Every cell secretes fully-formed bile; every cell stores up, or may store up, glycogen. So it is with the secretory alveoli of the pancreas, if we consider the islands of Langerhans as having no connection with the alveoli; one cell is just like another; all apparently perform the same work; each is a unicellular pancreas. (See p. 638.)

Paralytic Secretion.-When the chorda tympani is divided, a slow 'paralytic' secretion from the submaxillary gland begins in a few 
hours, and continues for a long time accompanied by atrophy of the gland. There is also a secretion of the same kind from the submaxillary on the opposite side, but it is less copious. This is called the 'antilytic' secretion, which is most pronounced in the first few days after the operation, and seems to be a transient phenomenon. It can be at once abolished by section both of the chorda and the sympathetic on the corresponding side, and is therefore due to impulses arising in the central nervous system. The cause of the paralytic secretion has net been fully made out. If within two or three days of division of the chorda the sympathetic on the same side is cut, the secretion is greatly diminished or stops altogether; and it is concluded that up to this time it is maintained by impulses passing along the sympathetic to the gland from the salivary centre, the excitability of which has been in some way increased by division of the chorda, possibly by some such degenerative process in the cells as the changes seen in cerebro-spinal motor cells whose axons have been divided (p. 858). This may also account for the antilytic secretion. But if section of the sympathetic is not performed for several days, it has no effect on the paralytic secretion, which at this stage seems to depend on local changes in or near the gland itself, leading to a mild continuous excitation of those nerve-cells on the course of the fibres of the chorda to which reference has already been made. Section of the sympathetic alone causes neither secretion nor atrophy, nor does removal of the superior cervical ganglion. The histological characters of the gland-cells during paralytic secretion are those of 'rest.'

Reflex Secretion of Saliva.-The reflex mechanism of salivary secretion is very mobile, and easily set in action by physical and mental influences. It is excited normally by impulses which arise in the mouth, especially by the contact of food with the buccal mucous membrane and the gustatory nerve-endings. The mere mechanical movement of the jaws, even when there is nothing between the teeth, or only a bit of a non-sapid substance like indiarubber, causes some secretion. The vapour of ether gives rise to a rush of saliva, as does gargling the mouth with distilled water. The smell, sight, or thought of food, and even the thought of saliva itself, may act on the salivary centre through its connections with the cerebrum, and make 'the teeth water.' A copious flow of saliva, reflexly excited through the gastric branches of the vagus, is a common precursor of vomiting. The introduction of food into the stomach also excites salivary secretion.

The researches of Pawlow and his pupils have shown that the salivary glands are not excited indifferently by everything which comes into contact with the buccal mucous membrane. A remarkable adaptation exists between the properties of food or foreign bodies introduced into the mouth and their effects upon the secretion of saliva. When solid dry food is given to a dog saliva is copiously poured out; much less is secreted when the food is moist.

Acids or salts induce an abundant flow, in order that they may be neutralized, diluted or washed out of the mouth. In this case a watery liquid, poor in mucin, flows from the mucous glands. Mucin is a lubricant to facilitate the swallowing of solid food, and 
here it could be of no use. When clean pebbles are put in the dog's mouth the animal may try to chew them, but eventually ejects them. Either no saliva or very little is secreted, since it could not aid in their expulsion. If, however, the very same stones are reduced to sand and again introduced into the animal's mouth, saliva is plentifully secreted to wash it out.

The serous and mucous salivary glands are not necessarily excited by the same food materials, and here again we can trace an astonishingly exact adaptation. A permanent parotid or submaxillary fistula can easily be made in a dog by freeing Stenson's or Wharton's duct from the surrounding mucous membrane for a little distance, bringing the natural orifice of the duct out through a small wound in the cheek, and stitching it in position there. When it is desired to collect saliva, the wide end of a funnel-shaped tube, whose stem is bent so as to hang vertically, can be attached by a little shellac of low melting-point to the skin around the orifice of the duct and at some distance from it, and on the narrow end can be hung a small graduated tube, into which the saliva drops. When fresh meat is given to the animal little or no parotid saliva is secreted, while a copious flow takes place from the submaxillary gland, mucin being required to lubricate it for deglutition, while water is not specially needed. But if the meat is in the form of a dry powder the parotid pours out a plentiful secretion, while the submaxillary also secretes a fluid relatively rich in mucin. The same difference is seen between fresh moist bread and dry bread. The afferent nerve-endings from which impulses are carried to the reflex centres (or the portions of the salivary centre) which preside over the various salivary glands must possess the power of very delicate selection as regards the kinds of stimulation by which they are affected. The mere relish of the animal for the different kinds of food plays but a small part. Most dogs display a much livelier interest in a piece of meat than in a piece of dry biscuit, yet it is the biscuit which excites the parotid to activity.

The sight of dry food causes an abundant flow of watery saliva from the parotid, and a flow of fluid rich in mucin from the submaxillary. Various uneatable substances, including substances which in contact with the mucous membrane of the mouth produce strong and disagreeable stimulation of it, and excite disgust, cause also, when viewed from a distance, secretion by all the salivary glands; but the submaxillary saliva, as ought to be the case for substances unfit for food, and therefore not destined to be swallowed, is poor in mucin. When the animal is shown pebbles and sand the phenomena are qualitatively the same as when they are put into its mouth - the glands remaining inactive in presence of the pebbles, but secreting plentifully at sight of the sand. In short, the same adaptation is observed in the case of the so-called psychical secretion as when the stimulating substances act directly 
upon the endings of the afferent salivary nerves in the buecal mucous membranc. It is further worthy of note that when the animal is hungry the psychical secretion is most copious and most easily obtained. After a full meal it cannot be excited at all. When food (or other exciting substance) is repeatedly shown to a fasting animal the reaction becomes each time weaker, and finally the glands cease to respond. A!l that is then necessary to restore the reaction is to put into the animal's mouth a little of the food (or other object). When it is now shown it at a distance the ordinary effect follows promptly. This indicates that the condition of the salivary centre exercises an important influ'nce upon the psychical secretion, its excitability to the weaker stimulus set up by the sight of the object being increased by the stronger reflex stimulation coming directly from the mouth. In the condition of saticty the inexcitability of the centre may be due to the action of foodproducts in the blood.

In most animals and in man the activity of the large salivary glands is strictly intermittent. But the smaller glands that stud the mucous membrane of the mouth never entirely cease to secrete, and the same is the case with the parotid in ruminant animals.

The centre is situated in the medulla oblongata, stimulation of which causes a flow of saliva. The chief afferent patls to the salivary centre are the lingual branch of the fifth and the glossopharyngeal; but stimulation of many other nerves may cause reflex secretion of saliva. In experimental reflex stimulation, the sole efferent channel seems to be the cerebral nerve-supply of the glands. After section of the chorda, no reflex secretion by the submaxillary gland can be caused, although the sympathetic remains intact.

It was alleged by Bernard that, after division of the chordolingual, a reflex secretion could be obtained from the submaxillary gland by stimulating the central end of the cut lingual nerve between the so-called submaxillary ganglion and the tongue, the ganglion being supposed to act as ' centre.' It has been shown, however, that this is not a true reflex effect, but is due to the excitation of certain (recurrent) secretory fibres of the chorda that run for some distance in the lingual, then bend back on their course and pass to the gland. It may be in part a pseudo- or axon-reflex (p. gr.3), elicited by excitation of efferent fibres, which send branches to some of the ganglion-cells.

The salivary centre can also be inhibited, especially by emotions of a painful kind-for instance, the nervousness which often dries up the saliva, as well as the eloquence, of a beginner in public speaking, and the fear which sometimes made the medieval ordeal of the consecrated bread pick out the guilty.

In rare cases the reflex nervous mechanism that governs the salivary glands appears to completely break down; and then two opposite conditions may be seen-xerostomia, or 'dry mouth,' in 
which no saliva at all is secreted, and chronic ptyalism, or hydrostomia, where, in the absence of any discoverable cause, the amount of secretion is pernanently increased. Both conditions are saicl to be more common in wonlen thitn in men.

The Influence of Nerves on the Gastric Glands.-I ike saliva, gastric juice is not secreted continuously, except in animals such as the rabbit, whose stomachs are never cmpty. 'The normal and most efficient stimulus is the eating of food and its presence in the stomach. Mechanical stimulation of the gastric mucous membrane with a non-digestible substance, such as a feather or a glass rod, causes secretion of mucus, but not of gastric juice. But the observations mentioned above on the difference of response of the salivary glands to different substances suggest that the local mechanical stimulation of the food on the gastric glands may be more effective. There is also at first thought much to indicate that the gastric glands are stimulated chenically in a more direct manner than the salivary glands by the local action of food substances reaching the cells by a short-cut from the cavity of the stomach, or in a more roundabout way by the blood. And it might be very plausibly argued that the gastric glands are favourably situated for direct stimulation, while the large salivary glands are not; and that the great function of saliva being to aid deglutition, an almost momentary, and at the same time a perilous act, it is necessary to provide by a nervous mechanism for an immediate rush of secretion at any instant, while it is not important whether the gastric juice is poured out a little sooner or a little later, and therefore it is left to be called forth by the more tardy and liaphazard method of local action. Nevertheless, on looking a little closer, we find that this does not exhaust the subject, and that the gastric secretion can be influenced by events taking place in distant parts of the body, just as the salivary secretion can. In a boy whose œsophagus was completely closed by a cicatrix, the result of swallowing a strong alkali, and who had to be fed by a gastric fistula, it was found that the presence of food in the mouth, and even the sight or smell of food, caused secretion of gastric juice (Richet).

Here there must have been some nervous mechanism at work. The secretion cannot have been excited by the direct action of absorbed food-products circulating in the blood-an explanation which might be given, though an insufficient one, of the secretion seen in an isolated portion of the cardiac end of the stomach during the digestion of food in the rest. The cfferent nervous channels through which these effects are produced have been defined by Pawlow's experiments on dogs. He first made a gastric fistula, then a few days afterwards divided the cesophagus through a wound in the neck, and stitched the two cut ends to the edges of the wound. After the animals had recovered, it was observed that when meat was given to them by the mouth, a copious secretion of 
gastric juice followed in five or six minutes, notwithstanding the fact that in this 'sham feeding ' the food immediately r'scaped from the opening in the upper portion of the divided resophagus. Much the same result was seen when the food was simply shown to the animal. Indeed, when a hungry animal is tempted with the sight of meat, the flow of gastric juice, always occurring after a latent period of five or six minutes, may be even greater than with sham feeding. Division of the splanchnic nerves had no effect on this reflex secretion, while it could not be obtained after division of both vagi below the origin of their cardiac and pulmonary branches, by which disturbance of the heart and respiration are avoided. Further, stimulation of the peripheral end of the vagus in the neck* caused secretion. These experiments show that secretory fibres for the gastric glands run in the vagi. It is probable that the vagi

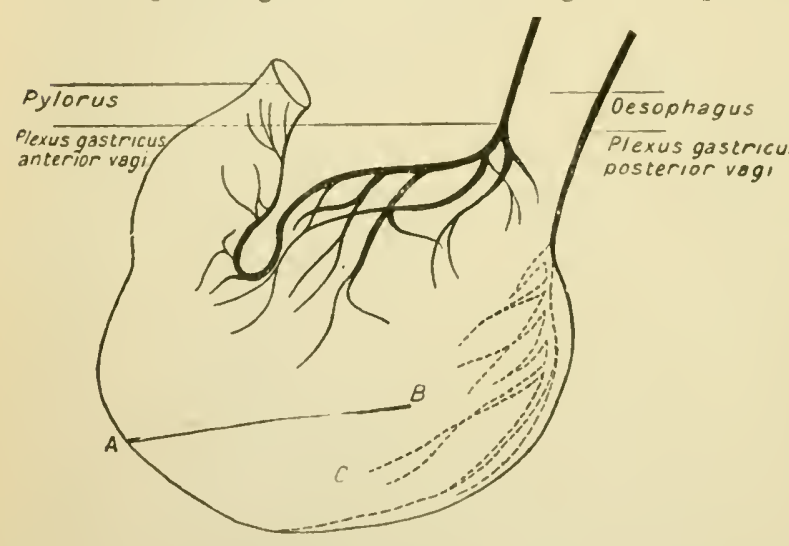

Fig. 161.-Pawlow's Stomach Pouch. AB, line of incision; C, llap for forming the stomach pouch. At the base of the flap the serous and inuscular coats are preserved, and only the mucous membrane divided, so that the branches of the vagus going to the pouch are not severed.

also contain efferent fibres which inhibit the gastric secretion. The excitation of the secretory fibres is not produced reflexly by the processes of mastication and deglutition as such. Dilute acid is the most powerful chemical stimulus for the buccal mucous membrane, and when it is introduced into the mouth of a dog with a double resophageal and gastric fistula, an abundant secretion of saliva at once ensues. But no matter how long the animal continues to swallow the mixture of saliva and acid, no gastric juice is formed. The same is the case in sham feeding with salt, pepper, mustard, smooth stones, and even extract of meat. It is the desire for food - the appetite, as we call it - and the feeling of satisfaction associaated with eating food that the animal relishes, which is the efficient cause of the gastric secretion in sham feeding. The more eagerly the dog eats, the greater is the flow of gastric juice.

* The nerve was not stimulated till a few days after the section, so as to allow the cardio-inhibitory fibres to degenerate. Otherwise the heart would have been stopped by the stimulation. 
Pawlow also performed the converse experiment. In dogs in which a pouch had heen isolated from the stomach and made to open to the exterior by the surgical procedure illustrated in Figs. I6I and 162 , he introduced into the large stomach, without the animal's knowledge, food of various kinds. This is best done in a sleeping dog. The secretion of gastric juice, both in the main stomach and in the pouch or miniature stomach, which is known in a great variety of conditions to present an exact picture of the process of secretion in the large, is markedly delaverl and scanty when it does appear. Bread and coagulated egg-white did not yield a single drop during the first hour or more. Raw flesh excited a secretion, but after an interval of fifteen to forty - five minutes, instead of five or six to ten, as in sham feeding. It was very scanty during the first hour (only onethird the normal amount), and possessed a very low digestive power. The importance of the psychical ele-

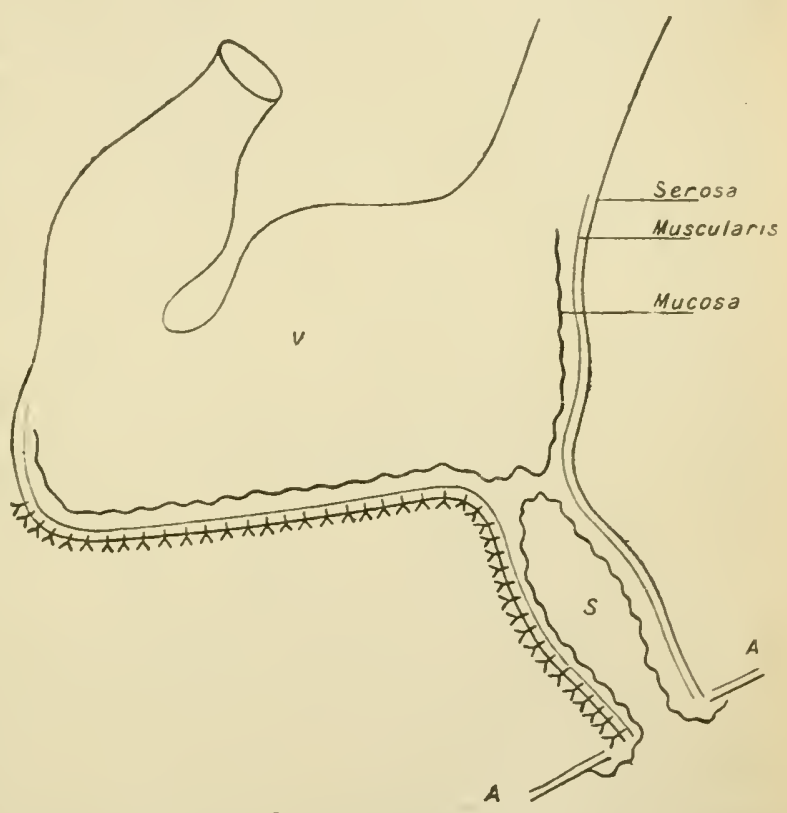

Fig. 162.-Pawlow's Stomach Pouch. S, the completed pouch; $\mathrm{V}$, cavity of stomach.

ment is shown by the fact that in one dog, which, after a weighed amount of meat had been introduced into its stomach (without its knowledge), received a sham meal of meat, the amount of protein digested after one and a half hours was five times greater than in another animal treated exactly in the same way, except that the sham meal was omitted. But even after division of the vagi, gastric secretion is still caused by the introduction of various substances into the stomach, especially water and meat extract. The active substances in the meat extract are, for the most part, insoluble in alcohol. Kreatin is inactive. It is in virtue of these substances that raw meat placed directly in the stomach causes some secretion after a 
time. Milk and gelatin solution are also direct excitants of gastric secretion apart from the water in them. Starch, fat, and egg-white are totally inert. After section of both vagi in dogs, no marked qualitative or quantitative changes have been observed in the gastric juice. The secretion caused by the presence of food in the stomach is still obtained when, in addition to the vagi, all other nerves which can possibly connect the central nervous system with the organ have been severed and the sympathetic abdominal plexuses have been destroyed (Popielski). We must therefore sugpose that the gastric glands, while normally under the control of a nervous mechanism in the upper portion of the cerebro-spinal axis whose efferent fibres run in the vagi, are also capable of being locally stimulated throug't the peripheral ganglia in the stomach walls or the chemical action of the products of digestion absorbed into the blood. Edkins showed that the injection of food substances or the products of their digestion (broth, dextrin, peptone) or of acid into the blood caused no secretion of gastric juice, while the injection of an extract of the pyloric mucous membrane, made by boiling it with water, acid, or peptone, excited a certain amount of secretion. He therefore concluded that the secondary secretion of gastric juice is determined, not by local stimulation of a reflex mechanism in the gastric wall, but by the production in the mucous membrane of the pyloric end of a chemical substance, the gastric secretin or gastric hormone, ${ }^{*}$ which is absorbed by the blood, and acts as an excitant to all the gastric glands. The cardiac mucosa was found incapable of forming this substance.

It is not to be imagined that the 'psychical ' secretion and the secretion called forth by the direct action of the food or loodproducts in the stomach perform independent offices. They can, in various instances, be shown to supplement each other. For example, not more than one-half or one-third of the gastric juice secreted during the digestion of bread or boiled egg-albumin can be ascribed to the psychic effect. Yet these substances, when introduced directly into the stomach, cause practically no secretion. We must suppose that during the digestion of the bread and albumin by the psychically secreted juice certain products analogols to those in the meat extract are formed, which act as chemical excitants of the local secretory apparatus. The psychic juice is inciispensable in this case to start the process, ' to set the stove ablaze,' as Pawlow puts it. In the case of meat it is not indispensable, since the meat can chemically excite the gastric glands; but it greatly hastens the process of digestion. These facts emphasize the importance of

* 'Hormone' (from ópuaw, I arouse or excite) is the name given to a sub stance which, carried by the blood from the place where it is formed, acts as a chemical messenger in exciting the activity of some more or less distant organ. The classical example is the pancreatic secretin which, manufactured in the intestinal mucosa, excites the secretion of the pancreatic juice. 
1)petite in digestion, a truism in treatment which thus receives for the first time a rational explanation. The influence of goodhumour upon nutrition, which experienc: has crystallized into the proverb ' Laugh and grow fat,' has also been sliown to dependin great part, at least-upon a beneficial action on the digestive functions, both motor and cliemical. The movements of a cat's stomach and intestines have becn observed to ccase when the animal became angry or excited by unpleasant emotions; and in a dog whose gastric glands were pouring out a copious psychical secretion in response to a sham meal, secretion stopped abruptly when the animal's wrath was awakened by what is probably to the normal dog the most specifically 'adequate' stimulus for the emotion of anger-the sight of a cat which he was restrained from chasing.

By means of experiments with the miniature stomach it has been further shown that cach kind of food has its own characteristic curve of gastric secretion. With flesh diet the maximum rate of sccretion occurs during the first or second hour, and in eacl of the first two hours the quantity of juice furnished is approximately the same. With bread diet we liave always a sharply-indicated maximum in the first hour, and with milk a similar one during the second or the third hour (Fig. I63). The juice secreted on different diets also differs in digestive power-i.e., in the amount of protein which a given quantity of it will digest in a given time. "Brearl juice' is much stronger in ferment than 'meat juice,' and 'meat juice' somewhat stronger than 'milk juice' (Fig. I64). But 'meat juice' has a higher aciclity than ' bread juice,' 'milk juice ' being intermediate. These differences do not necessarily indicate that the gastric mucous membrane responds in a specific way to each kind of food substance, as suggested by Pawlow. They may depend on several circumstances, and particularly on this-that the quantity, though not the quality, of the psychical or 'appetite" juice is related to the relish with which the animal eats the food. The products formed in the digestion of the different foods by the psychical juice may therefore be different in nature and amount, and thus the quantity of the gastric hormone which determines the secondary secretion may vary with the food.

The young mammal, like the adult, secretes gastric juice before the food reaches the stomach. In puppies from one to eighteen days old sham feeding (sucking the teats of the mother after an œsophageal fistula has been made in the younger animals and a double œsophageal and gastric fistula in the older) causes a liquid with the properties of gastric juice to gather in the stomach. This power, then, is a congenital one. The individual does not gain it by experience; it comes into the world with him (Cohnheim).

The Influence of Nerves on the Pancreas.-Like the stomach, the pancreas receives secretory fibres through the vagus. These are 
probably connected with a reflex centre in the medulla oblongata. It has long been known that when the medulla is stimulated a flow of pancreatic juice is occasionally set up, or is increased if already going on. The same is truc when the vagus is stimulated in the ordinary way in the neck. But the experiment often failed, for

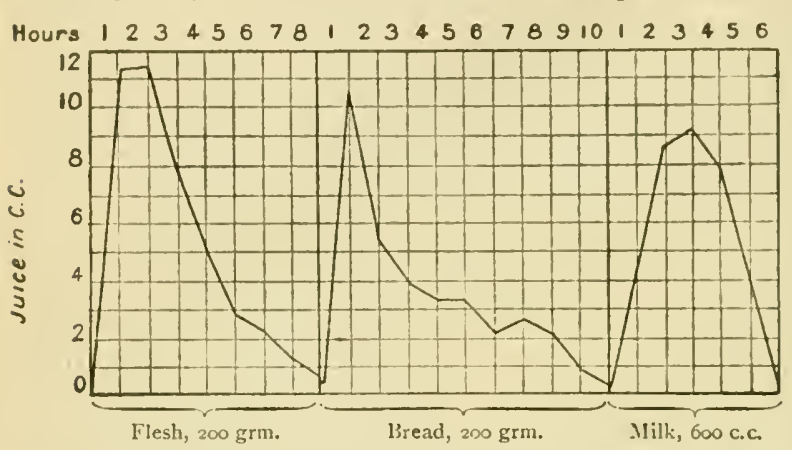

Fig. I63.- Rate of Secretion of Gastric Juice with Diets of Meat, Bread, and Milk (Pawlow). the pancreas is peculiarly susceptible to circulatory disturbances, and stimulation of the bulb or the vagus may interfere with the bloodflow through the gland by exciting its va so-con-

strictor fibres or causing inhibition of the heart. These disturbing influences may be avoided, as Pawlow has shown, by stimulating the vagus, three or four days after dividing it, with slowly-recurring stimuli (induction shocks or light blows from a small hammer worked by an electro - magnet at the rate of about one in the second). The secretory fibres are still susceptible of excitation, while the cardio-inhibitory fibres, which degenerate more rapidly, are almost or altogether in-

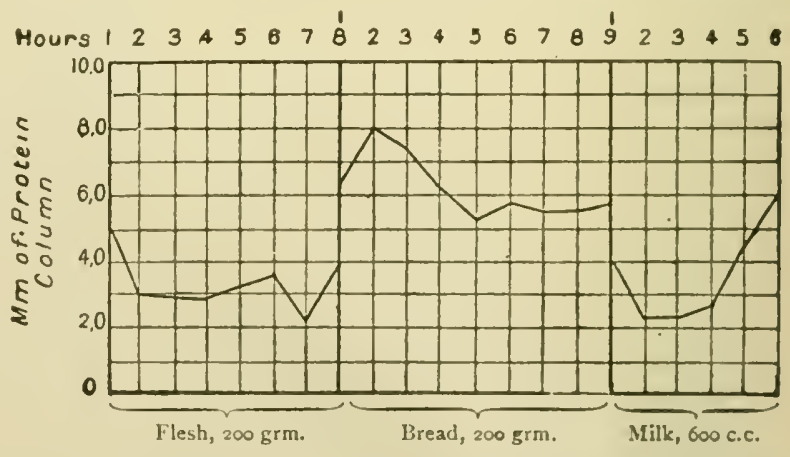

Fig. 164.-Digestive Power of Gastric Juice (Pawlow). The digestive power of the juice, as measured by the length of the protein column digested in Mett's tubes, is represented hour by hour, wth diets of flesh, bread, and mulk.

excitable, and the vaso-constrictors are but little affected by these slow rhythmical stimuli, which excite the secretory nerves (p. I75). A pancreatic fistula has previously been established by excising a small portion of the duodenal wall containing the opening of the pancreatic duct, closing the intestine by sutures, and 
stitching the orifice of the duct into the abdominal wound. On stimulation of the vagus the juice will begin in two to three minutes to drop from a cannula in the duct, and will continue to flow for several minutes after cessation of the stimulus. The sympathetic also contains secretory fibres for the pancreas. Efferent fibres which inhibit the secretion have been also discovered in the vagus. Their presence may be most clearly demonstrated when that nerve is stimulated during the flow of pancreatic juice excited by the introduction of dilute acid into the duodenum. Stimulation of the central end of the vagus and of the other nerves is capable of reflexly inhibiting the pancreatic secretion. Painful im. pressions have a strong inhibitory influence. This is one of the reasons why inany observers failed to detect the secretory nerves. The inhibition caused by vomiting is probably due to impulses ascending the vagus. It is possible that through these nervous channels the pancreatic secretion is affected by the psychical conditions connected with eating and the desire for food, just as in the case of the gastric secretion; but our information on

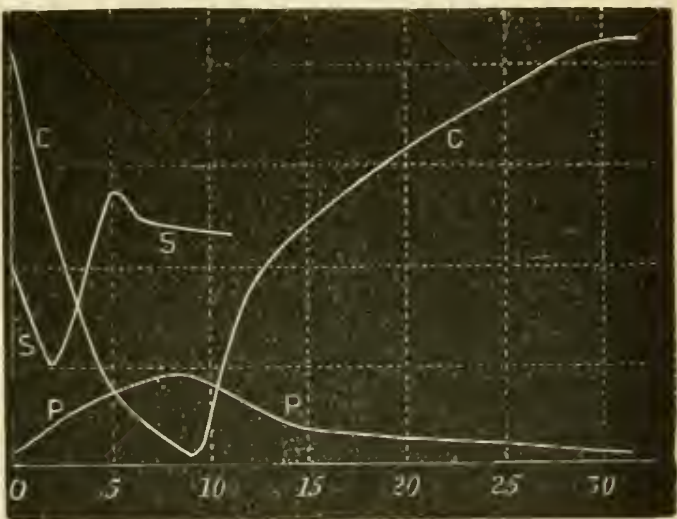

Fig. 165.- Secretion of Pepsin. C shows the quantity of pepsin(ogen) in the mucous membrane of the cardiac end of the stomach at different times during digestion; $P$, the quantity of pepsin(ogen) in the mucous membrane of the pyloric end; $\mathrm{S}$, the quantity of pepsin in the secretion of the cardiac glands. The numbers inarked along the horizontal axis are hours since the last meal. About five hours after the neal, $S$ reaches its maximum. From the very beginning of the meal $\mathrm{C}$ falls steadily down to the tenth hour, and then begins to rise-i.e., the glandcells of the cardiac end of the stomach become poorer in pepsin(ogen) as secretion proceeds.

this subject is scantier and less precise. A flow of juice may undoubtedly take place within three or four minutes after food is taken, but it is not quite certain whether this is not determined by the passage of some of the acid gastric contents into the duodenum.

Secretin.-We have already referred to the fact that pancreatic secretion is excited by the presence of acid in the duodenum. The mechanism of this action is of great interest. Two or three minutes after the introduction of 0.4 per cent. hydrochlo:ic acid into the duodenum, pancreatic juice begins to flow. A similar effect is seen when the acid is placed in the jejunum, but not when it is injected 
into the lower part of the ilemm. It is obtained as strongly and as promptly from an isolated loop of intestine when all the nerves passing to it have been cut, and the solar plexus extirpated, and also after the administration of atropine, which paralyzes the endings of secretory nerves elsewhere. The secretion accor lingly does not depend upon a local reflex mechanism, with its afferent endings in the intestinal mucous membrane, lut upon some substance which is carried to the pancreas by the blood, and acts directly upon its cells. This substance is not the acid, for the injection of 0.4 per cent. hydrochloric acid into the blood produces no effect upon the pancreas. It has been shown by Bayliss and Starling that the exciting substance is a diffusible body of low molecular weight, probabiy of organic natuse, but not a protein, which they call secretin. It is soluble in alcohol or alcohol and ether, and is not destroyed by boiling. It is produced in the mucous membrane of the jejunum or duodenum on exposure to dilute hydrochloric acid. Extracts of mucous membrane so treated cause a copious pancreatic secretion, and a smaller secretion of bile, when injected in small quantities into the blood of animals in which no such secretion is taking place, but have no influence on any other gland. At the same time the arterial blood-pressure falls somewhat. The substance which produces the fall of blood-pressure is different from secretin, since acid extracts of the lower end of the ileum, which have no effect on the flow of pancreatic juice. diminish the blood-pressure. A precursor of secretin, called pro-secretin, exists in the intestinal mucous membrane, and can be extracted from it by physiological salt solution. It dnes not affect the pancreatic secretion. By boiling or by the action of acid secretin is split off from it. Pro-secretin is most abundant in the duodenum, and diminishes as we pass down the intestine.

Secretin is very widespread in the animal kingdom. In the monkey, dog, cat, rabbit, man, ox, slicep, pig, squirrel, goose, tortoise, salmon, dog-fish, and skate evidence of its presence has been obtained. The secretin of one animal will excite a flow of pancreatic juice in an animal of a different kind as well as in one of the same kind. In normal digestion secretin is formed under the influence of the acid chyme, not in the stomach, but after it has passed into the duodenum. The passage of the chyme through the pylorus, as previously mentioned (p. 327), is regulated by the reaction of the duodenal contents, as well as by the consistence of the gastric contents. So long as the liquid in the duodenum is acid, the pylorus remains closed. As soon as the first small portion of acid chyme ejected from the stomach has been neutralized by the increased secretion of the pancreatic juice and the outpouring of bile from the gall-bladder in response to the stimulus of the acid, the pylorus opens again.

According to Pawlow, certain food substances, notably fat, and 
water stimulate the pancreatic secrition, and with great promptness, even before any acid has been produced in the stomach, and therefore before any can have passed into the duodenum. Possibly this effect is elicited through the long reflex patlis already described as ru'ning in the vagi or through a local nervous mechanism, which, although it does not take part in the excitation of the pancreatic secretion by acid, may yet exist for the performance of other offices. It is more probable, however, that it is due to the passage of some of the gastric contents through the pylorus; for when oil is introduced into the small intestine, it causes the production of secretin, although, unlike dilute acid, it is quite ineffective in forming secretin when rubbed up with the scraped-off mucous membrane. That sccretin acts on the pancreatic cells in a different way from the secretory nerve fibres contained in the vagus is indicated by the difference in the characters of the juice secreted under the influence of the two mechanisms. The nervous secretion is thick and opalescent, rich in enzymes, including trypsin in the active form, and proteins, but its alkali content is low. Like other secretions excited through nerves, it is inhibited by atropine. The chemical secretion due to secretin, is thin and watery, rich in alkalies, poor in proteins and in enzymes, and among the latter, trypsin occurs only in the inactive form (Sawitsch).

The pancreatic, like the gastric, juice is said to vary as regards its digestive properties with the

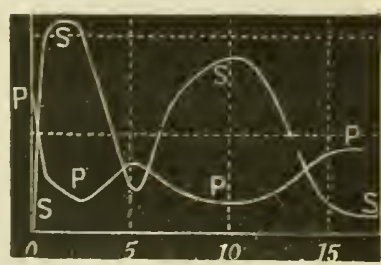

Fig. 166.- Rate of Secretion of Pancreatic Juice. S shows the variation in the rate of secretion of the pan. creatic juice in a dog; $P$, the varia. tion in the percentage of solids in the juice. It will be seen that the maxima of $\mathrm{S}$ fall at the same time as the maxima of $P$. The numbers along the horizontal axis are hours since the last meal. nature of the food. On a diet of bread the juice is very poor in fat-splitting ferment, while on a diet of flesh it is richer, and on a diet of milk richest of all. With bread the juice is relatively rich in amylolytic ferment. When we take the quantity of the juice as well as its strength in ferments into consideration, it is stated that bread occasions the secretion of a juice with a greater quantity of proteolytic ferment than either milk or meat, although it is relatively dilute (Fig. I69). The vegetable proteins require more ferment to digest them than proteins of animal origin. There is no more evidence that the adaptation of the pancreatic juice to the nature of the food is due to a specific sensibility of the duodenal mucosa to the various food-stuffs than there is in the case of the adaptation of the gastric juice. If the volume of the chyme and its acidity are related to the nature of the food, then the amount of secretin formed, and therefore the intensity of secretion in the 
pancreas, will be similarly related. The one apparently proved example of specific adaptation of the pancreatic juice has not stood the test of a critical examination. It was asserted that in dogs fed for some days with food containing lactose (milk) the ferment, laclase, is present in that secretion, while the pancreatic juice of dogs whose food is free from lactose does not contain lactase. The adaptation of the pancreas to lactose was supposed to be achieved through some substance produced by the action of lactose on the

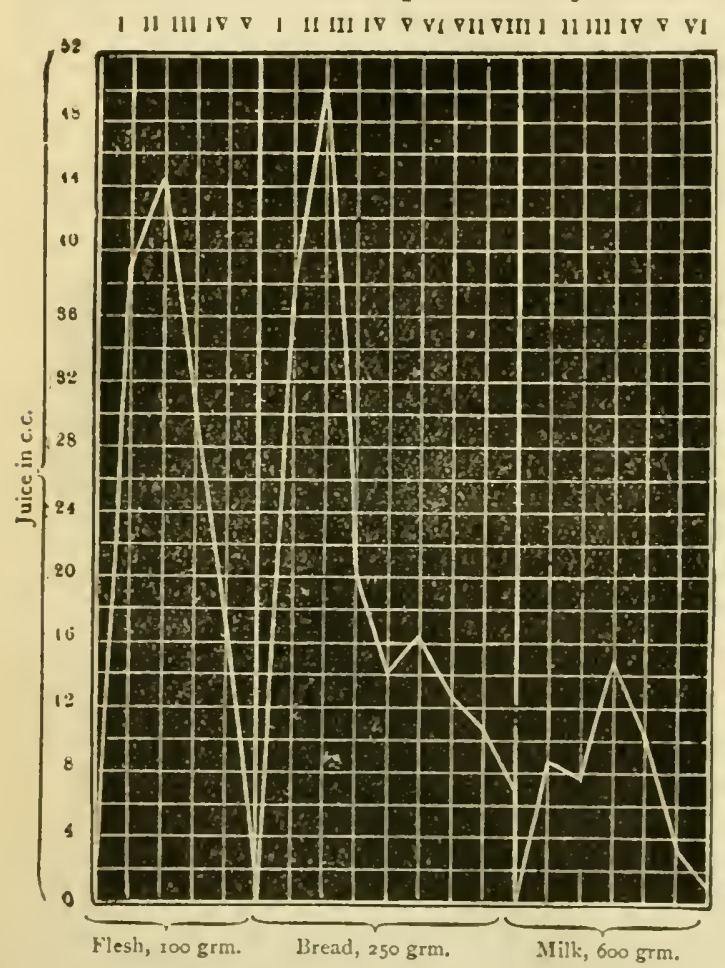

Fig. 167.-Secretion of Pancreatic Juice with Different Diets (Pawlow). The hours are in roman numerals. intestinal mucous membrane, which plays the part of a specific chemical stimulus to the pancreatic cells or their secretory nervous mechanism, causing them to form lactase. But it has been conclusively shown that when dogs are fed with lactose for weeks no lactase appears in the pancreatic juice (Plimmer).

The natural secretion of pancreatic juice is by no means so intermittent as that of saliva. In the rabbit the pancreatic, like the gastric, juice flows continuously. In the dog it begins almost as soon as

food is taken, rises in two or three hours to a maximum, then falls till the fifth or sixth hour, after which it may mount again somewhat, and then, gradually diminishing, ultimately stops (Figs. I66, I67). During normal activity the bloodvessels of the gland are dilated. But under experimental conditions the increased secretion caused by secretin is accompanied sometimes by an increase and sometimes by a diminution in the blood-flow, and secretion may continue for some time after complete cessition of the circulation, 
while the increased consumption of oxygen which goes hand in hand with the increased secretion is also independent of the blood-supply (May, Barcroft and Starling). This shows how far the secretory process is from a mere mechanical filtration, although it does not follow that, under normal conditions, a decreased blood-flow ever does accompany an increased secretion. There is one difference between the normal secretion of pancreatic juice and of saliva which may still be mentioned: the pressure of the latter in the submaxillary duct may, as we have seen, greatly exceed the arterial blood-pressure, without reabsorption and consequent œdema of the gland occurring; but the secretory pressure of the pancreatic cells is very low, not more than a tenth of that of the salivary glands. Edema begins before a manometer in the duct shows a pressure of $20 \mathrm{~mm}$. of mercury, the secreted fluid passing very easily into the lymph spaces.

The mutual relations of the spleen and pancreas have formed the subject of numerous inquiries. Some authors maintain that the spleen plays an important rôle in the elaboration of the proteolytic ferment of the pancreas, forming a substance which we may call pro-trypsinogen, since it is supposed to be carried in the blood to the pancreatic cells, and changed by them into trypsinogen. There is some evidence that extracts of the spleen prepared from it when congested during digestion exert a favourable influence on the proteolytic power of the pancreas (Mendel). And there is no doubt that the spleen, like other organs, contains an intracellular enzyme which can aid in the digestion of protein. The products of the action

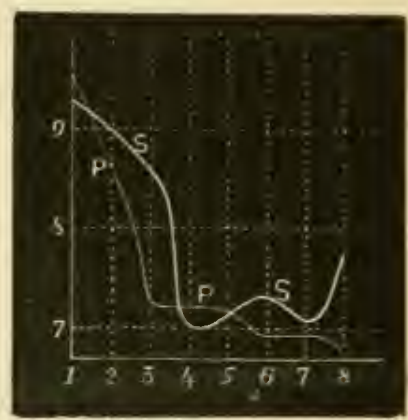

Fig. 168.- Rate of Secretion of Bile. S shows how the rate of secretion of bile falls in a dog when a biliary fistula is first made, and the bile thus pre. vented from entering the intes. tine; P shows the fall of the per. centage of solids. The numbers along the horizontal axis are quarters of an hour since bile began to escape through the tistula. The numbers along the vertical axis refer only to curve $S$, and represent the rate of secretion in arbitrary units. in an acid medium of this enzyme are the same as those formed by trypsin in an alkaline medium (Leathes). But this is not enough to prove that the spleen has any special relation to pancreatic digestion.

The Influence of Nerves on the Secretion of Bile.-Although bile is secreted constantly, it only passes at intervals into the intestine. For the liver in many animals, unlike every other gland except the kidney, has in connection with it a reservoir, the gall-bladder, in which its secretion accumulates, and from which it is only expelled occasionally. We have therefore to distinguish the bile-secretion from the bile-expelling mechanism. To study the rate of secreting of bile (Fig. I68), a fistula of the gall-bladder can be established. 
But to learn the function of bile in digestion it is more important to know when and at what rate it enters the intestine. For this purpose a fistula is made by cutting the nat ural orifice of the common bile-duct with a piece of the surrounding mucous membrane out of the intestine and transplanting it upon the serous coat, where it is sutured. The loop of intestine, with the orifice of the duct facing outwards, is then stitched into the abdominal wound, where it is allowed to heal. Of course, since a circulation of the bile-acids takes place-i.e., an absorption from and re-excretion into the intestine-the formation of that juice cannot proceed upon absolutely normal lines when the bile no longer enters the duodenum. The only condition under which fistula bile could have the same composition as normal bile would be that in which as great an amount of bile-acids is introduced into the gut as escapes through the fistula. A circulation of a smaller proportion of the bile-pigments is also probable. A circulation of the biliary cholesterin is denied by some observers (Stadelmann) but affirmed by others. It is certain that cholesterin is of importance in the body, and if the supply of sterins (p. 570) in the food is insufficient it is to be supposed that some of the biliary cholesterin would be used over again.

Of the direct influence of nerves, either on the secretion of bilc or on its expulsion, we have scarcely any knowledge, scarcely even any guess which is worth mentioning here. It is true the secretion of bile may be distinctly affected by the section and stimulation of nerves which control the blood-supply of the stomach, intestines, and spleen, for the quantity of blood passing by the portal vein to the liver depends upon the quantity passing through these organs, and the rate of secretion is diminished when the blood-supply is greatly lessened. In this way stimulation of the medulla oblongata, the spinal cord, or the splanchnic nerves stops or slows the secretion of bile by constricting the abdominal vessels; and the same effect can be reflexly produced by the excitation of afferent nerves.

The right splanchnic nerve contains inhibitory with some motor fibres, and the vagi (especially the left) contain motor fibres for the gall-bladder. Probably its contraction takes place naturally in response to reflex impulses from the mucous membrane of the duodenum, for the application of dilute acid to the mouth of the bileduct causes a sudden flow of bile,* and the acid contents of the stomach, when projected through the pylorus into the intestine, have a similar effect. But, in addition, as we have seen, the secretin formed will cause an increase in the rate of secretion of the bile. In studying the effect of secretin it is necessary to obtain it free from bile-salts, since these cause of themselves an increased secretion of bile. When this is done by dissolving out with alcohol any bile-salts which may be present in the extract of intestinal mucous membrane,

* This result seems to be difficult to realize experimentally. Bainbridge and Dale could not elicit reflex contraction of the gall-bladder (in anæsthetized animals) in this way. 
a solution of the residue containing the secretin still evokes a rapid secretion of bile. The fact that the same hormone excites the formation both of pancreatic juice and bile is obviously related to that common action of the two juices in digestion on which we have already dwelt.

When food passes into the stomach, there is at once a sharp rise in the rate of secretion of bile. A maximum is reached from the fourth to the eighth hour - that is, while the food is in the intestine. There is then a fall, succeeded by a second smaller rise about the fifteenth or sixteenth hour, from which the secretion gradually declines to its minimum. Upon the whole, the curves of secretion of pancreatic juice and bile show a fairly close correspondence, except that the latter is more nearly continuous. But when we compare the curves 1epresenting the rate at which the bile actually enters the intestine with the curve of pancreatic secretion (Fig. I69), we are struck by their almost absolute parallelism. This lends additional support to the conclusion deduced from their chemical and physical properties, that in digestion they are partners in a common work.

While the rate at which bile passes into the intestine seems to be influenced by digestion much in the same way as the rate of pancreatic secretion, the details are as yet less exactly known. In the fasting animal no bile enters the gut. When food is taken, the flow begins after a definite interval, which varies for the different kinds of food. As
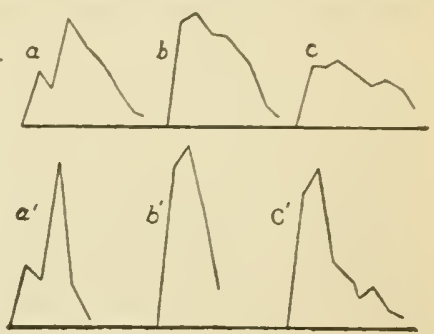

Fig. I69.-Pancreatic Juice and Bile (Pawlow). The upper curves repre. sent the hourly rate of pancreatic secretion, and the lower the rate at which the bile enters the intestine; $a, a^{\prime}$, milk diet; $b, b^{\prime}$, meat; $c, c^{\prime}$, bread. Only the general form of the curves is to be compared. The scale of the ordinates of the various curves was not the same.

long as digestion lasts bile continues to escape, but both the quantity and quality depend upon the nature of the food. Water, raw egg-white, and starch paste, whether given by the mouth or introduced directly into the stomach of a dog, cause no flow of bile. But fat, the extractives of meat, and the products of digestion of egg-white produce a copious discharge. This discharge may be determined by the relatively large amount of acid chyme passed through the pylorus when proteins are digested in the stomach and the stimulus to the formation of secretin occasioned by the presence of this chyme or of fatty material in the duodenum. In the case of fat a further favourable influence on the secretion of bile is the absorption of bile-salts which accompanies the absorption of the fatty acids and soaps produced in fat digestion. Bile-salts stimulate the secretion of bile, including bile-salts thems slves. An increased 
flow of bile-salts into the intestine accelerates the splitting of fats by the pancreatic juice, and therefore the absorption of bile-salts acting as solvents for, or chemically united to, the fatty acids and soaps. A circle analogous to the 'vicious circle' of the logicians, but constituting a physiological adaptation of most potent virtue in the digestion of fats, is thus established. Not only is the quantity of bile poured into the intestine increased on a diet rich in fat, but it is said that a given amount of it aids the fat-splitting action of the pancreatic juice more powerfully than if the diet were poor in fat. This may depend upon an increase in the concentration of the bilesalts in bile secreted when a large amount of fat is ingested. But it is well to recognize that we do not at present know with any great exactness the mechanism by which the rate of secretion and expulsion of bile and the properties of that juice are influenced by digestion. It has been conjectured that the first abrupt rise may be started by reflex nervous action, and that later on secretin and, in the case of fat digestion, bile-salts may directly excite the hepatic cells.

The pressure under which the bile is secreted is higher than the pressure of the portal blood, and therefore the liver ranges itself with the high-pressure salivary glands rather than with the low-pressure pancreas. But although the biliary pressure is high relatively to that of the blood with which the secreting cells are supplied, it is absolutely low, the maximum being no more than $25 \mathrm{~mm}$. of mercury:* This is a point of practical importance, for a comparatively slight obstruction to the outflow, even such as is offered by a congested or inflamed condition of the duodenal wall about the mouth of the duct, may be sufficient to cause reabsorption of the bile through the lymphatics. and consequent jaundice. Of course, complete plugging of the duct by a biliary calculus is a much more formidable barrier, and inevitably leads to jaundice, just as ligature of a salivary duct, in spite of the great secretory pressure, inevitably causes oedema of the gland.

The Influence of Nerves on the Secretion of Intestinal Juice. -As to the influence of nerves on the secretion of the succus entericus, our knowledge is almost limited to a single experiment, and that an inconclusive one. Ioreau placed four ligatures on a portion of the small intestine, so as to form three compartments separated from each other and from the rest of the gut. The mesenteric nerves going to the middle loop were divided, and the intestine returned to the abdomen. After some time a watery secretion was found in the middle compartment, little or none in the others. This is a true 'paralytic' secretion, and not a mere transudation depending simply on the vascular dilatation caused by section of the vaso-

- In the dog, cat, and monkey the average maximum pressure at which as much bile is secreted as is taken up from the bile-paths by the portal lymphatics is about $300 \mathrm{~mm}$. of bile. The highest pressure recorded was $373 \mathrm{~mm}$. of bile in a cat (Herring and Simpson). 
constrictor nerves, for it has the same composition and digestive action as normal succus entericus obtained from a fistula. The secretion begins about four hours after section of the nerves, goes on increasing for about twelve hours, and then rapidly diminishes, so that after about two days the midille loop, as well as the other two, will be found empty. The interpretation usually put upon the experiment is that nerves which normally inhibit the local secretory mechanism have been divided. But there is no real proof of the existence of such nerves.

The same adaptation is seen in the secretion of the succus entericus as in the secretion of the other digestive juices, and the adaptation is naturally most striking in regard to those points in which the intestinal juice is peculiar. While mechanical stimulation of the stomach is ineffective as regards the secretion of gastric juice, mechanical stimulation of the intestine, as by the contact of a cannula, produces a free flow of succus entericus. The reaction is a localized one, the secretion only taking place from the portion of the mucous membrane stimulated. This fact acquires significance when we reflect that the food moves very slowly in the intestine, and a secretion could be of use only at the points where the food happened to be. The juice secreted in response to mechanical stimulation is poor in enterokinase. But if a little pancreatic juice be put into the intestine, and left there for some time, the juice afterwards secreted is rich in enterokinase.

Summary.--Here let us sum up the most important points relating to the secretion of the digestive juices. They are all formed by the activity of gland-cells originally derived from the epithelial lining of the alimentary canal. The organic constituents or their precursors (including the mother-substances of the jerments) are prepared in the intervals of rest-absolute in some glands, relative in others-and stored up in the form of granules, which during activity are moved towards the lumen of the gland tubules, and there discharged.

The nerves of the salivary glands are, as regards their origin, $(a)$ cerebral, (b) sympathetic; the former group is vaso-dilator, the latter (usually) vaso-constrictor; both are secretory. Secretion of saliva depends strictly on the nervous system. That nerves influence the gastric and pancreatic secretions is also made out. The psychical secretion is of greater importance for the saliva and gastric juice than for the pancreatic juice. The direct action of secretin (produced in the intestinal mucous membrane by the influence of the chyme) is the most characteristic factor in pancreatic secretion. As regards the intestinal glands and the liver, it has not been proved that their secretive actirity. is under the control of the nervous system, except in so far as the latter may indirectly govern it through the blood-supply, although various circumstances suggest the probability of a more direct action. All the digestive juices show a certain adaptation to the nature of the food, 
although it has not becn demonstrated that this is due to a specific sensibility of the mucous membranes for each lind of food-stuff. The action of one juicc on the secrelion of another is also of great significance. Thus, the water of the saliva directly excites a flow of gastric juice when it reaches the stomach; the acid of the gastric juice excites a flow of pancreatic juice when it reaches the duodenum; and the pancreatic juice excites the intestinal mucous membrane to the production of enterokinase, the most characteristic constitucut of the succus entericus. In all the glands the blood-flow is increased during activity; in some (salivary glands) this is knowen to be caused through vaso-motor nerves. In the salivary glands clectro-motive changes accompany the active state, and more heat is producd. Both in the salivary glands and the pancreas it has been shown that much more carbon dioxide is given off, and much more oxygen used up, during secretion than during rest. In the other glands we may assume that the same occurs. This is one proof that work is done in the separation or manufacture of the constituents of the various secretions.

\section{Section VI.-Survey of Digestion as a IIhole.}

Having discussed in detail the separate action of the digestive secretions, it is now time to consider the act of digestion as a whole, the various stages in which are co-ordinated for a common end. The solid food is more or less broken up in the mouth and mixed with the saliva, which its presence causes to be secreted in considerable quantity. Liquids and small solid morsels are shot down the open gullet without contraction of the constrictors of the pharynx, and reach the lower portion of the osophagus in a comparatively short time ( $\frac{1}{10}$ second); while a good-sized bolus is grasped by the constrictors, then by the œsophageal walls, and passed along by a more deliberate peristaltic contraction.

Chemical digestion in man begins already in the mouth, a part of the starch being there converted into dextrins and sugar (maltose), as has been shown by examining a mass of food containing starch just as it is ready for swallowing (p. $45^{6}$ ). This process is no doubt continued during the passage of the food along the œsophagus.

The first morsels of a meal which reacl the stomach find it free from gastric juice, or nearly so. They are alkaline from the admixture of saliva; and the juice which is now beginning to be secreted, in response to the psychical excitement, and reflexly through the presence of the food and the water of the saliva in the stomach, is for a time neutralized, and amylolytic digestion still permitted to go on. For 20 to 40 minutes after digestion has begun there is no free hydrochloric acid in the stomach, although some is combined with proteins, and during this period the ptyalin of the swallowed saliva will be able to act even better than in the mouth, being favoured by 
a weakly acid reaction. Incleed, for a time, as the meal goes on, the successive portions of food which arrive in the stomach will find the conditions more and more favourable for amylolytic digestion. But as the acidity continues to increase, the activity of the ptyalin will first be lessened, and ultimately abolished; and, upon the whole, a considerable proportion of the starches must usually escape complete conversion into sugar until they are acted upon by the pancreatic juice. This is particularly the case with unboiled starch, as contained in vegetables which are eaten raw; and, indeed, we know that sometimes a certain amount of starch may escape even pancreatic digestion, and appear in the fieces. Meanwhile, pepsin and hydrochloric acid are being poured forth; the latter is entering into combination with the proteins of the food; and before the end of an ordinary meal peptic digestion is in full swing. The movements of the pyloric end of the stomach increase, and eddies are set up in its contents, which carry the morsels of food with them, and throw them against its walls. In this way not only are the contents thoroughly mixed, and fresh portions of food constantly brought into contact with the gastric juice secreted mainly in the more passive cardiac end, but a certain amount of mechanical disintegration is brought about. This is aided by the digestion of the gelatin-yielding connective tissue which holds together the fibres of muscle and the cells of fat, and the digestible structures in vegetable tissue which enclose starch granules. Such nucleo-proteins as come into contact with the gastric juice will be split up and the proteins digested to peptone. The globin of the blood pigment will undergo the same change, while the hæmatin is not much affected. If milk has formed a portion of the meal, the caseinogen will have been curdled soon after its entrance into the stomach, bv the action of the rennet ferment alone (see p. 353) when the milk has been taken at the beginning of digestion before the gastric contents have become distinctly acid, by the acid and ferment together when it has been taken later. The caseinogen and other proteins of milk, like the myosinogen and other proteins of meat, and the globulins, albumins, and other proteins of bread and of vegetable food in general, are acted upon by the pepsin and hydrochloric acid, yielding ultimately peptones; while variable quantities of these proteins and of the acid-albumin and proteoses derived from them may escape this final change, and pass on as such into the duodenum. In the dog, indeed, a very large proportion of a meal of flesh has been found to be digested to the peptone stage while still in the stomach, leaving for the juices that act on it in the intestine only its further hydrolysis to amino-acids, etc. But we may safely assume that, in the case of a man living on an ordinary mixed diet, a good deal of the food proteins passes through the pylorus chemically unchanged, or having undergone only the first steps of hydration. For, even a few minutes after food has been 
swallowed, especially liquid food or water, the pyloric sphincter may relax and allow the stomach to propel a portion of its contents into the intestinc; and such relaxations occur at intervals as digestion goes on, although it is not for several hours (three to five) that the greater portion of the food reaches the duodenum. During this period the acidity has at first been constantly increasing, although for a time the liydrochloric acid has combined, as it is formed, with the proteins of the food. Then comes a stage where the hyclrochloric acid has so much increased that, after combining witl all the proteins, some of it remains over as free acid. After a time the total acidity begins to fall, the partially digested proteins continually passing on through the pylorus, while a considerable proportion is so fully digested as to be absorbed by the gastric mucous membrane itself. Thus, in one experiment on the digestion of meat in a dog, it was found that 30 per cent. was absorbed in the stomach, while to per cent. passed through the pylorus as peptone, over 20 per cent. as undissolved or soluble protein (acid-albumin), and a little more than 8 per cent. as proteose (Tobler). The large proportion of peptone is noteworthy, as indicating some kind of selective passage of the different digestive products from the stomach into the duodenum. For the gastric contents contain plenty of proteose, although only traces of peptone. The total 'titratable acidity' goes on diminishing till the third or fourth hour, the proportion of free to combined acid continuing, nevertheless, to rise, since nearly all that is now secreted remains free. In addition to a certain amount of protein, small quantities of soluble and easily diffusible substances, like sugars and some of the organic crystalline constituents of meat-e.g., kreatinmayalso be absorbed into the blood by the gastric mucous membrane.

The substances which reach the duodenum are-(I) The greater part of the fats. The partial digestion in the stomach of the envelopes and protoplasm of the cells of adipose tissue, and of the protein which keeps the fat of milk in emulsion, prepares the fats which are not split up by the gastric juice for what is to follow in the intestine. (2) All the proteins which have not been carried to the stage of peptone, and much peptone. (3) All the starch and dextrins-and glycogen, if any be present-which have not been converted into sugars, and probably a portion of the sugars. (4) Nucleins, hæmatın, cellulose, and other substances not digestible, or digestible only with difficulty, in gastric juice. (5) The constituents of the gastric juice itself, including pepsin. Most of the pepsin is soon destroyed in the unfavourable environment of the intestinal contents. But it has been shown that a certain amount of active pepsin may be present for a time in the intestine, even in the free condition, and still more when enclosed in the interior of masses of protein which protect it, and which still continue to be digested by it. This is particularly true of certain materials, like elastin and connective tissue, which are 
more readily hydrolysed by pepsin than by trypsin. The ptyalin of the saliva has becin already destroyed in the stomach.

It must be remembered that all this time, even from the beginning of digestion, a certain amount of pancreatic juice has been fincling its way into the duodenum in response first perhaps to the psychical excitation, and later to that action of the acid chyme on the intestinal mucous membrane which has been described. In the duodenum its trypsinogen is becoming activated to trypsin by the enterokinase of the intestinal juice. The secretion of bile, too, has quickened its pace, the gall-bladder is getting more and more full as the meal proceeds and gastric digestion begins, and some of the bile may very soon escape into the intestine. The pylorus opens occasionally for a moment whenever the small portions of chyme which at this stage are beginning to pass through have been sufficiently neutralized by the pancreatic juice and bile, although it is not necessary that the reaction should become actually neutral. When the acid chyme, a greyish liquid, turbid with the débris of animal and regetable tissues - with muscular fibres, fat globules, starch granules, and dotted ducts-gushes throngh the pylorus and strikes the duodenal wall, the muscular fibres of the gall-bladder contract, and sudden rushes of bile take place from the common duct. By-and-by as bile and pancreatic juice continue to be poured out, the reaction in the duodenum becomes less acid and even weakly alkaline. The observations purporting to show changes in reaction of the intestinal contents at different levels made with indicators like litmus, phenolphthalein, methyl orange, etc., have lost much of their value since the introduction of physico-chemical methods for measuring the hydrogen-ion concentration. However, properly chosen colour indicators can still be employed for estimating the acidity of the gastric contents, at least with sufficient accuracy for most clinical purposes. It must be remembered that the differences in true reaction at different stages of intestinal digestion and at different levels of the gut below the duodenum are slight. There is never a great preponderance either of hydroxyl or of hydrogen ions between the point at which the pancreatic juice and bile are mingled with the gastric chyme and the lower part of the ileum.

In the duodenal contents of adult human beings a hydrogen-ion concentration of 0.00000002 (or $2 \times 10^{-8}$ ) normal has been found by the gas chain method (McClendon) and about the same in the intestinal contents of dogs (Auerbach and Pick). This is a slightly alkaline reaction, the hydrogen-ion concentration of pure water being about five times as great $\left(0^{\circ}\right.$ Oooooor, or $\left.\mathrm{I} \times \mathrm{IO}^{-7}\right)$. In the stomach, of course, a very different state of affairs is found. The hydrogen-ion concentration rises in the course of $I$ to 3 or + hours after a meal to a maximum which is very considerable. In a series of patients, including a number suffering from gastric disorders, the hydrogen-ion concentration ranged from 0.03 to 0.00000007 (or $7 \times 10^{-8}$ ). The lowest concen- 
tration is practically the same as that of water. In other words, in this patient the gastric content-after the test meal were neutral, and it woull be impossible for peptic digestion to proceed. It must be distinctly noted that the acidity of the gastric contents during the digestion of test meals is not the same thing as the acidity of the pure gastric juice. Observations on juice obtained from a case of gastric fistula without admixture with saliva showed that in 'hunger' juice, where continuous secretion was going on, the hydrogen-ion concentration saried from $(0 \cdot 05$, to 0.100 normal in several samples collected on diferent days (Menten).

This question of reaction has significance in two ways: in the first place thr reaction determines whether a given ferment shall be destroyed or not by another ferment or by the alkalinity or acidity of the medium. Thus pepsin can be destroved by the alkali of the pancreatic juice, enterokinase and trypin by the hydrochloric acid of the gastric juice, trypsinogen by the pepsin and hydrochloric acid. Trypsin has no destructive effect on enterokinase or trypsinogen (Mellanby). Secondly, the reaction affects the activity of this or that ferment on the food substances. The optimum hydrogen-ion concentration for peptic digestion is relatively high $(0.02$ to 0.03 normal). When it is decreased to 0.000 n normal the rate of digestion is only one-half to one-fifth as rapid. The high concentration of hydrogen-ions in the gastric contents of healthy persons is clearly advantageous, and the low concentration in the gastric contents in cases of hypoacidity clearly disadrantageous. Trypsin acts best in a medium which contains more hydroxyl than hydrogen ions. When the alkalinity is diminished it becomes less active, although it is not entirely inhibited even by an acid reaction until the hydrogen-ion concentration reaches about $0.000 I$ (or I $\times \mathrm{IO}^{-4}$ ) normal. (See footnote, p. I I39.)

In the intestine it is possible that trypsin may perform its work in a medium which is sometimes acid; and although the cause of the acidity and the character of the medium are far from being the same as in the gastric juice, it is obviously an advantage that the chief proteolytic ferment should be able to act upon the proteins in all parts of the intestine and at every stage oi intestinal digestion whether the reaction is acid or alkaline. The proteins of the chyme are all carried by the trypsin to the stage of peptone, and the peptone, even in perfectly normal digestion, is further split up into amino- and diamino-acids by the trypsin and by the erepsin of the succus entericus.

In the lower portions of the small intestine bacteria of various kinds are present and active; and it is not unlikely that even throughout its whole length a certain range of action is permitted to them, checked by the acidity of the chyme, though scarcely by the feeble antiseptic properties of the bile.

The lower end of the small intestine is not cut off by any bacteriaproof barrier from the large intestine, in which putrefaction is con- 
stantly going on. It has been actually shown that small particles, such as lycopodium spores, suspended in water, soon reach the stomach when injected into the rectum. So that micro-organisms, aided by the antiperistalsis of the colon, may be able to work their way above the ileo-colic sphincter and valve, even against the downward peristaltic movement of the small intestine. But even if this were not the case, a few barteria or their spores, passing through the stomach with the food, would be enough to set up extensive changes as soon as they reached a part of the alimentary canal where the conditions were favourable to their development. Indeed, from the time when the first micro-organism enters the digestive tube soon after birth, it is never free from bacteria; and their multiplication in one part of it rather than another depends not so much on the number originally present to start the process, as on the conditions which encourage or restrain their increase.

A certain amount of already emulsified fats is broken up into their fatty acids and glycerin in the stomach, unemulsified fats entirely by the fat-splitting ferment of the pancreatic juice. The acids will form soaps with alkalies wherever they meet them in the intestinal contents, or even in the mucous membrane. A portion of those soluble soaps may be immediately absorbed; the rest will aid in the emulsification of the fats not yet chemically decomposed, and thus greatly hasten the fat-splitting action of the pancreatic juice. The phosphatides are in all probability acted upon in the alimentary canal much in the same way as the fats. Lecithin is decomposed by pancreatic and intestinal juice into fatty acids and glyceryl-phosphoric acid, and cholin is liberated. As regards the behaviour of the sterins of the food little is known, but it is not unlikely that their esters are split up, and the sterins thus set free as well as those originally free in the food may then be absorbed, in part at least, without further change. The starch and dextrin which have escaped the action of the saliva are changed into maltose by the amylase of the pancreatic juice, and the maltose into dextrose by the maltase of the same secretion and of the succus entericus.

The succus entericus, in addition to its important functions already mentioned, aids as an alkaline liquid in lessening the acidity of the chyme and establishing the reaction favourable to intestinal digestion. It will convert into monosaccharides any cane-sugar, maltose, or lactose, which may reach the intestine; but it cannot be doubted that some cane-sugar may be absorber by the stomach, after being inverted by she hydrochloric acid of the gastric juice or by inverting ferments taken in with the food, or on its way through the gastric walls.

Upon the whole no great amount of water is absorbed in the small intestine, or at least the loss is balanced by the gain, for the intestinal 
contents are as concentrated in the doulenum as in the ileum. But as soon as they pass beyond the ileo-caccal valve water is rapidly absorbed, and the contents thicken into normal faces, to which the chief contribution of the large intestine is mucin, secreted by the vast number of goblet cells in its Lieberkiilın's crypts.

Bacterial Digestion.-So far we have paid no special attention to other than the soluble ferments of the digestive tract, although we have incidentally mentioned the action of the lactic acid bacilli on carbo-hydrates and of the fat-splitting bacteria on fats. It is now necessary to recognize that the presence of bacteria is an absolutely constant feature of digestion; and although their action must in part be looked upon as a necessary evil which the organism has to endure, against the consequences of which it has to struggle, and to which in all probability it has to a great extent adapted itself, it is not unlikely that in part it may be ancillary to the processes of aseptic digestion. But bacteria are not essential (in mammals, at any rate, living on milk), as some have supposed. For it has been shown that a young guinca-pig, taken by Caesarean section from its mother's uterus with elaborate aseptic precautions, and fed in an aseptic space on sterile milk, grew apparently as fast as one of its sisters brought up in the orthodox microbic way. The alimentary canal remained free from bacteria (Nuttall and Thierfelder). On the other hand, chickens hatched from sterile eggs and kept in a sterile enclosure lived, indeed, for a time, but did not thrive in comparison with the control animals, and died at latest after eighteen days (Schottelius). It is probable that the difference in the results is to be attributed to the difference in the food, purely vegetable food requiring the aid of bacteria for its proper digestion, especially for the decomposition of the cellulose, while an easily-digestible food like milk does not.

Among the more important actions of bacteria on the protein food-products in the intestines may be mentioned the formation of indol, phenol, and skatol, the first having tyrosin for its precursor, and being itself after absorption the precursor of the indican in the urine; the second being to a small extent thrown out with the faces, but chicfly absorbed and eliminated by the kidncys as an aromatic compound of sulpluric acid; the third passing out mainly in the freces.

The view put forward by Metchnikoff, that in the putrefactive bacteria of the intestine the body carries within itself the seeds of premature decay, owing to the harmful effects of absorbed products of decomposed protein, cannot be looked upon as established, although certainly the prophylaxis suggested by him (the increase of the lactic acid content of the intestine by the addition of sour nilk, butter-milk, etc., to the diet) might well be a useful morlification of the dietetic habits of many persons, especially if associated 
with a reduction in the total amount of protein consumed. That the intestinal contents may include substances capable of inducing severe toxic symptons if absorbed unchanged scarcely needs proof. Filtered extracts of fieces from normal persons made with salt solution cause, when injected in small amounts into the circulation of dlogs, a fall of blood-pressure which may be speedily recovered from or may be quickly fatal according to the specimen (Fig. I70).

The large intestine is the chosen haunt of the bacteria of the alimentary canal; they swarm in the fieces, and by their influence, especially in the cxcum of herbivora, but also to some extent in man, even cellulose is broken up, the final products comprising certain fatty acids, such as butyric, acetic and valerianic acids, carbon dioxide and marsh gas. A cellulose-dissolving enzyme of great activity is, present in the hepatic secretion of the snail, which rapidly produces sugar from that polysaccharide. Dextrose is also formed when it is hydrolysed by dilute acid. Apart from the importance of solution of the cellulose in facilitating the action of the digestive juices on the starch and other nutrient materials enclosed by it, it can be assumed that some of the intermediate products of its hydrolysis by the bacteria-e.g., bodies analogous to the dextrins which appear in the hydrolysis of starch-can be acted on by the ferments of the succus entericus and the pancreatic juice, so as to form dextrose, which on absorption then takes its place in the carbo-hydrate metabolism just as if it had been derived from starch. In the herbivora the contribution thus made to the nutrition of the animal may be of considerable importance; in omnivora it is not negligible. In man as much as to per cent. of the cellulose of young vegetables is said to be capable of assimilation. In carnivorous animals, however, it appears that cellulose when taken in the food is quantitatively excreted in the freces. In addition to the action of the intestinai flora on cellulose, certain of the bacteria of the alimentary canal affect some of the other carbo-hydrates in a not unimportant way. Dextrose, for instance, can be decomposed into two molecules of lactic acid, according to the equation

$$
\mathrm{C}_{6} \mathrm{H}_{12} \mathrm{O}_{6}={ }_{2} \mathrm{C}_{3} \mathrm{H}_{6} \mathrm{O}_{3} \text {. }
$$

This is called the lactic acid fermentation, and is due to a special bacillus.

Another micro-organism splits up dextrose into butyric acid, carbon dioxide and hydrogen, the so-called butyric acid fermentation, according to the equation

$$
\mathrm{C}_{6} \mathrm{H}_{12} \mathrm{O}_{6}=\mathrm{C}_{4} \mathrm{H}_{8} \mathrm{O}_{2}+2 \mathrm{CO}_{2}+2 \mathrm{H}_{2} \text {. }
$$

The contents of the large bowel are generally acid from the products of bacterial action, although the wall itself is alkaline.

Fæces.-In addition to mucin, secreted mainly by the large 
intestine, the face's consist of indigestible remnants of the fond, such as elastic fibres, spiral vessels of plants, and in general all vegetable structures chiefly composed of cellulose. They are culoured with a pigment, stercobilin, derived from the bile-pigments. Stercobilin is julcntical with urobilin, which forms a common, though not an

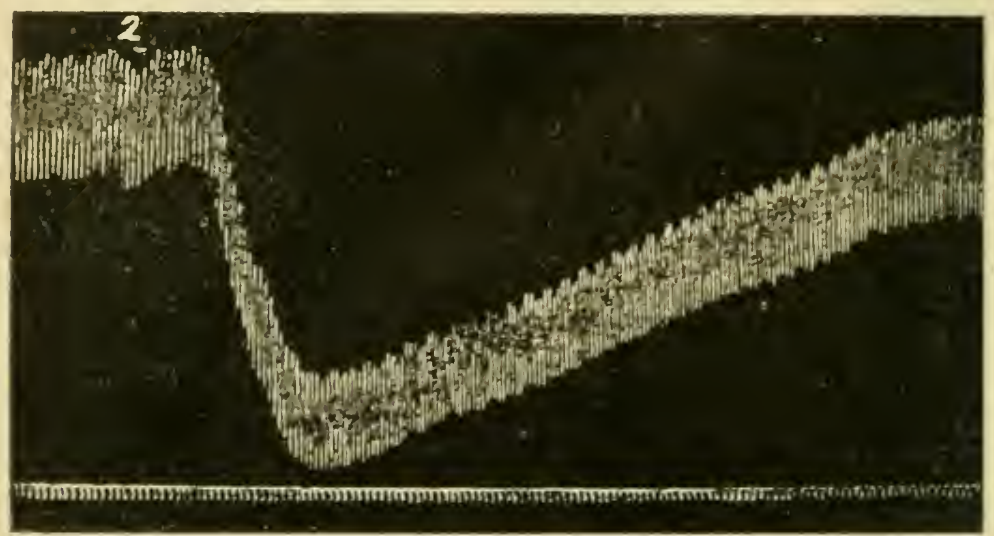

Fig. 170.-Effect of Extract of Fxces on Blood-Pressure. The extract was injected at 2. Time-trace, seconds.

invariable, constituent of bile itself. A portion of it is absorbed by the intestine and then excreted in the urine, the urobilin in which is often much increased in fever ("febrile' urobilin). No bilirubin or biliverdin occurs in normal fæces, although pathologically they may be present. The dark colour of the fæces with a meat dict is due to hrmatin and sulphide of iron, the latter being formed by the action of the sulphuretted hydrogen which is constantly present in the large intestine on the organic compounds of iron contained in the food or in the secretions of the alimentary canal. A small amount of altered bile-acids and their products is also found; and in respect to these, and to the altered pigments, bile is an excretion. And although its entrance into the upper instead of the lower end of the intestine, the ascertained importance of its function in digestion, and the fact that the greater part of the bile-salts is reabsorbed, show that in the adult it is very far from being solely a waste product, the equally cogent fact, that the intestine of the new-born child is filled with what is practically concentrated bile (meconium), proves that it is just as far from being purely a digestive juice. Skatol and other bodies, formed by putrefactive changes in the proteins of the food, are also present in the fieces, and are responsible for the frecal odour. Masses of bacteria are invariably present, and often make up a very considerable proportion of the total frecal 
solid: (). the inorganic substances in fieces the numerous crystals of triple phosphate are the most characteristic. When the diet is too large, or contains too much of a particular kind of food, a considerable quantity of digestible material may be found in the frecesc.g., muscular fibres and fat. But it should be remembered that under all circumstances the composition of the facces differs from that of the food. The intestimal contribution is always an important one, althongh relatively more important with a flesh than with a vegetable diet. The purin bases normally found in human faces come both from the food directly and from the metabolism of the tissues. They are increased in amount on a dict rich in purin bodies (such as meat extract or thymus), but are also formed on a diet like nilk, from which purin bases cannot be obtainer. An interesting constituent of faeces on which light has recently been thrown, especially by the researches of Gardner, is the so-called coprosterin (dihydrocholesterin), which appears to be produced from cholesterin by reduction, probably under the influence of bacteria, and perliaps also from the phytosterins of vegetable food. 


\section{CHAPTER YII}

\section{ABSORPTION}

\section{Section I.-Preliminary Physico-Chemical Data.}

Imbibition, or molecular imbibition, is the term applied to the entrance of liquid into a colloid, without the loss of its properties as a solid, when no preformed capillary spaces are present. The entrance of water into a piece of gelatin, or an epidermic scale, is an example of molecular imbibition. Most animal and vegetable tissues possess this property, which is believed to be of importance in such physiological processes as absorption, secretion, and the excretion of water from the lungs and skin. The process by which liquid passes into a solid with preformed capillary spaces-e.g., a sponge-is sometimes spoken of as capillary imbibition.

Diffusion.-When a solution of a substance is placed in a vessel, and a layer of water carefully run in on the top of it, it is found after a time that the dissolved substance has spread itsclf through the water, and that the composition of the mixture is uniform throughout. The result is the same when two solutions containing different proportions of the same substance, or containing different substances, are placed in contact. The phenomenon is called diffusion. The time required for complete diffusion is comparatively short in the case of a substance like hydrochloric acid or sodium chloride, exceedingly long in the case of albumin or gum. In both it is more rapid at a high temperature than at a low.

Osmosis.-If the solution be separated from water by a membrane absolutely or relatively impermeable to the dissolved substance, but permeable to water, water passes through the membrane into the solution. This phenomenon is called osmosis. E.g., a membrane of ferrocyanide of copper, nearly impermeable to cane-sugar, can be formed in the pores of an unglazed porcelain pot by allowing potassium ferrocyanide and cupric sulphate to come in contact there. If the pot is filled with, say, a r per cent. solution of cane-sugar, closerl by a suitable stopper, connected to a manometer, and then placed in a vessel of water, water passes into it till the pressure indicated by the manometer rises to a certain height. With a 2 per cent. solution it reaches twice this heiglit, and in general the osmotic pressure, as it is called, is in any solution proportional to the molecular concentration* of the solution,

* The molecular concentration is strictly defined as the number of grammes of the dissolved substance in a litre of the solution divided by the gramme-molecular weight. The gramme-molecular weight, or gramme molerule, is the number of grammes corresponding to the molecular weight. Thus, the gramme-molecular weight of sodium cliloricle ( $\mathrm{NaCl}$ ) is $55^{\circ} 36$ grammes, and of cane-sugar $\left(\mathrm{C}_{12} \mathrm{H}_{22} \mathrm{O}_{11}\right), 342$ grammes. 
or, in other words, to the number of molecules of the di-solved substance in a given volume of the solution. If in this sentence we substitute 'gaseous pressure' for 'osmotic pressure,' and ' gas ' for 'solution,' we have a statement of Boyle's law. which asserts that the pressure of a gas is propurtional to its density. Indeed, it luis been shown that the osmotic pressure of the dissolved substance is the sitme as the pressure that would be excrted by a gas, say hydrogen, if all the water were removed, and a molecule of hydrogen substituted for each molecule of the substance, or as would be exerted by the substance itself if, after r'moval of the solvent, it could be left as a gas filling the same volume. And the osmotic pressure of a solution of one substance is the same as that of a solution of any other substance which contains in a given volume the same number of molccules of the dissolved substance. In other words, the osmotic pressure is not dependent on the nature, but on the molecular concentration, of the substance. The analogy of the laws of osmotic to those of gaseous pressure becomes still more obvious when it is added that the osmotic pressure of a substance with any given molecular concentration is proportional to the absolute temperature; and that when a solution contains more than one dissolved substance the total osmotic pressure is the sum of the partial osmotic pressures which each substance would exert if it were present alone in the same volume of the solution.

The osmotic pressure of a solution may reach an enormous amount. Thus, a $\mathbf{I}$ per cent. solution of cane-sugar has a pressure at $0^{\circ} \mathrm{C}$. of $493 \mathrm{~mm}$. of mercury. A ro per cent. solution of cane-sugar would have an osmotic pressure of more than six atmospheres, and a 17 per cent. solution of ammonia a pressure of no less than 224 atmospheres. The manner in which the phenomenon known as osmotic pressure is developed is not definitely known. One theory attributes it to the attraction between the dissolved molecules and the molecules of the solvent on the other side of the membrane. The most commonly accepted view is that the osmotic pressure is due to the kinetic energy of the moving molecules. Where the molecules are hindered from passing a bounding membrane, the pressure exerted by their impacts on the boundary is greater than where the membrane is easily permeable, because in the latter case many of the molecules pass through, carrying with them their kinetic energy. The pressure must be still less when a dissolved substance diffuses freely into water; but however small it may become, it is in the same force which gives rise to the osmotic pressure of the molecules of the dissolved substance that the cause of diffusion must be sought. Recently interest in the nature of the membrane itself as an important factor in osmosis has been revived (Kahlenberg, Armstrong, etc.). There are many facts which indicate that in physiological processes the affinity of the dissolved substances for, or their solubility in, the cell envclopes or the cytoplasm plays an important rôle. 
It is as yet impossible or at least very difficult to directly measure the osmotic pressure with accuracy by means of a semi-permeable membrane. Recourse is therefore had to indirect methods, especially one which elepends on the fact that the freczing-point of a solution is lower than that of the solvent, salt water, e.g., freezing at a lower teniperature than fresh water. The amount by which the freezing-point is lowered depends on the molecular concentration of the clissolved substance, to whicl, as we have seen, the osmotic pressure is also proportional. When a gramme-molecule of a substance is dissolved in water, and the volume made up to a litre, the freezing-point is lowered by $1.80^{\circ} \mathrm{C}$.; the osmotic pressure is $22 \cdot 35$ atmospheres ( $16,986 \mathrm{~mm}$. of mercury). It is therefore easy to calculate the osmotic pressure of any solution if we know the amount by which its freczing-point is lowered. A I per cent. solution of cane-sugar, for example, would freeze at about $-0.054^{\circ} \mathrm{C}$. Its osmotic pressure $=\frac{0.054}{1.68} \times 16,986=493 \mathrm{~mm}$. of mercury.

A convenient apparatus for making freezing-point measurements is shown in Fig. I7I. The details of the method are given in the Practical lixercises, p. $5 \geq 9$.

The osmotic pressure of different solutions may also be compared by observing the effect produced on certain vegetable and animal cells. When a solution with a greater osmotic pressure than the cell-sap (a hyperisotonic solution) is left for a time in contact with certain cells in the leaf of Tradescantia discolor, plasmolysis occurs-that is, the protoplasm loses water and shrinks away from the cell-wall. If the osmotic pressure of the solution is lower than that of the colonred cell-sap (hypoisotonic solution), no shrinling of the protoplasn takes place. By using a number of solutions of the same substance but of different strength, two can be found, the stronger of which causes plasmolysis, and the weaker not. Between these lies the solution which is isotonic with the cell-sap-that is, has the same molecular concentration and osmotic pressure. The strength of an isotonic solution of some other substance can then be detcrmined in the same way with sections from the same lcaf.

Animal cells (red blood-corpuscles) may also be employed, the liberation of hamoglobin or the swelling of the corpuscles, as measured by the laxmatocrite ( $p .27)$, being taken as evidence that the solution in contact with them is hypoisotonic to the contents of the corpuscles. Here we may suppose that the impacts of the molecules of the salts of the corpuscle on the inside of its envelope, not being balanced by similar impacts on the outside, tend to distend it, and thus to create it potential vacuum for the surrounding water, which accordingly enters. If the corpuscles shrink, the solution is hyperisolonic to their contents. But since the cells are much more permeable to certain substances than to others, this method does not always yicld trustwortly results.

Electrolytes.- We have said that the osmotic pressure is proportional to the concentration of the solution, lut this statement must now be qualified. For certain compounds, including all inorganic salts and many organic substances, the osmotic pressure decreases less rapidly than the theoretical molecular concentration as the solution is diluted. The cxplanation is that in solution some of the molecules of these bodies are broken up into simpler groups or single atoms, called ions. Each ion exerts the same osmotic pressure as the moleculc dicl before. The proportion between the average number of these dissociated molecules and of ordinary molecules is constant for a given concentration of the solution and a given temperature. But as the solution is diluted, the proportion of dissociated molecules becomes greater. The bodics which 
behave in this way are electrolytes-that is, their solutions conduct a current of electricity: bodies which do not exhibit this behaviour do not conduct in sulution. And there are many reasons for believing that the dissociation of the electrolytes is the essential thing in electrolytic conduction. We may suppose that in a solution of an electrolyte-sodiun chloride, for instance-a ccrtinin number of the molecules fall asunder into a kation $(\mathrm{Na}+))^{*}$ carrying a clarge of positive electricity, and an anion ( $\mathrm{Cl}-$ ), carrying an equal negative eharge. These clectrical charges, it must be remembered, are not created by the pissage of a current through the solution. We do not linow low they arise, but the ions must be supposed to be electrically charged at the nionent when the molecule is broken up. And the ions of ditterent substances must cacl be supposed to carry the same quantity of electricity. But since they are all wandering freely in the solution, no excess of negative or of positive electricity can accumulate at any part of it-in other words, no difference of potential can exist. When electrodes connected with a voltaic battery are dipped into a solution of an electrolyte, a difference of potential, an electrical slope, is established in the liquid, and the positively charged kations are compelled to wander towards the negative pole, the negatively charged anions towards the positive pole. In this way that movement of electricity which is called a current is maintained in the solution. It is clear that the greater the number of ions, and the faster they move in the solution, the greater will be the quantity of electricity carried to the electrodes in a given time, when the difference of potential between the electrodes, or the stecjuess of the electric slope, remains constant. In other words, the specific conductivity of a solution of an electrolyte varies as the number of dissociated molecules in a given volume and the speed of the ions. It increases up to a certain point with the concentration, because the absolute number of dissociated molecules in a given volume increases. The molecular conductivity - that is, the conductivity per molecule, or, strictly, the ratio of the specific conductivity to the molecular concentration-increases with the dilution, because the relative number of dissociated molecules, as compared with undissociated, increases. At a certain degree of dilution the molecular conductivity reaches its maximum, for all the molecules are dissociated. The ratio of the molecular conductivity of any given solution to this maximum or limiting value is therefore a measure of the proportion between the number of dissociated, and the total number of molccules. The molecular conductivity of the salts dissolved in the liquids of the animal body, for the degree of dilution in which they exist there, is such that we must assume them to be for the most part dissociated.

Surface Tension.-This is a property of surfaces which is typically illustrated in such instances as a globule of mercury, a drop of water on a greasy slide, or a drop of oil suspended in a liquid with which it does not mix. The tendency of sucl drops to assume the spherical form when not large enough to be distorted by gravity is due to the fact that the surface layer is under a certain tension in virtue of which it strives to contract and to render the surface of the drop as small as

* It has been shown that the chemical atoms themselves are not homogeneous, but are all built up of simpler particles and possess a certain structure. All atoms, e.g., contain electrons, minute particles charged with negative electricity. The number of electrons in an atom appears to be not far from half its atomic weight. Thus in the carbon atom there are 6 electrons, in the oxygen atom 8 , and in the hydrogen atom probably only $\mathbf{r}$. There is evidence that the electrons in the atom are divided into groups or rings one within another (Thomson). 
possible, just as if it were a stretched elastic membrane. The cause of this tension is to be sought in the mutual attraction exerted by molecules which are very close to each other. This molecular pull is enormously strong. It lias been calculated, for instance, that the so-called internal pressure which is due to it is in the case of water not less than 23,000 atmospheres. In the interior of the drop each molecule, being surrounded by other molecules, is pulled by this attractive force cqually in all dircetions-that is to say. on the whole it is not pulled at all, since the pulls of all the surrounding molecules balance each other. At the free surface, on the contrary, the molecules are pulled towards the surface, but not away from it, and the pull of the molecules below the surface layer is not balaneed by the pull of molecules above it. The resultant tension on this layer is the surface tension. Changes in the amount of this surface tension in the case of a given liquid can be produced by bringing gases, solids, or other liquids into contact with the surface layer-tliat is, by bringing molecules of other substances so near the surface molecules of the liquicl that they can attract them, and so to a greater or less degree, depending upon the nature of the substances, balance the attraction of the molecules beneath the surface of the drop. Another way in which the surface tension can be altered is by changing the temperature. The higher the temperature, the greater is the average velocity with which the molecules of the liquid are moving, and the greater the average distance batween the molecules (expressed as the expansion of the liquid). Increase of temperature therefore causes the molccules, through the kinetic energy of heat imparted to them, say, from an external source, to repel each other, and to that extent counteracts their mutual attraction. Accordingly, at the surface the tension, which, as stated, depends upon the excess of this attraction acting towards the interior of the drop, will be diminished. When the temperature is diminished the surface tension will increase. The surface tension can also be altered by altering the electrical charge on the surface. An instance of this is described on another page in connection with the capillary clectrometer (p. 702). In such ways, then, the surface tension at the interface where the cells lining the intestine come into contact with the contents of the gut or witl the tissue lymph, or at the interfaces within the cells where solid and liquid ' phases' come into contact with each other or where different liquids touch, may undergo alterations in either direction. If the tension of the surface is altered, the surface energy or power of doing work inherent in the existence of this tension will, of course, be altered too. In this way the energy, or a portion of it, which is unquestionably expended in absorption may be supplied ultimately at the expense of the chemical encrgy of cell constituents or of food substances on their way through the cells, by means of which the original surface tensions are restored. It has been surmised that changes of surface tension may also be concerned in the secrition of glands, in muscular contraction (p. 744 ), and other functions (Macallum, Bernstein, ctc.).

Adsorption.-Connected with the peculiar propertics of surfaces referred to in the last paragraph are certain phenomena spolien of as adsorption phenomena. Adsorption is typically seen when a solid in such a form that the surface is greatly increased $(e . g$. , a fine powder or a colloidal solution in which the substance is suspended in the form of exccedingly small particles) is placed in contact with a gas or a solution. There occurs a diminution in the concentration of the gas or the dissolved substance, and a correspending accumulation of it on the surface of the solid. Equilibrium is rapidly established, and the 
charasteristic thing about adsorption is that at the equilibrium-point the concentration of the dissolverl substance (or the gas) on the surface is immensely greater than in the general mass of the solution. The concentration on the surface can, indeed, be increased by increasing the concentration of the solution, but in a far smaller proportion. According to the thermodynamic law enunciated by Willard Gibbs, substances which diminish the surface tension must tend to accumulate at the surface, and substances which increase the surface tension must tend to diminish in concentration at the surface. If a small quantity of a substance diminishes the surface tension at a given surface more in proportion than a larger quantity, not only will there be an accumulation of the substance at the surface, but this will be proportionally greater for small than for larger concentrations of the substance in the solution. This characteristic feature of adsorption may thus depend entircly on surface forces due to the conditions under which the attraction of the molecules for each other acts at the surface. It has not been shown, however, that chemical forces due to the interaction of the electrically charged ions are not also concerned. What is especially important to point out is that in the tissues of the body there is a great development of surfaces. The cell walls or cell envelopes come into contact with the tissue lymph or the contents of the digestive tube or the secretions in the alveoli of glands on the one side, and the cell contents on the other, and constitute in the aggregate an immense surface. The surfaces separating the nuclei from the cytoplasm, and, above a!l, the surfaces of the particles of the contents of cytoplasm and nuclei suspended in colloid solution, offer prodigious opportunities for such surface phenomena as adsorption.

\section{Section II.-Mechanisui of Absorption.}

In the preceding chapter we have traced the food in its progress along the alimentary canal, and sketched the changes wrought in it by digestion. We have next to consider the manner in which it is absorbed. Then, for a reason which has already been explained, instead of following its fate within the tissues, until it is once more cast out of the body in the form of waste products, it will be best to drop the logical order and pick up the other end of the clue-in other words, to pass from absorption to excretion, from the first step in metabolism to the closing act, and afterwards to return and fill in the interval as best we can.

Comparative.-And here, first of all, it should be remembered that the epithelial surfaces, through which the substances needed by the organism enter it, and waste products leave it, are, physiologically considered, outside the body. The mucous membranes of the alimentary, respiratory, and urinary tracts are in a sense as much external as the fourth great division of the physiological surface, the skin. The two latter surfaces are in the mammal purely excretory. Absorption is the dominant function of the alimentary mucous membrane, but a certain amount of excretion also goes on through it. The pulmonary surface both excretes and absorbs, and that in an equal measure. But it is by no means necessary that the surface through which oxygen is taken in and gaseous waste products given off should be buried deep in the body, and communicate only by a narrow channel with the exterior. 
In the frog the skin is largely an absorting as well as an excreting surface; oxygen passes freely in through it, just as carbon dioxide passes seely out. In most fishes, and many other gill-bearing animals, the whole gaseous interchange takes place through surfaces immersed in the surrounding water, and therefore distinctly external. In certain forms it lias even been shown that the alimentary canal may serve conspicuously for absorption and excretion of gascous, as well as liquid and solid substances. Still lower down in the animal scale, the surface of a single tube may perform all the functions of digestion, absorption and excretion. Lnwer still, and even this tube is wanting, and everytling passes in and out through an external suriace pierced by no permanent opænings.

Indeed, even in man the functions of the various anatomical divisions of the phrsiological surface are not quite sharply marked off from each other. Though gaseous exchange goes on far more readily through the pulmonary membrane than anywhere else, swallowed oxygen is easily enough absorbed from the alimentary canal and carbon dioxide given off into it; and to a small extent these gases can also pass through the skin. Though water is excreted chicfly by the skin, the pulmonary and the urinary surfaces, and on the whole absorbed chiefly from the digestive tract, there is no surface which in the twenty-four hours pours out so much water as the mucous membrane of the stomach. Under normal conditions, it is true, by far the greater part of this is reabsorbed in the intestine, yet in diarrhœa, whether natural or caused by purgatives, the intestines themselves may, instead of absorbing, contribute largely to the excretion of water. Again, although the solids of the excreta are normally given off in far the greatest quantity in the urine and faces (only part of the latter is truly an excretion, since much of the fæees of a mixed diet has never been physiologically inside the body at all), yet salts and traces of urea are constantly found in the sweat, and salts and mucin in the excretions of the respiratory tract. Further, although the solids and liquids of the food are usually taken in by the alimentary mucous surface, it is possible to cause substances of both kinds to pass in through the skin; and a certain amount of absorption may also take place through the urinary bladder. So that really it may be considered, from a physiological point of view, as more or less an accident that a man should absorb his food by dipping the villi of his intestine into a digested mass, rather than by dipping his fingers into properly prepared solutions, as a plant dips its roots among the liquids and solids of the soil; or that he should draw air into organs lying well in the interior of lis thorax, instead of letting it play over special thin and highly vascular portions of his skin; or that the surface by which he excretes urea should be buried in his loins, instead of lying free upon his back.

It has been already explained that, although digestion is a necessary preliminary to the absorption of most of the solids of the food, we are not to suppose that all the food must be digested before any of it begins to be absorbed. On the contrary, the two processes go on together. As soon as any peptone, or, at least, any amino-acids, have been formed from the proteins, or any dextrose from the starch, they begin to pass out of the alimentary canal; and by the time digestion is over, absorption is well advanced.

Even in the mouth it has already begun, although the amount of 
absorption here is quite insignificant, and it is continued with greater rapidity in the stomach. Here a not inconsiderable part of the proteins - at least, in the easily digested form of animal fooda certain amount of the sugar representing the carbo-hydrates and diffusible substances like alcohol, and the extractives of meat, which form an important part of most thin soups and of becf-tea, are undoubtedly absorbed. Water is very sparingly taken up by the stomach. It is in the small intestine that absorption reaches its leight. The mucous membrane of this tube offers an immense surface, multiplied as it is by the valvulre conniventes, and studded with inmumerable villi. Here the whole of the fat, much sugar, proteose and peptone, or rather the products of the further action of the ferments of the intestine on these derivatives of the native proteins, and certain constituents of the bile are taken in. In the large intestine, as has been already said, water and soluble salts are chicfly absorbed.

What now is the mechanism by which these various products are taken up from the digestive tube, and what paths do they follow on their way to the tissues?

Theories of Absorption.-Not so very long ago it was supposed by many that the proccsses of diffusion, osmosis and filtration offered a tolerably complete explanation of physiological absorption. At that time the dominant note of pliysiology was an eager appeal to chemistry and physics to 'come over and help it'; and as new facts were discovered in these sciences they were applied, with a confidence that was almost naïve, to the problems of the animal organism. The phenomena of the passage of liquids and dissolved solids through animal membranes, upon which the work of Graham had cast so much light, seemed to find their parallel in the absorptive processes of the alimentary canal. And when digestion was more deeply studied, facts appeared which seemed to show that its whole drift was to increase the solubility and diffusibility of the constituents of the food. But as time went on, and more was learnt of the phenomena of absorption and the powers of cells, these crude physical theories broke down, and discarded 'vitalistic' hypotheses began once more to arouse attention. Then came the investigations of De Vries, Van 'T Hoff, and others in the domain of molecular physics, which gave to our notions of osmosis the precision that was wanted before its relation to many physiological processes could be profitably discussed. At the present time it must be admitted that we possess no full explanation of absorption, none which is much more tnan a confession of ignorance, and does not itself need to be explained. Yet some progress has been made at least in defining the boundary between what is clearly known and what is still dark, and in showing that familiar pliysical processes are not without influence. Some physiologists, impressed with the vast progress of physics and chemistry, believe that it will eventually become possible to explain on mechanical and chemical principles all the peculiar phenomena which we observe in the passage of substances through the walls of the alimentary canal. As an aid to the framing of practical working hypotheses this attitude has everything in its favour. Others, taking account of the number and nature of these peculiarities, oppressed with the perennial paradox of vital action, incline to the less sanguine view, 
that after all physical explanations have been exhausted, the real secret of the cell will still lurk in some ultimate 'vital' property of structure or of function and still elude our search. The only sense in which this attitude can be said to be a useful one is that it presents a standing protest against the acceptance of superficial 'physical ' explanations mercly because they' are physical. Botl the optimists and the pessimists, the arlherents of the physical and the adherents of the vitalistic hypothesis, have unfortunately, as a rule talien up an extreme position, as if their theories were mutually exclusive. They agree, however, in admitting that the phenomena of absorption are essentially connected with the cells that line the alimentary canal, and not with any more or less inert ' cement' substance between them. But the one school must confess what the other proclaims, that while the processes carried on in these cells are definite, well ordered, and evidently guided by laws, these laws have as yet for the most part denied themselves to the modem physiologist, with chemistry in one hand and physics in the other, as they denied themselves to his predecessor, equipped only with his scalpel his sharp eyes. and his mothcr-wit. So that in the present state of our knowledge all we can really say is that, while absorption is certainly aided by physical processes, like osmosis and diffusion, possibly by physical processcs like imbibition, and is very likely not unrclated to the molecular properties of surfaces (surface tension, adsorption), it is at bottom the work of cells with a selective permeability which we do not fully understand, or at least which we cannot as yet ex: in in terms of known physical processes acting through a mombrane of known physico-chemical structure.

Thus, dissolved substances pass with equal ease in either direction through an ordinary diffusion membrane, but in genteral they pass, with the water in which they are dissolved, more readily out of the intestine than into it. This normal direction of the strum is still maintained for a considerable time after stoppage of the circulation, prosided that the intestine is kept in good conditionfor example, by being suspended in well-oxygenated blood. Water or solutions of sodium ihloride or sugar disappear from the lumen. And this is not due to mere imbibition by the intestinal wall, but the liquid is actually transported across it. The theory that liquids might be taken up from the gut by imbibition, and the water then mechanically removed by the blood flowing on the other side of the imbibing cells, is incompatible with this experiment (Cohnheim). Nor is it necessary that difierences of concentration of the dissolved substances on the two sides of the absorbing intestinal membrane, which would permit osmosis and diffusion to go on, should exist. When the excised intestine of a holothurian was filled with seawater and suspended in the same sea-water, its contents continued to diminish in bulk for hours or entirely disappeared. Here a liquid identical in composition and concentration with the external liquid was moved in a definite direction across the wall of the intestine from the lumen to the exterior surface. In like manner, when a piece of intestine from a newly-killed rabbit is stretched across a vessel of salt solution so as to divide it into two separate compartments, the solution continues for a while to leave the compartment 
to which the mucosa is turned, and to accumulate in the other. In the cases mentioned the transportation of the liquid depends upon the survival of those properties of the mucous membrane which characterize its elements as living cells. For when the cells that line the intestine are injured or destroyed, or subjected to the action of certain poisons, absorption from it is dininished or abolished. In the dead intestine the characteristic set of the tide out of the lumen across the mucosa can no longer be observed. It must be remarkel further, in this connection, that in its normal state the mucous nembrane does not take up indiscriminately all kinds of diffusible substances, or absorb those which it does take up in the direct ratio of their diffusibility. Nor does it reject everything which does not diffuse. Albumin, for example, which does not pass through dead animal membranes, is to a certain extent taken up from a loop of intestine without change. Cane-sugar (after inversion) and dextrose are absorbed more rapidly than their velocity of diffusion would indicate, when compared with inorganic salts. Glauber's salt diffuses in water fifteen times as fast as canesugar, but cane-sugar is absorbed from the intestines ten times faster than Glauber's salt. The velocity of absorption is different even for simple stereoisomeric sugars-i.e., sugars whose molecule, with the same number of atoms combined in the same way, has a different form (Nagano). Nor is there any clear relation between the rate of absorption of the various sugars and their osmotic pressure. Dextrose and cane-sugar are always absorbed in greater amount than lactose from solutions of the same osmotic pressure. Indeed, as we shall see, lactose is practically not taken up at all as such (p. 445), and in concentrated solutions may even cause a reversal of the normal movement of water, and act as a purgative. Even the water, organic and inorganic solids of the serum of an animal, are absorbed from a loop of its intestine when the blood-pressure in the capillaries of the intestinal wall is considerably greater than the pressure in the cavity of the gut. Since the serum in the intestine and the plasma in the capillaries must be isotonic, and practically identical in chemical composition, the absorption cannot be due to ordinary osmosis or diffusion. Nor can it be due to filtration, since the slope of pressure is from the capillaries to the lumen of the gut (Reid). It is therefore extremely difficult to reconcile this experiment with any purely physical theory of absorption. The same investigator, summing up the result of careful experiments on the absorption of weak solutions of glucose, concludes that, ' with the intestinal membrane as normal as the experimental procedure will permit, phenomena present themselves which are as distinctly opposed to a simple physical explanation as those previously studied in the absorption of serum.'

There is also evidence that even during the absorption of liquids 
which undergo no chemical changes in the gut-e.g., salt solutions of different kinds and different concentrations-chemical energy must be transformed, and on no mean scale, in the intestinal mucosa, for the consumption of oxygen and the production of carbon dioxide by the intestine is markedly increased (Brodie and Vogt).

It may be taken, then, as quite certain that in absorption from the alimentary canal an essential factor is the activity of the living cells of the mucosa, which, in some way at present unknown, maintain the 'set of the tide' from the lumen to the bloodvessels (or lymph spaces), whether the slope of concentration of the dissolved substances favours or opposes it, or when the concentration is the same on both sides of the membrane. It is even probable that this action of the cells is much the most important of all the factors involved. It would be highly misleading, however, to assume that, because this is so, other factors-osmosis, diffusion, possibly even filtration due to differences of pressure caused by the intestinal movements or the contractions of the muscular fibres of the villi (p. 443)-are of necessity negligible. On the contrary, these other factors cannot be adequately taken account of, nor can there be any possibility of assigning to them their proper value until it is recognized that their influence in absorption from the digestive tract is never under ordinary conditions expressed as a simple and uncomplicated effect, such as may be observed in experiments with dead membranes, but, on the contrary, is constantly overlaid, thwarted, or totally reversed, by the special action of the cells. For this reason the discussion of the mechanism of absorption under the time-honoured captions of 'mechanical or physical theory' versus 'physiological or vital theory,' as if the process must of necessity be purely 'physical ' or purely ' vital,' has lost interest. It may be confidently assumed, indeed, that just because the physiological factor is so dominant, the familiar physical forces must often appear to exert a smaller influence than is really the case, and that, could we disentangle the currents which they create and sustain from that steady drift of material out of the lumen of the gut maintained at the expense of its chemical energy by the still unknown machinery of the cells, we should be impressed with the magnitude rather than the insignificance of their total effect.

The following attempt by Höber to analyze on these lines an old experiment of Heidenhain, which the latter observer had interpreted as showing that diffusion and osmosis play no essential part in absorption from the alimentary canal, is of interest in this connection. A loop of small intestine was tied off at both ends, but its circulation was not otherwise interfered with. Solutions of sodium chloride of different strengths were introduced into the loop, and in each observation after fifteen minutes the contents of the loop were recovered and analyzed for the chloride. 


\begin{tabular}{|c|c|c|c|c|c|}
\hline \multicolumn{3}{|c|}{ Introduced. } & \multicolumn{3}{|c|}{ Recovered. } \\
\hline C.C. & $\begin{array}{l}\text { Percentage of } \\
\mathrm{NaCl} \text {. }\end{array}$ & $\begin{array}{l}\text { Total } \mathrm{NaCl} \text { in } \\
\text { (irammes. }\end{array}$ & C.C. & $\begin{array}{l}\text { Percentage of } \\
\mathrm{NaCl} \text {. }\end{array}$ & $\begin{array}{l}\text { Total } \mathrm{NaCl} \text { in } \\
\text { Grammes. }\end{array}$ \\
\hline 120 & 0.30 & $0 \cdot 36$ & 1 8 & 0.60 & $0 \cdot 108$ \\
\hline 120 & 0.50 & $0 \cdot 60$ & 35 & $0 \cdot 66$ & $0 \cdot 230$ \\
\hline $1 \times 7$ & $\mathrm{I} \cdot \mathrm{Oo}$ & $I \cdot I 7$ & 75 & $0 \cdot 90$ & 0.670 \\
\hline I 20 & $x \cdot 46$ & $1 \cdot 75$ & rog & $\mathrm{I} \cdot 20$ & $1 \cdot 310$ \\
\hline
\end{tabular}

Here it is seen that, from the markedly hypotonic solutions of the first two observations, sodium chloride was absorbed, of course, along witl much water. But from the strongly hypertonic solution of the last observation some water was also taken up, instead of water passing into it from the blood. The suggested explanation of these and other data yielded by the experiment is that an osmotic stream of water out of the loop into the blood is established on introduction of the hypotonic solutions, which raises their percentage of salt, while in the case of the hypertonic solution a diffusion stream of sodium chloride is established in the same direction, and its salt content falls. The volume of the hypotonic solutions in the loop rapidly diminishes because the osmotic current conspires with the normal 'physiological' drift from the lumen outwards. In this drift both salt and water are involved, as if the cells were filters which maintained through expenditure of their own energy a slope of hydrostatic pressure from free surface to depth. The hypertonic solution diminishes only slowly in bulk, because the 'physiological' current out of the lumen is opposed by the osmotic stream of water into the lumen. Nevertheless, even the hypertonic solution is gradually absorbed, because the pull of the cells-the suction, if it may be so expressed, of the cellular pump-is powerful cnough to overcome the osmotic current and to force water up the slope into the blood or into the tissue liquid, whose osmotic pressure is not much more than half that of the solution.

\section{Permeability of the Intestinal Epithelium and Lipoid-Solubility} of Absorbed Substances.-If the cells of the intestinal mucosa are to move materials out of the lumen of the gut, it is obvious that these materials must first be able to enter the cells. The ease or difficulty with which different substances arc absorbed may therefore depend upon the degree in which the cells are permeable for them. The question of the factors on which the permeability of cclls depends has already been discussed to some extent in the case of the coloured blood-corpuscles. A famous theory attributes the degree of permeability of erythrocytes and many other animal and plant cells to given substances to the degree of the solubility of these substances in the lipoids which are supposed to form the essential constituents in the outer layer or envelope of cells (Overton) Substances which are readily soluble in lipoids are supposed to gain an easy entrance by going into solution in the envelope; those which are insoluble in lipoids are checked at the boundary. Attempts have been made to apply this theory to the explanation of selective 
absorption by the intestine, but without much success. The very fact that the theory is held to apply to practically any cell greatly circumscribes at the ontset its power of dealing with cells like those lining the intestine, which are adapted to absorb nutrient materials for all the body, and must necessarily differ in their permeability from cells adapted for some limited function involving only a limited and specialized nutritive exchange. Thus sodium chloricle practically does not penetrate the red blood-corpuscles, the muscle fibres, and many other tissue clements. It is a lipoid-insoluble substance, and the lipoid theory says that this is the reason why it penetrates these cells with such difficulty. But sodium chloride must and does penetrate the intestinal mucosa, and with considerable ease, in order that the body, especially its extracellular liquids, may obtain a sufficient supply of this indispensable material. It is still a lipoid-insoluble substance, and pays no heed to the lipoid theory at all. It is perfectly true that some substances-e.g., cthyl alcohol-which are much more soluble in lipoids than sodium chloride, are also even more readily absorbed from the intestine. It has been stated also that, as regards the velocity of their absorption, the three alcohols, glycerin, erythrite, and mannite, are related to each other in the same way as in regard to their lipoid-solubility. There is, of course, some reason for this, and also some reason why ethyl alcohol is taken up more easily than salt, but we do not know that it has anything to do with lipoid-solubility. If there is a lipoid layer at the free ends of the cells covering the villi, it is very possible that a substance soluble in lipoids may be able to enter cells which would otherwise have denied it entrance. It may even inflict temporary or permanent injury on the cells in doing so, and may thus be taken up in greater amount than by normal cells, and this possibility has to be reckoned with in giving a pliysiological value to experiments with materials essentially foreign to the intestine, and to which it cannot have developed any adequate adaptation. For the essential food materials it is quite certain that, apart from any general relations of cell envelope and environing liquids which are common to the intestinal and to other cells, special relations of an adaptive nature have been developed between the intestinal cells and the very special liquids, elsewhere unknown in the body, with which they come in contact in the lumen of the gut. It is unlikely that the mucosa has developed a special adiptation for lipoidsoluble food materials; it must have developed an adaptation for such food materials, lipoid-soluble or not, as have been offered to it through countless ages, and as are necessary for the nutrition of the organism.

But if it be true that the action of the columnar epithelimm of the intestinal mucous membrane in the absorption of the food is in the main a process of selective secretion such as is found in glandular 
organs, an action which we nay perhaps clescribe as making use of purely pliysical processes, but not mastered by them, the possibility must be admitted that in the cells of endothelial type which line the serous cavities, the lymphatics, the bloodvessels, the alvenli of the lungs, and the Bowman's capsules of the kidney (p. $48 \mathrm{~g})$, the element of secretion may be less marked, and more overshaclowed by the pluysical factors. And it may very plausibly be urged that changes of considerable physiological complexity can only be wrought on substances that have to pass through a cell of considerable depth, while a mere film of protoplasm suffices for, and indeed farours, mechanical filtration and diffusion. We have already seen (p. 261), in the case of the lungs, that whatever the complete explanation may be of the gaseous exchange which takes place through the alveolar membrane, physical diffusion undoubtedly plays an important part. We shall see, too (p. 500), that in the case of the kidney the endothelium of the Bowman's capsule, although by no means devoid of selective power, does seem to have allotted to it a simpler task than falls to the share of the 'rodded 'epithelium.

Absorption from the Peritoneal Cavity.-Further, it has been stated that interchange between blood-serum, circulated artificially in the vessels of dogs and rabbits which have been dead for hours, and liquids introduced into the peritoneal cavity, is essentially the same as in the living animal, and can be explained on purely physical principles (Hamburger). But there is one experiment, at any rate, which is certainly difficult so to explain-viz., the absorption from the peritoneal cavity of sodium chloride solution isotonic with the blood-serum, an absorption which goes on with considerable rapidity. Starling has supposed that this is due to the circumstance that the proteins of the serum exert osmotic pressure, the peritoneal membrane being almost or altogether impermeable for them in comparison to its permeability for the salt solutions. In consequence, water passes into the bloodvessels from the peritoneal cavity. The solution thus becomes more concentrated as regards sodium chloride, some of which accordingly enters the blood by diffusion, and so on. But even isotonic serum is absorbed from the peritoneal cavity, and it seems to savour of special pleading to suggest, as has been done, that this takes place through the lymphatics, and not at all through the bloodvessels.

Up to a certain point an increase in the intraperitoneal pressure favours absorption, but beyond this it hinders it by interfering with the circulation. The removal of a portion of the fluid in this condition facilitates the absorption of the rest - a fact which has long been applied in the operation of tapping. Ligation of the thoracic duct has little effect on the fate of liquids injected into serous cavities, since the bloodvessels play the chief part in their absorption, just as strychnine, when injected under the skin-ic., into 
the lympl spaces of areolar tissue-is taken up by the blood and does not appear in the lymph.

But even if we admit that substances can pass, by physical processes alone, from serous cavities into the blood, and from the blood into serous cavities, this has little bearing upon the question of intestinal absorption. For we can hardly put anything into the peritoneal carity which is not forcign to it. It was never intended to come into contact with the lundred and one solutions, extracts, suspensions, and what not, which the industrious experimenter has offered to its unsophisticated endothelium. It cannot possibly have developed any high degree of 'selective' power. In the intestine everything is different. The mucosa is adapted to come into contact with an inmense variety of materials, all kinds of foodsubstances mingled with many kinds of refuse, the products of the action of numerous digestive ferments, and of a vigorous and varied bacterial flora. All these it has to sift and try. It cannot fail to have properties which suggest a severe and searching selection.

The difference between a serous cavity and the intestine is well illustrated by the follcwing experiment. in which the changes in the composition of a hypotonic ( 3 per cent.) solution of dextrose introduced into the peritoneal sac and into a loop of intestine respectively were compared (Cohnheim).

\begin{tabular}{|c|c|c|c|}
\hline & Introduced. & After & Recovered. \\
\hline $\begin{array}{l}\text { Peritoneum - } \\
\text { Intestine - - }\end{array}$ & $\begin{array}{l}50 \text { c.c. } \\
44,\end{array}$ & $\begin{array}{l}90 \text { mins. } \\
25, \text {, }\end{array}$ & $\begin{array}{l}\text { I9.5 c.c., containing I per cent. } \\
\text { dextrose and } 0.55 \text { per cent. } \\
\mathrm{NaCl} \text {. } \\
\text { I9 c.c., containing } 3.8 \text { per cent. } \\
\text { dextrose and } 0.04 \text { per cent. } \\
\mathrm{NaCl} \text {. }\end{array}$ \\
\hline
\end{tabular}

Here the water and sugar are both taken up from the intestine and the peritoneal cavity; but while the sugar concentration in the serous sac falls markedly, as ought to be the case if the sugar is diffusing into the blood along the slope of concentration. the perecntage of sugar in the intestine actually increases. Still more striking is the fact that sodium chloride accumulates in the peritoneal liquid in a concentration obviously tending to equality with that of the blood, as would happen if the peritoncal lining were a dead diffusion membranc. On the other hand, practically no sodium chloride passes into the lumen of the gut.

Closely connected with the question of ahsorption from and secretion (or transudation) into the serous cavities is the question of the factors concerned in the formation of the lymph (which will be considered in the next chapter), eren although recent researches throw grave doubt on the common view that these sacs are merely expanded lymph spaces, and indicate that the liquid found in them has a different origin from lymph. 


\section{Section III.-Absorption of the Various food Substances.}

Absorption of Fat-How the Fat gets into the Intestinal Epithelium. -It has been already mentioned that fat is split up in the intestine into the corresponding alcolıols (mostly glycerin) and fatty acids, but it has been a subject of discussion whether it all undergoes this change or only a portion of it. The common view has long been that the greater part of the fat escapes decomposition, and, after cmulsification by the soaps formed from the liberated fatty acids, is absorbed as neutral fat by the epithelial cells covering the villi. If an animal is lilled during digestion of a fatty meal, these cells are found to contain globules of different sizes, which stain black with osmic acid, are dissolved out by ether, leaving vacuoles in the cell substance, and are therefore fat (Fig. I72). It has always been difficult to explain how droplets of emulsificd fat could get into the interior of the epithelial cells, although, perhaps, no more difficult than to explain the passage of living tubercle bacilli from the contents of the intestine into the chyle of the thoracic duct - a fact which has been clearly demonstrated (Ravenel). The fat is certainly contained within the cells, and not between them. When fat is found in the cement substance between the cells, it has been mechanically squeezed out of them by the shrinking of the villi in

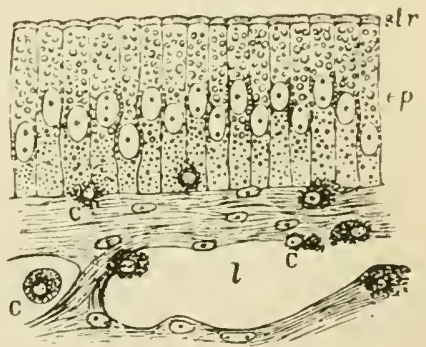

Fig. 172.-Mucous Membrane of Frog's Intestine during Absorp. tion of Fat (Schäfer). ep, epithe. lial cells; str, striated border; $C$, lymph corpuscles; $l$, lacteal.

preparation. This difficulty is obviated if we suppose that the whole of the fat is split up in the intestine, the products being absorbed in solution, the glycerin as such, and the fatty acids either as soaps or in the free state, or partly free and partly saponified. If this is the true theory-and the evidence of its truth has of late years been continually growing-neutral fat must again be built up in the epithelial cells from the absorbed glycerin and the fatty acids or soaps. Now, it has been shown that when an animal is fed with fatty acids they are not only absorbed, but appear as neutral fats in the chyle of the thoracic duct, having combined with glycerin in the intestinal wall; and the epithelial celis contain globules of fat, just as they do when the animal is fed with neutral fat. Further, it is known that fat-splitting goes on in the alimentary canal to a much greater extent than would be necessary merely for the formation of a quantity of soap sufficient to emulsify the whole of the fat in the food. Indeed, at certain stages of digestion most of the 
fatty material, both in the small and large intestine, has been found to consist of fatty acicls. The reversibility of the raction under the influence of lipase, which has already been alluded to, does not enter into the question so far as fat-splitting in the intestine is concerned, for the products of the reaction can be absorbed as quickly as they are formed. To clinch the matter, it has been proved that when mixtures of paraffin and fat, which can be emulsified in a watery solution of sodium carbonate, are eaten, the paraffin is completely excreted with the freces, while the greater part of the fat is absorbed. And fatty substances which are not casily split up and saponified (for example, lanolin, the fat of sheep's wool, a mixture of compounds of fatty acids with isocholesterin, a substance closely related to cholesterin and allied bodies) are not absorbed even when they are easily emulsificd. Even fats with a melting-point far above the temperature of the body can be absorbed after being split up. The palmitate of cetyl alcohol, the chief constituent of spermaceti, melting at $53^{\circ} \mathrm{C}$., was absorbed to the extent of I5 per cent., 85 per cent. being excreted in the faces. It appeared as palmitin in the chyle of a human being flowing from a fistula, the palmitic acid having becn absorbed as sucl, or as a sodium soap, and having then united with glycerin to form the neutral fat, palmitin.

Some observers have endeavoured to show that the fat is absorbed without change by introducing into the intestine fat stained with dyes, such as alkanna red or Sudan III., whicl are insoluble in water. The stained fat was found in the epithelial cells of the villi, in the lacteals, and, in the case of a patient suffering from cliyluria, in the urine. But this evidence is not conclusive, for it lias been shown that the pigments might easily have been absorber after decomposition of the fat, since, although insoluble in water, they are soluble in fatty acids, and therefore to some extent in the intestinal contents, and readily pass into the lymph.

As already pointed out, the bile plays an important part in the solution of the fatty acids, which. may form loose compounds with the amide group of the bile-acids. In these loose combinations, soluble in water, the fatty acids can be absorbed from the intestinal contents (Pflüger). In whatever way the fat which can be seen in the epithelial cells during absorption of fat gets into tlicm, it must be carefully noted that there is no quantitative proof that it represents all or even the greater part of the absorbed fat. So far as microscopic observations go, much of the fat may pass through the mucosa in the form of soluble decomposition products without appearing in particulate form in the epithelium.

How the Fat gets out of the Intestinal Epithelium.-I-encocytes have been asserted to be active agents in the absorption of fat. They have been described as pushing their way between the epithelial cells, fishing, as it were, for fatty particles in the juices 
of the intestine, and then travelling back to discharge their cargo into the lympl. This view, however, is erroneous. But, although the lencocytes do not aid in the absorption of fat from the intestine, they may take up a certain amount of it from the epithelial cells, and convey it through the spaces of the network of adenoid tissue that occupies the interior of the villus, to discharge it into the central lacteal, where it mingles with the lympl and forms the socalled molecular basis of the chyle. It has been supposed that a part of the fat may reach the lacteal in another way. The contraction of the smooth muscular fibres of the villus and the peristaltic movements of the intestinal walls, which alter the shape of the villi, alter as well the capacity of the lacteal chamber, and so alternately fill it from the lymph of the adenoid reticulum, and empty it into the lymphatic vessel with which it is connected. By this kind of pumping action the passage of fat and other substances into the lymphatics may be aided. There is, however, no proof that all the fat accumulated in the intestinal epithelium leaves it without further change. It is quite as probable that the lipase which is known to be contained in the cells again hydrolyses the fat, or a portion of it, and that the constituents then pass out into the lymph, or even in part into the blood. In the dog most of the fat goes into the lacteals, and thence by the general lymph-stream through the thoracic duct into the blood. And in man the chyle collected from a lymphatic fistula contained a large proportion of the fat given in the food (Munk). But this bare statement would be misleading if we did not add that the fat taken in can never be entirely recovered in the chyle collected from the thoracic duct. A small fraction of the deficit might be accounted for as fat directly used up for the nutrition of the intestinal wall itself. But even after ligation of the thoracic and right lymphatic ducts a large proportion of a meal of fat ( 32 to 48 per cent.) is absorbed from the intestine, obviously by the channel of the bloodvessels, since the fat-content of the blood increases up to, it may be, six times the highest a mount present in the blood of fasting animals. The statement that only fatty acids can be absorbed under these conditions is erroneous (Munk and Friedenthal).

A dog normally absorbs 9 to $2 \mathrm{I}$ per cent. of the fat in a meal in three to four hours; 21 to 46 per cent. in seven hours; and 86 per cent. in eighteen hours (Harley). After excision of the pancreas the absorption of fat is hindered, though not abolished. More fat, indeed, can be recovered from the intestine than is given in the food. This at first sight paradoxical result is explained by the well-established fact that a certain amount of fat is normally excreted into the intestine.

Mechanism of Fat Synthesis in the Intestinal Mucosa.-As to the manner in which the synthesis of the fat in the intestinal epithelium 
is accomplished, the most fascinating theory is that which attributes it to the reversed action of lipase, possibly the very same lipase as originally split it up in the intestine. The reversibility of the action of various enzymes under changed conditions, especially changes in the relative concentration of the bodies concerned in the reaction, has been previously mentioned. It has been shown, e.g., that the pancreas, intestinal mucous membrane, lymph glands, etc., and even cell-free extracts of these organs have the power of synthesizing the ester ethyl butyrate from butyric acid and cthyl alcohol (p. 338), as well as the power of decomposing the ester into the fatty acid and the alcohol. Moore, however, states that in the case of ordinary fats the synthesis takes place in the intestinal wall only in situ, and while the circulation is going on. In the intestinal mucosa the greater part of the fatty acid is already combined with glycerin as neutral fat, although considerable quantities of free fatty acid are also present. In the lymph coming directly from the mesenteric glands practically the whole of the fatty acids are in the form of neutral fat.

An additional, and in some respects even more remarkable, illustration of the synthesizing powers of the intestinal wall is the discovery of Munk, already referred to (p. 44I), that fatty acids given by the mouth appear in the lymph of the thoracic duct as neutral fats, having somewhere or other, in all probability on their way through the epithelium of the gut, been combined with glycerin.

Since, however, the amount of neutral fat recovered from the thoracic duct is not equivalent to more than one-third of the fatty acids given, it has been suggested that this synthesis of fat is only apparent, and that the whole of the fat which appears in the chyle after a meal of fatty acids comes from the fat excreted into the intestine (Frank), which is increased when fatty acids are given by the mouth. But the suggestion is more ingenious than the evidence advanced in its support is convincing. And, as we have seen (p. 443), a part of the deficit may be accounted for by absorption directly into the bloodvessels.

In concluding our review of the absorption of fat, certain gencral considerations which have a close relation to the question may be alluded to. There is some reason to think that the lipuses are enzymes less finely adjusted to minute differences in the structure of the fats on which they act than other digestive ferments- $e g$., maltase or lactase, to details in the chemical structure of their substrates. If this be so, a very few lipases, or even a single one. may suffice to accomplish all the enzymatic changes which occur in the fats both in the lumen of the intestine and in all the various tissue cells. At the same time the possible variation in those decomposition products which constitute the 'building-stones ' of the fats is less than in the case of, say, the proteins. Two consequences 
follow as regards the absorption of fat: (I) Each cell may be capable of dealing with the original neutral fats of the food, and of adapting them to its needs by decomposing and resynthesizing them so far as is necessary in its own substance. In this case it would not be necessary for assimilation by the cells that the fats should be completely split or even split at all in the alimentary canal, however important this might be for their absorption from its lumen. (2) If it were necessary for absorption that decomposition of the fats should take place in the lumen of the digestive tube, the whole of the fat, or at any rate such portion of it as was not at once needed, might without disadvantage for the tissues be resynthesized after absorption. It is not difficult to see that it might even be advantageous that not only the relatively fixed reserve in the fat cells, but also what might be termed the floating or circulating reserve constituted by the emulsified fat in the blood should be in the insoluble form of neutral fat.

Absorption of Carbo-Hydrates.-Carbo-hydrates are normally absorbed from the alimentary canal only in the form of monosaccharides, such as dextrose, levulose or fructose, and galactose, but especially dextrose. These monosaccharides are readily formed from polysaccharides like starch and dextrin, and the disaccharide maltose, which they yield on digestion with amylase, as well as from disaccharides like cane-sugar and lactose, by the ferments already studied. That, as a matter of fact, the hydrolysis in the intestine must convert practically all the carbo-hydrate into monosaccharides before absorption, can be shown in various ways. The ferment lactase, while present in the small intestine of all young mammals, is regularly absent in some mammalian groups in the adult. In other species, including man, it is found in some adults, but not in all. In birds and other animals below the mammals, it has not hitherto been found at any age. It has been surmised that these differences depend upon the presence or absence of lactose (milk) as a regular constituent of the food. (But see p. 4 Io.) If, now, lactose is introduced into a loop of intestine in an animal which does not possess lactase-e.g., an adult rabbit-it is not apsorbed, but remains in the lumen till it is at last decomposed by bacterial action. In animals in which lactase is present the lactose is rapidly absorbed. Maltose is easily taken up from the intestine because of the action of the ferment maltase, which is the most widely spread of all the inverting ferments. The dextrose formed by the maltase is so rapidly absorbed that none, or only traces, of it can be detected in the contents of the intestinal loop. But if absorption be interfered with by injuring the intestine, maltose disappears, and the dextrose produced from it accumulates in the lumen. The reason for the discrimination exercised by the intestinal mucosa in favour of the monosaccharides becomes apparent when an attempt is 
made to circumvent it by injecting the sugars parenterally-i.e., into subcutaneous or intramuscular connective tissue, into a scrous sac, or directly into the blood. Cane-sugar and lactose so introduced are excreted mnchanged in the urine. Dextrose, levulose, and galactose are used up in the body, and some maltose likewise, thanks to the presence of maltase in the blood and tissues. The cells of the body in general will burn only monosaccliaricles, and not di- or poly-saccharides. Galactose and fructose are probably first converted into dextrose before being ntilized by the tissues, a change which can also be readily incluced in the test-tube. Therefore the intestine admits the simple, but rejects the more complex sugars. It is only in the presence of abnormally great quantities or abnormally great concentrations of the sugars which are not directly ntilizable that they are to a certain extent taken up unaltered, to be for the most part quickly excreted as such (p. 540). In like manner we have seen that the native proteins can, so to speak, force their way by storm through the intestinal mucosa when offered to it in exceptionally large amount. The sugar absorbed from the intestine passes normally into the rootlets of the portal vein, not into the chyle, for no increase in the quantity of that substance in the contents of the thoracic duct takes place during digestion, while the sugar in the portal blood is increased after a starchy meal. The blood of the portal vein of a dog in the fasting condition contained 0.2 per cent. of dextrose. During absorption of a meal rich in carbo-hyclrates it contained as much as 0.4 per cent. In the lymph issuing from the thoracic duct the amount was the same in both conditions-viz., 0.16 per cent. In a case of lymph (chyle) fistula in a human being, where almost all the lymph from the digestive tract escaped through the fistula, out of roo grammes of carbo-hydrate taken (50 grammes starch and 50 grammes sugar), only $\frac{1}{2}$ gramme, or not I per cent. of the sugar corresponding to the carbo-hydrates of the food, could be recovered in the chyle. But when a large amount of a dilute solution of sugar is introduced into the intestine, some of it is taken up by the lacteals.

Absorption of Water and Salts.-The main channel for absorption of these is the bloodvessels of the intestine. As much as 3 to 5 litres of water can be absorbed in a day in the intestine of a healthy man, exceptionally even 6 to Io litres, without the faces altering their normal consistence. Absorption of the water and dissolved salts may theoretically take place either through the epithelial cells (intraepithelial absorption), or between the cells (interepithelial absorption). According to Höber, most metallic salts (silver, mercury, lead, bismuth, copper, manganese, etc.) are absorbed interepithelially, while iron salts form an exception, and pass into the epithelial cells. The distinction between interepithelial and intra-epithelial absorption does not rest upon an absolutely sure foundation. Yet 
it is probable that everything which is useful in the nutrition of the body is taken up by the cells, while such substances as metallic salts which are foreign to the organism, and are denied entrance into the cells, may pass in small amomnt between them, their passage being perhaps associated with more or less injury to the interstitial substance. The vigilant selection exercised by the mucosa is well illustrated by the facts that, although manganese and iron are chemically so closely related, iron, which is necessary for the formation of the blood-pigment, is absorbed in immensely greater amount than manganese; and that chlorides, especially sodium chloride, are readily taken up, sulphates with difficulty. Iron is absorbed by the bloodvessels, but also to some extent by the lacteals. From the blood it is carried to various organs, especially the spleen and liver. There is reason to believe that the eosinophile leucocytes take some share in its transportation.

It was supposed by Bunge that only organic compounds of iron could be absorbed, and that the undoubted benefit derived from the administration of inorganic iron compounds, such $\Sigma$; ferric chloride, in chlorosis, was due not to their direct absorpion, but to their shielding the organic compounds from the attack of the sulphuretted lyydrogen in the intestine (p. 424). But this theory has been shown to be inconsistent with the facts. For instance, after the administration of salts of iron, the iron in the blood, liver, spleen, and other organs increases, but there is no accumulation of iron in the liver of an animal to which salts of manganese have been given, although these are equally decomposed by sulphuretted hydrogen.

Absorption of Proteins.- The proteins of the food or their digested products also pass directly into the blood-capillaries which feed the portal system. For it has been shown that after ligature of the thoracic duct protein substances are still absorbed from the intestine, and the urea corresponding to their nitrogen appears in the urine. The total nitrogen in the chyle flowing from a fistula of the thoracic duct in a man was not found to be increased during the digestion of protein food. The quantity of chyle escaping in a given time was also unaffected, whereas during the digestion of fats it was greatly augmented (-Munk).

Although a certain amount of egg-albumin, serum-albumin, alkalialbumin, and other native or slightly altered protein substances can be absorbed as such by the small, and even by the large, intestine, there is no evidence that, under ordinary conditions, this mode of absorption is of any practical importance in nutrition, although in another relation it may possess a certain interest (p. 32). For when native proteins, with the possible exception of the serum proteins from an animal of the same species, are introduced ' parenterally,' so that they do not reach the tissues by way of 
the alimentary canal, they behave in a very different manner from the same proteins when given by the mouth. One notable difference is that the parenterally administered proteins give rise in general to the formation of antibodies-e.g., specific precipitins (p. 3I). This is not the case when they are administered per os, unless, like raw egg-white, which, as already mentioned (p. 4n 4 ), evokes no secretion of gastric juice, they remain long undigested in the alimentary canal, when an amount sufficient to cause the production of precipitins may eventually be absorbed unaltered. This has also been shown by means of the anaphylactic reaction. Secondly, they are not, as a rule, utilized in the metabolism of the body, or are utilized very incompletely. Egg-albumin, for instance, when injected into the blood, is excreted in the urine. It has been previously pointed out that the various proteins differ remarkably not so much in the kinds as in the relative quantities of the aminoand diamino-acids which can be obtained from them (p. 2). This is unquestionably one important reason why the food proteins arefor the most part, at any rate-so thoroughly hydrolysed before absorption. Another may be that it is easier for the irtestine to take up the small molecules of the decomposition products than the large colloid aggregates of the original protein solutions.

So far as the first reason is concerned, the degree of decomposition need not be the same for all the food proteins, although all must be decomposed, for even among the proteins the products of whose hydrolysis do not exhibit qualitative differences, no two have hitherto been discovered which show the same quantitativ: relations among the 'building-stones.' A new house has to be built $f_{1}$ om the materials of an old one. How far the work of demolition must be carried will depend upon the difference between the plans of the two houses. Sometimes the main part of the old building may be saved, and only the wings require reconstruction. In like manner it is conceivable that the central group or nucleus of the molecule of a given food protein may be identical with that of a given body protein, and that only the side-chains may be so different that they must be broken up and reconstructed. Or, again, the whole architectural plan of the new house may be so distinct from that of the old that the only feasible method is to completely' demolish the latter, and then to use the individual bricks in the new construction; just as a protein in the food may differ so radically from a tissue protein into which it is to be transformed that it must be decomposed into the simplest products of proteolysis before the reconstruction of the molecule can begin. It is not known what the minimum degree of hydrolysis is which will permit of effective absorption and utilization. But it would seem that it must be very complete. Even a body so simple in comparison with the proteins as the tripeptide (p. 2) alanyl-glycyl-tyrosin, con- 
taining only three 'building-stones,' can only be changed into the tripeptide alanyl-tyrosyl-glycin by first hydrolysing it into its three components and then synthesizing these afresh. On the other hand, in obtaining the tripeptide glycyl-tyrosyl-alanin from alanylglycyl-tyrosin, the dipeptide group glycyl-tyrosin can remain unlecomposed, and it is only necessary to split alanin off and link it up to the dipeptide to obtain the clesired glycyl-tyrosyl-alanin (Abderhalden). There can be no doubt that by far the greater part, if not the whole, of the proteins of the food is first changed into proteoses and peptones. But proteose and peptone are absent from the blood, and, indeed, when injected into the blood they are excreted in the urine. When injected in larger amount, they pass also into the lymph, from which they gradually reach the blood again, and are eventually, as before, eliminated by the kidneys. The clear inference is that if they are absorbed as such from the alimentary canal, they must be changed in their passage through its walls. The fact that a portion at any rate of the peptones and proteoses is decomposed into amino-acids, etc., in the lumen of the intestine has been already alluded to. It is certain that this portion is a very large proportion of the whole, although the question how much, if any, of the peptone passes as such from the lumen into the mucosa must still be left undecided. It is true that along with amino-acids peptones are always found in the intestine during the digestion of protein, and the quantity of amino-acids actually present in the lumen at any moment may De small in proportion to the quantity of peptone. But this is precisely what is to be expected if the peptone as such is incapable of absorption. For the easily absorbed amino-acids will disappear from the gut as fast as they are formed, leaving behind the peptone for further hydrolysis. The fact that all the amino-acids which the proteins are capable of yielding can be detected in the contents of the intestine, including even those which appear late and, as it were, reluctantly in artificial digestion, is a proof that the decomposition of the protein goes fast and far in the alimentary canal. If it is not complete, if some of the partially hydrolysed protein is taken up by the mucous membrane in the form of peptones or possibly even of proteoses, it would seem that this is similarly decomposed by the action of ercpsin in the intestinal wall.

It has been stated that during the digestion of a protein meal the mucosa of the stomach and intestine contains proteose and peptone, while none is present in the muscular coat or in any other organ. They rapidly disappear from a portion of the mucous membrane kept at a temperature of about $40^{\circ} \mathrm{C}$. outside of the body, and their disappearance is due, not to their regeneration into serum proteins, as was once supposed, but to their decomposition by the erepsin. We must suppose that the serum and organ proteins are 
built up from the products of this decomposition. But whether the mucosa of the alimentary tract is especially a seat of the synthesis is unknown (p. 573). On a priori grounds, it is at least equally probable that it occurs in all the cells of the body, each one building up for itself the particular kind of protein which it needs. The direct way of testing the question would be to examine the blood coming from the intestine during the absorption of proteins, and to determine quantitatively any changes which might have occurred in the nitrogenous constituents. But the flow of blood through the intestine is so great, the absorption of the digestive products so gradual, and their removal from the blood by the tissues, in all probability, so rapid, that there is no reason for surprise that till lately the results of such determinations were ambiguous. Leathes, however, showed some time ago that when peptone, proteose, or the final products of tryptic digestion are introduced into a ligated segment of a dog's small intestine, there is always, when absorption occurs, an increase in the nitrogenous substances in the blood, in the form of compounds which are not precipitated by tannic acid, and therefore are neither native proteins nor proteose. Urea accounts for about one-half of the increase; the rest he considered to represent probably amino-acids and similar substances. Quite recently it has been conclusively demonstrated by improved methods that the digestion of protein is associated with an increase of non-protein nitrogen in the blood, due, there is every reason to believe, to aminobodies derived from the hydrolysed protein (Folin and Denis, Van Slyke, Abel). This proves for the mammal what had been deduced by Cohnheim for a much lower form from experiments made on the intestines of certain octopods, which, when excised and suspended in the oxygenated blood, will live for many hours. A solution of peptone was introduced into the isolated intestine, and after twenty loours the crystalline products, lencin, tyrosin, lysin, and arginin, were found in the blood. In the intact animal none of these bodies could be detected in the blood (Cohnheim). The inference was that protein in these animals is absorbed in the form of amino-acids, etc., which are then carried to the tissues and utilized there. In the mammal the same thing appears to be true. For the increase in the amino-acids during digestion of proteins occurs not only in the portal blood, but in the blood of the general circulation. So that, although a part of the absorbed amino-bodies may be removed by the liver, a portion at least is a vailable for the tissues in general. That the tissues actually take up such decomposition products of proteins is indicated by the fact that during and after the digestion of protein in a loop of intestine the non-protein nitrogen of the tissues is increased (Folin). It may be that some of the proteose and peptone are regenerated by a shorter process, and without having heen further split up, but of this, too, there is no definite 
proof. The regeneration, wherever it occurs, must presumably take place in cells, and the only available cells in the digestive mucous membrane are those which line the tube, or the leucocytes which wander between them. Accordingly, both have been credited witl the power of absorbing (and perhaps transforming) these substances, but the balance of evidence is in favour of the epithelial cells. $\mathrm{IV}^{\circ} \mathrm{e}$ cannot, however, as in the case of the fat, single out any particular tract of epithelium as alone engaged in the absorption (and possibly in the resynthesis) of the products of the digestion of the proteins. In all likelihood the cells covering the villi are actively concerned, but there is no valid reason for denying a share to the general lining of the stomach and small intestine, even perhaps including the Lieberkühn's crypts or intestinal glands, which morphologically form a kind of inverted villi. It is, indeed, true that the crypts do not take part in the absorption of fat, for no granules blackened by osmic acicl occur in them during digestion of a fatty meal. But this is a ground for attributing to them other absorptive functions rather than for altogether denying to them a share in absorption, unless, indeed, we assume that the secretion of the succus entericus engrosses the whole activity of this extensive sheet of cells. Even within physiological limits distension of the gut causes the crypts to become shorter and broader, by a process of partial unfolding which permits a greater part of their epithelium to come into contact with the intestinal contents. In extreme distension they may be completeiy smoothed out.

The extraordinary efficiency of the small intestine in digestion and absorption is shown by the fact that, after removal of even 70 to $S_{3}$ per cent. of the combined jejunum and ileum in dogs, the metabolism is not necessarily much affected. On a diet poor in fat the animals absorb as much of the fat as a normal dog, although a smaller proportion when the diet is rich in fat. It has been generally stated that it is never permissible to remove more than one-third of the small intestine in man. But in one case $2 \frac{3}{4}$ metres was resected, or quite one-half, and the patient recovered. Even the large intestine, which possesses Lieberkühn's crypts, but no villi, is able to absorb not only peptones and sugar, especially monosaccharides like dextrose, but also fats and native proteins. And although these are powers which can be rarely exercised to any great extent in normal digestion, they form the physiological basis of the important method of treatment by nutrient enemata. The observation already mentioned (p. 33I), that considerable quantities of food administered by the rectum can pass through the ileo-colic sphincter and valve into the lower part of the ileum, thanks to the antiperistaltic movements of the large intestine, indicates that an important part of the preliminary digestion and of the absorption of enemata may occur in the small intestine. But remnants of the 
proteolytic, amylolytic, fat-splitting, and inverting ferments which have done their work in the small intestine are passed on into the large, and may be demonstrated in its contents. Doubtless these are able to act upon food substances which may have escaped complete digestion and absorption in the higher parts of the alimentary canal, as well as upon food substances injected into the rectum.

Summary.-With the proviso that in the case of the fats the statement may in the present condition of our knowledge be somewhat 'diagrammatic,' we may sum up in a few words the chief points in the absorption of the food materials. All the fats must bc split in order to be absorbed in soluble form from the intestine, but need not be split in the lumen of the gut in order to be ntilized by the cells. For this reason they are to a great extent resynthesized to neutral fat after absorption, and find their zeay into the blood, mainly by way of the lymph, in particulate form. Proteins could perhaps to a small extent be absorbed as such, but must be thoroughly hydrolysed in order to be utilized by the tissues, and also in order to be freely taken up from the gut. Carbo-hydrates in certain forms (monosaccharides) are capable without change of being both freely absorbed from the intestine and thoroughly utilized by the cells; only the more complex carbo-hydrates need to be hydrolysed in order to be absorbed, but all above the monosaccharides must be hydrolysed to monosaccharides in order to be utilized. The substances which eventually circulate in the blood in solution reach it through the gastro-intestinal capillaries; the substances which eventually circulate in the blood in particulate form reach it through the lymphatics.

\section{PRACTICAL EXERCISES ON CHAPTERS VI. AND VII.}

r. Contraction of Isolated Intestines in Ringer's Solution.-Arrange a good-sized water-bath (a water-tight garbage-can holding 20 litres will do) so that the temperature of the water is kept at $37^{\circ}$ to $38^{\circ} \mathrm{C}$. For this a gas regulator is most convenient, and also a stirring arrangement worked by a small motor. But if ncither of these is available, a student, by a little care, can easily keep the water at the required temperature by raising and lowering the gas flame and stirring occasionally by hand. In the bath, support (a) a stock bottle of Ringer's solution (footnote, p. 66), (b) a wide-mouthed bottle containing Ringer for the reception of the stock of intestine, (c) a small cylinder for segments of intestine whose contractions are to be recorded. (c) is conveniently made in different sizes by cutting down glass T-picces. One which holds 4 or 5 c.c. is convenient. The bottom is plugged with a rubber cork in which is fastened a hook. In the side-picce is fixed by a rubber cork a glass tube ending in the cylinder in a narrow orifice. This is connected with the oxygen-supply, conveniently obtained under constant pressure from a small gas-container which is periodically replenished from all oxygen cylinder. A separate oxygen cylinder is connected witl a tube passing to the bottom of $(b)$. A lever with two arms is arranged on the same stand as $(c)$, so that it can be thrown on and off a slow-moving drum by a single movement of the stand.

All being ready, a rabbit is killed by being struck on the back of the 
head. The small intestine is immediately removed. It may be cut between double ligitures into several pieces for this purpose. The contents are rapidly washed out by a strean of warm Ringer, and the pieces placed in $(b)$, through which oxygen is kept bubbling. The pieces are conveniently supported in the liquid by threads fixed by the cork of the bottle. There is a hole in the cork for the escape of the oxygen. The movements of the intestines in $(b)$ can be studied very well by inspection. Or a separate length of intestine may be kept for this purpose, the contents not being removed, but prevented from cscaping by ligatures at each end. This can be most easily observed in a sliallow dish of warm Ringer. Or a separate experiment can be made in which the whole alimentary canal of a rabbit is carefully removed and examined in oxygenated Ringer's solution.

A segment of intestine about 2 or $2 \frac{1}{2} \mathrm{~cm}$. in length is now cut off onc of the pieces. A small ring of platinum or aluminium is tied to a point on the circumference of one end of the preparation by a sill thread passed through the wall. The other end is canght by a serve. fine at a point exactly corresponding to the attachment of the ring, so that the pull of the contracting longitudinal muscle should be in the straight line joining these two points. The serre-fine has attached to it a thread with a hook on the other end. In preparing the intestinal segment it lies on a plate of glass above a vessel of warm water. The small cylinder $(c)$ is now partially filled with warm Ringer's solution. The ring is graspecl by fine forceps, and made to engage with the hook at the bottom of the cylinder, care being taken not to injure the preparation in the process. The cylinder is then fastened on its stand and lowered into the bath. The thread is connected by its attached hook to the lever, and oxygen allowed to bubble slowly and regularly through the cylinder. Very soon rhythmical contractions begin (Fig. I 73), and continue for a long time. The effect on these contractions of abolishing, reducing, or increasing the oxygen-supply may first be studied.

2. Effect of Blood-Serum on the Contractions of Intestinal Segments. -While a tracing is being taken as in I, fill a small bent pipette with serum already warmed in the bath, pass the point of the pipette down to the bottom of the cylinder without interfering with the preparation, and allow the serum to flow in till the Ringer's solution is displaced. Almost at once the lever will begin to rise, indicating strong tonic contraction. The increase of tone lasts for some time, but can soon be removed on washing the preparation with Ringer. This is most easily done, while the drum is stopped, by introducing pipetteful after pipetteful of Ringer's solution into the cylinder in the way described, allowing the liquid to overflow into the bath. The subsequent addition of serum causes a renewed increase of tone, and this may be many times repeated.

Determine the greatest dilution of the serum which still produces a distinct effect upon the intestinal segment.

3. Action of Epinephrin (Adrenalin) on Intestinal Segments.-Proceed as in 2 , but use various dilutions of adrenalin chloride instead of serum. They must be freshly prepared. Instead of increase of tone, inhibition of the movements and decrease of tone will be obtained (Fig. I73).

This cxperiment may be performed at another stage in the course (p. 720$)$.

4. Quantitative Estimation of Ferment Action.-For pepsin an easy method, although not a very accurate one, of estimating the rate at which the fibrin disappears is to use fibrin stained with carmine. As solution goes on, the dye colours the liquid more and more deeply, and by comparing the depth of colour at any time with standard solutions of carmine, we get the quantity of dye set free, and therefore of fibrin 
cligested. This metlod cannot be used for trypsin. A much locter method is that of Mett (p. 343). Fluid egg-wlite is sucked up into fine glass tubes (of $\mathrm{I}$ to $2 \mathrm{~mm}$. Dore). The tubes are then heated in a bath at $95^{\circ} \mathrm{C}$. For use sliort pieces ( $\mathrm{I}$ or $2 \mathrm{~cm}$. long) are cut off and placed in 1 or 2 c.c. of the liquid to be tested, the whole being kept at $38^{\circ}$ to $40^{\circ} \mathrm{C}$.

For amylolytic ferments where rapid work is required, glass tubes filled with tinted starch paste may be used in the same way as the Mett's tubes. A more accurate metliod, and yet a rapid and convenient one, is based upon the time which is necessary in order that the iodine reaction with starch may just disappear when a standard starch solution

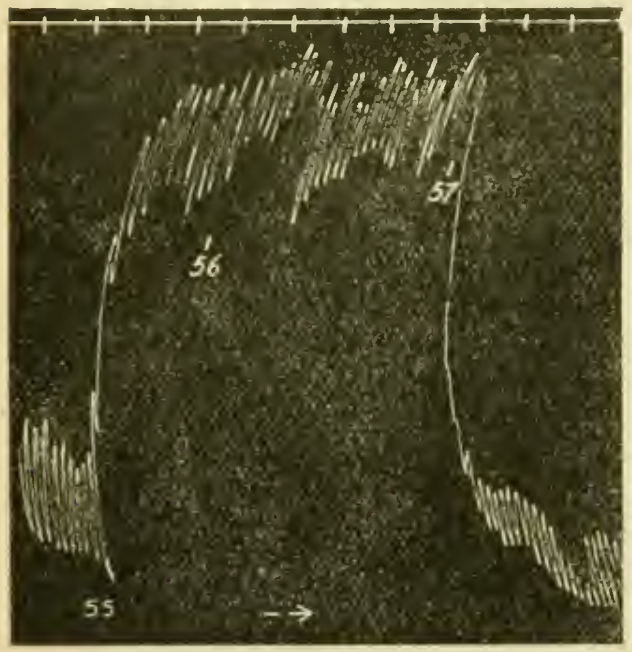

Fig. 173.-Effect of Serum and Adrenalin on Con. tractions of a Segment of Intestine. Rabbit's intestine contracting in Ringer's solution. At 55 the Ringer's solution was replaced by dog's serum, and this at 56 by adrenalin $(x: 5,000,000)$ in serum. At 57 this weak adrenalin solution was replaced by a stronger one $(x: 500,000)$ in serum. Time-trace, half-minutes. (Reduced to half.) is digested with a dilution of the ferment solution at $40^{\circ} \mathrm{C}$.

5. Saliva-Collection and.Microscopic Examination of Saliva.-Cliew a picce of paraffin-wax, or inlable ether or the vapour of strong acetic acid. The flow of saliva is increased. Collect it in a porcelain capsule. Examine a drop under the microscope. It may contain a few flat cpithclial scales from the mouth and a few round granular bodies, the salivary corpuscles, the granules in which often show a lively, dancing movement (Brownian motion). Filter the saliva to free it from air-bubbles, and perform the following experiments:

(a) Test the reaction with litmus paper. It is usually alkaline. An acid reaction may indicate that bacterial processes are abnormally active in the mouth.

(b) Add dilute acetic acid. A precipitate indicates the presence of mucin (p. 344). Filter.

(c) Add a drop or two of silver nitrate solution to the filtrate from (b). A precipitate insoluble in nitric acid, soluble in ammonia, proves that chlorides ar: present.

(d) Add to anotlier portion a few drops of dilute ferric chloride slightly acidulated with dilute hydrochloric acid, and the same quantity to as much distilled water in a control test-tube. A reddish coloration is obtaincd, duc to the presence of sulphocyanic acid, which is combined with potassium and other bases in the saliva. The colour is discharged by mercuric chloride. Or, a drop of saliva may be allowed to fall from the mouth on a test-paper (preparecl by soaking filter-paper with a dilute starch solution, containing a little iodic acicl, and allowing it to dry in the air). The paper is coloured blue by the union of the 
starch with iodine set frec from the iodic acid by the acticn of the sulplo)cyanic acid.

(e) Take some boilcd starch mucilage, and test it for reducing sugar by Trommer's test (p. Io). If no sugar is found, take three testtubes, label them A, B, C, and nearly half fill cach with the boiled starch. To A add a little saliva,* to B some saliva which has been boiled, to $C$ a little saliva which hiss been neutralized, and as much $0 \cdot+$ per cent. hydrochloric acid as has been taken of the mucilage, so as to make the strength of the acid in the mixture 0.2 per cent., a proportion well below that of the gastric juice. Put the test-tubes into a waterbath at $40^{\circ} \mathrm{C}$. In a few minutes test the contents for reducing sugar. Abundance will be found in $A$, none in $B$ or $C$. In $B$ the ferment ptyalin has been destroyed by boiling; in $\mathrm{C}$ its action has been inhibited by the acid. If the test-tubes have been left long enough in the batl, no blue colour will be given by $\mathrm{A}$ on the addition of iodine, but a strong blue colour by $\mathrm{B}$ and $\mathrm{C}-i . e .$, the starch will have completely disappeared from $\mathrm{A}$.

(f) Put some starch in a test-tube, add a little saliva, and hold in the hand or place in a bath at $40^{\circ} \mathrm{C}$. On a porcelain slab place several separate drops of dilute iocine solution. With a glass rod add a drop of the mixture in the test-tube to one of the drops of iodine at intervals as digestion goes on. At first only the blue colour given by starch will be seen; a little later a violet colour, due to the presence of erythrodextrin in addition to some unaltered starch. A little later the colour will be reddish, the starch having disappeared and the erythrodextrin reaction being no longer obscured. Later still no colour reaction will bc obtained, the erythrodextrin having undergone further changes, and only sugar (maltose, isomaltose, and perhaps a trace of dextrose) and achroodextrin-a kind of dextrin which gives no colour with iodinebeing present.

(g) Put two pieces of glass tubc filled with tinted starch paste (p. 454) into separate test-tubes. Cover one with 3 c.c. and the other with 6 c.c. of saliva. The saliva must all be taken from the same stock, and must not be collected separately. Put in a bath at $38^{\circ} \mathrm{C}$., and when a fair amount of digestion has taken place in each, measure the length of the column digested, and determine the relation between the amount digested in the two tubes (p. 342).

(h) Dilute 2 c.c. of saliva with distilled water up to 20 c.c., and filter. Take six test-tubes of the same width, and label them A, B, C, etc. Measure into A 3 c.c. of the diluted saliva, into B 2 c.c., into C I.3 c.c., into $\mathrm{D} \mathrm{o.9}$ c.c., in to $\mathrm{E}^{\circ} 6 \mathrm{c} . \mathrm{c}$., and into $\mathrm{Fo}_{4}$ c.c. Thus a series is obtained in which each tube contains (approximately) two-thirds as much ferment as the one it follows. Add distilled water to tubes B to F, sufficient to make up the volume in each to 3 c.c. Place the tubes in a beaker of iced water; add to each Io c.c. of a I per cent. solution of boiled starch previously cooled in iced water, and sliake so as to mix the contents. Each tube now contains starch in uniform concentration, and ferment in varying concentration. The low temperature prevents digestion till all the tubes are ready. Now put the tubes simultaneously in to a waterbath at $40^{\circ} \mathrm{C}$. for half an hour, and then back again into iced water to prevent furiner digestion. Move them about in the iced water to cool rapidly. Fill up the tubes with distilled water nearly to the top. add a drop or two of iodine solution to each, and mix uniformly. The tubes to which the smallest amounts of saliva were added will probably

* As it filters slowly, unniltered saliva may be used for Experiments (e), $(f)$. and (i). 
still show a distinct blue colour, while those at the other end of the scries will be brown or yellow, and the intermediate tubes bluish-violet. Suppose D is the last tube still slowing a bluish tint, then in the next higher tube, C, all the starch has been hydrolysed at least to dextrinthat is, I.3 C.C. of the ten-times diluted saliva, or 0.13 of the original saliva, has been sufficient to change all the starch in ro c.c. of the I per cent. solution. With another specimen of saliva the same result might be reached in tube $E$, containing an amount of ferment equal to that in 0.06 c.c. of the original saliva. We could then conclude that the diastatic power of the second saliva was about twice as great as that of the first. A closer approximation can now be made by setting up two fresh tubes ( $\mathrm{C}$ and E respectively for the two salivas) and determining the time required for the blue reaction with iodine to disappear, taking out a drop from time to time and testing on a porcelain slab.

(i) Put a little distilled water into a porcelain capsule, and bring the water to the boil. Now put into the mouth some boiled starch paste, and move it about as in mastication. After half a minute spit the starch out into the boiling water. Divide the water into two portions. Test one for sugar, and the other for starch. Repeat the experiment, but keep the starch two minutes in the mouth. Report the result.

(j) Starch solution to which saliva has been added is placed in a dialyser tube of parchment-paper for twenty-four hours. At the end of that time the dialysate (the surrounding water) should be tested for sugar and for starch. Sugar will probably be found, but no starch. If no reaction for sugar is obtained, the dialysate should be concentrated on the water-bath, and again tested.

6. Stimulation of the Chorda Tympani.-(I) Having previously studied the anatomy of the mouth and submaxillary region in the dog by dissecting a dead animal (Fig. I74), put a good-sized dog under morphine. Set up all induction-machine for a tetanizing current (p. 200), and connect it with fine electrodes. Fasten the dog on the holder, give ether if necessary, and insert a cannula in the trachea (p. 202). Then make an incision 3 or 4 inches long through shin and platysma muscle, along the inner border of the lower jaw, beginning about the angle of the mouth, and continuing backwards towards the angle of the jaw. Such branches of the jugular vein as come in the way may be generally pushed aside, but if necessary they may be doubly ligated and divided. Feel for the facial artery, so as to be able to avoid it. Divide the digastric muscle about its anterior third, and clear it carcfully from its attachments; or, without dividing it, pull it outwards with a hook. The broad, thin mylo-hyoid muscle will now be seen with its motor nerve lying on it. Divide the muscle about its middle at right angles to its fibres, and raise it carefully. The lingual nerve will be seen emerging from under the ramus of the jaw. It runs transversely towards the middle line, and then, bending on itself, passes forwards parallel to the larger hypoglossal nerve. In its transverse course the lingual will be seen to cross the ducts of the submaxillary and sublingual glands. These structures having been identified, the lingual nerve is to be ligatured before it enters the tongue and cut peripherally to the ligature. Then a glass cannula of suitable size is to be inserted into the submaxillary duct (the larger of the two), just as if it were a bloodvessel (p. 63). A short piece of narrow rubber tubing is carefully slipped on the end of the cannula. The lingual is now to be lifted by means of the ligature, and traced back towards the jaw till its chorda tympani branch is seen coming off and running backwards along the duct. The chordo-lingual nerve (Fig. I6o, p. 392) is then to be cat centrally to the origin of the chorda tympani, which can now be 
easily lair on electrodes by means of the ligature on the lingual. On stimulating the chorda. the flow of saliva through the cannula will be increased. The current need not be very strong. If the flow stops after a short time, it can be again caused by renewed stimulation after a brief rest. A quantity of saliva may thus be collected, and the experiments already made with human saliva repeated.

(2) Expose the rago-sympathetic nerv in the neck on the same side; ligature it; clivide below the ligature; and note the effect produced by stimulation of the upper end on the flow of saliva.

(3) Set up another induction-machine, and connect it with electrodes. Stimulate the chorda, and note the rate of fow of the saliva. Then, whilc the chorda is still bcing excited, stimul te $_{\text {te }}$ the vago-sympathetic, and observe the effect. If the experiment is successful, finish by stimulating the chorda for a long time. Then harden both sub-

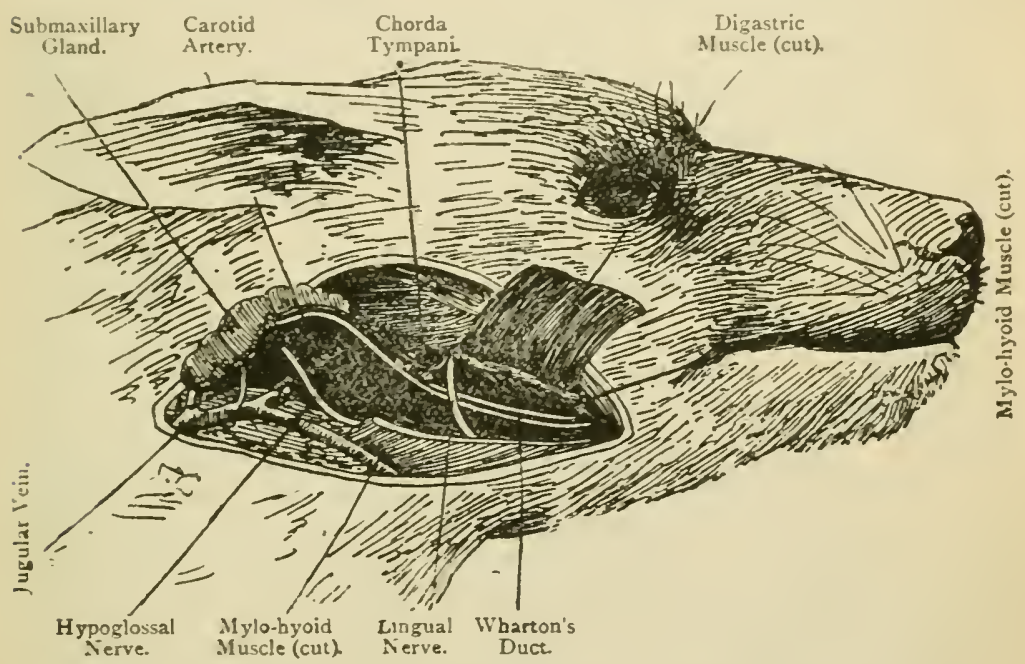

Fig. 174.-Dissection for Stimulation of Chorda Tyinpani (after Bernard).

maxillary glands in absolute alcohol, make sections, stain with carmine, and compare them.

7. Effect of Drugs on the Secretion of Saliva.-(I) Proceed as in 6 (I), but, in addition, insert a cannula into the femoral vein (p. 2 Is). On the cannula put a short piece of rubber tubing, filled with 0.9 per cent. salt solution and closed by a small clamp. or a small piece of glass rod, or a pair of bulldog forceps. While the chorda is being stimulated inject into the vein ro to 15 milligrammes of sulphate of atropine by pushing the needle of a hypodermic syringe through the rubber tube. This will stop the flow of saliva, and abolish the effect of stimulation of the clorda. Sec whether the sympathetic is also inactive, and report the result.

(2) Now empty the cannula in the submaxillary duct by means of a feather, and fill it with a 2 per cent. solution of pilocarpine nitrate by means of a fine pipette. Fill also the short rubber tube attached to the cannula, and close it again. Compress the tube, and so force into 
the duct a small quantity of the solution. Open the tube. Secretion of saliva will again begin, and stimulation of the chordit will again cause an increase in the flow. But after a few minutes the action of the atropinc will reassert itself, and the flow will stop. Renewed secretion may be caused by a fresh injection of pilocarpine.

8. Gastric Juice-(a) Preparation of Artificial Gastric Juice.-Takc a portion of the pig's stomach provided, strip off the mucous membrane (except that of the pyloric end, which is relatively poor in pepsin), cut it into small pieces with scissors, and put it in a bottle with roo times its weight of 0.4 per cent. hydrocliloric acid. Label and put in a bath at $40^{\circ} \mathrm{C}$. for three hours, and then in the cold for twelve hours. Then filter.

(b) Take another portion of the mucous membrane, cut it into pieces, and rub up with clean sand in a mortar. Then put it in a small bottle, cover it with glycerin, label, and set aside for two or three days. The glycerin extracts the pepsin.

(c) Take five test-tubes, A, B, C, D, E, and in eacli put a little washed and boiled fibrin or a small cubc of coagulated egg-white. To A add a faw drops of glycerin extract of pig's stomacll, and fill up the test-tube with 0.4 per cent. hydrochloric acid. To B add glycerin extract and distilled water; to $C$ glycerin extract and $\mathrm{I}$ per cent. sodium carbonate; to $\mathrm{D} 0.4$ per cent. hydrochloric acid alone; to E glycerin extract which has been boiled, and 0.4 per cent. hydrochloric acid.

Put up another set of five test-tubes in the same way, except that a few drops of a watery solution of a commercial pepsin are substituted for the gly'cerin extract. Label the test-tubes $A^{\prime}, B^{\prime}, C^{\prime}, D^{\prime}, E^{\prime}$.

Into another test-tube put a little fibrin (or an cgg-whits cubc), and fill up with the filtered acicl extract from $(a)$. Label it F. Place all the test-tubes in a tumbler, and set them in a water-bath at $40^{\circ} \mathrm{C}$. Put a piece of a Mett's tube (p. 343) into each of two test-tubes, and add I 5 c.c. of 0.4 per cent. hydrochloric acid. To one tube add 5 drops and to the other ro drops of the same filtered glycerin extract of gastric mucous membrane. Put the tubes in the bath, and when digestion is distinct at the ends of both tubes measure the length of the column digested in each. What is the relation between the two (p. 342)? The experiment can be repeated with the hydrochloric acid extract of the mucous membrane.

After a time the fibrin (or egg-white) will have almost completely disappeared in $\mathrm{A}, \mathrm{A}^{\prime}$, and $\mathrm{F}$, but not in the other test-tubes. Filter the contents of $\mathrm{A}, \mathrm{A}^{\prime}$, and $\mathrm{F}$ into one dish.

(d) Test the filtiate for the products of gastric digestion:

(a) Neutralize a portion carefully with dilute sodium hydroxide. A precipitate of acid-albumin may be thrown down. Filter.

$(\beta)$ To a portion of the filtrate from $(a)$ add excess of sodium liydroxide and a drop or two of very dilute copper sulphate. A rose colour indicates the presence of proteoses or peptones. The cupric sulphate must be very cautiously added, because an excess gives a violet colour, and thus obscures the rose reaction. If still mere cupric sulplate be added, blue cupric liylroxide is thrown down, and nothing can be inferred as to the presence or the nature of proteins in the liquid.

$(\gamma)$ Heat another portion of the filtrate from $(a)$ to $30^{\circ} \mathrm{C}$, and add crystals of ammonium sulphate to saturation. A precipitate of proteoses (albumoses) may be obtained. Filter off. 
(8) Adel to the filtrate from $(\gamma)$ a trace of cupric sulphate and excess of soclinm hydroxide. A rose colour indicates that peptones are present. More sodium liyclroxide must be added than is sufficicnt to break up all the ammonium sulphate, for the biuret reaction requires the presence of free fixed alkali. A strong solution of the sodium hydroxide slould therefore be used, or the stick canstic soda. The addition of ammonium sulphate will cause the red colour to disappear; so will the addition of an acid. Sodium hydroxide will bring it back. Ammonia does not affect the colour.

(e) To some milk in a test-tube add a drop or two of rennet cxtract. and place in a bath at $40^{\circ} \mathrm{C}$. In a short time the milk is curdled by the rennin. (Sec p. 353)

9. (1) To obtain Normal Chyme.-Inject subcutancously into a dog: one and a lialf lours after a meal of minced meat and water, $2 \mathrm{mg}$. of apomorphinc. Half of one of the ordinary tabloids is enough. Collect the vomit.

(2) To obtain Pure Gastric Juice.-If the laboratory possesses a dog with Pawlow's double osopliagcal and gastric fistula, the juicc may be obtained in large amount by sham feeding with meat (p. 402). If not, proceed as follows: Put a fasting dog under ether, and fasten on the holder. Clip the hair and shave the skin in the middle line below the sternum. Nake a longitudinal incision through the skin and subcutaneous tissue from the xiphoid cartilage downwards for 3 or 4 inclies. The linea alba will now be secn as a whitc mesial streak. Open the abdomen by an incision through it. Pull over the stomach towards the right, and stitch it to the abdominal wall, open it, and insert a stomach cannula (Fig. I75). Make an incision through the serosa and muscularis. Doubly ligate and divide any vessels exposed in the

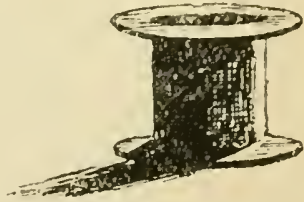

Fig. 175.-Stomach Cannula. submucosa. Then make an opening in the nucosa of sufficient sizc to just admit the gastric cannula. This will go into a smaller opening if it is provided with a nick in the flange which enters the stomach. Be careful to prevent blood from getting into the stomacli. Immediately stitch the wound in the stomach over the flange of the cannula, but do not pass the stitches through to the internal surface of the mucosa. Suture the muscles and skin separately. Then stitch up the wound in the abdomen. Wash out any stomach contents with warm water. Put a cork in the cannula, and cover the animal with a cloth. The following experiments may now be performed: Expose both vagi in the neck. Connect two pairs of electrodes with the secondary coil of an inductorium arranged for single shocks. By means of a key in the primary stimulate the nerves with slow rhythmical induction shocks at the rate of about one a second. Continue the stimulation for fifteen minutes, collect any juice that may have been secreted, and apply the tests in (3). If secretion is slow, a little distilled water may be put into the stomach, and the vagus stimulation repeated. Mechanical stimulation of the mucous membrane with a feather causes no secretion of acid gastric juice, but may cause a secretion of alkaline mucus.

(3) (a) Test the reaction to litmus of the chyme obtained in (I), and of the pure juice obtained in (2).

(b) Test their proteolytic powers by putting in a bath at $40^{\circ} \mathrm{C}$. for two hours two test-tubes containing respectively filtered chyme and 
fibrin, ancl gastric juice and fibrin. The fibrin will be digested in both. Estimate the proteolytic power quantitatively by Mett's tubes (p. 454 ).

(c) Add a few drops of the cliyme and gastric juice to milk in two test-tubes, and place them in a bath at $40^{\circ} \mathrm{C}$. Repeat $(c)$ after neutralizing the liquids.

(d) Examine a drop of the unfiltered cliyine under the microscope. Partially digested fragments of the food will be seen-muscular fibres or fat cells. Filter, and proceed as in $8(d)(p .458)$.

(4) Test the filtrate from the cliyme and the gastric juice for lactic acid by Uffelmann's test or Hopkins's test (p. $82 \mathrm{I}$ ), and for hydrochloric acid by Günzburg's reagent.

Uffelmann's Test for Lactic Acid.-The reagent is a dilute solution of carbolic acid to which dilute ferric chloride has been added till the colour is bluish (say a drop of a r per cent. ferric chloride solution to 5 c.c. of a I per cent. carbolic acid solution). The blue colour of the inixture is turned ycllow by lactic acid, but not by dilute hydrochloric acid. Since Uffelmann's test is given also by phosphates, alcohol, and sugar, which may sometimes be present in the stomach contents, it is best to shake the gastric contents with etlier, dissolve the ethereal cxtract in water, and then make the test on the watery solution.

Gunzburg's Reagent for Free Hydrochloric Acid in Gastric Juice is made by dissolving 2 parts of phloroglucinol and I part of vanillin in 30 parts by weight of absolute alcoliol. A few drops of the reagent are added to a few drops of the filtered gastric juice in a small porcclain capsule, and the whole evaporated to dryness over a small bunsen flame. If free hydrochloric acid is present, a carmine-red residue is left. If all the hydrochloric acid is united to proteins in the stomacl contents, the reaction does not succeed. It is also hindered by the presence of leucin.

Io. Pancreatic Juice.-(a) Take a piece of the pancreas of an ox or dog which has been kept twenty-four hours at the temperature of the laboratory, and make a glycerin extract in the same way as in the case of the pig's stomach in $8(b)$. Put in a small bottle, and set aside for a day or two.

(b) Put a little boiled fibrin into each of six test-tubes, A, B, C, D, E $F$. To $A$ add a few drops of glycerin extract of pancreas, and fill up with a $\mathrm{I}$ per cent. sodium carbonate solution; to $\mathrm{B}$ add glycerin cxtract and distilled water; to $\mathrm{C}$ glycerin extract and excess of $0^{\circ} 05$ per cent. hydrochloric acid; to $D$ I per cent. sodium carbonate alone; to $E$ I per cent. sodium carbonate in which a few drops of glycerin extract of pancreas have becn previously boiled; to $F$ glycerin extract and excess of 0.2 per cent. hydrochloric acid.*

Set up six test-tubes, $A^{\prime}, B^{\prime}, C^{\prime}, D^{\prime}, E^{\prime}, F^{\prime}$, in the same way, but substitute a few drops of a solution of commercial pancreatin for the glycerin extract. Set up two test-tubas as in exp?riment 8 (p. 458) with Mett's tubes. Put all the test-tubes in a tumbler, and place in a bath at $40^{\circ} \mathrm{C}$. The fibrin will be gradually eaten away in $\mathrm{A}$ and $\mathrm{A}^{2}$. by the action of the trypsin, but will not swell up or become clear before disappearing, as it does in dilute hydrochloric acid with glycerin

* With hydrochloric acid of different strengths the rapidity of digestion of boiled fibrin by glycerin extract of log's pancreas (1 volume of extract to 25 of acid) was found about the same for $0^{\circ} 3$ and $0^{*} 17$ per cent. acid; mucli less for $0^{\circ} 08$ per cent., while in $0^{\circ} 04$ per cent. acid there was practically no digestion at all. In $0_{4} 4$ per cent. acid digestion took place more rapidly than in $0^{\circ} 08$ per cent., but much less rapidly than in $0^{\circ} 17$ per cent. In acid of all strengths digestion was markedly slower than in I per cent. soclium carbonate. 
extract of stomach. Filter the contents of these test-tubes. Neutralize the filtrate witl dilute acid; a precipitate will consist of alkali-albuniu. If sucl a precipitate is olotained, filter it off and test the filtrate for proteoses and peptones as in $8(d)(p \cdot 458)$. Some digestion, and perloaps a considerable amount, may also have taken place in $F$ and $F^{\prime}$; less or none at all in Cand $C^{\prime}$; and none in the other iest-tubes (pp. 359, 420).

(c) Add a few drops of the glycerin extract to a test-tube containing starch mucilage, which has been previously found free from reducing sugar. Put in a bath at $40^{\circ} \mathrm{C}$. After a short time abundance of reducing sugar will be found, owing to the action of the ferment, amylopsin, or pancreatic amylase.

(d) Mince thoroughly a good-sized piece of fresh pancreas, and shake 11p well with three or four times its bulk of water. Put 5 c.c. of fresh cream into a test-tube, then ro c.c. of the extract, a few drops of chloroform to prevent the growth of bacteria, a few drops of litmus solution, and if necessary enough of very dilute sodium hydroxide to just render the colour distinctly blue. Shake up, and divide the mixture into two portions, A and B. Boil one portion (B), and place the test-tubes at $\mathrm{fc}^{\circ} \mathrm{C}$. Examine from time to time. The blue colour will disappear in $A$, owing to the formation of fatty acids from the neutral fats, and sodium hydroxide must be added to it to restore the colour. In B the tat-splitting ferment has been destroyed by boiling, and fat-splitting will not occur. Probably a distinct result will not be obtained for several hours, and it will be best to leave the tubes in the incubator overnight.

(e) If the laboratory possesses an animal with a pancreatic fistula, the following experiment may be done by a limited number of students with fresh pancreatic juice* collected from the fistula. Take five testtubes, A, B, C, D, E. Add 5 c.c. of pancreatic juice to each tube. Boil $\mathrm{E}$, and then cool it. Put into $\mathrm{A}$ and $\mathrm{B}$ small pieces of heat-coagulated egg-white, into $\mathrm{C}$ a little starch mucilage, and into $\mathrm{D}$ and $\mathrm{E} 5$ c.c. of fresh cream. Add further to B a scraping of the mucous membrane of the upper part of the small intestine which has first been washed free of contents. To $\mathrm{D}$ and $\mathrm{E}$ add a drop or two of litmus solution, and, if necessary, enough of dilute sodium hydroxide to just establish a blue colour. Then put the test-tubes at $40^{\circ} \mathrm{C}$., and examine after a time. No digestion will have taken place in $\mathrm{A}$, because the pancreatic juice, as secreted, does not contain active trypsin. In B digestion may take place, because the enterokinase in the intestinal mucous membrane will activate the trypsinogen to trypsin. In $C$ and $D$ there will be evidence of the production of reducing sugar and fatty acids respectively, since the pancreatic juicc, as secreted, contains active amylase and steapsin. E will be unchanged unless by bacterial action.

(f) Leucin and 7yrosin.-As examples of amino-acids formed when pancreatic digestion of proteins (fibrin or casein, e.g.) is allowed to go on for some days, $\uparrow$ leucin and tryosin may be isolated. Add brominewater by drops to 5 c.c. of the digest; a pink colour indicates tryptophane. If the 'digest' be neutralized, then filtered, and the filtrate concentrated and allowed to stand, a crop of tyrosin crystals will separate out, since tyrosin is only slightly soluble in watery solutions of neutral salts. These crystals having been filtered off, the proteoses (albumoses) and peptones can be precipitated together by alcohol, and

* A considerable flow of pancreatic juice can be obtained from a dog with a pancreatic fistula by injecting intravenously an extract of intestinal mucous membrane containing secretin (p. 407).

$\dagger$ A little chloroform is added to prevent bacterial growth. 
afterwards separated, if that is desired, by redissolving the precipitate in water and throwing down the proteoses by saturation with ammonium sulplate. The alcoholic filtrate will contain any leucin that may be present, since that body is moderately soluble in alcohol, as well as traces of tyrosin, which, however, is much less soluble in this medium. On concentration, crystals of both substances will be obtained. Tyrosin crystallizes characteristically from animal liquids in beautiful silky needles united into sheaves, leucin in the form of indistinct fatty-looking balls, often marked with radial strix and coloured with pigment (Figs. I 86 and I 87, p. 488).

Tests for Tyrosin by Mörner's Test.-Put a small quantity of tyrosin into a test-tube. Add about 3 c.c. of the reagent, * and heat gradually and gently to the boiling-point. A green colour is obtaincd.

II. Bile.-(a) Test the reaction of ox bile. It is alkaline to litmus.

(b) Add dilute acetic acid. A precipitate of bile-mucin (really nucleo-albumin) falls down. Some of the bile-pigment is also precipitated. Filter. (Pig's bile contains more of the mucin-like substance than ox bile.)

(c) Put a little of the filtrate from (b) or of the original bile into a porcclain capsule, add a drop or two of a dilute solution of cane-sugar, and mix with the bile. Then add a few drops of strong sulphuric acid, and stir; then a few drops more of the sulphuric acid, stirring all the time. A purple colour appearing at once, or after gentle heating. shows the presence of bile-acids (Pettenkofer's reaction). The bile may be diluted before the addition of the sulphuric acid. In this case a greater amount of the acid must be added. Examine the purple liquid in a test-tube with a spectroscope (p. 74). Dilute the liquid with water, adding some sulphuric acid to partially clear up the precipitate caused by the water. Two absorption bands are seen, one to the red side of $\mathrm{D}$, and the other, a stronger and broader band, over and to the right of $\mathrm{E}$. When only a very small amount of bile-salts is present, the reaction is made more sensitive if a solution of furfuraldehyde ( $\mathbf{r}$ to 1,000 ) is used instead of cane-sugar.

(d) Hay's Sulphur Test.-Sprinkle a little sulphur (in the form of the fine powder known as flowcrs of sulphur) on the surface of some bile in a small beaker or deep watch-glass. The sulphur will soon sink to the bottom. Repeat with water; the sulphur will float. The reaction is due to the diminution of the surface tension produced by the bile-acids, and succeeds also in a solution of bile-salts. The test is very sensitive. But in stomach contents, vomit, or stools, it rarely gives good results, since alcohol or acetic acid is often present in the gastric liquid, and phenol and its derivatives in intestinal contents, and all of these cause such an alteration in the surface tension that the sulphur sinks. Ether, chloroform, turpentine, benzine and its derivatives, anilin and soaps, also vitiate the test in the same way.

(e) Add ycllow nitric acid (containing nitrous acid) to a little bile on a white porcelain slab. A play of colours, beginning with green and running through blue to yellow and ycllowish-brown, indicates the presence of bile-pigment (Gmelin's reaction). The reaction may also be obtained by putting some yellow nitric acid into a test-tube, and then running a little bile from a pipette on to the surface of the acid. The play of colours is seen at the surface of contact. Where the bilepigment is present only in traces, some of the liquid may be filtered

* The reagent for this test is prepared by mixing thoroughly 1 volume of formalin, 45 volumes of distilled water, and 55 volumes of concentrated sulphuric acid. 
through white filter-paper, and the test applied by putting a drop of the nitric acid on the palper.

(f) Cholesterin or Cholesterol (Fig. I76)-Preparation.-Extract a powdered gall-stone (preferably a white one) with hot alcoliol and ether in a test-tube. Heat the test-tube in warm water, not in the frec flame. Put a drop of the extract on a slide. Flat crystals of cholesterin, often clipped at the corners, separate ont. (a) Carcfully allow a drop of strong sulphuric acid and a clrop of dilute iodine to run under the covergliss. A play of colours-violet, blue, green, red-is seen.

$(\beta)$ Evaporate a drop of the solution of cholesterin in a small porcelain capsule, add a drop of strong nitric acid, and heat gently over a flame. A yellow stain is left, which becomes red when a drop of ammonia is poured on it while it is still warm.

(y) Dissolve a little cholesterin in chloroform. Add an equal bulk of strong sulphuric acid, and shake gently. The solution turns red and the subjacent acid shows a green fluorescence.

( $)$ Put a drop or two of water in a watch-glass, and add a drop of an cthereal solution of cholesterin. The cholesterin is precipitated, and will not dissolve in the water even on heating. Repeat the observation with bile instead of water. The cholesterin dissolves in the bile.

$(\mathrm{g})$ To a little of the filtrate from a peptic digest (e.g., Abrin which has been digested for twenty-four hours with pepsin and hydrochloric acid) add some bile. A precipitate is thrown down, which is redissolved in excess of the bile (p. 370).

(h) Shake up a little bile with some rancid olive-oil in a test-tube. An emulsion is formed. Repeat the experiment with the same quantities of bile and oil, but use perfectly fresl oil. Compare the stability of the two emulsions, allowing the tubes to stand together for a while.

(i) To some starch mucilage, shown to be free from sugar, add a little bile,

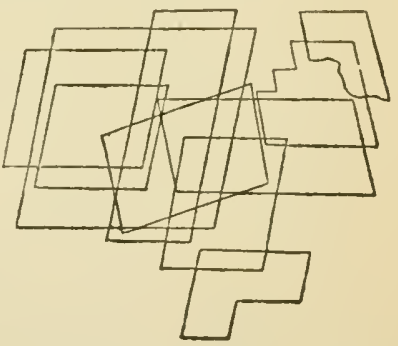

Fig. I76.-Crystals of Cholesterin (Frey).

and place in a batl at $40^{\circ} \mathrm{C}$. After a time test for reducing sugar. Report the result. Bile often has a slight diastatic power.

(j) To demonstrate the Presence of Iron in the Liver Cells.--Steep sections of liver in a solution of potassium ferrocyanide, and then in dilute hydrochloric acid. Or a $1 \cdot 5$ per cent. solution of potassium ferrocyanide in 0.5 per cent. hydrochloric acid may be used. (The iron may previously be fixed in the tissue by hardening it in a mixture of alcolool and ammonium sulphide.) The sections become bluish from the formation of Prussian blue. A fine-pointed glass rod or a platinum lifter should be used in manipulating them. A steel needle cannot be employed. Mount in glycerin or Farrant's solution, or (after dehydrating with alcohol and clearing in xylol) in xylol-balsam. Blue granules may be seen under the microscope in some of the hepatic cells. Sections of spleen may also be examined for this reaction.

I2. Microscopical Examination of Fæces.-Examine under the microscope the slides provided. Draw, and as far as possible determine the nature of, the objects seen $($ p. +24$)$.

I3. Absorption of Fat.-(a) Feed a rat or frog with fatty food; kill the rat in three or four hours, the frog in two or three days. Immediately after killing the rat open the abdomen, carefully draw out a loop of intestine, and look through the thin mesentery. The white 
lacteals will probably be scen ramifying in the mesentery. They appear white on account of the presence of globule's of fat in the chyle with which they are filled. Strip off tiny pices of the mucous membrine of the small intestine, and steep them in $\frac{1}{2}$ per cent. solution of osmic acid for forty-eight hours. 'Then tease fragments of the mucous membrane in glycerin and examine under the microscope. To preserve the specimens take off the glyeerin with blotting-paper and mount in Farrant's medium, which is a preservative glycerin mixture. Other portions of the mucous membrane may be liardened for a fortnight in a mixture of 2 parts of Müller's fluid and I part of a I per cent. solution of osmic acid. Sections are then made with a freezing nicrotome after embedding in gum. No process must be used by which the fat would be dissolved out (Schäfer). (See Fig. I72, p. 441.)

(b) Feed a cat with 30 grammes of butter stained a deep red witl the dye Sudan III. After five hours anæsthetize the animal with ether, insert a cannula in the caroticl artery, and obtain a sample of blood. Defibrinate the blood, and separate the scrum by the centrifuge. If digestion and absorption of the fat have procecded normally, the serum will contain numerous fat droplets, and will be tinged pink by the dye, which can be dissolved out of it by shaking up with ether. On opening the abdomen it will be seen that the mucous membrane of the small intestine, as far down as the fat lias reached, is stained pink, and that the lacteals in the mesentery are also pink. Observe whether any. of the pigment has passed into the urine

14 . $^{*}$ Time required for Digestion and Absorption of Various Food Substances.-Feed three dogs, A, B, and C, which have previously fasted for twenty-four hours, with a meal containing starch (proved to be free from sugar), lard, and meat.

(I) After fifteen minutes inject subcutaneously into A 2 c.c. of a o. I per cent. solution of apomorphine. Note the time which elapses before the animal vomits. Collect the vomit.

(a) Examine a little of it under the microscope, and make out fat globules, muscular fibres and starch granules. The latter can be recognized by their being coloured blue by a drop or two of iodine solution.

(b) Filter the chyme, mixing it, if necessary, witl a little water, and test it as in $8(d)\left(p .45^{8}\right)$ for the products of digestion of proteins. In addition, test for starch, dextrin, and reducing sugar.

(2) One and a quarter hours after the meal inject apomorphine into $\operatorname{dog} B$, and proceed as in (I).

(3) Two and a half hours after the meal inject apomorphine into $\operatorname{dog} C$, and proceed as in (I). Compare the results from the three specimens of cliyme.

I 5. ${ }^{*}$ Quantity of Cane-Sugar inverted and absorbed in a Given Time.Take three dogs, A, B and C, which have fasted for twenty-four hours. The animals should be about the same size. Feed $\mathbf{A}$ and $B$ with roo c.c. of a standard solution of cane-sugar (about a 20 per cent. solution), or as much more as they will take. If the dogs have been kept without water for a day they will more readily take the sugar solution. Or it may be given through a tube passed into the stomach, and in this case a larger quantity of sugar can be given. A gag consisting of a piece of wood with a hole in the middle of it, through which the tube is passed, must first be secured in the dog's moutl. Feed C with

- Experiments 14 and 15 are conveniently done in a class by assigning each of the three animals to a separate set of students. The contents of the stomach and intestine are divided into three portions, so that each set has a sample from each dog. 
jo grammes of powelered cane-sugar mixed with larl, the muxture being rolled into little balls.

(I) After it quarter of an lour put A tunder chloroform or the A.C.E. mixture, and fasten it on a holder. Kill tle animal with chloroform, open the abdomen, tic the cesophagus, place double ligatures on the pyloric end of the stomach and the lower end of the small intestine, and divide between tliem. Cut out the stomach and intestine; wasli their contents into separate vessels, and test tli. riaction with litmus paper. Add water and rub up thoroughly. Filter. Wash the residue repeatedly with small quantities of water, and pass all tlie washings througl the filter. Milke up each of the two filtrates to 200 c.c.

(a) Test the filtrales from the contents of the stornach and intestines qualitatively fol dextrose by 'Trommer's (p. 10) or Fehling's (p. 526) and the plicnyl-hydrazine test (p. 525).

(b) If no reducing sugar is present, add to 20 c.c. of cach filtrate 1 c.c. of hyclrochloric acid, boil for half an hour, and again test for reducing sugar. If it is now found, some cane-sugar is present.

(c) If reducing sugar is found, estimate its annount as dextrose by Fehling's solution (p. 527) in a measured quantity of the original filtrate of the gastric or intestinal contents before and after boiling with one-twentietl of its volume of hydrochloric acid.

(d) Estimate in the same way the amount (as dextrose) of the invert sugar in the standard solution of cane-sugar after inversion, and before inversion if it gives the qualitative test for reducing sugar before it has been boiled with acid.

From the data obtained (and taking 95 parts of cane-sugar as equal to roo parts of (lextrose) calculate the amount of cane-sugar absorbed, left unchanged, and inverted, though not absorbed.

(2) One and a half hours after the meal anzesthetize B, and proceed as in (I).

(3) Two hours after the meal proceed in the same way with C. But in addition observe the lacteals in the mesentery, by gently lifting up a loop of intestine immediately after opening the abdomen. If the absorption of the fat has begun, they will be easily visible, as a network of fine milk-white vessels. Also examine the gastric and intestinal contents with the microscope for fat globules. Compare your results on the amount of sugar obtained from the three animals. Probably much more unabsorbed sugar will be found in $\mathrm{C}$ than in $\mathrm{B}$, as the lard hinders it from being dissolved.

I6. Auto-Digestion of the Stomach.-In some of the previous experiments the stomach of an animal lilled during digestion should be removed from the bocky after double ligation of œsophagus and duodenum, and placed in a water-batl at $40^{\circ} \mathrm{C}$. After several hours the contents should be washed out and the mucous membrane examined. It may be entirely eaten away in paits. 


\section{CHAPTER VIII}

\section{FORMATION OF LYMPH}

Different Kinds of Lymph. - We ought to distinguish the lymph as we collect it from the great lymplatic trunks, not only from the liquids of the serous cavities, but still more sharply from the liquid which fills the multitudinous clefts and spaces of the tissues. It is now pretty definitely established that the tissue spaces do not conmunicate by actual passages with the lymphatic vessels, but that the latter form everywhere a closed system like the blood-vascular system, the lymph capillaries merely lying in the tissue spaces (Sabin, etc.). This conception entails a radical change in the current views of lymph production. If the lymphatics form a closed system, the lymph cannot be actual tissue fluid, but only tissue fluid modified by its passage through the walls of the lymph capillaries, just as tissue fluid is not actual blood-plasma, but plasma modified by its passage throggh the walls of the blood capillaries as well as by exchange with the tissue elements.

Although it is customary to speak of the lymph obtained from the lymphatic vessels as if it were perfectly homogeneous, there is no experimental ground for supposing that the lymph from different tracts, or the tissue liquid in contact with the cclls of different organs, or even the tissue liquid in contact with one and the same cell at different parts of its periphery, has a uniform composition, or even a uniform molecular concentration. There are, indeed, certain general considerations which show that this cannot be so. Still less can it be assumed that the serous cavities, although they come into relation with lymphatics and bloodvessels in their walls, are analogous to colossal tissue spaces or even to expansions of the closed lymphatic system, or that the liquids contained in them, normally in scant amount, are simply tissue, or if not tissue, then simply lymphatic lymph. The cerebrospinal fluid, which bathes the external surface of the central nervous system and fills its cavities, and the special liquids of the eyeball - the aqueous humour and the liquid of the vitreous liumour-although no doubt, in addition to their other functions, they may in some degree minister to the nutrition of the tissues with which they are in contact, arc 
as regards their composition and mode of formation scarcely more closely allied to lymph than sweat is. They are almost free from protein, and are secieted by special structures - the choroid plexus and the uveal epithelium-quite different from any that can be concerned in the formation of ordinary lymph.

It is very true these liquids are not blood, but tliat is scarcely a sufficient reason for calling them lymph, else we might classify sweat or even milk as lymplı also. If a term is desirable to indicate that they have certain relations with lymph, they might perhaps be spoken of as lymplioid secretions. It may be that the essential difference in the chemical composition of these lymphoid secretions and lymph-the practical absence of protein-is related to the difference in the manner of their formation. The uveal and choroidal epithelial cells, interposing the depth of their columns or cubes between the blood and the free surface at which the liquid escapes, may well be suited to hinder the passage of the protein molecules which find their way with greater ease through the thin endothelium of the capillary wall into the tissue spaces, and from these into the lumen of the closed lymphatics (see p. 439). Nevertheless, we shall recognize later on in the slomeruli of the kidney an instance of blood capillaries but little pervious to proteins, and there are several other facts which show that the capillaries may differ considerably in different organs in the readiness with which they permit the various constituents of the plasma to pass through their walls. Further, in discussing the mechanism by which lymph is formed, we shall see reason to doubt whether mechanical filtration, due to differences of hydrostatic pressure on the two surfaces of the capillary endothelium, has much, if anything, to do with the passage either of protein or of the other constituents of the lymph from the lumen of the capillaries into the tissue spaces. At first glance, indeed, such a process would seem to be admirably fitted to explain the fact that, while lymph differs but little from bloodplasma in the proportions of its other constituents, it is at most no more than half as rich in protein. For there are many filters which allow substances in ordinary solution and their solvent to pass through without alteration in their relative proportions, while substances like proteins in colloid solution are kept back to a greater or less extent.

Factors concerned in Lymph Formation.-The teaching of Ludwig, that lymph is formed by the filtration, and in a minor degree by the diffusion, of the constituents of blood-plasma through the walls of the capillaries into the tissue spaces, was based on such facts as the increase in the tissue liquid of a limb or organ which occurs when the exit of blood from it by the veins is hindered, or when the quantity of the circulating liquid is increased by the injection of blood or salt solution. It was first seriously called in question by 
Heidenhain, who advanced the theory that lymph is secreted by the endothelium of the blood capillaries. One of Heidenhain's strongest arguments in favour of his secretion theory was the existence of substances which, when injected into the blood, increased the flow of lymph from the thoracic duct of the dog without affecting appreciably the arterial pressure. He divided these socalled lymphagogues into two classes: (I) Substances like peptone, extracts of the head and liver of the leech, extract of crayfish muscle, egg-albumin, etc., which cause not only an increase in the rate of flow, but an increase in the specific gravity and total solids of the lymph; (2) crystalloid substances, like sugar, salt, etc., which cause an increased flow of lymph more watery than normal.

Starling has showin that, although the lymphagogues of the second class do not raise the arterial pressure, they do, by attracting water from the tissues and thus causing hydremic plethora (an excess of blood of low specific gravity), bring about a marked rise of venous, and therefore, what is the important thing for lymph filtration, of capillary pressure. But it can be demonstrated that vaso-dilatation with increase of capillary pressure is not in itself sufficient to increase the formation of lymph. We have seen, e.g. (p. I79), that when the chorda tympani nerve is stimulated in the dog the arterioles of the submaxillary gland are dilated, and no doubt the pressure in the capillaries is increased. No increased flow of lymph, however, takes place from the submaxillary lymphatics during even prolonged excitation of the chorda, nor do the lymph spaces of the gland become distended (Heidenhain). In the horse also the spontaneous flow of lymph from the quiescent parotid is not appreciably altered by excitation of the secretory nerves of the gland or by pilocarpine (Carlson). There is cvery reason to believe that during active secretion of saliva tissue liquid is really formed from the blood in increased amount, and that it is from the tissue spaces that the gland-cells directly obtain the increased supply of water and other substances necessary to sustain the increased secretion. But a balance is maintained between the production of tissue liquid and its removal by the gland-cells. When the gland is quiescent, the small amount of tissue liquid normally formed from the blood capillaries for the nutrition of the cells is balanced by, upon the whole, an equal amount of lymph secreted from the tissue spaces into the lymph capillaries.

We may say, indeed, that the closed lymphatic system has for its great function the regulation of the quantity and quality of the tissue liquid. In glands with an external secretion increased irrigation of the tissue spaces from the blood does not as a rule lead to increased flow of lymph, because the surplus fluid is required to form the secretion. In other organs, however, such as the muscles and the ductless glands, it is probable that the augmented irriga- 
tion rendered necessary by functional activity is always associated with an accelerated flow of lympl, which rarries off the surplus liquid, including a portion of the waste products. It is probable that an important factor in the production of aedema may be the derangement of the meclianism, whatever it is, through which the adjustment of the rate of formation of tissue liquid to that of lymphatic lymph is achieved. But it must be remembered that in all the organs the blood capillaries not only supply materials to the tissue spaces, but take up materials from them. Indeed, there are facts which indicate that in general water and dissolved substances pass more easily and in greater amount back from the tissues into the blood than into the lymphatics. So that, while the lymphatics constitute an accessory drainage system, the bloodvessels irrigate the tissues and drain them as well. A consequence of this, as well as of the great difference in the capacity of the different tissues for storing water, is that the amount of tissue lympl formed can never be estimated from the amount of lymphatic lymph leaving an organ. Thus the flow of lymph from the limbs at rest is very small in comparison with the flow from the abdominal viscera, which constitutes the great bulk of the lymph passing along the thoracic duct. This, however, does not prove that very little liquid passes out of the limb capillaries, for the chief tissue of the limbs, the muscles, possesses a far greater storage capacity for water than the intestines and digestive glands. In the one case we have a field whose soil takes up a great deal of water and is not easily saturated; in the other a field whose soil soon becomes water-logged and refuses to take up any more. With the same supply from the irrigating ditch, little or no water may drain off at the foot of the first field, a great deal at the foot of the second.

Where the main lymphatics are themselves blocked by mechanical pressure or by inclusion in a ligature, the balance is, of course, grossly upset by the failure of the outflow of lymph to keep pace with its formation. Where an obstruction on the course of the veins is responsible for the cedema, the lymphatic outflow, to be sure, is not directly interfered with. But the nutrition and the respiration of the vascular walls themselves, including the endothelium of the capillaries, necessarily suffers from the insufficiency of the blood-flow, and the crippled capillaries may very well become abnormally permeable for water, salts, and the other constituents of lymph. And while ordinary mechanical filtration may not be a factor, or a very unimportant one, in the passage of liquid through the normal capillary wall, it may become far more effective when it acts on a damaged wall. The tissue cells also suffer from lack of oxygen and nutritive material, and from the accumulation of waste products, including acid substances, which cause them to take up and to hold more water than normal. Even in the absence 
of changes in the mechanical conditions of the blood and lymph circulations, alterations in the tissues must be recognized as among the causes of œdema (Fischer). Thus it is clear that the interpretation of such an apparently simple experiment as the production of vedema by the ligation of a vein needs great care. Whatever it proves, it may be said with confidence that it does not prove that the increased capillary pressure is the direct cause of the œdema.

A mere increase in the capillary blood-pressure does not of itself accelerate the formation of tissue liquid from the blood any more than that of lymph from the tissue liquid, as is shown by the fact that, when the chorda tympani is stimulated after injection of a dose of atropine sufficient to prevent all salivary secretion, there is neither œdema of the gland nor increase in the flow of lymph from it, although the arterioles are as widely dilated as before. When the blood-pressure is greatly increased in the anterior portion of an animal by clamping the aorta, or in the whole animal by continued stimulation of the cut medulla oblongata or the splanchnic nerves, the blood does not alter in concentration in the least, showing that no sensible increase in the passage of water into the lymph has occurred. After division or embolism of the medulla oblongata, and consequent paralysis of the vaso-motor centre and general vascular dilatation, it is stated that the injection of sodium chloride produces an increase in the lymph-flow as great and as durable as in the normal animal, and which can continue even after death (Pugliese). The action of the first class of lymphagogues, which cannot be explained as the consequence of an increase of capillary pressure, because the pressure in the capillaries is not consistently increased, and may even in the case of some of these lymphagogues be diminished, is attributed by Starling to an injurious effect on the capillary endothelium (and especially on the endothelium of the capillaries of the liver, since nearly the whole of the increased lymph-flow comes from that organ), which increases its permeability. But it is not easy to distinguish an increase of permeability produced by lymphagogues from an increase of secretory activity of the endothelial cells.

Hamburger, too, has brought forward results which it is difficult to reconcile with a theory of filtration even for the second class of lymphagogues. Further, Heidenhain has shown that some time after injection of a crystalloid substance, like sugar, into the blood, a greater percentage of the substance may be found in the lymph than in the blood. Now, when a mixture of crystalloids and colloids is filtered through a thin membrane, the percentage of crystalloids in the filtrate is never, at most, greater than in the original liquid. And although Cohnstein states that, if time enough be allowed, the maximum concentration of sodium chloride in the lymph, after intravenous injection, becomes approximately the 
same as the maximum in the blood, this fact loses its weight as an argumcent in favour of the filtration hypothesis when we remember that, according to Asher, all the solids of the lymph are markedly increased when even small quantities of crystalloids are injecterl into the veins. Lpon the whole, then, it may be conclucled that up to the present it has not been shown that filtration due to the excess of pressure in the capillaries over that in the lympl spaces is an effective factor in the formation of lympl. For is it at all easier to explain lymph formation as a matter of pure osmosis or diffusion. Iazarus-Barlow found, for example, that in the great majority of his experiments the injection of a concentrated solution of sodium chloride, dextrose or urea into a vein was followed, not by an initial diminution in the outflow of lymph (as might have been expected if the exchange of water between the blood and the tissue spaces, and between the tissue spaces and the lymph capillaries, was regulated solely by differences in osmotic pressure), but by an immediate increase. And Carlson has shown that the osmotic pressure of lymph coming from the active salivary glands, as measured by the freezing-point method, may, under chloroform or ether anæsthesia, be distinctly less than that of the blood-serum. Water must therefore be passing from a liquid of higher to one of lower osmotic concentration.

Nevertheless, it would be erroneous to assume that because osmosis and diffusion have not been shown to satisfactorily account for all the phenomena of lymph formation, they exert no influence upon it. It is probable, indeed, that their action is fully as important as in absorption from the alimentary canal, although, as in absorption, it is often overlaid and always modified by the specific permeability of the blood-capillary walls, the lymph-capillary walls, and the tissue cells in general, in virtue of which they exert an action upon the quantity and composition of the lymph analogous to the action exerted in a higher degree by the cells of the digestive glands upon the quantity and composition of the liquids passing into their ducts.

It is not difficult to illustrate the fact that phenomena of osmosis and diffusion emerge, although not of course in such purity as in physical experiments, when we study the interchange between the blood and the tissue liquids. If, for example, a hypertonic solution of sodium chloride is injected into the blood, water rapidly passes from the tissues to the blood as it would through a semipermeable membrane, and the blood becomes diluted. At the same time sodium chloride leaves the blood and passes into the tissues, as it would do by diffusion from a place of higher to a place of lower concentration. But after this has gone on for some time, and the concentration of the blood in salt has sunk, it may be, to that in the lymph, salt still continues to pass out of the blood, and the excess of water also leaves the bloodvessels till the osmotic pressure of the blood has again become normal. When isotonic and even hypotonic solutions of sodium chloride are injected, salt also 
leaves the blood and enters the lympl, although it ought not to do sn by diffusion, while water, which might pass from the hypotonic bloorl in to the lymph by osmosis, moves in the same direction from the blood to which the isotonic salt solution has been added. Regulative mechanisms, in short, exist which tend, with, but also without, the co-operation of diffusion and osmosis, and even, so to say, in their tectl, to bring back the quantitative and qualitative composition of the blood to the normal. Exactly similar phenomena are witnessed when the equilibrium is upset from the other side by the injection of salt solutions into the subcutaneous tissue or the intramuscular connective tissue. Hypotonic sodium chloride solution injected into the subconjunctival connective tissue quickly loses water and gains sodium chloride, as it ought to do if under tle influence of osmosis and diffusion, and hypertonic salt solutions gain water. But eventually hypertonic, hypotonic, and isotonic solutions, and even scrum itself, are completely absorbed, which could not occur in the presence of diffusion and osmosis alone. Sometimes in dropsy it appears that the œedema liquid is absorb:d when the patient is put on a diet free as far as possible from salts. The suggestion is that the regulative mechanism which tends to keep the molecular concentration of the blood and lymph approximately constant provides that as the salt content of the body falls, which it does through continucl excretion of salts in the urine, water is eliminated in corresponding amount.

The Contribution of the Tissue-Cells to the Lymph.-So far we have considered the passage of the lympli constituents, on the one land through the endotliclium of the blood capillaries into the tissue spaces; on the other, from the tissue spaces through the endothelium of the lymph capillaries. But it is not to be supposed that the liquid lying in clefts, partly bounded by blood capillaries, partly by lymph capillaries, partly by tissue-cells, should be affected solely by the first two. The third anatomical element must contribute something to, or withdraw something from, the tissue liquid, and may thus play a part in the formation of lymph from the latter. The recent researches of Asher and his pupils have raised the question of the relation between the physiological activity of the organs, and especially of the glands, and the formation of the lymph. They conclude that the common doctrine that lymph is simply a diluted blood-plasma is erroneous. Lymph, they say, far from being a mere filtrate or even a secretion from the blood, is formed by the activity of the organs, and may actually be absorbed by the blood from the tissue spaces. In fact, according to their view, the intravenous injection of lymphagogues, both crystalloid and colloid, only causes an increased flow of lymph in so far as it leads to increased glandular secretion. But this generalization has had only a short-lived vogue, and one by one some of the main results which seemed to support it have been disproved or shown to be capable of interpretation in a different sense. For example, it was stated that secretin causes a flow of lympl from the lymphatics of the pancreas, as well as a flow of pancreatic juice. But it has been shown that the increased production of lymph is not due to the 
secretin at all, but to lymphagogue substances, including proteose, extracted with the secretin from the intestinal mucous membrane. A solution of secretin can be prepared which causes a considerable increase in the secretion of pancreatic juice and bile, but no augmentation whatever in the flow of lymph from the thoracic duct. Again, it was asserted that peptone, a noted lymphagogue, produces a great increase in the biliary secretion. It has been demonstrated, however, that the action of the peptone is merely to cause expulsion of the contents of the gall-bladder by the mechanical effect of the swelling of the liver, and not at all to stimulate the liver-cells to form more bilc. For it produces no effect on the flow of bile if the gall-bladder be emptied or the cystic duct tied before the injection (Ellinger). That active salivary secretion is not accompanied by increased lymph-flow from the lymphatics of the salivary glands has been mentioned above. Nevertheless, we may safely assume that the activity of the organs does make a contribution to the lymph-to its solids, if not in any important degree to its watercontent, although to say that they alone are concerned in its formation, to the exclusion of the capillaries, is altogether an over-statement. The waste-products of the tissues pass into the lymph, and possibly, as Koranyi suggests, may, by increasing its molecular concentration, cause the passage of some water into it from the blood. Or the decomposition of the large protein molecules, which in tissue metabolism are breaking down into numerous smaller molecules, may entail an increase of osmotic pressure in the cells themselves, which in turn may lead to withdrawal of water by the cells from the tissue liquid. The osmotic pressure of the liquid may thus rise, and water may pass into the tissue spaces from the blood. The molecular concentration of lymph (except in anæsthetized animals as mentioned above) is in general somewhat greater than that of blood-serum-e.g., in one observation $\Delta$ of serum was $0.605^{\circ} \mathrm{C}$, and of lymph $0.610^{\circ} \mathrm{C}$. For the solid tissues, the freezing-point of which, however, cannot be as satisfactorily determined as that of liquids, the following values of $\Delta$ were obtained: Brain, $0.65^{\circ}$; muscle, $0.68^{\circ}$; kidney, $0.94^{\circ}$; liver, $0.97^{\circ}$; while for blood it was $0.57^{\circ}$ (Sabbatani).

To sum up, we may say that while the physical processes of filtration, osmosis and diffusion may play a part in the passage of water and solids through the walls of the blood capillaries, as well as from the tissue-cells into the tissue spaces, and from these spaces into the lymph capillaries, there is much which they leave unexplained, and which at present, for the want of a more precise term, we must attribute to secretory activity.

Influence of Nerves on Lymph Formation.-In one instance it appears to have been shown that lymph may be formed under the influence of sccretory nerves. In the males of certain 
aquatic birds erection is due to the filling of the corpus cavernosum. not with blood, but with lymph. The lymph is secreted rapidly by the so-called bodies of Tannenberg when certain sympathetic nervefibres are experimentally stimulated, and passes into the corpus cavernosum, which swells up. If a small incision is made in the corpus, a large quantity of clear lymph, whicl clots slowly on standing, escapes. There is a simultaneous vasodilatation. After erection, the lymph is rapidly and completely reabsorbed (Eckhard, Müller).

Although no definite lymph-secretory nerve-fibres have as y't been discovered in mammals and for ordinary tissues, it is possible that they exist (Sihler). As already pointed out, the same volume of liquid as escapes into the ducts of the active submaxillary gland must, upon the whole, pass out of the blood capillaries. On what principle shall we distinguish one only of these processec as physiological secretion? They begin together when the chorda tympani is stimulated. A drug which paralyzes secretory nerve-endings abolishes both effects. The simplest explanation is that the chorda contains secretory fibres which influence the formation both of saliva and of the tissue liquid from which it is recruited; and, so far as this consideration goes, it is just as logical to consider the increase in the supply of tissue liquid as the cause of the increase in the flow of saliva as to consider the increased salivary secretion as the cause of the increased flow of liquid into the tissue spaces. The increased flow of liquid may be brought about either by an action of the nerve on the gland-cells, causing them to produce a hormone, which then effects the blood capillaries (Carlson), or by a direct action on the capillary endothelium. The advantage to cells engaged in the active secretion of saliva of being immersed in an abundant bath of tissue liquid is obvious.

The post-mortem flow of lymph, which may continue in some cases long after complete cessation of the circulation-for an hour after injection of dextrose to produce hydræmic plethora; for as much as four hours after injection of extract of the strawberry, which is a.lymphagogue of Heidenhain's first group (Mendel and Hooker)-is a phenomenon whose relation to normal lymph formation has not been definitely settled.

It ought to be remembered in this whole discussion that the epithelium of ordinary glands derives its supplies of material from the tissue lymph. The vicissitudes of blood-pressure affect it only in a secondary and indirect manner. On the other hand, the endothelial cells of the capillaries are in direct contact with the blood. And it is interesting to observe that in this respect the glomeruli of the kidney and the alveoli of the lungs (if the endothelial lining of Bowman's capsule and the alveolar membrane are assumed to be complete) takt a middle place between the glands proper and the quasi-glandular capillaries. 


\section{CHAPTER IX}

\section{EXCRETION}

WE have now followed the ingoing tide of gaseous, liquid, and solid substances within the physiological surface of the body. There we leave them for the present, and turn to the consideration of the channels of outflow, and the waste products which pass along them. In a body which is neither increasing nor diminishing in mass the outflow must exactly balance the inflow; all that enters the body must sooner or later, in however changed a form, escape from it again. In the expired air, the urine, the secretions of the skin, and the freces, by far the greater part of the waste products is eliminated. Thus the carbon of the absorbed solids of the food is chiefly given off as carbon dioxide by the lungs; the hydrogen, as water by the kidneys, lungs and skin, along with the unchanged water of the food; the nitrogen, as urea by the kidneys. The fæces in part represent unabsorbed portions of the food. A small and variable contribution to the total excretion is the expectorated matter, and the secretions of the nasal mucous membrane and lachrymal glands. Still smaller and still more variable is the loss in the form of dead epidermic scales, hairs, and nails. The discharges from the generative organs are to be considered as excretions with reference to the parent organism, and so is the milk, and even the fœtus itself, with respect to the mother.

Excretion by the lungs and in the fæces has been already dealt with. All that is necessary to be said of the expectoration and the nasal and lachrymal discharges is that the first two generally contain a good deal of mucin, and are produced in small mucous and serous glands, the cells of which are of the same general type as those of the mucous and serous salivary glands. The lachrymal glands are serous like the parotid; and the tears secreted by them contain some albumin and salts, but little or no mucin. The sexual secretions and milk will be best considered under reproduction (Chap. XIX.), so that there remain only the urine and the secretions of the skin to be treated here. 


\section{SECTION I.-EXCRETION By THE KIDNEYS-ThE ChEMISTRY OF THE URINE.}

Normal urine is a clear yellow liquid acid to litmus and similar indicators, but nearly neutral or very weakly acid in the physicochenical sense (p. 24). The average specific gravity is about I020, the usual limits being Io 15 and 1025, although when water is taken in large quantities, or long withheld, the specific gravity may fall to I005, or even less, or rise to I035, or even more. The quantity passed in twenty-four hours is very variable, and is especially dependent on the activity of the sweat-glands, being, as a rule, smaller in summer when the skin sweats much, than in winter when it sweats little. The average quantity for an adult male is 1,200 to I,600 c.c. (say, 40 to $50 \mathrm{oz}$.).*

Composition of Urine.-This is very closely related to the quantity and quality of the food. Hence it is impossible to speak of a definite normal composition of the urine. It is essentially a solution of urea and inorganic salts, the proportion of the latter being generally about I. 5 per cent., or double the usual amount of physiological liquids. Besides urea, there are other nitrogenous bodies in much smaller quantity, such as ammonia, uric acid, and the allied purin bases, hippuric acid, and kreatinin. Some of these at least are products of the metabolism of proteins within the tissues. And besides the inorganic salts there are certain organic bodiesindoxyl, phenyl, pyroliatechin, skatoxyl-united with sulphuric acid, which are primarily derived from the products of the putrefaction of proteins witlin the digestive tube.

Folin has published analyses of 'normal' urines from six persons. weighing from 56.6 to 70.9 kilos (average 63.4 kilos), who were kept for seven days on one standard uniform diet. The diet consisted of 500 c.c. of milk, 300 c.c. of cream (containing I 8 to 22 per cent. of fat), 450 grammes of eggs, 200 grammes of Horlick's malted milk, 20 grammes of sugar, 6 grammes of sodium chloride, water enough to make the whole up to two litres, and 900 c.c. of additional water. The ingredients contained II9 grammes of protein, about $\mathrm{I}_{4} 8$ grammes of fat, and 225 grainmes of carbo-hydrates. The average results of all the determinations are given in the following table:

* The average quantity of urine varies not only with the season, but also with the habits of the person, especially as regards the amount of liquid taken. The average for seventeen healthy (American) students, whose urine was collected for six to eight successive days in winter, was I, I66 c.c. The highest average in any one individual for the observation period was $1,4^{8} 7$ c.c. (for seven days), and the lowest 743 c.c. (for eight days). The greatest quantity passed in any one periol of twenty-four hours was 2,286 c.c. (by the individual whose average was the highest). The smallest quantity passed in twenty-four hours was 650 c.c. (by the individual whose average was the lowest. 


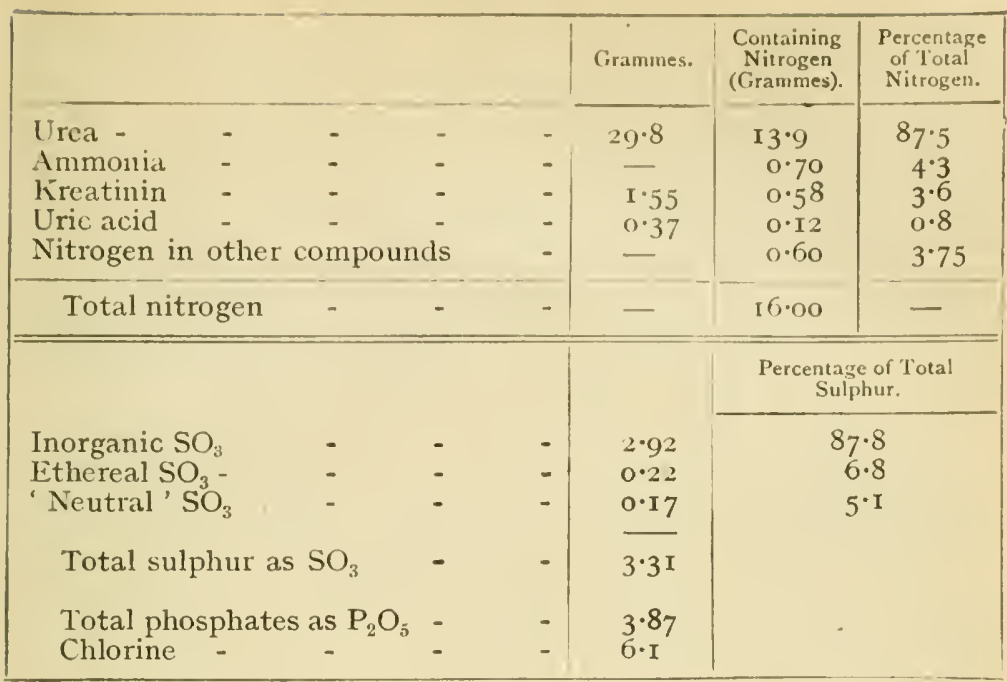

Titratable acidity in c.c. of decinormal acid - 6ry f mineral, 304 .

Indican (Fehling's solution $=$ I00* $^{*}$ - $\quad-77$

Volume of urine - - - - I430 c.c.

The great influence of diet on the composition of the urine is illustrated in the following table. Urine I. was obtained from a man weighing 87 kilos on the standard protein-rich diet described above. Urine II. was obtained from the same person on a diet very poor in protein ( 400 grammes of starch and 300 c.c. of cream), containing only about I gramme of nitrogen, as against 19 grammes in the first diet.

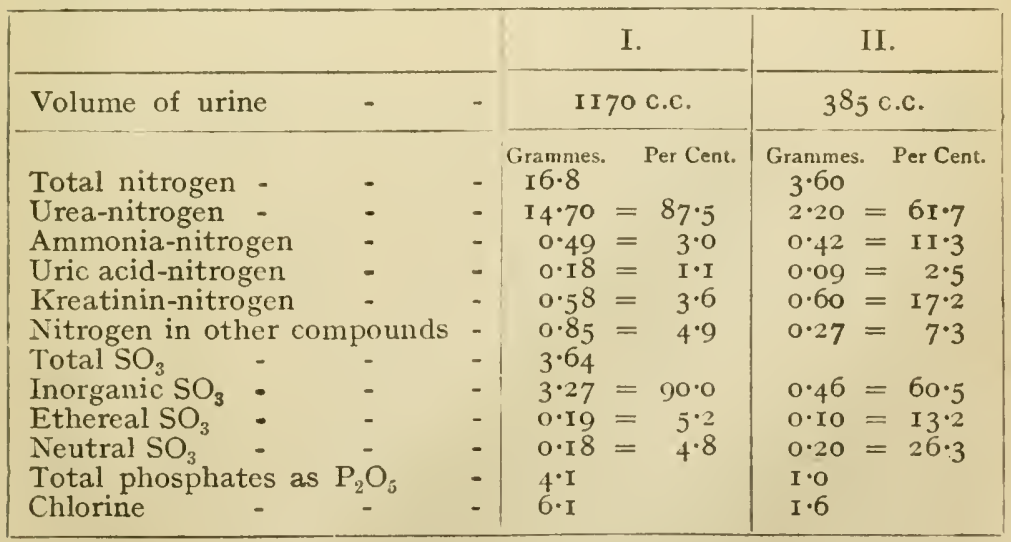

Titratable acidity in c.c. $\frac{\mathrm{N}}{\mathrm{I}}$ acid $-805\left\{\begin{array}{l}\text { mineral, } 398 \\ \text { organic, } 407\end{array} 324\left\{\begin{array}{l}\text { mineral, I2 } 3 . \\ \text { organic, } 20 \mathrm{I}\end{array}\right.\right.$ Indican (Fehling's solution = Ioo) - 120

* The indican is given in arbitrary units, the indigo blue being obtained from the urine and then estimated colorimetrically, using Fehling's solu. 
The titrable acidity of urine (see p. 25) is chiefly due to the acid (monobasic) pliosphate's, such as acid sodium phospliate $\left(\mathrm{NaH}_{2} \mathrm{PO}_{4}\right)$, but in an important degrec also to organic acids. According to Folin, indeed, the organic aciclity may be more than lialf the total acidity. Normally the acidity diminishes distinctly, or even gives place to alkalinity, luring digestion, when the acid of the gastric juice is being secreted. This is sometimes fancifully denominated the alkaline tide. After a fast, as before breakfast, the opposite condition, the acid tide, occurs.

The acidity varies with the quantity of vegetable food in the diet. The urine of herbivora and vegetarians is alkaline, and is either turbirl when passed, or on standing soon becomes turbid from precipitated carbonates and phosphates of earthy bases, while that of carnivora and of fasting herbivora, which are living on tlueir own tissues, is strongly acid and clear. Normal human urine may deposit urates soon after discharge, as they are more soluble in warm than in cold water. They carry down some of the pigment, and therefere usually appear as a pink or brick-red sediment. When urine is allowed to stand after being voided, what is generally described as ' acid fermentation' occurs. The acidity gradually increases; acid sodium urate is produced from the neutral urate, and comes down in the form of amorphous granules, while the liberated uric acid is deposited often in 'whetstone 'crystals, coloured yellow by the pigment (Fig. 177). Calcium oxalate may also

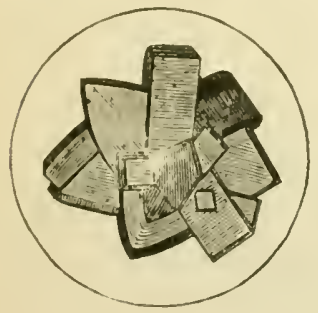

Fig. 177.-Uric Acid

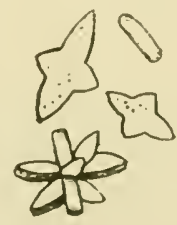

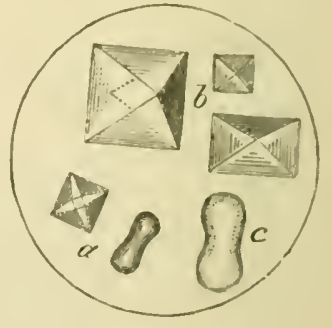

Fig. I78.-Calcium Oxalate

be thrown down as 'envelope,' $a, b$, or less frequently, 'sand-glass' crystals, $c$ (Fig. I 78). If the urine is allowed to stand for a few days, especially in a warm place, or in a place where urine is decomposing, the reaction becomes ultimately strongly alkaline, owing to the formation of ammonium carbonate from urea by the action of micro-organisms (Micrococcus urea, Bacterium urea, and others) which reach it from the air, and produce a soluble ferment urease, in whose presence the urea is split up with assumption of water. Thus:

$$
\underset{\substack{\mathrm{NH}_{2} \\ \mathrm{Crea}^{-}}}{\mathrm{NH}_{2}}+2 \mathrm{H}_{2} \mathrm{O}=\underset{\text { Ammonium carbonate. }}{\mathrm{O}}=\frac{\mathrm{O} . \mathrm{NH}_{4}}{\mathrm{O}}
$$

This is a reaction of considerable interest, for the reverse reaction occurs when blood containing ammonium carbonate is circulated timrough the liver, the ammonium carbonate being converted into urea with loss of water. The en\%yme urease is present in higher plants than bacteria and fungi. A solution containing it, conveniently prepared from the soy bean, can be used for the quantitative estimation of urea, and this is probably the casiest and most accurate method.

tion as a standard. Fehling's solution is employed because it is a blue liquid of a definite depth of tint already prepared in every physiological laboratory. 
The sulstances insoluble in alkaline urine are thrown fown, the cleposit contitining ammonio-magnesic or triple phosphate, formed by the union of ammonia with the magnesium plosphate present in frish urine, and precipitated as clcar erystals of 'knife-rest 'or 'coffin-lid ' sliape (Fig. I79), along witl amorphous carthy phosphates, and often acid ammonium urate in the form of dark balls occasionally covered with spines (Fig. 182). Calcium phosplite (CaHPO 4 ) is anotler phosplate fomm in serliments depo-iter from alkaline or faintly acid urine. It is usually amorphous, but sometimes in the form of long prismatic crystals arranged in star fashion, and hence spoken of as stellar phospliate (Fig. I8I). It is not pigmented.

It is only in pathological conditions that the alkaline fermentation takes place within the bladder. The reaction of the urine cean readily.

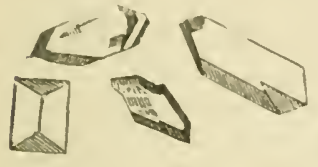

Fig. 179.-Triple Phosphate.

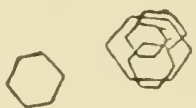

Fig. 180.-Cystin.

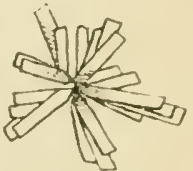

Fig. I81.-Stellar Phos. phate Crystals.

be made alkaline by the administration of alkalies, alkaline carbonates, or the salts of vegetable acids like malic, citric, and tartaric acid, which are broken up in the body and form alkaline carbonates with the alkalies of the blood and lymph. It is not so easy to increase the acidity of the urine, although mineral acids do so up to a certain limit. If the administration of acid be pushed farther, ammonia is split off from the proteins, and is excreted in the urine as the ammonium salt of the acid.

Determination of the Acidity.-A titration method is described in the Practical Exercises (p. 5r5). In speaking of the reaction of blood, it has alrcady been mentioned (p. 25) that we cannot deternuine by titration the true acidity or alkalinity of a liquid in the physico-chemical sense-i.e., the concentration of the dissociated hydrogen and hydroxyl ions respectively. E.g.. when we titrate equal quantities of decinormal* acetic acid and decinormal hydrochloric acid with decinormal potassium hydroxide, using, say, phenolphthalein as the indicator, nearly the same volume of the potassium hydroxide solution will be needed to neutralize each acid. Yet it can be shown by physico-chemical

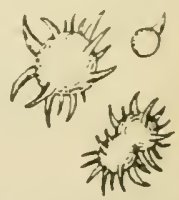

Fig. I82. - Ammonium Urate (after Nilroy). methods that the acetic acid in the strength used is only dissociated to the extent of a little more than $r$ per cent., while about 80 per cent. of the hydrochloric acid is dissociated. The concentration of the hydrogen ions is therefore eighty times as great in the hydrochloric as in the acetic acid solution. What we determine by the titration is not the true acidity, but the total amount of hydrogen which can be replaced by metal. The concentration of the hydrogen ions in normal urine is very small, on the average only about 0.003 milli-

* A normal solution of a substance contains in a litre a number of grammes of the substance equal to the number which expresses its equivalent weight -a decinormal (usually written $\underset{\mathbf{N}}{\mathrm{N}}$ ) solution one-tenth of this amount, a centinormal one-hundredth, etc. Thus, a normal solution of potassium hyclroxide contains 56 grammes of $\mathrm{KOH}$, and a decinormal solution $5^{\circ} 6$ grammes in 1,000 c.c. 
gramme in the litre, or about thirty times as much as is present in the purest distilled water. Urine departs about as much from neutrality in the one direction as blood does in the other.

Urea, $\mathrm{CO}\left(\mathrm{NH}_{2}\right)_{2}$, is the form in which by far the greater part of the nitrogen is under ordinary conditions discharged from the body. Its amount is as important a measure of protein metabrlism as the quantity" of carbon dioxide given out by the lungs is of the oxidation of carbonaceous material. Yet a glance at the table on p. 477 shows that, when the total protein metabolism is greatly reduced by diminishing the protein in the food, the relative as well as the absolute amount of nitrogen climinated as urea suffers a great diminution. The relative amount of the other nitrogenous urinary constituents, especially of the kreatinin, is markedly increased. The significance of this difference is alluded to in spealing of the kreatinin content of urine, and will have to be again considered under Protein Metabolism. Urea is soluble in water and in alcohol, and crystallizes from its solutions in the form of long colourless necdles, or four-sided prisms with pyramidal ends. It can be easily prepared from urine. Urea can also be obtained artificially by heating its isomer, ammoniuin cyanate $\left(\mathrm{NH}_{4}-\mathrm{O}-\mathrm{CN}\right)$, to $100^{\circ} \mathrm{C}$. This reaction is of great historical interest, as it forms the final step in Wöhler's famous synthesis of urea, the first cxample of a complex product of the activity of living mitter being formed from the ordinary materials of the laboratory. Heated in watery solution in a sealed tube to $180^{\circ} \mathrm{C}$, urea is entirely split up into carbon dioxide and ammonia, a change which can also be brought about, as already mentioned. by the action of micro-organisms. Nitrous acid, hypochlorous acid, and salts of hypobromous acid carry the decomposition still farther, carbon dioxide, nitrogen, and water being the products of their oxidizing ar tion on urea. Thus: $\mathrm{CO} .2\left(\mathrm{NH}_{2}\right)+3 \mathrm{NaBrO}=3 \mathrm{NaBr}+2 \mathrm{H}_{2} \mathrm{O}+\mathrm{CO}_{2}+\mathrm{N}_{2}$. This reaction is the basis of the hypobromite method of estimating the quantity of urea in urine (Practical Exercises, p. 519).

Ammonia.- The ammonia in urine is united with acids in the form of salts. Its formation from proteins is determined, as we shall sec later on, by the necessity of neutralizing certain acids produced in metabolism-e.g., those derived from the sulphur and phosphorus of the proteins, or acids adminisiered experimentally. According to some observers, the percentage amount of the total nitrogen in the urine in the form of ammonia remains the same whether the food be rich or poor in protein (Schittenhelm. ctc.), but others state that when the protein is reduced there is a relative increase in the ammonia-nitrogen (see table on p. 477) (Folin).

Uric acid $\left(\mathrm{C}_{5} \mathrm{H}_{4} \mathrm{~N}_{4} \mathrm{O}_{3}\right)$ exists in large amount in the urine of birds. The excrement of serpents consists almost entirely of uric acid. In both cases it is mainly in the form of acid anmonium uratc. In contrast to urea, uric acid is very insoluble, rcquiring 1,900 parts of lot and I5,000 parts of cold water to dissolve it. In man and mannuls the quantity is comparatively small in healtl, but is increased after a meal containing material (e.g., thymus gland) rich in nucleins, from the nucleic acid of which purin bodies are derived, or substances containing purin bases in the free state-e.g., hypoxanthin in meat. In mammals the amount of 11 ric acid excreted depends little, if at all, upon the quantity of protein in the food, but a great deal upon the quantity of purin bodlies, whether free or combined. When nitrogenous food is omitted altogether, the absolnte quantity of uric acid is diminished, but the proportion of the total nitrogen of the urine climinated is uric acid is increased, since the 'endogenous' uric acid (p. 596) still continues to be formed and cxcreted. 
The purin bases (sometimes called the nuclein bases, the alloxuric bases, or the xanthin bases) are a group of substances allied to vric acid, and including, besides xanthin itself, hypoxantlin, guanin, adenin, and other boclies. They exist in very small anonnt in urine, but, like uric acid, are increased in amount by the ingestion of nuclein-containing substances. The greater part of the purin bases produced in the body is transformed into uric acid; it is only the untransformed residue which appears in the urine. An interesting fraction of the purin bases in the urine which is not related to the nuclein metabolism is composed of the so-called licteroxanthin, derived from caffeine in the coffee and tea, $l$-methylxanthin, derived from theobromine in the cocoa, and paraxanthin, derived from theophyllin in the tea, consumed as beverages.

Hippuric acid $\left(\mathrm{C}_{9} \mathrm{H}_{9} \mathrm{NO}_{3}\right)$ occurs in consigerable quantity in the urine of herbivora (Practical Exercises, p. 52.4); in the urine of carnivora and of man only in traces; in that of birds not at all. Its amount is much more dependent on the presence of particular substances in the food thas that of the other organic constituents of urine. Anything which contains benzoic acid, or substances which can be readily changed into it (sucl as cinnamic and quinic acids), causes an increase of the hippuric acicl in urine. In fact. one of the best ways of obtaining the latter is from the urine of a person to whom benzoic acid is given by the mouth; the sweat

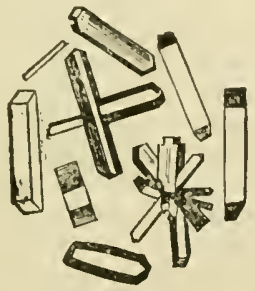

Fig. I83.-Creatin.

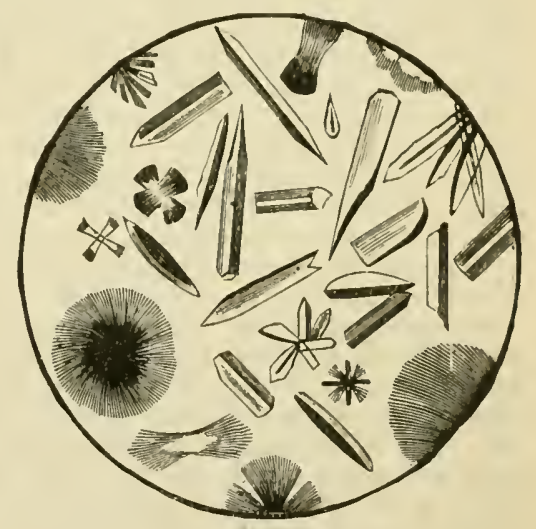

Fig. I84.-Creatinin-Zinc.Chloride.

may also in this case contain a trace of hippuric acid. Chemically it is a conjugated acid formed by the union of benzoic acid and glycin.

Amino-Acids. - The only amino-acid hitherto detected with certainty in normal urine is glycin.

Oxalic acid is always present, although in very small amount. Some of it comes from the oxalates of the food, but a portion of it arises in the metabolism of the tissues, probably from the decomposition of uric acid. It is known that outside of the body uric acid may be made to yield oxalic acid. Calcium oxalate crystals are often seen in urinary sediments.

Creatinin $\left(\mathrm{C}_{4} \mathrm{H}_{7} \mathrm{~N}_{3} \mathrm{O}\right)$. - Creatinin is the anhydride of creatin (Fig. I 83). Its fornulua differs from that of creatin only in possessing the elements of one molecule of water less; and creatinin can be obtained by boiling creatin with dilute sulphuric acid. From its alcoholic solution it crystallizes in colourless prisms. Creatinin forms crystalline compounds with various acids and salts. One of the best known of these is creatinin-zinc-chloride, formed on the addition of zinc chloride to an alcoholic or watery solution of creatinin, often in the shape of beautiful thick-set rosettes of ncedles (Fig. I $8_{+}$). A por- 
tion of the urinary creatinin is derived from the creatin of the meat taken as food. But this is not its only source, for on a nieat-free diet and in starvation creatinin is still excreted. The absolute guantity in the urine on a meat-frce clict is constant for one and the same individual, although different in different persons, and independent of the total anount of nitrogen eliminated. Hence on a diet poor in protein the percentage of the total nitrogen excreted as creatinin is much greater than on a protein-rich cliet, as shown in the table on p. 477. So constant ts the quantity that a determination of the creatinin may be used as a clieck upon the complete collection of the urine.

Carbo-hydrates are normally present in human urine, but only in very small amounts. Three are known with certainty-dextrose, isomaltose, and the so-called animal gum or urine dextrin. Glycuronic acid $\left(\mathrm{C}_{6} \mathrm{H}_{10} \mathrm{O}_{7}\right)$, a body which can be derived from dextrose, is constantly present in small amount as a conjugated acid, paired with phenol or indoxyl. It gives Fehling's test, and thus may casily be mistaken for sugar. Glycuronic acid becomes coupled very easily with a large variety of substances, including many drugs, and care must be taken after the administration of camphor, chloral hydrate, chlorcform, nitrobenzol, etc., not to confound the largely increased excretion of conjugate glycuronates in the urine with glycosuria. The yeast test will turn out negative if the reduction is due to glycuronic acid, and the polarimeter will show rotation to the right if it is due to dextrose. The total quantity of carbo-hydrates, including glycuronic acid. excreted in the urine of the twenty-four hours has been estimated at 2 to 3 grammes. The quantity of dextrose in normal human urine is about 0.02 per cent., or about one-fifth of the proportion in blood.

Proteins, mainly serum-albumin, are also found in normal urine in minute quantities, on the average about 0.0036 per cent. (Mörner).

Pigments of Urine.-The pigments of urine have not hitherto been exhaustively studied; but we already know tliat normal urine contains several, and pathological urines probably additional, pigmentary substances. The best-known pigments in normal urine are urochrome, the yellow substance which gives the liquid its ordinary colour; uroerythrin, the pink pigment which often colours the deposits of urates that separate even from healthy urine; and urobilin, which, as has been already stated, is identical with the freal pigment stercobilin, and occurs not only in many febrile conditions, but also in cases with no fever, sucl as functional derangements of the liver, dyspepsia, chronic bronchitis, and valvular diseases of the heart. The urobilin of urine represents, mainly at least, the portion of the stercobilin which is not excreted with the frecs, but absorbed from the intestine into the blood. The urobilin in normal urine only exists in small amount in the fully-formed condition, most of it being present as a chromogen or mother-substance (urobilinogen), which by oxidation, as on standing exposed to the air, is converted into urobilin. On the addition of ammonia and zinc chloride to a solution of urobilin a beautiful green fluorescence is obtained, and the solution now shows an absorption band between $b$ and $F$. Urobilin and urochrome are related substances, but the exact nature of the relation has not been settled. There is some evidence that a portion of the urobilin of urine is not derived from the intestine. but manufactured probably in the liver. In hunger urobilin is still excreted in the urine, although in greatly reduced amount. During menstruation it is markedly increased, both in fasting and in normally fed individuals. Urorosein is a red pigment which is produced from its chromogen by the action of mineral acids-e.g., strong lydrochloric acid-in the presence of an oxidizing agent, especially nitrites. 
The pigments of the blood and bile and some of their derivatives are of common occurrence in the urine in disease. Hamatoporphyrin has not only been fomnd in some patlological conditions, but is constantly present in minute traces in normal urine. Certain drugs-e.g., sulphonal -cause an increase in its amount. In paroxysmal hemoglobinuria, methemoglobin, mixed with some oxyhamoglobin, is found in the urine in large amount; and it is worthy of note that it is not formed in the urine after secretion, but is alrealy present as such when it reaches the bladder.

In the rare condition termed alkaptonuria, a body, alkapton, now known to be identical with homogentisinic acid, $\mathrm{C}_{6} \mathrm{H}_{3} \cdot(\mathrm{OH})_{2} \mathrm{CH}_{2}$. COOH, a dioxyphenylacetic acid, is present. The urine becomes dark brown on the aldition of an alkali, or simply on exposure to air. It gives Feluling's test for sugar. The substance has relations to the aromatic imnino-acids tyrosin and phenyl-alanin, and when either of these is given to a person suffering from alkaptonuria, the amount of alkapton excreted is increased. We may suppose, therefore, that in this condition the normal decomposition of these products of proteolysis is interfered with.

Ferments. - The urine contains traces of proteolytic and amylolytic ferments (Fig. 185). These may be easily separated from it by putting a little fibrin, which has the power of fixing (adsorbing) enzymes, into the urine.

Of the inorganic constituents of urine the most important and most easily estimated are the chlorine, phosphoric acid, and sulphuric acid.

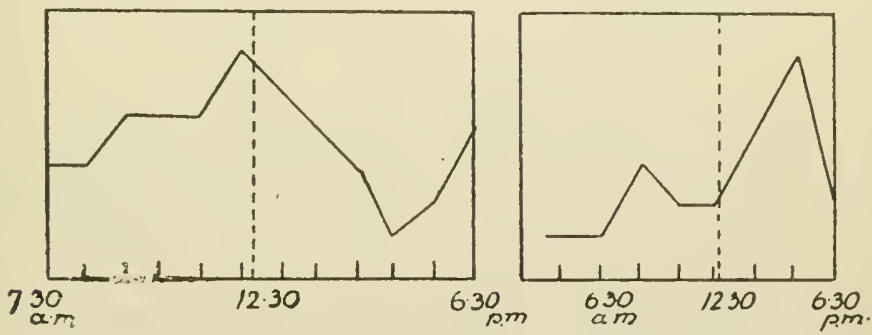

Fig. 185.-Pepsin in Urine. Diastatic Ferment in Urine.

At Different Times of the Day (Hoffmann).

Chlorine.--Nuch the greater part of the chlorine is united with sodium, a smaller amount with potassium. The chlorides of the urine are undoubtedly to a great extent derived directly from the chlorides of the food, and have not the same metabolie significance as the organic, and even as some of the other inorganic constituents. But it is noteworthy that in certain diseased conditions the chlorine may disappear entirely from the urine, or be greatly diminished-e.g., in pneumonia, and in general in cases in which much material tends to pass out from the blood in the form of effusions (p. 5 I 5 ).

Phosphoric Acid.-The phosphoric acid of the urine is chiefly derived from the phosphates of the food, but must partly come from the waste products of tissues rich in phosphorus-containing substances, such as lecithin and nuclein. The phosphoric acid is united partly with alkalies. especially as acid sodium phosphate, and partly with earthy bases, as phosphates of calcium and magnesium. The earthy phosphates are precipitated by the addition of an alkali to urine, or in the alkaline 
icrmentation. In some pathological urines they come down when the carbon dioxide is driven off by heating; a precipitate of this sort differs from heat-coagulated albumin in being readily soluble in acids (Practical Exercises, p. 524). A small amount of phosphorus may appear in the urine in a less oxidized form than phosphoric acid.

Sulphuric Acid.- This is only to a slight extent derived from readyformed sulphates in tlie food. The greater part of it is formed by oxidation of the sulphur of proteins. About nine-tenths of the sulphur in normal urine is present as inorganic sulphates, mainly those of potassium and sodium. Of the other tenth, a portion is represented by ethereal sulphates, and the remainder by the so-called 'neutral' sulphur, including the sulphur associated with the pigment urochrome. A small amount of sulphur occurs in less oxiclized forms than sulphates in such compounds as the sulphocyanide, which is probably, in part but not entirely, derived from that of the saliva, and ethyl sulplide, a substance witl a penctrating odour, which appears to be a constant constituent of dog's urine (Abel).

Thiosulphuric acid $\left(\mathrm{H}_{2} \mathrm{~S}_{2} \mathrm{O}_{3}\right)$ occurs almost constantly in cat's urine, often in dog's. It is not free, but combined with bases.

The ethereal sulphates are compounds in which the sulphuric acid is united with aromatic bodies (indol, phenol, etc.). Such are potassiumphenyl-sulphate $\left(\mathrm{C}_{6} \mathrm{H}_{5} \mathrm{KSO}_{4}\right)$, potassium-kresyl-sulphate $\left(\mathrm{C}_{7} \mathrm{H}_{7} \mathrm{KSO}_{4}\right)$, potassium-indoxyl-sulphate $\left(\mathrm{C}_{8} \mathrm{H}_{6} \mathrm{NKSO}_{4}\right)$, potassium-skatoxyl-sulphate $\left(\mathrm{C}_{9} \mathrm{H}_{8} \mathrm{NKSO}_{4}\right)$, and two double sulpliates of potassium and pyrocatechin. The formation of potassium indoyxl sulphate may be thus represented: Indol, $\mathrm{C}_{6} \mathrm{H}_{4} / \mathrm{NH}$. CH on absorption from the intestine is changed into indoxyl, $\mathrm{C}_{6} \mathrm{H}_{4} / \mathrm{CH}$.OH.CH, which $+\mathrm{SO}_{2} / \mathrm{OH}$ (potassium bydrogen sulphate) yields $\mathrm{SO}_{2} / \mathrm{OC}_{8} \mathrm{H}_{6} \mathrm{~N}$ (potassium indoxyl sulphate) $+\mathrm{H}_{2} \mathrm{O}$. The 'pairing' of these aromatic bodies with sulphuric acid renders them innocuous to the organism. Most of the compounds are present in greater amount in the urine of the horse than in the normal urine of man. But in disease the quantity of indican in the latter may be much increased; and to a certain extent it must be looked upon as an index of the intensity of putrefactive processes in the intestine and of absorption from it. Munk made the observation that in the urine of a starving dog the phenol-forming substances are absent, while in the urine of a starving man they are present in abnormally large amount. The indigo-forming substances (indican), on the other land, are in hunger excreted in considerable quantity by the dog, and not at all by man (Practical Exercises, p. 518). According to Folin, the indoxyl potassium sulphate or indican of the urine is not to any appreciable extent related to protein metabolism, but for the most part to the putrefaction of protein in the intestine. The indoxyl-potassium sulphate taken by itself may therefore afford a rough index of the intensity of the intestinal putrefactive processes. On the other hand, the total ethereal sulphuric acid cannot be taken as an index of the extent of the putrefaction, for, although absolutely diminished, it is increased relatively to the total excretion of sulphur on a diet poor in protein, or even protein-free (sec tables on p. 477).

Phenol and kresol can easily be obtained from horse's urine by mixing it with strong hydrochloric acid and distilling. These aromatic bodies pass over in the distillate. Pyrocatechin remains behind, and can be extracted by ether. It gives a green colour with ferric chloride, which becomes violet on the addition of sodium carbonate. 
The sulplum of the inorganic sulphates is the fraction of the total sulphur which fluctuates in proportion to the total protein metabolism. In this regard it follows the variations in the urea. It represents 'exogenous' metabolism. The neutral sulphur occupies a position analogous to that of the creatinin: the smaller the amount of protein in the food, and the smaller therefore the total protein decomposed, the larger is the fraction which the neutral sulplur forms of the total sulphur. The neutral sulphur accorlingly represents endogenous metabolisn. The ethereal sulphur takes an intermediate position in this regard, but upon the whole it also becomes a more prominent fraction of the total sulphur when the food contains little or no protein. The ethereal sulphates are therefore not entirely derived from the putrefaction of protein.

Carbonates of sodium, ammonium, calcium, and magnesium occur in alkaline urine. Their source is the carbonates and the vegetable organic acids of the food. In acid urine a certain amount of carbon dioxide is present, although not firmly united with bases, so that most of it can be pumped out.

So-called Physico-Chemical Analysis of Urine.-The freezing-point of urine has often been determined to obtain a measure of the molecular concentration, which with the total quantity of urine secreted in a given time was erroneously assumed to afford an index of the work done by the kidney. Clinically the method is of little use, but for certain physiological questions freezing-point determinations are of value and are sometimes combined with determinations of the electrical conductivity, by which we obtain an approximate measure of the number of dissociated ions in unit volume, mainly the inorganic salts. Normally, $\Delta$ has a higher value for urine than for blood-i.e., the molecular concentration of the urine is higher than that of the serum. But when large clraughts of water are taken $\triangle$ may be lower for urine than for blood, and in general it varies within far wider limits (from 0.1 I $5^{\circ}$ to $2.546^{\circ} \mathrm{C}$, according to Köppe). The following table from Kövesi and Roth-Schulz shows the changes in $\Delta$ under the influence of water:

\begin{tabular}{|c|c|c|}
\hline Time. & Urine in C.C. & $\Delta$ \\
\hline Io to 2 & 240 & $I \cdot 80$ \\
\hline 2 to 6 & 255 & $1 \cdot 72$ \\
\hline 6 to 10 & I6I & I.93 \\
\hline Io to 2 & I3I & $2 \cdot 18$ \\
\hline 2 to 6 & 160 & $2 \cdot 23$ \\
\hline 6 to $I D$ & I 20 & $I \cdot 9 I$ \\
\hline II to I2 & I.S litres 'Salvator' water taken & - \\
\hline I 2 to 12.30 & 500 & $0 \cdot 12$ \\
\hline I 2.30 to I & 444 & $0 \cdot I I$ \\
\hline I to 1.30 & $44^{2}$ & $0 \cdot 10$ \\
\hline I. 30 to 2 & 46 & $0 \cdot 7 S$ \\
\hline 2 to 2.30 & 45 & $I \cdot 30$ \\
\hline
\end{tabular}

The Urine in Disease--Although, strictly speaking, a truly pathological urine has no place in physiology, the line which separates the urine of health from that of disease is often narrow, sometimes invisible; while the study of abnormal constituents is not only of great importance in practical medicine, but throws light upon the physiological processes 
talking place in the kiclney, and upon the general problems of metabolism. Even in health the ciantity of the urinc, its specific gravity, its acidity, maty vary within wide limits. A hot day nay increase the secretion of sweat, and correspondingly diminish the secretion of urine, and the deficiency of water may lead to a deposit of brick-red urates. A meal rich in frisit or vegetables may render the urine alkiline, and its alkalinity. may determine a precipitate of earthy phosphates. But neither the scanty acid urine with its sediment of urates, nor the alkaline urine with its sediment of phosphates, comes into the category of pathological urines; the deviation from the normal does not amount to discase. The maximum deviation from the line of health is the total suppression of the urine. If this lasts long, a train of symptoms, of which convulsions may be one of the most prominent, and which are grouped under the name of uræmia, appears. It length the patient becomes comatose, and death closes the scene. Suppression of urine may be the consequence of many pathological conditions, but there is one case on record in the human subject which, in effect, though not in intention, belongs to experimental physiology. A surgenn diagnosed a floating lidney in a woman. With a natural impatience of loose odds and ends of this sort, he offered to remore it, and in an evil hour the patient consented. The surgeon, a perfectly slilful man, who acted for the best, and to whom no blame whatever attached, carried the kidney to a well-known pathologist for examination. The latter, to the horror of the operator, suggested, from the appearance of the organ, that it was the only kidney the woman possessed. This turned out to be the fact. Not a drop of urine was passed. Apart from this ominous symptom, all went well for seven or eight days; but then uramic troubles came on, and the patient dicd on the eleventh or thirtcenth day after the operation. The necropsy showed that her only kidney. had been taken away.

In disease the urine may contain abnormal constituents, or ordinary constituents in abnormal amounts. Of the normal constituents which may be altered in quantity, the most important are the water, the inor. ganic salts, the urca, the uric acid, and the aromatic substances.

Water.-A marked and persistent diminution in the quantity of urine-that is to say, practically in the water, with or without an increase in the specific gravity-is suggestive of disorganization of the renal epithelium. In some infective diseases the kidney is liable to be secondarily involved, its secreting cells being perhaps crippled in the attempt to eliminate the bacterial poisons. In the form of parenchymatous or tubal nephritis which so frequently complicates scarlet fever, the quantity of urine has in some cases fallen to 50 or 60 c.c. in the tiventy-four hours.

In chronic interstitial nephritis ('granular kidney'), on the other hand, where the structural changes in the tubules are, for a long time at least, comparatively circumscribed, the quantity of urine is often increased and of low specific gravity. In chese cases the increase in the blood-pressure, associated with hypertrophy of the heart. may be a factor in the exaggerated renal secretion. In diabetes mellitus the quantity of urine is greatly increased, perhaps in some cases because more urea is excreted than normal, and urea acts as a diuretic, perhaps also because the elimination of sugar draws with it an increased excretion of water to hold it in solution. Although a specific gravity as low as I002 has been seen in healthy persons (after copious potations), the persistence of a density below IO IO should suggest hydruria. W'atson mentions the case of a boy with diabetes insipidus, who voided in twenty-four hours 9 or ro pints (5 to 6 litres) of urine with a specific gravity of roo2. On the other hand, while the specific gravity has been 
occasionally observed to mount in health to at leist 1036, its persistence at 1025 or 1030 (or anything above this, especially if the urine is pale and apparently dilute, should suggest diabetes mellitus.

Inorganic Salts.- The changes in the quantity of the inorganic constitucnts of the urine in clisease are not, in the present state of our knowledge, of as much importance as the changes in the organic constituents. The chlorides are diminished in most acute febrile discases and may even totally disappear from the urine, and their reappearance after the crisis is, so far as it goes, a favourable symptom. In most cases in which the quantity of the urine is markedly lessened, all the inorganic substances are diminished in amount.

Urea.-The quantity of urea is, as a rule, increased in fever, either absolutely or in proportion to the amount of nitrogen in the food. In the interstitial varieties of kidney disease the ura is usually not diminished, but when the stress of the change falls on the tubules (parenchynatous nephritis), it is distinctly decreased-it may be even to one-twentieth of the normal.

Uric acid is diminished in the urine in gout (perhaps to one-ninth of the normal), not only during the paroxysms, but in the intervals. It accumulates in the blood and tissues, and, as sodium urate, may form concretions in the joints, the cartilage of the ear, and other situations. Watson relates the case of a gentleman who used to avail himself of his resources in this respect by scoring the points at cards on the table with his chalky knuckles. In leukæmia the quantity of uric acid and purin bases in the urine is greatly increased, not only absolutely, but also in proportion to the urea. As much as $4 \frac{1}{2}$ grammes of free uric acid, in addition to about $\mathrm{I} \frac{1}{2}$ grammes of ammonium urate, has been found in a urinary sediment in a case of leukremia.

The aromatic bodies, of which indoxyl may be taken as the type, are increased when the conditions of disease favour the growth of bacteria in the intestine-e.g., in cholera, acute peritonitis, and carcinoma of the stomach. A marked increase in the amount of the indican in the urine may, as far as it goes, be taken as an indication that the bacteria are gaining the upper hand in the intestinal tract; a marked diminution is usually a sign that the battle has begun to turn in favour of the organism (Practical Exercises, p. 5I7). Tryptophane, a substance which we have already recognized among the products of the tryptic digestion of proteins, has been shown to be a precursor of indol, which is formed from it under the influence of bacteria. When tryptophane is injected into the cæcum of rabbits, the indican in the urine is markedly increased. Putrefactive processes in other parts of the body than the intestine may also increase the indican in the urinee.g., a collection of putrid pus in the pleural cavity.

Abnormal Substances in Urine.-Sugar, proteins, the pigments of bile and blood, or their derivatives, are the most important abnormal substances found in solution in the urine. Normal urinc, as has been stated, contains a trace of dextrose, but so little that it cannot be detected by ordinary tests, and for practical purposes it may be considered absent. Dextrose is the sugar found in the urine in diabetes. In the urine of nursing mothers lactose may be present. Pentoses, sugars with five carbon atoms in the molecule (instead of six, as in the hexoses, of which group dextrose is a member), may also occasionally occur in urine. Pentoses give the ordinary reduction tests for sugar, and yield osazones, but do not ferment with yeast. Various plants contain pentoses, and when these are eaten the pentoses are excreted in the urine, but in cases of true pentosuria they originate in the body, possibly from nusleo-proteins. The condition has not the same sinister significance as diabetes. Specific toxic substances produced by bac- 
terial action lave been iemonstrated in the mine in certain discases. Red blood-corpuscles and leucocytes (pus corpuscles, white bloodcorpuscles, mucous corpuscles) are the chief organized deposits; but spermatozoa may occasionally be found, as well as pathogenic bacteriac.g., the typloid bacillus; and in disease of the kidney casts of the renal tubules are not uncommon. These tube-casts may be composed clicily of red blood-corpuscles, or of leucocytes, or of the epithelium of the tubules, sometimes fattily degenerated, or of structureless protein, or of amyloid substance. Abnormal crystalline substances, such as the amino-acids, leucin (Fig. 186), tyrosin (Fig. 187), and cystin (Fig. I 80 ), may be on rare occasions found in urinary sediments; but generally the unorganized deposits of pathological urine consist of bodies actually contained in, or obtainable from, the normal secretion. but present in exeess, either absolutely, or relatively to the solvent power of the urine. Cystin is of interest because of its relations to the sulphur of the protein molecule $(\mathrm{p} .360)$. It is not found in the normal organism. It very occasionally forms calculi in the bladder. Tlere are individuals who constantly pass as much as one-fourth of all the sulphur in the form of cystin, without any other symptoms.

Various amino-acids are present in solution in the urine in many pathological conditions. Of these the least soluble are leucin and tyrosin, and this is the reason why they are most easily detected. A general reaction for amino-acids is their precipitation as sparingly soluble compounds ( $\beta$-naphthalinsulphones) by $\beta$-naphthalinsulphochloride in the presence of an alkali (sodium hydroxide). In acute yellow atrophy of the liver leucin and tyrosin have been found in large amounts in the liver itself, as well as in the urine. In phosphorus poisoning these amino-acids, as well as glycocoll, have been detected in the urine, and there is no doubt that other amino-acids, arising from the decomposition of proteins, are also present in such conditions.

Sugar.-In diabetes mellitus, although the quantity of urine is usually much increased, its specific gravity is above the normal; and this is duc chicfly to the presence of sugar (dextrose), which generally amounts to I to 5 per cent., but may in extreme cases reach ro or even I 5 per cent., more than half a kilogramme being sometimes given off in twentyfour hours.

The name of the tests for dextrose is legion. They are mostly founded on its reducing action in alkaline solution. Hydrated oxide of bismuth (Boettcher), salts of gold, platinum and silver, indigo (Mulder). and a host of other substances, are reducad by dextrose, and may be used to show its presence. The reduction of cupric salts (Trommer),

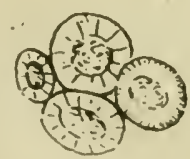

Fig. 186.-Leucin Crystals.

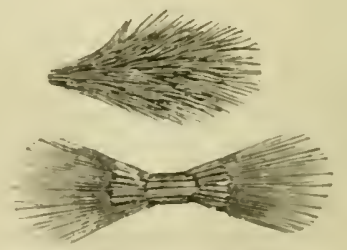

Fig. 187.-. Tyrosin Crystals.

fermentation by yeast, and the formation of crystals of plicnyl-glucosazone are the best established tests. (Sce Practical Exercises, p. 5 r 7.)

Proteins.-Serum-albumin and serum-globulin are the proteins most commonly found in pathological urine. Both are coagulated by heating the urine, sliglitly acidulated if it is not already acid, or by the addition 
of strungr nitric acid in the cold. Proteoses (albumoses) are also occasionally present, c.g., in the disease called 'osteomalicia' and in conditions associated with the formation and especially with the decomposition of pus. They may be recognized by the tests given in the Practical Exercises (p. 525). It is doubtful whether the presence of true peptone has as yet been satisfactorily made ont.

The presence of bile-salts may be slown by Hay's test or Pettenkofer's test (p. 402).

The pigments of blood and bile may be detccted by the characters described in treating of these substances; the spectrum of oxyhæmoglobin, or methæmoglobin, or any of the other derivatives of hæmoglobin, with the formation of hæmin crystals, would afford proof of the presence of the former, and Gmelin's test of the latter. The red blood-corpuscles, seen with the microscope, are the most decisive evidence of the presence of blood, as leucocytes in abundance are of the presence of pus. It should be remembered that pus in the urine of women has sometimes no significance except as showing that there has been an admixture of leucorrlical discharge from the vagina. (See Practical Exercises, pp. 74, 531.)

\section{Section II.-The Secretion of the Urine.}

We have now to consider the mechanism by which the urine is formed in the kidney from the materials brought to it by the blood. And here the same questions arise as have already been discussed in the case of the salivary and other digestive glands: (I) Are the urinary constituents, or any of them, present as such in the blood? (2) If they do exist in the blood, can they be shown to be separated from it by processes mainly physical or mainly 'vital '-in other words, by ordinary filtration, diffusion and osmosis, or by the selec. tive action of living cells? In the case of the digestive juices it has been seen that some constituents are already present in the blood, but that physical laws alone, so far as we at present understand them, cannot explain the proportions in which they occur in the secretions, or the conditions under which they are separated; while other constituents--and these the more specific and important -are manufactured in the gland-cells.

In the kidneys the conditions seem at first sight favourable to physical separation, as opposed to physiological secretion. Urine has been described as essentially a solution of urea and salts, and both are ready formed in the blood. The arrangement of the bloodvessels, too, suggests an apparatus for filtering under pressure.

Bloodvessels and Secreting Tubules of Kidney.-The renal artery splits up at the hilus into several branches, which pass in between the Malpighian pyranids, and form at the boundary of the cortex and medulla vascular arches, from which spring, on the one side, interlobular arteries running up into the cortex between the pyramids of Ferrein, and, on the other, vasa recta running down into the boundary layer of the medulla (Fig. I88). The interlobular arteries give off at intervals afferent vessels. Each of these soon breaks up into a glomerulus or tuit of vascular loops, which gather themselves up again into a single efferent vessel of somewhat smaller calibre than the afferent. The glomerulus is fitted into a cup or capsule (of Bowman), which is closely 


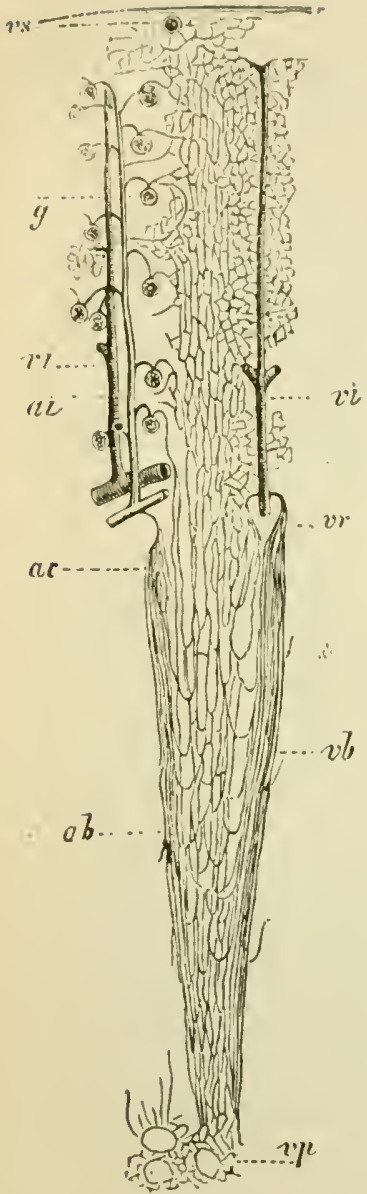

Fig. 188.-Diagram of Bloodressels of Kidney (Klein, after Ludwig). ai, interlobular artery; vi, interlobular vein; g. glomerulus, to which an afferent artery is seen coming from the interlobular artery, and from which an efferent artery proceeds to break ul into a capillary network surrounding the renal tubules; vs, vena stellata; ar, arterix recta; $v b$, leash of ven $x$ recta ; wp. vascular network round ducts at apex of a papilla.

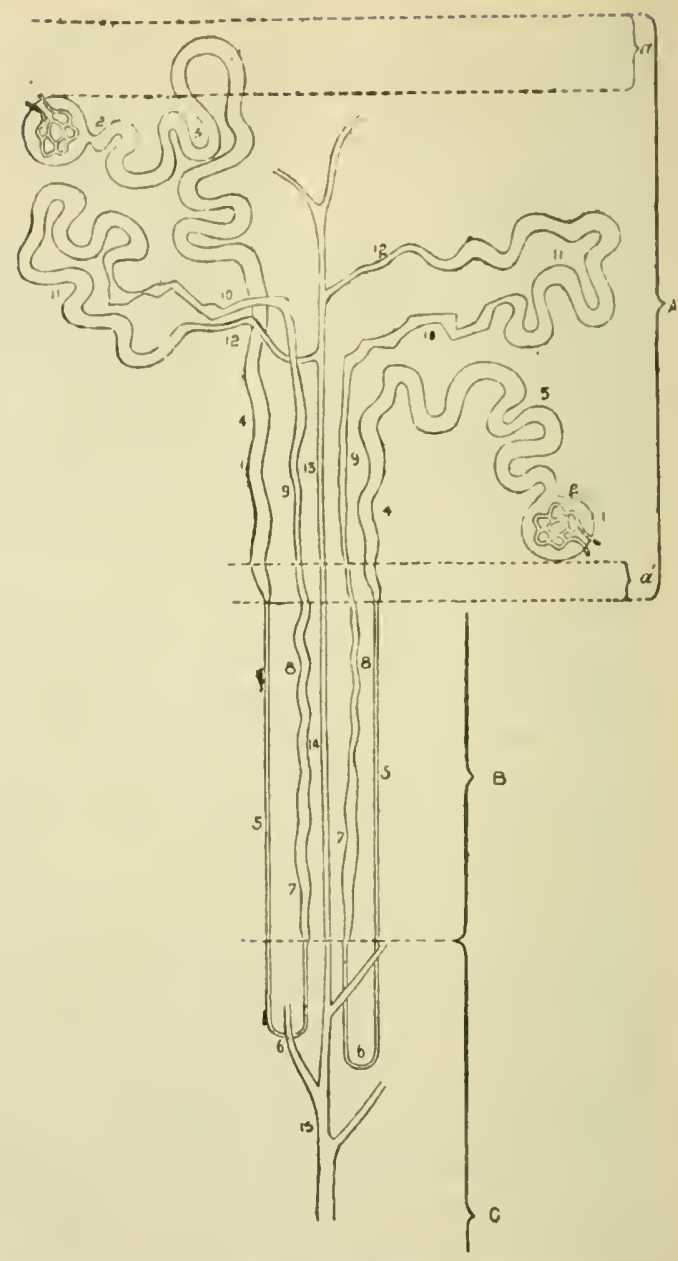

Fig. 189.-Diagram of Renal Tubule (Klein). A. cortex; $a$, layer of cortex immediately under capsule containing no Malpighian corpuscles; $a^{\prime}$, inner layer of cortex devoid of Malpighian corpuscles; B, boundary layer; C, papillary zone of medulla; 1, Bowman's capsule; 2 , neck of cap. sule; 3, proximal convoluted tubule; 4. spiral tubule; 5, dęscending part of Henle's looptubule; 6 , the loop; 7,8 , and 9 , ascending limb of loop-tubule; Io, irregular tubule; II, distal convoluted tubule; 12 , junctional tubule; I $_{3}$. collecting tubule in a medullary ray or pyra. mid of Ferrein; 14, collecting tubule in the boundary layer; 15. large collecting tubule ending in a duct of Bellini. 
reflected over it, except where the afferent and efferent vessels pass through, and forms the beginning of a urinary tubule. If we suppose the tuft prshed into the blind end of the tubule so as to indent it, it will be easily understood that the single layer of flattened 6 pithelinm reflected on the glomerulus is continuous with that lining the capsule, which in its turn is continuous with the epithelial layer of the rest of the urinary tubule. This lias been divided by histologists into a number of parts which it is mnecessary to enumerate here, further than to say that the urinary tubule proper begins in the cortex in Bowman's capsule and the proximal convoluted tubule (with its continuation, the spiral tubule), and ends in the cortex with the distal convoluted tubule, the connection between the two being made by a long loop (Henle's) with a descending and an ascending limb (Fig. I 89). Between the ascending limb and the distal convoluted tube is interposed the zigzag tubule. The tubule throughout its length is bounded by a bascment membrane lined by a single layer of epithelium, which differs in its character in different parts of the tubule

The distal convoluted tube joins by means of the short connecting tubule one of the straight tubules which form the pyramids of Ferrein or medullary rays in the cortex, and which run down into the medulla, always uniting into larger and larger tubes as they go, until at length they open as ducts of Ballini on the apex of a papilla. The two convoluted tubules (with the spiral and zigzag tubules) are lined by similar epithelial cells with gramular contents, and the tendency of the granules to be arranged in rows perpendicular to the basement membrane gives them a striated or 'rodded' appearance (Fig. I90). The granules are eosinophile (p. I 7), which is also a character of the granules of other secreting cells. Towards the lumen the cells may show a brush of processes, looking like cilia, but in mammals these are not motile. The ascending part of Henle's loop also has cells of the same general character, with numerous granules, although the ' rodding' may not be so distinct. We shall see directly that the morphological resemblance is the index of a function.l likeness. The blood-supply of the tubules, especially of the convoluted portions, is exceedingly rich, the efferent vessels of the glomeruli breaking up around them into a close-meshed network of capillaries, from which the blood is collected into interlobular veins running parallel to the interlobular arteries between the pyramids of Ferrein. The straight tubules of the medulla are also surrounded by capillaries given off from straight arteries (arteriæ rectx) running down into it partly from the arterial arches and partly from efferent vessels of the glomeruli nearest the boundary layer, the blood passing away by straight veins (vena rectæ) which join the larger veins accompanying the arterial arches. The greater part of the blood going through the kidney has to pass through two sets of capillaries, one in the glomeruli, the other around the tubules. Even the portion of it which does not go through the glomeruli has for the most part a long route to traverse in narrow arterioles and venules to and from its capillary distribution. And the mean circulation-time through the kidncy has been found to be longer than that through most other organs (p. 137).

Theories of Renal Secretion.--To come back to our problem of the nature of renal secretion, the anatomical structure of the kidney might be expected to throw light upon the question. And, indeed, it was on a purely histological foundation that Bowman established his famous 'vital' theory of renal secretion. Impressed with the 
resemblance between the renal epithelium and the epithelial cells of other glands, and with the distribution of the bloodvessels in the kidney, he came to the conclusion that the characteristic constituents of urine, including urea, were secreted from the blood by the tubules. To the Malpighian bodies he assigned what lie doubtless considered the humbler office of separating water from the blood for the solution of the all-important solids. To Ludwig, on the other hand, with his whole attention fastened on the mechanical factors by which the flow of urine could be influenced, the tubules

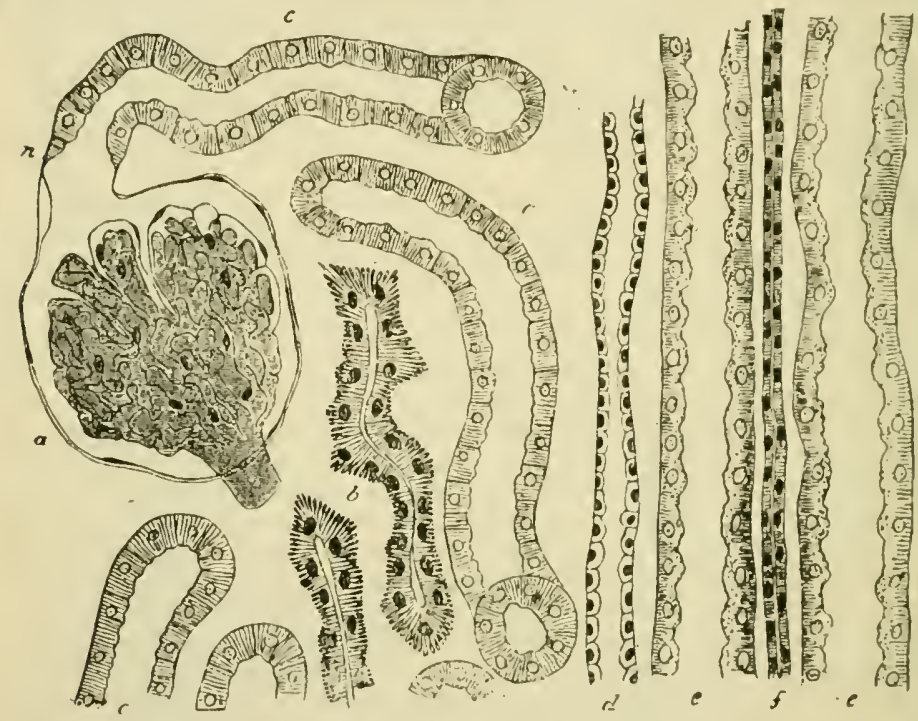

Fig. 190.-From a Vertical Section of Dog's Kinlney to show the Structure of Different

Portions of the Renal Tubule (Ḱlein). a, Bowman's capsule enclosing glomerulus, the capillaries of which are arranged in lobules separated by a little connective tissue. The capsule and glomerulus together constitute a Malpighian body or corpuscle; $n$, neck of capsule; $c, c$, convoluted tubules, cut in various directions; $b$, irregular or zigzag tubule; $\boldsymbol{d}, e$, and $f$ are straight tubules, which take part in the formation of a medullary ray or pyramid of Ferrein; $d$. collecting tubule; $e, e$, spiral tubule; $f$, narrow part of ascending limb of Henle's loop-tubule; $b, c$, and $e$ are lined with rodded epithelium.

seemed of secondary importance, while the glomeruli appeared a complete apparatus for filtering urine from the blood into Bowman's capsule. He saw that the efferent vessel was smaller than the afferent; that it was therefore easier for blood to come to the glomerulus than to get away from it, and that the pressure in the capillaries of the tuft must be higher than in ordinary capillaries, because the resistance beyond them in the comparatively narrow efferent vessel, and especially in the second plexus, is greater than the resistance beyond a single capillary network. And experimental investigation soon showed him that the rate at which urine 
was formed could be greatly influenced by clanges in the bloodpressure.

On such considerations, Ludwig founded the ' mechanical ' theory of urinary secretion, which, altlough in a much modified form, still divides with the 'vital' theory the allegiance of pliysiologists. It is impossible here to enter in detail into a controversy that has extended over more than half a century and produced an extensive literature. The result of the discussion has been, in our opinion, to establish in its essential principles the 'vital ' theory of Bowman, or at least to show that no purely physico-chemical theory as yet constructed will account for all the facts.

Ludwig supposed that the wrine, qualitatively complete in all its constituents, was simply filtered through the glomeruli, the work lone in this filtration being performed entirely at the expense of the energy of the heart-beat represented as lateral pressure in the vessels of the tufts. But as the proportion of salts, and especially of urea, is very far from being the same in urine as in blood, it had further to be assumed that the liquid which passes into Bowman's capsule is exceedingly dilute, and that absorption of water, and perhaps of other constituents, takes place in its passage along the renal tubules. This process of reabsorption he pictured as a purely physical diffusion between the dilute urine in contact with the free ends of the epithelial cells lining the tubules and the much more concentrated lymph with which their deep ends are bathed. The great length of these tubules, as compared with those of most other glands, might indeed seem to indicate a long sojourn of the urine in them, and the probability of important changes being caused in its passage along them. But if we consider the immense length (60 to $70 \mathrm{~cm}$.) of the seminal tubules and of their gigantic ducts (epididymis 6 metres), where, of course, absorption of water on a large scale is out of the question, it will be granted that little can be built upon the mere length of the renal tubules. On the other hand, the salivary glands, where there are no glomeruli, secrete as much water as the kidneys are supposed to filter; and the pancreas, whose capillaries form the first of a double set, and therefore in this respect correspond to the renal glomeruli, secretes less water than the liver, whose capillaries correspond to the low-pressure plexus around the convoluted tubules of the kidney. So that deductions drawn from the anatomical relations of the bloodvessels are not in this case of much value, unless supported by physiological results.

It is somewhat unfortunate that systematic writers have fallen into the habit of discussing the mechanism of urinary secretion as if the Ludwig theory and the Bowman theory presented an exact antithesis, as if the one offered a complete ' mechanical ' explanation of a process, which the other viewed as entirely 'vital,' and therefore withdrawn from physical explanation.

IVe need not concern ourselves here with the historical develop- 
ment of this discussion. Three main questions require our attention:

I. Is there any evidence that reabsorption actually occurs in the tubules? If reabsorption on an important scale does take place, it follows at once that there must be a difference of function between the two parts of the renal apparatus, through which urinary constituents pass in opposite directions.

2. But if there is no reabsorption, or none of importance, it may still be asked whether, the direction of movement of the urinary constituents through the glomeruli and the tubular epithelium being the same, some quantitative or qualitative difference in their activity may not exist, certain constituents, e.g., passing mainly or exclusively through the one or the other.

3. When these questions have been settled, we are in a position to consider the nature of the process by which the urinary constituents find their way from the blood into the lumen of the capsules and the tubules, or, if there is reabsorption, out of the tubules into the lymph and blood again, and to see whether or no -it can be entirely explained on mechanical and physico-chemical principles.

The Question of Reabsorption from the Tubules.-That some absorption can take place from the kidney when the pressure in the ureter is abnormally raised need not be doubted, and when substances like potassium iodide or strychnine are introduced into the ureter or the pelvis of the kidney under these circumstances, they can speedily be detected in the blood. When the ureter pressure (in dogs) is only slightly increased, instead of evidence of reabsorption, we obtain evidence of increased secretion. The volume of urine, the total quantity of sulphate in the urine when sodium sulphate is injected into the blood as a diuretic, and the total amount of reducing sugar when phlorhizin is injected, are all greater on the obstructed than on the normal side. These facts are quite opposed to the idea that filtration and reabsorption are important factors in the preparation of normal urine (Brodie and Cullis). Changes in the blood-flow through the kidney have nothing to do with the results, since the small increase in pressure in the ureter was shown not to affect the rate of flow of the blood. The attempt has been made to decide whether absorption normally ocrurs by removing as much of the tubules as possible, and seeing whether the character of the urine is altered. In rabbits the whole or a large portion of the medulla has been excised from one kidney and the other then extirpated. From the mutilated kidney two or three times as much urine was said to flow as was secreted by a control rabbit operated on in the same way, except for the removal of the renal medulla (Ribbert). The conclusion was drawn that the greater quantity of urine escaping was due to the smaller opportunity for reabsorption of the water. But experiments men- 
tioned in Chapter XI. suggest a different interpretation of these observations. And Boyd, who repeated Ribbert's work, obtained quite - lifferent results after partial removal of the medulla. He found it impossible to remove the whole. So that hitherto the direct method of eliminating the tubules has left the matter where it was.

Some light has been tlırown on this question, by taking advantage of the anatomical fact that the kidney of batrachians, and, indeed, that of fishes and ophidia as well, has a double blood-supply. The renal artery gives off afferent vessels to the glomeruli; the vena advehens, or renal portal vein, breaks up, like the portal vein in the liver, into a plexus of capillaries surrounding the tubules, and there seems to be no communication between the two vascular systems.

By tying all the arteries going to the kidneys in frogs the circulation through the glomeruli can be completely cut off, while ligation of the renal portal vein does not affect the blood-supply of the glomeruli, though markedly interfering with that of the tubules. Gurwitsch has found that, after ligation of the renal portal vein of one kidney in (male) frogs, the flow of urine from that kidney is much diminished as compared with the other. He argues that if reabsorption of dilute urine filtered through the glomeruli takes place in the tubules, the opposite result ought to be obtained, since the glomeruli are not affected, while any absorptive power of the tubules must be crippled or abolished.

Experiments on the Excretion of Pigments by the Kidney.-In connection with the second question, and also incidentally with the first, the results of experiments on the distribution of pigments in

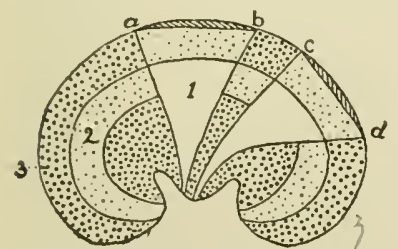

Fig. I9r.-Diagram of Distribution of Pigment in Kidney after Injection into Blood. The cortex between $a$ and $b$ and be. tween $c$ and $d$ was cauterized before the injection. In the blank wedge-shaped portions, I, there was no pigment. In the zones shaded like 2 there was some pigment, but no so much as in the areas shaded like 3 .

the kidney after their injection into the blood have often been appealed to. Heidenhain injected indigo-carmine into the blood of rabbits, and after a variable time killed them, cut out the kidneys, and flushed them with alcohol, in which the pigment is insoluble. His results were as follows: (I) When the spinal cord was cut before the injection in order to reduce the bloodpressure, the blue granules were found in the 'rodded' epithelium of the convoluted tubules and the ascending limb of Henle's loop, and in the lumen of the tubules, but nowhere else. Bowman's capsules contained no pigment. The renal cortex was coloured

blue. (2) When the spinal cord was not cut, the pigment was found in the medulla and pelvis of the kidney, as well as in the cortex, but always in the lumen of the tubules, and not in the epithelium, except in the situations mentioned.

(3) If a portion of the cortex 
of the kidney had been cauterized with nitrate of silver before injection of the pigment, the spinal cord being left intact, a wedge of the renal substance, corresponding to this area, remained coloured only in the cortex, although the rest was blue in the medulla also. The 'rodded' epithelium was filled with blue granules as before (Fig. I9I).

(I) shows that the epithelimm is capable of excreting some substances at least. (2) appears to show that when the blood-pressure is normal water is poured out from some part of the tubule, and washes the pigment separated by the 'rodded ' cpithelium down towards the papille. (3) suggests that it is through the glomeruli that most of the water passes. For cauterization has not destroyed the power of the epithelium to excrete pigment. and therefore, presumably, would not have destroyed its power to excrete water if it possessed this power in any great degree; and the glomeruli and their capsules are the only other part of the renal mechanism which can have been affected. It must be carefully noted that these experiments do not prove that urea is secreted by the tubular epithelium. Indeed, after section of the cord no accumulation of urea takes place in the kidney (Cushny). It would be equally crroneous to conclude from this that the cells of the tubules do not secrete urea. For the reduction of the blood-supply may have rendered them incapable of doing so.

When pigments are injected into the dorsal lymph-sac of a frog without interference with the renal circulation, they are found plentifully in the lumen of the convoluted tubules, and also in the epithelial cells lining them. The suggestion has been made that the pigments have been absorbed by the cells from the lumen, and not excreted by them into it. And certainly pigments soluble in the cytoplasm or in the substances that form the envelopes of cells, and therefore capable, like methylene blue, of staining them during life, might be taken up by the renal epithelium if excreted into the tubules by the glomeruli, and might cause staining of them, particularly, of course, of the free ends of the cells next the lumen. But this suggestion is inadmissible, since, on injection of the same pigments after ligation of the renal portal vein, the convoluted tubules contain little or no pigment in their lumen. And when the urinary flow is stopped on one side in mammals by teinporary compression of the renal ariery, the corresponding kidney takes up fully as much carmine as its fellow (Carter). There is no doubt that not only pigments capable of ' vital staining,' like methylene blue, but also pigments which do not stain living cells, are taken up from the blood (or lymph) by the epithelial cells, and, lying in vacuoles in their cytoplasm, are transported towards the lumen, and there extruded. It is not the solubility of the pigments in lipoids, and therefore their solubility in the supposed lipoid envelope of the cells, which determines whether they shall be excreted. The degree in 
which they are capable of being presented to the cells in non-colloid solution appears to solle extent to be a determining factor. The pigments not taken up are lighly colloidal (Gurwitsch, Höber). Shafer has recently confirmed Heidenluain's statements as to the place of excretion of indigo-carmine. When leuco-indigo-carmine (a colourless reduction-product of indigo-carmine) was injected, the blue oxidized substance was found in the lumen of the convoluted tubules and in the collecting tubules, but not at all in the Bowman's capsule. The cells of the convoluted tubules were colourless, because they kept the pigment in its reduced condition, and it only became oxidized in the lumina of those parts of the tubules whose contents, according to Dreser, show an acid reaction. On oxirlation by peroxide of hydrogen the cells of the convoluted tubules became faintly green, but the Bowman's capsule remained colourless. This can only be explained on the assumption that the leuco-product of the pigment was excreted by the cells of the convoluted tubules.

But these cells are far from taking up all pigments indifferently. Some pigments are extruded mainly by one part, others mainly by another part, of the renal tubule, and some even by the glomeruli, as shown long ago for ammonium carminate. The glomeruli, however, are in general far less active in this regard than the epithelial cells, and the fact that the latter pick out from the blood such substances as these foreign pigments, which pass through the Malpighian tufts unchallenged, renders it likely that the tubules also exercise a special function in the secretion of the normal constituents of urine. More direct evidence of this is not wanting, for Bowman saw crystals of uric acid in the epithelium of the convoluted tubules of birds. Heidenhain found that urate of soda injected into the blood of a rabbit is excreted by the epithelium of the convoluted tubules and the ascending part of Henle's loop, just as is the case with indigo-carmine. And Nussbaum's experiments, although not quite conclusive, have made it probable that in the frog urea is actually separated by the epithelium of the tubules. They were founded on the anatomical peculiarity in the renal circulation of the frog already mentioned. By tying the renal arteries in that animal, he thought he could at will stop the circulation in the glomeruli, and he found that after this was done there was no further spontaneous secretion of urine. But when urea was injected intravenously the secretion of urine again began, urea being eliminated by the kidneys, and water along with it. Sugar, peptone, and egg-albumin, injected into the blood, no longer passed into the urine, even when the secretion was excited by simultaneous injection of urea, although they readily did so when the arteries were not tied. He concluded that the Malpighian corpuscles have the power of excreting water, sugar, peptone, and albumin, while the epithelium of the tubules excretes urea as well as water.

Beddard has confirmed Nussbaum's statement that when all the 
arteries going to the kidney are tied the glomeruli are completely and permanently deprived of blood. The spontaneous secretion of urine is totally stopped, as Nussbaum found, but only in threc experiments out of eighteen was it possible to start the secretion by injection of urea. The epithelium of the tubules degenerated and desquamated after complete ligation of all the renal arteries, showing that it requires some arterial blood as well as the venous blood from the renal portal to maintain its vitality. The degeneration of the epithelium can be prevented by keeping the frogs in an atmosphere of oxygen after ligation of the arteries. In six such frogs, in wrich the complete elimination of the glomeruli was controlled by subsequent injection, secretion of urine followed the injection of urea, alone or in combination with dextrose, phlorhizin, or di-sodium hydrogen phosphate $\left(\mathrm{Na}_{2} \mathrm{HPO}_{4}\right)$. In all the cases the urine contained urea, chlorides, and sulphates, and was acid to phenolphthalein. In one case after injection of urea and dextrose, and in another after urea and phlorhizin, the urine reduced Fehling's solution, and therefore presumably contained dextrose (Beddard and Bainbridge). When the frog's kidney is perfused in situ with oxygenated salt solution a certain flow of urine takes place. Substitution of non-oxygenated saline markedly slows the flow (Cullis).

Apparently, then, the tubules have the capacity to secrete practically all the constituents of urme, and when the flow of urine is small, probably most of it comes from the tubules. When, as in the diuresis produced by salt solutions, large quantities of water and salts have to be rapidly excreted, the bulk of the liquid comes from the glomeruli, but also by a process of secretion.

Lindemann has endeavoured to exclude the glomeruli in mammals by injecting oil through the renal artery. After a short time, according to him, the oil emboli clear away from practically all parts of the kidney except the glomeruli, which remain plugged. If indigo-carmine be subsequently injected into the blood, it is not only taken up from it by the embolized kidney as well as by a normal one, but is excreted. The quantity of urine is much diminished, and its specific gravity increased, but its composition is not essentially altered. He infers that the tubules are in a high degree independent of the glomeruli as an apparatus for the secretion of urine. Iore conclusive observations have lately been reported in which the tubules were eliminated by producing an artificial nephritis in rabbits by the subcutaneous injection of sodium tartrate. Tartrates act mainly upon the tubules, causing no, or a much smaller, effect upon the glomeruli. After the intravenous infusion of a solution containing sodium chloride and urea during pronouncerl tartrate nepluritis, all the chlorine appears in the urine within fortyeight hours, but little, if any, of the urea. In the light of the histological findings, this is interpreted to mean that under normal conditions chlorides and water are passed through the glomerular 
mechanism, and urea through the convoluted tubules (Underhill, Wills, and coldschmidt). These results constitute a direct and striking confrmation of the Bowman hypothesis.

$A$ s regards our first two questions, we may conclude that there is no good eitidence that reabsorption of zeater or other constituents of the urine in the renal tubules plays an important part in the preparation of that secretion. Many facts farour the conclusion that the glomeruli and the renal epithelium act as distinct, although, of course, mutually supplementary mechanisms, the glomeruli separating the larger portion of the water and salts, the epithelium the larger portion, if not the whole, of the characteristic organic constituents.

As regards the third question, it is now generally admitted, even by those who uphold a modified 'mechanical' theory, that if the urine is originally separated from the blood by filtration at the expense of the energy of the heart-beat represented by the pressure of the blood in the glomeruli, the reabsorption in the tubules cannot be attributed to simple diffusion, but must be a selective process analogous to absorption in the intestine and entailing the expenditure of a large amount of work at the expense of the food materials or the protoplasm of the epithelial cells. Every attempt at a strictly mechanical explanation breaks down for the kidney, as for other glands.

The practical absence from urine of the proteins and sugar of the blood under normal circumstances, and the elimination by the kidney of egg-albumin, peptone, and other bodies when injected into the veins, show a selective permeability inexplicable by reference to any known structural or physico-chemical property of the renal epithelium or the glomeruli, but precisely the kind of thing which the physiologist has, without being hitherto able to explain it, learnt to associate with the activity of living cells. Urea and dextrose, both highly diffusible substances, circulate side by side in the bloodvessels of the kidney. The one is taken and the other left. The urea is a waste-product of no further use in the economy. The sugar is a valuable food-substance. The kidney selects with unerring certainty the urea, of which only 4 parts in ro,ooo are present in the blood, but rejects the sugar, of which there is three times as much. The theory that the dextrose of the blood or a part of it is combined with substances in the colloid state, and not in ordinary solution, has been advanced from time to time as an explanation of the practical impermeability of the kidney for this sugar under normal conditions. But no proof of the truth of this hypothesis has ever been given. On the contrary, there is good evidence that all the dextrose which is estimated in blood by analytical methods is in the free condition. For instance, dextrose easily escapes from blood circulating in the vivi-diffusion apparatus previously described (p. 48). And when the plasma of shed blood is placed in a dialyser tube of animal membrane surrounded by a liquid 
in which dextrose is dissolved in exactly the same concentration as that determined in the plasma by the ordinary chemical methods, the contents of the dialyser neither lose nor gain dextrose. Now, the plasma ought to gain sugar by diffusion if a portion of the dextrose in it exists in a combination which prevents its diffusion, just as it does gain dextrose when the liquid outside the dialyser contains sugar in greater concentration than the plasma (Michaelis and Rona).

Egg-albumin injected into the blood passes through the renal circulation side by side with the serum-albumin of the plasma. Both are indiffusible through membranes, and to the physical chemist the differences between them may appear superficial and minute. But the kidney does not hesitate for an instant. A large part of the egg-albumin is promptly excreted as a foreign substance; the serum-albumin passes on untouched.

Not only does the kidney exercise a power of qualitative selec. tion; it also takes cognizance of the quantitative composition of the blood. So long as there is less sugar in the plasma than about $I \cdot 5$ to 2 parts in $I, 000$, it is refused passage into the renal tubules. But when this limit is passed, and the proportion of sugar in the blood becomes excessive, the kidney begins to excrete sugar, and continues to do so till the balance is redressed.

The advocates of the theory of filtration through the glomeruli under the influence of the difference of hydrostatic pressure in the capillaries and in the lumen of the capsules have made their firmest stand on the excretion of the inorganic constituents of the urine. They have laid stress particularly on the fact that the hydræmic plethora caused by intravenous injection of salts is accompanied by diuresis. It is true that the direct introduction of water into the blood, or its attraction from the lymph-spaces when the osmotic pressure of the blood is increased by the injection of substances like urea, sugar, and sodium chloride, may cause a condition of hydramic plethora, and that this plethora may sometimes be associated with an increase of pressure in the capillaries in general, and therefore in the vessels of the Malpighian tuft. It may also be admitted that such an increase of pressure might be accompanied by an increased filtration of water and salts into Bowman's capsule. Even in the excised kidney, after the vital activity of its cells may be presumed to have ceased, filtration of the most varied solutions occurs when the organ is perfused with them through the renal artery. The liquid which escapes from the ureter always has the same composition as the perfusion fluid (Sollmann). It would certainly appear unlikely that the glomerular epithelium should make no use whatever for the furtherance of its task of the difference of hydrostatic pressure on its two surfaces. It is in taking advantage of such circumsta ces for the promotion of its specific work up to the point at which they cease to favour it that a great part of the true 
secretory activity of cells may be supposed to consist. When we see a barge passing through a lock, and being gradually lifted to the proper level by the inrush of water, we never dream of saying that the whole thing is an affair of the laws of hydrostatics. IVe know that the part played by the lock-keeper, the opening and closing of the gates and sluices at the proper time, is all-important, although he does not lighten by one ounce the weight which the water must lift. He uses the head of water for a specific purpose -namely, to lift the barge. In like manner it is to be expected that the glomerular epithelium, when the difference of pressure on its two surfaces is increased by hydræmic plethora, will use the increased facility of filtration to rapidly excrete a portion of the water. But who will believe that the addition of a tumbler of water, absorbed from the alimentary canal, to $f$ or 5 litres of blood circulating in a system of vessels whose capacity can and does vary within wide limits, should cause in the capillaries of the kidney an increase of pressure exactly proportional to the increase in the elimination of water in the urine, lasting for the same time and disappearing at the moment when the normal composition of the blood is restored? Nor is it easier to explain on any mechanical hypothesis how it is that in a starving animal the quantity of inorganic substances eliminated in the urine drops almost to zero, while the proportional amount in the blood and tissues is little, if at all, affected. In a rabbit rendered poor in sodium chloride by feeding it with salt-free food, the injection of a solution of sodium chloride isotonic with the blood produces no diuresis for a considerable time, but, on the contrary, a diminished flow of urine, while a similar solution injected into the veins of a rabbit previously fed with salted food causes an immediate and considerable diuresis. When small quantities of isotonic solutions of various salts are injected, those not normally present in the blood produce a greater diuresis than normal constituents. Sodium chloride, which is present in normal plasma in greater amount than any other salt, causes the smallest diuresis of all (Haake and Spiro).

Such facts suggest that the secreting cells of the kidney are stimulated or inhibited by the contact of blood or lymph in which the normal constituents are present in too great or in too small amount, and that the intensity of the action is proportional to the degree of deficiency or excess. The greater the velocity of the circulation in the kidney, the more effective will be the stimulation produced by any given substance present in excess, and therefore the greater the total amount of it eliminated in a given time. For in making the round of the renal circulation the concentration of the substance in any given portion of blood will fall less, and therefore the average stimulation exerted by it during the round will be greater the faster the blood flows. It is quite in agreement with this that when plethora is occasioned by transfusion of blood there is little 
or no cliuresis, although the increase of artejial, capillary, anr? venous presirure, and the dilatation of the kidney, are evident. For the rapid passage of liquid out of the versels would lead to at great increase in the relative propurtion of cor puscles to plasmathat is to say, to an abnormal condition of the blood. On the other hand, when plethora is produced by injection of serum diuresis occurs (Cushny). This, again, is what we should expect, since the elimination of the superfluous liquid will restore the normal proportion. The diminished viscosity of the blood (p. 23) produced by the excess of serum will aid the flow through the lidncy and therefore increase the diuresis, while in the case of the plethora produced by injection of blood the elimination of liquid will at once increase the viscosity, diminish the velocity of the renal flow, and tend to lessen diuresis.

There is, then, little more reason to assume that the copious flow of urine which follows the absorption of a large quantity of water is due to a mere process of filtration than there is to believe that filtration, and not selective secretion, is the cause of the gush of saliva which precedes vomiting, or the sudden outburst of sweat on sudden and severe exertion. In addition, there are the positive proofs already mentioned that the 'rodded' epithelium of the tubules, which no one supposes to be abandoned more to mere physical influences than the epithelium of the salivary glands, plays a part in the secretion of some of the urinary constituents.

Cushny has recently stated more clearly than had previously been done a theory which he designates as the 'modern theory' of urine formation. He assumes that blood-plasma is filtered through the glomeruli under the hydrostatic pressure of the blood, only the colloid proteins being kept back. The filtrate contains the non-colloid constituents approximately in the proportions in which they exist in plasma. It is therefore very poor in urea and very rich in sugar as compared with urine. In the tubules some of the constituents, which he terms 'thresholr bodies' are reabsorbed. These are the substances like sugar, the sodium and chlorine ions, etc., which are only excreted when they exceed a certain threshold value in the plasma. Other constituents of the filtrate, like urea, are not reabsorbed. These are called 'no-threshold bodies,' and are excreted in proportion to their absolute amount in the plasma. The cells of the tubules are supposed in some way at present unknown to take up from the filtrate the threshold bodies, and alway's in a definite concentration-namely, that in which they normally exist in blood. As Cushny puts it, the filtrate is deproteinized plasma, from which 'Locke's fluid' (p. 66) is reabsorbed by the tuluule cells. Apparently he thinks it simpler to make the assumption that the renal cells are organized to absorb from the lumen of the tubules a solution of invariable composition, leaving a variable residue to be excreted, than to make the assumption, underlying the Bowman-Heidenhain theory, that they are organized to leave behind in the blood or lympl an invariable resiclue by absorbing from them a solution of variable composition. In reality, however, the two assumptions are precisely on the same footing: they are equally 
simple or equally abstruse. For a cell which is able to take up 'Locke's fluid' from a deproteinized plasma is able in that very act to reject any constituent which does not belong to 'Locke's fluid, as well as an excess of any constituent which does belong to it. What is rejected and its amount are quite as important, if the final product is to lic constant, as what is accepted and its amount. If we are willing to attribute a power of this kind to the free end of a tubule cell we need not slurink on the ground of added complexity from investing the attached end of the cell with the power of refusing passage to 'Locke's fluid' from the plasma or the lymph while accepting crystalloid constituents like urea which do not belong to it, as well as an excess of any constituent which does belong to it. There is nothing more 'occult' abuut a ccll which bars out soclinm chloride till it exceeds 0.9 per cent. in the fluid offered to it, and then lets the surplus through, than there is about a cell which takes up sodium chloride from the fluid offered to it till it has amassed a concentration of 0.9 per cent., and then bars out the surplus.* In like manner, the financial organization required by a Government to take from a citizen in taxes the surplus of his income above one hundred pounds would be of the same general nature as that required to take from him his whole income, returning him one hundred pounds to live on. If a machine could manage the one operation, a mandarin would not be needed for the other.

It is impossible in this place to go further into the discussion of the reabsorption theory so ably presented by Cushny. In the absence of definite evidence of reabsorption on the great scale required by the theory, it remains simply a working hypothesis, which is all that can be claimed at present for any theory of urine formation. One of the objections always urged against filtration (with reabsorption) theories has been the enormous amount of liquid which must under certain conditions be poured into the renal tubules. Heidenhain calculated that in a man no less than $7^{\circ}$ litres of liquid per day must be filtered through the glomeruli in order that the urea found in the urine may be obtained from a glomerular filtrate containing 0.05 per cent. of urea. This is probably a moderate estimate, and considerably larger amounts of filtrate in proportion to body and kidney weight have been deduced from data obtained in animals. It has been argued by advocates of the reabsorption theory that equally great quantities of lymph containing urea in the small concentration in which it exists in the plasma must be poured out around the attached ends of the tubule cells on the hypothesis of direct secretion. This argument, however, is based upon an erroneous conception of the manner in which exchange between the blood and the tissues proceeds. There is no reason to suppose

* The reabsorption theory is perhaps inferior to the 'direct secretion' theory in one point. If a solution of constant composition is alway's absorbed from the glomerular filtrate, the smaller the concentration in the filtrate of any substance capable of being taken up by the cells, the greater will be the proportion of it absorbed; and the greater its concentration in the filtrate, the smaller will be the proportion of it absorbed. On the direct secretion theory, the greater the concentration of a substance in the blood or lymph in contact with the cells, the more of it will pass into the cells and be excreted by them. The 'modern' theory which sets out by saying to the Bowman-Heidenhain theory, 'Stand by thyself, come not near to me, for I am more physical than thou,' thus abandons a physical process, diffusion, which its rival utilizes. The fact is that the simplification attained by postulating filtration as the first stage in urine formation has to be paid for in the reabsorption. The boat having shot the rapids in the glomeruli with next to no physiological expense has straightway to be more or less painfully locked a certain distance upstream. 
that the exchange between blood and tissue lymph is mainly accom. plished by filtration from the capillaries of vast quantities of liquid containing the crystalloid constituents and the gases in the concentration in which they exist in plasma. On the contrary, the dissolved substances are currently and no doubt correctly assumed to move to a great extent by diffusion through the capillary walls (perhaps with a certain amount of active intervention of the endothelial cells) and across the thin sheets of tissue lympl on their way to and from the cells. In other words, they move mainly through the water and not with the water. An attempt to explain the gaseous exchange between the blood and the tissues as a matter purely of filtration of plasma containing dissolved gases, and not at all of diffusion of the gases, would lead to curious results. There is no more reason to believe that urea passes from the blood to the boundary of the tubule cells by a filtration process independent of diffusion, and therefore entailing the irrigation of the cells with a very large amount of lymph, than there is to believe that when 100 c.c. of arterial blood loses ro c.c. of oxygen in passing through the capillaries, this is accomplished by filtration into the lymph spaces of 4,000 c.c. of plasma containing 0.25 C.C. of oxygen in 100 c.c. (p. 252).

As to the nature of the mechanism set in motion, and the series of events that take place as the constituents of the urine journey from the interior of the bloodvessels to the lumen of the tubules, we know no more than in the case of other glands. This alone is clear, that the separation of the urine from the blood implies the performance of a large amount of work by the kidney. A token of the intensity of the metabolic effort required is the marked increase in the absorption of oxygen which occurs during diuresis. In one experiment the oxygen absorbed by a dog's kidneys was I I per cent. of what would have been used up by the entire animal under normal conditions.

The mere fact that urine differs in its quantitative composition from blood-plasma is sufficient to show that work must be done in its separation from the blood. Although the amount of work cannot be calculated from the difference in the osmotic pressures of the two liquids, a comparison of the freezing-points affords qualitative evidence of the performance of work by the kidney. For average urine, the value of $\Delta$ is several times as great as for the plasma. Blood-plasma freczes at $-0.55^{\circ}$ to $-0.05^{\circ} \mathrm{C}$. (average, $-0.6^{\circ}$, corresponding to an osmotic pressure of $5,662 \mathrm{~mm}$. of mercury, or about 75 metres of water). Human urine has been found to freeze at $-\mathrm{I} \cdot 38^{\circ}$ to $-2 \cdot \mathrm{II}^{\circ} \mathrm{C}$. (average, - I. $\mathrm{S}^{\circ} \mathrm{C}$., corresponding to an osmotic pressure of about $17,000 \mathrm{~mm}$. of mercury, or 225 metres of water). For highly concentrated urines, the depression of the freezing-point may be considerably greater. Even when the freezing-point is found in very dilute urines to be approximately the same as in the blood-plasina, work may still have been done in the separation of the urine, because although the total molecular concentration may be the same in the two liquids, the concentration of each of the substances in solution may be different. For 
example, even in the most dilute urine, the concentration of urea will in general be much greater than in the blood. It is of interest in connection with the work performed by the kidney that when the flow of urine is increased by diuretics like caffeine or sodium sulphate, which cause the secretion of urine with a very different crystalloid composition from that of the plasma, more oxygen is used up, whereas the diuresis caused by the injection of Ringer's solution where the urine and plasma do not differ materially in the amount or kind of the non-protein constituents, is accomplished practically without change in the oxygen consumption of the kidney.

Significance of the Glomeruli.-What is the significance of the peculiar arrangement of the glomerular bloodvessels, if the epithelium of the capsules has secretive powers like that of ordinary glands? It is difficult to believe that these unique vascular tufts have not a near and important relation to the renal function; but it is by no means clear what that relation is. The sccretion of water, and even its rapid secretion, is not at all bound up with any set arrangement of bloodvessels. Gland-cells all over the body secrete water under the most varied conditions of blood-pressure, although a comparatively high pressure is upon the whole favourable to a copious outflow.

But the kidney has perhaps other functions than excretion (Chapter XI.). And it may be that the simplest part of the latter process, the elimination of water and salts, is largely thrown upon the Malpighian corpuscles, as a physiologically cheaper machine than the epithelium of the tubules, which is left free for more complex labours. These may include not only the separation of nitrogenous metabolites, but also synthetic processes possibly concerned in the regulation of protein metabolism. One characteristic synthesis, the union of benzoic acid and glycin to hippuric acid, has already been referred to. As will be shown later (p. 580 ), it takes place mainly, in some animals perhaps exclusively, in the kidney. The epithelium of the glomerulus, being a less highly organized and less delicately selective mechanism than that of the convoluted tubules, may more easily respond to increase of bloodpressure by increased secretion. At the same time, placed as it is at the last flood-gate of the circulation, where the escape of anything valuable means its total loss, the glomerular epithelium may be endowed with a general power of resistance to transudation, which renders a comparatively high blood-pressure a necessary condition of its acting at all. And as a matter of fact water ceases to be secreted by the kidney long before the blood-pressure in the glomeruli can have fallen below that which suffices for the highest activity of the liver. Perhaps, however, the high minimum pressure required ( 30 to $40 \mathrm{~mm}$. of mercury in the dog) is merely the necessary consequence of the long and difficult path which most of the blood going through the kidney has to take, and, that a sufficient blood-flow cannot be kept up with less. It may be, too, that the comparatively small surface of the glomeruli, restricted in order to leave room for the more highly organized parts of the renal mechanism, entails the more intense and concentrated activity which the high blood-pressure renders possible, and the simplicity of work and organization renders harmless.

An obvious result, and perhaps an important one, of the peculiar arrangement of the bloodvessels of the kidney is that the renal tubules proper are shielded from an excessive blood-pressure by the interposition of the glomeruli as a block. This may be either because the epithelium of the tubules would not perform its work so well under a 
high blood-pressure, or because there would be a danger of substances which ought to be retained being cast out into the urine. In this connection it is interesting to note that the spccific constituents of urine are separated by epitlelimm surrounded by capillaries of the second orcler, and therefore with a smaller blood-pressure, than exists in the capillaries of most glands, while the same is true of bile, anotlies (practically) protein-free secretion.

The maximum secretory pressure in the kidney, as shown by a manometer tied into the divided ureter, is about $60 \mathrm{~mm}$. of mercury in the dog, or less than half that of saliva. If the escape of the urine is opposed by a greater pressure than this, or if the ureter is tied, the kidney becomes oedematous. Whether the odema is due to reabsorption of urine or to the pouring out of lymph owing to the pressure of the dilated tubules on the veins has not been definitely settled. It has been already pointed out that there is no necessary relation between the blood-pressure in the capillaries of a gland and its secretory pressure; and, so far as this goes, water might just as well be secreted at a pressure of $60 \mathrm{~mm}$. of mercury from the low-pressure blood of the second set of renal capillaries as from the high-pressure blood of the glomeruli. By obstruction the molecular concentration of the urine is diminished to half or three-quarters of the normal.

The Influence of the Circulation on the Secretion of Urine.Although the activity of no organ in the body is governed more by the indirect effects of nervous action than that of the kidney, no proof has been given of the existence of secretory fibres for it comparable to those of the salivary glands. All the clianges in the rate of renal secretion which follow the section or stimulation of nerves can be explained as the consequences of the rise or fall of local or general blood-pressure, and of the corresponding variations in the velocity of the blood in the renal vessels.

The best way to study variations in the calibre of the renal vessels is the plethysmographic method, and the oncometer of Roy is a plcthysmograph adapted to the kidney (Fig. I92). It consists of a mital capsule lined with loose membrane, between which and the metal there is a space filled with oil. The two halves of the capsule open and shut on a hinge; and the kidney, when introduced into it, is surrounded on all sides by the membrane, the vessels and ureter passing out through an opening. The oil-space is connected with a cylinder also filled with oil, above which a piston, attached to a lever, moves. The lever registers on a drum the changes in the volume of the kidney-i.e., practically the changes in the quantity of blood in it, and therefore in the calibre of its vessels. A still better oncometer is that of Schäfer, in which air is employed instead of oil.

Nerves of the Kidney.-Both vaso-constrictor and vaso-dilator fibres for the renal vessels, but most clearly the former, have been shown to leave the cord (in the dog) by the anterior roots of the sixth thoracic to second lumbar nerves, and especially of the last three thoracic. They run in the splanchnics, and then through the renal plexus-around the renal artery-into the kidney. The vaso-constrictors predominate, 
so that the general effect of stimulation of the nerve-roots, the splanchnics, or the renal nerves is shrinking of the kidney, with diminution or cessation of the secretion of urine. But slow rhythmical stimulation of the roots causes increase of volume, the scanty dilators being by this method excited in preference to the constrictors.

The renal nerves, entering at the lilum, branch repeatedly, so as to form a wide-meshed plexus around the arteries, and accompany them even to their finest ramifications in the cortex. Coming off from the nerves surrounding the arteries are fine fibres which are distributed to the convoluted tubules. Some of them terminate in globular ends, others in fine threads that pass through the menbrana propria (Berkely).

Section of the renal nerves is followed by relaxation of the small arteries in the kidney, and consequent swelling of the organ. The flow of urine is greatly increased, and sometimes albumin appears in it, the excessive pressure in the capillaries (particularly in thos:
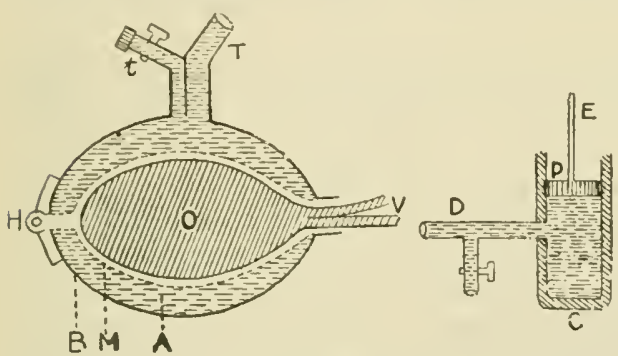

Fig. I92.-Diagram of Organ-Plethysmograph or Oncometer. B, metal box in two halves open. ing on the hinge $\mathrm{H}$; $\mathrm{M}$, thin membrane; $\mathrm{A}$, space filled with oil; $\mathrm{O}$, organ enclosed in oncometer; $\mathrm{V}$, vessels of organ; $t$, tube for filling instrument with oil; $T$, tube connected with $D$, which opens into cylinder $\mathrm{C} ; \mathrm{C}$ is also filled with oil; $\mathrm{P}$, pis. ton attached by $\mathrm{E}$ to a writing lever. of the glomeruli) being supposed to favour the escape of substances to which a passage is refused under normal conditions.

An experiment which is sometimes quoted as a decisive test of the relative importance of changes in the rate of flow, and in the pressure of the blood within the glomeruli, is that of tying the renal vein. This undoubtedly does not lower the intraglomerular pressure - on the contrary, it must increase it-but the secretion of urine stops. If the venous outflow from the kidney is only partially interfered with, the flow of urine is immediately diminished, but the administration of a diuretic like potassium nitrate causes an increase. It is more than likely that in these experiments the secretion stops or slackens not because a high blood-pressure, but because an active circulation is its necessary condition. When the blood stagnates in the kidney the natural stimulus to the renal apparatus speedily disappears owing to the elimination of the urinary constituents to the neutral or indifferent point (p.5 $\mathrm{OI}$ ). The experiment, however, is not perfectly conclusive. For few glands can go on performing their function after the circulation has ceased. The kidney must be able to feed itself in order to continue its work. Above all, it needs oxygen; and it might be urged that if the blood in the glomeruli could be kept at the normal standard of arterial blood, secretion might still go on after ligation of the renal vein. 
According to Ludwig, indeed, the flow of urine stops, in spite of continued filtration through the glomeruli, because the swelling of the veins in the boundary layer compresses the tubules, and may even obliterate their lumen. There is no conclusive experimental evidence, however, and no a priori probability, that the obstruction so produced is sufficiently sudden or sufficiently complete to cause instant and total cessation of the flow. It is even less justifiable to conclude from the experiment that the liquid part of the urine is, at any rate, not separated by the epithelium of the tubules, since the blood-pressure in the capillaries around the tubules must rise very greatly after ligature of the vein, and yet secretion is stopped. It might equally well be argued that the renal epithelium normally secretes water under a low blood-pressure, but is disorganized under the excessive and entirely unaccustomed pressure which follows the closure of the vein.

It is not only through nerves directly governing the calibre of the vessels of the kidney that the rate of urinary secretion can be affected. Any change in the general blood-pressure, if not counteracted by, still more if conspiring with, simultaneous local changes in the renal vessels, may be followed by an increased or diminished flow of urine; and the law which explains all such variations, or at least serves to sum them up, is that in general an increase in the rate of the blood-flow through the kidney is followed by an increase in the rate of secretion. It will be remarked that this is the converse of the great law, of which we have already seen so many illustrations, that functional activity increases blood-flow. It is probable that this law holds for the kidney as well as for other organs, but that the influence of activity on blood-supply is subordinated to that of blood-supply on activity, while in most tissues, as in the muscles, the opposite is the case. It is evident that an increase in the blood-flow would favour the secretory activity of the renal cells, since the average concentration of the blood presented to them as regards those constituents which they select would remain relatively high in its circuit through the kidney. The 'stimulus ' to secretion would, therefore, be relatively intense.

Destruction of the medulla oblongata (i.e., of the vaso-motor centre), or section of the cord below it, diminishes the secretion of urine, because the arterial pressure is lowered so much as to overcompensate the dilatation of the renal vessels, which the operation also brings ${ }^{\circ}$ about. If the blood-pressure falls below $40 \mathrm{~mm}$. of mercury, the secretion is abolished. Stimulation of the medulla or cord also lessens the flow of urine by constricting the arterioles of the kidney so much as to over-compensate the rise of general bloodpressure, caused by the constriction of small vessels throughout the body.

If the renal nerves have been cut, stimulation of the medulla 
oblongata increases the urinary secretion, because now the rise of general blood-pressure is no longer counterbalanced by constriction of the renal vessels. $A_{n}$ increase in the urinary flow can be produced in the rabbit by a lesion in a part of the funiculi teretes, which can be reached in the floor of the fourth ventricle (Eckhard), perhaps by destroying the portion of the vaso-motor centre governing the renal nerves, while the rest remains uninjured, or is even stimulated, and thus keeps up or even increases the general bloodpressure. There is either no glycosuria, or it is very slight.

Section of the splanchnic nerves causes a fall of arterial pressure, which is, however (in animals like the dog, in which compensation soon takes place), more than balanced by the simultaneous dilatation of the renal vessels, and therefore for some time the flow of urine is increased, but not so much as when the renal nerves alone are cut. In the rabbit there is no increase. On the other liand, stimulation of the splanchnics stops the urinary secretion, because the general rise of pressure is not enough to make up for the constriction of the renal vessels.

Diuretics are substances that increase the flow of urinc. Some of them act mainly on the circulation, as by increasing the general bloodpressure, others mainly by a direct influence on the secreting mechanism. Digitalis is a representative of the first class; urea and caffein belong to the second. The action of digitalis is to strengthen the beat of the heart, which is at the same time somewhat slowed, and to constrict the arterioles. Both effects contribute to the increase of pressure. The accompanying diuresis, seen practically only in cardiac disease with dropsical effusions, is due to the improvement of the renal circulation and the absorption of the œdema fluid. Caffein, when injected into the blood, affects the pressure but little. It causes dilatation of the renal vessels after a passing constriction, and an increase in the flow of urine after a temporary diminution. The vascular dilatation is not the chief reason for the diuretic effect, for the latter is still obtained when the vaso-motor mechanism has been paralyzed by chloral hydrate, and even after the secretion of urine has been stopped by the fall of pressure consequent on section of the spinal cord. Caffein, therefore, acts directly on the renal epithelium. When these cells are asphyxiated by temporary clamping of the renal artery, caffein after removal of the clamp causes no diuresis, while the injection of Ringer's fluid still causes a secretion of urine possessing the same crystalloid composition as the plasma, just as it would have done if perfused through the excised kidney. The action of urea, potassium nitrate, and saline diuretics such as sodium sulphate is probably also a direct action on the secreting structures, although some have supposed that their primary effect is to cause vaso-dilatation in the kidney.

Summary.-Our knowledge of renal secretion may be thus summed up: The water and salts of the urine are chiefly separated by the glomeruli; the process is not a mere physical filtration, but a true secretion. Substances like sugar, peptone, egg-albumin, and hamoglobin, when injected into the blood, are probably excreted mainly by the glomeruli; and so is the sugar of diabetes. Urea, uric acid, and presumably the other organic constituents of normal urine, with 
a portion of the weater and salts, are excreled by the physiological activity of the 'rodded' epithelium of the renal tubules. The rate of secretion of urine rises and falls with the pressure, and still more with the velocity, of the blood in the renal vessels. No secretory nerves for the kidney have been found; the effects of scction or stimulation of nerves on the secretion can all be explained by the changes produced in the renal blood-flow. Some dirtretics act by increasing the blood-flow, others directly on the epithelium of the tubules or the glomeruli.

\section{SECTION III.-EXPULSION OF THE URINE.}

Micturition.-The urine, like the bile, is being constantly formed; although secretion varies in its rate from time to time, it never ceases. Trickling along the collecting tubules, the urine reaches the pelvis of the kidney, from which it is propelled along the ureters by peristaltic contractions of their walls, and drops from their valvelike orifices into the bladder. When this becomes distended, rhythmical peristaltic contractions are set up in it, and notice is given of its condition by a characteristic sensation, which is perhaps aided by the squeezing of a few drops of urine past the tonically contracted circular fibres that form a sphincter round the neck of the bladder, and into the first part of the urethra. The desire to empty the bladder can be resisted for a time, as can the desire to empty the bowel. If it is yielded to, the smonth muscular fibres in the wall of the viscus are thrown into contraction. This is aided by an expulsive effort of the abdominal muscles. The sphincter vesica is relaxed; and the urine is forced along the urethra, its passage being facilitated by cliscontinuous contractions of the ejaculator urinæ muscle, which also serve to squeeze the last drops of urine from the urethral canal at the completion of the act.

Regurgitation into the ureters is to a great extent prevented by their compression between the mucous and muscular coats of the bladder, where they run for more than half an inch before opening at the posterior angle of the trigone. But it has been shown that a certain amount of back flow can take place. Small bodies like (liatoms suspended in water and pigments dissolved in it have been found in the pelvis of the kidney, the renal tubules, and even the circulation after being injected into the bladder.

The pressure in the bladder of a man may be made as higl as Io $\mathrm{cm}$. of mercury during the act of micturition; about half this amount is due to the contraction of the vesical walls alone, the rest to the contraction of the abiominal muscles. A pressure of I6 to $26 \mathrm{~mm}$. of mercury is required to open the sphincter of a rabbit's bladder in life.

Although the whole performance seems to us to be completely voluntary, there are facts which show that it is at bottom a reflex 
series of co-ordinated movements, that can be started by impulses passing to a centre in the spinal cord from above or from belowfrom the brain or from the hadeler. In dogs, with the spinal cord divided at the upper level of the lumbar region, micturition takes place regularly when the bladder is full, and can be excited by such slight stimuli as sponging of the slin around the anus (Goltz). Here, of course, the act is entirely reflex; and the centre is situated at the level of the fifth lumbar nerves. The efferent nerves of the bladder, like those of the rectum, come partly from the cord directly through the sacral nerves, and partly through the lumbar synipathetic chain (second to sixth ganglia). The sacral fibres are connected with nerve cells in the hypogastric plexus, and the sympathetic, partly at least, in the inferior mesenteric ganglia. This anatomical coincidence acquires interest in view of the striking pliysiological similarity between the processes of micturition and defrecation, a similarity which is emphasized by the fact that the latter is almost invariably accompanied by the former. An important difference, however, is that the will can far more readily set in motion the machinery of micturition than that of defæcation; a man can generally empty his bladder when he likes, but he cannot empty his bowels when he likes.

Sometimes in disease, and especially in disease of the spinal cord, the mechanism of micturition breaks down; the bladder is no longer emptied; it remains distended with urine, which dribbles away through the urethra as fast as it escapes from the ureters. To this condition the term incontinence of urine is properly applied.

Reflex emptying of the bladder, without an act of will or during unconsciousness, is not true incontinence. The involuntary micturition of children during sleep, for example, is a perfectly normal reflex act, although more easily excited and less easily controlled than in adults. Section either of both nervi erigentes, or of both hypogastrics, is never followed by more than quite temporary dis. turbance of function of the bladder in dogs, both male and female. In a few days the urine is normally passed. In bitches the same is true when both pairs of nerves are divided. But in male dogs true incontinence of urine follows section of the four nerves, as well as intense tenesmus due to paralysis of the lower part of the large intestine.

\section{SECTION IV.-EXCRETION BY THE SIIN.}

Besides permitting of tle trifling gaseous interchange already referred to (p. 299), the skin plays an important part in the elimina. tion of water by the sweat-glands.

Sweat is a clear colourless liquid of low specific gravity (I003 to) I006), consisting clirefly of water with small quantities of salts, 
neutral fats, volatile fatty acids, and the merest traces of proteins and urea. It is acid to litmus except in profuse sweating, when it may become neutral or even alkaline. It is secreted by simple gland-tubes, which form coils lined witl a single layer of columnar epithelium, in the subcutaneous tissue, with long ducts running up to the surface through the true skin and epidermis. Unless collected from the parts of the skin on which there are no hairs, such as the palm, it is apt to be muxed with sebum, a secretion formed by the breaking down of the cells of the sebaceous glands, which open into the hair follicles, and consisting chiefly of glycerin and cholesterin fats, soaps, and salts. Sebum is probably of considerable importance for maintaining the normal condition of the hair and skin.

Although it is only occasionally that sweat collects in visible amount on the skin, water is always being given off in the form of vapour. This invisible perspiration leaves behind it on the skin, or in the glands, the whole of the non-volatile constituents, which may be to some extent reabsorbed; and since even the visible perspiration is in large part evaporated from the very mouths of the glands in which it is formed, the sweat can hardly be considered a vehicle of solid excretion, even to the small extent indicated by its chemical composition.

The total quantity of water excreted by the skin, and the relative proportions of visible and invisible perspiration, vary greatly. A dry and warm atmosphere increases, and a moist and cold atmosphere diminishes the total, and, within limits, the invisible perspiration. Visible sweat-given the condition of rapid heat-production in the body as in muscular labour-is more readily deposited on freely exposed surfaces when the air is moist than when it is dry. The air in contact with surfaces covered by clothing is never far from being saturated with watery vapour. Here, accordingly, a comparatively slight increase in the activity of the sweat-glands suffices to produce more water than can be at once evaporated; and the excess appears as sweat on the skin, to be absorbed by the clothing without evaporation, or to be evaporated slowly, as the pressure of the aqueous vapour gradually diminishes in consequence of diffusion. The power of imbibition (p. 426 ) of water by the various layers of the skin diminishes as wc pass outwards, and the cells of the epidermis are characterized by the rapidity with which they return from a condition of excessive imbibition to their normal state. This constitutes a protective mechanism against excessive loss of water. When the skin is thoroughly moistened, its degree of imbibition is three times the normal.

The quantity of sweat given off by a man in twenty-four hours varies so much that it would not be profitable to quote here the numerical results obtained under different conditions of tempera. 
ture and humidity of the air (but see p. 69r). It is conough to say that the excretion of water from the slin is of the same order of magnitude as that from the kidneys: a man loses upon the whole as much water in sweat as in urine. But it is to be carefully noted that these two channels of outflow are complementary to each other; when the loss of water by the skin is increased, the loss by the kidneys is diminished, and vice versa.

The Influence of Nerves on the Secretion of Sweat.-The sweatglands are governed directly by the nervous system; and though an actively perspiring skin is, in health, a flushed skin, the vascular dilatation is a condition, and not the chief cause of the secretion. Stimulation of the peripheral end of the sciatic nerve causes a copious secretion of sweat on the pad and toes of the corresponding foot of a young cat, and this although the vessels are generally constricted by excitation of the vasomotor nerves. Not only so, but when the circulation in the foot is entirely cut off by compression of the crural artery or by amputation of the limb, stimulation of the sciatic still calls forth some secretion. As in the case of the salivary glands, injection of atropine abolishes the secretory power of the sciatic, while leaving its vaso-motor influence untouched; and pilocarpine increases the flow of sweat by direct stimulation of the 'ndings of the secretory nerves in the glands.

That the sweating caused by a high external temperature is normally brought about by nervous influence, and not by direct action on the secreting cells, is shown by the following experiments. One sciatic nerve is divided in a cat, and the animal put into a hotair chamber. No sweat appears on the foot whose nerve has been cut, but the other feet are bathed in perspiration. Similarly, a venous condition of the blood (in asphyxia) causes sweating in the feet whose nerves have not been divided, but none in the other foot; and stimulation of the central end of the cut sciatic has the same effect. All this points to the existence of a reflex mechanism; and it is certain that asphyxia acts by direct stimulation of the centre or centres. The vaso-motor centre is at the same time stimulated, and the bloodvessels constricted, as in the cold sweat of the death agony. Fear may also cause a cold sweat, impulses passing from the cerebral cortex to the vaso-motor and sweat centres.

It is probable that a general sweat-centre exists in the medulla oblongata, but its position has not been exactly determined nor even its existence definitely proved. On the other hand, it is known that in the cat there are at least two spinal centres, one for the fore-limbs in the lower part of the cervical cord, and another for the hind-limbs where the dorsal portion of the cord passes into the lumbar. That this latter centre does not exist or is comparatively inactive in man is indicated by the following case: A man fell from a window and fractured his backbone at the fiftl dorsal vertcbra. The lower half of the liody was paralyzed for a time. but recovered. Ultimatcly, however the 
paralysis returned; and shortly before his death (twenty-one years after the accident) it was noticed that a copious perspiration broke out several times on the upper part of the body, while the lower portion remained perfectly dry. If there is any functional spinal centre in man, it appears to lie above the fifth spinal segment. For it was seen in a professional diver who fractured his neck at that level, and lived three months after the accident, that sweat frequently appeared on parts of the body above the lesion, but never below. At the autopsy the whole thickness of the cord, except perhaps a small portion of the anterior columns, was found destroyed. Of course, it may be that in man the spinal centres, although normally active, lose their function for a long time after such severe injuries to the cord, owing to the condition known as shock.

The secretory fibres for the fore-limbs (in the cat) leave the cord in the anterior roots of the fourth to ninth thoracic nerves. They pass by white rami communicantes to the sympathetic chain, in which they reach the ganglion stellatum, where they are all connected with nervecells. Then, as non-medullated fibres, they gain the brachial plexus by the grey rami, and run in the median and ulnar to the pads of the feet. The fibres for the hind-limbs leave the cord in the anterior roots of the twelfth thoracic to the third or fourth lumbar nerves; pass by the white rami to the sympathetic ganglia, in which they form connections with ganglion cells; then, as non-medullated fibres, run along the grey rami, and are distributed to the foot in the sciatic.

The evidence of the direct secretory action of nerves on the sweatglands is singularly striking and complete, in contrast to what we know of the kidney. In the latter, blood-flow is the important factor; increased blood-flow entails increased secretion. In the former, the nervous impulse to secretion is the spring which sets the machinery in motion; vascular dilatation aids secretion, but does not generally cause it. It would, however, be easy to lay too much stress on this distinction, for in the horse the mere dilatation of the bloodvessels of the head after section of the cervical sympathetic has been found to be accompanied by increased secretion of sweat, and urinary secretion can certainly be affected by the direct action of various substances on the secretory mechanism, independently of vascular changes. But the broad difference stands out clearly enough, and the reason of it lies in the essentially different purpose of the two secretions. The water of the urine is in the main a vehicle for the removal of its solids; the solids of the sweat are accidental impurities, so to speak, in the water. The kidney eliminates substances which it is vital to the organism to get rid of; the sweat-glands pour out water, not because it is in itself hurtful, not because it cannot pass out by other channels, but because the evaporation of water is one of the most important means by which the temperature of the body is controlled. In short, urine is a true excretion, sweat a heat-regulating secretion. No hurtful effects are produced when elimination by the skin is entirely prevented by varnishing it, provided that the increased loss of heat is compensated. A rabbit with a varnished skin dies 
of cold, as a rabbit with a closely-clipped or slaven skin does; suppression of the secretory function of the skin has nothing to do with death in the first case any more than in the second (p. 299).

\section{PRACTICAI EXERCISES ON CHAPTER IX.}

Urine.

For most of the experiments human urine is employed-in the quantitative work the mixed urine of the twenty-four hours. Urine may also be obtained from animaIs. In rabbits pressure on the abdomen will usually empty the bladder. Dogs may be taught to micturate at a set time or place, or kept in a cage arranged for the collection of urine. Or a catheter may be used (p. 7 r 6 ).

1. Specific Gravity.-Pour the urine into a glass cylinder, and remove froth, if necessary, with filter-paper. Place a urinometer (Fig. r93)

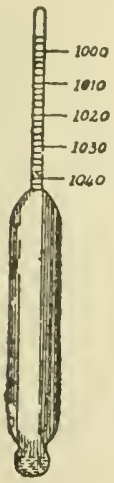

Fig. 193.-Urinometer. in the urine, and see that it does not come in contact with the side of the vessel. Read off on the graduated stem the division which corresponds with the bottom of the meniscus. This gives the specific gravity.

2. Reaction.-(a) Test with litmus-paper. Generally the litmus is reddened, but occasionally in health the urine first passed in the morning is alkaline. Sometimes urine has an amphicroic reaction-i.e., affects both red and blue litmus-paper. This is the case when there is such a relation between the bases and acids that both acid and 'neutral' (dibasic) phosphates are present in certain proportions. The acid phosphate reddens blue litmus, and the 'neutral' phosphate turns red litmus blue.

(b) Titratable Acidity.-To 25 c.c. of urine add $\mathrm{I}_{5}$ to 20 grammes of powdered potassium oxalate, and one or two drops of a I per cent. solution of plienolphthalein. Shake the mixture rapidly for a minute or two, and then titrate with decinormal sodium hydroxide at once (while still cold from the solution of the oxalate) till a faint pink colour remains permanent on shaking. The potassium oxalate is added to counteract the tendency of the calcium present in urine to form basic phosphates, which would be precipitated, and the acidity of the urine thus increased (Folin).

3. Chlorides--(a) Qualitative Test.-Add a drop of nitric acid and a drop or two of silver nitrate solution. The nitric acid is added to prevent precipitation of silver phosphate. A white precipitate soluble in ammonia shows the presence of chlorides. The precipitate appears to be incompletely soluble in ammonia, since the ammonia brings down a small precipitate of earthy phosphates.

(b) Quantitative Estimation.-The quantitative estimation of the chlorine in urine without previous evaporation and incineration is best made by one of the modifications of Volhard's method. It depends upon the complete precipitation of the chlorine combined with the alkaline metals, and also of sulphocyanic acid, by silver from a solution containing nitric acicl in excess; and avoids the error introduced into simpler methods, like Mohr's, by the union of some of the silver with other substances than chlorine. A given quantity of a standard solution of 
silver nitrate (more than sufficient to combine with all the chlorine) is added to a given volume of urine. The excess of silver is now estimated by means of a standard solution of anmonium sulphocyanide, which precipitates the silver as insoluble silver sulplocyanide. A fairly strong solution of the double sulplate of iron and ammonium (known as ironammonia-alum) is taken as the indicator, since a ferric salt does not give the usual red colour with a sulphocyanide so long as any silver in the solution is uncombined with sulplocyanic acid. The iron-ammoniaalum forms the red salt, ferric sulplocyanicle, when any cxeess of ammonium sulphocyanide is present, but it does not react witl silver sulphocyanide.

The standard solution of silver nitrate can be made by dissolving 29.063 grammes of pure fused silver nitrate in distilled water and making up the volume of the solution accurately to I litre. The solution should be kept in the dark. One c.c. of this solution corresponds to 0.0 I gramme $\mathrm{NaCl}$ or 0.00607 gramme $\mathrm{Cl}$.

The standard solution of ammonium sulphocyanide is prepared as follows: Dissolve 13 grammes of pure ammonium sulphocyanide $\left(\mathrm{NH}_{4} \mathrm{CNS}\right)$ in a litre of distilled water. Measure with a pipette into a beaker 20 c.c. of the standard silver nitrate solution, and add 5 c.c. of the iron alum solution and 4 c.c. of pure nitric acid (specific gravity I.2). Fill a burette with the sulphocyanide solution, and run it into the silver nitrate solution until a faint permanent red tinge is obtained. Note the number of c.c. of the sulplsocyanide solution required, and then dilute the solution till 2 c.c. of the sulphocyanide solution correspond exactly to I c.c. of the silver solution, so as just to allow of the end reaction with the iron solution being seen, and no more.

To carry out the method, put Io c.c. of urine, which must be free from albumin, in a stoppered flask, with a mark corresponding to Ioo c.c. or a graduated cylinder. Add 50 c.c. of water, 4 c.c. of pure nitric acid (specific gravity $\mathbf{I} \cdot 2$ ), and I5 c.c. of the standard silver solution; shake well, fill with water to the mark, and again shake. After the precipitate has settled, filter it off. Take 50 c.c. of the filtrate, add 5 c.c. of the solution of iron-ammonia-alum, and run in from a burette the standard solution of ammonium sulphocyanide until a weak but permanent red coloration appears.

Suppose $x$ c.c. of the sulphocyanide solution are required, then the chlorine in Io c.c. of urine evidently corresponds to (I $5-x$ ), o. or gramme $\mathrm{NaCl}$

4. Phosphates-(I) Qualitative Tests.-(a) Render the urine alkaline with ammonia. A precipitate of earthy phosphates (calcium and magnesium phosphates) falls down. Filter. The filtrate contains the alkaline phosphates. To the filtrate add magnesia mixture.* The alkaline phosphates (sodium, potassium, or ammonium phosphates) are precipitated as ammonio-magnesic or triple plosphate. (b) Add to urine half its volume of nitric acid and a little molybdate of ammonium, and heat. A yellow precipitate of ammonium phospho-molybdate sliows that phosphates are present. This test is given both by alkaline and earthy phosphates.

(2) Quantitative Estimation.-The quantitative estimation of phosphoric acid in urine is best done volumetrically, by titration with a standard solution of uranium nitrate, using ferrocyanide of potassiun as the indicator. Uranium nitrate gives with phospliates, in a solution containing free acetic acid, a precipitate with a constant proportion of

* Magnesium chloride I 10 grammes, ammonium chloride I fo grammes, ammonia (specific gravity $0^{\circ} 91$ ) 250 c.c., and water 1,750 c.c. 
phosphoric acid. As soon as there is more uranim in the solution than is required to combine witl all the phosplioric acid, a brown colour is given with potassium ferrocyanide, duc to the formation of uranium ferrocyanide. In carrying out the method, 5 c.c. of a mixture of acetic acid and sodium acetate (there are Io grammes of sodium acetate and 10 grammes of glacial acetic acid in 100 c.c. of the mixturc) are added to 50 c.c. of urine, which is then heated in a beaker on the water-bath almost to boiling. The standard uranium solution (which contains 35.5 grammes of uranium nitrate in the litre, and I c.c. of which corresponds to 0.005 gramme $\mathrm{P}_{2} \mathrm{O}_{5}$ ) is now run in from a burette, until a drop of the urine gives, with a drop of potassium ferrocyanide solution, on a porcclain slab, a brown colour. Uranium acctate may be used instead of uranium nitrate, but the latter keeps best. When uranium acetate is cmployed it is not necessary to add the sodium acctate mixture.

5. Sulphates-(I) Qualitative Test.-Add to urinc a drop of hydrochloric acid and then a few drops of barium chloride. A white precipitate comes down, showing that inorganic sulplates are present. The hydrochloric acid prevents precipitation of the phosphates.

(2) Quantitative Estimation of the Sulphates (Inorganic and Ethereal). -Add to 50 c.c. of albumin-free urine in a 200-c.c. Erlenmeyer flask 5 c.c. of a 4 per cent. potassium chlorate solution and 5 c.c. of strong hydrochloric acid, and boil the mixture to break up the ethereal sulphates. In five to ten minutes it becomes perfectly colourless. While it continues to boil, 25 c.c. of a ro per cent. solution of barium chloride are added by drops, at such a rate that it takes about five minutes to add this quantity. The flask is now put on the water-bath for one-half to one hour, till the precipitate has settled. Then filter through an ash-free filter. Wash the precipitate on the filter for lialf an hour with hot water. During the first twenty minutes of the washing, at intervals of a few minutes, substitute hot 5 per cent. ammonium chloride solution for the water. At the end of the half-hour's washing, as soon as the water has run through the filter, fold up the latter and press it gently between dry filter-papers to remove a portion of the water. Then place the filter in a weighed porcelain crucible. Pour into the crucible 3 or 4 c.c. of alcohol, and ignite it, to dry and partially burn the filter-paper. Then incinerate till all the carbon is burned off, cool, and weigh. From the weight of the barium sulphate, the sulphuric acid in 50 c.c. of urine is easily calculated $\left(\mathrm{SO}_{4}\right.$ in I gramme of barium sulphate, 0.4II87 gramme) (Folin).

(3) Quantitative Estimation of the Sulphuric Acid united with Aromatic Bodies (Aromatic or Ethereal Sulphates).--Put 200 c.c. of the same urine as used in (2) into a beaker. Add roo c.c. of Io per cent. barium chloride solution in the cold. Let stand for twenty-four hours. Then decant off the clear supernatant liquid, and filter it. Measure I5o c.c. of the clear filtrate, corresponding to roo c.c. of the urine, into a 400 -c.c. Erlcnineyer flask. Add Io or I 5 C.c. of concentrated hydrochloric acid and Io to I5 c.c. of 4 per cent. potassium chlorate. Heat the mixture to boiling, and proceed as in (2). From the weight of the barium sulphatc, the ethereal sulphuric acid in roo c.c. of urine can be calculated. Deducting this from the quantity per Ioo c.c. of urine obtained in (2), we get the amount of inorganic sulphuric acid per Ioo e.c. (Folin).

6. Indoxyl (contained in the urine as indican, the potassium salt of indoxyl-sulphuric acid) can be oxidized into indigo, and so detected and estimated.

A qualitative test is the following: Ten c.c. of horse's urine is mixcd with Io c.c. of Obermayer's reagent (pure concentrated hydrochloric acid containing 2 to 4 parts of ferric chloride in 1,000 ), and shaken well 
for a minute or two; a bluish colour appears if, as is generally the case, incloxyl is present, indigo $\left(\mathrm{C}_{16} \mathrm{H}_{10} \mathrm{~N}_{2} \mathrm{O}_{2}\right)$ being formed by the oxidizing action of the ferric chloride on the indoxyl, the compound of which with sulphuric acid has becn broken $u p$ by the hydrochloric acid. The urine must be free from albumin. In performing the test in liuman urine, which contains a smaller quantity of the indigo-forming substance, the faint blue liquid should be shaken up with a few drops of chloroform. The latter takes up the colour, which is thus rendered more crident. If there is difficulty in obtaining the reaction, the urine may first be decolorized by precipitating it with acctate of lead, avoiding excess. The precipitate is filtered off, and the test then applied to the clear filtrate. The skatoxyl of urine can also be oxidized to indigo, but it is present in far smaller amount. The average quantity of indigo obtained from a litre of horse's urine is about I 50 milligrammes; from a litre of human urine, not a twenticth of that amount.

For comparative quantitative determinations the method of Folin may be used. One-hundredth of the twenty-four hours' urine is taken. In this the indigo is developed by the addition of an equal volume of Obermayer's reagent (p. $5 \mathrm{r} \cdot \mathrm{7})$, and the indigo-blue dissolved by means of 5 c.c. of chloroform. The chloroform solution is then compared colorimetrically with Fehling's solution. This can be done by putting the indigo solution and 5 c.c. of the Felling's solution respectively into small test-tubes of equal calibre, and comparing the depth of tint. If the Fehling's solution is stronger than the indigo solution, run water into the former from a pipette, graduated in tenths of a c.c., sliaking up after each addition, till equality of tint has been reached. If the indigo solution has a stronger blue colour than the Feliling's solution, dilute a measured amount of it first of all with such a quantity of chloroform (say an equal volume) as will make its tint distinctly weaker than that of the Felhling's solution. Then dilute the Fehling's solution with water, as before, till the tint is the same. From the amount of dilution the quantity of indigo can be expressed in arbitrasy units, taking Fehling's solution as Ioo. Thus, if I c.c. of water must be added to the 5 c.c. of Fehling's solution, the indican can be expressed as $\frac{100}{\frac{5+1}{5}}=\frac{500}{6}=83$. The comparison can be made more accurately by a colorimeter, if one is available. To determine the absolute amount of indigo obtained, comparison must be made with a standard solution of indigo.

7. Urea-(I) Decomposition of Urea.-Heated dry in a test-tube, it glves off ammonia. The residue contains biuret. which, when dissolved in water, gives a rose colour with a trace of cupric sulphate and excess ut sodium hydroxide (or of the hydroxides of certain other metals of the alkalies and alkaline earths (p. 8). Some proteins-peptones and alb 1 moses-in the presence of the same reagents, give a similar colour, the so-called biuret reaction.

(2) Quantitative Estimation-Marshall's Urease Method. (According to Van Slyke).-One-half c.c. of urine is measured into the bottom of tube A (Fig. I94). Five c.c. exactly of a 0.6 per cent. solution of $\mathrm{KH}_{2} \mathrm{PO}_{4}$ is then run in from a burette, and $\mathrm{I}$ c.c. cxactly of a 10 per cent. watery solution of the dry urease powder. The phosphate is added as a 'buffer' to maintain the enzyme action near its maximum rate, by preventing the development of alkalinity as the production of ammonium carbonate goes on. The solutions in the tube are well 
mixed, a drops of caprylic alcohol are added to prevent foaming during the subse(juent aeration (by Folin's method, p. 52 I), and the stopper bearing the acrating tubes is put into place. Let stand twenty minutes at room temperature of $15^{\circ}$, or fifteen minutes at $20^{\circ} \mathrm{C}$. or above, for cormplete decomposition of urea. This time must not be shortened unless more enzyme is used, but no harm is done if it is longer. Meanwhile measure into tube 1325 c.c. $N$ hydrochloric or sulplutic acid, s drop of 1 per cent. sodium alizarine sulphonate indicator, and 1 drop caprylic alcolol, and connect the tubes as sliown in Iing. 19.4. After the twenty minutes for decomposition of urea las elapsed, the air current is passed for half a minute to sweep into $B$, a small amount of ammonia which has escaped into the air space of $\mathrm{A}$ during the decomposition. Then $A$ is opened, and $\&$ to 5 granımes potassium carbonate added to liberate the ammonia from the ammonium carbonate formed. Now let the air current pass gently through the tubes for the first minute and then rapidly until all the ammonia has been brought into the acid in $B$. The time necessary for this will depend upon the speed of the air current (varying from five minutes to half an hour, according to the efficiency of the pump or vacuum by which the air is moved) and should be letermined once for all. The excess acid in $B$ is now titrated with $\frac{\mathrm{N}}{\overline{5} 0}$ sodium hydroxide. The

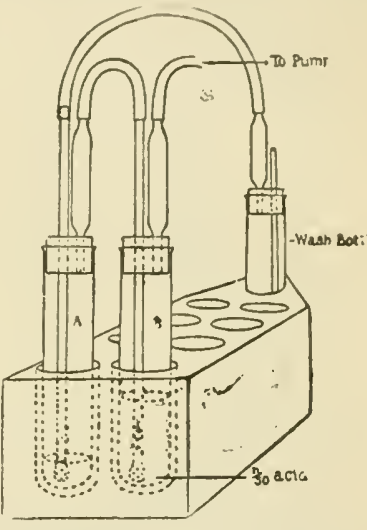

Fig. I94--Apparatus for determining Urea Content by means of Urease. (After Van Slyke.) weight of nitrogen contained in the urea of 100 c.c. of urine is $(25-x) \times 0.056$, where $x$ is the number of c.c. of the sodium hydroxide solution necessary to neutralize the acid. Included in the urea nitrogen is the nitrogen of any ammonia originally present in the urine. This can be determined separately, if desired, by putting 5 c.c. of urine into A without urease, adding the carbonate at once, and then aerating through the acid in $B$. The acid neutralized in this case is multiplied by $0 \cdot 005^{6}$ to give the ammonia nitrogen in 100 c.c. of urine, and this being deducted from the previous result, the net urea nitrogen is obtained.

The method as above described is adapted for urine containing not more than 3 per cent. urea, which is about the maximum found in human urine. Cat's or dog's urine should first be diluted to reduce the urea below 3 per cent.

Clinical Urease Method by Direct Titration of Urine.-Two 5 c.c. portions of urine are put into flasks $a$ and $b$ of 200 to 300 c.c. capacity, and diluted with distilled water to about roo to I25 C.c. One c.c. of Io per cent. urease is added to flask $a$, and a few drops of toluene to each. The flasks are allowed to remain, well stoppered, at room temperature over night, then titrated to a distinct pink colour with $\mathrm{N}$ hydrochloric acid, using methyl orange as indicator. From the amount of hydrochloric acid needed for $a$ is deducted the amount needed for $b$; and also the amount previously determined to be necessary to neutralize the alkalinity of the enzyme solution. The remaining number of c.c. multiplied by 0.6 gives the urea in grammes per litre of urine, since 
i c.c. of $\mathrm{N}$ hydrochloric acid is equivalent to 3 milligrammes of urea. Instead of the dried urease preparation, an extract of finely powdered soy beans can le made by mixing 25 grammes of the puwder with $25^{\circ}$ c.c. of distilled water, and allowing it to stancl with occasional shaking for an hour. Twenty-five c.c. of ${ }_{10}^{N}$ lydrochloric acid are then added, and the mixture allowed to remain a few minutes longer (best at about $35^{\circ} \mathrm{C}$.). The mixture is filtered, treated with a few dirops of toluene, and preserved for use in a stoppered bottle. It must be made

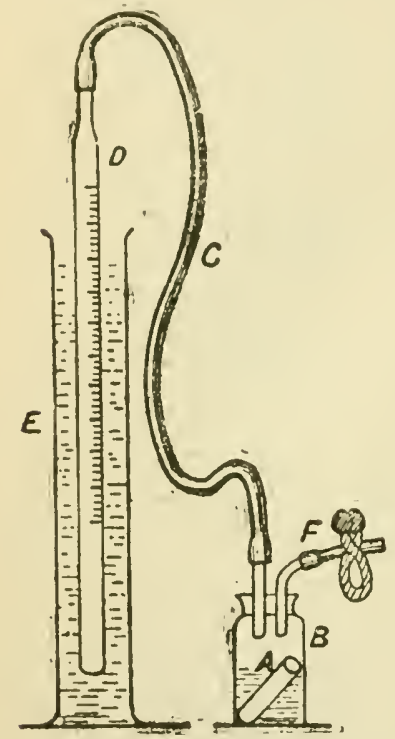

Fig. I95.- Hypobromite Method of estimating Urea. A, glass thimble; $\mathrm{B}$, bottle, through the rubber cork of which pass $t w 0$ short glass tubes, one connected by the rubber tube $C$ with a burette $D$, and the other armed with a short piece of rubber tube F. F is provided with a pinchcock. The burette is supported on a stand, and immersed in water contained in the glass cylinder E. up fresh after a few days as it does not licep long. The solution is alkaline to metlyyl orange, and 2 c.c. (the quantity used in the above clinical cletermination of urea) generally re(puires about 0.3 c.c. of $\mathrm{N}$ hydrochloric acid for neutralization.

A less exact method which is very rapid, and is therefore much used in clinical determinations, is the Hypobromite Method. The urea is split up by sodium hypobromite (p. 480 ), and the carbon dioxide being absorbed by the excess of sodium hydroxide used in preparing the hypobromite, the nitrogen is collected over water in an inverted burette. It is easy to calculate the weight of urea corresponding to a given volume of nitrogen measured at a given temperature and pressure. The nitrogen of urea is $\frac{45}{80}$, or $\frac{7}{15}$ of the whole molecular weight. Now, I c.c. of $N$ weighs, at 760 millinetres of mercury and $0^{\circ} \mathrm{C}$, 0.00125 gramme. Therefore, 1 c.c. of $N$ corresponds to 0.00 I $25 \times 15=0.00268$ gramme urea. Suppose, now, that 1 c.c. of urine was found to yield so c.c. of $\mathrm{N}$ measured at $17^{\circ} \mathrm{C}$. and $75^{\circ}$ millimetres barometric pressure. Since a gas cxpands $\frac{7}{73}$ part of its volume at $0^{\circ}$ for every degree above $0^{\circ}$, we must correct the apparent volume of nitrogen by multiplying by $27: 3$. Since the volume of a gas is inversely proportional to the pressure, we must further multiply by isol. Thus we get $10 \times \frac{273}{20} \times \frac{70}{80}=$ $201-9.29$ C.c. as the volume of the nitrogen reduced to $0^{\circ} \mathrm{C}$. and $760 \mathrm{milli}-$

metres of nercury. Nultiplying this hy $0 \cdot 00268$, we get 0.0249 gramme urea for 1 c.c. urine, which for a daily yield of 1,200 c.c. would correspond to $20 \cdot 88$ grammes urea.

As a matter of fact, however, it luas been found that there is always a cleficiency of nitrogen-that is, a given quantity of urea vields less than the estimated a monnt of gas. I gramme of urca in urine, instead of giving off 37.3 c.c. of nitrogen, gives only $35+$ c.C. at $0^{\circ} \mathrm{C}$. and 760 millimetres pressure. We must therefore take 1 c.c. of $I$ as correspond- 
ing to 0.00282 gramme, insteald of 0.00208 gramme urea. But it is affectation to make this correction if, as is seldom done in hospitals, the temperature is not taken into account.

A convenicnt apparatus is shown in lig. 195. In B placc ro c.c. of a solution made by adding bromine to ten times its volume of $4^{\circ}$ per cent. sodium hydroxicle solution. Mix 5 c.c. of urine with 5 c.c. of water. ['ut 5 c.c. of the mixture into the thimble $A$, which is then set in the small bottle B. The cork is now carefully fixed in B, and the tube $F$ being open, the level of the water in the burette is read off. The pincheock having been closed, the bottle $B$ is now tilted so that the urine in the thimble is gradually mixed with the hypobromite solution, and the nitrogen given off is added to the air in the burette and its comnections. The level of the water in the burette is therefore depressed. When gas ccases to be given off, and a short time has been allowed for the whole to cool, the tube is raised till the level of the water is once more the same inside and out. The level is again read off; the clifference of the two readings gives the volume of nitrogen at the temperature of the air and the barometric pressure. In order that the temperature of the water may be the same as that of the air, the cylinder should be filled a considerable time before the observations are begun.

S. Estimation of the Ammonia in Urine (Folin's Method).-Ammonia is liberated by addition of a weak alkali (sodium carbonate). Then the ammonia is driven out at ordinary temperature by a strong current of air and taken up in decinormal acid, which is then titrated with decinormal alkali.

The apparatus employed consists of-(1) A cylinder of about $45 \mathrm{~cm}$. diameter, with a rubber stopper through which pass two glass tubes. One of the tubes goes nearly to the bottom of the cylinder, and the other end is connected, through a $\mathbf{U}$-tube filled with cotton, with a tube containing sulphuric acid. The second tube is cut off short below the rubber cork, and its other end is connected, through a $\mathbf{U}$-tube containing cotton, with a sulphuric acid tube (or with two in series). (2) A water-pump to draw or force air through the apparatus (600 to 700 litres in an hour).

Put into the first sulphuric acid tube 25 c.c., into the second ro c.c. decinormal acid and some water; into the cylinder 25 c.c. of filtered urine, 8 to io grammes sodium chloride, 5 to io c.c. of petroleum or toluol to prevent foaming, and last of all I gramme dried sodium. carbonate. At once close the cylinder and allow a strong stream of air to pass through the apparatus. At a temperature of $20^{\circ}$ to $25^{\circ}$ (room temperature), and using 600 to 700 litres of air an hour, all the ammonia is in the sulphuric acid in one to one and a half hours. The contents of the sulphuric acid tubes are put into a beaker and titrated with decinormal alkali, using lacmoid (litmoid) or rosolic acid as indicator. Deduct the number of c.c. of alkali used from the number of c.c. of the decinormal acid originally taken, and multiply the remainder by $1 \cdot 7034$ to get the quantity of ammonia in milligrammes. The method can be employed also for albuminous urine.

9. Estimation of the Total Nitrogen.-It is sometimes more important to determine the total nitrogen of the urine than the urea alone. This is conveniently done by Kjeldahl's method (or some modification of it), which can also bc applied to the estimation of the nitrogen in the fæces, or in any of the solids or liquids of the body. It depends on the oxidation of the nitrogenous matter (or, rather, in the case of urine, mainly its hydrolysis) in such a way that the nitrogen is all represented 
as ammonia. The ammonia is then distilled over, collected and esti. mated, and from its amount the nitrogen is easily calculated. In urine the method can be carried out by adding to a measured quantity of it (say 5 c.c.) four times its volume of strong sulphuric acid, and boiling in a long-necked flask (capacity 200 c.c.), after the addition of a globulc of mercury (about O.I c.c.), which hastens oxidation and obviates bumping. A part of the mercuric sulphate formed remains in solution: the rest forms a crystalline deposit. The heating should continue for half an hour, or until the liquid is decolourized. It should be kept gently boiling. This completes the process of oxidation; and the next step is to liberate the ammonia from the substances with which it is united in the solution, and to distil it over. Dilute the liquid with water, after cooling, up to about i 50 c.c., and pour into a larger longnecked flask. Add enough of a solution of sodium hydroxide (specific gravity about $\mathrm{I} \cdot 25$ ) to render the liquid alkaline, avoiding excess, as this favours bumping. The proper quantity can be found by determining beforehand how much of the alkali is needed to neutralize the acid used for oxidation, and a little more than this amount should be added. Twenty c.c. of strong sulphuric acid needs about 75 c.c. of 40 per cent. sodium hydroxide to neutralize it. Bumping may further be prevented by the addition of a little granulated zinc. Shake the flask two or three times. Add also about 12 c.c. of a concentrated solution of potassium sulphide (I part to I $\frac{1}{2}$ parts water), which favours the setting free of the ammonia from the amino-compounds of mercury. that have been formed during oxidation. Commercial "liver of sulphur" will do quite well. Immediately connect the distilling-flask with a worm or Liebig's condenser, and distil the ammonia over into 50 c.c. of standard (decinormal) sulphuric acid (sce footnote, p. 473) contained in a flask into which a glass tube connected with the lower end of the worm dips. Heat the distilling-flask at first gently, then strongly, and boil for three-quarters of an hour, or until about two-thirds of the liquid has passed over. Then lift the tube out of the standard acid, and continue the distillation for two or three minutes longer. The ammonia is now all united with the standard acid, a certain amount of which is left over. By determining this amount we arrive at the quantity combined with ammonia, and therefore at the quantity of ammonia. Fill a burctte with a decinormal solution of potassium or sodium hydroxide. Add a little methyl-orange solution to the standard sulphuric acid, to serve as indicator. Then run in the potassium or sodium hydroxide till the pink tinge gives place to a permanent but just recognizable yellow. Let $x$ be the number of c.c. run in. Since I c.c. of any decinormal solution is equivalent to I c.c. of any other, $x$ represents also the number of c.c. of the standard sulphuric acid left uncombined with ammonia; and $50-x$, the quantity combined with ammonia. Then, I c.c. of decinormal sodium or potassium hydroxide being equiralent to I c.c. of decinormal ammonium hydroxide, and I c.c. of decinormal ammonium hydroxide containing 0.00 It $^{\prime}$ gramme nitrogen, we get $(50-x) \times 0.00 I_{4}$ as the quantity of nitrogen in 5 c.c. of urine.

Instead of mercury, potassium sulphate and copper sulphate may be added to the sulphuric acid in order to aid the decomposition in the first stage of the estimation. About 3 grammes of potassium sulphate and 1 gramme of copper sulphate are added to 5 c.c. of urine, and then 5 c.c. of sulphuric acid. The liquid is gently boiled for an hour, or until it is quite clear. The neutralization and distillation are conducted as before, the proper quantity of sodium hydroxide being determined in advance. No potassium sulphide is added, but a small quantity of 
talc may be put in to prevent bumping. Instead of metliyl orange, 'alizarin red,' which is bright red in tlee presence of the slightest trace of alkali, nay be used.

10. Uric Acid-(1) Qualitative Test for Lric Acid-Murexide Test.A small quantity of uric acid or one of its salts is heated with a little dilute nitric acid. The colour of the residue left by evaporation becomes yellow, and then red, and on the addition of ammonia changes to decp purple-red. Potassium or sodium hydroxide changes the yellow to violet. In the reaction alloxantin is formed by oxidation of the uric acid. When ammonia acts on alloxantin it is clianged into purpuric acid, and this into its ammonium purpurate, the purple-red substance called murexide. Tlius:

$$
\underset{\text { Alloxantin. }}{\mathrm{C}_{8} \mathrm{H}_{6} \mathrm{~N}_{4} \mathrm{O}_{8}+\mathrm{NH}_{3}}=\underset{8}{\mathrm{C}_{8} \mathrm{H}_{5} \mathrm{~N}_{5} \mathrm{O}_{6}+2 \mathrm{H}_{2} \mathrm{O} .}
$$

The reaction is also given by theobromine (dimetliylxanthin), an alkaloid in cocoa, and theine or caffeine (trimethylxantlin), an alkaloid in tea and coffee, which are also purin derivatives (p. $48 \mathrm{I}^{\prime}$ ).

(2) Quantitative Estimation - Folin's Modification of Hopkins's Method.-The chief rcagent is a solution of 500 grammes ammonium sulphate, 5 grammes uranium acetate, and 60 c.c. Io per cent. acetic acid, in 650 c.c. of water.

One hundred and fifty c.c. of urine is measured into a tall, narrow beaker or a cylinder, and $37^{\frac{1}{2}}$ c.c. of the reagent added. If enough urine is a vailable, 200 c.c. of urine and 50 c.c. of reagent are to be used. Allow the mixture to stand without stirring for about half an hour. The uranium precipitate las then settled, and the clear supernatant liquid is removed by siphoning or decantation. One hundred and twenty-five c.c. of this liquid is measured into another beaker, 5 c.c. of strong ammonia added, and the mixture set aside till next day. The precipitate is then filtered off, and washed with ro per cent. ammonium sulphate solution until the filtrate is quite or nearly free from chlorides. The filter is then removed from the funnel, opened, and the precipitate rinsed back into the beaker. Enough water to make about roo c.c. is added, and the precipitate is then dissolved by means of 15 c.c. concentrated sulphuric acid, and at once titrated with $\frac{N}{20}$ (one-twentieth normal) potassium permanganate solution (made by dissolving $\mathbf{r} \cdot 58 \mathbf{I}$ grammes of the permanganate in a litre of water), each c.c. of which corresponds to 3.75 milligrammes of uric acid. The very first pink coloration, extending through the entire liquid on the addition of two drops of permanganate solution, marks the end point. A correction of 3 milligrammes, owing to the solubility of ammonium urate, is added to the result.

I I. Creatinin.-Qualitatively, creatinin may be recognized in very small amounts by Weyl's test. A few drops of a dilute solution of sodium nitro-prusside are added to urine, and then dilute sodiun hydroxide drop by drop. A ruby-red colour appears, which soon turns yellow. If the urine is now strongly acidified with acetic acid and heated, it becomes first greenish and then blue. Enough acid must be added to more than neutralize the alkali.

Another test which has been made the basis of a quantitative method by Folin is Jaffé's test. A little urine (say 5 c.c.) is put in a test-tube, and then a solution of picric acid in water. The mixture is rendered alkaline by the addition of potassium or sodium hydroxide solution, and a reddish colour is produced, which turns yellow on the addition of 
acid. A similar red colour is given by dextrose, but not unless the solution is heated.

Quantitative Estimation of Creatinin by Folin's Method.-It depends upon the comparison of the colour which creatinin gives witl picric acid in an alkaline solution with that of a standard solution of potassium bichromate. Ten c.c. of urine is measured into a 500 c.c. measuringflask; I5 c.c. of a saturated picric acid solution (containing about I 2 grammes per litre) and 5 c.c. of a ro per cent. solution of sodium hydroxide are added. The mixture is allowed to stand for five minutes. Then water is added up to the 500 C.c. mark, and the flask shaken to mix uniformly. Samples of the liquid are then at once compared colorimetrically with a half-normal solution of potassium bichromate containing 24.55 grammes per litre. The colour of the urine does not introduce a scnsible error on account of the great dilution. For cxact work the comparison must be made with a good colorimeter. It has been found experimentally that, when 10 milligrammes of creatinin are present in 500 c.c. of a solution made as described, a layer of the solution $8 \cdot 1$ millimetres in thickness has the same depth of tint as 8 milliinctres of the bichromate solution. Suppose it takes 9 millimetres of the urine-picrate solution to equal 8 millimetres of the bichromate, then the ro c.c. of urine contains $10 \times \frac{8 \cdot 1}{9}=9 \cdot 0$ milligrammes of creatinin.

12. Hippuric Acid.-From horse's or cow's urine hippuric acid is prepared by evaporating to a small bulk, and adding strong hydrochloric acid. The crystalline precipitate is washed with cold water, then dissolved in hot water, and filtered hot. Hippuric acid separates out from the filtrate in the cold in the form of long four-sided prisms with pyramidal ends. Heated dry in a test-tube, the crystals melt, and benzoic acid and oily drops of benzonitrile, a substance with a smell like that of oil of bitter almonds, are formed.

\section{ABNORMAL SUBSTANCES IN URINE.}

3. Proteins-(I) Qualitative Tests. - (a) Boil and add a few drops of nitric acid. A precipitate on boiling, increased or not affected by the acid, shows the presence of coagulable proteins (serum-albumin or globulin). A precipitate of earthy phospliates sometimes forms on boiling. It is distinguished from a precipitate of proteins by dissolving on the addition of acid.

(b) Heller's Test.-Put some nitric acid in a test-tube. Pour carefully on to the surface of the acid a little urine. A white ring at the junction of the liquids indicates the presence of albumin or globulin. If much albumose is present, a white precipitate, which disappears on heating, may be formed. When this test is performed with undiluted urine, uric acid may be precipitated and cause a brown colour at the junction. A similar ring may be found in the absence of proteins when the test is made on the urine of a patient who has been taking copaiba. In very concentrated urine a white ring of nitrate of urea may be formed. A coloured ring is frequently seen, owing to the oxidation of certain chromogens of urine.

(c) Filter some urine, and add to the filtrate its own volume of acetic acid. A precipitate may indicate mucin or nucleo-albumin. If any is formed, filter it off, and add to the filtrate a few drops of potassium ferrocyanide. A white precipitate shows the presence of proteins.

(d) Test for Globulin in Uvine. - Serum-globulin probably never 
occurs in urine apart from scrum-albumin. It may be detected thus: Make the urine alkaline with ammonia, let it stand for an hour, and filter. Half saturate the filtrate with anmonium sulphate-i.e., add to it an equal volume of a saturated solution of ammonium sulphate. Scrum-globulin is precipitated, serum-albumin is not.

(e) Test for Albumose in Urine (Albumosuria).-Coagulable proteins are removed by boiling the urine (acidulated if necessary), and filtering off the precipitate if any. The filtrate is ncutralized. If a further precipitate falls down it is filtered off, the clear filtrate is heated in a bcaker placed in a boiling water-bath, and there saturated with crystals of ammonium sulphate. A precipitate indicates that albumoses (protcoses) are present. A slight precipitate might possibly be due to the formation of ammonium urate. A further test may be performed on the original urine if it is free from coagulable proteins, or on the filtrate after their removal. Add a drop or two of pure nitric acid. If albumoses are present, a precipitate, is thrown down which disappears on heating, and reappears on cooling the test-tube at the cold-water tap.

(2) Quantitative Estimation of Coagulable Proteins (Serum-Albumin and Globulin)-(a) Gravimetric Method.-Heat 50 to roo c.c. of the urine to boiling, adding a dilute solution ( 2 per cent.) of acetic acid by drops as long as the precipitate seems to be increased. Filter through a weighed filter. Wash the precipitate on the filter with hot water, then with hot alcohol, and finally with ether. Dry in an air-bath at I I ${ }^{\circ} \mathrm{C}$., and weigh between watch-glasses of known weight.

(b) Esbach's Method.-Esbach's reagent is made by dissolving Io grammes of picric acid and 20 grammes of citric acid in boiling water ( 800 or 900 c.c.), and then making up the volume to a litre. The so-called albuminimeter is simply a strong glass tube graduated and marked in a certain way. Fill the tube up to the mark $U$ with the urine. Then add the reagent up to the mark $\mathrm{R}$. Close the tube with the rubber cork, and invert it a dozen times without shaking. Set the tube aside for twenty-four hours, then read off the graduation on the tube which corresponds with the top of the precipitate. The figures indicate the number of grammes of dry protein in a litre of the urine. Suppose the top of the sediment is at 4 , this will indicate 4 grammes per litre, or 0.4 per cent. The method is of some clinical importance, owing to its simplicity, although it is, of course, not very accurate.

4. Sugar-(I) Qualitative Tests-(a) Trommer's Test (p. Io).-It is to be remarked that some substances present in small amount in normal urine reduce cupric sulphate-e.g., uric acid (present as urates) and kreatinin-but although a normal urine may thus decolourize the copper solution, it rarely causes so much reduction that a yellow or red precipitate is formed, as is the case in diabetic urine. Glycuronic acid (p. 482) also reduces cupric salts, as does alcapton or homogentisinic acid, a substance found in rare cases in disease (p. 483).

(b) Fehling's Test.-Fehling's solution (p. 526) is brought to the boil in a test-tube, a little of the urine then added, and the change of colour notcd. Benedict's modification of Fehling's solution may also be used. It has the advantage that it keeps indefinitely, and therefore is always ready for use, and is also said to be more delicate.

(c) Phenyl-Hydrazine Tesi.-This test depends upon the fact that phenyl-hydrazine forms with sugars such as glucose (dextrose), maltose, isomaltose, etc., but not with cane-sugar, characteristic crystalline substances (phenyl-glucosazone, phenyl-maltosazone, etc.) which can be recognized under the microscope, and are distinguished from each other by melting at different temperatures. Phenyl-glucosazone 
$\left(\mathrm{C}_{18} \mathrm{H}_{22} \mathrm{~N}_{4} \mathrm{O}_{4}\right)$ melts at $205^{\circ} \mathrm{C}$. To perform the test for dextrose in the urine, proceed thus: Put 5 c.c. of urine in a test-tube, add I decigramme of hydrochlorate of plenyl-hydrazine and 2 decigrammes of sodium acetate. It is sufficiently accurate to add as much phenyl-hydrazine as will lic on a sixpence (or a dime) and twice as much sodium acetate. Heat the test-tube in a boiling water-bath for half an hour. Then cool at the tap and examine the deposit under the microscope for the yellow phenyl-glucosazone crystals (Fig. 196). Sometimes the osazone precipitate is amorphous. If this should be the case, the precipitate, if no crystals can be seen, must be dissolved in hot alcohol. The solution is then diluted with water and the alcohol boiled off, when the osazone,
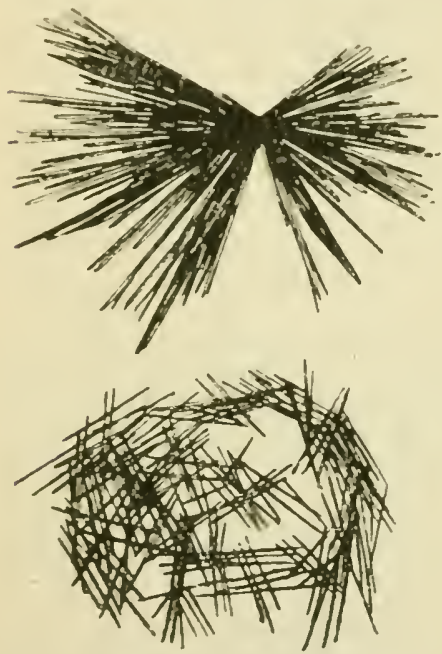

Fig. 196.-Phenyl-Glucosazone and Phenyl-Maltosazone Crystals (Mac. leod). The phenyl-glucosazone crystals are in the upper part of the figure, the phenyl-maltosazone in the lower. if any be present, will crystallize out. Very minute traces of sugar can be detected in this way (as little as o.I per cent. in urine). Often in normal urine yellow crystals are deposited during the first fifteen minutes' heating. They must not be mistaken for glucosazonc. They probably consist of a compound of glycuronic acid and phenyl-hydrazine. They are changed as the heating goes on into an amor. phous brownish - yellow precipitate (Abel).

(d) The Yeast Test is an important confirmatory test for distinguishing the fermentable sugars from other reducing substanzes, but it is not very delicate, and will with difficulty detect sugar when less than 0.5 per cent. is present. It can be performed thus: A little yeast (the tablets of compressed yeast do very well) is added to a test-tube half filled with urine. The test-tube is then filled up with mercury, closed with the thumb, and inverted over a dish containing mercury. The dish may be placed on the top of a water-bath whose temperature is about $40^{\circ} \mathrm{C}$. After twenty-four hours the sugar will liave been broken up into alcohol and carbon dioxide. The latter will liave collected above the mercury in the test-tube, and the former will be present in the urine. The tests for sugar will eitler be negative or will be less distinct than before. A control test-tube containing water and yeast should also be set up, as impurities in the yeast sometimes yield a small amount of carbon dioxide. Specially-constructed tubes are also often used for performing the test.

(2) Quantitative Estimation of Sugar in Uvine--(a) Volumetrically, the sugar can be estimatcd by titration with Fehling's solution. As this does not keep well, two solutions containing its ingredients should be kept separately and mixed when required. Solution I.: Dissolve 34.64 grammes pure cupric sulphate in distilled water, and make up the volume to 500 c.c. Solution II.: Dissolve 173 grammes Rochelle salt in 400 c.c. of water, add to this 51.6 grammes sodium lyydroxicle, and make up the volume with water to 500 c.c. Keep in well-stoppered 
bottles in the clark. For use, mix together equal volumes of the two solutions. Ten c.c. of this mixture is reduced by 0.05 gramme dextrose. To estimate the sugar in urine, put so c.c. of the mixture into a porcelain capsule or glass flask, and dilute it four or five times with distilled water. Dilute some of the urine, say ten or twenty times, according to the quantity of sugar indicated by a rough determination. Run the diluted urine from a burette into the Fehling's solution, bringing it to the boil each time urine is added, until, on allowing the precipitate to settle, the blue colour is scen to have entirely disappeared from the supernatant liquid. The observation of the colour must be made while the liquid is still hot. Benedict's modification of Fehling's solution* may also be employed.

Suppose that Io c.c. of Fehling's solution is decolourized by 20 c.c. of the ten-times diluted urine. Then 2 ¿.c. of the original urine contains 0.5 gramme dextrose. If the urine of the twenty-four hours (from which this sample is assumed to have been taken) amounts to 4,000 c.c., the patient will have passed $0.05 \times 2,000=100$ grammes sugar, in twenty-four hours.

(b) The polarimeter affords a rapid and, with practice, a delicate means of estimating the quantity of sugar in pure and colourless solutions, but diabetic urine must in general be first decolourized by adding lead acetate and filtering off the precipitate. What is measured is the amount by which the plane of polarization of a ray of polarized light of given wave-length (say sodium light) is rotated when it passes through a layer of the urine or other optically active solution of known thickness. Let $a$ be the observed angle of rotation, $l$ the length in decimetres of the tube containing the solution, $w$ the number of grammes of the optically active substance per c.c. of solution, and $(a)_{\mathrm{D}}$ the specific rotation of the substance for light of the wave-length of the part of the spectrum corresponding to the $\mathrm{D}$ line (i.e., the amount of rotation expressed in degrees which is produced by a layer of the substance I decimetre thick, when the solution contains I gramme of it per c.c.). Then $(a)_{D}= \pm \frac{a}{w l}$. In this equation $a$ and $l$ are known from direct measurement; $(a)_{\mathrm{D}}$ has been determined once for all for most of the important active substances, and therefore $w$ is easily calculated. For dextrose $(a)_{D}$ may be taken as $52.6^{\circ}$. It varies somewhat with the concentration, but for most investigations on the urine these variations may be neglected.

It is not possible to describe here the numerous forms of polarimeter that are in use. Those constructed on what is called the "half-shadow" system (Fig. 197) give sufficiently satisfactory results. A half-shadow polarimeter consists, like other polarimeters, of a fixed Nicol's prism (the polarizer), and a nicol capable of rotation (the analyzer). In addition, there is an arrangement which rotates by a definite angle the plane of polarization in one-half of the field, but not in the othere.g., a small nicol occupying only half of the field. In the zero position of the analyzer, both halves of the field are equally dark. The solution to be investigated is placed in a tube of known length, the ends of which

* It contains I 7.3 grammes of cupric sulphate, $173^{\circ}$ o grammes of sodium citrate, $100^{\circ} 0$ grammes of anhydrous sodium carbonate made up with distilled water exactly to one litre. In making the solution the citrate and carbonate are dissolved with the aid of heat in about 600 c.c. of water, and then made up to about 800 c.c. The cupric sulphate is dissolved in about Ioo or 150 c.c. of water and added to the other solution, the whole being then made up to a litre. 
are closed by glass dises secured by brass screw caps. The glass discs must be slicl on, so as to exclude all air. The tube having bec $n$ introduced between the polarizer and analyzer, the sharp vertical line which indicates the division between the two half-ficlds is focussed with the eye-piece, and then the analyzer is rotated till the two halves are again equally shadowed. The angle of rotation, $a$, is read off on the graduated are, which is provided with a vernier.

Pentoses reduce Felling's solution, but do not give the yeast test. They give the following cliaracteristic tests, which may be performed with gum arabic, a substance containing arabinose, one of the pentoses:

(I) Phloroglucin Reaction.- IVarm in a test-tube some pure concentrated hydrochloric acid to which an equal volume of distilled water has been added. Add pliloroglucin until a little remains undissolved.

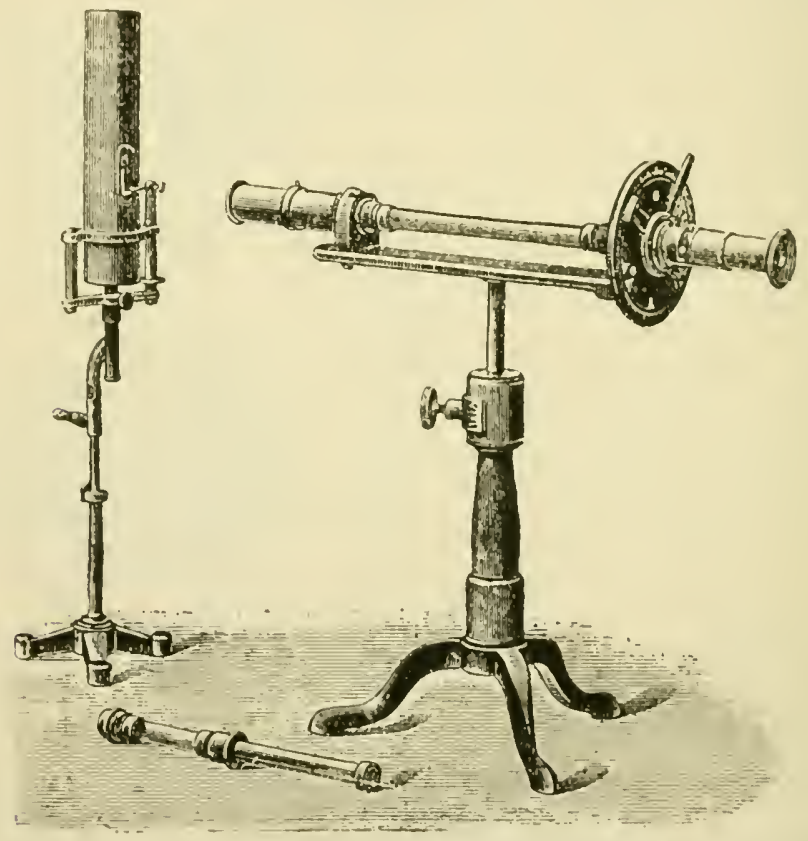

Fig. 197.-Mitscherlich's Polarimeter. (Half-shadow instrument.) (Simple form.)

Add a small quantity of gum arabic, and keep the test-tube in a waterbath at $100^{\circ} \mathrm{C}$. The solution becomes cherry-red, and a precipitate gradually separates, which may be dissolved in anyl alcoliol. The solution shows with the spectroscope a band between $\mathrm{D}$ and $\mathrm{E}$.

(2) Orcin Reaction.-Use orcin instead of pliloroglucin in (I). The solution becomes reddish-blue on warming, and slows a band between $\mathrm{C}$ and $\mathrm{D}$, near $\mathrm{D}$. The colour quickly changes from violet to bluc, red, and finally green. A bluish-green precipitate separates, which is soluble in amyl alcohol. Glycunonic acid gives all the above reactions of pentoses.

Bile-Salts (Hay's Test).-Put a little finely-divided sulpliur, in the form of flowers of sulplur, on the top of a glass of urine. If bile-salts are present the sulphur will sink to the bottom. If there are no bile- 
salts it will float on the top. The difference is due to an alteration in the surface tension of the urine produced by the bile-salts. We must exclude the presence of acetic acid, alcohol, ether, chloroform, turpentine, benzine and its derivatives, phenol and its derivatives. anilin and soaps, all of which also cause such an alteration in the surface tension of urine that the sulphur sinks to the bottom. The urine should be fresh, and if it has to be licpt it should be preserved from decomposition by cyanide of mercury, which does not alter the surface tension. The reaction has the great advantage over other tests of being easily carried out at the bedside.

Acetone-(r) Legal's Test (Rothera's modification).-To 5 to ro c.c. of the acetone-containing urine add enough ammoninm sulphate crystals to form a layer at the bottom of the test-tube, then 2 or 3 drops cf a fresh 5 per cent. solution of sodium nitro-prusside and $I$ to 2 c.c. of strong ammonia. The development of a colour like that of permanganate of potassium, often in the form of a ring a little above the undissolved salt, indicates the presence of acetone. The reaction must not be declared negative till half an hour has elapsed. The colour slowly fades.

(2) Where there is doubt as to the presence of acetone, it is best first to distil it over. Put 250 to 500 c.c. of the urine suspected to contain acetone into a litre flask. Add a few c.c. of phosphoric acid; connect the flask with a worm, and distil over the urine into a small flask. For qualitative tests it is best to collect only the first 20 to 30 c.c., as most of the acetone is contained in this. Test the distillate for acetone by (I) or by

Lieben's Test.- To a few c.c. of the distillate in a test-tube add a few drops of solution of iodine in potassium iodide, and then sodium or potassium hydroxide. A precipitate of yellow iodoform crystals (sixsided tables) is thrown down if acetone be present. Examine them under the microscope. On heating, the odour of iodoform may be recognized. If the precipitate is amorphous it may be dissolved in ether (free from alcohol), which is allowed to cvaporate on a slide, when crystals may be obtained.

Determination of the Freezing-Point of Urine.*--Study Beckmann's apparatus shown in Fig. I 7 I, P. 427. Note the large thermometer D graduated in hundredths of a degree centigrade. It is inserted through a rubber cork into the inner test-tube $\mathrm{A}$. A platinum wire, $\mathrm{F}$, bent at the lower end into a circle or a spiral, which passes easily up and down between the bulb of the thermometer and the tube, serves to stir the urine. The thermometer must be so supported by the rubber cork that the bulb is in the axis of the tube and a centimetre or two from the bottom of it. The side-piece $E$ on the tube $A$ is not absolutely necessary, but it is convenient for 'inoculating ' the urine with a crystal of ice at the proper time. A passes through a rubber cork into a shorter and wider outer glass tube B. The space between $A$ and $B$ serves as a badly conducting mantle, which prevents too rapid cooling of the contents of $\mathrm{A}$. B passes through a hole in the metal or wooden cover of a strong glass jar, $\mathrm{C}$, which contains the freezing mixture. B should fit the hole so tightly that it does not bob up out of the mixture when $A$ is removed. In $C$ is a stirrer, $G$, of strong copper wire, the end of which passes through the lid. This serves to stir up the freezing mixture from time to time.

Pulverize some ice by pounding it in a strong wooden box with a heavy piece of wood. Take the inner tube with the thermometer out of the apparatus. It is convenient to take the thermometer out of the

* This is not often of clinical value, but it affords an opportunity for the student to practise a method of great importance in physiology. 
tube, and to hang it up carefully on a stand by means of a fine flexible copper wire passing through the eye. The rubber cork can be taken out with the thermometer, and the platinum wire also, the bent free end of the latter supporting it in the cork, or it may be fastened temporarily to the thermometer stem by a small rubber band, which is slirl up over the cork when the thermometer is reinserted. Tube A can be set temporarily in a specially heavy test-tube 1ack. Remove the lid of $\mathrm{C}$, and with it tube $\mathrm{B}$. Now put ice and salt alternately into $\mathrm{C}$ until it is nearly full. mixing them up well. Add some cold water from the tap till the stirrer G can move frecly up and down in the mixture. For very exact work the temperature of the freezing mixture must not be more than a few degrees below the freczing-point of the liquid which is being examined. Put on the lid, and immerse tube B. Into A, which must be perfectly clean, put enough pure distilled water to fully cover the bulb of the thermometer. and introduce the latter. For ordinary purposes distilled water previously boiled to cxpel the carbon dioxide. and then cooled in a stoppered flask, is sufficiently pure. Inmerse A directly in the freezing mixture through the hole by which $\mathrm{G}$ comes out, or through a separate hole (not shown in the figure) till some ice hats formed in the water. Take $\mathrm{A}$ out of the mixture, wipe it with a cloth, and hold the lower part of it in the hand till nearly the whole of the ice has melted. If there is a cakc of ice at the bottom see that it is displaced by the platinum stirrer. A trace of ice being still left flodting in the water, place $A$ in $B$, and allow the temperature to fall to a few tenths of a degree below the freezing-point you cxpect to get, as determined by a previous rough experiment. The freezing mixture is stirsed up occasionally. The meniscus of the thermometer is to be carefully followed, as it goes on falling, by means of a weak hand lens. Now stir the water in A briskly. Suddenly it will be seen that the mercury begins to rise. Keep stirring with the platinum wirc. and read off the maximum height of the mercury, at which it is stationary for some time. The temperature can be estimated between the graduations to thousandths of a degrec. Take out A, and observe the finc icc crystals in the water. Heat $A$ in the hand as before till nearly all the ice has disappeared; then replace $A$ in $B$, and make another freczingpoint determination. A third one may also be made, and the mean of the three readings taken.

Take out the thermometer, and dry it and the platinum wire with clean filter-paper, or dip them in some of the urine, which is then thrown away. Dry A or rinse it with urine. Then make a determination of the freezing-point of the urine in the same way as was done with the water. The freezing-point of the urine will lic much lower on the scale.

Instead of freezing the liquid first and then leaving a little ice in it when $A$ is placed in B, A may be put into B before any ice has formed. Cooling is then allowed to go on with gentle stirring to a few tenths of a degrec below the anticipated freczing-point. A small crystal of clean dry ice is then introduced through the side-picce on a clean splinter of wood or the loop of a cooled platinum wire, the end of which passes through a piece of cork, by which it is held to prevent conduction of heat. The platinum stirrer can be raised to receive the crystal. The liquid is now vigorously stirred; freezing occurs, and the observation is made as before.

Instead of the above method, the liquid may first be cooled directly in the freezing mixture, but not so much that ice forms. A is then put in $\mathrm{B}$, and cooling allowed to go on while it is being stirred. When it has been undercooled to a certain extent-i.e., cooled below its freezingpoint-the vigour of the stirring is increased. Ice forms suddenly, as before, and the temperature rises to the freezing-point. With urine 
this metlod is sufficiently satisfactory, but it is not usually casy to get freezing of the distilled water till the undercooling is considerable, and it has been shown that this introduces some error.

Suppose the freezing-point of the distilled water on the scale of the thermometer was $5 \cdot 2.45^{\circ}$ and that of the urine $3.625^{\circ}$, the value of $\Delta$ for the urine is $1.620^{\circ}$. Since for most purposes it is sufficient to fix the second decimal point, much smaller and less expensive thermometers than the ordinary Beckmann may be employed.

In the same way the freezing-point of blood-serum (or blood), bils, and other physiological liquids can be determined.

Systematic Examination of Urine.-In examining urine, it is convenient to adopt a regular plan, so as to avoid the risk of overlooking anything of importance. The following simple scheme may serve as an example; but no routine should be slavishly followed, the object being to get at the important facts with the minimum of labour. More extensive information must be sought in the treatises on examination of the urine for clinical purposes.

I. Anything peculiar in colour or smell? If the colour suggests blood, examine witl spectroscope, hæmin test, guaiacum tes (pp. 76, $272)$; if it suggests bile, test for bile-pigments by Gmelin's test (p. $\left.f^{(j)}\right)$, and for bile-salts by Pettenkofer's test (p. 462) and by Hay's test (pp. $+62,528)$.

2. Reaction.

3. Sediment or not? Sediment may be procured by letting the urine stand in a conical glass, or in a few minutes by the centrifuge. If the appearance of the sediment suggests anything more than a little mucus, examine with the microscope. The sediment may contain organized or unorganized deposits.

Organized Sediments.- (a) Red blood-corpuscles (considerably altered if they have come from the upper part of the urinary tract).

(b) Leucocytes. A few are present in health. A large number indicates pus. When pus is present the sediment may be white to the naked eye.

(c) Epithelium from the bladder, ureters, pelvis of the kidney or the renal tubules. A few squamous epithelial cells from the urethra are always present in normal urine.
(d) Tube casts.
(e) Spermatozoa (occasional).
(f) Bacteria.
(g) Parasites (rare).
(h) Portions of tumours (rare).

\section{Unorganized Sediments.}

\section{IN ACID URINE.}

Uric Acid.-Crystals coloured brownish - yellow with urinary pigment. Various shapes, especially oval 'whetstones,' rlombic tables, and elongated crystals, often in bundles (Fig. I 77).

Urates.-Usually amorphous, in the form of fine granules, often tinged with urinary pigment, sometimes brick-red. Soluble on heating. On addition of acids (including acetic acid) they dis-
IN ALKALINE URINE.

Triple Phosphate.-Clear, colourless, coffin-lid or knife - rest crystals. Also deposited in the form of feathery stars (Fig. I 79).

Calcium Hydrogen Phosphate ('stellar' phosphate), $\mathrm{CaHPO}_{4}$ - Crystals often wedge-shaped and arranged in rosettes. May also occur in a dumb-bell form. (A phosphate of calcium is also occasionally seen in weakly acid urine.) (Fig. 181, p. 479.) 


\section{Unorganized Sediments (continued) -}

IN ACID URINE.

solve and uric acid cystals appear in their place. Acid urate of sodium and of ammonium occasionally found in the crystalline form (rosettes of needles).

Calcium Oxalate.-Octahedral, 'envelope' crystals, not coloured. Insoluble in acetic acid. Soluble in hydrochloric acid (Fig. I78, p. 478).

Cystin. - Hexagonal plates. Rare (Fig. I8o, p. 47).

Leucin and Tyrosin (Figs. I 86, 187 , p. 485).-Rare. Also found in alkaline urine, but rarely.

Triple Phosphate. - Sometimes found in weakly acid urine.

4. Specific gravity.

5. Quantity of urine in twenty-four hours. If the quantity is abnormally large and the specific gravity high, test for sugar.

6. Inorganic constituents not generally of clinical importance, but in special diseases they should be examined-e.g., chlorides in pneumonia.

7. Normal organic constituents. Sometimes quantitative estimation of urea or total nitrogen in fever, and in diabetes and Bright's disease.

8. Chemical examination for abnormal organic constituents, especially albumin and sugar.

Albumin.-(I) Heat to boiling some of the urine in a test-tube. A precipitate insoluble on addition of a few drops of acetic acid consists of coagulable protein. A precipitate soluble in acetic acid consists of earthy phosphates.

(2) Heller's test. Put some strong nitric acid in a test-tube and run on to it some urine. A white ring indicates protein.

A very rough quantitative estimation may be made by the method of Esbach (1). 525$)$.

Sugar.-(I) Trommer's test. ' (Felıling's solution may be used.) It the result is indecisive-

(2) Phenyl-hydrazine test (p. 525).

(3) In case of doubt confirm by yeast test.

A quantitative estimation may be made with Fehling's solution or the polarimeter. 


\section{CHAPTER X}

\section{METABOLISM, NUTRITION AND DIETETICS}

WE return now to the products of digestion as they are absorbed from the alimentary canal, and, still assuming a typical diet containing carbo-hydrates, fats, and proteins, we have to ask, What is the fate of each of these classes of proximate principles in the body? what does each contribute to the ensemble of vital activity? It will be best, first of all, to give to these questions what roughly qualitative answer is possible, then to look at metabolism in its quantitative relations, and lastly to focus our information upon some of the practical problems of dietetics.

\section{Section I.-Metabolism of Carbo-Hydrates-Glycogen.}

The carbo-hydrates of the food, passing into the blood of the portal vein in the form of dextrose, are in part arrested in the liver, and stored up as glycogen in the hepatic cells, to be gradually given out again as sugar in the intervals of digestion. The proof of this statement is as follows:

Sugar is arrested in the liver, for during digestion, especially of a meal rich in carbo-hydrates, the blood of the portal contains more sugar than that of the hepatic vein. Popielski, on the basis of experiments in which he fed with known quantities of sugar dogs whose inferior vena cava and portal vein had been united by an Eck's fistula, and determined the amount of sugar which passed into the urine, estimates the quantity of sugar kept back by the liver at from 12 to 20 per cent. of the whole. In the liver there exists a store of sugar-producing material from which sugar is gradually given off to the blood, for in the intervals of digestion the blood of the hepatic veins contains more dextrose ( 2 parts per 1,000 ) than the mixed blood of the body or than that of the portal vein (about I part per I,000). When the circulation through the liver is cut off in the goose, the blood rapidly becomes free, or nearly free, from sugar (Minkowski). And a similar result follows such interference with the hepatic circulation as is caused by the ligation of the three chief arteries of the intestine in the dog, even when the animal has been previously made diabetic by excision of the pancreas (p. 636). 
The nature of the sugar-forming substance is made clear by the following experiments: (I) A rabbit after a large carbo-hydrate meal, of carrots for instance, is killed and its liver rapidly excised, cut into small pieces, and thrown into acidulated boiling water. After being boiled for a few minutes, the pieces of liver are rubbed up in a mortar and again boiled in the same water. The opalescent aqueous extract is filtered off from the coagulated proteins. No sugar. or only traces of it, are found in this extract; but another carbo-hydrate, glycogen, a polysaccharide giving a port-wine colour with iodine and capable of ready conversion into sugar by anylolytic ferments, is present in large amount. (See Practical Exercises, p. 7I5.)

(2) The liver after the death of the animal is left for a time in situ, or, if excised, is lept at a temperature of $35^{\circ}$ to $40^{\circ} \mathrm{C}$., or for a longer period at a lower temperature; it is then treated exactly as before, but no glycogen, or comparatively little, can now be obtained from it, although sugar (dextrose) is abundant. The inference plainly is that after death the hepatic glycogen is converted into dextrose by some influence which is restrained or destroyed by boiling. This transformation might theoretically be due to an unformed ferment or to the direct action of the liver-cells, for both unformed ferments and living tissue elements are destroyed at the temperature of boiling water. It has been clearly shown that the action is brought about by a diastatic enzyme, which some writers call glycogenase, for it readily occurs when the minced liver is mixed with chloroform water, and chloroform kills all living tissues. Although blood contains a diastase in small amount, the change does not depend essentially upon this, since the glycogen also undergoes hydrolysis (glycogenolysis) to dextrose when all the blood has been washed out of the organ. Ixmph also contairs a diastase, but there is evidence that the post-mortem glycogenolysis is chiefly due to an enzyme contained in the hepatic cells (an endoenzyme) (Macleod). The diastases in the blood and lymph seem to be 'discards' of the tissues which are on the way to destruction or elimination (Carlson). The post-mortem change is to be regarded as an index of a similar action which goes on during life: sugar in the intact body is changed into glycogen; glycogen is constantly being changed into sugar. There is no reason to cloubt that here, too, the hydrolysis is effected by the endo-enzyme. It might be supposed, indeed, that the adjustment of the two processes glycog':nesis and glycogenolysis is simply a matter of the alteration of the equilibrium in a reversible reaction (p. 338), according to whether the dextrose content of the blood tends to rise or fall. If the concentration of dextrose in the blood is increased, more dextrose might be expected to 'diffuse 'into the hepatic cells, whose content of dextrose in proportion to glycogen would increase till the equilibrium was restored by the conversion of the excess of 
sugar into glycogen. Contrariwise, a diminution in the dextrose content of the blood might be expecterl to lead to diffusion of dextrose ont of the liver-cells, and a conse(puent acceleration of the liydrolysis of the glycogen. We have already learnt, lowever, that in physiology - above all, perhaps, in the physiology of the glands - simple 'explanations are usually suspect. And when we come to study those conditions in which, as a consequence of the derangement of the mechanisms which regulate the carbo-hydrate metabolism, sugar appears in the urine, it will be seen that the matter is more complicated. For one thing, the nervous system seems to take a hand in the regulation, and where the nervous system takes a hand things are generally doing which the experienced physiologist does not expect to be simple. We may be certain, as in the case of the intracellular proteolytic ferments, that the vital action of the hepatic cells is a most important factor in controlling the rate of production of the ferment, and therefore its concentration in relation to that of the substrate and the rate at which it works.

(3) With the microscope, glycogen, or at least a substance which is very nearly akin to it, which very readily yields it, and which gives the characteristic port-wine colour with iodine, can be actually seen in the liver-cells. The liver of a rabbit or dog which has been fed on a diet containing much carbo-hydrate is large, soft, and very easily torn. Its large size is due to the loading of the cells with a liyaline material, which gives the iodine reaction of glycogen, and is dissolved out by water, leaving empty spaces in a network of cellsubstance. If the animal, after a period of starvation, has been fed on protein alone, less glycogen is found in the shrunken livercells; if the diet has been wholly fatty, little or no glycogen at all may be found. Glycogen can even be formed by an excised liver when blood containing dextrose is circulated through it.

A fact of great interest recently demonstrated is that in arimals where the mobilization of the hepatic glycogen is accelerated (as in rabbits after puncture of the medulla), glycogen in the form of granules and small masses can be seen in the blood capillaries (or rather sinusoids) between the liver cells, and in the sublobular veirs (Huber and Macleod). Chemical evidence has also isen obtained of the presence of a glycogen-like polysaccharide in the blood leaving the liver.

Formation of Glycogen from Protein.-In the liver-cells of the frog in winter-time a great deal of this hyaline material-this glycogen, or perhaps loose glycogen componnd-is present; in summer, much less. The difference is remarkable if we consider that in winter frogs have no food for months, while summer is their feeding-time; and at first it seems inconsistent with the doctrine that the hepatic glycogen is a store laid up from surplus sugar, which might otherwise be swept into the general circulation and excreted by the kidneys. It has been found, hovever, that 
the quantity of glycogen is greatest in autumn at the beginning of the winter-sleep, and slowly diminishes as the winter passes on, to fall abruptly with the renewal of the activity of the animal in the spring. The glycogen present at any moment is, therefore, believed to be a residue, which represents the excess of glycogen formed over glycogen used up; and the amount is larger in winter, not because more is manufactured than in summer, but because less is consumed. It is possible, indeed, to produce the "summer " condition of the hepatic cells merely by raising the temperature of the air in which a winter frog lives; at $20^{\circ}$ or $25^{\circ} \mathrm{C}$. glycogen disappears from its liver. Conversely; if a summer frog is artificially cooled, a certain a mount of glycogen accumulates in the liver. The meaning of this seems to be that at a low temperature, when the wheels of life are clogged and metabolism is slow, some substance, probably dextrose, is produced in the body from proteins in greater amount than call be used up, and that the surplus is stored as glycogen; just as in plants starcl is put by as a reserve which can be drawn upon-which can be converted into sugar-when the need arises. That carbo-hydrates may be formed from proteins (or their constituent amino-acids) has been shown in various waysfor example, by feeding dogs with almost pure protein (casein) after the production of permanent glycosuria by removal of the pancreas (p. 636). To induce the animal to take the casein it had to be mixed with a certain amount of butter, or serum, or meat extract. The amount of sugar excreted was much more than could possibly have come from the glycogen originally present in the animal's body, computing it on the most generous scale (4I grammes per kilogramme of body-weight, according to Pfliger), or from free carbohydrate present in traces in the food, or as prosthetic groups (p. 2) in the ingested protein. That the source of the sugar was protein and not fat was indicated by the fact that when the amount of protein food was increased, the dextrose and the nitrogen excreted increased proportionally (see also p. 535).

Glycogen-Formers.-As true glycogen-formers in the higher animals - that is, compounds whose elements (particularly the carbon) actually enter into the composition of the glycogen molecule-may be mentioned such substances as proteins (including gelatin), the fermentable sugars, and glycerin. In the case of proteins it is, of course, not the entire molecule which is transformed bodily into glycogen, but amino-acids yielded by them, or dextrose derived from the amino-acids. The liver is of itself capable of dealing only with the dextrose, and not with the amino-acids. At least, when the isolated liver (of the tortoise) is perfused with blood containing amino-acids no increase in the glycogen of the liver occurs. When glycerin is added to the blood the glycogen content of the liver is very distinctly increased. Glycerin is a trivalent alcrinol $\left(\mathrm{C}_{3} \mathrm{H}_{5} \mathrm{O}_{3}\right)$ whose aldehyde, obtained from it by gentle 
oxidation, is glycerose $\left(\mathrm{C}_{3} \mathrm{H}_{6} \mathrm{O}_{3}\right)$, a substance with the typical propertics of a sugar. In the laboratory it has been shown that two molecules of glycerose can be combined to form one molecule of sugar of the hexose type with six carbon atoms $\left(\mathrm{C}_{6} \mathrm{H}_{12} \mathrm{O}_{6}\right)$. A similar transformation is accomplished in the liver, and then a number of the monosaccharide molecules $\left(\mathrm{C}_{6} \mathrm{H}_{12} \mathrm{O}_{6}\right)$ are condensed with loss of water to form glycogen. Thus, $n\left(\mathrm{C}_{6} \mathrm{H}_{12} \mathrm{O}_{6}\right)-n \mathrm{H}_{2} \mathrm{O}=\left(\mathrm{C}_{6} \mathrm{H}_{10} \mathrm{O}_{5}\right) n$. Since glycerin is a normal product of the hydrolysis of fats, the possibility that the fats of the food may contribute through their glycerin component to glycogen formation must be admitted. The monosaccharides dextrose, levulose, and galactose gave a similar result, while the disaccharides cane-sugar and lactose caused no increase in the glycogen of the perfused liver, since the liver contains no ferment capable of splitting them into monosaccharides. And although the first step in the linking of the monosaccharide molecules would seem to be the formation of a disaccharide such as maltose, the glycogen molecule must apparently be built up from single 'bricks,' the monosaccharides, and cannot be constructed from bricks which are already coupled in pairs, the disaccharides. Of course, since the disaccharides are hydrolysed in the digestive tube to simple sugars, they are to be reckoned with the true glycogenformers, for in the intact body they are presented to the hepatic cells in the form of monosaccharides. It is probable that levulose and galactose are first changed into dextrose.

By the action of alkalies such structurally related sugars can easily be transformed into each other. Thus dextrose is an aldehyde of an alcohol with six carbon atoms, and levulose the corresponding ketohexose.

By oxidizing the alcohol we get an aldehyde or a ketone, according to whether a primary alcohol group $\left(\mathrm{CH}_{\mathbf{2}} \mathrm{OH}\right)$ or a secondary group (CH.OH) is oxidized, with the loss of two atoms of hydrogen. The aldehyde is characterized by the presence of the group $\mathrm{C} / \mathrm{H}$, the ketone by the group $\mathrm{CO}$. Both the aldehyde and the ketone are sugars, and since each contains six carbon atoms, they are both sugars of the group known as 'hexoses.' Dextrose, being not only a hexose but an aldehyde, may be called an 'aldohexose,' and levulose, being not only a hexose but a ketone, a ' ketohexose.'

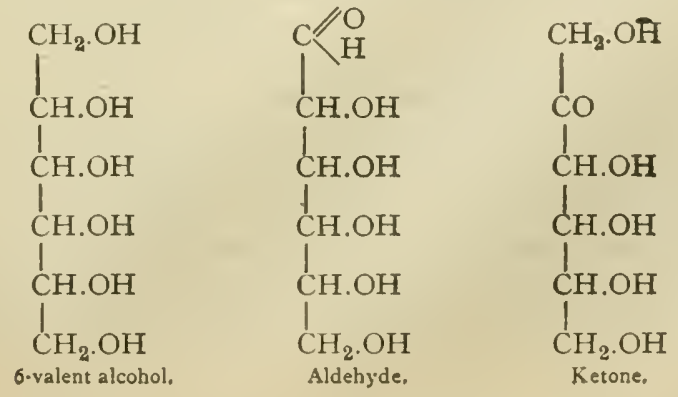


That levulose can be clianged into dextrose in the body is indicated by the observation that after extirpation of the pancreas in dogs the administration of levulose is followed by an increase in the excretion of dextrose mearly equal to the amount of levulose ingested. It is also stated that, when the surviving liver of a normal $\log$ is perfused with a suspension of washed blood-corpuscles to which levulose has been added, dextrose accumulates in the blood and levulose disappears from it.

It has not hitherto been proved that the fatty acid component of the food fats can be converted into glycogen. But a fatty acid, propionic acid, is capable of complete transiormation into dextrose when given either by the mouth or subcutaneously to dogs under the influence of phlorhizin (Ringer). Many other bodies are known to influence the formation of glycogen by 'sparing' substances which are true glycogen-producers, but their carbon does not actually take its place in the glycogen molecule. It has been shown that proteins can directly form glycogen or sugar apart from carbohydrate groups contained in the protein molecule. For the proteins of meat, gelatin, and casein are capable of forming 60 per cent. of their own weight of dextrose in diabetic metabolism, and even the end-products of pancreatic digestion of meat yield so much sugar that the greater part of it must have come from the amino-bodies, and not from a sugar-group in the protein. When given to dogs with total phlorhizin glycosuria (p. 55I), glycin and alanin are completely, glutamic and aspartic acids in great part (corresponding to about three carbon atoms of their respective molecules), converted into dextrose (Lusk and Ringer), and there is no reason to doubt that when such substances are produced by hydrolysis of protein in the normal body, and are not all utilized in rebuilding the bioplasm, a portion of the surplus, after deamidization, can be transformed into glycogen.

Extra-Hepatic Glycogen.-While the liver in the adult (containing as it does from 2 to Io per cent. of glycogen, or even, with a diet rich in sugar or starch, more than I 8 per cent.) may be looked upon as the main storehouse of surplus carbo-hydrate, depots of glycogen are formed, both in adult and fœtal life, in other situations where the strain of function or of growth is exceptionally hearyin the muscles of the adult $(0.3$ to 0.5 per cent. of the moist skeletal muscle, or on a carbo-hydrate regimen 0.7 to 3.7 per cent.), in the placenta, in many developing organs in the cmbryo (muscles, lungs, epithelium of the trachea, œsophagus, intestine, ureter, pelvis of kidney, and renal tubules). The fotus, however, is not, compared with the adult, especially rich in glycogen. In the adult under favourable circumstances the absolute amount of glycogen in the muscles may be several times greater than that in the liver, and 
usually the hepatic glycogen makes up considerably less than half the total glycorren of the body. That the muscles do not derive their glycogen by the migration of hepatic glycogen, but can themselves form it from (lextrose, has been shown by injecting that sugar subcutaneously into frogs after excision of the liver. The muscle glycogen was found to be increased.

Function and Fate of the Glycogen.-The glycogen store of the liver fulfils a different function from that of the muscles. This is indicated by the fact that when dogs, after being put on a given diet for two or tluree days, are starved for a time, and then put again on the original diet, the hepatic and the muscular glycogen behave differently at first during the period of re-alimentation. While glycogen accumulates in the liver in greater quantity than under normal conditions of nutrition, in the muscles it at first accumulates much less rapidly than normally. This is entirely in accordance with the view that the hepatic glycogen store has for its great function the regulation of the sugar content of the blood in the interest of all the tissues, while the glycogen store of the muscles and other tissues is mainly in the interest of their own nutrition and a source of energy for their own work. This does not imply that, when sugar is being absorbed in quantities too great for the liver to deal with after the current needs of the tissues have been satisfied, they do not add to their glycogen reserves. There is every reason to suppose that they do so, and thus act as a subsidiary regulating mechanism, although a less elastic one than that supplied by the liver. A third way in which a portion of the surplus sugar can be stored is in the form of fat.

When a fasting dog is made to do severe muscular work, the greater part of the glycogen soon disappears from its liver. When a dog is starved, but allowed to remain at rest, the glycogen still markedly diminishes, although it takes a longer time; and at a period when there is still plenty of fat in the body, there may be only a trace of hepatic glycogen left. The glycogen which is usually contained in the skeletal muscles also diminishes very rapidly in the first days of hunger, but the heart contains the normal amount of glycogen at a time when the proportion in the skeletal muscles has sunk to $\frac{1}{10}$ to $\frac{1}{30}$ of the normal.

These facts have been taken to indicate that glycogen and the sugar formed from it are the readiest resources of the starving and working organism, for the transformation of chemical energy into heat and mechanical work. To borrow a financial simile, the fat of the body lias sometimes been compared to a good, but rather inactive security, which can only be gradually realized; its organ-proteins to long-date bills, which will be discounted sparingly and almost with a grudge; its glycogen, its carbo-hydrate reserves, to consols, which can be turned into money at an lour's warning. Glycogen, on this view, is especially 
drawn upon for a sudden demand, fat for a steady drain, tissue-protein for a life-and-death struggle. While there may be some such difference in the tenacity with which the different kinds of reserve material are liclu back from consumption when the floating supplies are wearing low, modern investigation tends to the conclusion that the interchangeability of the various groups of nutritive substances is greater than had been supposed, and that in the long-run the cells-in normal circumstances at least-never work without dextrose, even after the glycogen store has been practically all consumed, but sccurc it from other sources.

Pavy has put forward the heterodox view that the glycogen formed in the liver from the sugar of the portal blood is never reconverted into sugar under normal conditions, but is changed into some other substance or substances, and he denies that the post-mortem formation of sugar in the hepatic tissue is a true picture of what takes place during life. But in spite of the brilliant manner in which he has defended this thesis, both by argument and by experiment, it must be said that the older doctrine of Bernard, which in the main we have followed above, is attested by such a cloud of modem witnesses that it seems to be firmly and finally established.

Fate of the Sugar-Glycolysis.-What, now, is the fate of the sugar which either passes right through the portal circulation from the intestine without undergoing any change in the liver, or is gradually produced from the hepatic glycogen? When the proportion of sugar in the blood rises above a certain low limit (about I. 5 or 2 parts per I,000), some of it is excreted by the kidneys (Practical Excrcises, p. 716).

A large meal of carbo-hydrates is frequently followed by a temporary glycosuria, but much depends upon the form in which the sugar-forming material is taken. We have seen that polysaccharides are quite incapable of absorption as such, and that they must be very completely hydrolysed in the lumen of the alimentary canal before their constituent sugars have any chance of reaching the blood. It is therefore not to be expected that the rapid absorption of such considerable quantities of sugar as would lead to its excretion should easily occur when the carbo-hydrate is in this form. Miura for example, after an enormous meal of rice (equivalent to $6 \% 4$ grammes of ash-and water-frce starch per kilo of body-weight), which, as he mentions, tasked even his Japanese powers of digestion for such food to dispose of, found not a trace of sugar in the urine. Dextrose, cane-sugar and lactose, on the other liand, when taken in large amount, were in part excreted by the kilneys, as was also the case with levulose and maltose in a dog (Practical Exercises, p. 7I7).* The amount of any carbo-hydrate which can be caten

* Twenty-four healthy students, whose urine had previously been shown to be free from sugar, ate quantities of cane-sugar varying from 250 grammes to $75^{\circ}$ grammes. The urine was collected in separate portions for twelve to twenty-four hours after the meal. In only three cases was reducing sugar found in the urine (by Fehling's and the phenyl-hydrazine test), and then merely in traces. In eight cases cane-sugar was found, and estimated by the polarimeter, and, after boiling with hydrochloric acid, by Fehling's solution. 
without the appearance of sugar in the urine is sometimes called the assimilation limit for that carbohydrate. The attempt has been made to fix the limit of tolerance of dextrose for normal persons and persons suffering from incipient diabetes, with the object of aiding in early diagnosis of that condition. But the limit varies with so many conditions, only some of which can be controlled, that such observations are not easily interpreted.

Except as an occasional phenomenon, glycosuria other than alimentary is inconsistent with health; and therefore in the normal body the sugar of the blood must be either destroyed or transformed into some more or less permanent constituent of the tissues. The transformation of sugar into fat we have already mentioned, and shall have again to discuss; it only takes place under certain conditions of diet, and no more than a small proportion of the sugar which disappears from the body in twenty-four hours can ever, in the most favourable circumstances, be converted into fat. The dextrose which is taken up from the blood by the tissues and there condensed to glycogen suffers sooner or later the converse change, in all probability under the influence of diastases or glycogenases produced in the cells, and reappears as dextrose to take its place in the cellular katabolism and begin the series of cleavages and oxidations by which its chemical energy is set free. Accordingly, it is the destruction of sugar which concerns us here, and there is every reason to believe that this takes place, not in any particular organ, but in all active tissues, especially in the muscles, and to a less extent in glands.

It has been asserted that the blood which leaves even a resting muscle, or an inactive salivary gland, is poorer in sugar than that coming to it; and the conclusion has been drawn that in the metabolism of resting muscle and gland sugar is oxidized, the carbon passing off as carbon dioxide in the venous blood. This is indeed extremely likely, for we know that, when the skeletal muscles of a rabbit or guinea-pig are cut off from the central nervous system by curara, the production of carbon dioxide falls much below that of an intact animal at rest; and the carbon given off by such an animal on its ordinary vegetable diet can be shown, by a comparison of the chemical composition of the food and the excreta, to come largely from carbo-hydrates. But, considering the relatively feeble metabolism of muscles and glands when not functionally excited, the large volume of blood which passes through them, the difficulty of determining small differences in the proportion of sugar in such a liquid, the possibility that even in the blood itself sugar may be

The greatest quantity of cane-sugar recovered from the urine was 8 grammes (7.92 grammes by Fehling's method and 8.29 grammes by the polarimeter); the highest proportion of the quantity taken which appeared in the urine was 2.5 per cent. When dextrose was found, cane-sugar was always present as well. 
destroyed, or that it may pass from the blood, without being oxidized, into the lymph, too much weight may be easily given to the results of direct analysis of the in-coming and out-going blood. And although the results of Chauveau and Kaufmann, obtained in this way, fit in fairly well with what we have already learnt by less direct, but more trustworthy, methods, such as the study of the respiratory exchange, they cannot be accepted as yielding exact quantitative information. They found that in one of the muscles of the upper lip of the liorse the quantity of dextrose used up during activity (feeding movements) was 3.5 times as much as in the same muscle at rest, and this corresponded with the deficit of oxygen in the blood entering the muscle, and with the excess of carbon dioxide in the blood leaving it. More dextrose was also destroyed in the active than in the passive parotid gland of the horse, but the excess per unit of weight of the organ was far less than in muscle. In dogs whose abdominal viscera have been removed, so that they constitute practically preparations composed of skeletal muscles it has been found that the amount of dextrose which disa ppears from Ioo grammes of blood per minute varies from 0.47 to $\mathrm{I} \cdot 8$ milligra nmes, the irregularities probably depending largely upon the irregular consumption by the muscles of the glycogen stored in them (Macleod and Pearce).

Intermediary Metabolism of Carbo-Hydrates.-Concerning the processes and the stages by which dextrose is destroyed in the tissues, we have no very exact information, and it cannot be definitely stated at present what share is taken by cleavage and what by oxidation, or rather through what intermediate products, formed, it may be, now by simple cleavage, now by oxidation, again by a combination of cleavage and oxidation, the dextrose molecule is finally resolved into carbon dioxide and water. It must be remembered that the synthetic powers of animal cells are now known to be very extensive. They build carbo-hydrates, fats, phosphaticles, and proteins, as well as destroy them, and at any of the earlier stages at any rate the degradation products of dextrose, or some of them, may be utilized in the construction of new compounds-for example, of fateither in the cells where they arise or elsewhere in the body. In like manner the decomposition of a molecule of dextrose begun in one cell or in one tissue may be consummated in another to which intermediate products are conveyed in the blood. In such ways it is obvious that the katabolic processes may be finely regulated both qualitatively and quantitatively in accordance with the specific wants of different organs and the intensity of their functional activity from time to time. It must be said, however, that at present there are few definitely ascertained facts to guide us in trying to form a scheme of the actual changes which occur in the intermediate katabolism of carbo-hydrates, and the sequence which 
they normally follow. Glycuronic acid has been previously mentioned as a substance occurring even in normal urine in small amount. It is very closely related to dextrose, as a comparison of their constitutional formulæ shows:<smiles>CC(O)(CO)CC(O)C(O)C(C=O)C(O)C(=O)O</smiles><smiles>CC(C)(C)CC(O)C(O)C(O)C(O)C(=O)O</smiles><smiles>CC(C)(C)C(O)C(O)C(O)C(=O)O</smiles>

Glycuronic acid agrees with dextrose in containing the characteristic aldehyde group $\mathrm{C} / \mathrm{O}$, but differs in that by oxidation two atoms of hydrogen in the primary alcohol group $\mathrm{CH}_{2} \mathrm{OH}$ have been replaced by one atom of oxygen. There is reason to believe that in the tissues glycuronic acid can be formed from dextrose in the same way, possibly through the mediation of an enzyme, and it may therefore represent a stage in the katabolism of sugar. But it is not known whether this is a normal transformation through which the whole or the greater part of the dextrose passes, or only a transformation involving a small part of the sugar under normal conditions. The appearance in the urine of large quantities of glycuronic acid, paired as already explained with various conpounds, in certain pathological states or after the administration of certain drugs (p. 48\%), might be explained either as the result of an increased production of that substance through a deflection of the normal trend of carbo-hydrate degradation, or as the result of a failure on the part of the cells to further transform the glycuronic acid in the quantities normally produced.

Lactic acid is the one intermediate stage in the decomposition of dextrose in the tissues whose importance seems to be definitely ascertained. The muscles and the liver have been proved to possess the power of producing lactic acid from dextrose obtained directly from the blood or from the hydrolysis of their own store of glycogen, and there is little doubt that this power is shared by many, perhaps by all, of the other organs. There is also good evidence that the lactic acid thus formed can be, and under normal conditions is, in large part oxidized so as eventually to yield carbon dioxide and water, although there is reason to believe that a portion of it may be utilized for the synthesis of more complex bodies.

The chemistry of the change or series of changes by which lactic acid is produced from dextrose and the end-products, carbon dioxide 
and water, from lactic acid has given rise to much discussion, and is not yet clearly known. The following scheme, based on the researches of Embden and others, and quoted from Abderlualden, illustrates one suggestion as to the course of the series of transformations, althougl it must be taken only as a diagram of the sequence of some of the possible stages. A molecule of dextrose is represented as giving rise to two molccules of glyceric aldehyde, eacli of which then yields a molecule of lactic acid. Each molecule of lactic acid, losing two atoms of liydrogen, becomes converted into a molecule of pyruvic acid, which by the loss of the elements constituting a molecule of carbon dioxide bccomes acetaldehyde or acetic aldehyde, and this by oxidation acetic acid, which is then oxidized to carbon dioxide and water. Thus-<smiles>CC(C)(C)C(C)(O)C(=O)O</smiles>

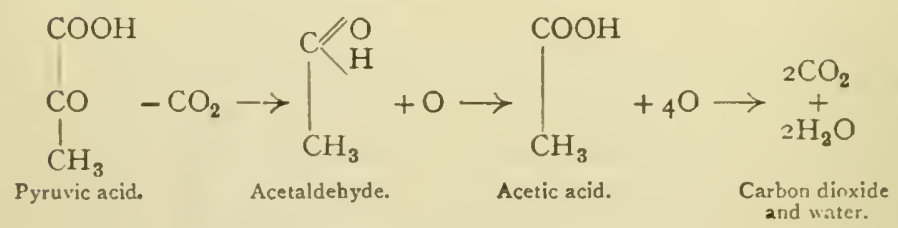

It has been shown that acetaldehyde and carbon dioxide are formed from pyruvic acid by the action of a ferment contained in ycast, and there is some evidence that a similar transformation may occur in the liver.

It is to be particularly remarked that according to this scheme the whole of the dextrose molecule is still represented in the lactic acid formed from it. Up to this stage no part of the molecule has been burnt. Nearly the whole of the cluemical energy-i.e., all but about 3 per cent. of it-is still available. For a gramme of lactic acid yields $3,66 \mathrm{I}$, and a gramme of dextrose 3,762 , small calories on complete combustion. The intermediate products of the decomposition may therefore be transported from the place of origin and utilized elsewhere with scarcely any loss of energy. Further, it is indicated in the scheme that the degradation process is not merely a series of cleavages and oxidations, but that these may be interspersed with stages of reduction. It is also clearly suggested that at certain points the metabolism may become recessive and syn- 
theses be started, which may go far to retrace the steps of the preceding katabolism in respect to a portion of the dextrose.

Thus, lactic acid can be retransformed into dextrose (Mandel and Lusk), and this, of course, into glycogen. Pyruvic acid can be changed into lactic acid by reduction, and dextrose can in this way be again produced. There is evidence also that under certain conditions pyruvic acid can yield dextrose in the organism by a different reaction, being changed into acetaldehyde, which can then undergo transformations leading back to dextrose (Ringer). The formation of fat from sugar may also start from some of the stages displayed in the scheme, for it is only a short step to obtain by the reduction of glyceric aldehyde its alcohol glycerin. And from acetaldehyde fatty acids can be derived.

Not only does lactic acid afford a point of contact between the metabolism of carbo-hydrates and that of fats-a junction, so to speak, where these two great metabolic currents cross each other, and where material originating in the one may be shunted into the other-but it also affords a point of junction and interchange with the current of protein metabolism. For example, the amino-acid, alanin, yields as a decomposition product a compound called methylglyoxal $\left(\mathrm{CH}_{3}\right.$. $\left.\mathrm{CO} . \mathrm{CHO}\right)$, a ketonic aldehyde, which by the assumption of a molecule of water can be changed into lactic acid. It may also be one of the intermediate stages in the decomposition of dextrose as a precursor of lactic acid, and one of the ways in which the conversion of amino-acids into dextrose is accomplished may be through this link. The presence of a ferment glyoxalase, or probably more than one ferment which rapidly changes methylglyoxal into lactic acid, has been demonstrated in tissue extracts and in leucocytes. The same change is effected when blood containing methylglyoxal is perfused through an excised surviving liver. The reaction can be reversed for methylglyoxal like lactic acid when given to an animal rendered diabetic by phlorhizin can be shown to vield dextrose, possibly being first converted into glyceric aldehyde. The conversion of methylglyoxal into lactic acid is also a reversible reaction, for in vitro, at any rate, lactic acid readily vields methylglyoxal (Dakin).

Pyruvic acid is another possible link. As has just been mentioned, it probably forms a stage in the decomposition of dextrose, and has, in addition, chemical relations on the one hand to certain of the aminoacids, especially to alanin, and on the other to glycerin and even to fatty acids. Thus-
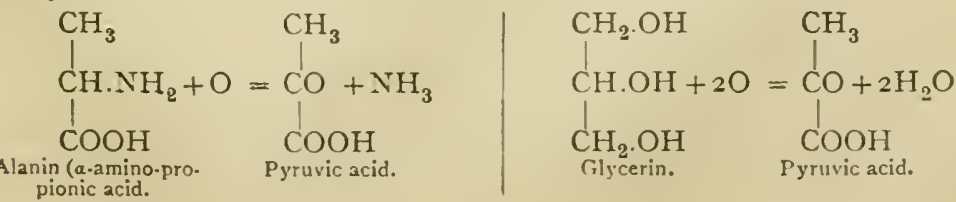
The following scheme, doubtless incomplete, illustrates a probalile chemical sequence through which the interconversion of alanin, lactic acid, methylglyoxal and dextrose may be brought about by reactions only involving the addition or removal of water or ammonia (Dakin):

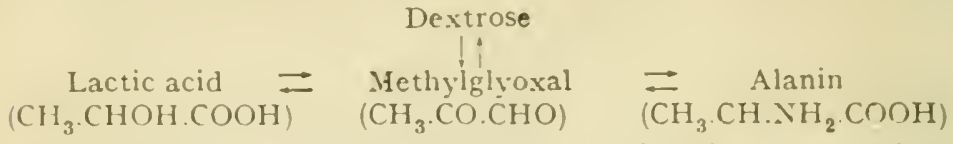

Since pyruvic acid can be reduced to lactic acid, any reaction in which it play's a part in the intermediary metabolism of carbohydrates or proteins can also be fitted into the scheme.

The more completely the various steps in the metabolism of the three great groups of food substances are unveiled, the more clearly does it appear that, far from being independent circuits, the three currents are constantly exchanging materials with each other.

It is to be supposed that in many of these transformations enzymes are concerned, although comparatively little is definitely known as to this. Normal blood itself has been credited with a ferment which has the power of destroying sugar (glycolysis). But with rigid aseptic precautions the loss of sugar, even in several hours, is small, and it is doubtful whether such a ferment exists. Even under the most favourable circumstances the quantity of dextrose which blood can destroy is so small a fraction of that which disappears in the same time in the body, that it is probably of no importance in carbo-hydrate metabolism (Macleod). On the other hand, Cohnheim stated that while no glycolytic ferment can be demonstrated in the pancreas, and only an exceedingly weak glycolytic action in muscular tissue (Brunton), by combining extracts of pancreas and extracts of muscles, distinct glycolysis, due to a ferment action, could be produced. He suggested that the glycolytic ferment is activated by another substance, as trypsinogen is activated by enterokinase (p. 372). This announcement aroused great interest, since it is known that the pancreas is intimately concerned in the metabolism of sugar. That sugar disappears under the conditions of Cohnheim's experiments has been confirmed by a number of observers. But his interpretation of his results has not been generally accepted. According to Levene and Mejer, the dextrose, far from being burnt, seems to be condensed to a polysaccharide, and can be recovered by hydrolysing this compound when the mixture is acted on by dilute acid. The action of the pancreas-muscle mixture is, therefore, not a true glycolysis. Indeed, of all the tissues investigated by Levene, leucocytes alone can be credited with a real glycolytic action. Excision of the pancreas in dogs causes permanent glycosuria (pancreatic diabetes) (v. Mering and Minkowshi), which is prevented if a portion of the pancreas be 
left (p. 636). Diabetes in man is known to be frequently associated with pancreatic lesions. Although much still remains obscure, the study of this pathological form of glycosuria and of the experimental glycosurias has thrown light upon the normal metabolism of carbohydrates and upon those regulative mechanisms whose breakdown is responsible for the excretion of sugar. It will be best to discuss the experimental glycosurias first, and to begin with the form which probably is better understood than any other, the so-called puncture glycosuria.

Puncture Glycosuria-Sugar-Regulating Mechanism.-An artificial and temporary glycosuria, in which the sugar in the urine undoubtedly arises from the hepatic glycogen, can be caused by puncturing the medulla oblongata in a rabbit-for example, at a level between the origins of the auditory nerves and the vagi. It is stated that a puncture of the thalamencephalon, or 'tween-brain (p. 850), produces the same effect. If the animal has been previously fed with a diet rich in carbo-hydrates-that is, if it has been put under conditions in which the liver contains much glycogen-the quantity of sugar excreted by the kidneys will be large. The immediate cause of the glycosuria is an increase in the sugar content of the blood (hyperglycæmia), an increase which is most pronounced in the blood of the hepatic vein. If, on the other hand, the animal has been starved before the operation, so that the liver is free, or almost free, from glycogen, the puncture will cause little or no sugar to appear in the urine, and the proportion of sugar in the blood will remain normal. That nervous influences are in some way involved in the mobilization of the glycogen reserve of the liver is shown by the absence of glycosuria if the splanchnic nerves, or the spinal cord above the third or fourth dorsal vertebra, be cut before the puncture is made. But sometimes these operations are themselves followed by temporary glycosuria, due, it is believed, to irritation of the same efferent nervous path whose elimination when the splanclunics are divided prevents the glycosuria. The simplest explanatis, n of the phenomena is that a 'sugar centre '-that is to say, a centre which has the important office of regulating the sugar content of the blood by governing the rate at which glycogen is built up and decomposed in the liver, as the salivary centre regulates the rate at which the constituents of saliva are formed and discharged-has been injured or irritated by the puncture. If a nervous centre does in fact preside over this internal secretion of the liver, it will, of course, be connected with efferent and afferent nerves. The former, as defined by the experiments alluded to, seem to be confined to the splanchnic nerves; the latter are believed to run especially, though not exclusively, in the vagus. Section of the vagi has no effect either in causing glycosuria of itself or in preventing the 'puncture' glycosuria, but stimulation of the central ends of these 
and of other afferent nerves may cause sugar to appear in the urine, although not, it is said, if precautions are taken to prevent any degree of asphyxia. Aspliyxia produces an increase in the sugar content of the blood, an increase in the flow of urine and glycosuria.

It has usually been assumed that this action of asphyxia is due to the effect upon the centre of blood over-rich in carbon dioxide (and other metabolic products) or impoverished as regards oxygen. But there is some evidence that the altered blood may also affect the liver-cells directly, or, what comes to the same thing in the long-run, that interference with the internal respiration of the hepatic tissue, operating, it may be, througl an increase in the concentration of the hydrogen ions, upsets the equilibrium of those intracellular reactions by which glycogen is formed from dextrose and dextrose from glycogen. In like manner it may be supposed that under normal conditions the rate of transformation of the hepatic glycogen into dextrose is adjusted to the dextrose content of the blood, not only by reflex nervous impulses passing through the sugar-regulating centre, but also by the direct influence of the dextrose itself circulating in the blood, upon whose concentration the reaction of the centre on the one hand and of the liver-cells on the other may in part depend. So that when the proportion of sugar in the blood tends to sink we may perhaps picture the centre as sending impulses to the liver which increase the rate at which the glycogen is hydrolysed; and when the proportion tends to rise, we may think of it as sending impulses which inhibit the hydrolysis, both effects being accentuated by the direct influence of the changes of concentration on the hepatic cells. Whatever the mechanism may be through which the puncture hastens the transformation of glycogen into dextrose in the liver, there is no evidence tliat the amount of the enzyme which hydrolyses the glycogen is increased. Whether the action of the cnzyme is favoured in some other way-e.g., by the production of a co-ferment or by some change in the condition of the glycogen which renders it more open to attack-is unknown.

Certain facts have recently been brought forward which go to show that the action of the splanchnic fibres on the liver is not a direct action, but that in some way or other the concomitant activity of the adrenal glands is essential. For if the adrenals liave been previously extirpated, the puncture does not cause glycosuria. It was at first thought that the reason for this was that the removal of the adrenals is itself followed by the disappcarance of glycogen from the liver, and, as lias been pointed out, the presence of glycogen in the liver is essential to the success of the puncture experiment. The matter, however, is not so simple. For although in certain animals-e.g., the dog-the liver does lose all its glycogen when the adrenals have been taken away, this is not the case in the rabbit, 
and yet in the rabbit also the urine remains free from sugar after puncture in the absence of the adrenal glands. In some way or other, then, the adrenals do intervene in the production of puncture glycosuria. The observation, which is easily confirmed, that the injection of adrenalin (or epinephrin) (p. 550) under the skin or into the blood, or into one of the serous sacs, does cause a pronounced increase in the sugar content of the blood, and the appearance of dextrose in the urine, secmed at first to supply the missing link in the chain of evidence. What could be simpler than the assunıt: $n$ that the splanchnic fibres stimulated in the puncture experinus were fibres going not to the liver, but to the adrenals, which ocrasioned an outpouring of adrenalin into the blood, and that puncture glycosuria was therefore merely a particular case of adrenalin glycosuria? It is known that excitation of the splanchnic nerves cail es the passage of adrenalin into the blood of the adrenal veins ( $\mathrm{p} 66 \mathrm{I}$ ). It is known that puncture of the medulla oblongata diminishes tle epinephrin content of the adrenal glands. The argument seen ed straightforward, and the adrenal hypothesis of puncture glycosi:ila triumphant. As soon, however, as the matter was put to the test of quantitative experiments, the hypothesis began to crumble. It was shown, for example, that during a stimulation of the splanchnic nerves sufficient to cause a decided increase in the dextrose content of the blood, a quantity of adrenalin was given off to the adrenal veins, which, when mingled with the rest of the blood on its way to the liver, could not possibly amount to more than one in a hundred million parts of blood, a concentration in which adrenalin, when introduced artificially into the blood-stream, produces no glycosuria whatever. Still more significant is the fact that, after destroying the hepatic plexus, stimulation of the splanchnic nerves causes no increase in the blood-sugar in spite of the increased output of adrenalin by the way of the adrenal veins. On the other hand, excitation of the hepatic plexus causes hyperglycæmia (Macleod and Pearce). It is not, then, a direct action on the liver of epinephrin secreted in response to stimulation of splanchnic fibres supplying the adrenal glands which is responsible for the increase in the dextrose content of the blood. The adrenals, however, play some part. For in their absence stimulation of the hepatic plexus is not followed by hyperglycæmia. But whether this is due to general derangement of the normal carbo-hydrate metabolism in their absence, or to the loss of some special influence on the liver, without which stimulation of the hepatic plexus is ineffective, is unknown.

Although several of the operations which lead to temporary glycosuria undoubtedly bring about changes in the hepatic circulation, it is as yet impossible to say whether vaso-motor effects contribute essentially to the result, or whether it is due entirely to nervous stimulation of the liver-cells, or to withdrawal of such 
stimulation or control (see also p. 509). There is some evidence that excitation of the uncut great splanchnic nerve (on the left side) in dogs nay cause hyperglycremia, diuresis, and glycosuria, even under conditions in which as far as possible circulatory cffects are climinated. Contrariwise, when in the puncture experiment on an unnarcotized animal the small instrument does not wound the medulla oblongata in the right place, a rise of blood-pressure duc to excitation of the vaso-motor centre may occur without any glycosuria. But absolute proof of the existence of glycogenolytic nerve fibres going to the liver-that is, fibres whose stimulation accelerates the hydrolysis of glycogen into dextrose (Macleod)-has not yet been brought forward.

Adrenalin Glycosuria.--In adrenalin glycosuria the sugar-content of the blood is increased. A given quantity of adrenalin introduced subcutaneously produces a more marlied hyperglycæmia and glycosuria than the same amount injected into a vein or into muscle. The best evidence is that the glycosuria is produced by some action on the liver, possibly through the excitation of sympathetic fibres controlling the production of dextrose from glycogen (Underlill and (losson), or by a direct effect on the hepatic cells, which hastens the normal transformation of glycogen into dextrose, or hinders the normal transformation of dextrose into glycogen. It has been stated that in the isolated surviving liver of the frog adrenalin causes the glycogen to be rapidly converted into dextrose. While this confirms the view that experimental adrenalin glycosuria is due to an action on the liver which increases the sugar-content of the blood, it does not necessarily show that the action is exerted directly on the hepatic cells without the intervention of nerve fibres. For the sympathetic nerve-cndings may survive a considerable time. The theory that epinephrin causes glycosuria by inhibiting the internal secretion of the pancreas, and that the condition is therefore a particular variety of pancreatic diabetes, is erroneous. Adrenalin glycosuria does not seem to be in any way related to true diabetes. The complete metabolism of dextrose is not interfered with. Indeed, a much larger proportion of the total heat produced cones from the destruction of sugar after the subcutaneous injection of epinephrin into dogs than in the normal animals (Lusk and Riche). If in spite of this glycosuria ensues, it is only because the carbohydrate reserve of the body is mobilized so rapidly that it cannot possibly be all consumed. Nor dacs epinephrin cause any increased production of sugar from protein or from fat. For in dogs rendered diabetic by phlorhizin and freed from glycogen by shivering, injection of epinephrin is not followed by an increase of either sugar or nitrogen in the urine (Ringer). After repeated injections of adrenalin, a tolerance for it is established, and glycosuria is no longer caused. 
Fhlorhizin Glycosuria, produced by subcutaneous injection of the glucoside phlorhizin, agrees with pancreatic, but differs from puncture diabetes in this, that it can be produced in an animal free from glycogen, and is accompanied by extensive clestruction of proteins. It differs from other forms of diabetes in being associated, not with an increase, but witl a diminution, in the sugar of the blood. This is best explained by supposing that the phlorhizin acts on the kidney in such a way as to increase the permeability of the glomerular epithelium for sugar, or (in terms of the secretion theory of urine formation) in such a way as to increase its sensitiveness to the stimulus of sugar circulating in the blood. The sugar is therefore rapidly swept out of the circulation, and this leads secondarily to an increased production of sugar to make good the loss. In addition, within certain limits there is a total inability on the part of the body to consume dextrose.

After the preliminary sweeping out of the sugar already in the body, a definite ratio is established between the dextrose and the nitrogen eliminated in the urine (dextrose : nitrogen : : 3 .6or 37.1 ). The sugar at this stage is produced entirely from proteins, and not at all from fat. It is a fact of considerable interest that, if small quantities of dextrose are now given, the amount of protein destroyed is reduced to some extent, although all of the dextrose is excreted, and none of it is burnt (Ringer). This supports the hypothesis of Landergren that in starvation some of the protein is metabolized for the formation of the indispensable dextrose, and that this fraction can be 'spared' by carbohydrate, though not by fat. The protein metabolized is so much increased under the influence of phlorhizin that it exceeds the starvation requirement by a greater amount than in pancreatic diabetes, perhaps because the diminished content of sugar in the blood constitutes a more insistent call upon the proteins to produce sugar. In pancreatic diabetes, where hyperglycæmia exists, there can at least be no reason for the formation of sugar from protein for the maintenance of the normal sugar-content of the blood, and it is interesting that in this condition the giving of dextrose does not seem to spare any protein (p. 606). The degree of intolerance for carbo-liydrates in pathological diabetes may be arrived at by putting the patient on a diet of protein and fat (rich cream, meat, butter, and eggs), and determining the ratio of dextrose to nitrogen excreted. If it is 3.6 or $3.7: \mathrm{I}$, intolerance is complete, none of the dextrose produced from protein being burned (Lusk and Mandel).

Glycosuria can be caused in many other ways than those already mentioned. Sometimes the action seems to be a direct one on the sugar-regulating centre-e.g., in concussion of the brain, occlusion and subsequent release of the arteries supplying the brain and cervical cord, and acute hæmorrhage. Carbon monoxide has a 
similar action owing to the deficiency of oxygen occasioned by it. Many drugs also cause glycosuria, including curara, morphine, strychnine, phosphorus, chloroform, ether, and other substances, some of which may' act on the 'sugar centre,' although others-c.g.. plospliorus and chloroform-are poisons which can affect the liver directly. It is probable that some of the experimental hyperglycamias are due to an associated acidosis. For when the hydrogenion concentration of the blood is increased the transformation of glycogen into dextrose in the liver is accelerated. The administration of alkali is said to have a beneficial influence upon the oxidation of dextrose in dogs after total or partial extirpation of the pancreas (Murlin and Kramer). Injection of water or physiological salt solution into the bile-ducts, or into the mesenteric veins, or of salt solution in considerable amount into the general circulation, is followed by glycosuria (Fischer, etc.). It is a mistake to apply the term diabetes to most of the forms of artificial hyperglycamia and glycosuria. The condition produced by removal of the pancreas, which will be returned to in Chapter XI., is, however, a true diabetes, a derangement of metabolism of the samo general nature as that which underlies human diabetes.

Diabetes Mellitus.-In the natural diabetes of man, as in all the forms of glycosuria mentioned, with the exception of that produced by phlorhizin, the immediate cause of the glycosuria is tlie increase of sugar in the blood. Instead of the I part per I,O00, or a little more or less, which constitutes the normal proportion in a healt ly man, in diabetes 3 or 4 parts, and in exceptional cases even 7 to $\mathrm{ro}$ parts per $\mathrm{I}, 000$ may be present. The riddle of diabetes is the cxplanation of this persistent hyperglycæmia. Innumerable hypotheses have been framed to account for this, but on the whole three possibilities have been emphasized: (I) Tliat the power of temporarily storing carbohydrates is deranged; (2) that the power of the tissues to utilize carbo-hydrates (i.e., eventually dextrose) is climinished or abolished; (3) that too much sugar is produced in the body. In addition, some writers have postulated a fourth factor to explain certain cases (of so-called ' renal diabetes ') - to wit, an increase in the permeability of the kidney's for sugar, as in phlorlizin glycosuria. Lest the student should be bewildered amongst all these theories, he should take note that while the second factor is now recognized as the essential one, there is some reason to believe that diabetes mellitus is not in every case a single pathological condition, but may comprise a group of such conclitions. Some cases may therefore present a pirture conforming closely to one or to another of the experimental glycosurias, but others a picture compounded of features characteristic of two or of several of these experimental conditions. 
It is possible that in some cases the sugar coming from the alimentary canal passes entirely or in too large amount through tl:c liver, owing to a deficiency in its power of forming glycogen. Bur although in certain cases of diabetes specimens of the hepatic cells, obtained by plunging a trocar into the liver, have been found free from glycogen, in others glycogen has been present. The muscles also are usually stated to be much poorer in glycogen than norma! muscles, but this might just as well be the case berause glycogen was being transformed into sugar with abnormal ease as because there was interference with glycogen formation. Indeed, it is saic that in the heart muscle of depancreatized dogs there is more glyccgen than in normal heart muscle. It must be carefully remembered that the amount of glycogen present in a tissue gives no information as to the rate at which it is being formed or decomposed. And if the cause of the supposed defect in glycogen-forming power be the absence of a glycogen-forming ferment, or its production in too small an amount, the same circumstance may occasion a too tardy: transformation into sugar of whatever glycogen happens to be present. In this case the sugar-regulating function of the glyccgen store would be equally lost, whether the storehouses were permanently filled with long-formed glycogen or only half-filled or empty.

In addition to an interference with the due and regulated storage of the surplus sugar as glycogen, it has usually been thought necessary for a rational explanation of the facts of diabetes, to assume that from some change in the tissues sugar has ceased to be a food for them, or is used up in smaller amount than in the healthy bodv. More and more the evidence points to this as the fundamental change both in the human disease and in experimental pancreatic diabetes.

Why the tissucs cannot decompose and utilize dextrose as they normally do, if it be really the case that they fail in this regard, is a question of great interest, but as yet no satisfactory answer can be given. It appears probable that the failure occurs at one or more of the earliest stages in the intermediate metabolism of carbchydrates (p. 542) or in the prelininary processes, whatever the $y$ may be, which, without profoundly altering the dextrose molecule, prepare it for the series of decompositions, in the course of which it cventually parts with all its chemical energy. For it has been shown that many of the products of the cleavage or oxidation of sugar, even those in which the decomposition has proceeded but a little way-e.g., glyconic and glycuronic acids (p. 543)-are completely utilized by the tissues of diabetics and of depancreatized dogs. And the derangement in the normal sequence of events, of whatever nature it may be, is not so deep-reaching as to prevent 
the retracing of the chemical steps by which sugar is synthesized from such derivatives of the proteins as a mino-acids ur their further degradation products. As to the actual cause of the alleger incapacity of the tissues to consume dextrose, the cliange has by sone been supposed to be the loss or diminution of a glycolytic ferment or a substance necessary for the activation of such a ferment. And although the sugar-destroying power of blood from diabetic patients, or from animals in which glycosuria has been caused by phlorhizin, is not at all inferior to that of healthy blood, it has been maintained that the intracellular glycolytic ferments, if such really exist, are much less active, especially in the more severe forms of the disease, which conform so closely in their clinical manifestations to the picture presented by the depancreatized animal. Nevertheless, up to the present all attempts to satisfactorily demonstrate for isolated tissues a loss or even a diminution in the capacity to utilize dextrose have broken down. In eviscerated dogs, for example-that is, in preparations consisting mainly of skeletal muscle-it has been found impossible to make out any deficiency as compared witlı normal animals in the amount of dextrose disappearing in a given time from the blood, even when the animals have been deprived of the pancreas as long as a week before the experiment, and therefore exhibit the condition of pancreatic diabetes in full intensity (Macleod and Pearce). This conclusion has been confirmed for the isolated heart-lung preparation.

As regards the hypothesis that an increased production of sugar from proteins, or it may be from fat, is the essential proximate cause of the hyperglycæmia and the glycosuria, there is no good evidence that this factor, acting by itself in the absence of a derangement of the regulative influence of the glycogen store, and in the absence of a derangement of the normal katabolism of dextrose, is ever responsible for pathological diabetes. But a secondary overproduction of sugar unquestionably occurs in many cases. The tissues, bathed as they are in liquids rich in dextrose, are nevertheless starving for sugar, if they cannot use what is offered to tlem and the body labours to avert the famine by increasing its production of sugar, the sugar-forming tissues being stimulated to their task either through nervous influences or by chemical messengers circulating in the blood.

In depancreatized dogs, and in dogs under the influence of phlorhizin, glycerin, given by the mouth, causes an increase in the excretion of sugar up to two or three times the original amount. The giving of fat does not increase the amount of sugar excreted, which, however, is increased by such substances as egg-yolk, which contain lecithin. These should accordingly be avoided in cases in which a strictly antidiabetic diet is desired. It is much more in:portant to exclude carbo-hydrates largely or entirely from the food, 
although oatmeal and potatoes are said to occupy an exceptirnal position, and have even been recommended as beneficial. Calcium chloride has been stated to diminish the sugar excretion in diabetes (Boiger), and it has a similar effect in certain of the artificial glycosurias (Brown, Fischer).

In many cases, even when carbo-liydrates are completely; or almost completely, omitted from the food, sugar, derived from the breaking-down of proteins, and possibly to some extent from fats, still continues to be excreted, although in smaller quantity. Other products formed or imperfectly transformed in the deranged metabolism, especially of fats, such as acetone, aceto-acetic acid, and oxybutyric acid (the so-called acetone bodies), may also appear in the urine (ketonuria), or, accumulating in the blood, may, by uniting with its alkalies, seriously diminish the quantity of carbon dioxide which that liquid is capable of carrying, and thus lead to the condition known as diabetic coma. The small amount of carbon dioxide in the venous blood may also be partly due to the hyperpnœa, marked by increased depth of the respiratory movements produced by stimulation of the respiratory centre by other substances than carbon dioxide. The increased rentilation causes a fall in the carbon dioxide pressure in the alveolar air, and therefore an increased elimination of that gas from the blood. This form of coma appears to be really in part an acid-poisoning comparable to the condition produced in animals by doses of mineral acids too large to be neutralized by the ammonia split off from the proteins. The administration of very large doses of alkalies (sodium bicarbonate, for instance, to the amount even of hundreds of grammes) has been recommended for the treatment of this serious complication, and in some cases it is successful in staving it off for a time. Often, however, in spite of a prolonged course of treatment, during which the urine has continued distinctly alkaline, fatal coma eventually occurs. The coma then is not merely a symptom of acidosis, but is also due to the specific toxic effects of the acids even when neutralized. Other toxic products may also be formed in the deranged metabolism. The appearance of the acetone bodies in diabetes presents a problem which cannot be said to have been as yet completely solved. Oxybutyric acid, from which aceto-acetic acid and acetone are easily derived (p. 567 ), seems to be one of the intermediate steps in the normal metabolism of fats. But whereas under ordinary circumstances it is readily oxidized in the body, in diabetes the power of the tissues to burn oxybutyric acid seems to suffer just as does the power to utilize dextrose. The suggestion that in diabetes the abnormally great consumption of fat entailed by the loss of availability on the part of the carbo-hydrates causes the intermediary metabolism of fats to be scamped, as it were, is not satisfactory. For many animals and some races of men dwelling 
in cold climates consume with impunity much greater quantities of fat than any diabetic ganism.*

\section{Section II.-The Metabolism of Fat.}

Chemistry of Fats. - The fats are compounds (esters) of an alcohol with fatty acids, and can be split, with assumption of water, into these constituents by the action of acids or alkalies or of enzymes (lipases). In the majority of the ordinary fats, and in all those which are of physiological importance (the triglyccrides), the alcohol is glycerin. The fatty acid components which may be united with the glycerin are very numerous, and the physical propertics of the different fats-e.g., their melting-points and solubilities-are closely related to the physical propertics of the corresponding fatty acids. Thus palmitic and stearic acids are solid at ordinary temperatures, and so are palmitin and stcarin, the glycerin esters or fats formed with these acids. Oleic acid, on the contrary, is fluid at the ordinary temperature, and the corresponding fat, olcin, is a liquid fat or oil. On the chemical sicle the fatty acids can be distinguished as saturated and unsaturated. The fatty acids of the series $\mathrm{C}_{n} \mathrm{H}_{2 n+1}$.COOI are saturated acids. Where $n$ is o we have formic acid, H.COOH; where $n$ is I, acctic acid, $\mathrm{CH}_{3} \cdot \mathrm{COOH}$; where $n$ is 2 , propionic acid, $\mathrm{CH}_{3} \cdot \mathrm{CH}_{2} \cdot \mathrm{COOH}$; where $n$ is 3 , butyric acid, $\mathrm{CH}_{3} \cdot \mathrm{CH}_{2} \cdot \mathrm{CH}_{2} \cdot \mathrm{COOH}$, and so on, each acid in the series differing from the one immediately preceding it in possessing an additional $\mathrm{CH}_{2}$ group. In the case of the higher members of the series these carbon chains become very long. In palmitic acid, for instance, $\mathrm{CH}_{3} \cdot\left(\mathrm{CH}_{2}\right)_{14} \cdot \mathrm{COOH}$, there are fourteen $\mathrm{CH}_{2}$ groups, and in stearic acid, $\mathrm{CH}_{3} \cdot\left(\mathrm{CH}_{2}\right)_{16} \cdot \mathrm{COOH}$, sixteen. Oleic acid, $\left.{ }_{8} \mathrm{H}_{17} \mathrm{H}_{17}\right\rangle \mathrm{C}=\mathrm{C}\left\langle\left(\mathrm{CH}_{2}\right)_{7} \cdot \mathrm{COOH}\right.$, is a representative of a series of unsaturated fatty acids whose gencral formula is $\mathrm{C}_{n} \mathrm{H}_{2 n-1}$. COOH. As the formula of oleic acid shows, the unsaturated fatty acids contain in their molecule two carbon atoms united by a double link, and one of these valencies can be occupied by halogens (e.g., chlorine) or by oxygen. Erucic acid, a fatty acid occurring in certain vegetable oils-for cxample, in rape oil-also belongs to this series, and linolic acid, found in linseed oil, to another serics of unsaturated fatty acids. Then there are the so-called oxyfatty acids, which in their turn comprise saturated and unsaturatcd acids. They differ from the ordinary fatty acids in containing one or more $\mathrm{OH}$ groups. Thus a dioxystearic acid, $\mathrm{C}_{17} \mathrm{H}_{33}(\mathrm{OH})_{2}$. $\mathrm{COOH}$, in which two of the $\mathrm{H}$ atoms in stearic acid are replaced by $\mathrm{OH}$, is found in castor-oil. It is clear, from the great varicty of the fatty acids, that by their union with glycerin (with loss of water) a vcry large number of different fats can be formed. Thus, when all the OH groups in the trivalent alcohol are replaced by palmitic acid we have tripalmitin; when they are replaced by stearic acid, tristearin; when they are replaced by oleic acid, triolcin: and so on. As a group such fats may be termed homo-acid fats, since all the $\mathrm{OH}$ groups are replaced by the same fatty acid. Thus-

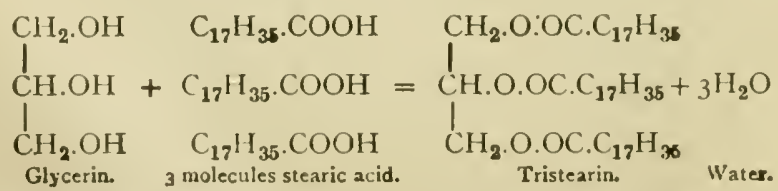

* The importance of controlling the diet, even to the extent of introducing periods of fasting, so as to keep the diabetic free from acidosis and glycosuria, has been recently emphasised (Allen). 
But it is not necessary that each $\mathrm{OH}$ group in the alcohol sliould le replaced by the same fatty acid, and when this does not occur we have letero-acid fats. For instance, one can be replaced by stenric acid, and the remaining two by palmitic acid, yielding a fat called 'stcarodipalmitin.' Conversely, one OH may be replaced by palmitic and two by stearic acid, forming palmito-distearin. Similarly, a dioleo-stearin (glycerin combined witl two molecules of oleic and one of stearic acid), and an oleo-distearin (glycerin combined with two molecules of stearic and onc of oleic acid) are known. Such compounds have been isolated from the fat of animals, and also formed synthetically. Again, each of the $\mathrm{OH}$ groups in the alcohol can be replaced by a different fatty acid.

It is obvious, then-and this is the point to which these chemical details are intended to lead up-that the number of different fats which the animal organism has at its disposal for concocting those varied mixtures designated as body fat is very great, and that there is room for a considerable degree of specificity in the fat stores of different animals, and it may be in the fat contained in different organs of the same animal, even if this specificity is not as marked as in the case of the proteins. It may be added, in connection with the composition of the body fat, that small quantities of free fatty acids and of glycerin may be present; but there is reason to believe that these are simply the surplus of raw materials which is about to be synthetized to neutral fat, or the surplus of decomposition products of the neutral fat which have not yet left the fat depots to take their place in the metabolism of the tissues.

The discussion of the metabolism of fat involves a study-(I) of the transformations and migrations of the food fat before it begins to be utilized; (2) of the possible production of fat from other constituents of the food; (3) of the processes and the stages by which fat, whatever its origin, undergoes katabolism to its end products. The fat of the food, passing along the thoracic duct into the bloodstream, is soon removed from the circulation, for normal blood contains only traces, except during digestion. Where does it go ? What is its fate?

Transformation and Migration of the Food Fat.-The presence of adipose tissue in the body might suggest a ready answer to these questions. The fat-cells of adipose tissue are ordinary fixed connective-tissue cells which have become filled with fat, the protoplasm being reduced to a narrow ring, in which the nucleus is set like a stone. It would, at first thought, seem natural to suppose that the fat of the food is rapidly separated by these cells from the blood, and slowly given up again as the needs of the organism require, just as carbo-lyydrate is stored in the liver for gradual use. And it has been found that a lean dog, fed with a diet containing much fat and little protein, puts on more fat, as estimated by direct analysis, or keeps back more carbon, as estimated by measurements of the respiratory exchange, than can be accounted for on the supposition that even the whole of the carbon of the broken-down protein corresponding to the excreted nitrogen 
has been laid up in the form of fat. Even with a diet of pure fatand with such a diet digestion and absorption are carried on under unfavourable conditions-more carbon is retained than can have come from the metabolism of the proteins of the body, as measured by the nitrogen given off in the urine and fæces: the fat passes rapidly from the blood into the organs, and especially into the liver (Hofmann, Pettenkofer and Voit). It is thus certain that some of the absorbed fat may be stored up as fat in the body.

This is borne out by the careful experiments of Munk and Lebedeff, who found that, when dogs are fed with excess of foreign fat (linseed oil, rape oil, mutton fat), a fat is laid down which is quite different from dog's fat, and has the greatest resemblance to the fat of the food. Thus, when rape oil, which contains a fatty acid, erucic acid, not found in animal fat, was given, erucic acid could be detected in the fat laid on. When the dogs were fed with mutton fat, whose melting-point is much higher than that of dog's fat, the fat laid on did not melt till it was heated to $40^{\circ} \mathrm{C}$. or more. When they were fed with linsced oil, the body-fat was found liquid even at $0^{\circ} \mathrm{C}$. We have already referred (p. 444) to the fact that neutral fat can be built up in the wall of the intestine from fatty acids given in the food. Munk has shown that fat formed in this way can also be laid down as body-fat. But besides the fat and fatty acids of the food, the fat of the body has other sources, and some of it is produced by more complex processes.

The fat of a dog consists of a mixture of palmitin, olein, and stearin. When a starved dog was fed on lean meat and a fat containing palmitin and olein, but no stearin, the fat put on contained all three, and did not sensibly differ in its composition from the normal fat of the dog (Subbotin). Stearin must, therefore, have been formed in some way or other in the body. If it was produced from the olein and palmitin of the food, the portion of these deposited in the cells of the adipose tissue must have undergone changes before reaching this comparatively fixed position. But there is conclusive evidence that fat may be derived from other sources, certainly from carbo-hydrates, and probably from proteins; and the stearin may have been formed from the carbo-hydrates or proteins of the food or tissues, and not directly from fat. And if the stearin was produced from proteins or carbo-hydrates, it is evident that the olein and palmitin might have been formed in this way too, the portion of the carbo-hydrate or protein devoted to this purpose being sheltered from oxidation by the combustion of the fats of the food. It is well known that not only neutral fats, but also fatty acids, exert such a 'protein-sparing ' action. It is possible also that the fat which is normally excreted into the intestine (p. 443), and which is perhaps derived from broken-down proteins, may be reabsorbed, and take its place among the fat 'put on.' 
At this point in the discussion it is necessary to remark that a distinction ought to be established between that store of surplus fat laid down in the connective tissue which, in order to avoid complicating the nuatter unduly, has hitherto been referred to as if it constituted the whole of the body-fat, and tle fat which is contained in greater or less amount in all the tissue cells. The fat contained in the tissue clements-e.g., in the liver cells-in the visible form of droplets, and which can be casily extracted from them by solvents such as chloroform, should also be distinguished from the fat which is so intimately incorporated or combined with the cell substance that it can only be extracted after this has been digested by the aid of proteolytic ferments or acids. The latter fraction of the body-fat is probably an integral and indispensable constituent of the protoplasm. Now, it is in the great fat depots of the subcutaneous tissue and the mesentery and omentum that variations in the proportions of the various fatty acids corresponding to variations in the nature of the food-fat are most easily produced, or, at least, most easily observed. These depots are laid down, not in the interest of the fat cells themselves, but to serve the purpose of a reserve of fat which may be drawn upon for the nutrition of the body as a whole, just as the glycogen store of the liver forms a general carbo-hydrate reserve. The free fat in the cells of the organs is superficially analogous to the glycogen reserves of such tissues as muscles and glands, and certain facts are known which inight be interpreted as indicating that this fraction of the body-fat, like the fat of the connective tissue, is not a definite and specific mixture of fats with an unvarying composition for each kind of animal, but a mixture whose composition can be made to vary by altering the nature of the fats in the food. On the other hand, the fat combined in the tissues appears to preserve a certain specificity which is independent of the fats supplied in the food. Thus, when dogs were fed with rape oil, and had accumulated considerable quantities of this fat of low melting-point in the subcutaneous and other fat depots, the fat combined in the organs remained in all respects the same as normal dog's fat. This was also the case with animals fed on fat of high melting-point, such as sheep's tallow (Abderhalden). Although the liver appears to have a special relation to the metabolism of fat, it is not known whether any particular organ is more than the rest responsible for the manufacture of this specific mixture of fats. It appears more probable that each cell has the power of forming for itself the characteristic fats from the crude materials represented by the food-fat directly absorbed from the tissue lymph, or the fat of the depots after it has been mobilized and has found its way again into the blood, or, finally, from other materials than fats, such as dextrose or some of its decomposition products.

Even in the case of the subcutaneous and similar collections of 
fat, it must be noted that upon the whole, under normal conditions, it is their specificity of composition rather than their dependence upon the composition of the fat mixture in the food which is the striking fact, and undue weight can casily be given to the results of feecling experiments where great quantities of quite foreign fats are administered. When sinall quantities of fats very far removed in their properties from the normal fat of an animal are given in the food, they are either completely utilized before reaching the fat depots, or transformed into normal body-fat, since no change whatever can be detected in the latter. If they have been utilized, then it may be that a corresponding amount of fat, formed, say, from dextrose, has been laid down in the fat stores. If this fat is formed from dextrose, it will, of course, be the kind of fat which the particular animal is accustomed to form from dextrose-that is, the fat characteristic of the animal. If the foreign fat is itself transformed into body-fat when given in small amount, this same feat can without doubt be gradually accomplished in the case of the surplus of foreign fat laid lown in the depots when a large quantity of it is given in the food.

Formation of Fat from Other Sources than the Fat of the Food(I) From Carbo-Hydrates. - It has been found that the addition of protein to a diet of fat, and especially to a diet of carbo-hydrate, in larger amount than is just necessary for nitrogenous equilibrium (p. 602), leads to a more rapid increase in the carbon deficit-that is, in the fat put on-than if the minimum quantity of protein required for nitrogenous equilibrium had been given. From this it is inferred that the carbonaceous residue of the broken-down protein is shielded from oxidation by the fat, and to a still greater extent by the carbo-hydrates, and so retained in the body as fat. And there is little doubt that the high repute of carbo-hydrates as fattening agents is in part due to their taking the place of proteins and fats in ordinary 'current' metabolism, and so allowing body-fat to be laid down from these. Voit, indeed, has gone so far as to assert that this is the only sense in which carbo-hydrates can be said to form fat, and that, in carnivorous animals at least, a direct conversion never occurs. But the experiments of Rubner have shown that in a dog fed with a diet rich in carbo-hydrates, and containing but little fat and no proteins at all, the carbon deficit was greater than could be accounted for by the proteins being broken down in the body and the fat of the food. In the pig and goose, too, the direct formation of fat from carbo-hydrates has been demonstrated.

For example, in an experiment by Tscherwinsky two young pigs of the same litter were taken. They wcighed respectively 7,300 grammes and 7,290 grammes. One was killed, and the amount of fat and nitrogen in its body directly estimated. From the nitrogen the maximum quantity of protein which could be present was calculatcd. The other pig was fed for four months with barley, which was analyzed. The pxcreta were also analyzed to determine the amount of unabsorbed 
fat and protein. At the cull of the tour nonths the pig was killed. It now we iglicd 24 kilogrammes, and containcd 2.52 kilogrammes protein and 9.25 kilogrammes fat. Subtracting the protein (o.0) liilogramme) and fat $(0.69$ kilogramme) originally present, 1.56 kilogrammes of protein and 8.56 kilogrammes of fat must lave becn put on. The anount of protein taken in the food was 7.49 kilogrammes, and of fat 0.66 kilogramme. Therefore, 5.93 kilogrammes of protein must have becu used 11 , and 7.90 kilogramines of fat laid on. At least 5 kilogrammes of this fat must have come from the carbo-hydrate of the food. Only a small amount of the fat put on could possibly have come from the protein.

The production of wax by bees, which used to be given as a proof of the formation of fat from sugar, is not decisive, for in raw honey proteins are present; and even when bees fed on pure honey or sugar manufacture wax, it may be derived from the broken-down proteins of their own bodies.

It is probable that in the formation of fats the carbo-hydrates are first split up to some extent, and that the fats are then constructed from their decomposition products, oxygen being lost in the process, since fat is much poorer in oxygen than carbo-liydrate. But the chemistry of the transformation as it takes place in the body is still imperfectly known, and all that can be done here is to indicate one or two of the ways in which chemists conceive that it may occur.

The formation of the glycerin component of the neutral fats from carbo-hydrates would appear to present little difficulty. In discussing the formation of glycogen from glycerin (p. 536), it was stated that two molcculcs of glycerose (glycerin aldehyde), a triose or sugar with three carbon atoms, can be condensed to form a hexose or sugar with six carbon atoms like dextrose, from the condensation or union of a number of molecules of which, with abstraction of water, glycogen is built up. The reaction can be worked equally well in the reverse direction - that is, from the hexose dextrose two molecules of glycerin aldeliyde can be formed, and then from each molccule of the aldehyde, by reduction, a molecule of the alcohol glycerin. As a matter of fact, it has been demonstrated that glycerin is produced when the corresponding aldehyde is bronght into contact with minced liver.

As regards the fatty acid components of the fats, it will be seen from the schematic representation of the katabolism of dextrose on p. 5.4 that acetic acid, a fatty acid, is represented at one of the stages as being formed by the oxidation of a molecule of acetaldehyde. Lactic acid is reprcsented in the same scheme as a previous stage in the decomposition of dextrose, and lactic acid can be convertcd into acetaldehyde and formic acid, the lowest of the same serics of fatty acids of which acetic acid is the next highest member. Thus:

$$
\underset{\mathrm{OH}_{3}>\mathrm{CH} . \mathrm{COOH}=}{\mathrm{CH}_{\text {Lactic acid. }}} \underset{\text { Acetaldehyde. }}{\mathrm{CH}_{3} \cdot \mathrm{C} / \mathrm{H}}+\underset{\mathrm{H}}{\mathrm{O}}+\mathrm{COOH}
$$

Aldelyydes (as well as ketones) have a great capacity for entering into reactions with other substances, and their molccules show also a marked tendency to combine with one another, forming new compounds by their condensation. Thus, from two molecules of acetaldehyde one 
molecule of aldol is formed, which by trausposition of certain groups, lecomes butyric acid, the fourth member of the fatty acid series of which acetic acid is the second member, and palmitic and stcaric acids, which form such important constituents of the ordinary body-fats, the sixteentli and eighteenth members respectively. By oxidation aldol becomes $\beta$-oxybutyric acid, which by further oxidation yiclds acetoacetic acid, compounds already referred to in connection with diabetes (p. 555). The following equations illustrate these reactions:

$$
\begin{aligned}
& \mathrm{CH}_{3} \cdot \mathrm{C} / \mathrm{O}+\mathrm{CH}_{3} \cdot \mathrm{C} / \mathrm{O}=\mathrm{CH}_{3} \cdot \mathrm{CH}(\mathrm{OH}) \cdot \mathrm{CH}_{2} \cdot \mathrm{C} / \mathrm{O} \\
& \text { Acetaldehyde. Acetaldehyde. Aldol. } \\
& \mathrm{CH}_{3} \cdot \mathrm{CH}(\mathrm{OH}) \cdot \mathrm{CH}_{2} \cdot \mathrm{CHO}=\mathrm{CH}_{3} \cdot \mathrm{CH}_{2} \cdot \mathrm{CH}_{2} \cdot \mathrm{COOH} \\
& \text { Aldol. Butyric acid. } \\
& \mathrm{CH}_{3} \cdot \mathrm{CH}(\mathrm{OH}) \cdot \mathrm{CH}_{2} \cdot \mathrm{CHO}+\mathrm{O}=\mathrm{CH}_{3} \cdot \mathrm{CH}(\mathrm{OH}) \cdot \mathrm{CH}_{2} \cdot \mathrm{COOH} \\
& \text { Aldol. } \quad \beta \text {-oxy'butyric acid. } \\
& \mathrm{CH}_{3} \cdot \mathrm{CH}(\mathrm{OH}) \cdot \mathrm{CH}_{2} \cdot \mathrm{COOH}+\mathrm{O}=\left(\mathrm{CH}_{3} \cdot \mathrm{CO}\right) \cdot \mathrm{CH}_{2} \cdot \mathrm{COOH}+\mathrm{H}_{2} \mathrm{O} \\
& \text { B-oxybutyric acid. Oxygen. Aceto-acetic acid. Water. }
\end{aligned}
$$

By reduction aceto-acetic acid is reconverted in to $\beta$-oxybutyric acid. Other aldehydes can react in similar ways, and thus many of the other fatty acids can be formed.

It may be added that acetone (another of the so-called acetone bodies which appear in the urine in diabetes nellitus) is casily obtained from aceto-acctic acid by the splitting off of carbon dioxide. Thus:

$$
\underset{\text { Aceto-acetic acid. }}{\left(\mathrm{CH}_{3} \cdot \mathrm{CO}\right) \cdot \mathrm{CH}_{2} \cdot \mathrm{COOH}=\underset{\text { Acetone. }}{\mathrm{CH}_{3} \cdot \mathrm{CO} \cdot \mathrm{CH}_{3}+\mathrm{CO}_{2}} \text { Carbon dioxide. }}
$$

Formation of Fat-(2) From Protein.-Dry protein contains on the average 16 per cent. of nitrogen and 50 per cent. of carbon, and urea contains 46 per cent. of nitrogen and 20 per cent. of carbon. Urea is therefore three times as riclı in nitrogen as the protein from which it is derived, but two and a half times poorer in carbon; and less than one-seventh of the carbon of protein will be eliminated in a quantity of urea sufficient to carry off all the nitrogen. It is probable that a portion of the remaining carbon may, after passing through various stages, take its place as the carbon of fat. We have seen that certain amino-acids derived from proteins can be converted into dextrose, and that dextrose can be converted into fat. So that the mere question whether carbon atoms or carbon chains originally present in protein molecules are ever capable of appearing in fat molecules can be straightway answered in the affirmative. But it is still in doubt whether amino-acids can be transformed into glycerin or into fatty acids, or into both, by processes which do not involve the production of dextrose from them. And in any case proof is required that the extent of the transformation, let the steps be what they may, is great enough to be satisfactorily demonstrated. In regard to this point it must be said that absolutely flawless experiments to prove the direct production of fat from protein seem still to be wanting. 
Phosphorus Poisoning and Migration of Fat.- in the ek, crinents of Batler, the amount of oxygen consuned and of carbon clioxide and nitrogen excreted was determinc 1 in starving dogs. Plosphorus, which, as is well known, causes extensive fatty changes in the organs, was then administered in small doses for several days. The excretion of nitrogen was doubled, the excretion of carbon dioxide and the consunption of oxygen diminished to one-half. When the animals dicd, in a few days, the organs were all found loadcd witl fat. In one case $42 .+$ per cent. of the solids of the muscles and 30 per cent. of the solids of the liver consisted of fat. This is much more than the normal amount. It was assumed that the fat could not have been simply transferred from the adipose tissue, since the dog lad been starved for twelve days before the phosphorus was given, and died on the twentictl day of starvation. Now, after such a period of hunger the amount of fat in the adipose tissue is greatly reduced. It was therefore concluded that the source of the fat could culy have been the broken-down protcin. Since the nitrogen excretion was increascd, while the carbon excretion was diminished, it was supposed that a residue ricl in carbon must have been split off from the proteins, and, remaining umburnt in the body, must have been converted into fat. Experiments of this kind are open to criticism on several grounds, but especially on this: that unless the fat-content of the whole body before the administration of the poison is known, it is impossible to be sure that the fat in a particular tissue has not been increased simply by the transportation of fat from some other tissue. It has been conclusively shown that migration of preformed fat does occur, and on an extensive scale, in pliosphorus poisoning. For example, a dog was fed for a time with sheep's tallow, and fat was laid down in its adipose tissue with the physical and chemical characters, not of dog's, but of sheep's fat. The animal was then poisoned with phosphorus, and the fat which accumulated in the liver examined. It also resembled sheep's fat, as it should have done had it migrated from the adipose tissue, and not dog's fat, as it might have been expected to do had it been formed in the hepatic cells from protein. The ease with which connective-tissue fat-i.e., food fat-migrates to the liver suggests, with other facts, that the liver has a special relation to the transformation of this fat into the fat of the organs. This 'organized ' intracellular fat differs in various ways from the fats of adipose tissue. Its 'iodine value' (p. 4) is higher (Leathes), and a large proportion of it consists of phosphatide lipoids (p. 57 I.)

The most convincing evidence that fat is not produced in increased amount under the influence of phosphorus has been obtained by determining by actual analysis the total fat in animals, then poisoning similar animals with phosphorus and again estimating the total fat. Far from being increased, the fat may even be decreased in the poisoned animals (Taylor, etc.). There is no ground, then, for the assumption that phosphorus and other substances, like arsenic, antimony, etc., which bring about so-called 'fatty degeneration ' of the organs, act by causing or accelerating the transformation of protein into fat. Yet there is good cvidence that they do accelerate the decomposition of protein, or at least interfere with its normal metabolisn, for after phosphorus poisoning amino-acids (leucin, tyrosin, glycin) appear in the urine. The observations of Lusk and his pupils indicate that phosphorus does not directly increase the amount of protein broken down, but does so indirectly, by favouring the conversion of the carbohydrate-like radicle of the protein molecule into leucin, tyrosin, and perhaps fat, and thcreby necessitating an increased consumption of protein.

A celebrated experiment, performed nearly forty years ago, was long 
supposed to furnish an absolute proof of the formation of fat from protein, under strictly physiological conditions, although in a humble form of animal life. Maggots were allowed to develop from the egg on blood containing a known anount of fat. The quantity of fat in tlie cggs was also known. After the maggots had grown, ten times as much fat was found in them as had been contained in the blood and eggs together. The trifling quantity of sugar in the blood was utterly inadequate to account for the fat, which, it was concluded, must therefore have come from the proteins of the blood (Hofmann). It can be objected to this experiment that no precautions were taken to prevent the growth of micro-organisms on the blood, and fat might have been formed by them from the proteins. Further, the fat estimations would scarcely pass muster according to the present standards.

The experiments of Pettenkofer and Voit, which were supposed to have demonstrated that in the higher animals also fat is formed from proteins under normil conditions, are in the same position. According to them, a dog fed for a time on a liberal diet of lean meat may go on excreting a quantity of nitrogen equal to that in the food, while there is a deficiency in the carbon given off. Or if the dog is not in nitrogenous equilibrium ( $\mathrm{p} 602$ ), but putting on nitrogen in the form of 'flesh,' the deficiency in the carbon given off may be too great in proportion to the nitrogen deficit to warrant the assumption that all the retained carbon lias been put on in the form of protein. In cither case, carbon in large amount can only come from the protcins of the food, and can only be stored up in the body in the form of fat. For lean meat contains but a trifling quantity of carbon in any other proximate principle than protein, and the non-protein carbon of the animal body is only to a very small extent contained in carbo-hydrates or other substances than fat.

Pflüger has criticized these experiments, and has shown that lean meat contains more fat than was supposed, and this is now generally admitted. He has endeavoured to show that the fat and glycogen in the meat given to the animals fully accounts for the carbon retained. Pflüger, indeed, takes up the position that the fat of the body comes cxclusively from the carbo-hydrates and fats of the food, and not at all from the proteins. But there is little doubt that in this he has gone too far, although his criticism has rendered it impossible any longer to a ppeal to Pettenkofer and Voit's results as good evidence on the other side.

If none of the supposed quantitative proofs of the conversion of proteins into fat which have hitherto been brought forward are free from flaw, the same is true of the alleged qualitative indications of its possibility and of its actual occurrence. The accumulation of fat between the hepatic cells caused by phlorhizin is, at the best, no better evidence tlian the accumulation within the cells in phosphorus poisoning. The formation of adipocere (a clicesy substance, rich in fatty acids united with calcium or ammonium), sometimes seen in dead bodies which have remained a long time under water or in moist graveyards, is largely, if not entirely, duc to the fat already present in the parts which have undergone the change, or to fat removed by the water from other parts of the body. If any portion of the adipocere represents fat formed from protein, this transformation may well be credited to the numerous microorganisms present, and throws no light upon the question of fat formation in the normal organism. The fat in the cells of the 
sebaccous glands, and of the mammary glands, may be produced from protein by a transformation of the cell-substance. But absolutely convincing proof is wanting. The old idea that the cells of these glands underwent a plyysiological process of transformation into fat analogous to the fatty degeneration of pathology, and then broke down bodily into the secretion, has bern long since disproved for milk formation, and is probably erronerus also as regards the secretion of sebum. The rule which experience has tauglit, that a woman during lactation requires an excess of proteins in her food corresponding not only to the proteins, but also to the fat given off in the milk, suggests such an origin for the milk-fat, but does not prove it. Other fat-containing secretions are the ear-wax formed by glands in the wall of the external auditory meatus, and the smegma formed by the glands of the prepuce, but nothing is known of the sources from which the fatty substances are derived.

The Intermediary Metabolism of Fat.-The meclianism and the stages of the transformation, including the migration, of fats is not well understood-indeed, not as well as that of the carbohydrates. Many of the tissues contain intracellular, soluble, fat-splitting ferments called lipases, especially the liver, the active mammary gland, and the intestinal mucosa. ITe have already seen that there is evidence that these lipases, like some other enzymes, have a reversible action. They are either fatsplitting or fat-forming ferments, according to the conditions (Kastle and Loevenhart). It is stated that the perfectly aseptic blood does not split ordinary neutral fats, although it contains a ferment which splits up monobutyrin (glycerin butyrate) into glycerin and butyric acid.

The question how the fat, after absorption from the intestine, passes from the blood into the cells, and how it is enabled again to pass out of the fat-cells when the needs of the tissues call for its mobilization, cannot at present be definitely answered. It is possible that just as fat is split in the lumen of the intestine before being absorbed, and then rebuilt in the epithelium, so it is split in the blood or in the lymph before being taken up by the fat-cells. The lipase in these cells would then be capable of synthetizing the glycerin and fatty acids to fat in their interior. When the fat is about to pass out of the cells in response to the call, of whatever nature it is, of the tissues for fat, it may again be split, resynthetized in the blood, and again hydrolysed for entrance into the tissue cells. Or it may be carried to the cells in the form of glycerin and fatty acids, or soaps, in such small concentration as to be harmless, and there built up again into the original fat, or transformed into other fats characteristic of the particular tissues, including the fatty acid components of the phosphatides, or utilized without synthesis into fat. An alternative hypothesis avoids this series of decomposi- 
tions and syntheses by assuming that the fat passes in the 1 orm of very fine droplets through the walls of the cells and of the capillaries. The reader will observe that we seem to be discussing again, and almost in the same terms, the question of the absorption of fat from the intestine. It is indeed at bottom the same question, and it miglit be argued that by analogy it should receive the same soliltion. Analogy, however, is a dangerous guide in such matters, and it is even more difficult to secure an unambiguous experimental test of the manner in which the internal migration of fat is accomplished than to secure the like for its absorption from the digestive tube.

As to the ultimate fate of the fat, from whatever source it may be derived, our knowledge may be compressed into very few sentences: Sooner or later it is split and oxidized to carbon dioxide and water, its energy being converted into heat or, directly or indirectly, into mechanical or other functional work; some of the fat absorbed from the intestine rapidly undergoes this change without entering the fat-cells of the adipose tissue. A portion of the fat may be changed into carbohydrates. This has been proved for the glycerin component; its possibility must be admitted for the fatty acids, but proof has not yet been given.

Of the intermediate stages by which the fatty acids are degraded into the simple end products but little is surely known. Included among these stages must be the compounds with which the formation of the acetone bodies (p. $5^{62}$ ) starts, if and in so far as their formation is a normal event which is merely unveiled by the dis. turbance of the ordinary course of the metabolism in diabetes. Among these intermediate stages must also be included, it is to be supposed, the compounds, whatever they may be, which act as connecting links between the currents of fatty acid and of carbohydrate metabolism, and witl which the transformation of fatty acids into carbo-hydrates commences, if this occurs at all.

According to the observations of Knoop, the saturated as well as some of the other series of fatty acids when oxidized decompose in a very characteristic way. As already remarked, these acids are made up of a larger or snialler number of $\mathrm{CH}_{2}$ groups forming a chain which at one end terminates with a carboxyl (COOH) group, and at the other with a $\mathrm{CH}_{3}$ group. The carbon atoms in the chain are designated by Greel: letters, $a, \beta$, etc., the $a$ position being that next the carboxyl group, the $\beta$ position onc remove from the carboxyl group, and so on. According to Knoop, the oxidation of the fatty acid chain takes place in such a way that the chain is shortened by the cutting off from the carboxyl end the $a \mathrm{CH}_{2}$ group along with the carboxyl group, while in place of the $\beta \mathrm{CH}_{2}$ group there is left a carboxyl group, an operation which might be fancifully compared to the naval manœuvre of breaking the enemy's line. Thus from caproic acid $\left.\underset{\epsilon}{\mathrm{C}} \mathrm{H}_{3} \cdot \mathrm{CH}_{2} \cdot \mathrm{CH}_{2} \cdot \mathrm{CH}_{2} \cdot\right|_{a} ^{\mathrm{C} \mathrm{I}_{2}} \cdot \mathrm{COOH}$, we get by oxidation butyric acid, $\mathrm{CH}_{3} \cdot \mathrm{CH}_{2} \cdot \mathrm{CH}_{2} \cdot \mathrm{COOH}$,

and water. It appears that the oxidation proceeds in two stages, the hydrogen of the $\beta$ group being first oxidized with formation of an 
oxyacid oxycaproic acid, $\mathrm{CH}_{3} \cdot \mathrm{CH}_{2} \cdot \mathrm{CH}_{2} \cdot \mathrm{CHOH} \cdot \mathrm{CH}_{2} \cdot \mathrm{COOH}$, which is then by further oxidation converted, with loss of two carbon atoms, into butyric acid. The oxidation process may then start afresh on the $\beta$ group of butyric acid. On the long carbon chains of the higher fatty acids this operation may be repeated again and again, the chain losing two atoms of carbon at each attack. If this represents what occurs in the normal metabolism, the groups cut off may then and there undergo the fate of the ships isolated by a successful application of the manœuvre allıded to, complete destruction-that is to say, oxidation to the end products carbon dioxide and water, a portion of the energy of the fatty acid being thus liberated at each oxidation of the $\beta$ group. Eventually a fatty acid or acids with very few carbon atoms will be left. There is some reason to think that acetic acid (and perhaps similar simple acids) may be one of the normal stages in the decomposition. Thus, butyric acid may first yield by oxidation of the $\beta$ group the oxyacid $\beta$-oxybutyric acid, $\mathrm{CH}_{3}$. CHOH.CH $\mathrm{CH}_{2} \cdot \mathrm{COOH}$, which by further oxidation of the $\beta$ group and the cutting off of the $\alpha$ and carboxyl groups would give $\mathrm{CH}_{3} \mathrm{COOH}$, or acetic acid.

If this is the general course of the oxidation of the fatty acids in the body, it is to be assumed that numerous intermediate stages 111represented in such a simple scheme may exist. Thus it is known, as has been mentioned more than once in other connections (p. 562), that $\beta$-oxybutyric acid by oxidation yields aceto-acetic acid, by losing from the $\beta$ group two atoms of hydrogen which unite with oxygen to form water. A molecule of aceto-acetic acid contains the elements of two molecules of acetic acid minus the elements of one molecule of water. It is therefore possible that aceto-acetic acid, if it is a normal stage in the katabolism of fatty acids, yields by its hydrolysis as a further step acetic acid, according to the equation

$$
\underset{\text { Aceto-acetic acid. }}{\mathrm{CH}_{3} \cdot \mathrm{CO} \cdot \mathrm{CH}_{2} \cdot \mathrm{COOH}}+\mathrm{H}_{2} \mathrm{O}=2\left(\underset{\text { Acetic acid. }}{\left.\mathrm{CH}_{3} \cdot \mathrm{COOH}\right)}\right.
$$

Aceto-acetic acid.

It is worth while, perhaps, to point out once more that even the relatively simple products now arrived at are not necessarily at once completely oxidized to their end products. That, it is to be assumed, will depend upon the needs of the organism. Acetic acid, for example, when added to blood and perfused through the surviving liver, can be transformed into aceto-acetic acid, and may thus become the starting-point of new syntheses.

The Liver and Fats. - The liver seems to play an important part in the metabolism of fat, as it does in the metabolism of carbo-hydrates and of proteins. It contains an oxidizing ferment, $\beta$-oxybutyrase (or $\beta$-hydroxybutyrase), which transforms $\beta$-oxybutyric acid into aceto-acetic acid (Dakin). This oxidation appears to occur in the normal as well as in the diabetic organism. The liver seems also to possess the power of transforming aceto-acetic acid into acetone, a reaction which does not involve an oxidation, and this may also be accomplished by means of an enzyme. But it is not at all likely 
that acetone forms a stage in the normal katabolism of the fatty acids or of the $\beta$-oxyacids derived from them. The importance of the liver in the metabolism of fats is further indicated by the extent of the migration of fat to that organ when the fat stores are mobilized in unusual amount (p. 503). The reason for this migration seems to be that the fats undergo preparatory changes which facilitate their utilization by the tissues. For example, there is evidence that saturated fatty acids are changed in the liver into unsaturated acids, which are then carried to the organs to be metabolized. The desaturation may serve the purpose of facilitating the rupture of the long carbon chains, or their capacity for entering into reactions witl other substances, at the points where double links exist between carbon atoms (p. 556).

Non-Nutritive Functions of Fat.-In connection with the metabolism of fat, it ought to be noted that, in addition to their value as reserve material for the nutrition of the body, the deposits of fat under the skin and in other situations perform important functions in protecting delicate structures from mechanical injury, in facilitating their movements upon each other, and in hindering the loss of heat. It would doubtless be a gross exaggeration to say that the mechanical and physical properties of the fat depots are as important in comparison to their chemical relations as is the case for the bones and ligaments, but it would be an error not less gross to consider them as of little account. It will even, perhaps, be ihought not unworthy of mention, from the point of view of the propagation of the race, that in the human species, at least, the amount and distribution of the cutaneous fat play a part of smm consequence in the aggregate of qualities which determine the physical attractiveness of the individual, especially of the female, although the standard in this regard varies widely in different communities.

It is perhaps partly because the fat depots have important mechanical functions that the fat reserve is far less mobile than the glycogen reserve. The semi-solid panniculus adiposus, the fatty tissue around the great nerve trunks, between the muscles, around the eyeball, on the soles of the feet, etc., possesses as a protective packing the good qualities of a water cushion with none of its disadvantages. But if the fat-cells were subject to sudden depletion, as the hepatic cells are-nay, in still greater degree, since they contain hardly any protoplasm-they would never serve for sucl a function. Of course, in the emergency of starvation, when even the glands and the muscles themselves are wasting, the fat reserves are necessarily mobilized, let their mechanical functions suffer as they may.

Obesity.-The proportion of the total mass of the body which is made np of fat varies greatly in different individuals, and often in the same individual at different stages in life. When the accumulation of fat 
passes beyond a certain point it causes obvious clianges in the contours of the body, and often some embarrassment in its movements. This condition is termed obesity. It is extremely difficult to say when the condition oversteps the pliysiological boundary and becomes actually patlological. Some individuals who are notoriously stout are noted also for their intellectual activity, and may not fall below the average even in the ordinary kinds of pliysical effort. It would be an exaggeration to speak of such persons as suffering from a disease. In other cases the pathological stanep is clearly imprinted upon the metabolic anomaly which leads to the overfilling of the fat depots. This is perhaps best illustrated in those cases of extreme obesity in children where, in spite of the intense metabolism associated with growth, with the restless muscular activity characteristic of that age, and with the relatively great surface through which heat is lost, great quantitics of fat continue to be put on. Muscular activity by itself is no certain antidote to or prophylactic against obesity, and it is a mistake to suppose that the condition is cxceedingly rare among manual workers sufficiently well paid to be able to gratify their tastes in the quality and quantity of their food. Statistics or rough estimates covering the whole of the hand-workers of a country throw no light on such a question, for few indeed are the lands where the masses of the people have such well-filled purses that they are able to nourish themselves according to their wishes.

While it is true that the great majority of normal individuals (although not all, since even in the fattening of stock for market some animals are rejected as bad feeders) can be compelled to lay on fat when overfed with fat and especially with carbo-hydrates, and prevented from taking mucli exercise or from losing heat freely, the most important factor in the excessive storing of fat by human beings leading a free life seems to be an anomaly in the metabolism which permits the machine to be run on less than the usual amount of fuel. From the point of view of thermodynamics the fat man, in very many instances at least, grows fat and fatter because his body is a machine whose 'efficiency' is greater than the normal-that is to say, a machine which is capable of doing a given amount of work and of keeping itself in repair with a food intake of smaller heat value than is usually needed. Whether this anomaly is to be considered a metabolic fault or a metabolic virtue depends largely upon the ease with which the intake is adjusted to the actual requirement of the body. If the adjustment is rendered accurate, the man with the anomalous tendency to put on fat, the adiposophil, ashe might be called, is in all probability just as well off in every pliysiolog ical sense on a smaller diet than a so-called normal individual of the same age, weight, and daily routine, on a larger quantity of food, and on this smaller diet he does not become fat. In this connection it may be recalled that, in speaking of the blood-flow in the hands and feet (p. I 27), which are in this relation to be regarded as essentially an "outcrop" of the cutaneous circulation, it was pointed out that some healthy persons have habitually small flows and a habitually cool skin which perspires little, in comparison with others living practically the sam e life. It was suggested that this difference in the blood-flow through the skin, which of course would correspond with a difference in the rate of heat loss, and therefore in the rate of heat production, may be correlated with a ditference in the intensity of the metabolism and the intake of food.

The difficulty of adjusting the appetite to the actual physiological requirement is perhaps the real anomaly in adiposophilia. Several factors seem to be involved in the group of sensations comprised under appetite and hunger (Chapter XVIII), and the onset and intensity of these sensations are unquestionably influenced by habit. The real 
question in many cases of obesity may be not why the metabolism is managed so parsimoniously-that is, in the physiological sense, so thriftily-but why the fat man or the man tending to become fat still experiences so strong a desire for food after he has eaten what in proportion to his metabolic wants is enough, whereas tle man with no tendency to obesity is no longer hungry after he lias eaten an amount of food sufficient for the requirements of his tissues. Is there here perhaps an anomaly in the nervous meclianism in virtue of which, for instance, the gastric hunger contractions are more readily initiated and less easily stilled than in the normal person? It is recognized that in the usually mucl more serious anomaly of the carbo-hydrate metabolism, diabetes mellitus, the nervous clement may be important. The influence of the loss of certain of the internal secretions on the deposit of fat will be alluded to in the next chapter.

In the treatment of obesity the factor of appetite and hunger control has to be specially kept in mind. Bulky but comparatively innutritious food, such as green vegetables, e.g., in the form of salads, should form an important constituent of the dietary, since the mere distension of the stomach staves off liunger. The total heat value of the food must be reduced gradually. Carbo-hydrates must be largely excluded, and also fats, although a certain amount of fat, say in the form of butter, is permissible and even beneficial as aiding in the passage of the food along the digestive tube. Alcoliolic beverages are in general contra-indicated, bccause alcohol, as an easily oxidizable substance, protects the carbo-hydrates and fats from oxidation, and perhaps also tecause the normal oxidative power of the tissues may be depressed by its habitual use. On the other hand, tobacco smoking, which has some power of inhibiting the gastric hunger contractions, may be permitted. Muscular exercise, cold baths, light clothing both during the day and at night, and a cool environment, are favourable to the reduction of fat by increasing the consumption of material and the loss of heat, just as a sedentary life in an overheated house in a person predisposed to obesity, and eating too much for his requirements, favours the putting on of fat. But if the appetite of the patient is allowed to govern the intake of food, the increased decomposition brought about by cxercise, etc., is very likely to be balanced by an increased ingestion, and no progress will be made.

Metabolism of Sterins or Sterols.-It has been previously stated that cholesterin appears to be the only representative of the sterins in the higher animals. Its source and function have been much discussed of late years. As to its source, there seems to be no reason to believe that any part of the cholesterin of the tissues is formed from decomposition products of ordinary fats, carbohydrates, or proteins. It is probably entirely derived from the cholesterins of animal, and the phytosterins of vegetable food. On this assumption, its metabolism, unlike that of the great groups of food substances, is carried on in a closed circuit. Evidence that it can be synthesized from other substances in the body is lacking. No increase in the cholesterin has been observed during the development of eggs, and the cholesterin content of growing chickens appears to correspond to the sterins taken in the food (Gardner). The portion of the cholesterin which is ingested in the form of esters 
is probably split, with liberation of the fatty acid, in the course of digestion. But if this be so, cholesterin esters are again formed in the tissues, for the cells and the blood contain both cholesterin esters and free cholesterin. While some cholesterin is excreted in the fieces (p. 423), there is evidence that a portion of the cholesterin of the bile may be reabsorbed, a ' circulation ' of cholesterin taking place analogous to the circulation of bile-salts. The appearance of cholesterin in the bile has been connected by some writers with the destruction of erythrocytes in the liver, or the conveyance of the products of their decomposition to that organ (p. 2I), but there are no means of distinguishing between the cholesterin set free from blood-corpuscles and that liberated from other cells. Since it is contained in all cells, every cell may be supposed to contribute something from time to time to the cholesterin excretion.

As to the office of the tissue-cholesterin, it can only be suggested that a substance so ubiquitous must be important. There is some evidence that cholesterin, free or combined, plays a part in conferring on the cells those peculiarities in their permeability upon which their functions, and indeed their integrity, depend. Free cholesterin, for instance, hinders the hæmolytic action of the saponins (p. 28), apparently by forming compounds with them Whether it or its esters are actually concentrated at the surface of the cell, and contribute to the formation there of the so-called 'lipoid' envelope, is not definitely known, although there are facts in favour of this idea.

Metabolism of Phosphatides.-The lecithins, which are the bestknown members of this class of compounds, have been already described (p. 366). They are built up of glycerin, fatty acids, phosphoric acid in the form of glyceryl-phosphoric acid, and a nitrogenous base cholin. There is some reason to think that the lecithins of the tissues are, in part at least, not free, but combined with proteins or with carbo-hydrates. Other bodies belonging to the phosphatide group are kephalin, a constituent of nervous tissue and of yolk of egg, cuorin found in heart muscle, etc.

It is probable, as stated in the chapter on Digestion, that the phosphatides of the food are hydrolysed in the alimentary canal with liberation of the glycerin, fatty acids, and the other components. It is not known whether they are resynthesized in the intestinal wall, but it is more probable that they pass directly to the tissues, where they can be utilized for building up the phosphatides of the cells. Cholin is found in small quantities free in the tissues, and also, it is said, in the blood-plasma. Glyceryl-phosphoric acid has also been obtained in small amount from various tissues. The other components of lecithin are, of course, never wanting, and there can be no doubt that the cells possess the power of reconstructing phosphatides from such materials. They can do 
more than this: they can prepare the 'building-stones' themselves. For even when the ingestion of plosphatides in the food is excluded, or the intake is so small as to be negligible, the formation of phosphatides in the body goes on apparently without check. An instance of this will be given on a future page in discussing experiments on the relative value of different proteins for nutrition and growth. A very striking observation has been recorded by IIcCollom, who fed three hens on a diet almost free from fat. In about three and a half months they laid fifty-seven eggs, containing over 9 per cent. of phosphatides. Calculation showed that here, first of all, fats or their components must have been constructed from carbo-hydrates. Then the nitrogenous component of the phosphatides (cholin in the case of lecithin, at least) must have been obtained from some source, possibly from an amino-acid by the addition of methyl groups $\left(\mathrm{CH}_{3}\right)$, of which cholin, OH. $\mathrm{H}_{2} \mathrm{C} \cdot \mathrm{H}_{2} \mathrm{C}-\mathrm{N} \underset{\mathrm{OH}}{-\mathrm{OH}_{3}} \mathrm{CH}_{3} \mathrm{CH}_{3}$ (trimethyl-oxyethylammonium hydroxide) contains three.

\section{Section III.-Metabolism of Proteins.}

Blood-Proteins.-The two chief proteins of the plasma, serum. globulin and serum-albumin, * must, as has been already pointed out, be recruited from proteins absorbed from the intestine and for the most part, at any rate, profoundly altered in its lumen and in their passage through the epithelium which lines it. Even when proteins are being actively absorbed, the plasma, after the blood-proteins have been separated, contains no substances which give the biuret reaction (p. 449). So that the peptones, which can be demonstrated in the intestinal contents, suffer great changes before or during their absorption. The physiological reasons for this alteration are in a measure known, and have already been alludect to in connection with the digestion of proteins. No doubt the far-reaching decomposition of the protein molecule may to some extent facilitate the absorption of protein food. No doubt also it is imperative that such comparatively slightly hydrolysed products as peptone, and particularly proteose, should not appear in quantity in the bloed, for when injected they cause profound changes in that liquid, one expression of which is the loss of its power of coagulation, and are rapidly excreted by the kidneys, or separated out into the lymph. But the passage of the food from the stomach is so gradual an affair, the quantity of digesting protein present at one time in any loop of intestine is so small, and the rush of blood which irrigates the

* It is probable that plasma contains a mixture of different albumins anc globulins. 
active mucosa is so large, that the concentration of peptone or proteose necessary to produce injurious effects could hardly in any case be realized. Again, there is no evidence that the simpler decomposition products of further hydrolysis are not in equal concentration as poisonous as proteose and peptone.

Apart from any influence which it may have in favouring absorption, the complete shattering of the protein molecule has a double significance. In the first place, as already pointed out, the foodproteins cannot be used directly in the upbuilding and repair of the protoplasm ( $p .448$ ), since the tissue-proteins differ from them and from each otlicr in the amount and nature of the amino-acids and other groups in their molecule (p. 2). Secondly, under ordinary dietetic conditions a surplus of nitrogen in the protein food has to be got rid of by being converted into urea without being built up into the tissue substance. Here we come upon the fundamental fact that the protcin katabolism is not a single uniform process. Two forms may be distinguished which are essentially independent in course and character. One kind varies extremely in its quantitative relations, according to the amount of protein in the food. Its chief end-products are urea, representing the nitrogen, and inorganic sulphates, representing the sulphur of the proteins. Since this form of katabolism, as we shall see directly, is not essentially connected with the life and nutrition of the living substance, it is termed exogenous. The other variety is practically constant in amount for one and the same individual, and independent of the quantity of protein in the food. Its characteristic end-products are creatinin and neutral sulphur. This form of protein katabolism is essentially an expression of the waste of the living substance itself, and is therefore spoken of as endogenous.

Some have supposed that the intestinal mucosa has as one of its special functions the resynthesis of a great part of the digestive decomposition products into the proteins of the blood-plasma. If this is the case, these proteins must be again decomposed in the cells of the various tissues in order that the 'building-stones' may be recombined to form the tissue-proteins. For the proteins of the organs are not the same as those of the blood, and the proteins of different organs differ characteristically from each other. The significance of the synthetic function of the intestinal wall would then lie in this: that from the varying mixture of anino-acids, etc., derived from the food-proteins an always uniform and suitable protein mixture (the blood-proteins) is fabricated for the feeding of the tissues. Experiments intended to test this hypothesis have hitherto yielded a negative result. No accumulation of protein in the wall either of the intestine $i n$ situ or of the isolated surviving intestine has been detected during absorption of the decomposition products of protein. An alternative assumption, and superficially at least a simpler one, is that no more extensive synthesis of proteins 
nccurs in the wall of the alimentary canal than is necessary for the needs of the tissues composing it, and, perliaps in addition, for the maintenance of the normal composition of the plasma, and that the decomposition products of the proteins are mainly absorbed as such. and pass in the blood to the tissues for which they are destined. If this is the case, the blood-proteins can no longer be looked upon as representing the main current of protein supply for the organs, but rather the store of protein material proper to the circulating tissuc blood itself, and which confers on it certain chemical and physiocliemical properties (e.g., the due degree of viscosity) necessary for its function. Slowly accumulated, under ordinary conditions, and slowly consumed, this protein store may, of course, be at the disposal of the organs in an emergency-for instance, in starvationor may be rapidly recruited from the organ-proteins, as after hemorrhage, just as in prolonged hunger the proteins of skeletal muscle may be utilized to feed the heart. That the blood-proteins can serve as nutritive material for the cells without undergoing digestion in the alimentary canal is well shown by the observations of Carrell and Burrows on the growth of isolated tissues in a medium romposed of clotted blood-plasma. But, as previously pointed out in another connection (p. 449), a portion, and probably a large portion, of the digested protein is absorbed from the intestine by the blood in the form of amino-acids. Considerable quantities of these compounds can be separated by dialysis from blood drawn off during the absorption of proteins or by the process of vividiffusion (p. 48) (Abel). Among these amino-acids, glycocoll, alaniı, glutaminic acid, and leucin, have been identified. While the quantity of amino-acids in the blood, which is very small in the fasting animal, is decidedly increased during protein digestion, it is probable that even in starvation amino-acids derived from the decomposition of the body-proteins are not entirely lacking. The normal concentration of amino-acids in the general blood of man and of the dog is about $0 . I$ per cent.-i.e., about the same as that of dextrose, and it may be nearly twice as great in the portal blood of dogs after a heavy protein meal. The amino-acids are very rapidly absorbed by the tissues from the blood, and can be demonstrated in muscles and other organs as free amino-acids. They accumulate there in much greater concentration tlan that in which they exist in the blood. Accordingly, the tissues take them up from the blood by some other process than simple diffusion (Van Slyke). It has been surmised that amino-acids constitute the form in which proteins are transported from tissue to tissue, as well as the form in which proteins are normally utilized by the cells.* Although this cannot be regarded as yet established, there is reason to believe that

- Recent experiments of Abel tend to rehabiiitate the old view that some protein is absorbed as proteoses, which can also be isolated from the tissues. 
the amino-acids play a great part in protein metabolism, perhaps as great a part as the dextrose does in the metabolism of the carboliydrates. There is some evidence that serum-albumin is more directly related to the proteins of the food than serum-globulin. And it is said that during starvation the albumin is relatively diminished, and the globulin relatively increased. It is, of course', not at all improbable that the plasma-proteins have a double source -organ-proteins on the one hand, food-proteins on the other. In any case, it is certain that serum-albumin and serum-globulin cannot be interchangeable without far-reaching decomposition, for their composition is very different. The globulin, e.g., yiclds glycocoll, but the albumin does not. That the plasma-protein mixture maintains a very constant composition in the face of wide variations in the composition of the food-protein is indicated by the following experiment:

A horse fed mainly on hay and oats was bled to the amount of 6 litres, and in the total protein of the serum the content of tyrosin and glutaminic acid was determined. In order to eliminate the influence of remains of the food in the digestive canal, nothing was given to the animal for a week. Then 6 litres of blood were again removed, and the tyrosin and glutaminic acid in the serum-protein again estimated. The horse was now fed with gliadin (one of the prolamins or alcoholsoluble proteins obtained from flour), a substance which contains 36.5 per ccnt. glutaminic acid and 2.37 per cent. tyrosin-that is, about the same amount of tyrosin as the serum-protein, but about four times as much glutaminic acid. The serum-protein was again analyzed for the two amino-acids after this diet. The results of one experiment are shown in the table:

\begin{tabular}{|l|c|c|c|c|}
\hline Normal, & $\begin{array}{c}\text { After 8 Days' } \\
\text { Hunger. }\end{array}$ & $\begin{array}{c}\text { After Feeding } \\
\text { with 1,500 } \\
\text { Grammes } \\
\text { Gliadin. }\end{array}$ & $\begin{array}{c}\text { After Feeding } \\
\text { again with 1,500 } \\
\text { Grammes } \\
\text { Gliadin. }\end{array}$ \\
\hline Tyrosin - - - & 2.43 & $2 \cdot 60$ & $2 \cdot 24$ & $2 \cdot 52$ \\
Glutaminic acid - & 8.85 & $8 \cdot 20$ & $7 \cdot 88$ & $8 \cdot 25$ \\
\hline
\end{tabular}

No increase in the glutaminic acid content of the serum-protein occurred, although, owing to the loss of blood, much new serum-protein must have been formed. If the amino-acids of the gliadin were used without change to build up the new serum-protein, three-quarters of the glutaminic acid must have been superfluous, and the nitrogen of this portion may have been straightway changed into urea and excreted. But the possibility that the glutaminic acid, or a portion of it, may have been changed into other amino-acids in the body cannot be excluded. In the case of some of the amino-acids it has been shown that such a transformation occurs (p. 613) (Abderhalden).

The high degree of independence of the food and body proteins is still more clearly exhibited in the table from Abderhalden on p. 570 , in which the proteins of milk are compared with some of the proteins which must be formed from them in the body of the suckling. The numbers represent percentages of the weight of each protein. 
Living and Dead Proteins.-Carried to the tissues, the decomposition products of the food-proteins, or the regencrated proteins of the plasina, which in ordinary language are still to be regarded as dead material, are built up into the living protoplasm, at any rate to the extent necessary to make good its waste. In this form they sojourn for a time within the cells, and then they become dead material again. The nature of this tremendous transformation has, of course, been the subject of speculation, but the truth is that we do not understand wherein the difference between a living and a dead cell, between a living and a dead particle in one and the same cell, really consists. All we know is that now and again a protein molecule or an aggregate of such molecules incorporated in the colloid mass which constitutes the protoplasm of a muscle-fibre, or a gland-cell, or a nerve-cell, must fall to pieces. Now and again a molecule of protein, hitherto dead (or perhaps, to speak more correctly, hitherto not a constituent of living protoplasm. since protoplasm is certainly more than protein), or a molecule of a particular amino-acid, or perliaps a polypeptide group intermediate in complexity between amino-acid and protein, coming witlin the grasp of the molecular forces or chemical affinities of the living substance, is caught up by it, talies on its peculiar motions, acquires its special powers, and is, as we phrase it, made alive. Each cell has the power of selecting and, if necessary, further decomposing or further synthesizing the protein materials offered to it; so that a particle of serum-albumin or a mixture of amino-acids may cliance to take its place in a liver-cell and lielp to form bile, while an exactly similar particle or mixture may furnish constituents to an endothelial scale of a capillary and assist in forming lymph, or to a muscular fibre of the heart and help to drive on the blood, or to a spermatozoon and aid in transferring the peculiaritics of the father to the offspring. And just as a tomb and a lighthouse, a palace and a church, may be, and have been, built with the same kind of material, or even in succession with the very same stones, so every organ builds up its own cliaracteristic structure from the common quarry of the blood.

\begin{tabular}{|c|c|c|c|c|c|c|c|c|c|c|c|}
\hline & & & 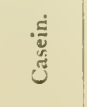 & 总总 & 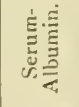 & 官产 & $\frac{\dot{\Xi}}{\frac{0}{0}}$ & 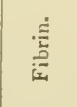 & 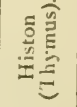 & 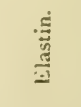 & 造 \\
\hline Glycocoll & - & - & 0 & o & o & $3 \cdot 5$ & 0 & $3 \cdot 0$ & 0.5 & $26 \cdot 0$ & $+\cdot 7$ \\
\hline Alanin & - & - & 0.9 & $2 \cdot 5$ & $2 \cdot 7$ & $2 \cdot 2$ & $4 \cdot 2$ & $3 \cdot 6$ & $3 \cdot 5$ & $6 \cdot 6$ & $1 \cdot 5$ \\
\hline Valin & - & - & $I^{\circ} \mathrm{O}$ & $0 \cdot 9$ & some & - & somc & $I^{\circ} \mathrm{O}$ & & $I \cdot O$ & $0 \cdot \frac{2}{9}$ \\
\hline Leucin & - & - & $10 \cdot 5$ & 19.5 & $20 \cdot 0$ & $18 \cdot 7$ & $29 \cdot 0$ & $1_{5} \cdot 0$ & I I. 8 & $21 \cdot 4$ & $7 \cdot 1$ \\
\hline Serin & - & - & $0 \cdot 2$ & - & $0 \cdot 6$ & - & 0.0 & some & - & - & 一 \\
\hline Cystin & : & - & 0.07 & - & $2 \cdot 3$ & 0.7 & $0 \cdot 3$ & $1-$ & - & - & $0 \cdot 6$ \\
\hline Asparagir & aic ac & & $I \cdot 2$ & $I \cdot O$ & $3 \cdot 1$ & $2 \cdot 5$ & 4.4 & $2 \cdot 0$ & - & - & 10.0 \\
\hline Glutamin & ic ac & & I I 'O & $10^{\circ} 0$ & $7 \cdot 7$ & $8 \cdot 5$ & $I \cdot 7$ & $8 \cdot 0$ & 0.5 & $0 \cdot 8$ & $3 \cdot 7$ \\
\hline Lysin & - & - & $5 \cdot 8$ & - & 一 & 一 & $+\cdot 3$ & 一 & $6 \cdot 9$ & - & 一 \\
\hline Arginil & - & - & +.8 & 一 & - & - & $5+1$ & 一 & 15.5 & $0 \cdot 3$ & 一 \\
\hline Phenylala & nin & - & $3 \cdot 2$ & $2 \cdot 4$ & $3 \cdot x$ & $3 \cdot 8$ & $t^{\circ}-2$ & $2 \cdot 0$ & $2 \cdot 2$ & $3 \cdot 9$ & - \\
\hline Tyrosin & - & - & +5 & $1 \cdot 0$ & $2 \cdot I$ & $2 \cdot 5$ & $1 \cdot 5$ & 3.5 & $5 \cdot 2$ & $0.3+$ & $3 \cdot 2$ \\
\hline Prolin & - & - & $3 \cdot 1$ & $4^{\circ} \mathrm{O}$ & $I \cdot O$ & $2 \cdot 8$ & $2 \cdot 3$ & $2 \cdot 5$ & $1 \cdot 5$ & $I \cdot 7$ & $3 \cdot 4$ \\
\hline Histidin & - & - & $2 \cdot 6$ & & - & & $1 I \cdot O$ & & $1 \cdot 5$ & 一 & \\
\hline
\end{tabular}


It is not any difference in the kind of protein offered them which determines the difference in structure and action between one organ and another. In this quarry alongsicle of the plasma proteins the tissue cells fund what is probably nore important for thcir individual nutrition, the building-stones of the shattered food-protein molecules. They are only under exceptional circumstances confronted with intact molecules of food-proteins. 'The body cells do not know what the kind of food was' (Abderhalden). In the case of the more highly developed tissues, at least, no mere change of food will radically alter the structure of the cells, nor even, as we have seen, the composition of the tissue proteins. A cell may be fed with different kinds of food, it may be overfed, it may be ill-fed, it may be starved; but its essential peculiarities remain as long as it continues to live. What may be called its organization, perhaps at bottom a more or less metaphorical expression for its cssential physico-chemical make-up, dominates its nutrition and function.

" We must assume that many of the enigmatical properties of living matter depend upon the activity of intact protein molecules. We can obtain some idea of the possible variety in the combinations of the "building-stones" of the proteins by recalling the fact that they are as numerous as the letters of the alphabet, which are capable of expressing an infinite number of thoughts. Every peculiarity of species and every occurrence affecting the individual may be indicated by special combinations of the "building-stones" - that is to say, by specific proteins. Consequently we may readily understand how peculiarity of species may find expression in the chemical nature of the proteins constituting living matter, and how they may be transmitted through the material contained in the generative cells, (Kossel). Add to the great variety of compounds rendered possible by the enormous number of permutations and combinations of the protein 'building-stones,'* the still greater variety rendered possible by the fact that the quantitative relations of given amino-acids may vary greatly in different proteins, and it will be scen what a practically infinite power of functional adjustment and reaction, correlated with a practically infinite variety of chemical clianges in the midst of which the cell still preserves its specificity through and through, may be conferred upon the living substance by its content of protein.

Some have supposed that the protein of the living substance is essentially different from dead protein, especially in possessing a characteristic instability, a prodigious power of dissociation and reconstruction. All the oider theories which attempted to explain this alleged difference require rivision in accordance with the newer chemistry of proteins, and speculations on the subject are probably in any case premature till the constitution of the proteins is thoroughly understood. In the meantime it is enough to say that the velocity of the reactions into which the proteins of living protoplasm or their constituent aminoacids may enter must depend upon intracellular conditions, which may vary rapidly and within wide limits. For example, enzymes may be present in greater or smaller concentration, or be activated and aided more or less powerfully by other substances, or by a more or less favourable chemical reaction of the medium. The protein itself, too, or such part of it as is ready for decomposition, may exist in a physical condition now more and again less favourable to the attack of the enzymes.

* Twenty different amino-acids, each used only once, but in a different order, would be capable of forming about $2,000,000,000,000,000,000$ (two thousand million times a thousand millions) of different polypeptides, all containing the various amino-acids in the same proportions (Abderhalden). 
It may not be superfuous at this point to again warn the reader that protoplasm and tissue-proteins are by no means synonymous. The pliysical, physico-chemical, and chemical changes involved in the katabolism of the colloid aggregates, including water, salts, phospliatides, sterins, and probably fats and dextrose as well as proteins, to wlich the term protoplasm is applied, may be many and complex before the individual proteins known to the chemist come face to face in the interior of the cells with the ferments which decompose them. On the other hand, it has not been proved that in the katabolic processes of the living substance isolated proteins ever form a stage. It may well be that without the complete decomposition of the protein molecules, or even without their complete detachment from the protoplasm, individual amino-acids or mixtures of amino-acids, or polypeptide groups, are cut out of the protoplasmic mass.

It is now necessary to follow, as far as is possible, the steps in the degradation of the body-proteins. Since there is reason to believe that these, like the food-proteins, are first split up into the amino-acids from which they were originally synthesized before undergoing further decomposition, a study of protein metabolism is to a great extent $\boldsymbol{v}$ study of the metabolism of amino-acids. In this study it is for most purposes impracticable, even if it were desirable, to distinguish between amino-acids directly derived from the food, and which have not yet been, and may never be, built up into tissue-proteins, and those clerived from the tissue proteins. There is nothing to indicate that the fate of a given amino-acid, once it has reached the blood, depends in the least upon its source. It may be said at once that the katabolism of the amino-acids is not a single and uniform process, one step in which inevitably follows another till the final end-products are reached. On the contrary, certain of the stages may become the starting-points of syntheses, which may lead back to the original or to another protein, or it may be to sugar or to fat. The extent of such syntliesis, and even in some legree the stage from which it starts, may be assumed to depend upon the needs of the tissues and the relative abundance of protein and of other foods.

Formation of Amino-Acids from Tissue-Proteins.-That aminoacids are formed in the metabolism of the cells and by the action of intracellular enzymes is indicated by the fact that proteolytic enzymes (proteases) are invariably present in the tissues, and can be obtained from them by appropriate methods--e.g., by subjecting the organ in a finely divicled state to a high pressure and collecting the expressed juice. Not only do unicellular organisms, like leucocytes, yeast cells, and bacteria, which must naturally depend upon themselves alone for all enzyuatic reactions, yield ferments which have the power of splitting proteins, peptones, and polypeptides into amino-acids, but their existence has been demonstrated in practically all the organs of the higher animals and man. When a piece of liver, e.g., is removed with aseptic precautions and kept at 
body-temperature, extensive auto-digestion occurs, and ammonia and other basic substances, glycin, and tryptophane, appear among the products. Tyrosin appears so early that it is scarcely possible to doubt that it must be a product of protein decomposition in the liver-cells under normal conditions-a decomposition which coulcl be observed also in the organ in situ were the circumstances as favourable. The circumstances are less favourable in an organ whose circulation is going on, becanse the amino-acids are removed by the blood as they are formed. Further, it is to be assumed that the regulation of the ferment action, which is a characteristic property of the normal cell, becomes feebler the longer it is witl. drawn from normal conditions. Similar autolytic processes have been observed in the spleen, muscle, lympli-glands, kiclneys, lungs, stomach wall (independently of pepsin), thymus, and placenta; also in pathological new growths like carcinoma, in the breaking down of which and in the removal of such exudations as occur in the alveoli in pneumonia, these proteolytic ferments seem to play a part. It is to be assumed that the syntheses of the proteins or their products, which are scarcely less characteristic of the tissue cells than the decompositions effected by them, are also due to the action of separate intracellular ferments or upon the reversed activity of the proteolytic ferments.

More direct proofs of the production of amino-acids in the tissues are not lacking. A rare condition known as cystinuria has been alluded to on a previous page (p. 489). Here there is a continuous excretion of the sulphur-containing amino-acid cystin in the urine. Sometimes the cystin is accompanied by other amino-acids, as leucin and tyrosin, a condition which might be called aminoaciduria. Cystinuria, while of course resulting from a.gross anomaly in metabolism, is of little clinical importance unless the sparingly soluble cystin should form calculi somewhere in the urinary tract. The most plausible explanation of the condition is that in the normal course of the metabolism each of the 'building-stones' of the proteins is sooner or later further decomposed by special ferments, and that for some reason the ferment which acts on cystin is absent, or if it is still produced, the conditions are more or less unfa vourable to its action. Unable to take its place in the metabolic current, except in so far as it can be utilized to form taurin, and therefore taurocholic acid-for this property it has not lostcystin becomes a chemical outcast in the body of the cystinuric individual, and is got rid of by the kidneys as an 'unemployable.' By comparing the amount of cystin excreted with the amount ingested in the food-proteins and with the (undiminished) amount contained in the tissues (especially the hair and the nails, since keratin is exceptionally rich in cystin), it has been shown that a portion of the cystin in the urine must have come from the tissues 
(Abderhalelen). ()bservations on animals in prolonged starvation afford additional evidence. The hair and nails continue to grow and to maintain the high cystin content, and taurocholic acid continues to be excreted. In like manner glycin continues to be produced and to unite with cholic acid to form the glycocholic acid of the bile.

There are other and more striking proofs that glycin can be formed in the body in large amount. For example, as already: stated (p. 48I), when benzoic acid is ingested it is not excreted as such in the urine, but coupled with glycocoll as hippuric acid. Thus-

$$
\underset{\text { Benroic acid. }}{\mathrm{C}_{6} \mathrm{H}_{5}} \cdot \mathrm{COOH}+\underset{\text { Glycocoll. }}{\mathrm{CH}_{2}\left(\mathrm{NH}_{2}\right)} \cdot \mathrm{COOH}-\mathrm{H}_{2} \mathrm{O}=\mathrm{C}_{6} \mathrm{H}_{5} \cdot \underset{\text { Hippuric acid. }}{\mathrm{CO}} \text {. } \mathrm{NH} \cdot \mathrm{CH}_{2} \cdot \mathrm{COOH}
$$

Benzoic acid, therefore, meets glycin in the body, and combines with it, as fatty acids meet glycerin and combine with it. Even starving animals fed with benzoic acid excrete large quantities of hippuric acid. Yet their tissues, as shown by analysis after death, yicld as much glycocoll as starving animals which have received no benzoic acid, and excreted little or no hippuric acid. Many other acids which are totally foreign to the body are, when ingested, paired in the same way with glycocoll and excreted in the urine. Even substances whose chemical nature does not permit of a direct union with the glycin are often altered by oxidation or reduction till they can unite with it, and then the coupling takes place, and the conjugated acid is eliminated by the kidneys. The paired or aromatic sulphuric acid which we have already recognized as a normal constituent of the urine affords another instance of this coupling. Cystein among the derivatives of proteins, and glycuronic acid (p. $4 \delta_{2}$ ) among the derivatives of carbo-hydrates, can also unite in the same way with numerous compounds. There is some evidence that the pliysiological significance of this process is that the toxicity of the foreign substances, or, as in the case of the aromatic sulphuric acid of the urine, of substances formed by bacteria in the intestine, or even produced in the metabolism of the tissues, is diminished by the pairing.

The place and manner of formation of hippuric acid have been investigated with the following result: If an excised kidney is perfused with blood containing benzoic acid, or, better, benzoic acid and glycin, hippuric acid is formed. Oxygen is required, for if the blood is saturated with carbon monoxide, or if serum is employed for perfusion, the synthesis does not take place. The kidney cells must be intact, for if a mixture of blood, glycin, and benzoic acid be added to a minced kidney immediately after its removal from the body, hippuric acid is produced, but not if the kidney has been crushed in a mortar. Nevertheless, there is some evidence that a 
ferment is concerned, and the known mechanism of similar reactions in the body scarcely permits the physiologist to acquiesce in any other explanation. It must not be forgotten that the urinary constituents which nust come into contact with the ferment when the kidney is crushed may injure or inhibit the enzyme. In herbivora hippuric acid cannot normally be detected in the blood; it is present in large quantities in the urine; it must therefore be manufactured in the kidney, not merely separated by it. In certain animals, as the dog, the kidney is the sole seat of the production of hippuric acid. But in the rabbit and the frog some of it must also be formed in other issues, for after estirpation of the kidneys the administration of benzoic acid causes hippuric acid to appear in the blood. It has, indeed, been recently shown that when the rabbit's liver is perfused with blood containing benzoic acid, hippuric acid is produced. The benzoic acid required for the normal excretion of hippuric acid comes mainly from substances of the aromatic group contained in vegetable food, but a small amount is produced in the body, since hippuric acid does not entirely disappear from the urine in starvation.

The differences which may exist in the metabolism of different groups of animals is well illustrated by the fact that in birds, when benzoic acid is given in the food, it unites not with glycin, but with ornithin, a derivative of arginin, forming not hippuric acid, but ornithuric acid (dibenzoyl-ornithin). A much more important instance of such a difference will be seen when we come to consider the formation of urea and uric acid.

The method by which the presence and the production of glycocoll in the body are demonstrated by coupling it with benzoic acid, and so saving it from decomposition and bringing it to excretion, can also be applied to other amino-acids. If instead of fishing with the bait benzoic acid we fish with a bait called brom-benzol or bromo-benzene $\left(\mathrm{C}_{6} \mathrm{H}_{5}, \mathrm{Br}\right)$, a substance derived from benzol by the substitution of an atom of bromine for an atom of hydrogen, we capture the amino-acid cystein in the form of a compound called mercapturic acid, produced by the union of brom-benzol with cystein and acetic acid, with oxidation and loss of water. In other words, when this substance is administered, mercapturic acid is excreted in the urine, the cystein, which is very unstable and readily changes into cystin (p. 360), being thus preserved from decomposition. Another instance in which amino-acids (ty'rosin and phenylalanin), which would normally be decomposed and so escape detection, come to the surface by being excreted in the urine, has a'ready been alluded to in connection with alkaptonuria (p. $4_{3}$ ). In this condition, which seems to have no serious significance so far as the well-being of the patient is concerned, not only does the taking of food containing the aforesaid amino-acids lead to ais 
increased cxcretion of homogentisinic acid, but even in starvation this substance still continues to appear in the urine. Since homogentisinic acid is undoubtedly formed from tyrosin and from phenylalanin, this observation constitutes a convincing proof that these amino-acids are produced from tissue-proteins. A striking illustration of the fact that the amino-acids of the tissues are not simply a store of reserve material derived directly from the alimentary canal is the failure of a period of starvation to make any impression upon their amount. They normally constitute 2 to 4 per cent. of the dry weight of the tissues, and if anything, tend somewhat to increase in the tissues of a starving animal.

Fate of Amino-Acids in the Body.-The problem of the katabolism of proteins is thus reduced to the question, What becomes of the amino-acids? Where, how, by what stages, and to what endproducts are they decomposed? Some possible or probable steps in their metabolic history have been already suggested in dealing with the intermediary metabolism of carbohydrates ( $\mathrm{p}, 54^{2}$ ). Something more will have to be said of the where and the how of their chemical degradation in treating of the place and manner of urea formation. As to the end-products by which they are represented in the final balance-sheet of tbe bodily economy, the answer is easy. The amino-acids, whatever intermediate stages they may pass through, whatever cleavages, oxidations, or reductions they may undergo, yield eventually carbon dioxide, water, and comparatively simple nitrogen-containing substances, which after further changes appear in the urine principally as urea, and in birds and reptiles as uric acid. When amino-acids are fed to mammals or introduced parenterally, a very large proportion of the nitrogen appears in the urine as urea. The same is true when, instead of simple aminoacids, polypeptides, like glycyl-glycin, alanyl-alanin, or leucyl-leucin, are given. When amino-acids are administered to birds, the great bulk of the nitrogen is excreted in the form of uric acid. Whether in mammals, and if so to what extent, uric acid is also one of the nitrogenous end-products of the decomposition of ordinary proteins or of the amino-acids which they yicld, are moot questions. In any'case, the most important and characteristic source of the uric arid in mammals and the other groups of animals whose chief nitrogenous end-product is urea, is not the ordinary proteins, but the nucleins which form constituents of the nucleo-proteins.

We have no definite information as to the production of water from the hydrogen of the tissues, except what can be theoretically deduced from the statistics of nutrition (p. 620). A few words will be said a little farther on about the production of carbon dioxide from proteins; we liave now to consider the seat and manner of formation of the nitrogenous metabolites. And since in man and the other mammals urea contains, under ordinary conditions, by far the 
greater part of the excreted nitrogen, it will be well to take it first.

Formation of Urea.-The starting-point of all inquiries as to the place of formation of urea is the fact that it occurs in the blood in

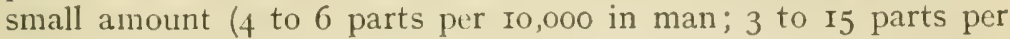
Io,000 in the dog), the largest quantity being found when the food contains most protein and at the height of digestion, the smallest quantity in hunger (Schöndorff). Evidently, then, some, at least, of the urea excreted in the urine may be simply separated by the kidney from the blood; and analysis shows that this is actually the case, for the blood of the renal vein is poorer in urea than that of the renal artery, containing only one-third to one-half as much. If we knew the exact quantity of blood passing through the kidneys of an animal in twenty-four hours, and the average difference in the percentage of urea in the blood coming to and leaving them, we should at once be able to decide whether the whole of the urea in the urine reaches the kidneys ready made, or whether a portion of it is formed by the renal tissue. Although data of this kind are as yet inexact and incomplete, it is not difficult to see that all, or most of, the urea may be simply separated by the kidney.

If we take the weight of the kidneys of a dog of 35 kilos at 160 grammes (E) th of the body-weight is the nean result of a great number of observations in man), and the average quantity of blood in them at rather less than one-fourth of their weight, or 35 grammes, and consider that this quantity of blood passes through them in the average time required to complete the circulation from renal artery to renal vein, or, say, ten seconds, we get about 300 kilos of blood as the flow through the kidneys in twenty-four hours. Even at 0.3 per 1,000 , the urea in 300 kilos of blood would amount to 90 grammes. Now, Voit found that a dog of 35 kilos body-weight, on the minimum protein diet ( $45^{\circ}$ to 500 grammes of lean meat per day) which sufficed to maintain its weight, excreted 35 to 40 grammes of urea in the twenty-four hours. If, then, the renal epithelium separated somewhat less than half of the 90 grammes urea offered to it in the circulating blood, the whole excretion in the urine could be accounted for, and the blood of the renal vein would still contain more than half as much urea as that of the renal artery. So that the whole of the urea in the urine may be simply separated by the kidney from the ready-made urea of the blood.

Another line of evidence leads to the same conclusion: that the kidney is, at all events, not an important seat of urea-formation. When both renal arteries are tied, or both kidneys extirpated, in a dog, urea accumulates in the blood and tissues; and, upon the whole, as much urea is formed during the first twenty-four hours of the short period of life which remains to the animal as would under normal circumstances have been excreted in the urine.

Where, then, is urea chiefly formed? The answer to this question is that, while some urea is probably produced from amino-acids in all the tissues, one organ is particularly associated with this function -namely, the liver. 
There is no reason to suppose that the hepatic cells, so far as the repair of their own protoplasm or the supply of energy for their own special work is concerned, require to metabolize particularly large quantities of amino-acids as compared, for instance, with the muscles. Glycin, however, they must have for the manufacture of glycocholic, and cystin for the manufact ure of the taurin of taurocholic acid. In addition, the liver is known to possess the power of utilizing amino-acids for the formation of dextrose and eventually of glycogen, and a portion of the surplus amino-acids of the food may be withdrawn from the blood of the portal vein for this purpose, just as the surplus of dextrose is withdrawn.

The liver contains a relatively large amount of urea, and there is strong evidence that it is the manufactory in which a great part of the nitrogenous relics of broken-down proteins, including aminoacids, reach the final stage of urea. This evidence may be summed up as follows:

(I) An excised 'surviving' liver forms urea from ammonium carbonate mixed with the blood passed through its vessels, while no urea is formed when blood containing ammonium carbonate is sent through the kidney or through muscles. Other salts of ammonium, such as the lactate, the formate, and the carbonate, undergo a like transformation in the liver. It is difficult, in the light of this experiment, to resist the conclusion that the increase in the excretion of urea in man, when salts of ammonia are taken by the mouth, is due to a similar action of the hepatic cells.

(2) If blood from a dog killed during digestion is perfused through an excised liver, some urea is formed, which cannot be simply washed out of the liver-cells, because when the blood of a fasting animal is treated in the same way there is no apparent formation of urea (v. Schroeder). This suggests that during digestion certain substances which the liver is capable of changing into urea enter the blood in such amount that a surplus remains for a time unaltered. These substances may come directly from the intestine; or they may be products of general metabolism, which is increased while digestion is going on; or they may arise both in the intestine and in the tissues. Leucin-which, as we have scen, is constantly, or, at least, very frequently, present in the intestine during digestioncan certainly be clianged into urea in the body. So can other amino-acids of the fatty series, like glycocoll or glycin, and aspartic acid, and it has been shown by perfusion experiments that this change can take place in the liver. Further, the blood of the portal vein during digestion contains several times as much ammonia as the arterial blood, and the excess disappears in the liver.

(3) During digestion the blood loses a greater proportion of its amino-nitrogen (amino-acids) in passing through the liver than in passing through other organs, as shown by the fact that the excess 
in the portal blood, as compared with the blood of the hepatic vein, is greater than the difference between the arterial and the vena cava blood. Now it has been proved that the liver does not store amino-acids to an appreciable extent as such, and therefore it must either have destroyed or condensed them into proteins. There is no evidence that the liver forms a store of reserve protein from aminoacids as it does of glycogen from carbohydrates, although this is possible. But there is good reason to believe that a portion at least of the amino-acids taken up by the liver is quickly broken down, and that urea is formed from them. For when amino-acids were injected into a vein in such amount that the content of amino-acids in all the tissues was considerably increascd, they disappeared far more rapidly from the liver than from muscle or kidney, and their disappearance from the liver was accompanied by an increasc in the urea content of the blood (Van Slyke).

(4) Uric acid-which in birds is the chief end-product of protein metabolism, as urea is in mammals-is formed in the goose largely, and almost exclusively, in the liver. This has been most clearly shown by the experiments of Minkowski, who took advantage of the communication between the portal and renal-portal veins (p. 385) to extirpate the liver in geese. When the portal is ligated the blood from the alimentary canal can still pass by the roundabout road of the kidney to the inferior cava, and the animals survive for six to twenty hours. While in the normal goose 50 to 60 per cent. of the total nitrogen is eliminated as uric acid in the urine, and only 9 to $I 8$ per cent. as ammonia, in the operated goose uric acid represents only 3 to 6 per cent. of the total nitrogen, and ammonia 50 to 60 per cent. A quantity of lactic acid equivalent to the ammonia appears in the urine of the operated animal, none at all in the urine of the normal bird. The small amount of urea in the normal urine of the goose is not affected by extirpation of the liver. And while urea, when injected into the blood, is in the normal goose excreted as uric acid, it is in the animal that has lost its liver eliminated in the urine unchanged.

(5) After removal of the liver in frogs, or in dogs which have survived the previous connection of the portal vein with the inferior vena cava by an Eck's fistula (p. 385), the quantity of urea excreted is markedly diminished, and the ammonium salts in the urine are increased. When the Eck's fistula is established and the portal vein tied, without any further interference with the hepatic circulation, the amount of urea in the urine is not lessened to nearly the same extent, evidently because the substances from which urea is formed still, for the most part, gain access to the liver through the hepatic artery and by means of the back-flow which is known to take place through the hepatic vein. Yet while in normal dogs the proportion of ammonia to urea in the urine is only $I: 22$ to $I: 73$, 
in dogs with Erk's fistula it rises to $I: 8$ to $1: 33$. If the animals are kipt on a diet poor in proteins, no symptems mav develop for a considerable time. But if much protein is given, characteristic symptoms, including convulsions, always appear. These may be produced by the saturation of the organism with ammonia compounds, which are formed from the proteins as in the normal animal, but which the liver, with its circulation crippled, is unable to cope with, and to completely change into urea, although the statement has been made that when ammonia or ammonium salts are injected into the blood larger quantities must be present to produce these symptoms tha' are found in animals with the Eck's fistula. Although the portal vein carries to the liver a greater supply of blood than the hepatic artery (about twice as much, according to Opitz and Macleod and Pearce), ligation of the latter causes a greater diminution in the ratio of the amount of urea to the total nitrogen in the urine than ligation of the former. While the blood of the hepatic artery is, of course, nearly saturated with oxygen. that of the portal vein is not half saturated, so thut the total quantity of oxygen transported to the liver by the hepatic artery is actually greater than the quantity transported by the portal vein. This indicates that a good supply of oxygen is an important factor in the formation of urea in the liver (Doven and Dufourt). But this is no proof that the process by which it is formed is an oxidation. The work of the liver, like that of other tissues, is no doubt deranged by lack of oxygen.

(6) In acute yellow atrophy, and in extensive fatty degeneration of the liver, urea may almost disappear from the urine, and leucin, tyrosin, and other amino-acids may appear in it along with a much larger amount of ammonia than normal. Here it may be supposed that the amino-acids and ammonia formed in the intestine in the digestion and absorption of proteins, perhaps also amino-groups formed in the tissues which would normally be culled from the blood by the hepatic cells for the manufacture of urea, pass unchanged through the degenerated liver, and are excreted by the kidney.

It would, however, be very easy to overdo this argument; for it is sometimes observed that in pathological and experimental conditions in which the liver has suffered severely considerable quantities of urea continue to be excreted. Urea does not entirely cuase to be produced even when the liver is removed; and it must again be pointed out that there is reason to believe that the formation of urea is not a function peculiar to the liver, but one shared probably with all tissues. The liver certainly does not arrest the whole of the amino-acids coming from the alimentary caual; for the nonprotein nitrogen in the muscles is distinctly increased during the absorption of amino-acids, and the muscular tissuc, even when freed from blood, contains some urea, which in all probability is formed 
there in the decomposition of amino-acids. Some writers, indeed, take the view that the muscles, containing as they do three-fourths of the proteins of the body, and utilizing as they appear to do a large proportion of the amino-acids of the food-protein, are more important seats of urea formation than the liver. Yet the fact that it is far easier to demonstrate this power-e.g., by perfusion experiments-for the liver than for such tissues as the muscles, renders it difficult to avoid the conclusion that in the preparation of an endproduct so important as that in which the great bulk of the nitrogen leaves the body, a certain degree of specialization has been developed, and that this preparation has been largely entrusted to a special organ. And, while it may be true that larger amounts of amino-acids are taken up and utilized by the muscles than by the liver under certain conditions, this does not show that amino-groups removed from the amino-acids in the muscles may not be largely transferred to the liver before being changed into urea. Further, the transformation of amino-acids into dextrose (and glycogen) may be assumed to entail a considerable absorption of amino-acids by the hepatic cells.

Processes by which Urea is formed.-In the case of only one of the amino-acids derived from proteins can urea be obtained by a simple process of hydrolytic cleavage. This is arginin ( $a$-amino- $\delta$-guanidin$n$-valerianic acid)-that is to say, normal valerianic acid,

$$
\mathrm{CH}_{3} \cdot \underset{\gamma}{\mathrm{CH}_{2}} \cdot \underset{\beta}{\mathrm{CH}_{2}} \cdot \underset{\alpha}{\mathrm{CH}_{2}} \cdot \mathrm{COOH} \text {, }
$$

in which an amino group is attached to the $\alpha$ carbon atom, while guanidin $\mathrm{NH}_{2}>\mathrm{NH}$ is attached to the $\delta$ carbon atom (p. 566).

When arginin is hydrolysed by barium hydroxide it yields urea and ornithin (diamino-valerianic acid), half of the nitrogen of the arginin appearing in each. Thus,

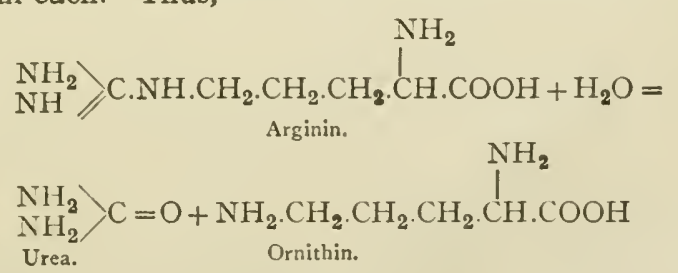

The amount of arginin, and therefore the amount of urea which can be artificially obtained in this way, varies extremely with the different proteins. Thus, salmin, a protamin (p. 2), prepared from the milt of salmon, yields $8+\cdot 3$ per cent. of its weight of arginin, while the casein of cow's milk yields only 4.8 per cent,, and gluten-fibrin, one of the proteins of wheat, only 3 per cent. In the body the hydrolysis of arginin to urea and ornithin is accomplished by the ferment arginase (Kossel and Dakin). This ferment is found in the liver, and also in many other organs. The urea formed in this way appears very rapidy in the urine. The ornithin itself is then more slowly transformed into urea. Since the ordinary food-proteins are poor in arginin, the 
amount of urea which can possibly be formed in mammalian metabolism by this process cannot be large, even if most of the arginin, as is the case when it is fed to an animal, is transformed into urea.

There is no reason to suppose that urea can be directly split off from the other amino-acids with which we are concerned. A comparison of their constitutional formula with that of urea (or with that of uric acid) shows that a more far-reaching decomposition must take place before products are obtained from which urea (or uric acid) can be formed. Urea has been artificially obtained from protein by oxidation with an ammoniacal solution of permanganate at body-temperature. When the protein is first split into its cleavage products, and these are then oxidized, a very large amount of urea is produced-e.g., as much as 3 grammes of urea from io grammes of glycin.

While these facts suggest possible ways of formation of urea in the borly, we cannot assume that what happens in the test-tube must happen in the tissues. The best cvidence is to the effect that in the borly the removal of the amino-group $\left(\mathrm{NH}_{\mathrm{g}}\right)$ in the form of ammonia from the amino-acicls is the essential step in the formation of at least a great part of the urea, which is then synthesized from ammonia and carbonic acid. The possibility exists that this cleaminization (or deamiclization) of the amino-bodies is the result of hydrolysis, or of oxidation, or of reduction, or of a combination of these processes. Evidence has been found that in the case of some of the amino-acicls the reaminization is associated not with hydrolysis in which hydroxyl is substituted for the $\mathrm{NH}_{2}$ group, but with oxidation (Neubauer). But this has not been shown to hold good for all amino-acids. In either case, however, whether the deaminization process is oxidative or hydrolytic the nitrogen is split off as ammonia, and it is to such ammonium compounds as have been already mentioned as being transformed into urea when circulated through an excised liver (p. $5^{84}$ ) that we have to look for the source of, at any ratc, a large portion, of the urea. Ammonia in the form of carbonate or carbamate is constantly found in the blood (p. 585). The excess of ammonia in the portal blood, which, however, is not admitted by all observers to be very large or very constant, has been interpreterl as indicating that a considerable decomposition of iamino-acids with liberation of the amino-groups occurs in the intestinal lumen or the intestinal wall. It is not established beyond doubt that ammonia is itself present in the protein molecule, or that its liberation in the hydrolysis of proteins can take place except at the cxpense of further decomposition of amino-bodies. It has been shown, however, that a great part of the ammonia in the blood is produced in the decomposition of protein in the digestive tube by putrefactive bacteria (Folin and Denis). This is a necessary part of the reaction by which phenol and indol are formed in the intestine.

It has been generally taught that the deaminization of the surplus of amino-bodies takes place chiefly in the liver, the extra nitrogen being thus 'shunted' out of the blood-stream before it has had a chance to reach the tissues. It would seem more advantagcous in the light of our present knowledge that a large and, so to say, a miscellaneous assortment of amino-bodies should be placed at the disposal of the tissues to facilitate the selection of those which are inclispensable. We have scen that tissues such as muscle can and do take up aminoacids when protein is digested in the intestine, and it is very probabie that they take up not merely the relatively small amount necessary to replace their wear and tear, but also a portion of the surplus, which after deaminization in the cells takes its place as a source of energy to drive the machine. The nitrogen in the form of ammonia may pass 
back into the blood, and may thus be carried to the liver for conversion into urea. It is not necessary, however, to suppose that all of the nitrogen must perforce make this journcy before being changed into urea. There is evidence that all the tissues sliare to some extent with the liver the power of forming urea just as they share with the liver the power of splitting off $\mathrm{NH}_{2}$ from the amino-bodies. It may be that the liver surpasses other tissues in its deamidizing power just as it seems to surpass other tissues in its power of transforming ammonium com. pounds into urea. But this does not prevent at least a considerable proportion of the amino-substances absorbed from the intestine from passing into the general circulation. It is of importance to remark that such hydrolytic clcavages as are associated with the splitting of protein into amino-acids, etc., only slightly reduce the available energy of the compounds. In so far as the liberation of the nitrogen from the amino-acids is also accomplished by hydrolytic clcavage (supposedly by a ferment desaminase), the residue, relatively rich in carbon, will still be available for yiclding to the body by its oxidation an amount of energy not much less than could be obtained from the original protein.

The combination of ammonia with carbon dioxide and the conversion of the carbonate into urea, perhaps through the intermediate stage of ammonium carbamate, does not require any oxidation. Thus,

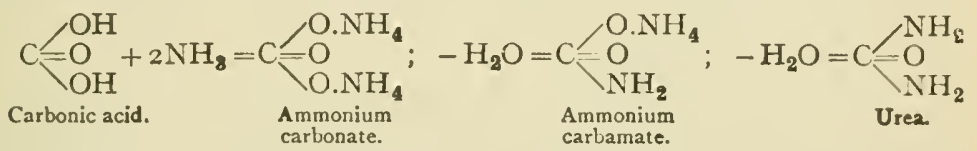

Another way in which some of the urea may be produced is by the direct formation of ammonium carbamate in the katabolism of aminoacids without the preliminary liberation of ammonia. By the loss of a molecule of water the carbamate would then become urea. But if, as there is every reason to believe, a part of the carbonaceous residue is converted into carbo-hydrate, a certain amount of oxidation must occur in the transformation.

Such compounds as guanin, sarkin or hypoxanthin, xanthin, uric acid, and creatin, used to be cited as among the possible intermediate substances between protein and urea. But while there is now complete evidence that the first three bodies can be and are converted into uric acid, there is nothing at all to indicate that they are stages on the way to urea. Uric acid is, indeed, very closely related to urea, and can be made to yield it by oxidation outside the body. Not only so, but it is, in part at least, excreted as urea when given to a mammal by the mouth and it replaces urea as the great end-product of nitrogenous metabolism almost wholly in the urine of birds and reptiles. But none of these things can be admitted as evidence that in the normal metabolism of mammals uric acid lies on the direct line from protein to urea. Creatin exists in the body in greater amount than any of these, muscle containing from 0.2 to 0.4 per cent. of it; and the total quantity of nitrogen present at any given time as creatin is not only greater than that of the nitrogen present in urea, but greater than the whole excretion of nitrogen in twenty-four hours. But although there are facts which indicate that creatin is an important derivative of the decomposed tissue proteins (p. 597) there is no evidence that it is related to urea formation.

Summary: Here may be summed up the most probable view of the normal decomposition in the body of the amino-acids, whether they undergo decomposition without being incorporated into the tissue proteins or proto- 
plasm, or are liberated as the protoplasm breaks down. The first step may be conccived of as the splitting off of the $N I_{2}$ gronp, which yields ammoniu. The ammonia is transformed into urea. The non-nitrogenous residue after removal of the amino-group differs according to the amino-acid. Some of the amino-acids, such as alanin, glycin, prolin and aspartic acid, vield substances which can be changed into dextrose in the body, as shown by. the increased amount of dextrose excreted by phlorhizinized animals when the amino-acids are fed to them.* Other amino-acids-e.g., lysin, hislidin and tyrosin, phenylalanin, etc., have such a chemical structure that the non-nitrogenous compounds derived from them cannot form dextrose. From certain amino-acids, such as histidin, phenylalanin, tyrosin and lencin, it has been shown that acetone bodies can be derived. These facts explain how sugar can be formed and the acidosis associated with the acetone bodies developed in diabetes, even when the diet consists of protein only or when the patient is living on his own tissues.

Formation of Uric Acid.-Uric acid, like urea, is separated from the blood by the kidneys, not to any appreciable extent formed in them. In birds, and often in man, it can be detected in normal blood. It is present in increased amount in the blood and transudations of gouty patients, in whose joints and ear-cartilages it often forms concretions. 'Chalk-stones' may contain more than half their weight of sodium urate.

As to the place and manner of formation of uric acid, it has already been stated that in birds, after extirpation of the liver, the uric acid excretion is greatly diminished, and that ammonium lactate appears instead in the urine. The simplest interpretation of this result is, that ammonia and lactic acid pass into the urine because they can no longer be utilized for the synthesis of uric acid. Chemical schemata can indeed be constructed, which show more or less plausibly how lactic acid, pyruric acid (p. 5+5) and other substances reacting with ammonia or with the urea derived from it (and birds form some urea) might yield uric acid. It has been further stated that when blood containing ammonium lactate is circulated through the surviving liver of the goose, an increase in the uric acid content of the blood occurs. As demonstrated by control experiments, this increase is too great to be due merely to the sweeping out of previously formed uric acid from the hepatic cells; also the feeding of lactic acid, pyruvic acid, and other organic acids leads to an increased output of uric acid. The story seems fairly complete, although criticisms have not been lacking. It has been suggested, for instance, that for some reason the loss of the liver leads to acidosis, an increased production of acids, especially lactic acid, in the organism; that ammonia, which would otherwise be employed in the formation of uric acid, is needed to neutralize these acids, and that the appearance of this ammonia in the urine is only a secondary consequence. of the elimination of the liver. The deficiency in the uric aciol

* In animals under the influence of phlorhizin sugar is formed from all substances capable of producing it in the organism. 
excreted, it is said, is therefore due, not to inability on the part of the remalning tissues to form uric acid, but to the absence of the ammonia which they require for its formation. This criticism, if it were admitted as against the current interpretation of such observations on the bird's liver, could scarcely be denied some validity as against the current interpretation of similar observations on the results of interference with the mammalian liver. It is therefore important to point out that there is still the same deficiency of uric acid when alkali is administered to neutralize the acids, although ammonia ought now to be available. There can be no question, then, that the liver in birds is the scat of an extensive synthesis of uric acid, and there is little doubt that ammonia compounds are essentially concerned in the process, whatever the rôle of the lactic or other acids may be. A similar synthetic formation of uric acid from ammonia and a derivative of lactic acid may take place in mammals, and probably exclusively in the liver, but it is of much less importance. Another way in which uric acid arises both in mammals and in birds is by the splitting and oxidation of nucleins. This is by far the most important mode of formation in mammals, as synthesis is the chief mode of formation in birds. In both groups of animals the oxidative production of uric acid takes place, not in any particular organ, but in the tissues in general, including the liver. It has been shown that when air is blown through a mixture of splenic pulp and blood, uric acid is formed from purin bodics already present in the spleen. When the quantity of these is increased by the decomposition of nucleins induced by slight putrefaction, the yield of uric acid is also increased. Uric acid is also formed by the perfectly fresh surviving spleen, liver, and thymus in the presence of oxygen, and the quantity is increased when purin bodies are artificially added.

Sources of the Uric Acid.-It is well established that in the bird it arises both from amino-acids derived from the hydrolysis of protein and from nuclein compounds and their derivatives in the food and tissues. The amino-acids constitute by far the greatest source of uric acid in these animals and in the reptiles, and it is practically certain that the course of the decomposition of the amino-acids and the form in which nitrogen is liberated from them in its transformation into this end-product are not essentially different from what obtains in the formation of urea in the mammal and the amphibian. This is sufficiently illustrated by the rôle played by ammonia and ammonia compounds in the production of uric acid in the birds and their congeners. In the mammal, the taking of food rich in nucleated cells, and therefore in nucleo-proteins and nucleins, the characteristic conjugated proteins of nuclei (thymus gland, pig's pancreas, and herring roe), or of food rich in purin bases (Liebig's meat extract), increases the quantity of uric acid in 
the urine. The increase is mainly clue to the production of uric acid from the nuclein substances of the food. But this is not the only source of the uric acid, since extracts of the thymus gland containing only traces of nucleins or nucleic acid cause, when injected, a characteristic increase in the uric acid excretion, just as the entire gland does when taken by the mouth. And during the period of increased nitrogen excretion occasioned by a meal containing protein, the increase in the uric acid occurs particularly in the hours immediately following the ingestion of the food, and does not last so long as the increase in the urea. Now, the nucleins of the food are comparativeiy little affected during the earlier stages of digestion (Hopkins and Hope). Whether in mammals any portion of the uric acid comes from amino-acids is still in doubt, but there are facts which indicate that a fraction of it may do so. We may conclude, therefore, that in the mammal, as well as in the bircl, a portion of the uric acid, although certainly a far smaller portion in the mammal, is derived from bodies other than the nuclein substances of the food-that is io say, from the nuclein substances of the tissues contained particularly in the cell-nuclei and probably from the ordinary proteins of both food and tissues. The portion derived Irom the proteins may be assumed to be that small fraction which has already been spoken of as synthetically formed.

Metabolism of the Nucleic Acids and Purin Bases.-Our knowledge of the metabolism of the nucleo-proteins and nucleins has been greatly augmented in recent years. When nucleo-protein is digested by gastric juice, a certain amount of protein is easily split off and hydrolysed to peptone and the other ordinary products of proteolysis. An insoluble residue of nuclein remains. This is acted upon with difficulty by gastric juice, although eventually an active juice will split it up also. By the action of pancreatic juice, or by heating with dilute acids, it is more easily hydrolysed, yielding a further quantity of protein along with nucleic acid. This second fraction of protein, which is split off with so much more difficulty than the first, undergoes proteolysis in the usual way. The resulting a mino-acids no doubt take their place in the general metabolism precisely like the amino-acids derived from ordinary proteins, and yield the same end-products. As regards the nucleic acid (or rather acids, since different nucleo-proteins contain different nucleic acids), pancreatic juice is practically inert, although succus entericus can effect a partial hydrolysis. For their complete decomposition more drastic treatment is required-namely, heating with hydrocliloric acid in a sealed tube. Thus treated, nucleic acids yield a number of components, out of which they may be assumed to be built up, as the proteins are built up out of amino-acids, etc. The characteristic components are purin bases (adenin, $\mathrm{C}_{5} \mathrm{H}_{3} \mathrm{~N}_{4} \cdot \mathrm{NH}_{2}$; guanin, $\mathrm{C}_{5} \mathrm{H}_{3} \mathrm{~N}_{4} \mathrm{O} . \mathrm{NH}_{2}$; hypoxantlin, $\mathrm{C}_{5} \mathrm{H}_{4} \mathrm{~N}_{4} \mathrm{O}$; and xanthin, $\left.\mathrm{C}_{5} \mathrm{H}_{4} \mathrm{~N}_{4} \mathrm{O}_{2}\right)$; 
pyrimidin bases (uracil, $\mathrm{C}_{4} \mathrm{H}_{1} \mathrm{~N}_{2} \mathrm{O}_{2}$; cytosin, $\mathrm{C}_{4} \mathrm{H}_{3} \mathrm{~N}_{2} \mathrm{O} . \mathrm{NH}_{2}$; thymin $\mathrm{C}_{4} \mathrm{H}_{3} \mathrm{~N}_{2} \mathrm{O}_{2} \cdot \mathrm{CH}_{3}$ ); phosphoric acid and a carbo-hydrate group.

Some of the nucleic acids contain all these components; they are sometimes spolien of as the true nucleic acids. In others ccrtain of the components are absent, and to these mucleic acids the name nucleotids has been applicd. The purin bases are always present. The carbohydrate group varies in different nucleic acids, being in some a liexose (p. 537), in others a pentose (p. 487). The pentose $d$-ribose is especially often met with. It is probable that the nucleotids are nerely simpler decomposition products of the true nucleic acids. Thus, inosinic acid, a nucleotid first isolated from meat extract, yields phosploric acid, $d$-ribose, and the purin base hypoxantlin. The nuclcotid guanylic acid found in the pincreas yields phosphoric acid, $d$-ribose, and the purin base guanin. There is cvidence that nucleic acids may be built up out of a number of nucleotid groups, and for this reason they have Lcen termed polynucleotids (Levene). The purin bases have a very close chemical relationship to uric acid, which, like them, is characterized by the possession of a group called the purin mucleus. For convenience of reference the atoms composing the purin nucleus are numbered, and the purin bodies are named with reference to the position of the carbon atom or atoms at which oxygen or the amino-group $\left(\mathrm{NH}_{2}\right)$ is introduced. Purin consists of the nucleus with $\mathrm{H}$ atoms introduced at the points shown in the constitutional formula. Adenin is a 6-aminopurin-i.e., purin in which $\mathrm{NH}_{2}$ replaces the $\mathrm{H}$ attached to $\mathrm{C}_{(0)}$. Guanin is 2-amino-6-oxypurin, $\mathrm{NH}_{2}$ being united with $\mathrm{C}_{(2)}$ and oxygen with $\mathrm{C}_{(0)}$ in purin. Uric acid is $2,6,8$-trioxypurin-i.e., purin in which oxygen is united to the carbon atoms 2, 6, and 8. Hypoxanthin is 6-oxypurin, oxygen being introduced at the position of $C_{(6)}$ in purin. By removal of the amino-group from adenin hypoxanthin is formed. Xanthin is 2,6 , dioxypurin, oxygen being introduced at $C_{(2)}$ and $C_{(6)}$ in the purin molecule. Xanthin can be derived from guanin in the same way as hypoxanthin from adenin.

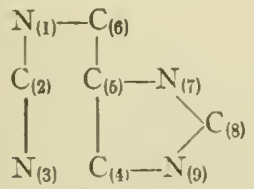

Purin nucleus.<smiles>C1=NC2NC=NC2N1</smiles>

Purin.

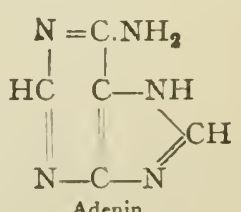

Adenin.

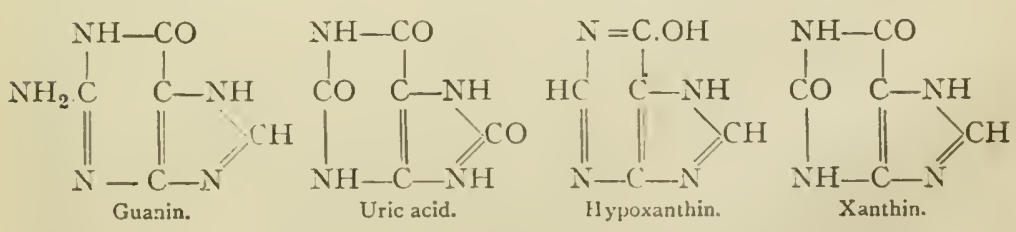

Besides the purin bases combined in the nuclein substances, purin bases and uric acid are widely spread in the tissues in the free state, although in very small amounts.

A portion of the intake of purin bodies is therefore ready formed, especially in the animal constituents of the food, and does not require the decomposition of nucleic acid for its liberation. The nuclei of vegetable cells contain nucleo-proteins, and accordingly can contribute to the purin intake. The most interesting contribution of vegetable 
origin has been previously alluded to (p. 481)-namely, the methyl purins forming the active principles of tea, coffce, and cocoa, caffein, or 1, 3, 7-trimethylxanthin $\left(\mathrm{C}_{8} \mathrm{II}_{10} \mathrm{~N}_{4} \mathrm{O}_{2}\right)$, theobromin, or 3, 7-dimethylxanthin $\left(\mathrm{C}_{7} \mathrm{H}_{8} \mathrm{~N}_{4} \mathrm{O}_{2}\right)$, and theophyllin, or $\mathrm{I}, 3$-dimethylxanthin $\left(\mathrm{C}_{6} \mathrm{H}_{8} \mathrm{~N}_{4} \mathrm{O}_{2}\right)$.<smiles>CN1C(=O)C2N=CC2N1C</smiles>
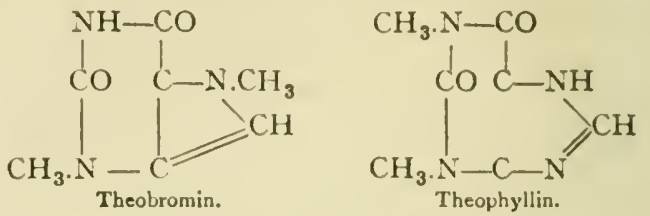

Nucleic acid, as stated, can be partially decomposed by the succus entericus, by means of a ferment called nuclease or, more accurately, nucleic-acidase. The groups into which it is split are nucleotids (see above). By another ferment, nucleotidase, a portion, at any rate, of the nucleotids is further decomposed to yield nucleosides, bodies of the glucoside class containing a compound of a purin base with the carbo-hydrate group of the nucleic acid, to which phosphoric acid is also coupled. Beyond this stage the hydrolysis of nucleic acid does not proceed in the intestine. The resultant products, probably along with unchanged nucleic acid, are absorbed, mainly at least, by way of the bloodvessels.

It will be well, however, to remember that our knowledge of the digestion of the nuclein bodies is still incomplete, and the natural tendency of the mind to think in diagrams is apt to give it greater precision than is justified by the facts; for example, it is known that even gastric juice is capable of liberating some of the phosphoric acid from nucleo-proteins.

In the tissues the absorbed products of the digestion of nucleic acids may be partially utilized without further decomposition for the synthesis of nucleo-proteins, to take the place of those which are destroyed in the metabolism of the cells; or they may be split completely into their components, and these resynthesized. Finally, and this fate is probably not long delayed in the case of the surplus of purin compounds contained in ordinary dietaries, both the purins of the fool and the purins arising from the waste of the tissues are for the most part converted into uric acid and excreted in the urine. Small quantities of purins leave the body in the fæces (p. 425). The phosphoric acid can be utilized not only for the building of nucleo-proteins, but for the synthesis of phosphatides. Eventually it is eliminated as phosphates in the urine. The carbo-hydrate groups, so far as they are not utilized in the synthesis of nucleic acids, may be supposed to undergo metabolism like other carbohydrates. The metabolic history of the pyrimidin bases has not been made clear.

Steps in Formation of Uric Acid.-As to the manner in which 
uric; acid arises from the nuclein substances, we may picture the process as taking place by the following steps: Certain orgins have been shown to contain ferments which split up nucleo-proteins into protein and nucleic acid. This nucleic acid, or nucleic acid arising in other ways in the metabolism of nuclein, and also any nucleic acid absorbed as such from the alimentary canal in the digestion of nuclein-containing substances, are then decomposed by another ferment, similar to or identical with the nuclease or nucleic-acidase previously encountered in the intestine. The resulting nucleotids are split up by a special ferment (nucleoticlase) so as to yield nucleosides. These are in turn decomposed by appropriate enzymes (nucleosidases), so that we finally arrive at the individual 'building-stones,' the nucleic acid molecule, phosphoric acid, the carbo-hydrate group, pyrimidin and purin bases, especially adenin and guanin. Then follows the action of ferments (adenase and guanase), which remove the amino-group from these purin bases, transforming adenin into liypoxanthin, and guanin into xanthin (Jones). The deaminization is associated with hydrolysis. Thus:

$$
\underset{\text { Adenin. }}{\mathrm{C}_{5} \mathrm{H}_{5} \mathrm{~N}_{5}}+\mathrm{H}_{2} \mathrm{O}=\underset{\text { Hypoxanthin. }}{\mathrm{C}_{5} \mathrm{H}_{4} \mathrm{~N}_{4} \mathrm{O}}+\mathrm{NH}_{3} ; \underset{\text { Guanin. }}{\mathrm{C}_{5} \mathrm{H}_{5} \mathrm{~N}_{5} \mathrm{O}}+\mathrm{H}_{2} \mathrm{O}=\underset{\text { Xanthin. }}{\mathrm{C}_{5} \mathrm{H}_{4} \mathrm{~N}_{4} \mathrm{O}_{2}}+\mathrm{NH}_{3} \text {. }
$$

$\mathrm{By}^{\circ}$ oxidation hypoxanthin is changed into xanthin and xanthin into uric acid, and the oxidation seems to be accomplished by a separate oxidizing ferment, xanthin oxidase, whose action may be thus represented:

$$
\underset{\text { Hypoxanthin. }}{\mathrm{C}_{8} \mathrm{H}_{4} \mathrm{~N}_{4} \mathrm{O}}+\mathrm{O}=\underset{\text { Xanthin. }}{\mathrm{C}_{6} \mathrm{H}_{5} \mathrm{~N}_{4} \mathrm{O}_{2}} ; \underset{\text { Xanthin. }}{\mathrm{C}_{5} \mathrm{H}_{5} \mathrm{~N}_{4} \mathrm{O}_{2}}+\mathrm{O}=\underset{\text { Uric }}{\mathrm{C}_{5} \mathrm{H}_{4} \mathrm{~N}_{4} \mathrm{O}_{8}} \text {. }
$$

Evidence of the existence of these ferments, and of their wide distribution, has been obtained by making experiments on the various substances mentioned with extracts of different tissues.

The portion of the uric acid which comes from the food (mainly from the purin bodies in it) is sometimes denominated the exogenous portion, while that which arises from the tissues is called the endeg. enous portion. The latter moicty, which generally amounts to about 0.6 gramme in the twenty-four hours, can be estimated by restricting the diet to articles of food free from purin bodies, such as bread, milk, cheese, eggs, and butter. It is stated that the endogenous uric acid remains practically constant in the same individual under constant conditions, and is unaffected by changes in the diet.

The total excretion of uric acid (and the other purin bodies) is by no means identical with the sum of the uric acid taken in as purin bases in the food and that produced in the body. A considerable destruction of uric acid (and other purin bodies) goes on in the body, and mainly in the liver. The quantity of endogenous uric acid excreted by the kidneys bears a certain ratio to the total zinount which has entered the circulation. This ratio varies much in different mammalian species. In man a full half is said to be 
excreted and about a half destroyed, being mainly changed into urees. Solle of the exogenous moicty is also broken down. When uric acid is heated in a sealed tube with strong hydrochloric acid, it is broken up into glycin, carbon dioxide, and ammonia. There are grounds for believing that a similar decomposition takes place in the body, and that the products are then transformed to urea in the liver.

The process of uricolysis, or destruction of uric acid, is usually attributed to a ferment called the uricolytic ferment, and it has been supposed that one of the factors in the production of gout may be a diminution in the amount or activity of this ferment. In some cases it is said to be entirely absent. It is doubtful, however, whether in man and the anthropoid apes the oxidizing enzyne, uricase or uricoxidase, which oxidizes uric acid to allantoin $\left(\mathrm{C}_{4} \mathrm{H}_{6} \mathrm{~N}_{4} \mathrm{O}_{3}\right)$, exists. In all other mammals hitherto investigateri it has been found in some of the tissues. In accordance with this, only a trace of allantoin is present in human urine and in the urine of the higher apes, while in the other mammals-for example, in the dog-a large proportion of the purin excretion assumes this form. It is probable that there is more than onc way in which uric acid may be decomposed in the body, and, if so, that there is more than one ferment concerned in its transformation. It would be well, therefore, not to speak of uricolysis as if it were synonymous with the well-ascertained process by which allantoin is formed from uric acid, and not to identify all enzymes which may take part in uricolysis with uricoxydase.

It is worthy of remark in this connection, as a further illustration of the differences which may cxist in the purin metabolism in different kinds of animals, that in man and the anthropoid apes the quantity of purin bases in the urine is small in proportion to the quantity of uric acid. In the pig, which is included among the animals that form allantoin from uric acid, the purin bases exceed the uric acid in anount, whereas in the dog, which likewise excretes allantoin, the purin bases exist in very small amount compared with the uric acid.

In concluding our consideration of the metabolism of the nucleic acids, the question may be raised whether it is related to the metabolism of the other substances-carbo-hydrates, fats, and proteinsin such a way that derivatives of nucleic acid can contribute to the formation of any of these, or derivatives of carbo-hydrates, fats, or proteins contribute to the formation of any of the components of nucleic acid. It has been already mentioned that the phosplioric acid can aid in the synthesis of plosphatides, and that the carbohydrate groups probably take their place in the ordinary carbohydrate metabolism. There is no evidence that the purin bases can take part or can yicld products capable of taking part in the formation of any of the other substances. The purin metabolism, so far 
as is known, moves in a closed circuit. Of the fate of the pvrimidin bases nothing is surcly known. Without doubt nucleic acid can be formed in the body when none is contained in the food. More than one source of the phosphoric acid and the carbo-hydrate are known and have been already pointed out, but how and from what materials the purin and pyrimidin base's are formed cannot yet be stated.

It has been sliggested that argirin and histidin constitute the raw material for the synthesis of the purin ring. Rats will grow when fed with an amino-acid mixture prepared by hydrolyzing caseinogen and lactalbumin, but they rapidly lose weight if arginin and histidin have been removed from the mixture, and the allantoin in the urine decreases. Restoration of the missing amino-acids causes growth to be resumed and a rise in the allantoin excretion to normal. This is true, although in lesser degree, even when one of these amino-acids is restored to the mixture, as they seem to be interconvertible (Ackroyd and Hopkins). Recently the synthesis of nucleosides has been accomplished in the laboratory (Fischer). It only needs the introduction of phosphoric acid in the appropriate way into the molecule to give nucleic acid.

The Significance of Creatin and Creatinin in Protein Metabolism. -A glance at the tables of composition of the urine (p. 477) will show that creatinin, as regards the quantity excreted, is a much more important product of nitrogenous metabolism than uric acid, standing, indeed, with the ammonia compounds, next in order to urea; but our information as to its source and significance is rery scanty.

Creatin is a-methylguanidin-acetic acid, and creatinin is dcrived from it by loss of the elements of water:

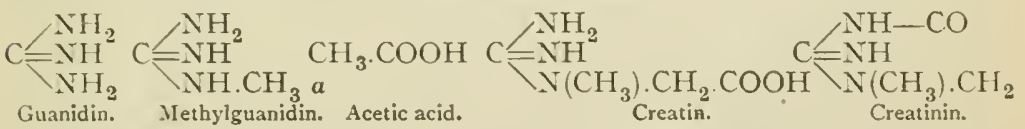

On heating with baryta-water creatin is decomposed, yielding urea, methylglycocoll or sarcosin, and other substances. It can be prepared synthetically from sarcosin and cyanamid. Thus:

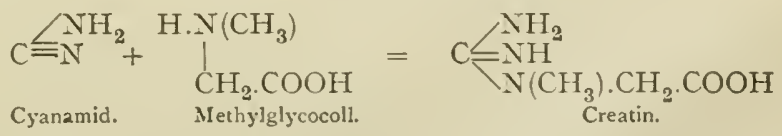

Creatin is found in considerable amount in muscular tissue (much less in children than in adults), and in traces in other tissues and in blood, which also contains small amounts of creatinin.

Creatinin can be so readily obtained from creatin outside the body that it is tempting to suppose that the portion of the creatinin 
of the wine which is not formed from the creatin in the fond is derived from the creatin of the muscles and other tissues, and many theories have been evolved to connect the creatinin of urine with the creatin of the muscles. But it is cloubtful whether there is any direct connection. The fact that persons who have been long il] and are fecble and wasted have a low creatin content in their muscles, and a low creatinin content in their urine relatively to the total nitrogen (Sliaffer) is, so far as it goes, in favour of some relationship between the two. The alleged absence of creatinin from muscle seemed to be opposed to the idea that the creatin store of the muscular tissue was an important source of urinary creatinin; for if a constant transformation of this kind was going on, traces of creatinin not yet absorbed by the blood might have been expected to be present in the muscles. Recently, however, it has been reported that small quantities of creatinin do exist in fresh muscle (4 to 8 milligrammes in 100 grammes of tissue), and that when the muscle is allowed to undergo autolysis the creatinin increases at a very uniform rate at the expense of the creatin. Added creatin experiences the same fate as the creatin originally present, while added creatinin inhibits the reaction, or even reverses it (Myers and Fine). A parallelism with the conversion of glycogen into dextrose in the liver easily suggests itself, and it is possible that we are here in the presence of a normal reaction which may account for at least a portion of the creatinin excretion. It is probable that both creatin and creatinin can undergo changes in the body, especially, in the liver, and it is possible that the products may be further utilized in metabolism. If this were so, the creatin store of the muscles would acquire new significance as a reserve of useful material with perhaps a long and varied metabolic career before it, and would not constitute merely a temporary depot of waste material whose metabolic history was ended, and which was waiting to be excreted. The novel view has recently been advanced that the living muscles contain little or no creatin, but that the creatin found on analysis is a post-mortem product originally constituting a part of the living protoplasm (Folin and Denis).

However this may be, the constancy of the creatinin climination on a meat-free diet (p. $4^{\$_{2}}$ ), and its complete independence of the changes in the total nitrogen excretion, show that it has a different significance in protein metabolism from the urea. Evidence is accumulating that it is especially in the metabolism of the organized or tissue protein that the product eventually excreted as creatinin arises; in other words, that it represents especially the nitrogenous waste connected with the wear and tear of the bodily machinery, while urea represents also, and under ordinary conclitions of dict chicfly, the nitrogen of the surplus amino-acids which are not utilized in the building of new or the repair of old tissue elements. The fact that the amount of creatinin cxcreted by different persons seems to be related to the weight of active tissue in the body, excluding fat, is in favour of this suggestion, and 
there is other evidence pointing in the same direction: for example in ordinary circumstances creatin is either absent from the urine or present in very small amount, except in young clildreu. When, however, the decomposition of tissue-protein is abnormally increased, as in starvation, in fevers, in women after delivery, while involution of the uterus and the associated destruction of a considerable mass of smooth muscle is taking place, creatin appears in larger quantities in the urine, perhaps because it can no longer be all converted into creatinin. Now, the increased excretion of creatin in starvation can be prevented by giving carbo-hychate food, which is known (p. 606) to lead to sparing of tissue-protein (Mendel and Rose). That the depletion of the body of carbohydrate is in some way related to the elimination of creatin is further shown by the fact that creatinuria is associated with diabetes and with the action of substances like phlorhizin, hydrazin, phosphorus, etc., all of which cause carbo-hydrate deficiency. Something more is involved, however, for under conditions of diet which produce acidosis (an increase in the hydrogen-ion concentration of the blood), creatin appears in the urine no matter how rich the food may be in carbo-hydrate. For example, a diet of oats and maize, which are typical acidproducing foods, causes creatinuria in rabbits, which promptly disappears when carrots, a base-producing food, are added (Underhill).

The statement that the content of the urine, in creatinin is increased by muscular work may indicate that the muscular machine wears out faster during activity than during rest, or perhaps only that alreadyformed creatin leaves the muscles in greater amount when the blood-flow in increased; but recent observations tend to show that this statement may require revision.

As to the manner in which creatin is changed into creatinin in the body, a highly suggestive fact is the presence of ferments in various organs which possess this power. Ferments also exist which can decompose both creatin and creatinin. The existence of such enzymes is presumptive evidence that the changes which they are capable of producing actually occur in the organism; but the seat of the changes if they do take place, and their metabolic significance, are unknown. Creatin when given by the mouth or injected into the blood does not cause any increase in the urinary creatinin, nor when administered in moderate quantities does it seem to be excreted as creatin. Like urea, creatinin, and amino-acids, it is taken up very rapidly by the muscles. Creatinin, on the other hand, when added to the food, causes an increase in the creatinin of the urine.

Intracellular Ferments-Autolysis.-As to the agencies by which the decomposition of the proteins is carried out in the cells, we have already spoken of the oxidizing cell ferments, or oxydases (p. 272). Reducing ferments, or reductases, are also known, and can be extracted from most organs, if not all. Like oxydases, they act in a weakly alkaline medium, causing in the presence of hydrogen such reductions as the formation of nitrites from nitrates. There is some evidence that one and the same ferment may act as an oxydase or a reductase according to the conditions. Recent researches have brought to light in addition hydrolytic intracellular ferments, which split up proteins very much in the same way as the proteolytic ferments of the digestive juices.

The significance of these autolytic enzymes in the normal metabo- 
lism of proteins has been already discussed (p. 578) ; indeed, so many of the chemical reactions of the body have been found to depend upon enzymes that modern physiology may at first thought seem almost to have reverted to the position of Van Helmont and his school in the seventeenth century, who resolved all difficulties by mumuring the magic word 'ferment.' No fewer than eleven ferments have been stated to be present and active in the liver alone -viz., a proteolytic and a nuclein-splitting ferment, a ferment which splits off ammonia from amino-acids, a milk-curdling ferment, a fibrin ferment, a bactericidal ferment, an oxydase, a lipase, a maltase, a ferment called glycogenase, which changes glycogen into dextrose, and an autolytic ferment. In the presence of such an array of enzymes the organs might seem to be little more than incubators in which the ferments do their work. It must not be supposed, however, that the intracellular ferments, whether they cause decomposition or synthesis, oxidation or reduction, work independently of what, for want of a better name, we must call the organization of the cell. We may be sure they are the servants and not the masters of the protoplasm, and that a drop of an extract containing intracellular ferments has very different powers from a living cell. 'It is not in the existence of the ferments, but in their combined action at the proper time and in the proper intensity, that the riddle of metabolism lies ' (Höber).

Summary.--At this point let us sum up what we have learnt as to the relation between the approximate principles of the tissues and the proximate principles of the food. Inside the body we recognize representatives of the three groups of organic food-substances in a typical diet-proteins, carbo-hydrates, and tats. But we should greatly err if we were to imagine that the three streams of foodmaterials have flowed from the intestines into the tissues each in its separate channel, neither giving to nor taking from the others. The fats of the body may, indeed, in part be composed of molecules which were present as fat in the food; but they may also be formed from carbo-hydrates, and probably from proteins. The carbo-hydrates of the body-the glycogen of the liver and muscles, the sugar of the blood - may undoubledly be derived from carbo-hydrates in the food, but they may also be derived from proteins and from fats (certainly from their glycerin constituent, perhaps from the fatty acids as well). The protcins of the body' come mainly', if not solely' from the proteins of the food. Although, of course, neither fats nor carbo-hy'drates can by themselies form protein, being deioid of nitrogen, it is possible that products arising in the intermediary metabolism of either may, by combining with nitrogenous groups, be tranfsormed into amino-bodies, which can then take part in the synthesis of proteins. In any case there is no doubt that both carbo-hydrates and fats can economize protcins and shield them from an oierhasty metabolism. 


\section{Section IV.-Statistics of Nutrition-The Income And Expenditure of tHe Body in Terms of Matter.*}

Preliminary Data.-The office of the food is to maintain the con. stituents of the body upon the whole in their normal proportions. A knowledge of the chemical composition of the body is, therefore, an important datum in the consideration of the statistics of its metabolism. The body of a man analyzed by Volkmann had the following composition:

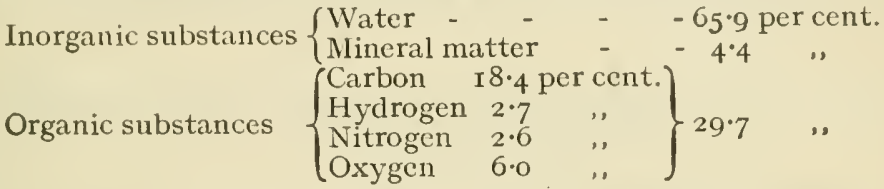

The muscles, the adipose tissue, and the skeleton form nearly fourfifths of the total body-weight in the adult. The following table shows the percentage amount of each of these tissues in a man, a woman, and a cliild (Bischoff):

\begin{tabular}{|c|c|c|c|c|}
\hline & & Man. & Woman. & $\begin{array}{l}\text { New-born } \\
\text { Child. }\end{array}$ \\
\hline Voluntary muscles & - & $4 I \cdot 8$ & $35^{\cdot 8}$ & $23 \cdot 5$ \\
\hline Adipose tissue - & - & I $8 \cdot 2$ & $28 \cdot 2$ & I 3.5 \\
\hline Skeleton - - & - & I $5{ }^{\circ} 9$ & I $5 \cdot$ I & I $5 \cdot 7$ \\
\hline Rest of body & - & $24 \cdot 1$ & $20 \cdot 9$ & $47 \cdot 3$ \\
\hline
\end{tabular}

The nitrogen is contained chiefly in the muscles, glands, and nervous system, and in the constituents of the connective tissues, which yield gelatin, various mucoids, and elastin. The ordinary proteins make up about 9 per cent. of the weight of the body, or 22 per cent. of its solids; the albuminoids or sclero-proteins (gelatin-yielding material, etc.) (p. 2) about 6 per cent. of the body-weight. Nitrogen exists in proteins to the extent of 16 per cent., so that the 6.5 kilos of protein of a 70 -kilo body contain about I kilo of nitrogen.

The carbon is contained chiefly in the fat, which forms a very large proportion of the water-free substance of the body, and in the proteins. A small amount is present as calcium carbonate in the bones. In the body of a strong young man weighing $68.6 \mathrm{kilos}$, Voit found the following quantities of dry fat in the various tissues:

\begin{tabular}{|c|c|c|c|c|c|c|c|}
\hline Adipose tissue & & - & - & - & - & \multicolumn{2}{|c|}{$8809^{\circ} 4$ grammes. } \\
\hline Skeleton & - & - & - & - & 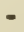 & $2617 \cdot 2$ & , \\
\hline Muscles & - & - & - & - & - & $636 \cdot 8$ & ", \\
\hline Brain and spin & al cord & - & - & - & - & $226 \cdot 9$ & " \\
\hline Other organs & - & - & - & - & - & $73 \cdot 2$ & $"$, \\
\hline & Cotal & - & - & - & - & 12363.5 & ". \\
\hline
\end{tabular}

equivalent to 18 per cent. of the whole body-weight, or 44 per cent. of the solids. In dry fat rather more than 75 per cent. of carbon is present, and in protein about 50 to 55 per cent.; so that while the fat of the body analyzed by Voit contained more than 9 kilos of carbon, only about a third of this amount would be found in the proteins.

* The income and expenditure of the body in terms of energy are considered in Chapter XII. 
In the fat there is, roughly speaking, 2 per cent. of hydrogen, in proteins only 7 per cent.; so that from three to four times as much liydrogen is contained in the fat of the body as in its proteins.

Oxygen forms about 12 per cent. of fat, and 20 to 24 per cent. of proteins; the protein constituents of the body, therefore, contain about as much of its oxygen as the fat.

Of the inorganic salts, calcium phosphate, $\mathrm{Ca}_{3}\left(\mathrm{PO}_{4}\right)_{2}$, is much the most abundant, owing to the large amount of it in bone, in the ash of which it is found to the extent of 83 per cent., along with 13 per cent. of calcium carbonate.

\section{Income and Expenditure of Nitrogen-The Nitrogen BALANCE-SHEET.}

Nitrogenous Equilibrium.-It is a matter of commoli experience that the weight of the body of an adult may remain approximately constant for many months or years, even when the diet varies greatly in nature and amount. And not only may the weight remain constant, but the relative proportions of the various tissues of the body, so far as can be judged, may remain constant too. Here it is evident that the expenditure of the body must precisely balance its income: it must lose as much nitrogen as it takes in, otherwise it would put on flesh; it must lose as much carbon as it takes in, otherwise it would put on fat. Or, again, the body may be losing or gaining fat, giving off more or less carbon than it receives, while its 'flesh' (its protein constituents) remains constant in amount, the expenditure of nitrogen being exactly equal to the income.* In both cases we say that the body is in nitrogenous equilibrium.

A starving animal or a fever patient, on the other hand, is living upon capital, the former entirely, the latter in part; the expenditure of nitrogen is greater than the income. A growing child is living below its income, is increasing its capital of flesh. In neither case is nitrogenous equilibrium present.

The starving animal, as long as life lasts, excretes urea, kreatinin, and other nitrogenous substances, and gives off carbon dioxide; but its expenditure, and especially its expenditure of nitrogen, is pitched upon the lowest scale. It lives penuriously, it spins out its resources; its glycogen goes, its fat goes, a certain part of its protein goes, and when its weight has fallen from 25 to $5^{\circ}$ per cent. it dies. At death the heart and central nervous system are found to have scarcely lost in weight; the other organs have been sacrificed to feed them. Fig. Ig9 sliows the percentage loss of weight and the proportion of the total loss which falls upon each of the organs of a cat in starvation (Voit).

* For long experiments extending over many days the nitrogen balance may be considered as practically the same as the protein balance, but this is not necessarily true of short periods of time, since the stock of nitrogen present in the body in other forms than proteins, although relatively small, is subject to variations. 
For the first day of starvation the excretion of urea in a dog or cat is not diminished; it takes about twenty-four hours for all the nitrogen corresponding to the proteins of the last meal to be eliminated. On the second day the quantity of urea sinks abruptly; then begins the true starvation period, during which the daily output of urea remains constant or diminishes very slowly until a short time before death, when it rapidly falls, and soon ceases altogether. An increase in the excretion may precede the final abrupt decline (premortal increase). This seems to indicate the time at which all the available fat has been used up, and after which protein is no longer 'spared' by the fat.* If the animal has little fat in its body to begin with, the rise in the urea excretion takes place even after the first few days. So long as the fat lasts the rate at which it is

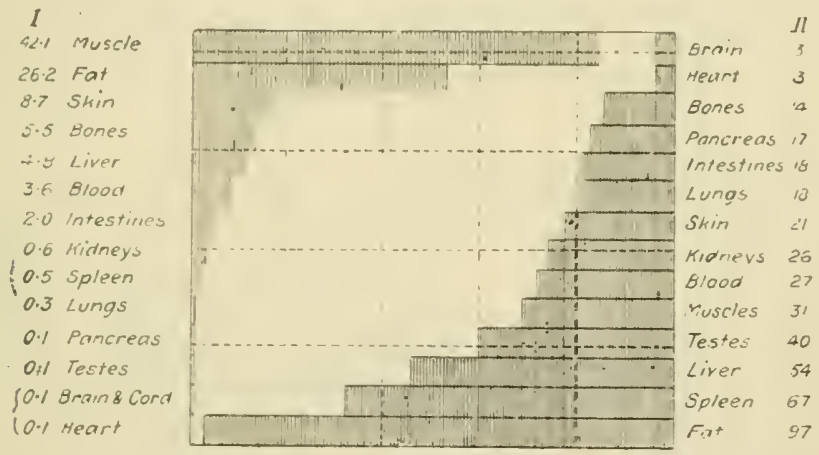

Fig. 198.-Diagram showing Loss of Weight of the Organs in Starvation. The numbers under $\mathrm{I}$. are the percentages of the total loss of body-weight borne by the various organs and tissues. The numbers under II. give the percentage loss of weight of each organ calculated on its original weight as indicated by com. parison with the organs of a similar animal killed in good condition.

destroyed-as estimated from the amount of carbon given off minus the carbon corresponding to the broken-down proteins-remains very nearly constant after the first day. The fat to a certain extent economizes the proteins of the starving body, but however much fat may be present, a steady waste of the tissue-proteins goes on. If non-nitrogenous food in the form of sugar is supplied to an otherwise starving animal, the premortal rise in the nitrogen excretion does not occur. By giving a sufficient quantity of sugar, or of sugar and fat, but practically no protein (so-called nitrogen starvation), the excretion of nitrogen may be reduced to one-third of its amount when no food at all is given. This is true both in animals

* If the animal has been for some time on a diet containing an abundance of proteins, several days may elapse before the constant excretion of urea is reached; if the previous diet has been poor in protein, the constant starvation output may be at once established. 
and man In this way the daily excretion of nitrogen in a man has been reduced to 4 grammès. It is a remarkable fact that while a mixture of carbo-hyclrate and fat will act just as well as carbohydrate alone in bringing about this reduction in the nitrogen output, fat without carbo-hydrate is much less effective. The hypothesis suggested by Landergren to explain this is alluded to on another page (p. 55I).

The results obtained on fasting men differ in some respects from those obtained on starving animals. In ten days of hunger, Cetti, a professional 'fasting man ' of meagre habit, excreted II 2 grammes nitrogen, or an average of II grammes a day. The excretion was least on the eighth, ninth, and tenth days-namely, about 9 grammes a day. On the third day it was higher than on the second, and almost as high on the fouth as on the third. A similar rise in the nitrogen excretion on the second day has been observed in other fasting men, but is either rare or absent in fasting dogs. The explanation apparently is that in the ordinary food of man there is a greater abundance of carbo-hydrates and fats, the protein-sparing action of which is most

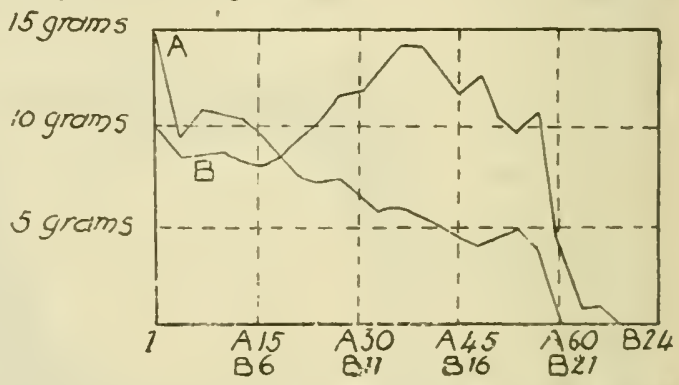

Fig. 299.-Excretion of Urea in Starvation. At is a curve representing the quantity of urea excreted daily by a fat dog in a starvation period of sixty days. B is the curve of urea excretion in a lean young dog in a starvation period of twenty-four days. Both are constructed from Falck's numbers, but in A only every third day is put in, in order to save space. The num. bers along the vertical axis represent grammes of urea; those along the horizontal axis days from the beginning of starvation.

pronounced at the very beginning of the starvation period. The quantity of chlorine and alkalies in the urine was also diminished, while the phenol was increased. The respiratory quotient sank to 0.66 to $0.69-$ even less than the quotient corresponding to oxidation of fats alone. The meaning of this, in all probability, is that some of the carbon of the broken-down proteins was laid up in the body as glycogen (Zuntz). In another professional fasting man (Succi) with a considerable amount of body-fat, the excretion of nitrogen was found to diminish continuously during a fast of thirty days, being less than 7 grammes on the tenth day. In another fast of twenty-one days by the same person it was a little less than 3 grammes on the last day. The surprisingly small nitrogenous waste in this case is perhaps to be accounted for by the proteinsparing action of the abundant body-fat. The nitrogenous metabo- 
lism has also been investigated during long-continued hypnotic sleep (Hoover and Sollmann). The results were very much the same as in an ordinary starvation experiment

It might be supposed that if an animal was given as mucl nitrogen in the food in the form of proteins as corresponded to its daily loss of nitrogen during starvation, this loss would be entirely prevented and nitrogenous equilibrium restored. The supposition would be very far from the reality. If a dog of 30 kilos weight, which on the tenth day of starvation excreted II.4 grammes urea, had then received a daily quantity of protein equivalent to this amountthat is to say, about 34 grammes of dry protein, or 175 grammes of lean meat-the excretion of nitrogen would at once have leaped up to nearly double its starvation value. If the quantity of protein in the diet was progressively increased, the output of urea would increase along with it, but at an ever-slackening rate; and at length a condition would be reached in which the income of nitrogen exactly balanced the expenditure, and the animal neither lost nor gained flesh.

In an experiment of Voit's, for instance, the calculated loss of flesh in a dog with no food at all was I 90 grammes a day. The animal was now fed on a gradually increasing diet of lean meat, with the following result:

\begin{tabular}{|c|c|c|}
\hline $\begin{array}{c}\text { Flesh in the } \\
\text { Food. }\end{array}$ & $\begin{array}{c}\text { Flesh used up in } \\
\text { the Body. }\end{array}$ & $\begin{array}{c}\text { Net Loss of } \\
\text { Body-flesh. }\end{array}$ \\
\hline 0 & 190 & I \\
250 & 341 & 91 \\
350 & 411 & 61 \\
400 & 454 & 54 \\
450 & 471 & 21 \\
480 & 492 & 12 \\
\hline
\end{tabular}

The loss of nitrogen in the urine and fæces is what was measured. Knowing the average composition of 'body-flesh' (muscles, glands, etc.), it is possible to translate results stated in terms of nitrogen into results stated in terms of 'flesh.' Muscle contains approximatcly 3.4 per cent. of nitrogen. Here, with a diet of 480 grammes of meat, the dog was still losing a little flesh; it would probably have required from 500 to 600 grammes for equilibrium. The results are graphically represented in Fig. 200, p. 607 .

The quantity of protein food necessary for nitrogenous equilibrium varies with the condition of the organism; an emaciated body requires less than a muscular and well-nourished body. The least quantity which would suffice to maintain in nitrogenous equilibrium the famous 35 kilo dog of Voit, even in very meagre condition, was 480 grammes of lean meat, corresponding to 16 grammes of nitrogen, or 35 grammes of urea-that is, about three times the daily loss 
during starvation. Fron this lower limit up to 2,500 grammes of meat a day nitrogenous equilibrium could always be attained, the animal putting on some flesh at each increase of diet, until at length the whole 2,500 grammes was regularly used up in the twenty-four lours. A further increase was only checked by digestive troubles. A man, or at least a civilized man, can consume a much smaller amount both absolutely and in proportion to the body-weight. Rubner, with a body-weight of 72 kilos, was able to digest and absorb over I,400 grammes of lean meat; Ranke, with about the same body-weight, could only use up I,300 grammes on the first day of his experiment, and less than $\mathrm{r}, 000$ grammes on the third. But whether the surplus of protein food above the necessary minimum is great or small, nitrogen equilibrium is eventually attained, and thereafter all the nitrogen of the food regularly appears in the excreta; the explanation of this fact will be considered a little later (p 609).

So much for a purely protein diet. When fat is given in addition to protein, nitrogenous equilibrium is attained with a smaller quantity of the latter. A dog which, with protein food alone, is putting on flesh, will put on more of it before nitrogenous equilibrium is reached if a considerable quantity of fat be added to its diet. Fat, therefore, economizes protein to a certain extent, as we have already recognized in the case of the starving animal. On the other hand, when protein is given in large quantities to a fat animal, the consumption of fat is increased; and if the food contains little or none, the bodyfat will diminish, while at the same time 'flesh' may be put on. The Banting cure for corpulence consists in putting the patient upon a diet containing much protein, but little fat or carbo-hydrate; and the fact just mentioned throws light upon its action.

All that we have here said of fat is true of carbo-hydrates. To a great extent these two kinds of food substances are complementary. Carbo-hydrates economize proteins as fat does, but to a greater extent, so that with an abundant supply of carbo-hydrate in the food the ninimum protein requirement can be forced down much below what is possible on a diet of protein and fat alone. Carbohydrates also economize fat, so that when a sufficient quantity of starch or sugar is given to an otherwise starving animal, all loss of carbon from the body, except that which goes off in the urea, kreatinin, etc., still excreted, can be prevented. Of course, the animal ultimately dies, because the continuous, though diminished, loss of protein cannot be made good. The fact that carbo-hydrates economize proteins so much more efficiently than fat indicates that sugar is essential in the bodily metabolism, so that when carbo-hydrates are absent from the food some of the protein must be broken down so as to yield eventually the compounds necessary for the formation of carbo-hydrate. It is probable, indeed, that purified proteins, 
absolutely free from admixture with carbo-hydrates, which, of course, is not the case with the natural protein foods, will not permanently suffice for nutrition, but that the protein must be supple-

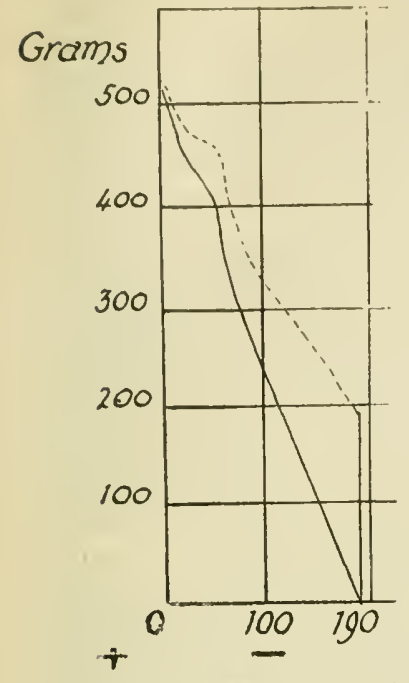

Fig. 200.-Curves constructed to illustrate Nitrogenous Equilibrium (from an Ex. periment of Voit's). The loss of flesh in grammes is laid off along the horizontal axis. The income and expenditure corresponding to a given loss are laid ofl (in grammes of 'flesh') along the vertical axis. The continuous curve is the curve of income; the dotted curve, of expenditure. With no income at all the expen. diture is rgo grammes; with an income of 480 grammes the expenditure is 492 and the loss 12 grammes. Nitrogenous equilibrium is represented as being reached with an income of about 530 grammes; here the two curves cut one another. Some flesh may be put on for a short time, some nitrogen may he stored up; but the excretion of nitrogen is soon adjusted to the new scale of supply, and the protein income is apparently spent as frcely as it is received. These facts were usually summed up in the tissues. It would appear, indeed, that fats are not absolutely indispensable either for maintenance or for growth. White rats liave been seen to grow normally over long periods with dietaries devoid of fat; for example, mixtures of the purified protein edestin (from hemp seed) or casein with starch, sugar, and 'proteinfree milk' freed from fat by extraction with ether (Osborne and Mendel). While in these experiments the food might not have been free from the so-called 'lipoids,' it has been demonstrated that an important group of substances of this class, the phosphatides, can be synthesized in the body, the necessary phosphorus being obtainable even from inorganic phosphates (McCollom).

Relation between Nitrogen excreted and the Quantity of Protein Food.-At this point we may consider a little more closely a phenomenon already alluded to, and to which much discussion used to be devoted by writers on metabolism. It has been stated that within the limits of nitrogenous equilibrium, which is the normal state of the healthy adult, the body lives up to its income of nitrogen; it lays by nothing for the future. In the actual pinch of starvation the organism, when its behaviour is tested by a comparison of the intake and excretion of nitrogen, appears to have become suddenly economical. When a plentiful supply of protein is presented to the starving body, it seems, judged by the same criterion, to pass at once from extreme frugality to luxury.

mented by a certain amount of carbo- 
dictum, often clignified as a 'law' of nitrogenous metabolism that: Consumption of protein is largely determined by supply (Practical Exercises, p. 720).

To explain this many hypotheses were invented. The famous theory of Voit assumed that the food-protein after absorption (the so-called 'circulating-protein') is carried to the tissues and taken up by the cells, where the greater part of it, without being incorporated with the protoplasm, is nevertheless acted upon, rendered unstable, shaken to pieces, as it were, by the whirl of life (by the intracellular enzymes we might now say less dramatically) in the organized framework, the interstices of wich it fills.

Pflüger, on the other hand, maintained that we have no right to draw a distinction between the consumption of organ- and circulatingprotein; that the whole of the latter ultimatcly rises to the height of organ- or tissue-protein, and passes on to the downward stage of metabolism only through the topmost step of organization. An increase in the supply of nitrogenous material in the blood must, on this view, be accompanied with an increased tendency to the break-up, the dissociation, as Pfluger put it, of the living substance. The actual organized elements, however, the existing cells, were not supposed to be destroyed; the building remained, for although stones were constantly crumbling in its walls, others were being constantly built in.

A much less plausible view was that the tissue clements themselves are short-lived; that the old cells disappear bodily and are replaced by new cells; and that the whole of the proteins of the food take part in this process of total ruin and reconstruction. Histological evidence, as soon as the methods of examining tissues with the microscope became sufficiently refined, told strongly against this idea. Although the cells of certain glands, such as the mammary, perhaps the mucous glands, and especially the sebaceous glands (p. 565), exhibit changes which, hastily interpreted, might seem to indicate that they break down bodily, as an incident of functional activity, no proof could be obtained of the production of new cells on the immense scale which this theory would require. The relatively small and constant amount of the endogenous metabolism indicates that the actual protoplasmic substance, the living framework of the cell, is comparatively stable; that it does not brcak down rapidly; and that only a small and fairly constant amount of food- or circulating-protein, or of the decomposition products of protein, is required to supply the waste of the organ-protein.

We have referred to these theories because there could scarcely be a more instructive instance of the way in which theories become obsolete with the advance of knowledge and of the way in which, with the advance of knowledge, a phenomenon which appcars an absolute ridule to one generation may become fairly intelligible to the ncxt, ferhaps childishly simple to a third. The student will not derive much lenefit from the perusal of this page should he fail to recognize that the hypotheses of the twentieth century are mortal too, and bound for the same bourne as those of the nineteenth.

It is apparent in the first place from our study of the metabolism of the proteins that the conclusion, ' consumption of protcin is proportional to supply, cannot be drawn from the equality of nitrogen intake and nitrogen output. The amino-acids derived from proteins, except that relatively small fraction employed in repairing the 
waste of the tissues, which in nitrogen equilibrium is exactly compensated for by a corresponding release of amino-acids or their equivalent from the cell-proteins, are indeed speedily deaminated and the nitrogen of the amino-groups excreted as urea (with ammonia compounds and creatinin). But, as we have seen, only a small proportion of the chemical energy of the amino-acids and only a small fraction of their carbon are liberated in this process. The carbon-containing residues are katabolized only to the extent required by the momentary needs of the tissues, any balance being stored as part of the reserve of carbo-hydrate or of fat. The body does not possess the means of storing surplus amino-acids as such or even in the form of proteins, except to the small extent corresponding to any increase which may occur in the body-protein when the food-protein is increased beyond the minimum required for nitrogen equilibrium. Why the organism has not developed the capacity to store large quantities of protein is, of course, an interesting question, but it need scarcely be discussed here. One obvious reason is that protein is not a suitable source, nor are aminoacids apparently a suitable source of energy for the tissues until they have been deaminated and have probably undergone further decomposition and transformation. Therefore they are decomposed at once and their available residue stored, if it is in any case to be stored, in the more available form of carbo-hydrate (or fat). Where the food-proteins differ greatly from the body-proteins in the proportions of the various amino-acids, there would be no object in storing a great surplus of those which are most plentiful in the food, if they were at the same time the scarcest in the tissues, or, in the case of gland-cells, the scarcest in the proteins which they manufacture for their secretions.

At any moment the magnitude of this non-utilizable surplus will depend upon the quantity of that one of the indispensable aminoacids which is present in the smallest amount. For the proper proportion must be preserved between the different 'stones 'out of which the molecule is built. When a single amino-acid is introduced into the body, it is at once changed into urea and excreted, since it cannot be utilized by itself for building up protein.

When the cells have once culled from the mixture circulating in the blood the amino-acids, a full supply of which they have most difficulty in obtaining, a residue, large or small, according to the quantity and quality of the protein intake, will be left, and this can only be utilized to supply energy or to add to the fat and carbohydrate stores. For these uses removal of the amino-group is an essential preliminary. The question whether the deamination of a large part of the amino-acids coming from the intestine takes place in the liver, so that the surplus nitrogen is shunted out of the main metabolic current at its very source, has been already touched upon 
(p. 588). Some writers conceive that in sucli a short-cut from protein to urea we have a kind of physiological safety-valve to protect the tissues from the burden of an excessive metabolism. And if by this is meant that it is advantageous to the tissues that a special mechanism should exist to eliminate a surplus of nitrogen which they do not require, and which they cannot store, and to present them with a residue which they can utilize, the conception is certainly correct. But there is no good evidence that in the presence of an over-abundant supply of amino-acids the endogenous protein metabolism would be essentially modified.

Relation of Nitrogenous Metabolism to Muscular Work.-This is another of those classical pliysiological problems which it is difficult to present properly apart from its historical setting. The general result of much experimental work and long-continued discussion is that when the work does not transgress what may be called 'normal limits,' the excretion of nitrogen is nearly independent of muscular work-that is to say, the quantity of nitrogen excreted by a man on a given diet is practically the same whether he rests or works. Before this was known it was maintained by Liebig that proteins alone could supply the energy of muscular contraction-that, in fact, proteins were solely used up in the nutrition and functional activity of the nitrogenous tissues, while the non-protein food yielded heat by its oxidation. As exact experiments multiplied, it was found that muscular work, the production of which is the function of by far the greatest mass of protein-containing tissue, had little or no effect upon the excretion of urea in the urine. More than this, it was shown that a certain amount of work accomplished (by Fick and Wislicenus in climbing a mountain) on a non-nitrogenous diet had double the heat equivalent of the whole of the protein consumed in the body, as estimated by the urea excreted during, and for a given time after, the work. On the assumption that all the urea corresponding to the protein broken down was eliminated during the time of this experiment, a part at least of the work must have been derived from the energy of non-nitrogenous material. And other experiments in which account was taken of the increase in the carbon dioxide given off (as conspicuous an accompaniment of muscular work as the constancy of the urea excretion), showed that during muscular exertion carbonaceous substances other than proteins - that is to say, fats and carbo-hydrates-are oxidized in gi eater amount than during rest.

So the pendulum of physiological orthodoxy came full-swing to the other side. Liebig and his school had taught that proteins alone were consumed in functional activity; the majority of later physiologists following Voit denied to the proteins any share whatever in the energy which appears as muscular contraction. The protcins, they said, ' repair the slow waste of the framework of the muscular machine, replace a loose rivet, a worn-out belt, as occasion may require: the 
carbo-hydrates and fats are the fuel which feeds the furnaces of life. the materal which, dead itself, is oxidized in the interstices of the living substance, and yields the cnergy for its work.'

Now, it is a singular circumstance, and full of instruction for the ingenuous student of science, that the facts which were supposed absolutely to disprove the older theory, and absolutely to establish its more modern rival, are now scen to do neither tlic one thing nor the other. The fact-and it is a fact-that the excretion of nitrogen is but little affected by muscular contraction, does not prove that none of the energy of inuscular work comes from proteins; the fact that, under certain conditions, some of the muscular energy must apparently come from non-nitrogenous materials, does not prove that these are the normal source of it all. The distinction had again been made too absolute. The pendulum must again swing back a little; and the experiments of Pflüger and his pupils were soon to set it moving.

In the first place, it is not perfectly correct to say that work causes no incrcase in the excretion of nitrogen; excessive work in man, and work, severe but not excessive, in a flesh-fed dog (Pflüger), do cause somewhat more nitrogen to be given off. On the first day of work the increase is always much less than on the second and third; and on the first and second rest days, following work, the elimination of nitrogen is still increased. After excessive exercise in man not only is the urea increased, but also the ammonia, kreatinin, and if the subject is in poor training, the uric acid and purin bases (Paton, Stockman, etc.). Moderate exercise causes no increase on the first day, but a slight increase on the second. The meaning of these facts seems to be that during muscular work the intensity of which does not exceed certain limits, the protein waste of the muscular substance itself is no greater than during rest. IVhen, however, the machine is ' speeded up' beyond a certain point the wear and tear is sensibly increased and an excess of tissue-protein is katabolized. There is no reason to suppose that the tissue-protein thus broken down will not yield energy for the muscular work by the oxidation of its non-nitrogenous residue, just as well as the surplus aminobodies derived from food-protein. The muscular machine has the peculiarity that it is constructed of combustible material; even the dust and the splinters, if we may so express it, which represent the wear and tear of the machine can be Eurnt in the furnace which keeps it going.

In the second place, even if the excretion of nitrogen were entirely unaffected by work, this would not prove that none of the energy of the work comes from proteins. For, as we have seen, it is after the nitrogen has been split off and converted into urea that the energy of a great part of the food-protein is developed by oxidation. Further, since the animal body is a beautifully-balanced mechanism which constantly adapts itself to its conditions, it is conceivable that it may, when called upon to labour, save proteins from lower uses to devote them to muscular contraction. In this 
case the excretion of nitrogen would not necessarily be altered; the proteins which, in the absence of work, would have been oxidized within the muscular substance or elsewhere, their energy appearing entirely as heat, may, when the call for protein to take the place of that broken down in muscular contraction arises, be diverted to this purpose.

In any case, there is no doubt that a dog fed on lean meat may go on for a long time performing far more work than can be yielded by the energy of fat and carbo-hydrates occurring in traces in the food, or taken from the stock in the animal's body at the beginning of the period at work. A large portion, and perhaps the whole, of the work, must in this case be derived from the energy of the proteins (Pflüger). On the otlier hand, it is well established that when fats and carbo-hydrates are present in sufficient quantity in the tissues or the food, they constitute the main source of the energy of muscular contraction (p. 772), and there is some evidence that of the two classes of food materials carbo-hydrates in the form of dextrose (or glycogen) is the material of election.

The outcome, then, of this famous controversy is essentially a compromise. Everybody now admits that the muscular machine can and does utilize predominantly any one of the great groups of food substances, be it carbo-hydrate, fat, or protein, when the dietetic conditions are such that only one of these is offered to it in large amount, the others being either absent or offered in small amount. To be sure, amino-acids are not the first choice, but if it must do so the muscle can make shift with them, and can indeed make them serve excellently well. When all the food substances are present in abundance, carbo-hydrate is favoured above fat, and fat above protein.

Experience has shown that the minimum quantity of nitrogen required in the food of a man whose daily work involves hard physical toil is higher than the minimum required by a person leading an easy, sedentary life. This is evidently in accordance with the view that protein is actually used up in muscular contraction; but it is not inconsistent with the opposite view. For the body of a man fit for continuous hard labour has a greater mass of muscle to feed than the body of a man who is only fit to handle a composingstick, or drive a quill, or ply a needle; and the greater the muscular mass, the greater the muscular wastc. But if an animal just in nitrogenous equilibrium on a diet of lean meat when doing no work is made to labour day after day, it will lose flesh unless the diet be increased. This must mean that some of the protein is being diverted to muscular work, and that the balance is not sufficient to keep up the original mass of 'flesh' (see p. 628).

Relative Value of Different Proteins in Nutrition-Synthesis of Amino-Acids. - The fact that the various proteins differ quantita- 
tively and qualitatively in respect to their amino-acids raises the question of the relative value of different proteins in nutrition. In this is involved the further question, whether the body can itsclf synthesize from other materials any of the amino-acids which may be deficient, or change one amino-acid into another. That the 'peptones' derived from a protein which is itself capable of permanently supplying the whole nitrogenous intake of the organism can be substituted for the protein scarcely needs demonstration, since it is known that the protein is converted into peptones in digestion. Nevertheless, this has been proved conclusively by feeding experiments with peptones. It was to be expected, too, leaving out of account all consideration of the means of overcoming the repugnance of animals to accepting such unnatural food substances, that the further products of protein hydrolysis, the amino-acids, etc., could be substituted for the original proteins when these were themselves adequate. For it is in the form of amino-acids or at most of such relatively simple polypeptide groups as may still hang together after complete digestion and absorption, that the nitrogenous food substances are normally offered to the tissues. Experimental demonstration of the feasibility of this substitution has also been obtained. The split products of meat, for example, will keep an animal in nitrogen equilibrium as well as the meat from which they are derived. But what happens when one or more of the aminoacids found in the proteins of the body are missing from the protein of the food? That the components of an amino-acid like arginin (ornithin and urea), into which it can be split not only by the possibly crude and violent methods of the chemical laboratory, but also by the delicate and precisely-adapted 'biological ' action of a special enzyme (arginase), should be able to replace the original amino-acid is a fact which does not greatly help towards an answer. For when these components, or ornithin alone, since urea is always present in the body, are given instead of arginin, the reversal of the enzyme reaction by which arginin is decomposed is all that is necessary for its synthesis, and the reversal of such a reaction is doubtless a very commonplace affair in tissue metabolism. The formation of one amino-acid from another, or from materials which do not originate exclusively from protein, is a different thing, and the answer to the question raised, so far as it can yet be given, is that the way in which the body deals with a deficiency in the protein 'building stones' depends upon the nature of the missing aminoacids. Thus, the phospho-protein casein does not yield glycin on hydrolysis; yet it has been shown that casein is a perfectly adequate or complete protein food capable of covering the whole nitrogen requirement of the body over long periods. The same is true of the cleavage products of casein which has been subjected to pancreatic digestion. In an animal fed on no other protein than casein, with 
suitable quantitics of carbo-hydrate and fat in addition, the glycin contained in certain of the body proteins must therefore have been produced in the body itself. We have already seen (p. 580 ) that for the synthesis of hippuric acid after the administration of benzoic acid, glycin is necessary, and the quantity of hippuric acid which can be thus produced is so great that it is impossible to suppose that it all comes from glycin preformed in the body or from glycin in the food substances. It may accordingly be taken as prored that the tissucs have the porrer of synthesizing at least this one of the amino-acids (amino-acetic acid), reckoned among the protein 'building stones.' It is said that if the casein has been hydrolysed by acid, the products will not preserve nitrogen equilibrium, perhaps because the acid has broken up all the polypeptides (p. 2), some of which the cells may reed as the starting-point of protein synthesis. This, however, is uncertain.

Lysin also appears to be capable of being synthesized in the body, and protein foods free from lysin, or containing only a trace of it, may yet be adequate for nutrition and growth. Prolin, too, is not indispensable, and this is of special interest, for the amino-acids hitherto mentioned as capable of being built up in the tissues are all more or less directly related to each other, being derivatives of the series of saturated fatty acids. The task of changing one of these into another in which the food is cleficient may, therefore, be considered a comparatively easy one. But prolin has no obvious relation to most of the other amino-acids.

It is a-pyrrolidin carboxylic acid,
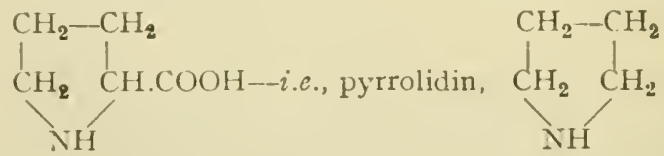

in which $\mathrm{H}$ in one of the $\mathrm{CH}_{2}$ groups is replaced by carboxy-i ( $\left.\mathrm{COOH}\right)$. It has been suggested that prolin may be formed in the body from glutaminic (amino-glutaric) acid, which by loss of a molecule of water can be made to yield a-pyrrolidon carboxylic acid. Thus,

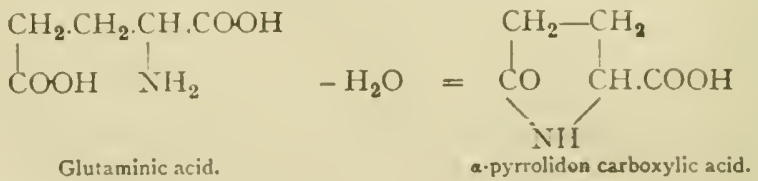

By reduction the latter compound might be changed into prolin.

With proteins deficient in certain other amino-acids a totally different result has been obtained. Gelatin, for example, contains most of the amino-acids and other groups which compose the body proteins, but tyrosin, cystin, and tryptophane are lacking in the 
gelatin molecule. \%ein, an alcohol-soluble protein or prolamin* derived from maize, yiclds no tryptophane, glycin, or lysin. Now, it is found that neither gelatin nor zein can replace the whole of the ordinary proteins in the food. When only enough protein is taken to prevent loss of nitrogen from the body, one-fifth of the necessary nitrogen can be supplied in the form of gelatin. When the food is much richer than this in ordinary protein, a correspondingly greater proportion of the protein can be replaced by gelatin. The surplus is not used in the endogenous metabolism of the cells (p. 573), but supplies energy to the body after the elimination of its nitrogen as urea, just as the surplus protein would do. Thus gelatin economizes protein in the same way that fat and carbohydrates do, but also to some extent in a different way by supplying ' building stones 'for the protoplasm. It is therefore an interesting question whether gelatin can fully replace protein when the missing substances are given in addition. Kauffmann has stated that his own nitrogen requirement ( 15.2 grammes) was almost completely covered by a mixture containing 93 per cent. of the nitrogen in the form of gelatin, 4 per cent. as tyrosin, 2 per cent. as cystin, and I per cent. as tryptophane, in addition to the same amounts of carbo-hydrate and fatty food as in the comparison diet, in which the nitrogen was supplied in the form of plasmon, a commercial preparation of casein.

Similar results have been reported in experiments on animals in which attempts have been made to 'complete' such inadequate proteins by addition of the missing amino-bodies, with fair but, according to Osborne and Mendel, not entirely satisfactory results. The converse experiment, in which an amino-acid such as tryptophane has been purposely eliminated from the food mixture, has also been tried, with the result that rapid deterioration in the condition of the animal ensued. It would seem, indeed, that whatever capacity the animal body may have for synthesizing certain of the amino-acids, this power does not extend to the cyclic compounds tryptophane, tyrosin, phenylalanin, and histidin, which must be supplied in the food. It has been suggested by Osborne that in this regard an essential difference exists between the animal and the plant, the latter alone being endowed with the function of ' cyclopoièsis,' or formation of substances of the cyclic type. It is not clearly understood as yet on what this difference really hinges, whether, as some have supposed, on the inability of the animal organism to form the appropriate fatty acid radicals, or on some

* The prolamins are so called because on hydrolysis they yield exceptionally large amounts of prolin (p. 360) and ammonia. They are insoluble in water and absolute alcohol, but soluble in 70 to 80 per cent. alcohol and in dilute acids and alkalies. Besides zein they include gliadin (from wheat and rye), hordein (from barley), and bynin (from malt). They are extraordinarily rich in glutaminic acid. hordein yielding more than any protein hitherto investigated (over $4^{\text {I }}$ per cent). 
other limitation of its chemical powers. While the cyclic (and heterocyclic) compound. cannot be replaced by' other 'Bausteine' of the proteins, they may to some extent replace each other. Thus it would seem that tyrosin can be replaced by phenvlalanin (Abderhalden).

While some of the food proteins like cascin are sufficient by themselves to supply all the amino-bodies necessary not only for the maintenance, but also for the growth of the body, and can accordingly be termed adequate or complete protein food substances, others, like gelatin, are insufficient by themselves to supply the protein required for mere maintenance, still less for growtli.
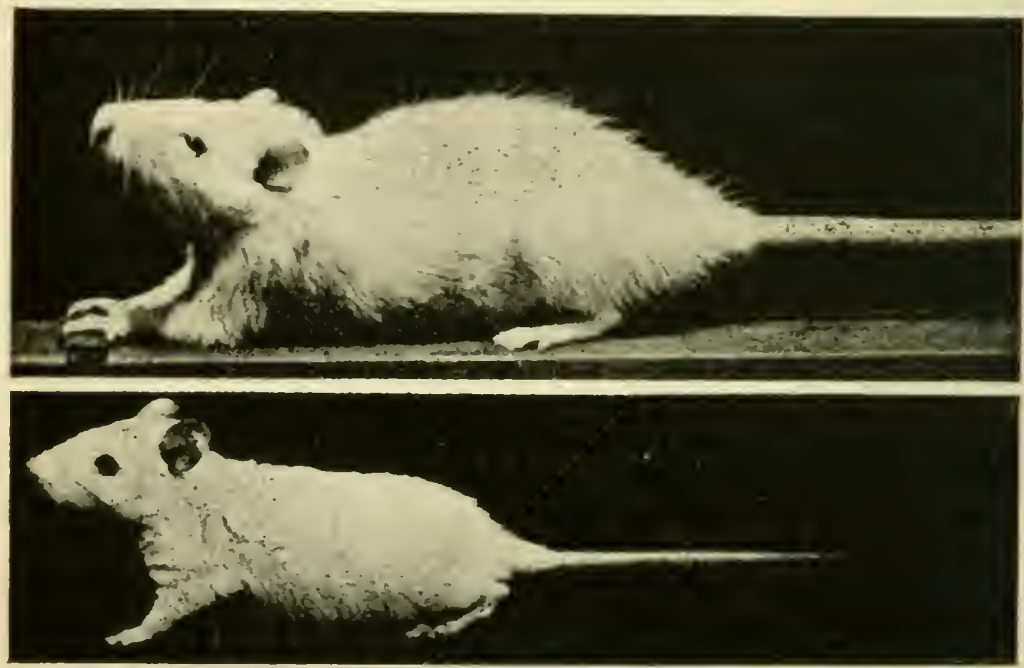

Fig. 20r.-Two Female Rats of the Same Age (r40 days). The upper one was fed on an ordinary diet, and is of the normal weight for its age. The lower one was fed on a diet composed of a mixture of isolated food-stuffs. Its weight is only equal to that of a normal rat thirty-six days old (Osborne and Mendel).

and mar be spoken of as inadequate or incomplete proteins. There is a third intermediate group. comprising proteins which suffice when given as the sole protein food to maintain the body for an indefinitely long period, and to repair the tissue waste without permitting growth of the animal to take place. Gliadin and hordein (see footnote, p. 6r5) are representatives of this group. The experiments of Osborne and Mendel with gliadin are of special interest. since this substance is very differently constituted from the ordinary food proteins, as well as from the tissue proteins of the animal body: While, as already stated, it yields very large quantities of glutaminic acid, prolin, and ammonia, it either contains no lysin 
and no glycin, or yields too little to be detected with certainty. It also yields comparatively little histidin and arginin. Now, it has been found that a dietary containing carbo-hydrate in the form of starch, fats in the form of lard, and inorganic salts, but no protein except gliadin* suffices to maintain adult rats in good condition for very long periods (up to 290 days), and also to maintain young rats in a stationary condition as regards growth, but in perfect health. The youthful appearance of the rats whose growth was thus inhibited was very striking, and corresponded with their size rather than with their age. The capacity for growth on a normal diet was apparently not in the least diminished; the growth processes simply romained in abeyance.

' In one rat, after a continuous suppression of growth lasting 277 days, when the animal was $31+$ days old - an age at which normally little or no growtl takes place-satisfactory growth was resumed on a suitable diet.' A still more remarkable experiment was the following: 'A male rat, kept for 154 days with gliadin as the sole protein in the food, was paired with a female also on the gliadin diet. At the end of 178 days on the gliadin diet she gave birth to four young, which were satisfactorily nourished by the mother, still on gliadin, during the first month of their existence. After a month three of the young rats were removed from the mother and put on diets of casein food (i.e., casein plus suitable proportions of carbo-hydrate, fat, and inorganic materials), edestin food and milk food respectively. The fourth was left with the mother. The fourth rat began to evince a failure to grow at about the period (thirty days) when young rats are wont to depend upon extraneous food.

The meaning of this last observation can only be that the young animal, when obliged to depend upon its share of the gliadin food left with the mother in place of the milk formed by the mother from this same gliadin food mixture, showed the typical failure to grow on a diet inadequate as regards the power of producing growth in respect to the protein contained in it. On the other hand, in the body of the mother this inadequate diet had been so transformed that not only had she maintained her body-weight and repaired

* Certain accessory substances of unknown nature, contained in some of the natural foods must also be supplied (p. 6,32$)$. Osborne and Mendel, for instance, gave their animals a certain amount of 'protein-free milk,' containing the salts of milk, but only traces of milk proteins. The important thing in the 'protein-free milk' is neither the slight residue of protein nor the salt, but an unknown substance, a so-called 'water-soluble vitamine.' When this was supplied in the 'protein-free milk' the animals were maintained on the artificial diet for very long periods, although they did not grow. It has since been rendered probable that in addition to the water-soluble vitamine, fatsoluble vitamine (IICCollom) is necessary for full growth of rats and the maintenance in health of the adults, upon otherwise complete artificial diets, for a great part of their natural life-span. Such fat-soluble vitamines may be supplied by replacing a part of the lard by butter-fat, egg-yolk fat, beef fat, or codliver oil. 
her tissue waste completely, but she had produced from it everything necessary for the development of the embryo rats up to full term, and after that everything necessary (in the form of milk) for their normal growth during the period of suckling. On the whole, a very large amount of body tissue in proportion to the original weight of the mother must have been formed or renewed in the 200 days or more during which the experiment continued, and during which gliadin was being steadily transmuted into tissue protein, and latterly into the proteins of milk as well, by what might almost be called a feat of chemical legerdemain. There must have occurred a synthesis "not only of the Bausteine (the "buildingstones ") deficient in the protein intake, but likewise of tissue and milk components like the nucleic acids (with their content of purins, pyrimidins, and organically combined phosphorus) and phosphoproteins like casein, etc., which were completely missing ' in the food.

It has been suggested that the bacteria of the alimentary canal, which, of course, are plant cells, may have and may exercise on a large scale the power of building up new amino-acids from a variety of materials in the intestinal contents, and that they may thus be synthesizing agents, thanks to which inadequate proteins may be reshaped to proteins adequate to the needs of the body. It is precisely, however, in the case of incomplete proteins like gelatin deficient in cyclical compounds, that the body fails to effect the necessary transformation in spite of the fact that plant cells are supposed to be specially capable of forming these compounds. In any case bacterial action would not explain why proteins like gliadin and hordein are only adequate for the renewal of tissue, and not for its growth. This points rather to the possibility that the processes by which the nitrogenous compounds degraded in cellular metabolism are replaced are not of the same character as the processes by which new nitrogenous complexes are built up into growing protoplasm. If, for instance, the protein molecule is not completely disrupted in ordinary metabolism, it will not need to be completely reconstructed, while in the formation of new tissue complete protein molecules will have to be synthesized. Incomplete proteins like gliadin may furnish building-stones adequate for repairing the house, but inadequate for building it from the foundations.

Income and Expenditure of Carbon-The Carbon Balance-Sheet.This division of the subject has been necessarily referred to in treating of the nitrogen balance-sheet, and may now be formally completed.

Carbon Equilibrium.-A body in nitrogenous equilibrium may or may not be in carbon equilibrium. It has been repeatedly pointed out that the continued loss or gain of carbon by an organism in nitrogenous equilibrium means the loss or gain of fat; and, since the quantity of fat in the body may vary within wide limits without liarm, carbon equilibrium is less important than nitrogen equilibrium. It is also less easily attained when the carbon of the food is increased, for the consumption of fat is not necessarily increased 
with the supply of fat or fat-producing food, and there is by no means the same prompt adjustment of expenditure to income in the case of carbon as in the case of nitrogen.

Carbon equilibrium can be obtained in a flesh-eating animal, like a dog, with an exclusively protein diet; but a far higher minimum is required than for nitrogenous equilibrium alone. Voit's dog required at least 1,500 grammes of meat in the twenty-four hours to prevent his body from losing carbon. For a man weighing 70 kilos, the daily excretion of carbon on an ordinary diet is 250 to 300 grammes. About 2,000 grammes of lean meat would be required to yield this quantity of carbon; and, even if such a mass could be digested and absorbed, more than three times the necessary nitrogen would have to undergo preliminary cleavage and excretion as urea or be thrown upon the tissues.

Not only may carbon equilibrium be maintained for a short time in a dog on a diet containing fat only, or fat and carbo-hydrates, but the expenditure of carbon may be less than the income, and fat may be stored up. But, of course, if this diet is continued, the animal ultimately dies of nitrogen starvation.

So far we have spoken only of the income and expenditure of carbon and nitrogen; and from these data alone it is possible to deduce many important facts in metabolism, since, knowing the elementary composition of proteins, fats, and carbo-hydrates, we can, on certain assumptions, translate into terms of proteins or fat the gain or loss of an organism in nitrogen and carbon, or in carbon alone. But the hydrogen and oxygen contained in the solids and water of the food, and the oxygen taken in by the lungs, are just as important as the carbon and nitrogen; it is just as necessary to take account of them in drawing up a complete and accurate balancesheet of nutrition. Fortunately, however, it is permissible to devote much less time to them here, for when we have determined the quantitative relations of the absorption and excretion of the carbon and nitrogen, we have also to a large extent determined those of the oxygen and hydrogen.

Income and Expenditure of Oxygen ard Hydrogen.-The oxygen absorbed as gas and in the solids of the food is given off chiefly as carbon dioxide by the lungs; to a small extent as water by the lungs, kidneys, and skin; and as urea and other substances in the urine and freces. The hydrogen of the solids of the food is excreted in part as urea, but in far larger amount as water. The hydrogen and oxygen of the ingested water pass off as water, without, so far as we know, undergoing any chemical change, or existing in any other form within the body. But it is important to recognize that although none of the water taken in as such is broken up, some water is manufactured in the tissues by the oxidation of hydrogen. 
We have already considered (p. 24I) the gaseous exchange in the lungs, and we have seen that all the oxygen taken in does not reappear as carbon dioxide. It was stated there that the missing oxygen goes to oxidize other elements than carbon, and especially to oxidize hydrogen. We have now to explain more fully the cause of this oxygen deficit

The Oxygen Deficit.-The carbo-hydrates contain in themselves enough oxygen to form water with all their hydrogen; they account for a part of the water-formation in the body, but for none of the oxygen deficit.

The fats are very different; their hydrogen can be nothing like completely oxidized by their oxygen. A gramme of hydroge $n$ is contained in 8.5 grammes of dry fat, and needs 8 grammes of oxygen for its complete combustion. Only a gramme of oxygen is yielded by the fat itself; so that if a man uses 100 grammes of fat in twenty-four hours, rather more than 80 grammes of the oxygen taken in must go to oxidize the hydrogen of the fat.

The proteins also contribute to the deficit. In Ioo grammes of dry proteins there are 15 grammes of nitrogen, 7 grammes of hydrogen, and 2 I grammes of oxygen. The carbon docs not concern us at present. The 33 grammes of urea, corresponding to roo grammes of protein, contains Is grammes of nitrogen, a little more than 2 grammes of hydrogen, and a little less than 9 grammes of oxygen. There remain 5 grammes of hydrogen and 12 grammes of oxygen. But 5 grammes of hydrogen needs for complete combustion 40 grammes of oxygen; therefore 28 grammes of the oxygen taken in must go to oxidize the hydrogen of 100 grammes of protein. Taking 140 grammes of protein as the amount in a liberal diet for a man, we get 39 grammes as the required quantity of oxygen. This, added to the 80 grammes needed for the hydrogen of the fat, makes a total of, say, 120 grammes, equivalent to about 85 litres of oxygen. A small amount of oxygen also goes to oxidize the sulphur of proteins.

With a diet containing less fat and protein and more carbo-hydrate, the oxygen deficit would of course be less.

The Production of Water in the Body.-One gramme of hydrogen corresponds to 9 grammes of water. In $x$ o grammes of proteins and roo grammes of fat there are, in round numbers, 22 grammes of hydrogen; in $35^{\circ}$ grammes of starch, 21.5 grammes. With this dict, 43.5 grammes of hydrogen is oxidized to water within the body in twenty-four hours, corresponding to a water production of 391 grammes, or 15 to 20 per cent. of the whole excretion of water. It lias been observed that during starvation the tissues sometimes become riclier in water, even when none is drunk. The only explanation is that the elimination of water does not keep pace with the rate at which it is produced from the hydrogen of the broken-down tissue-substances, or set free from the solids with which it is (physically ?) united.

Inorganic Salts.-The inorganic salts of the excreta, like the water, are for the most part derived from the salts of the food, which do not in general undergo decomposition in the body. A portion of the chlorides, however, is broken up to yield the hydrochloric acid of the gastric juice. Within the body some of the salts 
are more or less intinately united to the proteins of the tissues and juices, some simply dissolved in the latter. The chlorides, phosphates and carbonates are the most important; the potassium salts belong especially to the organized tissue clements, the sodium salts to the liquids of the body; calcium phosphate and carbonate predominate in the bones. The amount and composition of the ash of each organ only change within narrow limits. In hunger the organism clings to its inorganic materials, as it clings to its tissueproteins; the former are just as cssential to life as the latter. In a starving animal chlorine almost disappears from the urine at a time when tliere is still much chlorine in the body; only the inorganic salts which lave been united to the used-up protcins are excreted, so that a starving animal never dies for want of salts.

Wlen sodium chloride is omitted as an addition to the food of man, the decomposition of protein seems to be slightly accelerated, but for a time, at least, there are no serious symptoms (Belli).

It is a general rule that purcly carnivorous animals do not desire salt, and the same is true of human beings living on a purely a nimal diet, while vegetable feeders eagerly seek it. On the other hand, when an animal, even a carnivore, is fed with a diet as far as possible artificially freed from salts, but otherwise sufficient, it dies of sallhunger. The blood first loses inorganic material, then the organs. The total loss is very small in proportion to the quantity still retained in the body; but it is sufficient to cause the death of a pigeon in thrce weeks, and of a dog in six, with marked symptoms of muscular and nervous weakness. A deficiency of lime salts causes changes particularly in the skeleton, although the nutrition of the rest of the body is also interfered with. These changes are of course most marked in young animals, in which the bones are growing rapidly. In pigeons on a diet containing very little calcium the bones of the skull and sternum become extremely thin and riddled with holes, while the bones concerned in movement scarcely suffer at all (E. Voit).

It is not indifferent in what form the calcium is taken, nor can it be replaced to any great extent by other earthy bases, as magnesium or strontium. Weiske fed five young rabbits of the same litter on oats, a food relatively poor in calcium. One of the rabbits received in addition calcium carbonate, another calcium sulphate, a third magnesium carbonate, and a fourth strontium carbonate. At the end of a certain time it was found that the skeleton of the rabbit fed with calcium carbonate was the heaviest and strongest of all, and contained the greatest proportion of mineral matter. Then came the rabbit fed with calcium suiphate. The animal which received only oats had the worstdeveloped skeleton; the condition of the animals fed with magnesium and strontium carbonates was but little better.

Milk as a Food.-Milk is a food rich in calcium and also in phos. phorus, a circumstance evidently related to the rapid development 
of the skcleton in the young child. As in the other natural foods, the calcium and phosphorus are partly in the form of organic compounds, united with the proteins, the calcium especially with caseinogen, and partly in the form of inorganic salts. Both of these elements are more easily assimilated by the body in the organic than in the inorganic form. The same is true of iron, which exists in organic combination in the bran of wheat, in the hæmoglobin of the blood and of muscular fibres, in the nuclei of most cells, vigutable and animal, and conspicuously in the nuclein compounds of the yolk of the egg. Attempts have been made to increase the amount of iron in hen's eggs by giving them food mixed with preparations of iron-e.g., iron citrate. An increase takes place, but only after a long time. Thus in one experiment roo grammes of egg-substance contained 4.4 milligrammes of $\mathrm{Fe}_{2} \mathrm{O}_{3}$ before the administration of the iron was begun; after fecding with iron for three and a half weeks the amount was 4.5 milligrammes, after more than two months 7.4 milligrammes; and after a year only 7.3 milligrammes. Although, as we have seen, inorganic iron can be absorbed, it is certainly the case that under ordinary conditions all the iron that the body receives or needs is taken in the form of organic compounds, since there is no inorganic iron in the natural food substances. Stockman, from careful estimations of the quantity of iron in a number of actual dietaries, finds that it only amounts to about 8 to ro milligrammes a day. He concludes that the greater part of it must be retained in the body and used over and over again.

Milk is poor in iron, but this does not hinder the development of the young child, except when it is weaned too late, when it is apt to become anæmic unless the milk is supplemented with a food rich in iron, such as yolk of egg. The explanation is that the fœtus, especially in the last three months of intra-uterine life, accumulates a store of iron in the liver and other organs; so that, in proportion to its body-weight, it is at birth several times richer in iron than the adult. This iron, of course, all comes from the mother, and the loss is not exactly balanced by the excess of iron in her food; certain of her organs, the spleen, for instance, though not apparently the liver, are impoverished as regards their content of iron.

\section{SECTION V.-DieteTiCs}

There are two ways in which we can arrive at a knowledge of the amount of the various food substances necessary for an average man: (a) By considering the diet of large numbers of people doing fairly definite work, and sufficiently, but not extravagantly, fede.g., soldiers, gangs of navvies, or plantation labourers; (b) by making special experiments on one or more individuals.

Voit, bringing together a large number of observations, concluded 
that an 'average workman,' weighing 70 to 75 kilos, and working ten hours a day, required in the twenty-four hours II8 grammes protein, 56 granmes fat, and 500 grammes carbo-hydrate, corresponding to about 18.8 grammes* nitrogen, and at least 328 grainmes carbon.

Ranke found the following a sufficient diet for himself, with a body-weight of 74 kilos:

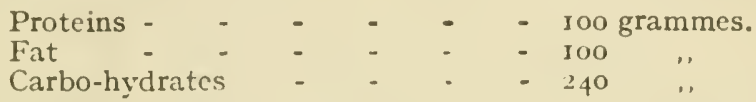

This corresponds to only 16 grammes nitrogen and, say, 230 grammes carbon.

A German soldier in the field receives on the average:

$\begin{array}{lllllll}\text { Proteins - } & - & - & - & - & - & \\ \text { Fat } & \text { I grammes. } \\ \text { Carbo-hydrates } & - & - & - & - & 462 & \text { ". }\end{array}$

representing about 24 grammes nitrogen and 340 grammes carbon. The average ration for four British regiments in peace-time contained I33 grammes protein, II5 grammes fat, and 424 grammes carbo-hydrate $(=3,400$ calories). But in addition the soldiers constantly obtained at their own expense a supper, generally conıprising meat (Pembrey). The Russian army war ration in the Manchurian campaign is said to have comprised 187 grammes protein and 775 grammes carbo-hydrate, but only 27 grammes fat $(=4,900$ calories). The diet of certain miners (Steinheil) and lumberers (Liebig) contained respectively 133 and II 2 grammes protein, II 3 and 309 grammes fat, and 634 and 69r grammes carbo-liydrates. The diet of a Japanese jinricksha man with a body-weight of 62 kilos contained 158 grammes protein, and its total heat value was 5,050 calories. The work of these men in running long distances with passengers is very laborious. They consume large amounts of fish, eggs, beef, and pork during their periods of rest, and large quantities of rice during their working periods (McCay). The diet of prize-fighters and of athletes in training is richer in protein than any of these. The members of two college football teams are stated to have consumed on the average 225 grammes protein, 334 grammes fat, and 633 grammes carbo-hydrates ( $=6,800$ calories). Caspari, from a study of the phenomena of training, concluded that continuous bodily work at a rate above the ordinary requires a large amount of protein ( 2 to 3 grammes a day per kilo of body-weight). But there seems to be a considerable difference between different individuals. So that a definite and typical diet for severe labour does not exist. And although perhaps the hardest physical work ever done in the world is to break athletic

* Taking the percentage of nitrogen in protein at 16. 
recorus, to cut and liandle timber, to mine:coal, and to make war, the diet on which these things are accomplished is very variable.

Recent observations tend to reduce the amount of protein considered necessary for a person under ordinary conditions. Sivén remained in nitrogen equilibrium, for a time at least, with an intake of only 0.07 to 0.08 gramme of nitrogen $(0.4$ to 0.5 gramme of protein) per kilo of body-weight, or not much more than one-third of the anount in Ranke's diet. It is obvious that the endogenous protein katabolism sets the limit below which it must be impossible permanently to reduce the allowance of protein. But it would be very hazardous to assume that this theoretical minimum limit corresponds with the permissible physiological limit. From experiments on men of various callings extending over many months, Chittenden has concluded that the average man eats at least twice as much protein as he really requires. We have already seen that the amount of nitrogen required to repair the actual waste of the tissues is comparatively small, and that with the ordinary amount of protein in the food a very large fraction of the total nitrogen is rapidly excreted as urea. There is no doubt, also, that many persons consume too much protein, at any rate in the form of animal food, and would feel better, work better, and probably live longer, if they restricted themselves in this regard. But there is no evidence that the digestion of such quantities of protein as the average healthy man eats, or the elaboration and excretion of the corresponding amounts of urea, 'strain' in the least the digestive apparatus, the liver, or the kidneys. And it may just as well be argued that it is advantageous that much more than the minimum protein requirement should be offered to the tissues, so that the appropriate amino-acids, even the scarcest of them, may be sure to be present in sufficient amount, rather than that the organs should be subjected to the unnecessary 'strain' of reconstructing some of the amino-acids themselves, supposing that they possess this power. In a question of this sort the immenorial experience and instinct of mankind cannot be lightly waved aside.

McCay points out that while Bengalis in I.ower Bengal subsist on food containing only about one-third the amount of protein in such a 'standard ' diet as Voit's (6 to 7 grammes of nitrogen a day), and may therefore be supposed to be immune from the dangers of an excessive protein metabolism, the large intake of carbo-hydrate rendered necessar $y$ by the poverty of the food in protein is associated with perhaps greater evils, among them a marked predisposition to diabetes and renal troubles. Their weight, chest measurement, and muscular development are inferior to those of other Asiatics living in the same climate, but with dietetic habits or economic conditions which ensure them a larger supply of protein. Thus the natives of Behar, with a larger intake of nitrogen, derived from wheat, and the natives of Eastern Bengal with a larger intake of nitrogen, derived 
from wheat and fish, are physically much superior to the rice-eating Bengalis of Lower Bengal, although all belong to the same race.

If we decide the matter merely on physiological grounds, we may say that for a man of 70 kilos, doing fairly hard, but not excessive, work, 15 grammes nitrogen and 250 grammes carbon are a sufficient allowance. The I5 grammes nitrogen will be contained in 95 grammes dry protein, which will also yield 50 grammes of the required carbon. The balance of 200 grammes carbon could theoretically be supplied either in $45^{\circ}$ grammes starch or in 260 grammes fat. But it has been found by experiment and by experience (which is indeed a very complex and proverbially expensive form of experiment) that for civilized man a mixture of these is necessary for health, although the nomads of the Asian steppes, and the herdsmen of the Pampas, are said to subsist for long periods on flesh alone, and a dog can live very well on proteins* and fat. The proportion of fat and carbo-hydrates in a diet may, however, be varied within wide limits. Probably no 'work' diet should contain much less than 40 grammes of fat, but twice this amount would be better; So grammes fat give about 60 gramnes carbon, so that from proteins and fat we have now got IIo grammes of the necessary 250 , leaving I.40 grammes carbon to be taken in about 3 Io grammes starch, or an equivalent amount of cane-sugar or dextrose. Adding 30 grammes inorganic salts, we can put down as the solid portion of a normal diet sufficient from the physiological point of view for a man of 70 kilos:

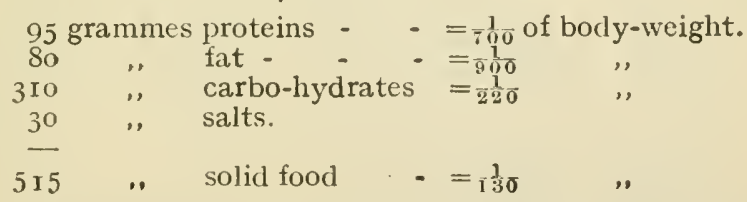

Now, knowing the composition of the various food-stuffs, we can easily construct a diet containing the proper quantities of nitrogen and carbon, by using a table such as appears on p. 626 .

Economic and social influences-prices and habits-and not purely physiological rules, fix the diet of populations. The Chinese labourer in a rice district, for example, is apt to live on a diet which no physiologist would commend. In order to obtain I5 grammes nitrogen or 95 grammes protein, he must consume more than I,500 grammes rice, which will yield 700 grammes carbon, or twice as much as is required. But if many of the Chinese labourers could not live on rice, or often on grains cheaper than rice, they could not live at all. The Irish peasant, in the days when the potato was his staple, was even in worse case; he would have been obliged to consume nearly 4 kilos of potatoes to obtain his I5 grammes nitrogen, while little more than half this amount would have furnished the 
necessary 250 grammes carbon. Of course a diet consisting, week in week out, entirely of potatoes or rice, would represent an extreme case, and no doubt the total nitrogen ingested would be considerably below the usual proportion. A certain amount of the necessary nitrogen is obtained even by the poorest populations, in the form of fish, milk, eggs, or bacon. A man attempting to live on flesh alone would be well fed as regards nitrogen with 500 grammes of meat, but nearly four times as much would be required to yield $25^{\circ}$ grammes of carbon. Oatmeal and wheat-flour contain nitrogen and carbon in nearly the right proportions ( $\mathrm{I} N: \mathrm{I}_{5} \mathrm{C}$ ), oatmeal being rather the better of the two in this respect; and the best-fed labouring populations of Europe still live largely on wheaten bread, while, one hundred years ago, the Scotch peasant still cultivated the soil, as the Scotch Reviewer the IIuses, 'on a little oatmeal.' But although bread may, and does, as a rule, form the great staple of diet, it is not of itself sufficient.

\begin{tabular}{|c|c|c|c|c|c|c|c|c|}
\hline & $\begin{array}{l}\text { Quantity } \\
\text { required } \\
\text { :o yield } \\
\text { is Grms. } \\
\mathbf{N} \text {. }\end{array}$ & $\begin{array}{l}\text { Quantity } \\
\text { required } \\
\text { to yield } \\
250 \mathrm{Grms} \text {. } \\
\text { C. }\end{array}$ & $\begin{array}{c}N \text { in } \\
100 \\
\text { Grms. }\end{array}$ & $\begin{array}{c}\mathrm{C} \text { in } \\
\text { 100 } \\
\text { Grms. }\end{array}$ & $\begin{array}{l}\text { Proteic } \\
\text { in } r 00 \\
\text { Grms. }\end{array}$ & $\begin{array}{l}\text { Fat in } \\
\text { to } \\
\text { Grins. }\end{array}$ & $\begin{array}{l}\text { Carbo- } \\
\text { hydrate } \\
\text { in too } \\
\text { Grms. }\end{array}$ & $\begin{array}{l}\text { Water } \\
\text { in roo } \\
\text { Grms. }\end{array}$ \\
\hline Chere * & & & & & & & & \\
\hline $\begin{array}{l}\text { (Gruyère) - } \\
\text { Peas (dricd) }\end{array}$ & $\begin{array}{l}300 \\
+30\end{array}$ & $\begin{array}{l}040 \\
700\end{array}$ & $\begin{array}{l}5 \\
3.5\end{array}$ & $\begin{array}{l}39 \\
35.7\end{array}$ & $\begin{array}{l}31 \\
22\end{array}$ & $\begin{array}{r}31 \\
2\end{array}$ & 55 & $\begin{array}{l}34 \\
15\end{array}$ \\
\hline Lean meat - & +40 & I 860 & $3 \cdot 4$ & I 3.5 & $2 I$ & $3 \cdot 5$ & - & 74 \\
\hline Wheat-flour & 650 & 625 & $2 \cdot 3$ & $39 \cdot 8$ & 12 & 2 & 70 & 15 \\
\hline Oatmeal- & $5^{\text {So }}$ & 620 & $2 \cdot 6$ & $4^{0 \cdot 3}$ & I 3 & $5 \cdot 5$ & 65 & 15 \\
\hline Eggs - - & 790 & 1700 & $1 \cdot 9$ & $14 \cdot 7$ & $11 \cdot 5$ & 12 & - & 75 \\
\hline $\begin{array}{l}\text { Maize } \\
\text { Wheat- }\end{array}$ & 810 & 610 & 1.85 & $40 \cdot 9$ & 10.5 & 7 & 65 & $I_{5}$ \\
\hline bread - - & 1200 & 1120 & $1 \cdot 25$ & $22 \cdot 4$ & 8 & $1 \cdot 5$ & 49 & 40 \\
\hline Rice - - & 1530 & 685 & 0.9 & $36 \cdot 6$ & 5 & I & $8_{3}$ & Io \\
\hline Milk - - & 2380 & 3540 & 0.6 & 7 & 4 & 4 & 5 & 85 \\
\hline Potatoes - & $375^{\circ}$ & 2380 & 0.4 & 10.5 & 2 & 0.15 & $2 I$ & 75 \\
\hline Good butter & 10000 & 360 & 0.15 & 69 & I & 90 & - & 8 \\
\hline
\end{tabular}

It is necessary to recognize that habit has much to do with the quantity as well as the quality of the food used by an individual or a community. Some concession may be made to custom in what is after all, not a purely physiological question, and in this country it is probable that 20 grammes of nitrogen and 300 grammes of carbon, while a liberal is not an excessive allowance, although it is certain that a man can maintain a normal body-weight and perform a normal amount of work on considerably less, in some cases even with advantage to his health.

We may take 500 grammes of bread and 250 grammes of lean meat as a fair quantity for a man fit for hard work. Adding

* A cheese manufactured from whole milk, curdled before the cream lias had time to rise, and therefore rich in fat. 
500 grammes milk, 75 grammes oatmeal (as porridge), 30 grammes butter, 30 grammes fat (with the meat, or in other ways), and 450 grammes potatoes, we get approximately 20 grammes nitrogen and 300 grammes carbon contained in 135 grammes protein, rather less than Ioo grammes fat, and somewhat over 400 grammes carbohydrates. Thus-

\begin{tabular}{|c|c|c|c|c|c|c|}
\hline & N. & c. & Proteins. & Fat. & $\begin{array}{c}\text { Carbo- } \\
\text { hydrates. }\end{array}$ & Salts. \\
\hline$(9 \mathrm{oz}$.) $250 \mathrm{grms}$. lean meat - & 8 & 33 & 55 & $8 \cdot 5$ & - & 4 \\
\hline (1 8 oz.) 500 grms. bread & 6 & I 12 & 40 & $7 \cdot 5$ & 245 & $6 \cdot 5$ \\
\hline (3 pint) 500 grms. milk & 3 & 35 & 20 & 20 & 25 & $3 \cdot 5$ \\
\hline (I oz.) 30 grms, butter & - & 20 & - & 27 & - & $0 \cdot 5$ \\
\hline (1 oz.) 30 grms. fat - & - & 22 & - & 30 & - & - \\
\hline (I6 oz.) 450 grms. potatoes - & I.5 & 47 & Io & 一 & 95 & $4 \cdot 5$ \\
\hline \multirow[t]{2}{*}{ (3 oz.) 75 grms. oatmeal } & $x \cdot 7$ & 30 & Io & 4 & $4^{8}$ & 2 \\
\hline & $20 \cdot 2$ & 299 & 135 & 97 & 413 & $2 I$ \\
\hline
\end{tabular}

This would be a fair ' hard work' diet for a well-nourished labourer. But the great clasticity of dietetic formulæ is shown in the following tables, which give the ration of the German soldier in peace and war and the minimum allowance per 'statute adult' prescribed in the British regulations concerning passenger-ships from Great Britain to America.

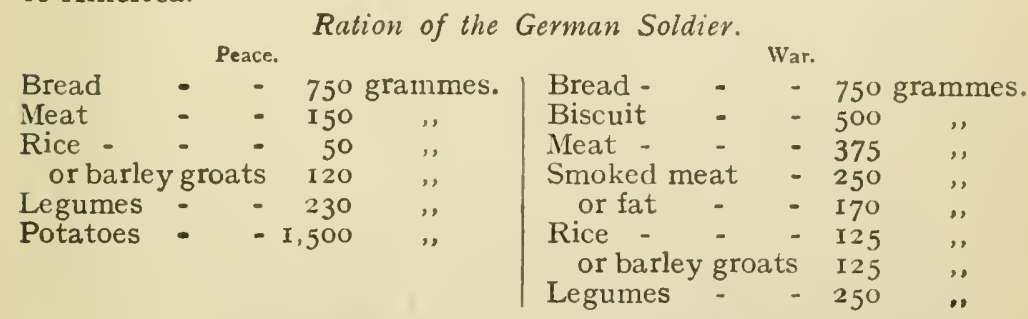

\section{Minimum Ration for Passenger Ships.}

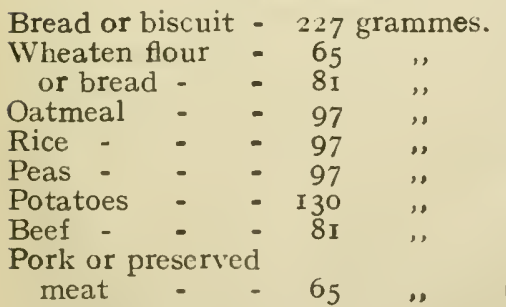

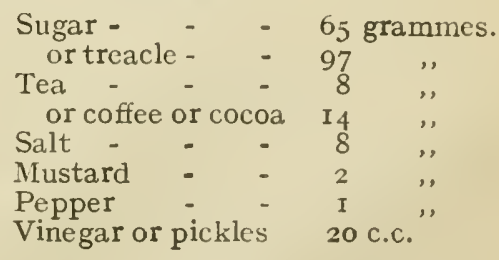

In prisons the object is to give the minimum amount of the plainest food which will suffice to maintain the prisoners in health. A 'hard work ' prison diet in Munich was found to contain 104 grammes proteins, $3^{8}$ grammes fat, and 52 I grammes carbo-hydrates; a 'no-work' diet, only 87 grammes proteins, 22 grammes fat, and 305 grammes carbohydrates. Here we recognize the influence of price; carbon can be much more cheaply obtained in vegetable carbo-liydrates than in 
animal fats; the cheapest possible diet contains a minimum of animal fat and proteins.

Many poor persons live on a diet which would not maintain a strong man, for an emaciated body has a smaller mass of flesh to keep up, and therefore needs less protein; it can do little work, and therefore needs less food of all kinds. A London needlewoman, according to Playfair, subsists, or did subsist forty years ago, on 54 grammes protein, 29 grammes fat, and 292 grammes carbo-hydrates. But this is the irreducible minimum of the deepest poverty, not so much in the protein content, perhaps, as in the very low heat equivalent ( 1,600 calories); and a woman, with a smaller mass of flesh and leading a less active life than a man, requires less food of all sorts. Even the Trappist monk, who has reduced asceticism to a science, and, instead of eating in order to live, lives in order not to cat, consumes, according to Voit, 68 grammes protein, I I grammes fat, and ${ }_{4} 69$ grammes carbo-hydrates: but manual labour is a part of iir discipline of the brotherhood, and this must be still above the lowest subsistence diet.

The question whether it is best to derive the proteins (and fats) of the food mainly from plants or mainly from animals is one which is never left to physiology alone to decide. But it has been definitely proved that vegetable proteins and vegetable fats are (when properly prepared) digested and absorbed as completely as those of animal origin, and play the same part in the metabolism of the body. Nor is there any difference in the basal metabohism (p. 686) of vegetarians and persons living on an ordinary diet.

A growing child needs far more food than its weiglt alone would indicate; the expenditure of organisms of different size in the same physiological condition is proportional not to the mass, but more nearly to the surface area. Now, speaking roughly, the cube of the surface of an animal varies as the square of the mass; when the weight is doubled, the surface only becomes $\sqrt[3]{4}$, or one and a half times as great. The surface of a boy of six to nine years, with a body-weight of I 8 to 24 kilos, is two-fifths to one-half that of a man of 70 kilos; and this would indicate that he should have about half as much food as the man. This is not all, the child is not in the same phvisiological condition as the adult. It is growing. Its income must exceed its expenditure, and by a far greater amount than the actual gain in weight, for growth is a physiologically expensive process. It needs many pounds of food to put on one pound of flesh. And in accordance with this it has been found that the basal metabolism of a child per unit of surface is decidedly greater than that of an adult (p. 697 ).

An infant for the first seven months should have nothing except milk. Up to this age regetable food is unsuited to it; it is a purely carnivorous animal. By careful observations on the amount of carbon dioxide and nitrogen excreted by a cliild nine weeks old, fed exclusively on its mother's milk, it has been shown that the absorption and assimilation of milk in the infant is very complete, over 9 I per cent. of the total energy being utilized; while an adult, taking as much milk as is necessary for the maintenance of nitrog- 
enous equilibrium, does not utilize at most more than 84 per cent. Human milk contains about 2 per cent. of protein (mainly cascinogen), 3 per cent. of fat, 5 or 6 per cent. of carbo-hydrate (lactose or milk-sugar), and from 0.2 to 0.3 per cent. of salts. Cow's milk contains about 4 per cent. of protein, 4 to 6 per cent. of fat, 4 per cent. of lactose, and 0.7 per cent. of salts. When given to infants it should, as a gemeral rule, be diluted with water, and some sugar sliould be added to it. Ass's milk has about the same amount of protein, lactose, and salts as human milk, but less than half as much fat. It is very well borne and very completely absorbed.

As to the place of water and inorganic salts in diet, it is neither necessary nor practicable to lay down precise rules. In most wellsettled countries they cost little or nothing; very different quantities can be taken and excreted without harm; and both economics and physiology may well leave every man to his taste in the matter. Salt is indeed for the most part used, not as a special article of diet, but as a condiment to give a relish to the food, just as a great deal more water than is actually needed is often drunk in the form of beverages. It is certain that the quantity of salt required, in addition to the salts of the food, to keep the inorganic constituents of the body at their normal amount, is very small. When the food is entirely animal, no additional salt is necessary. A 3o-kilo dog obtains in his diet of 500 grammes of lean meat only 0.6 gramme sodium chloride, and needs no more. An infant in a litre of its mother's milk, which is a sufficient diet for it at six to nine months, gets only 0.8 gramme sodium chloride. The Hereros in Damaraland, who are physically one of the finest races in Africa, do not use salt (Réclus). In this they resemble other tribes in different parts of the world who eat no vegetable food, for example the Kirghiz, who live on meat and milk, and the Todas, a pastoral tribe in Southern India, who are ignorant of the use of vegetable foods and know nothing of salt (McCay). Bunge has explained the difference between the flesh and the vegetable feeder by showing that the proportion of potassium and sodium salts in the food is a factor in determining the quantity of sodium chloride required. A double decomposition takes place in the body between potassium phosphate and sodium chloride, potassium chloride and sodium phosphate being formed and excreted; and the loss of sodium and chlorine in this way depends on the relative proportions of potassium and sodium in the food. In most vegetables the proportion of potassium to sodium is much greater than in animal food, so that vegetablefeeding animals and men as a rule desire and need relatively great quantities of sodium chloride. But it is stated that the inhabitants of a portion of the Soudan use potassium chloride instead of sodium shloride, obtaining the potassium salt by burning certain plants which leave an ash poor in carbonates, and then extracting the residue with water and evaporating (Dybowski). A beef-eating 
English soldier in India consumes about 7 grammes ( $\frac{1}{4}$ oz.), a vegetarian Sepoy about I 8 grammes ( oz.), of common salt per day.

Stimulants.-Wine, beer, tea, coffec, cocoa, etc., belong to the important class of stimulants. Some of them contain small quantities of food substances, but these are of secondary interest. In beer, for example, there are not inconsiderable amounts of proteins, dextrin, and sugar. But I4 litres of beer would be required to yield I5 grammes nitrogen, and Io litres to give 250 grammes carbon; and nobody, except a German corps student, could consume such quantities. The minimum nitrogen requirement, however, as well as the necessary heat value, could theoretically be covered by 6 or 7 litres of good German beer.

In some cocoas there is as mucl as 50 per cent. of fat, 4 per cent. of starch, and I3 per cent. of proteins; and in the cheaper cocoas much starch is added. Still, a large quantity of the ordinary infusion would be needed for a satisfying meal. Frederick the Creat, indeed, in some of his famous marches dined off a cup of chocolate, and beat combined Europe on it; but his ordinary menu was much more varied and substantial.

Alcohol.-The great social and hygienic evils connected with the abuse of alcohol, as well as its applications in therapeutics, render it necessary, or at least permissible, to state a little more fully, though only in the form of summary, some of the chief conclusions that may be drawn as to its action and uses.

(I) In small quantities alcohol is oxidized in the body, a little of it, however, being excreted unchanged in the breath and urine. A certain amount of protein is saved from decomposition when alcohol is taken, just as when fat or sugar is taken. For example, the addition of I30 grammes of sugar to the daily food of an individual caused a 'sparing' of 0.3 gramme nitrogen. The substitution of 72 grammes alcohol for the sugar caused 0.2 gramme nitrogen to be spared (Atwater and Benedict). Alcohol is therefore to some extent a food substance, although it is not, under ordinary circumstances, taken for the.sake of the energy its oxidation can supply, but as a stimulant.

(2) There is no reason to suppose that this energy cannot be utilized as a source of work in the body. Indeed, a certain amount of alcohol may be normally formed in the tissues as one of the intermediate products in the oxidation of sugar. Heat can certainly be produced from it, but this is far more than counterbalanced by the increase in the heat loss which the dilatation of the cutaneous vessels caused by alcohol brings about.

(3) It is a valuable drug, when judiciously employed, in certain diseases-e.g., pneumonia and puerperal insanity (Clouston).

(4) Alcohol is occasionally of use in disorders not amounting to serious discase-e.g., in some cases of slow and difficult digestion. In these cases it may act by increasing the flow of certain of the digestive sccretions, as saliva and gastric juice. This effect seems to more than counterbalance the retarding influence which, except when well diluted, it exerts on the chemical processes of digestion.

The action of alcohol on the secretion of gastric juice has been studied in a dog with a double gastric and œsophageal fistula. Before or during a sham meal of meat, alcohol diluted with water was given as 
an enema. After the enema the quantity of hydrochloric acid secreted increased in about the same proportion as the quantity of juice, but the pepsin was diminished, reaching a minimum after three-quarters to one and a quarter hours. The increase in the total quantity of the juice and in the acid over-compensated the moderate diminution in the digestive power, so that the net result was beneficial (Pekelharing). But it must be remembered that strong alcoholic beverages, when mixed with the gastric juice, and therefore when taken by the mouth, retard the proteolytic action, so that any favourable effect on the secretion of the juice may easily be lost in the subsequent digestion, unless the alcohol is dilute (Chittenden and Mendel). The action of alcohol introduced into the rectum on the gastric secretion is both reflex and direct.

(5) Alcohol is of no use for healthy men.

(6) Alcohol in strictly moderate doses,* properly diluted and especially when taken with the food, is not harmful to healthy men, living and working under ordinary conditions.

(7) Modern experience goes to show that in severe and continuous exertion, coupled witl exposure to all weathers, as in exploring expedi. tions, alcohol is injurious, and it is well known that it must be avoided in mountain climbing.

The drastic restrictions placed upon the use of alcoholic beverages in most of the belligerent countries during the present war, although adopted for economic as well as for physiological reasons, do not favour the view that the efficiency of the fighting man, or of the man who works at high pressure to equip and supply him, is increased by alcohol. But it would be quite erroneous to conclude that because alcohol, as such, may not at all improve the working or staying power of men accustomed to use it moderately, it can be suddenly and completely prohibited without detriment to their work. The factor of habit and its influence on efficiency has been far too little considered in connection with this question. The man who craves his wonted glass of beer, even if he does not resent its withdrawal, will almost certainly suffer in efficiency for a while, although the beer in itself may not have aided him in his wOR.

Alcohol in small doses, when given by the stomach or (in animals) injected into the blood, causes stimulation of the respiratory centre and increase in the pulmonary ventilation. In man, this increase usually amounts to 8 to 5 per cent., but is occasionally much greater. But the limit which separates the favourable action of the small dose from the hurtful action of the large, is easily overstepped. When this is done, and the dose is continually increased, the activity of the respiratory centre is first diminished and finally abolished. In dogs, for instance, after the injection of considerable quantities of alcohol into the stomach, death takes place from respiratory failure, and the breathing stops while the heart is still unweakened (Fig. 85, p. r91). This is the final outcome of a progressive impairment in the activity of the centre, of which the slow and heavy breathing of the drunken man represents an earlier stage.

Tea, coffee, and cocoa are more suitable stimulants for healthy persons, because they are less dangerous than alcohol, and they leave no unpleasant effects behind them. But it should be remem-

* Not more than $\mathrm{I} \frac{1}{2} \mathrm{oz}$. of absolute alcohol, corresponding to about $4 \mathrm{oz}$. of whisky, or 2 to 3 wineglasses of sherry or port, or a pint of claret, or a couple of pints of light beer in 24 hours. 
bered that there is no stimulant which is not liable to be abused. It has been shown by ergographic experiments (p. 750) that, lik? alcohol, tea, coffee, maté, and cola-nut, which all contain the alkaloid theine or caffeins, restore the power of performing muscular work after exhaustion, but only if food has been recently or is simultaneously taken.

Vitamines.-Certain substances, although neither in the ordinary sense foods nor condiments, seem to be necessary for the maintenence of health, for in circumstances in which these cannot be obtained for long periods so-called 'deficiency diseases,' such as scurvy, are liable to occur. Scurvy used to be the scourge of the sailing-ship in the days when fresh meat, and particularly fresh vegetables and fruits, were unobtainable on long voyages. It has long been known that it is prevented by the use of lime or lemonjuice, in which citric and a trace of malic acid are contained, and it used to be thought that it was the organic vegetable acids which were the important thing. Recent researches have shown, however, that scurvy is only one of a group of diseases, including beriberi, and probably pellagra, rickets, and others which are induced by deficiency in the food of certain substances minute in amount but essential to proper nutrition.

The importance of such accessory components in the food has been already pointed out, in discussing the influence of artificial diets upon the growth and maintenance of animals (p. 617). These substances are sometimes termed 'vitamines.' But their chemical nature is imperfectly known, and there is no evidence that the bodies which exert the beneficial influence belong to the same chemical group.*

McCollom prefers to designate provisionally the two substances or groups of substances essential, in addition to a diet of purified proteins, carbohydrate, fats and inorganic salts, for growth and maintenance of rats, as 'fat-soluble $A$ ' and 'water-soluble $B$,' rather than to use the unsatisfactory term vitamines. The first is soluble in fats and is abundant in butter-fat, egg-fat, and ether extract of kidney. It is also found in considerable amount in the leaves of plants, but is represented in the seed in too small amount to supply the needs of a young animal. The second, which is soluble in water and in alcohol, is plentifully present in wheat, wheat germ, maizc, alfalfa leaves, cabbage, and in a number of foods of animal origin.

One representative of the important food constituents in question is a basic substance separated by Funk from the polishings of rice, and named by him, ' vitamine.' 'Polished rice is rice deprived of the outer coats by modern milling processes, and the polishings are the coats which have been removed. Since the introduction of steel rollers instead of the primitive millstones which used to crush the whole grain, beri-beri, a disease characterized by inflammatory and degenerative changes in the

- It might be better in the present state of our knowledge to avoid giving those bodies a name which may easily mislead. They might possibly be provisionally spoken of as " vitines," a term involving no assumption as to their chemical nature, and implying only their importance in the nutritional processes associated with the life (and growth) of the tissues. 
peripheral nerves (peripheral neuritis) ald consequent paralysis, has greatly increased anong the rice-enting Japancse. 11 Bengal, although much rice is eaten, there is practically no beri-beri, as country rice and not the highly polished variety is consumed. When birds-e.g.: pigeons-are fed on polished rice, polynemritis similar to that scen in human beri-beri is produced, and both in man and in birds the condition is quickly cured by reverting to rice prepared according to the old-fashioncd methods, or by adding the polishings or an alcoholic cxtract of them containing the essential substance, or the isolated base itself.

The addition of various legumes to the diet, or alcoholic extracts of these, will produce the same beneficial effect (Mccay). Potatoes, carrots, fresh vegetables, lime and other fruit juices, also certain animal foods, such as fresh milk, fresh meat, and yolk of egg, are all valuable, in addition to their ordinary nutritive constitucnts, for their content of vitamines. Ycast contains them in exceptionally large amount, and it is possible, though not proved, that such fermented liquors as beer, or some varieties of it, may derive some part of their value from these substances liberated both from the yeast and the barley and not destroyed in the process of brewing.

The addition of yeast to artificial diets accelerates the growth of young rats. Yeast also prevents and cures polyneuritis developed in birds by a diet of polished rice. It is not known whether the growthpromoting component is identical with the anti-neuritic one or a different substance.

Since vitamines exert so great an effect on nutrition and growth, it might be expected that their absence would tell on those glands of internal secretion which appear to be concerned in the metabolism of growth. As a matter of fact, it has been found that in pigeons suffering from the typical deficiency disease beri-beri, certain of these glands show marked changes. The thymus gland, normally very large and persistent in these birds, can be caused to atrophy completely by a diet of polished rice. Changes also occur in the pituitary, and decided atrophy in the testes and ovaries (Funk and Douglas).

In bringing this chapter to a close, it may be useful to point out again that indispensable as the data of physiology are for intelligent and fruitful criticism of the dietetic habits of individuals or communities, they must be applied with due regard to the other factors. Nothing is more sure than that a certain minimum number of calories must be supplied to an average man of given weight and age, living and working under definite conditions, in order that his body may be maintained in health. It is also well made out that a certain balance, which, however, is by no means rigidly fixed, between the great groups of food-stuffs is of the essence of a good dietary. Yet of two rations each containing the proper number of calories, and the conventional proportions of protein, carbohydrate and fat, one may be good and sufficient, and the other so gravely deficient that malnutrition or disease may follow the use of it. Where natural foods are obtainable in abundance and variety, there is seldom danger of a lack of the indispensable accessories. This danger increases when economic or social conditions lead to an excessive use 
of 'artificial' foods. The factor of safety, however, is very large and the dietary of an individual or the dietetic habits of a nation may be changed from top to bottom, if the change is scientifically effected, without serious detriment, at least for a long time. The greatest experiment of this kind in the history of the world is now being worked out over a large part of Europe. 


\section{CHAPTER XI}

\section{INTERNAL SECRETION-ENDOCRINE GLANDS}

IT is long since Caspar Friedrich Wolff expressed the idea that each single part of the body, in respect of its nutrition, stands to the whole body in the relation of an excreting organ,' and thus emphasized the importance of substances produced by the activity of one kind of cell for the normal metabolism of another. But it is only in recent years that it has become possible to illustrate this mutual relation by any large number of experimental facts.

Certain of the substances taken in from the blood by the liver find their way, after undergoing various changes, into the biliary capillaries, and are excreted as bile; certain other substances, such as sugar and the precursors of urea, are taken up by the hepatic cells, transformed and sometimes stored for a time within them, and then given out again to the blood. Bile we may call the external secretion of the liver, glycogen and-urea constituents of its internal secretion. In one sense it is evident that all tissues, whether glands in the morphological sense or not, may be considered as manufacturing an internal secretion. For everything that an organ absorbs from the blood and lymph it gives out to them again in some form or other except in so far as it forms or separates a secretion that passes away by special ducts. But it is usual to employ the term only in relation to organs of glandular build, whether provided with ducts or not. Typical endocrine* glands (that is, glands producing an internal secretion) are the adrenals, thyroid, parathyroid, pituitary, thymus, etc. For convenience the action of extracts of some other tissues, such as nervous tissue, will also be considered here, although there is no reason to suppose that they form any specific internal secretion.

The capacity of manufacturing internal secretions of high importance can neither be attributed to all glands with ducts nor denied to all other organs. For the salivary, mammary, and gastric glands may be completely removed without causing any serious effects, while death follows excision of the, so far as mere bulk is concerned, apparently insignificant masses of tissue in the ductless thyroid, parathyroid, suprarenal or pituitary bodies.

* From $\xi \nu \delta o \nu$, within, and $x \rho l \nu \omega$, I separate. 
It is known that in the case of the liver the internal secretion is more important than the external, for an animal cannot survive without its liver, while it may be but little afferted by the continuous escape of the bile through a fistulous opening.

Pancreas.-The internal secretion of the pancreas is also indispensable. For when the pancreas is excised death follows in many species of animals, and especially in carnivorous animals; and in man severe and ultimately fatal diabetes is often associated with pancreatic disease, while the mere loss of the pancreatic juice through a fistula does not necessarily shorten life, although the absorption of fat is seriously interferc d with.

The ultimate cause of death seems to be a profound disturbance of metabolism, of which the most significant token is the increased proportion of sugar in the blood, and its speedy appearance in the urine-in dogs always within twenty-four hours following total removal of the organ. Associated with the glycosuria is an increase in the quantity of the urine (polyuria), excessive thirst (polydipsia), and a ravenous appetite (polyphagia accompanied by intense hunger contractions of the stomach-Luckhardt), in spite of which the animal becomes more and more emaciated-in short, the classical symptoms of a severe type of pathological diabetes in man, but, of course, far more acute in their onset, and far more rapid in their progress towards the inevitable end. Dogs rarely survive more than two or three wecks, the immediate cause of the rapidly fatal result being perhaps the extensive suppuration which is apt to ensue on slight and practically unavoidable superficial injuries. The resistance of the tissues to bacterial invasion and their tendency to spontaneous healing are reduced by the overloading of the blood and tissue liquids with sugar. Even when carbo-hydrates are excluded from the food, or when no food at all is given, sugar continues to be excreted in large amounts. The destruction of proteins is increased. It is a significant fact that glycosuria does not appear or is only transient when the pancreas is partially removed, so long as a comparatively small fraction of the gland (one-unuarter or onefifth) is left. Even when such a remnant is dislocated from its original position, care being taken not to interfere with its circulation, and sutured in the peritoneal cavity or, indeed, under the skin, the animal remains in good health. In the dog this operation can be practised on the lowest part of the descending division of the pancreas, which is not united with the duodenum, but lies frce in the mesentery. Removal of the fragment of pancreas is followed by the whole train of symptoms associated with total extirpation of the organ.

When the portion of pancreas left is smaller than is sufficient to completely prevent diabetes, only one-eighth of the total mass of the gland, for instance, the symptoms come on more gradually than 
after total extirpation, and the rescmblance to human diabetes becomes closer. The animals rapidly deteriorate if supplied with too much carbohydrate, while they may be kept alive for a long time in good health if the limit of their tolerance for carbohydrates is not exceeded (Thiroloix, Allen et al.).

Although as yet we are ignorant of the precise manner in which the pancreas influences the metabolism of the body, it is impossible to doubt, in view of the facts we have mentioned, that, like the liver, in addition to carrving on the exchanges necessary for the preparation of the ordinary or esternal secretion, the gland has other important relations with the circulating flusds, giving to them or taking from them substances on the manufacture or destruction of which the normal metabolic processes depend. It has bcen suggested that the pancreas neutralizes or renders harmless some toxic substance formed elsewhere in the body, the action of which produces glycosuria. But no evidence of the existence of any such substance has been obtained, and the transfusion into a norma dog of blood from a depancreatized animal, which ought to be laden with the hypothetical toxic material, does not cause glycosuria. It is niuch more probable that the hyperglycæmia on which the glycosuria depends is caused by the absence of something normally producer by the pancreas, and which is indispensable for the due regulation of the sugar-content of the blood. This something, as already pointed out in discussing pathological diabetes, may be necessary to regulate the transformation of sugar into glycogen, or eventually, it may be, into fat, so that too great a surplus of sugar does not remain unchanged; or to regulate the transformation of slycogen into dextrose, and prevent too hasty and too extensive action by the glycogenase; or to regulate the production of sugar from sources other than the carbo-hydrates; or, finally, to regulate and to aid in the normal utilization of the sugar in the organs (p. 553).

While the liver contains less than the normal content of glycogen, its power to form glycogen is certainly not abolished. On the contrary, there is some reason to think that a great deal of this reserve carbo-hydrate may be synthesized in the diabetic organism, and that the comparative poverty of the hepatic cells in glycogen may be due to rapid glycogenolysis, despite the hyperglycamia, in response to the insistent demand for sugar on the part of the tissues, which in the midst of plenty are hungry for dextrose on account of their inability to utilize it, or some of its decomposition products, in the normal way. It has indeed been shown by numerous experiments that interference with the formation or with the hydrol$y$ sis of glycogen, although it may be a factor, is not of itself sufficient to explain pancreatic glycosuria.

Failure in the katabolism of dextrose, as already mentioned 
(p. 554), has been asserted by some observers and ctenied by others. A great production of sugar from proteins (i.e., from amino-acids) has been demonstrated, but it is quite possible that just as much is produced from this source in the normal organism, although here its formation is masked by a corresponding utilization.

The clearest evidence that the pancreas produces something of high importance in carbo-hydrate metabolism has been obtained by experiments in which animals were united in such a way that substances could pass from one to the other (parabiosis, see Chap. XIX.). When two young dogs were so united and the pancreas then removed from one, no glycosuria followed. The internal secretion of the remaining pancreas was sufficient for both (Forschbach). In like manner the removal of the pancreas from pregnant bitches not far from full term caused no glycosuria or very little till the pups were born, when the usual train of events associated with pancreatic diabetes ensued. Obviously the pancreatic tissue of the embryos in the uterus supplied the mother with the indispensable secretion (Carlson and Drennan).

The question has often been raised why it should not be possible to supply animals or human beings suffering from pancreatic deficiency with the missing material by administering pancreas or pancreatic extracts. Hitherto, however, little if any success has attended attempts of this kind, perhaps because the active substance or substances are very easily destroyed. This has been all the more disappointing, as in the case of the internal secretion of the thyroid the so-called 'substitution therapy' has been brilliantly successful (p. 649).

The seat of the internal secretion of the pancreas seems to be the very vascular epithelioid tissue which is peculiar to this gland, and occurs in islands between or imbedded in the alveoli (islands or islets of Langerhans) (Schäfer). Fur animals survive the complete atrophy of the ordinary secreting epithelium caused by the injection of paraffin into the ducts, and no sugar appears in the urine. The islets remain intact. When a portion of the pancreas is separated from the rest, and its duct ligated, it undergoes extensive atrophy, a tissue remaining which is apparently composed of enlarged islands of Langerhans and remains of pancreatic ducts. If the rest of the gland is now removed, no glycosuria occurs, even when considerable quantities of dextrose are injected. But when the atrophied remnant is also removed, typical pancreatic glycosuria at once ensues (IV. G. MacCallum).

As further evidence that the islets have a different function from the pancreatic alveoli may be cited the statement that in teleostean fishes, in which the islands are so large that they can be separated from the rest of the tissue, the cells of the islets, instead of containing an amylolytic ferment like the alveolar cells, contain a glycolytic 
ferment, or at least possess the power of destroving sugar. Yet the question of the significance of the islets can hardly be considered settled, although, as previously mentioned, the supposed experimental basis of the theory that they do not differ essentially from the alveolar tissue, but are formed by certain changes in the arrangement and properties of the alveolar elements, appears to have collapsed under the criticism of Bensley and others. Far from being interchangeable with the cells of the acini, the islet cells present definite and permanent criteria by which they can be sharply distinguished from the alveolar epithelium. At least two types of islet cells may be identified by their staining reactions, the so-called $A$ and $B$ cells (Lane). The B cells are the most abundant in all the islets, and many of the small islets are composed of them. In the guinea-pig, on account of the great size of some of the islets, and because many of them are situated in the interstitial tissue (between the acini), it is not difficult to pick out an islet, isolate it from the surrounding tissue, and examine it in serum or salt solution. The cells are crowded with very fine granules exhibiting Brownian movement. In the fresh preparation the granules of the A cells cannot be distinguished from those of the $B$ cells. Both varieties stain intensely with neutral red and other dyes, and the islet tissue can in this way be easily differentiated from the tissue of the acini. By differences in their staining reactions and certain properties of their nuclei, which need not be gone into here, the two varieties of islet cells can be identified. The important point for our purpose is that by an appropriate histological technique the islet tissue can be studied in all the functional vicissitudes of the gland. When this is properly done, it is not found that there is any close connection between the secretory activity of the cells of the acini and the islets. Nor is there any evidence that the amount of islet tissue in the pancreas is ever affected by the formation of new islets out of acini. On the other hand, it seems that the islets, or the great majority of them, consist of epithelial cells which are in direct continuity with the pancreatic ducts, and that after removal of a portion of the pancreas, establishing an insufficiency of islet tissue, new islets can be developed from the duct epithelium, in addition to the increase by interstitial growth in the size of islets already existing (Bensley, etc.). If the islets are connected with the ducts, the possibility may be admitted that they yield something to the external secretion of the pancreas as well as to its internal secretion. But if this be so, there is no improbability in the idea that the alveolar epithelium, which is undoubtedly mainly concerned in the preparation of the pancreatic juice, may also contribute something to the internal secretion of the gland. While, then, the importance of the pancreas in carbo-hydrate metabolism is certain, and the dependence of this function upon an internal 
secretion is highly probable, it is not yet definitely settled whether this secretion is formed in the organ as a whole, or only in the islets. That lesions of the pancreas nay be concerned in pathological diabetes is well established, and it is of interest in connection with the question we have just been discussing that in a certain number of cases the changes observed have been in the islands (Opic). And in diabetes accompanying cirrhosis of the liver, which has usually been considered to depend upon the hepatic changes, it has been shown that in many, if not all, of the cases the pancreas is also affected by a growth of connective tissue outside the acini (Steinhaus). Some authors, indeed, have gone so far as to say that in all cases of diabetes mellitus there is disease of the pancreas, but of this there is no evidence.

Ligation, or the establishment of a fistula, of the thoracic duct, causes glycosuria in dogs. It is possible that this is really a mild form of pancreatic diabetes, due to interference with the supply of the internal secretion of the pancreas, or of that part of it which reaches the blood by the lymph-stream (Tuckett).

Pflüger long maintained that it is not the removal of the pancreas, as such, but the soction of certain nerves running into or through it from the duodenum, which is the cause of the glycosuria. For, according to him, when these nerves are divided or the duodenum removed while the pancreas remains untouched, the result is the same as if the pancreas itself had been excised. He imagined that these nerver are 'antidiabetic'-that is, in some way oppose the production of sugar-while nerves coming from the so-called 'sugar centre' in the bulb (the centre assumed to be affected in the puncture experiment) favour sugar production. Between these the normal balance is struck in health; it is the upsetting of this balance by the crippling of the duodenal fibres which is at the bottom of 'pancreatic' diabetes. But it has been shown that this hypothesis is without foundation. Although in frogrs renoval of the duodenum cloes cause a certain degree of glycosuria, this is not the case in dogs. And it has been demonstrated clearly in the dog. by special experiments, that section of all the old connections of a clislocated remnant of the pancreas does not cause permanent glycosuria, whereas removal of the pancreatic tissue does.

Sexual Organs.- The influence of castration in preventing the development of the sexual characters, and especially the physical and psychical changes that normilly occur at puberty, is also due to the loss of the internal secretion of the generative glands, and does not appear to depend at all upon the loss of nervous impulses arising in these organs. In Herdwick sheep an outstanding sexual difference is the presence of horns in the males, their absence in the females. Removal of the testes from ram lambs arrests further growth of horns forthwith and at any stage of development. The retention of the epididymes, provided that the testes proper are removed, does not alter the result of castration in tle least. The removal of one testicle slows horn growth without arresting it (Marshall and Hammond). In partially castrated cocks it has been 
seen that, so long as a portion of one testicle remains, the male characters are preserved, but after removal of this residue the comb and wattles wither in a few weeks (Hanau). At the breedingtime the muscles of the forearm of the brown land frog (Rana fusca) become hypertrophied in the male, so that it can more tightly hold the female. At the same time the balls of the toes increase in size, and become covered with a peculiar black growth. After the breeding season these sccondary sexual characters disappear. If the male frog is castrated, the periodic return of these phenomena does not occur, but the presence of one testicle suffices for their development on both sides. When pieces of testicle from normal frogs are introduced under the skin of the castrated frogs, the phenomena occur just as if the animals had not been castrated (M. Nussbaum).

Many facts indicate that the internal secretion is not furnished by the proper reproductive eiements (those which form the spermatozoa), but by the interstitial cells of Leydig, which are distributed in groups throughout the substance of the testes between the seminal tubules. The spermatogenic cells may be atrophic or absent, as in cryptorchids, or after ligation of the vas deferens (Bouin and Ancal); or they may be destroyed by X-rays, without interfering with the development of the secondary sexual characters. Steinach has shown that when the testes of a young rat or guinea-pig are transplanted to another part of its body (the peritoneal cavity or subcutaneous tissue), the animal develops all the secondary sexual characters at the proper time. The penis grows to the normal size. The seminal vesicles and prostate levelop in the ordinary way, and yield a plentiful secretion. Sexual desire and potency appear in due season, and in normal or, in not a few cases, indeed, increased intensity. Yet histological examination shows that not a single spermatocyte or spermatid (Chapter XIX.) has developed, while outside the seminal tubules the interstitial cells form large masses which much surpass in size the interstitial islands of the normal testis. Similar clanges are observed, though with less certainty and after a longer interval, when the vas deferens is ligated, a method often recommended and occasionally practised for the sterilization of the human male. On account of the influence, thus demonstrated, of the interstitial cells in producing the sexual development observed at puberty, Steinach designates these cells collectively as the 'puberty gland.'

When the ovaries of a young female rat or guinea-pig are transplanted into the peritoneal cavity or under the skin of a previously castrated male animal of the same kind (preferably, to facilitate accurate comparison, a male of the same litter), only the interstitial cells survive (in about half the cases). There is this difference in the fate of the ovary and the testis when auto-transplanted,* that the generative elements of the former, the Graafian follicles, with the ova contained in them, generally develop as well as the large interstitial cells rich in protoplasm lying in the stroma, which cells appear to constitute

- An auto-transplant or auto-graft is a portion of tissue transplanted into another part of the same animal's body. A homœo-graft is a portion of tissue transplanted into the body of another individual of the same species; a heterograft is a portion of tissue transplanted into an animal of a different species. 
the female puberty gland. The strict isolation of the frmale puberty gland is inly realized in those cases in which by some accident of healing the stroma of the transplanted ovary maintains itself while the generative elements disappear. In these cases the influence of the ovary on the development of the sexual characters is the same as when the reproductive elements proper persist and grow.

A more general influence of the sexual organs on metabolism seems also to be well established. The cxact experiments of I.ocwy and Richter on the metabolism of bitches before and after castration throw light upon the changes which follow that operation, and afford decisive proof that they are connected with the absence of substances specific to the ovary. They conclude that in the castrated animal the oxidative energy of the cells is lessened. The oxygen consumption sinks, even although protein is laid on and the total amount of active tissue thus increased. Under certain circumstances this specific diminution of metabolism may be balanced by conditions which cause an increase in the metabolism. The lessening of the oxidative power is due to the loss of ovarian substance, for the administration of an extract of the ovary (oophorin) not only neutralizes it, but actually causes an increase in the gaseous metabolism to far above the original amount, while it has no effect on the metabolism of the uncastrated animal. It is not the decomposition of proteins, but of non-nitrogenous substances, which is accelerated. Oophorin also brings about a notable increase in metabolism in the castrated male dog, while, curiously enough, extract of testicle causes only a small increase, due to a basic substance, spermin $\left(\mathrm{C}_{5} \mathrm{H}_{14} \mathrm{~N}_{2}\right)$, which can be isolated from the testicle. But the orchitic extract is not without influence in other ways. It certainly increases the capacity for muscular work (Zoth and Pregl), as tested by the ergograph (p. 750), and this distinct physiological action is sufficient to encourage the hope that it may possess some therapeutic value, although far from what has been claimed for it by its more enthusiastic advocates. The only constituent of extracts of the testicle made with salt solution which causes any pronounced effect on the blood-pressure when injected into the circulation is a nucleo-protein, the most plentiful of the protein substances. The pressure falls, mainly owing to inhibition of the heart, but partly through vaso-dilatation in the splanchnic area (Dixon).

The testicles also influence the growth of the bones. In eunuchs and in young men with atrophy of the testicles a tendency has been observed for the long bones to go on growing far beyond the usual period. This has been shown by the Röntgen rays to be due to delay in the ossification of the epiphyses. The same has been observed in animals, and is supposed to be caused by the loss of 
some substance normally formed in the testicle which influences the metabolism of the bones and the deposition of the bone salts.

I temporary diminution in the hamoglobin and in the number of the ervthrocytes has been observed in castrated bitches, an observation which, so far as it goes, is in favour of the view that an insufficient internal secretion of the ovaries is the cause of the form of anamia known as chlorosis.

While these effects on general metabolism and nutrition, as well as the influence on the development of the sexual characters, are probably to be ascribed to changes in the internal secretion of the interstitial cells, there are facts which indicate that other elements may be concerned. For example, evidence has been brought forward that the corpus luteum is a gland with an internal secretion, whose function is connected with menstruation and with the implantation of the ovum and the subsequent growth of both ovum and uterus in pregnancy (Born, Fraenkel) (Chapter XIX.). Removal of the corpora lutea occurring during the first half of pregnancy causes abortion.

Thymus.-Our knowledge of the function of the thymus is very incomplete. Even its histological structure, and especially the source and nature of its cellular elements, have long been, and still are, the subject of controversy. It is developed as a pair of diverticula, mainly from the ventral part of the third branchial cleft, and to a slight extent from the fourth. These pouches grow downwards into the thorax. At this stage the organ is a purely epithelial structure. Soon connective tissue and bloodvessels begin to grow into it, the two halves coalesce in the middle line, and the thymus becomes transformed by degrees into a structure with a general resemblance to a big lymph gland, and consisting mainly of small cells like lymphocytes. Some observers believe that these cells are true lymphocytes, derived from the mesoderm, which have migrated into and displaced the earlier epithelial tissue. Others maintain that the resemblance is merely superficial, and that they are simply epithelial cells diminished in size and altered in shape, but derived from the original epithelium by repeated division, and remaining epithelial to the end. Any theory of the function of the thymus must needs depend largely upon the view adopted as to its structure. For if it is in fully developed state merely a large collection of lymphocytes, it would appear quite unlikely that it should possess functions very different from those of other collections of lymphocytes. On the other hand, if the essential elements in the organ are epithelial, they may well, like the epithelial elements of the thyroid or of other glands with an internal secretion, be concerned in the elaboration of substances which exercise an important influence upon nutrition and growth. On the whole, the best histological evidence seems to favour the view that the thymus cells are 
different frum the cells of lymph glands. Chemical differences also exist. For example, nuclein substances characteristic of the nuclear framework of the true glands are much more abundant in the thymus than in lymph glands.

After a period of further development, which varies in duration in different aninuals, the organ undergoes involution. In mammals (including inans) the thymus does not completely disappear in the adult. Islands of thymus tissue are found at all ages among the fat by which the bulk of the organ is replaced. In certain diseases, as exophthalmic goitre, myxoedema and Addison's disease, an actual regeneration of the involuted thymus may occur. It is tisually stated that in man the thymus begins to diminish in size about the end of the seconcl year, but the careful observations of Hammar indicate that this is incorrect. According to him, the organ continues to grow till puberty is reached, weighing on the average 13 grammes at birth, 37 grammes at eleven to fifteen years, 25 grammes at sixteen to twenty years, and only 6 grammes at sixty-six to seventy-five years. Besides this involution with age, great changes in the size of the thymus may occur at any time under the influence of toxic substances or of deficient nutrition. In starvation, even in the first threc days of hunger, the weight of the thymus in rabbits has been observed to shrink to one-half, and cluring prolonged underfeeding even to one-thirtieth, of the normal (Jonson). The opposite effect, namely, cessation of the involution process, or even new formation of thymus tissue, may also occur, leading to the presence of an unusually large so-called persistent thymus in the adult -the so-called status thymicus, or status lymphaticus.

The point most clearly established in the physiology of the thymus seems to be its relation to the gonads or sex glands. It is well known that in castrated animals the thymus is larger and persists longer than in entire animals. In bulls and unspayed heifers the normal atrophy of the thymus, which begins after the period of puberty, is greatly accelerated when the bulls have been used for breeding, and when the heifers have been pregnant for several months. There is a reciprocal influence of the thymus on the testicles, and removal of the thymus before the time at which it naturally atrophies is followed by a more rapid growth of the testes (in guinea-pigs) (Paton). The remarkable atrophy or involution of the gland at puberty is a striking indication of this relation. That the nexus between the thymus and the gonads is chemical and not nervous is shown by the fact that when the thymus is autografted under the skin (a situation in which grafts readily take and grow after removal of the main portion of the gland in sexually immature rabbits) these grafts behave in the same way as the gland in situ, showing earlier involution both in the male and in the female when the animals are allowed to breed (Marine and Manley). The relation of the thymus to the growth of bones is less well established, but according to some observers extirpation of the gland retards their calcification.

In young mammals the loss of the thymus is said to cause transient disturbances of nutrition, as temporary decrease in the number of all varieties of leucocytes, and a diminished resistance to the pusforming micrococci, probably connected with the relatively feehle leucocytosis (or increase in the number of leucocytes) by which the animals react to the infection. In the frog the thymus persists throughout life. Yet the removal of it is not fatal if precautions against infection be taken. The contention that the thymus is indispensable for 
life in mammals, and that its removal is always followed by a characteristic cachexia (KIose) las been overthrown by later experiments on the effects of extirpation of the gland (Pappenlicim, Howland, etc.). The thymus of young mammals can be readily anto-grafted into the subcutaneous or subperitoneal tissues. Such grafts involute at sexual maturity with the main thymus. Homoo-grafts are not permanently successful in mamnals, except that they take and survive for two or three weeks (Marine and Manley).

The chief effect of intravenous injection of extract of human or ox thymus is a lowering of blood-pressure; but there is nothing specific in this, a similar effect being given by thyroid extract and the cxtracts of many other tissues. The heart may be at the same time accelerated. When thynus substance is fed to tadpoles, growth is markedly stimulated and metamorphosis delayed (Gudernatsch), the opposite of the effect produced by thyroid substances. This direct cvidence supports the observations on mammals above referred to that removal of the thymus hastens sexual differentiation; that delayed scxual differentiation, as in myxoedema, lymphatism, etc., is associated with enlarged thymus; and that castration delays the involution of the thymus.

Thyroids and Parathyroids. - The thyroid consists of two lobes connected by an isthmus across the middle line in man and some animals, but often separate. In the neighbourhood of the thyroid, or embedded in its tissue, are certain bodies called parathyroids, consisting of solid columns of epithelial cells. The number and situation of the parathyroids are not constant. As a rule, there are four in mammals, two on each side, but this number is subject to variations in different individuals of the same species. The variability in their anatomical relations to the thyroid is of greater significance. For much of the uncertainty in which the whole question of the symptoms following extirpation of the thyroids was until lately involved arose from ignorance or insufficient recognition of this variability.

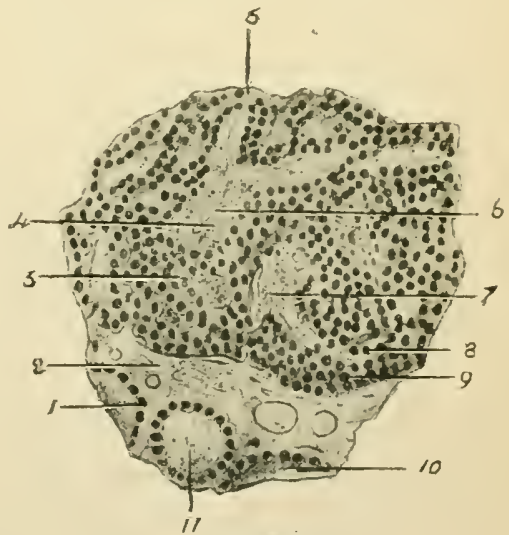

Fig. 202.-Parathyroid (Vincent and Jolly). A small portion of parathyroid of cat embedded in thyroid tissue. It consists for the most part of solid columns of epithelial cells $(3,5,8)$ with strands of vascular connective tissue (6). A thyroid vesicle (r I ) and portions of two others ( $r, 10)$ are seen in the lower part of the figure, separated from the parathyroid by a fibrous capsule (2). 4, 7, bloodvessels; 9 , lower boundary of the parathyroid tissue. $(x$ 50o.)

In most animals the inferior, anterior, or external pair of para. thyroids is more or less distinctly separated from the thyroid. The separation is especially evident in the herbivora, in the monkey, 
and in man, and this pair of parathyroids is much larger than the ( $t$ 'eer. In carnivorous animals, as the dog and cat, the anterior pair of parathyroids is closely adherent to the thyroid capsule. The superior, posterior, or intemal pair, both in herbivora and carnivora, is always very closely associated witli the capsule of the thyroid, and frequently embedded in the substance of the gland. The consequence of this arrangement is that in the older experiments the chief masses of parathyroid tissue were much more likely to escape removal with the thyroid in the case of herbivorous than in the case of carnivorous animals.

But even in one and the same species considerable variations may exist. It is easy to see, then, that in removing the thyroid the parathyroids would sometimes be completely removed as well, while at other times all or some of the parathyroid tissue would he spared. Add to this that sporadic masses of thyroid tissue (accessory thyroids), often existing as far down as the root of the aorta (always, indeed, in certain animals-e.g., the dog), must necessarily be spared in the most complete thyroidectomy, and it will cease to excite surprise that the symptoms and pathological changes described after that operation should have been so various and so contradictory. We know now that the parathyroids are perfectly distinct organs from the thyroid in development, in structure, in function, and in the consequences of their removal. The parathyroids, for instance, contain no iodine, while iodine is a characteristic constituent of the thyroid. Nor do the parathyroids show any compensatory hypertrophy when the thyroid alone is cxcised, or any changes which would indicate a definite relation to, still less, an active participation in, the pathological processes occurring in the thyroid in goitre. This does not mean, however, that there are no points of contact between the functions of the two glands. The more the matter is probed, the more clearly does it appear that none of the organs is quite independent of the rest, and the reciprocal relations of the ductless glands are probably of exceptional im. portance. But the premature attempts which have been made, in the absence of a sufficiency of exact data, to represent their mutual influcnce by crude schemata, have retarded lather than advanced our knowledge, and need not be referred to here.

Parathyroidectomy.- Total extirpation of the paratlyyroids is followed by a train of acute symptoms, ending fatally, as a rule in from one to ten days. The typical nervous symptoms following the operation are those of 'tetany' (tetania parathyrcopriva), and the tetany which used to be included among the consequences of removal of the thyroid is now known to be due to the simultaneous excision of the parathyroids (Kocher). A cat, after the combined operation, is perfectly well on the first day. On the second day a curious shaking of the paws is seen, tremors of central origin soon 
appear, and increase in severity, until at length they culminate in general spasmodic attacks. Even when the animal is at rest the fore-legs tend to be flexed, while the hind-legs are extended, and this attitude is exaggerated in the convulsions. In tlic later stages unconsciousness is associated with the onset of the convulsions. Similar results follow excision of the parathyroids alone in dogs. Although the tetany is the most striking symptom, it is only one token of a profound general disturbance of nutrition. The pulse-rate and the rate of respiration are markedly increased. There is fever and profuse salivation, with dilatation of the stomach and duodenum, duc to the loss of muscular tonicity. In the intervals between attacks the tonus returns to the normal. The secretion of the gastric juice, pancreatic juice, and bile are interfered with (Carlson, etc.). The excitability of the vaso-constrictor mechanism is said to be increased. The exact significance of these symptoms is unknown. It has been suggested that the loss of the parathyroid function is in some way associated with an angmentation of the irritability of the whole sympathetic system (Huskins). The administration of calcium conipletely relieves the symptoms, and by its use death ma: be long er perhaps indefinitely postponed (W. G. MacCallum). The mode of action of the calcium has not been made clear as yet. It does not seem to be so efficacious in rabbits as in dogs (Arthus).

A suggestive fact is the increased amount of granidin, methyl and dimethyl-guanidin in the urine of dogs after parathyroidectomy (Koch). Recently the hypothesis has been put forward that the parathyroids regulate the metabolism of guanidin in the body and by doing so, probably exercise a controlling influence on the tone of the muscles. In support of this, it is pointed out that in tetania parathyreopriva there is a marked increase in the amount of guanidin and methylguanidin in the blood, as well as in the urine. This is also true of the urine of children suffering from the disease known as idiopathic tetany, in which the parathyroids are supposed to be implicated (Paton, et a?.). Symptoms and metabolic changes like those in experimental and pathological tetany are said to be caused by guanidin and methyl-guanidin.

Thyroidectomy.-The symptoms that follow removal of the thyroid alone are perfectly different. The metabolic disturbance is eventually, in most animals, not less far-reaching than that which ensues when the parathyroids are alone excised. But it is far more chronic, reveals itself by totally distinct changes, is not amenable to calcium, and is completely corrected by the administration of thyroid substance. While no animals which have been examined survive the total removal of the parathyroids, certain speciese.g., the goat-are but slightly affected by thyroidectomy, and survive indefinitely. In man, before the consequences of thyroidectomy were known, the whole gland was not infrequently excised for goitre. If the parathyroids happened also to be completely involved in the operation, death quickly followed. But where only 
the thyroid itself, or the thyroid plus the small internal pair of parathyroids, was extirpated, the condition called cachexia strumipriva was observed to supervene. The symptoms resemble those of the disease known as myxœedema, in which the characteristic anatomical change is an increase (a hyperplasia) of the connective tissue in and under the true skin. Newly-formed connective tissue always contains an excess of mucoids, and for this reason in the early stages of myxodema there is somewhat more than the usual a mount of these substances in the subcutaneous tissue. The skin is dry, and the hair falls off. The features are swollen and heavy, and movements clumsy and trembling. As the disease progresses the mental powers deteriorate too; the patient becomes stupid and slow, and perhaps, at last, imbecile. When the gland is so affected in early life that extensive atrophy of the true secreting tissue occurs, a peculiar condition of idiocy (cretinism) results.

In animals there is a great difference in the results of total excision of the thyroids, both between different groups and between different individuals of the same group. In young animals the symptoms come on more rapidly and are more severe than in old. Monkeys develop symptoms resembling those of myxœedema.

The older descriptions of the very acute onset of the symptoms and the quickly fatal result in carnivorous animals were vitiated by the circumstance that, for the anatomical reason already alluded to, the parathyroids were also involved in the operation. Nevertheless, the consequences of complete removal of the thyroid proper are in general more serious in the carnivora than in the herbivora. Muscular weakness soon becomes marked; the tissues waste, the temperature becomes subnormal, and this is associated with changes in the heat regulation (p. 699).

If a portion of the thyroid be left, or an autograft be made in any part of the body, these effects are permanently obviated. Homœografts, although they take, rarely survive and function. Working with rabbits, Marine and Manley have found that thyroid autografts take and grow when placed in any tissue, spleen and boue marrow being the least favourable tissues, while the sex glands and the adrenals are probably the most favourable. The extent of the growth of thyroid autografts depends on the age of the animal, being more marked in young animals, on the amount of the thyroid gland removed, and on the administration or withholding of iodine. Thyroid homœegrafts rarely succeed. In a series of 507 thyroid homoeografts in rabbits, 92 per cent. underwent absorption in ten to thirty days; 6 per cent. remained active from two to six months before being absorbed: and 2 per cent. were permanent and behaved in all respects like autografts. The outlook for the therapeutic application of thyroid grafts in the treatment of thyroid insufficiencies is therefore not hopeful, unless some means is discovered to overcome the power of the host to destroy foreign proteins. It has been established that a hetero-thyroid graft does not even temporarily succeed. The alien thyroid cells are destroyed by cytolysins (p. 3i) in the scrum and tissue liquids of the animal. 
When a small part of a thyroid is left, it may undergo great hypertrophy, and the same is truc of the accessory thyroids. The administration of extracts of the thyroid glands or the glands themselves by the mouth brings about a cure, permanent so long as the thyroid treatment is continued, in cases of myxoedema in man, and prevents the development of the symptoms in animals, or removes them when they have appeared. The same is truc of a compound rich in iodine, the so-called thyroiodin, which has been extracted from the organ. Under this treatment the total metabolism, which in myxoedema is below the normal, is markedly increased. This is partly due to an increase in the metabolism of protein. An increase in the destruction of protein is also caused in normal persons and in normal animals by feeding with thyroid or with thyroid preparations. The excretion of nitrogen, carbon dioxide, and phosphoric acid, and the intake of oxygen, are augmented. But in spite of increased appetite the body-weight falls off, and diarrhœe is often caused. For these reasons the usc of thyroid preparations to reduce weight in cases of obesity, without evidence of thyroid insufficiency, is a dangerous remedy. For while a fat man can very well spare a great deal of his fat, he cannot spare much of his tissue-protein. That the gland exerts in some way an important influence on the metabolism of proteins is also indicated by other facts. The question whether the thyroid or parathyroid is, in addition, concerned in the carbo-hydrate metabolism is at present the subject of discussion, but the data are so contradictory that it would not be advisable to enter into the matter here.

The ready response of the thyroid by hyperplasia or involution to changes in the nutritive conditions is one of its most striking characteristics, and further illustrates the significant rôle which it plays in the chemical activities of the body. The thyroid swells and shrinks almost as easily and under almost as great a variety of conditions as the spleen. One of the most interesting of the physiological changes is the hypertrophy and sometimes hyperplasia of the gland, which is a normal accompaniment of menstruation and pregnancy. This is a classical instance of a definitc inter-relation of endocrine functions. Although known to the ancients in its crudest manifestations, the underlying physiology of the condition is as yet only slightly understood. A pathological change of great interest, because of the careful manner in which it has been studied, is the endemic goitre (sometimes erroneously termed 'carcinoma') of brook trout kept under artificial conditions in hatcheries. Marine has shown that this depends upon overfeeding with unsuitable food (such as livers of cattle, pigs, or sheep), overcrowding, and insufficiency of water-supply, and that the goitre can be readily cured or prevented by changing the conditions in these respects. Similar results have been obtained in mammals fed exclusively with meat. 
Thus, lion cubs at the Zoological Gardens in London on a diet ronststing only of raw meat developed rickets and goitre, as did puppies fed with meat, lungs, liver, or heart, and nothing else, whereas when milk, bread, and bone were added to meat the puppies grew normally (Marine). A meat dict caused hyperplasia of the thyroid in rats (Cla!mers Watson). The relation of the disease known as 'xophthalmic goitre to the thyroid has been much debated. The best evidence is against the hypothesis that the symptom complex is of thyroid origin. The increascd thyroid activity partially accounts for the increased metabolism cliaracteristic of the discase; but this is secondary and symptomatic. All attempts to produce

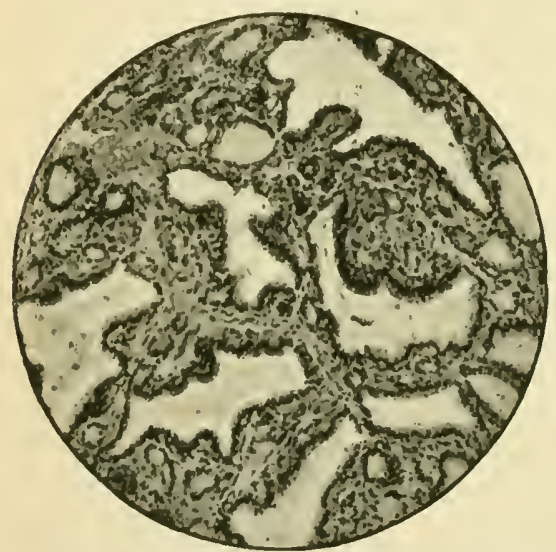

Fig. 203.-Microphotograpl of Active Thyroid Hyperplasia from a Case of Exophthalmic Goitre (Marine). The characteristic changes in the hyperplastic gland-the infoldings and plications of the alveolar epithelium, the great reduction in the colloid, and the increase in the stroma-are shown. anything resembling the pathological condition by the administration of large amounts of thyroid or of thyroid products have failed. Nor has it ever been shown that the changes in the gland are the primary cause of the syndrome. Indeed, no specific anatomical or chemical changes have as yet been demonstrated in the thyroid in this condition. The thyroid gland of $\mathrm{ex}$ ophthalmic goitre has the sime action on animals and on patients suffering from exophthalmic goitre as any other thyroid gland with like iodine content (Marine).

The relations of indine to the gland itself, and the modifications in its structure and function determincd by the giving or withholding of iodine, recently studied by Marine, are of great interest. In all animals, so far as examined, the normal throid contains iodine. The amount is varialnle, but the minimum percentage of iodine necessary, if the normal histologiral structure is to be maintained, is quite constant for a given species. So also the highest percentage of iodine associated with any degree of active hyperplasia (developing goitre) is always below the normal minimum of $0^{\circ}$ I per cent. of the dried gland as shown by Marine in the dog, sheep, man, and other mammals. As active hyperplasia of the thyroid (goitre) (Fig. 203) develops, the iodine content of the gland, both relative and absolute, decreases, until in extreme legrees of the condition there may be no demonstrable iodine present at all. 
Since the iocline is contained in the colloid as an indine-poretein compound, the generalization may be made that in the thyroid the iodine varies directly with the amount of colloid, and inversely with the degree of hyperplasia. The administration of any iodinccontaining substance to animals with actively hyperplastic thyroids (goitres) results in a rapid storage of iodine in the gland, and quickly (in two to three wecks in dogs) induces a listological cliange, the end stage of which is the so-called colloid goitre (Fig. 204). This is an involution to the normal histological structure (Fig. 205), so far as this is possible in a gland which has once undergone liyperplasia. This storage of

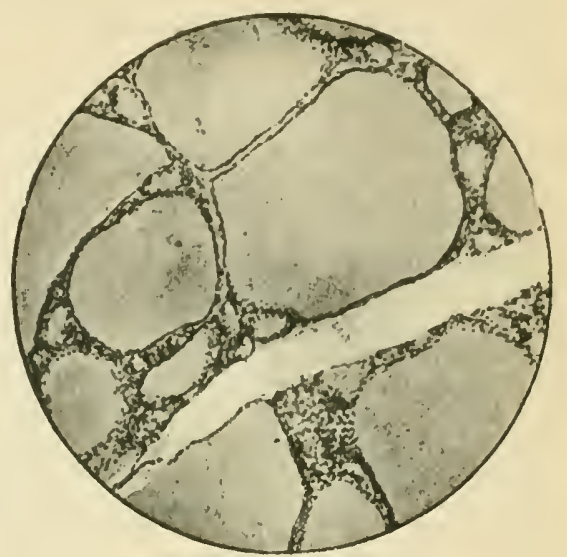

Fig. 204.-Microphotograph of a Colloid Gland (Goitre) (Marine). The effect of administration of iodine is shown in the return towards the normal structure from a preceding active hyperplasia, such as is shown in Fig. 203.

iodine in the thyroid is of unusual interest because it affords the best opportunity for the quantitative study of all essential inorganic constituent of the body. Iron is another such substance,

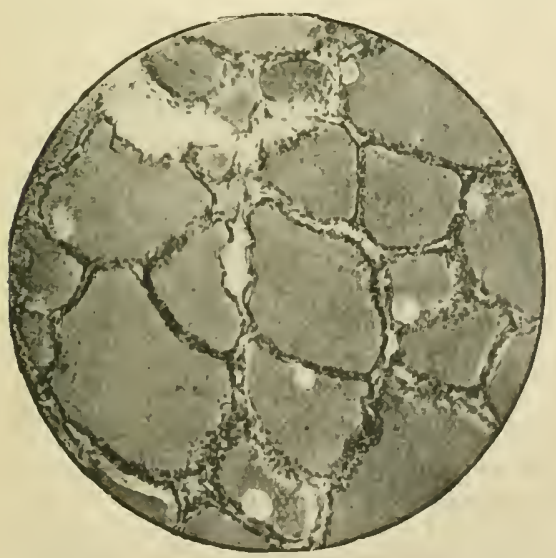

Fig. 205.--Nlicrophotograph of Normal Human Thyroid (Marine). but it is impossible to follow its absorption quantitatively with the same ease and accuracy, since it is so widely' distributed. Iodine is contained in the thyroid, and to all intents and purposes in the thyroid alone. And the thrroid is so situated andtomically that it is a simple matter to remove a portion of it for examination. The extraordinary affinity of the thyroid for iodine and its salts has been demonstrated both in vivo and in vitro. There is little difference whether the iodine salt is injected into the circulation of the living animal, or perfused through the excised surviring gland, so long as the gland is not 
already saturated with iodine. The greatest quantitative absorption takes place in those glands with the smallest iodine content, or. what comes to the same thing, those glands with the most marked hyperplasia.

Thus, a dog's thyroid (in early hyperplasia) weighing 16 grammes, and containing 0.54 milligramme of iodine per gramme of the dry tissue was perfused for thirty minutes with a mixture of blood and Ringer's solution to which was added 5 milligrammes of potassium iodide. The gland was then washed out thoroughly with Ringer's solution to remove remaining iodide. It was now found to contain I'38 milligramme of iodine per gramme of dry tissue. Ten minutes after the injection of 50 milligrammes of potassium iodide into the femoral vein of a dog, from which one thyroid lobe had been removed as a control, the remaining lobe was excised and all loose iodide removed by thorough washing. The iodine in it was then determined and found to be $1 \cdot 3 \mathrm{~S}$ milligramme per gramme of the dry tissue, as conpared with 0.79 milligramme in the control lobe, an increase of 75 per cent. Only traces of iodine can be demonstrated in other organs under such conditions, even when they have not been washed. Even in five minutes, an absorption of iodine of practically the same magnitude has been shown to occur. So that the thyroid has the power of taking up considerable amounts of iodine almost instantaneously. While, however, the tine required for the absorption of iodine by the thyroid from the blood, and the binding of it in such a way that it cannot be washed out, is so short as to be measured in minutes, the time required for the elaboration of the iodine into the active iodinecontaining substance, which exhibits the specific action of the gland, is much greater. In dogs, the earliest period at which an increase in the amount of active iodine could be made out was eight hours after the injection of an iodine salt into the circulation.

Summary :-The physiological significance of iodine in the thyroid may be summed up as follows: Iodine is absolutely essential for the normal actirity of the gland. The amount in the gland at any given time represents the store or reseri'e. Iodine prevents spontaneous hyperplasia (goitre) in all mammals, and also the compensatory hyperplasia which follow's partial removal of the thyroid. It exercises a curatice effect on actice hyperplasias. It is present in an active and an inactive form. The inactive form represents the newly acquired iodinc, which is usually a iery small fraction of the total content, but may undergo a great temporary increase after the administration of iodine. Accordingly, the physiological and therapeutic activity of thyroid substance appears in general to "ary with the total amount of iodine in it, although in reality with the amount which exists in the specific componnd. The nature of this compound is unknown.

No other body has been shown to possess its specific activating effect on metabolism. It is sometimes spoken of as the iodine-containing hormone. Kiendall has been able to separate it both from its protein combination and from the inactive indine by alkaline hydrolysis of the thyroid, and to obtain it in a crystalline form containing about 60 per cont., by weight, of iodine. 
The unost sensitive and accurate test object for estimating the pharmacological activity of thyroid substance, or of its hydrolytic products, is the tadpole. Its growth is hindered and its differentiation accelerated by the active iodine compound, so that the limbs grow and the tail atrophies, while the tadpole renains small (Gudernatsch). By means of this test, it has been possible to demonstrate the presence of active and inactive iodine in the gland, and to estimate the rate at which the thyroid can claborate the active iodine-containing substance from the inactive iodine (Figs. 206, 207).

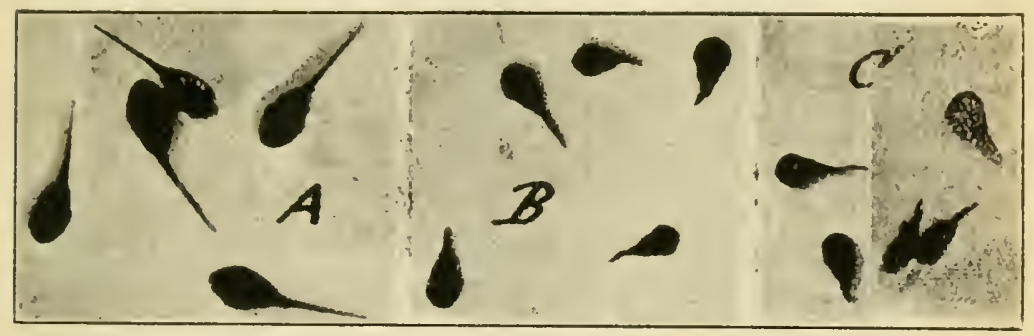

Fig. 206.-Tadpoles after seven days feeding: A, with iodine-free hyperplastic lamb thyroid in 50 milligramme doses on alternate days; $\mathrm{B}$, with sheep thyroid hydrolytic product (containing 17.9 milligrammes of iodine per gramme) in ro milligramme doses on alternate days; $\mathrm{C}$, with ox thyroid hydrolytic product (containing 14.8 milligrammes of iodine per gramme) in ro milligramme doses on alternate days. On the other days all the tadpoles received fresh liver. In A, normal growth similar to control. Great emaciation with differentiation in $B$ and $C$ (Rogoff and Marine).

While the precise rôle played by the thyroid in the economy remains obscure, it is evident that in most animals and in man its secretion is of great importance, whether it be solely the quasiexternal secretion of 'colloid,' with its specific iodine-containing substance that collects in its alveoli and slowly passes out of them by the lymphatics, or perhaps, in addition, some other substance, which, like the glycogen of the liver, never finds its way into the lumen of the gland-tubes at all. It may also be admitted that, by aiding in the maintenance of the normal level of general nutrition, particularly that of the central nervous system, the ability of the organism to cope with toxic substances introduced from the outside or manufactured in the body is favoured. There is, however, no evidence that an actual destruction or neutralization of toxic substances occurs in the gland itself.

It is uncertain whether the secretion of the thyroid is influenced by nerves. Section of most of the nerves entering the gland does not obviously alter its activity. Thyroid tissue may be transplanted into any part of the body, and there exhibit all the morphological, chemical, and functional changes seen in the gland in situ. Transplantation in the same nerve field as the original thyroid has no advantage over transplantation into any other region of the body (Marine and Manley). This is an indication that specific secretory nerves are not indispensable for thyroid activity. 
It has long been known that vaso-motor fibres for the dog's thyroid run up in the cervical sympathetic to the superior cervical ganglion, and

Sample.

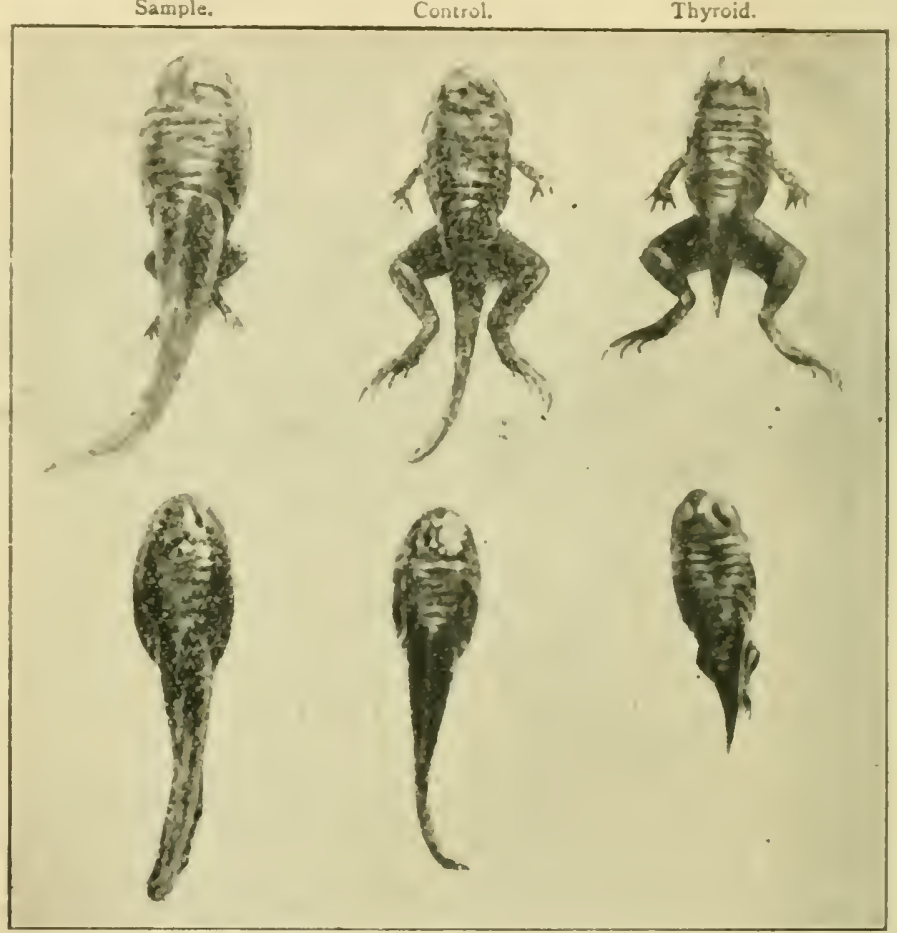

Fig. 20\%-Effect of Desiccated Human Thrroid on Tadpoles of Different Age-

The upper row shows the older, the lower row the younger tadpoles. The older ones had posterior leg buds slightly over 2 centimetres long, but no forelegs. The younger tadpoles had just visible posterior leg buds and no foreleg buds. The ind viduals of each agt were alike at the beginning of the experiment as to size at $d$ condition of development. One of each age was killed in tormalin at the beginning of the experiment (designated as 'Sample' in the figure). Another of each ah. was used for control, and fed fresh liver every second day. A third specinen I each age (under 'Thyroid' in the figure) was fed 50 milligramme- of desiccat d human thyroid every second day, alternating with fresh liver. The thyrnicl showed the condition of multiple adenoma and contained 4.3 milligrammes of iodine per gramme of the dry weight. The difference at the end of the experiment between the thyroid-fed and control tadpoles of the younger set is especially seen in the atrophy of the tail and the appearance of the hind-leg - in the former. In the older set, at each stage in the experiment differentiation was more advanced in the thrroid-fed specimen. This is still the case at the end, especially in the much greater atrophy of the tail. The difference is less than in the younger set, simply. because even the control is approaching the end of the metamor. phosis (Graham).

thence to the lobe of the same side. These were first discovered by the effect presluced by their stimulation on the thyroid circulation time (p. I35). Some evidence of the existence of secretory fibres lat- 
been brought forward by Asher and Flack. They compared the excitability of the depressor nerve, and also the effect on the blool-pressure of the intravenous injection of adrenalin, before and during stimulation of the thyroid nerves. They conclude that, when all the other conditions remain unchanged, both the effect of excitation of the depressor and the effect of adrenalin are greater during stimulation of the thyroid nerves than shortly before it without such stimulation. The difference is really connected with the internal secretion of the thyroid, since it is not obtained if the thyroids are previously extirpated, and injection of thyroid extracts influences the result exactly in the same way as stimulation of the thyroid nerves. Similar indirect evidence has been obtained by other observers (Oswald, I.evy!

Adrenal Bodies.-It had been observed by Addison that the malady which now bears his name, and in which certain vascular changes, with muscular wealness, anæmia, and pigmentation or 'bronzing ' of the skin, are prominent symptoms, was associated with disease, usually tuberculous, of the adrenal bodies, commonly. called in human anatomy the 'suprarenal capsules.' This clinical result was soon supplemented by the discovery that extirpation of the adrenals in animals is incompatible with life (Brown-Séquard). Our knowledge of the functions of these hitherto enigmatic organs was extended by the experiments of Oliver and Schäfer, who investigated the action of extracts of the adrenals (of calf, sheep, dog, guinea-pig, and man) when injected into the veins of animals. The arteries are greatly contracted, and this mainly through direct action on the vaso-motor nerve-endings or some structure intermediate between them and the smooth muscle of the vessels, but partly through the vaso-motor centre. The blood-pressure rises rapidly, although the heart may be inhibited through the vagus centre. The heart is at the same time directly stimulatec, so that, although it beats slowly, the beats are stronger than before. When the vagi are cut the action of the heart is markedly augmented, and the arterial pressure rises enormously (it may be to four or five times its original amount). Stimulation of the depressor is of no avail in combating this increase of blood-pressure. The generalization may be made that the active principle of the medulla-epinephrin or suprarenin, also called adrenin*-acts upon all plain muscle and gland-cells that are supplied with sympathetic nerve-fibres, and the result of the action, whether augmentation or inhibition, is the same as would be produced by stimulation of the sympathetic fibres going to the muscle or gland in question. Yet it is not through excitation of these fibres that epinephrin acts, for its effect is even more pronounced when the nerve-fibres have been caused to degenerate, in the case of the pupillo-dilator fibres, e.g., by excision of the superior cervical ganglion. Nor is the effect a direct one on

* It is advisable to restrict the term 'adrenalin' to the well-known commercial preparation of the active substance. 
the muscular fibres. For smonth muscle which is not, and never has been, in functional union with sympathetic nerve-fibres is indifferent to adrenalin (Elliott). It seems, then, to act on some structure intermediate between the nerve and the muscle, but so related to the latter that it continues to live so long as it is in connection with the muscle-fibre. Instead of a definite histological structure, the seat of the action may be a special 'receptive' substance at the myoneural junction. Thus adrenalin causes marked diminution of tone in the small intestine, with disappearance of the peristalsis and pendulum movements. The same effect is produced on an isolated loop of intestine immersed in Locke's solution, and the action is therefore local. The drug is effective in a dilution of $I: I 0,000,000$, or even in much greater dilution. A similar effect has been observed on the stomach. The vessels of the conjunctiva are constricted by local action when an extract of the capsulcs is dropped into the eyc, a fact which has proved of valuc in ophthalmological practice. Inhibition of the contraction of the stomach, intestine, urinary bladder. and gall-bladder; contraction of the uterus, vat deferens, and seminal vesicles; dilatation of the pupil and retraction of the nictitating membrane; stimulation of the salivary and lachrymal secretions, are among its actions (Langley).

Meltzer has shown that the dilatation of the pupil caused by the intravenous injection of adrenalin is distinct, though fleeting, in cats, less marked in rabbits. Subcutaneous injection has no effect. Instillation of the drug into the conjunctival sac is without effect on the pupil in the normal rabbit's eye, but causes dilatation if the superior cervical ganglion has been removed.

The influence of adrenalin in increasing the sugar content of the blood, and thus causing glycosuria, has been previously discussed (p. 550). A new and interesting action has been added to the already long list of the effects of adrenalin, by the discovery that small doses (0.0o I milligramme per kilo of body-weight) injected intravenously, and larger doses injected subcutaneously into cats shorten the coagulation time of the blood to one-half or one-third of its previous cluration, probably by stimulating the liver to greater activity in discharging some substance or substances concerned in clotting (Cannon). The injection of adrenalin into the blood is said to cause a temporary improvement in the response to stimulation of a fatigued muscle still in connection with the circulation, and this improvement does not seem to be entirely due to augmentation of the blood flow (Gruber, Cannon and Nice).

Methods for the detection and the assay of epinephrin in the small quantities in which it can only be supposed to be present in physiological liquids have been based upon certain of these actions. Such, for example, is the extraordinary power of this active principle that a dose of one-millionth of a gramme per kilo of bodly-weight is sufficient to cause a distinct effect upon the heart and bloodvessels (a rise of pressure of $\mathrm{I}_{4}$ millimetres of mercury) when injected into the veins of a mammal. The reaction is rendered more constant, although less delicate, when the brain is previously destroyed and the animal used as a spinal preparation. In pithed cats the assay can be accurately 
performed to o'or milligramme. Anotlere delicate, and for certain purposes a convenient, reaction for the detection and the physiological assay of epinephrin is the perfusion test on the legs of frogs already allucled to $(1), f^{(j)}$. The dilatation of the pupil in the excised eyeball of the frog, the contraction of stretched artery rings (1). (36), the increase in the tone of isolated segments of the uterus of rabbits, and the diminution in the tone of isolated segments of rabbits' intestine (Practical Exercises, p. 453), have also been employed as pliysiological tests.

$A$ clilute solution of adrenalin chloride is used in medicine as a styptic, and for reducing congestion in accessible parts. 'The intense local and'nia which it causes when given subcutaneously or by the mouth is onc reason, perliaps the most important, for the slow absorption on which depends the absence of its general effects, inclnding that on the blood-pressure, when it is administered in this way.

Function of Epinephrin. - The striking effects produced by adrenalin have naturally led to the assumption that the function of epinephrin in the body must be important. But there is perhaps no chapter of recent physiology in which the rash application of qualitative data to what is esscntially a quantitative problem has led to greater errors. Because adrenalin in a certain amount and concentration raises the bluod-pressure when injected intravenously (a fact certainly of some pharmacological interest), it has been assumed that the naturally secreted epirephrin must be an important factor in the maintenance of the normai vascular tone and arterial pressure. From this assumption it was only a short step to the announcement that the blood in cases with permanently increased blood-pressure (so-called arterial hypertonus, etc.), and even the urine contained truly incredible concentrations of epineplirin. Because the injection of what in proportion to the normal output of epinephrin must be designated as monstrous doses of adrenalin causes an increase in the sugar of the blood and the appearance of sugar in the urine, it has been supposed by many that all the experimental hyperglycemias and glycosurias (p. 547) are directly due to increased liberation of epinephrin from the adrenals. The fallacy of giving a physiological value to the details of the pharmacology and toxicology of a substance produced in the body or known to exist in the blood in the absence of information as to its amount and condition would have been very' eviclent in the case, for example, of anmonium or potassium, and nobody would liave dreamt that the effects produced by massive injections of anmonium or potassium compounds of various kinds would inform us of the rôle played by the ammonium and potassium compounds circulating in normal blood. The whole question of the function of epinephriy turns, therefore, upon the quantity which can possibly be present in the systemic arteries or capillaries. Direct assays of such blood by trustworthy observers using the inost delicate methods available have hitherto yielded negative results, both in health and in disease. This is due to the great dilution and rapid removal or oxidation of the epinephrin known to be given off by the adrenal glands.

That epinephrin is continuously liberated into the blood from the adrenals is readily shown by tving off a long segment of the inferior vena cava in sucli a way that only the adrenal veins discharge into it. The cava pocket is temporarily clipped above the level of the orifices of the adrenal veins and allowed to fill with adrenal blood. When the blood is now released into the circulation by removing the clip, a rise of blood-pressure (Fig. 208) is produced, beginning after an intervai sufficient for the blood from the pocket to pass round to the systemic arterioles. Another method is to derve the cffect of the released blood 
in causing dilatation of the pupil and retraction of the nictitating membrane of the eye of a cat from which the superior cervical ganglion on one side lias been removed a week or two before the experiment. It is well known that after this procedure these reactions are elicited by adrenalin with great ease.

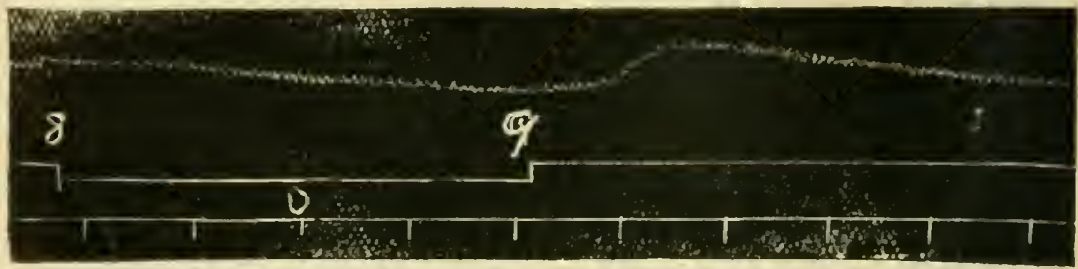

Fig. 203. - Showing Spontaneous Liberation of Epinephrin in a Cat. 8 to 9, Pockst. Epinephrin rise on release. Time-trace half-minutes ( $\frac{3}{10}$ reduction).

Or the adrenal vein blood can be collected directly from a cannula in the cava pocket, and then tested on rabbit's uterus and intestinc segments (Fig. 209).

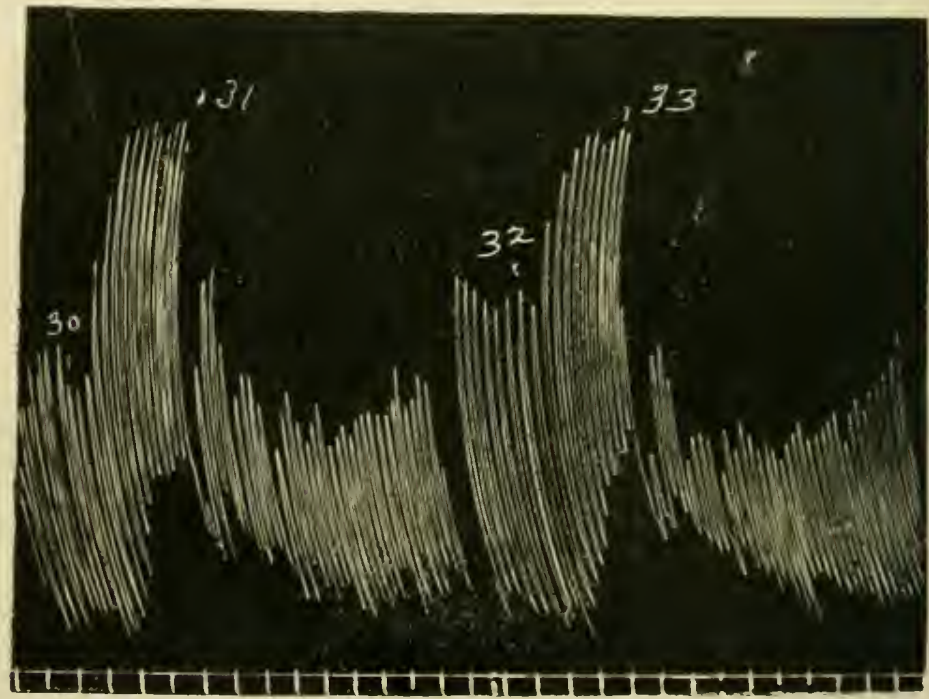

Fig. 200.-Intestine Segment Tracings with Adrenal Blood from a Cat. At 30, Ringer's solution was replaced by indifferent (jugular vein) blood, and this at $3 I$ by a specimen of adrenal vein blood collected during asphyia. At 32 , Ringer's solution was replaced by jugular blood, and this at 33 by a specimen of adrenal vein blood colleeted previously without asplyxia. The bloods were diluted with eight volumes of Ringer's solution The concentration of epinephrin in the specimens is seen to be about the same. Time, half-minutes (reduced to two-thirds).

The amount of epinephrin in the blood can be assayed by comparing the effects produced by it with those produced by known amounts of adrenalin. In such ways it has been shown that in cats the amount of 
epinephrin given off per minute varied from o 00 I milligramme to $0^{\circ} 002$ milligramme. All the epinephrin seems to be contained in the plasma and none in the corpuscles (ligs. $210,21 \mathrm{r}$ ).

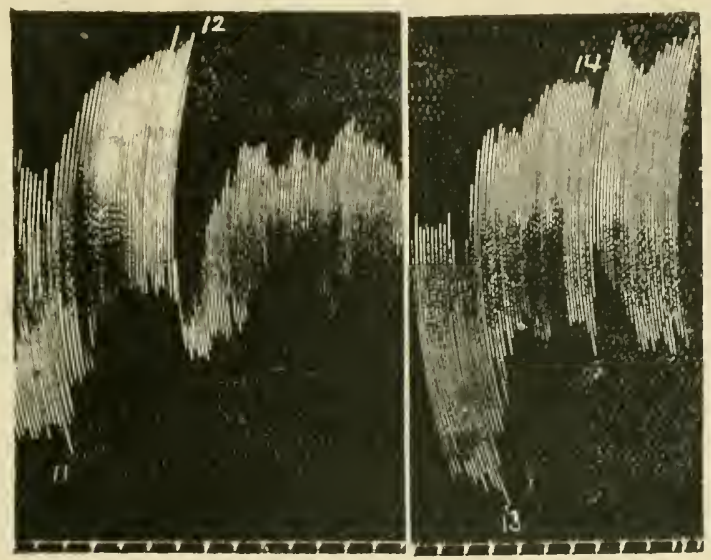

Fig. 2 10.-Comparison of Effect of Adrenal Vein Blood and its Sediment on Rablit's Intestine Segments. II, Ringer replaced by indifferent blood, and this at I2 by adrenal vein blood. I3, Ringer replaced by sediment of indifferent blood, and this at 14 by sediment of adrenal vein blood. The bloods and sera were diluted with 2 volumes Ringer. Time-trace, half-minutes (reduced to one-half).

If we suppose that a cat, weighing 2.5 kilos, and containing 200 c.c. of blood was giving off o.001 milligramme of epinephrin per minute, the concentration which this output would maintain in the mixed blood, supposing that there was no loss in passing through the lungs and up to the systemic capillaries, would be at most only $I: 400,000,000$ or I : 500,000,000. For at least 400 or 500 c.c. of blood would pass through
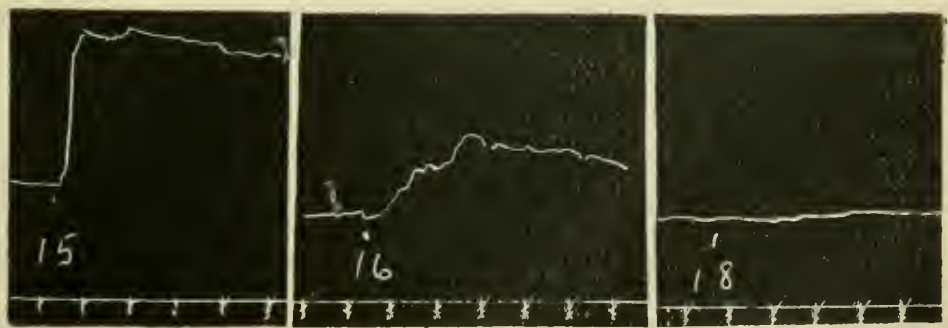

Fig. 2xr.-Effect of Adrenal Blood (16), Serum (15), and Sediment (18), on Rabbit's Uterus Segment. All the specimens were diluted with 5 volumes Ringer.

the heart in one minute. Now, with such concentrations of adrenalin no known reaction has ever been demonstrated in the normal intact animal. Of course, in the cava pocket experiments described above, the concentration in the blood will be much greater because a relatively considerable quantity of epinephrin-containing blood is accumulated in the pocket and then suddenly discharged. 
The function of the spontaneously liberated epinephrin, once it has entered the circulation, is accordingly involved in doubt. The common view that it exerts an important physiological action upon the sympathetic system, contributing especially to the maintenance of the normal blood-pressure is opposed to all the best evidence. Whatever influence the relatively small quantities of adrenalin discharged from time to time may have upon the nutrition of the smooth or of other muscles, it is quite unlikely that such amounts are continuously entering the blood as injection experiments have shown to be necessary for the maintenance of even a small excess of blood-pressure. Statements which connect the increased bloodpressure in such conditions as clironic nephritis with hypertrophy of the chromaffin tissuc (p. 665), an increased adrenalin production, and an increased adrenalin content of the blood, must be received with scepticism. The so-called experinental arteriosclerosis produced by repeated injections of adrenalin into the blood of rabbits throws little light upon the question, for the vascular changes, in so far as they lave not been confounded with similar lesions occurring spontaneously in a considerable proportion of rabbits, differ from those observed in pathological arteriosclerosis (M. C. Hill).

Certain facts indicate, indeed, that doses of adrenalin consicleral,ly smaller than those which give the effects described above, ancl usually considered the 'normal' effects, produce a reversal of the reaction, very small doses causing, for instance, a diminution of blood-pressure (Moore and Purington), an increase in intestinal tonus and peristalsis, and a diminution in the tone of uterine segments. The actions associated with these small doses are more likely to be the normal actions than those associated with the larger doses, and the normal action of adrenalin, if it is a continuous action, would therefore more probably be to inhibit than to cxcite the sympathetic mechanism. But there is no cvidence that even quantities of this order of magnitude are continuously discharged. The continuous introduction of epineplirin at a very slow rate produces no demonstrable effect at all, and the sudden ligation of all the adrenal bivodvessels has not the slightest influence upon the blood-pressure until the lapse of a period far greater than would be required for the destruction or removal-a quite rapid processof any epinephrin already present in the blood or tissucs (Trendelenburg, Hoskins). The injection of a quantity of adrenalin sufficient to cause and to long sustain even a minimal increase of blood-pressure creates conditions highly hazardous to life or incompatible with it-namely, complete inlibition of the musculature of the gastro-intestinal tract.

The only effect of interference with the passage into the blood of the spontaneously liberated epinephrin which has hitherto been demonstrated is upon a mechanism renclèred abnormally sensitive-namely, 
the iris of the cat's eye after removal of the superior cervical ganglion. When the pupil has been dilated by epineplurin, the dilatation passes off sooner if the adrenal vein blood is prevented from entering the circulation. This shows that the clilatation of the pupil must have been in part sustained by the epinephrin continuously entering the blood from the adrenal veins.

The suggestion that enough epinephrin may be continuously liberated to exert an influence upon the nutrition and metabolism of the sympathetic system, or of the myoneural junction, which is necessary for normal cxitability, without ever rising to the threshold of actual excitation, is a mere hypothesis, and a hypothesis which does not seem easily reconciled with the result of Hoslins, that after ligation of the a.drenals the excitability of the vaso-motors remains absolutely undiminished.

It has been supposed by some that although usually liberated in an amount too small to cause demonstrable effects, epinephrin can be discharged at a greatly increased rate under certain conditions-for example, under emotional stress (the so-called emergency function of epinephrin). There is no evidence, however, that such outbursts of epinephrin ever occur, and experimentally it is by no means easy to produce decided alterations in the rate of discharge.

Whatever the function of epinephrin may be, it is not indispensable for life and health. For cats after removal of one adrenal and section of the nerves which govern the epinephrin discharge from the other, not only survive indefinitely, but show no apparent differences from normal animals, although, when the nerve section is complete, no epinephrin can be detected by the most delicate tests in the adrenal vein blood, and the possible concentration in the arterial blood cannot at most amount to I : Ino thousand millions.

Nervous Mechanism Controlling the Liberation of Epinephrin. - The spontaneous liberation of epinephrin from the adrenals is entirely dependent upon nerve fibres, reaching the glands from the lower dorsal and upper lumbar portions of the sympathetic chain. The splanchnics form the most important path. When in the cat the fibres coming to a semilunar ganglion from the sympathetic, including the splanchnics, are cut, the secretion of epinephrin by the corresponding adrenal into the blood comes at once to an end. It is not resumed when the animal is allowed to survive. When the peripheral ends of the cut splanchnics are stimulated electrically, secretion is caused, and the amount of epinephrin discharged per minute may exceed that spontaneously liberated (Figs. 2I2, 2I3).

This was first indicated by the experiments of Dreyer, who collected blood from the adrenal vein of a dog during splanchnic stimulation, and caused a rise of blood-pressure in another dog by injecting the blood. The effect of stimulation of the splanchnic can be demonstrated very clearly by the eye reactions already alluded to. After the interval required for the blood to pass from the adrenals to the eye, dilatation of the pupil and retraction of the nictitating membrane are seen to occur. In this way it has been shown that 


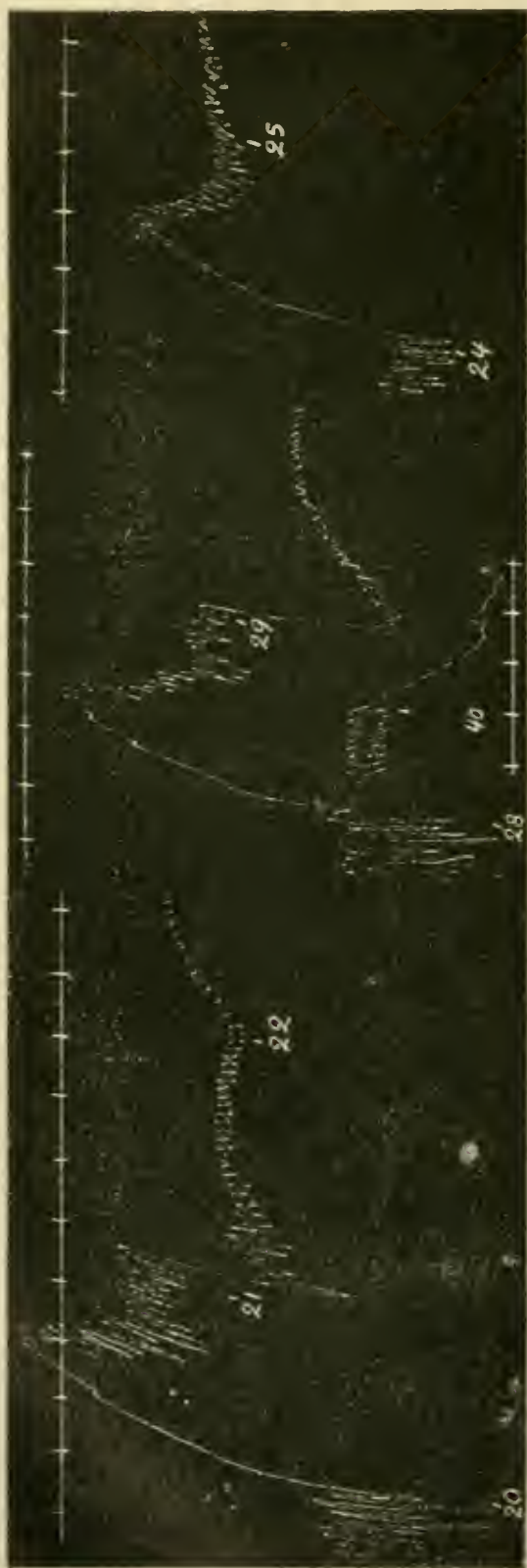

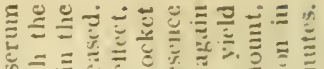

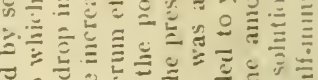

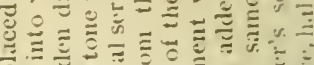

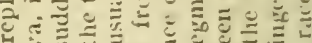

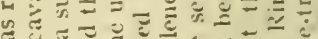

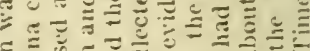

풀

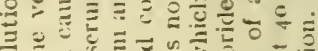

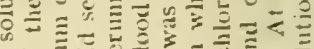

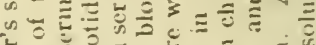

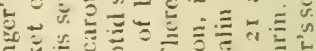

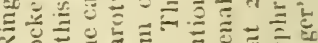
$\angle 0$

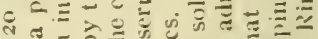
$\because-2=0$

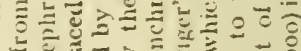
.

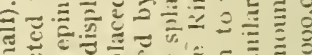

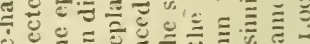

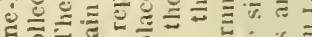

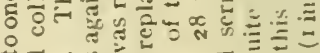

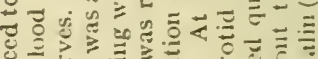

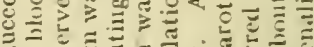

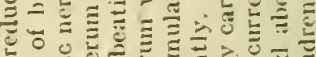

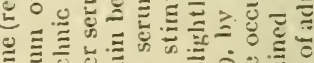

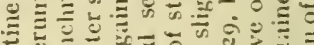

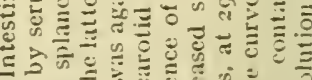

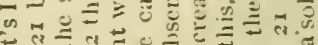

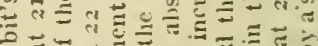

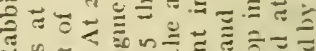

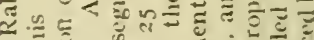

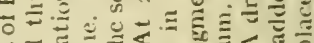

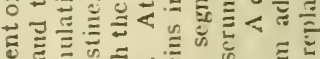

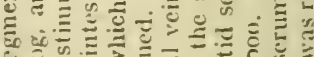

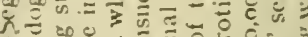

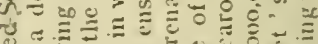
สั

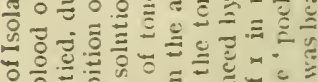

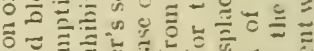

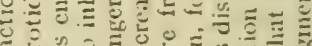

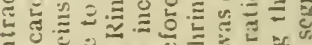
亏气 $1=\Xi \mathrm{J}$

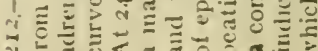
ปี $\frac{3}{2}$ 
the latent period of the secretion is so short that the reaction follows the commencement of stimulation of the nerve after a time-interval sensibly the same as when it is elicited by the corresponding amount

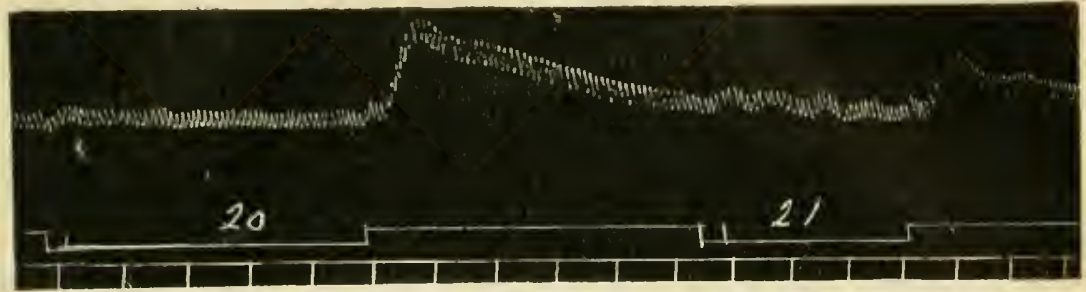

Fig. 2I3.-20, Pocket Experiment with Stimulation of Right Splanchnic in Abdu men after Section of Both Splanchuics. 2I, repetition of 20 , but with a shorter ably greater than after 2 r. Time-trace, half-minutes ( $\frac{2}{5}$ reduction).

of adrenalin injected at the level of thə adrenal veins. Even when the glands have ceased to respond to stimulation of the nerves, epincphrin can still be liberated into the blood by massaging the g'unds with the fingers (Fig. 2I4).
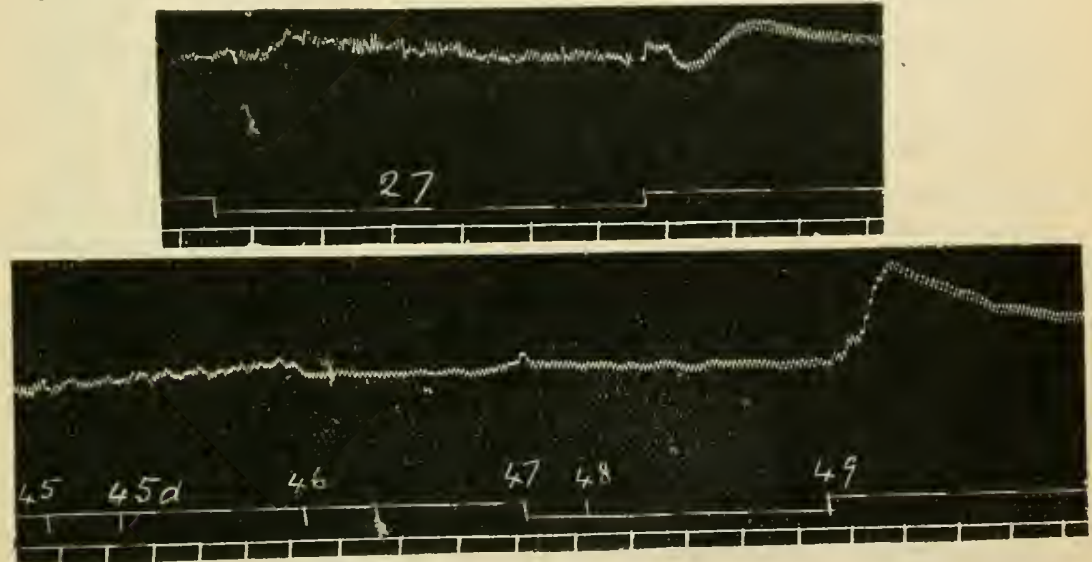

Fig. 214.-Showing the Effect of Massage in Liberating Epinephrin from the Left Adrenal whose Nerves had been Cut Five Weeks before. 27, cava pocket with massage, the left adrenal vein being open to the pocket. 47 to 49 , pocket with massage, the left adrenal vein having been closed at 45 and opened at 48 , after closure of the pocket. Massage begun at 45-A, stopped at 46 . Blood-pressure tracing from carotid artery. Time-trace, half-minutes (reduced to $\frac{3}{5}$ ).

A centre for epinephrin secretion seems to exist in the upper part of the thoracic region of the spinal cord. After section of the cord in the cervical region, even as low as the level of the last cervical segment, the secretion persists, but it is abolished when the cord is divided at the third or fourth dorsal segment. 
The relation of the nervous system to the adrenal medulla lias been furtler illustrated by comparison of the amount of epinephrin which can be extracted from the two adrenals when the nerves of one have been cut. Linder the influence of anæsthetics and some other drugs, bacterial toxins, such as diphtheria toxin, etc., the epinephrin store is markedly diminished in the gland whose nerve supply has been left intact as compared with the other.

For example, in a cat the fibres coming from the sympathetic to the left semilunar ganglion, including the left splanchnic nerves, were cut, and the animal allowed to recover. Several days later when it is known that equality of epinephrin load in the two adrenals would have been restored, morphin was administered to the animal, and after a few hours it was killed, and the epinephrin in the aclrenals assayed. The left gland contained 0.28 milligramme, the right, 0. I I milligramme. If the animal had been suddenly killed without morphin, the two ac.renals would have been found to contain equal amounts of epinephrin. Therefore the deficiency in the right gland must have been due to the fact that it was not protected by section of its nerves.

A similar deficiency has been found in the unprotected gland after administration of a drug, $\beta$-tetrahydronaphthylamine, after surgical operations (post-operative deficiency), and in many other conditions. IThen the animal recovers the deficiency is gradually made good, the adrenal having the power of forming and storing epinephrin up to a cc-tain point in the absence of its nerve supply, although it is unable to liberate it into the blood.

There is no foundation for the statement that emotional disturbances, as fright or anger, cause a depletion of the epinephrin store of the adrenals.

Summary :-The existence of a nerious mechanism through which the gland is stimulated to secrete epinephrin into the blood can therefore be considered as definitely established. It is the function of the epinephrin, once it has cntered the circulation that is iniolied in doubt. Although it is highlv improbable that the concentrations necessary for direct stimulation of simpathetic endings eier exist in the general mass of the blood, it is quite possible that a 'sensitizing 'infuence is exerted by epinephrin upon the sympathetic peripheral mechanisms which renders them more susceptible to nerie impulses originating in other ways. The possibility of a more direct action of epinephrin upon the chemistry of carbo-hydrate metabolism, particularly upon glycogenoly'sis or even ipon gly'cogen formation has becn emphasized by some uriters. But glycogen can be formed abundantly, and certain experimental hyperglycamias which depend upon the rapid transformation of glycogen into dextrose can be produced after the epinephrin secretion of the adrenals has been abolished.

Chemistry and Formation of Adrenalir.-It has been shown (Stolz, Dakin) that adrenalin $\left(\mathrm{C}_{9} \mathrm{H}_{13} \mathrm{NO}_{3}\right)$ is a dioxyphenyl-ethanol-methy. lamin,

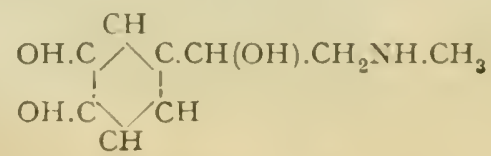


It has been prepared synthetically, and in the body appears to be formed, probably by the introduction of a metliyl $\left(\mathrm{CH}_{3}\right)$ group, from a compound arising from an aromatic amino-acid (tyrosin or pheny]alanin). While the natural adrenalin rotates the plane of polarization to the left, the artificial substance is optically inactive. This is because it consists of equal parts of lavo-rotatory and dextro-rotatory adrenalin. The artificial adrenalin has approximately half the effect of the natural on the blood-pressure, from which it may be inferrerl that the dextrorotatory isomer has only a very slight pressor effect. The left and right rotatory moieties have been separated. The former has exactly the same power of raising the blood-pressure as the natural adrenalin, the latter only $\frac{1}{1}$ to $1=$ as much. Practically the same proportion holds when the power of the two isomers in producing glycosuria is compared. This constitutes important corroboration of the view already referred to (p. 550), that adrenalin glycosuria is calsed by an action on the sympathetic system, for the effect on the blood-pressure is known to be thus produced (Cushny).

It is in the medulla of the adrenals that the cpinephrin is formed. The medullary cells contain a substance which gives a yellow or brown stain with chromic acid or chromates, and for this reason the cells are called chromaffin or chromaphil. Similar cells are found elsewhere in the body-e.g., within the sympathetic ganglia, and also strung out in clumps along the course of the abdominal aorta below the level of the adrenal glands (Vincent). These outlying masses of chromaffin tissue appear to contain epinephrin, or a substance with sinilar physiological actions, so that the formation of this compound seems to be a property common to chromaphil tissue, no matter what its situation may be. A remarkable fact, and one calculated to induce caution in assigning a physiological function to epinephrin, is that the so-called parotid gland of a Jamaican toad secretes it in a concentration not inucli short of 5 per cent. (Abel).

Function of the Adrenal Cortex.-The function of the cortical cells is obscure, but there is evidence that they, and not the chromaffin cells of the medulla, are concerned in the production of the internal secretion, whatever its nature may be, the loss of which leads so speedily to death on removal of the adrenals. For example, the period of survival after this operation is practically unaffected by the continuous intravenous administration of adrenalin, although the loss of the medulla might be supposed to be compensated in this way. Still more convincing is the fact already referred to that after removal of one adrenal and denervation of the other in cats, epinephrin ceases to be discharged in detectable amount, and yet the animal survives in good health. In Addison's disease adrenalin is likewise powerless.

The weakness (asthenia) of the skeletal, and to some extent of the cardiac, musculature which is characteristic of Addison s disease, is produced experimentally within a few hours by ligation of both adrenals, and all the evidence goes to show that it is the cortical and not the medullary region which is related to this disease. When the adrenals are not completely extirpated, compensatory hypertrophy of the remaining portions may occur, and the animal survive indefinitely. In such cases it has been found that the hypertrophy is confined to the cortex.

In animals like the rabbit in which accessory adrenals are not uncommon, when these are present complete removal of both adrenals does not cause death. Now, the accessory adrenals consist of cortical substance without medulla. Portions of the adrenal are readily grafted under the skin, but only the cortex survives and grows. 
The functional difference between cortex and medulla is easily understood when we reflect that the morplological history of the two tissues is quite different. The medulla is developed from cells which push their way into the gland from the rudim ints of the sympathetic ganglia at that level, and is therefore of ectulermic origin. The cortes is derivel from the same mesolermic structure which gives rise to the kidneys and genital organs.

Embryologically, the cortical cells are related to the interstitial cells of the ovaries and of the testes, and like these are characteri\%ed by their richness in lipins (or lipoids). There is some evidence of an interrelation between the thyroid and all these groups of sex celly (interstitial cells of ovary and testes, cortex of adrenal). lemoval of a large part of the arlrenals in the rabbit causes slight though definite hypertropliy of the thyroid and lymphoid hyperplasia. This is also seen in Addison's discase in man.

Pituitary Body or Hypophysis. - In the pituitary body two parts essentially different in origin and function may be distinguished: (I) The large anterior lobe, or pars anterior, consisting of epithelial cells, many of which are filled with granules of the type seen in glandular epithelium, and abundantly provided with bloodvessels; (2) the smaller posterior or nervous lobe, or pars nervosa, also called the infundibular portion, consisting chiefly of neuroglia, the whole connected witl the floor of the third ventricle by a stalk called the infundibulum.

A further subdivision of the epithelial portion is made into the anterior lobe proper and the pars intermedia or intermediate lobe, consisting of epithelial cells, less granular and less richly supplied with bloodvessels than those of the pars anterior. The pars intermedia forms an epithelial investment of the pars nervosa, almost completely. surrounding it and throwing out offshoots of epithelial cells into its substance, which is also invaded by colloid material secreted by the cells of the intermediate lobe. The differences in the structure of the anterior and posterior lobes of the pituitary body correspond to a difference in their development. The anterior lobe is leveloped (in man in the fourth week of intra-uterine life) from an ectodermal pouch (Rathke's pouch), which is purhed up from the roof of the buccopharyngeal cavity towards the micl-brain. The posterior lobe is developed from an extension of the neural ectoderm, which grows backwards as the infundibular process till it mects and blends with that portion of the buccal pouch which gives rise to the pars inte.media. The pars intermedia is separated from the anterior lobe proper by a cleft which represents what is left of the lumen of Rathke's pouch. In connection with the interpretation of the results of experiments on removal of the pituitary body, it is of consequence to remember that a residue of the same epithelium which develops into the anterior lobe appears alway's to get cut off in the vault of the pharynx, constituting the so-called pharyngeal hypophysis, and consisting of a cord of cells identical with those of the anterior lobe (Haberfeld). Embryonic 'rests' of hypophyseal tissue are also often found in the dura of the sella turcica, in which the pituitary body lies, and in the body of the sphenoid bone. Cells of the intermediate lobe also run up the stalk of the infundibulum, and even stretch for a little distance along the floor of the third rentricle. Add to this the formidable nature of the 


\section{Hy pojhisis}

operations required for the extirpation of the hypophysis from its sheltered position within the skull, and it will not be womilered at that cumplete harmony has nut been attained as to the cunsequences of its removal. The best evidence at present is to the following effect:

When the pituitary body is completely removed, deatl speedily and invariably ensues, in dogs, on the average within twenty-four to fortr-eight hours. Puppies often live as long as two or three weeks. The much longer periods of survival occasionally witnessed are due to failure to remove some small portion of the hipopliyseal epithelium. On the day after the operation the animals may be able to walk about, to eat and drink, and may show an interest in their surroundings. The temperature, pulse, and respiration at this time may be normal. Soon, lowever, they become lethargic, then comatose, with characteristically incurved spine, slow respiration, with long-drawn inspiration, a feeble pulse, perfectly limp muscles, and often a subnormal temperature, and the appearance of sugar in the urine. This deep coma passes into death, with no perceptible transition, and without a struggle (Paulesco, Cushing). The ablation of a part of the cortical substance of the anterior (epithelial) lobe of the hypophysis is compatible with permanent survival, and gives rise to no symptom of disorder. The same is true when only the posterior lobe is removed. This does not seem to be followed by any recognizable symptoms. In some animals, however, kept under observation for long periods after partial removal of the anterior lobe, a marked tendency to accunulate fat has been noted, accompanied by hypoplasia of the generative organs in adults or the persistence of the infantile condition in immature animals. On the other hand, complete removal of the anterior lobe causes death, just as if the whole gland had been taken away. Of all the structures included in the pituitary body, the most important from the functional point of view appears to be the superficial layer of the anterior lobe.

Mere separation of the stalk of the hypophysis may produce effects sometimes as serious as those of total removal of the gland, probably owing to the disturbance caused in the circulation. It is stated, indeed, by some observers that the vulnerable point is the base of the infundibulum, and that if this is not injured extirpation of the hypophysis is not incompatible with continued existence, and that in adult animals the resultant changes are only slight, although much more pronounced, especially as regards the disturbances in metabolism and development in young animals. It has been asserted that the pituitary undergoes (compensatory ?) hypertrophy after thyroidectomy. Some observers have accordingly assumed a similarity of function for these organs. It has even been stated that the production of colloid material by the cells of the pars intermedia is increased, and that colloid accumulates in the 
nervous portion of the posterior lobe. But this colloid, whateres its function may be, is very different from that of the thyroid alveoli, for the (sheep's) pituitary contains no iodine after extirpation of the thyroid any more than before (Simpson and Hunter). And in man pathological changes (tumours) in the pituitary borly are associated, not with myxoedema, or other disease connected with changes in the thyroid, but frequently with another condition, called acromegaly, in which the bones of the limbs and face, especially the hands and feet and the lower jaw, become hypertrophied.

Another condition often associated with tumours of the pituitary is gigantism-a condition occurring before the normal growth of the bones is completed, and resulting in a great increase in the length of the bones both in the limbs and the trunk.

\section{Action of Intravenous Injection of Extracts of the Pituitary.-} The effects on the vascular system of intravenous injection of extracts of the pituitary gland are also very different from those caused by thyroid extracts. The posterior lobe, or infundibular body, including the pars intermedia, contains two active substances, one pressor and the other depressor. The former is soluble in salt solution, but insoluble in absolute alcohol and ether; while the latter is soluble in salt solution as well as in alcohol and ether. The pressor substance (obtained in fairly pure form in the preparation called pituitrin, and in still greater concentration in a preparation to which the name hypophysin has been given) causes a rise of blood-pressure, due partly to constriction of the arterioles and partly to an increase in the force of the heart-beat, both of whirh are brought about by direct action. This rise of pressure lasts for a considerable time, and is sometimes accompanicd by a slowing of the heart. A second dose injected before the effect of the first has passed off is inactive; and this clistinguishes the pituitary from the suprarenal extract. Associatcd with the pressor effect is an increase in the flow of the urine. Whether this is due to a separate diuretic substance, as some maintain, has not been definitely settled.

Indeed, the factors on which the diuretic action depends are complex, and sometimes in spite of an increase of general arterial pressure, and of the volume of the kidney, there is no increased flow of urine. In diabetes insipidus pituitrin has been found to produce precisely the opposite effect-namely, diminution in the excessive urinary flow: The pressor substance, unlike adrenalin, directly stimulates smooth muscle fibres (especially the arteries, uterus, anl splecn) irrespective of their innervation (Dale).

Many other points of difference exist between the pressor principles of the infundibular body and the adrenal medulla. For example. pituitrin constricts the pupil and diminishes the fluw of saliva from the submaxillary gland, whereas adrenalin dilates the pupil and causes an increased flow of saliva. Hypophysin and other preparations of the posterior lobe have been employed to stimulate the uterine contractions in obstetrical practice, but their use does not seem to be free from 


\section{Wh pophysis}

danger. When injected intramuscularly, regular and poucrful contractions of the uterus arc excited in two or three minutes at any stage in parturition. Another effect of extracts of the infundibular body is on the mammary gland. An increased flow of milk follows the injec-

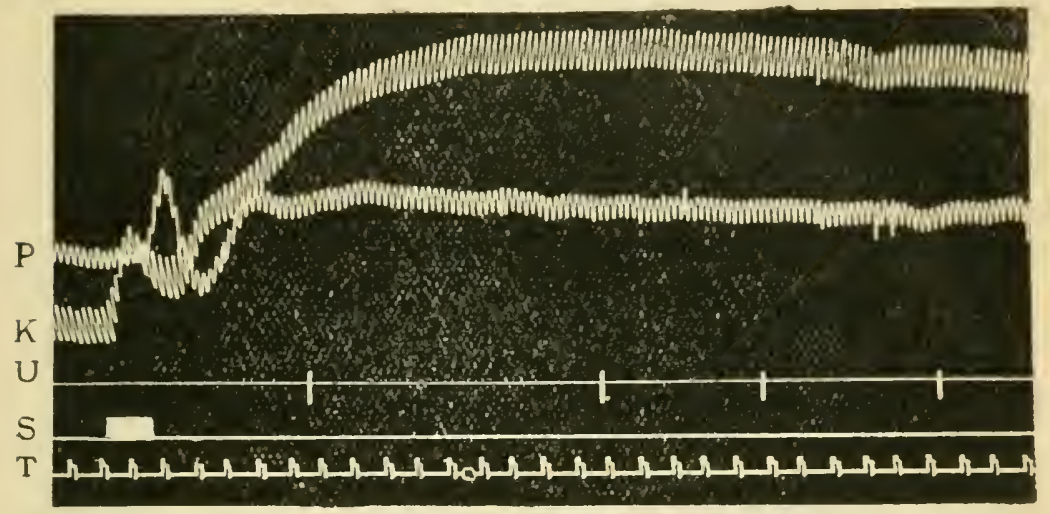

Fig. 215.-Action of Extract of Infundibular Lobe (Pars Nervosa of Ox Pituita1y). $\mathrm{P}$, Carotid pressure; $\mathrm{K}$, kidney volume; $\mathrm{U}$, drops of urine; $\mathrm{S}$, signal showing point at which pituitary extract was injected; $T$, time-trace in ro-second intervals. $\mathrm{T}$ is also the zero of the blood-pressure (Hering).

tion of pituitrin (Simpson). But it is in doubt whether this is due to increased secretion and not solely to stimulation of the smooth muscle of the organ with expulsion of milk already formed. The depressor substance produces a marked fall of blood-pressure, even when it is injected during the rise of pressure caused by an injection of the pressor

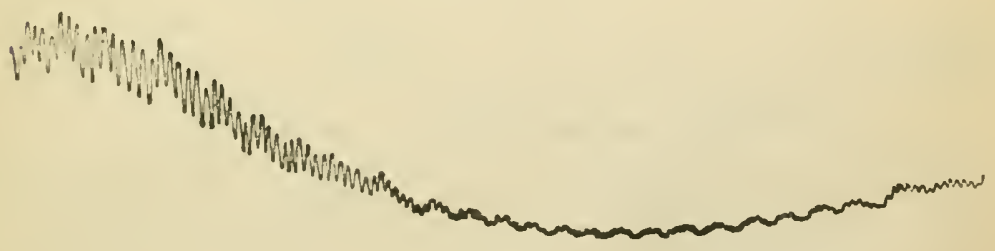

Fig. 2r6.-Action of Extract of $\mathrm{H}_{y}$ pophyseal Lobe of Pituitary on the Blood-Pressure (W. IV. Hamburger). The signal line at the top shows the time and length of injection of the saline extract into the blood. Time-trace (at bottom) shows second intervals. The figure is to be read from left to right.

substance. The anterior lobe, or hypophysis, also contains a depressor substance. Intravenous injection of a saline extract causes a distinct fall of blood-pressure, accompanied usually by acceleration and weakening of the heart (Fig. 2I6). A second injection immediately following the first produces no change in the pressure (W. W. Hamburger). 
An active substance which has been termed 'tcthelin,'* because of its iufluence on erowth, has becu separated from the anterior lobe. On intravenous injection into rabbits, it causes a slight fall of blood pressure and ro diuesis. Its effects are therefore quite different from those

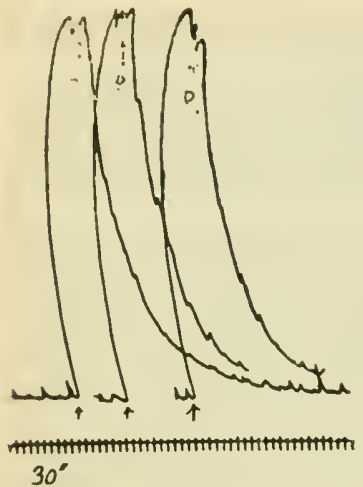

Fig. 217 - - Action of Infundibular lixtract upon Virgin Uterus of Guinea-Pig. The same dose was applied Ihree times in succession to an isolated segment, at the points marked by the arrows at the bottom of the curves. After each application the segment was washed with Ringer's solution at $R$. 'The tracings have bcen made to overlap. (Reduced to onehalf.) (Dale.) elements of the gland, to loss of its internal secretion, or to its manufacture in excessive amount. There is evidence that the colloid secretion of the pusterior lobe, probably formed by the epithelial cells of the pars intermedia, passes through the nervous portion to enter the infundibulum and the third ventricle of the brain, where it breaks down in the cerebro-spinal fluid (Hering). And it has been suggested that in virtue of the action of the hormones (p. 404) in this secretion on the vascular system in general, and on the renal cells and the renal circulation in particular, the posterior lobe constitutes a mechanism for the control of the secretion of urine. But this suggestion is still in the realm of hypothesis. Some support is given to it by the observation that continued irritation of the posterior lobe by a plug of the gutta-percha compound used by dentists for temporarily filling tecth introduced through the flowr of the sella turcica caused permanent polvuria in dogs, analogous to the diabetes insipidus seen in man (Mattlews).

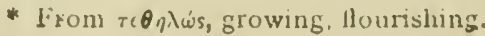


Pineal Gland.- Extracts of the pincal gland injected into the circulation have ro effect other than that due to the inorganic constituents of the calcareous concretions or 'brain sand,' which are its characteristic feature. Since in early life the organ has al glandular structure which is later replacel by fibrous tissue, it has been supposed that it nay exercise some function in connection with growth. But so far the plyysiology of the pineal borly is practically a blank sheet, or, at best, a budget of contradictory statements from which nothing certain can be decluced. Thus in two of the most recent papers, each based on a large number of careful cxperimesis, one author concludes that removal of the gland in male guinea-pigs is associated with hastened development of the sexual organs, and in fenales with a tendency to breed earlier than the normal controls (Horrax). The other observer finds that feeding young guinca-pigs with pineal tissic from young animals determines an carlier sexual maturity than normal, and increases the rate of growth of the body- (11cCord).

The alleged influence of the invasion of the gland in young children by pathological growths in accelerating the development of the skeleton and reproductive organs, which has been supposed to indicate that it normally exerts a restraining or regulative influence on this development, is at present purely fanciful.

Kidney.-The experiments of Bradford, which seemed to indicate that the kidney, in addition to its function as an excretory organ, plays an important, and indeed indispensable, part in protein metabolism, possibly by forming something of the nature of an internal secretion,

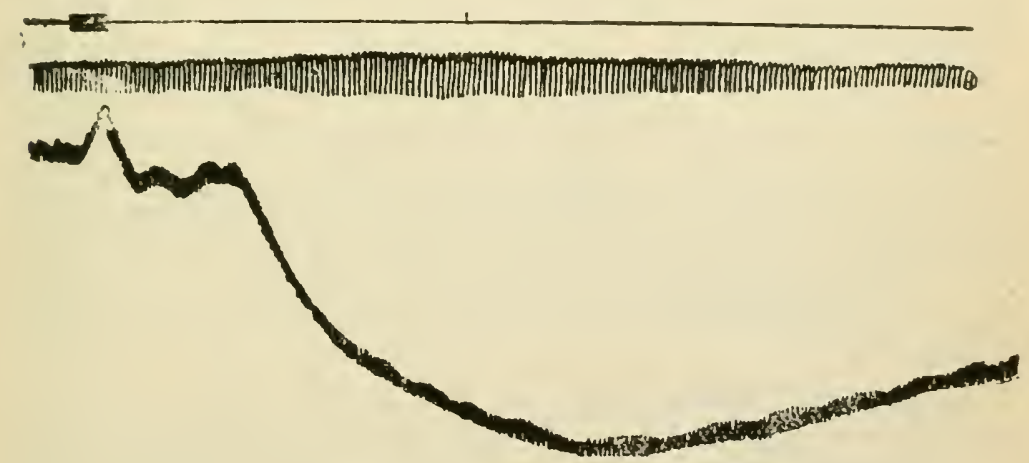

Fig. 2I8.-Effect of Bıne-Mlarrow on Blood-Pressure. Intravenous Injection of Saline Extract. Vagi Intact. The uppermust line is a signal trace showing the time and length of injection. Beluw this is the record of the respirat ry movements, and lowest the blood-pressure tracing. To be read from lett to right.

have not been confimed. He stated that, when the half or two-thirds of one kidney and the whole of the other have been removed from a dog by successive operations, death ensues, although the quantity both of water and urea excreted by the fragment of renal substance that remains is far above the normal. In spite of the increased elimination of urea, that substance was said to accumulate in the tissues, showing that the destruction of protein was increased-a conclusion which seemed to derive support from the wasting of the animal. It has since been shown that an increased output of nitrogen is not of constant occurrence, and only talies place under the same conditions as in starvation (p. 603). As a matter of fict, the animals waste and die 
within a few day' or weeks largely lecallse they refuse to eat. Polyuria (increase of urine beyond the normal) does not necessarily occur. It is well known that when only one kidney is extirpated the other hypertrophies, and no ill-effects ensue.

The statement that extracts of the kidney when injected into the vins of an animal cause a rise of arterial blood-pressure, essentially through direct action on the peripheral vaso-motor mechanism, is of consiclerable interest, for it may possibly have some bearing on the rise of pressure and comsequent hypertrophy of the heart associated with certain renal discalses. But there is nut as yet sufficient evidence that the hypothetical pressor substance, to which the name 'renin' has been given, in any scuse represents an internal secretion of the kidney:

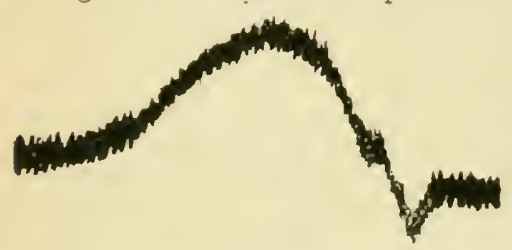
The pressor substance (so-called 'urohypertensine') which can be extracted by ether from normal human urine (Abelous) is probably only excreted by the kiclney, and perhaps arises from the putrefaction of proteins in the intestine. for it lias been shown that in the putrefaction of (horse-) meat bases

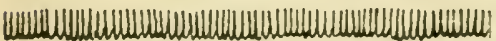
are formed which, when injected intravenously, cause a rise of blood-pressure. The most active Fig. 219.-Injection of Extract of Bone- of these is a body known as IIarrow with the Vagi Cut. To be read $p$-hyclroxy phenylethylamine, from left to right.

formed from tyrosin (Barger and Walpole). Whether the pressor (vaso-constrictor) substance which appears to be liberated from the platelets when blood is shed, and may therefore be presumed to be more slowly liberated from such platelets as normally break down in the circulating blood, has any relation to the pressor substance of urine is unknown. It is also quite uncertain whether, as has been stated by some observers, extracts of the kidney or blood from the renal vein stave off for a time the onset of the uramic symptoms that follow removal of both kidneys or ameliorate them when they have already appeared.

Spleen.-The spleen does not produce an internal sccretion necessary to life, for it can be removed both in animals and in man, without the development of serious symptoms. Its bloodforming and blood-destroying functions (p. 22) are taken on by other structures (particularly the red bone-marrow).

The most definite changes following splenectomy are transient anæmia, increased resistance to hæmolytic agents, and an increase in the content of cholesterol in the blood (Pearce). These effects are more pronounced in the young. This is intelligible if the spleen normally plays a considerable part in the destruction of worn-out erythrocytes with liberation of hamoglobin, the source of the bilepigment. The formation of the bile-pigment is said to be interfered with, and its amount reduced by more than 50 per cent. (Pugliese).

The spleen can be auto-transplanted readily into the subcutaneous tissues of animals. There is a great differenen in the behaviour of 
such transplants arcording to the age of the animal (Marine and Janley). In young rabbits, the transplants grow rapidly if the spleen is removed at the time of transplantation, while in sexually mature rabbits they do not grow and often undergo gradual absorption. Likewise, transplants which have grown rapidly in young animals decrease in size after adult life is reached, indicating that the function of the spleen is more necessary in young than in older animals; or that its function is more easily and completely assumed by otlier tissues, probably the bone marrow, in adults. A further and very suggestive fact, is that a subcutaneous spleen graft which has 'taken,' but is not growing, in a young animal whose spleen lias not been removed, can be made to grow by removal of the spleen. These results indicate that splenic insufficiency is a necessary condition of growth of a splenic transplant, just as thyroid insufficiency is a necessary condition of growth of a thyroid transplant. The stimulus to growth in the one case, as in the other, must be assumed to be a chemical stimulus transmitted through the blood, and not dependent upon the nervous system.

The salivary glands may be extirpated without any sensible change being produced in the normal metabolism. It has been stated however, that the secretion of the gastric juice is diminished. It has been supposed that this may be due to the absence of a hormone (p. $\left.f^{\circ} 4\right)$ normally produced in the salivary glands. A temporary increase in the gastric secretion is said to be caused when extracts of the glands of normal dogs are injected into the veins or into the peritoneal cavity of dogs deprived of their salivary glands (Hemmeter). But later experiments contradict the theory of the existence of a hormone in the salivary glands, which stimulates the secretion of gastric juice. The average rate of secretion into a Pawlow gastric pouch $(\mathrm{p} .403)$ was not diminished in dogs after extirpation of the glands (Swanson.)

Extracts of nervous tissue (sciatic nerve, white matter of brain, and spinal cord, but especially grey matter of brain) cause, on injection into the veins, a decided fall of arterial blood-pressure, which soon passes off, and can be renewed by a frcsh injection. The fall of pressure is due to direct action upon the hloodvessels of a depressor substance in the extracts, and not to the action of vaso-motor nerves. It can be obtained after section of the vagi.

Extracts of muscular tissue also cause a distinct though transient fall of pressure, but not so great a fall as in the case of extracts of nervous tissue. Saline decoctions of other tissues (testis, kidney, spleen, pancreas, liver, mucous membrane of stomach and intestine, lung, and mammary gland) all produce a fall of blood-pressure (Osborne and Vincent). The same is true of bone marrow (Brown and Guthrie; Figs. 218, 219). It must be repeated that there is no evidence that these depressor substances are specific internal secretions in the same sense as epinephrin. 


\section{CHAPTER XII}

\section{ANIMAL HEAT}

From the earliest ages it must have been noticed that the bodies of many animals, and particularly of men, are warmer than the air and than most objects around them. The 'vulgar opinion' of Bacon's time, 'that fishes are the least warm internally, and birds the most,' if it does not imply a very extensive knowledge of animal temperature, at least shows that the fundamental distinction of warm and cold-blooded animals, which is to-day more accurately expressed as the distinction between animals of constant temperature (homoiothermal) and animals of variable temperature (poikilothermal), had been grasped, and was even popularly known. Since that time the accumulation of accurate numerical results, and the advance of physical and physiological doctrine, have given us definite ideas as to the relation of animal heat to the metabolic processes of the body. It is impossible to understand the present position of the subject without an elementary knowledge of the science of heat. For this the student is referred to a textbook of physics. All that can be done here is to preface the physiological portion of the subject by a few remarks on the physical methods and instruments employed:

\section{Section I.-Thermometry and Calorimetry.}

Temperature.-Two bodies are at the same temperature if, when placed in contact, no exchange of heat takes place between them. They are at different temperatures if, on the whole, heat passes from one to the other, and that body from which the heat passes is at the higher temperature. It is known by experiment that if two bodies of different temperature are placed in contact, heat will pass from one to the other till they come to have the same temperature. If, then, we have the means of finding out the temperature of any one body, we can arrive at the temperature of any other by placing the two in contact for a sufficiently long time, under the proviso that the quantity of heat necessary to bring the temperature of the first body, which may be called the 'measuring' body', to equality with that of the second is so small as not to make a sensible difference in the latter. This is the principle on which thermometric measurements depend. A mercurial thermometer consists of a quantity of mercury ordinarily contained in a thin glass bulb, the cavity of which is continued into a tube of very 
fine bore in the stem. Like nost other substances, mercury expands when the temperature rises, and contracts when it sinks, and the amount of expansion or contraction is shown by the rise or fall of the inercurial column in the stem of the thermometer. The point at which the meniscus stands when the bulb is immersed in melting ice or ice-cold water is, on the centigrade scale, taken as zero; the point at which it stands when the thermometer is surrounded by the steam rising from a vessel of boiling water is taken as roo degrees. The intermediate portion of the stem is divided into degrees and fractions of degrees. When, now, we measure the temperature of any part of an animal with such a thermometer, we place the bulb in contact with the part until the mercury has ceased to rise or fall. We know then that the mercury has ceased to expand or contract, and therefore that its temperature is stationary, and presumably the same as that of the part. It is to be noted that we have gained no information whatever as to the amount of heat in tlie body of the animal. We have only observed that the mercury of the thermometer when its temperature is the same as that of the given part expands to an extent marked by the division of the scale at which the column is stationary. And we know that if the mercury rises to the same point when the thermometer is applied to another part, the temperature of the latter is the same as that of the first part; if the mercury rises higher, the temperature is greater; if not so high, it is less. The thermometer, then, only informs us whether heat would flow from or into the part with which it is in contact if the part were placed in thermal connection with any other body of which the temperature is known. In other words, the temperature is a measure of the heat 'tension,' so to speak; and difference of temperature between two bodies is analogous to difference of potential between the poles of a voltaic cell (p. 724), or to difference of level between the surface of a mill-pond and the race below the wheel.

The temperature of an animal is measured in one of the natural cavities, as the rectum, vagina, mouth, or external ear, or in the axilla, or at any part of the skin. For the cavities a mercury thermometer is nearly always used; the ordinary little maximum thermometer is most convenient for clinical purposes. The temperature of the skin may be measured by an ordinary mercury thermometer, the outer portion of the bulb of which is covered by' some badly conducting material. An uncovered thermometer, heated nearly to the temperature expected, will also give results sufficiently accurate for most purposes, especially if the bulb is flat or in the form of a flat spiral, which can be easily applied to the surface. A theoretically better method, but more laborious in practice, is the use of a thermo-electric junction, or a resistance thermometer formed of a grating cut out of thin lead-paper or tirfoil (Fig. 220). This is especially useful for comparing the temperature of two portions of skin. The temperature of the solid tissues and liquids of the body may also be measured or compared by the insertion of mercurial or resistance thermometers or thermo-electric junctions ( $p$. 764).

Calorimetry. - The quantity of heat given off by an animal is generally measured by the rise of temperature which it produces in a known mass of some standard substance. Sometimes, however, as in the icecalorimeter of Lavoisier and Laplace, and the ether calorimeter of Rosenthal, a physical change of state-in the one case liquefaction of ice, in the other evaporation of ether-is taken as token and measure of heat received by the measuring substance, the number of units of heat corresponding to liquefaction of unit mass of ice or evaporation of unit mass of ether being known. The unit generally adopted in the measurement of heat is the quantity required to raise the temperature 
of a kilogramme of water $\mathrm{I}^{\circ} \mathrm{C}$., which is called a caloric, ${ }^{*}$ or kilocalorie or large calorie. The thousandth part of this, the quantity neederl to raise the temperature of a gramme of water by $I^{\circ}$, is termed a small calorie or millicaloric or gramme-caloric.

In the calorimeters which have been chiefly used in physiology either water or atir lias been taken as the measuring substance. The simplest form of water calorimeter is a box with double walls, the space between which is filled witl a weiglied quantity of water. The animal is placed inside the ressel, and the temperature of the water noted at the leginning and end of the experiment. Suppose that the quantity of water is $I 0$ lilos, and that the temperature rises $I^{\circ}$ in thirty minutes, then the amount of heat lost by the animal is Io calories in the half-liour, or 480 calories in the twenty-four hours; and if the rectal temperature is unchanged, this will also be the amount of lieat produced.

Here we assume (I) that all the heat lost by the animal has gone to heat the water and none to lieat the metal of the calorimeter; (2) that none has been radiated away from the outer surface of the latter. The first assumption will seldom introduce any sensible error in a prolonged physiological experiment; but it is very easy to determine by a separate observation the water-equivalent of the calorimeter-that is, the quantity of water whose temperature will be raised $I^{\circ}$ by a quantity of heat which just suffices to raise the temperature of the metal by $r^{\circ}$ (p. 721). Then the water-equivalent is added to the quantity of water actually present, and the sum is multiplied by the rise of temperature. If the temperature of the room is constant, as will be approximately the case in a cellar, any error due to interchange of heat between

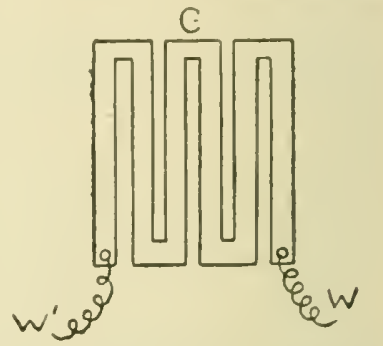

Fig. 220.-Resistance Thermometer for measuring Temperature of Skin. G, grating of lead. yaper, attached to a cover.slip, and mounted on a holder; W. $W^{\prime \prime}$, wires to the Wheatstone's bridge. An increase of tem. perature causes an increase in the resistance of the lead. The balance of the bridge is thus dis. turbed. By experimental grad. uation the temperature value of the deflection, or of the change of resistance that balances it, is known (p. 725).

the calorimeter and its surroundings may be eliminated by making the initial temperature of the water as much less than that of the air as the final temperature exceeds it. Then if the loss of heat by the animal is uniform, as much heat is gained during the first half of the experiment by the calorimeter from the air as is lost by it to the air during the last half. Or, without lowering the temperature of the water, the amount of heat lost by the calorimeter during an experiment may be previously determined by a special observation, and added to the quantity calculated from the observed rise of temperature. Or, finally, two similar calorimeters may be used, one containing the animal and the other a hydrogen flame, or a coil of wire traversed by a voltaic current, which is regulated so as to keep the temperature the same in the two calorimeters. From the quantity of liydrogen burnt, or electricity passed, the heat-production of the animal can be calculated.

In Atwater's great respiration calorimeter (Fig. 22I) both the heat production and the respiratory excliange are measured. The heat produced by the person in the calorimeter is carried away from it by a

* The student should carefully note that when the term 'calorie' is used without qualification, a large caloric, i.e., r,oou gramme-calories, is meant. 
stream of water llowing through the chamber in a series of tubes, the temperature within the calorimeter bejng kept constant by regulating the temperature and velocity of the entering stream of water. The quantity of the escaping water and the incerce in its temperature are measured, and the heat-production can then be calculated. The apparatus consists of a chamber in which a human being can live for several days and nights. A stream of air is supplied, and the chemical changes producel in this are investigated in the manner alreaciy described (p. 2. $\left.f^{\prime \prime}\right)$.

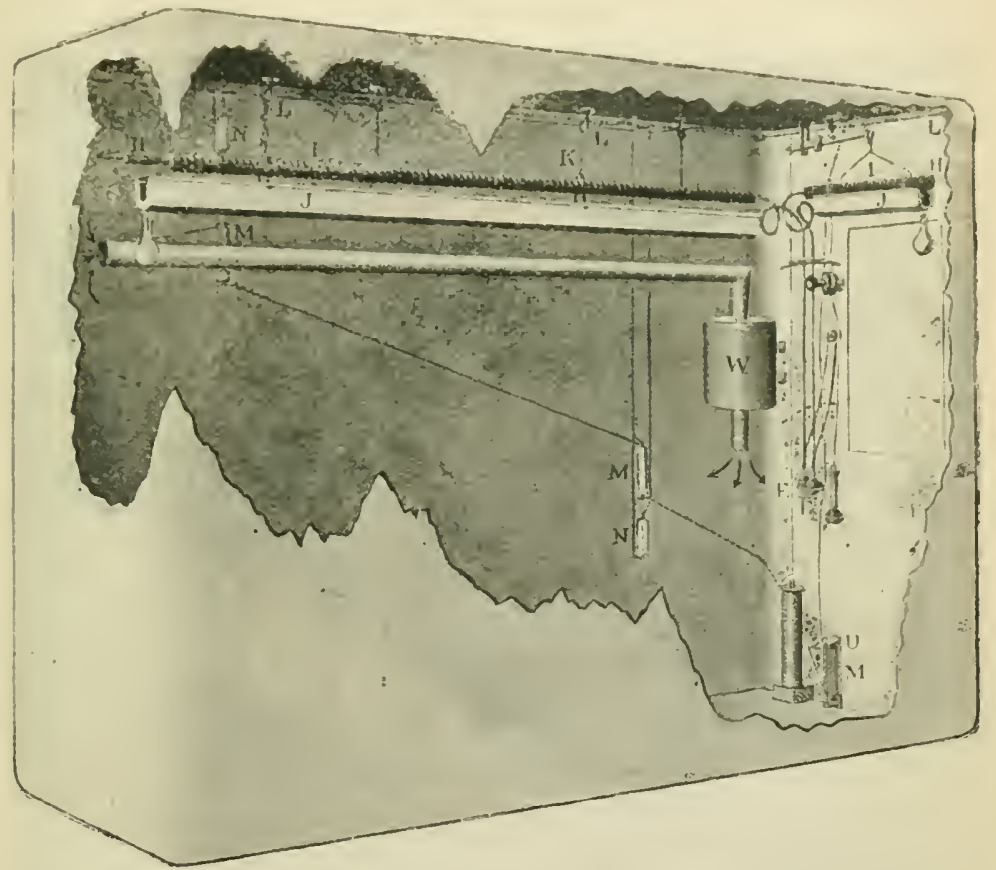

Fig. 221.-Respiration Calorimeter (Atwater). Interior of chamber. A corner ( $\mathbf{f}$ the inner copper wall is supposed to be taken away. The ventilating air-current enters the chamber at the lower end of $\mathrm{W}$, and leaves the chamber through the long tube fastened above $\mathrm{W}$. The copper tubes $\mathrm{H}, \mathrm{H}$ are surrounded by copper dises I, I, fastened on them like a string of beads to increase the surface. These tubes constitute the arrangement through which the stream of water flows which removes the heat formed in the chamber. J, J are copper troughs which receive the water dropping from $\mathrm{H}, \mathrm{H}$. M, MI, M are electrical thermometers which show the temperature of the chamber; $\mathrm{N}, \mathrm{N}$, similar thermometers which show the temperature of the copper wall.

This calorimeter, improved in various ways, has served as the model for all subsequent constructions used for calorimetrical studies in man. . large amount of work of fundamental importance, both on normal and diseased persons, has already been carried out, especially by Benedict and by Lusk with their numerous co-workers, and our quantitative knowledge of the energy transformations in the body has been notably extended. 
All the morlern respiration calorimeters permit the direct estimation not only of the carbon dioxide produced, but also of the oxygen consumed. This allows the amount of heat to be calculated indirectly (so-called indirect calorimetry), as well as measured directly. For if we know how much of the oxygen taken in goes to oxidize protein, fat and carbohydrates, respectively, we can calculate the amount of heat procluced in the body with a given consumption of oxygen. The amount of protein oxidized is easily obtained from the nitrogen excretion. The respiratory quotient (corrected for the influence of the protein metabolized) informs us, with certain limitations, as to the proportions of fat

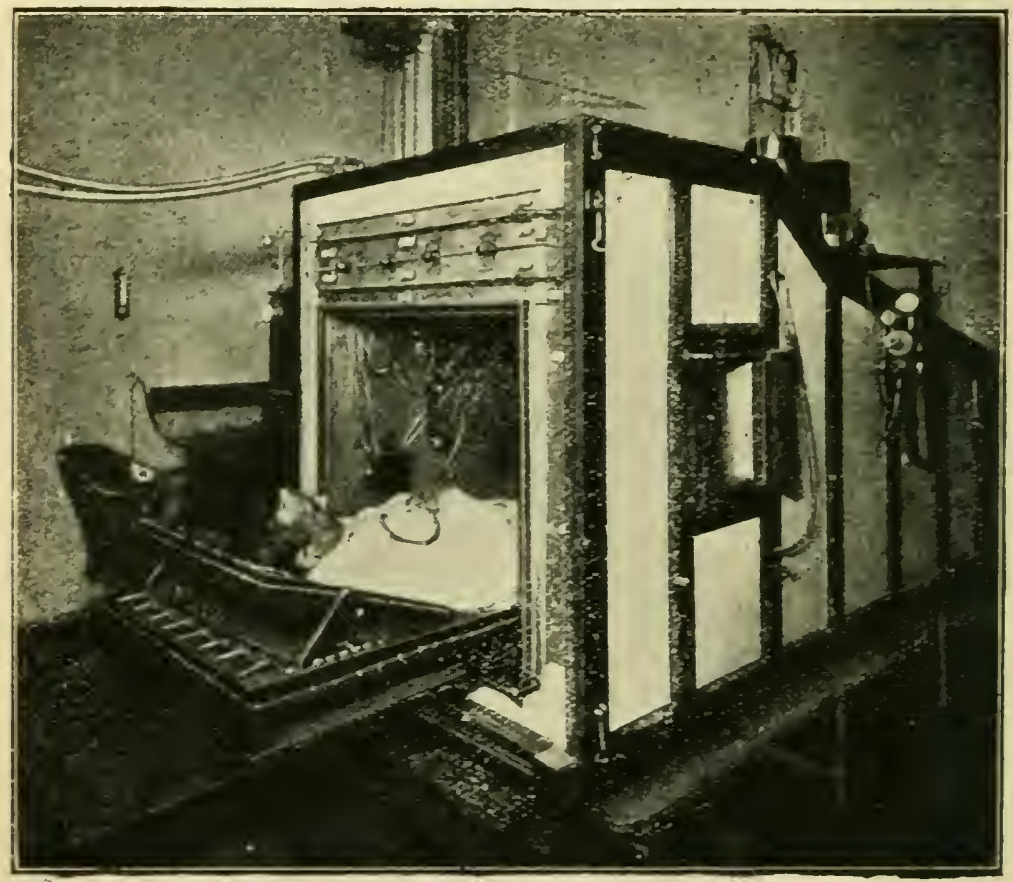

Fig. 222.-Clinical Respiration Calorimeter. The patient is shown on the bed half way in. The tube of a stethoscope strapped over the beart is seen. Coiled up on the wall is the (electric resistance) rectal thermometer not yet inserted. To the right of this is shown a telephone for communlcating with the patient if necessary (Lusk, et al.).

and carbohydrates being consumed. Thus, a respiratory quotient of $0 \cdot 70$ represents the oxidation of fat alone, a quotient of $I$ 'oo the oxidation of carbohydrate alone (p. 24 ).

If the respiratory quotient is intermediate between these extreniese.g., $0.8_{5}$, it would represent the oxidation of fat and carbohydrate in such a proportion that 49 per cent. of the number of calories produced are due to the burning of carbohydrate, and 51 per cent. to the oxidation of fat. With this respiratory quotient, the absorption of I litre of oxygen would indicate that $4, \$ 63$ gram-calories had been produced in the body. In a similar way the amount of heat produced can be calculated, 
although in general with less accuracy, from the amount of carbon dioxide given off. With careful technique, the results obtained by indirect agree closely with those obtained by direct calorimetry, which is tantamount to saying that the law of conservation of energy holds in the animal body (Rubner).

Air calorimeters have sometimes been used for physiological purposes, but at piesent they have little more than historical interest. A diagram of one is shown in Fig. 223. Such calorimeters are really thermometers with an immense radiating surface, for only a small proportion of the heat given off by the animal goes to heat the measuring substance. The heat required to raise the temperature of a litre of air by $\mathrm{I}^{\circ}$ is very small in comparison with that required to raise the tem-

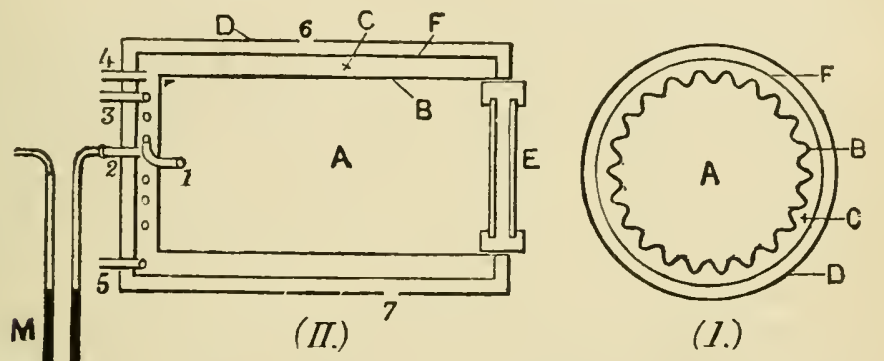

Fig. 223.-Air Calorimeter: (I.) Cross-Section; (II.), Longitudinal Section. A, cavity of calorimeter for animal; B, copper cylinder corrugated so as to increase the radiating surface; $\mathrm{C}$, air space enclosed between $B$ and a concentric copper cylinder $F$; $C$ is air-tight, and is connected by the tube 2 with the manometer $M$. The other end of the manometer is connected with an exactly similar calorimeter, in which a hydrogen flame is burnt in the space corresponding to A, or in which the air in $A$ is heated by a coil of wire traversed by an electrical current. The flame or current is regulated so as to keep the coloured petroleum or mercury in the manometer $\mathrm{M}$ at the same level in both limbs; the amount of heat given off to the one calorimeter by the flame or current is then equal to that given off by the animal to the other. $D$ is an external cylinder of copper or tin perforated by holes $(6,7)$ at intervals. The purpose of it is to prevent draughts from affecting the loss of heat from $F ; 4,5$, are tubes through which thermometers can be introduced into $\mathrm{C}$; I is the terminal of a spiral tube, which is coiled in the end portion of the air space $C$. The sections of the coils are indicated by small circles. The other end of the spiral tube is 3 ; through this tube air is sucked out, and so the proper ventilation of the animal is kept up. The object of the spiral arrangement is that the air aspirated out of A may give up its heat to the air in $\mathrm{C}$ before passing out. $\mathrm{E}$ is a door with double glass walls.

perature of a litre of water by the same amount. Hence a given quantity of heat raises the temperature of an air calorimeter much more than that of a water calorimeter of the same dimensions; and the loss of heat to the surroundings being proportional to the elevation of temperature, in the water calorimeter the chief part of the heat is actually retained in the water, while in an air calorimeter the greater portion passes through the air space, and is radiated away. When the amount of heat lost by the calorimeter becomes equal to that gained from the animal, the 'steady' reading of the instrument is taken, and from this the heat-production can be deduced by an experimental graduation of the apparatus. The one advantage of an air calorimeter is that it follows more closely rapid variations in the heat-production of the 
animal, or, to speate more correctly, in the heat loss. It should be carefully noted that in calorinetry what is directly measured is the quantity of lieat given ont by the animal, not the quantity produced. The two puantities are iclentical only when the temperature of the animal has remained unchanged throughout the experiment. If the temperature has fallen, the quantity of heat produced is equal to the quantity measured by the calorimeter minus the rifference between the quantity in the animal at the beginning and at the end of the observation. This clifference is ecual to the average specific heat of the animal multiplied by its weight and by the fall of temperature. It can be approximately found by multiplying the weight (in kilogrammes or grammes) by the fall of rectal temperature (in degrees), since the average specific heat of the body is not very difficult from that of water, and the specific heat of water is taken as unity.

The differential micro-calorimeter of $A$. V. Hill is the most accurate apparatus for measurısg very small quantities of heat-e.g., the heat given off by resting muscles, or by muscles during the process of heat rigor, or in such reactions as the souring of milk by the lactic acid bacillus. It consists of a pair of Dewar flasks (ordinary therinos or vacuum bottles), in which loss of heat to the surroundings is greatly hindered by exhausting the air from the space enclosed by the double wall of the flask. The bottles are packed in sawdust in cylindrical tins. The tissue or liquid experimented with is put into one flask, and water into the other (control) flask, and the amount of the water is adjusted so that the change of temperature of each flask due to conduction and radiation to or from the outside is the same. Loss of heat is thus automatically eliminated, and from the excess of temperature of the experimental over that of the control flask the heat production in the former can be calculated. The temperatures are measured by thermoelectric junctions of an alloy called constantan and copper, one junction bcing in each flask. To economize time in making such observations, a number nf pairs of flasks can be simultaneously employed. The apparatus is so sensitive that a liberation in ten hours of $I$ grammecalorie per gramme of contents of the flask can be followed and estimated with an error not exceeding 3 per cent.

Body-Temperature.-All the higher animals (mammals and birds) have a practically constant internal temperature (fowl $4 \mathrm{I}^{\circ}$ to $44^{\circ} \mathrm{C}$., mouse $37^{\circ}$ to $38^{\circ}, \operatorname{dog} 38^{\circ}$ to $39^{\circ}$, man $37^{\circ}$ in the rectum), but a few hibernating mammals, such as the marmot, are homoiothermal in summer, poikilothermal during their winter sleep. In the lower forms the body-temperature follows closely the temperature of the environment, and is never very much above it (frog $0.5^{\circ}$ to $2^{\circ}$ above external temperature). Both in a frog and in a pigeon heat is evolved as long as life lasts; lut per unit of weight the amplibian produces far less than the bird, and loses far more readily what it does produce. The temperature of the frog may be $25^{\circ} \mathrm{C}$. in June and $5^{\circ}$ in January. The structure of its tissues is unaltered, and their vitality unimpaired by such violent fluctuations. But it is necessary, not only for health, but even for life, that the internal temperature (the temperature of the blood) of a man should vary only within relatively narrow limits around the mean of $37^{\circ}$ to $38^{\circ} \mathrm{C}$. 
Why it is that a comparatively high temperature sho'ld be needed for the full physiological activity of the tissues of a mammal, while the, in many respects, similar tissues of a fish work perfectly. althougl perhaps more sluggishly, at a much lower temperature, is not quite clear. Nor do we know the precise significance of that relative constancy of temperature in the warm-blooded animal, which is as important and peculiar as its absolute height. The higher animals must possess a superior delicacy of organization, hardly revealed by structure, which makes it necessary that they should be shielded from the shocks and jars of varying temperature that less highly cndowed organisms endure with impunity. T,eaving the discussion of the local differences and periodic variations of the temperature of warm-blooded animals to a future page, let us consider now the mechanism by which the loss of heat is adjusted to its production, so that upon the whole the one balances the other.

\section{Section II.-Income and Expenditure of the Body in Terms OF ENERGY.}

Heat-Loss.-Heat is lost (I) from the surfaces of the body by radiation, conduction, and convection; (2) as latent heat in the watery vapour given off by the skin and lungs; and (3) in the excreta. Even in the bulky excrement of herbivora a comparatively trifling part of the total heat is lost. The second channel of elimination is much more important; the first is in general the most important of all.

The loss of heat by direct radiation from a portion of the skin or clothes, or from hair, fur, or feathers covering the skin, may be measured by means of a thermopile or a resistance radiometer (bolometer). The latter instrument is similar in principle and allied in construction to the resistance thermometer used in measuring superficial temperatures, and already described (Fig. 220, p. 676). It may consist of a grating of lead-paper or tinfoil fixed vertically in a small box which protects it from draughts. The box lias a sliding lid, which is kept closed till the moment of the observation, when it is withdrawn and the portion of skin applied to the opening at a fixed distance ( 5 to $10 \mathrm{~cm}$.) from the grating. The intensity of radiation depends on the excess of temperature of the radiating surface over that of the surroundings, as well as on the nature of the surface. The uncovered parts of the skin (face and hands in man) radiate more per unit of area than the clothes or hair; and the warm forehead more than the comparatively cool lobe of the ear or tip of the nose. When a man is sitting at rest in a still atmosphere, pure radiation plays a greater, and conduction and convection play a smaller, part in the total loss of heat from the skin than when he is walking about or sitting in a draught. The more rapidly the air in contact with the skin and clothes is renewed, the lower, other things being equal, the temperature of the radiating surfaces is kept, the greater is the loss of heat by conduction to the adjacent portions of air, and the smaller the loss by radiation to the walls of the room, the furniture, and other surrounding objects. It is probable that, under 
the most favourable conditions, the amount of heat lost from the surface by true radiation does not exceed the amount lost by conduction and convection.

The loss of heat by evaporation of water from the skin can be calculated if we know the quantity of water so given off. For a gramme of water at the ordinary temperature (say $15^{\circ}$ C.) needs 0.555 calorie to convert it into aqueous vapour at the average temperature of the skin. If we take the average quantity of water excreted as sweat in twentyfour hours as 750 c.c., this will be equivalent to a heat-loss of 4 16.25say, in round numbers, 400 larg 2 calories.

The quantity of heat given off by the lungs may be also deduced from calculation, the data being ( $I$ ) the weight, temperature, and specific heat of the expired air, and (2) the excess of water it contains in the form of aqueous vapour over that contained in the inspired air. Helmholtz calculated the quantity of heat needed to warm the air expired by a man in twenty-four hours from an initial temperature of $20^{\circ}$ to body-temperature, at 70 calories, and that required to evaporate the water given off by the lungs at 397 , making the total heat-loss by the lungs in these processes from 400 to 500 calories. A certain amount of heat is also absorbed in connection with the escape of the carbon dioxide. The reason why a great deal more water and therefore more heat is not given off by the lungs with their enormous surface, and the high degree of imbibition (p. 426) of the epithelium of the alveoli, is

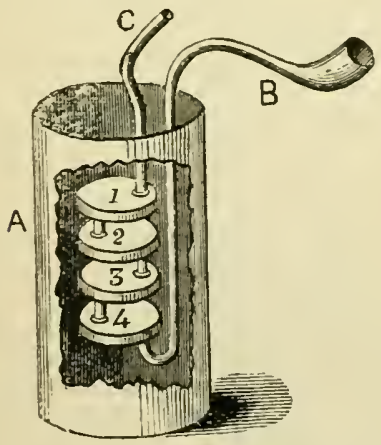

Fig. 224. - Calorimeter for Measuring Heat given off in Respiration. B, copper tube with mouthpiece, connected with the thin brass capsule $4 ; 4$ is connected with a similar capsule 3 by a short tube, which passes out from it at the side opposite to that at which B enters; 2 and $\mathbf{I}$ are similar capsules. From I an outlet tube $C$ passes off. The whole is set in a copper cylinder A filled with water. A piece is supposed to be cut out of $\mathrm{A}$ in order to show the cap. sules. A is placed in another wider copper cylinder.

that the air is already saturated with aqueous vapour, or nearly so, before it reaches the alveoli. By direct calorimetric observations it was found that a man of 70 kilos weight gave off in normal breathing, with an air-temperature of $12^{\circ}$ to $15^{\circ} \mathrm{C}$., from $35^{\circ}$ to $45^{\circ}$ calories. Forced respiration, as might be expected, increased the amount often to double or even treble. A diagram of a calorimeter for measuring the heat given off in respiration is shown in Fig. 224. (Seo Practical Exercises, p. 72I.)

The following table gives an analysis of the heat-loss of an average man. It must be understood that the figures are only approximate. In round numbers we may say that two-thirds of the heat-loss is due to radiation, conduction, and convection, and one-third to the evaporation of water. 


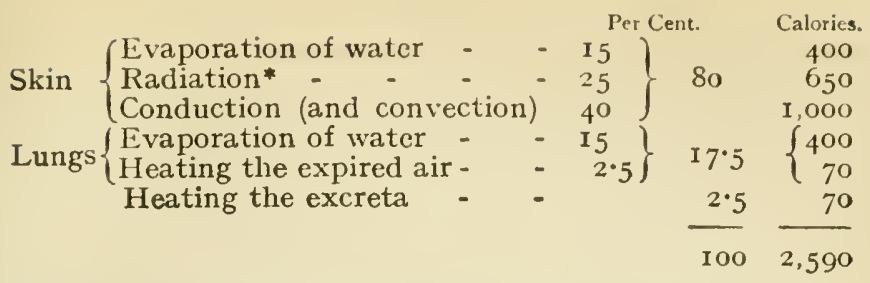

In the rabbit, according to Nebelthau, the heat lost by evaporation of water is about 16 per cent. of the whole, or about half the proportion in man, according to the above calculation. This is not surprising when we reflect that the rabbit does not sweat, and drinks comparatively little. water.

Sources of the Heat of the Body-Heat Production.-Some heat enters the body as such from without - in the food, and by radiation from the sun and from fires. The ultimate source of all the heat produced in the body is the chemical energy of the food substances. For this reason, the distinction between much of the subject matter of this chapter and that of Chapter $\mathrm{X}$. is scarcely even a formal one. Whatever intermediate forms this energy may assumewhether the mechanical energy of muscular contraction; the entrgy of electrical separation by which the currents of the tissues are produced; the energy of the nerve impulse; or the energy, be it what it may, which enables the living cells to perform their chemical labours-it all ultimately, except so far as external mechanical work may be done, appears in the form of heat. As already pointed out (p. 544), the fraction of the total energy liberated in the processes of hydrolytic cleavage is comparatively small. Most of the heat is set free in the oxidative processes which accompany or follow the hydrolytic changes.

Thus the energy-value of a gramme-molecule (p. 426) of maltose, cane-sugar, or lactose is a little more than 1,350 calories; that of the two gramme-molecules of dextrose formed by hydrolysis of the maltose is $1347^{\circ} 4$ calories; that of the gramme-molecule each of dextrose and levulose formed from the cane-sugar, $1349^{\circ} 6$; and that of the grammemolecule each of dextrose and galactose formed from the lactose, $1343^{.6}$ calories. That is to say, the hydrolysis of these disaccharides to monosaccharides, which is the first step in their metabolism, is accomplished with the liberation of very little heat. The same is true of the splitting of the fats and proteins. The dried residue of a filtered pancreatic digest was found to yield, when burned in the calorimetric bomb, only ro per cent. less heat than the same weight of dry meat. Much the greater part of this deficiency was accounted for by the leucin and tyrosin which had crystallized out, and the derivatives of higher fatty acids in the meat, as these would be removed from the digest by filtration.

It has been shown that the law of the conservation of energy holds for the animal body; in other words, there is a practically exact

* The relative amounts lost by radiation and conduction cannot be accurately fixed. The pronortion is extremely variable. 
agreement between the potential energy of the food and the kinetic energy into which it is transformed in the body both during rest and during work. This kinetic energy is represented by the heat given off plus the heat-equivalent of any mechanical work done (Atwater). In other words, the food, whether it is burned in a calorimeter to simple end-products like carbon dioxide and water, or more slowly oxidized in the body, yields the same amount of heat, provided always that in both cases it is entirely consumed, and that no work is iransferred to the outside. In the body the combustion of carbo-hydrates and fats is complete; but the nitrogenous residues of the proteins-urea, uric acid, etc.-can be further oxidized, and the remnant of energy which they yield must be taken into account in any calculation of the total heat-production founded on the heat of combustion of the food substances. Fron, careful experiments, it has been found that a gramme of dry protein (egg-albumin), when burned in a calorimeter, yields 5.735 calories of heat, a gramme of dextrose $377+2$, and a gramme of animal fat 9.500 calories (Stohmann).

\begin{tabular}{|c|c|c|c|c|c|}
\hline Heat-equivalent of $\mathrm{I} g$ & & imin & & - & $5 \cdot 735$ \\
\hline Albumin (minus urea & uc & m it) & - & - & 4.949 \\
\hline Cane-sugar & - & - & - & - & 3.955 \\
\hline Kreatin (water-free) & - & - & - & - & $4 \cdot 275$ \\
\hline Starch & - & - & - & - & I 82 \\
\hline
\end{tabular}

In applying such results to the calculation of the heat-production of the body, it is not sufficient to deduct from the heat of combustion of the proteins the heat which the residual urea would yield if fully oxidized. For other incompletely oxidized products arise from proteins when consumed in the body, and Rubner has shown, by actually determining the heat of combustion of the urine and fæces, that the real equivalent of a gramme of albumin is at most only $+4+20$ calories. The heat-equivalent of our less liberal specimen diet $(p .625)$ will be approximately:

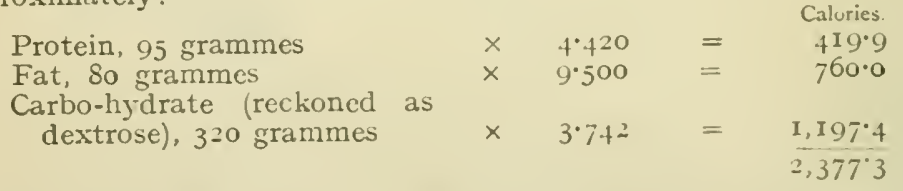

The heat-equivalent of the more generous specimen dict (p.027) would be 2,878 calories.

But this is the diet of a man doing a fair day's work, and to get the quantity of energy" which actually appears as heat, the heat-equivalent of the mechanical work performed must be deducted. A fair day's work is about I 50,000 kilogramme-metres-that is, an amount equal to the raising of $I_{50} 000$ hilogrammes to the heiglit of a metre. Now, a kilogramme-degree or calorie of heat is equivalent to 425.5 kilogramme-metres of work, and a kilogramme-metre to $\frac{I}{425.5}$ calorie. The heat-cquivalent of the day's work is, therefore, 150 , ooo $\times \frac{1}{425.5}=$ 352 calories. Deducting this from the heat-equivalent of the food, 
we get in romud 11 mubers 2,520 large calories as the heat given off on the more liberal cliet. This corresponds fairly well with the calculated lieat-loss (p. 681).

The table on this jagc, based on the direct calorimetric observations of Atwater and Benclict, show's the average heat-production in a large number of experiments ou several individuals at rest and doing measured amounts of work, with a stationary bicycle, for instance. This was connected with a small dynamo, which transformed the greater part of the work into electrical energy. The electrical energy in its turn was changed into heat, the current passing through a lamp.

The heat-production cluring the honrs of sleep, in the second niglit period, is much less than in the waking hours of rest, and of cource enormously less than in the hours of work. After work the lieat production in the period of sleep is only a little greater than after rest.

As already indicated (p. $68_{3}$ ), it is permissible to calculate the heatproduction from the diet, and Rubner has done this for various classes of men, reducing everything to the standard of a body-weight of 67 kilos. The fasting man, of 67 kilos body-weight, produces 2,303 calories in the twenty-four hours. The class of brain-workers, represented by physicians and officials, produce only a little more heat than the fasting man, viz., 2,445 calories. The second class, represented by soldiers (presumably in time of peace) and day-labourers (probably of a cautious and conservative type), work up to 2,868 calories. The third class, composed of men who work with machines and other skilled labourers, attain a heat-production of 3,362 calories. The fourth class, typified by miners (who are engaged, usually by the piece and not by the day, in severe and exhausting toil), produce as much as 4,790 calories. In the fifth and last class, represented by lumberers and other out-of-door labourers (who, in addition to excessive exertion, have often to face intense cold), the heat-production rises to 5,360 calories. The diet of ordinary prisoners in Scotland, doing light work, chiefly of a sedentary character, was found to correspond to $3, I_{5}$, and that of convicts on ' hard labour' to 3,707 calories. It is a fair presumption that in Scotch prisons the total heat value supplied is not excessive. From the general agreement of calculated results with actual measurements we can safely conclude that most healthy adults produce between 2,000 and 3,000 large calories (35 to 40 per kilo of body-weight) on a 'rest' day, or a day of light labour, and between 3,000 and 4,000 (45 to 6o per kilo of body-weight) on a day of hard manual work.

\begin{tabular}{|c|c|c|c|c|c|c|c|c|c|c|}
\hline & \multirow{3}{*}{ 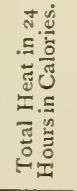 } & \multicolumn{5}{|c|}{ Heat eliminated per Hour. } & \multicolumn{4}{|c|}{$\begin{array}{l}\text { Percentage of Total Heat } \\
\text { in } 24 \text { Hours. }\end{array}$} \\
\hline & & \multicolumn{2}{|c|}{ Day-time. } & \multicolumn{2}{|c|}{ Night-time. } & \multirow{2}{*}{ 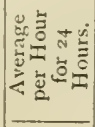 } & \multicolumn{2}{|c|}{ Day-time. } & \multicolumn{2}{|c|}{ Night-time. } \\
\hline & & $\begin{array}{l}7 \text { a.m. } \\
\text { to } \\
\text { r p.m. }\end{array}$ & $\begin{array}{l}\text { I p.m. } \\
\text { to } \\
7 \text { p.m. }\end{array}$ & $\begin{array}{l}7 \text { p.m. } \\
\text { to } \\
\text { I a.m. }\end{array}$ & $\begin{array}{c}\text { r a.m. } \\
\text { to } \\
7 \text { a.m. }\end{array}$ & & $\begin{array}{l}7 \mathrm{a} . \mathrm{m} . \\
\text { to } \\
\text { I p.m. }\end{array}$ & $\begin{array}{l}\text { I p.m. } \\
\text { to } \\
7 \mathrm{p} . \mathrm{m} .\end{array}$ & $\begin{array}{l}7 \mathrm{p} . \mathrm{m} . \\
\text { to } \\
\mathrm{x} \text { a.m. }\end{array}$ & $\begin{array}{l}\text { I a.m. } \\
\text { to } \\
7 \text { a.m. }\end{array}$ \\
\hline $\begin{array}{l}\text { Rest experi- } \\
\text { ments - }\end{array}$ & 2,262 & $106 \cdot 3$ & $104^{\circ} 4$ & $98 \cdot 3$ & $67 \cdot 9$ & $94 \cdot 3$ & $28 \cdot 2$ & 27.7 & $26 \cdot I$ & $18 \cdot 0$ \\
\hline $\begin{array}{l}\text { Work experi- } \\
\text { ments - } \\
\text { Heat-equiva- }\end{array}$ & 4,225 & $23 I \cdot 7$ & $235^{\circ} 6$ & $\operatorname{II} S \cdot I$ & $78 \cdot 4$ & $166 \cdot 6$ & 一 & - & - & 一 \\
\hline $\begin{array}{l}\text { lent of work } \\
\text { Total for work }\end{array}$ & 451 & $5^{8 \cdot 5}$ & $56 \cdot 8$ & - & - & - & 一 & 一 & - & - \\
\hline experiments & 4,676 & $290 \cdot 2$ & $292 \cdot 4$ & IIS I I & $7^{8 \cdot 4}$ & $194 \cdot 8$ & $37 \cdot 5$ & $37 \cdot 2$ & $15 \cdot 2$ & $10 \cdot I$ \\
\hline
\end{tabular}


W7 at has been already said in connection with standard dietaries (p. 624) indicates that the work of the world might possibly be accomplished as well with a smaller transformation of energy in the human machine, at least in the more prosperous countries, and that in the body, as in an engine, more careful 'stoking' might result in a saving of fuel. It is extremely improbable, however, that any argument of this sort will have much effect upon the deep-rooted dietetic habits of mankind.

In any case it must be carefully remembered that the question of the minimum amount of protein necessary in a permanent diet is quite distinct from the question of the minimum heat value of the diet for a man of given body-weight doing a definite amount of work under definite conditions. Whether the protein allowance be scanty or liberal, the total heat value cannot be permanently reduced below a certain minimum depending on the work done, the climate, and other conditions.

Basal Metabolism.-The total metabolism of a normal man or animal as measured by the heat-production is so greatly affected by the taking of food, by the kind as well as the quantity of the food, by the amount and nature of the work done, even by the position of the body, that for many puiposes it is necessary to simplify the conditions in order to disentangle the various factors. The greatest degree of simplification which is practicable is attained when the observations are made upon a subject a considerable time (at least twelve, but better eighteen hours) after the last meal, in the recumbent posture, and in a condition of rest as absolute as possible. The metabolism under those conditions is termed the basal metabolism. In 80 normal men, the basal metabolism, indirectly calculated from the calorific value of the oxygen consumed, was found to vary from 0.8 to 1.3 calories per kilo of body-weight, and from $28^{\circ} 9^{\text {to }} 39^{\circ}$ o calories per square metre of surface per hour (Benedict). The average heat-production per square metre of surface was $34^{\circ} 7$ calories per hour (or calculated on surface measurements made by newer methods, 4o calories per hour). This agrees very closely with the average obtained by direct determination of the heat with the 'bed' calorimeter ( $\mathrm{D}$ u Bois).

Specific Dynamic Action of the Food-Stuffs.-The old idea that the increase in the metabolism above the basal level which follows the ingestion of food is chiefly due to increased work of the alimentary canal in digestion and absorption has been disproved. A large meal of meat given to a dog may cause the heat-production to be nearly doubled. This phenomenon is spoken of as the specific dynamic action of the food-stuffs. It is especially well marked in the case of the proteins. The best evidence is that the action is due to some kind of stimulation of the protoplasm by decomposition products of the amino-acids; in the case of alanin, for instance, probably lactic acid produced in the process of deaminization. It is not merely the oxidation of the aminoacids themselves which is mainly responsible for the increase in metabolism. For it has been found tliat glycoco!l and alanin when fed to phlorhizinized animals, in which they do not yield energy by oxidation, being excreted as sugar and urea, still exert the specific dynamic action, and increase the heat-production (Lusk).

The Seats of Heat-Production.-We have already recognized the skeletal muscles as important seats of heat-production. A frog's muscle, contracting under the most favourable conditions, does not 
convert at most more than one-fourth or one-fifth of the energy it expends into mechanical work; at least three-fourths or four-fifths of the energy appears as heat. The muscies of mammals and of man in the intact body work, upon the whole, more economically than the excised frog's muscles at their maximum efficiency. Under the best conditions-that is, when the work is moderate and not too

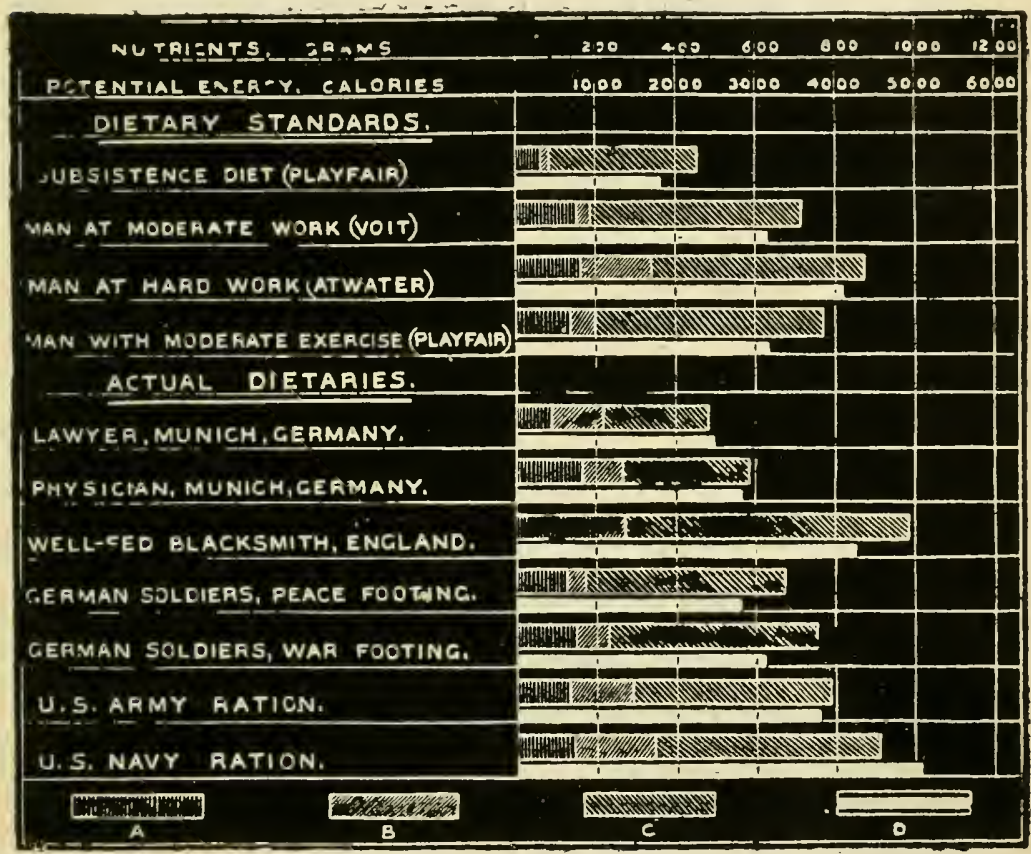

Fig. 225.-Diagram showing the Heat-equivalent of various Dietaries. A, proteins; B, fats; C, carbo-hydrates; D, heat-equivalent.

rapidly done-about one-third of the chemical energy expended may be transformed into mechanical work, and only two-thirds into heat (Zuntz). In hard work three-quarters of the energy may be changed into heat; but even then the-efficiency of the muscles far outstrips that of the best steam-engines, which convert only an eighth of the total energy into work.

Notwithstanding the splendid efficiency of the muscular machine, the gaseous metabolism easily rises during muscular work to five times, and in severe labour to nine times its resting value, although persons inured to toil work more economically than amateurs. In one of Atwater's 'severe work' experiments the work done in twenty-four hours had a heat-equivalent of $I, 482$ calories (equal to 
vier 630,000 kilogramme-metres). The total heat-production (in. cluding the equivalent of the work) was $9,3 I+$ calories. It is not difficult to show that the greater part of the metabolism and heatproduction of a man doing ordinary work is accounted for by the contraction of the voluntary and involuntary muscles.

Even in muscles completely at rest metabolism goes on, and some heat is produced. In resting frogs, newts, and snalies, the rate of heatproduction per gramme of tissue is about 0.5 gramme-caloric per hour with a room temperature of $20^{\circ} \mathrm{C}$., or about a third of that of a resting man (Hill). Of course, this must be above the heat-production of muscles of these poikilothermal animals absolutely at rest. For not only must the active heart and glands contribute something, but the muscles of frogs lying huddled in a micro-calorineter not only maintain the normal tomus, but are certainly liable to contract actively from time to time. By analyzing the gases of the arterial and venous blood, Zuntz compared the oxygen consumption and carbon dioxide production in the hind-legs of dogs when the sciatic and anterior crural nerves were divided and intact. In both cases the muscles were at rest in the ordinary sense. But in the second experiment the central "tonus' (p. 9I7) was preserved, while in the first it was abolished. In one experiment in which the nerves were intact the oxygen consumed amounted to $\mathrm{I} \cdot 22$ c.c., and the carbon dioxide produced to I.32 c.c., per kilo of tissue per minute. In the experiment in which the nerves were severed, the corresponding numbers were $0.68 \mathrm{c.c}$. for the oxygen, and 0.63 c.c. for the carbon dioxide. Although it is probable, from the results of Chauveau and Kauffmann already referred to (p. 27I), that these figures are too low for the normal resting muscle, they still demonstrate that, even in the absence of innervation from the central nervous system, the metabolism, and therefore the heat-production of the muscles, are by no means negligible; 0.68 c.c. of oxygen per minute corresponds to $40.8 \mathrm{c} . \mathrm{c}$. per hour, or more than one-tenth of the oxygen consumption per lisilo per hour of a fasting dog lying at rest (Zuntz).

If the work of the heart is taken as 16.600 kilogramme-metres in twenty-four hours (p. I 38 ), the total heat produced by this organ will be equivalent (on the assumption that it converts one-third of its energy into work) to about 50,00o kilogramme-metres, or not mucl less than 20 calories, since, practically, the whole work is expended in overcoming the friction of the vessels, and finally appears as heat. Enough energy is transformed in twenty-four hours in the heart of the colonel of a regiment of $x, 000$ men to lift the whole regiment to the height of the mess-table, if it could be all changed into mechanical work. Barcroft and Dixon have calculated tlie energy of the heart's contraction on the assumption that it is derived from the oxidation of a carbo-hydrate by the oxygen absorbed by the organ. They concluded that the energy set free in the heart of a dog weighing $x 2$ kilos corresponds on the average to 7.86 kilogramme-metres per minute, which is equivalent to $26 \cdot 6$ calories in twenty-four hours. Allowing for the fact that the heart of a small animal pumps more blood in proportion to the body-weight than the heart of a large animal (p. 139), this result agrees very well with that deduced from the work of the heart. The work of the inspiratory muscles may be reckoned at 13,000 kilogramme-metres, equal to $30^{\circ} 5$ calories, and the heat produced by them at, say, 90 calories. In sum, the muscular work of the circulation and 
respiration is responsible for the production of about 210 calories (without including the heat produced by the smooth muscle of the bronchi and bloodvescels), or nearly one-twelfth of the total production of a man doing ordinary labour.

The glands, and then the central nervous system, rank after the muscles, though at a great distance, as seats of heat-production. The liver and brain (?) are the hottest organs in the body; and that this is not altogether due to their being well protected against loss of heat is shown, in the case of the liver, by the excess of temperature of the blood of the hepatic over that of the portal vein. In view, however, of the exaggerated importance which some have given to these organs as foci of heat-production, it may be well to point out that although many of the chemical changes in the animal body are undoubtedly associated with the setting free of heat (exothermic reactions), other, and not less weighty and characteristic, reactions may cause the absorption of heat (endothermic reactions); and it is possible that some of the syntheses which many of the tissues are capable of performing may be included in this latter category. For example, when urea is decomposed so as to yield ammonium carbonate (p. 478), heat is set free. We must assume that if ammonium carbonate were transformed into urea in the liver, an equal amount of heat would be, on the whole, absorbed. So that the heat-production of an organ may depend, not only upon the quantity, but also upon the quality, of its chemical activity. In all the tissues, including the muscles, it is necessary to assume that some of the energy transformed is expended in so-called ' restitution' processes-that is, in replenishing the store of nutritive material within the cells and in building up the protoplasm. Claude Bernard observed an excess of $0.6^{\circ} \mathrm{C}$. in the temperature of the blood of the hepatic vein over that of the portal during hunger, and as much as $I \cdot 6^{\circ}$ at the height of digestion, although at the beginning of digestion the portal blood was the hotter by $0.4^{\circ}$. But such observations, like the corresponding ones on the salivary glands, are open to many errors, and when we consider the enormous tide of blood which during digestion sets through the portal system, we shall look with suspicion upon results that announce a difference of more than a small fraction of a degree in the temperature of the incoming and outgoing blood of the liver. Probably not less than 200 litres of blood pass in twenty-four hours through the liver of a 2-kilo rabbit. If the temperature of this blood is raised even onetenth of a degree in its passage through the hepatic capillaries, this would correspond to a heat-production of 20,000 small calories, or one-tenth of the whole heat produced in the animal.

In the case of the brain there is some evidence, obtained by com. parison of the gases of blood taken from the carotid and from the venous sinuses (torcula Herophili), that the metabolism is feeble as compared 
even with that of resting muscles (Hill). Nor is it possible to demon. strate any marked or constant increase when the cerebral cortex is roused to such an active discharge of impulses as leads to general epileptiform convulsions. The rise of temperature of certain regions, especially the occipital portion, of the scalp, which some observers have stated to take place during mental activity, cannot be due to conduction of heat from the brain through the skull. It might be caused by vaso-motor changes in the scalp, associated with corresponding changes in related areas of the cortex. The alleged increase in the temperature of the brain during intense psychical activity, sometimes to $0.2^{\circ} \mathrm{C}$, or $0.3^{\circ} \mathrm{C}$. above the rectal temperature (Mosso), may also, if genuine, be due to vascular changes. And if we remember low large a proportion of the central nervous system is made up of nervefibres, in which no sensible production of heat has ever been demonstrated, it will not appear surprising if even a considerable increase in the metabolism of the really active elements should fail to make itself felt.

\section{Section III.-Regulation of Temperature or Thermotaxis.}

What, now, is the mechanism by which the balance is maintained in the liomoiothermal animal between heat-production and heatloss? In answering this question we have to recognize that both of these quantities are variable, that a fall in the production of heat way be compensated by a diminution of heat-loss, and an increase in the loss of heat balanced by a greater heat-production.

The loss of heat from the surfaces of the body may be regulated both by involuntary and by voluntary means. It is greatly affected by the state of the cutancous vessels, and these vessels are under the influence of nerves. A cold skin is pale, and its vessels are contracted. In a warm atmosphere the skin is flushed with blood, its vessels are dilated, its temperature is increased; an effort, so to speak, is being made by the organism to maintain the difference of temperature between its surface and its surroundings on which the rate of heat-loss by radiation and conduction depends. A still more important factor in man, and in animals like the horse, which sweat over their whole surface, is the increase and decrease in the quantity of water evaporated and of heat rendered latent. It is owing to the wonderful elasticity of the sweat-secreting mechanisin, and to the increase of respiratory activity and the consequent increase in the amount of watery vapour given off by the lungs, that men are able to endure for days an atmosphere hotter than the blood, and even for a short time a temperature above that of boiling water. The temperature of a Turkish bath may be as high as $65^{\circ}$ to $80^{\circ} \mathrm{C}$. Blagden and Fordyce exposed themselves for a few minutes to a temperature of nearly $127^{\circ} \mathrm{C}$. Although meat was being cooked in the same chamber by the heat of the air, they cxperienced no ill-effects, nor was their body-temperature even increased. But a far lower temperature than this, if long con- 
tinued, is dangerous to life. During the 'hot waves' not infrequently experienced in summer in the United States, hundreds of persons have died within a few days from the excessive heat. It is stated that during the unusually hot summer of 1819 the temperature at Bagdad ranged for a considerable time between $108^{\circ}$ and $\mathrm{I} 20^{\circ} \mathrm{F}$. $\left(42^{\circ}\right.$ to $49^{\circ} \mathrm{C}$.), and there was great mortality. A much higher temperature may be borne in dry air than in air saturated with watery vapour. A shade temperature of $100^{\circ} \mathrm{F} .\left(37^{\circ} 7^{\circ} \mathrm{C}\right.$.) in the dry air of the South African plateaux is quite tolerable, while a temperature of $85^{\circ} \mathrm{F} .\left(29.4^{\circ} \mathrm{C}\right.$.) in the moisture-laden atmosphere of Bombay may be oppressive. The reason is that in dry air the sweat evaporates freely and cools the skin, while in moist air, although according to Rubner the loss of heat by radiation and conduction is increased, the loss of heat by evaporation of sweat is diminished in a still greater degree. In saturated air at the bodytemperature no loss of heat by perspiration or by evaporation from the pulmonary surface is possible; the temperature of an animal in a saturated atmosphere at $35^{\circ}$ to $40^{\circ} \mathrm{C}$. soon rises, and the animal dies. In animals like the dog; which sweat little or not at all on the general surface, the regulation of the heat-loss by respiration is relatively more important than in man.

The observations of Boycott and Haldane in a deep mine, in the incubating-room of a laboratory, and in a Turkish bath illustrate the important influence of the humidity of the air. In still air the bodytemperature rose above normal when the wet-bulb thermometer rose above $3 \mathrm{I}^{\circ} \mathrm{C} .\left(88^{\circ} \mathrm{F}.\right)$, and it remained normal whatever the external temperature might be so long as the reading of the wet-bulb thermometer did not exceed that level. The more the wet-bulb thermometer rose above $3 \mathrm{I}^{\circ}$ the more rapid was the increase in the body-temperature. In moving air a greater degree of humidity could be borne without increase in the body-temperature, which did not occur till the temperature shown by the wet-bulb thermometer exceeded $35^{\circ} \mathrm{C}$. The great increase in the evaporation of sweat when the temperature of the air is high is shown by the observation that on a warm day (dry bulb, $79^{\circ} \mathrm{F}$.; wet bult, $67^{\circ} 5^{\circ} \mathrm{F}$.) the average loss of moisture from the body was I,8I6 grammes for four soldiers during a march of seven miles, while on a cold day (dry bulb, $45^{\circ} \mathrm{F}$.; wet bulb, $3^{\circ} \mathrm{F}$.) it was only 4I9 grammes during the same march by the same men (Pembrey).

The winter fur of Arctic animals is a special device of Nature to meet the demands of a rigorous climate, and combat a tendency to excessive loss of heat. The experiments of Hösslin, and the experience of squatters in Australia, go to show that even domesticated animals have a certain power of responding to long-continued changes in external temperature by changes in the radiating surfaces which affect the loss of heat. It is said that in the hot plains of Queensland and New South Wales the fleeces of the sheep show a tendency to a progressive decrease in weight. And Hösslin found that a young dog exposed for eighty-eight days to a temperature 
of $5^{\circ} \mathrm{C}$. developed a thick coat of fine woolly hairs. Another dog of the same litter, exposed for the same length of time to a temperature of $31.5^{\circ}$ to $32^{\circ} \mathrm{C}$, had a much scantier covering. The increased protection against heat-loss in the case of the 'cooled' dog was not sufficient fully to compensate for the lowered external temperature. The metabolism-that is to say, the heat-production - was also increased. And although the food was exactly the same for both animals in quantity and quality, the dog at $5^{\circ} \mathrm{C}$. put on less than half as much fat in the period of the experiment as the ' heated ' dog, but the same amount of 'flesh.'

The voluntary factor in the regulation of the heat-loss is of great importance in man. Clothes, like hair and other natural coverings, retald the loss of heat from the skin chiefly by maintaining a zone of still air in contact with it, for air at rest is an exceedingly bad conductor of heat. A man clothed in the ordinary way has two or three concentric air-jackets around him. The air in the intervals between the inner and outer garments is of importance as well as that in the pores of the clothes themselves; and it is for this reasons that two thin shirts put on one above the other are warmer than the same amount of material in the form of a single shirt of double thickness. When a man feels himself too hot and throws off his coat, he really removes one of the badly conducting layers of air, and increases the rate of leat-loss by radiation and conduction. At the same time the water-vapour, which practically saturates the layer of air next the shin, is allowed a freer access to the surface, and the loss of heat by the evaporation of the sweat becomes greater. The power of voluntarily influencing the heat-loss must be looked upon in man as one of the most important means by which the equilibrium of temperature is maintained. In the lower animals this powe also exists, but to a much smaller extent. A dog on a hot day puts out its tongue and stretches its limbs so as to increase the surface from which heat is radiated and conducted. The mere placing of a rabbit on its back, with its legs apart, may cause in an hour or two a fall of $\mathrm{I}^{\circ}$ to $2^{\circ} \mathrm{C}$. in the rectal temperature. The power of covering themselves with straw or leaves, of burrowing and of forming nests, may be included among the voluntary means of regulation of the heat-loss possessed by animals. A man opens the window when he is too hot, and pokes the fire when he feels cold. Both actions are a tribute to his status as a homoiothermal animal, and illustrate the importance of the voluntary element in the mechanism by which his temperature is controlled.

The production of heat, like the loss, is to a certain extent under voluntary control. Rest, and especially sleep, lessen the production; work increases it. The inhabitants of the tropics, human and brute, often tide over the hottest part of the day by a siesta; and it is as natural, and as much in accordance with physiological 
laws, that a man overpowered by the heat should lie down, as it is that he should walk about and stamp his feet or clap his hands on a cold winter morning. In the one case a diminution, in the other an increase, in the heat-production is aimed at by a corresponding change in the amount of muscular contraction. The quantity and quality of the food also influence the production of heat. The Eskimo, who revels in train-oil and tallow-candles, unconsciously illustrates the experimental fact that the heat of combustion of fat is high; the rice diet of the ryot of the Carnatic, with its low heatequivalent, seems peculiarly adapted to the dweller in tropical lands. But it would be easy to attach too much weight to considerations such as these. The Arctic hunter eats animal fat, and the Indian peasant vegetable carbo-hydrate, not only because fat has a high and carbo-hydrate a low heat-equivalent, but because in the climate of the Far North animals with a thick coating of badly-conducting fat are plentiful, and vegetable food scarce; whereas in the river-valleys of India Nature favours the growth of rice, and religion forbids the killing of the sacred cow.

The production of heat is also controlled by an involuntary nervous mechanism, through which the 'chemical ' regulation of the bodytemperature is achieved, as the 'physical' regulation is accomplished by the nervous mechanisms that control the circulation, the sweat-glands, and the respiratory movements. It is a matter of everyday experience that cold causes involuntary shiveringinvoluntary muscular contractions-the object of which seems to be a direct increase in the heat-production. But besides this visible mechanical effect, the application of cold to a warm-blooded animal, when not carried so far as to greatly reduce the rectal temperature, is accompanied by a marked increase in the metabolism, as shown by an increased production of carbon dioxide and consumption of oxygen. In cold-blooded animals like the frog the metabolism, on the other hand, rises and falls with the external temperature; there is no automatic mechanism which answers an increased drain upon the stock of heat in the body by an increased supply. Or, in the light of recent experiments, we ought rather to say that, although the rudiments of a heat-regulating mechanism may exist in such animals as the frog, the newt, and even the earthworm (Vernon), it is only able to modify to a certain extent the effects of changes of external temperature, not to balance or even override them, as in the homoiothermal animal. In resting frogs and snakes the rate of heat-production, as measured by the micro-calorimeter, increases two to three times when the temperature rises $10^{\circ} \mathrm{C}$. (Hiil). The warm-blooded animal loses its heat-regulating power when a dose of curara sufficient to paralyze the voluntary muscles is given. A curarized rabbit, kept alive by artificial respiration, reacts to changes of external temperature like the cold-blooded frog. Now, the only action of curara adequate to account for this 
effect is its power of paralyzing the motor innervation, and so cutting off from the skeletal muscles impulses which in the intact animal would have reached them. The excitation by cold of the cutaneous nerves, or some of them, which in the unpoisoned animal is reflected along the motor nerves to the muscles, and causes the increase of metabolism, is now blocked at the end of the motor path; and the muscles, the great heat-producing tissues, are abandoned to the direct influence of the external temperature (Pflüger).

How is it, then, that nervous impulses from the skin produce in the intact animal their effect upon the chemical processes in the muscles? We know that the heat-production of a muscle is greatly increased when it is caused to contract; but it has not hitherto been possible by artificial stimulation to demonstrate that any chemical or physical effect is produced in a muscle by excitation of its motor nerve unless as the accompaniment of a mechanical change. When the gastrocnemius of a frog poisoned with not too large a dose of curara is laid on a resistance thermometer (p. 783), and its nerve stimulated from time to time as the curara paralysis deepens, heating of the muscle is observed as long as, and only as long as, there is any visible contraction. The gaseous metabolism of a rabbit immersed in a bath of constant temperature may sink by as much as 30 to 40 per cent. when curara is given. One obvious cause of this is the complete muscular relaxation. And the whole secret of the regulation of the heat-production might be plausibly supposed to lie in the bracing effect of cold upon the skeletal muscles and the relaxing effect of heat. Indeed, in man it has been observed that exposure to moderate cold causes no metabolic increase when shivering is prevented by a strong effort of the will (Loewy). Nevertheless, the explanation is inadequate in the case of small animals, such as guinea-pigs, rabbits, and cats; for very great changes in the metabolism may be brought about by external cold without any outward token of increased muscular activity. In a man also a fall in the external temperature from $23^{\circ}$ to $15^{\circ} \mathrm{C}$. caused a certain increase in the output of carbon dioxide (from 27.9 to $32 \cdot 3$ grammes per hour), although no shivering was observed. As the temperature of the air is lowered, the point is soon reached at which shivering can no longer be suppressed, and then it is neither practicable nor perhaps very important to distinguish clearly the portion of the increased heat-production associated with the visible muscular contractions and the portion due to quickened muscular metabolism without contraction. Lefèvre found that in man a marked increase in the heat-loss, such as is caused by immersion for a considerable time (one to three hours) in cold water (at a temperature of $7^{\circ}$ to $15^{\circ} \mathrm{C}$.), was accompanied by a great increase in the production of heat, so that the axillary temperature fell comparatively little-e.g., only $\mathrm{I}^{\circ} \mathrm{C}$. during a stay of three hours in a bath at $15^{\circ} \mathrm{C}$. With short periods of immersion, a characteristic 
reaction occurs after the person comes out of the bath. The rectal temperature falls to a minimum, which is reached in twenty to thirty minutes after exit from the bath, and then gradually returns to normal. This fall of internal temperature is due to the heating of the superficial portions of the body at the expense of the central portions. By training, the fall of temperature is greatly lessened, the heat-regulating mechanism acquiring, so to speak, with practice, greater promptitude and precision of adjustment.

It must be admitted, then, that the metabolic changes normally. going on in the resting muscles may be reflexly increased-especially. in the smaller homoio-thermal animals - without the usual accompaniment of mechanical contraction, ${ }^{*}$ and that such an increase of 'chemical tone' is an important means by which the temperature is regulated. It is possible that other organs besides the muscles may be concerned, though not to a sufficient extent to secure the due regulation of temperature during curara paralysis. It is obvious that in man, whose environment is so much under his own control, a mere automatic regulation is less required than in the inferior animals, and that a regulative power, if present in rudiment, would tend to ' atrophy ' by disuse, or, at all events, to become less sensitive to slight changes of temperature. In the larger animals. again. mere bulk is an important safeguard against any sudden change of internal temperature. To reduce the temperature of a horse or an elephant by $\mathrm{I}^{\circ}$, a considerable quantity of heat must be lost, while a very slight loss would suffice to cool a mouse by that amount. Not only so, but the surface by which heat is lost is greater in proportion to the mass of the body in small than in large "nimals. The power of rapidly increasing the heat-production to meet a sudden demand is, therefore, far more important to the mouse than to the horse; and the fact (p. 628) that the metabolism of an animal varies approximately as its surface, and not as its mass, $\uparrow$ is an illustration of the nice adjustment by which heat-

* Increased tonus of the muscles might, however, account for a portion of the increased heat-production.

$\dagger$ The relation between mass (M) and surface (S) in man is approximately given by the equation $\frac{S \cdot \sqrt[3]{M}}{M}=K$, and the relation between surface, mass, length of body (L), and circumference of chest (C) just above the nipples in the 'mean' position of respiration, by the equation $\frac{S \sqrt[6]{M^{4} \cdot L^{4} \cdot C^{2}}}{M L C}=K^{\prime} . M$ is expressed in grammes, $\mathrm{S}$ in square centimetres, $\mathrm{L}$ and $\mathrm{C}$ in centimetres. $\mathrm{K}$ is a constant whose mean value is $12 \cdot 3$, and $K^{\prime}$ a constant whose mean value is 4.5 (Meeh).

It has been shown that this formula gives somewhat too high results. In any case, a formula of this nature is accurate only for objects of similar shape. Many other formulæ have been proposed. A more accurate 'height-weight' formula is $\mathrm{A}=\mathrm{W}^{0.425} \times \mathrm{H}^{0.725} \times \mathrm{C}$, where $\mathrm{A}$ is the surface area in square centimetres, W, the weight in kilograms, and $\mathrm{C}$, a constant $7 \mathrm{I} \cdot 84$. Another method is based on the principle of multiplying the length by the average cir. 
equilibrium is maintained. There is reason to believe that at a temperature equal to that of the human body, the heat-production of a frog per unit of mass would equal that of a man or other large mammal, although it would be far less than that of a small homoiothermal animal. This is in favour of the view that in the larger mammals a nervous regulation of the intensity of the metabolism is not of prime importance.

Relations between Heat-Production, Surface Area, and Blood-Flow.The following table slows how close is the agreement in the heatproduction per unit of surface for animals of different species and very different body-weight:

\begin{tabular}{|c|c|c|c|c|c|c|c|}
\hline & & & & & \multirow{2}{*}{$\begin{array}{l}\text { Weight in } \\
\text { Kilos. }\end{array}$} & \multicolumn{2}{|c|}{ Calories produced in 24 Hours. } \\
\hline & & & & & & Per Kilo. & $\begin{array}{l}\text { Per Sq. Metre } \\
\text { of Surface. }\end{array}$ \\
\hline Horse & - & - & - & - & $44^{\mathrm{I}}$ & II $\cdot 3$ & $94^{8}$ \\
\hline Man & - & - & - & - & $6+3$ & $32 \cdot I$ & 1,042 \\
\hline Dog & - & - & - & - & $15^{\circ} 2$ & $5 I \cdot 5$ & 1,036 \\
\hline Rabbit & (withou & ears) & - & - & $2 \cdot 3$ & $75^{\circ} \mathrm{I}$ & 917 \\
\hline Fowl & - & - & - & - & 2 & $7 \mathrm{I} \cdot 0$ & 943 \\
\hline Mouse & - & - & - & - & $0 \cdot 018$ & $212 \cdot 0$ & I, I 88 \\
\hline
\end{tabular}

The relation between heat-production and surface area has been reinvestigated by modern methods. Some observers, notably Benedict, are inclined to deny that any simple proportion exists between the basal metabolism and the area of the skin. They consider that the magnitude of the metabolism is related rather to the mass of active protoplasm, which is a different quantity from the weight of the body, the fatty tissues-e.g., contributing largely to the body-weight, but little to the netabolism. On the other hand, Lusk, Dn Bois, and their fellow-workers, believe that when the surface area is accurately calculated there is no reason to doubt the hitherto accepted view of a proportionality betwcen heat-production and area of surface. It must be remembered that it is only for subject.s in the same general physiological state that the proportionality is supposed to hold. Nobody imagines, for instance, that a patient with exophthalmic goitre will have the same heat-production per unit of surface as a normal individual. Nor can an infant be cxpected to be exactly comparable with an adult, since it cliffers so much not only in the proportions of the body and the relative size of the various organs, but also in its metabolism. It has been found, as a matter of fact, that the heat-produc-

cumference or width of each of the characteristic parts of the body. Constants have been worked out for each part by comparison of the actual area of a mould applied to the skin with the data obtained by the measurements. This so-called 'linear formula' does not involve the body-weight, and gives good results with bodies of very different size and shape (Du Bois). Benedict has also developed a photographic method of obtaining the surface area. The main interest of such measurements lies in their bearing upon the relation between the metabolism and the body-surface. 
tion per square metre of body-surface, which is low at birth, increases rapidly during the first y'ar, reaches a maximum between the ages of one and six, then falls steeply until the age of twenty, and thereafter very slowly. The lasal metabolism of boys of twelve or thirtcen years old per unit of surface is 25 per cent. higher than that of the adult.

The next table, calculated by Rubner from the quantity of tissueprotein and fat consumed, gives the relative intensity of heat-production in fasting logs of different sizes: and along with it is given for comparison some of the results on the output of the heart in logs of different weight, obtained by the method of injecting salt solution into the ventricle described on p. 139.

\begin{tabular}{|c|c|c|c|}
\hline $\begin{array}{l}\text { Weight in } \\
\text { Kilos. }\end{array}$ & $\begin{array}{c}\text { Calories per Kilo } \\
\text { per Hour. }\end{array}$ & Weight in Kilos. & $\begin{array}{l}\text { Output per Kilo } \\
\text { per Second in c.c. }\end{array}$ \\
\hline $3 I$ & I. $5^{8}$ & $27 \cdot 89$ & $I \cdot 92$ \\
\hline 24 & $\mathrm{I} \cdot 70$ & I $8 \cdot 20$ & $2 \cdot 3 \mathrm{I}$ \\
\hline 20 & I. 87 & I 2.82 & $3 \cdot 28$ \\
\hline I 8 & I. 92 & I I 68 & $3 \cdot 56$ \\
\hline Io & $2 \cdot 55$ & $10 \cdot 32$ & 3.86 \\
\hline 6 & $2 \cdot 84$ & $7^{\cdot 16} 6_{5}$ & $4^{\circ} 13$ \\
\hline 3 & 3.78 & $4^{\circ} 975$ & $5 \cdot 36$ \\
\hline
\end{tabular}

\begin{tabular}{|c|c|c|c|c|}
\hline $\begin{array}{l}\text { Number of } \\
\text { Dugs. }\end{array}$ & $\begin{array}{c}\text { Range of Weight in } \\
\text { Kilos. }\end{array}$ & Average Weight. & $\begin{array}{l}\text { Average Output } \\
\text { per Second in c.c. }\end{array}$ & $\begin{array}{l}\text { Average Output } \\
\text { per Kilo per } \\
\text { Second. }\end{array}$ \\
\hline 3 & $34^{\circ} 5$ to $27^{\circ} 9$ & $3 I \cdot 5$ & $75 \cdot 7$ & $2 \cdot 40$ \\
\hline 8 & I $8 \cdot 2,$, I I.7 & $I_{4} \cdot 3$ & $46 \cdot 6$ & $3 \cdot 25$ \\
\hline 3 & $10 \cdot 3,9 \cdot 3$ & $9 \cdot 8$ & $34^{\cdot} 8$ & $3 \cdot 55$ \\
\hline 2 & $8 \cdot 4,, \quad 7 \cdot 2$ & $7 \cdot 8$ & $28 \cdot 8$ & $3 \cdot 69$ \\
\hline 2 & $6 \cdot 5, \quad 5^{\circ}$ & $5 \cdot 7$ & $25 \cdot 2$ & $4 \cdot 42$ \\
\hline
\end{tabular}

It is obvious that the two quantities, heat-production (or loss) and heart-output, vary in the same general way. The reason is clear. Oxygen must be absorbed by the lungs in proportion to the heat produced, and where more oxygen is to be absorbed, more blood passes through the lungs to take it up.* It is interesting to inquire whether,

* For simplicity, the possibility that the coefficient of utilization of the oxygen-carrying capacity of the blood (i.e., the quantity of oxygen absorbed by a litre of blood during its passage through the lungs divided by the total quantity of oxygen which it can take up) may vary, is disregarded. This possibility would imply that the average oxygen content of the venous blood coming to the right side of the heart varied in animals of different size, more oxygen being abstracted, for example, from the blood in passing through the tissues of small animals than of large. The greater the utilization of the oxygen the smaller would the quantity of blood passing through the lungs require to be. There is no evidence, however, that such differences exist between animals of the same species of different size. although it has been suggested that a higher coefficient of utilization coupled with a proportionately smaller heart output may be one way in which training diminishes the difficulty and discomfort of hard muscular effort. 
in the smaller animals the contact of the alveolar air with the relatively increased quantity of blood is obtained by a similar increase in the area covered by capillaries (or in what is doubtless approximately proportional to this area, the mass of the lung tissue), or by a shortening of the pulmonary circulation time (p. r37). The answer is that the pulmonary circulation time is markedly shortened as the size of the animal diminishes, although not in proportion to the diminution in weight, but rather in proportion (roughly speaking) to the square root of the surface. This could not be the case if the area of the pulmonary surface bore the same proportion to the area of the skin as the output of the heart does. For in this case the vascular capacity of the lungs (or the quantity of blood contained in them) would be proportional to the heart output, and the time required for the blood discharged by the right ventricle to displace the whole of the blood contained in the lungs at any given moment-that is to say, the average pulmonary circulation time-would be the same for animals of all sizes. But it may very well be the case if the vascular capacity of the lungs (or the mass of the lung tissue) decreases more rapidly than the surface decreases, say in proportion to the body-weight. At first thought it might appear advantageous that the area of the surface through which oxygen is absorbed should be proportional to the area through which the heat produced in the oxidations of the body is chiefly eliminated, so that the greater the necessary heat loss, the greater should be the facilities for absorption of oxygen. We have, however, already seen (p. 251) that the blood, even when it passes through the pulmonary capillaries at its maximum speed, has still sufficient time to practically saturate itself with oxygen. Within the limits where this holds good there would be no advantage in increasing the relative size of the lungs rather than in increasing the linear velocity of the blood passing through them-that is, diminishing the pulmonary circulation timeand on general principles it may be assumed that a larger pulmonary reservoir than is necessary for the maximum possible intake of oxygen would not be provided. For if the pulmonary reservoir holds an excessive amount of blood, some other tissues must have too little. It has been stated, indeed, that in animals of different size in the same species the total quantity of blood in the body is a function not of the body-weight, but of the surface, so that the smaller animals have a relatively larger amount of blood (p. 56). If this be so, it is probably related to the greater intensity of metabolism and the greater loss of heat from the surface of small animals, which entails a greater circulation through the skin. This greater cutaneous circulation means the permanent withdrawal of blood from the rest of the body, which can be compensated by a corresponding increase in the total circulating mass. The fact that the blood contained in the living skin must form a substantial fraction of the total blood, and a fraction relatively more important in the smaller than in the larger animals, would of itself help to establish a relation between surface area and total quantity of blood. The greater volume of the blood will contribute to the increased output of the heart in the smaller animals. In this connection the fact already alluded to more than once may be again emphasized, that it is not the quantity of blood contained in an organ or an organism, but the quantity passing through the capillaries in a given time, which is the important thing for its function, in the case of the lungs for the function of the gaseous exchange, in the case of the skin for the regulation of the heat-loss. 
There is reason to suppose that the average amount of blood contained in the cutancous vessels, as well as the average ainount flowing through them per unit of time, is greater in proportion to the total area of the skin in animals like man, witlı a skin devoid of fur and well supplied with sweat glands, tlian in animals well protected by liair and with few sweat glands. For in the latter the cutaneous circulation cannot take as great a sliare in the regulation of the heat-loss as in the former, although, of course, the nutrition of the hair itsclf requires a certain supply of blood. The relatively insignificant proportion of the total blood usually assigned to the skin of such an animal as the rabbit (p. 56) probably gives quite an erroneous idea of the proportion in the skin of a living man. It must be remembered that such determinations as have been carried out liave been made on dead animals whose skin under ordinary conditions contains less blood than during life.

Rubner has found that animals abundantly fed do not show so much change in the production of heat when the external temperature is varied as starving animals, perhaps because the thicker coat of subcutaneous fat so steadies the rate at which heat is lost that it becomes easy for the vaso-motor mechanism alone to hold the balance between loss and production. In well-fed animals it is the heat-loss which is chiefly affected, and it may be that this has something to do with the explanation of Loewy's results on man (p. 694).

Lorrain Smith discovered the interesting fact that after removal of the thyroid glands (in cats), the heat-production, as measured by the amount of carbon dioxide given off, is more sensitive to changes of external temperature than in the normal animal.

Effect of Excessive Loss of Heat-Varnishing of the Skin.-It must not be imagined that the production of heat can be increased indefinitely to meet an increased heat-loss. The organism can make considerable efforts to protect itself, but the loss of heat may casily become so great that the increase of metabolism fails to keep pace with it. The internal temperature then falls, and if the fall be not checked, the animal dies. A mammal, when cooled artificially to the temperature of an ordinary room ( $15^{\circ}$ to $20^{\circ} \mathrm{C}$.), does not recover of itself, but may be revived by the employment of artificial respiration and hot baths, even when the rectal temperature has sunk to $5^{\circ}$ to $10^{\circ} \mathrm{C}$. If the skin of a rabbit be varnished, and the air which it is the function of the fur to maintain at rest around it be thus expelled, the animal dies of cold, unless the loss of heat is artificially prevented. If, without varnishing at all, the greater portion of the skin of a rabbit or guinea-pig be closely clipped or shaved, similar phenomena are observed. Prevented from covering itself with straw, the animal dies, sometimes in twenty-four hours. The radiation from the skin, as measured by the resistance-radiometer (p. 68I), is greatly increased; the animal shivers constantly, and the rectal temperature falls. Placed in a warm chamber before the temperature in the rectum has fallen below $25^{\circ}$, the animal recovers perfectly. If the fall is allowed to go on, it dies. If it is kept from the first in the warm chamber, no fall of temperature occurs. When the increased loss of heat is less perfectly compensated-when, for example, the animal is left at the ordinary temperature, but supplied with sufficient straw to cover itself, or allowed to crouch among other animals - a curious phenomenon may sometimes be seen. The rectal temperature, which lias fallen sharply during the operation, remains subnormal (as much as $2^{\circ}$ to $3^{\circ}$ below the ordinary temperature) for a time (a week or more), and then 
gradually rises as the coat again begins to grow. The meaning of this seems to be that the power of regulating the temperature by increasing the metabolism is overtasked by the removal of the natural protective covering, unless the escape of heat is artificially diminished. When the loss of the fur is entirely compensated, no fall of temperature occurs; when it is not compensated at all, the animal cools till it dies; when it is partially compensated, the increased metabolism may only suffice to maintain a temperature lower than the normal, although constant muscular contractions (shivering) are brought in to supplement the efforts of the regulative chemical processes.

Hitherto we have only spoken of a reflex regulation of the heatproduction called into play by external cold. It might be supposed - and, indeed, has often been assumed - that heat would lessen the metabolism, as cold increases it; and thero are indications that in the smaller animals this is the case, although the influence of heat seems to be much smaller than the influence of cold. But neither experimental results nor general reasoning have as yet shown that in man, either in the tropics (Eykman) or in the north temperate zone (Loewy), the chemical tone is diminished by a rise of external temperature much above the mean of an ordinary English summer, apart from the effect of the muscular relaxation which heat induces. In a man, indeed, at rest in a hot atmosphere, the production of carbon dioxide and consumption of oxygen are, if anything, greater than at the ordinary temperature. The regulation of temperature in an environment warmer than the normal seems, in fact, to be brought about more by an increase in the loss than a decrease in the production of heat. Evaporation from the skin and lungs is an automatic check upon overheating as important as the involuntary increase of metabolism upon excessive cooling.

Nervous Mechanism of Thermotaxis.-While the skeletal muscles, and perhaps the glands, are at one end of the reflex arc by which the impulses pass that regulate the temperature through the metabolism, we are as yet ignorant of the precise paths by which the afferent impulses travel, of the nerve-centres to which they go, and even of the end-organs in which they arise. There are nerves in the skin which minister to the sensation of temperature (Chap. XVIII.). A change of temperature is their 'adequate' and sufficient stimulus; and it is a tempting hypothesis that these are the afferent nerves concerned in the reflex regulation of temperature - that impulses carried up by them to some centre or centres in the brain or cord are reflected down the motor nerves to control the metabolism of the skeletal muscles, and down the vaso-motor nerves to control the loss of heat from the skin.

It is more than doubtful, however, whether the whole chemical regulation can be attributed to such stimuli. For it has been found that the relation between heat-production and extent of surface in animals 
(guinea-pigs) of different size is unaltered when the air temperature is made so nearly the same as that of the skin that the temperature nerves can hardly be supposed to be excited.

There is some evidence that the bioplasm-the living substance-of different animals, even when the external conditions are the same, may differ specifically in the average intensity of metabolism to which it is pitched. When exposed to a temperature about equal to that of warmblooded animals, the grcen lizard (Lacerta viridis) and the bull-frog. which live in the temperate zone, and for which a temperature of $37^{\circ} \mathrm{C}$. is highly abnormal, double their heat-production, and soon die. Tropical poikilothermal animals, such as the alligator, also double their heat-production, but the highest values reached are only one-half that of the lizard at $25^{\circ} \mathrm{C}$. Apparently the bioplasm of the tropical animals has adapted itself to a high external temperature, and works very economically even at the highest temperatures (Krehl).

Heat Centres.-It is known that certain injuries of the central nervous system are related to disturbance of the heat-regulating mechanism. Injury to various portions of the cortex cerebri in the dog and other animals, and lesions of the pons, medulla oblongata and cord in man, may be followed by increase of temperature. When the spinal cord is cut below the level of the vaso-motor centre, the increased loss of heat from the skin due to dilatation of the cutarieous vessels masks any increase of the heat-production which may possibly have taken place, and the internal temperature falls; but if the loss of heat is diminished by wrapping the animal in cotton-wool the temperature may rise. From such phenomena it has been surmised that certain 'ccntres' in the brain have to do with the regulation of temperature by controlling the metabolism of the tissues; that they cause increased metabolism when the internal temperature threatens to sink, diminished metabolism when it tends to rise. The cutting off, it is said, of the influence of the 'heat centres' by section of the paths leading from them allows the metabolism of the tissues to run riot, and the temperature to increase. But diversity of opinion still reigns as to the existence or, if they exist, as to the precise location of the nervous centres which preside over this function. It is stated by some workers that animals deprived of the cerebral hemispheres, the corpora striata and one of the optic thalami, still maintain to a remarkable degree a body temperature independent of their environment, but that when both optic thalami have been removed they become poikilothermal (Krehl, etc.). The greatest mass of evidence points, however, to the corpora striata as structures more intimately concerned in temperature regulation than any other. Puncture of the median portion of the corpus striatum in the rabbit by a needle thrust through a trephine hole in the skull is followed in a few hours by a rise of temperature in the rectum $\left(\mathrm{I}^{\circ}\right.$ to $\left.2^{\circ}\right)$, and still more in the duodenum, which is normally the hottest part of the body in this animal. The heat-production, the respiratory exchange 
and the nitrogen excretion are increased. These phenomena mav last for several days (Ott, Richet, Aronsohn, and Sachs), and are due to stimulation of the portions of the brain in the immediate neighbourhood of the injury. Electrical stimulation of this region has a similar effect. When the temperature has returned to normal, a fresh puncture may again cause a rise.

As to the manner in which these centres are excited, there is some evidence that, in addition to any influence exerted on them by afferent nerves, they are capable of being directly affected by the temperature conditions of the blood passing through them, as well as by numerous drugs. Thus it is stated by Barbour and Wing that direct application of cold to the region of the corpus striatum, especially its caudate nucleus, in rabbits causes a rise in the rectal temperature, associated with shivering and consequent increase of heat-production in the contracting muscles, and with peripheral vaso-constriction and consequent diminution in the heat-loss. The application of warmth has the opposite effect: the peripheral bloodvessels dilate, the animal becomes quiet, and the rectal tem perature falls. *

Some observers hold that the chief seat of the increased metabolism in the puncture fever is the skeletal muscles, others the liver. The question turns largely upon the success of the puncture experiment after the previous administration of curara on the one hand, and of strychnine on the other. For curara cuts out the motor innervation of the skeletal muscles, and strychnine convulsions exhaust the store of hepatic glycogen. Certain investigators have found that after an adequate dose of curara no puncture fever can be obtained, and they locate the increased metabolism associated with the fever in the muscles. Others maintain that even after curara the puncture is followed by fever, but is not followed by fever if strychnine has first been given. They accordingly conclude that the rapid combustion of the glycogen (or the dextrose derived from it) is the primary factor in the increased metabolism. It may be pointed out, however, that neither experiment is a crucial test. For if strychnine reduces the liver glycogen, it also reduces the glycogen of the muscles. And if in the puncture fever the liver glycogen is transformed into dextrose more rapidly than usual, the dextrose is probably in great part used up in the muscles more rapidly than usual, eiso it would appear in the urine. The effect of strychnine on the puncture fever, then, is no proof that the muscles are not essentially concerned in it. On the other hand, the alleged absence of the fever after curara is not sufficient to show that the muscles are alone concerned. For curara causes a lowering of the body-temperature, which, if it be not overcompensated, may

* Other observers, however, have failed to confirm these results (Cloetta and Waser). 
mask the fever. The positive result of the puncture in curarized animals, which some observers say they have obtained, would, if animals, which some observers say they have obtained, would, if confirmed, be important evidence that the primary effect is not on the muscles, or, at least, not solely on them, but would not prove that it is on the liver. That the liver is concerned, however, is more directly indicated by the fact that during the puncture fever the liver continues to be what it is under normal conditions, the warmest organ in the body, warmer than the blood in the root of the aorta by about $\mathrm{I}^{\circ} \mathrm{C}$. The most probable conclusion is that the increased production of heat in this form of experimental fever is due to an increased metabolism of carbo-hydrate (glycogen) both in the liver and in the muscles.

Temperature Regulation in Hibernating Animals.-The behaviour of hibernating mammals, such as the marmot, dormouse, hedgehog, and bat, is of interest in connection with the temperature regulation. In the active waking state these animals are homoiothermal, but in profound winter sleep they are poikilothermal, the body-temperature rising and falling with that of the air. The rectal temperature may be as low as $2^{\circ} \mathrm{C}$. There is an intermediate state in which the animal is partially awake, though inactive, and its temperature is much below the normal, but considerably above that of its environment. In this condition it has an imperfect thermotaxis, something like that of an ordinary mammal (including the human infant) in the period of immaturity, immediately after birth. When the hibernating mammal awakes the rise of temperature is enormous and abrupt. The temperature of a dormouse rose in an hour from $13.5^{\circ} \mathrm{C}$. to $357^{\circ} \mathrm{C}$., and that of a bat in fifteen minutes from $17^{\circ} \mathrm{C}$. to $34^{\circ} \mathrm{C}$. (Pembrey).

Fevers.-Fever is a pathological process generally caused by the poisonous products of bacteria, and characterized by a rise of temperature above the limit of the daily variation (p. 7I2). It is further associated with an increase in the rate of the heart and the respiratory movements, and a diminution in the alkalies and carbon dioxide of the blood. The total excretion of nitrogen is increased, at least in proportion to the amount of protein ingested, indicating an increase in the consumption of tissue-protein. The distribution of the nitrogen among the urinary constituents is altered. The anmonia (in the form of ammonium salts of organic acids), the uric acid, and to a smaller extent the creatinin (Leathes), are in. creased, while the urea is relatively decreased, even when its absolute amount is greater than normal. Creatin, which is not normally present in urine, unless the food contains it, may also appear in fever (Shaffer). It has been suggested that the proximate cause of fever is the action of bacterial poisons or of other substances on the 'heat centres,' and that antipyretics, or drugs which reduce the temperature in fever, do so by restoring the centres to their normal 
state, by preventing the development of the poisons, aiding their elimination, or antagonizing their action. In favour of this view, it has been stated that when the basal ganglia are cut off, by section of the pons, from their lower nervous connections, fever is no longer produced by injection of cultures of bacteria which readily cause it in an intact animal, while antipyrin has no influence upon the temperature (Sawadowski). And while it is almost certain that some pyrogenic or fever-producing agents-cocaine, e.g.-act indirectly, through the brain or cord, it is quite possible that others affect directly the activity of the tissues in general, just as some antipyretics or fever-reducing agents, such as quinine, act immediately upon the heat-forming tissues, so as to diminish their metabolism, while others, like antipyrin, affect them through the nervous system. Quinine has no influence upon 'puncture' fever in rabbits. A still more important action of antipyrin, and the group of antipyretics to which it belongs, is the increase in the heatloss which they bring about by the dilatation of the bloodvessels of the skin. This effect is also produced through the nervous system.

Fever is a condition so interesting from a physiological point of view, and of such importance in practical medicine, that it will be well to consider a little more closely the possible ways in which a rise of temperature may occur. It must not be forgotten that the febrile increase of temperature is always accompanied by other departures from the normal, and that all the fundamental febrile changes may even, in certain cases, be present without elevation, and even with diminution of temperature. But here we have only to do with the disturbance of the normal equilibrium between the loss and the production of heat; and it is evident that any of the five conditions illustrated in the diagram (Fig. 226) may give rise to an increase of temperature. It is not necessary to discuss whether cases of fever can actually be found to illustrate every one of these possibilities. It is probable that not infrequently diminished loss and increased production may be both involved; and it ought to be remembered that the healthy standard with which the heat-production of a fever patient should be compared is not that of a man doing hard work on a full diet, but that of a healthy person in bed, and on the meagre fare of the sick-room. When this is kept in view, the comparatively low heat-production and respiratory exchange which have sometimes been found in fever cease to excite surprise. But in any case, no mere change in the absolute quantities of heat formed and lost is sufficient to explain the febrile rise of temperature; there must be a change in the relative proportion. That an increase in heat-production is not of itself enough to produce fever is proved by the fact that severe muscular work, which in- 
creases the metabolism more than higlı fever, only causes in a healthy man a rise of about $\mathrm{I}^{\circ} \mathrm{C}$. in the rectal temperature. When the work is over, the temperature comes rapidly back to normal. The essence of the change in fever is a derangement of the mechanism by which in the healthy body excess or defect of average metabolism, or of average lieatloss, is at once compensated and the equilibrium of temperature maintained.

This derangement only lasts as long as the temperature is rising. When it becomes stationary at its maximum we have again adjustment, again equality of production and escape of heat; but the adjustment is now pitched for a higher scale of temperature. A rough analogy, so far as one part of the process is concerned, may be found in the behaviour of the ordinary gas-regulator of a water-bath. It can be 'set' for any temperature. That temperature, once reached, remains constant within narrow limits of oscillation; but the regulator can be equally well adjusted for a higher or a lower temperature. It is,

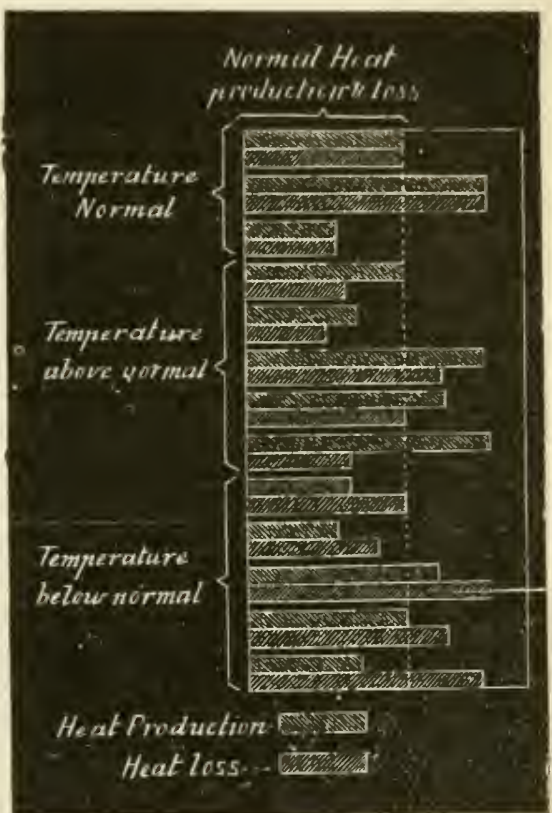

Fig. 226.-Diagram to show the Possible Relations between Heat-Production and Heat-Loss in Fever.

however, important to note that the equilibrium is more unstable in fever than in health, so that changes of external temperature more easily depress or increase the temperature of a fever patient than of a healthy man.

Rosenthal has concluded from calorimetric observations that, in the first stage of fever, while the temperature is rising, there is always increased retention of heat. Maragliano actually found evidence, by means of the plethysmograph, that the cutancous vessels are at this stage constricted, and that the constriction may even precede the rise of temperature. The blood-flow in the feet in cases of typhoid fever investigated by the calorimetric method (p. I22) was not found to exceed the normal flow, and was usually decidedly below the normal. Hyperexcitability of the vaso-constrictor mechanism of the peripheral parts, especially of the skin, 
was present. All these observations lend support to the famous 'retention' theory of Traube. It has been suggested that the significance of the increased action of the cutaneous vaso-constrictor mechanism in typhoid fever is that the peripheral vaso-constriction is a compensatory arrangement which secures for the organs mainly suffering from the infective process an increased flow of blood to combat the infection. On this hypothesis the rise of temperature in so far as it depends upon diminished loss of heat is a secondary. phenomenon inevitably following the redistribution of the blood, and unavoidable except by a corresponding diminution in the total metabolism. In the great majority of cases the production of heat is also increased, on the average by 20 to 30 per cent. of the normal production of a resting man. The increase may be much greater during the chill which ushers in so many infections on account of the muscular contractions in shivering. During the period of rising temperature the production of heat is not necessarily increased. At the height of the fever there is often, though apparently not always, an increase in the heat-production. After the crisis, while the fever is subsiding, the rate at which heat is lost rises sharply.

As to the explanation of the increase of metabolism in fever, and especially of the increased metabolism of tissue-protein, various views have been held. Some have gone so far as to say that the increase is merely the consequence, not the cause, of the rise of temperature. But the rebutting evidence which has been brought against this view is strong and, indeed, overwhelming. It is perfectly true that, when the temperature of the body is artificially raised by preventing the free loss of heat for a sufficient time (so-called physiological fever), the destruction of protein is augmented. A fasting dog whose temperature was increased to $40^{\circ}$ or $4 \mathrm{I}^{\circ} \mathrm{C}$. for twelve hours eliminated 37 per cent. more nitrogen than when the body-temperature was normal. But this increase in the protein metabolism could be entirely prevented by giving the animal a sufficient amount of carbo-hydrate. Similar results have been obtained in man. The carbon dioxide excretion and oxygen absorption are, of course, also markedly increased. But the increase in the nitrogen excretion is often much greater in fever than any increase which can be brought about by artificially raising the temperature of a healthy individual by means of hot baths. A typhoid patient was found to lose Io.S grammes of nitrogen a day (corresponding to $3 \mathrm{IS}$ grammes of muscle) during eight days of fever (F. Müller). A portion of the loss of nitrogen on the routine fever regimen may be due to the fact that the ordinary typhoid patient is really on a semi-starvation diet, the heat-equivalent of which is not much more than half his heat-production. Yet it has not been found possible to completely prevent the loss of 
nitrogen by putting the fever patient on a diet rich in protein, or on a diet containing a moderate amount of protein with a large quantity of fat and carbo-hydrate, even when the total heat-value of the diet is much in excess of the 32 or 33 calories per kilo of bodyweight which corresponds to the heat-production of a resting man. Another suggestive fact is that the excessive excretion of nitrogen does not run parallel with the rise of temperature in fever, but is often most marked after the crisis. During the stage of defervescence an enormous amount of urea is sometimes given off. In a case of typhus, in the mixed urine of the third and fourth days after the crisis, 110 less than I6o granımes of urea was found (Naunyn), or nearly three times the normal amount for a man on full diet. Again, when fever is caused by the injection of bacteria or their products, the increase in the carbon dioxide eliminated and oxygen consumed occurs even when the temperature is prevented from rising by cold baths. It seems perfectly clear, then, that the increase of metabolism is, in many cases at least, a primary phenomenon of fever. Its course and incidence, falling as it does so largely upon the proteins, the steady loss of tissue nitrogen, and the inability of the tissues to recoup their losses from the protein of the food or to shield their own protein by burning more carbo-hydrate or fat, all suggest that the cells are poisoned by toxic products of the infective process. The poisoned bioplasm falls an easy prey to the hydrolysing and oxidizing agents always present in the tissues. It breaks down more rapidly and builds itself up more slowly than normal bioplasm. This increased, and to some extent perverted, metabolism, far from being occasioned by the febrile temperature, is quite probably the cause of the thermo-regulative upset which we call fever. For Mandel has shown-(I) that one of the purin bases (xanthin) causes fever in monkeys; (2) that the purin bases in the urine are increased both in infective fevers and the so-called aseptic or surgical fever-that is, in cases where the temperature rises after such injuries as extensive crushing of tissues without infection. There is a constant relation between the height of the fever and the quantity of purin bases excreted. The source of the purin bases in aseptic fever is presumably the autolysis of the injured tissue, from which they pass into the blood without being oxidized to uric acid. The xanthin fever can be prevented by salicylates, though not by antipyrin.

It has been very generally admitted that the chief seat of excessive metabolism in fever is the muscles; but U. Mosso has stated that cocaine fever-the marked rise of temperature produced by injection of cocaine-can be obtained in animals paralyzed by curara. This, even if true, would not support the conclusion that a "nervous fever '-that is to siy, a fever due solely to increased metabolism in the nervous system-exists; for in a curarized animal a large 
amount of 'active' tissue (glands, leart, smooth muscie) still remains in physiological connection with the brain and cord. But, as a matter of fact, in an animal under a dose of curara sufficient to completely paralyze the sheletal muscle cocaine causes no appreciable rise of rectal temperature; and this is strongly in favour of the view that the fever produced in the non-curarized animal is comnected with cxcessive muscular metabolism.

Significance of the Increased Temperature in Fever.-It remains to ask whether the rise of temperature is anything more than a superficial and, so to speak, an accidental circumstance. The question has already been raised in discussing the changes in the circulation in fever (p. 706). The orthodox vicw for many ages lias undoubtedly been that the increase of temperature is in itself a serious part of the pathological process, a symptom to be fought with and, if possible, removed. And, indeed, it is not denied by anyone that the excessive rise of temperature seen in some cases of febrile discase (to $43^{\circ} \mathrm{C}$, or even to $45^{\circ}$ ) is, apart from all other changes, a most imminent danger to life, unless, as is sometimes the case (in influenza, e.g., where a temperature of $44^{\circ}$ has been observed), the high temperature lasts only a short time. Experimental heat paralysis, a conclition in which all voluntary and reflex movements are abolished, is produced in frogs by raising the internal temperature to about $3 t^{\circ} \mathrm{C}$. On cooling, the animal recovers. A similar condition can be induced in mammals, but, of course, at a higher temperature. The central nervous system succumbs before the peripheral structures. The superior cervical ganglion in the cat or rabbit loses the power of transmitting nerve impulses at $50^{\circ} \mathrm{C}$. But some evidence has been brought forward, mostly from the field of bacteriology, to support the iclea that in infective processes the rise of temperature is of the nature of a protective mechanism, that the fever is, indeed, a consuming fire, but a fire that wastes the body, to destroy the bacteria. The streptococcus of erysipelas, for example, does not develop at $39^{\circ}$ to $40^{\circ} \mathrm{C}$., and is killed at $39^{\circ} 5^{\circ}$ to $4 \mathrm{I}^{\circ} \mathrm{C}$, and erysipelas infections in rabits are less virulent if the body-temperature be artificially raised. Anthrax bacilli, kept at $42^{\circ}$ to $43^{\circ} \mathrm{C}$. for some time, are attenuated, and when injected into animals confer immunity to the disease. Heated for several days to $4 \mathrm{I}^{\circ}$ to $42^{\circ} \mathrm{C}$, pnemmococci render rabbits immune to pneumonia, and in rabbits in which 'puncture ' fever has been induced pneumococcus infections run a milder course. These bacteriological results are supported to a certain extent by clinical experience. For it has been observed that a cholera patient with distinct fever has a better chance of recovery than a case which shows no fever. But too much weight orght not to be given to isolated facts of this sort, and adverse evidence can be produced both from the laboratory 
and the hospital. For although hens are immune to anthrax under ordinary conditions, but can be infected by inoculation when artificially cooled, frogs, equally immune at the temperature of the air, become susceptible when artificially lieated. And it is impossible to deny that the use of cold baths in typhoid fever is sometimes of remarkable benefit. This benefit, however, while very unlikely to be connected with any directly unfavourable action of the reduced body-temperature on the growth of the bacilli, may perhaps be due, in some part at least, to an increase in the cutancous vaso-constriction which helps to send through the infected intestine a more copious stream of blood.

\section{Section IV.-Distribution of Heat-Temperature}

TOPOGRAPHY.

The great foci of heat-formation-the muscles and glands-would, if leat were not constantly leaving them, in a short time become: much warmer than the rest of the body; while structures like the bones, skin, and adipose tissue, in which chemical change and heatproduction are slow, would soon cool down to a temperature not much exceeding that of the air. The circulation of the blood insures that heat produced in any organ shall be carried away and sp edily distributed over the whole body; while direct conduction also plays a considerable part in maintaining an approximately uniform temperature. The uniformity, however, is only approximate. The temperature of the liver is several degrees higher than that of the skin, and the temperature of the brain several degrees higher than that of the cornea. The blood of the superficial veins is colder than that of the corresponding arteries.

The crural vein, for example, carries colder blood than the crural artery, and the external jugular than the carotid. The heat produced in the decper parts of the regions which they drain is more than counterbalanced by the heat lost in the more superficial parts. When loss of heat from the surface is sufficiently diminished by an artificial covering. or prevented by the protected situation of any organ with an active metabolism, the venous blood leaving it is warmer than the arterial blood coming to it. The temperature of the blood passing from the levator labii superioris muscle of the horse during mastication may be sensibly higher than that of the blood which feeds it; the blood in the vena profunda femoris, and in the crural vein of a dog with the leg wrapped in cotton-wool, is warmer by $0 \cdot 1^{\circ}$ to $0 \cdot 3^{\circ}$ than of the crural artery. The difference is due to the heat produced in the muscles, and it ought to be of this order of magnitude. The quantity of blood in a 7 -kilo dog is about $\frac{1}{2}$ kilo; $\frac{1}{4}$ of this, or $\frac{1}{8}$ kilo, is in the skeletal muscles, and the average circulation-time through them may be taken as ten seconds. Six times in the minute, or 360 times in the hour, $\frac{1}{8}$ kilo of blood passes through the muscles, and is heated on the average by $0.2^{\circ}$. This represents a heat-production of about $\frac{360}{8} \times \frac{2}{10}$, or 9 calories per hour. Now, the total heat-production of a 7 -kilo dog is about 
Io calories per hour, of which somewhat less than one-half is formed in the skeletal muscles.

The blood of the inferior vena cava at the level of the kidneys may be ${ }^{\circ} \cdot \mathrm{I}^{\circ}$ colder than that of the abdominal aorta, but is warmer than the blood of the superior cava. The right heart, therefore, receives two streams of blood at different temperatures, which mingle in its cavities. A controversy was long carried on as to the relative temperature of the blood of the two sides of the heart; but the researches of Heidenhain and Körner have shown that a thermometer passed into the right ventricle through the jugular vein stands, as a rule, slightly higher than a thermometer introduced through the carotid into the left ventricle. The method gives not so much the temperature of the blood in the two cavities as that of their walls. The thin-walled right ventricle is heated by conduction from the warm liver, from which it is only separated by the diaphragm, while the left ventricle loses heat to the cooler lungs. The difference of temperature is not caused by cooling of the blood in its passage through the pulmonary capillaries, for even when respiration is suspended, a difference of temperalure between the two sides of the heart is found. Under ordinary circumstances, the inspired air is already heated almost to body-temperature before it reaches the alveoli. But, while this is the case, a fall of less than $77^{\circ}$ in the temperature of the blood passing through the lungs would account for all the heat lost by the expired air. If lialf of the loss took place in the upper air-passages, less than $10^{\circ}$ would be sufficient. A slight difference of temperature in the blood of the two ventricles might be caused, even in the absence of respiration, by the heat developed in the cardiac muscle itself during contraction, a large proportion of which must be conveyed by the coronary veins into the right heart.

The surface temperature varies between rather wide limits with the temperature of the environment. The temperature of cavities like the rectum, vagina, and mouth, and of secretions like the urine, approximates to that of the blood in the great vessels or the heart, and undergoes only slight clianges. An increase in the velocity of the blood causes the internal and surface temperatures to come nearer to eacli other, the former falling and the litter rising. When the loss of heat from a portion of the surface is prevented, the temperature of this portion approaches the internal temperature. For this reason a thermometer placed in the axilla approximately measures the internal temperature, and not that of the skin; and a thermometer in the groin of a rabbit, and completely covered by the flexed thigh, may stand as ligh as, or, it is said, even higher than, a thermometer in the rectum (Hale White). The temperature in the mouth is not a very reliable inclex of the deep temperature of the body, especially in cold weather or after excrcise. as it is apt to be infuenced by the inspired air. The moutlinust, of course, be kept closed during the measurement. On the average its temperature is about the same as that of the axilla, and $0.4^{\circ} \mathrm{C}$. below that of the rectum. The rectal temperature is $0 \cdot 2^{\circ}$ or $0.3^{\circ}$ above that of the urine. In point of accuracy rectal observations are the best, and next to them determinations of the temperature of the stream of urine. The latter method, although subject to obvious limitations, is rapid and free from the danger of conveying infection to the person (Pembrey).

The surface temperature is a rough index of the rate of heat-loss: the internal temperature, of the rate of heat-production. A normal skin temperature and a rising rectal temperature would probably indicate increased production of heat; an increased rectal temperature, 
in conjunction with a diminisled surface temperature, as in the cold stage of agne, might be due either to diminished heat-loss while the lieat-production remained normal, or to diminished lieat-loss plus increased heat-production.

The following tables illustrate the differences of temperature found in the body. It should be remembered that the numbers are not strictly comparable with each other; the temperature of the mammals in which direct observations have been made on the blood is not exactly the same as that of man, the temperature of the dog, for example, being a little (about $\mathrm{x}^{\circ} \mathrm{C}$.) higher. Then in the same animal there is $n o$ very constant ratio between the temperature of the blood in two ressels or of the skin at two points. Fiven in the same vessel the temperature may vary with many circumstances, such as the velocity of the stream, and the state of activity of the organ from which it comes. Apart from physiological variations, experimental fallacies sometimes cause a want of constancy, especially in measurements of blood temperature. The insertion of a mercurial thermometer into a vessel is very likely to obstruct the passage of the blood; and if the blood lingers in a warm organ, it will be heated beyond the normal. In man the blood-temperature in the arteries at the wrist has been estimated indirectly by the calorimetric method of measuring the blood-flow in the hand (p. I22), probably with greater accuracy than would be attainable by the direct insertion of a thermometer, were this permissible. The temperature of the calorimeter is determined at which it neither imparts heat to the blood nor gains heat from the blood. On the assumption that the heat-production of the resting hand is negligible for this purpose,* the temperature so fixed will be that at which the blood enters the hand-i.e., the temperature of the arterial blood at the wrist.

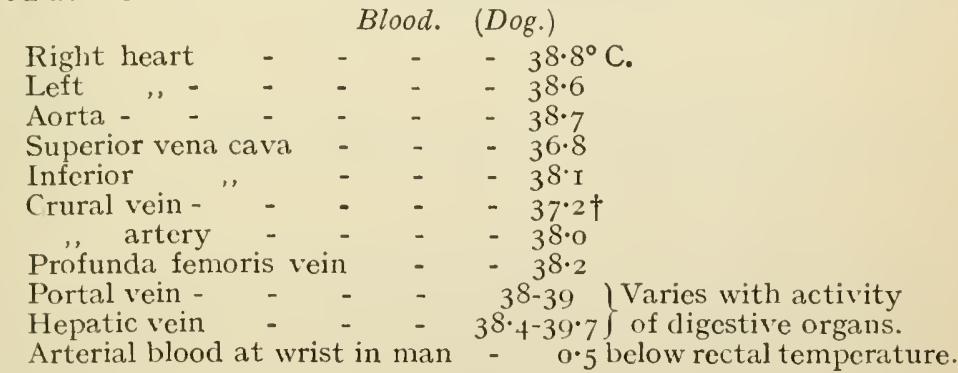

* Since, of course, some heat is produced in the hand even at rest, although doubtless less per unit of weight than in the resting body as a whole, the arterial blood temperature as thus determined must be somewhat too high. No error is caused by this in the calculation of the blood-flow in the hand (p. 122); for while the factor $T-T^{\prime}$ in the denominator is somewhat too great, the corresponding quantity of heat produced in the hand is included in $\mathrm{H}$ in the numerator.

$\dagger$ The following numbers were obtained (in an anæsthetized dog whose rectal temperature had fallen $2^{\circ} \mathrm{C}$.) for the temperature of the walls of the crural artery and vein, as measurcd by an electrical resistance thermometer.

Leg of dog lightly wrapped in wool.

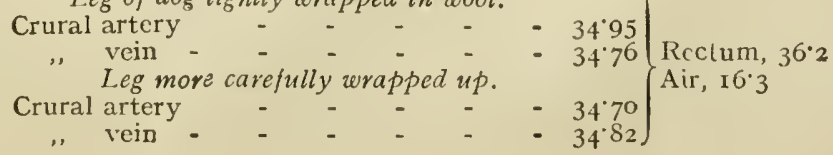




\section{Tissues.}

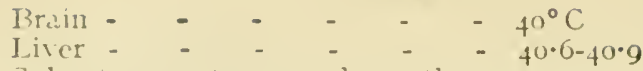

Subcutaneous 11s:ue $2 \cdot 1$ lower than that of subjacent muscles (man).

$\left.\begin{array}{llll}\text { Anterior chamber of eyc } & - & - & 31 \cdot y \\ \text { Vitreous humour } & - & - & 36 \cdot 1\end{array}\right\}$ (rabbit).

\section{Cavities. (Man.)}

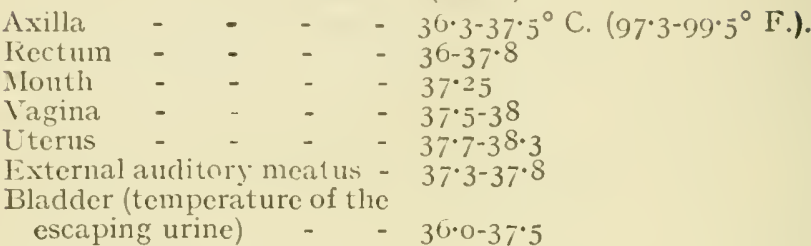

\section{Respiratory Passages. (Horse.)}

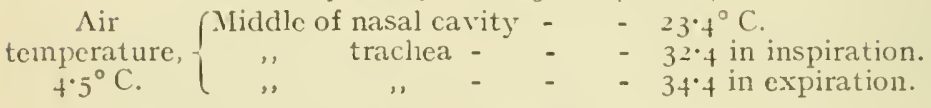

Natural Surfaces.

Cheek (boy, immediately after running) - $36.25^{\circ}$

(Iin) (Anterior surface of forearm - - $\quad$ - 33.5-34.4

Posterior ," $\quad$, - - - $\quad-34^{\circ} 0$

Room Skin over biceps - - - $-35^{\circ}$

$17^{\circ} 5^{\circ}$, immediately below xiplroid cartilise - $34^{\circ} 7$

over sternum - $\quad-\quad-\quad-\quad-\quad 3_{3}^{\circ}$

On liair (boy) - $\quad$ - $\quad$ - $\quad$ - $\quad$ - 30.0

Under hair over sagittal suture (boy) - 33.7-34.0

Shared skin of neck (rabbit) - - $\quad-36.5$

On liair ., ., $\quad$, $\quad$ - $\quad$ - $\quad$ - $31 \cdot 5$

"between eyes , b $\quad$ - $\quad$ - $\quad$ - 30.7

Artificial Surfaces.

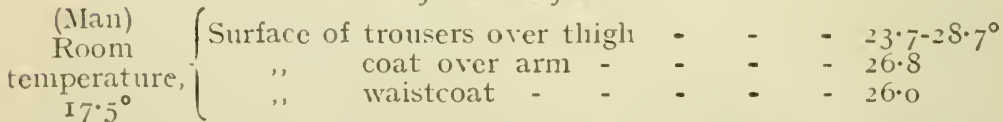

Normal Variations in the Temperature.-The internal temperature, as has been already said, is not strictly constant. It varies with the time of day; with the taking of food; with age; to some extent with violent changes in the external temperature, such as those produced by hot or cold baths; and possibly with sex. On the average the range of variation in the temperature of the rectum or urine of a healthy man is from $36.0^{\circ} \mathrm{C} .\left(96 . \mathrm{S}^{\circ} \mathrm{F}\right.$.) to $37^{\circ} \mathrm{S}^{\circ} \mathrm{C}$. $\left(100 \cdot 0^{\circ} \mathrm{F}\right.$.).

In the monkey a very distinct and constant diurnal variation has been observed, and the range is much wider than in man (as much as $5^{\circ} t^{\circ} \mathrm{F}$.), the maximum falling between $\sigma$ and $S$ p.m. and the minimum between 2 and + a.m. (Simpson). 
The daily curve of temperature shows a minimum in the early morning, between two and six o'clock $\left(36.3^{\circ} \mathrm{C}\right.$.), and a maximum in the evening, between five and eight o'clock $\left(37^{\circ} 5^{\circ} \mathrm{C}\right.$.) (Fig. 227). The daily range in health may be taken as a little over $\mathrm{I}^{\circ} \mathrm{C}$., or about $2^{\circ} 1^{1}$. In fever it is generally greater, but the maximum and minimum fall at the same periods; and it is of scientific, and also of practical, interest that the early morning, when the temperature and pulse-rate are at their minimum, is often the time at which the flagging powers of the sick give way. From two to six o'clock in the morning the daily tide of life may be said to reach low-water mark. Even in a fasting man the diurnal temperature curve runs its course, but the variations are not so great. The taking of food of itself causes an increase of temperature, although in a healthy man this rarely amounts to more than half a degree. The rise of temperature is certainly due in part to the increased work of the alimentary canal, but it is in the main

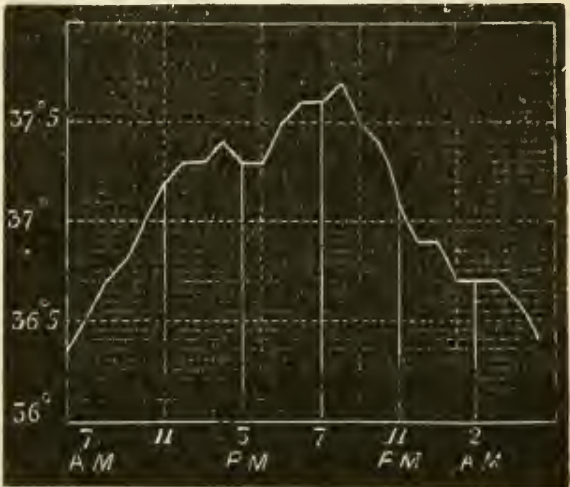

Fig. 227.-Curve showing the Daily Variation of Body-Temperature. connected with the increase of metabolic activity which the entrance of the products of digestion into the blood brings about. The heat-production is especially increased by proteins.

A dog weighing 15.3 kilos, the heat-production of which was 22.3 calories during an hour previous to feeding, was given $\mathrm{I}, 200$ grammes of meat at noon. The heat-production rose to 36 calories in the 2 nd hour, and 42 calories in the $3 \mathrm{rd}$. It remained above 40 calories per hour

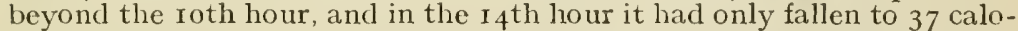
ries, to reach 25 calories in the 2 Ist hour. On the whole, the increase in heat-production ran parallel with, and was proportional to, the increase in the excretion of nitrogen (Williams, Richie and Lusk). The relatively unimportant share taken by the increased work of the gastro-intestinal tract in the augmentation of the metabolism is illustrated by the fact that a high rate of heat-production was maintained till the $14^{\text {th }}$ hour, even although by this time three-quarters of the nitrogen corresponding to the food protein had been eliminated in the urine, and the work of digestion and absorption must have been largely completed.

The rise of temperature during digestion is gradual, the maximum being reached during the fourth hour, or even later.

The cause of the daily variation of temperature has been much liscussed. There is no doubt that several factors are concerned, among the most important being the variation in the amount of contraction of the skeletal muscles and the influence of food. Mus. 
cular exercise is capable of causing a considerable rise in the temperature of the rectum and urine, to $38.5^{\circ} \mathrm{C}$. (101.3. $\mathrm{F}$ ) or even $38.9^{\circ} \mathrm{C}$. $\left(102^{\circ} \mathrm{F}\right.$.) without producing any feeling of distress. Other unknown influences seem also to be involved, as is shown by the fact that in persons who work at night and sleep during the day the curve of temperature, although greatly altered, is not reversed. Recent observations on this subject are those of Benedict. By means of a resistance thermometer in the rectum, readings were

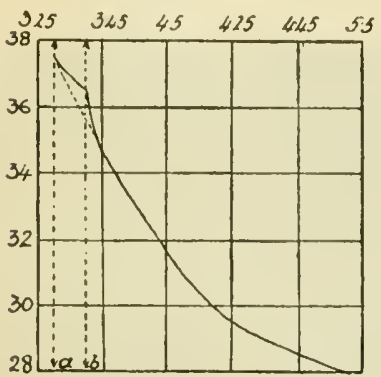

Fig. 228, - Curve of Cooling after Death: Guinea - Pig. Time marked along horizontal, and temperature along vertical axis. At $a$ ether and chloroform given to kill animal; death, as indicated by stoppage of the heart, took place at $b$. The dotted line shows the course the curve would have taken if death had occurred at the moment the anæsthetics were given. Air of room $x 7 \cdot 6^{\circ}$. taken usually every four minutes. With such a thermometer no disturbance of the person's sleep is necessary to obtain a reading. He can sit without discomfort in any position, walk about the room (returning to the observer's table for the observations), and even ride a stationary bicycle. Even years of night-work do not clim. inate the tendency to a fall of temperature at night, a minimum in the early morning, and a morning rise.

As to the relation of age and sex to temperature, it is only necessary to remark that the mean temperature both of the young child and of the old man is somewhat higher than that of the vigorous adult; but a point of more importance is the relative imperfection of the heat-regulation in infancy and age, and the greater effect of accidental circumstances on

the mean temperature. Thus, old people and young children are specially liable to chills, and a fit of crying may be sufficient to send up the temperature of a baby. In infants an hour or two old the temperature may be as low as $34^{\circ} \mathrm{C} .\left(93^{\circ} 2^{\circ} \mathrm{F}\right.$.) or $33^{\circ} 0^{\circ} \mathrm{C}$. $\left(9 \mathrm{r}^{\circ} 4^{\circ} \mathrm{F}\right.$.) even when they are fully clothed in a room at $15^{\circ} \mathrm{C}$. $\left(59^{\circ} \mathrm{F}\right.$.). It rises gradually during the first day or two, but shows marked variations. On the fifth day after birth, c.g., the rectal temperature ranged from $36^{\circ} 2^{\circ} \mathrm{C} .\left(97^{\circ} 16^{\circ} \mathrm{F}\right.$. $)$ to $33^{\circ} 5^{\circ} \mathrm{C} \cdot\left(92^{\circ} 3^{\circ} \mathrm{F}\right.$. $)$ in a child weighing $5 \frac{1}{2}$ pounds (Babak). The temperature of women is generally a little higher than that of men, and is also somewliat more variable. A fall of temperature, rarely amounting to more than $I^{\circ} \mathrm{F}_{\text {, }}$ is associated with the menstrual period.

After death the body cools at first rapidly, then more slowly (Fig. 2.8). But occasionally a post-mortem rise of temperature may take place. In certain acute diseases (like tetanus) associated with excessive muscular contraction this has been especially noticed: in boclies wasted by prolonged illness it does not occur. Nearly an 
hour after death, in a case of tetanus, the temperature was found to be $45^{\circ} 3^{\circ}$, while before death it was $44^{\circ} 7^{\circ}$ (Wunderlich). In dogs a slight post-mortem rise may be demonstrated, especially when the body is wrapped up; but when an animal has been long under the influence of anrsthetics no indication whatever of the phenomenon may be obtained. The explanation of post-mortem rise of temperature is to be found: (I) In the continued metabolism of the tissues for some time after the heart has ceased to beat, for the cell dies harder than the body. (2) In the diminished loss of heat, due to the stoppage of the circulation. (3) To a small extent in physical changes (rigor mortis, coagulation of blood) in which heat is set free.

\section{PRACTICAL EXERCISES ON CHAPTERS X., XI., AND XII.}

I. Glycogen*-(I) Preparation.-(a) Cut an oyster into two or three pieces, tlirow it into boiling water, and boil for a minute or two. Rub up in a mortar with clean sand, and again boil. Filter. Precipitate any proteins which have not been coagulated, by adding alternately a drop or two of hydrochloric acid and a few drops of potassio-mercuric iodide so long as a precipitate is produced. Only a small quantity of these roagents will be required, as the greater part of the proteins has been already coagulated by boiling. Filter if any precipitate has formed. The filtrate is opalescent. Precipitate the glycogen from the filtrate (after concentration on the water-bath if it exceeds a few c.c. in bulk) by the addition of four or five times its volume of alcohol. Filter off the precipitate, wash it on the filter with alcohol, and dissolve it in a little water. To some of the solution add a drop or two of iodine; a reddish-brown (port wine) colour is produced, which disappears on heating, returns on cooling, is removed by an alkali, restored by an acid. Add saliva to some of the glycogen solution, and put in a bath at $40^{\circ} \mathrm{C}$. In a few minutes reducing sugar (maltose) will be found in it by Trommer's test (p. Io).

Note that dextrin (erythrodextrin) gives the same colour with iodine as glycogen does. Dextrin is also precipitated by alcohol, but a greater proportion must be added to cause complete precipitation. Glycogen is completely precipitated by saturation with magnesium sulphate or ammonium sulphate, so that the filtrate no longer gives the reddish colour with iodine. A pure solution of erythrodextrin is not precipitated. On the addition of a drop or two of a solution of basic lead acetate to a solution of glycogen in distilled water, a precipitate forms immediately. When the same reagent is added to a solution of dextrin in distilled water there is no immediate precipitate. Maltose is formed when dextrin is digested with saliva.

(b) Cut another oyster into pieces, throw it into boiling water acidulated with dilute acetic acid, and boil for a few minutes. Rub up in a mortar with sand, boil again, and filter. Test a portion of the filtrate with iodine for glycogen. Precipitate the rest with alcohol, filter, dissolve the precipitate in water, and test again for glycogen. On boiling some of the opalescent solution for a few minutes after the addition of a few drops of sulphuric acid the opalescence disappears, and

* For the quantitative estimation of glycogen in organs, Pflüger's method is the best. The organ is minced and heated with strong (6o per cent.) potassium hydroxide. The glycogen is precipitated with alcohol, and then, after hvdrolysis with hydrochloric acid, estimated as dextrose. 
when the solution has been neutralized witl sodium hydroxicle it gives Trommer's test, owing to the hydrolysis of the glycogen into dextrose.

(2) Deeply etherize a dog or rabbit five hours after a meal rich in carbo-hydrates-e.g., rice and potatoes in the case of the dog, carrots in the case of the rabbit. Fasten it on a lolder. Clip off the hair over the abdomen in the middle line. Make a mesial incision through the skin and abdominal wall from the ensiform cartilage to the pubis. The liver will now be rapidly cut out (by the demonstrator) and divided into two portions, one of which will be (distributed among the class and) treated as in $(a)$ or $(b)$; the other will be kept for an lour at a temperature of $40^{\circ} \mathrm{C}$., and then subjected to process $(a)$ or $(b)$. Little, if any, sugar and much glycogen will be found in the portion which was boiled immecliately after excision. Abundance of sugar will be found in the portion kept at $4^{\circ} \mathrm{C}$; ; it may or may not contain glycogen.

2. Catheterism.- In many physiological experiments it is necessary to obtain urine from the bladder by means of a catheter. It is possible to pass a fine rubber catheter into the bladder of a male dog. A larger one is easily: passed in a male rabbit, and a still larger in a bitch, which is often used for experiments on metabolism. Even in the bitch the opening of the urethra lies entirely concealed within the vagina, much deeper than the cul-de-sac in the mucous membrane. into which the beginner usually tries to force the catheter. For a first attempt the animal should be etherized and fastened on a holder. The little or index finger of the left hand is passed into the vagina till the symphysis pubis can be felt. A little below this is the opening of the urethra. With the right hand the point of a catheter of suitable calibre is directed along the finger, and after a little 'guess and trial' it slips into the bladder, its entrance being announced by the escape of urine. A glass tube drawn out to a sufficiently small calibre and bent near the point is the easiest form of catheter to pass in a bitch. The point must, of course, be rounded in the flame. The insertion of the catheter is much facilitated by the use of a speculum.

When the bitch is to be used in a long series of experiments an operation is sometimes performed first of all to render the urethral orifice more accessible.

3. Glycosuria.-(I) (a) Weigh a dog (female by preference) or rabbit. Fasten on a holder, and etherize. Insert a glass cannula into the femoral or saphena vein of the dog, or into the jugular of the rabbit (p. 214). Fill a burette with a 2 per cent. solution of dextrose in physiological salt solution, connect it with the cannula by means of an indiarubber tube, taking care that there are no air-bubbles in the tube, and slowly inject as much of the solution as will amount to $\frac{1}{2}$ or $\frac{3}{4}$ grm. of sugar per kilo of body-weight. Tie the vein, remove the cannula, and in half an hour evacuate the bladder by passing a catheter, hy pressure on the abdomen, or, if both of these methods fail, by tapping the bladder with a trocar pushed through the linea alba (suprapubic puncture). In an hour again draw off the urine. Test both specimen : for sugar.

In this experiment the opportunity may also be taken to demonstrate that egg-albumin. when injected into the blond, is excreted by the kidneys, a filtered solution containing the albumin of one egg and sugar in the quantity mentioned being injected.

The catheter may be inserted before the injection is begun, and the bladder evacuated. After the injection the urine that drops from the catheter may be collected in test-tubes, first every two minutes, and then, as soon as sugar is found, every ten minutes. Determine the interval between injection and the appearance of the first trace of sugar and albumin. If a sufficient amount of urine is obtained, the 
quantity of sugar in successive specimens may be estimated and com. pared. The rate of thow of the urine as measured by the number of (rops falling from the catheter may also be estimated from time to time in order to determine whether diuresis is taking place.

If a rabbit is used for this experiment, the sugar solution may be injected into the ear vein. The vein is caused to swell up by pressing on it with the finger and thumb, and the hypodermic needle is then inserted towards the heart.

(u) Instead of collecting the urine by a catheter in the bladder, the abdomen of the dog may be opened, and a cannula tied into each ureter. The two cannula are then connected by short rubber tubes with a glass $\mathbf{Y}$-piece, on the stem of which a test-tube is ticd for collecting the urine. Replace the test-tube by a fresh one from time to time. The urine already in the bladder is removed by pressure or by a trocar, and tested for sugar, since the anasthetic itself may cause a certain amount of glycosuria. Test the samples of urine obtained from the ureters for sugar, and in those in which it is present estimate its amount. Note also any changes in the rate of secretion of urine, and any abnormal constituents, as albumin.

(2) Phlorhizin Glycosuria.-Dissolve $\frac{1}{4}$ grm. of phlorhizin in warm water, and inject it subcutancously into a rabbit. Obtain a sample of the urine at the end of two hours, by pressure on the abdomen with the thumb or by passing a catheter, and test for sugar. If none is present, wait some time longer, and again test the urine.

This experiment can also be performed without risk on man. One grm. of phlorhizin has been injected twice a day without disturbing the individual. Nuch sugar is found in the urine, but it disappears the lay after the administration of phlorhizin is stopped. The phlorhizin may also be given by the mouth, but more is required, and it is not very easily absorbed, and often causes diarrhœa (v. Mering).

(3) Alimentary Glycosuria.-The urine having been tested for sugar for two successive days, and none being found--

(a) A large quantity of dextrose is to be taken in the form which is most agreeable to the student some hours after a meal. The urine of the next twenty-four hours is to be collected and measured. A sample of it is then to be tested for reducing sugar by 'Trommer's and the phenyl-hydrazine test. If any sugar is found, the reducing power of a definite quantity of the urine is to be determined by titration witl Fehling's solution (p. 526).

(b) Instead of dextrose use cane-sugar and proceed as in $(a)$. But estimate the reducing power of the urine $(a)$ before and $(\beta)$ after boiling with hydrochloric acid (p. 465).

(c) A large meal of rice and arrowroot, sweetened with as much dextrose as the observer can induce himself to swallow, is to be taken, and the urine treated as in $(a)$.

(d) A large number of sweet oranges may be eaten.*

t. Estimation of the Sugar in Blood-Method of Lewis and Benedict, somewhat modified. - The blood must be used immediately after being clrawn. When only a single sample is required it can be obtained by puncturing a vein with a large-sized hypodermic ncedle. If a number of students have to be provided with blood an animal may be killed by decapitation. Long anzesthetization is to be avoided, as this causes hyperglycæmia. To take blood from a vein, place a few crystals of potassium oxalate in the tip of a 2 c.c. pipette, and having punctured the vein with a needle, draw up blood into the pipette to a little above the

* These experiments may be distributed among the class so that each student does one. 
mark. Allow the blood to run back just to the mark, and immediately discharge the contents of the pijette into a large test tube (about $5^{n}$ c.c. capacity) containing 8 c.c. of distilled water. Shalke until hamolysis is complete. Then add 15 c.c. of saturated aqueous solution of pure picric acid; shake thoronghly to precipitate the proteins of the blood, and filter through a small filter paper. Into each of two long narrow test tubes (capacity about 25 c.c.), which have been marked accurately with a file at 10 c.c., measure 7 c.c. of the filtrate, add 2 c.c. of thie saturated solution of picric acid and 1 c.c. of a to per cent. solution of anhvolrous sodium carbonate. A duplicate determination will thus be made. The test tubes are placed in an antoclave (according to Pearce's modification of the method), and the pressure gradually brought up to 20 to 25 pounds to the square inch $(2 \cdot 5$ kilogrammes to the square centimetre), and lept at this level for twenty-five minutes. Picramic acid is formed by the reducing action of the sugar, and from the amount of picramic acid estimated by a colorimeter the amount of sugar is deduced. Let the antoclave cool down till the pressure is zero, remove the tubes and allow them to cool to room temperature. Sufficient water is now added to each tube to bring the volume back to the ro c.c. mark, and after shaking the contents are filtered through cotton into the (Duboscq) colorimeter bottles. The rletermination is made by comparison with a solution containing a known amount of pure clextrose which has been carried through the same process, or with a picramic acid standard solution.*

A solution of pure dextrose may be used as a stanclard instead of the picramic acid, as follows: Into a test tube place + c.c. of a solution, corresponding to 0.56 milligramme lextrose ; adld 5 c.c. of the saturated picric acid solution, and $\mathrm{I}$ c.c. of o per cent. solution of anhydrous sodium carbonate. Place in the autoclave and proceed as above described, bringing the final volume again to 10 c.c., and filtering through cotton into one of the colorimeter bottles.

Calculation :

Reading of standard $\times$ mgm. dextrose in standard $)={ }^{m g m}$. dextrose per Reading of unknown $\times$ c.c. blood used $j=$ c.c. blood.

The amount of blood taken for each of the duplicate determinations is $\Sigma^{7} \times 2$ c.c. $=0.56$ c.c. The standard $=0.56$ milligramme dextrose in Io c.c., so that the second fraction on the left sicle of the equation becomes unity, and the reading of the stanclard clivided by the reading of the unknown gives at once the number of milligrammes of dextrose in 1 c.c. of blood.

5. Milk. - (1) Examinc a drop of fresh cow's milk with the microscope. Note the fat globules of varions sizes.

(2) Determine the specific gravity of the milk with a liydrometer (lactometer). Then centrifugalize some of the milk to separate the cream, which rises to the top of the tubes. Remove the cream and determine the specific gravity of the skimmed milk. It will be found to have increased, since the fat is of lower specific gravity than the rest of the milk. Normal cow's milk has a specific gravity of $\mathrm{I}, 02 \mathrm{~S}$ to $\mathrm{I}, 034$, skimmed milk $1,033^{\circ}$ to $1,0,37$.

* Picramic acid, 50 milligrammes, anhydrous sodium carbonate, roo milligramme's, and distilled water to make up I,Ooo c.c. Dissolve the sodium carbonate in about 50 c.c. of water, add the picramic acid and dissolve it with the aid of heat. When cooled to room temperature add enough water to make $r, 000$ c.c. Picramic acid obtained on the market varies in the amount of colour produced in the preparation of this solution. The standard should therefore be compared with a solution of pure dextrose which has been standard. ized by another method, and then treated by the Lewis and Benedict method. 
(3) lest the reaction of the nilk to litmus-paper. It is slightly alkaline.

(4) (a) l'ut Io c.c. of milk in a test-tule, and nearly fill it up with water. Add strong acetic acid drop by drop. A precipitate of caseinogen is thrown lown which entangles the fat, and carries it down mechanically along with it. Filter off the precipitate. Keep the filtrate for $(b)$. Wash the precipitate with water, scrape a portion of it off the filter, and add to it some 2 per cent. sodiun carbonate solution. The caseinogen (lissolves, while the fat remains in suspension. The solution gives the colour reactions for proteins ( $p .8)$.

(b) Test some of the filtrate (p. 10) for lactose. Add dilute sodium carbonate solution to another portion till it is only slightly acid. Boil, and lactalbumin is coagulated. Remove the lactalbumin by filtering. and test this filtrate for eartly (i.e., calcium and magnesium) phosphates by adding a few drops of ammonia, which precipitates them as a slight cloud.

(c) To 5 c.c. of milk add an equal volume of saturated ammonium sulphate solution. The caseinogen is precipitated, entangling the fat. Filter off. The filtrate may be used to test for lactalbumin by boiling. The addition of water to the precipitate of caseinogen (and fat) on the filter causes the cascinogen to dissolve, as it is soluble in weak salt solutions. Caseinogen can also be precipitated by saturating milk with sodium chloride or magnesium sulpliate.

(5) To 5 c.c. of milk add a couple of drops of 20 per cent. sodinm or potassium hydroxide, and then a few c.c. of ether. Shake up. The ether dissolves the fat, and the opacity of the milk diminishes. Take off the ether with a pipette, evaporate away most of it on a water-bath, and place a drop or two of the remainder on a filter-paper. A greasy stain is left, slowing the presence of the fat of the milk, or butter.

(6) Clotting of Milk.-(a) To a few c.c. of milk in a test-tube add a few drops of rennet. Place the tube in a bath at $40^{\circ} \mathrm{C}$. In a short time a clot or curd is formed, consisting of casein, which is derived from the caseinogen. The fat is entangled in the clot. On standing some time the clot contracts, and exudes the whey. Boil some of the whey after slight acidulation with acetic acid; the lactalbumin and wheyprotein are coagulated. Test another portion of whey for proteins by one of the general protein tests (p. 8)-e.g., the xanthoproteic.

(b) Repeat (a) but use rennet which has been previously boiled. The milk is not curdled, because the ferment has been inactivated by boiling.

(c) To Io c.c. of milk add 3 c.c. of I per cent. potassium oxalate. Divide the oxalated milk into three portions- $\mathrm{A}, \mathrm{B}$, and $\mathrm{C}$. To $\mathrm{A}$ add a few drops of rennet, to B I c.c. of 2 per cent. calcium chloride solution and a little rennet, and to $\mathrm{C} I$ c.c. of 2 per cent, calcium chloride solution alone. Put the tubes at $40^{\circ} \mathrm{C}$. Clotting will occur in $\mathrm{B}$, but not in $\mathrm{A}$ or $\mathrm{C}$.

6. Cheese.-(I) Rub up some finely-grated cheese in a mortar with 2 per cent. sodium carbonate solution. Filter. The filtrate contains casein, which can be precipitated by adding lilute acetic acid by drops to a portion of the filtrate. The precipitate is soluble in excess of the acid. With another portion of the filtrate perform some of the general protein tests (p. 8).

(2) Shake up some finely-grated cheese in a dry test-tube with ether. Take off the ether with a pipette, and evaporate on a water-bath till only a few drops remain. With a glass rod put a drop of the ether on a piece of filter-paper. A greasy spot is left, showing that fat is present.

7. Flour.-(r) Mix some wheat-flour with a little water into a stiff dough. Let it stand for a few minutes at body-temperature to facilitate the formation of gluten. Wrap a piece in cheese-clotl, forming a kind 
of bag, and knead it with the fingers in a capsule of water. Tho starch grains cone throngli the cheese-cloth. Pour the water into a beaker. It is opaque, and on standing the starch grains sink to the bottom. (a) Test for starch with the iodine test, and also examine microscopically. The grains are round, witl a central hilum, and are smaller than those of potato starch (p. II). (b) Test for sugar by Trommer's test (p. 10). None is present unless tlie flour has been made from inferior grain in which some germination has taken place.

(2) Go on lineading the dough till no more starch comes through. The sticky mass which remains in the bag is a protein called gluten, which is formed from certain globulins and other proteins in the flour on addition of water. Oatmeal, gronnd rice, and other grains poor in gluten-forming globulins do not form dougl when mixed with water. Suspend some of the gluten in water in a test-tube, and apply to it the general protein colour tests (p. 8).

8. Bread.-(I) Rub up a small piece of the crumb of a stale loaf in a mortar with water. Strain through cheese-cloth. The fluid which passes through contains starch grains. (a) Filter it, and test a portion of the filtrate for dextrose by Trommer's test. A positive result is obtained. Test another portion with iodine for erythrodextrin. (b) Test a portion of the residue of the bread which has not passed through the cheese-cloth for protein by the general protein tests-e.g., the xanthroproteic or Millon's tests.

(2) Repeat (I) using the crust of the bread. Both dextrose and erythrodextrin are present in the cold-water extract, but the dextrose is less plentiful than in the crumb, having been converted into caramel in the baking. The sugar and dextrin are formed from the starch of the flour by the ferments of the yeast employed to make the bread rise.

9. Variations in the Total Nitrogen (p. $52 \mathrm{I}$ ) and in the Quantity of Urea excreted, with Variations in the Amount of Proteins in the Food.The student should put himself, or somebody else if he can, for two days on a diet poor in proteins, then (after an interval of forty-eight hours on his ordinary food) for two days on a diet rich in proteins. A suitable table of diets will be supplied. The urine should be collected on the six days of the period of experiment, on the day before it begins, and on the day after it ends. Small samples of the mixed urine of the twenty-four hours for each of these eight days should be brought to the laboratory, and the quantity of urea determined by the hypobromite method. The volume of the urine passed in each interval of twentyfour hours being known, the total excretion of urea for the twenty-four hours can be calculated, and a curve plotted to show how it varies during the period of experiment.* If sufficient time is available, the experiment will be made still more instructive by determining the total nitrogen in each sample in addition to the urea. A curve showing the variation in the total nitrogen can tien be plotted on the same pajer as the urea curve, and a table calculated giving the percentage of the total nitrogen contained in the urea for each day of the experiment.

10. Action of Epinephrin (Adrenalin).-Several experiments to illustrate this are given in the Practical Exercises following otlier chapters, but may equally well be performed here. (See Experiment 8, p. 66; Experiment 3. p. 453:)

* In 17 healthy students the average amount of urea excreted in twenty four hours on the ordinary diet was $29^{\circ} 5^{\text {I }}$ grammes (minimum i9.35 grammes maximum 46.01 grammes); on a diet poor in protein, average $20^{\circ} 75$ grammes (minimum $9^{\circ} 52$ grammes, maximum $32^{\circ} 86$ grammes); on a diet rich in protein, a verage $3^{8 \cdot 8}$ grammes (minimum $23^{\circ} 26$ grammes, maximum $67^{\circ} 82$ grammes). 
11. * Measurement of the Quantity of Heat given off in Respiration.This may be done approximately as follows: Put in the inner copper vessel, A, of the calorineter shown in Fig. $22+($ p. 682) a measured quantity of water sufficient to completely cover the series of brass dises. Place $\mathrm{A}$ in the wide outer cylinder, the bottom of which it is prevented from touching by pieces of cork. The outer cylinder hinders loss of heat to the air. Suspend a thermometer in the water tlirough one of the holes in the lid. In the other liole place a glass rod to serve as a stirrer, Read off the temperature of the water. Put the glass tube connected with the apparatus in the mouth, and breathe out through it as regularly and normally as possible, closing the opening of the tube with the tongue after each expiration and breathing in through the nose. Continue this for five or ten minutes, taking care to stir the water frequently. Then read off the temperature again. If $\mathrm{W}$ be the quantity of water in C.C., and $t$ the observed rise of temperature in degrees Centigrade, Wt equals the quantity of heat, expressed in small calories (p. 675), given off by the respiratory tract in the time of the experiment, on the assumptions $(x)$ that all the heat has been absorbed by the water, (2) that none of it has been lost by radiation and conduction from the calorimeter to the surrounding air. Calculate the loss in twenty-four hours on this basis; then repeat the experiment, breathing as rapidly and deeply as possible, so as to increase the amount of rentilation. The quantity of heat given off will be found to be increased. $\dagger$

In an experiment of short duration (2) is approximately fulfilled. As to (I), it must be noted that in the first place the metal of the calorimeter is heated as well as the water, and the water-equivalent of the apparatus must be added to the weight of the water $(p .676)$. The water-equivalent is determined by putting a definite weight of water at air temperature $\mathrm{T}$ into the calorimeter, and then allowing a quantity of hot water at known temperature ' $T$ ' to run into it, stirring well, and noting the temperature of the water when it has ceased to rise. Call this temperature $T^{\prime \prime}$. Enough hot water should be added to raise the temperature of the calorimeter about $2^{\circ} \mathrm{C}$. The quantity run in is obtained by weighing the calorimeter before and after the hot water has been added. Suppose it is $m$. Let the mass of the cold water in the calorimeter at first be $M$, and let $M^{\prime}=$ the mass of water which would be raised $\mathrm{r}^{\circ} \mathrm{C}$. in temperature by a quantity of heat sufficient to increase the temperature of all the metal, etc., of the calorimeter by $I^{\circ}$-in other words, the water-equivalent of the calorimetor.

The mass $m$ of hot water has lost heat to the amount of $m$ ( $\left.\mathrm{T}^{\prime}-\mathrm{T}^{\prime \prime}\right)$, and this lias gone to raise the temperature of a mass of water $M$, and metal equivalent to a mass of water $\mathrm{M}^{\prime}$, by $\left(\mathrm{T}^{\prime \prime}-\mathrm{T}\right)$ degrees. $\therefore m\left(\mathrm{~T}^{\prime}-\mathrm{T}^{\prime \prime}\right)=\mathrm{M}\left(\mathrm{T}^{\prime \prime}-\mathrm{T}\right)+\mathrm{M}^{\prime}\left(\mathrm{T}^{\prime \prime}-\mathrm{T}\right)$. Everything in this equation except $\mathrm{M}^{\prime}$ is known, and $\therefore \mathrm{M}^{\prime}$, the water-equivalent of the calorimeter, can be deduced, and must be added in all exact experiments to the mass of water contained in it.

Secondly, all the excess of heat in the expired over that in the inspired air is not given off to the calorimeter, for the air passes out of it at a slightly higher temperature than that of the atmosphere. At the beginning of the experiment this excess of temperature is zero. If at the end it is $\mathrm{I}^{\circ} \mathrm{C}$., the mean excess is $0.5^{\circ} \mathrm{C}$. Now, when respiration

* This experiment is given as an example of a simple calorimetric measurement, which can be easily performed with sufficient accuracy by students, and involves the essential principles of such determinations.

t The average heat-loss by the lungs for $5 \mathrm{I}$ men (calculated for the 24 hours) was 312,000 small calories for normal, 919,000 for the fastest, and 195,000 for the slowest breathing. 
is carried on in a room at a temperature of $10^{\circ} \mathrm{C}$., the expired air has its temperature increased by nearly $30^{\circ} \mathrm{C}$. About of of the heat given off by the respiratory tract in raising the temperature of the air of respiration would accordingly be lost in such an experiment. But since the portion of the heat lost by the lungs which goes to heat the cxpired air is only $\frac{1}{6}$ of the whole heat lost in respiration (p. 682), the error would only amount to $\frac{1}{3+0}$ of the whole, and this is negligible.

"Thirdly, the air leaves the calorimeter saturated with watery vapour at, say: $10.5^{\circ}$, while the inspired air is not saturated for $10^{\circ} \mathrm{C}$. Now, the quantity of heat rendered latent in the evaporation of water sufficient to sisturate a given quantity of air at $40^{\circ} \mathrm{C}$. (the expired air is saturated for body-temperature) is six times that required to saturate the same quantity of air at $10^{\circ}$. If, then, the inspircd air is half saturated, the ertor under this head is $r$, or $8 \frac{1}{2}$ per cent. If the inspired air is three-quarters saturated, the error is 24 , or about 4 per cent. If the air is fully saturated before inspiration, as is the case when it is drawn in througl a water-valve (Fig. 229) by a tube fixed in one nostril, the only error is that due to the slight excess of temperature of the air leaving the calorimeter over that of the inspired air. The latent heat of the aqueous vaponr in saturated air at $10.5^{\circ} \mathrm{C}$. is about $\frac{x}{20}$ more than the latent heat of the aqueous vapour in the same mass of saturated air at $10^{\circ} \mathrm{C}$., or about $T^{?}$ of of the latent heat in saturated air at $40^{\circ}$. The error in this case would therefore be under I percent. Tlee tubes must be wide and the bottle large.

12. In the observations on the blood-flow in the hands (Experiment 31, p. 219) data on the quantity of heat given off by the hands when immersed in water at a given temperature have already been obtained. Additional data slould be got by putting the liand into the calorimeter witlout previous immersion in the bath, and comparing the heat given off during the period when the hand is acquiring the temperature of the calorimeter with that given off when the steady state has been reached. Different calorimeter trmperatures should be employed. It will be found that as the calorimeter temperature is diminished the

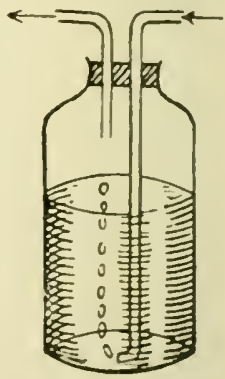

Fig. 229. - Bottle arranged for Water-Valve. quantity of heat given off may be increased although the blood-flow is diminished, each gramme of blood passing through the hand giving off more heat the lower the calorimeter temperature.

The quantity of heat lost by the hand, at a given temperature of the calorimeter, per square centimetre of skin surface can be calculated. If no special instrument for measuring the area of irregular surfaces is available, the surface of the hand can be arrived at approximately by covering it with strips of gummed paper of known breadth, and noting the length used to cover the whole hand up to the lower level of the styloid process of the ulna. Or an old thin glove which fits the hand can be cut off at this level and weighed. As large a piece as possible of regular shape is then cut from the glove, weighed, and its area deduced by measuring it with a rule. The ares of the whole glove, on the assumption that it is of uniform thickness, is thus known. Or, without cutting the glove, it may be laid flat on a piece of paper, an outline of it traced, and the paper cut out. The weight of the paper cut out is compared with tliat of a piece of paper of known area, and its area deduced. Dbviously this is approximately equal to balf the surface of the hand. 


\section{CHAPTER XIII}

\section{THE PHYSIOLOGY OF THE CONTRACTILE IISSUES}

\section{Section I. - Preliminary Observations - Physical axd TECHNICAL DATA.}

In all the great functions of the body muscular movements play an essential part. The circulation and the respiration, the two functions most immediately essential to life, are kept up by the contraction and relaxation of muscles. The movements of the digestive canal, the regulation of the blood-supply to its glands and to all parts of the body, and that immense class of movements which we call voluntary, are all dependent upon muscular action, which, again, is indebted for its initiation, continuance, or control, to impulses passing along the nerves from the nerve-centres. Hitherto we have not gone below the surface fact, that muscular fibres have the power of contracting, either automatically, or in response to suitable stimuli. In this chapter and the two next we shall consider in detail the general properties of muscle, nerve, and the other excitable tissues.

Lying deeper than the peculiarities of individual muscles, muscular tissue has certain common properties-physical, chemical, and physiological. The biceps muscle flexes the arm upon the elbow, and the triceps extends it. The external rectus rotates the eyeball outwards. The intercostal muscles elevate the ribs. The sphincter ani seals up by a ring-like contraction the lower end of the alimentary canal. These actions are very different, but the muscles that carry them out are at bottom very similar. And it cannot be doubted that the functional differences are due entirely, or almost entirely, to differences of anatomical connection, on the one hand with bones and tendons, on the other with the nerve-cells of the spinal cord and brain. The common properties in which all the skeletal muscles agree are the subject-matter of the general physiology of striated muscle.

The cardiac muscle differs more, both in structure and in function, from the skeletal muscles than these do among themselves; the smooth muscle of the intestines and bloodvessels still more. But every muscular fibre, striped or unstriped, resembles every other 
muscular fibre more than it does a nerve-fibre or a gland-cell or an epithelial scale. The properties common to all muscle make up the general physiology of muscular tissue.

A nerve-fibre is at first sight very different from a muscular fibre. It has diverged more widely from the primitive type of undifferentiated protoplasm, but it retains, in common with the musclefibre, susceptibility to stimulation, or excitability, the capacity for growth, and to a limited extent the capacity for reproduction; and while it has lost the power of contraction or contractility, it has developed in a higher degree than any other tissue the power of conducting the excited state. This inheritance of primitive properties, retained alike by both tissues, is the basis of the general physiology of muscle and nerve.

The electrical organ of Torpedo or Malapterurus is intermediate in some respects between muscle and nerve, and has properties common to both. In the gland-cell the chemical powers of native protoplasm have been specialized and developed. Contractility has been, in general, entirely lost; but excitability remains. The idea that certair common endowments find expression in the action of muscle, nerve, electrical organ, gland, etc., in the midst of all their apparent difierences, is the basis of the general physiology of the excitable tissues.

It is impossible to understand the general physiology of muscle and nerve without some acquaintance with electricity. It would be out of place to give even a complete sketch of this preliminary but essential

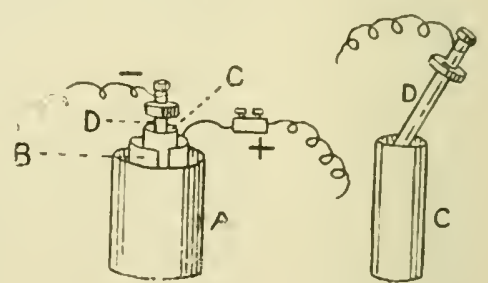

Fig. 230.-Daniell Cell. A, outer vessel; B, copper; C, porous pot: D. zinc rod; $\mathrm{D}$ is supposed to be raised a little so as to be seen.

knowledge here; and the student is expressly warned that in this book the elementary facts and principles of physics are assumed to be part of his mental outfit. But in describing some of the electrical apparatus most commonly used in the study of this portion of our subject, and which are employed in the Practical Exercises, it may be useful to recall some of the plyysical facts involved.

Batteries. - The Danicll cell is perhaps better suited for physiological work than any other voltaic element, although for special purposes Grove, Leclanché, bichromate of potassium or dry batteries may be employed (p. 197). Storage batteries or current from the street supply may also be used.

Inside the Daniell cell the current (the positive electricity) passes from zinc to copper; outside, from copper to zinc. The copper is called the positive, the zinc the negative, pole. When the current is passed through a tissue, the electrode by which it enters is termed the anode, and that by which it leaves the tissue the kathode. The anode is, therefore, the electrode connected with the copper of the Daniell's cell; the kathode is connected with the zinc.

Potential-Current Strength-Resistance.-We do not know what in reality electricity is, but we do know that when a current flows along 
a wire energy is expended, just as energy is expended when water flows from a ligher to a lower level. Many of the phenomena of current electricity can, in fact, be illustrated by the laws of flow of an incom. pressible liquid. The difference of level, in virtue of which the flow of liquid is maintained, corresponds to the difference of electrical level, or potential, in virtue of which an electrical current is kept up. The positive pole of a voltaic cell is at a higher potential than the negative. When they are connected by a conductor, a flow of electricity takes place, which, if the difference of level or potential were not constantly restored, would soon equalize it, and the current would cease; just as the flow of water from a reservoir would ultimately stop if it was not replenished. If the reservoir was small, and the discharging-pipe large, the flow would only last a sliort time; but if water was constantly being pumped up into it, the flow would go on indefinitely. This is practically the case in the Daniell cell. Zinc is constantly being dissolved, and the chemical energy which thus disappears goes to maintain a constant difference of potential between the poles. Electricity, so to speak, is continually running down from the place of higher to the place of lower potential, but the cistern is always kept full.

The difference of electrical potential between two points is called the electromotive force: and from its analogy with difference of pressure in a liquid, it is easy to understand that the intensity or strength of the current-that is, the rate of flow of the electricity between two points of a conductor-does not depend upon the electromotive force alone, any more than the rate of discharge of water from the end of a long pipe depends alone on the difference of level between it and the reservoir. In both cases the resistance to the flow must also be taken account of. With a given difference of level, more water will pass per second through a wide than through a narrow pipe, for the resistance due to friction is greater in the latter. In the case of an electrical current, a wire connecting the two poles of a Daniell's cell will represent the pipe. A thick sliort wire has less resistance than a thin long wire; and for a given difference of potential, of electric level, a stronger current will flow along the former. But for a wire of given dimensions, the intensity of the current will vary with the electromotive force. The relation between electromotive force, strength of current, and resistance were experimentally determined by $\mathrm{Ohm}$, and the formula $\mathrm{C}=\frac{\mathrm{E}}{\mathrm{R}}$, which expresses it, is called Ohm's Law. It states that the current varies directly as the electromotive force, and inversely as the resistance.

For the measurement of electrical quantities a system of units is necessary. The common unit of resistance is the ohm, of current the ampere, of electromotive force the volt. The electromotive force of a Daniell's cell is about a volt. An electromotive force of a volt, acting through a resistance of an ohnt, yields a current of one ampère. But the current produced by a Daniell's cell, with its poles connected by a wire of I ohm resistance, would be less than an ampère, because the internal resistance of the cell itself - that is; the resistance of the liquids between the zinc and the copper-must be added to the external resistance in order to get the total resistance, which is the quantity represented by $\mathrm{R}$ in Ohm's Law.

Measurement of Resistance.-.To find the resistance of a conductor, we compare it witl known resistances, as a grocer finds the weight of a packet of tea by comparing it with known weights. The Wheatstone's bridge method of measuring resistance depends on the fact that if four resistances, $\mathrm{AB}, \mathrm{AD}, \mathrm{BC}, \mathrm{CD}$, are connected, as in Fig. $23 \mathrm{I}$, with each 
other, and with a galvanometer $G$, and a battery, F, no current will flow through the galvanometer wi.en $\frac{A B}{A D}=\frac{B C}{C D}$.

In making the measurement, a resistance box, containing a large number of coils of wire of different resistances, is used. The resistances

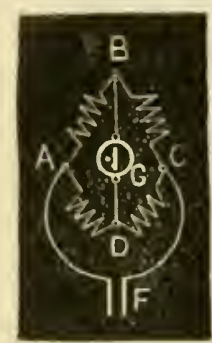

rig. 23r. -Wheat. stone's Bridge. corresponding $10 \therefore B$ and $A D$ may be made equal, or may stand to each other in a ratio of $1: 10,1: 100$, etc. Then, the mnknown resistance being $C D$. BC is arljusted by taking piugs out of the box till, on closing the current, there is either no deflection, or the deflection is as small as it is possible to make it with the given arrangement.

Galvanometers. - A galvanometer is an instrument used to rletect a current, to determine its dircetion, and to measure its intensity. Since, by Ohm's law, clectromotive force, resistance, and current stręngth are connected together, any one of them may be measured by the galvanometer. A galvanometer of the kind ordinarily used in physiology consists essentially of a small magnet suspended in the axis of a coil of wire, and free to rotate under tlie influence of a current passing through the coil. The most sensitive instruments possess a small mirror, to which the magnet is rigidly attached. A ray of light is allowed to fall on the mirror, from which it is reflected on to a scale; and the rotation of the mirror is magnified and measured by the excursion of the spot of light on the scale. The method of reading by a telescope can be applied to any mirror galvanometer, $B$ and is often extremely convenient in physiolngical work. Sometimes a small scale is fastened on the mirror itself, and ohserved directly through a low-power microscope.

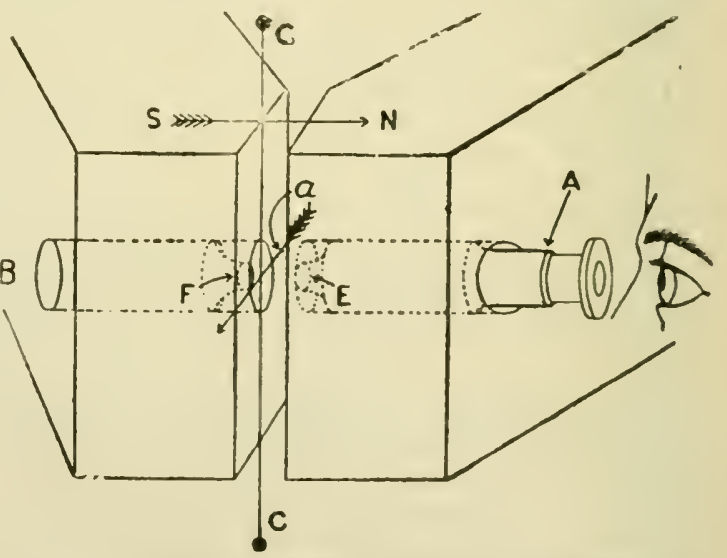

Fig. 232.-Diagram of String Galvanometer. The string or fibre $\mathrm{CC}$ is stretched belween the poles of a puwerful electromagnet. When a current passes down the string it is deflected in the direction of the largo arrow a-r.e., al right angles to the magnetic field NS. When tho current is reversed, the siring moves in the opposite direction. The movements of the string can be observed by a microscope, A (objective E), passing through a hole bored through the centre of the magnet poles. For obtaining records a source of light is placed at $\mathrm{B}$ and concentrated on the fibre by a condenser, $F$, and the movements of the shadow are recorded by pholography.

In the d'Arsonval galvanometer the current passes through a small coil of fine wire suspended in the field of a strong magnet. When the current passes the coil is deflected, carrying with it a small mirror at tached to the suspending filament. \& great advantage of this galvanometer in many-situations is that it is unaffected by neighbouring currents. 
The string galvanometer of Einthoven has peculiar merits for certain physiological purposes. It consists of a silvered quartz-or glass-fibre stretched in a very strong magnetic field. When traversed by a current

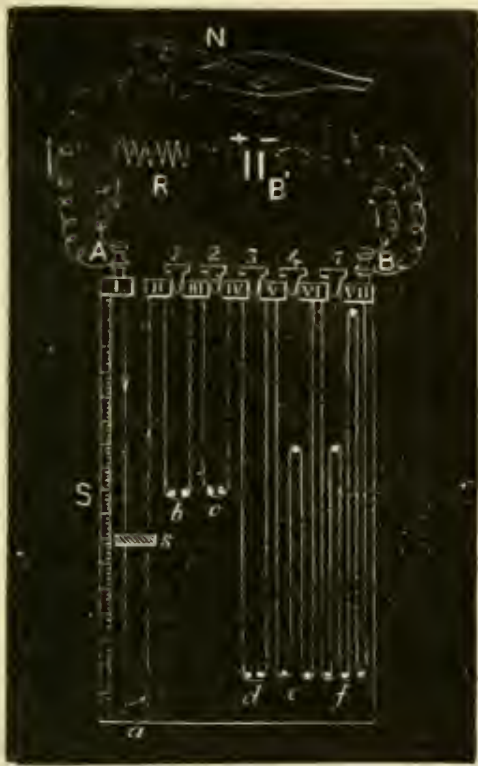

Fig. 233-Diagram of Rheocord (after Du Bois-Reymond's Model). the fibre is deflected, and by means of a heam of light the deflection is greatly magnified (Fig. 232).

$A$ rheocord is an instrument by means of which a current may be divided, and a lefinite portion of it sent through a tissue (Fig. 233).

A compensator is simply a rheocord from which a branch of a current is led off, to balance or 'compensate' any electrical difference in a tissue, like that which gives rise to the current of rest of a muscle, for example (Fig. 23t).

An electrometer is an instrument for measuring electromotive force-

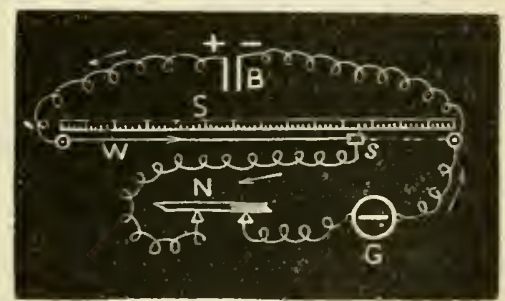

Fig. 234--Compensator.

Description of Fig. 233: I to VII are pieces of brass connected with the wires $a$ to $f$ in such a way that, by taking out any of the brass plugs $I$ to 5 , a greater or less resistance may be interposed between the binding-screws $A$ and $B$. The two wires $a$ are connected by a slider $s$, filled with mercury or otherwise making contact between the wires. The current from the battery $B$ divides at $A$ and $B$, part of it passing through the rheocord, part through $\mathrm{N}$, the nerve, muscle, or other conductor which forms the alternative circuit. When a sufficient resistance $R$ is interposed in the chief circuit to make the total strength of the current independent of changes in the resistance of the rheocord, the strength of the current passing through $N$ will vary inversely as the resistance of the rheocord. When all the plugs are in, and the slider close up to $\mathrm{A}$, there is practically no resistance in the rheocord, and all the current passes across the brass pieces and plugs to $B$, and thence back to the battery. As $s$ is moved father away from $A$, the resistance of the rheocold is increased more and more, and the intensity of the current passing through $\mathrm{N}$ becomes greater and greater. The scale $\mathrm{S}$ show's the length of wire interposed for any position of $s$, and this gives a rough measure of the fraction of the current passing through $N$. When plug $I$ or 2 is taken out, a resistance equal to that of the two wires $a$ is interposed; plug 3 , twice that of $a$; plug 4 , five times; plug 5, ten times.

Description of Fig. $234: W$ is a wire stretched alongside a scale $S$. A battery B is connected to the binding-screws at the ends of the wire. A pair of unpolarizable electrodes are connected, one with a slider moving on a wire, the other through a galvanometer with one of the terminal binding-screws. In the figure a nerve is shown on the electrodes, one of which is in contact with an uninjured portion, the other with an injured part. The slider is moved until the twig of the compensating current just balances the demarcation current of the nerve and the galvanometer shows no deflection. 
that is, dıfferences of electric potential. Lippmann's capillary clectrometer las been much employerl in physiology. A simple form, suitable for students working in a class where a considerable number of copies of the instrument is needed, can be conveniently mate as follows: A glass tube is drawn out to a capillary at one end and filled with mercury. The tube is inserted into a small glass bottle,* and fastened in its neck by a cork or a plug of sealing-wax which does not quite fill the opening, so that the interior of the bottle is still in communication with the external air. The upper end of the tube is connected by a sliort piece of rubber tubing with a glass $\mathbf{T}$-tube as in Fig. 235 . The bottle is partially filled with 5 to 10 per cent. sulphuric acid, inder which the capillary dips. By means of a small reservoir made from a piece of glass tubing filled with mercury, and commected with the stem of the T-tube, a little mercury is forced through the capillary so as to expel the air in it. When the pressure is lowered again, sulphuric aciol is drawn up, and now lies in the capillary in contact with the meniscus of the nercury. A platinum wire fused through the tube, or simpiy inserted through its upper end, dips into the mercury. Another, passing through the cork, or, better, fused through the bottom of the bottle, makes contact with the sulphuric acicl through some mercury. The bottle is fastened on the stage of a microscope, the capillary brought into focus, and the meniscus adjusted by raising or lowering the reservoir. When the platinum wires are connected with points at different potential, a current begins to pass though the instrument, and the meniscus of the mercury in the capillary tube, where tle current density is the greatest, becomes polarized by the ions separated from the sulphuric acid at the surface of contact between the acid and the mercury, so that the meniscus is no longer in equilibrium in the tube. The surface tension $(p .+2 g)$ is diminished when the direction of the current is from mercury to acid (mercury at a higher potential than acid), and is no longer able to counterbalance the hydrostatic pressure of the mercury. The meniscus therefore moves down in the tube. With the opposite diection of current (mercury at a lower potential than acid) the surface tension is increased, and the meniscus moves up. The polarization develops itself almost instantaneously, and thus an clectromotive force is at once established in the opposite direction to that between the points connected with the electrometer, and equal to it so long as the external electromotive force is not sufficiently great to cause continuous electrolysis of the acirl that is, so long as it is below about 2 volts. The external current is therefore at once compensated, and after the first moment no current passes through the instrument, which is accordingly not a measurer of current, but of clectromotive force.

Induced Currents.-When a coil of wire in which a current is flowing is brought up suddenly to another coil, a momentary current is developed in the stationary coil in the opposite direction to that in the moving coil. Similarly, if instead of one of the coils being moved a current is sent through it, while the other coil remains at rest in its neighbour-

* A parallel-sided bottle is best, as it gives the clearest image of the meniscus. But it is rasiest to make a cylindrical bottle from a piece of wide glass tubing, and to insert a platinum wire into it before closing it at the bottom in the blow-pipe flame. The tube can then be firmly fastened with sealing-wax in a dejression in a piece of wood the wire being bronght out through a hole in the wool. Once the instrument is arranged there is little clance of the capillary getting broken, and there is very little craporation of the acicl. 
hool, a transient oppositely-directed current is set up in the latter. When the current in the first coil is broken, a current in the same direction is induced in the otler coil.

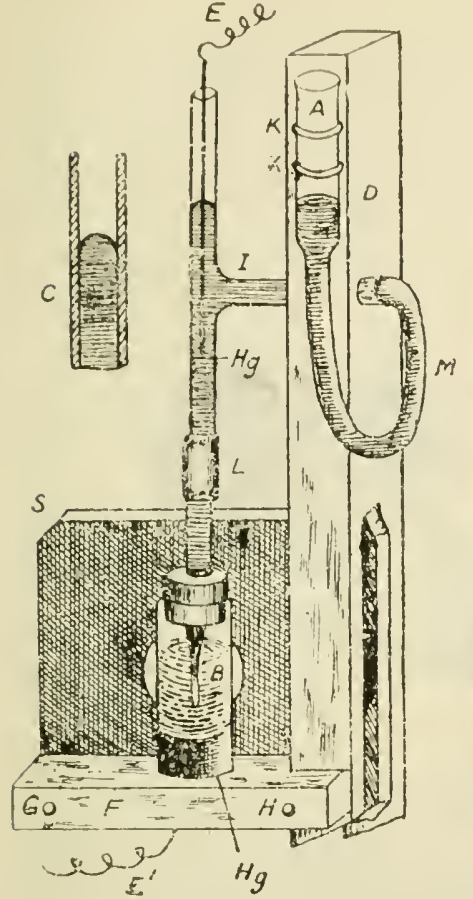

Fig. 235.

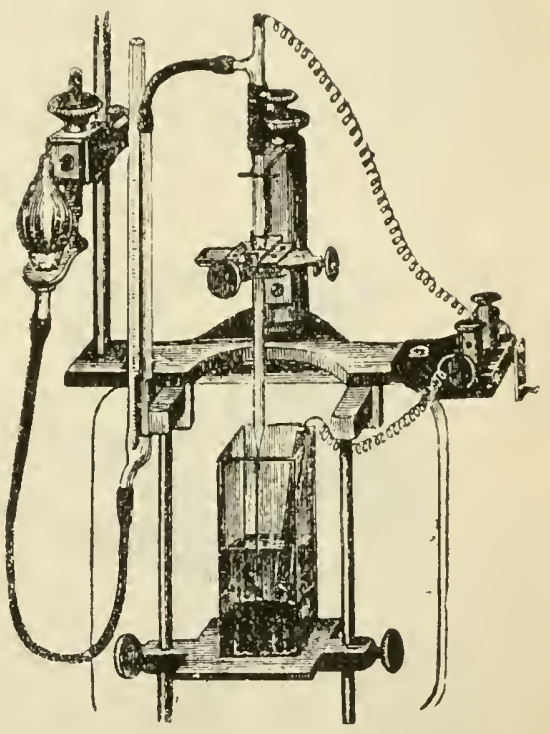

Fig. 236 .

Fig. 235.-A Simple Capillary Elec:rometer. B. bottle containing sulphuric acid; $H g$, mercury; $E, E^{\prime}$, platinum wires. $E$ dips into the mercury in the vertical tube, and $E^{\prime}$ is fused through the bottom of $B$, so as to make contact with the mercury in $B$, the other end of it passing out through a small hole in the wooden platform $F$, on which $B$ rests. $F$ is fastened to the stage of the microscope $S$ by a pin, $G$, passing through one of the clip-holes, and to the wooden upright $D$ by the pin $H . D$ fits tightly over the microscope stage, but cun be moved laterally a little so as to bring the capillary into the middle of the field. I, stem of glass T-tube passing through a hole in $D$. $L$, rubber tube connecting the capillary point with the vertical portion of the T-tube. $A$ is a reservoir containing mercury connected by the rubber tube $M$ to $I$. A can be raised or lowered by sliding it in the clips $K$. C, magnified poition of the capillary tube showing the meniscus.

Fig. 236.-Capillary Electrometer (after Frey).

Du Bois-Reymond's Sledge Inductorium (Fig. 237).-This consists of two coils, the primary and the secondary, the former having a comparatively snall number of turns of fairly thick copper wire, the latter a large number of turns of thin wire. The object of this is that the resistance of the primary, which is connected with one or more voltaic 
cells, may not cut down the current too much; while the currents induced in the secondary, having a ligh electromotive force, can readily pass through a high resistance, and are directly proportional in intensity to the number of turns of the wire.

By means of various bincling-serews and the electro-magnetic interrupter or Neef's hammer, shown in the figure and explained below it, the current can be made once in the primary or broken once, or a constant alternation of make and break can be kept up. We can thus get a single make or break shock in the secondary, or a series of shocks, sometimes called an interrupted or faradic current. Such a series of stimuli can also be got by making and breaking a voltaic current at any given rate.

A 'self-incluced ' current can also be obtained from a single coil; for instance, from the primary coil alone of the induction apparatus. The

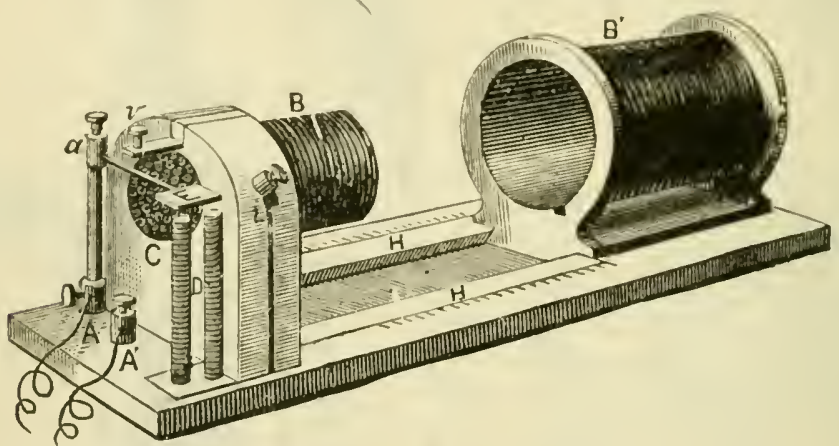

Fig. 237.-Du Bois-Reymond's Inductorium. B, primary, B', secondary, coil, H, guides in which $\mathrm{B}^{\prime}$ slides, with scale; 1 , electro-magnet; E, vibrating spring; $i$, wire connecting wire of $\mathrm{D}$ to end of primary; $v$, screw with platinum point, connected with other end of primary; $\mathrm{A}, \mathrm{A}^{\prime}$, binding-screws, to which are attached the wires from battery. $A^{\prime}$ is connected with the wire of the electro-magnet $D$; and through it and $i$ with the primary.

reason of this is, that when a current begins to flow through any turn of a coil of wire it induces in all the other turns a current in the opposite direction, and, when it ceases to flow, a current in the same direction as itself. The former current, 'the make extra shock,' being in the opposite direction to the inducing current, is retarded in its development, and reaches its maximum more slowly than 'the break extra shock.' But, as we shall see, the suddenness with which an electrical change is brought about is one of the most important factors in electrical stimulation, and therefore the break extra shock is a much more powerful stimulus than the make. Owing to these self-induced currents, the stimulating power of a voltaic stream may be much increased by puttung into the circuit a coil of wire of not too great reristance.

The self-induction of the prinary also affects the stimulating power of the currents induced in the secondary; the shock induced in the secondary by break of the primary current is a stronger stimulus than that caused at make of the primary. 'The reason is that with a given distance of primary and scconclary, and a given intensity of the voltaic current in the primary, the abruptness with which the induced current 
in the secondary is developel depends upon the rapidity with which the primary current reaches its maximum at closing, or its minimum (zero) at opening. Now, the make extra current retards the development of the primary current, while in the opened circuit of the primary coil the current intensity falls at once to zero.

The ineruality between the make and break shocks of the secondary coil can he greatly reduced by means of Helmholtz's wire. Connect one pole of the battery with $v$ (Fig. 237), and the other with $A^{\prime}$. Join $A$ and $\lambda^{\prime}$ by a sliort, thick wire. With this arrangement the primary circuit is never opened, but the current is alternately allowed to flow through the primary, and short-circuited when the spring touches $\tau^{\prime}$. The 'make now corresponds to the sudden increase of intensity of the current in the primary when the shori-circuit is removed, and the 'break' to its sudden decrease when the sliort-circuit is established. ln botl cases self-induced currents are developed, and therefore both shocks are weakened. But the opening stimulus is now slightly the weaker of the two, because the opening extra shock has to pass th-ough a smaller resistance (the short-circuit) than the closing extra shock (which passes by the battery), and therefore opposes the decline of current intensity on short-circuiting more than the rlosing shock opposes the increase of current intensity on longcircuiting through the primary.

By means of wires connected with the terminals of the secondary coil, and leading to electrodes, a nerve or muscle may be stimulated. It is usual to connect the wires to a short-circuiting key
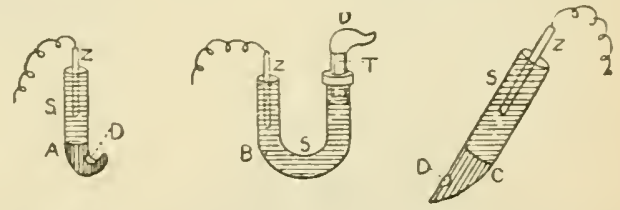

Fig. 238. - Unpolarizable Electrodes. A, hook. shaped; B, U-tubes; C, straight; D, clay in contact with tissue; $S$, saturated zinc sulphate solution; $Z$, analgamated zinc wire. (Fig. 240), by opening which the induced current is thrown into the tissue to be stimulated. For some purposes the electrodes may be of platinum; but all metals in contact with moist tissues become polarized when currents pass through them-that is, have decomposition products of the electrolysis of the tissues deposited on them. And as any slight chemical difference, or even perhaps a difference of physical state, between the two electrodes will cause them and the tissues to form a battery evolving a continuous current, it is often desirable to use unpolarizable electrodes.

Unpolarizable Electrodes.-Some convenient forms of these are represented in Fig. 238. A piece of amalgamated zinc wire dips into saturated zinc sulphate solution contained in the upper part of a glass tube. The lower end of the tube may be straight, but drawn out so as to terminate in a not very large opening, or it may be bent into a hook, in the bend of which a hole is made. Before the tube is filled with the zinc sulphate solution, the lower part of it is plugged with china clay made up with physiological salt solution. The clay just projects through the opening, and thus comes in contact with the tissue. When these electrodes are properly set up, there is very little polarization for several hours, but for long experiments, U-shaped tubes, filled with saturated zinc sulphate solution, are better. The amalgamated zinc dips into one limb, and a small glass tube filled with clay, on which the tissue is laid, into the other. 
Pohl's Commutator or Reverser (Fig. 239) consists of a block of paraffin or wood witl six mercury cups, each in connection with a bindingscrew (not shown in the figure). Cups $I$ and 6 and 2 and 5 are connected by copper wires, which cross each other without touching. The bridge consists of a glass or vulcanite cross-piece $a$, to which are attached two wires bent into semicircles, each connected with a straight wire dipping into the cups 3 and + respectively. With the bridge in the position shown in the figure, a current coning in at 4 would pass out by the wire connected with $I$, and back again by that connected with 2 , in the direction sliown by the arrows. When the bridge is rocked to the other side so that the bent wires dip into 5 and 6 , the direction of the current is reversed. The cross-wires may be taken out altogetler, and the commutator used to send a current at will through either of two circuits, one connected with $I$ and 2, and the other with 5 and 6 .

Du Bois - Reymond's Short circuiting Key.-A cheal) and convenient form is shown in Fig. 240.

Time-Markers - Electric Signal.-It is of importance to know the time relations of many physiological phenomena which are graphically recorded; for

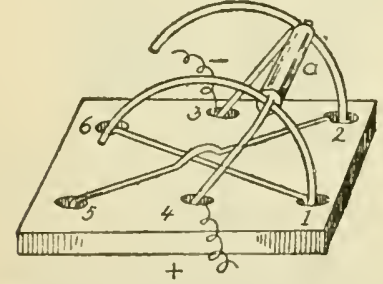

Fig. 239.-Pohl's Commutator.

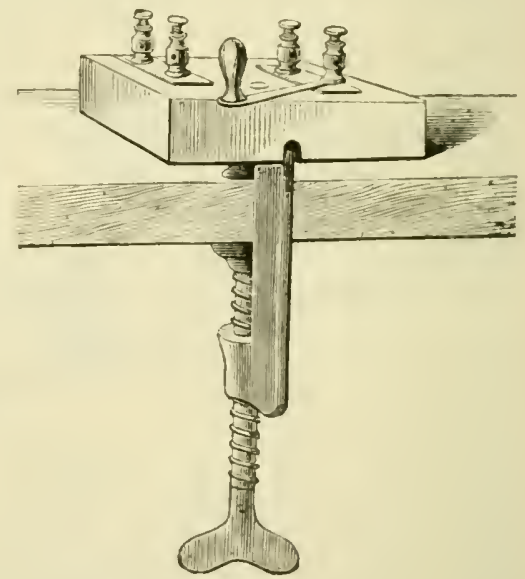

Fig. 240.--Short-Circuiting Key.

example, the contraction of a skeletal muscle or the beat of a heart. For this purpose a tracing showing the speed of the travelling surface in a given time is often taken simultaneously with the record of the movement under investigation. For a slowly-moving surface it is sufficient to mark intervals of one or two seconds, and this is very readily done by connecting an electro-magnetic marker (such as the electric signal of Deprez) with a circuit which is closed and broken by the seconds pendulum of an ordinary clock or a metronome (Iig. 88, p. I95). Special clocks have also been constructed which permit of the time intervals being varied. For shorter intervals a tuning-fork is used, which makes and breaks a circuit including an electromagnetic marker, or writes on the drum directly by means of a writing-point attached to one of the prongs.

Amœboid movement (p. I6) is the most primitive, the least claborated form of contraction. The maximum velocity of the movement has been reckoned at 0.008 millimetre a second. Stimu- 
lation with the constant current or induction shocks causes the whole of the pseudopodia to be drawn in. This illustrates a universal property of protoplasm, excitability, or the power of re sponding to certain influences, or stimuli, by manifestations of the peculiar kind which we distinguish as vital or physiological. Many unicellular organisms and the chicf varieties of the white bloodcorpuscles possess the power of amœboid movement; and we have already dwelt upon some of the important functions fulfilled by such movement in the higher animals and in man. A great distinction between this kind of contraction and that of a muscular fibre is that it takes place in any direction.

Cilia.-Cilia possess a higher and more specialized grade of contractility. They are very widcly distributed in the animal kingdom; and analogous structures are also found in many low plants, such as the motile bacteria.

In the human subject ciliated epithelium usually consists of several layers of cells, the most superficial of which are pear-shaped, the broad end being next the surface, and covered with extremely fine processes, or cilia, about $8 \mu$ in length, which are planted on a clear band. It lines the respiratory passages, the middle ear and Eustachian tube, the Fallopian tubes, the uterus above the middle of the cervix, the epididymis, where the cilia are extremely long, and the central cavity of the brain and spinal cord.

Ciliary motion can be readily studied by placing a scraping from the palate of a frog or a small portion of a gill of a fresh-water mussel under the microscope in a drop of physiological salt solution. The motion of the cilia is at first so rapid that it is impossible to make out much, except that a stream of liquid, recognized by the solid particles in it, is seen to be driven by them in a constant direction along the ciliated edge. When the motion has become less quick, which it soon does if the tissue is deprived of oxygen, it is seen to consist in a swift bending of the cilia in the direction of the stream, followed by a slower recoil to the original position, which is not at right angles to the surface, but sloping streamwards. All the cilia on a tract of cells do not move at the same time; the motion spreads from cell to cell in a regular wave. The energy of ciliary motion may be considerable, although far inferior to that of muscular contraction. The work which cilia are capable of performing can be calculated by remoring the membrane, fixing it on a plate of glass, cilia outwards, putting weights on the glass plate, and allowing the cilia, like an immense number of feet, to carry it up an inclined plane. Bowditch found in this way that the cilia on a square centimetre of mucous membrane did nearly 7 gramme-millimetres of work per ninute (equal to the raising of 7 grammes to a height of a millimetre). 
Since the cilia in the respiratory tract all lash upwards, they must play an important part in carrying up foreign particles taken

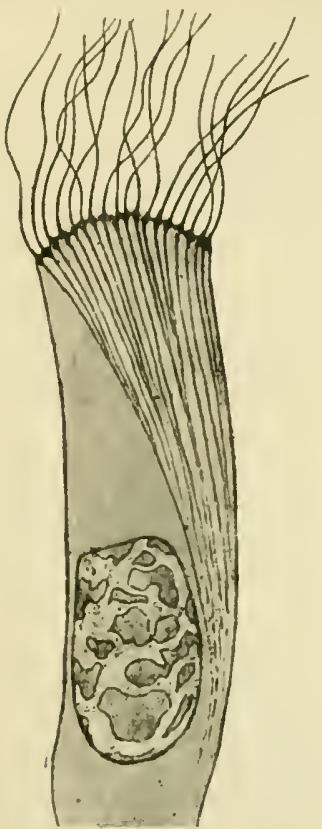

Fig. 24r.-Ciliated Cell (M. Heidenhain). From a 'liver duct' of the garden snail $\times 2,500$. in with the air, and the mucus in which they are entangled, as well as pathological products. Engelmann found that the energy of ciliary motion increases as the tomperature is raised up to about $40^{\circ} \mathrm{C}$, , after which it

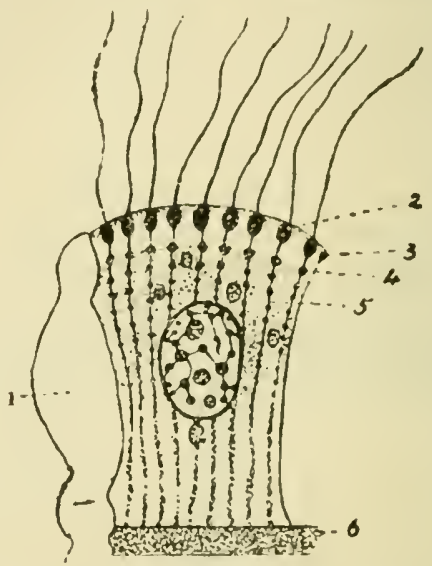

Fig. 242.-Ciliated Cell (Schneider). From a flatworm (Planocerafolium). $I$, space between two adjoining ciliated cells; 2, basal bodies; 4 . inner granule; 5, 'cilia roots'; 6 , boundary layer.

diminishes quickly. Over-heating causes cilia to come to rest, but if the temperature has not been too high, and has not acted too long, they recover on cooling, thus exhibiting the phenomena of heat standstill which we have already studied in the heart.

It is not well understood in what way the contraction of the cilia depends upon their connection with the body of the ciliated cell. Very few cases occur in which cilia have the power of independent motion when severed from the cell-body. It has been observed in certain low forms of animals that cilia which have been broken off from the cell are still able to contract when a small portion of the substance of the cell-body at the point where the cilium is attached to the cell, the so-called basal piece, or basal body (Fig. 242), has come off along with them. In other forms isolated cilia can contract in the absence of anything corresponding to the basal piece. It cannot, therefore, be said that continuity with the basal piece is absolutely necessary. Nor is it known what significance for the ciliary movements is possessed by the long fibrillæ, called the ' roots of the cilia,' which in some animals 
run down through the cell from the basal bodies (ligs, 241, 242). In some worms and mollnscs ciliated cells are supplied with nerve-fibres, but this has not been demonstrated for the higher animals.

\section{Shction II.-Physical Properties and Stimulation of Muscle.}

Since most of our knowledge of the general physiology of muscle has been gained from striped muscle, in what follows we always refer to ordinary skeletal muscle, unless it is otherwise stated. The sartorius and the gastrocnemius are the classical objects for experiments on striated muscle. For smooth muscle the adductor muscle of Anodon, the fresh-water mussel, a ring cut from the middle portion of the frog's stomach, the rabbit's ureter and uterus, and the cat's bladder, have been most used.

Physical Properties of Muscle-Elasticity.-All bodies may have their shape or volunie altered by the application of force. Some require a large force, others a small force, to produce a sensible amount of distortion. The elasticity of a body is the property in virtue of which it tends to recover its original form or bulk when these have been altered. Liquids and gases have only elasticity of volume; solids have also elasticity of form. Most solids recover perfectly, or almost perfectly, from a slight deformation. The limits of distortion within which this occurs are called the limits of elasticity, and they vary greatly for different substances. Living muscle has very wide limits of elasticity; it may be deformed-stretched, for example-to a very considerable extent, and yet recover its original length when the stretching force ceases to act.

The extensibility of a body is measured by the ratio of the increase of length, produced by unit stretching force per unit of area of the cross-section, to the original length of a uniform rod of the substance.

If $e$ is the extensibility, $e=\frac{l s}{\mathrm{LF}}$, where $l$ is the increase of length, $\mathrm{L}$ the original length, $s$ the cross-section, and $F$ the stretching force. Suppose we wish to compare the extensibility of two substances. Let $A$ and $B$ be strips or rods of the substances, the length of $A$ being $500 \mathrm{~mm}$., that of $\mathrm{B} \mathrm{I}$, ooo $\mathrm{mm}$.; the cross-section of $\mathrm{A}$, I $100 \mathrm{sq}$. mm., of $\mathrm{B}, 200 \mathrm{sq} . \mathrm{mm}$. Let the elongation produced by a weight of $\mathrm{I}$ kilo be $10 \mathrm{~mm}$. in each, then the extensibility of $\mathrm{A}$ is $\frac{\mathrm{IO} \times 100}{500 \times I}=2$; and that of $B$ is $\frac{10 \times 200}{1,000 \times I}=2$; that is, the substances are equally extensible. Young's modulus of elasticity, or the coefficient of elasticity, is the quotient of the deforming force acting on unit area of the given body by the deformation produced (within the limits of elasticity). In the above example it is $\frac{F}{s} \div \frac{l}{L}$, that is, $\frac{L F}{l s}$, the reciprocal of the extensibility $e$. For steel the coefficient of elasticity is very large, for muscle small. Or, as we may otherwise express it, living muscle within its limits of elasticity is very extensible; a small force per unit area of cross-section of a prism of it will produce a comparatively great elongation. The extensibility. however, diminishes continually with the elongation, so that equal increments of stretching force produce always 
less and less extension. If, for instance, the sartorious or semi-membranosus of a frog be connected with a lever writing on a blackened surface, and weights increasing by equal amounts be successively attached to it, the recording surface being allowed to move the same distance after the addition of each weight, a series of vertical lines, representing the amount of each elongation, will be traced. When the lower ends of all the vertical lines are joined, a smootl curve with the concavity upwards is obtained (Fig. 243). This is a property common to living and dead muscle and to other animal structures, such as arteries. Marey's method, in which the weight is continuously increased from zero and then continuously decreased to zero again by the flow of mercury into and out of a vessel attached to the muscle, gives directly the curve of extensibility.

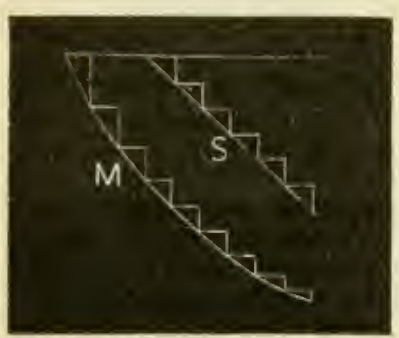

IFig. 243 -Curves of Extensibility. M, of muscle; $\mathrm{S}$, of an ordinary inorganic solid.

The elongation of a steel rod or other inorganic solid is proportional within limits to the extending force per unit of cross-section; and a curve plotted with the weights for abscissa and the amounts of elongation for ordinates would be a straight line. But this is not a fundamental clistinction between animal tissues, and the materials of unorganized nature, as some writers seem to suppose. For when the slow after-elongation which follows the first rapid increase in length in the loaded, excised muscle is waited for, the curve of extensibility comes out a straight line (Wundt), and within limits this is also the case for human muscles in the intact body. And although a steel rod much more quickly reaches its maximum elongation for a given weight when loaded, and its original length when the weight is removed, than does a muscle, time is required in both cases, and the difference is one of degree rather than of kind. When muscle (striated or smooth) is not stretched beyond the limit of physiological relaxation, the amount of stretching is proportional to the weight, and the same is true of all the simple tissues of the body (Haycraft).

Dead muscle is less extensible than living, and its limits of elasticity are much narrower. In the state of contraction the extensibility is increased in excised frog's muscle. When fatigue comes on after many excitations, the after-elongation becomes more pronouncel, but the return after unloading is very incomplete. Donders and Van Mlansveldt have found that contraction causes little difference in the muscles of a living man, although fatigue increases the extensibility.

The great extensibility and elasticity of muscle must play a considerable part in determining the calibre of the vessels, and in lessening the shocks and strains which the heart and the vascular system in general are called upon to bear, and must contribute much to the smoothness with which the movements of the skeleton are carried out, and immensely reduce the risk of injury to the bones as well as to the muscles themselves, the tendons and the other soft tissues. And not only is smothmess gained, but economy also; for a portion of the energy ot a sudden contraction, which, if the muscles were less extensible and elastic, might be wasted as heat in the jarring of bone against bone at the joints, is stored up in the stretched muscle and again given out in its elastic recoil. The skeletal muscles, too, are even at rest kept slightly on the stretch, braced up, as it were, and 
ready to act at a moment's notice without taking in slack. This is shown by the fact that a transverse wound in a muscle 'gapes,' the fibres being retracted, in virtue of their elasticity, towards the fixed points of origin and insertion. Smooth muscle, as we meet it in the hollow viscera, is lighly (listensible and elastic, as is suited to organs whose capacity is continually varying within wide limits (Fig. 244).

In the further study of muscle it is necessary first of all to consider the means we have of calling forth a contration-in other words, the varions kinds of stimuli.

Stimulation of Muscle.-A muscle may be excited or stimulated either directly or through its motor nerve. It is usual to classify stimuli as electrical, mechanical, chemical, or thermal. Electrical stimuli are by far the most commonly employed, and will be discussed in detail. A prick, a cut, or a blow are examples of mechani-
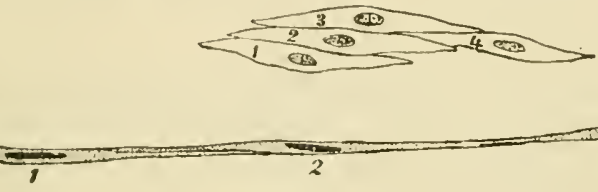

Fig. 244.-Extensibility of Smooth Muscle (Griitzner). The upper group of four cells $(x$ to 4 ) is from a hollow organ, whose walls are contracted, and its lumen almost abolished; the under group represents the saine fibres when the organ is full. The fibres are longer and somewhat darker. They are also dibplaced soinewhat along each other.

cal stimuli. The action of a fairly strong solution of common salt or of a dilute solution of a mineral acid is usually described as chemical stimulation. But in considering the excitation of nerve (p. 783 ) we shall see that physical changes are often mixed up with so-called chemical stimulation. The contraction caused is not a single brief iwitch, as is the case with a not too severe mechanical excitation, but a sustained contraction or a tetanus. Sudden cooling or heating acts as a stimulus for muscle, but thermal stimulation is somewhat uncertain. It is not quite settled whether the contraction which can be obtained from a muscle when it is subjected to brief local heating - to a 'thermic shock,' as some writers prefer to say (e.g., by the momentary glow of a platinum wire below but not touching it) -is an ordinary muscular contraction, or a physical, although transient, contracture analogous to that caused by certain drugs (Waller). Smooth, like striped, muscle is susceptible to electrical, mechanical, thermal, and chemical stimulation. In addition, in certain situations it can be excited by light (photic stimulation), as in the case of the excised iris of fish and amphibia. In all artificial stimulation there is an element of sudden or abrupt change, of shock, in other words; but we cannot tell in what the 'natural' or 'physiological' stimulus to muscular contraction in 
the intact body really consists, nor how it differs from artificial stimuli. All we know is that there must be a wide difference, and that our methods of excitation must be very cructe and inexact imitations of the natural process.

Direct Excitability of Muscle.-The famous controversy on the existence of independent 'muscular irritability' has long been forgotten, and has no further interest except for the antiquaries of science, if such exist. The direct excitability of muscle in the modern sense is not quite the same as the 'muscular irritability,' the cliscussion of which occupied Haller and his contemporaries. What the modern physiologists have been called upon to decide is whether muscular fibres can be caused to contract except by an excitation that reaches them through their nerves. In this sense there can exist no doubt that muscle is directly excitable, and some of the proofs are as follows:

(I) The ends of the frog's sartorius contain no nerves, yet they respond to direct stimulation. (2) Certain chemical stimuliammonia, for instance-excite muscle but not nerve. (3) When the motor nerves of a limb are cut they degenerate, and after a certain time stimulation of the nerve-trunk causes no muscular contraction, while the muscles, although atrophied, can be made to contract by direct stinulation. (4) Finally, there is the celebrated curara experiment of Claude Bernard, which is described in a somewhat modified form in the Practical Exercises, p. 8II. A ligature is tied firmly round one thigh of a frog, omitting the sciatic nerve; then curara is injected, and in a short time the skeletal muscles are paralyzed. That the seat of the paralysis is not the contractile substance of the muscles itself is shown by their vigorous response to direct stimulation. The 'block' is not in the nervetrunk, nor above it in the central nervous system, for the ligated leg is often drawn up-that is, its muscles are contracted-although the poison has circulated freely in the sacral plexus and the spinal cord. Further, if the nerve of the ligated leg be prepared as high up above the ligature as possible, where the curara must undoubtedly have reached it (just above the ligature the nerve has been isolated and the circulation in it more or less interrupted), stimulation of it will cause contraction of the muscles of the limb; while excitation of the other sciatic is ineffective.

It can be also shown, by means of the negative variation or current of action (p. 824), that a nerve-trunk on which curara has acted remains excitable, and capable of conducting the nerveimpulse. The conclusion, therefore, is that the curara paralyzes neither nerve-fibre nor the contractile substance of the muscular fibre, but some link between the two. If the assumption be made that the efferent medullated nerve-fibres within the muscle, since 
they are anatomically similar to those in the nerve-trunk till near their termmations, are similarly affected by curara-and it is a justifiable assumption-the seat of the curara paralysis must either be the nerve-ending or some mechanism, pliysiological if not anatomical, interposed between the nerve-ending and the contractile substance. Now, Langley has shown that the contractions caused by the local application of dilute nicotine solution to points of the skeletal muscles of the frog, both in normal muscles and in muscles whose motor nerves and nerve-endings have degenerated after section of the nerves, are prevented by curara. He tliere-

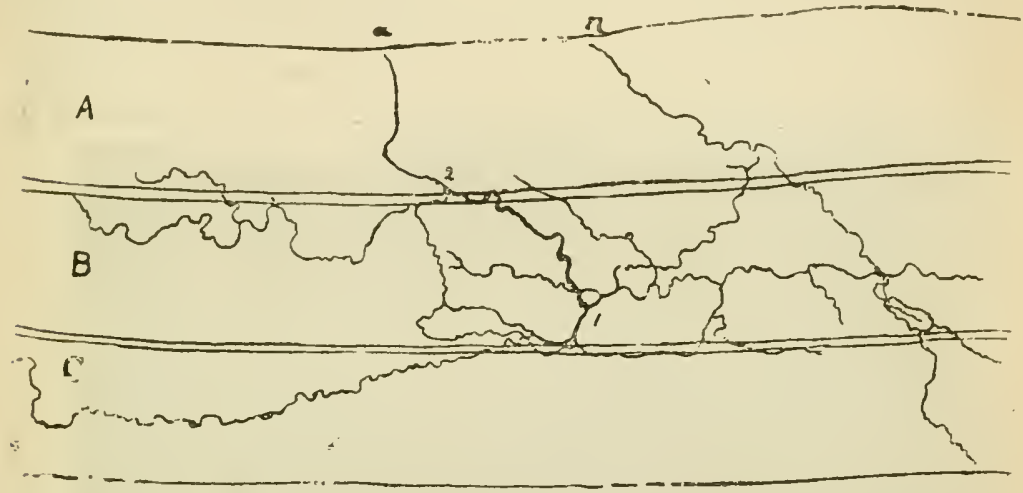

Fig. 245.-Frog's Motor Nerve-Ending (Wilson). A, B, C, three muscle-fibres. The medullated nerve $a$ loses its medullary sheath and breaks up on B at I. It gives off at 2 a large non-medullated branch, which also breaks up on B. The nerveendings send ultraterminal fibrillæ to A, B, and C, some of which were seen to e:ıd in small knobs. A separate non-medullated nerve, $n$, is shown, which forms a sinall plexus on B, one fibre of which penetrates to a lower plane than the other, and ends by forming a knob under the sarcolemma.

fore concludes that, since nicotine produces its effects by a direct action on muscle, and not by an action on nerve-endings or on any special structure (such as the protoplasmic mass or 'sole.' at the nerve-ending in many animals) interposed between the nerve and the muscle, no such special structure existing in the frog (Fig. 245), curara must also act directly on the muscle. But obviously curara does not paralyze the general contractile substance of the muscle, else the curarized muscle would not contract on direct stimulation. Langley accordingly assumes that, in addition to the contractile or ' general' substance, 'receptive' substances exist in the fibre, through which the excitation is transferred to the contractile substance when the motor nerve is stimulated. He pictures these receptive substances as 'side-chains ' of the contractile molecule, in accordance with Elirlich's theory of immunity (p. 3I), and distinguishes those in the neighbourhood of the nerve-ending 
from those present throughout the muscle fibre. Both the slow local tonic contraction and the quick, brief conducted contractions or twitches set up in a muscle fibre by nicotine, but especially the latter, are much more easily elicited in that part of it which lies under the nerve-ending than elsewhere. Indeed, the position of the nerve-endings in the superficial fibres of a muscle can be ascertained by observing the points which respond most readily to nicotine. Nicotine and curara, etc., are supposed to combine with the receptive substance, which is then in both cases rendered incapable of being affected by nerve impulses. In the case of nicotine an additional action results from the combination with the receptive substance-viz., the change in the contractile substance which leads to contraction. Curara paralyzes the transmission of the excitation from the motor nerves to smooth muscle-the muscles of the

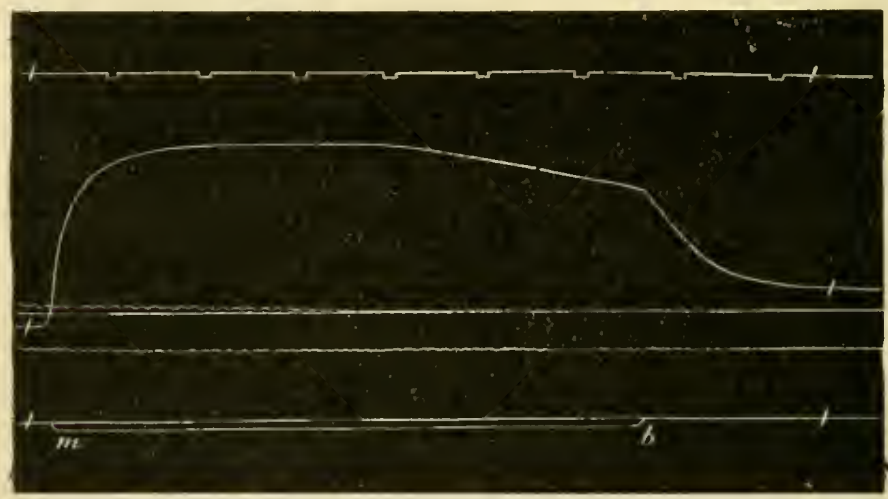

Fig. 246.-Tonic Contraction of Muscle during Passage of Constant Current. Two sartorius muscles of frog connected by pelvic attachments. Current from I2 small Daniell cells in series passed through their whole length. Current closed at $m$, opened at $b$. Time trace, two-second intervals.

bronchi, for instance-with much greater difficulty than to ordinary skeletal muscle, and the same is true of the inhibitory nerves of the heart.

The action of curara gives us the means of stimulating muscle directly; when electrical currents are sent through a non-curarized muscle, there is in general a mixture of direct and indirect stimulation, for the nerve-fibres within the muscle are also excited. Induced currents stimulate nerve more readily than muscle. Voltaic currents may excite a muscle whose nerves have degenerated, while induced currents are entirely without effect.

For direct stimulation, a curarized frog's sartorius or semi-membranosus is generally used on account of their long parallel fibres. For indirect excitation, a muscle-nerve preparation, composed of a frog's gastrocnemius with the sciatic nerve attached to it, is commonly employed, as it is easy to isolate the muscle without hurting its nerve. 
Stimulation by the Voltaic Current.-While the current continues to pass through a nerve without any sudden or great change in its intensity, there is no stimulation, and the muscle connected with the nerve remains at rest. The same is true of striated muscle when a weak current is passed directly through it. But in muscle the constancy of the rule is more and more frequently broken by exceptional results as the currest is strengthened, a state of permanent contraction being very apt to show itself during the whole time of flow (Wundt) (Fig. 246). Above a certain intensity of current a greater or less degree of permanent contraction is invariably produced. This is sometimes called the 'closing tetanus.' It is, however, not a true tetanus, but a tonic contraction, which is strongest in the neighbourhood of the kathode, and does not spread far from it. A similar condition, the so-called galvanotonus, is normally seen in human muscles when they or their motor nerves are traversed by a stream of considerable intensity. Under certain conditions, too-e.g., when a strong current is allowed to flow for a comparatively long time through a. muscle-the muscle remains contracted after the opening of the current (so-called ' opening or Ritter's tetanus '). Smooth muscle is excited to contraction even when a voltaic current is very gradually passed into it and slowly increased, and again when it is caused very gradually to disappear. But striped muscle is not stimulated under

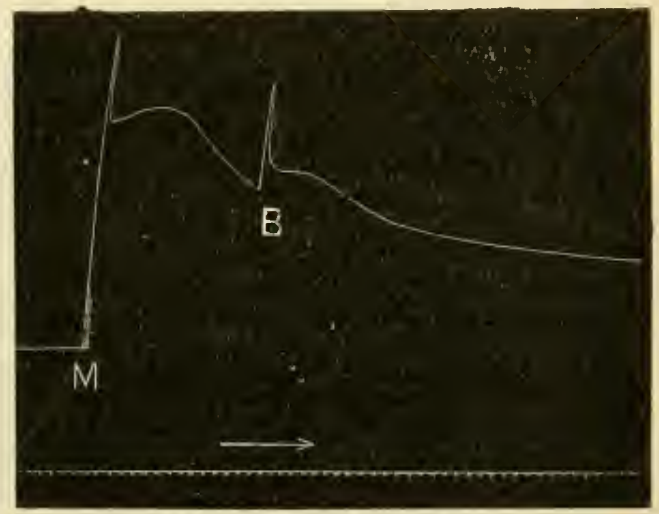

Fig. 247.-Tonic Contraction during and after Flow of Voltaic Current. Curve from frog's gastroc. nemius. At $\mathrm{M}$ constant current closed, at B broken. Contracture continues after opening of current. Time trace, two-second intervals. these conditions.

For nerve, and with these qualifications for muscle, too, the law holds that the voltaic current stimulates at make and at break, but no: during its passage. Or, generalizing this a little, since it has been shown that a sudden increase or decrease in the strength of a current already flowing also acts as a stimulus, we may say that the voltaic current stimulates only when its intensity is suddenly and sufficiently increased or diminished, but not while it remains constant.*

When a strong current is closed through a muscle there is an inmediate sharp contraction (initial contraction). The muscle then promptly relaxes, but incompletely. When the current is opened, there is another contraction (Fig. 247). The force of the initial contraction, as measured by the resistance necessary to prevent it, is greater than that of the tonic contraction which follows it.

A second law of great theoretical importance is that of polar stimulation. At make the stimulation occurs only at the kathode; at break only at the anode. This is true both for muscle and nerve, but it is most

* This law of Du Bois-Reymond has been questioned by Hoorweg and others. It seems to need modification, but the subject cannot be discussed here. 
directly and simply demonstrated on muscle. A long parallel-fibred curarized muscle is supported about its middle; the two ends, which hang down, are connected with levers writing on a revolving drum, and a current is sent longitudinally through the muscle. It is not difticult to see from the tracings that at make the lever attached to the kathodic end moves first, and that the other lever only moves when the contraction started at the kathode has had time to reach it in its progress along the muscle. Similarly, at break the lever connected with the anodic end moves first. The law of polar excitation holds both for striated and for smocth muscle. Not only is there no excitation of unstriped muscle at the anode on closure of the current, but a previously existing contraction disappears. For skeletal muscle the make is stronger than the break contraction. It has not been proved that this is the case for smooth muscle.

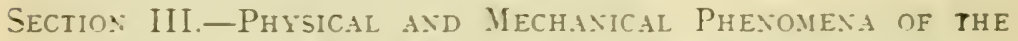 MLESCLLAR CONTRACTION.}

When a muscle contracts, its two points of attachment, or, if it be isolated, its two ends, come nearer to each other; and in exact proportion to this shortening is the increase in the average crosssection. The contraction is essentially a change of form, not a change of volume. The most delicate observations fail to detect the smallest alteration in bulk (Ewald). Living fibres kept contracted by successive stimuli can be examined under the microscope; or fibres may be 'fixed ' by reagents like osmic acid, and sometimes a very good opportunity of studying the microscopic changes in contraction is given by a group of fibres in which the "fixing" reagent has caught a wave of contraction, and, so to speak, pinned it down. It is then seen that the process of contraction in the fibre is a miniature of that in the anatomical muscle. The individual fibres shorten and thicken, and the sum-total of this shortening and thichening is the muscular contraction which we see with the naked ere. The phenomena of the muscular contraction may be classified thus: (I) Optical, (2) Mechanical, (3) Thermal, (4) Chemical, (5) Sonorous, (6) Electrical. (5) will be treated under 'Voluntary Contraction'; (6) in Chapter IV.

(I) Optical Phenomena-Microscopic Structure of Striped Muscle.The structure of striped muscle has long been the enigma of histology: and the labours of many distinguished men have not sufficed to make it clear. On the contrar, as investigations have multiplied, new theories, new interpretations of what is to be seen, hare multiplied in proportion, and a resolute breviry has become the chief duty of a writer on elementary physiology in regard to the whole question.

The muscle-fibre, the unit out of which the anatomical muscle is built up, is surrounded by a structureless membrane. the sarcolemma. The length and breadth of a fibre vary greatly in different situations. The maximum length is about $4 \mathrm{~cm}$; ; the breadth may be as much as $70 \mu$ and as little as Io $\mu$. When we come to analyze the musclefibre and to determine out of what units it is built up, the difficulty. begins. The fibre shows alternate dim and clear transverse stripes, and 
can actually be split up into discs by certain reagents. It also shows a longitudinal striation, and can be separated into fibrils. Some have supposed that the dises are the real structural units which, piled end to end, make up the fibre. The fibrils they consider artificial. This view is erroneous. It seems certain that the fibres are built up from fibrils ranged side by side, and that the discs are artificial. The contents of the muscle-fibre appear to consist of two functionally different substances, a contractile substance, and an interstitial, perliaps nutritive, non-contractile material of more fluid nature. The contractile substance is arranged as longitudinal fibrils embedded in interfibrillar inatter (sarcoplasm). In a muscle impregnated with chloride of gold the interfibrillar matter appears as a network.

Schäfer has described the contractile elements of the muscle-fibre (Figs. 248, 249) as fine columns (sarcostyles), divided into segments

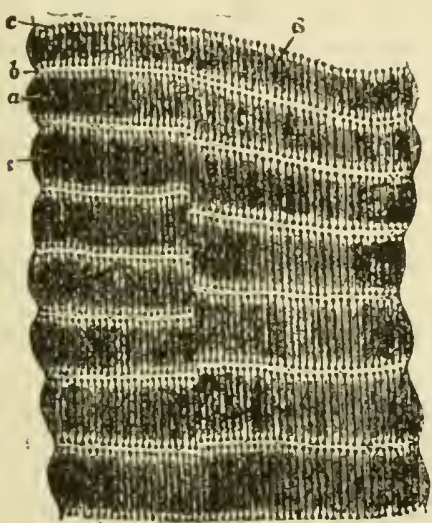

Fig. 248.-Living Muscle of Water. Beetle (highly magnified) (Schäfer). $s$, sarcolemma; $a, \operatorname{dim}$ stripe ; $b$, bright stripe; $c$, row of dots in bright stripe, which appear to be the enlarged ends of rod-shaped particles, $d$, but in reality represent expansions of the interstitial substance (sarcoplasm). (sarcomeres) by thin transverse discs (Krause's membranes), occupying the position of the middle of each light stripe. Each sarcomere contains a sarcous element (a portion of the dark stri $\hat{\mathrm{p}}$ ) with a clear substance at its ends, filling up the

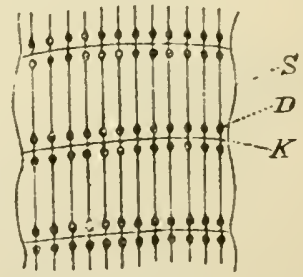

lig. 249.-Portion of Leg Muscle of Insect, treated with Dilute Acetic Acid (Schäfer). S, sarcolemma; $D$, dot-like enlargement of sarcoplasm; K. Krause's membrane. The sarcous elements have been swollen and dissolved by the acid.

space between the sarcous element and Krause's membrane, and constituting a portion of the light stripe. The sarcous element is itself louble, and if the fibre be stretched, the two portions separate at a line wlich runs transversely across the middle of the dim stripe (Hensen's line). Scliäfer considers that the appearance of longitudinal fibrillation in the sarcous elements is due to the presence in them of fine longitudinal canals or pores.

The Krause's membrane of the individual fibrils is scarcely ever visible in an intact mammalian fibre, and the apparent line in the clear stripe of an intact fibre is an optical appearance due to interference of light. Kühne, who was fortunate enough to find one day a small nematode worm moving in the interior of a fibre, saw it pass along the fibre with perfect freedom, ignoring Krause's membrane. Possibly, however, it was moving in the sarcoplasm, the fibrils being simply pushed aside. 
Crangesduring Contraction-Theories of Contraction.-Incontractions: according to Schäfer, the clear substance between Kirause's membrane and the sarcous element passes into the canals, which are open towards Krause's membrane, but closed towards Hensen's line. The sarcous element therefore swells $11 \mathrm{p}$, and the sarcomere is shortened. In the extended muscle the clear substance leaves the pores of the sarcous clement, and accumulates in the space between it and Krause's membrane. The sarcomere is thits lengthened and narrowed. Iinile the cxistence of Schäf(r's pores is not admitted by all ol servers, there is a pretty general agreement that the sarcomere, like the cytoplasm of an amoboid cell, does consist of two substances, one of which (the liyaloplasm of the cell, the clear material of the sarconere) interpenetrates; the other (spongioplasm of the cell, substance of the sarcous element); and that in relaxation the clear fluid passes from the sarcous clement to the ends of the sarcomeres, whereas in contraction it passes in the reverse direction into the sarcous elements. Whether the fluid passes into and out of the meshes of an actual network, or along actual physical pores in the sarcous element, or whether it is transferred by some process like molecular imbibition (p. 426), need not be discussed here, since it is not definitely known. The fundamental question by what process the transference is determined when the muscle is excited also remains unsettled. So far as is known at present, it is probable that the mechanical energy of the contracting muscle must be derived from the transformation of chemical energy into one of three forms: energy associated with osmotic processes, energy associated with imbibition, and energy associated with changes of surface tension. It is not clifficult to see that a sudden increase in the osmotic concentration in the sarcous element, due to the breaking up of large molecules or colloid aggregates into small molecules, or the libcration of electrolytes from the colloids, might lead to the rapid passage of water into it from the bright bands. A sudden change of permeability of the sarcous elements for dissolved substances in the clear fluid would have a similar effect. The same is true of a change in their power of imbibition. But, according to Bernstein, it is scarcely to be supposed that the extraordinarily rapid movement of water molecules which must occur in contraction can be accounted for either by osmosis or by imbibition. A more plausible theory is that the surface tension-say between the substance of the sarcous element and the clear fluid-is altered. That the shortening of the muscle in fatigue (p. 749) and rigor (p. 774), as well as its shortening in normal contraction, is due in some way to the liberation of metabolic products, esperially lactic acid, is a theory of some standing, and fresh evidence in its favour has been recently supplied. Tlus it has been pointed ont that the course of leat production in the active muscle, and its relation to the time of the mechanical response, and the development and time relations of the electrical change which precedes that response, can be very naturally explained on the supposition that the liberation of lactic acid on or ncar some surface in the contractile substance is an essential factor in the contraction (Mines, etc.). It is known that in the prescuce of acid on the surface of certain colloid structures shortening occurs (Fischer and Strietman).

The substance of the sarcous element which forms the dark stripe is cloubly refracting, and therefore rotates the plane of polarization, but the clear substance of the light stripe is singly refracting. When an uncontracted fibre is viewed with crossed nicols, the dim stripe accordingly appears bright in the otherwise dark field. In the contracted fibre the doubly refractive material remains in the stripe which 
is dim in ordinary light. There is no transference of it, but, according to most writers, the bands which are dim in ordinary light increase in size by the transference of liquid from the isotropous band.

Diffraction Spectrum of Muscle.-When a beam of white light passes through a striced muscle, it is broken up into its constituent colours, and a series of diffraction spectra are produced, just as hapfens when the light passes through a diffraction grating (a piece of glass on which are ruled a numter of fine parallel equidistant lines). The nearer the lines are to each other, the greater is the displacement of a ray of light of any given wave-length. It lias accordingly been found that when a. muscular fibre contracts, the amount of displacement of the diffraction spectra increases. At the same time the whole fibre becomes more transparent.

(2) Mechanical Phenomena.-The muscular contraction may be graphically recorded by connecting a muscle with a lever which is moved either by its shortening or by its thickening. The lever writes on a blackened surface, which must trave. a.t a uniform rate if the form and time-relations of the muscle curve are to be studied, but may be at rest if only the height of the contraction is to be recurded. The whole arrangement for taking a muscletracing is called a myograph (Fig. $28 \%, \mathrm{p}, 8 \mathrm{I} 2)$. The duration of a 'twitch' or single contraction (including the relaxation) of a frog's muscle is usually given as about one-tenth of a second, but it may vary considcrably with tempcrature, fatigue, and other circumstances. It is measured by the vibrations of a tuning-fork written immediately below or above the muscle curve. when the muscle is only slightlyweighted, it but very gradually reaches its original length after contraction, a period of rapid relaxation being followed by a period of "residual contraction," during which the

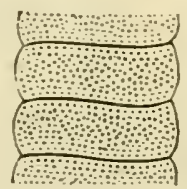

1

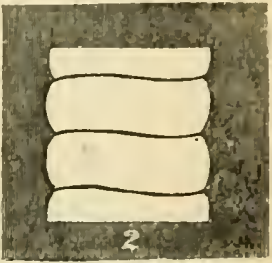

Fig. 250.-Living Muscular Fibre (from Geotrupes stercorarius). I, in ordinary; 2, in polarized light. (Van Gehuchten.) In living muscle (at least in fibres which are not extended) in contrast to dead muscle after treat. ment with reagents, the doubly refracting or anisotropous substance is present in the greater part of the fibre; and with crossed nicols the position of the singly refracting or isotropous material is indicated only by narrow iransverse black lines or rorrs of dark dots.

descent of the lever towards the base-line becomes slower and slower, or stops altogether some distance above it. The duration of the contraction of smooth muscle evoked by a single momentary stimulus is much greater than that of stiiped muscle (two to seven seconds for the rabbit's ureter; five to fifteen seconds for the cat's nictitating membrane; one to two minutes for the frog's stomach).

Latent Period.- If the time of stimulation is marked on the tracing, it is found that the contraction does not begin simultaneously with it, but only" after a certain interval, which is called the latent period.

This can be measured by means of the spring myograph (Fig. 25I) or of the pendulum myograph, a pendulum which in its swing carries a smolied plate against the writing-point of a lever connected with a muscle. The carrier of the recording plate opens, at a definite point in its passage, a key in the primary coil of an induction machine, and so causes a shock to be sent through the muscle or nerve, which is connected with the secondary. The precise point at which the stimulus is thrown in can be marked on the tracing by carefully bringing the 
plate to the position in which the key is just opener, and allowing the lever to trace here a vertical line (or, ratlier, an are of a circle). The portion of the time-tracing between this line and a parallel line drawn through the point at which the contraction begins gives the latent period.

Helmlioltz measured the length of the latent period by means of tlie principle of Poulllet, that the deflection of a nagnet by a current of given strength and of very short duration is proportional to the time during which the current acts on the magnet. He arranged that at the moment of stimulation of the muscle a current should be sent through a galvanometer, and should be broken by the contraction of the muscle the moment it began. In this way he obtained the value of 100 second for the latent period of frog's muscle. The tendency of

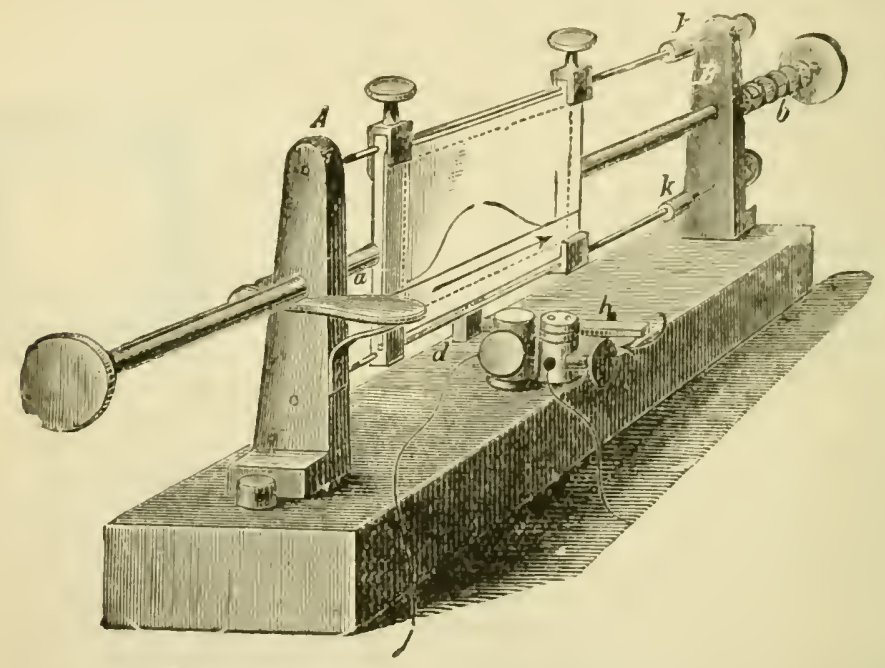

Fig. 25r.-Spring Myograph. A, B, iron uprights, between which are stretched the guide-wires on which the travelling plate $a$ runs; $k$, pieces of cork on the guides to gradually check the plate at the end of its excursion, and prevent jarring; $b$, spring, the release of which shoots the plate along; $h$, irigger-key, which is opened by the pin $d$ on the frame of the plate.

later observations has been to make the latent period shorter. Burdon Sanderson found that the change of form begins in unweiglited or very slightly weighted muscle with direct stimulation in 1000 second after, and the electrical change (p. 824) simultancously with, the excitation. It is known that the apparent latent period depends upon the resistance which the muscle has to overcome in heginning its contraction.

The maximum slsortening, or 'height of the lift,' depends upon the length of the muscle, the direction of the fibres, the strength of the stimulus, the excitability of the tissue, and the load it has to raise.

In a long muscle, other things being equal, the absolute shortening, and therefore the maximum height of the curve, will be greater than in a short muscle; in a muscle with fibres parallel to its length-the sartorius, for instance-it will be greater than in a muscle like the gastrocnemius, with the fibres directed at various angles to the long axis. For stimuli less than maximal, the absolute contraction increases 
with the strength of stimulation, and a given stimulus will cause a greater contraction in a muscle witl a given excitability than in a muscle which is less excitable. Under ordinary experimental conditions ..t least, weak stimuli cause a smaller contraction than strong. not only because each stimulated fibre contracts less, but because a smaller number of fibres are excited (p. I 55 ). The objects used for the stucly of muscular contraction contain many fibres, and it is not in

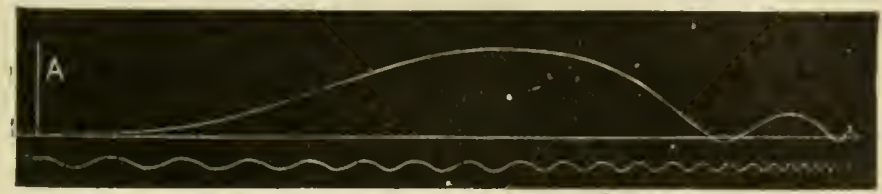

Fig. 252.-Curve of a Single Muscular Contraction or Twitch taken on Smoked Glass with Spring Myograph and photographed. Vertical line A rrarks the point at which the inuscle was stimulated; time tracing shows ${ }_{1 \frac{1}{10 \pi}}$ of a second (reduced).

general possible to distribute the stimulus equally to all. This is true for smooth muscle as well as for striped. Finally, increase of the load per unit of cross-section of the muscle diminishes above a certain limit the 'height of the lift.'

Influences which affect the Time-Relations of the Muscular Contraction.-Many circumstances affect the form of the muscle curve and its time-relations.

(a) Influence of the Load-Isotonic and Isometric Contraction.-The first effect of contraction is to suddenly stretch the muscle, and the more the muscle is loaded the greater will this elongation be. So that at the beginning of the actual shortening part of the energy of contraction is already expended without visible effect, and has to be recovered from the elastic reaction during the ascent of the lever.

The contraction of a muscle loaded by a weight which is not increased or diminished during the contraction is said to be isotonic, for here the tension of the muscle is the same throughout, and its lengtl alters. When the muscle is attached very near the fulcrum of the lever, so that it acts upon a short arm, while the long arm carrying the writing-point is prevented from moving much by a spring, the muscle can nnly shorten itself very slightly; but the changes of tension in it will be related to those in the spring, and therefore to the curve traced by the writing-point. Such a curve is called isometric, since the length of the muscle remains almost unaltered. In the body muscles usually contract under conditions more nearly allied to those of the isometric than to those of the isotonic contraction.

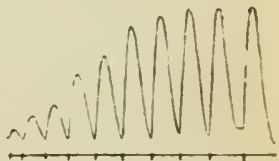

Fig. 253. - Contrac. tions of Smooth Muscle: Cat's Bladder (C. C. Stewart). Stimulated with progressively stronger induction shocks. The lowest line is the time trace (ro-second intervals). Immedi. ately below the mus. cular contractions are marked the points at which the stimuli were thrown in.

The work done by a muscle in raising a weight is equal to the product of the weight by the height to which it is raised. Beginning with no load at all, it is found that the weight can be increased up to a certain limit without diminishing the height of the contraction; perhaps the height may even increase. Up to this limit, then, the work evidently increases with the load. If the weight is made still greater, the con- 
traction becomes less and less, but up to annther limit the increase of weight more than compensates for the diminution of 'lift,' and the work still increases. Beyond this, further increase of weight can no

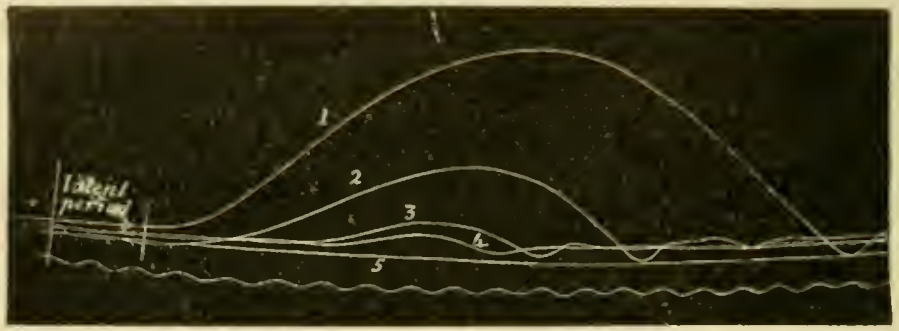

Fig. 254.-Infiuence of Load on the Form of the Muscle Curve. I, curve taken with unloaded lever: 2, 3, 4, weight successively increased; 5, abscissa line: time trace, Ito second (reduced).

longer make up for the lessening of the lift, and the work falls off till ultimately the muscle is unable to raise the weight at all.

The 'absolute contractile force' of an active muscle may be measured by determining the weight which, brought to bear upon the muscle at

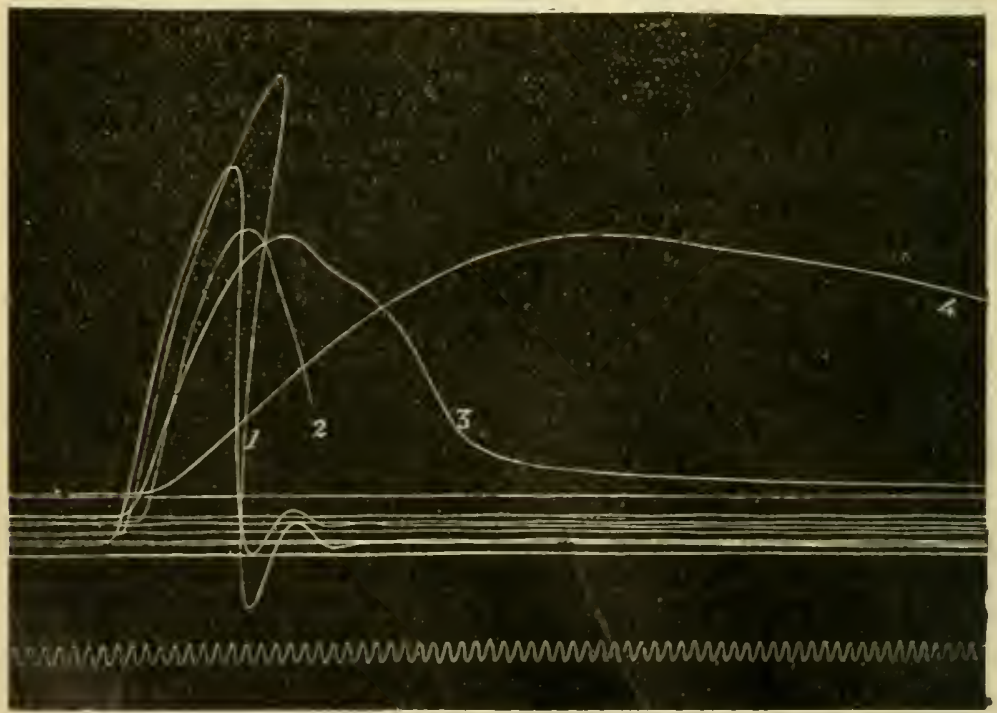

Fig. 255.-Influence of Temperature on the Striated Muscle Curve. 2, air tempera. ture; $1,25^{\circ}-30^{\circ} \mathrm{C} . ; 3,7^{\circ}-10^{\circ} \mathrm{C}$; 4 , ice in contact with muscle. The fifth curve was taken at a little above air temperature.

the instant of contraction, is just able to prevent shortening without stretching the muscle. It, of course, depends, among other things, on the cross-section of the muscle. During the contraction the absolute force diminishes continually, so that a smaller and smaller weight is 
stifficient to stop any further contraction the more the muscle has a!ready shortened before it is applied. At the maximum of the contraction the absolute force is zero. Hence a muscle works under the most favourable conclitions when the weight decreases as it is raised, and this is the case with many of the muscles of the bocly. I) nring flexure of the forearm on the clbow, with the upper arm horizontal, a weight in the hand is felt less and less as it is raised, since its moment, which is proportional to its distance from a vertical line (lrawn through the lower end of the humerus, continually diminishes.

(b) Influence of Temperature on the Muscular Contraction.-Increase of temperature of the muscle up to a certain limit diminishes the latent

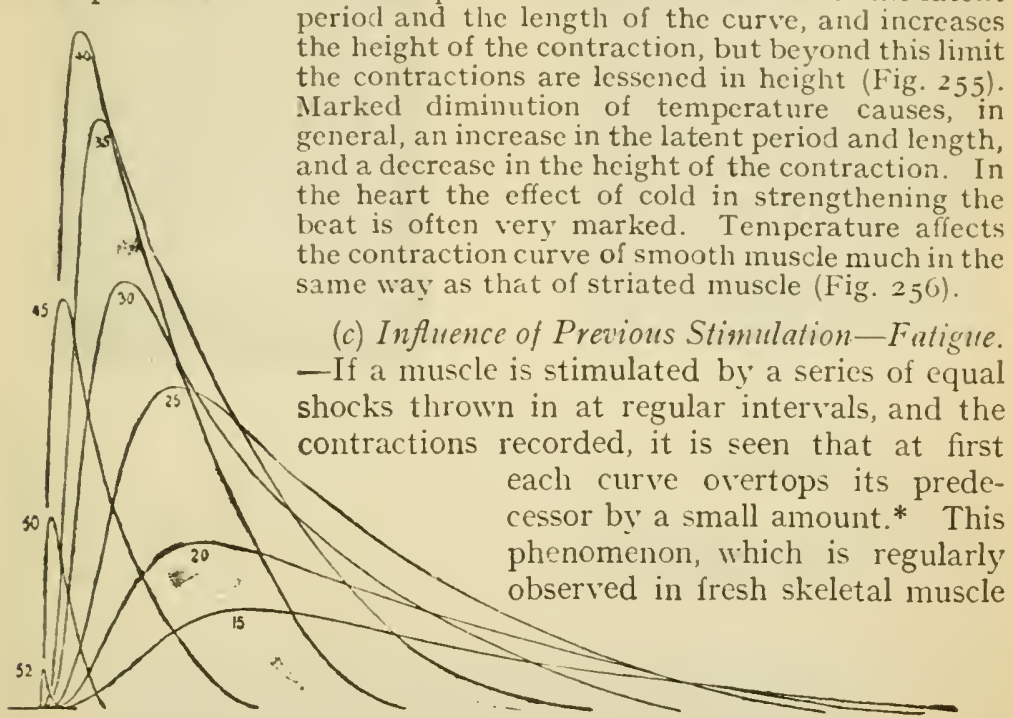

Fig. 256.- Influence of Temperature on the Smooth Nuscle Curve: Cat's Bladder (C. C. Stewart). Contractions at different temperatures with the same strength of stimulus. The temperatures (Centigrade) are marked on the curves.

(Fig. 260), although it was at one time supposed to be peculiarly a property of the muscle of the heart (Fig. 26I), is called the staircase,' and seems to indicate that within limits the muscle is benefited by contraction and its excitability increased for a new stimulus. Soon, however, in an isolated preparation, the contractions begin to decline in height, till the muscle is at length utterly exhausted, and reacts no longer to even the strongest stimulation (Figs. 258, 259, 28S).

A conspicuous feature of the contraction-curves of fatigued muscle is the progressive lengthening, which is much more markid in the descending than in the ascending prriods; in other words,

* Guthrie has recently described an interesting series of phenomena illustrating the influence of previous stimulation on the irritability of muscle. Under given conditions, for example, strong stimulation increases the response of skeletal muscles to subsequent single stimuli. it may be, by hundreds per cent. 
relaxation becomes more and more difficult and imperfect (Fig. 288 . p. 8I4). In smooth muscle (cat's bladder or ring from frog's stomach) fatigue can be very easily demonstrated in the same way, and the curves present similar features, with the exception that, instead of becoming longer in fatigue, the successive contractions become shorter.

It is by no means so easy to fatigue a muscle still in connection with the circulation as an isolated muscle. But even the latter, if left to itself, will to some extent recover, and be again able to contract, although exhaustion is now more readily induced than at first.

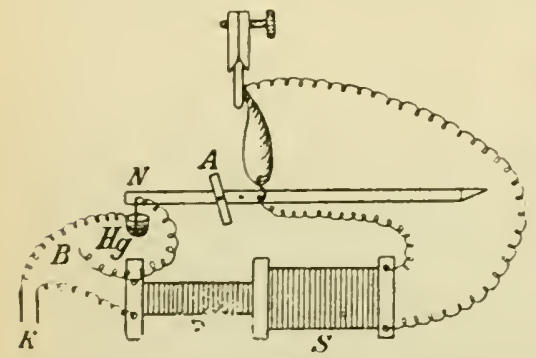

Fig. 25--Automatic Muscle Interruptor.

K, battery; P, primary; S, secondary coil; $A$, axis of lever; $\mathrm{N}$, needle; $\mathrm{Hg}$, mercury cup.

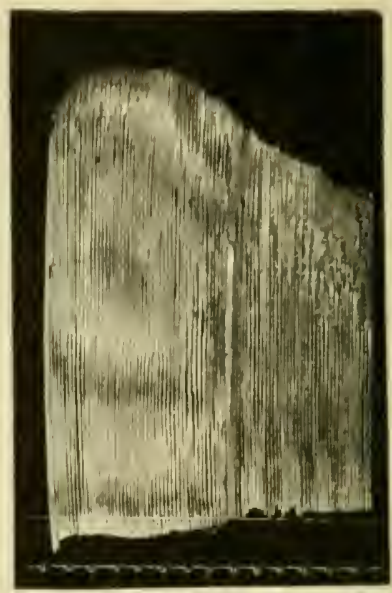

Fig. 258.-Fatigue Curve of Muscle: Frog's Gastrocnemius. The arrangeinent with which the curve figured was obtained was a so-called auto. matic muscle interruptor (Fig. 25?). A wire on the lever is made to close and open the primary circuit of an inductorium, the inuscle or nerve being connected with the secondary. Every time the needle touches the mercury the muscle is stimulated automatically.

In man, muscular fatigue can be studied by means of an arrangement called an ergograph (Fig. 262). A record of successive con-

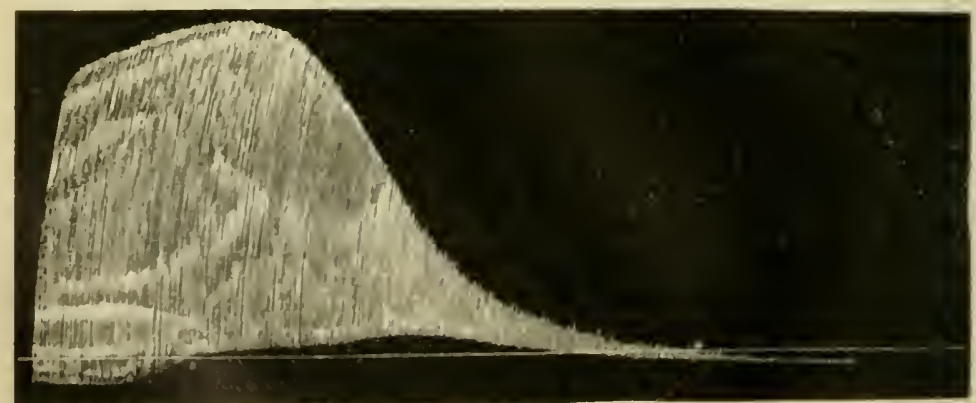

rig. 259.-Fatigue Curve taken on a Slowly-moving Drum (reduced to Half): Frog " Gastrocnemius. Excited through the sciatic nerve by maximal shocks once it six seconds. 
tractions, say, of one of the flexor muscles of a finger, in raising a weight (isotonic mothod) or in deforming a spring (isometric method) is taken on a drum. When the contrations are repeated every second, or every half-serond, distinct cridence of fatigue is secn on the tracing after a longer or bhorter period, according to the conditions.*

IThat is the cause of muscular fatigue? An exact answer is not possible in the present state of our knowledge, but we may fairly conclude that in an isolated preparation it is twofold: (I) Waste products, among which some are so directly related to the onset of fatigue as to deserve the name of 'fatigue substances,' are formed by the active muscle faster than they can be removed, oxidized or otherwise decomposed. (2) The material necessary for contraction is used up more quickly than it can be reproduced or brought

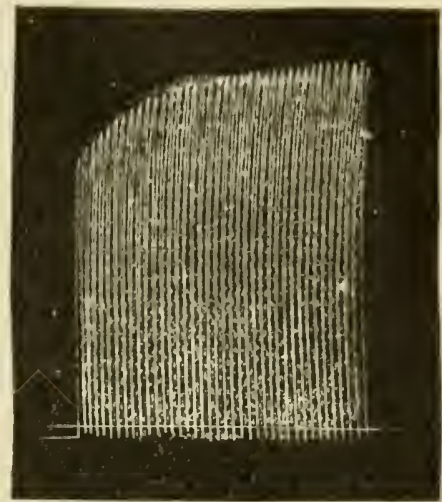

Fig. 260.- 'Staircase' in Skeletal Muscle: Frog. Stimulation by an automatic arrangement.

to the place where it is required. That the accumulation of fatigue products has somcthing to do with the exhaustion is shown by the firct that the muscles of a frog, exhausted in spite of the continuance of the circulation, can be restored by blecding the animal, or washing out the ressels with physiological salt solution, while injection of a watery extract of exhausted muscle into the bloodvessels of a

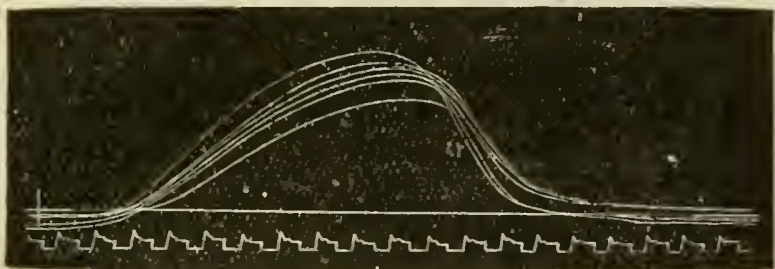

Fig. 261.- 'Staircase' in Cardiac Muscle. Contractions recorded on a much more quickly moving drum than in Fig. 260. The contractions were caused iy stimulating a heart reduced to standstill by the first Stannius' ligature (p. 199). The contractions gradually increase in height.

curarized muscle renders it less excitable (Ranke). This observer supposed that it was specially the removal of the acid products of contraction which restored the muscle. Such acid products as carbon dioxide and lactic acid, or the lactates which it may form with bases in the blood, lymph or tissues, when they act on muscle

* Recent observations (by Ryan and Agnew) with improved methods have emphasized the necessity for care in the interpretation of ergographic records. This caution is timely, inasmuch as in modern war the question of the relation of fatigue to industrial practice acquires first-class importance. 
in more than a certain concentration, produce the same effects on its powe's of contraction as are produced by fatigue, and tlere is some reason to suppose that lactic acid is the most influential of the fatigue substances. In smaller concentration, on the contrary, they increase the excitability of the muscle, and, according to lece, the phenomenon of the "staircase' is due to the augmenting action of these, and perhaps other fitigue substances, before they have accumulated sufficiently to cause fatigue.

The lack of oxygen holds a conspicuous place among the conditions which permit an excessive accumulation of fatigue substances, and may contribute also to the failure of the processes normally going on in the muscle which replenish the store of materials needed for contraction. An isolated muscle is necessarily an asphyxiated muscle, and the favourable action of an atmosphere of oxygen on restoration of its contractile power after exhaustion

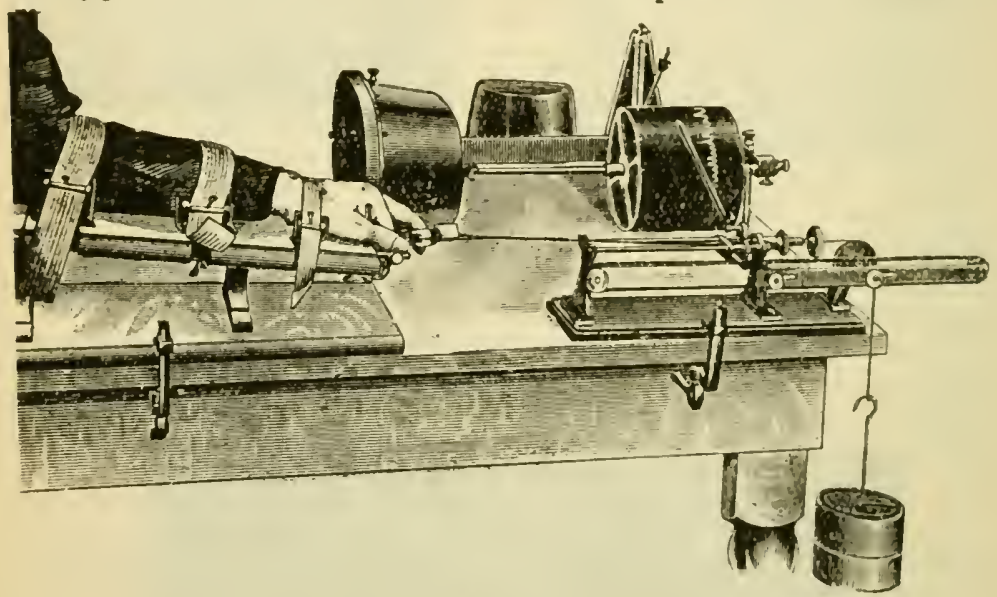

Fig. 26i2.-Ergograph (Mosso's, modified by Lombard).

(Fig. I23, p. 269) shows that asphyxia is itself an important factor in the onset of fatigue. Injection of arterial blood, or even of an oxidizing agent like potassium permanganate, into the vessels of an exhausted muscle causes restoration (hronecker). The depletion of the available store of carbo-hydrate in the form of glycogen (and dextrose) seems to be another factor in fatigue, although not the chief direct cause of the phenomena associated with that condition.

Seat of Exhaustion in Fatigue.-When a fatigued muscle responds no longer to indirect stimulation, it can still be directly excited. The seat of exhaustion must therefore be either the nerve-trunk or the nerve-endings. It is not the nerve-trunk which is first fatigued, for this still shows the negative variation (p. $S_{24}$ ) on being excited. And if the two sciatic nerves of a frog or rabbit be stimu- 
lated continuously with interrupted currents of equal strength, while the excitation is prevented from reaching the muscles of one limb till those of the other cease to contract, it will be found that when the 'block' is removed the corresponding muscles contract vigorously on stimulation of their nerve. The passage of a constant current through a portion of the nerve or the application of ether between the point of stimulation and the muscles may be used to prevent the excitation from passing down (p. 813). Or a dose of curara just sufficient to paralyze the motor innervation may be given to a rabbit, and the animal kept alive by artificial respiration. The sciatic is now stimulated for many hours. As soon as the influence of the curara begins to wear off, the muscles of the leg contract.

The possible seats of fatigue caused by voluntary muscular contraction are (I) the muscle; (2) the nerve-endings (or the receptive substances in the muscles, p. 739); (3) the nerve-trunk; and (4) the path of the voluntary motor impulses in the central nervous system, which includes the pyramidal cells in the motor region of the cerebral cortex (p. 875), the fibres of the pyramidal tract, and the motor cells in the anterior horn of the spinal cord.

The two weak links in this chain appear to be the motor nerveendings and the muscles. The nerve-fibres, whether peripheral or central, are certainly the strongest link. Ergographic experiments have hitherto yielded results too discordant to justify any very definite statement as to the point at which the chain suaps in complete fatigue, if, indeed, it al vays necessarily breaks at the same point. The muscles and motor en.lings appear to be always affected. The position of the nerve centres, including the synapses (p. 852), is in doubt. That the synapses easily lose their power of conducting nerve impulses under the influence of repeated excitations is indicated by the experiments of Sherrington on fatigue of reflex mechanisms in which two or more afferent paths can cause discharge along a common efferent path (p. 902). When excitation of one of the afferent paths has ceased to be effective, the reflex contractions can still be obtained on exciting the other. In this case the motor neuron from cell-body to nerve-ending and the muscle are eliminated as the seats of the fatigue block. IVhether the temporary loss of conduction in this case is comparable to the fatigue of muscle, or is a perfectly different phenomenon (' pseudo-fatigue' of Lee), scarcely bears on our present question. For if 'pseudofatigue ' of afferent synapses can cause a reflex to miss fire, this at least shows that the conductivity of the synapse is very easily affected by repeated excitation, just as it is known to be very easily affected by anæmia. The fact that a muscle, completely fatigued by direct electrical stimulation, can still be voluntarily contracted, has been supposed to indicate that the voluntary excitation is more effective 
than any artificial stimulus. But the alternative explanation that the electrical stimuli cannot be applied to a muscle in situ, so as to cause uniform excitation, and therefore uniform fatigue, of all the fibres of the muscle, is more probable (Hough).

It has been shown that the injection of the blood of an animal exhausted by running or other muscular effort into the circulation of a normal animal produces in the latter all the symptoms of fatigue. Here the fatigue-producing substances will have the opportunity of acting on both the central and the peripheral mechanisms. There are reasons for believing that the fatigue process is fundamentally the same in different tissues. The fatigue substances produced in
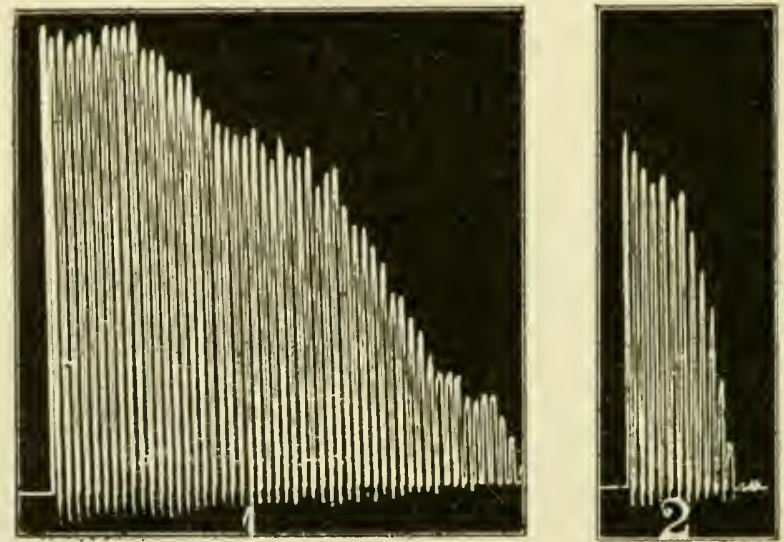

Fig. 2j3.-Influence of Mfental Fatigue on Muscular Contraction. I, series of contractions of flcxors of middle finger before, and 2 , series of contractions imme. diately after, a period of three and a half hours' hard mental work. In both cases the muscles were stimulated directly every two seconds by an electrical current, and callsed to raise a certain weight till temporary exhaustion occurred. In the first series fifty-three contractions were found possible, in the sccond only twelve (Maggiora).

muscle, and not immediately eliminated or transformed during active muscular exertion, may therefore very well be a factor in inducing fatigue of the central nervous mechanisms in addition to the formation of fatigue products, and the using up of necessary material in these mechanisms themselves. Conversely, active and long-continued mental exertion may occasion muscular fatigne (Fig. 263). The sensation of fatigue is alluded to in Chapter XVII,

(d) The Influence of Drugs on the Contraction of Muscle.-The total work which a muscle can perform, its excitability and the absolute force of the contraction, may all be altered either in the plus or the minus sense by drugs. But in conneetion with our present subject those drugs which conspicuously alter the form and time-relations of the muscle-curve have most interest. Of these veratrine is especially: 
important. When a small quantity of this subsiance is injected below the skin of a frog. spasms of the voluntary muscles, well marked in the limbs, cone on in a few minutes. These are attended with great stiffuess of movement, for while the animal can contract the extensor muscles of its legs so as to make a spring, they relax very slowly, and some time elapses before it can spring again. If it be killed before the reflexes are completely gone, the peculiar alterations in the form of the muscle-curve caused by veratrine will be most marked. The poisoned

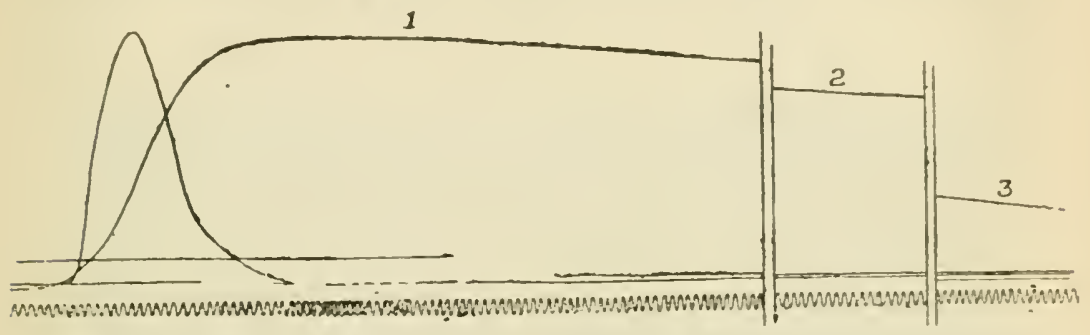

Fig. 264.-Veratrine Curve compared with Normal: Frog's Gastrocnemius. The tuning-fork marks hundredths of a second. Between $I$ and 2 a portion of the tracing corresponding to one and a half seconds has been cut out, and between 2 and 3 a portion corresponding to one second. The veratrine curve does not show a peak. At 3 it has not yet fallen to the base-line.

muscle, stimulated directly or through its nerve, contracts as rapidly as a nornal muscle, while the height of the curve is about the same, but the relaxation is enormously prolonged (Fig. 204). This effect scems to be to a considerable degree dependent on temperature, and it may temporarily disappear when the muscle is made to contract several times without pause. Barium salts, and, in a less degree, those of strontium and calcium, have an action on muscle similar to that of veratrine. Sometimes the curve shows a peak (Fig. 265), due to a rapid descent of the lever for a certain distance. This is followed by a slow relaxation. The peak appears to be analogous to the initial contraction when a strong voltaic current is passed tlirough a muscle, and the rest of the curve to the tonic contraction.

(e) The individuality of the muscle itself has an influence on the muscle-

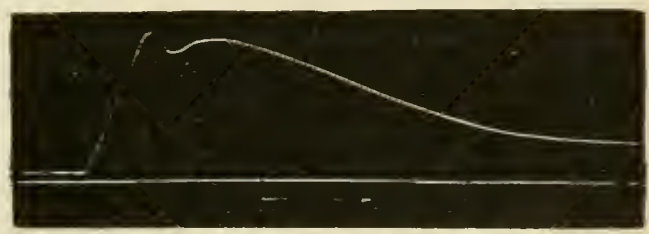

Fig. 265.-Veratrine Curve: Frog's Gastrocnemius. The curve shows a peak, the lever falling a little before the sustained contraction begins. curve. Not only do the muscles of different animals vary in the rapidity of contraction, but there are also differences between the skeletal muscles of the same animal.

In the rabbit there are two kinds of striped muscle, the red and the pale (the semitendinosus is a red, and the adductor magnus a pale muscle), and the contraction of the former is markedly slower than that of the latter. In many fishes and birds, and in some insects, a similar difference of colour and structure is present.

Even where there is no distinct histological difference, there may be great variations in the length of contraction. In the frog, for instance, the hyoglossus muscle contracts much more slowly than the gastroc- 
nemius. The wave of contraction, which in frogs' striped muscle lasts only about $0^{\circ} 07$ second at any point, may last a second in the forceps muscle of the crayfish, though only half as long in the muscles of the tail. In the muscles of the tortoise the contraction is also very slow. The muscles of the arm of man contract more quickly than those of the leg.

Summation of Stimuli and Superposition of Contractions.-Hitherto we have considered a single muscular contraction as arising from a single stimulus, and we have assumed that the muscle has completed its curve and come back to its original length before the next stimulus was thrown in. We live now to inquire what happens when a second stimulus acts upon the muscle during the contraction caused by a first stimulus, or during the latent period before the contraction has actually begun; and what happens when a whole series of rapidly-succeeding stimuli are thrown into the muscle.

First let us take two stimuli separated by a smaller interval than the latent period (p. 745). If they are both maximal-i.e., if each by itsclf would produce the greatest amount of contraction of which the nuuscle is capable when excited by a single stimulus-the second has no effect whatever; the contraction is precisely the same as if it had

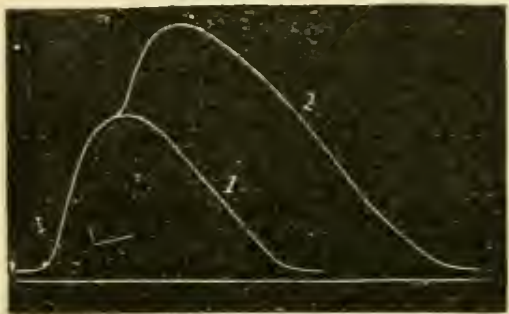

Fig. 256.- Superposition of Contractions. $I$ is the curve when only one stimuius is thrown in; 2 , when a second stimulus acts at the time when curve I has nearly reached its maximum height. never acted. But if they are less than maximal, the contraction, although it is a single contraction, is greater than would have been due to the first stimulus alone; in other words, the stimuli have been summed or added to each other during the latent period, so as to produce a single result.

Next let us consider the case of two stimuli separated by a greater interval than the latent period, so that the second falls into the muscle during the contraction procluced by the first. The result here is very different: traces of two contractions appear upon the musclecurve, the second curve being that which the second stimulus would have caused alone, but rising from the point which the first had reached at the moment of the second shock (Fig. 266). Although the first curve is cut short in this manner, the total height of the contraction is greater than it would have been had only the first stimulus acted; and this is true even when botl stimuli are maximal. Under farourable circumstances, when the sccond curve rises from the apex of the first, the total height may be twice as great as that of the contraction which one stimulus would have caused $(\mathrm{p} .8 \mathrm{r} 6)$. It is worthy of note that striated muscle has no power of summation of subminimal stimuli each of whicl is just too weak to cause contraction. No matter how rapidly they are thrown in, the muscle remains at rest. It is otherwise with smooth muscle. Stimuli which are singly ineffective cause contraction when repeated.

Tetanus. - Not only may we have superposition or fusion of two contractions, but of an indefinite number; and a series of rapilly following stimuli causes complete tetanus of the muscle, which remains contracted during the stimulation, or till it is exhausted (Fig. 267). 
The meaning of a complete tetanus is readily grasped if, beginning with a series of shocks of such rapidity that the muscle can just completely relax in the intervals between successive stimuli, we gradually increase the frequency (p. 8I6). As this is done, the ripples on the curve become smaller and smaller, and at last fade out altogether. The maximum height of the contraction is greater than that produced by the strongest single stimulus; and even after complete fusion has been attained, a further increase of the frequency of stimulation may cause the curve still to rise.

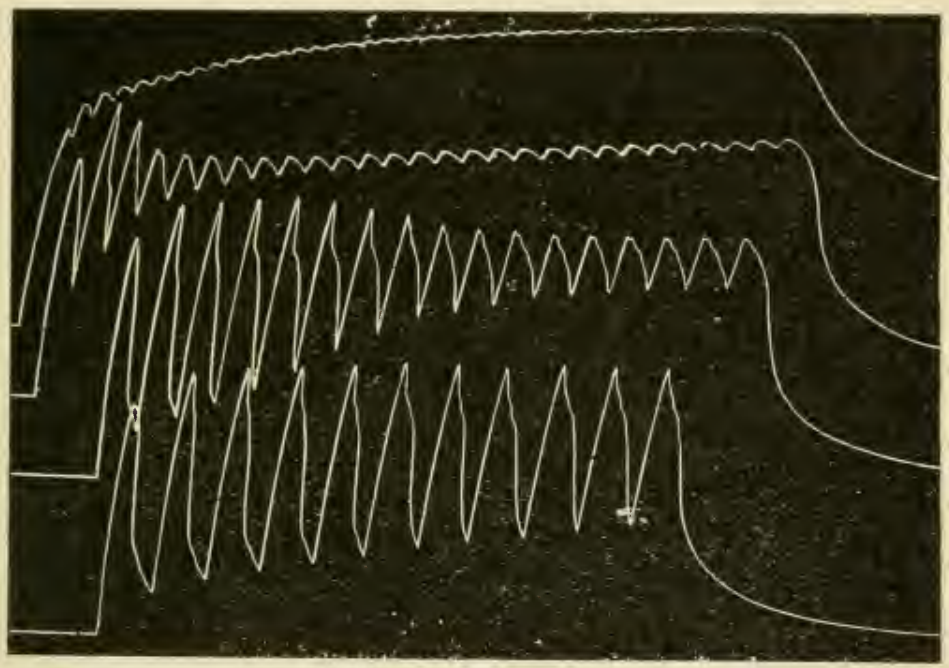

Fig. 267.-Analysis of Electrical Tetanus (reduced to $\frac{2}{3}$ ). Four curves showing the effect of increasing frequency of stimulation of the frog's gastrocnemius through its nerve. In the lowest curve the frequency is such that the muscle relaxes almost completely between the successive contractions. In the uppermost curve, with a frequency more than three times greater, the contractions are almost completely fused. In all the curves the fusion becomes more nearly coniplete as stimulation goes on, owing to the slower relaxation of the fatigued muscle.

It is evident from what has been said that the frequency of stimulation necessary for complete tetanus will depend upon the rapidity with which the muscle relaxes; and everything which diminishes this rapidity will lessen the necessary frequency of stimulation. A fatigued muscle may be tetanized by a smaller number of stimuli per second than a fresh muscle, and a cooled by a smaller number than a heated muscle. The striped muscles of insects, which can contract a million times in an hour, require 300 stimuli per second for complete tetanus, those of birds Ioo, of man 40 , the torpid muscles of the tortoise only 3. The pale muscles of the rabbit need 20 to 40 excitations a second, the red 
muscles only io to 20 ; the tail muscles of the crayfish 40 , but the muscles of the claw only 6 in winter and 20 in summer. The gastrocnemius of the frog requires 30 stimuli a second, the hyoglossus muscle only half that number (Richet). The frequency of stimulation necessary for complete tetanus of unstriped muscle is much less than for striped muscle. Smooth tetanus of a band of muscle from the frog's stomach was obtained with strong opening induction shocks at the rate of $I$ in 5 seconds.

There appears also to be an upper limit beyond which a series of stimuli becomes ton rapid to produce complete tetanus, and at which an interrupted current acts like a constant current, causing a single twitch at its commencement or at its end, but no contraction during its passage. This limit does not depend upon the frequency of stimulation alone; the intensity of the individual excitations, the temperature of the muscle, and probably other factors, affect it. For Bernstein found that with moderate strength of stimulus tetanus failed at about $25^{\circ}$ per second, and was replaced by an initial contraction; with strong stimuli at more than 1,700 per second, tetanus could still be obtained. Kronecker and Stirling saw tetanus even with , ,ooo shocks a second. Kries in a cooled muscle found tetanus replaced by the simple initial twitch at 100 stimuli per second, although in a muscle at $38^{\circ} \mathrm{C}$. stimulation of ten times this frequency still caused tetanus. Einthoven, exciting the nerve of a frog's nerve-muscle preparation with extremely: frequent oscillatory condenser discharges, observed tetanus up to even a million vibrations a second, if the current intensity was at the same time very greatly increased (to more than 16,000 times the intensity needed with a constant current). These results are not really so discordant as they appear; for it is known that with electrical stimulation the number of excitations is not necessarily the same as the nominal number of shocks. By applying a telephone to a muscle excited through its motor nerve, it has been shown that the pitcls of the note produced by the tetanized muscle corresponds exactly to the rate of excitation up to a certain frequency. This frequency is about 200 per second for frog's and about 1,ooo per second for mammalian mirscle under the best conditions. If the rate of excitation is still further increased, there is no corresponding increase in the pitch. Therefore, some of the stimuli are now producing no effect- 'falling flat,' so to speak (Wedensky). A physical reason for this is the overlapping of the make and break shocks (Erlanger and Garrey); and a physiological reason, the alterations of conductivity and excitability, which even very brief currents leave behind them (Sewall), and which we shall have to discuss in another chapter.

It is only while the actual shortening is taking place that a tetanized muscle can do external work. But, although during the maintenance of the contraction no work is done, energy is nevertheless being expended for the metabolism of a muscle during tetanus is greater than during rest, and, among other changes, lactic acid is produced. There are great differences in the ease with which different muscles can be exhausted by tetanus. For example, the muscles which close the forceps of the crayfish or lobster have, as everyone knows, the power of most obstinate contraction. Richet tetanized one for over seventy minutes, and another for an hour and a half, before exhaustion came 
on, while a tetanus of a single minute exhausted the musces of li.e crayfish's tail. The gastrocnemius of a summer frog kept up for twelve minutes, and a tortoise muscle for forty minutes.

Continuous stimulation is not always necessary for the production of continuous contraction; in some conditions a single stimulus is sufficient. A blow with a hard instrument may cause a dying or exhausted, and in thin persons even a fairly normal, muscle to pass into longcontinued contraction. This so-called 'idio-muscular' contraction seems to depend, in part at least, on the great intensity of the stimulus, lt can sometimes be obtained in the frog's gastrocnemius, partic larly in spring after the winter fast. It is not a tetanus and is not propagated along the muscular fibres, as an electrical tetanus is, but remains localized at the spot where it arises. Similar non-tetanic contr, ctions have already been mentioned, such as the tonic contraction during the passage of a strong voltaic current and the sustained veratrine contraction. Ammonia causes also a long but non-tetanic contraction, and this, too, does not spread when the substance has acted only on a porticn of the muscle. The contraction force of all these tonic contractions, as measured by the resistance necessary to overcome or prevent them, is less than the contruction force in electrical tetanus (Sclienck).

The rate at which the wave of muscular contraction travels may be measured by stimulating the muscle at one end, and recording, by means of levers, the movements of two points of its surface as far apart from each other as possible. Time is marked on the tracing by ineans of a tuning-fork, and the distance between the points at which the two curves begin to rise from the base-line divided by the time gives the velocity of the wave. Another method is founded upon the measurement of the rate at which the negative variation ( $p .824)$ passes over the muscle, this being the same as the velocity of the contraction-wave. In frog's muscle it is about three metres a second, or six miles an hour. Rise of temperature increases, fall of temperature lessens it. When a numscle is excited through its nerve, the contraction springs up first of all about the middle of each muscular fibre where the nerve-fibre enters it, and then sweeps out in both directions towards the ends. But so long is the wave, that all parts of the fibre are at the same time involved in some phase or other of the contraction.

The wave of contraction in unstriped muscle lasts a relatively long time at any given point, and in tubes like the intestines and ureters, the walls of which are largely composed of smooth muscle arranged in rings, the wave shows itself as a gradually-advancing constriction travelling from end to end of the organ. There is no evidence that the contraction of smooth muscular fibres is discontinuous-that is, :omposed of summated contractions like a tetanus; it appears to be a sreatly-prolonged simple contraction. An artificial stimulus, mechanical or electrical, causes, after a long latent period, a very definitelylocalized contraction in a rabbit's ureter, which slowly spreads in a peristaltic wave in one or both directions along the muscular tube. Here, as in the cardiac muscle, the excitation passes from fibre to fibre, while in striped skeletal muscle only the fibres excited directly or through their nerves contract. That the rhythmical contraction of the heart is not a tetanus has already been seen. It is a simple contraction, intermediate in its duration and other characters between the twitch of voluntary muscle and the contraction of smooth muscle. The contraction both of unstriped and of cardiac muscle is lengtlened and made stronger by distension of the viscera in whose walls they occur, just as a skeletal muscle contracts more powerfully against resistance. 
Voluntary Contraction.-There is evidence that the voluntary contraction is a tetanus. One of the strongest buttresses of the theory of natural tetanus has been the muscle-sound, a low rumbling note which can be heard by listening with a stethoscope over the contracting biceps, or, when all is still, by stopping the ears with the fingers and strongly contracting the masseter and the other muscles concerned in closing the jaws.* Discovered about ninety years ago, first by Wollaston and then by Erman, half a century passed away before it was investigated more fully by Helmholtz. The latter observer, confirming the results of his predecessors, put down the pitch of the sound at 36 to 40 vibrations per second. He found, however, that little vibrating reeds with a rate of oscillation of about I9. 5 per second were more affected when attached to muscle thrown into voluntary contraction, than those that vibrated at a smaller or a greater rate. He therefore concluded that the fundamental tone of the muscle corresponded to this frequency, although, since such a low note is not easily appreciated, the sound actually heard was really its octave or first harmonic (p. 310).

The objection has been brought forward that the resonance tone of the ear also corresponds to a vibration frequency of 36 to 40 a second. In other words, this is the natural rate of swing of the elastic structures in the middle ear, the rate they will most easily fall into if set moving by an irregular mixture of faint, low-pitched tones and noises, and not compelled to vibrate at some other rate by a distinct sound of definite pitch. Now, this resonance tone might be elicited by a quivering muscle if, among many diverse rates of oscillation of different portions of its substance, the rate of 36 to 40 a second anywhere appeared, and the note corresponding to the real rate of vibration of the muscle as a whole might be overpowered. Or, even if there were no regular rate of vibration of the whole muscle, but, instead, a series of irregular tremors or pulls due to irregularities in the contraction, connected with a want of co-ordination of all the fibres (Haycraft), the ear might from time to time pick out of the turmoil of feeble aerial waves those corresponding to its resonance tone, just as a tuning-fork or a piano-string attuned to a particular note would catch it up amid a thousand other sounds and strengthen it.

But while this renders it highly probable that the resonance of the ear contributes to the production of the muscle-sound, and shows that we cannot from the pitch of the muscle-sound alone deduce the rate at which the muscle-substance is vibrating, it does not invalidate Helmholtz's objective observations with the oscillating reeds.

And several observers (Schäfer, Horsley, v. Kries) have noticed periodic oscillations, at the rate of Io or $\mathbf{I} 2$ per second, in the curves taken from muscles (Fig. 268), contracted voluntarily against a small resistance. When the resistance is greater, the rate may be as much as $I 8$ or 20 a second, and in quick and rapidly

* In order that a muscular sound may be produced there must be a certain abruptness in the contraction. Thus, the slowly-contracting smooth muscles (lo not produce a sound, nor the slowly-contracting heart-muscle of coldbloorled animals. 
repeated movements of the fingers even 40 a second. In habitual movements, such as those employed by a man in his trade, the tremors are much less coarse than in unaccustomed movements. For this reason the tremors of the left hand are greater than those of the right in executing a movement usually made with the latter (Eshner). In disease these tremors are often increased-e.g., in the clonic convulsions of epilepsy-but the frequency is the same.

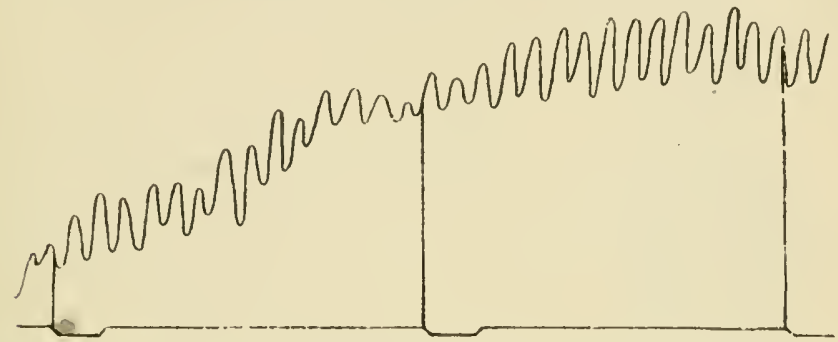

Fig. 268.-Vibrations of Contracted Arm Muscles (Griffiths). The arm was stretched out, holding a weight of about 6 kilos.

Similar vibrations, and at about the same rate, are seen in curves traced by muscles excited through stimulation of the motor areas of the surface of the brain. Since this rate remains the same whether the motor cortex, the corona radiata, or the spinal cord is excited, and, unlike the rate of response to excitation of peripheral nerves, is independent of the frequency of stimulation (so long as the rate of stimulation is greater than IO or I 2 a second), it has been supposed to represent the rhythm with which impulses are discharged from the motor cells of the cord (Fig. 269). It is probable that the cortical centres discharge at about the same rate, for not only is it impossible to articulate more rapidly than eleven syllables per

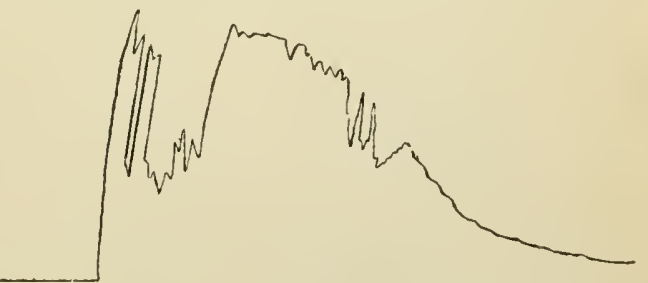
second, but it is impossible to reproduce the act of articulation in thought at a greater rate than this (Richet). But while this rate of IO or I2 a second does seem to represent a fundamental rhythm of the central discharge, there are facts which indicate that upon this relatively slow rhythm a quicker rhythm is superposed. In other words, each of these discharges is itself discontinuous, and made up of a number of separate impulses. 
Thus, according to Piper, the total number of simple discharges, each associated with an electrical change in the muscle, as recorded by the string galvanometer, is 47 to 50 a second. The rhythm of strych. nine tetanus in the frog is about 8 to 12 per seconcl. By means of the capillary electrometer (p. 729) large electrical oscillations at this rate can be demonstrated, each of which represents a sliort tetanic spasm, as is slown by the fact that a number of smaller electrical oscillations are superposed upon the large ones (Sanderson). The electrical changes suggest that each discharge causes a simple contraction much more prolonged thian the twitch of a directly stimulated muscle. This removes the difficulty of understanding how such al small number as Io contractions per second could be smoothly fused, and indicates that even the sinortest possible voluntary movement, which can be executed in $\frac{1}{10}$ to $\frac{1}{20}$ of a second, is not caused by a single inpulse, but is a tetanus. For these brief movements the frequency of oscillation, as shown by the action currents, is the same as for sustained contractions. The electrical changes in the voluntarily contracted muscle seem to differ in amplitude or abruptness from those produced in experimental tetanus. For secondary tetanus (p. 833) is not caused by muscle in voluntary contraction. But this is also the case with the ather prolonged contractions caused by continuous artificial stimulation-e.g., Ritter's tetanus (p. 74I) and the contraction produced by sodium chloride or ammonia. We need not hesitate to conclude, then, that the voluntary contraction is discontinuous, in the sense that it is not a perfectly smooth and uniform tonic contraction, although we still lack a decisive proof that it is maintained by a strictly intermittent outflow of nervous energy, and not by a continuous outflow causing a sustained contraction, which, it may be, remits and is reinforced at intervals. The apparent discrepancies as to the rate of discharge in the results obtained by different observers, and by different inetliods, far from exciting distrust of them all, really lend support to the idea of a fundamental and fairly constant rhythm in the outflow as soon as it is recognized that the higher rates are approximately multiples of the lower. Thus, the number deduced by Helmboltz from the experiment of the springs is twice the lowest rate calculated from graphic records of the contraction. The rates corresponcling to the musclesound and to the frequency of the electrical oscillations are about four times this number. Now, in a vibrating elastic body like a contracting muscle, a simple mathematical relation of this sort might be expected to appear when determinations of the rate of oscillation and of accompanying periodic changes are made by methods varying in principle and in delicacy. For instance, an arrangement suited to record and to count coarse vibrations could not be expected to give the same result as an arrangement suited to record and count fine vibrations. But if both the coarse and the fine vibrations were related to a fundamental rhythm, a simple proportion might be expected to exist between the two sets of results.

(3) Thermal Phenomena and Transformation of Energy in the Muscular Contraction.-When a muscle contracts, its temperature rises; the production of heat in it is increased. This is most distinct when the muscle is tetanized, but has also been proved for single contractions. The change of temperatule can be detected by a delicate mercury or air thermometer; and, indeed, a thermometer thrust among the thigh-muscles of a dog may rise as much 
as $I^{\circ}$ to $2^{\circ} \mathrm{C}$. Wlien the muscliss are thrown into tetanus. In the isolated muscles of cold-blooled animals the increase of temperature is much less; and thermo-electrical methods, which are the most delicate at present known, liave generally been used for its detection and measurement.

They depend upon the fundamental fact of thermo-electricity, that in a circuit composed of two metals a current is set up if tle junctions of the metals are at different temperatures.

Where no very fine differences of temperature are to be measured, a single thermo-junction of German silver and iron, or copper and iron, is inserted into a muscle or between two muscles. But the electromotive force, and therefore the strength of the thermo-electric current, is proportional for any given pair of metals to the number of junctions, and for licate measurements it may be necessary to use several connected together in series. A thermopile of antimony-bismuth junctions gives a stronger current for a given difference of temperature than the same number of German silver-iron couples, but from its brittle nature is otherwise less convenient.

The direction of the current in the circuit is such that it passes through the heated junction from bismuth to antimony and from copper or German silver to iron. Knowing this direction, we are aware of the changes of temperature which take place from the movements of the mirror of the galvanometer with which the pile is connected. In the thermopiles employed in the recent extensive investigations of Hill the alloy constantan is coupled with iron, the electromotive force of this combination being exceptionally great.

The muscle which is to be excited is brought into close contact with one junction or set of junctions, the other set being kept at constant temperature. The image will now come to rest on the scale; and excitation of the muscle will cause a movement indicating an increase of temperature in it, the amount of which can be calculated from the deflection. In one form (Fig. 270) the thermopile con-

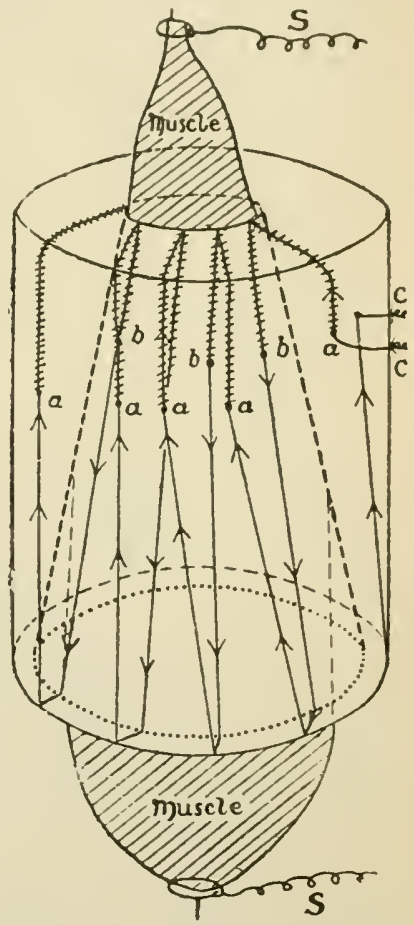

Fig. 270.-Conical Thermopile containing Gastrocnemius Mus. cle Reversed. C, copper leads to galvanometer; $S$, stimulating wire. The straight lines indicate iron, the crossed lines constan. tan, the external junctions em. bedded in the ebonite frame being at $a$, the internal junc. tions, $b$, in contact with the muscle. stitutes a hollow cone, in which a muscle can be arranged so as to eliminate largely the errors due to differences of temperature of the muscle, or to the "slip" of the contracting muscle over the junctions.

In this way Helmholtz observed a rise of temperature of $0.14^{\circ}$ to $0.18^{\circ} \mathrm{C}$. in excised frogs' muscles when tetanized for a couple of minutes. 
Heidenhain, with a very delicate pile, found a rise of $0.00 \mathrm{r}^{\circ}$ to $0.005^{\circ} \mathrm{C}$. for a single contraction of a frog's muscle. On the assumption that the pile had time to take on the temperature of the muscle before there was any appreciable loss of heat, this would be equal to the production by every gramme of muscle of a thousandth to five-thousandtlis of a gramme-caloric (p. 676) of heat. From Fick's observations we may take about three-thousandths of a gramme-calorie as the maximum production of a gramme of frog's muscle in a single contraction.

Hill has shown that in the case of the single contraction or twitch the evolution of heat may be so rapid as to be practicilly instantaneous, indicating that it depends upon some sudden 'xplosive" chemical reaction; or, on the other hand, under certain conditions

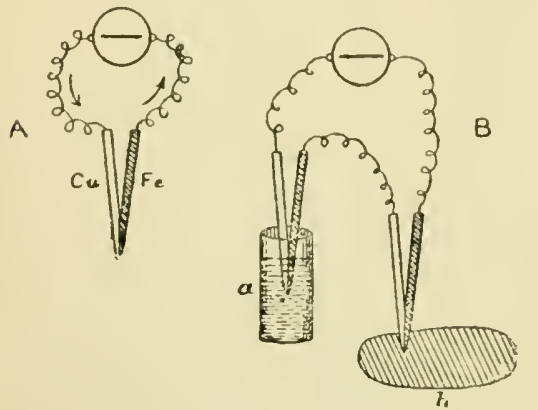

Fig. 271.-A, a single copper-iron thermotectric couple; 13, two pairs, one inserted in to the tissue $b$, the other dipping into water in a beaker $a$. The temperature of the water may be adjusted so that the galvanometer show's no deflection. The temperature of the tissue is then the same as that of the water. it may last as long as two seconds -that is, from four to ten times as long as the contraction itself. In the absence of oxygen, when the muscle is left, for instance, in an atmosphere of hydrogen, the heat production becomes markedly prolonged. When abundant oxygen is supplied, the duration of the discharge of leat is decreased. In a tetanus the evolution of heat lags behind the excitation, and the discharge associated with each stimulus is not complete till 0.5 to 2.5 seconds after the stimulus. In a prolonged complete tetanus the heat production corresponding to the first tenth of a second of excitation is far greater than that corresponding to the second tenth of a second, and so on, until eventually a uniform discharge of heat, at a rate much smaller than the initial rate, is reached. When frogs' muscles are rapidly stimulated indirectly (through the nerves) till fatigue has occurred, the maximum value of the heat evolved approximates to 0.9 gramme-calorie per gramme of muscle, about 70 or 80 per cent. being liberated in the first two minutes (Peters).

A fact of great significance in regard to the relation of the reaction upon which the heat production depends and the mechanical conditions in the active muscle is that the production of heat is determined by the length of muscular fibres existing at the time when the heat is being evolved. From this it has been assumed that the production of heat in active muscle is a surface effect, and 
not an effect taking place uniformly throughout the musele substance and related accordingly to the volume or mass of the muscular substance (Blix). Much evidence has been accumulated in favour of this hypothesis. For example, a muscle contracting isometrically (p. 747) produces more heat the greater is the initial tension (the more it is stretched at the begining of the excitation)-that is, the greater its length during contraction (Heidenhain). When a muscle is allowed to shorten in a tetanus, the heat production as compared with that of an isometric contraction of the same duration, and evoked by the same strength of stimulus, is diminished by as much as 40 per cent.

Relation between the Development of Mechanical Energy and Heat Production in Active Muscle.-There is no simple relation between the external work done in a nuuscle twitch and the heat set free. The efficiency of the muscular machine, as estimated by the proportion of the work done to the total energy degraded, varies with a number of factors-e.g., the load, the number of fibres excited, and the intensity of the excitation of each fibre, the two latter factors depending upon the strength of the stimulus.

The greater the resistance, so long as the muscle can overcome it so as to do its utmost amount of external work,* the larger is the proportion of energy which appears as work, the smaller the proportion which appears as heat. For every muscle, under given conditions, there is a certain load which can be raised more advantageously than any other; but even in the most favourable case, an excised frog's muscle never does work equal to more than $\frac{1}{4}$ of the heat given off. Generally the ratio is much less, and may sink as low as $\frac{1}{25}$. In the intact mammalian body the muscles work somewhat more economically than the excised frog's muscles at their best; for both experiment and calculation show (p. 687) that in a normal man under the most favourable conditions as much as $\frac{1}{3}$ of the energy is converted into work. According to Zuntz and Katzenstein, 35 per cent. of the total energy appeared as muscular work in climbing a mountain, and in bicycling only 25 per cent. Movements which have been much practised are more economically performed than unaccustomed ones, and this explains the superior efficiency of the muscles concerned in climbing, for no movements can possibly be more familiar than those concerned in locomotion. So far as this indication goes, it would seem that in the treatment of obesity unfamiliar, and therefore physiologically expensive, forms of exercise should be recommended, in so far, of course, as they do not injuriously react upon the general condition, especially upon the circulation.

* This statement, based on experiments with excised frog's muscles, is not, of course, inconsistent with the fact mentioned on p. 687 , that in the intact body the fraction of the energy transformed into heat is greater in hard than in moderate work. 
When a inuscle, excited by maximal stimuli, is made to lift continuonsly increasing weights, both the work clone and the lieat given out increase up to a certain limit. The muscle, as it were, burns the candle at botli ends. The heat-production reaches its maximum somewliat sooner than the work.

It is certain that when work is done by a muscle an equivalent amount is subtracted from its sum-total of energy, and under proper conditions this can be actually demonstrated by the deficiency in the heat-production. This is done by means of a contrivance called a work-adder. It consists of a wheel, the rotation of which raises a weight attached to a cord wound round its axle. Tle muscle acts on the periphery of the wheel, and by rotating it raises the weight a little at each contraction. At the end of the contraction the wheel is prevellted from moving back by a catch. The work done in a series of contractions is calculated from the total height to wlich the weight has been raised. Suppose a frog's gastrocnemius is made to contract a certain number of times while attached to the work-adder, and that simultaneously the heat-production is measured by means of a thermopile. Let $H$ represent the heat actually produced, and $h$ the heat equivalent of the work done. Now let the muscle be disconnected from the adder and made to raise the same weight, directly attached to it, by a series of contractions elicited in precisely the same way as the previous ones, except that the weight is allowed to fall with the muscle when it relaxes after each contraction. Here heat corresponding to the external work disappears from the muscle during the contraction just as in the first experiment, but this heat is returned to the muscle during the relaxation, since on the whole no external work is done. The heat produced in the second experiment is found, as a matter of fact, allowing for unavoidable errors, to be equal to $H+h$.

According to Hill, the true 'efficiency' of the muscle is not the rutio $W / H$, where $H$ is the external work and $H$ the total heat liberated, but $T / H$ where $T$ is the maximum increase of tension set up during the twitch when the muscle is contracting isometrically. This fraction $T / H$ is constant whatever be the initial tension, the number of fibres excited, or the strength of excitation of each fibre. For the theory of the muscular contraction the tension auring an isometric muscle twitch, which represents the potential energy suddenly developed in consequence of the excitation, is accordingly 11 uch more important than the height of the contraction, which is related to the work actually done. The essential thing in muscular contraction may be the abrupt developinent of this tension through a chemical reaction which liberates certain substances at some membrane or surface in the muscle. The potential energy once in being may or may not be transformed into work, and if so transformed the change may be accomplished economically or wastefully, according to the conditions of the contraction. The ratio $T / H$ decreases in fatigue, and with the time during which the muscle has been deprived of its blood-supply. Hill has calculated the absolute value of the heat-production in tetanus of a sartorius or semimembranosus muscle of the frog. This quantity, reckoned per centimetre of length of the muscle, per gramme weight of the tension developed and per second of maintenance of the tension, is relatively constant at about 0.000015 gramme-calorie. Including the recovery processes of oxidation following the contraction the total heat-production would amount to about $0 \cdot 000025$ calorie. The potential energy possessed by a muscle of a length of a centimetre when naintaining a tension of a gramme is about $0.00000+$ calorie. So that to maintain this state of potential energy six or seven times as much energy must be liberated 
per second. The maintenance of prolonged tension is, therefore, from the point of view of the mechanical result, an exceedingly wasteful process, with a very low efficiency in comparison with the higll efficiency in a rapid twitch. This enables us to see how important a part in heatproduction, and therefore in temperature regulation, the tonus of muscle and the prolonged contractions of shivering may possess (p. 695).

We are as yet in the dark as to the precise relation of the energy which appears as heat and of that which is converted into work. The ultimate source of both is, of course, the oxidation (and cleavage) of the food substances. It was at one time a favourite theory that in a muscle, as in a heat-engine, the chemical energy is first converted into heat, and part of the heat then transformed into work. There is no evidence that this is the case. It is, indeed, impossible that such differences of temperature can exist as would be compatible with the known efficiency of the muscular machine. Hypotheses based on the assumption that the chemical energy is immediately changed into work, perhaps through the production of surface effects, have met with increasing favour, but data are as yet too few for the formulation of any really satisfactory theory. The close relation between the heat-production and the formation of lactic acid in contraction which have been shown to exist, is a suggestive fact whose full significance will only be revealed by further investigation. The restitution processes by which the original state of the muscle is restored after contraction are, of course. intimately related to those concerned in the actual shortening; but unless we know how, and in consequence of what chemical or physical changes, the equilibrium of the resting muscle has been disturbed, we cannot know how, or in consequence of what chemical or physical changes, it is restored.

\section{Section IV.--Chemical Phenomena of the Muscular CONTRACTION.}

The composition of dead mammalian muscle of the striped variety may be stated, in round numbers, as follows, but there are considerable variations, even within the same species:

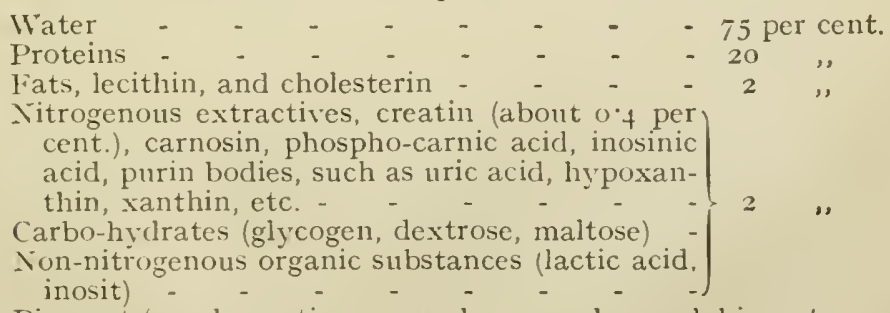

Pigment (myohamatin or myochrome, a hæmoglobin not precisely identical with that of blood).

Inorganic substances less than I per cent. (chlorides, carbonates, phospliates, and sulplates of potassium, sodium, iron, calcium, magnesium). Potassium is absent from the nuclei (Frontispiece) 
Of the nitrogenous extractives, creatin (p. 597) and carnosin are present in greatest quantity, muscle containing $0 \cdot 2$ to $0^{\circ} 4$ per cent. of kreatin. Cirnosin $\left(\mathrm{C}_{9} \mathrm{H}_{14} \mathrm{~N}_{4} \mathrm{O}_{3}\right)$ is a substance with basic propertics, and can be split up into histidin and $\beta$-amino-propionic acirl, an amino-acid not identical with alanin (or "-amino-propionic acid), but having the $\mathrm{NH}_{2}$ coupled to the $\beta$ instead of the a carbon atom (p. 566 ).

There is more water in the muscles of young than of old anime is (v. Bibra), and more in tetanized than in rested muscle (Ranke). The fats are variable in amount, and belong to a small extent to the actual muscle-fibres. For even when the visible fat is separated with the utmost care, nearly I per cent. of fat still remains (Steil).

The glycogen content varies extremely in different muscles and in the same muscle under different nutritive and functional conditions. Thus, in one and the same dog the biceps brachii.contained 0.17 and the quadriceps femoris 0.53 per cent. In dogs on a diet rich in carboliydrate and protein the percentage in the whole skeletal musculature ranged from 0.7 to $3 \cdot 7$, and in the heart from $0 \cdot 1$ to $1 \cdot 2$. The average for human muscles has been given as 0.4 per cent. In lean horse-flesh Pflüger found 0.35 per cent. of glycogen, but no sugar. The total nitrogen was 3.21 per cent. of the moist tissue. The lactic acid of inuscle and other tissues is the $d$-lactic acid, which rotates the plane of polarization to the right. By the action of certain bacteria on canesugar l-lactic acid is obtained, which is left rotatory. The optically inactive fermentation lactic acid is obtained by the fermentation of lactose.

Smooth muscle is somewhat richer in water than the striated variety from the same species, because skeletal muscle is richer in fat. Glycogen is either absent or present only in traces in the smootl muscle (of the stomach and bladder). Lactic acid, creatin, and creatinin are also found in much smaller amount than in striped muscle (Mendel and Saiki). As in striated muscle, hypoxanthin is the conspicuous purin base occurring in the free form-i.e., obtainable in inuscle extracts. The most remarkable difference in the quantitative relations of the inorganic constituents is that in striated muscle potassium preponderates over sodium and magnesium over calcium, whereas in the smooth variety this relation is reversed.

It would be natural to expect that the proteins, which bulk so largely among the solids of the dead muscle, and which are so obviously important in the living muscle, should be affected by contraction. But up to the present time no quantitative difference in the proteins of resting and exhausted muscle has ever been made out. The quantity of creatin (and creatinin) is said by some authorities to be increased. The following chemical changes have been definitely established. In an active muscle-

(a) More carbon dioxide is produced. (b) More oxygen is consumed. (c) Lactic acid is formed. (d) Glycogen is used up. (c) The substances soluble in water diminish in amount; those soluble in alcolıol increase.

Production of Carbon Dioxide and Consumption of Oxygen during Contraction.-This subject has already been dealt with in part in connection with tissue respiration (p. 266). The fact that muscular exercise increases the carbon dioxide output and the oxygen absorption at the pulmonary surface, shows that oxidation processes involving ultinately the combustion of carbon-containing substances 
are associated with the activity of the muscular tissue, but does not of itself prove that the final steps of the oxiclation occur in the: muscles themselves. This lias been demonstrated, lowever, by observations on isolated muscles. When well supplied with oxygen, these, in adclition to the stock of carbon dioxide in solution, in the form of carbonates and in other combinations, which they possess at the moment of isolation, continue to produce carbon dioxide, and this production is markedly increased by stimulation. The best evidence is to the effect that only preformed carbon dioxide is given off by isolated muscles in the absence of oxygen. They can go on contracting indeed, as previously stated, in an atmosphere of liydrogen or nitrogen, and may seem to be producing carbon dioxide, but the increased output appears to be due simply to an accelerated decomposition of already cxisting carbonates, or perhaps other combinations in which carbonic acid is loosely held, brought about by lactic acid, which in the absence of oxygen, is not transformed as it is under normal conditions, and accumulates in the muscular substance, uniting with bases, and thus displacing carbonic acid.

Formation of Lactic Acid-Reaction of Muscle.-To litmus-paper fresh muscle is amphicroic-that is, it turns red litmus blue and blue litmus red. This is due, partly at least, to the plosphates. Monophosplate (tribasic phosphoric acid, $\mathrm{H}_{3} \mathrm{PO}_{4}$, in which one hydrogen atom is replaced, say, by sodium or potassium) reddens blue litmus, while diphosphate (where two hydrogen atoms are replaced) turns red litmus blue. Litmoid (lacmoid) differs from litmus in not being affected by monophosphates. Diphosphates turn red litmoid blue, and so does fresh muscle, which has no effect on blue litmoid. A cross-section of fresh muscle is about neutral (sometimes faintly acid) to turmeric paper, which is turned yellow by monophosphates. A muscle which has entered into rigor or has been fatigued by prolonged stimulation is distinctly acid to blue litmus and to brown turmeric, reddening the former and turning the latter yellow, but does not affect blue litmoid.

Perfectly fresh resting muscle excised with avoidance of all unnecessary manipulation contains very little lactic acid (as little as 0.02 per cent. expressed as zinc lactate). Mechanical injury, heating, and chemical irritation cause a marked increase in the amount. Under anacrobic conditions-in an atmosphere of hydrogen, for instance-lactic acid is spontancously developed in the resting muscle so long as irritability persists, but not longer. In air, which for even small excised muscles corresponds to a partial asphyxia, there is a small increase in the lactic acid, but its production is very slow in comparison with that in the hydrogen atmosphere. In pure oxygen not only is there no accumulation of lactic acid for a long time after excision, but a portion of the amount originally present in the resting excised muscle disappears. The same is true of the lactic acid formed in a muscle fatigued by stimulation when it is afterwards placed in an atmosphere of pure 
oxygen. There is no doubt that the production of lactic acid in functional activity and its transformation into other substances are procesees that go on also in the muscles of the intact body. The formation of the acid in the excised muscle, far from being a sign of death, is an index of the 'survival ' of a process by which it is normally formed, as the accumulation of it is an index of the crippling, in the absence of oxygen, of a mechanism by which it is normally transformed.

The lactic acid which accumulates in the excised muscle in rigor and activity does not remain free, since blue litmoid paper is not reddened as it would be by free lactic acil. It causes a repartition of the bases at the expense of the sodium carbonate and disodium phosphate, the latter being changed into monophosphate, which, in part at least, accounts for the acid reaction to turmeric (Röhmann). It is of great interest that this oxidative transformation of lactic acid only occurs in muscle whose structure is so far preserved that its irritability is not lost. In minced or triturated nuscle it does not take place.

The relations between the heat production, the formation of carbon dioxide, and the production of lactic acid indicate that liberation of lactic acid from some precursor is an essential stage in the sudden, 'explosive' reaction or series of reactions which precedes and induces the mechanical response to stimulation. This stage takes place whether oxygen be present or absent, and it seems to be accompanied by a considerable liberation of energy, at the expense of which alone the anaerobically contracting muscle works. It is most probable that the liberation of lactic acid follows the same course in the nuscle abundantly supplied with oxygen, although it has not been shown that oxidative processes, resulting in the formation of carbon dioxide, do not contribute also at this stage to the energy which is transformed into the mechanical effect. But while in the absence of oxygen the reaction stops at the formation of lactic acid, when oxygen is available the cycle is completed by restitution processes which lead to the disappearance of the lactic acid either by restoration to its original position in the precursor from which it is derived, or perhaps in the case of a portion of the lactic acid to its combustion to carbon dioxide and water. For these restitution processes oxygen is essential, and it is to be supposed that the energy required for the rebuilding of the lactic acid precursor, or, to speak more generally, for the restoration of the muscle to its original state in readiness for a fresh contraction, is derived largely from oxidations in which carbon dioxide makes its appearance.

The Precursor of Lactic Acid.-What material is the lactic acid formed from? There are reasons for thinking that lactic acid is an intermediate substance which in metabolism serves as a link between 
the products of protein decomposition and carbo-liydrates, and between carbo-hydrates and fat. From what we know of the production of lactic acid both outside the body and in the intestine from carbo-hydrates, it might seem a most plausible suggestion that in the active muscle it comes from glycogen.

Glycogen is the one solid constituent of muscle which has been definitely proved to diminish cluring activity. It accumulates in a resting muscle, especially in a muscle whose motor nerve has been cut; but rapidly disappears from the muscles of an animal made to do work while food is withheld; or from the muscles of an animal poisoned by strychnine, which causes violent muscular contractions. But the best evidence points the other way-e.g., in rigor mortis lactic acid is produced just as in muscular contraction. Nay, more, the amount of lactic acid (as much as 0.5 per cent. cxpressed as zinc lactate) produced in full heat rigor (at $40^{\circ}$ to $45^{\circ} \mathrm{C}$.) is constant for similar excised muscles. This 'acid-maximum' is the same when fresh muscle is at once put into rigor; or when fatigue is first induced, with formation of lactic acid, before rigor; or, finally, when the lactic acid of the fatigued muscle is caused to disappear under the influence of oxygen, and heat rigor is then brought about in the muscle (Fletcher and Hopkins). Yet in rigor mortis the quantity of glycogen is unaltered (Boehm). Further, under certain conditions an excised muscle is capable of producing a quantity of lactic acid much greater than could be derived from the glycogen contained in it.

An indirect argument against the view that the lactic acid precursor is glycogen has been based by Hill on the results of his studies on the heat production of surviving muscle. From the amount of heat evolved, he calculates that the precursor of lactic acid must have a heat value ro per cent. greater than that of lactic acid. Now, the heat of combustion of dextrose is only about 3 per cent. more than that of lactic acid. He concludes that the precursor which yields lactic acid is a body of greater energy than dextrose. This, of course, does not preclude the possibility that the complex, whatever it is, from which lactic acid is liberated, contains a carbohydrate group. But it would not be profitable to pursue these speculations at present. The facts just mentioned suggest that it is the same precursor which yields the lactic acid developed with the onset of rigor. Further evidence of the close relations betwcen the chemical changes occurring in contraction and those occurring in rigor will be developed in considering the latter phenomenon.

The Substances metabolized in Muscular Contraction.-If the liberation of lactic acid were assumed to be the immediate cause of the mechanical changes in muscular contraction, if the nature of the body which yields lactic acid were known, and if it were proved, which is far from being the case, that the whole of the energy con- 
cerned in initiating and carrying out the mechanical effect is derived from the decomposition of this precursor, the question would still confront us, What are the materials at the expense of the energy of which the muscle is restored to its original condition ready for another contraction? If the lactic acid is used over and over again, it is indeed the metabolism of these substances which will be chiefly represented in the waste products given off by the muscle; the lactic acid complex will merely represent a chemical machine through which the energy of these other substances is transformed into mechanical energy, and they will constitute the ultimate source of energy of the muscular contraction. In this sense the muscular glycogen, whether it yields lactic acid or not, is almost certainly one source of energy for the active muscle, being converted into dextrose, of course, before utilization. Dextrins and maltose, the intermediate products of this decomposition, have been detected in muscle, more maltose, indeed, than dextrose being present (Osborne), since the dextrose is rapidly oxidized. Glycogen cannot be the only source of muscular energy, for its amount is too small.

For example, the heart of an average man, which weighs 280 grammes, contains about 60 grammes of solids, and among these certainly not more than I gramme of glycogen. In twenty-four hours it produces r 20 calories of heat (pp. 138,688 ), equivalent to the complete combustion of a little less than 30 grammes of glycogen. To supply this amount, the wliole store of glycogen in the heart would have to be used and replaced every fifty minutes. But the accumulation of glycogen is immensely slower in the muscles of a rabbit made glycogen-free by strychnine, and therefore we have to look around for some otler source of energy to supplement the glycogen. We have already brought forward evidence (p. oio) that, under ordinary circumstances, not a great deal, at any rate, of the energy of muscular contraction comes from the proteins. Of carbo-hydrates, the only one except the glycogen of the heart muscle which is at all adequate to the task of supplying so much energy is the dextrose of the blood. The quantity of blood passing through the coronary circulation has been estimated at 30 c.c. per Ioo grammes of cardiac muscle per minute (Bolir and Henrigues). which would be equivalent for an average man to about 120 litres in twenty-four hours. This quantity of blood will contain at least I20 grammes of dextrose, and about 32 grammes will suffice to supply all the heat produced by the heart. There is no reason to suppose that this dextrose must first be changed into muscular glycogen, which only represents a certain amount of reserve carbo-hydrate. Of proteins a little less than 30 grammes would be needed, of fat a little more than I 2 grammes. We see, therefore, how intense must be the metabolism that goes on in an actively contracting muscle. On any probable assumption as to the source of muscular energy, a quantity of material equal to half of its solids must be used up by the lieart in twenty-four hours. Or, to put it in another way, the heart requires not less than two-fifths of its weight of ordinary solid food in a day. The body as a whole requires so to ${ }_{i j}^{1}$ of its weight.

The general conclusions to which physiologists have been led as to the relative importance of the different food substances for 
muscular work have been previously given (p. 6I2), and need not be repeated here. It may be added that the various food substances yield muscular energy in isodymamic relation. In other words, a given amount of muscular work requires the expenditure of approximately the same quantity of chemical energy, whether it comes almost entirely from protein, or chiefly from carbo-liyclrates, or chiefly from fat. Some observers have stated that the taking of even a comparatively small quantity of sugar vastly increases the capacity for muscular work as measured by the ergograph (p. 750). But although it is not to be doubted that sugar is under normal circumstances one of the most important substances used up in muscular contraction, the claim that sugar is, par excellence, the food for muscular exertion has not yet been made out.

Physico-Chemical Conditions of Muscular Contraction.-For excised fresl muscle $\Delta(\mathrm{p} .+27)$ has been estimated at $0.68^{\circ} \mathrm{C}$. But this is probably higher than in the living body, for after excision waste products, with their relatively smasl and numerous molecules, are still for a time produced, and are no longer removed by the blood. In salt solutions isotonic with the muscle substance-e.g., for the frog's gastrocnemius at room-temperature a 0.75 per cent. solution of sodium chloride-the resting muscle neither gains nor loses water for some hours. The active muscle behaves quite differently. When a muscle immersed in isotonic salt solution is tetanized, water enters it, leading to an increase in weight and a diminution in specific gravity (Ranke, Loeb, Barlow). The same occurs even when blood is circulated through active muscles, the blood becoming poorer in water (Ranke). This may be explained by the increase of osmotic pressure in the muscle substance which must accompany the decomposition of large molecules into small. As fatigue progresses, a movement of water in the reverse direction occurs, and the muscle rapidly loses water. Exposure of the fatigued muscle for a sufficient time to an atmosphere of oxygen restores the osmotic properties of the resting muscle. Striking differences have also been demonstrated in the behaviour of resting and fatigued muscle to hypotonic solutions or water. Hales observed long ago that, on injecting large quantities of water into the bloodvessels of a dog, so as to replace the blood, marked swelling of the muscles occurred. This physiological fact is well known to the pork-butchers in China, who have given it a practical, if not a very praiseworthy, application in sophisticating their product by increasing its weight (MacGowan).

So long as the muscular fibres are uninjured they are permeable or impermeable for exactly the same compounds as other animal and vegetable cells. All substances easily soluble in media like ether or olive oil readily penetrate them (Overton). To most salts they are relatively impermeable, as is shown by the fibres retaining their oiginal volume in isotonic solutions of them. In particular, they cannot easily take up or retain the salts of the blood-plasma, otherwise the observed qualitative differences-e.g., the preponderance of potassium in the muscle and sodium in the plasma-could not be maintained. There are facts which indicate that temporary changes in the permeability to ions, not only of muscular fibres, but also of nerve fibres and other excitable structures, are concerned in their stimulation. Potassium salts after a time seem to produce an effect upon frog's muscle, which alters its permeability so that it takes up water from hypertonic solutions. 
Calcium salts have the opposite effect (Locb). Sodium (and in a minor degree lithium) salts have a peculiar relation to the contraction of skeletal muscle, for which they appear to be indispensable. Yet sodium chloride produces a paralyzing action on the frog's motor nerve-endings, so that after perfusion with a solution of that salt stimulation of the motor nerve callses no contraction, or with a slighter degree of paralysis contraction only after a long interval. The efiect can be counteracted by solutions containing calcium salts (Locke, Cushing).

Rigor Mortis.-When a muscle is dying, its excitability, after perhaps a temporary rise at the beginning, diminishes more and more until it ultimately responds to no stimulus, however strong. The loss of excitability is not in itself a sure mark of death, for, as we have seen, an inexcitable muscle may be partially or completely restored; but it is followed, or, where the death of the muscle takes place very rapidly, perhaps accompanied, by a more decisive. event, the appearance of rigor. The muscle, which was before soft and at the same time elastic to the touch, becomes firm; but its elasticity is gone. The fibres are no longer translucent, but opaque and turbid. If shortening of the muscle has not been opposed, it may be somewhat contracted, altlough the absolute force of this contraction is small compared with tliat of a living muscle, and a slight resistance is enough to prevent it. The reaction is now distinctly acid to litmus. This is rigor mortis, the deatl-stiffening of muscle.

An insight into the real meaning of this singular and sometimes sudden change was first given by the experiments of Kiihne. He took living frog's muscle, freed from blood, froze it, and minced it in the frozen state. The pieces were then rubbed up in a mortar with snow containing I per cent. of common salt, and a thick neutral or alkaline liquid, the 'muscle-plasma,' was obtained by filtration. This clotted into a jelly when the temperature was allowed to rise, but at $0^{\circ} \mathrm{C}$. remained fluid. The clotting was accompanied by a cliange of reaction, the liquid becoming acid. An equally good, or better, metlood is to use pressure for the extraction of the plasma from the frozen fragments of muscle. A low temperature is essential, otherwise the plasma will coagulate rapidly within the injured muscle. A similar plasma can be expressed from the skeletal muscles of warm-blooded animals (Halliburton), and with greater difficulty from the heart.

When the muscle, after exhaustion with water, is covered with a solution of a neutral salt, a 5 per cent. solution of magnesium sulphate or Io per cent. solution of ammonium chloride being the best, ccrtain proteins are extracted which clot or are precipitated much in the same way as the muscle-plasma obtained by cold and pressure; and the process is hastened by keeping them at a temperature of $40^{\circ} \mathrm{C}$.

In the extracts of mammalian muscle three chicf proteins are present: paramyosinogen (v. Fürth's myosin), coagulating by heat at $47^{\circ}$ to $50^{\circ} \mathrm{C}$.; myosinogen (v. Fürth's myogen), coagulating at $55^{\circ}$ to $60^{\circ} \mathrm{C}$., usually about $5^{\circ}$ ); and serum-albumin, coagulating about $73^{\circ}$. The 
serum-albumin belongs to the blood and lympln, and is not a constituent of the muscle-fibre. The most recent work on the subject is that of Botazzi, who obtained muscle juice without the addition of water or salt solutions, by rubling muscles up with sand, and then subjecting the triturated material to a pressure of many atmospheres. He finds that, leaving out of account the serum-albumin, muscle juice contains only one protein in solution, and this corresponds upon the whole in its properties to myosinogen. A second protein, and only these two have been proved to exist in muscle juice, is not in solution, but in the form of very fine granules revealed by the ultramicroscope. 'This corresponds in a general way to paramyosinogen. Botazzi supposes that it represents the substance of the muscular fibrils. The granules show a tendency even at the ordinary temperature to agglutinate and to be precipitated. The process is hastened by dilution witl water, removal of the salts by dialysis, addition of acids, and the agglutination and precipitation are accomplished very rapidly at $45^{\circ}$ to $55^{\circ} \mathrm{C}$., giving rise to 'heat coagulation.' The protein in solution (myosinogen) is insoluble in distilled water when thoroughly freed from salts, and is precipitated by dialysis, but not so easily as paramyosinogen. The total proteins in the juice obtained by pressure varied from 5.3 to 9.5 per cent., a great deal of the muscle protein being, of course, left in the residue. The granules (paramyosinogen) constituted from a third to two-thirds of the protein in different experiments, and the true proportion must have been considerably higher, since on account of their small size the loss in separating them by filtration was great. The 'myosin' precipitate, which rapidly forms in muscle-plasma at body temperature, is sometimes called the muscle-clot, and the liquid which is left the muscle-serum, but it would probably be better to avoid these terms, as they suggest an analogy with the coagulation of blood-plasma, which is apt to be misleading. A similar precipitate or clot seems to be formed in the interior of the muscular fibres in natural rigor and in the rapid rigor produced by heating a muscle to a little above the bodytemperature. But in natural rigor the whole of the paramyosinogen and myosinogen do not undergo the change, since a certain amount of these substances can as a rule be extracted from dead muscle by saline solutions. Thus, in rabbit's muscles, before the onset of rigor mortis, 87.3 per cent. of the total protein was found to be soluble in Io per cent. ammonium choride solution, and only 12.7 per cent. coagulated; while after rigor had occurred, $7 \mathrm{I} \cdot 5$ per cent. was coagulated, and only $28 \cdot 5 \mathrm{per}$ cent. remained soluble (Saxl). It is not known whether in the living muscle paramyosinogen and myosinogen exist as such. It has, indeed, been stated that, if a tracing is taken from a muscle which is gradually heated, it first shortens at the temperature of coagulation of paramyosinogen, and then again at that of myosinogen, and that in frog's muscle there is an additional shortening at $40^{\circ}$, the temperature at which in extracts an additional heat precipitate occurs. The conclusion has been drawn that these substances must be present as such in the living fibres, and that the successive shortenings are mechanical plenomena due to their heat coagulation. Similar shortenings have been described in nerve and liver tissue at about the temperatures at which the proteins in extracts of these tissues are coagulated by heat. But Meigs has shown that the supposed correspondence is far from being exact, and that muscles whose proteins have been already coagulated in a mixture of alcohol and salt solution still show the typical shortening on being heated. The heat shortening is, therefore, dependent on some other process than àgregation of the particles of coagulable protein. 
Certain analogies between rigor and muscular contraction were early pointed out. In botl there is (I) shortening; (2) heat-production; (3) formation of lactic acid; (4) discharge of carbon dioxide; (5) electrical changes. As regards the production of lactic acid, there is reason to believe that the process is fundamentally the same as in contraction, and the study of rigor, especially of certain of the artificially induced forms - e.g., heat rigor-in relation to the liberation of lactic acid, carbon dioxide, and heat, has thrown liglit upon the changes normally occurring in muscle. Another analogy might be forced into the list by anyone who was determined to see only rigor in contraction: the rigor passes off as the contraction passes off, although the 'resolution' of a rigid muscle takes days, the relaxation of an active muscle a fraction of a second. The disappearance of rigor is not dependent on putrefaction; it takes place when growth of bacteria is prevented (Hermann). Possibly it is connected with autolytic processes due to intracellular ferments (p. 599).

Why does coagulation of myosin occur at the death of the muscle? 'To this question no clear answer can be given. Some have looked on the process as analogous to the clotting of blood when it is shed, and it has even been suggested that just as a fibrin ferment is developed when the leucocytes and blook-plates begin to die, a myosin ferment, which aids coagulation, is developed in dead or dying muscle. But no proof has been given of the existence of such a ferment. And it is easy to make too much of the apparent analogy between the clotting of muscle and the clotting of blood, for there are differences as well as resemblances. For instance, the addition of potassium oxalate does not prevent coagulation of muscle extracts, as it does of blood and blood-plasna. If the development of lactic acid in the muscle is not the primary cause of the coagulation which constitutes the essential feature of rigor mortis, it seems to be closely related to it. For when excised muscles are abundantly supplied with oxygen, no lactic acid accumulates in them, and the final loss of excitability of the muscle is not followed by rigor. In any case, direct precipitation of hitherto unclotted muscle proteins may be induced by the acid, or the acid salts formed in its presence. Deficiency of oxygen is associated with the occurrence of rigor mortis, as it is with the accumulation of lactic acid, and a developing rigor can be abolished by oxygen, and its onset long or indefinitely delayed. When strict aseptic technique is observed an excised sartorius muscle of the frog may remain irritable in sterile Ringer's solution, even without oxygenation, for as long as three weeks (Mines).

Various influences affect the onset of rigor. Fatigue hastens it; heat has a similar effect; the contact of caffeine, chloroform, and other drugs causes most pronounced and immediate rigor. 
Blood applied to the cross-section of a muscle first stimulates the fibres with which it is in contact, and then renders them rigid. But it is to be remembered that normally the blood does not come into direct contact even with the sarcolemma, much less with its confents.

The effect of heat is of special interest. A skeletal muscle of a frog, like the gastrocnemius, if dipped into physiological saline solution at $40^{\circ}$ or $4 \mathrm{I}^{\circ} \mathrm{C}$. goes into rigor at once; the frog's heart requires a temperature $3^{\circ}$ or $4^{\circ}$ ligher; the distended bulbus aortæ can withstand even a temperature of $48^{\circ}$ for a short time. An excised mammalian muscle passes into immediate rigor at $45^{\circ}$ to $5^{\circ}$. In heat rigor the reaction of the muscle becomes strongly acid owing to the formation of lactic acid, and the evolution of carbon dioxide is also increased.

The total discharge of carben dioxide in heat rigor induced at $40^{\circ}$ amounts to 35 to 40 c.c. per 100 grammes of muscle. An additional I 5 to 20 per cent. is obtained on heating to $75^{\circ} \mathrm{C}$. to completely coagulate the proteins, and a further $I_{5}$ to 20 per cent. on heating to about $100^{\circ} \mathrm{C}$. When a muscle is scalded by being suddenly immersed in boiling salt solution, lactic acid is not formed, but carbon dioxide to the amount of 60 to 70 per cent. is discharged. An excised muscle kept in oxygen for many hours, during which it has diseharged several times as much calbon dioxide as is ever liberated by heating, still yields the normal discharge on heating whether to $40^{\circ} \mathrm{C}$. or to $100^{\circ} \mathrm{C}$. On the other hand, previous survival in an anaerobic atmosphere (of nitrogen) reduces greatly or abolishes the yield of carbon dioxide at $40^{\circ} \mathrm{C}$., although not that at $100^{\circ} \mathrm{C}$., the sum of the carbon dioxide given off to the atmosphere of nitrogen and that given off on heating to $100^{\circ} \mathrm{C}$. being about equal to the total amount which would have been discharged by a freshly-excised muscle on heating first to $40^{\circ} \mathrm{C}$. and then to $100^{\circ} \mathrm{C}$. If acid is added to a fresh muscle at about $0^{\circ} \mathrm{C}$. even more carbon dioxide is liberated than in heat rigor, while the yield of lactic acid is, even after many hours, very little increased above the normal amount for fresh resting muscle. When the acidified muscle after the discharge of the carbon dioxide is now heated to $40^{\circ} \mathrm{C}$., the yield of lactic acid is increased, but only traces of carbon dioxide are given off.

From these and similar observations, Fletcher concludes that the carbon dioxide discharged during heat rigor at $40^{\circ} \mathrm{C}$. is pre-existent carbon dioxide set free from carbonates or other compounds by the lactic acid known to be produced in heat rigor. The carbon dioxide discharged at $75^{\circ}$ and $100^{\circ} \mathrm{C}$. he regards as held by muscie colloids or in combination with amino-acid groups. These results tend to discredit the 'inogen' theory (p. 270), with its assumption that 'intramolecular oxygen' is stored away in the muscle, which was largely based upon erroneous observations on the discharge of carbon dioxide from heated muscles. According to this theory, carbon dioxide and lactic acid were supposed to arise from a common precursor into which oxygen had been previously introduced. 
The production of heat in heat rigor is also of great interest. Hill has shown that it amounts to from 0.6 to $I \cdot 0$ gramme calorie per gramme of muscle. Of this no more than $0^{\circ} 0_{j}$ calorie can be due to the heat of neutralization of lactic acid by the sodium bicarbonate in the musile, with which it reacts as soon as it is liberated. The rest of the heat is associated with the chemical reaction by which lactic acid is formed from its precursor, a reaction which, there is every reason to believe, is the same as that which occurs in muscular contraction. The heat production can only be due in very slight degree to the physical alteration (clotting or precipitation) of the muscle proteins.

The so-called rigor caused by water, which is not a true rigor, causes no increase in the carbon dioxide given off. Chloroform, on the other hand, produces a marked increase in the carbon dioxide production, and this is evidently related to its action in hastening the onset of rigor. Rigor mortis is to some extent influenced by the nervous system, for section of its nerves retards the onset of rigor in the muscles of a limb. Ante-mortem stimulation of the peripheral ends of the vagi, even with currents too weak to cause a perceptible effect upon the heart-beats, prolongs the period of spontaneous contraction and the irritability of the ventricles after death, and retards the onset of rigor (Joseph and Meltzer). Cold rigor is obtained when frog's muscles are cooled to $-15^{\circ} \mathrm{C}$. The muscles remain perfectly translucent. They do not recover their irritability on thawing, but if cooled only to $-7^{\circ} \mathrm{C}$. they recover (Folin).

In a human body rigor generally appears not earlier than an hour, and not later than four or five hours, after death. In exceptional cases, however, it may come on at once, and the annals of war and crime contain instances where a man has been found after death still holding with a firm grip the weapon with which he had fought, or which had been thrust into lis hand by his murderer (so-called cataleptic rigor). It is related that after one of the battles of the American Revolutionary War some of the dead were found with one eye open and the other closed as in the act of taking aim. A high temperature favours a rapid onset; a body wrapped up in bed will, other things being equal, become rigid sooner than a body lying stripped in a field. Muscular exhaustion, as we have said, is another favouring condition: hunted animals and the victims of wasting diseases go quickly into rigor. It is a rule, but not an invariable one, that rigor, when it comes on quickly, is short, and lasts longer when it comes on late. All the muscles of the body do not stiffen at the same time; the order is usually from above downwards, beginning at the jaws and neck, then reaching the arms, and finally the legs. After two or three days the rigor disappears in the same order. The position of the 
limbs in rigor is the same as at death; the muscles stiffen without any marked contraction. This can be strikingly shown on a newlykilled animal by cutting the tendons of the extensors of one foot and the flexors of the other; when natural rigor comes on, the feet remain just as they were. If heat rigor, however, is caused, the one foot becomes rigid in flexion and the other in extension; and the contraction-force is considerable, although not so great as that of an electrical tetanus in a living muscle.

The Possibility of Recovery of Muscles after Rigor.-When the circulation in the hind legs of rabbits is interrupted by compression or ligation of the abdominal aorta (Stenson's experiment), the muscles lose their excitability, but speedily recover, if they have not been deprived of arterial blood for too long a time, when the blood is again allowed to reach them. A longer interruption of the circulation leads not only to total inability to respond to stimulation, but also to rigor, and most observers are agreed that, as regards the skeletal muscles at least, this is the irrevocable end of excitability. Brown-Séquard, indeed, stated that after the full development of rigor in the rabbit's muscles (Stenson's experiment), and also in the hand of an executed criminal through which an artificial circulation was established, recovery ensued. But probably the rigor was incomplete or did not involve all the fibres. In heart muscle the conditions appear to be somewhat different, and Heubel has alleged that rhythmical contractions of the frog's heart can be restored by filling its cavity with blood, after rigor has been caused by heat and in other ways, and we have already seen that the same is true of the mammalian heart atter the onset of rigor. Excised frog's muscles which have undergone rigor mortis become less stiff when exposed to an atmosphere of oxygen. 


\section{CHAPTER XIY}

\section{NERVE}

THE voluntary movements are originated by efferent or outgoing impulses from the brain, which reach the muscles along their motor nerves. The involuntary movements and the secretions are in many cases able to go on in the absence of central connections, but are normally under central control. Afferent impulses are continually ascending to the cord and brain from the skin, joints, bones, muscles, and organs of special sense like the eye and the ear. Everywhere the connection between the nervous centres and the peripheral organs, and between different parts of the central nervous system, is made by nerve-fibres. Those which run outside the brain and cord are called peripheral nerve-fibres to distinguish them from the intracentral fibres of the central nervous system itself.

In this chapter we propose to consider certain of the general properties of nerve-fibres. Most of our knowledge of these properties has been derived from experiments on the peripheral, and particularly the peripheral motor nerves; but there is every reason to believe that the main results are true of all nerve-fibres, afferent and efferent, peripheral and central.

What we call nerve-fibres were known and named, and many important facts in their physiology discovered, long before their true morphological significance was recognized. The researches of recent years have slown that every nerve-fibre is, as regards its essential constituent the axis-cylinder, a process of a nerve-cell. The nerve-cells, each of which, including all its processes, may be conveniently termed a neuron, are the essential elements of the nervous system. The cellbodies of most of the neurons are situated in, or in close relation to, the spinal cord and the brain, and therefore the detailed description of them will be reserved till we come to treat of the central nervous system (see p. 850 and Figs. 328 to $34^{\circ}$ ). It is enough to say here that in general a nerve-cell gives off two kinds of processes: (i) one or more dendrites or protoplasmic processes, which repeatedly bifurcate like the branches of a tree into thinner and thinner twigs, and extend only for a relatively short distance from the cell-body; (2) an axis-cylinder process or axon, which as a rule runs for a considerable distance without altering its calibre, and either gives off no branches (as in the peripheral nerves) or only a comparatively small number of lateral twigs (col- 
laterals). Ultimatcly the axis-cylinder process and its collaterals, if it has any, end by breaking up into a brush, a plexus or a feltwork or basketwork of fibrils. The axons of different nerve-cells vary greatly' in length. Some terminate within the grey matter of the brain or spinal cord not far from their origin; others run in the whitc tracts of the central nervous system or in the peripheral nerves for half the height of a man. All cxcept the shortest axis-cylinder processes become clothed at a little distance from the cell-body with a protective covering, which continues to invest them (and their collaterals) throughout the rest of their course, disappearing only when they begin to break up at their terminations. An axis-cylinder process (spoken of simply as the axis-cylinder, when considered apart from the nerve-cell) constitutes, with its covering, a nerve-fibre.

The axis-cylinder is the essential conducting part of the fibre, for it is present in every ncrve-fibre, running from end to end of it without break, and towards the periphery it is alone present. It is made up of fine longitudinal fibrils embedded in interstitial substance (Fig. 329, p. 85 I). Sucl a fibrillar structure is best shown after treatment of the nervefibres with certain reagents, although it is certain that it exists preformed in the living fibres.

\section{Section I.-The Nerve-Impulse or Propagated Disturbance: itS INITIATION AND CONDUCTION.}

So far as we know, the only function of nerve-fibres is to conduct impulses from nerve-centres to peripheral organs, or from peripheral organs to nerve-centres, or from one nerve-centre to another. In the normal body these impulses never, or only very rarely, originate in the course of the nerve-fibres; they are set up either at their peripheral or at their central endings. By artificial stimulation, however, a nerve-impulse may be started at any part of a fibre, just as a telegram may be dispatched by tapping any part of a telegraph wire, although it is usually sent from one fixed station to another.

Nature of the Nerve-Impulse. - What the nerve-impulse actually consists in we do not know. All we know is that a change or dis. turbance of some kind, of which the most evident token is an electrical change, passes over the nerve with a measurable velocity, and gives tidings of itself, if it is travelling along efferent fibresthat is, out from the central nervous system-by the contraction or inhibition of muscle or by secretion; if it is travelling along afferent fibres-that is, up to the central nervous system-by sensation, or by reflex muscular or glandular effects.

Whether the wave which passes along the nerve is a wave of chemical change (such, to take a very crude example, as runs along a train of gunpowder when it is fired at one end), or a wave of mechanical change, a peculiar and most delicate molecular shiver, if we may so phrase it, or a shear in a definite direction along the colloidal substance of the axis-cylinder (Sutherland), there is no absolutely definite experimental evidence to decide. An 
electrical change accompanies the nerve-impulse travelling at the same rate, and although this is to be distinguished from the impulse itself, there is little doubt that the latter is essentially connected with a disturbance of the electrical equilibrium of the nervesubstance.

An attempt has been made to settle the question by determining the temperature coefficient of the velocity of conduction of the impulsei.e., the quantity which measures the cliange of velocity for a given change of temperature. For most pliysical processes the quotient velocity at $\mathrm{T} n+10^{\circ}$

velocity at T $n$, where $\mathrm{T} n$ is any given temperature, is not greater than $\mathbf{1} \cdot 2$, while for frog's sciatic nerve the temperature coefficient for the most part lies between 2 and 3 (Snyder). The mean value of a large number of observations is $I^{\circ} 79$, with $\mathrm{T} n=8^{\circ}$ to $9^{\circ} \mathrm{C}$. (Lucas). For the pedal nerve of the giant slug the mean value of the temperature coefficient is 1.78 (Maxwell). In other words, while for the majority of physical processes an increase of $10^{\circ} \mathrm{C}$. increases the velocity of the process by at most one-fifth, the same increase of temperature increases the velocity of conduction of the nerve-impulse by four-fifths, or even more. While it is true that it may not be entirely safe to apply such a criterion to a biological process which need not be either entirely chemical or entirely physical, and very likely is a complex one, the suggestion, so far as it goes, is undoubtedly in favour of the chemical hypothesis.

That chemical changes go on in living nerve we need not hesitate to assume; and, indeed, if the circulation through a limb of a warmblooded animal be stopped for a short time, the nerves lose their excitability. Even the nerves of cold-blooded animals gradually become inexcitable and incapable of conduction when placed in an oxygen-free medium, as the oxygen already contained in the tissue is exhausted. The excitability and conductivity of the nerve are restored by oxygen. It is clear, then, that even a resting nerve requires oxygen, and it can be shown that the loss of function is accelerated by stimulation in the absence of oxygen. But the metabolism is very slight compared witl that in muscle or gland. Until recently even in active nerve no measurable production of carbon dioxide had ever been observed, nor, in fact, had any chemical difference between the excited and the resting state ever been unequivocally made out. However, it has been announced that by the aid of an extremely delicate method of estimating small quantities of carbon dioxide, a measurable production of carbon dioxide can be detected even in resting frogs' nerves, and that this production is increased two to three times on stimulation (Tashiro). This result is somewhat puzzling in view of the fact that neither in coldblooded nor in mammalian nerves is there any sensible rise of temperture during stimulation. With the apparatus shown in Fig. 272 (an electrical resistance thermometer or bolometer whose use depends upon the fact that the electrical resistance of a metallic conductor varies with its temperature) an increase even of $0.0003^{\circ} \mathrm{C}$. in the temperature of the sciatic nerves of dogs could not be detected during tetanization. Rolleston failed to find evidence of a rise of even $0.0002^{\circ} \mathrm{C}$. in frog's nerves during stimulation. And according to the latest investigation with a more suitable and mucl more sensitive thermo-clectric arrangement, the passage of a single nerve impulse along a frog's nerve cannot be associated with an increase of temperature in the nerve of even the hundredth million of a degree (A. V. Hill). The difficulty of inducing fatigue in nerves under ordinary conditions has been considered a strong 
support of the physical nature of the conduction process. Nevertheless, it is possible to slow by special methods that nerve can be temporarily fatigued, although it recovers very rapidly. When a medullated nerve is stimulated, a brief period ensues during which it refuses to respond to a second stimulus. This refractory period is normally very short-not more than $\mathrm{c} \cdot 0 \mathrm{O} 2$ second for the frog's sciatic. But it can be greatly piolonged by cold, asphyxia, or anasthesia, especially by the alkaloid yolimbine (Tait and Gunn), and when the refractory period is thus prolonged, fatigue phenomena are readily induced by stimulation. And while the nerves of warm-blooded animals at body temperature and those of cold-blooded animals at about $32^{\circ} \mathrm{C}$. can hardly be shown to undergo fatigue when tetanized in atmospheric air, fatigue phenomena are easily elicited when the temperature is lowered even although air is supplied (Thörner).

Stimulation of Nerve.-With some differences, the same stimuli are effective for nerve as for muscle (p. 737); but chemical stimulation is not in general so easily obtained. The so-called thermal

Fig. 272. - Electrical - Resistance Thermometer (Natural Size) as used for investigating heat-pro. duction in mammalian nerves is situ. A, a piece of hard rubber in the hook-shaped part of which the fire platinum wire $P$ is fixed, and covered with insulating varnish; c. c, thick copper wires connected with $P$, fastened in grooves, and covered with paraffin. Above they end in contact with the small binding posts, $\mathrm{B}_{1}, \mathrm{~B}_{2} . \quad \mathrm{B}$ is a hard rubber sliding piece, with a slot $s$. When B is in position the screw, $a$. projects througli the slot. By a nut on this screw B is fixed on A when the nerve has been arranged in the groove.

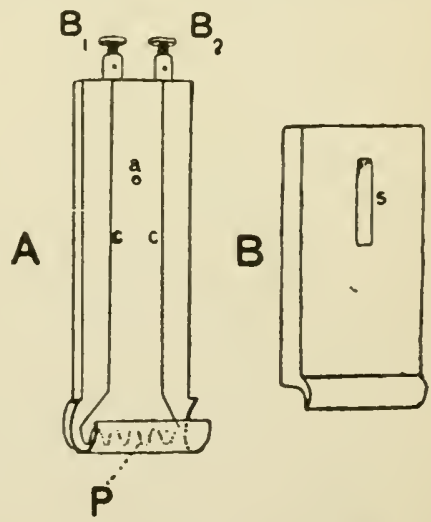

stimulation is not a real stimulation due to the sudden change of temperature. The irregular contractions of the muscle caused by the local application of heat to the nerve are dependent on desicca. tion of the nerve.

Chemical Stimulation.--When hyper-or hypotonic solutions are employed, the withdrawal or entrance of water may be an important factor.

For salts which penetrate the fibres with equal difficulty this factor can be eliminated by applying them as isotonic solutions. There is evidence that chemical stimulation proper, as distinguished from the stimulation produced by changes in the water content of the fibres by osmosis, is connected with the electrical charges on the dissociated ions of the salts (p. 428). Electrical stimuktion, indeed, may only be a variety of chemical stimulation (Loeb, Mathews, etc.).

Mechanical Stimulation may be applied to a nerve by allowing a small weight to fall on it from a definite height or by permitting mercury to frop upon it from a vessel with a fine outflow tube. A regular tetanus may. thus be obtained. Tigerstedt found that the smallest amount of work spent on a frog's nerve which would suffice to excite it was a little less 
than a grimme-millimetre-that is, the work done by a gramme falling through a distance of a millimetre, or (taking an erg as cquivalent tn ot gramme-centimetre) about roo ergs. No doubt a great part of this is wasted, as a much smaller quantity of work done by a beam of light on the retina or by an electrical current on an isolated nerre, bot $\mathrm{l}_{1}$ of which may be supposed to act more directly on the excitable constituents, suffices to cause stimulation. Thus, the work done by the minimal, natural or specific, stimulus for the retina in the form of green light may be as little as $\frac{\mathrm{I}}{\mathrm{IO}^{\mathrm{g}}} \mathrm{erg}$ (S. P. Langley), or only one-ten-thousandmillioneth part of the minimum work necessary for mechanical stimulation. Again, with electrical stimulation (closure of a voltaic current, or condenser discharges) it has been shown that an amount of work equal to $\frac{\mathrm{I}}{\mathrm{IO}^{4}} \mathrm{erg}$ may be enough to cause excitation of a frog's nerve. This is ten thousand times as great as the minimal luminous stimulus, but a million times less than the minimal mechanical stimulus.

The laws of electrical stimulation for nerve are essentially the same as those we have already discussed for muscle (p. 74I). The roltaic current stimulates a nerve, as it does a muscle, at closure and opening. During the flow of the current, so long as its intensity remains constant. there is as a rule no excitation, or at least none which is propagated along the nerve, so that the muscles supplied by it remain uncontracted. But under certain conditions-for example, when the nerve is more excitable than usual (as is the case with nerves taken from frogs which have been long kept in the cold) - a closing tetanus may be scen while the current continues to pass through the nerse, and an opening tetanus after it has ceased to flow, just as when the current is led directly through the muscle. Sensory nerve-fibres, too, are stimulated by a voltaic current during the whole time of flow. Induction shocks are relatively more powerful stimuli for nerve than the make or break of a voltaic current. The opposite, as we have scen, is true of muscle; and, upon the whole, we may say that muscle is more sluggish in its response to stimuli, and is excited less easily by very brief currents, than nerve is. An apparent illustration of this difference is the fact that the nervous excitation has no measurable latent period, while muscular excitation has. But it is quite possible that, if the conditions of experiment were as favourable in nerve as in muscle, a sensible latent period might be found here too.

In neric as in muscle, strength of stimulus and intensity of response correspond within a fairly wide range. when we take the height of the muscular contraction or the amount of the negative variation (p. S2f) as the measure of the nervous excitation. Summation of stimuli, superposition of contractions, and complete tctanus, are caused by stimulating the muscle through its nerve, just as by stimulating the muscle itsclf (p. 756$)$.

Excitability of Nerve.-It has usually been stated that the excitability of frog's nerve, as measured by the muscular response to stimulation, is increased by risc of temperature, and diminished by fall of temperature. It has, however, been shown that this increase of excitability is only apparent, and due to the strengthening of the current by diminution of the resistance, since the resistance of all animal tissues, like that of electrolytic conductors in general, diminishes as the temperature rises (Gotch). IThen precautions 
are taken to kecp the current intensity the same at the various temperatures compared, it is found that cooling of a (frog's) nerve, even to $5^{\circ} \mathrm{C}$., increases the excitability for currents of long duration (several hundredths of a second). It has, indeed, been sliown both for muscle and for nerve that the cooler tissue requires a smaller current strength for its excitation when the current is of long duration. With brief currents this effect is masked, either partially or completely, by the greater increase of current strength needed in the case of the cooler tissue to compensate for a given decrease in duration (p. 789$)$ (Lucas and Mines). This is the reason that for induction shocks or voltaic currents of short duration, the excitability of the nerve seems to be increased by a rise of temperature (up to about $30^{\circ} \mathrm{C}$. in the case of frog's nerve), and diminished by cooling.

Drying of a nerve at first increases its excitability; and the same is true of separation of a nerve from its centre. In the latter case the increase of irritability begins at the proximal end of the nerve, and travels towards the periphery. As time goes on, the excitability diminishes, and ultimately disappears in the same order (Ritter-Valli Law). At a certain stage it may be found that a given stimulus causes a smaller and smaller contraction the farther down the nerve-that is, the nearer to the muscle-it is applied. On this was based the now abandoned ' avalanche theory,' according to which the impulse continually unlocked new energy as it passed along the nerve, and so gathered strength in its course like an avalanche. It is now known that no material change takes place in the intensity of the excitation while it is being propagated along a normal uninjured nerve. For instance, experinents on the phrenic nerve, in its natural position, and with all its connections intact, have shown that with a given strength of stimulus the amount of contraction of the diaphragm is the same whether the nerve be excited in the upper, middle, or lower portion of its course. In the above experiment on the isolated, and therefore injured, nerve, the contraction varies in height with the distance of the point of stimulation from the muscle, not because the excitation grows as it travels, but because it is already greater at the moment when it sets out from a point near the central end of the nerve than at the moment when it sets out from a point near the muscle.

Electrotonus.-Although the constant current does not, unless it is very strong or the nerve very irritable, cause stimulation during its passage, it modifies profoundly the excitability and conductivity of the nerve. In the neighbourhood of the kathode the excitability is increased (condition of katelectrotonus), while around the anode it is diminished (anelectrotonus). Immediately after the opening of the current these relations are for a bricf time reversed, the excitability of the post-kathodic area (area which was at the kathode during the flow) being diminished, and that of the post-anodic 
increased. In the intrapolar area there is one point the excitability of which is not altered. This indifferent point, as it is called, shifts its position when the intensity of the current is varied, moving towards the kathode when the current is increased, towards the anode when it is diminisled.

It is only under certain defunite conditions that these phenomena, first described by Pflïger, appear in their purity and uncomplicated by other changes. The nerve should be quite fresh, the current a weak or at most a moderately strong one, and the stretch of nerve enuployed should be as far as possible from the cross section, and from the cross sections of branclies. The middle region of the frog's sciatic nerve is the best. When all these conditions are fulfilled, the whole stretch of nerve in katelectrotonus-i.e., the part on both sides of the kathode and at the kathode itself shows an increased stimulation effect, the more pronouncel the nearer to the kathode tlie point of stimulation. This condition, however, only lasts an instant. Then the excitability begins to sink sharply first at the kathode, then on both sides of it, till

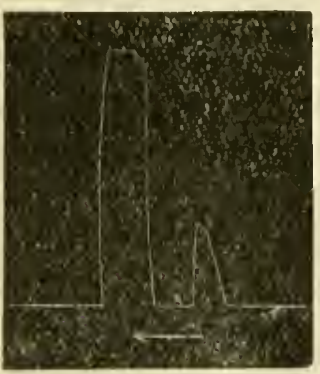

Fig. 273.-- Katelectrotonus. IVeak tetanus of muscle (the right-hand elevation), greatly intensified in katelectrotonus of the inotor nerve (the left-hand clevation). it ultimately becomes decidedly less than the initial excitability. This secondary depression of excitability, always most marked at the very kathode, is just as con-

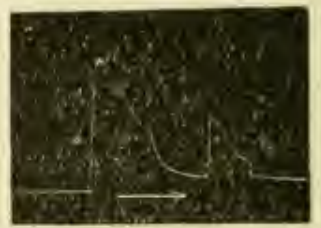

Fig. 2-4.-Anelectrotonus. Strong tetanus of muscle (left-hand cle. vation), lessencd in strength by anelectrotonic condition of the mo. tor nerve (right-hand elevation).

stant a phenomenon as the preliminary increase. The stronger the current the more profomel is the depression, the more quickly it is developed, and the greater is the distance to which it spreads along the nerve. IVith a certain strength of current the depression appears so rapidly that the preliminary increase of excitability may be completely missed. When the current is epened the excitability quickly increases again, but with strong currents it may remain depresserl for a while. At the anode clianges in the reverse direction may be observed, although they are less pronounced than at the kathode. Thus at the anode during the passage of the current the initial depression of the excitability tends to give place to an increase (Werigo).

These statements liave been made on the strengtl of experiments in which the height of the muscular contraction was taken as the index of the excitability of the nerve at any given point. It is difficult, however, to disentangle the effects of alterations in the excitability from the effects of alterations of conductivity-i.c., ef the power of a portion of the nerve to conduct an impulse set up elsewhere. Whether these two properties are distinct or not is a question which will be considered a lilile later on. 13ut it is perfectly elear that in deducing conclusions 
as to the effect produced on a nerve by excitation at a given point from the resultant effect on the muscle to which the nerve is attached (or on a galvanometer or electrometer if we are following the effect by means of the electrical changes), we must know whether the change set up in the nerve at the point of excitation can pass freely along the nerve to the muscle or to the point at which it is led off to the galvanometer. Now, changes of conductivity are certainly produced in a nerve by the constant current, which even outlast its flow. For all currents above a certain strength the conductivity at the kathode and in its neiglbourhood is eventually diminisled, and with currents still only moderately strong the block deepens into impassability. The conductivity at the anode is, during this stage, higher than at the kathode, so that at the time of full kathodic block tloc nerve-impulse still passes through the region around the positive pole. IVith still stronger currents the conductivity here, too, diminishes, until the anode as well as the whole intrapolar region is blocked. After the opening of the current, the relation between kathodic and anodic conductivity is reversed, for now the postkathodic region conducts the nerve-impulse relatively better than the post-anodic. It will be seen that these changes of conductivity upon the whole run parallel to the (secondary) changes of excitability, depression of excitability corresponding to depression of conductivity, and vice versa. With the relatively strong currents required to produce decided

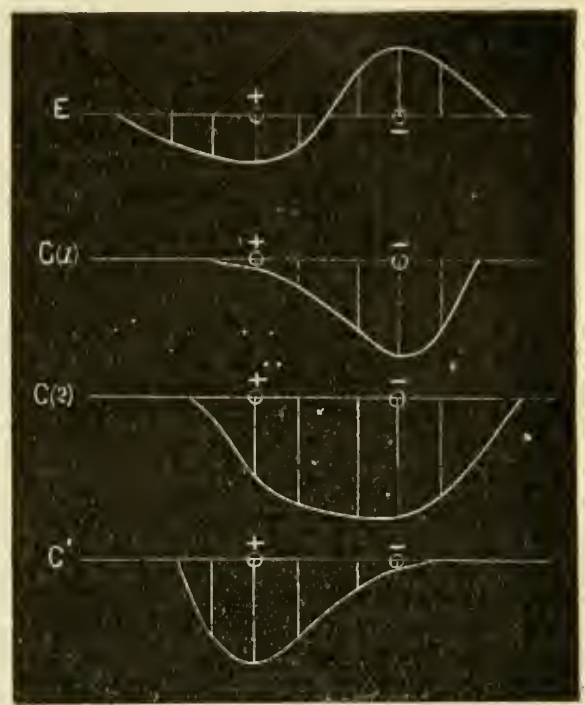

Fig. 275.-Diagram of Changes of Excitability and Conductivity produced in a Nerve by a Voltaic Current. E, changes of excitability during the flow of the current, according to Pfliiger. These are seen most typically with the weaker currents. In particular the increased excitability at and around the kathode when the current is strong very quickly gives place to depression. The ordinates drawn from the abscissa axis to cut the curve represent the amount of the change. $C(I)$, changes of conductivity found shortly after the closure and during the flow of a moderately strong current. Conductivity greatly reduced around kathode; little affected at anode. $\mathrm{C}(2)$, changes of conductivity during flow of a very strong current. Conductivity reduced both in anodic and kathodic regions, but less in the former. $C^{1}$, changes of conductivity just after opening a moderately strong current. Con. ductivity greatly reduced in region which was formerly anodic; little affected in region formerly kathodic. effects on the conductivity, any preliminary change in the same sense as the (so-called primary) effects on the excitability (increase at kathode, decrease at anode) might be expected to be fleeting, and ther efore less easy to detect.

The above facts serve to explain the manner in which the effects of stimulation of a nerve with the constant current vary with the strength 
and direction of the stream. These effects, so far as the contraction of the muscles supplied by the nerve is concerned, have been formulated in what has been someivhat loosely termed the law of contraction. In this formula the direction of the current in the nerve is commonly distinguished by a thoroughly bad but now ingrained phraseology, as ascending when the anode is next the muscle, and descending when the katlode is next the muscle.

\begin{tabular}{|c|c|c|c|c|c|}
\hline \multirow{2}{*}{ Current. } & & \multicolumn{2}{|c|}{ Ascending. } & \multicolumn{2}{|c|}{ Descending. } \\
\hline & & II. & b. & II. & E. \\
\hline Weak & - & C & - & C & 一 \\
\hline Medium - & - & C & C & C & C \\
\hline Strong - & - & - & C & C & - \\
\hline
\end{tabular}

Here M means 'malie,' B, 'break,' of the current; C means 'contraction follows.'

The explanation gencrally given is as follows: Wherever there is an increase of excitability sufficiently rapid and sufficiently large, stimulation is supposed to take place; where there is a fall of excitability. stimulation does not occur. Accordingly, at closure the kathode stimulates-the anode does not; while at opening, the anode, at which the depressed excitability jumps up to normal or more, is the stimulating pole; the kathode, at which it declines to normal or under it, is inactive.

With a weak current, (I) contraction only occurs at make, and (2) the diection of the current is indifferent. The explanation of the first fact is that the make is a stronger stimulus than the break, and when the current is weak enough the break is less than a minimal stimulus. No sensible change of conductivity is caused by weak currents, which suffices to explain (2).

With a 'medium' current, contraction occurs at make and break with both directions. Here the break excitation is effective as well as the make. With anode next the muscle (ascending current), there is, of course, nothing to frevent the opening excitation, which starts at the anode, from passing down the nerve and causing contraction; and since there is no block around the anode or in the intrapolar region with 'medium' currents, there is nothing to keep the closing (kathodic) excitation from reaching the muscle too. With the kathode next the muscle (descending current), the closing excitation, which starts from the kathode, has no reigon of diminislied conductivity to pass through, nor has the opening (anodic) excitation, for the kathodic block, caused by moderately strong currents, is removed as soon as the current is broken.

With ' strong' currents there are only two cases of contraction out of the four, just as with 'weak,' but for very different reasons. There is a break-contraction with ascending, and a make-contraction with descending current. With ascending current the anode is next the muscle, and the break-excitation starting there has nothing to hinder its course. The make-excitation, althcugh as strong or stronger, has to pass through the whole intrapolar region and over the anode, and here the conductivity is depressed and the nerve-impulse blocked. With descending current the kathode is next the muscle, and there is no hindrance to the passage of the make-excitation. The break-excitation, however, has to traverse the whole intrapolar region, and this does not 
at once, after a strong current, become passable. The break-excitation. accordingly, cannot get through to the muscle.

A formila similar to the law of contraction has been shown to hold for the inhibitory fibres of the vagus (Donders). 'inhibition' being substituted for 'contraction.' There is also some evidence that a similar law obtains for sensory nerves.

It is not difficult to see that with currents of bricf duration the break follows so quickly on the make that interference of their opposed cffects may occur. This is the reason-or, at least, one reason-why, ibove a certain frequency. a muscle or nerve ceases to respond to all of a series of rapidly recurring electrical stimuli (p. 758). It is also the reason why, with single very bricf stimuli, a greater current intensity must be employed in order to cause excitation than when the duration of the stimulating current is greater (Woodworth, Lucas).

The Law of Contraction for Nerves 'in Situ.' - When a nerve is stimulated without previous isolation-in the human body, for instance, through electrodes laid on the skin-the current will not enter and leave it through definite small portions of its sheath, nor will it be possible to make the lines of flow nearly parallel to each other and to the long axis of the nerve, as is the case in a slender strip of tissue when there is a considerable distance between the electrodes.

On the contrary, when, as is usual in electro-therapeutical treatment, a single elcctrode-say, the positive-is placed over the position of the nerve, and the other at a distance on some convenient part of the body, the current will enter the nerve by a broad fan of stream-lines cutting it more or less obliquely, and pass out again into the surrounding tissues; so that both an anode (surface of entrance) and a kathode (still larger surface of exit) will correspond to the single positive pole. Similarly, the single negative elcctrode will correspond to an anodic surface where the now narrowing

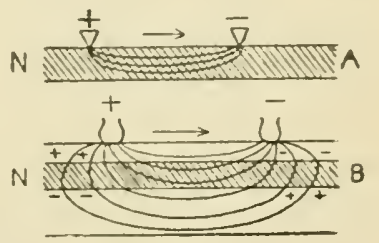

Fig. 276.-Diagram of Lines of Flow of a Current passing through a Nerve. A, an isolated nerve; $B$, a nerve insitu. Secondary anodes $(t)$ are formed where the current re-enters the nerve below the negative elec. trode after passing through the tissues in which it is embedded and secondary kathodes ( - ) where the current passes out of the nerve into the surrounding tissues below the positive elec. trode.

sheaf of lines of flow enters the nerve, and

a smaller kathodic surface, where they emerge. Even if the two electrodes were on the course of the nerve, the stream-lines would still cut it in such a way that each electrode would correspond both to anode and kathode (Fig. 276).

It is impossible under these circumstances to take account of the direction of a current in a nerve, or to connect divection with any specific effect. When we place one of the electrodes over the nerve and the other at a distance, the law of contraction only appears in a disguised form; for since a kathode and an anode exist at each pole, there is, with a current of sufficient strength ("strong current'), excitation at each, both at make and break. The negative make contraction is, however, stronger than the positive, for the excitation corresponding to the latter arises at the secondary kathodic surface, where the sheaf of current-lines spreading from the positive electrode passes out of the nerve. Now, this is much larger than the primary kathodic surface, through which the narrow wedge of stream-lines passes to reach the negative electrode, and the current density at the latter is accordingly much greater. The 
positive break-contraction is, for a similar reason, stronger than the negativic.

With a 'weak' current, the only contraction is a closing one at the kathode; with a 'medium ' current there are both opening ard closing contractions at the positive pole, and a closing but no opening contraction at the negative (Practical Exercises, p. 846).

Conductivity of Nerve.-The disturbance which is called the nerve-impulse, once set up, is propagated along the fibres. Are the changes in the nervous substance involved in the initiation of the disturbance at a given point identical with those involved in its transmission from one point to the next, or are they different ? This is a question which has been much discussed, and many attempts have been made to prove that the two processes can be dissociated by acting on nerves with substances like carbon dioxide, ether, and alcolıol, which gradually suspend their functions, by cutting nerves off from the circulation and aliowing them to die gradually, by depriving them of oxygen and in other ways. Many of the results obtained from such experiments seem at first sight to be favourable to the view that the local change is different from the propagated disturbance. Nevertheless, careful examination of the results on which such statements are based indicates that none of them supplies a crucial test of the question at issue. For example, when a stretch of frog's sciatic nerve is treated with ether or another of the narcotics which act on nerve, and the strength of stimulus determined which is necessary to elicit a contraction when applied to an untreated portion more remote from the muscle than the narcotized area, this strength is found, for some time after the application of the narcotic, to be just the same as it was previous to the application. 'The conductivity' of the narcotized stretch appears to be unaltered. On the othar hand, the stimulus, when applied within the narcotized region, must be strengthened, and the narcotic appears to have diminished the 'excitability' of the nerve. When the narcotic has acted for a longer time, the reverse effect appears. No stimulus, however strong, applied to the central non-narcotized stretch will cause a contraction, the 'conductivity' having been apparently totally abolished by the narcotic, whereas a strong stimulus applied in the narcotized region will still cause a contraction, showing that 'excitability' still remains. As to the facts there is general agreement; it is their interpretation which is in doubt. Now, it has been shown that in passing along a narcotized nerve the propagated disturbance diminishes in proportion to the length of nerve which it has to traverse. Accordingly in the second stage of narcosis the failure of the stimulus applied to the upper part of the nerve to elicit a contraction is explained most naturally as due to the extinction of the disturbance, which must pass through the whole narcotized region, whereas the disturbance set up by stimulation in that region succeeds in reaching 
the muscle, since it has a shorter stretch of narcotized nerve to traverse. This experiment, then, in reality affords no proof that excitability and conductivity can vary inclependently. Facts are also known, to which allusion need not be made here, but whicls greatly modify the ordinary interpretation of the experinental results obtained in the first stage of narcosis, and upon the whole it may be said that these direct methods of determining the question have failed to yield a satisfactory answer. Indirect evidence exists, lowever, that the local process initiated by stimulation is not cuite the same as the process involved in the propagation of the disturbance (Lucas). Thus, a brief current too weak to set up a propagated disturbance, nevertheless causes some change at the point of stimulation, since a second current, also too weak to be effective by-itself, will, when thrown in a short time after the first, cause a disturbance which is propagated along the nerve. There is good reason to believe that the change produced by the first current is not the same in kind as that produced by the second, only weaker, but that it is inherently different in quality. Above all, it is a local change incapable of being itself propagated, but constituting the necessary prelude to the starting of the propagated disturbance. Lucas has called this preliminary local effect the "local excitatory process.'

There are many facts which indicate that the capacity of different functional or anatomical groups of nerve-fibres for responding to stimulation and for conducting the nerve-impulse can be differently affected by one and the same influence. For example, pressure abolishes the conductivity of sensory fibres sooner than that of motor fibres.

Cocaine locally applied to a nerve diminishes or abolishes its conductivity, according to the dose. It exercises a selective action as regards nerve-fibres of different kinds, picking out and paralyzing sensory fibres before motor; vagus fibres conducting upwards before those conducting downwards, vaso-constrictors before vaso-dilators, and broncho-constrictors before broncho-dilators (Dixon).

The conduction or propagation of a definite disturbance or impulse is a phenomenon not confined to nervous tissue. It is also characteristically seen in muscle, although there the mechanical effect which constitutes the normal response to the arrival of the propagated disturbance obtrudes itsclf and tends to divert attention from the latter. It is unlikely that the conduction process in muscle should be essentially different from that in nerve, and in muscle, as in nerve, there is evidence that it is associated with only a small, perhaps not cven a detectable, liberation of heat. The main heat-production in muscle is essentially a feature not of conduction, but of contraction. Conduction in muscle can be completely dissociated from the contraction process in various ways. For example, if a portion of a muscle is immersed for a time in distilled water, so-called water rigor cnsues, and the altered muscle has lost the power of contraction. It will nevertheless conduct the impulse which on reaching the unaltered part of the muscle causes it to contract normally.

Double Conduction.-When a nerve (or muscle) is stimulated artificially, the excitation runs along it in both directions from the 
point of stimulation; so that nerve-fibres which in the intact body are afferent can conduct impulses towards the periplery and efferent fibres can conduct impulses away from the periphery. In the normal state, however, double conduction must seldom occur, for efferent fibres are connected centrally, and afferent fibres peripherally, with the structures in which their natural stimuli arise. In general, too, an impulse, if it did pass centrifugally along an afferent fibre, would not give any token of its existence, for the peripheral organ would not be able to respond to it; and there is no ground for assuming that the central meclianisms connected with afferent fibres are better fitted to answer such foreign and unaccustomed calls as impulses reaching them along normally efferent nerves. There is good evidence that muscular excitation is no carried over to the motor nerve-fibres; in other words, the rave of action flows from the nerve to the muscle, but cannot be got to flow backwards. Excitation of the central end of an efferent (anterior) spinal root is not transferred to the corresponding afferent (posterior) root, the connection between the efferent and afferent neurons presenting the character of a physiological 'valve,' which permits impulses to pass only in one direction. We have seen that vaso-dilator impulses possibly pass out to the limbs over fibres which, morphologically speaking, are afferent fibres (p. I8I). And we shall see that a nutritive influence is exerted over the afferent fibres of the spinal nerves by the ganglion cells of the posterior root ganglia (p. 796), an influence which must spread along these fibres in the opposite direction to that of the normal excitation.

The best proofs of double conduction in nerves, with artificial stimulation, are: (I) The propagation of the negative variation or action current in both directions. This holds for sensory as well as for motor fibres, as du Bois-Reymond slowed on the posterior roots of the spinal nerves of the frog and the optic nerves of fishes. (2) Stimulation of the posterior free end of the electrical nerve of Malapterurus (p. 84I) causes discharge of the electric organ, although the nerve-impulse travels normally in the opposite direction. (3) If the lower end of the frog's sartorius is split into two, gentle stimulation of one of the tongues causes contraction of individual fibres in the other. This is supposed to be due to conduction of the nerve-impulse up a twig of a nerve-fibre distributed to the one tongue, and down another twig of the same fibre going to the other tongue. A similar experiment can be done on the gracilis of the frog. This muscle is divided by a tendinous inscription into two parts, each supplied by a branch of a nerve which divides after entering the muscle. Stimulation of either twig is followed by contraction of both parts of the muscle (Kühne).

Bert's much-quoted experiment on the rat is valueless as a proof of double conduction. He caused union of the point of the tail with the tissues of the back, then divided the tail at the root, and found that stimulation of what was now the distal end caused pain. From this he concluded that the sensory fibres of the 'transposed' tail conducted in the direction from root to tip. But the conclusion is not warranted, for sensation disappeared in the tail after the section, and did not 
return till some months later, when the nerve-fibres, after degenerating, woull :alve been replaced by new sensory fibres growing down from the $d_{i} \cdot 7$ nerves (Ranvier). For a similar reason the so-called union of the ripheral end of the cut liypoglossal nerve (motor) with the central end of the cut lingual (sensory) proves nothing as to double conduction, nor as to the possibility of motor nerves taking on a sensory function. lior while sensation is after a time restored in the affected portion of the tongue, this is due to the growth of sensory fibres from the central stump of the lingual down through the degenerated hypoglossal, and not to the conduction upwards of sensory impulses by the motor fibres of the latter.

Every fibre of a nerve is physiologically isolated from the rest, so that an impulse set up in a fibre runs its course within it, and does not pass laterally into others (law of isolated conduction). In connection with this physiological fact there is the anatomical fact that nerve-fibres do not normally branch in the trunk of a peripheral nerve. (But see p. 802.) It has, liowever, been shown that bifurcation of nerve-fibres may occur in the spinal cord (Sherrington). The axis-cylinder of a peripheral nerve-fibre only begins to branch where complete isolation of function is no longer required, as within a muscle. The experiment of Kühne on double conduction, mentioned above, shows that an excitation set up in one twig or one fibril of an axis-cylinder which has branched can spread to the rest.

Velocity of the Nerve-Impulse.-We have said that the nerveimpulse travels with a measurable velocity. It is now time to describe how this has been ascertained (p, 818). For motor fibres the simplest method is to stimulate a nerve successively at two points, one near its muscle, the other as far away from it as possible, and to record the contractions on a rapidly-moving surface (pendulum or spring myograph) (p. 746). The apparent latent period of the curve corresponding to the nearer point will be less than that of the curve corresponding to the point which is more remote, by the time which the impulse takes to pass between the two points. The distance between these points being measured, the velocity is known. Helmholtz found the velocity for frog's nerves at the ordinary temperature of the air to be a little under, and for human nerves, cooled so as to approximate to the ordinary temperature, a little over 30 metres per second. For observations on man the contraction curves of the flexors of one of the fingers or of the thumb may be recorded, first with stimulation of the brachial plexus at the axilla, and then with stimulation of the median or ulnar nerve at the elbow. Probably at the same temperature there is little difference in the rate of transmission in the nerves of warmblooded and cold-blooded animals, but temperature has a considerable influence (p. 782).

By cooling a frog's nerve Helmholtz reduced the rate to $\frac{1}{1 \pi}$ of its value at the ordinary temperature. In the human arm he found a variation from 30 to 90 metres per second, according to the temperature, 50 metres 
being about the normal rate. This is greater than the speed of the fastest train in the workl. According to Piper's recent measurenents the velocity in human medullated nerve is even greater than Helmholtz concluded, about 120 metres a second under ordinary conditions. The rate is inclependent of the intensity of the excitation. The velocity with which the negative variation is propagated (p. 824$)$ is the same as that of the nerve-impulse.

In sensory nevves there is no reason to believe that the velocity of the nerve-impulse differs from that in motor nerves, but experiments on man really free from objection are as yet wanting.

The usual method is to stimulate the skin first at a point distant from the brain, and then at a nuch nearer point. The person experimented on, as soon as he feels the stimulation, makes a signal, say, by closing or opening with the hand a current connected with an electric timemarker, writing on a moving surface. There is, of course, a measurable interval between the excitation and the signal, and this being in general longer the more remote the point of stimulation is from the brain, it is assumed that the excess represents the time taken by the nerve-impulse to pass over a length of sensory nerve equal to the difference in the length of the path. But there is this difficulty, that the propagation of the impulse from the point of stimulation to the brain is only onc link in the chain of events of which the signal marks the end. The impulse has first to be transformed into a sensation, and then the will has to be called into action, and an impulse sent down the motor nerves to the hand. And while the time taken by the excitation in travelling up and down the peripheral nerve-fibres is probably fairly constant, the time spent in the intermediate psychical processes is very variable.

\section{Section II.-Chemistry, Degeneration, and Regeneration of Nerve.}

Chemistry of Nerve.-Our knowledge of this subject is still scanty; and most of what we do know has been obtained from analyses, not of the peripheral nerves, but of the white matter of the central nervous system

Proteins are present, especially in the axis-cylinder. The proteins of nervous tissue include two globulins, one coagulated by heat at $47^{\circ} \mathrm{C}$., the other at $70^{\circ}$ to $75^{\circ} \mathrm{C}$, and a nucleo-protein coagulating at $56^{\circ}$ to $60^{\circ} \mathrm{C}$.

Very important constituents are certain substances soluble in organic solvents, like benzol and ether, and comprising cholesterin, certain phosphatides (kephalin and lecithin), and certain cercbrins or cerebrosides. The cerebrins are glucosicles containing nitrogen, but no phospliorus, and they yicld a reducing sugar (galactose) on hydrolysis. In the nervous tissue there is also present, according to some authorities, a compound called protagon. Others consider it a mere mixture of pliosphatides and cerebrosides. The lipoids of nerve-fibres belong largely to the medullary sheath, but they are not confined to it, since non-medullated nerves also yield a considerable quantity of lipoids ( $\mathrm{I} \cdot 5$ per cent. of the solids as against 46.6 per cent. for medullated nerves). Nonmedullated nerves (splenic nerves of the ox) are distinguislied from medullated nerves (human sciatic) by the high proportion of their total lipoids constituted by the phosphatides (kephalin and lecithin) and cholesterin. Thus, in non-medullated fibres 47 per cent. of the lipoid 
extract consisted of cholesterin, and 23.7 per cent. of keplalin; while in the medullated fibres cholesterin made up only 25 per cent. of the extract, and kephalin $\mathbf{1 2 . 4}$ per cent. On the other hand, the cerebrosides are present, both relatively and absolutely, in much larger quantity in medullated than in non-medullated nerves. In both varietics of fibres kephalin, and not lecithin, is the chicf phosphoruscontaining body (Falk). The medullary sheath further contains a kind of network of a peculiar resistant substance, neurokeratin. The neurilemma consists of substances insoluble in dilute sodium hydroxidc. Gelatin is obtained from the connective tissue which binds the nervefibres together. There may also be ordinary fat in the meshes of the epineurium connecting the bundles. Small quantitics of xanthin, hypoxanthin, and other extractives, can also be obtained from nerve. According to Halliburton's analyses, the water in sciatic nerves amounts to $65^{\circ} \mathrm{I}$ per cent., and the solids to $34^{\circ} 9$ per cent. The proteins make up 29 per cent, of the solids.

For an analysis of the white matter of the brain, see Chapter XVI.

Nerve-cells contain no potassium, according to Macallum; and this is true both of the dendrites and the axons. In medullated nerves, how. ever, potassium compounds are present external to the axons, chiefly at the nodes of Ranvier (Frontispiece) and in the neurokeratin framework of the sheath.

The only chemical difference between living and dead nervous tissue which has been made out with any degree of certainty is that the former is neutral or faintly alkaline, and the latter acid, in reaction to such indicators as litmus. This is especially true of the grey matter of the central nervous system, although the white matter also is often found acid. The change of reaction is due to the accumulation of lactic acid. Such a change has not hitherto been clearly demonstrated in peripheral nerves, either after death or after prolonged stimulation. The (nonmedullated) splenic nerves of the dog, even after stimulation for six hours, never became acid (Halliburton and Brodie).

Degeneration of Nerve.-Nerve-fibres are 'bound in the bundle of life' with the nerve-cells from which their axis-cylinders arise; the connection between cell and axon once severed, the nervefibre dies inevitably. This is an illustration of a general law that no portion of a cell can live once it is separated from the nucleus. We shall see later on that changes also occur in the nerve-cell whose axon has been divided from it, although they are of a different nature (rather a slow atrophy than an acute degeneration), and do not necessarily lead to the destruction of the cell. We must regard the neuron not only as a morphological unit, a single cell from nucleus to remotest end-brush, but also as a functional and nutritive unit, the fortune of any portion of which is not indifferent to the rest. Thus, when a man's arm is amputated the arm fares worse than the man, for the arm dies. But the man is not unaffected. He lives, but he suffers much temporary disturbance and some permanent loss. What is left of him is not quite the same as it was. The acute changes that occur in severed nervefibres are most conveniently studied in the peripheral nerves, although essentially similar phenomena take place also in the fibres of the central nervous system. 
A spinal nerve is composed of efferent fibres whose cells of origin are in the grey matter of the anterior horn, and afferent fibres whose cells of origin are in the posterior root ganglion. When such a nerve is cut below the junction of its roots, muscular paralysis and impairment of sensation at once follow in the region supplied by the nerve; but for a time the nerve remains excitable to direct

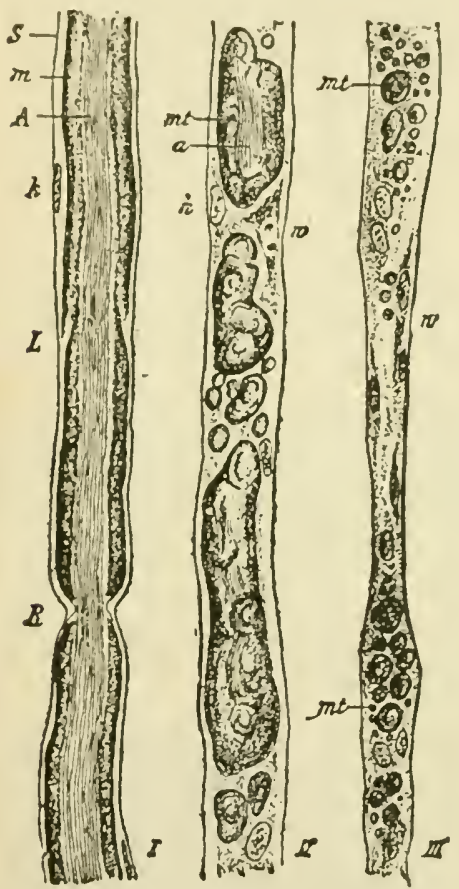

Fig. 277.-Degeneration of Nerve-Fibres after Section (Barker, after Thoma). I, normal fibre; II, degenerating fibre; III, further stage of degeneration; $S$, neurilemma; $m$, medullary sheath; $A$, axis-cylinder; L, Lantermann's line or cleft; R, node; $m t$, drops of myclin ; $a$. remains of axis.cylinder; $w$, prolifera. ting cells of neurilemma. stimulation. The excitability gradually diminishes, and in a few days is completely gone. If portions of the nerve distal to the lesion are examined at different periods after section, a remarkable process of degeneration (commonly spoken of as Wallerian degeneration) is seen to be going on. In the medullated fibres this begins on the second or third day with a swelling of the axis-cylinder, which breaks up into detached pieces (fragmentation), and assumes a granular appearance. The medullary sheath also undergoes fragmentation at the lines of Lantermann, and a little later separates into clumps and droplets of myelin. The nuclei under the neurilemma increase in size, proliferate by mitosis, and insinuate themselves between the fragments of the medullary sheath and axis-cylinder, which ultimately disappear, leaving the nerve-fibre represented only by a kind of mummy of connective tissue, in which the neurilemma with its abnormally numerous nuclei can still be recognized. The fragmentation of the myelin sheath is not dependent upon the proliferation of the muclei, since it occurs also in nerves removed from the body and kept under conditions in which the nuclei do not proliferate (Feiss and Cramer). The protoplasm around the nuclei of the neurilemma also increases in amount, and undergoes other changes, which will be more particularly referred to in describing the regeneration of nerve. The degenerative process begins near the cut end, and extends gradually to the peri- 
pliery, and more rapidly in warm- than in cold-blooded animals. At any rate, that is the interpretation generally given to the fact that at a given period after section the changes-especially the breakingup of the myelin-are more pronounced near the proximal end of the peripheral stump. In a mammal degeneration is far advanced in a fortnight, although the last remnants of the myelin may not be absorbed for months. In the degenerated nerve (cat's sciatic) the percentage of phosphorus undergoes a diminution from about the third day. About the eighth day the loss of phosphorus-i.e., of the phosphatides (lecithin, kephalin)-is markedly accelerated, coinciding with the appearance of a strong Marchi* staining reaction. By the twenty-ninth day the degenerated nerve is practically devoid of phosphorus. A progressive increase in the water and a diminution in the total solids also culminate about the same time (Mott and Halliburton). In the portion of the nerve-fibre still connected with the nerve-cell the degeneration only extends as far back as the next node of Ranvier, and seems to be due to the direct effect of the injury. In non-medullated fibres, such as the fibres arising from the cells of the superior cervical ganglion (Tuckett), the degeneration is confined to the axis-cylinders. It begins in
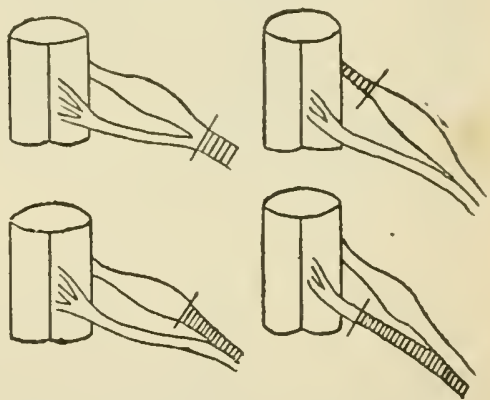

Fig. 278.-Degeneration of Spinal Nerves and their Roots after Section. The shading shows the degenerated portions. about twenty-four hours after section, and the loss of excitability and conductivity is complete by the fortieth hour.

It follows from what has been said as to the position of the cells of origin of the root fibres of the spinal nerves that section of the anterior root causes degeneration on the peripheral, but not on the central side of the lesion. $\dagger$ Only the anterior root fibres in the mixed nerve degenerate. Section of the posterior root above the ganglion causes degeneration of the central stump, but not of the portion still connected with the ganglion, nor of the posterior root fibres below the ganglion or in the mixed nerve. Section of the posterior root below the ganglion causes degeneration of the fibres of the root below the section and in the mixed nerve, but not above it.

* The chief constituents of Marchi's solution are potassium bichromate and osmic acid. It stains medullated nerve-fibres black in the earlier stages of degeneration.

$\dagger$ A few fibres in the peripheral stump of the anterior root do not degenerate, and a few fibres in the central stump do. These are the 'recurrent fibres, whose course is described on p. 892 . 
Regeneration of Nerve.-Degeneration of nerve is followed, if its divided ends are not kept artificially apart, by a process of regeneration, already distinct under favourable conditions in from three to four weeks after the section, and indeed in some cases commencing as early as the second week. This consists in the outgrowth of new axis-cylinders, in the form of fine fibres, from the ends of the divided axis-cylinders of the central stump of the nerve. These push their way into and along the degenerated fibres, uitimately acquire a medullary sheath, and develop into complete nerve-fibres, restoring first sensation, and later 'on voluntary motion, to the paralyzed part. The process needs several months for its completion, even in warm-blooded animals. It takes place under the influence of tha nucleated portion of the neuron (the cell-body), and is never completed if the peripheral and central portions of the nerve are permanently separated by a substance through which the new axis-cylinders cannot grow or by a gap too wide for them to bridge over. When the cut ends of the nerve are carefully sutured together, the conditions for complete and speedy regeneration are rendered more favourable-a fact which finds its application in the surgical treatment of injured nerves. The cycle of chemical changes described in the degenerating nerve is retraced in the reverse order. In the cat's sciatic the first sign of the return of the phosphorus was seen with the beginning of the normal myelin reaction about the sixtieth day after section. At the one-hundredth day the phosphorus content was almost as great as that of the normal nerve (a little under I per cent. of the solids for the regenerated, as compared with a little over I per cent. for the normal nerve).

It is not as yet well understood how the regenerating fibres are directed in their growth, so that they join their centres to the appropriate end-organs without mistake. That they have a high capacity for finding their way is indicated by the results of cross-suturing such nerves as the median and ulnar-i.e., of uniting the central end of the one with the peripheral end of the other. Howell and Huber found that after this operation in the dog, both co-ordinated voluntary motion and sensation returned in large measure in the parts supplied by the nerves. Here the motor fibres of the median nerve must, of course, have made connection with muscles previously supplied by the ulnar, being guided to them along the nerve-sheaths of the latter. Doubtless the old nerve-sheaths serve to some extent as mechanical guides by offering to the new axons a path of least resistance. And when a nerve-trunk containing motor and sensory fibres is simply crushed so as to destroy all physiological continuity, but is not cut, no distortion of the motor and sensory 'patterns ' of the nerve-in other words, no 'straying' of the fibres from their old paths-can be detected on regeneration. When the 
nerve is cut and then sutured, a certain amount of distortion of the pattern is inevitable. The mechanical apposition of central and peripheral stumps is, of course, much more nearly perfect in the crushed nerve than in the cut nerve, however exact the suturing may be (Osborne and Kilvington). Yet, even after crushing or ligation of nerves, or after section and suturing, the regenerating fibres do not pass straight through the scar tissue from the central to the peripheral stump, but cross and mingle, apparently in the most inextricable confusion (Feiss) (Fig. 279). This is che to the proliferation of cells in the scar which run in all directions, and show no signs of following the

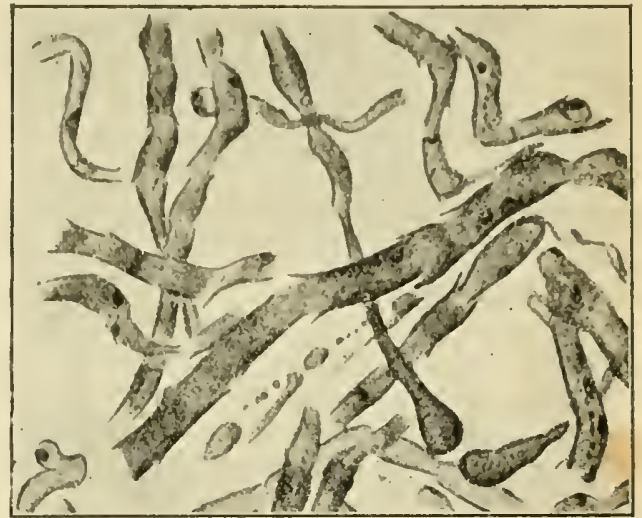

Fig. 279.--Regenerating Fibres crossing in the Scar after Ligation of a Dog's Sciatic Nerve I65 Days previously. Weigert-Palstain. Drawn under oilinmersion (Feiss).

parallel arrangement of the nerve-sheaths in the central or the distal segment. These being formed before the regenerating nerve-fibres, the latter must necessarily grow also in all direc-

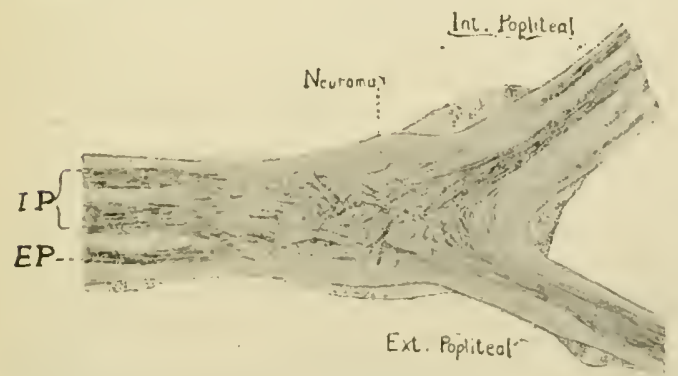

Fig. 280.-Semidıagrammatic Representation of Longi. tudinal Section through Neuroma or Scar produced by ligating the Sciatic Nerve with Catgut, and crushing it with a hæmostat just above its Division into the External and Internal Popliteal Nerves. Weigert-Pal preparation (Feiss). tions in the scar. This, however, is a local phenomenon. Beyond the scar the arrangement of the regenerating axiscylinders recovers its regularity, and the amount of distortion of the nerve pattern, as indicated by histological exa mination and functional tests, is by no means so great as the complete effacement of the pattern in the scar might appear to promise. That the degenerated peripheral stump directs the growth of the axons from the central stump in some other than a merely mechanical way is evident from the experiments of Langley on 
regeneration of the cervical sympathetic in the cat after section below the superior cervical ganglion. The nerve contains fibres of various functions which reach it from the upper thoracic nerves. The anterior roots of the first and third thoracic nerves supply the cervical sympathetic mainly with fibres which end in the ganglion around cells that give off dilator fibres for the pupil. The fibres connected with the cells in the ganglion which send vaso-motor fibres to the vessels of the ear are for the most part contained in the anterior roots of the second and fifth thoracic nerves; and the fibres connected with the cells that give origin to the pilo-motor fibres for the hairs of the face and neck in the anterior roots of the fourth to the seventh. Stimulation of any one of the upper thoracic roots accordingly causes a specific effect, which, according to Langley, is in general the same after regeneration as before section of the cervical sympathetic. We must assume, therefore, that each regenerating fibre seeks out either the ganglion cell with which it was originally connected, or one belonging to the same class. No mere mechanical guidance of the growing axons by the old neurilemmas will suffice to explain this selective growth. It is necessary to postulate, in addition, an attraction of a chemical or physicochemical nature (chemiotaxis), dependent upon a specific relation between the new axons and the scaffolding of the peripheral stump or the ganglion cells. But it is not possible at present to form any very precise conception of the properties on which the chemiotactic phenomena depend. And the specificity is not an absolute one. Under certain conditions these pre-ganglionic nerve-fibres (that is to say, nerve-fibres running from the spinal cord to end around the sympathetic ganglion cells) can form connections with nerve-cells of a different class-e.g., pupillo-dilators with cells whose axons end in the erector muscles of the hairs. Further, after section of the sympathetic above the superior cervical ganglion, the postganglionic nerve-fibres (i.e., the fibres coming off from the cells of the ganglion) may also, if the opportunity be favourable during regeneration, exchange their old end-organs for new ones; pilomotor fibres, for instance, finding their way into the iris and becoming pupillo-dilators. After excision of the superior cervical ganglion, the cervical sympathetic does not recover its function. Accordingly the pre-ganglionic fibres cannot form direct functional connection with the post-ganglionic fibres, but can become connected with them only indirectly through the ganglion cells. Nor can efferent post-ganglionic fibres achieve regenerative union with a cerebrospinal (somatic) motor nerve, although they can themselves regenerate, as has been shown, e.g., in the case of the vaso-constrictors of the limbs. On the other hand, union easily takes place between pre-ganglionic fibres and efferent somatic fibres, and vice versa. For example, the cervical sympathetic can unite with the phrenic 
nerve, and ciuse contraction of the diaphragm, or wilh the decurnent laryngeal nerve, and cause movement of the vocal cords, of with the spinal accessory; and cause contraction of the stermo-mastoid muscle. Conversely, the phrenic nerve, when mited with the cervical sympathetic, can, when stimulated, produce the usual effects observed on exciting the latter nerve (Langley and Androrson).

Central and Autogenetic Theories of Regeneration.-Although the establishment of connection with the central and of the cut nerve is necessary for complete regeneration, it must not be supposed that no share whatever is taken in the process try the peripheral stump. Even while it remains completely isolated from the central nervous system, changes occur which are often described as the third or final stage of degeneration, but which are more correctly interpreted as forming a stage in the regenerative cycle. Spindle-shaped cells or fibres with elongated nuclei make their appearance, produced by the proliferation of the nuclei of the primitive sheath already described, and the increase of the protoplasm in which these nuclei are embedded. These so-called axial strand fibres or this fibrillated protoplasm may appear long before the remains of the degenerated axis-cylinder and myelin sheath have been completely removed. It is generally acknowledged that in the adult they do not develop beyond this, so long as the peripheral portion of the nerve remains completely isolated, but neither do they disappear even after a very long interval. When strict precautions against union with other nerve-trunks were taken, the radial nerve of an adult cat was found in this resting-stage nearly a year and a half after division, and the same was true after two years and a half in a nerve divided in a human being. The fibres are incapable of being excited or of conducting nerve impulses. The precise relation between these axial strand fibres of the peripheral stump and the myelinated fibres found there after regeneration has been much debated. All are agreed that nerve-fibrils sprout from the central stump, and the weight of evidence is in farour of the long-accepted view that it is by the growth of these fibrils along the peripheral stump that the new axons are formed, and that all the changes in the distal portion of the nerve, however important for directing and perhaps sustaining the growth of the central fibrils, are subsidiary to this. But some maintain that the outgrowing central fibrils mect and unite with corresponding fibrils sprouting from the peripheral stump, and that the new axiscylinders arise from the fibrils of the axial strand. It is said that very shortly after being brought into connection with the central portion of the same or of another nerve by careful suturing the spindle cells begin to lengthen, and form non-medullated fibres, like those of the sympathetic. Four weeks after union the afferent fibres, although still non-medullated, are capable of being stimulated 
mechanically and (dextrically, and of ronducting impulses towarls the centre. in about eight weeks they becene medullated, but at first are of small calibre (Ilead and I Iam). Bethe, tle most strenuous defenter of the inherent regencrative power of the isolated peripheral stump (antegenctic theory), has even stated that complete regeneration occurs in voung animals in nerves cutircly separated from their centres. There is no doubt that this result is due to some error of technicpue or of interpretation. The controversy turns largely upon the precautions jurlged necessary to prevent the ingrowth of central fibres. And while it is comparatively easy to make sure, by removing a large part of it, that the central end of the nerve under observation shall remain completely unconnected with the peripheral cul, it is often a mattre of the greatest difficulty to prevent the union of the distal stump with central inbes from other sources-e.s., from the nerves cut in the wound. Many of the results which seemed to favour the autogenetic theory were certainly tue to this cause.

The most conclusive evidence in favour of central and against autogenetic regeneration, because the most direct and uncomplicated, has been afforded by the demonstration that the development of axis-cylinders occurs in itro in a suitable plasmatic medium, in the absence of any other clements than the nerve-cells from which they arise (Harrison). This observer, worling with the medullary plates of tadpoles, in which the nerve-cells originate in the embryo. showed further that peripheral nerves do not develop when the nerve-centres are removed, and that the sheath-cells of Schwann are not essential to the growth of axis-crlinders, since in their absence the latter continue to grow and reach their normal length. It has also been proved tlat nerve-fibres grow out from pieces of the cerebellum and spinal ganglia (Fig. 28I) of young mammals when cultivated on clotted plasma ontside of the body (Fig. 337. p. 857 ). Many fibres sprouting ont from the spinal ganglia attain a length of more than half a millimetre in forty-eight hours, and their growtl need not be accompanied by eithre neuroglia or comective tissue. When portions of normal peripheral nerve are incubated in plasma no growth of axis cylinders is ever observed, nor does the Mallerian degeneration occurring in peripheral nerves incubated in Ringer's solution take place (1: $\mathrm{g} .2 \mathrm{~S} 2$ ). On the other hand, if nerves in which Wallerian degeneration has been produced by section are placed in plasma on the fifth day after section or later, growth of the elements of the slieath of Schwann may be seen, again unaccompanied by any growth of axis cylinders (Ingebrigtsen).

A fact of great physiological interest, and also of practical importance, in commection with the anastomosis of nerves for the relicf of certain forms of paralysis, is the bifurcation of axons in regeneration, when the conclitions are such that the axoms of the central stump are offererl more than me path along which to regenerate. If, for instance, a limb nerve-trunk contining motor fibres is cut, and its central end 
sutured both to its own distal end ambl to the distial end of an adjacent nerve-trunk, the sum of the nerve-fibres in the two distal trinks after regeneration has occurred is greater than the number of fibres in the central stump (Kilvington). That this is clue to splitting of axons is

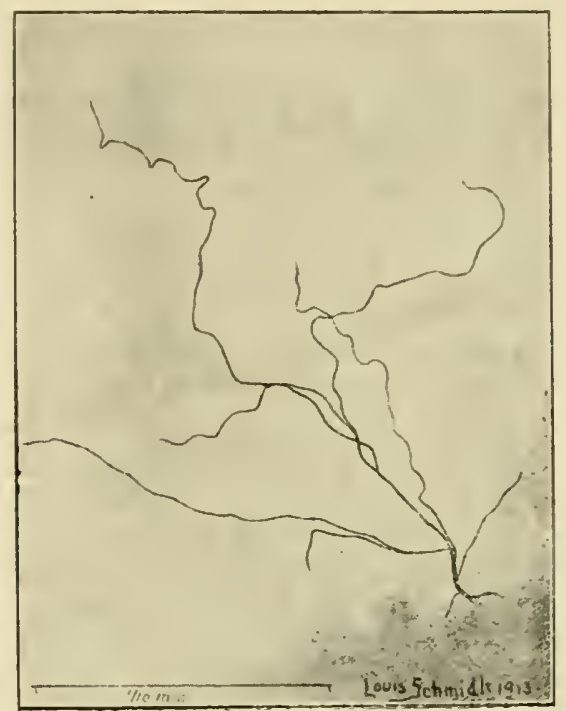

Fig. 28r.-Two-day-old Culture of Spinal Ganglia of a Guinea Pig Six Days Old (Ingebrigtsen).

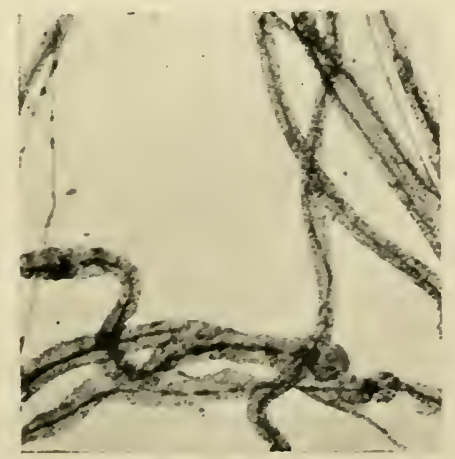

Fig. 282.-Microphotograph of Nerve Fibres from the Sciatic Nerve of a Rabbit, incubated in Plasma for Twelve Days. Hardened in formalin and stained with hæmatoxylin. No trace of Wallerian degeneration (Ingebrigtsen).

shown by the fact that an axon reflex (p. 913) can be elicited on dividing one of the distal trunks and stimulating its central end after complete separation of the proximal or parent stem from the central nervous system. Even when the second path offered to the regenerating motor axon is a sensory path, bifurcation of the axon occurs, one branch 
passing down along the previous motor path to its proper muscular termination, and the other passing down the sensory path. Although there is no evilence that efferent fibres can unite with afferent fibres, a degenerated afferent path can therefore serve as a chemiotactic scaffolding or guide for the growth of regenerating motor axons, though not such an efficient one as a degenerated motor patli. Sensory fibres, however, cannot regenerate along inotor paths or make functional union with the receptive substance of skeletal muscle.

Regeneration of the fibres of the central nervous system either does not in general occur, or is exceedingly difficult to realize. This lends support to the doctrine of the importance of the neurilemma in regeneration, since its elements are scantily developed in the fibres of the brain and cord (p. 8ho). Regeneration of the fibres which procecel from the cells of the spinal ganglia along the postcrior roots into the cord may take place after the roots have been cut, so that the normal reflexes through the respiratory, carliac, and vaso-motor centres may be once more obtained.

Degeneration of Muscle.-Experimental section or, in man, tranmatic division or compression of a nerve leads not only to its degeneration, but ultimately, if regeneration of the nerve does not take place; to degeneration of the muscles supplied by it as well. The muscle-fibres dwindle to a quarter of their normal diameter; the stripes disappear; the longitudinal fibrillation fades out; and at length only hyaline moulds of the fibres are left, filled, and separated by fatty granules and globules and surrounded by engorged capillaries. Amidst the general decay, the muscular fibres of the te'minal 'spindles' with which the afferent nerves of muscles are comnecterl alone remain unchanged (Sherrington). Certain diseases of the cord which interfere with the cells of the anterior horn cause degeneration of motor nerves, and ultimately of muscles. The motor nerve-endings degenerate sooner than the sensory. Both may, under suitable conditions, regenerate (Huber).

Reaction of Degeneration.-Muscles whose motor nerves have been separated from their trophic centres show, when a certain stage in degeneration has been reached, a peculiar behaviour to electrical stimulation, called the 'reaction of degeneration.' To the constant current the muscles are more excitable, and the contraction slower and more prolonged thin normal. When a current, either constant or induced, is passed through a normal muscle, the muscular fibres maly be stinulated either directly, or indirectly through the intramuscular nerves. Under ordinary conditions the nerves respond more readily than the muscular fibres, especially to momentary stimuli like induction shocks, and theretore the so-called direct stimulation of uncurarized muscle is as a rule an indirect stimulation. When the muscle is curarized and the nerves thus eliminated, the excitability to induced currents is found to be diminished. The same is the case in a muscle which exhibits the reaction of degeneration after section of its motor nerve, only the loss of excitability to induced currents is greater, and may even be complete. The common statement that the closing anodic contraction is stronger than the closing kathodic-the opposite of the ordinary law-is subject to so many exceptions that it has no diagnostic value. The nerves are inexcitable either to constant or induced currents. The reaction of degeneration is only obtained from paralyzed muscles when the paralyzing lesion is sitnated in the cells of the arterior 
lorn trom which the motor nerves take origin, or below that level. Accordingly, it is sometimes of use in localizing the position of a lesion. For instance, a group of muscles might be paralyzed by a lesion in the grey matter of the lurain or in the nerve-fibres connecting this with the grey matter of the anterior horn of the cord, or in the grey matter of the anterior horn itself, or in the peripheral nerve-fibres leading from this to the muscles. In the first two cases the reaction of legeneration would be absent, although the muscles, if the lesion was of long standing, would be atrophied to some extent; in the last two there would be acute atrophy of the muscles, and the reaction of degeneration would be obtaincd.

Trophic Nerves.-There is no question that nerves exert a very important influence upon the nutrition of the parts supplied by tliem, in influencing the specific function of those parts. So that in this sense all nerves are trophic rerves. The fact that the proper nutrition of nerve-fibres and strialed muscular fibres is dependent on their connection with nerve-cells las been by some writers generalized into the doctrine that all tissues are provided with 'troplic' nerves, which, apart from any influence of functional activity, regulate the nutrition of the organs they supply. But the evidence for this view, when weighed in the balance, is found wanting; and it may be said that up to the present no unequivocal. proof. expcrimental or clinical, has ever bcen given of the existence of specific trophic fibres, anatomically distinct from other efferent or afferent nerves.

It is true that in various diseases and injuries of the nervous system nutritive changes in the skin, and sometimes in the bones and joints, are apt to appear. But it is very difficult in such cases to disentangle the effects produced by accidental injuries acting on structures whose normal sensibility is lost or lessened, or whose circulation is deranged, from true trophic changes. The most that can be said is that there is some evidence that the power of the skin to resist injury, and the capacity of recovering from it, are diminished by interference with its nerve-supply, so that a large sore may result from a trifling lesion, and healing may be slow and difficult. Experimentally it has been found that division of the trigeminus nerve within the skull is sometimes followed by cloudiness of the cornea, going on to ulccration, and ultimately inflammation and destruction of the eyeball. Ulcers also form on the lips and on the mucous membrane of the mouth and gums; and the nasal mucous membrane on the side corresponding to the divided nerve becomes inflamed. But in this case the sensibility of the eye is lost, and reflex closure of the eyelids ceases to prevent the entrance of foreign bodies. The animal is no longer aware of the contact of particles of dust or bits of straw or accumulated secretion with the conjunctiva, and makes no effort to remove them. The lips, being also without sensation, are hurt by the teeth, particularly as the muscles of mastication on the side of the divided nerve are paralyzed, and decomposed food, collecting in the mouth, and inhaled dust in the nose, will tend still further to irritate the mucous membranes. There is thus no more need to assume the loss of unknown trophic influences in order to explain the occurrence of the ulcerative changes than there is to explain the production of ordinary bed-sores, bunions or corns on parts peculiarly liable to pressure. And, as a matter of fact, if the eye be 
artificially protected, after section of the trigeminal nerve, the ophthalinia either does not occur or is much relayed.

In man, too, a case has been recorded in which both the fiftli and the third nerves were paralyzed. The eye was still shielded by the contraction of the orbicularis oculi supplied by we seventh nerve, as well as by the drooping of the upper eyelid that accompanies paralysis of the third. It remained perfectly sound for many months, till at length the tumour at the base of the brain which had affected the other nerves involved the seventh too. The eye was now no longer completely closed; inflammation came on, and vision was soon permanently lost (Shaw). In another case a patient lived for seven years with complete paralysis of the fifth nerve, yet the eye remained free from disease and sight was unimpaired (Gowers).

The so-called 'trophic' effects following division of both vagi we have already discussed (p. 286) so far as they are concerned with the respiratory system. The degenerative changes sometimes seen in the heart are perhaps due to its being overworked in the absence of nervous restraint on its functional activity. The uutritive alterations in muscles and salivary glands after section of motor and secretory nerves seem to depend in part on functional and vaso-motor changes. In the paralyzed muscles nutrition is not only interfered with in consequence of their inactivity, as would be the case even if the paralysis wera due to a lesion above the level of the anterior cornual cells, but the already poorly nourished fibres are continually pressed upon by the capillaries, which are dilated owing to the division of the vaso-motor nerves. The degeneration may also be in part ascribed to tlie loss of a reflex tonic influence exerted on the muscles by the spinal cord, through the ordinary motor nerves (p. 91 7 ). When all allowance has been made for these factors, the rapid and characteristic degeneration of the striated muscles, after their connection with the central nervous system is severed, is still inexplicable, except on the assumption that their nutrition is specially related to the integrity of their efferent nerves. In other words, it is necessary to suppose, not, indeed, that distinct trophic nerves exist for the muscles, but that an influence or impulses, which can be termed trophic or nutritive, do normally pass out to them from the spinal cord along their motor nerves.

Section of the cervical sympathetic in young rabbits and dogs increases the growth of the ear and of the hair on the same side. But it is impossible to separate these consequences from the vaso-motor paralysis; and the same is true of the hypertrophy following section of the vaso-motor nerves of the cock's comb and of the nerves of the bones. After section of the superior laryngeal the vocal cord on the side of the section is at once rendered motionless, and remains so, but the muscles, notwithstanding their inaction, do not degenerate. And Mott and Sherrington have found that, although section of the posterior roots in monkeys is followed after a time (three weels to three months) by ulceration over certain portions of the foot, no corresponding lesions occur in the hand. They believe, therefore, that the lesions are not due to the withdrawal of a reflex trophic tone, but are accidental injuries in positions specially exposed to mechanical or microbic insults.

One of the best examples of interference with the proper nutrition of a part produced by a lesion in the nerves supplying it is an eruption (herpes zoster), limited to the skin supplicd by the nerve-fibres coining from one or more spinal ganglia, and depending on an (infectious) inflammatory change in the ganglia. It has been suggested that the vesicles are formed either because the passage of afferent impulses normally concerned in the nutrition of the skin is interfered with or 
because the skin is bombarled by antidromic (p. I81) impulses discliarged irom the inflamed ginglia. But an alternative hypothesis is that a toxine spreads out along the nerves from the ganglia, just as in tramatic tetanus the toxine is known to pass in the opposite direction along the nerves from the seat of injury to the central nervous system.

Classification of Nerves.-Omitting the group of 'trophic' new ves, and the even more problematical 'thermogenic' fibres (which some have supposed to presicle over the production of heat, and thereiore to assist in the regulation of the temperature of the body, but of whose existence as clistinct and specific nerve-fibres with no other function there is not the slightest proof), peripheral nerves may be classificd as follows:

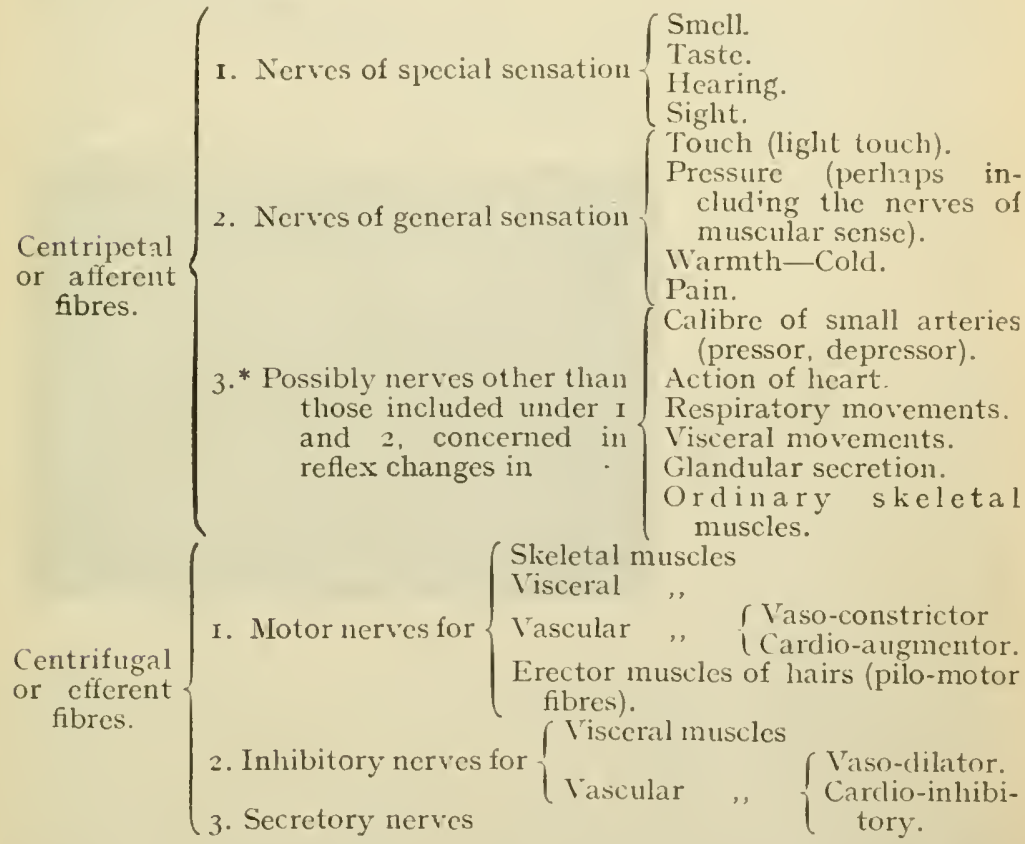

* It is not known whether the afferent pertion of a reflex are is alu'ays composed of fibres included in the first two categories, although undoubtedly in some cases it is.

PRACTICAL EXERCISES ON CHAPTERS XIII. AND XIV.

I. Difference of Make and Break Shocks from an Induction Machine. -Connect a Daniell or other cell B (p. 724) with the two upper bindingscrews of the primary coil $P$, and interpose a spring key $\mathrm{K}$ in the circuit. Connect a pair of electrodes with the binding-screws of the secondary coil (Fig. 283). 
Electrodes can he very simply made by pushing copper wires through two glass fubes, filling the cnels of the tubes with sealing-wax and binding them together with waxed thread. The projecting points may be filed, and the nerve laid directly on them, or they may be tipped with small pieces of platinum wire soldered on.

(a) Push the secondary away from the primary, until no shock can be felt on the tongue when the current from the battery is made or broken with the key. Then bring the secondary gradually up towards the primary, testing at every new position whether the slock is perceptible. It will be felt first at break. If the sccondary is pushed still further up, a shock will be felt both at make and at break. From this we learn that for sensory nerves the break slsock is stronger than the make. The same can easily be demonstrated for motor nerves and for muscle.

(b) Smoke a clrum and arrange a myograph, as shown in Fig. 287. But omit the brass picee $F$, and do not connect the primary through the drum, as there shown, but connect it as in Fig. 283 . Pith a frog (brain and cord), and make a muscle-nerve preparation.

To make a Muscle-Nerie Preparation.-Hold the frog by the hind legs back upwards; the front part of the body will hang down, making an angle with the posterior portion. Witl strong scissors divicte the backbone anterior to this angle, and cut away all the front portion of the body, which will fall down of its own weight. Make a circular incision at the level of the tencio Achillis, and another at the lower end of the femur, through the skin. The sciatic nerve must now be dissected out, as follows: Remove the skin from the thigh, and, loolding the leg in the

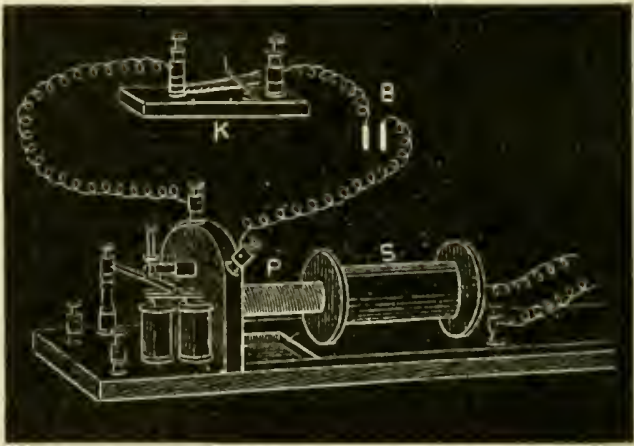

Fig. 283.-Arrangement of Coil for Single Shocks.

left hand, slit up the fascia which connects the external and internal groups of muscles on the back of the thigh. Complete the scparation with the two thimbs. Cut through the iliac bone, taling care that the blade of the scissors is well pressed against the bone. otherwise there is danger of severing the sciatic plexus. Now divide in the middle line the part of the spinal column which remains above the urostyle. A piece of boue is thus obtained by means of which the nerve can be manipulated without injury. Seize this jicce of bone with the forceps, and carefully free the sciatic plexus and nerve from their attachments right down to the gastrocnemius muscle, taking care not to drag upon the nerve. The muscles of the thigh will contract, as the branches going to them are cut. This is an instance of mechanical stimulation. Now pass a thread under the tendo Achillis, tie it, and divide the tendon below it. Strip up the tube of skin that covers the gastrocnemius, as if the finger of a glove were being taken off. Tear through the loose connective tissue between the muscle and the bones of the leg, and divide the latter with scissors just beiow the knec. Cut across the thigh at its middle.

Fix the preparation on the cork plate of the myograph by a pin passed 
through the cartilaginous lower end of the femur, and attach the thread to the mpright arm of the lever by one of the holes in it. Hang not fir from the axis by means of a hook a small leaten weight (5 to In grammes) on the arm of the lever which carries the writing-point, and move the myograph plate or the muscle-nerve preparation until this arm is just horizontal. Fasten the electrodes from the secondary coil on the cork plate with an indiarubber band; lay the nerve on them; and cover both muscle and nerve with an arch of blotting-paper moistened with physiological salt solution, taking care that the blottingpaper does not tonch the thread. Or put the preparation in a moist chamber* (Fig. 322, p. S+3). Muscle troughs of various kinds may also

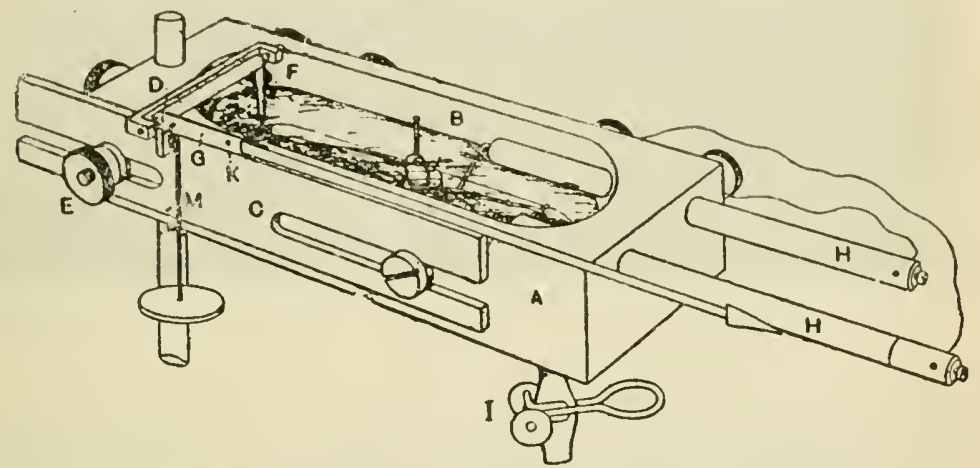

Fig. 28.,-Lucas's Muscle Trough. A, trough made of hard rubber; B, a hard rubber boss with a bole drilled in it to receive the pin which fastens the gastrocremius preparation; $\mathbf{H}, \mathbf{H}$, electrodes cased in hard rubber except at the ends, which in the trough carry platinum wires; $\mathrm{C}$, a brass plate mounted on one side of the trough, carrying a lever with a vertical arm F ending in a hook, which is attached by a loop of thread to the tendon of the preparation; $G$, the writing arm of the lever; $K$, M, holes in $G$ for loading the muscle. $C$ can be slid horizontally by means of the slots in it, and clamped by the screw E. 1, tube for running off the solution.

be used, which permit immersion of a muscle (or nerve) in Ringer's solution. A convenient form is shown in Fig. 284 , but a trough sufficient for the purposes of the student can be easily improvised in any laboratory. Adjust the writing-point to the drum. Begin with such a distance between the coils that a break contraction is just obtained on opening the key in the primary circuit, but no make contraction. The lever will trace a vertical line on the stationary drum. Read off on the scale of the induction machine the distance between the coils, and mark this on the drum. Now allow the drum to move a little, still kecping the writing-point in contact with it; then push up the secondary coil I centimetre nearer the primary, and close the key. If there is a

* Porter's moist chamber is found in many laboratories, and is very convenient. It consists of a porcelain plate around which runs a groove. A bellshaped glass cover, which can be lifted off at will, rests in the groove. The femur of the muscle-nerve preparation is fixed in a small clamp, composed of a split screw on which moves a nut. By means of the nut the clamp is tightened on the femur. The gastrocnemius hangs vertically down, the thread on the tendo Achillis passing through a hole in the porcelain plate to a lever separately supported on the same stand as the moist chamber. A piece of wet blotting-paper fixed inside the cover keeps the air in the chamber saturated. 
contraction, let the drum move a little before opening the key again, so that the lines corresponding to make and break may be separated from each other. If there is still no contraction at make, go on moving the secondary up, a centimetre (or less) at a time, till a make contraction appears. When the coils are still further approximated, the make may become equal in height to the break contraction, both being maximal-i.e., as great as the muscle can give with any single shock (Fig. 285).

(c) Attach a thin insulated copper wire to each terminal of the sccondary. Loop the bared end of one of the wires through the tendo

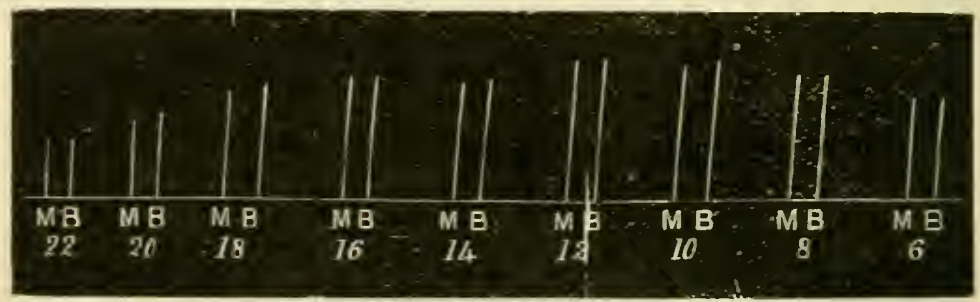

Fig. 285.-Contractions caused by Make and Break Shocks from an Induction Machine. M, make, B, break, contractions. The numbers give the distance between the primary and secondary coils in centimetres.

Achillis, and coil the other round the pin in the femur, so that the shocks will pass through the whole length of the muscle. Repeat the experiment of $(b)$, with direct stimulation of the muscle.

2. Stimulation of Nerve and Muscle by the Voltaic Current.-(a) Connect a Daniell cell throngh a key with a pair of electrodes on which the nerve of a muscle-nerve preparation lies. Observe that the muscle contracts when the current is closed or broken, but not during its passage.

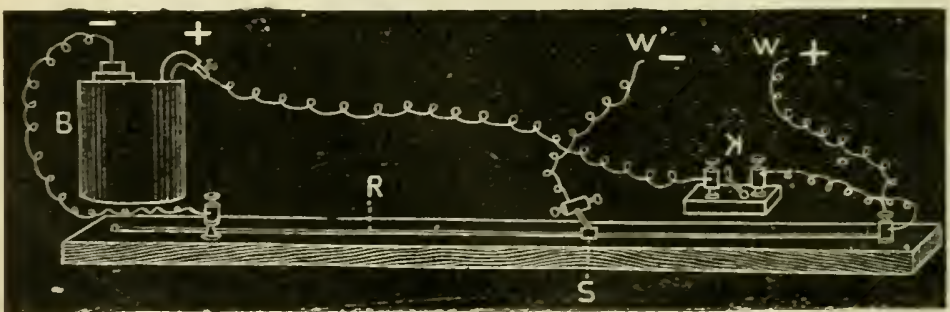

Fig. 286. Simple Rheocord arranged to send a Twig of a Current tlirough a Muscle or Nerve. B, battery; R, rheocord wire (German silver); S, slider formed of a short piece of thick indiarubber tubing filled with mercury; $K$, spring key; $\mathrm{W}, \mathrm{W}^{*}$, wires connected with electrodes.

Connect the cell with a simple rheocord, as shown in Fig. $280^{\circ}$, so that a twig of the current of any desired strength may be sent through the nerve. As the strength of the current is decreased by moving the slider $\mathrm{S}$, it will be found that it first becomes impossible to obtain a contraction at break. The current must be still further reduced before the make contraction disappears, for the closing of a galvanic stream is a stronger stimulus than the breaking of it. The break or make con- 
traction obtained by stimulating a newve with an induction macline must not be confused with the break or niake contraction caused by the voltaic current. In the case of the induction macline, the break or make applies merely to what is done in the primary circuit, not to what happens to the current actually passing through the nerve. The current induced in the secondary at make of the primary circuit is, of course, both made and broken in the nerve-made when it begins to flow, broken when the flow is over; the shock induced at break of the primary is also made and broken in the nerve. And although make and break of the actual stimulating current come very close together, the real make, here, too, is a stronger stimulus than the real break.

(b) Repeat $(a)$ with the muscle directly connected to the cell by thin copper wires, or, better, unpolarizable electrodes (p. 73I).

3. Ciliary Motion.-Cut away the lower jaw of the same frog, and place a small picce of cork moistened with pliysiological salt solution (o.75 per cent.) on the ciliated surface of the mucous membrane covering the roof of the mouth. It will be moved by the cilia down towards the gullet. Lay a small rule, divided into millimetres, over the mucous membrane, and measure with a stop-watch the time the piece of cork takes to travel over ro millimetres. Then pour salt solution heated to $30^{\circ} \mathrm{C}$. on the ciliary surface, rapidly swab with blotting-paper, and repeat the observation. The piece of cork will now be moved more quickly than before, unless the silt solution has been so hot as to injure the cilia.

4. Direct Excitability of Muscle-Action of Curara.-Pith the brain of a frog, and prevent bleeding by inserting a piece of matcl. Expose the sciatic nerve in the thigh on one side. Carefully separate it, for a length of half an inch, from the tissues in which it lics. Pass a strong thread under the nerve, and tic it tightly round the limb, excluding the nerve. Now inject into the dorsal or ventral lympli-sac a few drops of a I per cent. curara solution. As soon as paralysis is complete, make two muscle-nerve preparations, isolating the sciatic nerves right up to the vertebral column. Lay their upper ends on electrodes and stimulate; the muscle of the ligatured limb will contract. This proves that the nerve-trunks are not paralyzed by curara, since the poison has been circulating in them above the ligature. The muscle of the leg which was not ligatured will contract if it be stimulated directly, although stimulation of its nerve has no effect. The ordinary contractile substance of the muscular fibres, accordingly, is not paralyzed. The seat of paralysis must therefore be some structure or substance physiologically intermediate between the nerve-trunk and the general contractile substance of the muscular fibres (p. 738).

5. Graphic Record of a Single Muscular Contraction or Twitch.-Pith a frog (brain and cord), make a muscle-nerve preparation, and arrange it on the myograph plate, as in I $(b)$. Lay the nerve on elcctrodes connected with the secondary coil of an induction macline arranged for single shocks. Introduce a short-circuiting key (Fig. 240, p. 732) between the electrodes and the secondary coil, and a spring key in the primary circuit. Close the short-circuiting key, and then press down the spring key with the finger. Let the drum off (fast speed); the writing-point will trace a horizontal abscissa line. Open the shortcircuiting key, and then remove the finger from the spring key. The nerve receives an opening shock, and the muscle traces a curve. Now adjust the writing-point of an electrical tuning-fork (Fig. 2S7), vibrating, say, roo times a second, to the drum, and take a time-tracing below the muscle-curve. Stop the drum, or take off the writing-point, the moment the time-tracing has completed one circumference of the drum, 
so that the trace may not run over on itself. Cut off the drum-paper, write on it a brief description of the experiment, with the time-value of
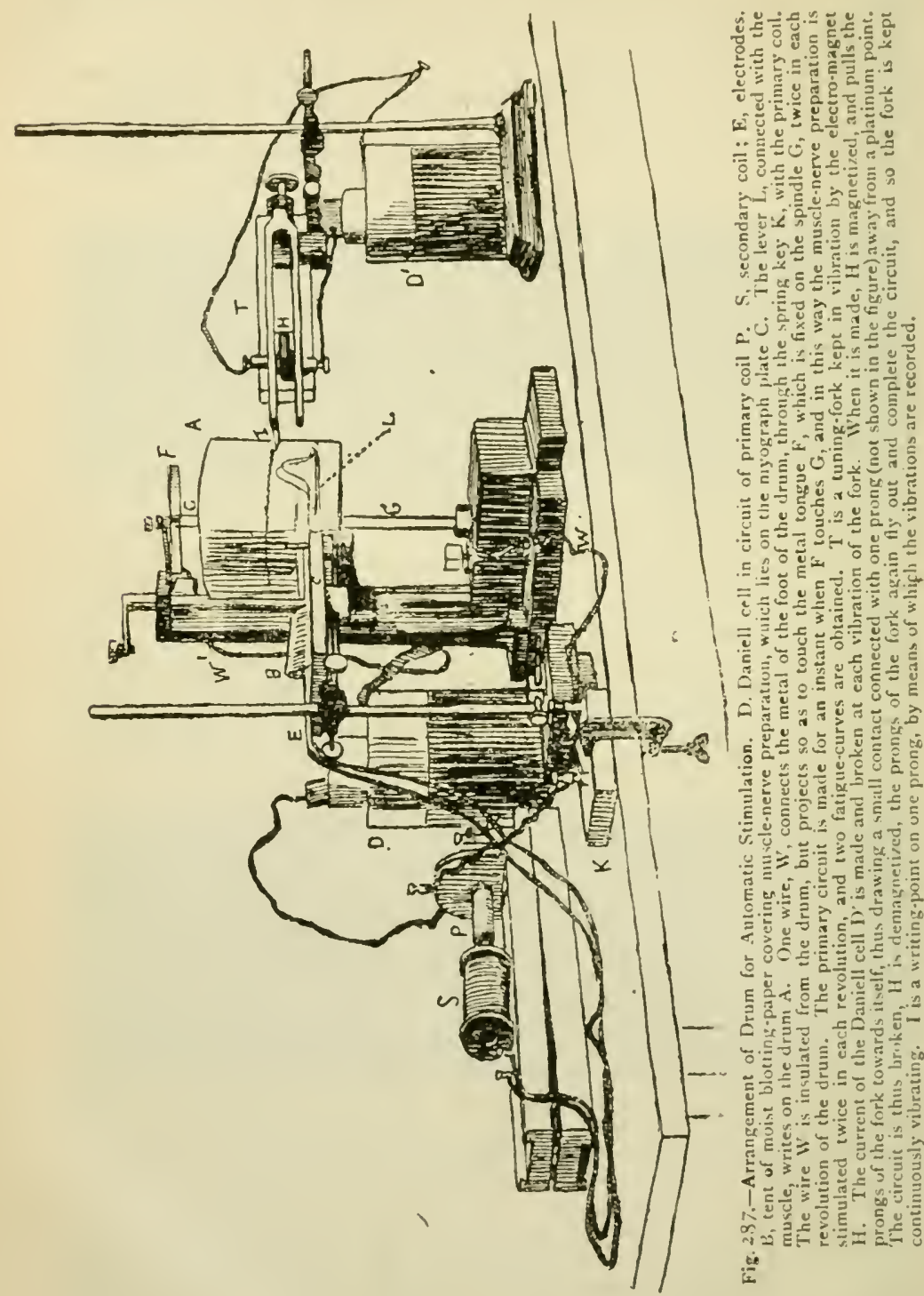

each vibration of the fork, the date, and the name of the make of the tracing, and then varnish it. An exactly similar tracing can be obtained by directly stimulating the inuscle (curarized or not). 
6. Influence of Temperature on the Muscle-Curve.-Pith a frog (brain and cord), make a muscle-nerve preparation, and arrange it on a myograph. Lay the nerve on electrodes connected through a shortcircuiting liey with the secondary coil of an induction machine, or connect the muscle directly with the key by thin copper wires. Take a Daniell cell, connect one pole through a simple key with one of the upper binding-screws of the primary coil, and the other pole with the metal of the drum. A wire, insulated from the drum, but clamped on the vertical part of its support, and with its bare end projecting so as to make contact with a strip of brass fastened on the spindle, is connected with the other upper terminal of the primary (Fig. 28\%). At each revolution of the drum the primary circuit is made and broken once as the strip of brass brushes the projecting end of the wire. The object of this arrangement is to ensure that when the writing-point of the myograph lever lias been once adjusted to the drum, successive stimuli will cause contractions, the curves of which all rise from the same point. Close the key in the primary, set the drum off (fast speed), open the short-circuiting key, and as soon as the muscle has contracted once, close it again. Now stop the drum, mark with a pencil the position of the feet of the stand carrying the myograph plate, take the writing-point off the drum, and surround the muscle with pounded ice or snow. After a couple of minutes brush away any ice which could hinder the movement of the muscle, rapidly replace the stand in exactly its original position, witl the writing-point on the drum, and take another tracing. Again take off the writing-point, and remove all unmelted ice or snow. With a fine-pointed pipette irrigate the muscle with physiological salt sohution at $30^{\circ} \mathrm{C}$, , and quickly take another tracing. Then put on a time-tracing with the electrical tuning-fork. Fig. 255. p. 748 , shows a series of curves obtained in this way.

7. Influence of Load on the Muscle-Curve.-Arrange everything as in 6. Take a tracing first with the lever alone, then with a weight of Io grammes, then with 50, I00, 200, and 500 grammes (Fig. 254, p. 748).

8. Influence of Fatigue on the Muscle-Curve.-Arrange as in 7 , but leave on the same weight (say 10 grammes) all the time. Place the nerve on the electrodes. Leave the short-circuiting key open. The nerve will be stimulated at each revolution of the drum, and the writingpoint will trace a series of curves, which become lower, and especially longer, as the preparation is fatigued. Two or four curves can be taken at the same time, if both ends of one or of two brass slips be arranged so as to make contact with the projecting wire at an interval of a semicircumference or quadrant of the drum (Fig. 287). (For specimen curve, see Fig. 288, p. 81 4.)

9. Seat of Exhaustion in Fatigue of the Muscle-Nerve Preparation for Indirect Stimulation.- When the nerve of a muscle-nerve preparation has been stimulated until contraction no longer occurs, the muscle can, under ordinary conditions, be made to contract by direct stimulation. The seat of exhaustion is, therefore, not the general contractile substance of the muscular fibres themselves. To determine whether it is the nerve-fibres or some structure or substance intermediate between them and the ordinary contractile substance of the muscle, perform the following experiments:

(a) Pith a frog; make two muscle-nerve preparations; arrange them both on a myograph plate, which has two levers connected with it. Attach each of the muscles to a lever in the usual way, and lay both nerves side by side on the same pair of electrodes. Cover with moist blotting-paper. The electrodes are connected with the secondary of an induction machine arranged for tetanus. With a camel's hair brush 
moisten one of the nerves between the electrodes and the muscle with a mixture of equal parts of ether and alcohol, diluted with twice its volume of water, to abolish the conductivity. Or put the mixture in a small bottle, in which dips a piece of filter-paper. The projecting end of the filter-paper is pointed, and the nerve is laid on the point. As soon as it is possible to stimulate the nerves without obtaining contraction in this muscle, proceed to tetanize both nerves till the contracting muscle is exhausted. If the other muscle begins to twitch during the stimulation, more of the ether mixture must be painted on the nerve. As soon as the stimulation ceases to cause contraction in the non-etherized preparation, wash off the mixture from the other nerve with physiological salt solution, and soon contraction may be seen to take place in

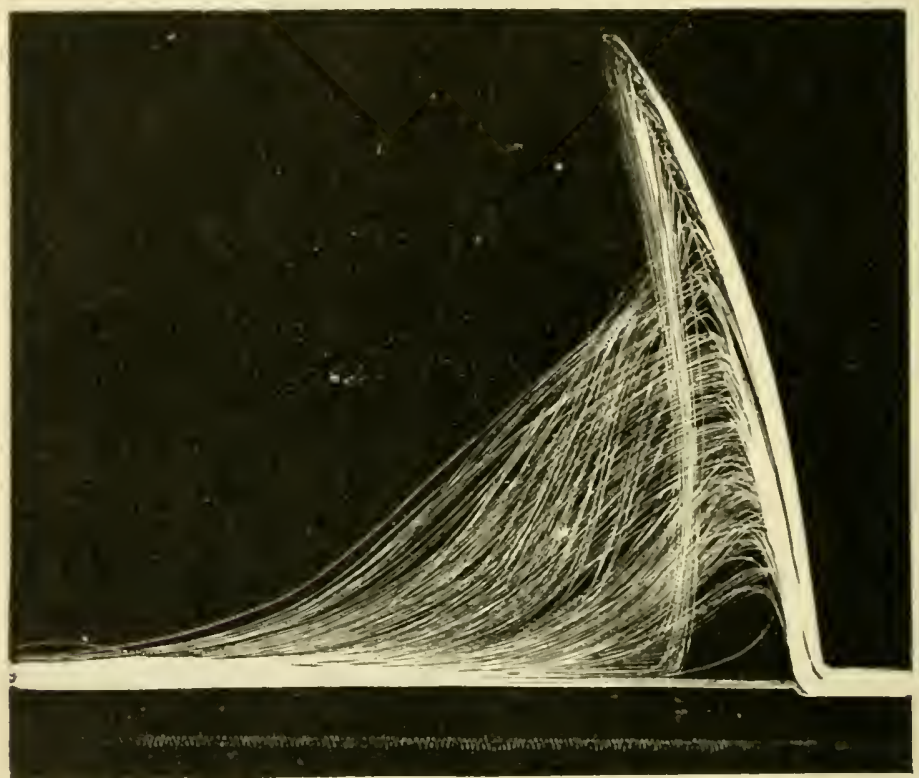

1:ig. 288,-Fatigue Curve of Skeletal Muscle: Gastrocuemius of Frog. Indirec 1 stimulation; taken with arrangement shown in Fig. 287 (p. 812). Time-tracing, $1 \frac{1}{00}$ of a second.

the muscle of this preparation. This shows that the nerve-trunk is still excitable. Now, both nerves have been equally stimulated, and therefore the exluaustion in the non-etherized preparation was not due to fatigue of the nerve-fibres, but of something between them and the contractile substance of the muscle.

Io. Influence of Veratrine on Muscular Contraction.- Arrange a (rum as in Fig. 287. Pith a frog (brain only), expose the sciatic nerve in one thigh, and isolate it for $\frac{1}{2}$ inch from the surrounding tissues. Pass under it a strong thread, and ligature everything except the nerve. Now iuject into the dorsal or ventral lymph-sac a few drops of $\alpha^{\prime} \mathbf{p}$ per cent. solution of sulphate of veratrine. In a few minutes make two inuscle-nerve preparations from the posterior limbs. First put the preparation from the unligatured limb on the myograph plate. Lay 
the nerve on electrodes connected through a short-circuiting ley with the secondary of in induction mathine arranged as in Fig. 287 . Put the writing-point on the (Irum and set it off (fast speed). Open the short-circuiting key till the nerve has been once stimulated, then close it again. The curve obtaned differs from a normal curve, in that the period of descent (relaxation) is exceclingly prolonged. Now connect the preparation from the ligatured limb with the lever, and take a tracing of a single contriction. I'ut on a time-tracing with the electrical tuning-fork (sec Figs. 264, 265, p. 755).

11. Measurement of the Latent Period of Muscular Contraction. (I) For this the drum must travel at a fister speed than usual. It is most convenient to use a drum rotated very rapidly by a cord atticlied to a falling weight or by the recoil of a stretched rubber band or spring. The arrangement for automatic stimulation described in Expcriment 6 (p. 813) maty be eniployed. Or an electro-magnetic signal may be connected in the primary circuit of the induction coil so that when the prinary is closed or opened the writing-point of the signal moves. Arrange the writing-point of the signal on the drum in the same vertical line as the writing-point of the muscle lever, and in the same line place the writing-point of a vibrating electric tuning-fork. The coil is adjusted for single opening shocks as in Experiment 5 (p. 8II). Pith a frog, and make a muscle-nerve preparation. Arrange it on the myograpli plate. The muscle, or the nerve very near the muscle, is to be excited by a single opening shock while the drum is moving. When the curve has been traced, the latent period is got by drawing a vertical line through the point at which the curve just begins to rise from the abscissa line, and another through the signal marli. The number of vibrations of the tuning-fork included between these two verticals gives the latent period.

Or (2) use the spring myograph (Fig. 25I, p. 746), raising it on blocks of wood. Smoke the glass plate over a paraffin flame, or cover it with paper, and smoke the paper. Connect the knock-over kev of the myograph with the primary circuit of an induction coil. Arrange a musclenerve preparation on the myograph plate. Place electrodes below the nerve as near the muscle as possible, and connect by a short-circuiting key with the secondary. Bring the writing-point in contact with the smoked surface of the spring myograph, so as to get the proper pressure. Sce that the writing-point of the tuning-fork is in the right position for tracing time. Then push up the plate so as to compress the spring, till the rod connected with the frame which carries the plate is held by the catch.

With the short-circuiting key closed, press the release and allow an abscissa line to be traced. Again shove back the frame till it is caught. Push home the rod by means of which the prongs of the tuning-fork are separated, and rotate it through $90^{\circ}$. Close the knock-over key, open the short-circuiting key, shoot the plate again, and a muscle-curve and time-tracing will be recorded. Again close the short-circuiting key, withdraw the writing-point of the tuning-fork, push back the plate, close the trigger key, then open the short-circuiting key, and, holding the travelling frame witl the hand, allow it just to open the knockover and stimulate the nerve. The writing-point now records a vertical line (or, rather, an arc of a circle), which marks on the tracing the moment of stimulation. The latent period is obtained by drawing a parallel line (or arc) through the point of the muscle-curve where it just berins to diverge from the abscissa line. The value of the portion of the tinle-tracing between these two lines can be readily determined, and is the latent period. 
12. Summation of Stimuli.-Arrange two knock-over keys on tho spring myograph at such a distance from each other that the plato travels frem one to the other in a time less than the latent period. Connect each tey with the primary circuit of a separate induction coil having a couple of Daniclls in it. Join two of the binding-screws of the secondaries together; connect the other two through a shortcircuiting key with electrodes, on which the nerve of a muscle-nerve preparation is arranged. Push up the secondaries till the break shocks ebtained on opening the two knock-over keys are maximal. Then shoot the plate as described in I 1 , first with one trigger key closed, and then with both. The curves obtained should be of the same height in the two cases, as a second maximal stimulus falling within the latent period is ignored by the nerve or muscle. Repeat the experiment with submaximal stimuli-i.e., with such a distance of the coils that opening of either trigger key does not cause as strong a contraction as is caused when the coils are closer. The curve will now be ligher when the two shocks are thrown in successively than when the nerve is only once stimulated. This shows that (submaximal) stimuli can be summed in the nerve. The same could be demonstrated for muscle (p. 756).

I3. Superposition of Contractions.-Smoke a drum arranged for automatic stimulation as in Fig. 287. Adjust the brass points with a distance of, say, I centimetre between them, so that a second stimulus may be thrown into the nerve at an interval greater than the latent period of muscle. Put two Daniells in the primary circuit. Lay the nerve of a muscle-nerve preparation on electrodes connected through a short-circuiting key with the secondary. Allow the drum to revolve (fast speed); open the short-circuiting key till both brass points have passed the projecting wire, then close it. Now bend back the second brass point, and take a tracing in which the first curve is allowed to complete itsclf. This will not rise as ligh as the second curve obtained when the two stimuli were thrown in. Repeat the experiment with varying intervals between the brass points-that is, between the two successive stimuli. Put on a time-tracing with the electrical tuningfork. (For specimen curve, see Fig. 206, p. 756.)

I4. Composition of Tetanus.-(a) Adjust a muscle-nerve preparation on a myograph plate, the nerve being laid on electrodes connected through a short-circuiting key with the secondary of an induction machine, the primary circuit of which contains a Danicll cell and is arranged for an interrupted current (Fig. 93, p. 200). The lever should be shorter than that used for the previous experiments, or the thread should be tied in a hole farther from the axis of rotation, so as to give less magnification of the contraction. Set the Neef's hammer going, let the drum revolve (slow speed), and open the key in the secondary. The writing-point at once rises, and traces a horizontal or perhaps slightly-ascending line. Close the short-circuiting key, and the lever sinks down again to the abscissa line. If it does not quite return, it should be loaded with a small weight. This is an example of complete tetanus.

(b) Connect the spring shown in Fig. 289 with one of the upper terminals of the primary coil, and the mercury cup with the other. Fasten the end of the spring in one of the notches in the upright picce of wood by means of a wedge, so that its whole length can be made to vibrate. Let the druin off, set the spring vibrating by depressing it with the finger, then open the key in the secondary. The muscle is thrown into incomplete tetanus, and the writing-point traces a wavy curve at a higher level than the abscissa line. Close the short-circuiting key, and the lever falls to the horizontal. Repeat the experiment with 
the spring fastenerl, so that only $\frac{3}{4}, \frac{1}{2}, \frac{1}{4}, \frac{1}{4}$ of its length is free to vibraic. The rate of interruption of the primary circuit increases in propurtion

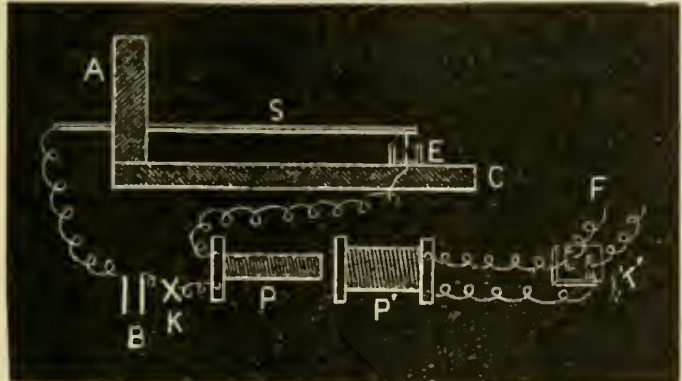

Fig. $289-$ Arrangement for Tetanus. A, upright with notches, in which the spring $S$ is fastened (shown in section); $\mathrm{C}$, horizontal board to which $\mathrm{A}$ is attached, and in a groove in which the mercury-cup $\mathbf{E}$ slides. The primary cnil $\mathrm{P}$ is connected with $\mathrm{E}$, and through a simple key, $\mathrm{K}$, with the battery $\mathrm{B}$, the other pole of which is connected with the end of the spring. The wires from the secondary coil, $\mathrm{P}^{\prime}$, go to a short. circuiting key, $\mathrm{K}^{\prime}$, from which the wires $\mathrm{F}$ go off to the electrodes. immediately after death from a cat, or, still better, from a clicken. Or a segment of rabbit's intestine may be employed as described on p. $45^{6}$. Use the arrangement described on p. 456 . In the case of the cat's œesophagus the ring should be taken from the lower half of the œsophagus, since the upper portion contains purely striated muscle. Obtain tracings of the rhythmical contractions on a slowlymoving drum (Fig. 290).

(2) Fix one end of a piece of cat's nesophagus, 2 to 5 centimetres long, to a muscleclamp in a moist chamber, and the other end to a lever writing on a drum. Conncet thin copper wires from the secondary coil of an inductorium with the two ends of the piece of œesophagus. Take

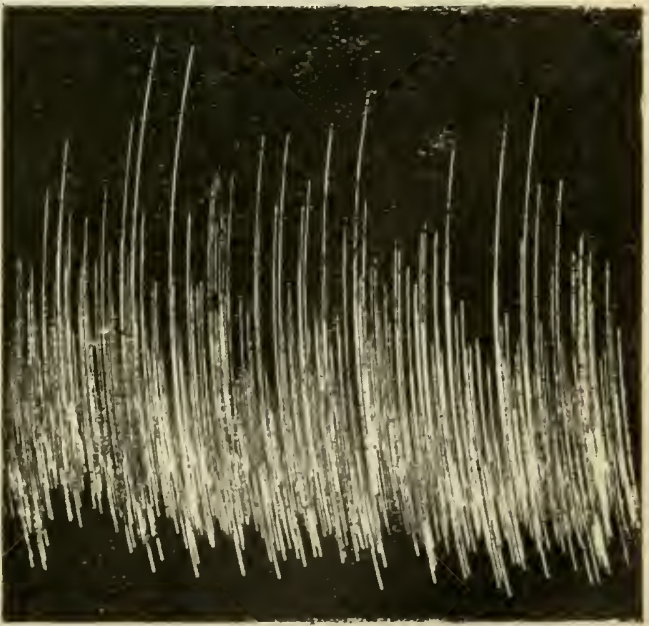

Fig. 290.-Rhythmical Contractions of CEsophagus of Chicken (Botazz:).

tracings to show $(a)$ the curve of a single contraction caused by a single make or break shock, with estimation of the latent period, as in Experiment I I, P. 81 5; (b) summation, as in Experiment 12, p. 816; (c) genesis 
of tetanus, as in lixperiment I4, p. $816 ;(d)$ the relations between strength of stimulus and amount of contration. For this last experiment the drum should be stationary while the contraction is being recorded, and should be allowed to move a little between successive contractions. Begin with the sccondary at such a distance from the primary that a contraction is just caused by a break sliock. Then gradually increase the strength of the stimulus (always using the break) till maximum contraction is obtained. The gradual increase in the response is very clearly seen with the osophageal preparation (Waller).

fior further experiments on the contraction of smooth muscle, sec pp. 66 and 4.57 .

16. Velocity of the Nerve-Impulse.-Use the spring myograph (Fig. 251,1$) .74^{6)}$ ) or a very rapidly rotating (Irum. Nake a musclenerve preparation from a large frog (preferably a bull-frog), so that the sciatic nerve may be as long as possible. Connect the kneck-over key with the primary circuit of an induction machine, which should contain

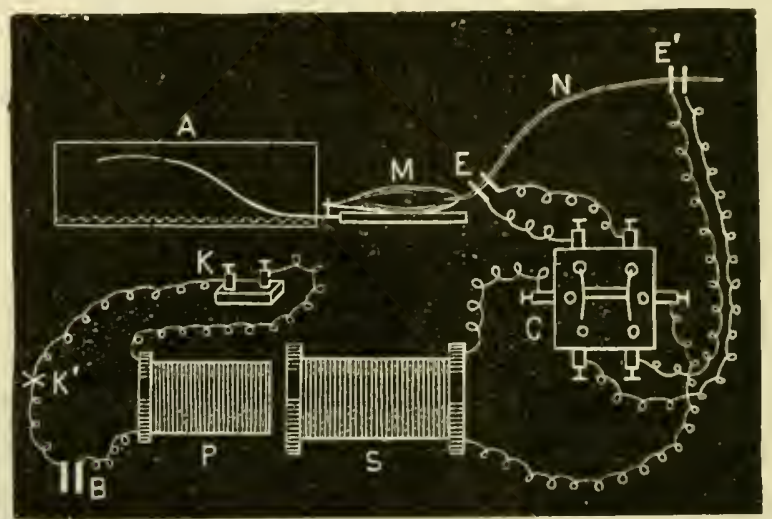

Fig. 291. Arrangement for Measuring the Velocity of the Nerve-Impulse. A. travel ling plate of spring miyograph; $M$, nuscle lying on a myograph plate: $N$, nerve lying on two fairs of electrodes, E and I'; C, Pohl's rommutator without cross. wires; $\mathrm{K}$, knock-over key of spring myograph (only the binding-screws shown); $\mathrm{K}^{\prime}$, simple key in primary circuit; $\mathrm{B}$, battery; $\mathrm{P}$, primary coil : $\mathrm{S}$, secondary coil.

a single Daniell cell. Arrange two pairs of fine electrodes under the nerve on the myograph plate, one near the muscle, the other at the central end. Conmect the electrodes with a Polll's commutator (without cross-wires), the side-cups of which are joined to the terminals of the secondary coil, as slown in Fig. 291. By tilting the bridge of the commutator the nerve may be stimulaterl at either point. Great care must be taken to keep the nerve in a moist atmosphere by means of wet blotting-paper or a moist chamber; but at the same time it must not lic in a pool of salt solution, as twigs of the stimulating current would in this case spread down the nerve; and we could never be sure that the apparent was always the real point of stimulation. The writingpoints of the lever and tuning-fork having been adjusted to the s.moked plate, as in II (p.815), the bridge of the Poll's commutitor is arranged for stimulation of the distal point of the nerve, the plate is slot with the short-circuiting key in the secondary closed, and an abscissa line and time-curve traced. Then the writing-point of the fork is removed and the plate again shot with the key in the sccondary open, and a 
muscle-curve is obtained. The commutator is now arranged for stimulation of the central end of the nerve, and another muscle-curve taken. Vertical lines are drawn through the points where the two curves just begin to separate out from the abscissa line. The intcrval between these lines corresponds to the time taken by the nerve-impulse to travel along the nerve from the central to the distal pair of clectrodes. Its value in time is given by the tracing of the tuning-fork. The length of the nerve between the two pairs of electrodes is now carefully measured with a scale divided in millimetres, and the velocity calculated (p. 793).

I7. Chemistry of Muscle.-Nince up some muscle from the hind-legs of a dog or rabbit (used in some of the other experiments), of which the bloodressels have been washed out by injecting $0^{\circ} 9$ per cent. salt solution through a cannula tied into the abdominal aorta until the washings are no longer tinged with blood. To some of the minced muscle add twenty times its bulk of distilled water, to a nother portion ten times its bulk of a 5 per cent. solution of magnesium sulphate. Let stand, with frequent stirring, for twenty-four hours. Tlien strain through several folds of linen, press ont the residue, and filter through paper. (I) With the filtrate of the watery extract make the following observations:

(a) Reaction.-To litmus-paper acid.

(b) Determine the tcmperatures at which coagulation of the various proteins in the extract takes place, according to the method described on p. 9.* Put some of the watery extract in the test-tube, and heat the bath, stirring the water in the beakers occasionally with a feather. Note at what temperature a coagulum first forms. It will be about $47^{\circ} \mathrm{C}$. Filter this off, and again heat; another coagulum will form at $56^{\circ}$ to $58^{\circ}$. Filter, and heat the filtrate; a third slight coagulum may be formed at $60^{\circ}$ to $65^{\circ} \mathrm{C}$., but this represents merely a residue of the myosinogen which was left in solution at the previous heating. A fourth precipitate (of serum-albumin) will come down at $70^{\circ}$ to $73^{\circ}$. Saturate some of the watery extract with magnesium sulphate; a large precipitate will be formed, showing the presence of a considerable amount of glcbulin. Filter off the precipitate and heat the filtrate; coagulation will again occur at very much the same temperatures as before, although the total amount of precipitate will be less. Note in particular that there is still some precipitate at $47^{\circ}$ to $50^{\circ}$. Paramyosinogen possesses some of the characters of both globulins and albumins, for it is partially but not entirely precipitated by saturation with magnesium sulphate, and is not precipitated by sodium chloride.

(2) (a) Test the reaction of the magnesium sulphate extract. It will usually be faintly acid to litmus.

(b) Heat some of it. Precipitates will be obtained at the same temperatures as in $(I)(b)$, but those at $47^{\circ}$ to $50^{\circ}$ and $56^{\circ}$ to $5^{8^{\circ}}$ will be more abundant. Of the two, that at $47^{\circ}$ to $50^{\circ}$ will usually be the larger when time is given for it to come down and the heating is gradual.

(c) Dilute some of the magnesium sulphate extract with three times, another portion witl four times, and another with five times, its volume of water in a test-tube, and put in a bath at $40^{\circ} \mathrm{C}$. Coagulation or

* It should be remembered that the temperature of heat-coagulation of any substance is by no means an absolute constant. It depends on the reaction, the proportion and kind of neutral salts present, perhaps on the strength of the protein solution and the manner of heating. A solution of egg-albumin, e.g., can be coagulated at a temperature much below $70^{\circ}$ when it is heated for a week. Small differences in the temperature of heat-coagula. tion, unless supported by well-marked chemical reactions, are not enough to characterize protein substances as chemical individuals. 
precipitation will occur in one or all of these test-tubes. To another test-tube of the extract diluted in the proportion which has given the hest 'muscle-clot' addl a few drops of a dilute solution of potassinm oxalate, and place in a bath at $40^{\circ}$. Coagulation occurs as before. Filter off the clot from all the test-tubes. The filtrate is the "muscleserum,' and yields a precipitate of serum-albumin at $70^{\circ}$ to $73^{\circ} \mathrm{C}$.

(3) Myosinogen, lilie other globulins, is insoluble in distilled water, but soluble in weak saline solutions. Saturation with neutral salts like sodium chloride and magnesium sulphate precipitates myosinogen, but not albumin, from its solutions; saturation with ammonium sulplate precipitates both. Verify the following reactions of myosinogen, using the original magnesium sulphate extract of the muscle:

(a) Dropped into water, it is precipitated in flakes, which can be redissolved by a weak solution of a neutral salt (say 5 per cent. magnesium sulphate).

(b) When a solution of myosinogen is dialyzed, it is after a time precipitated on the inside of the dialyzer as the salts pass out.

(c) If a piece of rock-salt is suspended in a solution, the myosin gradually gathers upon it, diffusion of the salt out through the precipitated myosin always keeping a saturated layer aronnd it.

(d) Saturate a solution containing myosinogen with crystals of magnesium sulphate, stirring or shaking at frequent intervals. The myosinogen is precipitated.

(e) Without adding any salt, simply shake a myosinogen solution vigorously; a certain amount of the myosinogen will be precipitated and the solution will become turbid. This reaction can also be obtained with solutions of other proteins, sucl as albumins (Ramsden).

Extracts qualitatively similar to those obtained from the muscles of a freshly-killed animal can be got from muscles that have cntered into rigor, but the quantity of the various proteins going into solution is less.

18. Reaction of Muscle in Rest, Activity, and Rigor Mortis.-(a) Take a frog's muscle, cut it across, and press a piece of red litmus-paper on the cut end; it is turned blue. Yellow turmeric paper is not affected.

(b) Immerse another muscle in physiological salt solution $(0.75$ per cent. for frog's tissues) at $40^{\circ}$ to $42^{\circ} \mathrm{C}$. It becomes rigid. The reaction becomes acid to litmus-paper, and also turns brown turmeric paper yellow.

(c) Plunge another muscle into boiling physiological salt solution. It becomes harder than in $(b)$.

(d) Stimulate another muscle with an interrupted current from an induction machine (Fig. 93, p. 200), till it no longer contracts. The reaction is now acid to litmus-paper. Brown turmeric paper may also be turned yellow.

(e) To demonstrate the formation of lactic acid in muscle in heat rigor or fatigue, perform the following experiment: Pith a frog, and afterwards leave it for half an hour at rest, so that the lactic acid produced in the movements connected with the pithing operation may disappear from the muscles. See that the circulation in the hind-limbs is not interfered with by pressure or flexion. Then remove both hindlimbs. Carefully, but rapidly, remove the muscles of one from the bones with as little manipulation as possible. Immediately place them in a small mortar cooled in ice, and containing some sand and 20 or 30 c.c. of ice-cold 95 per cent. alcohol, and quickly grind them up. Produce heat rigor (p. 777) of the muscles of the other hind-limb, or fatigue them with induction-shocks, and then grind them up under alcohol in the same way. Filter the alcoholic extracts. and then 
evaporate them to dryness on the watcr-bath. Rub up the residues with a few c.c. of hot water. Add to each aqueous extract a small quantity (say a decigramme) of finely powdered charcoal. Then heat each extract to boiling in a test-tube, and filter. Evaporate the filtrates to dryness, and apply.

Hopkins's Reaction for Lactic Acid.-The reagents required are (I) a very dilute alcololic solution of thiophene $\left(\mathrm{C}_{4} \mathrm{H}_{4} \mathrm{~S}\right.$ ) (10 to 20 drops in Ioo c.c.); (2) a saturated solution of copper sulphate; and (3) ordinary strong sulphuric acid.

Have ready a glass beaker containing water briskly boiling. Place about 5 c.c. of strong sulphuric acid in a test-tube, with I drop of the copper sulphate solution.* Add to the mixture a few drops of the solution to be tested, and sliake well. $\dagger$

(In the case of the muscle extracts the dry residues are dissolved in the 5 c.c. of strong sulphuric acid, the acid transferred to test-tubes, and the test procecded with by the addition of the copper sulphate solution, ete.)

Now place the test-tube in the boiling water for one to two minutes. Then cool it well under the cold-water tap, and add 2 or 3 drops of the thiopliene solution from a pipette. Replace the tube in the boiling water, and immediately observe the colour. If lactic acid is present, the liquid rapidly takes on a bright cherry-red colour, which is only permanent if the test-tube be cooled immediately after its appearance. The tube should always be cooled as described, before addition of the thiophene, as the gradual appearance of the colour on re-warming makes the test more delicate.

(The extract of the resting limb generally gives a negative, that of the other a strongly positive, reaction.)

* The copper sulphate is added to hasten the oxidation that follows.

$\dagger$ For practice use a I per cent. alcoholic solution of lactic acid. The test cannot be applied directly to material which chars with the strong sulpluuric acid used. In this case preliminary extraction of the lactic acid is necessary. Alcohol should be used as the solvent, or if ether is employed it must first be well washed to remove aldehyde-yielding products, since the colour-change is due to an aldehyde reaction with thiophene. 


\section{CHAPTER XV}

\section{ELECTRO-PHYSIOLOGY}

A LJTTLE more than a hundred years ago the foundation both of electro. pliysiology and of the vast science of voltaic electricity was laid by a cliance observation of a professor of anatomy in an Italian garden. It is indeed true that long before this electrical fishes were not only popularly known, but the slıock of the torpedo had been to a certain extent scientifically studied. But it was with the discorcry of Galvani of Bologna that the epcch of fruitful work in electro-physiology began. Engaged in cxperiments on the effect of static and atmospheric electricity in stimulating animal tissues, he happened onc day to notice that some frogs' legs, suspended by copper hooks on an iron railing, twitched whencver the wind brought them into contact with one of the bars (p. 842). He concluded that electrical charges were developed in the animal tissues themselves, and discharged when the circuit was completed. Volta, professor of physics at Pavia, fixing his attention on the fact that in Galvani's experiment the metallic part of the circuit was composed of two metals, maintained that the contact of these was the real origin of the current, and that the tissues served merely as moist conductors to complete the circuit; and after a controversy lasting for more than a decade, he finally clinched lis argument by constructing the voltaic pile, a series of copper and zinc discs, every two pairs of which were separated by a disc of wet cloth, or paper moistened with salt solution. The pile yielded a continuous current of electricity. 'So,' said Volta, 'it is clear that the tissue in Galvani's experiment only acts the part of tlie cloth.' Galvani, however, had shown in the meantime that contraction without metals could be obtained by dropping the nerve of a preparation on to the muscle (p. 842); and it soon began to be recognized that both Galvani and Volta were in part right, that the tissues produce electricity, and that the contact of different metals does so too. Although it is curious to note how completely the growth of that science of which Volta's discovery was the germ has oversliadowed the parent tree planted by the hand of Galvani, yet animal electricity has been deeply studied by a large number of observers, and many interesting and important facts have been brought to light.

Since it is in muscle and nerve that the phenomena of electrophysiology are seen in their simplest expression, and have been chiefly studied, we shall develop the fundamental laws with reference to muscle and nerve alone, and afterwards apply them to other excitable tissues.

I. All points of an uninjured resting muscle or nerve are approximately at the same potential (or iso-electric). In other words, if any 
two points are connected with a galvanometer by means of unpolarizalsle electrode's, little or no current is indicated. (Although it is scarcely pussible to isolate a muscl. witlout its showing some current, the more carefully the isolation is performed, the feebler is the current; and between two points of the inactive, uninjured ventricle of the frog's heart no electrical difference has been found. Frogs' nerves kept ten to twenty hours after excision in physiological salt solution to which a little calcium salt and frog's blood have been added, are absolutely iso-electric.)

2. Any uninjured point of a resting muscle or nerve is at a different potential from any injured point. The difference of potential is such that a current will pass through the galvanometer from uninjured to injured point and through the tissue from injured to uninjured point (current of rest, or demarcation current, or injury response) (Fig. 292).

3. Any unexcited point of a muscle or nerve is at a different potential from any excited point, and any less excited point is at a different

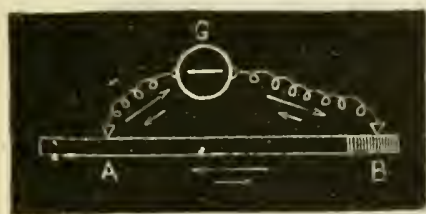

Fig. 292.-A, uninjured, B, injured, portion of nerve; $G$, galvanometer. The large arrows show direction of demarcation current or current of rest, the sinall arruws direction of negative variation or action current.

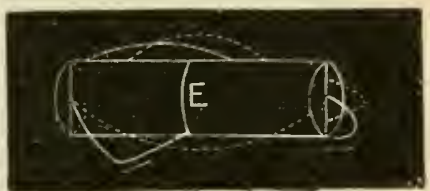

Fig. 293. - Diagram of Currents of Rest in a Regular Muscle, or Muscle Cylinder. E, equator. The dotted lines join points at the same potential, between which there is no current.

potential from any more excited point. The difference of potential is such that a current will pass through the galvanometer to the excited from the unexcited or less excited point (action current, or negative variation, or excitatory electrical response).

It has been customary in physiological writings to speak of the electrical change in injured or active tissue as a negative one, because when the tissue is led off to a galvanometer the current passes from the galvanometer to the injured or excited portion of the tissue. It may be called with greater precision 'galvanometrically negative.' It is in this sense that we shall employ the term.

The best object for experiments on the demarcation current is a straight-fibred muscle like the frog's sartorius. If this muscle be taken. and the ends cut off perpendicularly to the surface, a muscle-prism or muscle-cylinder is obtained (Fig. 293). The strongest current is got when one electrode is placed on the middle of either cross-section, and the other on the 'equator'-that is, on a line passing round the longitudinal surface midway between the ends. The direction of this current is from the cross-section towards the equator in the musele. If the electrodes are placed on symmetrical points on each side of the equator, there is no current. 
Current of Action, or Negative Variation.-When a muscle or nerve is excited, an electrical change sweeps over it in the form of a ware. Suppose two points, A and B (Fig. 294), on the longitudinal surface of a muscle to be connected with a capillary electrometer (p. 729), the movements of the mercury being photographed on a travelling surface-for example, a pendulum carrying a sensitive plate. I.et the muscle be excited at the end, so that the wave of excitation will be propagated in the direction of the arrow. The wave will reach $A$ first, and while it has not yet reached $B$, A will

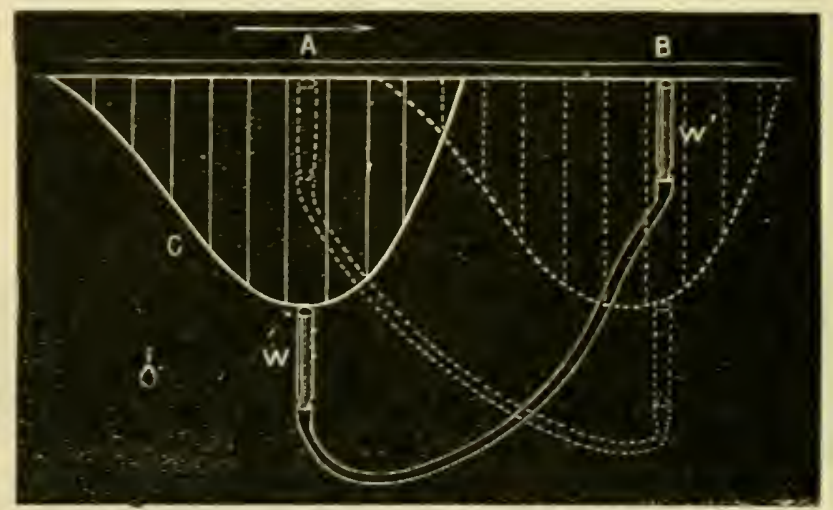

Fig. 291.--Jiagram to illustrate Propagation of the Electrical Change ilung an Active Muscle or Nerve. Suppose AB to be a horizontal bar representing the muscle or nerve. Let $\mathrm{C}$ be a curved piece of wood representing the curve of the electrical change at any point. Let $W, W^{\prime \prime}$ be two glass cylinders connected by a flexible tube, the whole being filled with water. Suppose the rims of the cylinders originally to touch $\mathrm{AB}$ at the points $\mathrm{A}$ and $\mathrm{B}$, and let them be movable only in the vertical direction. The level of the water being the same in both. there is no tendency for it to flow from one to the other. This represents the resting state of the tissue when $\mathrm{A}$ and $\mathrm{B}$ are symmetrical points. Now let $\mathrm{C}$ be moved along the bar at a uniform rate The cylinder $W$, being free to move down, but not borizontally, will be displaced by $C$, and, if it is kept always in contact with its curved margin, will, after describing the curve of the electrical variation, come again to rest in its old position at $\mathrm{A}$. B will do the same when $\mathrm{C}$ reaches it. But since $C$ reaches $A$ before $B$, the level of the water in $B$ will at tirst be higher than that in $A$, and water will flow from $B$ to $A$ as the current lluws through the galvanometr. This will correspond to the time during which the point of the tissue represented by $A$ would be galvanometrically negative to a point represented by B. Later on, when $C$ has reached the position sluwn by the dotted lines, the level of the water in $A$ will be higher than that in $\mathbf{B}$, and $\mathrm{d}$ flow will take place in the opposite direction to the tirst flow. This corresponds to a second phase of the electrical variation.

become negative to $B$. If there is a resting difference of potential between $A$ and $B$, this will be altered, the new and transitory difference adding its(if ilgebraically to the old. When the wave reaches $B$, it may already have passed over $A$ allogether, and $B$ now becoming negative to $A$, there will be a movement of the meniscus of the electrometer in the opposite direction. This is called the 
diphasic current of action. If the wave has not passed over $\mathrm{A}$ before it reaches $\mathrm{B}$, as would in general be the case in an actual experiment, there will be first a period during which $\mathrm{A}$ is relatively negative to B (first plrase); this will end as soon as B has become iso-electric with $\mathrm{A}$, and will be succeeded by a period cluring which $\mathrm{B}$ is relatively negative to $\mathrm{A}$ (second phase). Since the wave takes time to reach its maximum, it is evident that a well-marked first phase will be favoured when the interval between its arrival at $A$ and at $B$ is long, for in this case A will have a clance of becoming strongly negative while $\mathrm{B}$ is still normal. Similarly, if A has again become normal, or nearly normal, before the maximum negative clange has passed over B, a strong second phase will be favoured. The heartmuscle, accordingly, where the wave of contraction, and its accompanying electrical cliange, move with comparative slowness, is better suited for showing a well-marked diphasic variation than skeletal muscle, and'still better suited than nerve. In the gastroc-

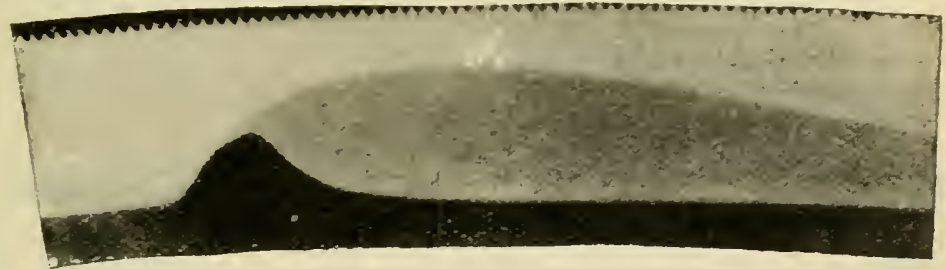

Fig. 295.-Photugraphic Electrometer Curves from Sartorius Wuscle (Sanderson). The darkly-shaded curve represents the diphasic variation of the uninjured muscle; the lightly-shaded curve the monophasic variation of the muscle after injury of one end. The toothed curve at the top is the time-tracing registered by photographing the prong of a tuning-fork vibrating five hundred times a second.

nemius muscle of the frog, when excited through its nerve, the electrical response begins about $\frac{4}{100 \pi}$ second, and the change of form of the muscle about $\frac{8}{1000}$ second, after the stimulation. It is believed that in a muscle directly excited the electrical change begins in less than $\frac{1}{1000}$ second, and the mechanical change in $\frac{4}{1000}$ second (Burdon Sanderson, Figs. 295-300).

When one electrode is placed on an injured part, the wave of action and of electrical cliange diminishes as it reaches the injured tissue; and if the tissue is killed at this part, it diminishes to zero; so that here the second phase may be greatly weakened or may disappear altogether, and we then have what is called a monophasic variation.

In this case the current of action can be demonstrated, even for a single excitation, but still better for a tetanus, with an ordinary galvanometer, which in general is not quick enough to analyze a diphasic variation with equal phases, and gives, therefore, only their algebraic sum - that is, zero. When the muscle or nerve is tetanized, the action current appears, while stimulation is kept up, as a permanent deflection representing the 'sum' of the separate effects. It is in the opposite direction to the current of rest, since the injured tissue, being less 
affected by the excitation, and therefore undergoing a smaller negative change than the uninjured, becomes relatively to the latter less negative. Appearing as a diminution or reversal of the current of rest, it was ealled the negative variation. The term negative is not used here in its electrical, but in its algebraic, sense, and merely as indicating the direction of the current with reference to that of the demarcation current. It is in this sense that 'negative variation' and the converse term, 'positive variation,' are used (pp. 838, 839) in speaking of the electrical changes produced in glands and in the retina by stimulation.

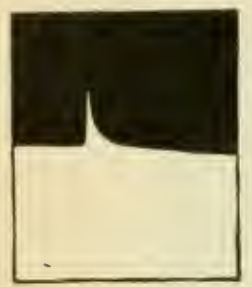

A.

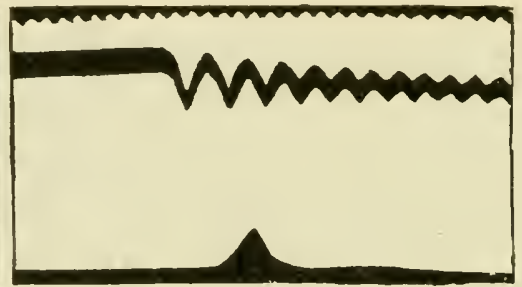

B.

Fig. 206.- 'Spike' (Diphasic Variation) of Uninjured Gastrocnemius (Sanderson).

A photographed on slow, B on fast-moving, plate.

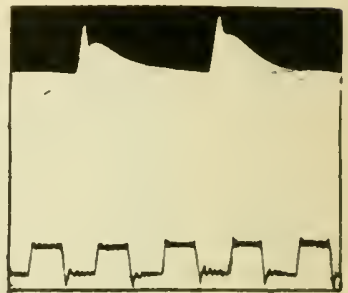

Fig. 297.-Variation of In. jured Gastrocnemius (San. derson). A 'spike' fullowed by a 'huinp.'

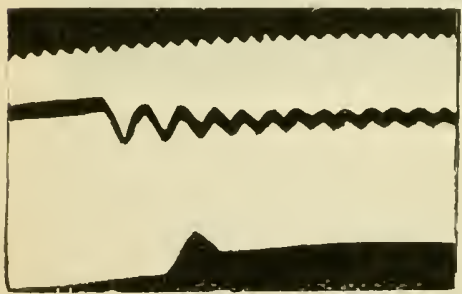

Fig. 298.-Variation of Injured Gas. trocnemius (Sanderson). The plate was moving ten times faster than in Fig. 297.

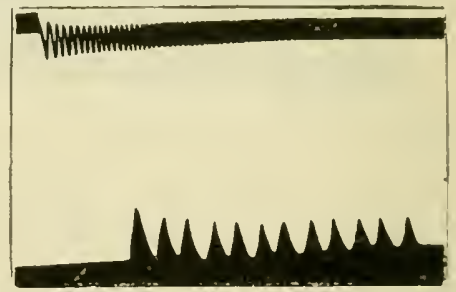

Fig. 299.-Variation of Uninjured Muscle excited Eighty-Four Tines a Second (Sanderson).

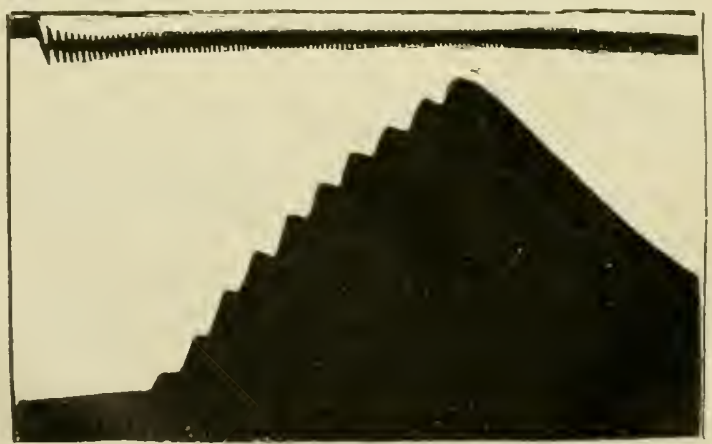

Fig. 300.-Curve of an Injured Muscle excited Sixty Times a Second (Sanderson). 
When the current of rest is compensated by a branch of an external current just sufficient to balance it and bring the galvanometer image back to zero (Fig. 234, p. 727), the action current appears alone in undiminished strength. This shows that the latter is not due to a change of electrical resistance during excitation, since such a change would equally affect current of rest and compensating current, and they would still balance each other. The action current is really due to a change of potential, which can be measured by determining what electromotive force is just required to balance it, and which may actually exceed that of the current of rest. Thus, Sanderson and Gotch obtained an average of 0.08 of a Daniell cell (the electromotive force of the Daniell would be about a volt) as the electromotive force of the action current due to a single indirect excitation of a vigorous frog's gastrocnemius, and about 0.04 Daniell as that of the current of rest. The electromotive force of the current of rest in the rabbit's nerve was found by du Bois-Reymond to be $0 \cdot 026$; Gotch and Horsley found the average for the cat 0.01 , and for the monkey only $0^{\circ} 005$.

That the fusion of the successive variations of a tetanized muscle, as seen with an crdinary galvanometer, is only apparent has been shown by means of the capillary electrometer or the string galvanometer. Even with a frequency of stimulation far beyond what is necessary for complete tetanus, each stimulus is answered by a movement of the meniscus (Figs. 299, 300). In nerve, also, each of two successive stimuli causes its appropriate electrical change when they are separated by an interval longer than a certain small fraction of a second. The precise interval at which the second stimulus ceases to be effective depends on the temperature of the nerve, being markedly increased by cold (Gotch and Burch).

The rate of propagation of the electrical change in muscle is the same as that of the mechanical change, and in nerve the same as that of the nervous impulse. The velocity of propagation of the diphasic variation along a fresh sartorius at $14^{\circ} \mathrm{C}$. was in one experiment 2.8 metres, in another at $\mathrm{r} 8^{\circ} \mathrm{C} ., 3.5$ metres (Sanderson). (See p. 759.) Lucas has pointed out that in strict accuracy what is observed is merely that the time interval separating contraction at one point of the muscle from contraction at another is equal to the time interval separating the electrical changes which occur at the same points. The facts observed do not formally prove that either the contraction or the electrical disturbance is propagated at all. So far as they go, some other perfectly distinct change may be propagated, which at all points of the fibre at which it arrives sets 11 p both the contraction and the electrical change. Such direct evidence, however, as we possess goes to show that it is the electrical disturbance which is the propagated one, and that this evokes the contractile disturbance.

There is ample evidence that the excitatory electrical response is a normal physiological phenomenon. In human skeletal muscles the current of action has been demonstrated by connecting a galvanometer with ring electrodes passing round the forearm, and throwing the muscles into contraction. A diphasic variation is thus obtained; and the electrical change travels with a velocity of as much as twelve metres per second, which is greater than the velocity in frogs' muscles. Electromotive changes are likewise associated with the beat of the heart. Action currents have also been detected in the phrenic ne"ves of living animals accompanying the respiratory 
discharge (Reid and Macdonald), in the vagi accompanying the movements of the lungs, in the œsophagus during swallowing, in the cutaneous sensory nerves in response to the ' adequate 'stimulus of pressure (Steinach), in the retina in response to the adequate stimulus of light, in glands during secretion, in the central nervous system during the passage of impulses along its conducting paths. Some of these will be further considered a little later on.

As to the interpretation of the facts we have been describing, and which are summed up in the three propositions on p. 823, two chief doctrines long divided the physiological world: (1) the theory of du Bois-Reymond, the pioneer of electro-physiology, and (2) the theory of Hermann. It was believed by du Bois-Reymond that the current of rest seen in injured tissues is of deep physiological import, and that the electrical difference which gives rise to it is not developed by the lesion as such, but only unmasked when the electrical balance is upset by injury. He looked upon the muscle or nerve as built up of electromotive particles, with definite positive and negative surfaces arranged in a regular manner in a sort of ground-substance which is electrically indifferent. The 'negative variation' he supposed to depend on an actual diminution of previously existing electromotive forces; and from this conception arose its historic name. Hermann and his school assumed that the uninjured muscle or nerve is 'streamless,' not because equal and opposite electromotive forces exactly balance each other in the substance of the tissue, but because electromo tive forces are absent until they are called into existence (by chemical changes) at the boundary, or plane of demarcation, be-

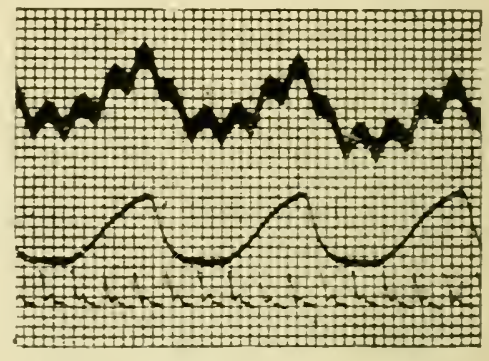

Fig 3or.-Upper Curve, Record of the Electrical Changes in the Vagus Nerve (so-called 'Electrovagogram '). taken with the String Galvanometer. The small waves on it are synchronous with the heart-beats, while the large waves are synchronous with the respiratory movements, the mechanical record of which constitutes the second curve (ascent, inspiratiol.). The lowest curve is a mechanical record of the pulse (Eintlioven).

tween sound and injured tissue. For this reason du Bois-Reymond's current of rest is called in the terminology of Hermann the 'demarcation ' current.

The newer theories, such as Macdonald's, have sought to take account of the recent developments of physical chemistry, and it is unquestionable that it is here the real explanation is to be found. There is little doubt that the electrical phenomena of the tissues are connected with the existence in them of membranes, en relopes, or sheaths, physiological if not always anatomical, which are relatively impermeable to certain ions. When such a sheath is injured, these ions, carrying with them their electrical charges, may be supposed to migrate with abnormal freedom through the injured part. A new distribution of electricity is thus establisbed in the tissue, and differences of potential depending upron differences in the concentration of the ions at different points are set up. Bernstein and Tschermak, from an investigation of the thermodynamic relations of bio-electrical currents, have come to the conclusior that they are analogous to the currents produced by so-called concentra- 
tion cells-i.e., arrangements of solutions of electrolytes of different concentration in contact with each other. Since the development of the new electrical condition depends upon the fundamental structure of the tissue, these modern views lead us back to du Bois-Reymond's doctrine of a pre-existing electrical equilibrium connected with the essential physiological properties of muscle or nerve. But instead of his electromotive elements and their definite arrangement, we have the ions and their definite relaticn to the semi-permeable membranes.

Relation between the Action Current and Functional Activity.Although the negative variation is so general an accompaniment of excitation, and is even within tolerably wide limits, in muscle and nerve at least, pretty nearly proportional to the strcngth of the stimulus, it is at present impossible to say definitely what the chemical or physical changes are which underlie it. Unquestionably the electrical changes are closely related to the excitatory process and to the functional activity of the tissues. In the case of nerve some writers, indeed, assume that the redistribution of potential asscciated with the excited state is identical with the nervous impulse, but the common view is that the negative varialion is an accompaniment of some other change which constitutes the propagated disturbance. There is at present no clear experimental evidence sufficient to decide the question. From time to time attempts have been made to show that the two processes can be dissociated, but none of the experiments so far reported are realiy crucial.

like the demarcation current, the action current and the excitation which accompanies it may be due to changes in the permeability of membranes or changes in the concentration of ccrtain ions.

Although the electromotive changes caused by excitation are much more transient than those caused by injury, everything suggests that there must be some deep analogy between the two conditions. Some have supposed that what may be called a subdued and more or less permanent excitation exists in the neighbourhood of the injured tissue, an excitation which, like some other forms, does not spread, and that this cxplains the similarity of electrical condition in activity and injury.

It is, of course, clear that energy must be transformed to produce an electromotive force capable of doing work. It may be assumed that this energy is ultimately derived from the stock of chemical energy in the tissue-substance. But whether in the final transformation the electrical phenomena are the expression of chemical changes or of physical (osmotic) changes, or of both, we do not know. In the case of muscle it is possible that the liberation cf lactic acid, which there are several reasons for regarding as essentially concerned in the initiation of the mechanical change, is associated in some way with the appearance of the negative variation. It is known that the latter, although it begins before the contraction, and very rapidly reaches its maximum, declines more gradually, so that it overlaps the mechanical change of form. This is particularly well seen in veratrinized muscles (p. 755), in which the electrical variation, like the contraction, is greatly prolonged (Garten).

Polarization of Muscle and Nerve.-We have already spoken of electrical excitation and of the changes of excitability caused by the passage of a constant current ( $p .785$ ). We are now to see that these physiological effects are accompanied by, and indeed very closely related to, more physical changes which the galvanometer or electrometer reveals to us. Since these throw light on the 
physical, and therefore ultimately on the pliysiological, structure of the tissues, they have been deeply studied, especially in nerve. There is no question that they depend upon the presence in the tissues of membranes presenting a relatively great resistance to the passage of ions. When a current is passed by means of umpolarizable electrodes (Fig. 238, p. 73I) through a muscle or nerve for several seconds, and the tissue connected to the galvanometer immediately after this polarizing current is opened, a deflection is seen indicating a current (negative polarization current) in the opposite direction.

This (negative) polarization. like the polarization of the electrodes seen after passage of a current throngh any ordinary electrolytic conductor, dilute sulphuric acid, e.g., depends on the liberation of ions (p. 429) at the kathode and anode. It is seen not only in muscle, nerve, and other animal tissues, but also in vegetable structures, and indeed, to a certain extent, in unorganized porous bodies soaked with electrolytes. In muscle and nerve, however, it is particularly well marked; and although it is not bound up with the life of the tissue, and may be obtaincd when this has become quite inexcitable, it is nevertheless dependent on the preservation of the normal structure, for a boiled muscle shows but little negative polarization.

When the polarizing current is strong, and its time of closure short, we obtain, on connecting the tissue with the galvanometer after opening the current, not a negative, but a positive deflection, indicatir.g a current in the same direction as that of the polarizing stream. This is really an action stream, due to the opening excitation set up at the anode (p. $7 f^{1}$ ). It is cnly obtained when the tissue is living, and is far more strongly marked in the anodic than in the katlodic region.

Suppose that the nerve in Fig. 302 is stimulated by the opening of the battery $B$, and that, immediately after, the nerve is connected with the galvanometer $G$ by the electrodes $E, E_{1}$. Suppose, further, that the shaded region near the anode remains more excited for a short time than the rest of the nerve, and we have seen (1). 787 ) that after the opening of a strong current there is a defect of conductivity, especially in the neighbourlioed of the anode, which would tend to localize excitation. An action current will pass through the galvanometer from $\mathrm{E}_{1}$ to $E$, and through the nerve in tlie same direction as the original stimulating stream. Under certain conditions a state of continuous excitation in the ancdic region of a nerve is shown by a tetanus of its muscle (Ritter's tetanus, p. 74I, and Fig. 303).

Electrotonic Currents. - If a current be passed from the battery through a medullated nerve (Fig 304 ) in the direction indicated by the arrows, while a galvanometer is connected with either of the extrapolar areas, as shown in the figure, a current will pass through the galvanometer, in the same direction in the nerve as the polarizing current, so long as the latter continues to flow.

These currents are called electrotonic (in the katlodic region katelectrotonic; in the anodic, ane?ectrotonic). The exact mode of their production is obscure. Similar currents can be detected in artificial models consisting of a good conducting core and a badly conducting envelope; for example, a platinum wire in a glass tube fillod witl saturated zinc sulpliate solution, or a zinc wire covered with cotton-wool soaked in salt solution. In such models it appears to be essential that there 
should be polarization (separation of ions) at the boundary between the core and the sheath-i.e., between the wire and the liquid, where the current passes from the one to the other.

A current led into the sheath tries, so to speak. to pass mostly by the good ccnducting wire. If this is not polarizable-if it is, e.g., a.

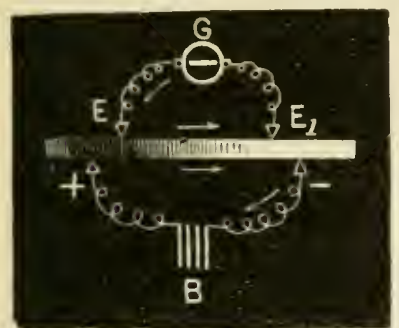

Fig. 302.-Diagram to slow Distribution of 'Positive Pularzation' after opening Polar. izing Current. B, battery; G, galvanometer. The dark shading signifies that the excitation to which the current causing the positive deflection after the opering of the polarizing current is due is greatest in the immediate neighbour. hood of the anode, and fades away in the intrapolar region. + indicates the anode, and - the kathode of the polariz. ing current.

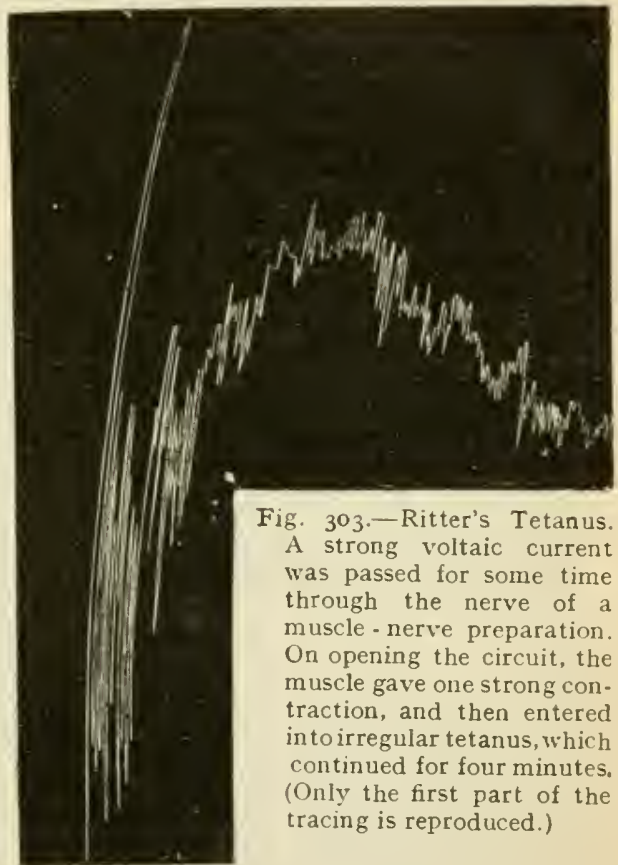

zinc wire surrounded by saturated zinc sulphate solution-there is little or no spreading of the current outside the electrodes: it passes at once into the core, and so on to the othe electrode. If, lowerer, there is polarization when the current passes from the liquid into the wire. as is the case in the platinum-zinc sulphate or the zinc-sodium chloride

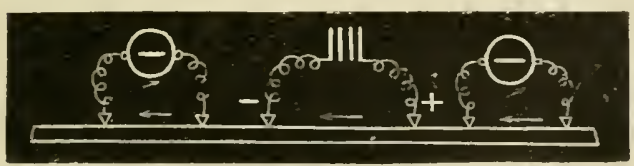

Fig. 304.-Diagram showing Direction of the Extrapolar Electrotonic Currents. + is the anode and - the kathode of the polarizing current. combinations, the stream spreads longitudinally in the sheath, since the polarization introduces a virtual resistance at the surface of the wire, in comparison with which the difference in resistance of an oblique and a direct transverse path through the liquid becomes small. It has been supposed that in medullated nerve a similar polarization takes place at the boundary between some part of the nerve-fibre which may be called a core, and another part which may be called a sheatl-for instance, between the axis-cylinder and the medullary sheath, or between the latter and the neurilemma. It is known that the electrical resistance of nerve in the 
transverse direction is much greater (five to seven times)* than the longitudinal resistance. Since a rapidly-established polarization would, by the ordinary methorls of measurement, appear as a resistance, this has been adduced as evidence of the great capacity of nerve for polarization by a current passing across the fibres. It is, however, probable. from what we know of the high electrical resistance of the physiological envelopes of such cells as the red blood-corpuscles (p. 26), that the great transverse resistance of nerve, and indeed the electrotonic currents, are due in part, if not wholly, to the true resistance of one or more of its envelopes (perhaps the medullary sheath). Examples of such differences of resistance even in the fluid constituents of one and the same animal structure are not wanting. For instance, the specific resistance of the yolk of a hen's egg may be three times greater than that of the white.

The electrotonic currents cannot spread beyond a ligature; they are stopped by anything which destroys the structure of the tissue; they are affected by various reagents. But this does not prove that they are other than physical in origin, for what destroys the structure of the tissue or modifies its molecular condition may destroy or diminish its capacity for polarization, or alter its electrical resistance.

There are, however, certain facts which indicate that physiological factors, as well as physical, are concerned. While the currents obtainerl from core-models show a general resemblance to the electrotonic currents of medullated nerve, there is one significant clifference: in the former the katelectrotonic and anelectrotonic currents are of equal intensity; in the latter the anelectrotonic preponderates. The most probable ex. planation is that the anelectrotonic current of medullated nerve is made up of two distinct electrical effects, one physiological in nature, the other dependent merely on the structure and physical properties of the fibres while the katelectrotonic current is wholly physical. It is in favour of this hypothesis that under the inflinnce of ether, which abolishes the physiological functions of nerve, the anelectrotonic current diminishes till it becomes equal to the kateletrotonic. Non-medullated nerves, in which the conditions for physical clectrotonus, if present at all, are only feebly developed, and which exhibit no katelcctrotonic current, or only' a very weak one, show an anelectrotonic current, which is abolished by ether, and secms to represent the physiological portion of the anelectrotonic current of medullated nerve.

A nerve may be stimulated by an electrotonic current producer in nerve-fibres lying in contact with it. A well-known illustration of this is the experiment known as the paradoxical contraction (Practical Excrcises, p. 841).

The current of action of a nerve can also stimulate another nerve when the cxcitability of both is greater than normal, as is the case in the nerves of frogs kept in the cold. This comes under the head of secondary contraction. But the best-known form of secondary contraction is where a nerve, placed on a muscle so as to touch it in two points (Fig. 305), is stimulated by the action-current of the muscle, and causes its own muscle to contract. A secondary tetanus

* Since a part of the current is conducted by the connective tissue and other structures lying between the nerve-fibres, and the longitudinal and transverse resistance of these tissues may be supposed equal, the disproportion between the longitudinal and transverse resistance of the nerve fibres them. selves is probably much gi'eater than this. 
can be obtained in this way by dropping a nerve on an artificially tetanized muscle. The beat of the heart causes usually only a single secondary contraction when the sciatic nerve of a frog is allowed to fall on it (p. 203). But when the diphasic variation is well marked, as it is in an uninjured heart, there may be a secondary contraction for each phasei.e., two for each heart-beat. Excitation of one muscle may in the same way cause secondary contraction of another with which it is in close contact.

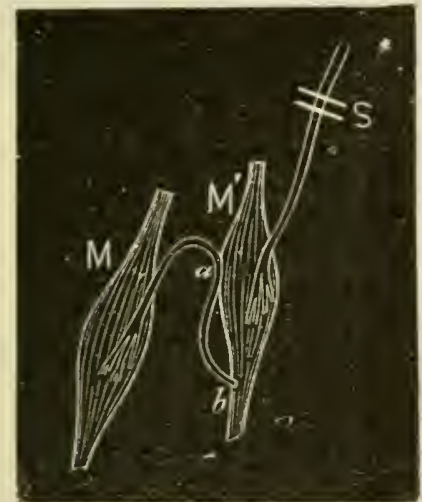

Fig. 305. - Secondary Contraction. The nerve of muscle M touches muscle $\mathrm{M}^{\prime}$ at $a$ and $b$. Stimulation of the nerve of $\mathrm{M}^{\prime}$ at $\mathbf{S}$ causes contraction of $M$.

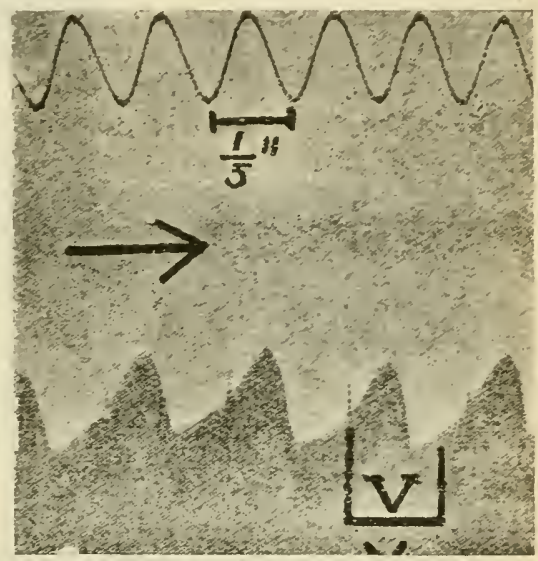

Fig. 306.-Electrometer Record from Rabbit's Heart (Gotch). The heart was exposed and beating in situ. Contarts, one on base of right ventricle, the other on right apex. The commencement of the beat is on the left-hand edge of the dark line $V$. The length of the dark line shows the duration of the beat. Upwaid movement signifies re!ative negativity (activity) of the part at or near the base contact. Time-trace at top, one-fifth second.

The electromotive phenomena of the heart and of the central nervous system are naturally included under those of muscle and nerve.

Heart.-Records of the electrical changes obtained with the capillary electrometer or string galvanometer from the exposed ventricles vary in certain details with the position of the two contacts. When one contact is on the base of the ventricles (in the rabbit) near the auriculo-ventricular groove, and the other on the apex (Fig. 306), for each beat of the ventricle the electrometer record shows (I) a sharp rise, indicating relative negativity (activity) of the base; (2) an equally sharp fall, indicating relative negativity at the apex; (3) a slower but marked rise, indicating an increase or a fresh development of relative negativity at the base; (4) a more rapid fall, which returns first slowly, then quickly, until (I) follows again (Gotch). The time between the beginning and the top of rise (I) is bəlieved to correspond to the time of transmission of the 
active state from the base to the apex. The rate of propagation on the rabbit's ventricle varies from I to 3 metres a second, according to the rate of the heart-beat. Such observations have been interpreted as indicating that the excitation, with its accompanying electrical change, begins at the base, then develops in the region of the apex, and finally involves the poition of the ventricles near the aorta and pulmonary artery, possibly extending even into the roots of these vessels. The full explanation of this seemingly erratic course of the excitation wave is doubtless dependent upon a full knowledge of the course and connections of the conducting system,

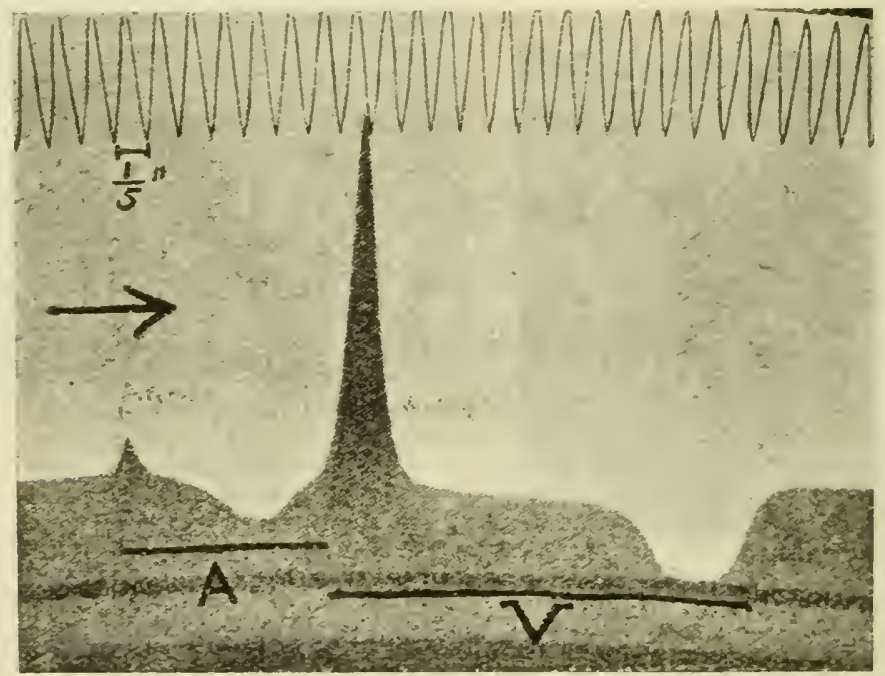

Fig. 307.-Electrnmeter Record from fortois? Heart (Gotcl.). Ore contact upon the sinus, the other on the apex of the ventricle. One complete beat shown. Upward movement signifies relative negativity of the sinus contact. The dark line $A$ shows the auricular efiect, and the dark line $V$ the ventricular effect. Time-trace at top, one-fifth second.

and this we do not possess as yet. The fact that the ventricle is originally developed from a tube with a venous and an arterial end, and that this tube later on becomes bent upon itself so that the two ends (the auricular or venous and the aortic or arterial) lie together at the base of the ventricle, probably affords the clue. It should be mentioned, however, that this explanation of the second rise of the ventricular curve (the T-wave, according to Einthoven's nomenclature, Fig. 3I3) is by no means universally accepted. Einthoven, who has worked with the string galvanometer, believes that between the first (R) and the second (T) ventricular waves the whole of the ventricle is in contraction, and that there is no difference of 
potential at this time between its various parts. The $\mathrm{T}$-wave he considers to be produced, when it is present, merely because the
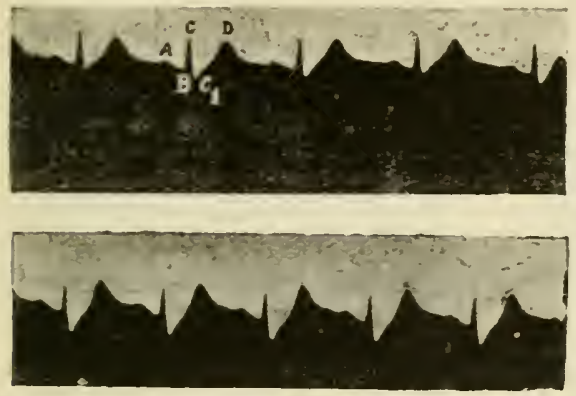

Fig. 308.-Electro-Cardiograms from Man (Capillary Electrometer) (Einthoven and Lint). Obtained from the same individual at rest (upper curve), and immediately after vigorous muscular exercise (lower curve). The elevations A, C, D, indicate negativity of base to apex; the notches $B$ and $C_{1}$, negativity of apex to base. this may be demonstrated by means of the capillary electrometer.

Einthoven and Lint then investigated the phenomenon on a large number of persons. From the photographic records of the movements of the meniscus they constructed the true electro-cardiographic curves* (Fig. 309), which express the actual changes in the potential difference between the two points led off. They distinguished in every one of these constructed electro-cardiograms five points or cusps, three of which indicate relative negativity excitated state does not disappear simultaneously over the whole ventricle.

In the ventricle of the frog and tortoise the same order of development of the negative change is seen, the base first becoming relatively negative, then the apex, and then the neighbourhood of the origin of the aorta (Fig. 307).

Under certain conditions the action current of the heart may stimulate the phrenic nerves, causing the diaphragm to contract synchronously with the heart.

The Human Electro-Cardiogram.-An electrical change
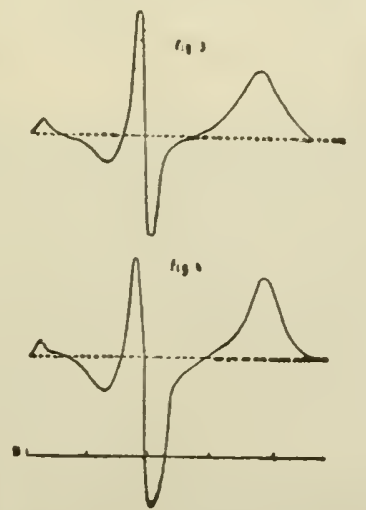

Fig. 309.-Constructed Elec. tro-Cardiograms from Man (Ein thoven and Lint). Time is laid off along the horizontal, and electromotive force along the vertical axis, the same space being allot-

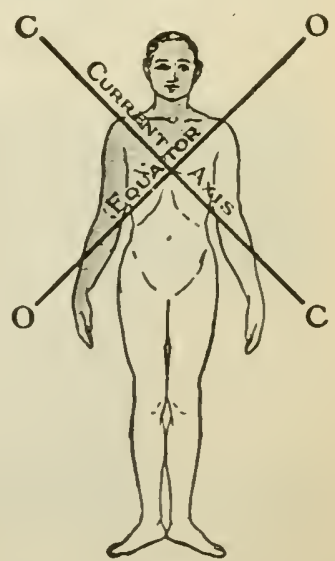

Fig. 3ro.-Illustrating the Position of Favourable and Unfavourable Leads for the Human ElectroCardiogram (Waller). ted to ten millivolts (i.e.,

* In all accurate work with the capillary electrometer such curves must be obtained by construction from the direct photographic records, which do not themselves give an absolutely true picture of the variations. 
of the base of the heart to the apex, and two negativity of the apex to the base. The capillary electrometer has now been superseded by the string galvanometer (1).726) for the investigation of the human electrocarliogram (Figs. 3II-3I5). But a sample of the records obtained by the former method (Fig. 3os), with the corresponding constructed
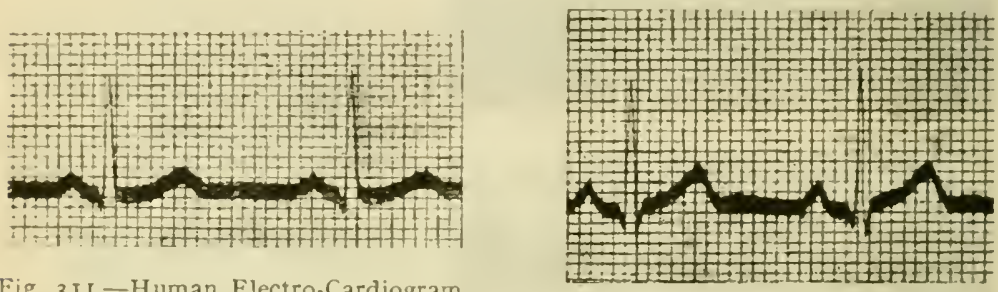

Fig. 3 I r.-Human Electro-Cardiogram (String Galvanometer) (Einthoven). Led off from the two hands. I $\mathrm{mm}$. of the abscissa corresponds to o.o second.

Fig. 3I2.-Human Electro-Cardiograin (String Galvanometer) (Einthoven). Led off from right hand and left foot.

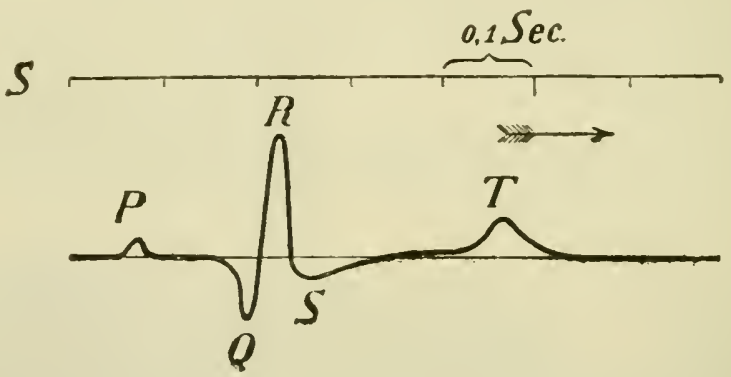

Fig. 313.-Schematic Representation of Electro-Cardiogram (String Galvanometer) (Einthoven). Five points are lettered at which the curve changes sign. P cor. responds to the auricular contraction; the other four are included in the ven. tricular cycle.

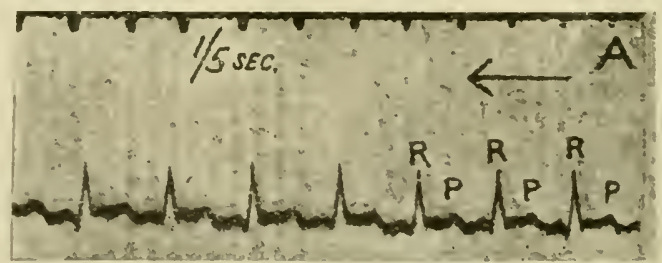

Fig. 314.-Electro-Cardiogram from Man (String Galvanometer) (Lewis). From a case of paroxysmal tachycardia. The heart-rate was 200 a minute. The upper notched line is the time-trace in one-fifth seconds.

electro-cardiograms, (Fig. 309) is reproduced for their historical interest. In their main features it is obvious that they agree with the records obtained by the string galvanometer. The electro-cardiograms are distinctly affected by exercise and by the position of the body, and very markedly in disease. The galvanometer may be connected with the two 
Irands, or, better, with the right hand and the left foot. The two feet are the most mifavourable combination. The reason is obvious from the direction of the long axis of the heart, which deternines the direction of the lines of flow of currents due to differences of potential hetween batse and apex (hig. 310 ).

Relation of the Waves of the Electro-Cardiogram to the Mechanical Events in the Cardiac Cycle. There is evillence that the excitation process with its associated clectrical change spreads over the auricle from the simus rode, followed at each point by the mechanical change or contraction, in such a manner that the portions nearer the node begin to relax before the more distal units have funished enntracting. When a tracing of the approximation of two clistant points of the auricle is taken, the total shortening represents the algebraic sum of the contractions and relaxations of all the muscular units between the two points.
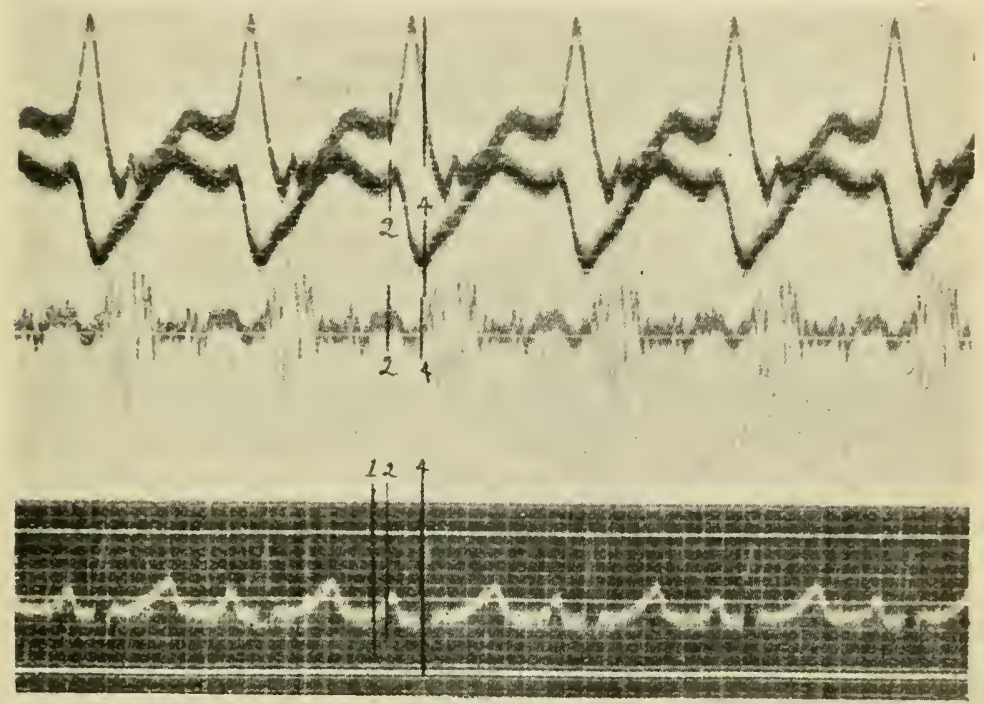

Fig. 315.-Upper curve, pressure in right auricle. Second curve from top, right auricular myogram; the down-stroke corresponds to contraction. Third curve, ventricular sounds. Bottom curve, electrocardiogram, lead II (left hind and right fore leg) (Wiggers).

Electrical effects obtained by leading off from the heart in any particular way must also be more or less complex resultants of the changes at different points. Certain general relations, however, have been established between the mechanical events and the electro-cardiogram. In Fig. 315 are shown simultancous records of the contraction of the auricle (auricular myogram), the intra-auricular pressure, the heart sounds and the electro-cardiogram (WViggers). The first electrical variation, commencing at 1 , is seen to precede the rise of pressure in the auricle, 2, by a definite interval (about $00^{\circ} 2 \mathrm{sec}$.), and the onset of the mechanical shortening, 3 , by a somewhat greater interval ( $0.03 \mathrm{sec}$.). The length of these intervals is, of course, not preciscly the same in different experiments.

The relation of the beginning of the rise of intra-auricular pressure and of the beginning of the mechanical systole of the auricles to the 
$P$ wave of the electro-cardiogram (the wave specially associated with the passage of excitation oser the auricle) is also slightly varialole. Koughly, the apex of the $P$ wave may be taken as indicating the onset of the auricular systole. The relation between the end of the auricular systole, 4 , and the electro-cardiogram, is still more variable, but in general it falls not far from the summit of the $R$ wave (a wave specially associated with the passage of the excitation over the ventricle). This shows that the auricular systole still contimues at a time when the excitation process has already made considerable progress in the ventricle. The mechanical contraction of the ventricle. as shown in Fig. 315, by the position of the vibrations corresponding to the first sound, follows very promptly the completion of the auricular systole.

Central Nervous System.- It was discovered by du Bois-Reymond that the spinal cord, like a nerve, shows a current of rest between longitudinal surface and cross-section, and that a current of action is caused by excitation. Setschenow stated that when the medulla oblongata of the frog was connected with a galvanometer, spontancous variations occurred which he supposed due to periodic functional changes in its grey matter. Goteh and Horsley have made experiments on the spinal cords of cats and monkeys. Leading off from an isolated portion of the dorsal cord to the capillary electrometer, and stimulating the 'motor' region of the cortex cerebri, they obtained a persistent negative variation followed by a serics of intermittent variations. This agrees remarkably with the muscular contractions in an epileptiform convulsion started by a similar excitation of the cortex, which consist of a tonic spasm followed by clonic or phasic (interrupted) contractions.

By means of the galvanometer, the same observers have made investigations on the paths by which impulses set up at different points travel along the cord. To these we shall have to refer again (p. 595).

Electrical Phenomena of Glands.-These have been studied with any care chiefly in the submaxillary gland and in the skin, although the

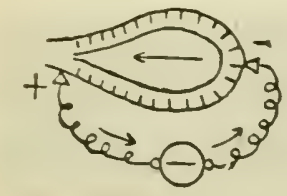

Fig. 316.-Current of Submaxillary Gland. liver, kidney; spleen, and other organs also show currents when injured. In the submaxillary gland the hilus is galvanometrically positive to any point on the external surface of the gland; a current passes from hilus to surface through the galvanometer, and from surface to hilus through the gland (Fig. 316). When the chorda tympani is stimulated with rapidly-succeeding shocks of moderate strength, there is a positive rariation-i.e., the hilus becomes still more positive to the surface. This variation can be abolished by a small dose of atropine.

Skin Currents.-So far as has been investigaterl, the integument of all animals shows a permanent current passing in the skin from the extermal surface inwards. This is feebler in skin which possesses no glands. In skin containing glands the current is chiefly, but not altogether, secretory. As such, it is affected by influences which affect secretion, a positive variation being caused by excitation of secretory nerves-e.g., in the pad of the cat's foot by stimulation of the sciatic. The deflection obtained when a finger of each hand is led off to the galvanometer, which was at one time looked upon as a proof of the cxistence of currents of rest in intact muscles, is due to a secretion current.

Of more doubtful origin is the current of ciliated mucous membrane, which has the same direction as that of the skin of the frog and the mucous membrane of the stomach of the frog and the rabbit-riz., from ciliated to under surface through the tissue, or from ciliated surface to cross-section, if that is the way in which it is led off. The current is 
strengthencel by induction shocks, by heating. and in gencral by infuences which increase the activity of the cilie. Some circumstances point to the golblet-cells in the menuloran: as the source of tlue current but, ou the whole, the balance of evidence is in favour of the cilia being the chicf factor (Engelmamm), although the mucin-secreting cells may be concerned, too. Electrical chaneses associated with secretion have been observed in the frog's tonguc on excitation of the glosso-pharyngeal nerve.

Eye-Currents.-If two unpolarizable electrodes connected with a galvanometer are placed on the cxcised eyc of a frog or rabbit, one on the cornea and the other on the cut optic nerve, or on the posterior surface of the cyeball, it is found that a current passes in the eye from optic nerve to cornea, the fundus of the cye being therefore negative as regards the cornea (Fig. 317 ). The current has the same direction if the anterior electrode is placed on the anterior surface of the retina itself, the front of the eycball being cut away, or if one electrode is in contact with the anterior and the cticr with the posterior surface of the isolated retina. There is nothing of spccial interest in this; but the important point is that if light be now allowed to fall upon the eye, or upon the isolated retina, characteristic electrical changes are caused. These are spoken of as the photoelectric reaction, and are best studied by means of the string galvanometer. The features of

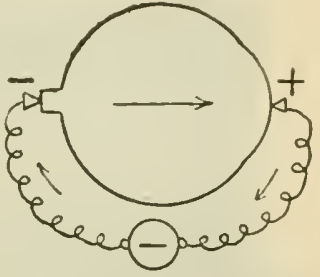

Fig. 317.-liye-Current. the curve representing the photo-electric reaction vary with the duration and intensity of the illumination and with the previous condition of the eye as regards illumination. A careful analysis of the curves obtained under different conditions supports the hypothesis that there occur in the eye three separate processes, which may for convenience be considered to dipend upcn the existence in the retina of three separate photo-chemical substances. When light of moderate intensity is allowed to act upon an eye which has not shorlly before been exposed

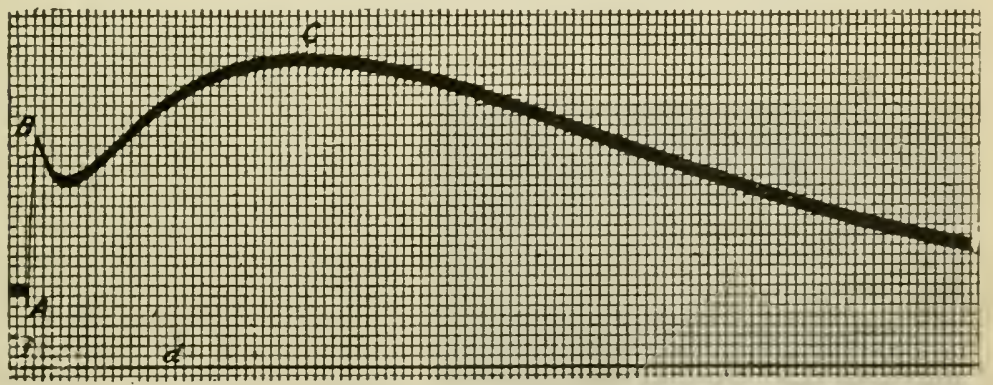

Fig. 3I8.-Photo-Electric Reaction of Frog's Eye (Einthoven and Jolly). The duration of the flash (of green light) was o*or second. The eye had been pre. viously in the dark. I millimetre of the abscissa corresponds to 0.5 second, I millimetre of the ordinate to Io microvolts. Curve to be read from left to right.

to strong light, a form of curve is obtaincd which seems to represent the combined reaction of the three substances (Einthoven and Jolly) (Fig. 318). After a latent period a small preliminary negative deflection $\mathrm{A}$ is observed (downward movement of the string). This is at once 
followed by a mivh larger upward movement (positive variations) in the same direction as the resting effect, the fundus becoming relatively more negative to the cornea than hefore. After the peak $B$ has been reached, the curve sinks first rapidly, then more gradually, but soon mounts again, and reaches a second maximum $C$, vlich is higher than $B$ (second positive variation). Finally, the curve descends to its original level.* The photo-clectric reaction is substantially the same in all vertebrate eyes hitherto investigated. In the cephaloped retina, too, the only important electrical cliange on jllumination is in the same direction as the resting effect.

The reaction depends 11 pon the retina alone, and docs not occur when it is removed. Bleaching of the visual purple does not much affect it, so that it is not connected with chemical changes in this substance. Its seat must be the layer of rods end cones, since in the

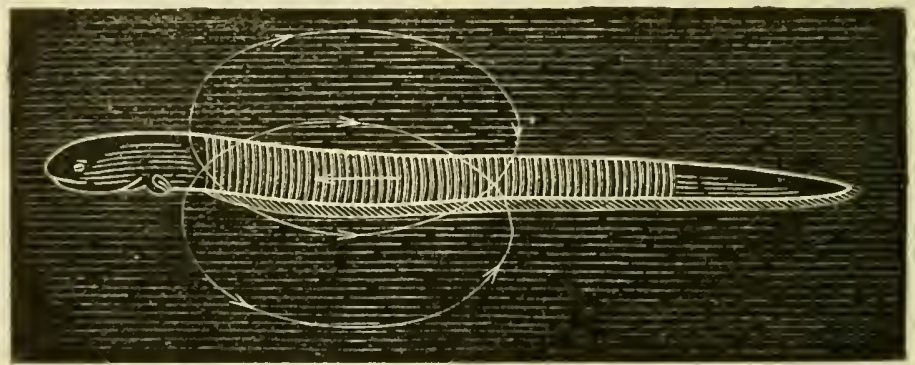

Fig. 3 19.-Diagram showing Direction of Shock in Gymnotus.

cephalopods the structure called the retina contains only this layer, the other layers of the vertebrate retina being represented in the optic nerve and ganglion (Beck). Of the spectral colours, yellow light causes the largest variation; blue, the least; but white light is more powerful than either (Dewar and McKendrick). (For'visual purple,' sec (hap. XVIII.)

Electric Fishes.-Except lightning, the shocks of these fishes were probably the first manifestations of electricity observed by man. The Torpedo, or clectrical ray, of the coasts of Europe was known to the Greclis and Romans. It is mentioned in the writings of Aristotle and

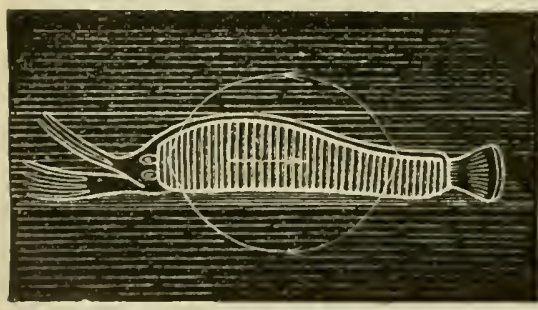

Fig. 320.-Diagiam showing Direction of Shock in Malapterurus. Pliny, and had the honour of being described in verse 1,500 years before Faraday made the first really exact investigation of the sliock of the Gymnotus, or clectric eel, of South America. Another of the electric fislies, Malapterurus clectricus, although found in many of the African rivers, the Nile in particular, and linown for ages, was scarcely investigated till fifty years ago.

In all these fishes there is a special bilateral organ immediately under the skin, called the electrical organ. It is in this that the sliock is developed. It consists of a series of plates arranged parallel to each other. To one side of each plate a branch of the electrical nerve supplying each lateral half of

* In the figure the last portion of the curve while it is still slowly descending has not been reprorluced. 
the organ is distributed, so that each half of the organ represents a battery of many cells arranged in serics.

In (iymnotus the plates are vertical, and at riglit angles to the long axis of the fish, and the nerves are distributed to their posterior surface; the shock passes in the animal from tail to head. In Malapterurus, although the direction of the plates is the same, and the nerve-supply is also to the posterior surface, the shock passes from head to tail.

In Torpedo, the plates or septa dividing the vertical hexagonal prisms of which each lateral half of the organ consists are horizontal; the nervesupply is to the lower or ventral surface; and the shock passes from belly to back through the organ. In all electric fishes the discharge is discontinuous; an active fish may give as many as 200 shocks per second.

The electrical nerve of Malapterurus is peculiar. It consists of a single gigantic nerve-fibre on each side, arising from a giant nerve-ccll. The fibre has an enormously thick sneath, the axis-cylinder forming a relatively small part of the whole; and the branches which supply the plates of the organ are divisions of this single axis-cylinder.

The electromotive force of the shock of the Gymnotus may be very considerable; and even Torpedo and Malapterurus are quite able to, kill other fish, their enemies or their prey. Indeed, Gotch has estimated the electromotive force of $\mathrm{I} \mathrm{cm}$. of the organ of Torpedo at 5 volts. Schönlein finds that the electromotive force of the whole organ may be equal to that of 3 I Danicll cells, or 0.08 volt for eacl, plate, and it is one of the most intercsting questions in the whole of electro-physiolıgy, how they are protected from their own currents. There is no doubt that the current density inside the fish must be at least as great as in any part of the water surrounding it, and probably much greater. The central nervous system and the great nerves must be struck by strong shocks, yet the fish itself is not injured; nay, more, the young in the uterus of the viviparous Torpedo

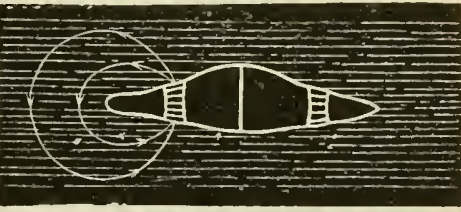

Fig. 32r.-Diagran showing Direc. tion of Shock in Torpedo.

are unharmed. The only explanation seems to be that the tissues of electric fishes are far less excitable to electrical stimuli than the tissues of other animals; and this is found to be the case when their muscles or nerves are tested with galvanic or induction currents. It requires cxtremely strong currents to stimulate them; and the electrical nerves are more casily excited mechanically, as by ligaturing or pinching, than electrically. In general, too, the shock is more readily called forth by reflex mechanical stimulation of the skin than by electrical stimulation. But that the organ itself is excitable by electricity lias been shown by Gotch. He proved that in Torpedo a current passed in the normal direction of the shock is strengthened, and a current passed in the opposite direction weakened, by the development of an action current in the direction of the shock. And, indeed, a single excitation of the electrical nerve is followed by a series of electrical oscillations in the organ, which gradually die away. The latent period of a single shock is about $\overline{1} \mathbf{0} 0$ second. The skate must be included in the list of electric fishes. Although its organ is relatively small, and its electromotive force relatively feeble, yet it is in all respects a complete electrical organ. It is situated on either side of the vertebral column in the tail. The plates or discs are placed transversely and in vertical planes. The nerves enter their anterior surfaces; the shock passes in the organ from anterior to posterior end. Gotch and Sanderson have estimated the maximum elcctromotive force of a length of $\mathrm{I} \mathrm{cm}$. of the electrical organ of the sliate at about half a volt. 
Whether the elcctrical organ is the homologue of muscle or of nerve. ending, or whether it is related to cither, has been much discussed. Our surest guide in a question of this sort is the study of development: and researches along this line have shown that there are two kinds of electrical organ, one being modified muscle (as in Gymnotus, Torpedo, and the skate); the other transformed skin-glands (as in Malaptcrurus). The scanty blood-supply of the electrical organs in comparison with that of muscle is noteworthy. In no case do bloodvessels enter the substance of the plates.

\section{PRACTICAL EXERCISES ON CHAPTER XV.}

I. Galvani's Experiment.-Pith a frog (brain and cord). Cut through the backbone above the urostyle, and clcar away the anterior portion of the body and the viscera. Pass a copper hook beneath the two sciatic plexuses, and hang the legs by the hook on an iron tripod. If the tripod has been painted, the paint must be scraped away where the hook is in contact with it. Now tilt the tripcd so that the legs come in contact with one of the iron feet. Whenever this happcns, the circuit for the current set up by the contact of the copper and iron is completed, the nerves are stimulated, and the muscles contract $(p, 822)$.

2. Make a muscle-nerve preparation from the same frog. Crush the muscle near the tendo Achillis, so as to cause a strong demarcation current. Cut off the end of the sciatic nerve. Then lift the ncrve with a small brush or thin glass rod, and let its cross-section fall on or near the injured part of the muscle. Every time the nerve touches the muscle a part of the demarcation current passes through it, stimulates the nerve, and causes contraction of the muscle (p. 822).

3. Secondary Contraction.-Make two muscle-nerve preparations. Lay the cross-section of one of the sciatic nerves on the muscle of the other preparation (Fig. 305, p, 8331. Place under the nerve near its cut end a small piece of glazed paper or of glass rod, and let the longitudinal surface of the nerve come in contact with the muscle bcyond this. Lay the nerve of the other preparation on electrodes connected with an induction machine arranged for single shocks, with a Daniell cell and a spring key in the primary circuit (Fig. 283, p. 808). On closing or opening the key both muscles contract. Arrange the induction machine for an interrupted current. When it is thrown into one nerve, both muscles are tetanized; the nerve lying on the muscle whose nerve is directly stimulated is excited by the action current of the muscle.

4. Demarcation Current and Current of Action with Capillary Electrometer. - (a) Study the construction of the capillary electrometer (Fig. 235, p. 729). Raise the glass reservoir by the rack and pinion screw, so as to bring the meniscus of the mercury into the field. Place two moistened fingers on the binding-screws of the electrometer, open the small key connecting them, and notice that the mercury moves, a difference of potential between the two binding-screws being caused by the moistened fingers.

(b) Demarcation Current.-Set up a pair of unpolarizable electrodes (Fig. 238, p. 731 ). Fill the glass tubes about one-third full of kaolin mixed with physiological salt solution till it can be easily moulded. To do this, make a piece of the clay into a little roll, which will slip down the tube. Then with a match push it down until it forms a firm plug. Next put some saturated zinc sulphate solution in the tubes, above the clay, with a fine-pointed pipette. Fasten the tubes in the holder fixed in the moist chamber (Fig. 322). Now amalgamate the small pieces of zinc wire (p. 197) which are to be connected with the binding-screws of the chamber. (Or use Porter's 'boot' electrodes. These are made of unglazed potter's clay. In use the leg of the boot is half-filled with 
saturated zinc sulphate solution, into which dips a thick amalgamated zinc wire. In the toot of the boot is a hollow (or well) which is filled with pliysiological salt solution and serves to keep the feet well moistened with the salt solution. The nerve is laid on the feet of the boots. When not in use the boots should be kept in pliysiological salt solution.)

The zincs are now placed in the tubes, dipping into the zinc sulphate. A piece of clay or blotting-paper moistened witl physiological salt solution is laid across the electrodes to complete the circuit between their points, and they are connected with the electrometer to test whether they have been properly set up. There ought to be little, if any, movement of the mercury on opening the side-key of the electrometer. If the. movement is large, the electrodes are 'polarized,' and must be set up again. The second pair of binding-screws in the chamber are connected with a pair of platinum - pointed electrodes on the one side, and on the other. through a short-circuiting key, with the secondary coil of an induction machine arranged for tetanus.

Next pith a frog (cord and brain), and make a muscle-nerve preparation. Injure the muscle near the tendo Achillis. Lay the injured part over one unpolarizable electrode, and an uninjured part over the

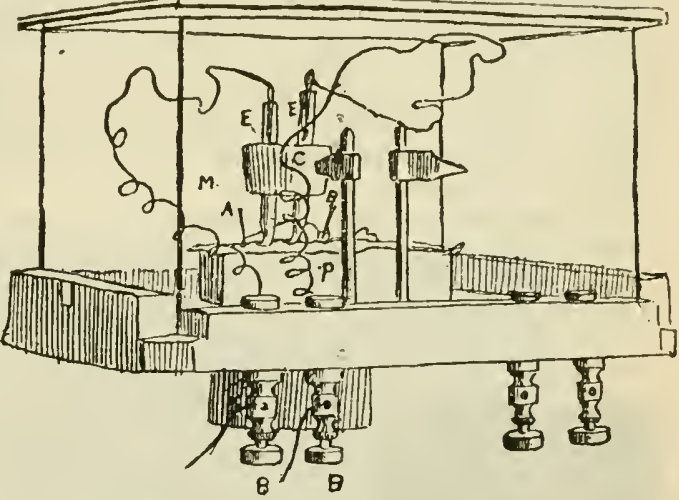

Fig. 322.-Moist Chamber. E, unpolarizable electrodes supported in the cork $\mathrm{C} ; \mathrm{M}$, muscle stretched over the electrodes and kept in position by the pins $\mathrm{A}, \mathrm{B}$, stuck in the cork plate $\mathrm{P} ; \mathrm{B}$, binding-screws connected with galvanometer or capillary electrometer. The other pair of binding-screws serves to connect a pair of stimulating electrodes inside the chamber with the secondary coil of an induction machine.

other. Put a wet sponge in the chamber to keep the air moist, and place the glass lid on it. Focus the meniscus of the mercury, and open the key of the electrometer; the mercury will move, perhaps right out of the field. Note the direction of movement, and, remembering that the real direction is the opposite of the apparent direction, and that when the mercury in the capillary tube is connected with a part of the muscle which is relatively positive to that connected with the sulphuric acid, the movement is from capillary to acid, determine which is the galvanometrically positive and which the negative portion of the muscle (p. 823).

(c) Action Current.-Now, without disturbing its position on the electrodes, fasten the muscle to the cork or paraffin plate in the moist chamber by pins thrust through the lower end of the femur and the tendo Achillis. Lay the nerve on the platinum electrodes. Open the key of the electrometer, and let the meniscus come to rest. This happens very quickly, as the capillary electrometer has but little inertia. If the meniscus has shot out of the field, it must be brought back by raising or lowering the reservoir. Stimulate the nerve by opening the key in the secondary circuit; the meniscus moves in the direction opposite to its former movement.

(d) Repeat $(b)$ and $(c)$ with the nerve alone, laying an injured part (crushed, cut, or overheated) on one electrode, and an uninjured part on the other. Of course, the nerve does not need to be pinned. 
('lean the unpolarizable clectrodes, and be sure to lower the reservoir of the electrometer; otherwise the mercury mat reach the point of the capillary tube and rum out.

In 4 a galvanometer (p. 726) may be used with advantage by students, if one is available, instead of the electrometer, the unpolarizable electrodes being connected to it through a short-circuiting key. The spot of light is brought to the midclle of the scale by moving the control-magnet; or if a telescope-reading is being used, the zero of the scale is brought by the same means to coincide with the vertical hair-line of the telescope. The short-circuiting key is then opened.

5. Action Current of Heart.-Pith a frog (brain and cord). Excise the heart, and lay the base on one unpolarizable electrode, and the apcx on the other, having a sufficiently large pad of clay on the tips of the electrodes to insure contact during the movements of the heart, or having little cups hollowed in the clav and filled with physiological salt solution, into which the organ dips. Connect the electrodes with the capillary electrometer and open its key. At each beat of the heart the mercury will move (p. $\left.\delta_{33}\right)$.

6. Electrotonus. - Set up two pairs of unpolarizable electrodes in the moist chamber. Connect two of them with a capillary electrometer (or galvanometer), and two with a battery of three or four small Daniell cells, as in Fig. 304. Lay a frog's nerve on the electrodes. When the key in the battery circuit is closed, the mercury (or the needle of the galvanometer) moves in such a direction as to indicate that in the extra-

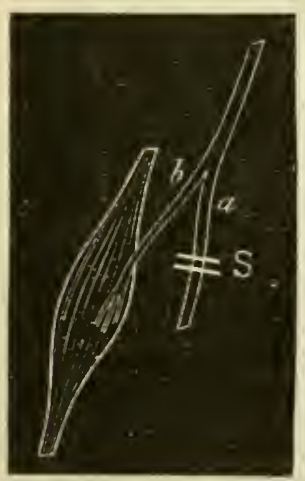

Fig. 323.-Paradoxical Contraction. polar regions parts of the nerve nearer to the anode are relatively positive to parts more remote, and parts nearer to the kathode are relatively negative to parts more remote. The direction of movement of the mercury (or galvanometer neerle) must be made out first for one direction of the polarizing current. Then the latter must be reversed, and the movement of the mercury (or needle) on closing it again noted (p $83^{\circ}$ ).

7. Paradoxical Contraction.-Pith a frog (brain and cord). Dissect out the sciatic nerve down to the point where it splits into two divisions, one for the gastrocnemius $b$, and the other for the peroneal muscles $a$. Divide the peroneal branch as low down as possible, and make a muscle-nerve preparation in the usual way. Lay the central end of the peroneal nerve on electrodes connected through a simple key with a battery of two Daniell cells. When the peroneal nerve is stimulater, the gastrocnemius muscle contracts. This result is not due to the current of action, for it is not obtained with mechanical stimulation of the nerve. But it is not the result of an escape of current, for if the peroneal nerve be ligatured between the point of stimulation and the bifurcation, no contraction is obtained. Tlie contraction is really due to a part of the electrotonic current set up in the peroneal nerve passing through the fibres for the gastrocnemius, where they lie side by side in the trunk of the sciatic.

8. Alterations in Excitability (and Conductivity) produced in Nerve by the Passage of a Voltaic Current through it.- Set up two pairs of unpolarizable electrodes in the moist chamber. Connect a battery of two or three Danicll cells, arranged in series through a simple key 
with the side-cups of a Polll's commutator with cross-wires in. Connect the commutator to one pair of the unpolarizable electrodes ("the polarizing electrodes '), as in lig. 324. The other pair of unpolarizable electrodes (" the stimulating electrodes ') are to be connected through a short-circuiting key with the secondary of an induction machine arranged for tetanus. A single Daniell is put in the primary coil. Pith a frog (brain and cord), make a muscle-nerve preparation, pin the lower end of the femur to the cork plate in the moist chamber, attach the thread on the tendo Achillis to the lever connected with the chamber through the hole in the glass provided for this purpose, and arrange the nerve on the electrodes so that the stimulating pair is between the muscle and the polarizing pair. By moving the secondary, seck out sucl a strength of stimulus as just suffices to cause a weak tetanus when the polarizing current is not closed. Set the drum off (slow speed), and take a tracing of the contraction. Then close the polarizing current with a Pohl's commutator so arranged that the anode is next the stimulating electrodes-i.e., the current ascending in the nerve. Again open the short-circuiting key in the secondary; the contraction will now be weaker than before, or no contraction at all may be obtained. Allow the preparation two minutes to recover,

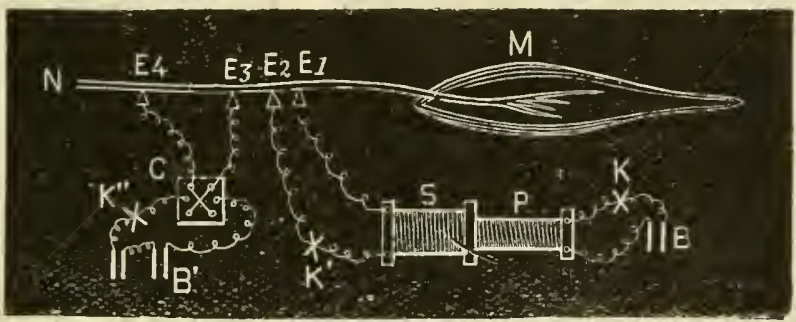

Fig. 3 24.-Arrangement for showing Changes of Excitability produced by the Voltaic Current. $\mathrm{M}$, muscle; $\mathrm{N}$, nerve; $\mathrm{E}_{1}, \mathrm{E}_{2}$, electrodes connected with secondary coil S; $E_{3}, E_{4}$, unpolarizable electrodes connected with Pohl's commutator (with cross.wires) C; B'. 'polarizing' battery; $\mathrm{B}$, 'stimulating' battery in primary circuit $\mathrm{P}$; $\mathrm{K}, \mathrm{K}$ "', simple keys; $\mathrm{K}^{\prime}$, short-circuiting key.

then stimulate again, as a control, without closing the polarizing current. If the contraction is of the same height as at first, close the polarizing current with the bridge of the commutator reversed, so that the kathode is now next the stimulating electrodes. On stimulating, the contraction will now be increased in height. (See Figs. 273. 274. p. 786.)

9. Pfüger's Formula of Contraction (p. 788 ). - To demonstrate this, connect two unpolarizable electrodes, through a spring key ind a commutator, with a simple rheocord (Fig. 286, p. 8 Io), so as to lead off a twig of a current from a Daniell cell. The unpolarizable elec. trodes are placed in a moist chamber. A muscle-nerve preparation is arranged with the nerve on the electrodes and the muscle attached to a lever. The effects of make and break of a weak current, ascending and descending, can be worked out with the simple rheocord. The effects of a medium current will probably be obtained with a single Daniell connected dircctly with the electrodes through a key. The effects of a strong current will be got when three or four Daniells are connected with the electrodes. Care must be taken to keep the preparation in a moist atmosphere, and more than one preparation may be needed to verify the whole formula. 
10. Formula of Contraction for (Human) Nerves in Situ.-Connect ejglet or ten try cells in series.* Commect one terminal of the battery to a large plitic electrode, and the uther to a small electrode, but h covered with cotton, flannel, or sponge, moistened with salt soluti n Include in the circuit a simple key for making or breaking the current, and a commutator for changing its direction at will. Leave the key open. Place the large electrode behind the shoulder (or on the back of the neck), and the small electrode over the ulnar nerve at the elbow between the internal condyle and the olecranon. Arrange the commutator so that the small electrode shall be the kathode. Close, and then open the key. If no contraction occurs at closing, the battery is too weak, and more cells must be added. If contraction occurs at closing, but not at opening, reverse the commutator, making the small electrode the anode, and observe whether contraction now occurs at closing, at opening, or at both. Note also the relative strength of the various contractions. If the current is 'weak,' the only contraction will be a closing one when the kathode is over the nerve. If the current is of 'medium" strength, a closing kathodic contraction and both opening and closing anodic contractions will be obtained. With ' strong' currents contractions will occur at closing and at opening, whether the kathode or the anode is over the nerve. The contractions will vary in strength, as described on p. 789 . To work out the different cases of the formula summarized in the table, the number of cells must be increased or diminished.

\begin{tabular}{|c|c|c|}
\hline Weak Currents. & Medium Currents. & Strong Currents. \\
\hline KCC & KCC & KCC \\
- & ACC & ACC \\
AOC & AOC \\
& - & KOC \\
\hline
\end{tabular}

The abbreviations KCC, ACC, are used respectively for kathodic closing contraction and anodic closing contraction; KOC, AOC, for kathodic opening contraction and anodic opening contraction. IKCC is stronger than $\mathrm{KOC}$, and $\mathrm{ACC}$ than $\mathrm{AOC}$. $\mathrm{KCC}$ is stronger than ACC, and AOC than KOC. Therefore, as the strength of the current is increased, in the case of normal tissues, $\mathrm{KCC}$ is first obtained, then $\mathrm{ACC}$, then AOC, and finally KOC.

II. Ritter's Tetanus.-Lay the nerve of a muscle-nerve preparation on a pair of unpolarizable electrodes connected through a simple key with a battery of three or four small Daniells. Connect the muscle with a lever. Pass an ascending current (anode next the muscle) for a few minutes through the nerve, and let the writing-point trace on a slowly-moving drum. When the current is closed there may be a single momentary twitch, or the muscle may remain somewhat contracted (galvanotonus) as long as the current is allowed to pass, or it may continue to contract spasmodically ("closing tetanus"). When the current is opened the muscle will contract once, and then immediately relax, or there may be a more or less continued tetanus (Ritter's or 'opening tetanus'). If opening tetanus is obtained, divide the nerve between the electrodes: the tetanus continues. Divide it between the anode and the muscle: the tetanus at once disappears. This shows that the seat of the excitation which causes the tetanus is in the neighbourhood of the anode (p. 83I).

* If the laboratory possesses a battery (with rheostat), such as is used by neurologists, the experiment is more conveniently performed with this. 


\section{CHAPTER XVI}

\section{THE CENTRAL NERVOUS SYSTEM}

IN other divisions of our subject we have been able to follow to a greater or less extent the processes which take place in the organs described. The chemistry and the physics of these processes have bulked more largely in our pages than the anatomy and histology of the tissues themselves. In dealing with the central nervous system, we must adopt a method the very reverse of this. Its anatomical arrangement is excessively intricate. The events which take place in that tangle of fibre, cell, and fibril are, on the other liand, almost unknown. So that in the description of the physiology of the central nervous system we can as yet do little more than trace the paths by which impulses may pass between one portion of the system and another, and from the anatomical connections deduce, with more or less probability, the nature of the physiological nexus which its parts form with each other and the rest of the body. And here it may be well to remark that, although for convenience of treatment we have considered the general properties of nerves in a separate chapter, there is not only no fundamental distinction between the central nervous system and the outrunners which connect it with the periphery, but obviously a central nervous system would be meaningless and useless without afferent nerves to carry information to it from the outside, and efferent nerves along which its commands may be conducted to the peripheral organs.

\section{Section I.-Structure of the Central Nervous System- Histological Elements.}

In unravelling the complex structure of the central nervous system, we avail ourselves of information derived (I) from its gross anatomy; (2) from its microscopical anatomy; (3) from its development; (4) from what we may call, although the term is open to the criticism of cross-division, its physiological and pathological anatomy.

Certain tracts of white or grey matter are differentiated from each other by the size of their fibres or cells. For example, the posteromedian column of the spinal cord has small fibres, the direct cere- 
bellar tract large fibres; the large pyramidal cells (giant cells or cells of Betz), in what we shall afterwards liave to distinguish as the 'motor area' (p. 950) of the cerebral cortex, are the cells of origin of fibres of the pyramidal tract subserving the volitional movements of the limbs and trunk. The pyramidal cells of the 'face area' are comparatively small. In general, an efferent or motor nerve-cell is larger the longer its axon is-e.g., the largest of all the pyramidal cells in the 'motor' region are found in the portion known as the 'leg area,' from which the pyramidal fibres have to pass all the way down the cord to the segments from which the spinal nerves going to the lower limbs arise.

The recent work of Brodman and of Camplell has shown that the cerebral cortex may be histologically differentiated into regions which correspond to a great extent to the various functional regions mapped out by physiological methods (p. 958).

The study of development enables us not only to determine the homology, the morphological rank, of the various parts of the brain and cord, but also, by comparison of animals of different grades of organization, sometimes to decide the probable function and physiological importance of a strand of nerve-fibres or a column of nervecells. It is of special value in helping us to differentiate the various areas of grey matter on the surface of the brain, and to trace the various tracts or paths into which the white inatter of the central nervous system may be divided. For the medullary sheath is not developed at the same time in all the tracts, and a strand of nerve-fibres in which it is wanting-e.g., the pyramidal tract (p. 878), which is the last of the spinal tracts to becone myelinated-is readily distinguished under the microscope.

Then, again-and this is what we propose to include under the fourth head-experimental physiology and clinical and pathological observation throw light not only on the functions, but also cn the structure, of the central nervous system. For instance, coraplete or partial section, or destruction by disease, of the white fibres of the cord or brain, or of the nerve-roots, or removal of portions of the grey matter, is followed by degeneration in definite tracts. And since, as we have already seen, degeneration of a nerve-fibre is caused when it is cut off from the cell of which it is a process, the amount and distribution of such degeneration teaches us the extent and position of the central connections of the given tract. Conversely, the cells in which a tract of nerve-fibres arises may sometimes be identified by the alterations in the chromatin (p. 859) and other changes which occur in them after section of their axons. Particularly in young animals, removal of a peripheral organ-an eye or a limb-or section of its nerves, may be followed by atroplyy of portions of the central nervous system immediately related to it.

'Softening' of a definite portion of the white or grey matter may also in certain cases be caused by depriving it of its blood-supply by the injection of artificial emboli, and the resulting degenerations may then be studied. For instance, fine particles like lycopodium spores are injected into the abdominal aorta between the origins of the renal and inferior mesenteric arteries. They are prevented by clamps from entering these vessels, and, passing through the lumbar arteries, stick in the branches of the anterior spinal artery, and cause softening mainly of the grey matter of the lumbar portion of the cord. When the abdominal aorta of a rabbit is temporarily compressed (for about an hour) below the origin of the renal arteries, the grey matter of the corresponding portion of the cord is so seriously injured that it and the fibres that arise from it degenerate, while the fibres whose cells of 
origin are unt situated in this part of the grey matter are not affected, or at least enmpletely recover.

Certain tracts may also be marked out by means of the electrical variation, which gives token of the passage of nervous impulses along then when portions of the central nervous system or periplicral nerves are stimulated (Horsley and Gotcli).

Development of the Central Nervous System.-Very carly in development (Fig. 325) the keel of the vertebrate embryo is laid down as a groove or gutter in the ectoderm of the blastodermic area (Chap. XIX.). The walls of this medullary' or 'neural' groove

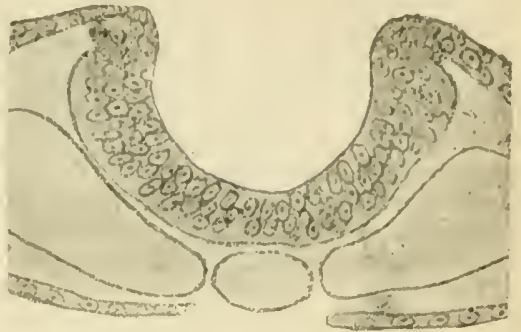

Fig. 325.-Formation of the Neural Canal at an Early Stage (Beard). grow inwards, and at length there

is formed, by their coalescence, the 'neural canal' (Fig. 326), which expands at its anterior end to form four cerebral vesicles (Fig. 327). Thus there is a continuous tunnel from end to $\mathrm{cnd}$ of the primary cerebro-spinal axis; and this persists as the central canal of the spinal cord and the ventricles of the brain, whose ciliated epithelium represents the ectodermic lining of the primitive neural canal. In the adult portions of the canal may become obliterated from an overgrowth of the lining cells, and the cilia are, if present at all, less distinct than in the child, and far less distinct than in the lower animals. From the wall of this canal is formed the cerebro-spinal axis, in which developing nerve-cells or neuro-
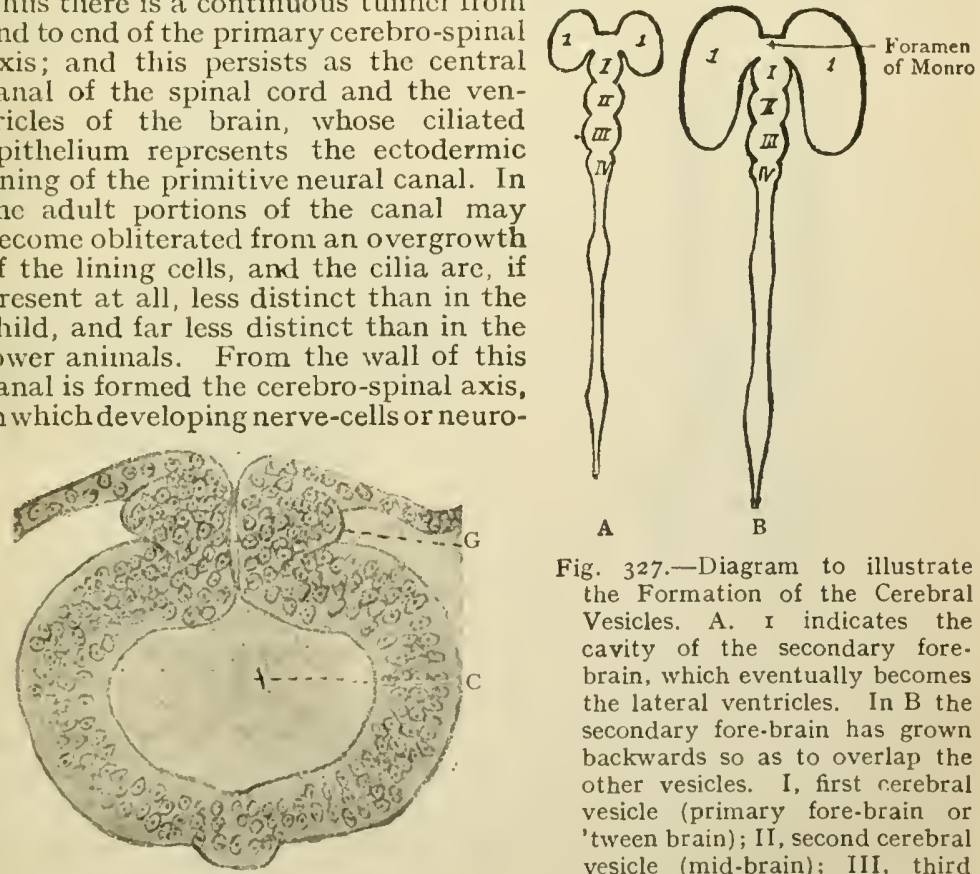

Fig. 327.-Diagram to illustrate the Formation of the Cerebral Vesicles. A. I indicates the cavity of the secondary fore. brain, which eventually becomes the lateral ventricles. In $B$ the secondary fore-brain has grown backwards so as to overlap the other vesicles. I, first cerebral vesicle (primary fore-brain or 'tween brain); II, second cerebral vesicle (mid-brain); III, third cerebral vesicle (hind-brain); IV. fourth cerebral vesicle (after. brain).

ig. 326. - Neural Canal at a Later Stage (Beard). C, neural canal; $\mathrm{G}$, posterior spinal ganglion.

blasts soon become differentiated from the supporting cells or spongioblasts, and wander outwards from the neighbourhood of the central cana] (Fig. 338) till their further progress is checked by the barrier of the mar- 
ginal veil, a closely-woven network or thicket, into which the processes of the spongioblasts break up at the outside of the primitive cerebro-spinal axis. Although the neuroblasts themselves are unable to penetrate the marginal veil, the axis-cylinder processes of some of them do so, and form the motor roots of the spinal nerves. The neuroblasts from which the fibres of the white columns of the cord are developed are apparently unable to send their axons through the marginal veil. They are accordingly forced to assume a longitudinal direction, and in this way the central grey matter becomes covered with a slieath of longitudinal white fibres. For a time only motor nerve-cells and the fibres connected with them are developed in the cerebro-spinal axis. The ganglia on the posterior roots arise from a scries of ectodermic thickenings or sprouts from the neural crest which runs along the dorsal aspect of the neural canal. These sprouts contain the neuroblasts which develop into the spinal ganglion cells with the posterior rootfibres. From each pole of each neuroblast a process grows out, one towards the periphery, which forms a peripheral nerve-fibre, the other centrally to connect the cell with the cord. From the after-brain (or myelencephalon) is developed the medulla oblongata or spinal bulb, from the hind-brain (or metencephalon) the cerebellum and pons, from the mid-brain (or mesencephalon) the corpora quadrigemina and crura cerebri. The fore-brain, or primary fore-brain (thalamencephalon), gives rise of itself only to the third ventricle and optic thalamus; but a secondary fore-brain (telencephalon) buds off from it and soon divides into two chambers, from the roof of which the cerebral hemisplieres, and from the floor the corpora striata, are derived. Their cavities persist as the lateral ventricles, which communicate with the third ventricle by the foramen of Monro. The olfactory tracts are formed as buds from the secondary fore-brain.

To complete the story of the development of the brain, it may be added that the retina is really an cxpansion of its nervous substance. A hollow process, the optic vesicle, buds out on each side from the primary fore-brain. A button of ectoderm, which afterwards becomes the lens, grows against the vesicle and indents it so that it becomes cup-shaped, the inner concave surface of the cup representing the retina proper, the outer convex surface the choroidal epithelium. The stalk becomes the optic nerve.

Histological Elements of the Central Nervous System.-The central nervous system is built up (r) of true nervous elements, (2) of supporting tissue. The nervous elements have usually been described as consisting of nerve-fibres and nerve-cells, but the antitliesis of a timehonoured distinction must not lead us to forget that the essential part of a nerve-fibre, the axis-cylinder, is a process of a nerve-cell, and the medullary sheath a structure whose integrity is intimately related to that of the axis-cylinder.* In strictness, the term 'nervecell' ought to include not only the cell-body, but all its processes, out to their last ramifications. Bnt the habit of spealing of the position of the cell-bodyt as that of the nerve-cell is so ingrained, that it seems better to continue the use of the latter term in its old signification, and to speak of the cell and branches together as a neuron (also spelled neurone).

* While the medullary sheath, like the axis-cylinder, seems to be as regards its nutrition under the control of the nerve-cell, and must therefore be looked upon as an integral portion of the neuron, although not essential for its development, the neurilemma in respect both of its nutrition and its develop. ment appears to be an independent structure.

$\uparrow$ Foster and Sherrington call the cell-body the perikaryon. 
The Neurons. $-A$ typical nerve-cell (Figs. 328, 330, 332) is a knot of granular protoplasm, containing a large nucleus, inside of which lies a lighly refractive nucleolus. A centrosome and attraction spliere (p. 5) have also been found in some nerve-cells, though not as yet demonstrated in all. Pigment may also be present, especially in old age. By certain methods of staining it may be slown that fibrils (neuro-fibrils) rum through the protoplasm of the cell, forming a feltwork in it, and entering the dendrites on the one hand and the axiscylinder process on the other (Figs. 328, 33I, 335). In the axiscylinders of nerve-fibres the fibrils (Fig. 329) appear to preserve their

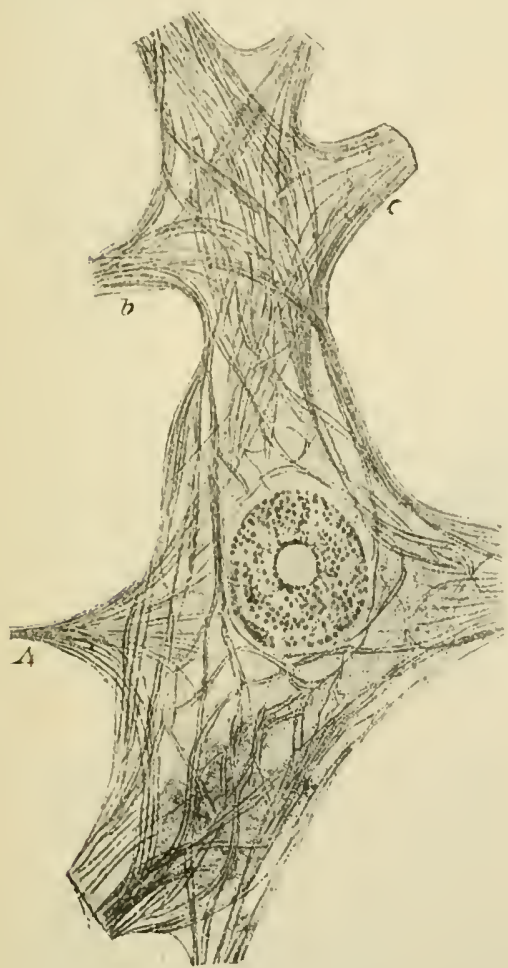

Fig. 328. - Anter:or Horn Cell from Man showing Fibrils (Bethe). identity down to the distribution of the fibre. In the ground substance between the fibrils lie round, angular, or spindle-shaped bodies (Nissl's bodies) which stain with basic dyes (Fig. 340).* Thesc bodies vary in appearance in different kinds of nerve-cells, and in the same nerve-cell under different conditions. According to Macallum. they contain organically combined iron. In a multipolar cell, like those

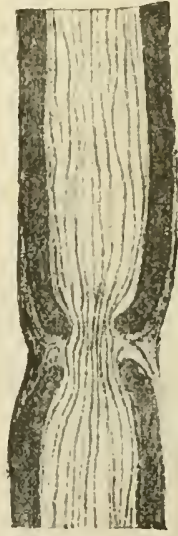

Fig. 329. - Medullated NerveFibre showing Fibrils of AxisCylinder (Bethe). The fibrils are seen passing, without interrup. tion, across a node of Ranvier.

in the anterior horn of the spinal cord, several processes-it may be five or six, or even more-pass off from the cell-body (Frontispiecc). The most complete pictures of them are given by preparations impregnated according to the method of Golgit (Figs. 330, 333). One of the pro-

* In Nissl's method the sections are stained in a solution of methylene blue, and decolourized in anilin-alcohol.

+ The method depends upon the deposition of mercury, or silver, in or around the cell-bodies and their processes in tissues which have been hardened in bichromate of potassium and then soaked in a solution of mercuric chloride or silver nitrate. In Pal's improvement of Golgi's method a solution of sodic sulphide follows the mercuric chloride. 
cesses of most nerve-cells is distinguished from the rest by the fact that it maintains its original dianıeter for a comparatively great distance from the cell, and gives off comparatively few branches. This process, which in favourable preparations can be traced on till it becomes the axis-cylinder of a nerve-fibre, is called the axis-cylinder process, or more shortly the axon. The few slender bral ches that come off from it, usually at right angles, are called collaterals. The collaterals consist essentially of one or more fibrils of the axon. Both the main thread of the axon and the collaterals end by breaking up into an arborescent system of fibrils or telodendrion. The telodendrions vary greatly in appearance from simple end-brushes to far-branching thickets, or such special cnd-organs as motor plates (Fig. 335) or muscular spindles. The rest of the processes of the cell, which are termed dendrites or protoplasmic processes, very rapidly diminish in cliameter, as they pass away from the cell by breaking up into

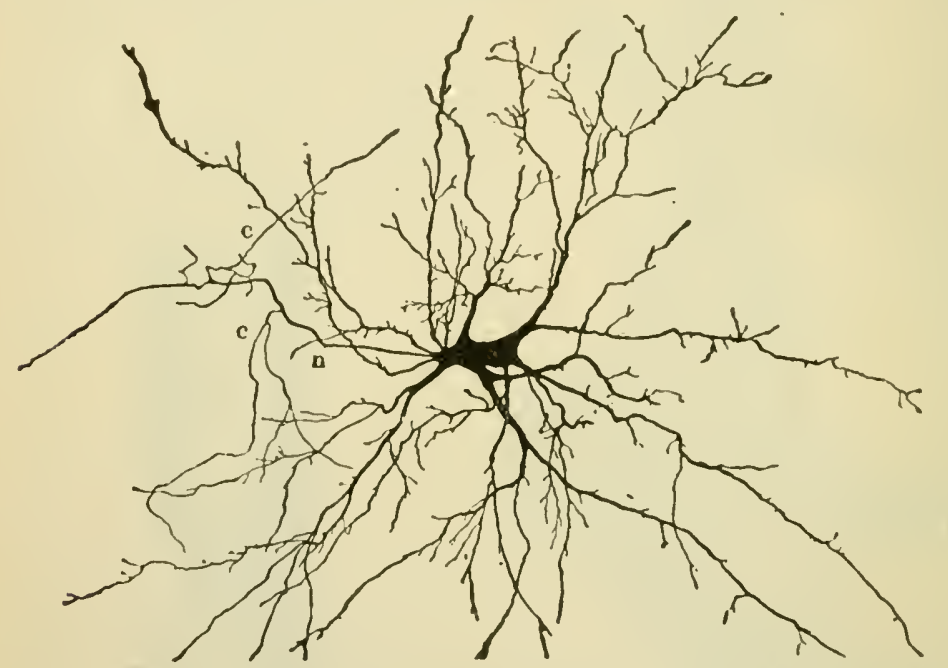

Fig. 330.-Multipolar Nerve-Cell: Golgi Preparation (Barker, after Kölliker). $n$, axon; $c$, collaterals.

fibrils like the branches of a tree. The Nissl bodies extend for some distance into the dendrites, but not into the axon. The dendrites of some cells, especially the pyramidal cells of the cerebral, and the Purkinje's cells of the cerebellar cortex, have small swellings, the so-called lateral buds or gemmules, on their course. Their significance is unknown. The dendrites terminate at a little distance from the cell, where they come into relation with the end-arborizations of the axons of other ncurous. In this way two or more neurons are linked together to form a nervous path. According to the view most commonly held (neuron hypothesis), the relation is not one of actual anatomical continuity, but the processes come so close together that nerve impulses are able to pass across from the terminal brush of the axon of one nervous element to the dendrites or cell-body of another. This kind of junction is called a synapse.

It has been suggested that the contact may be rendered more or less close through amœboid movements of the dendrites, and that in this 
way the nervous impulse may be switclied like a railway-train from one path to another. But therc is no experimental basis for this somewhat crucle, if fascinating, hypothesis. Sherrington has suggested that the presence of a 'membrane' at the synapse may limit the conduction and determine its direction. Some membrancs, such as frog's skin, are known to possess a so-called irreciprocal permeability for certain substances, permitting them to pass more easily in one direction than the other, and it is conceivable that a membrane at the synapse might have a similar action in respect to the movement of ions concerned in the propagation of the nervous excitation. Whatever

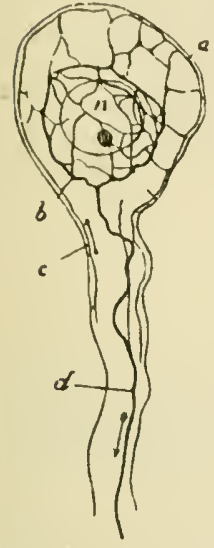

d.

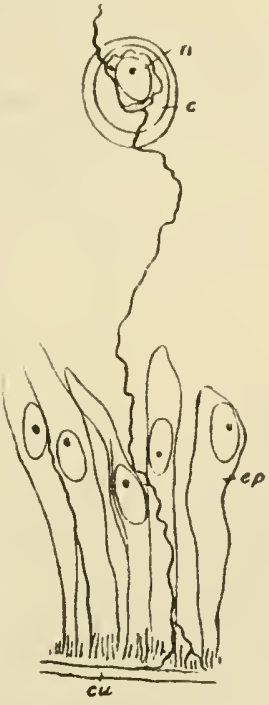

$B$.

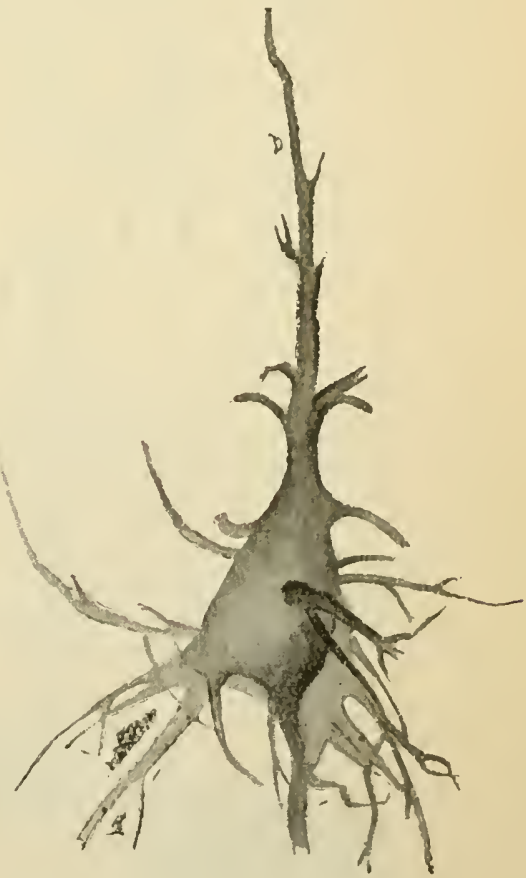

Fig. 332.-Large Pyramidal Cell of Cerebral Cortex (Barker, after Bech. terew). $a$, axon; $b$, dendrite.

the nature of the relation between two superposed neurons may be, it does not permit the conduction of nerve-impulses indiscriminately in both directions. For instance, stimulation of the central end of the posterior root of a spinal nerve causes an electrical response (p. 824) in the anterior root of the same segment, while no electrical change is produced in the posterior root by stimulation of the anterior. We shall see later on (p. 872) that some of the fibres of the posterior root and their collaterals end by arborizing around the dendrites of the cells of the anterior horn. The excitation is, therefore, able to pass from the 
telodendrions of the posterior root-fibres through the dendrites of the anterior horn cells towards their cell-hodies, but not in the opposite

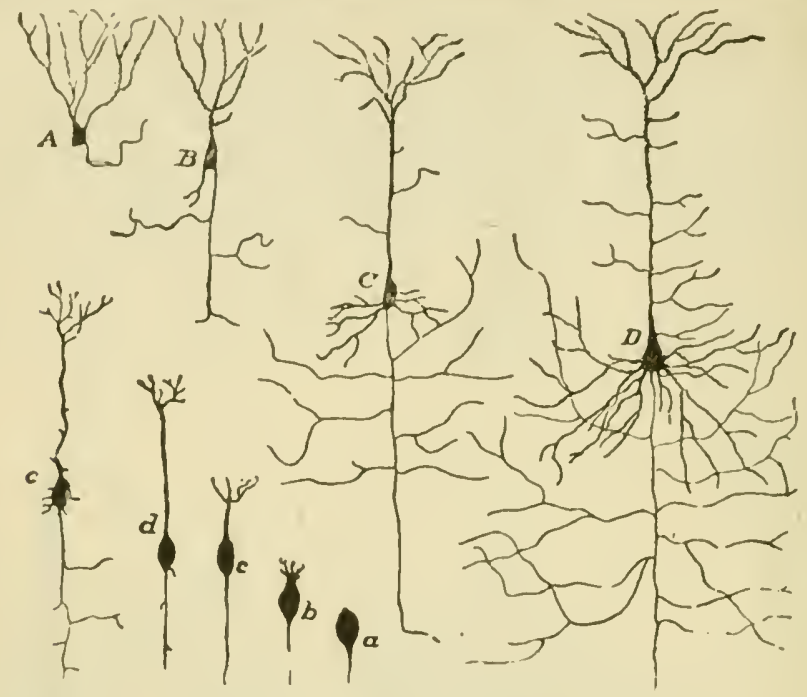

Fig. 333.-a-e shows the development of the pyramidal nerve-cells of the cerebra] cortex in a typical mammal; $a$, neuroblast with commencing axon; $b$. dendrites appearing; $d$, commencing collaterals. A-D shows the different degree of complexity in the fully-developed pyramidal cells in different vertebrates: $A$, frog; B, lizard; C, rat; D, man (Donaldson, after Ramón y Cajal).

direction, and in general the direction of conduction is from the dendrites towards the cell-body.

Some investigators believe that the fibrils already spoken of as forming a felt-work in the protoplasm of the nerve-cell may run right

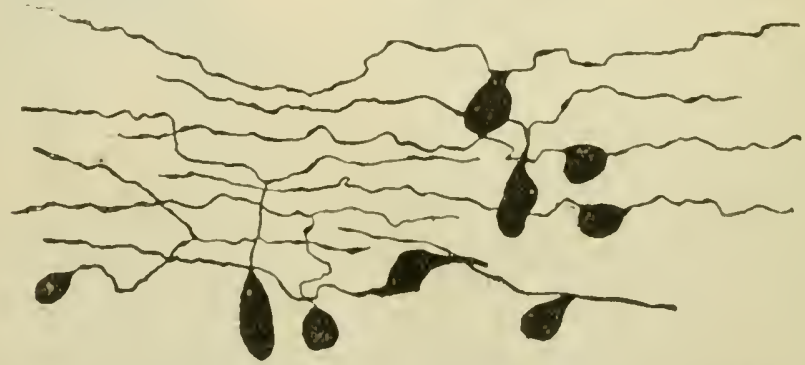

Fig. 334.-Cells from the Gasserian Ganglion of a Developing Guinea.Pig. The originally bipolar cells are seen changing into cells apparently unipolar. The same process occurs in the cells of the spinal ganglia (Van (iehuchten).

through from one ccll to another, thus constituting an actual anatomical connection between the neurons, and that such a connection may be established also by fibrils which do not enter the cells at all, but run in the intercellular substance of the grey matter. Such a continuity of 
fibrils from cell to cell has been demonstrated in some of the invertebrates - e.g., in annelids (Fig. 33I)where previously the best examples of strictly isolated neurons were supposed to be found (A pathy). The supporters of the theory of continuity look upon the cell-body as mercly necessary for the nutrition of the nerve-net, but deny that it is necessary for the conduction of nerve-impulses. If this is the case, it is obvious that the neurons can no longer be considered as functional units in which the law of isolated conduction of nerve-impulses (p. 793) holds good. Nor is it by any means so easy to understand as on the neuron hypothesis such facts as the strict limitation of Wallerian degencration to the boundaries of the neurons directly affected, or the strict limitation of the silver reduction in Golgi preparations to single neurons. It is, of course, true that the simplicity and order introduced by the neuron hypothesis into our conceptions of the nervous conduction paths by no means prove its accuracy. Yet they are reasons for not lightly abandoning it, and it has recently been corroborated by important new evidence on the growth of nerve-cells on artificial media outside of the bodi; (p. 802; Fig. 337, p. 857).

Varieties of Neurons. Nearly all the nerve-cells of the cerebro-spinal axis agree with the cells of the anterior horn in the possession of an axon and one or more dendrites, although sometimes the dendrites are scanty in number and

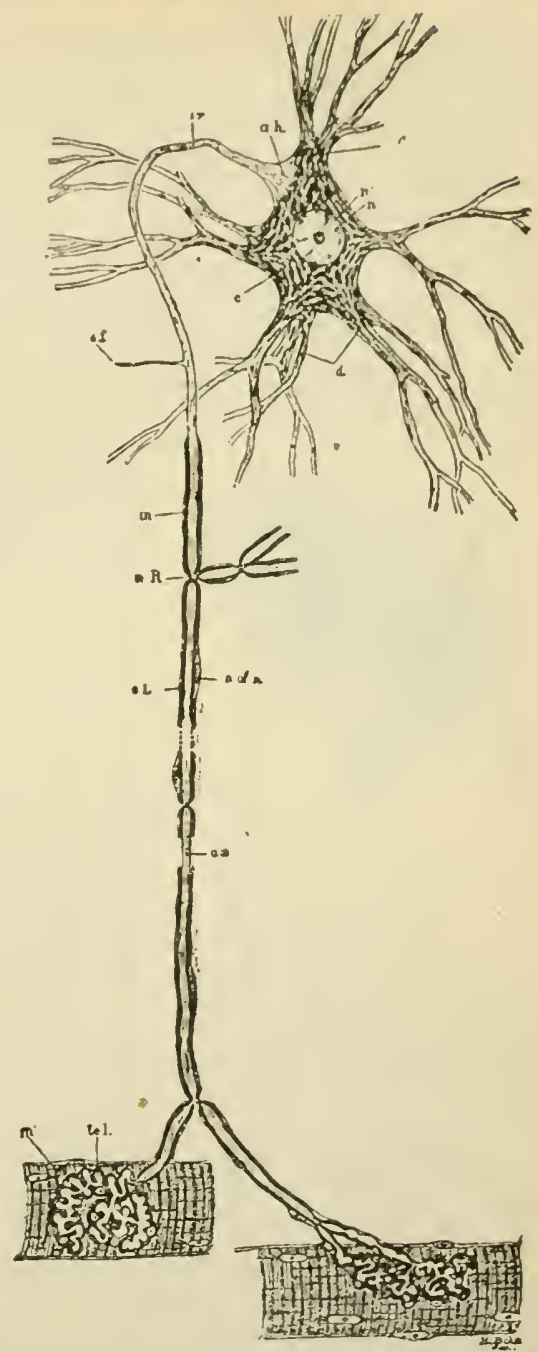

Fig. 335.-Scheme of Lower Motor Neuron (Barker). $a, h$, axon-hilluck (the portion of the cell from which the axon comes off), containing no Nissl bodies, and showing fibrillation; $a x$, axis-cylinder or axon; $m$, medullary sheath, outside of which is the neurilemma; $c$, cell. substance (cytoplasin), showing Nissl bodies in a lighter ground substance; $d$, protoplasmic processes or dendrites containing Nissl bodies; $n$, nucleus; $n^{\prime}$, nucleolus; $n, R$, node of Ranvier; $s, f$, side fibril; $n$ of $n$, nuclcus of the neurilemma : $t e l .$, motor end-plate; $m^{\prime}$, striped mus:lc-fibre; $s, I$, incisure. 
insignificant in size. In the cerebral cortex the typical cells are of pyramidal shape. From the base comes off the axon, and from the angles dendritic processes, a particularly massive dendrite proceeding from the apex of the pyramid towards the surface of the brain.

Sometimes an axon, instead of ending in an arborization which comes into relation with the dendrites of another nerve-cell, or, as is more frequently the case, with the dendrites of more than one cell, breaks up into a sort of basket-work of fibrils surrounding the cellbody. The cells of Purkinje, for instance, in the cerebcllum are surrounded by such pericellular baskets (Fig. 336). The cells of the spinal ganglia have two axons, which in the embryo arise one from each end of the bipolar cell, but in the adult, in all vertebrates except some fishes, are connected to the cell by a single process (Fig. 334). It has been commonly held that the unipolar cell with a single T-shaped

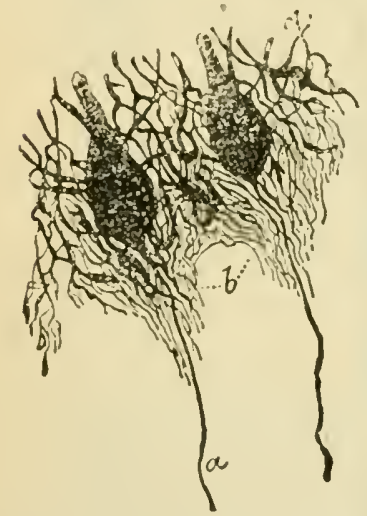

Fig. 336.-Pericellular Baskets (Schäfer, after Cajal). Two cells of Purkinje from the cerebellum are seen sur. rounded by end ramifications forming a basket-work, $b$, de. rived from the branching of axons of small nerve-cells in the molecular layer; $a$, axon. process is developed from a bipolar cell, which grows towards one side, so that the two processes come together and fuse. Such observations as that of Harrison on the bifurcation of the growing end of the main process of isolated nerve-cells cultivated in vitro suggest an alternative and a simpler explanation-viz., that the T-sliaped process is derived from the splitting of a single chief process. If this be the case, one of the original processes at the poles probably undergoes a retarded development or disappears, since the great majority of the spinal ganglion cells with the $\mathrm{T}$-shaped process appear to have no dendrites. Another kind of cell which seems undoubtedly to be of nervous nature is the 'granule-cell.' Granule-cells are much smaller than the nerve-cells we have been describing. Their processes are much less easily followed, but all appear to give off an axon and several dendrites. They contain a relatively large nucleus ( 5 to $8 \mu$ in diameter), with only a mere fringe of cell-substance. The nucleus, unlike that of a large nerve-cell, stains decply with hæmatoxylin. Some parts of the grey matter are crowded with these granule-cells-e.g., the nuclear layer of the cerebellum and the substantia gelatinosa, or substance of Rolando, which caps the posterior hoin in the cord. In other parts they are more thinly scattered, but probably they are as widely diffused as the large nerve-cells proper, and no extensive area of the grey matter is wholly without them.

Although there are several varieties of granules (Hill), they all agree in this, that their axons run a comparatively slort course, and never, or rarely, pass beyond the grey matter. Another kind of neuron which is also confined to the grey matter, and is typically seen in the cortex of the cerebrum and cerebellum, presents the peculiarity of an axo:l which branches into an intricate network immediately after coming off from the cell (cell of Golgi's second type). Unlilie the long axon of the typical large nerve-cell, the axis-cylinder process of this Golgi cell remains unmedullated.

The sympathetic ganglion cells are developed from immature neuro- 
blasts thit migrate, in the course of development, from the rudiments of the spinil ganglia, and gathering in clumps form the ganglia of the sympathetic chain (His). They agree in general with the cells of the cerebro-spinal axis in possessing an axon and one or more, commonly several, dendrites, although a few of theın are devoid of dendrites. The great majority of the axons remain unmedullated, but a few acquire a very fine medullary sheath.

The epithelium lining the central canal of the cord and the ventricles of the brain lias also been considered by some as of nervous nature. The fact that the decp ends of the cells are continued into processes which pierce far into the grey substance has been supposed to lend weight to this opinion, but there is no good ground for it.

Growth of Neurons. - The growth of a neuron from origin to completion is a comparatively slow process in the higher animals. Early in fotal life (about the third or fourth week in man) certain round germinal cells make their appearance amid the columnar ectodermic cells surrounding the neural canal. From their division are formed, in the first months of embryonic life, the primitive nerve-cells or neuroblasts. These soon elongate and push out processes, first the

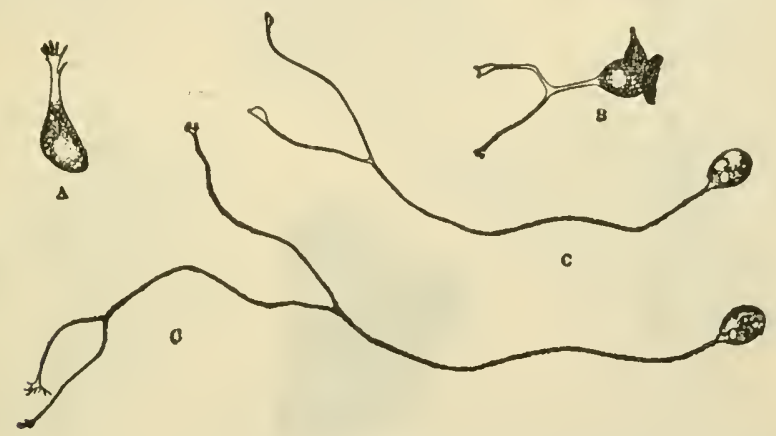

Fig. 337.-Isolated nerve-cells from the spinal cord of a tadpole growing in clotted lvmph. A, B, C, are cells in different stages of growth. The lower view of C was drawn under the microscope $4_{4}^{3}$ hours later than the upper (Harrison).

axon or axons, and then the dendrites (Fig. 333). The formation of the axons from the nerve-cell is most clearly followed in isolated cultures (Fig. 337). As development goes on, the cell-body grows larger, and the processes longer and more richly branched. The axon and its collaterals, when it has any, in the case of the great majority of the nervous elements of the brain and cord, ultimately acquire a medullary sheath, although, as we have said, the time at which medullation is completed varies in different groups of elements, and in some nervous tracts it is even wanting at birth. At birth, too, the branches of many of the cells are less numerous, and the connections between different nervous elements therefore less intimate than they will afterwards become. For many years the processes, and particularly the axons, continue not only to grow longer, but to grow thicker as well. The cell-body also enlarges, and the quantity of material in it that stains with basic dyes increases. In the growing (lumbar) spinal ganglia of the white rat the increase in volume of the largest cellbodies is very closely correlated with the increase in area of the crosssection of the nerve-fibres growing out of them. The cross-section of the axis-cylinder is, and remains, almost exactly equal to the area 
of the medullary sheath (Donaldson). Even after puberty is reached the anatomical organization of the nervous system may still continue to advance, although at an ever-slackening rate, and the finishing touches may only be given to its architecture in adult life. In old age the nervous elements decay as the body does. The cellbody diminishes in size; the stainable material lessens in amount; vacuoles form in the protoplasm and pigment accumulates; the nucleus shrinks; the nucleolus is obscured or may disappear altogether. At the same time the processes of the cell, and especially the dendrites, tend to atrophy (Fig. 339).

Nutrition of the Neuron.-We have already seen that when an axon is cut off from its cell-body, it and its medullary sheath, when it possesses one, undergo a rapid degeneration. It was long supposed

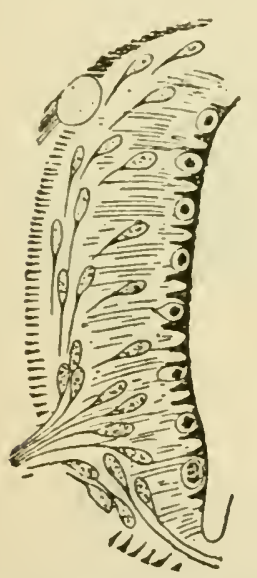

Fig. 338. - Section through Half of Neural Tube (Barker, after $\mathrm{H}$ is). The pear. shaped neuroblasts are seen migrating out. wards. The axons of some of them are seen pushing their way out through the marginal veil as the anterior root of a spinal nerve.
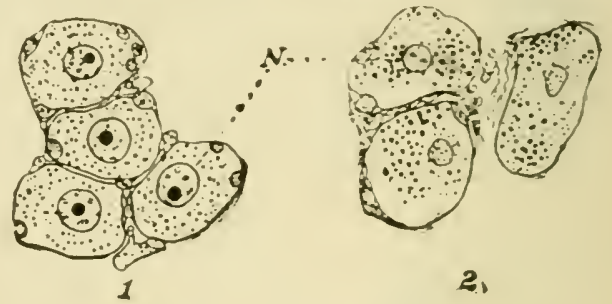

2.

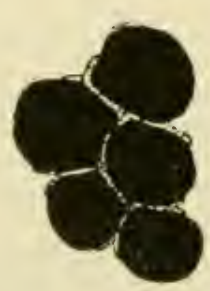

3.

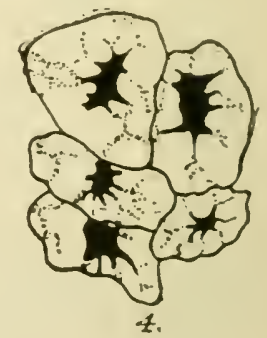

Fig. 339.-I, spinal ganglion cells of a still-born male child; 2 , of a man ninety-two years old $(X 250)$ $-N$, nuclei; 3 . nerve-cells from the antennary ganglion of a honey-bee just emerged in the perfect form; 4 , of an old honey-bee. The nucleus is black in the figure. In 3 it is very large, in 4 it is shrunken and the cell-substance contains vacuoles (Hodge).

that no change took place in the nerve-cell. The researches of recent years have shown that not only does loss of the specific function and trophic influence of the cell-body affect the nutrition of the axon, but loss of function of the axon reacts on the cell-body. In many cases at least, when a nerve-fibre is divided from its cell, characteristic changes are produced in the latter and in its dendritic processes, and they are scarcely less rapid, although usually less profound, and far more transient than the degeneration in the peripheral portion of the nerve-fibre. The cell-body and the nucleus swell. Many of the Nissl bodies (Fig. 340) disintegrate, and are rednced to a finely granular condition. After a time much of the disintegrated chromatic substance disappears altogether. The nucleus inay be displaced to one 
side of the cell. Certain changes in the neurofibrils of the cell may accompany the clianges in the chromatin. In rabbits after division of the facial nerve the alterations in its nucleus of origin have been found to reach a maximum in about three weeks, after which there is a tendency to recovery on the part of the majority of the cells, even when regeneration of the nerve has been prevented by cutting out a portion of it. Some of the cells may completely atrophy and disappear. Similar changes liave bcen found by Warrington in the motor cells of the anterior horn after section of the posterior (dorsal) spinal roots. Since in this case no anatomical injury has been inflicted on the motor neurons, it has been surmised that the cause of the alterations is the loss of impulses which normally reach them along their dendrites. In short, we may say, with Marinesco, that the functional and anatomical integrity of the neuron depends on the integrity of all its constituent parts, and of the neurons which carry to it functional excitations-i.e., excitations connected with its proper physiological work. The neuron, in fact, lives by its function, or, in common language, by doing its work. Yet the anatornical tokens of mere disuse, as in the motor cells

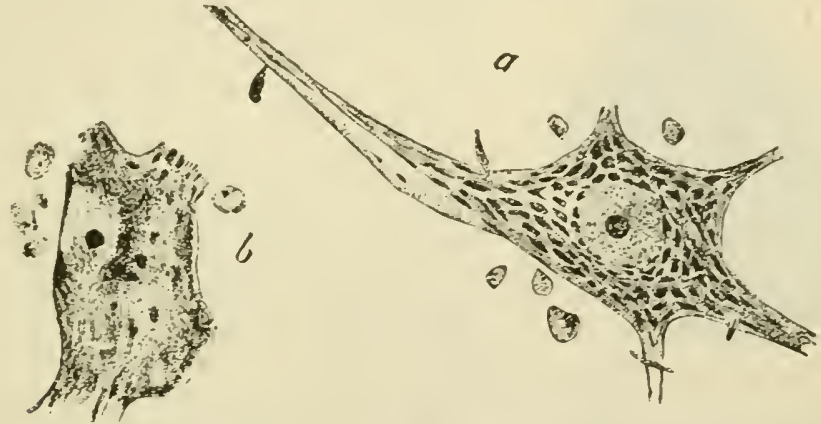

Fig. 340.-Cells from the Nuclei of the Oculo-Motor Nerves of the Cat Thirteen Days atter Division of the Root-Fibres on one Side: Nissl's Stain (Barker, after Flatau). $a$, normal cell from side on which the roots were not cut; $b$, cell from side operated upon. Only a few Nissl bodies are present in $b$, and the nucleus is displaced to one side of the cell.

of the anterior horn after division of the cord at a higher level, are less distinct than those which follow section of the axon. Therefore it must be concluded that the latter, although not indispensable for the nutrition of the cell as the cell is for the axon, exerts an influence upon it. Similar changes in the chromatin may also be produced in nervecells by a period of anæmia, in extensive superficial burns, in tetanus caused by the injection of bacterial cultures, in acute alcoholic poisoning, in fatigue, and in other ways. According to Wright, the inhalation of ether or chloroiorm (in dogs) so alters the chromatic substance that it loses its affinity for aniline dyes. In long-continued anæsthesia the nucleus is also affected, while the nucleolus is the last part of the cell to suffer. A greater alteration occurs in the cells in the three hours between the sixth and ninth hours of anæsthesia than in the five hours between the first and sixth. Although the changes are transitory, the cells, after a narcosis of nine hours, being practically normal in fortyeight hours, they indicate that the duration of safe surgical anæsthesia. has a limit measured by hours.

It is probable that the alterations in the chromatic substance should 
not be looked upon as the token of any specific lesion; they are the common structural response of the cell to injurious influences of the most varied nature.

Grey and White Matter.-Nerve-cells are the most distinctive his. tological feature of the grey nervous substance. Sown thickly in the cerebral cortex, the basal ganglia, the floor of the fourth ventricle, and the cervical and lumbar enlargements of the cord, they are scattered more sparingly wherever the grey matter extends. They also occur in the spinal ganglia, and their cercbral homologues (such as the Gasserian ganglion), in the ganglia of the sympathetic system, and the sporadic ganglia in general. But wide as is their distribution, and great as is the size of the individual cells, some of which have a diameter of $140 \mu$, or even more, they yet make up but a small portion of the whole of the central nervous substance, the total weight of the 9,000 millions of nerve-cell bodies in the human brain being less than 27 grammes (Donaldson). And although it is not to be wondered at that objects so notable when viewed under the microscope should liave struck the

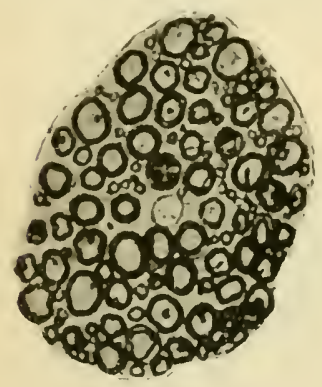

Fig. 34x.-Transverse Section of a Bundle of NerveFibres from the Anterior (Ventral) Root of the First Coccygeal Nerve of the Cat (Dale). The great differ. ence in the diameter of the fibres is well shown. The small fibres are vaso-motor. imagination of pliysiologists, it is probable that the very high powers which it is so common to attribute exclusively to them are, in part at least, shared with the network or feltwork formed by their processes.

The grey matter, in addition to this exceedingly delicate feltwork of non-medullated fibres and filaments representing the dendrites and such axons and collaterals as terminate within itself, contains also, as may be seen in preparations stained by Weigert's method,* great numbers of exceedingly fine medullated fibres, many of which are the collaterals of fibres that are passing out to the white matter.

Only medullated nerve-fibres are met with in the white matter of the cerebro-spinal axis. They are commonly stated to be devoid of a neurilemma (or neurolemma), and in the sense that there is no continuous separate membranous sheath corresponding to the sheath of Schwann of the peripheral medullated fibres this is correct. Sheath cells, however, are present, and form a reticulum around each fibre in the meshes of which myelin is contained. In diameter the medullated fibres of the white matter vary from $2 \mu$ to $20 \mu$. In Malapterurus electricus the fibre in the cord which supplies the electrical organ is of immense size; and in the anterior column of many fishes may also be seen a single gigantic fibre on each side with a diameter of nearly roo $\mu$. It cannot be said that any relation between the functions of neurons and the calibre of their axons has been definitely established. Many afferent fibres, it is true, are small-this is notably the case with the fibres of the posterior column, and many motor fibres are large. But the distinction can by no means be generalized, for the fibres of the direct cerebellar tract (p. 866), which certainly are afferent, are amongst the largest in the spinal cord; and the vaso-motor fibres, which pass from the cord by the anterior (ventral) roots (Fig. 34I) into the sympathetic, are smaller than the fibres of the posterior column. Even the motor

* Weigert's is a special method of staining the medullary sheath with hæmatoxylin. 
nerve-fibres of striated muscles vary considerably in diameter, those of the tongue, $\varepsilon . g$. being smaller than those of the muscles of the limbs. lurther, the medullated fibres of the brain are, without reference to function, in general finer than the fibres of the cord. As a rule the fibres whose course is the longest are the thickest, but the rule is often broken. For example, the average dianeter of the fibres going to the thigl of the frog is greater than that of the fibres going to the lower part of the limb (Dumn). The cause of these differences in the size of nerve-fibres is quite unknown. It is more likely to be morphologicai than physiological.

Supporting Tissue.--The protective membranes of the central nervous system consist of ordinary connective tissue derived from the mesoderm. The supporting framework which interpenctrates the nervous substance consists of a peculiar form of tissue derived from the ectoderm, and called neuroglia. The whole cerebro-spinal axis is wrapped in four concentric shcaths. Next the walls of the bony hollow in which it lies is the dura mater. Next the nervous substance itself, following the convolutions of the brain and the fissures of the cord, and giving off bloodvessels to both, is the pia mater. Between the dura and the pia, separated from the latter by a jacket of cerebro-spinal fluid, is the double layer of the arachnoid. The comparatively coarse septa that run into the nervous substance as if coming off from the pia mater are the main beams in the scaffolding of non-nervous material with which that substance is interwoven, and by which it is supported. The interstices are filled in by a thick-set feltwork of interlacing neuroglia fibres, which lie close against the small glia cells. In preparations impregnated by the Golgi method many of the neuroglia fibres appear to be processes running out from the attenuated cell-body like the arms of a microscopic crab or spider. But this is a deceptive appearance, as has been shown by means of special methods in which the neuroglia fibres are alone stained (Weigert, Huber, etc.). They generally lie in close contact with, or embedded in, the protoplasm of the neuroglia cells, from which they have become differentiated structurally and chemically, but sometimes they may detach themselves entirely from the cells and lie frec in the intervening tissue. The neuroglia is present in greatest abundance in the grey matter immediately surrounding the central canal of the cord and the ventricles of the brain (the ependyma, as it is called), from which long neuroglia fibres pass out radially, giving off branches on their course, and ending in little knobs or enlargements attached to the pia mater.

\section{Section II.-General Arrangement of the Grey and White Matter in the Central Nervous System.}

(I) Around the central canal, as we have seen, a tube of grey matter sheathed with white fibres is developed. This tube, from optic thalamus to conus medullaris, may be conveniently referred to as the central grey axis or stem, which, in the lowest vertebrates-e.g., fishes--is much the most important part of the central nervous system.

(2) On the outer surface of the anterior portion of the neural axis, but not in the part corresponding to the spinal cord, is laid down a second sheet or mantle of cortical grey matter. Between 
this and the primitive grey stem are interposed $(a)$ the sheath of white fibres that clothes the latter, and connects its various parts, and (b) a new development of white matter (corona radiata, cerebellar peduncles), which serves to bring the cortex into relation with the primitive axis, and through it with the rest of the body.

Although there are histological and developmental differences between the cerebral and the cerebellar cortex, we may, for some purposes, classify them together as cortical formations. An's we may also include under this head the corpora striata, which, although for descriptive purposes generally grouped with the optic thalami and the other clumps of grey matter at the base of the brain, as the basal ganglia, are to be regarded as cortical in character. As we. mount in the vertebrate scale the cortex formation of the secondary fore-brain and hind-brain acquires prominence.

In other words, the grey matter developed in the roof of the cerebral vesicles I. and III. (Fig. 327) (the grey matter of the cerebral and cerebellar cortex) comes to overshadow the superficial grey matter hitherto present only in the roof of vesicle II. (in the corpora bigemina). And this cortex formation becomes larger in amount, and, in the case of the cerebral grey matter, more richly convoluted, the higher we ascend, until it reaches its culmination in man. As the anterior cerebral vesicles develop, they spread continually backward, until at length the ccrebral hemispheres cover over, and almost completely surround, the primary fore-brain and the mid-and hind-brains, so that the anterior portion of the primitive stem comes, as it were, to be invaginated into the second wider tube of cortical grey matter. This development of the cortical grey substance is accompanied with a corresponding development of nerve-fibres, for an isolated nerve-cell (apart, of coursc. from possible embryonic rudiments which have not undergone complete development) is no more conceivable than a railway-station the track from which leads nowhere in particular, or a harbour on the top of a hill.

But it is to be particularly observed that the new formation does not supplant the old, but works through and directs it. The neuroblasts of the cortex do not throw out their axons to make direct junction with muscles and sensory surfaces. Such junction the cortex finds already established between the primitive cerebro-spinal axis and the periphery. It joints itself on by nerve-fibres to the cells of the central stem; and we have reason to belicve that no single axon in an ordinary spinal or cranial nerve* runs all the way from the periphery: to the cortex, and no axon of a cortical nerve-cell all the way from the cortex to the periphery, but that the connection is made by a chain of at least two neurons, the cell-body of one of which is situate in this primitive grey tube.

The fibres from the cortex of each cerebral hemisplucre (corona radiata), radiating out like a fan below the grey matter, are gathered together into a compact leash as they sweep down through the isthmus of the brain in the internal capsule, to join the crura cerebri. The cortex of each cerebellar hemisphere, and the ribbed pouch of grey

* The olfactory and possibly to some extent the optic nerves are exceptions to this statement. Their relation to the cortex, as is easily understood from the manner of their development (p. 85o), is different from that of the other nerves. 
matter, known as the corpus dentatum, which is buried in its white core, are also connected by strands of fibres with the central stem and the cerebral mantle. The restiform body or inferior peduncle brings the cerebellum into communication with the spinal corrl. The superior peduncle by one path, and the middle peduncle by another, connect it with the cerebral cortex. A great transverse commissure, the corpus callosum, unites the cerebral hemispheres across the middle line, while transverse fibres, that break through the middle lobe or worm, form a similar though far less massive junction between the two hemispheres of the cerebellum.

The fibres of the nervous system may be divided into (I) fibres connecting the peripheral organs with nerve-cells in the central grey axis; (2) fibres connecting nerve-cells in this central axis with cells in the external or cortical grey tube; and (3) fibres linking cortex with cortex, or central ganglia with each other. In the third group are included $(a)$ fibres which connect portions of the cortex on the same side (association fibres); (b) fibres which connect portions on opposite sides of the middle line (commissural fibres); (c) fibres which connect the central grey matter at different levels-e.g., the propriospinal or endogenous fibres of the cord. Our first task is, therefore, to trace the peripheral nerves to their cells of origin or centres of reception* in the nervous stem. And although there is reason to believe that the whole of the peripheral nerves, cerebral and spinal (with the exception of the olfactory and optic, which are rather portions of the brain than true peripheral nerves), form a morphological series, it will be well to begin with the spinal nerves, since their motor and sensory fibres are gathered into different and definite roots, whose course within the cord is, in general, more easily traced than the course of the cerebral root-bundles within the brain.

\section{Section III.-Arrangement of the Grey and White Matter in THE SPINAL CORD.}

The grey matter of the spinal cord is arranged on each side in a great unbroken column of roughly crescentic section, joined with its fellow across the middle line by a grey bar or bridge, which springs from the convexity of the crescent, and is pierced from end to end by the central canal. The anterior horn of the crescent, although it varies in shape at different levels of the cord, is, in general, broad and massive, in comparison with the slender and tapering posterior horn. In the lower cervical and upper dorsal region a moulding or projection, forming a lateral horn, springs from the fluted outer side of the grey substance. Within the grey matter nerve-cells are found, sometimes so regularly arranged that they form veritable cellular or vesicular strands. Of these the best

* The centre or nucleus of recption of a nerve contains the nerve-cells around which its axons terminate; the nuclcus of origin of a nerve contains the cells from which its axons arise. 
marked are-(I) The tract or tracts made up by the cells of the anterior horn (Fig. 342), which practically run from end to end of the cord, swell out in the cervical and lumbar enlargements, where the cells are very numerous and of great size (7o $\mu$ to $140 \mu$ in diameter), and contract to a thin thread in the thoracic region, where they are relatively few, scattered, and small. In the enlargements there are several groups of these cells corresponding with the segments

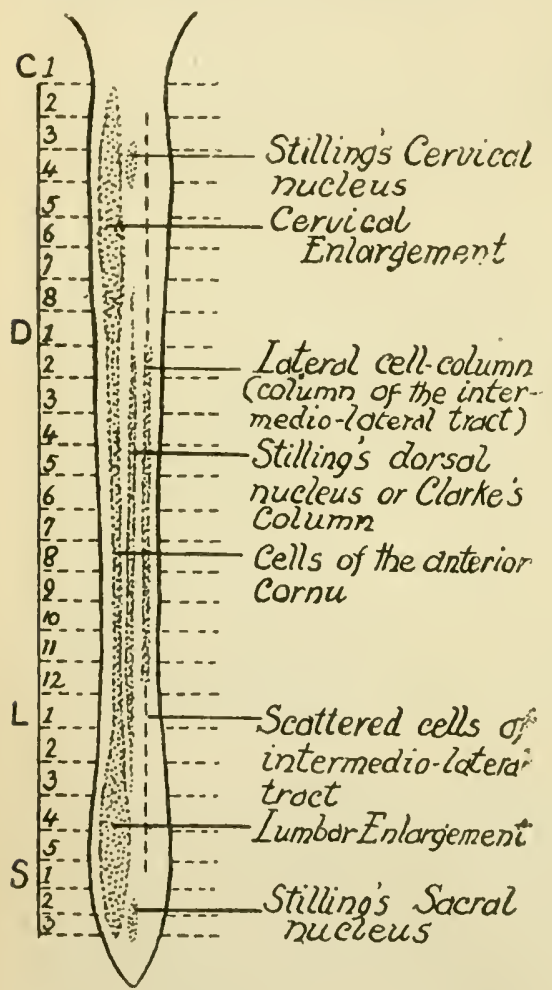

Fig. 342.-Diagram of Grey Tracts of Cord. of the limbs, the movements of the hand, forearm, and upper arm being each represented by a group in the cervical, and those of the foot, leg, and thigh by groups in the lumbar swelling. In the rest of the cord only two well-marked groups of cells are present in the anterior horn, a mesial and a lateral. (2) Clarke's column, whose cells, mostly of good size and somewhat rounded in outline, are situated at the inner side of the root of the posterior horn just where it joins on to the grey cross-bar. It gradually increases in size from above downwards, usually appearing first at the level of the seventh or eighth cervical nerve, attaining its maximum development at the eleventh or twelfth dorsal and disappearing altogether, as a continuous strand, at the level of the second or third lumbar nerves. Scattered nerve-cells, however, constituting the socalled cervical and sacral nuclei of Stilling, are frequently found occupying the same position towards the upper and lower ends of the cord, and may be looked upon as isolated portions of Clarke's column. (3) A tract of small cells called the intermedio-lateral tract, lateral cell column, or lateral horn, situated at the outer edge of the grey matter, about midway between the anterior and posterior horn.s. It is best marked in the thoracic region, up to about the second thoracic segment, although in the corresponding situation there are scattered cells in the lumbar swelling and the cervical 
cord. There is reason to believe that the axons of cells of the intermedio-lateral tract, which pass out as small medullated fibres in the anterior roots, form the preganglionic segments of the efferent vascular and visceral nerves (p. 185). (4) The cells of the posterior horn, which, although numerous, are smaller than those of the anterior horn. Throughout the whole cord, however, two small groups of cells may be distinguished, one on the lateral side of the horn, about its middle, and the other on the mesial side, a little in front of-i.e., ventral to - the edges of the substance of Rolando. Both of these groups are broken up by the passage through then of bundles of fibres which form a network, and they are therefore called respectively the group of the lateral and the group of the posterior reiicular formation.

The white matter of the cord is anatomically divided by the position of the nerve-roots and the anterior and posterior fissures
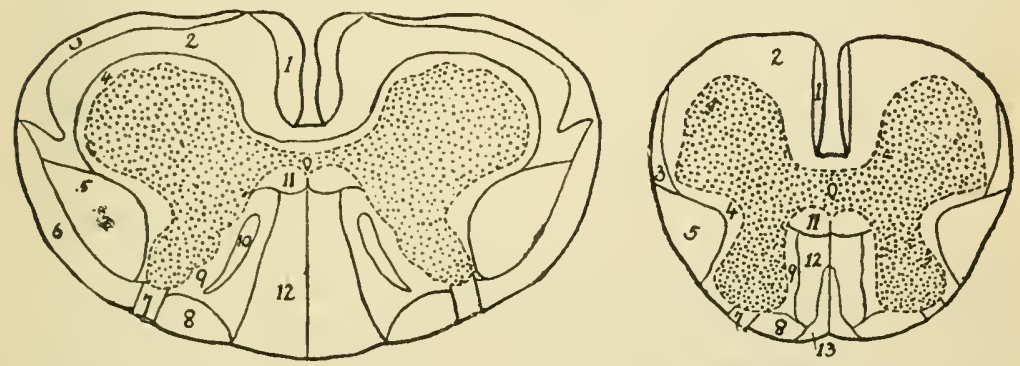

Fig. 343.-Diagrammatic Section of the Spinal Cord in the Cervical and Lumbar Enlargements, to show Tracts of Fibres (Starr). I, antero-median column; 2, autero-lateral column; 3, ascending antero-lateral or Gowers' tract; 4, marginal tract (ground bundle, consisting of short endogenous fibres); 5, lateral or crossed pyramidal tract; 6 , direct cerebellar tract; 7 , tract of Lissauer; 8 , external portion, and 9, root zone, of Burdach's column; Io, comma tract; Ir, pos. terior commissural tract; I2, Goll's column; I3, septo-narginal tract.

into three columns on each side: the anterior, lateral, and posterior columns. The first two, since they are not separated by a perfectly definite boundary, are often grouped together as the antero-lateral column. In the cervical region it may be seen with the microscope that the posterior white column is almost bisected by a septum running in from the pia mater towards the grey commissure. The inner half is called the postero-median column, or column of Goll; the outer half the postero-external column, or column of Burdach (Fig. 343). No localization of any of the other conducting paths in the cord is possible by gross anatomical examination; but by means of the developmental method and the method of degen sration the columns of Goll and Burdach can be followed throughout the cord, and several similar areas can be mapped out. We shall only mention those that are physiologically the most important. 
When the spinal cord is divided, and the animal allowed to survive for a time, certilin tuacts are picked out by the degeneration of their fibres, although in every degenerated tract some fibres remain unaffected. We may distinguish the tracts that degenerate above the lesion (ascending degeneration) from those that degenerate below the lesion (descending degeneration).

Ascending Tracts.-Above the lesion degeneration is found both in the posterior and the antero-lateral columns. Immediately above the section nearly the whole of the posterior column is involved. Higher up the degeneration clears away from Burdach's tract, and, shifting inwards, comes to occupy a position in the column of Goll. In the antero-lateral column two degenerated regions are seen, both at the surface of the cord, one a compact, sickle-shaped area extending forwards from the neighbourhood of the line of entrance of the posterior roots, and the other an area of scattered degeneration, embracing many intact fibres, and completing the outer boundary of the column almost to the anterior median fissure. The compact area is called the dorsal or direct cerebellar tract, or tract of Flechsig (or the fasciculus cerebello-spinalis), the diffuse area the antero-latcral ascending tract, or tract of Gowers, or ventral cerebellar tract (or the fasciculus antero-lateralis superficialis). * The dorsal cerebellar tract is distinguished by the large size of its fibres. It is only distinct in the dorsal and cervical regions of the cord. The tract of Lissaner, or posterior marginal zone, is another small ascending tract at the onter sicle of the tip of the posterior horn. It is made up of fine fibres from the posterior roots which soon pass into the posterior column,

Descending Tracts.-When the cord is divided, say, in the upper dorsal or cervical region, the following tracts degenerate below the lesion:

(I) A small group of fibres close to the artero-median fissure, which has received the name of the direct pyramidal tract-pyramidal lecause higher up in the medulla oblongata it forms part of the pyramid; direct, because it does not cross over at the decussation of the pyramids, but continues down on the same side. In the standard anatomical nomenclature it is termed the fasciculus cerebrospinalis anterior. The direct pyramidal tract is only present in man and the higher apes.

(2) A tract of degenerated fibres in the posterior part of the lateral column. This is the lateral or crossed pyramidal tract (or the fasciculuscercbro-spinalis lateralis), and is much larger than the direct. In the medulla it also lies within the pyramid, but, unlike the direct pyramidal tract, it crosses to the opposite side of the cord at the decussation. The pyramidal tracts are also called cortico-spinal to indicate their origin and termination.

* Some writers employ the terms dursal and ventral spino-cerebellar tracts. 
(3) A tract of scattered degeneration lying along the margin of the corcl in the anterior portion of tho antero-lateral column, and partly overlapping the tract of Gowers. It is called the antero-lateral descending tract, or tract of Loezenthal, or the vestibulo-spinal tract.

(4) The prepyramilal (or rubro-spinal) tract, or Monaliow's tract (also called the fasciculus intomedio-lateralis), lying immediately in front of the crossed pyramidal tract.

(5) A small, conmma-shaped island of degeneration (comma tract) can be followed downwards for a short distance in the middle of Furdach's column. It is only seen in the cervical and upper thoracic regions.

Less well known descending tracts are--

(6) The olivo-spinal and thalamico-spinal tracts (or Helweg's bundle) in the anterclateral column opposite the head of the anterior horn. This tract does not pass down beyond the lower cervical region. The olivo-spinal tract appears to consist of fibres running down from the olivary body into the cord, while the thalamico-spinal tract is made up of descending fibres originating in the optic thalamus. This is an important tract in the lower vertebrates, but not in man.

(7) The tract of Marie in the anterior column is chiefly a continuation into the cord of

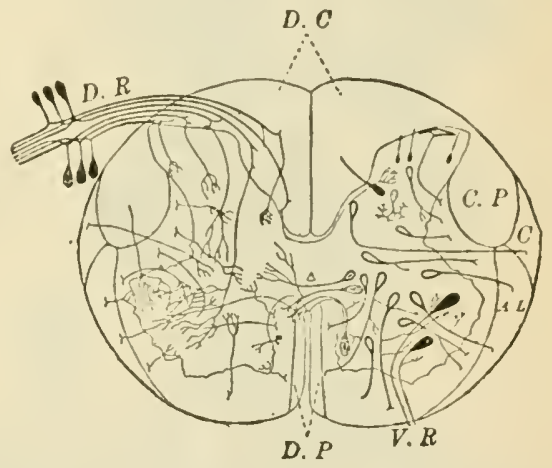

Fig. 344.-Scheme of Cross-Section of Spinal Cord (Donaldson, after Lenhossek). On the left side only the afferent fibres are shown; the efferent fibres and the spinal cells on the right side. D.R., posterior (dorsal) root; V.R. anterior (ventral) root: C.P., crossed pyranidal fibres; $C$., direct cerebellar tract; A.L.. antero-lateral tract; D.C., posterior columns.

the posterior longitudinal bundle, one of the conspicuous tracts of the brain-stem or upper portion of the cerebro-spinal axis (p. 885). It contains both ascending and descending fibres.

When we have deducted the long ascending and descending tracts which have been described, there still remains in the antero-lateral column a balance of white matter unaccounted for. This white substance, which does not degenerate for any great distance either above or below a lesion, is called the antero-lateral ground-bundle, and lies chiefly in the form of an incomplete ring around the grey matter. For descriptive purposes it is sometimes distinguished as the anterior ground-bundle (or fasciculus anterior proprius) in the anterior column, and the lateral ground-bundle (or fasciculus lateralis proprius) in the lateral column. It is believed to consist of fibres (endogenous or proprio-spinal fibres) which run only a comparatively short course in the cord, and serve to connect nerve-cells at different levels. Some of these endogenous fibres are ascending, others 
descending. The septo-marginal bundle consists also largely of fibres which begin and end in the cord (proprio-spinal fibres). Some endog-

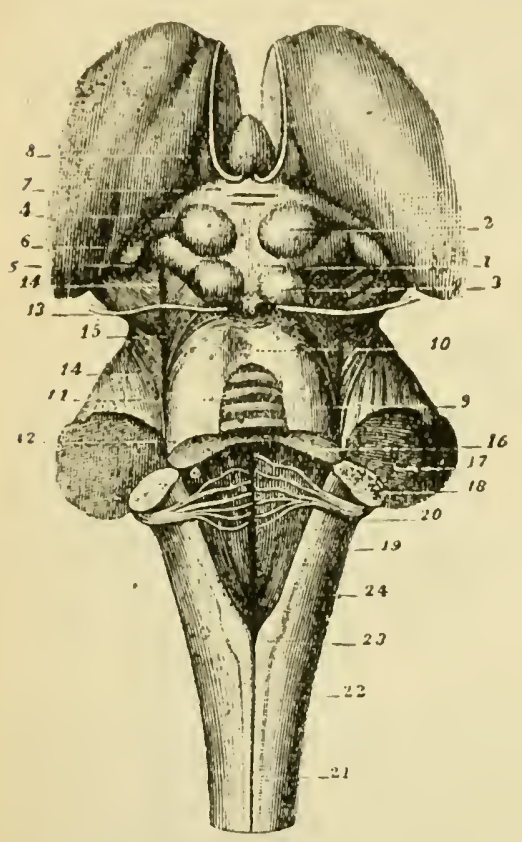

Fig. 345.-Medulla Oblongata, Pons and - Corpora Quadrigemina (Dorsal or Pos. terior View) (Sappey). I, corpora quadrigemina; 2, nates; 3 , testes; 4 , anterior brachium uniting the nates to the lateral geniculate body; 5 , posterior brachium uniting the testes to the internal geniculate body $6 ; 7$, pos. terior commissure; 8 , pineal gland pulled forward to show nates; 9, superior peduncle of the cerebcllum; ro, I1, 12, valve of Vieussens; 13, trochlear nerve; 14, lateral sulcus; 15, fillet; 16 , superior, 17 , middle, and 18 , inferior, peduncle of the cerebellum; I9, floor of fourth ventricle; 20 , audi. tory nerve; 21 , spinal cord; 22, posteromedian column, continued in the me. dulla as the funiculus gracilis; 23 , the clava, the continuation of the funiculus gracilis.

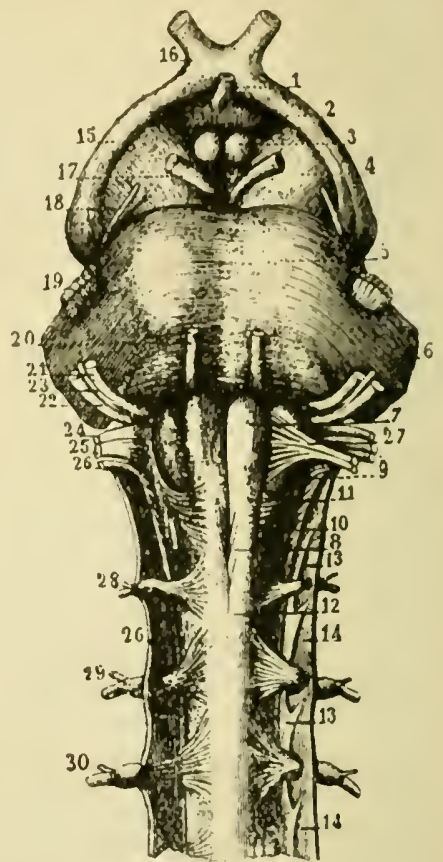

Fig. 346.--Medulla Oblongata, Pons and Crura Cerebri (Ventral or An. terior View). I, infundibulum; 2, tuber cinereum; 3, corpus man. millare; 4 , cerebral peduncle or crus cerebri; 5 , pons; 6 , middle peduncle of cerebellum; 7, pyramid; 8 , decus. sation of pyramids; 9 , olive; 10, tu. bercle of Rolando; II, external arcuate fibres; I 2, upper end of cord; 13. ligamenium denticulatum; I4. dura mater of cord; 15 , optic tract; 16, chiasma; 17 , third nerve; 18 , fourth nerve; 19. fifth nerve; 20. sixth nerve; 21 , seventh nerve; 22 . eighth nerve; 23 , nerve of Wrisberg (portio intermedia), which unites with the facial; 24, glosso-pharyn. geal nerve; 25 , vagus; 26 , spinal accessory; 27, hypoglossal; 28, 29. 30, first, second, and third pairs of cervical spinal nerves.

enous fibres may also be intermingled with the fibres of certain of the long tracts, both in the antero-lateral and posterior columns, and 
Sherrington has shown (in the dog) that long proprio-spinal fibres passing down in the lateral column connect the upper with the lower parts of the cord (1) gos).

The next question which arises is: How are the long tracts connected below-i.e., with the periphery-and above-i.e., with the higher parts of the central nervous system? The answer to this question, partly derived from clinical records and partly from experimental results, is in the case of some of the tracts unexpectedly full and minute, though meagre in regard to others. But to render it intelligible it is necessary, first of all, to describe briefly-

\section{Section IV.-Arrangement of Grey and White Matter in the Upper Portion of the Cerebro-Spinal Axis.}

In the medulla oblongata the grey and white matter of the spinal cord is rearranged, and, in addition, new strands of fibres and new nuclei of grey substance make their appearance. Of these nuclei the most conspicuous is the dentate nucleus of the inferior olive, which, covered by a crust of white fibres, appears as a projection on the antero-lateral surface of the medulla. In front of the olive, between it and the continuation of the anterior median fissure, is another projection, the pyramid, which looks like a prolongation of the anterior column of the cord, but is made up of very different constituents. Dorsal to the olive is the restiform body or inferior peduncle of the cerebellum, and behind the restiform body lie two thin columns, the funiculus cuneatus, which continues the postero-external column of the cord, and the funiculus gracilis, which continues the pos-

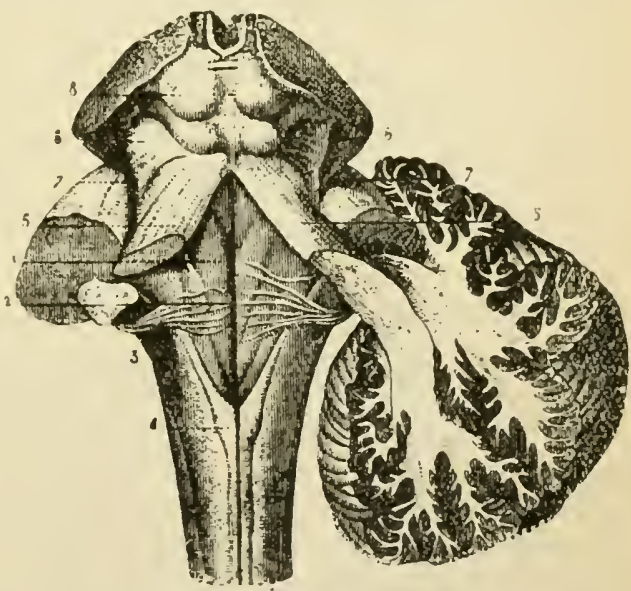

Fig. 347.-Medulla Oblongata and Cerebellum, with Fourth Ventricle (Hirschfeld). I, mesial groove of floor of ventricle running down to the calamus scriptorius; 2 , striæ acustica; 3 , inferior peduncle of the cerebellum; 4, clava; 5 , superior peduncle crossing the inferior and passing to its internal side; 7,7 , lateral sulcus; 8 , corpora quadrigemina. tero-internal column. In these funiculi are contained collections of small or medium-sized nervecells termed respectively the nucleus cuneatus and the nucleus gracilis. The rearrangement of the constituents of the cord is due mainly to two causes: (I) The opening up of the central canal to form the fourth ventricle, and the folding out, on either side, of the grey matter which lies posterior to it in the cord; (2) the breaking up of the grey matter of the anterior horn by strands of fibres as they swcep through it from the lateral pyramidal tract to take up a position in the pyramid of the opposite side (decussation of the pyramids), and a little higlier up by 
fibres passing across the middle line from the gracile and cuneate rucle (sensory decussation or decussation of the fillc $t$ ). The mosaic of grey and white matter formed in tle medulla by the interlacing of longitudinal and transwerse fibres with each other and with the relics of the anterior horn, is called the reticular formation (formatio reticularis). It occupies the anterior and lateral portions of the bulb behind the pyranids and olivary bodies, and is continued upwards in the dorsal portion of the pons and crura cerebri, and downwards for a little way into the upper part of the cervical cord.

The cerebro-spinal axis passes up from the medulla through the pons, encircled and traversed by the transverse pontine fibres derived from the middle cerebellar peduncle or commissure, which enclose everywhere between them numerous collections of nerve-cells (nuclei pontis). Enlarged by the accession of many of these fibres which come from the cortex of the cerebellum on the opposite side, as well as of fibres from the nuclei of the cranial nerves that take origin in this neighbourhood (fifth and eighth), the central nervous stem bifurcates above the pons into the two divergent crura cerebri. From each crus a great sheet of fibres passes up between the optic thalamus and the caudate nucleus of the ccrpus striatum on the one hand, and the globus pallidus of the lenticular nucleus on the other, as the internal capsule, from which they are dispersed, in the corona radiata, to the cerebral cortex. Both in the upper part of the pons and in the crus a ventral portion, or crusta, containing the fibres of the pyramidal tract, and a dorsal portion, or tegmentum, can be distinguished, the line of separation being marked in the crus by a collection of grey matter, called from its usual, though not invariable, colour the substantia nigra (Fig. 352). A portion of the tegmentum is continued below the optic thalamus.

\section{Section V.-Connections of the Long Paths of the CoRd.}

Coming back now to our question as to the connections of the long tracts of the cord, let us consider, first of all,

The Connections of the Postero-Median and Postero-External Columns.-When a single posterior root is divided, say, in the dorsal region, between the cord and the ganglion, its fibres, as we have already seen (p. 797), degenerate above the section. Since the cellbodies of these neurons lie in the ganglion, if a series of microscopic sections of the spinal cord be made, well-marked degeneration will be found at the level of entrance of the root on the same side of the cord, while below that level there will be only a few degenerated fibres in the comma tract. Immediately above the plane of the divided root the degeneration will be confined to Burdach's column and to its external border. Higher up it will be found in the internal portion of Burdach's and the external rim of Goll's column. Still higher up the degenerated fibres will be confined to the posteromedian column; the postero-external will be free from degeneration.

When a number of consecutive posterior roots are cut, the whole of the postero-external column in the sections immediately above the highest of the divided roots will be found occupied by degenerated fibres, while Goll's column may be free from degeneration, or 
degenerated only at its outer border. Higher up degeneration will be found to have involved the whole of the postero-median column, and to have cleared away altogether from the postero-external. The degeneration in the column of Goll may be traced along the whole length of the cord to the medulla, although the number of degenerated fibres diminishes as we pass upward. The explanation

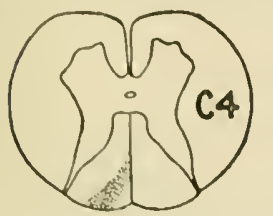
of these appearances is as follows: It may be scen in preparations of the cord impregnated by Golgi's method that the fibres of the posterior roots soon after their entrance into the cord divide into two processes, one of which runs up and the other down in the posterior column, or in the adjoining portion of the posterior horn.

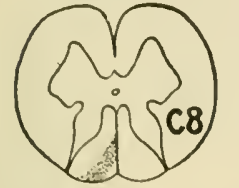
From both of these collaterals are given off at intervals to the grey matter. The descending branclies run downwards only for a short distance, and the degeneration in the comma tract seen after section of the cord is due
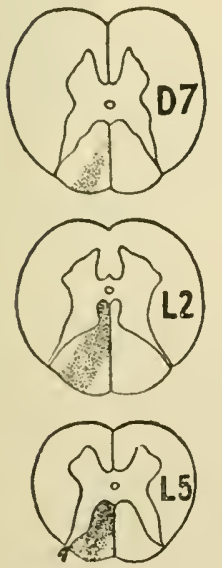

Fig. 348.-Diagrams of Degeneration at Different Levels in the Cord after Sec. tion of a Number of Posterior Roots of Nerves forming the Lumbo-Sacral Plex. us (Mott).

to the division of these branches. Many of the ascending branches pass up for a short distance in the postero-external column, sweeping obliquely through it to gain the tract of Goll. In this tract some of them run right on to the medulla oblongata, to end by arborizing among the cells of the nucleus gracilis. Other fibres, both of Goll's and of Burdach's tract, end at various levels in the cord,

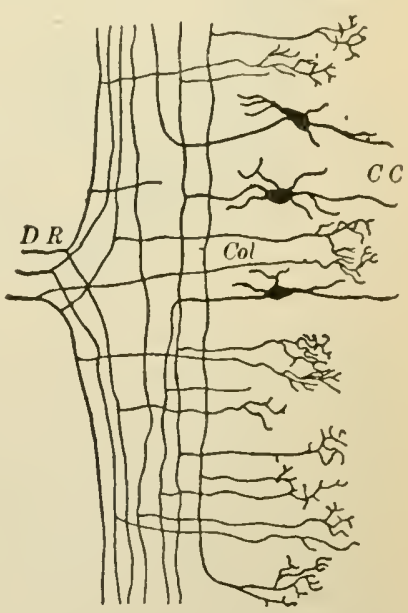

Fig. 349.-Branching of Posterior Root-Fibres in Cord (Donald. son, after Cajal). Collaterals, $\mathrm{Col}$, are seen coming off from the two main branches of the root-fibres, $D R$, and ending in arborizations. $C C$, cells in the grey matter of the cord, whose axons also give off collaterals.

their collaterals, and ultimately the main branches themselves, coming into relation with nerve-cells in the grey matter. When the cervical posterior roots are cut, many of the degenerated fibres remain in Burdach's column up to the medulla, where they terminate 
in the nucleus cuncatus. In the posterior column, then, the numerous fibres of the posterior roots which do not end in the spinal cord are arranged in layers, the fibres from the lower roots being nearest the median fissure (in the postero-median column), and those from the higher roots farthest away from it (in the postero-external column. Thus, in a section through the upper cervical region Goll's column is almost entirely composed of fibres from the posterior limb, while the column of Burdach consists of fibres from the anterior limb. Other collaterals from the posterior root-fibres, and many of the main root-fibres themselves, run into the anterior horn and terminate in arborizations around its cells; some pass into the posterior horn, and doubtless come into relation with its scattered cells and, in the dorsal region, with the cells of Clarke's column. Some of the posterior root-fibres and their collaterals also form synapses with the cells of the intermedio-lateral tract. Other collaterals and probably some axons cross the middle line in the anterior and posterior commissures and end in the grey matter of the opposite sicle.

Connections of the Direct or Dorsal Cerebellar Tract.-Since the dorsal or direct cerebellar tract does not degenerate after section of the posterior nerve-roots, but does degenerate above the level of the lesion after section of the spinal cord, the nerve-cells from which its axons arise must be situated somewhere or other in the cord. Now, it has been observed that the vesicular column of Clarke first becomes prominent in the lower dorsal region, and that in this same region the direct cerebellar tract begins. Atrophy of the cells of Clarke's column has sometimes in disease been shown to accompany degeneration of the direct cerebellar fibres. After an experimental lesion of these fibres in animals, some of the cells of the vesicular column show the changes in the Nissl bodies and the other changes which we have already described as occurring in nerve-cells whose axons have been cut. After two or three months these cells may be found almost completely atrophied (Schäfer). Finally, axiscylinder processes have been seen sweeping out from Clarke's column into the direct cerebellar tract (Mott). The evidence, then, is complete that the cells of origin of this tract are in Clarke's column. Clarke's cells are surrounded by arborizations, some of which, as previously stated, represent the terminations of posterior root-fibres and of their collaterals. The neurons whose axons run in the dorsal cerebellar tract are therefore the second link in an afferent path. The direct cerebellar tract runs right up to the cerebellum through the restiform body, without crossing and without being further interrupted by nerve-cells. The restiform body ends partly in the dentate nucleus of the cerebellum, partly in the vermis, and among the fibres which end in the vermis are those of the direct cerebellar tract. In the dorsal cerebellar tract there is a definite stratification 
of the fibres: the fibres from the lowest segments of the cord lie outermost; beneath these come fibres from the lowest thoracic segments, then fibres from the higher thoracic segments; and, internal to all, fibres from the topmost thoracic and lowest cervical segments.

Connections of the Antero-Lateral Ascending Tract.-According to Schäfer, the axons of this tract are probably connected with cells situated in the middle and posterior parts of the grey crescent, mainly

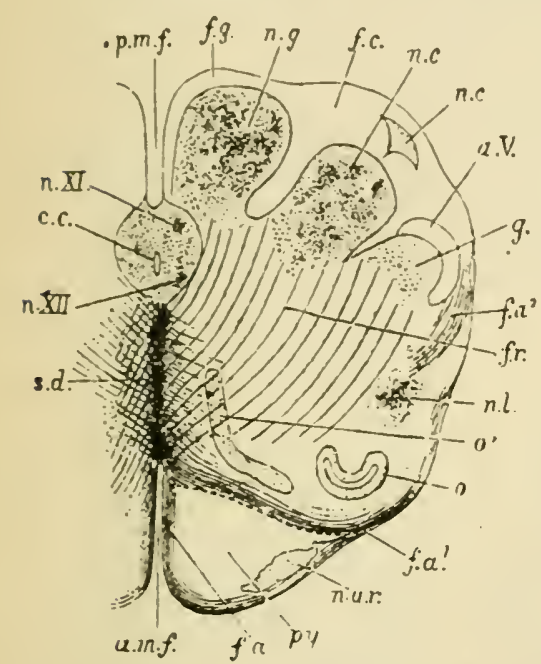

Fig. 350.-Transverse Section of Medulla Oblongata at the Level of the Decussa. tion of the Fillet (Halliburton, after Schwalbe). a.m.f, anterior, and p.m.f, posterior, median fissure; $f . a$ and $f . a^{2}$, external arcuate fibres; $f . a^{\prime}$, internal arcuate fibres becoming external; n.a.r, nuclei of arcuate fibres; $p y$, pyramid; $o, o^{\prime}$, lower end of nucleus of olive; f.r, formatio reticularis; $n . l$, lateral nucleus; $n . g$, nucleus gracilis; $f . g$, funiculus gracilis; $n . c$, nucleus cuneatus; $n . \iota^{\prime}$, external cuneate nucleus; $f . c$, funiculus cuneatus; g, substance of Rolando;c.c, central canal surrounded by grey matter; $n . X I$, nucleus of spinal accessory; $n . X I I$, of hypoglossal; $a . V$, ascending root of fifth nerve; s.d, the decussation of the fillet, or superior decussation.

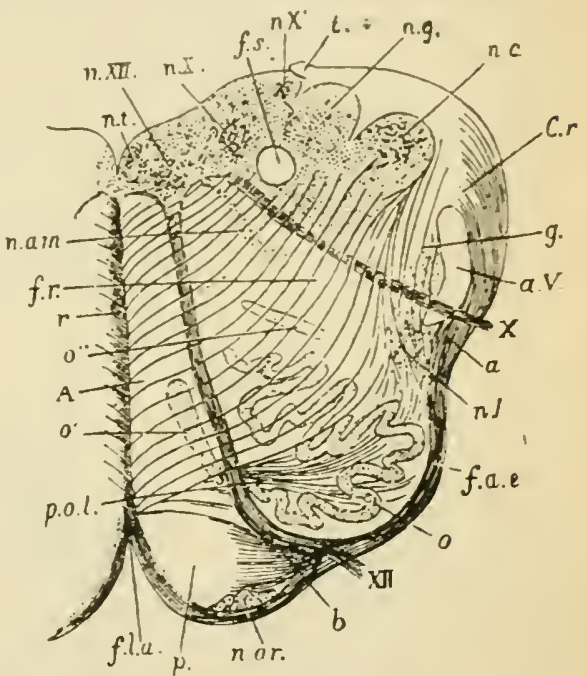

Fig. 35 - Transverse Section of Medulla Oblongata at about the Middle of the Olive (Schivalbe). f.l.a, anterior median fissure; n.a.r, arcuate nucleus; p., pyramid; n.XII, hypoglossal nucleus; $X I I$, root bundle of hypoglossal nerve coming off from the surface; at $b$ it runs between the pyramid and the dentate nucleus of the olive, 0 ; f.a.e, external arcuate fibres; $n . l$, lateral nucleus; $a$, arcuate fibres going to restiform body c.r, partly through the substantia gelatinosa $g$, partly superficial to the ascending root of the fifth nerve a. $V ; X$, root-bundle of vagus; $n . X, n . X^{\prime \prime}$, two portions of vagus nucleus; $f . r$, formatio reticularis: $n . g$, nucleus gracilis; $n . c$, nucleus cuneatus; $n . t$, nucleus of the funiculus teres; n.am, nucleus ambiguus; $r$, raphe; $o^{\prime}, o^{\prime \prime}$, accessory olivary nucleus; p.o.l, peduncle of the olive.

on the opposite side of the cord, although also on the same side. None of the fibres of the tract can come directly from the posterior nerve-roots, since no degeneration is seen in it on section of the roots alone.

The antero-lateral ascending tract passes up through the medulla, where some of its fibres perhaps form synapses with the cells of the 
lateral nucless. a collection of grey matter in the lateral portion of the spinal bulb. But its main strand runs on unbroken through the medullat, in front of the restiform body, and behind the olive, and after reaching the upper part of the pons bends back over and in company with the superior peduncle as the ventral spino-cerebellar bundle, to end in the worm of the cerebellum (Fig. 363).

$A$ few fibres of Gowers' tract may pass by the middle peduncle to the opposite cerebellar hemisphere. Some of its fibres do not go to the cerebellum at all. One group can be followed to the corpora quadrigemina (spino-tectal fibres), and another by way of the tegmentum of the crus cerebri to the optic thalamus (spino-thalamic fibrss).

Through the relay of the gracile and cuneate nuclei, the posterointernal and postero-external columns of the cord are further connected on the one hand with the cerebrum, and on the other with the cerebellum. The cells of the nuclei give off fibres (internal arcuate fibres) which, sweeping in wide arches across the nesial raphe to the

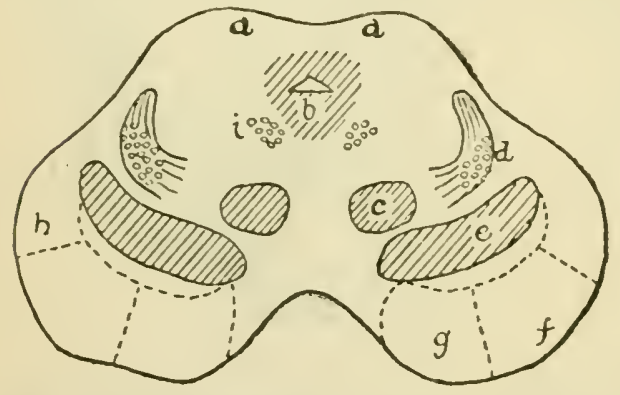

Fig. 352.-Diagrammatic Transverse Section of Crura Cerebri and Aqueduct of Sylvius. $a$, an. terior corpora quadrigemina $b$, aq̨ueduct; $c$, red nucleus; $d$, fillet; $e$, substantia nigra; $f$, pvramidal tract in the crusta of the crura cerebri: g. fibres from frontal lobe of cerebrum: $h$, fibres from temporo-occipital lobe; $i$, postcrior longitudinal bundle.

opposite side, take up a position behind the pyramid in the tract of the fillet, a bundle of fibres which becomes more compact, and therefore more distinct, as it passes brainwards. Receiving fibres from other sources on its way, and also giving off fibres, it runs upwards through the dorsal or tegmental portion of the pons. In the midbrain it divides into two portions, the lateral fillet, also called the lower fillet or fillet of Reil, and the intermediate, also called the upper fillet. The lateral fillet contains mainly fibres arising in the cochlear nucleus of the auditory never, and ends in grey matter of the posterior corpus quadrigeminum, and partly in the mesial geniculate bocly. It appears to be a path for the conduction of auditory impulses. The intermediate fillet contains chiefly the fibres that come off from the gracile and cuncate nuclei, but is enlarged by the accession of fibres from the sensory muclei of the cranial nerves. It terminates in the lateral nucleus of the optic thalamus by forming synapses with nerve-cells, whose axons, passing through the posterior limb of the internal capsule and the corona radiata, continue the afferent path to the cerebral cortex.

Not all of the axons from the cells of the cranial sensory nuclei run 
in the fillet. Many of them occupy a position in the reticular formattion of the tegmentum dorsal to the fillet as they pass through the pons and mid-brain to end in the thalamus and the region below it (sub-thalamic region). From the sensory nucleus of the fifth nerve a separate bundle of fibres ascends to the thalamus, in the tegmentum of the mid-brain lateral to the posterior longitudinal bundle.

Connections of the Pyramidal Tracts.- When the cortex in and in front of the fissure of Rolando is destroyed by disease in man, or removed by operation in animals, it is found that in a short time degeneration has taken place in the fibres of the corona. radiata which pass off from this area. The degeneration can be followed down through the genu and the anterior two-thirds of the posterior limb of the internal capsule (Fig. 353) and the crusta of the cerebral peduncle of the corresponding side into the medulla oblongata . Below the decussation of the pyramids it is found that the degeneration has involved the two pyramidal tracts, and only these - the crossed pyramidal tract on the side opposite the cortical lesion, the direct pyramidal tract on the same side -and that the crosssection of the two degenerated tracts goes on continually dimin-

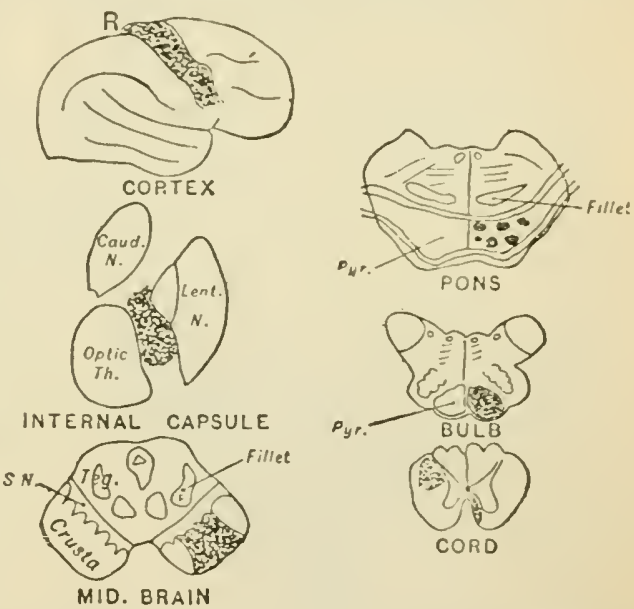

Fig. 353.-Pyramidal Path (after Gowers). Degenera. tion after destruction of the 'motor' area of the right cerebral hemisphere. The degenerated areas are indicated by the shading.

ishing as we pass down the cord. (We overlook for the moment, in the interest of simplicity of statement, the fact that some degenerated fibres are found in the crossed pyramidal tract on the same side as the lesion.) This is proof positive that the cell-bodies of the neurons whose axons run in these tracts are situated in the cerebral cortex. They have indeed been identified with certain of the large pyramidal cells (the so-called giant cells or cells of Betz) in the cortex of the "motor' region in front of the Rolandic fissure (p. 950). For after division of the motor pyramidal fibres in the upper cervical region of the cord (in monkeys) changes in the chromatin (so-called chromatolysis) and atrophy of these large cells occur. The same has been found to be true in man in cases where the cord was injured by fracture of the spine in such a way as to interrupt the tract (as well as other tracts) 
completely and permanently, without entailing death for a considerable time (Holmes and May). The fact that after destruction of the cortex or the path in its course the degeneration below the lesion does not spread to the anterior roots shows that at least one relay of nerve-cells intervenes between the pyramidal fibres and the rootfibres. The results both of normal and morbid histology enable us to identify the cells of the anterior horn as the cells of origin of the axons of the anterior root-fibres. For

(I) Axis-cylinder processes have been actually observed passing out from certain of the so-called motor cells of the anterior horn to become the axis-cylinders of the anterior root.

(2) In the pathological condition known as anterior poliomyelitis, the cells of the anterior horn degenerate, and so do the anterior roots of the affected region, the motor fibres of the spinal nerves, and the muscles supplied by them.

(3) As already mentioned (p. 858), comparatively transient but decided changes occur in the anterior horn cells on section of the corresponding anterior roots.

(4) An enumeration* has been made in a small animal (frog) of the cells of the anterior horn and of the anterior rcot-fibres, and it has been found that the numbers agree in a remarkable manner. From all this it cannot be doubted that most, at any rate, of the cells of the anterior horn are connected with fibres of the anterior rcot. But since the number of fibres in the pyramidal tracts (about 80,000 in each half of the human cord) falls far short of the number of fibres in the anterior roots (not less than 200,000 in man on each side), it is necessary to suppose either that one pyramidal fibre may be connected with several cells or that all the anterior root-fibres are not in functional connection with the pyramidal tract.

There is no reason to assume any such connection in the case of the fine medullated root-fibres arising in the lateral lorn and going to the visceral and vascular muscles.

While there is no doubt that anterior root-fibres and pyramidal fibres of the brain and cord form segments of the same nervous path, the connection between the pyramidal fibres and the cells of the anterior horn has not yet been anatomically demonstrated. Many of the pyramidal fibres pass into the grey matter between the anterior and posterior horns or near the base of the posterior horn. The anterior horn cells are surrounded by arborizations. Some of these are probably the terminations of axons whose cell-bodies are sit uated in the posterior horn, others the terminations of posterior root-fibres or their collaterals. Many of them very likely represent the end arborizations of pyramidal fibres or their collaterals. Some observers, however, suppose that the pyramidal fibres do not come into immediate relation with the anterior horn cells, but that another neuron is intercalated between them and the cells.

The pyramidal fibres are unquestionably paths for voluntary motor impulses passing down from the cortex to the cord. But

- Such enumerations can be made with great accuracy from photographs of sections of the nerves (Hardesty, Dale). (See Fig. 341, p. 360.) 
they are not the only cortico-spinal efferent paths, and in many animals they are not even the most important paths for voluntary movements. It is the more skilled and delicate movements which the pyramidal tract subserves in man, and it is these movements which are permanently lost when the tract is destroyed. The size of the path is proportioned to the degree of development of the brain. Thus, it is larger in the monkey than in the dog, larger in the anthropoid apes than in the lower monkeys, and larger in man than

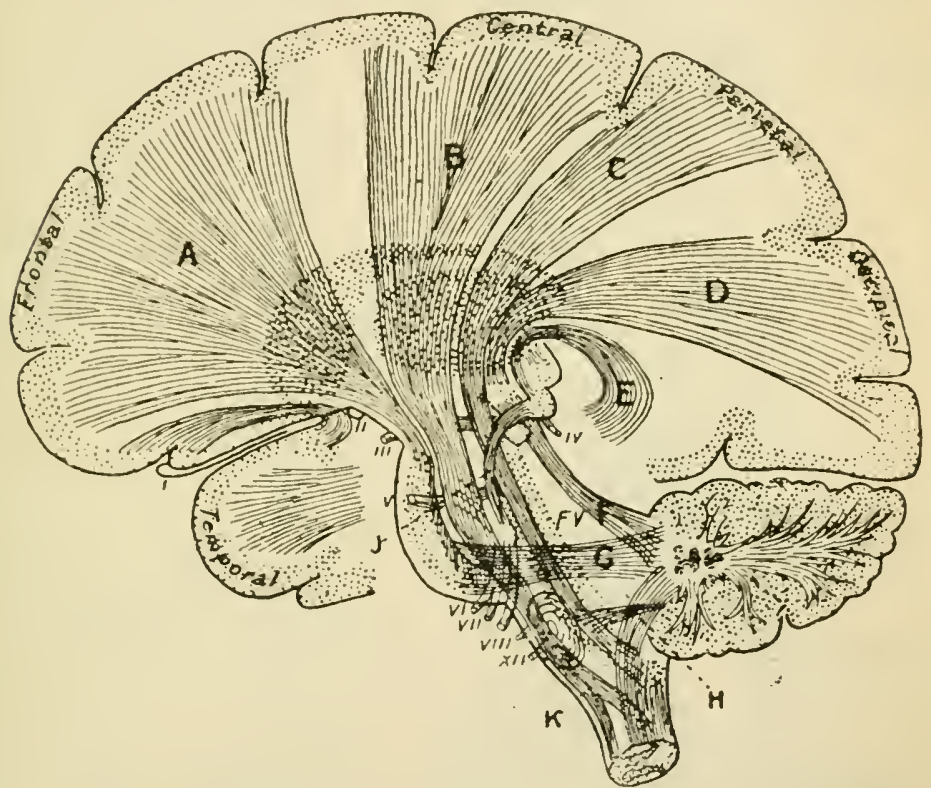

Fig. 354.-Paths from Cortex in Corona Radiata (Starr). A, tract from frontal con volutions to nuclei of pons and so to cerebellum; B, motor pyramidal tract, $C$, afferent tract for tactile sensations (represented in the diagram as separated from $B$ by an interval for the sake of clearness); D, visual tract; $E$, auditory tract; F, G, H, superior, middle, and inferior cerebellar peduncles; J, fibres from the auditory nucleus to the posterior corpus quadrigeminum; $\mathrm{K}$, decussation of the fyramids in the bulb; FV, fourth ventricle. The roman numerals indicate the cranial nerves.

in even the highest of the apes. In the lower mammals it is exceedingly small. While in man the pyramidal tracts constitute nearly I2 per cent. of the total cross-section of the cord, they make up little more than I per cent. in the mouse, 3 per cent. in the guinea-pig, 5 per cent. in the rabbit, and nearly 8 per cent. in the cat. In some mammals, as the rat, mouse, guinea-pig, and squirrel, the pyramidal tracts lie, not in the antero-lateral, but in the posterior columns. In vertebrates below the mammals the pyramidal system does not exist as a collection of neurons which send their axons with- 
out interruption down from the cortex to the cord. In bircls, e.g., after the removal of a hemisphere, the degeneration does not extend below the micl-brain (Boyce).

\section{Section VI.-Paths From and to the Cortex.}

Thus far we have been able to map out two great paths from the cerebral cortex to the periphery-one efferent, the other afferent.

(I) The great efferent or motor pyramidal path, which, starting in the cortex in front of the fissure of Rolando, where its axons give off numerous collaterals to the grey matter soon after emerging from the cells, and sweeping down the broad fan of the corona radiata,

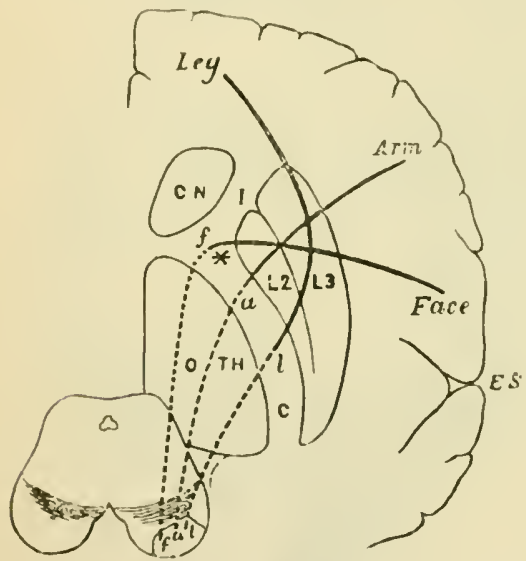

Fig. 355.-Motor Pyramidal Tracts (Diagrammatic) (Halliburton, after Gowers). The convolutions are supposed to be cut in vertical transverse section, the internal capsule, I, $C$, and the crus in horizontal section. O, TH, optic thalamus; CN, calldate nucleus; $L_{2}$ and $L_{3}$, middle and $e x$. ternal portions of lenticular nucleus; $f, a, l$. fibres from the face, arm, and leg areas of the cortex respectively; E, S, Sylvian fissure. The genu or knee of the internal capsule is indicated by the asterisk. passes through the narrow isthmus of the internal capsule into the crusta of the crus cerebri, and thence into the pons (Figs. 354, 355). At this level, the fibres destined to make connection with the motor nuclei of the cranial nerves in the grey matter underlying the aqueduct of Sylvius and the fourth ventricle terminate. Most of these fibres decussate to make physiological connection with nuclei on the opposite side, but some join nuclei on the same side. The question whether they arborize directly around the cells of the motor nuclei or make junction with them through another intercalated neuron is precisely in the same position as the corresponding question for the spinal pyramidal path (p. 876). On their way through the pons they send off collaterals to the nuclei pontis, as they do higher up to the grey matter of the basal ganglia of the cerebrum and the substantia nigra, and the path may be continued to the motor nuclei by axons arising here. There is no proof, however, that this is the case. The rest of the pyramiclal fibres run on into the pyramid of the bulb, where the greater part (usually about go per cent.) of the fibres decussate, appearing in the cervical cord as the massive crossed 
pyramidal trat of the opposite side. A few (usmally about io per eent.) remain on the sane sicle as the slender direct pyramidal tráct. The size of this tract varies much in different individuals, and it is occasionally absent. Its brealth constantly diminishes as it proceeds down the cord, and it disilppears before the midcle of the thoracic region is reached, its libres continually decussating across the anterior white conmissure and plunging into the opposite anterior horn. They either end among its cells, or, passing through it, reinforce the crossed pyramidal tract. The fibres of this crossed tract are, in their turn, continually passing off into the grey inatter to make connection (p. 876) with the cells of the anterior horn, whose axis-cylinder processes enter the anterior roots of the spinal nerves. The losses which it suffers as it descends the cord may be in some slight degree compensated by the bifurcation of some of its fibres (geminal fibres), but ultimately the whole tract forms synapses with cells in the grey matter, and divindles away as the lumbar region is reached(Fig. $3+3$ ). A certain number of the pyramidal fibres do not decussate either in the bulb or in the cord. These are called homolateral fibres. They run down in the lateral pyramidal tract, and are represented by the fibres that degenerate in that

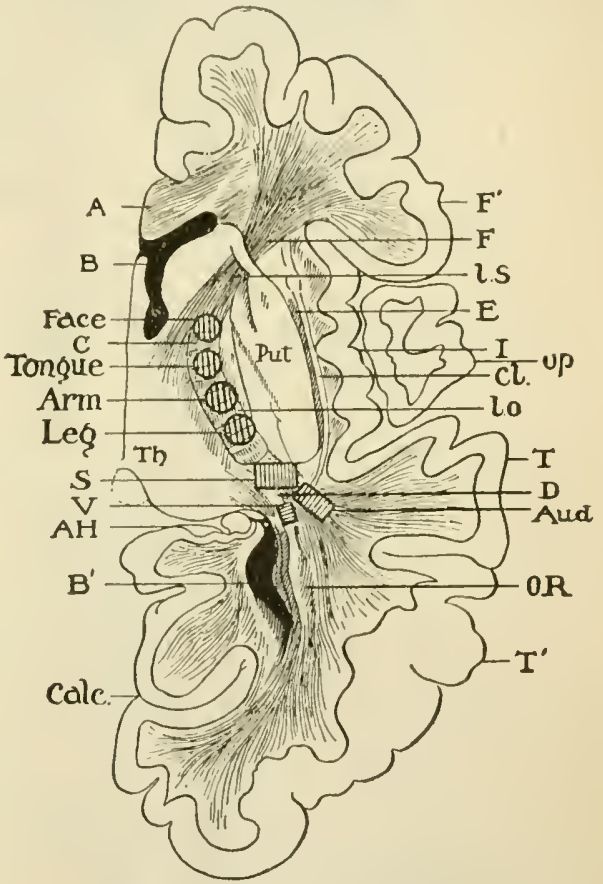

Fig. 356.--Horizontal Section tnrough the Right Hemisphere to show the Constituents of the Internal Capsule (von Monakow). A, knee of corpus callosum; $\mathrm{B}$, anterior, $\mathrm{B}^{\prime}$, posterior, horn of lateral ventricle; $C$, knee of internal capsule; $\mathrm{S}$, sensory fibres; V, visual tract; AH, Ammon's horn; Calc, calcarine fissure; T, first, T', second, temporal convolution; OR, optic radiation; $\boldsymbol{A} u d$, auditory tract; $D$, retrolenticular region of internal capsule; lo, lenticulo-optic division of internal capsule; $\mathrm{Cl}$, claustrum; op.. operculum; I, island of Reil; E, external capsule; $l s$, lenticulo-striate division of internal capsule; $F$, fibres from frontal lobe: $F^{\prime}$, inferior part of third frontal convolution; T\%, optic thalamus; Put, putamen.

tract after a lesion in the 'motor' area of the same side (p. 875)This would explain the escape in hemiplegia (paralysis of one sicie 

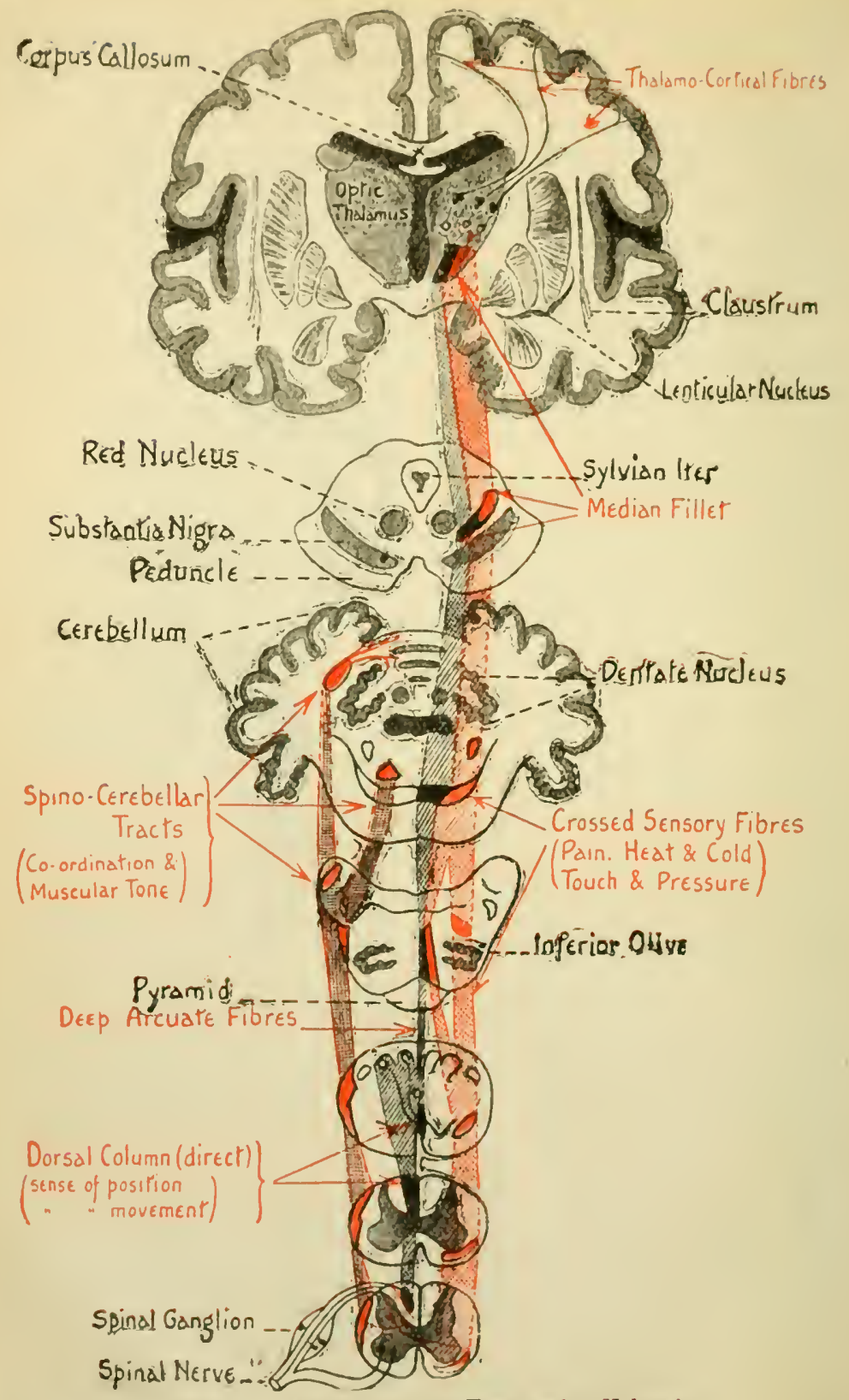

Fig. 357.-Ascending Nerve Tracts (after Holmes). 
of the body) of those muscles which are accustomed to work with the corresponding muscles on the opposite side-e.g., the respiratory muscles, these being innervated to some extent from both cerebral hemispheres.

(2) A great afferent or sensory path by which some at least of the impulses carried up through the posterior roots of the spinal nerves, after passing through various relays of nerve-cells, reach the cortex of the cerebellum; or the upper portions of the central grey tube, the corpora quadrigemina and optic thalamus; or, finally (through the tegmentum and the posterior limb of the internal capsule behind the motor fibres), the cerebral cortex itself.

The efferent pyramidal path from the cortex to the periphery is broken by at most two relays of nerve-cells-those intercalated cells to which reference has already been made (p. 876), if they really exist, and the motor cells of the anterior horn. The afferent path to the cerebral cortex is interrupted by at least three relays with axons of considerable length. One of the cells is situated in the ganglion on the posterior root, another in the medulla oblongata, a third in the optic thalamus; and on some of the routes another, or even more than one, is intercalated between the medulla and the cortex (Fig. 357).

The Internal Capsule.-We have already recognized the pyramidal tract and the afferent tegmental path as constituents of the internal capsule. The cranial fibres of the pyramidal tract occupy mainly the genu or knee, the spinal fibres the posterior limb as far back as the posterior border of the lenticular nucleus (Fig. 356).

The fibres from the various motor areas are to a certain extent arranged in order in the capsule, those for the eyes and head lying farthest forward, those for the leg farthest back, while the fibres going to the face, arm and trunk occupy intermediate positions. The separation, however, is far from complete, the fibres of neighbouring regions being considerably intermixed (Hoche). As the tracts pass downwards the intermingling becomes continually greater (Simpson and Jolly) (Figs. 358, 359). The afferent fibres from the thalamus to the cortex, which we have described as the last segment of the afferent tegmental path, lie in the posterior part of the posterior limb. But here again there is no absolutely sharp line of demarcation. Some motor fibres are intermingled with the sensory in the posterior part of the capsule, for lesions of this region produce a certain degree of paralysis as well as anæsthesia on the opposite side of the body. A pure capsular hemianæsthesia-that is, a loss of sensation on the opposite side due to a lesion in the internal capsule and unaccompanied by motor defect-does not appear to exist. Accordingly the common statement that the efferent (motor) path occupies the anterior two-thirds, and the afferent (sensory) path the posterior third of the posterior limb of the internal capsule, while true in a general sense, is not strictly correct. 
The destination of the afferent fibres of the internal capsule lias not been definitely settled. There is no doubt that they pass up to the convolutions around the fissure of Rolando (central convolutions), and there is reason to believe that some of them terminate in the 'motor' region in front of that fissure, althongh many of the fibres concerned in tactile sensations seem to end in the ascending parietal convolution.

But we have not yet exhausted the constituents of the internal

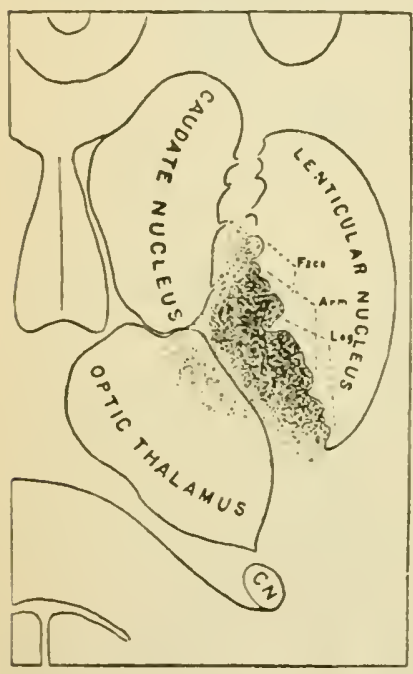

Fig. 358.-Pyramidal Tract in In. ternal Capsule (Simpson and Jolly). Horizontal section through right cerebral hemisphere, cutting fibres of internal capsule transversely at an upper level a little.below the upper surface of the lenticular nucleus. The extent of tl:e degeneration following destruction of the whole of the right 'motor' cortex, except the 'head and eyes. area (in one of the lower mon. keys), is shown. Nots over. lapping of fibres from face, arın. and leg areas, as shown by experiments in which one or other of these areas was alone removed. capsule. Two great cones of fibres sweep down into it, one from the frontal, the other from the occipital and temporal portions of the cerebral cortex. The first passes through its anterior limb, the second behind the sensory path in its posterior limb. The cells of origin of the frontal fibres are known, and those of the occipital and temporal fibres are supposed, to be situated in the cortex. They are therefore efferent fibres as regards the cortex (cortifugal). Running on through the crusta of the cerebral peduncle (Fig. 352), the frontal tract

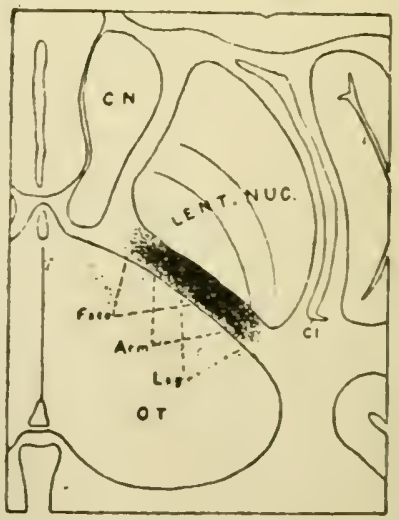

Fig. 359.-Pyramidal Tract in Internal Capsule at Lower Level (Sumpson and Jolly). CN, head of caudate nucleus; OT, optic thalanus; $\mathrm{Cl}$, claustrum.

internal, the occipito-temporal external, they end in the grey matter of the pons, and serve as one segment of an extensive connection between the cerebral and the cerebellar cortex of the opposite side, the other segment being formed by neurons whose cell-bodies are situated in the pons, and whose axons, crossing 
the midclle line, pursue their course through the middle cerebellar peduncle, to terminate in the superficial grey matter of the cerebellum. It is evident that the junction of the cerehral cortex with this pontine grey matter, through and into which so many nervetracts pass, multiplies the number of possible routes by which impulses may travel between one part of the brain and another. The corpus callosum forms a mighty link between the two cerebral hemispheres. And intertwined in the corona radiata with the callosal fibres are other systems, of which it is especially necessary to mention the afferent (cortipetal) fibres that join the optic thalamus with nearly every part of the cerebral cortex. Such fibres pass from the cells of the grey matter of the thalamus to the frontal and parietal

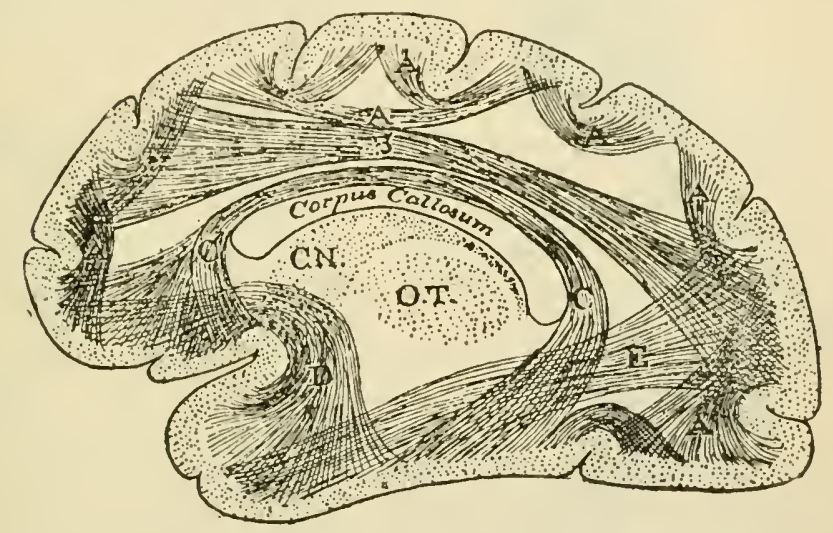

Fig. 300.-Association Fibres (after Starr). Cerebral hemisphere seen frum the side A, A, association fibres between adjacent convolutions; B, between frontal and occipital lobes; C, cingulum, connecting frontal and tersporo-sul henoidal lobes; D. uncinate fasciculus between frontal and temporal regions; $\overline{\mathrm{E}}$, inferior longitudinal bundle between occipital and temporo-sphenoidal lobes; O.T., optic thalamus; C.N., caudate nucleus.

regions through the anterior border of the internal capsule in front of the frontal fibres previously described as running in the anterior limb of the capsule to the pons; and from the thalamus to the occipital region through the extreme posterior border of the internal capsule, behind the occipital fibres that proceed to the pons. The fibres that connect the thalamus with the occipital cortex are spoken of as the optic radiation. Some of the fibres of the optic radiation, however, proceed, not from the thalamus, but from the anterior corpus quadrigeminum and the lateral geniculate body. The thalamus is also connected with the cortex of the temporal lobe, with the cerebellum, and through the fillet with the posterior part of the tegmental system, the medulla oblongata and the spinal cord (p. 874). Fibres also pass from the inner and deeper part of the thalamus to the lenticular nucleus of the corpus striatum. The 
thalimus must be regarded as a great sensory centre through which afferent impulses stream on their way to all parts of the cortex.

The fibres which connect the cerebral cortex with lower levels of the central nervous system are sometimes grouped together as projection fibres in contradistinction to the commissural and association fibres. A large proportion of the fibres of the corona radiata are projection fibres, including efferent groups (pyramidal tract, fronto-

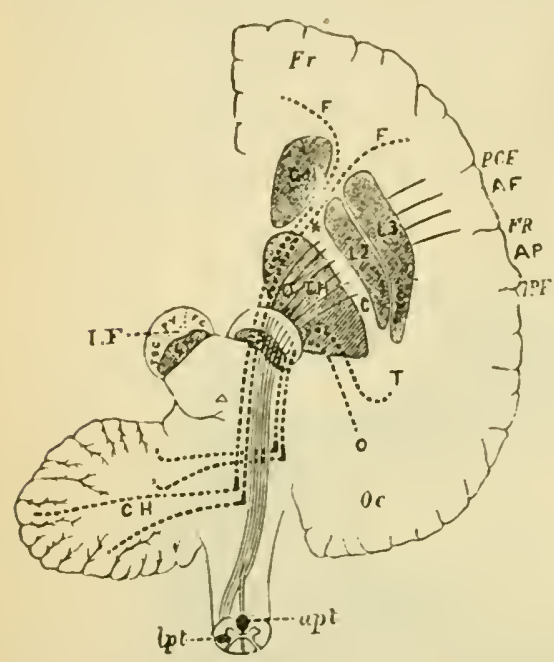

Fig. 36r.-Fibres connecting Frontal and Temporo-Occipital Lobes with Cercbellum, etc. (Diagram) (after Gowers). Fr, frontal, Oc, occipital lobe; interrupted lines indicate fibres (TOC) connecting cerebellum and temporo-occipital cortex, and fronto-cere. bellar fibres (FC). On left side the position of these two groups of fibres and of motor (pyramidal) tract, PY, in the crus, is indi. catcd by letters. The pyramidal tract is seen on the right passing down from the Rolandic arca through postcrior limb of internal capsule IC (the genu or knec of which is indicated by asterisk) to decussate in the bulb. AF, ascending frontal convolu. liou; AP, ascending parietal convolution; $F R$, fissure of Rolando; IPF, intraparietal fissure; PCF, precentral fissure; $l p l$, crossed pyramidal, apt, direct pyramidal tract.

pontine fibres, temporopontine fibres) and afierent groups (the fillet system, thalamo-cortical fibres from the grey matter of the thalamus to the cortex, including the optic radiation).

SeCtion VII.-CONNECTIONS of Brain Stem with CORD - Connections of CereBELLUM.

Connections of the VestibuloSpinal or Antero-Lateral Descending Tract. - The main origin of these fibres is the nuclcus of Deiters, a collection of large multipolar nerve-cells in the floor of the fourth ventricle near the inner auditory nucleus. This nucleus constitutes an important intermediate station between the cerebellum and the cord. Its cells give off axons which pass into the posterior longitudinal bund!c of the bulb and pons, mostly to the bundle of the same side, but partly into that of the opposite side. Here the fibres bifurcate into an ascending brancl, which passes up to the oculo-motor nucleus, and a descending (vestibulo-spinai) branch, which passes down. wards to the spinal cord and enters the antero-lateral cicscending tract. The fibres of this tract ultimately pass into the anterior horn. where most of then cnd by arborizing amongst the cclls of the hoin. Higher up corresponding fibres from the posterior longitudinal bundle arborize in the crinial motor nuclei.

Connections of the Rubro-Spinal Tract.-These filsres, as the name given to the bundle implies, originate in the red nucleus and run down into the cord. A little distance from their cells of origin they cross the 
median line, and then pass down first in the tegmentum, and below that in the lateral column of the bulb and cord. On their way they come into relation with the motor nuclei of the cranial nerves, and in the cord with the cells of the anterior horn. It is obvious that in contrast to the projection fibres the fibres of the rubro-spinal, vestibulo-spinal. olivo-spinal, and thalamico-spinal tracts (p. 867) and the pesterior longitudinal bundle connect the brain stem or cerebral axis with the cord, or clifferent levels of the brain stem with each other.

Connections of the Grey Matter of the Cerebellum with the Periphery and other Parts of the Central Nervous System. - Numerous as are the nervous ties of the ccrebral cortex, those of the grey matter of the cerebellum are, in proportion to its mass, still morc extensive, particularly as regards afferent fibres, and perliaps not less important.

Speaking broadly, we may say that the restiform body or inferior peduncle connects chiefly the dentate nucleus and the grey matter of the worm with the spinal cord and medulla oblongata, and through them with the periphery. The fibres which it receives from the direct

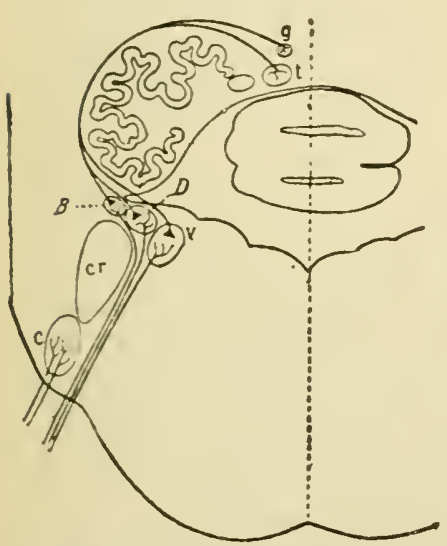

Fig. 362.-Direct Sensory Cerebellar Path of Edinger. $D$, Deiters' nucleus; $v$, median nucleus of auditory nerve; $t$, nucleus of the roof; $g$, nucleus globosus.

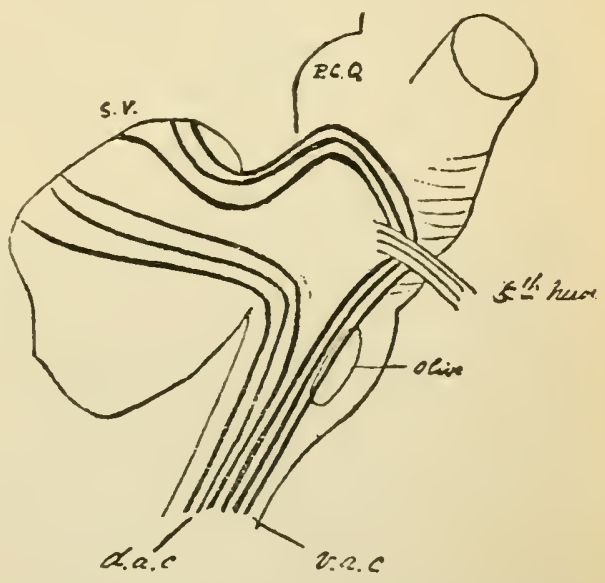

Fig. 363.-Diagram of Dorsal and Ventral Spino Cerebellar Tracts entering Cerebellum (Motı). P.C.Q., posterior corpora quadrigemina; s.v., superior vermis of cerebellum; a.a.c., v.a.c., dorsal and ventral ascending cerebellar tracts.

cerebellar tract (dorsal spino-cerebellar tract) of its own side it carries to the worm. These fibres occupy the outer portion of the peduncle. The fibres which reach the restiform body from the olivary nucleus of the opposite, and also in smaller numbers from that of the same side, run mainly to the hemisphere. All these fibres are afferent in relation to the cerebellum (cerebello-petal). An uncrossed afferent connection also exists between the cerebellum and the restibular branch of the auditory nerve, through certain of its nuclei of reception, and also between it and the nuclei of other cranial nerves, such as the trigeminus and the vagus. The fibres pass up in the inner portion of the inferior peduncle (direct sensory cerebellar path of Edinger, Fig. 362) to the nucleus of the roof (nucleus tecti) and nucleus globosus. Some efferent fibres (cerebellofugal) also run down from the cerebellum in the inferior reduncle, including fibres from the nucleus tecti of the opposite side which are on their way to the medulla oblongata. 
The middle peduncle is in the main a link between the cerebellar cortex and the cerebral cortex of the opposite side, through the relay of the pontine grey matter. Most of the fibres in it are afferent in rela. tion to the cerebclium, their cells of origin being situated in the nuclei of the pons, and sending their axons across the middle line to end in the cerebellar cortex.

The superior peduncle connects chiefly the dentate nucleus of one side with the cortex of the opposite cerebral hemisphere through the red nucleus of the tegmentum of the crus cerebri and the optic thalamus on the opposite side. The great majority, or perhaps all, of its fibres are efferent fibres as regards the cerebellum-i.e., their cells of origin lie in the dentate nucleus. Running upwards and forwards in the superior

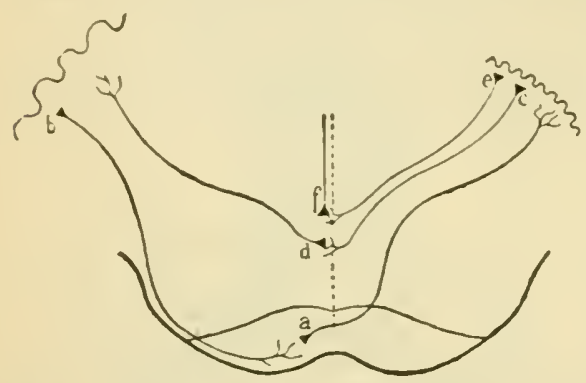

Fig. 364.-Paths of Middle Cerebellar Peduncle (Mingazzini). The scheme indicates the afferent and efferent paths which run through the middle cerebellar peduncle, connecting cerebellum with opposite side of cerebrum. $a$, fibre coming from a cell in the nuclei pontis and going to the cere. bellar cortex; $b$, fibre from a cell in cortex of opposite cerebral hemisphere making connection in the pons with $a$ ( $a$ and $b$ together constitute an afferent path to the cerebellum); $c$, a fibre springing from a Purkinje's cell in the cerebellar cortex and making connection in the pons with a cell $d$, which sends its axon to the cerebral cortex of the opposite side. $c$ and $d$ constitute an efferent path from cerebellum to opposite cerebral hemisphere; $e, f$, represent a path coming from the cerebellar cortex, which crosses the middle line in the pons, and then ascends till it loses itself in the formatio reticularis.

backwards along its mesial border to the worm. Since the cortex of the cerebellum is linked to the dentate nucleus, the superior peduncle affords an indirect connection between it and the cerebral cortex. Through the restiform body afferent impulses pass up to the cerebellum. From the cerebellum they may proceed to the cerebrum. So that the path by the restiform body, dentate nucleus, and superior peduncle may form an altemative route for afferent impressions ascending from the periphery to the great brain - a path broken by at least four relays of nerve-cells. The cerebellar hemisphere may be connected by an efferent path through the nucleus of Deiters and the descending antero-lateral tract with the motor roots of the same side. Another efferent path (from the dentate nucleus) may be constituted by the fibres of the superior peduncle and Monakow's bundle.

peduncle towards the midbrain they cross the middle quadrigemina, and then bifurcate into ascending The ascending branches end mainly in connection with cells in the red nucleus, but some of them pass on to the optic thalamus, with which cells of the red The thalamus, as we have seen, is in its turn extensively connected with the cerebral cortex and the red nucleus (by the efferent tract of Mlonakow) with the grey matter of the cord.
The descending branches of the fibres of the superior peduncle, entering the reticular formation of the pons, pass down, it is said, to make connection with the motor nuclei of the The tract of Gowers, as previously stated, comes into relation with the su. perior peduncle, passing 
We have purposely omitted to enumerate other patls by which the various tracts of grey matter in the brain are brought into com. munication with each other, and our knowledge of such connections is no doubt far from complete. When we add that not only are the cerebral hemispheres united by many ties to the subordinate portions of the cerebro-spinal axis and to each other, but that cortical areas of one and the same hemisphere are in communication by short connecting loops of ' association ' fibres (Fig. 360), it will be seen that the linkage of the various parts of the central nervous system is extremely complex; that an excitation, blocked out from one path, may have the choice of many alternative routes; and that the apparent simplicity and isolation of the pyramidal tracts must not be allowed too far to govern our views of the possibilities open to a nervous impulse once it has been set going in the labyrinth of the nervous network. Nor is it only by the main channel of the axiscylinder that nervous impulses can be conducted, they can also pass along the collaterals. And the actual route taken by a given impulse is determined not only by anatomical relations, but also by molecular conditions, particularly in the terminal fibrils of the axons, collaterals and dendrites, and in the substance, if such a substance there be, which intervenes between the end arborizations of a neuron and the dendrites or cell-bodies of the neurons with which they lie in contact. So that a road open at one moment may be closed at another. (See p. 902.) We may suppose that the greater the number of connections between the cells of the central nervous system, the greater is the complexity of the processes which may be carried on within it. And, indeed, comparison of the brains of different animals shows that it is not so much by excess in the number of nerve-cells as by the increased complexity of linkage, that a highly-developed brain differs from a brain of lower type; the higher the brain, the more richly branched are the dendrites and the terminations of the axons and their collaterals, and, therefore, the greater is the number of possible paths between one nerve-cell and another.

\section{Section Vili.-Functions of the Central Nervous System- (I) The Spinal Cord (including the Medulla Oblongata).}

Much of our knowledge of the functions of the central nervous system and of its divisions has been gained by the removal or destruction of more or less extensive tracts of nervous substance, or the cutting off of connection between one part and another. But it is well to warn the reader at the very outset that in no other part of physiology is such caution required in making deductions as to the working of the intact mechanism from the phenomena which manifest themselves after such lesions. 
In the first place, every operation of any magnitude on the brain or cord is inmediately followed by a depression of the functional power of the nervous tissue distal to the lesion, a depression which may extend fir from the actual seat of injury and manifest itself by various phenomena, which are grouped together under the name of 'sliock,' better terued spinal or cerebro-spinal shock, to distinguish it from the cardiovascular or surgical slock already mentioned (p. 192). Thus, when the spinal cord of a dog is divided, e.g., in the dorsal region, all power, all vitality, one might almost say, seems to be for ever gone from the portion of the body below the level of the section. The legs hang limp and useless. Pinching or tickling them calls forth no reflex movements. The vaso-motor tone is destroyed, and the vessels gorged with blood. The urine accumulates, overfills the paralyzed bladder, and continually dribbles away from it. The sphincter of the anus has lost its tone, and the facces escape involuntarily. And if we were to continue our observations only for a short time, a few hours or days, we should be apt to appraise at a very low value the functions of that part of the cord which still remains in connection with the paralyzed extremities. But these symptoms are essentially temporary. They are the immediate results of the section; they are not permanent 'deficiency' ' phenomena. And if we wait for a time, we shall find that this torpor of the lower dorsal and lumbar cord is far from giving a true picture of its potentialities; that, cut off as it is from the influence of the brain, it is still endowed witl marvellous powers. If we wait long enough, we shall see that, although voluntary motion never returns, reflex movements of the hind-limbs, complex and co-ordinated to a high degree, are readily induced. A few months after transection of the cord it is easy to show that, although the dog cannot use the hind limbs for continuous progression, the machinery for executing the appropriate movements exists in the cord. When, for example, the animal is held in the proper position and given a slight push forwards, it may take two or three steps before its legs give way. The regulation of the movements necessary for the maintenance of equilibrium cannot be achieved when the control of the higher centres is eliminated. In water, where the problem of the maintenance of the normal position is solved by the mechanical properties of the medium, the dog can use the hind-legs in swimming. The tone of the resting muscles below the lesion is even somewhat greater than normal. Vaso-motor tone also comes back. The functions of defrecation and micturition are normally performed. Erection of the penis and ejaculation of the semen take place in a dog. A man with complete paralysis below the loins and destitute of all sensation in the paralyzed region has been known to become a father (Bracliet). Pregnancy carried on to labour at full term has been observed in a bitch whose cord was completely divided above the lumbar enlargement. The severity and duration of spinal shock are greater in the monkey than in the dog, in man than in the monkey, and in the whole mammalian group than in the lower vertebrates. The mechanism of its production has been much discussed, and will be referred to on another page (p. 912).

We cannot doubt that the spinal cord takes an important share in the recovery of function after shock. But liere again it would be erroneous to conclude that everything is due to the cord. For Goltz and Ewald have been able to keep dogs alive for long periods after preliminary section of the cord in the cervical region and subsequent removal of large portions of it. They find that even after destruction of the limbar and sacral regions of the cord the external sphincter of the anus, striped and even voluntary muscle though it be, regains its 
tone, although it is temporarily lost after the first cervical section. The blader ultimately recovers the power of emptying itself spontancously and at regular intervals. A pregnant bitch in which the lumbar enlargenent and the whole cord below it to the cauda equina had been removed, and therefore all the nerve-roots supplying fibres to the uterus cut, whelped in a normal manner, and the corresponding mammary glands behaved exactly as the rest. Digestion went on as usual when practically nothing of the cord except its cervical portion was left. Certain vaso-motor phenomena were also observed which suggest that the sympathetic system, independently of the cerebrospinal system, is itself possessed of regulative powers (p. 183).

Secondly, we must not run into the opposite error, and assume, without proof, that all the functions which the brain or cord is capable of manifesting under abnormal circumstances are actually exercised by either when, under ordinary conditions, it is working along with and guiding, or being guided by, the other. For example, in many animals certain of the reflex powers of the cord are, if not increased, at all events more freely exercised when the controlling influence of the higher centres has been cut off than when the central nervous system is intact.

Thirdly, there is another class of phenomena which we must make allowance for, and perhaps more frequently in the case of pathological lesions in man than in experimental lesions in the lower animals. This is the class of 'irritative ' phenomena. The irritation set up by a bloodclot or a collection of pus, or in any other way, in a wound of the grey or white matter, may cause a stimulation of nervous tracts by which. for a time, the 'deficiency' effects of the lesion may be masked.

In the fourth place, we must not hastily conclude that, when no obvious deficiency seems to follow the removal of a portion of the central nervous system, the function of that portion must necessarily be of such a nature as to give rise to no objective signs. For there is reason to believe that, to a certain extent, the function of one part may, in its abserice, be vicariously performed by another.

Bearing in mind the cautions we have just been emphasizing, we may broadly distinguish between the functions of the cord (including the bulb) and those of the brain proper by saying that the cord is essentially the seat of reflex actions, the brain the seat of automatic actions and conscious processes. But neither of these conceptions is entirely correct. Both err by defect and by excess. The brain, it is true, is pre-eminently automatic. The movements which are started in the grey matter of the cerebral cortex are pre-eminently voluntary (p. 946), but we cannot deny to the brain the possession of reflex powers as well. The movements in which the only nerve centres concerned are those of the spinal cord are above all reflex (p. 897). But some of its centres, and especially those lying in the medulla oblongata-e.g., the respiratory centre-are, much as they are influenced by afferent impulses, capable of discharging automatic impulses too. And while consciousness is certainly bound up with integrity of the brain, and in all the higher mammals is associated with cerebral activity alone, it has been plausibly maintained that the spinal cord, even of such an animal as the frog, may also be endowed with something which might be called a kind of hushed consciousness. Whether this be so or not for the frog, with its 
distinct though relatively ill-developed cere, ral hemispheres, it would seem by no means innlikely in the case of fishes and animals below them, which are practically devoid of a cerebral cortex altogether.

The functions of the spinal cord may be classified thus:

I. The conduction of impulses set up elsewhere-either in the brain or at the periphery.

2. The modification of impulses set up elsewhere (reflex action).

3. The origination of impulses (?).

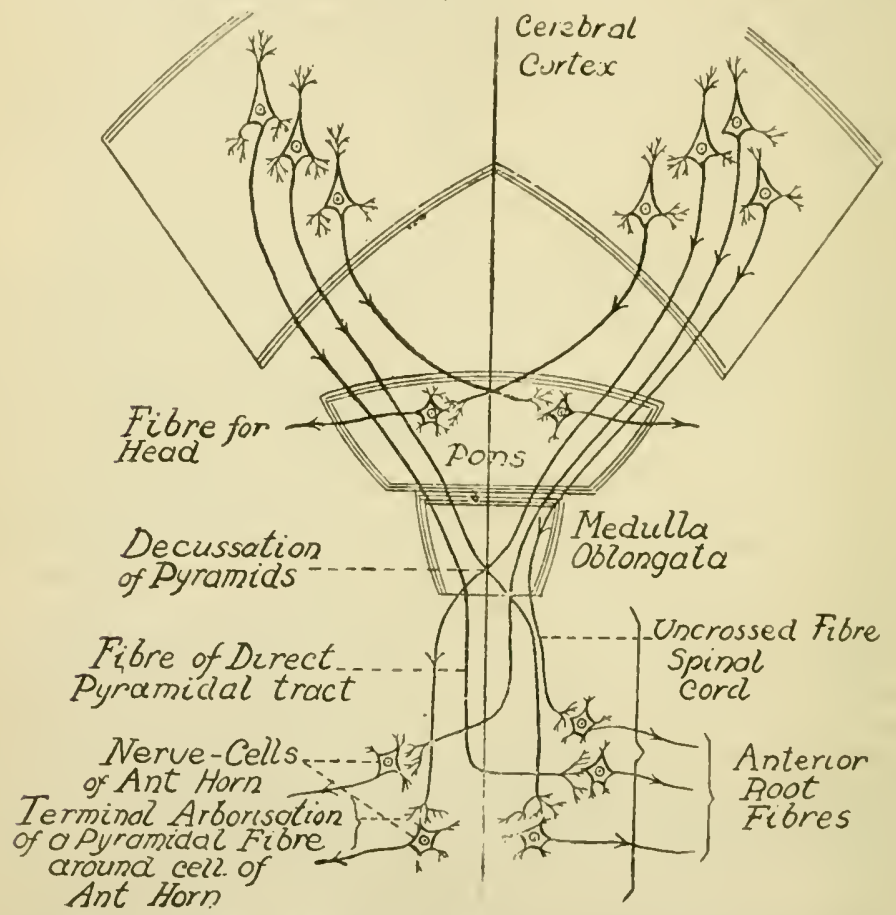

Fig. 365.- Some Possible Paths of Efferent Impulses in the Central Nervous System (Schematic). Details are omitted from the scheme. For instance, each pyra. midal fibre is represented as arborizing around one anterior cornual cell only, and no collaterals are shown. The hypothetical intercalated neurons between the pyramidal fibres and the anterior horn cells $(p, 876)$ are not shown.

I. Conduction of Nervous Impulses by the Cord.- The old controversy as to whether the white fibres of the spinal cord are directly excitable has long been settled in the affirmative.

The inquiry was complicated by the presence of the spinal roots, which, since the experiments of Cliarles Bell, have been known to be capable of excitation by artificial stimuli. But at length the difficulty was overcome in this way: The posterior (dorsal) portion of several 
segments of the cord with the attached posterior roots and the grey matter was excised. Long strands of the white matter of the anterior (ventral) portion of the cord were isolated, and laid on electrodes, and contractions of muscles were seen to follow stimulation, even when the anterior roots nearest the stimulating electrodes had been cut, and every precaution taken to avoid escape of current on to the distant anterior roots of the nerves supplying the muscles. Indeed, apart from direct experimental evidence, the fact that the white fibres of the brain are universally admitted to be excitable by artificial means would be of itself almost sufficient to decide the question, for we know of no essential difference between the cerebral and the spinal fibres. But the conditions must rarely occur under which direct stimulation of white fibres in their course is possible in the intact body; and the only impulses with which we need concern ourselves here are those that reach the conducting paths from grey matter in the cord itself or in the brain, or from the peripheral organs.

What sort of impulses do the various tracts of the spinal cord conduct ? For the dorsal or posterior roots this question was first fully answered by Magendie; for the ventral or anterior roots, although with a certain degree of ambiguity, by Sir Charles Bell. Bell observed that when, in an animal just killed, he mechanically stimulated the anterior roots, muscular contractions were obtained at each touch of the forceps. He concluded that the anterior roots are motor and sensory, while the posterior roots are 'vegetative 'i.e., connected with the functions of the viscera, the so-called 'vegetative' organs. But although he is often credited with the discovery of the functions of the posterior roots as well, he was not the first to make the decisive experiment necessary to show that they are the conductors of sensory impulses. It was after Magendie's discovery that only a portion of the nerves are sensitive, and that there are nerves ' which are like tendons, aponeuroses, or cartilages in insensibility,' that Bell formulated the law that the anterior roots are purely motor, the posterior purely sensory. This law, often termed Bell's Law, is more correctly denominated the MagendieBell Law.

When the posterior roots are divided, loss of sensation occurs in the region to which they are distributed. If only one root is cut, the loss of sensation is never complete in any part of the skin; and Sherrington has found that the cutaneous areas of distribution of consecutive nerve-roots are not perfectly independent, but to some extent overlap. Stimulation of the peripheral end of the divided posterior root has no effect. Stimulation of the central end gives rise, if the animal be conscious, to evidences of pain, and other signs of the passage of afferent impulses-e.g., a rise in blood-pressure. The latter may also be observed when the animal is anæsthetized.

Referred Pain.-The posterior roots contain sensory fibres not only for the skin, but also for the deeper structures and the viscera. The afferent fibres reach the viscera by the sympathetic, the vagus, and the pelvic nerves or nervi erigentes. Clinical observations have thrown 
much light upon the distribution of the visceral fibres and their relation to the cutaneous sensory nerves. It has long been known that in clisease of an internal organ the pain is often referred to some superficial part. It has now been demonstrated that each organ is related to a more or less definite region, or more than one region, of the skin. In disease of the organ there is in this area increased excitability (hyperalgesia) or tenderness to slight mechanical stimuli, and often also increased excitability for heat or cold, and the reflexes elicited by stimulation are exaggerated (Head, Dana).

The bond of connection appears to be the origin from the same spinal segments of the autonomic* sensory fibres of any viscus and the sensory supply of the correspending cutaneous area. The common anatomical origin seems to carry with it a physiological correlation, either because the irritation of the visceral fibres spreads in the cord to the somatic afferent fibres which enter the corresponding segments, or because of some action higher up in the cerebral centres, the nature of which will be best considered along with the general topic of the localization of sensory impressions (Chapter XYIII.).

Recurrent Sensibility.-Although muscular contraction is the most conspicuous event that follows stimulation of the peripheral end of an anterior nerve-root, it is by no means the only one. It is frequently observed, though not in all kinds of animals, that here, too, pain is caused. That this pain is not due to the muscular contraction is proved by the fact that it can still be elicited when the nerve-trunk is divided between the junction of the roots and the periphery. The real explanation of the phenomenon is that certain fibres from the posterior roots ('recurrent fibres,' see footnote on p. 797) bend up for some distance into the anterior roots, and then turn round again and pursue their course to their peripheral distribution in the mixed nerve, or run on in the motor roots to supply. the sheath surrounding them (nervi nervorum), and even the membranes of the spinal cord.

The afferent impulses that enter the cord along the posterior roots have the choice of many paths by which they may reach the brain. The following are a few of the routes which they may follow:

(I) They may pass directly up through the postero-median column. If they take this route, their course will be first interrupted by nervecells in the gracile or cuneate nuclei in the medulla oblongata. Thence they may find their way across the middle line by the arcuate fibres of the upper or sensory decussation, and, sweeping along the fillet and the sensory path in the hinder part of the posterior limb of the internal capsule, finally arrive at the cerebral cortex. Between the gracile and cuncate nuclei and the cortex they pass through nerve-cells in the optic thalamus.

(2) They may pass up by the direct cerebellar tract and restiform body to the grey matter of the cerebellar worm. If they take this route their course will be interrupted very soon after their entrance into

* Langley uses the term autonomic nervous system to inclucle the nerve supply of heart muscle, all unstriated muscle, and all gland cells in the body. It embraces, in addition to the sympathetic, cranial alutonomic fibres in several of the cranial nerves and sacral autonomic fibres in the nervi erigentes (see Chapter XVII.). 
the cord in the cells of Clarke's column. Since the superficial grey matter of the vermis is connected by association fibres with the dentate nucleus, and the dentate nucleus by the superior peduncle with the opposite cerebral hemisphere, this is also a possible path to the great brair.

(3) They may reach the antero-lateral ascending tract of the same side through its cells of origin in the spinal grey matter, and, passing through the medulla and pons to the superior peduncle of the cerebellum, enter the grey matter of the superior worm.

(4) They may cross the middle line, after entering the cord, through axons or collaterals (p. $87^{2}$ ) which run in the anterior and also in the posterior conmissure, enter one of the ascending tracts on the other side-e.g., the tract of Gowers-and continue without further decussation up to their central destination.

(5) They may spread from neuron to neuron in the tangle of the grcy matter itself, and pass out again at a different level into one of the white tracts on the same or on the opposite side of tlic cord.

Efferent impulses from the brain may travel-

(I) Through the direct or crossed pyramidal tract.

(2) From one side of the cerebral cortex to the other, and then down the pyramidal tracts corresponding to that side (?).

(3) From the frontal part of the ccrcbral cortex, through the anterior limb of the internal capsule to the grey matter in the pons, and thence to the cercbellum by its middle peduncle.

(4) Fron the occipital or temporal cortex, in the hinder rim of the internal capsule, to the pontine grey matter, and through the middle peduncle to the cerebellum. From the cerebellum they may possibly pass down to the nucleus of Deiters and thence along the antero-lateral descending tract to the anterior horn of the cord, and indirectly to the periphery.

All the paths enumerated, as well as others to which it would be tedious to formally refer, and which the ingenuity of the reader may profitably be employed in constructing for himself, from the data already given, are to be looked upon as possible channels for the passage of impulses between the brain and the periphery. But it must be distinctly pointed out that what is certain is in this case much more limited than what is possible. Among the efferent paths it is certain that the pyramidal tracts are conductors of voluntary motor impulses, and that in most individuals the great majority of such impulses decussate in the medulla oblongata, only a small minority in the cord. For a lesion involving the pyramidal tract above the decussation of the pyramids causes paralysis of the opposite side of the body, a lesion below the decussation paralysis of the same side. It is certain that when one pyramidal tract has been destroyed, in many animals at least, the resulting paralysis is soon recovered from, at any rate to a great extent, and it is possible that in this case the motor cortex on the side of the lesion has placed itself again in communication with the paralyzed muscles through its commissural connections with the opposite hemisphere. This, however, is not the only alternative, for, as already pointed out, the pyramidal tracts are not the only cortico-spinal paths which can 
subserve volitional movements, and division of the anterior portion of the antero-lateral column may cause deeper and more permanent paralysis than division of the pyramidal tract.

In the dog total section of the pyramids is not followed by complete paralysis of voluntary movements, and stimulation of the cortical motor areas can still elicit characteristic movements. It is obvious that impulses emanating from the cortex can reach the motor nuclei of the cord by other routes than the long pyramidal fibres, possibly by paths with several segments, of which, for example, the rubro-spinal tract (p. 867) may be one. Just as an important business house may find it useful or indispensable to supplement or replace the common telegraph service by private wires in the interest of more prompt and satisfactory communication with its principal correspondents, while still utilizing the ordinary channels to some extent, so the higher brains may be supposed to have developed more and more the direct service of the pyramidal tract to tighten the grip of the cortex upon the motor nuclei of the cerebrospinal axis, while still availing themselves, although in diminishing degree as their evolution proceeds, of the more primitive indirect paths.

Decussation of the Sensory Paths.- On the other hand, it is certain that pathological or traumatic lesions, apparently involving the destruction of one lateral half of the cord in man and experimental jemisections in some mammals, are followed by symptoms which suggest that some kinds of sensory impulses decussate chiefly in the spinal cord-viz., diminution or loss of sensibility to pain and to changes of temperature on the opposite side below the level of the lesion, with little or no impairment, and often increase of sensibility (hyperæsthesia) on the same side. Tactile sensibility is lost on the side of the lesion, and likewise the muscular sense.

The first general description of this symptom-complex was given by Brown-Séquard. On the basis of clinical observations in man, he came to the conclusion that unilateral lesions of the cord, equivalent approximately to a semisection, are associated with muscular paralysis below the level of the lesion on the same side, and loss of cutaneous sensibility on the opposite side, whule on the side of the lesions there may be an augmentation of sensibility. He interpreted these facts as meaning that the sensory path decussates soon after its entrance into the cord. The sensory path from the left side is therefore spared by a lesion of the left side of the cord, but interrupted by a lesion of the right side of the cord. The left and right motor paths, having already decussated in the bulb, are cut by lesions in the left and right halves of the cord respectively. Long afterwards Brown-Séquard saw cause to retract this interpretation of the facts observed by him, but the majority of subsequent observers have considered his original hypothesis more satisfactory than his later ones. While it may be true that in man it has not been rigidly demonstrated that the symptoms are associated with a clean-out lesion precisely limited to one-lialf of the cord, clinical observation has on the whole tended to confirm the view that an 
important portion of the sensory path decussates in the cord. But it is a curious circumstance that experimental pliysiologists have for the most part obtained contradictory results. Thus Mott, working with $1110 n k e y$, found that the different kinds of sensation, far from being abolished, are as a rule impaired in a smaller degree on the side opposite to the semisection than on the same side, while Ferrier and Turner obtained on the whole a contrary result, and one that corresponded closely with Brown-Séquard's original description. The discovery that no ascending degencration, or only a trifling amount, is to be found on the opposite side of the cord, eitlier after semisection or after division of posterior roots, does not of itself enable us to decide the question. For while this latter fact shows that few or none of the afferent fibves cross the middle line to enter the long conducting paths before being interrupted by nerve-cells, it by no means proves that afferent impulses do not decussate in the cord. The long paths of the posterior column, indeed, do not decussate below the level of the bulb. The dorsal and ventral spino-cerebellar tracts are also, in the main at least, uncrossed spinal paths. A portion of the afferent impulses must therefore be carried up to the cercbrum and the cerebellum without decussating in the cord. But nobody can tell how massive a link between the two halves of the cord may be formed by the grey matter and the endogenous fibres of the white columns and their collaterals. We know that some afferent impulses do decussate far below the level of the medulla. For, (I) A part of the action current (p. 838) crosses the middle line and ascends in the opposite half of the cord when the central end of one sciatic is stimulated (Gotch and Horsley). (2) Crossed reflex movements are possible; and when excitation of the central end of the sciatic is followed by contraction of the muscles of the opposite fore-limb, the afferent impulses must either decussate in the lumbar cord, and then run up on the opposite side to the level of the brachial plexus, or must ascend on the same side and cross over somewhere between the plane of the sciatic and the brachial nerve-roots. The only other hypothesis on which crossed reflex action can be explained-but a hypothesis for which there is not a tittle of evidence-is that the afferent impulse always acts on the few motor cells whose axis-cylinder processes pass over to the opposite side, and there enter anterior nerve-roots. But while, for these reasons, it cannot be denied that some afferent impulses decussate in the cord, it would be an error to conclude that all do so in any animal, or that all animals are in this respect alike. It is indeed extremely probable that in different species of animals, and even in individuals of the same species, there are considerable differences in the extent of the sensory decussation in the cord, just as there are in the extent of the motor decussation in the bulb. In some animals the greater part of the sensory path may decussate in the cord; in others the greater part may decussate in the bulb, or higher up. The lack of agrcement in the experimental results may be due partly to this cause. When it is further remembered how difficult it sometimes is to interpret the account which a man gives of lis sensations, and to recognize precisely the degree and nature of sensory defects produced by disease in the human subject, it will not be thought surprising that experiments on animals, from the time of Galen onwards, should have yielded evidence which, although perhaps now at length tending to a definite: result, is still unfinished and in part conflicting.

If, leaving them out of account, not as valueless but as still difficult of interpretation, we attempt to draw any general conclusion 
from the clinical observations which, however imperfect, are in such questions our surest guide, it can only be this, that in man some of the sensory impulses, and particularly those connected with the culaneous sensations of pain and temperature, decussate, in part at least, in the cord. But there is also evidence that tactile afferent impulses, including those coming from the muscles and related to the muscular sense, and, it may be, some of the impulses associated with pain, decussate, not in the cord, but in the bulb.

The Paths for Different Kinds of Sensory Impressions.-If this is the state of our knowledge where the problem is merely to determine the crossing-place of afferent impulses which are certainly known to cross, it is only to be expected that we should be still more in the dark as regards the routes by which different kinds of afferent impulses thread their way through the maze of conducting paths in the neural-axis to reach their planes of decussation and gain the 'sensory crossway' in the internal capsule. Some authors have indeed cut the Gordian knot by assuming that any kind of sensory impression may travel up any afferent path. Direct stimulation of a naked nerve-trunk, it has been argued in favour of this view, gives rise to a sensation of pain; stimulation of the skin in which the end-organs of the nerve lie gives rise to a sensation of touch or a sensation of temperature, according as the stimulus is a mild mechanical or a thermal one, the contact of a feather or of a hot test-tube. Why, it has been asked, should we imagine that the difference in the result of stimulation depends on a difference in the - nerve-fibres excited, and not on a difference in the lind of impulses set up in the same nerve-fibres? This is a question which we shall have again to discuss. But apropos of our present problem, we may say that there is very clear proof from the pathological side that a limited lesion in the conducting paths of the central nervous system may be associated with defect or total loss of one kind of sensation, while all the other kinds remain intact. And there seems no other tenable hypothesis than that in such cases the pathological change has picked out a particular group of fibres, either collected in to a single strand or scattered among unaltered fibres of different function. For example, in syringo-myelia, a condition in which cavities are formed in the grey matter of the cord secondary to a new growth of the neuroglia surrounding the central canal, a frequent symptom is the loss in a certain region of sensibility to pain and to changes of temperature, while tactile sensibility is unaffected (dissociation of sensations). Again, in locomotor ataxia, a disease in which inco-ordination of movement and derangement $\mathrm{f}$ the mechanism of equilibration are prominent symptoms, degeneration in the posterior column of the cord is a most constant lesion. And there is strong evidence that afferent impulses from muscles and tendons, which either give rise to impressions belonging to the group of tactile sensations, or produce no effect in consciousness, ancl which, according to the most widely accepted doctrine, serve as the basis of the muscular sense, and play an important part in the maintenance of equilibrium (p. 94I), pass up in the posterior column. It may also conduct tactile impressions from the skin. A case has been observed where a man received a stab which divided the whole of one side of the cord and the posterior column of the other side. Sensibility to touch was lost on botly sides of the body below the level of the injury: sensibility to pain only on the side upposite to the main lesion. In another case, in which some small syphilitic tumours (gummata) in 
the lateral colurnn on the left side caused marked degeneration in the left direct cerebellar tract, the tract of Gowers, and the crossed pyramidal tract, without affecting the posterior columns, tactile sensibility was only slightly impaired in the opposite leg, while the sensibility for pain and temperature was much enfeebled. In the left leg, which was paralyzed, there was slight hyperesthesia. These observations indicate that impressions of pain and temperature pass up in the antern-lateral column, either in the tract of Gowers, or in the direct cerebellar tract, or in both (Déjerine and Tlionzas).

But it does not follow that they cannot ascend by other peths as well. It appears, indeed, that the grey matter of the cord, or, rather, shert cudogenous fibres arranged in series in the antero-lateral column so as to connect the grey matter at different levels, constitute such a path, and that impulses which give rise to pain can be propagated along a cord in which hardly a vestige of white substance remains uncut. In man the path for pain and temperature impressions along these short endngenous fibres seems to be mainly or entirely a crossed path. The afferent paths for such vaso-motor reflexes as are elicited by stimulation of the central end of the sciatic ascend in the lateral column, and the impulses largely cross the middle line in the cord. 'The posterior columns have nothing to do with the conducticn of painful impressions, for division of them causes not anæsthesia, but rather hyperæsthesia, while if they are left intact, and the rest of the cord, including the grey matter, divided, the animal is insensitive to pain below the level of the lesion. Just as man differs from lower animals in the completeness with which certain of the sensory impressions decussate in the cord, so differences exist in the degree of localization of the different kinds of impressions in particular tracts. One of the outstanding differences is that in animals it seems to be easier for a still intact path to be substituted for a severed path as a conductor of impulses which normally traverse the latter. The rapidity with which sensation is restored below the lesion after semisection of the cord in animals is an illustration of this. Another difference, which can be explained in the same way, is that a sharply-marked dissociation of sensations-retention of tactile sensibility, for example, with loss of sensibility to pain or to pain and temperature changes-either cannot be produced experimentally in animals, or is very difficult to realize.

The impulses which descend the cord give token of their arrival at the periphery by causing either contraction of voluntary muscles, or contraction of the smooth muscular fibres of arteries, or secretion in glands. They all pass down in the antero-lateral column, but the path of the voluntary impulses in the pyramidal tracts is the best known and most sharply defined.

2. Modification of Impulses set up elsewhere (Reflex Action).The spinal cord, although it is a conductor of nervous impulses originating elsewhere, is by no means a mere conductor. Many of the impulses which fall into the cord are interrupted in its grey matter. Some of the efferent impulses proceeding from the brain are perhaps modified in the cord, and then transmitted to the muscles. Some of the afferent impulses are modified, and then transmitted to the brain; some are modified, and deflected altogetherinto an efferent path. These last are the impulses which give rise to reflex effects. A reflex action has sometimes been defined as an action carricd out in the absence of consciousness: not necessarily, bowever, in the 
absence of general consciousness, but in the absence of consciousness of the particular act itself. But the term is now more correctly used so as to embrace all kinds of actions which are not directly voluntary, whether the individual is conscious of them or not. For cxample, when the sole of rhe foot is tickled, the leg is irresistibly and involuntarily drawn up by reflex contraction of its muscles; yet the person is perfectly cognizant both of the movement and of the sensation which accompanies the afferent impulsc. Many reflex actions usually associated with sensations procecd normally when consciousness is entirely in abeyance; during sleep most of the ordinary reflexes can be clicited.

Anatomical Basis of Reflex Action.-Since the esscnce of reflex action is that the arrival of afferent impulses in the spinal cord or brain causes the discharge of efferent impulses, there must be some connection between the incoming and the outgoing nerve-fibres. In unicellular animals, such as amœba, there is no differentiation of any special nervous or conducting path. A stimulus applied at one point may cause contraction anywhere. Even in the lowest multicellular animals or metazoa-e.g., in the sponges-there is no special nervous tissue. In some species of liydra, however, many of the surface or ectodermic cells (p. 6) possess decply-placed contractile or muscular processes, and stimulation of the surface cells is followed by contraction of these processes. We may imagine that the first beginnings of an actual nervous system may have arisen by a further differentiation of such an ectodernic cell into a receptive portion at the surface, a deeper contractile portion, and an intermediate strand of protoplasm connecting the two, and capable of conducting the excitation from surface to muscular proccss. In such a reflex arc the nervous link would consist only of the conducting strand analogous to the nerve fibre joining the receptive or sensory, element to the contractile element, and the distinction betwecn afferent and efferent fibre would not exist. When development lias gone a step further, and the neuro-muscular process is interrupted by a sccond epithelial cell transformed into a nerve-cell, the afferent fibre enters one pole and the efferent fibre leaves the other pole of the same cell. It is this condition which we actually find when the nervous system first emerges in the animal scale as an unmistakably differentiated structure-namely, in the Cœelenterates in such forms as the jellyfishes. Here the three types of cell, receptive or sensory cell, reactive or central cell, and motor or contractile cell, are connected together by conducting paths or nerve-fibres. In a simple reflex action three events can be distinguished: stimulation of a receiving mechanism, conduction of the cxcitation, and the consequent reaction or end-effect. The receiving mechanism or receptor may consist of ordinary sensory nerve-endings in the skin, or of special sense-endings, as in the retina or internal ear. The conducting mechanism or conductor in all except the very simp!est nervous systems is made up of at least two neurons, one the afferent portion of the reflex arc connected with the receptor, the other the efferent portion of the arc, connected with the organ, sometimes termed the effector organ-a muscle, e.g., or a gland-which accomplishes the end-effect. The transference of the excitation from the afferent to the efferent neuron takes place across the intervening synapie. The simple isolated reflex arc, as thus described, although a convenient abstraction, corresponds but little to anything which actually exists in one of the higher animals. With increasing cons- 
plexity of organization the nervous impulse passing up an afferent fibre is in general offered, instead of a single efferent path, a choice of many potential routes when it reaches the spinal corl. IVe have previously (p. 872) described the course taken by the fibres of the posterior roots on entering the cord. It is obvious that through the main fibres and their collaterals an extensive connection-partly direct, partly by the link of intermediate neurons-is established with the motor cells on both sides of the cord. But the facts of pirysiology demonstrate an even ampler connection than the mere anatomical study of the distribution of the root-fibres would suggest. Indeed, the phenomena of strychnine-poisoning seem to show that every afferent fibre is potentially connected with the motor mechanisms of the whole cord, or at least with a very large proportion of them. For in a frog under the influence of this drug, stimulation of the smallest portion of the skin will cause violent and general convulsions, which are unaffected by destruction of the brain, but cease at once on destruction of the cord (p. 904 ).

In an unpoisoned reflex frog-that is, a frog in which interference with the single spinal reflexes has been prevented by section of the bulb or destruction of the brain-the movements resulting from stimulation of a given receptive area are by contrast surprisingly limited, localized, and constant. Thus, a harmful stimulus of a certain intensity applied to a toe will elicit time after time a raising of the leg-in other words, an excitation of muscles whose motor nerves arise from cells in the same region of the cord into which the afferent fibres from the receptive skin area enter. The localization of the reflex is in this case without doubt dependent upon the fact that the connections of the afferent fibres with the group of efferent neurons in question are more direct and more intimate than with any other group. This anatomical isolation of a given reflex arc is never complete, but so far as it goes it may be assumed to be constant and incapable of variation. Under normal conditions the anatomical isolation is always supplemented by a physiological isolation, which is susceptible of variation in the direction either of increase or of diminution.

It is therefore a question of great interest how the isolated conduction of the impulses in a given reflex arc, in so far as it depends upon the physiological condition of the arc and of its connections, is normally achieved. The best answer which can at present be given is that it is not equally easy for a reflex excitation to pass across all the synapses which are potentially open to it, and that a lowering of the resistance of the synapses in the favoured path is probably quite as important a factor in the isolation as an increase of the resistance in those which are to be barred. Following the path of least resistance, the excitation traverses the synapse or synapses which it is easiest for it to break through. What property of the synapse is associated with resistance to the passage of the impulse is unknown. But it is a variable property, and when a general reduction in the resistance is produced, as by strychnine or tetanus toxin, an excitation impressed upon a single afferent path may force a great many synapses normally impervious to it.

While it is convenient in a preliminary survey to speak of the resistance to spreading of the excitation in the cord being diminished by strychnine or by tetanus toxin, we shall see presently that more than this is involved (p. 903).

Principle of the Common Path.--In considering the architecture of the cerebro-spinal nervous system as a basis of reflex action, one feature is of such importance as to deserve special mention. The 
afferent neurons, running from the receptive surfaces to the centres, constitute each for its own receptive point.a 'private ' path which can only be used by impulses arising at that point, and not by impulses arising at any other point. Through its central connections an afferent neuron from a single point may be put into communication with numerous efferent neurons, and thus with numerous and distant effector organs (muscles or glands). Conversely, the efferent portion of a single reflex arc can convey reflex excitations originating in numerous and distant receptive fields. It is the sole path which all efferent impulses-let them originate where they may -must use to reach the end-organ in question. It is therefore not a private but a public path, and may be termed in this relation the final common path (Sherrington).

The existence of the common path is of great importance in understanding the manner in which reflexes are compounded together, a problem absolutely fundamental in nervous co-ordination. One consequence of the existence of a common path is that when, among the receptors which may use it, two are simultaneously stimulated which, when separately excited, produce opposite effects upon the affector organ, only one of the effects is produced. In other words, impulses which produce the two opposed effects can be successively, but cannot be simultaneously, sent along the common path. Thus, 'excitation of the central end of the afferent root of the eighth or seventh cervical nerve of the monkey evokes reflexly in the same individual animal sometimes flexion at the elbow, sometimes extension. If the excitation be preceded by excitation of the first thoracic root, the result is usually extension; if by excitation of the sixth cervical root, it is usually flexion. Yet though the same root may thus be made to evoke reflex contraction of the flexors or of the extensors, it does not evoke contraction in both flexors and extensors in the same reflex response. . . The flexor-reflex, when it occurs, seems, therefore, to exclude the extensor-reflex, and vice versa. Either the one or the other results, but not the two together' (Sherrington). It is obvious that this is an advantageous arrangement. An algebraical summation of the opposed effects by the common path would result in a useless action which was neither effective flexion nor effective extension, a compromise and not a co-ordination. The conditions which determine which of two or more competing reflexes shall obtain possession of the final common path are considered on p. 902.

Rôle of the Receptor in Reflex Action.-The rôle of the receptor in the reflex are is above all to sift out from the various kinds of impressions impinging upon the receiving surface the particular kind to which the appropriate response is the reflex action in question. As will be pointed out in greater detail in the study of the special senses, each kind of afferent end-organ has become adapted to a 
special, or, as it is termed, an 'adequate' stimulus, so that it is easily affected by this, and with difficulty or not at all by other modes of stimulation. Thus, light is the adequate stimulus of the end-organ of the optic nerve, heat that of the end-organs of the nerves by which we perceive the sensation of warmth, mechanical pressure that of the nerves by which we perceive the sensation of pressure. Other kinds of stimuli are either entirely inactive or much less effective in evoling the particular sensory response. There is every reason to believe that the receptor in the reflex arc occupies the same position in regard to adequate stimuli as it does when it functions as a sense-organ.

Sherrington has shown that the different kinds of nerve-endings in one and the same area of the skin (in the dog) must be assumed to possess totally different spinal connections, since the movements elicited by stimuli suitable for one form of nerve-ending are quite different from those elicited by stimuli suitable for another.

The 'extensor-thrust' is a reflex obtained in the hind-leg of the $\mathrm{dog}$, and characterized by a brief, strong extension at the hip, knee, and ankle. It is only elicited by a certain kind of mechanical stimulation, best in the spinal dog-i.e., in a dog whose brain has been destroyed or severed from the cord some time before-by pushing the tip of the finger between the plantar cushion and the pads of the toes. The stimulus is similar to that which normally liberates the reaction -namely, the pressure of the ground on the sole of the foot during locomotion. The reflex cannot be obtained by electrical stimulation or by any kind of direct stimulation of afferent nerve-trunks. The same is true of the pinna-reflex in the cat-i.e., the backward crumpling of the ear elicited by squeezing or tickling its tip. The scratch-reflex,* a scratching movement of the hind-foot, is much more easily elicited in the spinal dog by mechanical stimulation (rubbing, tickling, or tapping) applied to the skin of the back behind the shoulder than by electrical stimulation, which often fails to evoke it at all. The puzzling fact that, according to surgical experience, many of the internal organs-e.g., the ureters and bileducts-can be handled, cut, and sutured without pain, while the passage of a renal calculus or a gall-stone may cause excruciating agony, becomes explicable in view of the apparently slight difference which sometimes distinguishes an adequate from an inadequate stimulus. Thus Sherrington has shown that very distinct reflex effects-e.g., a rise of blood-pressure-can be obtained by sudden distension of the bile-duct by the injection of salt solution into its lumen. Distension is here the adequate form of mechanical stimulation, and it is the form induced by the passage of a calculus, while nerve-cutting, although a mechanical stimulus, is not an adequate one.

* The scratch-reflex is very easily obtained in cats during resuscitation after a period of cerebral anæmia. 
Characteristic Properties of the Reflex Arc.-Conduction in reflex ares shows certain peculiarities when compared with the conduction in nerve-trunks already studied (p. 78I): (r) The direction of the reflex conduction cannot be reversed. There is an absolute block on the passage of impulses backwards through a synapse. (2) The velocity of conduction over the whole reflex arc is much smaller than over a nerve-trunk of equal length. Botl of these differences depend mainly on the fact that the impulses must be transmitted from one neuron to another, and very likely on a fundamental property of the synapse. The delay or ' lost time ' in the discharge of the efferent impulses which constitute the reflex response to the excitation of an affererst path increases with the complexity of the response-that is, with the number of neurons and therefore of synapses involved in it. (3) The reflex arc is easily fatigued, easily affected by deprivation of oxygen and by drugs, in comparison with the nerve-trunk. This difference is due to the portion of the arc in the grey matter, including the synapse or synapses. Fatigue expresses itself by an increase in the degree of block or resistance to the passage of impulses along the arc. (4) The reflex end-effect may much outlast the stimulus - in other words, a marked 'after-discharge 'is characteristic of reflexes. The more intense the stimulus which liberates the end-effect, the greater is the duration of the afterdischarge. For example, the 'crossed extension reflex' (extension at the knee, ankle, and hip, produced in the spinal dog by stimulation of the skin of the opposite or contralateral hind-limb), when provoked by a stimulus of more than a certain intensity, may outlast the stimulation by ten or fifteen seconds, and the after-discharge may be stronger than any other part of the reflex (Sherrington). (5) A succession of impulses may easily pass along a reflex arc when one of the series would fail to pass (temporal summation). This does not occur in a nerve-trunk. The first stimulus, though itself unable to produce the reflex effect, facilitates the action of succeeding stimuli, so that summation of the impulses occurs in the cord (Stirling). A stimulus-e.g., a make-induction shock, far too weak to produce the scratch-reflex when applied once only to a point of that area of skin from which the reflex is normally elicited - has been seen to cause the reflex after more than forty shocks had been delivered at the rate of eighteen per second. The facilitation of the passage of an impulse by the previous passage of impulses along the same reflex path recalls a somewhat similar phenomenon already alluded to in connection with the conduction of the propagated disturbance in nerve-fibres (p. 79I), although in the case of the reflex arc the effect, it may be supposed, is exerted upon the fields of conjunction, including the synapses, between the different neurons. There is reason to believe that summation in the reflex arc is mainly achieved by the removal of block or resistance. The phenomenon of 
facilitation is probably of great importance in the acquirement of new reactions and in rendering these acquisitions stable. It is probably one of the main physiological foundations of habit, and therefore of education. In this connection it is important to note that the very same repetition of stimuli which leads to facilitation leads to fatigue when the stimuli are applied in too rapid succession. (6) The rhythm and intensity of the reflex end-effect correspond much less closely with the rhythm and intensity of the stimulus than in nerve-trunks. (7) The phenomena of refractory period (p. I55), inhibition and 'shock,' are much more conspicuous in the reflex arc than in nerve-trunks.

Inhibition in Reflex Action.--Special emphasis must be laid upon the part played by inhibition in reflex actions. For the proper carrying out of many reflex movements it is necessary not only that the appropriate effector organ, the appropriate muscle, or group of muscles, should be caused to contract at the proper time, but that their contraction, or that of other muscles, should be diminished or abolished by inhibition, or even rendered for a certain period inpracticable by the establishment somewhere in the reflex arc of a refractory state, which is itself a phenomenon of inlibition. There is good evidence that this is a central inhibition-i.e., it depends on some process occurring in the spinal portion of the reflex arc.

As an example of the numerous class of reflexes in which the excitation of certain muscles is accompanied by the inhibition of their antagonists (reciprocal inhibition), we may take the 'flexion reflex,' the flexion at the knee, hip, and ankle of the hind-limb readily elicited in the spinal dog by ' nocuous' or harmfu, stimuli (such as a prick, a strong squeeze, chemical agents, or excessive heat), or by electrical stimuli applied to the skin of the limb or of any afferent nerve of the limb.

Sherrington has shown that when the legs of the animal are so prepared that only the flexors can act on one knee, and only the extensors on the other, stimulation of symmetrical points on the two sides in the area of skin (receptive field) from which the flexion reflex can be evoked causes contraction (excitation) of the flexors and simultaneous relaxation (inhibition) of the tone of the extensors. The same is true when corresponding afferent nerve-twigs are stimulated on the two sides. From this it is inferred that each of the nerve-fibres from the receptive field of the reflex divides in the cord into two sets of end-branches (e.g., collaterals)-a set which produces excitation when it is stimulated, and another set which produces inhibition.

Reversal of Reflexes.-The difference in action is specific in the sense that no mere change in the kind or intensity of stimulation affects it. Yet there are facts which show that the specificity is not absolutely immutable, and that a change of conditions in the spinal cord may permit excitation of a given group of muscles to be pro- 
duced by the stimulation of an afferent path which is primarily inhibitory for them. One of the most striking illustrations of this possibility is seen in the action of strychnine. Stimulation of the internal saphenous nerve below the knee-say in a dog after removal of the cerebrum-is known always to produce inhibition of tl.e portion of the quadriceps extensor whose contraction causes the knee-jerk.

If now the animal be poisoned by a small dose of strychnine, stimulation of the nerve causes no longer reflex relaxation, but reflex contraction of the muscle. This fact indicates that the essential action of strychnine is something different from a mere reduction of the resistance to the spread of impulses in the cord (Sherrington). Tetanus toxin produces a similar effect, though more slowly.

The reversal of the depressor reflex on the blood-pressure has been previously alluded to (p. I88). A different type of reversal, and one of most interest in connection with the co-ordination of reflexes, is illustrated by such observations as the following: The extensor thrust is only obtained by the adequate form of stimulation described on p. 9or, when the hind-limb is in a condition of flexion. When the leg is passively extended at the time when the stimulus is applied, the response is not the extensor thrust, but flexion of the leg and thigh (direct flexion reflex). The passive assumption of a condition of flexion at the knee and thigh appears, accordingly, to favour the extensor reaction (Sherrington). The observations of Magnus have shown that such relations are general; for example, the reaction is usually extension when the opposite posterior limb is flexed at the time of stimulation, and flexion when the opposite leg is extended. In the spinal cat, stimulation applied to the tail, especially near the root, elicits always a stroke towards the side on which at the time of stimulation the muscles are extended. The phenomenon is dependent upon the integrity of the afferent nerves of the passively extended or flexed muscles whose position influences the reflex, and of the afferent nerves of the tendons and fascia related to them. The condition of the reflex centres is in some way influenced by impulses conducted along those afferent paths.

Not only is the tone of the extensors diminished or abolished during the activity of the flexors, but the contraction of the knee extensors evolved by striking the patellar tendon, which is called the knee-jerk, either fails to appear, or appears but feebly, when the flexion reflex is simultaneously elicited, even when the mechanical antagonism of the flexor contraction has been eliminated by previously detaching the flexors from the knee.

The Knee-Jerk. - This is sometimes termed a pseudo-reflex. For certain authorities believe that the mechanism by which it is produced is different from that concerned in the reflex blinking of the eyelid, or the reflex retraction of the testicle, or the drawing-up of 
the foot when the sole is tickled. The knee-jerk is obtained in undiminished strength when the nerves of the ligamentum patella have been divided. It is therefore not a reflex movement caused by stimulation of afferent nerves coming from the tendon, and the name 'tendon-reflex 'is clearly a misnomer. But that it is related in some way or other to afferent impulses is certain, for division of the posterior roots that enter into the anterior crural nerve abolishes the knee-jerk. The phenomenon, accorcling to these authors who deny that it is a true reflex, comes under the head of what is called inyotatic irritability - that is, it depends on mechanical stimulation of the slightly-stretched muscle by the pull of the tendon when it is struck. It is necessary for this stimulation that the muscle should be to a certain extent tonically contracted. So that when the afferent fibres are interrupted, or the grey matter of the cord disorganized, and the reflex tone abolished, the knee-jerk disappears. The strongest objection to considering it an ordinary reflex is the shortness of the interval which elapses between the tap and the jerk, which, according to some observers, is not much greater than the latent period of the quadriceps muscle for direct electrical stimula tion, as measured under the ordinary conditions of its contraction. There is no doubt that the interval is very brief, although somewhat conflicting results have been obtained by different observers for the corrected latency - - that is, the period between stimulus and response minus the latent period of the muscle. In man this period seems to be about 0.02 second. In a dog with divided spinal cord the interval was found to be $0.0{ }_{4}$ to 0.02 second (Applegarth); in the rabbit only 0.008 to 0.005 second (WValler and Gotch). Recent observations in which the electrical response of the muscle as recorded by the string galvanometer was employed instead of the contraction have yielded results not very different from those obtained by the older methods, o.oII to o.0I5 second according to Synder. Taking account of the newer observations on the velocity of the nervous impulse (p. 793), it would appear that the interval is not really too short to prevent the knee-jerk from being classified as a true reflex contraction, although a very brief one.* The reinforcement of the knee-jerk is referred to under another heading (p. 9II). It is admitted that, in addition to the direct stimulation of the muscle on the same side, the tendon-tap may cause also a true reflex knee-jerk on the opposite side, the interval between tap and contraction being about $\frac{1}{8}$ second.

Spread or Irradiation of Reflex Action.-As the strength of the stimulus which has been evoking a given reflex movement is increased, the reflex effect becomes more and more extensive, spreading out or irradiating in various directions. If, for example, the reflex

* There is really now no good reason for regarding the knee-jerk as anything else than a true reflex. The term pseudo-reflex and other terms implying that the knee-jerk is not a reflex should therefore be dropped. 
in question is the flexion reflex elicited by stimulation of the plantar surface of the hind-foot in the spinal animal, increase of the stimulus will cause, in addition to flexion of the same hind-foot, extension of the opposite hind-limb, then in the homonymous fore-limb (i.e., the limb on the same side) extension at the elbow and retraction at the shoulder, then certain definite movements, the details of which need not detain us here, in the opposite fore-limb, and ultimately also definite movements of the head and tail (Sherrington). Obviously there is a certain orderliness in the spread of the reflexes; they follow a certain regular march; the irradiation in the tangle of the spinal paths is not an indiscriminate one. The same fact emerges quite as clearly when other reflexes are studied in a similar way; and certain laws or rules which define the spread of the impulses in spinal reflexes have been deduced. For descriptive purposes, in dealing, with reflex action, it is convenient to consider each lateral half of the cord as divisible into regions each related on the one hand to a certain area of the receptive surface (skin), and on the other to certain muscles. Such regions are those of the neck, including the pinna (cervical), the fore-limb (brachial), the trunk (thoracic), the hind-limb (crural), and the tail (caudal). According to their relation to these regions the spinal reflexes can be classified as 'short' or 'long.' The short spinal reflexes are those in which the muscular response takes place in the same region as the application of the stimulus. The long reflexes are those evoked when the stimulus is applied to the receptive field of one region, and the response occurs in the musculature of another region. For the short reflexes Sherrington has given a number of rules, which may be stated as follows: (I) The closer together their spinal segments, the easier is it for stimulation of a given efferent root to excite reflex contractions of muscles supplied by a given afferent root. (2) For each afferent root there exists in its own spinal segment (of course, on its own side of the cord) a refiex motor path of as low a threshold (i.e., as easily set into action) and of as high potency (i.e., producing as great a reflex effect) as any open to it anywhere. It has been shown that the afferent nerves of a skeletal muscle are derived from the spinal ganglion corresponding to the segment of the cord containing its motor cells. (3) Motor mechanisms for the skeletal musculature lying in the same region of the cord, and in the selfsame spinal segment, show markedly unequal accessibility to the local afferent chamnels as judged by the reflex contractions produced. For example, the reflex contraction of the flexors of the knee on the stimulated side, and of the extensors of the opposite knee, is in many animals much more easily elicited than contraction of the extensors of the homonymous and the flexors of the contralateral (i.e., opposite) side. This, however, is not because the last-named extensors and flexors are really incapable of being reflexly affected through the afferent fibres of the corresponding spinal segments, but 
hecause the reflex effect produced by them is in this case not contraction, but inhibition. (4) The groups of motor cells contemporaneously discharged by spinal reflex action innervate synergic muscles (muscles which act in the same direction in effecting a harmonious movement), and not antergic muscles (which antagonize each other).

This disproves the old idea that the movements, caused by excitation of an efferent spinal root, are co-ordinated synergic movements, since at many joints the flexors and extensors both receive motor fibres from one and the same root, and stimulation of the root must simultaneously excite antagonistic muscles. 'The collection of fibres in a motor spinal root does not represent a reflex figure-i.e., a number of simple reflexes occurring simultaneouslynor does the receptive field of a reflex correspond with the distribution of an afferent root.'

(5) It follows from (I), (2), and (4) that the spinal reflex movement which can be elicited in and from any one spinal region will exhibit much uniformity even when the exciting stimulus is applied at different and distant points within the receptive field. The flexion reflex of the hind-limb, e.g., will have the same general character-i.e., flexion of each of the three main joints-whatever part of the surface of the limb is stimulated. Yet the flexion movement will be strongest at the joint whose flexors are innervated by motor cells situated in a spinal segment near the entrance of the afferent fibres from the stimulated skin area.

For the long spinal reflexes it is less easy to deduce definite rules, for they can be less easily and constantly evoked than the short reflexes. The so-called laws of spread formulated by Pflüger for the long spinal reflexes, and based mainly on observations made in the brainless frog and on clinical records in cases of spinal lesion in man, need not be stated here. For Sherrington has shown that they require serious modification. Especially is this true of Pfluger's fourth law, that the reflex irradiation spreads always more easily up in the direction of the medulla oblongata, so that stimulation of a fore-limb does not cause reflex contraction of a hind-limb, although excitation of a hind-limb may cause movement of one or both forelimbs. This law does not hold in the mammal. As a rule, indeed, irradiation takes place more easily down than up the cord. Excitation of the skin of the pinna easily causes reflex movements of the limbs, while the reverse is rare. Reflex movements of the hindlimb in the spinal animal are more easily evoked by stimulation of the fore-limb than movements of the fore-limb by stimulation of the hind. It is easier for the irradiation to cross the cord from hind-limb to hind-limb than to pass up from hind- to fore-limb; but it is often easier for irradiation to occur down the cord from fore- to hind-limb than across the cord from one fore-limb to the other. Afferent channels from the skin of the shoulder, through 
which the scratch-reflex is discharged (in the dog), are freely connected with efferent paths to the muscles of the hip, knee, and ankle by an uncrossed path descending the lateral column (Sherrington). In cats, after temporary occlusion of the cerebral circulation, which throws the brain out of gear, it is easy to elicit movements of the hind-legs by pinching the fore-paws or the skin of the upper part of the body. The scratch-reflex can also be very readily evoked, and in great intensity, by stimulating the pinna, and is not confined to the side stimulated. In anæmia of the brain and (cervical) cord and subsequent resuscitation, homolateral reflexes (i.e., on the same side as the stimulus) are submerged later and emerge sooner than contralateral reflexes whose centres lie in the area which was rendered anremic (Pike, Gutlirie, and Stewart).

Co-Ordination of Reflexes. - The co-ordination or orderly combination of muscular actions for the production of appropriate and harmonious movements is one of the most important functions of the central nervous system. Both the brain and the cord take a share in this co-ordination. The rôle of the brain will be considered later on, but it is essential to recognize now that many of the movements which the brain directs represent spinal reflexes already synt hesized, compounded, or co-ordinated in a very high degree. This is the reason why, in the spinal animal, the inexperienced observer may sometimes be startled by the apparently 'purposive character' of a reflex movement-the scratch-reflex in the dog or cat, e.g., or the extensive reflex movements of the hind-legs of a brainless frog when the skin is pinclied or painted with dilute acid, so plainly directed to the seat of irritation. When a drop of acid is applied to the flank of such a frog, it will attempt to wipe it off with the foot which is situated most conveniently. If this foot be held, it will use the other. These reactions are necessarily purposive in character, since they have been evolved with reference to the advantage of the organism as a whole. They are the sort of complex reactions which the intact animal would have had to improvise by the combination of a considerable number of simple movements when it was executing such defensive reactions, with the conscious purpose of escaping from the irritant, were they not already present as purposive reflexes in the ready-made condition.

In the combining of reflexes we may distinguish between simultaneous combination-i.e., the combination of reflex actions taking place at the same time-and successive combination-i.e., the combination of reflexes in such a way that they follow each other in an orderly sequence. The facts already mentioned in speaking of irradiation afford a partial explanation of the co-ordination of reflexes by simultaneous combination. The movements are orderly and harmonious because the spread of the reflexes is not indiscriminate, but follows a definite 'march,' determined partly by the 
anatomical relations of afferent and efferent patlis, partly by the varying resistance of the synapses or other structures whose properties fix the threshold value of the excitation by which an arc can be forced. In general it is not enough that the channel of the final common paths (p. 899) to the muscles whose contraction produces the reficx movement should be thus open to the afferent arcs that elicit the movement; they must be closed to other afferent arcs which might disturb the reflex. Not only so: there is evidence that very frequently the final common paths are, so to say, more widely opened to the afferent ares in question by the 'reinforcing' or 'facilitating' influence of allied, though it may be distant, afferent arcs, which are simultaneously excited (p. 9II). Further, the final common paths to antagonistic muscles must also be temporarily closed. The closing of these central connections, or rather the raising of their threshold sufficiently to bar the impulses from passing through the door, is an inhibitory phenomenon. Excitation of the desired movements and inhibition of antagonistic movements go hand-in-hand in the simultaneous combination of reflexes. It is obvious that if a movement is to be efficiently executed, it cannot be the result of a compromise between competing reflexes. A segment of a limb can be either flexed or extended, but cannot at the same time undergo both flexion and extension. In the interests of an effective movement, one or the other must give way utterly. The reflex which eventually prevails makes a clear field for itself by inhibiting all other reflexes which do not co-operate with it. For example, if the receptive area of skin from which the scratch reflex is elicited be stimulated and a painful stimulation be at the same time applied to the foot, we do not obtain a mixed scratch and flexion reflex, which would result in a confused and ineffective combination of movements, but a pure scratch or flexion reflex, as a rule the latter.

The successive combination of reflexes is well illustrated by the contraction of the œesophagus in deglutition. First one portion of the tube and then the next below are involved in the reflex action. The combination consists in the orderly sequence. The manner in which this is secured in this class of reflex action has been luminously discussed by Sherrington, * but details cannot be given here. While only allied reflexes-i.e., such as mutually reinforce and therefore harmonize with each other-can be simultaneousiy com. bined, and antagonistic reflexes cannot, both allied and antagonistic reflexes can be successively combined. An example of the successive combination of allied reflexes is the series of scratch reflexes caused by a parasite travelling across the receptive field of the reflex. An example of the successive combination of antagonistic

* 'Integrative Action of the Nervous System,' to which work the advanced student is referred. 
reflexes is afforded when either the scratch reflex or the flexion reflex is induced and caused to interrupt the other while it is proceeding. The transition-e.g., from flexion to scratch reflex-is made without any period of confusion. Thus, if the scratch reflex has been induced and is being executed, and the foot is then painfully stimulated, the scratch reflex immediately ceases, and the flexor reflex takes its place. When the flexor reflex has terminated, the scratch reflex may be resumed. The same holds good for other antagonistic reflexes. In many cases the avoidance of confusion is due to the inhibition of the first reflex, or often to inhibition of the set of muscles which were active in the first reflex combinect with excitation of their antagonists (so-called interference). It is obvious that this is an adaptation of great importance.

Influence of the Brain on the Spinal Reflexes.-The spinal reflexes call be influenced by inspulses descending from the higher centres. For $(a)$ it is a matter of common experience that a reflex movement may be to a certain extent controlled, or prevented altogether by an effort of the will, and it is worthy of remark that only movements which can be voluntarily produced can be voluntarily inhibited. (b) Long-continued muscular contractions may be caused in animals after removal of the cerebral hemispheres by stimulation of afferent nerves-for example, by scratching the mucous membrane of the mouth in a 'brainless' frog or Necturus. (c) By stimulation of certain of the higher centres reflex movements which would otherwise be elicited may be suppressed or greatly delayed. If the cerebral hemispheres are removed from a frog, and one leg of the animal dipped into dilute acid, a certain interval, the (uncorrected) reflex time, will elapse before the foot is drawn up (p. 996). If, now, a crystal of common salt be applied to the optic lobes, or corpora bigemina, or the upper part of the spinal cord, and the experiment repeated, it will be found either that the interval is much lengthened or that the reflex disappears altogether. Stimulation of the optic lobes relaxes the reflex sexual embrace of the male frog when it is present. From such experiments it has been concluded that centres which can inhibit the spinal reflexes are situated in the thalamus, the corpora bigemina and the medulla oblongata of the frog. In mammals, also, there is evidence of the existence of mechanisms in the brain, the excitation of which diminishes the reflex excitability of the cord. For example, stimulation of the frontal convolutions in the dog causes a diminution in the height of reflex contractions of the limbs. Strong stimulation of an afferent nerve may abolish or delay a reflex movement which is being elicited through other receptors. (d) If such inhibitory mechanisms exist, it is to be supposed that elimination of the brain will render it easier to elicit reflexes from the cord. Experiment shows that this is actually the case. An animal like a frog responds 
to stimuli by reflex movements more readily after the medulla oblongata las been divided from the spinal cord or the brain re. moved. In the dog the scratch reflex is elicited more easily after removal of the cerebral cortex or its elimination by cerebral ancmia. In the guinea-pig, after extirpation of the cortex of one hemisplere, the scratcli reflex is more readily evoked on the side of the lesion (Brown).

That the brain exerts more than a merely inhibitory influence on the production of reflex movements is suggested by many facts. The knee-jerk, for example, is increased or 'reinforced' if an instant before the tendor is struck the patient makes a voluntary movement or is acted on by a sensory stimulus (Bowditch and Warren). In health it varies in strength with many circumstances which affect the activity of the central nervous system as a whole (Lombard, etc.). It often disppears in pathological lesions, situated high up in the cord in man, and is markedly impaired after high section of the cord in dogs. In hemiplegia (paralysis of one side of the body, caused by disease in the brain) the cutaneous reflexes on the paralyzed side may sometimes be absent for years. Some observers have even gone so far as to say that under normal conditions the so-called spinal reflexes are really cerebral-in other words, that the afferent impulses run up to the brain and there discharge efferent impulses, which pass down to the motor cells of the anterior horn and cause their discharge. It may be admitted that there is no physiological ground for supposing that the afferent impulses which have to do with the reflex contraction of the muscles of the leg when the sole is tickled, stop short at the motor cells of those spinal segments from which the efferent nerves come off, while the afferent impulses which have to do with the sensation of tickling pass up to the brain. The probability is that under ordinary circumstances such afferent impulses pass up the cord in long afferent paths, as well as directly towards the motor cells along those fibres of the posterior roots and their collaterals which bend forward into the anterior horn at the level of their entrance into the cord. And the only question is whether, as a matter of fact, the spinal motor cells are most easily discharged by the impulses that reach them directly, or by the impulses that come down to them by the roundabout way of the brain, and the efferent fibres that connect it with the cord. It is evident that the answer to this question need not be the same for all kinds of animals. It may well be that in the higher animals, in which the cortex has undergone a relatively great development, the spinal motor mechanisms are more easily discharged from above than from below, while in lower animals the opposite may be the case. When the cord is cut off from the brain. the afferent impulses may overflow more easily into the spinal motor cells since their alternative path is blocked. In the frog, where 
there is already a beaten track between the posterior root-fibres and the cells of the anterior horn, this overflow may be established immediately after section of the cord, and may of itself lead to an exaggeration of the reflexes. In animals like the dog a longer time may be necessary before the unaccustomed route from the end arborizations of the afferent axons and their collaterals to the dendrites or the bodies of the motor cells becomes natural and easy; in man a still longer interval may be required. Moore and Oertel have made a careful comparative study of reflex action after complete section of the cord in the cervical or upper dorsal region, and conclude that the spinal reflexes in the higher animals are far more dependent on the upper portions of the central nervous system than in the frog.

Spinal Shock.-The phenomena of spinal shock and its varying severity in different animals may be accounted for by the rupture of the paths nornially used in the reflexes. The theory that the shock is due to an inhibition set up by the mechanical injury is untenable. For shock affects only the portion of the central nervous system clistal (or aboral) to the lesion. When a dog is allowed to live after transection of the cord in the lower cervical region till shock lias been recovered from, a second transection distal to the first is followed by only slight and very transient depression of the reflex power, although the direct effect of the second injury ought, of course, to be as great as that of the first. Finally, according to Sherrington, the condition of the spinal reflex arcs in shock differs from the condition caused by inhibition, and resembles rather a general spinal fatigue in which conduction along the arc and especially across the synapses is difficult and uncertain. This condition is supposed to be due to the loss of a "tonic ' influence of higher centres, assumed to be necessary for the maintenance of the normal conductivity of the arc. These cranial centres, if they exist, or, at least, the most efficient of them, must be assumed to be situated distal to the cerebral cortex, probably in the pons or mid-brain. For section just behind the pons causes much more severe shock than removal of the cerebral hemispheres.

Peripheral Reflex Centres. -Tlie question whether any reflex centres exist outside of the spinal cord and brain, and especially in the sympathetic ganglia, has been the subject of a lengthy controversy. That the spinal ganglia cannot act as reflex centres is generally acknowledged, and it is not difficult to see that, for anatomical reasons, this must be so. A reflex arc must, so far as we know, in all highly-organized animals include at least two neurons. There is no proof tlat an afferent impulse can ascend an axon to a cell-body and there excite an cfferent impulse, which, descending the same axon in a separate set of fibrils, gives rise to a reflex contraction, or a reflex secretion. Now, the cells of a spinal ganglion represent the original neuroblasts from which the posterior root-fibres grew out as processes towards the cord on the one side and the periphery on the other. A sensory fibre passing into the ganglion makes connection with a cell by a T-sliaped junction and pasecs on its course again. No afferent fibres run from the nerve-trunk into the ganglion, to end in arborizations around the ganglion cells, and no efferent fibres arise from nerve-cells in the ganglion to pass out into the trunk. For although a slightly greater number of medullated fibres of small calibre is found in a spinal nerve-trunk immediately 
distal to the junction of the roots than in both roots taken together, this appears to be due to the passage into the nerve (from the grey ramus communicans) of medullated fibres which end in the bloodvessels or other tissue of the ganglion (Dale). Here it is evident that there is no possibility of a complete reflex arc. Inclecl, it is not certain that the normal afferent impulses pass through the borlies of the spinal ganglion cells at all. For (I) a negative variation can be observed in the josterior roots above the ganglia on stimulation of the trunk of a fr g's sciatic nerve more than two days after the death of the animal, when the ganglion cells may be supposed to have completely lost their vitality, and when no reflex negative variation can be detected in the central stump of a severed anterior root on excitation of the sciatic or the corresponding posterior root. Such a reflex action current is normally obtainable from a fresh preparation. (2) When the blood-supply of the postcrior root-fibres ancl the ganglion is cut off without killing the frog, the nerve impulsc is still conducted by the fibres, as is shown by the reflex movements elicited on stimulation of tlie central end of the sciatic, at a time when the nerve-cells show marked histological alterations. (3) Prolonged excitation of the posterior roots or the mixed nerve causes no noticeable microscopical clianges in the ganglion cells (Steinach).* (4) The application of nicotine to a spinal ganglion docs not hinder the passage of impulses through the corresponding afferent fibres. If it acts on spinal ganglion cells as it does on sympathetic ganglion cells (p. I 82), this must be because the impulses do not require to traverse the ganglion.

Axon-Reflexes.-In the ordinary sympathetic ganglia, $\uparrow$ also, it is doubtful whether the anatomical foundation for a reflex arc exists, and the most careful physiological experiments have failed to connect them with any reflex function. Sokownin, indeed, observed that stimulation of the central end of the hypogastric nerve caused contractions of the bladder, and he considered these movements to be reflex, the centre being the inferior mesenteric ganglion. Langley and Anderson have also found that when all the nervous connections of the inferior mesenteric ganglion, except the hypogastric nerves, are cut, stimulation of the central end of one hypogastric causes contraction of the bladder, the efferent path being the other hypogastric. In addition, they have observed an apparent reflex excitation of the nerves which supply the erector muscles of the hairs (pilo-motor nerves) through other sympathetic ganglia. They believe, however, that in neither case is the action truly reflex, but that it is caused by stimulation of the central ends of motor fibres, which come off from the spinal cord, and in passing through the ganglion give off collateral branches to some of its cells. In the case of the inferior mesenteric ganglion the spinal fibres passing down in the left hypogastric would send branches to arborize around ganglion cells which give origin to fibres of the riglit liypogastric, and vice versa. When the central end of the left hypogastric is stimulated the excitation is conducted up the spinal fibres, and so reaches their branches, and, through the ganglion cells, the sympathetic fibres of the right liypogastric, which convey it to the muscles of the bladder (see sartorius or gracilis experiment of Kühne, p. 792). Other examples of such axon-reflexes exist.

* Hodge obtained changes. In such experiments it is necessary that thr ganglion should not be directly excited by electrotonic currents or escape of the stimulating current.

† The ganglion cells of Auerbach's and Meissner's plexus in the intestine are not of ordinary sympathetic type, and, as has been previously pointed out, it is probable that they, or some of them, are true reflex centres for the stomach and intestines. 
Reflex Time.-When a reflex movement is evoked, a measurable period elapses between the application of the stimulus and the commencement of the movement. This interval may be called the uncorrected reflex time or the latent period of the reflex. A part of the interval is taken up in the transmission of the afferent impulse to the reflex centre, a part in the transmission of the efferent impulse to the muscles, a part represents the latent period of muscular contraction, and the remainder is the time spent in the centre, or the true reflex time. Ordinarily this time, though absolutely short, is relatively so great that the total latent period of a reflex is much longer than when a similar length of nerve-trunk is interposed between the point of application of the stimulus and the muscle. When the conjunctiva or erelid is stimulated on one side both eyelids blink. This is a typical reflex action reduced to its simplest expression, and the true reflex time is correspondingly short-only about $\frac{1}{20}$ second $\left(5^{\circ} \sigma^{*}\right)$. An additional $\frac{1}{100}$ second $($ ro $\sigma)$ is consumed in the passage of the afferent impulse along the fifth nerve to the medulla oblongata, of the efferent impulse from the medulla to the orbicularis palpebrarum along the seventh nerve, and in the latent period of the muscle. When a naked nerve, like the sciatic, is stimulated, the true reflex time is reduced to $\frac{1}{100}$ to $\frac{7}{50}$ second. As estimated by 'Türck's method (p. 996), the uncorrected reflex time is greatly lengthened, it may be to several, or even many, seconds. For here it is evident that the time taken by the acid to soak through the skin and reach the nerve-endings in strength sufficient to stimulate them is included. But even when the peripheral factors remain constant, the central factor may vary. With strong stimulation, e.g., the reflex time is shorter than with weak stimulation. With weak stimuli the latent period of the flexion reflex in the dog is usually $60 \sigma$ or I20 $\sigma$. It may even be as long as $200 \sigma$. With strong stimuli it may be as little as $30 \sigma$. Even $22 \sigma$ has been seen, which is little more than for nerve-trunk conduction. Fatigue of the nerve-centres delays the passage of impulses through them; and strychnine, while it increases the excitability of the cord, also lengthens the reflex time.

Reflexes in Disease.-In order that a reflex action may take place, the reflex arc-afferent nerve, central mechanism. and efferent nervemust be complete; and, in fact, a whole series of simple reflex movements exists, the suppression, diminution, or exaggeration of which can be used in diagnosis as tests of the condition of the reflex arc. It is customary to divide these into superficial reflexes, elicited from receptive fields on the surface of the body (extero-ceptive fields), and deep reflexes, elicited from receptors in the depth of the organism (proprioceptice fields). especially in the muscles and the tendons and joints connected with them. The extero-ceptive reflexes are normally excited by extraneous stimuli acting on the surface from the environment. The proprio-ceptive reflexes are normally excited by changes (muscular

* $\sigma=0.00$ I second. 
contractions) occurring in the body itself, which changes are in turn isually initiated by excitation of surface receptors by the environment. Examples of superficial reflexes are the plantar reflex (the drating-up of the foot when the sole is tickled), the cremasteric reflex (retraction of the testicle when the skin on the insicle of the thigh just below Poupart's ligament is stroked, especially in boys), the gluteal, abdominal, epigastric. and interscapular refices (contraction of the muscles in those regions when the skin covering them is tickled). The behaviour of the toes, especially of the great toe, is of considerable diagnostic importance. Normally, on tickling the sole, the toes are flexed towards the planta; but when a lesion of the pyramidal tract exists, as in hemiplegia, there is dorsal instead of plantar flexion, most marked in the case of the great. toe, and the toe moves more slowly than in the healthy person (Babinski's sign). This is an instance of reversal of a reflex owing to the elimination of the influence of the cortex. In an epileptic fit there is said to be a temporary reversal of the same reflex, indicating probably that the cortex is temporarily eliminated in consequence of fatigue due to intense and prolonged discharge. In children during the first few months of life stimulation of the sole causes normally a dorsal flexion of the big toe, this reversal of the normal reaction of the adult persisting until the pyramidal path has attained functional completion (p. 8.8). During sleep the reversed reaction (dorsal flexion) is still obtained for a time. Examples of deep reflexes are the knee-jerk (a sudden extension of the leg by the rectus femoris and vastus medialis components of the quadriceps muscle when the ligamentum patella is sharply struck), the heel-jerk or foot-jerk (a movement of the foot caused in most healthy persons, though not in all, by tapping the tenclo Achillis), and the periosteal radial reflex (a movement of flexion and slight pronation of the forearm and hand elicited by tapping

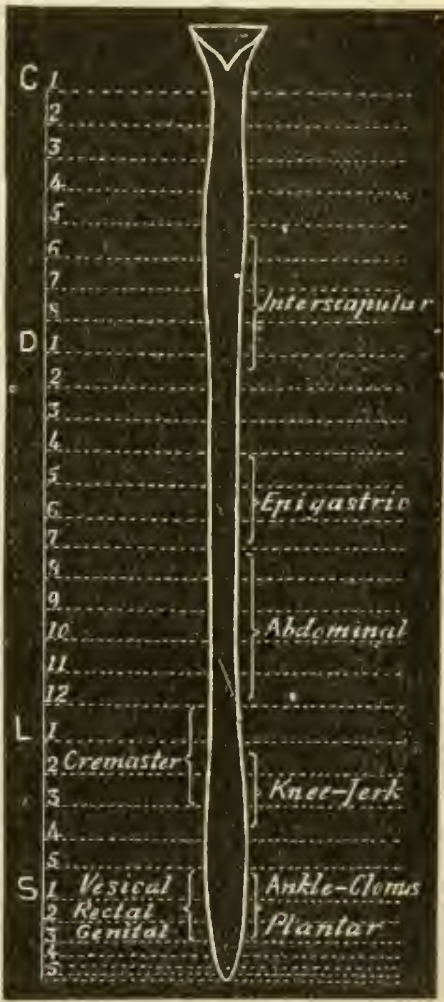

Fig. 366.-Diagram of Reflex Centres in Cord (after Hill). the lower end of the radius). The jaiv-jerk (a movement of the lower jaw when, with the moutl open, the chin is smartly tapped) and ankle-clonus (a series of spasmodic movements of the foot, brought about by flexing it sharply on the leg) are phenomena of the same class, which can be elicited only in disease. Any condition which impairs the conducting power of the afferent or efferent fibres of the reflex are necessarily diminishes or abolishes the reflex movement, even if the central connections are intact. E.g., in locomotor ataxia the disappearance of the knee-jerk is one of the most important diagnostic signs. This disease involves the posterior roots and the fibres that continue them in the posterior 
column. The anterior nerve-roots are perfectly healthy. The grey matter of the cord-at least, in the earlier stages of the disease -is unaffected. The weak link in the chain is the afferent path. Where the presence of the knee-jerk is doubtful, it is necessary to search for the most favourable position of the limb for eliciting it before determining that it is absent. The patient may be made to clasp his hands tightly at the moment of the tap to reinforce the jerk (p. 9II). In anterior poliomyclitis (p. 876) the afferent link is intact, but the other two are broken, and the reflexes also disappear. Certain lesions which partially cut off the spinal cord from the higher centres without affecting the integrity of the spinal reflex arcs increase the strength of reflex movements and the facility with which they are called forth. In primary spastic paraplegia (a paralysis of the legs and the lower portion of the body), which is associated with degenerative changes in the lateral columns, the deep reflexes are all exaggerated. But according to the best authorities, a lesion amounting to total transection of the cord in man abolishes all reflexes below the lesion. In the monkey the kneejerk may be tried for in vain for weeks after section of the cord in the midcle of the thoracic region, whereas in the rabbit it can be obtained ten to fifteen minutes after the transection. The position of the centres in the cord for the various reflex movements is shown in Fig. 366.

3. The Origination of Impulses in the Spinal Cord (Automatism).An action known to be caused or conditioned by afferent impulses is called a reflex action. There is some evidence that the reflex centres are continually in a state of activity, and are not simply roused to activity by the arrival of the afferent impulses, which discharge the reflex. Their condition, when not discharging, seems to represent a state of balance between excitatory and inhibitory influences, a so-called 'innervation equilibrium,' which can be upset in one direction or the other, according to the intensity of the antagonistic influences. A physiological action is termed automatic when it depends upon a nervous outflow which seems to be spontaneous, in the sense that it is not brought about by any evident reflex mechanism, or, in other words, is not discharged by afferent impulses falling into the centre where it arises, althougl it may be determined by substances in the blood. Automatic actions being thus defined in a negative manner by the defect of a quality, there is always a possibility that some day or other it may be demonstrated that any given action which at present seems automatic in its origin depends on afferent impulses hitherto unnoticed. As a matter of fact, the supposed proofs of spinal automatism have in more than one case vanished with the advance of knowledge, and as the domain of purely automatic action has been narrowed, that of reflex action has extender!, until the controversy as to the boundaries between the two seems not unlikely to be ended by the absorption of the automatic in the reflex. And as we seem almost driven to conclude that from the anatomical standpoint the nervous system is essentially a vast collection of looped conducting paths, each with an afferent portion, an efferent portion, and connections between them formed by the end arborizations of the axons and 
their collaterals, the dendrites and the ecll-bodies, so it may be that no strict physiological automatism really exists either in cord or brain, that every form of plyysiological activity-muscular movement, secretion, intellectual labour, consciousness itself-would cease if all afferent impulses were cut off from the nervous centres. Assuredly no neuron is entirely isolated from other neurons. The more the nervous system is investigated, the deeper grows the conviction of its essential solidarity, the more clearly it displays itself as a single mechanism, the most distant parts of which are intricately knit together. But there are certain gromps of actions so widely separated from the most typical reflex actions that, provisionally at least, thcy may be distinguished as automatic. Such are the voluntary movements, and certain involuntary movements, like the beat of the heart. And we may proceed to inquire whether the spinal cord has any power of originating movements or other actions of this high degree of automatism.

Muscular Tone or Tonus-Postural Reflexes.-It is generally stated that so long as a muscle is connected with the spinal segment from which its nerves arise, it is never completely relaxed; its fibres are in a condition of slight tonic contraction, and retract when cut. This tonus has been clearly demonstrated for some muscles, though not for all. If a frog whose brain has been destroyed is suspended so that the legs hang down, and one sciatic neive is cut, the corresponding limb may be observed to elongate a. little as compared with the other, owing to a slight relaxation of the flexors. At one time this tone of the muscles was supposed to be due to the continual automatic discharge of feeble impulses from the grey matter of the cord along the motor nerves. But it has been proved that if the posterior roots of the limb be cut, its tone is completely lost, although the anterior roots are intact. So that the tone of the skeletal muscles in this classical experiment depends on the passage of afferent impulses to the cord, and must be removed from the group of automatic actions and included in the reflexes. The subject is considered here and not with the ordinary reflexes, partly to emphasize by a notable example the gradual abridgenent of the supposed automatic functions of the cord, which has been already referred to, but partly because these reflexes have certain peculiarities which distinguish them from the reflexes manifested by morements. It has since been shown that this reflex tonus of skeletal muscle is a postural reflex-i.e., a reflex contraction of muscles associated with the maintenance of the posture of a part or of the animal as a whole. It can be readily demonstrated in the decerebrate mammal that the tonus of the extensor muscles of the limbs is a reflex sustained through the afferent fibres of the muscles themselves, including those from the tendons and the neighbouring joints (the proprioceptive system) and not through the cutaneous nerves. It 
is obviously in accord with the interpretation of the tonus as a postural reflex that in the mammal the extensor limb muscles should exhibit the phenomenon and the flexors not, while in the frog it is the reverse. For it is the extensors which are concerned in the reflex standing posture so characteristic of the mammal, while the flexors of the hind limbs are especially concerned in the squatting posture so characteristic of the frog.

The postural reflexes differ in no essential way from the reflexes previously studied, except that they are directed to the maintenance of posture and not to the production of movement. Reflex postural tonus has also been demonstrated for other gro:ıps of skeletal muscles, of which the most important are the neck muscles concerned in the position of the head. The reflexes in this instance originate not only through the afferent fibres of the muscles themselves, but also from the otic labyrinth, in discussing the functions of which the subject must be returned to. But it may still be mentioned here, and it shows how intricate is the correlation of the postural reflexes to one another, that not only does the labyrinth influence the postural contraction of the extensors of the limbs clirectly-i.e., through afferent fibres from the labyrinth itself, but also inclirectly through the changes produced in the posture of the head and neck-i.e., through the afferent fibres of the neck muscles (Magnus and de Kleijn). Even when the head of a normal rabbit is passively moved so that its position relatively to the body is altered, the postural tonus of the muscles of the extremities and even of some of the muscles of the trunk, especially those connected with the lumbar vertebræ, is definitely affected.

A remarkable property of a muscle which exhibits postural reflex tonus is that its tension remains approximately unaltered, even when its length is greatly changed by placing the joint on which it acts in different postures. Within a wide range it is able to counterbalance just the same extending force whatever the length of the active muscle may be (Practical Exercises, p. 1000). This property is dependent upon the integrity of the reflex arcs. A muscle which is not under the influence of its nerve centre reveals no trace of this peculiarity, which, however, is possessed by the smooth muscle of viscera-e.g., the urinary bladder and the stomach. It has been shown that these hollow organs can hold very different quantities of liquid without any great change of tension. With a larger volnme of contents, indeed, the tension may be even less than with a smaller volume. It is obvious that this property must play an important part in regulating the pressure within such viscera, so that in the case of the bladeler-e.g., the pressure may not rise prematurely to the point at which the desire to micturate is aroused. The arljustment of the tonus of these smooth muscles does not depend altogether upon the central nervous system, for it is still exhibited, although not so amply, by the excised living stomach.

Other characteristics of the reflex postural contraction are the long periods for which it usually enclures without apparent fatigue, and what is probably associated with this relative mufatiguability, the slight expenditure of energy by which it is maintained (Roaf).

it is probable that the tone of such visceral muscles as the sphincters of the anus and bladiler has a reflex element analogous to postural contraction, and possible that the same is true of the tone of the smooth muscular fibres of the blondvessels on which the maintenance of the mean blood-pressure so largely depends $\left(p .18_{5}\right)$. 
Trophic Tone.--The degencrative changes that occur in muscles, nerves, and other tissues when their connection with the central nervous syistem is interrupted have been already referred to (p. 8n.t). It is possible to explain these changes in some cases withont the assumption that tonic impulses are constantly passing out from the brain and cord to control the nutrition of the peripheral organs; and we have seen that there is no real evidence of the existence of specific trophic fibres. But the degeneration of muscles after section of their motor nerves is difficult to understand, except on the hypothesis that impulses from the cells of the anterior horn influence their nutrition. The only question is whether these are the impulses to which muscular tone is due, and therefore reflex, or different in nature and automatically discharged. Now, degeneration of a muscle is not usually caused, or at least not lor a long time, by intermption of its afferent nerve-fibres, as in locomotor ataxia, or after section of the posterior nerve-roots (Mott and Sherrington). We can hardly suppose that in any case the troplic influence of the cells of the spinal or sympathetic ganglia to which all other reflex powers have been denied, is of reflex nature. And there is, indeed, more evidence in favour of trophic tone being an automatic action of the cord than for any of the other tonic functions hitherto considered.

The evidence for respiratory automatism upon which the spinal cord has been chiefly credited with truc automatic action has previously been given (p. 284).

The 'Centres' of the Cord and Bulb.-We have frequently used the word 'centre' in describing the functions of the spinal cord, but the term, although a convenient one, is apt to convey the idea that our knowledge is far more minute and precise than it really is. When we say that a centre for a given physiological action exists in a definite portion of the spinal cord, all that is meant is that the action can be called out experimentally, or can normally go on, so long as this portion of the cord and the nerves coming to it and leaving it are intact, and that destruction of the 'centre' abolishes the action. For example, a part of the medulla oblongata on each side of the middle line in the floor of the fourth ventricle above the calamus scriptorius is so related to the function of respiration that when it is destroyed the animal ceases to breathe. But this respiratory centre-- the 'nœud vital' of Flourens - does not correspond in position with any definite collection of grey matter, although it includes the nuclei of origin of several cranial nerves, and forms an important point of departure for efferent, and of arrival for afferent, fibres connected with the respiratory act. Its destruction involves the cutting off of the impulses constantly travelling up the vagus to modify the respiratory rhythm, and of the impulses constantly passing down the cord to the phrenics and the intercostal nerves. And just as the traffic of a wide region can be paralyzed at a single blow, by. severing the lines in the neighbourhoor of a great railway junction, or more laboriously, though not less effectually, by separate section of the same tracks at a radius of a hundred miles, so destruction of the respiratory centre accomplishes by a single puncture what can be also 
performed ly section of all the respiratory nerves at a distance from the medulla iblongata. But while nobody speaks of the destruction of a ' centre ' when a reflex action is abolished by division of the peripheral nerves concerned in it, there is a tendency, when the same effect is brought about by a lesion in the brain or cord, to invoke that mysterious name, and to forget that the cerebro-spinal axis is at lcast as much a stretch of conducting paths as a collection of discharging nervous mechanisms.

It is, perhaps, a profitless task to enumerate all the so-called centres in the bulb and cord. In addition to the great vaso-motor, respiratory, cardio-inhibitory and cardio-augmentor centres in the bulb, which perhaps, have more right than the rest to be regarded as distinct plysiological mechanisms, if not as definitely bounded anatomical areas, there have been distinguished ano-spinal, vesico-spinal, and genito-spinal centres in the lumbar cord, a cilio-spinal centre for rlilatation of the pupil in the cervical cord, and in the medulla centres for succzing, for coughing, for sweating, for sucking, for masticating, for swallowing, for salivating, for vomiting, for the production of general convulsions, for closure of the eyes, for the secretion of tears, and even a 'diabetes' or 'sugar' regulating centre $(\mathrm{p} .5+7)$. It has been recently shown that in the cat a region of the dorsal cord between the last cervical and the third dorsal segments is capable of sustaining the spontancous liberation of epinephrin from the adrenal glands after the cord has been divided higher up.

\section{Section IX.-The Cranial Nerves.}

Unlike the spinal nerves, which arise at not very unequal intervals from the cord, the nuclei of the cranial nerves, with the exception of the olfactory and optic, are crowded together in the inch or two of grey matter of the primitive neural axis in the immediate neighbourhood of the fourth ventricle and the Sylvian aqueduct. Of these nuclei some are the end nuclei or 'nuclei of reception ' of sensory fibres-that is to say, collections of nerve-cells around which the sensory fibres break up into terminal arborizations. Such are the sensory nuclei of the fifth, the nuclei of the eighth, and the sensory nuclei of the glossopharyngeal and vagus nerves (Figs. 367 , 368). The nuclei of origin of the motor fibres lie, upon the whole, in two longitudinal rows-a median row, which consists of the nuclei of the third and fourth nerves in the floor of the aqueduct, and those of the sixth and twelfth nerves in the floor of the fourth ventricle; and a lateral row comprising the motor nuclei of the fifth, seventh, tenth, and eleventh nerves. The clumps of grey matter which make up these nuclei may be considered as homologous with the grey matter of the ventral or anterior (including the lateral) horn of the spinal cord; and the motor fibres of the nerves themselves as homologous with the anterior spinal roots. Without going further into the thorny subject of the homologies of the cranial and spinal nerves, we may point out that while all the spinal nor contain both efferent and afferent fibres, some of the cranial norv 
are purely efferent, some purely afferent, and others mixed. So that if we are to look upon tl:e motor nerves as the homologues of

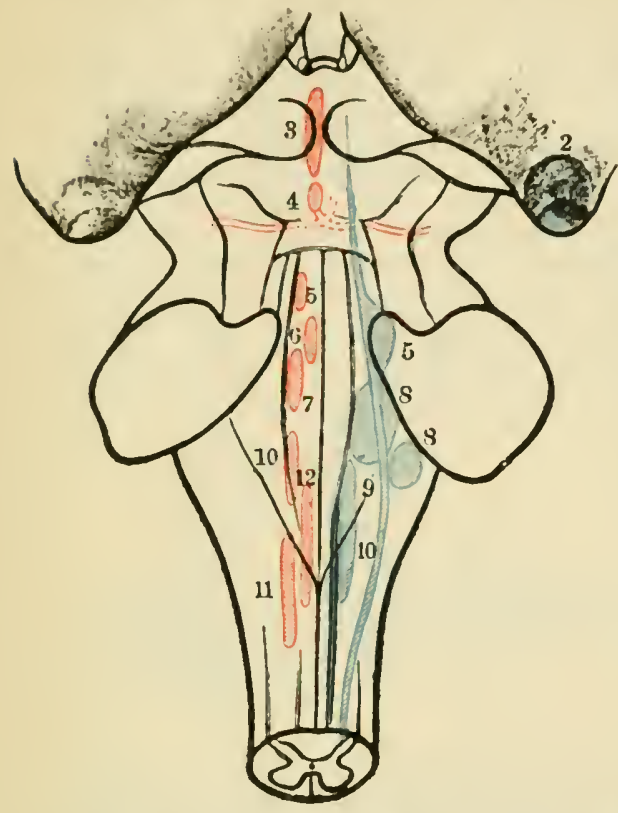
lice ventral roots, the dorsal (posterior) root-fibres corlesponding to them must be represented in the other cranial nerves. Thus, the sensory portion of the mixed fifth nerve, and the purely afferent auditory nerve, inust be supposed to contain fibres corresponding to several dorsal roots.

Fig. 367.-Nuclei of Cranial Nerves (Toldt). Motor red, sensory blue. The numbers correspond to the cranial nerves.

The first or olfactory nerve consists of fine fibres, each of which is a process of an olfactory cell (Fig. 369). The olfactory cells, which are really peripheral nerve-cells, lie among the epithelial cells in the olfactory region of the Schneiderian membrane, the common lining of the nostrils. Each olfactory cell gives off two processes, a short

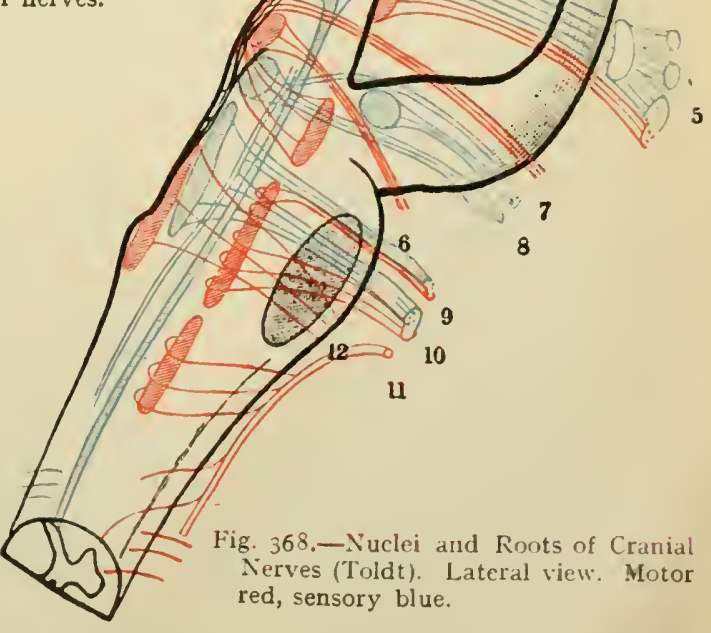
onc, representing a dendrite, which runs out to the surface of the mucous membrane, and a longer but more slender process, representing an axon, 
which as a fibre of the olfactory nerve pierces the cribriform plate of the ethmoid bone, and plunges into the olfactory bulb.

In the olfactory bulb at least four layers can be distinguished-(1) on the surface, beneath the pia mater, the layer ef entering ollactory nerve-fibres; (2) the layer of olfactory' glomeruli, peculiar structures, each of which is made up of an intricate basket-like arbusization formed by an elfactory nervi-fibre, or, it may be. more than one, and a brush-like arborization belonging to a dendrite of one of the mitral cells of the next laver; (3) the molecular or mitral layer, which contains a number of lirge nerve-cells called, from their most common shape, mitral cells, along with smaller nerve-cells ("granules') and neuroglia; (4) the nuclear layer, containing numerous small nerve-cells or ' granules 'intermingled with white fibres. 'The mitral cells give off axons, which pass through the fourth layer, and then as fibres of the olfactory tract to the grey matter of the hippocampal region of the brain. The course of the inipulses from the olfactory mucous membrane to the brain is shown in Fig. 369. The olfactory tract, as it runs back, divides into portions

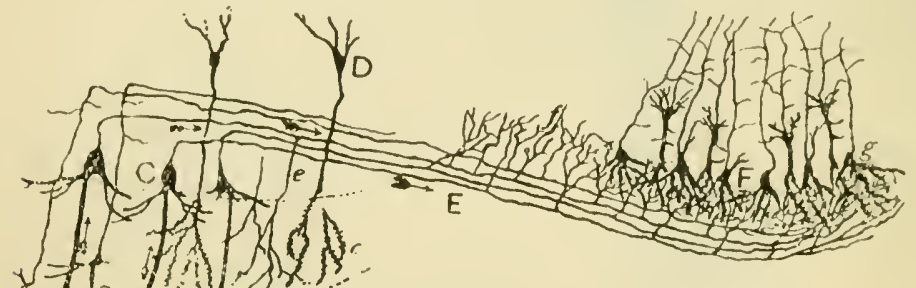

Fig. 369. - Scheme of the Olfactory Nervous Apparatus (Cajal). A, olfactory cells; B, glomeruli; C, mitral cells; 1), olfactory granule cell; E, lateral root of olfactory tract; 1 , cortex of brain in the region of the uncinate gyrus; $a$, small cell of mitral layer; $b$, brush of dendrite of a mitral cell ending in a glomerulus; $c$, thorns or spines on the processes of an olfactory granule; $e$, collateral coming off from the axon of a mitral cell; $f$, collaterals ending in the molecular layer of the uncinate gyrus; $g$. pyramidal cells of the cortex; $h$, supporting epithelial cells of the olfactory inucous membrane.

called its 'roots.' Of these the lateral is the most important, and it terminates in the hippocampal and uncinate gyri of the same side. Fibres of the olfactory tract are also connected cither directly or through the relay of another neuron with the opposite side of the brain, especially the opposite uncinate gyrus. The anterior commissure contains numerous fibres, which connect the hippocampal regions of the two sides. Other central connections of the olfactory tract exist, but sone are imperfectly known. The name "rhinencephalon' is given to the portions of the brain concerned with the sense of smell. Disturbances of smell sensation may be caused by lesions in any part of the rhinencephalon, and also by changes in the olfactory mucous membrame and olfactory fibres; but the symptoms do not obtrude themselves, and are doubtless often overlooked. Excessive stimulation of the olfactory nerve by exposure to a strong odour has been said to cause complete and permanent loss of smell.

The second or optic nerve contains mainly afferent fibres, which arise from the ganglion cells of the retina, and terminate ly forming synapses with nerve-cells in the lateral or cxterna! gr niculate body, the 
pulvinar (or posterior portion) of the optic thalamus, and the anterior corpus quadrigeminum. In young animals all these structures undergo atrophy after cxtirpation of the eyeball. The visual path is continued from the pulvinar and the external corpus geniculatum by the axons of these nerve-cells, which proceed in the optic radiation (1). 883) to the occipital cortex. The fibres which pass from the retina to the anterior corpus quadrigeminum are distinguished by their small size, and probably constitute the path of the impulses which cause contraction of the pupil when light falls on the retina. The reflex are is schematically shown in Fig. 370, where optic nerve-fibres are represented as forming synapses with cells in the anterior curpus quadrigeminum whose axons pass to the nucleus of the third nerve and arberize around

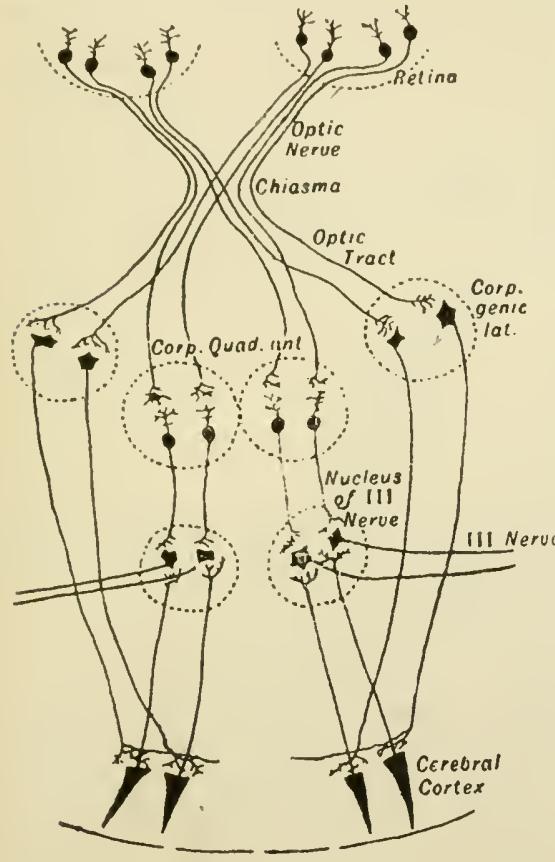

Fig 370.--Scheme of the Visual Path (after Schäfer). some of its cells (Figs. 354, 3 j6, and 370 ). At the chiasma the fibres of the optic nerve decussate, partially in man and some mammals, as the rabbit, dog, cat, and monkcy, completely in animals whose visual field is entirely independent for the two eyes, as in fishes and birds. In man the fibres for the nasal halves of both retina cross the middle line at the chiasma, those for the temporal halves do not. This does not mean, however, that exactly half of the optic nerve-fibres decussate. The number of uncrossed fibres is smaller than that of crossed. The chiasma also contains fibres in its posterior portion, which extend from one optic tract to the other, but are not connected with the retina or the optic nerves. They are commissural fibres which connect the two mesial geniculate bodies across the middle line, and are called Gudden's commissure. A sufficiently extensive lesion involving the occipital cortex on onc side, or the posterior portion of the optic thalamus, or the optic tract, causes hemianopia* or defect of the visual field on the side opposite to the lesion, with blindness of the corresponding halves of the two retinæ. Thus, a lesion equivalent to complete section of the right optic tract would cause blindness of the nasal lialf of the left, and of the temporal half of the right eye, and the left half of the field of vision would be blotted out-the patient would be unable, with his eyes directed forwards, to see an object at his left. Such a complete hemianopia is much rarer in disease of the cortex than in disease of the

* The terms ' hemiopia,' 'hemianopia,' ' hemianopsia,' are used with reference sometimes to the blind side of the retinæ, but ordinarily to the half of the visual field which is deficient We shall always use the word 'hemianopia' in the latter sense. 
optic tract. A lesion-e.g., a tumour of the pituitary body -involving the whole of the optic nerve in front of the chiasma, would cause complete blindness in the corresponding eye. Sometimes in disease of the optic nerve vision is not totally destroyed in the eye to which it belongs, but the field is narrowed by a circumference of blindness. In this case the pathological change involves the circumferential fibres of the nerve. When the chiasma is affected by disease, a very frequent symptom is bitemporal hemianopia, blinclness of the nasal halves of the retina, with loss of the outer or temporal half of each ficld of vision. The optic nerve and tract contain a few efferent fibres for the retina, whose cell-bodies have not yet been certainly located.

The third nerve, or oculo-motor, arises from an elongated mucleus, or a series of nuclei, containing large nerve-cells in the floor of the Sylvian aqueduct below the anterior corpora quadrigemind. The rootbundles coming off from the most anterior of the nuclei carry fibres that innervate the ciliary muscle, and thus have to do with the mechanism of accommodation, and also fibres that innervate the sphincter muscle of the iris, and thus cause contraction of the pupil when light falls on the retina. Both groups of fibres terminate by arborescing around sympathetic cells in the ciliary ganglion, from which the path to the (unstriated) ciliary and sphincter muscles is continued by post-ganglionic fibres. Farther back in the oculo-motor nucleus arise the motor fibres for four of the extrinsic muscles of the eycball and the eievator of the upper eyelid. In the dog these fibres come off in the following order, from before backwards; internal rectus, superior rectus, levator palpebra superioris, inferior rectus, inferior oblique. Most of the fibres of the third nerve arise from nerve-cells on their own side of the midalle line, but a certain number decussate to enter the nerve of the opposite side.

Complete paralysis of the third nerve causes loss of the power of accommodation of the corresponding eye, dilatation of the pupil by the unopposed action of the sympathetic fibres, diminution of the power of moving the eyeball, ptosis, or drooping of the upper iid, external squint, and consequent diplopia, or double vision.

The fourth or trochlear nerve arises from the posterior part of the same tract of grey matter which gives origin to the third nerve. It supplies the superior oblique muscle. Paralysis of the nerve causes internal squint when an object below the horizontal plane is looked at, owing to the unopposed action of the inferior rectus. There is also diplopia on looking down. Unlike the other cranial nerves, the two trochlear nerves decussate completely after they emerge from their nuclei of origin.

The fifth or trigeminus nerve appears on the surface of the pons as a large sensory root and a smaller motor root. Its deep origin is more extensive than that of any of the other cerebral nerves, stretching as it does from the level of the anterior corpus quadrigeminmm above to the upper part of the spinal cord below. Its sensory root, in fact, seems to include the sensory divisions of several motor cranial nerves.

The motor root arises partly from a nucleus (principal motor mucleus) in the floor of the fourth ventricle below the pons, partly from large round nerve-cells lying at the sicle of the grey matter bounding the aqueduct of Sylvius all the way from the anterior quadrigeminate body to the point at which the motor root is given off accessory or superior motor nucleus).

The fibres of the sensory root have their cells of origin in the Gasserian ganglion, whence they pass into the pons. Here they bifurcate into ascending and descending branches. The ascending branches end in the principal sensory mucleus, a collection of grey matter at the sicle of 
the principal motor nucleus. The descending branches, turning downwarls into the medulla oblongata, terminate in a long tract of scattered cells, constituting with the fibres the so-called spinal root, and extending from the level of the second cervical nerve through the medulla oblongata and the pons, where it is continued in to the principal sensory nucleus. The afferent path is continued by the axons of cells of the sensory nuclei (or nuclei of reception) of tinc nerve, many of which cross the middle line and cuter the intermediate fillet of the opposite side, and also the special ascending bundle going to the thalamus. Some of the axons do not decussate, but ascend in the fillet of the same side.

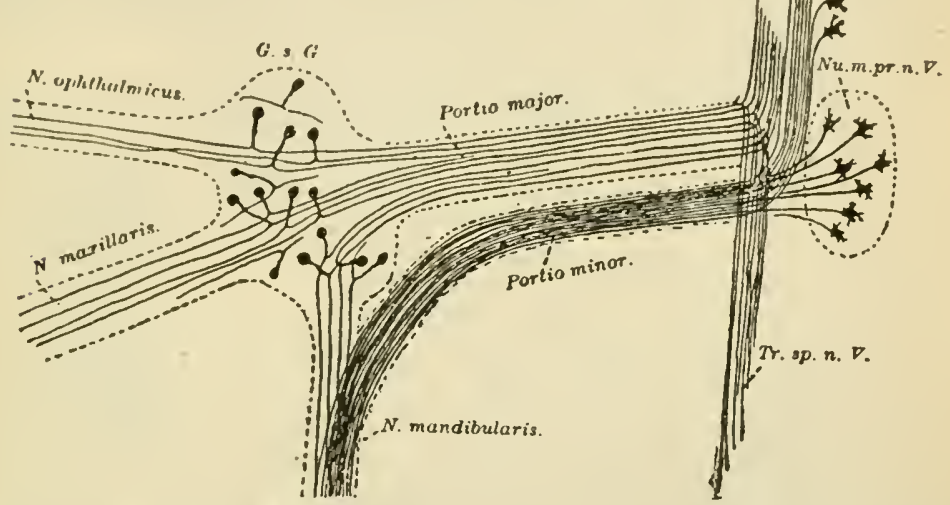

Fig. 37r.-Scheme of Motor and Sensory Neurons of Trigeminus (Gehuchten). G. s. G., Gasserian gangtion; $N u, m . m . u$. V., nucleus of the descending root; $N$ u. m. pr. n. V., chief motor nucleus of the fifth nerve; Rad. desc. mes. $n$. V.. accessory motor nucleus, sometimes called the descending root; Tr. sp. n. V.. tractus spinalis, or spinal root of the fifth.

The motor fibres of the fifth nerve supply the muscles of mastication and the tensor tympani. The sensory fibres confer common sensation on the face, conjunctiva, the mucous membranes of the mouth and nose, and the structures contained in them, and, according to Gowers, special sensation, through branches given off to the facial and glosso-pharyngeal nerves, on the organs of taste.* Complete paralysis of the nerve causes

* It should be stated that some physiologists believe that the glosso-pharyn. geal is the nerve of taste, and that none of the taste fibres go to the sensory nuclei of the fiftl nerve. The majority hold that the glosso-pharyngeal supplies the posterior third, and the chorda tympani and lingual the anterior two-thirds of the tongue with gustatory fibres. The removal of the Gasserian ganglion and the adjacent portion of the fifth nerve for severe and persistent neuralgia, has afforded opportunities to test this question. But, unfortunately the results described by various observers do not agree, some finding that taste is unimpaired, others that it is abolished. Gowers states that the gustatory sensations may persist for some time after the operation, although ultimately (in two or three weeks) they disappcar. It has been shown by Harris that when alcohol is injected into the third division of the fifth nerve at the foramen ovale or into the Gasscrian ganglion, immediate loss of taste develops in a very large proportion of cases in the anterior half of the tongue on that side. Taste fibres are therefore certainly present in the fifth nerve at this level. 
loss of movement in the muscles of mastication, sometimes impaired hearing, and loss of common sensation in the area supplied by it. Los: or imparment of taste in the corresponding half of the tongue is also often seen in disease involving the sensory root, althongh not in affections of the trunk of the nerve, since the taste fibres leave it near its origin (Cowers). Both taste and touch are lost in the monkey in the anterior two-thirds of the tongue after intracranial section of the trigeminus (Slerringtou).

Vaso-motor clianges are occasionally, and 'trophic' changes frequently, observed in clisease of the fifth nerve. The trophic disturbance is most conspicuous in the eyeball (ulceration of the cornea, going on, it may be, to complete disorganization of the eye). These effects are partly due to the loss of sensation in the eye, with the consequent risk of lamage from without, and the unregarded presence of foreign bodies and accumulation of secretion within the lids (p. 805).

The sixth or abducens nerve takes origin from a nucleus in the floor of the fourth ventricle at the level of the posterior portion of the pons. It supplies the external rectus muscle of the eyeball. Paralysis of it causes internal squint. It is generally described as a purely motor nerve, but this is incorrect. It has been shown that the sixth, as well as the third and fourth, cranial nerves contain a considerable number of afferent fibres, whose receptive nerve-endings are situated in the recti and obliqui muscles and their tendons.

The motor fibres of the seventh or facial nerve arise from a nucleus in the reticular formation of the medulla oblongata, and running up some distance into the pons. They supply the muscles of the face; and when these are greatly developed, as in the trunk of the elephant, the nerve reaches very large proportions. Since the fibres which connect the cerebral cortex with the nucleus decussate about the middle of the pons, a lesion above this level which causes hemiplegia paralyzes the face on the same side as the rest of the boly-i.e., on the side opposite the lesion. But the paralysis is confined to tiue muscles of the lower portion of the face, and affects especially the muscles about the mouth. Sometimes the pyramiclal tract and the facial nerve, or nucleus, are involved in a common lesion. In this case paralysis of the face is on the side of the lesion, and is total, while the rest of the body is paralyzed on the opposite side. Paralysis of the seventh nerve is more common than that of any other nerve in the body. It is often caused by an inflammatory process in the nerve itself (neuritis). The symptoms of complete facial palsy are very characteristic. The face and forehead on the paralyzed sicle are smooth, motionless, and devoid of expression. The eye remains open even in sleep, owing to paralysis of the orbicularis palpebrarum. A smile becomes a grimace. An attenpt to wink with both eyes results in a grotesque contortion. The mouth appears like a diagonal slit in the face, its angle being drawn up on the sound side, and the patient cannot bring the lips sufficiently close together to be able to blow out a canclle or to whistle. Liquids escape from the mouth, and food collects between the paralyzed buccinator and the teeth. The labial consonants are not properly prononnced. Taste may be lost in the anterior two-thirds of the tongue when the nerve is injured above the exit of the gustatory fibres in the chorda tympani, but not when the lesion is in the nucleus of origin, or anywhere above it. Hearing is sometimes impaired because the auditory and facial nerves, lying close together for part of their course, are apt to suffer together, but perhaps also because the stapedius muscle is supplied by the seventh. 
The seventh nerve is not purely motor. From the cells of a ganglion on it corresponding to a spinal ganglion (the geniculate genglion) afferent fibres arise, which pass in the pars intermedia or nerve of IVrisberg into the pons between the seventh and eighth nerves, and there bifurcate into ascending and descending branches, like other afferent fibres originating in ganglia of the spinal type. The descending branches enter the fasciculus solitarius, and end by arborizing around nerve-cells in the upper part of that bundle. The peripheral axons of the nerve-cells in the geniculate ganglion enter the large superficial petrosal nerve and the chorda tympani, in which they, or some of them. perlaps represent taste fibres.

The eighth or auditory nerve enters the medulla oblongata by two roots (a dorsal and a ventral), one of which passes in on each side of

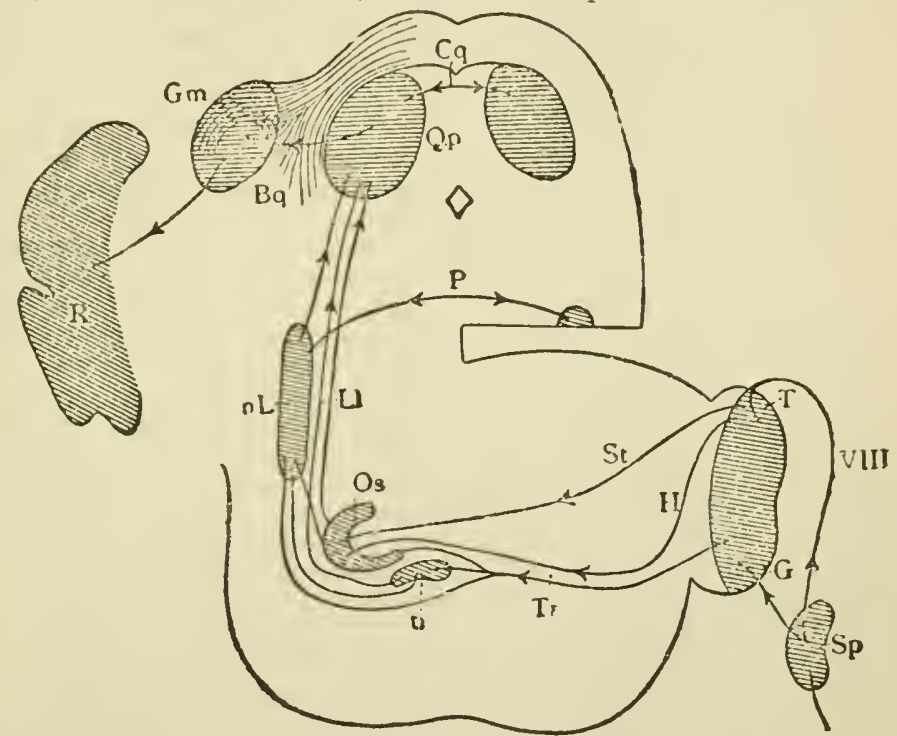

Fig. 372.-Scheme of Path of Auditory Impulses (Lewandowsky). Sp, ganglion spirale; G, accessory nucleus; T, acoustic tubercle; Tr, trapezium; H, Held's fibres; St, striæ acustica; tr, trapezoid nucleus; Os, upper olive; LI, lateral fillet, with its nucleus, $\mathrm{nL}$; P, commissure of the lateral fillets; Qp, posterior corpora quadrigemina, with $\mathrm{Cq}$, their commissure, and $\mathrm{Bq}$, the prachia; $\mathrm{Gm}$, mesial or internal geniculate body; $R$, cerebral cortex.

the restiform body. The cells of origin, both of the dorsal and of the ventral root, are situated in the internal ear, the former in the ganglion spirale, or ganglion of Corti, which is embedded in the bony spiral of the cochlea, the latter in the ganglion vestibulare, or ganglion of Scarpa, which lies in the vestibule. These cells correspond to the ganglion cells on the posterior root of a spinal nerve, but, unlike them. they remain, even in mammals, bipolar throughout life. Their central processes form the axons of the eighth nerve. Their peripheral processes are distributed in the case of the clorsal root to the organ of Corti, in the case of the ventral root to the semicircular canals and the vestibule. For this reason the dorsal root is often called the cochlear division, and the ventral root the vestibular division of the auditory 
nerve. And the cochlear and vestibular roots are physiologically as well as anatomically listinct. For the cochlea subserves the function of hearing, the semicircular canals and vestibule the function of equilibration. As they enter the inedulla oblongata, the fibres of the dorsal root bifurcate. Of the two branclies, one is considerably thicker than the other. Many of the thicker branches terminate by arborizing around the cells of the accessory auclitory nucleus, whose position is indicated by a swelling on the ventral surface of the restiform body at the junction of the dorsal and ventral roots; but some pass over the restiform body to end in another nucleus (lateral nucleus), also indicated by a swelling (tuberculum acusticum) lying over the restiform body. The nerve-cells of the accessory nucleus and the acoustic tubercle, therefore, constitute nuclei of reception for the dorsal root-fibres. The more slender branches of the cochlear rootfibres run downwards for some distance before breaking up into fibrils.

The path to the higher parts of the brain is continued by the axons of nerve-cells in the accessory nucleus and the acoustic tubercle. The fibres from the accessory nucleus pass into the trapezium, a mass of transverse fibres lying in the pons behind the pyramidal fibres. In their course through the trapczium some of the fibres terminate around the cells of the nucleus of the trapezium, others run into the superior olive of the same side, and end there; but most of them cross the middle line, and enter the trapezoid nucleus and superior olive of the opposite side, where many of them terminate. Others, however, run through those nuclei and pass into the lateral fillet, to end in its nucleus or in the posterior corpora quadrigemina. The path of the fibres which terminate in the nuclei of the trapezium, superior olive, and lateral fillet, is continued by another relay of fibres, which link them also to the posterior corpora quadrigemina. The axons of the cells of the acoustic tubercle enter for the most part the strice acustica, a series of prominent strands that run transversely across the floor of the fourth ventricle. Passing across the raphe, they join the fibres from the accessory nucleus on their way to the superior olive, and accompany them into the lateral fillet, which terminates in the grey matter of the posterior corpus quadrigeminum. We must assume, from clinical and experimental data, that the dorsal root is ultimately connected with the first or first and second temporo-sphenoidal convolutions on the opposite side. From the posterior corpora quadrigemina the auditory path to the convolutions seems to run in the brachium to the internal or mesial geniculate body, whence it is continued in the posterior extremity of the internal capsule.

The fibres of the ventral root of the eighth nerve, better termed the vestibular nerve, after entering the medulla oblongata, pass to a nucleus called the principal nucleus of the vestibular division. Here each bifurcates into a descending and an ascending branch. The descending branches running down in the medulla terminate at different levels around cells in the principal nucleus, and the grey matter continued down from it (descending vestibulur mucleus). The ascending branches run up on the inner side of the restiform body towards the nucleus of the roof (nucleus tecti) in the cerebellar worm. On their course they enter into relation through their collaterals with the nucle of Deiters and Bechterew. The nucleus of Deiters, as already stated, sends fibres into the posterior longitudinal bundle. Through ascending branches of these fibres a communication is established with the nuclei of the third and sixth nerves, and through descending branches that pass into the antero-lateral descending tract of the cord with the anterior horn cells. It is obvious that through these connections 
which link the vestibnle with the cerebellum, the nuclei of the motor nerves of the cyeball and the nutor cells of tha cord, the nucleus of Deiters has an important relation to the co-ordination of those movements mainly concerned in equilibration. Nothing is known of the connections of the vestibular nerve with the cercbrum. Two prominent symptoms may be associated with disease of the auditory nerve(a) disturbance or loss of hearing; (b) loss or impairment of equilibration.

The ninth or glosso-pharyngeal nerve comprises botl sensory and motor fibres-sensory for the posterior third of the tongue and the mucous membrane of the back of the moutl, motor for the midile constrictor of the pliarynx and the stylo-pharyngeus. It also contains the nerves of taste for the posterior third of the tongue. The efferent fibres arise from a nucleus (molor nucleus of the glosso-pharyngeal) a little posterior to the facial nucleus. The afferent fibres take origin from unipolar cells in ganglia of spinal type connected with the nerve (ganglion petrosum and ganglion supcrius). Entering the medulla. oblongata, the central processes of these cells bifurcate into ascending and descending branches. 'l'heir peripheral processes pursue their course as the axons of sensory fibres to the structures to which the nerve is distributed. The ascending branches terminate in a nucleus (principal nucleus of the glosso-pharyngeal) beneath the floor of the fourth ventricle. The descending branches, as well as similar branches from the pars intermedia of the seventh nerve and from the afferent fibres of the vagus, form a bundle called the fasciculus solitarius (sometimes termed the descending root of the facial, vagus, and glosso-pharyngeal). It can be traced to the lower boundary of the spinal bulb. Along the mesial border of the fasciculus solitarius are strung out the somewhat scattered nerve-cclls (descending nucleus of facial, vagus, and glosso-pharyngeal), around which the descending branches arborize. At its upper end the grey matter of the fasciculus solitarius is cortinuous with the principal nuclei of the glosso-pharyngeal and vagus.

The tenth nerve, or vagus, also contains both motor and sensory fibres. The efferent fibres arise partly from the nucleus ambiguus or ventral uucleus of the vagus, a collection of large nerve-cells situated in the reticular formation, and extending from a point a little below the facial nucleus to a point a little above the lower limit of the medulla oblongata, where it becomes continuous with the column of cells from which the spinal fibres of the eleventh nerve take origin. A second nucleus of origin for efferent vagus fibres is constituted by the upper part of the dorsal accessory-vagus nucleus, a collection of rather small cells extending from a little below the lower margin of the pons to nearly the level of the first cervical nerve.

The afferent fibres of the vagus arise from unipolar cells in ganglia connected with the nerve (ganglion jugulare, ganglion nodosum). In the medulla oblongata they bifurcate, like other fibres coming off from the cells of ganglia of spinal type. The ascending branches, which are short, terminate in the upper sensory or principal mucleus, and the descending branches, which are long, in the cells of the fasciculus solitarius, just as in the case of the glosso-pharyngeus.

The motor fibres of the vagus are partly derived from the accessory, whose internal branch joins the vagus not far from its origin. The distribution of the nerve is more extensive than that of any other in the body. The œesopliagus receives both motor and sensory branches from the œsopliagcal plexus. The pharyngeal branch of the vagus is the chief motor nerve of the pliarynx and soft palate (including the tensor palati). The supcrior laryngeal branch is the nerve of common sensation for the larynx above the vocal cords, and the motor nerve 
of the crico-thyroid muscle. The inferior or recurrent laryngeal supplies the rest of the laryngeal muscles, and the sensory fibres for the mucous membrane of the trachea and the larynx below the glottis. The superior laryngeal contains afferent fibres, stimulation of which gives rise to coughing, slows respiration, or stops it in expiration. Reflex movements of deglutition are also caused. The vagus supplies the lungs both witi motor and sensory filaments through the pulmonary plexus. The motor fibres when stimulated cause constriction of the bronchi; excitation of the afferent fibres causes reflex changes in the rate or depth of respiration. The cardiac branches contain inlibitory fibres probably deriverl from the spinal accessory, and depressor fibres which pass up in the vagus trunk (dog), or as a separate nerve to join the vagus or its superior laryngeal branch or both (rabbit). The gastric and intestinal branches contain both motor and sensory nerves for the stomach and intestines. The sensory are probably large medullated fibres $(7 \mu$ to $9 \mu)$. The afferent vagus fibres from the stomach carry up impulses which excite the action of vomiting. Lesions of the vagus, its nuclei of origin, or its branclies, are associated with many interesting forms of paralysis and other symptoms. Paralysis of the pharynx is generally caused by disease of the nucleus in the medulla. From its anatomical relation to the nuclei of the glosso-pharyngeal and hypoglossal, it will be easily understood that these nerves are often involved in localized central lesions along with the vagus. But the fact that in progressive bulbar palsy (glosso-labio-laryngeal paralysis) - a condition characterized by progressive paralysis and atropliy of the muscles of the tongue, lips, larynx, and pharynx-the orbicularis oris and other muscles of the mouth and chin are paralyzed, while the rest of the muscles supplied by the facial remain intact, might seem to indicate that in system discases it is not so much anatomical groups of nervecells which are liable to simultaneous degeneration and failure, as physiological groups normally associated in particular functions. Such functional groups of cells, occupied with the same kinds of labour at the same times and under the same conditions, might be supposed to take on a similar bias or tendency to degeneration-a tendency not indicated, it may be, by any structural peculiarity, but traced deep in the molecular activity of the cells. There is no foundation for the view that the lips are involved in progressive bulbar palsy because the fibres of the facial which supply them arise from the liypoglossal nucleus, any more than for the idea that the upper part of the face escapes because its motor fibres, while reaching it in the seventh nerve, really arise from the oculo-motor nuclcus (Bruce). Difficulty in swallowing is the chicf symptom of pharyngeal paralysis. The symptoms of laryngeal paralysis have been already described under "Voice" (p. 316). Tachycardia, or a permanent increase in the rate of the heart, has been stated to occur in certain cases of paralysis of the vagus, caused by disease or accidental interference; and a persistent slowing of the respiration has been occasionally attributed to the same cause. But it is difficult to reconcile many of these cases with experimental results, for in most of them the lesion only involved one vagus; and in animals section of one vagus has no permanent effect on the rate of the heart or of the respiratory movements.

Destruction of the nerve near its origin has been sometimes found associated with disappearance of the food-appetites, hunger and thirst. and it has been assumed that this was due to loss of afferent impulses from the stomach. But clinical testimony is by no means unanimous on this point, and experiments on animals sliow that other factors are involved in these sensations (sce Chapter XVIII.). 
The eleventh or spinal-accessory nerve contains only cfferent fibres. The cells of origin of its spinal portion lie in the lateral horn of tho cord, from about the level of the first to the fiftl or sixth cervical nerves. The bulbar portion, sometimes called the bulbar accessory, arises from the lower two-thirds of the dorsal accessory-2agus nucleus, from about the level of the first cervical nerve up to the level of the tip of the calamus scriptorius. The accessory portion of the nucleus lies behind and to the side of-i.e., dorso-lateral to-the central canal; the upper or vagus portion is inore laterally placed in the floor of the fourth ventricle. Soon after the junction of its bulbar and spinal portions, the nerve divides into two branches, an internal and an external. The external branch, containing the spinal fibres, passes out to supply the trapczius and sterno-mastoid muscles with motor fibres. The internal branch, containing the bulbar fibres, passes borlily into the vagus.

The twelfth or hypoglossal nerve is exclusively an efferent nerve. Its nucleus of origin is an elongated collection of large nerve-cells extending throughout approximately the lower two-thirds of the bulb close to the median line and parallel to it. It contains the motor supply of the intrinsic and extrinsic muscles of the tongue and of the thyro- and genio-hyoid. Paralysis of it causes deficient movement of the corresponding half of the tongue. When the tongue is put out, it deviates towards the paralyzed side, being pushed over by the unparalyzed genio-hyoglossus of the opposite side, which is thrown into action in protruding the tongue.

\section{Section X.-Functions of the Central Nervous System-} (2) The Brain.

The paths by which the various parts of the central nervous system are connected with each other and with the periphery have been already described, and we have completed the examination of the functions of the spinal cord and medulla oblongata. The events that take place in the upper part of the central nervous stem and in the cortex of the cerebellum and cerebrum now claim our attention.

From very early times the brain has been popularly believed to be the seat of all that we mean by consciousness-sensation, ideation, emotion, volition. And he who loves to trace the roots of things back into the past may see, if he choose, running through the whole texture of the older speculations a belief that the brain does not act as a whole, but is divided into mechanisms, each with its special work-a foreshadowing, often in grotesque outlines, of the doctrine of localization so widely held to-day. But until comparatively recent times, cerebral physiology remained a kind of scientific terra incognita; and no notable additions were made for a thousand years to the doctrines of Galen. Even to-day the utmost limit of our knowledge is reached when in certain cases we have connected a particular movement or sensation with a more or less sharply-defined anatomical arca. How the cerebral processes that lead to sensations and movements, to emotions and intellectual acts, arise and die out; what molecular changes are associated with them; above all, how the molecular changes are translated into consciousness-how, for example, it is that a series of nerveimpulses from the optic radiation flickering across the labyrinth of the 
occipital cortex should light up there a visual sensation-these are questions tc which we can as yet give no answer, and the answers to some of which must for cver remain hidlen from us.

Functions of the Upper Part of the Central Stem and Basal Ganglia. - Tle function of the pons is sufficiently indicated by its name. Ilic grey matter so plentifully scattered, especially in its ventral portion, may cxercise a not unimportant influence on the impulses that traverse it. But on the whole its main office is to provide a bridge along which impulses may travel between other portions of the nervous system. We have already scen that many of its transverse fibres arising from the cells of the pontine grey matter, and then crossing the middle line to the opposite micldle peduncle, are the cerebellar segments of commissural arcs connecting the ccrebral with the opposite cerebellar hemispheres. The cerebral segments of these arcs are the corticopontine fibres originating in the prefrontal, temporal, and occipital portions of the cercbral cortex, and passing through the corona radiata, internal capsule, and crura cerebri, to end in the nuclei-pontis. Many fibres and collatcrals of the pyramidal tract also terminate herc. On the dorsal aspect of the pons in the floor of the fourth ventricle are the nuclei of origin (or reception) of the fifth, sixth, and seventh cranial nerves. Various reflex centres are situated in this region-e.g., that for the closure of the eyelids, when the conjunctiva is stimulated.

The posterior corpora quadrigemina and internal geniculate bodies are connected with the cochlear division of the auditory nerves, and form important stations on the auditory path to the cortex.

The anterior corpora quidrigemina and the lateral corpora geniculata are connected with the optic tracts. Their development is arrested after extirpation of the eycball in young animals, and they may therefore be assumed to be concerned in vision, although the size of their homologues, the optic lobes or corpora bigemina, in animals bclow the rank of mammals (birds, reptiles, amphibians), does not secm to be related to the development of the organs of sight. Proteus and the Hag-fish, e.g., have large optic lobes, rudimentary eyes and optic tracts. The optic nerve, the anterior corpus quadrigeminum, the nucleus of the oculo-motor nerve in the wall of the Sylvian aqueduct, and' the filsres which it carries to the iris, form a reflex arc for the contraction of the pupil to light, as represented in Fig. 370, p. 923.

The functions of the optic thalami have not been fully defined either by experiment or pathological observation, except in so far as they can be deduced from their connections. I.ying as they do in the isthmus of the brain, begirt by the great motor and sensory paths, it is to be expected that lesions of the thalami should affect also the internal capsule, and give risc to the symptoms of motor and sensory paralysis. But it is questionable, whether any definite defect of motor power or common sensation has ever been unequivocally associated with a lesion restricted to the thalami. The most constant features of the so-called thalamic syndrome (or symptcm-complex) are partial loss of sensibility, especially to tactile impressions, and of the muscular sensc on the opposite side, with some degree of inco-ordination and disorder, though little, if any, actual paraly'sis of voluntary movements. These plicnomena are accuunted for by the extensive connections of the thalami. Each of the thalamic nuclei is linked with a definite cortical region in such a way that destruction of the cortical area in young animals or human beings lcads to degeneration of the corresponding nucleus. Some of the fibres connectirg the cortex (and the corpus striatum) with the thalamus end in the thalamic grey matter, and are therefore efferent with resvect to the cortex (corticofugal). It is, however, the 
afferent paths to the cortex with which the thalami are specially related as centres of relay. The fibres of the upper fillet carrying afferent impulses up from the opposite posterior column of the cord to the cerc. brum end in the grey matter of the thalamus, as does the central path of the afferent fibres of the opposite fifth nerve. The posterior portion of the thalamus, or pulvinar, forms part of the central visual apparatus; for $(a)$ it is found to be undeveloped in animals from which the eycballs have been removed soon after birtl; $(b)$ a portion of the optic tract is certainly connected with it; $(c)$ in some cases of atrophy of the occipital cortex, which, as we shall see, is undoubtedly a central area for visual sensations, atrophy of the pulvinar has also been noticed; $(d)$ a lesion of the pulvinar may give rise to hemianopia (p. 923).

Hicmorrhage into the caudate or lenticular nucleus of the corpus striatum often causes hemiplegia, but this is frequently due to implication of the internal capsule. It is said, however, that lesions presumably confined to the lenticular nucleus cause paralysis or paresis of the limbs or face, which is less severe than that produced by lesions in the internal capsule. Experimental lesions in dogs and rabbits are stated to be followerl by disturbances of the heat-regulating mechanism and rise of temperature.

Certain structures belonging to the primary fore-brain which have now lost some or all of thcir functional importance may nevertheless be mentioned as milestones in the march of development. The pineal body is made up of the vestiges of the unpaired mesial eye of such animals as the ancient labyrinthodonts, which resembled the eye of invertebrates in having the retinal rods directed towards the cavity instead of towards the circumference of the eyeball. In many living forms. especially in certain lizards, this pineal or parietal eye is found in a more perfect condition, though covered by a thin membrane. The ganglia habenule, two small collections of nerve-cells, one of which is situated at the posterior part of each thalamus, are supposed by some authorities to represent the optic ganglia of this cyclopean eye. They are less prominent in man than in many of the lower animals. The infundibulum is probably what remains of the gullet of the ancestors of the vertebrates. The pituitary body is in a different category. It is now known that, far from being a useless vestigial remnant, it has a lighly important function (p. 667). It consists of two portions, the anterior lobe, or hypophysis, derived from the buccal cavity, the posterior lobe, or infundibular body, from the primary fore-brain.

Functions of the Cerebellum.-The elaborate pattern of the arbor vitæ, the appearance given by the branched laminæ in a section of the cerebellum, excited the speculation of the old anatomists. A structure so marvellous must be matched, they thought, with functions as unique. At a time when the discoveries of Galvani and Volta were fresh, and the world ran mad on electricity, the hypothesis of Rolando, that 'nerve-force' was generated by the lamellæ of the cerebellum as electrical energy is gcnerated by the plates of the voltaic pile, ridiculous as it now appears, was not unnatural. The speculation of Gall, who connected the cerebellum with the development of sexual emotions and the action of the generative mechanisms, was based on no fact. It has been definitely disproved by the observations of Luciani, who found that a bitch deprived of its cerebellum showed all the phenomena of heat or 
' rut,' was impregnated, whelped at full term in an entirely normal manner, and manifested the maternal instincts in their full intensity. Flourens put forward the doctrine that the cerebellum is an organ concerned in the co-ordination of movements, and especially the maintenance of equilibrium, supporting his conclusions by an elaborate series of experiments. Notwithstanding the very large amount of experimental and clinical study which has been devoted to the cerebellum since the time of Flourens, our actual knowledge

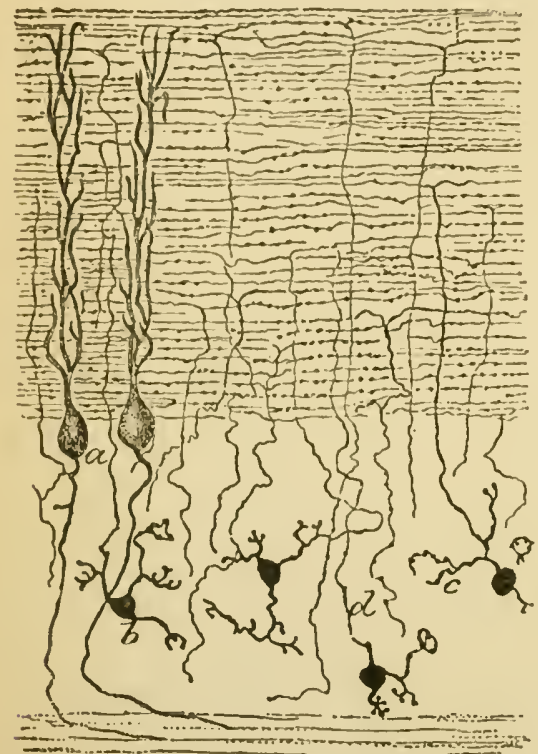

Fig. 373.-Cerebellar Cortex: Section in Direc. tion of Lamina (Cajal). a, Purkinje's cell; $b$, granule cell in inner laycr; $c$, dendrite of a granule cell; $d$, axon of a granule passing into the molccular layer, where it bifurcates into two fine longitudinal branches (Golgi's method).

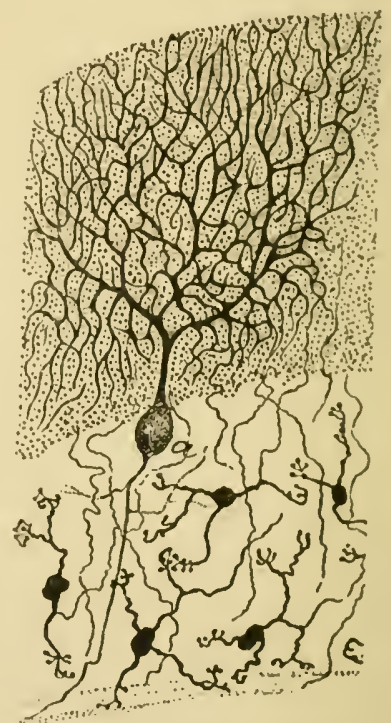

liig. 374 -Cerebellar Cortex : Section acruss a Lamina (Cajal). a, Purkinje's cell; the numerous dots in the molecular layer represent cross-sections of the bifur. cated axons of the granule cells (Crolgi's method).

of its functions lad not until recently greatly advanced beyond the point then reached. Some of the more modern authorities restrict its influence entircly to the actions on which equilibration depends; others extend it to all volitional movements. Luciani looks upon it as "an organ which by processes tliat do not awaken consciousness exerts a continual strengthening (reinforcing) action upon the activity of all other nerve-centres.' Sherrington conceives of the cerebellum as the head ganglion of the proprio-ceptive systemi.c., of the system of neurons whose receptors lie not on the surface, 
but in the deeper parts of the body (labyrinth of ear, muscles, tendous, joints, viscera, etc.) (p. gI t). After removal of the whole ccrebellum (in the dog or monkey), there is at first rigillity and tonic spasm of certain muscles, which contribute to the difficulty of coordinating tlicir movements. When this stage las passed, the muscles all over the body, but especially those of the loins and hindlimbs, and those which fix the head, are weaker than normal (asthenia), are deficient in tone (atonia), and contract with a peculiar want of steadiness (Luciani). When one lateral half of the cerebellum is removed, the symptoms affect especially the muscles on the same side. In extensive lesions of the cerebcllum in man what has been noticed is a marlicd inability to maintain the upright posture, giddiness, a staggering gait, twitching movements of the eyes (nystagmus), tremor accompanying voluntary movementsin a word, a general breakdown of the co-ordinating machinery (asynergy or asynergia), and especially of the part of it concerned in the movements necessary for locomotion, and for the maintenance of the equilibrium of the body-the so-called cerebellar ataxia. There is no sensory paralysis and none of voluntary movement such as lesions of the cerebral cortex produce, nor is there any psychical disturbance. In cases of congenital defcct of the cerebellum, the power of walking, and even of standing, may be late in being acquired, and imperfect. But it is remarkable what great deficiencies in the cerebellar substance are often compensated for when established early in life, so that even cases of marked atrophy or lack of development have sometimes been recognized for the first time at the necropsy.

The connections of the cerebellum with other parts of the central nervous system and with the periphery corroborate the direct results of experiment. For, in addition to the visual impressions, the most important afferent impulses concerned in equilibration are those from the semicircular canals and vestibule of the internal ear, the muscles, tendons, joints, etc., and certain portions of the skin, such as that of the soles of the feet. And the cercbellum, as we have seen (p. 885), is linked with all of these, and has besides an extensive crossed connection through the middle and superior peduncles with the opposite cerebral hemisphere. The importance and extent of this crossed connection with the great brain is illustrated by the facts that in disease atrophy or deficient development of one cerebellar hemisphere is associated with a similar condition of the opposite cerebral hemisphere, and that a lesion in onc-half of the cerebellum affects chiefly the co-ordination of the movements of the same side of the body-that is to say, of the side connected with the opposite cerebral hemisphere.

We do not as yet know the full significance of this extraordinarily free communication of the grey matter of the cerebellum with every part of the central nervous system. But it is evident that by the broad 
lighway of tice restiform body, or the cross-country routes from cere. bral cortex to cercbellum, impulses may reach it from cvery quarter; while impulses passing out from it along its peduncles may influence the motor discharge either indirectly through the Rolanclic cortex and the pyramidal tract, or more directly tlirough the antero-lateral descending spinal path that brings it into relation with the nuclei of origin of the motor nerves. It is an organ so connected that is suited to talic cognizance of the multitudes of afferent impressions concerned in the co-ordination of movements and the maintenance of equilibrium, and to regulate the outflow of efferent impulses in correspondence with the inflow of aflerent.

Sherrington points out that all the modern theories of cercbellar function harmonize with his conception of the cerebellum as the head ganglion of the proprio-ceptive systcm ( $p$. 934). The most influential of the proprio-ceptive organs being the labyrunth, the central organ of the whole proprio-ceptive mechanism is built up over the central connections of the labyrinth. Thither converge connecting (internuncial) paths from the central endings of proprio-ceptive neurons in all segments of the body (from joints, muscles, tendons, ligaments, viscera, etc.). Thus a central organ is devcloped, which varies in size and complexity in different kinds of animals according to the complexity of their habitual move. ments.

\section{Afferent Im-} pulses concerned in Equilibration and Orientation. -This is a convenient place to consider a little more in detail the nature and

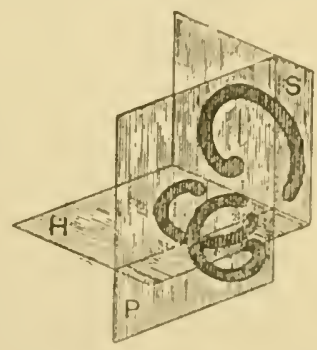

Fig. 375.-The Semicircular Canals (Diagrammatic) (after Ewald). H, horizontal or external; S, superior; P, pos. terior. The two horizontal canals lie in the same plane. The plane of the superior vertical canal of one side is parallel to the plane of the posterior vertical canal of the opposite side.

peripheral sources of some of the most important afferent impressions concerned in equilibration and orientation.

(I) Afferent Impulses from the Semicircular Canals.-The semicircular canals are three in number, and lic nearly in three mutually rectangular planes: the cxternal canal in the horizontal plane, the superior canal in a vertical longitudinal plane, and the posterior canal in a vertical transverse planc. Each canal bulges out at one end into a swelling, or ampulla, which opens into the utricular division of the vestibule (Figs. 375, 455). The other cxtremities of the superior and posterior canals join together, and have a common aperture into the utriclc, but the undilated end of the cxternal or horizontal canal opens separatcly. The utricle and the semicircular canals are thus connected by five distirct orifices. The greater part of the internal surface of the membranous canals, utricle and saccule, is lined by a single layer of flattencd cpithclium. But at or.e part of each ampulla projects a transverse ridge, the crista acustica, covered not with squamous, but with long columnar cpithclium. Hair-like processes (auditory hairs) 
are borne by some of the colmmnar cells, between which lie more elongated fibre-like supporting cells. The latirs project into a mucuslike mass, sometimes containing otoconial, or crystils of calcium carbonate. The ampulle, like the rest of the membranous labyrinth, is filled with a watery fluid called endolympl. The utricle and saccule have each a somewhat similar but broader elevation, the macula acustica, covered with epithelium and hair-cells of the same character, and the hairs project into a similar mass in which otoconia are constantly present. In some animals, as fishes, the calcareous matter in the utricle and saccule forms masses of considerable size (otoliths). Fibres of the auditory nerve end in arborizations around the bodies of the hair-cells of the macula and cristæe acustice. We have already seen that it is the ventral or vestibular division of the nerve which is; especially related to the restibule (p. 927).

There is very strong evidence that the semicircular canals are concerned, not in hearing, but in equilibration. A pigeon from which the membranous canals have been removed still hears perfectly well so long as the cochlea is intact, but exhibits the most profound disturbance of equilibrium. If the horizontal canal is destroyed or divided, the pigeon moves its head continually from side to side around a vertical axis; if the superior canal is divided, the head moves up and down around a horizontal axis. The power of co-ordination of movements is diminished, but not to the same cxtent in all kinds of animals. Thrown into the air, the pigeon is helpless; it cannot fly; but a goose with divided semicircular canals can still swim. The condition is only temporary, even when the injury involves the three canals on one side; but if the canals on both sides are destroyed, recovery is tardy, and often incomplete. In mammals the loss of co-ordina-

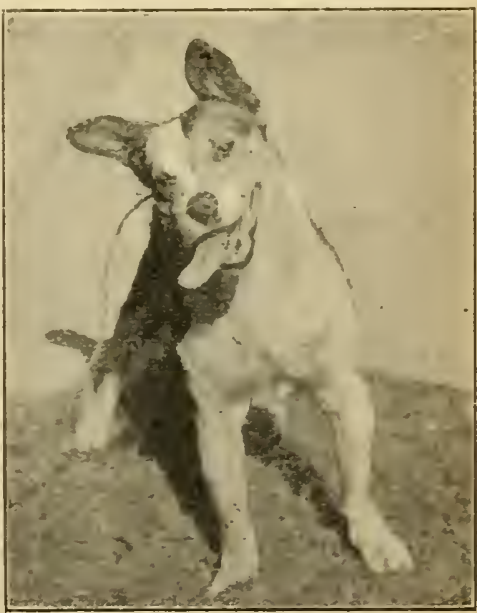

Fig. 376. - Dog Twenty - two Months after Destruction of Right Labyrinth (Wilson and Pike). tion is less than in birds, although at first the animal is unable to walk properly, easily falls over to the injured side, and goes continually in a circle turning towards the side of the lesion. Movements of the eyes, the direction of which depends on the canal destroyed, take to a large extent the place of movements of the head. Torsion of the head towards the side of the injury is, however, pronounced and permanent (Wilson and Pike) (Fig. 376). Speaking generally, the eyes are deviated to the side of the lesion, and exhibit tlie phenomenon of nystagmus. 
This is composed of two pliases, a slow movencut towar.]s the injured side and a quick jerk back towards the uninjured side. The nystagmus is synchronous in both eyes, and is constantly present for some days after the operation. There is reason to believe that the slow (leviation is due to excitation arising in the labyrinth and conveyed through the vestibuiar nuclei to the oculomotor nuclei by way of the posterior longitudinal bundle. The slow movement can still be obtained after removal of the cerebral cortex. The (juick return jerk is different in its origin, since it is abolished by the removal of the cerebral hemisphere on the side to which the eyes are moved in the slow deviation-i.e., the sicle of the cerebrum from which the efferent impulses concerned in pulling the eyes back to the primary position of equilibrium arise.

In spealing of the postural reflexes it was stated that evidence had been obtained of the widespread influence of the labyrinth on the tonus of the skeletal muscles. The effects on the postural tonus of the limb muscles were separated into two components, one due to the labyrinth directly, and the other to impulses passing along the afferent nerves of the neck muscles whose postural tonus is itself affected from the labyrinth. These phenomena have been demonstrated especially by Magnus and his pupils, in mammals like the rabbit, cat, and dog, and to a certain cxtent in man. They studied the effects of excitation of the labyrinth as well as the effects of its extirpation. To excite the labyrinth they employed such 'adequate' stimuli as are generated when the position of the licad is altered with refcrence to the vertical. Nithout going into the details of this elaborate work, it may be said that it has placed in a clear light the manner in which the posture of the head and of the neck reacts upon the postural contractions of the limbs. The strange attitudes so often seen after injury or discase of the labyrinth are to a great cxtent the consequences of the abnormal postures assumed by the neck.

In the frog, in which no direct influence of destruction of the labyrinth on the tonus of the extremities has been established, the effects of the altered neck posture on the postural tonus of the limbs have also been clearly demonstrated. On the injured side the extremities, especially the anterior limb, are flexed and adducted, the extremities of the other side extended and abducted; the head and vertebral column are rotated to the injured side (Ewald). All that is necessary to abolish the clifference in the posture of the limbs on the two sides is to divide the posterior roots of the spinal nerves supplying the neck muscles (Fiig. 377).

The effects of destructive lesions of the labyrinth have their counterpart in the phenomena caused by stimulation; cxcitation of a posterior canal, for example, in the pigeon causes movements of the head from side to side.

Lee's results in fishes are, on the whole, of similar tenor. Mechanical stimulation of the ampulla in the dogfish, by pressing on them with a blunt necdle, calls forth characteristic movements of the 
eyes and fins, and electrical stimulation of the auditory nerve causes movenents compounded of the separate movements obtained by stimulation of the ampulle one by one. J.ee concludes that the semicircular canals are the sense-organs for dynamical equilibrium (i.e., equilibrium of an animal in motion), and the utricle and saccule for statical equilibrium (i.e., equilibrium of an animal at rest).

The evidence from all sources points strongly to the conclusion that afferent impulses are actually set up in the fibres of the auditory nerve, through the hair-cells, by alterations of piessure or by streaming movements of the endolymph when the position of the head is changed. Rotation of the head to the right may be supposed to cause the endolymph in the right external canal, in virtue of its inertia, to lag behind the movement, and to press upon the anterior surface of the ampulla. The disorders of movement after lesions of the canals may be explained as the result of the withdrawal of certain of these afferent impulses, and the consequent overthrow of

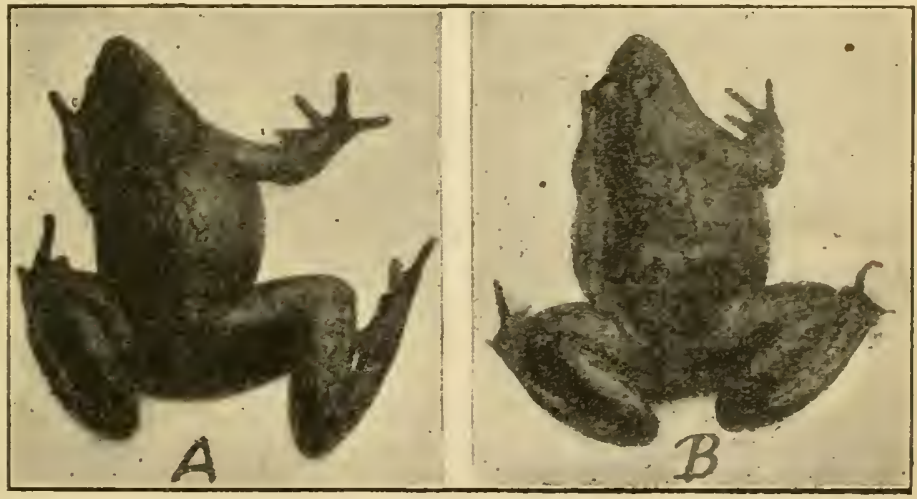

Fig. 377.-A, Frog after Extirpation of the Left Labyrinth, showing the Difference in the Posture of the Limbs on the Two Sides. $B$, the Same Frog after Section of the Posterior Roots of the Second Pair of Spinal Nerves. The head and vertebral column remain rotated toward the side of the lesion, but the legs are now held symmetrically.

that equipoise of excitation necessary for the maintenance of equilibrium. An experiment of Kreidl on a crustacean (palæmon) has made it probable that the otoliths by their weight may mechanically affect the hair-cells, and so increase their sensitiveness to changes of position. This animal has the peculiarity that in moulting the inner lining of the otocysts, in which the otoliths lie and which open to the exterior, are shed along with the otoliths. When moulting is over, the animal by means of its claws conveys fine sand grains into the otocysts, where they function as otoliths. Kreidl placed the animal after moulting upon finely powdered iron, 
some of whicl was conveyed into the otocyst instead of sand. It was now found possible to obtain definite reactions from the animal in the presence of a magnet, which, of course, tended to attract the ferruginous otoliths, and so to alter their position with reference to the hairs. The way in which the animal clanged its position in response to the magnet could be satisfactorily accounted for on the liypothesis that normally the contact of the otoliths with the hairs is altered under the influence of gravity when such changes of position occur. Even in man there is evidence of the existence of some mechanism not depending on the muscular sense or on impressions passing up the channels of ordinary or special sensation, by which orientation (the determination of the position of the body in space) is rendered possible. For a man lying perfectly still, witl eyes shut, on a horizontal table which is made to rotate miformly, can not only judge whether, but also in what direction, and approximately through what angle, he is moved. The phenomena of pathology afford weighty additional testimony in favour of the cquilibratory function of the senicircular canals. For many cases of vertigo arc associated with changes in the internal ear (Mènière's disease). And while nearly every normal individual becomes dizzy when rapidly rotated, 35 per cent. of deaf-mutes are entirely unaffected (James), and the proportion scems to be mucl higher among congenital deaf-mutes. Kreidl and Bruck, too, have found that abnormalities of locomotion and equilibration are much more common in cleaf-and-dumb clildren than in others. Now, in these cases the rlefect is usually in the internal car.

Summary.-We must conclude, then, that the co-ordination of muscular moicments necessary for cquilibrium is achieved in some contre or contres to which afferent impulses pass from the internal ear by the vestibular branch of the auditory neric, and from which cfferent impulses pass out to the muscles. If this centre or one of these contres is situated in the corcbellum, the efferent path is, as already suggested, partly an indirect one (perhaps by commissural fibres to the Rolandic area, and then out along the pyramidal tract), or more probably to lower centres, which control such massive co-ordinated movements as those concerned in walking and the maintenance of the normal attitude, and thence out along ccrtain tracts that connect the thalamus to the spinal cord (p. 886).

The influence of the labyrinth on the eye movements through its connection with the oculomotor nuclei in the mid-brain has been previously mentioned as a factor in the phenomena which follow: its destruction or stimulation. It would, however, be crroncous to assume that either the cercbcllum or the labyrinth is essential to the crude maintenance of that postural muscular tonus which is the reflex foundation of the act of standing. For in the dog and cat the posture persists after removal of the fore-brain, the mid-brain back to the 
hinder edge of the posterior corpora quadrigemina and the labyrinth, being supported partly through spinal centres and partly through pre-spinal centres mainly in the region of the pons, but partly in the bulb (Sherrington). But it is none the less true that the act of standing is aided and regulated in important ways by contres in the mid-brain and cerebcllum through afferent impulses originating in the labyrinth and elserehere-e.g., in the muscles.

Evald has made an observation which illustrates the peculiar relation of the semicircular canals to the muscular system-namely, that the labyrinth (in rabbits), influences the course of rigor mortis in the striped muscles. Rigor does not come on so soon on the side from which the labyrinth has been removed.

(2) Afferent Impressions from the Muscles.--Muscles are richly supplied with afferent fibres, for about half of the fibres in the nerves of skeletal muscles degencrate after section of the posterior roots beyond the ganglia (Sherrington). Various kinds of impressions may pass up these nerves: (a) Impressions giving rise to pain, as in muscular cramp and in experimental excitation of even the finest muscular nerve-filament; $(b)$ impulses causing a rise of blood-pressure; $(c)$ impulses which are not associated with a distinct impression in consciousness, but which enable us to localize the position of the limbs, head, eyes, and other parts of the body; $(d)$ impulses which inform us as to the extent and force of muscular contraction, and seem to underlie the so-called muscular sense. It is the last two kinds-if, indeed, they are distinct-which must be concerned in equilibration. In locomotor ataxia such impressions are blocked by degeneration in a part of the afferent path (p.9I5), and disorders of equilibrium are the result.

(3) Afferent Impressions from the Skin.-Of the various kinds of impulses that arise in the nerve-endings of the skin, only those of touch and pressure seem to be concerned in the maintenance of equilibrium. When the soles of the feet are rendered insensitive by local anæsthesia or by cold, and the person is directed to close his eyes, he staggers and sways from side to side. The disturbance of equilibrium in locomotor ataxia must be partly attributed to the loss of these tactile sensations, for numbness of the feet is a frequent symptom, and the patient asserts that he does not feel the ground. An interesting illustration of the importance of afferent impulics from the skin in the maintenance of equilibrium is afforded by the behaviour of a frog deprived of its cerebral hemispheres. Such a frog will balance itself on the edge of a board like a normal animal, but if the skin be removed from the hind-legs, it will fall like a log.

Localization of Function in the Cerebellum.-In birds and lower vertebrates the cerebellum is only represented by the worm. Yet in many of these animals the same characteristic disturbances follow its removal as in the higher animals where the cerebellar hemispheres have recome so prominent. Indeed, it was mainly on the pigeon that 
lilourens made his classical experiments. At first the pigeon can neither fly nor feed itself. When it attempts to walk, extensor spasms of the legs come on, and it falls, wildly struggling and apparently panic-stricken, to the ground. The power of flight is soon regained, but for a long time the animal is unable to perch, the legs and talons stiffening in rigid extension as it attempts to alight.

In the higher animals stinulation of certain parts of the worm and lateral lobe causes conjugate movements of the cyes towards the same side, both eyes being turned to the right-e.g., when the cercbellum is stimulated to the right of the middie line. Inhibition of movement can also be elicited from the organ. Excitation of the cercbellar cortex

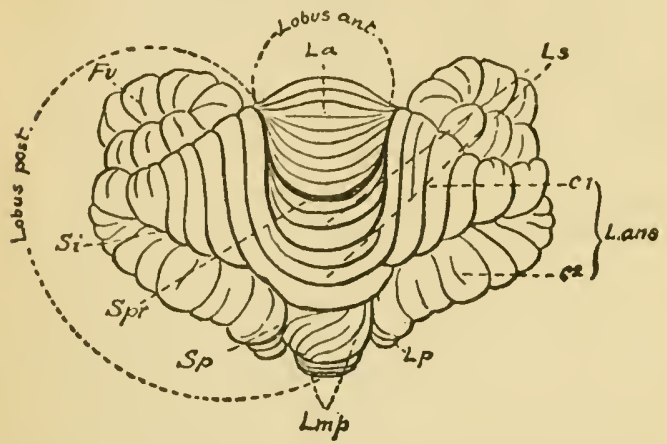

Fig. 378.-Scheme of Dog's Cerebellum (Dorsal View), according to the Anatomical Division of Bolk (after van Rynberk). La, lobus anterior, which is separated from the larger posterior lobe by the deep primary fissure (sulcus primarius), $S p r, L s$, lobulus simplex; Si, sulcus intercruralis; $C^{1}$, crus primum; $C^{2}$, crus secundum; $L . a n s$, lobulus ansiformis; $L p$, lobulus paramedianus; $L m p$, lobulus medianus posterior; $F v$, formatio vermi. cularis (pars tonsillaris); $S p$, sulcus paramedianus. for some distance outwards from the line of junction of the superior worm with the lateral lobe in animals which exhibit tonic contraction of extensor muscles after excision of the cerebral hemispheres (decerebrate rigidity or acercbral tonus, as it is called) causes immediate relaxation of the rigid muscles of the neck, tail, and especially the anterior limb, particularly on the same side. The relaxation of the extensors may be accompanicd by contraction of the antagonistic flexors - for example, relaxation of the triceps and contraction of the biceps (Horsley and Löwenthal). But this call scarcely be considered a reaction specific to the cerebellum. For Sherrington, who finds that the tonus or spasm is largely due to centripetal impulses coming from the rigid limb, has been able to inhibit it by stimulation of various other regions, including the portion of the cerebral cortex in front of the fissure of Rolando (p. 955).

The confusion which so long reigned in regard to cercbellar localization has in great measure been cleared up by recent physiological work following on a more accurate anatomical mapping of the lobes and lobules of the cerebellum in accordance with their genetic relations (Elliott Smitl, Bolk) (liigs. 378, 379). The classical description of the mammalian cerebellum as consisting of a median lobe or vermis and two hemispheres lias been abandoned.by the modern investigators in this field. They divide the organ into two chief lobes, an anterior and a posterior, separated by the deep and constant primary fissure. 
Following this scheme, van Rynberk has obtained satisfactory rvidence of lucalization of function. Thus the lobulus simplex constitutes a centre for the neck muscles, and the climination of its influence by excision leats to movements of the head (so-called hearl nystagmus). The anterior extremity is represented by a centre in the crus primum, and the posterior extremity by a centre in the crus secundum, of the ansiform lobule of its own side, and injury in the region of these centres is associated with abnormal movements of the corresponding fore and hind fuot respectively. Extirpation of a lobulus paramedianus causes rolling movements of the body around its long axis or bending of the body to one side, and this centre is connected with the muscles of the trunk. A general scheme of the localizations in the mammalian cerebellum is given in Fig. 379.

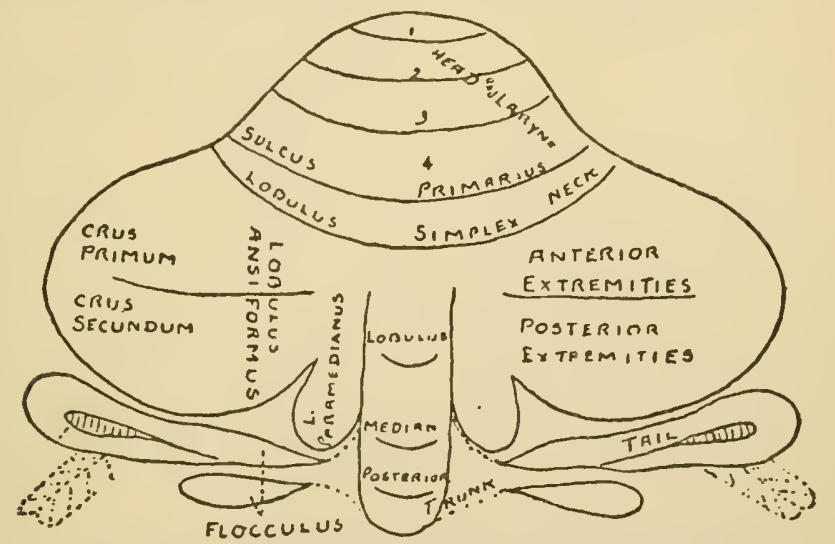

Fig. 379.-Bolk's Scheme of the Mammalian Cerebellum. On the right half, certain localizations are indicated (Mills after Von Bechterew).

It must be remarked that the areas connected with the coordination (synergia) of the muscles concerned in particular movements cannot be so sharply localized on the surface of the cerebellum as the areas connected with the discharge of the movements can be localized on the surface of the cerebrum. It is stiji an open question whether in the function of these centres only the cortex of the lobules is concerned, or in addition the corresponding portions of the central nuclei of the eerebellum. These observations are supported by other facts. For example, microscopical studies have shown that definite regions of the cerebellar cortex are especially connected with definite levels of the spinal cord. Further, the lobulation of the cerebellum in mammals keeps pace with the increase in complexity of the voluntary motor apparatus of the whole body, and the variations in the degree of development of definite lobules are related to the variations in the anatomical and physio- 
logical development of the corresponding groups of muscles. All this fits in well with the idea that the cerebellum is a great reflex mechanism standing in intimate relation on the one hand to numerous afferent paths (skin, muscles, labyrinth, etc.), and on the other

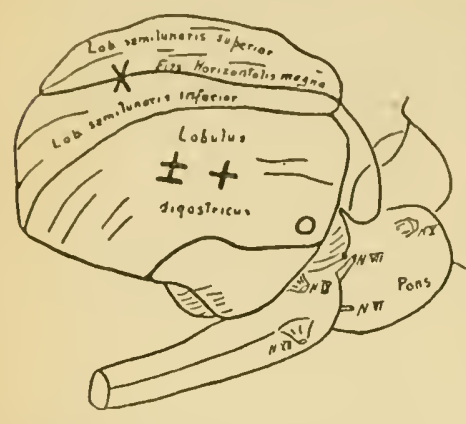

Fig. 380.-Localizations on the Inferolateral Aspect of the Human Cerebellum (Barany). $X$, is a centre for the tonus of the muscles abducting the right arm; $\mathrm{O}$, a centre for the tonus of the muscles concerned in adduction of the right hand ;,$+ a$ centre for the tonus of the muscles adducting the right arm; \pm , a centre for the tonus of the muscles adducting the right hip. N.V, N.VI, N.VII, $N . I X, N . X I I$, are cranial nerves.

to the voluntary muscles. It is the precise nature of the influence exerted by it upon the latter whicl is in doubt, whether an augmenting sthenic influence, as Luciani supposes, or a co-ordinating infuence, as Flourens assumed, or a combination of these.

Progress has recently been made in utilizing the results of experimental and clinical study of the cerebellum for the localization of circumscribed cerebellar lesions in man (Barany).

The centres for the limbs are situated in the cortex of the hemispheres, those for the right extremities in the right semilunar (superior and inferior) and digastric lobules; those for the left extremities in the corresponding positions on the left cerebellar hemisphere.

Forced Movements.-We have incidentally mentioned that in fishes injuries to the semicircular canals may give rise to movements which seem to be beyond the control of the animal, and which have consequently received the name of 'forced movements.' It may be added that when the internal ear of a Necturus (one of the tailed amphibia) is destroyed on one side, rapid movements of rotation around a longitudinal axis are observed. The animal spins round and round apparently without voluntary control, purpose, or fatigue. The direction of rotation is towards the side of the lesion, the observer being supposed to look down upon the animal as

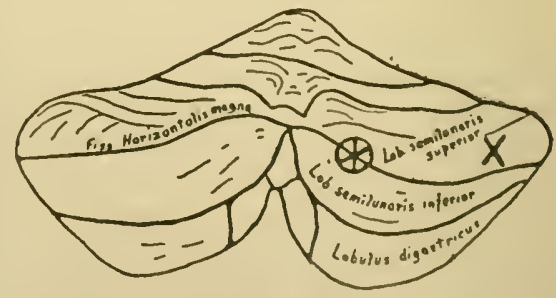

Fig. $38 \mathrm{r}$-Localizations on Posterior Aspect of Human Cerebellum (Barany). $\otimes$, a centre for the tonus of the muscles concerned in the movements of the right arm downwards; $x$, same as in Fig. $3^{80}$. it lies in its normal position. After a time it becomes quiescent; but the forced movements can be again produced by pinching or exciting it in other ways. Similar rolling movements, and in the same direction, have been observed in 
mammals after cxtirpation of one labyrintls. They are elicited with special easn in the rabbit. In this animal it has becn slown by stmlying the movements by the aid of the cincmatograple that the rolling movenents are really movenuents of progression (running and springing), which, on accomnt of the changes in the position of the head and neck, and in the tonus of the muscles, lead to a spiral rotation of the body, in which the forward movement is small in proportion to the rotation.

In man, too, during the passage of a galvanic current through the head by electrodes applied just behind the ears, a tendency to move the head towards the anode is experienced. The person may resist the tendency; but if the current be strong enough his resistance will be overcome; he will execute a forced movement. When the head turns towards the anode the eyes move in the same direction, and then undergo jerking movements towards the kathode. There is at the same time a fecling of vertigo. Complex as such an experiment is, involving as it does stimulation of so many structures within the cranium, there is reason to believe that it is the excitation of the semicircular canals, or their ccrebellar connections, that is responsible for these forced movements. For when the experiment is performed on a pigeon, forced movements are caused so long as the membranous canals arc intact, but not after they have been destroyed (Ewald). The observation of Rawitz, that the peculiar rotatory movements of the so-called Japanese dancing mice are associated with marked anatomical peculiarities in the labyrinth, is another fact in favour of the connection of the canals with the maintenance of equilibrium and the sense of rotation. So is the relation between the degree of development of the canals in different species of birds and the degree of agility in the co-ordination of then movements (Laudenbach).

But forced movements may also follow injuries (especially unilateral) to many portions of the brain-e.g., the pons, crus cerebri, posterior corpora quadrigemina, corpus striatum, even the cerebral cortex, and above all the cerebellum. The movements are of the most various kinds. The animal may run round and round in a circle (circus movement); or, with the tip of its tail as centre and the length of its body as radius, it may describe a circle with its head, as the liand of a clock does (clock-hand movement); or it may rush forward, turning endless somersaults as it goes. Intervals of rest alternate with paroxysms of excitement, and the latter may be brought on by stimulation. In man forced movements associated with vertigo have becn sometimes seen in cases of tumour of the cerebellum-e.g., involuntary rotation of the body in tumour of the middle peduncle. No entirely satisfactory explanation of these forced movements has been given. They are evidently connected with disturbance of the mechanism of co-ordination, leading to a loss of proportion in the amount of the motor discharge to muscles or groups of muscles accustomed to act together in executing definite movements. For instance, in circus movements the muscles of the outer side of the body contract more powerfilly than those of the inner side, and the animal is therefore constrained to trace a circle instearl of a straight line, the excess of contraction on the outer side being analogous to the acceleration along the radius in the case of a point moving in a circle.

In connection with the consideration of the mechanism of cquilibra. tion, a short account of the muscular actions concerned in the maintenance of the erect posture so characteristic of man, and of those concerned in locomotion, is subjoined lierc:

Standing.-In the upright posture the body is supported chiefly by 
non-muscul.ur structures, the bones and ligaments. But muscles also play an essential part, for it is only peculiarly-gifted individuals, like some of the fishermen of the Nortl Sea, who can go to sleep on their feet, and a dead body cannot be made to stand crect. The condition of equilibrium is that the perpendicular dropped from the centre of gravity to the ground slould fall within the base of support-that is, within the area enclosed by the outer borders of the feet ard lines joining the toes and heels respectively. The centre of gravity alters its position with the position of the body, which tends to fall whenever the perpenclicnlar cuts the ground beyond the base of support.

In the comfortable and natural erect position the centre of gravity of the head is a little in front of the vertical plane passing through the occipital condyles, and as much as 4 centimetres in front of the vertical plane passing tlirough the ankle-joints. A certain degree of contraction of the muscles of the nape of the neck is required to balance it. When these muscles are relaxed, as in sleep, the head must fall forwarel, and this is the reason wliy Homer or any lesser individual nods. In animals which go upon all-fours none of the weight of the liead bears directly upon the occipito-atloid articulation; its support by muscular action alone would be an intolerable fatigue, and the ligamentum nuchre is specially strengthened to hold it up.

The vertebral column is liept erect by the ligaments and muscles of the back. The centre of gravity of the trunk lies almost vertically over the horizontal line joining the two acetabula, but the centre of gravity of the whole body is about the level of the third sacral vertebra, and a little more than 4 centimetres in front of the vertical plane passing through the ankle-joints. Equilibrium is maintained by contraction of the muscles of the back and of the legs. By means of the muscular sense, and the tactile sensations set up by the pressure of the soles on the ground, alterations in the position of the centre of gravity, and consequent deviations of the perpendicular passing through it, are detected, and adjustment of the amount of contraction of this or the other muscular group is promptly made.

In standing at 'attention' the liecls are close together, the legs and back straightence to the utmost, and the head erect; the weight falls equally ujon botli legs, but the advantage may be more than counterbalanced by the muscular excrtion associated with this more ornamental tlian uscful position. In 'standing at case,' practically tlie whole weight is supported by one leg, the perpendicular from the centre of gravity passing through the linec and ankle-joint. The centre of gravity is brought over the supporting leg by flexule of the borly to the corresponding side, and comparatively little muscular cffort is required. The other foot rests lightly on the ground, the weight of the leg itself being almost balanced by the atmospheric pressure acting upon the air-tight and air-free cavity of the hip-joint. The light touch of this foot varies slightly from time to time, so as to maintain equilibrium.

When the head or arms are moved, or the body swayed, the centre of gravity is correspondingly displaced, and it is by such movements that tiglit-rope dancers continue to keep the perpendicular passing throngh it always within the narrow base of support.

In sitting, the base of support is larger than in standing, and the equilibrium therefore more stable. The easiest posture in sitting without support to the back or feet is that in which the perpendicular from the centre of gravity passes through the horizontal line joining the two tubert ischii.

Locomotion.-In walking, the legs are alternately swung forward 
and rested on the ground, With most persons the swinging foot first strikes the ground by the heel; then the sole comes down, the heel rises, the leg is extended, and, with a parting push from the toe, the leg again swings free. By this manœuvre the body is raised vertically, tilted to the opposite side, and also pushed in advance.

The forward swing of the leg is only slightly, if at all, due to muscular action; it is more like the oscillation of a pendulum displaced belind its position of equilibrium, and swinging through that position, and in front of it, under the influence of gravity. For this reason the natural pace of a tall man is longer and slower than that of a short man; but it may be modified by voluntary effort, as when a rank of soldiers of different height keeps step. The lateral swing of the body is illustrated by the everyday experience that two persons knock against each other when they try to walk close together without keeping step. In step both swing their bodies to the same side at the same moment, and there is no jarring. Even in the fastest walking on level ground there is a short time during which both feet touch the ground logether, the one leg not beginning its swing until the other foot has begun to be set down. In running, on the other hand, there is an interval during which the body is completely in the air, while in walking uphill or in carrying a load the one foot is not raised until the other has becn firmly planted.

Functions of the Cerebral Cortex.-When an animal, like a frog, is deprived of its cerebral hemispheres, the power of automatic voluntary movement appears to be definitively and entirely lost. The animal, as soon as the effects of the anæsthetic and the shock of the operation have passed away, draws up its legs, erects its head, and assumes the characteristic position of the normal frog at rest. So close may be the resemblance, that if all external signs of the operation have been concealed, it may not be possible for a casual observer to tell merely by inspection which is the intact and which the 'brainless ' frog. 'The latter will jump if it be touched or otherwise stimulated. It will croak if its flanks be stroked or gently squeezed together. It will swim if thrown into water. If placed on its back, it will promptly recover its normal position. But it will do all these things as a machine would do them, without purpose, without regard to its environment, with a kind of 'fatal' regularity. Every time it is stimulated it will jump, every time its flanks are squeezed it will croak, and, in the absence of all stimulation, it will sit still till it withers to a mummy, even by the side of the water that might for a while preserve it.

A Necturus, without its cerebral hemispheres, will, like the frog, refuse to lie on its back. On stimulation it moves its feet or tail, or its whole body; but if not interfered with, it lies for an indefinite time in the same position. Its gills are seen to execute rhythmic movements, which never stop, and rarely slacken, except for an instant, when some part of the skin, particularly in the region of the head, is mechanicilly or clectrically stimulated. The normal Necturus, on the other hand, lies for long periods with its gills at 
perfect rest, and when stimulated, moves for a considerable distance. Alter a time-two montlis or more-it is true the brainless frog, if it be kept alive, as may be done by careful attention, will recover a certain portion of the powers which it has lost by removal of the cerebral hemispheres; and, indeed, the longer it lives, the nearer it approximates to the cundition of a normal frog. A brainless frog has been seen to catch flies and to bury itself as winter drew on. A fish even three days after the destruction of its cerebrum has been seen to dart upon a worm, seize it before it had time to sink to the bottom of the aq̣uarium, and swallow it. Even in the pigeon the loss of the hemispleres, which at first induces a state of profound and seemingly permanent lethargy, is to a great extent compensated for, as time passes on, by the unfolding in the lower centres of capabilities previously dormant or suppressed. A brainless pigeon has been known to come at the whistle of the attendant and follow him through the whole house.

In the mammal the removal of the whole or the greater part of the cerebral hemispheres at a single operation is uniformly and specdily fatal; cven rabbits or rats, which bear the operation best, survive but a few hours. During those hours they manifest phenomena similar to those observed in the bird and the frog. In the dog the entire cortex has been removed piecemeal by successive operations. In this case, of course, the change in the condition of the animal is more gradually produced, and an opportunity is afforded for a certain recovery of function in the intervals between the operations. On the whole, however, as might be expected, from its greater intellectual development, recovery is more imperfect in the dog than in the bird, much more imperfect than in the frog. But even in the dog wonderful resources lie hidden in the grey matter of the central neural axis, and are called forth by degrees to replace the lost powers of the cerebral cortex. It is truc that a brainless dog is a less efficient animal than a brainless fish, or even than a brainless frog; but in favourable cases, even in the dog, the movements of walking may still be carricd out with tolerable precision in the absence of the cerebral hemispheres. The animal can swallow food pushed well back into the mouth, although it cannot feed itself. Stupid and listless as it is compared with the normal dog, it seems to be by no means devoid of the power of experiencing sensations as the result of impressions from without, or of carrying on mental operations of a low intellectual grade. Goltz had a dog which lived more than a year and a lualf practically without its cerebral hemispheres, and another which lived thirteen weeks. He believes that they had lost understanding, reflection, and memory, but not sensation, special or general, nor emotions and voluntary power. Their condition may be best described as one of general imbecility. Hunger and thirst are present. They experi- 
ence sitisfaction when fed, become angry when attacked, see a very bright light, avoid obstacles, hear loud sounds, such as those produced by a fog-liorn, and can be awakenced by them. They are not completely deprived of sensations of taste and touch. But it ought to be remembered that the interpretation of the objective signs of sensation in animals is beset with difficulties; and although everybody admits the accuracy of Goltz's description of what is to be seen, his interpretation of the facts has been severely criticized, particularly by H. Munk.

Difficult as it is to keep dogs alive after removal of the greater part of the cercbrum, the problem is far more difficult in monkeys. In some recent experiments, however, in which the hemispheres were removed by two successive operations from Macaque monkeys, one of the animals survived for as long as twenty-six days the excision of the second hemisphere, and others lived a week. The movements of the extremities were much affected. The animals seened to be in a sleepy condition with the eyclids closed, and most of them made no movements which could be interpreted as voluntary movements during the first days after loss of the second hemisphere. Sounds caused the appropriate reflex movements of the ears. The pupil was constricted by light. The animals cried in the usual manner when subjected to painful stimuli, but the ordinary facial accompaniments of pain were absent.

In man the destruction of considerable masses of brain-substance, particularly if gradual, is not necessarily fatal. How great a loss is compatible with life cannot be exactly stated. It depends to a large extent on the position of the lesion. But it is possible that one cerebral hemisphere may be rendered functionally useless without immediately putting a term to existence. In the fœtus, lowever, no portion of the great brain is absolutely indispensable for life and movement. An anencephalous foetus (in which the brain has remained undeveloped) may be born alive, and live for a short time.

IVe see, then, that homologous organs are not necessarily, nor indeed usually, of the same physiological value in different kinds of animals. A loss which perhaps hardly narrows the range of the psychical, and certainly restricts only to a slight extent the physical powers of a fish, impairs in a marked degree the voluntary movements of a dog, still more those of a monkey, in addition to cutting off from it a great part of its intellectual life, and is in man incompatible with life altogether. It would be easy, however, to exaggerate the difference in the importance of the cerebrum to the higher and the lower animals. After all, it remains a striking fact that monkcys and dogs can live in the absence of the ccrebral hemispheres, and to the remnant of the central nervous system which enables such highly organized animals to support life must still be regarded as a very wonderful and complex mechanism. Perhaps. indeed, it is not too much to say that the real lesson of these experiments on the 
extirpation of the cercbrum is not so much the supreme importance of the hemispheres of the great brain in the higher animals as the manifold and fundamental functions which still reside in the basal ganglia. The cerebral cortex secms to have taken over and extensively developed and adapted some of those primitive powers of lower portions of the nervous axis, but without depriving the older formations of the capacity for reassuming them in some degree when the cortex has been destroyed.

The results of the removal of the entire cercbral hemispheres help us to fix their position as a whole in the plyysiological hicrarchy. A more minute analysis shows us that the cerebral cortex itsclf is not homogeneous in function, that certain regions of it have bcen set

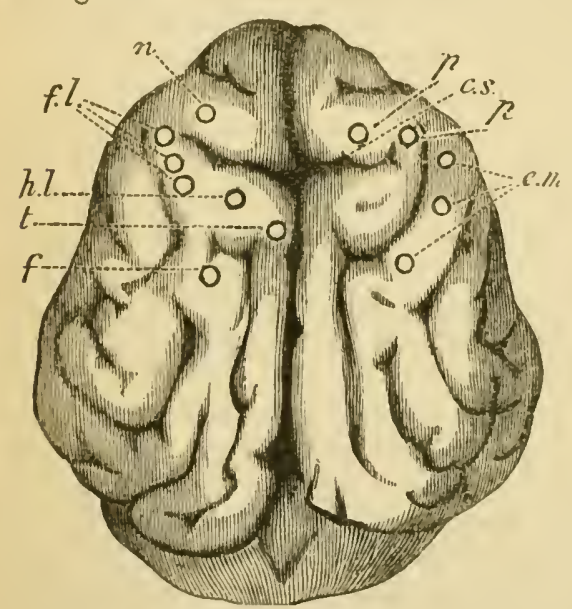

Fig. 382.-Mintor Areas of Dog's Brain. $n$, neck; $f . l$, fore-limb; h.l., hind-limb; $t$, tail; $f$, face; c.s., crucial sulcus; e.m., eye movements; $p$, dilatation of the pupil in both eyes, but especially in the opposite eye. All the areas are marked in the figure only on the left side except the eye areas, whose position, to avoid confusion, is indicated on the right hemisphere.

was that the cerebral cortex is inexcitable to artificial stimyilicians no visible response can be obtained from it. The great names of Flourens and Magendic stood sponsors for this error, and repressed research. In 1870 , however, Hitzig and Fritsch showed that not only was it possible to elicit muscular contractions by stimulation of the cortex of the brain in the dog with voltaic currents, but that the excitable area occupied a definite region in the neighbourhood of the crucial sulcus or sulcus centralis, which runs out over the convexity of the hemispheres nearly at right angles to the longitudinal fissure. In this region they were further able to isolate several distinct areas, stimulation of which was followed by movements respectively of the head, face, neck, hind-leg, and fore-leg (Fig. 382). This was the 
starting-point of a long series of researches by Ferrier, Munk, Horsicy. Scliäfer, Heidenhain, and many others, on the brains of monkeys as well as dogs-researches which have formed the basis of an exact cortical localization in the brain of man, and liave enriched surgery with a new province. In these later experiments the interrupted current from an induction machine has been found the most suitable form of stimulus (sec Practical Exercises, p. Iool), especially when one electrole only is placed on the cortex and the otlier on some indifferent part of the body-e.g., in the rectum (unipolar stinulation), a pro-

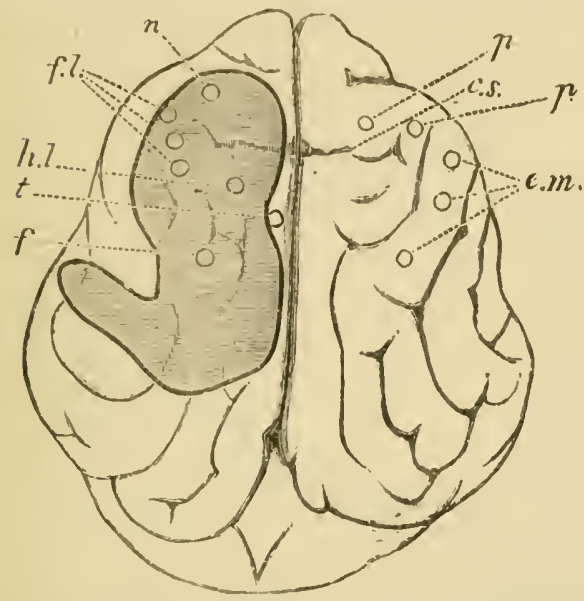

Fig. 383.-Dog's Brain with Lesion. A portion of the cortex indicated by the shaded area was destroyed by cauterization. The symp. toms were complete blindness of the opposite eye (in this case the right); weakness of the muscles of the limbs and of the neck on the right side; slight weakness of the limbs on the left side. When the animal walked, there was a tenclency to turn to the left in a circle. In eating or drinking, the head was turned to the left, so that the mouth was oblique, and the right angle of the mouth was lower than the left. The tail movements were normal, and there was no deviation of the tail to one side. cedure which permits of finer localization than when both electrodes are applied to the brain (bipolar stimulation).

'Mutor' Areas. *-These have been localized with great care (both by stimulation and by removal of portions of the cortex) in the brains of the higher apes (gorilla, orang, and chimpanzee) by Sherrington and Grünbaum, and there can be no doubt that the results, in their general outlines at least, can be applied to the human brain. These obscrvers employed the so-called unipolar method of stimulation.

The 'motor' region includes the whole length of and the whole of the free width of the precentral or ascending frontal convolution, and dips down to the bottom of the central sulcus (fissure of Rolando in man), but does not extend behind the sulcus. It extends also into the depth of all the fissures, so that the hidden part of the excitable area probably equals, perhaps exceeds, the part which is free on the surface of the hemisphere. The anterior limit of the 'motor' field is not quite

* Since the so-called 'motor' area, as is now well known, is really sensorimotor, and a region having to do purely with the discharge of motor impulses does not exist, it would be better to call it the sensori-motor, or, following Bastian's suggestion, the kinæsthetic area. Probably, however, the alteration of a term so long sanctioned by custom in physiological writings would lead to confusion. Accordingly, in what follows the word ' $m$ )tor' will be retained, but to show that it is used in a special sense it will be enclosed in quotation marks. 
sharp, but shades off somewhat gradually into inexcitable cortex. The sulci in this region cannot be considered to represent physioslogical boundaries, and they vary so much in these higher brains, that they can easily prove fallacious landmarks. On the mesial surface of the hemisphere the 'motor' area does not extend quite to the calloso-marginal fissure.

Within this area are localized movements of the leg and arm and their various joints, of the head, face, mouth, tongue, ear, nostril, and vocal cords, of the neck, chest, and abdominal wall, of the pelvic floor, and the anal and vaginal orifices.

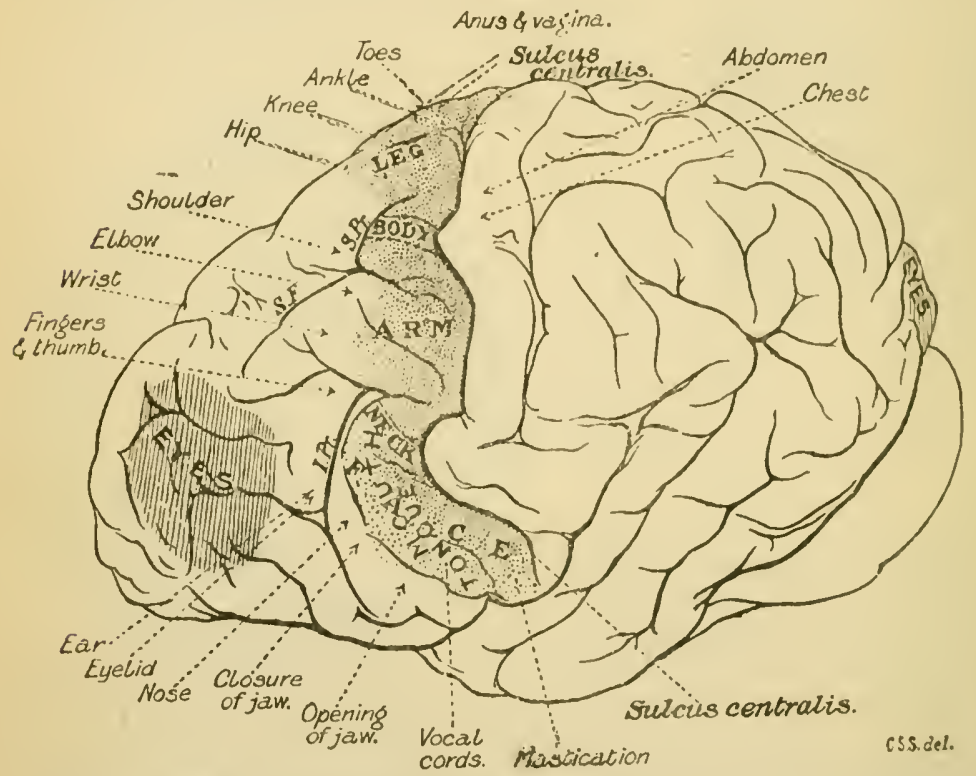

Fig. 384,- 'Motor' Area of Cortex of Chimpanzee (Grünbaum and Sherrington). Lateral aspect of the hemisphere.

The arrangement of the various regions follows very closely the order of the cranio-spinal nerves, which supply them, but the organs whose nerves come off lowest down are represented highest up in the ' motor' area. Figs. $3 \$ 4,385$ will make this clear. In the frontal region, isolated from the motor' area by a strait of inexcitable cortex, lies an area the stimulation of which causes conjugate deviation of the eyes. But the reaction differs from that obtained on excitation of the 'motor' area proper in front of the Rolandic fissure.

It is to be particularly noted (I) that within the larger areas, such as those of the arm and leg, smaller foci can be mapped off which are related to movements of the separate joints-thus, in the leg area, the hip, knee, and ankle-joints, and the great toe, are repre- 
sented by separate and special centres; (2) that stimulation of any onc of these areas leads, not to contraction of individual nuscles, but to contraction of muscular groups which liave to do with the cxecution of definite movements.

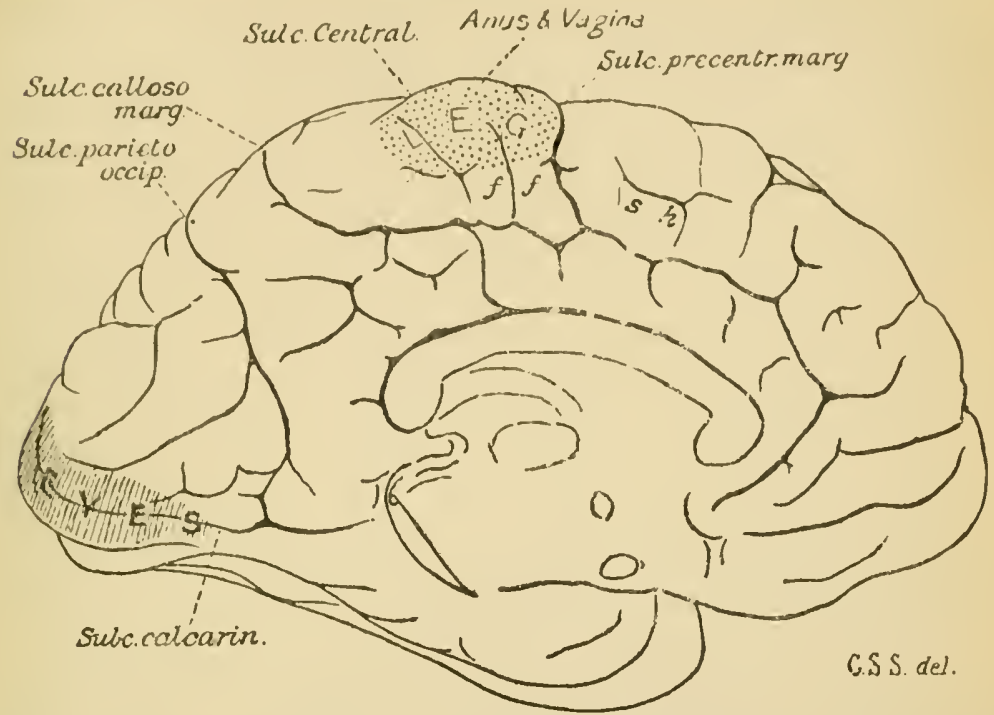

Fig. 385.- 'Motor' Area on Mesial Surface of Hemisphere: Brain of a Chimpanzee (Troglodytes Niger) (Grüubaum and Sherrington). Left hemisphere: mesial surface. The extent of the 'motor' area on the free surface of the hemisphere is indicated by the black stippling. On the stippled area ' $L E G$ ' indicates that the movements of the lower limb are represented in all the regions of the 'motor' area visible from this aspect. The minuter subdivisions in this area overlap each other so much that no attempt is made to distinguish them in the diagram. 'Anus and vagina' indicates the position from which perineal movements call be primarily elicited. Sulc. central. = central fissure; Sulc. calcarin. $=$ calcarine fissure; Sulc. parieto occip.=parieto-occipital fissure; Sulc. calloso marg.=callosomarginal fissure; Sulc. precentr. marg. = precentral marginal fissure. The single italic letters mark spots whence, occasionally and irregularly, movements of the loot and leg $(f f)$, of the shoulder and chest $(s)$, and of the thumb and fingers $(h)$, liave been evoked by strong faradization. The shaded area marked 'EYES' indicates a field of free surface of cortex which, under faradization, yields conjugate movements of the eyeballs. The conditions under which these reactions are obtained separates them from those characterizing the 'motor' area.

The Stability of the Reactions obtained by Stimulating Cortical Points. -The question whether stimulation of a 'motor' area or point invariably causes the same mavements, when it causes any movements at all, has been recently investigated by Graham Brown and Sherrington. They observed the contractions of two isolated antagonistic muscles acting on the clbow-joint (in monkeys) after elimination of all the other muscles of the arm and shoulder by section of their 'motor' nerves, when a point on the area of the cortex in which the movements of the elbow are represented was excited by the unipolar method. They find that a cortical point which has given flexion at the elbow, sometimes on 
investigation the next day, inay give the opposite result of exteision of the elbow. Fien within short intervals reversal of the reaction clicited from one and the same point may be secn. They do not question at all the general regularity of the results which such cortical points give when investigated by suitable methods after sufficient intervals of rest, and on which the current statements as to the reactions clicited from the various 'motor' areas are based. But they see in the influcnce of transient excitation cither of the point itsek or of other more distant points in modifying or reversing the reaction an inclication that one of the functions of the cortex may be the carrying out of such phemomena of reversal, a function which may play some part in the co-ordination of voluntary movements. In this conncction it may be remarked that a phenomenon analogous to the "facilitation " already described for the reflex arc is also exhibited by the motor cortex. If a motor reaction-e.g., the movement of flexion at the clbow, as studied in a pair of isolated antagonistic muscles in monkeys, is elicited by stimulation of the appropriate cortical area, and a second cortical stimulation is made to follow the first, the reaction evoked by the second stimulus is greater than that following the first, provided that the interval of time between the two is not too long. If the first stimulus is so chosen that it is just below the strength necessary to evoke a movement, a second or third stimulus, which also by itself would fail to cause a reaction, may do so. Not only so, but stimulation of one cortical point, say in the area which gives flexion of the elbow, may raise the excitability of ncighbouring points in that area (so-called 'secondary facilitation '), so that a stimulus which previously did not elicit the flexion movement from these points may now do so.

Inhibition from the Cortex.-Contraction is not the only effect on the muscles which can be elicited by stimulating the cortex. Cortical inhibition of tonus and of active contraction is just as cliaracteristic, though not so obvious a result. There is abundant eridence of reciprocal innervation of volitional movements from the cortex. When, e.g., the part of the arm area which presides over extension of the elbow is stimulated (in the monkey), it can be shown that the biceps relaxes as the triceps contracts. In like manner, stimulation of the appropriate part of the leg area will cause along with contraction of the extensors of the hip relaxation of such flexors as the psoasiliacus and the tensor fascia femoris. Such observations are most easily made when, in a certain stage of narcosis, the limbs. instead of hanging limp, assume a position of tonic flexion, especially at the clbow and hip. Under other conditions the position of tonic extension of a joint may be assumed, and then it can be shown that excitation of the appropriate focus for flexion of that joint will cause simultaneous contraction of the flexors and relaxation of the extensors.

The observer cannot fail to be struck with the general resemblance between these cortical reactions and their co-ordination and the co-ordinated bulbo-spinal reflex movements previously studied. There are, however, certain differences which place the cortical reactions upon a higher level. One of the most important is the part played by visual, auditory, and pure ' touch ' stimuli in cliciting 
cortical motor responses-c.g., "the closure of the hand, pricking of the ear, opening of the eyes, and turning of the head in the direction of the gaze' (Sherrington). The facility of response to stimuli acting from a distance through the distance-receptors, such as those of the retina and labyrinth, is one of the great characteristics of the cerebrum as an organ concerned in movements, and helps to place the 'motor' cortex at the helm, since these distancereceptors control more than others the slieletal musculature as a whole. Spinal reflex movements are mainly such as are elicited by harmful (nocuous) stimuli (protective reflexes), or through the sexual skin nerves, or from the visceral afferent fibres, or such as are concerned in the chicf movements of locomotion.

Decerebrate Rigidity is a phenomenon closely related to the inhibitory function of the cerebral cortcx. It is a condition of prolonged spasm of certain groups of skeletal muscles (especially the retractor muscles of the head and neck, the elcvators of the jaw and tail, and the cxtensors of the elbow, knec, shoulder, and hip), supervening on removal of the cerebral hemispheres by transection anywhere in the mid-brain or in the posterior part of the thalamus, and favoured by suspending the animal in the rertical posture. If the afferent roots belonging to one of the rigid limbs are severed, it at once becomes flaccid, while the other limbs remain rigid. The tonus is therefore reflex through the local afferent nerves, and, to be more precise, through those that supply the decp structures (joints, muscles, etc.). The centre must be situated somewhere between cerebrum and spinal bulb, since section of the bulb abolishes the rigidity. It is not apparently in the cerebellum. It is noteworthy that the muscles mainly involved in decerebrate rigidity arc those which are much more easily inhibited than excited from the ' motor' cortcx, and also in the local spinal reflexes. After removal of the cerebrum, the mechanism which maintains their tonic contraction has free play. Sherrington points out that this mechanism sustains the steady muscular tension necessary to preserve against the force of gravity the attitude or posture of the body. According to him, decerebrate rigidity is simply ' reflex standing.' When the transient spinal reflex or the transient cortical effect breaks in upon this tonic contraction-e.g., in locomotion-inhibition of the contracted extensors accompanies contraction of the flexors (see also p. $9+2$ ).

Removal of a single 'motor' region leads to paralysis of the corresponding limb, or part of a limb, on the opposite side. For example, after extirpation of the hand area the hand is for a few days practically useless and apparently powerless. In a few weeks, however, it recovers remarkably, so that it is once more used in climbing or in conveying food to the mouth. It is an important question in what way this recovery is brought about. If the whole 
of the corresponding area in the opposite hemisphere is now removed, a similar paralysis occurs in the other hand, but the hand whose ' motor' area was first extirpated remains entirely unaffected by the second lesion. On the contrary, the first hand is used more freely and more adroitly than before the second operation, probably because the animal needs to use it more. The second hand recovers eventually, like the first. If when this has taken place the remaining part of the arm area from which the hand area was first excised be removed, neither hand is apparently affected, although there is severe paralysis of the shoulder and slighter paralysis of the elbow on the side opposite to the lesion, which is again largely recovered from. The recovery of the hand movement cannot therefore be attributed to the taking on of the function of the corresponding

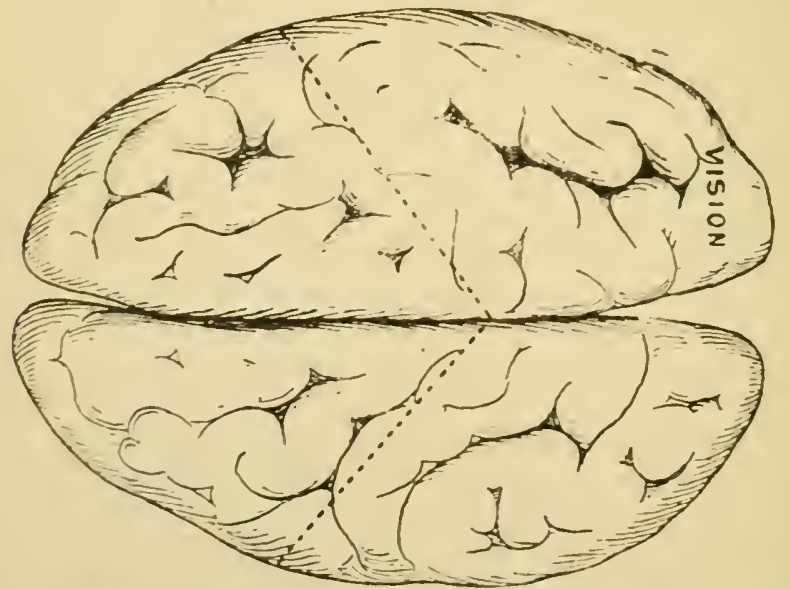

Fig. 386.-Cerebral Cortex Man (seen from Above). The front of the brain is towards the left. The dotted line shows the position of the fissure of Rolando, as fired by Thane's rule (p. $9^{6} 3$ ).

' motor' area either by the opposite hand area or by the adjacent ' motor' cortex of the same hemisphere. According to some authorities, the recovery is due to the representation of the upper limb in the post-central gyrus (ascending parietal convolution in man) acting through fibres that descend from this gyrus to the optic thalamus, and thence through the rubro-spinal tract, which runs to the spinal cord (p. 867).

Removal of the whole of the 'motor' cortex of one hemisphere, in such animals as this operation has been performed on, causes paralysis of movement on the opposite side of the body. The paralysis is less marked in the case of bilateral muscles that habitually act together than in the case of those which ordinarily act alone. Thus the muscles of respiration and the muscles of the trunk in 
general are, although perhaps weakened, never completely paralyzed. This is an indication that each member of such functional pairs of muscles is innervated from both hemispheres; and this physiological deduction is supported by the anatomical fact already referred to, that after removal of the 'motor' cortex, or injury to the pyramidal tracts in the internal capsule or crus, some degenerated fibres (homolateial fibres) are found in the crossed pyramidal tract on the side of the lesion (p. 875).

In the dog after a time the paralysis may more or less completely disappear. In the monkey restoration is less complete.

Some interesting observations have been made on a monkey, which was carefully watched for eleven years after the removal by two operations of the cortex of the greater portion of the frontal and parietal lobes on the left side. The character of the animal: which had been studied for months before the operations, was entirely unaffected. All its traits remained unaltered. There was no loss of memory or intelligence. On the other hand, disturbances of movement on the right side were very noticeable up till its death. It learned again to use the right limbs in locomotion; but, although they were not markedly weaker than those of the left side, their movements had a certain clumsiness, which was associated with a permanent diminution in the sensibility of the skin of these limbs. Muscular sensibility was also lessened. In acts requiring the use only of one hand, the right was never willingly employed, and it evidently cost the animal a great effort to use it in such movements, but by special training it learnt again to give the right hand when asked for it, and to make use of it for other purposes. The movements with which the 'motor' areas are concerned are essentially skilled movements, and we may suppose that it is more difficult for a monkey to educate again a centre for such complex and elaborate manœuvres as are performed by its hand than for a dog to regain normal control of the comparatively simple movements of its paw. In man in cases of hemiplegia, when the patient lives for some time, a certain amount of recovery usually takes place, especially in young persons, in the paralyzed leg, but much less in the paralyzed arm.

In the lower monkeys the 'motor' area was formerly stated to extend behind the sulcus centralis into what in man would be called the ascending parietal convolution (post-central gyrus), and also to be more extensively represented on the mesial surface of the hemisphere than in the higher apes. Such observations, however, require to be reinterpreted in view of the results of Sherrington and Grünbaum, especially as they were carried out by the bipolar method of stimulation, with both electrodes on the cortex. This method does not admit of such strict localization of the stimulus as the unipolat method. The most recent work with the unipolar method has 
indicated that in the lower apes also excitation of the gyrus postcentralis does not cause movements (C. and O. Vogt).

It is in the light of the results obtained in monkeys, and by the aid of histological, embryological, clinical, and pathological observations, that the 'motor' areas in man have to a great extent becn mapped out.

The histological differentiation of the various cortical regions recently demonstrated by Brodmann and by Campbell are of especial interest (Figs. $3^{8} 7-39 \mathrm{I}$ ). It has long been customary to divide the cortex into layers, although the number and the boundaries of these layers are somewhat arbitrarily fixed. Brodmann distinguishes six layers: (I) A zonal or peripheral layer, containing many nerve-fibres and neuroglia cells, but few nerve-cells; (2) a layer containing 'granules' ancl small pyramidal cells (external gramular layer); (3) a layer of medium and large pyramidal cells (pyramidal layer); (4) a layer of small irregular

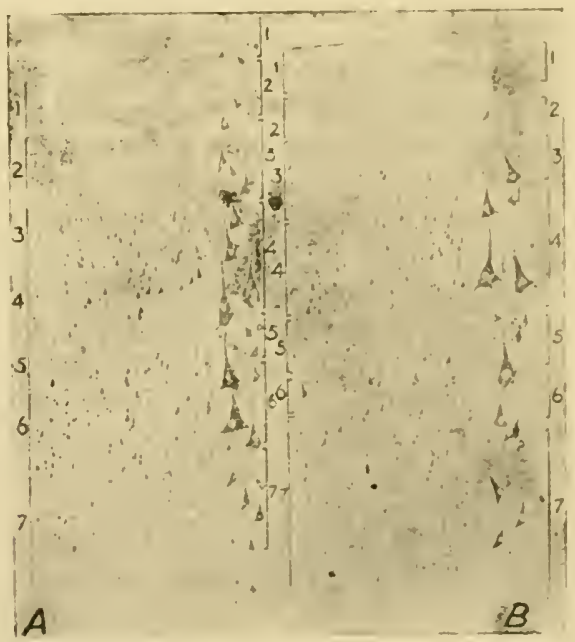

Iig. 387.-Cell-Lamination of Gyrus Postcentralis (Campbell). $A$, just behind upper end of fissure of Rolando; $B$, from the posterior edge of the gyrus (intermediate postcentral area of Campbell).

cclls (internal granular or stellate layer); (5)-a 'ganglionic' layer, containing the largest pyranidal cells (deep large py ramids); (6) a layer (lamina mulliformis) of spindle-shaped or polymorphous cells. These layers vary in their structural details, and especially in their relative development in animals of different rank in the mammalian scale, in one and the same animal at dlifferent periods in its embryonic and extra-uterine growth, and also in different parts of the cortex in an adult animal of given species. The, region in front of the central sulcus (fissure of Rolando), e.g., i:; characterized by the presence of the giant pyramids of Betz, which give origin to the pyramidal fibres going to the trunk and limbs (Fig. 388). Nore than forty years ago 
Betz, when first describing these large pyramidal cells, suggested that they were related tosome motor function, and this view was a few years later more clearly developed by Lewis, the real pionecr in cortical localization by the histological method. He mapped out the motor area in man by this method, and lis results differ surprisingly little from those of Campbell obtained by a much more claborate technique. He pointed out that arcas containing the giant pyramicls corresponded with a number of the areas defined as motor areas by the experimental physiologists by means of electrical excitation. Definitc proof of the connection of the Betz cells with the pyramidal fibres was not obtainel till much later. Some of the facts which have cstablished such a connection are alluded to on a previous page (1). 875). Other characters than the size, shape and distribution of the nerve cells

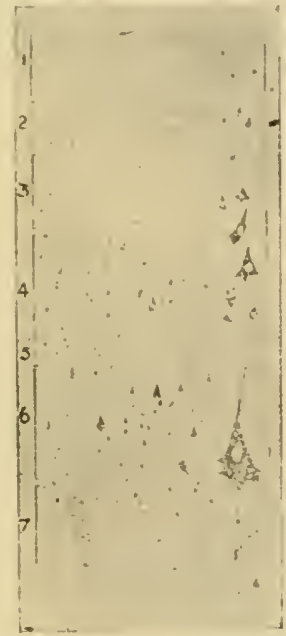

Fig. $3 \$ S$ - Cell-Lamination of Gyrus Precentralis (Campbill). From the nortion of the gyrus immediately in front of the central sulcus (Campbell's precentral area in Figs. 390, 39I).

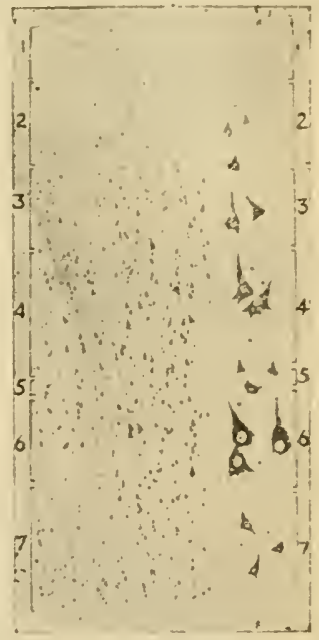

Fig. 389.-Cell-Lamination of Gyrus Precentralis (Campbell). From anterior part of the gyrus (Campbell's intermediate precentral area in Figs. 390,39 I).

have also proved of value in differentiating histologically the various regions of the cortex-for cxample, the differences in the diameter and the number of the medullated nerve fibres which run vertically into and out of the grey matter. Although the method has yielded valuable and suggestive results, it would be misleading to state that the agreement with the experimental and pathological findings is anything more than a general one. Discrepancies in detail are not lacking, and this might be expected. Histological differences, after all, arc only rough critcria for the differentiation of function.

Although the results are less definite, the work of Flechsig on the time of development of the medullary sheath of the fibres in the various cerebral convolutions has also contributed to our knowledge of localiza- 


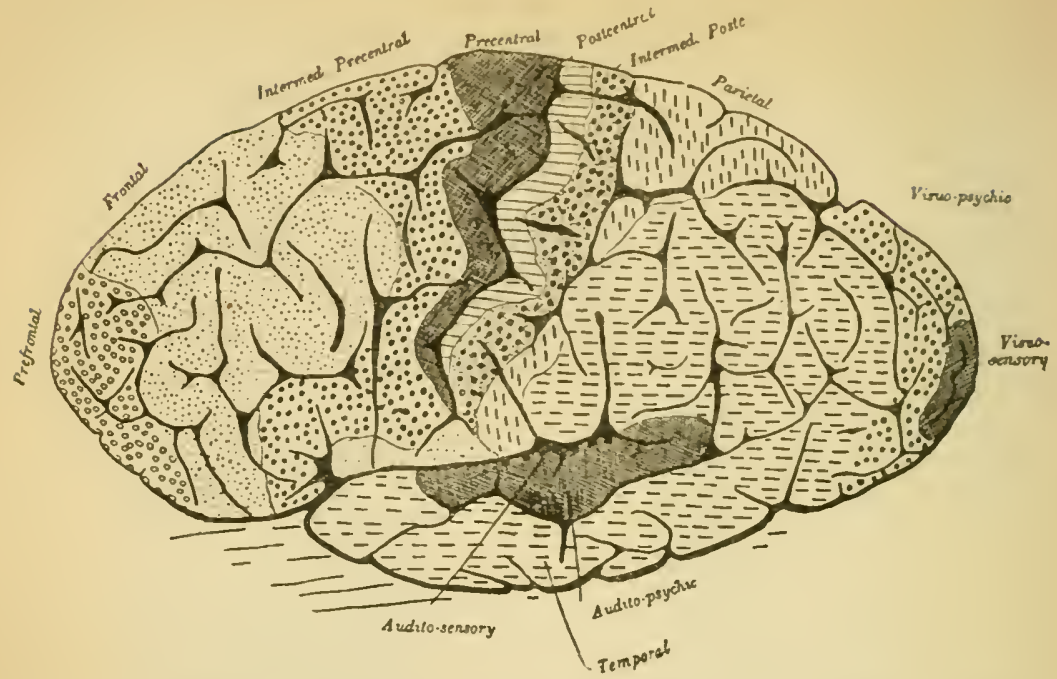

Fig. 390.-Structurally Differentiated Cortical Areas (Campbell). External surface of hemisphere (human brain).

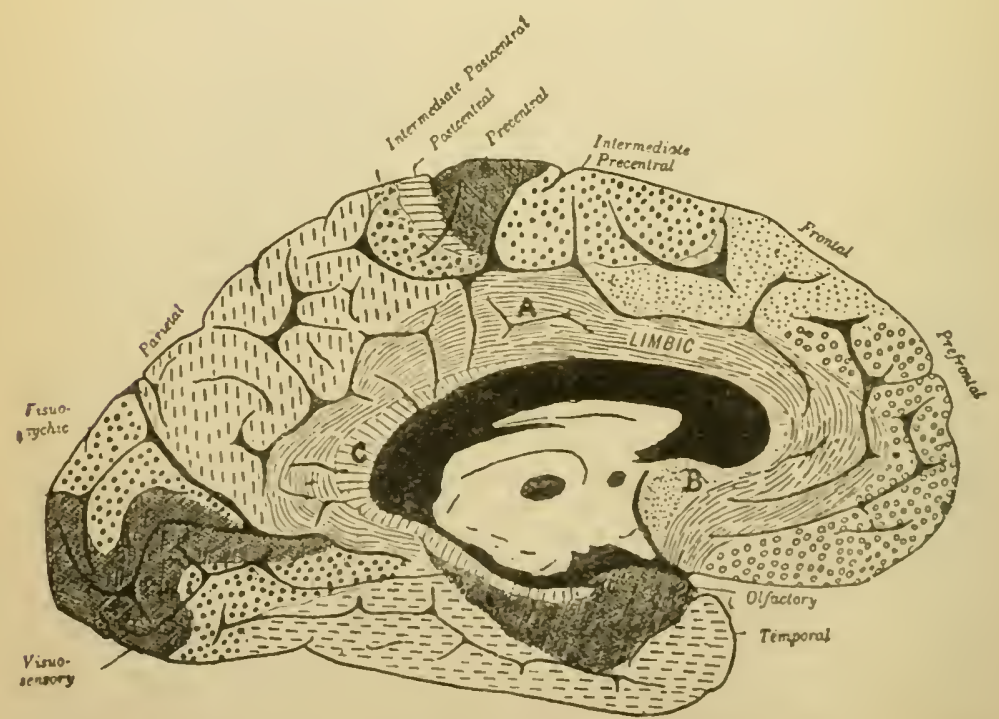

Fig. 391.-Structurally Differentiated Cortical Areas (- 2502boil; Mesial surface of hemisphexe (humar, brain). 
tion in the cortex. In the development of a neuron four stages can bo listinguished: (1) Cells without processes; (2) the appearance of processes, first the axon and then the dendrites; (3) the formation of col-

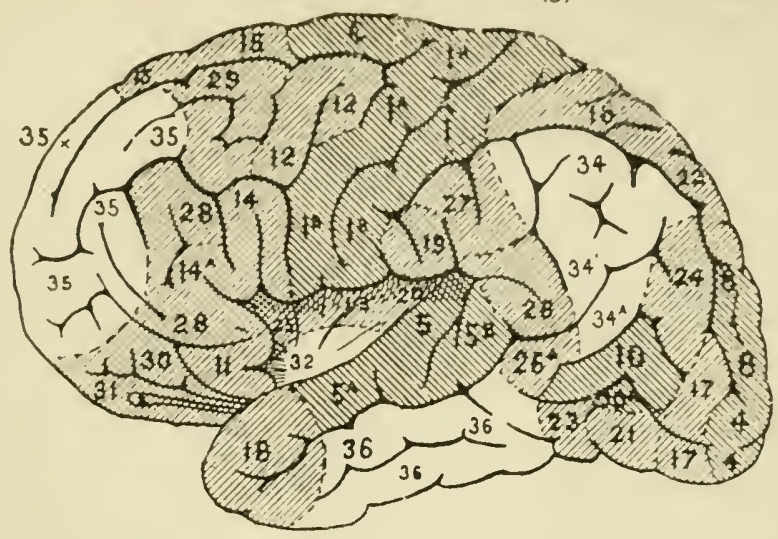

Fig. 392.-Flechsig's Developmental Zones (after Flechsig). Outer surface of human cerebral hemisphere. Primary zones (I-IO), darkly shaded; intermediate zones (II-3I), less deeply shaded; terminal zones $(32-36)$, unshaded.

laterals; (4) myelination or the formation of the medullary sheath (Fig. 333, p. 854).

Myclination occurs in the cerebral convolutions in a regular order. In some areas the fibres mzy be medullated three months before birth,

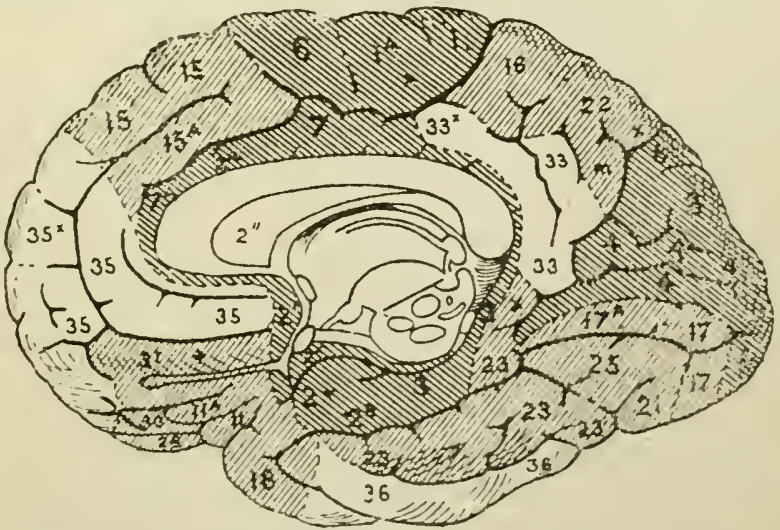

Fig. 393.-Flechsig's Developmental Zones (after Flechsig). Inner surface of human cerebral hemisphere.

in others not till six months later. For instance, the Rolandic and olfactory regions, the calcarine purtion of the occipital lobe associated with vision, and the portion of the temporal lobe associated with hearing, are plentifully provided with medullated fibres a short time after birth, at any rate before the first month, whereas the remaining regions of the cortex are completely, or almost completely, free from 
such fibros. In this way Flechsig has distinguished thirty-six cortical fields (Figs. 392, 393), which he divides accurding to the time of inyelination into three groups:

I. Primary fields, ten in number, which are well provided with myelinated fibres at birth. They include the cortical centres for the various sensations and also the 'motor' area. They are connected especially with the so-called projection fibres. Thus, the cutaneous and muscular sense is assumed to be represented in field $\mathbf{I}$, the sense of smell in field 2 , of vision in 4 , and of hearing in 5 . From field 1 arise the fibres of the pyramidal tract, chicfly from the ascending frontal convolution, while the sensory fibres from the skin and muscles end mainly in the ascending parietal. This is an illustration of what Flechsig considers a general rule for these primary fields-viz., that each primordial sensory region is connected both with an afferent (cortici-petal) and with an efferent (cortici-fugal) tract. From the visual area (4), e.g., arises a tract which proceeds mainly to the anterior corpus quadrigeminum.

2. Terminal fields (32 to 36 in the figures) which become myelinated late, the process not beginning until at least a month after birth.

3. Intermediate fields (I I to $3 \mathrm{I}$ ) which become myelinated earlier than the terminal, but later than the primary. They and the terminal fields constitute par excellence association centres, which furnish fibres (association fibres) connecting the centres represented in the primary ficlds-e.g., such fibres as must be continually conveying impressions from the visual centre to the 'motor' cortex when the hand is sketching a landscape. It may also be considered a function of these association centres to store up the memories of previous sense impressions. Flechsig divides the association centres represented in the terminal fields into-(I) The great anterior association centre in the frontal lobe in front of the 'motor' area; (2) the great posterior association centre in the parieto-temporal region; (3) the smaller middle or insular association centre, which coincides with the island of Reil, an area which, according to Sherrington and Crünbaum, is totally 'inexcitable' as regards the production of movement in the anthropoid apes. These association centres are foci, from which issue and to which come the long association paths. The reader must bear in mind that Flcchsig's conclusions as to the functions of his very numerous areas are in mary cases hypothetical, and can only be accepted when corroborated by other methods. We are far from being able at present to subdivide the functions of the cortex so minutely as is suggested by his map.

Clinical and Pathological Observations in man agree, upon the whole, with wonderful precision with the results of experiments on animals; and, indeed, before any experimental proof of the minute and elaborate subdivision of the cortex had been obtained, Broca had already, from the phenomena of the sick-bed and the postmortem room, located a centre for speech in the left inferior frontal convolution (but see p. 97 I), and Hughlings Jackson had associated pathological lesions of the Rolandic area with certain cases of epileptiform convulsions.

An extensive hæmorrhage involving the Rolandic area of the cerebral cortex, or an embolus blocking the middle cerebral artery; causes paralysis of the opposite side of the body. An embolus of a branch of the middle cerebral artery causes paralysis of the muscles, 
or rather movements, represented in the area supplied by it. A tumour causes symptoms of irritation, motor or sensory-convulsions beginning in, or sensations referred to, the parts represented in the regions on which it presses. In connection with the localization of lesions in the 'motor' area of the cortex, and operative interference for their cure, the cortex has been frequently stimulated in man. There is no doubt that the 'motor' region corresponds closely in position to that of the higher apes. It does not include the postcentral gyrus, for stimulation of this convolution with such strengths of current as are permissible evokes no movements, while movements are readily elicited from the precentral gyrus (Horsley, etc.). In exposing the 'motor ' region, or any particular part of it, the exact position of the fissure of Rolando becomes important; and Thane has given the following simple method for fixing it: The point midway between the point of the nose and the occipital protuberance is fixed by measuring the distance with a tape. The upper end of the fissure of Rolando lies half an inch bebind this middle point. The fissure makes an angle of $67^{\circ}$ with the longitudinal fissure (Fig. 3S6). The minor fissures are so inconstant as to afford no safe guidance in the localization of a given area. This must be delimited by stimulation.

Sensory Functions of the Rolandic Area.--There are many proofs that the 'motor' region is not a purely motor, but a sensori-motor, or kinesthetic, area. Histological and embryological studies on the course of the sensory paths, as already pointed out, support this conclusion. It has also been mentioned that, according to Goltz's observations (p. 948), removal of the Rolandic cortex causes defects of sensation as well as of movement. In man, in connection with operations on the brain, still better evidence has been obtained. In two cases Cushing was able to elicit tactile sensations by electrical stimulation of the gyrus postcentralis (ascending parietal convolution), and the sense of muscular movement by electrical stimulation of the gyrus precentralis. In a very careful study of a case in which he removed the upper limb area of the right hemisphere in a boy for violent convulsive movements of the whole of the left arm, Horsley came to the conclusion that the precentral gyrus in man is the seat of representation of (I) slight tactile sensation (after the operation appreciation of the lightest tactile stimuli was lost); (2) topognosis-i.e., appreciation of the localization in space of the point touched; (3) muscular sense; (4) stereognosis, or the power of recognizing the form of objects touched and handled; (5) paine.g., that caused by a pin-prick; (6) volitional movement. The postcentral gyrus in man appears to be the seat of a similar sensory representation, but as its relation to the efferent impulses concerned in volitional movements is less decided than that of the precentral gyrus, so its relation to afferent impulses, both from the skin and the 
deeper structures, is better marked. From the field of experiment further evidence of the sensori-motor nature of the 'motor' region is forthcoming.

(I) It has been found that if the posterior roots of the nerves supplying one of the limbs be cut in a monkey, all the most delicate and skilled movements of the limb are either greatly impaired or totally abolished (Mott and Sherrington). The limb is not used for progression or for climbing, but hangs limp, and apparently helpless, by the side of the animal. That this condition is not due to any loss of functional power by the peripheral portion of the motor path may be assumed, since the anterior roots remain intact. That it is not due to any want of capacity on the part of the 'motor' centres to discharge impulses when stimulated may be shown by exciting the cortical area of the limb-either electrically or by inducing epileptic convulsions by intravenous injection of absinthe -when movements of the affected limb take place just as readily as movements of the sound limb. The cause of the impairment of voluntary motion, then, can only be the loss of the afferent impulses which normally pass up to the brain, and presumably to the ' motor cortex. When only one sensory nerve-root is cut, no defect of movement can be seen; and this is evidently in accordance with the fact previously mentioned (p. 89r), that complete anæsthesia of even the smallest patch of skin is never caused by section of a single posterior root. And that it is the loss of impulses from the skin which plays the chief part is shown by the fact that after division of the posterior roots supplying the muscles of the hand or foot, which only partially interferes with the sensory supply of the skin, joints, sheaths of tendons, etc., movement is unimpaired; while section of the nerveroots supplying the skin, those of the muscles being left intact, causes extreme loss of motor power.

(2) If a strength of stimulus be sought which will just fail to cause contraction of the muscular group related to a given motor area, and a sensory nerve, or, better, a sensory surface (best of all, the skin over the corresponding muscles), be now stimulated, contraction may occur-that is to say, the excitability of the motor centres may be increased. This shows that the 'motor' region is en rapport not only with efferent, but also with afferent fibres, that it receives impulses as well as discharges them.

The same experiment is a proof that the results of excitation of the motor cortex are due to stimulation of the grey matter, and not, as might be objected. of the white fibres of the corona radiata. It is undoubterly possible to excitc these fibres by electrodes directly applied to the motor cortex, but in the lattcr case the current has to be made shonger than is sufficient to excite the grey matter alone. Further evidence is afforded by the following facts: (a) The "period of deldy '-that is, the period which elapses between stimulation and contraction-is greater by nearly $5^{\circ}$ per cent. when the cortex is stimu- 
lated than when the white hbres are directly excited. (b) Morphine greatly increases the period of delay for stimluation of the cortex, and at the same time renders the resulting contracticns more prolonged than normal, while the results of direct stimulation or the white fibres are much less, if at all, affected. (c) Stimulation of the grey matter, when separated from the subjacent white matter by the knife, but left in position, is without effect unless the strength of stimulus be increased, although twigs of the current ought, of course, to pass into the corona radiata as easily as before. Perfectly definite movements can, lowever, be excited or inhibited by stimulating definite spots in the corona radiata, and even in the internal capsule. This simply means that in these positions the fibres representing these movements are not yet intermingled with fibres representing other movements.

\section{Sensory Areas-VisualCen-} tres.-In the occpital lobe in animals an area of considerable extent has been found, destruction of which causes hemianopia (p. 923). Thus, if the right occipital cortex is destroyed, the right halves of the two retinæ are paralyzed, and the left half of the field of vision is a blank. There is conjugate deviation of the head and eyes to the same side as the lesion-in other words, the animal turns its head and eyes to the right. Destruction of this region on both sides causes complete blindness. When the same region is stimulated, the eyes and head are turned to the left-that is, there is conjugate deviation to the opposite

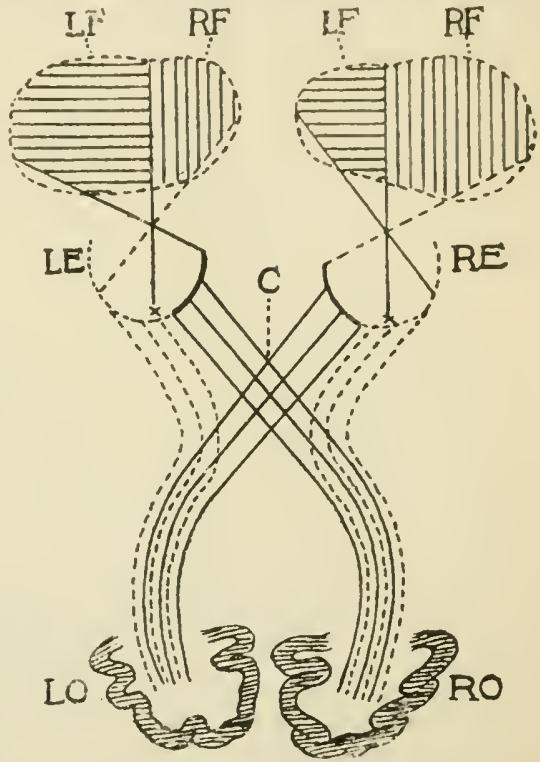

Fig. 394.-Diagram of Relations of Occipital Cortex to the Retinæ. RO, LO, right and left occipital cortex; RE, LE, right and left retina; C, optic chiasma; RF, LF, right and left visual fields. The continuous lines passing back from the retinæ to the occipital cortex represent the crossed, the broken lines the uncrossed, fibres of the optic nerves and tracts. For the sake of simplicity the intermediate stations on the visual path in the anterior corpora quadri. gemina, lateral geniculate bodies, and pulvinar are not represented in the diagram. For these connections, see Fig. 370, p. 923.

side. In the higher monkeys the eye movements can be elicited only from the extreme posterior apex of the occipital lobe and from its calcarine region, and then not easily. The movements differ from those produced by stimulation of the area for eye movements in the frontal lobe. They are not so certain, their latent period is longer, and a stronger stimulus is required to evoke them. It cannot be 
doubted that the occipital region is concerned in vision, and it is a very natural suggestion that the movements are the result of visual sensations in the excited occipital cortex. The right occipital lobe is concerned with vision in the right halves of the two retinx (Figs. 370 and 3 44). Now, under normal conditions, a visual image would be cast on the two right retinal halves by an object placed towards the left of the field. The movements of the head and eyes to the left may therefore be plausibly explained as an attempt to look at, and a rotation towards, the supposed object.

The pathological evidence is very clear that disease of the occipital lobe, especially of the cuneus, a triangular area on its mesial surface, causes liemianopia in man. A limited lesion may cven be associated with an incomplete hemianopia, and cases have been recorded in which colour hemianopia (blindness of the corresponding halves of the two retinæ for coloured objects) co-existcd with normal vision for white light. The precise limits of the occipital visual area are still disputed. It probably occupies, in addition to the cuncus, the lingual lobule and a portion of the external aspect of the occipital lobe. The question of the projection of the retina upon the visual cortex-i.e., the question whether each retinal area is represented in a definite cortical areahas given rise to much debate. The representation of the fovea centralis, the area of most distinct vision, has aroused especial interest. It has been asserted that a circumscribed area in the region of the calcarine fissure is the centre for the fovea (Henschen). But it is totally opposed to this view that extensive lesions of the occipital cortex, even on both sides, do not, except in rare cases, cause total blindness in the foveal region, although peripheral vision is destroyed. On the other hand, in no case has a purely cortical lesion been found associated with blindness confined to the fovea (Monakow). The fibres of the optic radiation which are on the path from the fovea are accordingly distributed diffusely to the visual cortex. Sometimes dimness of vision in the whole of the opposite eye (crossed amblyopia), and not hemianopia, is caused by a lesion of the occipital cortex. It seems impossible to explain this and other facts without postulating the existence of more than one visual centre; and it has been supposed that in the angular gyrus and the neighbouring region a higher visual centre exists which is connected with the lower occipital centres for the two halves of the opposite eye. Thus, the right angular gyrus would be in connection witl the part of the right occipital cortex which has to do with vision in the nasal half of the left eye, and with the part of the left occipital cortex which has to do with vision in the temporal half of that cye. This higher centre, which perhaps functions as a storehouse of visual memories, probably corresponds to the structurally differentiated area (visuo-psychic area of Campbell), as the lower centre corresponds to his structurally differentiated visuo-sensory area (Figs. 39r, 392).

Auditory Centre.-On the outer surface of the temporo-sphenoidal lobe, mainly in the first temporal convolution, lies an area associated with the sense of liearing. Stimulation in the region of the first temporal convolution may cause the animal to prick up its ears on the opposite side. Destruction of this area on both sides is followed by complete and irremediable loss of hearing. If it is destroyed only on one side, there is partial deafness of the opposite 
ear, and also to some extent of the ear on the same side. This is graduaHy recovered from. If it is destroyed on the left side there is also the peculiar condition called 'word-deafness,' which will be referred to directly (p. 972). In deaf-mutes the first temporal convolution may be atrophied. There is evidence that the posterior corpora quadrigemina and the mesial geniculate body form an inferior relay on the route between the fibres of the auditory nerve and the temporal cortex. There are indications that within the auditory area so-called 'musical centres ' exist-that is, an orderly
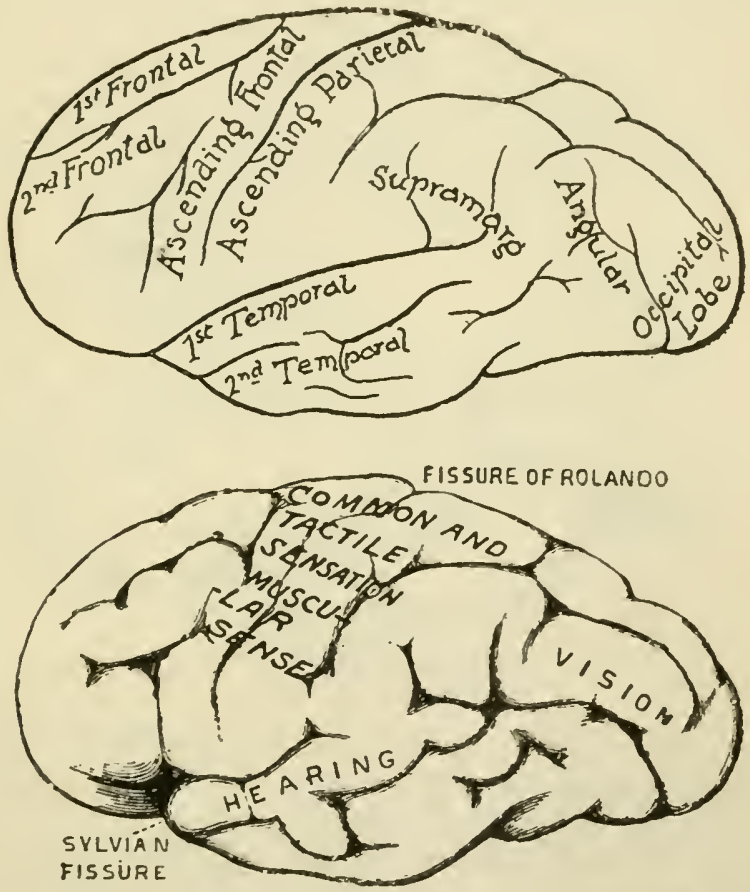

Fig. 395.-Lateral View of Left Hemisphere with Sensory Areas: Man. The front of the brain is towards the left.

arrangement of the cell-bodies of the neurons that have to do with the perception of pitch, so that a limited lesion may cause deafness to notes of a particular pitch when it is situated on one part of the area, and deafness to notes of a different pitch when it is situated elsewhere (Larionow).

Centre for Smell.-As to the position of the centre for smell, direct experiment on animals cannot teach us much, for if the outward tokens of visual and auditory sensations are dubious and fuctuating, still more is this the case with the signs of sensations of smell. A 
further source of fallacy is the fact that other sensations than those of smell are caused by stimulation of the mucous membrane of the nose. Substances like anmonia, for example, affect entirely the endings of the trigeminus, which is the nerve of common sensation for the nostrils. Pathological and clinical evidence would be of great value, but it is as yet scanty, and of itself indecisive. Some cases of epilepsy have been reported in which the attack was heralded by smells for which there was no objective cause. At necropsy the uncinate gyrus was found diseased. So far as it goes, such evidence supports the view derived from the anatomical connections of the olfactory tracts, that the centre for smell is situated in the uncinate gyrus on the mesial aspect of the temporal lobe, for the olfactory track may be traced into this region. In animals with a very acute sense of smell, this gyrus is magnified into a veritable lobe, called from its shape the pyriform lobe; from its supposed function, the

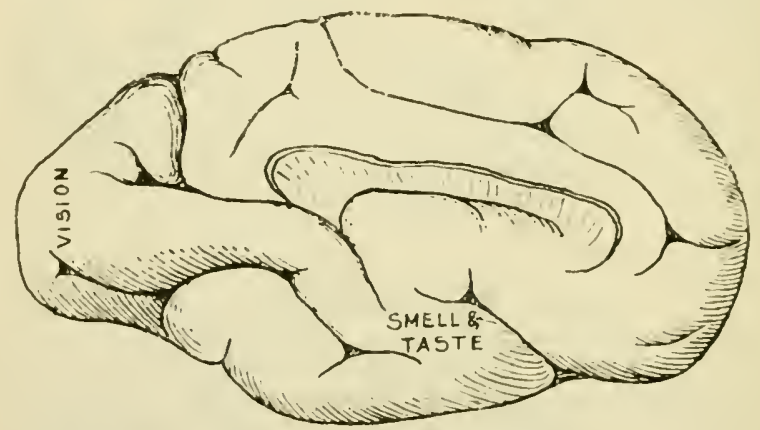

Fig. 396.-Sensory Areas of Mesial Surface of Human Brain. The front of the brain is towards the right.

rhinencephalon. The centre for taste is supposed to be situated in the same region as the centre for smell (in the hippocampal convolution posterior to the uncinate gyrus).

Ordinary and Tactile Sensations, including the muscular sense, have been located in the Rolandic area (p. 963); and there are good grounds for believing that afferent fibres from the joints, the muscles and their accessory structures and the skin terminate here in arborizations which come into contact either with the motor pyramidal cells, or with intermediate cells which link them to the pysamidal cells.

Our knowledge of the localization of this gromp of sensations is still musatisfactory, largely because it has litherto been necessary to rely almost exclusively npon extirpation experiments, and it is difficult to determine in animals the existence uf a local defect of sensation due to a limited cortical lesion. A promising new method, the application of strychnine to limited regions of the cortex, secms likely to aid in illuminating this lark corner of cerebral physiology (de Barenne). changes in cutaneous and deep sensibility of the fore-limbs of the cat 
are produced-c.g., by applying strychuine to at zone including the gyrus sigmoideus anterior, the frontal part of the gyrus sigmoideus posterior, the fromtal part of the gyrus suprasylyins and the midelle thirl of the gyrus ectusylvius anterior. The zone for the head almost completely overlaps the fore-limb zone. The zone for the hind-limb occupies the frontal half of the gyrus marginalis.

As regards the cutancous sensibility lecalized in these zones, both sides of the body secm to be represented in the cortex of one hemisphere, but the opposite side the most. The disturbances of deep
sensibility caused by the application of strychnine to the cortex of onc hemisphere are confined to the bones, tendons, and muscles of the opposite side of the body. The remainder of the region of the cortex

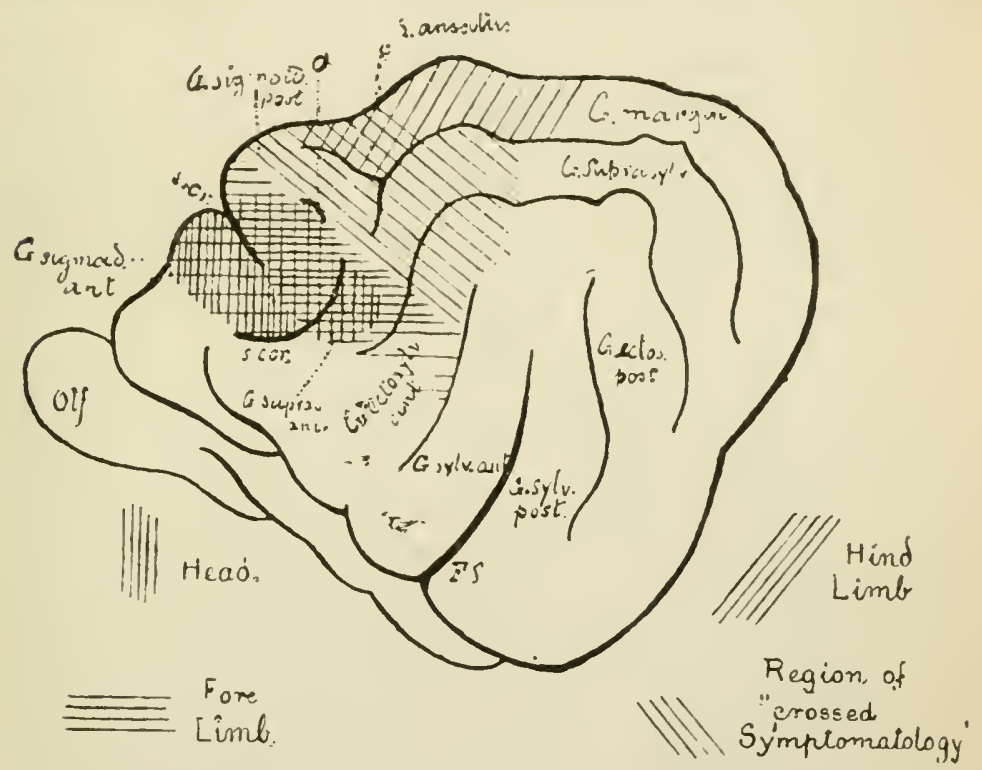

Fig. 397.-Zones for Cutaneous and Deep Sensibility on the Convexity of the Left

Cerebral Hemisphere of the Cat (Dusser de Bareme). The chief gyri and sulci are indicated. s.cr., Crucial sulcus; F.S., fissure of Sylvius; $\alpha$, the 'compensatory ansate fissure' of Campbell.

which responds to strychnine by sensory changes is called by de Barenne, the zone of 'crossed symptomatology,' because the altered or increased sensitivity of the skin is manifested in the fore-limb of the same side, and the hind-limb of the opposite side. He suggests that the sensory mechanism in this area may be related to the movements of progression. In so far as these sensory zones overlap the motor area a common sensori-motor cortical zone may be said to exist.

Aphasia. - Worts are, at bottom, arbitrary signs by which certain ideas are expresied. The power of intelligent communication by spoken or written language may be lost: (1) by paralysis of the muscles of articulation or the muscles which guide the pen: (2) by inability to hear or see the spoken or written word-i.e., by deafness or blindness; (3) by inability to comprehend the meaning of spoken or written language, although sensations of hearing and sight may not be abolished 
-that is to say, by inability to interprel the auditory or visual symbols by which idcas are conveyed; (4) by inability to clothe ideas in words, although the words may be present in the patient's consciousness, and the ideas conveyed by specch or writing may be comprehended. Neither (I) nor (2) is considered to constitute the condition of aphasia: (3) represents what is called amnesia, or sensory aphasia : (4) is aphasia in the ordinary restricted sense, or motor aphasia.

Motor aphasia may be divided into two varieties-subcortical or pure motor aphasia, and cortical, or Broca's aphasia. In tice subcortical type the patient understands speech and writing perfectly, and is able to write normally; but he cannot speak spontaneously or read aloud, or repeat words when requested to do so. He may know quite well wliat to reply in answer to a question, but the worls necessary to cxpress his meaning do not come to him. In Broca's type of aphasia, which is the most common, the patient may understand spoken and written words-often imperfectly, it is true-but he is unable to speak spontancously, to repeat words spoken to him, and to read aloud. Unlike the person suffering from the subcortical type of motor aphasia, he has difficulty in reading by the eye without articulation, and in writing spontaneously or to dictation. There is often or always some intellectual deficiency. The gradations in the loss of the expressive factor in speech may be infinite. A patient may sometimes sing a song without a single slip in words or measure, and yet be unable to speak or write it. In a case recorded by Larionow an aphasic could speak only one syllable, 'tan,' but could sing the 'Marseillaise.' In certain cases the change is confined to loss of the power of spontaneous speech, and the patient may be able to read intelligently. Sometimes he can express his ideas in speech, but not in writing (agraphia). Sometimes the loss is restricted to certain sets of ideas. For example, a boy was injured by falling on his head. Typical symptoms of motor aphasia developed, but the power of dealing with ideas of number was not interfered with, and the boy continued to learn arithmetic as if nothing had happened. Proper names and nouns are more easily lost than adjectives and verbs. Motor aphasia is generally accompanied by paralysis, frequently transient, of voluntary movement on the right side, sometimes amounting to complete hemiplegia, but more often involving the right arm alone. This association is generally explained by the proximity of the inferior frontal convolution to the motor area of the arm, and their common blood-supply. It has already been stated that since Broca it has been generally assumed that in most persons the inferior frontal convolution on the left side is concerned in the expression of ideas in spoken or written language. It is even said that oratorical powers have been found associated with marked development of this convolution (as in the case of Gambetta, the French statesman). It is the cortical or Broca's type of motor aphasia which has been supposed to be associated with a lesion in the left inferior frontal convolution. The portion of the convolution concerned is the posterior extremity, where it borders on the fissure of Sylvius, and it either completely coincides with or largely overlaps the centre for the movements of the tongue, lips, and larynx concerned in articulation. The failure, however, does not lie in the articulatory mechanism. The patient uses the same muscles of articulation, without any marked impairment of function, for chewing and swallowing his food. It is only when the corresponding area in the right inferior frontal convolution, or the path from it to the internal capsule, is also destroyed, that articulation is greatly and permanently interfered with.

The question obviously presents itself why it is that motor aplasia is 
commonly due to a lesion in the left hemisphere alone. The answer to this question is supposed to be partly supplied by the important and curious observation that in left-lianded individuals damage to the right inferior frontal convolution may cause aphasia. In the right-handed man the motor areas of the left hemisplicre may be supposed to be more highly educated than those of the right hemisphere. The movements of the right side which they initiate or control are stronger and more delicate and precise than those of the left side. It is only necessary to assume that this processs of specialization, of selective training, has been carried on to a still greater extent in the left frontal convolution, that in most men the speech-centre there has taken upon itself the whole, or the greater part, of the labour of clothing ideas in words, leaving to the right centre only its primitive but undeveloped powers. In lefthanded persons the speech-centre on the right side may be supposed to share in the general functional development of the right hemispherc. That great capabilities are lying dormant in the right speech-centre of the ordinary right-lianded individual is indicated by the fact that after complete destruction of the left inferior frontal convolution the power of speech may be to a considerable extent, though slowly and laboriously regained; and it is said that this second accumulation may be swept away, and without remedy, by a second lesion in the right inferior frontal convolution. But frail is the tenure of life in a person who has twice suffered from such a lesion; and we do not know whether recovery might not take place to some extent even after destruction of both inferior frontal convolutions, if the patient only lived long enough.

Recently Marie lias reopened the whole question of the relation of aphasia to lesions of the inferior frontal convolution. He believes that the so-called Broca's area has nothing to do with aphasia in the proper sense of the term-i.e., it is not a cortical area concerned in 'internal' speech processes, or in which motor or kinæsthetic 'speech memories' are stored-but simply a 'motor' area for the movements of articulation. He maintains that there is but one form of true aphasia-the aphasia of Wernicke-which has for its basis a lesion of the so-called zone of Wernicke (the supramarginal and angular gyri, and the posterior portions of the first and second temporal convolutions). This, according to him, is the true speech-centre. The symptom-complex known as Broca's a phasia, which everybody admits to exist as a distinctly characterized clinical condition, is due, he says, to a double lesion. One lesion causes aphemia (loss of the power of co-ordinating the movements needed in the articulation of words without actual paralysis of the muscles), and the other the disturbance of internal speech, and the difficulty of reading and of writing, which constitute the true aphasia. According to Marie, the lesion which causes the aphemia is not ever situated in Broca's convolution, but somewhere in a rather badly defined region, which he denominates the lenticular zone, since it includes the lenticular as well as the caudate nucleus, in addition to the external and internal capsules and the cortex of the island of Reil. It would be out of place to enter more minutely here upon such controversial matters. The conclusion which emerges most definitely from the discussion is that Broca's localization was based upon a very narrow foundation, and must probably be modified.

It is generally recognized that in almost all cases of aphasia in which the brain has been studied after death, some lesion of association fibres has been present, and not merely a cortical lesion. Interference with the association fibres causes confusion in the processes of association which are so important in mental activity, and defects of intelligence are therefore commonly observed in aphasia. 
A so-called temporary aphasia may occur without any structural change in the speech-centre-for exampic, during an attack of migraine. In children it nay even be caused by some comparatively slight irritation in the digestive tract, such as that due to the presence of a tapeworm.

In the anthropoid apes no evidence of the existence of any ' speechcentre,' even distantly foreshadowing the human, has been obtained by stimulating the inferior frontal convolution on either side. No movements, and particularly no movements connected with vocalization, are elicited.

Sensory Aphasia.-In typical motor aphasia spoken and written words convey to the patient their ordinary mcaning. They call up in his mind tlie usual sequence of ideas, but the chain is broken at the speech-centre, and the outgoing ideas cannot be clothed in words. The expressive factor in speech is deranged. In sensory aphasia the perceptive factor in speech is deranged. In ordinary sensory aphasia (Wernicke's, or cortical sensory aphasia) the patient cannot understand spoken or written language, but, far from being unable to speak, he often babbles incessantly. He may string together a scries of words, each correctly articulated, but having no meaning, or may utter a jargon not composed of known words at all. Instead of the words which he lesires to use to express his meaning, he may use others having a similar sound (paraphasia). Damage to two regions of the brain has been found associated with this condition: ( $I$ ) the middle part of the first and second temporal convolutions, (2) inferior parietal convolutions and the angular gyrus in the neighbourhood of the occipital visual centre. When the temporal region is alone affected, it is the spoken word that is missed, the written that is understood (word-deafness). When, as occasionally happens, the lesion is confined to the occipital region, spoken language is perfectly understood, written language not at all (word-blindness). It is the left hemisphere which is affected in right-handed persons, the right hemisplere in left-handed persons. Sensory, like motor aphasia, may exist in any degree of completeness, from absolute word-deafness or word-blindness, in wlich no spoken or printed word calls up any mental image, to a condition not amounting to much more than a marked absence of mind or unusual obtuseness. Motor and sensory aphasia may be present together. In well-marked cortical word-deafness speech is always interfered with to some extent. In so-called pure word-deafness (subcortical sensory a phasia) the paticnt may be perfectly capable of rational specch. He may talk to himself or on a set topic with fluency and sense, may write intelligently, and understand what he reads; but he may be mable to understand a single word spoken to him, or to repeat words when asked to do so.

Cortical Epilepsy.-Disturbed action of the motor centres may take the form either of depression or of increased excitability. The former will be associated with partial or complete paralysis of the movements represented in the area, the latter by abnormally intense or prolonged discharge leading to the condition called cortical epilepsy-that is, epileptic attacks associated with cortical lesions. Among these are the cases of so-called Jacksonian cpilepsy-a condition claracterized by the fact that the scizure does not begin by general, but by local, convulsions. They may remain confined to a single limb, or to one side of the face, or to one side of the body. So long as the convulsions are not general, consciousness need not be lost. Or a seizure beginning as Jacksonian inay spread so as to involve the whole body, in whicli case the symptoms become identical with those of ordinary epilepsy, including the loss of consciousness. It has been found possible in some cases to localize the 
position of the lesion from the part of the body in which the fit, or the aura (the sensation or group of sensations peculiar to each case, which precedes and announces the attack) begins. For example, if the convulsions commence with a twitching of the right thumb and extend over the arm, or if the aura consists of sensations beginning in the thumb, there is a strong presumption that the seat of the lesion is the part of the arm-area known as the 'thumb-centre' in the left cercbral hemisphere. It is the seat of the convulsion at its commencement, not the regions to which it may afterwards spread, that is important in diagnosing the position of the lesion. For just as strong or long-continucd electrical stimulation of a given 'centre' of the 'motor' cortex may give rise to contractions of muscles associated with other 'centres,' so the excitation set up by localized disease may spread far and wide from its original focus, involving area after area of the 'motor' region first in the one hemisphere and then in the other. The part of the body to which a sensory aura is referred is as significant an indication of the seat of the discharging lesion as is the part of the body which first begins to twitch. This is one of the proofs that the 'motor' region is not a purely motor area. Disturbed action of the sensory areas on the cortex may, as in the case of the motor regions, take the form either of deficiency or of excitation. Excitation expresses itself by hallucinations, the person having the impression of a sight, a sound, a smell or taste, or one or other of the cutaneous sensations in the absence of the related objects.

Seat of Intellectual Processes-Association Areas.-When we have deducted from the cortex of the hemisphere the whole Rolandic region and the sensory centres, there still remains a large territory unaccounted for. Considerable portions of the occipital, parietal, and temporal lobes, nearly the whole of the island of Reil and the greater part of the frontal lobe anterior to the ascending frontal convolution are 'silent areas,' and respond to stimulation by neither motor nor sensory sign. They correspond to the association centres previously referred to. They are connected with the sensory and motor areas and with each other, but are not directly connected by projection fibres with the lower parts of the central nervous system, as the motor area, for example, is by the pyramida] path. By a process of exclusion it has been supposed that, in addi. tion to, or partly in virtue of, their associative function, they are the seat of intellectual and psychical operations. It is supposed that the sensations aroused in the various sensory areas by the impulses received from the sense organs are linked in the association areas into complex perceptions. For instance, when an orange is taken into the hand, the visual sensation of a yellow body, the tactile sensation of a smooth round body, and perhaps the olfactory sensation characteristic of an orange, are collected from the sensory areas, connected and combined, or synthesized in an association area to the concept of an orange. Somewhere in the association areas it is to be supposed is stored the memory of past experiences. The intellectual function has been more particularlv, assigned to the frontal lobes, and with great probability, although 
we have little real knowledge to guide us to a decision. Extensive destruction and loss of substance of the prefrontal region may sometimes occur without any marked symptoms. But usually there is restriction of mental power, or it may be loss of moral restraint. Thus in the famous 'American crowbar case,' an iron bar completely transfixed the left frontal lobe of a man engaged in blasting. Although stunned for the moment, he was able in an lour to climb a long flight of stairs, and to answer the inquiries of the surgeon. Finally, he recovered, and lived for nearly thirteen years without either sensory or motor deficiency, except that he suffered occasionally from epileptic convulsions. But his intellect was impaired; he became fitful and vacillating; profane in his language and inefficient in his work, although previously decent in conversation and a diligent and capable workman.

Flechsig supposes that his great anterior association centre in the frontal lobe is concerned in the retention of the memory of all conscious bodily experiences, especially those connected with voluntary acts. The great posterior association centre he imagines to be cngaged in the formation and collection of ideas of external objects and of the 'word pictures ' which represent them, and with the preparation of speech in respect of the thoughts to be expressed and the form of expression, the office of the Broca's area (but see p. 97I) being to execute the mechanical part of the process by transforming these thoughts into actual spoken words. This posterior association centre may be looked upon as the seat of intellect in the narrower sense, as the anterior is of will and fecling.

The experiments of Franz on the relation of the cerebral association areas, and especially the frontal area, to certain acquired habits are of interest. Cats were allowed to acquire certain habits involving simple mental processes, and then it was seen how these were affected by cortical lesions. After bilateral extirpation of the frontal lobes (the area anterior to the crucial sulcus) newly-formed, but not long-standing. habits are lost. This cannot be due to shock, since other brain lesions are not followed by loss of the habits. Extirpation of one frontal area usually causes a partial loss of newly-acquired habits, or, rather, a slowing of the association process leading to unusual delay in the execution of the movements connected witl the habit. Habits once lost after removal of the frontal lobes may be relearned.

The influence of psychical events upon bodily functions is well known. and has been more than once illustrated in preceding pages. The converse question of the influence of bodily states upon psychical event: has also been raised, especially in connection with the gencsis of emotion. Some psychologists assume that the bodily changes associated with such emotions as grief, fear, rage, or love, are not evoked as a consequence of the emotions, but that the bodily changes follow directly the perception of the exciting fact-e.g., a spectacle which causes fear or rage, "and that our feeling of the same changes as they occur is the emotion" (James). Sherrington, however, has shown that in dogs in which, by transection of the vagi and the spinal cord, all sensation of viscera, skin, and muscles bchind the level of the shoulder was eliminated, no obvious emotional defect was caused. Notwithstanding the immense abridgment of the field of sensation, anger, joy, fear, disgust (as on being offered dog's flesh, which most dogs refuse to eat), were as marked as ever, and were evoked by the same objects as bcfore the operation. 
When the afferent field is still more restricted, as in the head of a dog grafted on the circulation of anuther dog by anastomosis of the bloodvessels, with precautions to avoid interruption of the blood-flow, not only docs the respiratory centre continue to discharge itself with a regular rhythm, but cortical volitional movements persist (Guthrie, Pike, and Stewart), and, so far as can be judged, sense perception, emotional, and even intellectual, processes continue. In one case the picture represented by the engrafted head was essentially the same as that presented by the head of the 'host' for over two hours. In a transplanted head from a younger dog in which the circulation had been interrupted for twenty-nine minutes, a remarkable return of cerebral function was observed (Guthrie).

Conditioned or Conditional Reflexes.-This is perhaps the place to refer briefly to certain phases of brain function which have been illustrated by the work of Pawlow, already alluded to in Chapter VI, on the so-called psychical secretions. This study lias led him to formulate some novel riews of the higher nervous activities. He has shown, for instance, that if food is presented to an animal, and at the same time the skin of the foot is stimulated electrically, the psychical secretion of saliva, which the mere sight or smell of the food brings about, becomes, when the experiment has been repeated for a sufficient number of times, associated with the electrical stimulus. It is then no longer necessary to offer the food in order to elicit the secretion; all that is required is to stimulate the foot. Even the nature of the stimulus may be altered, cutting or burning the foot being substituted for the electrical excitation; the secretion is still obtained. Pawlow has termed such reactions conditioned or conditional reflexes, because, unlike the ordinary spinal and bulbar reflexes which are always obtainable and are therefore spoken of as unconditioned reflexes, the conditioned reflexes depend upon the establishment of certain conditions, such as the associated influence of some stimulation of the sense organs or of psychical excitation. He concludes from his extensive studies on this subject that an important function of the central nervous system, and especially of the cerebrum, in addition to its power of integrating or correlating nerve impulses, is that of dispersing impulses, producing new types of activity and new reflexes.

Localization of Function in the Central Nervous System.--Let us now consider a little more closely the real meaning of this localization of function. Scattered all over the grey matter of the primitive neural axis, and, as we have seen, over the grey mantle of the brain as well, are numerous 'centres' which seem to be related in a special way to special mechanisms, sensory, secretory, or motor. The question may fitly be asked whether those centres are really distinct from each other in quality of structure or action, or whether they owe their peculiar properties solely to differences in situation and anatomical connection. It is clear at the outset that the nature of the work in which a centre is engaged must be largely determined by its connections. The kind of activity which goes on in the vaso-motor centre in the bulb, for example, may in no essential respect differ from that which goes on in the respiratory centre. The calibre of the bloodvessels will alter in response to a change of activity in the one because it is anatomically con- 
nected with the muscular coat of the bloodvessels. The rate or depth of the respiratory movements will alter in response to a change of activity in the other, because it is connected with muscles which can act upon the chest-walls.

Experiments on the anastomosis of nerves afford a very interesting illustration of the determining influence of their peripheral connections on the function of nerve-fibres. It has, in fact, been shown that the central end of any efferent somatic fibre-i.e., any fibre running from the central nervous system and ending in striated muscle - can make functional connection with the peripheral end of any other efferent fibre of the same class, whatever be the normal actions produced by the two fibres. Advantage has been taken of this in surgery. lor instance, in a case of severe facial (motor) tic the facial nerve was divided, and its peripheral end united with a portion of the fibres of the spinal accessory. The voluntary movements of the face, after regeneration had occurred, were normally carried out through impulses descending the spinal accessory. In cases of local paralysis, due to destruction of anterior horn-cells (anterior poliomyelitis), restoration of movement has also been obtained by connecting the motor nerve of the paralyzed muscles to a portion of a nerve coming off from an uninjured region of the cord. But the possibilities of this procedure have been exaggerated

By such operations it has been possible to transpose motor areas on the cerebral cortex associated with the flexion and extension of a particular joint, so that the part of the cortex which originally caused flexion after the nerve anastomosis causes extension, and vice versa. When the nerves supplying a group of muscles of the dog's fore-limb are eliminated, the nerves of the antagonistic group may be used to supply both groups, and co-ordinated movements may be restored, although this does not occur so rapidly as when the nerves supplying the two groups are simply cut and crosssutured (Kennedy). However, the limitations of this method ought to be recognized. Before any anastomosis of nerves can be made, good fibres must first be destroyed. Under favourable circumstances these may all regenerate and find their way to the structures they are intended to innervate. When regeneration is complete, the number of fibres capable of functioning will at best be the same as before the operation, and may easily be considerably less. The benefit, whatever it is, will be associated solely with the redistribution of the fibres. There is reason to think that the closer to the cell of origin a nerve is injured or divided, the less is the chance of restoration, and Feiss has found that after lesions in the cord or the spinal roots neither the anatomical pattern of the affected nerves nor their functional power is much affected by subsequent nerve anastomosis.

The central end of any efferent somatic fibre can also make 
functional union with the peripheral end of any of the efferent fibres which run from the rentral nervous system and end in ganglion cells (pre-ganglionic fibres), and the central end of any pre-ganglionic fibre can do the sime with the peripheral end of any efferent somatic fibre (Langley and Anderson). For instance, Langley divided (in cats) the vagus nerve and the cervical sympathetic. The peripheral end of the former degenerated, of course, below the section, and the peripheral (cephalic) end of the latter degenerated above the section, up to the terminations of its axons in the superior cervical ganglion. The central end of the cut vagus was subsequently sutured to the peripheral end of the cut sympathetic. After a time the vagus-fibres grew along the course of the degenerated sympathetic up to the ganglion, where some of them formed arborizations around the ganglion cells. It was now found that stimulation of the vagus produced the effects usually caused by stimulation of the cervical sympathetic-for example, dilatation of the pupil and constriction of the bloodvessels of the head and neck. From these experiments it follows that the functions of the various groups of fibres in the cervical sympathetic do not depend on anything peculiar to the fibres; any fibre which can make connection with one of the ganglion cells that send axons to the dilator muscle of the iris will, when stimulated, act as a pupillodilator fibre, just as well as a cervical sympathetic fibre. Other instances of the same law have already been given in connection with the regeneration of nerves (p. 80o).

Functional union does not take place between efferent somatic fibres (or pre-ganglionic fibres) and post-ganglionic fibres-i.e., fibres arising in peripheral ganglia, and ending in smooth muscle and glandular tissue; e.g., the cervical sympathetic after excision of the superior cervical ganglion does not unite with the fibres leaving the anterior end of the ganglion in such a way that stimulation of it can produce any of the effects normally produced through these fibres. No proof lias been given that afferent fibres can unite with efferent fibres or efferent with afferent.

Afferent fibres of one nerve can unite with afferent fibres of another nerve, but there is not sufficient evidence to show whether fibres concerned in one sensation can unite with fibres concerned in another.

The localization of function in the cerebral cortex has been likened to the localization of industries in the multiplex commercial life of the modern world. The barbarian household in which cloth is woven and worked into garments; sandals, or moccasins cobbled together; rough pottery baked in the kitchen fire, and all the rude furniture of the lodge fashioned by the hands which built it, and which rest beneath its roof at night-this state of things where centralization has not yet begun, it has been said, is a picture of what goes on in the undeveloped brains of the frog, the pigeon, and the rabbit. The 'diffusion' of industries which is characteristic of a primitive state has given place among the 
must highly civilized men to extreme centralization and concentration. Manchester spins cotton and Liverpool ships it. Chicago handles wheat and pork that have been produced on the prairies of Iinnesota and lllinois. Amsterdam cuts diamonds. Munich brews beer. Lyons weaves silk. New York and London are centres of finance. This, it is said, is the picture of the highly specialized brain of a monkey or a man. But ingenious and alluring though such analogies are, they do not rest upon a sufficient basis of fact. Indeed, the more deeply the structure and function of the central nervous system are studicd, the more clearly does its essential solidarity appear, the more clearly does it emerge as an organized co-ordinated system, not an aggregate of separate mechanisms jumbled together for convenience of storage in the protected craniospinal cavity.

It has never been shown-nor is it likely that the proof will soon be forthcoming-that there is any difference whatever in the physical, chemical, or psychical processes which go on in the various centres of the 'motor' cortex. It may be supposed, indeed, that the so-called sensory areas of the cortex differ more widely in their internal activity. from the 'motor' areas than the latter do among themselves, and that the activity of the anterior portion of the brain, the portion which has been credited par excellence with pyschical functions, differs in kind, not merely in degree, from that of all the rest. But, as we have just seen, even the 'motor' areas have sensory functions. A cast-iron physsiology' may explain this by the assumption of 'sensory' as well as 'motor" cells in the Rolandic area, and may find support for such an assumption in the well-known fact that the large pyramidal cells wliose axons form the pyramidal tract make up but a small proportion of the total number of pyramidal cells in this region, which, besides, contains numerous cells of Golgi's second type (p. 356). And although it may be true that the tactile sensations constituting the so-called body-sense are represented mainly not in the motor region itself, but in the adjacent gyrus postcentralis posterior, to the Rolandic fissure (p. 95I), there is nothing to contradict the supposition that the discharge of encrgy from the most circumscribed motor area or element may be accompanied with consciousness. And, indeed, some writers have supposed that sich a consciousness of, or even conscious measurement of, the discharge from the 'motor' areas is the basis of the muscular sense (Bain, Wundt).

So far, at least, as the 'motor' region and the grey matter immediately around the neural canal are concerned, the analogy of an electrical switch-board connected with machines of various kinds might be more correct. Touch one key or another, and an engine is set in motion to grind corn, or to saw wood, or to light a town. The difference in result lies not in any difference of material or workmanship in the switches, but solely in the difference in their connections.

Grey matter in the upper part of the precentral convolution is excited, and the muscles of the leg contract. Grey matter on the lower part of the convolution is excited, and there are movements of the face and mouth. Grey matter in the medulla oblongata is excited, and the salivary glands pour forth a thin, watery fluid, poor in proteins, and containing an amylolytic ferment. Another portion of grey (?) matter in the medulla is thrown into activity, and the pancreatic ducts become flushed with a thickersecretion, relatively rich in proteins and in ferments which act on proteins, starch, and fat. Here, too, there is a variety in result according as one or another nervous switch is closed; here, too, the variety is due, not to essential differences in the structure of the actirity or the nervous centres, but to their conncction, by nervous 
vaths, with peripehral organs of different kinds. There is, indeed, a specialization, a localization, of function, but the localization is at the periphery, the specialization is in the peripheral organs.

It may be asked whether, if this is the case for the periplieral organs of efferent nerves, the converse does not hold true for the afferent nerves -in other words, whether the localization here is not at the centre. And that there is in some clegree a central localization of sensation may be considered proved by the well-known clinical fact, already referred to, that sensations of various kinds may be produced by pathological changes in the cortex. For example, a tumour involving the upper part of the temporal lobe may give rise to epileptiform convulsions preceded by an auditory aura, a sound, it may be, resembling the ringing of bells; a tumour involving the occipital region may cause a visual aura, and so on. Central sensory localization is the fundamental idea of the old doctrine of the specific energy of nerves, which, in modern phraseology, expresses the fact that excitation of the central end of a sensory nerve by various kinds of stimuli causes always - or at least very often-the particular hind of sensation appropriate to the nerve. The observation so frequently made in surgery before the days of anzesthetics, that wher the optic nerve was cut in removing the eyeball the patient experienced the sensation of a flash of light, * was long looked upon as the strongest prop of the law of specfic energy, and well illustrates the meaning of the term. Here a mechanical excitation of the optic fibres in their course gives rise to the same sensation as excitation of the retina bv the natural or homologous or adequate stimulus of lights Since a similar mechanical stimulus applied to the auditory nerve gives rise to a sensation of sound, and, applied to the trigeminal nerve, to a sensation of pain, many physiologists have assumed that the impulses set up in the auditory nerve when sound impinges on the tympanic membrane do not differ essentially from those set up in the optic nerve when a ray of light falls upon the retina, or from those set up in the fifth nerve by the irritation of a carious tooth, or from those set up in certain fibres of the cutaneous nerves when a warm body comes in contact with the skin. Since the results in consciousness are very different, this assumption has necessitated the further conclusion that somewhere or other in the central nervous system there exist organs that are differently affected by the same kinds of afferent impulses-in other words, that sensory localization is at the centre. On this view, the viscual areas in the cortex respond to all kinds of stimuli by visual sensations; the auditory areas by sensations of sound, and so on.

But while it cannot be doubted that special sensory regions exist in the grey matter of the brain, where the afferent paths concerned in the different kinds of sensation end, it has not been proved that the nerveimpulses which travel up the various paths are absolutely similar until they have reached the centres, and there suddenly become, or produce, sensations absolutely different. There is, indeed, evidence of a certain amount of sensory specialization at the periphery. For example, when an ordinary nerve-trunk is touched, the resultant sensation is not one of touch. If there is any sensation at all, it is one of pain. Heating or cooling a naked nerve-trunk gives rise to no sensations of temperature. When the ulnar nerve is artificially cooled at the elbow, the first effect is severe pain in the parts of the hand supplied by the nerve. The pain disappears somewhat abruptly as cooling goes on, and is succeeded by gradual loss of all sensation in the ulnar area of the hand; but the cooling of the nerve-trunk does not give rise to any sensation of cold (Weir Mitchell). Stimulation of the receptors or end-organs is normally

- It is said that this is not always the casa 
essential in order that sensations of touch and temperature should bo experienced. Although as previously stated, one great function of the receptor is to lower the threshold of the adequate stimulus, and thus to render the afferent neuron more easily excited by an adequate stimuius than by any other, it may also serve to impress a particular rhythm or other character upon the nerve impulse, so that the afferent impulses may be to some extent differentiated beforc they reach their centres. One reason, then, why excitation of the temporal cortex by impulses falling into it along the a uditory nerve-fibres calises a sensation different from that caused by impulses reaching the occipital cortex through the fibres of the optic nerve may be a difference in the nature of the impulses. If this were the only reason, it would follow that were it possible to physiologically connect the fibres of the optic radiation with the temporal cortex, and those of the temporal radiation with the occipital cortex, sights and sounds would still be perceived and discriminated in a normal manner, although now the integrity of the occipital lobe would be bound up with the perception of sound, the integrity of the temporal lobe with visual sensation. This state of affairs would correspond to complete specialization for sensation in the peripheral organs, complete absence of specialization in the centres. On the other hand, it is conceivable that, after such an ideal experiment, sound-waves falling on the auditory apparatus might cause visual sensations, and luminous impressions falling on the retina sensations of sound. This would correspond to complete specialization of sensation in the centres, complete absence of specialization at the periphery. A third possibility would be that the 'transposed ' centres, responding at first feebly or not at all to the new impulses, might, by slow degrees, become more and more excitable to them. This would correspond to a peripheral specialization, combined with a tendency to development oi central specialization. And, indeed, it is not easy to conceive in what way, except as the result of differences in the nature of impulses coming from the periphery, specialization of sensory areas in the central nervous system could have at first arisen.

Degree of Localization in Different Animals.-Before leaving this subject, two points ought to be made clear: (I) The degree of localization of function in the cortex goes hand in hand with the general development of the brain. In man and the monkey, the motor localization is more elaborate than in the dog-that is to say, a greater number of movements can be associated with definite cortical areas. In the rabbit, whose 'motor' centres have been particularly studied in recent years by Mann and Mills, localization is still less advanced than in the dog. Towards the bottom of the mammalian group certain ' motor' areas can still be demonstrated, though they are rather ill-defined, for instance in the hedgehog (Mann), opossum (Cunningham), and ornithorhynchus (Martin). In general the movements of the anterior limb are easier to obtain than those of the posterior. In birds Mills found no evidence of the existence of any ' motor' centres.

(2) Areas of the same name (homologous areas) in different groups of animals do not necessarily have the same function-that is, in the case of the 'motor' areas, are not necessarily associated with the same movements. Taking the position of the centre for the 
orbicularis oculi as a test, Ziehen has come to the conclusion that in the anthropoid apes and in man this centre has been pushed forward by the encroachment of the centres behind it, and especially of the visual centre, the arm centre, and the speech centre, which have undergone a great functional development.

Acquisition of Co-ordination of Voluntary Movements. - The co-ordination of movements has already been alluded to in connection with the spinal reflexes. No fundamental distinction can be drawn between the co-ordination of reflex and of voluntary movements, but the conscious and often long-continued efforts necessary to acquire mastery over the latter lends to their co-ordination a special interest. The new-born child brings with it into the world a certain endowment of co-ordinative powers; it has inherited, for example, from a long line of mammalian ancestors the power of performing those movements of the cheeks, lips, and tongue, on which sucking depends; perhaps from a long, though somewhat shadowy, race of arboreal ancestors the power of clinging with hands and feet, and thus suspending itself in the air. Many movements, such as walking and the co-ordinated muscular contractions involved in standing, and even in sitting, which, once acquired, appear so natural and spontaneous, have to be learnt by painful effort in the hard school of (infantile) experience, and this despite the fact that in these movements the voluntarv co-ordination mechanism makes use to c great extent of a motor machinery already existing in the cord and capable of discharging well co-ordinated reflexes. * In addition to such fundamental movements, most people consciously learn, and are willing to confess that they have learnt, to execute a considerable number of co-ordinated movements with the arms, and especially with the fingers. Some part even of the extreme dexterity of jaws, tongue, and teeth displayed by a hungry school-boy, in a minor degree, perhaps, by a hungry mouse, is the result of the much practice, entailing at first some conscious effort, which maketh perfect. The exquisite co-ordination of the muscles of the eyeball, which we shall afterwards have to speak of, and the no less wonderful balance of effort and resistance, of power put forth and work to be done, of which we have already had glimpses in studying the mechanism of voice and speech, become to a great extent the common property of all fully-developed persons. But the technique of the finished singer or musician, of the swordsman or acrobat, and even the operative skill of the surgeon, are in large part the outcome of a special and acquired agility of mind or bod - in virtue of which highly-complicated co-ordinated movements are promptly determined on and immediately executed.

With such special and elaborate movements it is impossible to occupy ourselves in a book like this. Their number may be almost indefinitely

* The question, how much is 'learned' in an act such as walking, and how much is already present at birth in the form of a co-ordinated mechanism in the nervous system, is not easy to answer, and indeed the answer may be different for different animals. It has been shown that even the unborn fœetus of the cat, when shelled out of the uterus into saline solution without interference with the circulation through the umbilical cord, can be made to execute unmistakable movements of progression under the influence of asphyxia, produced by pressure upon the cord. It may also execute such movements spontaneously. Grahan Brown, to whom we owe these facts, concludes that the co-ordination of the mechanism concerned in walking is already developed during intra-uterine life, and is not 'learned' after birth. 
extended, and their nature almost infinitely varied, by the needs and training of special trades and professions. It will be sufficient for our purpose to sketch in a few words the mechanism of one or two of the most common and fundamental co-ordinations of muscular effort. passing over the rest with the general statement that the more refined and complex movements are in general brought about, not by the abrupt contraction of crude anatomical groups of muscles, but by the contraction of portions of muscles, perhaps even single fibres or small bundles of fibres, while the rest remain relaxed. 'The excitation may' gradually wax and wane as the different stages of the movement require. Antagonistic muscles may be called into play to balance and tone down a contraction which might otherwise be too abrupt.

Many interesting illustrations of this process of 'give and take' between opposing inuscles have been reported, especially by Sherrington. Some have been already alluded to in discussing reflex movements (p. 903). One or two additional observations may be given here. In the cortex cerebri, as we shall see (pp. 95 I, 965), there is an area in the frontal region, and another in the occipital region, stimulation of which gives rise to conjugate deviation of the eyes-that is, rotation of both eyes - to the opposite side. Sherrington divided the third and fourth cranial nerves in monkeys - say on the left side. The external rectus, which is supplied by the sixth nerve, caused now by its unopposed contraction external squint of the left eye. When either of the cortical areas referred to, or even the subjacent portion of the corona radiata, was stimulated on the left side, both eyes moved towards the right, the left eye, however, only reaching the middle line-that is, the position in which it looked straight forward. The same thing was observed when the animal, after complete recovery from the operation, was caused to voluntarily turn its eyes to the right by the sight of food. Here an inhibitory influence must have descended the fibres of the abducens, the only nervous path connected with the extrinsic muscles of the left eye, and the relaxation of the left external rectus must have kept accurate step with the contraction of the right internal rectus. Hering has made an exhaustive analysis of the co-ordinated movements concerned in opening and closing the hand in monkeys. These movements can be produced by stimulation of the cortex or the internal capsule, but not by stimulation of the anterior spinal roots. When the hand is opened the muscles that open it are excited, and those which close it are inhibited from the cortex.

Reaction Time.-Just as in a reflex act a certain measureable time (reflex time) is taken up by the changes that occur in the lower nervous centres, so we may assume that in all psychical processes the element of time is involved. And, indeed, when the interval that elapses between the application of a stimulus and the signal which announces that it has been felt (reaction time) is measured, it is found that for the cerebral processes associated with the perception of the simplest sensation and the production of the simplest voluntary contraction it is longer than the time which the spinal centres require for the elaboration of even complex and co-ordinated reflex movements. Suppose, e.g., that the stimulus is an induction shock applied to a given point of the skin, and that the signal is the closing of the circuit of an electro-magnet, then, if both events are 
automatically recorded on a revolving drum, the interval can be readily determined. It is evident that this includes, not only the time actually consumed in the central processes, but also the time required for the afferent impulse to reach the brain, and the efferent impulse the hand, along with the latent period of the muscles. The time taken up in these three events can be approximately calculated, and when it is subtracted, the remainder represents the reduced or corrected reaction time-that is, the interval actually spent in the centres themselves. This is by no means a constant. It is influenced not only by the degree of complexity of the psychical acts involved, and the mental at titude of the person (whether he expects the stimulus or is taken by surprise, whether he has to choose between several possible kinds of stimuli and respond to only one, etc.), but it varies also for different kinds of sensation, for the same sensation at different times, and, as is recognized in the personal equation of astronomers, in different individuals. For sensations of touch and pain it may be taken as one-ninth to one-fifth, for hearing one-eighth to one-sixth, and for sight one-eighth to onefifth of a second. So that the proverbial quickness of thought is by no means great, even in comparison with that of such a gross process as the contraction of a muscle (one-tenth of a second). Nor is it the case that the man ' of quick apprehension' has always a short reaction time, or the dullard always a long one, although in all kinds of persons practice will reduce it.

\section{Section XI.-Fatigue and Sleep-Hypnosis.}

Sleep and Fatigue.-Certain gland-cells, certain muscular fibres, and the epithelial cells of ciliated membranes, never rest, and perhaps hardly ever even slacken their activity. But in most organs periods of action alternate at more or less frequent intervals with periods of relative repose. In all the higher animals the central nervous system enters once at least in the twenty-four hours into the condition of rest which we call sleep. What the cause of this regular periodicity is we do not know. It is accompanied by changes in the microscopical appearance of the nerve-cells. Thus, Hodge found differences between the cells of certain portions of the cerebral cortex in birds, and of certain ganglia in the honey-bee after a long day of work and after a night's rest. Mann, Lugaro, and other observers, found similar differences in the cells of the cerebral cortex and the anterior horn, and Dolley in the Purkinje's cells of the cerebellum in dogs fatigued by muscular exercise as compared with rested dogs (Fig. 398). 
Arcording to bolley, there is, as a result of continned activity, at first a stearly increase of the basic cloromatic matcrial. This increase affects first the extra-nuclear chromatin, the Nisst substance, which, according to the most modern view, is really nuclear substance distributed through the cytoplasm and functions as such (foldschmidt). The size and number of the granules are increased, and some of the chromatic material is diffused throughout the cytoplasm, as indicated by diffuse staining. Then the intranuclear chromatin also undergoes an increase, and the size of the cell is increased troo. In morlerate activity the change goes no farther. At this stage the cell is hyperchromatic-i.e., as compared with a normal resting cell it contains an

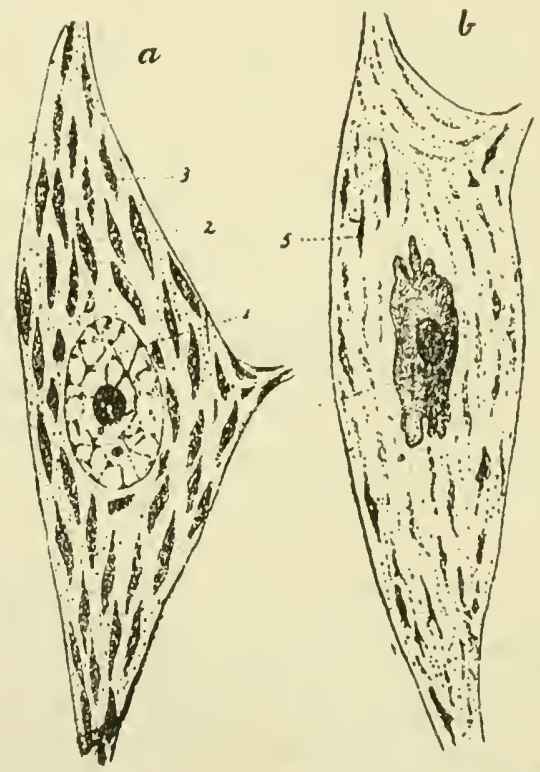

Fig. 398.-Effect of Fatigue on Nerve-Colls (Barker, after Mann). Two motor cells from lumbar cord of dog fixed in sublimate and stamed with toluidin blue. a, from rested dog; 1, pale nucleus; 2, dark Nissl spindles; 3 , bundles of nerve fibrils. $b$, from fatigued dog; 4 , dark shrivelled nucleus; 5 , pale spindles.

excess of chromatin. The production of chromatin having reached the maximum of which the nucleus is capable, and functional activity, which entails the using up of the extranuclear chromatin, still continuing, the total chromatin content begins to diminish, first in the nucleus, through the passage of its chromatin into the cytoplasm to recruit the Nissl substance, then in the cytoplasm as well. Accompanying the disappearance of the chromatic material there is diminution in the size of both cell and nucleus, but especially of the nucleus, so that the normal proportion between volume of cell and volmme of nucleus (nuclensplasnat relation of Hertwig) is disturbed in favour of the cytoplasm. Both cell and mucleus become irregular in outline or crenated. Later 
on, and, it would secm, rather abruptly, swelling of the nucleus and, after some time, of the evtoplasm vecurs. This is due to oedema, and may be taken to indicate an upset of their normal osmotic relations. 'The earlier occurrence of uelema in the nuclens leads to another change in the nucleus-plasma relation, which is now clisturber in favour of the nucleus. In the measure in which fatigue progresses the extranuclear chromatic material continues to be used up, and, in spite of its replenishment from the nucleus, it almost or entirely vanishes from the cytoplasm. Then follow's what is perhaps a 'last effort' on the part of the nucleus to supply the cytoplasm, in the form of a discharge of chromatic substance, which forst masses itself around the outside of the nuclear membrane, and thence gradually diffuses into the cytoplasm. With the using up of this supply all the basic chromatic material of the cell, except that in the karyosome (nucleolus), is exhausted. Finally, this too is rielded up to the cytoplasm, and with its consumption there remains a totally exhausted cell, deroid of basic chromatin and incapable of recuperation.

According to Pugnct, even in extreme fatigue, as when dogs were caused to run forty to nearly sixty miles in a special apparatus, the changes varied greatly in degree in different cortical cells, from mere diminution of the chromatic substance to complete disappearance of it and such disintegration of the cell as must have precluded its recovery; had the animal been allowed to live. Many, and indeed most, of the cortical cells were quite unaffected. Histological alterations may also be caused in sympathetic ganglion cells by prolonged artificial stimulation of the nerves connected with the ganglia. Experiments on fatigue changes in the cclls of the spinal ganglia after electrical excitation of the posterior root-fibres are less decisive, some observers having obtained positive, others negative, results (p. 9I3).

Theories of the Causation of Sleep.-(I) Some have suggested that sleep is induced by the using up of substances necessary for the functional activity of the neurons-e.g., the stored-up or intramolecular oxygenor by the action of the waste products of the tissues, and especially lactic acid, when they accumulate beyond a certain amount in the blood, or in the nervous elements themselves.

(2) Others have looked for an explanation to vascular changes in the brain, but so far are the possible causes of such changes from being understood, that it is even yet a question whether in sleep the brain is congested or anæmic. Certain writers have settled this question by the summary statement that when the brain rests the quantity of blood in it must be supposed to be diminished, as in other resting organs. But this is a fallacious argument. For when the whole body rests, as it does in sleep, it has as much blood in it as when it works; in sleep, therefore, if some resting organs have less blood than in waking life, othcr resting organs must have more; and it is the province of experiment to decide which are congested and which anæmic. In coma, a pathological con. dition which in some respects has analogies to profound and long. continued sleep, the brain is congested, and the proper elements of the nervous tissue presumably compressed. And artificial pressure (applied by means of a distensible bag introduced through a trephine hole into the cranial cavity) may cause not only unconsciousness, but absolute anæsthesia. But it is possible that this artificial increase of intracranial pressure may produce its effects by rendering the brain anæmic, and it has been actually observed that the retinal vessels, as seen with the ophthalmoscope, and the vessels of the pia mater exposed to direct observation in man by disease of the bones of the skull, or in animals by operation, shrink during sleep. Statements to the contrary may be due 
to neglecting the influence of difference of position in thie sleeping and waking states. In sleeping children the fontanelle sinks in, an indication that the intracranial pressure is reluced. Observations with the plethysmograpl have shown that the arm swells in sleep, and shrinks when the sleeper awakes, or even when he is subjected to sensory stimuli not sufficient to arouse him-e.g., a tune played by a musical-box (Howell). The tone of the vaso-motor centre is therefore diminished, and the arterial pressure falls during slecp. But a fall of gencral arterial pressure is usually accompanicd by a diminution of the quantity of blood passing through the brain. So that the balance of evidence is in favour of the view that sleep is associated with a certain degree of cerebral anamia.

As to the nature of the relation between the two conditions, it has been suggested that the anamia is produced by fatigue of the vasomotor centre, which causes it to relax its grip upon the periplueral bloodvessels, and that the condition of the cortical nerve-cells whicls we call sleep is directly produced by the lack of blood. But there does not appear to be any good reason for believing that the vaso-motor centre is more susceptible of fatigue than the higher cerebral centres. On the contrary, it is probable that the bulbar centres are less delicately; organized and more resistant than the higher centres. In any case, if the cerebral nerve-cells 'go to sleep' because their blood-supply is diminished, ought we not to look for a similar cause for diminished activity of the vaso-motor centre? Or if the answer is made that the activity of the vaso-motor cells is directly lessened by fatigue, or by the cessation of external stimuli, why should not this be the case also for the cortical cells? It can be shown by means of the sphygmomanometer (p. I I 4 ) that the fall of arterial pressure is not essentially connected with sleep, but is produced by the bodily rest and warmth which accompany. it. Further, even a great diminution in the supply of blood going to the brain is not necessarily followed by sleep. For example, both carotids and both vertebral arteries may frequently be tied in dogs at the same time without producing any symptoms, the anastomosis of the superior intercostal arteries with the anterior spinal artery providing a sufficient channel for the blood absolutely required by the brain. Monkeys after ligation of both carotids may be most alert and active. To produce sopor in animals the cortical circulation must be reduced almost to the vanishing-point, and to a far greater degree than ever occurs in sleep (Hill). We must, therefore, conclude that although sleep is normally, associated with some ancmia of the brain, it is not directly caused by it. The cortical centres go to sleep because they are 'tircd,' or because the stimuli which usually excite them have ceased, and not because their blood-supply is diminished.

(3) The idea that the dendrites are contractile, and by pulling themselves in, and thus breaking certain nervous chains, cause sleep, is a mere theory, unsupported by any real evidence. The same is true of the notion that the fibrils of the neuroglia insinuate themselves into the 'joints,' by which one neuron comes into contact with another, and, acting as insulating material, block the nerve-impulses.

In general, the depth of sleep, as measured by the intensity of sound needed to awaken the slceper, increases rapidly in the first hour, falls abruptly in the second, and then slowly creeps down to its minimum, which it reaches just before the person awakens. As to the amount of sleep required, no precise rules can be laid down. It varies with age, occupation, and perhaps climate. An infant, whose main business is to grow, spends, or ought to spend, if mothers were wise and feeding-bottles clean, the greater part of its time in sleep. The man, whose main 
business it is to work with his hands or brain, requires his full tale of eight hours' sleep, but not usually more. The dry and exlilarating air of some of the inland portions of North America, and perhaps the plains of Victoria and New Soutl Wales, incites, and possibly enables a newcomer to live for a considerable period with less than his ordinary amount of slecp. Idiosyncrasy, and perhaps to a still greater extent 'habit, have also a marked influence. The great Napoleon, in his heyday, never slept more than four or five hours in the twenty-four. Five or six hours or less was the usual allowance of Frederick of Prussia throughout the greater part of his long and active life.

Hypnosis is a condition in some respects allied to natural slumber; but instead of the activity of the whole brain-or perhaps we should rather say, the whole activity of the brain-being in abeyance, the susceptibility to external impressions remains as great as in waking life, or may be even increased, while the critical faculty, which normally sits in judgment on them, is lulled to sleep. The condition can be induced in many ways-by asking the subject to look fixedly at a bright object, by closing his eyes, by occupying lis attention, by a sudden loud sound or a flash of light, etc. The essential condition is that the person should have the idea of going to sleep, and that he should surrender his will to the operator. In the hypnotic condition the subject is extremely open to suggestions made by the operator with whom he is en rapport. He adopts and acts upon them without criticism. If, for example, the hypnotizer raises the subject's arm above his head, and suggests that he cannot bring it down again, it stays fixed in that position for a long time without any appearance of fatigue; or the whole body may be thrown, on a mere hint, into some unnatural pose, in which it remains rigid as a statue. Suggested hemiplegia or hemianæsthesia, or paralysis of motion and sensation together or apart in limited areas, can also be realized; and surgical operations have been actually performed on hypnotized persons without any appearance of suffering. If, on the other hand, the operator suggests that the subject is undergoing intense pain, he will instantly take his cue, writhing his body, pressing his hands upon his head or breast, and in all respects behaving as if the suggestion were in accord with the facts. If he is told that he is blind or deaf, he will act as if this were the case. If it is suggested that a person actually present is in Timbuctoo, the subject will entirely ignore him, will leave him out if told to count the persons in the room, or try to walk through him if asked to move in that direction. What is even more curious is that the organic functions of the body are also liable to be influenced by suggestion. A postage-stamp was placed on the skin of a hypnotized person, and it was suggested that it would raise a blister. Next day a blister was actually found beneath it. The letter $\mathrm{K}$, embroidered on a piece of cloth, was suggested to be red-hot. The left shoulder was then 'branded' with it, and on the right shoulder appeared a facsimile of the $\mathrm{K}$ as if burnt with a hot iron. The secretions can be increased or diminished, subcutaneous hæmorrhages, veritable stigmata, * can be caused, and many of the 'miracles ' of Lourdes and other shrines, ancient and modern, repeated or surpassed by the aid of hypnotic suggestion. Hypnotism has also been practically employed in the treatment of various diseases, and particularly in functional derangements of the

* I.e., bleeding spots on the skin generally corresponding to the wounds of Christ. In the well-known case of Louise Latour, which excited great interest in France in I 868, blisters first appeared; they burst, and then there was bleeding from the true skin. The probable explanation is that she concentrated her attention on these parts of her body and so influenced them. perhaps by causing congestion through the vaso-motor centre. 
nervous system. But care and judgment are necessary on the part of the operator, and although as a rule there is no difficulty in putting an end to the condition by a suitable suggestion, it is said that in rare instances grave mischances have occurred. There seems to be no ground for the opinion that women are more easily hypnotized than men. Out of more than a thousand persons, Liébault found only seventeen absolutely refractory.

\section{Section Xil.-Size of Brain and Intelligence-Circulation} in and Resuscitation of Central Nervous System after ANAMIA-CheMistry OF Nervous ACTIVITY-CEREBRO-SPINAI. Fluid.

Relation of Size of Brain to Intelligence.-While it is the case that some men of great ability have had remarkably heavy and richly convoluted brains, it would seem that in general neither great size nor any other obvious anatomical peculiarity of the cerebrum is constantly associated with exceptional intellectual power. In the animal kingdom, as a whole, there is undoubtedly some relation between the status of a group and the average brain development within the group. But that this is a relation which is complicated by other circumstances than the mere degree of intelligence is sufficiently shown by the fact that a mouse has more brain, in proportion to its size, than a man, and thirteen times more than a horse ; while both in the rabbit and sheep the ratio of brain-weight to bodyweight is nearly twice as great as in the horse, in the dog only half as great as in the cat, and not very much more than in the donkey. The following tables, too, which illustrate the weight of the brain in man at different ages, show that, although we might give 'the infant phenomenon' an anatomical basis, we shoukd greatly overrate the intellectual acuteness of the average baby if we were to measure it by the ratio of brain to body-weight alone.

\begin{tabular}{|c|c|c|c|c|}
\hline \multicolumn{3}{|c|}{ Age. } & \multicolumn{2}{|c|}{ Brain.weight. } \\
\hline I & year & $\cdots$ & $88_{5}$ & grm. \\
\hline 2 & years & $\cdots$ & 909 & ," \\
\hline 3 & ", & .. & $\mathrm{I}, 07 \mathrm{I}$ & " \\
\hline 4 & ," & . & I,099 & " \\
\hline 5 & ", & . & 1,033 & ", \\
\hline 6 & ," & $\cdots$ & $1, I+7$ & , \\
\hline 7 & ," & .. & $\mathrm{I}, 20 \mathrm{I}$ & " \\
\hline
\end{tabular}

\begin{tabular}{|c|c|c|c|c|}
\hline 8 & $\begin{array}{l}\text { Age. } \\
\text { years }\end{array}$ & $\cdots$ & $\begin{array}{l}\text { Brain-we } \\
\text { I, } 045\end{array}$ & $\begin{array}{l}\text { eight. } \\
\text { grm. }\end{array}$ \\
\hline Io & , , & .. & I, 3I 5 & ", \\
\hline I I & ,. & . & $\mathrm{I}, \mathrm{I} 68$ & ," \\
\hline I 2 & ," & . & $I, 286$ & , \\
\hline I 3 & ,. & $\cdots$ & 1,505 & ", \\
\hline I 4 & ," & . & 1,336 & , \\
\hline 15 & ", & . & $\begin{array}{c}x, 4^{14} \\
-\mathrm{BISC}\end{array}$ & chOFF \\
\hline
\end{tabular}

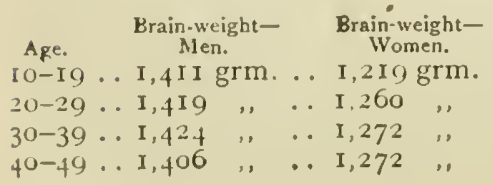

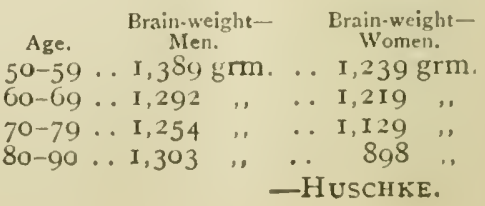


In some small birds the ratio is as high as I : I2, in large birds as low as I : I, 200; in certain fishes a gramme of brain has to serve for over 5 kilos of body. As a rule, especially within a given species, the brain is proportionally of greater size in small than in large animals. It is to be supposed that quality as well as quantity of brain substance is a potent factor in determining the degree of mental capacity.

The Cerebral Circulation.--The arrangement of the cerebral bloodvessels has certain peculiarities which it is of importance to remember in connection with the study of the diseases of the brain, many of which are caused by lesions in the vascular system-hremorrhage, or embolism. lour great arterial trunks carry blood to the brain, two internal carotids and two vertebrals. The vertebrals unite at the base of the skull to form the single mesial basilar artery, which, running forward in a groove in the occipital bone, splits into the two posterior cerebral arterics. Each carotid, passing in through the carotid foramen, divides into a middlle and an anterior cerebral artery; the latter runs forward in the great longitudinal fissure, the former lies in the fissure of Sylvius. A communicating branch joins the middle and posterior cerebrals on each side, and a short loop connects the two anterior cerebrals in front. In this way a hexagon is formed at the base of the brain, the so-called circle of Willis. While the anastomosis between the large arteries is thus very free, the opposite is true of their branches. All the arteries in the substance of the brain and cord are 'end-arteries '- that is to say, each terminates within its area of distribution without sending conmunicating branches to make junction with its neighbours. The consequence of these two anatomical facts is: (I) that interference with the blood-supply of the brain between the heart and the circle of Willis does not readily produce symptoms of cerebral anæmia; (2) that the blocking of any of the arteries which arise from the circle or any of their branches leads to destruction of the area supplied by it. Nearly all dogs recover after ligation in one operation of both carotids and both vertebral arteries. In monkeys both carotids may, as a rule, be safely tied, and one carotid in man. If, in addition to the two carotids, one vertebral be ligated at the same time in the monkey, sopor results, and this is generally followed by extensor rigidity, coma, and death in twenty-four hours. In one case a monkey survived this triple ligation, but became demented. The motor paralysis and rigidity were much greater than in the dlog. In the condition of partial anæmia the cortex is more excitable than normal, but the excitability disappears at once when the anæmia is rendered completc (Hill).

The basal ganglia are fed by twigs from the circle of Willis and the beginning of the posterior, middle, and anterior cerebral arteries. Of these the most important are the lenticulo-striate and lenticulo-optic branches of the middle cerebral, which are given off near its origin, and run through the lenticular nucleus into the internal capsule, and thence to the caudate nucleus and optic thalamus respectively. The chief part of the blood from the circle of Willis is carried by the three great cerebral arteries over the cortex of the brain. The white matter, with the exception of that in the immediate neighbourhood of the basal ganglia, is nourished by straight arteries which penetrate the cortex. The middle cerebral supplies the whole of the parietal as well as that portion of the frontal lobe which lies immediately in front of the fissure of Rolando and the upper part of the temporal lobe. The rest of the 
frontal lobe is supplied by the anterior cerebral, and the occipita! lobe, with the lower part of the temporal lobe, by the posterior cerebral. The medulla oblongata, cerebellum, and pons are fed from the vertcbrals and the basilar artery before the circle of Willis has been formed.

Resuscitation of the Central Nervous System after Total Anæmia. -Complete temporary anæmia of the brain and upper cervical cord can be produced in most cats by passing temporary ligatures around the innominate artery and left subclavian proximal to the origin of the vertebral artery. Artificial respiration is maintained through a tube passed through the glottis. The eye reflexes disappear very quickly, and a period of high blood-pressure immediately follows the occlusion. A fall of pressure succeeds, due to vagus inhibition of the heart, and this is followed by a second rise after the vagus centre succumbs to the anæmia. Respiration stops temporarily (in twenty to sixty seconds) after occlusion; then follows a series of strong gasps, and finally cessation of all respiratory movements. The blood-pressure slowly falls to a level which is then maintained approximately constant for the remainder of the occlusion period. The anterior part of the cord and the encephalon lose all function; no reflexes can be elicited from this part of the central nervous system. The intra-ocular tension is much reduced, and the cornea is characteristically wrinkled.

When the cerebral circulation is restored by releasing the vessels, the general arterial pressure soon begins to rise if the period of occlusion has not overstepped the limit of successful cardio-vascular resuscitation. The respiration returns suddenly, the time of return depending on the length of the occlusion and on other factors. The respiratory rate, at first slow, soon becomes normal, and then more rapid than normal. The eye-reflexes reappear more gradually; the intra-ocular tension increases, and the shrunken cornea becomes smooth and hard. The anterior part of the cord recovers its functions gradually; the reflexes connected with it return, first the homonymous, then the crossed. A short period of quiet follows; then spasms of the skeletal muscles appear, gradually increase in severity and extent, and terminate in $(a)$ death, $(b)$ partial, or (c) complete recovery. In partial recovery, disturbances of locomotion, such as walking in a circle, paralysis, apparent dementia or loss of intelligence, and loss of sight or hearing, may be observed. Voluntary movements of the head, neck, shoulders, and fore-limbs have been seen eight minutes after release from an occlusion of six minutes. Blindness has been observed without loss of the pupillary light reflex. In this case the visual cortex would seem to have suffered more than the lower centres, an illustration of a general rule. Complete recovery is rare after total anæmia lasting as much as fifteen minutes, and has not been observed after an anæmia of twenty minutes. Ten to fifteen minutes of total anæmia represent 
the linit beyond which recovery of the brain, and therefore successful resuscitation of the aninal, cannot be expected.

Chemistry of Nervous Activity.-Of this we are practically ignorant. The percentage composition of the solids and the percentage of water in the brains of three persons of different ages are exhibited in the following table (W. Koch):

\begin{tabular}{|c|c|c|c|c|c|c|c|}
\hline & \multirow{2}{*}{$\frac{\begin{array}{c}\text { Child } 6 \text { Weeks } \\
\text { (Brain } 640 \text { Grms.) }\end{array}}{\text { Whole brain. }}$} & \multicolumn{3}{|c|}{$\begin{array}{c}\text { Child } 2 \text { Years } \\
\text { (Brain } 1,100 \text { Grms.). }\end{array}$} & \multicolumn{3}{|c|}{$\begin{array}{c}\text { Adult } 19 \text { Years } \\
\text { (Brain } x, 670 \text { (irms.). }\end{array}$} \\
\hline & & Grey. & White. & $\begin{array}{l}\text { Whole } \\
\text { Brain.* }\end{array}$ & Grey. & White. & $\begin{array}{l}\text { Whole } \\
\text { Brain.t }\end{array}$ \\
\hline Proteins & $4^{6 \cdot 6}$ & $4^{8 \cdot 4}$ & $3 r \cdot 9$ & $40 \cdot 1$ & $47 \cdot 1$ & $27 \cdot 1$ & $37 \cdot I$ \\
\hline Extractives .. & I $2 \cdot 0$ & Io.o & $5 \cdot 9$ & $8 \cdot 0$ & $9 \cdot 5$ & $3 \cdot 9$ & $6 \cdot 7$ \\
\hline Ash $\quad \ldots \quad \ldots$ & $8 \cdot 3$ & $5^{\cdot 8}$ & $3 \cdot 2$ & 4.5 & $5 \cdot 9$ & $2 \cdot 4$ & $4 \cdot I$ \\
\hline $\begin{array}{c}\text { Lecithins and } \\
\text { kephalins }\end{array}$ & $24 \cdot 2$ & & $26 \cdot 3$ & & & & \\
\hline Cerebrins $\quad \ldots$ & $\begin{array}{r}242 \\
69\end{array}$ & $\begin{array}{r}24.7 \\
8.6\end{array}$ & $17 \cdot 2$ & $\begin{array}{l}25.5 \\
12.9\end{array}$ & $\begin{array}{r}23 \cdot 7 \\
8.8\end{array}$ & $\begin{array}{l}31.0 \\
16.6\end{array}$ & $\begin{array}{l}27 \cdot 3 \\
12 \cdot 7\end{array}$ \\
\hline Lipoid $\mathrm{S}$ as $\mathrm{SO}_{4}$ & $0 \cdot 1$ & $0 \cdot 1$ & 0.5 & $0 \cdot 3$ & 0.1 & 0.5 & $0 \cdot 3$ \\
\hline Cholestcrin & $I \cdot 9$ & $2 \cdot 4$ & $15^{\circ} \mathrm{O}$ & $8 \cdot 7$ & $4 \cdot 9$ & $18 \cdot 5$ & I I $\cdot 7$ \\
\hline Water.. & $88 \cdot 78$ & 84.49 & $76 \cdot 45$ & $80 \cdot 47$ & $83 \cdot 17$ & $69 \cdot 67$ & $76 \cdot 4^{2}$ \\
\hline
\end{tabular}

The next table shows the variations in the content of water, solids, and protein in different parts of the nervous system (Halli. burton):

\begin{tabular}{|c|c|c|c|c|c|c|}
\hline & & & & $w_{\text {ater. }}$ & Solids. & $\begin{array}{l}\text { Percentage of Pro. } \\
\text { teins in Solids. }\end{array}$ \\
\hline \multicolumn{2}{|c|}{ Cerebral grey matter } & $\ldots$ & $\ldots$ & $83 \cdot 5$ & $16 \cdot 5$ & $5 I$ \\
\hline \multicolumn{2}{|c|}{ Cerebral white matter } & . & .. & $69 \cdot 9$ & $30 \cdot 1$ & 33 \\
\hline Cerebellum & . & $\ldots$ & $\ldots$ & $79 \cdot 8$ & $20 \cdot 2$ & 42 \\
\hline Spinal cord as & whole & . & $\ldots$ & $71 \cdot 6$ & $28 \cdot 4$ & $3^{x}$ \\
\hline Cervical cord & . & . & . & $72 \cdot 5$ & $27 \cdot 5$ & $3 I$ \\
\hline Dorsal cord & .. & . & .. & $69 \cdot 8$ & $30 \cdot 2$ & 28 \\
\hline Lumbar cord & .. & .. & . & $72 \cdot 6$ & $27 \cdot 4$ & 33 \\
\hline Sciatic nerves & . & . & $\ldots$ & $65 \cdot 1$ & $34^{\circ} 9$ & 29 \\
\hline
\end{tabular}

The grey matter of the cerebrum in the adult contains $8 \mathrm{I}$ to S6 per cent. of water, the white matter 68 to 72 per cent., the brain as a whole $8 \mathrm{I}$ per cent., the spinal cord 68 to 76 per cent., and the peripheral nerves 57 to 64 per cent. In the fœt us more water is present $(92$ per cent. in the grey and 87 per cent. in the white matter).

The superior richness of the grey matter in proteins and the preponderance of water in it are the chief chemical peculiarities which distinguish it from the white matter. That it should have 
a ligh protein content is easily understood, for the protoplasmic structures, the nerve-cells, are situated in the grey matter. But that the most important functions should have their seat in a tissue containing only i 4 to I 9 per ecent. of solicls is surprising, and slould warn us that the water is no lest significant a constituent of living matter than the solids, and that it is not the mass of the solid substances in a tissue which is the essential thing, but the whole colloid complex, which cannot be constituted witlout the water.

Fresh nervous tissues are alkaline to litmus, but become acid soon after death. No change of reaction has been detected during activity.

That oxygen is used up during cerebral activity is certain, and when the brain is coloured witl methylene bluc, by injecting it into the circulation, any spot of it which is stimulated loses the blue colour, the pigment being reduced. But if the animal is so deeply narcotized that it does not respond to stimulation, the change of colour does not occur.

Cholin (p. 366), a substance which can be derived from lecithin, is believed to represent one of the maste products of nervous activity. Exceedingly small traces of it are present in normal cerebro-spinal fluid, and in certain diseased conditions of the brain, as in gencral paralysis, the quantity is said to be notably increased, indicating an increased decomposition of lecithin. The fatty acid constituent of lecithin is liberated in degenerating nerve, giving rise to the reaction with osmic acid (p. 797). Some writers assert that this increase in the cholin can be used as a test to distinguish organic nervous disease from that which is purely functional. But the matter is in dispute.

Cerebro-spinal Fluid. The cercbro-spinal fluid, which lills the ventricles of the brain and the central canal of the cord, is continuous with that contained in the subarachnoid space throush the foramen of Magendie, an opening in the piece of pia mater that helps (c) roof in the fourth ventricle. It is secreted in part by the cubical colls covering the choroid plexus, a fold of pia mater which projects into each lateral ventricle. Extracts of clioroid plexus, when injected intravenously, increase the rate of secretion.

This action is dependent upon the presence of some substance in the choroid plexus, which, however, is not a specific product of the activity of the plexus, since extracts of the brain produce the same effect. It may therefore be some product of the metabolism of the brain which passes to the choroid plexus and stimulates secretion by the epithelium. The substance is removed from the fluid by filtration through a Clamberland filter, and is therefore probably of high molccular weiglit. It is probable that variations in the rate of secretion of the cercbro-spinal fluid by the choroid plexus are more influcntial in governing the intracranial pressure than variations in 
the arterial and venous pressures. The idea that the cranial contents constitute a lixed quantity, without the power of contraction or expansion, can no longer be maintained (Dixon and Halliburton). A graphic record of the rate of secretion of the cerebro-spinal fluid in the dog may be obtained by inserting a hollow needle through the occipito-atlantoid ligament into the great subaraclunoid cistern, and allowing the liquid to fall upon a drop-counter writing on a drum (Fig. 399). It is not always possible, however, to be certain by this method that the rate at which the fluid escapes represents

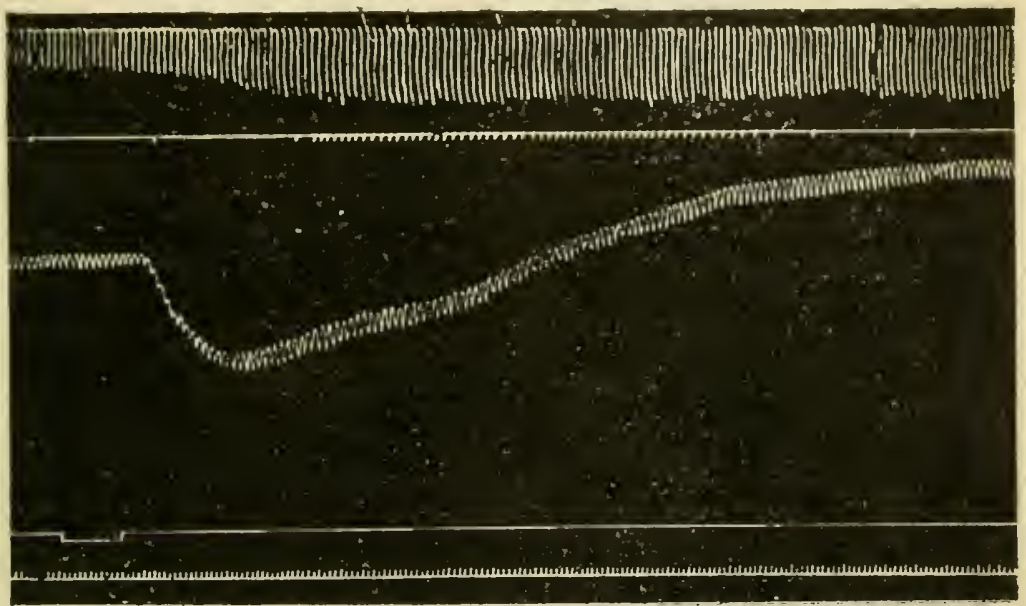

Fig. 399.- Influence of Extract of the Posterior Lobe of the Pituitary upon the Flow of Cerebro-Spinal Fluid through a Hollow Needle inserted into the Cisterna Magna through the Occipito-Atlantoid Ligament in a Dog. The animal was anzesthetized by a constant method, insufflation of ether into the trachea. The uppermost curve is respiration; the next, drops of cerebro-spinal fluid; the next, arterial blond pressure; the fourth, signal line showing the point at which $50 \mathrm{mg}$. of a dried extract of posterior lobe was injected into a vein. The signal line is also the zero of blood pressure. The bottom trace is the time in seconds (Weed and Cushing).

accurately the rate at which it is formed. A more exact method appears to be the introduction of the needle into the third ventricle (Fig. 400).

Cerebro-spinal fluid can easily be obtained in man by lumbar puncture with a hypodermic needle sufficiently long to enter the subarachnoid space in the spinal canal. The point usually selected for the puncture is between the fourth and fifth lumbar vertebræ. The normal pressure of the fluid is such that it trickles out by drops, but in disease it is sometimes so high that it spurts out in a steady stream. An examination of the fluid, especially for leucocytes or bacteria, is of great diagnostic value in certain conditions. Normally it is a thin, clear, watery fluid, faintly alkaline in reaction 
to litmus, and with a specific gralvity of absut rosof to Ino7. It contains the ordinary salts, but more putassium than sodium, unlike other body tluids; a very small amount of protein (globulin)usudlly about O.I per cent. - and a little dextrose (Nawratzki). It: composition is evidently different from that of ordinary lymph. Only a few lrmphocrtes are present in health, but in some diseases (as in general paralysis of the insane, tabes, and cerebro-spinal syphilis) a marked increase occurs. In acute cercbro-spinal meningitis numerous polymorphonuclear leucocytes are found, which are absent from the normal fluid.

The depression of the freezing-point $(\Delta)$ usually lies between $0.60^{\circ}$ and $0.05^{\circ} \mathrm{C}$. In a case of livdrocephalus it was $0.65^{\circ} \mathrm{C}$. Normally, cerebro-spina! fluid is somewhat hypertonic to the blood-

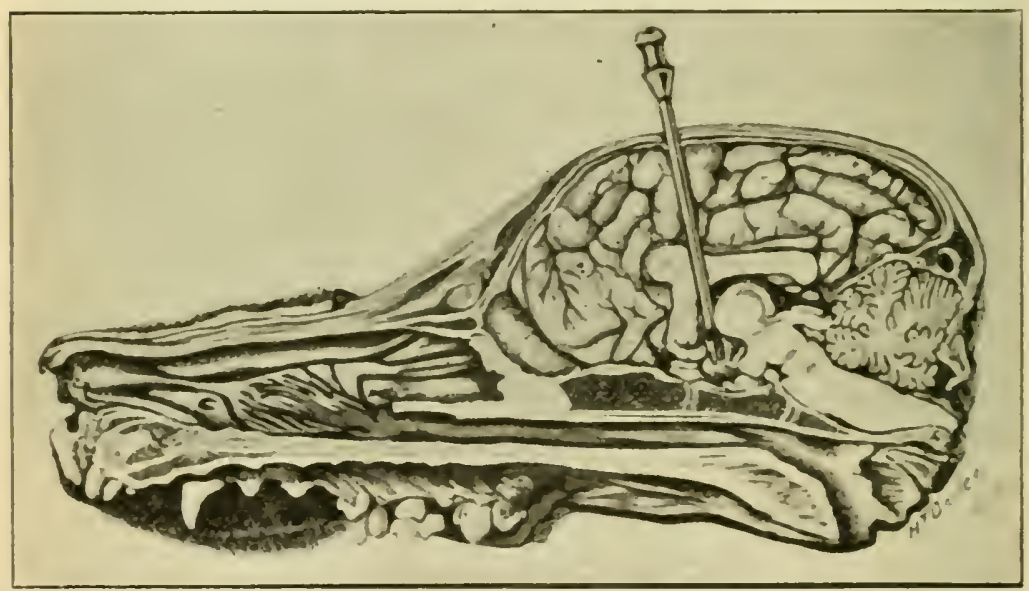

Fig. 400 - Sagittal Section of Dog's Skull, showing the Needle introduced into Third Ventricle to tap Cerebri-Spinal Fluid (Weed and Cushing).

serum. In injury of the cribriform plate of the ethmoid bone and also in some cases where there is no traumatic injury, the fluid iseapes from the nose, and the rate of its formation can thus be ascertained. In one case it was found to be as much as 2 c.c. to nearly + c.c. in ten minutes.

\section{PRICTICAI. EXERCISES ON CHAPTER XIT.}

I. Section and Stimulation of the Spinal Nerve-Roots in the Frog. - (a) Select a large frog la bull-frug, if pusible Pith the brain. Fasten the frog, belly down, on a plate of cork. Wake an incision in the mildle line nier the spinoirs procesese of the lowest three or four vertebre. spparate the muscles from the vertebra! arches, and with strong sclsiors (1) wen the spinal canal, taking care not to injure the corll by pasing the bla le of the scisors to deeply. Extend the opening upwards till two 
or three posterior roots come into view. Pass fine silk ligatures under two of them, tic, and divide one root central to the ligature, the other peripheral to it. Stimulate the central end, and reflex movements will occur. Stimulate the peripheral end; no effect is produced. Now cut away the exposed posterior roots and isolate and ligature two of the anterior roots, which are smaller than the posterior. Stimulate the central end of one: there is no effect. Stimulation of the peripheral end of the other causes contractions of the corresponding muscles.

(b) Stimulation of the roots may be repeated on the mammal, using the dog employed for the experiment on the motor areas ( $\mathrm{p}$. IOOI): Place tue alll11al, belly down, and insert a good-sized block of wood between it and the board at the level of the lumbar vertebra of the spine. Divide the skin and muscles on either side of this region till the laminæ of the vertebræ are exposed. Snip through them with strong forceps, and open the spinal canal, exposing a length of cord corresponding to three or four vertebræ. Ligate and stimulate the roots as in $(a)$.

2. Reflex Action in the 'Spinal' Frog.--Pith the brain of a frog, destroying it down to the posterior third of the medulla oblongata. (r) Note the position of the limbs immediately after the operation, and again thirty to forty minutes later. Its hind-legs possess tone, and are drawn up against the flanks. The animal can still execute certain co-ordinated movements-e.g., pulling away its leg if a toe is pinched. The power of maintaining equilibrium is lost. If placed on its back, it lies there. When thrown into water it sinks usually without any attempt at swimming. Verify the following facts, using mechanical stimulation (pinching the toes or skin of the leg): (a) If the stimulus provokes muscular movements only on one side of the body, this is usually on the same side as the stimulated point. (b) When the stimulus causes reflex movements on both sides of the body, the stronger contractions are on the side to which the stimulus was applied.

Determine whether it is easier to obtain movement of a portion of the body innervated from a region of the cord above the level of the stimulated nerves or below that level.

(2) With electrical stimuli (using a coil arranged for single shocks determine if reflex movements are elicited by a single induced shock applied to the skin. Verify the fact that a series of slocks is more efficient, the effects of the separate stimuli being summated in the reflex centres.

(3) To test the effect of thermal stimuli, dip the leg into a beaker of warm water. Vary the temperature of the water, using a series of beakers with water at $10^{\circ} \mathrm{C}$, $15^{\circ} \mathrm{C}, 20^{\circ} \mathrm{C}$, , etc., above the temperature of the room. Place the leg for a moment in each, and determine which is the most efficient stimulus. Immediately on withdrawing the leg from each of the hot-water beakers immerse it in a beaker of water at room temperature. Finally, dip the leg into a beaker of cold water, and heat it gradually to a teinperature at which a reflex was previously obtained. Probably it will not be elicited by the gradual warming.

(4) 'Purposive' Movements. - Touch the skin of one thigh with blotting-paper soaked in strong acetic acid. The leg is drawn up, and the foot moved as if to get rid of the irritant. If the leg is held, the other is brought into action. Immerse the frog in water to wash a way the acid.

(5) Spread (Irradiation) of Reflexes.-Gently stimulate a toe or a small spot on the flank with weak induction shocks or weak mechanical stimuli, and note the reflex effect obtained. Then go on gradually increasing the strength of stimulation without increasing the area of the field stimulated, and observe the extent and order of spread of the reflex movements.

3. Reflex Time.-Pass a hook through the jaws. Holding the frog by 
the hook, dip one leg into a dilute solution of sulphuric acid $(0.2$ to 0.5 per cent.), and note with the stop-watch the interval which elapses before the frog draws up its leg ('Türck's inetliod of determining the reflex tine). Wash the acid off with water.

Determine how the reflex time varies with the strength of the stimulus. This can be done by using various strengths of acicl. The refiex time will be shorter the stronger the stimulus up to a certain point. Compare the reflex time of movements on the same side of the body as the point of application of the stimulus and on the opposite side.

4. Inhibition of the Reflexes.-(I) Destroy the corebrum of a frog. Dip one leg into dilute sulphuric acid as in 3 , and estimate the reflex time. Then apply a crystal of common salt to the upper part of the spinal cord. If the opening made for pithing the frog is not large enough to enable the cord to be clearly seen, enlarge it. Again dip the leg in the dilute acid. It will either not be drawn up at all, or the interval will be distinctly longer than before.

(2) Expose the viscera, including the heart, taking care not to injure the cardiac nerves. Tap the intestines sharply $w$ ith the handle of a scalpel many times in succession. The heart is inhibited.

(3) Tie strings tightly around both fore-legs of a normal frog. Place the animal on its back; it does not turn over. The hind-legs may be pulled about in various ways without the frog turning over into its normal position. The reactions concerned in the maintenance of equilibrium are inhibited. Remove the strings. The animal cannot be made to lie on its back except by force.

5. Spinal Cord and Muscular Tonus.-Destroy the brain of a frog. Isolate the gastrocnemius, and cut away the bone below the knee. Isolate the sciatic nerve without injuring it. Remove the muscles from the femur, cut the bone and fix it in a clamp for graphic recording. Connect the tendon with a lever, weighted with 5 to 10 grammes. Take a base line. Destroy the spinal cord, or cut the sciatic and again take a base line. The length of the muscle is slightly altered.

6. Spinal Cord and Tonus of the Bloodvessels.-Destroy the brain of a frog. Arrange the web of the foot on the stage of a microscope, and note the calibre of the bloodvessels in the field. Destroy the cord, and observe the change in their calibre. They will dilate.

7. Action of Strychnine.-Pitl a frog (brain only). Inject into one of the lympli-sacs three or four drops of a o.1 per cent. solution of strychnine. In a few minutes general spasms come on, which have intermissions, but are excited by the slightest stimulus. The extensor muscles of the trunk and limbs overcome the flexors. Destroy the spinal cord; the spasms at once cease, and cannot again be excited.

8. Mammalian Spinal Preparation (Sherrington).*-Deeply anæsthetize a cat with ether. Insert a cannula into tlie trachea (p. 202), and continue the anæsthesia through this. Expose and ligate both common carotids. Make a transverse incision through the skin over the occiput, and extend it laterally behind the ears. Pull back the skin so as to expose the neck muscles at the level of the axis vertebra. Feel for the

- A similar preparation can be used for certain experiments on the circulation (Crile, Guthrie). For these, as well as for the study of many reflexes, a good preparation is obtained by occlusion of the cerebral blool-supply in cats (without decapitation). Even a human 'spinal preparation' is capable of executing reflex movements. The 'lurkomans are stated to have decapitated their prisoners and inmediately placed on the neck a hot metal plate, which seuled up the cut vessels. The (reflex) movements, which are clescribed as very lively were then watched with an interest, it is to be supposed, not wholly scientific. 
ends of the transverse processes of the atlas, and divide the miscles (lown to the bone just behind these processes. Now start artificial respiration (1).202), or sooner if necessary. Notch the spinous process of the axis with bone forceps. Pass a strong thick ligature by a sharpcnded aneurism needle close under the body of the axis, and tie it tightly in the gruove left by the incision behind the transverse processes of the atlas and the notch made in the spinous process of the axis. This compresses the vertebral arteries where they pass from transverse process of axis to transverse process of atlas. Pass a sccund strong, ligature under the trachea at the level of the cricoid cartilage and include in it the whole neck, except the trachea, but at present only tie a single loop on it. Now decapitate the animal with a large knife (an amputating knife) passed from the ventral aspect of the neck through the occipitoatlantal space, severing the cord just behind its junction with the bulb. At the moment of decapitation tighten the ligature round the neck and complete the knot. Destroy the head. If there is oozing of blood from the vertebral canal, arrest it by raising the neck somewhat above the level of the body. The carcass must be kept warm by placing it on a metal box or table containing hot water, and the air used for artificial respiration must also be warmed, as by passing it through a coil of rubber tubing immersed in a water-bath which is kept lot. Stitch the skin-flaps together so as to cover the cut end of the spinal cord and the other structures cut in decapitation. By this procedure the spinal cord is usually severed about 4 millimetres behind the point of the calamus scriptorius. Although the blood-pressure remains low, reflexes employing the skeletal muscles can be fairiy well elicited for hours. Study on the preparation the reflexes described in the text (pp. 90 I, 904)-e.g., the flexion reflex of the hind and fore limb, as elicited from the skin, or one of the afferent nerves of the limb-the crossed extension reflex of hind and fore limb, the scratch reflex.

(I) Scratch Reflex.-(a) Evoke the reflex by rubbing the skin of the neck behind the pinna. The scratching movements are in the hind-leg of the same side. Record them on a drum, on which is also written a time-tracing in seconds. The record can be obtained by tying a piece of tape, not too tightly, round the foot, leg, or thigh, and connecting this by a thread with a lever. The thread is passed over a pulley below the lever, so that its pull may be exerted at right angles to the axis of rotation of the lever. The lever is attached to a light spring or a rubber band, which is stretched when it moves in one direction, and in recoiling brings it back again to its position of rest at the end of the contraction. If the reflex is not easily evoked, it can be facilitated by producing a slight degree of asphyxia by temporarily clamping the respiration tube. Some time must elapse after the decapitation before a fair scratch reflex can be expected. It is usually sufficiently well marked within an hour.

(b) While the reflex is occurring, stimulate with an interrupted current the central stump of the popliteal nerve of the opposite hind-limb. The scratch reflex may be cut short by inhibition. Also, during the stimulation of this nerve the reflex may be incapable of being elicited till the excitation of the inhibitory afferent nerve is stopped.

(2) Flexion Reflex.-(a) Stimulate with a weak interrupted current the skin of some part of the hind-limb-say one of the toes. The flexion reflex of the hind-limb on the same side may be evoled-i.e., a flexion movement at the knee, hip, and ankle. Record the movements of one of the joints or of flexor muscles after severing them from their insertion.

(b) Stimulate with a weak interrupted (faradic) current the central stump of one of the nerves of a hind-limb-say the peroneal nerve. The flexion reflex of the same limb may be elicited. Record the move- 
ments. Now produce temporary asphyxia by clampine the respirition tube, and repeat the stimulation at half-minute intervals. The refex will be increased by the asphyxia. Do not interrupt the respiration for more than two or three minutes, and immediately start it if the heart, which can be felt through the chest, begins to weaken.

(3) Elicit the knee-jerk, as described in the text (p. $9 \circ$. ). It is generally exaggerated.

(1) By the unipolar method (p. 951) stimulate with a point electrorle one lateral half of the cross-section of the cervical cord exposed in decapitation. The large electrode is placed on a shaved part of a forearm. Varions effects may be clicited according to the point of the cross-section stimulated-e.g., stepping and scratch movements of the hind-limbs. Other facts mentioned in the text in regard to spinal reflexes can be verified on this preparation.

9. Decerebrate Cat Preparation (Miller and Sherrington).-This preparation, which must be made by the demonstrator, differs from the spinal preparation described in 8 , in that the plane of the section is considerably higher, passing through the posterior part of the midbrain 'entering the posterior colliculi (posterior corpora quadrigemina) near their hinder end and emerging about a millimetre anterior to the front eclge of the pons.' Many reactions can be conveniently studied on this preparation, some of which cannot be obtained with the spinal preparation. The respiratory movements usually go on without interruption, and reflexes whose centres are situated in the bulb, such as the swallowing reflex, can be elicited. The circulation is well maintained, and the preparation can be used for a number of the experiments given after Chapters III. and IV.

Under deep anæsthesia (chloroform and ether), the carotids are temporarily clamped opposite the uppermost tracheal cartilage, and the eat is then placed on the decerebrator (Fig. fo I) in the prone position, with its neck on the upper edge of the neck-block.

The interparictal suture is exposed by an incision through the scalp from beyond the coronal in front to the lambdoid ridge and supraocipital protuberance behind. A distance of 30 millinetres is then measured off from the coronal suture backward along the interparictal and the point is marked by notching the longitudinal median ridge of bone. The head is placed between the prongs of the yoke with the tongue-guard of the yoke-plate inside the mouth above the tongue, which it protects. The chin lies in the hollow of the yoke under the yoke-plate, the lower canines covered by the plate. The pelvis lies on the pelvic platform, $P$, the hind limbs hanging freely. The head is pushed down, the tongue guard in the mouth, until the embayed end of the yoke-plate on either side of the tongue-guard meets the anterior edge of the coronoid process of the lower jaw. The hook attached to the leather cord is fixed in a loop of string previously tied transversely through the upper lip, and the snout is drawn firmly down by the cord, securing the head in position. The cord is fastened to a cleet on the under surface of the neck-block. The nose-piece is now slid up so as to engage and support in its notel the apex of the muzzle, and is fixed by the serew-clamp. A knife consisting of a planing-blade mounted in a wooden handle is used for the decerebration.*

* The blade is 12.5 centimetres wide, a centimetres ligh, and + millimetres thick. It is bevelled on one face, the hevelled edge heing 2 centimetres (lecp). The cutting edge dres net extend the whole width of the blade, but stops short at 1.5 centinctres from each lateral edge. The mid-wiclth of the blale is marked by an engraved line on the frunt of the knife. 
The operator, standing on the lelt sicle of the anmal, applies the mirtpoint of the sharp edge of the blade, bevelled side forward, to the point notehed in the interparietal ridge, the width of the blade being kept truly at right angles to the mediun plane of the head. The knife thus heli in the left hand is kept vertical, or nearly so, and is rirected so that the plane of the blade if continued downward through the

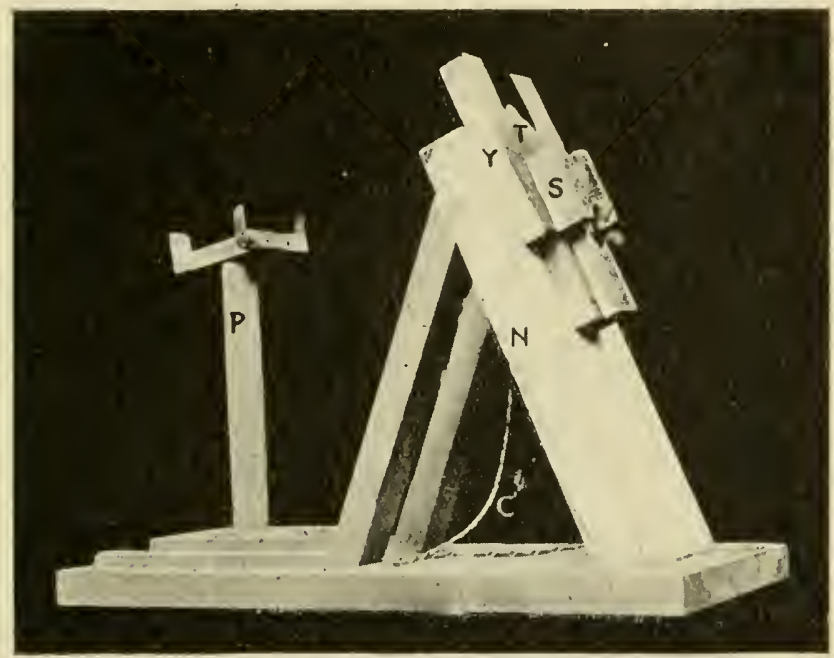

Fig. 40r.-Cat Decerebrator (Miller and Sherrington). $N$, wooden neck-block, mounted firmly on a strong base-board. $N$ is inclined at $22^{\circ}$ to the vertical and supported by two stout wooden props meeting it somewhat below its top at an angle of $44^{\circ}$. The top edge of the neck-block (cut at right angles to the face of the block) is therefore at an angle of $22^{\circ}$ to the horizontal. $Y$, yoke, a $\mathbf{Y}$-shaped fork of wood with one of the prongs projecting 6 centimetres beyond the top of the neck-block, the other prong truncated nearly to its base. A $Y$-shaped steel plate (the yoke-plate) is screwed to $Y$. It is shaped somewhat like the wooden yoke, but its two side prongs are of equal length, and between them projects a shorter prong, $T$, the tongue-guard. $S$ is a $T$-shaped wooden nose-piece, adjustably attached to the front of the steel-covered yoke. In the upper border of the cross-piece of the $T$, is a $\mathbf{V}$-shaped notch. A slot in the stem of the $T$ allows the $T$-block to be slid up or down on the steel yoke-plate, and it can be fixed at any position by a thumb-screw. $C$, a leather cord passing through a hole picrcing neck-block, yoke and yoke-plate. The top cnd of the cord, issuing from the hole in the mid-line of the yoke-plate, carries a short strong hook. The point of the notch in the nose-piece slides up to and slightly beyond this hole. $P$, a light single-pillared platform, somewhat saddle-shaped at the top, which can be slid on the baseboard nearer to or farther from the ncck-block.

head would meet a horizontal line engraved on the side-prongs of the yoke-plate. This line is at the level of the free end of the tongueguald. A light blow is then struck with a mallet on the top of the handle of the knife, sufficient to engage the edge in the skull in the proper direction-i.e., toward the horizontal line on the metal plate. Then with a couple of heavier blows the head is severed in the desired plane. 
The preparation is then remored from the apparatus, the neck being held laterally belintel the wings of the atlas to control the vertebral arteries. It is placed on the cxperiment table with the neck well raised by a string passed through the skin over the occiput, to restrain any hicmorrlage. Some cotton is packed across the cut surface of the midbrain. Bleeding soon ceases, and in two or three minutes one carotid can be unclamped, arteries in the masseteric regions being tied if necessary. In two minutes more the clamp can be removed from the other carotid.

10. With the preparation described in 9, study the swallowing reflex, evolied -e.g., by the application of water by drops to the pre-epiglottidean sinus between the base of the tongue and the epiglottis, or by allowing water to drop into the pharynx. Dilute alcohol (one part of ethyl alcohol added to four parts of water) is even more effective than water; oil much less effective.

11. Reflex Postural Tonus (Decerebrate Rigidity).-Study the distribution of the tonus in the decerebrate cat prepared as in $a-c . g$. in the cxtensor muscle of the knee (the vasto-crureus), the gastrocnemius, scmimembranosus, triceps, supraspinatus, etc. Isolate the knce cxtensor by paralyzing all the other muscles of both hind limbs by nerie section. The vasto-crureus still maintains its postural reflex contraction. To observe the right vasto-crureus place the animal on its left side. Begin with the knce nearly at full extension and determine what weight, attached to the tibia and pulling it backward by a cord fastened over a pulley-i.e., tending to flex the knee-is just counteracted by the postural action of the muscle. Now forcibly flex the knee nearly. to the full, bencling it steadily and not too quickly, so that the movement occupies a couple of seconds. Apart from a slight partial return towards extension, and this not always, the limb remains in the new position. Although the length of the vasto-crureus is now greater than before, the weight needed to counterbalance its pull is practically. the same. That is to say, the muscle has assumed a new postural length without any sensible change of tension. This is the so-called 'lengthening reaction' of the posturally contracted muscle. The 'shortening reaction' can be obtained by repeating the observations in the reverse order-i.e., starting with the knee in nearly full flexion (Sherrington).

12. Reflexes in Man.-Study systematically on a fellow-student and on yourself the chicf reflexes described in the text (p. 914), cspecially-

The Knee-Jerk.-(1) Elicit the jerk in the usual way by striking the ligamentum patellie and observe its height. Then cause the patient to make a strong voluntary movement (squeezing the hands together or clenching the jaws) at the moment when the tendon is struck, and note whether the height is increased by ' reinforcement.'

(2) Attacli a suitable recording apparatus to the foot of a person sitting with his legs over the edge of a table, and record the jerks clicited by taps made as uniform in strength as possible. A small hammer worked by an electro-magnet or a spring night be employed for this purpose. Compare the records obtained when the jerk is elicited while the person is squeczing his hands together with those previously obtained. The influence of mental activity, especially of excitement or irritation (opportunities of studying such physical states occasionally offer themselves in plyysiological laboratories) in increasing the height of the knee-jerk may also be verified (Lombard).

13. Excision of Cerebral Hemispheres in the Frog (Fig. f02).Inasthetize a frog lightly by putting it under a bell-jar or tumbier 
witl a small piece of cotton-wool soaked in ether. Put very little ether (1) the cotton, and leave the frog only a very short tinie under the bell-jar. Then, holding it in a cloth, make an incision through the skin over the skull in the mesial line. Witl scissors open the cranium about the position of a line drawn at a tangent to the posterior borders of the two tympanic membranes. Remove the roof of the skull in front of this line with forceps, scoop out the cerebral hemispheres, and sew up the wommd. Is soon as the animal has recovered from the ether, the phenomena described at p. 947 should be verified. The frog will swim when thrown into water, will refuse to lie on its back, and will not fal! if the board on which it lies be gradually slanted. Iet the frog live for a lay, keeping it in a moist atmosphere; then expose the brain again, determine the reflex time by Türck's method: apply a crystal of common salt to the optic lobes, and repeat the observation. The reflex movements will be cornpletely inhibited or delayed. Remove the salt, wash with physiologiral salt solution, excise the optic lobes, and see whether the frog will now swim.

14. Excision of the Cerebral Hemispheres in a Pigeon.-Feed a pigeon for two or three lays on dry food, etherize it by holding a piece of cotton-wool sprinkled with ether over its beak, or inject into the rectum gramme chloral liydrate. The pigeon being wrapped up in a cloth, and the head held steady by an assistant, the feathers are clipped off the head, an incision made in the middle line through the skin, and the flaps reflected so as to expose the skull. Cut through the bones with scissors,

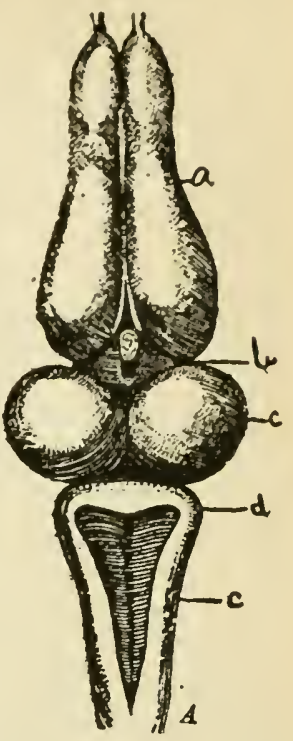

Fig. 402.-Brain of Irric (after Steiner). $\boldsymbol{a}$, cerebral hemispheres; $b$, position of optic thalami; $c$, optic lobes; $d$, cerebellum; $e$, medulla obiongata; $\mathbf{A}$, upper end of spinal cord. and make a sufficiently large opening to bring the cerebral hemispheres into view. They are now rapidly divided from the corpora bigemina and lifted out with the handle of a scalpel. The bleeding is very free. but may be partially controlled by stuffing the cavity with the vegetable fibre known as Pengavar Djambi, which should be removed in a few minutes, the wound cleansed with iodoform gauze wrung out of physiological salt solution at $50^{\circ} \mathrm{C}$, and sewed up. Study the phenomena described on p. 948.

15. Stimulation of the Motor Areas in the Dog.-(a) Study a hardened brain of a dog, noting especially the crucial sulcus (Fig. 382, p. 950), the convolutions in relation to it, and the areas mapped out around it by Hitzig and Fritsch and others. (b) Inject morphine under the skin of a dog. Set up an induction-coil arranged for tetanus, with a singie Daniell in the primary circuit. Connect a pair of fine but not sharp. pointed electrodes tlirough a short-circuiting key with the secondary. Fasten the dog on the holder, belly down, and put a large pad under the neck to support the head. Clin the hair over the scalp. Feel for the condyles of the lower jaw, and join them by a string across the top of the head. Connect the outer canthi of the eyes by another thread. The crucial sulcus lies a little behind the mid-point between these two lines. Now give the dog ether, make a mesial incision through the skin down 
to the bone, and reflect the flaps on either side. Detach as much of the temporal muscle from the bone as is necessary to get room for two trephine holes, the internal borders of which must be not less than t inch from the middle line, so as to avoid wounding the longitudinal sinus. Carefully work the trephine through the skull, taking care not to press heavily on it at the last. Raise up the two pieces of bone with forceps, connect the holes with bone forceps, and enlarge the opening as much as may be necessary to reach all the "motor' areas. At this stage only enough ether should be given to prevent suffering. Now unbind the hind-and fore-limbs on the side opposite to that on which the brain has been exposed, apply blunt electrodes successively to the areas for the fore- and hind-limbs, and stimulate." The 'unipolar' method of stimulation (p. 95 I) may also be employed. Contraction of the corresponding groups of muscles will be seen if the narcosis is not too deep. Movements of the head, neck, and eyelids may also be called forth by stimulating the 'motor' areas for these regions. Stimulation in front of the crucial sulcus may also cause great dilatation of the pupil, the iris almost disappearing. The dilatation takes place most promptly and is greatest on the opposite side, but the pupil on the same side is also widened. Even after section of both vago-sympathetic nerves in the neck, a slow and slight dilatation, greatest perhaps on the same side, may be caused by cortical stimulation. Repeat the whole experiment on the opposite side of the brain. In the course of his observations the student will perhaps have the opportunity of seeing general epileptiform convulsions set up by a localized excitation. They begin in the group of muscles represented in the portion of the cortex directly stimulated. After the convulsions have been sufficiently studied, they should be again induced, and the stimulated 'motor' area rapidly excised during their course. In some cases this will be followed by immediate cessation of the spasms. (c) The same animal can be used for stimulation of the spinal nerve-roots, as described in Experiment I (p. 995).

- it is not necessary to remove the dura mater. 


\section{CHAPTER XVII}

\section{THE AUTONOMIC NERVOUS SYSTEM (THE SYMPATHETIC AND ALLIED NERVES)}

THE cfferent fibres of the body can be divided into two classes: (I) Those which supply multinuclear striated muscle (skeletal muscle); (2) those which supply other structures (smooth muscle, heart muscle, glands). The second group is called 'autonomic,' to indicate that it possesses a certain independence of the central nervous system, although this independence is far from absolute. The autonomic fibres arise from four regions of the central nervous system: (I) The mid-brain; (2) the bulb; (3) the thoracic and upper lumbar cord; (4) the sacral portion of the cord. All autonomic fibres after issuing from the central nervous system end sooner or later by forming synapses around nerve-cells of sympathetic type, by whose axons the path is continued to the peripheral distribution. The autonomic path accordingly comprises two neurons, the fibre which arises from the brain or cord being termed the "preganglionic,' and that which arises from the sympathetic ganglion the 'postganglionic' fibre.

The autonomic fibres originating in the mid-brain emerge in the oculo-motor nerve, and form synapses with cells in the ciliary ganglion, which in turn send fibres to the ciliary muscle and the constrictor muscle of the iris (pp. 923, I024), The bulbar autonomic fibres emerge in the seventh, ninth, and tenth cranial nerves. Those in the vagus include inhibitory fibres for the heart muscle, motor and inhibitory fibres for the smooth muscle of the alimentary canal from the osophagus to the descending colon, and for the muscles of the trachea and lungs, and secretory fibres for the gastric glands and the pancreas. The sympathetic ganglion cells with which these preganglionic fibres form synapses have not always been definitely located, but lie near or in the tissue supplied (p. $18 \mathrm{I}$ ). The autonomic fibres in the seventh and ninth nerves supply the mucous membranes of the mouth and nose with vaso-dilator and secretory fibres. The preganglionic portion of the path terminates in such ganglia as the submaxillary and sublingual (p. 39I) and the sphenopalatine and otic ganglia. 
The part of the autonomic system which originates in the middle region of the spinal cord (in the cat from the first thoracic to the fourth or fifth lumbar nerves) is the sympathetic proper. The course of the fibres has already been described in connection with the vaso-motor nerves (p. I8I). Among the fibres may be men-

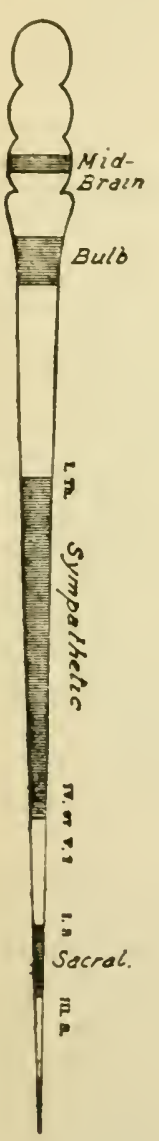

Fig. 403.- Diagram showing the Cen. tral Origin of the Autonomic Fibres (Langley). tioned the dilators of the pupil, the augmentors ef the heart, motor (viscero-motor) and inhibitory fibres for the smooth muscle of the alimentary canal, sweat-secretory, pilo-motor and vasoconstrictor fibres. The preganglionic fibres issue from the cord in the anterior roots, and leave the corresponding spinal nerve in the white ramus communicans, which connects it with the corresponding ganglion of the lateral sympathetic chain. A fibre may either end in this ganglion by forming a synapse, or it may run up or down in the chain for some distance before terminating. Some of the preganglionic fibres: particularly the vaso-constrictors for the abdominal and pelvic viscera, do not end in the lateral chain at all, but, issuing from it still as medullated fibres, terminate in one of the prevertebral ganglia-e.g., cœliac ganglion, inferior mesenteric ganglion-from which postganglionic fibres proceed to the viscera, as previously described (p. 332). The postganglionic fibres arising from cells of the lateral ganglia return as non-medullated fibres in grey rami communicating to the spinal nerves, and are distributed with them to the head, limbs, and the superficial parts of the trunk.

The autonomic fibres arising from the sacral region of the cord emerge as preganglionic fibres in the anterior roots of the second to the fourth sacral nerves, from which they pass to the pelvic nerve (nervus erigens) (pp. I81, 332). They comprise vaso-dilator fibres for the rectum, anus, and external genitals, motor (viscero-motor) fibres for the smooth muscle of the descending colon, rectum, and anus, inhibitory fibres for the smooth muscle of the anus, and the muscles of the external genitals, motor fibres for the bladder, etc. The preganglionic fibres terminate by forming synapses with sympathetic ganglion cells in the pelvic plexus, or in the neighbourhood of the organs which they supply. From these ganglion cells the postganglionic fibres arise. 
Action of Nicotin and Adrenalin on the Autonomic System.The action of nicotin upon the sympathetic ganglion cells, or the link between them and the preganglionic fibres, which has been taken advantage of in tracing the course of the autononic fibres, has already been described (p. 182). The special relation of adrenalin or epinephrin to the sympathetic, although not to the rest of the autonomic system, has also been alluded to (p. 655).

Functions of the Autonomic System.-The functions of the autonomic nerves are sufficiently defined by the enumeration of the peripheral organs with which they are connected. It is obvious that they preside over functions for the most part withdrawn from the control of the will, the so-called vegetative functions, like the heart-beat, the tone of the bloodvessels, the movements of the alimentary canal and of the uterus, the erection of the hairs, and the secretion of sweat. It is no doubt advantageous that these functions should be withdrawn from voluntary control, and this withdrawal is, we may assume, secured either by the absence of anatomical connections between the regions of the cortex connected with voluntary movements and the ganglion cells in the cerebrospinal axis from which the preganglionic fibres arise, or by the existence of a high threshold of resistance in such paths as exist. There is no anatomical or physiological reason why autonomic fibres should not carry impulses which would elicit voluntary movements were such impulses once shunted on to an autonomic path, and certain autonomic fibres do innervate structures which are under voluntary control-e.g., the fibres to the ciliary muscle of the eye and those to the urinary bladder. The power of voluntarily accelerating the heart possessed by some individuals (p. I 72) is a further instance showing that the general rule is broken by exceptions. 


\section{CHAPTER XVIII}

\section{THE SENSES}

HITHERTO we have been considering from a purely objective standpoint the organs that compose the body, and their work. The student has been assumed to be in the little world - the ' microcosm '-of organization which he has been studying, but not of it. He has listened to the sounds of the heart, seen its contraction, felt it hardening under his fingers; but we have not inquired as to the meaning or the mechanism of this hearing, seeing, and feeling. We have now to recognize that all our knowledge of external things comes to us by the channels of the senses, and, like the light that falls through coloured windows on the floor of a church, is tinged, and perhaps distorted, in the act of reaching us.

The Senses in General.-The old and orthodox enumeration of 'the five senses' of sight, hearing, touch, taste, and smell, must be augmented by at least two more, the senses of pressure and temperature. The so-called temperature sensations are themselves divisible into two groups of quite distinctive quality, sensations of warmth and sensations of cold. The power of appreciating the amount of a muscular effort; the power of localizing the various portions of the body in space ; the sensations of pain, tickling, itching, hunger, and thirst; the sensations accompanying the generative act, etc., can certainly be no longer lumped together in the omnium gatherum of 'common sensibility.' They are more appropriately regarded as separate senses subserved by special nerves, and perhaps connected with definite centres. In the development of a simple sensation we may distinguish three stages: the stimulation of a peripheral end-organ, the propagation of the impulses thus set up along an afferent nerve, and their reception and elaboration in a central organ.

We do not know in what manner a series of transverse vibrations in the ether when it falls upon the eye, or a series of longitudinal vibrations in the air when it strikes the ear, excites a sensation of light or sound. We can trace the ray of light through the refractive media of the eyeball, see it focussed on the retina, lead off the current of action set up in that membrane, which, doubtless, gives token of the passage of nervous impulses into and up the optic nerve. We can even follow the nervous impulses to a definite portion of the cortex of the occipital lobe, and determine that if this is removed no sensation of sight will result from 
any excitation of retina or optic nerve. And it is fair to conclude that in some mamner this part of the cerebral cortex is essential to the production of visual sensations. But in what way the chemical or physical processes in the axis-cylinders or nerve-cells are related to the psychical change, the interruption of the smooth and unregarded flow of consciousness which we call a sensation of light, we do not know. To our reasoning, and even to our imagination, there is a great gulf fixed between the pliysical stimulus and its psychical consequence; they secm incommensurable quantities; the transition from light to sensation of light is certain, but unthinkable.

Each kind of peripheral end-organ is peculiarly suited to respond to a certain kind of stimulus. The law of 'adequate' or 'homologous' stimuli is an expression of this fact. The 'adequate' stimuli of the organs of special sense may be divided into (I) vibrations set up at a distance without the actual contact of the object - e.g., light, sound, radiant heat; (2) changes produced by the contact of the object-e.g., in the production of sensations of taste, touch, pressure, alteration of temperature (by conduction). Midway between (I) and (2) lies the adequate stimulus of the olfactory end-organs, which are excited by material particles given off from the odoriferous body and borne by the air into the upper part of the nostrils.

The end-organs of the special senses all agree in consisting essentially of modified ectodermic cells, but they occupy areas by no means proportioned to their importance and to the amount of information we acquire through them. The extent of surface which can be affected by light in a man is not more than $20 \mathrm{sq} . \mathrm{cm}$.; the endings of both nerves of hearing taken together do not at most expand to more than $5 \mathrm{sq} . \mathrm{cm}$.; the olfactory portion of the mucous membrane of the nose has an area of not more than ro sq. $\mathrm{cm}$.; the sensations of taste are ministered to by an area of less than $50 \mathrm{sq} . \mathrm{cm}$.; the end-organs of the senses of pressure, touch, and temperature are distributed over a surface reckoned by square metres. As the physiological status of the sensory end-organs rises, their anatomical distribution tends to shrink. The organs of comparatively coarse and common sensations are widely spread, intermingled with each other, and seated in tissues whose primary function may not be sensory at all. Even the nerve-endings of the sense of taste are not confined to one definite and circumscribed patch, but scattered over the tongue and palate; and both tongue and palate are at least as much concerned in mastication and deglutition as in taste. The olfactory portion of the nasal mucous membrane, although a continuous area with fairly distinct boundaries, is still a part of the general lining of the nostril. The epithelial surfaces which minister to the supreme sensations of sight and hearing-the retina and the sensitive structures of the cochlea-are the most sequestered of all the sensory areas, as the organs of which they form a part are, of all the organs of sense, the most highly specialized in function, and anatomically the most limited. But al though hidden in protected hollows, they are endowed, either in virtue of their own movements or of those of the head, with the power of receiving impressions from every side, and their actual size is thus indefinitely multiplied. 


\section{Section I.-Vision.}

Physical Introduction.-Physically, a ray of light is a series of dis. turbances or vibrations in the luminiferous ether. which radiates out from a luminous body in what is practically a straight line. The ether is supposed to fill all space, including the interstices between the molecules of matter and the atoms of which those molecules are composed. Suppose a bar of iron to be gradually heated in a dark room. In the cold iron the molecules are moving on the average at a relatively slow rate, and the waves set up in the ether by their vibrations are comparatively long. Now, the long ethereal vibrations do not excite the retina, because it is only fitted to respond to the impact of the shorter waves: and, indeed, the long waves are largely absorbed by the watery media of the eye. As the temperature of the iron bar is increased, the molecules begin to move more quickly, and waves of smaller and smaller length, of greater and greater frequency, are set up, until at last some of them are just able to stimulate the retina, and the iron begins to glow a dull red. As the heating goes on the molecules move more quickly: still, and, in addition to waves which cause the sensation of red, shorter waves that give the sensation of yellow appear. Finally, when a high temperature has been reached, the very shortest vibrations which can

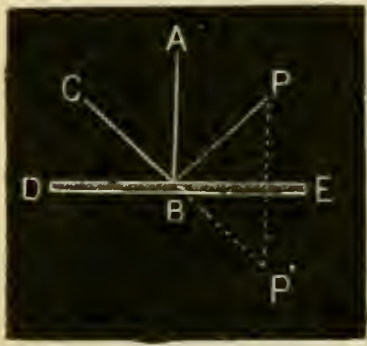

Fig. 404.-Reflection from a Plane Mirror. affect the eye at all mingle with the medium and long waves, and the sensation is one of intense white light.

We have said that a ray of light trave's in a straight line, and the direction of the straight line does not change as long as the medium is homogeneous. But when a ray; reaches the boundary of the medium through which it is passing, a part of it is in general turned back or reflected. If the second medium is transparent (water or glass, e.g.). the greater part of the ray passes on through it, a smaller portion is reflected. If the second medium is opaque, the ray does not penetrate it for any great distance; if it is a piece of polished metal, e.g., nearly the whole of the light is reflected; if it is a layer of lampblack, very little of the light is reflected, most of it is absorbed.

Reflection.- The first law of reflection is that the reflected ray, the ray which falls upon the reflecting surface (incident ray), and the normal to the surface, are in one plane. The second law is that the reflected ray makes with the perpendicular (normal) to the reflecting surface the same angle as the incident ray. A corollary to this is that a ray perpendicular to the surface is reflected along its own path.

Reflection from a Plane Mirror.-Let a ray of light coming from the point $\mathrm{P}$ (Fig. 404) meet the surface $\mathrm{DE}$ at $\mathrm{B}$, making an angle PBA with the normal to the surface. The reflected ray $B C$ will make an equal angle $A B C$ with the normal; and the eye at $C$ will see the image of $P$ as if it were placed at $\mathrm{P}^{\prime}$, the point where the prolongation of $\mathrm{BC}$ cuts the straight line drawn from $\mathrm{P}$ perpendicular to $\mathrm{DE}$. This is the position of an ordinary looking-glass image.

Reflection from a Concave Spherical Mirror.-A spherical surface may be supposed to be made up of an infinite number of infinitely small plane surfaces. The normal to each of these plane surfaces is the radius of the sphere, and the reflected ray makes with the radius at the point of 
incidence the same angle as the incident ray. Let D (Fig. 405 ) be the middle point of the mirror, and $\mathrm{C}$ its centre of curvature-i.e., the centre of the sphere of which it is a segment. Then CD is the principal axis, and any other line through $C$ which cuts the mirror is a secondary axis. When the mirror is a small portion of a sphere, rays parallel to the principal axis are focussed at the principal focus $F$ midway between $C$ and $D$; rays parallel to any secondary axis are focussed in a point

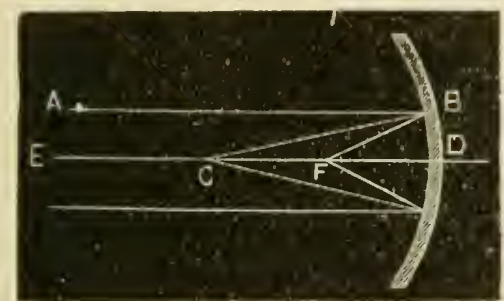

Fig. 405.-Reflection tion a Concave Spherical Mirror.

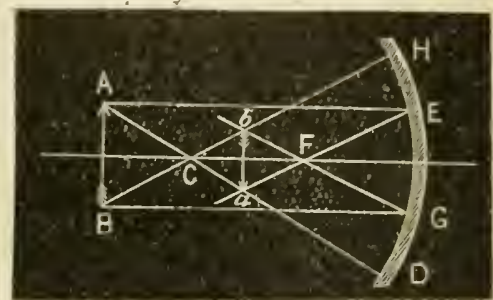

Fis. 406.-Formation of Real Inverted Image by a Concave Spherical Mirror.

lying on that axis; and rays diverging from a point on any axis are focussed in a point on the same axis.

These facts afford a simple construction for finding the position of the image of an object formed by a concave mirror. Let $\mathrm{AB}$ be the object (Fig. 406). Then the image of $\mathrm{A}$ is the point in which all rays proceeding from $A$ and falling on the mirror, including rays parallel to the principal axis, are focussed. But the ray AE, parallel to the principal axis, passes after reflection through the principal focus $F$, therefore the image of A must lie on the straight line EF. If any secondary axis ACD be drawn, the image of A must lie on ACD. It must therefore be the point of intersec. tion, $a$, of EF and ACD. Similarly, the image of $B$ must be the point of intersection, $b$, of GF and $\mathrm{BCH}$. The image $a b$ of an object $A B$ farther from the mirror than the principal focus is real and inverted. The Purkinje-Sanson image reflected from the concave anterior surface of the vitreous humour (Fig. 42I)

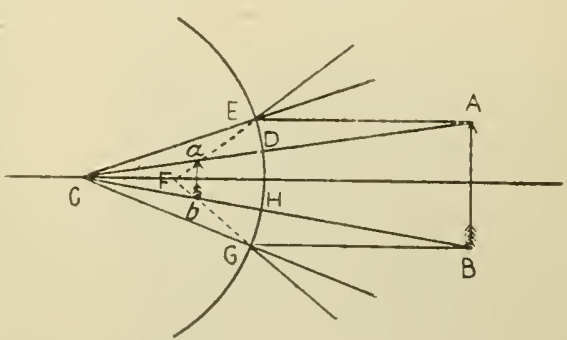

Fig. 407.-Formation of Image by a Convex Mirror. is an example.

After reflection from a convex mirror, rays of light always diverge, and only erect, virtual images are formed-i.e., images which do not really exist in space, but which, from the direction of the rays of light, we judge to exist. The position of the image of an object AB (Fig. 407) may be found by a construction similar to that for refection from a concare mirror. The image of a flame reflected from the anterior surface of the cornea or lens is erect and virtual. It diminishes in size with increase in the curvature or convexity of the reflecting surface (Fig. 42I).

Refraction.-A ray of light passing from one medium into another has its velocity, and consequently its direction, altered. It is said to 
be refractecl. The fust law of refraction is that the refracted ray is in the same plane as the incident ray and the normal to the swiface. The second law is that the sine of the angle of incidence has a constant ratio (for any given pair of media) to the sine of the angle of refraction. The angle of incidence is the angle which the ray makes with the normal to the surface, separating the two merlia; the angle of refraction is the angle made with the normal in the second medium. This ratio is called the index of refraction between the two melia. For purposes of comparison, the refractive index of a substance is usually taken as the ratio of the sine of the angle of incidence to the sine of the angle of refraction of a ray passing from air into the substance.

When a ray strikes a surface at right angles, it passes through without suffering refraction. When a ray passes from a less dense to a denser medium (e.g., from air to water), it is bent towards the perpendicular.

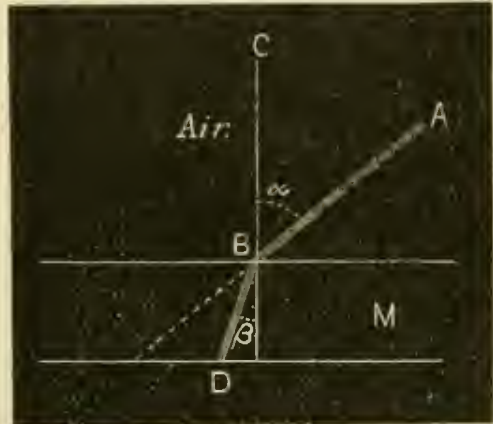

Fiz. $40^{2}$ - - Refraction at a Plane Surface $\mathrm{AB}$ is the incident; $\mathrm{BD}$, the refracted ray; $C B$, the normal to the surface. When the ray passes from air into another medium, the refractive index of the latter is the fraction $\frac{\sin \alpha}{\sin \beta}$.

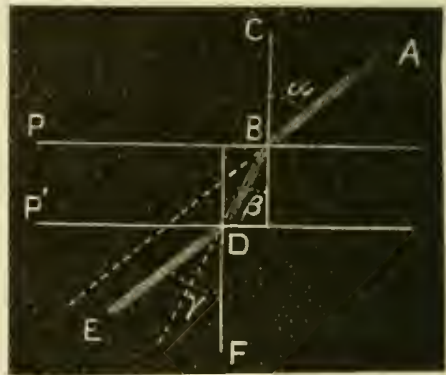

Fig. 40).- Refraction by a Medium bounded by Parallel Planes, $\mathrm{P}$ and $\mathrm{P}^{\prime}$. The ray ABDE issues parallel to its original direction; CB, FD, normals to $\mathrm{P}$ and $\mathrm{P}$; $\boldsymbol{\alpha}$, angle of incidence; $B, \gamma$, angles of refraction.

When it passes from a more dense to a less dense medium (as from water to air), it is bent away from the perpendicular.

When a ray passes across a medium bounded by parallel planes, it issues parallel to itself; in otler words, it undergoes no refraction (Fig. 409).

Refraction and Dispersion by a Prism.-The beam of light is bent towards the normal $N$ as it passes across $B A$ and away from the normal $N^{\prime}$ as it passes across BC (Fig. $\left.4 \mathrm{IO}\right)$; at both surfaces it is bent towards the base of the prism AC. At the same time the light suffers dispersion - that is, the rays of shorter wave-length are more refracted than those of greater wave-length. The deviation of any given ray is measured by the angle which the refracted ray makes with its original direction. The amount of dispersion produced by a prism is measured by the difference in the deviation of the extreme rays of the spectrum. The dispersion produced by a given substance is proportional to the difference of its refractive indices for the extreme rays.

Refraction by a Biconvex Lens. - A straight line $\mathrm{ABC}$ passing through the centres of curvature of the two surfaces of the lens is called the principal axis. A point $C$ lying on the principal axis between the 
two centres of curvature, and possessing the property that rays passing through it do not suffer refraction, is callerl the optical centre of the lens. Any straight line, DCl, passing throngh the optical centre, is a secondary axis. Ritys of light proceeding from a point in the principal axis are focussed in a point on that axis. IVhen the rays procect from an infinitely distant point in the principal axis-i.e., when they are parallel to it-they are focussed in $F$, the principal focus. Similarly rays parallel to, or procceding from, a point in a sccondary axis are focussed in a point on that axis; but if the focus is to be sharp, the angle between the secon(iary and the principal axis must not be so large as is indicated in Fig. $41 \mathrm{I}$.

Formation of In1. age by Biconvex Lens (Iiig. 4 I2).-Let AB be tlie object; then if $A H D$ be the path

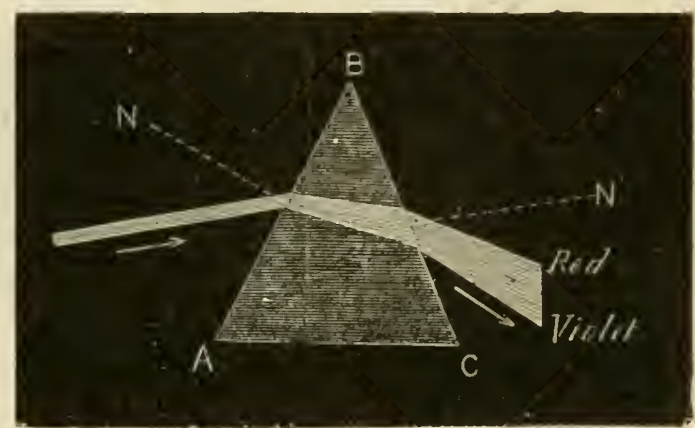

Fig. f I0. - Refraction and Dispersion by a Prism. of a ray from $A$ parallel to the principal axis, the image of $A$ will be the intersection of the straight line $D F$ and the secondary axis passing through A. Similarly, the imagc of $B$ will be the intersection of $G F$ and the secondary axis $B C$. Where $A B$ is farther from the lens than the principal focus, the image $a b$ is real and inverted. This is the case with the image of an external object formed on the retina. When the object is nearer than the principal focus, the image is virtual and direct.

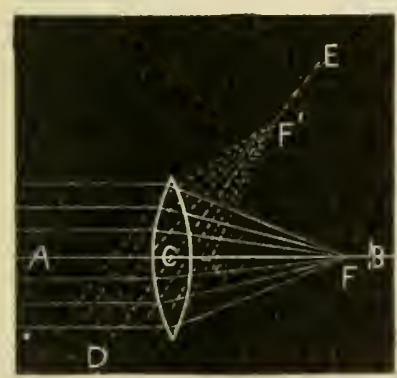

Fig. 4 II. - Refraction by a Biconvex Lens.

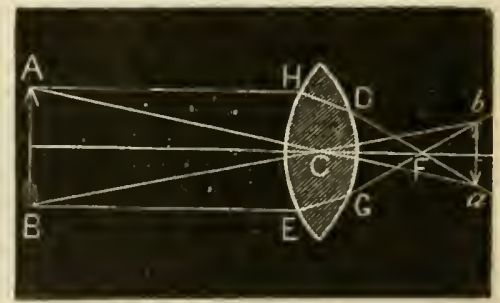

Fig. 4r2, - Formation of Image by Biconvex Lens.

The image formed by the objective of a microscope when the object is in focus is real and inverted; the ocular forms a virtual erect image of this real image.

Refraction by a Biconcave Lens (Fig. 4I3).-Parallel rays are rendered divergent by the lens; there is no real focus; but if the rays are prolonged backwards they meet in the virtual focus $F$, from which they appear to come when received by the eye through the lens.

Formation of Image by Biconcave Lens (Fig. 4I 4).-Let AB be the object. Let AHDI be the path of a ray from any point $A$ of the object 
parallel to the principal axis. Produce DI backwards (dotted line); it will pass through the principal focus $F$. Through $\Lambda$ draw the secondary axis $\mathrm{AC}$. The image of $\mathrm{A}$ must lie both on $\mathrm{AC}$ and on IDF-i.e., it must be the intersection, $a$, of these straight lines. Similarly, the image of $\mathrm{B}$ is $b$, the intersection of $\mathrm{KGF}$ and $\mathrm{BC}$. The image is virtual and erect.

Absorption.- No substance is perfectly transparent; in addition to what is reflected, some light is always absorbed. In other words, in

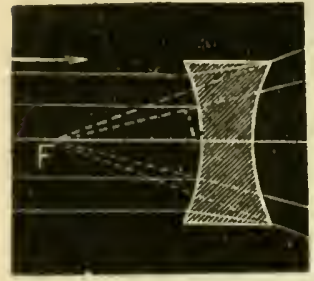

Fig. 4 I3.-Refraction oy a Biconcave Lens.

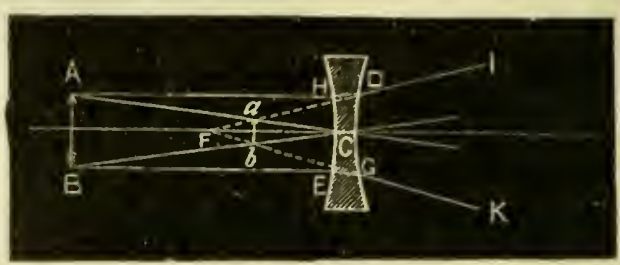

Fig. 4I4.-Formation of Image by Biconcave Lens.

passing through a body some of the light is transformed into heat, a portion of the energy of the short, luminous waves going to increase the vibrations of the molecules of the medium, just as a wave passing under a row of barges or fishing-boats set them swinging and pitching, and so imparts to them a certain amount of energy, which is ultimately changed into heat by friction against the water, and against each other, and bv

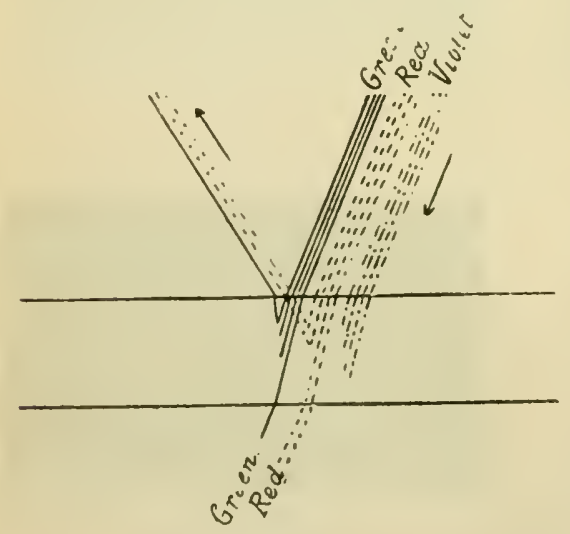

Fig. 415.-Diagram to show Connection of Body Colour with Selective Absorplion.

the straining and rubbing of the cluains at their points of attachment. Some bodies absorb all the rays in the proportion in which they occur in white light; whether looked at or looked through, they a ppear colourless or white. Other substances absorbcertain rays by preference, and the a mount of absorption is proportional to the thickness of the layer. The colours of most natural bodies are due to this selective absorption. Even when looked at in reflected light, they are seen by rays that have penetrated a certain way into the substance and have then been reflected; and, of course, a smaller number of the rays which the body specially absorbs are reflected than of the rays which it readily transmits, for more of the latter than of the former reach any given depth. This is called 'body colour'; and such substances have the same colour when seen by reflected and by transmitted light. The colour of hæmoglobin is due to the absorption of the violet and many of the yellow and green rays, as is shown by the position of the absorption bands in its spectrum (p. 51). In Fig. +15 the violet rays are represented as being totally absorbed before passing through the substance. Some of the grecn rays are reflected, some 
transmaitted, some absorbed. The red rays are supposed to be mostly reflected and transmittel, only $(0)$ a slight extent abiorbed. The colour of sucl a substince, both wlicn louked at and when looked through, would therefore be that due to a mixture of red light with a smaller quantity of green. Then there is another class of substances which owe their colour to selective reflection. Certain rays only are reflected from their surface, and the light trinsmitted through a thin layer is complementary to the reflected light--that is, the reflected and transmitted ray's together would nake up white light. These bodies have what is called 'surface colour,' and include metals, various aniline dyes, and other substances.

Comparative. - Many invertebrate animals possess rudimentary senseorgans, by means of which they may receive certain luminous impressions. It is true that the mere sensation of light is not in itself sufficient for the exact appreciation of the form and situation of surrounding objects. But even the closure of the eyelids does not prevent a person of normal eyesight from distinguishing differences in the intensity of illumination.

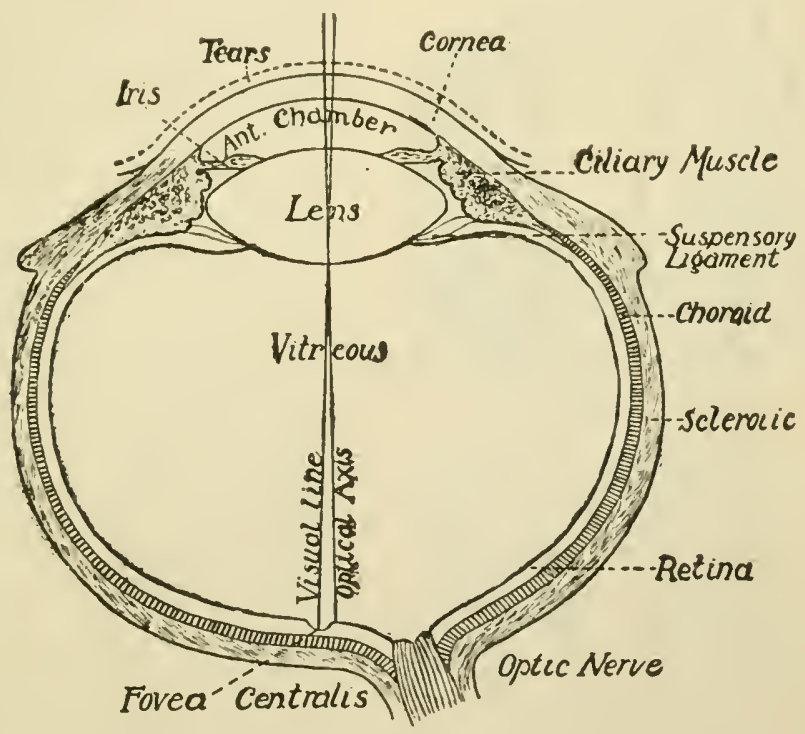

Fig. 4 I f.-Diagrammatic Horizontal Section of the Left Eye.

And it is possible that many of the humbler animals may, through the pigment spots which are often called eyes, or perliaps, as in the earthworm, by means of end-organs more generally diffused in the skin, attain to some such dim consciousness of light and shadow as will enable them to avoid an obstacle or an enemy, to seek the sunny side of a boulder or the obscurity of an overhanghing ledge of rock. But the indispensable condition of distinct vision is that an image of each part of an object should be formed upon a separate portion of the receiving or sensitive surface. This condition is, to a certain exentt, fulfilled by the compound eyes of some of the higher invertebrates (insects, e.g.). Here rays from one point of the object pass through one of the funnelshaped elements of the compound eye, and rays from another point 
through another. Rays striking obliquely on the facets are stopped by the opaque partitions between them. In the Cephalopods we find that this compound type of eye has already been abandoned; the single system of curved refracting surfaces so characteristic of the vertebrate eve has made its appearance; and the formation of a clean-cut image of the object on the retina, with the excitation of a sharply-bounded area of that membrane, follows as a geometrical consequence from the theor ${ }^{\circ}$ of lenses.

We have to consider (I) the mechanism by which an image is formed on the retina, and (2) the events that follow the formation of such an image and their relations to the stimulus that calls them forth.

Structure of the Eye.-The eye may be described with sufficient accuracy as a spherical shell, transparent in front, but opaque over

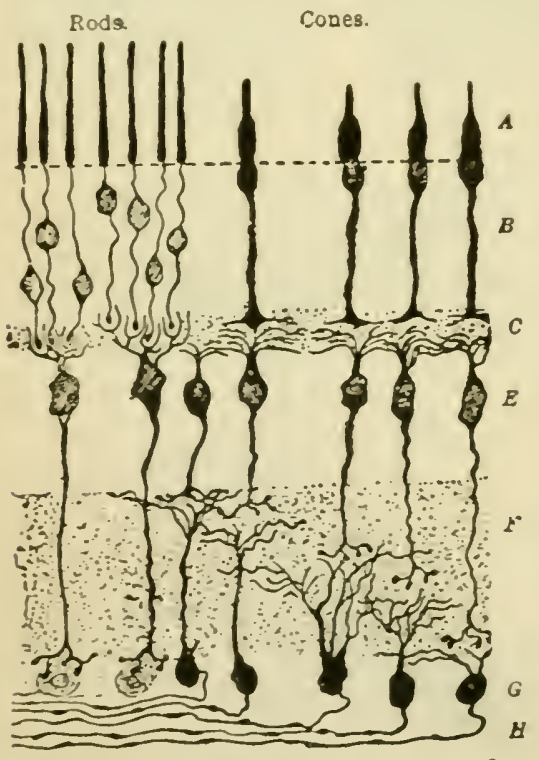

Fig. 417.-Diagram of Siructure of Retina (after Cajal). $H$, layer of nerve-fibres; $G$, layer of ganglion cells; $F$, internal mole. cular layer; $E$, internal nuclear layer; nuclear layer; external limiting membrane; $A$, layer of rods and cones. $C$, external molecular layer; $B$. external

the posterior five-sixths of its surface, and filled up with a series of transparent liquids and solids. The shell consists of three layers concentrically. arranged, like the coats of an onion: (I) An external tough, fibrous coat, the sclerotic, the anterior portion of which appears as the white of the eye. In front this external layer is completed by the transparent cornea. (2) A vascular layer, the choroid, which, in the restricted sense of the term, ends in front in a series of folds or plaits, the ciliary processes. The choroid contains a greater or smaller quantity of the black pigment melanin. The ciliary processes abut on the outer boundary of the iris, which may be looked upon as an anterior continuation of the choroidal or middle coat of the eyeball. Between the corneosclerotic junction and the anterior portion of the choroid is interposed a ring of unstriped muscular fibres, the ciliary muscle. (3) The inner or sensitive coat, termed the retina (Fig. 4I?). This covers the choroid as a delicate membrane, extending to the ciliary processes, where it ends in a toothed margin, the ora serrata. The optic nevie forms a kind of stalk to which the eyeball is attached. Its point of entrance at the optic disc is a little nearer the median line than the antero-posterior axis which nearly passes through the centre of a small depression, the fovea centralis, situated in the middle of the macula lutea, or yellow spot. From the optic disc sometimes called the optic 
papilla) the optic nerve spreads over the retina as a layer of nonmedullated fibres, separated from the interior of the eyeball only by the internal limiting membrane. This so-called membrane is formed by the exprancled feet of the fibre's of Müler, wlich run like a scaffolding or framework through nearly the whole thickness of the retina, terminating at the outer limiting membrane. External to the latyer of nerve-fibres is the stratum of large ganglion cells, whose axons they are; next to this the inner molecular layer, or inner synapse layer, made up largely of the branching dendrites of these cells. The fifth layer is the inner granular or nuclear layer, containing many fusiform (bipolar) 'granule' cells which send out axons into the fourtl, and dendrites into the sixth, or outer molecular layer, and are thus connected with the ganglion cells of the third layer on the one hand, and with the terminations of the rod and cone fibres of the seventh or outer nuclear layer on the other. The arborizations of the axons of these bipolar cells are situate at different levels in the internal molecular layer. The bipolar cells connected with the rod fibres send their axons right through the internal, mole. cular layer to arborize around the bodies of the ganglion cells, whereas the axons of the bipolar cells connected with the cone fibres ramify about the middle of the layer (Fig. 417). The seventh stratum receives its name from the large number of nuclei which it contains. These belong to structures continuous with the rods and cones of the ninth layer, which is divided from the seventh by the exterral limiting membrane. Each rod is prolonged into the external nuclcar layer as a fine fibre, which has on its course a swelling containing a nucleus, and terminates (in mammals) in a fine knob in the external molecular layer among the dendrites of the bipolar cells. Each

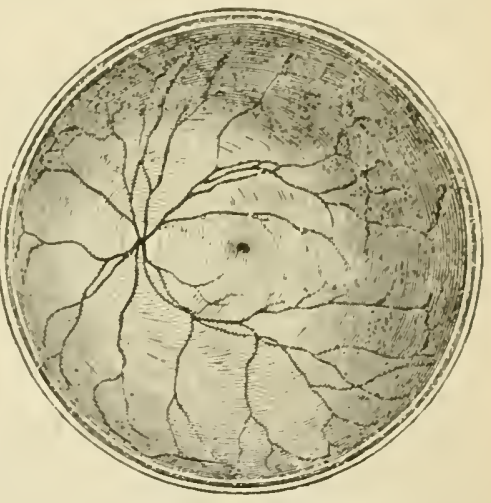

Fig. 418.-Retinal Bloodvessels (Henle). The arteria centralis is seen issuing from the optic dics and branching over the retina. The shaded area in the middle of the figure represents the yellow spot with the fovea centralis in its centre. cone of the rod and cone layer is directly prolonged into a nucleated enlargement in the external nuclear layer. From this enlargement a fibre (cone fibre), of considerably greater calibre (in mammals) than the rod fibre, passes into the external molecular layer, where it forms an arborization, which comes into relation with the arborization of the dendrites of a bipolar cells. At the fovea centralis the rods are entirely absent, and the other layers of the retina greatly thinned; over the optic disc neither rods nor cones are present. The disc is pierced by the retinal bloodvessels (Fig. 4i 8).

External to the rods and cones is a sheet of pigmented epithelial cells of hexagonal shape, belonging to the choroid, but remaining attached to the retina when the latter is separated, and therefore often reckoned as its most external layer.

A little belind the cornea and anterior to the retina is the lens, enclosed in a capsule, and attached to the choroid by the suspensory ligament, or zonule of Zinn. The iris hangs down in front of the lens like a diaphragm, with a central hole, the pupil. Incorporated in the 
stroma or frameworli of the aris are lwo arrangements of smooth muscular fibres, which confer on it the power of adjusting the size of the pupil. One of these-the sphincter pirpilla-consists of a well-clefined band of concentric fibres surrounding the margin of the pupil. The other-the dilator pupillic-is less sharply differentiated. It is represented by radial bundles of elongated, spindle-shaped cells running in from the ciliary border of the iris towards the pupil. Between the iris and the posterior surface of the cornea is the anterior chamber of the eye. filled with the aqueous humour. Between the iris and the anterior surface of the lens lies the posterior chamber, which is rather a potenial than an actual cavity. The space between the lens and the retina is accurately occupied by an almost structureless semi-fluid mass, the vitreous humour, enclosed by the delicate hyaloid membrane, which in front is reflected over the folds of the ciliary processes, and blends with the suspensory ligament of the lens. The at tachment of the suspensory ligament is rendered firmer by the connection of this part of the hyaloid membrane to a circular fibrous portion of the vitreous. Around the edge of the lens is left a space, the canal of Petit.

Chemistry of the Refractive Media.-The aqueous humour is a perfertly colourless, watery liquid, of slightly allialine reaction to litmus. The specific gravity is about 1008 , and the total solids about I per cent. (If the solids the inorganic salts (mainly sodium cliloride) constitute much the largest portion. A very small amount of protein (o.0 I to $0.0+$ per cent.) is present, also a little dextrose (0.05 per cent.). and minute traces of urea and other substances. The liquid of the vitreous humour has a very similar composition, except that it contains a mucin-like body, hyalomucoid, to the anount of 0.06 to $0 \cdot 1$ per cent. A similar mucin-like substance is present in the cornea. The freezingpoint of both liquids is a little lower than that of blood-serum, $\Delta$ being about $0 \cdot 6^{\circ}$.

The lens is far richer in solids than the aqueous and vitreous humours with wlich it is in contact ( 30 to 35 per cent of solids, 60 to 65 per cent. of water). The salts, with small quantities of lecithin and cholesterin, inake up about I per cent. ; the balance of the solids consists of proteins. The physical alterations, with production of turbidity, which occur in the lens, and presumably in its proteins, when water enters or leaves it in too great amount through imbibition or osmosis, are of importance in connection with the etiology of cataract. The anatomical and physiological integrity of its capsule is a prime factor in the maintenance of that ligh degree of transparency which is necessary for the function of the lens. Cataract can be experimentally induced by injuring the capsule. In like manner the cornea is protected against injurious changes in its water-content (normally about 80 per cent.) and consequent turbidity by the cpithelium, which separates it from the tears, and the endothelium, which separates it from the aqueous humour.

Secretion of the Intra-ocular Liquids. - The aqueous humour is secreted by the uveal epithelium covering the ciliary processes, and to some extent by that covering the iris. As it is continually secreted, so it is continually absorbed, the absorbed constituents finding their wav eventually into the vein or venous sinus called the canal of Schlemm and the bloodvessels of the iris and ciliary processes. The source of the liquid of the vitreous body is also the uvea. While the intra-ocular liquids differ from ordinary lymph, there is no reason to donbt that they are secretions which contribute to the nutrition of those transparent structures of the eye which are not, ancl, on account of their function. cannot be supplied with bloodressels. Their most obvious use is tc 
maintain the proper intra-ocular pressure on which the gcometrical figure of the cyeball, and therefore its efficiency as an optical instrument, depend. The balance between secretion and absorption is accurately adjusted in licalth, but in disease it may be upset, as in glaucoma, where the intra-ocular tension is so much increased as to interfere with the circulation, and injuriously affect the nutrition and function of the retina. Experimentally, occlusion of all the arteries supplying the head causes a rapid fall of tension, and the cornea becomes wrinkled and slack to the touch. On restoring the circulation after not too long an interval, the tension gradually returns to normal, and then becomes markedly hypernormal, even when the general arterial pressure is still low. This is probably due to the crippling of the elements which secrete and absorb the intra-ocular fluids, or of the capillary walls, so that a proper adjustment can no longer be attained, as happens in a tissue rendered odematous by temporary antemia. Where asphyxia of the eyeball is avoided or is brief the intra-ocular pressure varies directly as the blood. pressure in the ocular vessels within a wide range (Henderson and Starling).

Refraction in the Eye-Formation of the Retinal Image.-The amount of refraction which a ray of light undergoes at a curved surface depends upon two factors-the radius of curvature of the surface, and the difference between the refractive indices of the media from which the ray comes and into which it passes. The smaller the radius of curvature, and the greater the difference of refractive index, the more is the ray bent from its original direction. A ray of light passing into the eye meets first the approximately spherical anterior surface of the cornea, covered with a thin layer of tears. Since the refractive index of the tears is much greater than that of air, the ray is strongly refracted here. The anterior and posterior surfaces of the cornea being practically parallel, and the refractive indices of the tears and aqueous humour being nearly equal, but little refraction takes place in the cornea itself. At the anterior and posterior surfaces of the lens the ray is again refracted, since the refractive index of the aqueous and vitreous humours is less than that of the lens. The following tables show the radii of curvature of the refracting surfaces and the refractive indices of the dioptric media, as well as some other data which are of use in studying the problems of refraction in the eye:

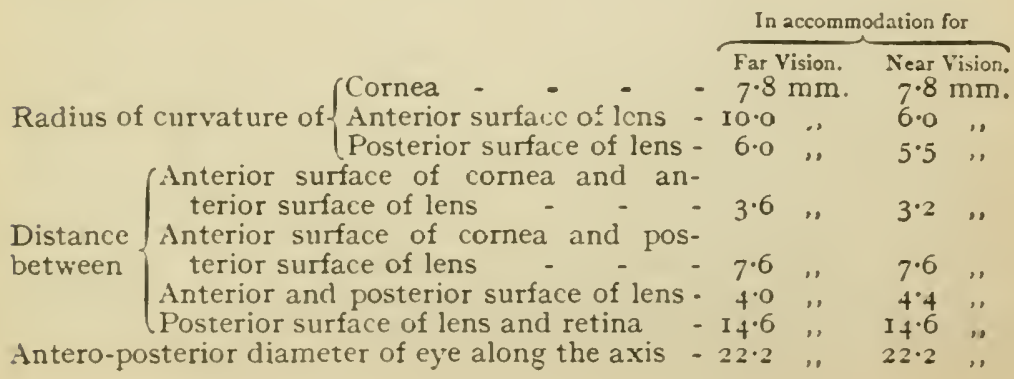




\begin{tabular}{|c|c|c|c|c|c|c|c|c|}
\hline \multicolumn{9}{|c|}{ efract } \\
\hline Air & - & - & - & - & - & - & - & $I \cdot O \omega O$ \\
\hline Cornea & - & - & - & - & - & - & - & $\mathrm{I} \cdot 377$ \\
\hline Aqueous & s humour & - & - & - & - & - & - & $I \cdot 336_{5}$ \\
\hline Vitreous & s humour & - & - & - & - & - & - & I. 3365 \\
\hline Lens (to & tal refrac & tive index) & - & - & - & - & - & I. 437 \\
\hline Water & - & - & - & - & - & - & - & $I \cdot 335$ \\
\hline
\end{tabular}

It will be seen that the refractive indices of the aqueous and vitreous humours are nearly the same as that of water. That of the lens differs for its various layers, the central core having a higher refractive index (I.4II) than the more superficial portions (I.388). Although such calculations are open to error, it has been computed

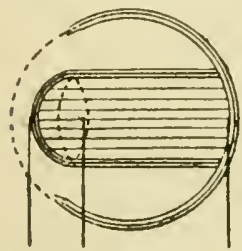

$S \mathrm{~N}$

Fig. 419.-The Rerluced Eye. $S$, the single spherical refracting surface, $2.2 \mathrm{~mm}$. be. hind the anterior sur. face of the cornea; $\mathrm{N}$, the nodal point, $5 \mathrm{~mm}$. behind $\mathrm{S} ; \mathrm{F}$, the principal focus (on the retina), $20 \mathrm{~mm}$. behind S. The cornea and lens are put in in dotted lines in the position which they occupy in the noimal eye.

liquids of the eye. The principal focus being supposed to lie on the retina, the position of the nodal point-i.e., the point through which rays pass without refraction-of such a 'reduced ' or 'schematic ' or 'simplified ' eye, and other constants, are shown in the following table. The single refracting surface would be situated behind the cornea and in front of the lens, at a rather smaller distance from the anterior surface of the latter than from the anterior surface of the former. The nodal point would be less than half a millimetre in front of the posterior surface of the lens (Fig. 4I9). The refractive index of the single transparent medium would be a little greater than that of water. 


\section{Reduced Eye-}

Radius of curvature of the single refracting surface

Index of refraction of the single refracting medium -

Antero-posterior diameter of reduced eye (distance of principal focus from the single refracting surface) -

Distance of the single refracting surface behind the anterior surface of the cornca

Distance of the nodal point of the reluced eye from (its anterior surface

Distance of the nodal point from the principal focus (retina) -

Knowing the position of the centre of curvature of the single ideal refracting surface-i.e., the nodal point of the reduced eye -all that is necessary in order to determine the position of the image of an object on the retina is to draw straight lines from its circumference through the nodal point. Each of these lines cuts the refracting surface at right angles, and therefore passes through without any deviation. "The retinal image is accordingly inverted and its size is proportional to the solid angle contained between the lines drawn from the boundary of the object to the nodal point, or the equal angle contained by

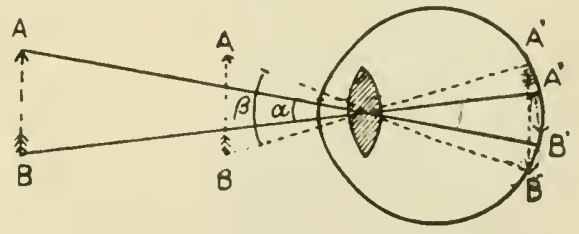

Fig. 420.-Figure to show how the Visual Angle and Size of Retinal Image varies with the Distance of an Object of Given Size. For the distant position of $\mathrm{AB}$ the visual angle is $a$, for the near position (dotted lines) $\beta$.

the prolongations of the same lines towards the retina. This angle is called the visual angle, and evidently varies directly as the size of the object, and inversely as its distance. Thus the visual angle under which the moon is seen is much larger than that under which we view any of the fixed stars, because the comparative nearness of the earth's satellite more than makes up for its relatively small size.

The dimensions of the retinal image of an object are easily calculated when the size of the object and its distance are known. For let $A B$ in Fig. 4'20 represent one diameter of an object, $\mathrm{A}^{\prime} \mathrm{B}^{\prime}$ the image of this diameter, and let $\mathrm{AB}^{\prime}, \mathrm{BA}^{\prime}$, be straight lines passing through the nodal point. Then $A B$ and $A^{\prime} B^{\prime}$ may be considered as parallel lines, and the triangles of which they form the bases, and the nodal point the common apex, as similar triangles. Accordingly, "if $\mathrm{D}$ is the distance of the nodal point from $A$, and $d$ its distance from $B^{\prime}$, we have $\frac{A B}{D}=\frac{A^{\prime} B^{\prime}}{d}$. Now, $d$ may approximately be taken as $15 \mathrm{~mm}$. Suppose, then, that the size of the moon's image on the retina is required. Here $\mathrm{D}=238,000$ miles, and $\mathrm{AB}$ (the diameter of the moon) $=2,160$ miles.

* Or a little more than that of the aqueous humour. 
Thus we get $\frac{2,160}{238,000}=\frac{A^{\prime} B^{\prime}}{15}$, or (say) $\frac{I}{I I 0}=\frac{A^{\prime} B^{\prime}}{I 5}$, from which $A^{\prime} B^{\prime}$ (the diameter of the retinal image $)=\frac{\mathrm{x}_{5}}{\mathrm{I} \mathrm{IO}^{\circ}}$, or about $\frac{1}{8} \mathrm{~mm}$.

A ship's mast $x 20$ feet high, seen at a distance of 25 miles, will throw on the retina an image whose height is $\frac{120 \text { feet }}{25 \text { miles }} \times 15 \mathrm{~mm}$., i.e., $\frac{\text { I } 20 \text { feet }}{5,280 \times 25 \text { feet }} \times I_{5} \mathrm{~mm}$, or $\frac{I}{I_{1} \text { I Oo }} \times I_{5} \mathrm{~mm}$., equal to $0.013 \mathrm{~mm}$, or I $3 \mu$ in size. This is not much larger than a red blood-corpuscle, and only four times the diameter of a cone in the fovea centralis, where the cones are most slender. In this calculation the effect of aberration (p. 1027) in enlarging the image has been neglected. This effect is, of course, proportionately greater for small and distant than for large and near objects; and it is doubtful whether the smallest possible image can be confined to an area of the retina of the size of a single cone.

Accommodation.-A lens adjusted to focus upon a screen the rays coming from a luminous point at a given distance will not be in the proper position for focussing rays from a point which is nearer or more remote. Now, it is evident that a normal eye possesses a great range of vision. The image of a mountain at a distance of 30 miles, and of a printed page at a distance of $30 \mathrm{~cm}$., can be focussed with equal sharpness upon the retina. In an opera-glass or a telescope accommodation is brought about by altering the relative position of the lenses; in a photographic camera and in the eyes of fishes and cephalopods, by altering the distance between lens and sensitive surface; in the eye of man, by altering the curvature, and therefore the refractive power of the lens. That the cornea is not alone concerned in accommodation, as was at one time widely held, is shown by the fact that under water the power of accommodation is not wholly lost. Now, the refractive index of the cornea being practically the same as that of water, no changes of curvature in it could affect refraction under these circumstances. That the sole effective change is in the lens can be most easily and decisively shown by studying the behaviour of the mirror images of a luminous object reflected from the bounding surfaces of the various refractive media when the degree of accommodation of the eye is altered. Three images are clearly recognized: the brightest an erect virtual image, from the anterior (convex) surface of the cornea; an erect virtual image, larger, but less bright, from the anterior (convex) surface of the lens; and a small inverted real image from the (concave) posterior boundary of the lens (PurkinjeSanson images). The second image is intermediate in position between the other two. It is possible with special care to make out a fourth image; but since it is reflected from the posterior surface of the cornea, at which only a slight change in the refractive index ocrurs, it is less brilliant than the first three. When the eye is accommodated for near vision, as in focussing the ivory point of 
the phakoscope (Practical Exercises), the corneal image is unchanged in size, brightness, and position. The middle image diminishes in size, comes forward, and moves nearer to the corneal inage. This shows that the curvature of the anterior surface of the lens has been increased-that is to say, its radius of curvature diminished - for the size of the image of an object reflected from a convex mirror varies directly as the radius of curvature. A slight change takes place in the image from the posterior surface of the lens, indicating a small increase of its curvature too. By means of a method founded on the observation of the changes in these images, and a special instrument callod an ophthalmometer which allows of their measurement, Helmholtz has calculated that during maximum accommodation, the radius of curvature of the anterior surface of the lens is only $6 \mathrm{~mm}$., as compared with ro $\mathrm{mm}$. when the eye is directed to a distant object and there is no accommodation. When the lens has been removed for cataract, fairly distinct vision may still be obtained by compensating for its loss by convex spectacles of suitable refractive power (Io diopters* for distant vision, and I5 diopters for the distance at which a book is usually held), but no power of accommodation remains. The person does indeed contract the pupil in regarding a near object, just as happens in the intact eye; the most divergent rays are thus cut off and the image made somewhat sharper, and there may appear to be some faculty of accommodation left. But the loss of the whole iris by operation does

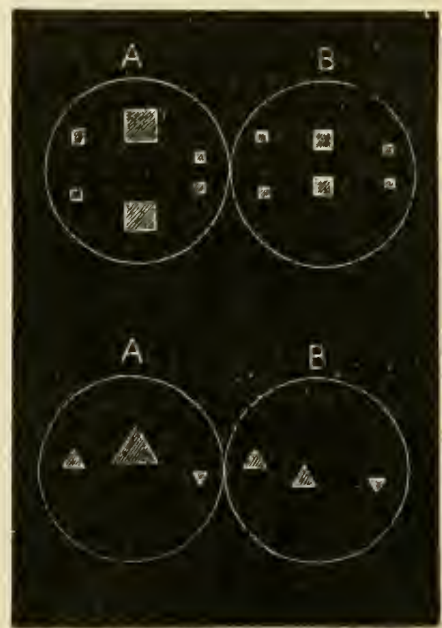

Fig. 421.-Purkinje-Sanson Images. $A$, in the absence of accommoda. tion; B, during accommodation for a near object. The upper pair of circles enclose the images as seen when the light falls on the eye through a double slit on a pair of prisms: the lower pair show the images seen when the slit is single and triangular in shape.

not affect accommodation in the least; the iris, therefore, takes no part in it. That no change in the antero-posterior diameter of the eyeball, caused by its deformation by the contraction of the extrinsic muscles, can have any share in accommodation, as has

* A diopter ( 1 D.) is the unit of refractive power generally adopted in measuring the strength of lenses, and corresponds to a lens of I metre focal length. A lens of 2 diopters $\left(2 D\right.$. ) has a focal length of $\frac{1}{2}$ metre, a lens of 4 diopters $\left(4 \mathrm{D}\right.$.) a focal length of $\frac{1}{4}$ metre, and so on. The diverging power of concave lenses is similarly expressed in diopters with the negative sign prefixed. Thus, a concave lens of I metre focal length has a strength of - I D.. and will just neutralize a convex lens of $1 \mathrm{D}$. 
been suggested, is clearly proved by the fact that atropine, which does not affect the action of these muscles, paralyzes the mechanism of accommodation. To the consideration of that mechanism we now turn.

The Mechanism of Accommodation.-While everybody is agreed that the main factor in accommodation is the alteration in the curvature of the lens, there is by no means the same unanimity as to the manner in which this is brought about. Helmholtz's explanation, which has long been the most popular, is as follows: In the unaccommodated eye the suspensory ligament and the capsule of the lens are tense and taut, the anterior surface of the lens is flattened by their pressure, and parallel rays (or, what is the same thing, rays from a distant object) are focussed on the retina without any sense of effort. In accommodation for a near object, the meridional or antero-posterior fibres of the ciliary muscle by their contraction pull forward the choroid and relax the suspensory ligament. The elasticity of the lens at once causes it to bulge forwards till it is again checked by the tension of the capsule.

The explanation of Helmholtz, although widely adopted in the textbooks, has not escaped question in the archives. Tscherning has put forward the view that when the ciliary muscle contracts, the suspensory ligament is pulled backwards and outwards. Its tension is thus increased, and the soft external layers of the lens are in consequence moulded upon the harder nucleus, so as to increase the curvature especially around the anterior pole. And Schoen, reviving a similar theory originated fifty years ago by Mannhardt, believes that the ciliary musile, in contracting, exerts pressure on the anterior portion of the lens, and so increases its curvaturc. He likens the process to the bulging of an indiarubber ball when it is held in both hands and compressed by the fingers a little behind one of the poles. It will be observed that in both of these theories the suspensory ligament is supposed to be stretched during accommodation, not relaxed as Helmholtz supposed. While they have certain advantages over the theory of Helmholtz, particularly in taking account of the presence of radial and circular as well as meridional fibres in the ciliary muscle, they do not agree so well with such experimental tests as have been applied, and therefore Helmholtz's explanation must still be regarded as the best.

It is supported by the observation of Hess that when the ciliary muscle has been very strongly contracted by eserine the lens can be observed to move about with each slight movement of the eye. The suspensory ligament must therefore be slackened by the contraction of the ciliary muscle. When atropine is applied the movability of the lens soon disappears, owing to paralysis of the ciliary muscle. These facts were first established in patients after iridectomy, but have also been demonstrated in the normal eyc. Even under the influence of gravity alone, without any movements of the eye, the lens sinks about t to $\frac{1}{3} \mathrm{~mm}$. in strong accommodation. An additional proof that the suspensory ligament is perfectly slack during accommodation is derived from the result of simultaneous measurements in animals of the pressure in the anterior chamber and in the vitreous. Even in strong accommodation no alteration occurs, althougl even slight contact with the outer surface of the eyeball or contraction of the external eye muscles causes 
a clistinct effect. In two cavities separated by a slack membrane no differences of pressure would be expected.

Anderson Stuart lays stress upon the function of those fibres of the suspensory ligament which are attached to the vitrcous body, and are put under tension by the contraction of the ciliary muscle, in anclioring the lens during strong accommodation. He believes that the liquid contents of the liyaloid canal move from its anterior to its posterior end in accommodation, and in the opposite direction when accommodation is relaxed, and that this movement tends to prevent strains in the vitreous.

In ceplalopods and fishes, which are normally short-sighted, accommodation for objects at a distance is effected by a movement of the lens towards the retina. In the fish's eye this is acconplished by the contraction of a special muscle, the retractor lentis. In amphibia and most snakes the lens is moved towards the corned and away from the retina by changes of intra-ocular pressure (Beer).

Innervation of the Ciliary Muscle and the Muscles of the Iris.-The ciliary muscle and the sphincter pupilla are supplied by autonomic fibres (p. I004), reaching them through the short ciliary nerves arising from the ciliary ganglion (Fig. 422). The preganglionic fibres take origin from cells in the anterior part of the oculo-motor nucleus in the mid-brain. Passing to the orbit in the third nerve, they reach the ciliary ganglion, and end there by forming synapses with some of its cells. The axons of these cells continue the path as post-ganglionic fibres in the short ciliary nerves. The dilator pupillæ is supplied by the long ciliary nerves coming from the ophthalmic branch of the fifth nerve.

The preganglionic dilator fibres pass out by the anterior roots of the first three thoracic nerves (dog, cat, rabbit), accompanied by vasoconstrictor fibres for the iris. Reaching the sympathetic chain through the corresponding rami communicantes, they traverse the first thoracic ganglion, the annulus of Vieussens, the inferior cervical ganglion, and the cervical sympathetic. They end by arborizing around some of the cells of the superior cervical ganglion, whose axons eventually arrive.at the Gasserian ganglion, and running along the ophthalmic division of the trigeminal to the eye, reach the iris by its long ciliary branches.

The exact origin of the dilator path in the brain has not been definitely settled. Some place it in the mid-brain, others in the bulb. There must be at least one neuron on the path central to the spinal neuron whose axon emerges from the cord as a preganglionic fibre. The lower cervical and upper thoracic portion of the spinal cord has received the name of the cilio-spinal region from its relation to the pupillo-dilator fibres. It must not be looked upon as a centre in any proper sense of the term, but rather as the pathway by which these fibres pass down from the bulb, and where they may accordingly be tapped by stimulation.

Stimulation of certain areas on the cortex of the frontal lobe of the cerebrum (p. 1002) causes slight dilatation of the pupil even after the sympathetic has been divided. This is due to inlibition of the pupilloconstrictor fibres in the third nerve.

Changes in the Pupil during Accommodation.- - It has been already mentioned that along with the alteration in the curvature of the lens a change in the diameter of the pupil takes place in accommodation. When a distant object is looked at, the pupil becomes larger; when a near object is looked at, it becomes smaller. Narrow- 
ing of the pupil is thus associated inth contraction of the ciliary muscle, and widening of the pupil with its relaxation.

This phy'siolc gical correlation has its anatomical counterpart; for the third nerve supplies both the iris and the eiliary muscle. Stimulation of the nerve within the cranium causes contraction of the pupil, while stimulation of certain portions of its nucleus in the floor of the third ventricle and the Sylvian aqueduct or of the short ciliary nerves (Fig. 422), which receive branches from the third nerve, or of the ganglion itsclf, is followed by that change in the anterior surface of the lens which constitutes accommodaticn (Hensen and Voelckers). This can be observed either through a window in the sclerotic in a dog or by following the movements of a needle thrust into the eyeball. $\mathrm{B}$. carefully localized stimulation near the junction of the aqueduct with

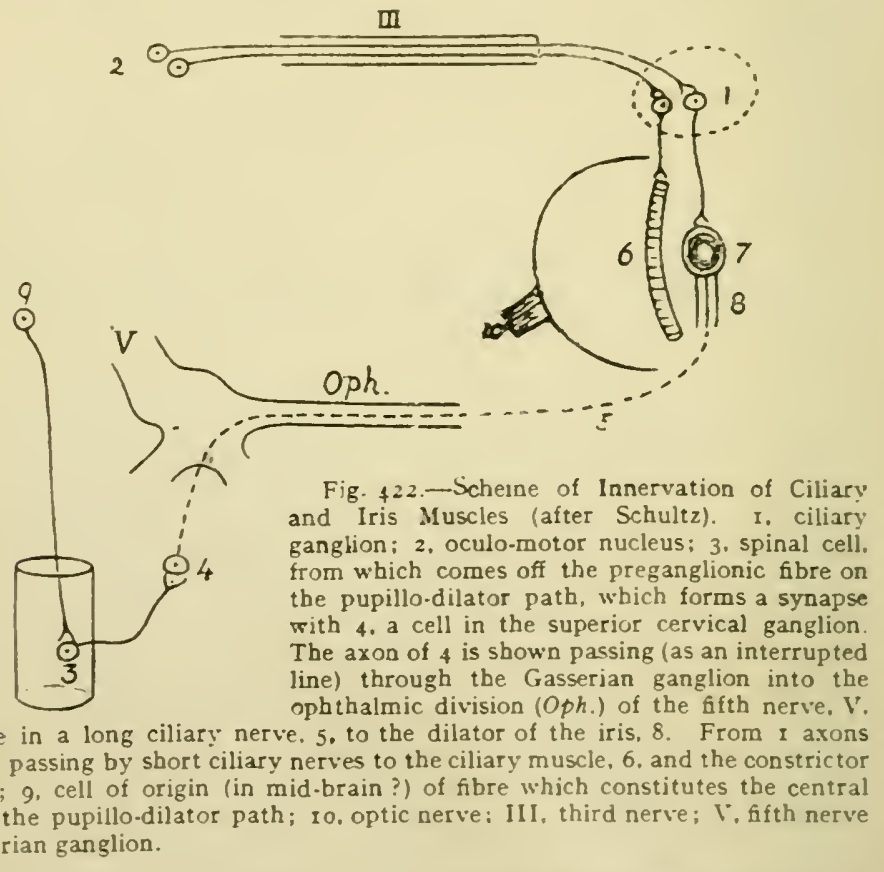

the third ventricle, it is possible to bring about the forward bulging of the lens without any change in the iris: but the normal and voluntary: act of accommodation cannot be disjoined from the corresponding alterations in the size of the pupil. Inward rotation of the eyes accompanies contraction of the pupil in accommodation, and the question may be raised whether the pupillary change is associated with the action of the extrinsic muscles of the eycball which cause convergence or with the action of the intrinsic muscles which determine the clanges in the curvature of the lens. It is usually considered to be associated with both. In any case, dicual convergence is not necessary for the reaction, since it may still bc obtained on accommodation when convergence is impossible on account of paralysis of the internal recti. 
Changes in the Pupil produced by Light.-It is not only by accommodation that the size of the pupil may be affected. In the dark it dilates, at first rapidly, then gratclually, and it maintains the width it has reached for several lours. This has been shown by taking photograplis of the eye with the magnesium flashlight. In this way the widt $l_{1}$ of the pupil is recorded before it has time to alter. Or a longer exposure to ultra-violet light, which affects the pupil but little, may be employed. When ordinary light falls upon the retina the pupil contracts, and the amount of contraction is roughly proportional to the intensity of the light. Contraction of the pupil to light is brought about by a reflex mechanism, of which the optic nerve forms the afferent and the oculo-motor the efferent path, while the centre is situated in the floor of the aqueduct of Sylvius. The relation of this centre to that which controls the changes in the pupil during accommodation has not as yet been sufficiently elucidated; but this we do know, that one of the paths may be interrupted by disease, while the other is intact. For in tabes (locomotor ataxia), and in dementia paralytica (general paralysis), the light-reflex sometimes disappears, while the constriction of the pupil in accommodation and convergence still takes place (Argyll-Robertson pupil). Artificial stimulaiion of the optic nerve has the same effect on the pupil as the ' adequate 'stimulus of light; and in many animals (including man), though not in those whose optic nerves completely decussate, there is a consensual light-reflex-i.e., both pupils contract when one retina or optic nerve is excited. This should be remembered in using the pupil-reaction as a test of the condition of the retina. For although the absence of contraction may show that the retina of the eye on which the light is allowed to fall is insensible (unless there is some physical hindrance to its passage, such as opacity of the lens or cataract), the occurrence of contraction does not exclude insensibility of the retina unless the other eye has been protected from the light.

Stimulation of the cervical sympathetic causes marked dilatation of the pupil, even when the third nerve is excited at the same time. The pupillo-dilator fibres do not act by constricting the bloodvessels of the iris. For dilatation of the pupil can be caused in a bloodless animal by stimulating the sympathetic. And even when the circulation is going on, a short stimulation of the sympathetic causes dilatation of the pupil without vaso-constriction, while with longer excitation the dilatation of the pupil begins before the narrowing of the bloodvessels. Nor does it seem possible to accept the view that the sympathetic fibres are inhibitory for the sphincter muscle of the iris. They act directly upon dilator muscular fibres. It has, indeed, long been known that in the iris of the otter and of birds a radial dilator muscle exists; and it has been 
shown by Langley and Anderson that in the iris of the rabbit, cat, and dog, the presence of radially arranged contractile substance, different it may be in some respects from ordinary smooth muscle, must be assumed. Both the constrictor and the dilator muscles of the iris are normally in a condition of greater or less tonic contraction, so that the size of the pupil at any given moment depends on the play of two nicely balanced forces. Reflex dilatation of the pupil through the sympathetic fibres is caused in man by painful stimulation of the skin, by dyspncea, by muscular exertion, and in some individuals even by tickling of the palms. In animals the stimulation of naked sensory nerves has the same effect. The 'starting of the eyeballs from their sockets,' which the records of torture so often note, is due to a similar reflex excitation of the sympathetic fibres supplying the smooth muscle of the orbits and eyelids.

Action of Drugs on the Function of the Intrinsic Eye Muscles.-The local application of atropine causes temporary paralysis of accommodation and dilatation of the pupil. When the third nerve is divided, the pupil dilates; it dilates still more when atropine is administered after the operation. Dropped into one eye in small quantity, atropine only produces a local effect; the pupil of the other eye remains of normal size, or somewhat constricted on account of the greater reflex stimulation of its third nerve by the greater quantity of light now entering the widely-dilated pupil of the atropinized eye. Even in the excised eye the effect of the drug is the same. Introduced into the blood atropine causes both pupils to dilate. Its action is to paralyze the endings of the oculo-motor fibres to the sphincter pupillæ and ciliary muscle. Other mydriatic, or pupil-dilating drugs, are cocaine. daturine, and hyoscyamine. Physostigmine or eserine, pilocarpine, and muscarine are the chicf miotics, or pupil-constricting substances. They also cause spasm of the ciliary muscle, and inability to accommodate for distant objects. They act by stimulating the structures (nerve-endings) (see pp. 182,739 ) which atropine paralyzes. The work of the mydriatics can be undone by the miotics. Thus the dilatation produced by atropine is removed by pilocarpine.

Functions of the Iris.-In vision the iris performs two chief functions: (I) It regulates the quantity of light allowed to fall upon the retina. The larger the aperture of a lens, the greater is its collecting power, the more light does it gather in its focus. In the eye, the area of the pupil determines the breadth of the pencil of light that falls upon the lens. If this area was invariable, the retina would either be 'dark from excess of light 'in bright sunshine, or dark from defect of light in dull weather or at dusk. In ordes that the iris may act as an efficient diaphragm it must be pigmented, and it is the pigment in it which gives the colour to the normal eye. The vision of albinos, in whose eyes this pigment is wanting, is often, though not invariably, deficient in sharpness. There is always intolerance of bright light; and the same is true in the condition known as irideremia, or congenital absence or defect of the iris. 
(2) Another, and perhaps equally inportant, function of the iris is to cut off the more divergent rays of a pencil of light falling upon the eye, and thus to increase the sharpness of the image. This leads us to the consideration of certain defects in the dioptric arrangements of the eye.

Defects of the Eye as an Optical Instrument.-(I) Spherical Aberration.-It is a property of a spherical refricting sufface that rays of linht passing through the peripheral portions are more strongly refracted than rays passing near the principal axis. Hence a luminous point is not focussed accurately in a single point by a spherical lens; the image is surrounded by fainter circles of light, the so-called circles of diffusion representing the rays which have not yet come to a focus, or having been already focussed have crossed and are now diverging. In the eye this spherical aberration is partly corrected by the inter position of the iris, which cuts off the more peripheral rays, especially in accommoctation for a near object, when they are most divergent. In addition, the anterior surfaces of the cornea and lens are not segments of spheres, but of ellipsoids, so that the curvature diminishes somewhat with the distance from the optic axis, and, thercfore, the re. fracting power as we pass away from the axis does not increase so rapidly as it would do if the surfaces were truly spherical. Further, the refractive index of the peripheral parts of the lens is less than that of its central portions.

(2) Chromatic Aberration.-All the rays of the spectrum do not travel with the same velocity through a lens, and are,

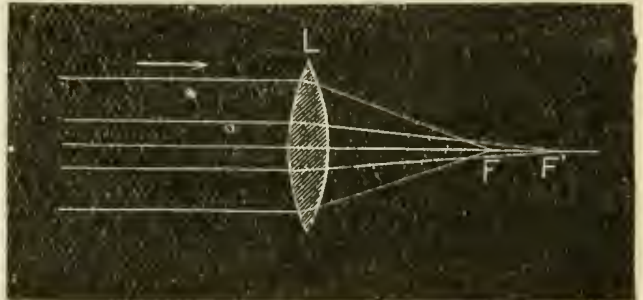

Fig. 423.- Spherical Aberration. Rays passing through the more peripheral parts of a biconvex lens $L$ are brought to a focus $F$ nearer the lens than $F^{\prime}$, the focus of rays passing through the central portions of the lens.

therefore, unequally refracted by it, the short violet rays being focussed nearer the lens than the long red rays. It was at one time supposed that this chromatic aberration, as it is called, is compensated in the eye; and it is said that this mistake gave the first hint that Newton's dictum as to the proportionality between deviation and dispersion was erroneous, and led to the discovery of achromatic lenses. But in reality the eye is not an achromatic combination; and the violet rays are focussed about $\frac{1}{2} \mathrm{~mm}$. in front of the red. Tluss, in Fig. 424 the white light passing through the lens is broken up into its constituents: the violet focus is at $\mathrm{V}$, and the red at $R$, behind it. A screen placed at $R$ would show not a point image, but a central point surrounded by concentric circles of the spectral colours, with violet outside. If the screen was placed at $\mathrm{V}$, the centre would be violet and the red would be external. For this reason it is impossible to focus at the same time and with perfect sharpness objects of different colours: a red light on a railway track appears nearer than a blue light, partly perhaps for the reason that it is necessary to accommodate more strongly for the red than for the blue, and we associate stronger accommodation with sliorter distance of the object, although other data are also involved in such a visual judgment. Whien we look at a white gas-flame through a cobalt glass, which allows only red and violet to pass, we see either a red flame, surrounded by a 
violet ring, or a violet flame surrounded by a red ring, according as we focus fir the red or for the violet rays. But the dispersive power of the eye is so small, and the capacity of rapidly altering its accommodation so great, that no practical inconvenience results from the lack of achromatism, which, however, may be easily demonstrated by looking at a pattern such as that in Fig. +25 at a distance too small for exact accommodation.

It is also reckoned among the optical imperfections of the eye (3) that the curved surfaces of the cornea and lens do not form a 'centred 'system - that is to say, their apices and their centres of curvature do not all lic in the same straight line; $(4)$ that the pupil is cccentric, being situated not exactly opposite the middle of the lens and cornea, but nearer the nasal side, and that in consequence the optic axis, or straight line joining the centres of eurvature of the lens and cornea, does not coincide with the visual axis, or straight line joining the fovea centralis with the centre of the pupil, which is also the straight line joining the centre of the pupil and any point to which the eyc is directed in vision. The angle between the optic and visual axis is about $5^{\circ}$ (Fig. 416 ).

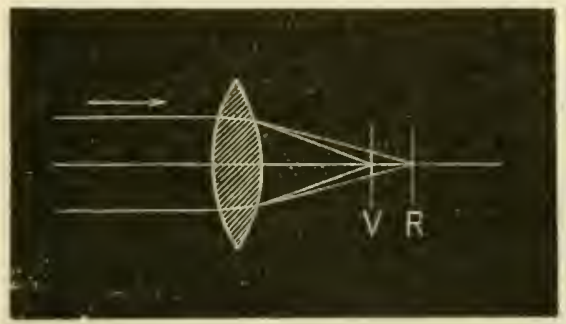

Fin. 424.-Chromatic Aberration. The violet rays are brought to a focus $V$ nearer the lens than $R$, the focus of the red rays.

(5) Muscre volitantes, the curious beadlike or fibrillar forms that so often flit in the visual field when one is looking through a microscope, are the token that the refractive media of the eye are not perfectly transparent at all parts; they seem to be due to floating opacities in the vitrcous humour. probably the remains of the embryonic cells from which the vitreous body was devcloped. (6) Lastly, it may be mentioned that slight irregularities in the curvature of the lens exist in all eves, so that a point of light, like a star or a distant street-lamp, is not seen as a point, but as a point surrounded by ray's (irregular astigmatism). In bringing this review of the imperfections of the dioptric media of the normal eye to a close, it may be well to explain that what are defects from the point of view of the student of pure optics are not necessarily defects from the freer standpoint of the plyysiologist, who surveys the mechanism of vision as a whole, the relations of its various parts to one another and to the needs of the organism it has to serve, the lnng series of developmental changes through which it has come to be what it is, and the possibilities, so far as we can limit them, that were open to evolution in the making of an eye. The optician may perhaps assert, and with justice, that he could easily have made a better lens than Nature has furnished, but the physiologist will not readily admit that he could have made as good an eye. 
While the defects hitherto mentioned are shared in greater or less degree by every normal eye, there are certain wther defects which either occur in such a comparatively small number of eyes, or learl to such grave disturbances of vision when they do occur, that they must be reckom d as alonormal comelitions. In the normal or emmetropic eye, parallel rays-and for this purpose all rays coming from an object at a distance: greater than 65 me:tres may be considered parallel-are brought to a focus on the retina without any effort of accommorlation. The distance at which objects can be distinctly seen is only limited by their size, the clearness of the atmosplere, and the curvature of the earth; in other words, the punctum remolum, or far-point of rision, the most distant point at which it is possible to see with distinctness, is practically at an infinite distance. When accommodation is paralyzed by atropine, only remote objects can be clearly seen. On the other hand, the normal eye, or, to be more precise, the normal eye of a middle-aged

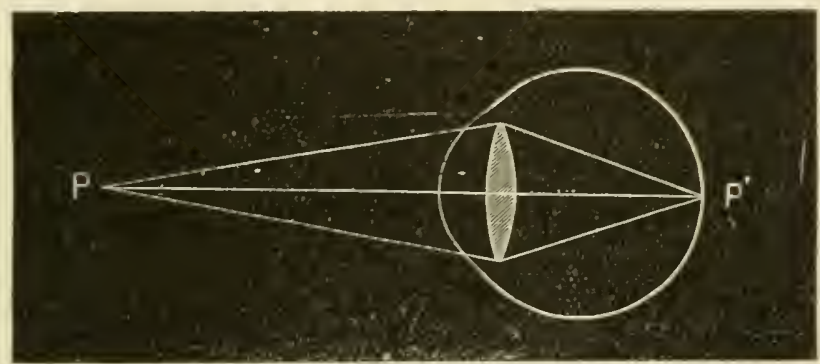

Fig. 426.- Refraction in the (Normal) Emmetropic Eye. The image P' of a distant point $P$ falls on the retina when the eye is not accommodated. To save space, $P$ is placed much too near the eye in Figs, $426,427$.

adult, can be adjusted for an object at a distance of not more than I $2 \mathrm{~cm}$. (or 5 inches). Nearer than this it is not possible to see distinctly; this point is accordingly called the punctum provimum or near-point. The range of accommodation for distinct vision in the enmetropic eye is from $12 \mathrm{~cm}$. to infinity.

Myopia, or short-sightedness, is generally due to the excessive length of the antero-posterior diameter of the eyeball in relation to the converging power of the cornea and the lens. Even in the absence of accommodation, parallel rays are not focussed on the retina, but in front of it; and in order that a sharp image may be formed on the retina the object must be so near that the rays proceeding from it to the eye are sensibly divergent-that is to say, it must be at least nearer than 65 metres - but as a rule an object at a distance of more than 2 to 3 metres cannot be distinctly seen. With the strongest accommodation the near-point may be as little as $3 \mathrm{~cm}$. from the eye. The range of vision in the myopic 
eye is therefore very small. The defect may be corrected by concave glasses, which render the rays more divergent. It is to be noted that many cases of internal squint in children are connected with myopia, the eyes necessarily rotating inwards as they are made to fix an abnormally near object. The treatment both of the squint and the myopia in these cases is the use of concave spectacles (Fig. 427). Myopia, although a condition that shows a distinct

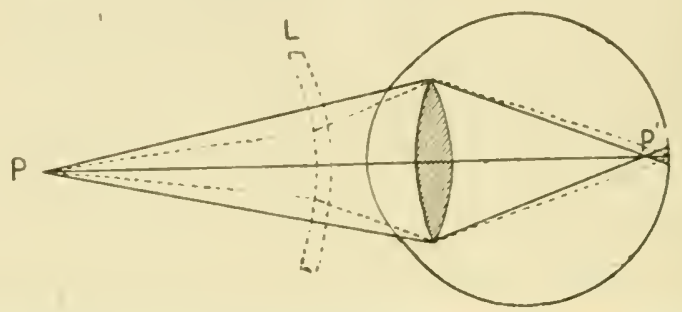

Fig. 427.- Ilyopic Eye. The inıage P' of a distant point $\mathrm{P}$ falls in front of the retina even without accornmodation. By means of a concave lens I, the image may be inade to fall on the retina (dotted lines).

hereditary tendency, is rarely present at birth; the elongation of the antero-posterior diameter of the eyeball develops gradually as the child grows.

In hypermetropia, or long-sighteduess, the eye is, as a rule, too short in relation to its converging power; and with the lens in the position of rest, parallel rays would be focussed behind the retina. Accordingly, the hypermetropic eye must accommodate even for

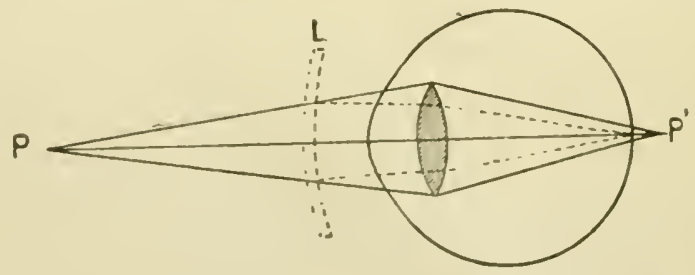

Fig. 428.--Hypermetropic Eye. The innage $P^{\prime}$ of a point $P$ falls belind the retina in the unaccommodated eye. By medns of a convex lens 1 , it may be focussed on the retina without accommodation (dorted lines).

distant objects, while even with maximum accommodation an object cannot be distinctly seen unless it is farther away than the near-point of the emmetropic ere. The far-point of distinct vision is at the same distance as in the emmetropic eye-viz., at infinitythe near-point is farther from the ey't. The defect is corrected by convex glasses (Fig. 428). Hypermetropia, unlike myopia, is present at birth. 
Presbyopia, or the long-sightedness of old age, is not to be confounded with hypermetropia. It is essentially due to failure in the power of accommodation, chiefly through weakness of the ciliary muscle, but partly owing to increased rigiclity and loss of elasticity of the lens. Inages of clistant objects are still formed on the retina of the unaccommodated eye with perfect sharpnessi.e., the far-point of vision is not affected. But the eye is unable to accommodate sufficiently for the rays diverging from an object at the ordinary near-point; in other worils, the near-point is farther away than normal. Convex glasses are again the remedy.

The near-point of distinct vision can be fixed in various waysanong others, by means of Scheiner's experiment (Practical Exercises, p. I I03). Two pin-holes are pricked in a card at a distance less than the diameter of the pupil. A needle viewed through the holes appears single when it is accommodated for, double if it is out of focus. The near-point of vision is the nearest point at which the needle can still, by the strongest effort of accommodation, be seen single.

Astigmatism.- It has been mentioned that slight differences of curvature along different meridians of the refracting surfaces exist in all eyes. But in some cases the difference in two meridians at right angles to each other is so great as to amount to a serious defect of vision. To this condition the name of 'astigmatism ' or 'regular astigmatism' has been given. It is usually due to an excess of curvature in the vertical meridians of the cornea, less frequently in the horizontal meridians; occasionally the defect is in the lens. Rays proceeding from a point are not focussed in a point, but along two lines, a horizontal and a vertical, the horizontal linear focus being in front of the other when the vertical curvature is too great, behind it when the horizontal curvature is excessive. The two limbs of a cross or the two hands of a clock when they are at right angles to each other cannot be seen distinctly at the same time, although they can be successively focussed. The condition may be corrected by glasses which are segments of cylinders cut parallel to the axis (Practical Exercises, p. II05).

The Ophthalmoscope.-The pupil of the normal eye is dark, and the interior of the eye invisible, without special means of illuminating it. But this is not because all the light that falls upon the fundus is absorbed by the pigment of the choroid, for even the pupil of an albino appears dark when the eye is covered by a piece of black cloth with a hole in front of the pupil. The explanation is as follows:

Let the rays from a luminous point, $\mathrm{P}$, be focussed by the lens, $\mathrm{L}$, at $\mathrm{P}^{\prime}$ (Fig. 429). It is plain that rays proceeding from $\mathrm{P}^{\prime}$ will exactly retrace the path of those from $P$ and be focussed at $P$. Now, the eye receives rays from all directions, and, when it is 
sufficiently well illuminated, sends rays out in all directions. The moment, however, that the observing eye is placed in front of the observed eye, the latter ceases to receive light from the part of the field occupied by the pupil of the former, and therefore ceasos to reflect light into it.

This difficulty is avoided by the use of an ophthalmoscopic mirror. The original, and theoretically the most perfect, form of

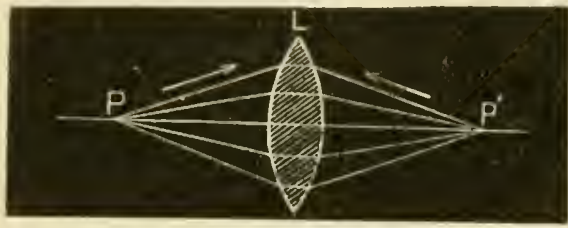

Fig. 429 . such a mirror is a plate, or several superposed plates, of glass, from which a beam of light from a laterally placed candle or lamp is reflected into the observed eye, and through which the eye of the observer looks (Fig. 430). But the illumination thus obtained is comparatively faint; and a concave mirror is now generally used. In the centre is a small hole or a small unsilvered portion of the mirror for the observer's eye. In the direct method of examination (Fig. 432), the mirror is held close to the observed eye, and an erect virtual image of

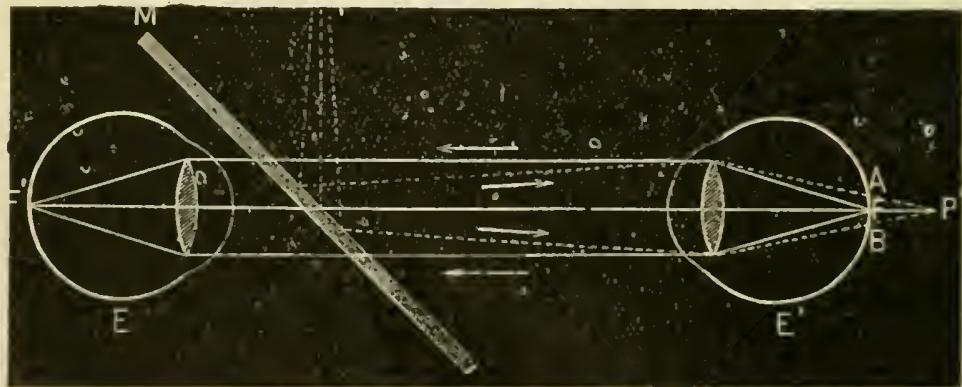

Fig. 430.-Figure to illustrate the Principle of the Ophthalmoscope. Rays of light from a point are reflected by a glass plate .II (several plates together in Helunholtz's original form) into the observed eye E: 1 . Their focus would fall, as shown in the figure, at $P$, a little behind the retina of $E^{1}$. The portion of the retina $A B$ is therefore illnminated by diffusion circles; and the ravs from a point of it $F$ will, if $F_{1}^{1}$ is emmetropic and unaccommodated, issne parallel from $F_{1}^{1}$ and be brought to a focus at $\mathrm{F}^{\mathrm{L}}$ on the retina of the (emmetropic and unaccommodated) ubserving eye E.

the fundus is seen. When the eye of the observer and of the patient are both emmetropic, and both eyes are unaccommodated, the rays of light proceeding from a point of the retina of the observed eye are rendered parallel by its dioptric media, and are again brought to a focus on the observer's retina.

If the observed eye is myopic, the rays of light coming from 
a point of the retina leave the eye, even when it is unaccommodated, as a convergent pencil; and the emmetropic non-accommodated eye of the observer must have a concave lens placed before it in order that the fundus may be distinetly seen.

When the observed eye is hypermetropic, the rays emerging from the unaccommodated eye are divergent, and a convex lens, the strength of which is proportional to the amount of hypermetropia, must be placed before the observer's unaccommodated eye if lie is to see the fundus distinctly. By accommodating, the observer can see the fundus clearly without a convex lens.

By this method errors of refraction in the eye may be detected and measured. The observer must always keep his eye unaccommodated, and if it is not emmetropic, he must know the amount of his short- or long-sightedness-i.e., the strength and sign of the lens needed to correct

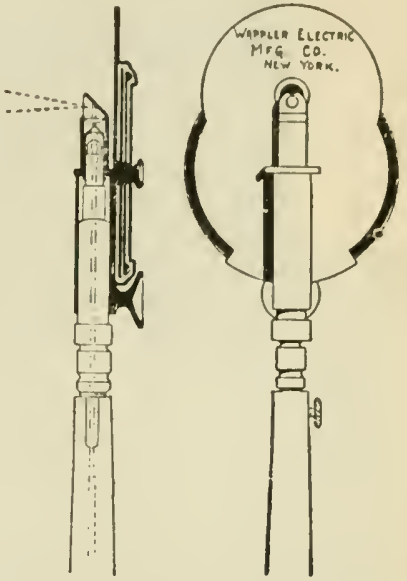

Fig. 431.-May's Electric Ophthalinoscope. his defect of refraction, and must allow for this in calculating the defect of his patient. Nonaccommodation of the eye of the latter can always be secured by the use of atropine.

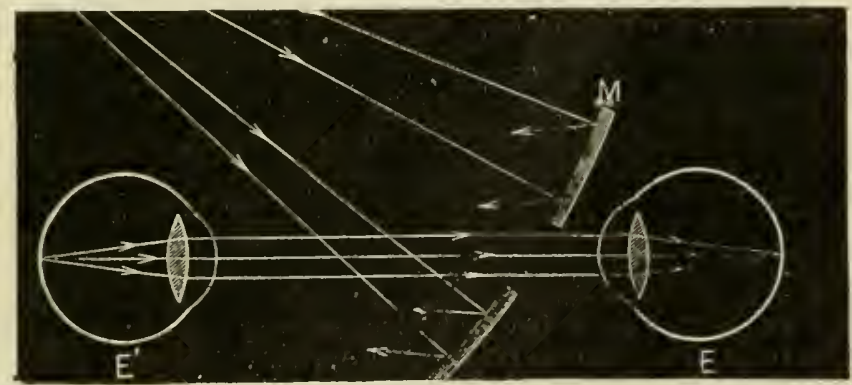

Fig. 432,-Direct Method of using the Ophthalmoscope. Light falling on the perforated concave mirror $M$ passes into the observed eye $E^{\prime}$; and, both $E^{\prime}$ and the observing eye $\mathrm{E}$ being supposed emmetropic and unaccommodated, an erect virtual image of the illuminated retina of $E^{\prime}$ is seen by $E$.

By the direct method of ophthalmoscopic examination, only a small portion of the retina can be seen at a time, and this is highly magnified. A larger, though less magnified, view can be got by the indirect metlood. The observed eye is illuminated as before, 
but the mirror and the observer's eye are at a greater distance (Fig. 435). Here the rays from a comsiluable portion of the retina are brought to at focus by a convex lens held near the eye of the plient, so as to form a real and inverted aerial inage of the

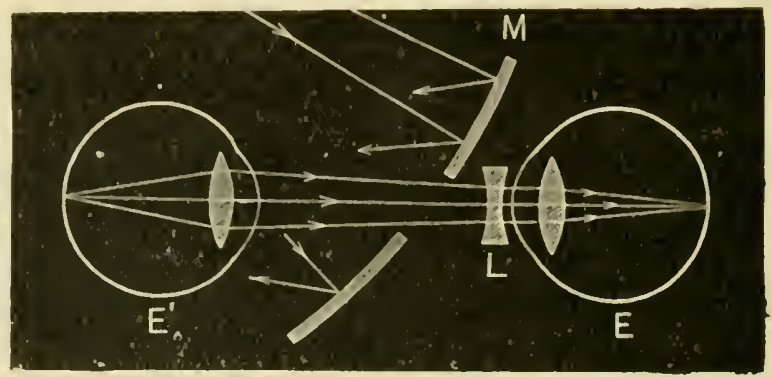

Fig. 433.- Use of the Oplithalmoscope (Direct Method) for testing Errors of Re. fraction in Myopic Eye. Ray's issuing from a point of the retina of $E^{\prime}$, the observed (myopic and unaccommodated) eye, pass out, not parallel, but convergent. They will therefore be focussed in front of the retina of the observing (unaccommodated) eye $\mathrm{E}$ if the lat ter is emmetropic. By introducing a concave l'ns $\mathrm{L}$ of suitable strength, however, a clear view of the retina of $\mathrm{E}^{\prime}$ will be (h)t ained, and the strength of this lens is the measure of the amount of myopia.

retina. This image is viewed by the observer at his ordinary visual distance. It is not necessary in this methocl that the observed eye should be ron-accommodated, although it is convenient as in

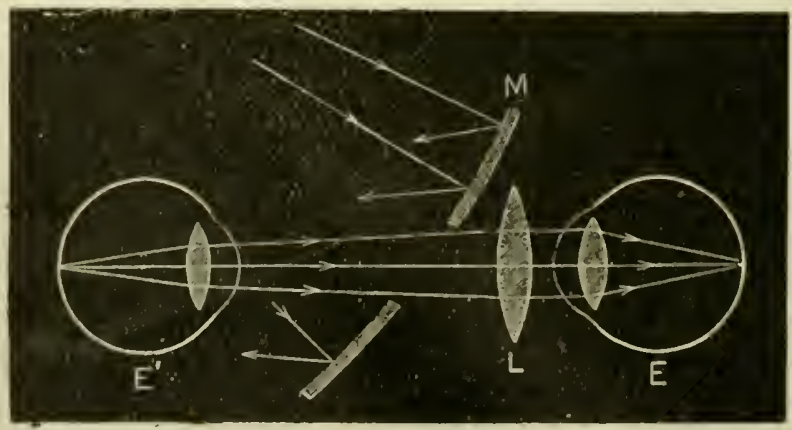

Fig. 434. Testing Errors of Refraction in Ilypermetropic Eye. Ray's from a point of the retina of $E^{\prime}$, the observed eye, issue divergent, and are focussed behind the retina of the observing (unaccommodated and emmetropic) eye $E$. The strength of the convex lens $\mathrm{L}$, which must be introduced ili front of $\mathrm{E}$ to give clear vision of the retina of $\mathrm{E}^{\prime}$, measures the degree of hypermetropia.

the direct method to cause dilatation of the pupil by atropine, which also relaxes the accommodation (Practical Exercises, p. II08).

Skiascopy.-To a great extent the ophthalmoscopic method of measuring errors of refraction has been replaced by the more modern 
method of skiascopy (sizadow test). It depends upon the followne observation: When one throws light from a little distance with a concave mirror into an observed "ge and then rotates the mirror slowly around the long axis of the handle, one sees that the pupil, which at first was completely illuminated, becomes dark from one side as if covered by a shadow. This shadow will move in the same direction in which the mirror is rotated or in the oppusite direction, according to whether the observer is farther from thr: observed eye than its far-point, or between the ey" and the far-point. If the observer is exartly at the far-point, no direction of movement of the shadow can be made out, but the pupil in its whole extent is

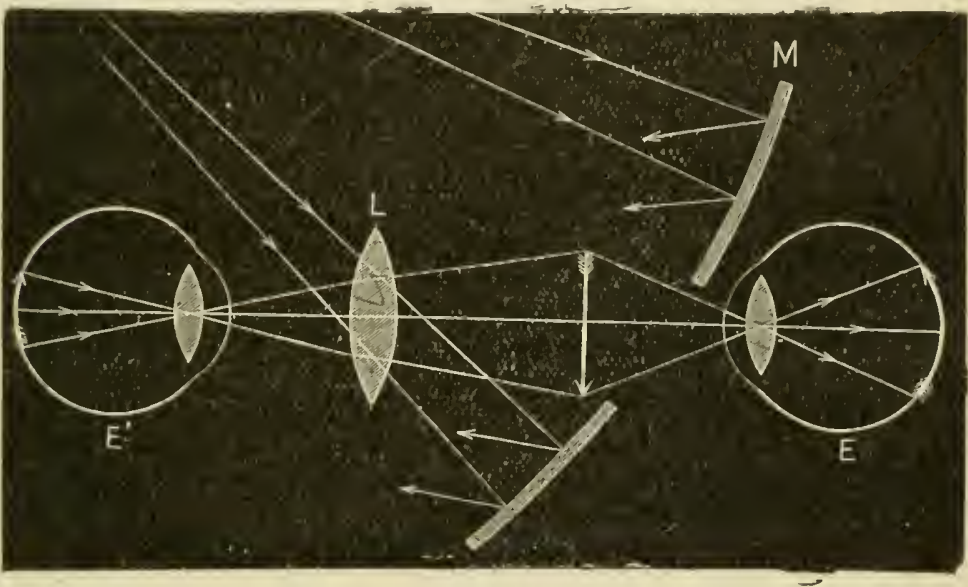

Fig. 435.-Indirect Method of using the Ophthalmoscope. The rays of light issuing from $E^{\prime}$, the observed eye, are focussed by the biconvex lens $L$, and a real inverted image of a portion of the retina of $\mathrm{E}^{\prime}$, magnified four or five times, is formed in the air between the lens and the observing eye $\mathrm{E}$. This image is viewed by $\mathrm{E}$ at the ordinary distance of distinct vision (Io to 12 inches). (The exaggeration of the size of the mirror makes it appear as if some of the rays from the lamp passed through the lens before being reflected from the mirror. This would not be the case in an actual observation.)

either illuminated or altogether dark. In this way the distance of the far-point of a myopic eye can be easily determined by a metre rule, and from this the degree of myopia. If the far-point is either too near, as in strong myopia, or too distant, as in weak myopia and emmetropia, or behind the observed eye, as in hypermetropia, it can be brought to a convenient distance by interposing suitable lenses. The observer then deternines the far-point exactly by moving his eye nearer to or farther from the observed eye, or, keeping his own eye fixed, by bringing the far-point of the observed eye to coincide with it by inserting lenses (Practical Exercises, PP. IIOg, II IO). 
The phenomenon depends upon the interruption which the light proceeding from the observed retina experiences first at the margin of the pupil of the observed eye, and then at the margin of the hole in the mirror or of the observer's pupil. When the mirror is rotated, an illuminated point of the observed retina will move in the opposite direction over the retina.* The light proceeding from this point when the observed eye is emmetropic is so refracted by the lens and cornca tlat it leaves the eye as a bundle of parallel rays in the direction of the image of the source of light (L') (Fig. 436). If the image of the flame reflected by the inirror is situated on the principal axis of the observer's eye, and if the pupils of observed and observer are of equal size, all the rays coming from the observed retina will fall on the observer's retina, and therefore the whole pupil of the observed eye will appear light. If the mirror is now rotated so that the image of the source of light moves away from the principal axis, and the illuminating rays are no longer in that axis, the illuminated point will move in the opposite direction from the principal axis, and the light returning from the pupil of the observed eye will again issue in the direction of the image of the

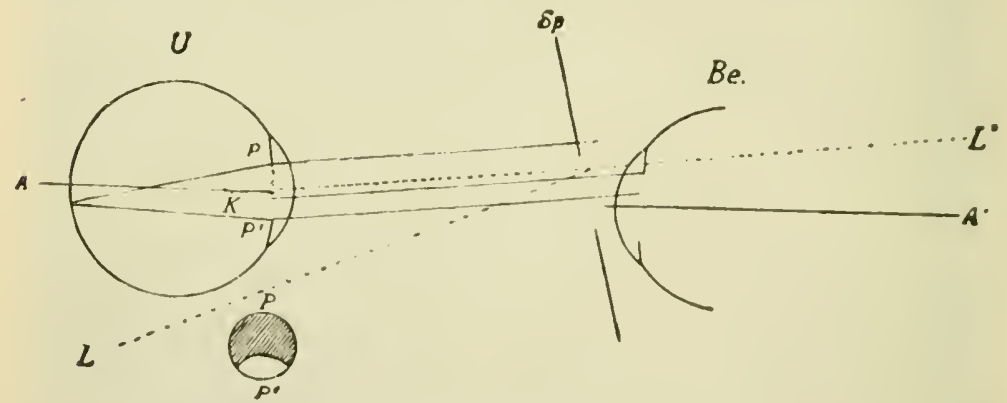

Fig. 436.-Path of Rays in Skiascopy (Snellen). $U$, observed eye; Be, eye of ob. server; $S p$, mirror; $L$, source of light; $L$, inage of the source of light; $A, A^{\prime}$. principal axis; $I^{\prime}, I^{\prime \prime}$, pupils.

source of light. It can then happen that none of the rays hit the observer's pupil, and the observed pupil will appear entirely dark. Or the direction of the rays may be such that a portion of them enters the observer's pupil, the rest being interrupted by its border. In this case the part of the observed pupil from whicl rays enter the observer's pupil will appear light, while the rest is dark. From Fig. 436 it can be seen that the light part of the observel pupil is on the opposite side of the principal axis from the image of the source of light. If, therefore, the image of the source of light moves to the right (by rotation of a concave mirror to the right, or rotation of a plane mirror to the left) the skiascopic appearance in the observed pupil moves to the left-i.e., in the opposite direction to the image of the source of light.

If the observed pupil is myopic-i.e., if its far-point is between the observer and the observed eye, rotation of the inirror so far from the principal axis that only a part of the rays issuing from the observed pupil enter the observer's eye, will cause the pupil to appear light only

- When a concave mirror is rotated to the right, the inverted real mirror image also moves to the right, and the illuminated point to the left. When a plane mirror is rotated to the right, the virtual mirror image inoves to tho left, and the illuminated point on the retina therefore to the right. 
on ane side, and on account of the crossing of the rays this illuminaterl portion will be on the same side of the principal axis as the image of the source of light (lig. +37). When the image of the source of light is moved to the right the light area of the observed pupil will also move to the right-i.e., with rotation of a concave mirror in the same direction as the image of the source of light, and with rotation of a plane mirror in the opposite direction (Snellen).

A method of photographing the retina in the living eye has also been employed as a means of investigating the fundus.

Single Vision with Both Eyes-Diplopia.-Scheiner's experiment shows that it is possible to have double vision, or diplopia, with a single eye when two separate images of the same object fall upon different parts of the retina. In vision with both eyes, or binocular vision, an image of every object looked at is, of course, formed on each retina, and we have to inquire how it is that as a rule these images are blended in consciousness so as to produce the perception of a single object; and

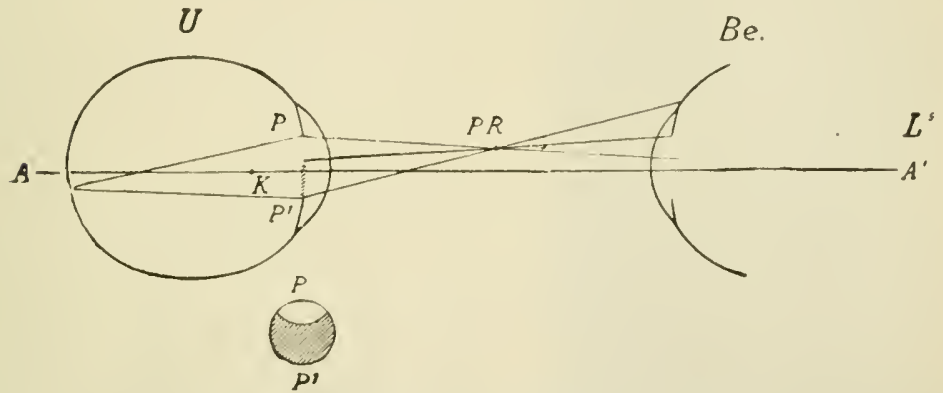

Fig. 437.- Path of Rays in Skiascopy (Myopic Eye) (Snellen). PR, far-point of observed eye. The other references are as in Fig. 436.

how it is that under certain conditions this blending does not take place, and diplopia results. Two clief theories have been invoked in the attempt to answer these questions: (I) the theory of identical points, (2) the theory of projection.

In regard to the second theory, we shall merely say that it assumes that in some way or other the retina, or, rather, the retino-cerebral apparatus, has the power of appreciating not only the shape and size of an image, but also the direction of the rays of light which form it, and that the position of the object is arrived at by a process of mental projection of the image into space along these directive lines. Where the directive lines of the two eyes cut each other, the two images coincide, and the object is seen single in the position of the point of intersection. The first theory we shall examine in some detail.

The Theory of Identical Points.-This theory assumes that every point of one retina 'corresponds' to a definite point of the other retina, and that in virtue of this correspondence, either by an inborn necessity or from experience, the mind refers simultaneous impressions upon two corresponding or identical points to a single point in external space. If we imagine the two retina in the position which the eyes occupy when fixing an infinitely distant object-that is, with the visual axes parallel-to be superposed, with fovea over fovea, every point of the one retina will be covered by the corresponding point of the other retina, so that identical points could be pricked through with a needle. 
But since the actual centre of the retina docs not corresjond with the forea centralis (1 ig. +10$)$, but lies nearer the nasal side, the nasal edge of the left retina will overlap the temporal edge of the riglit, and the nasal edge of the right will overlap the temporal edge of the left; so that a part of each retina lias no corresponding points in the other.

The adherents of this theory claim, and with justice, that a small object so situated that its image must be formed on corresponding points of the two retina docs, as a rulc, appear single, and, what is even more striking, that a phosplene, or luminous ring produced by pressing the blunt end of a pencil or the finger-nail on a point of the globe of one cye (which Newton compared to the circles on a peacock's tail), is not doubled by pressure over the corresponding point of the other eye, although two circles are seen when pressure is made upon points which do not correspond. If in rotating the eyes one eye is prevented by pressure with the finger from following the movement of the other, there is double vision. When strabismus or squinting is produced by paralysis of the thirel (p. 924) or the sixth cranial nerve (p. 920), it is accompanied by diplopia, until in course of time the mind icarns to disregard one of the images. In some cases of squint the double images are never completely suppressed, but a new abnormal form of visual localization is developed, which, however, very seldom permits any accurate judgment of depth. In strabismus it is obvious that the two images of an object cannot fall on corresponding points.

But it is also a fact that, under cortain conditions, images situated on corresponding points may not, and that images not situated on corresponding points may, give rise to a single impression. For example, if one of the closed eyes be held slightly out of its ordinary position by the finger, pressure on identical points of the two eyes gives rise to two separate phosphenes. And some of the phenomena of stereoscopic vision (p. Io39) show. clearly that images falling on points not strictly corresponding may give a single impression; while we do not habitually see double, although it is certain that the images of multitudes of objects are constantly falling on points of the retinæ not anatomically "identical."*

The question therefore arises, How is it that we do not see these double images? This is one of the difficulties of the theory of identical points. The following is a partial cxplanation: (I) The images of objects in the portion of the field most distinctly seen-that is, the portion in the immediate neighbourhood of the intersection of the visual lines, or the part to which the gaze is directed-are formed on identical points; and by rapid movements the eyes fix successively different parts of the field of view. (2) Vision grows less distinct as we pass out from the centre of the retina, and we are accustomed to neglect the blurred peripheral images in comparison with those formed

- In every fixed position of the eyes, the objects whose images fall on corresponding points will be arranged on certain ilcfinite lines or surfaces which vary with the direction of the visual axis and to which the name of horopter, or point-horopter. has heen given. For most eyes when directel to the horizon - that is, with the visual axes parallel-the loropter is practically the horizontal plane of the ground, so that all objects within the field of vision, and resting on the ground, fall upon corresponding points, and are seen single. When the eyes are directed to a point at such a distance that the lines of vision are sensibly convergent, the horopter consists (I) of a straight line drawn through the fixing-point and at right angles to a plane passing through the fixing-point and the two visual lines (visual plane); (2) of a circle passing through the fixing-point and the nodal points of the two eyes (the famous horopteric circle of Miuller). 
on the forea. (3) When the images of an object do not fall on iulentica" points, one of the points on which they do fall maty be occupied with the images of other objects, some of which may be so lonlly marked as to enter into conflict with the extra image and to suppress it. (4) Lastly, the pliysiongiral 'identical po:s.t' is not a geometrical point, but an area which increases in size in $\tau_{4}$ a more peripheral zones of the retina, and can also he increased by practice; and images wlich lie wholly or in chief part within two corresponding areas practically coincide.

Stereoscopic Vision.-Althougl the retinal image is a projection of external objects on a surface, we perceive not only the length and breadth, but also the depth or solidity of the things we look at. When we look dircetly at the front of a building, the impression as to its form is the same whether one or both eyes be used, although with a single eye its distance cannot be judged so accurately. But when we view the building from such a position that one of the corners is visible, we obtain a more correct impression of its depth with the two eyes. This is partly due to the fact that to fix points at different distances from the eyes the visual lines must be made to converge more or less, and of the amount of this convergence we are conscious through the contraction of the muscles which regulate it. But there is another element involved. When the two eyes look at a uniformly-coloured plane surface, the retinal image is precisely the same in both. But when the two eyes are directed to a solid object (say a book lying on a table) the picture former on the left retina differs slightly from that formed on the right, for the left eve sees more of the left side of the book, and the right eye more of the right side.

That there is a close connection between uniformity of retinal images and impression of a plane surface on the one hand, and difference of retinal images and impression of solidity on the other, is proved by the facts of stereoscopy. It

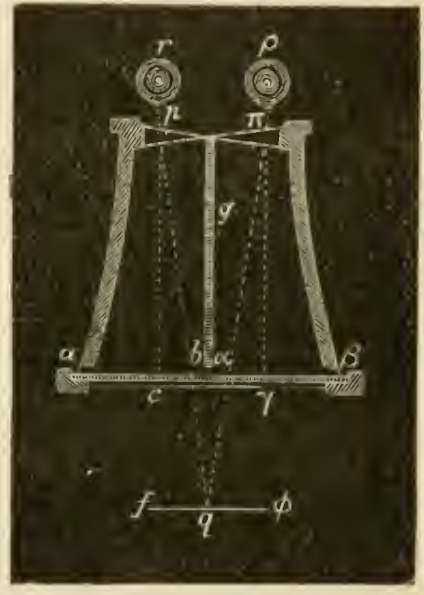

Fig. 438.-Brewster's Stereoscope. $p$ and $\pi$ are prisms, with their re. fracting angles turned towards each other. The prisins refract the rays coming from the points $c, \gamma$ of the pictures $a b$ and $a \beta$ so that they appear to come from a single point $q$. Similarly, the points $a$ and $\phi$ appear to be situated at $f$, and the points $b$ and $\beta$. at $\alpha$. is evident that if an exact picture of the solid object as it is seen by each eye can be thrown on the retina, the impression produced will be the same, whether these images are really formed by the object or not. Now, two such pictures can be produced with a near approach to accuracy by photographing the object from the point of riew of each eye. It only remains to cast the imagc of each picture on the corresponding retina, while the eyes are converged tc the same extent as would be the case if they were viewing the actual object. This is accomplished by means of a stereoscope (Fig. 438).

It is found that the resultant impression is that of the solid object. It is impossible to reconcile this with the doctrine of strictly identical geometrical points. A pair of identical pictures gives with the stereoscope not the impression of a solid, but of a plane surface. If the 
relative position of any two points differs in the two pictures, the blended picture las a correspondling point in relicf. So great is the delicacy of this test that a good and a bad banknote will not blend under the stercoscope to a flat surface, and the method may be actually used for the detection of forgery.

When the pictures are interchanged in the stereoscope so that the image which ought to be formed on the right retina falls on the left, and that which is intended for the left eye falls on the right, what were projections before become hollows, and what were hollows stand out in relief. The pseudoscope of Wheatstone is an arrangement by which each eye sees an object by reflection, so that the images which would be formed on the two retine, if the object were looked at directly, are interchanged, with the same reversal of our judgments of relief.

Visual Judgments.-We say judgments of relief; for what we call seeing is essentially an act that involves intellectual processes. As the retina is anatomically and developmentally a projection of the brain pushed out to catch the waves of light which beat in upon the organism from every side, so, physiologically, retina, optic nerve, and visual nervous centre are bound together in an indissoluble chain. We cannot say that the retina sees, we cannot say that the optic nerve sees-the optic nerve in itself is blind-we cannot say that the visual centre sees. The ethereal waves falling on the retina set up impulses in it which ascend the optic nerve; certain portions of the brain are stirred to action, and the resulting sensations of light springing $u p$, we know not where, are elaborated, we know not how (by processes of which we have not the faintest guess), into the perception of what we call external objects-trees, houses, men, parts of our own bodies, and into judgments of the relations of these tlings among themselves, of their distance and movements.

A child learns to see, as it learns to speak, by a process, often unconscious or subconscious, of 'putting two and two together.' The musical sounds united and terminated by noises which make up the spoken word 'apple' are gradually associated in its mind with the visual sensation of a red or green object, the tactile sensation of a smooth and round object, and the gustatory and olfactory sensations which we call the taste or flavour of an apple. And as it is by experience that the child leams to label this bundle of sensations with a spoken, and afterwards with a written, name, so it is by experience that it learns to group the single sensations together, and to make the induction that if the hand be stretched out to a certain distance and in a certain direction-i.e, if varions muscular movements, also associated with sensations, be made-the tactile sensation of grasping a smooth round body will be felt, and that if the further muscular movements involved in conveying it to the moutl be carricd out, a sensation agrecable to the youthful palate will follow. At length the child comes to believe, and, unless lie happens to be specially instructed, carries his belief with hin to his grave, that when he looks at an apple he sees a round, smootl, tolerably hard body, of definite size and colour; while in reality all that the sense of sight can inform him of is the difference in the intensity and colour of the light falling on his retina when he turns his head in a particular direction.

An interesting illustration of the rôle of experience in shaping our visual judgments is found in the sensations of persons born blind and relieved in after-life by operation. A boy between thirteen and fourteen years of age, operated on by Cheselden, 
thought all the objects he looked at touched his eyes. He forgot which was the (log and which the cat, but catching the cat (which he knew by feeling), he lookerl at her steadfastly and said, "So, puss, I shall know you another time." Pictures seemed to him only parti-coloured planes; but all at once, two months after the operation, he cliscovered they represented solids.' Nunnely, perhaps rementering the dictum of Diderot, that 'to prepare and interro-

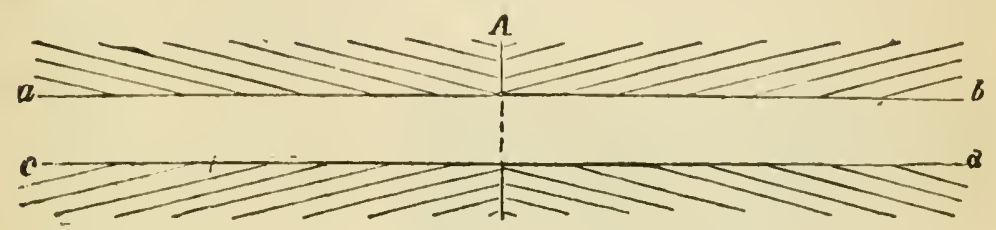

lFig. 439.-11lusion of Harallel Lines (Hering).

gate a person born blind would not have been an occupation unworthy of the mited talents of Newton, Des Cartes, Locke, and Leibnitz,' made an elaborate investigation in the case of a boy nine years old, on whom he operated for congenital cataract of both eyes, and, what is of special importance, instituted a set of careful experiments and interrogations before the operation, so as to gain lata for comparison. Objects (cubes and spheres) which before the operation he could easily recognize by touch were shown him afterwards, but although "he could at once perceive a difference in their shapes, he could not in the least say which was the cube and which the sphere.' It took several days, and the objects had to be placed many times in his hands before he could tell them by

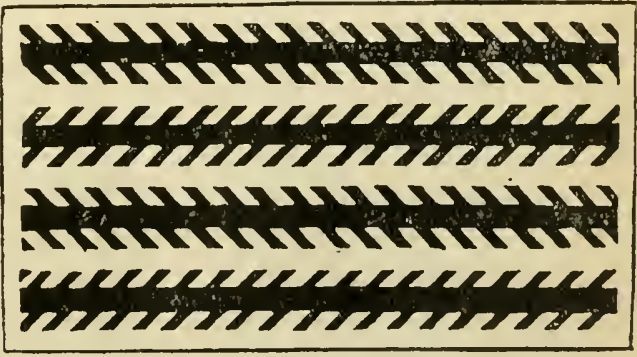

Fig. 4 ^.,-Illusion of Parallel Lines (Zöllner). the eye. "He said everything touched his eyes, and walked most carefully about, with his hands held out before him to prevent things hurting his eyes by touching them.'

Many other illustrations might be given of the fact that 'seeing ' is largely an act of reasoning from data which may sometimes mislead. Thus in Figs. 439 and 440 the long horizontal lines are really parallel, but do not appear so owing to the confusion of judgment produced by the short sloping lines. In Fig. 44.I the spaces covered by $A, B$, and $C$ are equal squares, but $A$ appears 
taller than B, and C smaller than either A or B. In the same figure the lines 1 ) and $E$ are of the same length, but E seems consiclerably longer than $\mathrm{D}$.

Illusions of movement are among the most interesting optical illusions. If two similar objects are momentarily shown to the eye in rapid succession and at points in space not separated by too great a distance, the illusion is produced that the first object has moved to the position of the second. Such illusions are the basis of the so-called ' moving pictures' shown by the cinematograpli. A series of instantaneous photographs of a morement are taken, recording the successive positions assumed by the moving body. When these are thrown on the retina in the same order and in rapid succession, an illusion of the original movement is produced.

The apparent size and form of an object is intimately related to the size, form, and sharpness of its image on the retina. We are,
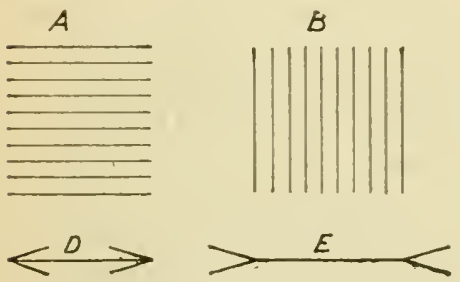

Fig. 44r.-Illusions of Space-Perception. therefore, able to discriminate with great precision the unstimulated from the excited portions of that membrane, especially in the forea centralis, and also the degree of excitation of neighbouring excited parts. But instead of localizing the image on the retina as we localize on the skin the pressure of an object in contact with it, we project the retinal image into space, and see everything outside the eye.

In vision, in fact, we have no conception of the existence of either retina or retinal image; and even the shadows of objects within the eye-for instance, an opacity or a foreign body in any of the refractive media-are referred to points outside it. Generally opacities in the vitreous humour are movable, in the lens not.

Purkinje's Figures. - As was first pointed out by Purkinje, the shadows of the bloodvessels in the retina itself, and even of the corpuscles circulating in them, although neglected in ordinary vision, may be recognized under suitable conditions, a conclusive proof that the sensitive layer must lie behind the vessels.

If a beam of sunlight is concentrated on the sclerotic as far as possible from the margin of the cornea, and the eye directed to a dark ground, the network of retinal bloodvessels will stand out on it. Another method is to look at a dark ground while a lighted candle, held at one sicle of the eye at a distance from the visual line, is moved slightly to anrl fro. In the first method, a point of the sclerotic behind the lens is illuminatel, and rays passing from it across the interior of the eyeball in everv direction cast shadows of the vessels of the retina on its sensi- 
tive layer. In the second method, the image of the flame formed on the retina by rays falling obliquely through the pupil becomes in the general darliness itself a source of light, by interrupting the rays from which the retinal vessels form shadows. The distance of the sensitive from the vascular layer may be approximately calculated by measuring the amount by which the shadows change ilieir position, when the position of the illuminated point of the sclerotic is altered. The nearer a vessel lies to the sensitive layer, the smaller must be the angle through whicls the apparent position of its shadow moves for a given movement of the spot of light. In this way it has been calculaterl that the sensitive layer is about 0.2 to $0.3 \mathrm{~mm}$. behind the stratum which contains the bloodvessels. This corresponds sufficiently well with the position of the layer of rods and cones, which all other evidence shows to be the portion of the retina actually stimulated by light. The shadows of the blood-corpuscles in the retinal vessels may be rendered visible by look: ing at a bright and uniformly illumina ted groun $\mathrm{s}_{\text {, }}$ like the milk glass shacie of a lamp or the blue sky, and moving the slightly separated fingers or a perforated card rapidly before the eye. From the rate of their apparent movement, Vierordt calculated the velocity of the blood in the retinal capillaries at 0.5 to $0.9 \mathrm{~mm}$. per second. One reason why the shadows of these intra-retinal structures do not appear in ordinary

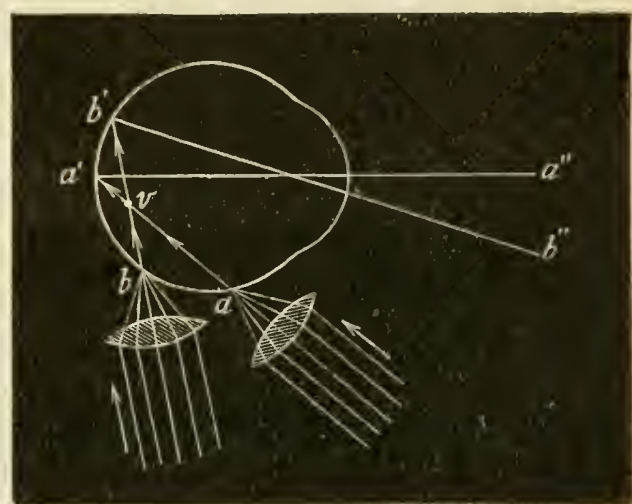

Fig. 442,- - Method of rendering the Retinal Bloodvessels visible by concentrating a Beam of Light on the Sclerotic. From the brightly-illuminated point of the sclerotic, $a$, rays issue, and a shadow of a vessel, $v$, is cast at $a^{\prime}$. It is referred to an external point, $a^{\prime \prime}$, in the direction of the straight line joining $a^{\prime}$ with the nodal point. When the light is shifted so as to be focussed at $b$, the shadow cast at $b^{\prime}$ is referred to $b^{\prime \prime}-i . e$., it appears to move in the same direction as the illuminated point of the sclerotic.

vision seems to be their sinall size. The retinal vessels are in reality only vascular threads; the thicliest branch of the central vein is not $15 \mathrm{~mm}$. in diameter. The apex of the cone of complete shadow (umbra) cast by a disc of this size, at a distance of $20 \mathrm{~mm}$. from a pupil $4 \mathrm{~mm}$. wide, would lie only $15 \mathrm{~mm}$. behind the disc - that is to say, the umbra of the retinal vessels would not reach the layer of the rods and cones at all, and only the penumbra, or region of relative darkness, would fall upon it.

When the eyes, after being closed for some time, are suddenly opened, the branches of the retinal vessels may be seen for a moment. This is especially the case after sleep; and a good view of the phenomenon may be obtained by looking at a white pillow or the ceiling immediately on awaking. If the eyes are kept open for a few seconds, the branching pattern fades away; if they are only allowed to remain open for an instant, it may be secn many times in succession. The main vessels appear to radiate out from a central point. But their actual junction there is not seen, since it lies in the optic disc or blind spot. 
The Blind Spot.-The fibres of the optic nerve are insensible to light; light only stimulates them through their end-organs. This can be proved by directing by mcans of an ophthalmoscope a beam of light upon the optic disc, where the true retinal layers do not exist. The person experimented on has no sensation of light when the beam falls entirely upon the disc; when its direction is shifted so that it impinges upon any other portion of the retina, a sensation of light is at once experienced. The blind spot is not recognized in ordinary vision, for (I) the two optic discs do not correspond.

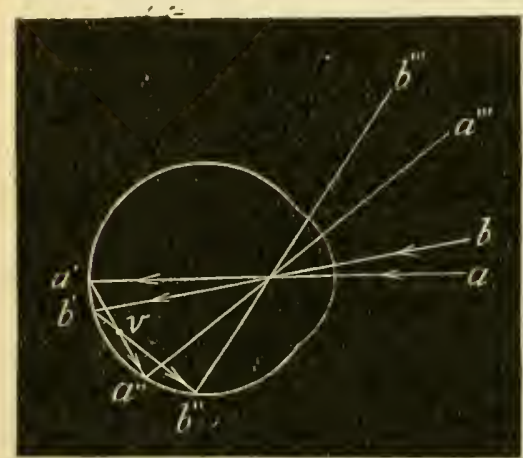

Fig. 443.-Method of rendering the Bloodvessels of the Retina visible by Oblique Illumination through the Cornea. Light from a candle at $a$ illuminates $a^{\prime}$, and rays proceeding from $a^{\prime}$ cast a shadow of the bloodvessel, $v$, at $a^{\prime \prime}$, which is referred to $a^{\prime \prime \prime}$. When $a$ is moved to $b$, the shadow on the retina moves to $b^{\prime \prime}$, and the shadow in the visual field of the illu. minated eye to $b^{\prime \prime \prime}$.
The left disc has its corre sponding points on a sensitive part of the right retina, and the right disc on a sensitive part of the left retina; and the consequence is that in binocular vision the objects whose images are formed on the corresponding points fill up the blind spots. (2) The optic disc does not lie in the line of direct, and therefore distinct, vision. The eye is constantly moving so as to bring the surrounding objects successively on the fovea centralis; and the gap which the blind spot makes in the visual field of a single eye is thus more easily neglected. In any case we ought not to see it as a

dark spot, for darkness is only associated witl the absence of excitation in parts of the retina capable of being excited by light. There is no more reason why the optic discs slould appear dark than there is for our having a sensation of darkness behind us when we are looking straight in front. And since the experience of our other senses - the sense of touch, for example-tells us that the objects we look at do not in general have a gap in the position corresponding to the part of the image that falls on the blind spot, we see, so to speak, across the spot.

By Mariotte's experiment, however, the existence of the blind spot can not only be demonstrated, but its size determined and its boundaries mapped ont. Let the left eye be closed, and fix with the right the small cross; then, if the eye be moved towards or away from the paper, keeping the cross fixed all the time, a position will be found in which the white disc disappears altogether. In this position its image falls on the blind spot (Fig. 444). 
Relation of the Rods and Cones to Vision.--We have more than once referred to the rods and cones as the sensitive layer of the retina. It is now necessary to develop a little more the evidence in favour of this statement. And at the outset, since the sensitive layer has been shown to lie behind the plane of the retinal bloodvessels, the only competitors of the rods and cones are the external nuclear layer and the pigmented epithelium. The nuclear layer may be at once excluded as a separate mechanism, since, as we have seen (p. IOI5), the portions of the rod and cone elements in it are continuous with the portions in the layer of the rods and cones proper. In the fovea centralis, where vision is most distinct, the nuclear layer becomes very thin and inconspicuous.

The layer of pigmented hexagonal cells, or at.least their pigment, cannot be essential to vision, for albino rats, rabbits, and men, in whose eyes pigment is absent, can see. In man and most mammals there are cones, but no rods in the yellow spot and fovea centralis; the relative proportion of rods increases as we pass out from the fovea towards the ora serrata. But this does not enabic us to

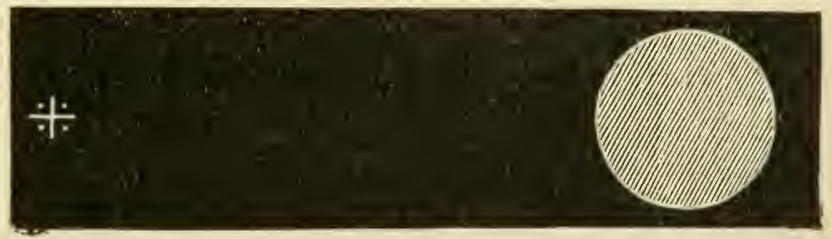

Fig. 444,-Mariotte's Experiment.

analyze the bacillary layer into sensitive cones and non-sensitive rods, for on the rim of the retina, which is still sensitive to light, there are only rods; in the bat and mole there are said to be no cones even in the yellow spot, in the rabbit very few. Reptiles possess only cones over the whole retinal surface, and birds, true to their reptilian affinities, have everywhere more cones than rods, as have also fishes.

One of the difficulties in the way of understanding how a ray of light can set up an excitation in a rod or cones is the transparency of these structures. An absolutely transparent substance-that is, a substance which would allow light to traverse it without the least absorption-would, after the passage of a ray, remain in precisely the same state as before; its condition could not be altered by the passage of the light unless some of the energy of the ethereal vibrations was transferred to it. But an absolutely transparent body does not exist in Nature; and it is not necessary to suppose that all the energy required to stimulate the end-organs of the optic nerve comes from the luminous vibrations. These may, and probably do, act by setting free energy stored up in the 
retina, just as the touch of a child's hand could be made to fire a mine, or launch a ship, or flood a province. Some have looked upon the transierse lamella into which the outer members of the rods and cones can be made to split as an arrangement for reflecting back the light to the inner members, and have compared them to a pile of plates of glass, which, transparent as it is, is a most efficient reflector. It is even possible, although here we are already treading the thin air of pure speculation, that the light may be polarized in the process of reflection, and that the rods and cones may be Icss transparent to light polarized in certain planes than to unpolarized light.

As to the nature of the transformation undergone by the ethereal vibrations in the rods and cones, various theories have been formulated. Some have supposed that the absorbed light-waves are transformed into long heat-waves, and that the endings of the optic nerve are thus excited by thermal stimuli. This hypothesis has so little eviclence in its favour that it is perhaps an unjustifiable waste of time eren to mention it. It is ruled out of court by the mere fact that the long radiations of the ultra red, filtered from luminous rays by being passed through a solution of iodine, and focussed on the eye by a lens of rock-salt, produce not the slightest sensation of light, alt hough they are by no means all absorbed in their passage through the dioptric media. Again, it has been suggested that the energy of the waves of light is first transformed into electrical energy, and that the risual stimulus is really electrical. In support of this view it has been urged that the passage of a voltaic current through the eye causes sensations of light, and that light, undoubtedly, causes (p. 839) an eiectrical change in the retina and optic nerve. But, as has more than once been pointed out, an electrical change is the token and accompaniment of the activity of the excitable tissues in general; and all that the currents of action of the retina show is that light excites the retina-a proposition which nobxly who can see requires an objective proof of, and which does not carry us very far towards the solution of the problem how that excitation is brought about. Then there is the photo-mechanical theory, according to which the pigmented epithelial cells of the retina, aftering their shape and volume under the stimulus of light, press upon the rods and cones, and thus mechanically stimulate them. Lastly, there is the photo-chemical theory, which supposes that some chemical change produced in the rods and cones under the influence of light sets up impulses in them which ascend the optic nerve. This is the most probable of all the theories, notwithstanding the fact that the discovery by Boll of the famous visual purple or rhodopsin, which at first secmed likely to place it upon a sure foundation, has lost its significance in this regard. But although the visual purple is not a photo-chemical 
substance through which the retinal elements are excited by luminous stimuli, it seems to fulfil an important function in adapting the retina-i.c., rendering it more sensitive-for vision in dim light. In any case, its discovery is jn itself so interesting and so suggestive as a basis for future work, that a short account of the properties of the substance camnot be omitted here.

Visual Purple.-If the eye of a frog or rabbit, which has been kept in the dark, be cut out in a dimly-lighted chamber or in a chamber illuminated only by red light, and the retina removed, it is seen, when viewed in ordinary light, to be of a beantiful red or purple colour. Exposed to bright light, the colour soon fades, passing through red and orange to yellow, and then disappearing altogether. The yellow colour is due to the formation of another pigment, visual yellow; the preceding stages are due to the intermixture of this visual yellow with the unchanged visual purple in different proportions. With the microscope it may be seen that the pigment is entirely confined to the outer segment of the rods, where it exists in most vertebrate animals. It may be extracted by a watery solution of bile-salts, and the properties of the pigment in solution are very much the same as its properties in situ; light bleaches the solution as it does the retina. Examined with the spectroscope, the solution shows no definite bands, but only a general absorption, which is very slight in the red, and reaches its maximum in the ycllowish-green. In accordance with this, it is found that of all kinds of mono. chromatic light the yellowish-green rays bleach the purple most rapidly, the red rays most slowly.

If a portion of the retina is kept dark while the rest is exposed to light, only the latter portion is bleached. And when the image of an object possessing well-marked contrasts of light and shadow (e.g., a glass plate with strips of black paper pasted on it at intervals, or a window with dark bars) is allowed to fall on an eye otherwise protected from light, the pattern

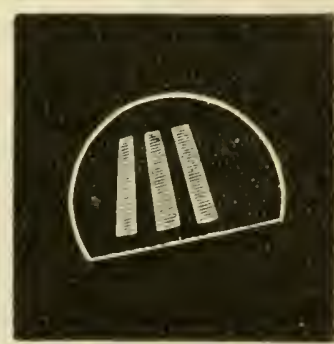

Fig. 445.-Optogram. Part of retina of rabbit, the eye of which had been directed to an illuminated plate of glass cov. ered with strips of black paper. of the object is picked out on the retina in purple and white. A veritable photcgraph or 'optogram' may thus be formed even on the retina of a living rabbit; and if the eye be rapidly excised, the picture may be 'fixed' by a solution of alum, and thus rendered permanent.

These facts certainly suggest that light falling on the retina may cause in some sensitive substance or substances chemical changes, the products of which stimulate the endings of the optic nerve, and set up the impulses that result in visual sensations.

The visual purple cannot itself be such a substance, for it is absent from the cones of all animals and the rods of some. Frogs and rabbits can undoubtedly see at a time when, by continued exposure to bright sunlight, the purple must have been completely bleached. And although the alleged absence of the pigment in the eye of the bat might seem to afford a ready explanation of the 
provirbial 'blindness' of that animal, such a hasty deduction would be at once corrected by the fact that birds with as sharp vision as the pigeon are equally devoid of visual purple, while in other nocturnal animals, like the owl, it is plentifully found. The most probable hypothesis of the function of the visual purple is indeed that which attributes to it the property, in virtue of its capacity for regeneration in the dark, of adapting the eye for night or twilight vision-in other words, of increasing the sensitiveness of the retina for faint light, especially of the shorter wave-lengths. If this is the case, it is precisely in nocturnal animals that we should expect to find it in large amount; and recently visual purple has been obtained from more than one species of bat (Trendelenburg). The fact that central vision (p. 1058) in which the rodless fovea is concerned is but little, if at all, susceptible of dark-adaptation, while peripheral vision shows a marked capacity of adaptation, agrees well with this hypothesis. We shall see later that there is some evidence that it is the mere perception of luminous impressions as such and of their intensity, without any distinction of quality or colour, with which the rods have to do. They are, then, on the hypothesis under discussion, elements concerned in achromatic sensations under conditions of feeble illumination (twilight vision). The cones are supposed on this theory to be more highly developed elements than the rods, their function being connected, especially with the perception of colour, but also with the perception of achromatic sensations under daylight conditions.

The pigmented retinal epithelium is undoubtedly sensitive to light, and has important relations to the formation of the visual purple. When the eye is exposed to light, black pigment migrates along the processes of the epithelial cells between the rods, even as far as the external limiting membrane. In the dark the pigment moves back again, and gathers around the outer portions of the rods, where the visual purple is being regenerated. That the central nervous system is not concerned in the pigment migration, or at least that it is not indispensable for it, has been shown in the larva of Amblystoma, one of the tailed amphibia. Optic cups were transplanted to various parts of the body, where they developed to form more or less perfect eves. The forward movement of the pigment in these transplanted eyes when exposed to light was fully as great as in the normal eyes. Contraction of the cones was also observed in them just as in the normal eyes. In the eye exposed to the light, the cones, whose expanded length is $25 \mu$ shortened by more than $4 \mu$ (Laurens and Williams). The precise meaning of the changes in the pigmented cells is obscure.

The pigmented epithelium is known to be concerned in the regeneration of the visual purple. When a frog is curarized, œelema occurs between the retina and the choroid, so that the former membrane is separated from the hexagonal epithelium. If the frog is now exposed to sunlight till the visual purple is bleached, and the retina then taken out and placed in the dark, no regeneration of the purple takes place. When the same experiment is repeated on a non-curarized frog, the visual purple is restored in the dark, and may be scen under the nnicrnscope in the rods. The only difference in the two experiments is that in the latter the pigmented epithelium adheres to the retina, and it 
must therefore have a hand in the regeneration of the pigment. Ever the visual purple of a retina from which the epithelimm has been cletached will, after being bleached, be restored if the retina is simply laid again on the epithelial surface. And it does not seem to be the black pigment of the licxagonal cells which is the agent in this restoration, for it takes place in the pigment-free retine of albino rabbits or rats. Even a retina. isolated from the pigmented cpithelium, and then bleached, may, to a certain extent, develop new visual purple in the dark. This is even true when it has been kept in the dark in a saturater solution of sodium chloride, and is then, after wasling with physiological salt solution, bleached by light. Here the regeneration of the pigment cannot be the result of vital processes, but must be clue to chemical changes in products formed from the original pigment by the action of light. No such regeneration takes place in a retina which, after having been bleached in situ, is removed without the pigmented epithelium and placed in the dark; and the only probable cxplanation of the difference is that in this case the photo-chemical substances from which visual purple can be formed have been absorbed into the circulation, and have so escaped.

The inner segments of the cones of certain animals (birds, reptiles, amphibia, and some fishes) contain globules of various colours, ranging over almost the whole spectrum, and inclucling, besides, the non-spectral colour, purple. The globules are composed chiefly of fat with the pigments (chromophanes, as they have been called) dissolved in it. The function of these globules is unknown. They cannot be concerned in colour vision, or, at least, they cannot be essential to it, for in the human retina they do not exist.

The yellow pigment of the macula lutea does not belong to the layer of rods and cones; it only exists in the external molecular layer and the layers in front of it; in the fovea centralis it is absent.

Time necessary for Excitation of the Retina by Light-Fusion of Stimuli.- Whatever the exact nature of retinal cxcitation may be, it is called forth by exceedingly slight stimuli. A lightning flash, although it may last only $\frac{1}{x, 000,000}$ th of a second, lasts long enough to be seen. A beam of light thrown from a rotating mirror on the eye stimulates when it only acts for $\frac{\mathrm{I}}{8,000,000}$ th of a second. The minimum stimulus in the form of green light corrcsponds, as we have already seen (p. 784 ), to a quantity of work equivalent to no more than $\frac{\mathrm{I}}{1 \mathrm{O}^{8}}$ erg-that is, about $\frac{\mathrm{I}}{1 \mathrm{O}^{10}}$ gramme-millimetre, or $\frac{\mathrm{r}}{10^{7}}$ milligramme-millimetre, which is the work done by $\frac{1}{10,000,000}$ th of a milligramme in falling through a millimetre; and it cannot be doubter that a portion even of this Lilliputian bombardment is wasted as heat. So quickly, too, is the stimulus followed by the response that no latent period has as yet ever been measured. It is certain, however, that there is a latent period, as surely as there is a latent period in the excitation of a naked nerve-trunk, although this also has never been experimentally detected. The analogies, in fact, between a muscular contraction and a retinal excitation are numerous and close. Like the muscle, the retina seems to possess a store of explosive material which the stimulus serves only to fire off. The retina, like the muscle, is exhausted by its activity, and recovers during rest. Like the muscle curve, the curve of retinal excitation rises not abruptly, but with a measurable slowness to its height, and when stimulation is stopped, 
takes a sensible time to fall again, the retinal impression outlasting the luminous stimulus by about one-eighth of a sccond. With comparatively slow intermittent stimuli the retinal, like the muscle curve, flickers 11 ) and down. When the rate of stimulation is increased, the steady contraction of the tetanized muscle is analogous to the fusion of the individual stimuli by the tetanized retina for retinn-cerebral apparatus) into a continuous sensation of light, such, e.g., as the bright 'trail ' of a falling star, or the fiery circle traced in the air when a fire. brand is rapidly whirled round. But the maximum retinal excitation which a stimulins of given strength can call forth depends much more closely upon the time during which the stimulus acts than the maximum contraction does upon the length of the muscular stimulus.

As the strength of the light increases in geometrical progression, the time during which it must act in order to produce its maximum effect decreases a pproximately in arithmetical progression (Exncr). For light of moderate intensity this time is about $\frac{1}{4}$ second. Since for complete fusion the stimuli must follow each other at a much more rapid rate than four in the second, the intensity of the resultant sensation is always less when a succession of similar stimuli are fused than when one of the stimuli is allowed to produce its maximum effect.

If the time of each stimulus is equal to the interval during which there is no stimulation, the sensation, when complete fusion has been reached, is the same as would be produced by a constant light of half the strength employed. And, in general, if $m$ be the proportion of the time during which the eye is stimulated by a light of intensity $I$, and $n$ the proportion of the time during which it is not stimulated, the resultant impression is the same as that which would be produced by an uninterrupted light of intensity $\left(\frac{m}{m+n}\right) I$. This is Talbot's law, which may be expressed without the aid of symbols thus: When a light of given intensity is allowed to act on the ey'e at intervals so short that the

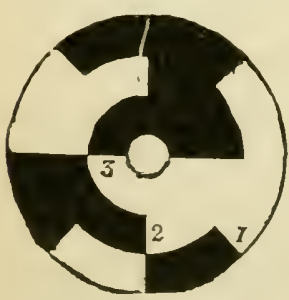

Fig. 446.-Disc for de. monstrating Talbot's law. impressions are completely fused, the resultant sensation is independent of the absolute length of each flash, and is proportional only to the fraction of the whole time which is occupied by flashes and to the intensity of the light. Talbot's law may be readily demonstrated by means of a rotating disc with alternate white and black sectors (Fig. 427 ), so arranged that the same proportion of the circumference of each of the thrce concentric zones is black.

When the rotation is sufficiently rapid to give complete fusion (say 20 to 30 times a second), the whole disc appears equally bright. However much the rate of rotation is now increased, no further change occurs. It has been shown that even for stimuli as short as the intervals of $-\frac{1}{1}$ th second, Talbot's law holds good. So that not only does a flash so inconceivably brief affect the retina, but it scts up changes which last for a measurable time. For intense stimuli Talbot's law ceases to be true: the field appears brighter than it should be (Grünbaum).

Two chief theories have been proposed to account for the fusion of intermittent retinal stimuli: (1) The persistence thcory, according to which the excitatory process in the retina remains for a short time at the maximum reached when the light ceases to act. Steady fusion is supposed to be obtained when the interval between successive stimuli does not exceed this time. (2) The theory of lick, who maintains that 
as soon as the light is withdrawn the retinal cxcitation begins to sink, at first rapiclly, then more gradually. As the rate of stimulation is increascel the time allowed for the decline of the excitation is, of course, correspondingly shortened, and nltimately the oscillations become so small that a continunus smooth scusation results. Fick's theory appears to explain the phenomena best.

The experiments of Charpentier have shown that the retina when stimulated has a natural tendency to enter into oscillations at the rate of about 36 in the second, so that the effect of a flash of light when it falls on a retinal area is not a single excitation which rises smoothly to its maximum and then declines smoothly to zero, but a scries of swings which die away like the vibrations of an elastic body. This may be demonstrated by slowly rotating a well-illuminated disc, one quadrant of which is white and the rest black, while the eye is kept fixed on the centre. A black band, or rather sector, running out from centre to circumference, will be seen in the white quadrant a little behind the border of it which first passes the eye. This band may be succeeded by one or more fainter black bands placed at regular intervais in the white portion of the disc. The explanation is this. At the moment when the image of the advancing edge of the white quadrant falls upon the retina it is excited, and we get the sensation of white. Then comes a swing in the opposite direction which gives rise to the first black band, and succeeding swings cause the other bands. The period of the oscillatory process can be calculated from the speed of the disc, and the distance of the first band from the edge of the white quadrant. The well-known fact that a single flash of lightning, or other intense stimulus, may appear is two flashes, finds its explanation in these retinal oscillations.

Colour Vision.-Besides differences in the distance, size, shape, and brightness of objects, the eye recognizes differences in their colour; and we have now to consider the physical and physiological differences on which these depend.

Colours may differ from each other-(I) In tone or hue, e.g., red, yellow, green. (2) In degree of saluration or fulness or purity, i.e., in the degree in which they are free from admixture with white light, e.g., a 'pale' or 'light' blue is a blue mixed with much white light, a ' deep or 'full ' blue with kittle or none. (3) In brightness or intensity, i.e., in the amount of the light coming from unit area of the coloured object. Thus, a 'dark' red cloth sends comparatively little light to the eye, a 'bright' red cloth sends a great deal.

When a beam of sunlight falls into the eye, a sensation of ' white light ' results. When a prism is placed before the eye, the sensation is entirely different; we see a spectrum running up from red through green to violet, with a multitude of intermediate shades, the eye being able to distinguish in the solar spectrum at least one thousand different hues (Aubert). What, then, has happened ? Physically, nothing mure has taken place than a rearrangement of the rays in the beam of white light. A few of them may have been lost by reflection, but upon the whole the beam is made up of exactly the same constituents as before; only the rays are now arranged in the precise order of their refrangibility, the more refrangible, which are also those of short est wave-length, being displaced more towards the base of the prism than the longer and less refrangible rays. In- 
stead of the long and short rays falling together on the same elements of the retina, as they did in the absence of the prism, they now fall, if proper precautions have been taken to secure a pure spertrum, in regular order from one side to the other of the portion of retina on which the image is tormed. The physical condition, then, of our sensations of the prismatic colours is, that rays of approximately the same wave-length should fall unmixed with other rays upon the retinal elements. Rays of a wave-length of $760 \mu \mu^{*}$ to $65^{\circ} \mu \mu$ give the sensation of red; from $65^{\circ} \mu \mu$ to $590 \mu \mu$, the sensation of orange; from $430 \mu \mu$ to $400 \mu \mu$, the sensation of violet, and so on. When rays of all these wave-lengths fall together, in the proportion in which they are present in sunlight, upon the same part of the retina, the resultant physiological effect is very different; we are no longer able to distinguish red, blue, green, etc.; we receive the single sensation of white light. The sensation is a simple one; in consciousness we have no hint that it has a multiple physical cause.

But we find further that it is not necessary for the sensation of white light that waves of every length present in the solar spectrum should be mixed. If rays of wave-lengths $675 \mu \mu$ (which acting alone produce the sensation of red) be mixed in certain proportions - i.e., be allowed to fall on the same part of the retina-with rays of wave-length $496 \mu \mu$ (which give the sensation of bluish-green), the resultant sensation is also that of white light. And an indefinite number of sets can be combined, two and two, so as to give the same sensation of white. Such colours are called complementary. The following are pairs of complementary colours:

Red and bluish-green.

Orange and cyan-bluc. $\dagger$

Yellow and ultramarine-blue.

Greenish-yellow and violet.

The green of the spectrum has no simple complementary colour; purple, a colour not present in the spectrum, but obtained by mixing light from the two spectral extremes-i.e., by mixing red and violet - may be considered complementary to it. Suppose now that one of a pair of complementary colours is added to the other in greater intensity than is required to give white, the resultant sensation is a colour which has a certain amount of resemblance both to white and to the colour present in excess. Thus, if the two colours are orange and blue, and the blue is present in greater intensity than is necessary to give white, the resultant colour is a whitish or pale blue, or, to use the technical phrase, an unsaturated blue. The more nearly the intensity of the blue rays in the mixed light approaches the proportion necessary to give white, the less saturated is the resultant colour; the greater the excess of blue, the more nearly does the resultant sensation approach that of the saturated blue of the spectrum. But any non-saturated spectral

* $\mu \mu$ is a symbol representing one-millionth of a millimetre.

$\uparrow$ Cyan-blue is a greenish-blue. 
colour produced by the mixture of two complementary colours may be equally well produced by the mixture of the corresponding spectral colour with a certain quantity of ordinary white light. And it is found that when two spectral colours which are not complementary are mixed together the resultant is not white, but a colour which may be matched by some spectral colour lying between the two (or by purple), either without addition or plus a larger or smaller quantity of ordinary white light. From all this it follows that the retina may be excited by an infinite nuniber of different physical stimuli, and yet the resultant sensation may be the same. This leads straight to the conclusion that somewhere or other in the retino-cerebral apparatus simplification, or synthesis, of impressions must take place; and we have to inquire what the simplest assumptions are which will explain all the phenomena. Now, it is not possible, from two spectral colours alone, to produce a sensation corresponding to all the others. By mixing three standard spectral colours, however, in various proportions, we can produce not only the sensation of white light, but that of every colour of the spectrum (and of purple). These statements are based on demonstrated facts obtained by very numerous experiments on colour mixtures. The hypotheses framed to explain the facts are to be carefully discriminated from the facts themselves.

Primary Colours.-The simplest assumption we can make, then, is that there are three standard sensations, and that either the retina itself can respond by no more than three distinct modes of excitation to the multiplex stimuli of the luminous vibrations, or that complex impulses set up in the retina are reduced to simplicity because the central apparatus is capable of responding by only three distinct kinds of sensation. Which three sensations we select as fundamental or primary is, to a certain extent, arbitrary. Fick chose red, green, and blue; most commonly red, green, and violet are accepted as the primary colours. Red, yellow, and blue, although so long considered the primary colours, from data yielded by the mixture of pigments, will not do; for no possible combination of them will produce either a pure green or white light.

The Young-Helmholtz Theory.-The theory which has been most widely accepted is that of Young, generally called, on account of its adoption and extension by Helmholtz, the Young-Helmholtz theory. Red, green, and violet are taken as the fundamental or elementary colour sensations. In its more modern form it assumes that in the retina, or in the retino-cerebral apparatus, there are three kinds of elements-(I) a substance or a component chiefly affected by light of comparatively long wave-length (red), to a less extent by light of medium wave-length (green), and to a still less extent by the shortest visible waves (violet); (2) a component mainly affected by medium, but also to a certain extent by long and short waves; (3) a component chiefly affected by the short vibrations, 
less by the modium, and still less by the long waves. The curves in Fig. 447 illustrate these relations.

The theory explains as follows the phenomena of colour-mixture referred to above. When all the rays of the spectrum act upon the retina together, the three components are alout equally affecterl, and this equal effect is supposed to be the condition of the sensation of white light. When the green of the spectrum alone falls on the retina, the 'green' component is strongly excited, the other two

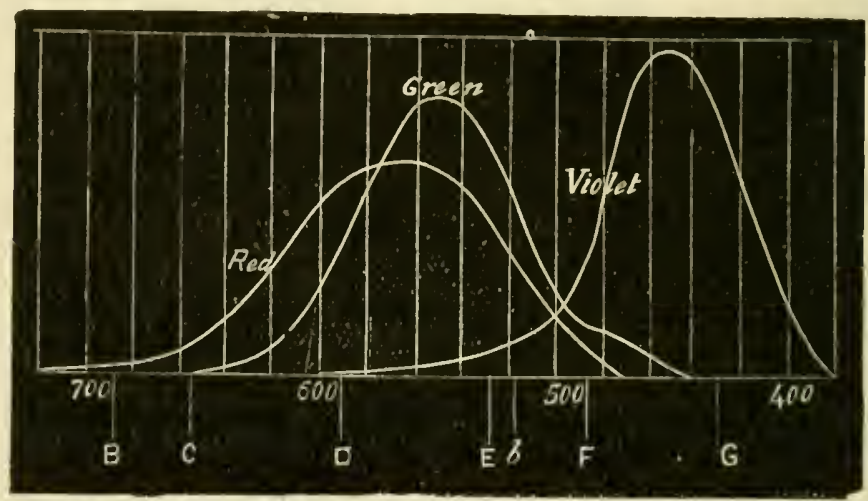

Fig. 447.-Curves of Excitability of Primary Sensations from Observations on Colour Mixtures (König). The numbers give wave-lengths of the spectrum in millionths of a millimetre.

only slightly; this is the relation between the amount of excitation in the three components which is associated with a sensation of spectral green. When two complementary colours, such as red and bluish-green, fall together on the same portion of the retina, the three components are excited in the relative proportions associated with the sensation of white light.

The colour triangle is a graphic method of representing various facts in colour-mixture (Fig. $44^{\circ}$ ).

The chicf points to be noted are the following: (I) On the curve

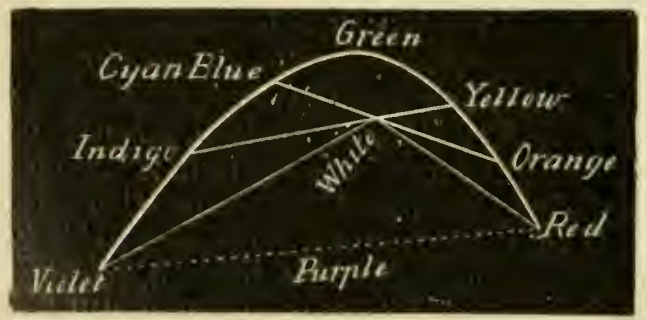

Fig. $44^{8}$.-Colour Triangle. the spectral colours are arranged at such dis. tances that the angle contained between straight lines drawn from the point marked 'white,' and intersecting the curve at the positions corresponding to any two colours is proportional to their difference in tone. (2) The distance of any point of the curve from the point marked 'white' is proportional to the stimulation intensity of the colour corresponding to it. (If the stimulation intensities of all the colours be represented by 
proportional weights lying at the corresponding points on the curve, the pont 'white' will be the centre of gravity of the system.) (3) The position of a colour produeed by the mixture of any pair of spectral colours is found by joining the corresponding points by a straight line. The mixed colour lies on this line at distances from the two points inversely proportional to the stimulation intensity of the two colours-i.e., it lies in the centre of gravity of the weights representing (he two colours. (4) It is a particular case of (3) that the complementary colours are situated at the points whese straight lines drawn through 'white' intersect the curve, since the point marliel 'white' is the centre of gravity corresponding to a pair of colours only when it lies on the straight line joining them. Fhus the orange and yellow lying between the red and green are mixtures of the red and green sensitions in different proportions; the cyan-bluc and indigo-blue are mixtures of the green and violet sensations. The purples, represented by a broken line, are not present in the sjectrum, and are mixtures of red and violet.

It is a point of great theoretical interest that on the Young-Helmholtz theory the pure spectral colours, although physically saturated (i.e., due to cthereal vibrations of a definite wave-length for each colour), ought not to be physiologically saturated, since they all affect the three components, although in different degrees. In other words, the red, let us say, of the spectrum ought not to be the purest or fullest red which it is possible to perceive. Now, it is found that this is really the case. If, for example, we look first at the bluish-green, and then at the red of the spectrum, the sensation of red is fuller or more saturated than if we had looked at the red directly. Similarly, if we look first at a small bluish-green square on a black ground, and then at a red ground, we see a more fully saturated square in the middle of the latter. The explanation, on the Young-Helmholtz thcory, is that the "green" component, being fatigued before the eye is turned upon the red, the latter colour no longer afferts it, or affects it less than it would otherwise do, and therefore the excitation is almost entirely confined to the red component in the area fatigued for green. This brings us to the subject of retinal fatigue, and the related phenomena of after-images and contrast.

After-Images.-We have seen that the retinal excitation always takes time to die a way after the stimulus is removed. If a white object is looked at, especially when the eye is fresh, for a time not long enough to cause fatigue, and the eye is then closed, an image of the object remains for a short time, diminishing in brightness at first rapidly, then more slowly. This is a positive after-image, and by careful observation it may, under certain conditions, be seen that the positive afterimage of a white object, of a slit illuminated by sunlight, for example, undergoes changes of colour as it fades, passing through greenish-blue, indigo, violet, or rose, to dirty orange. On the Young-Helmholtz theory this is explained by the supposition that the excitation does not decline with the same rapidity in the three hypothetical components. If the object is looked at for a longer time, or if the eye is fatigued, a dark or negative image may be seen upon the faintly-illuminated ground of the closed eyes: but negative after-images may be more easily obtained when the eye, after being made to fix a small white object on a black ground, is suddenly turned upon a white or neutral tint surface.

Here Helmholtz supposed the portion of the retina on which the image of the object is formed to be more or less fatigued. And this fatigue will extend to all three kinds of fibres; so that white light of a given intensity will now cause less excitation in this part than in the rest of the retina. It is easy to understand that the negative afterumage of a coloured object will be seen, upon a white ground, in the 
complementary colour, for the components chiefly excited by the latter will lave been least fatigued. The negative after-images seen when the eye, after receiving the positive impression, is turned upon a coloured ground, vary with the colour of the object and ground in a manner which has been explained as due to fatigue of one or other component. It is difficult, however, to reconcile the fatigue hypothesis of the afterimage witl all the facts. Hering supposes that the retina is not passively fatigued, but that a metabolic change is set up in it which is of the opposite kind to that caused by the original excitation (see p. 1057$)$.

The phenomena of negalive after-images are often included together as examples of successive contrast, the name implying mutual influences of the portions of the retina (or retino-cerebral apparatus) successively stimulated. We have now to consider simultaneous contrast, often spoken of simply as contrast.

Contrast.--A small white disc in a black field appears whiter, and a small black disc in a white field darker, than a large surface of exactly the same objective brightness. A dise with alternate sectors of white and black, so arranged that the proportion of white to black increases in each zone from centre to circumference, when set in rotation, ought, by Talbot's law, to show sharply marked and uniform rings, of which each is brighter than that internal to it. But each zone appeurs brightest at its inner edge, where it borders on a zone darker than itself, and darkest at its outer edge, where it borders on a brighter zone. A plausible explanation of this is based on the assumption that in the neighbourhood of an excited area of the retina, as well as within the area itself, the excitability is diminished; and the same explanation has been extended to the contrast phenomena of coloured objects. A small piece of grey paper, e.g., is placed on a green sheet. The grey patch appears in the complementary colour of the ground-viz., pink or rose-red (Meyer). The red colour is much stronger if the whole is covered with translucent tracing-paper. Here we may suppose that the fatigue of the substance or component chiefly affected by the ground colour spreads into the portion of the retina occupied by the image of the grey paper; the white light coming from the latter, therefore, affects mainly the component connected with the sensation of the com. plementary colour.

The curious phenomenon of coloured shadows is also an illustration of contrast. They may be produced in various ways. For example, when a lamp is lit in a room in the twilight, before it has yet grown too dark, the shadows cast by opaque objects on a white window-blind are coloured blue. The yellow light of the lamp overpowers the feeble daylight which passes through the blind, and the general ground is yellowish; but wherever a shadow is thrown it appears of a bluish tint in contrast to the yellow ground. Here the only illumination the eye receives from the region occupied by the shadow is the feeble daylight. Falling upon an area in which the component chiefly affected by yellow rays is more or less fatigued, it causes a sensation of the complementary colour. As darkness comes on, the shadows become black, for now practically no light at all comes from them.

Helmholtz looked upon simultancous contrast as a result of false judgment, and not of a change of excitability in parts of the retina bordering on the actually excited parts. For the sake of perspective, it will be worth while to apply this theory by way of illustrating it, to the explanation of the case of contrast we liave just been considering, from the other point of view, in Meyer's experiment. Helmholtz's explanation of this experiment is as follows: When a coloured surface is 
covered with translucent paper, the latter appears as a colourcd covering spread over the ficld. The inind docs not recognize that at the grey patch there is any breach of continuity in this covering; it is therefore assumed that the greenish veil extends over this spot too. Now, the grey seen through the translucent white paper is objectively white-i.e., sends to the eye the vibrations which together would give the sensation of white light. But with a gicen veil in front of it, this could only happen if the really grey patch was the colour complementary to green - that is, rose-red. The mind, therefore, judges falsely that the patch is red. Hering has severely criticized this theory of Helmholtz as to false judgments; and the weight of evidence certainly seems to be in favour of the view that simultaneous, like successive, contrast is due to the influence of one portion of the retina, or retino-cerebral apparatus, on another.

Hering's Theory of Colour Vision.-The Young-Helmholtz theory of colour vision has not met with universal acceptance. The bestknown rival theory is that of Hering, who takes his stand upon the fact that certain visual sensations (red, yellow, green, blue, white, black) do appear to us to be fundamentally distinct from each other. while all the rest are obviously mixtures of these. Accepting these six as primary sensations, he assumes the existence in the visual nervous apparatus of substances of three different kinds, which may be called the black-white, the green-red, and the blue-yellow. Like all other constituents of the body, these substances are broken down and built up again-in other words, undergo disassimilation and assimilation, destructive and constructive metabolism. The sensations of black, of green, and of blue he supposes to be associated with the constructive, and the sensations of white, of red, and of yellow with the destructive, processes in the three substances. The blackwhite substance is used up under the influence of all the rays of the spectrum, but in different degrees; the smaller the quantity of light falling on the retina, the more rapidly is it restored, and the more intense is the sensation of black. The green-red substance is built up by green rays, broken down by red. The blue-yellow substance is destroyed by yellow rays, restored by blue. A prominent difference between this and the Young-Helmholtz theory, and, so far as it goes, an advantage, is that Hering's theory attempts to assign a direct objective cause for the visual sensations of white, black, and yellow, as well as for red, green, and blue, instead of making the sensations depend upon the magnitude of the stimulation process. When any of the visual substances are consumed at one part of the retina, they are supposed to be more rapidly built up in the surrounding parts, and in this way many of the phenomena of simultaneous contrast receive an easy and natural explanation. The same is true of the simpler phenomena of after-images or successive contrast. But in applying the theory to the more complicated phenomena difficulties soon emerge, which, to say the least, are not less formidable than those connected with the Young-Helmholtz 
theory. Neither theory, in short, can be considered more than a partially successful attempt to grapple with a very complex mass of facts. Each, lowever, las been fruit ful in leading to the discovery of new facts-a great merit in a scientific hypothesis.

Sensibility of Different Parts of the Retina-Perimetry.-The perception of colours, like the perception of white light, is not equally distinct over the whole retina. We have repeatedly had occasion to refer to the fovea centralis as the region of most distinct vision; but it would be a mistake to suppose that it is therefore necessarily more sensitive than the rest of the retina. As a matter of fact, when the minimum intensity of white light which will cause an impression at all is determined for each portion of the retina, it

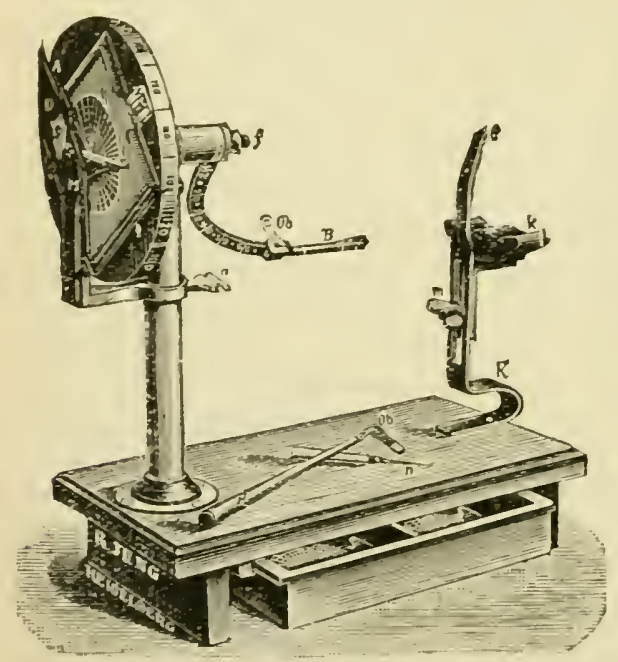

Fig. 449. - Priestley Smith's Perimeter. K, rest for chin; $O$, position of eye; $O b$, object, white or coloured, which slides on the graduated arc $B$; $f$, point fixed by the eye. is found that the fovea centralis requires a somewhat stronger stimulus than the zone immediatcly surrounding it. Objects only a little brighter than the general ground on which they lie-e.g., very faint stars-are best seen when the eve is directed a little to one sidc. This has been attributed to the absence of visual purple from the fovea, in accordance with the theory previously alluded to that the visual purple acts as a mechanism which 'adapts' the retina for the perception of light of varying intensity. But, with this exception, the sensibility of the retin diminishes steadily from centre to periphery, both for white and for coloured light.

When the eye is fixed, and the visual field-that is, the whole space from which light can reach the retina in the given position, or, what comes to the same thing, the projection of the visual field on the retina by straight lines passing through the nodal pointcxplored by means of a perimeter (Figs. 449,450 ), it is found that, under ordinary conditions, a white object is seen over a wider field than any coloured object, a blue object over a wider field than a red, and a red over a wider field than a green object. The exact shape, as well as size, of the visual field also differs somewhat for 
different colours. In disease of the retina, or of the visual path between it and the cortex, or of the visual cortex itself, the abridgment of the field for white and for monochromatic light as mapped out by observations with the perimeter is often of value in diagnosis. Although it has been shown by Aubert and others that monochromatic light of considerable intensity can be perceived over the whole retina, yet it may be said that the retinal rim is even then relatively and, under ordinary conditions, absolutcly colour-blind. This and

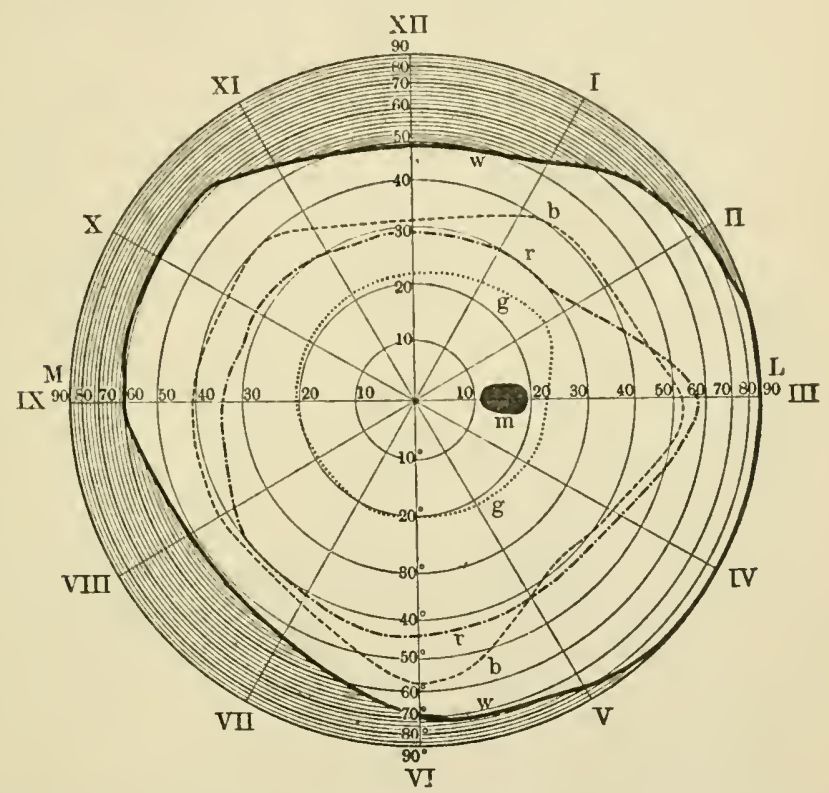

Fig. 450--Perimetric Chart of Right Eye (after Hirschberg). The numbcrs repre. sent degrees of the visual field measured on the graduated arc of the perimeter. $w$, boundary of field for white object; $b$, for blue; $r$, for red; $g$, for green; $m$, blind spot; M, medial, and L, lateral side of the field of vision. The Roman ilumbers represent twelve meridians of the retina, each making an angle of $30^{\circ}$ with the next. They fix the 'longitude' of any point in the fieid. The concentric circles indicated by Arabic numbers represent angular distances from the fixation point in the planes of these meridians. They give the 'latitude ' of any point.

other facts have given rise to the theory (p. I047) that the rods, which are alone present at the ora serrata, are concerned in achromatic vision (under conditions of dark adaptation), the cones in colour vision as well as in achromatic vision (under daylight conditions).

This brings us to the subject of colour-blindness, a phenomenon of great interest in its theoretical as well as in its practical bearings.

Colour-Blindness. - A considerable number of persons (about 4 per cent. of all males, but only one-tenth of this proportion of 
females) are deficient in the power of distinguishing between certain colours. They are said to be colour-blind; but the term must not be taken to signify that they are absolutely devoid of colour-sensations. A very small minority of the colour-blind appear to have but one sensation of colour tone, everything appearing as white, grey, or black (total colour-blindness, sometimes called monochromatic vision). All colours are confused by them, but differences of brightness are correctly appreciated. Probably the totally colour-blind person receives somewhat the same impressions from a coloured picture as the normal person does from a reproduction of the samc picture in black-and-white. There are close rescmblances between the vision of the totally colour-blind eye and that of the normal eyc adapted hy resting in the dark for twilight vision. The fovea is relatively, and in some cases absolutely, insensitive to light, while the peripheral portion of the retina is normal, or nearly normal, in this regard. This is the foundation of the theory that in total colour-blindness the cones are devoid of their normal functions, and that the hypothetical mechanism for twilight vision (the rods) is functioning alone. In another condition (night-blindness, or hemeralopia) it is sometimes assumed that the other mechanism (that of the cones) which is adapted for daylight vision, and has little power of dark-adaptation, is alone acting. But it cannot be said that this has been proved.

The rest of the colour-blind are dichromatic-i.e., their colour reactions seem to correspond only to two of the fundamental colour sensations of the normal person and their combinations, in addition to white. Of the dichromates a very few confuse blue with yellow. The great majority are unable to distinguish between red and green. The condition will be most easily understood by considering some of the extraordinary mistakes which may be made by the colourblind, without necessarily leading them to suspect that there is anything abnormal in their vision. Thus, to quote the words of a distinguished writer on this subject, himself a sufferer from the deficiency: 'A naval officer purchases red breeches to match his blue uniform; a tailor repairs a black article of dress with crinson cloth; a painter colours trees red, the sky pink, and human cheeks blue.' The shoemaker, Harris, the discoverer of colour-blindness, picked up a stocking, and was surprised to hear other people describe it as a red stocking; it seemed to him only a stocking. The celebrated Dalton was twenty-six years of age before he knew that he was colour-blind. He matched samples of red, pink, orange, and brown silk with green of different shades; blue both with pink and with violet; lilac with grey.

When the condition of vision in dichromates is tested by means of the spectrum, it is found that they fall into two classes: (1) A class (of grecn-blind) by whon the whole of the spectrum from red to yellow is described as yellow of different degrees of brightness (intensity); the green 
appears as a pale yellow with a grey or white band in its mirlst; while the violet end is seen as different shades of blue. (2) A class of (redblind) whose whole spectrum, from red to green, is seen as green of different intensities, the extreme red being entirely invisible. The violet end is blue, as in ( $I)$, and there is a band of white or grey near the blue end of the green.

Sir John Herschell explained Dalton's peculiarity of vision on the hypotliesis that be only possessed two, instead of three, primary sensations.

On the Young-Helmholtz theory, the missing sensation is supuosed to be either red or green. At the intersection of the curves that represent the violet and green sensations (Fig. 447), the red-blind individual will see what he describes as white-viz., the sensation produced by the stimulation of the only two components he possesses. Similarly, at the interscction of the red and violet curves the green-blind person will see what is white to him.

Those who have attempted to explain colour-blindness on Hering's theory have usually assumed that the colour-blind possess the blueycllow, but lack the green-red visual substance. So that on this theory there should be no difference between red-blindness and green-blindness. But v. Kries, in a study of twenty cases of congenital partial colour-blindness, brings forward strong evidence that the red-green blind can be divided, as regards the comparison of red (lithium) and orange (sodium) light, into two sharply-separated groups-a result which is, so far as it goes, in favour of the Young-Helmholtz theory, and agairist the theory of Hering.

The observations of Burch on temporary colour-blindness produced by placing the eye behind a transparent coloured screen and focussing a beam of strong sunlight on it, lend additional support to the former theory. Thus, if a spectrum is looked at after green-blindness has been induced by exposure of the eye to green light, the red portion of the spectrum seems to pass into the blue, and no intermediate green band is seen. If the eye is exposed to yellow light it becomes temporarily blind not only for yellow, but also for red and green. This is in favour of the assumption of the Young-Helmholtz theory that the sensation of yellow is caused when the retinal elements concerned in the production of the sensations of red and green are simultaneously stimulated. It is, however, equally difficult to reconcile some of the phenomena of colourblindness with the Young-Helmholtz theory. Anomalies and defects of colour-sensation are common accompaniments of pathological lesions of the visual apparatus, and can be produced by various drugs, as by abuse of tobacco. But colour-blindness, in its true sense, is congenital, often hereditary; the colour-blind are 'born, not made.' And although the condition cannot be cured, it is of great importance that it should be recognized in the case of persons occupying positions such as those of engine-drivers, railway-guards, and sailors, in which coloured lights have to be distinguished. For, while it is true that the sensations which red and green lights give the colour-blind are far from being identical (Pole) under favourable conditions, it is precisely when the conditions are unfavourable-as in a fog or a snow-storm-that the capacity of distinguishing them becomes invaluable (Practical Exercises, p. I I I 2 ).

Irradiation. - The phenomenon known as irradiation was first described by Kepler, who gave as an example the appearance known as the 'new moon in the old moon's arms,' where the crescent of the new moon seems to overlap and cmbrace the unilluminated portion of the luner disc. A white circle on a black ground (Fig. 45I) appears, in 
a good light, to be larger than an exactly equal black circle on a white ground. The explanation is as follows: Owing to the aberration of the refractive media of the eye (p. 1027), all the rays proceeding from the luminous object are not brought accurately to a focus on the retina, and the image is surrounded by diffusion circles (p. ro28) which encroach upon the unilluminated boundary. Physically these represent a weaker illumination than that of the image proper, and therefore the latter ought to stand out in its real size as a brighter area surrounded by weaker lialoes. That this is not the case, and that the image is projected in its full brightness for a certain distance over its dark boundary, is due to the fact that the eye does not recognize very small differences of brightiness. When the accommodation is not perfect, the diffusion circles are, of course, much wider, and irradiation is better marked when the object is a little out of focus.

The Movements of the Eyes.-That the eyes may be efficient instruments of "vision, it is necessary that they should have the power of moving independently of the head. An eye which could not move, though certainly better than an eye which could not see,
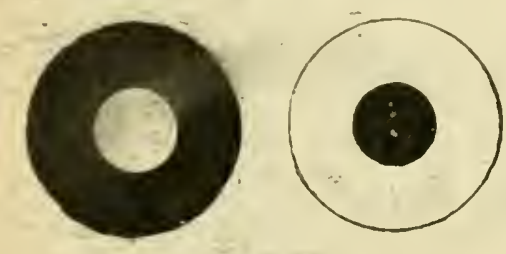

Fig. 45I: would yet be as imperfect after its kind as a ship which could run before the wind, but could not tack. The mere fact that the angle between the visual axes must be adapted to the distance of the object looked at renders this obvious; and the beauty of the intrinsic mechanism of the eyeball has its fitting complement in the precision, delicacy, and range of movement conferred upon it by its extrinsic muscles.

Not only are movements of convergence and divergence of the eyeballs necessary in accommodating for objects at different distances, but without compensatory movements of the eyes it would be impossible to avoid diplopia with every movement of the head; for the images of an object fixed in one position of the head would not continue to fall on corresponding points of the retinæ in another position.

All the complicated movements of the eyeball may be looked upon as rotations round axes passing through a single point, which to a near approximation always remains fixed, and is situated about $\mathrm{I} \cdot 77 \mathrm{~mm}$. behind the centre of the eye.

The position which the eyeballs take up when the gaze is directed to the horizon, or to any distant point at the level of the eyes, is called the primary position. Here the visual axes are parallel, and the plane passing through them horizontal. While the head remains fixed in this position, the eyeballs can rotate up or down around a horizontal axis, or from side to side around a vertical axis; or upwards and inwards, downwards and outwards, downwards and inwards, and upwards and outwards around oblique axes, which always lie in the same plane as the vertical and horizontal axes of rotation-i.e., in the vertical plane 
passing through the fixed centre of rotation. These facts, spoken of coliectively as Listing's law, and first deduced by him from theoretical considerations, were afterwarls proved experimentally by Heimholtz and Donders. It nccessarily follows from l.isting's law (and this is, indecd, another way of stating it) that in moving from the primary position into any other, there is no rotation of the cycball round the visual axis-no wheel-movement, as it is called.

A true rotation of the cye round the visual axis loes, lowever, occur when the eves are converged as in accommodation for a near object, each cycbali rotating towards the temporal sidc. This is especially the case when the eyes are at the same time converged and directed downwards; and the rotation may amount to as much as $5^{\circ}$. When the head is rolled from side to side, while the cyes are kept fixed on an object, a slight compensatory rotation of the eycballs takes place against the direction of rotation of the head. The amount of rotation of the eyes is relatively greater for small than for large movements of the head (eye $5^{\circ}$ for head $20^{\circ}$; eye $10^{\circ}$ for head $80^{\circ}$-K ïster).

The Extrinsic Muscles of the Eyes.-The eyeball is acted upon by six muscles arranged in three pairs, which may be considered, roughly speaking, as antagonistic sets. These are the internal and external recti, the superior and inferior recti, and the superior and inferior obliqui.

Although the movements of the eye have been very fully studied, and are, upon the whole, well understood, our knowledge of the manner in which any given movement is brought about, and of the exact action of the muscles which take part in it, is by no means as copious and precise. From the nature of the case, the greater part of what we do know lias been inferred from the anatomical relations of the muscles as revealed by dissection in the dead

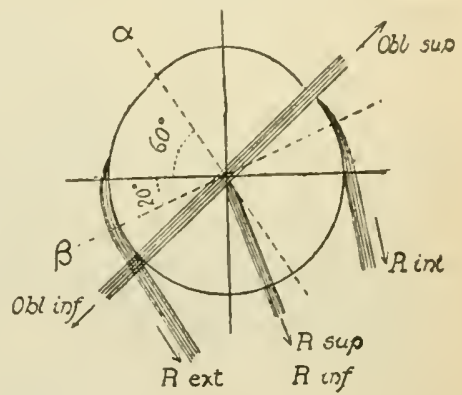

Fig. 452.-Horizontal Section of Left Eye. Arrows show direction of pull of the muscles. The axis of rotation of the external and internal recti would pass through the inter. section of $a$ and $\beta$ at right angles to the plane of the paper. body rather than gained from actual observation of the living eye. A plane, called the plane of traction, is supposed to pass through the middle points of the origin and insertion of the muscle whose action is to be investigated, and through the centre of rotation of the eyeball. A straight line drawn at right angles to this plane through the centre of rotation is evidently the axis round which the muscle when it contracts will cause the eye to rotate, provided that the fibres of the muscle are symmetrically distributed on each sicle of the plane of traction. The axes of rotation of the antagonistic pairs almost, but not completely, coincide with each other. The common axis of the external and internal recti practically coincides with the vertical axis of the eyeball (Fig. 452) in the primary posi- 
tion. The ere is turned towards the temple when the external rectus alone contracts, towards the nose when the internal rectus alone contracts. The common axis of the superior and inferior recti, $\beta$, lie's in the horizontal visual plane in the primary position, but makes an angle of about $20^{\circ}$ with the transrerse axis, its inner end being tilted forwards. The conseruence is that contraction of the superior rectus turns the eye up, and contraction of the inferior rectus turns it down, but both movements are also comsbined with a slight inward rotation. The common axis of the oblique muscles, $\alpha$, makes an angle of $60^{\circ}$ with the transverse axis, the outer end of it being the most anterior. The direction of traction of the superior oblique is, of course, given not by the line joining its bony origin and its insertion, but by the direction of the portion reflected over the pulley. When the superior oblique contracts alone, the eycball is rotated outwards and downwards; the inferior oblique causes an outward and upward rotation. None of the common axes of rotation of the pairs of muscles, except that of the external and internal recti, lies in Listing's plane. Now, as we have seen that every movement which the eye, supposed to be originally in the primary position, can execute may be considered as a rotation round an axis in this plane, it is clear that every movement, except truly transverse rotation, must be brought about by more than one pair of muscles. For vertical rotation, the inward pull of the superior rectus is antagonized by a simultaneous outward pull of the inferior oblique; for downward rotation, the inferior rectus and superior oblique act together. In oblique movements, a muscle of each of the three pairs is concerned. The effect on the eyeball of simultaneous contraction of certain pairs of muscles nay be summarized thus:

External rectus (outward) + internal rectus (inward) $=$ none.

Superior rectus (upward and inward) + inferior oblique (upward and outward) = upward.

Inferior rectus (downward and inward) + superior oblique (down ward and outward) $=$ down ward.

\section{SECTION II.-HEARING.}

The transverse vibrations of the ether fall upon all parts of the surface of the body, but only find nerve-endings capable of giving the sensation of light in the little discs, which we call the retinx. So the much longer and slower longitudinal waves of condensation and rarefaction which are being constantly originated in the air or imparted to it by solid or liquid bodies that have been themselves set vibrating fall upon all parts of the surface, but only produce the sensation of sound when they strike upon the tiny mechanism of the internal ear.

But just as the ethereal vibrations, and especially those of greater wave-length, are able to excite certain cnd-organs in the skin which have to do with the sensation of temperature, so the sound-waves, when sufficiently large, are also capable of stimulating certain cutaneous 
nerves and of giving rise to a sensation of intermittent pressure or thrill. This is readily perceived when the finger is immersed in a vessel of water into which dips a tube connected with a source of sound, or when a vibrating bell or tuning-fork is touched. So far as we know, what takes place in the ear is essentially similar - that is to say, a mechanical stimulation of the ends of the auditory nerve, but a stimulation which acts through, and is graduated and controlled by, a special intermediate meclianism.

As the visual apparat us consists of a sensitive surface, the retina, which contains the end-organs of the optic nerve and of dioptric arrangements which receive and focus the rays of light, the auditory apparatus consists of the sensitive end-organs of the cochlear division of the eighth nerve and of a mechanism which receives the sound-waves and communicates them to these.

Physiological Anatomy of the Ear.-At the botton of the external auditory meatus lies the membrana tympani, a nearly circular membrane set like a drum-skin in a ring of bone, and separating the meatus from the tympanum or middle ear. Its external surface looks obliquely downwards, and at the same time somewhat forwards, so that if prolonged the membranes of the two ears would cut each other in front of, and also below, the horizontal line passing through the centre of each (Figs. 453,454 ).

The tympanum contains a chain of little bones stretching right across it from outer to inner wall. Of these the malleus, or haminer, is the most external. Its manubrium, or handle, is inserted into the membrana tympani, which is not stretched taut within its bony ring, but bulges inwards at the centre, where the handle of the malleus is attached. The stapes, or stirrup, is the most internal of the chain of ossicles, and is inserted by its foot-plate into a small oval opening-the

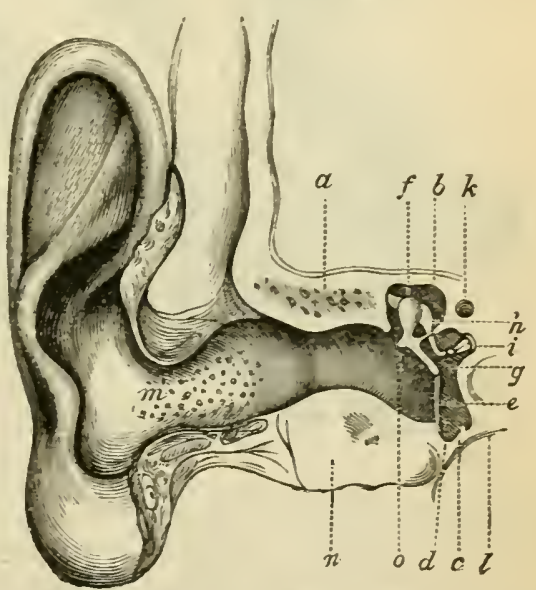

Fig. 453.-The Ear. 1 $m$, external meatus; $f$, head of malleus; $o$, short process of malleus; $g$, handle of malleus; $h$, incus; $i$, foot of stapes in oval foramen; $e, t y m$. panic membrane.

foramen ovale-on the inner wall of the tympanic cavity. A membranous ring-the orbicular membrane-surrounds the foot of the stapes, helping to fill up the foramen and attaching the bone to its edges. The inner surface of the foot of the stapes is in contact witl the perilymph of the internal ear. The incus, or anvil, forms a link between the malleus and the stapes. The auditory ossicles, as well as the whole cavity of the tympanum, are covered by pavement epithelium.

The tympanum is not an absolutely closed chamber; it has one channel of communication with the external air-the Eustachian tube -which opens into the pharynx. By the action of the cilia lining this tube the scanty secretion of the middle ear is moved towards its pharyngeal opening, which, usually closed, is opened when a swallowing 
movement occurs. Its function is to keep the pressure in the mirldle car approximatcly that of the atmosphere. In a balloon ascent an excess of pressure is established on the internal surface of the tympanic membranc. In the air-lock of a caisson when the air is being compressed the cxcess of pressure is on the external surface of the membrane. The feeling of uncomfortable tension is relicved in both cases by swallowing movements which allow the pressure in the tympanum to adjust itself to that in the pharynx. In catarrh of the naso-pharynx the orifice may be occluded, and this is accompanicel by impairment of hearing and a disagrecable sensation of tension in the car, owing to absorption and consequent rarefaction of the air in the tympanum.

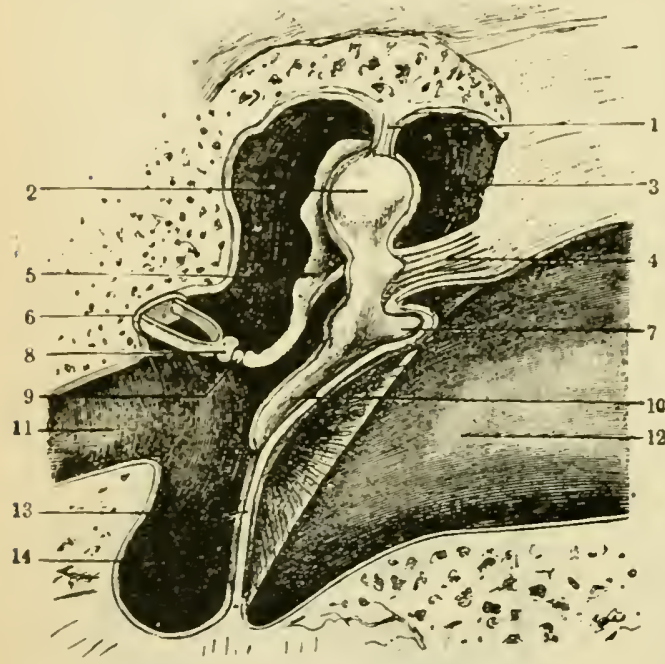

Fig. 454--Tympanum of Left Ear, showing the Ossicles (Morris). I, superior, and 4, external, ligament of malleus; 2 , head; 7 , short process, and Io, manubrium or handle, of malleus; 5 , long process of incus, terminating in 9 , the os orbiculare; 6 , base, and 8 , head, of stapes; II, Eusthchian tube; $\mathbf{I} 2$, external auditory meatus; I3, membrana tympani; 3 . upper, and 14 , lower, part of tympanum. The patient instinc. tively makes efforts which increase the pha. ryngeal pressure from time to time so as to open the tube.

The loosely - jointed chain of ossicles is steadied and its move. ments directed by ligaments and by the ten. sion of its terminal membranes. It forms a kind of bent lever by which the oscillations of the membrana tympani are transferred to the mem. brane covering the oval foramen, and at the same time reduced in size. Two slender muscles, the tensor tympani and stapedius, contained in the tympanic cavity, are also connected with and may act upon the ossicles. The former lies in a groove above the Eustachian tube, ard its tendon, passing round a kind of osseous pulley (processus cochleariformis), is inserted into the handle of the mallcus; the stapedius is lodged in a hollow of the inner bony wall of the tympanum. Its tendon is attached to the neck of the stapes near its articulation with the incus. This inner wall is pierced not only by the oval foramen, but also by a round opening, the fenestra rotunda, which is closed by a membrane to which the name of secondary membrana tympani is sometimes given.

The internal ear consists of the bony labyrinth, a series of curiously excavated and communicating spaces in the substance of the petrous portion of the temporal bone, filled with a liquid called the perilymph, in which, anchored by strands of connective tissue, floats a corresponding series of membranous canals (the membranous labyrinth), filled with a liquid called endolymph. The labyrinth of the internal ear is 
divided into three well-marked parts: the cochlea, the vestibule, and the semicircular canals (lig. 455). The cochlea, the most anterior of the threc, consists of a convoluted tube which coils round a central pillar, the columella or modiolus, like a spiral staircase. The lamina spiralis projects from the modiolus and divides the tube into an upper compartment, the scala vestibuli, and a lower, the scala tympani (Fig. 456). The part of the lanina next the modiolus is of bone, but it is completed at its outer edge by a membiane, the lamina spiralis membranacea, or basilar membrane. The scala tympani abuts on the fenestra rotunda, and its perilymph is only separated from the air of the tympanic cavity by the membrane which closes that opening. At the apex of the cochlea the lamina spiralis is incomplete, ending in a crescentic border, so that the scala tympani and the scala vestibuli here communicate by a small opening, the helicotrema. The scala vestibuli conmunicates with the vestibule, and the vestibule with the semicircular canals, so that the perilympls of the entire labyrinth forms a cuntinuous sheet separated from the cavity of the middle ear by the

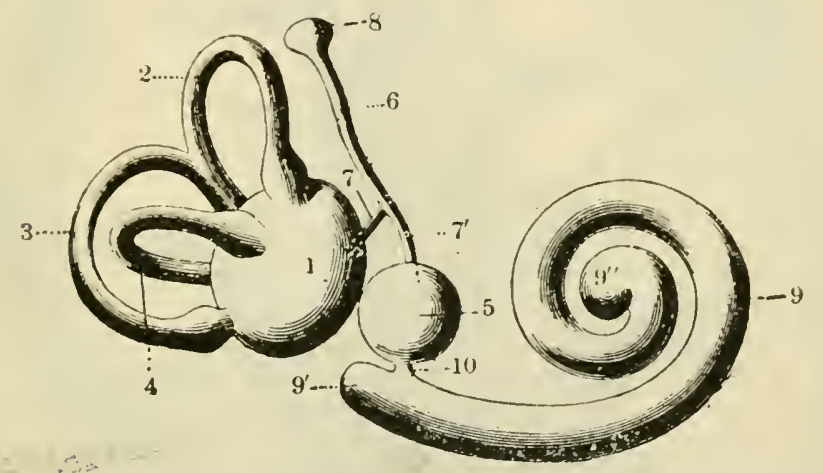

Fig. 455--Diagram of Right iMembranous Labyrinth (after Testut). I, utricle; $2,3,4$, superior, posterior, and horizontal semicircular canals; 5, saccule: 6 , ductus endolymphaticus arising by two branches, $7,7^{\prime} ; 8$, saccus endo. lymphaticus; 9, canalis cochlearis (canal of the cochlea) ending at $9^{\circ}$, and $9^{\prime \prime}$; ro, canalis reuniens.

structures that fill up the round and oval foramina. In the membranous labyrinth, and in it alone, are contained the end-organs of the auditory nerve. The membranous portion of the cochlea is a small canal of triangular section, cut off from the scala vestibuli by the membrane of Reissner, which stretches from near the edge of the bony spiral lamina to the outer wall (Fig. 457), to which it is attached by the spiral ligament. The canal has received the name of the scala media, or canal of the cochlea. The membrane of Reissner forms its roof. Its floor is composed ( $\mathrm{r}$ ) of the projecting edge of the spiral lamina, called the limbus, and (2) of the basilar membrane. The most conspicuous constituent of the basilar membrane is a layer of stiff, parallel, transparent fibres arranged radially-i.e., in the direction from limbus to spiral ligament. They are embedded in a homogeneous material. Below the cochlear canal ends blindly, but communicates by a sidechannel with the portion of the membranous vestibule called the sacculc, which in its turn communicates with the utricle by the $Y$-shaped origin of the ductus endolymphaticus. In to the utricle open the three 
semicircular canals, the endolymph of which has, therefore, free com munication with that of the vestibule and cochlea. But although the semicircular canals and vestibule belong anatomically to the internal ear, and are supplied by branclies of the auditory nerve, we have no positive proof that in the higher animals, at least, they are in any way concerned in hearing; and since experiment has assigned them a definite function of another kind (p. 936), we shall not consider thein

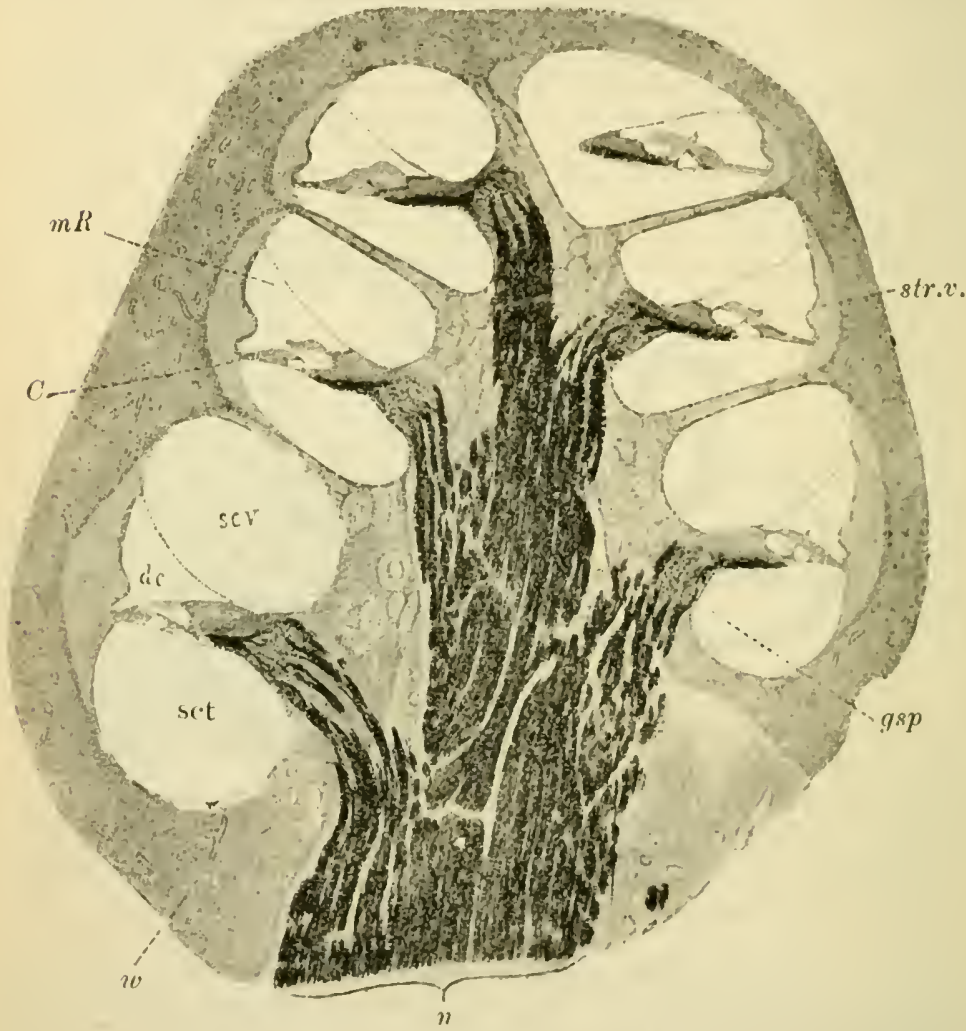

Fig. 456.- Longitudinal Section through the Cochlea of a Cat (Schåfer, after Sobotta) $\times 25$. dc, canal or duct of cochlea; $s c v$, scala vestibuli; scl, scala tympani; $w$, bony wall of cochlea; $C$, organ of Corti; $m R$, Reissner's membrane; $n$, fibres of cochlear nerve; gsp, ganglion spirale; str.v., stria vascularis.

further in this connection. The scala media contains the organ of Corti, which (Fig. 458) consists of a series of modified epithelial cells planted upon the basilar membrane. The epithelial cells are of three kinds: (1) supporting epithelial cells; (2) the pillars or roits of Corti, in two series (inner and outer), sloped against each other like the rafters of a roof, and covering in a vault or tunnel which runs along the whole of the scala media from the base to the apex of the cochlea; (3) the haircells, around which the fibres of the auditory nerve arborize. These last are columnar epithelial cells, surmounted by hairs. They are 
arranged in several rows, one row lying just internal to the inner line of pillars, and several rows cxternal to the outer line of pillars. Between the outer hair-cells are supporting cells (cells of Deiters). A thin membrane, the reticular lamina or membrana reticularis, composed of ficldle-shaped rings or plialanges, covers the hair-cells, and through openings in it the hairs project. A thicker membrane, the membrana tectoria, springiug from the edge of the osseous spiral lamina near the attachment of Reissner's membrane, forms a kind of canopy over both pillars and hair-cells. The outer wall of the canal of the cochlea is clad by cubical epithelium covering a membrane richly supplied with bloodvessels (stria vascularis). The fact that the hair-cells of Corti's organ are connccted with the fibres of the cochlear division of the

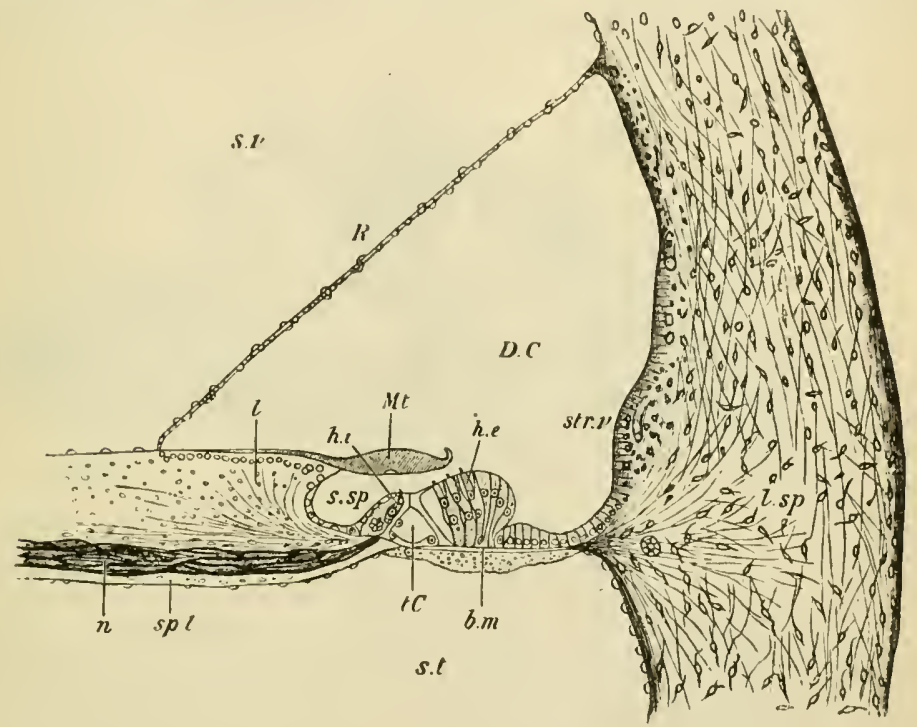

Fig. 457--Vertical Section of the First Turn of the Cochlea (after Retzius). D.C canal of cochlea; $t C$, tunnel of Corti; b.m, basilar membrane; h.i, h.e, internal and external hair-cells; $M t$, membraua tectoria; s.sp, spiral groove; str.v, stria vascularis; $s p . l$, spiral lamina; $n$, fibres of the cochlear nerve; $l$, limbus laminæ spiralis; $R$, Reissner's membrane; s.v, scala vestibuli; s.t. scala tympani; $l . s p$. spiral ligament.

auditory nerve, and its elaborate structure, suggest that it must play a peculiar part in auditory sensation. Comparative anatomy shows us that the cochlea is the most highly developed portion of the internal ear, the last to appear in its evolution, and the most specialized. It is absent in fishes, which possess only a vestibule and one to three semicircular canals. It first acquires importance in reptiles, but attains its highest development in mammals; and there is every reason to believe that it is the terminal apparatus of the sense of hearing.

Functions of the Auditory Ossicles.-The anatomical arrange ments of the middle ear suggest that the tympanic membrane and the chain of ossicles have the function of transmitting the soundwaves to the liquids of the labyrinth; and observation and experi. 
ment fully confrm this idea. Tracings of the movements of th: ossicles have been obtained by attaching very small levers to them, and their movements have been directly observed with the microscope. Even in man it may be shown, by viewing the membrane through a series of slits in a rapidly-revolving disc (stroboscope), that it vibrates when sound-waves fall on it.

When the handle of the malleus moves inwards, rotating around itl axis which may be supposed to pass through its neck, its head moves in the opposite direction. The joint between that bone and the incus is thus locked, on account of the shape of the articular surfaces. The long process of the incus, constituting the second

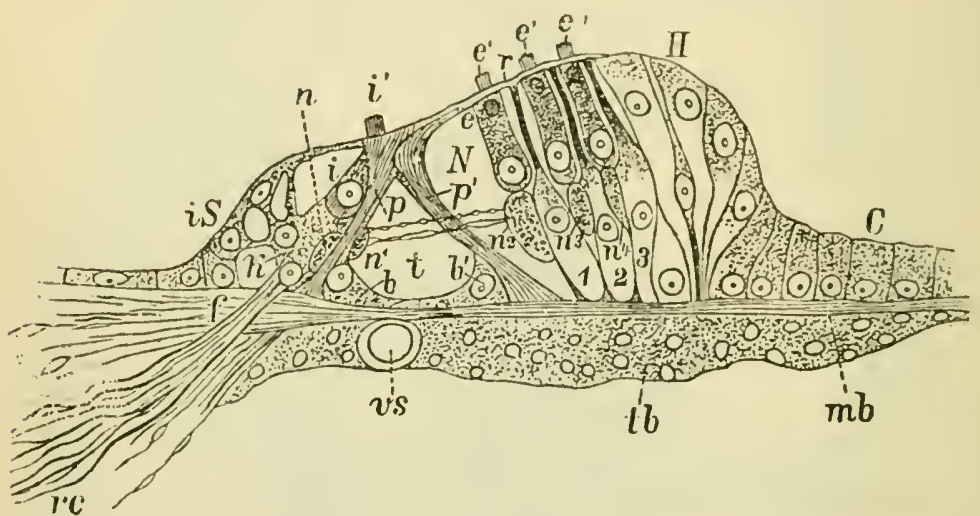

Fig. 458.-Organ of Corti (Barker, after Retzius). $m b$, basilar membrane; $t b$, its tympanal covering; $v$ s, bloodvessel (vas spirale); $r c$, incdullated distal processes of bipolar nerve-cells in the ganglion spirale, passing in to arborize arourd the hair-cells; is, epithelial cells continuous with the cpithelium of the sulcus spiralis internus; $p$, inner pillar of Corti, with its basal cell, $b ; p^{\prime}$, outer piliar with its basal cell, $b^{\prime} ; 1,2,3$, supporting cells of Deiters, whose processes run up to be attached to the lamina reticularis, $r ; H$, Hensen's supporting cells; C, cells of Claudius: $i$, internal hair-cell with its hairs, $i^{\prime}$ (the upper part of the hair-cell is concealed by the head of the inner pillar of Corti); $c$, external hair-cell; $c^{\prime}$, hairs of three external hair-cells; $n, n^{1}$, to $n^{4}$, cross-sections of the spiral strand of cochlear nerve-fibres.

portion of the bent lever, passes inwards, carrying with it the stapes, which is attached to it by an almost rigid joint, and the stapes is pressed into the oval foramen. Since the long process of the incus is about one-third shorter than the handle of the malleus, the excursion of the point of the former is correspondingly smaller than that of the latter, but at the same time more powerful. When the tympanic membrane passes outwards, the handle of the malleus and foot of the stapes do the same. But the joint now unlocks, and excessive outward movement of the stapes, which miglit result in its being torn from its orbicular attachment, is prevented. The ossicles vibrate en masse. It is only to a trifling extent that sound 
can be conducted through them to the labyrinth as a molecular vibration; for when they are anchylosed, and the foot of the stapes fixed immovably in the foramen ovale, as sometimes recurs in disease, hearing is greatly impaired.

Of course, every vibration of the tympanic membrane must cause a corresponding condensation and rarefaction of the air in the middle ear; and this may act on the membrane closing the fenestra rotunda, and set up oscillations in the perilymph of the scala tympani. That this is a possible method of conduction of sound is shown by the fact that, even after closure of the oval foramen, a slight power of hearing may remain. But under ordinary conditions by far the most important part of the conduction takes place viâ the ossicles. And when it is remembered that the tympanic membrane is about thirty times larger than that which fills the oval foramen, it will be seen that the force acting on unit area of the foot of the stapes may be much greater than that acting on unit area of the membrana tympani, and that the mode of transmission by the ossicles is a very advantageous method of transforming the feeble but comparatively large excursions of the tympanic membrane into the smaller but more powerful movements of the stapes. The average excursion of the membrane of the oval foramen does not at most amount to more than $\mathrm{O}_{4} \mathrm{O}_{4}$ millimetre. Even the so-called cranial conduction of sound when a tuning-fork is held between the teeth or put in contact with the head, which was at one time supposed to be due solely to direct transmission of the vibrations through the bones of the skull to the liquids of the labyrinth or the end-organs of the auditory nerve, has been shown to take place, in great part, through the membrana tynjpani and ossicles; the vibrations travel through the bones to the tympanic membrane, and set it oscillating. So that this test, when applied to distinguish deafness caused by disease of the middle ear from deafness due to disease of the labyrinth or the central nervous system, may easily mislead, although it enables us to say whether the auditory meatus is blocked-by wax, e.g.-beyond the tympanic membrane.

A membrane like a drum-head has a note of its own, which it gives out when struck, and it vibrates more readily to this notc than to any other. It would evidently be a serious disadvantage if the tympanic membrane, whose office it is to receive all linds of vibrations, and respond to all, had a marked fundamental tolle which would be continually obtruding itself among other notes. The difficulty is obviated by the damping action of the ossicles and the liquids of the labyrinth on the movements of the membrane, which in addition is not stretched, but lies slackly in its bony frame, so that when the handle of the malleus is detached from it, it retains its shape and position.

The tensor tympani, when it contracts, pulls in wards the handle of the malleus, and thus increases the tension of the tympanic membrane. The precise object of this is obscure. It has been suggested that damp. 
ing of the movements of the auditory ossicles is thus secured. Another theory is that the increased tension of the membrane renders it more capable of responding to higher tones, and that the muscle thus acts as a kind of accommodating mechanism. But Hensen has observed that the tensor only contracts at the beginning of a sound, and relaxes again when the sound is continued; and this is difficult to reconcile with either of these hypotheses. The muscle is normally excited reflexly through the vibrations of the membrana tympani, but some individuals have the power of throwing it into voluntary contraction, which is accompanied by a feeling of pressure in the ear, and a harsh sound. The function of the stapedius is unknown. Its contraction would tend to press the posterior end of the foot-plate of the stapes deeper into the foramen ovale, and cause the anterior end to move in the opposite direction; but it is not easy to see how this would affect the action of the auditory mechanism.

The tensor tympani is supplied by the fifth nerve through a branch from the otic ganglion; the stapedius is supplied by the seventh. Paralysis of the fifth nerve may be accompanied with difficulty of hearing, espccially for faint sounds. When the seventh nerve is paralyzed, increased sensitiveness to loud sounds has been observed.

We have already recognized the organ of Corti, particularly the hair-cells, as a sensory epithelium which constitutes the terminal apparatus of the cochlear nerve. The adequate stimulus of the auditory receptors is the periodic changes of pressure in the endolymph. But there are various opinions as to how these vibrations are transmitted to the hair-cells, and as to how the vibrations of the hair-cells are translated into nerve impulses in the auditory fibres. The pillars of Corti, the basilar membrane, and the membrana tectoria, have in turn been regarded as the structures immediately set into vibration by the changes in the endolymph. The case for the tectorial membrame is perhaps the most plaisible, for its position renders it most capable of acting on the hairs. Others have supposed that the hairs of the hair-cells are directly affected by the endolymph. Some, despairing of further analysis, content themselves with the conclusion that the organ of Corti vibrates as a whole. Some of these theories will be again referred to in considering what is the greatest problem of the physiology of hearing, viz.:

The Perception of Pitch-Analysis of Complex Sounds.-As the eye, or, ratlier, the retina plus the brain, can perceive colour, so the labyrinth plus the brain can perceive pitch. The colour-sensation produced by ethereal waves of definite frequency depends on that frequency; and upon the frequency of the aerial vibrations depends also the pitch of a musical note. But there is this difference between the eyc and the ear: that while the sensation produced by a mixture of rays of light of different wave-length is always a simple sensation - that is, a sensation which we do not perceive to be built up of a number of sensations, which, in other words, we lo not analyze-the ear can perceive at the same time, and distinguish 
from each ot lier, the components of a complex sound. When a number of notes of different pitcl are souncled tngether at the sane distance from the ear the disturbance which reaches the membrana tympani is the physical resultant of all the disturbances produced by the individual notes, and it strikes upon the nembrane as a single wave. ' $A$ single curve describes all that the ear can possibly hear as the result of the most complicated musical performance. ... In the complicated sound the variations of the pressure of the air are more abrupt, more sudden, less smooth, and less distinctly periodic than they are in softer, purer, and simpler sound. But the superposition of the different effects is really a marvel of marvels ' (Kelvin). The ear or brain must, therefore, possess the power of resolving the complex vibrations into their constituents, else we should have a mixed or blended sensation, and not a sensation in which it is possible to distinguish the constituents of which it is made up. Several hypotheses have been proposed to explain this physiological analysis of sound, on the assumption that the analysis takes place in the labyrinth. The most important, in spite of certain defects, is still that of Helmholtz.

Helmholtz attempted to explain the perception of pitch on the assumption that in the internal ear there exists a series of resonators, each of which is fitted to respond by sympathetic vibration to a particular note, while the others are unaffected; just as when a note is sung before an open piano it is taken up by the string which is attuned to the same pitch and ignored by the rest. Let us suppose that a given fibre of the auditory nerve ends in an organ which is only set vibrating by waves impinging on it at the rate of 100 a second, and that the end-organ of another fibre is only influenced by waves with a frequency of 200 a second. Then, on the doctrine of 'specific energy' (according to which the sensation caused by stimulation of a nerve depends not on the particular kind of stimulus but on the anatomical connection of the nerve with certain nerve centres), in whatever way the first fibre is excited, a sensation corresponding to a note with a pitch of roo a second will be perceived. Whenever the second fibre is excited, the sensation will be that of a note of 200 a second, or the octave of the first. If both fibres are excited at the same time the two notes will be heard together. Now, Hensen actually observed that in the auditory organs of some crustaceans, the hair-like processes of certain epithelial cells can be set swinging by waves of sound, and, further, that they do not all vibrate to the same note unless the sound is very loud. In the lobster there are between four and five hundred of these hairs, varying in length from $\mathrm{I} 4 \mu$ to $740 \mu$; and in some insects, such as the locust, similar hairs, also graduated in length, exist.

To gain an anatomical basis for his theory, Helmholtz supposed first of all that the pillars of Corti were the vibrating structures, 
and that, directly or through the hair-cells, their mechanical vibrations were translated into impulses in the auditory nerve-fibres. But apart from the fact that their number is too small (about 3,000 ) to allow us to assign one rod to each perceptible difference of pitch, and their dimensions too similar to permit of the requisite range of vibration frequency, it was pointed out that birds do not possess pillars of Corti-a fact which was decisive against the assumption of Helmlioltz, since nobody denies to singing-birds the power of appreciating pitch. Helmholtz accordingly, choosing between the remaining possibilities, gave up the pillars of Corti, and adopting a suggestion of Hensen, substituted the radial fibres of the basilar membrane as his hypothetical resonators. These are more adequate to the task imposed on them, since their range of length is far greater ( $4 \mathrm{I} \mu$ at the base to $495 \mu$ at the apex of the cochleaHensen); and the elaborate structure of Corti's organ certainly suggests that some one or other of its elements may be endowed with such a function. Experimentally, too, it has been shown that destruction of the apex of the cochlea causes loss of appreciation of low notes, and destruction of the base loss of appreciation of high notes, which agrees with Helmholtz's view. But while the theory of peripheral analysis of pitch tends upon the whole to be strengthened as evidence gathers, it is possible that the analysis is accomplished in some other way than by sympathetic resonance.

Ewald has developed a theory according to which each note causes the basilar membrane to vibrate throughout its whole extent in such a way that stationary waves are produced in it, like the Chladni's figures seen on a metal plate strewed with sand when it is set into vibration. The pattern of the movement, the 'sound-picture,' will be different for each tone, since the interval between the waves will be different. The hair-cells and auditory fibres of particular parts of the organ of Corti will therefore be stimulated by the pressure of the membrane, or escape stimulation, according to the position of the stationary waves with reference to them for each note. In this way each soundpicture will be printed, so to speak, upon the sensitive terminal apparatus of the auditory nerve, as a letter is printed upon a piece of paner by a type. The corresponding excitation pattern-i.e., the particular distribution of cochlear fibres stimulated-is supposed to be associated in consciousness with the appreciation of the pitcli of the particular note. Ewald has endeavoured to support his theory by showing that fine membranes of the dimensions of the basilar menibrane do yield very distinct sound-pictures for different simple tones as well as for complex tones. These can be observed with the microscope and photographed (Fig. 459).

One of the best-known theories of central analysis may be conveniently labelled the 'telephone theory,' in accordance with the simile used by Rutherford. He supposed that the organ of Corti (or at any rate the hair-cells) is set in to vibration as a whole by all audible sounds, and that its vibrations are translated into impulses in the auditory nerve, which are the physiological counterpart of the aerial waves and the waves of increased and diminished pressure in the liquids of the labyrinth to which they give rise. Thus, a sound of 100 vibrations a second would start 100 impulses a second in the auditory 
nerve; a loud sound would set up impulses more intense than a fecble sound; and a complex wave, which is the resuitant of several somuds of different vibration-frequency, would also in some way or other stamp the impress of its form on the allelitory excitation wave; just as in a telephone every wave in the air causes a swing of the vibrating plate, and thus sets up a current of corresponding intensity and duration in the wires. This theory evidently abandons the doctrine of specific encrgy for the particular case of the analysis of pitch, for it assumes that differences of auditory sensation are related to differences in the nature of the impulses travelling up the auditory nerve, and not inerely to differences in the anatomical connections (peripheral and central) of the auditory nerve-fibres. It is unsatisfactory becausc it takes no account of the remarkable and suggestive structure of the telephone plate -i.e., of the organ of Corti-and gives nohint of how the analysis is accomplished in the central organ.

The range of hearing is very great. The highest audible tone corresponds to 30,000 to 40,000 vibrations a second, the lowest to about 30. Between thesc limits as many as 6,000 variations of pitch can be perceived.

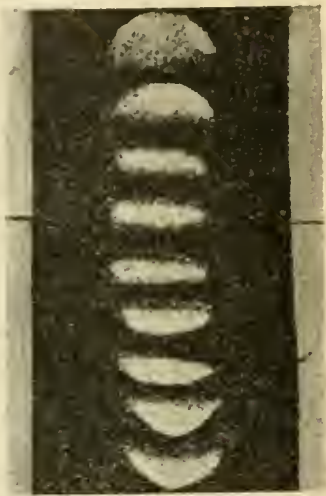

Fig. 459.- Photograph of a Sound-Picture (Ewald).

Wien has elaborately investigated the question how the sensitiveness of the ear varies for tones of different pitch. A tone of $5^{\circ}$ vibrations a second, in order to be just heard, must have an intensity corresponding to about roo million times as much energy as is needed for a tone of 2,000 vibrations. It is only on the extraordinary sensibility of the ear for the range of tones used in ordinary speech that the possibility of understanding speech depends when the circumstances are unfavourable-e.g., at a great distance, or in the presence of much stronger accompanying noises.

\section{Section III.-SMEll AND TAste.}

Smell was defined by Kant as 'taste at a distance'; and it is obvious that these two senses not only form a natural group when the quality of the sensations is considered, but are closely associated in their physiological action, especially in connection with the perception of the flavour of the food. Their intimate relation is further indicated by the fact that the cortical areas in which smell and taste are represented lie close together or overlap each other on the gyrus hippocampi and uncus (p. 968). The olfactory endorgans in the mucous membrane of the upper part of the nostrils, the so-called regio olfactoria, have been already described (p. $92 \mathrm{I}$ ). In cases of anosmia, in which the olfactory nerve is absent or paralyzed, smell is abolished; but substances such as ammonia and acetic acid, which stimulate the ordinary sensory nerves (nasal branch of fifth) of the olfactory mucous membrane, are still perceived, though not distinguished from each other. In fact, the so-called pungent odour of these substances is no more a true smell 
than the sense of smarting they produce when their vapour comes in contact with a sensory surface like the conjunctiva, or a piece of skin desoid of epidermis.

It was at one time believed that odoriferous particles could not be appreciated unless they were borne by the air into the nostrils; but this appears not to be the case, for the smell of substances dissolved in physiological salt solution is distinctly perceived when the nostrils are filled with the liquid; and fish, as every line-fisherman knows, have no difficulty in finding a bait in the dark. The odoriferous substances, even when air-borne, are dissolved in the nasal secretion before they can affect the olfactory end-organs, and it may be due to the peculiarities of this solvent that it is so difficult to imitate a normal stimulation of the olfactory organs by solutions experimentally introduced into the nose (Parker).

The substances which can affect the olfactory mucous membrane can be divided into four groups:

I. Those which act only on the olfactory nerves, the odours proper.

2. Substances which act at the same time on olfactory nerves, and on nerves of common sensation (tactile nerves)-e.g., acetic aciol.

3. Substances which act at the same time on the gustatory nerves.

4. Substances which act only on the nerves of common sensation (tactile nerves) - e.g., carbon dioxide.

Zwaardemaker has classified the pure odours as follows:

(1) Ethereal odours, as those of fruits; (2) aromatic odours, as of camphor or bittcr almonds; (3) fragrant odlours, as of flowers; (4) ambrosial odours, as of amber or musk; (5) garlic odours, as of onion, garlic, asafotida; (6) empyreumatic, or burning odours, as of burnt coffee or tobacco smoke; (7) caprylic or goat odlours, as of sweat; (8) repulsive odours, as the odour of the disease oziena; (9) nauseating odours, as of faces or putrefying material.

The most interesting form of inadequate stimulation is electrical excitation of the olfactory mucous membrane, which causes a sensation like the smell of phosphorus. The sensation is experienced at the kathode on closure and the anode on opening. As to the manner in which the multitudinous adequate stimuli excite the olfactory nerves, we can only suppose tlat they act as chemical stimuli. Smell and taste are pre-eminently the 'chemical' senses, as sight and hearing are pre-eminently 'physical' senses. But little is known of the relation between the chemical constitution or physical properties of substances and the quality of the odoriferous sensation which they excite, although Haycraft has pointed out some interesting relations between the atomic weights of certain elements and their power of exciting odours. The number of distinct odours which can be perceived is so great that it is scarcely conceivable that each is subserved by special olfactory fibres. Marked changes occur in disease, and all odours need not be affected to the same extent. Some may be almost normally perceived, while relative or complete loss of smell exists as regards others. These and other facts have given rise to the idea that there are several groups of olfactory fibres, each concerned in the appreciation of a particular odour or group of odours. Yet it has not proved possible to reduce them to a limited number of funclamental odours and their combinations.

Acuteness of smell may be measured by arrangements called olfactometers. Zwaardemaker's olfactometer consists of a piece of indiarubber tubing fitted inside a glass tube, through which air is drawn 
into the nostrils. Another glass tube just fitting the rubber tube is pushed insicle it, so as to cover a portion of it. The minimum amount of surface of the indiarubber tube which must be left exposed so that the smell of the rubber may be perceived is a measure of the acuteness of smell. To investigate other odours tubes of the corresponding odorous substances can be constructed.

Taste.-The sense of taste is not so strictly localized as the sense of smell. The tip and sides of the tongue, its root, the neighbouring portions of the soft palate, and a strip in the centre of the dorsum, are certainly endowed with the sense of taste; but the exact limits of the sensitive areas have not been defined, and, indeed, vary in different individuals.

The nerves of taste are the glosso-pliaryngeal, which innervates the posterior part of the tongue, and the lingual, which supplies its tip (see p. 925). The end-organs of the gustatory nerves are the tastebuds or taste-bulbs, which stud the fungiform and circumvallate papillæ, and are most characteristically scen in the moats surrounding the latter. They are barrel-like bodies, the staves of the barrel being represented by supporting cells; each bud encloses a number of gustatory cells with fine processes at their free ends projecting through the superficial end of the barrel. They are surrounded by the end arborizations of the fibres of the gustatory nerves. Taste-buds are also found on the posterior surface of the epiglottis and in the larynx. It has been suggested that these form the afferent end-organs of a reflex apparatus which guards the glottis against the entrance of food in deglutition (Wilson). Epithelial buds, different from the olfactory elements, also occur in the olfactory region of the nasal mucous membrane. It is possible that the so-called nasal taste-e.g., the sweet taste caused by chloroform when aspirated in not too small an amount through the nose-depends upon these buds.

As to the properties in virtue of which sapid substances are enabled to stimulate the gustatory nerve-endings, we know that they must be soluble in the liquids of the mouth, and there our knowledge ends. An attempt has been made by various authors to connect the taste of such bodies with their chemical composition, but researches of this kind have not hitherto yielded much fruit. The number of distinct qualities of taste sensation is considerable, but by no means so great as the number of qualities of olfactory sensations, and they are more easily reduced to a few primary or fundamental sensations. Sapid substances have generally been divided into four classes as regards the fundamental sensations produced by them-viz.: (I) Sweet, (2) acid, (3) bitter, (4) saline. All taste sensations seem to be combinations of these, or combinations of one or more of them with olfactory sensations, or with sensations due to excitation of the ordinary sensory nerves of the tongue.

Sweet and acid tastes are best appreciated by the tip, and bitter tastes by the base, of the tongue. Differences have been detected between individual papillie in their power of reaction to sapid substances which produce one or other of the fundamental sensations. Of 125 fungiform papillæ tested with solutions of tartaric acid, sugar, and quinine, 27 gave no sensation of taste. Tartaric acid evoked 
its acid taste in 91 of the remaining $9 S$, sugar its sweet taste in 79 , and quinine its bitter taste in 7 i; 12 reacted only to tartaric acid. and 3 only to sugar (Ohrwall). Such facts indicate, although they do not definitely prove, the existence of specific receptors for each of the fundamental taste sensations-i.c., gustatory end-organs, which are easily excited by an adequate stimulus (acid, e.g., in the case of an 'acid' taste-bud), with difficulty or not at all by an inadequate stimulus.

The form of inadequate stimulation most investigated is that produced when a constant current is passed through the tongue. An acid taste is experienced at the positive, and an alkaline or bitter taste at the negative pole; and this is the ease even when the current is conducted to and from the tongue by unpolarizable combinations, which prevent the deposition of electrolytic products on the mucous membrane (p. 731). The sensations are due to stimulation of the gustatory end-organs and not of the nerve-trunks.

Normal lymph, which bathes these end-organs, does not excite any sensation of taste, but when the composition of the blood is altered in disease or by the introduction of foreign substances, tastes of various: kinds may be perceived. Sometimes this may be duc to the stimulation of substances excreted in the saliva; but in other cases it seems that, without passing beyond the blood and lymph, forcign substances may excite the gustatory nerves.

Flavour cmbraces a group of mixed sensations in which smell and taste are both concerned, as is shown by the common observation that a person suffering from a cold in the liead, which blunts his sense of smell, loses the proper flavour of his food, and that some nauseous medicines do not taste so badly when the nostrils are held.

In common speech, the two sensations are frequently confounded with each other and with tactile sensations. Thus the 'bouquet' of wines, which most people imagine to be a sensation of taste, is in reality a sensation of smell; the astringent 'taste' of tannic acid is not a taste at all, but a tactile sensation; the 'hot' taste of mustard is no more a true sensation of taste than the sensation produced by the same substance when applied in the form of a mustard poultice to the skin.

As already remarked, the substances which affect the olfactory endorgans in air-breathing animals, like those which affect the gustatory end-organs, must eventually go into solution before causing stimulation. The most striking distinction between the two senses is the astonishingly small concentration in which substances can elicit sensations of smeli, as compared with sensations of taste. Thus ethvl alcohol is a stimulus for both smell and taste, but it can be recognized by smell in a dilution 24,000 times greater than the dilution necessary for taste (Parker).

\section{SECTION IV.-CutANEOUS AND INTERxil SExSATIONS.}

Under the sens: of touch it was at one time usual to include a group of sensations which differ in quality-and that in some instances to as great an extent as any of the sensations which are universally cousidered as separate and distinct-but agree in this, that the cnd-organs by which they are perceived are all situated in the skin, the mucous membranes, or the subcutaneous tissue. They are more correctly designated 'cutaneous sensations.' Such are the common tactile sensations-including pressure, tickling, and itching - and the sensations of temperature, or, more correctly, 
of change of temperature, or of warmth and cold. The sensation of pain, although it cannot be absolntely separated from these, ought not to be gromped along with them. It is called forth by the stimulation of afferent nerve-fibres in their course; and it may originate, under certain conditions, in internal organs which are devoid of tactile sensibility, and the functional activity of which in their normal state gives rise to no special sensation at all. The peculiar sensation associated with voluntary muscular effort, to which the nanie of the muscular sense has been given, also deserves a separate place; for although it may in part depend on tactile sensations set up through the medium of end-organs situaterl in muscle, tendon, or the structures which enter into the formation of the joints, other elements are, in all probability, involved.

The simplest form of tactile sensation is that of mere contact, as when the skin is lightly touched with the blunt end of a pencil. This soon deepens into the sensation of pressure if the contact is made closer; and eventually the sense of pressure merges into a fecling of pain. Most physiologists agree that in the skin itself four fundamental qualities of sensation are represented-touch in the restricted sense (the sensation elicited by light contact), warmth, cold, and pain. Pressure is mainly a sensation connected with the stimulation of structures deeper than the skin-e.g., the sensation of contact is abolished in cicatrices where the true skin has been destroyed, while sensibility to pressure persists - although the sensation of light pressure may be to some extent represented in the skin itself in association with touch. In a somewhat diagrammatic

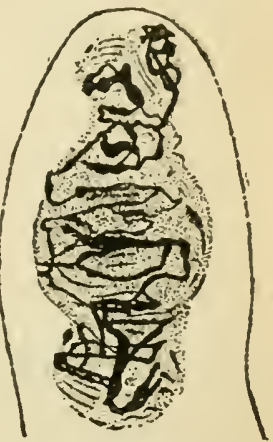

Fig. 460,--Tactile Cor. puscle from Skin of Finger (Smirnow). (Golgi preparation.) The winding and in. tersecting black lines are the non-medullated endings of the one or more nervefibres that enter the ccrpuscle. sense it may be said that the surface of the skin is divided into a great number of very small areas, each of which is related especially to one or other of the four fundamental sensations. Areas concerned in one sensation are everywhere mingled with areas concerned in the others. By appropriate methods it has been found possible to determine the existence on the skin of the trunk and limbs of not less than 30,000 'warm-spots,' which always react to stimulation by a sensation of warmth; $25^{0}, 000$ ' cold-spots,' which react by a sensation of cold; and half a million touch-spots, whose specific reaction is a sensation of touch. It is more difficult to localize definitely bounded ' pain-spots,' partly because of the very rich supply of pain-fibres to the akin. Yet there is reason to believe that pain, like touch, warmth, and cold, is subserved by separate 
receptors. The simplest assumption which will satisfactorily account for the distribution of the four fundamental cutaneous sensations is that the skin is supplied with four kinds of nervefibres, anatomically as well as functionally distinct. Some fibres minister to the sensation of cold, others to that of warmith, others to that of touch, and others still to pain. And just as stimulation of the optic nerve gives rise to a sensation of light, so stimulation of any one of the cutaneous nerves gives rise to the specific sensation proper to the group to which it belongs. The existence of different forms of sensory end-organs in the stin and other tissues (tactile or touch-corpuscles, corpuscles of Pacini, end-bulbs of Krause, etc.) points in the same direction. The end-organs of the touch sensations are believed to be the ring-like arrangements of non-medullated nerve-fibres encircling the hair-follicles, and in parts of the skin devoid of hairs the corpuscles of Meissner (v. Frey)

Touch-spots can easily be demonstrated by touching the skin lightly with some small object such as a hair. The most exact quantitative observations have been made by means of $v$. Frey's hair asthesiometer. This consists of a handle in which hairs of different diameter can be fixed. The area of the cross section of each hair is measured under the microscope, and the pressure necessary to bend it is determined by pressing it upon the scale-pan of a balance. The pressure in milligrammes, divided by the cross section in square millimeters, gives the pressure per square millimetre, which, according to v. Frey, permits hairs to be chosen so as to give a uniform intensity of stimulation or a variable intensity, according to the object of the investigation. Many observers, however, believe that it is more accurate to take no account of the pressure per unit of area, but to graduate the hairs according to the total pressure neederl to bend them. When touch-spots ascertained in this way are excited, by an inadequate stimulus-e.g., an alternating current of minimal strength, applied by the unipolar method through the head of a pin as an electrode-they still respond by their characteristic or spccific reaction-namely, a sensation of touch-in the case supposed, a vibrating sensation like that caused by a tuning-fork in contact with the skin. In the spaces between the touch-spots the sensation produced by the same strength of current, or even by a weaker current, is not one of touch, but a painful pricking sensation which has no vibratory character, but is permanent as long as the current lasts.

The spots most sensitive to touch lie close to the hairs on their "windward' side-i.e., on the side away from which they slope. The minimum pressure necessary to evoke a sensation of contact is not the same for every portion of the skin. The forehead and palm of the hand are most sensitive. According to Lombard the cutaneous pres. sure and tickle sensations called out by delicate mechanical stimuli (hairs, etc.), rlo not arise from the same spots.

If two points of the skin are touched at the same time there is a double sensation when the distance between the points exceeds a certain minimum, which varies for different parts of the sensitive surface.

Practice increases the acuity of touch for the two points test. Even in a few hours it may be temporarily quadrupled on some parts of the skin. Since at the same time it is increased in the corresponding part of the opposite sicle of the body, it is argued that the modification takes place in the central nervous system, not in the end-organs themselves. 
liew of the internal organs are supplied with tactile nerves. The mucous membrane of the alimentary canal from the upper end of the aesophagus to the junction of the rectum with the anal canal is insensitive to tactile stimulation (Hertz). The movements of a tapeworm in the intestines are not recognized as tactile sensations, nor the movements of the alimentary canal during digestion, nor the rubbing of one nuscle on another during its contraction.

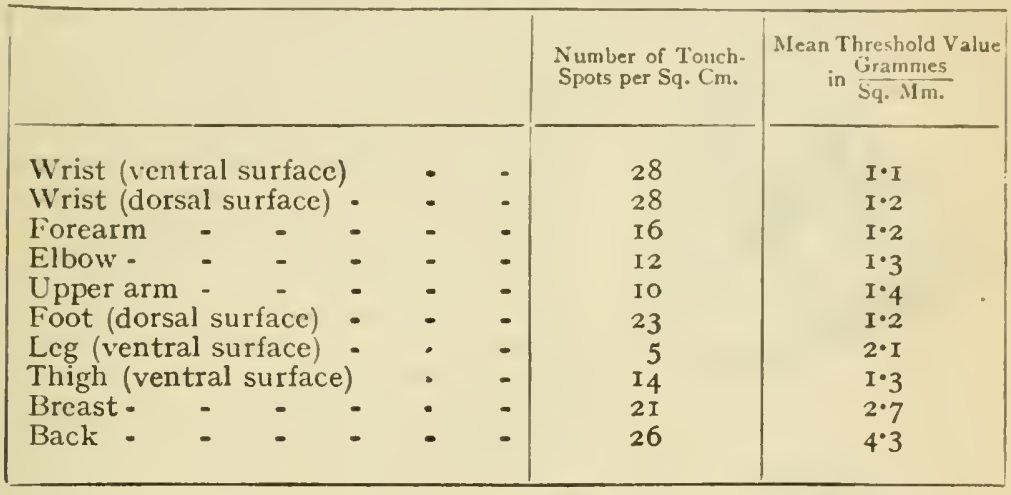

(Kiesow).

Pressure is only perceived when it affects two neighbouring areas to a different degree. Thus, the atmospheric press ure, bearing uniformly on the whole surfacc of the body, causes no ser sation; we are so entirely unconscious of it that it needed the inspiration of genius to discover it, and the persistence of genius to force the discovery on the world. When the finger is dipped in a trough of mercury at its own temperature, no sensation is perceived except a feeling of constriction at the surface of the liquid. The perception of light pressure and of the form and size of objects in contact with the skin is believed to be due to the touchspots. Deep pressure, however, is appreciated, not by the skin, but through sensory end-organs in deeper structures-probably, e.g., Pacini's corpuscles and the muscle-spindles (Fig. 47 I, p. I096).

\begin{tabular}{|l|l|}
\hline & $\begin{array}{l}\text { Distance at which Two Points } \\
\text { can he distinctly felt, in Mm. }\end{array}$ \\
\hline Point of tongue - - & I.I \\
Palmar surface of third & \\
phalanx of finger - & $2 \cdot 2$ \\
Dorsal surface of third \\
phalanx of finger -
\end{tabular}

Sensations of Warmth and Cold.- When a body colder or hotter than the skin is placed on it, or when heat is in any other way 
withdrawn from or imparted to the cutaneous tissues with sufficient abruptness, a sensation of cold or warmth is experienced. And when two portions of the skin at different temperatures are put in contact, we feel that, relatively to one another, one is warm and the other cold. But it is worthy of remark that it is only difference

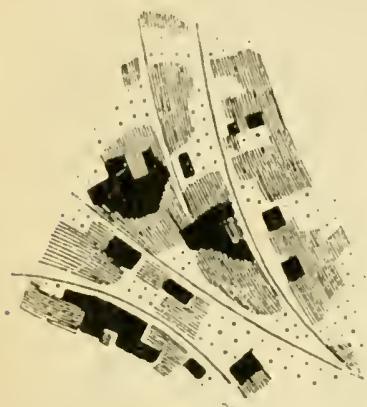
of temperature (or, perhaps, rather the rate at which heat is being gained or lost by the skin), and not absolute height, which we are able to estimate by our sensations. Thus, a hand which has been working in ice-cold water will feel water at $10^{\circ} \mathrm{C}$. as warm; whereas it would appear cold to a warm hanci.

Blix, Goldscheider, and others have shown that the whole skin is not endowed with the capacity of distinguishing temperature, but that the temperature sensations are confined to minute areas scattered over the cutaneous surface. The great majority of these are 'cold' spots-i.e., respond to stimulation only by a sensation of cold - while a smaller number are - warm 'spots, and respond only by a sensation of warmth (Fig. 46r). These spots can be mapped out by bringing into contact with the skin small pieces of wire at a temperature a few degrees above or below that of the skin. With such mild stimuli a response can generally be obtained only from one kind of spot-that is, the cold wire stimulates only the cold and not the warm spots, and vice versa-but with much more intense thermal stimulisay, temperatures of $45^{\circ}$ to $5^{\circ}$ C.not only do the warm spots respond with the appropriate sensation, but the cold spots respond with a sensation of cold. This is well seen when a beam of sunlight is focussed successively on a warm and a cold spot. Inadequate stimuli (mechanical and electrical) also evoke the specific response of warmth from warm spots, and of cold from cold spots.

When the hand is put into water at the temperature of the skin, and the water slowly heated, the warm spots are at first alone stimu- 
lated, and the sensations of lukewarm and then of warn are experienced. When the temperature of the water reaches $45^{\circ} \mathrm{C}$, , the quality of the sensation changes to 'lot.' At a still higher temperature the sensation becomes painful or burning. The most probable explanation of these facts is mentioned below (p. I084).

It is not only of physiological interest, but of practical importance, that most mucous membranes are in comparison with the skin but slightly sensitive to changes of temperature. Only towards the ends of the alimentary canal, in the mouth, pharynx, osophagus, and anal canal, is it possible to elicit warmth or cold sensations. There is some difference of opinion whether a blunted sensibility appears in the stomach also. The uterus, too, is quite insensible to moderate heat; and hot liquids may be injected into its cavity at a temperature higher than that which can be borne by the hand, without causing inconvenience-a fact which finds its application in the practice of gynæcology and obstetrics. It is, indeed, obvious that in the greater number of the internal organs the conditions necessary for stimulation of temperature nerves, even if such were present, could hardly ever exist.

It has already been mentioned that changes of external temperature exert a remarkable influence on the intensity of metabolism (p. 693), and it has been supposed that this is brought about by afferent impulses travelling up the cutaneous nerves. We have also seen that for certain kinds of stimuli the excitability of nerve-fibres is increased by cooling (p. $7^{84}$ ). It is possible that this is the case for the fibres in the skin which are concerned in the regulation of the production of heat, and it has been suggested that this fact may have a bearing on the reflex regulation of temperature (Lorrain Smith).

Pain Sensations. - While the cold and the warmth spots are irregularly distributed over the skin in more or less compact groups, and the touch sensations are intimately associated with the hair follicles, the pain spots are more uniformly spread, and at the same time set closer together. In parts of the body where but one of these elementary forms of general sensibility is present, as in the central parts of the cornea and in the dentine and pulp of the teeth, it is always pain.

In certain situations pain and temperature sensibility are found together, but not touch-e.g., at the margin of the cornea and on the conjunctiva.

In general, the skin is far more sensitive to pain than the deeper structures. The most painful part of an operation is generally the stitching of the wound. The cutting of healthy muscle causes no pain. In an operation in which an artificial connection was established between the stomach and the small intestine (gastro-enterostomy), and in which no anæsthetic was administered, the only pain of which the patient complained was produced by the incision in the skin (Senn). This, however, does not prove that the abdominal viscera are devoid of pain nerves, for it has been shown in animals that exposure of the intestines, etc., as in laparotomy, leads to a rapid depression (exhaustion ?) of the sensibility for pain 
(Kast and Meltzer). In the intact animal and human being painful impressions can unquestionably be excited in the viscera by adequate stimuli (p. 90I). Thus, the spasmodic contraction of the intestines and stomacl causes the intense pain of colic and gastralgia. Labour is an example of a strictly physiological function which is the occasion of severe pain. It would appear from the observations of Hertz that the only immediate cause of true visceral pain, as distinguished from referred pain (p. 89I) is distension acting on the muscular coat of hollow organs and on the fibrous capsule of solid organs. The sensation of pain in the alimentary canal is due to a more rapid or a greater distension than that which constitutes the adequate stimulus for the sensation of fulness. Visceral sensibility seems to be exaggerated in such conditions as hypochondriasis, neurasthenia, and anzmia. Tissues normally insensible, or, rather, but slightly sensible, to pain may become acutely painful when inflamed.

The question has been raised whether the sensation of pain can be caused by excessive stimulation of the nerves of common tactile sensibility, or of the nerves that subserve the sensations of coolness and warmth. It is true that when the skin is lightly touclied in the region of a touch-spot with a small object at its own temperature the sensation is one of pure touch. As the pressure is increased, a sensation of pressure, quite distinct from that of contact, may be felt; and if the pressure is still further increased, a sensation of pain may be elicited. It seems to be quite clearly made out that the pressure sensation in this case is due not to excessive stimulation of the touch-nerves, but to stimulation of the specific pressurenerves when the threshold is reached. The most natural explanation of the pain sensation is that it, too, is due to excitation of the nervous apparatus for pain. Similarly (as was stated on p. I082), if the skin is raised to higher and higher temperatures, the response is at first a pure sensation of warmth, increasing in intensity without changing its quality. When a certain temperature (about $45^{\circ} \mathrm{C}$.) is exceeded, the sensation changes to ' hot,' either because a pain element is now added to the pure thermal sensation, or because the cold spots are now stimulated as well as the warm spots, and mingle their specific response (cold sensation) with that of the warm spots. Further increase of the temperature will cause distinct pain, the sensation assuming a burning character. When a cold spot is tested with decreasing temperatures, an analogous series of sensations is run through, the pure sensation of coolness eventually giving place to cold, intense cold, and finally pain. Here, also, it is simplest to assume that the pain sensation is caused not by excessive stimulation of warm or cold spots, but by excitation of the specific painspots. In any case, there is no doubt that afferent 'pain' fibres exist which are anatomically distinct from the fibres of tactile and 
of temperature sensations. For the conducting paths in the spinal cord are not the same for tactile and for painful impression. And in certain cases of disease sensibility to pain may be lost, while tactile sensations are still perceived; or, on the other hand, pain may be felt in cases wliere tactile sensibility is abolished. Loss of temperature sensation, however, is usually accompanied by loss of sensibility to pain. When a nerve is compressed, the sensibility of the tract supplied by it disappears for cold sooner than for warmith.

Pain has been defined as 'the prayer of a nerve for pure blood.' The idca is not only true as poctry, but, with certain deductions and limitations, true as physiology; that is to say, pain, as a rule, is a sign that something las gone wrong with the bodily machinery; freedom from pain is the normal state of the healthy body. Physiologically, pain acts as a danger-signal. It points out the seat of the mischief, and even, in certain cases, by compelling rest, favours the process of repair. Thus, the surgeon has sometimes looked upon pain as 'Nature's splint.' But, as a matter of fact, a certain amount of pain occurring at intervals is not incompatible with high health; and probably nobody, even when accidents and indiscretions of all kinds are avoided, is entirely free from pain for any considerable time. Sometimes, indeed, the mere fixing of the attention on a particular part of the body is sufficient to bring out or to detect a slight sensation of pain in it; and it is matter of common experience that a dull continuous pain, like that of some forms of toothache, is aggravated by thinking of it, and relieved when the attention is diverted.

As to the sensations of tickling and itching, it is enough to say that physiologists are not agreed whether they represent specific sensibilities subserved by special nerves distinct from those of touch and pain, or merely modifications or mixtures of these sensations.

Phenomena observed after Section of Cutaneous Nerves.-The innervation of the skin can be explored not only by appropriate stimulation of the normal skin, but by study of the defects or alterations of sensibility which follow section of a cutaneous nerve, and which may be observed at different stages in its regeneration. In recent years this has proved a fruitful method, especially in experiments made by skilled observers in whom one or more cutaneous nerves were intentionally divided.

An extensive investigation was made by Trotter and Davies. They divided at different times, extending over more than a year, no fewer than seven of their own cutaneous nerves, including the internal saphenous at the knee, the great auricular, three divisions or branches of the internal cutaneous of the arm just below the elbow, and a branch of the middle cutaneous of the thigh. The operations were purposely done at such intervals as would allow the experience gained in investigating one area to be applied to others. About a quarter of an inch was cut out of each nerve, and the ends then sutured together. 'In each 
case the area of skin supplied by the nerve slowed defects in seven distinct functions: four sensory-namely, sensibility to touch, cold, heat, pain-and three motor-namoly, vaso-motor, pilo-motor, sudo-motor (sweat-secretory). The sensory changes showed is central area of profound loss, an area of inoderate extent surrounding

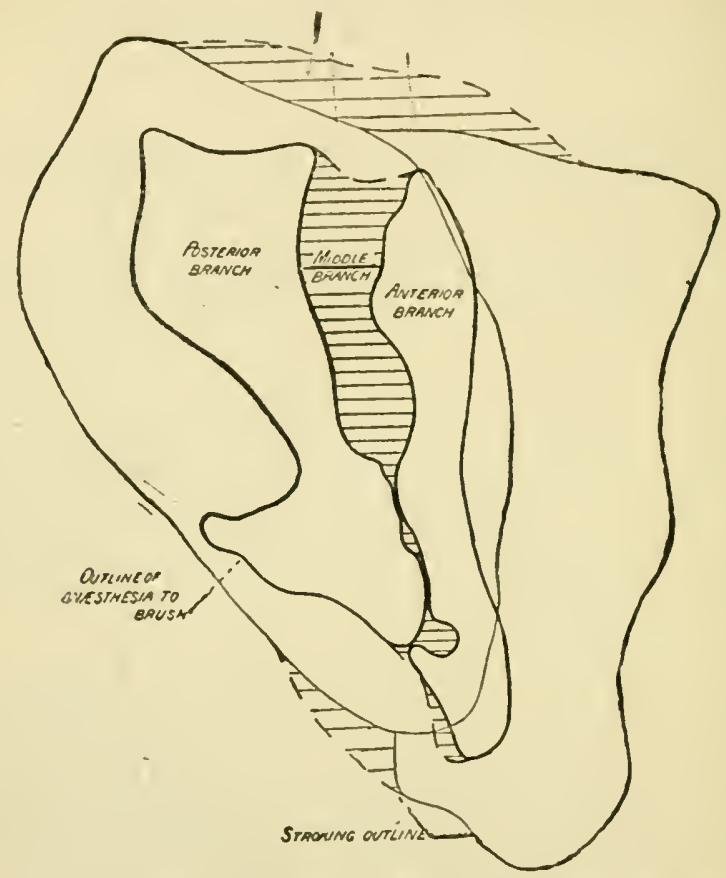

Fig. 463.-Areas of Altered Sensibility produced by Section of all Three Branches of the Internal Cntaneous Nerve of the Left Forearm (Trotter and Davies). (Reduced by Two-thirds.) The thick lines show the areas of anxsthesia to the brush. The thick continuous lines enclose the areas of the anterior and posterior branches. The thick broken line and heavy shading mark the area of the in. crease in anæsthesia which followed section of the middle branch. The thin lines show the areas of minimal hypoxsthesia-i.e., the 'stroking ontline.' The complete oval outline is the 'stroking outline' which followed section of the pos. terior branch. The large addition to the oval on the right of the diagram show's the increase in the 'stroking ontline' which followed section of the anterior branch. The thin broken line and fine shading show the additions to the "stroking out. line 'produced by division of the middle branch.

this of partial loss, and a large area in which a qualitative change could be alone detected.' The maximal extent of cliange, and therefore the outer boundary of this third area, can be mapped out by getting the subject to determine by light, stroking touches the area which feels in any way unnatural when he touches it himself. The most common feeling is that the skin has become smoother at 
the boundary as the stroking linger crosses it, coming from the normal skin. This area is always much larger than the area included in it, in which by quantitative methorls-e.g., the use of at very fine camel's-hair brush, or more exactly by the v. Frey lairsthe sensibility to touch can be shown to be diminislied (region of hyporesthesia to touch) (Fig. 462 ).

For a variable distance within the 'stroking outline' the hypo-

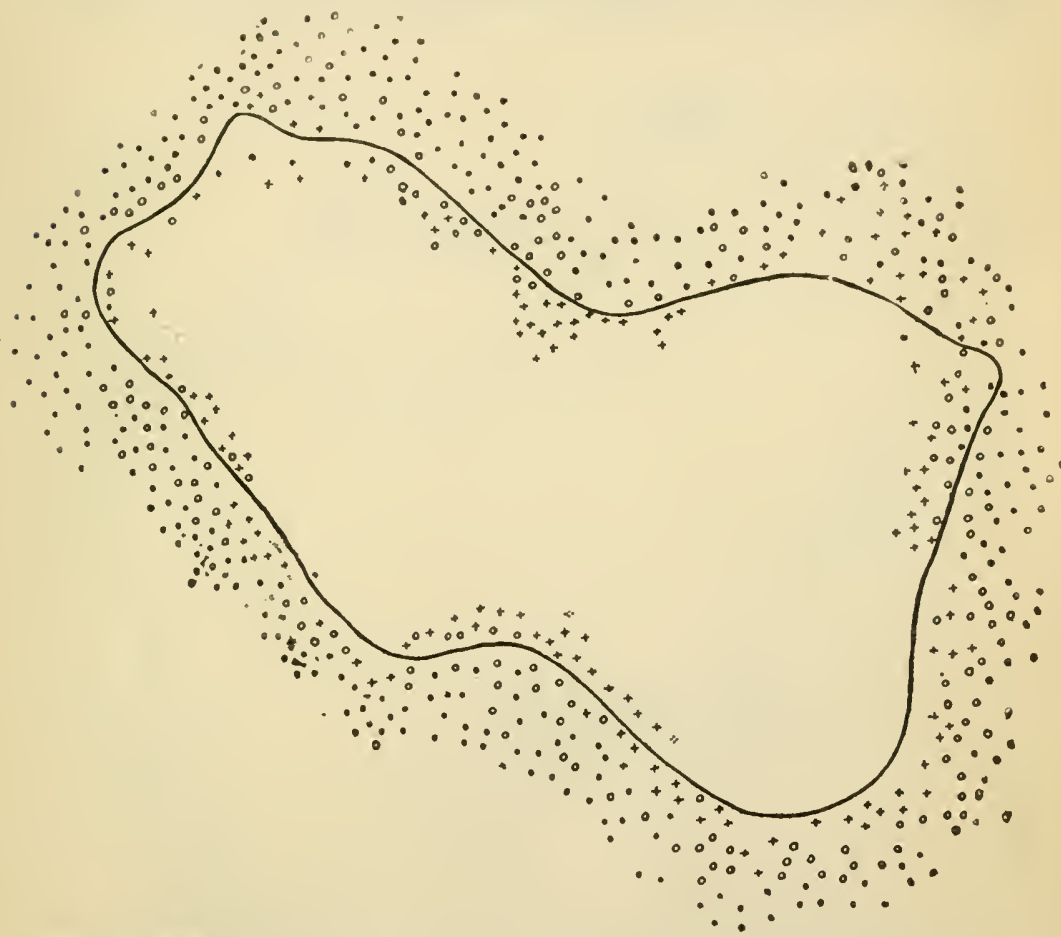

Fig. 463.-Middle Cutaneous: Left Thigh (Trottcr and Davies) (reduced by Onethird Linear). Twenty-six days after section. Results of examination with $\mathrm{v}$. Frey hairs. Touch spots marked - responded to hair of 280 milligrammes' pressure; those marked o to hair of 800 milligrammes; and those marked + to hair of 2,280 milligrammes. The continuous line marks the limit within which there was anæsthesia to the camel's-hair brush.

æsthesia for tactile stimuli is so slight that it cannot be detected with the brush or with cotton-wool, or even with the v. Frey hairs. Like those of normal skin, 90 per cent. of its hair-bulbs respond to a hair exerting a pressure of 70 milligrammes, and the remaining Io per cent. to hairs exerting a pressure of $I_{4} 0$ or 280 milligrammes. Inside this zone of minimal hyporsthesia the defect of sensibility 
rapidly increases as we pass inwards, each line of hair bulbs re. quiring a heavier pressure than the line external to it, till at last $3 \frac{1}{2}$ or 4 gramme's pressure is necded to cause a sensation of touch, and inside oi this line of hairs the skin does not respond at all (Fig. 463).

For thermal sensibility there is also a region of complete anæsthesia and a region of partial anæsthesia. The best way of out-

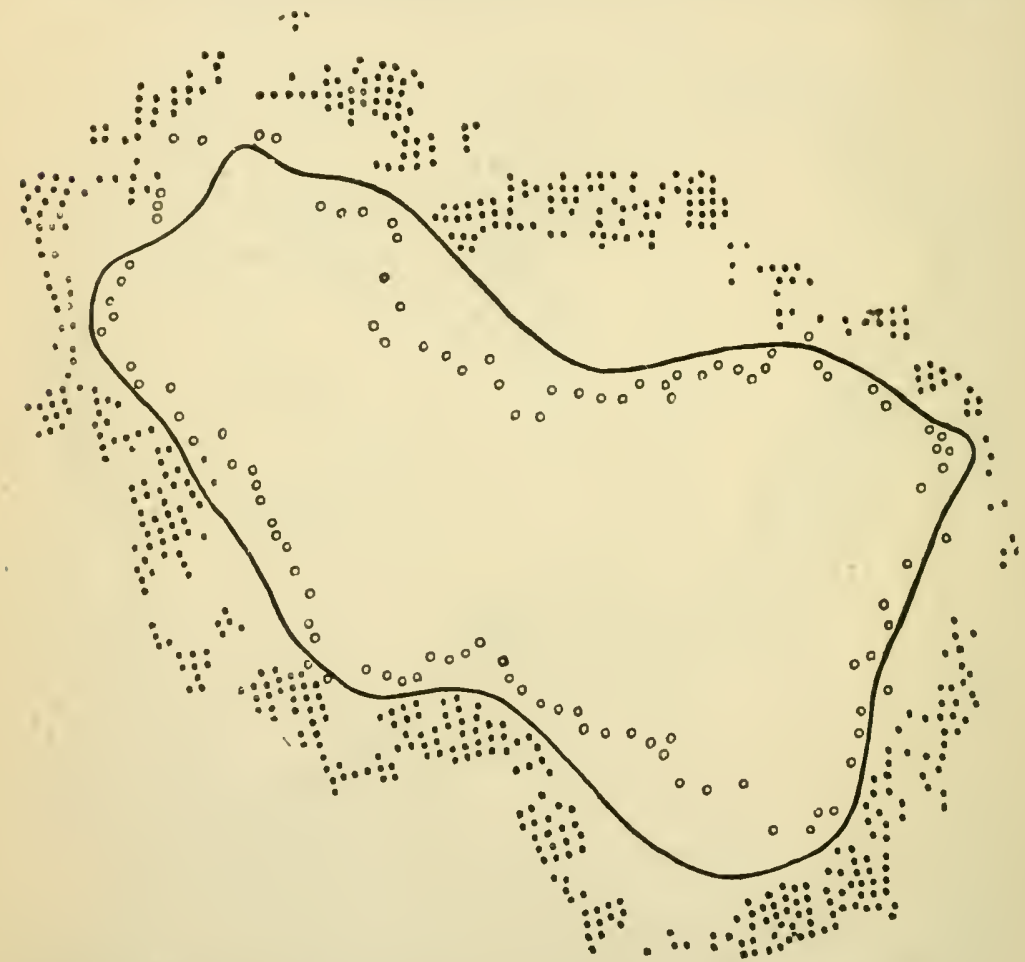

Fig. 464.-Middle Cutaneous: Left Thigh (Trotter and Davies). Twenty-one days after section. Results of examination with temperature of $0^{\circ} \mathrm{C}$. On spots marked - stimulus was felt as cold; on spots marked o it was felt as cool. The blank area is that of thermal anæsthesia. The continuous outline marks the limit within which there was anæsthesia to the camel's-hair brush.

lining these is the use of a temperature of $0^{\circ} \mathrm{C}$. as the stimulus (Fig. 464).

Outside the zone of complete thermal anzsthesia there is a region in which temperature sensations are distinctly elicited, but do not possess the normal intensity, the temperature of $0^{\circ} \mathrm{C}$., for example, being felt only as cool, and not as cold. The outer limit of this region is the line at which the temperature of $0^{\circ} \mathrm{C}$. is first felt as 
we work inwards from the normal skin to yield the sensation of cool instead of cold. Similarly, the outer limit of thermo-hypoaesthesia can be determined by using a high temperature $\left(50^{\circ} \mathrm{C}\right.$. $)$. It is the line at which the sensition of hot yielded by the normal skin gives place to the sensation of warm. The two boundaries correspond closely when allowance is made for the separate grouping of cold and warmth spots on the normal skin.

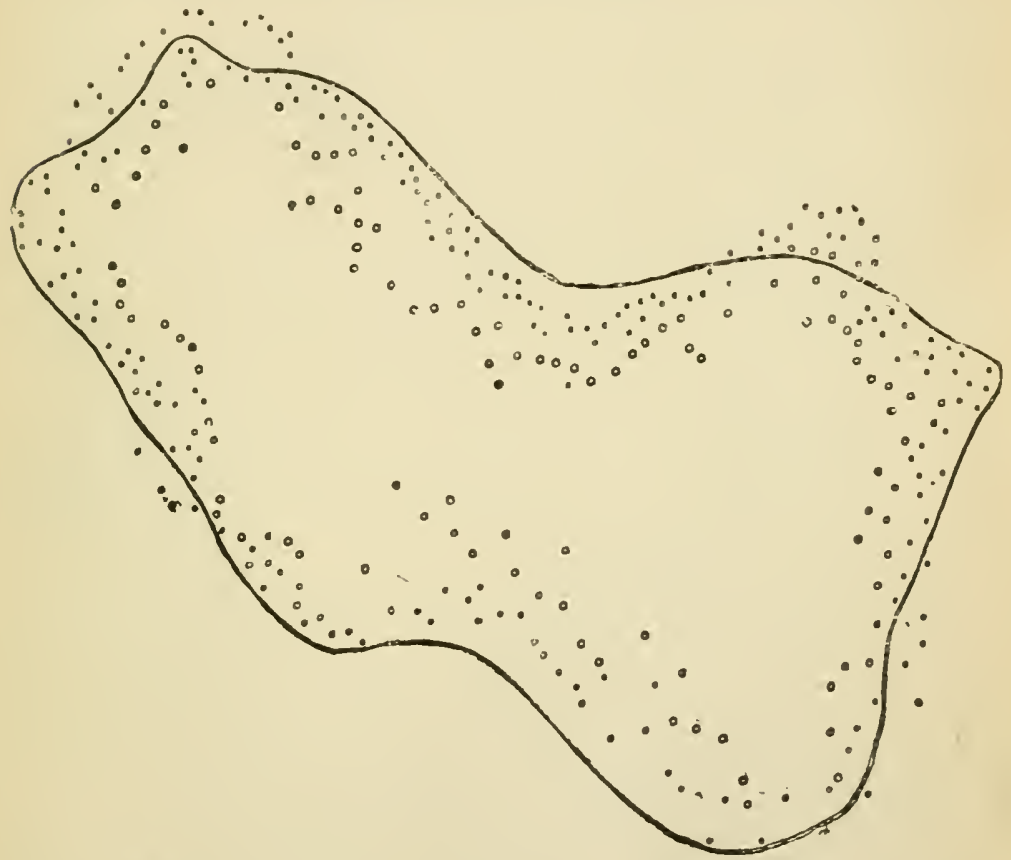

Fig. 465.-Middle Cutaneous (External Branch): Left Thigh (Trotter and Davies). Twenty-three days after section. Results of examination with algometer (aii arrangement by which a needle is pressed against the skin by a hair whose pressure value has been determined). Spots marked - reacted by sensation of pain to pressure of r,860 milligrammes (normal threshold); spots marked o re. quired 2,280 milligrammes. The continuous line marks the area within which there was anæsthesia to the camel's-hair brush.

The investigation of the sensibility of the skin areas for painful stimuli is complicated by the fact that during a certain period, from about the second to the sixth week after division of the nerve, hyperalgesia (increased sensitiveness to painful impressions) may appear. This, however, does not seem to be a consequence of any sensory loss, but ratlier a complication due to an irritative change. When this is taken account of, it is found that the defect of sensibility to pain after nerve section resembles the defects of sensi- 
bility to touch and temperature, showing a contral area of absolute anasthesia surrounded by a zone of partial loss, which is slight towards the outer boundary, but increases as we pass inwards (Fig. 405).

After section of a nerve function is recovered only as a result of regeneration. This is true of all the sensory functions of the skin and of the pilo-motor and sudo-motor functions. Vaso-motor tone in the affected area is restored much sooner than the other functions. This rapid recovery probably depends upon a local compensatory mechanism, and not upon regeneration of the vaso-notor fibres. Recovery of all the functions dependent upon regeneration begins about the same time, and this recovery progresses over the area at about the same rate for all, although the rate at which they progress towards normal acuity is different.

Sensibility to touch probably appears a little carlier than sensibility to cold and pain. Yet the recovery of touch does not progress so fast, and for a while a given zone of the recovering area remains hyporsthetic (less sensitive than normal) to touch, while to cold and pain it soon becomes even hypersensitive. The most remarkable peculiarities of a recovering area are: (I) This qualitative change, in virtue of which cold, pain, and the pain element of heat are intensified, while touch is little altered, ak hough more difficult to elicit; (2) the reference of sensations, not to the point stimulated, but to distant parts of the area.

- When a spot which has developed this peripheral reference is touched, onc of two possibilities may occur: either the touch is felt locally, and is referred as well, or nothing is felt locally, and the touch is felt in the area of peripheral reference. The region in which the referred touch is felt is always at the edge of the most peripheral part of the aniesthesia,' perliaps more than a foot away fron the spot actually touched. The peripheral reference of cold is even more striking, particularly in the remarkable intensity of the referred sensation.

Pcripheral reference occurs also with pain. "The referred pain shows three well-marked qualities: it is, proportionately to the stimulus, very intense; it does not reproduce a normal sensation with the exactitude found in the case of touch or cold, but has a special quality of strangeness and unpleasantness, such as no pinprick on normal skin can give; finally, it produces an almost irresistible desire on the part of the subject to rub or scratch the region in which it is felt.' As recovery proceeds the local sensory response becomes more distinct, and the abnormal quality of botli local and referred sensations fades. But 'while peripheral reference is the earliest phenomenon of recovery, it persists until recovery is so far advanced that hypoesthesia is scarcely detectable by any quantitative methods.' 
The work of lleat, who was the pioneer in this methol of investigation, must also be mentioned. He found that when the median nerve was divided in his own arm, recovery of sensation began with the restoration of sensibility to pain and to extreme degrees of heat and cold: but the hand still remained for a time ats insensitive as before to such stimuli as slight touch. In the parts which lawl regained their sensibility to severe stimuli, like pricking and extremes of heat and cold, the sensition radiated widely, was referred toremote parts, and could not be accurately localized. This form of semsibility Head calls protopathic. As the nerve recovered further, a second form of sensibility appeared, associated witl accurate focalization of cutaneous stimuli and discrimination of two compass points. J,ight touch and moderate degrees of heat and cold could now be again appreciated. This form of sensibility he terms epicritic. A third form of sensibility (deep sensibility) was investigated after complete division of the radial and external cutaneous nerves at the elbow. The radial half of the arm and back of the hand became totally insensitive to cutancous stimuli, but retained their sensibility to pressure or to any stimulus which deformed the subcutancous structures, as well as their power of localization of such stimuli. The afferent fibres upon which this deep sensibility depends must rum with the motor nerves. According to Head, the other two forms of sensilvility (protopathic and cpicritic) also depend on two separate systems of nerves, of which the protopathic is the older in the phylogenetic sense, and has a wider distribution. It is assumed that the protopathic fibres regenerate more easily and speedily than the epicritic or than the motor nerves of voluntary muscle. The protopathic fibres are supposed by Head to exert a trophic influence. A part deprived of its nerve-supply is liable to injuries, and the sores so produced heal slowly. But as soon as 'protopathic' sensibility returns to the part, they heal rapidly, cven in the absence of all epicritic sensation. The intestine is described as possessing 'protopathic,' but not 'epicritic,' sensibility-i.e., it reacts to extremes of heat and cold, but not to moderate heat and cold or light touch.

Quite recently an elaborate study of the loss and return of the shin sensations after section of a cutaneous nerve has been made by Boring. His work is distinguished from that of all previous observers by the fact that he brought to his task the training of a professional psychologist, and that he studied in detail for fifteen months the area of skin with whose innervation he intended to interfere. Section of the nerve chosen (the anterior branch of the internal cutaneous nerve in the forearm) caused anresthesia and hypoasthesia of a relatively small area of skin (on the volar aspect of the forearm near the wrist), so that it was possible to make an intensive study of $i t$, and the observations were continued for more than I,000 days after the operation. Points on the affected area were identified by reference to a series of points tattooed on the skin represented by crosses in Fig. 466.

The sensations studied, in addition to those of warmth and cold, were the four qualities which appear upon mechanical stimulation of the skin: Contact, cutaneous pressure, subcutaneous or deep pressure and pain. Boring uses the terms 'tickle-contact,' 'penetrating pressure,' ' dull pressure,' and 'sharpness ' for these quali- 
ties. The localization of the sensations and the power of discriminating two points were also investigated.

The general tendency during recovery was from ancesthesia or hi poiesthesia through decreasing degrees of hyporest hesia to normal. IV armth and cold, however, passed from hypoesthesia through a stage of hyperiestlesia on their way to normal. Pain, pressure, and cold approaclied normality at about the same rate, but in comparison with these the return of warmth sensation was much delayed

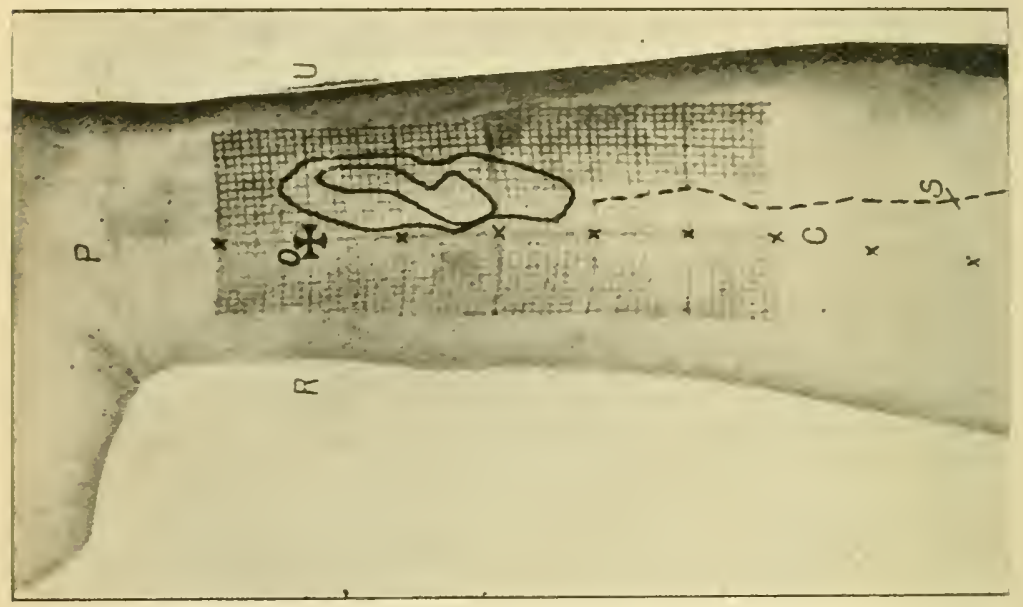

Fig. 466. - Volar Aspect of Left Forearm, showing Affected Region in Outline. The larger area was that marked off by the suliject with the camel's hair brush as insensitive. The inclosed smaller area is that which was marked off when the experimenter manipulated the brush, and the subject with closed eyes reported when he felt anything at all. This smaller area was taken as the region of greatest change in sensitivity, and the experiments on lucalization and the discrimination of two points were mainly made within this area. The dotted line shows the approximate course of the nerve, divided at $S$. The horizontal and vertical lines dividing the area into small squares were impressed by a rubber stamp on the skin, to facilitate identification of points. The pusition of each point stimulated was fixed with reference to these rectilinear co-ordinates, the tattooed point represented by the Maltese cross being taken as the origin, and the distances in $\mathrm{mm}$. nneasured in the central (C), peripheral $(P)$, radial $(R)$, and ulnar (U) directions (Boring).

For all four the distribution of sensitivity over the skin was irregular and patchy, and no definite boundaries could be drawn. In general, however, immediately after disision of the nerve the central zone of the affected area was practi ally anæsthetic as regards cutaneous sensation. This was surrounded by a zone of decreased sensitivity. In the return of sensibility the outer zone preceded the inner. No new modes or qualities of sensation were observed at any time, although ' a number of unusual sensory complexes, which might be described by an untrained observer as new sensations, were noted. 
The most striking single experience was the intense algesic cold, which occurred when the skin was hyperæsthetic to cold.' Deep sensibility to pressure and pain was not altered. Localization of pressures (of $20 \mathrm{gm}$.) and discrimination of two pressures (twopoint discrimination) remained unaffected.

This study on the whole confirms that of Trotter and Davies. However, the larger outer or third area defined by the so-called 'stroking outline' of 'Trotter and Davies is probably not an area of sensory abuormality at all, but merely an area in which a physical change in the skin, due, e.g., to some interference with the action of the sweat glands, is appreciated by the stroking finger. This follows from the fact that it can be mapped out approximately by an observer who strokes it with his own finger without asking the subject to report his sensations. The results of Boring's investigation are quite opposed to the most essential of Head's conclusions. No evidence was found in favour of Head's distinction between epicritic and protopathic sensibility, and weighty evidence against it. There does not seem to be any real necessity in the observed facts for introducing so revolutionary a conception of the nervous system. Nor is it possible to uphold the distinction in any thoroughgoing fashion for all structures. For instance, in abdominal operations performed under local anæesthesia it has been seen that the parietal peritoneum is quite insensitive to touch, pressure, and temperature stimuli, including extreme temperatures (Ramström), while pain is caused by traction on it. Its sensibility is therefore neither purely epicritic nor purely protopathic in Head's sense. In like manner the mucous membrane of the mouth, in which sensibility only to touch and temperature is present, conforms entirely to neither type. Its sensibility is not alone epicritic, since it responds to extreme temperatures, nor is it purely protopathic, since a pin-prick produces no painful sensation. These terms and the theory associated with them should be dropped.

Localization of Cutaneous Sensations.- We not only perceive the quality and estimate the intensity of sensations of touch, warmth, cold pain, etc., but are able, more or less accurately, to localize the part of the body from which the sensory impressions come. In other words, two impressions from different parts of the body, although identical in quality and intensity, are nevertheless stamped with a distinctive sonething, which may be called the local sign. This power of localization is not equal for all portions of the body nor for all kinds of sensations. It is best developed for touch (in the restricted sense), and all the varieties of common sensation are better localized on the skin than in any of the deeper structures. The precise mechanism of the localization is unknown. But we must suppose that each peripheral area is 'represented ' in the brain, so that the arrival of afferent impulses from it affects particularly the related cerebral area. The brain, therefore, so to speak, associates excitation of a given cerebral area with stimulation of the corresponding peripheral area, and thus not only recognizes the quality and quantity of the resultant sensation, but also localizes it; just as a waiter who watches the bell-indicator not only learns how a bell has been rung, whether once or twice, peremptorily or languidly, but also in which room it has been rung. If, to pursue the illustration a little farther, he is aware that two rooms are connected with one bell, but that one of the rooms is scarcely ever occupied, he associates the ringing of the bell with a summons from the other room even when it happens to be rung from the usually vacant room. In like manner 
the brain secms to connect the arrival of scneory impulses from the internal organs, which have few sensory fibres, and these perhaps not often stimulated, with excitation in a related cutanesus regiun, from which it is constantly receiving sensory impressions. The fact already mentioned $(p, 8,2)$, that in disease of ititernal organs the pain is re. terred to some portion ol the skiti, mily le thus explamed.

An attempt has been mate to explatin certain illusions of touch on the theory that just as an olject is recognized as single by the eye when its images fall on corresponding prints of the two retina (p. I037). so all object is recognized as single by tle fingers when it comes into contact with eorresponding points or rather areas of the skin. These are the areas which experience has taught us are in contact with an wbject when it is held in the natural way. When now a single olject is made, by placing the fingers in an unnatural position, to touch areas which conde ordinarily be touched at the same time only by two (1) by threc objects, we experience the sensation of contact with two or with three objects.

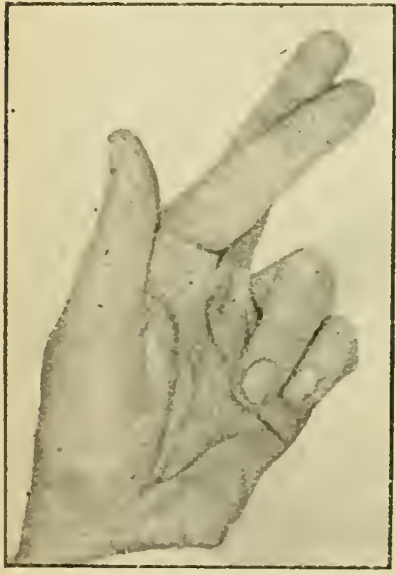

liig. 467.--Illusion of Touch of Aristotle. A small roject placed between the index and middle fingers, crossed as shown, is felt as two objects (Wassenaar).

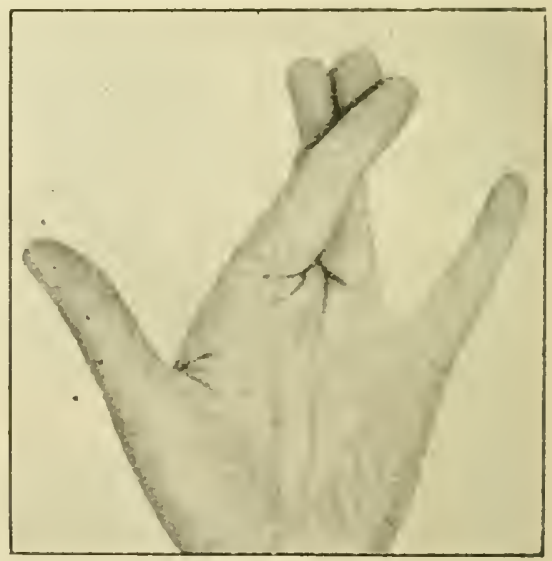

Fig. $468 .-$ An Olject placed in crittact witl the Index, Midclle and Ring Fingers, crossed as shown, is felt as three objects (II:assenar).

It is through the localization of touch sensations that the size and form of objects in contact with the skin are perceived in the absence of other than the cutancous sensations, and especially in the absence of risual and muscular sensations (stereognosis).

Muscular Sensations (Muscular Sense), etc.-Sometimes, although rather loosely, grouped together as muscular sensations, are a number of forms of sensation of which our linowledge is much less accurate than it is in the case of the fundamental skin sensations. Among these may be mentioned especially (1) the sensations by which the position in space of the body as a whole or of particular parts is recognized in the absence of visual sensations; (2) the sensations associated with movements, passive as well as active: (3) the scnsations associated 
with resistance to movement. In none of these groups are we dealing with purely muculat semsitions; cutaneous tactile sensations and pressure sensations clicited from other structures than muscles are also involved.

Voluntary mosendar movements are accompanied with a peculiar sensation of effort, graduated accorling to the strength of the contraction, and affording datis from which a julgment as to its amonnt and direction may be formed.

It has been shown, however, that when pressure sensations are eliminated or reduced to a minimum by enclosing the arm, held horizontally, in a rigid apparatus suc'? as that shown in lig. fog, the moments of rotation of a weight* fastened at different distances from the shoulder can be discriminated with great exactness. The sensation of effort is therefore an independent sensation (v. Frey).
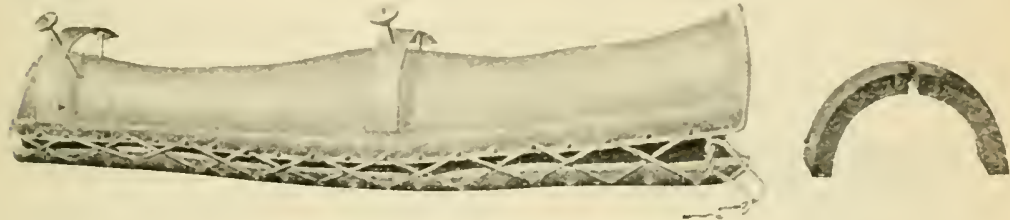

Fig. 469.-Apparatus for Arm for Testing Muscle Sense. At the right is shown a lead weight which can be placed on either of the hoops of the apparatus (v. Frey).

Some writers have supposed that this so-called muscular sense does not depend upon afferent impulses at all, but that the nervous centres from which the voluntary impulses depart take cognizance, retain a record, so to speak, of the quantity of outgoing nervous force; that the cffort which wc feel in lifting a heavy weight is an effort of the cells of the motor centres from which the groups of muscles are innervated, and not of the muscles themselves.

But although this fceling of central effort or outflow (we can hardly say of central fatiguc) may be a factor, it cannot be doubted that the brain is kept in touch with the contracting muscle by impulses of various kinds which reach it by different afferent channels.

The corpuscles of Pacini, which exist in considerable numbers in the neiglibourhood of joints and ligaments, and in the periosteum of bones,

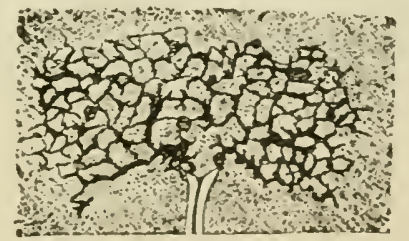

Fig. 470.-Nerve-Ending in Tendon near the Insertion of the Muscular Fibres (Golgi).

would scem well fitted to play the part of end-organs for the tactile sensations caused by the movements of flexion, extension, or rotation of one bone on another, which form so large a portion of all voluntary

* With the arm horizontal the moment is the product of the weight by its distance from the shoulder joint (see p. 749 ). 
muscular movements. And it has been stated that paralysis of these bodies in the limbs of a cat by section of the nerves going to them causes a characteristic uncertainty of movenent which suggests that something necessary to normal co-ordination has been taken away. Tendons also possess afferent nerve-fibres, which terminate by breaking up into reticulated end-plates (Fig. 470). We have already seen that the skeletal muscles possess numerous afferent fibres (p. 94I). Some of these must be nerves of ordinary sensation. For, although when a muscle is laid ba , in man and stimulated electrically, the sensation does not in general amount to actual pain, it is capable, under the influence of strong stimuli, of taking on a painful character. And nobody who has felt the severe and sometimes almost intolerable pain of muscular cramp would be likely to deny the existence of sensory muscular nerves. But after deducting these, we must assume that a

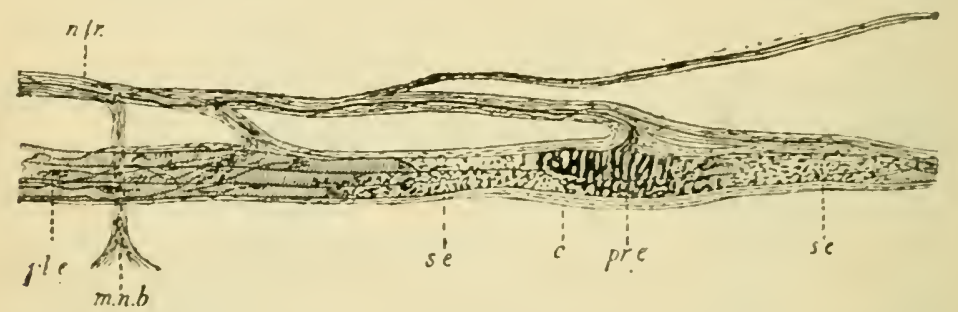

Fig. 448.-Muscle Spindle (after Ruffini). c, sneath of the spindle; n.tr., trunk of nerve, which sends fibres through the sheath into the spindle, where they form endings (pr.e., s.e., pl.e.) of various kinds; m.n.b.. bundle of motor fibres.

large proportion of the afferent nerves of muscle have other functinns, and among them may be the conveyance of impulses connected with the muscular sense. The muscle-spindles or neuro-muscular spindles (Fig. 47 I), peculiar structures which occur in large number in most of the skeletal muscles, and have been carefully studied by Huber, Sihler, Ruffini, and other observers, are the terminations of many of the semsory fibres. They are long narrow bodies, with a thick sheath of connective tissue cnclosing fine striped muscular fibres. Medullated nerve-fibres enter the spindle, and there, dividing into branches and losing their medullary sheath, form endings of various linds around and betwcen the muscular fibres. It is possible that in contraction of the muscles the nerve-fibres in the spindies are compressed. and thus mechanically stimulated.

In the spinal cord these impulses are conducted up through the posterior column; and, although less is known as to the paths they follow in the higher parts of the central nervous system, it is certain that there is some afferent bond of connection between the cortical motor areas and the muscles which they control (p. 963).

Tactile sensations set up in the slin or mucous membrane lying over contracting muscles may also help the nervous motor mechanism in appreciating and regulating the amount of contraction; but the fact that, in anxesthesia of the mucous membrane covering the vocal cords produced by cocaine, the voice is not at all impaired, shows that muscular contractions of extreme nicety can be carried on without any such aid.

Sensations of Hunger and Thirst.-These are representatives of the group of interior sensations. As Tiedemann pointed out long 
ago, at least two elements are involved in the somewhat vague sensation of hunger: the: local sensation of emptiness in the stomach, and the general sensations of malaise, depression, and weakness. There is some evidence that the general sensations are-in part at, least-dependent upon the state of the stomach. But it would appear that - at any rate, during prolonged deprivation of food-a general condition of the tissues may exist which can arouse in consciousness the sensation of hunger, even after the stomach has been amply filled. Thus a patient with a fistula in the upper part of the small intestine constantly suffered from hunger in spite of the enormous quantities of food consumed. The stomach always felt full, but as most of the food escaped from the fistula, the tissues continued to be starved, and the general sensation of hunger remained (Hertz). In diabetes the same thing may be observed. On the other hand, it was noted by Carlson and one of his pupils that after a fast of five days practically all of the mental depression and some of the feeling of weakness disappeared during the first meal. He therefore concluded that the depression of the central nervous system was essentially a reflex condition, depending probably on afferent impulses from the digestive tract, rather than a result of deficiency of nutrient material in the blood. Complete recovery from the bodily weakness, however, did not take place till the second or third day after breaking the fast.

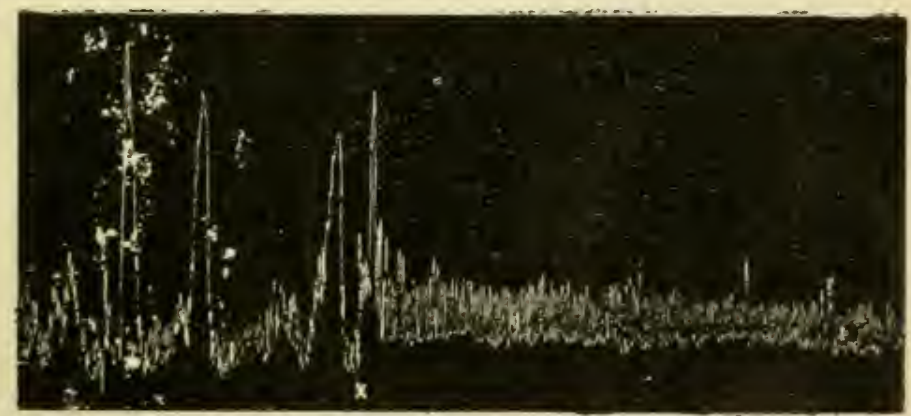

Fig. 472.-Commencement of Gastric Hunger Contractions (the Large Elevations in a Man. At $x$ she belt was tightened and the hunger contractions inhibited. To be read from left to right (Carlson and Lewis).

An important factor in the local sensations associated with hunger is the strong periodical contractions of the empty stomach, which have been shown to coincide with the hunger pains (Cannon and Washburn).

Carlson was able in observations on a man with a permanent gastric fistula to confirm this coincidence Even when the empty stomaclı was artificially caused to contract by distending it with a 
balloon, the man experienced a typical humger pain. During his own five dilys' fast Carlson recorded these contractions by means of a small balloon attached to a rubber tube, which was swallowed and allowed to remain in the stomach. The tube was connected to a recording apparatus. It was found possible to go to slecp with the balloon in the stomach, and to obtain a record all thrungl

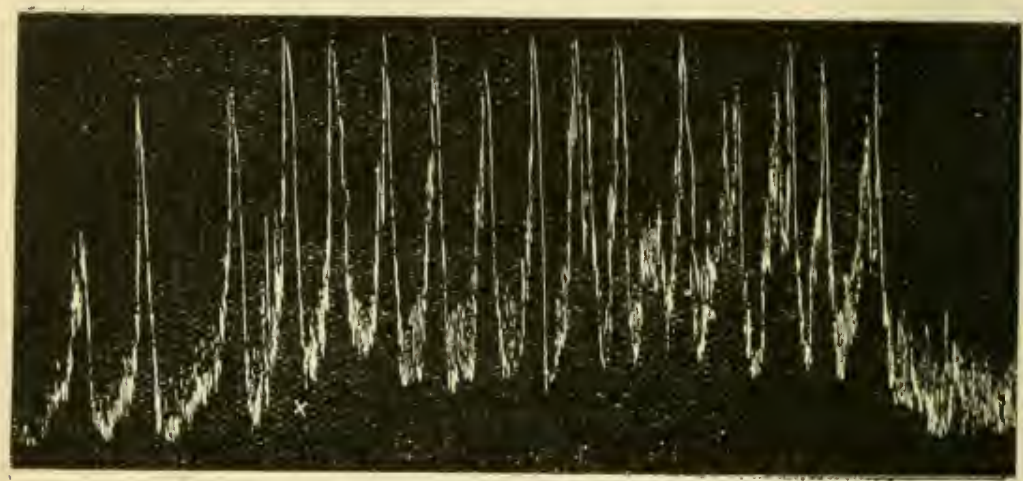

Fig. 473.-Gastric Hunger Contractions in a Man at a More Advanced and Intense Stage than in Fig. 472 . Tightening of the belt at $x$ did not stop the ron. tractions which ran the usual course to their termination. To be read fruml left to right (Carlson and Lewis).

the night. After the first day of starvation the hunger sensation referred to the epigastrium was almost continuous, and did not wholly disappear during the intervals between the periods of vigorous gastric contractions. This feeble continuous hunger sensation was obviously associated with the increased tonus and the more or less continuous, although weak, rhythmical contractions that correspond to the periods of relative quiescence of the empty stomach during prolonged starvation. The precise manner in which the hunger contractions of the stomach arouse the pangs or pains of hunger remains in doubt. Since the sensation has a specific character, it is to be supposed that it is subserved by a special sensory apparat us with receptors in the stomach. The vagi do not seem to be concerned. But the gastric contractions during digestion of a meal notoriously do not cause such sensations, and therefore it has been suggested that the nervous mechanism associated with the local sensation of hunger becomes more and more excitable in the absence of food, until at last the threshold is reached at which the stimulus connected with the hunger contractions becomes effective. It comes to the same thing to say that the presence of food in some way inhibits the discharge which leads to the sensation. This, 
howerer, is only another way of saying that the true explanation is still to seek.

Carlson was unable to confirm the common statement that hunger disappears after the third day of starvation, although there was certainly some decrease in the sensation of hunger, and especially in appetite, on the fourth and fifth days. As has been often shown, the deprivation of food for long periods, or even till death, when water is allowed, is not associated with acute suffering.

Appetite is distinguished from liunger by those observers who have studied the question most precisely, but of the physiological basis of the sensations that constitute appetite we know even less than we do of the physiological basis of hunger. The taking of food blunts the appetite, as it stills hunger. Fasting evokes both. Yet during a prolonged fast, appetite, the desire for food and the pleasure in the thought or at the sight of it, may disappear, or be much lessened, while the hunger pangs are still sharp. The smell and taste of agreeable food and the mental representations of these sensations are elements in appetite, and even the associations connected with the time and place of a customary meal and with those who share it. But there is a gastric element as well: the mere filling of the stomach apart from the passage of nutrient material into the blood helps to satisfy the appetite; the emptying of the stomach in the course of digestion seems of itself to take a part in restoring the appetite for the next meal. To what extent, if at all, the gastric element in the sensation of appetite is dependent upon the same meclianism as the gastric element in hunger is unknown.

Some have supposed that the same stimulation which, when its intensity is sufficiently increased, causes gastric hunger pains, causes in smaller intensity a milder hunger sensation, which is the gastric factor in appetite. According to Carlson, a factor in appetite is the memory process of removal of hunger pangs by feeding, and he assumes that the revival of such memories in consciousness depends upon the condition of the alimentary canal, and is inhibited when the stream of afferent impulses from the viscera is altered by changes in the motility or ecretory activity of the gastro-intestinal tract. He believes that the secretion of the so-called appetite gastric juice, in man at least, although clearly demonstrated on a case of gastric fistula during mastication of palatable food, does not possess the great importance attributed to it by Pawlow (p. $\left.4^{\circ} 4\right)$, since normally there is a continuous secretion of gastric juice in the absence of food in the stomach and of psychical stimulation, and this is sufficient to initiate gastric digestion, and therefore to insure a sufficient gastric secretion. The vagi do not seem to contain fibres concerned in the sensations of hunger or appetite. After section of these nerves, dogs, when they survive some time, eat ravenously, although the food is often regurgitated.

Thirst.-This is a sensation, referred chiefly to the pharynx, and certain of the sensory nerve fibres of this region, supplied by the 
glosso-pharyngeal nerve, may be assumed to be specifically related to it. Conder ordinary conditions the sensation is elicited through the afferent nerves of the pharynx when the mucous membrane becomes dry, as when dry or salt food is eaten, or dry and dusty air inhaled, and local moistening of the area in question gives temporary relief, even when no water is swallowed. When water is long withheld, the water-content of all the tissues sinks, and a more intense and distressing thirst, which cannot be allayed in any way except by the ingestion of water, ensues. Probably in this case afferent impulses originating in many organs, and conditioned in some way by the abnormally low water-content of the blood and tissues, as well as a more direct action of the loss of water upon the (unknown) centre in which the sensation is represented, are responsible.

Relation of Stimulus to Sensation.-It is impossible to measure sensation in terms of stimulus. All that we can do is to compare differences in the intensity of stimuli and differences in the resultant sensations, or, in other words, to compare stimuli together and to compare sensations together. And when we determine the amount by which a given stimulus must be increased or diminished in order that there may be a just perceptible increase or diminution in the sensation, it is found that (with certain limitations) the two are connected by a simple law: Whatever the absolute strength of a stimulus of given kind inay be, it must be increased by the same fraction of its amount in order that a difference in the sensation may be perceived (sometimes called IVeber's law). Thus, a light of the strength of one standard candle must be increased by 160 th candle, a light of 10 candles by 100 , and in light of 100 canclles by a candle, in order that the eye may perceive that an increase has taken place, just as the weight necessary to turn a balance increases with the amount already in the pans. The fraction varies for the different senses. It is about $\frac{1}{10 \pi}$ for light, $\frac{1}{3}$ for sound. But it would appear that Weber's law does not hold for the pressure sense, nor for the other senses above and below certain limits. liecliner, making various assumptions, has thrown Weber's law into the form $y=k \frac{\log x}{x_{0}}$, where $y$ is the intensity of sensation, $x$ the intensity of stimulation, $x_{0}$ the smallest intensity of stimulus which can be perceived (liminal intensity), and $k$, a constant. This so-called psycho-physical law of Fechner states that the sensation varies as the logarithm of the stimulus. But Fechner's law has been subjected to serious criticism. and the subject cannot be further pursued here. 
PRACTICAL IEXERCISES ON CHAPTER XVIII.

VISION.

1. Dissection of the Eye.-'l he student may profitably refresh his memory on the anatomy of the eye by dissecting a fresli eye - that of a large animal like an ox is preferable, but the eye of a shecp or dog may also be used. The eyc is removed from the orbit by cutting through the conjunctiva where it is reflected on to the eyelids, carefully severing the extrinsic muscles and scooping the eycball out of the mass of loose connective tissue and fat in which it is embedded, and which serves as a cuslion to protect it from injury during its movements. Observe the transparent cornea in front, blending at its posterior border with the opaque sclerotic, which is covered by a layer of conjunctiva reflected from the lids. On clearing the fat cautiously away, the tendinous insertions of the external or extrinsic muscles of the eycball into the anterior part of the sclerotic will be seen. Identify the various muscles (p. Io63).

lumerse the eye in water in a small glass dish, with the cornea uppermost. The interior can now be seen, because the refractive index of the cornea being nearly the same as that of water, the light is only very slightly refracted there. The same effect is produced when a cover-slip is placed over the cornea in the air; a plane surface being substituted for the curved anterior surface of the cornea, its refraction is abolished. Observe in the fundus of the eye the optic disc, eccentrically placed in the retina, and the retinal vessels radiating out from it. A portion of the fundus shows brilliant iridescent colours in many animals (the tapetum lucidum). This portion is abruptly bounded by a line a little above the optic disc. The appearance is due to a peculiar arrangement of the connective-tissue (including elastic) fibres in this part of the choroid.

Pinch up with forceps a small portion of the sclerotic a little posterior to its junction with the cornea, and clip it away with fine, bluntpointed scissors, being careful not to penetrate the choroid layer, which lies immediately beneath the sclerotic. Extend the incision through the sclerotic backwards, and then transversely, and peel off strips of the sclerotic from behind forwards. The lower surface of the sclerotic (the so-called lamina fusca) is dark, owing to the presence in it of the same pigment which is so abundant in the choroid coat. Go on removing the sclerotic piecemeal until a considerable area of the dark choroid layer is exposed with the ciliary nerves passing forward on its surface towards the iris. One or other of the long ciliary arteries may also be seen coursing between the sclerotic and choroid if the sclerotic happens to have been removed at its position. On the anterior part of the choroid may be observed some pale fibres passing backwards from the corneo-sclerotic junction. They are the meridional fibres of the ciliary muscle (p. 1022).

The eye being immersed in water, remove cautiously with the forceps and scissors the portion of the choroid exposed. The retina is now seen as a pale membrane, transparent when quite fresh, but becoming whitish soon after death. Cut through sclerotic, choroid, and retina about halfway round the eyeball, a little posterior to the corneo-sclerotic junction. The vitreous humour will bulge out. Since its refractive index is nearly the same as that of water, it is scarcely observed when immersed, and the interior of the eye can be easily seen through it.

The optic disc can now be again studied, with the stump of the optic 
nerve entering it and the retinal vessels piercing the disc. In the centre of the retina is the yellow spot.

In the anterior portion of the eycball note the crystalline lens, and at its circumference the radiating folds of the choroid called the ciliary processes. Closely covering the ciliary processes, the anterior border of the retina forms the ora serrata, a plaited arrangement like an oldtime ruff.

Now complete the separation of the anterior and posterior portions of the cycball. Remove the vitreous humour, noting that it is attached to the ciliary processes and the posterior surface of the capsule of the lens by its enveloping membrane, the hyaloid membrane. With scissors snip through the corneo-sclerotic junction at one point down to the border of the lens, and observe the suspensory ligament passing from the ciliary body chiefly towards the anterior surface of the lens, where it blends with the lens capsule. Open the anterior chamber of the eye by an incision through the cornea in front of its junction with the sclerotic. It is filled with the clear, watery, aqueous humour. Note the pigmented iris projecting in front of the lens.

Remove the sclerotic and cornea for some distance along their line of junction, using gentle pressure with the edge of a fine knife to separate the junction from the attached border of the iris. The ciliary muscle, forming a pale, narrow ring around the eye at the corneo-sclerotic junction will be thus exposed. Its external surface is closely adherent to the sclerotic, and its internal blends with the ciliary body. The circumference of the iris is attached at its anterior border. Posteriorly it passes into the choroid.

Take out the lens and observe the curvature of its anterior and posterior surfaces. Determine which has the greater curvature. In the excised eye the lens will, of course, be in the condition of relaxed accommodation.

2. Formation of Inverted Image on the Retina.-Fix the eye of an ox or of a dog or rabbit, after careful removal of part of the posterior surface of the sclerotic, in one end of a blackened tube, with the cornea in front. A tube made by rolling up a piece of thick brown paper will do. Place a candle in front of the eye. Look through the other end of the tube, and obscrve the inverted image of the candle formed on the retina. Nove the candle until the image is as sharp as possible. Now bring between the candle and the eye a concave lens. The image becomes blurred, the candle must be put farther away to render it distinct, and perhaps no position of the candle can be found which will give a sharp image. If the lens is convex, the candle must be brought nearer, and a sharp image can always be formed by lringing it near enough. If both a convex and a concave glass be placed in front of the eye, they will partially or wholly neutralize each other. Instcad of the candle a window may be looked at. If the eye of an albino rabbit can be obtained, it is not necessary to remove a part of the sclerotic.

3. Helmholtz's Phakoscope (Fig. 474).-This instrument is cmployed in studying the changes that take place in the curvature of the lens during accommodation. It is to be used in a dark room. A candle is placed in front of the two prisms $\mathrm{P}, \mathrm{P}^{\prime}$. The observer looks through the hole $B$; the observed eye is placed at a hole opposite the hole $\mathrm{A}$. The candle or the observed eye is moved till the observer sees three pairs of images, one pair, the brightest of all, reflected from the anterior surface of the cornea; another, the largest of the tluree, but dim, reflected from the anterior surface of the lens; and a third pair, the smallest of all, reflected from the posterior surface of the lens (Fig. $4^{2} \mathbf{I}$. 
p. 1021). The last two pairs can, of course, only be scen within tlie pupil The observed eye is now focussed first for a distant object (it is cnough that the person should simply leave his eye at rest, or imagine he is looking far away), and then for a near object (an ivory pin at A). During accommodation for a near ohject no change takes place in the size, brightness, or position of the first or third pair of images; therefore the cornea and the posterior surface of the lens are not altered. The midlde images become smaller, somewhat brighter, approacl each other, and also come nearer to the corneal images. This proves $(a)$ that the anterior surface of the lens undergoes a change; $(b)$ that the cliange is increase of curvature (diminution of the radius of curvature), for the virtual image reflected from a convex mirror is smaller the smaller is its radius of curvature. (The third pair of images really

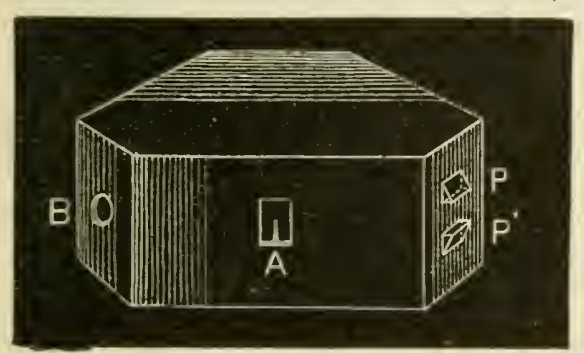

Fig. 474.-Phakoscope. undergo a slight change, such as would be caused by a small increase in the currature of the posterior surface of the lens ; but the student need not attempt to make this out.)

4. Scheiner's Experiment. Tro small holes are pricked with a needle in a card, the distance between them being less than the diameter of the pupil. The card is nailed on a wooden holder, and a needle stuck into a picce of wood is looked at with one eye through the holes. When the eye is accommodated for the needle, it appears single; when it is accommodated for a more distant object, or not accommodated at all, the needle appears double. The two images approach each other when the needle is moved away from the eye, and separate out from each other when it is moved towards the eye. When the eye is accommodated for a point nearer than the needle, the image is also double; the images approach each other when the needle is brought closer to the eje, and move away from each other when it is moved away from the eye. If while the needle is in focus one of the holes be stopped by the finger, the image is not affected. When the eye is focussed for a greater distance than that of the needle, stopping one of the holes causes the image on the other side of the field of vision to disappear; if the eye is focussed for a smaller distance, the image on the same side as the blocked hole disappears (Fig. 475). To determine the near-point of distinct vision (p. 1029) the card may be mounted vertically on a cork, and this fastened by a rubber band to the end of a foot-rule. Nove a needle, also inserted vertically into a cork, along the rule, beginning at the end farthest from the eye, until with the strongest effort of accommodation it is seen double. Then push it back slightly to the point at which, again with maximum accommodation, it is just scen single. Repeat the measurement with a needle mounted horizontally. If regular astigmatism is present, the distances will not be the same. Most eyes have slight regular astigmatism.

In myopic persons the far-point of distinct vision can also be determined by Schciner's experiment. The needle being left on a shelf at the level of the eye, the person walks away from it backwards, regarding it all the time through the perforated card, till it is no longer seen single. 
5. Kuhne's Artificial Eye.-This is an elongated box provided with it glass leys to represent the crystalline, and a ground-glass plate to represent the retina. The hox is filled with water to which a little cosin has been added. The water must be perfectly clear. If the tap-water is turbid it should be filtered or allowed to settle, or distilled water should be used. A beam of sunlight or electric light, or. in case these are not available, a beam from an oil stercopticon, is made to pass through the box. Many of the facts of vision can be illustrated by means of this picce of apparatus. The modification of it introduced by Lyon is very convenient.

(a) Let the rays of light pass through an arrow-shaped slit in a piece of cardboard. An inverted image of the arrow is formed on the retina. Move the retina ncarer to or farther from the lens to malic the image sharp. In the eye of man and of most animals, accommolation is not brouglit about by a change in the distance of retina and lens, but by a change of curvature in the lens.

(b) Remove the lens. The focus is now far behind the retina. This illustrates the state of matters after the lens has been removed for cataract. The arrow can again be sharply focussed on the retina by putting a convex lens in front of the artificial eye. But this must be much weaker than the lens which has been renoved, for if the latter be placed in front of the eye, the image is formed a little behind the cornea.

(c) Replace the lens. Nove the retina so far back that the image is focussed in front of it. This is the condition in the myopic eye. Put a

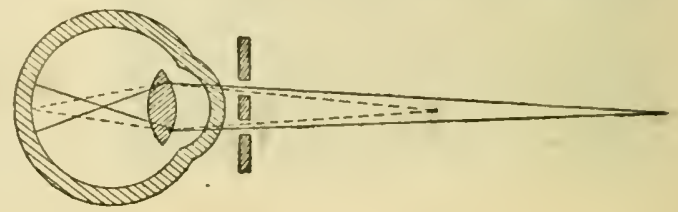
weal concave lens in

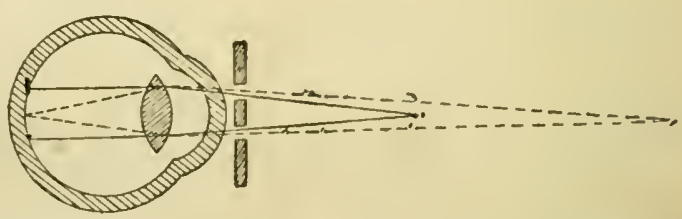

Fig. 475.-Scheiner's Experiment. In the lower figure the eye is focussed for a point farther away than the needle, in the upper for a nearer point. The con. tinuous lines represent rays from the needle, the inter. rupted lines rays from the point in focus.

front of the cye; the image now falls more nearly on the retina. Move the retina forward so that the focus is behind it. This corresponds to the hypermetropic eye. Put a weak convex lens in front of the eye to correct the defect.

(d) Observe that a plate with a hole in it, placed in front of the eye, renders an indistinctly focussed image somewhat sharper by cutting off the more divergent peripheral rays.

(e) Fill with water the chamber in front of the curved glass that represents the cornea. The focus is now behind the back of the eye altogether. Refraction by the cornea is liere abolished, as is the case in vision under water. An additional lens inside the eye, or a weaker one in front of it, corrects the defect. Fishes have a much more nearly spherical lens than land animals, and a flat cornea.

(f) Fill the hollow cylindrical lens with water, and place it in front of the artificial eye. The eye is now astigmatic. A point of light is focussed on the retina, not as a point, but as a line. The vertical and 
horizontal limbs of a cross cut ont of a piece of cardloard and placed in the path of the benus of light cannot be hoth focussed at the same time.

6. Astigmatism (Regular).-(1) Look at a fignre showing a number of lines radiating horizuntally, vertically, and in intermediate directions from a common centre. First fix the figure at such a distance that one can comfortally accommoditc. If astigmatism is present, all the lines cannot be seen with egual distinctness at the same time, but they can

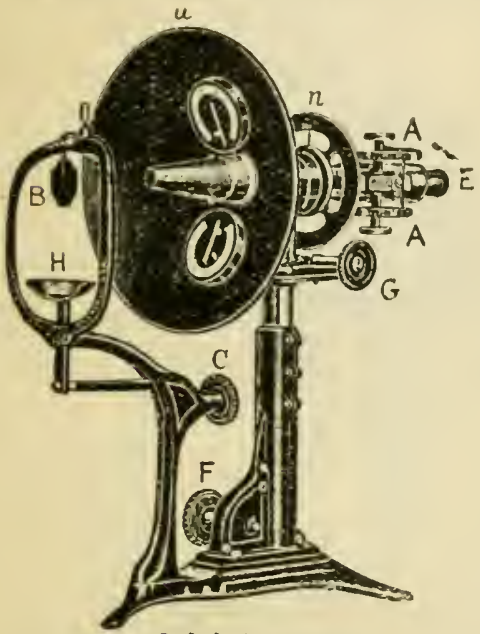

Fig. 476.-Ophthalmometer, as seen from behind the Patient. B, blind for covering the eye not being ex. amined; $\mathrm{H}$, chin-rest; $\mathrm{A}, \mathrm{A}$, graduated discs on which radii of curva. ture of the cornea in various merldians are read off or their equivalent in diopters; E, eye-piece of telescope; $\mathrm{C}$, milled head for raising and lower. ing chin-rest; $F$, milled head for adjusting height of the ophthalmoineter, and $\mathrm{G}$ for moving it horizon. tally back and forth; $n$, graduated clisc for giving the rotation of the vuter tube of the telescope and the black disc $u$. In $u$ are seen the two illuminated mires.

forming a perfect cross with the longer ones (Fig, 478), and the adjustable pointer on the left-hand disc is made to coincide with the stationary one and a reading taken. Now rotate $d$ through 90 degrees; the long axial lines of the images will be in alignment without further adjustment. But if the cyc is astigmatic, the short lines will not (Fig. 479). By. rotating $A$, the short lines are made to coincide, so that a perfect cross is again formed, and the graduation is read. The difference between this and the previous reading-i.e., the difference between the two pointers-gives the difference in the curvature of the cornea in the two meridians. The images of circles which form the outer portion of the mires are oval in ordinary astigmatism.

all be successively accommodated for. Next, bring the figure to the nearpoint of clistinct vision for the loria)ntal and neighbouring lines. Probably the vertical lines will be blurred horizontal by any effort of accommodation. If the eye is distinctly astigmatie, the difference will be marked.

(2) Use the Ophthalmometer. - A convenient form is shown in Figs. 476 and 477 .

Raise or lower the chin-rest till the upper bar of the head-rest is just above the patient's eyebrows, his head being exactly vertical. The eye not to be examined is covered with the blind. The patient looks steadily into the opening of the tube with his eye wide open. The height of the instrument having been adjusted, a clear mage of the mires is obtained by ssing. The tube is then turned zontally slightly to right or left until the two images of the mires are Rotate the outer tube (Fig. 477, d) until the long meridian lines of the images are exactly in line with each other. If there is no astigmatism, tions; if there is astigmatism, at only two positions. An axis having thus been obtained, the graduated disc (Fig. 476, $A$ ) on either side of the tube is rotated until the shorter lines or spurs of the images also unite, 
7. Spherical Aberration.-Close one cye, and bring a small olject (a pin or the point of a pencil) towards the other 'ye till it becomes blured. Interpose between the object and the cye a card perforated by a small liole. The object becomes more distinct owing to the cutting off of the peripheral rays (p. 1027).

8. Chromatic Aberration.- Look at Fig. 424 (p. ro28) from a distance too small for perfect accommodation, and verify the facts given in the description of the figure.

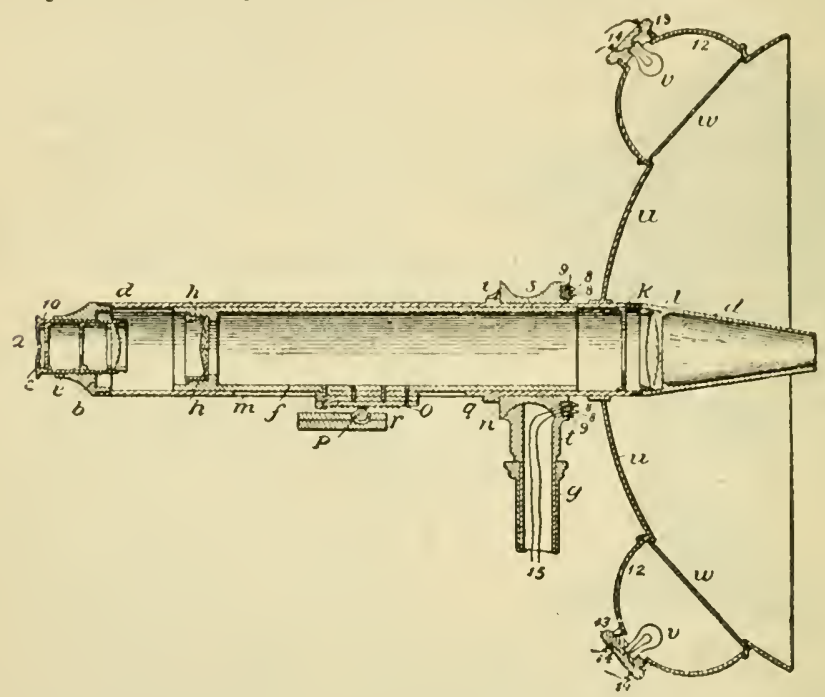

Fig. 477.-Vertical Section of Ophthalmometer. $d$, outer tube of the telescope rotating in sleeve or collar s (supported by standird $t$, which is swivelled in tubular support, $g) ; k$, diaphragm; ro, eye-piecc with lenses $a$ and $b ; n$, a station. ary disc, borne on collar $s$, graduated to indicate angle of rotation of $u$, a black concave disc rotating with tube $d$, and having fixcd in it two illuminated figures (or mires), $w, w$, whose images reflected from the cornea are observed; $i$ is a pointer carried on the tube $d$ which shows on the graduated arc the amount of rotation; I 2, I2, hemispherical shells containing small incandescent lamps for illuminating the translucent mires. The lamps are connected with wires running in the hollow stem $t$ : $f$ is the inner tube of the telescope carrying the double prism, $h, h$. By means of the rack 0 , projecting through the slot $m$, and engaged by the pinion $p, f$ is moved back and forth in the outer tube, tlius approximating or separating the corneal images of the mires. On the axis of $p$ is a milled head for turning it, and two duplicate discs graduated with a scale showing the radii of curvature of the cornea in millimetres, and another scale sliowing their equivalent in diopters.

9. Measurement of the Extent of the Field of Vision.-Use the perimeter slown in Fig. 449 (p. I0 58 ).

(x) For White Light.-Fix in the holder, Ob, on the graduated are, a small piece of white paper, and put one of the charts supplied with the instrument at the back of the wheel which revolves with the are. The observations can be recorded on this chart. The patient rests his chin on $\mathrm{K}$ and adjusts one eye against $\mathrm{O}$. This eye is kept fixed on the mark at $f$ during the whole period of observation, and the other eye 
is covered. The are is placed in a definite position, and the white object gradually moved from the end of the arc until the person annomnces that he can just see it. The angle at which this occurs is read off and recorded on the chart. The are is then rotated into a new position and the observation repeated. A line is drawn through all the points thus obtained, and this constitutes the boundary of the field of rision (Fig.450).

If the position of each point is inserted on the chart, a point above the horizontal plane passing through the visual axis being placed

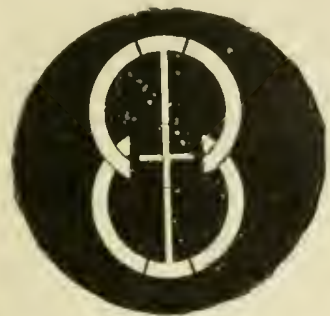

Fig. 478 .

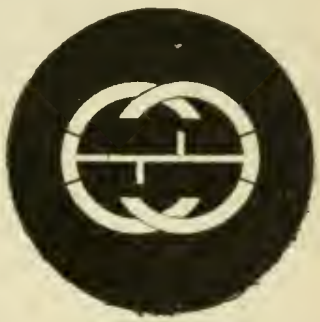

Fig. 479 .

below it, and a point to the right of the vertical plane being moved to the left, we obtain a map of the sensitive portion of the retina. Usually perimeters are arranged to do this automatically.

(2) Repeat the mapping of the field, using coloured papers (red, green, and blue) instead of white.

Io. Mapping the Blind Spot.-Make a black cross on a piece of white paper attached to the wall, the centre of the cross being at the height of the eye in the erect position. Stand about 12 inches from the wall,

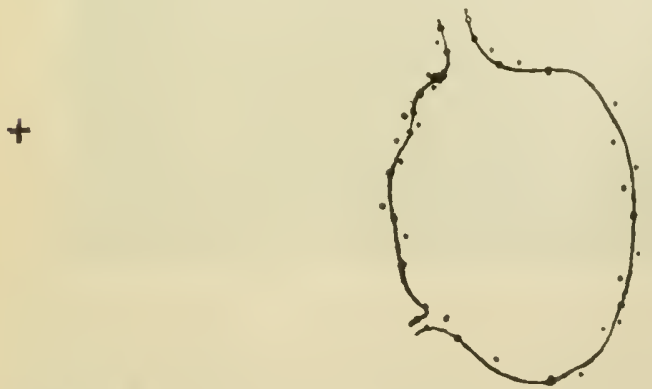

Fig. $480,-$ Map of Blind Spot (reduced by One-half).

Right eye. Distance of eye from paper 12 inches.

Its marked out (Fig. 480! Its shape is not the same in all eyes (Fig. 48I). Its size and distance from the fovea centralis can be calculated from the construction given in Fig. 420 (p. IOI9).

I I. The Macula Lutea, or Yellow Spot.-(I) After closing the eyes for a minute or two, look with one eye through a strong solution of chrome alum in a clear glass bottle with parallel sides. Hold the bottle between the eye and a white screen or a white cloud. An oval rosecoloured spot will be seen in a greenish field. The pigment of the sellow spot absorbs the blı.e and green rays. 
(2) Keep the eye closed for a short time. Then direct it to a surface illuminated by a weak blue light. A dark l, lue or almost black spot (Maxwcll's spot), corresponding to the macula, is seen in the visual field, owing to the absorption of the blue rays.

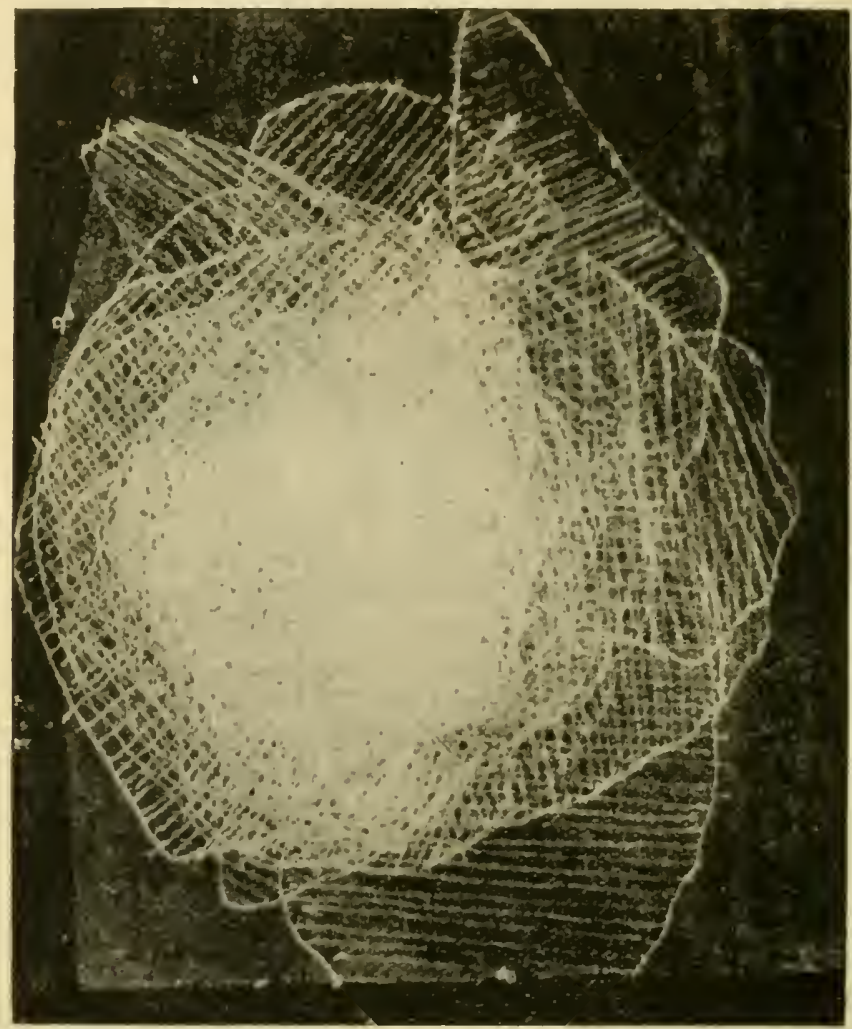

Fig. $48 \mathrm{r}$-Composite picture of Blind Spot (not reduced). The blind spot of the right eye was mapjed by 31 men, the eye being always at a distance of 12 inches from the paper. The inaps were then superposed. The amount of wbite at any point of the figure is intended to correspond to the number of maps which overlapped at that point. Although the inechanical process of reproduction gives rather an iniperfect view of the composite map, the area in the centre of the figure where the white is most continuous, and which represents the shape of the majority of the blind spots, evidently bears a general resemblance to the outline in Fig. $4^{80}$.

12. Ophthalmoscope-(I) Human Eye(p. Io31).-Let $A$ be the observer, and $B$ the person whose eye is to be examined. A and $B$ are seated facing each other. Suppose that the right eye of $B$ is to be examined. Close to the left ear of $B$ is a lamp on a level with his eyes: the room is otherwise dark. For a clinical examination, the pupil should be dilated by putting into the eye a drop of a 0.5 per cent. 
solution of atropine sulphate, but this is not indispensable for the experiment.

(a) Direit Method.- I takes the mirror in his right hand, and, holding it close to his own eye, looks through the central hole, and throws a bean of light into B's eyc. $\Lambda$ red glare, the so-called ' reflex' from the choroidal vessels, is now seen. A then brings the mirror to within 2 or 3 inches of B's eyc, kecping lis own eye always at the aperture. $A$ and $B$ both relax their accommodation, as if they were looking away to a clistance. If both eyes are enmetropic, the retinal vessels will be seen. B should now look away past the little finger of A's right hand. This causes slight inward rotation of B's eye, and brings into view the white optic disc with the central artery and vein of the retina crossing it.

(b) Indirect Melhod.-A takes the mirror in his riglit hand to exanine B's right eye, places his own eye behind the aperture as before at a distance of about i 8 inches from $\mathrm{B}$, and throws a bcam of light into B's eye. Then A takes a small biconvex lens in his left liand, and places it 2 or 3 inches in front of B's eyc, keeping it steady by resting his-little finger on B's temple. A now moves the mirror until he secs the optic disc.

(2) Examine a rabbit's cye by the direct and indirect method. Dilate the pupil by a drop or two of atropine solution.

For practice, before cloing (I) and (2) the student should cxamine an artificial 'eye' by both methods, so as to get a clear view of what represents the retina. A substitute for the artificial eye may be made by unscrewing the lower lens of the eyepiece of a microscope, and fastening in its place a piece of paper witl some printed nuatter on it. The letters must be made out with the ophthalmoscope.

The opportunity should also be taken to observe the eye of an anæsthetized animal by the simple cover-glass method mentioned in I (P. IIOI): A round cover-glass is slipped under both eyclids and so held in position on the cornea. The fundus of the eye can now be clearly seen, including the optic disc and retinal, vessels. The instillation of a little cocaine into the eye of a rabbit will produce local anxsthesia sufficient to permit the experiment.

13. Skiascopy or Retinoscopy.-The simplest method is as follows: The observer places himself at a distance of a metre from the observed eye, which he illuminates by a bcam reflected from a concave ophthalmoscopic mirror held in front of his eye. The accommodation of the observed eye is relaxed. If, now, when the mirror is rotated no direction of movement of the shadow or the light area (p.ro35) can be made out, the pupil becoming all at once dark throughout its whole extent when the mirror is rotated in one direction, and all at once light throughout its wholc extent when the mirror is rotated in the opposite direction, the observer is in the far-point of the observed eye. Since the far-point is at the distance of a metre, there is in this case myopia amounting to one diopter. If, however, the light area moves in the same direction as the rotation of the concave mirror, the far-point of the observed eye lies between the observer and the observed eye, so that the myopia amounts to more than one diopter. The precise degree of myopia can be estimated by interposing biconcave lenses of different strength until the far-point is made just $r$ metre.

If the light area moves in the opposite direction to the rotation of th: mirror, the far-point is more than a metre distant, and therefore the onserved eye is emmetropic or hypermetropic, or myopic to a degree less than a diopter. The lens, convex or concave, can now be sought out which will just bring the far-point to a metre, and from the strength 
of it, minus one diopter, tlie refraction can be estimated. Suppose, for iustance, that a convex lens of two diopters is required, then liypermetropia of one diopter exists.

In order to facilitate tlie introduction of the various lenses, instru-

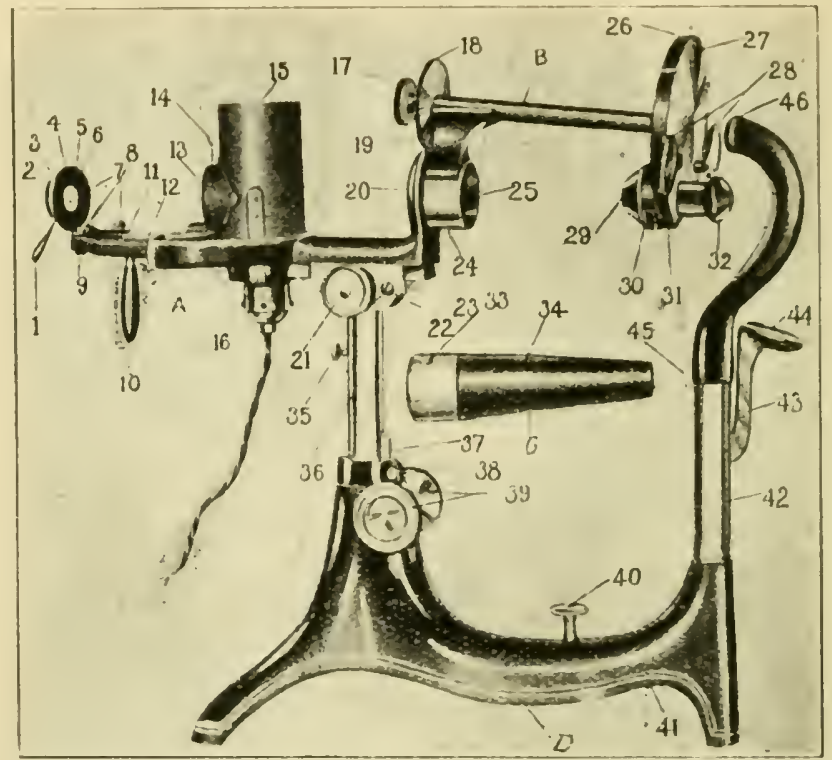

Fig. 482.-Geneva Retinoscope and Ophthalmoscope. A, frame of instrument; B, retinoscope attachment; C, ophthalmoscope attachment; D, base; r, mirror handle; 2 , clip to hold the proper lens to correct the abnormality of refraction of observcr or patient when viewing the retina with the ophthalmoscope; 3 , scale indicating the meridian of handle and pointer; 4 , ring in which mirror cup rotates; 6 , mirror; 7 , mirror spring for reflecting the light to a given point; 8 , screws for adjusting mirror; 9 , screw for holding light and ring 4 in position; 10 , handle for swinging A from side to side; 13, opening in iris diaphragm, controlled by handle $14 ; 15$. lamp hood; 17 , knurled handle for rotating disc containing the full diopter lenses; 18 , handle for rotating the disc containing the fractional lenses (white numbers indicate plus lenses, and red minus lenses); 20, opening through which observer looks when adjusting the retinoscope to the patient's cye; 21 , pinion for advancing or retracting instrument; 24 , bracket ring of retinoscope attachment $B$, which is slipped over ring 25 when putting retinoscope attachment into place; 28 , clips for 'fogging' lenses through which the patient looks to relax accommodation; 29, opening through which the pupil is viewed in retinoscopy; 30, opening containing clip in which extra leuses may be inserted when required, or the defect is over 8 diopters; 32 , patient's eye-cup; 33 , ring of ophthalmoscope attachment $\mathrm{C}$, which telescopes over $25 ; 34$. ophthahmoscope tube: 35 , binding-screw which holds the instrument in a fixcd position when retinoscope is being used; 37 , rack to raise and lower the instrument; 40 , handle controlling height of chin-rest $44 ; 46$, forelicad-rest.

ments called skiascopes or retinoscopes may be used, one of which is shown in Fig. 482 .

I 4. Pupillo-dilator and Constrictor Fibres.-(a) Set up an induction machine arranged for tetanus, and connect a pitir of electrodes through 
$z$ short-cincuiting key with the sccondary. Etherize a cat by putting it into a large vessel with a lid, slippiug into the vessel a piece of cottonwool soaked with ether, and waiting till the movements of the animal inside the vessel have ceased. Then quickly put the cat on a holder and maintain anesthesia with ether. Expose the vago-sympathetic in the neck (pp. 203, 212); the carotid is taken as the guide to it. I.igature the nerve and cut below the ligature. On stimulating the upper (cephalic) end, the pupil of the corresponding eye dilates.

Carefully separate the sympathetic from the vagus, and repeat the observation on the former. The result on the pupil is the same.

(b) Observe in the eye of a fellow-stuclent, or, by means of a lookingglass, in your own eye, that when light falls on one eye both pupils contract.

(c) Observe that when the eye is accommodated for a near object the pupil contracts, and that it dilates when a distant object is looked at.

I5. Colour-Mixing.-(a) Arrange a red and a bluish-green disc on one of the steel discs of the colonr-mixing apparatus shown in Fig. 48., so that a part of each is seen. On another arrange a violet and a yellow disc, and on the third an orange and a blue disc. By adjustment of the proportions of the two colours a uniform grey can be obtained from each of these combinations (complementary colours) when the discs are rapidly rotated.

(b) Mix two colours that are not complementary-e.g., blue and redgrey or white cannot be obtained by any adjustment of proportions; the result is always a mixed colour, the precise hue depending on the amount of each ingredient.

(c) Take papers of any three colours from widely-separated parts of the spectrum-e.g., blue, green, and red-and arrange them on one of the rotating dises. By varying the proportions, white (grey) can be produced, and any other coloured paper fastened on another of the rotating discs can be matched by adding white to the three colours.

16. After-Images-(I) Positive.-(a) Rest the eyes for two or three minutes by closing them, or by going into a dark room. Then look for an instant at a bright object, a window or an incandescent lamp. and at once close the eyes again. A bright positive after-image of the object looked at will be seen.

(b) Look at an incandescent lamp through a coloured glass as in $(a)$. The positive after-image will appear in the same colour as the glass.

(2) Negative After-Image.-(a) Look at an incandescent lamp for thirty seconds, and then direct the eyes to a white surface. The after-image of the filament will appear dark.

(b) Look at the lamp through a coloured glass for thirty or forty seconds, and then close the eye or look at a white ground. The afterimage of the filament will appear in the complementary colour of the glass. If the glass was red, for instance, the after-image will be greenish.

(c) Look at a white square on a dark ground for thirty seconds, then quickly cover the field with white paper. A dark square will be seen on the white ground.

(d) Repeat (c) with coloured squares. The after-image of the square will be in the complementary colour.

Contrast.-Perform Meyer's experiment (p. 1056).

I7. Retinal Fatigue.-Fix the eye steadily on a portion of a printed page a considerable distance away. Note that the print soon becomes blurred. Wink the eye; the short rest causes a notable recovery of the retina.

18. Visual Acuity.-Draw on a white card a series of vertical black lines I millimetre thick, and separated from each other by a distance 
of a millimetre. Set the card wp in a good liglit, and walk backwards from it till the individual lines just fail to be discriminated. Measure the distance from the card at which this occurs, and calculate the size of the retiual image (p. Io19).

I9. Colour-Blindness.-Spread out Holmgren's coloured wools on a shect of white filter-paper in a good light. Do not mention the colours of any of the wools, but (I) ask the person who is being tested to pick out all the wools which seem to him to match a pale pure green wool (neither yellow green nor blue green), which is lianded to lim. He is not to make an cxact match, but to pick out the skeins which

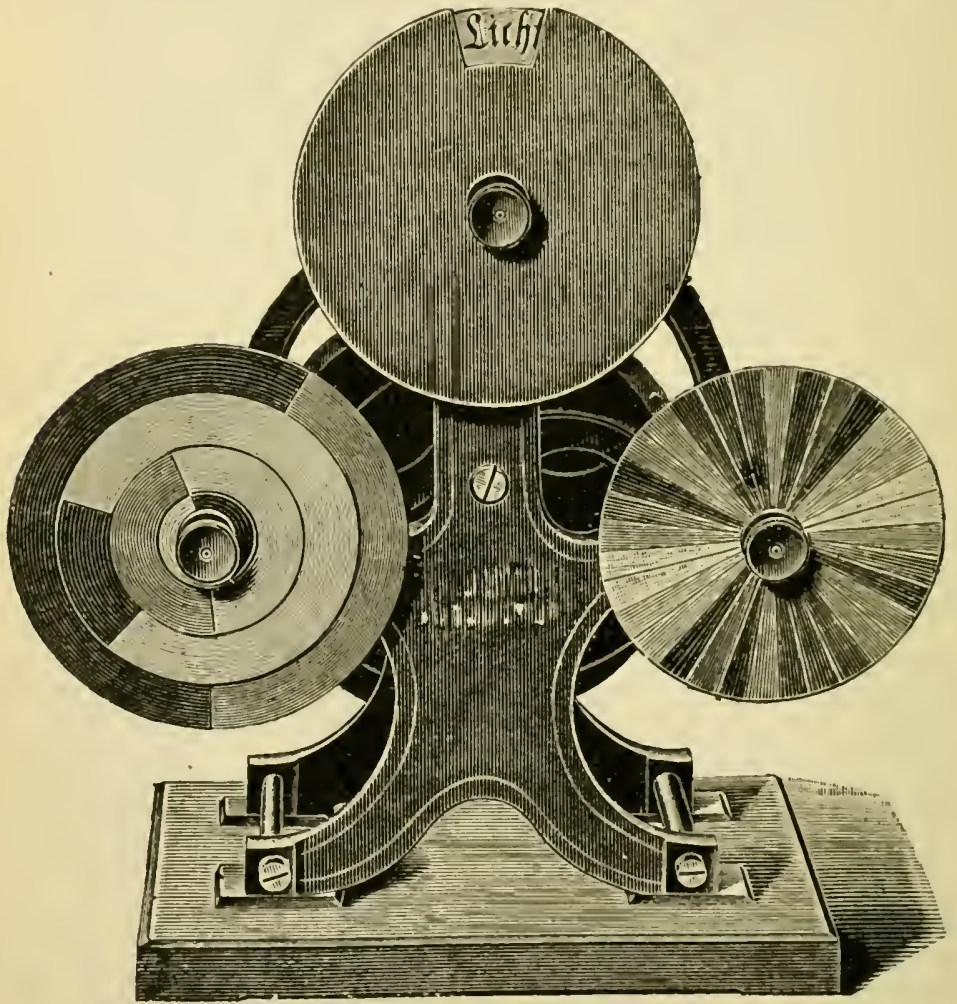

Fig. 483.-Apparatus for Colour-Mixing.

scem to liave the same colour. If he makes any mistakes, by selecting, e.g., in addition to the green skeins, any of the 'confusion colours, such as grey, grcyish-yellow, or blue wools, there is some defect of colour discrimination. To determine whetler the person is red or green blind, tests (2) and (3) are then made. (2) five lim a medium purple (magenta) wool, ancl ask lim to pick ont matcles for it. If he is redblind, he will selert as matilies to it only blues and violets, as well as other purples. If he is green-blind, he will select only greens and greys. (3) The third test is a red wool. In selecting matches for this, the red-blind will choose (with reds) greens, greys, or browns less bright 
than the test. The green-blind will choose (with reds) greens, greys, or browns which are brighter than the test.

It must be remembered that the results of tests with the coloured wools need not be precisely the same as those with coloured lights, and that when there is a discrepancy between the two the test with the coloured lights should be accepted; for it is usually the normal perception and discrimination of coloured lights which has practical importance.

20. Talbot's Law.-TRotate a disc one sector of which is black and the

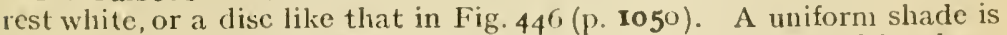
produced as soon as a speed of about 25 revolutions a second lias been attained, and this is not altered by further increase in the speed.

21. Purkinje's Figures.-(a) Concentrate a beam of sunlight by a lens on the sclerotic at a point as far as possible from the corneal margin, passing the beam througl a parallel-sided glass trough filled with a solution of alum to sift out the long lieat-rays. The eye is turned towards a dark ground. The field of vision talies on a bronzed appearance, and the retinal bloodvessels stand out on it as a dark network, which appears to move in the same direction as the spot of light on the sclerotic. A portion of the field corresponding to the yellow spot is devoid of shadows (p. 1043 ).

(b) Direct the eyes to a dark ground while a flame held at the side of the eye, and at a distance from the visual line, is moved slightly to and fro. A picture of brancling bloodvessels appears. This experiment is performed in a dark room.

(c) Immediately on awaking look at a white ceiling for an instant; a pattern of branched bloodvessels is secn. If the eye be at once closed, and then opened with a blinking movement, this may be observed again and again. Ultimately the appearance fades away.

\section{HEARING, TASTE, SMELL, TOUCH, ETC.}

22. Monochord.-Study by means of the monochord, a stretched string with a movable stop, the relation between the pitch of the note given out by a vibrating string, and its length and tension.

23. Beats. - Cause two tuning-forks of nearly equal pitch to vibrate at the same time. Malie out the beats, and count their number per second.

24. Sympathetic Vibration.-Take three tuning-forks mounted on resonators. Let two of them be of the same pitch. Strike one of these; the other is thrown into sympathetic vibration, and continues to give out a note after the first is quickly stopped by touching it. The third fork is unaffected.

25. Determine by means of Galton's whistle the pitch of the highest audible tone.

26. Cranial Conduction of Sound.-When a tuning-fork is held between the teeth, a part of the sound passes out of the ear from the vibrating membrana tympani; if one ear is closed, the sound is heard better in this than in the open ear. If the tuning-fork is held between the teetl, till, with both ears open, it becomes inaudible, it will be licard for a short time if one or both cars be stopped; and when in this position the sound again becomes inappreciable, it can still be caught if the handle be introduced into the auditory meatus.

27. Taste.-(I) Apply to the tongue by means of a camel's-hair brush a solution of quinine ( $I$ to $I, 000$ ), sodium chloride ( $I$ to 200), cane-sugar ( $\mathrm{I}$ to 50 ), and sulphuric acid (I to I.000). Determine at what part of the tongue the strongest sensations are elicited by each. 
(2) Prepare a series of solutions of sulphuric acid of gradually increasing strength, beginning with a $\frac{M}{2,000}$ solution (a two-tiousandth gramne-molecular solution) (p. 426,. Put into the month, after previous rinsing with distilled water, 4 or 5 c.c. of one of the solutions of the acid, beginning with the weakest, and determine at what con. centration of the $\mathrm{H}$ ions the acid taste first appears, rinsing out the mouth after cach observation. Repeat the experiment with solutions of hydrochloric acid, and determine whether the threshold value is the same.

A similar comparison of the necessary concentration of the $\mathrm{OH}$ ions can be made with solutions of sodium hydroxide and potassium hỹdroxide.

(3) Connect two sliort pieces of platinum wire with the copper wire from the poles of a Daniell or dry cell. Apply one platinum wire to the inner surface of the lip and the other to the tip of the tongue. Reverse the poles. Note the difference in the sensation according to whether the anode or the kathode is on the tongue.

28. Smell.--(I) Pass a current through the olfactory mucous membrane by connecting one electrode with the forehead and the other by means of a small piece of sponge or cotton-wool soaked in physiological salt solution with one nostril. An odour like that of phosphorus will be perceived.

(2) To distinguish between Taste and Smell.-Use a solution of cloveoil in water which can just be distinguished from water when it is placed on the tongue by means of a camel's-hair brush. Close the nostrils, and determine whether the clove-oil can now be detected.

29. Touch and Pressure.-(I) Prepare a number of hair asthesiometers by fastening hairs of different thicknesses to small wooden handles about 3 inches long by means of sealing-wax. Hairs as straight as possible should be chosen, or straight portions of hairs. The hair is to be fastened on one end of the piece of wood at right angles to the long axis of the handle, so that about an inch of the hair projects to one side. Determine the pressure value of each hair by pressing it down upon the scale of a balance till it is slightly bent, and obscrving the greatest weight in the other scale which it will lift. Mark the number in milligrammes on the handle. In this way, when a hair is placed at right angles to a point of the skin, and pressure exerted on it till it begins to bend, the intensity of the touch stimulus-i.e., the pressure exerted on the skin-is definitely measured, and by using hairs of different pressure values the threshold value of the stimulus for any touch area-i.e., the pressure which just gives the sensation of light touch-can be determined (p. 1081 ).

(a) Using the back of the hand, note how light a touch of the asthesiometer applied to the end of a hair suffices to elicit a sensation of touch, as compared with a part free from hairs. The hairs diminish the threshold of the stimulation by acting as levers, whose short arm presses against the nerve-endings surrounding the hair-follicles, while the stimulating weight acts on the long arm. When the skin is shaved the threshold is always raised.

(b) Shave an area on the back of the hand, and make out the relation of the touch-spots to the hair follicles. Each hair has an especially sensitive touch-spot just on the 'windward' side of the follicle (p. roso). I'sing esthesiometers of different pressure values, determine the threshold value for the shaved area. Outline an area of a square centimetre on the skin, and determine the number of touch-spots, using first a hair of the threshold value, and then going over the area 
again witl a hair of a decidedly higher pressure value. The threshold value for many parts of the hairy skin is obtained with a hair which bends at 70 milligrammes. Repeat the determinations for other skin areas, such as the back of the upper arm, the palm of the hand, the anterior surface of the leg, the chest, the back, and the cheek, forchead, and lips.

It is well that the subject should be blindfolded during the examination of the skin areas. He slould understand by preliminary practice what the sensation of light touch is, the perception of which he is to indicate. With strong aesthesiometer hairs the pricking sen. sation due to stimulation of pain-spots must be discriminated from touch sensation. When the two sensations are elicited together, the touch sensation is momentary, and the subject must be alert to letect it immediately on stimulation. The pain sensation develops more slowly, but lasts longer and becomes much more conspicuous than the touch sensation, which accordingly is apt to be submerged by it in consciousness.

(2) Touch the skin with a blunt point (at or about skin temperature). With light contact the sensation is that of simple touch. On increasing the pressure, the quite distinct sensation of deep pressure is perceived.

(3) Touch a portion of skin with a camel's-hair brush of ordinary size, pressing on it till the hairs of the brush begin to bend. The first sensation of simple contact gives place to a sensation of pressure. Repeat with a camel's-hair brush of the finest hairs half a centimetre in length, cut away till its cross section is only half a millimetre in diameter at the base. Probably a pure sensation of touch, without any pressure element, will be obtained when the brush is applied so as just to bend the hairs.

(4) Find the least distance apart at which the points of the asthesiometer compasses can be recognized as two when applied to the back of the hand, the forearm, upper arm, forehead, finger-tips, or tip of the tongue. Both points of the compasses must be placed on the skin at the same time, and the same pressure applied to both. The subject must not see the points.

(5) Time Discrimination of Touch.-Touch the prong of a vibrating tuning-fork lightly with the tip of the finger. The taps of the prong on the skin do not blend into a continuous sensation even when the fork vibrates several hundred times per second.

30. Temperature Sensations.-For the investigation of these, pieces of thick copper wire, filed at one end to a blunt point, and fixed by the other in a small wooden handle, may be used. They can be heated in a sand-bath or in a beaker of water to the desired temperature, or cooled in cold water or in ice. Or a metal tube drawn out at one end, through which water at the required temperature can be passed before use, may be employed. Another device is a metal cylinder ending in a point, and filled with water at the given temperature.

(I) On the dorsal side of the hand outline an area of skin with a pen or a coloured pencil. Divide this into areas of 4 square millimetres. Go over the area with a wire or cylinder at a temperature of about $40^{\circ} \mathrm{C}$, and determine the extent and position of the spots which on contact yicld a sensation of warmth, marking them on the skin by ink-dots, or mapping them on ruled paper. Then repeat the exploration with points at a temperature of about $15^{\circ} \mathrm{C}$., and map the spots which yield a sensation of coolness. Now note whether a warm spot touched with a point at $15^{\circ} \mathrm{C}$, , or a cold spot touched with a point at $40^{\circ} \mathrm{C}$, yields any temperature sensation. 
(2) Touch the skin with a test-tube containing water at $50^{\circ} \mathrm{C}$., and agatin with a test-tube containing ice. Do the sensations differ in any way from those of pure warmth and coolness? Repeat (1) with temperatures of $50^{\circ}$ and $0^{\circ}$, and note whether there is any difference in the quality of the scusations yiclded by the warn and cold spots. When a cold spot is touched with a point at al temperature of $50^{\circ}$, or a warm spot with a point at a temperature of $0^{\circ}$, is any sensation obtained? If so, what?

(3) Apply successively to one and the same portion of the skin testtubes containing water at $50^{\circ}, 45^{\circ}, 40^{\circ}, 35^{\circ}, 30^{\circ}, 25^{\circ}, 20^{\circ}, 15^{\circ}, 10^{\circ}$, $5^{\circ}$, and $0^{\circ}$ (ice), and determine the sensations excited in each case. The contact should only be momentary, so as not to cause extensive and lasting change of temperature of the skin. Note that there is a certain range of temperature above and below that of the skin within which no sensation of heat or cold is given.

(4) Take three beakers of water at $20^{\circ}, 30^{\circ}$, and $40^{\circ} \mathrm{C}$. respectively. Place a finger of one hand in the coldest beaker, a finger of the other hand in the warmest, until no definite temperature sensations are felt by either finger. Plunge both fingers into the beaker at $30^{\circ} \mathrm{C}$, and temperature sensations will be perceived.

(5) Temperature Discrimination.-Find the least perceptible differcuce in temperature between two beakers of water at about $0^{\circ} \mathrm{C}$. Repeat the experiment with two beakers of water at about $30^{\circ} \mathrm{C}$., and again with two beakers of water at about $55^{\circ} \mathrm{C}$. Use the same liand. Expose the same amount of surface to ihe water.

(6) Compare the acuteness of the temperature sensations of the skin and the mucous membrane of the mouth, touching a given portion of skin and then a portion of mucous membrane with tubes containing water at various temperatures.

3I. Pain.-(I) Using a pin, explore a cutaneous area to determine whether every point of the skin yiclds the painful sensation of pricking. kispecially compare the result of stimulating the region in the immediate neighbourhood of the hairs with the spaces between hairs. Discriminate the touch sensation given by the light contact of the pin-point from the painful impression caused when the pressure is increased. Note that the touch element is more evanescent than the pain element.

(2) With strong $v$. Firey hairs determine the pressure at which the sensation of touch passes into that of pain.

(3) Compare the sensibility to pin-priclis of the mucous membrane within the mouth with that of the skin.

32. Having deternined the systolic blood-pressure in one arm of a fellow-student (p. I 13 ), release the pressure in the cuff, then raise the hand in the air so as to empty the arm of blood, and while it is still raised, get up a pressure in tlie cuff equal to the systolic pressure. Lower the hand and maintain this pressure by squeczing the bulb occa. sionally. Be careful not to increase the pressure above the systolic pressure. There is no disadvantage in letting it drop 5 to ro millimetres below systolic pressure from time to time. Now compare the acuity of the sensations of contact, pressure, warmth, cold, and pain in the anomic and the normal hand, always on symmetrically placed areas of the two hands. Repeat the comparison at intervals till a definite difference is found, and note the sensition for which the acuity first diminishes. Do not prolong the experiment unduly. If the subject experiences discomfort, the pressure in the aumlet is to be at onco released. 


\section{CHAPTER XIX}

\section{REPRODUCTION}

Regeneration of Tissues.-In lower forms of animals and in all or most plants, the power of regeneration is much grcater than in the higher animals and in man. A newt can reproduce an amputated toe, and every tissue - skin, muscle, nerves, bone-will be in its place. After extraction of the crystalline lens in triton larvie, a new lens is formed from the iris epithelium. Artificial mouths surrounded by tentacles can be formed in Cerianthus, an animal belonging to the same group as the sea-anemons, merely by making a cut in the body-wall and preventing it from closing. In an Ascidian, too (Cynone intestinalis), artificial openings in the branchial sac, surrounded by numerous pigmented points similar to the eye-spots around the natural mouth and anus, have been produced (Loeb). A classical example of regeneration in invertebrates is that of the rays of starfishes after amputation. According to some observers, this massive regeneration, as well as other instances of the regeneration of complicated organs in invertebrates, depends, in some degree at any rate, upon the nervous system.

Even in the higher animals regeneration of tissues is a common phenomenon. Since cells are constantly dying within the body, they must be constantly reproduced. In some tissues the process by which this is accomplished is more evident, and therefore better known, than in others. The most highly-organized tissues are with difficulty repaired, or not at all. The epidermis is always wearing away at its surface, and is being constantly replaced by the multiplication of the cells of the stratum Malpighii. In the corneous layer we have only dead cells; in the Malpighian layer we have every histological gradation from squames to columns, and every physiological gradation from cells which are about to die to cells that have just been born. The corpuscles of the blood undoubtedly arise at first, and are recruited throughout life, by the proliferation of mother-cells. The gravid uterus grows by the formation of new fibres from the old, and by the enlargement of both old and new. A severed muscle is generally united only by connective or scar tissue, but under favourable conditions a complete muscular 
splice' may be formed. A broken bone is regenerated by the proliferation of cells of the periosteum, which become bonecorpuscles. Gland cells-e.g., liver cells-are atso, under certain circunstances, capable of regencration. Some of the most striking instances of new formation of glandular tissurs hate already been mentioned in connection with the growth of grafts of certain of the endocrine organs (e.g., thyroid, adrenal cortex), when a physiological insulficiency has been created by removal of the greater portion of the organ (p. 648). There is no evidence that the influence of the nervous sy'stem is a factor. It is doubtful whether there is any new formation of nerve-cells in the adult organism, but peripheral nerve-fibres which have been destroyed by accident or operation are readily regenerated, and the end-organs of efferent nerves may' share in this regeneration.

Thus, in a sense, reproduction is constantly going on within the bodies even of the ligher animals. But since the whole organism eventually dies, as well as its constituent cells, a reproduction of the whole, a regeneration en masse, is required.

A cell of the stratum Malpighii can only, so far as we know, reproduce a similar cell, and this is characteristic of cells that have undergone a certain amount of differentiation, especially in the higher animals. The fertilized ovum, on the otler hand, has the power of reproducing not only ova like itself, but the counterparts of every cell in the body. And this is only the highest development of a power which is in a smaller degree inherent in other cells in lower forms. Plants and the lowest animals are far less dependent upon reproduction by means of special cells. A piece of a Hydra separated off artificially or by simple fission becomes a complete Hydra, as was shown by Trembley a century and a half ago. A cutting from a branch, a root, a tuber, or even a leaf of a plant, may reproduce the whole plant. It is as if each cell in these lowly forms carried within it the plan of the complete organism, from which it built up the perfect plant or animal.

Reproduction in the Higher Animals. - In regard to the sccretions of the reproductive glands, all that is necessary to be said here is that, unlike other secretions, their essential constituents are living cells. The spermatozoa in the male have, indeed, diverged far from the primitive type. Certain cells (spermocytes) in the tubules of the testicle divide, each forming two daughter spermocytes. Each of the daughter spermocytes in turn divides, so that four cells (spermatids) are formed from each spermocyte. In the final division which produces the spermatids a reduction of the chromosomes (p. II22) occurs, so that the spermatid possesses only one-half the number characteristic of the somatic cells of the species. The spermatids elongate and become spermatozoa, the head of the latter representing the nucleus of the former; and it is this nucleus (with the 
middle piece originally containing the male centrosome and attraction sphere, p. 5) which is the essential contribution of the male to the reproductive process. The tail of the spermatozoon is simply, from the physiolngical point of view, a motile arrangement, whose function it is to carry the mucleus of the male element, freighted with all that the father can transmit to the offipring, into the neighbourlood of the female reproductive element or ovim. After the spermatozoon has penetrated the ovum its tail disappears, being probably absorbed. The function of the accessory reproductive glands, the prostate, the seminal vesicles, and Cowper's gland, are not well understood. But the spermatozoa in the act of ejaculation are mixed with the secretions of these glands, and therefore it is to be supposed that they are of importance. When the prostate and the seminal resicles are removed in white rats, the female is no longer fertilized, although the sexual power of the male is unaltered. The testes apparently develop spermatozoa in the normal manner, but for some reason they either do reach the ovum or do not react with it normally if they do reach it. When the testes are removed from a young animal, the development of the prostate is interfered with; in an adult animal the gland atrophies.

The ovum also begins as a typical cell with nucleus (germinal vesicle), nucleolus (germinal spot), centrosome and attraction sphere, and it forms, by its repeated subdivision, all the cells of the foetal body. But, except in some (parthenogenetic) forms, it never awakens to this reproductive activity till fecundation has occurred; and fecundation essentially consists in the union of the male with the female element, or rather in the union of the male and female nuclei.

From time to time a ripe Graafian follicle, overdistended by its liquor folliculi, bursts on the surface of the ovary and discharges an ovum. It is probable that in the majority of mammals (e.g., the cow, mare, sow, sheep, and bitch) ovulation, or the discharge of the ovum, occurs spontaneously during œstrus (period of heat). In others (e.g., the rabbit, ferret, and cat), it scems only to take place as a result of copulation. Whether sexual intercourse has any influence upon ovulation in women can hardly be considered as settled. The common opinion is that most ova are discharged spontaneously at the time of the menstrual period, but some writers take the view that the discharge bears no relation to menstruation. Only one orum seems to be shed each month. It was formerly believed that the frayed or fimbriated end of the Fallopian tube, rising up fingerlike from the dilatation of its bloodvessels, grasps the ovum. But it is more than doubtful whether this occurs. it is more probajie that the orum is first discharged into the pelvic cavity, and is guided to the orifice of the Fallopian tube, not necessarily that of its own side, by the movements of the cilia around the orifice, and then passed slowly along the tube by the downward 
lashing cilıa which line it. Probably the oxum takes as a rule eight or ten days to reach the nterus, and it is during this time that fertilization takes place. If not impregnated, it soon perishes amid the secretions of the uterus- how soon has be'n matter of discussion, and can hardly be considered as settled. If, however, impregnation nccurs, the oitum penetrating the superficial epithelium into the subepithelial conuective tissue becomes fixed in one of the crypts or pouches of the utrine mucous membrane (decilua serolina), which grows round it as the decidua reflexa. The Graafian fullicle, after the discharge of the ovum, fills up with blood, and a cellular structure, the corpus lutemm, is developed in its interior from cells in the wall of the follicle. In the absence of impregnation the corpus lutenm begins to (lisappear before the next menstrual period, and is spoken of as a false corpus luteum. But when pregnancy occurs, it continues to grow till the fourth or fifth montl of pregnancy, and is called a true corpus luteum.

Menstruation.- In the mature female, from puberty, the age at which the reproductive power begins (thirteenth to fifteenth year), on till the time of the menopause (fortieth to fiftieth year), at which it ceases, an ovum-or it may be in some cases more than one-is discharged at regular intervals of about four weeks. This discharge is accompanied by certain constitutional symptoms and local signs that last for a variable number of days. The temperature of the body diminishes somewhat, rarely more thin $I^{\circ} F^{1}$, and there is also a slight fall in the pulse-rate. The genit:। organs are congested, and a quantity of blood, which varies in different individuals, but is usually not over 50 c.c., is shed. If more than 60 c.c. is lost, the flow is copious. Over roo c.c. it is abnormally great (G. HoppeSeyler). At the same time, the whole or a portion of the mucous membrane of the uterus is cast off.

As to the physiological meaning of this menstruation, as it is called, opinion is divided. Two chief theories have been proposed to account for it, both of which agree in considering the plienomenon to be comnected with a preparation of the uterus for the reception of the ormm. But according to the theory of Pfluger, the mucous membranc is stripped off (by a process analogous to the ' $\mathrm{f}$ eshening or paring of the indurated edges of a wound by the surgeon, in order that union may occur when they are brought together) on the chance, so to speak, that an impregnated on'um may arrive. On the alternative theory, this change takes place because the ovum has not been impregnated, and the bed prepared for it not being required, the swollen and congested uterine mucous membrane undergoes degeneration, and is in part cast off (Reichert, Williams, etc.). However this may be, it is now pretty generally agreed that the degenerative process involves only the superficial portion of the mucosa, and not its whole tlickness. 
The process of menstruation, and the nutrition of the genital organs, especially the uterus, are intimately dependent upon the ovaries. There is good evidence that the influence is exerted through an internal secretion formed by some portion of the ovarian sub stance. When, for instance, the ovaries of young animals (guineapigs) are removed from their normal situation and transplanted to a distant part of the body, the external genitals, vagina, and uterus undergo the normal development instead of being arrested in their growth, as is the case when the ovarics are removed altogether. The removal of the ovaries in adult animals leads to fibrous degeneration of uterus and Fallopian tubes. On the other hand, removal of the iterus has no effect on the development of the ovaries in a young animal, and does not cause degeneration of the ovaries of an adult animal. In monkeys, in which a menstrual flow comparable to that in the human female occurs, it was found that menstruation took place after the ovaries had been transplanted from their original seat, and the flow stopped when the transplanted ovaries were removed. The view has been put forward that the important part of the ovary for these functions is the corpus luteum, which is considercd to be a gland with an internal secretion (Born). This secretion seems to be connected with the implantation of the ovum and the subsequent growth of both ovum and uterus. According to Fraenkel, the absence of the corpus luteum prevents implantation. The experiments of Marshall and Jolly also indicate that the corpus luteum forms some substance, which exerts an action on the uterine mucosa, during the earlier stages of pregnancy. When the ovum has not been fertilized the corpus luteum brings about menstruation. Where fertilization has occurred it prepares the uterus for the implantation of the ovum. It is generally considered that as regards their origin there is no difference between the true and the false corpora lutea.

The mode of origin of the corpus luteum has given rise to much discussion. Two chief views have been put forward: (I) That it is a structure derived from certain large epithelium-like cells (theca cells) in the connective-tissue wall (theca) of the discharged follicle (v. Baer, et al.); (2) that it is developed from the follicular epithelium (membrana granulosa) (Sobotta, et al.). The first view seems to be best established. The theca cells multiply and grow into the cavity of the discharged follicle. Their yellowish colour is due to the presence in them of lipoid droplets.

The influence of the ovary on the formation of the decidua has been illustrated in a very interesting way by the investigations of L. Loeb on the artificial production of deciduomata. He has shown that if a number of incisions are made into the uterus of a rabbit or guinea-pig within a certain interval after the œstral period (period of heat), a structure with the histological characters of the decidua 
develops at each wound. Impreguation does not appear to be a necessary factor, nor even contact of the ovum with the uterine mucous membrane. On the other hand, ovulation, the discliarge of an ovum or ova, or at any rate the condition of the ovary associated with this discharge, seems to be indispensable. For extirpation of the ovaries in a large number of guinea-pigs prevented the formation of deciduomata from wounds of the uterus made at the most favourable period after copulation. The uterus then appears to have an inherent power of responding to such a stimulus as a mechanical injury by the production of a decidual structure, but only under the influence of the ovary. The ovarian factor is probahly not nervous but chemical, some specific substance which acts on the uterus being liberated periodically in connection with the sexual rhythm.

Development of the Ovum.-Before fecundation, and apparently as a preparation for it, the ovum is the seat of remarkable changes, similar upon the whole to those seen in the mitotic or indirect division of ordinary cells.* They have been most fully studied in the eggs of certain invertebrate animals.

The division of the cell is initiated by changes in the centrosome and attraction sphere. The centrosome divides into two daughter centrosomes. These take up a position one at each pole of the nucleus. Each claughter centrosome is surrounded by a system of radiating lines or filaments, which are less conspicuous than the chromatin filaments of the nucleus, since they do not stain as these do. Meanwhile the nuclear membrane and the nucleoli disappear, or at any rate become indistinguishable from the rest of the chromatin skein. The skein breaks up into chromosomes, the number of which is constant for a given species, but is not the same in all species of animals.

The daughter centrosomes or astrosoheres are united by meridional achromatic fibres, which form a spindle running through the nucleus from one pole to the other. The chromosomes arrange themselves at right angles (equatorially) to the spindle, and then each chromosome divides longitudinally into two. The halves of the chromosomes now pass toward their respective centrosomes, being perhaps guided by the fibres of the spindle. It results from this that two daughter nuclei are formed, each with the same number of chromosomes as the original nucleus, although with only half the amount of chromatin. Tlie cytoplasm divicles also, so that the parent cell is now represented by two daughter cells. In ordinary cell division the two daughter cells are of equal size, but in the division of the ovum which occurs before fertilization the two resulting cells are very unequal. The large cell continues to be known as the ovum; the small one is the first polar body. After extrusion of the first polar body the ovum again divides unequally. A new spindle forms, and a second polar body, again much the smaller of the two daughter cells, is cast off. There is a difference, howeve; between the process of division which gives rise to the first and that which gives rise to the second polar body. In the case of the latter a so-called reduction-division occurs; the chromosomes do not split longitudinally, but half of the original number pass into each daughter nucleus. As to the significance of these changes there has been much discussion. It is agreed that the result of the process is the expulsion

* For figures illustrating the changes, see any good textbook of Histology. 
of a portion of the cloromatin, the ovinn now pussessing only half the original number of chromosomes, although nearly all the original cytoplasm. In fertilization the original number is restored by the male clement when it arrives and penetrates the ovum. For in the final cell division by which the mature spermatozoon is formed the chromosomes of its nucleus are also, after two divisions esseritially similar to those occurringin maturation of the ovum, reduced to half the normal number.

The two reduced nuclei in the fertilized ovum are spoken of as the male and female pronuclei. By their union a single nucleus is formed with the number of chromosomes normal to the species.

An enormous amount of interesting work has been done with the view of illustrating the connection of the complicated phenomena described with the structure of the ovum. Only a bare reference to one or two of the experiments is possible here. Driesch and Hertwig find tliat the nucleus can be made artificially to change its place with reference to the yolk, without hindering the development of a normal animal. Lillie has shown that centrifugalization of the eggs of annelids, although it markedly alters the distribution of the yolk and other substances, does not affect the form of cleavage. The polar bodies appear in the position which they would normally occupy. In other words, no redistribution of the granules or nucleus affects the polarity of the egg, which therefore is a function or property of the ground substance of the protoplasm. The whole of the protoplasm, however, is not necessary for complete development. Even in Amphioxus, the lowest of the vertebrates, the eggs have been broken up by shaking, and a complete animal evolved from as little as one-eighth of an ovum. If the separation was incomplete a kind of Siamese twins, or even triplets, could be obtained (Wilson and Mathews). Nor is it always indispensable that both pronuclei should be present.

Parthenogenesis. - Attempts have been made to separate the constituents of spermatozoa which are essential to fertilization. From the sperm of a sea-urchin a substance can be extracted by strongly hypotonic salt solutions, containing ether, which acts as a powerful fertilizing, agglutinating, and cytolyzing agent upon the eggs. It is soluble in dilute acid, and is probably identical with a fertilizing agent called oöcytase present in blood-serum (Robertson). Whatever it is that the spermatozoon supplies, the process of fertilization can in certain forms be started artificially in the absence of spermatozoa or any of their constituents. The studies of Loeb and his pupils on artificially induced parthenogenesis are of special importance. When the unfertilized eggs of the sea-urchin are exposed for one or two minutes to 50 c.c. of sea-water, to which 3 or + c.c. of decinormal acetic acid has been added, the majority of the eggs form the membrane characteristic of the entrance of the spermatozoon. When these eggs are afterwards exposed for thirty to forty minutes to IOO c.c. of sca-water, to which If or I5 c.c. of a strong solution of sodium chloride (two and a half times the strength 
of a normal solution, or about I $_{4} \cdot 6$ per cent.) has been added, those of the (ggs which lave formed membranes levelop into swinming larvas that rise to the surface. These larvas develop into perfect sea-urchin larvas or 'plutei' as fast as the larvas of eggs fertilized with sperm. It has lately been shown that pricking of the unfertilized egg of a frog with a needle suffices to induce normal development of the egg (Bataillon). These parthenogenetic frogs have been successfully reared, and apparently are all males (Loeb). These observations have an important bearing on the question of the determination of sex. In the frog it would seem that the eggs are all alike, since in the absence of a spermatozoon only one sex (the male) is developed. The male frog is hetero-gametic for sex-i.e., there are two kinds of spermatozoa, one with and the other without a sex chromosome. If a spermatozoon of the former type enters an egg, a female is produced. If a spermatozoon of the latter type, or no spermatozoon at all, enters an egg, a male is produced.

It is impossible to enter here into a discussion of the factors which determine sex. While due weight must be given to such morphological distinctions as sex chromosomes in the gametes (or sexual reproductive elements), evidence has been adduced that the fundamental factor may be chemical and metabolic pecularities in the gametes. In pigeonse.g., -in which the female is the hetero-gametic sex, producing two kinds of eggs-it has been shown that the egg which is to develop a female is characterized by a lower metabolism, a lower percentage of water and a higher total content of fat and phosphorus or of phosphatides, than the egg which is to develop a male. There are indications that by changing these chemical conditions sex can be to some extent controlled (Whitman, Riddle).

The facts of parthenogenesis show that it is not absolutely necessary for development that the ovum should have the normal number of chromosomes restored. It can develop with half the number, the chromosomes of the female pronucleus being sufficient for growth, although, of course, in this case for a growth uninfluenced by the properties of the male element. In like manner it is stated that portions of the maturated. ovum devoid of a nucleus can undergo development if penetrated by a spermatozoon, the chromosomes of the male pronucleus being sufficient for growth.

Formation of the Embryo.- - Vot till all these crents liave caken place -extrusion of the two polar bodies, or maturation; penetration of the spermatozoon, and blending of its head (the male pronucleus) with the remnant of the nucleus of the ovum (female pronucleus), or fecundation -not till then does the ovum begin the process of repeated division by which the whole body is reproduced. The fused or segmentation nucleus divides in to two, each containing the normal number of chromosomes derived from the splitting of those contributed by both the male and female elements. It is belicved that the division takes place in such a way that both male and female chromosomes are represented in each nucleus. The cytoplasm being also cleft by a corresponding furrow: 
two complete nucleated cells make their appearance. These divide in turn, till at length (in the mammal) the cmbryo is represented by a hollow sphere or vesicle, with a cellular crust. During division the upper or outer cells have always been larger than the inner and lower, and have multiplied more rapidly: and thus it comes about that the hollow sphere of large cells encloses a mass of smaller cells, along with remnants of broken-down yolk and of fluid derived by absorption from the contents of the uterus. The smaller cells continue to multiply and arrange themselves as a lining to the sphere already formed, so that in a short time it becomes double, and we have already differentiated two of the primary embryonic layers - the ectoderm, also called the epiblast, or superficial, and the endoderm, also called the hypoblast, or deep layer. The whole sphere is callcd the blastoderm, or the blastodernic vesicle.

IThile this inner shell of endodermic cells is gradually creeping on to completion, there appears at a part where it is already fully formed a small opaque whitish disc, the germinal area or embrynal shield. This represents the stocks on which the framework of the embryo is to be laid down. The area elongates; at its posterior end appears a thickened line, the primitive streak, soon furrowed by a longitudinal groove, the primitive eroove, that marks the direction in which the long axis of the future embryo will lie, but is not itself a permanent line in the building, and ultimately vanishes. The appearance of the primitive streak is the signal that a rapid proliferation of the cells of the germinal area, and especially of the ectoderm, has begun; and this goes on until a third layer is formed, intermediate in position to the original two, and therefore named the mesoderm. While this is pushing its way over the germinal area and into the rest of the blastodermic vesicle, the ectoderm in front of the primitive streak rises up in two lateral ridges, enclosing between them the medullary groove. The medullary groove is the beginning of the cerebro-spinal axis; its walls first come to overhang the furrow, and then to coalesce; and the medullary groove has now become the neural canal. Immediately under it the mesoderm forms a rod of cells, the notochord, which is the forerunner of the vertebral column; around this the bodies of the vertebræ are afterwards developed from cubical masses of mesodermic cells, arranged in pairs alon: the notochord, and called the protovertebre. The rest of the mesoderm, running out on each side from the protovertebræ, splits into two layers, an uppey or somatic layer, which unites with the ectoderm, forming with it the somatopleure, and a lower or splanchnic layer, which unites with the endoderm to form the splanchnopleure. Between the somatopleure and the splanchnopleure is a space called the colom, or pleuro-peritoneal cavity (Fig. 485). The layer of ectoderm which envelops the whole (termed the trophoblast, from its nutritive function), in conjunction with the underlying mesoderm, represents the prechorion, the early stage of the chorion.

Up to the present, apart from the enclosure of the neural canal, all this formative activity is buried beneath the surface of the blastoderm, and has not showed itself by any external token; the embryo still appears as a portion of the germinal area, and lies in its plane. But now a pocket, or crease, or moat, beginning at the head as the head-fold, then pushing under the tail, gradually creeps round and undermines the whole embryo, which is raised above the general level, and, as it were, scooped out from the rest of the blastoderm; till at length it lies on the latter, scmething like an upturned canoe, enclosing a tube, complete in front and behind, but still open in the middle, where it communicates with the interior of the yolk-vesicle. Since this tube has been formed by the tucking in of the three ancestral layers of the blastoderm, it follows that it is lined by endoderm, supported externally by the splanchnic sheet 
of mesoderm. So that now the body consists of a dorsal tube the neural canal), essentially of ectodermic origin, a ventral tube (the alimentary canal), essentially of endodermic origin, and between the two a massive double layer of mesodermic tissuc, which contributes supporting elements to both. At this point it mav be well to empluasize the fact that this embryological distinction of the three primitive layers has a deep and fundamental meaning, and corresponds to a physiological distinction that endures throughout life. The endoderm, the lowest layer in position, may also be described as the lowest in the physiological hierarchy. It furnishes the epithelial lining of the alimentary canal from the beginning of the osophagus to near the end of the rectum, as well as the epithelium of the organs which arise from diverticula of the primitive intestine-viz., the digestive glands (with the exception of the salivary glands), the lungs, and the passages leading to them, the thyroid, and the greater part of the thymus gland in its primitive condition before tha lymphoid tissue derived from the mesoderm has as yet grown into it. According to some authorities, the notochord is also derived from the endoderm.

Upon the whole, it may be said that the tissues of endodermic origin are essentially concerned in chemical labours, in the absorptirn of food material and excretion of waste products. The mesodermic tissues are essentially concemed in mechanical labour; they are the tissues of movement and of passive support. The ectodermic tissues are at the top of the pyramid; they govern the rest.

From the mesoderm arise the muscles, the entire vascular system, with its blood-and lymph-corpuscles, the bones and connective tissues; and the Wolffian body and its appendages, which are the predecessors of the genital glands and ducts, and of the chief portion of the renal apparatus.

The ectoderm forms the epidermis and its appendages, the epithelial end-organs of the nerves of special sense, and the nervous system, cerebro-spinal and sympathetic. The salivary glands and the mucous lining of the mouth and anus are developed from the ectoderm, which is indented to meet the intestinal canal and give it access to the exterior at either end.

It is not possible here to trace in detail the development of all the organs of the embryo. Its nutrition and metabolism not only distinctly belong to the physiological domain, but, carried on as they are under conditions that seem so strange, and even so bizarre, to one acquainted only with adult physiology, are calculated to throw light on the metabolic processes of the fully-developed body. And they cannot be understood without reference to the peculiarities of the vascular system in fœtal life. These we shall accordingly describe, but for further details as to the anatomy of the embryo the student is referred to some standard anatomical textbook, such as Quain's 'Anatomv.'

Development of the Connections between ine Embryo and the Uterus. - In the first period of its development the ovum, nestling in the pouch formed by the decidua serotina and reflexa, is fed from the maiernal blood and tissues directly, without the mediation of fotal bloodvessels, through the finger-like processes or villi with which its extemal layer, the zona pellucida, becomes studded. At the earliest stage at which a human ovum has been studied after implantation it is already enveloped by a thick ectodermic covering (the trophoblastic envelope), consisting of two layers of cells, one unquestionably of fortal origin, the so-called cells of Langhans, and the other the syncytium, the origin of which is assigned by some authorities to the ovum, by others 
to the maternal tissues. The trophoblastic covering is everywhere in contact with the maternal blood, which, pushing its way in to the trophoblast at intervals, divides it into columns. Later on the fœetal mesoderm grows into these, and so the primary chorionic villi are formed. It is not till after the first three weeks that bloodvessels make their way into these villi, although the mesolerm of the fotus begins to enter the villi about ilie end of the first, or the beginning of the sccond, week. The scanty yolk of the human ovum is totally inadcquate to supply it with nutriment for the time that elapses before the bloodvessels are developed, and food substances must be obtained from the maternal liquids by imbibition, osmosis, diffusion, or filtration, aided, perhaps, by more special absorptive processes on the part of the fotal tissues. Soon the heart appears as a tube (at first double), formed by cells belonging to the splanchnic layer of the mesoderm. It begins to pulsate in the chick as early as the middle of the second day, although it as yet contains neither nerve-cells nor fully-formed muscular fibres. In the mammal pulsation is late in making its appearance, in man about the beginning of the third week. A bloodvessel grows out from the anterior end of the heart and divides into two primitive aortic arches. from each of which a vessel (omphalo-mesenteric cr vitelline artery) runs out in the mesoderm covering the umbilical vesicle, or yolk-sac. The blood is returned to the heart by the vitelline veins coursing in on the walls of the vitelline duct. In this way the store of nutriment in the umbilical vesicle of the chick, which is the only solid or liquid food it receives or needs during the whole period of development, is tapped, and a regular channel of supply established. Oxygen is at the same time absorbed through the porous shell; but later on this respiratory function is taken over by the second or allantoic circulation. In the mammal the circulation on the umbilical vesicle is of much less consequence, for the quantity of material left over after the formation of the blastoderm is exceedingly small; it is only with a few days' provision in its haversack that the embryo starts out on its developmental march. And the vitelline vessels deriving their further supply of food and oxygen from the tissues of the mother in contact with the ovum cease to be of use as soon as the second and more perfect placental circulation is established, and soon shrivel up and disappear, as the umbilical vesicle shrinks.

The second circulation of the embryo is developed in connection with a remarkable offshoot from the hind-gut called the allantois, which, before the fifth day in the chick and during the second week in man, pushes its way out between the somatic and splanchnic layers of the mesoderm-i.e., in the pleuro-peritoneal cavity-and grows through the 
umbilicus, carring blcorlvescels along with it in its mesodermic layer. Sill carlicr, and, mdecd, while the cmbryo is being separated nff from

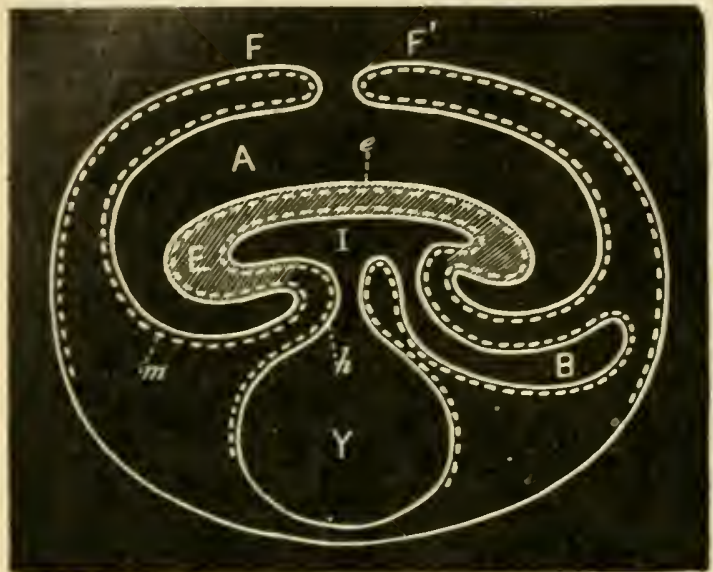

Fig. 495 -Diagram to illustrate Formation of Amnion and Allantois. A, cavity of true amnion; $F, F^{\prime}$, folds about to coalesce and complete the amniotic cavity; $m$, meso. dermic layer of amnion; B, allantois; $I$, intestinal cavity of embryo; Y, yolk-sac; $h$, endodermic layer; $c$, ectodermic layer of embryo. The embryo is the shadec por. tion in the middle of the figure. $E$ is placed over the head region. No attempt is made to delineate its actual form. The mesoderm is represented by the interrupted line. -ed for the rest of its intra-uterine life by the umbilical cord alonc. floats freely within it. The amniotic fluid acts as a water-jacket or cushion, to brcak the force of the inevitable shocks and jars transmitted from the mother to the foetus and from the foctus to the mother. To some extent, in addition, it may serve as a nutritive fluid, for substances can pass from the blood of the mother into the amniotic fluid, and the rmniotic fluid can be swallowed by the fretus. This is shown by the fact that sodium sulphindigotate, when injected into the maternal circulation, is found in the amniotic fluid and in the alimentary canal of the foetus, although not in any of the foctal tissues. Fine lanugo hairs froni the fotal skin have also bcen found in the meconium.

The precise origin and manner of formation of the amniotic flund have not been cottled. It is probably in the main a maternal secretion or transudation. But something is contributed by the foetus in the form of renal, and perhaps of skin, secretions. The fluid is poor in solids. Its maximum content of protein, reached during the first half of pregnancy, is only 0.7 per cent. I ater on it diminishes, and at full zerm is only one-tenth of this amount. The specific gravity is 1000 to Ioog. Its osmotic concentration, as measured by the depression of the freezing-point, is less than that of the mother's blood-serum.

The allantois, gruwing out at the umbilicus, in the manner described, insinuates itsclf between the true and false amnion, and soon blends with the latter. For a time the secretion of the primitive kidneys continues to be poured in to the cavity of the allantois, so that it serves 
in part as an excretory organ, while in the bird it also performs the function of respiration; and in the mammal both fool and oxygen are carried by its vessels to the foetus during the greater part of intrauterine life. But later on the outgrowth atrophies and disappears, all except its origin from the alimentary canal, which dilates and persists as the urinary bladder, and its bloodvessels, which grow in the form of tufts or loops into the chorionic villi. The vessels are fed by two umbilical arteries which arise from the hypogastric arteries and run out at the umbilicus on the allantois. The blood is returned by an umbilical vein, whose further course we shall have soon to trace. The shrivelled stalk of the allantois, projecting through the umbilicus, takes part, with its bloodvessels, in the formation of the umbilical cord, which contains also the remains of the yolk-sac and is clothed externally by a layer of the amnion. Continuous with the umbilical cord, and stretching from the umbilicus to the urinary bladder, is a portion of the allantois which is represented in extra-uterine life by a thin cord-like structure, the urachus. The vascular tufts of the chorion, which at first cover the whole surface of the ovum and suck up food and oxygen from decidua serotina and reflexa alike, disappear in the region of the reflexa, hypertrophy all over the serotina - that is, where the ovum is in actual contact with the uterine wall-and this part of the chorion is now distinguished as the chorion frondosum. The giant villi of the chorion frondosum push their way into the thickened decidua serotina, and ultimately penetrate into the great capillaries or sinuses of the uterine mucous membrane. At the same time the tissue of the villi external to the vessels recomes reduced to a mere film, so that, except for a thin covering of decidual cells, the fœtal vessels are bathed in maternal blood. By this interweaving of decidua and chorion frondosum is formed the placenta, which for the rest of intra-uterine life acts as the great respiratory, alimentary, and excretory organ of the fœetus.

Exchange of Materials in the Placenta. - The maternal blood, as it streams through the colossal capillaries of the decidua, gives up to the fœtal blood oxygen and food substances and receives from it carbon dioxide and in all probability urea. It is true that the blood in the uterine sinuses is not itself fully oxygenated; it is not bright red arterial blood. But it yet contains more oxygen, and oxygen at a higher partial pressure (p. 247), than the purest blood of the fœet us, and is, therefore, able to part with some of the surplus to the dark stream of oxygen-impoverished blood brought by the umbilical arteries to the placenta. Thus, it has been found that while the blood of the umbilical artery of the fotus of a sheep had 47 volumes per cent. of carbon dioxide, and only $2 \cdot 3$ of oxygen, that of the umbilical veins had 6.3 volumes of oxygen, and only 40.5 of carbon dioxide (Zuntz and Cohnstein). In the exchange of gases between the placental and the fotal blood the same general features present themselves as in the external and internal respiration of the mother, with this difference, that the exchange of oxygen is neither between air and hæmoglobin, as in the lungs, nor between hæmoglobin and tissue elements, as in the organs; but between maternal and fotal hæmoglobin, of course, through the mediation of the maternal and fœtal plasma. There is no reason to suppose that the mechanism 
of the exchange is essentially different from that of the more familias forms of respiration. Diffusion of the gases from places of higher to places of lower tension unquestionably plays an important part. But this does not exclude the possibility of a more active process of some other kind, although there is at present no direct evidence of such a gaseous secretion as has been previously discussed in connection with pulmonary respiration (p. 262). The presence of oxydases in the placenta does not throw any light on the question. For there is no proof that they act in transferring oxygen from the one circulation to the other, and oxydases are found in the most diverse tissues. Their significance for the combustion processes of the body has already been alluded to (p. 27I).

Salts soluble in water, including not only those necessary for nutrition, like sodium chloride, but many foreign salts, pass readily from the placenta to the foetus, and in general more easily the lower their molecular weight. Such salts as potassium iodide, e.g., when injected into the maternal circulation, appear in the foet us in a very short time. On the other hand, colloidal solutions-e.g., of silver or silicic acid-do not pass over at all. It is of practical importance that substances like chloroform, ether, and other narcotics, and alkaloids likemorphineand scopolamine, when administered in obstetrical practice, may find their way from the mother to the child, although more slowly and more capriciously than the salts. While diffusion and osmosis assuredly take part in the passage of materials from the placenta to the fotus, there is no more reason to conclude that the whole exchange, even for the salts, depends upon such simple physical processes than there is in the case of the exchange between any one of the maternal tissues and the maternal blood. The essential similarity of placental and intestinal absorption, to take one instance, is seen in the mechanism by which the fœt us gains the iron required for the development of its hæmoglobin. The hæmoglobin of the mother appears to be the most important source of this iron. Erythrocytes in all stages of decomposition can be found in contact with the chorionic villi, and even in the epithelium covering the villi. These corpuscles come partly from extravasations in the maternal portion of the placenta, but it is possible that the villi also possess the power of hæmolyzing intact corpuscles in the crrculating placental blood. Iron can be demonstrated by microchemical reactions in the epithelial cells of the chorionic villi as fine granules, which increase in size towards the base of the cells. As we pass deeper into the villus towards its central bloodvessel, the granules again diminish in size. The picture is very like that seen in the absorption of iron from the intestine. And if the microchemical picture is practically the same, the process by which the iron is absorbed is not likely to be fundamentally different in the two cases (p. 44\%). 
The same is true of the passage of fat across the placenta. Fat can always be demonstrated micredemically in the chorionic villi. The most superficial layer of the villi is free from visible fat droplets. They increase in mmber towards the base of the epithelial cells. As in the case of the intestine, these appeatrances asree well with the view that the fat is split before being absorbed by the villi, and undergoes resynthesis in the (p)it]relium. That, as a matter of fact, fat passes from the mother to the foctus is shown by the observation that when pregnant guinea-pigs were ferl with a foreigin fat from cocoanuts), the characteristic fatty acicl (lauric acid) was found in the foetus. This, however, does not exclude the possibility that the fotus may form fat in its own tissues from carbo-hydrates, and perliaps from proteins, as it is destined to do in extra-uterine life.

Among the carbo-hyclrates the passage of dextrose from the maternal to the foetal blood has been experimentally demonstrated A specially interesting proof is afforded in cases where the mother suffers from diabetes mellitus. In one case in which the mother, during diabetic coma, was delivered of a stillborn child, the blood of the child contained $2 \cdot 2$ per cent. of sugar, its urine $5 \cdot 24$ per cent., and the amniotic fluid 0.47 per cent. The blood of the mother had a sugar content of 0.8 per cent., and her urine a content of 6.94 per cent. The sugar of the maternal blood is not the only source of the carbo-hydrates of the foetus. The glycogen store of the placenta is to be regarded as a second source, which is rendered available on conversion into dextrose by the placental diastatic ferment. This store of easily available food material is especially important in the early stages of development of the ovum before a circulation has been established in the villi. In the youngest ova investigated the decidual covering has been found rich in glycogen.

While it is to be supposed that the products of the hydrolytic decomposition of proteins can be absorbed by the foetal blood in its passage through the placenta, to be synthesized to the appropriate tissue proteins in the fœtal organs, there is evidence that certain proteins can be taken up without change. In this connection it must be remembered that the mother is much more closely related to the foetus as regards her protein composition than any ordinary protein food can be to an animal in extra-uterine life. In some respects, indeed, the fœtus may be considered, especially, perhaps, in the first stages of its development, as a part of the mother, an additional, although very complex, organ rather than an independent organism.

The blood of the umbilical artery, although far from the level of the ordinary arterial blood of the mother as regards its gaseous content, is yet the best the foetus ever gets; and by a series of contrivances it is assured that this best should go first to the most important parts-the liver, the heart, and the head-while the legs 
and most of the abdominal organs have to put up with an inferior supply: This is brought about mainly by the existence of three s!ort-cuts for the blood, which disappear in the adult circulation, the luctus venosus, the ductus arteriosus, and the foramen ovale.

The blood of the umbilical vein, rich in oxygen for foetal blood, passes partly through the circulation of the liver, but a part takes the route of the ductus venosus, and empties itself into the inferior vena cava. The latter gathers up the more or less vitiated blood from the inferior extremities and the renal and hepatic reins, and pours its mixed, but still fairly oxygenated cuntents into the right auricle. By means of the Eustachian valve, the jet coming from the mouth of the inferior vena cava is directed into the left auricle through the foramen ovale in the inter-auricular septum. There it is joined by the trickle of blood which is creeping through the unexpanded lungs. The left ventricle propels its contents through the aorta, and thus a large part of this comparatively pure or second-best blood is sent to the head and upper extremities. It returns in a ritiated state by the superior vena cava into the right auricle, and owing to the position of the Eustachian valve and the direction of the current, it Hows now, not through the foramen ovale, but into the right ventricle. Thence it is driven through the pulmonary artery, but only a small quantity of it finds its way through the lungs; the main stream is short-circuited through the ductus arteriosus, and mingles with the contents of the thoracic aorta below the origin of the cephalic and brachial vessels.

We may now give something more of precision to the statements that different parts of the body receive blood of different quality: and it is possible roughly to divide the organs in this respect into four categories: (I) The liver, which partakes both of the best and the worst, the purified blood of the umbilical veins and the vitiated blood of the intestines and spleen; (2) the heart, head, and upper limbs, which receive the blood from the inferior extremities and kidneys, mixed with the pure blood of the venous duct; (3) the legs, trunk, intestines, and kidneys, which are fed chiefly by the off-scourings of the cephalic end, mitigated, however, by a proportion of mixed blood from the inferior cava; (4) the lungs, which receive only a feeble stream of unmixed venous blood.

These peculiarities of the embryonic circulation are in obvious correspondence with the physiological events taking place in the fœtal body. The liver is not only the greatest gland in the embryo. as it continues to be in the adult, but its activity seems to dwari that of all the other glands put together, and is in striking contrast with the functional torpor of the lungs. From the third month of intra-uterine life the secretion of bile begins and the intestines gradually fill with meconium, of which the principal constituent is bile. Accordingly the liver is most lavishly supplied with blood, 
while the lungs are stinted. And since the liver has, as we have already learnt, other and, in the adult at least, even more important labours than excretion, a large portion of the blood it receives is of the best quality: it enters the gland comparatively rich in oxygen, and passes out comparatively poor; while the lungs, which have to be nourished only for their own sake, and are of no use whatever till the child is born and respiration has begun, must be content with the poorest fare-with the crumbs that fall from the table of fotal nutrition. The full-fed cephalic end of the embryo grows far more rapidly than the half-starved inferior extremities, and the head of the new-born child is large in proportion to the rest of the body.

Metabolism of the Embryo.-There are some other points in the physiology of intra-uterine life which call for remark; and, to sum up in a few words the grand distinction between fœtal and adult life, we may say that growth is the keynote of the former, work (functional activity) of the latter. Thus, the muscles at an early period in their development, long before any glycogen can be found in the liver, become the seat of an accumulation of glycogen, which, since it cannot be used up in contraction as in the adult muscles, seems to be intimately connected with their own growth, and perhaps also with the growth of other tissues. It is true that the fœtal tissues as a whole, including the muscles, are not richer, as used to be taught, but poorer in glycogen than adult tissues, and therefore the old doctrine that the fœtal glycogen fulfils a special 'formative' function in the development of the tissues, has lost its experimental basis. Nevertheless, there is a parallelism between the growth of the fœetus and its glycogen content. In cases where the growth of the fœtus has been spontaneously arrested, the percentage amount of glycogen in its organs has been found to be diminished out of proportion to the diminution in weight. A similar retardation of development can be produced by repeatedly injecting phloridzin into the mother, and thus reducing the glycogen store of the fœt us (Lochead and Cramer). Probably, then, the fœeta! glycogen assists the growth of the embryo, which is known to be accompanied by an intense carbo-hydrate metabolism, by furnishing a store of easily oxidized material for the nutrition of the developing tissues. When the muscles have been formed, their glycogen is still consumed in growth, and their functional powers lie dormant, but for the infrequent and feeble movements, generally regarded as reflex, but possibly to some extent originated in the cerebral cortex, which give the mother the sensation of 'quickening.' It is only late in development that the embryonic liver takes on its glycogenic function. In the earlier stages it is entirely free from glycogen. ' It is an interesting illustration of that exact adaptation of means to ends which so constantly impresses the investigator of the animal 
mechanism that the ferment which converts glycogen into dextruce (glycogenase) is also either eutirely absent from the liver early in gestation, o1 present only in traces; and that as the glycogren-forming and glycogen-storing functions of the organ increase in importance, it becomes richer in glrcogenolytic ferment. It cannot be drubted that the glycogen found in the placenta is also deposited there in the interest of the rapidly growing fotal tissues, perhaps as a kind of current account on which they can operate at any moment of emergency, when the more distant maternal reserves cannot be drawn upon in time. The glyrogen is formed in the placenta, probably from the dextrose of the maternal blood. By means of a glycogen-splitting ferment, which can be extracted by glycerin from the placenta, the glycogen appears to be reconverted into dextrose for absorption by the faetus. In the earlier period of gestation the placenta seems to perform vicariously the glycogenic function of the liver, and as the glycogen content of the liver increases in the later stages of intra-uterine life, that of the placenta diminishes proportionally.

The excretory glands of the embryo, except the liver, scarcely awaken to activity during foctal life. Urine may indeed be somelimes found in the bladder at birth, but it is often absent. It is a dilute urine, with a molecular concentration only about lialf as great as that of the blood, and although a portion of the amniotic fluid, which contains traces of urea and salts, in addition to small quantities of albumin, may be secreted by the renal tubules, and find its way through the still open urachus int o the amniotic sac, this contribution cannot imply more than a slight degree of glandular action. Under certain experimental conditions, however, it can be largely increased. Thus, extirpation of the kidneys in a pregnant animal causes an increase in the amount of amnjotic fluid (hydramnios) through the stimulation of the foetal kidneys to increased activity by the passage of the unexcreted urinary constituents of the mother's blood into that of the foctus. After the injection of phloridzin into the fnetus sugar las been found in abundance in the amniotic fluid, although the injection of that drug into the mother causerl no such effect. On the other hand, after injection of sodium sulphindigotate into the circulation of the fotus in the sheep, the foetal kidneys contained particles of the pigment, while tlu: amniotic fluid remained uncoloured. Long before full term the sebaceous glands have begun their work by the secretion of the vernix caseosa, an oily matcrial which covers the skin and serves to protect it from the continual irritation of the fluid in which the embryo floats.

The nervous system is even less active than the glandular tissues, and nut more active than the muscles. There is evidently no scope for the exercise of the special senses. Psychical activity of every 
kind must be at its lowest ebb. Conscionsness, if it exists at all, must be dull and muffled. And if motor impulses are discharged from the cortex, the psychical accompaniments of such discharge are doubtless widely different from those which we associate with voluntary effort.

It is a remarkable fact that this functional calm, broken only by the beat of the heart, is accompanied by a relatively intense metabolism of the same order of magnitude as that of the adult. In the hen's egg at all stages of development the consumption of oxygen and production of heat (per kilogramme and hour) are the same as in the adult hen. The oxygen consumption and carbon dioxide production of pregnant guinea-pigs were determined before and during compression of the umbilical cord of a fœtus, and a distinct diminution was observed when the respiratory exchange of the fotus was eliminated. From the results of a number of observations it was calculated that the carbon dioxide produced by the mother was 462 c.c., and by the fœtus 509 c.c. per kilogramme of bodyweight per hour (Bohr and Hasselbach). A similar comparison between women before and during pregnancy never showed any dininution in the respiratory exchange reckoned on the unit of bodyweight in the pregnant condition. In one case, indeed, and that the most exactly observed, there was an increase in pregnancy. Now, in the pregnant woman a considerable part of the increase of body-weight is due to the amniotic fluid, in which, of course, metabolism does not go on. It is evident, then, that in the human foctus also the intensity of metabolism is at any rate not of a lesser order of magnitude than in the mother, in spite of the much smaller amount of muscular contraction taking place. The heat production of mother and child together has been directly estimated in several cases in a respiration calorimeter provided with a bed just before parturition and just after it. After parturition the heat production of the mother was also separately determined. From the difference it was concluded that the heat production of the child per kilogramme of body-weight per hour is approximately two and a half times that of the mother under the same conditions. (Carpenter and Murlin.)

The fœtal heart beats at the rate of about Ifo times a minute at full term. The blood-pressure in the umbilical artery of the mature embryo (sheep) varies from 60 to so mm. of mercury; but at the beginning of the aorta it will be more. The pressure in

* It has not been finally determined whether the rate of the heart varies with the size or, what probabiy comes to the same thing, with the sex of the fœtus. As we have seen, the variation of the rate in the adult with the size of the body is associated with a corresponding variation in the metabolism and heat-loss, which are proportionally greater in a small than in a large animal. If this is a causal connection we should not expect that in the cmbrin in utero, where the conditions as regards heat-loss are entirely different, such a relation should exist, at any rate within the same species. 
the pulmonary trunk must be about equal to that in the aorta, since the comparatively short and easy circuit through the lungs does not as yet exist; and in accordance with this equality of pressure (of work to be dione) is the equality of thickness (of working power) in the walls of the two sides of the heart.

Suppose, now, that the embryo contains 60 grammes of blood for every kilo of body-weight, and that the whole of the blood passes through the circulation in twenty seconds. Then in twenty-four hours $259^{\circ} 2$ kilos of blood will be forced through the heart for every kilo of body-weight against a pressure of, say, $80 \mathrm{~mm}$. of mercury, or I metre of blood. This is equivalent, in round numbers, to 260 kilogramme-metres of work, or 0.6 calories. Now, taking the total heat-production of the heart at three times the equivalent of its mechanical work, we get $\mathrm{I} \cdot 8$ calories per kilo of body-weight in twenty-four hours (see p. 676 ), or about $\frac{1}{20}$ of the heat-production of a resting adult.

Such movements of the skeletal muscles as occur cannot account for any large proportion of the total metabolism, since they are executed in a medium (the amniotic fluid) of nearly the same specific gravity as that of the body, and therefore require the expenditure of a very limited amount of energy. The ordinary functional activity of the embryo, then, is quite incapable of accounting for the intensity of the fœetal metabolic processes. Still less can it be due to an active combustion in the tissues to compensate for a rapid loss of heat, for the fotus lies sheltered in the uterus as in a thermostat at its own temperat ure, and can lose practically no heat unless its temperature be kept a little above that of the maternal blood. The only remaining explanation of the magnitude of the fortal metabolism is that the growth processes are associated with a large amount of oxidation (and cleavage).

Notwithstanding the intensity of metabolism in the embryo, not only is even the purest blood, as has already been stated, far from saturated with oxygen, but the relative proportion of hæmoglobin, the oxygen-carrier, is less than in the adult; and although constantly increasing in amount from the moment of its first appearance, it is still somewhat deficient, even at full term, but leaps sharply up at birth. At an early period of development the embryo also contains much more water than the adult; the specific gravity of its tissues increases as development goes on.

The remarkable vitality of the fotus, and its resistance to asphyxia, are related not to the feebleness of its metabolism, but to the comparatively slight excitability and high endurance of nervous centres like the respiratory, vaso-motor, and cardio-inhibitory. Even when totally deprived of oxygen, as by pressure on the umbilical cord during delivery, the child does not perish in the two or 
three minutes which decide the fate of the aspliyxiated adult; nor are the convulsions, rise of blood-pressure, and slowing of the heart-beat associated with aspliyxia in the latter, so readily induced, nor premature and fatal efforts at respiration easily excited in utero. But although in such a case the embryo behaves as a separate organism, governed by its own laws, there are circumstances in which it becomes merely a part of the mother and participates in her fate. Thus, the stream of oxygen which normally passes from the maternal to the foetal blood is turnerl back if asphyxia threatens the mother; the blood of the umbilical arteries, instead of being purified in the placenta, loses the little oxygen it holds to the blood of the uterine sinuses, and the tissues of the embryo are impoverished to support the metabolism of the maternal organs. In the same way, the phenomena of starvation have taught us that the nutrition of the organism is not subject to the rules of red tape. In normal circumstances the flow of nutriment follows definite lines: the blood feeds the tissues through its intermediary, the lymph, and recoups itself from the contents of the alimentary canal. But when the normal sources of nutrient material fail, the body falls back upon its stores. The organs immediately necessary to life are kept, as far as possible, on full diet; organs of secondary importance have to be content with half-rations; organs less important still are drawn upon for supplies.

Parturition.-The period of gestation is abruptly closed about 280 days after the last menstruation, usually in what would have been the tenth intermenstrual period had menstruation been occurring. There is necessarily a consiclerable variation in the time when reckoned in this way, since the cessation of the menses merely announces that conception has occurred some time after the last period. It may even be disputed whether the fertilized ovum corresponds to the last menstruation or to the first absent period. Parturition, or the expulsion of the fotus, is accomplished by periodical contractions, the 'pains' of labour, at first confined to the uterus. Soon the os uteri begins to soften and dilate, the walls of the vagina become congested, and its secretions are augmented. The uterine contractions increase in frequency and force, and are now accompanied by reflex contractions of the abdominal muscles, and, if the woman is not anæsthetized, also $b_{i}$ voluntary contractions of these and of other muscles, which can increase the intra-abdominal pressure. The uterine contractions can be initiated and modified by impulses coming from the central nervous system by way of the extrinsic nerves of the organ. It is known, e.g., that the gravid uterus can be excited to contraction by the stimulation of various sensory nerves. Powerful mental impressions, such as fright, may bring on premature labour. Conversely, sudden cessation of labour pains during parturition is not uncom- 
1monly ols rind to be prorluced by emotiunal disturbances-for instance, the entrance of a stranger into the room. Yet the con1 ractions of the uterus are not essentially dependent upon extrinsic impulses. For not only do rhythmical contractions occur, but the whole process of parturition has been seen to take place in a uterus whose nerves have all been cut. Even the excised uterus may be kept alive for as long as forty-eight hours, and may go on executing periodical contractions when its bloodvessels are perfused with such an artificial fluid as Locke's solution, or, indeed, when it is simply immersed in the oxygenated solution (Kurdinowski) (Practical Exercises, p. I I 47 ).

It is a question of great interest how the uterine contractions are started so abruptly at full term after so long a period of quiescence. It can hardly be that the increasing mechanical distension of the uterus, tolerated for so many months, should suddenly, in an hour, beconne intolerable. For if the fotus dies before full term it is expelled without reference to the bulk which the uterus has reached. It is more likely that some chemical change associated with the completion of intra-uterine development, a change which leads, perhaps, to the production of some specific substance in the placenta or the foetus, is the determining event. The placenta is a structure whose function is strictly linited to the term of intra-uterine development. The fœtus is to live on, and so is the mother. May it not be that the placenta or essential elements in it are timed to die, or to begin to die, at full term, and that in their death or degeneration the substance or substances are produced which start, and later sustain, the uterine contractions? And may not the contractions of the utelus, by exciting its afferent nerves, or through the pressure of the foetus the afferent nerves of the vagina, in turn evoke the associated reflex contractions of the abdominal walls? These are questions which have been asked, but not as yet satisfactorily answered. It has also been suggested that a hormone formed in the mammary gland at full term stimulates the uterus and thus brings on labour.

At birth, great changes take place in the fotal circulation, and these are intimately connected with the commencement of the respiratory activity of the lungs. The canses of the first respiration are: (I) The increasing venosity of the blood circulating in the bulb, which stimulates the respiratory centre when the umbilical cord has been cut 0 : tied and the placental circulation thus interfered with; $(2)$ the stimula. tion of the skin by the air, which, as we have scen, acts reflexly upon the respiratory centre. That both of these factors may be involved is shown by the fact that either compression of the umbilical cord alone, or exposure of the foctus by opening the uterus of an animal without interference with the circulation, lias been observed to be followed by attempts at breathing. Once distended, the lungs never again completely collapse-not even after death, nor when the chest is opened. The aspiration caused by the elevation of the cliest-walls in 
inspiration (for the respiration of the newborn child is mainly costal) sucks blood into the thorax, and expands the vessels of the lungs for its reception; and in the measure in which the blood passing through the pulmonary trunk finds an easy way througl the lungs, the quantity which takes the route of the cluctus arteriosus diminishes. The pulmonary veins, and consequently the left auricle, are better filled; and the increasing pressure on this side of the septum tends to oppose the passage of the blood through the foramen ovale, to approximate its valve, and to close its orifice.

By the second or third day the ductus arteriosus has usually become obliterated. The umbilical arteries and veins and the ductus venosus become impervious som after the interruptios of the placental circulation. The vein and venous duct remain in the adult as the round ligament of the liver, the arteries as the lateral ligaments of the bladder.

Although from birth onwards the young mammal oltains its oxygen and gets rid of its carbon dioxide through its own pulmonary surface instead of through the placenta, it still lives, as regards its food proper, on the tissues of the mother, and that in as literal a sense as when it drew its supplies directly from the maternal blood.

Milk. -The milk secreted during the first few days of each lactation, the colostrum, as it is called, indeed may represent in part the fragments of cells lining the alveoli of the mammary glands, which have undergone a fatty change and been bodily broken down. The colostrum corpuscles are leucocytes filled with fat globules taken up from the contents of the alveoli. The chief chemical difference between colostrum and ordinary milk is the greater richness of the former in protein. It has been supposed that it is of special importance for the nutrition of the suckling, perhaps in virtue of the enzymes contained in it, and it is said that young animals bear artificial feeding much better if they have been allowed to suckle the mother for the colostrum.

In addition to the fat, which when milk is allowed to stand rises to the top as cream, milk contains a considerable quantity of caseinogen, to whose coagulation, under the influence of the lactic acid produced from the lactose, or milk-sugar, by certain bacteria, spontaneous curdling is due. Another protein, lact-albumin (Halliburton), a large amount of water, and some inorganic salts, are the most important of its remaining comstituents. Recently the presence of small amounts of phosplatides intimately associated with the protein constituents, and possibly combined with them as 'lecith-albumins' has been shown (Osborne and Wakeman). The molecular concentration (p. 426) of milk, as measured by its freezing-point, is almost exactly the same as that of blood-serum. Its electrical conductivity varies extremely, since it depends on the quantity of fat present, the fat globules, like the bloodcorpuscles, being practically non-conductors. The hydrogen-ion concentration of fresh cow's milk has been found to vary from $0 \cdot 18.10^{-6} \mathrm{~N}$ $\left(P_{\mathbf{B}}=6 \cdot 75^{*}\right)$ to $0.25 \cdot 10^{-6} \mathbf{N}\left(P_{\mathbf{H}}=6 \cdot 60\right)$ in the great majority of specimens.

* Instead of expressing the hydrogen-ion concentration as a fraction of a normal solution, it is more convenient for many purposes to take as a measure of the concentration the logarithm of this number, omitting the negative sign, represented by the s; mbol $\mathrm{P}_{n}$. 
This is distinctly greater than the hydrogen-ion concentration of blood. $-0 \cdot 10^{-8}\left(\mathrm{P}_{11}=7 \cdot 2\right)$ to $2 \cdot 10^{-8}\left(\mathrm{P}_{11}=7 \cdot 7\right)$. During the course of remmin action there is no change in the hydrogen-ion contentration of milk (Milroy').

The inorganic composition of milk is particularly interesting when compared witl that of the blood on the one hand and that of the -ucling on the other. Thus, 100 grammes of ash from each source give the following values for the rabbit ( $\Lambda$ bderhalden):

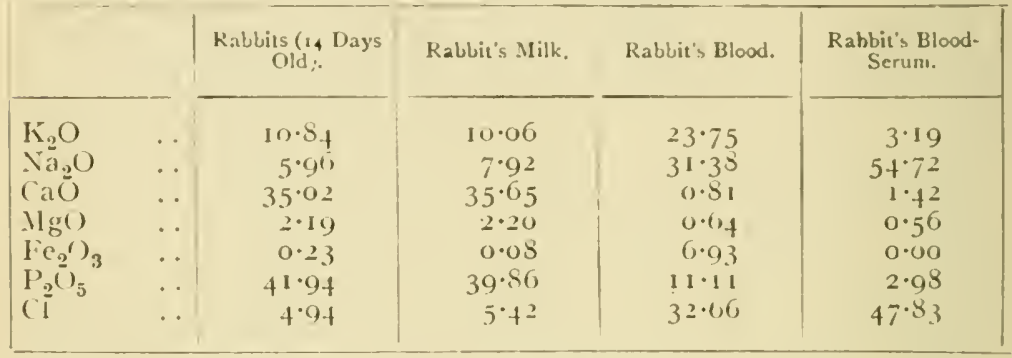

The richness of the milk (and of the suckling) in calcinm, phosphorus, and magnesium, as compared with the serum, is to be especially remarked. This is, of course, essential for the development of the bones. Whereas sodium predominates greatly over potassium in the serum, the opposite is the case in the milk (and the suckling). This is connected with the development of the tissue cells, which are richer in potassium than in sodium. The high chlorine content of the serum is in sharp contrast with the relative poverty of the milk in that element, which preponderates in the tissue liquids and is relatively scanty in the cells.

In addition to substances susceptible of chemical analysis, milk contains enzymes like those present in blood-serum, including oxydases and various hydrolytic ferments (proteolytic, diastatic, and perhaps lipolytic). It is now universally acknowledged that mother's milk is superior for the feeding of the infant to any artifirial substitute, and one factor in this superiority may be the presence of ferments specifically adapted for the digesticn of the human suckling. More important is the practical sterility of the human milk and the necessarily finer adaptation of its quantitative and qualitative composition, particularly the closer relationship of its proteins with those of the child. In addition, there is some evidence that the maternal milk contains immune bodies (antibodies) which may increase the resistance of the suckling to infections.

However this may be, there is no question that much of the high infant mortality associated with the industrial conditions of our great cities could be prevented if breast-feeding were carried out by every mother physically capable of it.

As to the manner in which milk is secreted, there is no doubt that its chief constituents are formed in the gland-cells. Caseinogen 
and lactose do not exist in the blood or lymph. The former is probably produced by an alteration in one or other of the serum proteins, the latter by a change in the dextrose of the blood. The fat of the milk may come partly trom the fat of the blood, but it mav also be formed in the gland-cel's from proteins, and carbohydrates. The precise manner in which the fat globules are extruded from the cells into the lumen of the alveoli is not clear, but there is $n o$ good ground for believing that the cells or their free ends break up bodily in the process.

Little is known as to the influence of the nervous system on the secretion of milk, and no definite secretory fibres have as yet been clearly demonstrated, although the fact that marked changes may be produced in the milk of nursing women as the result of emotional disturbances indicates that such nerves do exist. There is reason to suppose that the stimulus for growth and development of the mammary glands may be distinct from the stimulus which causes increased secretion. Some observers lean to the opinion that milk secretion is governed in an important degree by hormones carried to the glands in the blood. The effect of pituitrin has already been alluded to (p. 669.).

Pregnancy is accompanied with vascular dilatation and hypertrophy of the mammary glands, but the mechanism by which these changes are produced is unknown. It is probable that they depend upon some internal secretion of the ovary or some other of the organs of reproduction. Pregnancy is not an absolutely indispensable condition, and therefore it would seem that the exciting substance, if any specific substance exists, is not a product of the fœtus or of the placenta. Intravenous injection of extract of placenta is said to cause an increase in the flow of milk in goats, and the deduction has been drawn that some 'internal secretion' of the placenta may be responsible for initiating the activity of the mammary gland at the time of parturition. But precisely similar phenomena are occasionally seen in animals which have not been impregnated and even in men. Humboldt relates the case of an Indian father, who so well understood the responsibilities of paternity, and was so capable of fulfilling them, that he suckled his child for five months on the death of the mother. Virgin bitches are frequently known to produce milk, occasionally even in quantity sufficient to rear pups, the flow occurring about the time when they would have whelped had they conceived during the previous ostrus. Bitches which after copulation have 'missed ' having pups have been known to produce so much milk, beginning at the time they were due to whelp, that they were able to rear litters of puppies belonging to other bitches. Mules, which are themselves sterile, may have enough milk to suckle a foal. The nipples of certain monkeys become swollen and congested at each menstruation 
(Heape), and in women some development of the mammary glands is often associated with the menstrual period. The stimulus to the development of the gland in these cases appears to be some change. correlated with oestrus, and cannot be a cliange correlated witl pregllancy.

Cultivation of Tissues outside of the Body.-Closely related to the marvellous power of growth of the fertilized ovum in the favourable nidus of the pregnant uterus, although, of course, incomparably inferior, is the power of growth and reproduction of isolated tissue cells in a suitable medium outside of the body. An instance of this has already been described in the case of nerve-cells (pp. 803, 857). Many other tissues have been successfully cultivated in sterile coagulated lymph or blood-plasma. Comnective-tissue cells grow very easily, and can apparently be preserved indefinitely in the living state. A strain of these cells, originally obtained from a fragment of the heart of an embryo chick which had been pulsating in vitro for Iof days, has been seen to proliferate rapidly outside of the organism for more than sixtecn montls, and after more than I9) passages into fresh media. At the end of this time the rate of proliferation of the connective-tissue cells was even greater than that of fresh connective tissue taken from an embryo cight days old. Extracts of tissues and tissue juices under certain conditions accelerate the growth of connective tissue from tliree to forty times, the growth being measured by the increase in area of the mimute pieces of tissue. This activating power is especially marked in extracts of embryos, of adult spleen, and of certain sarcomas. This is noteworthy as the great characteristic of malignant tumours is their indefinite power of growth. The activating substance is unable to pass through a Chamberland filter (Carrel). Cultures of adult tissue have a smaller power of persistent growth. In the majority of cases growth in plasma without the addition of a stimulating tissue extract ceases after three or four generations (Walton).

The time of survival of tissues at low temperatures under conditions which do not encourage growth is also a matter of considerable interest, both from the physiological and the practical point of view, since living sterile tissue is required for a number of surgical operations-for example, slin for grafting. Skin lias been successfully grafted after being kept two to seven weeks in cold storage, but after a longer period there were many failures. Embryonic chick and rat tissues live longest at about $6^{\circ} \mathrm{C}$., but not more than twenty days under the most favourable conditions, according to 1.ambert.

Transplantation of Tissues.-Besides the growth and regeneration of tissues or organs, the simple displacement of them from their rormal situation and their implantation in a new enviromment have 
been studied. Normally, a migration of tissue elements is only' witnesed in the adult in the case of cells moving with the circulating liquids, or endowed with the power of amceboid movement. Under pathological conditions fragunents of tissue, such as tumour cells, may be carried by the blood or lympl to distant parts, and, settling there, may undergo development (forming metastases). In the embrvo the slow migration of tissue elements is a process which is responsible for some of the anatomical pecularities of the adult. The migration of the ovum from the ovary is the starting-point of the process of reproduction. The artificial displacement of tissues within the body of one and the same animal (anto-transplantation or autografting) can be successfully accomplished in the case of most normal organs and tissues, and also in the case of most tumours. Instances have already been given in speaking of the endocrine functions of the ovary, thyroid, spleen, thymus, etc. (Chap. XI.). A small piece of tissue or sometimes a small organ is simply inserted in its new situation without provision for the immediate establishment of a circulation. Necrosis of the central portion occurs, but the peripheral zone soon becomes vascular, the graft 'takes' anrl under suitable physiological conditions grows. Hetero-transplantation, or grafting between animals of different species, does not succeed. Homoco-transplantation, or grafting from one animal to another of the same species, is in the case of normal tissues successful only in rather rare instances. In most cases, although some of the homøe-grafted tissue may remain alive for some time, or even begin to grow, its growth is soon checked, and it is eventually absorbed. Certain tumours, however, can be readily grafted from one animal to another of the same species, and can live and grow in the new environment.

The difference in the fate of auto- and homcografts illustrates in a striking way the important chemical and metabolic differences which cxist, not only between different species, but between individuals of the same species. In general, a piece of tissue from a rabbit is treated as an 'unclean' thing, which must eventually be cast out, not only when it is introduced into the body of a dog, but when it is introduced into the body of another rabbit. It is unable to adapt itself to its new environment, and soon perishes. But a bit of thyroid, of adrenal cortex, or of uterus, may easily settle down as a successful colonist in very outlandish places within the body of the animal to which it belongs. An invasion of lymphocytes, and an ingrowth of fibroblasts, causing development of bands of connective tissue, have been regarded by some observers as the immediate causes of the failure of homøografts to grow. It is fully as probable, however, that the lymphocytes gather around and invade the graft, and that the connective tissuc trabcculie appear in it, because it has already been injured by the antibodies of the host, or, if not by specific antibodies, then simply by exposure to the more or les; altered metabolic conditions, which it is unable to facc successfully: The difference betwcen the autograft and the homøograft has not yet been sufficiently taken account of by surgeons, e.g., in connection with 
transfusion of blood. They have slowly learnt that in this case hetero. grafting for we can truly call transfusion the grafting of the fluid tissue blool) is not permissible, and nolody now allows sheep's blood to pass into the veins of a man. But the danger to the host of repeated and massive lomoo-transfusions is only beginning to be recognized by the men who have the power to "bind and to loose "veins and arteries.

Some normal organs, e.g., the ovary, are more readily homoeografted than others. Guthrie has shown that heis whose ovaries have been interchanged are capable of laying eggs. When the hens were impregnated and the eggs hatched out the colour characters of the resulting offspring secmed to have been influenced, not only by the hen to which the ovary originally belonged, but also by the hen to which it had been transferred.

Young liave also been obtained from guinea-pigs, whose ovaries had been replaced by ovaries from other gninea-pigs. Eighteen months after interchange of the ovaries in two sister puppies, it was shown by histological examination that the engrafted ovaries contained numerous normal Graafian follicles, as well as corpora lutea. Statements are on record of successful ovarian homceografts even in women.

Another point of great interest in connection with homoeografting is that a small number of individuals of a species may constitute more favourable hosts than the great majority for a tissue from another

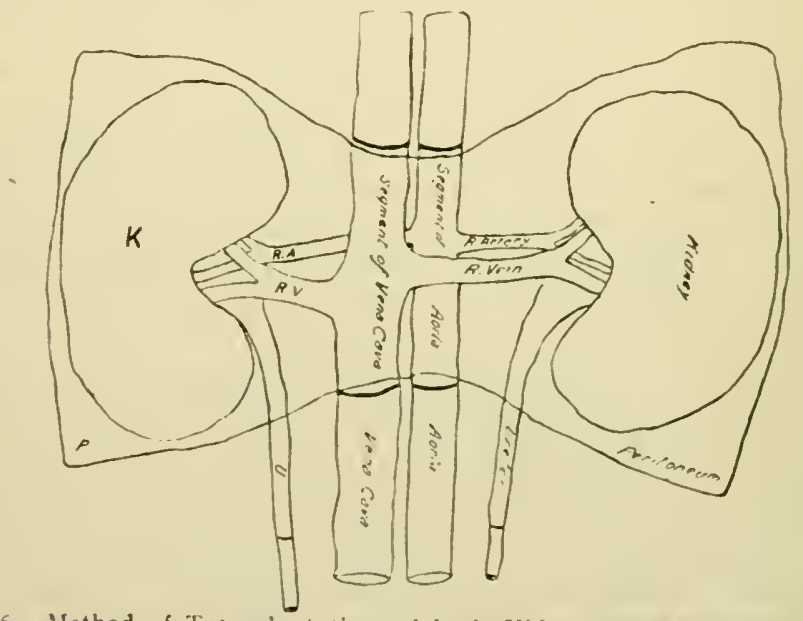

Fig. 486.- Method of Transplantation (of buth Kidneys) in Mass (after Guthrie). Segments of the inferior vena cava and abdoninal aorta are removed with the kidney's and renal vessels, and interposed in the course of the vcna cava and aorta of another animal, according to the method of Carrel and Guthric.

individual of the same species. For instance, a rabbit's thyroid cannot as a rule be successfully grafted into another rabbit, even when thyroid deficiency has been caused by removal of the greater part of the thyroid from the host. But if a large number of rabbits are tried one will occasionally be found in which thyroid homeo- 
grafts succeed (Marine and Manley). It is not as yet known what the circumstances are which so modify the usnal adverse conditions that a homoegraif can take, grow and permanently survive.

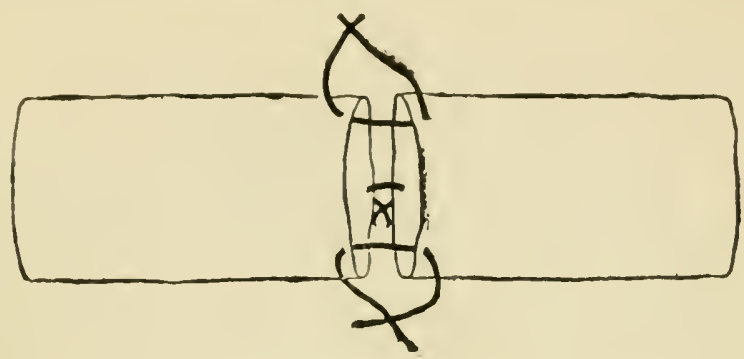

Fig. 487 . - Suturing Bloodvessels: Prelininary lixation of Ends of Divided Vessels (after Guthrie). Three fixing ligatures arc placed at equidistant points on the circunference of the cut ends, each ligature being passed through corresponding points of the two vessels. The cuds of the vessels are approximated by drawing on the ligat ures, which are then tied, and the margins of the vessels sewed together by continuous stitches in the intervals between the fixing ligatures, as in Fig. $\$ 88$ (Carrel's method).

But there is reason to believe that the solution of this problem would be a long step towards answering the inmensely important practical question what the conditions are which permit the development of malignant tumours.

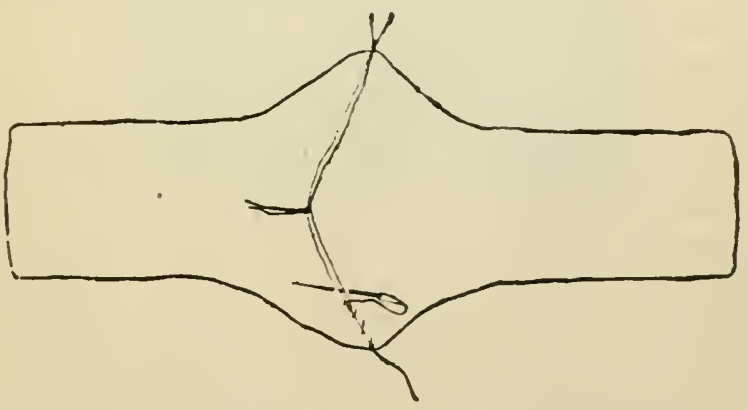

Fig. 488.-Suturing Bloodvessels: Method of approximating Idges a id putting in Continuous Suture (after Guthrie). The needles are very tnt ca nbric sewingneedles, and the threads single strands of Chinese twist silk or human hair. Needles and threads are sterilized in paraffin-oil. (Method of Carrel and Guthrie.)

Transplantation of organs may also be done with anastomosis of bloodiessels. The main ressels of the engrafted organ are sutured to suitable arteries and veins in the 'host,' so that the circulation is at once effective. Consequently there is practically no limit to the size of the grafts. The kidney. spleen. and even a limb, have 
been transplanted in this way from one dog to another. Although from the operative point of view successful, the homoer-transplants do not permanently survive. Reimplantation of organs, however, in one and the same animal with suturing of the bloodvessels has often been successfully performed. Such orgaus as the kidney live and function after reimplantation, and the operation is now a recognized physiological method of insuring that an organ has been completely disconnected from the central nervous system, since even the nerves running in with the bloodvessels must have been cut. It has been shown that a reimplanted kidney suffices to maintain a dog in complete health for an indefinite period after the removal of the other kidney.

In the case of structures like the large bloodve'sels, which perform mainly a passive mechanical function, homøo-transplantation succeeds. Segments of arteries preserved in cold storage for a few days or even weeks, and even portions of arteries fixed by formaldehyde, have been transplanted so as to take the place of segments removed from arteries of living animals, and have continued to function perfectly for long periods. Portions of veins have also been used to fill up gaps in arteries. Even heteroplastic vascular grafts have been found to succeed, portions of dog's arteries, e.g., grafted into a cat, and portions of rabbit's, cat's, or human arteries grafted into a dog. Doubtless the favourable result is largely due to the fact that the mechanical function of the large arteries can be discharged even by a dead tube of the requisite strength, and with the smooth interior presented by a dead endothelial lining (Carrel, Guthrie).

Parabiosis.- Not only may an organ or a portion of tissue from one individual be engrafted on another, but two individuals may be so united that a greater or smaller degree of physiological intimacy is produced between them. Occasionally, as in the famous Siamese twins, an anomaly of development results in such close anatomical union of the circulatory and other systems that in certain respects the two individuals constitute almost a single organism, and cannot be separated by surgical interference. A less intimate union can be established experimentally by opening the peritoneal cavities of the two animals, and suturing the skin and connective tissue together so as to permit of permanent communication. Pairs of animals living in this condition (socalled parabiosis) have been utilized for the study of certain questions in immunity. White rats lave been kept alive in parabiosis for as long as thirty-four days in order to test the question whether destructive antibodies for cancer are present in the circulation (Rous), since it has bcen slown that circulating antibodies easily pass trom one to the other of such a pair of animals (Ehrlich). 
One of each pair of rats had a glowing tumour produced by transplantation, while the other had been prosed resistant to the same type of tumour. No evidence of the passage of an antibody was found in this case.

\section{PRACTICAL EXIERCISES.}

1. Contractions of Isolated Uterine Rings.-Kill a female adult rabbit by striking it at the back of the neck. A rabbit which is not pregnant, or only at the beginning of pregnancy, shomld be selected. Open the abdomen, and carefully remove the uterus. While separating the organ from the broad ligament and vagina, support the horns of the uterus on soft threads. Ligature the vagina before cutting through

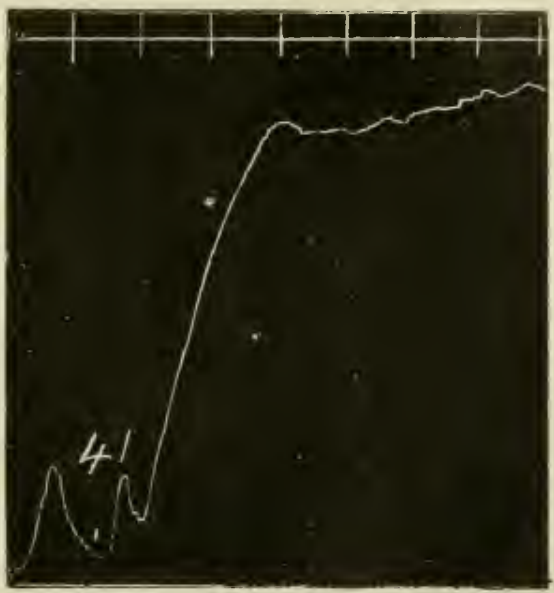

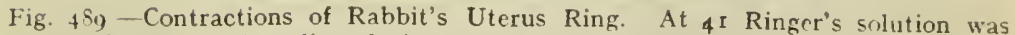
replaced by adrenalin solution, $r: 1,000,000$. Time-trace, half-minutes.

it, and cut below the ligature, which can then be used to manipulate the uterus. Do not pinch the uterus with forceps, and handle it as little as possible. At once place it in Ringer's or 'Tyrode's solution* (p. 200), kept at body temperature $\left(38^{\circ} \mathrm{C}\right.$.) in a small beaker immersed in a water-bath, as in the experiment on the contraction of isolated intestine ( $\mathrm{p} .452$ ). Cut a ring of tissile about $\mathrm{I} \frac{1}{2}$ centimetres in width from one of the horns. Tic a loop with a fine silk thread at each end of a diameter of the ring, pinching up a little of the external coat to do so with fine forceps. Make the arrangements necessary for recording contractions of the circular fibres of the ring while it is immersed in a glass cylinder in the bath, as in Experiment 1, p. 452. Comnect another segment

* Tyrode's solution contains $0.8 \mathrm{gm}$. $\mathrm{NaCl}, 0.02 \mathrm{gm}$. $\mathrm{KCl}, 0.02 \mathrm{gm} . \mathrm{CaCl}_{2}$ $0.0 \mathrm{Im}$. $\mathrm{IgCl}_{2}, 0.005 \mathrm{gm} . \mathrm{NaH}_{2} \mathrm{PO}_{4} 0^{\circ .1} \mathrm{gm}$. $\mathrm{NaHCO}_{3}$ and $0.1 \mathrm{gm}$. dextrose in roo c.c. of water. 
longitudinaly to a lever as in that experiment, and make all the arrange. ments mentioned there. After a longer or shorter interval spontancous rhythmical contractions of the uterus ring connence. As soon as they are well established, and while the contractions are being recorded on a very slow drum, replace the Ringer's solution by serum, defibrinated blood, blood prevented from coagulating by citrate solution (p. 66), or hirudin, or by plasma, and note the effect. The serum or plasma may be cliluted to a known amount with Ringer's or 'Tyrode's solution before

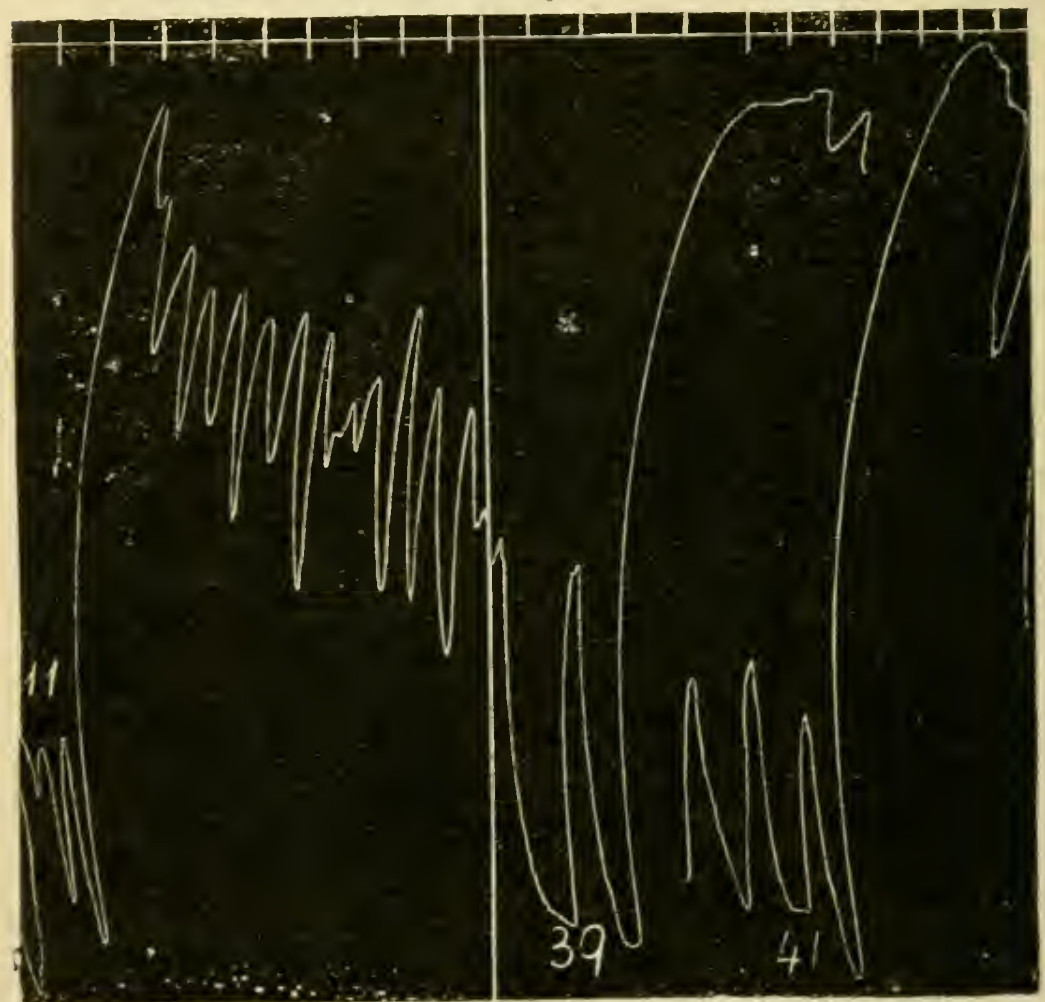

Fig. 490.-At I I Ringer's solution was replaced by citrate plasma. At 39 Ringer s solution was replaced by hirudin plasma; at 4 I by the corresponding hirudin serum.

application to the segment. Wish away the serum or plasmat thoroughly with Ringer's solution. Replace the Ringer's solution by adrenalin solution $(1: 10,000,000)$. Note whether the tone of the ring (as shown by its permanent shortening) or the rate and strength of the contractions are increased. While a tracing is being tilken repeat the observation, adding a larger proportion of adrenalin. Determine in what concentration a distinct effect is produced. A suffecient number of utcrus rings can be obtained from one animal for a considerable number of experiments. 
2. Comparison of Changes of Tone Produced in Uterus Segments by Different Concentrations of Adrenalin. lint this the utelus of a virgin rabbit (full-grown or nearly so), is best, as it is alvantageous that the spontaneous contractions should be absent or fecble. Starting always with the segment* in Ringer's or Tyrode's solution replace the solution by adrenalin in different dilutions ( $I: 10,000,000,1: 50,000,000$, I: $\mathrm{r}, 000,000$, etc.), washing off the adrenalin thoroughly with the Ringer's solution. Compare the effect of serum or defibrinated blood obtained from the rabbit itself or from some other laboratory animal with that of a known solution of adrenalin.

If blood collected from the adrenal veins of an animal is available it should be compared with blood from the same animal taken from the
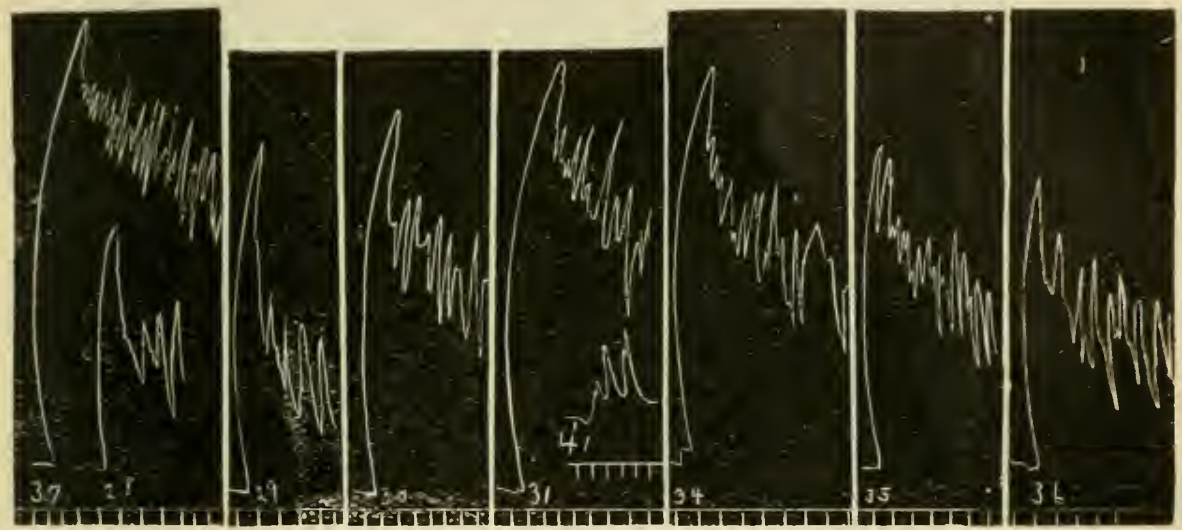

Fig. 49r.-Action on Rabbit's Uterus Segment of Blood Specimens from the Adrenal Veins (of a Dog) with Different Concentrations of Epinephrin, and Comparison with Adrenalin added to Blond. At 28 Ringer's solution was replaced by the second adrenal specimen; at 29 by the third adrenal specimen; at $30 \mathrm{by}$ the fuurth adrenal specimen; at $3 \mathrm{I}$ by the fifth adrenal specimen; at 37 by the sixth adrenal specimen; at $4 \mathrm{I}$ hy jugular vein blood. All bloods diluted with 15 volumes Ringer's solution. At 34 adrenalin in jugular blood ( $1: 2,000,000)$; at 35 adrenalin in jugular blood ( $1: 3,000,000)$; at 36 adrenalin in jugular blood ( $1: 4,000,000)$ replaced Ringer's solution. The adrenalin bloods after being made up to the concentrations mentioned, were diluted with I5 volumes of Ringer's solution before application to the segment. (Reduced to one-half.)

general circulation (jugular vein or carotid artery). The tone-increasing power of the adrenal vein blood will be greater than that of the indifferent blood, becausc both the serum and the epinephrin will act in the same sense (liig. +9I).

3. Partition of Adrenalin between Serum and Corpüscles.-Add to roo c.c. of dog's blood 0.5 c.c. of the $I: 1,000$ solution of adrenalin. Centrifuge a portion of the mixture to obtain clear serum. Centrifuge another portion of the dog's blood to which adrenalin has not been alded. 'Test on rabbit's uterus segments and also on intestine seg-

* It is lest to arrange the segment to record the longitudinal shortening. 
ments (p. 453) the defibrinated blood, the serum and the sediment of corpuscles from the original blood and from the blood to which arlenalin was added. The adrenalin action of the sediment will be much less than that of the serum or of the blood (Fig. 492).

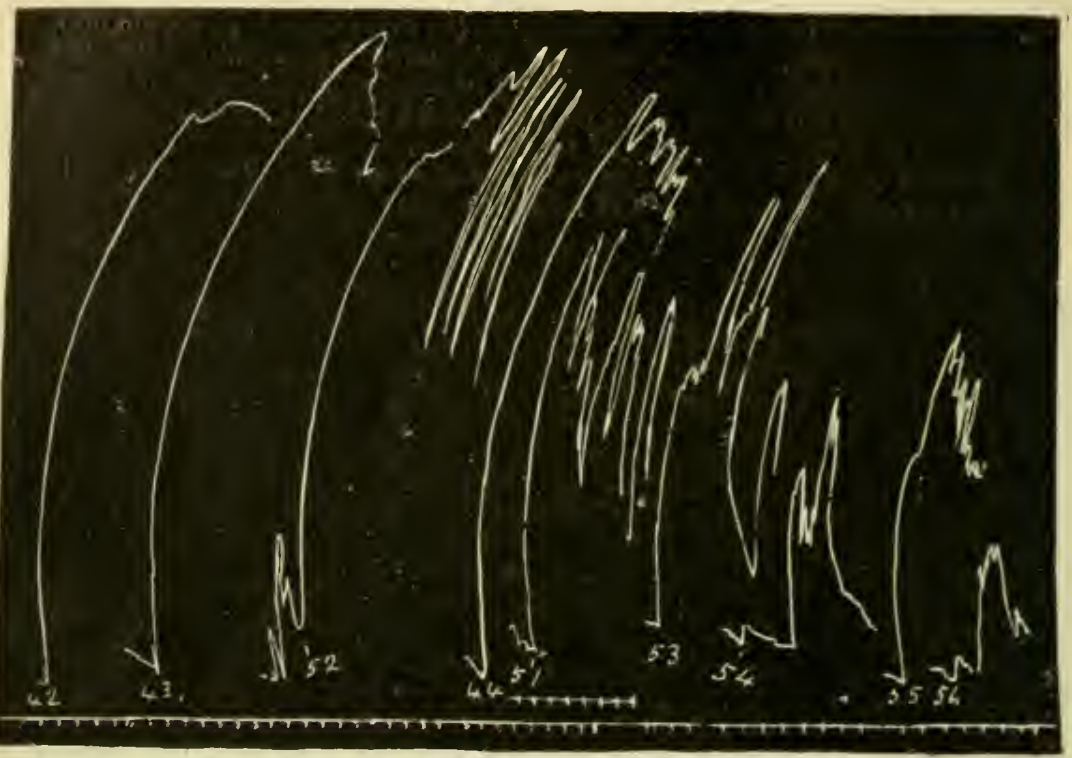

Fig. 492.-Action of Adrenalin Defibrinated Blood, Serum and Sedinent on Rabbil's Uterus Segments. At 42 Ringer's solution was replaced by adrenalin blood serum (I part in 6 parts of Ringer's solntion); at 43 by the same serum ( $I$ in II); at 44 by the same serum ( $\mathrm{I}$ in 16 ); at 52 by the same sermm ( $\mathrm{I}$ in 24); at $5 \mathrm{I}$ Ringer's solution was replaced by adrenalin blood ( 1 in 24); at 5.3 by adrenalin hlord sediment ( $\mathrm{I}$ in 6 ); at 54 by the adrenalin blood sediment ( 1 in 24 ); at 55 by the ordinary defibrinated blowl ( 1 in (6); at 56 by the ordinary defibrinated blood (I in 24). (Reduced to one-half.) 


\section{APPENDIX A}

C MIPARISON OF METRICAL IVITH ENGLISH MEASURES.

Measures of Length.

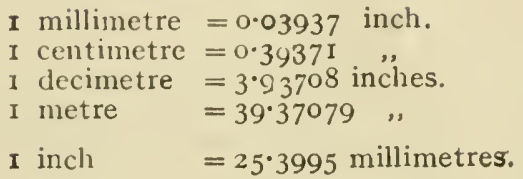

Measures of Weight.

$\begin{array}{ll}\text { I gramme } & =\text { I } 5 \cdot 432349 \text { grains. } \\ \text { I kilogramme } & =2 \cdot 20462 \text { I } 3 \text { pounds. } \\ \text { I ounce } & =28 \cdot 3495 \text { grammes. } \\ \text { I pound } & =453.5926 \quad \text { ". }\end{array}$

Measures of Volume.

I cubic centimetre $=0.061027$ cubic inch.

I litre ( $\mathrm{I}, 000$ cubic centimetres) $=61 \cdot 027052$ cubic inches.

$$
\begin{aligned}
& =\mathrm{I} \cdot 760773 \text { English or } 2 \cdot 1 \mathrm{r} \\
& \text { American pints. } \\
& =0.22009668 \text { gallon. }
\end{aligned}
$$

I cubic inch $=$ I6.386I759 cubic centimetres.

I cubic foot $=28 \cdot 3$ I 53 II 9 cubic decimetres (or litres).

I pint $=0.567932$ litre.

I gallon $=4 \cdot 5+34579$ litres.

\section{Measures of Work.}

I kilogrammetre =about $7 \cdot 24$ foot-pounds.

I foot-pound $\quad=0.138 \mathrm{I}$ kilogrammetre.

I (kilo)calorie of heat $=425.5$ kilogrammetres of work.

Temperature Scales.-To convert degrees Fahrenheit into degrees Centigrade, subtract 32 , and multiply the remainder by 5 . To convert degrees $C$. into degrees $F$., multiply by $\frac{y}{3}$, and add 32 to the result. 


\title{
A PPENDIX B
}

\section{BIBLIOGRAPHY.}

\author{
CHAPTER I. \\ INTRODUCTION.
}

Colloids.-Becriold, Die liolloide in Biologie und Medizin, Dresden, I913. VAN Bemmelex, Die Absorption (colloids), Dresden, Igro. Botazzi, Archivio di Fis., Igoy, 7, 579. Buxtox and RAlle. Hofmeister's Beit., Igo8, 11, 479. Field and Texaue, J. Exp. Med., I 907, 9, 222 (electric charge of native proteins and agglutinins). HARDY, J. Phy*s., I905-6, 33, 251 (colloidal solution). HEARD, J. Phỹs., I9I 2, 45, 27. HōBER, Hofmeister's Beit., I9oS, 11, $6_{4}$ (neutral sall actions). PAI'LI, Hofmeister's Beit., I $906,7,531 ; 3,225$. ROBERTSON (T. B.), Die physikalische Chemie der Proteine, Dresden, igi 2. Schriver, Proc. Roy. Soc., Igio, B 83, 96. Walpole, J. Phys., I91 3, 47, p. xiv.

Coagulation of Proteins.-Brglia, Arch. Internat. de Physiol., I9Io, 10, 224. Chick and Martix, J. Phys., I910, 40, 404; 19II, 43, I; 1912, 45, 6i, 26i. IItrRay (C.), Bioch. J., I906, 1, I67. RaMsdex, Proc. Roy. Soc., I903, B 72, I 56 (surface layers of solutions and suspensionsmechanical cnagulation). Sutheriaxd, J. Phỹs., Igri, 42, p. vii.

Structure and Aggregate State of Protoplasm.-Chambers, Am. J. Phys., I 917,43 , r. JEXSEx, Pflüger's Arch., I901, 8y, 36r. HARDY. J. Phy's., I899, 24, I58, 2n?. Kite, Am. J. Phys., I913, 32, i 46. Mathews, Biol. Bull., Igo6, 11, I 4 i. Rhumbler, Z. f. allg. Phys., I902, 1, 279. SClïfer, Qu. J. Exp. Phys., I $910,3,285$. SCHExck, P'flüger's Arch., Igoo, 81,584. IIACALLUM (A. B.). J. Phys., I905, 32, 95 (distribution of $K$ in cells).

Reaction of Protoplasm.-Barratt, Brit. Med. J., June I8, I904. p. I4I3. Hevdersox (L. J.) and Black (O. F.), Am. J. Phys., 1907, 18, 250.

Protoplasmic Movement.-Jexsex, Ergeb. d. Physiol. (Bioph.), I902, I. KüHNe, Z. f. Biol., I $897,35,43 ;$ ib., I $898,36,425$. SснENCK, PHüger's Arch., I $897,66,2+1$ (oxygen and protoplasmic movement).

Differentiation and Specificity of Starches.-REICHERT (E. T.), Carnegie Instit. Pub., I913 (Washington).

Nucleus Plasma Relation.-Howard (W. T.). J. Exp. Med., I9oS, 10, 207.

Combinations of Proteins and Inorganic Substances.-LOEB ( J.), Am. J. Phys., r $900,3,327$ (ion-protein compounds). RORFRTSON (T. B.), Ergeb. d. Physiol., 1910, 216 . Mass (G.), Chemistry of the Proteids, London, Igof. PAuli and Haxdowskr, Hofmeister's Beit., IgoS, 11, ${ }^{\text {I }} 5$.

Plant Proteins.-Osborie (T. B.), Ergeb. d. Phys., I9ro, 47; Science, rgos, $28,41 \%$ 
CH.IPTER II.

\section{THE CIRCULATING LIQUIDS OF THE BODY.}

Bl'CKMAStER (G. A.), The Morpholugy of Niormal and Pathological Blood, Lonclon, 1906.

Erythrocytes.-DEETJEN, Vircliow's Arch., I901, 165, 282 (envelope). MEYIR, Arch. Int. Med., I91 $4,14,94$ (colour index). Ali3RECHT, Zentr. f. Physs., 1905, 19, II) (envelope). Meves, Anat. Anzeig., 23, 212 (structure). Preskixd, Am. J. Pliys., I903, 8, $4 \mathrm{I} 4$ (action of acids and acid salts). Lölisik, Arch. Mikros. Anat., 1907. 71, 129 ('membrane' of). Rous ind TURner, J. Exp. Med., I916, 23, 219,239 (living erythrocyles in vitro). SCHÄFle. Anat. Anzeig., Iyn5, 26 (structure). WERER and SUCHARD, Arch. de Pliys., ISSo, 12, 52 I (rouleaux). SteWART (G. N.). Am. J. I'hy"s., I 902, 8, i is (envelope of Ncchurus corpuscles).

Blood Formation and Regeneration.-Foot, J. Exp. Med., 1913, 17, 43 (bone-marrow in villo). HALL and EuBANK, ib., I896, 1, 656. Noll, Ergeb. d. Phys. (Bioch.), I903, 433. Malassez, Arch. de Phys., 1882, I. Pearce (R. M.) Et al., J. Iixp. Med., I9I 2, 16, 758, 769, 780 (spleen). Seenan, Ergeb. d. Phys. (Bioch.), r904. 12. Woolley, J. Lab. Clin. Med., I916, 1, 3+7 (in foctus). OPIE, J. Exp. Med., 1905, 7, 759. SeEMass, Ergel. d. Pliys. (Bioch.), I g04, 30.

Blood at High Altitudes.-ABDERHALDEN, Z. f. Biol., 1902, 43, 125. 443; Pflüger's Arch., I 905, 110, 95. BÜRKER, $i b ., 1904,105,480$. JAQUET, Arch. Exp. Path. Pharm., I900, 45, i. Schneider and Havens, Am. J. Phys., I91 5, 36, 380 . Henocoue, Arch. de Phy.s., 1889, 7 Io. Gaule, Pflüger's Arch., I902, 89, i i9. Gurllemard and Moog, J. Phys. Path. Gén., I907, 9, 17; ib., I910, 12, 869.

Destruction of Erythrocytes in the Body.-BAIN, J. Phys., 1903, 29, 352 (role of liver and spleen). Findlay, ib., I910, 40, 445 (hamolysis in the liver?). KYES, Internat. Monatsch. Anat. Phys., I9I 4, 31, 543 (in birds). Rous and Robertson, J. Exp. Med., I91 $7,25,651,665$.

Variation in Number of Erythrocytes.-Bootin and PERRY, Am. J. Phys., I9I 5, 37, 378. HAWk, ib., I $90_{4}, 10,38+$ (effect of exercise). BÜRKER, Pflüger's Arch., 1905, 107, 426 (techirique). Dowss and EDDY, Am. J. Phys., $1917,43,415$ (influence of secretin). HASSELBalch and HEYERDAHL, Skand. Arch. Phys., Igo8, 20, 289 (some physical causes of variation). WARD, Am. J. Phy.s., i 90, 11,394. Wells (J. J.) and Sutton (J. E.). Am. J. Phys., I915, 39, $3 \mathrm{r}$ (blood counts in frog, turtle and mammals). (For influence of Adrenalin see Chapter Xil.)

Permeability of Erytbrocytes.-DE, BoER, J. Pliys., r9I7, 51, 2 I I (influence of respiration on exchange (f $\mathrm{SO}_{4}$ between corpuscles and plasma). HAMBURGER, Osmotischer Druck und Ionenlehre; Bioch. Z., I9I5, 71, $46+$ (influence of osmotic pressure and the permeability problem); Z. f. Phy: ikal. Ch., I909, 69, 663 (for Ca ions). Hö.3ER, I'füger's Arch., 1904, 102, r 96; Oppenheimer's Handb. d. Bioch., I9o8, 2, I. MAsiNG, Pflüger's Arch., 1913, 149, 227 (glucose). MANwaring and Kusama, Soc. Exp. Biol. Med., I916, 13, I73 (for protein). Ronovy, liolloid. Chem. Beihefte, I9I6, 8, 337, 377 (Physiol. Abstracts, 1917, 2, 178, I79). SPIRo and HENDERSON, Bioch. Z., I 908,15 , I 44 (influence of $\mathrm{CO}_{2}$ ).

Osmotic Relations of Erythrocytes.-BA.NG. Bioch. Z., I909, 16, 255. Eykuas, Pfluger's Arch., I 897, 68, 58 (permeability). Hedix, $i b ., 68$, 229 (permeability). HöBER, ib., I904, 102, 196 (ion-permeability). GRYNS, $i b ., 1896,63,86$; ib., I905, 109, 289. HAMBCRGER, Z. f. Biol., $1897,35,252,289$ (respiratory exchange and volume of crythrocytes). Koravyr and Bexce, Pflüger's Arch., 1905, 110, 532 (action of $\mathrm{CO}_{2}$ ). KOEPPE, $i b_{,}, 1897,6 \%, 189$. MOORE and ROAF, Bioch. J., 1907, 3, 55. Scott (F. H.), J. Phys., 191 j, 50, 128. Stewart (G. N.), J. Phys., I $000-1,26,470$. 


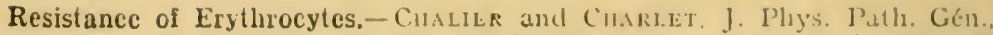

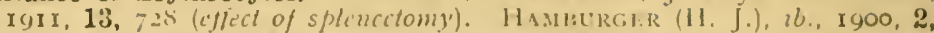

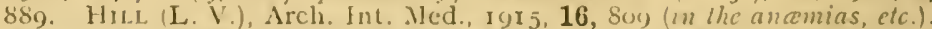
Musser and likumbhiar, J. Am. Med. Ass., I916, 67, 180,1.

Hæmolysis.-ARrhisius, Bioch. Z., rgo8, 11, I6r; Eig(h. d. Phys., 1908, 48o. BasifoleD, Arch. de Pharmacodyn., 8, 101; 9, 15 I (bloodimmunity). BAUMGarTen, Bioch. Z., I9o8, 11, 2 I (osmological theory). BORDET, Anm. Instit. Pastenr, Igoo, 14, 257; BORDET and GAY, ib., I908, 22 (hamolytic sera). Eilricn, Proc. lioy. Soc, I 9o6, B 66; Berl. Klin. Woch. (numerous papers from 1899 onwards). FLexNer and Nogecur, J. Exp). Med., 6, 277; Kiyes, Berl. Lilin. Woch., ryo3, Nos. 42, 43; Bioch. Z..,

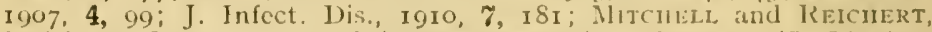
Smithson. Contrib., ISS6, 9 (venom lamoly'sis). Gutnkr: (C. C.), $\Lambda \mathrm{m}$. J. Phys., 1903, 8, 4tr ; J. Lab. Clin. Med., r917, 3, S7; GrTIIRIE and LeE, Soc. lixp. Biol. Med., Ior t. 11, I 49 (laking by hipertonic solutions). JOBLING and BULL, J. Exp. AIed., I91 3, 17, 61 (velation of immune serum lipase to hamolysis). likat's and Levaditr, IJandl). d. 'Iech. u. Method. d. Inmunit ätsforschung, 1, 57; 2, 903. MICPIEURAN, J. Exp. Med., 1913, 18, 527 (acids and hamoly'sis). MANIVARING, Irit. Med. J., 1906, 2, I542; J. Inf. Dis., 1907, 4 (physical chemistry of hamolytic sermm); J. I3iol. Ch., 1907, 3, 387 (quantitative methods). MIELtzER and WELCH, J. Phys., I $88.4-5,5,255$ (shaking). Nogucur, Bioch. Z., I907, 6, 185. TraubE, Bioch. Z., Igo8, 10, 37I; Pflüger's Arch., I904, 105, 5łI, 559 (lipoids and hamolysis). PESKIND (S.), Am. J. Plyss., 1904, 12, 184 (ether laking). ROAF, Q. J. Exp. Phys., I9r2, 5, r32 (laking by alkali, hypotonic NaCl, heat). Stewart (G. N.), J. Pharm. Exp. Ther., Igog, 1, 49 (mechanism of hemolysis); An. J. Phys., 1902, 8, 103 (nucleated corpuscles); ib., 1903, 9, 72 (influcnce of cold) ; ib., 190.4, 11, 250; 12, 363 (hamolysinogenic and agghetininogenic action of laked curpuscles); J. Mled. Res., 1902, 8, 268; J. Phys., r900-r, 26, 470. WASSErMand, Immune Scra (translated by Bolduan).

Cytolysins.-Lambert, J. Exp. Med., I913, 19, 277. Taylor, J. Biol. Ch., I $908,5,31 x$.

Leucocytes.-Busch and VAN Bergex, J. Afed. Res., 1902, 8, 408; ib., 1903. 10, 250 (diffcrential connt in dog and cal). DEETJ EN, Arch. f. Phy's, 1906 4 or (division of human leucocyles in vitro). Evass (F. A.), Arch. Int. Med., r916, 18, 692 (mononuclear). Harny and Wesiruok, J. Phys., I 895, 18, 490. Hamburger (H. J.), Arch. f. Phys., I yoz, Sipp. Bd., Ir9 (permcability of loucocytes). Kantmack and Hardy, J. Phys., I $894-5,17,81$ (wandering cells of mammalia). Hlow'E and HAWK, Am. J. Phys., I9I2, 30, 174 (differential count during fasting). JiHRLICH and LAzarus, The Histology of Blood, Normal and Pathological. Ross (H. C.), J. Phys., I 905, 37, 327 (death of lencocytes). WALKER (C. E.), 1'roc. Roy. Soc. (Lond.), 1906, 3 78, 53; ib., 1907, 79, 491, 195 (life histor)").

Blood-Platelets.-Brown (IV. H.), J. Exp). Ned., I9I3, 18, 275 (hislogenesis). BürkER, Pflüger's Arch., r904, 102, 36 (in coagulation). IBUNTING, J. Exp. Mled., 1909, 11, 54I. BAYNE-Jones, Am. J. Phys., IgI 2, 30, 74 (prothrombin and thromboplastin). DEETJEN, Z. f. Ply'siol. Ch., Igog, 63, I. DukE, Arch. Int. Med., r913, 11, I00; J. Am. Mcd. Ass., I915, 65, I600 (cause of variations in platclel count). EMMEL (V. L.). J. Med. Res., I917, 37, 67 (crythrocytic origin). LEE and MINOT, J. Am. Mled. Ass., Apr. 2I, I9I 7 , I II (significance).-LE SOURD and PAGNiez, J. Phy"s. Path. Gén., I909, 11, I (in coagulation); ib., I9I 2, 14, I167 (in regencration of blood). Minot, Arch. Int. Nled., I917, 19, Io62 (in purpura, elc.). Zucker and STEWART, Zentralb. f. Phy's., 1913, 2\%, 85 (platelcts and the vasoconstrictor property of scrum). ISEMP and CALHOUN, Am. J. I'llys, IgoI, 5, iv. Wrigit (J. H.) and Mlinot, J. Exp. Med., IoI 7, 26, 395. \%uCker (T. I⿳.), Soc. Exp. Biol. Med., 191., 11, 60 (platclets and clolling).

Viscosity of Blood.-Burton-Opitz, J. Exp. Mled., IgoG, 8, 57, 2q0; J. Phys.,

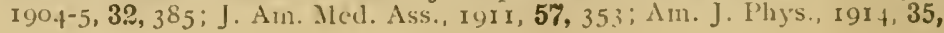


51. 265. DENMING and W.ATSUN, ['ruc. Roy. Suc., 1yor, 13 78, 328.

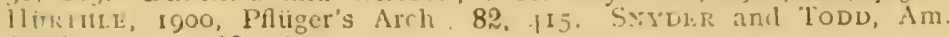
J. Phys., I9II, 28, I6I.

Reaction of Blood.-Aller, Am. J. Phys., Iyo7, 19, I. ClLleN, J. [Biol.

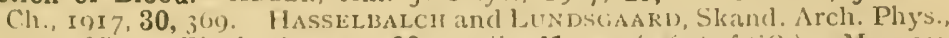

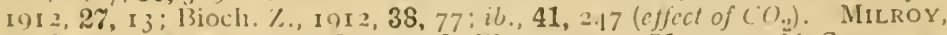

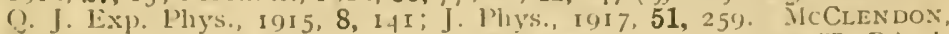
f. Biol. Ch., I0If, 24, 519; ib., 25, orig (mothod). ['ARSOSS (T. R.), J.

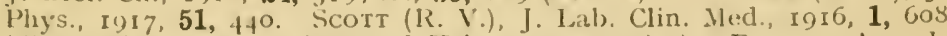
(dissociation curve as index of $H$-ion concentration). PETERS, Am. J. Phys., 19r7, 43, II3 (CO $)$. Levy, Rowntree and Marriot, Arch. Int. Med., I915, 16, 389. Robertson, J. Biol. Ch., 1909, 7, 351.

Regulation of Reaction.-Hevderson (L. J.) and Black (O. F.), Am. J Phy's., r9os, 21, t20. Henderson, $i b ., 21,173,427 ; i 6$., ryo $7,18,250$; Ergeb. d. Physs., ryo9, 25t; J. Biol. Cl., I909, 7, 29. MOORE and Bigidand, Bioch. J., I009, 5, 32. MCClendon ET AL., J. Biol. Chem., 1917, 31, 519. lROBLRTSON, ib., 1909, 6, 3:3

Relative Volume of Corpuscles and Plasma.-Bleibtreu, Pflüger's Arch., I 892,51 , I $51 ; i b .$, IS'95, 60, 405. CAPPS, J. Med. Res., I903, 10, 367 (volume index). FRan NCKEL (1.), \%. f. Klin. Med., I904, 52, 470; STEWART (G. N.), J. Phys., I S99, 24, 356 (electrical resistance method). HEDIN, Pil ü., er's Arch., I $95,60,360$; KOEPPE, ib., 1905, 10\%, I87 (hematocrit). LARRABEE, J. Med. Res., I9I I, 24, I5 (volume index).

Electrical Conductivity of Blood.--Brünings, Pfü̈rer's Arclı., I903, 100, 393. HöBer, Physikal. Chem. d. Zelle. Hamburger, Osmotischer Druck. Frank. Am. J. Phys., 1905, 14, 466 (during coagulation). Oker-Blon, Pfï̈rer's Arch., i 900, 81, i67. StewArt (G. N.), J. Phys., 1899, 24, 2 I I. Wilson (T. P.), Am. J. Phys., r905, 13, r39; Bioch. J., $1907,2,377$ (in coagulation).

Blood-Coagulation.-Artuus, J. Physs. Path. Gén., I gor, 3, 887 (fluoride); i6., I902, 4, I, 28I; Arch. de Phys., I890, 22, 79. Collinivood and Mácllailon, J. Phys., igr 2, 45, i i9. Cramer and Pringle, Q. J. Exp. Phys., 1913, 6, 1; J. Phys., 1912,45 , p. xi. Delezexne, J. Phys. Path. Gén., I $897,333,347$ (bivd's blood). IIowell, Am. J. Pliys., I9I I, 29, i 87 (antithrombin); ib., I9I 4,35, I 43 (ultramicroscope); $i b .$, I9I6, 40, 526 . Loeb (L.), Hofmeister's Beit., I 907,9 , 185 . LOEB and FileISCHER, Bioch. Z., I910, 28, I69 (coagulins). Mellanis, J. Phys., I908, 38, 28. Pick and SpIro, 'L. Physiol. Ch., I goo, 31, 235 (proteoses). Morawitz, Ergeb. d. Phys., 1905, 307; Deutsch. Arch. Klin. Mled., 1903-4, pp. I, $215,432$. REICHERT, J. Exp. Med., I905, \%, I 73 (second coagulalion). RETTGER, Am. J. Phys., I 909, 24, to6. Dastre and Floresco, Arch. de Phys., 1896, 402. STUebel, P'flüger's Arch., I91 +, 156, 361 (ullramicroscope).

Coagulation Time.-ADDIs, Q. J. Exp. Phy's., 1908, 1, 305. BüRkER, Pfüger's Arch., I9I 3, 149, 3 I 8. CANnon and GRAY, Am. J. Phys., I9I 4, 34, 232. CANion and MeNDENHALl, ib., I91 4, 34, 243 (adrenalin effect). Simpson and Rasmussen, Q. J. Exp. Phỹs., inio. 10, r 59.

Calcium and Coagulation.-Akthus, Arch. de Phys., 1896, 47; ib., I $897,219$. Ar'stin and PePper, Arch. Int. Med., I9I3, 11, 305. ADdis, Q. J. Exp. Med., 1909, 2, r 49. BE.1RD, J. Phy's., I917, 51, 294. Hess, Soc. Exp. Biol. Med., I9r6, 13, 59. Goddird, Im. J. Phys., r914, 35, 333. Morawirz, Hofmeister's Beit., I904, 4, $38 \mathrm{r}$. Ricur, An. J. Phys., r917, $43,37 \mathrm{I}$.

Anticoagulants.-Fraxz (F.), Arch. Exp. Path. Pharm., 1903, 49, 342 (hivudin). HAYCRAFT, ib., r88.1, 18, 209 (leech extract). LeE and VinCENT, J. Med. Res., 19I5, 32, 445 (anaphylaxis and lecch extract). Mellanby (J.), J. Phys., 1909, 38, 4f I (venoms). Minot, Am. J. Phys., I9I5, 39, I I (chloroform). PRINGLe and TAIT, J. Phys., I910, 40, p. xxxv.; ib., I9I I, 42, p. xxxviii. Sinttuck, Arch. Int. Med., ror7. 20, I67 (profein intorication). 
Proteoses and Blood-Coagulation.-Artius and HuBl: Arch. de Phys., I896, 857. DASTRE, and liLORESCO, ib., I897, 210 . DLLLLENNL, ib., I895, 8, $655 ; i b ., 1 S 98,568$ (velation of liver). GLEY and I'ACHON, ib., 1896, 715 (liver). PICK and SPIRo, Z. Physiol. Clı., Iyoo, 31, 235. ScirmidTMUÜLheIs, Arch. f. Pliys., isSo, 33.

Fibrin, Fibrinogen.-Howell, Am. J. Phys., IyI6, 40, 526 fiurin-gel and theories of gel-formationt. Morawitz, Oppenlieimer's Handb. d. Bioch., ii., 2,60 .

Origin of Fibrinogen.-Goodpasture, Am. J. Phys., 1914, 33, 70. NATHews (A. P.), ib., I 899, 3, 53. MEEK, ib., 1912, 30, IGI (liver). OPIE, BARKFR and Dochez, J. lixp. Ned., 1911, 13, 162. Winpple, Am. J. Phys., I $91.1,33,50$.

Thrombin, etc.-Collinwoov and MAcMalon, J. Phys., I9I3-I 4, 47, 44 (thrombin and antithromlin). GASSER, Am. J. Phys., I9I7, 42, 378 (significance of prothrombin and thrombin in serum). HOWELL, Am. J. Pliys., IyI4, 35, 474 (prothrombin). DRINkER (C. K. and K. R.), ib., I II), 41,5 (prothrombin from bone-marrow). MINOT and DENNy, Arch. Int. Med., I9I 6, 17, Iо I (prothrombin and antithrombin factors in coagulation). Ricil, Am. J. Phys., I9I 7, 43, 549 (metathrombin). MellanBy (J.), J. Pliys., Ior 7, 51, 396 (vate of formation from prothrombin).

Antithrombin.-Dexiny and Minot, $\Lambda$ m. J. Phys., I915, 38, 233. Hess, J. Exp. Med., I915, 21, 338.

Thromboplastic Substances in Coagulation.-Howell, Am. J. Phys., I9I2, 31, I. MACRAF and SCHNACK, ib., I913, 32, 211 (action in clotting). ICLean, ib., I916, 41, 250; ib., I9I 7, 43, 580 (cephalin).

Intravascular Coagulation.-DAvis, An. I. I'hy's, I9II, 29, I6o. HalliBURTON and Brodie, J. Phy's., I 89., 17, I 35. MUdGE, Proc. Roy. Soc. (Lond.), 1907, B 79, 103. Pickering, J. Phys., 1896, 20, 3 Io (albinos). IV'RigitT (A. E.), J. Pliys., I $891,12,184$.

Coagulation in Invertebrates.-ALSBERG and ClARK, J. Biol. Ch., I9o8, 5, 323 (limulus). LOEв (L.), Bioch. Z., I910, 24, 478 . TAIt, Q. J. Exp. Phys., I $910,3,1$.

Blood-Proteins.-Briggs, J. Biol. Ch., I915, 20, 7. Cullex and VAx Slyke, Soc. Exp. Biol. Med., I9I6, 13, 197 (melhods). EPstein, J. Exp. Med., I913. 17, 444. Porges and SPIRo, Hofmeister's Beit., Igo3, 3, 277 (globulius). ROBERTSON, J. Biol. Cl., I912, 13, 325. THOMpson, ib., I 915,20 , I. Wells, $i b .$, I91 $3,15,37$. WOOLSEY, ib., I9I3, 14, 433. Hammarsten, Ergeb. d. Phys. (Bioch.), 5902, 330. Mellanby (J.), J. Phys., I907-8, 36, 288.

Blood-Lipoids.-BLooR, J. Biol. Ch., I9I6, 25, 577 (man). BRown (E. W.), Am. J. Phys., 1899, 2, 306 (cholesterol in birds). Joslin, Bloor and Gray, J. Am. Med. Ass., I917, 69, 375; SEo, Arch. Exp. Path. Pharm., I909, 61, I (diabetes).

Cholesterol in Blood.-Bloor, J. Biol. Ch., 1916, 24, 227; ib., 1917, 29, 437. Csonka, ib., 1916, 24, 431. Gorhay and MYERS, Arch. Int. Med., I9I7, 20,599. WEston and línt, J. Med. Res., I912, 26, 531.

Blood-Fat.-Bloor, J. Biol. Ch., I9I 4, 19, I. Terroine, J. Phys. Path. Gén., $1914,16,212$.

Serum Ferments.-Bronfenbrenner and Scott, Soc, Exp. Biol. Med., I915, 12, I 37. Jobling, Petersen and Eggstein, J. Lah. Clin. Med., 1916, 1, 172; J. Exp. Med., I91 5, 22, 1 29, 508. SLOAN, Am. J. Phys., 1915, 39, 9. CARLSON and Luckiiard, Am. J. Pliys., I co8, 23, 148. GOULD and CARLSON, ib., I9I I, 29, I65; OTTEN and GALLOWAY, ib., 19I0, 26,347 (relation of pancreas to sernm diastase). Kisa, ib., I9I 4, 35, 301. VAN DER ERVE, ib., 191 I, 29, I82 (diastatic enzymes in serum).

Vasoconstrictor Property of Serum.- - TKINSON and IitzPATRICK, Soc. Exp. Biol. Med., Ior2, 9, 49. Cusmis and Guns, J. Pharm. Exp. Ther., IOI $3,5,1$ (action of serum on perfused heart). O'Coxior (J. MI.), Arch. 
Jixp. l'ath. Pharm., I912, 67, r95. STEWART (H, A.) and HARVEY (S. (.). J. Exp. Med., 1012, 16, I03. Stewart (G. N.) and 7,icklek, 1. Exp. Aled., Ior 3, 17, 152. TAtum, J. Plarm. Exp. Ther., 191 2,4, i 5 .

Anaphylactic or Protein Shock.-Avisirson and Scuulrz, Soc. Exp. Biol,

Mled., 1910, 7, 32. AUEr and Robinson, J. Exp. Med., 1913, 18, +50, 5.5). EISENBRE and PEARCE, J. Pharm. Exp. Ther. I012, 4, 21. Joliling, PETERSEX and Eciciser, J. lixp. Med., 1015.22, for (ferment achons). MANWARING and CrowE, J. 1 mmunity, I917, 2,517; VOFGTLIN and I3ERNIEIM, J. Pharm. Exp. Ther., I9I I, 2, 507; WEIL, J. Immunity, I $) 17,2,525$ (relation of liver to). WEIL, ib., I9I $7,2,129$ (the vasomotor depression in).

Hænoglobil and Derivatives.-BARCroft and Hill, J. Phys., IoIo, 39, 4I I (nature of oxyhamoglobin, molecular weight). JlÜFNER and GANSSER, Arcli. f. Phys., 1907, 209 (molecular weight). Jisciner (H.), Ergeb. d. l'hys., I916, I85, 791 (blood and bile pigments). I1ALDANE, J. Phys., I) oo-1, 26, 497 (colorimetric delermination); ib., ISos, 22, 29S (methamoglobin). Hartery, J. Phys., 1907, 36, 02 (sulphhamoglobin). AlsBera; and Clark, J. Biol. Ch., I910, 8, I; AlsberG, ib., I915, 23, 495 (hamocyanin). MENzIES, J. Phys., I $895,17,402$ (methamoglobin). MILROY (J. A.). J. Phys., I909, 38, 38+ (hamochromogen); ib., I909, 38, 392; MeNzIES, $i b .$, I9I 4,49 , p. iv. (hamatin). LAIDLAw, ib., 1904, 31, $46+$ (synthesis of homatin). REICHERT, Am. J. Phys., I903, 9, 97. REICHERT and Brown, Soc. Exp. Biol. Med., I907-8, 5, 66; Proc. Am. Phil. Soc. (Philadelphia), Iyo8, 47, 298. FriEboEs, Pflüger's Arch., 1903, 98, $43+$ (hemoglobin crystals).

Quantity of Blood.-Dougl.ss, J. Phys., I905-6, 33, 493; ib., I910, 40, 472. Haldane and Siitil, J. Pliys., i 899-I900, 25, 33 I ; Smith (J. Lorrain), ib., p. vi. (CO method). DREYER (G.), RAY and WALKER, Skand. Arch. Phys., I9I 3, 28, 299. KeIth, Rowntree and Geraghty, Arch. Int. Med., I9I 5, 16, 547. Piescir, Z. Exp. Path. Ther., I909, 6, 380. Zuntz and PlesCH, Bioch. Z., I908, 11, 47.

Vividiffusion.-Abel, Rowitree and Turner, J. Pharm. Exp. Ther., I9I4, 5, 275. ROHDE (A.), J. Biol. Ch., I9I 5, 21, 325 (ammonia of the circulating blood).

Lymph.-Carison, Greer and Luckhardt, Am. J. Phys., I908, 22, 9 I (chlorides). Carison, Woelfel and Powel, ib., I9II, 28, I 76 (local hamodynamic action of tissue metabolites). GREEN (J. R.), ib., I9Io, 26, 68; Rous, J. lixp. Med., I908, 10, 537 (leucocytes). Davis and Petersen, J. Exp. Med., I9I 7, 26, 693 (ferments). Howell, Am. J. Phys., I9I $4,35,483$; Lussky, ib., 25, 354 (coagulation). Hughes and CARLsor, Am. J. Phys., I908, 21, 236 (hamolytic action). LuckHardT, ib., I9I0, 25, 345 (conductivity compared with serum).

Chyle.-Hall (IV. S.), J. Am. Med. Ass., I9Io, 55, 388. Hamill, J. Phys., i 906-7, 35, I 5 I. Paton (D. NökL), J. Phys., iS9o, 11, i I I. Sollmann, Am. J. Phys., 1907, 17, 487 .

\section{CHAPTER III.}

\section{THE CIRCLLATION OF THE BLOOD AND LYMPH.}

CARLSON, Biol. Bull., I905, 8, I 23 (comparative phy'siology of invertcbrate hearl). MacWillia., J. Phys., I 885, 6, I92 (heart of eel); ib., I888, 9, I07 (rhythm of mammalian heart). NUKADA (S.), Die Automatic und Koordination des Herzens, Tokyo, I9I 7 (limulus heart). RoY and ADAMI, Phil. Trans. Roy: Soc., I892, 183, I 99 (phy'siology and pathology of heart). TIgerstedt, lireislauf. WIGgers, The Circulation in Health and Diseaso, 1915 . 


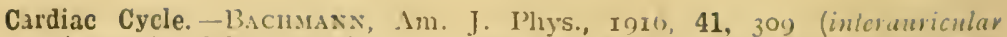
internal). Wiggers, ib., In16, 40, 218 (anicular mugram); ib., Iyto,

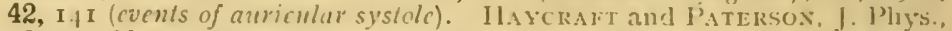
I $895-6,19,496$ (changes in hearl's shape and position); ib., 262 (papillary wuscles). TUNNiclifFe, $i b ., 1890,20,51$ (diaslolc).

Heart Sounds. -BATTAERD, I Hart, 19I5. 6, I 21 (graphic ;esearches). TBRIDGMAN, Heart, I9I 5, 6, 4 I. Einthoves, Pfliiger's Arch., 1907, 120, 31. Sewall, Phila. Monthly Med. J., Sep., i 899 (papillary muscles). TulYir, Arcli. Int. Med., I909, 4, 297 (third heart-sound). Wiggers and DE.x, Am. J. Phys., I917, 42, +70 (nature of time relations).

Registration of Heart Sounds.-CREnore and MEARA, J. Exp. Med., IgII, 13, oif (micrograph). Eixtiovex, Pfliger's Arch., 1907, 117, 46r. Holowixski, Arch. de I'hys., IS96, 823. HürThle, Pflüger's Arch., I $895,60,263$. Weiss (O.), Phono-kardiogramme (Jena, I909). IVIGGERS and DEAN, Am. J. Mcd. Sci., I917, 153, 666.

Heart Rate.-BAINBRIDGE, J. Phys., I9I 5, 50, 65 (influence of venous filling on rate). BuchaNax (F.), ib., 1910, 40, p. xlii (in hibernation); ib., I9os, 37, p. Ixxix (in mouse); Sci. Progress, July, r91o (in vertebrates). Loeb and EWALD, 13ioch. Z., I9ז3, 58, 177 (heartorate as function of the lemperalure).

Function of Pericardium.-Barnard (H. L.), J. Phys., 1898, 22, p. xriii. KíNo (l.) , ib., I9I5, 50, r.

Endocardiac Pressure: Auricle.-Wiggers, Am. J. Phys., I91.4, 33, I3. ZWALUWENirtrg and AGNEW, Heart, I91 2, 3, 3. 3.

Endocardiac Pressure: Ventricle.-FraNk (O.), \%. f. Biol., i $S_{9}$, $35,478$. FraxçoIs-Frank, Arch. de Phys, r890, 22, 395: ib., I893, 25, 83. Porter, J. Exp. Med., i $S 96,1,296$. Tigerstedt, Skand. Arch. Phys., 1912, 28, 37; ib., 1914, 31, 241. Wiggers, Am. J. Phys., 1914, 33, 382.

Coronary Circulation.-Barbour and Princi, J. Exp. Mld., I9I5, 21, 330 (epinephrin). Guthrie and PIKE, Science, I906, 24, 52 (coronary pressure and action of heart). HYDE, Am. J. Phys., 1, II 5 (dilation of ventricles and coronary flow). LANGENDORFF, Phüger's Arch., 1899, 78, 423 (isolated heart). Magrati and IEENnedr, J. Exp. Med., 1897, 2, I 3 (coronary circulation and ventricular beat). MARKWALDER and STARLING, J. Phys., 191 3. 47, 275. Miller and MAtruews, Arch. Int. Med., r 909, 3,476 (obstruction of left coronary). PORTER, J. Exp. Med., $1895,1,46$ (closure of coronaries); Am. J. Phys., 1, I.t5 (infucnce of bcat on coronary flow).

\section{PULSE.}

Arierial Pulse.-Dawson (P. M.), Am. J. Phys., r9I 7, 42, 6r, 3 (pulsc íclocity). FRANK (O.), Z. f. Biol., 1899, 3\%, 483 (mathematical); ib., 1905, 46, 44 . Hewlet , Arch. Int. Med., I9I4, 14, 609 (reflection of primary wave). Hill, BARNARD and SequeIRa, J. Phys., IS97, 21, I 47 (effect of venous fressure). HOORWEG, Pflüger's Arch., I 905, 110, 59 S (peripheral reflection). HürTILE, Pflüger's Arch., I $890,4 \%$, I 7 (origin of secondary waves). Lewis (T.). J Phys., 1906, 34, 41 4. Lohmax, Pflüger's Arch., 1904. 103, 632 (dicrotic wave). MacWilliam, liesson and Melvis, Heart, I@I 3 , 4, 393 (conduction of pulse wave). Tigerstedt, Eirgeb. d. Phys., 1909, 593. WigGers, J. Am. Med. Ass., I915, 64, I +53 (contour of normal pulse).

Venous Pulse.-Cushny and Grosh, J. Am. Mled. Ass., 1907, 49, I 254. Evixg, Am. J. Phys., I914, 33, I58. Erster, J. Ixp. Med., I911, 14, 594; ib., 1910, 12, 257. F'REDÉRICQ, Zentralb. f. Phy"s., 190S, 22, 297. MICQUEEN and FAlconer, J. Phys, 191 4, 48, 292 (" $C$ 'wave). Morrow, Brit. Ned. J., Oct. 27, and Dec. 22, I006; Pflïger's Arch., 1900, 79, $44^{2}$ (velocity). Wiggers, J. Am. Med. Ass., I9I 5, 64, I $4 S_{3}$.

Cardiopneumatic Movements.-HARris, J. Plyys., 1905, 32, 495 . HAYCRAET, ib., I891, 12, 426. Meltzer, Am. J. Phys., ISg8, 1, i 7 . Stewart (G. N.), Arcli. f. Phys., I912, 460. 


\section{BLOOD-PRESSURE.}

Arterial Blood-Fressure.-13kooks, Heart, roro, 2, 5 (tracings frour quicscent

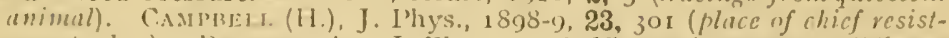
ance to flow). 1).11:son, Am. J. Phys., 1906, 15, 211 (pressure al different points of arterial tree). lirank (O.), Z. f. Biol., I907. 50, 281 (clustic

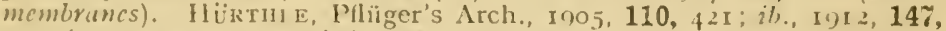
509 (pressure and velocity). MACCRAKEN and WERnless, J. Pharm, Exp. Therilp., I') r 7, 9, 305 (overcoming clolling). Prlcurer, Am. J. Phy's., I915, 38, 209 (membrane manometcr curves). RiDdLe and MATTIEIVS, Am. J. P'hyss, Ino7, 19, 108 (birds). WIragers, ib., I9r.f. 33, r (pressure curve in pulmonary artery). Wood (H. C.), ib., I899, 2, 352 (traube waves). Wooley, J. Lab. Clin. Med., I9 5 $5,1,203$ ( $\mathrm{CO}_{2}$ and blood-pressure).

Action of Proteoses on Blood-Pressure (and Coagulation).-ChitTenden, Mendel and Henderson, Am. J. P'hys., x $899,2, \mathrm{I}+2$. Pearce (R. M.) and EISEnBREy, Arch. Int. Med., I9I0, 6, 2 I 8 . Thompson, J. I'hys., I $\$ 96,20,455 ;$ I $\$ 99,24,37 \%$ UNderinll, Am. J. Phy"s., I903, 9, 345. Zuxz, Arch. Int. de Plyys., I9Ix, 11, 73. See also under Chapter if. (proteoses and coagulation).

Arterial Blood-Fressure in Man.-Boracif and MArks, Arch. Int. Med., I9r 3, 11, 45 ; ib., I91.t, 13, 6.48. Elliott (B. L.), Am. J. Phys., r 9 r 7, 42, 290 (effect of occlusion of bloodvessels). ERLANGer and HOOKER, Johns Hopkins Hosp. Rep., 190 $4,12, \mathrm{r} 45$. Hill and Rowlands, Heart, I $9 \mathbf{I}_{2}$, 3, 219 . Lombard, Am. J. Phys., rgr 2, 29, 335 (skin). McCurdy, Am. J. Phys., Igor, 5, 95 (exercise). Müller (F.), Harvey Lecture, New York, 1907. Rolleston, Heart, I $912,4,8_{3}$ (in aortic incompetence). TAUSSIG, Arch. Int. Med., I9I $3,11,5+2$.

Measurement of Blood-Pressure in Man.-FRANK (O.), Tigerstedt's Handbuch d. physiol. Methodik, 2, Abth. 4, 2 I6. Macleod, J. Lab. Clin. Med., I 9 I $5,1,62$, I $3 S$ (vésumé).

Auscultatory Method.-Brooks and Luckhardt, Am. J. Phys., I 9 I6, 40, 49. ErLANGer, $i b ., 40,83$. Foley, Coblentz and SNyDer, $i b ., 40,35$. Hill (L.), Heart, 1909, 1, 73. Hooker and Southworth, Arch. Int. Med., I9I 4, 13, 384. Kilgore, Arch. Int. Med., r915, 16, 927. MACIVrlliam and Melvin, Heart, I9I 4, 5, 153. IVArfield, Arch. Int. Mied., I912, 10, 258. Wrggers, J. Am. Med. Ass., I915, 64, I485 (segment capsules, optical record).

Oscillatory Method.-ErLANger, Johns Hopkins Hosp. Rep., r9o.f, 12, 53; Am. J. Phys., I908, 21, p. xxiv. Killgore, Arch. Int. Med., r915, 16, 893.

Venous Pressure.-Burton-Opitz, Am. J. Phys., r903, 9, 198. Hooker, ib., ror $4,35,73 ; i b .$, r 9 r $6,40,43$. v. Recklinghausen, Arch. Exp. Path. Pharm., r9o6, 55, 470. Selvall, J. Am. Mled. Ass., 1906, 47, 1279.

\section{RATE OF PERIPHERAL BLOOD-FLOW.}

Burtox-Opitz, Am. J. Phys., I9r $4,36,64$ (carolid); Quart. J. Exp. Phys.,

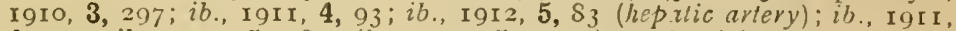
4, I 3 ; ib., rgr 2, 5, rS9; ib., r9ז 2, 5, 309 (portal vein). Brodie and Russell, J. Phys., I905, 32, p. xIvii.

Blood-Flow in Man.-Ediunds, Am. J. Phys., 1907, 18, 29 (influence of drugs on velocity). Irrank (O.). Z. f. Biol., IS9S, 37, I (velocity, Pitot's tubes). Hewlett and VAN Zwaluwensurg, Heart, 1909, 1, S7 (arm); Am. J. Med. Sci., I913, 145, 656; Arch. Int. Med., rgr r, 8, 59r. Hougir and Ballantyne, J. Bost. Soc. Med. Sci., June, IS99 (skin, influence of lemperature). Macleod, J. Lab. Clin. Med., r9r6, 1, 359 (vésumé). Macleod and Pearce, Am. J. Phys., rgr 4, 35, 87 (liver). Miatas, J. Am. Med. Ass., r914, 63, r 44 r (collatcral circulation). ShIELds, J. Exp. Ned., I896, 1, 7 I (effect of odours and mental work). STEwaRT (G. N.), Heart, I9I I, 3, 33 (hands); Am. J. Phys., I9I r, 28, I go (forced breathing); Arch. Int. Med., rgI 2, 9, 706 (pressures on upper arm); J. Exp. Med., I9I3. 
18, $35+(f e c t) ; i b ., 18, \operatorname{Ir}_{3}$ (ancmias); ib., I915, 22, 694 (colluteral circulation after ligation of innominate). v. KRIES, Z. Exp. Path. Therap., r9I, 9, 453 (arteries in man).

Rate of Blood-Flow in Veins.-Burton-Opitz, Am. J. Phys., r903, 7, 435; ib., Pflüger's Arch., I907, 121, I 50 (stromuhr); ib., I908, 121, I 56.

Circulation Time.-Herisi, Vicrordt, Vicrordt's Plyysiologic, r87I, p. I 47. LAsiglois and Deseours, J. I'hys. Path. Gén., Iyl 2, 14, 282, I II 3 (pulmonary). STEWART (G. N.). J. Phys., r893, 15, I (electrical and methylene blue methods).

Heart Output.-Bornstein (A.), Z. Exp. Path. Ther., rgi , 9, 382 (gasometric method in man). Cowl, Arch. 1. Physs, 1900, $56+$ (Rüntgen cardiographs in man). Dawson and Gorhan, J. Exp. Mled., I9os, 10, 48.4 (pulse pressure as index). Geseli. (R.), Am. J. Phys., Iyr5, 38, tof. HENDERSON (Y.) and BARRINGER, Am. J. l'hys., I 9 r 3, 31, 288 (conditions determining oulput). HEXDERSON ( $\mathrm{I}^{\circ}$.) and PRINCE, ib., I9I 4.35 , ro6 (the oxygen pulse and the systolic discharge). KNowlton and STARLING, J. Phys., I912, 44, 206 (influence of temperature and blond-pressure on performance of isolated heart). MURLIN and GREER, Am. J. Phys., I9I4. 33,253 (velation of heartrate to respiratory metabolism). KRoGn and LivdHARD, Skand. Arch. Phys., rgr 2, 27, 10o (gasometric method in man). Krogh, ib., 27, 126, 227. Patterson, Piper and Starling, J. Phys., I914, 48, 465 (oulput depends on inflow). Plescri, Z. Exp. Path. Ther., r $909,6,380$ (in mulu). STEWART (G. N.), J. Phys., I 898, 22, I 59 (dog).

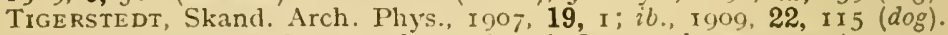
WoLf, Arch. Int. Med., I9I I, 8, 463 (cat, influence of temperature).

Pulmonary Circulation.-FüHNer and Starixng, J. Phys., I9I3, 47, 286. Plesch, Z. f. Exp. Path. Ther., rgr 3, 13, r65. Tigerstedt, Ergeb. d. Phys. (Bioph.), I903, 528; Skand. Arch. Phys., 1907, 19, 23 I. WIGGERs, Am. J. Phys., rgr 2, 30, 233. Wood (H. C.), Am. J. Phys., r $902,6,28_{3}$; J. Exp. Med., I9r $x, 14,326$.

\section{ORIGIN OF HEARTBEAT.}

Sino-Auricular Node.-Corn, Kessel and Masox, Heart, I9l 2, 3, 3 I r, 34 r. EYSTER and MEEK, Arch. Int. Hed., 1916, 18, 775 (conduction of excitation from sino-auricular node). FLACK, J. Phys., I9 10, 41, 6f. LEllis and OPPENHEIMER (B.S. and A.), 'Heart, 1910, 2, 1 47 (locution of pacemaker). MaCleod, J. Lab. Clin. Med., 1916, 1,263 (origin and spread of impulse -résumé). MEEK and Eyster, Am. J. Phys., 1914, 34, 368 (location of pacemaker); Heart, 1914, 5, 119, 227; Am. J. Phys., 1917, 42, 6II; Am. J. Phys., r916, 39, 291. Scilomovitz and CHASE, Am. J. Phys., 19r 5. 41, II 2 (paccmaker in turlle's heart). Moorhouse, ib., I91 2, 30, 358; ib., r91 3, 31, 421. OPPENhemer (B. S. and A.), J. Exp. Med., I91 2, 16, 6r 3 (nerves in sino-auricular node). Sansum, Am. J. Phy's., I9I 2, 30, 4II (extra systoles caused by stimulation of node).

Cause of Heart Beat.-Carlson, Am. I. Phys., I904, 12, 67,17 I (limulus). CARLSON and MEEK, ib., r9o8, 21, 1 (cmbryonic heart rhythm in limulus). Cyox, Pflüger's Arch., I906, 113, III. DALE (D.) and TrACKER, J. Phys., I9I + 47, 493 (H-ion concentration). ERLANGER, Harvey Lecture, New York, I9r2-I3. Greese (C. IV.), Am. J. Phys., I 899, 2, 82 (blood salls). Herixg, Pflüger's Arch., 1907, 116, I 43. KRONECKER, Z. f. Biol., I 896, 34, 529.-LANGENDORFF, Lirgeb. d. Phys., I902, 317 ; ib., I905. 76\%. LAKE, J. Phys., I9I6, 50, 364 (growth of heart tissue in vitro). MARTIN (E. G.), Am. J. Phys., I9I2, 30, IS 2 (ventricular tonus and causation of beat); Am. J. Phys., 1913,32, 165 (blood salts). P'ORTER (W. T.), J. I'hys., I $\$ 97,2,39$ I.

Extra Contraction and Compensatory Pause.- Carlson, Am. J. Phy:s., rgos, 21, I9 (limulus); ib., I907, 18, 7I (mechanism of refractory period); ib., 1 $906,16,67$ (excitation of heart during different phases of beat). Cusmix: Heart, 19x 2, 3, 257 (stimulation of isolated ventricle). Cusuny and 
IAtTuEWS, J. Phys., I897, 21, 2I 3 (effects of elcclvical stimnelution of mammalian heart). HirscuFelder and EYster, Am. J. Phys., I907, 18, 222. LANGeNDORFF, lirgeb. d. Phys. (Bioph.), 1902, 28: (physiological properties of heart muscle). MülLER (l'k.), Harvey Lecture, New York, 1907 (extra systoles). Sciultz (IV. H.), An. J. Phy's., I'jo8, 22, I33 (refractory period). WreNkriacir, Arch. f. Phys., I903, 57 (compensatory paltse). WOODWorTII (R.S.), Am. J. Phys., 1902, 8, 2 I 3 (refractory pcriod and compensatory pause).

Heart Muscle Strips.-Erlaxger, Am. J. Physs, I910, 27, 87 (auricle). MARTIN (E. G.).ib., I901, 11, I03 (tervapin ventricle).

\section{CONDUCTION OF CARDIAC EXCITATION AND CONTRACTION.}

Atrio-Ventricular Conduction System : Auriculo-Ventricular Bundle.BARKER and HIRSCIIFELDER, Arch. Int. MIed., I9O9, 4, I93. CARLSON, Am. J. Phyrs., I908, 21, I (limulus). Coнs, Heart, Iyo9, 1, I6- (auriculonodal junction). Cullis and Dixon, J. Phys., I9II, 42, I56. ENGelMANx, Pflüger's Arch., I 894,56, I $49 ;$ I $895,61,275 ;$ I $890,62,5+3$. Erlaiger, Am. J. Phys., I 909, 24, 375 ; Arch., Int. Med., I9I 3, 11, 334 ; ib., I9I 2, 30, 395 (physiology of Purkinje tissue). FrédericQ. Arch. Int. de Phys., I9r2, 11, 405, 478. Gault, Am. J. Phys., I9I7, 43, 22 (lurtle, influence of vagus and sympathetic). HERIxG. Pflüger's Arch., I $905,108,267 ; 10 \%, 99$. Howell, J. Am. Med. Ass., I 9oG, 46, Nos. 22, 23. KeIth, Lancet, Mar. 5, I904. KENr, J. Phys., I893, 14, 233; Quart. J. Exp. Phys., I913, 7, 193; Proc. Roy. Soc., I914, B 87, I98. LAurexs, Am. J. Phys., I 9I6-I 7, 42, 89, 5 I 3 (turlle). Lewis, WhIte and MeAKINS, Heart, I9I 4, 5, 289. LEWIS, ib., I9I 3, 5, 2 I (vate in dog's auricle). PRINCE and Ceraci, Heart, igi 5, 6, I67. Saltzmax, Skand. Arch. Phys., 1908, 20, 233 (papillary muscles). TAWARA, "Das Reizleitungssystem des Saïgethierherzens," Jena, I906; Zentralbl. f. Phys., xgo5, 19, 70. IVhite and Kerr, Heart, I9I7, 6, 207 (whale). Wilson (J. G.), Proc. Roy. Soc., I 909, B 81, I5I (nevves of atrio-ventricular bundle).

Heart-Block.-BACHмA.x, Arch. Int. Med., I909, 4, 238 (strophanthin). BuchaxA. (F.), J. Phys., 42, p. xix (dissociation of auricles and ventricles in hibernation). CHRISTIAX, Arch. Int. Med., I9I 5, 16, 34 I (digitalis). Erlaxger, J. Exp. Med., I905, 7, 676; $i b .$, I906, 8, 8. ERLANGer and BlackMax, Heart, I910, 1, I77. EYster and MeEk, Arch. Int. Med., I9I7, 19, II (sino-auricular and sino-ventricular block). EYSTER and Evaxis, ib., I9I5, 16, 832. GARREY, Am. J. Phys., I9I2, 30, 283 (compression of cardiac nerves of limulus). GESELL, ib., 1916, 40, 267.

Fibrillation in Heart.-GARREy, Am. J. Phys., I908, 21, 283 (infuence of cardiac nerves on); $i b ., 19 I_{4}, 33,397$. Guxs (J. A.), Heart, I9I5, 5, I (rale). Levy (A. G.), J. Phys., I9I 4, 49, 54. MacWillia., J. Phys., I887, 8, 296. PORTER, Am. J. Phýs., I 898, 1, 7 I (recovery of heart from).

Influence of Temperature on the Heart.-CARLSO:, Am. J. Phys., I906, 15, 207. Evass (C. L.), J. Phys., I9I7, 51, 9 I (mechanism of acceleration by warmth and adrenalin). LANGENDORFF, Ergeb. d. Phys., (Biophys.), I903. 5I 7. STEWART (G. N.), J. Phys., I 892, 13, I I9 (heal slandstill).

Isolated Mammalian Heart.-Barcroft and Dixox, J. Phys., 1906-7, 35, I82 (gaseous metabolism). Cushisy and Guxs, J. Pharm. Exp. Therap., I9I 3, 5, i ; MANiWARING, MeiNhard and Denhart, Soc. Exp. Biol. Med., I9I6, 13, I 73 (effect of serum). Evans (C. L.). J. Phys., I9I 4, 47, 407 (effect of glucose on gaseous metabolism). Guthrie and Pike, Am. J. Phys., I907, 18, I+ (relation of activily to pressure in coronary'). GORHAM and MIORRISON, ib., I 9 IO , 25, + I 9 (action of blood proteins). LA.icieNDORFF, Pflïger's Arch., 1895, 61, 291; $i b ., 1897,66,355 ; i b ., 1898,70,473$. Locke and Rosexhers, J. Phys., 1907, 36, 205 (consumption of dextrose). NEUKIRCII and RoNA, Pflüger's Arch., I912, 148, 285 (consumption of sugars by). Porter (IV. T.), Am. J. Phys., IS98, 1, 5 I I. WAller and ReId, Phil. Trans. (Roy. Soc., Lond.), I\$87, B 178, 215 (action of excised heart). 


\section{RELATION OF SALTS TO HEART ACTION.}

Jienedict (S. R.), Am. J. Phys., igos, 22, I6; Carlson, ib., 16, 22 ; EGgers (H. E.), ib., I907, 18, 6.1 (non-electrolytes). Botazz1, Arch. de Plhysiol., i 896, 882 (potassium). Burridgie, O. J. lexp. Pliys., I9I5, 8, 303 ( $\mathrm{NaCl}$ ): $i b ., 8,33$ I (Ca salls and alkalies). CLARK (A. J.). J. Phys., I9I 3-I 4, 47, 106 (ions and lipoids). Howell, Am. J. Phys., 1901, 6, i 8 г ( $N a, K$ and Ca). LANGeNDORli, lirgcb. d. Phy*s. (Bioch.), 1902, 312 (chemical conditions of heart-beat). Lingle (D. J.), Am. J. Phys., I goo, 4, 265 (nonelcctroly'las); $i b ., 1902,8,75((\mathrm{NaCl}) ; i b ., 1905,14,433$. Loen (J.). Am. J. Phys., I9oo, 3, $38_{3}$; Phluger's Arch., I900, 80, 229 ( $C$ a and $K^{\prime \prime}$ ). Mines, J. Phys., I9I 3, 46, Is8 (electrolytes); ib., igI t, 42, 309. MARIIN (E. G.), Am. J. Phys., I9I3. 32, I65 (blood salts). MAXWEll and IILL, Am. J. Phys., I902, 7, 109 (Ca and free oxygen). Ringer (S.), J. Phys., I 895, 18, 425 (antagonism between $\mathrm{Ca}$ salts and $\mathrm{Na}, K$, and $\mathrm{NH}_{4}$ salts). IVALlER (M. D.), J. Phys., I9I.4, 48, p. xlvi (electrolyle concentrations and frequency).

$\mathrm{CO}_{2}$ and Heart.-Bayliss, J. Phys., I900-I, 26, p. xxxii. CathCart and ClARK, $i b$. I II 3, 47, 393. JERUSALEM and STARLING, ib., I9Io, 40, 279. Keтcham, King and Hooker, Am. J. Phys., I9I2, 31, 64.

Resuscitation of Heart.-CRILE and Dolley, J. Exp. Med., I 906, 8, 7 I 3 (after ancesthetics and asphyxia). ERLANGER, ib., I9I2, 16, 452 (sinus stimulation as a factor). GUNN (J. A.) and MARTIN (P. A.), J. Pharm. Exp. Ther., I9I 5, 7, $3 \mathrm{I}$ (intraspinal medication and massage). LiEmp and Gardner, Iledical News, 83, i 84 (after chloroform). Kuliabro, Pflüger's Arch., i 903, 97, 539. Langendorfa, Ergeb. d. Phys., 1905, 769. Pike, Guthrie and Stewart, J. Exp. Med., I908, 10, 37 I.

Heart Nerves (Cardio-Regulative Nerves).-Carlso, Ergeb. d. Phys., I909, 37 I (comparative physiology of heart nerves and heart ganglia in invertebrates). Clark (G. H.), Heart, I9I 3, 4, 379 (influence of temperaine). CoIn, J. Exp. Med., I912, 16,732 (differences between the two vagi in dog). CUllis and TRIBE, J. Phys., I91 3, 46, I +1 (distribution of nerves in heart). Cyon, Die Nerven des Herzens (Berlin); Les Nerfs du Cour, Paris, I 905. Dogiel and Archangelsixy, Pflüger's Arch., I906, 113, I. Engelanan, Arch. f. Phys., I900, 31 5; ib., I902, Supp. Bd., I, 103, t+3 (effects of vagus on rate, force, clc.). Garrex, Anı. J. Phys., I9I 2, 30, 451 (effects of vagi in heart-bloch); ib., I9I I, 28, 33 (tumle). Gaskeld, J. Phys., IS83, 4, +3 (tortoise); Phil. Trans. (Roy. Soc., Lond.), I 882, 993 (frog). HARRINGTON (D. W.), Am. J. Phys., x 898, 1, 383 (guinca pig). Hough (T.). J. Phys., 1895, 18, I61 (cscape of hart from vagus inhibition). HUNT (R.). J. Exp. Med., IS97, 2, I51; Am. J. Phy's., I 899, 2, 395 (relation of accelerator to inhibitory nerves). LANGENDORFF, Ergeb. d. Phys. (Bioph.), I 902, 263 (heart muscle and intracardial innervation). LEwIS (T.). Heart, I9I 4, 5, 247 (vagal stimulation and antrio-ventricular rhythm). МЕEK and EYSTER, Am. J. Phys., 1912, 30, 27 I (electrical changes in heart during vagus stimulation). PATON (D. N.), J. Phy's. I912, 45, Io6 (birds). ScrilomoVitz, Eyster and MEEK, Am. J. Phys., I9I5, 37, I 77 (relation of nodal lissuc to inhibitory nerves). Sewell and Donaldson (F.), J. Phys., I SSo$\mathrm{S}_{2}, 3,357$ (influence of inlracardiac pressure on vagus action). STEWART (G. N.). J. Phys., I892, 13, 59; Z. f. Biol., I91 3, 59, 53 I (influence of temperalure of heart on aclivity of heart nerves); $\mathrm{Am}$. J. Phys., 1909, 24, $34 \mathrm{I}$ (lortoise vagus). WIGcERs, Am. J. Pliys., 1916, 42, I33 (influence of vagus on "fractionate" contraction of right auvicle).

Accelerators.-CYOx, Pfinger's Arch., Iyo6, 113, III. FFÉEERICQ (H.), Arch. Internat. de Phys., I913, 13, II5. HERING (II. E.), Pllüger's Arch., 1905, 10\%, 125 (direct action on mammalian ventricle).

Action of Accelerated on Quiescent Heart.-CARLSON, $\mathrm{Am}$. J. Phÿs, I 904 , 12, 55 (molluscs). HERING, Pflüger's Arch., 1906, 115, 35t (mammals). STEWART (G. N.). J. Pliys., IS92, 13, 83, 90 (amphibia). 
Voluntary Acceleration of Heart.-FAvili, and Wute, Heart, 19.7, 6, I 75. VAN DE VE1.DE, J'lligiger's Arch., I $897,66,-32$.

Cardio-Inhibitory Centre and Reflexes.-BANBRIDGE, J. Phy's., I9r4. 48 , 332. EYSTER and Hookne, Am. J. Pliys., 1908, 21, 373; FilenNE and BIBERFIELD, Pflüger's Arch., r909, 128, 443 (efject of inereased blond. pressure). Hookrer, $\Lambda$ m. J. Physs., I 908, 19, 417 (reflex accelcration independently of cardio-inhibitory centre?). MILlER and BowMan, An. J. Phy's., r9i 5, 39, i 49. Wertieimeis and Meyer, Arch. de Phys., i 890, $28+$ (influence of deglutition on heart-rhythm).

Relation of Salts to Inhibition.-BRINE (13. M.), $\Lambda \mathrm{m}$. J. Phys., I91 7. 44, I 7 I ;

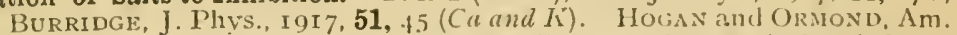
J. Phys., I91 2, 30, Io5 (Ca). Howell, ib., I906, 15, 2 so (salls of blood). Howell and DUKE, ib., I908, 21, 5 I (output of $K$ in inhibition); ib., I908, 23, 174 (accelerators and $C a, K$ and $N$ metabolism of isolated hcart); J. Phys., I $906-7,35$, I 3 (isolated mammalian hearl). LANGENDORFF and HUECK, Ptlūger's Arch., I 903, 96, 473 iCa). Loeb (J.), J. Biol Ch., I906, 1, 427 ( $M g$ and $C$ a on contractions of jellyfish). Miartin (E. G.). Am. J. Phys., I 904, 11, $370(\mathrm{KCl})$.

\section{VASOMOTOR NERVES.}

Bowditch and Warren, J. Phys., 7,4 I 6 (of limbs). DRINkER (C. K. and K. R.), Am. J. Phys., I 9I6, 40, 5 I 4 (of bone-marrow). Gaskell (W. 1I.), J. Phys., I 878-9, 1, 262 (vasomotors of muscles). LANGLEy, J. Phys., ISOI, 12, 345, 375 (course of sweat and vasomolor fibres of cat's foot) ; ib., I $11,41,4 S_{3}$ (frog's foot).

Vasomotors of Intestine.-Bunch (J. L.), J. Phys., I 899, 24, 72. BurtoxOpitz, Am. J. Phys., I9I 5, 35, 203 (duodenum). Hallion and FrançoisFranck, J. Phys. Path. Gén., I896, 478, 493.

Vasomotors of Lungs.-Brodie and Dixon, J. Plyys., I904, 30, 476. Fraxicols-Franck, Arch. de Phys., i 896, y 78. JACkson, J. Pharm. Exp. Ther., I9I 3, 4, 291. Krogh, Zentralbl. f. Physiol., 20, 802 (lortoise). Langlois and Desbouis, J. Phys. Path. Gén., r9r 2, 14, 282. Plunier, ib., I904, 655. Tigerstedt, Ergeb. d. Phys. (Bioph.), I903, 571. Trise (E. M.), J. Phys., I 914 , 48, I 54 . Wiggers, J. Pliarm. Exp. Ther., I $909,1,3.1 \mathrm{I}$.

Cerebral Vasomotors.-BAyliss and HrLl, J. Phys., I $895,18,334$. Dixon and Halliburton, Q. J. Exp. Phys., I9io, 3, 3 I 5. Gulland (G. L.), J. Phys., I895, 18, 36I; Huber (G. C.), J. Comp. Neurol., I899, 9, I; Hunter(W.), J. Phys., 1900-r, 26, 465 (histological). Hill and Macleod, $i b ., 26,394$. JENSEN, Pflüger's Arch., I904. 103, I 71, r96. WVigGers, Am. J. Phys., I905, 14, 452;ib., I907, 20, 206; ib., I 908, 21, 454; J. Phys., 1914,48 , 109 .

Vasomotors of Heart.-Brodie and Cullis, J. Phys., I9I I, 43, 3r 3. Dagiel and Archangelsix, Pflüger's Arch., I907, 116, 482. PORTER, Am. J. Phys., I 912,29 , p. xxxi (method). WVIGGERs, ib., I 909, 24, 391.

Vasomotors of Veins (Veno-Motors).-Bancroft, Am. J. Phys., I \$98, 1, 477 (hind limb). Bayliss and Starling, J. Phys., I 894, 1\%, 120; BurtonOpItz, Q. J. Exp. Phys., I9I3, 7, 57; Am. J. Pliys., I9I 4, 36, 325; EDMUNDS (C. W.), J. Pharm. Exp. Ther., I9I 5, 6, 569 (portal vein). FRANÇOIS-FRANCK and HALLION, Arch. de Phys., I897, 434 (liver). Henderson (Y.), Am. J. Phys., I91 7, 42, 589 (veno-pressor linechanism).

Vasomotor Mechanism.-Asher, Ergeb. d. Physs. (Biopliys.), I902, 346. BAYliss, ib., 1906, 319 . COTTON, SLADE and Lewis, Heart, I917, 6, 227 (contractile power of capillaries). EDWARDS (D. J.), Am. J. Phy.s., 1916, 35, ${ }_{5}$ (compensalory phenomena during splanchnic stimulation). Hooker, An. J. Phys., I9I I, 28, 36I (chemical regulation of vasculartone). PORTER and NewBurgh, ib., I9I $f, 35$, I (in pnemmonia). Porter and Turner, ib., I $915,39,236$ (¿'asotonic and vascrefiex mechanism). 
Vasomotor Centres.-Matirson, J. Pliys., IgI I, 42, 283; I'ORTER (W. T.), Am. J. I'hy"s., IyI 3,31 , p. xxix (functional relation of ncrve colls in); $i b$., I9I 5, 36, fI 8 (vasotonic and vasoreflex centre). PORTER and CLARk, ib., 1908, 21, p XV (difference belwcen bulbar and spinal vasomolor cells). PORTER and STOREY, ib., 1907, 18, I8 I (effect of injuries of brain). RANsox, ib., I9I6, 42, I (chief vasoconsiviclor centre). SOLLMaNin and PILCHER, ib., I910, 26, 233 (effect of sciatic stimulation and curara).

Vasodilators.-BAylrss, J. Phys., 1900, 26, I73; ib., I902, 28, 276 (hind limb. antidromic impulses). BERNARD (CL.), Liquides de l'organisme, 2, 269 (chorda). Carlson (A. J.), Am. J. Phys., I907, 19, 408; IIcLean (F. C.). ib., 22, 279 (vasodilators to submaxillary in cat's cervical sympathetic). ECKHARD, Beiträge, 3, I25；4, (g9 (mervi evigentes). KENDALL and Luchsinger, Pflüger's Arch., I 876, 13, I97 (difference between dilators and constrictors after nerve section).

Depressor.-BAyliss, J. Phys., I908, 37, 26. Cron, Pllüger's Arch., I9oI, 84, 30.4. PORTer and Beyer, Am. J. Phys., I9Oo, 4, 283. RANSON and Billingsley, Am. J. Phys., igi6, 42, 9. Sewall and Steiner, J. Phys., I885, 6, I62. Sollmann and Pilcher, Am. J. Physi, i 912 , 30, 369 (response of vasomotor centre 10). Tigerstedt, Skand. Arch. Phys., I908, 20, 330. TscinRwinsky, Zentralb. f. Phys., 9, 777; 10, 65.

Vasomotor Reflexes.-Henderson (V. E.) and Lozwi (O.), Arch. Exp. Path. Pharm., I905, 53, 56 (vasodilator excitation). Hunt, J. Phys., I 895, 18, 38 I (fall of blood-pressure on stimulation of afferent nerves). LANGLEY, J. Phys., I91 2, 45, 239 (effect of strychinine). MARTin and LACEY, Am. J. Phys., I9I4, 33, 2 I 2 (vasomotor reflex thresholds). MARTin and MENDENHALL, $i b$., I 9I 5, 38, 98 (vasodilator response to sensory stimulation). MARtin and Stiles, $i b$., I9I6, 40, I94 (vasomotor summations). PORTER, ib., I907, 20, 399 (uniform stimuli with blood-pressure at different levels); ib., I910, 2\%, 276 (velations of afferent impulses to vasomotor centres). PORTER and MARKS, ib., I908, 21, 460 (effect of hemorrhage). PORTER and Richardson, $i b$., 1908, 23, I3I. Stewart (G. N.), Heart, I9I I, 3, 76 ; StEwART and LAFFer, Arch. Int. Med., I913, 11, 365; Stewart and (VALKER, ib., 11, 383 (elicited by wamth and cold in man). VINCENT and Caneron, Q. J. Exp. Phys., I9I 5, 9, 45.

Influence of Asphyxia on Circulation.-Hill and FLACK, J. Phys., 1908, 37, 77 (on circulation and respiration). MacWillian, J. Phys., I90I-2, 27, 337 (asphyxia and cardiac failure). Mathison, ib., I II , 42, 28,3 (medullary centres); $i b ., 1910,41,416$. Sollmann and PIlcher, Am. J. Phy's., I9I I, 29, 100 (reaction of vasomolor centre to asplyxia).

Pressor Amines.-Abelous and BArdier, Compt. Rend. Soc. de Biol., Mar. I 7 , I906; DALE and DixON, J. Phys., I909, 39, 25 (formed in putrefaction). BAEHR and Pick, Arch. Exp. Path. Pharm., I9I6, 80, I6I (point of attack). BAIN (W.), Q. J. Exp. Phy's., I9I 4. 8, 229 (pressor bases in wrine). DAlE and LAIDLAw, J. Phys., I9I I, 43, I82; LAidlaw, Bioch. J., I911, 6, I.fI (action). WALPOLE, J. Phys., I 909, 38, 2.13.

Depressor Amine ("Vasodilation").-BARGER and DALE, J. Phy"s., I9I I, 41, 499: Mellanby and 'TwOrT, ib., 1912, 45, 53 (in intestine cuall).

Intracranial Pressure.-Cushing (H.), Johns Hopkins Hosp. Bull., Sep., I gor. Eyster, Burrows and Essick, J. Exp. Med., I909, 11, 489.

Influence of Gravity on Circulation.-Hill (L.), J. Pliys., I 895, 18, I 5. HilL and BARNARD, ib., I 897, 21, 323. SALATIle, Compt. Rend., I $877,85,145$.

\section{SHOCK.}

BAINBRIDGE and Bullen, Lancet (London), I9I7, 2, 5 I. RAYliss (IV. MI.), Arch. Med. Belges, I9I 7, 70, 793 (Physiol. Abstracts, r9I8, 2. (i25) (treatment by intravenous injections). Jirlanger and Woodratt, J. Am. Med. Ass., I9I 7, 69, I 4 Io; IERLANGER, GeSELl, GASSER and ELLIOTT, ib., I9I 7 , 69, 2089. Guthrie, ib., 1917, 69, 1 394. JENdersoN (У.), Am. J. Phys., 
I908, 21, 126; ib., Inon, 23, 3.45; ih., r910, 27, 152; HENDERSON and ScARBrolgil, ib., Iylo, 26, 260 (acupnia and shock). HENDERSON, ib., I $90,25,3$ IO, 355 ; IIENDERSON, PRINCE and IIAGgard, J. Am. Med. ASS., I () $17,69,965$. JANEWAY and JACKSON, Soc. Exp. Biol. Mled., I9I 5. 12, I1)3; J. Am., Med. Ass., Oct. I6, 1915. 37 I. LyoN and SWARTz, Soc. lixp. Biol. Med., r910, 7, I 39. Métzer, Arch. Int. Med., 1908, 1, 57 I. Murrison and Hooker, Am. J. Pliys., 1915, 37, 86. Pike and Coomis, J. Am. Med. Ass., 19I7, 68, I892, PORTER, Bost. Med. Surg. J., I9I7, 17\%, 320. PORTER, MARKS and SWIFT, Am. J. Pliys, 1907, 20, 441. PORTER and QUinbr, Am. J. Phys., I908, 20,500. SEelig and JOSEPH, J. Lab. Clin. Med., I916, 1, 28 3. SimoNDs, J. Am. Med. Ass., r91 7, 69, 883 .

Transfusion.-BotAzzI and JAPELLI, Bioch. Z., I90\$, 11, 33 I (physico-chemical properties of blood and lympl after). CARLSON and GINSBURG, Am. J. Phys., I I) 5, 36, 280 (influence on hyperglycamia of pancreatic diabetes). CRILE (G. Wi.), Soc. Exp. Bial. Med., I906, 4, 6. Haskins (H. D.), J. Biol. Ch., $1907,3,32 \mathrm{I}$ (effect on $N$ metabolism). OTTENBERG and Thilminer, J. Med. Res., I9I5, 33, 2I3. Rabens, Am. J. Phys., I9I 4. 36, 29 + (influence on kidneys).

Lymph Hearts.-Agel and TunNer, J. Pliarm. Exp. Ther., I9I4, 6, 9I (action after cardiectomy). LANGEXDORFF, Pflüger's Arch., 1906, 115. 533. MOORE (A.), Am. J. Phys., I 90I, 5, 87 (influence of ions); ib., 5, 196 (spinal centres). PRIEstley (J.). J. Pliys., I878-9, 1, I, I9 (older literature). Tschermak, Pflüger's Arch., I907, 119, I65 (spinal innervation).

Channels in Liver Cells communicating with Blood Capillaries.-SCḦ̈FER, Anat. Anzeig., 1902, 21, I 8 . Herring and Simpson, Proc. Roy. Soc., I $906, \mathrm{~B} 78,455$.

\section{CHAPTER IV.}

\section{RESPIRATION.}

Blood in Lungs.-Kixo, J. Phys., I917, 51, I 54 .

Respiratory Movements.-BAGlioxi, Ergeb. d. Phy"s., IgII, 526. Eyster, AUSTRIAN and FixgSLEY, Am. J. Phys., I 907, 18, + I 3 (temporary occlusion of aorla). FITz (G. W.), J. Exp. Med., I $896,1,677$. DU BoIs-REYMOXD (R.), Ergeb. d. Phys. (Bioph.), I902, 377 (mechanics of respiration). GuTHRIE and Pike, Am. J. Phys., I906, 16, 475; 1907, 20, 45 (effect of change in blood-pressure on). LUNDSGAARD and VAN SLYKE, J. Exp. Med., I9I $8,27,65$ (lung volume).

Temperature of Expired Air.-Loewy and Gerhartz, Pflüger's Arch., I9I4, 155,231 .

Artificial Respiration and Resuscitation.-SchäFER, Lancet, May 30, I903; Trans. Roy. Mled.-Chir. Soc., London, 1904, 86; Proc. Roy. Soc. (Edin.), I904, 25, 39 (in the apparently drowned). STEWART (G. N.) and PIKE (F. H.). Am. J. Phys., 1907, 19, 328 (resuscitation of respiratory and other bulbar centres, with reference to their antomatism).

Respiratory Dead Space.-Douglas and Haldane, J. Phys., I9I 2, 45, 235. Haldane (J. S.), Am. J. Phys., I9I 5, 38, 20. HeNderson (Y.) ET al., Am. J. Phys., I9I 5, 38, I. Krogh (A.) and Livdhard (J.). J. Phys., I $917,51,59$; $i b .$, I9r 3, 47, 30. PEARCE (R. G.) and Hoover (D. H.), Am. J. Phys., I9I 7, 44, 391.

Alveolar Air.-Hovgh (T.), Am. J. Phys., I9 I 2, 30, IS (alveolar air in muscular exercise). Haldane and Priestley, J. Phys., 1905, 32, 225. Henderson (Y.) and MORRISS (IV. H.), J. Biol. Ch., I9I7, 31, 2I7. KROGH and

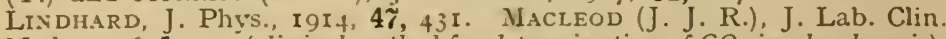
Med., I $916,1,522$ (clinical method for determination of $\mathrm{CO}_{2}$ in alveolar air). Pearce (R. G.), Am. J. Phys., I9I7, 44, 369. VAx SlYke, StilliaA and Cullex, J. Biol. Ch., I9I7, 30, for (alveolar $\mathrm{CO}_{2}$ and plasma bicarbonate). 
Respintory Gaseous Exchange.-Brindet and llomaxis. An. J. Pliys. 1)1 1, 28, 2u. B1.Nen] I (b. G.), u., ivo9, 24, :15 (uppurutus). Bris:-

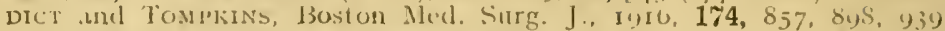
(apparatus for clinical usc). CARPlixtek, Carnegie lnstit. Pub., No. 2 i6, 1915 (methouls in man). JagUrT, Ergeb. d. Phys. (Bioch.), 1902, 157. Plimbey, J. Phys., igor, 2\%, 66 (marmol). - Wolf and Hele, J. Phys., $1914,48, \cdot 28$ (decerebrate unimal).

Respiratory Quotient.-Benedict, Emmes and Riche, Am. J. Phys. I9I, 27,383 (influence of preceding diel on). LUSK, Arch. Int. IJed., 1915, 15, 939 (diabetes).

\section{BLOOD GASES.}

Barcroft, J. Phys., I908, 37, I2; Barcroft and Higgins, ib., I911, 42, 5I2; BARCROFT and ROBERTS, ib., I9IO, 39, 420 (differential melhod). BUCKMASTER, J. Phys., 1917,51 , I64 (relations of $\mathrm{CO}_{2}$ in llood). BuCkmaster and Garnner, J. Phys., I9Io, 40, 373 (gas pumps); ib., I9Io. 41, wo (gases of arlerial and venous blood); ib., 1912, 43, for (nitrogen). COOKE and BARCROFT, J. Phys., I9I 4,47 , p. xxxv (percentage saluration of $\mathrm{O}_{2}$ in arlerial blood in man). FRIEDMAN (E. D.) and JACKSON (H. C.). Arch. Int. Med., I9I $7,19,767\left(\mathrm{CO}_{2}\right.$ of blood and alveolar air in obstructed respiration). Haldane, J. Phys., I $898,22,465$ (analysis). Krogir, Skand. Arch. Phys., I goS, 20, 259 (microlonometer). MurLin, EdelmaNe and KRAMER, J. Biol. Ch., 19r3, 16, 79 (after clamping abdominal aorla

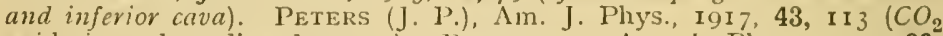
acidosis and cardiac dyspnoca). RAsmussen, Am. J. Phys., 1915, 39, 20; ib., 1916, 41, I62 (blood gases in hibernation).

Oxygen Teusion of Arterial Blood.-Haldane and Smiti, (J. L.), J. Phys., 1 896, 20, 497. Krogly, Skand. Arch. Phys., 1910, 23, 252. OSBORNie (W. A.), J. Pliys., 1907, 36, 48. Scott (R. WV.), Am. J. Phys., 1917, 44, r96 (decerebrale cat).

CO, Tension.-BootıBy and SAndrord, Am. J. Phy's., I9I6, 40, $5+7$ verrous blood, rest and work). Christiaxsen, Douglas and Haldane, J. Phys., I $914,48,244$. HeNderson (Y.) and PRINCE, J. Biol. Ch., I9I 7, 32, 325 (venous blood).

Oxygen Dissociation Curves (of , Blood and Oxyhæmoglobin).-BARCrofT and CAMIS, J. Phys., rgog, 39, I.43. BARCROFT and ORIBLL, ib., I9Io, 41, 355 (influence of lactic acid). BARCROFT, ib., I9II, 42, it (altitude). BARCROTT and KING, ib., I907, 39, 374 (lemperature). BoIIR, HASSELBALCH and Krogir, Skand. Arch. Phys., I9o6, 16, toz (infuence of $\mathrm{CO}_{2}$ ). Douglas and Haldane (J. S. and J. B. S.), J. Phys., 1912, 44, 275 (combinalion of $\mathrm{Hb}$ with $\mathrm{CO}_{2}$ and $\mathrm{O}_{2}$ ). IlüreNer, Arch. f. Phys., I9O1, Supp. Bd., is 7 (dissociation of oxyhamoglobin). Mathison, J. Phys. I $911,43,347$ (influence of aciels on reduction of arterial blood).

Oxygen Capacity of Blood.-BARCROFT and Burn, J. Physs., I9I3. 45, 493. BURN, $i b .$, I $913,45,482$. DOUGLAs, $i b ., 1910,39,453$ (afler hamorhage): ib., $1910,40,+72$ (tolal $\mathrm{O}_{2}$ capacity and blood volume at different altitudes). Haldane, ib., 25, 295 (jerricyanide melhod). Hürner, Arch. f. Plyys., I 894, I $30 ;$ il., I903, 2 I 7 ( $\mathrm{O}_{2}$-capacily of blood pigmenl). Priters, J. Pliys., $1912,44,131$.

Carbon Monoxide.-Grenant, Arch, de Phys., I898, 3I5, 434 (absorption).

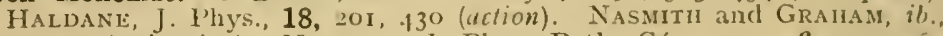
35, 32 (poisoning). Niclot'x, J. Phys. Path. Gén., I9I4, 6, I 45, IG.4 (absorption).

Chemical Regulation of Respiration.-BAYliss and Starlixg, Ergeb. d. Pliys., r 906,669 . Camplezl, Douglas, Haldane and Horson, J. Phys., 1913, 46, 301. Gasser and Loevenhart, J. Pharm. Exp. Ther., I9I $4,5,239$ (decreased $O_{2}$ ). GoRdon and HALDANE, J. Phys., Igog, 38, 420. Haldane and Poultow, ih., Ino8, 37, $390\left(\mathrm{O}_{2}\right.$ definency). Haldane and Priestley, ib., Iy05, 32, 225. Hoviri, An. J. Phys., 
1911, 28, 3(u) (beathing a confuned rolume of (iir). HII.L and FLACL, J. I'hys., igo8, 3\%, 77 (excess of $\mathrm{CO}_{2}$ and want of $\mathrm{O}_{2}$ ). PETLRS, Am. J. I'liys., I017, 44, s.t (H-ion concentrition of blood). Scort (R. WV.), Am. J. Phys., 1917,44 , igf $\left(\mathrm{CO}_{2}\right.$ and $\mathrm{H}$-ion concentration in decerebrate cat $)$.

Nervous Mechanism of Respiration.-BARKr, J. Phys., I I 13, 45, 473 (afferent impressions). Borutrau, Ergeb. d. Phys. (Biopliys.), $1902,403$. DEAson and Roвb, Am. J. Phys., I9II, 28, 57 (paths in cord). GAD. Arch. f. P'hys., ISSo, I. Gruber, Am. J. Phys., 1917, 42, 450. Nice, Am. J. Pliys., I9I 4, 33, 20, (thresholds for respiratory reflex). Frörulcu. Plligger's Arch., 1906, 113, 133 (climination of vagus without stimulation). IIALDANE and MAVRogordato, J. Phys., I? I 6, 50, p. xli (vagus regulation). LotW (A.), Pfligger's Arch., r888, 42, 273. LrWANDowskr, Arch. 1. Phys., 1Si76, 195. Melizer, Arch. f. Phys., 1892, 340. Nicolaides, Airh. f. Phys., 1907, 68. PORTER (II'. T.), J. Phys., IS9.1-5, 17, 455 (phrenic puth). l'ORTER and TURNER, Am. J. Phys., I1) 13, 32, 95 (reflex resfirutory movements). STIEWAT (G. N.), ib., I907, 20, 407. STEWART and PIKk, ib., I907, 19, 328; ib., 20, 6 I (resuscitation of nervous respiratory mochanism).

Respiratory Centre.-BAglioni, Ergeb. d. Phys., I9II, 588 (automatisin of). Boruttad, ib., I904, 89. Brown (T. G.). J. Phys., I9I 4, 48, p. xxxii (a respiratory tract in mid-brain). Hooken, J. Pharm. Exp. Ther., IgI 3. 4, 443 (perfusion in frog). LAQUEUR and VERZÁR, Pfliger's Arch., I9r2, 143,395 (specific action of $\mathrm{CO}_{2}$ on). LOEWY, $i b ., \mathbf{I} 888,42,245$ (repetition of MARCKWALD's experiments on paraffin injecion [Z. f. Biol., I89o, 26, 259]).

Bronchi.-Aвbotr, J. Med. Res., I912, 26, 513. Dixon and IBronie, J. Phys., I903, 29, 97; Dixon and Ransom, J. Phys., 1912, 45, 4r3 (innervation). DU BoIs-Reyaiond (R.), Ergeb. d. Phys. (Bioph.), I9O2, 396 (altcrations in calibre). Henderson (V. E.) and TAYlor, J. Pharm. Iixp. Ther., I9IO, 2, I53 (secretion). JACKSON, ib., I9I4, 5, 479 (drugs).

Dyspncea.-Athanasiu and Carvallo, Arch. d. Phys., I 898, 95 (heat dysproea). LEWIS, RYFFEL, IVOLF, COTtON and BARCROFT, Heart, IgI5, 5, 45 (dyspneea in cardiac and renal patients). Moornouse, Am. J. Phys., I9II, 28, 223 (heat dyspnœa).

Apnca.-Bootury, J. Phys., I912, 45, 328. Fod, Arch. di. Fis., IyI , 9, 453. Githens and Meltzer, Soc. Exp. Biol. Med., rgi4, 12,64. Milnoy, Q. J. Exp. Phys., I9I 3, 6, 373. Mosso, Arch. Ital. de Biol., 40, I. PEMBREY and PITTS, J. Phys., I S99, 24, 305 (respiration in hibernation). Scarbrough and Henderson, Am. J. Phys., I9ro, 25, p. xiii.

Cheyne-Stokes Respiration.-Barbour, J. Pharm. Exp. Ther., I9I 4, 5, 393 (morphine). CLARK (A. J.) and HAMIll, ib., 357 (circulatory changes in). Douglas, J. Phys., I9I0, 40, 454 (at high altitudes). Erster, J. Exp. Med., I $906,8,565$. Fulton, Heart, I 91 5. 6, 77. Gordon and Haldane, J. Plyys., I 909, 38, 401 . Mackenzie and Cushny, $i b ., 1907,36$, p. xiii. Penibrey, Beddard and French, ib., I906, 34, p. vi. Pembrey and Allen, J. Phys., 1905, 32, p. xviii. Pollock, Arch. Int. Med., I9I2, 9, 406.

Mechanism of Gas Exchange in Lungs.-Boothby and BERRy, Am. J. Phys., r9I 5, 37, 433; Christiansen and Haldane, J. Phys., I9r 4, 48, 273 (influence of distension of lmings). Dixon and Ranson, J. Pharm. Exp. Ther., $1914,5,539$ (effect of allered vascular conditions in lungs). DOUGLAS and HALDANE, J. Phys., I9I2, 44, 305 (causes of $\mathrm{O}_{2}$ absorption in lungs). Evans and Starling, J. Pliys., 1913, 46, 4I3 (oxidation in lungs). Haldane and Lorrain SMith, - ib., I898, 22, 231, 307. HARTRIDGe, ib., 8.912, 45, I $70\left(O_{2}\right.$ secretion?). KROGH (M.), ib., I9I 5, 49, 27 I (diffusion throitgh lungs).

Swim Bladder.-BAglioni, Z. allg. Phys., I9TO, 11, I45. JAECER, Pfliger's $\mathrm{Arc}^{\mathrm{H}}$., I $903,94,65$. 
Tissue Respiration.-13atthlli and Sters, J. Plyys. Path. Gén., I907, 9, r,

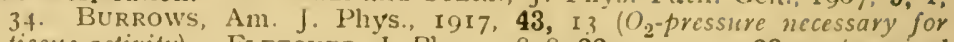
tissue activity). Fletciler, J. Phys., 1898, 22, 10; 1902, 28, $5+$ (survival respiration of muscle). JAckson (D. E.). J. Lab. Clin. Med., 1916, 2, I 45 (action of drugs on rale of $\mathrm{O}_{2}$-consumption). MANN and GAGE, J. Phys., 1912, 45, p. ix (muclei and melabolism, particularly in blood). NewaA, Am. J. Pliys., I 906, 15, 37 I (limulus heart). VERNon, J. Phys., I 909,39, I 49 (action of poisons); ib., I $907,36,8$ r (perfusion experincnls); ib., 19I0, 40, 295 (tortoise heart). HARRIS (D. F.), J. Biol. Ch., 19I5, 23. 469 (time required for reduction of oxyhcemoglobin in vivo). LESSER, Ergeb. d. Physs., rgo9. 712 (life withoul oxygen). JACKARD, Am. J. Phỵs., I $90 \%, 18,164$, ib., 1908, 21, 2 ro (resistance to lack of oxygen).

Reducing Power of Tissues.-HarRIS and IRVINE, Bioch. J., 1906, 1, 355. Herter, Am. J. Phy's., Igo 4,12, I $28,457$.

Gaseous Metabolism of Organs.-BARCROFT and Brodie, J. Phy's., 1905, 32, I $8 ; i b ., 33,52$ (killney). BARCROFT and DixoN, ib., I 9O6-7, 35, I82 (heart). BARCROFT and SHORE, ib., 1 $912,45,296$ (liver). BARCROFT and STARLING, $i b ., 1904,31,496$ (pancreas). BARCROFT and MÜLLER, ib., I91 2, 44, 259 (submaxillary gland). Boycort, ib., I905, 32, 343 (intesline). Evass, ib., I9I2, 45, 2I 3 (heart and lungs). HILL and NABARRo, ib., I895, 18, 2 IS (brain and muscle). LANGLEY and ITAGAKI, ib., I9I 7, 51, 202 (oxygen use of denervated muscle). Neruax, ib., I912, 45, I 88 (suprarenal). PEARCE and CARTER, Am. J. Pliys., I9I5. 38, $35^{\circ}$ (influence of vagus on gaseous metabolism of kidney). VERZÁR, J. Phy's., I912, 44, $2 .+3$; Ergeb. d. Phys., r916, I (muscle).

Experiments Bearing on Mechanism of Oxidations in the Body.-Bunzel, J. Biol. Ch., rga9, 7,157 (mechanism of oxidation of glucose by bromine). Cushny, Arcl. Exp. Path. Ther., Supp. Bd., 1908, I 26 (action of oxidizing salts). Dakix, J. Biol. Ch., r907, 3, 57 (simple aliphatic substances). Guthrie (F. Y.), Sac. Exp. Biol. Med., I910, 7, I 52 (modification of tissue oxidation in vitro). HARDEN and MACLEAN, J. Phys., r9II, 43, 34 (oxidation of isolated tissues). Mathews and MICGUIGas, Am. J. Phys., 1907, 19, I 99 (cxidizing power of cupric acetale solutions). MCCLENDON, J. Biol. Ch., I915, 21, 275 (oxidizing power of oxychamoglobin and erythrocytes). UXDERHILl and Closso.j, Am. J. Pliy.s., I905, 13, $35 \mathrm{~S}$ (methylene blue, elc.). Usur, Pflüger's Arch., I912, 147, noo (measurement of tissue oxidations in vitro).

Oxidizing Ferments.-Abel $\phi U$ S and BiARNès, Arch. de Phys., I896, 3 Ir; ib., I 898, 664; $i b ., 1897,277$. BATtelli and Sters, Ergeb. d. Phys., I 9 10, 10,$531 ; i b ., 1912,12,96$, I97. BERTRA.ND, Arch. de Phys., r896, 23 (laccase). Bunzel, J. Biol. Ch., 1916, 24, 9 I (mode of action); ib., 1916, 28, 315 (velation to $H$-ion concentration); ib., 24, 103 (plants). KAstlf, Hyg. Lab. Bull., No. 59, Dec., rgog. Kastle and PORCH, J. Biol. Ch., I 908,4 , 301 (peroxidase reaction of milk). Lillie, J. Biol. Ch., I913, 15, 237 (formation of indophenol at nuclear and plasma membranes of frogs' blood-corpuscles). LOevenhart and Grove, J. Pharm. Exp. Ther., I9II, 3, ror. Moore and Whitley, Bioch. J., 1909, 4, 136. REed, J. Biol. Ch., I9I 5, 22, 99 (rôle in respiration); $i b ., 1916,27,299$ (relation to $\mathrm{H}$-ion concen:ration).

Catalases.-AMBERG and WINTERNitz, J. Biol. Ch., I9II, 10, 295 (sea-urchin eggs). Arkis, Am. J. Phys., I $917,42,603$. Battell 1 and StFRs, Ergeb. d. Physiol., r9ro, 531. Burce, An. J. Phys., 1917, 43, 545; 44, 75, 290; ib., 1916, 41, I53 (muscles). BURGE, IKEN.NEDY and NEILL, Am. J. Physs., I $917,43,433$ (effect of thyroid feeding). Evass, Bioch. J., 1907, 2. 133 (blood). Loew, Pfüger's Arch., 1909, 128, 560 ; (T. S. Dept. Agric. Rep., No. 68, rgor. MENDEL and LEAVENWORTH, Am. J. Phys., I 9o8, 21, 85 (tissues). Simffer, Am. J. Plyys., 1905. 14, 299. Winternitz and MIELOY, J. Exp. Med., I908, 10, 759 (blood). WINTERNitz and Pratr, J. Exp. Ned., r910, 12, 1 , ir 5 (blood). Wisteriitz and. Rogers, ib., 1910, 12, I2,755 (eggs). 
Reductases. - Harris (D. F.), J, Biol. Ch., I9I5, 22, 53j. Harkis and

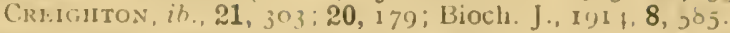

Bioluminescence (Phosphorescence).-HARvEY, Am. J. Phy's., Igr 5, 37, 230; ib., I) I6, 41, 449, 45t; ib., 1917, 42, 318 ; J. Biol. Ch., I917, 31, 31 . KASTLE and MCDERMOTt, Am. J. Phys., 1yIO, 27, I22. Moore, Bioch. J., 1908,4 , I.

\section{COMPRESSED AND RAREFIED AIR.}

Caisson Illness.-Boycotr and DAMANT, J. P'hy's., x907-8, 36, p. xiv. HA. and IILL, $i b .$, I905-6, 33, pp. v, vi, vii. HILL and MACLEOD, $i b ., 1903$, 29, 382, 492. HILl and Gren.wivood, Proc. Roy. Soc., Igo6, 13 77, 442; I $707, \mathrm{~B} 79,2 \mathrm{I}, 284 ; 1908, \mathrm{~B}$ 80, I2. TWort and Hill, J. Phys., I9Io, 41, p. v.

Effects of Atmospheres Rich in Oxygen.-BENEDICT and HIGGiss, Am. J. Phys., I9x I, 28, I (effect of $\mathrm{O}_{2}$-rich mixtures on man). BULlort, J. Phys., I904, 31, 359 (action of $\mathrm{O}_{2}$ at low and high pressure on the cornea). HILL and MACLEOD, Proc. Roy. Soc., B \%0, 455, 465 (effects of an almosphere of $\mathrm{O}_{2}$ on respiratory exchange and circulation in mouse and frog). HrLl and F LACK, J. Phys., I910, 40, 347 (effect of oxygen inhalation on muscular work). IKARSNer, J. Exp. Med., I9I6, 23, I49. KARSNer and Ash, J. Lab. Clin. Med., I $917,2,254$. Lorrain Silitil, J. Phys., I 899, 24, I 9 (pathological effect of increased $\mathrm{O}_{2}$ tension). Mosso, Arch. de Phys. et Path., I878, 10, 76 (action of compressed air).

Influence of High Altitudes.-BARCROFT, J. Phys., I9II, 42, 44; ib., I9I3, 46, p. xxx. Bartlett, Am. J. Phys., i904, 10, I 49. Boycott and HALdANe, J. Phys., 1908, 37, 355 (low atmospheric pressures). Colinheim, Ergeb. d. Phys. (Bioch.), I903, 6I2. Douglas, Haldine, Henderson and Schneider, Proc. Roy. Soc. (Lond.), I9I3, B 85. Durig and Zuxiz, Arch. f. Phys., 1904, Supp. Bd., 417. HUEPPE, Pfï̈zer's Arch., I903, 95, 447. Mosso and MARro, Arch. Ital. Biol., I903, 39, 387. SchNeider et al., Am. J. Phys., I908, 23, 90; ib., I914, 34, 29; ib., I916, 40, 380 (circulation). Tissot, J. de Phys. Path. Gén., I910, 12, 520 ; ib., I 9I I, 13, 75 (mountain sickness). WARD, J. Phys., I908, 3\%, 378. ZUNTZ and v. Schrörter, Arch.f. Phy's., 1902, Supp. Bd., 430, 436 (balloon).

Influence of Respiration on Circulation.-GollA and Syses, J. Phys., igr6, 50 , p. xxxii (cerebrospinal pressure and respiratory movements). HENDERSON and BARRINGER, Am. J. Phys., I9I3, 31, 399 (respiration and heart output). SNYDER, Am. J. Phys., I9I 5, 87 , I04 (causes of respiratory
change in heart rate).

Respiratory Waves in Blood-Pressure.-ERLANGer and Festerling, J. Exp. Med., 191 2, 15, 370. LEwIS (T.). J. Phys., I908, 37, 21 3, 233. SNydER, Am. J. Phys., I 91 5, 36, 430. WIGgers, Am. J. Phys., I 9 I I, 35, I 24 (in pulmonary artery).

Cutaneous Respiration.-Barratt, J. Phys., I 897, 21, I92 $\left(\mathrm{CO}_{2}\right.$ and $\left.\mathrm{H}_{2} \mathrm{O}\right)$; ib., 24, II (vamished skin). BонR, Skand. Arch. Phys., I9oo, 10, 74 (frog). Reid and Hambley, J. Phys., I 895, 18, 4 I I (CO $\mathrm{CO}_{2}$ frog).

\section{CHAPTER V.}

\section{VOICE AND SPEECH.}

Fleeming Jenkin and Ewing, Trans. Roy. Soc. (Edin.), I879, 28, 745 (vowe sounds). GKÜrZNER, Ergeb. d. Physiol. (Bioph.), 1902, 466 (voice and speech). Hermann, Pfü jer's Arch., 1901, 83, I, 33 (consonants); ib., 86, 92 (vowel sounds). MICKENDRICK, Trans. Roy. Soc. (Edin.), 37, Pt. 4. Meyer, The Organs of Speech. Paulsen, Pflüjer's Arch., r 895,61 , 407 (the singing voice of children); ib, I899, 74, 570 (pitch of the voice). Russell (J. S. R.), Proc. Roy. Soc., I 892 , 102 (innervation of glottis). SCRIPTLRE, Study of Speech Curves, Carnerie Instit. Pub., Washington, I906. 


\section{MOVEMENTS OF ALIMENTARY CANAL.}

CANNon, Mechunical liactors of Digestion, New Jurk, 1911; J.A.M.A., 1903. 40,749 .

Deglutition.-Botazzi, J. Phys., I899, 25, i57 (neress). Caxion, Am. J. I'liy:.. 1907, 19, 436 (cosophageal peristalsis after vagotomy). CANso. and MUSER, $i b .$, I $898, \mathbf{1}, 435$. KAHN, Arch. f. Phys., I 903, Supp. Band., 380 (nerves); $i b ., 1906,355,362$. Kisiser, Arch. Néerland de. Phy's., I 9 7 7, 1, I 48. Meltzer, J. Exp. Med., 1 897, 2, 153; Soc. Exp. Biol. Med., I906, 3, 52 (veflexes); Brit. Med. J., Dec. 22, I906 (vagus reflexes); Soc. Lxp. Biol. Med., 1907, 4, 35 (secondary peristalsis of cesophagus). MILler and Sinerisigton, Q. J. Exp. Phys., I915-I6, 9, I 47 (reflex deglutition in decerebrate animal). Srulis, Am. J. Phys., I90I, 5, 338 (rhythmic activity of cesophagus). STUART (T. P. ANDERSON), J. Phys., I907, 35, $44^{6}$ (epiglottis).

Stomach Movements.-Auer (J.), Am. J. Phys., 1908, 23, I65 (rablit). CANNox (IV. B.), ib., $1911,29,250$ (nature of gastric perislalsis); ib., 29, 267 (receptive relaxation). CARLSON, ib., 1912, 31, 151; ib., 191 3. 32, 245 (cmfty stomach of man). HERT\%, O. J. Med., Igro, 3, 373 (man). Roux and BALthAzARD, Arch. de Physiol., I 897,85 ( $x$-rays).

Innervation of Stomach.-AUER (J.), Am. J. Pliys., I gro, 25, 334. BRUxiMEIER and CARLSON, ib., I9I5, 36, rgi (veflexes from intestinal mucosa to simmach). Cannon, ib., I906, 17, 420 . LANGLle J. Phys., I 895-9, 23, to7 (ivihibitory fibres in z'agus). MAY (W. P.), J. Phys., I90., 31, 260. Rogers, Am. J. I'liys, I9I 7, 42, 605 (r.jlex control of gastric vagus tonc).

Sensibility of Stomach.-CARLSON and BRAAFLAADT, Am. J. Phys., I9I5, 36, 153. HERTz, COOK and Schlesincier, J. Phys., I908, 37, 48 I (stomach and intestines). Miller, J. Phys., I910, 41, 409.

Pylorus Control.-Boldyer1, I'flüger's Arch., I907, 121, I 3. CAxiox, An. J. Phys., 1904, 12, 387; ib., I907, 20, 283; ib., 1908, 23, 105 (acid control). CAthcart, J. Phy's., I91 , 42, 433. Morse, Am. J. Phys., I $16,41,439$ (acid control). Hedblom and Canson, Am. J. Med. Sci., Oct., 1 yog.

Movements of Intestines.-Alvarez, Am. J. Phys., I9I 4, 35, I 77; ib., I9I 5 , 37, 267 (rhythm of segments from different parts of iniestine); J. Am. Med. Ass., I91 5, 65, 383. BAYLISS and StaRling, I. Phys., I900, 26, 107, 125. Cannon, Am. J. Phys., I 902, 6, 251 ; 191 I, 29, 238 ; 1912,30 , I I 4 (tonus and antiperistalsis); J. Am. Med. Ass., I91 2, 59, I (large intestine); Arch. Int. Ned., I9I 1, 8, 47 (importance of lonus). JilloTt and BARCLAYSari II, J. Phys., 1904, 31, 272; Hertz and Newton, J. Phys., I9I3-1.4. 47, 57; Lyman, Am. J. Phys., 19r 3, 32, 6 I (colon). Hasbleton, Am. J. Phys., I9I 4, 34, 44 (movements of villi). HeRTz, J. Phys., I9I 3, 47, 54 (ileo-colic sphincler). Magivus, I'flüzer's Arch., I904. 102, I 23, 349; ib., 103, 515, 525; ib., I $906,111,152$ (strviring intestinc). MELTZER and AUER, Am. J. Phys., 1907, 20, 259 (peristaltic rush).

Innervation of Intestine.-Buncir, J. Phys., I $898,22,357 ;$ ib., I $899,25,22$. Cannon, Am. J. P'hys., 1906, 17, 429. Langley and Magnus, J. Phys., I $905^{-6}, 33,34$.

Defæcation.-Charles, Brit. Med. J., Sept. 30, I 899; Frankl-I focuwart and 1'1.0̈HL1Cı, Pfüger's Arch., I900, 81, $\{20$ (tomus and innervation of anal sphincters). Illktz, Sensibility of the Alimentary" Canal, InI I.

Vomiting: Emetics.-l3Rouks and Luckinard, Am. J. Plyys., I9r 5. 36, IOf (lood-pressure during vomiting). EGcLESTON and HATCIER. J. Pharm. Exp. Ther, I91 5, 7, 225. MAgnts, Ergeb. 1. Plyysiol., I903, 645. Miller (F. R.), Píügr's Arch., I9I2, 143, I; Am. J. Physs., I9I 5, 38, 2 to (cardiac inhibition during vomiling). 


\section{CHEMICAL PHENOMENA OF DIGESTION.}

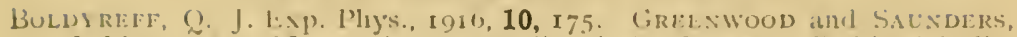
J. l'hys., Isuf. 16, If (proto om digestion). Loxiox, \%. I'hysinl. C'l. 1905, 45, 381: ib., I006, 4\%, jos (protein digestion); ib., 1urs, 51, 2.11; ib.. I90S, 56, 512 (curtuhydrate digestion). PAwiow, Work of the Digestive Cilandis, London, I910; lirgeb. d. Pliysiol. (Bioch.), I902, zio (phy'siological surgery of digestive canal). Sildw, Am. J. I'hys., I I I 3. 31, 139 (in birds-chick).

Catalysis. Catalysers. BRE I) Anorganische Fermente; Bioch. 7.., I907, 6. 253. BHERG and (iIEs, J. Biol. Clı., I906, 2, 189 (ions und catalysis). Brow. (O. H.), Am. J. Plyys., 1905. 13, 427. EuLER, \%. f. Physiol. Ch., Ino5, 45, 420. KASTLE and LOEVkNART, Im. Chem. J., $1903,29,397$. 5(13. LOEVirilart, Aul. J. P'hys., 1905, 13, I 7 I $\left(H_{2} \mathrm{U}_{2}\right)$. Neilsow and Tierri, Am. J. Pliys., I905, 14, 2 fS. DAkIN, J. Biol. Ch., r9o9, 7, 49 (catalytic action of amino-acids, etc.). TAYLOR, ib., 7, 49; ib., I910, 8, 503 .

\section{ENZYMES OR FERMENTS.}

Arustroyg (H. E. and E. F.), Proc. Roy. Soc. (Lond.), I907, B 79, 360 (nature of enzymes). ARMSTRONG and ORMEROD, ib., B 78, 376 (lipase). BAlliss, Nature of Enzyme Action, r9o8; J. Pliys, r913. 46, 236; ib., I9I 5, 50, 85 (enzyme action). BEARX and CRAMER, Bioch. J., I907, 2, I 7 (zymoids). BREdig, Ergcb. d. Phys. (Bioch.), I902, I 34. Bunzel, J. Biol. Ch., r9I 5, 20,697 (alfalfalaccase). Buchner, Arch.f. Phys., rgo6, $5 t^{8}$ (in micro-organisms). Croft HILl, Brit. Med. J., June 20, I903 (reversibility of maltasc action). CoLE (S. W.), J. Phys., I90 1, 30, 28I; MAtheWS and GlexN, J. Biol. Cl., I9I r, 9, 29; Osgorne (IV. A.), Z. f. Physiol. Ch., I899, 28, 399 (invertase). FALK (K. G.), J. Biol. Ch., I9I7. 28, 389; VAN SLYKE and Cullen, J. Biol. Ch., I9I 4 . 19, I 4 (molle of action of urease and of enzymes in general). FISCHER (E.), Ber. Deutsch. Chem. Ges., 28, I 429; Zentralb. Physiol., 10, I 7 (configuration and enzyme action). EulER, Ergeb. d. Phys., I 907, 239 (synthesis by ferments); ib., I87 (chemistry). MUTCH, J. Phys., I912, 44, I76 (histozym). MICHAELIS and EIIRENREICH, Bioch. Z., I908, 9, 283 (adsorption analysis of ferments). PAVY and Bywaters, J. Phys., igro, 41, i6s. PeTers, J. Biol. Ch., I908, 5, 367 (adsorption of diastase and catalase). Porter (A. E.), Q. J. Exp. Phys., I9IO, 3, 375 (inactivation of enzymes). OPPENHEIMER, Ferments and their Actions, translated by Mitchell. SöRENSON, Bioch. Z., I907, 7, 45; ib., I909, 22, 352.

Action of Substances on Ferments.-Bièrry, J. Phy's. Patlı, Gén., I9I 2, 14, 253 (electrolytes in diastatic actions). $\operatorname{COLE}(\mathrm{S} . \mathrm{IV}$.), J. Phys., I90.t, 30, 202 (acid on saliva). Morse, J. Biol. Ch., I915, 22, I 25 (halogens). NinsoN and Brown, Am. J. Phys., Igof, 10, 225, 335 (ions). Quixix, J. Biol. Ch., I909, 6, 53 (hydroxyl-ion concentration and diastatic hydroly'sis).

Enzyme Syntheses.-Bradley and Kellersierger, J. Biol. Ch.. I912, 13, 425 (diastase and starch). BRADLEY, ib., I912, 13, +3 I (lactase of mammary gland). TAYLOR (A. E.), ib., rgo8, 3, 87 (trypsin and protein).

Proteolytic Enzymes.-CATHCART, J. Phys., I905, 32, 299 (spleen enzyme). DAKIN, ib., I903, 30, 84 (kidney enzyme). EFFroxt, Biochemical Catalysis in Life and Industry, Proteolytic Enzymes (translated by Prescott), I9I7. FRANKEL, J, Biol. Ch., I9I6, 26, 3 I (action on purified proteins). Hedis, J. Phys., rgof, 30, 155, r 95. LEVENE and STooker, Am. J. Phy's., 1904, 12, I (interaction of). IIOBER, J. Biol. Ch., I9I r, 10, 9 (method). OPIE and BARKER, J. Exp. Med., I907, 9, 207 (lencoprotease). SHACKLES and MeLIzER, Am. J. Phys., r909, 25, 8 I (effect of shaking). ROAF, Bioch. J., I908, 3, I 88 (colorimetric method). SloAv, Am. J. Phys., I9I7, 42, 568 (origin of proteolytic ferments).

Lipases.-Arthus, J. Phys. Path. Gén., I902, 4, 56, 455 (lipasc of blood). BOLdrREFF. Z. Physiol. Ch., I $900-7,50,394$ (lipase of intestinul juice). BRADLEY, J. Biol. Ch., I9Io, 8, 25 I (lipase reactions). ConNsteis, 
Ergeb. d. Phys. (Bioch.), r904, 194. FALk, J. Biol. Ch., I917. 31, 97. HULL and Killiox, J. Biol. Cli, I917, 32, I 27 (gastric lipase). JoevexHART, J. Biol. Ch., rgo6, 2, 391; JRoseviners, J. Pliys., r9 10, 40, p. xiv (co-cnzyme of lipase). LOEvenilart, J. Biol. Cli., I9o6, 2, 427 (identily of lipases); Am. J. Phys., I 902, 6, 33 I (lipase and fal molabolism). I.ONDON, Z. f. Phys. Cli., I 906-7, 50, 125 . JObLING, EgGSTEIN and PETERSEN, J. Lixp. Med., 1915, 22,707 (serum csterase). MENDEL and LEAVENWORTH, Am. J. Pliys., 1908, 21, 95 (lipase in embryonic lissues). MEL1.A.NiY and Woolley, J. Phys., 191., 48, 287; Siraw-Miackexzie, J. Phys., 1915. 49, 216 ; D1: SouzA, J3ioch. J., 1916, 10, 108 (pancreatic lipase). QUINAN, I. Med. Res., 1915, 32, 45 (lissue esterases). vo. HEss, J. Biol. Ch., I9I I. 10, 38 I (rclation of pancreas to blood and lymph lipase).

Antiferments.-Bayliss, J. Phys., I9I2, 43, 455 (anti-emulsin). CAтHCART, J. Phy's., r904, 31, 497 ; Jobling and Petersen, J. Exp. Med., I9I4, 19, 459; WeIl, Arch. Int. Med., rgro, 5, rog (sermm antilrypsin). EULER, Lirgeb.d. Physiol., I 907, 229. Hasill, J. Phy's., I 906, 33, 479. Jobling and Pietersen, J. Lixp. Med., I9I 4, 20, 452 (baclevial). LANGenskJöLd, Skand. Arch., I91 4, 31, I. WE1NLAND, Z. f. Biol., 1902, 43, 86; 44, I (tape-worm).

\section{SALIVARY DIGESTION.}

Cannon and Day, Am. J. Pliys., I903, 9, 396 (in stomach). Maxwell, 13ioch. J., I91 5, 9, 323 (salivary and gastric digestion).

Diastase in Saliva.-CARLSON and Ryan, Am. J. Phys., 1908, 22, I (cat). Evass, J. l'lys., I9I 2, 44, I9I (amylo-clastic action of saliva). CHITTENDEN and Richards, Am. J. Phys., 1898, 1, 46r (man). MENDEL and Underiill., J. Biol. Ch., I907, 3, I35 (dog). Palmer, Am. J. Phy's., I $916,41,483$ (ox). Seymour, Am. J. Phys, I9I $7,43,577$ (horse).

Secretion of Saliva.-Asier and Cutter, Z.f. Biol., I900.40,535. BArCrort, J. Phys., 1900, 25, 479 (movement of water in secretion). BUxCH, J. Phy's. 1907, 36, I (changes in volume of submaxillary during activity). CARLSON and McLean, Am. J. Phys., 1908, 20, 157 ( $O_{2}$ supply and secretion). Carlson, Greer and Becht, Am. J. Phys., I jo7, 20, y 80 (blood-supply and character of saliva). CARLSON and CRITTENDEN, ib., 1910, 26, I69: RYAN (J. G.), ib., I909, 24, 234 (ptyalin concentration). DEMOOR, Arch. Internat. de Phys., I9I3, 13, I87. Grüxisur (O. F. F.), J. Phys., I $898,22,385$ (vesistance to secretion and percentage of salls). HENDERSON (V. E.). J. Pharm. Exp. Ther., 1910, 2, I (action of drugs). LANGLEX, J. Phys., $1885,6,71 ; i b ., 1888,9$, 55; ib., 1890, 11, 123 (paralytic secrelion); 1916, 50, p. xxvi (trophic secielory fibres). JAPELLI, Z. f. Biol., 1906, 48, 398 (physico-chemical conditions). LATHMER and WARREN, J. Exp. Med., I 897, 2, 465 (zymogen of plyalin). MATHEWs (A. P.), Am. J. Phys., I901, 4, 482 (atropin). PAwlow, Ergeb. d. Physiol., I 904. 177 (psychical secretion); Arch. Internat. de Phys., 1004. 1, 119. IVerthilmer and BAttez, J. Phys. Path. Gén., I9I 4, 6, 438.

Salivary Glands and Gastric Juice.-Hemmeter. Bioch. Z., I908, 11, 238. Swasson, Am. J. Phy's., I9I7, 43, 205. HydE, Z. f.'Biol., I897, 35, 459 (Oclopus macropus).

\section{GASTRIC DIGESTION.}

Gastric Juice.-Carlson, Am. J. Phys., I9I 5, 37, 50 (sccretion in man). CARLSON, IIAGAR and ROGERS, $i b ., 1915,38,248$ (chemistry of nomal humangastricjuice). Cornneim and Soetreer, Z. f. Physiol. Ch., Iyoz-3. 37, 467; Girelin, Pflüger's Arch., 1904, 103, 6 I 8 (secreition of new-born). EDKINS, J. Phys., I906, 34, I33; EDKINS and TWEEDY, ib., I909, 38, 263 (chemical mechanism of gastric secretion). HAMBURGER (W. W.), J.EXp. Med., $191 \mathrm{I}, 14,535$ (pepsin and so-callcd antipepsin). HARVEY (B. C. H.), Am. J. Anit., 1907, 6, 207 (gastric glands). PORTER (A. E.), J. Phys., I9II, 42, 389 (pepsin and rennet are independent). ROGERs, RAHE, J.Allelit and HACKETt, Am. J. Phys., rgi6, 39, 345 (effect of organ extracts on gastric secretion). 
Quantitative Relations of Pepsin Action.-Hurpert (and Sochrz). Pfliger's Arch., 1900, 80, 470. Nisilso. and Bowsor, Arch. Int. Med., Iy13. 11, 395. SPRigGs, Z. \{. Phigsiol. Ch., 1902, 35, 465.

$\mathrm{HCl}$ of Gastric Juice.- Fifisc, J. Plıyss. Pattl. Gén., I908, 10, toog (reactions for). LUSK and liERRIS, $\lambda_{n 11}$. J. Phys., IS98, 1, 277 (inversion of cane sugur by). Palmik, Bioch. J., 1906, 1, 398 (in curcinoma). Sü̈zrst, Skand. Arch. Ply's., 189.5, 5, 277 . W'Eislaxd, Z. f. IBiol., 19ro, 55, 58 (in shark). Widdicombe (J. H.), J. Phys., I902, 28, I 75 (digestion of cane sugar).

Acidity of Gastric Juice.-Menten, J. Biol. Ch., $1915,22,34$ (in man). Michaelis and Davidosoln, Z. Exp. Path. Ther, 19 ro, 8, 39.9. TAxgl, Ptlïger's Arch., roou, 115, 64.

Formation of $\mathrm{HCl}$ of Gastric Juice.-Bensley and HARvey, Trans. Chicago Path. Soc., I9r 3, 9, 22 I. BERGEIM, Soc. Exp. Biol. Med., Ig14. 12, 23. Bexratir and SAcis, Pflïger's Arch., I905. 109, 466. lioeppe, Phüger's Arch., I 896, 62, 567. Lieberman,, Phüger's Arch., I891, 50, 25. Osborne (T. B.), Am. J. Phys., igor, 5, r8o. Monti, Archivio di Fisiol., I9I3, 11, I 55 (function of the delomorphic cells). vON RIIORER, Pflüger's Arch., 1905, 110, 416. WESENER, ib., I 899, 77, 483.

Acidity of Gastric Contents.-Fowler, BERGEIM and HAwk, Soc. Exp. Biol. Med., r9I6, 13, 58 (indicators). McClendon, Am. J. Phys., I9I5. 38, I9I (adults and infants, acidity in stomach and duodenum). MOORE, Alexander, Kelly and Roaf, Bioch. J., 1906, 1, 274.

Protein Digestion in Stomach.-Tobler, Z. f. Physiol. Ch., I905, 45, I $8_{5}$. ZuNz (E.), Ergeb. d. Phys., 1906, 622, 663. CHITtEnden, MIENDEL and JAckson, Am. J. Pliys., 1898, 1, I64 (action of alcohol on digestion).

Proteoses, Albumoses.-Chittenden and Hartwell, J. Phys., I89i, 12, I2. HASLAM, $i b ., 1907,36,164$ (deutero-albumose). KÜHNE and CHITENDEN, Z. f. Biol., ISS 4,20, I 1, 409 .

Digestion of Nucleins.-Amberg and Joses, J. Biol. Ch., I9 I I, 10, 8I. LEVENE and MEDIGRECEANU, $i b ., 9,375$.

Chymase (Rennin).-BAxg, Z. f. Physiol. Ch., I904-5, 43, 358. Bosworth, J. Biol. Cl., 1913, 15, 231 ; ib., 191 4, 19, 397. BuRGe, Am. J. Phys., I912, 29, 330. Ediunds, J. Phys., I 896, 19, 466. Fuld., Ergeb. d. Physiol. (Bioch.), I902, 468. Hawk, Am. J. Phys., I903, 10, 37. KENt, J. Phys., I 1 I , 43, p. xxiv. Leary and Sheir, J. Biol. Ch., I 91 7, 28, 393. Locke, J. Exp. Med., I 897,2 , 493. Loevenhart, Z. f. Physiol. Ch., 1904, 41, r 77. Mellanby, J. Phys., I91 2, 45, 345. Pawlow and Parastschak, Z. f. Physiol. Ch., I904, 42, 4 I 5. PORTER (A. E.), J. Phys., I 91 I, 42, 389. TAÝlOR (A. E.), J. Biol. Cl., 1908, 5, 399. WARREN (J. W.), J. Exp. Med., ז $897,2,475$.

\section{PANCREATIC JUICE.}

BAYliss and Starling, J. Phys., I904, 30, 6I. Boldyreff, Q. J. Exp. Phys., I9I + 8, I; i6., I916, 10, 175; Ergeb. d. Physiol., I9I I, I21, I56. BRADLEY, J. Biol. Ch., I909, 6, I33 (human). DE ZILwA, J. Phys., 1904, 31, 230 (composition). Fischer (E.) and Abderhalden, Z. f. Physiol. Ch., I907, 51, 264 (action on polypeptides). IVELKER and FALLS, J. Biol. Ch., I9I 7 , 32,509 (infuence of pancreatic digestion on non-colloidal $N$-content of s(rum).

Enzymes of Pancreas.-BAyliss, J. Phys., I907, 36, 22 I (causes of rise of electrical conductivity in trypsin action). EDKINS, ib., I891, 12, 2 I8 (action on casein). HEDIN (S. G.), ib., 1905, 32, 468; ib., 1906, 34, 370 (trypsin action). Magnus, Z. f. Physiol. Ch., 1906, 48, 376 (sy'nthetic bile acids and pancreatic fat-splitting). MAYs (K.), ib., 1907, 51, 182. Mellaxby and Worley, J. Phys., I9I 3, 47, $33 \mathrm{~S}$ (trypsin, tmpsinogen, enterokinase); ib., I9I3, 46, I 59 ( $C a$ and generation of trypsin from try'psinggen). ROBERTSON (T. B.). J. Biol. Ch., I908, 5, 3 I (role of alkali in hydrolysis of proteins by trypsin). TERROINE and SCHAEFFER, J. Phys. 
Path. Gén., I9IO, 12, $\delta 84,965$ (irypsin and erepsin). TI:RROINE, ib., I9I I 13, S37 (saponifying ferments); ib., I9I 3, 15, II25. II 48 (digestion and alsorplion of fiil). TERROINE and WEILI, ib., I912, 14, 437 (starch digesion). VERNON, J. Phys., 1901, 26, 405 (trypsin); ib., I9I0, 47, 325 (trypsinogen autocatalysis); ib., 27, I 7t (pancreatic rennin and diastase); ib., I yo2, 28, 448 (zymogens); ib., 1904, 30, 330. Zuxz, Arch. Internat. Physiol., I9II, 13, I9I (action on proteins and protcoses). RACIIFORD, J. Phys., I 899, 25, 165 (influence of bile); Am J. Phys., 1899, 2, 483.

Secretion of Pancreatic Juice.-Von ANrle, J. Phys., I9I4, 49, I; ib., I9I6, 50, 42 I (vagus). CAMUS and Gley, Arch. Internat. Phys., I9r 3, 13, I02 (pilocarpin). Herring and Simpson, Q. J. Kixp. Phys., Igog, 2, 100 (secretory pressure). MAY (O.), J. Phys., 190. 30, too (secretion and blood supply). Rogers, Rahe, Fawcett and Hacklts, Am. J. Phys., I9I6, 40, I 2 (effect of organ extracts). WERTHIEIMER and BOULIE, Arch. internat. Phys., 1912, 12, 247.

Secretin.-Bayiss and Starling, Ergel). d. Physiol., 1906, 670; ib., 693 (chemical nature of hormones); J. Phys., 1903, 28, 325; ib., 29, I7t. Bainbridge and Beddard, Bioch. J., 1906, 1, 429 ; livans, J. Phys., I91 2, 44, 46I (secretin and diabetes). Delezeñis, J. Phys. Path. Gén., I9I2, 521, 540. Dixon and Hamill, J. Phy's, Igog, 38, 314 . Hédon, Arch. Internat. Phys., I912, 12,485. IVERTheimer and LEPAge, J. Phys. Path. Gén., I901, 3, 689, 708 .

Adaptation in the Digestive Juices.-Bayliss and Starling, Ergeb. d. Physiol. I 906, 681; J. Phys., I 904, 30, 6.t. Plimmer (R. H. A.). J. Pliys., 1906, 34, 93; ib., 1906, 35, 20 (adaptation of pancreas to lactose).

\section{BILE.}

Chemistry of Bile.-BRAND, Pflïger's Arch., I902, 90, 49I; Rosenbloom, J. Biol. Ch., I9r3, 14, 24 I (human bile). Fischer (H.), Ergeb. d. Phys., I9I6, I85 (bile and blood pigment). Heynsius and CAMPBEL., Pflüger's Arch., I 871, 4, 497; HAycraft and Scofield, Z. f. Physiol. Ch., i S9o, 14, 73; Stewart (G. N.), Studies Physiol. Lab., Univ. Manchester, I891, 2OI (bile-pigment spectra). Hammarsten, Ergeb. d. Physiol., 1905. 1. OKADA, J. Phys., I9I 5, 50, I I 4 (reaction). GARDNER and KNox, J. Phys., 1907, 36, p. ix (cholesterol in bile). Hammarsten, Z. f. Physiol. Ch., I904-5, 43, I 27 ; Lewis, Bioch. J., I908, 3, I I9; Schryver, J. Phys., 1912, 44, 265 (bile-salts). Sellards, J. Exp. Med., I909, 11, 786 (reaction with blood-serumn).

Bile and Bile-Pigment Formation.-Whipple and Hooper, Am. J. Phys., 1916, 40, 332; ib., I91 7, 42, 256, 544; ib., 1917, 43, 258, 275, 290; J. Exp. IIed., 1913, 1\%, 612 (from hemoglobin outside the liver).

Bile and Bile Pigment Secretion.-Barbéra, Arch. Ital. Biol., I q02, 38, 447. Bayliss and StakliNG, Ergeb. d. Physiol., 1906, 677. Doro: and Dufourt, Arch. de Phy's., I 896, 587; ib., I897, 562. DAsTre, ib., I89o, Soo. Erger, Z. f. Biol., I9I 5, 66, 229 (vagus influence). Hooper and Whipple, Am. J. Pliy's., I917, 42, 264, 280 (influence of bile). OKaDA, J. Phys., I915, 49, 457. Pfarf and BALCh, J. Exp. Mled., I897, 2, 49. Pugliese, Arch. Ital. Biol., I902, 38, 257. Simpson (S.), Soc. Exp. Biol. Med., I910, 7, 8.

Eck's Fistula.-Bersheim and Voegris, J. Pharm. Exp. Ther., 1909, 1, 463. CARrel and Guthrie, Compt. Rend. Soc. Biol., June 30, I906, IIOA (simple method). Hawk, Am. J. Phys.. I908, 21, 259. Herrick (F. C.), J. Exp. Med., I905, 7, 751. SWert, J. lixp. Med., I905, 7, I63.

Absorption of Bile, Icterus.-HARlis;, Proc. Roy. Soc., 1892, (paths). Harold, Pepper and Perri, J. Jixp. Med., i9i5, 22, 675. Herring and Simpsos, Proc. Roy. Soc., I907, B 79, 5 I 7 (secretory presswre and absorption). PEARCE (R. M.) ET AL., J. Exp. Med., I912, 16, 758, 769. 7So (hamolytic jaundice and the spleen). SUTHFRLA. BD, Bocli. J., I906, 1, 364. Voegtirn and BernieiM, J. Pharm. Exp. Ther., I9I I, 2, 455. 
WertheIMER and LFPArre, Arch, de Phys., I897, $3^{\text {ij }} 3$ (paths of alsorp. tion); ib., I Sos, 3.3t; J. I'lyges. P'ath. Gén., 18.99, 1, 2.59. Whipple and

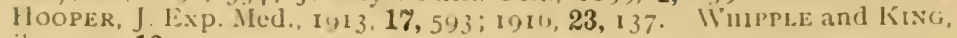
ib., $1911,13,115$.

Toxicity of Bile.-lirotmingma and Misot, J. Med. Res., IgI 2. 27, 79).

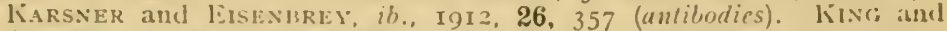
Stewart (H. A.), J. Exp. Med., I gog, 11, 573. Mleltzk and Salast, ib., 1906, 8, 127. TAтUs, J. Biol. Ch., 1910, 27, 243 (influence on autolysis).

Bile Circulation.-Stadelann, Z. f. Biol., I897, 34, I. Wertueimir, Arch. de Phys., I892. 577.

Innervation of Gall-Bladder.-BAINBRIDGE and DALE, J. Pliys., I905. 33, I 38. l'REESF, Johns Hopk. Hosp. BuIl., 16, June, I905. LIEB and MC WHORTER, Soc. Lxp. Biol. Med., I915, 12, 102.

Bile and Bile-Salts in Digestion.-Chittexden and AlrRo, Am. J. Phys., ISgS, 1, 307 (influence on pancreatic proteolysis). DE JoNGE, Arch. Néerland. Physiol., Ig1 7, 1, 182. Kingsbury, J. Biol. Ch., I917. 29, 367. Lofvexhart and SOUder, ib., I906-7, 2, 4I5. PFlÜger (E.), Pflüger's Arch., 1902, 90, I. RACiford, J. Phys., IS9I, 12, 93.

\section{INTESTINAL JUICE.}

Bayliss and StaRlisg, Eirgeb. d. Physiol., ig06, 678 (chemical reflexes). Hamburger and Hekma, J. Phys. Path. Gér., I902, 4, 805; ib., I904, 6, 40 (man). MExdel, PAüger's Arch., I $896,63,425$ (paralytic secretion). Mosenthal, J. Exp. Med., I9I I, 13, 3 I 9. PREgl, Pfliger's Arch., I 895. 61, 359 (sheep). Salaskin, Z. Physiol. Ch., I902, 35, 4 I9 (dog).

Ferments.-Blood (A. F.), J. Biol. Ch., I9Io, 8, 2 I5; Connheim, Z. Physiol. Ch., I 906, 47, 286; I902, 35, I 34; 36, I 3, 244; REED and STAHL, J. Biol. Ch., I9II, 10, ro9; VernoN, J. Phys., I905. 33, 8 i (erepsin). Hamill, J. Phys., 1905-6, 33, 476; Mellanby and Woolley, ib., I91 2, 45, 370 (enterokinase).

Reaction of Intestinal Contents.-MCClendoN, SHEdLov and Thomson, J. Biol. Ch., I $917,36,269$ (ileum). MlOorE and BERGIs, Am. J. Phys., I $900,3,316$.

Digestibility of Foods.-Bryant and Milner, Am. J. Phys., I903, 10, 8I (vegetables). Frank, J. Biol. Ch., I9I I, 9, f63 (egg-white). LasGworthy and Holnes, U. S. Dept. Agric. Bull., I $917,505,50 \%$; Langworthy, $i b .$, I915. 310; SMITH, Miller and HAwk, J. Biol. Ch., I9I 5, 23, 505 (fats). Rockwood, J. Biol. Ch., I910, 8, 327 (flour).

Bacteria of Alimentary Canal.-Baldwis, J. Diol. Ch., r909, 7, 37. Cushisg and Livingood, Johns Hopkins Hosp. Rep., 9, 5+3. GERHARd, Ergeb. d. Physiol. (Bioch.), 1904, 107. HerTER, Harvey Lect., Yew York, Nov. 3, Ig06. Herter and KEndall, J. Biol. Ch., I909, 6, 499; ib., I $909,7,203$ (influence of diet on intestinal flora). KENDALL, J. Mled. Res., I9I I, 25, Ir7. Kranizin, J. Phys. Path. Gén., i91 I, 13, 689: J. Physi., I9I6, 50, 391; Nuttall and Thierfelder, Arch. f. Physiol., I 895, 559; Schottelius, Arch. f. Hygiene, 34 (aseplic digestion).

Fæces.-Elis and Gardier, Proc. Roy. Soc., I9I2, B 86, I3 (excretion of cholesterol).

\section{CHAPTER VII.}

\section{ABSORPTION.}

Adsorption.-BARRETT and EDIE, Bioch. J., I907, 2, 443. BAYliss, ib., I906, 1, 175. Hedrs, ib., I907, 2, II2 (of enzymes). Hofmas., Zentr. f. Phys., I9IO, 24, 805. Michaelis and RoNa, Bioch. Z., I9OS, 15, I96. ROBERTSON, J. Biol. Ch., I 908, 4, 35. VA.N SlYKE, $i b .$, I $908,4,259$.

Diffusion.-Dexis (IV.), Am. J. Phys., I906, 17, 35 (salts of blond). Flexier and Nortchi, Soc. Exp. Biol. Jled., I $906,3,66$. Hedis, Pflüger's Arch., I 899, 78, 205. HöBER, ib., r898, 71,624 (in intestinal absorption); ib., 
IS99, 74, 225. MLYiR, Hofmeister's Beit,, Ino6, 7, 393. REID (IV.), J. Phys,, 1897, 21, 85; MINes, ib., IyI , 42, p. xxviti (method). VoigrI.Ä.NDER, \%. f. Pliysikal. Ch., I 889, 3, 316 .

Imbibition.-Fiscuer and MoOre, Am. J. l'hys., I907, 20,330. HENDERSON, PALMER and BECHT, J. Pharms. Exp. Ther., IyI 4, 5, 449 (H-ion concentration). Ostwald (1T.), Phïger's Arcl1, I905, 108, 563; 109, 277; ib., I $906,111,58 \mathrm{I}$.

Surface Tension.-Beard and Cramer, Proc. Roy. Soc., 1915, B 88, 575 (and ferment action). CRAnER, ib., B 88, 584 (and cell metabolism). MiCALLUM (A. B.), Ergeb. d. Physiol., Iyı I, 11, 598; Proc. Roy. Soc., I 91 3, B 86, 527. TrAUBE, Pflïger's Arcli., I $904,105,559$ (surface tension and vital phenomena).

Mechanism of Absorption.-Munk, Ergeb. d. Phys. (Bioch.), I902, 296. Overton, Nagel's IIandb. d. Physiol., Bd. ii., 2, 744 . Scott and Denis, Am. J. Phys., I91 3, 32, i (dog-fish). Hamburger, Arch. f. Phys., 1896, 302,428 (intra-intestinal and intra-abdominal pressure and absorption).

Absorption from Intestine.-BRODIE and VOGT, J. Phys., I910, 40, I 35 (gaseous exchange of intestine and absorption). BRODIE, CULLIS and HALLIBURTON, ib., I910, 40, I 73. CoHNiein, Z. f. Biol., I 896, 33, 9; ib., 37, 443; 38, 419; 39, 167. Dakin, J. Biol. Ch., I908, 4, 437 (isomeric substances). HaNzLiK and Collins, J. Pharm. Exp. Ther., I9I 3, 5, i 85 (alcohol). London ET Al., Z. f. Physiol. Ch., I906, 49, 324, 328. WAllace and Cushny, Am. J. Phys., 1898, 1, 4 I1. ReAch, Pflïger's Arcli., I901, 86, 247 (comparative absorption in large and small intestine). REID, J. Phys., 1 898, 22, p. Ivi.; ib., I900-I, 26, 436 (nerves and absorption); ib., I 896, 20, 298. HeIdeniain, Pfüger's Arch., I $894,56,579$ (sermm). RAVENEL and Hammer, J. Med. Res., rgi i, 24, 5 I 3 (bactevia). Coleman and Gephart, Arch. Int. Med., I915, 15, 882 (in fever).

Absorption from Peritoneum.-Adler and Meltzer, J. Exp. Med., I896, 1, 482. Buxton, J. Med. Res., 1907, 16, I7. FLEISHER and LOEB, J. Exp. Med., I9Io, 12, 288, 487, 5 Io. MELtzer, J. Phys., I $898,22,196$. Shipley and Cunnixghal, Am. J. Phys., i916, 40, 75. Starling, J. Phys., I 898 , 22, p. xxiv. Wells and Mendel, $\Lambda \mathrm{m}$. J. Phys., I 907,18 , I 56.

Absorption from Connective l'issue.-HAMBURGer, Bioch. Z., 1907, 3, 359. MELTZER and AUER, J. Exp. Nled., I 905, 7, 59; I 9 I I, 13, 328 (intramuscular tissue). Starling, J. Phys., i 895-6, 19, 312.

Absorption by Skin (Frog).-MAxwelL, Am. J. Phys., I913, 32, 286.

Absorption of Fat.-BLoOR, J. Biol. Ch., I9I 2, 11, 429; I9I3, 15, I05; I9I3. 16, 5 I 7; I9I 5, 23, 3I7. HARLEY, J. Phys., IS95, 18, I (effect of pancreatectomy). Greene and Skaer, Am. J. Phys., I9I3, 32, 358. Greene, $i b$. I9I 2, 30, 278 (salmon). MIENDEL and BAumanN, J. Biol. Ch., I9I5, 22, I65 (from stomach). McCluRE, VINCENT and PRATr, Am. J. Phys., I9I 7, 42, 596 (pancreatectomy); J. Exp. Med., I9I7, 25, 381. MOORE and Rockwood, J. Phys., I 897, 21, 58 . Munk, Zentr. f. Phys., 13, 657 ; 14, I 21, I 53, 409; 15, 297. Plant, Am. J. Phys., I908-9, 23, 65. PflüGer (E.), Pflüger's Arch., I900, 82, 303; 1902, 88, 299, 43I. MENDEL, Am.J. Phys., 1909, 24, 493 (stained fat); J. Biol. Ch., I91 2, 13, 7 I. Whitehead, Am. J. Phys., I909, 24, 294; ib., 1910, 25, p. xxviii (stained fat).

Absorption of Cholesterol.-CORPER, J. Exp. Med,, I9I5. 21, I79. Fraser and Garden, Proc. Roy. Soc., r9o9, B 81, 230. Leiman, J. Biol. Ch., I9I 3, 16, 495. MUeller, J. Biol. Ch., I9I6, 27, 463.

Absorption of Proteins.-Caticart and Leathes, J. Phys., I905-6, 33, 462. Cohnheim, Z. f. Physiol. Ch., I902, 35, 396, 4 IG (octopods); ib., I906, 49, 64. Folin and Denis, J. Ijol. Ch., I9I 3. 14, 153; ib., I $912,12,253$ (large intestine). MENDEL, Am. J. Phys., I 899. 2, I 37 (paths). MUNK. Zentralb. f. Phys., I $907,11,585$ (paths). Re1D, J. Pliys., I $895-6,19,240$ (peptones). Nolf, J. Phýs. Path. Gén., r907, 9, 925.957. Salaskin, Z. f. Physiol. Ch., I 907, 51, I67 (stomach). Folin and Lymax, J. Biol. Ch., I91 2, 13, 389 (stomach); ib., I912, 12, 259. LoxdoN, Z. f. Pliysiol. Ch., I $909,62,4 t^{8}$. 
Albumoses in Tissues and Blood.-ABEL, PIxcoffs and Rouller, Am. J. I'hy's., I917, 44,320. EMnIEx and Kxoop, Hofmeister's Beit., I903, 3, I 20. Laxgster., ib., 3, 373. Schum, ib., r904, 4, 453.

Abscrption of Sugars. Hedov, Arch. de Pliarmacod., 1900, 7, rG3. MUsk, Arch. f. Pliys., 1 suo, $37^{6}$ (paths for sugar, protein, fat). REAcit, Arch. Exp. Path. Pharm., 47, 231 (Uy rectum). REID, J. Phys., Igor, 26, 427; I 902, 28, 2q1. Ruimaxis and N.gAvo, Pfüger's Arch., I 903, 95, 533.

Absorption of Iron.-ABderilatden, 7. f. Biol., Igoo, 39, II 3. I93. Bunge, Z. f. Physiol. Ch., I 898, 25, 30. Mall (II'. S.), Arch. f. Phy's., I896, 49; ib., I89.4 455. MEYl: Ergeb. d. Phys., I 906, 698. Sattler, Arch. Jixp. Path. Pharm., Iy05, 52, 326.

\section{CHAPTER VIII. \\ FORMATION OF LYMPH.}

Lymphatic System.-Јов, Anat. Record, I9I5, 9. MacCallua (W. G.), Arch. f. Anat. (u. Physiol.), I 902, 273 (velation of lymphatics to connective tissue). Sabin (F. R.), Ergeb. d. Anat. u. Entwick., I9I3; Am. J. Anat., I904, 3 , I 83 (origin of lymphatic system).

Lymph Formation.-Asher and BArbéra, Z. f. Biol., I 898, 36, I54. ASHer and Besch, Z. f. Biol., I900, 40, 333. Asher, Z. f. Biol., I 899, 37, 26 r. Asher and Gies, Z. f. Biol., I 900, 40, I 80. BAinbridge, J. Phys., igo6, 34, 275 (post-mortem); J. Phys., I900-I, 26, 79 (submaxillary gland); ib., 28, $20+$ (liver); ib., 32, I (pancreas). Braxde and Carlson, Am. J. Phys., 19, $22 \mathrm{I}$ (lymphagogues and agglutinins in semm and lymph). Cuttat-Galizka, Z. f. Biol., r9i I, 56, 309 (post-mortem). Cohnstein, Pliüger's Arch., I $895,59,350,508$; ib., I 895, 62, 58; Arch. f. Phys., I 896, 379. Carlson, Greer and Becht, Am. J. Phys., 1907, 19, 360 (salivary glands); ib., 22, rot (lymphagogue action of lymph). DixoN (R. L.), J. Exp. Med., I91 2, 16, I39. Ellixger, Ergeb. d. Phys. (Bioch.), I902, 355. Hamblrger (H. J.), Arch. f. Phys.. IS95, 364 (résumé of Heidenhain's view) ; ib., I897, I32. JAPELLI, Z. f. Biol., I9I0, 53, 319. LAzARUsBARlow, J. Phys., I 895-6, 19, 4 I 8 (osmosis and filtration). LEATHES, J. Phys., 19, I. MeNdel and Hooker, Am. J. Phys., 19o2, 7, 380 (strawberry extract). MExdel, J. Phys., 19, 227. MÏ̈lleR, Pflüger's Arch., 1908, 122, 455 (Tannenberg bodies). OpIE, J. Exp. Med., I9I 2 , 16, 831 (lymph formation and cedema of liver). PUGLiesi, Arch. Ital. Biol., I902, 3S, 422. ScotT (F. H.), J. Phys., I9I5-I6, 50, I 59; Scotr, Herman and SNell, Am. J. Phys., r9i 7, 44, 3 r 3 ; Scott, Am. J. Phys., 19r 7, 44, 29S. Sihler, J. Exp. Med., I 900, 5, 493. Starlixg, J. Phys., I 894-5, 17, 30; Wertheimer, J. Phys. Path. Gén., I906, 8, 806 (work of glands and lymph formation). YANAGAuA, J. Pharm. Exp. Ther., I9I6, 9,75 .

EEdema.-Bolto., Proc. Roy. Soc., 1907, B \%9, 267 (by obstruction of vence cave and portal). Fischer (M.), Edema, I9Io. Fleisher, Hoyt and Loeb (L.), J. Exp. Med., I909, 11, 29 I. LAzarus-Barlow, Phil. Trans. Roy. Soc., I 894, 185, 779 (accompanying passive congestion). MELTZER, Amer. Medicine, I904, 8. Miller (J. L.) and Matrhews (S. A.), Arch. Int. Med., I909, 4, 356 (pulmonary). Moore (A. R.), Pflüger's Arch., I9I 2, 14\%, 28; Am. J. Phy.s., I9Ij, 3\%, 220. PEARCE (R. M.), Arch. Int. Med., I 909, 3, 422. Woolley, J. Lab. Clin. Med., I9I6, 1, 267 (nevous influences and).

\section{CHAPTER IX \\ EXCRETION. COMPOSITION OF URINE.}

Bouchez, J. Physs. Path. Gén., I9I2, 14, 46, 74. CAMerer, Z. f. Biol., I904, 45, I; Folix, Am. J. Phys., I905, 13, 45, 66 (nomal urines). CATHCART, Bioch. Z., I907, 6, IO9 (in stavealion). 
Total Nitrogen Excretion in Normal Persons.-BARRiNGer (T. B. and B. S.), Am. J. Phys., I910, 27, i I9.

Urea in Urine.-Marsiall, J. Biol. Ch., I9I 3, 14, 2 $33 ; i b ., 15,495$; VAN SLYKE

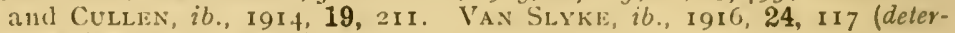
mination by wrease method).

Ammonia in Urine.-Folin and BELL, J. Biol. Cl., 1917, 29, 329 (colorimetric deterimution). FolIN and MACALLUM, ib., I91 2, 11, 523 (determination).

Acidity of Urine.-Henderson (L. J.) and PAlaler, J. Biol. Cl., I9r2, 13, $393 ; i b ., 14,81$. Henderson and SPIRo, Biocli. \%., I908, 15, 105.

Phenols.-DuBin, J. Biol. Ch., I916, 26, 69 (phy'siology of). Folin and Dexis, ib., 507 (velative excretion of, by hidney's and intestine).

Uric Acid Excretion.-Benedict and Hitcricock, J. Biol. Ch., 19I5, 20, 6r9; Folin and DExis, ib., I9I2, 13, 363, 469 (colorimetric estimation of uric acid in urine). HANZI.IK and HAWK, ib., I908, 5, 355 (normal men). LeAtues, J. Phys., Igo6, 35, I25 (dintrual and nocturnal variations). MENDEL and Brown, J. Am. Med. Ass., I907, 49, 896 (rate in man). MeNdel and Stehle, J. Biol. Cl., I9I5, 22, 2 I 5 (rúle of digestive glands in excretion of endogenous). Rockwood, Ain. J. Phys., 1904, 12, 38 (endogenous). Wells (H. G.), J. Biol. Ch., IyI6, 26, 3 I9 (accumulation in tissues in suppression of urine).

Alkaptonuria.-Dakin, J. Biol. Ch., I9II, 9, I5I. GarRod and Hartley, J. Phys., 1907, 36, I 36. GARROD and HELE, ib., I905-6, 33, I98. GARROD and Clarke, Bioch. J., I907, 2, 2 I 7. RAwold and WARren, J. Biol. Ch., I 909, 7, 465. Schulz, Jirgeb. d. Physiol. (Bioch.), I903, I80.

Bile-Salts in Urine.-ALLEN, J. Biol. Ch., I91 5, 22, 505 (Hay's surface tension method). Grünbaum, J. Phys., 1904, 30, p. xxvi.

Bile-Pigments in Urine.-Hammarsten, Skand. Arch. Phy's., 9, 3 r 3. Jolles (A.), ib., 10, 338; Pflüger's Arch., I 899, 75, 446. Musk, Arch. f. Phys., I 898, 361. Scrulz, Ergeb. d. Physiol. (Biocli.), I903, I 74.

Proteinuria.-Caneron and Wells, Arch. Int. Med., 1915, 15, 74; Cloetra, Arch. Exp. Path. Pliarm., 1899, 42, 453 (origin of the proteins). Folin and Denis, J. Biol. Ch., I9I 4, 18, 273 ; MARsiall, Banks and Graves, Arch. Int. Med., r 916, 18, 250 (estimation of the proteins). Sikes, J. Phys., 1905-6, 33, IOI (the globulin of "albuminous" urine).

Amino-Acids in Urine.-Benedict and Murlin, J. Biol. Ch., I9I3, 16, 365 (determination of amino-acid nitrogen). Forssner, Z. f. Phy'siol. Ch., 1906, 47, 15. Hall (I. WV.), Bïocli. J., I906, 1, 241. LeVene and Van SLYKE, J. Biol. Ch., I9I2, 12, 301; VAN SLYKE, ib., I9I 3, 16, 125 (estimation). Мчтсн, J. Phys., I914, 49, p. ii.

Pentosuria.-Levene and LA Forge, J. Biol. Ch., I9 $3,15,48$ r ; $i b ., 19$ I 5.18 , 3r9 (note on a case). Neuberg, Ergeb. d. Physiol. (Bioch.), I904. 373 (physiology of pentoses and glycuronic acid).

Hømatoporphyrin in Urine.-GARROD, J. Phys., IS92, 13, 598; ib., I894, 15, I 08; ib., I 894-5, 17, 349. Schulz, Ergeb. d. Physiol., I903, I62.

\section{SECRETION OF URINE.}

Cushny, The Secretion of Urine, London, 1917; J. Phys., I901-2, 27, 429; J. Pliys., I9I7, 51, 36 (excretion of urea and sugar). BAINRRIDGE and BEDDARD, Bioch. J., I906, 1, 255 (secretion by renal lubules in frog). Batwiridge, Mienzies and Collins, J. Phys., I $914,48,233$ (urine formation in frog). BARCROFT and STRAUB, $i b$., I9I0, 41, I 45 . BARCROFT and PIPER, ib., I9I5, 49, p. xiii (in decerebrate animals). BEDNARD, ib., 1902, 28, 20 (ligation of venal arteries in frog). BorD, ib., 1902.28, 76 (extirpafion of renal medulla). BRODIE and Cullis, ib., r9o6, 34, 224. BRODIE and IIAckenzie (J. J.), Proc. Roy. Soc., InI 4. B 87, 594 (changes in glomeruli and tubules accompanying activity). BRoDIE, ib., 87, $57 \mathrm{I}$ (glomerular function). Cow (D.). J. Phys., I9I4, 48, I. Cúlis (C.). J. Phỹs., 1906, 34, 250; ib., 1908, 37, p. xvi (frog). Fouss and DeNIs, 
J. Biol. Ch., I915, 22, 32 I (selective activity of human hidney'). Haskixs, Am. J. Phys'., 1904, 10, 362; Hatciler and Sollmasx, ib., Ig03, 8, I 39 (an sull-hunger). vox lửrt11, Ergeb. d. Pliysiol. (Bioch.), 1902, 395 (urine secretion of lower animals). KNowlton (li. P.). J. Phys., 1911, 43, 2Iu (influence of colloids). IsAacs (R.). Am. J. Phys., I9I 7, 45, 7I (reaction of kidney colloids and renal function). LINDEMANN, 7. f. Biol., I90I, 42, I6I (elimination of glomeruli). GEsell, Am. J. Phys., I9I3, 32,70 . HoOKER, $i b .$, I9IO, 27, 24 (relation of pulse pressure to renal secretion). Macallum and Benson, J. Biol. Ch., I909, 6, 87 (composition of dilute renal excrctions). Magnus, Arch. Exp. Path. Pharm., I9or, 45, 20 I (in plethora). Quinby. Am. J. Phys., 191 7, 42, 593; J. Exp. Med., I $910,23,535$ (denervated kidncy). Ricliards and Plavt, Am. J. Phy's., I91 $7,42,592$ (blood-pressure und urine formation). Sollass.s. Am. J. Physi, I902, 8, I55. Sollmanx and Brown, J. Exp. Mell., 6, 207 (injection of egg-allumin, ctc.). Sollmann, Am. J. Phys., 1905, 9, 425 (chlorides of urinc). DE SouzA, J. Phys., 1900, 26, I39 (effects of venoris obstruction). SPIRO and VogT, Jirgeb. d. Physiol. (Bioch.), Igo2, 4 I4. SPIRo, Arch. Exp. Path. Pharm., 1898, 41, I 48 (action of artificial concentration of the blood). Sharpe, Am. J. Phys., I912, 31, 75 (in birds). Starling, J. Phys., I $899,24,3$ I 7 (glomerular function). UNderhill, Wells and Goldschmidt, J. Exp. Med., I913, 18, 347 (renal secretion during tartrate nephritis).

Perfusion of Excised Kidneys.-Richards and Plant, J. Pharm. Exp. Ther., 1915, 7, 485. Solliann (T.), Am. J. Phys., 1905, 13, $241 ; i b ., 1907$, 19,$233 ; i b ., 1908,21,37$. Willia Ms, $i b ., 1907,19,252$.

Excretion of Pigment by the Kidney.-CARTER (W. S.), J. Am. Med. Ass., Nov. 2 I, I903, p. I 248 . GuRwITSCH, Pflüger's Arch., I 902, 91, 7I. HöBER and Kexipner, Bioch. Z., I908, 11, I05. Höber and Köxigsberg, Pflïger's Arch., I905, 108, 323. Shaffer (G. D.), Am. J. Phys., I908, 22, 335. SobIÈranski, Pflüger's Arch., I903, 98, I 35 .

Acid Secretion of Kidney.-Cushny, J. Phy's., I904, 31, IS8. DReser, Hofmeister's Beit., I905, 6, I 78. HENderson and PAl.Mer, J. Biol. Ch., I9I 4, 17, 305. HeNderson (L. J.), ib., I9I I, 9, 403. Treva., J. Phys., I $916,50,265$.

Reabsorption from Kidney.-Addis and Shevky, Am. J. Phys., I91 7, 43, $36_{3}$ (relurn of urea from kidney to blood). HENDERSON (V. E.). J. Phys., I $905-6,33,175$.

Saline, Diuresis.-Cushny, J. Phys., r902, 28, 43x. Sollyann, Am. J. Phys., I903, 9, 454. Thompson (IV. H.), J. Phys., I899-I900, 25, 487. WinFIELD, ib., I $912,45,182$ (osmotic pressure of blood and urine during diuresis by Ringer's fuid). YAGI and KURODA, ib., I9I 5, 49, I62.

Tests of Renal Function.-Mosenthal, J. Am. Med. Ass., I9i6, 67, 933; Arch. Int. Med., I915, 16, 733. PePper and Austin, Am. J. Med. Sci., I913, 145, 254. Rowntree and Geraghty, J. Pharm. Exp. Ther., I9Io, 1, 579; Arch. Int. MIed., I9I2, 9, 284 (phenolsulphonephthalein test).

Urea Excretion.-AMBARD, J. Pliys. Path. Gén., I9ro, 12, 209; AMBARD and WVEILl, ib., I9I 2, 14, 753; MICLEAN, J. Exp. Med., I9I 5, 22, 2 I 2 (numerical laws of renal secretion of urea and $\mathrm{N}$ ( $\mathrm{CCl}$ ). ADDIS and WATANABE, J. Biol. Ch., I91 6, 24, 203; $i b ., 27,249 ; i b .$, I91 7, 29, 38 I, 399 (vate of urea excretion). MEXDEL and LEWIS, J. Biol. Ch., I9I3, 16, 19, 37, 55 (rate of nitrogen excretion as influenced by diet factors).

INephrectomy, etc.-Herter and WAKEMaN, J. Exp. Med., I 899, 4, I 77. Jacksox and SaIki, Arch. Int. Med., I9I 2, 9, 79. MacNider, J. Medi. Res., 19I I, 24, 425 (ligation of one branch of renal artery). PILCHER (J. D.). J. Biol. Ch., I9I $3,14,3$ S9 (N-excretion after ligation of branches of renal artcries).

Innervation of Kidney.-ASHER and PEARCE, Z. f. Biol., I9I 3, 63, $83 ;$ Zentralb. f. Physiol., I $913,27,584$ (secretory fibres). Burtox-Opitz, Am. J. Phys., I9I6, 40, 437; J. Exp. Med., I9I I, 13, 308. BurToN-OpITz and LeCAs, 
Pllüger's Arch., 1909, 127, I 43, I48. BRADrOKD (J. R), J. Phys., I 889, 10,358 (vusomotors). Jost (IV.), Z. f. Biol., I9I $, 64,4$ I. DE SouzA, J. Phys., I 900,26 , I 39.

Innervation of Bladder.-BARrixgtox, Q. J. Exp. Phys., I9I5. 9, 26. ELLIOT, J. I'ly*s., I905-6, 33, p. xxix. Kxowltox, ib., IyI1, 43, 9ा. FAGGE, ib., I902, 28, 304. I.ANGLEY, $i b ., 1901-2,27,252 ; i b ., 1910,40$, p. Ixii; ib., 10II, 43, I 25. OTr, Zentralb. f. Physiol., r 89.5. 335 (centre). Stewart (C. C.). Am. J. Phy:s., I 899. 2, I82; ib., Igoo, 3, I. Waddell, J. Pharm. Exp. Ther., 191 7, 10, 243 (drugs).

Excretion by Skin.-BENEDICT (F. G.). J. Biol. Ch., 1905, 1, 263 (nitrogenous matter). Schwexkexbecher and Spitta, Arch. Exp. Path. Pharm., I907, 56, 2S4 ( $\mathrm{SaCl}$ and N). TAYLOR (A. E.), J. Biol. Ch., IyI I, 9, 2 I (nitrogen, sulphur, phosphorus).

Sweat.-Biggs, J. Med. Res., I 9 I I, 24, 285 ; CAMERER, Z. f. Biol., I9or, 41, 27 (chemistry of sweat). O'CoNior (J. MI.). J. Phys., I9I 5, 49, II3 (infuence of temperature on sweat secretion). WENDE and Biscri, J. Am. Med. Ass., I $909,53,207$ (localized facial sweating following olfactory stimuli).

CH.APTER X.

\section{METABOLISM, NUTRITION AND DIETETICS.}

Lusk The Science of Nutrition. Schryver, Bioch. J., I906, 1, 123 (chemical dysamics of animal nutrition).

\section{CARBOHYDRATE METABOLISM.}

DAkis and Didiey, J. Biol. Ch., 1913, 14, 555. Johasison, Skand. Arch. Phy's., I 908,21 , I. Woodyatr, Harvey Lecture, New Iork, I9I5-I6 (intermediate carbohydrate metabolism).

Dextrose.-Davis, Am. J. Phys., I9I 7, 43, 514 (intravenous injection). MACleod, J. Lab. Clin. Med., I91 7, 2, i 2 (utilization). KileINer, J. Exp. Med., I9II, 14, 274 (excretion in slomach and inlestine). JANXEY and Csovka, J. Biol. Ch., I 9 I 5, 22, 203 (glucose formation from body proteins). MATHEW's (A. P.), J. Biol. Ch., I 909, 6, 3 (sponfaneows oxidation of sugars). Lusk, Am. J. Phys., I908, 22, I 74. RiNGer, J. Biol. Ch., I91 2, 12, 5 II; StIles and Lusk, Am. J. Phys., I 903, 9, 380 (formation from amino-acids). MACLEOD and FULK, Am. J. Phys., I9I 7, 42, I93 (retsntion of dextrose by liver and muscles).

Glycogen.-Botazzi and D'ERRICo, Pflüger's Arch., 1906, 115, I59 (physicochemical experiments). Cremer, Ergeb. d. Phys. (Bioch.), I902, $\mathrm{SO}_{3}$ (physiology of gly'ogen). HUBER and MACLEOD, Am. J. Phys., I9I 7, 42, 619; MaCleod, Soc. Exp. Biol. Med., 191 7, 14, 124; liavfian, Compt. Rend. Soc. Biol., I 895. 317 (glycogen in bloodvessels of liver). LANGSTEIx, Ergeb. d. Phys. (Bioch.), I 904.453 (formation of carbohydrate from protein). LUSK, Am. J. Phys., I9II, 27, 427 (cold baths and glycogen content). MACLEOD and PeARCE, Am. J. Pliys., I9II, 27, 34I; ib., I9I5, 38, 425 (sugar-retaining power and glycogen content of liver). PFLËGER (E.). Pfliger's Arch., I 902, 91, I19; ib., I903, 96, I (monograph on glycogen); ib., I903, 93, I65 (directions for estimation); ib., I904, 103, I69 (abbrevialed method). Rusk, Soc. Exp. Biol. Med., 1912, 12, 21 (comparison of chemical and microchemical methods).

Glycogenesis.-EPSTEIN and BAEIIR, J. Biol. Chem., IOI6, 24, I 7 (infuence of phlorhizin). HATCHER and WOLF, ib., 1907, 3, 25 (formation of glycogen in muscle). MacLEOD, Soc. Exp. Biol. Med., I9I6, 13. Itig (influence of alkali). McGuigan, J. Pharm. Exp. Ther., I9I6, 8, 40 i (influence of atropin and pilocarpin). MCDANELL and UNDERHILL, J. Biol. Ch., TOI 7, 29, 255 (relation of diet to glycogen content of liver). PFLÜGER (E.). Pfluger's Arch., 1907. 120, 253 (in starvation) 
Glycogenolysis.-BAxe, Bioch Z., I913, 49,40, 8I; MACrEod and IRUri. Am. J. Phys., I yOS, 22, 397 (splanchnic stimulation). MACLEOD, ib. 22, 373 (glycogenolylic fibres in splanchnic?). MACLEOD and PEARCE, ib., I9I I, 28, 403 (glycogenase). NeILso. and Terry, ib., I905, 14, I05. PAVY, J. Phys., I 898, 22, 391. PATox (D. N.). J. J'lny's., I 899, 24, 36 (sugar formation in liver). TAYLOR, J. Biol. Ch., 1908, 5, 315.

Blood-Sugar Content Methods.-Firc (R.), Arch. Int. Med., I9I4, 14, I33 (Bang and Bertrand methods comparcd). LEWIS and BEXEDICT (S. R.), J. Biol. Chein.. I I j, 20,6I. MACLEOD, J. Lab. Clin. Med., I916, 1, 445 (resume). IIcGuiga.i and Ross, J. Biol. Ch., I91 7, 31, 533 (Benedict and Bertrund methods compared). Mlorris, J. Lab. Clin. Med., I9I6, 1, 208, 252. MYers and Balley, J. Biol. Ch., I9I6, 24, I47. PEARCE, ib., I $915,22,525$. Scott (E. L.), Am. J. Phys., I9I 4, 34, 271. Shaffer, J. Biol. Ch., I91 4, 19, $285,297$.

Blood-Sugar Content.-Epsteix and Aschner, J. Biol. Ch., 1916, 25, 151. (psychic and sensory stimuli). Gramas, J. Phys., I9I6, 50, 285. KRAMER and CofFis, J. Biol. Ch., I9I6, 25, 423 (psychic and sensory stimuli). IIACLEOD and WEDD, ib., I9I4, 18, 447 (in hepatic vein). McGuigax, Am. J. Phỵs., I9I6, 39, 4 So. LeE and Scott, ib., I9I 7, 40, 456. MACLEOD, ib., I9O9, 23, 278 (asphyxia). MACleod and PeARCE, ib., I9I 5, 38, qI5. MCDA.VELL and U.XDERHILl, J. Biol. Ch., I9I 7, 29, $227,233,265$ (alkali). RoNA and Michaelis, Bioch. Z., 1908, 14, 476; ib.. 1909, 16, 60 (partition between corpuscles and plasma) Ross and McGuigas, J. Biol. Ch., I9I5, 22, 407 (ether anasthesia).

Glycalysis.-CohxheIM, Z. Physiol. Ch., I 903, 39, 336; ib., 1904, 42, tor; ib. I905, 43, 547; ib., I 906, 47, 253; TUCketr, J. Phys., I9I0, 41, 89; Claus and EMBDEN, Hofmeister's Beit., I905, 5, $214 ; i b ., 1906,6,343$; LEVENE and Meyer, J. Biol. Ch., I9I I, 9, 97; ib., I9I2, 11, 3+7; MCGuigar, Am. J. Phys., I908, 21, 35 I (muscle and pancreas). LEVENE and MEYER, J. Biol. Ch., I9I2, 11, 353 (action of tissues on glucose); ib., I912, 11, 36. I (leucocytes).

Blood Glycolysis.-Arthus, Arch. de Phys., I891, 425. Lépine, J. Biol. Ch., I9I 3, 16, 559. MACLEOD, J. Biol. Ch., I9I3, 15, 497. MACleod and Pearce, Am. J. Phys., I9I 4, 33, 378. PAVy and Siat, J. Phys., I901-2, 27, 451. Mackexzie (G. MI.), J. Exp. Med., I9I 5, 22, 757.

\section{HYPERGLYCEMMIAS AND GLYCOSURIAS.}

Brows (O. H.), Am. J. Phys., I904, 10, 378 (effect of $\mathrm{Ca}$ on glycosuria). EDIE, Bioch. J., I906, 1, 455 (excess of $\mathrm{CO}_{2}$ in air). HENDERSOX and UXDERHILl, Am. J. Phys., I9II, 28, 275 (acapnia and glycosuria). MACLEOD, Harvey Lecture, New York, February If, I9I f. MicGuigas and Brooks, Am. J. Phys., I907. 18, 256. PAVY and GodDEN, J. Phys., I 9 II, 43, I99.

Salt Glycosuria.-Burneti, J. Biol. Ch., I908, 5, 351. Fischer (M.), Pflïger's Arch., I $905,106,80 ; 109$, I; Univ. of Calif. Pub., Dec. 24, 1903; ib., Feb. 15, I904. MCDANELL and UNDERHILL, J. Biol. Ch., I9I 7. 29, 273. UNDERHILL and KLEINER, ib., I908, 4, 395.

Adrenalin Glycosuria.-BLtu, Pfūger's Arch.. I902, 90, 6I 7 . HerTer and WAKEMAN, Virchow's Arch., 169, 479. MCDANELl and UNDERHILL, J. Biol. Ch., I $917,29,245$. MACleOD and PeArCe, Am. J. Phys., i 9 I 2, 29, 4 I9 (adrenals and sugar production by liver). PETERS and GEYELIN, J. Biol. Ch., I9I 7, 31, 47I. PAION (D. N.). J. Phys., I903, 29, 2S6; ib., 1905. 32, 59. Pollak, Arch. Exp. Path. Pharm., I909, 61, 149. UNDERHill and Closson, Am. J. Phys., 1906, 17, 42 . VOSBURGH and Richards, Am. J. Phys., 1903, 9, 35.

Relation of Adrenals to Experimental Hyperglycæmias.-K.AN, Pflüger's Arch., I9I I, 140, 209; ib., I9I 2, 144, 25I, 396; ib., I9I 2, 146, 576; Schwarz, Pflüger's Arch., I9I0, 134, 259; Wertheiner and BATtez, 
Arcli. Interuat de Physs., 1910, 9, r40, 363; Nisur, Arch. Lxp. I'dth. Pharm., 1yog. 61, 186 (puncture hyperglycamia). STEWART and lrucurs, An. I. Ihys., I017, 44, 543 (asphyxia and ancesthesia).

Puncture G ycosuria.-BERxARD, Leçons sur le systeme nerveux, t. 1, 440. ECkHAll, Z. f. Biol., 1903, 44, 407.

Alimentary Hyperglycæmia and Glycosuria.-BAILEY, Soc. Lixp. Biol. Med. I916, 13, 153. Hofmeister, Arch. Exp. Path. Pharm., I889, 25, 240 (assimilation limits for sugars). MIrURA, Z. f. Biol., I 898, 34, 281. TAYLOR (A. E.) and Hulton, J. Biol. Ch., 1916, 25, I73; Wilder and Sa.vsun, Arch. Int. Med., I917, 19, 3 II (sugar tolerance).

Emotional Hyperglycæmia and Glycosuria.-CANion, SHOHL and WRIGH Am. J. Phys., igi I, 29, 280, Folix, Dexis and Silllie, J. Biol. Chem., 191 4, 17, 519. UNDERHILL, ib., I91 7, 29, 279.

Ether Glycosuria.-Kisis, Moyle and Haupt, J. Exp. Med., 1912, 16, I78. Grube, Pflüger's Arch., I911, 138, GoI. Joss and HAWK, Arch. Int. Med., 191 4, 14, 779. Seelig, Arch. Exp. Path. Pharm., 1905, 52, 481.

Phlorhizin Diabetes.-CATHCART and TAYLOR, J. Phys., I910, 41, 276. LEVENE, $i b ., 1894,17,259$. PAVY, BRODIE and SiAv, ib., 1903, 29, 467. UNDERHILL, J. Biol. Ch., I912, 13, I5 (mechunism of). D.AKIx and Dudley, J. Biol. Ch., 1914, 17, $15 \mathrm{l}$ (jate of alanin). Lusk. Am. J. Med. Sci., I9I7, 153, 40 (intermediary metabolism); Am. J. Phys., I 908, 22, I 74 (sugar production from glutamic acid). II INDEL and LUSK, Am. J. Phy's., Iyo3, 10, 47 (respiration cxperiments). UICDERMotT and LUSk, il., Igoo, 3, 139 (phosphorts poisoning and phlorhizin). IREILLY, Nolan and Lusk, ib., $1898, \mathbf{1}, 395$. StIles and Lusk, ib., 1903, 10, 67 (action of phlorhizin). Rixger, J. Biol. Ch., 1914, 1\%, 107 (theory of diabeles, acidosis). Woodyatr and SAxstir, J. Biol. Ch., I9I 5, 21, I (nurcotics in); ib., 1916, 24, 23 (determination of utilizable carbohydrates in foods).

Pancreatic Diabetes.-Carlson and Drennax. Am. J. Phys., I9I I, 28, 39I; CARLSON and Ginsburg, ib., 1915, 36, 21\%; CARLSON, ORR and JONEs, J. Biol. Ch., I914, 17, 19 (infucnce of pregnancy). AL'ER and KLEINER, Soc. Exp. Biol. Med., 1917, 14, I51 (coagulation of pancreas in silu). Epsteix, J. Biol. Chem., 1916, 24, Homaxs, J. Med. Res., I915, 33, I; Thirolorx, Arch. de Phys., I 892, 24, 716 (diabetes after partial pancreatectomy). Evans (C. L.), J. Phys., 1912, 44, 461 (fate of secretin in). HÉ Dox, Arch. Internat. de Physs., I9I I, 11, I95; ib., I913, 13, 4; J. de Phys. Path. Gén., I91 2, 14, 907. Miskowski, Pflüger's Arch., I906, 111, 13; Arch. Exp. Path. Pharm., 1905, 53, 331. MIUrLin and Kramer, J. Biol. Ch., 1916, 27, 517 (influence of alkali). MURLIN and SweEt, ib., I916, 28, 261 (influence of gastrectomy). UXDERHILL and FINE, ib., I9I I, 10, 27I (inhibition of pancreatic diabctes). ScotT (E. L.), Am. J. Phys., I $912,29,306$ (influence of pancreatic cxiract). TUCketr, J. Phys., 1910, 41, 88. Woonyatt, J. Biol. Chem., I91 5, 20, I29.

Sugar Consumption in Diabetic Tissues.-CLARK (A. H.). J. Exp. Med., I I 7. 26, $72 \mathrm{I}$ (surviving heart and pancreas in sugar metabolism). CRUICKSHANK, J. Phys., 1913, 47, I (glycogen in normal and diabclic animals). CRUICKSHANK and Patterson, $i b$, $1914,47,381$; MaClean and SMEDley, $i b$. 1912, 45, 470; PATtersoN and StARLING, ib., 1913.47, 137; liNowltoN and StARLiNG, $i b ., 1912.45$, I 6 (sugar consumption in diabctic heart). Hoagland and MaNsfield. J. Biol. Ch., igr 7, 31, 50 i (muscle). Murlin, J. Biol. Ch., I913, 15, 365 (respiratory cxchange in depancreatized animal). Macleod and PEARCE, Am. J. Phy's. 1913,32, I8t (normal and depancreated eviscerated dogs). Mir.Ne and Peters, J. Med. Res., I9I 2, 26, 415 (glycolytic power of blood and tissues in diabetic organism). MYERS and Killian, Am. J. Phys., I $917,42,582$ (diastatic activity of blood $2 n$ dialetes). Starling, Fvass and Lovatt. J. Phys., $1914,49,67$ (respiratory exchange in diabelic heart). STEWART (H. A.), J. Exp. Med., 1910, 12, 59 (perfus od human heart). 
Diabetes Mellitus, Itra N. Studes concening Cifycosuria and Dabeles,

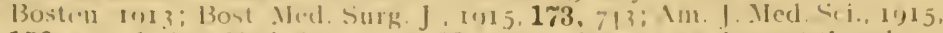

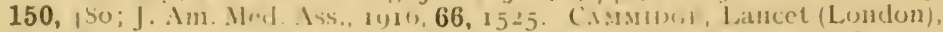

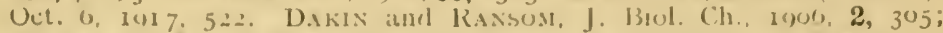

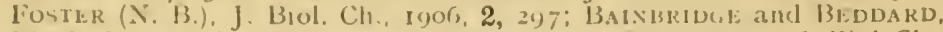
Bioch. J., 1906, 1, . 29 (trealment with sectolin). GRliswild, J. Biol. Ch., InIt, 18, II 5 (glucose from cilric ucid); ib., 1913, 16, 375 (flucose from

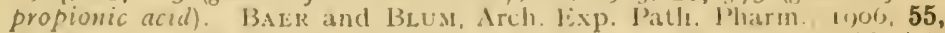
$87 ;$ ib., 1907,56, 92; ib., 1010, 62, 120 (decomposition of fally ucids in). BENEDICT (1:. (j) and Josins, Carnegie Instit., Pul, 136, Igro (melabolism in dicibcles mellitus). BA1NBRIDG: and BEDDARI), Bioch. J., $1907,2,89$ (diastalic ferments $i n$ ). CiEpliarT, AUn, Du bols and Lusk, Arch. Int. Ned, I91 7, 19, 908 (mclabolism). JANNEY. Arcli. Int. Med., Ig16, 18, 584 (sugar from protein). Josus, Arch. Int. Med., I9I5. 16, unj (carbohydrate ntilization). MACLEOD (J. J. R.), Diabetes: Its Pathological Physiology, London, I9I 3; J. Am. Aled. Ass., I910, 55, 2133 (experimental diabeles and diabetes mellitus). Lusk, Arch. Int. .Ied., igo9. 3, I (metabolism in). Moorhouse, Patterson and Stephenson, Bioch. J., 1915, 9, I 7I (metabolism in experimental diabetes). Josś. J. de Phys. Path. Gén., I9or, 3,792; 4, 12S (polaloes in). NurLix and CRAVER, J. Biol. Ch., I916, 28, 289 (sodium carbonale action). vow Noordex, Diabetes Mellitus, its Pathological Chemistry and Treatment. iVoodyat, J. Am. Med. Ass., I9 6, 66, I910 (acidosis). Magnus-Levy, Arch. Exp. Path. Pharm., I goI, 45, 389 (acidosis). OpIE, J. lixp. Med. I900, 5, 527 (islets). Cecil, J. Exp. Mled., 1909, 11, 266; RExile and Fraser, Bioch. J., I906, 2, 7 (islels). Pavy, Physiology of the Carbohydrates; Lancet (London), Nov. 21, 28 and Dec. 12, I908. MoorE, EDIE and ABRAM, Bioch. J.. 1906, 1, 446. RINGER, FRANKEL and Jones, J. Biol. Ch., 1913, 14, 525; ib., 14, 539; 1914, 18, 81 (sugar formation in diabetic organism).

Diabetes Insipidus.-Christie and StEWARt, Arch. Int. Med., I9I 7, 20, ro. Fitz, ib., I9I4, 14, 706. Herrick (J. B.), ib., I912, 10, I. Mlattiews (S. A.), ib., I $915,15,45 \mathrm{I}$.

Diabetic Coma.-Beddard, Pembrey and Spriggs, J. Phys.. igof. 31, p. xliv.; ib., I908, 37, p. xxxix. Magnus-Levy, Arch. Exp. Path. Pharm., 1899, 42, I 49. Poultox, J..Phÿs., I9I 5, 50, i.

Acidosis,-Fitz and VAN Slyke, J. Biol. Ch., I9I 7, 30, 389. VA. Slyke, ib., I9I 7, 30, 289. Srullmas and VAN SLYke, J. Biol. Ch., I9I 7, 30, 457 (diabeles). Woolley, J. Lab. Clin. Med., $1916,1,712$ (résumé). PAlaer and VAN SLYKe, J. Biol. Ch., I917, 32, 499 (alkali retention and alkali reserve).

Acidosis and Respiration.-MACLFOD, J. Lab. Clin. Med., I9 I 5, 1, I97 (methods). Kennaway, Pembrey and Poulton, J. Phys., I913-14, 47, p. x. Peabody, Arcli. Int. Med., I9I $4.14,236 ; i b ., 1915.16,955$. Peters, Am. J. Phys., I9I 7,43 , I I 3 .

Acidosis, Experimental.-Fitz, Alsberg and Hexderson, Am. J. Plyys., I907, 18, I I 3. Hirscirfelder, J. Am. Med. Ass., igi 6, 6\%, isiji.

Acidosis in Nephritis.-Bostock, Z. Physiol. Ch., I9I3, 84, 468. Goto, J Exp. Med., 1917, 25, 693. Marriott and Howlaxd, Arch. Int. Med., I9I6, 18, 708. SzILI, Pfluger's Arch., I909, 130, 134 .

Acetone Bodies.-Folin, J. Biol. Ch., I907, 3, I 77; lolms and Desis, ib., r9I 4 , 18, 263: Rothera, J. Phys., 1908, 3\%, 49I; Scott-Wilson, J. Phys., I9II, 42, 44t (eslimulion in urine). EMBDEN and MARX. Hofmeister's Beit., 1908, 11, 3 I 8 (acelone formalion in liver).

Aceto-Acetic Acid, Formation and Decomposition in Body.-Dikis and DUdLEY, J. Biol. Ch., I9I3, 16, 5 I 5 . WAKEMAX and DAKiN, J. Biol. Ch., I) 10. 8, 105. EMBDEN et Al., Hofmeister's Beit., 1908, 11, 323; Bioch. Z., I gOS, 13, 262. Wilder, J. Biol. Ch., I9I 7, 31, 59. 
Oxybutyric Acid.-Dikis, J. Biol. Ch.. I910, 8, 97 (formation in body from acelo-acelle acid). WAKEMAN and D.Kin, ib., 1909, 6, 373 (decomposition by liver enzymes).

Glyoxal in Intermediary Metabolism.-DAk1: and DLDLEY, J. Biol. Ch., I914. 18,$29 ; i b ., 1913,14.123 ; i b ., 15,+63 ; i b .16,505$ (glyoxalase).

Pyruvic Acid in Intermediary Metabolism.-DAKIN and JANey, J. Biol. Ch., 1913. 15, I 77 (relations loglucose). LEVENE and MEYLR, ib., I9I $4,17,+43$ (action of leucocytes and kidney lissue on). RINGER (A. I.), 2L., I9I 3, 15, 145 (intermediary metabolism of alanin); ib., 1914, 17, 281 (fate of pyru2zc acid).

Glycuronic Acid.-Ñeuberg, Ergeb. d. Phys. (Bioch.), 1904, 373. Austin, J. Med. Res., I905, 14, I8I (production in dog).

\section{METABOLISM OF FAT.}

Bloor, J. Blol. Ch., I916, 24, 447. DAkin, ib., 1908, 4, 419; ib., 5, 1 73, 303; ib., 1909, 6, 203, 221, 235 (oxidation of phenyl deriva!lues of fally acids). DAKIN and DUDLEY, ib., I913, 14, I 55; DAKIN and WAKEMAN, $2 b .$, I9I I. 9, 329 (intermediary melabolism). FISCHER (M.), Fats and Fatty Degeneration-Emulsions, I91 7. Leathes, Problems in Animal Metabolism (London, I906). Livene and Allen, J. Biol. Ch., 1916, 27, 433 (oxidation of branched chain fatty acids). LYMA.N. J. Biol. Ch., I91 7, 32, I 3 (effect of palmitic acid, elc., on depot fat). PORGES, Ergeb. d. Phys., 1910, I (decomposition of fatty acids). MURLIN and Lisk, J. Biol. Ch., I9I5. 22, 15 (influence of fat on heal production).

Fat Formation.-Leathes, Ergeb. d. Pliys., I909, 8, 356; Moore (B.), Proc. Roy. Soc. (Lond.), B 72, I34; TAYLOR, Univ. Calif. Publ., I90f (synthesis of fat in body). McClexios, J. Biol. Ch., 1915. 21, 269 (from protein). PFlüger (E.), Pflüger's Arch., I 899, 77, 52 I (against ' 'oit on fat formation from prolein). MorGLLIS and PRATT, Am. J. Phys.. 1913, 32, 200; RUBNer, Z.f. Biol., 1886, 22, 272 (from carbohydrate). PEMBREY, J. Phys., I901-2, 27, 407 (respiratory exchange during fat deposition). ROSENFELD, Ergeb. d. Phys. (Bioch.), I 902, 651; ib., 1903.50. SMEDLEy (I.), J. Phys., 1912, 45, p. xxv. (synthesis of fatty acids from carbohydrate). RUTTAN and Marshall, J. Biol. Ch., I9I 7, 29, 3 I 9 (adipocere).

Migration of Fat.-MENDEL and DANiels, J. Biol. Ch., 1912, 13, 71 (slained fal). Krauss and Sommer, Hofmeister's Beit., 1902, 2, 86; Wells (H. G.). Z. Phys. Ch., 1905, 45, 4I 2 (in phosphorus poisoning).

Liver and Fats.-CORPER and MotIRAM, J. Phy's., I912, 45, 363; ib., 1915. 49, 157; ib., I9I 4, 49, 23 (fatty acids in liver in pregnancy and lactation). JACKSON and PEARCE, J. Exp. Med., I 907, 9, 578 ( $\mathrm{fals}$ and lipoids in liver necrosis). Leatues, Lancet (Lond.), Feb. 27. 1909: Arch. Exp. Path. Pharm. Supp. Bd., I908, 327. RAPER, J. Biol. Ch., I913. 14, I 77. Patox (D. X.), J. Phys., I 895-6, 19, 167.

Obesity.-Croftan (A. C.), J. Am. Med. Ass., r9o6, Sept. 1 5.820 (dieletics of). Fol1N and DExis, J. Biol. Ch., 1915, 21, IS3 (starration and obesity with reference to acidosis). VON WilleBRA.TD, Skand. Arch. Phys., I908, 20, I 52 (metabolism in).

Phosphatides.-Hatal, Am. T. Phys., 1903, 10, 57 (lecithin and growth). IICOllua, Halpin and DrescheE, J. Biol. Ch., I9I2, 13, 219 (synthesis of lecithin in hen). NERKING. Bioch. Z., 1908, 10, 193 (distribution of lecithin in the body').

Lipoids, Lipins.-BAwg, Ergeb. d. Phys., 1907, 136; ib., 1909. 463 (cell lipoids). HANES. J. Exp. Med., I9I2. 16,512 (lipoid metabolism in chick). MACARtIUR and LUCKetT, J. Biol. Ch., 1915, 20, 161 (lipins in nutrition). McCollum and Davis, J. Biol. Ch., 1913, 15, 167 (necessily of lipins in growth). 


\section{METABOLISM OF PROTEINS.}

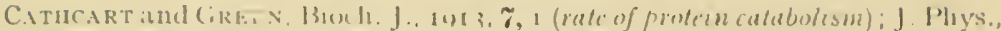

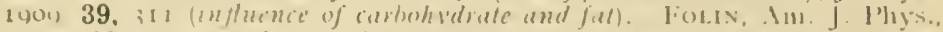

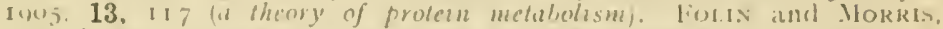

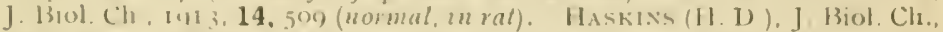

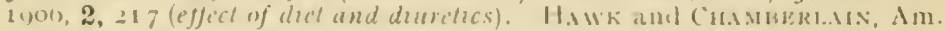

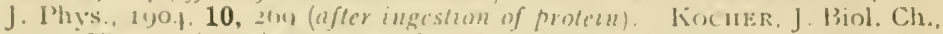
Ioto, 25, 57 I (sparing uchon of corbohydrate). JANiver, J. Biol. Ch., Ii) $15.20,321$ (velution of prolezus to glucose). 1.ANistres, lirgeb. d.

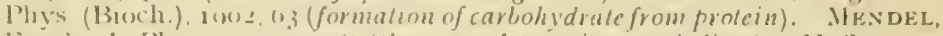

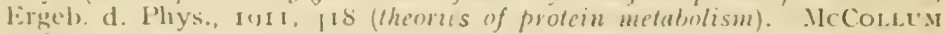
and HOACiLAND, J BHol. Ch., I1)1, 16, 2(y), 317 (effects of fat and acids on endogenous N'-metabolism). J's rox (1. N.). J. Phys., 1905-6, 33, I (Folin's

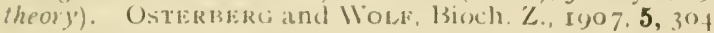

Nitrogen Estimation.-DAKIN and DUDLEY, J. Biol. Ch., I9I.4. 17, 275; KUTACHER and STEQDEL, Z. Phy's. Ch., InO3. 39, I 2 (Kijeldahl method). FOLIN and DENIS, I. Biol. Ch., Iolo, 26, +73 (trinc); il., 26, 19! (nonprotein natrogen of blood) (by' Nessleriation). P'EARCE (K. G.). J. Libl). Clin. Med., 1010, 2, 1,30 (review).

Non-Protein Nitrogen in Blood. Folri and Dexis, J. Biol. Ch., 1916, 26, f9I. Foster (N. 13.). Irch. Int. Med., 1915. 15, 351). PEPPER and Austin, J. Biol. Ch., 1915, 22, si. Grienwald, J. Biol. Ch., 19i6, 21, 61. WELKER and lialls, ib., 1916, 25, 567. TAYLOR and HLLTO:, ib., $1915,22,63$.

Amino-Acids.-Dakin, J. Biol. Ch., 1905, 1, I 7 I; ib., Iyo8, 4, 63; Desis, J. Biol. Ch., 1911, 9, 365; ib., 1911, 10, 73 (oxidation of amino-acids). Folin, J. Am. IIed. Ass., igi 7, 69, r zog (résumé). ABDERHALDEN ET A... Z. Phys. Ch., 1906, 47, 391; ib., 48, 557; ib). 51, 311, 323 (decomposition of amino-acids in the body). HARDIACi and MACLEAX, J. Biol. Ch., 1916, 25, 337 (ninhydrin reaction). Folin and DENIs, J. Biol. Ch., 1912, 12, 1.I ; WISHART (1I. 13.), ib., I915. 20, 535 (amino-acids in blood and lissues). HARdiNg and MACLEAN, ib., 1916, 24, 503; VAN SLYKE, ib., 1912, 12, $275 ; i b .$, I913, 16, I21; ib., 19I5, 23, to 7 (amino-acids, amino-nitrogen, estimation of). OSBORNE and JoNes, An. J. Phys., I0IO, 26, 212 (amino-acids in proleins). OSBORNE and MENDEL, J. Biol. Ch., I914, 17, 325 (amino-acids in nutrition and growth). VAN SLYKE, Arch. Int. Med., I9I $7,19,56$ (amino-acids in physiology, and palhology, résumé). VAs SLYKE and MEYER, J. Biol. Ch., I912, 12, 399; ib., 1913, 16, 197, 23 I (amino-acid nitrogen of blood). GrökGY and ZUNz, J. Biol. Ch., 1915, 21, 5 I (amino-acid nitrogen in blood).

Intermediary Metabolism of Amino-Acids.-DAkis, J. Biol. Ch., 1913, 14, 321. Greexwald, J. Biol. Ch., 1916, 25, 8I; Lusk, Z. Physiol. Ch., 1910, 66, IO6; RINGER, J. Biol. Ch., 1912, 12, 223 (production of dextrose from amino acids). Janney, J. Biol. Ch., I915, 22, I9I; CsonkA, J. Biol. Ch., I915, 20, 539 (rate of melabolism of amino-acids). LEVENE and MEYER, Am. J. Phys., 1909, 25, 214 (climination of nitrogen, elc., after administration of some amino-acids). MACLEOD, J. Lab. Clin. Med., 1915, 1, II 2 (résumé). Lusk, J. Am. Chen.. Soc., 1910,32, 671 (fate of a mino-acids in organism). MatThews (S. A.) and Nelson, J. Biol. Ch., I91 4, 19, 229 (fale in muscular lissue). WAKEMAX and DAKIN, ib., I9I1, 9, 139. VAN SLYKe and Merer, J. Biol. Ch.. I9I 3, 16, 213 ; Woelfel, Am. J. Phys., I91 2, 29, p. xxxviii (locus of transformation of absorbed amino-acids).

Arginine Metabolism.-DAKIN, J. Biol. Ch., I907, 3, 435; KosskL and D. KIN, Z. Physiol. Ch., 1904, 41, 32 I (arginase). Thompson, J. Phys., 1905, 32, I $37 ; i b ., 1905-6,33,106 ; i b ., 1917,51,111,347$.

Tryptophane.-HopkiNs and COLE, J. Pliys.. 1903, 29, 451 (constitution). WILLCOCK and HopkiNs, J. Phy.s., 1906 35, SS (in nutrition). DAKIN, J. Biol. Ch., I906, 2, 289 ; Hopkixs and Cole, Proc. Roy. Soc. (Lond.), 


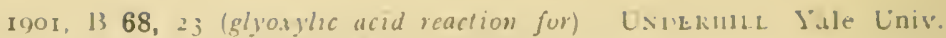

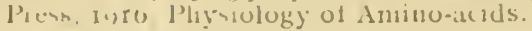

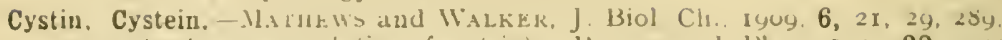

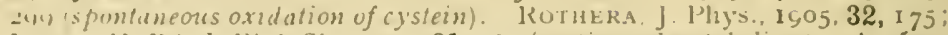
LEWIS (11. 13.), J. Biol. Ch., IgI 7. 31, 363 (cy'stin and metalolism). ABDERHALUEX and SCHITTENIELM, Z. Physiol. Ch., I9O5. 45, fus; GARROD and Hurtley, J. Phys., I906, 34, 2r 7. Alsberg and Folis, Am. J. Phýs., Iyo5, 14, 5t: Hele, J. Phys., I909, 39, 52 ; Simox, Z. Pliysiol. Ch., rgo5. 45, 357 ; Milliams and Molf, J. Biol. Ch., I9or, 6, 33\%; Wolf and SHAFFER, J . Biol. Cl., Igos, 4, +39 (protein metaioolismincy'stinuria).

Hippuric Acid Synthesis,-Epsteix and Boоkмax, J. Biol. Ch., Igri, 10, $353 ; i b$. I I I 2, 13, I I ; ib., I $914,1 \%, 455$ (formation of glycocoll in the body). liixisBCRI and BELL, ib., I9I 5, 20, 73 (in tartrate neplintis); ib., IgI5. 21, 297 (after nephrectomy). LEmis (H. B.). ab.. I91 + 17, 503 (on glycocoll-free diet) ; ib., I9I +. 18, 225 (afterbenzoate in man). PARKER and LUSK, Am. J. Phys., ryoo, 3, foz; R1xger, J. Biol. Ch., ryi I, 10, 327 (maximum prodiction). RAIzIss and Dribix, J. Biol. Ch., IyI $5,21,331$.

Lactic Acid in Intermediary Metabolism -. A $\rightarrow$ ER and JACKSox, Z. f. Biol., I9OI, 41, 393. LEVENE and MEYER, J. Biol. Ch., IgI 3, 14, I 49, 55 I (lactic acid from carbohydrales). MACLEOD and Hoover (D. H.). Am. J. Phys., I9I 7. 42, 460 (lactic acid production in blood after injection of aikali). MACLEOD and IVEDD, J. Biol. Ch., I914, 18, $4+7$ (sugar análactic acid in liver blood). MAxDEL and Lusk, Am. J. Phys., I906, 16, I29; RxFFEL, J. Plys., I9o9, 39. p. xxix (lactic acid formation in man).

Urea in Blood.-Folis and Dexis, J. Biol. Ch., I912, 11, 527; ib., I916, 26, 505. LEWIS and KARR, ib., I9I6, 28, I 7 . ICLEAN and SELLING, ib.. $191+19,31$. MARSHALL, $i b ., 1913,15,487$.

Ammonia in Blood.-FiskE and KARSNER. J. Biol. Ch., I9I 4, 18, 38 I (destruclive lesions of liver and reduced ammonia in blood). FoLix and DExis, ib., I9I 2, 11, I6I, 527 J JCoBsoN (C.), ib., I9I 4. 18, I 33; An. J. Phys.. I9IO, 26, 407. SALASKIX ET AL., Z. Physiol. Ch., 1902, 35, 246, 552. TAYLOR and RIXGER, J. Biol. Ch., IyI3, 14, to7 (ammonia in protem metabolism).

Urea Formation.-Bostock (G. D.), Bioch. J., Igr I, 6, 388 (deamidization). DJYON and DLFot'RT, Arch. de Phys., ISy8. 522 (ligation of hepatic artery and portal vein). FiskE and KARSNER, J. Biol. Ch., Igr 3, 16, 399 (perfusion of liver with ammonimm carbonate and with glycocoll). Fiske and SUMLIER, ib., IgI 4, 18, 285 ; JANSEN, i6., I9I 5, 21, 557 ; SALASKIN. 7.1. Pliysiol. Ch. ISgs, 25, I 28 (from amina-acids in liver). Folix and DExIs. J. Biol. Ch.. IgI 2, 12, I 4 I. HoAglaxi and MANSFIELd, ib., I91 7, 31, ${ }_{487}$ (muscular tissue and urea formation). MACLEOD and HASkINS. ib., I906, 1, 3 I9 (carbamates). MAtтHEWS and Mrller, ib. I9I $3,15,87$ (liver circulation and nitrogen melabolism). HALSEY, Z. Physiol. Ch., IS98, 25, 325 (precursors of urea). JAcoræ (M.), Ergeb. d. Phys. (Bioch.), F902.532. TAYLOR and LEwIS, J. Biol. Ch., I9I j. 22, I 7 (predominance of liver). WAKEMAX and DAKIx, J. Bjol. Ch., I9II, 9, 327 (relationship belieen urea and ammonimm salls). VA. SLYKE, CLLLEX and MCLEA.', Soc. Exp. Biol. Med., I915, 12, 93 (liver).

Purin Metabolism.-Ackroyd and Hopkixs, Bioch. J., I916, 10, 55 I (arginin and histidin as possible precursors of purins). BURIAN and SCHUR, Pfinger's Arch., I900, 80, 2fI; ib., I901, 87, 239; ib., 1903, 94, 273 (in max). HuNTER (A.). J. Biol. Ch., I9I 4,18 , IO7; HUNTER and GIVENS, 16., I9r2, 13, 37I; ib., 19I4, 17,37 (monkey). Huxter, GIVExs and

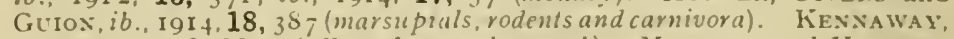
J. Phys., 1908, 38, i (effect of muscular work). MACleod and HASkiss. J. Biol. Ch., Igo6, 2, 23 I (man). Wels. (H. G.). J. Biol. Ch., I909. 7, I 7 I (monkey); J. Lab. Clin. Med. I910, 1, I64.

Purin Enzymes.-Joxes (IV.), Z. Physiol. Ch., Ioo5. 45, $\mathrm{S}_{4}$ (guanase in spleen). CALDWELl and VELLS, J. Biol. Ch., 191 4, 18, 15,;it.. 19, 279. Loxi. ib., I9I3. 15, tf9 (adenase). ROHDE and JoxEs, ib., I 909, 7, 237. 
Allantoin.- Ilentek (A), J. Blul. Cir, 1917, 28, 3(oy (in blood). Givens,

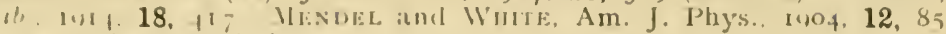
(Serduction in the body).

Uric Acid in Blood.-BENIEDICT (S. R.), J. Biol. Ch., IoI5, 20, 533 (ox and chicken). DENIS, J. Biol. Cli, 1015, 23, I +7 (effert of ingested purins). Fixe and ('nacke, J. Pharm. lixp). Ther, 1914, 6, 219 ; Jolin and Denis, J. Biol. Ch., I913, 14, 20) (uvic ucid, urea and non-protein nitrogen in blood). Folds and Denis, Arch. Int. Med., I915, 16, 33. Slemons and BOGERT, J. Biol. Ch., twז7, 32, o3 (maternal and fatal blood).

Uric Acid Metabolism.-Caticart and Leatues, Proc. Roy. Soc. (Lond.), 1907, $1379,54 \mathrm{I}$ (relation bctween uric acid output and heat production). Croftan, Phüger's Arch., Igo8, 121, 377. BENEDICT (S. R.). J. Lab. Clin. Med., I9I6, 2, 1. RaIziss, DuBIN and RiNger, J. Biol. Ch., IgIt, 19, +73 (endogenous). WiEner, lirgeb. d. Phys. (Bioch.), I902, 555; ib., I y03. 377 (in pathology).

Uric Acid Formation.-Cathcakt, Kennaway and Leathes, Q. J. Med., Igo8, 1, 4I0; STEHLE, Soc. Exp. Biol. Nled., 1915,12 , I 48 (origin of endogenous). JEROME, J. Phys., 1898, 22, I 46 (influence of diet). Milroy (T. H.), J. Pluys., I903, 30, 47 (in birds). I'LIMMer, DiCK and LieB, ib., I909, 39, gS (origin). TAYLOR and Ross, J. Biol. Ch., I9I4, 18, 5 I 9 (influence of protein intake).

Uricolysis.-FINE (M. S.), J. Biol. Ch., I9I 5, 23, 47 I (non-destructibility of uric acid in man). Schittenhelm, Z. Physiol. Ch., 1905, 45, I6I (uricolyticferments). TAYLOR and ADOLPH, J.Biol.Ch., I9I 4, 18,521. TAYLOR and Rose, J. Biol. Ch., I9I3, 14, 419; Wiechowski, Arch. Exp. Path. Pharm., I 909, 60, I 85 (in man).

Metabolism of Nucleins.-A bderhalden and Schittenhelm, Z. f. Physiol. Ch., 1906, 47, 452 (formation and decomposition of nucleic acids in the body). JoNes (W.), J. Biol. Ch., I908, 5, I (identity of nucleic acids of thymus, spleen and pancreas). Jones and Austrian, Z. Physiol. Ch., I906, 48, i io; Schittenhelm and Schuid, Z. Physiol. Ch., i 906-7, 50, 30 (ferments of nuclein metabolism). JoNes (W.), J. Biol. Ch., I9II, 9, I69 (des amidases). JONES, ib., I9I1, 9, I29; LEVENE and LA FORGE, ib., 1912, 13, 507; LeVene and MEdigReceanu, ib., I9II, 9, 65, 389 (nucleases). MCCollum, Am. J. Phys., 1909, 25, 120 (nuclein synthesis in the body). Milroy and Malcolm, J. Phys., i 898-9, 23, 2 I 7 (metabolism of nucleins); ib., I899, 25, 105 (in leucocythamia). SWEET and LeVENE, J. Exp. Med., I $907,9,229$ (nuclein metabolism with Eck's fistula). Givens and HuNTER, J. Biol. Ch., I915, 23, 299 (sodium nucleate).

Creatin and Creatinin Metabolism.-Folin, J. Biol. Ch., I914, 17, 469 (determination in urine); ib., 1914, 17, 475 (determination in blood, milk, and tissues). FOLIN and DENIS, ib., I9I $4,17,487$ (content of blood); ib., I 914 , 17, 493 (in relation to metabolism). FOSTER (N. B.) and FISHER, ib., I9I I, 9, 359 (with Eck's fistula). MellanBy (E.). J. Phys., I907-8, 36, $4+7$. MENDEL and ROSE, J. Biol. Ch., I 9 I I, 10, 2 I 3 (róle of carbohydrates). Graham and Poulton, J. Phys., 1914, 48, p. liii (in stavvation). Shaffer, Am. J. Phys., 1908, 23, I (in health and disease): J. Bil. Ch., I9I 4, 18, 525. Towles and Voegtuin, J. Biol. Ch., igi I, 10, 479 (function of liver). WOLF, ib., I9II, 10, 473 .

Creatinin.-Benedict and MYERs, Am. J. Phys., I907, 18, 377; TrACY and CLARK, J. Biol. Ch., I9I 4, 19, I 5 (elimination in woman). AMBERg and Morrill, J. Biol. Ch., Igo 7, 3, 3 I I (in new-born infant). LEVENE and Kristellar, Am. J. Phys., 1909, 24, 45 (factors regulating output in man). MYers and Fine, J. Biol. Ch., I9I $4,17,65 ;$ ib., I9I 5, 21, 383 ; SCOTT (E. L.) and Spohs, Am. J. Phys., I9I 7. 42, 600 (in muscles). PAlner, J. Biol. Ch., I9I 4, 19, 239 (basal metabulism and creatinin elimination). RINGER and RAIZISS, J. Biol. Ch., IOI $4,19,487$ (excretion of creatinin with creatin-free diet). RоскwоoD and VAN EPPS, Am. J. Phys., r907, 19, 97 (influence of drugs on elimination of creatinin). 
Creatin: Creatin Content of Muscle.-Brow: and Catjcart, Bjoch. J., Igor,

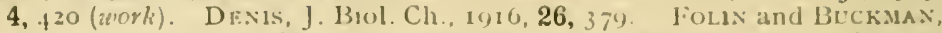

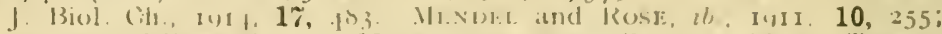

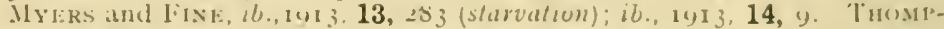
son, J. Pliys., IgI (1, 50, p. lisi (excitation of molor nerve).

Creatin: Creatin Estimation.-BAumaxi: and Hixis, J. Biol. Ch., IyI6, 24, 439. BAUMANN and INivaldSEx, ib., I9I6, 25, I95. Bl:NFuICt (S. R.). ib., I91 t, 18, I II. JAXXEY and BLATHFRWICK, ib., I9I 5. 21, 507. ROSE, ib., I $912,12,73$. WALPOLE, J. Phys., I91 1, 42, 301

Creatin: Creatinuria.-DExIs and MlNoT, J. Biol. Ch., Iyl 7, 31, 56I (normal adulls). FFolis and DENIS, $i b ., 1912,11,253$ (children). LiRALSE and Cramer, Q. J. Exp. Phys., I9IO. 3, 289; ib., IyI 4,293 (in women). ROSE, J. Biol. Ch., I9I 7, 32, I (women). MCCRUDDEX and SARGEXT, ib., I9I6, 24, 423. TAÝLOR (A. E.), ib., I9I5, 21, 663 .

Creatin : Creatin Metabolism. - BAvMANn and MARKER, J. Biol. Ch., I9I5, 22, t9 (origin of creatin). BENEDICT (S. R.) and OSTERBERG, ib., I9I 4,18 , Iy5 (origin of urinary creatin). NCCOLLLM and STEENBOCh, ib., IyI2. 13, zoy (groreing pig). MYERS and lixis, ib., I9I5, 21, 377 (fale of creatin administered to man). PATOx and MAckik, J. P'hys., IgI2, 45, II 5 (liver). Ross, Dimmit and Cheethas, J. Biol. Ch., 1916, 26, 339 (protein feeding and creatin elimination in fasting man). RowE, Am. J. Phy's., I912, 31, I69 (crealin-splitting enzyme). TWOKT and MELlanBy, J. Phys., 1912, 44, 43 (creatin-destroying bacilli in intestine).

Autolysis.--Bostock, Bioch. J., I912, 6. Bradley, J. Biol. Clı., I9I6, 25, 201. BRADLEY and TAYLOR, ib., 25, 261 (effect of reaction); ib.. I91 : 29, 28 I (effect of bile). JACksox (H. C.), J. Exp. Medl., I goy, 11, 55. Dоснеz, J. Exp. Mled., I9Io, 12, 666 (liver). ЈАсову, Ergeb. d. Phy's. (Bioch.), I902, 213 . Levexe, An. J. Phys., I904, 11, $437 ; i b ., 1904,12$, 276. LANe-Clayton and Schrrver, J. Phys., I904, 31, I69. BexsoN and Wells, J. Biol. Ch., 1910, 8, 6I ; Stewart (G. N.), J. Exp. Med., 1899. 4, 235. (autolysis studied by physico-chemical methods). MEXDEL and Leave.iWorth, Am. J. Phys., igos, 21, 69 (embryo). Morse, J. Biol. Ch., 1916, 24, 163; 1917, 30, 197 (reaction); 1917, 31, 303 (spleen); Am. J. Phys., 1915, 36, 145 (in involntion): J. Lab. Clin. Med., I91 7, 2, 500 (résumê). Tatra, J. Biol. Ch., 1916, 27, 2ł3. Schryver, Bioch. J., 1906, 1, 123. Schryver, J. Phys., 1905. 32, I59; Wells and Bexson, J. Biol. Ch., 1907, 3, 35 (influence of thyroid).

Protein Metabolism after Removal of Portions of Intestine.-CARREL, MEYER and LEVENE, Am. J. Phys., I9IO, 25, $439 ; i b ., 26,3(69$. ERLANGER and Hewlett, $i b ., 1902,6$, 1. LeV1X, MANSON and LeVENE, ib., 1909, 25, 231. UNDERHILL, $i b ., 1911,27,366$.

Assimilation of Protein.-Abderhalden, Funk and Loxdox. Z. Pliysiol Ch., I $907,51,269$. Pringle and Cramer, J. Phys., Igo\$, 37, i 5 S.

Parenterally Introduced Substances.-Austin and EISENBREY. Arch. Int Med., I $12,10,305$ (utilization of serum). Mlichallis and Roxa, Pfliger's Arch., 1907, 121, I63 (protein). Hocis, J. 13iol. Ch., IoI 4. 18, 485 ;

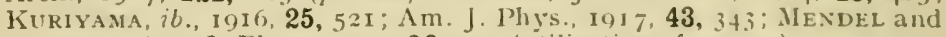
KLEINER, Am. J. Pliys.. 1910, 26, 396 (utilization of sucrose).

\section{INORGANIC SALTS.}

Calcium.-Bergeim, Stewart and HAwk, J. Exp. Med., I9I 4. 20, 225 (Ca metabolism after thyoparathyoidectomy). Lrisax, J. Biol. Cl., I915. 21, 5.5 (estimation in urine and faces); ib., I $19 \mathrm{I} 7,29$, I6y; ib.. 30, I (in blood). MiALColi (J.).J. Pliys., 1905, 32, I 83 (Ca and Mg relationship). MEXDEL and Bexedict (S. R.). Am. J. Phys., 1909. 25, 23 (Ca excretion). Nelson and Williams, J. Biol. Ch.. I916, 28,231 ( $\mathrm{C}$ a in urine and faces). PAtTr. son, Bioch., J. Igo8, 3, 39 (Ca melabolism). 


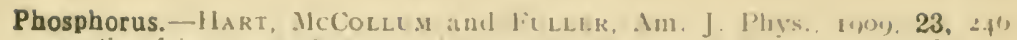

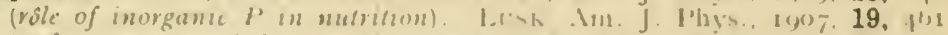

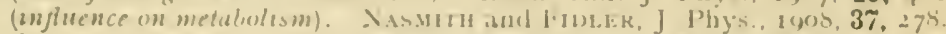

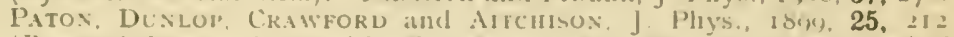

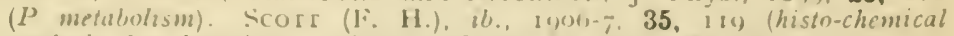

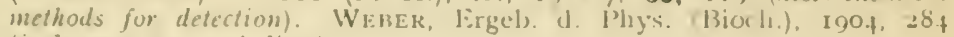
(influence on melabolism).

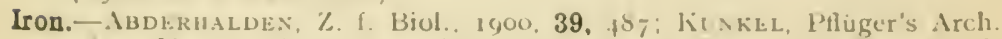
15y5, 61, 595 (blood-formation). Diris anel P'LARCL, J. Exp. Med. I9I 7. 25, i, (distrabution in ancmua). MAcsuldes (A. B.), J. Phys

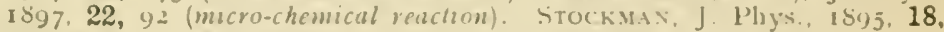

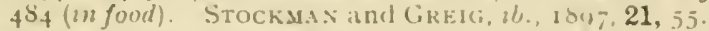

Metabolism, Quantitative Data.-BENenict and Rotil, J Biol. Ch., I115.

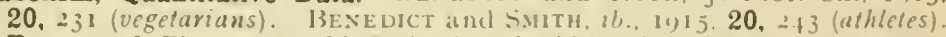

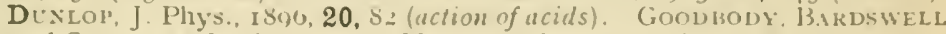

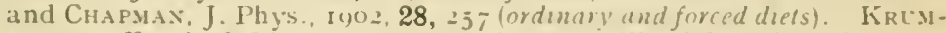

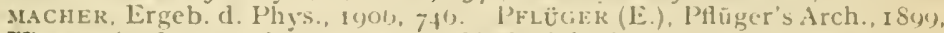
77,425 (mfluence of juantuly and kind of food). RLBNER. Gesetze res Energieverbrauchs. SPECK, Ergeb. (1. I'hy's. (Bioch.), IUO3. I. WEBER, ib., I904, 233 (influence of various suhsiances on metabolism). Zuxtz, Pflüger's Arch., I90j, 95, 192; Arch. f. Phys., IS95, 378 (work and metabolismi).

Basal Metabolism.-Ack and Di Bols, Arch. Int. Med., I9r 7, 19, 8 40 (dwarfs and legless men); $i b$. I I) 7.19, S23 (old men). BE.NEDICT, EMMES, ROTII

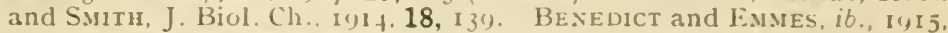
20, 253 (men and women). GEPHART and DU Bols. Arch. Int. Med., I $915,15,8_{3}$ (effects of food). MEANs, J. Med. Res., Iy I 5, 32, I21; Arch. Int. Med., IyI0, 17, 70.1 (obesity).

\section{DIETETICS.}

Bexedict (F. G.), Am. J. Phys., I906, 16, 409. McCAy, The Protein Element in Nutrition. ChITtexdex, The Nutrition of Man, N. Y.. I907; Physiological Economy in Nutrition, 1905. MACLEOD, J. Lab. Clin. Med., 19I $7,2,7+3$ (résume, economic readjustment of dietaries). MExDEL, Changes in Food Supply and their Relation to Nutrition. Yale Univ: Press, 1916. KNigt. Pratt and LANGworthy, U.S. Dept. Agric. Bull., 223, IgIo (dietary studies in public institutions). PEMBREY and Parker, J. Phys., Igo7-S, 36, p. xlix (food of the soldier). Volt (C.), Physiologie des Stoffwechsels, I8S1. Wilsox and RathBU:, J. Am. Med. Ass., 1916, 66, I 760 (dielary at. ‥ Y. Municipal Sanitarium). SIVÉ.., Skand. Arch. Phys., I9OI, 11, jos; CASPari, Arch. f. Phys., I901, 323 (minimum protein requirement). TAYLOR (A. E.), Univ of Calif. Pub., July 30. Igot (ash-free diet). RLBNER, Z. f. Biol., I901, 42, 26I (energy value of food of human beings). LITTLE and HARRIS, Bioch. J., 1907, 2, 230 (vegelarians).

Inanition.-BExedict, X. Y. Med. J., Sept. 11, I907; Proc. Nat. Acad. Sci., I $915,1,228$. CATHCART and FalleEtT, J. Phys., I907, 36, 27. Givess, Soc. Exp. Biol. Med. I $917,14,149$ ( $\mathrm{C}$ and IIg metabolism). GreExe, Am. J. Phys., I9I T. 42, wou (changes in composition of muscle). HoOVER and Solliaxi, J. Exp. Med., IS97, 2, 105 (hypnosis). Howe, Mattill and Hawk, J. Biol. Ch., I912, 11, IO3. KAUFMAXx, Z. f. Biol., I901, 41, 75. LEwIs (H. B.). J. Biol. Ch., 1916, 26, o1 (S and N climination). MeYERs, J. Med. Res., I9I 7, 36, 5 I (morphological changes). SHERwis and HAWk, J. Biol. Ch., 1912, 11, I6g (putrefaction in intestine). Vort, Z. f. Biol., I901. 41, 167, 502; ib.. 1905, 46, 167 (diminution in weight of organs). WVeber, Ergeb. d. Phys. (Bioch.), 1902, 702. Woelfel, J. Biol. Ch., $1909,6,189$ (transfer of protein).

Milk and the Suckling. - Abderhaldex, Z. Physiol. Ch., 1Sys-19, 26, fy8 (ash of suckling lnd ash of milk). CAMERER, Z. f. Biol., I g,oo, 39, 37 (physiology of suckling). LAxGstelx, Ergeb. d. Phys., 1905, 85i (energy 
halance of suckling). OPPEXHEIMER, \%. f, Biol., 1yo1, 42, 147 (food requircment and body surface of sucliling). RUBNER and HEUBNILR, Z. f. Biol., I Sus, 36, I (feeding of suckling). Sikt:s (A. W.). J. Phy's., I900, 34, 404 ( $\mathrm{C} a$ and I' of human milk).

Influence of Diet on Growth and Nutrition. GARDNER and LAUDER, PrOC. Roy. Soc., I GI 4. B 87, 220; MURLLER, J. Biol. Ch., 1915, 21, 23 (cholesterol and growth). MENDEL, Jirgeb. d. Physiol., I9It), 102; Harvey Lecture, New York, I9I f-I 5. McCollum, Simmonds and Fitz, J. Biol. Ch., 1916, 27, 33 (fat-solwble $A$ and reter-soluble $B$ and the grouth-promoting properties of milk). MENDEL and OSBORNE, J. Lab. Clin. Med., I9I6. 1, 2 I I (resumé). McCollum and Divis, J. Biol. Ch., I9I5, 23, 231 (essential factors in diet during growth). OSBORNE and MENDEL, J. Biol. Ch., I9I $2,12,8$ I (growth on fal-free food); ib., 12, +73 (gliadin in nutrition): $i b ., 13,233$ (maintenance experiments with isolared proteins); ib., I9I 4. 18, 95 (suspension of growth); ib., 1915, 20, 351 (comparative value of certain proteins in growth, and the problem of the protein minimum); $i b$., I) I 5, 23, 439 (resumption of growth); ib., I916, 25, I (anino-acid minimum for maintenance and growth). ROBERTSON (T. B.), J. Biol. Ch., I9I6, 25, 035 (cholesterol and growth); ib.. 25, $4+7$ (lecithin). Watson (C.), Lancet (Lond.). July 21, I906, I45; Dec. 8, 1585 (excessive meat); Proc. Iroy: Soc. (Edin.), 1905-6, 26, 87 (varying diets). WATSON and HUNTER, J. Phys., I 906, 34, I I I.

Vitamines, Accessory Factors in Dietaries.-Dacmand and Halliburton, J. Phys., 19I 7, 51, 235 (nutritive value of margarine and butter substitutes). Funk (C.), Ergeb. d. Phys., I913, I24; J. Biol. Ch., 1916, 25, 409 (exclusive diet of oats); J. Phys., 1913, 46, I 73 (chemistry of vitamine from yeast and vice polishings); FUNK and MACALlUM, J. Biol. Ch., I9I6, 27, 5 I (lavd and butter-fat in growth); ib., I9I5, 23, 4I3 (nature of growth-promoting substances). Hopkins (F. G.), J. Phys., 1912, 44, 425. McCollum, Srmsonds and Fitz, Am. J. Phys., 1916,41, 36I (fat-solwble A). OSBORNE and MENDEL, J. Biol. Ch., I916, 24, 37 (butter-fat).

Polyneuritis, Beriberi.-Funk and DJuglas, J. Phys., I914, 47, 475 (relation of beriberi to endocrine glands). McCollum and KenNedy, J. Biol. Ch., I9I 6, 24, 491; $i b .$, I91 2, 45, 75. STEENBOCK, Am. J. Phy's., I91 7, 42, 610 (antineuritic substances from egg-yolk).

Pellagra.-Chittenden and Underhill, Am. J. Phys., igr 7.44, I3. McColIUM and SIMMONDS, J. Biol. Ch., 1917, 32, I I. VEDDER, Arch. Int. Med., I916, 18, I 37 .

Experimental Scurvy.-JACksox (L.) and Moore (J. J.). I. Infect. Dis., 19I6. 19, 478 .-McCollum and Firz, J. Biol. Ch., Y9I $7,31,229$.

\section{CHAPTER XI}

\section{INTERNAL SECRETION.}

Biedl, Innere Sekretion. Sciäfer, The Endocrine Organs, I9I6. Vincent (S.). Internal secretion of the ductless glands, Ergeb. d. Phys, I9Io, $45 \mathrm{I} ; i b ., 19 \mathrm{I}, 2 \mathrm{I} 8$. KoJrma (M.), Q. J. Exp. Phys., 19 1 7, 11, 255 (relations between endocrine glands). IIANN (F.C.), Am. J. Phys., 1916. 41, I 73 (ductless glands and hibernation).

\section{PANCREAS.}

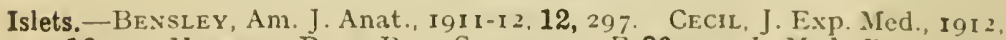
16, I. Homans, Proc. Roy. Soc., I9I 3, B 86, 73; J. Med. Res., I9I 4. 30, 49. Miline and Peters, J. Med. Res., I9I 2, 26, 405 (alrophy of pancreas after occlusion of duct). MacCaluem (IV. G.). Johns Hopkins Hosp. Bull., Sep. 19, I909. Pratt and MURPHY, J. lixp. Med., I9I3. 17, 252 (rransplantation of pancreas in spleen). V'INEXT and THOMPSON,

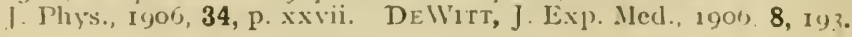


Internal Secretion of Pancreas. DRI:NNAN, Am. J. Phys. I911, 28, 396 (presence of. 1 h hlood). Hinon, Arch. Internat. de Pliys, Inı3. 13, 255 (pancreatic diabetos). (Fior ablditional references see Clibpter X.)

\section{SEXUAL ORGANS.}

Bayliss and STARLiNe, Eirgel). d. I'hys., Inor, ob. (chemical correlations of sexual organs). CARMICHALL and MARsilal, J. Phys., 19o8, 36, 43 I (compensatory hypertrophy of ovary). Dixos (IV. F.). J. Phys., 1900-I, 28 , 21f (action of orchitic extracts); ib., 1100, 25, 35t); Rosenileis, ib., 1017.51 , p. vi (spermine). HANE, J. Exp. Med., I91 1, 13, 338; STEINACH, Pfluger's Arch., 1912, 144, 71 (interstitul cells of leydig and internal secretion of testicle). Lipscuürz, J. Phys., IgI 7, 51, 283. Lowwy. Ergeb. d. Phys. (Bioch.), 1903. I 30. Marshall. and Jolly, Q. J. Exp. Phys., 1oos, 1, II 5 (heteroplastic iransplantation of ovaries). OLiver (J.). J. Phys., I9I 2,44, 355 (internal secretion of ovary). WHELLON and SHIPLex, Am. J. Phys., 1916, 39, 394 (effects of testicular transplants on vasomotor irritability)

Castration. Hexderson (J.), J. Phys, Ig0f, 31, 222 (castration and the thymus). Livincoston, Am. J. Phy's., InIU, 40, I53 (effect on pitulary). Marshall and Hammond, J. Pliys., I9I t 48, i 7 i (effect on horn growth). McCrudden. J. Biol. Ch., IgOy. 7, I 85 (effect on metabolism). Mor(ias. Soc. Exp. Biol. Med., I915, 13, 3 I (effect on feuthers). Simpson and Marshall, Q. J. Exp. Phys., 1908, 1, 257 (stimulation of nevvi erigentes after castration).

\section{THYMUS.}

Goodal. J. J. Phys., I 905, 32, inI (effect of castration). Hewer, J. Phys., I9I 4. $47,+79$ (effect of thymus feeding on reproductive organs). MARINE and Mavley, J. Lab. Clin. Med., I9I 7, 3, +8 (grafting; velation of thymus to sexual malurity). PA ron, J. Phy's, I905, 32, 28 (thymectomy and growth of sexual organs); ib., IOI I, 42, 267 (relation of thymus and sexual organs to growth). PATON and GOODALL, ib., I904, 31, 49. PAPPENHEIMER, J. Exp. Mled., I I I $4,19,3$ I $9 ; i b .$, I9I $4,20,477$; PARK (E. A.), ib., I9I 7, 25, I 29; ViNCENT (S.), J. Phys., 1904, 30, p. xvi. (results of extirpation). Semian, Ergeb. d. Phys. (Bioch.), 1904, 45. Vixcent (S.), ib., I9I I, 303 .

\section{PARATHYROID.}

Arthus and Schafernann, J. Phys. Path. Gén., I9IO, I 77 (paralhyroideclomy and Ca salts in rabbit). CaMIs, J. Phys, I909, 39, 73; MeIgham, J. Phy's., I9I 7. 51 (action of guanidin on muscle). Cooke (J. V.). J. Exp. Mled., I9I0, 12, 45 ( $C$ a and. $\mathrm{Mg}$ exeretion); 2b., I9I I, 13, 439 (N metabolism after parathyroidectomy'). HALSTED, Am. J. Med. Sci.. I907, 134, I; J. Exp. lled., 1909, 11, 1 75; ib., I $912,15,205$ (grafts). Hoskrss and WHEELON, Am. J. Phys., I9I $4,34,263$ (vasomotor irritability after parathyroidectomy). JOSEPH and MELTZER, J. Pharm. Exp. Ther., I9I 1, 2, 36I (action of $\mathrm{NaCl}$ after parathyroidectomy). Koch (W. F.), J. Biol. Cls., I912, 12, 313 (methyl guanidin in urine); ib., 191 3, 15, +3 (toxic bases in urine after parathyroidectomy); J. Lab. Clin. Med., I9I6, 1, 299 (physiology of parathyroid). MARINE (D.), Soc. Exp. Biol. Med., 191 t, 11, 11 i (hyperplasia in fowls). P'ATON ET AL. Q. J. Exp. Phys, I9I 7 10. THOMPSON (F. D.). Phil. Trans. Roy. Soc. (Lond.), I9IO, B 201, gI (thyroid and parathyroid in vertebrates). Wilsox, Steakss, Thorston and Jasing, J. Biol. Ch., 1015.23, I 23 (excretion of acids and ammonia after parathyroidectomy).

Parathyroid Tetany.-CakLson, An. J. Phys., I91 2, 30, 309 (digestive tract in). CARLSON and JACKSON, ib., I9I 1, 28, I33 (nature of tetany). GREENWALD, J. Biol. Ch., I9I6, 25, 223. Haskixs and Gerstexiberger, J. Exp. Med,, I 91 I, 13, 3I ( C a metabolism in infantile tetany). KEeton, Am. J. Phys., I $914,33,25$ (secretion of gastric juice during the tetany). MacCallun (IV. G.), Med. News. Oct. 31 , Ino3; ib., I905, 86, 625 (gastric and duocienal. dilatation in); Soc. Exp. Biol. Micd, I912, 9, 23 (seat of action in tetany). 
MacCallum and Vomethix, J. Exp. Med., Inon. 11, is (Ca in); J Pharm. Exp). Ther., IoIr, 2, fir (infuence of qurious salts $i n$ ). WAC-

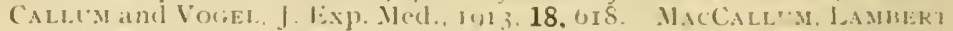
and Voribl. J. Fixp). Med.. Ior \& 20, I jo (remoeal of (" from the hood hy"

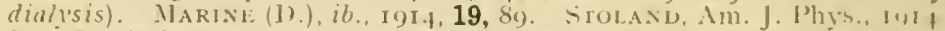

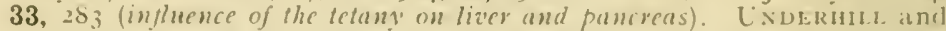
BlatUERWICK, J. Biol. Ch., 19I, 19, In (infuence of dextrose and ca lactate on blood-sugar and (cluny).

\section{THYROID.}

ASHER, PHüger's Arch., IOII, 139, 502 (history and methods). BAYLISS itnd STARIING, Ergeb. d. Phys., I got), 683 (chemical correlutions of throoid). V. FüRTH, ib., IgO9, 9. 52. ; OsWALD, I'fligger's Arch, I6It, 164, 506 (thyroid and the circulation). VINCENT (S.), lirgel). d. Plyys., 1911, 233; VIXClit and Jolly, J. Pliys., Igob, 34, 295 (functions of thyroid and parathyroid)

Results of Thyroidectomy.-GLEY, Arch. de Phys, I Sraz, fl- (rabbit); ib.,

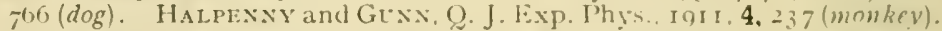
Hoskins and Morris, Soc. Exp. Biol. Med, iol 7,14, if (amphibia). Palmer, Am. J. Phys., 19I 7 42, 572 (pig). Simpsox, (). J. Exp. Jhys.,

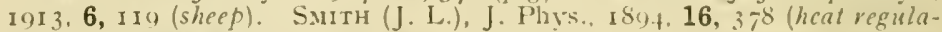
tion). TAtuM (A. L.). J. Exp. Med., 1913.17, 136 (experimentalcretinism).

Morphological Changes in Thyroid. 11ALsted (IV. S.). Trans. Ass. Amer. Physicians, 1913; Johns Hopkins Hosp. Rep.. I896. 1 (compensatory hypertrophy). MLARINE (D.). J. Exp. Med., I 91 f, 19, $37^{6}$ (involution of active hyperplasia in brook (rout). MARINE and LENHART, Arch. Int. Med., 1909. 4, 253, f $40 ;$ MARINE and W'ILLIAMS, ib., I908, 1, $3 \$ 9$ (relation of iodine to thyroid structure). WaTson (C.), J. Physs., 1905, 32, p. xvi; $i b .$, I906, 34, p. xxix; ib., I907, 36, p. i (changes in, on meat diet).

Influence of Thyroid on Metabolism.-CARLsox and JAcobsox, Am. J. Phys., 1910, 25, to3: JAcobson (C.), J. Biol. Ch., 191 4, 17, I33 (blood-ammonia after thyroidectomy). Cramer and KRAC'se. Proc. Roy. Soc., IOI3. B 86, $55^{\circ}$ (carbohydrate metabolism and the thyroid). HoNTER (1.), Q. J. Exp. Phys. IgI \& 8, 23 (thyro-parathyroidectomy and . metabolism in sheep).

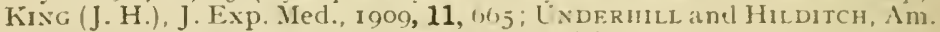
J. Pliys., I909, 25, 66; KuriYald, Am. J. Phys., I9I 7, 43, +81 (carbohydrate metabolism after thyroidectomy). ICC $\mathrm{I}^{\mathrm{R} D Y}$ (J.), J. Exp. Iled., I 909, 11, 798 (thyroidectomy and alimentary glycosuria).

Thyroid Feeding.-CARLsox, Rooks and IICKE, Am. J. Phys., I 912,30 , I 29 (attempts to produce hyperthyroidism). CRAMER and MCCAL. Q. J. Exp.

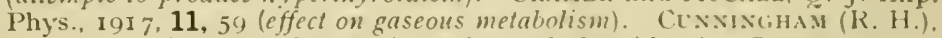
J. Exp. Med., I sos. 3, Itj (experimental thyroidism). GLDERNATSCH, Am. J. Phys., 1915, 36, 3 go (in rats). Hewitr (J. A.). Q. J. Exp. Phys.. I9I. 8, I I3, 207 (influence of small amounts of thyroid and anterior lobe of pituitary on metabolism). KURIYAMA. J. Biol. Ch., I91 7, 33, I9.3 (storage and mobilization of liver glycogen in).

Active Constituents of Thyroid.-BArMANx. \%. f. Physiol. Ch., rSo5-6, 21, 3 I9 (thyroidin). CALDWELL, Am. J. Phys., I (1) 2, 30. 42 (intravenous injection of thyroid pressure liquid). CARLSOS and llOELFE, ib.. IOIO. 26, 32 (internal secretion of thyroid). FAWCETT. ROCit RS, RAHE and BEFBE,

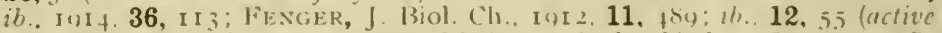
principle in thyoid and suprarenal hefore and after birth). Grainam (A.). J. Exp. Medl, Io16, 24, 345; LexHART, ib., lit.5, 22, -30 ; ROGOFF and IAarise, J. Pharm. Exp. Ther., I') I6, 9, 57 (relution of effect on tadpoles to amount of iodine). Gidirsarscu, Arch. Entwick. Mech. d. (Org.,

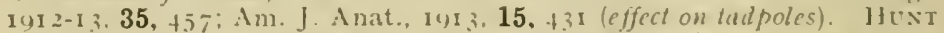
and SEmELL, J. Phatrm. Exp. Ther., roio. 2, 15 (threotropic iodine

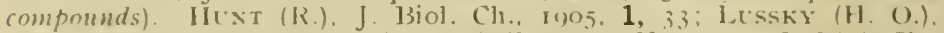
Am. J. I'lyys., 1912, 30, 63 (acelo-nitrile tesi). KexdaLi, J. Bjol. Ch., 
IuI.5 20. 50 s (product of allialine hedrolysis of throid). Liscin (F. C.).

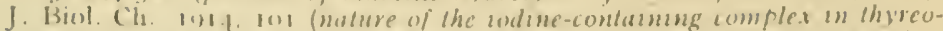

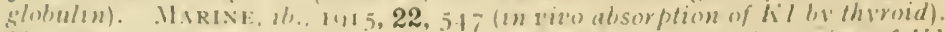

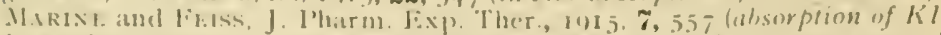
by perfused thyroul). MARI: for clabosation of the adane-containing sudstance). Mokss. (.18.), J. Biol. ('h., 1u1.1. 19, t21 (the principle accelerating involution in tadpoles). Oswazd, Arch. lixp. Path. J'harmo, woy, 60, il 5 (iodothwin). Pick

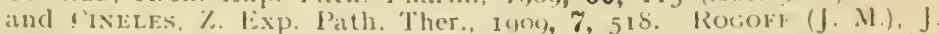

Pharm. Jixp. Ther., 101 7, 10, 199 (talpole method for standardization of thyroid preparations.

Iodine Content of Thyroid.-ALDRIC11, Am. I. PhYS., 11)1 2, 31, I 25. FENGER, J. Biol. Ch., IOI3. 14, 397 (l and P' in felul thyroids). HINTEk (A.). ib. 1910, 7, 321 (estimation). II'NTER ind sisusos, ib, 1015, 20, I11 (influenee of diel of marine alga). WATts, Am. J. I'hys., Iy15. 38, 3511 (iodine content and bloodflow through thyoid).

Innervation of Thyroid.--Secretory Nerves?-AsHER and FACK, \%. f. Biol

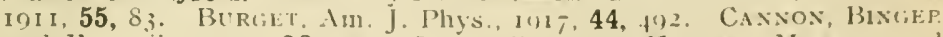
and FiTz, ib., I914, 36, 303. LEVY, ib., I1) $6,41,492$. MANLEY and Marine, Soc. Exp. Biol. Med., 1915, 12, 202. Marine, Rogoff and Stewart, Am. J. Pliys, ig I 8, 45, 268. Rahe, Rociers, Fawcett and 13EEBE, $i b, 1916,34,72$.

Vasomotors of Thyroid.-liraxcols-liraxck and Halliox, J. de Phys. Path. Gén., I908, 10, 472. SteWAR'T (G N.). J. Phys., I893, 15, 79.

Thyroid Grafts.-Hesselberg. (C.). J. Exp. Med.. 1015, 21, Ith 4 (auto- and homeo-grafts in guinea pig). MANLEY and MARINE, J, An. Med. Ass., 191 $6,67,260$.

\section{ADRENALS.}

Epinephrin (Adrenalin, Suprarenin).-ABEL (J. J.), Am. J. Phys., Igor, 5, p. $v ; i b .$, I903.8, p. xxix; AlDRICII, $i b .$, I $901,5,457 ; i b ., 7,359 ;$ v. FüRTH,

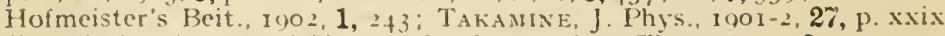
(isolation). ABEL and MACHT, J. Pharm. Exp. Ther., I91 2, 3, 327, 334 (in toad's venom). EMBDEE and $\checkmark$. FüRTII, Hofmeister's Beit., 1904, 4, t2I. Weiss and Harris, Pflïger's Arch., 1904, 103, 510 (destruction in the body'). Ewiss and LAIDLAw, J. Physs, I1)IO, 40, 275 (formation). Fusk, J. Phys., I911, 43, p. iv̈; GREer and Wells, Arch. Int. Med., I909, 4, 29I (absent in hipernephroma).

Action of Suprarenal Estract and Adrenalin.-Aule and Gates, J. Exp. Iled., I91 7, 26, 201; JACksox, J. Pharm. Exp. Ther., r91 2, 4, 59 (on lungs). BaIxBridge and Treiax, J. Phys., 1917, 51, 460 (on liver). Barbour and Kleiner, J. Pharm. Exp. Ther., 1915, \%, $5+1$ (on vagus). BARCROFT and PIPER, J. Phys., I9I 2, 44, 359 (on gaseous metabolism of submaxillary'). BotazzI, D'ENRICO and JAPELLI, Bioch. Z., I908, 7, 43 I (on saliva and urine secretion). Browx (E. D.), J. Pharm. Exp. Ther., I9I6, 8, I95. BURKET. Am. J. Phys., I912, 30, 382 (on blood-pressure). CaNxon and Gray. Am. J. Phys., I9I t, 34, 232; Grabfield, Am. J Phys., 1916, 42, f6 (on coagulation). CAxxox and NICE, ib., I91 $3,32,44$ : GRUBER, ib.. I9I t. 33, 335; ib., r 9I $7,43,530$ (on muscular fatigue).

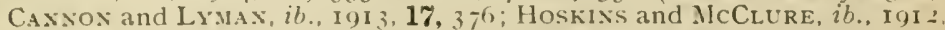
30, I92; Mloore and Piristor, Pfliger's Arch., I900, 81, 483; Am. J. Phys., 1900, 3, p. xy (depressor action of minimal doses). Cusuxy, J. Phys., 1908, 3\%, 130; ib., 1909, 38, 259 (adrenalin isomers). Evass and OGAWA, ib., 1914, 4\%, $t^{6}$ (on gaseous metabolism of heart). EDMuxs. Am. J. Phys., 18, 129 (on blood-velocily). Elliott, J. Phys., I905. 32, foI. Fleisher and Loeb, J. Exp. Med., I9Io, 12, 2SS (on absorption). Githens, J. Exp. Med., I9 7. 25, 323. Golla and Symes, J. Pharn. Exp. Ther., I913, 5, 87 (on uronchioles). Grxx. Q. J. Exp. Phys., I913. 7, 75 (on hearl). Guxw and Chavasse, I'roc. Roy. Soc., 1913, (on veins). Harold, Nierensteix and Roaf, J. Phys., iylo, 41, 3os. Hartmas: 
and Fraser, Am. J. Phy's., 19I\%, 44, 353 (vasodilatution). Hoskins, GUNNING and BerRY, Am. J. Phys., I9I6, 41, 513 (on circulation in limb). Hoskixs and McCltre, Mrch. Int. Med., IO)I2, 10, $3+3$ (blood-pressure). Hoskins, Am. J. I'hy's., 1915, 36, +23 (adrenals and sympalhetic irritability). Lamson, J. Pharm. Exp. Ther., I916, 8, 167; Lamson and Keith, ib., 247 (polycythamia). LANgley, J. Phys., 1901, 27, 237. LEWANDOW'skr, Arch. f. Phys., 1899, 97 (smooth muscle, especially eye). MIGuigan and Mostros, J. Pharm. Exp. Ther., 1913, 4, 277; Magnus, Pflüger's Arch., 1905, 108, 48 (on intestine strips). MANiN and MCLACHLIN, J. Pharm. Exp. Ther., I9I 7, 10,25I (on pancreatic secretion). MEEK and Eyster, Am. J. Phys., I 1915, 38, 62 (heart rate). Meltzer (S. J. and C.), Am. J. Phys., I 903, 9, 252 (subcutaneous injection). Meltzer and Ater, ib., 1904, 11, 28, to (paradoxical pupil dilatation): ib., 11, 449 (frog's pupil). NICE, ROCK and COLRTRIGHT, ib., I9I $4,34,326$ (on respiralion). Oliver and SchäFer, J. Phys., 1895, 18, 230. PARK, J. Exp. Med., 191 2, 16, 558 (bronchi). Pilcher and Sollmaxi, J. Pharm. Fxp. Ther., 1915, 6, 339 (on vasomotor centre). TAKayasu, Q. J. Exp. Phys., 1916, 9, 347 (on skeletal muscle). Vincext (S.), J. Phys., I 898, 22, i I I.

Determination of Epinephrin.-Folix, CANnox and Dexis, J. Biol. Ch., $1912,13,477$; SEIDELl, ib., I9I3, 15, 197 (colorimelric). Ellot, J. Phys., 1912,44, 374 (pithed cat); ib., I913,46, p. xi.

Epinephrin Content of Adrenals.-Elliott, J. Phys., I91 2, 44, 374. MCCORD, J. Biol. Ch., I9I 5, 23, .435 (feetus). RITCHIE and BRUCE, Q. J. Exp. Phys., I9II, 4, I27 (effect of diphtheria toxine). SEIDELl and FEXiger, U.S. Hyg. Lab. Bull., 100, 1914 . p. 57. STEWART and Rogoff, J. Exp. Med., I $916,24,709$.

Epinephrin in Blood (and Tests for).-BARbour and Prixce, J. Exp. Med., I9I 5. 21, 330; BARBOUR, ib., 1912, 15, 403; JANEWAY and PARK, $i b$. I9I2, 16, 54I; PARK, ib., I9I 2, 16, 532 (coronary ariory test). DALE and Laidlaw, J. Phys., I912, 45, I; J. Pharm. Exp. Ther., 191 2, 4, 75 (guinea pig uterus test). JACKSON (D. E.), Am. J. Phys., 1909, 23, 26 (disappcarance after injection). Läwen, Arch. Exp. Path. Pharm., Iyo4, 51, 4I 5 (frog perfusion test). MEYER, Z. f. Biol., 1906, 48, 352; PARK and JANEWAY, Soc. Exp. Biol. Med., I91 2, 9, 51 (artery ring test). Schultz (IV. H.), J. Pharm. Exp. Ther., 1909, 1, 291 (critique of iests). STEwart (G. N.), J. Exp. Nied., 19II, 14, 377 (biological tests-rabbit intestine and uterus segments); ib., I912, 15, 517 (absence of detcctable epinephrin in venous blood); ib., I91 2, 16, 502 (comparison of plasma and serum on intestine segments). STEWART and Rociofr, J. Pharm. Exp. Ther., I917, 9, 393 (partition between corpuscles and plasma). STEWART and ZUCKER, J. Exp. Med., I913, 17, 152, I 74 (artery ring, frog perfusion, rabbit intestinc and uterus compared). TRENDelexburg, Arch. Exp. Path. Pharm., $1915,79,154$.

Epinephrin Secretion and Adrenal Innervation.-ASHER. Z. f. Biol., 1912. 58, 274; Pflüger's Arch., 1917, 166, 372. CAssos, AUrk and Briger, J. Pharm. Exp. Ther., I $912,3,379$ (nicotine). DREYER, Am. J. Phys., I 899. 3, 203. ElLIOTT, J. Phys., I (912, 44, 374; ib., I91 3, 46, 285 (innervation) : $i b ., 1914,49,38$ (some results of excision of adrenals). Hoskins and MCPEEK, J. Am. Med. Ass., IgI 3, 60, 1777 (massage of udrenals). JosEPu and MeLtzer, Am. J. Phys., IgI 2, 29, p. xxxiv (splanchnic stimulation). O'Consor, Arch. Exp. Path. Pharm., 1912, 67, 195; ib., 68, is.3. Stewart, Rogoff and Gibson, J. Pharm. Exp. Ther., I910, 8, 20,5 (splanchnic stimulation). STEWART and Rociofl, it.. 1926, 8, 47i) (spontaneous libcration); $i b ., 1917,10,49$ (asphyxia) ; J. Exp. Med., 1917. 26, 637 (effect of sensory stimulation); ib., 191 $7,26,61$. (spinal contre for epinephrin secretion): J. Pharm. Exp. Ther., 1917, 10, I (indispensability of epinephrin?); Am. J. Phys., 1917, 44, I+9 (velation of libcration of epinephrin and adrenal blood-flow).

Adrenals and Metabolism.-EvmtNDs, J. Pharm. Iixp. Ther., I9II, 2, 559; IIAN, Arch. Int. Med., I9I 5, 16, 781 ; PENBERTON and SWEET, ib., I9I2, 
10. 169 (relation to pancreas). I.'sk and KiснE, 46., 1914, 13, 673;

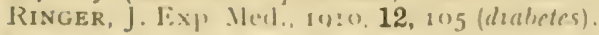

Disseminated Chromaftin Tissue,-13HEDl, and Wikst., Pthliger's Arch., Iyo2, 91. 434. FILK and MA(LEOD, Am. J. PhyS., II) 40, 40, 21. GASKELL (J. F.). J. Physs, 1112. 44,59) (distribution of medullary tissue in petromyzon).

\section{PITUITARY.}

BlaIk BELL, Q. J I:xp). Phys., r9I 7, 11, 77 (experimental operations). Cusmini: (H.). The pituitary body and its disorders, Philadelplua, 1912; Johus Hopkins Hosp. Bull., 1910, 21, I2T (experimental hypophysectomy). ․ Cron, Phiger's Arch., rgon, 81, 207; J. I'hy's. Path. Gén., 1909, 11, 259. Goetsch (E.), Q. J. Med., 1914 , 7, 173 (review). Herrixc (P. T.). 8. J. Exp. Phys., 1908, 1, 121 (histology); ib., 19:3, 6, 73 (comparative matomy and physiology); $i b ., 1914,8,26,7$ (activity of pars intermedia and pars nervosa); $i b ., 1914,8,245$ (origin of active material of posterior lobe). Pavlesco, J Phy's. Path. Gén., I907, 9, 4ti. SChäfer, Proc. Roy. Soc., 1909, B 81, 4t2 (functions). SiMPSOx and HuNTER, Q J. Exp. Phys., I9IO, 3, I 21 (relation between thyroid and pituitary). VINCENT (S.). Ergeb. d. Phys., I9I 1, 309; Practitioner, Jan, I915 (general review).

Influence on Growth and Metabolism.-Aldeich, Am. J. Phys., 1912, 31, 94 (feeding to rats). BENEDICT and HoMANS, J. Mled. Res., I 9 I 2, 25, 409 (melabolism after hypophysectomy). Cushing and GOETSCH, J. Exp. Med., I9I5, 22, 25 (hibernation and the pitwitary). Malcolm (J.). J. Phys., 1903, 30, 270. Maxivell, Univ. of Calif. Pub., 1916, 5, 5; PEARL (R.), J. Biol. Ch., 1916, 24, 123 (feeding pituitary to fowl). MILler and LEWIS, Arch. Int. Med., I91 2, 9, 60I (glycosuria after injection of extracts). ROBERTSON (T. B.), J. Biol. Ch., 1916, 24, 397, 409; ROBERTSON and Delprat, $i b .$, I $917,31,567$; Schmidt, J. Lab. Clin. Med., I $917,2,719$ (tethelin). SCHÄFER (E. A.), Q. J. Exp. Phys., I91 2, 5, 203 (effect of ovarian, pituilary, and thyroid tissue on growth of rats). WULzEN (R.), Am. J. Phys., 1914, 34, 127 (growth in birds).

Action of Extracts,-ADdis and BARvetr, Soc. Exp. Biol. Med., 1916, 14, 49 (on urea secretion by kiduey). AUER and Meltzer, J. Pharm. Exp. Ther., IyI 3. 4, 359 (pituitrin on depressor action of vagus). DALE, Bioch. J., I909. 4, 427. HAMBURger (WV. WV.), Am. J. Phys., 1904, 11, 282; ib., 1910, 26, I 7 (anteriorlobe). Howel, J. Exp. Med., I $898,3,245$ (posterior lobe). Hoskins and Means. J. Pharm. Exp. Ther., InI 3, 4, 435 (pituitrin diuresis). KIng and StOland, Am. J. Phys., 1913, 32, to5 (action on renal activity). NICE, ROCK and COtrTRIGHT, Am. J. Phys., I9I4, 35, I94 (on respiration). PATON and WATsON, J. Phys.. I912, 44, 4 I 3 (on circulalion of bird). Pilcher and Sollmaxy, J. Pharm. Exp. Ther., I $915,6,403$ (on vasomotor centre). SchäfER and HerRING. Phil. Trans. Roy. Soc. (Lond.), I 906, B 199, I (on kidney). WADDEll, Am. J. Phy's., I $916,41,529$ (on frog's cesophagus).

Formation and Secretion (?) of Activo Substance,-CarLsoN and MARTiN, Am. J. Phys., I9II, 29, 64 (question of infundibular secretion in cerebrospinal fluid). Cow (D.). J. Phys., 1915.49,367. FExger, J. Biol. Ch., 1915. 21, 283; ib. I916, 25, ti 7 (composition and activity of pituitary). KEETON and BECHT, Am. J. Phys., I91 5.29, IO9 (stimulation of piluitary). IICCORD, J. Biol. Ch., I9I 5, 23, 435 (active substance in falus). RABENis and Lifschitz, Am. J. Phys., I9I 4. 36, 47 (innervation). WEED and Cushing, ib., I9I5, 36, 77 (effect of pituitary extract on its secretion). (For action of pituitary extract. on milk secretion, see Chapter XIX.)

\section{SPLEEN.}

Splenectomy.--Goldscruint and Margot, J. Exp. Med., 1915. 22, 319 ; MeNDel and Gibsox, Am. J. Phys., 190\%, 18, 201 ( $N$ metabolism in man). Richet, J. Physs. Path. Gén., I9I 2, 14, USy; ib., 1913, 15, 579 (effects on nutrition). 
Blood Changes after Splenectomy. - KARSNER and PEARCE, J. Exp. Med.

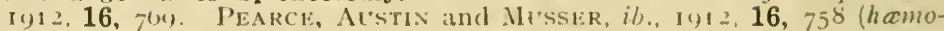
lytic jaundice). OrR. J. Lab. Clin. Med., 191 7, 2, 605.

Spleen and Blood Formation.-DONHAlste, J. Exp. Merl., Igos, 10, 559.

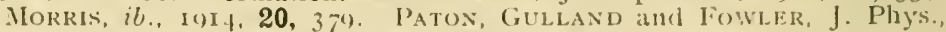
I903, 28, 82. SEEMANN, Ergeb. (1. Phy"s. (Bioch.). 1004, 30.

Spleen and Hæmolysis.-LEVIx, J. Med. Res., 8, I16. KirUMBHAAR and MI USSER. J. Exp. MIed., I9I6, 23, 97. KRUMHIHANR, ib., I9I 4, 20, I08. Paton and Goodall, J. Thys., IgO3, 29, fI1. P'EARCE, AUSTIN and KRUmbliAAR, J. Exp. Med., IOI 2, 16, 363. P'EARCE and PEET, J. Exp. Ned., I $913,18,494$.

Relation of Spleen to Pancreas.-HERzEN, J. Phys. P'ath. Gén., Iyoz, 4, 625; Phliger's Arili., igoi, 84, i 5 . Mendel and IRettger, Am. J. Phys., $1902,7,387$.

Spleen Innervation.-LANGLEy, J. Phys., I896, 20, 223. SCHÄFER and MOORE, ib., 20, I

Spleen Grafts.-MANLEY and MARINE, J. Exp. Med., I917, 25, 619; Soc. Exp. Biol. .Ied., $1917,14,123$

Effects of Reduction of Kidney Substance. BAINBRIDGE and BEDDARD, Proc. Roy. Soc., I907, B 79, 75. BARRINGton, Heart, 1915, 6, I63.

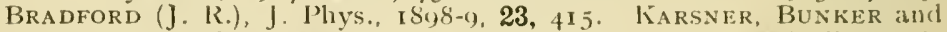
Grabfield, J. Exp., Mecl. I915, 22, 54t. Pearce (R. M.), ib., igos, 10, 632. SAMPSON and PEARCE, ih., 1908, 10, 745 .

Effect of Kidney Extracts on Blood-Pressure.-PEARCE (IR. M.). J. Exp. Med. I $909,11,430$; Arch. Int. MIed., I $912,9,362$. TIGFRSTEDT and BERGMAN, Skand. Arch. Phys., I 898, 8, 223 (pressor substance in kidney extracts).

Pineal Body.-v. Cyox, Pflüger's Arch., I903, 98, 327. Daxdy, J. Exp. Med. I91 5, 22, 237 (extirpation). FexGer, J. Am. Ned. Ass., I9I6, 17, 1835 (composition and activity). Horrax, Arch. Int. Med., 1916, 17, 60\%. MICCORD, J. Am. MIed. Ass., 1915, 65, 5 I 7. JORDAN and EYSTER, Am. J Phys., 19II, 29, Ir5 (extracts). OTr and Scotr, Soc. Exp. Biol. Med. I $912,9,6+$ (extracts).

Salivary Glands (Removal).-SCHÄFER and Mloore, J. Phys., 19, p. xiii.

Organ Extracts.-BRow and JosEPH, J. Phys., I go6, 34, 282. CAMPBELL (J. A.). Q. J. Exp. Pliys., I9I I, 4, I (on bloodvessels). IFaWCett, Rahe, Hackett and Rogers, Am. J. Phys., r9I5. 39, I5t (on smooth muscle). Josepir (D. R.), J. Exp. Med., I907, 9, (oo (on blood-pressure). MILler (J. L. and E. M.), J. Phys., I9I I, 43, 2,2.

Bone-Marrow Extracts. Brow: and Guthrie, Am. J. Phys., I905, 14, 328 ; Browx and Joser'H, ib., I $) 00,16$, I 10.

Extracts of Nervous Tissue.-Halliburton, J. Pliys., I goo-I, 26, 229. OSBORNE and VINCENT, $i b$., I 899-I900, 25, 283. SCIḦFER and MoORE, $i b$. IS96, 20, I. SCHÄFER and VinceNt, 25, 87. Viscent and Cramer, $i b$. I $904,30,1+3$.

\section{CHAPTER XII}

\section{ANIMAL HEAT.}

Calorimetric Observations. ATwATER, Eregel, d. 1'hys. (Biocli.). I BENEDICT, and CARPENTER, Carnegie Instit. l'ul)., 123, 1910 (respiration calorimeters for man); ib., Pub. 126, Io10 (metaholism of nomal men). Du BoIs, J. Am. Med. Ass., I9I 4, 63, 827,830 (tolal energy requirement in disease); Arch. Int. Mecl., I9I6, 17, 887 (children). D'ARsonvar, Arch. de Phys., r89o, oro, $78 \mathrm{r}$. Hill (A. V.). J. Pliys., I I)1 I, 43, 379 (coldblooded animals): ib., IOI2-I3, 45, 261 (micro-calorimeler). HILL (A. V.

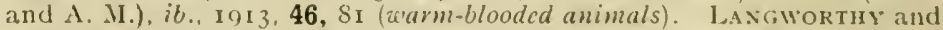
IIILNer, Yearbook, IT.S. Dept. Agric., IuIo (respiration culorimeler). Lefìvre. J. Phys. Path. Gén., ryou, 2, 259. Le'sk, Arch. Int. Iled., 


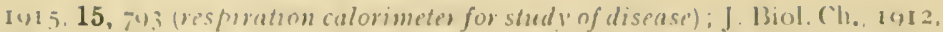

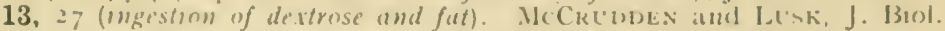

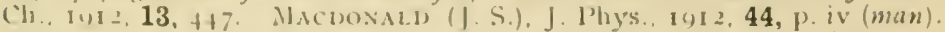
Wikl and Hoomlek, Ans. J. Dis. Children, Int5, 9, gl (infanis) MurLix and L,usk, J. Isiol. Ch., Iol5, 22, 15 (ingestion of ful). Plinbody. MEYER and Du BuIs, Arch. Int, Mcoll. I0It, 17, oso (basal metabolism in cardio-renul diseuse). Ricul: and SoDErstrom, Arch. Int. Med., IGI5, 15, 905. Williasts. J. Biol. Clt., 1912, 12, 317 (small calorimeter). WilliaMs, Riche and Lusk, ib., I9I2, 12, 3.9 (ingestion of meat).

Speciflc Dynamic Action of Foodstuffs.-Lusk, J. [3iol. Ch., I915, 20, 555. Surface Area.-BExedict (F. (i.), Am. J. Phys., 1916, 41, 275. Du Bors (D. and E. F.), Arcli. lint. .lecl., $1915,15,868 ; i b ., 1916,17,863$.

Surface Area and Metabolism (Heat Production).- Bexenict (1: G.). Am. J. Phys., IgIt, 41, 2()2. CEPllart and Du Bols, Arch. Int. Med., I9It, 17, 902. MleANS (J.H.). J. Biol. Ch., 1915, 21, 263.

Heat Production in Various Organs (and Functions).-13AYliss and HiLL (L.). J. Phys., I894, 16, 35 I (salivary glands). Chauveal, Arch. de Phys., I887, 2:9 (thermodynamics of muscular work). HenLitzkA, Archivio di Fis., I9I2, 10, 50I; SXYder, Am. J. Phys., 19I7, 44, 42I (heart). Hil. (A. V.). J. Phys., 191 2, 43, 433; Rollestox (H. D.), ib. I $890,11,208$; Stewart (G. N.), ib., I Sul, 12, fog (nerve). Mosso (A.). Proc. Roy. Soc., I 892 , B 51, 83 (brain). REICHERT (E. T.), Am. J. Phys., I 900, 4, 397 (digestion). ReID (W'.). J. Phys., I 895, 18, p. xxxi (glands). Vernon, ib., 1894, 17, 277 (velation of respiratory exchange to temperature).

Effect of Various Conditions on Heat Production and Loss (Climate).-LEe, EDmuxds, ET Al., Soc. Exp. Biol. Med., I915, 12, 72 (humidity, etc.). LEFÈV'RE, Arch. de Phys., ISOS, 085 (death by cold); J. de Phy's. Path. Gén., I903, 5, 783 (radiation). MACLEOD (J. J. R.), Am. J. Phy's., 1907, 18, I (hmmidily). MaSje, Virchow's Arch., I 887, 107; Stewart (G. N.), Studies, Physiol. Lab. Iniv. Nanchester, I891, 1, IOI (loss by radiation). IICClure and SaUer, Am. J. Dis. Children, 1915, 9, 490 (effect of clothing). Osborne (IV. A.). J. Phys., I9IO-I 1, 41, 345; ib., 1915, 49, I33 (climate). PEMBREY and PITTS, J. Phys., I 899, 305 (hibernating animals). RUBNeR, Arch. f. Hygiene, I891, 11, 208; Nature, Nlay 28, I891, 666 (dry and moist air); Die Gesetze (les Energieverbrauchs, r902. WALLER (A. D.), J. Phys., I893. 14, p. xxy (calorimetry by surface thermometric and hygrometric dala).

Varnishing the Skin.-BAB̊́K, Pflïger's Arch., I905, 108, 389. La Ulaví, Arch. de Phỹs., I8y7, 302. StEwART (G. N.). Studies, Physiol. Lab. Univ. Manchester, 1891, 1, 122. Wixtersitz, Arch. Exp. Path. Pharm., I $894,33,286$.

Effect of Cold (Hibernation).-CAMeroN and Brownlee, Q. J. Exp. Phys., I9I3, 7, I I5; CAMERON (A. T.), ib., I9I5, 8, 34I; HARRIS, J. Phys., I9Io, 40, p. Jiii (on frog). MerzBacher, Ergeb. d. Pliys. (Bioph.), I9o4, 2 I 4 (general physiology of hibernation). Srmpsos (S.), Soc. Exp. Biol. Med., 1913. 10, I8o (hibernation and external temperature). Simpson and Herrisg, J. Phys., 1905, 32, 305 (cold narcosis).

Heat Paralysis.- Веснт, Am. J. Phy.s., 1908, 22, 456 (heat paralysis in nerve tissue). Camerox and Brownlee, Q. J. Exp. Plyys., 19r 5, 9, 247 (death temperature in frog). EvE, J. Phys., r9oo-1, 26, I 9 (superior cervical sympathetic ganglion). HallibtrTox, Q. J. Exp. Physiol., I9I5-IG, 9, r93 (death temperature of nerve). MAYER, Am. J. Phys., I9I 7. 44, 58 r.

Body Temperature.-Dansixt (G. C. C.), J. Physs., I906, 35, p. v (goal). Frothisghay and MInot, Am. J. Phys., I $912,30,430$ (rabbit). LazarusBarlow, Lancet (London), i 895, Oct. 26 (month). PeMbreY and Nicol, J. Phys., I 8us-9, 23, 386 (deep and surface temperatures). Simpsox (S.), Proc. Roy. Soc. (Edin.), I9I 2, 32 (I), I Io (seasonal changes); ib., 1907-S, 28 (2), G6I (fishes). Woodhead (G. S.). J. Phys., I 898-9, 23, p.xv (rest and zeork, in horse). YouxG (W. J.), ib., 1915, 49, 202 (Europeans in tropics). 
Diurnal Variation of Body Temperature.-BF.enICT (IF G.), Am. J. Phys.,

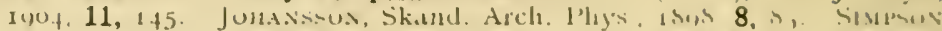
(S.), J. Phy's., Iyoz, 28, p. xxi (monkey). Simpos and Giliskilin. ib., 1905, 33, 225 (nocturnal birds, etc.). Toulouse and Pléros. J. Phy's. Path. Gén., I907, 9, 425 (inversion of daily routine).

Temperature Topography.- HeIDENHAIx and KökNeR, Pflüger's Arch., I 8 j, 4, 558 (right and left ventricles). Kunkel (A.), Deutsch. Med. Zeit., I 887; Stewart (G. N.), Studies, Physiol. Lab. Lniv. Manchester, 1, I891, ro. (surface temperatures in man). LEFEVRE. Arch. de Phys., I 898, 254 (thermal :opography in cooling by baths). RA_rCKEN and TIGERSTEDT, Skand. Arch. Phys., I909, 21, 80 (stomach).

Thermotaxis.-BABḰK, Pflūger's Arch., I902, 89, I5ł (new-born). BoycotT and HALDANE, J. Phys., 1905-6, 33, p. xii (high external temperature). Chsuveau, Compt. Rend., 136 (13), p. 792 ; ib. (I 4 ), pp. 847.852 (animal thermostat); Zentralb. f. Phys., 1903, 17, 391. Colosa.xtr, Pfluger's Arch., IS 71 , 14, 92. HALE White, Lancet (London), I 897 (June I 9, 26; July 3, I0). HÁRI, Pflüger's Arch., I909, 130, 90 (chemical regulation in mammals). Johansson, Skand. Arch. Phys., I904, 16, 85. Lefèvre, J. Pliys. Path, Gén., rgor, 3, p. I. Penirey and Hale White, J. Phys., I895-6, 19, 477 (hibernation). PEMBREY, GORDON and WARREN, ib., I $694-5,17,33$ I (chick before and after hatching). PEMBREY, ib., I895. 18. 363 (young animals). RICHET, Arch. de Phys,, I 893, 25, 312 (shivering)

Heat Centres.-Aronsons, Virchow's Arch., 169, 505; Aronsons and Citron, Z. Exp. Path. Ther., I9I0, 8, I3 (puncture fever). BArbour and WIng, J. Pliarm. Exp. Ther., I9I 3. 5, IO5 (application of drugs to centres); Barbour and Prince, ib., I9I4, 6, I; CloftTa and WASEr, Arch. Exp. Path. Pharm., I9I 4, 77, I6 (local heating of centres). KENNAwAY and PENBREy, J. Phys., I $912,45,82$ (effect of section of spinal cord on temperature and metabolism). MEAxs, AUB and DU Bols, Arch. Int. Med., $1917,19,832$ (effect of caffein on heat puncture). OrT (1.). J. Nerv. Ment. Dis., N. Y., I 884 . Penbrey, Brit. Mled. J., I897 (2), 883. ReIChert, Univ. Pann. Med. Mag., Mar., I893, Feb., I894; J. Am. Med. Ass., I902. 38, I 3. Sachs and Greex, Am. J. Phys., I9I 7, 42, 603. Hale Wiite, J. Phys., I 89o, 11, I; ib., т891, 12, 233.

Fever--BABÁ, Pfüger's Arch., I904, 102, 320. BURnftT and MARTix, Soc. Exp. Biol. Mled., 1916, 13, I +1. CARPENTER and Benedict, Am. J. Phys., 1904, 24, 203. Colemax and Du Bors, Arch. Int. Med., I9I4, 14. I68 (high-caloric diet in typhoid). LeATHEs, J. Phys., I907, 35, 205 (nitrogen, creatin, and uric ecid excretion). LoEw' Ergeb. d. Phys. (Bioch.), 1904. 339. MANDEL, Am. J. Phys., 1907, 20, t39 (xanthin); ib., I904, 10, 452 (alloxuric bases in aseptic fever). OTt and Coll.Mar, J. Phys., I887, 8, 216 (pyrexial agents); OTt and Scort, J. Exp. Med., 1907, 9,671 (metabolism). Rosexthal, Zentralb. f. Phys., 1888, 635; Dentsch. Med. Woch., I 888, No. 8. Sharpe and Simox, J. Exp. Med, I YI $4,20,282$ (nitrogen excretion). STEWART (G. F.), ib., I9I3, 18, 372 (bloodflom in extremities in fever).

\section{CHAPTER XIII.}

\section{THE PHYSIOLOGY OF THE CONTRACTILE TISSUES.}

Cilia.-Lillie (R. S.), Am. J. Pliys., I901, 5, 56; 1902, 7, 25; 1906, 16, Sy (influence of ions). MAXWell, ib., I905, 13, I 54. MEYER, Soc. Fip. Biol. Med., I909, 7, I3. PARKER, Am. J. Phÿs., Ig05, 13, I; 14, I. PǗtTer, lirgeb. d. Phys. (Bioph.), IчO3, I. SCḦ̈FER, Anat. Anzeig., Iy04, 24, Nos. I9 and 20; I yo5, 26, 5 I 7 .

Smooth Muscle.--Botazzi, Pflüger's Arch., I906, 113, I36. Botazzi and GRt'NBAly, I. Physs, 1899, 24, 5 I (aesophagus of load and auricle of torluise). GrützNer. Ergeb. d. Plyys., suo4. I2. LEWIS (M. R. and W. H.), Am. J. Pliys, IษI , 44, 57 (smooth muscle cells in cultures). 


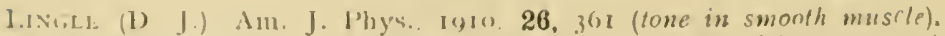

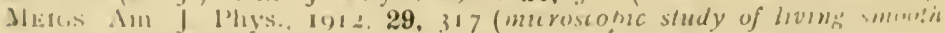

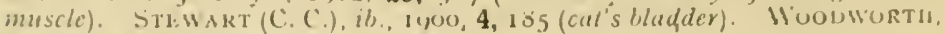
2b, 1 sing, 3, 26.

General Physiological Properties of (Diaphragm) Muscle-LeE, Guextritr and Mrelexer, Am. J. P'lyss, 1916, 40,446. Botazzi, Rend. Acad, dei Lincei, 1915 (numerous papers).

Elasticity of Animal Tissues,- HAYCralt, J. Pliys., 1904, 31, 392.

Muscle Contraction-Theories.- BERsstein, P'flüger's Arch., I909, 128, I 36 ; il., 1915, 162, 1. BER, (IV.), थ6. 1913, 149, I95 (Zuntz's theory). ENcielmain, Arch. I. I'hys., Iyo 7,25 (contractility and double refraction). BukNert (T, C.). J. Biol. Ch., I007, 2, I95 (influence of temperature on contractiluty, and its relation to chemical reaction velocity). EWALD, I'flüger's Arch., I $887,41,215$ (volume unaltered in contrection). GARREY, Am. J. I'lys., 1905, 13, is6 (twitchings produced by sall solutions). L.ANciley, J. Phys., Igo7-8, 36, 3i7; 1008, 37, 165, 285; 1 yo9, 39, 235 (receptive swbslances). LombarD and ABßotT, Am. J. Phys., 1907, 20, I (contraction of individual muscles of frog's thigh). LUCAs (K.). J. Phys., I905-6, 33, 125 (graduation of activity in skeletal muscle); ib., 1904, 30, 443 (infuence of tension). McDougale, J. Anat. and Phys., 1897, 31, 410 ; ib., I898, 32, 187. MaCdOnald (J. S.). J. Phys., I908, 37, p. xxv. Meigs, Am. J. Phys., 1905, 14, I38. Schäfer, Q.J. Exp. Phys, I910, $3,6_{3}$.

Action of Substances on Muscle-Permeability of Cells.-Asher, Bioch. Z., I908, 14, I. CARLIER, Brit. Med. J., Sept. I6, I9I I (various salts). Hamburger (H. J.), Bioch. Z., 1908, 11, +43; Osmotischer Druck, etc. Harvey (E. N.), J. Exp. Zool., I911, 10, 507; Am. J. Phys., 1913, 31, 335. JOSEPH and Meltzer, Am. J. Phys., I9II, 29, I ( $\mathrm{Na}$ and $\mathrm{Ca}$ ). KITE, ib., I9I5, 37, 282 (permeabitity of internal cyloplasm). LILlie (R. S.), ib., I9I I, 28, I97 (velation of stimulation and conduction to permeability changes). Locke (F. S.), Pflitger's Arch., 1893, 54, 501 ( $\mathrm{NaCl}$ solutions): J. Exp. Med., I S95, 1, 630 (ether). LOEB (J.), see J. Biol. Ch., 23 to 32 , for 13 merous papers. MATHEW's and Lovglellow, J. Pharm. Exp. Ther., I910, 2, 201 (dyes). McCleNdon, Am. J. Phys., 1912, 29, 302 (increased permeability of muscle to ions during contraction); ib., 1915. 38, I 73 (ancesthetics and permeability). MeIGs and ATwOOD, ib., 1916. 40, 3 I $\left(\mathrm{K}^{\circ} \mathrm{Cl}\right)$. Millikix and Stiles, Am. J. Phys., I905, 14, 359 ( $\mathrm{Na}$ or Li ions). MINEs and DALE (D.), J. Phys., I9II, 42, p. XXix (acids). Osterhout, J. Biol. Ch., I9I4, 19, 335, 493 (effect of alkali and acid). Overton, Ergeb. d. Phys. (Bioch.), I902, 749 (anilin dyes); Pfluger's Arch., 1902, 92, I 5 (osmotic protherties of miscle); ib., 92, 346; ib., I904, 105, I 7 G ( $\mathrm{N}$ a or Li tons in coniraction). RoAF, Bioch. J., I906, 1, 88 (ladpole). Robertson (T. B.), J. Biol. Ch., 1908, 4, I. Stiles, Am. J. Phy's., 1903, 8, 269 (Ca and $K$ on smooth muscle tone). Zoethout, $i b$. , 1902, 7, 199 ( $K$ and $C a$ on striated muscle); ib., 1904, 10, 373 (electrolytes).

\section{EXCITATORY PROCESS IN MUSCLE (AND NERVE).}

Biedermann, Ergeb. d. Phys. (Bioph.), I902, I 21 ; ib., 1909, 26 (the excitable lissues). Erlanger and Garrey, Am. J. Phys., 19I4, 35, 377; Martin (E. G.), Am. J. Phys., r908, 22, 61, 116; ib., 1911, 28, 49; 1910, 27, 226 (faradic stimulation): Measurement of Induction Shocks, New York, I9I2. Hill (A. V.), J. Phys., I910, 40, I 70. LapicQue, J. Phys. Path. Gén., 1903, 5, 843, 991; ib., 1907, 9, 568, 619; $i b ., 1909,11$, 1009, 1035; ib., I $910,12,601,624$; ib., I9II, 13, 42 . Lillie (R. S.). Am. J. Phys., I916, 41, 126. LuCAS (K.). J. Phys., I 908, 37, 459 (rale of developinent of excitution): ib., 1907, 36, 253 ; ib., I910, 40, 225; ib., 1909. 39, 331 (refractory period); ih.. 1908, 37, p. xxx (Nernst's Theory applied); Proc. Roy. Soc. (Lond.), I912, B 85, 495 (process of excilation in nerve and muscle). Lucas and Mines, J. Phys., 1907-8, 36, $33+$ (lempera! tre). 


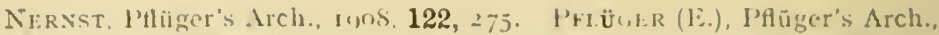

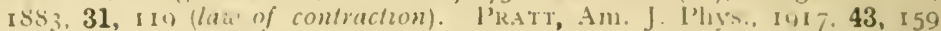
(excitation of microscopic areas). Wlidexsky, l'fluger's arch., Ieput. 100, 5. MOolley, J. Plysis., 1902, 37, 122 (temperature coeficicul of conduction). ZoEthour, Am. J. Phys., 1yo2. 7, 320 (contact irnitabilaty).

Threshold Stimuli.-GrabFleld and Martix, Am. J. Phys., I913, 31, 300. GRUBER, ib., I9I5, 37, 259 (cervical sympathctic). MARTIN, GRACE and MCGuiRe, J. Pharm. Exp. Ther., 1015, 6, 527 (influence of drugs). MьNDenuale, Am. J. Pliys., 191 $4,36,57$ (autonomic).

Relations of Salts and Ions to Muscular Contraction.--BURRIDGE, J. Plyys., I9I I, 42, 359 (polassium). GUenther, Am. J. Phys., 1905, 14, 73 $\left(\mathrm{KCl}\right.$, NaCl and $\left.\mathrm{CaCl}_{2}\right)$. Locke, J. Phys., 1yo5, 32, p. xiii ( $\mathrm{K}$ and . Va action on indirect excilability). Zoeruout. An. J. Phys., 1909, 23, 37t ( $\mathrm{NaCl}$ and $\mathrm{CaCl}_{2}$ influence on $\mathrm{K}$-concentration).

- All or None' in Muscular Contraction.-Eisexibrger, Am. J. Pliys., I9I 7. 45, 44 (minimal contraction). Lucas (K.), J. Phys., 1gog, 38, II 3. Pratt, Am. J. Pliys., I9I 7, 44, 5 I 7.

Summation.--ADRIAN and LUCAS, J. Pliys., I9I2, 44, 68 (of propagaled disturbance in nerve and muscle). Lucas (Ki.), ib., 1910, 39, for (of inadequate stimuli). MINes (G. R.), ib., I9I3, 46, I (of contractions).

Staircase Phenomenon ('Treppe').-LEE (F. S.), Am. J. Pliy's., 1907, 18, 267. LeE and Harvey, Soc. Exp. Biol. Mrd., I910, 7, 135.

Tetanus.-BAsler, Pflüger's Arch., 190 f, 105, 3łf. HofMAxx, $i b ., 1903,93$, I $86 ; i b ., 95,4 \delta_{4} ; i b ., 1904,103,291$. KRONECKER and STIRLING, J. Phys., I $878-9, \mathbf{1}, 384$. Sewall (H.), J. Phys., I 888, 9, 92.

Tonus Rhythms in Striated Muscle.-CAxion and Gruber, Am. J. Phys., I916, 42, 36. SAMOJLOFF, Arch. f. Phys., 1907, I 45. Storey, Am. J. Phys., 1904, 12, 75. Wedensky, Arch. de Phys., IS91, 23, 59,

Voluntary Contraction.-Clegiors and StewART (C. C.), Am. J. Pliys., igor, 5, 281 (inhibition time). FOA., Z. f. Allg. Phys., 1911, 13, 35 (rhythm of motor impulses). HaYcraft, J. Phys., I $890,11,352 ; i b .$, I 898,23 , I (rapid voluntary movements). HARRIS. J. Phys., 189,-5, 17, 315 (lime relations in man). GRIFFITHS, $i b ., 1888,9,39$ (rhyihm). LOMBARD (IV. P.), J. Phys., I $892,13,1$.

Fatigue.-BURRIDGE, J. Phys., 19I0, 41, 285 (chemical fictors). CRJDER and Robinson, Am. J. Phys., I9I6, 41, 376 (frog's muscle). Forbes (A.). Am. J. Phys., 1912, 31, IO2 (reflex faligue). FRANZ (S. I.), ib., 1900, 4, 348. GrUBER, ib., I9I3, 32, 22I, 438. LEE (F. S.), ib., I907, 20, I 70 (fatigue substances); J. Am. Mled. Ass., Nay 19, 1906. LEE and ArovioVitch, Soc. Exp. Biol. Med., I9I7, 14, I53 (fatigue toxin?). LeE and Everinghas, Am. J. Phys., 1909, 24, $38+$ (psendo-fatigue of cord). Lombard (II: P.). J. Phys., 1893, 14, 97. Piper. Arch. f. Phys., Iyog. 49I. RYAN and AGNew, Am. J. I'hys, 1917, 42, 599. STOREY, Am J. Phys., I903, 8, 355. SCineNCK. Pnïger's Arch., I900, 82, 384 . WoodWORTH, Am. J. Phys., 1901, 5, p. iv.

Ergograph, Ergometer.-BExedict and EmMes, Ain. J Phy's., I915, 38, 52. HALL (W. S.). $i b ., 1902,6$, p. xxiii. Hovgh (T.), $i b ., 1901,5,240 ; i b$. I902, 7, 76. LOMBARD, ib., 1902, 6, p. xxiv. MARTIN (C. J.) J. Phys., I9I4, 48, p. xv.

Lactic Acid and Muscular Work.-Feldman and Hili. (L.), J. Phys., I9I I, 42,439 (influence of oxygen). I'LETCHER, ib., I9II, 43, 286; ib., I9II-I2, 45,$286 ; i b$. I $913,47,361$. FLETCHER and HOPKINS, $i b ., 1906-7,35,247$.

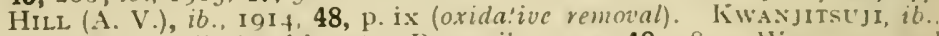
I9I6, 50, 312 (isolated hearl). ROAF, ib., I914, 48, 380 . WINFIELD and Hopkiss, $i b ., 1915,50, \mathrm{p} . \mathrm{v}$ (influence of pancrcatic extracts).

Source of Energy of Muscular Contraction.-Dunlop, PATON, STOCKMAN and Macadam, J. Phys., I 898, 22, 88 (muscular exercise and melabolism). Frentzel, Pflüger's Arch., I897, 68, 191. K'RuMmacher, Z. 1. Biol., 
Is(e), 33, IOS; PETTENKOFER and Volt, ih. I866, 2, 538 (prolein melabolism not incrised). PHIÜuER (L.). Pflüger's Arch., I 89 I, 50, 98.

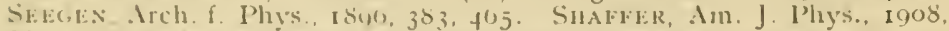
22. +t.5 (dminished muscular achvity and protein melabolism). SPECK, Arch. 1. Phys, Ibo5. foj.

\section{ENERGY TRANSFORMATIONS IN MUSCULAR WORK.}

A.XDERSON and Li'sk, J. Biol. Cls., I9I 7, 32, $12 \mathrm{I}$ (diel and body condition and heat production in muscular work). BExEdct and CatuCaRT, Carneg. Instit. Pub., 19I3. Bevedict and Ilurschauser, Carneg. Pub., No. 231,

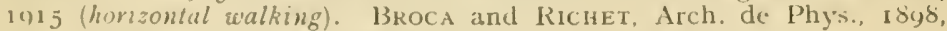
2.5. Berg, DU BoIs-Reymoxd (R.) and Zunz, Arch. f. Phys. Supp. Bd., I9of, 20 (bicycling). Chatveau, Arch. de Phys., I897, 229. 261 . Johaxisox, Skand. Arch., Phys., I901, 11, 273; ib., 1903, 14, 60 (CO.2 outpul). LEE and ScOTT, Am. J. Phys., I9I $6,40,486$ (action of temperalure and humidity on working power of muscles). MacdonaLD (J.S.). J. Phys., I 914 , 48, p. xxxiii (efficiency in man). REACH (F.), Bioch. Z., I908, 14, 430 .

Heat Production in Muscular Contraction.-Blix, Skand. Arch. Phys., I 902 , 12, 52. Evass and Hill, J. Phys., I9I 4, 49, Io (velation of length to tension and heat production). FRANk (O.), Ergeb. d. Phys., I90 t 348 (thermodynamics of mus .llar contraction). Hill (A. V.), J. Phys., 19I1, 42, I (position of heal production in the sequence); ib., 1912, 44, 466; ib. I9I $3,47,305 ; i b .$, I91 3, 46, 435 (mechanical efficiency); ib., I910, 40, 359 (contracture and tonus). PETERS, J. Phys., I9I3, 4\%, 243 (in fatigue and relation to lactic acid); ib., I9I $3,46,2 S$ (recovery processes). SNYDER, Am. J. Phys., I9 i 4, 35, 340 (smooth muscle). Weizsacker, J. Phys., i 9 I 4 , 48, 396.

Effęcts of Muscular Exercise.-BARAch, Arch. Int. Med., I910, 5, 382 (severe exertion, Marathon race). COOK and PeMbrey, J. Phys., I $913,45,429$. GASSER and MEEK, Am. J. Phýs., I 9 I 4. 34, 48 (mechanism of heart acceleralion). Hill (L.) and FlACK, J. Phys., I9Io, 40, 3tj (O, inhalation). Hotgh, Am. Phys. Educ. Rev., April, r909. HYDE, Root and C.urL, Am. J. Phys., 191 7, 43, 37 (athlete and nori-athlele). KRoGH and LiNDHARD, J. Phys., I9I $3,4 \%$, I 2 (regulation of respiration and circulation in work). Lowsley, Am. J. Phys., I9II, 27, 446 (blood-pressure and heart-rate). MLARTix, GRUBer and LANiAx, Am. J. Phys, I9I f, 35, 2 I (body temperature and pulse-rate). SCHNEIDER and HAVExs, ib., I9I5, 36, 239 (blood changes).

Denervated and Regenerating Muscle.-LANiGLEY, I9I 7, 51, 377.

Rigor Mortis.-Folin (O.), Am. J. Phys., I 903, 9, 37t (cold rigor). LATHAM, Bioch. J., I908, 3, 193 (lactic acid and $\mathrm{CO}_{2}$ formation). JosepH and Meltzer, J. Exp. Med., I 909, 11, Io, 3 I f; Am. J. Phys., I909, 25, I I3; MacWillia., J. Phys., I90I-2, 27, 336 (rigor of heart). MLLtzer and AUer, J. Exp. Med., IgoS, 10, 4.5 (influence of $\mathrm{C} a$ and Mg). WixterSTEIx, Pflüger's Arch., I907, 126, 225.

Heat Rigor. Burridge and ScotT. J. Phys., I9I 2, 44, p. iii. Fletcher and BROW., ib., I9I f, 48, I 77 ( $\mathrm{CO}_{2}$ production). VERson, ib., I $899,24,239$. Vroomas, Bioch. J., I90 $7,2,303$.

Water Rigor.--Brooks (C.). Am. J. Phys., I906, 17, 2 I 8 (conduction and contraciion in). IIEIGs, J. Phys., I 909, 39, 385.

\section{CHEMISTRY OF MUSCLE.}

BotazzI (F.). Bioch. Bull., I9I3, 2, 379 (physical chemistry of muscle-plasma). Botazzi and Quagliariello. Arch. Internat. Phys., I $912,12,234.289$, 409 (proteins); Arch. Ital. de Biol., I91 3. 60, 255. HalliblrtoN. J. Phys., I\$S7, 8, I33; Biochemistry" of .Iuscle and Nerve. vox FüRTH, Ergeb. d. Phys. (Bioch.), 1902, I10; ib., 1903, 575; Arch. Exp. Path. Pharm., I 895. 
36, 231. JANAEY, J. Biol. ('h, 19I1, 25, 177, 185 (tolal proteins). LeE. scort and Colvis, Am. J. Phys., 1916, 40, 176. NeIcis, Am. J. Plyys

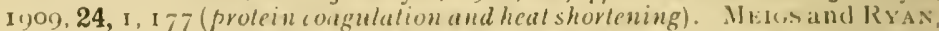
J. Biol. Ch., ryiz, 11, for (ash of smoolh muscle). OSBORNE and ZOBEL, J. Phys., 1903, 29, I (sugars). Raxsom (F.). J. Phys., I9IO, 40, I (enzymes). Salki, J. Biol. Ch., igo8, 4, 483. Stewart and Sollmann, J. Phys., 1899, 24, 427 (proteins). URANO, Z. f. Biol., 1908, 51, 483 (salts). VINCENT (S.) and Lewis, J. Phys., I901, 26, 445 (proleins and heat shorlening).

\section{CHAPTER XIV.}

\section{NERVE.}

The Nerve Impulse or Propagated Disturbance.-BRANIELL and LicAs, J. Phys., 1911, 42, 495 (refractory period and propagated dislurbancc). MATHEWS (A. P.), Science, 1902, 15, 41); ib., 1903, 17, 729; MAXWELL. (S. S.), J. Biol. Ch., 1907, 3, 359; Strosg, J. Phys., 1899-Igoo, 25, 427; Sutherland, Am. J. Phys., 1905, 14, $112 ; i b ., 1906,17,297 ; i b ., 1909$, 23, 115 (theories).

Chemistry of Nerve.-Alcock and Lyxcil, J. Phys., 1907, 36, 93; ib., I910, 39,402 (salis). Halliblrton, Ergeb. d. Phys., 1905, 24. Macallum (A. B.), Proc. Roy. Soc., 1906, B 77, 165. Macdoxald (J.S.). J. Phy's., 1907, 36, pp. iii, xvi (chlorides). TASiliro, Am. J. Phys. 1913,32, 107. 1 37: TAshiro and A dams, Am. J. Phys., $1914,34,405$; J. Biol. Ch., 1914. 18, 329; WAllek (A. D.), J. Phys., 1896, 19, p. i (CO, production in nerve). TyöRNER, Pflüger's Arch., I9I $4,156,2.53$ ( $0_{2}$ requirement of nerves).

Fatigue of Nerve.-Brodie and Halliburton, J. Phys., 1903. 28, i8I (nonmedullated). Scott (F. H.), J. Pliys., 1906, 34, 1.5. TAIT (J.) and GuNN (J.A.), (. J. Exp. Phys., I908, 1, I9I (yohimbinized nevve). TA1T, J. Phys., $1906.34, \mathrm{p}$. xxxw. (cooling and fatigue).

Excitation and Conduction in Nerve.-ADRIAN, J. Phys., 1914, 47, 400; ib., I913.46, 3s+ (all-or-none principle); ib., I9I0, 50, 345 (vecovery). Biedermani, Ergeb. d. l'hys. (Bioph.), Igoz, ifl. Carnot, J. Phys. Patl. Cién., Ig1 2, 14, 737. GaD and SAWYer. Arch. 1. Phýs., i 889, 350 ( $\mathrm{CO}_{2}$ and alcohol). GRLBER, Am. J. I'hys., 1913,31, +1 3 (nerve block). Laxilitey, J. Phy's., 19oi, 2\%, 226; Proc. Roy Soc, 1900, 13 78, i 7o (nerve cndings). Liliti (K. S.), Anı. J. Phy's., 1915, 37, 3f8. Lucas (K.). J. Phys., IgI 1, 43, th (Iransference of impulse from nevve 10 muscle); ib., $1913,46,470$ (alcoinl). LicAs (K.), Conduction of the Nervous Impulse (revised hy F. D. Adrian), London, I917. MIEEK and LeEPER, Am. J. Phys., 1911, 27, 3o8 (pressure). MAYlik, Am. J. Phys., 1916, 39, 375; ib., 1917,42, foy. Srales (IV. L.). J. Phys., 1917, 51, p. xix (swrvival of mammalian neve). WALlek, J. Phys., 1917, 18, p. xlv (anasthetics). WEDENSKY, I'flugger's Arch., 190o, 82, I34 (fundamental properties of nerves under cerluin poisons).

Temperature and Excitability of Nerve.-ADRIAN, J. Phys., IyI $4,48,453$ (lemperalure coefficient of refractory period). Borcots, ib.. 1902, 27, 458 . Gotch and Macdonald, ib., I8g6, 20, 247. TaIt, Q. J. lixp. Phy's, igio, 3, 221 (refractory phase and clectrical change); ib., 1you. 2, 157 (vefraciory phase in yohimbinizal neve).

Electrotonus.-Lokis (J.), Pflüger's Arch., 1907, 116, 193; Univ. of C:blif. P'ub. 1905. 3, 9. Hermaxx and Tschitscikin, P'füger's Arch., is S99. 78, 53 (inexcilability of kalhode with cerlain current slrenglhs). PFLÜCER (E.).

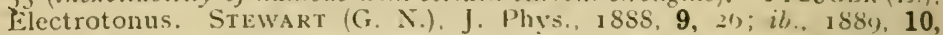
$45^{8}$ (depression of conduclivily al kalhoile as compared wilh anode wilh cerlctn current strenglhs). WERIcio (B.). l'tlïger's Arch., 1901, 84, 547 (depressive action at hathode).

'Chemical' Stimulation of Nerves.-GutıRIE and LEE, Soc. lixp. Biol. Med., IyIf. 11, I.f' (sensory). GrürzNler, Pflüger's Arch., Idy4, 58, 69. 


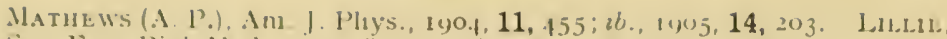
Soc. Jixp. Biol. Med suro, 7, 190. Lolen and liwald, J. Biol. Ch., 1010,

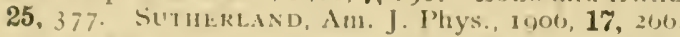

Velocity of Nerve Impulse.-ADriAn, J. Phÿ., I114, 48, 13. CARLsos, Aml. J. Plyys., Ino f. 10, for. Hermax, Ptliger's Arch., 1902, 91, I 89. JrinkiNs and CARLson, J. Comp. Neurol. Iyo3, 13, 25y; Am. J. Phys.

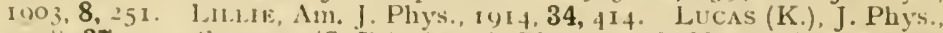
$1908,37,112$; SNYDER (C. D.), An. J. Phys., 1908, 22, I 79) (temperalure coetficient of velocity of nerve conduction); ib., 1911, 28, 167 (temperalueve coefficients of velocities of varions physiological actions). MCCLENDON, J. Biol. Ch., 191 7, 32, 275 (effect of stretching). Nicolat, Phluger's Arch. I (joI, 85, o5 (olfaclory nevec of pike). PIPER, ib., 1908, 124, 59; ib., 1909, 127,474 (in man)

Degeneration of Nerves. Jikiss and CRAMER, Proc. Roy. Soc., I912-13, B 86, II9. LANGley, J. Pliys., InOg, 38, 504 (nerve endings). T'CKETT, J. Phys., I806, 19, 20,7 (non-medullaled fibres).-VAan Genuchtes, Le Nevraxe, $1703,1,1,263 ; i b ., 1905,7,203$.

Regeneration of Nerve. - BaEk, Dawson and Marshall, J. Exp. Med., I 899, 4, 29 (dorsal root-fibres in cord). Bline. Pflüger's Arch., I907, 116, 385 . BRUCE and DAWson, Trans. Roy. Soc., lidinburgh, 1913, 48,697. CAJAL. Compt. Rend. Soc. de Biol., 1905. 120 (against auloregeneration). CLARK (E)., J. Comp. Neurol., 1914, 24, (1). FeIss, Q. J. Exp. Phy's., 1913, 7, 31. Harrison (R.), Anat. Record., Dec., Iyo8; Ingebrigrsen, J. Exp. lled., 1913, 17, 182; ib., 1913, 18, +12 (in vitro). HEAD, Brit. Med. J., May 27, I905. HuBER, J. Lab. Clin. Med., I9I 7, 2, 837 (operative treatmenl of peripheral neves); Am. J. Phy's., 1900, 3, 339 (degeneration and regeneration of nerve endings in muscle). LANGLEY and ANDERSON, J. Phys., 1904. 31, +18 (on aulogenetic regeneration); ib., 31, 365 (union of different kinds of fibres). LANGLEy, J. Phys., 1900, 25, 417 (preganglionic filves); $i b ., 1898,22,215$; Killvington and OsBorne, J. Phys., I906, 34, 207 (postganglionic fibres). MleEk, Am. J. Phys., 1910, 25, 367; 1911, 28, 352 (myenteric nerves). Мотt, Halliburton and Edmunds, Proc. Roy. Soc., 1906, B 78, 259, 529, (no autoregenevalion). OSBORNE and Kilvington, J. Phys., Igo8, 37, I; ib., 1909, 38, 268 (axon bifurcation); Brain, 1910, 33, 261 (central nevvous response to peripheral nevous distortion). SCHÄFER and lieiss, Q. J. Exp. Phys., 1916, 9, 329 (cervical sympathetic and vagus). Tucketr, J. Phys., 1903, 29, 303 (vagus).

Anastomosis of Nerves.-Budiett and Greex, Am. J. Phys., I9oo, 3, I 5 (vagus and hypoglossal). Cunvingiham, ib., x $898,1,239$. ErLanger, ib., 1905, 13, 372 (spinal nerve and vagus). IiEIss, Q. J. Exp. Phys., I912, 5, I, 399. Howell and Huber, J. Pliys., I 892, 13, 335. Kennedr, Proc. Roy. Soc., I9I 4, B 87, 33 I (nerve anaslomosis). LANGLEy, J. Phys., $1904,31,365$ (phrenic and cervical sympathetic); ib., 1898, 23, 240 (vagus and cervical sympathelic). LANGLEY and ANDERSON. J. Phys., 1904, 30, 439 (union of a cervical nerve with superior cervical ganglion).

Trophic Nerves.-Brkelss and Jassinski, Zentralbl. f. Phys., 12, 345. Jacobsox (C.), Am. J. Phys., I910, 26, 413. Mlarinesco and Serieux, Arch. de Phys., I 893, 25, 455. Turner (IV. A.), Brit. Med. J., Nov. 23, 1895 .

\section{CHAPTER XV.}

\section{ELECTRO-PHYSIOLOGY.}

Biedermans, Elecktrophysiologie; Ergeb. (1. Phys. (Bioph.), 1902, 120; ib., 1903, 103. Du Bors-REYsond, Untersuch, über thierische Elektricität.

Relation of Permeability of Cell Envelopes to Ions and Electrical Phenomena of Tissues.-Bernstein, Pflüger's Arch., 1902, 92, 521 . BRŰNINGs, $i b$., I $903,98,241 ; 100,367$. HöBER, $i b ., 1905,106,599$. LILLIE (R. S.), Am. J. Phys., 191 4, 34, $414 ; i b ., 1915,37,3+8 ; i b$., I916, 41, 126 (conditions 
of physiological conduction in irritable tissucs). MAcuonsto (J. S.), Thompson Yates lab. Rep., Laiv., Liverpoul, 1yo2, 4, 213. NERxs, (iö tinger Nichrichte Math. Plıss. Kil., 1899,1 left 1 ('ésumé in Höjer's l'hysikal. Chem. d. Zell. u. Geweb. 3rd Edit., I91 1, +5t) (theory of stimulation). STEWART (G. N.), J. Bost. Soc. Med. Sci., June 3, 1897 ; J. Phys., IS94, 24, 2 I 2 .

Electrolytes and Cells.- CKozlek, Am. ). Phys., 1y16, 39, 2y7 (sensory stimulation). LOEB and CATtell, J. Biol. Ch.. 1915. 23, 41 (diffusion of potassium). LokB (J.), Am. J. Phys., I9o2, 6, 41 ; Pflüger's Arch, 1902, 88, us; ib., 1902, 91, 248; ib., 1905, 10\%, 252; Bioch. Z., 1907, 5. 351 ; MAнHE's (A. P.), Am. J. Phys., 1904, 12, 49 ("toxic and antitoxic effects of ions)

Current of Injury (Demarcation Current, Resting Current).-BERNSTE1N and 'TSCHERMAK, Pflüger's Arch., I904, 103, 67. BERNSTEIN, ib., I9O6, 113, 605. BrüNiNGs, ib., 1904, 101, 201. CYBulski, Arch. Intermat. de Phys., I91 2, 11, f I8. HẼze, Pflüger's Arch., I902, 92, 451. HERMANX, Z. f. Biol., I91 2, 58, 28 I. HARDY, J. Phys., I913, 47, Io\&. Hö3ER, P'fluger's Arch., 1909, 126, 331; ib., I910, 136, 333; STEWART (G. N.), Am. J. Phys., I903, 9, 75 (effect of saponin on resting current of muscle and nerve). LOEB and BEUTNER, Science, 1912,35 , 912 ; Bioch. Z., 1912, 44, 303; ib., I $914,59,195$. Macdonald (J. S.), Proc. Roy. Soc., I90o, B 67, $310,315$. 325; SowTON and MACDONALD, ib., B 71, 282, 472 (nerve). MATHEWS (A. P.), Am. J. Phys., I903, 8, 294 (electrical polarity in hydroids).

Action Current of Muscle.-Bersste1n, Pflüger's Arch., 1902, 89, 289) (relation to work). Buchaxas, J. Phys., I901-2, 27, 95 (persistent contractions). Hoffmans, Arch. f. Pliys., 1909, $3+1$ (voluntary contraction). Licas (K.), J. Phys., I 909, 39, 207 (relation to propagation of excitation). SANDERSON (J. B.). J. Phys., $1898-9,23,325$. SiYder, Am. J. Phys., I9I 3, 32, 336 (twitch).

Action Current of Nerves.-Bernste1n, Pflüger's Arch., 1897, 67, 349. Alcock and SEemAxi, Pflüger's Arch., 1905. 108, 426; J. Phy's., I905, 32, p. xxx; Lewandowsky, Pfliger's Arch., 1898, 73, 288 (vagus in respira. tion). Macdonald (J. S.), J. Phys., i 899, 24, p. xxvi (vagus). MícDONALD and ReID, J. Phys., ISyS, 23, IOO (phrcuic). II Aller and Sowton, Proc. Roy. Soc., Oct. 31, I go3; J. Pliys., I S9S, 22, I (effect of poisons on). Medexsky, J. de Phys. Path. Gén., I9o3, 5, I 42 (telcphone).

Polarization of Nerves.-Du Bors-REYMond, Sitzungsber. d. Kgl. Preuss. Akad., 1883, 1 (secondary electromolive phenomcna). HERMANN, 'Hïger's Arch., I879, 19, 416; ib., I $881,26,246 ; i b ., 1884,33,103$; Handbuch. d. Physiologie, Band 2, i878. LAPICQUE and PETETiN, J. Phys. Path. Gén., 1910, 12, 696. Locke (F. S.), J. Exp. Med., 1896. 1, 6jo (effect of ether). Sciwakrz, Pfliger's Arch., I911, 138, 487 . StewART (G. N.). Proc. Roy. Soc. (Edin.), Jan. 2 I, I 889; J. Phys., I 850, 10, 45 S. WAlleR (A. D.), J. Phys., I 895, 19, p. vii; ib., 20, p. xi ; ib., 21, p. vi; ib., 22, p. i (electrotonic curreuts).

String Galvanometer.-E1NrHoven, Pflüger's Arch., I9o9, 130, 2S7. SAMoJlorf, Arch. f. Phys., I910, 477. Sxyder, Am. J. Phys., I()I 3, 32, 329

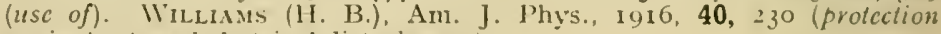
against external electrical disturbances).

Action Current of Heart (Electrocardiogram).-DALE and MiNes, J. Phys., $1913,46,319$ (nerve stimulation). lirAsir, J. Exp. Ned., 1915, 22, 2(1)2. Gotch, Heart, 1910, 1, 235. Jolly, Q. J. Exp. Pliys., I915, 9, 9. MeEk and Eyster, Am. J. Phys., I912, 30, 271 (vagus stimulation); ib., 1912. 31, 31. Mines, J. Phys., I914, 47, 19 (vagus). Pike (F. H.), Am. J. Phys., 1916, 40, 433 (stimulation of phrenic by). SAxDERsox and PAGE, Proc. Roy. Soc., is 78,401 . SYMEs, J. Phys., 1905, 32, 1). Ixxi (heartnerve stimulation). WMLLER, Phil. Trans. Roy. Soc., ISS9, B 169. VIGgers, Arch. Int. Mled., 1917, 20, 93. 


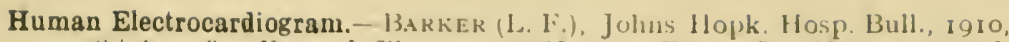
$35^{\circ}$ (résume). [3t'l.L. J. I'hy's., I911, 43, p. v: Fank (G.), Heart, 19ז2, 4, 147 (electro- und phono-cardiograms). Eistnovi.N R.T Al.., Pflüger's Arch., I $900,80,134 ; i 6 ., 1903,99,+72 ; i b .14013,149,+8,05 ; i b ., 1913,150$, ErSTER and MEEK, Arch. Iut. Med., 1013.11, 20.4; lORBES and RAPPLEYE, Am. J. Phys, lor $7,42,22 S$ (effect of temperature). GodDand, Arch. lint. Med., 1015, 16, 633. Krumismar and JENks, Heart, 1917, 6, I89

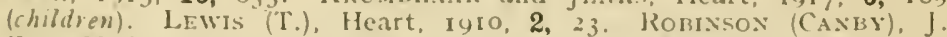
Exp. Med., 1912, 16, 201; Arch. Int. IIed., I9r6, 18, 830. Walier. Lancet, 1913. May 24 and 3 I (general review); Arch. d. Phys., I 8 gro, 22, I 6 ; J. Phys., ro1 4 48, pp. xvii, xl. Wrimams, Am. J. Physe, I91 35,292 .

Gland Currents.-BRADFORD (J.R.), J . J'hys., 1 888, 9, 287 (submaxillary gland).

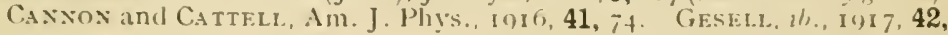
541 .

Eye Currents.-DAy, An. J. Phys., r915, 38, 369. Listhovex and Jolly, O. J. Exp. Phys., roos, 1, 373. Gotch, J. Plyys., 1903, 29, 388; ib., 1904,

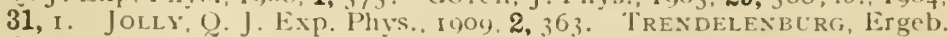
d. Phys., IyI I, 21. WAller, Q. J. Exp. Pliys., 1909, 2, foI.

Electric Fishes.-DU BoIs-REyoxd, Ges. Abhand., 2, woI (Malapterurus); Sitz. d. Berl. Akad., March 1, $388_{3}$; July I6, I 885 (torpedo). Gorch and Burch, Phil. Trans. Roy. Soc., i Syf, B 187, 347 (Malaplenurus). SA.NDERsox and Gorcn, J. Phys., 1858, 9, 137 (skate).

Galvanotropism.-ABвотT and LifE, Am. J. Phys., I908, 22, 202 (bacteria). Bancroft (F. W.), Lniv. Cálif. Pub., 1904, 1905, 1906 (paramecium). Dale (H. H.), J. Phys., I900-I, 26, 291; GARREY (W. E.), Am. J. Phys., 1900, 3, 291; PEARl (R.), Am. J. Phýs, 1900, 4, g6 (infusoria). Hadley (P. B.), ib., 1907, 19, 39 (lobster larve). LOEB and BUDGETt, Pflüger's Arch., I 897, 65, 518 (theory). Mil.ter (F. R.), J. Phys., 1906-7, 35, 2 I 5 (crayfish). MOORE and Goodspeed, Lniv. Calif. Pub., IgII. MoORE and Kellogi, Biol. Bull., ro16, 30, I, I .

\section{CIIAPTER XVI.}

\section{THE CENTRAL NERVOUS SYSTEM.}

VoN BEchterew, Funktionen der Nervencentra, Jena, I9I I. Doxildoson (H. H.), The Rat, I9I5 (statistical data). TAN GEHuchtex, Anatomie du Système Nerveux. Schäfer and Symingtox, Quain's Anatomy. 3. Pt. I (numerous references). SHERRINGTON, Integrative Action of the Nervous Sýstem, New York, rgo6. VAs Rijnberk, Arch. Néerland, de Phys., 191 7, 1, 19S (organization of nerions system).

Nerve-Cells.-Doxaldsox (H. H.), J. Comp. Neurol., I899, 9, I 4 I; Am. J. Phys., I 900, 4, p. vi (size of cell body and axon). GoMez and PIKE, J. Exp. Med., 1909, 11, 25; Tuскетт, J. Phys., 1905, 33, 77 (effects of ancemia). línvixgton, J. Phys., I903, 28, +26 (snake venom). STEWART (C. C.), J. Exp. Med., I $896,1,623$ (alcohol poisoning). Warrixgtox, J. Phys., $1898-9,23,112 ; 24,+64 ; 1899-1900,25,+62$ (alterations after section of nerve fibres).

Spinal Ganglia.-KRamer (S. P.), J. Exp. Med., 1907, 9, 3 I4 (function). SteINACH, Pflüger's Arch., I 899, 78, 291 (centripetal conduction).

Enumeration of Fibres in Spinal Roots.-DALE, J. Phy.s., I S99, 25, I 98 (dorsal roots, cat). Dixis. J. Comp. Neurol., I 909, 19, 685; HARDESTY, ib., I 899 , $9,64$. Ingbert, $i b ., 1903,13,53 ; i b ., 1904,14,209$ (man). HaraJ, ib., 13, 177 (in growing rats).

Paths in Central Nervous System.-Browx-Ś́guAR, Arch. de Phys., I 889 , $t_{f}$ (sensory). BRTCE (N.). Q. J. Exp. Phys., I9I0, 3, 391 (Gower's tract). liRASER (E. H.), J. Phys., I9OI-2, 27, 372; 1902, 28, 366 (.Monakow's bumle). Raxsos and Brlusgseer, Am. J. Phys., rgro, 40, 571; 
RANSON and VoN HEss, ib., IgI5, 38, I 28 (pain and affirent vasomotor paths). SCHÄFER, Q. J. Exp. Ityys., Iy[0, 3, 355 (paths for volitional impulses). Simpsox and Jolly, Proc. Roy. Soc. (Edin.), 190r-7, 27, 281 (degenerations after lesions of motor cortex in monkeys).

\section{SPINAL CORD.}

Donaldson and Davis, J. Comp. Neurol., 1903, 13, I9 (area of cross-sections). FRIFDexthal, Arch. f. Phys., 1905, I27; Goltz and Ewald, PAluger's Arch., I S96, 63, 362 (excision of part of cord). SHERRINGTON, J. Phy's., I 897, 21, 209; DALE, ib., I $901-2,27,3-50$ (efferent fibres in dorsal roots). SHERRINGTON, ib., 27, 360 (spinal roots and dissociative ancesthesia). TREXDELEXBuRG, Ergeb. d. Phys., 1910, 454 (comparalive physiology' of $\operatorname{cord}$ ).

Spinal Preparation.-Guthrie (C. C.), Z. bicl. Tech. Meth., I9II, 2, I38 (mammal and bird). ROAF and Sherrixgtox, Q. J. Exp. Phys., 1910, 3, 209. Sherringtox, J. Phys., 1909, 38, 375 (cat).

Decerebrate Preparation.-Browx (T. G.), Q. J. Exp. Phys., 1914, 7, 293. 345 (reflexes). Forbes and Sherrixgtos, Am. J. Phys., I91 $4,35,367$ (acoustic reflexes). Miller and Sherringtox; Q. J. Exp. Phys.. Igro, 9, 147 (decevebrator). WEED, Am. J. Phys., I9I7, 43, I3 I (reactions in decerebrate kittens).

Reflexes.-Baglioni, Z. f. Allg. Phys,, I912, 40, I6o (cutaneous reflexes). Brows (T. G.), Ergeb. d. Phys., I913, 279; O. J. Exp. Phys., 1914, 8, 155, I93 (progression). Brooks, Am. J. Phys., 1910, 27, 212 (effect of lesions of dorsal rools). FORBES and GREGG, Am. J. Phys., I91 5,39 , I $=$ (strength of stimulus and reflex response); ib., I915. 37, I 8 (flexion reflex). LANGLEY, J. Phys., I900, 25, $36+$ (axon reflexes). MAYHEw, J. Exp. Iled., I $897,2,35$ (time of reflex winking). MOORE and OERTEL, Am. J. Phys., I $900,3,45$ (reflexes after high section of cord). PAlliow, Ergeb. d. Phys.. I9I1, 345 (conditioned refiexes). PORTER (E. L.). Am. J. Phys.. 1913. 31,223 (reflex arc in asphyxia); ib., I $917,43,497$ (variations in irritability of reflex arc). SHERRINGTON and LASLETT, J. Ply ys., I $903,29,58$ (interconnection of spinal segments). SHerRIsGtox, Ergeb. d. Phys., 1905. 797 (co-ordination of refiexes, common path); J. I'lyys., 1904, 30, 37 (spinal refiex and quality of culaneous stimulus); ib., 1910, 40, 28 (fexion reflex, crossed extension reflex); ib., i906, 34, 1; Q. J. Exp. Phys., 1910, 3, 213 (scratch reflex): Proc. Roy. Soc., I907, B 79,337 ; $1908,80,53$; 190:). 81, 249; 1913. 86, 219 (reciprocal innervation of antagonistic muscles). SIMPSON and HERRING, J. Phys., 1905, 32, 305 (cold narcosis and reflexes).

Knee-Jerk.-Bowditch and WARrex, J. Phys., I 890, 11, 25. Frasz, Am. J. Insanity, 190y, 65, +71. JoLL1, Q. J. Exp. Phys., 191 $1,4,67$. Lombard, j. Phys., 1889. 10, I 22 . Sherrisgtos, J. Phys., I892, 13, 669 (posterior roots concerned). SXYDER, An. J. Phys., I9Io, 26, 474. Stewart (P.), J. Phys., I 898, 22, o1. Waller, ib., I 89o, 11, 384.

Reversal of Reflexes.-Brown (T. G.), Ergeb. d. Phys., 1913, 430. KixowlTOX and MOORE, Am. J. Phys., I9I\%, 44, tyo (reversal of reciprocal inhibition in earth-worm). SHERRINGTON and SOWTON, J. Phys., 19I1, 42, 383 (chloroform and reversal); Proc. Roy. Soc., I 911 , B 83, 435.

Inhibition in Nervous Reactions.-Brow:-SequRD, Arch, de Phys., I889, 21, 751. Hering, Ergeb. d. Phys. (Bioph.), I902, 503. Forbes (A.), Q. J. Exp. Phys., 1912, 5, 149 (reflex inhibition). MicDoctall, Brain, 1903, 102 (2), p. I 53. PAWLow, Richet's Livre Jubilaire, 1912, 325. YERKES, J. Comp. Neurol., 1904, 14, I 24 (frog). SHERrixcitoN, J. Phys. 1907,36 , I 85 (effect of strychnin); ib., I91 3. 47, 196; Q. J. Exp. Phys. 1908, 1, 67 (combination of excitation with inhibition).

Spinal Shock.-F1ke (F. H.), 1912, Am. J. Phys., I91 2, 30, 436; Q. J. Exp. Phys., I9I3, \%, I. PORTER and .ILHLberG, Am. J. Phy's., I900, 4, $33+$ (inhilition after injury of cord ?). 


\section{CEREBELLUM.}

HERRICK (C. J.), J. Comp. Neurol., 1895, 5, u6 (hislogenesis); ib., 191 4, 24, 1 (Necturus). Liciaxi, Ergeb. (l. Phy's. (Biopli.), 1904, 259. Wilsos: and PIKl: Anı. J. Pliys., Iy $6,41,571$ (lesions).

Localization. BirRiv, Wicn. Kilin. Wóch., 1912, No. 52. BLACK, J. Lab.

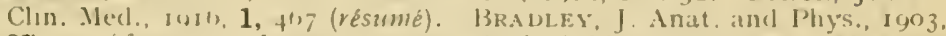
37, 221 (fissures). LEWANDOWSKY, Arch. f. Phys, 1903, 129. MEYlER,

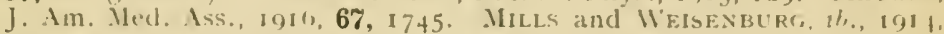

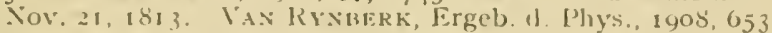

Labyrinth and Equilibration.-ALEXANDER and KinfiDe, Pflüger's Arch., $1902,89,+75$ (galvanic reaction in deaf mules). BeritofF, Q. J. Exp. Phys., Io10, 9, I09 (labyrinthine reflexes in decerebrate preparation). BÄÁxY, Z. f. Sinnesphysiol., Igof, 41 (2), 37. Cyox, Pflüger's Arch., 1goo, 79, 2I ; Arch. f, Hhys., 1897, 29 (vestibule and space perception). EWALD (J. R.), Pfligger's Irch., I 890, 52I (labyrinth and rigor). FISHER and

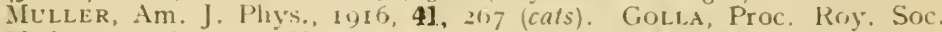
Med., Iy12, 5, 123. Hexsex, PAtiger's Ir(h., 189., 74, 22 (slatocysts). KREIDL, Ergeb. d. P'hy's., Iyon, 572 (vestibular apparatus). LEE (F. S.), Am. J. Phys., $180 \mathrm{~s}, 1, \mathrm{I} 28$ (cal and lateral line in fishes). Lro.s (E. P.). ib., Iyoo, 3, 86; 4, 77 (compensulory movements in fishes). MAXWELL.

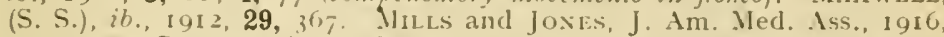
67. 1296. PARKER, Bull. L'S. Bur. Fisheries, 1yog, 29, t5 (influence of eves, ears, elc.. on movements of dog fishy. PRINCE, Am. J. Phy's., I gi 7 . 42, 308 (killens). Wilsox and Plke, Phil. Trans. Roy. Soc. (Lond.), 1912, B 203, I 27 Arch. Int. Med., I914, 14, 911 . YFRKes, Am. J. Phys. I907, 10, p. xviii, Zотн, Pflüger's Arch., 1901, 86, 147 (ear of dancing mouse).

Postural Reflexes, Decerebrate Rigidity.-BRown (T. G.), J. I'hy's., 19 I5, 49, I 80 (plastic flexor lonus in monkey). СовB, BAILEY, and HOLTz, Am. J. Phys., I9I 7, 44, 239 (extensor rigidity). FRöHLIC» and SHERRiNGTos, J. Phys., 1903, 28, It (path of inhibilory impulses). MAGXt's and DE KLE1JN, Pflüger's Arch., I9I3, 154, I63, I $78 ; i b ., 1915,160,+29$ (postural reflexes); ib., I9I 4, 159, 218 (frog). Mills (C. K.), J. Am. Med. Ass., I9I6, $67,{ }_{4} 85$ (probleins of cerebral tone). ROAF, Q. J. Exp. Phys., I9I 3, 5, 3 I $\left(\mathrm{CO}_{2}\right.$ output in decerebrate rigidity). SHERRINGTON, 13rain, Jy15, 38, I9I (postural activity of musclc and nevve); (). J. Fixp. Ihys., 1909, 2, 109. $\mathrm{J}$. Phys., I898, 22, 319 (deccrehrate rigidity and reflex co-ordination of movements). VAa RijNBERK, Arch, Néerland. de Phys., 1917, 1, 762 (muscular tonus and decerebrate rigidity). WEED, J. Phys., 19I f, 48, 205.

Muscular Tonus.-Birsett (T. C.), Soc. Exp. Biol. Mel., I915, 12, 177 (skelelal muscle). HookER, Am. J. Phys., 1912, 31, ti (influence of $\mathrm{CO}_{2}$ and $\mathrm{O}_{2}$ on tone in hloodvessels and alimentary canal). LINGLE (D. J.), Am. J. Phys., 1910, 26, 361 (plain muscle). MotT and SHerrington, Proc. Roy. Suc., I $895,5 \%, 48$ (influence of sensory neves on movements and nutvition of limbs). SHERRINiTON, J. Phys., isof-5, 17, 21 I (nevves of muscles).

Co-ordination of Movements.- BEevor, Ergeb. ll. l'hys., ryog, 326. Du BolsREYMO:D (R.). Arch. f. Phy'., Supp. Bd., 1900, 327;ib., 1902 , Supp. Bd., 27. HerING and SHERrixgtox, Pflüger's Arch., I $\$ 97,68,222$ (inhibition of voluntary muscles from corlex). MAcists, ib., 1909, 130, 211, 253; ib., I9I0, 134, 545; SHERRINitos, P'Toc. lioy. Soc, 1905. B 76, 160, 269 (reciprocal innerition).

Basal Ganglia and Mid-Brain.-Bkow: (T., G.), J. Plyys, 1915, 49, 195, 208 (is progression learnt?). HERRICK (C. J.), J. Comp). Neurol., 19I7, 28, 215 (mid-brain and thalumns of Necturns).

\section{CEREBRUM.}

Baglioxi, Zentralb. f. Phys., I90 I, 14, 97 ; MAxwell (S.S.). J. Biol. Ch., i907, 2 , I83 (chemical stimulation). Vo. BECHTEREW. Arch. f. Phys., I902, $26+$ (corlical secretory centres); ib., 1905, 207 (urine and sweal centres); 
ib., 1905, 525 (influence of cortex on sexual glands). BRow's (T. G.), Q. J. lixp. Pliys., Ig10, 3, I39 (removal of cortex of one hemisphere); J. P'hys., I9I t 48, pp. xxx, xxxiii (post-centrul gyrus). Campible, Brit. Med. J. Feb. 6, InO.1 (histological differentiation, summary). FilecusI(; Lancet, Oct. I9, 1901, I027; Vogt., J. Phys. Path. (ié11., I900, 525 (myelination).

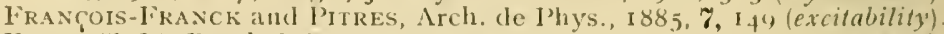

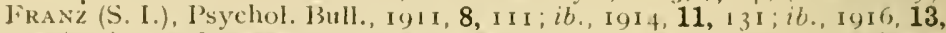
I. 9 (review and summary); J. Am. Med. Ass., 19o6, 47, Ifot (association areus in monkeys). Golrz, Pfliiger's Arch., 1892, 51, $57^{\circ}$; ib., 1899, 76, fII (brain extirpations, monkey). HERRICK (C. J.), J. Animal Behav., 1913, 3, 222 (origin of cerebral corlex). Holmes (G.), J. Phys., I9or, 2\%, I (histology of Goltz's 'trainless dng '). K'ARPLus and KREIDL, Arch. f. Phys., 10I4. 155 (extivpation of hemispheres in monkeys). VON MoNakow. Ergeb. d. Phys. (Bioph.), t9o2, 53.1; ib., 19oł, 100 (localization). MotT, Schuster and ballibtrton, P'roc. Roy. Soc., 19I0, 13 82, izf (cortical lamination). Rollett, Pllüger's Arch., rgoo, 79, 303; 80, 638 (historical, especially ou Gall's doctrines).

Frontal Lobes.-Bolton, Brain, 26, 2 I 5. Franz, Am. J. Phy's., I902, 8, I (frontal lobes and sensori-motor habits); Arch. of P'sychol., Mar., 1907. No. 2 (functions). HERRICK (C. J.), Science, 1910, 31, 7 (intelligence and its organs). McDouciall, Brain, I901, 24, 577 (seat of psycho-physical processes).

Motor Areas. -von Bechterew, Arch. f. Phys., i 899, Supp. Bd., 543 (man); ib., 1 yoo, 22 (sensory functions of motor area). BROWN and SIIERRINGTON, J. Phys., 1911, 43, 209 (baboon); ib., 1913, 46, p. xxii (recovery after lesions). Brown (T. G.), Q. J. Exp. Phys., I9I 5, 9, 82, 10I, II 7, I3 I ("facilitation' in motor cortex in monkey). CunNinginam, J. Phys., I 898 , 22, 204 (opossum). Franz (S. 1.), Psychol. Monographs, April, 1915. GRÜnHAUM and SHerrinctox, Proc. Roy. Soc., I90I, 13 69, 206; ib., 72, I52 (higher apes). HITzIG, Brit. Med. J., Dec. 1, Igoo. LEYTON and Sherrington, Q. J. Exp. Phys., I9I 7,11 , 135 (excitable cortex of chimpanzee, orang-utan and gorilla). RuSsele (J. R.). J. Phys., I 89-5, 17, 378 (eye-movements). Simpson and King, Q. J. Exp. Phys., igir, 4, 53 (sheep). Sснӓrer, J. Phys., I898-9, 23, 310 (sensory function). ZIEHEN, Arch. f. Phys., I899, 158 (velation between position and function in motor region).

Sensory Localization in Cerebral Cortex.-Dusser DE Barienne (J. G.), D. J. Exp. Phys., 1916, 9, 355; Arch. Néerland. de Phys., 1916, 1, I5. voN BECITEREw, Arch. f. Phys., I905, 53; 1912, 33; BLACK, J. Comp. Neurol., 1913, 23, I93; Hitzig, Arch. f. Psychiat., 35, 385; Sharker, Lancet, May 22,1897, I399; VitzoU, Arch. ie Ihys., I 893, 678 (visual centres). Franz, Am. J. Phys., 1911, 28, 308 (occipitallobes). Larionow, Pfliger's Arch., 1899, 76, 6os (auditory centre, music). MUNk, Zentralb. f. Phys., 9, $77^{\circ}$ (tactile sensibility).

Fatigue of Nerve Cells (Centres).-CARLSON, Am. J. Phys., 1903, 2, 3 f I (retina). Dolley, ib., 11909, 25, 151; J. Med. Res., 1909, 21, 95; ib., I 9 I I, 25, 285 (Purkinje cells); ib., I913, 29, 65. EVE, J. Phys., I \& $66,20,334$. Hodis, J. Phys., 1894-5. 17, 129 . Kociner, J. Am. Med. Ass.. 1916, 67, 278. Legendre and Piéron, J. Phys. Path. Gén., I9I1, 13, 519. Levr:, J. l'hys., 1900-1, 26, $210 ; i b ., 1903,28$, I. PUginet, J. Phys. Path. Gél1., 1901, 3, is 3 .

Sleep.-Brush and FAYERWEATHER, Am. J. Phys., 1901, 5, I99 (changes in blood-pressure). Brodmann, J. Psych. Neurol., 1, io; Zentralb. f. Physiol., 16, 3 10. GODDARD, J. Conip. Newrol., iso8, 8, 245 (movements of nerve cells). Howris, J. Exp. Ned., I897, 2, 313 (plethysmograms in). WALDes, Am. J. Phys., 19oo, 4, I 24 (hypnotic sleep).

Cerebral Circulation.-Brown (E. D.), J. Pharm. Exp. Ther, Io) 6, 8, I85. CANNON (W. B.), Am. J. Physs., I go 2, 6, 9 I (cerebral pressure after trauma). GRƯNiBAUM and SHERRINGTON, Brain, 25, 270 (apes). IHILL (L.), Phil. Trans. Roy. Soc., 1900, B 193, 6) (ligation of cevebral arteries). MotT 
and HiLt, J. Plives, isys-y), 23, p. xix; ih., lyon, 34, p. iv (histological

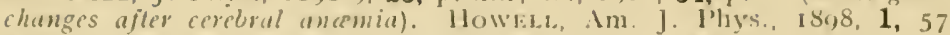
(effect of high arterial pressure). LikaMl:k, J. lixp. Iled., I912, 15, 348 (function of circle of 11 illis). Jexsien, Pfliiger's Arch., 1905, 107, 81.

Resuscitation of Central Nervous System, PIKE, (iUTukIE and STEWART, 3. Exp. Med., Hos, 10, t90; Am. J. Hiys., apos. 21, 3.50 (reflex excita-

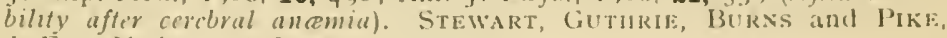
J. Exp. Med., Loo6, 8, 2So.

Chemistry of Central Nervous System. Cirs., J. Biol. Cl., Ig07, 2, I 59 (cerebron). HATAI, Anı. J. PHos., Juol, 12, is (effect of partial starvation).

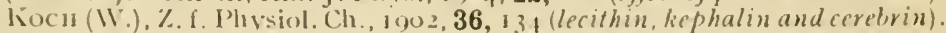

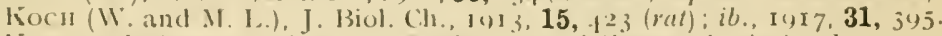

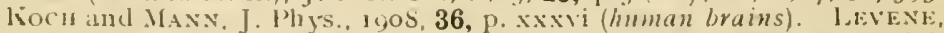
1. Biol. Ch., I912, 13, f63 (sulphatide). LEVENE and WEst, J. Biol. Ch.. I9I 7, 31, 635, (4), LEVENL, ib., I913. 15, 359 (cerebrosides); ih.. I9I6, 24, I (kephalin). Rosexherm, Bioch. J., I9I3, 7, 604; ib., I9I , 8, IIO (galactosides). ГЕВß, J. Phy ys., I906, 34, Iо6 (cholesterol).

Cerebrospinal Fluid.-Blumextial, Ergeb. d. Phys. (Bioch.), 1902, 285. CARTER (WV. S.), Arch. Int. Med., I9I 2, 10, +25 (intraspinal injection of ringer's solution). DIXON and HALliBURTON, J. Phys., I9I3. 47, 2 I 5 (secretion); ib., I 9 I 4, 48, I 2S, 3 I 7 (pressure); ib., I 916, 50, I 98 (circulation). FRAZIER and PEET, Am. J. Phys., I9I $4,35,268$ (formation and circulation); ib., IGI5, 36, fot. Kiraner (S. P.), New York Med. J., Mar. I6, I912 (circulation). SPInA. Pfliger's Arch., I 899, 76, 204; ib., I900, 80, 370; $i b .$, I 90 r, 83, I 20, fi5. Thomson, Hall and Halliburton, Proc. Roy. Soc., B 64, 3+3 (man). WEED and Cushise, Am. J. Phys., I915, 36, 77.

Chemistry of Cerebrospinal Fluid.-Carlson and MARTIN, Am. J. Phys,, rgi I, 29, 64 (pituitary secretion in?). FELTON, HUSSEY and BAYNE-JONES, Arch. Int. Med., I9I 7, 19, Io85. Fine and Myers, Soc. Exp. Biol. Med., I9I6, 13, I 26 (non-protein N). HALverson and Bergeim, J. Biol. Ch. I9I7, 29, 3.37 (calcium). Hurwitz and Tranter, Arch. Int. Med., 1916, 17, 828. KAHN and NEAL, Soc. Exp. Biol. Med., ig16, 14, 26. Kramer (S. P.), Brain, igi I, 34,39. Krause and Corveille, J. Lab. Clin. Med., I916, 1, 685; Nawra Tski, Arch. f. Phys., 1897, I 56 (sugar in). MYYeRs, J. Biol. Ch., 1909, 6, I I5; Rosexbloon, Arch. Int. Med., I9 I 4. 14, 536 (potassium). Weston, J. Med. Res., I9I 7, 35, 367 (reaction). WWoods, Arch. Int. Med., I915, 16,577 (nitrogen).

\section{CHAPTER XVII.}

\section{THE AUTONOMIC NERYOUS SYSTEM.}

Autonomic System.-ANderson (H. K.). J. Phys., 1902, 28, 499 (regeneration of sympathetic). Brown and SherRington, Q. J. Exp. Phys., igri, 4, I93 (pilomotor system). Burton-OpITz (R.). Am. J. Phys., 19I6, 41, I03; ib., I9I $7,42,+98$ (depressor function of thoracic sympathetic). DASTRE. and MORA T, Arch. de Phys., I S82, 337 (vasodilatators in cervical sympathetic). EDWARDS (D. J.). Am. J. Phys., I914, 33, 220 (sympathetic nevvous system in the turtle). Gaskell, J. Phys., 1886, 7, 1. Herring (P. T.), J. Phys., 1903, 29, 282 (spinal origin of cervical sympathetic). LAXGLEY, Ergeb. d. Phys. (Bioph.), 1903. 818 (autonomic sy'stem of vertebrates). LANGLEX, J. Phys., 1894-5, 17, 296 (secretory and vasomotor fibres of cat's foot): ib., I 896, 20, 5.5 (medullated fibres of the sympathetic system). LAxGLEY, J. Phys., I899-1900, 25, th) 8 ; $i b$. I904, 31, $2+4$ (commissural fibres in sympathetic sy'stem). LA.NGLEY and ANDERSOX, J. Phys., I895-6, 19, 7 I. 372 (innervation of pelvic and adjoining viscera); ib., 17, I77 (hypogastric neves). Laviley and Orbeli, J. Phys., I9IO, 41, 450; ib., I9II, 42, I 3 (sympathetic and sacral autonomic system of amphibia). MELTZER (S. J. and C..). Am. J. Phys., 1903. 9, 57 (r'asomotor neres of rabbit's ear). 


\section{CHAPTER XYII.}

\section{THE SENSES.}

Helmalotz, Physiologische Uptik. Helmoltz, Tonempfindungen (translated by Ellis). Mïllek. Ergeb. d. Phys. (Bioch.), 1903, 26) (psychophysical methods).

\section{VISION.}

Eye Liquids.-Hexderson (E. E.) and Starling, J. Phys., 1904, 31, 305 : Proc. Roy. Soc., I9or, B 77, 294 (intraocular pressure and secretion). HuL and FLACK, $i b, 1912, \mathrm{~B}$ S5, 439 (secretion of aquenus). WESSELY Ergeb. d. Phys.. 1905. 5h,5.

Accommodation.- BARRETt, J. Phys., I855,6, 46. BeER, Pflüger's Arch. $\mathrm{IS}_{44}, 58,523 ; i b ., 1897,67,5+1 ; 69,507 ;$ Science, Nor. 18, 1904. ErxTHoves, Ergeb. d. Phy's. (Bioph.), 1902, 680. Hess, Arch. f. Ophthal. 42, 288 (velocity of accommodation). SCHOEx, Pfliger's Arch., I895, 59, 427. Sxellex, Ergeb. (l. Phys. (Bioph.), 1904,339 (skiascopy). Stlart (T. P. Astdersos), J. Phys., 1904, 31, 38. Tschersisg, Arch, de Phys., 1802, 158;ib. 1894, 40; 1895, 158, 181; J. Phy's. Path. Gén., 1899, 312.

Iris. ANDERSOX (H. K), J. Phys., Iyoh, 33, : $55^{(1,}+4_{4} ; i b ., 1904,30,15,290$; MELTZER and AUER, Am. J. Phys., 1904, 11, 28 (paradoxical dilatation of pupil). COBB (P.). Am. J. Phys., 1914, 36, 335 (pupil diameter and viswal acuity). Guthrie and RYAx, Science, 1910, 31, 395 (effect of asphyxia on pupil). GRU'NeRT, Zentralb. f. Phys., IS99, 12, 406. SCrü̈FER, Q. J. Exp. Phy's., 1908, 2, 287 (dilator pupilla in man).

Eye Movements and Innervation.-Dodge, Am. J. Phys., 1903, 8, 30 HofMas., Ergeb. d. Phys. (Bioph.), I903, 799; 1906, 599. SHERriNGTON. J. Phys., 1896, 17, 27; ib., 1916, 50, p. xlvi (apparatus to illustrate I.isting-Donders la $\left.i^{\prime \prime}\right)$. Zотн, Nagel's Handbuch der Physiologie.

Retina.-Dittler, Pflüger's Arch., 1907, 117, 295 (contraction of cones). Ferree nd Raxd, Am. J. Phys., 1912, 29, 398; Ha.ssell, Philadelphia Iled. J., Oct., I899; HAYCRAFT (J. B.), J. Phys., Ig10, 40, 492 (blind spot). LACRExS and Williams, Soc. Exp. Biol. Med., I9I6, 13, I83 (movements of retinal elements). TRENDELENBCRG, Ergeb. d. Phys., 19II, 1. TSCHERMAK, Firgeb. d. Phys. (Bioph.), 1902, 605 (functions of rods and cones).

Colour Vision.-Calkivs, Arch. f. Phys., 1902, Supp. Bd., 244. Edridge. GREex, J. Phys., 1915, 49, 265. EXNer, Zentrall, f. Phys., 1903, 17, 24 (Young-Helmholtz system). Fraxkin (Mrs. C. L.), Z. f. Psych. u. Phys. d. Sinn., 1892, 4; Psychol. Review, 1894, 1890, 1899 . HARTRIDGE, J. Phys., 1912, 45, p. xxix (yellow sensation). HERIxG (E.), Pfliger's Arch., 1888, 42, 330.

Contrast-After-images.-Edridge-Greex, J. Phys., 1912. 43, p. xxviii ; 45. p. xix (simultaneous colour contrast); ib. I01 3,46, i $80 ; 47, p$. i. (after-images). HARTRIDGE, J. Phy's., I1915, 50, t7 (contrast). TSCHERisk, Ergeb. d. Phys. (Bioph.), 1903,726 (contrast and irradiation).

Colour-Blindness.-Collix and NAGEL, \%. f. Sinnesphysiol., 1906, 41, it (violet blindness). Foridge-Grees, Nature, Feb. 10, 1910, p. +29 (tests); J. Phys., 1912, 43, p. xxxiv; Colour Blindness atnd Colour Perception, London. GRUNERT, Graefe's Arch. f. Ophthal., 56, 132. Hess and Herisg, P'fliger's Arch., I89s, 71, Ioj. Hess, \%. f. Psycll. u. Physiol. d. Sinn, 29, 99. VoN líries, ib., 32, 113 . Pedde, Trans. Roy. Soc. (Edin.), I896, 501. Pole (II.), Contemporary Review, May, IS82. SicHEXCk, Pflïger's Arch., 1907, 118, I29.

Intermittent Stimulation of Retina. CHARPENTIER, Arch, de Phys., I Sa6. $0 \rightarrow 7$ (retinal oscillations). SCHExick (li.), Plfüger's Arch, , 1uon, 112, 202. SHERRIXGTON, J. Phys., $1597,21,33$ (reciprocal action in retina). StEWART (G. N.), Trans, Roy. Soc. (Vidin.). July I6, ISSS, t45 (colow 
phenomena afteruards described jor Benham's (op). BinWLL.., P'roc. Roy.

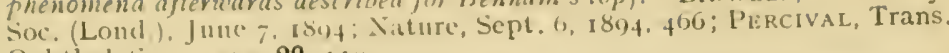
Ophthal. sic.. 140u, 29, 111 .

Talbot's Law. I31'R(11, J. J'hys.. I $898-9,23$, p. vii (and ficker). GrünBacm (O. F. F.). I. Phys., Iso8, 22, 43.3. MARB1, Pflïger's Arch., 1903, 9\%, 335. Pakkik and Patten, Am. J. Plyys, I912, 31, 22. Stewart (G. N.), Trans. Roy. Soc. (Edin.), July' 16, I 888, 14 I (for very short stimuli).

Heliotropism (Phototropism).-DAVENPORT and CANNoN, J. Phy', I897, 21,

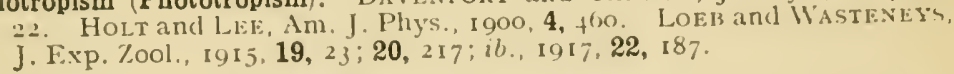

\section{HEARING, SMELL, TASTE.}

Hearing.-Bernoullli, Phïger's Arch., 1910, 134, 633. Ewald (J. R.), ib. 1895, 59, 258; ib., 1903, 93,485; WUNDT, ib., I895, 61, 3.39 (lativinth and hearing). EWALD, $i b ., 1899,76,1+7$ (sound picture theory). H1:NSEN, Ergeb. d. Phys. (Bioph.), I902, 847. Kolmar, il., I911,372 (endapparatus of eighth nerve and its significance). Ji. TER KUILE, Pfliger's Arch., I900, $79,146,484$; MEYer (N1.), ib., 1900, 81, 6I (theory of hearing). MckeNDRICK, Nature, June 15, I S99, I63 (perception of musical tone). PARKER (G. H.). U. S. Fish Conmission Bull., 1903, 45; Am. Naturalist, 37, I $\delta_{5}$ (hearing in fishes). WIтTEMAACK, Pfliger's Arch., 1907, 120, 249 (support of Helmholtz's resonance theory). ZWAardenaker, Arch. f. Phys., Ig05. supp. Bd., $12+$ (sound-pressure in Corti's organ as the stimulus).

Smell and Taste.-Cushixg (H.), Am. J. Phys., 1903, 8, p. xxvii; Gowers, J. Phys., 1903, 28, 300; Harris (IV.). J. Am. Med. Ass., I9I4, 63, I 725 (course of taste fibres). PARKER and STABLER, Am. J. Phys., 1913. 32, 230 (distinctions between smell and taste). WWAARDEMAKER, Ergel). (l. Phys. (Bioph.), 1902, 896 (smell); ib., r903, 699 (taste); Arch. f. Phys. 1907. Suppl. Bd., 59; ib., 1908, $5 \mathrm{I}$

\section{COMMON SENSIBILITY.}

Cutaneous Sensations.-Barker (L. F.), J. Exp. Med., I896, 1, I (localized sensory paraly'sis). BORING, Q. J. Exp. Phys., i 916,10 , I ; HEAD, Rivers and SHerren, Brain, 110, Ioo; HeAd and SHERrex, Brain, 110, II Trotter and Davies, J. Phys., I909, 38, I 34; (Brodmann's) J. f. Psych. Neurol., 1913, 20, 102 (section of culaneous nerves in man). BucH (M.), Arch. f. Phys., Igor, I. Lombard (W. P.), Am. J. Phys., 191 3, 31, p. xv; Stanley Hall and Albin, Am. J. Phys., 1897, 9, io (tickle-sense). CARr, Psychol. Review, 19x6, 23, 252 (Head's theory'). Elo and NicolaI, Skand. Arch. Phys., I910, 24, 26 (warmth sense). FRANz and RUEDIGER, Am. J. Phys., 1910, 27, t5 (effect of local anasthetics). Frasz, J. Comp. Teurol. Psychol., 1909, 19, I07 (pressure sense). VON FREY, Z. f. Biol., 191 3-I4, 63, 207, 3.35; J. Am. Ned. Ass., 1906, 47, 145; Frgeb. d. Phys. 1913, 96; $\mathrm{MAY}^{2}$ (P.), ib., 1909, 657 (culaneous sensations). NAcieL, Handbuch d. Physiol., 3, 703 (tickling and itching): YeRkes (R. I1.), Pflüger's Arch., 1905, 10\%, 207 (facilitation and inhibition of tactile stimuli in the frog).

Sensibility of Internal Organs.-HERTz, The Sensibility of the Alimentary Canal, igI I. Kast and Meltzer, Med. Rec., Dec. 29, I go6.

Muscular Sense--vox Frey, Z. f. Biol., I914, 63, I29; 64, 203. Lashley, Am. J. Phys., I917, 43, I 69 (accuracy of movement in absence of excila.
tion from moving organ).

Localization of Sensations.-BRowx (T. G.) and StEWART (R. M.), Brain, I $916,39,348$. LANGSTROTh, Arch. Int. Med., I9I 5, 16, I 49 (referred pain. Head's zones of hyperalgesia).

Hunger and Appetite.-CANxon and Washburs, Am. J. Phys., I9I , 29, $+t^{1}$; Carlson (A. J.). Am. I. Phys., 1912-1 3, 31, 151, 175, 21 2; ib., 1913. 32,$369 ; i b ., 191+, 33,95 ; 34,155$ (nervous control of hunger contractions). CARLsur, Control of Hunger in Health and Disease, Chicago, 1916. 
CARLSON and LEWIS, Am. J. I'liys., r91 $4.34,1+9$ (influence of smoking). CARLSOX, ORR and ICGRATH, ib., 33, I IO. (IINSBLRG, TUMPOWSKI and CARLSOX, J. Ams. Mled. Ass., 1915, 64, I $\$ 22$ (hunger in injants after feeding). LUCKHARDT, Am. J. Fhys., I9I 5, 39, 335 (dreaming). LickHARDT and CARLSOS, ib., IU1 $4,36,37$ (chemical conirol). MEYER and CARLSOX, ib.,

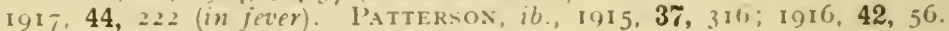
ROGERS and HARDT, $26 ., 1914,38,274$

CHAPTER XIX. REPRODUC'TION.

Buriax, Ergeb. d. Physs. (Bioch.), 1904,$84 ; i b ., 1901,-68$ (chemistry of spermalozoa). Marshall (F. H. A.), Physiology of Reproduction, Lumclon, 19 Io.

Ovary.-Carmichare and Marshall, Proc. Roy. Soc., 1907, 13 79, 387 (correlation of ovary and uterus). Guthrie (C. C.), J. Exp. Zool., igob, 5. $5^{f)} 3$ (transplantation of oraries in chickens); Science, Nov., Igorg (guineapig graft hybrids); ib.. I yI , 33, 8I 6 (influence on offspring of engrafled ovarian tissue). L.oEb (L.), Soc. Exp. Biol. Med., 1916, 13, I62 (cychc changes); J. Am. Med. Ass., 191 7, 69, 236 (velation of oz ary to uterus and mammary gland). Rosenbloom, J. Biol. Ch., I9I2, 13, 5 II (lipins of otary and corpus (uteum).

Corpus Luteum.-Axcel, J. de Phys. Path. Gén., I9I1, 13, 31. Bourx and AxCEL, ib., I910, 12, I6. ITAGaki, Q. J. Exp. Plyss. IyI 7, 11, I (influence of exiracts on plain muscle). Loeb (L.), J. Am. Iled. Ass., 19o6, Feb. Io, 1906 ; Anat. Record, I908, 2, z40. Marshall, Q. J. Exp. Micros. Sc., I905, 49, isg (development, review). Novak, J. Am. Med. Ass, I9I6, 67, I 285 . OTt and Scott, Soc. Biol. Exp. Med., 191 2, 9, 64 (extracts); ib., I914, 12, tj (action on mammary glands). PEARL and SURFACE. J. Biol. Ch., IgIt. 19, 263 (effect on ovulation).

Uterus.-BARRY (D. T.), J. Phys., I9I5, 50, 259 (uterus contractions and ovarian extract). CLSHXY, J. Phys., I go6, 35, I (movements). BARIBOLR and COPENHAVER, Soc. Exp. Biol. Med., I916, 13, 1.59 (cerebral control of uterus ?). lítroinowski, Arch. Internat. de Phys., ico $4.1,359$; Arch. f. Phys., rgoł, Supp. Bd., 323 (isolated uterus mozements). LoEB (I.). J. Am. Med. Ass., I908, 50, I897; Arch. f. Entwick. (1. Org., 1909. 27, 39 (production of deciduomata). Oтт and Scotт, J. Fxp. Med., Iyou 11, 326 (action of glandular extracts on uterus contractions).

Menstruation-CEstrus,-Hamoxid and MLarshall, Proc. Roy. Soc., Iyl 4 . $\mathrm{B} 87,+22$ (correlation between ovaries, werus, and maminary glands). HeApe, Brit. Iled. J., Dec. 24, I 898 (monkeys). Kisci(J.) Am. J. Phys., $1914.34,203$ (cardio-vascular and temperature variations in women). LokB (L.), Biol. Bull.. 19I4. 27, I (correlation betiecen cyclic changes in ulerus and ovaries). Marshall and Ruxcimax, J. Phys., I9I 4, 49, i 7 (ovary and eestrus). Marshall and Jolly, Phil. Trans. Roy. Soc., Inos. 198, $99($ dog $)$.

Fertilization.-Fischer and Ostwald, Pflüger's Arch., 1905, 106, 229 (physico-chemical theory). GorhaM and Towrer, Am. J. Plivs.. I903, 8, I75. LOEB (J.). Pflüger's Arch., I904, 103, 257; ib., 104, $325 ;$ inis. of Calif. Pub., I903-7; Harvey Lecture, New York, 191 I; Am. Yaturalist, I $15,49,257$ (conditions determining entrance of spermatozoon); Arch. 1 . Entw. d. Org., I914, 11, 301; Science, Nor, 6, I9It (ultrariolet light). LyoN (F. P.), An. J. Plyys. I909, 25, 199 (catalase). ILoRGAX (T. H.), Science, Feb. IS, I SoS. Nèmec, Fertilization Processes, Berlin, 19 In. MAtheWs (A. P.), Am. J. Phýs, I902, 6, 216; ib., I907, 18, S9. RORERTsox (T. B.), Soc. Exp. Biol. Med.. I I 2,9 , go (öocituse); J. Biol. Ch.,

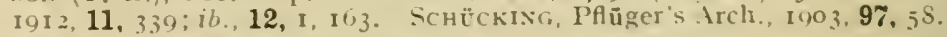

Artificial Parthenogenesis.-Fisciar (.M. H.), Am. J. Pliys., I q02, 7, 30I:

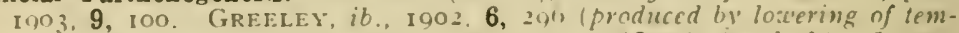

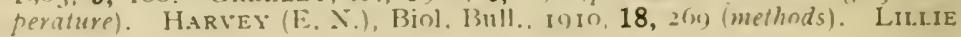


(R. S.), An. J. Phys. swo7, 18, p. xvi (by rasing temperature). Lotb (J.), In. J. Phys., Iono, 4, I 78,123 ; Dic Chemisthe kintwickelungserre-

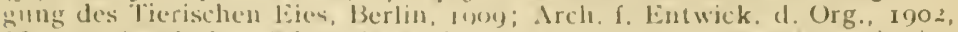

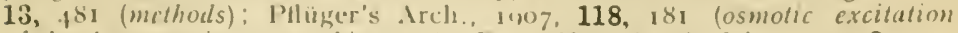
of development in seu-urchin cggs); Proc. Nal. Acal. Sci., Iynt, 2, 3 I 3 (sex of parthenogenetic frogs). MATuLW: (A. I'.), IIn. J. I'hys., Igou, 4, $3+3$; ib., 19oz, 6, 1+2 (by' incehanical excilation). McClexdos, Am. J. Phys., Iy1 2, 29, 29s (in vertebrates). Morciax (1. H.), J. Exi. Zoul., I904, 1, I35 (self-fertilization induced by urtificial means)

Sex. Doxcaster, Determination of Sex, Cambridge, IgI 4 . Ridde, Science, 1917, 46, 19. Wilsox (L. B.), Soc. Lxp. Biol. Mecl., Igo5-6, 3, 19.

Pregnancy.-Hasselbalci, Skind. Arch. Phys., 1912, 27, I (respirutory l.xchange). MLrLis, Am. J. Pliss., I010, 26, I3t (energy metabolism of pregnant dog); ib., 1910, 27, 177 (nitrogen bulance). MURLIx and BAILEY, Arch. Int. Med., I $4 I_{3}, 12,255$ (protein metabolism).

Abderhalden Reaction.-BOLdyefF, J. Phys., I917, 51, p. xxii. BRONFEx13RENNER, J. Lab. Clin. Med., I915, 1, 79. WELls (H. G.), ib., 1, 175. IVIllia.Ms and Pearce, Soc. Exp. Biol. Med., 1913, 10, 73. VAx SlykE Er AL., J. Biol. Ch., 1915. 23, 377.

Development of Egg.-Abderhaldex, Phlüger's Arch., 1915, 147, 99 (compounds which infuence development). BABAK. Pflüger's Arch., 1y05, 109, $7^{3}$ (central nervous system and metumorphosis of frog). BRow: (O. H.). An. J. Phys., I 905, 14, 354 (permeability of egg). EAvEs, J. Phys., Igio. 40, +51 (fat transformations in eggs in development). HARVEY. J. EXp. \%ool., I910, 8, 355 (membrane formation). Hultox, J. Biol. Ch., igi 6 , 25,227 (ferment production in response to introduction of placenta). HYDE (1. H.), Anı. J. Pliys, Igo 4, 12, $2 f^{\top}$ (differences of potential in developing eggs). Lrogh, Z. f. Allg. Phys., IgI 4, 16, 163 (temperature and rate of embryonic development). LOEB (J.), Biol. Bull., IgI 5, 29, Io3 (membrane formation). LOEB and WASTExEYS, Bioch. Z., I9I I, 3\%, for; J. Biol. Ch., IyI3. 14, 355, +59; ib., I9I5, 21, I 53 (infuence of bases on development and oxidations in sea-urchin eggs). Lillie (R. S.), Am. J. Phys., I916. 40, 2+9;ib., 1917, 43, 43 (permeability). Lyox, An. J. Phys., I9o4. 11, 5-(vhythms in cleavage). LyoN and SHAckelL, J. Biol. Ch., Ig09. 7, 37I. (antolysis of fertilized and unfertilized eggs). MATHEW's (A. P.), J. Biol. Ch., I9I 3, 14, 465 (chemical difference between sea-urchin and starfish eggs), McClexdox, Am. J. Phys., I912, 31, 131 (effect of alkaloids); ib., I915. 38, 163 (devclopment and permeability). MELTzER. Am. J. Phys., 1903, 9, 246 (effect of agitation). Mloore (A. R.), J. Biol. Ch., I9I7, 30, 5 (cytolysis in echinoderm eggs). MALcolı (J.), J. Phys., I9o 1, 27, 356 (composition of egg-yolk). MExdel and LEAVExworTH, Am. J. Phys., 1)08, 21, 77 (changes in purin, cholesterol, etc., content in development). IRIDDLE (O.), Am. J. Phys., IyI6, 41, fog (metabolism of egg-yolk in incubalion). RIDDLE and BASSETT, it., 41, 425 (effect of alcohol on size of egg-yolk). RoBertsox, Soc. Exp. Biol. Med., 191 2, 9, 61 (sea-urchin cggs). SHACKELL, Science, IgII, 34, 573 (phosphorus metabolism in cihinoderm eggs).

Metabolism of Embryo.-Joxis and A[srRiAx, J. Biol. Ch., t907, 3, 227 (muclein ferments). LOCHEAD and CRAMER, Proc. Roy. Soc., 1908, B 80, 263 (glycogenic changes in placenta and fectus). MEXDEL and LEAVENwORTH, Am. J. Phys, 1907, 20, 117 (glycogen in embryo). MExDEL and IITCHELL, ib., 20, \&I (inverting enzymes of alimentary tract in embryo); ib., 20, 97 (enzymes in purin metubolism). WELls and CorPer, J. Biol. Ch., 1909, 6, 469 (purin metabolism of foctus and placenta). ZuxTz and Hasselbalch, Skand. Arch. Phys., I900, 10, I 49 (CO production in embryo).

Placenta.-CHARRIx, Arch. Ae Phys.. I byS, 703 (transmission of toxins from fotus to mother). FAMULExER, J. Infect. Dis., I912, 10, 332 (transmission of immunity from mother to offspring). FENGER, J. Biol. Ch., 1917, 29, 
I") (composition). LEDERER and P'FIBRAM, I'fluger's Arch, IyIO, 134, 53 I (relation of placenta and mammary gland). LöB and 11 Icicus, Biocls. Z., toog, 22, 310 (enzymes). Lors (1.). J. Am. Med. Ass., 1you, 53, $1+71$ (maternal placentu und function of corpusluteum). ROSENHEIM, J. Plnys. $1000,38,337$ (pressor principles from placenta). WVRTHEIMER and MEYER, Arcli. de Phys., 1890, I93; 1891, 204 (exchanges between mother and fotus).

Parturition.-HeAly and Kastre, Soc, Exp. Biol. Med., 1912, 9, 48 (intcrnul sccretion of mammary gland as a factor).

Chemistry of Milk.-Milroy, Bioch. J., IgI5, 9, 217 (veaction). Olsox, J IBiol. Ch., I908, 5, 26I (proteins). OSBORNE and WAKEMAN, ib., 19I 5, 21, 539; ib., I916, 28, I (phosphatids). RAuDNITz, Ergeb. d. Phys. (Bioch.), I 903, I93. VAN SLYKE and Bosworth, J. Biol. Ch., 1 gi 4, 19, 73 (casein); ib., 19I5, 20, 135; ib., 1916, 24, 173 (goal's milk).

Human Milk.-Bosworth, J. Biol. Ch., 1915, 20, 707; Bosworth and VAN SLYKE, ib., I 916,24, I 87. HAMMETT, ib., I9I 7, 29, 381 (after parturition). Holt, Courtĩey and Fales, Am. J. Dis. Child., 19I 5, 10, 229 (salls). MEIGS and MARSH, J. Biol. Ch., 1913, 16, I47 (human and cow's). SikEs, J. Phys., 1906, 34, 404 ( $P$ and $C a)$.

Colostrum.-St. Exgle, Ergeb. d. Phys., I y11, 11. Kastle and Healy, Soc. Exp. Biol. Med., I9I 2, 9, 44; WoobWARD, J. Exp. Med., I897, 2, 2 I 7 (chemistry).

Secretion of Milk.-BASC1, Ergeb. d. Plyss. (Bioch.), 1003, 320.-CASPARI, Irch. f. Phys., I 899, Supp. Bd., 267 (source of milk fat). Bowex, J. Biol. Cin., I9I5, 12, I I (passage of food and falty acids imlo mammary glands). CAMPBELL (J.A.), Q. J. Exp. Phys., I9I 3, 7, 35 (chemistry of mammary gland). GAINEs, Am. J. Phys., I9I 5, 38, 285 (lactation). HAMmond (J.), Q. J. Exp. Phys., I9I 3, 6, 311 ; Hill and Simpsox, ib., 191 4-1 5, 8, I03, 377 (pituitary extract). HAMMETT and MCNeILE, J. Biol. Ch., I9I7, 30, I 45 (effect of placenta). Hill and Simpson, Am. J. J'hys., I9I4, 35, 361;ib., $1915,36,3+7$ (effect of piluitary extract). MARSHALL and lírkNess, Bioch. J., 1000,2 , I; PATON and CATHCART, J. Phys., 1911, 42, 17y; PORCHER, Arch. Internat. Phys., 1909, 8, 356 (laciose formation). MACKENzIE (lí.), Q. J. Exp. Phys., I 91 I , 4, 305; SCHÄFER and MACkENZIE, Proc. Roy. Soc., I911, 13 84, 16 (action of animal extracts). MIOORE and PARkER, Am. J. Phy's., 1900, 4, 231) (laclose formation). OTt and ScotT, Soc. Exp. Biol. Med., I912, 9, 63; Scil̈̈rer, Q. J. Exp. Phys., i 91 3, 6, I 7 (effort of pituilury and corpus luteum); ib., I915, 8, 379 (pituitary extract).

Correlation of Mammary Gland with Other Sexual Organs.-Bied and KöxIcistzis, Z. Exp. Patl. Ther., I9I0, 8, 358 (hormone which excites mammary gland in pregnancy). HEAPE, J. Phys., IgOo, 34, p. i. LANIClaypon and Starling, Proc. Roy. Soc, 1906, B 77, 505. Loeb (L.) and HesselberG, J. Exp). Med., 1917, 25, 285, 305 (corclation in cycle of uterus, ovaries, and mammary gland). O'DonoghuE, J.Phys., I91 1, 43, 1). xvi (corpus luteum and mamniary gland).

Growth.-CrameR, J. Pliys., I916, 50, 322 (the biochemical mechanism of growth). HATAI, Am. J. Phys., 1907, 18, 309 (rat). LoEB (J.), Science, I015, 41, I 69 (simplest constituents required for growth in an insect). ROBERTSON (T. B.), Anl. J. Pliys., 1915. 37, 1. 74 (pre-and post-natal growth of mail). OsBORNE and MENDEL, see Chapt. $\mathrm{X}$.

Anastomosis of Bloodvessels.-CARrel. (A.) and Guturie (C. C.), J. Mm. Med. Ass., 1906, Nov. I7, p. I6 45 (kidney transplantation); Surg. Gyn. Obst. I $906,2,266$ (veins). CARKEL, J. Exp. Med., I910, 12, I 39; ib., 1912,15 , $388 ; i b ., 16$, I 7 (aorta). GutHRIE, Heart, 1910,2 , I I 5 (survival of engrufted organs). CARrel, J. Exp. Med., 1yo7, 9, 226; Guturie, Am. J. Phy̌s.. IgO7, 19, 482 (hetero-transplantation of bloodvessels). Gurrure, Bloodvessel Surgerv, London, 1912. CARREL, Johns Hopk. Bull., 1906, 17, 231) (surgery of bloodvessels). Cilthrik, J. Am. Med. Ass., I908, 50, 1035. 
Transplantation of Oigans.-C, АкRLL, J. Exp. Med., 1910, 12, 140 (remote

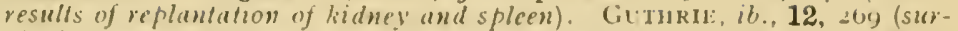
vival of engrafted ovaries and iesticles). Gunkik and LeE, J. Am. Med. Ass., 1915, 64, 1823 (ovarian transplantation). Genarit, Arch. Int. Med., Joro, 5, 23z (kidney, perfused or not). MURHuY (J.13.), J. Iixp. Med., $1913,17,482$ (iranspluniability of tissues to the embryo of foreign species); $i b ., 191+, 19,513 ; i b ., 19,181$ (ultimale fate of mammalian lissue implanted in chick embryo). Losis and ADDisox, Arch. f. Lintwick. d. Org., 1009, 27, 73 (hetero-plastic shin transplantation). LoEB (L.), J. Am. Med. Ass., 1915, 64, 725 (changes in chemical environment and growth of tissues); J. Med. Res., I9r7, 37, 229 (kidney tissue). (See also Chapter Xi.)

Tissue Cultures.-Battseld, J. Exp. Med., 1915, 21, 455 (origin of a fibrous lissue in cultures of adull frog lissues). CARREL and BURROW's, J. Exp. Iled., 1911, 13, 387; LAMHERT, ib., $1916,24,367$ (techrique); ib., 13, 416 (thyroid). CARREL. J. Exp. Jed. 1913, 18, 287 (connective tissue); ib., 17, If (activation of growth of connective tisste) ; ib., 1912, 15, 516; EBELING, $i b ., 1913,17,273$ (permanent life of tissues outside of the organismi). INGEBRIGTSEN (R.). J. FXXp. Med.. 1913, 18, 112 (regeneration of axis cylinders in vitro); ib., 1010, 23, 251 (life of peripheral nerves in plasma). LOEB (L.), Unte:such. über U mwandlungen 1 . Thätigkeiten in d. Geweben, Chicago, 1897. LOSEE and EBELING, J. Exp. Med., 191 4, 19, 593 (human tissue). LAlBt (R. A.). J. Exp. Med., 1913.18, to6 (infuence of temperature and medium on survival of embryonic tissue in vitro). LEWIS (MI. R. and W. H.). Am. J. Phys., 1917, 44, 57 (contraction of smooth muscle cells in tissue cullures). NEWMANN and Burrows, Am. J. Phys., 1917. 42, 597 (effect of amino-acids and peptones on growth of cells $i$ uitro). WALTON, J. Exp. Med., 1915, 22, 194 (production of substances inhibiting cell-growth); ib., $1914,20,554$ (effect of tissue extracts on growth in vitro). WEIL, J. Med. Res., 1912, 26, 159 (tissue cultures in vitro). UHLENHuTH, J. Exp. Med., 191 $5,22,76$ (skin); ib., I91 $4,20,614$

Parabiosis.- Rots, Soc. Exp. Biol. Med., 1909, 7, 8 (test for circulating anti. bodies in cancer). 


\section{N D E X}

* References to the Practical Excrcises are in black figures.

ABDOMINAL breathing, 230

inuscles in expiration, 229

Abducens, or sixth nerve, 926

Aberration, chronatic, $1027,1063,1106$ spherical, 1027, 1063, 1106

Absorption, $\$ 26$

and lipoid solubility, 437

coefficient of gases, 247

comparative phy"siolog! of, 43 I

factors concerned in, 435

from the peritoneal cavity, 439

from the stomach, 433

gas exchange in intestine, 436

in large intestine. $45 \mathrm{I}$

intra-and inter-epithelial. 446

of bile.constituents in jaundice, $38_{4}$

of cane-sugar, 436.464

of carbo-hydrates, 445

of fat, path of, $4+3,463$

of gases in blood. 250

of iron, 447

of light, roI 2

of proteins, $4+7$

of the food. 43 I

physical introduction to, 426

theories of, $\$ 33-436$

of water and salts, $\$ 22,446$

osmosis and diffusion in, 435

parenteral, $4+6$

Acapnia and blood-pressure, $1 \delta_{4}$

and mountain sickness, $29^{9}$

and shock, 192

Acceleration of heart by sipping water, 171, 210

Accelerator nerves of hcart, I57, I62, I 66, I97, 199

Accessory auditory nucleus, 928

dietetic factors, 617

vagus nucleus, 929

Accommodation, 1020

mechanism of, 1022

pupil in, 1023

Acerebral tollus, 942,955

Acetaldehyde, 544.562

Acetone. 562

and katabolism of fatty acids, 567
Acetone in diabetes, 529,555

in urine, 529

Aceto-acetic acid, 562, 567

in diabetes, 555

A.C.E. mixture, 63

Acetic acid. 556

Acid albumin, $352.45^{8}$

Acidity of gastric juice, 350,4 I 7

Acidosis, 24, 252, 555

Acrolein, 12

Action currents, $S_{23}, 5_{24}, 830,842$

and functional activity, 825

diphasic. 825

double conduction of, 792

electromotive force of. 827

in polarized nerves, $\delta_{3} 1$

in voluntary" contraction. 762

monophasic, 825

of central nervous systern, $8_{3} \varepsilon$

of eye. 839

of glands, 83 i

of heart. $58.533 \cdot 53.5,844$

of imuscle, 823.843

of phrenic nerves, 827

of skin, $S_{3} s$

of spinal cord. 838.849 .895

of vagus, 828

of veratrinized inuscles, 829

propagation of, 824

rate of propagation of varia.

tion, 827

reflex, 913

thcories of, 825

Adunkiewicz's reaction ot prutein, 8

Adaptation of digestive juices (n) food. $37 \pi, 398,+05,109.414$

of retina, $1047.105^{\circ}$

Addison's disease and adrenals, 655

Adenin, 451,592,593

Adequate stimuli, 901,1007

Adipocere, 564

Arliponsophilia. 563

Aririnal hodirs. 655 epinephrin cuntent of, 6,64 relatiun of, to coagulation, 45 secretory nerves of, $66 \mathrm{I}$ 
A'renal cortex. function of isis

Adrenalin, action of, o 1 artervisis; 66 , 216

on coagulation time. 45.056 on hcart. 655

on ncrve"endings, $18_{2}, 6.55$

on pupil. 656 .

on sympathetic. 65. 5 . 1005

on vaso-motors, 173. 179,655

on veins, is:

action of illall dor's of, 660

arterio-sclerosis, 060

artiticial. 665

a-say of, 453.656

hiological tests for, 46,656

chemistry of, 664

formation of. 665

furetion of, 657

glycosuria, 550

secretory influence of nerves on, $66 \mathrm{t}$

tdsorption, 430

terotonometer, 258

fisthesiometer, compasses, 1115

Frey's hair, Io\$o, I II 4

Afferent impulses, decussation of, 894

paths, 892 scheme of. 850

After-brain (myelencephalon), 850

After-images, 1055, I III

Agglutination, 30,7 I

by foreign serum, $7 \mathbf{I}$

Agglutininogens, 31

Agraphia. 970

Alanin, 2, 360, 574, 590

formation of dextrose from, 538

Alanyl-glycyl-tyrosin +49

Albinos, intravascular clotting in, +3

Albuminates or derived albumins, 9

Albuminoids, 2

Albumins, 2,9

heat-coagulation of, 9

in urine, $488,497,497.524,525$

Al bumoses, 3

action of, on blood-pressure, I 73,215 on coagulation. $37,+5$

in peptic digestion. 352

tests for, in urine. 525

Albumosuria. 489.525

Alcrohol. 630

action of, on respiratory centre, I9I, 631

on gastric secretion, 630

in diet, 630

poisoning, blood-pressure curve in,

I9l

precipitation of proteins by: 8

Alcohols, relation of, to carbohydrates, 3

Aldehydase. 272

Aldehydes. relation of. to carbo-hỵdrates, 3. $5+3.5+4.5+5.56 \mathrm{I}$

Aldohexoses. $5+3$
Aldorl, 562

Alsomileter.tosin

Alimentary cillal. anatoing of. 319 leigth of. 320

tinue of pasadye through. 331

glycusistia. 511,717

Alkali-albumin. :0, 46I

Alkalinity of blood, etc., titratalste. 25

Alkaptonuria, 4 \$3, 5 ki

Allantoin. excretion of. 596

Allantois, 1128

"All-or-nothing" law, I 54

Alloxurir bodies, $4^{S t}$

Alveolar air, partial pressure of gases in. 241,263

Amblyopia after occipital lesion, 966

Arnboceptors. 29,73

Amide-nitrogen in proteolysis. 360

Anuino-acetic acid, 2

Amino-acids, 1, 360. 352.+17,562

absorption of, $449 .+50$

changes in liver. $5^{\beta_{t}}$

chemical nomenclature of, 360

conversion of, into glycogen and dextrose, $536,538,590$

formation of. from tissue-proteins, 578

of urea from, $582,5 s_{4}, 586,588$, 624

in blood, 574

in liver diseases, 586

in phosphorus poisoning, 563

in urine, 481,485

metabolism of. $5^{32}$

synthesis of, by bacteria. 6 I 8

Amino-bodies, deaminization of, $5^{33}$

Amino-succinic acid. 360

Amino-valerianic acid. 360

Ammonia, action of, on muscle, 759

impermeability. of lungs for, 240

in proteolysis. 360

in the blond. source of. 585.588

after Eck's tistula, $5 S_{5}$

in urine. 480,521

reflex in hibition of heart by, rาo. 210

Ammonium salts, fomation of urea from. 584.585

sulphate, precipitation of protein. by: 8, 525

Amnesia. 970

Amnion. 1128

Amniotic fluid. I I28, I I 34

Amoeba, 6, 16, 732

Amoboid movements. 732

Amp ire, 725

Ainvlase, $3+5.3+6$

pancreatic, 35 S, 362,46I

salivary. $3 \$ 6,+17,455$

Amyl nitrite, action on pulse, 106 formation of methemoglnbin by. 53 
Anylulytic stage of gastric digestion, 34 i., $356,+17$

Amylepsin, $35^{8}, 362,461$

intluence of bile on. 370

Anabolic changes in living matter, 6

Anacrotic pulse, y 06

An.estluesia by A.C.k. inixture, 63 bychloral.217

by chloroform (Ciréhant's method).

\section{2}

by morplia. 63, 201

by pressure on brain, $9^{85}$

by urethane. 217

for animals, 63

Anaphylaxis, 32

Anastomosis of nerves, 975

of bloodvessels. I $1+5$

Anelectrotonus, $786,844,845$,

Angular gyrus and vision. 966

Animal board. 201, 214

Animal heat, 674

Animals, localization in different, 980

Anions, 429

Ankle-clonus, 9 Is

Annulus of Vienssens, 157, I62, I 79

Anode, +29.724

Anterior commissure, 922,893

lorn, cells of, $858,864,876$

connections of, 376

roots, 797,876

Antern.lateral ascending tract, $\$ 66,873$. 886 connections of, 873

descending tract, 867

connections of, $88_{4}$

ground bundle, 867

Antibodies, 3 I, 64 i

Antidiabetic diet, 55 \&. 556

'Antidromic' nerve impulses, I 8 I, 792. Bo7

Antiferments, $339,300,343$ in intestinal parasites, 390

Antigens, 3 I

Antikinase, $44.39 \mathrm{I}$

Antilytic secretion, 398

Antimony and protein metabolism, 563

Antiperistalsis, $330,332,421$

Antipyretics, 704

Antithrombin. $38,39,42,44$

Antitrypsin. 343.390

Antrum pvlori. 326.327

Aorta, effect of compression of, 204

Aortic insufficiency, effect of, on pulse, 106

noteh. 96

stenosis, effect of, on pulse. Io7

valves, $87,96,206$ and dicrotic wave, 104

Apex-beat, 20, 201, 207

Apex preparition of heirt, 14.3 .194

Aphasia, 468

Aphasial. Broca's, n7o
Aphasia, motor, 970

sensory. 970,972

subcortical, 972

termporary,, 772

Wernicke's; 971

Aphemia. 97 I

Apnckit. $28_{3}, 300$

vagi, 284

vera. 283

Apocodeine, action of, on vaso-motors, I 73

Appetite, rog?

juice, 1099

Aqueduct of Sylvius. 874

Aqueous humour. $5^{8}$ composition of, +66 secretion of, ro16

Arachnoid. 86 I

Arachnolysin, action of, on erythrocytes, 28

Arcuate fibres, internal, 873

Arginase, 587

Arginin, 360,587

Argyll-Robertson pupil, 1025

Arhythmia, respiratory, 287

Aromatic sulphates in urine. $4^{8}+5^{17}$

Arsenic and protein metabolism. 563

Arterial blood-pressure, amount of, 112

Arteries, blood-pressure pulse in. 109 structure of, 82

to insert cannule into, 63

tonc of, 185,918

Artery rings, action of adrenalin on, 216 ation of serum on, 46

Arterioles, resistance in, I 20

Arteriosclerosis, velocity of pulse in, ron

Artificial respiration, 202, 230 with oxygen, 204

Ascending degenerition, 866

Aspartic acid. 360

Asphria, $178,283,187$

condition of hituroglobin in. 5 I

effect of, on circulation, I>2, I87. 213

glyeosuria caused by, $5 t^{8}$

in the foetus, I 137

inlluence of, on blond-pressure, 213

Assimilation limits for carbohydrate. 540 . 551,637

Association areas. 973 centres, 962.973 fibres, 883.885

Asthma, spasmodic, and bronchial muscles, 287

Astigmatism, irregular. I028 regular, 1031, 1062, 1105

Astrospheres, I I 22

Atrio-ventricular bundle. See Auriculo. ventricular bundli.

Atropine, action of. on heart, I66, I99 on nerveecells, is z on pupil, 1026 
Atropille, action of, on saliviry secre. tion, 392, 396, 457

Attraction spliert. $5.851,1079$

in nerve-cells, 851

Auditury centre, 1,25, 060 nerve, 427. 10(1)

cochlear division of, 928

vestibular br.mch of, $1, \geq 8$

ussicles, 1000, 10(x)

path, scheme of. 927

Aucrhach's plexus, 319.330

Augmentatinn of heart-beat, 157, 172, I99

nature of. 167

primary. 150

seeondar 5, I 50

Augmentor nerves. effect of, on quiescent heart, 168

Aurd, 973

Auricular canal, 8 I

tibrillation, 15 I

llutter, 151

pressure curve, $9^{8}$

Auriculo-ventricular bundle. 81,135 , I 3 i. 147

pulse-tracings in disease of, $I+9$ junction, stimulation of, 198

1lode, $8 \mathrm{I}, \mathrm{I}+7$

valves, 89,206

11zoment of opening of, $9^{6}$

A uscultation of breath-sounds, 231, 304 of heart-sounds, 207

of lungs, 304

Auto-digestion of stomach. 389,465

Autogenetic theory of nerve regeneration, $80 \mathrm{I}$

Autolysis. 578

Automatic actions of spinal cord, 916

Autonomic nervous systein, 892, 1003 functions of, 1005

Avalanche theory, 785

Aviator's sickness, 299

Axial strand fibrils. 80 I

Axis-cylinder or axon, $781,852,855$

bifurcation of, $\mathrm{SO}_{2}$

fibrils in. 85 I

growth of, in cultures, 803857 .

Axon-retlexes, $400,792,913$

Babinski's sign, 9 I 5

Bacteria and digestion, 422,423

in faces, 424

in intestine, $3+3,423.618$

synthesis of amino-acids by, 6 Is

Bactericidal action of gastric juice. 356

Balloon ascents, deaths in. 293

Baryta. absorption of carbon dioxide by. 240

Basal ganglia. 932 metabolisın, 686

Basilar membrane, 1067, 1070

Bat's wing, contractile vessels of, $S$ I, I 80
B.tteries, 197, 724

13ials (hearing), III3

l3eamonot on digestion. 349

Burhle.rews muchens, y 28

13.ckmanu's apparatus, 427.529

Bu llows recorder. 302

13e-ll's experiments on uerver-touts, 8nt

Belt recorder for respiration. 233

Beneelict estimation of blood sugat, 717

Benzoic acid, 48 I, 580

Beri-beri caused by polished rice. 632 thynus in, 633

Bert on double conduction in nerves. 702 on effects of oxygen at high pressure, 297

Bety cells. $8_{4} 8,875,959$

Bichromate cell, 197

Bidder's ganglia, If I

Bilc. 363-370 absorption of, 384,412 acids, 365,462 circulation of, $4 \mathrm{I}_{2}$ formation of, $3_{4}$ Hay's test for, 462 Pettenkofer's reaction, 366,462 adaptation of, to food. 4 I 3 and absorption of fat. 269 and pancreatic juice, adjuvant action of. 367

and surface tension. 369.462

as an excretion, 424

composition of, 363

curve of secretion of, 413

digestive functions of. 367

formation of, $3^{8} 4 \cdot 3^{85}$

freezing-point of. 387

gases of, 267.366

in emulsification of fats, 367

influence of nerves on secretion of, 4 II

inhibition of heart by, I72

mucin. 364

pigments, $364.385,462$

circulation of, 412

Gmelin's test for. 365,462

production of, in liver. $3^{85}$

relation of, to spleen, 672

precipitation of gastric digest by, 370

quantity of. 367

rate of secretion of, $+13,+19$

reactions of. 462

reinforcing action of, 368

sults, 365

artion of, on blood, 28, 70

decomposition of. 365

in urine. Hay's test. 528

influence of, on secretion of bile. $4^{13}$

secretion of, influence of nerves on, 4 I I

influence of secretin on, 408,412 
Bile, secretory pressure of, 414 spectrum of, 365

Biliary fistula, 369,412

Bilirubin, 364

Biliverdin, 364

Bioplasm. See Protoplasn, and Living matter

Bipular ganglion cells. $\times 5$ (s

Bird's blood, coagulation of $36,+3$

Biuret reaction. 8, 459

Bladder, 5 Io pressure in, in micturition. 510

Blastoderin, i I 25

Blind spot, 1044, :064, 1107 mapping the. 1064

Blond. bird's, $36^{\circ}$ carbon dioxide content of, 26,255 chemical composition of, 47 circulation of, 80 coagulation of, 33.62 composition of, 49 -corpuscles, coloured, composition of, 50 osmotic resistance of. 73

crenation of, 16

destruction of, 2 I

dextrose in, 50, 540.717

enumeration of. 18,67

formation of, in embryo. 20

gaseous metabolism oi. 252

life-history of, 20

in pernicious anamia, 21

red, 15

destruction of, 2 I origin of, 20

rouleaux formation of, 16 shadows or ghosts of, 70 size of, 15

structure of, 15

white. See Leucocytes

distribution of, 56,1 is

- electrical conductivity of, 26.68

flow, calorimetric method of mea. suring, 122

in different organs, 127

in feet, 127

in hands, 126 measurement of, 219

functions of. 50

gases of, $245 \cdot 266$

cstimatim by ferricyanide, 250 quantity of, 250

teusion of. $25^{8}$ results, 26 I

guaiacum test for, 76

kinetic and potential energy of cir. culating, I in

laking of. 70

measurement of velocity of, 120-124 morphology of. 14

opacity of. 70

pigment. microscopic test for. 78
Blood pigment, preparation of, 73

plasma, proteins of, 47,572

relative volume of, 27,68

plates, 18

platelets and coagulation, 39, 40

anticoagulants and preservation of, 40

disintegration of, 40

functions of, 62

precipitin test for, 31

quantity of, 55

in lungs, 224

which may be lost, 19I

reaction of, 24,62

regeneration of, 22

relative volnumes of corpuscles and plisma, 27,68

serum, electrical conductivity of, $26,27,68,356$

freezing-point of, 386

specific gravity of, 26,62

sugar in, 47, 499, 502, 533,540, 547

$549,551,552,636,657,717$

temperature of, 680,711

testing for adrenalin in, $656,65 \%, 662$

tests for, $45-47$

titratable alkaliuity, 25

vaso-constrict or property of shed, 45 velocity of, 11 - 127

in arteries, I 19,125

in capillaries, 120,130

in veins, 120,134

measurement of, $120,122,219$

vessels, suturing, 1144

viscosity of, 23, 502

volume of corpuscles and plasma 27,68

why it does not clot in the vessels, 45

Blood-pressure, arterial. measurement of, IOT

and acapnia, I 84

curves with elastic manometers, 93, I11

with mercurial manometers, I 1 O I 1 2, 210

effect of clianges of posture on, r 90 , 213

of extracts of bone-marrow on, $6>1,6>3$

of freezing the cord on, i 84

of luamorrhage and transfusion

OII, InI, 214

of kiduey on, 6 ; I

of inuscular exercise on, I I 5, 213

of nervous tissue on, 673

of peptone on, 173,215

of pituitary on, 668

of protenses on, 173,215

of suprarenal on, $171,216,655$

of testicle on, 642

of thymus on, 645 
Blood-pressurc, estimation of the arterial, 210,213

lactors which madintain, $\mathrm{t}$ เ

fill of, in slex'p, ysito

fiydrontatic and hydrolvinamic clements, 1 (x)

in capillaries, $1,30,1,3$

influeuce of respiration on, $28, y$

in man, influence of exercise ( )11, 115 , measurement if, bu stethuscope, I I 3,213

in pulmowary arters, $1 \mathrm{It}$

in right and left ventricles, $t 16$, i 38

incan arterial, ron, 1 i 2

measuremest of, Iog, 2 I I

permanemt element iu, I I

systolic and diastolic, I 13

tracings, $165,184,186,187,188,191$, $210,213,216,237,279,250$

Blood-pump, $2+9$

Blood-serum, freezing-point of, 386

Bloud-supply, regulation of, I so

Bloodvessels, anastomosis of, $114+$

rhythunically contractile, si, I 75 , I so structure of, 82

tone of, Is 4,996

Bods, composition of, 6 or

Bohr, on blood-gases, 254, 261, 264

Bolometer, $675,78_{3}$

Bone-marrow and blood-formation, 20, 22

action of extracts of, $6,-1,673$

Bones, composition of, 601,621 infuence of pituitary un, 670 of testicles on, $6 f^{2}$

Boot' electiodes, 842

Brain, anæmia of, 986 chemistry of, 99 I circulation in, 959) condition of, isolated, 975 in sleep, 9 I 3

developinent of, $8+9$

functions of, 93 I

heat-production in, 689

influence of, on spinal reflexes, gro, IOOI

respiratory changes in volume of, 295

resuscitation of, 990

size of, at different ages, 988 and intelligence, 988

temperature of, 7 I 2

volume, respiratory variations in, 295

Bread, 720

Breast-feeding, superiority of, II fo

Breath, holding, 234

Brocd's area, 971, 974 aphasia, 970

Brouchi, 223

movements of, in respiration, 231 nerves of, 287
Bronchi,al breathing, 23 I

mon-clen, innervation of, 287

Browebuncupe, 2.31

Browni,all nution, 454

Brown-Siepuard's symdrones, sig.t

Brumerer's glandi, 380,38 ;

"Buffy" cuilt, 3y

Burdach's tract, $A 60$ commections of, 870

Burdon-Sindersun on negative variation, $825,82 f$

Burus, superficial, death from, 299

Buttronitk diet, $\$ 22$

Butyric acid, 56? fermentation, $\$ 23$

Cachexia strumipriv $ı, 648$

Cincum, nerves of, 332

Caffeine, $4^{81}, 583,594,632$ as diuretic, 50 ()

Caisson disisise, 217

Calcium and bone formation, 62 I deficievcy of, $62 \mathrm{I}$

relation of, to heart-beat, I52, 200

Calorimeter, air, 6-1) differential micm-, 680

respiration, $240,6-6,6-8,721$

Atwater $s, 6,6$

water equivalent of, 72 I

Calorimetric method for blood-flow in hands, 219

for vaso-motor reflexes, I 88,22 I

Calorimetry, 675, 72I

Campbell, visuo-psychic area of, 966

Cancer, gastric juice in, 350

Cane-sugar, absorption of, $435,445,464$ inversion of, $\mathbf{1 0}, 339$

by gastric juice, 356

tests for, I I

Canuula, three-way, 212

gastric, 459

to put into an artery, 63

trachea, 202

vein, 2 I 5

Capillaries, blood-pressure in, I $3 \mathbf{I}$

clianges of calibre of, 173

circulation in, $120,129,193$

pulse in, $\mathbf{r} 3 \mathbf{I}$

resistance in, $120, I 30$

total cross-section of, I30

velocity of blood in, I 30

Capillary electrometcr, $728,729,842$

Caproic acid, 566

Capsule, internal. See Iuternal capsule

Carbo-hydrates, absorption of, $\$ 45$

.nount of, in standard diet, $623,6 \geq 5$ assimilation limits, $540,551,637$

composition of, $\mathbf{1}, 3$

constitution of, 3

intermediary, metabolisın of, 542

in diabetes, 553

inetabolism of, 533 
Cirbu-hydrates,Molisch's test for, II necessity of, in mutrition, (10)7 pasisage of, through placenta, 113 I protein-sparing action of, 606 reactions of, 10

tests for, 10

tolerance after pancreatectomy, 637

Carbon balance-sheet, 618 ę)uilibrimu, 6I 8

dioxide, action of, on respiratory centre, $28 \mathrm{r}$

and blosd-flow in heart, $1>8$

distribution in blood, 255

estimation of, 240,305

excretion, influence of forced respiration on, 245

mechanism of, 264

in alveolar air, 241

in blood, condition of, 257

in $f(x \cdot t a l$ blood, 129

influence on hamoglobin, 255

in seruml, 255, 256

in renous blood, tension of, 261

partial pressure of, in alveoli, 261,264

in blood, 259, 26 I

in tissues and liquids, 272,273

production, Haldane's appara-

tus for measuring, 304, 305

pruduction of, in different animals, 244

in museular work, 242

in relation to body-weight, 244

in rigor, 270,777

tension of, in tissues, 272

wishing out of 245,283

monoxide method for quantity of blood, 56

necisary quantity in diet, 625

Carbonic acid and urea formation, $5 \mathrm{~S}_{9}$ oxide hitmoglobin, 53,74

Cardiac cyele, 85

death, 1 ;

impulse, 00, 208

nerves, 15\%, 162, 196, 198, 203

sound, , 6

sphincter, 320

Cardio-augmentor centre, 169

Cardiogram, 91, 207

inversion of, 01

Cardiugraph, 90, 208

(ardio-inhilotory and augmentor centres, 160 tome of, I 69

Cardionseter, 1.39

(ardioplonogram, 88

(ardiopuemmatic movements, 128, 303

Carmosin in mucle, 767

Cistill, 35. as adequate protein, 613
Cascinogen, 354, 719

Castraticu, coffects of, 640,642 (1) thymus, 644

Catalase, 76, 271

Catalysers, 339

Cathetrism, 76

Catheter, pulmoniry, 259

Cells, structure of, 4

Celhiluse, digestion of, 123

Central canal of cord, 841 , 86 I urey axis, $86 \mathrm{I}$

nervous system, action currents of, 838

arrangement of white and giey matter in, $86 \mathrm{I}$

development of, 84')

functions of, 887

gencral arrangenent of, 861

histological elements of, $850-86 \mathrm{I}$

localization of function iv, 1) $>5$

methuxls of study of, 848 structure of, 847

Centre for smell, 967

for taste, 068

of gravity of body, 946

thumb, 973

Centres, cardio-inhibitory and augmentor, I 69

heat, 701

'motor, of cortex, $100 \mathrm{r}$

Imusical, 967

of cord and bulb, 9 in

spusory, of cortex, 965-968

vaso-motor, I $82-185$

Centrifuge, 64

Centrosome, 5, 851

in the ovum, 1122

Cerebellar ataxia, 935

Cerebellum, anatomical division of, 942 commections of, 885,935

functions of, 1133

inferior peduncle of, 885

linealization of function in, 242-944

middle peduncle of, 886

peduncles of, $\$ 85$

structure of, 1/34

superior pedumcle of, 856

worm of, $874,885,1) 42$

Cerebral anxmia, $190,786,089,900$ stimulation of vagus after, 187

circulation, 989

cortex, and respiration, 276 clinical aud pathological ubservations on, 962

development of, $\$ 62$

functions of, 947

histological differentiation of, 1258

inhibition from, 954 
Cerebral curtex, lavers of, u 58

localization of function in, 45 I

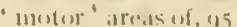

sencerty a:las of, 1, 105

st.1bility of reatetion $11,0,53$

homispheres, ixcision (1), 1) $47-(1)$ (1)

frog, 947,1000

pif(') (1) (1)

localization, flechsig's areats, 101 peduncle, 88.2

vesicles, sifi)

Cerphrins, 704,041

(eretrur-spinal fluid, $58,460,992$

lisplacelutent of, 295

secretion of, 90.3

(erebrum, effects of remoxal of, $\left(9+7^{-1}\right)+9$

Cervical svmpathetic. Sec Sympathetic

(halk-stones, 487 , 51)0

(herese, 626, 719

Chenical regulation of respiration, $28 \mathrm{I}$

'Chemical tone, 695

Chemiotaxis, 61

in nerve regeleration, 800

Cheyne-Stokes respiration, 287, 300

Chiasma, optic, 923

Child, food requirement of, 628

basal metabolisn of, 628,686

gaseous exchange in, 243

(hloral, andesthesia by, 190, 213

Chlorides, estimation of, 515

Chloroform anesthesia, inhibition in, I 70 passage of, through placenta, 1130

Chulesterol or cholesterin, $4,47,570,794$, () 9 I

circulation of, 571

in bile, $360,4^{12}$

reactions of, 463

Cholic acid, 365,366

Cholin, 4, 421,571

Cholohæmatin, 365

Chorda tympani, I 79, 391-393, 456 stimulation, of, 456

Chordo-lingual triangle, 39 I

Chorion, I 128

Choroidal epithelium, I016, $104^{8}$

Choroid plexus, 902

Cliromaftin cells, $66_{5}$

Chromatin, 5 changes in, in nerve-cells, $98_{3}, 984$ extranuclear, 984

Cliromogen, $4 \mathrm{~S}_{2}$

Chromophanes, ro4c

Chomosomes, 6, 1122

Chrisotoxin, action of, on bluod-pressure, 173

Chyle, I4, 58, 443 composition of fistula, 58

Clivine, 326 passage of, through pỵlorus, 327, 408

to obtain normal, 459

Cbymosin (i'ide Rennin), 353
Ciliil, 7.33

III)

wo)k (k)te by, 73.4

Ciliary gitnglion, 92.4

Imisele, 12. 1, 1022

111.1.1.5, 103.3

processis, $101+$

and secretion of aumeous liuแum 1016

Cincmatograph, 2.15, [1].2 2

Cireulating blond, nuicruscup)ic cxansinatii)!1 of, 193

liquids, 14

(ircul.ıtin)n, changes in, at birth, I 138

comparative, 80

cross, through brain, 284

gencral view of, 8 I

in brain, 980

in capillaries, 129,131

in the embryo, I 138

in the frog's web, I 5, I93

in the lungs, 137, 223

in the tadpole, I93

influence of posture on, 190, 2I3

of linuph, I92

time, I $35-138$

electrical method, I 35-I.37

Hering's method, 135

methylene blue method, 137 , 217

pulmonary, 137, 698

Circus movements, 945

Citrates and coagulation, 37

Clarke's column, 864

connections of, 872

Coagulated proteins, reactions of, 9

Coagulation of blood, 42, 62

action of fluorides on, 37

of citrates on, 37

birds, 36

calcium in, 36

factors in, 42

hirudin and, 37

influence of platelets in, 37 of proteoses on, 37 of tissue extracts on, 36

influences restrainiug, 42

intravascular, 42

leech extract and, 38

manganese and, 37

of crayfish, 40

of Limulus, 40

peptones and, 37

relation of adrenals to, 45

liver to, 44

studied with ultranicroscopc, 39

of 1 inuph, 57

temperature, to deternine, 9

Congulins, $+\mathbf{I}$

Coal-gas poisoning, 53. 74

Cobra-venom and coagulation, 43 
Cocame, action of, nit nerves, 791 on pupil, I026

$$
\text { fever, 7o. }
$$

Conclilea, $1227,106 \%, 1008,1009$

Cuchlear ront of eighth nerve, 874,928

Cicoa, 594, 630,63i

(o-cuzymes or co-ferments, $343,368,548$

cinffee, $594,631,632$

Cola-intit, 632

Cold sensations, 1041,1082 , 1084, III5 after section of cutaneuus nerves, 1088 , $109 \mathbf{I}$ paths for, 896

Collaterals, $-8 \mathrm{I}, 852$ of posterior ruot fibres, $S 7 I$

Colun, movenents of, 330, 333 innervation of, 332

Colustrum, I 139

Colour, body and surface, I0I2 blinduess, 1059, III 2 temporary, ro6I

mixing, 1052, IIII

triangle, 1054

vision, $105 \mathrm{l}$

Hering's theory of, I057

Young-Helmholtz theory of, 1053

Cinloured shadows, 1056

Colours, complenrent ary, I 052, I068, I I I I primary, I 053

Coma, curgestion of brain in, 985 diabetic, 555

Cimmna tract, 867,871

Commissural fibres, 863,893

(immnon path, principle of the, 899

Commutator, Pohl's, 732

Compensator, 727

Compensatory pulse of heart, 155,156

Complement, $2 S, 72$

Conplemental air, 235, 303

Complementary colours, 1052, II II

Condesised air, effects of breathing, 295

Conditioned reflexes, 975

Cunduction, double, in nerve, 791

irreciprocal, 792

isolated, law of, 793

loss of heat by, $68 \mathrm{I}$

Conductivity, molecular, $\$ 29$ of nerve, $-86,700$

anæsthetics and, $7 \% 0,8 \mathrm{I} 4$

effect of temperature un, 7 I

electrical currents un, 756 , $\tau$; 7,844

specific, of electrulytes, 429

congu-red as test for aciels, 35 I

Conjugate deviation, 96.5

Conjugated proteins, 2

Conservation of energy, law of, in budy, $6-5,68_{3}$

Consonants, 313

Contractiosi, formulit of, $7 \$ 8,845$ for nerves in silu, ; 9
C.meran tinn, law of, for human uerves, 846 paraduxical, $\$ 32,844$

secondart, 832,842

Contractions, superposition of, 756,816

Contrast (vision), s050

Co-ordination of movement- $y+5, y 51$

of reflexes, gos

Core models, and electrutunic currents, 831

Corneal, radius of curvature of, 1017

Corneal reflex, "st

Corona radiatit, $862,8,5,8 \$_{3}$ path from cortex in, 877

Corpora Arastii, $\$ 7$

quadrigeniua, $874,881,032$ and respiration, $2 ; 6$

anterior, 1)23, 433 pusterior, 428,432

striata, $850,862,870$ and temperature regulation, zor

Corpuscles and plasma, relative volume, 27, 68. Sec Blood

Corpus callosum, $863,88_{3}$

dentatum, 863

luteum, 643, I I 20, II 2 I

and menstruation, I 12 I

hæmatoidin in, 385

internal secretious of, 643,1078 , I 12 I

origin of, 112 I

striatum, $850,862,870$

Cortex of brain, functions of, 247

'motor' areas of, 1951

serisory areas of, 965

Corti, ganglion of, 927

orgals of, 927, 1067, 1069, 1074

Cortical epilepsy, 972

grey matter, 861

Costal breathing, 229

Cutton seed, 36I

Coughing, 288

Cranial conduction of sound, $1071,11 \mathbf{I}_{3}$ nerves, 920

bifurcation of afferent fibres, $924,925,926,928$

homologies of, 92 I

nuclei of, 920

Crayfish blood, clotting of, to

Cream, 718

Creatill, $48 I, 703$

Creatin and creatinin in protein meta. [ulisill, 597

in inuscle, -68

Creatinin, 481,523

excretion aud inusculat wurk, 6It

in fever, 703

source of urinary, 598

Crenatin, 16

Crista acustica, 936

Crosis circulation through brain, 284

Crossed pyramidal tract, $S 66,8,5,879$

connections of, $\$ 75$ 
Crowbar cace, Anerican, 971

Crura cerchi, $870,87+$

Cinstil, 8;0

Cultivation of tissues untsiele the buxly, I $1+2$

Cimeate funiculus, s6u uncleus, 860

relation of, to fillet, 874

Cuncus and visinn, 960

Cuorin, 57 r

Curara, i 82

action of, on gaseous excluange, 2.44 $5+1$

on heat production, 694, 702

on nerve-endiugs, I 52

on skeletal muscle, 730,8 I I

on voniting, 336

Curdling of milk by rennin, $353,354,459$, 7 ro

Current intensity and stimulation, $74 \mathrm{r}$, 784

of action. See Action current

of rest. See Demarcation current

Cushny's views on urine secretion, 502

Cutaneous burns, death from, 299

excretion, 511

ncrves, section of, 1085

respiration, 299

sensations, ro78

Boring's experinents, $109 \mathrm{I}$ cortical centres for, 969

localization of, $\mathrm{IOg}_{3}$

Trotter and Davies' experiments, ros5

Cybulski's arrangement (velocity of blood), 123

Crelic compounds, $615,616,618$

Cyclopoiesis, 6 I $_{5}, 6$ I6, 6 I 8

Cystein, 360, 365, 581, 582

Cysteinic acid, 366

Cystinuria, 366, 488, 579

Cytolysins, 31

Cytoplasm, 5

Cytosin, 593

Dancing mice, labyrinth in, 945

Daniell cell, r97, 724

Daphnia, Metchnikoff's researches on, 60

Dark-adapted ey'e, r 047 , I04'), I 058

Daturine, action of, on pupil. 1026

'Dead space,' respiratory, 2,36

Deaf-inutes, atrophy of temporal convolutions in, 967

equilibration in, 9.0

Decerebrate rigidity, 917, 942, 955, 1000

preparation, 998

Decerebrator, 999

Decidua, 1076, 1083 absurption of, by leucocytes, 60 artificial production of, 1078

Decinormal solutions, 479,522
D.cussation of afferent impulses, 804

of efferent impulses, 8y 3

if tillet, $8>0$

s) optic nerve, $, \geq 3,4,66$

if pyraunids, $869,875,878$

of sensory patlis, 89.4

D. fideation, 332

1) eficiency diseases, 617,632

phrinuenat after nervous !esions, 883

Degeneration of nuscle, 80.4 of nerves, 795,797 chemistry of, 798

of spinal roots, 797

reaction of, 804

Deglutition, 322

centre, 325

lierves of, 325

reflex, 1000

sounds, 324

'Deiters' nucleus, $88_{4}, 886,893,928$

Delirium cordis, 151,205

Denuarcation current, 823,842 electromotive force of, 826 theories of, 828

Dendrites, 852 anceboid movenents of, 852 and sleep, $9^{86}$

Dentate nucleus, $863,86 y, 886$ of olive, 860

Depressor nerves, I $70,173,185,186$ pressor action of, 188

reflex, reversal of, 904 in cerebral anituia, 158

Descending degeneration, $866,8>5$

Deutero-proteose, 3, 10

Developinent of embryo, I I 24 of ovum, 1122

Dextrins, 3, II $347,455,7$ I5 formed in salivary digestion, 347,455 tests for, II, $7 \mathbf{I 5}$

Dextrose, $3,47,338,347,455,525,5.34$ 537

estimation of, in urine, $\mathbf{5 2 5}$

in blood, $47,50,446,499,500,540$, 552

estimation of, 7 I7

in lymph, 446

ratio to nitrogen in urine iu diabetes, 55 I

tests for, I0, 488

Trommer's test for, 10

Diabetes, 546,552

dextrose-nitrogen ratio in, 55 I

diet iı, 554, 556

inellitus, 552

uxygen consumption in, 242

pancleatic, $546,554,636$

phlorhizin, 551, 717

reaction of blood in, 24

respiratory quotient in, 242

sugar-destroying power of blood in, 554 
1)i.1betic corma, 555

Diapedesis, 61, 194

Diaphragm in respiration, 226, 228, 275 recording mevements of, 2.33

1)iastases, 87, 3.4. $370,38.4,5.34$

l)iastase, salivary, 3.1.

1)iastole of heart, 87

Dichromatic vision, 1060 , I I I 2

Dicrotic wave of pulse, 104, 11 I

Dietaries, st:undard, $623,625,627$

1)ictetics, 622

Diet in diabctes, 554,556

Diffusion, 426 circles, 1021,1062 of gases, 246 through lungs, 264

Digestion as a whole, 416 and absorption, time required for, 464

bacteria and, 422,423

changes in acidity of gastric contents in, 4 I 8

clicmical phenomena of, $336-374$

comparative, 3 I 8

gascous exchange during, 243

heat-production in, $7{ }_{3}$

in intestines, 4 I8

in stomach, 46

mechanical phenomena of, 321

of carbo-hydrates, $356,3^{62}, 416$

of fats, $355,363,367,461,463,464$

of proteins, $351,35^{8}, 417,45^{8}, 460$

significance of, $32 \mathrm{I}$

time required for, 418,464

Digestive glands, microscopical changes, 374

juices, adaptation of, to food, 36y), $398,404,410,415$

process of formation of, $38_{3}$

protection of mucous menbranc from, 380

summary, 415,416

organs in different animals, 318

Digitalis, diuretic action of, 509

Dilator of pupil, 1025

Diopter, 1021

Diphasic variations, 825

Diphtheria toxin, action of enzymes on, 372

Diplopia, 924, 1037

Direct cerebcllar tract, 866,$8 ; 2,892$ connections of, 872

pyramidal tract, 866,$8 ; 5,8>8$

Disaccharides, 3,371 absorption of, 445

Jischarge of ventricle, periud of, 87,97 Dispersion in cye, 1028

Dissuciation of oxyhanoglobiu, 253,273

Diuresis ly salts, 509

Duretics, 500

Double concluction in nerve, 792

D)romograph, 122 bulcitc, 3

1)undenum, digestion in, 417 glycusuria after remes:al of, 6.10 reaction of contents of, \& 9

l) Ira inater, 861

1) $\mathrm{s}$ puc:a, 28,3

heat, 283,302

respiratory quotient in, $24 I$

liar, anatomy of, 1065

ossicles of, 1065,1060

functious of, $106 y$

resonance tone of, $8,5,-60$

lichidnase, 53

lick's listula, $385,5^{85}$

Ectoderm, 6, 1124

Ectoplasın, 4

Edestin, 607

tryptic digestion of, 36I

Effector organs, 898

Efferent inpulses, decussation of, 869 , $875,878,893$

paths of, 893

schelne of, 800

Egg-albumin, absorption of, 4.7 amino-acids in, I

excretion of, $448,500,7$ I 6

reactions of, 9

ligus, iron in, 622

Elurlich's triacid stain, I 7

lighth nerve. Sef Auditory nerve

lilasticity of muscle, 735

lilectric fishes, 840 sigual, 732

Electrical conductivity of blood, 26, 68 of gastric juice, 387

of injlk, I I 39 of serum, 69,386

urgin, 841

response. See Action current

lilectro-cardiogram, human, $8_{35}-838$

Electrodes, to make, 808 unpolarizable, 731,842

Electrolytes, 428

Elcctrometer, 727 capillary, $728,729,826,842$

Electromotive force, 725,827

Electrous, 429

Electrotonic alterations of excitability and conductivity, $,-56,844$

currents, 830,844

Electrotonus, $-85,844$

Eleventh nerve, 931

Eumbli, artiticial, 848

Embryo and uterus, connections be. tween, 1126 asplyxis in, 1137

circulation in, I 126,1 I 38

develupment of, 1122 , 1 1 26

formation of the, 1124

gases of blood in, 1120

Hly cogen in, 538, I I 31,1 I 33 
Einbryo, heat-proluction in, I 35 inverting 111/9mes in, 371

liver in, 1132

Inetalulisin of, 1133

physislegl of, 1125, I 129

Einetic: $336,459,464$

linnutropic ere, 1021)

limotims, genesis of, 1771

Emulsitication, 12, 367

Eumulsiu, 338, 3.40

Endocardiac pressure, 02,114 anomnt of, in ventricle, 92, 94 curves if, () $3,1,6,1) 7$ measurement of, 93 negative, Io I

Findocrine glands, 635

linduderm, 6, I 125

Eudo-enzymes, 337

Endingenous fibres of cord, $863,867, \mathrm{~S}_{97}$ metabolism of proteius, 573

Endoplatull, +

Endothermic reactions, 689

Enemata, 331, 451

Energy of food, influence of hydrolysis on, $6 S_{3}$

law of conservation of, in body, 679 , $6 \mathrm{~S}_{3}$

lingrafting, 641,1142

Enterokinase, $372,+15$

nature of, 37.3

Enzymes. Sec Ferments

Ependyma, 86 I

Eipiblast. Sec Ectoderm

Epicrit ic sensibility, $\operatorname{Iog} 1$

Epiglottis, 323

Epilepsy, cortical, 972

Jacksonian, 972

produced by absinthe,, 64

Epinephrin, 66, 173, 655. Sec Adrenalin function of, 657

indispensability of, 657

partition of, in blood, 659 , I I 49

secretion of, $66 \mathrm{I}$

testing for, 656

Equilibration and afferent impressions from muscles, 1) 4 I

from skin, $9+1$

and orientation, afferent impulses concerned in, 935, 936

cerebellum and, 934

Deiters' nucleus and, 929

in dog, 937

in dogfish, 938

in pigem, 937

muscular nerves and, 94 I

postural reflexes and, 1) 38

semicircular canals and, 928, 921, $936-9+1$

skin and, $9+1$

Lircction centre, 183

Erepsill, 37 I

Ergograph, $642,750,752,813$
Frgot and blond-pressure, 173

lirucic acicl, $55^{6}, 55^{8}$

irvtliroblasts, 21

Erythrocytes, 15. Sec Blood-corpuscles, redi

milumeration of, 19,67

Ealies of, $250-257$

lif(-) history of, 21$)$

Eirvthredextrin, 11, 347, 455, 7 I5

lisibach's methond of estinnting allumuin,

525

liserinc, action of, on accommodation, 1022

on pupil, I026

lither, action of, on blund-curpuscles, 28 , 21), 70

lithyl butyrate, synthesis of, by lipase, $3.3^{8},+47$

lindiometer, 249

linglobulin, $4^{8}$

Eustaclian tube, 297, 1065

ville, II.32

Evaporation, loss of heat $\mathrm{hr}, 682$

E.xcitalility, a property of living matter, 7 and conductivity, voltaic current and, 787,844

direct, of muscle, 738,8 I I

of nerve, effect of temperature on, 784,790

electrical currents on, 758 , 785,844

Excitable tissues, the, 724

Excretion, 475

Exogenous metabolisun of proteins, 573

Exothermic reactions, 689

Expectoration, 475

Expiration, 226, 229

duration of, 234

forced, 230

Expired air, composition of, 239-241, 305

Extensibility of muscle, 736

Extension reflex, crossed. 902, 997, 998

Extensor thrust reflex, the, 901

Extero-ceptive reflexes, 014

Extra contraction of heart, 155

systoles, in man, I 55, I 56

Exudation, inflammatory, 61

Eye, action currents of, 83 ? artificial, Kïlne's, I 104 clemistry of refractive media, ror 6 compound of insects, $1_{0} 13$

defects of, 1027

developunent of, $S_{5} \mathrm{O}$

dissection of, I IOI

extriwsic muscles of, 1063

licyuids, 1 oI 6 secretion of, 1016

movenuents of, 1062

nerves of, 1023 , 1024

optical constants, IOI 7

pupillo-constrictor fibres, I023, II I 0

dilator fibres, I023, I I IO 
liye, reluced, rorg

refraction in, sor 7

retinal fatigise, I055, III2

structure of, $10 \mathrm{I}$, I IOI

visual acuity, IIII

Facial nerve, 926

union of, with accessury, 976 palsy, 926

Facilitation of reflexes, ")nin

ill motor curtex, 054

Yixces, action of extracts of, $\$ 24$

hacteria in, 424

composition of, +23

microscopical examination of, 463

udour of, +24

lasting men, metabolism in, 604

lunger sensations in, 1006 6

Fat, absorption of, 44,463

influenced by bile, $36 y$

smount of, in staildard diet, 623,625 , 627

chemistry of, 556

composition of, I

cxcretion of, into intestine. $4+3$

formation of, from carbo-hydrates, 560

protein, $56 \mathrm{r}$

intermediary metabolism of, 565

ucling-point of, 12

metabulism of, 556

liver and, 567

migration of, $557,563,568$

in phosphorus polsoning, 563

mobilization of, 565

non-mutritive function of, 568

passage out of, intestinal epithelium, $4+2$

storing of, 558

synthesis of, in intestinal mucosa, $4+3,444$

Fatigue, changes in nerve cells, s59, 983

influence of, on muscular contractions, 749,813

on muscle-curve, 813

muscular, cause of, $75 \mathrm{I}$

of muscle-nerve preparation, seat of cxhaustion in, 752,813

seats of, in voluntary contraction, 753

Fats, cullstitution of, 3,556

tests for, II

Fatty acids, absorption of, $4+3$ and glycogen formation, $53^{8}$ decomposition of, in body. 566 formation of, from carbu-hydrate, 561

sinthesis of, to fat, 444 tests for, I 2

Fechner's law, i ioo

Fcbling is solution, 526
Fohling's sulution, Benedict's muditica. tion of, 527

test for sugar, 525

Ferment action, quantitative estimation of, 342,453

cellulnse-di-iolving, 423

mude it action of, 339,341

reverible actinn of, 339

Fermentation, butyric acid, 423

lactic acid, 423

Ferments, 336

autolytic, 599

in the liver, 600

intracellular, 337

list of, 345

specificity of, 340

Fever, aseptic, 7o

caused by cocain, 704

by xanthin, 707

changes in urine in, 703

derangement of heat regulation in, 705

metabolism in, 706

nervous, jo8

production of heat in, 704

'puncture,' 7or

'retention theory,' 706

significance of, 708

vaso-muturs in, 706

Fibrillary contraction, 151, 205

Fibrin-ferment, 34. Sec Thrombin

formation of, 35

preparation of, 65

librinogen, 34

production of, in liver, 35

Fibrino-globulin, 47

Fick's theory of fusion of visual stimuli, 1050

Fillet, $8 \%+$, sit

Flavour, Io-s

Flechsig's developmental cortical zones, $06 \mathrm{I}$

Flexion reflex, yo3, 997

Flour, 7 I9

Flow of liquids, with intermittent pressure, is

Fluoride plasma, clotting uf, $3^{\mathrm{s}}$

Flnorides, action of, un coagulation, i), 64

F' (1'tal heart, I I 35

Folin's method of estimating ammonia. 52 I

creatinin, 524

indican, 518

uric acid, 523

Fond substances, 320

Fond-, isudynanic relations of, -73 specific dynanic action of, 0.06

Foramen of Monro, $\$ 50$

Forced movenuents, 944

lore brain, 350

Formaldehvde reaction for protcius, 8 
Formatio reticularis, 870

Formic acid, 556

lormula of contraction. Sie Law of contraction, 845

Freezing-point, determination of, 529

Frey's asthesiometer, I080

Frog, heart of, anatony, 194

to pith a, 193

vagus, dissection of, 196 stimulation of, 198

web of, circulation in, I93

Iunction, localization of, in cortex, 975

Jiundus of stomach, 326

Funiculus cuneatus, 869 gracilis, 860

Gall-bladder, 411,419 reflex contraction of, 412

Gall-stone, pain caused by, $90 \mathrm{r}$

Galvani's experiment, 822,842

Galvanometers, 726 string, 727,836

Ganglia habenulax, 933 of posterior roots, development of, 850

Ganglion cells, sympathetic, 856

spirale, 927

vestibulare, 927

Gaskell's method (heart), I95

Gas-pump, 249

Gaseous exchange in lungs, mechanism of, 263

Gases, absorption coefticient of, 247

diffusion of, 246

of blood, 245

extraction of, 249

quantity of, 250

tension of, 258

of muscle, 265,269

partial pressure of, 247

tension of, 248

in tissues, 267

Gasserian ganglion, development of, 854

Gastric digestion, rôle of $\mathrm{HCl}$ in, 353 testing for products of, $45^{8}$

glands, influence of nerves on, 401

juice, 349 secretory changes in, $378 \cdot 38 \mathrm{I}$

acidity of, 350

antiseptic function of, 356

artificial, $45^{8}$

Beaumont's work on, 349

chemistry of, 350

digestion of proteins by, 35I, $45^{8}$

electrical conductivity of, 387

freezing-point of, 387

hydrochloric acid of. 350

in cancer, 350

influence of substances on secretion of, 403,405

lactic acid in, 35 I
Gastric juice, nilk-curdling action of, 353

psychical secretion of, 404

to obtain pure, 459

secretion, for

Gelatin as a fond, 614

from uerves, 7y5

tests for, 9

Geminal fibres, 879

Gemmules, 852

Geniculate bodies, 923

Giant pyramids of Bet 2,959

Gialluzzi, crescents of, 375

Gibbs, thermodynamic law, 431

Glands, action currents of, 838 heat-production in, 689

Gliadin, feeding with, 617

Globin, 54

Globulins, tests for, 9

Glomerulus, function of, 492, 493, 495, 497,505

Glosso-pharyngeal nerve, 929 and taste, 925

Glottis, in voice production, $308,3 \mathbf{I} 2$

Gluco-proteins, 2

Glucose, ro. See Dextrose

Glutamic or glutaminic acid, 360,575

Glycerine, r, $363,366,536,556$

formation of, from carbo-hydrates, 561

of glycogen from, 536

tests for, 12

Glycerose, 537

Glycery-phosphoric acid, 366, 42 I, 571

Glycin or glycocoll, I, $360,576,580,581$

Glycocholic acid, 365

Glycogen, 3, 523

and lactic acid, production in muscle, 771

as reserve material, 539

extra bepatic, $53^{8}$

formation of, from protein, 535, 538

formers, 536

function and fate of, 539

in liver, 534

in liver capillaries, 535

in muscle, $76 \%, 768$

preparation of, 7 I5

Glycogenase, 600

Glycogenolytic nerve fibres, $5+9$

Glycolysis, 540

pancreas and, $5+5$

Glyconic acid, $5+3$

Glycosuria, after injection of sugar into the blood, 7 I 6

adrenalin, $55^{\circ}$

alimentary, 540,717

caused by drugs, 552

phlorhizin, 551, 717

produced by asphyxia, 548

puncture, 547

relation of adrenals 10,548 
Gilycuronic acid, $47,482,526,543$

Glyoxal (methyt-), conversion into lactic aci 1,545

Goitre, exophthaluic, 650 of brook trout, 649

Golgi's method, 851

Goll, column of, 865,866 connections of, 870

Gont, $48 \%, 590$

Gowers, tract of, 860

Graafian follicles in ovarian grafts, $64 \mathrm{I}$

Gracile funiculus, 860

Gracilis experiment of Kühne, 913

(irafting tissues, 641,1142

(iramme-molecular weight, 426

' (iranule-cell,' 856

Ground bundles of cord, 807

Growth, foods adequate for, 614-6r8

Guaiconic acid and oxydases, 272

Ginaiacun test for blood, 76,272

Guanidin and parathyroid, 647

(juanin, 592, 593

Gudernatsch on influence of thyroid on tadpoles, 653

Giinzburg's reagent for hydrochloric acid, 460

Gymnotus, 841

(iyrus postcentralis, stimulation of, 963 precentralis, 963

Hamatachometer, I $2 \mathbf{I}$

Hiematin, 54 acid, 54.75

alkaline, 54,75

Hæmatoerite, 27,68

Hæuatoidin, 385

Hamatopoiesis, 20

Hæulatoporphyrin, 55, 76 in urine, 433

Hæmin, 55, 79

Hamochromogen, 54, 76

Haunglobin, composition of, 50 crystallization of, 52

crystals, preparation of, 73

curves of clissociation, $253-255$

influence of earbon dioxide on, 255

intracorpuscular crystallization of, 52

quantitative estimation of, 76

spectroscope examination of, 74

spectrum of, $5 \pi, 53$

Hxmoglobinometer, 76

Humoglobinuria, paroxysmal, 483

H:enolysis, 28, 7I

by foreigu serumı, ; 7 I

unechanism of, 29

Haxuoneter, 77

Himophilia, coagulation in, 45

Hæmorrhage and transfusion, influence of, on blood-pressure, I 1 I, 2 I4 quantity of blood which may be lost in, 1 i 1
Hair-cells of internal ear, 937

Haldane's apparatus for $\mathrm{CO}: 304$

for $\mathrm{H}_{2} \mathrm{O}$ and $(\mathrm{O}), 305$

Harmonics, 310

Hay's test for hill--salts, 528

Head, transplanting of, 975

Hearing, 106.4

analysis of complex sounds, 1072

beats, III3

cranial conduction of sound, ro7r, III3

monochord, III3

perception of pitch, 1073

range of, 1075

sympathetic vibration, 313, III 3

theories of, 1074

Heart, action current of, $8,33,844$

' all or nothing ' law of, 154

apex, preparation of, 194

arrangement of fibres in, 78

augmentor nerves of, $157,162, x 65$, 168,198

auricular flutter, 15 I

automatism of, 142

beat, $85,194,201$

cause of, 141

chemical conditions of, 152

neurogenic and myogenic hypothesis of, 142

standstill, action of augmentor nerves in, 168

conduction and co-ordination in, 146

conductivity of, $x_{42}$

excitability of, $\mathrm{I}_{42}$

extra systoles of, 155

extrinsic nerves of, 156

'fractionate' contraction of, 163

ganglion cells of, 171,165

inupulse of, 90

inhibitory nerves of, $157,162,164$,

\section{$198,203,212$}

inhibition of, 156,167

intrinsic nerves of, 141, 157

uusscle, action of inorganic salts on, 198

of limulus, $x+3$

output of, 139

pacemaker of, 143

pause of, 97

perfusion of, 153,205

pressure in, variations of, 97

refractory period and extra contraction of, 155

resuscitation of, 153

ruythmicity of, $1+2$

sunuds of, $88,207,837$

source of energy of contraction of, 772

suction action of, ror

temperature in, 710

tonicity of, 142 
Heart tracings, $164,171,194,203,837$ sinsultaneous, from auricle and ventricle, $160,161,196$

valves of, $86,204,206$

work done by, 138

lleat centres, jor

coingulation, 9, 8 I 9

distribution of, 700

equivalents of food substances, 684

given off in respiration, measure. ment of, 68z, 72I

liberated in cleavage of food substances, $08_{3}$

loss, 68 ।

regulation of, involunt:ary, 690

voluntary, 692

production, amount of, 683

and work, 684

effect of curari on, 694

is brain, 689

in digestion, 686, 713

in fever, 704

in glands, 680

in heart, 688

in muscles, 687

in rigor, 778

of different classes of workers, 685,687

relation of, to muscular work, 764,765

regulation of, involuntary, 693 voluntary, 6y 2

seats of, 686

surface and blood-flow relations, 600

rigor. See Rigor, heat, 777,820

sources of, in body, $68_{3}$

standstill of heart, I 59, I94

Heller's test for albunin, $5 \mathbf{2 4}$

Helnholtz's theory (vision), 1053

(vowel sounds), 3 I 4

(pitch perception), I073

Hemeralopia, I060

Hemianasthesia, capsular, 88 I

Hemianopia, 923, 965

Hemibilirubin, 365

Hemiplegia, reflexes in, 9 I I

Hering's theory of colour vision, 1057

Herpes zoster and trophic nerves, 806

Hexoses, 3, 537

Hibernating animals, temperature regulation in, 703 respiratory quotient in, 241

Hiccup, 288

Hippuric acid, 524, 580, $6 I_{4}$ formation, 580

Hirudin and coagulation, 37

Histidin, 54,360

Histones, 2

Holingren's wools, I I I2

Homogentisinic acid, 483,525
Homoiothermal animals, production of carbont dioxide in, 244

thermotaxis ilı, $6 g 0$

Homo-lateral fibres, 87y

Homblogous stimuli, 1007

Hormunes, to

Humidity of air and heat regulation, 691

Hunger sensation, 930, 1096

IIydriemic plethora, 462, 500

Hydrocele fluid, 39

coagulation of, 35

Hvdrochloric acid in gastric juice, 350

formation of, 379,380

Hydrogen, income and expenditure of, () I )

Hydrogen ion concentration of blood, 24

and respiratory centre, 282

of duodenal contents, 419

of gastric contents, 420

of nilk, I I 39

Hydrolysis, 2

Hidrostomia, 401

Hyoscyamine, action of, on pupil, 1026

Hyperglycxmia, 500, 540

adrenalin, $55^{\circ}$

alimentary, 5.40

asphyxia, 548

in diabetes, 552

pancreatic, 546,636

puncture, 547

relation of adrenal to 543

Hyperisotonic solutions, 428

Hypermetropia, 1030

Hyperpnoed, 283

Hypoglossal nerve, 93 I

Hypoisotonic solutions, 428

Hypophysin, 668

Hypoplyysis. See l'ituitary body

Hypoxanthin, 589, 592, 593

Identical points, theory of, 1037

ldio-nuscular contraction, 759

Heu-cacal valve, 331

colic sphincter, $33 \mathrm{I}$

Illusions, optical, IO4 I

Inbibition, 426

Indican, 484,517 estimation of, 5 I 8

Indol, formation of, in intestine, 422

Indophenyloxydase, 272

Induction machine, arranged for tetanus, 200

arranged for single shocks, 808

Inductorium, 730

lnfundibulum, infundibular body, 666 , 933

Inhibition from the cortex, 954

in reflex action, 903

nature of, 167

of heart, 156

Inorganic salts. See Salts 
Inosinic acic, 767

Inosit,,-67

Inspiration, 226

forced, 230

muscles of, $226,2: 27$

Intellectual processes, seat of, 973

Intelligence, size of hrail and, 985

Intermedio-lateral $\mathrm{rract}, 31 \mathrm{~h}_{4}, \mathbf{9 6 5}$

Internal capsule, $\$ 8$ I

froutal fibres in, 832

occipito-tenuporal fibres in, 332

respiration, 265

secretion of adrenal, 655

of kidney, $6 ; 1$

of ovaries, 6.41

of pancreas, 6.36

of parathyroid, 646

of pituitary bodv, 666

of pineal gland, $6 ; 1$

of spleen, 672

of testicles, 640

of thymus, 643

of thyroid, 645,617

Intestinal epithelium, permeability of, $+37$

juice, adaptation of, to food, 4 I 5

collection of, 370

composition of, $37 \mathrm{I}$

influence of nerves on, 4 I 4

segments, effect of blood-serum on contractions of, 453

action of epinephrin on, 453,657 658

Intestine, large, absorption in, $422,45 \mathrm{I}$ inovements of, 330

Intestines, absorption of water in, 421 contraction of isolated, $45^{2}, 330$

digestion in, 4 I 8

movements of, 328

nerves of, 331,332

reaction of contents of, 419,420

resection of, 45 I

Intraocular tension, 1017

Intrathoracic pressure, 236,237

Invertase, 338,339

in intestinal juice, $37 \mathrm{I}$

Inversion of carbo-hydrates, 356

Iodine, influence of, on thyroid, 650-654

Ions, 429

Iris, effect of stimulation of sympathetic on, 1023,1110

functions of, 1026

in accomınodation, 1023

local mechanism of, 1025

nerves of, 1023

Iron, absorption of, 447

in $\mathrm{rgg} 5,622$

in liver cells, 463

in milk, 622

Irradiation, 106I

Island of Reil, 973

Islets of Langerhans, 638
Isodynamic relation of frod substances 773

Isinnaltose, $335^{\circ}$

Isotonic and isonetric contraction, 747 solutions, 428

Itching, seusation of, toss

Jacksonian epilepsy,,$i^{2}$

Japanese dancing mice, labyrinth of, 945

Jaundice, $384,+14$

colour of stools in, 369

hematogenic, $3^{85}$

Jaw-jerk, 9I5

Karyokinesis, 5

Kiaryosome. See Nucleolus

Katabolism. See Metabolism

Kephalin, 42, 571, 794, 201

Ketohexoses, 537

Ketones, 537

Kidney, bloodvessels and tubules of, 489

excretion of pigments by, 495

formation of hippuric acid in, 580

gas exchange of, 266,504

internal secretion of, 671

nerves of, 504

tubules of, 490,492

Kinasthetic area, 963

hinee-jerk, 904, 9 I 5

reinforcement of, 9 I I

Kreatin and kreatinin. See Creatin and Creatinin

Kinne's artificial eye, I I04

Labyrinth, 927, 1067

and equilibration, 936,938

and postural reflexes, 918

Laccase, 272, 337

Lachrymal glands, 475

1.actalbumin, 7 I9

Lactase, $338,340,371,410$

Lacteals, 463

absorption of fat by +43

Lactic acid and heat-production in muscle, 76 ;

as stage in decomposition of, dextrose, $5+3$

fermentation, +23

formation of, in muscle, -69

Hopkins's reaction for, 82 I

in netabolism, $54+, 5+5$

in nervous tissue, -795

in stomach, 350

precursor of, in inuscle, 7 ;o

l'ffelinann's test for, 460

Laking of blood, 28. Sec Hanolysis

Landergren's hypothesis, 55 I

I.angerhans, islets of, $6{ }_{3} \mathrm{~s}$

J.anolin, and fat absorption, $\$ 42$

l.arynx, action of nuscles of, 303 and voice proxluction, $30 \%$ 
Latent period of nuscular contraction, 745,815

Lateril gremud-bundle, 807 mucleus of buth, $8 ;$;

Law of contraction, 788,845

Lecithin, $1,4 \pi, 360,571,794,1801$ digestim of 42 i

1.eclanchic battery, 198

lecele extract and coagulation, 38

Left-hauded poople, aplsatia in, 97 7

Ligal's test for acetone, 529

I. (i)

Lcucucytes, chenistry of, 55 eusinoplsile, I7 number of, 1 ? urigin of, 22 pulymorphonuclear, 17 transitional, I 7 varieties of, $[7$

Leucucytosis, In

Leukicmia, 19

Levatores custarum, action of, in respiration, 227

Lcvulose, 356,538 formation of glycugen from, 537

Liebcn's test for acctone, 529

Lieberkiuhn's crypts, $370,374,375$ functions of, 451

Line salts, deficiency of, changes caused by, $62 \mathrm{I}$

Limulus heart, $\mathrm{I}+3$

Linolic acid, 556

Lipase, $45^{\circ}, 33^{5}$ gastric, 355 pancreatic, 363 reversible action of, 338,444

Lipoids, I, 3

Lipoid-solubility of absorbed substances, 437

Liquids, flow of, 83

Lissauer, tract of, 866

Listing's law, 1063

Liver and amino-acids, 584 and coagulation, 44 and deamidization, 588 and glycogen formation, 533, 535 and metabolism of fat, 567 and urca formation, 58.1 cells, iron in, 385, 463 teinperature, 6819, 712

Living matter, chemical composition of, I

functions of, 6

structure of, 4

Living test-tube experinent, 34

Localization in different animals, y8o of function' in cortex, 975

Locomotion, 946

Locomotor ataxia, disappearance of knee-jerk in, 915

Lungs, area of, 224 auscultation of, 231,304
L.ung ", 1, woxl-supply of, 223 eirculation time of, 224 mechanism of gats "xchange in, 263 yuantity of blexel in, 22.4

Lymph, coniposition of 57 different kinds of, 466 tluw, factors in, 192 formation, and activity of organs, 472

factors concerned in. 407

influence of nervers on, 473

freezing-point of, 473

hearts, 192, 193

post-mortem flow of, 174

pressure of, Io) 2

ritte of flow of, 192

Lyuphatagogues, 468

Lymphatic circulation, 192 glands, 192

Lymphatics, valves of, 192

Lyinphoblasts, 22

Lymphocites, i 7

Lysin, 360

synthesis of, in body, 6ra

Macula lutea, 975, 1107

Magendic-Bell law, $89 \mathrm{I}$

Makc and break shocks, 807

Malapterurus electricus, 8 to nerve of electrica! organ, 850

Maltase, 340,345

in intestinal juice, $37 \mathrm{I}$

Maltose, absorption of, 145

Mammary glands, fat formation in, 565, $1 \mathrm{I}+\mathrm{I}$

line, go

Manganese salts and coagulation, 37

Mannite, 3

Manometer, 92

differential, 96

Hirthle's clastic, 93

maximum and mininum, 92

optical, 93

with side-tube, 212

Marchi staining reaction, 797

Margiual veil, $850,85^{\circ}$

Marie, tract of, 867

Mariotte's expcriment, I0.44, II07

Wast cells, 18

Mastication, 32 I

Maté, 632

Mitiwell's spet (visiont), I 107

Mrcunimu, 42.4

Mediastinum, 22.5

Medulla oblongata, structure of, 869

'Medullary" groose, 8\$9 sheath, developinent of, 959

Megakaryocytes, I 8

Megaloblasts, z I

Meissner's plexus, 320

Ménicre's disease, 970

Menstruation, I 20 
Mercapturic acid, 5is

Mraencephalou, $x$ 50

Metabrlism, 6

based, fiaf,

in fever, jurs

in siarsation, 602

intermediary, rif carbu-hydrates, 542 of fat, 565

of amints-acids, 5 52

of nucleic acids and purin bases, $5^{\prime 92}$ of phosphatides, $5 ; 1$

of proteins, $5: 2$

and muscular work, 610

in starvation, 603

of sterins, 5,0

relation of, to surface, 695,696

Mcta-proteins, 3

Mctencephalun, 850

IIcthamuglubin, 53,75

Methylene blue, behaviour of, in tissues, $13 \div, 218,056$

Ifethylglyoxal, 545

Methyl orange as indicator, $\mathbf{5 2 2}$

Metronume, 195

Mett's tubes, $3+3,454$

Micrublasts, 2I

Microtonometer, 259

Micturition, 5 Io

centre, 5II, 9I 5, 920

Nilk, I I 39

as a foud, $6 \geq 1$

chemi-tro of, 629,718 , II 33

clotting uf, 353.719

dige - tion of, $353,+17$

hydrogen ion concentration of, 1139 secretion of, II

Millun s reagent, 8

Mitusis, 5

Mitral valve, 56,90

Moist chamber, 809,843

Velecular concentration, $\$ 26$

Molisch - test for carbo-bydrares, in

Monlakis tract; 86 ;

Misnuchurd, $\$ 113$

Mfonosaccharide:, absorption of, +45

formation of glycogen from, 537

Monro, foramen of, $\delta 50$

Morphine, quantity of, for dugs, 63

Morpholugy of the blood, 14

. Iotor aphasia, 97o

'Motur' areas, $95 \mathrm{I}$

Mountain sickness, 298

Mureturents, forced, 744

Mucin in lule, 364.462 ill -aliva, $34+, 454$

Mucuus gland, secretory changes in, $38 \mathrm{I}$

Ifüller's experiment, 296

Mu=carine, action of, on heart, 164,199

.Mu=cle, action of curara un, 738,811

action of nicotine on, 73 ?

anaerubic contraction of, 269,306 , 770
Muscle, arrangement for tracings, 812 chemistry of, 767,819

conpesitiun of, 767

contraction of, $i+3$

theories of, 744

influence of load on, 747.813

of temperature oll, $\left.74^{\prime}\right), 813$

degeneration of, bos

diffraction spectrun, $7+5$

direct excitability of, $73 \%, 81 \mathrm{I}$

direct stimulation of, $i \neq 0$

efticiency of, -65

clasticity of, 736

extensibility of, 736

fatigue of, $7+9,813$

calise of, 751

seats of, $752,753,813$

formation of lactic acid in, $75 \mathrm{r},-63$ 820

gases of, 270

general physiolugy of, $; \geq 3$

heat-pruduction in, $656, ; 62,764$

'idio-muscular' contraction of, 750

nerve preparation, 808

fatigue of, 752,813

of eyes, extrinsic, $\mathrm{IO}_{3}$

argen consumption of, 269

permeability of, 773

physical properties of, 735

reaction of, 769,820 ,

receptive substances of, 739

respiration of, 269

smooth, contraction of, $i+5, i 47,817$

wave of contraction in, 759

spindles, $\mathrm{SO}_{4}$

stimulation, 737

structure of, 742

trough, 809

Muscular contraction and lactic acid, 767 , $7 j 0$

changes during, 744

chemical phenomena of, 767

$\mathrm{CO}_{2}$ production in, $270,-68$

duration of, 745

graphic recurd of, $8 \mathrm{II}$

heat-production in, $762,-763$

in absence of oxygen, 269,306 , $7 ; 0$

influence of fatigue, $7+9,812$

of load on, $7+7,813$

of inental fatigue on, 754

of previous stimulation , $\mathrm{n}$, $7+4,813$

of temperature on, $i 49$,

813

of veratrine, on 754,814

isutunic and isometric, 747

Iatent period of, $7+5,815$

mechanical phenumena of, $i \neq 5$

uptical phenomena of, $7 \notin 2$

oxygen consumption in, 703 
Muscular contraction. phỵ-ico-chemical conditions of, 773

rate of wise of, $75 \%$

relation between mechanical energy and heat-production, $\div 65$

relation of glycogran tu, 771

substances nutululized in, 771

superposition of, 750,816

theories of, 744

time relations of, 747

voluntary, 760

work done in, 747

sensations, I O1) 4

tissue, actinul of extracts of, 673

ture, $917,938,1000$

work and nitrogenuus metabolism, 610

source of energy of, 611,767 , 771

Musical centres, 967

Mrelencephalon, s5o

Myelin sheath, fragineutation of, 796

Myelination of tracts at difierent times, yor

Myeloblasts, 22

Myocardiograph, 162, 203

Myograph, I95, 745, 812

spring, 746,815

Myohamatin, 767

Myosin, 820

Myosinogen, 820

Myxœdema, 648

Negative variation. Sec Action current Nerve, carbon dioxide production in, 782

chenical changes in, 782

chemistry of, 794

conductivity of, 790 anasthetics and, 814

degeneration of, 795 phosphorus in, 797

cudings, action of drugs on, 182

excitability of, 784 did conductivity of, 786

tibres, medullated, 781,860 structure of, $78 \mathrm{I}, 85 \mathrm{I}$

heat-production in, 782

impulse, nature of, $78 \mathrm{I}$

velucity of, 793,818

temperature cuefficient of, 782

muscle preparatiun, 808

pdttern of, 799

polarization of, 829

propagated disturbance of, $78 \mathrm{r}$

regeneration of, 798

autogenetic, 802

chemiotaxis in, 800

stimulation of, 783

trunks, cooling of, 979
Nerves, allastemusis uf, $79,9,976$

classification of, 807

posteriur roots, section of, 7リ7, 964

preganglionic, 185,865

specitic energy of, 979)

trupluic, 805

Nerse-cells, growth in vitro, 802, 803,856 , 857

effect of anamia on, 859

anxesthetics on, 85 \%

division of axons on1, 745, 859

fatigue on, $859,983,984$

of (jolgi's second type, 856

Nervi erigentes, $180,332,334$

Nervous activity, chemistry of, 782,795 , 901

Nervous system, autonomic, 1003 development of, in different animals, 898

tissue, action of extracts of, 673

Nicural canal, 849

groove, $8+9$

Neuroblasts, $8+9,858$

Neuroglia, 86 I

Neurokeratin, 795

Neurons, $85 \mathrm{I}$

growth of, 857

liutrition of, 8,8

scheme of lower motor, 855

varieties of, 855,856

Nicotine, action of, on ganglion cells of heart, 166

on skeletal inuscle, 740

on sympathetic, I82, I005

effect of, on nerve-cells, 182,913

Night-blindness, ro6o

Nissl substance, 851,984

Nitrogen balance-sheet, 602

necessary quantity of, in diet, 623, 624,625

total, estimation of, $52 \mathrm{I}$

variation of, with protein in food, 720

Nitrogenous equilibrium, o0z protein necessary for, 605

metabolism and muscular wurk, 610

Norlcucin, 360

Normal solution, 479

Normoblasts, 2 I

Nuclease, 594

Nucleic acids, chemistry of, 593 inetabolisin of, 51)2

Nuclco-proteins, I, 2, 47

digestion of, 417,592

Nucleosides, synthesis of, 597

Nucleotidase, 594

Nuclcotids, 593

Nucleus, 5

globosus, 885

importance of, for cell, 795

tecti, 885,928 
Oatmeal as a food, 626 in diabetes, 555

Obesity, 568

Banting cure for, 606

treatument of, 570

()ecipital lesions, 966

Ocul(1-mutor nerve, 92 4, 1)82, 1023

Udours, classification of, 1070

(Visophaysts, contractions of, 323,817 pulse, 99

Ohim's law, 725

Oleic acid, 556

Ulein, 4

Olfactometer, 1076

Olfactory bulb, g22 mejve, $92 \mathrm{I}$

Olivo-spisal tract, 867,885

Uncometer, 507

Oophorin, 642

()phthalinometer, 1021, I I05

Oplithalmoscope, 1031, I 108 (iclleva, I I IO

()psonins, 61

Optical illusions, 1041

Uptic lobes, 932 and inlubition of refiexes, 950, 996

ner'c, 922 radiation, 883,923 thalanus, 883,932

Orcin redetion for pentoses, 528

Orientation, mechanisin of, 936

Urnitlıiı, 587, 613

Usinosis, 426

and diffusion, in lympl formation, 471

Osinotic pressure, 426

resistance of coloured corpuscles, 73

Ossicles, auditory, 1066, 1069

Otoliths, 937

Output of heart, I 39

Ovaries, internal secretion of, $64 \mathrm{I}$

Ovary, influence on metabolism, 6.42

Orertones, 310,313

Ovun, development of the, 1122

Oxalates, action of, on coagulation, $3 \%, 64$ in urinary sediments, 478,532

Uxilic acid, 481

Uxidation, seats of, 265

Oxidative process, nature of, 271

Oxidizing ferments or oxydases, 271,272 , 306

Oxybutyric acid, 555, 562, 567

Oxydases, $18,76,271,306$

Oxygen absorption, mechanisn of, 263

capacity of bloed, 25 I

cuefficient of utilization of, 697

consuluption of, 242, 243

in different animals, 245

of different tissues, 268,271

deficit, 620

distribution of, in blood, 252
Oxygen, incone and expenditure of, $6 \mathrm{rg}$ partial pressure of, in alveoli, 24 I 261,263

passage from blum into tissues, 266 tension in blool, 258, 261, 262

Oxintic cells, $375,378,380$

Oxyprolin, 360

Pain, in internal organs, gux, 1093 referred, 80 I

sensátions, 1085, 1089, III6 paths for, 806

Pancreas and glyonlysis, 546 and spleen, mutua! relations of, 411 influence of ucrves on, 4u5, 407 internal secretion, 636

islet tissue of, 638 removal of, in preguancy, 638 secretory clianges in, 376

Pancreatic diabetes, 546,636 juice, adaptation of, to lactose, 4 10 and bile, adjuvant action of, 367,368

artificial, 460

composition of, 358

ferments of, $358-363,460$

treezing-point of, 386

rate of secretion of, 110

secretion, influence of food on, 409

Panticulus adiposus, 568

Parabiosis, 638 , I 146

Paradoxical coutraction, 832,844

Paraglobulin, $\$ 8$

'Paralytic' secretion of intestinal juice, 414

of saliva, 397

Paraphasia, 972

l'arasterual line, 90

Parathyroid, effects of removal of, 646 location of, 645

Parenteral absorption of proteins, 33

Parotid, secretory change's in, 375,376

Paroxysual tachycardia, electro-cardiogram, 836

l'arthenogencsis, 1123

l'artial pressure of gases, 248

l'arturition, I I 37

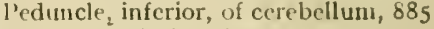

Pellagra and vitamines, 632

P'elvic nerves, 179,332

Pendulum movencints of intestines, 328

l'eugavar Djambi as styptic, rool

l'entoses, 528, 593

l'entusuria, 487

Peptases, 361

Peptides, 2

Peptones and coagulation, 37 tests for, Io

Percussion, 232

Perfusion of heart, 205

Perikaryon, 850 
Perimetrs, 1048

Periodic breathing, production of, 287 , 300

Peripleral reflex centres, 1912

P'rictialsis, $324,326,321), 3,30$

Peritoneal cavity, absorption from, 439

Peruxydase, 76

l'ersistence theory (visjon) 1050

Perspiration, visible and invisible, 512

Pettenkofer's test for bile acids, 366,462

l'lagocytosis, 59

I'hakoscope, Helmholtz's, 1021, I 103

Phenol, excretlon in starvation, 604

Phenyl-alanin, 2, 352, 360

Phenyl-hydrazine test for sugar, 525

Phlebograin, roi

I'hlorhizin glycosuria, 551, 7 I7

Phloroglucin reaction for pentoses, 528

Phosphocaruic acid, 767

Phospliates, estimation of, 516

Pliosphate triple, sediments, 479, 53I

I'hosphatides, 57 I, 794

digestion of, 421

metabolisin of, 571

synthesis of, 597,607

Phospho-proteins, 2

Phosphorus in degenerated nerve, 797, 798

in milk, 621

poisoning and fat migration, 563

Photo-electric reaction of eye, 839

Phrenic nerves and respiration, 275

afferent fibres in, 284

anastomosis of, with sympathetic, 801

Phytosterins, 366, 570

Pia mater, 86i

Pilocarpine, action of, on nerve-endings, I 82

effect of, on heart, I66, 199

on salivary secretion, 457

on pupil, 1026

Pilo-motor nerves, I 80, 913, 1004

Pineal gland, 671, 933

Piotrowski's test, 8

l'iston recorder, 233

Pitch, 310

Pithing a frog, 193

Pitot's tubes, I2I

Pituitary body, 666, 933

action of extracts of, 668

functions of, $6 \% 0$

removal of, 667

Pituitrin, testing for, 670

Placenta, excliange of materials in, $x 129$

liantar reflex, 9.5

Plasma, proteins of, 49

Plasmine of Denis, 34

H'asınolysis, 428

Patelets and coagulation, 37

l'lethysmograph, 128

Plethy'smographic tracings, 129, 2 ro
Pleural cannula, 234

Perikiluthermal animals, production of carlon dioxide in, $2+4$

heat in, Gon 3

Poicruille's laws of flow, 85

space, 15, I93

Polar stinulation, law of, 74 r

l'olarimeter, 528

l'olarization of muscle and nerve, 829

Polygraph, 103

tracings, 209

l'olynorphonuclear lencocrtes, I7

l'olypeptides, 2, 352, 361, 578

Polysaccharides, $3,347,348,535,537$

Polss, 932

comlections of, 883

grey matter of, 883

Jost-central gyrus, 963

Posterior longitudinal bundle, 885

Justerior root ganglia, developinent of, s 50

Post-sphygmic interval of heart, 37

Postural reflexes, 917 , 1000

Potassium in muscle, 767 salts, influence of, on heart-beat, I 53 , 200

Potential, electrical, 725

l'recentral gyrus, 963

Precipitius, 3 I

Prepyramidal tract, 867

Presphygmic interval of heart, 97

Pressor nerves, 173

bases, 672

Pressure sensations, I O4I, I08I, II I4

blood-, measurement of arterial, 109 I I 2,2 IO

in man, $113-116,213$

influence of exercise, I 5

Primary colours, 1053

Prisms, roso

Proferment, 343, 351, 358, 376, 382

Prolamins, 615

Prolin, 352, 360

Propionic acid, 556

transformation into dextrose, 538

Proprio-ceptive fields, 9 I 4 spinal fibres, 868

Pro-secretin, 408

Protamins, 2 and coagulation, 44

Protejus, I absorption of, $4+7$

"building stones" of, $5 \% 6$

cleavage of, and absorption, $4+3$ products of, 300

colour reactions of, 8 complete and incomplete, 6 I $3_{3}$

consumption determined by supply, 607

digestion of, $351,359,361,370,417$ formaldehyde reaction, 8 
Proteins, formation of glycogen from, 536 of sugar from, $53^{8}$

general reactions of, 7

Heller's te'st for, 524

in nutrition, relative value of different, 612

living and dead, 576

metabolism of, 572

necessary amount of, 623,624

of tissues, specificity of, $32 \mathrm{I}, 575$

parenteral absorption of, 33

precipitation reactions of, 8

specific dynamic action of, 686

synthesis of, 573,612

temperature of coagulation, 9

Protein-sparing action of fat, 606 of carbo-hydrates, 606

Proteoses, 3 action of, on coagulation, 37,63 on clotting, 44

(and peptones), influence on bloodpressure, 173, 215

formed in gastric digestion, $352,45^{8}$ action of erepsin on, 371 of trypsin on, 362

in blood and tissues, 574

in urine, 489,525

secondary, I3

tests for, 10

Protlironbin, 36

Protoplasm, structure of, 4

Prozymogen, $343,351,358,382$

granules, 376

Pseudo-globulin, 49 -podia, 17

reflexes, 904

Psychical cecretion, 399, 404, 1099

Ptyalin, 346, 4I6, 455

'Puberty gland,' 642

Pulmonary catheter, 259

circulation, 223

ventilation, 235

regulation of, $28 \mathrm{I}$

Pulse, anacrotic, ro6

arterial, ro1

curve, variation in form of, ro6

effect of anyl-nitrite, 208

exercise on, 208

frequency of, 107, 210

tracings, 103, 209

venous, 99, 100, 101, 131, 209

wave, rate of propagation of, 108

Pulsus alternans, $\mathbf{1 5 5}$

bigeninus, 155

Pulvinar, 923, 933

'P'uncture' fever, 701

glvcosuria, 547

Pupillo-dilator fibres, 1023, 1025, 1110

Purin bases in urine, 481 metabolism of, 592

bodies, chemnistry of, 593

excretion of, 594
Purkinje fibres of heart, 147

Purkinje's figures, 1042, I II3

l'utrefaction in intestine, 422,588

of proteins, action of products of, 672

Pyloric sphincter, relaxation of, 327,418

Pyramidal cells, 853, 875, 958, 959

development of, 854

path in internal capsule, 878,882

tracts, 866

connections of, 875

in different animals, 877

Pyramids, decussation of, $869,875,8 ; 8$

Pyrimidin bases, 593, 595, 597

l'yruvic acid, $544,545,5+6$

Quadratus lumborum, action of, in respiration, 227

Radiation from the skin, $68_{1}, 68_{3}$

Radiometer, 68I

Raffinose, 340

Rarefied air, effects of breathing, 295, 208

Rations, soldiers', 623, 627

Reaction of blood, 24

of degeneration, 804

of gastric juice, 420

of intestinal contents, $4 \mathbf{1 9}$

of milk, $\mathbf{I} \mathbf{3} 9$

of urine, 479

- Receptive' substances, 182, 739

Receptor in reflex action, rôle of, 900

Receptors, 898

Reciprocal relation of vasomotors, 186

Recurrent sensibility, 892

Referred pain, 891

Reflex action, anatomical basis of, $8 y 5$ extensor thrust, 904

flexion, 903, 904, 997

inhibition in, 903

irradiation of, 905

of spinal cord, 897

scratch, 901, 902, 997

arc, fatigue of, 902

isolated conduction of impulses in, 899

peculiarities of conduction in, 902

properties of, 902

refractory state in, 903

cardiac death, $1>1$

centres in cord, 915

peripheral, 912

inhibition of heart, 170,210

time, $9 \mathrm{I}_{4}$

Reflexes, axon, 803, 913

cominon patli of, 899

co-ordination of, 908

effect of strychnine on, 899

tetanus toxin on, 899

facilitation of, 909

in disease, 9 I 4 
Reflexes, influence of brain on spinal, 910 , 996

in henijplegia, 911

inhilis ion of antagunistic, 9 Io

long spiral, no7

pessural, , 1 7, 1000

reinforcement if, iा I, 998

reversial of, $18 x, 903,204$

role of the receptor in, 9oo

short spinal, gue

simultaneous, combination of, 908

successive combinations of, 208,909

superficial. 914

vain-motor, 185,212

Refractory perind of heart, 155

Regeneration, of nerves, 798

autogenetic theory; Sot

bifurcation of axous in, 802

of tissues, 1117

Reil, island of, 973

Renal calculus, pain caused by, $90 x$

Rennin, 353, 459, 719

function of, 35.4

Reproduction, I I 7

in higher animals, III 8

Reserve air, 2.36, 303

Resistance, electrical, 724

measurement of, $>25$

of blood, 26,68

Resonance, 232, 310, 314

Respiration, accessory phenomena of, 231 afferent nerves of, $2,6,300$

and blood-pressure, II 1,289

and nervous system, 274

artificial, 202, 230

by insufflation of oxygen, 204 236

influence of, on blood-pressure, 292

breaking-point in, 234

calorimeter, $240,6-6$

chemical regulation of, $2 S \mathrm{I}$

chemistry of, 239

comparative, 222

cutalleous, 299

efferent nerves of, 274

external, 225

forced, influence on carlon dioxide. excretion, $234,245,282,2 S_{3}, 300$ frequency of, 234

heat given off in, $68 \mathrm{r}, 682,63_{3}, 721$

in condensed and rarefied air, 295

infuence of cutaneous nerves un, 280 of muscular exercice on, 250 of superior laryngeal nerve on, 278,301

of vagi on, $2 \% 6,300$

internal, 222,265

mechanical phenomena of, 225

methods in chemistry of, 239,305 , 306

of muscle, 269
Respiration, regulation of, 276,281 types of, z2n

Respiratory apparatus, physiolngical atuatony of, 22.3

capacity, 236,303

centre, 274

action of carlen diuxide on, 281 , $2 \mathrm{X}_{2}$

of deficiency of oxygen on, $28 \mathrm{I}$

automaticity of, 284

spinal, 285

' dead space,' 236

exclange, $239,242,243,305$

gravimetric method, 2f', 306

Zuntz's method, 239

movements, tracings of, $233,2-5$, $279,280,289,301,302$

i: man, 233, 259, 301

recording of, 233,301

pressure, 238,304

quotient, $2+1$

sounds, 231

traciugs, $233,278,279,280,289,301$, 302

Restiform body, $885, S_{92}$

Resuscitation of heart, I 53

of central nervous system, 990

scratch reflex in, 908

Reticular formation, 865,870

Retina, development of, 850

epithelium, pigmented, 1048

fatigue of, 1049 , I 055 , I 056 , I I I I

formation of image $01,1017, I 102$

intermittent stimulation of, 1050 ,

I I 3

seusibility of different parts, 1058

time needed for excitation, 1049

Retinoscope, Geneva, II 10

Retinoscopy, I034, I109

Reverser, current, 732

Rheocord, 727,810

Rhinencephalon, 922, 968

Rhodopsin, 10.46

Ribose, 593

Right-handed people, aphasia in, 971

Rigor, heat-, production of carbon dinxide in, 270,777

Inortis, 774

and inuscular contraction, 776

production of carbon dioxide in, 270,777

of lactic acid in, 776 , 208

removability of, 779

time of onset of, 776,778

production of heat in, $\rightarrow>8$

Ringer's solution, 66

Ritter's tetanus, $S_{31}, 846$

Ritter- - alli law, 785

Riva-Rocci apparatus for blood-pressure, I 3,213 
Rotandic area of cortex, 9.51 sencory function of, 963 fissure, 875

Thane's rule, for position of, 956,963

Röntgen rays for study of gastro-iutes-

tinal movennents, 327,328

Roulcaux formation, 16

Rubro-spinal tract, 867 connections of, 884

Saliva, action of, in stomach, 348,417 amylotytic action of, 346,454 antilytic secretion of, 398 chemistry of, 344,454

effect of drugs on secretion of, 392 , 394,457

freezing-point of, 387

functions of, 346

paralytic secretion of, 397

psichical secretion of, 399

reflex secretion of, 398

Salivary centre, 400

glands, cranial nerves of, 39I, 394, 396,456

extirpation of, 673

oxygen consumption in, 266 , 268

sccretory changes in, 375,382

secretory pressure in, 393

sympathetic nerves of, $39 \mathrm{I}, 394$, 397,457

trophic-secretory fibres of, 395

Salt hunger, $38_{1}, 621$

solution, physiological, 194

Salts, absorption of, $44^{6}$

in diet, $620,625,629$

in foud, rôle of, in mutrition, 625

Saponification, II, 367, 421

Saponir, laking of blood by, 70

Sarkin, 589

Scalene imuscles in respiration, 227

Scheiner's experiment (vision), I031, I103

Schiitz's law of ferment actiou, 342

Sclero-proteins, 2

Scratch reflex, $90 \mathrm{I}$

in resuscitation, 908

Scurvy and deficiency in diet, 6,32

Sealing of wounded vessels, $4^{6}$

Sebacems glands, fat formation in, 565

Sillum, 512

Secondary contraction, $203,833,842$

Siecretin, fo7

Secretion, internal, 63.5

Segmentation of fond in intestine, 329

senucircular canak and equilibration, 1) 30

Semilunar valves, 89 and dicrotic wase, 105

inoment of closure of, 96

Semisection of cord, effects of, int

Sensation, relation of stimulus to, 1100
Sencations, cold, ros2

eutinewis, 10,8

heat, soki

hunger, 1096

inuscular, 1094

or dinary, cercbral localization of, 963

pain, 1083

tactile, cerebral localization of, 968 , 969

thirst, I 096

toluch, I078, I080

Sensibility, recurrent, 797, 892

Sensori-motor area, 963

Sensory aphasia, 972

areas, visual centres, 923, 965

functions of Rolandic area, 963

paths, decussation of, 894

in internal capsule, 88 $\mathrm{I}$

scheme of, 880

Septo-marginal bundle, 868

Serin, 360

Serratus posticus, action of, in respiration, 227

Serum, action of, on artery rings, 46,66 albumin, 47,65, 575,819

coagulation of, 47

composition of, 47,65

globulin, $34,47,65,575$

immune, 31

inorganic salts of, 49

proteins, $49,65,572$

Sexual organs, internal secretion of, 640

Sham feeding, qoz

Shock, anaphylactic or protein 32 spinal, 888,912

surgical or vascular, 192

Sighing, 288

Sino-auricular node, $\delta_{1}$

Sinus venosus, 8o, 81 , I + I, I94 stimulation of, 198

Skate, electrical organ, $8+1$

Skitul, 424 formation of, in intestine, 422

Skatoxyl in urine, 494.518

Skiascopy, I034, I I09

skin, action currents of, $\delta_{3} 8$ effects of varnishing, 2179, 699 excretion by, 5 I I

respiration by, 299

sensations, $10-8$

Smetl, 1075, III4 centre for, 922, 967

Sum ruth muscle, composition of, 768

Sinahe-venom, effect of, on coagulation 43

Sileczing, 288

Sinlutions, scheme for testing, I3

Sorbite, 3

Sipecitic energy of nerves, 479 dynamic action of forcts, 0.86

Speech, 312 uervous mechanism of, 315 
Spermin, 642

Sphincter ani, 320, 333

c.rrliar, $330,324,331,335,336$

ilco-culic, 33I, 335, 421

pylori, 320, 326, 327, 3.3 I, 335, 418

vesicae, 5 to

Sphygmograph, Marey's, 103

Dudgeon's, 208

Sphygmographic tracings, I03, 104, 209

Splyginomanometer of Erlanger, IIt

of Hill and Barnard, 1 i 5

Spinal acccssory nerve, 9.3 1 united with fasial, 976

cord and bulb, ceitres of, 9r9

automatism of, 916

conduction of nervous impulses by, 890

consciousness in, 889

effects of transection of, 888

grey matter of, 863

reflex action of, 897

scheme of cross-section of, 867

section of, to show tracts, 865

semisection of, effects of, 894

ganglia and reflexes, 912

cells of, 854

development of, 850

fatigue of, 913

roots, degeneration of, 797

function of, sor

shock, 888,912

Spinotectal fibres, 874

Spino-thalamic fibres, 874

Spirograph of Fitz, 233,300

Spirometer, 235,303

Splanchnic nerves and adrenals, $66 \mathrm{I}$

and hyperglycamia, 547,550

and intestines, 332

and kidneys, 506

vasomotors in, 177

Spleen and hxmatopoiesis, 2 I

and pancreas, mutual relations of, 411

functions of, 672

grafting of, 672

proteolytic enzyme of, 4II

Spongioblasts, $8+7$

Staircase phenomenon, 156,749

Standing, 945

Stannius' experiment, 144, 194, 199

Starch, tests for, II

Starvation, excretion of urea in, 604 loss of weight of organs $\mathrm{ill}, \mathrm{OO}_{3}$ metabolism in, 604 protein metabolism in, 602

Stasis, 61, I03

Steapsin, $35^{8}, 363,46 \mathrm{x}$

Stearic acid, $556,55^{8}$

Stercolbilin, 424

Stereognusis, 963

Stereoscopic vision, I039
Sterins, or stemols, 366

digustion of, $4 \geq$ I

metilumisı of, $5 \% 0$

Stilling, cervical and sacral nuclei of, SG

Stimulants in diret, 6.30

Stimulation by voltaic corrent, $74 \mathrm{I}, 784$,

\section{0,845}

chemical, 737,783

law of polar, $741,810,845$

recording beginning and end of, 201

Stimuli, adcquate, 901, 979, 1007

different kinds of, 737

sumination of, 756,816

Stimulus, relation of, to selisation, I roo

Weber's law, I roo

Stokes-Adams disease, 150

Stomach, absorption of proteins by, 418 auto-digestion of, 388,465

digestion of fat in, $355,+21$

excision of, 357

movements of, 326

nerves of, 331, for

pouch, 403

protection from gastric juice, 388

Stria acusticit, 928

Striped muscle, structure of, 742

Stroinuhr, 120

Strychnine and reversal of reflexes, 904 effect of, on reflexes, 899

in localization of sensory cortical zones, 968

Substantia nigra, 870

Sillostrate, 338

Succus entericus, 370. See Intestinal juice

Suckling, food requirement of, 628

Sugar and muscular work, 54 r, 772

constitution of, 537

consumption of, in diabetes, 554

destruction of, $5+1$

formation of, from amino-acids, 538 , 545,590

from fatty acids, $53^{8}, 545,566$

in blood, $47,50,446,499,500,540$, 552,717

intermediary metabolism of, 542

in urine, tests for, 488,525

regulating centre, 548

mechanism, 547

tolerance, 540, 551, 637

Sulphates in urine, 484 estimation of, 5 I7

Sulphocyanide in saliva, $3+6,454$

Summation of stimuli, 756, 8I6

Superposition of contractions, 756,8 I6

Supplemental air, 236,303

Suprarenal capsules and coaguation, 45 extract, effect of, on blood-pressure, I 73, I 77, 216, 655

Suprarenals, 655. See Adrenals

Suprarenin. See Adrenalin and Epinephrin 
Surface and mass of body, relation be(werll, 6055

heat-production and bloxl-flow, relations of, 606

phenomena and absorption, 43 I

tension, $\$ 29$

and muscular contraction, 744

Surgical shock, 192

Suturing bloodvessels, I 145

Sweat-centre, 513

composition of, 5 II

function of, 514

quantity of, 512

secretion of, influence of nerves on, $5 i 3$

Swim-bladder, secretion of gases in, 265

Sympathetic cardiac fibres of, in frog, 1 57, 196, 199

cervical, vaso-motor fibres in, 175 ganglion cells, action of nicotine on, 182

nervous system, 1003

vibration, 314, 760, I073, III3

Synapse, 852

resistance of, 899

Syntheses in the body, 542, 570, 571, 573 , $578,579,580,591,597$

Systoles, extra, I 55

Tachycardia, 930

Tactile sensations, 1079

cortical centres for, 968 paths for, 8,6

Tadpole test for thyroid active substance, 653

Talbot's law, 1040, III3

Tambour, 91 for respiratory movements, 233 Marey's, 208

Taste, I077, III3 centre for, 968 nerves of, 925

Taurin, 365,579

Taurocholic acid, 365,462

Tea, 594, 630

Tears, 475

Teeth, 320

Teginentum, 870, 874

Telencephalon, 850

Telodendrion, 852

Temperature, 674 daily curve of, 712 discrimination, I I 6

in cavities of heart, 7 ro

in mouth, 712

in rectum, 712

normal variations in, 712

of blood, 7 II

of body, 680 measurement of, 675

of brain, 689, 712

of coagulation, 9
Temperature of different parte, 711,712 of skin, 676,712

post-mortem rise of, $71+$

regulation, 690

influence of curara on, 603

in hibernating animals, 703

sensalions, 1082, 1088,1092 , I1 15 paths for, 8,6

topography, 709

Tension of carbon dioxide in blood, 261 , 264

of gases, 24 ?

in alveoli, 241,263

of nxygen in blood, $253,26 \mathrm{I}$

measurement of, 258

surface. See Surface tencion

Testes, interstitial cells of, 6,41

Testicle, action of extracts of, 642

Testicles, internal secretion of, 640

Tetanizing current, arrangement for, 200

Tetanus (electrical), composition of, 756 , 816

Ritter's, $8_{31}, 8_{46}$

secondary, 203, 833,842

toxin effect of, on reflexes, 899

Tetany, after parathyroidectomy, 646

Tethelin, 670

Thalamico-spinal tract, 867,885

Thane's method for position of fissure of Rolando, 956, 963

Theine, $481,583,594,632$

Theobromine, $481,583,594,631$

Theophyllin, $48 \mathrm{I}, 594$

Theory, Hering's, of colour vision, 1057 of identical points, 1037

loung-Helmholtz, 1053

Thermo-electric junction, $675,680,763$

Thermogenic nerves, -80

Thermometer, electrical resistance, 676 , 677,783

maximum, 675

Thermometry, 674

Thermopile, 763

Thermotaxis, 690

Thirst, sensation of, 930, 1099 .

Thiry's fistula, 370

Thrombin, 35

formation of, 36

nature of action on fibrinogen, 41 preparation of, $+\mathrm{I}$ specificity of, 40

Thrombocytes. See Blond plates

Thrombogen, 36 solirces of, 38 specificity of, 40

Thrombokinase, 36 preparation of, 65 sources of, 38,40 specificity of, to

Thromboplastic substances, 4 I

Thrombo-regulative mechanism, 43 
Thrombosis, 45

T!rombotaxis, 42

Thunb centre, 973

Thymin, 593

Thymus, involution of, 644

grafting of, 645

lymphocytes of, $6+3$

relation of, to sexual glands, 644 relluvial of, $6+5$

Thyroid and heat-production, 650,699 changes with meat diet, 650 grafting of, 648

hyperplasia of, $6 \neq$ ?

influence of iodine on, 650

of nutritive conditions on, 64 ?

iodine in, 652

infuence on tadpoles, 653 nerves of, 653

Thyroidin, 649

Thyroids, effects of excision of, 647 , 648

Tickling, sensation of, 1078, I080

Tidal air, 235

measurement of, 303

Timbre of sounds, 310

of vowels, 3 I 3

Time-markers, 195, 732

Tissue extracts and coagulation, 36 respiration, 265,269

Tissues, cultivation of, outside the body, I 42

gas tensions in, 267

transplantation of, $6+1$, II 42 .

Tone of muscles, 917

vaso-motor, $18_{4}$

Tonsils, 320

Topognosis, 963

Torpedo, 840

Torricelli's theorem, $8_{3}$

Touch, 1079, I080, III4 spots, 1080

Trachea, to put a cannula in, 202

Tracheal cannula, 201

Tracts in spinal cord, 865 myelination of, $96 \mathrm{I}$

Transfusion, influence on blood-pressure, I9I, 214

Transplantation of tissues, $64 \mathrm{I}, \mathrm{II}_{42} 2$

Traube-Hering curves, 294

Tricuspid valve, 86,96

Trigeminus nerve, 924

Tripalmitin, 556

Tristearin, I, 556

Trochlear nerve, 924

Trommer`s test, for sugar, 10, 525

Trophic nerves, $80_{5}$ tone, 919

Trypsin, $3+5,358,359,411,+18,420$, 460

Trypsinogen, $35^{8}, 372,377,3^{89} 9,391$

Truptophane, $2,360,48 \%, 615$

'Twitch,' $7+5,8 \mathrm{II}$
Tympanum, 1065

Tyrosin, 2, 360, 462, 488, 575, 576

.Worner's test for, 462

Tyrosinase, 272

Uffelinann's test for lactic acid, 460

Ultra-microscope, coagulation studied by, 39

Uncinate gyrus, 968

Uracil, 593

Lrea, in blood, 47, 499 estimation of, 518,520

excretion of, by kidney, $492,496,497$, 498,502

in tever, 706,707

in starvation, 604

premortal rise in starvation, 603

formation of, 583

processes of formation of, 588

Urease, 478

method of estimating urea, 518

Uric acid, $48 \mathrm{I}, 523$

destruction of, 595

exogenous and endogenous, 595

formation of, 585,590

in gout, 487,590

sources of, 59 I

Uricolysis, 595, 596

Uricoxydase, 596

Urine, acidity of, $478,479,515$

albumose in, 509, 525, 572

alkapton in, $483,58 \mathrm{I}$

amino-acids in, $48 \mathrm{I}, 488,579$

ammonia in, $480,519,521$

bile-pigments in, $433,53 \mathrm{I}$

bile-salts in, $489,528,53$ I

carbo-hydrates in, 482

chlorides in, $477,483,515$

composition of, 476

creatinin in, 481,523

cystin in, $488,532,579$

ethereal sulphates in, 484,517

expulsion of 510

ferments in, 483

freezing-point of, 485,529

hamatoporphyrin in, $4 \$_{3}$

hippuric acid in, 481, 524

in disease, 485,524

in fever, 703

incontinence of, 5 II

indican, indoxyl in, $484,487,517$

oxalic acid in, $478,481,532$

phenol in, 484,604

phosphates in, 483,516

physico-chemical analysis of, $4 \$ 5$, 529

pigments of, 482

proteins in, $482,+88,524$

purin bases in, 481

reabsorption of, from the tubules, 494,502 
Urine, secretion of, 489

He-idenhain's experiments on, 495

influence of circulation on, 506 of nerve's on, 508

Nussbaum's experiments on, $49^{8}$

pigments, by the kidney, 495

theories of, 491,502

work done by kidney in, 50.4

secretory pressure of, 506 ,

sediments of $, 478,479,4 \times 8,531$

skatoxyl in, 484,518

specific gravity of, 476,515

sugar iu, $487,488,526$

sulphates in, 484,517

systematic cxaminations of, 531

temperature of, $7 \mathrm{I} 2$

total nitrogen in, $477,52 \mathrm{I}$

urea in, $450,487,518$

uric acid in, 4 xu, $487,523,531$

Urobilin, $365,424,482$

C'robilinogen, 365

l'rochrome, 482

'roerythrin, +82

'Lrohypertensine,' 672

Urorosein, 482

Uterus rings and segineuts, isolated, II47

Utricle, 936, 1067

Vago-sympathetic nerve, in frog, I+I, 196, 198, 199

Vagotomy, death after double, 286

Vagus, stimulation during resuscitation after cerebral anxinia, 8 87

in manmals, 162, 164, 203, 212

inspiratory and expiratory fibres in, 276-279

negative variation of, 277,828

nerve, functions of, 929

of frog, dissection of, 196

relation of, to respiration, 276

stimulation of, in dog, 203, 212

Valin, 360 in $\mathrm{frog}, 198$

Valsalva's experiment, 296

Valves of veins, 82

semilunar, moment of closure, 96

Varnishing the skin, 299, 514, 69?

Vaso-constrictor and vaso-dilator nerves, 173

differences in excitability of, 175 property of shed bluod, 45

Vaso-dilator filbres, 179

in cervical sympathetic, 176

V'aso-dilators of chorda tympani, I79, 392

of nervi erigentes, 179,180

V'aso-motor centres, 182 anatomical relations of, I 85 natire of tone of, $18_{4}$ spirual, 183
Vaso-motor nerves, $173,175,176$

cruturse of, 181

in cervical sympathetic, 175

in splanchnic, 177

in trigeminus, 170

of brain, $17($ s

of extremitics, 177

of heart, I 78

of lungs, 179

of inuscle, 178

of veins, 180

reciprocal relations of, I 36

reflexes, 185,212

studied by calorimetric method: I 88,22 I

tone, 184

Vaso-luotors, methods of investigating, 174

V'eins, action of adrenalin on, I 8 I

circulation in, 132

facturs crncerned in flow in, 133

pressure in, 132

measurement of, in man, 133

pulse in, IUo, I 31

vasu-mutor nerves of, I 80

velucity of blood in, 134

Vella's fistula, 370

Velocity of blood, I 17, 127

in capillaries, 130

in veins, 134

measurement of, I20

Vena portie, vaso-motor nerves of, I 80

Venous blood, tension of carbon idoxide iil, 261

pressure-curve, $9^{8}, 100$

pulse, $99,100,131,134$

tracing, 100,209

Ventilation, 242

pulmonary, 235, 28I

Ventricle, suction action of, IOI

Ventricular pressure-curve, 94

Veratrine, action of, on inuscle, 754,814

Vesicular murnur, 23 I

Vestibule, paths from, 928

Vestibulo-spinal tract, 867 connections of, $88_{t}$

Visceral pain, 891,901

Visuo-psychic area of Campbell, 966

Vision, after-images, 1055

apparent size of objects, 1042

astigmatism, 103I, I I05

blind spot, I044, I 107

colour, I O5 I

Hering's theory, I057

mixing, 1053, I I I I

triangle, I054

Young-Helumbltz. theory, I053

colour-blinduess, I 054), I I 12

comparative anatumy, 1013

contrast, 1056

duration of stimuli, I 049

lick's persistence theory, I050 
Vismu, IInlungren's wouls, III2

irradiation, $106 \mathrm{r}$

l.inting's l.1w, 1063

Maxwell's spett, 1107

medsurement of ficld uf, in58, 1106

oplithalmometer, 10:1, 1105

ophthalmoscope, 1031, 1108

perinetro; 1058,1106

P'urkinje's figures, 1042, III3

relation of reds and anes to, 1045

skiascopy, 1034, I109

stereoscupic, I039

Talbot's law, 1050, 1113

theory of identical points, 1037

Visual acuity, 111I

centres, 365

path, $883,1 / 23,966$

purple, 840,1047

lital capacity, 236,303

litanines, 617,632

Vitreous humour, 466 , InI 6

livi-diffusion apparatus, 48

Vucal cords, 307

in voice pruduction, 308

movements of, in respiration, 3 II

paralysis of, 316

Voice, 307

air-pressure necessary for, 309

falsetto, 312

in children, 310

nervous mechanism of, 315

bultaic current, alterations in excitability and conductivity by, $785-788,844-846$

stimulation by, $741,784,810$

Volume pulse, 127

Voluntary contraction, 760

electrical changes in, 762

fatigue in, 753

movements, acquisition of, $98 \mathrm{I}$

Vomiting, 335

centre, 336
Voniting, induced by aponorphine, 336 , 459,464

Vinwel cavities, 3 I 4

formation, theories of, 314

Vincls, timbre of, 313

Warmeth, sensations of, 108 I

Water, absorption of, $+t^{6}$ in diet, (52) production of, in the body, 620

Weber's law (stimuli), nuo

Wernicke, aphasia of, $97 \mathrm{I}$ zone of, 971

Wharton's duct, 391 insertion of cannulis in, 456

Wheatstone's bridge, 726

Whey protein, 354, 719

Word-blindness, 972 -deafness, 967,972

Wounded vessels, sealing of, $\neq 6$

Ainthin, $48 \mathrm{I}, 589,592,5133$ fever, 707

Nantho-proteic reaction, 8

Acrostomia, 400

Yawning, 288

least and vitamines, 633 test for sugar, fio, 526

Yelluw atrophy, acute, and excretion of amino-acids, 586 spot, 975,1107

Yohimbine, action on refractory period in nerve, 783

vaso-dilator action on submidxillary, 39) 2

Young-Helnholtz theory of coluur vision, 1053

Zein, as a food, 6 I 5

Zoosterius, 366,570

Zymase, 337

Zymogen, 351, 356, 382, 383, 389 granules, 376 




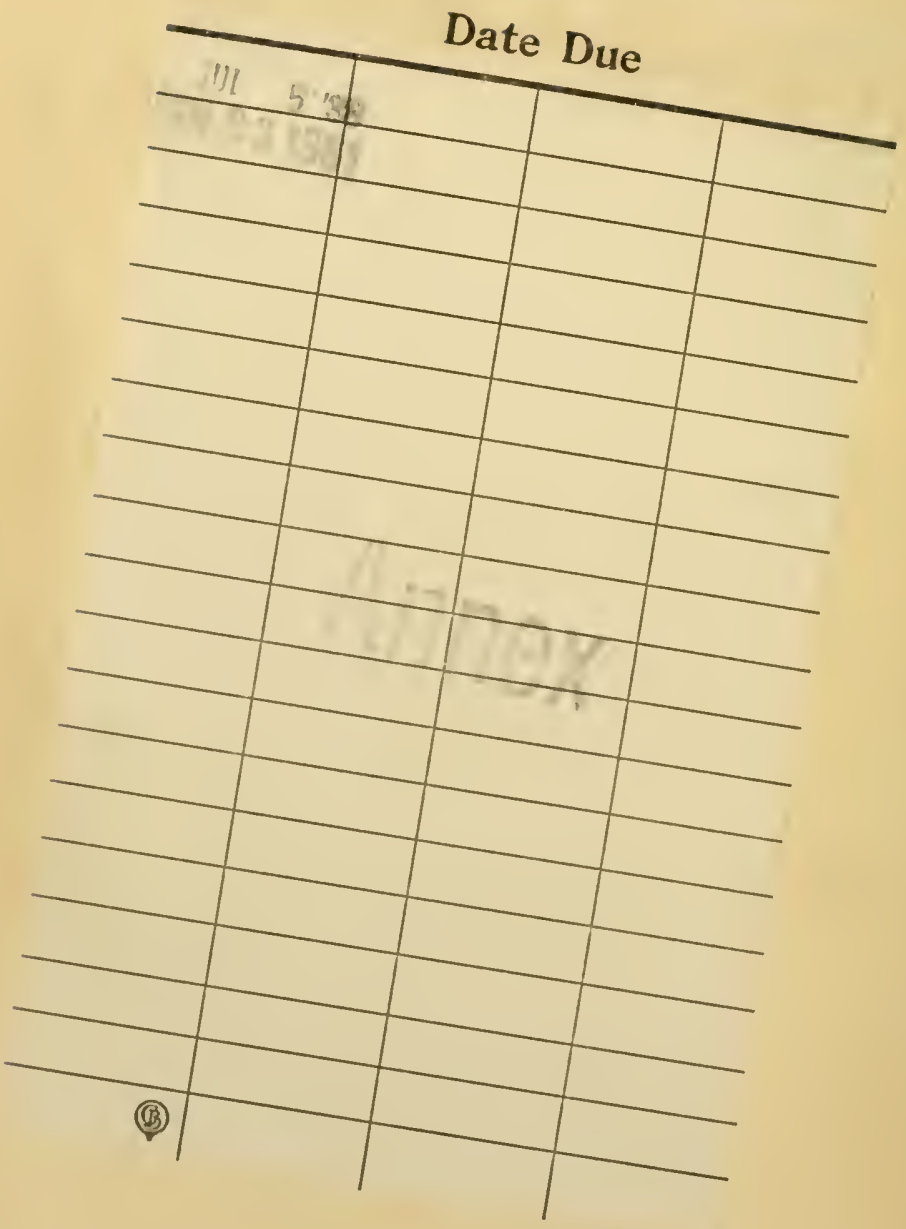




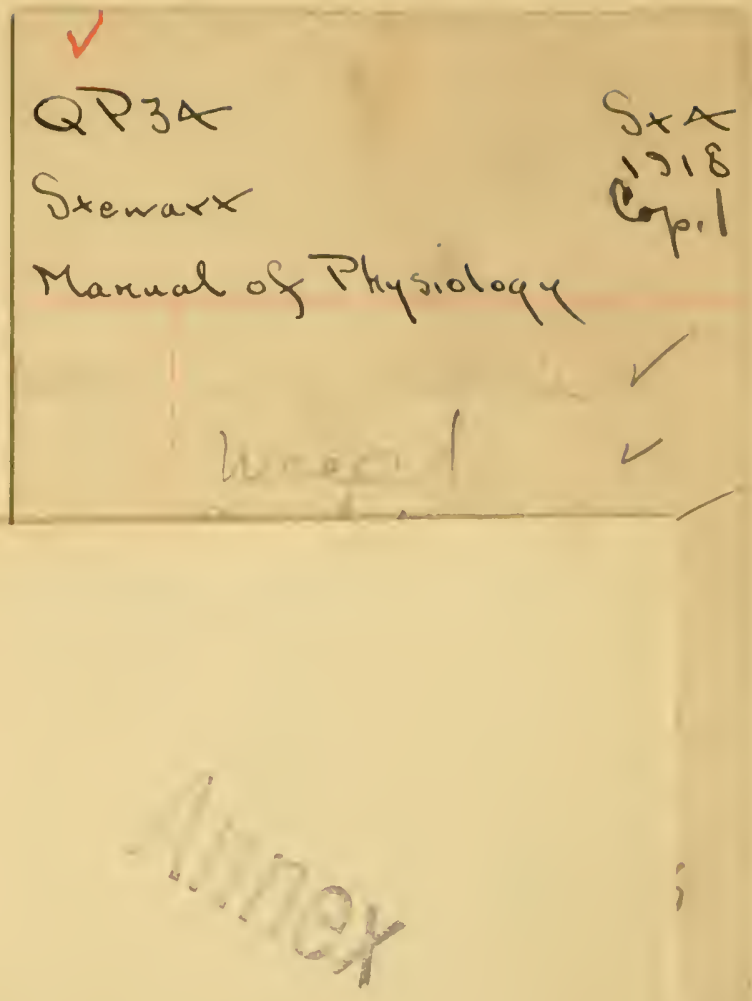




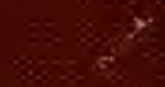

